\title{
Al110】 170159
}

\section{NATL INST OF STANDARDS \& TECH R.I.C.}

\section{PUBLICAIIONS OF THE NATIONAL BUREAU OF STANDARDS}

U.S. DEPARTMENT OF COMMERCE NBS SPECIAL PUBLICATION 305/SUPPLEMENT 13

\section{CATALOG}

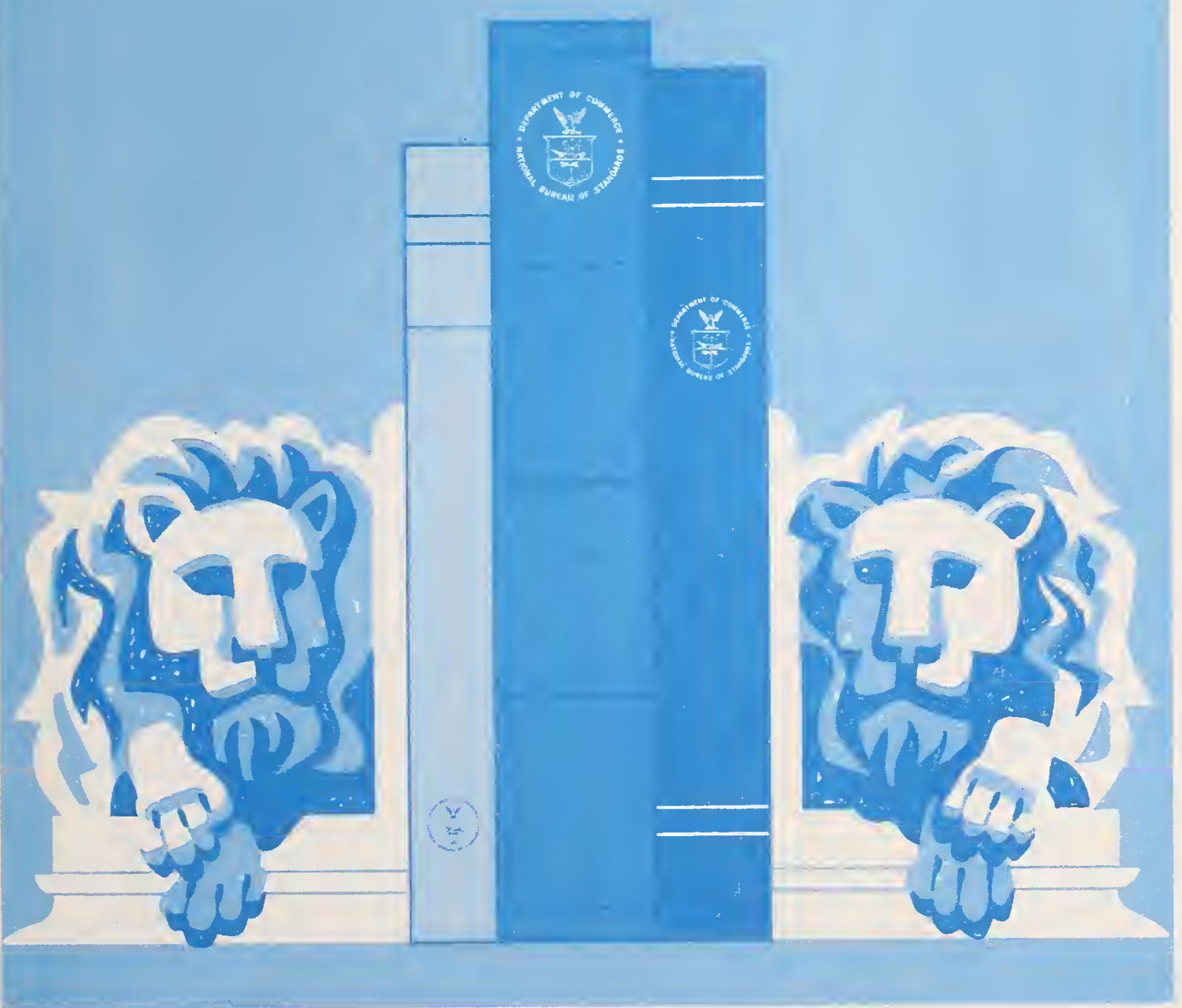

$=Q C$ minnom 100

.057

iNo, 305

SUPPL, 13

1982

C. 2 


\section{NATIONAL BUREAU OF STANDARDS}

The National Bureau of Standards' was established by an act of Congress on March 3, 1901. The Bureau's overall goal is to strengthen and advance the Nation's science and technology and facilitate their effective application for public benelit. To this end, the Bureau conducts research and provides: (1) a basis for the Nation's physical measurement system, (2) scientific and technological services for industry and government, (3) a technical basis for equity in trade, and (4) technical services to promote public safety. The Bureau's technical work is performed by the National Measurement Laboratory, the National Engineering Laboratory, and the Institute for Computer Sciences and Technology.

THE NATIONAL MEASUREMENT LABORATORY provides the national system of physical and chemical and materials measurement; coordinates the system with measurement systems of other nations and furnishes essential services leading to accurate and uniform physical and chemical measurement throughout the Nation's scientific community, industry, and commerce; conducts materials research leading to improved methods of measurement, standards, and data on the properties of materials needed by industry, commerce, educational institutions, and Government; provides advisory and research services to other Government agencies; develops, produces, and distributes Standard Reference Materials; and provides calibration services. The Laboratory consists of the following centers:

\section{Absolute Physical Quantities ${ }^{2}$ - Radiation Research - Chemical Physics - Analytical Chemistry - Materials Science}

THE NATIONAL ENGINEERING LABORATORY provides technology and technical services to the public and private sectors to address national needs and to solve national problems; conducts research in engineering and applied science in support of these efforts; builds and maintains competence in the necessary disciplines required to carry out this research and technical service; develops engineering data and measurement capabilities; provides engineering measurement traceability services; develops test methods and proposes engineering standards and code changes; develops and proposes new engineering practices; and develops and improves mechanisms to transfer results of its research to the ultimate user. The Laboratory consists of the following centers:

Applied Mathematics - Electronics and Electrical Engineering ${ }^{2}$ - Manufacturing Engineering - Building Technology - Fire Research - Chemical Engineering ${ }^{2}$

THE INSTITUTE FOR COMPUTER SCIENCES AND TECHNOLOGY conducts research and provides scientific and technical services to aid Federal agencies in the selection, acquisition, application, and use of computer technology to improve effectiveness and economy in Government operations in accordance with Public Law 89-306 (40 U.S.C. 759), relevant Executive Orders, and other directives; carries out this mission by managing the Federal Information Processing Standards Program, developing Federal ADP standards guidelines, and managing Federal participation in ADP voluntary standardization activities; provides scientific and technological advisory services and assistance to Federal agencies; and provides the technical foundation for computer-related policies of the Federal Government. The Institute consists of the following centers:

Programming Science and Technology-Computer Systems Engineering.

'Headquarters and Laboratories at Gaithersburg, MD, unless otherwise noted;

mailing address Washington, DC 20234.

${ }^{2}$ Some divisions within the center are located at Boulder, CO 80303. 


\section{Publications of the National Bureau of Standards 1981 Catalog \\ A Compilation of Abstracts and Key Word and Author Indexes}

Betty L. Burris and Rebecca J. Morehouse, Editors

Technical Information and Publications Division

National Bureau of Standards

Washington, DC 20234

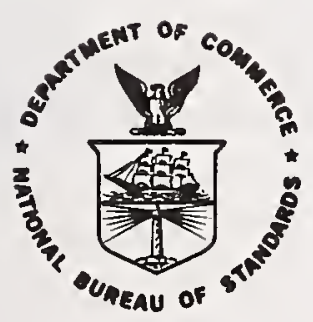

U.S. DEPARTMENT OF COMMERCE, Malcolm Baldrige, Secretary NATIONAL BUREAU OF STANDARDS, Ernest Ambler, Director

Issued May 1982 


\section{Library of Congress Catalog Card Number: 48-47112}

National Bureau of Standards Special Publication 305 Supplement 13

To Accompany National Bureau of Standards Special Publication 305 and its Supplements 1 through 12 Nat. Bur. Stand. Spec. Publ. 305 Suppl. 13, 474 pages (May 1982)

CODEN: XNBSAV 


\section{CONTENTS}

A Guide to Users of This Publication

NBS Publication Program

1. Introduction

2. Periodicals.

3. Nonperiodicals

4. Purchase Procedures and Document Availability

4.1 NBS Periodical Subscription Rates....

4.2 Prices for Nonperiodicals.

4.3 Former NBS Reference Publications

4.4 Announcements of NBS Publications

4.5 Depository Libraries in the United States

4.6 U.S. Department of Commerce District Offices

5. Titles and Abstracts of NBS Publications, 1981

5.1 Journal of Research

5.2 Journal of Physical and Chemical Reference Data.

5.3 DIMENSIONS/NBS, article titles only

5.4 Monographs

5.5 Handbooks

5.6 Special Publications.

5.7 Applied Mathematics Series

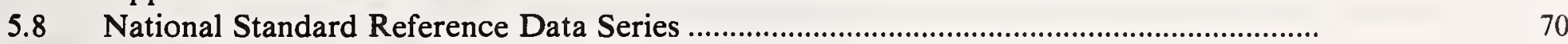

5.9 Building Science Series

5.10 Federal Information Processing Standards Publications ............................................

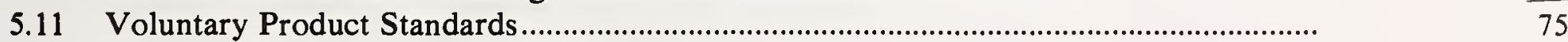

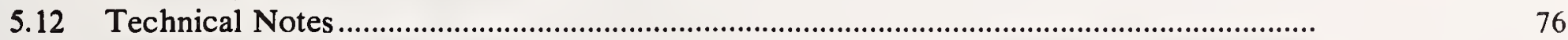

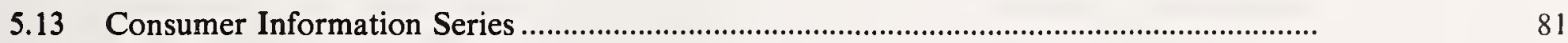

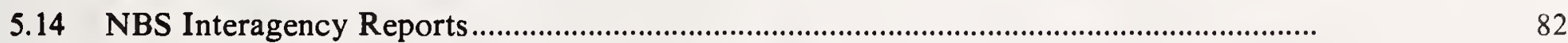

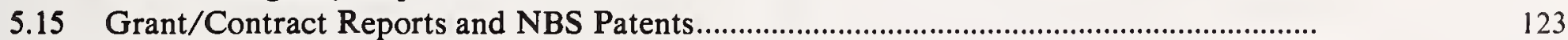

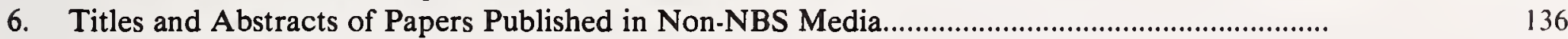

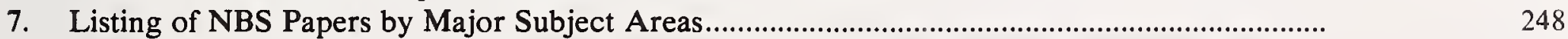

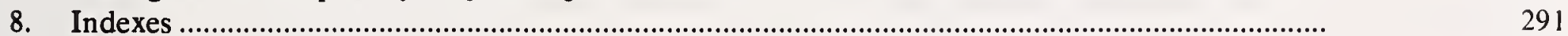

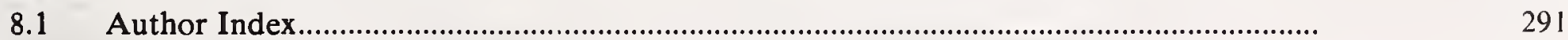

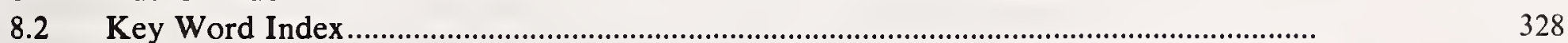

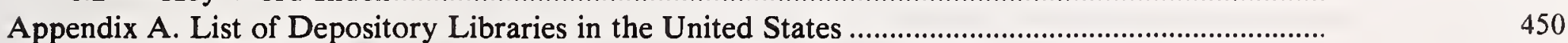

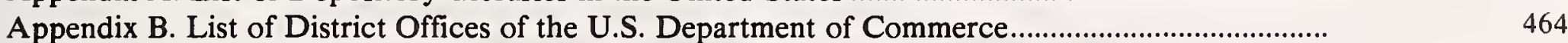




\section{A GUIDE TO USERS OF THIS PUBLICATION}

In addition to the usual author index, a subject index is provided in the form of a permuted key word index. In this type of index the key words in each publication or paper are arranged by shifting each group of key words along the horizontal printing line so that each key word in turn has an opportunity to appear alphabetically. The user is thus able to locate papers of interest through the subject-related words in the key word index.

The index symbols used in the author and key word indexes are explained in the following three tables. These tables also give the pages on which the abstracts of the various publication series begin.

\section{SYMBOLS FOR NBS PUBLICATIONS}

A. Symbols for the Periodicals

\begin{tabular}{|c|c|c|c|c|c|c|}
\hline \multicolumn{3}{|c|}{ Index Symbol } & \multicolumn{3}{|c|}{ Issue Date } & $\begin{array}{l}\text { Page } \\
\text { Number }\end{array}$ \\
\hline $\begin{array}{l}\text { NBS Journal } \\
\text { of Research }\end{array}$ & $\begin{array}{l}\text { l. } 86 \\
\text { s. } 86 \\
\text { s. } 86 \\
\text { s. } 86 \\
\text { s. } 86 \\
\text { s. } 86\end{array}$ & $\begin{array}{c}\text { No. } \\
1 \\
2 \\
3 \\
4 \\
5 \\
6\end{array}$ & \multicolumn{3}{|c|}{$\begin{array}{l}\quad \text { 1981-Bimonthly } \\
\text { January-February } \\
\text { March-April } \\
\text { May-June } \\
\text { July-August } \\
\text { September-October } \\
\text { November-December }\end{array}$} & $\begin{array}{r}9 \\
9 \\
10 \\
10 \\
11 \\
12\end{array}$ \\
\hline \multirow{2}{*}{\multicolumn{3}{|c|}{$\begin{array}{l}\text { Journal of Physical } \\
\text { and } \\
\text { Chemical Reference Data }\end{array}$}} & \multicolumn{2}{|c|}{ Index Symbol } & Issue Date & $\begin{array}{c}\text { Page } \\
\text { Number }\end{array}$ \\
\hline & & & $\begin{array}{l}\text { Vol. } \\
\text { JPCRD } 10 \\
\text { JPCRD } 10 \\
\text { JPCRD } 10 \\
\text { JPCRD } 10\end{array}$ & $\begin{array}{l}\text { No. } \\
1 \\
2 \\
3 \\
4\end{array}$ & $\begin{array}{l}1981 \\
1981 \\
1981 \\
1981\end{array}$ & $\begin{array}{l}13 \\
13 \\
14 \\
15\end{array}$ \\
\hline & \multicolumn{2}{|c|}{ Index Symbol } & \multicolumn{3}{|c|}{ Issue Date } & $\begin{array}{c}\text { Page } \\
\text { Number }\end{array}$ \\
\hline DIMENSIONS/NBS ${ }^{*}$ & $\begin{array}{c}\text { Vol. } \\
\text { DIM/NBS } 65 \\
\text { DIM/NBS } 65 \\
\text { DIM/NBS } 65 \\
\text { DIM/NBS } 65 \\
\text { DIM/NBS } 65 \\
\text { DIM/NBS } 65 \\
\text { DIM/NBS } 65 \\
\text { DIM/NBS } 65\end{array}$ & $\begin{array}{r}\text { No. } \\
1 \\
2 \\
3 \\
4 \\
5 \\
6 \\
7 \\
8\end{array}$ & \multicolumn{3}{|c|}{$\begin{array}{l}\quad 1981-10 \text { issues per year } \\
\text { January/February } \\
\text { March } \\
\text { April } \\
\text { May/June } \\
\text { July } \\
\text { August } \\
\text { September } \\
\text { October }\end{array}$} & $\begin{array}{l}16 \\
16 \\
16 \\
16 \\
16 \\
16 \\
17 \\
17\end{array}$ \\
\hline
\end{tabular}

*Publication discontinued with October 1981 issue. 
B. Symbols for the Nonperiodicals

\begin{tabular}{l|l|c}
\hline \multicolumn{1}{c|}{ NBS Nonperiodical Series } & \multicolumn{1}{c}{ Index Symbol } & Page Number \\
\hline Monographs & Monogr. & 18 \\
Handbooks & H & 19 \\
Special Publications & SP & 20 \\
Applied Mathematics Series & AMS & 69 \\
National Standard Reference Data Series & NSRDS & 70 \\
Building Science Series & BSS & 71 \\
Federal Information Processing Standards & FIPS PUBS & 73 \\
Publications & VPS & 75 \\
Voluntary Product Standards & TN & 76 \\
Technical Notes & CIS & 81 \\
Consumer Information Series & NBSIR & 82 \\
NBS Interagency Reports & GCR and/or NBS Patent & 123 \\
Grant/Contract Reports and Patents &
\end{tabular}

C. Symbols for the Papers Published in Non-NBS Media

\begin{tabular}{l|c|c}
\hline \hline NBS Papers Published in Non-NBS Media & Index Symbol & Page Number \\
\hline $\begin{array}{l}\text { Professional Journals, Books, Book Chapters, } \\
\text { Proceedings, etc. }\end{array}$ & 5-digit numbers, 19962-20774 & 136 \\
\hline
\end{tabular}

SAMPLE

ENTRIES

FOLLOW 


\section{CATALOG ENTRIES: HOW TO READ THEM}

\section{Abstracts-Sample Entries}

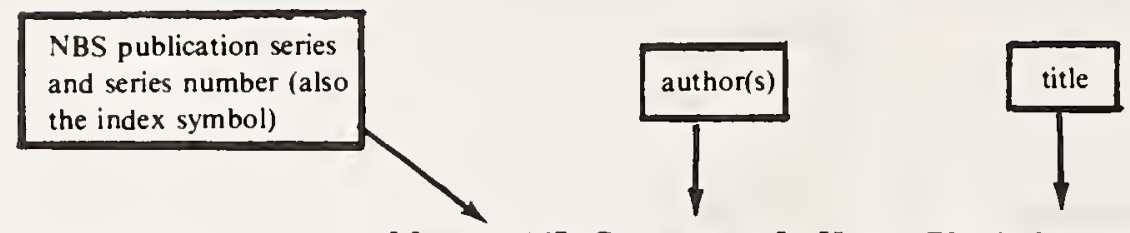
\begin{tabular}{|l|l} 
Monogr. 167. Gevantman, L. H., ed. Physical properties data for \\
rock salt. Nat. Bur. Stand. (U.S.) Monogr. 167; 1981 January. \\
number or
\end{tabular}$\quad \begin{aligned} & 288 \text { p. SN003.003.02166-1, \$12.00. }\end{aligned}$ NTIS number

key words

(as indexed in

this catalog)
Key words: chemical; electrical; geological; magnetic; mechanical; monograph; optical; physical; properties data; radiation damage; rock salt; sodium chloride.

Rock salt and pure sodium chloride properties data are assembled into a single source. The properties covered include geological, mechanical, optical, thermal, radiation damage, electrical, magnetic, chemical, and physical. A concerted attempt has been made to present the best data consistent with their availability in the literature. Recommended values for data are given where possible. A brief discussion of measurement techniques is included for each property.

\section{Example of NBS-published paper}

arabic number index symbol

key words (as indexed in this catalog)

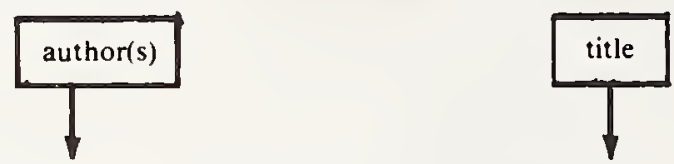

19996. Celotta, R. J.; Pierce, D. T. Sources of polarized electrons, Adv. At. Mol. Phys. 16, 101-157 (1980).

Key words: electron gun; electron polarization; electron spin; polarized electrons; spin; spin polarization.

Sources of spin polarized electron beams are described and compared. Included are discussions of the basis of the polarizing effect, the operating characteristics, features, and applicability of each source. A discussion of relevant electron optical theory and a table comparing available sources are also presented. bibliographic citation: publisher or place of publication, Vol./No./pages, date of publication 
in alphabetical order

Chen, P. T.; Hill, J. E.; May, W. B., Jr.; Richtmyer, T. E.; Elder, J.;

Tibbott, R. L.; Yonemura, G. T.; Hunt, C. M.; BSSI33.

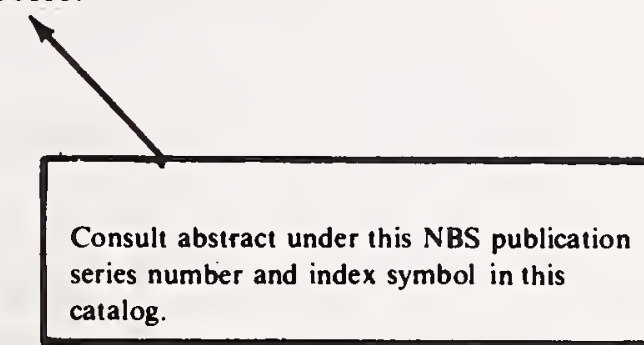

\section{Example of NBS-published paper}

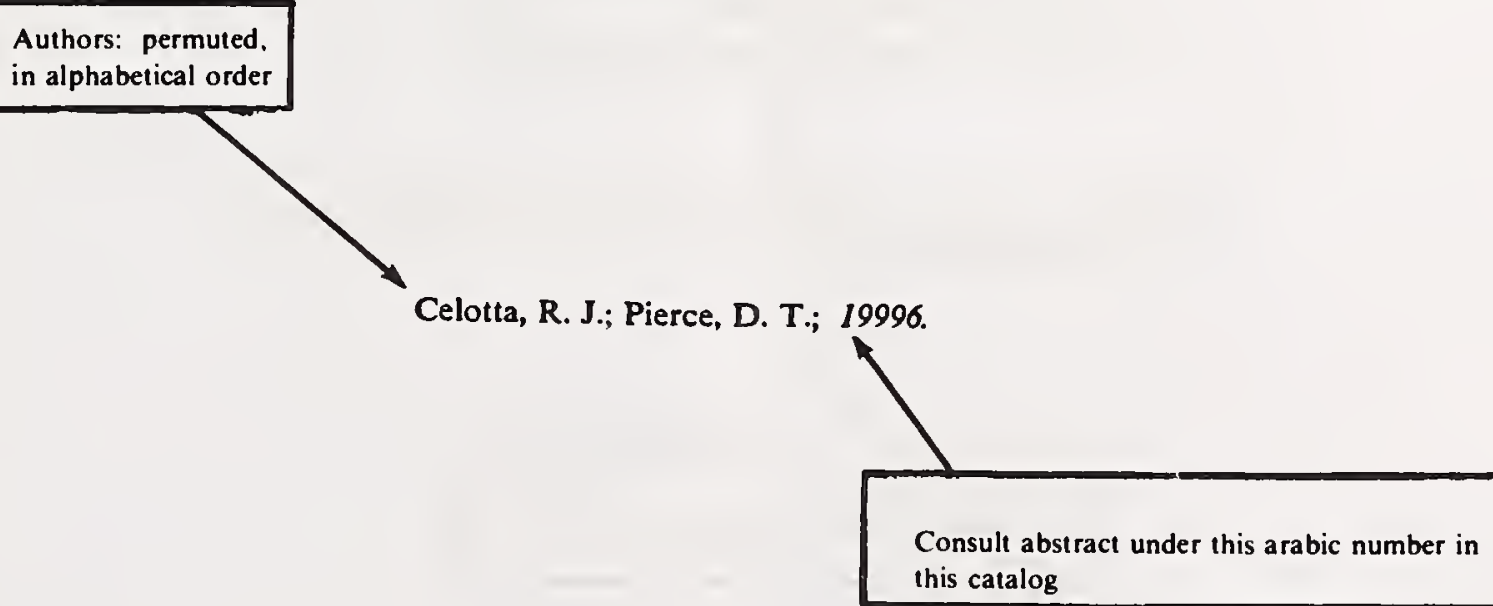

Example of NBS paper published in non-NBS media 


\section{Key Words-Sample Entries}

Key Words: permuted, each word or phrase appears

in alphabetical order

chernical; electrical; geological; magnetic; meclıanical; monograph;

optical; physical; properties data; radiation damage; rocl sall;

sudium clıloride; Monogr. 167.

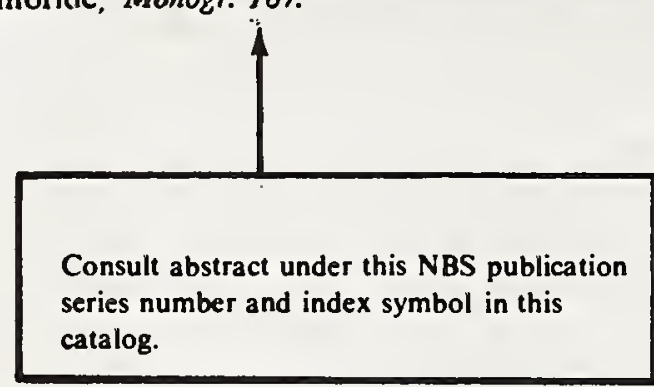

\section{Example of NBS-published paper}

Key Words: permuted, each word or phrase appears in alphabetical order

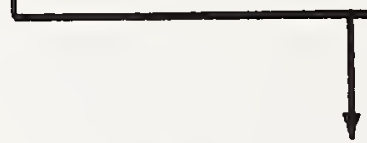

clectron gun; electron polarization; electron spin; polarized electrons; spin; spin polarization; 19996.

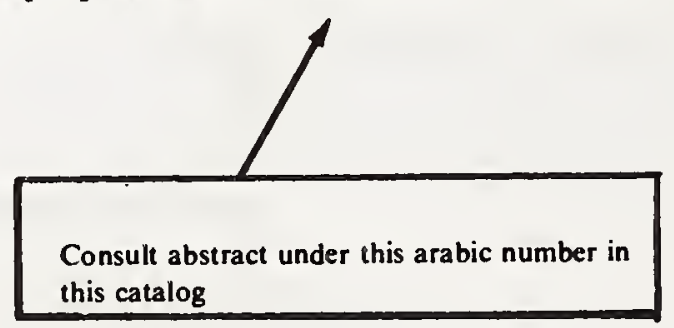

\section{Example of NBS paper published in non-NBS media}




\section{NBS PUBLICATION PROGRAM}

\section{INTRODUCTION}

The formal publications of the National Bureau of Standards are the primary way of communicating the results of NBS programs to its varied technical audiences and the general public. Publications are a major end product of the Bureau's efforts. These take the form of the Bureau's 3 periodicals, its 12 nonperiodical series, and articles in the journals of professional organizations and technological associations.

In 1981 , over 59,736 pages were published by NBS in 1767 papers. These appeared in the Bureau's own publications series and in non-NBS journals, books, and proceedings. Also included, to complete the record, are those NBS papers published prior to 1981 but not reported in previous issues of this annual catalog.

This annual catalog, Publications of the National Bureau of Standards, lists the 1981 output of papers documenting the results of the Bureau's current programs. The various media in which these papers appeared are described in sections 2 and 3.

\section{PERIODICALS}

Journal of Research-The Journal of Research of the National Bureau of Standards reports NBS research and development in those disciplines of the physical and engineering sciences in which the Bureau is active. These include physics, chemistry, engineering, mathematics, and computer sciences. Papers cover a broad range of subjects, with major emphasis on measurement methodology and the basic technology underlying standardization. Also included from time to time are survey articles on topics closely related to the Bureau's technical and scientific programs. As a special service to subscribers each issue contains complete citations to all recent Bureau publications in both NBS and non-NBS media. Issued six times a year.

Board of Editors for 1981:

Churchill Eisenhart, Executive Editor (Mathematics)

John W. Cooper (Physics)

Vacant (Chemistry)

Andrew J. Fowell (Engineering)

Joseph O. Harrison (Computer Science)

Howard J. M. Hanley (Boulder Labs.)

DIMENSIONS/NBS-This magazine has been discontinued with the October 1981 issue.

The table of contents for issues published in 1981 are listed in section 5.3 .
Journal of Physical and Chemical Reference Data (JPCRD)_This Journal is published quarterly by the American Chemical Society and the American Institute of Physics for the National Bureau of Standards. The Journal provides critically evaluated physical and chemical property data, fully documented as to the original sources and the criteria used for evaluation. Critical reviews of measurement techniques assess the accuracy of available data in a given technical area. The principal source for the Journal is the National Standard Reference Data System (NSRDS). The Journal is not intended as a publication outlet for original experimental measurements normally reported in the primary research literature, nor for descriptive or primarily theoretical review articles. (See also sec. 5.8 National Standard Reference Data Series.)

\section{NONPERIODICALS}

Monographs-major contributions to the technical literature on various subjects related to the Bureau's scientific and technical activities.

Handbooks-recommended codes of engineering and industrial practice (including safety codes) developed in cooperation with interested industries, professional organizations, and regulatory bodies.

Special Publications-include proceedings of conferences sponsored by NBS, NBS annual reports, and other special publications appropriate to this grouping such as wall charts, pocket cards, and bibliographies. Special subject-matter subseries include Semiconductor Measurement Technology (SP400-), Standard Reference Materials (SP260-), Precision Measurement and Calibration (SP300.), Law Enforcement Technology (SP480.), and Computer Science and Technology (SP500- ).

Applied Mathematics Series-mathematical tables, manuals, and studies of special interest to physicists, engineers, chemists, biologists, mathematicians, computer programmers, and others in scientific and technical work.

National Standard Reference Data Series-provides quantitative data on the physical and chemical properties of materials, compiled from the world's literature and critically evaluated. Developed under a worldwide program coordinated by NBS, under the authority of the National Standard Data Act (Public Law 90-396). This series supplements the JPCRD described in section 2. 
Building Science Series-disseminates technical information developed at the Bureau on building materials, components, systems, and whole structures. The series presents research results, test methods, and performance criteria related to the structural and environmental functions and the durability and safety characteristics of building elements and systems.

Federal Information Processing Standards Publications (FIPS PUBS)-publications in this series collectively constitute the Federal Information Processing Standards Register. The Register serves as the official source of information in the Federal Government regarding standards issued by NBS pursuant to the Federal Property and Administrative Services Act of 1949 as amended, Public Law 89.306 (79 Stat. 1127), and as implemented by Executive Order 11717 (38 FR 12315, dated May 11, 1973) and Part 6 of Title 15 CFR (Code of Federal Regulations).

Voluntary Product Standards-developed under procedures published by the Department of Commerce in Part 10, Title 15, of the Code of Federal Regulations. The standards establish nationally recognized requirements for products, and provide all concerned interests with a basis for common understanding of the characteristics of the products. NBS administers the program as a supplement to the activities of the private sector standardizing organizations.

Technical Notes-studies or reports complete in themselves but restrictive in their treatment of a subject. Analogous to monographs but not so comprehensive in scope or definitive in treatment of the subject area. Often serve as a vehicle for final reports of work performed at NBS under the sponsorship of other government agencies. Special subject-matter subseries include Optical Radiation Measurements (TN594- ) and Self Calibrations Manual for Optical Radiation (TN910- ).

Consumer Information Series-practical information, based on NBS research and experience, on areas of interest to the consumer. Easily understandable language and illustrations provide useful background knowledge for shopping in today's technological marketplace.

NBS Interagency Reports-a special series of interim or final reports on work performed by NBS for outside sponsors (both government and non-government). In general, initial distribution is handled by the sponsor; public distribution is by the National Technical Information Service (NTIS), Springfield, VA 22161, in paper copy or microfiche form.

\section{Grant/Contract Reports and NBS Patents-}

Grant/Contract Reports are prepared by non-NBS persons or organizations working under grant or contract from the National Bureau of Standards. Those contract reports not incorporated into the formal NBS publication series are available directly from the National Technical Information Service, Springfield, VA 22161, in paper copy or microfiche unless otherwise stated. When ordering a report from NTIS you must order it by the "COM, PB, AD, or N" number as indicated.

Patents are obtained on NBS inventions with high commercial potential, to establish Government ownership of the patent rights. The patents are then made available for the grant of nonexclusive licenses to all qualified applicants. A limited exclusive license may be granted under a particular patent if it appears some period of exclusivity is necessary as an incentive for the investment of risk capital. For information on licensing any of the NBS-held patents, write to the Office of the Legal Adviser, National Bureau of Standards, Washington, DC 20234.

Papers Published in Non-NBS Media-reflect significant contributions by NBS authors and are cited annually in this catalog. Citations, key words, and abstracts for these papers are also published bi-monthly in the NBS Journal of Research.

\section{PURCHASE PROCEDURES AND DOCUMENT AVAILABILITY}

Most publications of the Bureau are available from the Superintendent of Documents, U.S. Government Printing Office, Washington, DC 20402. You may also order through the U.S. Department of Commerce District Office nearest you (see app. B).

Microfiche copies of all recent NBS publications, and paper copies of many nonperiodicals, may be ordered through the National Technical Information Service, U.S. Department of Commerce, Springfield, VA 22161.

FIPS PUBS, NBS Interagency Reports (NBSIR's), and Grant/Contract Reports (GCR's) are available only from the National Technical Information Service, Springfield, VA 22161.

Copies of patents may be obtained from the U.S. Patent and Trademark Office, Washington, DC 20231, for 50 cents each.

Photoduplicated copies of many NBS publications can be purchased from the Library of Congress. For full information concerning this service, write to the Photoduplication Service, Library of Congress, Washington, DC 20540.

How to Make Remittances to the Superintendent of Documents. Remittances for publications should be mailed to Superintendent of Documents, U.S. Government Printing Office, Washington, DC 20402, by money order or check. Master Card and VISA are also accepted. Be sure to give your credit card number and expiration date on all orders. Postage 
stamps will not be accepted. Publications cannot be mailed before remittances are received. Foreign remittances should be made either by international money order, draft on an American or Canadian bank, or UNESCO coupons.

The letter symbol, publication number, full title of the publication, and SD stock number MUST be given when ordering. The Superintendent of Documents allows a discount of 25 percent on orders of 100 or more copies of one publication, when mailed to a single address.

Persons who make frequent purchases from the Superintendent of Documents may find a deposit account convenient. Deposits of $\$ 50$ or more are accepted, against which orders may be placed without making individual remittances or first obtaining quotations. Order forms are furnished for this purpose. After the order has been processed, the order itself is returned, showing the publications supplied, explanations regarding those not sent, the amount of charge, and the balance on deposit.

No charge is made for postage on documents sent to points in the United States and its possessions. In computing foreign postage, the charge is approximately one-fourth of the current selling price of the publication. The charge is to cover special handling required to comply with customs and international mailing regulations.

How to Make Remittances to NTIS. Orders for publications purchased from the National Technical Information Service (NTIS) must be accompanied by postal money order, express money order, or check made out to the NTIS and covering total cost of the publications order. NTIS also accepts charges to American Express, VISA, or Master Card. You may also establish an NTIS deposit account by contacting them for this service. All inquiries or orders should be addressed to: National Technical Information Service, Springfield, VA 22161.

SD and NTIS order forms are included at the end of this publication for your convenience in ordering.

\subsection{NBS PERIODICAL SUBSCRIPTION RATE}

Periodical
Journal of Research of the National
Bureau of Standards:
Paper covers ..............................
Bound volume (1 volume per year),
blue buckram ..................................
NOTE_Send order, with remittance, to Superintendent of Documents, U.S. Government
Printing Office, Washington, DC 20402.
${ }^{1}$ United States and its possessions.
${ }_{2}^{2}$ Foreign price includes the cost of the publication and postage.
${ }^{3}$ Prices of the bound volumes vary. The Superintendent of Documents will furnish prices on
request.

\subsection{PRICES FOR NONPERIODICALS}

Prices are subject to change without notice, and the prices charged on your order will be those in effect as of the date your order is processed. Except for the Federal Information Processing Standards Series (FIPS) and the National Bureau of Standards Interagency Reports (NBSIR's), which are only available by purchase from the National Technical Information Service, Springfield, VA 22161, publications may be ordered from the Superintendent of Documents, U.S. Government Printing Office. SD order forms are included at the end of this publication.

For availability and price of Patents see section 4.

Your nearest depository library may still have a copy of out-of-print NBS publications. (See sec. 4.5.) Many NBS publications may be purchased from the National Technical Information Service. (See sec. 4.)

\subsection{FORMER NBS REFERENCE PUBLICATIONS}

Certain NBS publications are out of print because they have been replaced or partially replaced by material issued by other organizations. NBS is able to offer the following information on some of these publications:

Circular 410, National Standard Petroleum Oil Tables. Information in this Circular has been incorporated in the American Edition-ASTM Petroleum Measurement Tables issued as PCN12-4125. 10-12 by the American Society for Testing and Materials, 1916 Race Street, Philadelphia, PA 19103. Available at $\$ 30,20$ percent discount to ASTM members. Tables 5 and 7 of the ASTM Petroleum Measurement Tables may also be purchased from the ASTM in separate reprint form at $\$ 2.25$ and $\$ 2$ per copy, respectively.

Circular 438, Static Electricity. The National Fire Protection Association, Batterymarch Park, Quincy, MA 02269, has issued a publication by the same title, available from them as NFPA Publication $77, \$ 6$.

Circular 499, Nuclear Data. Replaced by Atomic Data and Nuclear Data Tables, published by Academic Press, 111 Fifth Avenue, New York, NY 10003. Available bimonthly for $\$ 78$.

Circular 547, Section 1, Precision Laboratory Standards of Mass Laboratory-Weights. Information in this Circular has been incorporated in the ANSI/ASTM E 617-78, Standard Specification for Laboratory Weights and Precision Mass Standards issued by the American Society for Testing and Materials, 1916 Race Street, Philadelphia, PA 19103, Attn: Sales, for \$4. 
Circular 576, Automotive Antifreezes. For information on this subject consult American National Standards Institute, 1430 Broadway, New York, NY 10018.

Circular 577 and Supplement, Energy Loss and Range of Electrons and Positrons. These have been superseded by NASA Special Publication 3012, available from the National Technical Information Service, Springfield, VA 22161 , as N65-12506, at $\$ 13.50$ hardcopy and N67. 14099 , at $\$ 7.50$ hardcopy and $\$ 4$ microfiche.

Miscellaneous Publication 179, American Standard Building Code Requirements for Minimum Design Loads in Buildings and Other Structures. The American National Standards Institute, 1430 Broadway, New York, NY 10018, has issued a publication on this subject. A vailable from them as A58.1-1972, at \$9.75.

Miscellaneous Publication 187, Directory of Commercial and College Laboratories. A new revised Directory of Testing Laboratories, issued as STP 333E, is published by the American Society for Testing and Materials, 1916 Race Street, Philadelphia, PA 19103, at \$15.

NBS Handbook 28, Parts 1, 2, and 3, Screw Thread Standards. Federal Government responsibility for screw thread standards has been transferred to the General Services Administration (GSA). Standards will be promulgated by the GSA as Federal Standard 28. Copies of Federal Standard $\mathrm{H} 28$ are available from: GSA Specifications, Bldg. 197, Washington Navy Yard, Washington, DC 20407 for $\$ 25.80$ per set. Telephone: 202-472-2205. Questions regarding administration of the program should be addressed to General Products Division (FREGM), General Services Administration (Federal Supply Service), Washington, DC 20406. Telephone: 703-557-7595.

Handbook 30, National Electrical Safety Code (also H81 and its Supplements and H110-1). All NBS publications on this subject have been superseded by National Electrical Safety Code, 1981 Edition, issued by the American National Standards Institute, 1430 Broadway, New York, NY 10018. Available from them as ANSI C2-1977, at \$9.75.

Handbook 46, Code for Protection Against Lightning. A United States of America Standards Institute Code for Protection Against Lightning (NFPA-78-1980) is available from the American National Standards Institute, 1430 Broadway, New York, NY 10018, at $\$ 5.75$, as ANSI/NFPA78-1980.

Handbook 48, Control and Removal of Radioactive Contamination in Laboratories. Reprints of this Handbook can be purchased as NCRP Report 8 at $\$ 6$ from NCRP Publications, P.O. Box 30175, Washington, DC 20014.

Handbook 49, Recommendations for Waste Disposal Phosphorus-32 and Iodine-131 for Medical Users.
Reprints of this Handbook can be purchased as NCRP Report 9 at $\$ 6$ from NCRP Publications, P.O. Box 30175, Washington, DC 20014.

Handbook 53, Recommendations for the Disposal of Carbon-14 Wastes. Reprints of this Handbook can be purchased as NCRP Report 12 at \$6 from NCRP Publications, P.O. Box 30175, Washington, DC 20014.

Handbook 55, Protection Against Betatron-Synchrotron Radiations up to 100 Million Electron Volts, February 26, 1954 has been combined with NBS Handbook 97. Available as NCRP Report 51, Radiation Protection Design Guidelines for $0.1-100 \mathrm{MeV}$ Particle Accelerator Facilities from NCRP Publications, P.O. Box 30175, Washington, DC 20014, at \$9.

Handbook 58, Radioactive Waste Disposal in the Ocean. Reprints of this Handbook can be purchased as NCRP Report 16 at $\$ 6$ from NCRP Publications, P.O. Box 30175, Washington, DC 20014.

Handbook 59, Permissible Dose from External Sources of Ionizing Radiations. Reprints of this Handbook can be purchased as NCRP Report 39 at \$8 from NCRP Publications, P.O. Box 30175, Washington, DC 20014.

Handbook 63, Protection Against Neutron Radiation up to $30 \mathrm{MeV}$. Reprints of this Handbook can be purchased as NCRP Report 38 at $\$ 9$ from NCRP Publications, P.O. Box 30175, Washington, DC 20014.

Handbook 65, Safe Handling of Bodies Containing Radioactive Isotopes. Reprints of this Handbook can be purchased as NCRP Report 37 at \$8 from NCRP Publications, P.O. Box 30175, Washington, DC 20014.

Handbook 69, Maximum Permissible Body Burdens and Maximum Permissible Concentrations of Radionuclides in Air and in Water for Occupational Exposure. Reprints of this Handbook can be purchased as NCRP Report 22 at \$6 from NCRP Publications, P.O. Box 30175, Washington, DC 20014.

Handbook 71, Specifications for Dry Cells and Batteries. Available as ANSI C18.1-1979 from the American National Standards Institute, 1430 Broadway, New York, NY 10018, at \$6.

Handbook 73, Protection Against Radiations from Sealed Gamma Sources (Supersedes H54). Reprints of this Handbook can be purchased as NCRP Report 40 at $\$ 8$ from NCRP Publications, P.O. Box 30175, Washington, DC 20014.

Handbook 74, Building Code Requirements for Reinforced Masonry. The American National Standards Institute, 1430 Broadway, New York, NY 10018 has issued a publication on this subject. Available from them as ANSI/NBS Handbook H74-1960 (R1970), at $\$ 4.50$. 
Handbook 75, Measurement of Absorbed Dose of Neutrons and of Mixtures of Neutrons and Gamma Rays. Reprints of this Handbook can be purchased as NCRP Report 25 at $\$ 6$ per copy from NCRP Publications, P.O. Box 30175, Washington, DC 20014.

Handbook 76, Medical X-Ray Protection Up to Three Million Volts. Now available as NCRP 33. Purchase from NCRP Publications, P.O. Box 30175, Washington, DC 20014, at $\$ 7$.

Handbook 80, a Manual of Radioactivity Procedures. Reprints of this Handbook are available as NCRP Report 58 , for paper copy at $\$ 14$, and buckram at $\$ 16$. For more information write to NCRP Publications, P.O. Box 30175, Washington, DC 20014.

Handbook 81 and Its Supplements, Safety Rules for the Installation and Maintenance of Electric Supply and Communication Lines (also H30 and H110-1). All NBS publications on this subject have been superseded by National Electrical Safety Code, 1981 Edition, issued by the American National Standards Institute, 1430 Broadway, New York, NY 10018. Available from them as ANSI C2-1977, at $\$ 9.75$.

Handbook 84, Radiation Quantities and Units. Reprints of this Handbook can be purchased as ICRU Report 33 at $\$ 8.50$ from ICRU Publications, P.O. Box 30165, Washington, DC 20014.

Handbook 85, Physical Aspects of Irradiation. Reprints of this Handbook can be purchased as ICRU Report 10b at $\$ 5.50$ per copy from ICRU Publications, P.O. Box 30165, Washington, DC 20014.

Handbook 86, Radioactivity. Reprints of this Handbook can be purchased as ICRU Report 10C, Radioactivity at \$4 from ICRU Publications, P.O. Box 30165, Washington, DC 20014.

Handbook 87, Clinical Dosimetry. Information in this Handbook has been incorporated in ICRU Report 23 at $\$ 8$ per copy; ICRU Report 24 at $8 \$$ per copy; and ICRU Report 29 at $\$ 10$ per copy from ICRU Publications, P.O. Box 30165, Washington, DC 20014.

Handbook 88, Radiobiological Dosimetry. Reprints of this Handbook can be purchased as ICRU Report 30, Quantitative Concepts and Dosimetry in Radiobiology at $\$ 10$ from ICRU Publications, P.O. Box 30165, Washington, DC 20014.

Handbook 89, Methods of Evaluating Radiological Equipment and Materials. Reprints of this Handbook can be purchased as ICRU Report $10 \mathrm{~F}$ at $\$ 3.50$ from ICRU Publications, P.O. Box 30165, Washington, DC 20014.

Handbook 96, Inspection of Processed Photographic Record Films for Aging Blemishes. Reprints of this
Handbook can be purchased as PH 1.28-1976 at $\$ 5.50$ from the American National Standards Institute, 1430 Broadway, New York, NY 10018.

Handbook 97, Shielding for High-Energy Electron Accelerator Installations. July 1, 1964, has been combined with NBS Handbook 55. Available as NCRP Report 51, Radiation Protection Design Guidelines for 0.1-100 MeV Particle Accelerator Facilities from NCRP Publications, P.O. Box 30195, Washington, DC 20014 , at $\$ 9$.

Handbook 102, ASTM Metric Practice Guide. Available as ANSI/ASTM E380-76 from the American National Standards Institute, 1430 Broadway, New York, NY 10018 , at $\$ 4$.

Handbook 110-1, National Electrical Safety Code. Part 1. Rules for Installation and Maintenance of Electric Supply and Communication Lines (also H30 and H81 and its Supplements). All NBS publications on this subject have been superseded by National Electrical Safety Code, 1981 Edition, issued by the American National Standards Institute, 1430 Broadway, New York, NY 10018. Available from them as ANSI C21977 at $\$ 9.75$.

Technical Note 938, Recommended Practice for the Use of Metric (SI) Units in Building Design and Construction, has been superseded by ASTM E621.78, Standard Practice of the Use of Metric (SI) Units in Building Design and Construction. It is available from the American Society for Testing and Materials, 1916 Race Street, Philadelphia, PA 19103, at \$4.

\subsection{ANNOUNCEMENTS OF NBS PUBLICATIONS}

The National Bureau of Standards and the agencies mentioned below regularly announce NBS publications:

DIMENSIONS/NBS. Discontinued October 1981. Issues published in 1981 are included in this catalog.

NBS Journal of Research. The Journal carries a listing of all NBS Publications as issued. See section 4.1 for subscription information.

NBS Publications Newsletter. Discontinued June 1981.

Monthly Catalog of United States Government Publications. Issued by the Superintendent of Documents, U.S. Government Printing Office, Washington, DC 20402. Annual subscription, with consolidated annual index, $\$ 90 ; \$ 112.50$ foreign.

Commerce Publications Update. Biweekly announcement of publications of the Department of Commerce. Lists titles and prices of National Bureau of Standards publi- 
cations, as well as those of other offices of the Department of Commerce. Contact the Editorial Policy and Review Division, Office of Publications, U.S. Department of Commerce, Washington, DC 20230, for information on how to receive this listing.

NBS Catalogs of NBS Publications. These catalogs list all NBS publications through December 31, 1981. The catalogs are available from the Superintendent of Documents, U.S. Government Printing Office, Washington, DC 20402, unless otherwise stated, or may be consulted in a library which maintains sets of National Bureau of Standards publications.

Circular 460: Publications of the National Bureau of Standards 1901 to June 30, 1947, 375 pages including subject and author indexes. Brief abstracts are included for the period January 1, 1941 to June 30, 1947.

Supplement to Circular 460: Publications of the National Bureau of Standards, July 1, 1947 to June 30, 1957. 373 pages, including subject and author indexes.....

Miscellaneous Publication 240: Publications of the National Bureau of Standards, July 1, 1957 to June 30, 1960. First NBS Catalog to include titles of papers published in outside journals 1950 to 1959,391 pages including subject and author indexes.....

Supplement to Miscellaneous Publication 240: Publications of the National Bureau of Standards published by NBS, July 1960 through June 1966; published by others, 1960 through 1965. 740 pages including subject and author indexes............

Special Publication 305: Publications of the National Bureau of Standards, published by NBS, July 1966 through December 1967; published by others, 1966-1967. 223 pages, including author and key word indexes.

Supplement 1 to Special Publication 305: Publications of the National Bureau of Standards, 1968 through 1969. 497 pages including author and key word indexes...

Supplement 2 to Special Publication 305: Publications of the National Bureau of Standards, 1970. 378 pages including author and key word indexes....

Supplement 3 to Special Publication 305: Publications of the National Bureau of Standards, 1971. 342 pages including author and key word indexes.

Supplement 4 to Special Publication 305: Publications of the National Bureau of Standards, 1972. 449 pages including author and key word indexes.

Supplement 5 to Special Publication 305: Publications of the National Bureau of Standards, 1973. 349 pages including author and key word indexes

Supplement 6 to Special Publication 305: Publications of the National Bureau of Standards, 1974. 523 pages including author and key word indexes

Supplement 7 to Special Publication 305: Publications of the National Bureau of Standards, 1975. 595 pages including author and key word indexes

Supplement 8 to Special Publication 305: Publications of the National Bureau of Standards, 1976. 728 pages including author and key word indexes.

Supplement 9 to Special Publication 305: Publications of the National Bureau of Standards, 1977. 601 pages including author and key word indexes.

Supplement 10 to Special Publication 305: Publications of the National Bureau of Standards, 1978. 679 pages including author and key word indexes...

Supplement 11 to Special Publication 305: Publications of the National Bureau of Standards, 1979. 615 pages including author and key word indexes.
Supplement 12 to Special Publication 305: Publications of the National Bureau of Standards, 1980. 634 pages including author and key word indexes ..............................................

Supplement 13 to Special Publication 305: Publications of the National Bureau of Standards, 1981. 000 pages including author and key word indexes.

Special Publication 535, Catalog of NBS Publications, 1966 . 1976. Volumes 1 (2 parts) and 2 (2 parts). Consolidated reprint of bibliographic citations, abstracts, and key words from NBS SP305, and its Supplements 1.8.

SP535, Volume 1

$\$ 23.75$

SP535, Volume 2

$\$ 21.25$

*Available by purchase from the National Technical Information Service, Springfield, VA 22161.

\subsection{DEPOSITORY LIBRARIES IN THE UNITED STATES}

The Superintendent of Documents, U.S. Government Printing Office, is authorized by law to furnish Government publications to designated depository libraries.

Under Provisions of Title 44 of the United States Code, certain libraries are designated depositories for Government publications. Through them, Federal Government documents are made available to residents of every State, the District of Columbia, Guam, Puerto Rico, and the Virgin Islands. Distribution to the libraries is made by the Superintendent of Documents.

It is sometimes impossible to obtain desired publications by purchase from the Superintendent of Documents. Stocks may have been exhausted or the document may be permanently out of print. The depositories provide a valuable service by keeping such publications permanently available. Every Government publication cannot be consulted at all depository libraries. Designated Regional Depositories are required to receive and retain one copy of all Government publications made available to depository libraries either in printed or microfacsimile form. All other libraries are allowed to select the classes of publications best suited to the interest of their particular clientele.

These libraries are now receiving selected publication series of the National Bureau of Standards for general reference use. Whether a given library has a copy of a particular publication can be determined by inquiring at the library.

\subsection{U.S. DEPARTMENT OF COMMERCE DISTRICT OFFICES}

U.S. Department of Commerce District Offices provide ready access at the local level to the services of the Department of Commerce and its reports, publications, statistical statements, and surveys. Most District Offices serve as official sales agents of the Superintendent of Documents, U.S. Government 
Printing Office, making available for purchase locally a wide range of Government business publications. The reference library maintained by each District Office contains many Government and private publications, periodicals, directories, reports, and other reference materials. 



\section{TITLES AND ABSTRACTS OF NBS PUBLICATIONS, 1981 5.1 PAPERS FROM THE JOURNAL OF RESEARCH OF THE NATIONAL BUREAU OF STANDARDS, VOLUME 86, JANUARY-DECEMBER 1981}

\section{January-February 1981}

Mandel, J. Fitting curves and surfaces with monotonic and nonmonotonic four parameter equations. J. Res. Nat. Bur. Stand. (U.S.). 86(1): 1.25; 1981 January.February.

Key words: curve fitting; empirical fitting; surface fitting.

This is a series of three papers in which methods are presented, with instructions on computational details, on the empirical fitting of tabulated data. Part I deals with fitting functions of a single argument; Part II with functions of two arguments; and Part III with functions of three or more arguments.

Jones, F. E. The refractivity of air. J. Res. Nat. Bur. Stand. (U.S.). 86(1): 27.32; 1981 January-February.

Key words: air density; index of refraction of air; refractivity of air; wavelength of light in air.

The air density equation of Jones, Edlén's dispersion formula for standard air, and Edlén's empirically-derived expressions for the effects of $\mathrm{CO}_{2}$ abundance and water vapor partial pressure on refractivity have been combined into a simplified equation for the refractivity of air, and estimates have been made of uncertainties in calculated refractivity. Under ambient conditions typical of metrology laboratories, the agreement between the simplified equation and Edlén's formulation is well within the uncertainty in each. The simplified equation is valid in the visible region.

Roth, C. The configurations $3 d^{n} 4 p+3 d^{n-1} 4 s 4 p+3 d^{n-2} 4 s^{2} 4 p$ in the first spectra of the iron group. J. Res. Nat. Bur. Stand. (U.S.). 86(1): 33 77; 1981 January-February.

Key words: iron group elements; least squares optimization; theoretical spectroscopy.

Energy levels and Lande g-factors for the configurations $3 d^{\pi} 4 p+$ $3 d^{n-1} 4 s 4 p+3 d^{m-2} 4 s^{2} 4 p$ in the first spectra of the iron group were calculated and compared with experimental values, in both general and individual treatments. The calculations were done in intermediate coupling taking into account explicity the interactions between configurations, as well as complete effective interactions of the core, and effective interactions $d-p$. Due to a successful starting point based on Hartree-Fock calculations for the Slater parameters, as well as the insertion of the effective interactions, considerable improvement was obtained compared to previous results.

On fitting 1537 levels using 67 free interaction parameters a mean error of $182 \mathrm{~cm}^{-1}$ was obtained. Altogether 3652 energy levels were calculated including all the levels for the configurations $3 d^{m-2} 4 s^{2} 4 p$ across the sequence. It was shown that all interaction parameters could be expressed either as linear functions, or linear functions with small quadratic corrections, of the atomic number. There was general qualitative agreement between the values of the parameters calculated using the semi-empirical method and those calculated using HartreeFock methods. There remained some isolated terms with large deviations. These are attributed to be due to the interactions with the configurations $(3 d+4 s)^{n} 5 p$, that were not considered explicitly in this analysis.

Tables comparing the experimental and calculated energy levels and Lande g-factors, as well as detailed analyses for each spectrum are given in another paper.

Malkus, D. S.; Fuller, E. R., Jr. An isoparametric finite element model for large-strain elastostatics. J. Res. Nat. Bur. Stand. (U.S.). 86(1): 79-109; 1981 January-February.

Key words: checkerboard pressure; elastostatics; finite elements; in-core solver; isoparametrics; Mooney-Rivlin material; nonlinear equation-solvers; penalty method; plane-stress; strain invariants; tensile-test specimen.

This paper describes a simple finite element model for large-strain elastostatics. The realization of the model in a small-scale computercode is described. The purpose of the model is to produce test problems for research on the application of penalty techniques in nonlinear elasticity. For this reason the code must balance the requirements of reasonable flexibility with those of computational economy. The current code employs multilinear isoparametric elements. The model is capable of generalization to a variety of element types. The solution method employed is that of incremental loading combined with the Newton-Raphson method. Symmetric, banded systems of equations are produced which are solved in-core. Two-and three-dimensional symmetric bodies which are isoparametric images of a reference "brick" may be modeled. An example comparing two- and three-dimensional models of a "dogbone"-shaped A.S.T.M. rubber tensile-test specimen is presented. The results shed some light on the nature of stress-concentrations which occur in specimens of this geometry.

\section{March-April 1981}

Younglove, B. A. Velocity of sound in liquid propane. J. Res. Nat. Bur. Stand. (U.S.). 86(2): 165-170; 1981 March-April.

Key words: adiabatic compressibility; liquid; pressure; propane; sound; specific heat ratios; speed; temperature; velocity.

Sound velocity measurements on liquid propane from 90 to $300 \mathrm{~K}$ and for pressures to $34 \mathrm{MPa}$ are reported. Also included are saturated liquid sound velocities from 90 to $290 \mathrm{~K}$. The data were combined with $P \rho T$ data to compute compressibility and specific heat ratio. Comparisons were made to computed values of sound velocity of Goodwin and to the data of Lacam.

Mahajan, B. M.; Galowin, L. S.; Kopetka, P. A. Models of quasisteady and unsteady discharge from plumbing fixtures. J. Res. Nat. Bur. Stand. (U.S.). 86(2): 171-179; 1981 March-April.

Key words: characteristics; discharge; drainage; flow; model; plumbing; quasi-steady; unsteady.

Modeling methods are developed to predict the discharge characteristics of simulated simplified configurations for plumbing fixtures connected to horizontal drain branch piping. Computations are carried out to illustrate several methods of determining the effect of various loss coefficients of the drain connection, pipe length, pipe diameter, and friction factor. Solutions are obtained for the case of a fixture with a constant head (continuous refill) and a falling head (emptying of a sink). Numerical solution of the non-linear differential equation for the falling head case was obtained by the Runge-Kutta method. Discharge characteristics are presented for a range of flows and pipe diameter-to-length ratios representative of plumbing installations. The feasibility of developing predictive models for hydraulic characteristics of interconnected plumbing fixture and drainage piping systems is shown. The variations of efflux rate with the drain pipe diameter, length, and slope obtained from the assumed models are similar in trend to the available experimental data.

Magin, R. L.; Mangum, B. W.; Statler, J. A.; Thornton, D. D. Transition temperatures of the hydrates of $\mathrm{Na}_{2} \mathrm{SO}_{4}, \mathrm{Na}_{2} \mathrm{HPO}_{4}$, and KF as fixed points in biomedical thermometry. J. Res. Nat. Bur. Stand. (U.S.). 86(2): 181-192; 1981 March-April.

Key words: biomedical thermometry; disodium hydrogen phosphate; fixed point; hydration temperature; potassium fluoride; salt hydrate; sodium sulfate; temperature calibration; temperature standard; thermistor; thermometer; transition temperature.

The hydrate transition temperatures of $\mathrm{Na}_{2} \mathrm{SO}_{4} \cdot 10 \mathrm{H}_{2} \mathrm{O}$ to $\mathrm{Na}_{2} \mathrm{SO}_{4}$, 
$\mathrm{KF} \cdot 2 \mathrm{H}_{2} \mathrm{O}$ to $\mathrm{KF}$, and $\mathrm{Na}_{2} \mathrm{HPO}_{4} \cdot 7 \mathrm{H}_{2} \mathrm{O}$ to $\mathrm{Na}_{2} \mathrm{HPO}_{4} \cdot \mathrm{H}_{2} \mathrm{O}$ were established using ACS grade salts as $32.374^{\circ} \mathrm{C}, 41.422^{\circ} \mathrm{C}$, and $48.222^{\circ} \mathrm{C}$, respectively. A simple and reliable procedure involving inexpensive materials was used to realize these transitions as temperature fixed points. Each transition temperature was attained within 30 minutes of hydrate initiation and remained constant to within $\pm 0.002^{\circ} \mathrm{C}$ for more than 10 hours if the mixture was stirred. The established transition temperatures were sensitive at the $0.001^{\circ} \mathrm{C}$ level to the amount of impurities, so the materials used should be of the highest quality available.

These systems fill a gap in the existing spectrum of temperature standards and should be useful in biomedical laboratories for calibrating thermometers.

Pearl, M. H.; Goldman, A. J. A game-theoretic model of inspectionresource allocation. J. Res. Nat. Bur. Stand. (U.S.). 86(2): 193-215; 1981 March-April.

Key words: inspection; mathematical model; regulation; theory of games.

This paper presents a generalization of a game-theoretic model, first described in an earlier paper, of the relationship between an inspectee who may decide to "cheat" or not, and an inspector whose task it is to minimize the expected gain that the inspectee achieves by cheating. When cheating is detected by the inspector, a penalty is assessed against the inspectee. The generalized model permits imposing a relationship between the level of the penalty to the inspectee when he/she is caught and the value to the inspectee of not being caught when he/she is cheating. The solution of the game takes on different forms depending on whether or not the inspector's resources are sufficient to make the detection of cheating likely.

\section{May.June 1981}

Driver, R. G.; Houck, J. C.; Welch, B. E. An intercomparison of pressure standards between LNE and NBS. J. Res. Nat. Bur. Stand. (U.S.). 86(3): 277.279; 1981 May-June.

Key words: effective area; intercomparison; piston gage; pressure; primary standard; transfer standard.

An intercomparison between a transfer piston gage used by the Laboratoire National d'Essais (LNE) and a primary standard piston gage of the National Bureau of Standards was performed over the range of pressure of 0.4 to $3.9 \mathrm{MPa}$. The agreement between the computation of the effective area of the LNE gage by the two laboratories was within $6.4 \mathrm{ppm}$ and the agreement between the average of the pressures generated by these two gages was within 3.3 ppm, well below the estimated uncertainty of either gage (NBS 30 ppm and LNE 24 ppm).

deCastro, C. A. N.; Roder, H. M. Absolute determination of the thermal conductivity of argon at room temperature and pressures up to $68 \mathrm{MPa}$. J. Res. Nat. Bur. Stand. (U.S.). 86(3): 293.307; 1981 May.June.

Key words: ambient temperature; argon; critical enhancement; hard sphere; hot wire; thermal conductivity; transient.

The thermal conductivity of argon at room temperature and for pressures up to $68 \mathrm{MPa}$ has been measured with a transient hot-wire technique in order to assess the accuracy of an instrument of this type. The data are presented for a nominal temperature of $300.65 \mathrm{~K}$ and comparison with other authors shows that our data is accurate to within \pm 1 percent, and it is the most accurate set of data for pressures above $35 \mathrm{MPa}$. Experimental evidence of a thermal conductivity enhancement near the critical density for a temperature about twice the critical temperature is herein reported. The experimental data were compared with the values predicted by the hard sphere model and it has been found that the theory gives values that are about 4 percent lower than the experimental ones in the density range $0-400 \mathrm{~kg} / \mathrm{m}^{3}$ and about 1 to 2 percent lower in the high density region $\quad 400-825 \mathrm{~kg} / \mathrm{m}^{3}$.

Graminski, E. L.; Parks, E. J. The effect of calcium carbonate on the stability of acid treated papers. J. Res. Nat. Bur. Stand. (U.S.). 86(3): 309.315; 1981 May.June.

Key words: alkaline reserve; cellulose stabilizers; paper deacidification; paper destabilization; paper permanence; $\mathrm{pH}$ history.

Exposure of kraft wood pulps to an acidic medium results in a destabilization of wood pulp. The degree of destabilization appears to depend on the concentration of acid the pulp is exposed to. The addition of calcium carbonate to acid destabilized pulp does not restore the pulp to its original stability. The absorption of alkali metals in $\mathrm{pH}$ dependent which could explain the destabilization of wood pulps when exposed to an acid medium. A number of questions arise about the merit of stabilizing degraded paper documents by deacidification with alkaline earth salts and the usefulness of an alkaline reserve in paper.

Shier, D. R.; Witzgall, C. Properties of labeling methods for determining shortest path trees. J. Res. Nat. Bur. Stand. (U.S.). 86(3): 317-330; 1981 May-June.

Key words: complexity; labeling; network; sequence list; shortest path; tree.

A number of labeling procedures for determining shortest paths in a network employ a sequence list in order to carry out the required steps systematically. This paper studies certain formal properties of such sequence lists. It is shown that the desirable property of branching out from nodes whose labels represent actual in-tree distances is assured for certain ways of managing the sequence list, but not for others. The relationship of this property to the computational complexity of various labeling procedures is also investigated.

\section{July-August 1981}

Brown, R. L. A method of calculating tunneling corrections for Eckart potential barriers. J. Res. Nat. Bur. Stand. (U.S.). 86(4): 357-359; 1981 July-August.

Key words: chemical rate constants; Eckart potential; potential barriers; quantum mechanical tunneling calculations; tunneling corrections.

A method is presented for the direct calculation of tunneling corrections for unsymmetrical Eckart type potential barriers. It is based on a modified 6-point Gaussian quadrature formula. Accuracy is better than 1 percent over a wide range of tunneling parameter values.

DeVoe, H.; Miller, M. M.; Wasik, S. P. Generator columns and high pressure liquid chromatography for determining aqueous solubilities and octanol-water partition coefficients of hydrophobic substances. $J$. Res. Nat. Bur. Stand. (U.S.). 86(4): 361-366; 1981 July-August.

Key words: generator column; hydrophobic; octanol; partition coefficient; propylbenzene; solubility; solute; water.

Generator columns packed with a solid support and loaded with a liquid organic phase make it possible to rapidly and conveniently equilibrate water with the organic phase. By coupling the generator column to an extractor column for high pressure liquid chromatographic analysis of the aqueous solution, errors from surface adsorption and loss to the atmosphere are avoided. Using this method, the mean values and confidence limits at a 95 percent confidence level of the aqueous solubility, $S$, and the octanol-water partition coefficient, $P$, of n-propylbenzene at $25^{\circ} \mathrm{C}$ were found to be 
$S=(4.32 \pm 0.02) \times 10^{-4} M$ and $\log P=3.720 \pm 0.003$.

Kilday, M. V. Enthalpies of solution of the nucleic acid bases. 6 . Guanine in aqueous $\mathrm{HCl}$ and $\mathrm{NaOH}$, and guanine hydrochlorides in aqueous HCl. J. Res. Nat. Bur. Stand. (U.S.). 86(4): 367.382; 1981 July-August.

Key words: calorimetry; $\mathrm{C}_{5} \mathrm{H}_{5} \mathrm{~N}_{5} \mathrm{O}$; density; enthalpy of solution; Gua. $\mathrm{HCl} \cdot 1 \mathrm{H}_{2} \mathrm{O}$; Gua.HCl.2 $\mathrm{H}_{2} \mathrm{O}$; Gua.2 $\mathrm{HCl}$; guanine (Gua); guanine hydrochlorides; heat capacity; nucleic acid base; solution calorimetry; thermochemistry; $\Delta C_{p}$ of reaction.

Values of the enthalphy of solution of well-characterized samples of guanine were obtained from measurements in an adiabatic solution calorimeter. Enthalpies of protonation were calculated for guanine.

Goldman, A. J. Player aggregation in noncooperative games, II. J. Res. Nat. Bur. Stand. (U.S.). 86(4): 383-391; 1981 July-August.

Key words: aggregation; equilibrium; game theory; mathematical economics; noncooperative games; total orders.

Conditions are given under which subsets of the players of a noncooperative game can be combined into "aggregate players" without changing the set of equilibrium-point solutions of the game. These conditions are shown to be the weakest possible ones with a certain specified kind of generality. "Approximate" versions of the results are also formulated and proven.

\section{September-October 1981}

Rainwater, J. C. Propagation of density fluctuations in nonuniform fluids: Simple models. J. Res. Nat. Bur. Stand. (U.S.). 86(5): 429.455; 1981 September-October.

Key words: damped wave motion; density fluctuations; diffusion; dynamic structure factor; initial value problem; nonuniform systems; one-dimensional models; Rayleigh-Brillouin scattering.

As a first step toward the understanding of Rayleigh-Brillouin scattering from a fluid with a temperature gradient, we analyze the initial value problem for certain prototype one-dimensional nonuniform systems. For sufficiently short times and localized initial pulses, it is not necessary to impose actual physical boundaries on the linearly nonuniform system. By a straightforward, though unconventional, application of Fourier and Laplace transform methods, explicit and physically reasonable solutions are constructed for the propagation of fluctuation pulses in space and time according to the nonuniform wave and diffusion equations and a special case of the nonuniform damped wave equation. The analog of the dynamic structure factor is also constructed for the latter two cases.

Roder, H. M. A transient hot wire thermal conductivity apparatus for fluids. J. Res. Nat. Bur. Stand. (U.S.). 86(5): 457.493; 1981 September-October.

Key words: helium; hot wire; nitrogen; pressure; temperature; thermal conductivity; transient.

A new apparatus for measuring the thermal conductivity of fluids is described. This is an absolute method utilizing a transient hot wire. Measurements are made with a $12.7 \mu \mathrm{m}$ diameter platinum wire at real times of up to 1 second. The data acquisition system includes a minicomputer and a digital voltmeter. The experimental core of the system incorporates a compensating hot wire in a Wheatstone bridge circuit. The cell containing the core of the apparatus is designed to accommodate pressures from 0 to $70 \mathrm{MPa}$ and temperatures from 70 to $320 \mathrm{~K}$. Oxygen was measured over a wide range of physical states including the dilute gas, the moderately dense gas, the near critical region, the compressed liquid states, and the vapor at temperatures below the critical temperature. Performance checks of the apparatus were conducted with nitrogen, helium and argon. Measurement of rare gases allows a direct comparison to the kinetic theory of gases through the viscosity. A second check looks at the variation of the measured thermal conductivity as a function of the applied power. The precision $(2 \sigma)$ of the new system is between 0.5 and 0.8 percent for wire temperature transients of 4 to $5 \mathrm{~K}$, while the accuracy is estimated at around 1.5 percent.

Pruitt, J. S.; Domen, S. R.; Loevinger, R. The graphite calorimeter as a standard of absorbed dose for cobalt-60 gamma radiation. J. Res. Nat. Bur. Stand. (U.S.). 86(5): 495.502; 1981 September-October.

Key words: absorbed dose standard; cobalt 60 gamma rays; electron density; graphite calorimeter; ionization chamber; scaling theorem; water phantom.

Absorbed dose to water in a cobalt-60 gamma-ray beam has been determined using a thick-walled graphite ionization chamber. The chamber was calibrated in a graphite phantom against a graphite calorimeter, and the graphite calibration factor was converted to a water calibration factor using published energy absorption coefficient ratios and a measured replacement factor. Comparisons between the graphite and water measurements were made at pairs of points that were scaled in position according to the ratio of electron densities, so that the photon spectra were the same for the two points in a given pair. Measurements performed in graphite over a wide range of phantom depths, field sizes, and source distances, showed that the calibration factor varies slowly with the phantom depth and field size, and probably has a negligible dependence on source distance. By comparison with the thick-walled chamber in a cobalt-60 gamma-ray beam, a secondary ionization chamber can be calibrated in terms of absorbed dose to water with an estimated uncertainty of about \pm 1 percent.

Bennett, H. S. Upper limits for the number of bound states associated with the Yukawa potential. J. Res. Nat. Bur. Stand. (U.S.). 86(5): 503-508; 1981 September-October.

Key words: Bargmann limits; bound-state estimates; Lieb limits; number of bound states; Schwinger limits; screened Coulomb potential; Sobolev's inequality; Yukawa potential.

The number of bound-state solutions of the Schrödinger equation for the screened Coulomb potential (Yukawa potential), $-(C / r)$ exp(-ar), occurs frequently in theoretical discussions concerning, for example, gas discharges, nuclear physics, and semiconductor physics. The number of bound states is a function of $(C / \alpha)$. Three upper limits for the number of bound states associated with the Yukawa potential are evaluated and compared. These three limits are those given by Bargmann, Schwinger, and Lieb. In addition, the Sobolev inequality states that whenever $(C / \alpha)<1.65$ no bound state occurs. This agrees to within a few percent of the numerical calculations of Bonch. Bruevich and Glasko. The Bargmann and Lieb limits and the Sobolev inequality are substantially easier to evaluate than the Schwinger limit. Among the three limits, the Schwinger limit gives the most restrictive limit for the existence of only one bound state and, therefore, is the best one to use for the approach to no binding, i.e., $1.65<(C / \alpha) \leqslant 1.98$. The Lieb limit is the best among the three when $(C / \alpha)>1.98$. The Bargmann limit is the least restrictive.

Francis, R. L. A "uniformity principle" for evacuation route allocation. J. Res. Nat. Bur. Stand. (U.S.). 86(5): 509.513; 1981 September-October.

Key words: building evacuation; mathematical optimization; network flow.

This paper establishes what might be called a "uniformity principle" for building evacuation problems. The principle may be stated as follows: given a building for which each occupant has reasonable access to every evacuation route, if the building is evacuated in minimum time, then the allocation of evacuees to routes is such that the route evacuation times are all the same. That is, there is a uniformity of route evacuation times. Also, analytical expressions 
for the minimum time to evacuate a building, and for the corresponding allocation of evacuees to routes, are obtained.

\section{November-December 1981}

Lin, C. C. Turbulence, plasma containment, and galaxies. J. Res. Nat. Bur. Stand. (U.S.). 86(6): 557.563; 1981 November-December.

Key words: density wave; galactic spirals; hydrodynamic instability and turbulence; plasma dynamics; spiral grand design; WASER; winding dilemma.

These three exciting areas of research, apparently disjointed in content, have similar basic mechanisms in common which can be described by the same mathematical principles, concepts, and methods. Scientific problems will be discussed in all three areas. Emphasis will be placed on galaxies, where observational data are plentiful for checking the theory. A unified mathematical approach applicable to all three areas will then be described.

Esaki, L. Highlights in semiconductor device development. J. Res. Nat. Bur. Stand. (U.S.) 86(6): 565-570; 1981 November-December.

Key words: diode; field-effect transistor; III-V compounds; IMPATT diode; integrated circuit; LASER; LED; photocell; solar cell; solid state; transistor; tunnel diode.

Following a brief description of early semiconductor history, the invention of the transistor and subsequent important events are presented in perspective, with emphasis on the role of semiconductor physics in device development.

Yu, H. Application of light scattering to polymers, liquid crystals, and biological systems. J. Res. Nat. Bur. Stand. (U.S.). 86(6): 571-590; 1981 November-December.

Key words: amphoteric latex; dynamic light scattering; light scattering; nematic liquid crystal; photo-receptor membrane vesicles; random coil polymers.

The applications of elastic and quasielastic light scattering techniques to polymers in dilute solution, thermotropic liquid crystals and biological membrane vesicles are presented. It is focused on how we extract specific structural features or dynamic processes of these condensed medium samples through the light scattering methods. The paper reviews the light scattering studies that were carried out at the author's laboratory in Wisconsin from 1973 until 1980.

Kuo, F. K. Computer networks-The ALOHA System. J. Res. Nat. Bur. Stand. (U.S.). 86(6): 591-595; 1981 November-December.

Key words: ALOHANET; ALOHA system; computer network; packet broadcasting; radio computer communication network; satellite communication.

The ALOHA System, an experimental UHF radio computer communication network, was developed at the University of Hawaii, 1970-76. In this survey paper, we give a general overview of packet communication techniques applied to computer networks. Then we discuss the concept of packet broadcasting and give a short description of the ALOHANET. Next, a discussion of the application of ALOHA techniques to satellite communications is presented. Finally, a short survey of present-day research and development efforts in packet broadcasting is presented.

Ely, J. F. An Enskog correction for size and mass difference effects in mixture viscosity prediction. J. Res. Nat. Bur. Stand. (U.S.). 86(6): 597.604; 1981 November-December.

Key words: corresponding states; Enskog theory; mass difference effects; mixture viscosity; size difference effects.

A method is presented which corrects the one-fluid conformal solution viscosity model for size and mass difference effects. This correction, which is based on the Enskog model for hard sphere mixtures, is empirical as applied to transport but has a rigorous basis in equilibrium theory. Comparisons of predictions and experimental viscosities for 24 binary mixtures are presented.

Brown, R. L. Rate constants for $\mathbf{H}$-atom transfer reactions by the BEBO method. J. Res. Nat. Bur. Stand. (U.S.). 86(6): 605.654; 1981 November-December.

Key words: absolute chemical rate; BEBO activation energy; bond-order-bond-energy; chemical rate constants; computer program; hydrogen atom transfer.

A detailed discussion of the calculation of rate constants for hydrogen atom transfer reactions based on the BEBO method is presented. Linear transition state models are used. A computer program using this method for determining rate constants is provided.

Colbert, J. C.; Xiheng, H.; Kirklin, D. R. Enthalpy of combustion of microcrystalline cellulose. J. Res. Nat. Bur. Stand. (U.S.). 86(6): 655 . 660; 1981 November-December.

Key words: alternative fuel; bomb calorimetry; cellulose; enthalpy of combustion; refuse-derived-fuel; test substance.

A test substance with characteristics and properties similar to those of cellulose-based solid waste products is needed to calibrate calorimeters and combustors which will be routinely burning these materials to determine their calorific values precisely for use in commerce. Microcrystalline cellulose was found to be a good calibrant for this purpose. The enthalpy of combustion of microcrystalline cellulose $\Delta \mathrm{H}_{c}^{\circ}$ at $\left(25^{\circ} \mathrm{C}\right)$, and its estimated uncertainty, was determined to be $-2812.401 \pm 1.725 \mathrm{~kJ} / \mathrm{mol}$ based upon the sample mass.

A calculated heat of wetting correction of $1.514 \mathrm{~kJ} / \mathrm{mol}$ was applied to the combustion data.

Stegun, I. A.; Zucker, R. Automatic computing methods for special functions. Part IV. Complex error function, Fresnel integrals, and other related functions. J. Res. Nat. Bur. Stand. (U.S.). 86(6): 661. 686; 1981 November-December.

Key words: argand diagram; complex error function; continued fraction; Dawson's function; FORTRAN subroutine; Fresnel integrals; key values; line broadening function; plasma dispersion function; Voigt function.

Accurate, efficient, automatic methods for computing the complex error function to any precision are detailed and implemented in an American Standard FORTRAN subroutine. A six significant figure table of erfc $z, e^{22} \operatorname{erfc} z$, and $e^{22} \operatorname{erfc}(-z)$ is included for $z$ in polar coordinate form with the modulus of $z$ ranging from 0 to 9 . The argand diagram is given for erf $z$. 


\subsection{PAPERS FROM THE JOURNAL OF PHYSICAL AND CHEMICAL REFERENCE DATA, VOLUME 10, JANUARY-DECEMBER 1981}

This journal is published quarterly by the American Chemical Society and the American Institute of Physics for the National Bureau of Standards. The objective of the Journal is to provide critically evaluated physical and chemical property data, fully documented as to the original sources and the criteria used for evaluation. Critical reviews of measurement techniques, whose aim is to assess the accuracy of available data in a given technical area, are also included. The principal source for the Journal is the National Standard Reference Data System (NSRDS). The Journal is not intended as a publication outlet for original experimental measurements such as are normally reported in the primary research literature, nor for review articles of a descriptive or primarily theoretical nature.

\section{Volume 10, No. 1}

Goldberg, R. N. Evaluated activity and osmotic coefficients for aqueous solutions: Bi-univalent compounds of zinc, cadmium, and ethylene bis(trimethylammonium) chloride and iodide. J. Phys. Chem. Ref. Data. 10(1): 1-56; 1981.

Key words: activity coefficient; cadmium; critical evaluation; electrolyte; ethylene bis(trimethylammonium) chloride; ethylene bis(trimethylammonium) iodide; excess Gibbs energy; osmotic coefficient; solutions; thermodynamic properties; zinc.

A critical evaluation of the mean activity and osmotic coefficients in aqueous solutions of eleven bi-univalent compounds of zinc and cadmium and ethylene bis(trimethylammonium) chloride and iodide at $298.15 \mathrm{~K}$ is presented. Osmotic coefficients were calculated from direct vapor pressure measurements, from isopiestic measurements and from freezing point depression measurements. Activity coefficients were calculated from electromotive force measurements on galvanic cells with and without transference. Given are empirical coefficients for three different correlating equations, obtained by a weighted least squares fit of the experimental data, and tables consisting of the activity coefficients of the compounds, the osmotic coefficients and activity of water, and the excess Gibbs energy of the solution as functions of the molality for each electrolyte system. The literature coverage is through the computerized version of Chemical Abstracts of September 1979.

Kestin, J.; Khalifa, H. E.; Correia, R. J. Tables of the dynamic and kinematic viscosity of aqueous $\mathrm{KCl}$ solutions in the temperature range $25-150^{\circ} \mathrm{C}$ and the pressure range 0.1-35 MPa. J. Phys. Chem. Ref. Data. 10(1): 57.70; 1981

Key words: aqueous solutions; compilation; potassium chloride; reference data; viscosity.

Tabulated values of the dynamic and kinematic viscosity of potassium chloride solutions are given. The tables cover the temperature range $25-150^{\circ} \mathrm{C}$, the pressure range $0.1-35 \mathrm{MPa}$ and the concentration range $0-5$ molal. It is estimated that the accuracy of the tabulated values is $\pm 1 \%$. The correlations from which the tables were generated are also given.

Kestin, J.; Khalifa, H. E.; Correia, R. J. Tables of the dynamic and kinematic viscosity of aqueous $\mathrm{NaCl}$ solutions in the temperature range $20-150^{\circ} \mathrm{C}$ and the pressure range 0.1-35 MPa. J. Phys. Chem. Ref. Data. 10(1): 71-88; 1981.

Key words: aqueous solutions; compilation; reference data; sodium chloride; viscosity.

Tabulated values of the dynamic and kinematic viscosity of aqueous sodium chloride solutions are given. The tables cover the temperature range $20-150^{\circ} \mathrm{C}$, the pressure range $0.1-35 \mathrm{MPa}$ and the concentration range $0-6$ molal. It is estimated that the accuracy of the tabulated values is $\pm 0.5 \%$. The correlating equations from which the tables were generated are given.

Gaur, U.; Shu, H. C.; Mehta, A.; Wunderlich, B. Heat capacity and other thermodynamic properties of linear macromolecules. $I$. Selenium. J. Phys. Chem. Ref. Data. 10(1): 89.118; 1981.

Key words: calorimetry; crystal; enthalpy; entropy; floor temperature; fusion; Gibbs energy; glass transition; heat capacity; linear macromolecule; melt; selenium.

The heat capacity of selenium from $0 \mathrm{~K}$ to $1000 \mathrm{~K}$ is reviewed using measurements on 20 samples reported in the literature. A set of recommended data for trigonal, monoclinic, glassy, and molten selenium is derived. Ring-chain equilibrium parameters are critically evaluated. Entropy, enthalpy, and Gibbs energy functions are calculated. Selenium is a model compound for a monoatomic, linear macromolecule. This paper is first in a series which will ultimately cover all heat capacity measurements on linear macromolecules.

Gaur, U.; Wunderlich, B. Heat capacity and other thermodynamic properties of linear macromolecules. II. Polyethylene. J. Phys. Chem. Ref. Data. 10(1): 119-152; 1981.

Key words: crystal; crystallinity; density; enthalpy; entropy; fusion; Gibbs energy; glass transition; heat capacity; linear macromolecule; melt; polyethylene.

The heat capacity of polyethylene from $0 \mathrm{~K}$ to $600 \mathrm{~K}$ is reviewed using measurements on 46 samples reported in the literature. The crystallinity dependence is evaluated critically and a set of recommended data for completely crystalline and amorphous polyethylene is derived. Entropy, enthalpy, and Gibbs energy functions are calculated. Polyethylene can serve as a standard material for the thermodynamic functions of a simple linear macromolecule. This paper is the second in a series which will ultimately cover all heat capacity measurements on linear macromolecules.

Martin, W. C.; Zalubas, R. Energy levels of sodium, Na I through Na XI. J. Phys. Chem. Ref. Data. 10(1): 153.196; 1981.

Key words: atomic energy levels; atomic ions; atomic spectra; electron configurations; ionization potentials; sodium.

Energy level data are given for the atom and all positive ions of sodium $(Z=11)$. These data have been critically compiled, mainly from published material on measurements and analyses of the optical spectra. We have derived or recalculated the levels for a number of the ions. In addition to the level value in $\mathrm{cm}^{-1}$ and the parity, the $\int$ value and the configuration and term assignments are listed if known. Leading percentages from the calculated eigenvectors are tabulated wherever available. Ionization energies are given for all spectra.

Corliss, C.; Sugar, J. Energy levels of nickel, Ni I through Ni XxVIII. J. Phys. Chem. Ref. Data. 10(1): 197-290; 1981.

Key words: atomic energy levels; atomic spectra; nickel energy levels.

The energy levels of the nickel atom in all of its stages of ionization, as derived from the analyses of atomic spectra, have been critically compiled. In cases where only line classifications are reported in the literature, level values have been derived. Electron configurations, term designations, $J$-values, experimental $g$-values, and ionization energies are included.

\section{Volume 10, No. 2}

Marshall, W. L.; Franck, E. U. Ion product of water substance, $0-1000^{\circ} \mathrm{C}, 1-10,000$ bars. New international formulation and its background. J. Phys. Chem. Ref. Data. 10(2): 295-304; 1981.

Key words: equation; formulation; high pressure; high temperature; IAPS; International Association for the Properties of Steam; ionization; ionization constant; ion product; review; water; water substance. 
This paper is the background for a new international formulation for the ion product of water substance (May 1980) issued by the International Association for the Properties of Steam. The ion product of water $\left(K_{\mathrm{w}}\right)$ is represented by an equation, based on density and two quadratic functions of reciprocal absolute temperature, for use from 0 to $1000^{\circ} \mathrm{C}$ and 1 to 10,000 bars pressure. The equation is believed to describe within \pm 0.01 units of $\log K_{\mathrm{w}}^{*}$ (where $K_{\mathrm{w}}^{*}$ equals $\left.K_{\mathrm{w}} /\left(\mathrm{mol} \mathrm{kg}^{-1}\right)^{2}\right)$ many of the measurements at saturated vapor pressure up to $200^{\circ} \mathrm{C}$, and to within \pm 0.02 units up to the critical temperature $\left(374^{\circ} \mathrm{C}\right)$. It also describes within the experimental uncertainty the several sets of measurements at high pressures and should provide values within \pm 0.05 and 0.30 units at low and high temperatures, respectively.

Fuhr, J. R.; Martin, G. A.; Wiese, W. L.; Younger, S. M. Atomic transition probabilities for iron, cobalt, and nickel (A critical data compilation of allowed lines). J. Phys. Chem. Ref. Data. 10(2): 305 $566 ; 1981$.

Key words: allowed transitions; cobalt; $f$-values; iron; isoelectronic sequence; line strengths; nickel; oscillator strengths; systematic trends; transition probabilities.

Atomic transition probabilities for about 5100 spectral lines of the elements iron, cobalt, and nickel in all stages of ionization have been critically evaluated and compiled. All available literature sources have been considered. Systematic trends along isoelectronic sequences have been exploited to predict oscillator strengths ( $f$-values) whenever no data were available in the literature. The data are presented in separate tables for each element and stage of ionization and are arranged according to multiplets and, where appropriate, also according to transition arrays and increasing quantum numbers. For each line the transition probability for spontaneous emission, the absorption oscillator strength, and the line strength are given, along with the spectroscopic designation, the wavelength, the statistical weights, and the energy levels (when available) of the upper and lower atomic states. In addition, the estimated accuracy and the literature reference are indicated. In short introductions which precede the tables for each spectrum, the main justifications for the choice of the adopted data and for the accuracy ratings are discussed. A general introduction contains additional details on the evaluation procedure.

\section{Volume 10 , No. 3}

Haas, J. L., Jr.; Robinson, G. R., Jr.; Hemingway, B. S. Thermodynamic tabulations for selected phases in the system $\mathrm{CaO}$. $\mathrm{Al}_{2} \mathrm{O}_{3}-\mathrm{SiO}_{2}-\mathrm{H}_{2} \mathrm{O}$ at $101.325 \mathrm{kPa}(1 \mathrm{~atm})$ between 273.15 and 1800 K. J. Phys. Chem. Ref. Data. 10(3): 575-670; 1981.

Key words: enthalpy; enthalpy of formation; entropy; equilibrium constant for formation; Gibbs energy function; Gibbs energy of formation; heat capacity; lime-alumina-silica-water; minerals; thermodynamic data.

The standard thermodynamic properties of phases in the limealumina-silica-water system between 273.15 and $1800 \mathrm{~K}$ at $101.325 \mathrm{kPa}$ (1 atm) were evaluated from published experimental data. Phases included in the compilation are boehmite, diaspore, gibbsite, kaolinite, dickite, halloysite, andalusite, kyanite, sillimanite, Ca-Al cliniopyroxene, anorthite, gehlenite, grossular, prehnite, zoisite, margarite, wollastonite, cyclowollastonite (=pseudowollastonite), larnite, $\mathrm{Ca}$ olivine, hatrurite, and rankinite. The properties include heat capacity, entropy, relative enthalpy, and the Gibbs energy function of the phases and the enthalpies, Gibbs energies, and equilibrium constants for formation both from the elements and the oxides. Tabulated values are given at $50 \mathrm{~K}$ intervals with the 2 -sigma confidence limit at $250 \mathrm{~K}$ intervals. Summaries for each phase give the temperature-dependent functions for heat capacity, entropy, and relative enthalpy and the experimental data used in the final evaluation.

Goldberg, R. N. Evaluated activity and osmotic coefficients for aqueous solutions: Thirty-six uni-bivalent electrolytes. J. Phys. Chem. Ref. Data. 10(3): 671-766; 1981.

Key words: activity coefficient; cesium; critical evaluation; electrolyte; excess Gibbs energy; lithium; osmotic coefficient; potassium; rubidium; sodium; solutions; thermodynamic properties

A critical evaluation of the mean activity and osmotic coefficients in aqueous solutions of thirty-five uni-bivalent electrolytes at $298.15 \mathrm{~K}$ is presented. The systems which have been treated are ammonium orthophosphate, guanadinium carbonate, 1,2-ethane disulfonic acid, $m$-benzene disulfonic acid, ammonium decahydroborate, and the unibivalent compounds of lithium, sodium, potassium, rubidium, and cesium. Osmotic coefficients were calculated from direct vapor pressure measurements, from isopiestic measurements and from freezing-point depression measurements. Activity coefficients were calculated from electromotive force measurements on galvanic cells without transference and from diffusion measurements. Given are empirical coefficients for three different correlating equations, obtained by a weighted least squares fit to the experimental data, and tables consisting of the activity coefficients of the compounds, the osmotic coefficients and activity of water, and the excess Gibbs energy of the solution as functions of the molality for each electrolyte system. The literature coverage is through the computerized version of Chemical Abstracts of September 1979.

Staples, B. R. Activity and osmotic coefficients of aqueous alkali metal nitrites. J. Phys. Chem. Ref. Data. 10(3): 767.778; 1981.

Key words: activity coefficients; alkali metal nitrite salts; aqueous; critical evaluation; $\mathrm{CsNO}_{2}$; electrolytes; excess Gibbs energy; $\mathrm{KNO}_{2} ; \mathrm{LiNO}_{2} ; \mathrm{NaNO}_{2}$; osmotic coefficients; $\mathrm{RbNO}_{2}$; solutions; standard reference data; thermodynamic properties.

A critical evaluation of the mean activity $\gamma_{ \pm}$, and osmotic coefficients, $\phi$, of aqueous alkali metal nitrites at $298.15 \mathrm{~K}$ is presented for the molality range from dilute to saturation. Osmotic coefficients were calculated from static vapor pressure measurements. A nonlinear least-squares program was used to fit $\phi$ data as a function of molality. Several equations describe the osmotic coefficient, the mean activity coefficient, and the excess Gibbs energy as a function of the square-root of molality for each salt. The scientific literature was covered through March 1979.

Staples, B. R. Activity and osmotic coefficients of aqueous sulfuric acid at 298.15 K. J. Phys. Chem. Ref. Data. 10(3): 779.798; 1981.

Key words: activity coefficients; aqueous; critical evaluation; electrolytes; excess free energy; free energy; ionic; osmotic coefficients; solutions; sulfuric acid; standard reference data; thermodynamic properties.

A critical evaluation of the mean activity coefficient, $\gamma_{ \pm}$, and osmotic coefficients, $\phi$, of aqueous sulfuric acid at $298.15 \mathrm{~K}$ is presented for the molality range of 0 to $28 \mathrm{~mol} \cdot \mathrm{kg}^{-1}$. Osmotic coefficients were calculated from direct vapor pressure measurements, from isopietic measurements or from freezing point depression measurements. Activity coefficients were calculated from electromotive force measurements of galvanic cells. A least-squares program was used to fit data from all sources using both $\phi$ and $\ln \gamma_{ \pm}$ as functions of molality. A nine parameter equation describes the osmotic coefficient, the mean activity coefficient, and the excess Gibbs energy as a function of the one-half power of molality. The scientific literature has been covered through January, 1979. 
Volume 10, No. 4

Wilkinson, F.; Brummer, J. G. Rate constants for the decay and reactions of the lowest electronically excited singlet state of molecular oxygen in solution. J. Phys. Chem. Ref. Data. 10(4): 809. 999; 1981.

Key words: chemical kinetics; data compilation; oxidation; photochemistry; rates; review; singlet oxygen; solution.

The available rate data on the reactivity and physical deactivation of molecular oxygen in its first electronic excited state $\left({ }^{1} \Delta_{g}\right)$ in liquid solution have been critically compiled. Where possible, relative rates reported in the literature have been normalized to standard values selected by a statistical analysis of the experimental data. Secondorder rate constants for the deactivation and chemical reaction of singlet oxygen are reported for 670 compounds. Additionally, pseudo first order rate constants $\left(k_{4}\right)$ for solvent deactivation of singlet oxygen are reported for 50 different solvents.

Gaur, U.; Wunderlich, B. Heat capacity and other thermodynamic properties of linear macromolecules. III. Polyoxides. J. Phys. Chem. Ref. Data. 10(4): 1001-1049; 1981.

Key words: crystal; crystallinity; density; enthalpy; entropy; fusion; Gibbs energy; glass transition; heat capacity; linear macromolecule; poly(oxymethylene)s; poly(oxyphenylene)s; poly(oxypropylene).

The heat capacity of poly(oxymethylene), poly(oxyethylene), poly(oxytrimethylene), poly(oxytetramethylene), poly(oxyoctamethylene), poly(oxypropylene), poly(oxymethylleneoxyethylene), poly(oxymethyleneoxytetramethylene), poly(oxy-1,4-phenylene), poly(oxy-2,6dimethyl-1,4-phenylene), poly(oxy-2,6-diphenyl-1,4-phenylene) and poly[oxy-2,2-bis(chloromethyl)trimethylene] is reviewed on the basis of measurements on 35 samples reported in the literature. The crystallinity dependence is critically evaluated and a set of recommended data for each polyoxide is derived. Entropy, enthalpy, and Gibbs energy functions are calculated. The data have been compared to that of polyethylene and the contribution of-O-group has been evaluated. This paper is the third in a series which will ultimately cover all heat capacity measurements on linear macromolecules.

Gaur, U.; Wunderlich, B. Heat capacity and other thermodynamic properties of linear macromolecules. IV. Polypropylene. J. Phys. Chem. Ref. Data. 10(4): 1051-1064; 1981.

Key words: atactic; crystal; crystallinity; density; enthalpy; entropy; fusion; Gibbs energy; glass transition; heat capacity; isotactic; linear macromolecule; melt; polypropylene; syndiotactic.

The heat capacity of polypropylene from $0 \mathrm{~K}$ to $600 \mathrm{~K}$ is reviewed on the basis of measurements on 20 samples reported in the literature. The crystallinity dependence is critically evaluated and a set of recommended data for completely crystalline and amorphous polypropylene are derived. The effect of tacticity on the heat capacity is also evaluated. Entropy, enthalpy and Gibbs energy functions are calculated. This paper is the fourth in a series of publications which will ultimately cover all heat capacity measurements on linear macromolecules.

Olson, W. B.; Maki, A. G.; Lafferty, W. J. Tables of $\mathbf{N}_{2} \mathbf{O}$ absorption lines for the calibration of tunable infrared lasers from $522 \mathrm{~cm}^{-1}$ to $657 \mathrm{~cm}^{-1}$ and from $1115 \mathrm{~cm}^{-1}$ to $1340 \mathrm{~cm}^{-1}$.J. Phys. Chem. Ref. Data. 10(4): 1065-1084; 1981.

Key words: infrared standards from $522 \mathrm{~cm}^{-1}$ to $657 \mathrm{~cm}^{-1}$; infrared standards from $1115 \mathrm{~cm}^{-1}$ to $1340 \mathrm{~cm}^{-1}$; infrared wavelength calibration; $\mathrm{N}_{2} \mathrm{O}$; nitrous oxide; spectroscopic constants of $\mathrm{N}_{2} \mathrm{O}$.

Tables of $\mathrm{N}_{2} \mathrm{O}$ absorption lines are given for use as wavelength calibration standards in two regions in the infrared. The absorption lines are calculated from spectroscopic constants which have been determined by fitting of selected data in the literature to appropriate equations. Hot band lines and lines of $\mathrm{N}_{2} \mathrm{O}$ containing less abundant isotopes of $\mathrm{N}$ and $\mathrm{O}$ are also given, along with relative intensities of all lines in order to provide patterns for correct identification of lines to be used as wavelength standards.

Ozier, I.; Gerry, M. C. L.; Robiette, A. G. Microwave spectra of molecules of astrophysical interest. XX. Methane. J. Phys. Chem. Ref. Data. 10(4): 1085-1095; 1981.

Key words: distortion dipole transitions; interstellar molecules; methane; microwave spectra; molecular parameters; radio astronomy; rotational transitions.

The available data on methane are critically reviewed for information applicable to radio astronomy. Molecular data such as rotational constants, centrifugal distortion constants, and the distortion electric dipole moment are presented for ${ }^{12} \mathrm{CH}_{4}$ and ${ }^{13} \mathrm{CH}_{4}$. Observed microwave, infrared-microwave double resonance, and molecular beam measurements of $(\Delta J=0)$ frequencies are tabulated along with experimental uncertainties which represent estimated $95 \%$ confidence limits. For ${ }^{12} \mathrm{CH}_{4}$, these data have been analyzed to predict all $Q$-branch rotational transitions for $J \leqslant 20$; the predictions are presented with $95 \%$ confidence limits which have been calculated from the analysis.

Sugar, J.; Corliss, C. Energy levels of cobalt, Co I through Co XxviI. J. Phys. Chem. Ref. Data. 10(4): 1097.1174; 1981.

Key words: atomic energy levels; atomic spectra; cobalt; cobalt energy levels; spectra.

The energy levels of the cobalt atom in all of its stages of ionization, derived from analyses of atomic spectra, have been critically compiled. In cases where only line classifications are reported in the literature, level values have been derived. Electron configurations, term designations, J-values, experimental g-values, leading percentages and ionization energies are included.

Mackay, D.; Shiu, W. Y. A critical review of Henry's law constants for chemicals of environmental interest. J. Phys. Chem. Ref. Data. 10(4): 1175-1199; 1981.

Key words: alkanes; aromatics; critical review, evaluated data; gases; halogenated hydrocarbons; Henry's law constants; liquids; pesticides, solids; solubility; vapor pressure.

The Henry's law constants (air-water partition coefficients) of hydrophobic organic compounds of environmental concern are reviewed. An outline of the thermodynamic principles which govern the relationships between vapor pressure, solubility and Henry's law constant for solid and liquid compounds is presented and experimental techniques for obtaining these quantities with the required accuracy are discussed. Vapor pressure, solubility, and Henry's law constant data are tabulated and reviewed for a total of 150 compounds in 12 tables consisting of gaseous, liquid and solid alkanes, cycloalkanes, alkenes, alkynes, monoaromatics, polynuclear aromatics, halogenated alkanes, alkenes and aromatics, and selected pesticides. 


\subsection{DIMENSIONS/NBS, ARTICLE TITLES ONLY}

This monthly magazine is published to inform scientists, engineers, businessmen, industry, teachers, students, and consumers of the latest advances in science and technology, with primary emphasis on the work at NBS.

DIMENSIONS/NBS highlights and reviews such issues as energy research, fire protection, building technology, metric conversion, pollution abatement, health and safety, and consumer product performance. In addition, DIMENSIONS/NBS reports the results of Bureau programs in measurement standards and techniques, properties of matter and materials, engineering standards and services, instrumentation, and automatic data processing.

\section{January-February 1981}

DIM/NBS 65(1): 1.28; 1981.

Key words: air pollutants; antenna coupling; electrical distribution; glass-liquidus; insulation; monitors; neutron diffraction, samplers; SRM's; time-domain calibrations; weights and measures; $x$-ray diffraction.

Insulators: Liquid, Solid, or Gas, E. Rudin

A Sample a Day ..., G. Porter

"Seeing" Is Believing, G. Porter

Glass-Liquidus Temperature Testing, M. Cellarosi

Changes in SRM Inventory, S. Rasberry

Antenna Coupling Analyzed, A. Yaghijian

Research Improves Time-Domain Calibrations, N. Nahman

Measurement Program for State Weights and Measures, H. Opperman

Conferences

Publications

News Briefs

\section{March 1981}

\section{DIM/NBS 65(2): 1.28; 1981.}

Key words: atomic ions; calibration; clock synchronization; Columbia River model; computer; insulation; lasers; powder data; progress; R-values; single-crystal; SRM's.

NBS Through the Years

Fishing on the Computer Frontier, E. Rudin

Measuring Through Thick and Thin, M. Heyman

Calibrating the FFTF, M. Baum

Planning for Future SRM's, S. Rasberry

NBS Develops New Clock-Synchronization Technique, D. Allan

Program Aids Critical Evaluation of Single-Crystal and Powder

Data, J. Stalick

Cooling Atomic Ions with Lasers, J. Bergquist

Conferences

Publications

News Briefs

\section{April 1981}

DIM/NBS 65(3): 1.24; 1981.

Key words: cement and concrete; cover plates; earth's tilt; electric; fire; fracture toughness; measurement; parameter; physics; solar collector; space charges; technical competence.

Swinging to the Earth's Tilt, F. McGehan

Testing for Technical Competence, E. Rudin

Physics at the Measurement Limits, M. Baum

New Parameter Proposed for Fracture Toughness, C. Smith

Study Evaluates Solar Collector Cover Plates, E. Rudin

Improved Modeling of Cement and Concrete, J. Clifton

Fire Development in Basement Rooms, J. B. Fang

Generation and Measurement of DC Electric Field with Space

Charges, M. Misakian
Conferences

Publications

News Briefs

\section{May-June 1981}

DIM/NBS 65(4): 1.28; 1981.

Key words: air pollution; automation; buildings; certified reference materials; computers; construction; data converter; mass comparator; radiation; radiocarbon; soft aluminum; windows.

Automation Research at NBS, J. Simpson

Reducing the Risks of Failure, R. Marshall

A New Twist on "Dating," G. Porter

Results of "Soft Aluminum" Study Announced, G. Porter

Industry Gas Suppliers to Produce CRM's, G. Porter

New Standard Radiation Source Possible, J. Roberts

New Mass Comparator is Quicker, Costs Less, R. Schoonover

Data Converter Calibration Service Announced, B. Bell

Conferences

Publications

News Biefs

\section{July 1981}

\section{DIM/NBS 65(5): 1.24; 1981}

Key words: copper; deterioration; electromagnetic radiation; film; industry; metals; productivity; research associate; scanning technique; spin dependent; SRM's; standards; tape.

Getting More for Less, G. Porter

Preserving the Past, E. Rudin

NBS Research Forges Strong Link with Industry, E. Rudin

New NBS Instrument Monitors Electromagnetic Radiation, F. McGehan

Verification Procedures for Federal Interface Standards, S. Lichtenstein

Hybrid Planar-Cylindrical Scanning Technique, A. Newell

Spin Dependent Mean Free Paths, R. Rendell

New SRM Aids Detection of PAH's

Copper "Benchmark" SRM's A vailable

Conferences

Publications

Newsbriefs

\section{August 1981}

\section{DIM/NBS 65(6): 1.24; 1981.}

Key words: atomic weight; coal; computers; drill failure; Faraday; firefighting; hot atom; industrial innovation; measurements; oils, PCB's; standards.

Firefighting in the Computer Age, J. P. Cohn

A Blueprint for the Future

A Faraday Keeps the Doctors Astray, M. Baum

NBS Develops Cheap, Effective Method to Predict Drill Failure, M. Baum

New Method of Probing "Hot" Atom Phenomena, S. R. Leone and F. Magnotta

Coal Slurry Level Monitor Developed, V. E. Bean and F. G. Long., Jr.

Measuring PCB's in Lubricating and Cooling Oils, S. N. Chesler, R. M. Parris, and W. E. May

Conferences

Publications

News Briefs 
DIM/NBS 65(7): 1-24; 1981.

Key words: evolution; fracture mechanics; gamma rays; inventors; molecular interactions; molecular processes; neomycin; neutron dosimeters; polymers; radiography; SRM's; stars; visual acuity.

Listening to the Stars, F. McGehan

NBS Inventors Are Honored, S. Webber

Charting the World of Radiography, J. Cohn

Fracture Mechanics Research Aims at Lowering Construction Costs, C. Smith

New Source for Testing Neutron Dosimeters, M. Baum

Molecular Processes Affecting Durability of Polymers Studied,

B. M. Fanconi

Neomycin B Studied, R. E. Botto and B. Coxon

SERS Used to Study Molecular Interactions, K. A. Bunding, R. A. Durst, and M. I. Bell

New Gamma-Ray SRM's Have Greater Lifetimes, SRM

Conferences

Publications

News Briefs

\section{October 1981}

DIM/NBS 65(8): 1.28; 1981.

Key words: alloy; carbon; computers; energy; fine structure; interface; materials; measurement; office building; phase diagrams; radioactivity; research; sediment; soil.

Playing the Numbers, G. Porter

Meeting the Critical Materials Shortage, J. Wachtman

Using Natural Materials to Measure Radioactivity, M. Baum

Alloy Phase Diagrams: "Going Public," M. Baum

LC/MS Interface Capability Extended, R. Christensen

NBS Study Completed on Energy Conserving Office Building, J. Hill

First Direct Measurement of Carbon Ground-State Fine Structure,

K. Evenson

Publications

Conferences

Index

News Briefs 


\subsection{MONOGRAPHS}

Major contributions to the technical literature on various subjects related to the Bureau's scientific and technical activities.

Monogr. 25, Section 18. Morris, M. C.; McMurdie, H. F.; Evans, E. H.; Paretzkin, B.; Parker, H. S.; Panagiotopoulos, N. C.; Hubbard, C. R. Standard $x \cdot$ ray diffraction powder patterns. Section 18-Data for 58 substances. Nat. Bur. Stand. (U.S.) Monogr. 25, Sec. 18; 1981 October. 110 p. SN003-003-02370-1.

Key words: crystal structure; densities; lattice constants; powder patterns; reference intensities; standard; $x$-ray diffraction.

Standard $\mathrm{x}$-ray powder diffraction patterns are presented for 58 substances. These experimental patterns, useful for identification, were obtained by diffractometer methods. The lattice constants were refined by least-squares methods, and reflections were assigned Miller indices consistent with space group extinctions. Relative intensities, calculated densities, literature references, and other relevant data are included.

Monogr. 162. Kerns, D. M. Plane-wave scattering-matrix theory of antennas and antenna-antenna interactions. Nat. Bur. Stand. (U.S.) Monogr. 162; 1981 June. 179 p. SN003-003-01995-0.

Key words: antenna-antenna systems; antenna measurements; antenna-scatterer systems; antenna theory; microwave antenna theory; scattering-matrix description of antennas.

This monograph is distinguished by the use of plane-wave spectra for the representation of fields in space and by the consideration of antenna-antenna (and antenna-scatterer) interactions at arbitrary separation distances. The plane-wave representation is eminently suitable for this purpose as well as for the expression of conventional asymptotic quantities of antenna theory, such as power gain, effective area, and polarization. The primary objective of the monograph is to facilitate the critical acceptance and proper application of antenna and field measurements techniques deriving more or less directly from the plane-wave scattering-matrix (PWSM) theory of antennas and antenna interactions. A secondary objective is to present some recent and some new theoretical results based on this theory. The three chapters of this monograph provide (a) an introduction to the basic theory and practice of microwave network analysis (which form an inescapable part of microwave antenna measurement expertise); (b) a thorough formulation of the PWSM theory of antennas and antenna-antenna interactions, including analytical techniques for derived measurement methods; and (c) recent and new theoretical results and analytical examples. Topics include: a convenient and attractive reformulation of the PWSM theory, some theory of minimum scattering antennas; theory of classes of completely solvable antenna-antenna interaction problems; and convergent, asymptotic expansions of transmission- and reflection-integrals in reciprocal powers of $r_{0}$, where $r_{0}$ is the magnitude of the relative displacement vector $r_{0}$ and the direction of $r_{0}$ is a parameter.

Monogr. 167. Gevantman, L. H., ed. Physical properties data for rock salt. Nat. Bur. Stand. (U.S.) Monogr. 167; 1981 January. 288 p. SN003.003.02166.1.

Key words: chemical; electrical; geological; magnetic; mechanical; monograph; optical; physical; properties data; radiation damage; rock salt; sodium chloride.

Rock salt and pure sodium chloride properties data are assembled into a single source. The properties covered include geological, mechanical, optical, thermal, radiation damage, electrical, magnetic, chemical, and physical. A concerted attempt has been made to present the best data consistent with their availability in the literature. Recommended values for data are given where possible. A brief discussion of measurement techniques is included for each property.
Monogr. 168. McCarty, R. D.; Hord, J.; Roder, H. M. Selected properties of hydrogen (engineering design data). Nat. Bur. Stand. (U.S.) Monogr. 168; 1981 February. 523 p. SN003-003-02296-9.

Key words: hydrogen; hydrogen computer codes; hydrogen design data; hydrogen gas; hydrogen graphs; hydrogen handbook; hydrogen liquid; hydrogen properties; hydrogen safety; hydrogen solid; hydrogen tables; hydrogen thermophysical properties.

The National Bureau of Standards has been engaged in the compilation, review, analytical and experimental derivation, and publication of hydrogen properties for over 20 years. The properties data presented herein are compiled largely from those accumulated data; of course, pertinent data and work of other researchers in the field are also included.

The general interests of scientists and engineers engaged in energy systems studies were given top priority in choosing the properties material presented in this book. Hydrogen systems cut across many energy related fields, e.g., nuclear fusion, magnetohydrodynamics, electrolysis and thermochemical decomposition of water, coal and shale derivative fuels, solar and wind power, ocean energy, geothermal processes, etc. It is generally conceded that hydrogen could be used to satisfy virtually all fuel requirements that are currently being met with natural gas and oil. To satisfy the demand for properties data over this broad spectrum of interests, we have attempted to provide comprehensive coverage of physical properties over a wide range of pressures and temperatures.

Thermophysical properties of liquid, liquid-vapor, vaporous, and gaseous hydrogen are presented in Chapter 1 and the solid-liquid, solid-vapor, and solid phase properties are compiled in Chapter 2. Ortho-para modifications of the hydrogen molecule and attendant property variations are considered in both chapters. Combustion and safety data, pertinent to hazard analysis of hydrogen systems, is collected in Chapter 3. Important miscellaneous properties are compiled in Chapter 4, data figures are compiled in Chapter 5, data tables are collected in Chapter 6, and Chapter 7 summarizes symbols, units, and conversion factors used throughout the book. 


\subsection{HANDBOOKS}

Recommended codes of engineering and industrial practice (including safety codes) developed in cooperation with interested industries, professional organizations, and regulatory bodies.

H44, 1982 Edition. Wollin, H. F., ed. Specifications, tolerances, and other technical requirements for weighing and measuring devices. Nat. Bur. Stand. (U.S.) Handb. 44, 1982 Edition; 1981 September. 205 p. SN003.003-02364-7.

Key words: length-measuring devices; liquid-measuring devices; measures; scales; specifications; taximeters; tolerances; volumemeasuring devices; weighing devices; weights.

Handbook 44 was first published in 1949, having been preceded by similar handbooks of various designations and in several forms, beginning in 1918. This 1982 edition was developed by the Committee on Specifications and Tolerances of the National Conference on Weights and Measures, with the assistance of the Office of Weights and Measures of the National Bureau of Standards. It includes amendments adopted by the 66th National Conference on Weights and Measures during its annual meeting in 1981. Handbook 44 is published in its entirety each year following the annual meeting of the National Conference on Weights and Measures.

The National Bureau of Standards has a statutory responsibility for "cooperation with the States in securing uniformity of weights and measures laws and methods of inspection." In partial fulfillment of this responsibility, the Bureau is pleased to publish these recommendations of the National Conference.

H130, 1982 Edition. Brickenkamp, C. S., ed. Model state laws and regulations. Nat. Bur. Stand. (U.S.) Handb. 130, 1982 Edition; 1981 September. 104 p. SN003-003-02369-8.

Key words: basic weights and measures law; method of sale of commodities; open dating; packaging and labeling; registration of service persons; unit pricing; weighmaster law.

This Handbook compiles the latest Model State Laws and Regulations adopted by the National Conference on Weights and Measures (NCWM). The compilation itself was approved by the NCWM in 1979, and this edition includes amendments adopted at the annual meeting in 1981. The NCWM in 1981 decided to date the editions of Handbook 44 by the year in which substantive changes to the handbook become effective. Therefore, changes adopted by the NCWM in July 1981 are printed in the 1982 edition of H.44 (the effective date of changes is January 1, 1982). In order to avoid confusion, the date of this and subsequent editions of Handbook 130 will also be the year following NCWM action.

H133. Brickenkamp, C. S.; Hasko, S.; Natrella, M. G. Checking the net contents of packaged goods. Nat. Bur. Stand. (U.S.) Handb. 133; 1981 June. 164 p. SN003-003-02331-1.

Key words: commodity inspection; compliance testing; net weight; package checking; packaged product inspection; prepackaged consumer goods; statistical sampling.

This handbook provides procedures for testing individual lots of packaged goods for conformance with legal requirements for the net quantity of contents using statistical sampling techniques. In its advisory capacity, NBS provides these methods as guidelines.

The handbook is divided into five chapters and seven appendices. The first chapter covers introductory material on package inspection. The second chapter contains procedures and concepts generic to package inspection including general sampling plans. The third, fourth, and fifth chapters detail test methods; Chapter 3 covers packages labeled by weight, Chapter 4 covers packages labeled by volume and Chapter 5 covers other package quantities. Appendices include instructions for taking a random sample and a compendium of Federal regulations referring to labeled net contents on packages.
H136. Eisenhower, E. H., Chairman, ANSI Subcommittee N43-3.5. American National Standard N432; Radiological safety for the design and construction of apparatus for gamma radiography. (ANSI N4321980). Nat. Bur. Stand. (U.S.) Handb. 136; 1981 January. 19 p. SN003-003-02293.4.

Key words: classification criteria; gamma radiography; national standard; performance tests; radiation source; safety standard.

This standard applies to the design and construction of apparatus used for industrial gamma radiography which employs radioactive material as the energy source. It establishes the criteria to be used in the proper design and construction of the various components to ensure a high degree of radiation safety at all times. This includes the classification and labeling criteria for the exposure device; and factors which should be considered in the design and construction of exposure devices, controls, and source assemblies. The testing procedures and equipment for the various classifications of the exposure devices and source assemblies are detailed. 


\subsection{SPECIAL PUBLICATIONS}

Include proceedings of conferences sponsored by NBS, NBS annual reports, and other special publications appropriate to this grouping such as wall charts, pocket cards, and bibliographies.

SP250, 1980 Edition. Kieffer, L. J., ed. Calibration and related measurement services of the National Bureau of Standards. Nat. Bur. Stand. (U.S.) Spec. Publ. 250, 1980 Edition; 1981 March. 105 p. SN003-003-02299-3.

Key words: calibration; measurement assurance; measurement services; standards; traceability.

This publication provides detailed descriptions of the currently available NBS calibration services, measurement assurance programs, and other measurement services. In addition, each section describing specific services contains references to additional publications giving even more detail about the measurement techniques and procedures used. This revised edition reflects the services available as of the second quarter of 1980. NBS Special Publication 250 was last issued in 1978. The Appendix to SP250 is reissued every six months (June and December). It lists current prices for the services described in this publication and the NBS points of contact (addresses and phone numbers) from whom additional information can be obtained.

SP260, 1981-83 Edition. Seward, R. W. NBS Standard Reference Materials Catalog-1981.83 Edition. Nat. Bur. Stand. (U.S.) Spec. Publ. 260, 1981-83 Edition; 1981 November. 122 p. SN003-003. 02382-5.

Key words: analysis; characterization; composition; materials; properties; research materials; standard reference materials.

This Catalog lists those Standard Reference Materials (SRM's) Research Materials (RM's), and Special Reference Materials (GM's) that are available from the National Bureau of Standards (NBS), and those that are soon to be available. The Catalog describes these materials as to their certified characterization, unit size, and type, as well as providing ordering information. Prices for these materials are listed separately in annual supplements to this Catalog.

SP260-70. Marinenko, R. B.; Biancaniello, F.; DeRoberts, L.; Boyer, P. A.; Ruff, A. W. Standard reference materials: Preparation and characterization of an iron-chromium-nickel alloy for microanalysis: SRM 479a. Nat. Bur. Stand. (U.S.) Spec. Publ. 260-70; 1981 May. 36 p. SN003-003-02328-1

Key words: austenitic stainless steel; electron probe microanalysis; Fe-Cr-Ni alloy; homogeneity testing; standard reference material.

An alloy of weight fraction 0.710 iron, 0.181 chromium and 0.109 nickel was characterized at the micrometer level of spatial resolution by means of electron probe microanalysis. This alloy designated SRM $479 \mathrm{a}$, is of suitable homogeneity for use as a standard in microanalytical techniques. There is no statistically significant variation in composition within specimens or from specimen to specimen. SRM 479a is supplied as a disk about $4.5 \mathrm{~mm}$ diameter by $0.8 \mathrm{~mm}$ thick.

SP260-71. Seward, R. W.; Mavrodineanu, R. Standard reference materials: Summary of the Clinical Laboratory Standards issued by the National Bureau of Standards. Nat. Bur. Stand. (U.S.) Spec. Publ. 260-7l; 1981 November. 176 p. SN003-003-02381-7.

Key words: chemical composition; clinical materials; standard reference materials.

This publication is a summary of the Clinical Laboratory Standards issued by NBS as Standard Reference Materials (SRM's). The material, composition, certification, use, and remarks concerning each of the SRM's described are presented in tabular form. Copies of the certificates for these SRM's are contained in the appendix for more detailed information.
SP260-72. Reeder, D. J.; Coxon, B.; Enagonio, D.; Christensen, R. G. Howell, B. F.; Paule, R. C.; Mandel, J.; Schaffer, R. Standard reference materials: SRM 900, antiepilepsy drug level assay standard. Nat. Bur. Stand. (U.S.) Spec. Publ. 260-72; 1981 June. 79 p. SN003-003-02329-9.

Key words: anticonvulsants; antiepilepsy drugs; blood analysis; chromatography, gas; chromatography, liquid; drug standards enzyme immunoassay; epilepsy; ethosuximide; mass spectrometry; nuclear magnetic resonance spectroscopy; phenobarbital phenytoin; primidone; reference material; serum base modification; serum matrix.

Recognition of the efficacy of monitoring the concentrations of therapeutic drugs in the blood of patients has revealed many needs for standardization of the laboratory tests used for such monitoring. The National Bureau of Standards was asked to provide a Standard Reference Material (SRM) consisting of three serum samples, each to contain four antiepilepsy drugs at different concentrations. The four drugs are phenobarbital, phenytoin, primidone, and ethosuximide. The SRM would fill a basic role for the achievement of accurate analysis to help ensure the reliability of analyses for these drugs.

The needs that had to be fulfilled to produce the SRM included: (1) analytical criteria for purity of the drugs; (2) serum to be used as a matrix for the drugs; (3) techniques for achieving homogeneity and stability of the SRM; and (4) two independent, highly accurate analytical methods for the certification.

This document describes development of methods and procedures used to produce and certify the SRM.

SP260-73. Interrante, C. G.; Hicho, G. E. Standard reference materials: A standard reference material containing nominally fifteen percent austenite. Nat. Bur. Stand. (U.S.) Spec. Publ. 260-73; 1981 December. 34 p. SN003-003-02386-8.

Key words: austenite in ferrite; austenite standard; electron microprobe; powder metallurgy; quantitative microscopy standard reference material; $x$-ray diffraction; $x$-ray fluorescence analysis.

This Standard Reference Material, SRM-486, is intended for the calibration of $\mathrm{x}$-ray diffraction equipment used in determining the amount of retained austenite in ferrous materials. It was produced using powder metallurgical techniques and known amounts of type 310 stainless steel powder (austenitic) and type 430 stainless steel powder (ferritic) to make a blend of 15 percent by weight (14.7 percent by volume) austenite in ferrite. From a larger blend of these powders, powder for 174 compacts were taken. Using 12 of these compacts, a calibration curve was established for the certification of the remaining compacts. The curve relates the nickel $x$-ray count rate of $\mathrm{x}$-ray fluorescence measurements to the volume percentage of austenite as determined by quantitative microscopy measurements of area percentage. The austenite content of this SRM can be related directly to the nickel count rate, because the nickel content of the austenitic powder (20.9 weight percent) is many times that of the ferritic powder ( 0.10 weight percent). The mean percentage austenite for 162 certified standards is 14.7 volume percent; this value exactly equals the volume percentage of austenite powder in the original blend. X-ray diffraction determinations of the austenite content are in good agreement with the certified content of austenite. The SRM may be used as an $x$-ray diffraction standard for austenite or in special cases as an x-ray fluorescence standard for nickel content.

SP304, Revised August 1981. Goldman, D. The modernized metric System-The International System of Units (SI). Nat. Bur. Stand. (U.S.) Spec. Publ. 304; 1981 August. 1 chart. SN003-003-02365-5.

Key words: International System of Units; measurement systems; metric system; modernized metric system; SI; weights and measures.

The seven base units of the modernized metric system (also called 
the International System of Units, abbreviated SI), two supplementary units, several derived units, a meter stick and, for purposes of comparison, a yardstick are all shown on this four-color wall chart. This edition contains the new definition of the base unit of luminous intensity, the Candela. Additional information shown includes a brief statement on the nature of the modernized metric system and its use in the United States, a table permitting conversion of commonly used units from SI to customary units and vice versa, and a table listing the names and symbols of decimal multiples and submultiples used with SI units.

SP304A, Revised August 1981. Goldman, D. Brief history of measurement systems with a chart of the modernized metric system. Nat. Bur. Stand. (U.S.) Spec. Publ. 304A; 1981 August. 4 p. SN003. 003-02366-3.

Key words: International System of Units; measurement systems; metric system; modernized metric system; SI; weights and measures.

The Modernized Metric System (also called the International System of Units) is made up of seven base units, two supplementary units, and many derived units. The chart describes this entire system, including details about the standards for each base unit and information on how the system is used.

The reverse side of the chart contains a brief history of measurement systems. The need for measurement began with primitive man, who used parts of his body and his natural surroundings for measurement standards and measuring instruments. As societies evolved, weights and measures became more complex. Two systems became predominant: the English system, rooted in the history and tradition of England; and the metric system, a scientifically based system using decimal notation. The metric system, with its inherent decimal advantages, gained widespread acceptance and is now the official measurement system in nearly all countries of the world.

SP305. Supplement 12. Burris, B. L.; Morehouse, R. J., eds. Publications of the National Bureau of Standards 1980 Catalog. A compilation of abstracts and key word and author indexes. Nat. Bur. Stand. (U.S.) Spec. Publ. 305, Suppl. 12; 1981 June. 634 p. SNO03. 003-02349-3.

Key words: abstracts, NBS publications; key words; publications.

This 12th Supplement to Special Publication 305 of the National Bureau of Standards lists the publications of the Bureau issued between January 1-December 31, 1980. It includes an abstract of each publication (plus some earlier papers omitted from Special Publication 305 Supplement 11), key word and author indexes; and general information and instructions about NBS publications.

SP330. Goldman, D. T.; Bell, R. J., eds. The International System of Units (SI). (Supersedes NBS Special Publication 330, 1977 Edition.) Nat. Bur. Stand. (U.S.) Spec. Publ. 330; 1981 December. 53 p. SN003-003-02380-9.

Key words: BIPM; CCU; CGPM; CIPM; metric; SI; translation.

This is a translation of the French "Le Système International d'Unités (SI)," 4th Edition. It is a revision of the 3d edition, published in 1977 and takes into account recent decisions of the General Conference on Weights and Measures (1979), of the International Committee for Weights and Measures $(1977,1978,1980)$ and revisions suggested by the Consultative Committee for Units (1978, 1980). Included are an exposition of a coherent system of units, a definition of supplementary units, a new definition of the base unit, the candela, and a new unit of dose equivalent, the sievert.

SP400-64. Thurber, W. R.; Mattis, R. L.; Liu, Y. M.; Filliben, J. J. Semiconductor measurement technology: The relationship between resistivity and dopant density for phosphorus- and boron-doped silicon. Nat. Bur. Stand. (U.S.) Spec. Publ. 400-64; 1981 May. 53 p. SN003-003-02320-5.

Key words: boron; capacitance-voltage technique; dopant density; electron mobility; Hall effect; hole mobility; Irvin curves; phosphorus; resistivity; semiconductor; silicon.

New data have been obtained for the resistivity-dopant density relationship for silicon doped with phosphorus or boron for dopant densities in the range $10^{13}$ to $10^{20} \mathrm{~cm}^{-3}$. For dopant densities less than $10^{18} \mathrm{~cm}^{-3}$, results were calculated from resistivity and junction capacitance-voltage measurements on processed wafers. For more heavily doped material, data were obtained from Hall effect and resistivity measurements on specimens cut from bulk silicon slices. These primary methods were supplemented for phosphorus-doped material by neutron activation analysis and a photometric technique and for boron-doped material by the nuclear track technique. For phosphorus-doped silicon the results of this work differ by 5 to 15 percent from the commonly used Irvin curve, always in the direction of lower resistivity for a given dopant density. For boron-doped silicon the results differ significantly from the $p$-type Irvin curve for boron densities greater than $10^{16} \mathrm{~cm}^{-3}$ with a maximum deviation of 45 percent at $5 \times 10^{17} \mathrm{~cm}^{-3}$ in the direction of lower resistivity for a given dopant density. Hole mobility values derived from the data are in reasonable agreement with the Wagner expression with a maximum discrepancy of 10 percent in the $10^{18} \mathrm{~cm}^{-3}$ range. Analytical curves were fitted to the resistivity-dopant density product as a function of resistivity and dopant density for temperatures of $23^{\circ} \mathrm{C}$ and $300 \mathrm{~K}$. Similar curves were obtained for the calculated carrier mobility as a function of resistivity and carrier density.

SP400-65. Mitchell, M. A.; Linholm, L. W. Semiconductor measurement technology: Test patterns NBS-28 and NBS-28A: Random fault interconnect step coverage and other structures. Nat. Bur. Stand. (U.S.) Spec. Publ. 400-65; 1981 March. 35 p. SN003-003. 02297-7.

Key words: electronics; interconnect; microelectronics; random fault; step coverage; test structures.

This report describes microelectronic test structures for detection of random faults in interconnect step coverage and associated process parametric and physical analysis test structures included in two test patterns, NBS-28 and NBS-28A, recently designed under the device test structure program at the National Bureau of Standards. Information about the geometry and application of the test structures in these two test patterns is provided for those who wish to fabricate or utilize the test patterns prior to their complete evaluation at NBS. Test pattern NBS-28 consists of a random fault test structure for measuring the integrity of metal interconnects stepping over polysilicon lines. The structure is a metal serpentine $0.93 \mathrm{~m}$ long with $8-\mu \mathrm{m}$ linewidths and spacings stepped over polysilicon lines with $8-\mu \mathrm{m}$ linewidths and spacings. The serpentine is divided into nine arrays containing $150,220,480,960,2880,5760,13440,28800$, and 62400 steps, respectively. Test pattern NBS-28A contains three similar, but reduced, step-coverage structures with $4-$, 2-, or $1-\mu \mathrm{m}$ linewidths and spacings. Test pattern NBS.28A also contains 37 other microelectronic test structures for providing process parameter or physical analysis information. Some of these are new structure designs which are now undergoing development and evaluation.

SP400-66. Linholm, L. W. Semiconductor measurement technology: The design, testing, and analysis of a comprehensive test pattern for measuring CMOS/SOS process performance and control. Nat. Bur. Stand. (U.S.) Spec. Publ. 400-66; 1981 August. 148 p. SN003-003. 02345-1.

Key words: integrated circuits; microelectronics; process validation wafer; random faults; silicon-on-sapphire; test pattern; test structure; yield.

A Process Validation Wafer (PVW) is a wafer containing only test patterns. One PVW accompanies a product lot during the fabrication process. Test patterns NBS-16 and NBS-26 are designed to be used on PVWs. They contain both process parameter test structures and random fault test structures. Eighteen NBS-16 PVWs were fabricated in a radiation-hardened silicon-gate CMOS/SOS process. These PVWs were tested on a high-speed computer-controlled dc test system. Test results from the process parameter test structures were used to establish the baseline electrical parameters for each product lot and to produce an eight-level gray scale wafer map for these parameters. Based on correlations of selected wafer maps, it was possible to identify specific yield-related process problems otherwise unknown to the manufacturer or user.

Test results from two random fault test structures were used to establish a statistically significant data base for identifying and evaluating major yield-limiting fault mechanisms in the process. Test 
results from a developmental random access fault structure and a gate dielectric integrity array are presented. The results are analyzed for selected PVWs and a major yield-limiting fault mechanism detected. A description of the test pattern and test results, including a drawing of each test structure, recommended test procedure, and design rules, are found in the appendices.

SP400-67. Schwarz, S. A.; Helms, C. R.; Spicer, W. E.; Taylor, N. J. Semiconductor measurement technology: The capabilities and limitations of Auger sputter profiling for studies of semiconductors. Nat. Bur. Stand. (U.S.) Spec. Publ. 400-67; 1981 September. 53 p. Available'from: NTIS; PB 82-120122.

Key words: Auger electron spectroscopy (AES); metal-oxidesemiconductor (MOS); secondary ion mass spectrometry (SIMS); semiconductor devices; sputtering; $x$-ray photoelectron spectroscopy (XPS, ESCA).

Materials characterization is a critical area in current silicon integrated circuit technology. Those techniques that are commonly used include Auger sputter profiling, X-ray photoelectron spectroscopy, secondary ion mass spectrometry, and Rutherford backscattering. All of these techniques have unique capabilities and limitations for studies of silicon device structures. In this paper, we describe the capabilities and limitations of Auger sputter profiling especially with regard to sensitivity, spatial resolution, depth resolution, and chemical state determination. Although much of the discussion centers on Auger sputter profiling, the results are also applicable to X-ray photoelectron spectroscopy and secondary ion mass spectrometry.

SP400-68. Carver, G. P.; Cullins, W. A. Semiconductor measurement technology: A manual wafer probe station for an integrated circuit test system. Nat. Bur. Stand. (U.S.) Spec. Publ. 400-68; 1981 May. 19 p. SN003-003-02319-1.

Key words: integrated circuit test methods; microelectronic test structure; probe station; semiconductor process control; test pattern; test structure; wafer chuck; wafer probe station.

This report describes the design of a manual wafer probe station for wafer-level testing of integrated circuits, test structures, and test patterns. The station includes a translatable vacuum chuck, a probe assembly which can be used for probe cards or individual micropositioner-mounted probes, and a microscope holder. Except for a few components which are commercially available, construction details are provided for all parts.

SP400-69. Schafft, H. A.; Ruthberg, S.; Cohen, E. C., eds. Semiconductor measurement technology: ARPA/NBS workshop V. Moisture measurement technology for hermetic semiconductor devices. Proceedings of the ARPA/NBS Workshop V held at the National Bureau of Standards; 1978 March 22-23; Gaithersburg, MD. Nat. Bur. Stand. (U.S.) Spec. Publ. 400-69; 1981 May. 202 p. SN003-003-02326-4.

Key words: analysis of moisture content; mass spectrometer measurement; moisture; moisture generators; moisture sensors; quality control; reliability of semiconductor devices; semiconductor devices.

The workshop, fifth in a series concerned with measurement problems in integrated circuit processing and assembly, served as a forum to examine present problems with the measurement of moisture in hermetic semiconductor devices. While moisture-induced failure modes and mechanisms had received considerable attention in the published literature and in meetings, the accurate and reliable measurement of the moisture content had not; yet, this lack of measurement of moisture reliability is a major obstacle to meaningful efforts to limit and control this pervasize contaminant. Manuscripts and summaries are provided of 19 talks, panel meetings, and group encounters on three major topics: mass spectrometer measurements of internal package moisture, moisture sensors, and package analysis and quality assurance. These proceedings include the following papers (indented):

SP400-69; 1981 May. 3.23. Moore, B. A. Mass spectrometer measurements at RADC.

SP400-69; 1981 May. 24-32. Hartley, J. L. A method for measuring the PPM moisture-sensing limitations of mass spectrometers in testing small packages.

SP400-69; 1981 May. 33-41. Merrett, R. P. A dynamic method of calibrating a mass spectrometer used for measuring the water content of semiconductor encapsulations.

SP400-69; 1981 May. 42. Pernicka, J. C. Moisture measurement by mass spectrometer.

SP400-69; 1981 May. 43-57. Haack, R. F.; Shumka, A. Microcircuit package gas analysis.

SP400-69; 1981 May. 58.68. Perkins, K. L. Comparison of mass spectrometric moisture measurements by different laboratories.

SP400-69; 1981 May. 69-74. Fong, V. The effects of assembly techniques upon the performance characteristics of $\mathrm{Al}_{2} \mathrm{O}_{3}$ in-situ moisture sensors.

SP400-69; 1981 May. 75.79. Bennewitz, P. F. Application considerations of solid state moisture chips for hybrids and associated calibration measurement techniques.

SP400-69; 1981 May. 80-81. Webster, H. L. Hysteresis and longterm repeatability of aluminum oxide humidity sensors.

SP400-69; 1981 May. 82.93. Messenger, C. G. Moisture standards and sensors.

SP400-69; 1981 May. 94-107. Merrett, R. P.; Sim, S. P. Assessment of the use of measurement of surface conductivity as a means of determining moisture content of hermetic semiconductor encapsulations.

SP400-69; 1981 May. 108-114. Senturia, S. D.; Huberman, M. G.; Van der Klott, R. Moisture sensing with the charge-flow transistor.

SP400.69; 1981 May. 115-121. Knight, C. W. T.; Korgav, A.; Pierron, E. A surface conductance technique for the evaluation of internal package environments.

SP400-69; 1981 May. 126-127. Hasegawa, S. Moisture sensors.

SP400.69; 1981 May. 128-132. Hartley, J. L. Parts-per-million watervapor generating system used to simulate moisture in small integrated circuit packages.

SP400-69; 1981 May. 133-142. Loucks, S. R.; Burnham, A. W. A total moisture analyzer for hermetic devices.

SP400-69; 1981 May. 143-152. Dun, H.; Mattes, B. L.; Stevenson, D. A. Analysis of water vapor in purified gas systems.

SP400-69; 1981 May. 153-158. Hickam, W. M.; Morgan, W. R. Mass spectrometric moisture analysis of hermetic semiconductor devices by acetylene conversion.

SP400-69; 1981 May. 159-178. Der Marderosian, A. Moisture in packages-A user's viewpoint.

SP400-69; 1981 May. 179-186. Thomas, R. W. Problems in specifying and measuring moisture content within èlectronic devices.

SP446-5. Raufaste, N.; Olmert, M., eds. Building technology project summaries 1980-1981. Nat. Bur. Stand. (U.S.) Spec. Publ. 446-5; 1981 July. 82 p. SNOO3-003-02343-4.

Key words: building research; building technology; codes; criteria; measurement methods; performance criteria; project summaries; technical bases.

The Center for Building Technology provides the technical and scientific bases for criteria and standards that improve the usefulness, safety and economy of buildings while conserving building materials and energy. The Center's activities support building technology programs of the Federal, State and local governments; assists design professions, building officials and the research community by developing design criteria that improve buildings; and assists manufacturers of building products by developing criteria for 
evaluating innovative building materials. This report summarizes the Center's projects for calendar year 1980-81. It enables idividuals to get a clear impression of CBT research activities.

SP457-5. Webber, S., ed. Building technology publications 1980Supplement 5. Nat. Bur. Stand. (U.S.) Spec. Publ. 457-5; 1981 June. 90 p. SN003-003-02332-9.

Key words: abstracts; building technology; Center for Building Technology; key words; publications.

This report presents NBS' Center for Building Technology (CBT) publications for 1980. It is the fifth supplement to NBS Special Publication 457, Building Technology Publications, and lists CBT reports issued during January 1-December 31, 1980. It includes titles and abstracts of each CBT publication and those papers published in non-NBS media, key word and author indexes, and general information and instructions on how to order CBT publications.

This publication is divided into three main sections. The first, Titles and Abstracts, provides the report title, author(s), date of publication, selected key words, and an abstract of each NBS publication and each paper published in an outside source. The Author Index cites CBT authors and their publication number which is listed in this supplement. The Key Words Index is a subject index, listing word summaries of the building research topics for each publication and paper. By selecting a main word or subject, the user is able to locate reports of interest through these subject-related words.

SP480-37. Rubin, A. I.; Howett, G. L. Emergency vehicle warning systems. Nat. Bur. Stand. (U.S.) Spec. Publ. 480-37; 1981 May. 25 p. SN003-003-02323-0.

Key words: conspicuity; effective intensity; emergency warning lights; flashing lights; lights, warning; sirens; sound level; warning light; warning signals.

The subject of visual and auditory warning devices (lights and sirens) for emergency and service vehicles is surveyed from a broad perspective. It is intended that this user guide should provide directly useful information at all levels from the selection of hardware to an understanding of the psychophysical factors determining the effectiveness of these devices. Topics covered include: the theory of warning signals; the present situation and the need for uniform national standards; suggested performance standards for warning light systems and for sirens, including the reasons for the principle requirements; recommendations for actions that can be taken to improve the signal effectiveness of emergency vehicles; an illustrated classification of the many types of emergency-vehicle warning lights; and brief summaries of some of the physical measurements that were made on a selection of lights and sirens.

SP480-40. Thornton, J. I.; Kraus, S.; Lerner, B.; Hendrickson, B. Paint solubility testing. Nat. Bur. Stand. (U.S.) Spec. Publ. 480-40; 1981 December. 21 p. Available from: NTIS; PB 82-177049.

Key words: automotive paints; household paints; sequential tests; solubility tests.

This report presents the results of a study to develop criteria for the characterization of paint through solubility testing. Experiments were conducted with common solvents to classify chemical reactivity with automobile and household paints. Seven categories of reactivity are defined, which form the basis for specifying the solubility of paints to various reagents under controlled conditions of time and temperature. The report also describes a novel solubility test procedure by which acrylic lacquers, organic-dispersed enamels, and water-based enamels can be distinguished through sequential testing, a characterization not previously accomplished with solubility testing.

SP480-41. Flores, A.; Eliason, L. K.; Wu, Y. C. Breath alcohol sampling simulator (BASS) for qualification testing of breath alcohol measurement devices. Nat. Bur. Stand. (U.S.) Spec. Publ. 480-41; 1981 July. 31 p. SN003-003-02336-1.

Key words: blood alcohol analysis; blood alcohol content; breath alcohol content; breath sampling; evidential breath testers; human respiratory characteristics; intoxication.

The blood alcohol content of an individual suspected of driving while intoxicated is often measured through the analysis of a breath sample using an evidential breath tester (EBT). Two factors determine the ability of an EBT to provide an accurate analysis of alcohol concentration: a) the capability of obtaining an appropriate breath sample, and b) the inherent accuracy of the analytical instrument. This report characterizes the relevant breath parameters and describes a breath alcohol sample simulator (BASS) that was developed as a means of reproducing accurate equivalents of human breath. The report discusses measurements made on human subjects to establish values for parameters the BASS must accommodate, and to demonstrate the validity of the BASS as a replacement for human drinking subjects with different levels of alcohol content and a range of physiological characteristics.

SP480-43. Brown, J. E. Hydrocarbon vapor standards for performance evaluation of combustible gas detectors. Nat. Bur. Stand. (U.S.) Spec. Publ. 480-43; 1981 October. 15 p. SN003-003-02371-0.

Key words: accelerants; arson; combustible gas; detectors; diffusion rates; diffusion tubes; gas chromatography; hydrocarbons; vapor detector; vapor generator; vapor standards.

Dynamic standards of hydrocarbon vapor were prepared for the calibration and performance evaluation of portable combustible gas detectors which are used in the investigation of suspected incendiary fire scenes. The standards contained vapors of aliphatic and aromatic compounds common to petroleum distillates, such as gasolines, that are most frequently employed as accelerants in arson fires. These standards were prepared from vapors evolving from hydrocarbon liquids contained in diffusion tubes into a constant flow of air.

The concentrations of the vapors were ascertained from gravimetrically determined diffusion rates of the hydrocarbons at three temperatures, 30,50 and $70^{\circ} \mathrm{C}$. Gas chromatographic analyses of the vapor-air mixtures generated at 30 and $50^{\circ} \mathrm{C}$ showed that the vapor concentrations are essentially constant within measurement periods of one to three days and from one period to another.

SP480-44. Taggart, H. E. Methods of suppressing automotive interference. Nat. Bur. Stand. (U.S.) Spec. Publ. 480-44; 1981 December. 15 p. Available from: NTIS; PB 82-165259.

Key words: automotive; EMI; ignition interference; suppression.

The purpose of this report is to review the sources of electromagnetic interference (EMI) within a vehicle so that the reader will have a basic understanding of the EMI problem, discuss the techniques that have been used successfully by the auto industry to suppress EMI, and suggest some newer techniques for suppressing EMI within an automobile.

The automotive manufacturers utilize several techniques to reduce EMI emanating from the vehicle. These techniques include, but are not limited to, resistor spark plugs, resistor spark plug cables, use of silicon grease in the distributor, use of capacitors as filters, placement of grounding straps at key locations, conductive fan belt discharge, and grounding in wheels to reduce tire static-charge build-up. If further reduction of EMI is needed to obtain full utilization of a mobile communication system, there are additional suppression techniques that can be employed to achieve this goal. These are listed. Most of these techniques are effective at frequencies from approximately 30 to $1000 \mathrm{MHz}$. Measurement results show that the EMI from a new production-line automobile, as measured in accordance with SAE Standard J55lg, can be reduced by an additional 10 to $15 \mathrm{~dB}$ by employing these additional suppression techniques. A measurement technique is described whereby the amount of degradation to a mobile narrowband FM receiver (used by law enforcement agencies) can be measured. This same technique can then be used as a tool to further reduce EMI from the vehicle.

SP480-45. Clifton, J. R. Penetration resistance of concrete-A review. Nat. Bur. Stand. (U.S.) Spec. Publ. 480-45; 1981 December. 22 p. Available from: NTIS; PB 82-177932.

Key words: concrete; dynamic properties; fiber-reinforcement; impact; impulsive loading; penetration resistance; reinforcing bars; stress waves.

The mechanisms accounting for the failure of concrete under impact and impulsive loads, and the factors controlling the resistance of concrete to such loads, have been reviewed. It was found that little is known concerning the damage mechanism within concrete in 
response to dynamic loads. Apparently, the dynamic tensile strength of concrete has an important effect on its impact and impulsive resistances. A model based on the propagation of stress waves, which appears to give some insight to important material variables is described.

Types of concrete which may merit evaluation for constructing structures subjected to dynamic loads are identified and discussed.

SP500-73. Federal Computer Performance Evaluation and Simulation Center. Computer science \& technology: Computer model documentation guide. Nat. Bur. Stand. (U.S.) Spec. Publ. 500-73; 1981 January. 56 p. SN003-003-02289-5.

Key words: documentation; manuals; models; simulation.

This document provides guidelines for preparing documentation for computer models. Recommended structures for four types of manuals providing model information for four different classes of audiences (managers, users, analysts, and programmers) is presented. This document specifies the content of sections and subsections for each type of manual. Manuals prepared using these guidelines will enable persons interested in a model to understand the capabilities and limitations of that model.

SP500-74. Houghton, R. C., Jr. Computer science \& technology: Features of software development tools. Nat. Bur. Stand. (U.S.) Spec. Publ. 500-74; 1981 February. 24 p. SN003-003-02295-1.

Key words: dynamic analysis; programming aids; software development; software engineering; software tools; static analysis; taxonomy.

Software tools are powerful productivity and quality aids that in many cases are not being used effectively. This report discusses an effort to lessen this problem by providing a formal way in which tools can be classified according to the features that they provide.

SP500-75. Adrion, W. R.; Branstad, M. A.; Cherniavsky, J. C. Computer science \& technology: Validation, verification, and testing of computer software. Nat. Bur. Stand. (U.S.) Spec. Publ. 500-75; 1981 February. 62 p. SN003-003-02289.6.

Key words: automated software tools; software lifecycle; software testing; software verification; test coverage; test data generation; validation; verification.

Programming is an exercise in problem solving. As with any problem solving activity, determination of the validity of the solution is part of the process. This survey discusses testing and analysis techniques that can be used to validate software and to instill confidence in the programming product. Verification throughout the development process is stressed. Specific tools and techniques are described.

SP500-76. Berg, J. L.; Graham, M.; Whitney, K., eds. Computer science \& technology: Database architectures-A feasibility workshop report. Nat. Bur. Stand. (U.S.) Spec. Publ. 500-76; 1981 April. 64 p. SN003-003-02305-1.

Key words: conversion; database; data description; data dictionary; data directory; data manipulation; DBMS; language; query; standards.

To help the decision maker evaluate the potential benefits and pitfalls in moving forward with database technology, the National Bureau of Standards organized two workshops whose results are presented in this report. The workshops, held in August 1978, explored the progress plan and potential pitfalls involved in specifying, designing, and implementing systems based on the ANSI/X3/SPARC framework and the CODASYL JOD languages specification. Workshop 1 investigated the general topic of data independence, and Workshop 2 examined supporting topics such as query languages, data dictionaries, and database conversion.

SP500-77. Cugini, J. V. Computer science \& technology: Specifications and test methods for numeric accuracy in programming language standards. Nat. Bur. Stand. (U.S.) Spec. Publ. 500-77; 1981 June. 42 p. SN003-003-02324-8.

Key words: computer arithmetic; conformance testing; numeric accuracy; programming language standards.
This publication formulates language-independent and machineindependent criteria for assessing the quality of floating-point arithmetic operations and functions. The criteria require that results be within the limits generated by perturbing the arguments or operands by a specified amount, and thus allow for the mathematical instability of some functions at certain arguments and also for the granularity of numeric representation inherent in digital machines. Automatic test methods derive naturally from the accuracy requirements. Model algorithms for testing are included.

SP500-78. Branstad, M. A.; Adrion, W. R., eds. Computer science \& technology: NBS programming environment workshop report. Nat. Bur. Stand. (U.S.) Spec. Publ. 500-78; 1981 June. 106 p. SN003-003. 02334-5.

Key words: development support systems; programming environments; software development; software tools; toolboxes.

In May of 1980, NBS hosted a workshop to assess the state-of-theart in programming environment technology and to determine the key questions and issues that must be addressed to use these techniques to improve software quality and productivity within the Federal Government. This document reports the results of the workshop.

SP500-79. Gray, M. M. Computer science \& technology: An assessment and forecast of ADP in the Federal Government. Nat. Bur. Stand. (U.S.) Spec. Publ. 500-79; 1981 August. 151 p. SN003-003-02368.0.

Key words: data base management systems; Federal contracts services; Federal Government computers; programming languages; software expenditures; software program life-cycle analysis.

This report summarizes the findings of the International Data Corporation (IDC) on the current status of Federal ADP and projects ADP trends in the Federal Government for 1979 through 1985. Hardware areas included are computers and related peripheral equipment such as magnetic tape drives, disk drives, terminals, printers, modems, multiplexers, and word processors. Other areas included are: an analysis of programming languages; analysis of software spending; analysis of installation workload by mode of operation and by application; implementation of data base management systems; scope of contract services in the Federal Government; and the state of software and software development in selected Federal installations.

SP500-80. Houghton, R. C., Jr., ed. Computer science \& technology: Proceedings of the NBS/IEEE/ACM software tool fair. Proceedings of the NBS/IEEE/ACM Software Tool Fair, held in conjunction with the 5th International Conference on Software Engineering; 1981 March 10-12; San Diego, CA. Nat. Bur. Stand. (U.S.) Spec. Publ. 500-80; 1981 October. 238 p. SN003-003-02362-1.

Key words: programming aids; software automation; software development; software engineering; software testing; software tools.

This document summarizes the presentations made by each demonstrator at the San Diego Tool Fair. The San Diego Tool Fair was a first-of-its-kind demonstration of software engineering tools at a major conference. Each summary includes a short description of the tool, a scenario of the demonstration, a list of references, background on the demonstrators, sample output, and a page of miscellaneous data obtained from the NBS Software Tools Database. The appendix provides a cross reference to the features of the tools. These proceedings include the following papers (indented):

SP500-80; 1981 October. 1-6. Berild, S.; Nachmens, S. CS4: A tool for the design and generation of database systems.

SP500-80; 1981 October. 7.9. Wirth, N.; Ohran, R. LILITH: A modula machine.

SP500-80; 1981 October. 10-14. Fenchel, R. S. SARA: A demonstration of the development of interactive software with integral help.

SP500.80; 1981 October. 15-20. Scherrer, D.; Martin, D.; Peterson, C. VIRTUAL OS: The software tools virtual operating system project. 
SP500-80; 1981 October. 21-24. Resendez, D. L.; Winchester, J. W. SCG: Structure chart graphics system.

SP500.80; 1981 October. 25-28. Resendez, D. L.; Winchester, J. W. DQM: Design quality metrics system.

SP500-80; 1981 October. 29-32. Austell, W. P., Jr.; Willis, R. R. AISIM: Automated interactive simulation modelling system.

SP500-80; 1981 October. 33-37. Miller, J. C.; Lyons, M. J. THE ENGINE: COBOL structuring engine.

SP500-80; 1981 October. 38-43. Callender, E.; Sayani, H. H.; Teichroew, D. PSL/PSA: Problem statement language/problem statement analyzer.

SP50 -80; 1981 October. 44-48. Callender, E.; Hartsough, C.; Kleine, H. SDDL: Software design and documentation language.

SP500-80; 1981 October. 49-57. Putnam, L. H. SLIM: A quantitative tool for software cost and schedule estimation.

SP500-80; 1981 October. 58-63. Levy, A. POD: Performance oriented design.

SP500-80; 1981 October. 64-68. Lycklama, H. PWB FOR VAX/VMS: Programmer workbench tools on VAX/VMS.

SP500-80; 1981 October. 69-75. Erickson, R.; Gerhart, S.; Lee, S.; Thompson, D. AFFIRM: A specification and verification system.

SP500-80; 1981 October. 76-82. Penedo, M. H. SARA: SARA as a tool for software design: Building-block modelling and composition.

SP500-80; 1981 October. 83-87. King, W. C. ARGUS/MICRO: ARGUS in the microcomputer environment.

SP500-80; 1981 October. 88-95. Stucki, L. G. DYNA: A tool from the ARGUS toolbox.

SP500-80; 1981 October. 96-102. Stucki, L. G. COMMAP: A tool from the ARGUS toolbox.

SP500-80; 1981 October. 103-110. Moy, S.; Nielsen, G.; Sanchez, R.; Wallace, T.; Mc Wethy, S. LOGICFLOW: A software design and analysis tool.

SP500-80; 1981 October. 111-116. Hoffman, R. H.; Loshbough, R. P.; Smith, R. W. SREM: Software requirements engineering methodology.

SP500-80; 1981 October. 117-121. Linden, N.; Yavne, M. SDP: A computerized tool for system design and maintenance.

SP500-80; 1981 October. 122-127. Hug, R.; Strelich, T.; Resnicow, M.; Ahola, R. SOFTOOL 80(B): A methodology and a comprehensive set of tools for software management, development, and maintenance.

SP500-80; 1981 October. 128-135. Henderson, J. B.; Miller, E. F., Jr. ITB: Interactive test bed.

SP500-80: 1981 October. 136-145. Henderson, J. B.; Miller, E. F., Jr.; Hirschberg, M. ISUS: Interactive semantic update system.

SP500-80; 1981 October. 146-151. Rosenbaum, J.; Early, C. FAME: Front-end analysis and modeling environment.

SP500-80; 1981 October. 152-158. Robillard, P. N.; Plamondon, R. SCHEMACODE: An interactive schematic pseudocode for program development, documentation, and structured coding.

SP500-80; 1981 October. 159-162. Huang, J. C. INSTRU: An automated software instrumentation system.

SP500.80; 1981 October. 163-166. Razouk, R. R. SARA: Controlflow analysis in SARA.

SP500-80; 1981 October. 167-173. Relles, N.; Price, L. A. ONLINE ASSIST: A user interface for online assistance.

SP500-80; 1981 October. 174-179. Barkley, J.; Powell, P. FTN.77 ANALYZER: FORTRAN 77 analyzer.
SP500-80; 1981 October. 180-185. Houghton, R. C., Jr.; Oakley, K. A. TOOLS DATABASE: NBS software tools database.

SP500-80; 1981 October. 186-189. White S. SRIMP: Software requirements integrated modeling program.

SP500-80; 1981 October. 190-193. Gilb, T.; Krzanik, L. AUTODBO: Automated design by objectives.

SP500-80: 1981 October. 194-196. Overgaard, M.; Gianetta, J. UCSD P.SYSTEM: A portable software development system.

SP500-80; 1981 October. 197-199. Thall, R.; Anderson, G. MSEF: The microprocessor software engineering facility.

SP500-80; 1981 October. 200-207. Saib, S. H.; Benson, J. P.; Gannon, C.; DeHaan, W. R. IFTRAN@: A preprocessor for FORTRAN.

SP500-80; 1981 October. 208-217. Saib, S. H.; Benson, J. P.; Gannon, C.; DeHaan, W. R. RXVP80ß: A software documentation, analysis, and test system.

SP500-81. Knoerdel, J. E. Computer science \& technology: A survey of standardization efforts of coded character sets for text processing. Nat. Bur. Stand. (U.S.) Spec. Publ. 500-81; 1981 September. 50 p. SN003-003-02355-8.

Key words: coded character sets; code extension techniques; code standards; control functions; information interchange; text communications; text processor.

As the desire to interchange documents among different text processing systems via communications increases, the incompatibilities which exist between those text processing systems become more and more apparent. One such incompatibility is that often the sending device and the receiving device use different bit assignments or coding schemes for their alphanumeric characters, special symbols, and control characters (i.e., different coded character sets). Considerable work has been done both nationally and internationally to standardize coded character sets. However, the knowledge of such standards efforts is not always widespread. Or, if there is familiarity with the standards efforts, the relationship of those efforts among various standards organizations is frequently not easy to perceive. The objective of this report is to describe the status of those standard coded character sets, with special attention to text processing systems. The report includes, first of all, a brief description of the major national and international standards organizations which develop code standards. Next, it describes the various code standards according to the following categories: basic code sets for information interchange, methods of augmenting those basic code sets, additional control characters to be used with the basic code sets, and code sets developed specifically for text communications. Finally, the summary of the report discusses a number of limitations which still exist when interchanging information via communicating text processors.

SP500-82. Hecht, H. Computer science \& technology: Final report: A survey of software tools usage. Nat. Bur. Stand. (U.S.) Spec. Publ. 500-82; 1981 November. 58 p. SN003-003.02378-7.

Key words: programming aids; software automation; software development; software engineering; software tools; software tools usage.

The state of the art regarding the development and use of software tools is presented. The information was gathered from an in-depth survey of 23 sites with Government and industry. A comparison is made among the levels of tool usage based on the size and type of programming group. The survey examines aspects that both promote or inhibit tool usage and provide requirements for the future.

SP500-83. Potter, T. W., ed. Computer science \& technology: Proceedings of the Computer Performance Evaluation Users Group (CPEUG) 17th meeting. "Increasing organizational productivity". Proceedings of the Computer Performance Evaluation Users Group (CPEUG) 17th Meeting; 1981 November 16-19; San Antonio, TX. Nat. Bur. Stand. (U.S.) Spec. Publ. 500-83; 1981 November. 320 p. SN003-003-02374-4.

Key words: benchmarking; capacity planning; chargeback systems; computer performance management; data base machines; end user productivity; human factors evaluation; information system management; office automation; performance management systems; resource measurement facility; simulation; 
supercomputers.

These Proceedings record the papers that were presented at the Seventeenth Meeting of the Computer Performance Evaluation Users Group (CPEUG 81) held November 16-19, 1981, in San Antonio, TX. With the theme, "Increasing Organizational Productivity," CPEUG 81 reflects the critical role of information services in the productivity and survival of today's organization, as well as such trends as increasing personnel costs, limited budgets, and the covergence of data processing, communications, and word processing technologies. The program was divided into three parallel sessions and included technical papers on previously unpublished works, case studies, tutorials, and panels. Technical papers are presented in the Proceedings in their entirety. These proceedings include the following papers (indented):

SP500-83; 1981 November. 7.14. Alexander, W.; Brice, R. Longrange prediction of network traffic.

Key words: computer networks; long-range forecasting; user behavior; workload forecasting.

A method of making long-range computer system workload predictions is presented. The method quantifies the effect of qualitative changes in computing by identifying assumptions and by considering the effect of a change on individual users. The method is illustrated by an example involving message traffic in a large computer network.

SP500-83; 1981 November. 15.19. Chandler, P. Functional grouping of application programs in a timesharing environment.

Key words: functional categories; resource oriented groupings; timesharing.

The ability to adequately describe the computing environment is important in the selection of timesharing services. A method is presented by which the user can functionally describe the workload in terms of requirements for processing, data input, and data output. Large numbers of programs can thereby be reduced to only eight functional categories which are sufficient to characterize the workload. Unsophisticated non-ADP oriented users who are unable to use traditional performance or resource oriented groupings can use this functional method satisfactorily.

SP500-83; 1981 November. 21-34. Wight, A. S. Cluster analysis in workload characterisation for VAX/VMS.

Key words: cluster analysis; workload characterisation.

Accurate models of computer system workloads are desirable for system modelling, performance evaluation studies, capacity planning, benchmarking, tuning and system selection. The use of cluster analysis for workload characterisation has received much attention recently. In this paper we explore the use of cluster analysis for characterisation of workloads on VAX systems running the VMS operating system. In a largely interactive environment we take as our basic workload unit the program or executable image invoked by a command from a terminal user e.g., file directory manipulation, editor, compiler, application program. We discuss some of the problems of cluster analysis and the possible uses of cluster descriptions.

SP500-83; 1981 November. 39-43. Mullen, J. W. Capacity management considerations.

Key words: business case; business elements; business problem; capacity management; capacity planning; disaster recovery planning.

Initiating an installation capacity management program required certain basic elements that must be in place in the installation to assure success of the program. These basic elements will be addressed with respect to their relationship to the business community and how this interaction should be developed in support of the capacity management/planning effort. An additional aspect of the capacity management program and function, capacity requirements in disaster recovery planning, will be outlined with respect to the aid that the capacity management function can provide to the disaster recovery planning effort.

SP500-83; 1981 November. 45-54. Penansky, S. G. Capacity considerations in disaster recovery planning.

Key words: capacity planning; contingency planning; disaster recovery planning.

Increasing dependence on data processing in the Federal Government and private industry has resulted in growing interest in recovery plans that minimize the impact resulting from a loss to an organization's data processing capability. Although disaster recovery planning is not generally the responsibility of an organization's CPE group, capacity considerations play a significant role in the development of a cost-effective recovery plan, and CPE practitioners are being asked to participate in the development of these plans.

This paper describes and discusses several aspects of the disaster recovery planning process in which capacity considerations should be addressed. The concepts of a "minimum configuration" and "recovery windows" are discussed. Capacity considerations involved in evaluating component recovery strategies based on reciprocal agreements, equipped recovery centers, recovery shells, and multiple facilities are presented. Finally, relationships between ongoing capacity planning and recovery plan maintenance activities are examined.

SP500-83; 1981 November. 59.65. Wilson, C. B.; Mohr, J. M.; Chu, C. M. Service reporting for the bottom line.

Key words: ADP installation management; cost-benefit; performance management; productivity; user service reporting.

The productivity of the end-users of an ADP installation is affected by the service being rendered by the installation, and by the anticipated service levels. Losses in productivity of the endusers can ripple outward in the form of losses in benefit to the organization from missed opportunities, etc., or in the form of increased cost to the organization to produce the same products or services. As the ADP installation becomes more critical to the success of the organization, the need to ensure the continuation of adequate service becomes more important. This paper describes the basis of an approach to user service reporting based on the fiscal aspects of ADP service. Such a system would provide the necessary information to various levels of organizational management to permit the assessment of ADP performance as it affects end-user productivity and the bottom line for the organization.

SP500-83; 1981 November. 67.70. Elsen, L. C. Automating the automation process.

Key words: automated automation; computer performance management.

SP500-83; 1981 November. 71-80. Archer, N. P. Methodology for analyzing computer user productivity.

Key words: end user performance; interactive systems; performance analysis; productivity analysis; text editors.

A methodology is proposed for the analysis of computer user productivity. The methodology is based upon a multi-layered level structure of the computer system in an interactive environment, where each lower level develops more detail of the interactive system. This structure enables a logical approach to the analysis of interactive systems, and productivity may be analyzed at any level, from the lowest primitive function up to 
the most aggregated system level. Here, considerations of productivity are considerably different in scope and direction, depending upon the level of detail. Examples are drawn from the area of text editing, which is having an increasingly strong effect on the performance of interactive systems.

SP500-83; 1981 November. 85.91. Hardgrave W. T.; Salazar, S. B.; Beller, E. J. III. Design of information systems using scenariodriven techniques.

Key words: database design; data dictionary; design; flowchart; information flow; information systems; interactive systems; requirements; scenarios.

This paper describes a technique for developing information systems using a scenario-driven design approach. The approach emphasizes client (that is, the user who is purchasing the system) participation in the design process. The first step is to develop a collection of "scenarios" which document the interaction between the computer and the human user. Using the scenarios, information-flow diagrams and database designs may be constructed. After the client has approved these documents, they can be used to establish disk capacity requirements and transaction rates, and finally to specify all hardware and software requirements. The primary advantage of this approach is that the scenarios provide a good indication of the ultimate usefulness and cost of the system. The client can review these documents and approve, modify, or reject the system design before any software is generated. This paper describes the scenarios, the information flow technique, and the database design approach using, as an example, a small business application.

SP500-83; 1981 November. 93-99. Edwards, S. R. Containing the cost of software maintenance testing-An examination of the quality assurance role within U.S. Army Computer Systems Command.

Key words: developmental center test; environmental test; field validation test; independent testing; quality assurance program; software change package; software maintenance testing.

This paper provides a brief introduction to the U.S. Army Computer Systems Command (USACSC) to include its various elements and their respective functions. The previous (prior to 1 Sept. 80) requirements for software maintenance testing within the Command are examined, with the greatest emphasis being placed on the specific allocation of the Quality Assurance Directorate's resources (manpower and computer) necessary to fulfill these requirements. Key points in this portion include an explanation of Developmental Center Testing (DCT), Environmental Testing (ENT) as an independent, third-party test, and Field Validation Testing (FVT). The evolution of third party testing within the Command is also discussed. Major points envisioned in the revised testing procedures which the Command and QAD are implementing are detailed including a discussion of the advantages and disadvantages of independent testing as perceived by various members of the academic community as well as by the individuals directly involved with this independent testing within USACSC. Conclusions reached as a result of weighing these advantages and disadvantages and their ultimate impact on the revised testing procedures are also examined. The underlying point is the anticipated increase in effectiveness of the Quality Assurance program obtained from the readjustment of resources, the gradual phasing out of the Environmental Test, and the subsequent increase in "up front" QAD involvement. Concluding comments encompass an overview of the methodology being developed to gather and evaluate feedback data on the success and/or shortcomings of the revised procedures. This feedback data includes reports of problems in fielded systems, especially immediately subsequent to the release of maintenance changes to this software, as well as requested changes to fielded systems.
SP500-83; 1981 November. 101-106. Pyper, W. R. Historical files for software quality assurance.

Key words: configuration management $(\mathrm{CM})$; cross reference; data structure; design, documentation; functional; historical file; keyword; life cycle management; requirements; software quality assurance (SQA); specification.

This paper summarizes a combination of ideas, adapted from software engineering and other disciplines, which provide insight into software life cycle management activities. This paper is written for an audience which may not be familiar with software engineering terminology, and some ideas and terminology are taken from related file maintenance and systems analysis fields. The impact of historical files, which represent the software development and testing activity, upon traditional software configuration management $(\mathrm{CM})$ and software quality assurance (SQA) is examined. The implementation of historical files is explored. Both advantages and disadvantages are indicated. The relationship to keyword cross-referencing is discussed.

SP500-83; 1981 November. 109-118. Giese, K. W.; Halstead, D.; Wyrick, T. F. A step-by-step approach for developing and implementing a DP charging system.

Key words: chargeback; chargeout; charging; charging system; DP cost accounting; DP cost allocation; pricing; standard costing.

Charging for data processing (DP) services refers to distributing the costs of providing DP services to the users who receive the services. The distribution of costs requires definition of the basic DP services, the resources used to provide the services, and the costs incurred to obtain and make use of the resources. A charging system is comprised of two subsystems: the rate-setting and the billing subsystems. The rate-setting subsystem incorporates procedures for forecasting the use and the total cost of each service and establishing the rate to be charged for each unit of service. The billing subsystem includes procedures for monitoring the use of services, applying the rate for each service unit to compute the total charge for the services each user receives, and reporting the charges to the user and pertinent accounting groups. Organizations may or may not collect funds to recover the costs of DP services.

The Federal Government has established policies that call for distributing the "full costs of operating DP facilities to users according to the service they receive." The National Bureau of Standards (NBS), assisted by the Federal Computer Performance Evaluation and Simulation Center (FEDSIM), is developing a set of Guidelines to assist Federal DP management in implementing the charging aspects of this policy. This paper describes a step. by-step methodology for the development and implementation of a charging system that will form the basis of the Guidelines. The material presented in this paper represents an overview of the preliminary results of the Guidelines development project and may change during the review of the draft Guidelines document. Selected technical and policy references are cited to encourage further investigation.

SP500-83; 1981 November. 121. Kelly, J. C. Session overview: Control of the information system resource through performance management.

Key words: computer system; data processing function; performance evaluation; performance management; productivity; system tuning.

Performance evaluation has expanded during the past few years to take on a more global view of the data processing function. Performance analysts can no longer limit their activities to system tuning. The issues have become broader than just the 
central hardware and software. Thus the shift in terminology from performance evaluation to performance management. Two trends have forced this change in emphasis. First, with the thrust toward on-line systems, the performance of systems has become visible to a wider and wider user community. Performance evaluation can no longer be confined to the back room. It is out in the open whether we like it or not. Second, the productivity of the entire organization depends more and more on the computer system. In many cases, corporate profits are directly related to computer performance.

SP500-83; 1981 November. 123-138. Vincent, D. R. Increasing system productivity with operational standards.

Key words: system inefficiencies; system productivity.

The user service levels achieved by a data center are dependent on three variables: volume, mix, and efficiency.

By isolating and tracking these elements, the data center can assign accountability to the user for their demands on the system as well as isolate system inefficiencies to be corrected by the data center. These methods also help to create the environment where data center and user can agree on the essentials of the delivery of computer services.

SP500-83; 1981 November. 143-156. Bays, W. N.; Kincy, W. P., $\mathrm{Jr}$. Universal skeleton for benchmarking batch and interactive workloads: UNISKEL BM.

Key words: benchmarks; interactive workloads; UNISKEL; universal skeleton.

The most important objective of the acquisition of new computer systems is to acquire the least expensive system or systems which have the necessary capability to meet workload and quality of service requirements.

The determination of "the necessary capability" may be accomplished by several methods; the most important and accurate method being the execution of performance benchmarks in a "Live Test Demonstration (LTD)."

Building benchmarks is time-consuming and costly. This is particularly true if the benchmarks are designed to represent accurately the batch and interactive workloads to be executed.

One solution would be a benchmark which could be adapted to represent any workload requirements relatively inexpensively. Such a benchmark (UNISKEL BM) is the subject of this paper.

SP500-83; 1981 November. 157-168. Cabrera, L. F.; Pâris, J. F. Comparing user response times on paged and swapped UNIX by the terminal probe method.

\section{Key words: terminal-probe method; UNIX.}

In this paper we present a comparison of user response times on paged and swapped versions of the operating system UNIX for the DEC VAX 11/780. The technique used was to construct a script that periodically evaluated the system's work-load and measured the system's response times to a set of benchmark programs.

These measurements, collected at two different sites, show that differences of responsiveness observed between the two systems depended much more on the workloads and the configurations than on the operating systems themselves.

Since we only used standard UNIX tools to build our scripts, they are highly portable and can be installed on any standard UNIX system in a matter of minutes without bringing the system down.

SP500-83; 1981 November. 169-177. Bennett, D. J. Practical application of remote terminal emulation in the Phase IV competitive system acquisition.

Key words: benchmark testing; Phase IV; remote terminal emulation; response time; terminal networks; workload sizing.

The Air Force's Phase IV program is the largest computer system acquisition ever attempted. It will replace the Air Force inventory of base supply UNIVAC 1050-II and base level support Burroughs B3500 computer systems. At the outset, a strategy was developed for expressing the workload requirements for the more than 200 systems to be replaced. Specific system throughput requirements were established. Along with this the decision had to be made how to validate the capabilities of the proposed systems to support the workload.

The point of this paper is to outline how the following questions were answered by the Air Force in the currently ongoing Phase IV acquisition: Given a real acquisition situation, how can we test effectively? What are the practical day-to-day decisions that must be made to support the selected testing strategy?

The end decision regarding the workload test approach was to use some tools from the classic benchmarking tradition, but to enhance their effectiveness with the use of remote terminal emulation. This determination was only a starting point for a number of implementation decisions that had to be made by the Air Force to support effective use of an emulator.

The current status of the Phase IV program is that contracts have been awarded to two contractors to compete for worldwide system replacement. The contracts require transition of five major standard Air Force on-line automated data systems (ADSs) and a small sampling of standard Air Force batch systems, and demonstration of contractor-proposed systems with the transitioned Air Force standard software. At the end of the contract, based on cost and evaluation of performance, one contractor will be selected for worldwide system replacement.

SP500-83; 1981 November. 181-189. Hodgins, B. D.; Cox, L. A., Jr. An evaluation technique for early selection of computer hardware.

Key words: instruction mix technique; performance prediction; sensitivity technique.

There is a need for a decision making tool for use early in the computer selection process. Such early selection tools are critical to the decision maker due to the environment in which the manager of large scale procurements is forced to operate.

The instruction mix technique was well known in the early years of computing. It was simple, easy to use and easy to understand. However, as the complexity of machines and support software grew, the technique was replaced by more sophisticated performance prediction tools. With certain modifications, this technique can continue to provide important information. By using a number of instruction mixes in a computer assisted environment, it is possible to find performance trends of hardware in the very early design stages. These trends can be used effectively to make selection decisions.

The instruction mix sensitivity technique as demonstrated here has the potential to aid the decision maker in evaluating the performance of a system prior to the actual existence or availability of that hardware without resorting to costly and time consuming techniques such as simulation or modeling.

SP500-83; 1981 November. 191-197. Rucks, A. C.; Ginter, P. M. Deficiencies in the process of procuring small computer systems: $\mathbf{A}$ case study.

Key words: computer procurement; proposal evaluation; RFP preparation; small systems.

The Latin phrase caveat emptor is nowhere more applicable than in the practice of acquiring computer systems for small business and small government entities. In a recent issue of Business Week, it was estimated that approximately 50 percent of the purchasers of small computer systems are dissatisfied with their systems. While many computer users are litigating vendors 
for redress of losses caused by insufficient data processing capability, this does not address the major failings of the process of procuring small systems. The small system procurement process produces a high rate of failure because many buyers inadequately analyze their data processing needs and fail to express these needs to potential vendors in a manner that will lead to the procurement of need satisfying computer systems.

SP500-83; 1981 November. 199.215. von Mayrhauser, A. K.; Haftka, R. T. Cost/performance comparisons for structural analysis software on mini- and mainframe computers.

Key words: performance comparison; program behavior; structural analysis.

Due to an ever-increasing selection of machines and widely different charging algorithms, the question often arises "on which machine does a program run most efficiently and/or cheaply." The program's behavior, i.e., its resource demands on the machine under consideration and their impacts on charges to be explored, as well as accuracy and correctness of the program's results and reliability of the computer service. Human factors influence decisions as well. We will discuss the selection of computer services from a user's point of view with an emphasis on structural analysis software. However, the data collection and evaluation procedure which analyses cost and performance relevant factors and their relationship with each other can also be applied to other software. The decision making process uses a set of automated tools which we developed.

SP500-83; 1981 November. 223-230. Drayton, P. A. Experiences with DASD, tape, and MSS space management using DMS.

Key words: DASD; data set; disk management system; migration; MSS; performance; space management.

This paper was written to share our experiences in the space management of DASD, Mass Storage and tape datasets in order to reduce DASD cost and improve the performance of the $1 / O$ subsystem. Included is a description of the situation which precipitated our turning to space management as a solution. Also included are the standards and procedures we introduced and the software products we utilized and wrote to service our needs. Finally the results that were achieved and some unforeseen problems that arose are documented.

SP500-83; 1981 November. 231-238. Silliman, K. E. DASD capacity planning 3350 DASD.

Key words: DASD I/O capacity; density of data reference; I/O capacity algorithms; 3350 DASD.

A pragmatic approach to Direct Access Storage Device (DASD) I/O capacity is viewed as the ability to do $I / O$ in terms of the number of $\mathrm{I} / \mathrm{O}$ operations and bytes of data transferred. Maximum and "average" I/O capacity algorithms are developed for 3350 DASD. Data transfer and density of reference capacity values are derived. Examples of capacity value usage are given.

SP500-83; 1981 November. 239-246. Atwood, J. W.; Yu, K. C. An analysis of a CDC844-41 disk subsystem.

Key words: CDC 844-41; computer system evaluation; disk subsystem configurations; input/output; modeling; rollin/rollout files; simulation.

In contrast with IBM control programs, input/output supervisor software for CDC systems frequently issues transfer requests for entire program images (e.g., when loading or rolling out a program). These program image transfers have substantially larger service times than regular input/output accesses. A detailed simulation model has been used to show that, for CDC systems, significant performance advantages normally accrue when the access path to the rollout files is disjoint from the path(s) used for regular input/output accesses. The system modelled has a 12 -spindle CDC 844.41 disk subsystem, which is shared between two CDC Cyber 170/750 central processors. Projections for a 20-spindle subsystem are also reported.

SP500-83; 1981 November. 251-258. Kelly, J. C.; Blaylòck, J. W. Tuning the facilities complex of UNIVAC $1100 \mathrm{OS}$.

Key words: facilities complex; performance parameters; UNIVAC; UNIVAC 1100 OS.

Performance of UNIVAC 1100 computer systems is largely determined by the way the operating system, $1100 \mathrm{OS}$, manages the hardware resources. Since workload and performance requirements are unique to a particular site, UNIVAC supplies an extensive set of performance parameters for tailoring the system. One set of parameters, those associated with the Facilities Complex are examined in detail. The Facilities Complex is responsible for managing mass storage (disks and drums). The parameters associated with the Facilities Complex are defined, performance hypotheses are stated, and sources of data for testing the hypotheses are discussed. This paper extends and updates work reported by Kelly and Route.

SP500-83; 1981 November. 259-265. Lin, W. K.; Tonik, A. B. Approximate evaluation of a bubble memory in a transaction driven system.

Key words: bubble memory; mathematical model; standard transaction; transaction driven system.

An approximate queueing model is built to evaluate the performance of a transaction-driven computer system with three levels of memory hierarchy, one of which is the bubble memory. The criterion of performance is the average transaction response time excluding the time spent waiting for transaction initiation because it does not measure the system performance, but the amount of transactions entering the system. The results are presented in a form which can be used by the system users to decide how much memory to include in the system configuration to achieve desired transaction throughput rate and average response time.

SP500-83; 1981 November. 271-276. Snow, A. P. Local integrated communication systems: The key to future productivity.

Key words: CATV systems; coaxial cable; data communications; fiber optics information systems; integrated communications; local area networks, microwave; video teleconferencing.

An overview of the types of local communication services required in typical large organizations is given. Such services as voice communication, data communications in support of Information Systems, Closed Circuit Television, Security Management, and Energy Management are discussed. Desirable characteristics of an integrated communications system that must provide a highway for these services are presented, with particular emphasis given to organizational productivity. Transmission media that are capable of forming the building blocks for an integrated communications system such as CATV, coaxial cable, microwave, fiber optics, and infrared/laser communication media are discussed in terms of the desired characteristics. Finally, an illustrative example of an integrated communication system is presented.

SP500-83; 1981 November. 277.290. Harrison, P. G. Performance analysis of a flow controlled communication network node.

Key words: analytic models; communication networks; flow control; Markov processes; performance prediction; queueing models; simulation; validation.

A Markov model is presented for a message processing node 
with batch arrivals, window size constraints and an additional flow control based on a credit allocation scheme to limit congestion effects. The model is sufficiently general for use in modelling many nodes which represent servers or sub-systems in any queueing network with congestion controls. Its principal application lies in the study of communication networks, in particular representing the network-independent flow control of messages between networks, which may have quite different message sizes and protocols, via a "gateway." No closed form analytic solution exists for the direct representation of the congestion control scheme, and the approach taken is to exploit its properties, approximately in some cases, to simplify the model, reducing the size of the state space sufficiently to permit direct, numerical solution of the balance equations. The credit allocation scheme is shown to be equivalent to the node's operation in different modes, each with its own buffer capacity. Great simplification is achieved in this way; credit control need no longer be modelled explicitly. The approximations made are suitably realistic to retain adequate representation of parameters, resulting in a good physical interpretation and accurate predictions. Accuracy is assessed by comparison with the results of explicit simulations of a selection of nodes of this type with various parameterizations. Finally, we suggest applications for the model in the assessment and comparison of performance under various congestion control schemes.

SP500-83; 1981 November. 293-302. Jun, E. T. Resource management in a distributed processing environment-Computer resource selection guidelines.

Key words: computer resource selection; cost; long range plan; system requirements; user requirements.

In an installation where computer resources of various types and sizes are either available or feasible, resource management and planning personnel have the responsibility for ensuring that user requirements are met in the most cost-effective manner. This paper is a tutorial on what considerations should be a part of the computer resource selection process for any given application.

SP500-83; 1981 November. 305.307. Cunningham, M. L. Office automation and productivity.

Key words: automation; information management; information technology; productivity; word processing.

SP500-83; 1981 November. 311-312. Conti, D. M.; Giese, K. W.; Kyriazi, C. N.; Trehan, R. ADP cost accounting \& chargeback.

Key words: chargeback; cost accounting; cost recovery.

There is no question that the ADP shop plays a vital role in the day-to-day operations and survival of most organizations. However, two distinct views still exist on the nature of the ADP shop within an organization. One view exists that the ADP shop is an overhead function that should provide free service to the users. Another view sees the ADP shop as a service center that should run on a profit and loss basis. An extension of this latter view is that the full costs of ADP should be accounted for and charged back to the users. However, whether or not these charges are actually recovered from the users, the mere reporting to the users of their fair share of the total ADP costs is believed to have a beneficial effect by increasing the users' awareness of and accountability for their use of ADP. Most recently within the Federal Government, OMB circular A.121 requires agencies to implement full cost accounting, and where appropriate, cost recovery. Examples of some of the costs to be accounted for under A.121 are software depreciation and space occupancy costs, in addition to the more traditional ADP cost elements.

Although many sites currently have some form of chargeback system, few account for all of the costs of ADP. For those organizations that are considering implementing a chargeback system, reluctance by both the ADP manager and the user community to make their activities more visible makes such an implementation more difficult.

SP500-83; 1981 November. 325. Artis, H. P. Measurement, modeling, and capacity planning: The secondary storage occupancy issue.

Key words: capacity planning; device architecture; secondary storage; space management.

SP500-83; 1981 November. 327.328. Berry, W. W. Microcomputers in office automation.

Key words: micro-computers; office automation.

SP500-83; 1981 November. 329. Sprung, J. G. Computer performance management in the ADP system life cycle.

Key words: computer performance; performance tools; resource availability data.

SP500-83; 1981 November. 331-334. Neuse, D.; Chandy, K. M.; Misra, J.; Berry, R. Simulation tools in performance evaluation.

Key words: analysis; performance evaluation; pictures; simulation; tools.

The selection of modeling tools for performance evaluation is discussed. Simulation and analytic tools are discussed and compared. A simulation system designed to accept computer readable, picture-oriented models is described.

SP500-83; 1981 November. 335. Sauer, C. H. Computational methods for queueing network models.

Key words: network models; product form; queueing models; system resources.

SP500-83; 1981 November. 337. Howard, P. C. Productivity in the development function.

Key words: development environment; development function; productivity.

SP500-83; 1981 November. 339. Conti, D. M. Acquisition benchmarking.

Key words: benchmarking; FIPS PUB 75; vendor performance.

Benchmarking has traditionally been more of an art than a science, primarily because of the previous lack of well-established procedures for constructing and testing benchmarks. As a tool for evaluating vendor performance during the competitive acquisition of computer systems, benchmarking has become an accepted practice. However, in spite of the widespread use of benchmarking, especially within the Federal Government, practitioners are still unaware of the sources of error that affect the ability of a benchmark to represent a real workload.

This tutorial will explore the purpose of benchmarking during the acquisition of computer systems, the sources of error inherent in the benchmark process, and ten steps to constructing and testing benchmark mixes. These steps have formed the basis for a recent NBS Federal Guideline in this area, FIPS PUB 75.

SP500-83; 1981 November. 341. Buschbach, T. R. Software conversion process and problems.

Key words: Federal Conversion Support Center; reimbursable services; software conversion.

This tutorial will provide users with a basic understanding of the software conversion process. The technical and management problems which arise during a conversion project will be discussed. A brief description of the Federal Conversion Support Center's capabilities to assist agencies in the conversion process 
will be given.

SP500-83; 1981 November. 343-348. Mullen, J. W. Resource measurement facility (RMF) data: $A$ review of the basics.

Key words: analysis; data elements; I/O; logical swapping; measurement; MVS; paging/swapping; TSO.

Analysis and understanding of RMF measurement data is a basic prerequisite to performance management in the MVS environment. A basic approach to RMF analysis is described for key measurement data elements often overlooked in the analysis process, how RMF measures these values, and some rules-ofthumb based upon the author's experience. Specifics in the areas of 1/O, paging/swapping, TSO, and analysis of cause and effect relationships of RMF measured data will be discussed.

SP500-84. Draper, J. M. Computer science \& technology: Costs and benefits of database management: Federal experience. Nat. Bur. Stand. (U.S.) Spec. Publ. S00-84; 1981 November. 106 p. SN003.003. 02376.1.

Key words: application development; computer software; cost/benefit analysis; database management systems; data management; program maintenance; requirements studies; software procurement.

The Federal Government has a large investment in a wide variety of database management systems (DBMS's) and in diverse applications using those systems. Data managers from eight Federal agencies report that they are pleased with the power, flexibility, and cost effectiveness of DBMS's, whether they use their system as sophisticated access software or make it the focal point of a large, integrated data processing system. While DBMS's may save an agency money in the long run, their major immediate benefits are almost always increased functionality and productivity, not reduced cost. Centralized data and high-level user languages reduce the time for developing and maintaining computer programs, and the latter enable nonprogrammers to use computers effectively.

The amount of cost/benefit analysis an agency needs before deciding to buy a DBMS increases with the complexity of the application. The experiences of the interviewed agencies, together with a structured list of cost/benefit parameters, should help Federal managers in understanding the potential value of DBMS technology and in defining their requirements for data management.

SP589. Free, G. M., ed. Eddy current nondestructive testing. Proceedings of the Workshop on Eddy Current Nondestructive Testing, held at the National Bureau of Standards; 1977 November 3.4; Gaithersburg, MD. Nat. Bur. Stand. (U.S.) Spec. Publ. 589; 1981 January. 153 p. SNO03-003-02287-0.

Key words: conductivity; defect detection; eddy current test; multifrequency; nondestructive testing.

The proceedings of the Eddy Current Nondestructive Testing Workshop held at NBS in November, 1977 contains papers related to all areas of eddy current testing. A historical overview of the discipline from its inception until the present is given. Other papers discuss the use of eddy current testing in the primary metals industry (both ferrous and nonferrous metals), the use of eddy currents for the sorting of metals and for defect detection, the state-of-the-art in eddy current instrumentation, and the use of signal processing in the analysis of eddy current signals. The development and use of eddy current standards is discussed as well as several of the newer areas of eddy current development, i.e., multifrequency and pulsed eddy current techniques. These proceedings include the following papers (indented):

SP589; 1981 January. 1-32. McMaster, R. C. The history, present status, and future developments of eddy current tests.

SP589; 1981 January. 33-37. Moyer, R. B. Eddy current testing:
Present and future applications in the ferrous metal industry.

SP589; 1981 January. 39-44. Burley, C. E. Eddy current standards in nonferrous metals.

SP589; 1981 January. 45-48. Betz, R. A. Eddy current inspection of gas turbine blades.

SP589; 1981 January. 49-55. Wehrmeister, A. E. Eddy current examination in the nuclear industry.

SP589; 1981 January. 57-61. Denton, C. J. Eddy current inspection systems for stream generator tubing in nuclear power plants.

SP589; 1981 January. 63-76. Reinhart, E. R. Use of round robin tests to determine eddy current system performance.

SP589; 1981 January. 77-79. McEleney, P. C. Eddy current testing in government.

SP589; 1981 January. 81-85. McFarlan, T. W. Current status and future directions of eddy current instrumentation.

SP589; 1981 January. 87-105. Libby, H. L. Multifrequency eddy current inspection techniques.

SP589; 1981 January. 107.111. Waidelich, D. L. Pulsed eddy current techniques for nondestructive evaluation.

SP589; 1981 January. 113-119. Weismantel, E. E. The introduction of signal processing techniques to eddy current inspection.

SP589; 1981 January. 121-132. Jones, A. Development of nonferrous conductivity standards at Boeing.

SP589; 1981 January. 133-136. Free, G. NBS eddy current standards program.

SP589; 1981 January. 137-141. Kahn, A. H.; Spal, R. D. Electromagnetic theory and its relationship to standards.

SP589; 1981 January. 143-147. Green, R. E. Discussion: New science directions and opportunities concluded from workshop sessions.

SP589; 1981 January. 149-154. Belecki, N. B. Discussion: New directions for standards concluded from workshop sessions.

SP593. Gallagher, J. W.; Van Blerkom, J.; Beaty, E. C.; Rumble, J. R., Jr. Data index for energy transfer collisions of atoms and molecules-1970-1979. Nat. Bur. Stand. (U.S.) Spec. Publ. 593; 1981 April. 346 p. SN003.003-02315.9.

Key words: atom; charge transfer; chemi-ionization; collisional ionization; cross section; detachment; energy transfer; excitation; ion; molecule; reaction rate; relaxation.

An index to sources of data describing processes which occur when atoms, molecules or ions collide with each other at energies between 0 to $10 \mathrm{keV}$ and gain or lose internal energy or change charge state is presented. The index facilitates retrieval of cross sections and reaction rate data for specific processes involving atomic or molecular species. It includes data published during the years 1970 through 1979. A comprehensive author list is included.

SP599. Wollin, H. F.; Barbrow, L. E.; Heffernan, A. P., eds. Report of the 65th National Conference on Weights and Measures 1980. Nat. Bur. Stand. (U.S.) Spec. Publ. 599; 1981 January. 319 p. SN003.003. 02286-1.

Key words: domestic standards; education programs; electronic devices; international recommendations; legal metrology; measurement assurance; metrication; model laws and regulations; packing and labeling; specifications and tolerances; technology 
transfer; weights and measures.

This is the proceedings of the 65th National Conference on Weights and Measures, sponsored by the National Bureau of Standards, held in Washington, D.C., June 22-27, 1980, and attended by State, county, and city weights and measures officials, and representatives of the Federal Government, business, industry, and consumer organizations. The publication includes papers presented by Conference officials and other top authorities from Government and industry in the United States and several foreign countries. Reports by the several standing and annual committees of the Conference are also included.

Major issues discussed at the National Conference included measurement science education, enforcement uniformity, national type approval, computers and microelectronics, new design and performance requirements for weighing and measuring technology, radiation measurements, metric conversion of retail gasoline dispensers, and weights and measures program evaluation. These proceedings include the following papers (indented):

SP599; 1981 January. 1-5. Vincent, C. H. Expectations for the eighties.

SP599; 1981 January. 6-9. Ambler, E. 1980-A diamond anniversary, A golden opportunity.

SP599; 1981 January. 14-24. Birkeland, K. Societal and technological demands upon legal metrology-A strategy for meeting increased national and international needs.

SP599; 1981 January. 25-33. Page, W. J., Jr. Sharing power, responsihility, and resources in the Federal System.

SP599; 1981 January. 34-38. Jordan, B. A university looks at measurement science education.

SP599; 1981 January. 39-44. Elengo, J. J., Jr. The scale manufacturers association report on scale tolerances.

SP599; 1981 January. 45-53. Vastagh, S. A. The role of the U.S. Metric Board in the voluntary conversion of gas pumps to metric measurement.

SP599; 1981 January. 54-63. Simila, K. J.; Kloss, C. Report by the special study group on enforcement uniformity.

SP599; 1981 January. 64-68. Pierce, W. J. Mechanisms for unification of state and local laws and regulations.

SP599; 1981 January. 69-84. Gerk, F. A. Western Weights and Measures Association.

SP599; 1981 January. 85-94. Young, W. M. Scale design and weighing trends in the eighties.

SP599; 1981 January. 95-101. Hodsman, G. F. Weights and measures regulations-The European scene in the eighties.

SP599; 1981 January. 102-109. Meyer, L. S. The consumer movement of the 1980 s - What we would like to see from weights and measures.

SP599; 1981 January. 110-114. Blasgen, M. W. Computers, microelectronics, and people.

SP599; 1981 January. 115-126. Muhe, W. Interdependence of metrology, standardization and quality assurance.

SP599; 1981 January. 127-137. Trask, R. P. Liquid hydrocarbon measurement in the decade of the eighties: The need for research and resourcefulness.

SP599; 1981 January. 138-146. Seiler, E. Type approval-A tool for measurement assurance in legal metrology.

SP599; 1981 January. 147-155. Parrott, M. W. Radiation
measurement-A challenge that calls for cooperative federal and state action.

SP599; 1981 January. 156-164. Eagle, J. W.; Little, A. D. The 1980s food marketplace-Demographics, lifestyles, and technology.

SP599; 1981 January. 165-178. Bijloo, A. C. The system of control of prepackaged products in the Netherlands.

SP599; 1981 January. 180-201. Vincent, C. H. Report of the committee on national measurement policy and policy and coordination.

SP599; 1981 January. 202-211. Offner, D. I. Report of the committee on laws and regulations.

SP599; 1981 January. 212.240. Guensler, D. A. Report of the committee on specifications and tolerances.

SP599; 1981 January. 241-248. Darsey, S. J. Report of the committee on education, administration, and consumer affairs.

SP599; 1981 January. 249-262. Greene, C. H. Report of the committee on liaison.

SP599; 1981 January. 263-270. Vincent, C. H. Report of the executive committee.

SP599; 1981 January. 271-272. Scott, T. W. Report of the resolutions committee.

SP599; 1981 January. 273.275. Simila, K. J. Report of the nominations committee.

SP599; 1981 January. 276. Gerk, F. A. Report of the auditing committee.

SP599; 1981 January. 277-280. Nelson, A. M. Report of the conference treasurer.

SP600. Porter, G. National Bureau of Standards 1980. Nat. Bur. Stand. (U.S.) Spec. Publ. 600; 1981 January. 40 p. SN003.003.02300. 1.

Key words: basic measurements and standards; building technology; calibration methods; computer standards and guidelines; energy research; environmental measurements; fire safety; industrial productivity; materials properties; research; standards; technology transfer.

National Bureau of Standards 1980 explains the Bureau's role as the central U.S. reference laboratory for the physical and engineering sciences. It also discusses briefly Bureau plans for its programs in electronics, automation, chemical processing, and materials research. Included in the report are highlights of recent accomplishments within the Bureau's National Measurement Laboratory, National Engineering Laboratory, and Institute for Computer Sciences and Technology. It describes more than 40 different projects in such areas as basic measurements and standards, energy research, environmental measurements, materials properties, industrial productivity, fire safety, building technology, calibration methods, and computer standards and guidelines. Also featured are summaries of NBS services to industry, other government agencies, and the public, as well as information on cooperative research programs and a directory of the names, titles, and phone numbers of NBS research managers.

SP601. Weinstein, A.; Gibbons, D.; Brown, S.; Ruff, W., eds. Implant retrieval: Material and biological analysis. Proceedings of a Conference held at the National Bureau of Standards; 1980 May 1.3; Gaithersburg, MD. Nat. Bur. Stand. (U.S.) Spec. Publ. 601; 1981 January. 772 p. SN003-003-02292-6.

Key words: analysis; biological; cardiovascular; implants; metals; orthopaedic; polymers; retrieval.

This book contains the proceedings of a conference on implant 
retrieval and analysis as well as a report on a workshop concerned with implant retrieval systems. Twenty-six invited papers that were presented at the conference are included. Four subject areas are specifically addressed: bulk phenomena, release phenomena, interface phenomena, and information utilization. Implants of both orthopaedic type and cardiovascular type were considered at the conference. Data on the failure modes of implants were presented. Specific consideration of biocompatibility problems was included. Several operating data/information systems were described in detail. Recommendations were made in the workshop concerned with uniformity and standardization of information systems dealing with implant retrieval data. These proceedings include the following papers (indented):

SP601; 1981 January. 3-28. Rose, R. M.; Crugnola, A. N.; Cimino, W. R.; Ries, M. D. The in-vivo performance of polyethylene components of total joint replacements.

Key words: acrylic cement; joint replacements; polyethylene components.

The polyethylene components of retrieved total joint replacements are investigated with the scanning electron microscope to assess the degree and causes of wear and other deterioration in vivo. The highly variable performance of the implants, ranging from no observable wear to disintegration of the articular surface, can be associated in some cases with the presence of particles of acrylic cement or bone abrading the articular surface. Severe wear in the absence of such abrasives may originate with the structure of the polyethylene itself, and may be caused by molecular weight degradation during processing: in such cases the primary damage loci are fusion defects in the molded or extruded material.

SP601; 1981 January. 29-129. Guidoin, R.; King, M.; Blais, P.; Marois, M.; Gosselin, C.; Roy, P.; Courbier, R.; David, M.; Noël, H. P. A biological and structural evaluation of retrieved Dacron arterial prostheses.

Key words: arterial prostheses; biodeterioration; polyethylene terephthalate (Dacron).

Pioneered in the 1950's, reconstructive procedures involving the implantation of synthetic blood conduits have become relatively common since the early 1970's. However, long term success is largely dependent on patient selection and surgical techniques as well as the design, the dimensions and the material chosen to repair the nature conduit. Polyethylene terephthalate (Dacron) tissue ingrowth fabric devices have accounted for the larger portion of commercial devices and are currently implanted in a rapidly broadening spectrum of patients, many of whom could potentially outlive the service lifetime of some of the newer prostheses. "In-vitro" observations and studies on explanted prostheses suggest that herefore unrecognized textile design and material biodeterioration considerations may have impact on long term prognostics of success. In this work, the results of an ongoing prosthesis recovery program encompassing more than 120 devices collected at autopsies and reoperations are discussed. The evaluation protocol addresses healing, textile geometry, product identification, dimensional stability of the fabric, morphological changes of the yarns and fibres, mechanical properties and chemical changes in the material with "in-vivo" residency time. The significance and the limitations of data derived from cardiovascular device recovery programs are also discussed.

SP601; 1981 January. 131-156. Plitz, W.; Griss. P. Clinical, histo. morphological and material related observations on removed alumina-ceramic hip joint components.

Key words: alumina-ceramic; artificial hip joints; endoprostheses; histo-morphological.

SP601; 1981 January. 157-174. Gibbons, D. F.; Buran, K. A.
Microscopic analysis of retrieved polymethylmethacrylate (PMMA) bone cement.

Key words: femoral component loosening; polymethylmethacrylate (PMMA); prosthesis interface.

Metallographically prepared sections from retrieved bone cement were examined with reflected light microscopy. Gross plastic deformation was observed in the femoral collar region and interdigitating bone regions. Deformation strain lines are also observed frequently associated with cracks in the cement. Plastic deformation of the bone cement under physiological stress states can therefore account for femoral component loosening. Cracks are also observed radiating from the corners of the medial edge of the femoral stem. The bone-trabeculae interface was observed to be closely adapted with no fibrous capsule interposed. The surface morphology of the cement with bone was demonstrated to range from smooth to porous. These data correlate well with previous scanning electron microscopic evidence. Bone debris from the surgical procedure was also observed. Abrasive wear of the bone cement at the prosthesis interface was observed which can account for the small PMMA particles observed in the tissues of the joint capsule.

SP601; 1981 January. 175.200. Yoganathan, A. P.; Harrison, E. C. Prosthetic heart valves: What have we learned from our clinical pathologic experience.

Key words: bio-prostheses; prosthetic heart valves; thromboembolism.

Some of our clinical and pathologic observations, and in vitro hemodynamic experiences with the Björk-Shiley, Starr-Edwards (ball valves), Smeloff.Cutter, St. Jude, Kay-Shiley and tissue bioprostheses are discussed. It is quite clear that at present there is no ideal prosthetic heart valve. The major problems that affect the mechanical valves are thromboembolism and tissue overgrowth, while the porcine valves are affected by structural degradation and poor hemodynamic performance in the small sizes.

The observed problems of thrombus formation, tissue overgrowth, red-cell destruction and damage to the endothelial lining of vessel walls adjacent to the valve prosthesis are directly related to the hemodynamics of the respective prostheses. It is our experience that all prosthetic heart valves that are presently commercially available are liable to thrombus formation and tissue overgrowth. Therefore, correlative studies such as described in this article should be conducted with all heart valve prostheses. Detailed pathologic studies on approximately 150 to 200 recovered prosthetic heart valves of different types are presently in progress.

SP601; 1981 January. 201-222. Taussig, L. M. Examination of orthopaedic implant failures.

Key words: fatigue corrosion; internal fixation devices; mechanical fatigue; metal prostheses; stress corrosion.

A study of 110 removed metal prostheses and internal fixation devices was conducted over a 26 -year period. The case histories accompanying each implant allowed the correlation of the metallurgical analyses with possible causes of failure. Statistical tables provide information regarding classifications of the implants by cause for removal, anatomical location, type of implant, fabrication method and alloy, fracture mode and location for various implants, and general patient information relative to the implants. The required metallurgical analysis (designed to comply with all ASTM specifications), consisting of non-destructive testing, semi-destructive testing, and in certain cases mechanical tests, is briefly described. The characteristics of different fracture modes, such as due to mechanical fatigue and fatigue corrosion, are discussed and illustrated by photomicrographs, and the origins of the failures presented when 
possible. The difference between stress corrosion and fatigue corrosion is also explained. The study utilizing metallurgical analysis, patient histories, and the physiological aspects surrounding the events prior to and after failure, indicates correlations between implant fractures and lack of bony union, fatigue forces, design deficiencies, surgical implantation techniques, and improper use of the implants. Experience in metallurgy and its application to the implant and its environment required for acceptable and proper retrieval analysis is emphasized.

SP601; 1981 January. 223.235. Wilkinson, R.; Shepherd, M. T. A study of inclusions in stainless steel implants and their effect on corrosion.

Key words: corrosion; stainless steel implants; wavelength dispersive $\mathrm{x}$-ray analysis.

A study of stainless steel implants nominally conforming to BS 3531 has been undertaken. Corroded areas have been examined using the SEM and inclusions visible in the corroded areas identified using energy dispersive $\mathrm{X}$-ray analysis. Inclusions present in a polished section of the component were also identified and measured to produce inclusion ratings which were then compared with the estimated corrosion. Some components were analysed using wavelength dispersive X-ray analysis and 5 screws were found deficient in nickel. Screws having low nickel were then compared with the others. The interrelationships of the different inclusions and relationships with corrosion are discussed and the case for more stringent metallurgical control considered.

SP601; 1981 January. 239-267. Willert, H. G.; Buchhorn, G.; Buchhorn, U.; Semlitsch, M. Tissue response to wear debris in artificial joints.

Key words: artificial joints; biomaterials; wear debris.

Macroscopic and microscopic examinations of the tissue from the joint capsule and the bone/cement-border of revised artificial joints are carried out. The tissue response to wear products of the biomaterials (CoCrMo-cast alloy, stainless steel-AISI $316 \mathrm{~L}$, Polyester, Polyethylene, silicone rubber and bone cement on acrylate base) is described and its connection with implant loosening is demonstrated. The particles are characterised and some clinical measurements of the wear rate of polyethylene are reported.

SP601; 1981 January. 269-282. Schoen, F. J.; Titus, J. L.; Lawrie, G. M. Materials degeneration causing late failure of mechanical heart valve prostheses: Problems and promise.

Key words: mechanical heart; valve prostheses; valvular dysfunction.

Materials degradation with resultant clinically significant valvular dysfunction has been an important late complication of cardiac valvular replacement with mechanical prostheses. Major mechanisms of failure are primarily related to either lipid absorption by silicone elastomers or abrasive wear of polymers. In a review of the necropsies of 99 patients dying late after cardiac valvular replacement, we encountered two cases of fatal valve deterioration, one involving each of the above failure modes. We examined by scanning electron microscopy and analytical surface profilometry seven contemporary heart valve prostheses recovered from this series, all of which had no clinical or pathological evidence of degeneration/dysfunction, and all of which contained pyrolytic carbon components and had been insitu for 30 months or longer. Results of this analysis suggest that progressive deterioration by abrasive wear of pyrolytic carbon heart valves will not be a significant problem.

SP601; 1981 January. 283-298. Anderson, J. M.; Barenberg, S.;
Hiltner, A. Prosthetic valve wear products-Embolization and systemic presentation.

Key words: aortic prosthesis; embolization; prosthetic heart valve failure.

An uncommon but potentially catastrophic mode of prosthetic heart valve failure is related to the inadequate durability of the materials used in the construction of the heart valve. The inadequate durability of these materials results in wear of the disc or ball occluders, the polymeric fabric covering the seat and struts and the metallic frame. This paper presents some of the common modes of wear of prosthetic heart valves with an emphasis on the embolization and systemic presentation of wear products. In particular, the identification in organs of ball occluder fragments from the Braunwald.Cutter aortic prosthesis will be presented.

SP601; 1981 January. 299-321. Brown, S. A.; Merritt, K. Metal allergy and metallurgy.

Key words: hip hemiarthroplasties; metal allergy; metal sensitivity; reactions to implants.

Metal allergy reactions result from sensitization of T.lymphocytes to metal ion-protein complexes. In the case of reactions to implants it is presumed that the metal ions as corrosion products complex with local tissue proteins. An in vitro test for metal sensitivity is described in which lymphocytes are challenged with metal chlorides in the presence of plasma proteins. The test can differentiate between patients with no sensitivity to nickel, cobalt, or chromium, sensitivity to one or more of the metals, and patients with allergic reactions to implants. The test results performed on 164 patients at the time of implant removal show that $37 \%$ of the patients in this group were reacting to their implants. The results of implant analysis and the blood test results are presented for cobalt-chrome hip hemiarthroplasties, stainless steel plates, and stainless steel nailplates. Specific cases are presented in which metallographic and SEM analysis of the implants are used to demonstrate correlations between implant degradation and the immune response.

SP601; 1981 January. 323.336. Williams, D. F.; McNamara, A.; Clark, G. C. F. Techniques for the examination of tissues in implant retrieval analysis.

Key words: atomic absorption spectrophotometry; EDAX analysis; enzyme histochemistry; histology; implant retrieval analysis; polarography; tissue response.

A detailed examination of the tissue response to implanted high purity metals has been undertaken. The methods used include conventional histology, enzyme histochemistry, transmission and scanning electron microscopy, EDAX analysis and elemental analysis by flame and flameless atomic absorption spectrophotometry and polarography. Details of these techniques are given in this paper with a summary of some of the results obtained in experiments and the applicability of the methods to the examination of tissue samples taken at implant retrieval.

SP601; 1981 January. 339-348. Levy, R. J.; Sanders, S. P.; Lian, J. B. Retrieval analysis of calcific degeneration of prosthetic tissue valves: The role of vitamin $K$-dependent processes and other regulatory mechanisms.

Key words: calcification; calcific degeneration; porcine xenograft valves; prosthesis failure; vitamin K-dependent.

Calcification of glutaraldehyde preserved porcine xenograft valves represents a major clinical problem affecting the use of these prostheses. Currently, there is no satisfactory explanation for this pathologic process. The present work explores the interrelationships of both clinical parameters and biochemical changes 
in the affected valve tissue. The results of the present study show that the prosthesis failure in our patient population was always associated with calcification, and this complication was noted to occur only in those patients under 15 years of age at the time of valve replacement. Amino acid analysis of calcified leaflet tissue revealed the presence of high levels of proteins containing the vitamin K-dependent, $\mathrm{Ca}^{++}$binding amino acid, gammacarboxyglutamic acid (Gla), in mineralized specimens, with no Gla present in non-calcified valve tissue. In addition, the $\mathrm{Ca}^{++}$binding amino acid O-phosphoserine was detected in relatively greater amounts in the mineralized specimens, compared to control. Calcified xenografts also demonstrated a relative reduction in collagen content (as evidenced by hydroxyproline analysis). The implications that vitamin $\mathrm{K}$-antagonism could be of benefit in treating or preventing prosthesis calcification is discussed.

SP601; 1981 January. 349-362. Parks, P. J. Prosthesis-related infections.

Key words: histological examinations; hydrocephalus; prosthesis related infections.

Thirty-one patients with prosthesis related infections have been identified. Seventeen patients treated for hydrocephalus with ventriclojugular, ventriculoatrial and ventriculoperitoneal shunts exhibited bronchopneumonia and/or evidence of septic emboli. An additional fourteen patients had evidence of direct infection of prostheses. In the later group, histological examinations in conjunction with positive bacterial or fungal cultures was superior to either technique alone in confirming an infectious process. The causative organisms were similar to those reported in other series on device-related infection, and are similar to those seen in immunosuppressed patients.

SP601; 1981 January. 363-393. Quijano, R. C. Study of 208 explanted bioprosthesis: Distribution of dysfunction patterns and assessment of durability at $\mathbf{1 0}$ years of use.

Key words: bioprosthesis; dysfunction patterns; valvular substitute.

As the HANCOCK bioprosthesis completed 10 years of clinical use as a human valvular substitute, specific dysfunction patterns have become evident in a very small percentage of the xenografts. Simultaneously, the durability of longevity of this porcine derived material extending over this period has been questioned. Studies at this laboratory have addressed both phenomena: dysfunction and durability of these bioprostheses. An explanted valve retrieval program has been ongoing at HANCOCK Laboratories Inc., for the past eight years and the observations and studies generated are the subject of this paper. A total of 208 explanted valves are analyzed and reported. The distribution of the modes of dysfunction show they may be due mainly to: 1) two distinct forms of mineralization, 2) unhealed prosthetic valvular endocarditis, 3) early uncomplicated thrombosis, and other minor reasons. A statiscal breakdown for each occurrence is given. Physical and biochemical studies on excised valves returned in nonfixative solutions give clues as to the nature of interstitium material reported elsewehere to be products of valve material degeneration. Together the findings indicate that the HANCOCK bioprosthesis demonstrates conservation of intrinsic pre-implant characteristics for up to 100 months in vivo, and a longer projected prosthetic durability in the absence of extrinsic factors.

SP601; 1981 January. 395-422. Simpson, J. P.; Geret, V.; Brown, S. A.; Merritt, K. Retrieved fracture plates: Implant and tissue analysis.

Key words: bone plates; cobalt-chrome-nickel-tungsten-iron-alloy; fretting corrosion; histologic analysis; stainless steel 316 LVM; titanium, titanium alloy.
A study was undertaken by AO/ASIF to investigate the suitability of different materials for bone plates for osteosynthesis. A total of 80 plates were retrieved together with clinical data, histologic data, and chemical analysis. The materials used were stainless steel 316 LVM, commercially pure tìtanium, titanium (Ti-6Al-4V) alloy, and a cobalt-chrome-nickel-tungsten-iron alloy as AO/ASIF compression plates and screws. The protocols used and the results of these first 80 cases are presented. The histologic analysis revealed the greatest differences occurring in the accumulation of lymphocytes, macrophages, and giant cells which were greatest with the cobalt alloy. The quantity of debris in the tissue was greatest with titanium. The chemical analysis revealed a wide scattering of values and the results are discussed. The examination of plates and screws revealed that stainless steel suffers fretting corrosion and that the amount of metal loss is less on the cobalt alloy, titanium, and the titanium alloy although significant corrosion was observed at the plate screw contact area for the cobalt alloy.

SP601; 1981 January. 423-447. Skinner, H.; Weinstein, A. M.; Clemow, A. J. T.; McPhillips-Meade, M.; Klawitter, J. J.; French, G. Corrosion, materials characteristics and local tissue reaction associated with osteosynthesis devices.

Key words: corrosion; osteosynthesis devices; tissue reaction.

A series of 112 internal fixation devices, comprising 75 bone plates and 37 hip nails, have been retrieved and subjected to mechanical bend tests. In addition, the severity of any corrosion of these devices was measured, together with their metallurgical condition.

Further, in seven cases, the reaction of the tissue adjacent to the removed implant was examined and graded upon an arbitrary scale. Results from these tests are presented and discussed.

SP601; 1981 January. 449-469. Weinberg, S. The evaluation of explanted umbilical vein grafts.

Key words: umbilical vein grafts; vascular prosthesis.

The evaluation of retrieved vascular graft samples is critical if the clinical function of a vascular prosthesis is to be adequately understood. During the past five years, 36 explanted human umbilical vein grafts have been evaluated after implant periods ranging from 1 minute to 42 months. The testing program included CST, ATR, SEM, EDAX, histopathology, TEM, and compliance studies. Critical surface tensions were stable and remained at the pre-implant value of about $25 \mathrm{dynes} / \mathrm{cm}$ for periods up to 42 months. Some lipid was identified within the graft using IR analysis, however, results indicate that this phenomena is primarily patient-related rather than graft-related. No calcification of any graft samples has been noted to date. The blood-contact surface of patent grafts is covered with protein, moreover, no neo-intimal proliferation has been seen within the graft material. Histological and TEM evaluations have demonstrated that the primary changes seen in a patent graft are: 1) a compaction of the graft, and 2) a decrease in cellularity with implant time. In general the graft has many characteristics similar to the ASV and excellent clinical results have been seen thus far.

SP601; 1981 January. 471-499. Clarke, I. C. Wear of polymeric prosthesis-Clinical reality, retrieved implants and laboratory predictions.

Key words: polyethylene acetabulae; polymeric prosthesis; wear.

Up to 17 years clinical experience with polymeric components of hip prostheses has shown that (a) polyethylene femoral balls have poor wear resistance, and (b) with one exception the major series have generally had no reoperations due to wear or fracture problems in polyethylene acetabulae. Average wear rates 
expected in up to 10 years use may vary from a low of 40 $\mu \mathrm{m} /$ year to a high of $200 \mu \mathrm{m} /$ year, (c) there at least 5 published reports, each describing between 2 and 6 cases of documented gross wear or fracture of retrieved polyethylene acetabulae, (d) the effect of acrylic cement on polyethylene wear rates is unclear, and (e) Delrin has also been used apparently successfully for 10 years in the Christiansen total hip.

Up to 9 years followup with tibio-femoral joints has not yet shown any wear revision problems. Retrieved polyethylene components feature more extensive disruption and deformation than in hips. Knee simulator studies indicated that contact stresses, volumetric and linear wear rates in various designs could vary by $\times 270, \times 20$ and $\times 110$, respectively. Patello-femoral results on implant performance are not yet available. Simulator studies of various patellar designs showed that volumetric and linear wear rates could vary $\times 20$ and $\times 210$ times, respectively. The clinical implications of these data may be worthy of concern. Concern was expressed that (a) the relatively large numbers of annual rates of implant exchanges seen nationwide in this country did not appear reflected in the collections of the current implant retrieval programs and (b) the public dissemination of the retrieved implant data seldom seemed effective.

SP601; 1981 January. 503-508. Flink, R. C.; Malette, W. Considerations concerning international standards for accumulating and reporting of implant experience with cardiac pacemakers.

Key words: cardiac pacemakers; international standards; reporting of implant experience.

SP601; 1981 January. 509-557. Hori, R. Y.; Lewis, J. L.; Wixson, R. L; Kramer, G. M. An analysis of 130 removed total hips and knees.

Key words: component loosening; debonding joint replacements; knee replacements.

Retrieval analysis is an efficient means of monitoring and evaluating the problems associated with total joint replacements. In 5 years, 130 prostheses have been collected from 8 hospitals in the Chicago area, including 75 hips and 52 knees. All study data are summarized and coded for computer analysis.

Total hip replacements are being removed at a relatively constant rate with no evidence of an increase in removal with time. In contrast, the majority of total knee replacements collected were removed within 3 years of the original surgery. Fractured and cracked components appear to be almost always associated with loosening. Unlike most other TJR components which usually debond at the bone-cement interface, femoral stems, when loosened, generally debond at the cementcomponent interface. Such debonding may ultimately lead to gross component loosening.

SP601; 1981 January. 559-572. Wright, T. M; Burstein, A. H. The method of implant retrieval anlaysis at the Hospital for Special Surgery.

Key words: implant retrieval analysis; joint prostheses; metallurgical analysis; prosthetic devices.

An implant retrieval analysis program was established at the Hospital for Special Surgery in December 1977, and since then over 1300 implants have been gathered and examined. The primary objective of the program is to identify problem areas in design and materials of implants with emphasis on total joint prostheses. All implants removed in the operating rooms at the Hospital and, in a few instances, at other institutions in the U.S.A. are included in the program. In addition, protocols have been established to retrieve whole joints (containing total joint implants) which have been removed en.bloc at autopsy or amputation. Examination of retrieved implants includes visual and light microscopy inspection, a review of the patient's radiographs and medical records, scanning electron microscopy of fracture and articulating surfaces, and metallurgical analysis where appropriate. Additional procedures, including examination of properties of bone and structure, are performed on the joints removed en-bloc. The biomechanical data obtained from the implant retrieval analyses are included in a data file of radiographic and medical history information for use in statistical analysis.

SP601; 1981 January. 573-613. Cook, S. D.; Weinstein, A. M. An interactive computer system for implant data retrieval/analysis.

Key words: cross-referencing capabilities; interactive computer system; orthopaedic implants.

An interactive computer system for the storage, retrieval and analysis of clinical and material characterization data associated with orthopaedic implants is described. The system consists basically of four independent modules. The essence of the system centers on the cross-referencing capabilities which are unlimited. The system has been designed for use by non-computer trained personnel.

SP601; 1981 January. 615-642. Schurman, D. J.; Burton, D. S.; Nagel, D. A.; McShane, D. A data retrieval system for implant failures in total joint replacement surgery.

Key words: ASP (Arthritis Surgery Proforma); data retrieval system; implant failures.

A prospective information system to measure arthritis surgery outcomes was developed cooperatively and over a period of years with the American Rheumatism Association Medical Information System and a national group of arthritis surgeons. The system called ASP (Arthritis Surgery Proforma) defines the essential minimum data for assessing surgical outcomes and is designed for use by multiple institutions in cooperative or independent studies.

To best implement the use of ASP, a Time-Oriented Record (TOR) is suggested. TOR allows for uniformity in data collection over time and uniformly among different investigators. The chart system itself can be modularized by sections such as history, physical exam, complications and $x$-ray findings. Data includes stratifying, intervention and outcome variables. A new diagnostic system was developed that is indexed by ICD code where it exists.

An advanced computer system in operation checks, stores, retrieves and analyzes data. This computer system known as TOD (Time-Oriented Database) is nationally accessible through local phone lines via TELENET.

Stanford's experience with 584 total hip or knee replacements is briefly defined for the 21 patients requiring revision surgery for non septic loose or broken prostheses. This group of revision patients specifically includes only those patients whose failed prosthesis were initially put in at Stanford. Failed prostheses tended to occur in: 1) non rheumatoids; 2) men more than women; 3) heavier patients especially women; 4) accelerate with time since implantation and; 5) in younger patients. Complications were described by specific product or manufacturer and identified to specific surgeon.

SP601; 1981 January. 643-653. Dobbs, H. S. Prediction of femoral component performance through implant retrieval information.

Key words: femoral component; loosening; metal-on-metal prostheses; metal-on-plastic prostheses.

The use of standard actuarial methods for the analysis of total hip replacement performance is demonstrated by means of a study of the survival of total hip replacements in patients operated on at the Royal National Orthopaedic Hospital. Two types of Stanmore cobalt chrome molybdenum prostheses were considered: (1) metal-on-metal prostheses of various 
developmental designs inserted between 1963.72 (173), (2) metal. on-plastic prostheses of one design inserted between $1969-72$ (248). The survival criterion was taken to be non-removal of the prosthesis and all cases not removed were regarded as survivors. The results indicate that for metal-on-metal prostheses the overall probability of survival was only $53 \%$ after 11 years and the average annual probability of removal irrespective of cause was $5.5 \%$. As expected, for metal-on-plastic prostheses the results were considerably better, the figures being $88 \%$ after eight years and $1.5 \%$ respectively. For both prosthesis types the predominant failure mode was loosening and for this failure mode the annual rate of removal increased as the follow-up time increased, suggesting that loosening is a wearing-out process. Femoral component fracture occurred less frequently, but also appeared to be a wearing-out process. Finally, the advantages of the survival method as compared with conventional methods are discussed, and it is demonstrated how the analysis can be used to predict the probability of survival at sometime in the future.

SP601; 1981 January. 655-666. Piehler, H. R.; Sloter, L. E. Il. Legal and regulatory implications of implant retrieval and analysis.

Key words: implant retrieval and analysis; liability trials; performance testing; regulatory enforcement.

Implant retrieval and analysis can provide valuable input into the decisions made by our courts and regulatory agencies. The procedures used by these bodies in reaching their decisions, however, often place restraints on the ways in which the retrieval and analysis are performed in the first place. Implant retrieval and analysis is but one method by which risk reduction can be accomplished, i.e., the development and utilization of a clinical and/or device-related data base. The polling of experts and fault/failure analyses are viable, even preferable, alternatives to achieve expenditious risk reduction in certain situations. A threshold question which must be answered by any court or regulatory agency prior to a compensatory or regulatory decision is whether a device flaw (production or design) did indeed cause the failure or malfunction. Procedural modifications to liability trials and regulatory enforcement which respond to this threshold question are described, as are performance evaluation procedures which follow from the answer to this question. Laboratory fatigue data and results from litigated cases are introduced to indicate that risk reduction can expeditiously be accomplished through performance testing, and conformance to the performance standards can reduce or eliminate liability exposure.

SP602. Albus, J. S.; VanderBrug, G. J.; Barbera, A. J.; Fitzgerald, M L.; Wheatley, T. E., eds. NBS/RIA robotics research workshop. Proceedings of a workshop held at the National Bureau of Standards; 1979 November 13-15; Gaithersburg, MD. Nat. Bur. Stand. (U.S.) Spec. Publ. 602; 1981 April. 54 p. SN003-003-02307-8.

Key words: Delphi Forecast; robot control; robotic assembly; robotics research; robotic standards; robot vision.

The NBS/RIA Robotics Research Workshop had two objectives: 1) To provide a forum for structured discussions between researchers in robotics and manufacturers and users of robot systems and 2) To develop a consensus forecast of future developments in sensors and control systems for industrial robots.

The 3-day workshop brought together 31 researchers, manufacturers, and users of industrial robots in order to determine the needs and priorities for future research in sensors and control techniques for industrial robots. There were no formal papers; instead, small group discussions and presentations and the preparation of a Delphi Forecast were used to address research needs and priorities.

SP603. Eisenhower, E. H. Requirements for an effective national ionizing radiation measurements program. $A$ report to the Congress by the National Bureau of Standards. Nat. Bur. Stand. (U.S.) Spec. Publ. 603; 1981 March. 139 p. SN003-003-02298-5.
Key words: beta rays; Federal and State laboratories; gamma rays; instrument calibrations; intermediate calibration laboratories; ionizing radiation measurements; measurement accuracies; neutrons; radiation dosimetry; radiation protection; radiation standards; $x$ rays.

This report was prepared for the Senate Committee on Commerce, Science, and Transportation in response to its recommendation that the National Bureau of Standards review in cooperation with the Conference of Radiation Control Program Directors the need for intermediate calibration laboratories for ionizing and nonionizing radiation. Conclusions relevant to the measurement of nonionizing radiation are presented in a separate report. This report is a description of the elements of an effective support system for ionizing radiation measurements, an evaluation of current needs in this area, a description of necessary measurement accuracies for specific applications, and a discussion of possible options to improve the support system. The focus is on institutional and technical actions needed to assure the accuracy of ionizing radiation measurements for the protection of workers and the general public.

SP604. Heinrich, K. F. J.; Newbury, D. E.; Myklebust, R. L.; Fiori, C. E., eds. Energy dispersive x-ray spectrometry. Proceedings of a Workshop on Energy Dispersive X-Ray Spectrometry held at the National Bureau of Standards; 1979 April 23-25; Gaithersburg, MD. Nat. Bur. Stand. (U.S.) Spec. Publ. 604; 1981 June. 441 p. SN003. 003-02333-7.

Key words: electron probe microanalysis; energy dispersive $\mathrm{x}$-ray spectrometry; germanium (lithium) detector; scanning electron microscopy; silicon (lithium) detector; spectral deconvolution; $\mathrm{x}$-ray analysis; $\mathrm{x}$-ray fluorescence.

This book is the formal report of the Workshop on Energy Dispersive X-Ray Spectrometry held at the National Bureau of Standards, April 23-25, 1979. The papers included in these proceedings cover a wide range of topics in the field: (1) Physics of detectors-Properties of silicon (lithium), germanium (lithium), intrinsic germanium, and mercuric iodide detectors are described and compared. (2) Windowless $\mathrm{x}$-ray detectors-The application of windowless energy dispersive $\mathrm{x}$-ray detectors to the measurement of soft $\mathrm{x}$-rays below $\mathrm{l} \mathrm{keV}$ is described, including special electronic techniques for processing pulses produced by low energy $\mathrm{x}$-rays. (3) Electronics for energy dispersive $x$-ray spectrometry-The signal processing chain is discussed with special emphasis on state-of-the-art multichannel analyzers. (4) Spectral processing and statisticsFundamental statistical considerations in spectral processing are described. A variety of curve techniques is discussed, including sequential simplex and least squares fitting with a digital filter. (5) Artifacts in energy dispersive $x$-ray spectrometry in electron beam instruments-Artifacts arising from the basic detection and amplification processes and instrument-related artifacts observed in electron beam instruments operating in the range $10-1000 \mathrm{keV}$ are described. (6) Applications-Applications of energy dispersive $x$-ray spectrometry are given in quantitative $\mathbf{x}$-ray microanalysis and $\mathrm{x}$-ray fluorescence analysis. These proceedings include the following papers (indented):

SP604; 1981 June. 1-4. Heinrich, K. F. J. The development of energy-dispersive $\mathrm{x}$-ray analysis.

Key words: Blodgett-Langmuir pseudo-crystals; Bragg crystal diffraction; energy-dispersive; $\mathbf{x}$-ray spectrochemical analysis.

Energy-dispersive $x$-ray analysis with gas proportional detectors has been used widely in $\mathrm{x}$-ray spectrochemical analysis. It was particularly useful in the detection of low-energy x-ray emission, before the commercial availability of the BlodgettLangmuir pseudo-crystals. With the development of solid-state $\mathrm{x}$-ray detectors of high resolution, and improvement in associated electronics and computers, the interest in energy-dispersive $\mathrm{x}$-ray 
analysis has increased enormously, to the point where it has replaced Bragg crystal diffraction spectrometry in many instances.

SP604; 1981 June. 5.34. Fink, R. W. Properties of silicon and germanium semiconductor detectors for $\mathbf{x}$-ray spectrometry.

Key words: collimation; deadlayer determinations; germanium; monoenergetic photons; partly-resolved doublets; silicon; tailing; $\mathrm{x}$-ray detectors.

The characteristics of silicon and germanium semiconductor $x$-ray detectors of various designs (disk, top hat, grooved, and ion implanted types) are reviewed, including discussion of sensitive area and depth, metallic- and dead-layers. The effects of electronic operating conditions and timing requirements on spectral peak shapes (tailing), resolution, and efficiency in singles and in coincidence measurements are reported. The response to monoenergetic photons is discussed, including second-order $\mathrm{x}$-ray spectral effects, such as the low-energy plateau, escape peaks, the continuum around the full-energy peak, summing and sum peaks, and effects of collimation. Various methods of measuring relative and absolute detector efficiencies for photons from about $200 \mathrm{eV}$ to $140 \mathrm{keV}$ in energy are summarized, and a useful table is presented of long-lived radioactive sources suitable for calibration of energy and efficiency curves of $x$-ray detectors, in which the current best values of halflife, photon energies, and the absolute number of photons emitted per 100 decays are tabulated. Finally, some recently reported advances in detectors, electronic systems, and a new method of unfolding partly-resolved doublets (e.g. $K a_{1}$ and $K \alpha_{2}$ lines) in coincidence spectra are summarized.

SP604; 1981 June. 35.44. Barbi, N. C.; Lister, D. B. A comparison of silicon and germanium $\mathrm{x}$-ray detectors.

Key words: germanium; semiconductor detectors; silicon; tailing; $\mathrm{x}$-ray spectroscopy.

In recent years, semiconductor detectors (germanium and silicon) have become widely used in the analysis of $x$-rays. $\mathrm{Si}(\mathrm{Li})$ detectors have been applied predominantly in the analytical range of 1 to $30 \mathrm{keV}$, while $\mathrm{Ge}(\mathrm{Li})$ and intrinsic germanium (IG) detectors have been applied in the analysis of higher energy photons. This devision of detector application with $\mathrm{x}$-ray energy is a consequence of the inherent characteristics of the two materials.

Germanium, due to its lower band gap energy, can theoretically exhibit superior resolution compared to silicon. For example, resolution at $5.9 \mathrm{keV}$ for a commercially available IG detector with an active area of $25 \mathrm{~mm}^{2}$ would be comparable to a $\mathrm{Si}(\mathrm{Li})$ detector with half the active area. However, the resolution advantage in germanium detectors is often countered by other material characteristics, and is therefore not realized throughout the spectrum. In particular, for photon energies less than $3 \mathrm{keV}$, peaks from the germanium detector exhibit severe tailing, due to incomplete charge collection, rendering the germanium detectors of little use in the analysis of Na through S.

As the photon energy increases from 3 to $\approx 20 \mathrm{keV}$, the IG performance becomes comparable to that of the $\mathrm{Si}(\mathrm{Li})$ detector, with the exception of the more complex escape peak effects in germanium. Above $\cong 30 \mathrm{keV}$, the IG detector has the advantage of better detection efficiency than the $\mathrm{Si}(\mathrm{Li})$.

The choice of detector for a particular application should be based on resolution and efficiency parameters for the analytical range of interest. A secondary, but practical, consideration is that IG detectors can be stored indefinitely at room temperature without degradation. Practical applications of the IG detector in $\mathrm{x}$-ray analysis will be discussed and a comparison of the detector performance to that of $\mathrm{Si}(\mathrm{Li})$ for these applications will be presented.

SP604; 1981 June. 45-59. Dabrowski, A. J.; Singh, M.; Huth, G.
C.; Iwanczyk., J. S. Room temperature energy dispersive $x$-ray spectrometry with mercuric iodide detectors.

Key words: energy resolution; mercuric iodide detectors; $x$-ray spectra; $x$-ray spectrometry.

The compound semiinsulator mercuric iodide is under development as a detector for room temperature operable, energy dispersive x-ray spectrometry. Energy resolution values approaching those characteristic of cryogenically cooled semiconductor detectors have been achieved. A miniature spectrometer consisting of an $\mathrm{HgI}_{2}$ detector and preamplifier and having a total volume of $300 \mathrm{~cm}^{3}$, has been fabricated. This does not even closely approach reduction in spectrometer size that is possible and much smaller designs are possible in the near-term future. Portable $x$-ray spectrometers are currently being designed for on-site, extra-laboratory applications such as detection of uranium in mines, or for microanalysis in space. An energy resolution of $420 \mathrm{eV}$ is currently obtained with a $7 \mathrm{~mm}^{2} \mathrm{HgI}_{2}$ detector for the $5.9 \mathrm{keV}$ x-rays of Fe-55. An improvement in resolution by $50-100 \mathrm{eV}$ is expected in the near future.

The various sources which contribute to noise and background in the $x$-ray spectra have been studied and compared to corresponding features of cooled $\mathrm{Si}(\mathrm{Li})$ and germanium detectors. X-ray spectra of many targets have been measured with mercuric iodide detectors. These spectra were generated either by charged-particle excitation of the sample, or by the method of $x$-ray fluorescence. Several potential applications have been outlined which are particularly suited to mercuric iodide $\mathrm{x}$-ray spectroscopy.

SP604; 1981 June. 61-70. Walter, F. J.; Stone, R.; Blackburn, D. H.; Pella, P. A. Standard techniques for measuring window absorption and other efficiency losses in semiconductor $x$-ray energy spectrometers.

Key words: dead-layer; SRM-477; window absorption; window thickness; window transmission; $\mathrm{x}$-ray detectors; $\mathrm{x}$-ray energy spectrometers.

A conceptual technique for measuring the relative window and dead-layer thickness on semiconductor $x$-ray energy spectrometers by monitoring the transmitted intensity of a series of lines from a standard target has been described previously. This technique, which utilizes secondary $x$-rays fluoresced from a standard glass by the Mn X-rays from decay of Fe-55, was never incorporated in a measurements standard because there was no suitably stable glass target whose composition was traceable and certifiable. Recently, the National Bureau of Standards has developed a glass, SRM-477, specifically for this application.

Results of tests on the sensitivity, repeatability, and reliability of the technique will be presented. The various contributions to dead and partially dead layers will be discussed. Other techniques used to verify the efficiency of semiconductor $x$-ray detectors which are also proposed for the new IEEE/ANSI/IEC standard will also be described.

SP604; 1981 June. 71-95. Russ, J. C.; Sandborg, A. O. Use of windowless detectors for energy dispersive light element $x$-ray analysis.

Key words: energy dispersive; light elements; soft $x$ rays; spectral distortion; windowless detectors.

Energy-dispersive $\mathrm{Si}(\mathrm{Li})$ detectors can be used to efficiently detect soft $\mathrm{x}$-rays in the $0.2-1.5 \mathrm{keV}$ (C $\mathrm{K}-\mathrm{Al} \mathrm{K}$ ) range by removing the $\mathrm{Be}$ window normally used to isolate the detector from the microscope vacuum. This requires some hardware changes to protect the detector from contamination, electrons, and light. Normal high energy performance of the same detector is not compromised in most cases. Analysis of elements in this range in concentrations from about 0.5 percent up is practical for many samples; examples and exceptions are noted. The spectrum 
appearance in this range is not easily handled by the techniques routinely employed at higher energies, and in any case conventional quantitative (ZAF) methods are not readily used for these elements.

SP604; 1981 June. 97-126. Musket, R. G. Properties and applications of windowless $\mathrm{Si}(\mathrm{Li})$ detectors.

Key words: electron excitation; energy-dispersive spectrometry; energy resolution; peak shapes; surface-layer analyses; ultra-thinwindowed (UTW) Si(Li) detectors; windowless detectors; $x$-ray transmission.

Windowless and ultra-thin-windowed $\mathrm{Si}(\mathrm{Li})$ detectors are useful for the energy-dispersive spectrometry of $x$-rays with energies $E_{x}$ above approximately $200 \mathrm{eV}$. Properties of such detectors are discussed with regard to the limits they impose on performance. In particular, considerations of detection efficiency, output linearity, energy resolution, peak shapes, vacuum conditions, and general requirements are presented. Recent developments have greatly increased the actual and potential applications of these detectors. Examples of applications to bulk, thin film, and surface-layer analyses using ion and electron excitation will serve to demonstrate the advantages and limitations of these detectors in contrast to beryllium-windowed detectors.

SP604; 1981 June. 127-139. Statham, P. J. The efficiency of Si(Li) detectors at very low photon energies.

Key words: dead layer; low x-ray energies; $\mathrm{Si}(\mathrm{Li})$ detectors.

The efficiency of a $\mathrm{Si}(\mathrm{Li})$ detector for low $\mathrm{x}$-ray energies is an important parameter in determining both the shape of the observed continuum and the sensitivity factors for "standardless" techniques such as the analysis of thin samples by the intensity ratio technique. This paper considers the factors which determine the efficiency and examines the possibilities for measurement and their limitations.

SP604; 1981 June. 141-164. Statham, P. J. Electronic techniques for pulse-processing with solid-state $x$-ray detectors.

Key words: pulse-processing; solid-state $x$-ray detectors; $x$-ray energy spectrometry.

This paper reviews the methods which have been proposed for quantifying the output of a solid-state detector and summarize the state-of-the-art with regard to noise and count rate performance for $x$-ray systems. Ancillary techniques which improve the performance of the basic pulse-processor such as pulsed excitation, pile-up rejection and spectrum stabilisation will also be discussed and the fundamental limitations will be analyzed.

SP604; 1981 June. 165-175. Lifshin, E.; Hayashi, S. R. Understanding multichannel analyzers.

Key words: multichannel analyzers; solid state detector.

The multichannel analyzer (MCA) is a basic component of any solid state detector system. It is responsible for the sorting of pulses from the main amplifier, the storage and retrieval of spectra and, in most modern computer-based systems, the various states of data reduction required for both qualitative and quantitative analyses. Over the past few years, the need for a detailed understanding of the inner workings of MCA's has diminished through the development of more user oriented systems where tedious tasks like counting dots, calculating energies from channel numbers and looking up peak positions in tables have been replaced by "push button controls." While on the surface this clearly leads to improved operator productivity, it is important to periodically examine the inner workings of such systems both from the point of view of obtaining a general understanding of what's going on as well as to assure oneself that the various compromises which have been made are really compatible with special purpose user needs. The intent of this paper is to provide a general overview of what's behind the buttons.

SP604; 1981 June. 177-191. Ryder, P. L. Statistical considerations of detectability limits and deconvolution in energy dispersive $\mathrm{x}$-ray spectrometry.

Key words: deconvolution; energy dispersive $x$-ray spectrometry; Fourier methods; Gaussian curves.

The first part of the paper considers theoretically the experimental errors due to counting statistics in the determination of line intensities by fitting Gaussian curves to the experimental values. The line positions and the background are assumed to be known, and the background level is taken to be constant over the relevant energy range. Expressions are derived for the standard deviations of the line intensities a) for a single line and b) for two overlapping lines. Numerical examples are given to illustrate the effect of line and background intensities on these errors. In the case of two overlapping lines, it is shown how the ultimate spectral resolution is limited by the statistical errors, which increase as the line separation decreases.

The second part of the paper is concerned with the effect of counting statistics on the deconvolution of unsmoothed ED spectra by Fourier methods, when no assumptions are made concerning the line positions or background spectrum. It is shown that a criterion for the resolution limit of two overlapping lines is provided by the requirement that the amplitude of the first maximum in Fourier spectrum of the line pair must be equal to or greater than the mean amplitude of the noise spectrum. In retransforming the Fourier spectrum the high-frequency noise must be eliminated by a suitable frequency filter. It is suggested that the filter shape should be that of the Fourier transform of the spectrometer function (in this case a Gauss curve). The consequences for computer evaluation of energy dispersion x-ray spectra are discussed.

SP604; 1981 June. 193.231. Schamber, F. H. Curve fitting techniques and their application to the analysis of energy dispersive spectra.

Key words: curve fitting; energy dispersive spectra.

Curve fitting is a widely used procedure in many areas of analysis and has become one of the dominant methods employed in the analysis of energy dispersive spectra. Despite this popularity, curve fitting methods are not universally understood by $\mathrm{x}$-ray analysts and there is thus the potential both for misapplication of the techniques and disagreement as to the interpretation of curve-fitted results.

Curve-fitting analysis can frequently produce spectacular results by extracting information which is not readily accessible by other techniques. Since the basic principles of the method are generally buried in the complexities of a computer program, the unfortunate impression is sometimes given that curve-fitting is a form of statistical alchemy which purports to extract unlimited information from minimal data. Enthusiasts have been known to make extravagant claims for the method, thereby confirming the worst fears of skeptics, who properly mistrust any technique which seems to offer "something for nothing."

The primary area of misunderstanding seems to be one which is addressed by the question: "What can curve fitting really do, and what can it not do?" It is the intent of this paper to deal with this primary question by discussing the basic theory and practice of curve fitting, at a common sense level and as it specifically applies to problems in extracting $x$-ray intensity information from energy dispersive spectra. In so doing, the following basic questions will be addressed: (1) What actually is meant when we speak of "curve fitting?" What are the underlying principles? (2) How does one actually implement a curve-fitting procedure? 
What are the basic algorithms and how do they differ? What are the practical considerations which determine which technique is most appropriate? (3) What kind of information does one have to supply to the procedure and what kind of information can one obtain? What is the tradeoff between information supplied and information extracted? (4) What are the practical problems encountered when one applies curve fitting methods to EDS spectra? How serious are these problems and how might one propose to deal with them? (5) What kind of confidence can one have in curve-fitted results? How can one estimate statistical uncertainty and how reliable are such estimates? Does the technique have the ability to generate grossly erroneous results, and if so, what precautions must be exercised in interpreting fitted results?

It is clearly impossible to deal completely with each of these topics-indeed, unqualified answers cannot be given to many of these questions. It is instead the intent of this paper to clarify and briefly explore these issues as they specifically relate to the suitability of curve-fitting techniques for practical analysis of $\mathrm{x}$-ray spectra.

SP604; 1981 June. 233-272. Fiori, C. E.; Myklebust, R. L.; Gorlen, K. Sequential simplex: A procedure for resolving spectral interference in energy dispersive $\mathrm{x}$-ray spectrometry.

Key words: detector; energy dispersive x-ray spectrometry; Gaussian profiles; lithium-drifted silicon $(\mathrm{Si}(\mathrm{Li})$ ) detector; $x$-ray microanalysis.

A method is described for the fitting of Gaussian profiles to the pulse-height distributions of overlapping $x$-ray peaks measured with an energy-dispersive detector. The desired result is to know the areas under individual $x$-ray peaks in a spectrum in which the peaks are not resolved. A sequential simplex procedure for selection of the parameters in a mathematical expression that describes a spectral peak is used. Starting and stopping criteria and a method to reduce the number of independent variables for the procedure are discussed, and a computer program is included. Examples of the method applied to computer generated peaks and to peaks obtained from a lithium-drifted silicon $\mathrm{Si}(\mathrm{Li})$ detector are provided.

SP604; 1981 June. 273-296. McCarthy, J. J.; Schamber, F. H. Least-squares fit with digital filter: A status report.

Key words: digital filter; filter-fit method; linear least-squares; $\mathrm{x}$-ray analysis.

The technique of incorporating digital filtering within a linear least-squares fitting procedure was initially conceived and reported in 1973. At this time, it was one of the very few x-ray analysis procedures which attempted to perform quantitative analysis on spectra containing complex peak overlaps. Since then, the method has been used extensively in a number of applications and continues to be a powerful analysis technique.

The continued success of the filter-fit method is more than a little surprising in view of its essential simplicity of concept. Unlike continuum-modeling which achieves quantitative accuracy by careful attention to the details of the $x$-ray production and measurement process, the filter-fit method revolves about a single empirical fact: the continuum (background) components in a spectrum are relatively "smooth" structures compared to the characteristic $x$-ray peaks and thus can be selectively suppressed by application of an appropriate digital filter operator. In view of the considerable success which has been achieved with this very simple concept, it seems clearly worthwhile to analyze why the method works as well as it does.

It is the purpose of this paper to review the current state of knowledge of the technique, based upon the past five years of experience in its application. In addition to reviewing the basic concept of the technique and its application to spectral analysis, the presentation will focus attention on the inherent strengths and limitations of the technique and areas for future improvement.

SP604; 1981 June. 297-313. Russ, J. C. Multiple least squares fitting for spectrum deconvolution.

Key words: digital filter; electron excited x-ray spectra; energydispersive $\mathrm{x}$-ray spectrum deconvolution; multiple least squares fitting; nuclear spectroscopy.

The multiple least squares fitting approach to energy-dispersive $\mathrm{x}$-ray spectrum deconvolution has been described by several authors and is used in some commercial ED software. It is also commonly used in nuclear spectroscopy. The fundamental assumption in this method is that the measured spectrum is a summation of spectra measured on each of the pure element standards. For the peaks this is often a good first approximation as we shall see.

SP604; 1981 June. 315.339. Fiori, C. E.; Newbury, D. E.; Myklebust, R. L. Artifacts observed in energy dispersive $x \cdot r a y$ spectrometry in electron beam instruments-A cautionary guide.

Key words: electron probe microanalysis; energy dispersive $\mathrm{x}$-ray spectrometry; microanalysis; qualitative analysis; quantitative analysis; scanning electron microscopy; spectral artifacts; transmission electron microscopy.

Microanalysis with the energy dispersive x-ray spectrometer on electron beam instruments is complicated by the existence of spectral artifacts which are introduced during the $x$-ray detection, amplification and display processes. The artifacts of the $x$-ray detection mechanism include absorption in the window and silicon dead layer, peak broadening, formation of parasitic peaks by escape of silicon $x$-rays, sum peaks due to pulse coincidence, and effects due to system deadtime. Pulse coincidence effects are exacerbated when high energy $(>30 \mathrm{keV})$ x-rays strike the detector. In the transmission electron microscope high energy $x$-rays cause pulse saturation to occur. Artifacts arising from interactions of the spectrometer with its environment include microphonics, electromagnetic interference, ground loops, and stray radiation in the sample chamber. Spectral artifacts influence the strategy from qualitative analysis since artifact peaks may be misassigned to a chemical element not actually present in the specimen.

SP604; 1981 June. 341-349. Williams, D. B.; Goldstein, J. I. Artifacts encountered in energy dispersive $x$-ray spectrometry in the analytical electron microscope.

Key words: analytical electron microscope; artifacts; energy dispersive $\mathrm{x}$-ray spectrometry; scanning transmission electron microscope.

The analytical electron microscope (AEM), consisting of an energy dispersive spectrometer interfaced to a scanning transmission electron microscope, produces its own particular spectral artifacts. These artifacts are due specifically to the use of high $\mathrm{kV}$ electrons and thin foil specimens. The detection of these artifacts, the identification of their source, and the minimization of their effects are discussed. It is concluded that an ideal microanalytical configuration for an AEM exists and that now it is possible to reduce the effect of spectral artifacts to the level where they do not limit quantitation of the $\mathrm{x}$-ray data.

SP604; 1981 June. 351-364. Zaluzec, N. J. Uncollimated fluorescing radiation in an analytical electron microscope $(100-1000 \mathrm{keV})$ : Sources and solutions.

Key words: analytical electron microscope; electron-optical column; energy dispersive spectrometer; uncollimated fluorescing radiation.

The interfacing of a solid state energy dispersive spectrometer 
(EDS) to the column of a modern transmission or scanning transmission electron microscope (TEM/STEM) can potentially provide an extremely powerful analytical tool for the microcharacterization of materials. Unfortunately, there are sometimes serious instrumental limitations involved in this seemingly straightforward modification of a TEM/STEM instrument into an analytical electron microscope (AEM) of the simplest kind. The limitations arise due to the presence of uncollimated radiation, consisting of both hard x-rays and electron tails, which is generated within the probe forming system of the microscope. In an unmodified instrument this radiation can reach the specimen area and thus produce characteristic $x$-ray emission by fluorescence of the specimen and its environment. Clearly, in any quantitative analysis scheme such an effect is undesirable. Fortunately, these complications can be minimized by judicious modification to the electron-optical column of the AEM system. The correction procedures employed in instruments with incident electron beam energies from 100 to $1000 \mathrm{keV}$ will be outlined and their effects on quantitative analysis discussed.

SP604; 1981 June. 365-379. Myklebust, R. L.; Fiori, C. E.; Heinrich, K. F. J. Spectral processing techniques in a quantitative energy dispersive $\mathrm{x}$-ray microanalysis procedure (FRAME C).

Key words: energy dispersive $x$-ray microanalysis; NBS theoretical matrix correction; $\mathbf{x}$-ray continuum.

The correction procedure FRAME $\mathrm{C}$ was developed for quantitative electron probe microanalysis with an energy dispersive detector. Algorithms incorporated to compute the background due to the $\mathrm{x}$-ray continuum and to correct for overlaps due to $\mathrm{x}$-ray peaks will be described in detail. The method employed to determine the detector efficiency for the $x$-ray continuum calculation will be discussed as well as the computation of the continuum.

SP604; 1981 June. 381-389. Brown, J. D. The effect of electron incidence angle on quantitative electron probe microanalysis.

Key words: electron incidence angle; electron probe microanalysis.

$\phi(\rho z)$ curves have been measured at 25 and $30 \mathrm{keV}$ electron energies and electron incidence angles from 60 to $90^{\circ}$. These data can be used to establish the effect of changing incidence angle on quantitative correction procedures and will be invaluable for comparison with Monte Carlo predictions. None of the factors currently used to correct for inclined electron incidence adequately compensates for this effect.

SP604; 1981 June. 391-415. Short, M. A.; Bonfiglio, S. Energy and wavelength dispersive $x$-ray fluorescence analysis of heavy elements using radioactive, secondary fluorescence and $x$-ray tube sources, and with germanium, silicon, and scintillation detectors.

Key words: catalysts automotive; energy dispersive analysis; heavy element fluorescence analysis; intrinsic germanium detectors; secondary target fluorescence analysis; source excited fluorescence analysis; $x$-ray fluorescence analysis.

Comparative analytical results are presented for the analysis of barium/lanthanum/cerium and osmium/iridium/platinum using both $\mathbf{K}$ and $\mathbf{L}$ series characteristic lines for four different excitation/detection systems. These systems include a radioactive source with an intrinsic germanium detector, a radioactive source fluorescing a secondary target with an intrinsic germanium detector, an $\mathrm{x}$-ray tube fluorescing a secondary target with a lithium drifted silicon detector, and an x-ray tube used in a conventional wavelength dispersive spectrometer. The two principal advantages of $\mathbf{K}$ lines of heavy elements are discussed: the relative simplicity of the $K$ spectra and the relatively great penetrating power of high energy $\mathbf{K}$ radiation. It is concluded that better resolution is obtained for $L$ series lines using wavelength dispersive spectrometry. An example of the high penetrating power is shown in the in-laboratory analysis for plantinum and lead on an automobile catalyst contained within a stainless steel can.

SP604; 1981 June. 417-439. Fiori, C. E.; Swyt, C. R. Energy dispersive detectors-A bibliography (1981).

Key words: bibliography; electron column instruments; energy dispersive detectors; scanning electron microscopy; x-ray spectrometry, review of scientific instruments.

This paper is an expanded and updated bibliography of references relating to energy dispersive detectors for electron column instruments with more complete cross-referencing by subject than the previous bibliography by the first author. The bibliography is not all-inclusive and is meant only to give the reader a starting point from which to become familiar with the large body of literature which exists on the topic of energy dispersive detectors. Papers from the more relevant journals, e.g., X-Ray Spectrometry, Review of Scientific Instruments, Scanning Electron Microscopy, etc., have been cited most heavily but a number of historical papers of general interest from many sources are also included. Both new citations and references from the previous bibliography are listed because of the nature of the workshop resulting in this volume.

SP605. Debelius, J. R. Standards committee activities of the National Bureau of Standards, 1980 highlights. Nat. Bur. Stand. (U.S.) Spec. Publ. 605; 1981 May. 102 p. SN003-003.02327.2.

Key words: annual report; committee participation; standards committees.

NBS uses a decentralized system for managing the participation of NBS representatives in outside standards committee activities. This type of management is governed by NBS policy; coordinated through standards offices in each NBS Major Operational Unit; and monitored and supported by the Office of Standards Information, Analysis, and Development (OSIAD).

This report summarizes NBS standards committee activities during calendar year 1980. It contains highlights of significant technical and individual contributions made by NBS staff, a description of NBS standards management and information activities, and a directory of standards committees on which NBS staff serve. (Supersedes NBS Special Publication 573 (March 1980).)

For further information on NBS standards activities, contact OSIAD, National Bureau of Standards, Technology Building, Room B166, Washington, D.C. 20234, (301) 921-2092.

SP606. Trechsel, H. R.; Launey, S. J. Criteria for the installation of energy conservation measures. Nat. Bur. Stand. (U.S.) Spec. Publ. 606; 1981 July. 203 p. SN003-003-02337-0.

Key words: automatic ignition devices; caulks; effectiveness; energy conservation; energy conservation measures; installation; insulation; oil burners; practices; safety; sealants; standards; storm doors; storm windows; vent dampers; water heaters; windows.

Standard installation practices were developed to assist in assuring the effectiveness and safety of energy conservation measures installed under the Residential Conservation Service (RCS). They serve as mandatory standards under RCS but are recommended guides for all installations of the covered materials and products. The criteria are being used by DoE to develop training manuals for installers, inspectors, and energy auditors.

Part I provides information on the intended use of the practices, outlines the RCS program, and discusses specific major technical and related issues that were considered in the development of the standards: moisture and building retrofit, attic ventilation, electrical wiring, recessed and surface-mounted fixtures, the use of diagnostic tools (infrared thermography, air change rate, and window air leakage 
measurements), and product certification.

Part II provides the actual practices together with commentary and additional recommendations. The products covered are loose-fill, batts and blankets, rigid foam boards, UF foam and reflective insulations, window devices, caulks and sealants, water heater insulation, oil burner replacements, and vent dampers.

SP607. Zimmerman, J. E.; Sullivan, D. B.; McCarthy, S. E., eds. Refrigeration for cryogenic sensors and electronic systems. Proceedings of a Conference held at the National Bureau of Standards; 1980 October 6-7; Boulder, CO. Nat. Bur. Stand. (U.S.) Spec. Publ. 607; 1981 May. 223 p. SN003-003-02310-8.

Key words: cryocoolers; cryogenic sensors; helium; refrigeration; superconducting devices.

This document contains the proceedings of a meeting of refrigeration specialists held at the National Bureau of Standards, Boulder, $\mathrm{CO}$, on October 6 and 7, 1980. Participation included representatives of industry, government, and academia. The purpose of the meeting was to discuss progress in the development of refrigeration systems which have been specialized for use with cryogenic sensors and electronic systems. The meeting focused primarily on the temperature range below $20 \mathrm{~K}$ and cooling capacity below $10 \mathrm{~W}$. The meeting was jointly sponsored by the International Institute of Refrigeration-Commission A 1/2, the Office of Naval Research, the Naval Research Laboratory, the Cryogenic Engineering Conference, and the National Bureau of Standards. These proceedings include the following papers (indented):

SP607; 1981 May. 3-14. Roubeau, P. M. A preview of unconventional suggested cooling processes and some practical problems related to small refrigerators.

Key words: adsorption; cryocoolers; cryostats; helium; refrigeration; Stirling cycle.

This paper describes two non-conventional means of refrigeration, perhaps out of reach of practical realization, whose purpose is to shed light on some properties of gases and adsorbants having great potential applications. An account is given of a variation of the Stirling machine and finally a sketch is made of a crossed cycles method independent of the refrigeration process used.

This descriptive part is sandwiched by overall considerations on heat losses, mainly by the cryostat necks and in general by any structures for which the non-heat conducting properties could, in fact, be an important criterion of success for cryocoolers. Concerning minicoolers which make use of a circulating coolant, the need for a dry and tight compressor is equally emphasized and some solutions are suggested.

SP607; 1981 May. 15-20. Guernsey, R. W.; Flint, E. B. Refrigeration requirements for superconducting computers.

Key words: cryogenic equipment; Josephson junction devices; superconducting computers; thermal contact; $4 \mathrm{~K}$ refrigeration.

High speed, low power Josephson junction logic and memory chips densely packed into a structure immersed in liquid helium may constitute the high performance computer of the future. In this paper we discuss chip cooling, input/output cable requirements, computer cryosystem design considerations, and refrigeration requirements.

Conventional gas expansion refrigeration should be suitable for medium to large size superconducting computers $(0.5$ to 10 watts). Small systems, such as a $25 \mathrm{~mW}$ future minicomputer, will require innovative refrigerator design that achieves high reliability at low cost.

SP607; 1981 May. 21.29. White, R.; Haskin, W. Development approaches for long-life cryo-coolers.

Key words: component development; contamination control', cryogenics; helium retention; long-life; refrigerators; reliability; rotary-reciprocating; turbo-Brayton; Vuilleumier.

Development of cryogenic refrigerators (cryo-coolers) capable of operating for over three years without maintenance is being sponsored by the Flight Dynamics Laboratory for several Air Force missions. Future cryo-coolers for superconducting devices will need similar high reliability and durability. Therefore, the general approaches used and the lessons still being learned will be described as they apply to all long-life cryo-coolers.

Highly reliable and durable cryo-coolers are developed in a carefully planned and controlled process. Required operating conditions will influence the selection of the basic type of cooler, but the selected concept must have an inherent long-life potential. The principal long-life cryo-coolers being developed by the Flight Dynamics Laboratory are the Vuilleumier (VM), turbo. Brayton and rotary reciprocating refrigerators. These machines either use gas film bearings to avoid rubbing contact or have lightly loaded bearings and seals with low wear rates.

Careful development is necessary to realize the long-life potential of the coolers. This includes detailed analysis of stress levels for all parts of the cooler, adequate quality controls, contamination control, and reliability analysis. Final verification of adequate durability, with acceptable cooler performance, is demonstrated by reliability growth in a test, analyze, and correct program.

SP607; 1981 May. 30-47. Horn, S. B.; Asher, M. S. Theoretical analysis of a 3-stage Stirling cycle cryocooler.

Key words: cryocooler; cryogenic; helium; low temperature; refrigerator; Stirling cycle.

Previous analyses of three stage coolers have neglected the real gas equation of state of helium. Those analyses have therefore neglected the compressibility effects that occur below $20 \mathrm{~K}$. This paper derives the thermodynamic performance of three stage Stirling type coolers using the real gas equation of state. An analysis of a small, efficient three stage cooler is given with the low temperature gas effects illustrated.

SP607; 1981 May. 48-56. Daney, D. E. Some thermodynamic considerations of helium temperature cryocoolers.

Key words: cryocooler; hybrid cryocoolers; refrigeration; regenerative cooler; regenerator; Stirling cooler; Stirling cycle.

Attempts to reach liquid helium temperatures with regenerative cryocoolers have been generally unsuccessful. The principal and well known cause of this failure is the decay of the regenerator matrix heat capacity at low temperature. As the regenerator effectiveness decreases, more and more of the refrigeration effect goes to the regenerator losses rather than to the load. A less well-recognized problem for some cryocoolers has been operation under adverse thermodynamic conditions. For example, operation at high pressure (40 bar) can decrease the calculated efficiency of the lowest stage by a factor of 10 or more-primarily because of the strongly negative Joule-Thomson effect of helium at low temperature and high pressure.

We first investigate the effect of the operating regime on the efficiency of helium temperature cryocoolers. Next, we propose two schemes of hybrid regenerative-counter flow cryocoolers as solutions to the problem of low helium-temperature regenerator heat capacity. Finally, we calculate the net mass flow through a typical hybrid regenerator-heat exchanger for one of the schemes that we propose.

SP607; 1981 May. 57-69. Ackermann, R. A. Dynamic analysis of a small free-piston resonant cryorefrigerator.

Key words: displacer and piston drives; linear electric motors; linear free-displacer cryorefrigerator; linear resonant machinery; phasor notation; thermodynamic and dynamic analyses. 
The development of small, cryogenic refrigerators is receiving increased attention through the use of linear resonant machinery which has the potential for increasing the reliability and efficiency of these devices. The use of linear machinery, however, presents a complex second dimension to the analysis of the system's dynamics and thermodynamics which are implicitly tied together through the pressure wave produced in the refrigerator. This paper describes the dynamics of a linear cryogenic refrigerator and presents the analytical development of the equations of motion through the use of phasor notation. Solutions for both the transient and steady-state motions are presented, and the conditions of stability and selection of key operating parameters such as frequency, piston-displacer amplitudes and phase angles are discussed.

SP607; 1981 May. 70-81. Radebaugh, R.; Linenberger, D.; Voth, $R$. O. Methods for the measurement of regenerator ineffectiveness.

Key words: cryogenics; Gifford-McMahon cryocoolers; heat capacity; heat transfer; helium; refrigerators; regenerators; Stirling cryocoolers; thermal conductance; Vuilleumier cryocoolers.

The regenerator ineffectiveness is usually the dominant heat loss term in regenerative-cycle refrigerators which operate below about $15 \mathrm{~K}$. Systematic measurements of regenerator ineffectiveness have never been done in this temperature range in spite of the fact that theoretical models are usually not adequate to fully describe and predict the thermal behavior of regenerators. The paper introduces the proper definition of regenerator ineffectiveness, which is valid for non-ideal gases and systems where a pressure wave exists. All parameters which influence the regenerator ineffectiveness are discussed. The concept of a temperature-dependent ineffectiveness is proposed which simplifies computations of regenerator ineffectiveness when the material properties are temperature dependent and which yields the average ineffectiveness when it is integrated between the lower and upper operating temperatures of the regenerator. Previous methods used and proposed for the measurement of regenerator ineffectiveness at cryogenic temperatures will be reviewed and their limitations discussed. A new method will be presented which for the first time will allow measurements of regenerator ineffectiveness at all phase angles between the mass flow rate and the pressure wave, and important consideration with non-zero regenerator volumes. Temperatures considered are those between 4 and $35 \mathrm{~K}$.

SP607; 1981 May. 82-92. Longsworth, R. C. Serviceable refrigerator system for small superconducting devices.

Key words: cryogenic refrigerator; Josephson technology; liquid helium; serviceable; superconducting device; thermal coupling.

The design of a refrigeration system for cooling small superconducting devices such as Josephson Technology computers which can be serviced while the superconducting device remains in a cold operating state is described. The refrigeration system incorporates new (passive) thermal couplings which permit the Displex ${ }^{R}$ expander and JT circuit to be warmed up, the expander serviced, and the JT circuit purged, while helium boils off at a relatively low rate from the bath that is cooling the superconducting device.

When connected to the $1.4 \mathrm{~L}$ test pot, a warmup-purgecooldown test was run. Helium was initially condensed in the pot by the refrigerator at the rate of $0.3 \mathrm{~L} / \mathrm{hr}$ then the refrigerator turned off. It took 7 hours to warm up and purge the refrigerator, followed by 3 hours to cool back down to $4 \mathrm{~K}$. Just a few minutes before reaching $4 \mathrm{~K}$, helium stopped venting and started to be drawn into the test pot indicating that slightly more than $1.4 \mathrm{~L}$ of helium was used during the service cycle.

SP607; 1981 May. 93-102. Flint, E. B.; Jenkins, L. C.; Guernsey,
R. W. Performance of a 1 watt $4 \mathrm{~K}$ cryosystem suitable for superconducting computer.

Key words: convective heat transfer; cryocooler; heat transfer by evaporating and condensing helium; helium refrigerator; superconducting computer; thermal couplings; thermal isolation.

Superconducting computers of the future will require closed cycle refrigeration systems capable of removing $0.5-10$ watts at $4 \mathrm{~K}$. The refrigerators must be reliable and the interface to the cryostat must allow continued computer operation during refrigerator shutdown. Such a system has been designed by $\mathbf{R}$. Longsworth and one unit has been built by Air Products. This cryocooler is being operated now in a test configuration that allows detailed study and analysis of the design. The results of these studies, including cool-down times, refrigeration capacity, and interface performance will be discussed and compared with the design calculations.

SP607; 1981 May. 103.115. Gasser, M. G.; Sherman, A.; Beale, W. Developments toward achievement of a 3.5 year lifetime Stirling cycle refrigerator for space applications.

Key words: clearance seals; cryogenics; hydrodynamic gas bearing; linear magnetic suspension; long lifetime; space application; Stirling cycle.

A major effort is underway at the NASA/Goddard Space Flight Center to develop a long life time Stirling cycle cryogenic cooler for spaceflight. The initial objective is a 3.5 year, 5 watts at $65^{\circ} \mathrm{K}$, cooler to be followed by a $12^{\circ} \mathrm{K}$ cooler. The previous paper, "Progress on the Development of a 3.5 Year Lifetime Stirling Cycle Refrigerator for Space," presented at the 1979 Cryogenic Engineering Conference, outlined the approach. During the past year, the design of a refrigerator with linear magnetic bearings has changed. The displacer rod no longer goes through the piston, resulting in a machine that is comprised of a compressor section and an expansion section bolted together. Each section has its own magnetic bearings, linear motor, displacement transducer (LVDT), and displacement volume. The phase angle and displacements of the two moving parts are electronically controlled, utilizing the signals from the LVDT's. Delivery of the engineering model magnetic bearing cooler is scheduled for March, 1981.

Work on the engineering model gas bearing cooler previously reported has ceased. However, an advanced concept for a gas bearing cooler is being studied.

Preliminary results indicate that the normal gas flow in a free displacer Stirling cycle cooler can be utilized to spin the displacer at a speed sufficient to form a self-acting hydrodynamic gas bearing with a clearance seal. The periodic gas flow from the cold end through the generator to the ambient end makes intermittent contact with a turbine located at the ambient end of the displacer. The induced spin is unidirectional.

SP607; 1981 May. 116. Benson, G. M.; Vincent, R. J. Thor cryocooler.

Key words: cryocooler; gas bearings; refrigeration; regenerator; resonant free piston; Stirling cycle.

A resonant, free-piston Stirling-cycle cryocooler driven by linear reciprocating synchronous electric motors and utilizing gas bearings and gas springs has been designed, and selected components tested. This hermetically-sealed, dynamically balanced design is based on ERG's previous work and has been engineered for long-life through use of: 1) non-contacting linear gas bearings and narrow clearance reciprocating seals, 2) brushless reciprocating electric motors with hermetically-sealed coil and core, 3) stationary external first-stage regenerator, and 4) solid-state controller. The design has been efficiency-optimized by 1) transferring heat nearly isothermally (to better approach Carnot efficiency), and 2) minimizing losses through use of 
advanced components of resonant free-pistons (which eliminate piston drive losses). The single-stage unit, designed to produce 10 watts at $65 \mathrm{~K}$, may be expanded to three-stages, using a novel design, for producing $4.2 \mathrm{~K}$. Designs and test results of selected components are described and compared with theoretical and computer modeled results.

SP607; 1981 May. 117-127. Urban, E. W.; Katz, L.; Hendricks, J. B.; Karr, G. R. A cryogenic system for the small infrared telescope for Spacelab 2.

Key words: cryogenics; infrared telescope; Spacelab; superfluid helium.

The cryogenic system for the small helium cooled infrared telescope for Spacelab 2 is described. The system consists of a $250 \mathrm{l}$ storage dewar containing superfluid helium at about $1.6 \mathrm{~K}$ and a separate experiment cryostat. The liquid helium is confined within the dewar in zero-g by means of a porous plug. The cold vapor leaving the plug surface is split into two, not necessarily equal, flows. One flow path is through heat exchangers which cool the radiation shields of the storage dewar and, therefore, control the liquid storage efficiency. The second flow path is through a flexible joint to the independent set of heat exchangers for cooling the infrared optical apparatus and radiation shields in the cryostat. By means of commandable valves on the dewar and cryostat vent lines, the fraction of total helium boiloff used for cooling the dewar radiation shields can be controlled, and the cooling available at the cryostat can be adjusted from zero flow rate to nearly $50 \mathrm{mg} / \mathrm{s}$ of helium gas at $2 \mathrm{~K}$. A number of unusual cryogenic problems which have been solved in the development of this complex system are discussed.

SP607; 1981 May. 127-135. Hartwig, W. H. Requirements for and status of a $4.2^{\circ} \mathrm{K}$ adsorption refrigerator using zeolites.

Key words: adsorption; compressor; cryogenics; molecular adsorption; refrigeration; zeolites.

Previous work by the author and his associates had demonstrated the feasibility of $77^{\circ} \mathrm{K}$ adsorption refrigerators using one $\mathrm{N}_{2} \mathrm{O}$ stage which provides the adsorption temperature $\left(185^{\circ} \mathrm{K}\right)$ for $\mathrm{N}_{2}$. The concept of adding an $\mathrm{H}_{2}$ stage has been examined theoretically and experimentally using zeolites and/or other molecular adsorbers. The results are described which shows the requirements for each stage and suggests that a reliable low-power $4.2^{\circ} \mathrm{K}$ non-mechanical refrigerator can be built with a considerable development effort and some additional research.

SP607; 1981 May. 136-140. Byrd, E. A.; Hansen, R. G. Cooling of squid devices by means of liquid transfer techniques for reduced helium consumption and enhanced temperature stability.

Key words: cryogenics; helium; infrared; SQUID; temperature sensors.

In an effort to establish a state-of-the-art SQUID device for both laboratory and field applications, a developmental effort has been undertaken by Naval Surface Weapons Center to utilize a liquid helium transfer system to perform cooling of SQUID devices in the temperature region of 4.5 to 16 Kelvin. Various techniques have been employed to enhance the temperature stability of the inherent system as well as to provide increased system portability and flexibility of application.

The specific refrigeration system used in this program has been further modified for expanded applications in the low temperature testing of infrared sensors, low noise amplifiers, and other devices.

This particular developmental effort has resulted in a system which allows the user to function in either a horizontal or vertical orientation. Operation had previously been limited to single axis orientation as a result of liquid cryogenic systems.

This paper presents a concept of utilizing state-of-the-art cryogenic systems to interface with diverse technical needs in the area of electro-optics, SQUID devices, and supporting electronic accessories.

SP607; 1981 May. 141-145. Daunt, J. G.; Heiden, C. A contamination free compressor for small scale Stirling refrigerators.

Key words: bellows; compressor; contamination; continuous operation; cryocooler; low power refrigerator; Stirling refrigerator.

Contamination of the working gas appears to be a major problem encountered in the design of reliable small scale Stirling cycle refrigerators for cooling cryoelectronic devices over long uninterrupted periods. Seals for the reciprocating shafts of such a refrigerator may be a source for contamination due to lubrication and possible small leaks. The use of bellows provides the potentiality for a hermetically sealed refrigerator. As a first step towards such a system, a bellows compressor was designed. For increased lifetime, mechanical load on the bellows is reduced significantly by keeping the differential pressure acting on the membranes at a low level. This is achieved by placing the bellows in the volume of a conventional piston compressor. Such a compressor system is tried in connection with a three stage plastic displacer unit. First results on the performance of the scheme are reported and discussed together with possible design variations that may lead to a further reduction of mechanical vibrations and magnetic interference of the cooling unit.

SP607; 1981 May. 146-153. Studer, P. A.; Gasser, M. G. A bidirectional linear motor/generator with integral magnetic bearings for long lifetime Stirling cycle refrigerators.

Key words: clearance seal; gas compressor; linear motor/generator; magnetic bearings; Stirling Cycle.

Linear motors are well suited to drive Stirling Cycle machines since they reduce the machine dynamics to straight line motion. The first device is being constructed as a dynamic balancer for the Stirling Cycle cooler, which is described in a companion paper at this conference. The motor concepts also applies to Stirling Cycle cryogenic coolers, and its advantages in this application are presented. The permanent magnets, pole pieces and drive coils, which form the stator, are stationary and external to the working fluid. The only moving part is the iron piston, which is not in physical contact with anything except the working fluid. It is magnetically suspended and positioned to within 10 microinches by differential capacitive sensors. This allows the clearance between the piston and cylinder to be small enough to form a clearance seal. The piston is also driven electromagnetically and its axial position sensed capacitively. Heat generated in the motor coils does not enter the working fluid. Gas springs allow a controlled resonant drive to minimize power. The same magnetic structure is used for both the drive function and the magnetic suspension. The electromagnetic design along with the sensing and control implementation are described in the paper. Preliminary test data is presented. Magnetic bearing performance characteristics are discussed.

SP607; 1981 May. 154-159. Little, W. A. Design considerations for microminiature refrigerators using laminar flow heat exchangers.

Key words: Joule-Thomson refrigerators; laminar flow heat exchanger; microminiature refrigerators.

Conventional counter flow heat exchangers are designed to operate with the fluid streams in turbulent flow. The resultant mixing of the fluid allows heat to be transferred from the walls of the exchanger to the body of the fluid. It, however, the dimensions of the exchanger are sufficiently small, enough heat may be transferred by conduction alone that operation in the 
laminar flow regime is possible. We discuss the design parameters of a microminiature Joule-Thomson cryogenic refrigerator operating in this regime. Such operation offers the advantages of simplicity of design and low noise. The latter is of particular advantage for the cooling of cryogenic sensors.

SP607; 1981 May. 160-163. Hollman, R.; Little, W. A. Progress in the development of microminiature refrigerators using photolithographic fabrication techniques.

Key words: cryogenic; Joule-Thomson; microminiature devices; photolithographic fabrication; refrigerators.

We report on progress in the development of fabrication techniques for the construction of microminiature cryogenic Joule-Thomson refrigerators using photolithographic means. A new technique for the fabrication of the devices from glass will be described. Consideration has been given to the design of refrigerators operating in this $50-200 \mathrm{mWatt}$ region at $77 \mathrm{~K}$ and $20 \mathrm{~mW}$ att below $20 \mathrm{~K}$. Recent results on their operation will be reported.

SP607; 1981 May. 164-172. Wade, L. A. Low temperature regenerators.

Key words: cryogenics; heat capacity; refrigeration; regenerators; specific heat.

A study was conducted to examine ways of enhancing the heat capacity of regenerators used in refrigerators for achieving refrigeration below $30^{\circ} \mathrm{K}$. It was found that, due to an increase in the energy of their surface vibrational modes, thin films, thin wires, and ultrafine particles will exhibit a higher specific heat than that which would be normally expected for a bulk sample of the same material.

Based on an idealized refrigerator performance model, it is predicted that a Stirling cycle refrigerator having the third stage of its regenerator replaced with an ultrafine particle $(22 \AA$ dia. lead particles) matrix whose void volume is partially filled with mercury, will deliver at a cooling temperature of $10^{\circ} \mathrm{K}, 2.75$ times as much refrigeration as one with a typical third stage regenerator made up of $0.005^{\prime \prime}$ dia. lead spheres.

SP607; 1981 May. 173-177. Zimmerman, J. E.; Sullivan, D. B.; Kautz, R. L.; Hobbs, R. D. Measurement of thermal properties of cryocooler materials.

Key words: composites; cryocoolers; plastics; refrigeration; regenerators; specific heat; thermal conductivity.

Materials used in several low-power cryocoolers are characterized by very low thermal conductivities and moderate to large heat capacities at low temperature. Consequently, thermal equilibrium times are inordinately long for measurements of thermal properties to be made by the usual quasi-steady-state methods. We have developed a method of measurement in which the thermal conductivity and the specific heat are derived from observations of the time-dependent temperatures at two or more points on a cylindrical sample in response to a step function of heat applied to one end of the sample. Some preliminary results have been obtained on G-10 epoxy laminated tubing. These transient measurements give results in much less time than the steady-state method, and have the additional advantage of being adaptable, with fair accuracy, to the cryocooler structure itself, or to a separate structure geometrically similar to the cryocooler.

SP607; 1981 May. 178-185. Tward, E. Gas heat switches.

Key words: cryogenics; gas adsorption; heat switch; refrigerators.

Thermally operated gas heat switches for use in refrigeration systems operating in the temperature range $4 \mathrm{~K}$ to $300 \mathrm{~K}$ have been designed and constructed. The switches are operated by a miniature adsorption pump which when heated supplies gas to the switch, turning it on, and when cooled removes gas from the switch, turning it off. The switches have been operated at $4 \mathrm{~K}$, $77 \mathrm{~K}$, and $300 \mathrm{~K}$ with $\mathrm{He}$ or $\mathrm{H}_{2}$ gas adsorbed on either charcoal or zeolite $13 \mathrm{X}$. Switching times as low as a second and switching ratios of 80 have been achieved in miniature devices whose total mass is 8 grams.

SP607; 1981 May. 186-194. Sullivan, D. B.; Zimmerman, J. E.; Ives, J. T. Operation of a practical SQUID gradiometer in a lowpower Stirling cryocooler.

Key words: cryocoolers; low temperature; magnetic gradiometer; refrigeration; SQUID; Stirling cycle; superconductivity.

A commercial $\mathrm{Nb}$ point-contact SQUID has been used in the construction of a simple magnetic gradiometer system supported by a split Stirling refrigerator. The magnetic interference generated by the cryocooler was sufficiently small to permit the acquisition (for demonstration purposes) of a reasonable magnetocardiogram. Preliminary investigation of the source of the residual refrigerator interference indicates that cyclic temperature variation is the largest single factor influencing the gradiometer output. A simple electronic temperature controller has been added to a different cryocooler to study temperature stabilization. Better than $1 \mathrm{mK}$ stability has been achieved at 8.5 K.

SP607; 1981 May. 195-209. Nisenoff, M. Current status of high temperature Josephson device technology.

Key words: closed cycle refrigeration systems; Josephson effects; proximity effect devices; superconducting devices; tunnel devices; weak links.

The fabrication of Josephson devices from refractory thin films with high superconducting transition temperatures $\left(T_{c}\right)$ should yield devices which are rugged and reliable, and capable of withstanding repeated thermal cyclings between room and cryogenic operating temperatures. If the onset of Josephson response could be raised to above $15 \mathrm{~K}$, the devices could be operated near $10 \mathrm{~K}$ at a reduced temperature of 0.6 where the temperature dependences of the relevant superconducting parameters are relatively weak. This temperature region is readily accessible using compact, energy-efficient closed cycle refrigeration systems.

A review of recent progress in the fabrication of various types of refractory high temperature Josephson devices is presented. Certain configurations require dimensional resolutions approaching 0.1 micrometer or less for successful operations. In other structures, there are problem areas in the growth of suitable thin films of the superconducting materials of interest and of suitable barrier layers and controlling the properties of the interfacial regions between these layers and the superconducting electrodes. Current progress and accomplishments are summarized and critical areas for future R\&D are identified. Based on recent progress, the prospects for preparing allrefractory Josephson devices with onset temperatures exceeding $15 \mathrm{~K}$ appears very promising.

SP608. Berry, S. A., ed. Research and innovation in the building regulatory process. Proceedings of the Fifth Annual NBS/NCSBCS Joint Conference Technical Seminar on Solar Energy and Energy Conservation; 1980 August 6; Denver, CO. Nat. Bur. Stand. (U.S.) Spec. Publ. 608; 1981 May. 201 p. SN003.003-02321-3.

Key words: ASHRAE 90-75; class K code; computer modeling; electrical design; energy audit; energy conservation; HVAC systems; performance standards; solar collector; solar energy; space heating and cooling; thermal storage.

The Proceedings of the Fifth Annual NBS/NCSBCS Joint 
Conference on Research and Innovation in the Building Regulatory Process contain 17 technical papers. This year's joint conference addressed solar energy and energy conservation.

These proceedings include papers on: Energy programs in the State of Colorado; Building energy performance standards concepts; State energy audits; Energy and building systems services; Solar energy and building codes. These proceedings include the following papers (indented):

SP608; 1981 May. 21.35. Emery, A. F.; Dorri, B.; Kippenhan, C. J.; Heerwagen, D. R.; Banken, G. Alternate optimal control strategies for residential HVAC systems.

Key words: cost savings; energy conservation; HVAC systems; innovation; mathematical model; optimization.

The optimal control of HVAC systems on an hourly basis is determined both by the thermal inputs which precede the hour under question and by future thermal effects. Most simulation programs treat only the past history. This paper describes results obtained with a special program which optimizes the use of the HVAC system in conjunction with natural and artificial lighting and with other thermal load schedules by considering such future effects.

The results indicate that substantial savings can occur through such a look-ahead optimization and that the extent of the needed future knowledge for most residential structures is in the neighborhood of a few hours. It is also shown that such an optimization is more valuable for the gradual changes associated with the usual weather than with rapid changes of internal thermal loads.

SP608; 1981 May. 37.56. Zackrison, H. B., Jr. Energy conscious electrical design.

Key words: electrical design; energy distribution; National Electrical Code; power factor correction; source energy; transformers.

Concern over the adequacy of future energy resources has led the Federal Government to require States to adopt energy conservation techniques for all new construction. Different approaches have been tried, such as Resource Utilization Factors, which consider how efficiently one source of energy can be converted into another (i.e., 3.04 Btus of heat energy are required to produce and transmit $1 \mathrm{Btu}$ of electrical energy to the point of use); and Resource Impact Factors, which consider the relative availability and renewability of a fuel.

The Department of Energy's current approach centers around the formulation of Building Energy Performance Standards, which prescribe energy consumption goals for various types of structures in different climates while allowing designers to achieve these goals in their own ways.

Thus, this paper will consider a number of electrical design techniques that contribute to minimizing energy consumption and maximizing the efficiency of electrical installations. Some of the techniques discussed also involve the source concept, as opposed to the boundary concept, of energy conservation: reducing the total energy expenditure required to construct buildings by minimizing the quantities of raw materials and finished products required.

SP608; 1981 May. 57.67. Kainlauri, E. O. Potential energy savings in buildings resulting from the NCSBCS code compared with BEPS.

Key words: building code; building envelope; comparison; compliance; energy budget; energy conservation; performance standards; Statement of Review; verification.

In the State of Iowa, under the present State Energy Conservation Building Code (National Conference of States on Building Codes and Standards [NCSBCS] Code), all buildings that are larger than 100,000 cubic feet must be submitted for State approval, with a statement from a registered architect or engineer verifying that the plans and specifications meet the code requirements. During the past 2 years, more than 100 design professionals and about as many building inspectors have completed a training course organized by the Architecture Extension of the lowa State University (ISU), and more than 400 building plans have been submitted to the Iowa Building Code Commission for approval. The required "Statement of Review" includes design conditions and overall U.values for exterior walls, roof/ceilings, floors, and the gross wall cooling thermal transfer value (OTTV).

During the 2 years since the code was introduced, some $\mathbf{4 1 2}$ buildings have been analyzed by the ISU Architecture Extension as to what degree the results compare with the corresponding code requirements. It was found that the results are encouraging. By an average, the $U_{0}$ wall figures are about 40 percent better than the allowable American Society of Heating, Refrigerating, and Air-Conditioning Engineers (ASHRAE) 90-75 standards (or the revised ASHRAE 90.1-75R), the roof/ceiling values about 15 percent, and the OTTV values more than 50 percent better.

For the purpose of comparison with the Building Energy Performance Standards (BEPS), the results have been classified in 17 categories in anticipation of BEPS categories (splitting office buildings into large and small categories). The results illustrate characteristically how buildings of various types may approach or even exceed BEPS values. Of course, the $U_{0}$-values by themselves cannot be compared directly with BEPS performance standards and energy budgets, but a close implication can be seen from values produced by approximation.

As a second phase of the research, the Architecture Extension has been checking sample building plans in various parts of the State, in order to verify the accuracy of statements submitted. So far, the statements have been proven accurate, with only minor computation errors. Later on, another check is planned inspecting those buildings for actual installations. Finally, energy audits are planned for verification of performance criteria. The ISU Building Energy Utilization Laboratory is also involved in studies of lowa buildings.

SP608; 1981 May. 69-81. Loxley, T. E. Solar and geothermal housing innovations via inverted cave construction system.

Key words: buildings; construction; energy conservation; ground coupled; hybrid solar energy system; inverted cave; low grade geothermal; radiant floor heating; space heating and cooling; thermal mass; walls; water source heat pump.

The cave offers many energy saving advantages, but the problems of underground construction severely limit its application. The author shows that modern materials can be used to build a house on grade as though it were a cave turned inside out. The result is a house of generally conventional appearance that functions like a cave.

The system consists of a highly insulated exterior wall and roof surrounding a central core of high thermal mass. Insulation extending to the base of the foundation isolates the house from outside temperature fluctuations. A concrete floor and central masonry wall then thermally couple the home interior to the underlying subsoil.

Reduced heating and cooling loads and the nature of the design introduce climate control options not possible with conventional construction. A piping loop placed directly beneath the slab floor permits the storage and circulation of water for use with a highly efficient water source heat pump. A modestly sized solar array is used in a simple, attractive arrangement to heat the loop water. This provides a radiant floor heating effect and boosts system efficiency.

Readily compatible with today's quality builders, this versatile system concept costs little more than current energy wasting construction.

SP608; 1981 May. 83.103. Forest, A. Basic human values and 
energy conserving lifestyles or the implications of California's innovative new Class $K$ code.

Key words: alternative; California; Class $\mathbf{K}$ code; conservation; discrimination; energy; United Stand.

This report stresses the energy conserving implications of California's innovative new "Class K" code provision for primitive architecture and alternative technologies. The philosophy of United Stand, proponent of the "Class K" code, is here highlighted in the interest of energy conservation, and owner-builders across America who wish to maintain basic human values and energy conserving lifestyles.

We now propose a similarly conceived national code that will specifically mandate the right of owner-builders to employ alternative technologies in their own homes-a new code which will spell our functional performance rather than prescribe specific technology; a code written briefly, and simply, to provide a rule of thumb, rather than repression; a code which will legalize and facilitate low-cost, low-technology, energy. efficient housing, rather than discriminate against it. We ask for a code specifying the right of homeowner-builders to turn "OFF" a portion of our Nation's energy - their own.

SP608; 1981 May. 105-112. Lord, D. Setting standards for building energy performance in Hawaii.

Key words: building energy performance; codes; energy conservation; energy policy; Hawaii; performance standards.

The State of Hawaii is unique in its energy supply and energy consumption patterns. Precariously dependent on imported petroleum, Hawaii has the potential for meeting much of its energy needs through the development of its solar, wind, geothermal, and ocean resources. These issues will play important roles in the formulation of policy in the statewide approach to energy conservation in buildings. The proposed Building Energy Performance Standards (BEPS) and the existing American Society of Heating, Refrigerating and AirConditioning Engineers (ASHRAE) 90.75 standard have raised questions among design professionals and at the State planning level about appropriate climatic criteria, design energy budgets and weighting factors for fuels. The climate of Hawaii was not represented in the Baseline Energy Study by the American Institute of Architects (AIA) Research Corporation and does not appear in the list of Standard Metropolitan Statistical Areas (SMSA) in the Notice of Proposed Rulemaking for BEPS. Weighting factors developed by the U.S. Department of Energy (DoE) for the various fuels used for lighting, cooling, and water heating seem to be inappropriate for Hawaii.

A modified BEPS approach is proposed which would probably offer advantages over the present ASHRAE 90.75 based building code. This would encourage the use of renewable energy and passive techniques in meeting design energy budgets and in reducing the State's dependence on imported fossil fuels.

SP608; 1981 May. 113-122. Anderson, J. M.; Evans, P. M.; Smith, R. L. A simplified low cost technique for evaluating building energy performance standards submittals.

Key words: BLAST; building energy performance standards; computer modeling; maintain accuracy; reduce cost; reduce input; standard evaluation technique.

The Building Energy Performance Standards propose a "Standard Evaluation Technique" based almost entirely on the modeling of energy performance by computer techniques. This modeling requires elaborate data, manipulation of complex data bases, large amounts of memory, and is feasible only with the latest in computer technology. Currently, where such technology is available, its use is prohibitively expensive.

A study was made on the University of Illinois CYBER 175 computer using an energy analysis program similar to the one required by BEPS. This study found, for the test case, that lines of input could be reduced by up to 77 percent, that the number of surfaces needed to describe the building could be reduced by 80 percent, and that the computer cost of running this analysis could be lowered by 75 percent. It was found that all of these reductions could be made and still maintain a high degree of accuracy (within six percent) as compared to the thorough base run.

The results indicate a large potential for the reduction of building code officials' time in preparing the computer input and for the reduction in cost for running such energy evaluation programs on large computers.

SP608; 1981 May. 123-132. Glickman, D. S.; Terry, D. L. Energy audits of public buildings in Illinois.

Key words: cost; construction; energy audit; energy conservation; Illinois; maintenance; public buildings; state government.

The Illinois Capital Development Board is a State governmental board which manages the construction ol $\$ 200-400$ million ol public buildings and related facilities per year.

This paper will discuss the technical experience gained in the energy audit program wherein approximately 277 public buildings have been audited and recommendations made to change operating and maintenance procedures and make construction changes. When implemented, the changes, estimated to cost $\$ 5$ million will save approximately $\$ 75$ million over a 15 year period.

Types of buildings audited to date include dormitories, schools, dietary facilities, hospitals, offices, steam generation plants, and general and mechanical stores. Approximately 10.3 million gross square feet (GSF) have been audited. The largest building audited was $444,000 \mathrm{GSF}$, the smallest $1500 \mathrm{GSF}$.

The cost of performing the audits is, on the average, repaid in 2 years by the savings realized from implementation of recommended operating and maintenance changes alone. This program was praised by Region V U.S. Department of Energy (DoE) Headquarters, Chicago, Illinois.

Also discussed will be the Technical Research Unit, its energy functions and goals.

SP608; 1981 May. 133-138. Kainlauri, E. O. A professional approach to class $A$ and school and hospital energy audits, with case studies.

Key words: certification; Class A energy audit; economic analysis; energy analysis; energy conservation; Federal programs; government buildings; hospitals; rate of return; schools; technical assistance.

The State of Iowa has probably one of the toughest but most appropriate programs of Class A Energy Auditor Certification. As a first requirement the applicant must be either a registered architect or engineer. Secondly, he or she must take a 3-day training course organized by an advisory committee composed of representatives from the professions and industry and from the Iowa State University (ISU), and finally pass an all-day test which includes a partial energy audit with instrumentation. The training courses have been given seven times since late 1978, and additional courses are scheduled in the future. Nearly 200 Class A Energy Auditors have so far "graduated." Special courses are also given on school and hospital energy auditing. The first part of this paper will deal with the training aspects and requirements.

The second part of the paper will concentrate on results of these audits which are both quantitative and qualitative. Several hundred energy audits on government buildings, schools, and hospitals have taken place, partly financed by Federal programs. Information on Iowa buildings is being computerized and analyzed.

At a recent case study workshop, specific aspects were highlighted, including a number of building environmental topics, decision making criteria, computer applications, audit procedures, 
cost of audits and energy conservation measures, and codes and standards. The impacts on finances, building energy consumption, personnel, and the profession were discussed. Means to improve results of future energy audits by sharing experiences was the main objective. The results are encouraging.

SP608; 1981 May. 139-157. McCarney, S. A simplified procedure for the use of solar energy in complying with the Colorado energy conservation standards for residential buildings.

Key words: BEPS; code compliance; code conflicts; energy conservation standards; solar energy; trainee performance testing.

This study reviews the history of the residential energy conservation building standards in Colorado. Attendant educational efforts are described. Results from performance testing of trainees are presented. Numerous sources have requested simplified code compliance methodologies. Presented is a simplified strategy for code compliance through the use of solar energy. Code conflicts with solar are identified. The effects of the Building Energy Performance Standards (BEPS) on solar use are briefly discussed.

SP608; 1981 May. 159-166. Gaines, R. S.; McLafferty, P. S. Solar systems facilitated by the proper application of existing building codes.

Key words: building codes; building officials; codes and standards; convective loops; greenhouses; heat exchangers; light and ventilation; solar systems; space heating and cooling; training programs.

The California Energy Commission (CEC) and the California Building Officials, Inc. (CALBO) have jointly developed a manual designed to assist local building officials in identifying, understanding, and completing a code analysis of solar systems presented to building departments for permit approval. Published by the International Conference of Building Officials (ICBO), the Solar Systems Code Review Manual provides both building departments and builders a set of clear guidelines to be followed by specifying the code sections which will be applied in the plan check and inspection processes. The manual is based on the rationale that the plumbing, structural, electrical, and mechanical components of both solar and conventional space conditioning and water heating systems are largely similar and can, therefore, be regulated by existing codes and standards. Several code items have been identified which, if rigidly enforced, could serve to inhibit the implementation of solar systems, and a process has been developed for resolving such problems without sacrificing important health and safety standards.

SP608; 1981 May. 167-172. Trant, B. S. Development of recommended requirements to code officials for solar heating, cooling and hot water systems.

Key words: building codes; building officials; consensus; construction; document; installation; recommended requirements; solar energy; solar systems.

Under a Department of Energy contract, the Council of American Building Officials has developed "Recommended Requirements for Code Officials on Solar Heating, Cooling and Hot Water Systems." The recommended requirements treat all the major code categories-building, electrical and mechanical/plumbing.

These requirements will serve a guide for building code officials to judge the health and safety aspects of solar systems and their proper installations. Where requirements exist in a major model code they are referenced with commentaries provided for technical backup or where further explanation is needed.

The development of the recommended requirements through the consensus process is described.
SP608; 1981 May. 173-178. Williams, S. A method for retaining an experimental attitude in solar codes.

Key words: deed; developers; energy wars; experimental code; field test; guideline; low income; non-code; policymaking; protestors; solar code; victim role.

The viability of the incipient solar age will in large part be determined by humankind's ability to learn new technologies. The basis of leaning is being able to experience the failure or success of one's ideas. For innovative structures, the most direct and readily available means of experiencing their workability is to live in them. Proposed solar building codes are based on our existing solar methodology, thereby leaving little room for a person wanting to experiment with a new idea on his/her own home.

In order to solve the problem of the code becoming a barrier to further development of solar technology, the author proposes recording on the property deed a notation stating that the property has a building whose solar system is not according to code.

A 2-year field trial of the Recommended Requirements to Code Officials on Solar Heating, Cooling, and Hot Water Systems, developed by the Council of American Building Officials, is suggested to enable the public and building inspectors time to acquaint themselves with it and work out any bugs.

SP608; 1981 May. 179-181. Wrenn, P. State Government roles in national solar collector labeling, certification, and rating.

Key words: certification; consumer protection; Interstate Solar Coordination Council; labeling procedures; national testing program; regulation; solar collector; standards; voluntary program.

Dr. Ron Doctor of the California Energy commission and Dr. David Block of the Florida Solar Energy Center initiated a program in late 1979 to encourage reciprocity between states in their regulations concerning solar collector certification and labeling. Florida and California had both instituted testing and certification programs at that time which were required for their tax credits for solar devices and used for other regulatory purposes. Also, the Solar Energy Industries Association (SEIA) and the Air-conditioning and Refrigeration Institute (ARI) had come up with two additional labeling and certification programs. Potential confusion and duplication of effort seemed to be developing so a meeting was called of 25 States active in solar energy matters.

Several meeting have now been convened and remarkable progress has been made toward the goal of a single national program for rating, labeling and certifying solar collectors, and eventually, for complete solar systems. The four programs now in existence (both State and industry) were represented, as well as about 30 State solar offices which have been considering implementing such programs.

At an April 1980 meeting there was a preliminary agreement that there should be a single national system, run by a non-profit corporation with a Board of Directors composed of individuals nominated by SEIA and ARI and representatives of the State Government consensus effort initiated by Dr. Block and Dr. Doctor.

This paper details progress to date and projects completion dates for a national testing program, labeling program, and rating program for solar collectors. An applications manual is also planned, as well as additional work on systems and eventually certification.

SP608; 1981 May. 183-194. Key, W. P.; Potter, T. Residential conservation service program solar model audit.

Key words: auditor training; energy conservation; program 
measure; Residential Conservation Service; Solar Model Audit.

The Residential Conservation Service (RCS) has been developed by the Department of Energy (DoE) as a requirement of the National Energy conservation Policy Act (NECPA). The goal of the program is to reduce non-renewable energy use in the Nation's homes through increased application of conservation methods and the use of solar and wind energy systems to displace conventional energy sources.

The DoE program includes development of a model audit to assist the States in preparation of the State plans for implementation of RCS. Oak Ridge National Laboratory managed the model audit development effort with the Solar Energy Research Institute (SERI) assigned responsibility for development of the solar and wind energy systems portions of the audit.

This paper discusses the solar model audit development and the potential impact for homeowners, utilities, and solar suppliers/contractors. An overview of the solar model audit reviews the regulation requirements pertinent to solar with emphasis on standards, warranties, and installation considerations. A discussion is presented on the program impact on utilities, especially with regard to identifying sufficient numbers of qualified auditors and the training of these auditors. The active and passive solar and wind energy portions of the audit are described including data bases used, assumptions for respective solar or wind system measures, and examples of the audit use on a field trial home.

SP608; 1981 May. 195-200. Heinemeyer, R. R. Building codes vs. the design of passive solar heat storage.

Key words: codes; concrete; masonry; prohibitions; reexamination; thermal storage; wood structure.

This paper explores the difficulties in complying with the major national building codes when designing thermal mass storage elements into a low-mass (wood frame), direct-gain passive solar building. Experience with one of the first premanufactured passive solar buildings in the United States (the Boise Cascade solar prototype house recently erected near Denver) is presented. Different strategies for the design of thermal mass elements and their acceptability to code administering authorities are discussed.

SP610. Knight, N. H., ed. Telecommunication technologies, networking and libraries. Proceedings of a Conference on Telecommunication Technologies, Networking and Libraries, held at the National Bureau of Standards; 1977 June 3; Gaithersburg, MD. Nat. Bur. Stand. (U.S.) Spec. Publ. 610; 1981 December. 90 p. SN003-003. 02347.7.

Key words: computer network interconnection; computer network protocol; digital transmission of information; electronic message transmission; image transmission; information networks; national bibliographic network; satellite communications; slowscan television; telecommunications policy; teleconferencing; videodiscs.

The conference provided an overview of current and developing technologies for digital transmission of image data that are likely to have an impact on the operations of libraries and information centers or provide support for information networking. Technologies reviewed include slow-scan television, teleconferencing, and videodisc. Other papers discuss technology and standards development for computer network interconnection through hardware and software, particularly packet-switched networks; computer network protocols for library and information service applications; the structure of a national bibliographic telecommunications network; and the major policy issues involved in the regulation or deregulation of the common communications carriers industry. These proceedings include the following papers (indented):
SP610; 1981 December. 1-8. Cotton, 1. W. Network architecture.

SP610; 1981 December. 9.12. Darby, L. F. Competition vs. monopoly in telecommunications.

SP610; 1981 December. 13-16. Johnson, R. B. Competition vs. monopoly in telecommunications.

SP610; 1981 December. 17.27. Avram, H. D. The National Bibliographic Telecommunications Network.

SP610; 1981 December. 28-38. Little, J. L. Progress in the development of computer network protocols for library and information service applications.

SP610; 1981 December. 39-52. Meyer, S. L. Telecommunications and the expansion of information services.

SP610; 1981 December. 53-60. Belden, T. G. Uses of teleconferencing in crisis and warning situations.

SP610; 1981 December. 61.65. Henderson, E. Interactive telecommunications via satellite.

SP610; 1981 December. 66-73. Lee, R. W. H. Telecommunication R\&D-Implications and support for library programs.

SP610; 1981 December. 74-79. Warnar, R.; Henderson, M.; Berger, P. Videodiscs-A fast retrieval, mass storage media for libraries.

SP611. French, J. C. New technology challenges metrology. Nat. Bur. Stand. (U.S.) Spec. Publ. 611; 1981 July. 90 p. SN003-003-02341-8.

Key words: calibration; electrical; electronics; engineering; hazards; measurement; metrology; National Bureau of Standards; power; radiation; semiconductor; technology.

Assurance of measurement quality to meet requirements for regulatory agencies, marketplace equity, and productivity and quality control in manufacturing and research is challenged by unprecedented demands for sensitivity, speed, precision, and accuracy over wide ranges of properties and signal characteristics. New approaches are needed. Examples of NBS response, principally in electrical and electronic engineering, are described.

SP612. Sherwood, G. B. Standard reference data publications 1964 1980. Nat. Bur. Stand. (U.S.) Spec. Publ. 612; 1981 November. 121 p. Available from: NTIS; PB 82-134362.

Key words: atomic and molecular properties; bibliographies; chemical kinetics; evaluated data; fluid properties; indexes; publication list; solid state; thermodynamic and transport properties.

This document provides a comprehensive list of the outputs of the National Standard Reference Data System (NSRDS) with author, materials, and property indexes for the years 1964-1980. NSRDS data centers prepare evaluated data bases of physical and chemical properties of substances. The program is managed by the National Bureau of Standards' Office of Standard Reference Data. Data bases are available in printed form, on magnetic tapes, and through online computer networks.

SP613. Clark, H. E. Requirements for an effective national nonionizing radiation measurement system. Nat. Bur. Stand. (U.S.) Spec. Publ. 613; 1981 June. 41 p. SN003.003.02335-5.

Key words: calibration; dosimetry; electric fields; electromagnetic interference; exposure history; instruments; magnetic fields; nonionizing radiation; regional calibration laboratories; standards; techniques.

The report provides a detailed assessment of the capabilities, 
limitations, and requirements of the National Nonionizing Radiation Measurement System. The priorities of these measurement requirements are assessed according to their ability to contribute (1) to the core competence of determining the electric and magnetic fields of a source or (2) to the associated capabilities for (a) generating and applying reference fields, (b) characterizing sources and reflectors, or (c) recording exposure histories. The report examines these measurement capabilities at all frequencies between dc and 300 $\mathrm{GHz}$. These measurement requirements are reviewed in the context of the overall Federal Program for Nonionizing Electromagnetic Radiation Safety. The report concludes that the need to develop and improve instrumentation, measurement standards, calibration services and standardized measurement techniques far outweighs the need to establish regional calibration laboratories at this time. The study was conducted with the assistance of the Conference of Radiation Control Program Directors, Inc. in response to a recommendation by the Senate Committee on Commerce, Science, and Transportation.

SP614. Meijer, P. H. E.; Mountain, R. D.; Soulen, R. J., Jr., eds. Sixth International Conference on Noise in Physical Systems. Proceedings of a Conference held at the National Bureau of Standards; 1981 April 6-10; Gaithersburg, MD. Nat. Bur. Stand. (U.S.) Spec. Publ. 614; 1981 September. 419 p. SN003-003-02353-1.

Key words: hot carrier noise; noise; noise in devices; noise measurement; quantum noise; $1 / \mathrm{f}$ noise.

This document contains the full text of papers submitted to the Sixth International Conference on Noise in Physical Systems. There are six categories of papers: Theory; Devices; 1/f Noise; Applications and Measurement Techniques; Quantum Noise; Hot Carrier Noise. These proceedings include the following papers (indented):

SP614; 1981 September. 3-11. van Vliet, K. M. Classification of noise phenomena.

SP614; 1981 September. 12-17. Landauer, R. Fundamental physical limitations of the computational process.

SP614; 1981 September. 18-21. Shlesinger, M. F.; Hughes, B. D.; Montroll, E. W. Fractal random walks.

SP614; 1981 September. 22-25. Frehland, E. Transport fluctuations around non-equilibrium steady states in discrete systems.

SP614; 1981 September. 26-29. Sancho, J. M.; San Miguel, M.; Katz, S.; Gunton, J. D. Multiplicative noise in stochastic differential equations: A numerical study.

SP614; 1981 September. 30-33. Mimaki, T.; Sato, H.; Tanabe, M. Level-crossing intervals of a Gaussian noise.

SP614; 1981 September. 34-37. Celasco, M.; Mazzetti, P.; Stepanescu, A. Study of disordered structures as a spatial noise problem.

SP614; 1981 September. 38-41. Buckingham, M. J. On the theory of ambient acoustic noise in the shallow ocean.

SP614; 1981 September. 42-46. Nougier, J. P.; Vaissiere, J. C.; Gasquet, D. Mathematical formulation of the impedance field method. Application to the noise of the channel of field effect transistors.

SP614; 1981 September. 47-50. Sautereau, J. F.; Graffeuil, J.; Martin, J. C. Time domain large signal noise modeling in microwave oscillators.

SP614; 1981 September. 51-53. Walma, A. A. Spectral superposition and irreversibility.

SP614; 1981 September. 54.56. Walma, A. A. Classical noise and non-linearity.
SP614; 1981 September. 57-60. Bergman, D. J.; Ben-Jacob, E.; Schuss, Z. Fluctuations and transition rates in time dependent dissipative steady states.

SP614; 1981 September. 63-66. Sodini, D.; Rigaud, D. A new approach to model channel "thermal" noise of the JG. Fet considered as an active $\mathrm{R}-\mathrm{C}$ line.

SP614; 1981 September. 67.70. Liu, S. T.; Huang, J. S. T. Noise and scaling properties of subthreshold MOSFETs.

SP614; 1981 September. 71-74. Cox, 'C. E.; Kandiah, K. The dependence of the low frequency noise of JFETs on device parameters and operating conditions.

SP614; 1981 September. 75.78. Kandiah, K.; Deighton, M. O.; Whiting, F. B. The characteristics of noise due to individual defects in JFETs.

SP614; 1981 September. 79.83. Mehta, H.; van Vliet, K. M. Noise due to fast surface states in MOSFETs.

SP614; 1981 September. 84-87. Graffeuil, J.; Sautereau, J. F.; Tantrarongroj, K. Low frequency noise in GaAs FET's at low drain voltages.

SP614; 1981 September. 88.92. van der Ziel, A. Thermal noise in the semiconductor regime of double-injection diodes.

SP614; 1981 September. 93-96. Aoki, K.; Miyamae, K.; Kobayashi, T.; Yamamoto, K. Photocurrent noise caused by impact ionization of neutral donors, and free and bound excitons in n-GaAs.

SP614; 1981 September. 97-99. Knott, K. F. Low energy electron beam investigation of planar transistors using scanning electron microscopy with particular reference to burst noise.

SP614; 1981 September. 100-104. Sikula, J.; Sikulova, M.; Vasina, P.; Koktavy, B. Burst noise in diodes.

SP614; 1981 September. 105-108. Alabedra, R.; Walma, A. A.; Maille, C.; Lecoy, G. Non-white multiplication noise in the dark current regime of $\mathrm{A} \mathrm{P}^{+} \Pi \mathrm{MPIN} \mathrm{N}^{+}$avalanche diode.

SP614; 1981 September. 109-112. Maille, C.; Alabedra, R.; Lecoy, G.; Savelli, M. Theory and experiment of avalanche noise in the weak multiplication regime.

SP614; 1981 September. 113-117. Gong, J.; van Vliet, K. M. Noise measurements on photo avalanche diodes.

SP614; 1981 September. 118-120. Blasquez, G.; Lavernhe, F. Marty, A.; Bailbe, J. P. Noise in heterojunction transistors.

SP614; 1981 September. 121-124. Touboul, A.; Walma, A. A.; Lecoy, G. Digital analysis of superimposed fluctuations on a pulse train.

SP614; 1981 September. 125-129. Sikula, J.; Cermakova, A.; Cermak, M.; Vasina, P. Noise in dielectric materials.

SP614; 1981 September. 133-142. Weissman, M. B. Survey of recent $1 / \mathrm{f}$ noise theories.

SP614; 1981 September. 143-146. Musha, T. 1/f fluctuations in biological systems.

SP614; 1981 September. 147-150. Scofield, J. H.; Darling, D. H.; Webb, W. W. 1/f noise in continuous metal films is not due to temperature fluctuations.

SP614; 1981 September. 151. Kilmer, J.; van Vliet, K. M.; 
Chenette, E. R.; Handel, P. H. Temperature response and correiation of $1 / \mathrm{f}$ noise in transistors.

SP614; 1981 September. 152. Black, R. D.; Weissman, M. B.; Fliegle, $F$. M. Lack of spatiai cross-correlation in $1 / f$ noise in chrome films.

SP614; 1981 September. 153-156. Pavlin, I.; Engelsberg, S. Temperature fluctuations in a steady state.

SP614; 1981 September. 157.160. Machlup, S. Earthquakes, thunderstorms, and other $1 / \mathrm{f}$ noises.

SP614; 1981 September. 161-164. Ngai, K. L.; Liu, F. 1/f diffusion noise.

SP614; 1981 September. 165-168. Ngai, K. L. Temperature dependence of the excess $1 / \mathrm{f}$ noise in metais.

SP614; 1981 September. 169.172. Bell, D. A. The roie of mobility in $1 / f$ noise.

SP614; 1981 September. 173-177. Jindal, R. P.; van der Ziel, A. A modei for $1 / \mathrm{f}$ mobility fluctuations in eiementai semiconductors.

SP614; 1981 September. 178-180. Zaklikiewicz, A. M. Common mechanism of $1 / f$ noise and burst noise.

SP614; 1981 September. 181-184. Bliek, L. M. 1/f voitage noise caused by scattering of eiectrons in metais.

SP614; 1981 September. 185.188. Pellegrini, B. Compiements of $1 / f$ noise theory.

SP614; 1981 September. 189.191. Uebersfeld, J.; Handel, P. H.; Gagnepain, J. J. Fluctuations of the reiaxation time as a source of $1 / f$ noise in macroscopic physicai systems.

SP614; 1981 September. 192-195. Maccone, C. 1/f noises and the Riemann-Liouvilie fractional integral/derivative of the Brownian motion.

SP614; 1981 September. 196.198. Handel, P. H.; Wolf, D.; van Vliet, K. M. Non-Gaussian ampiltude distribution of thermal noise in resistors with $1 / \mathrm{f}$ noise.

SP614; 1981 September. 199.201. D'Amico, A.; Cappuccio, G.; Petrocco, G.; Fotinos, C.; Stochino, G. 1/ $\mathrm{f}^{\mathrm{a}}$ noise behavior in infrared photoconductive poiycrystaliine thin flims of $\mathrm{Pb}_{\mathrm{x}} \mathrm{SN}_{1-\mathrm{x}} \mathrm{Te}$.

SP614; 1981 September. 202-205. Prudenziati, M.; Morten, B.; Masoero, A. Temperature dependence of $1 / f$ noise in thick-flim resistors.

SP614; 1981 September. 206-209. Jones, B. K. Excess conductance noise in silicon resistors.

SP614; 1981 September. 210-212. Amberiadis, K.; van der Ziel, A.; Rucker, L. M. 1/f noise in gate-controiled impianted resistors.

SP614; 1981 September. 213.216. Krusin-Elbaum, L.; Voss, R. F. Low frequency voitage noise in smali area Josephson junctions.

SP614; 1981 September. 217.220. van den Berg, R. J.; de Vos, A.; de Goede, J. Concentration fluctuations in smali voiumes of ionic soiutions.

SP614; 1981 September. 221.225. Min, H. S. 1/f noise in MOS transistors in ohmic region under strong inversion.

SP614; 1981 September. 226-229. Park, H. S.; van der Ziel, A. 1/f noise modeis in MOSFETs.

SP614; 1981 September. 230-233. Gentil, P.; Mounib, A. 1/f noise in short channei MOSTs.

SP614; 1981 September. 234-235. Hiatt, C. F.; van der Ziel, A.; Zijlstra, R. J. J. Theoretical interpretation of low $1 / f$ noise in JFETs.

SP614; 1981 September. 236-239. Suh, C. H.; van der Ziel, A.; Jindal, R. P. 1/f noise in GaAs MESFETs.

SP614; 1981 September. 240-243. Kleinpenning, T. G. M. 1/f noise of hot carriers.

SP614; 1981 September. 244-247. Vandamme, L. K. J.; Dik, L. S. H. Bias-temperature treatment, surface state density, and $1 / \mathrm{f}$ noise in MOSTs.

SP614; 1981 September. 248-250. Sikula, J.; Vasina, P.; Koktavy, B.; Chobola, Z. 1/f noise in Schottky barrier diodes.

SP614; 1981 September. 253.259. Ulrich, B. T. Josephson junctions, piasma physics, and eiectron temperatures.

SP614; 1981 September. 260-268. Blalock, T. V.; Shepard, R. L Survey, appiications, and prospects of Johnson noise thermometry.

SP614; 1981 September. 269-271. Klempt, G.; Storm, L. Determination of the thermodynamic temperature of fixed points by means of a highiy accurate noise thermometer.

SP614; 1981 September. 272.275. Pickup, C. P. A precision noise thermometer for temperature scale studies.

SP614; 1981 September. 276-282. Zijlstra, R. J. J.; Westera, W Eiectro-acoustical noise.

SP614; 1981 September. 283.287. Swinney, H. L.; Roux, J. C.; King, G. P. Experimental studies of noise in a chemical reaction and in a fluid flow.

SP614; 1981 September. 288.291. Gollub, J. P. Dynamical noise in tunnel diode osciliator systems.

SP614; 1981 September. 292-297. Giffard, R. P.; Michelson, P. F.; Taber, $R$. C. Nolse in resonant gravitational wave detectors.

SP614; 1981 September. 298.301. Bonifazi, P.; Bordoni, F.; Pallottino, G. V.; Pizzella, G. Measurement of the Brownian noise of a harmonic osciliator with mass $\mathbf{M}=389 \mathrm{Kg}$.

SP614; 1981 September. 302-304. Blasquez, G. Two-port noise characterization by means of a feedback method.

SP614; 1981 September. 305.308. Gasquet, D.; Vaissiere, J. C.; Nougier, J. P. New method for wide band measurement of noise temperature of one-port networks at high pulsed bias.

SP614; 1981 September. 309.313. Difilippo, F. C.; Pineyro, J. J. Appiication of the theory of random matrices to a reactor noise probiem.

SP614; 1981 September. 314-316. D'Amico, A. Burst noise measurements on op amp's by R. F. SQUID.

SP614; 1981 September. 317.323. Grosse-Nobis, W. Domaln walis in ferromagnetic materiai and irreversibie magnetization processes.

SP614; 1981 September. 324-327. Bertotti, G.; Fiorillo, F.; Sassi, $M$. P. Barkhausen noise and domain structure dynamics in $3 \%$ SiFe single crystais.

SP614; 1981 September. 328.331. Bertocci, U. Noise in electrochemical systems.

SP614; 1981 September. 332.334. Haemmerli, A.; Janata, J.; Brophy, J. J. Ion noise in ISFETs.

SP614; 1981 September. 335.338. Corti, M. Quasielastic light 
scattering from macromolecules and micelles.

SP614; 1981 September. 339-342. Miraldi, E.; Trossi, L.; Valabrega, P. T.; Oldano, C. Frank elastic constant ratios evaluation in MBBA by intensity measurement of the scattered light.

SP614; 1981 September. 343-345. van der Meulen, J. P.; Zijlstra, R. J. J.; van Kooten, J. J. The determination of elastic constants of nematic liquid crystals from noise measurements of scattered laser light.

SP614; 1981 September. 349-354. Tucker, J. R. Quantum noise limited detection in tunneling devices at millimeter wavelengths.

SP614; 1981 September. 355-358. Leggett, A. J. Quantum tunnelling and noise in SQUIDs.

SP614; 1981 September. 359-363. Koch, R. H.; van Harlingen, D. J.; Clarke, J. Quantum noise in Josephson junctions and SQUIDs.

SP614; 1981 September. 364-368. van der Ziel, A. Quantum noise effects at high frequencies and low temperatures.

SP614; 1981 September. 369-372. Tesche, C. D. A thermal activation model for the DC-SQUID.

SP614; 1981 September. 373-375. Kurkijarvi, J. Ultimate sensitivity of an AC-SQUID.

SP614; 1981 September. 376-380. Ben-Jacob, E.; Schuss, Z. The mean lifetimes of the meta-stable states of the DC-SQUID and its I-V characteristics.

SP614; 1981 September. 381.383. Ben-Jacob, E.; Bergman, D.; Carmeli, B.; Nitzan, A. External field effect on particle diffusion above a potential barrier.

SP614; 1981 September. 384-387. Dirks, H. M.; Beckstette, K. F.; Heiden, C. Flux flow noise during inhomogeneous vortex-motion. Dependence on DC-velocity field.

SP614; 1981 September. 388-390. Beckstette, K. F.; Dirks, H. M.; Heiden, C. Flux flow noise during inhomogeneous vortex-motion. Magnetic field and temperature dependence.

SP614; 1981 September. 391-394. Knoedler, C. M.; Voss, R. F. Horn, P. M. Vortex noise in two dimensional superconducting films.

SP614; 1981 September. 397-405. Nougier, J. P. Modeling noise of devices working under hot carrier conditions.

SP614; 1981 September. 406-408. Bareikis, V.; Galdikas, A.; Miliusyte, R.; Viktoravicius, V. Noise of hot holes in Ge due to predominant inelastic scattering.

SP614; 1981 September. 409.413. Bosman, G.; Zijlstra, R. J. J.; van $R$ heenen, A. Hot carrier transport noise in p-type silicon.

SP614; 1981 September. 414-416. Reggiani, L.; Brunetti, R.; Jacoboni, C. Monte Carlo calculation of hot-electron noise in Si at $77 \mathrm{~K}$.

SP615. Lederer, P. S. Sensor handbook for automatic test, monitoring, diagnostic, and control systems applications to military vehicles and machinery. Nat. Bur. Stand. (U.S.) Spec. Publ. 615; 1981 October. 450 p. SN003-003-02372-8.

Key words: ATE; automatic test equipment; calibration; data acquisition; durability; dynamic calibration; environmental testing; evaluation; measurand; reliability; sensor; transducer.

The Sensor Handbook is intended as a guide for those who design, specify, use, and test military automatic test equipment containing sensors. The handbook addresses measurands and principles of measurement, data acquisition, sensor calibration and testing, environmental considerations, stability, durability, reliability, and error assessment. Sensor manufacturers and sensor calibration and evaluation resources are included, as is an annotated bibliography. The handbook is based largely on the present, proved state-of-the-art. Possible future trends are briefly discussed. The handbook is addressed to the general engineer, system designer, or manager with an engineering background. It does not provide the highly detailed technical information needed by the measurement engineer, although ample references are included for further study.

SP616. Gass, S. I., ed. Validation and assessment of energy models. Proceedings of a Symposium held at the National Bureau of Standards; 1980 May 19-21; Gaithersburg, MD. Nat. Bur. Stand. (U.S.) Spec. Publ. 616; 1981 October. 248 p. SN003-003-02373-6.

Key words: assessment; composite models; data quality; energy models; mathematical models; model confidence; model credibility; policy models; sensitivity analysis; validation.

The Symposium on Validation and Assessment of Energy Models, held at the National Bureau of Standards (NBS), Gaithersburg, MD (May 19-21, 1980), was sponsored by the Energy Information Administration (EIA), of the Department of Energy (DOE), Washington, DC. The symposium was organized by NBS' Operations Research Division with a two-fold agenda: (1) to summarize the recent ideas and advances of model validation and assessment that have been applied to DOE energy models, and (2) to hold workshops on key open questions that are of concern to the validation and assessment research community. Speakers addressed current and future practices, the EIA model validation program, model structure and data, and model credibility. Full-day workshop sessions were held on the following topics: validating composite models, the measurement of model confidence, model structure and assessment, sensitivity and statistical analysis of models, and model assessment methodologies. This volume documents the symposium proceedings and includes the formal papers presented, discussant comments, panel discussions and questions and answers, and summaries of the issues and conclusions reached in the workshops. These proceedings include the following papers (indented):

SP616; 1981 October. 7-24. Wood, D. O. Energy model evaluation and analysis: Current practice.

SP616; 1981 October. 25-41. Greenberger, M. Humanizing policy analysis: Confronting the paradox in energy modeling.

SP616; 1981 October. 43-47. Hoffman, K. Remarks on Wood and Greenberger papers.

SP616; 1981 October. 49-63. Richels, R. Building good models is not enough.

SP616; 1981 October. 65-77. Lady, G. M. Quality control for analysis.

SP616; 1981 October. 79.86. Hausman, J. A. Validation and assessment procedures for energy models.

SP616; 1981 October. 87-89. Jackson, R. H. F. Energy model assessment procedure development project.

SP616; 1981 October. 93-116. Freedman, D. Are energy models credible?

SP616; 1981 October. 117-120. Glassey, C. R.; Greenberg, H. J. Model credibility: How much does it depend upon data quality?

SP616; 1981 October. 139-147. Kydes, A. S.; Rubin, L. Workshop report 1-Composite model validation.

SP616; 1981 October. 149-158. Joel, L. S.; Maybee, J. S. Workshop report 2-The measurement of model confidence.

SP616; 1981 October. 159-169. Holloway, M. L. Workshop report 
3-Model standards to aid assessment.

SP616; 1981 October. 171-181. Harris, C.; Hirshfeld, D. Workshop report 4-Sensitivity and statistical analysis of models.

SP616; 1981 October. 183-200. Gruhl, J.; Wood, D. O. Workshop report 5-Model assessment methodologies. Part A: Enumeration of validation methodologies.

SP616; 1981 October. 201-203. Woods, T. J. Workshop report 5-Model assessment methodologies. Part B: Model assessment methodologies.

SP616; 1981 October. 217.242. Goldman, A. J. Reflections on modeling and modeling assessment.

SP618. Brinckman, F. E.; Fish, R. H., eds. Environmental speciation and monitoring needs for trace metal-containing substances from energy-related processes. Proceedings of the DoE/NBS Workshop held at the National Bureau of Standards; 1981 May 18-20; Gaithersburg, MD. Nat. Bur. Stand. (U.S.) Spec. Publ. 618; 1981 November. 336 p. Available from: NTIS; PB 82-132770.

Key words: bacterial mobilization; biogeochemical cycles; biological uptake; cellular toxicity-essentiality mechanisms; chromatography; element-selective speciation; environmental monitoring; fossil fuels; metal(loid) complexes; organometallic compounds; trace element standards; waste cycling.

This book presents the Proceedings of the DoE/NBS Workshop on Environmental Speciation and Monitoring Needs for Trace MetalContaining Substances from Energy-Related Processes held at the National Bureau of Standards, Gaithersburg, MD, on May 18-20, 1981. The Workshop was sponsored by the Office of Health and Environmental Research, DoE, and the Office of Recycled Materials, NBS.

The volume contains refereed papers submitted by 24 invited speakers, along with substantially complete text of the discussion following the papers, edited by the Co-chairpersons. The Proceedings address three major topic areas forming the overall objective of the Workshop: (1) what are the general and specific types of metal- or metalloid-containing substances occurring in energy-related process materials such as coals, oil shales, and waste products? (2) what are the current status and future prospects of element- and compoundspecific measurement methods suitable for speciation of trace (ppm, $\mathrm{ppb}$ ) metal- and metalloid-containing substances transmitted to the environment by energy-processing or waste cycling technologies? (3) for what toxic molecular forms of speciated process effluents should biological dose-response data be generated to assure reliable environmental impact and monitoring measurements? Main questions focused on providing assurance that the speciation methods (or their development) fit basic bioassay criteria along with appropriate standard reference materials for meeting quantitative process and waste monitoring or control needs. These proceedings include the following papers (indented):

SP618; 1981 November. 1-8. Stephens, R. D. Laboratory characterization of complex environmental contamination.

Key words: availability; environmental contamination; extraction tests; fossil fuels; laboratory characterization; metals; mining industry; public health impacts; residues; toxic elements.

A major scientific task before us is that of assessing the environmental and public health impacts resulting from the generation and disposal of large volumes of residues containing toxic elements. These residues, which emanate primarily from fossil fuels and the mining industry, contain toxic elements in widely varying concentrations and which naturally occur geologically. Questions of impact, therefore, generally center on what concentrations of specific elements pose a hazard to the environment, and what is the "availability" of specific elements for environmental contamination. Environmental availability relates to the basic chemistry of the elements. Impact assessments considering these factors shall be discussed with several current laboratory tests illustrated.

SP618; 1981 November. 9.20. Yen, T. F. The role of metalheteroatom complexes in fossil fuel production.

Key words: fossil porphyrins; fuel conversion; fuel extraction; heterocyclics; metal chelates; metallo-complexes; origin of pollutants; pollutant properties; synfuel.

Many metal-containing organic species of biological origin are present in the precursors of fossil fuel deposits which have undergone geological transformations through biostratinomy (change after death) and taphonomy (fossilization). The reactions of these molecules during extraction, recovery, conversion, refining, and combustion are anticipated to alter the composition and chemical nature of the fuel end-product and the pollutant byproducts. Chemical modification, decomposition, and resynthesis of complexes and the consequent structural changes of the ligands together with the donor atom-metal interactions are possible. The unique nature of nitrogen, sulfur, and oxygen bonding to various metals, before and after pyrolysis and carbonization, for coal, heavy oil, oil shale, and tar sands is discussed. Studies in this vast unexplored area are anticipated to provide insight into the planning of strategies for monitoring methodology and control technology.

SP618; 1981 November. 21-38. Filby, R. H.; Sandstrom, D. R.; Lytle, F. W.; Greegor, R. B.; Khalil, S. R.; Ekambaram, V.; Weiss, C. S.; Grimm, C. A. Chemistry of trace element species in coal liquefaction processes.

Key words: arsenic; chromium; coal hydrogenation; cobalt; liquefaction; mercury; nickel; process waters; selenium; solvent refined coal; titanium; trace elements.

A combination of chemical and physical techniques has been used to investigate trace element species in Solvent Refined Coal (SRC) solid and liquid products and in important waste streams. Neutron activation analysis, atomic absorption spectroscopy, and liquid chromatography have been used to obtain information on possible trace element species present in solid SRC I and liquid SRC II products derived from coal. EXAFS has been used to characterize Ti species in coal, SRC I, and SRC II liquefaction residues. The chemistry of volatile species of $\mathrm{Hg}$, As, and Se in the SRC processes is discussed in relation to their concentration in waste streams. The formation of metal-organic complexes by elements such as $\mathrm{Ti}, \mathrm{V}$, etc., during coal hydrogenation is discussed in relation to their chemical forms in coals.

SP618; 1981 November. 39-53. Jenne, E. A. Speciation of aqueous contaminants-Role of the geochemical model.

Key words: aqueous speciation; computerized; geochemical model; heavy metals; redox couples; trace elements.

A combination of accurate, selected analytical determinations and a competent geochemical model constitutes the only possible means of achieving a sufficiently thorough and reliable aqueous speciation of waters for the evaluation of societal risks resulting from anthropogenic inputs from energy related technologies. Thorough aqueous speciation is essential to adequately estimate the thermodynamic activity of those solute species having biological availability, hence toxicity, as well as high sorption potential. A reliable calculation of the distribution of species requires analytical determination of: 1) the quantity of each element in true solution; and 2) the amount of quantitatively and/or environmentally important aqueous species which are in disequilibrium. Because redox couples are rarely at equilibrium, the quantity of each redox state of multiple valence-state elements must be analytically determined. Due to the: 1) very numerous organic compounds which may be present, 2) the absence of 
Gibbs free energy and enthalpy data defining the association of these organic compounds with trace and major elements, and 3 ) the lack of competence of speciation-solubility models for organic ligands, the quantity of organic, trace-element complexes must, at the moment, be addressed analytically.

SP618; 1981 November. 54.64. Craig, P. J.; Rapsomanikis, S. Biogeochemical cycles of metals and metalloids-The role of methyl-cobalamin and some other chemical routes to methylation in the environment.

Key words: environment; GC.MS; heavy metals; lead; mass spectrometry; mercury; methylation.

The evidence for the role of methylcobalamin, $\mathrm{CH}_{3} \mathrm{CoB}_{12}$, as the proximate source of the methyl group in heavy metal methylation is discussed. For metals in low or zero oxidation states other methyl donors are suggested. Chemical routes to methylation of already partially methylated species are detailed, particularly for the $\left(\mathrm{CH}_{3}\right)_{3} \mathrm{M}^{+}$species $(\mathrm{M}=\mathrm{Sn}, \mathrm{Pb})$.

SP618; 1981 November. 65.80. Chau, Y. K.; Wong, P. T. S. Some environmental aspects of organo-arsenic, lead, and tin.

Key words: alkylleads; alkyltins; chemical speciation; GC-AAS techniques; LC.AAS techniques; organoarsenics; organometals; partition coefficients; toxicity.

Organometals and organometalloids such as alkylleads, alkyltins, organoarsenics have been found in environmental samples. Apart from anthropogenic sources, amazingly, some of these compounds are synthesized by biotic and abiotic processes under environmental conditions. These compounds also undergo complex chemical and biological interactions including, for example, methylation, transmethylation, hydrolysis, photolysis, degradation, etc. For these studies, speciation identification and analysis are prerequisites. The toxicity of organometals towards aquatic biota is closely related to the nature of the organic groups. Generally, toxicity increases with increasing carbon chain length, and also with the degree of alkylation on the metal nucleus. The possibility of using octanol/water partition coefficients of a number of alkyltin compounds to predict toxicity is discussed.

SP618; 1981 November. 81.92. Manahan, S. E.; Fraley, D. M.; Vogt, C.; Yates, D. Environmental chemical investigations of trace element mobilization in synthetic fuels manufacture.

Key words: coal; environmental chemistry; shale oil; synthetic fuels; trace elements.

The principles of environmental chemistry may be applied to predict pollution problems from synthetic fuels manufacture, enabling corrective action to be taken at the design stage. One of the environmental concerns in synthetic fuels manufacture is the mobilization in soluble and/or volatile forms of trace elements originally present in the solid fossil fuel feedstock. Some of the conditions that may obtain during various coal conversion or shale oil retorting operations conductive to production of mobile trace element species are a hydrogen-rich reducing atmosphere, high carbon monoxide pressures, presence of aromatic compounds, and presence of chelating molecules. It is important to determine if mobile trace element species are produced; it is equally important to determine if they are not produced to avoid unnecessary concern over nonexistent environmental problems. This chapter describes studies of potential mobilization of trace elements from coal pyrolysis, simulated in situ coal gasification, and shale oil retorting.

SP618; 1981 November. 93.104. Sievers, R. E.; Conditt, M. K.; Stanley, J. S. Speciation of organic compounds and trace elements in oil shale wastewaters.

Key words: alkylpyridines; dissolved organic compounds; gas chromatography/mass spectrometry; hydrophobic bases; metal complexation; nitrogen bases; oil shale; retort water; speciation; trace metals.

Oil shale wastewaters are complex mixtures containing a large number of trace elements and organic ligands present at potentially significant concentrations. Speciation of the trace elements in these wastes is necessary in order to effectively evaluate possible environmental impacts. Speciation methods should include size fractionation measurements for trace elements and organic ligand identifications to fully account for possible complexes, both stable and labile. Ultrafiltration of oil shale wastewater has indicated that most trace elements exist as simple hydrated molecules or as labile complexes with organic ligands. Complexing measurements have shown that oil shale wastewaters contain significant concentrations of organic ligands that are capable of forming complexes with trace elements. The organic compounds that have been identified in this work as important ligands include substituted pyridines, quinolines, and anilines. The complexing ability measurements have indicated a correlation with the nitrogen base content in oil shale wastewaters from three different and distinct operations. Specific compounds, some of which form complexes, are toxic, and are biorefractory, have been identified.

SP618; 1981 November. 105-119. Olsen, K. B.; Wilkerson, C. L.; Toste, A. P.; Hayes, D. J. Isolation of metallic complexes in shale oil and shale oil retort waters.

Key words: chromatography; direct current plasma; hydrophilic; hydrophobic; metal complex; porphyrin; shale oil; speciation; trace metals.

This paper describes and discusses methods applied to the fractionation of trace metals in shale oil by silica gel and alumina chromatography and the fractionation of retort waters by $C_{18}$ partition chromatography. Direct current plasma emission spectroscopy was used to analyze $\mathrm{Fe}, \mathrm{Ni}, \mathrm{Cu}$, and $\mathrm{Mo}$ in the oils and $\mathrm{B}, \mathrm{Zn}, \mathrm{Mo}, \mathrm{Fe}$, and $\mathrm{Mn}$ in the waters. Instrumental neutron activation analysis (INAA) was used for the determination of arsenic in the oils. In the silica gel separation most of the metals eluted in two fractions corresponding to the moderately polar and highly polar fractions of the oil. In contrast, nickel eluted in the slightly polar fraction of the oils. Further separation of the nickel-enriched fraction on alumina yielded a fraction rich in nickel-porphyrin type compounds, one of which was tentatively identified as Abelsonite porphyrin. Following $C_{18}$ Sep-pak fractionation of the retort waters, boron and manganese remained associated with the aqueous, or hydrophilic, fraction. Portions of the zinc, molybdenum and iron partitioned in the hydrophobic organic fraction of the retort waters.

SP618; 1981 November. 120.133. Risby, T. H. The use of mass spectrometry for metal analysis.

Key words: bioavailability; element speciation; gas chromatography; ionization processes; liquid chromátography; mass spectrometry.

The measurement of the bioavailability and speciation of trace elements is extremely important for the assessment of potential health effects which may occur as the result of exposure to emissions from energy-related processes. This paper presents the current state-of the-art in the use of mass spectrometry for trace analysis and also suggests areas for future research. Different methods of sample ionization are compared and contrasted and the advantages of various methods of sample introduction are discussed. Representative analyses are presented in order to exemplify the various conclusions which have been presented in this publication.

SP618; 1981 November. 134-145. Braman, R. S.; Shelley, T. J. 
Diffusional discrimination and reversible chemisorption for speciation in air.

Key words: air analysis; diffusion; hollow tubes; mercury; preconcentration.

Hollow air sampling tubes having chemisorbing interior coated walls offer several attractive features useful in preconcentration for air analysis. A high discrimination of gaseous versus particulate forms is achieved based upon the much greater diffusional activity of molecules. Since the preconcentration of gases is achieved without the use of filters, particle-gas interconversions are avoided. The dynamics of such a system have been described mathematically and form the basis for accurately determining the diffusion coefficients, and hence the molecular form of gases in air, even at sub-ppb concentrations. Several different selective chemisorbing surfaces can be used for studies of mercury, arsines, sulfur compounds, and nitrogen compounds in air.

SP618; 1981 November. 146-162. Anderson, M. A. Kinetic and equilibrium control of interfacial reactions involving inorganic ionic solutes and hydrous oxide solids.

Key words: adsorption; desorption; hydrolyzable cations; hydrous oxides; kinetics; protolyzable anions; thermodynamics.

Environmental ecosystems are largely heterogeneous with most chemical and biochemical reactions occurring at a solidliquid interface. The availability of chemical species for biological assimilation and/or physical transport is governed by factors that influence the kinetic and equilibrium controls of this partitioning. This paper addresses the current state-of-the-art models that describe interfacial reactions of inorganic ionic solutes. Discussion is divided into equilibrium versus kinetic controls over interfacial reactions. Experimental data is compared with the prediction of current models and inadequacies are assessed.

SP618; 1981 November. 163-172. Brown, T. F.; Caster, D. M.; Brown, S. D. Speciation of labile and quasi-labile metal complex systems using the Kalman filter.

Key words: cadmium-NTA; convolution voltammetry; electrochemistry; Kalman filter; metal speciation; modeling; semiderivative; stability constants; voltammetry; zinc-NTA.

One approach used in the study of trace metal speciation is the measurement of metal-complex stability constants, which are then combined with models of the metal systems under study to predict the species present. The electrochemical techniques used to measure metal complex species have the advantage of sensitivity and specificity, but suffer a number of limitations. For voltammetric techniques, these include requirements of excess ligand and rapid kinetics. We have developed a new approach to voltammetric studies of metal complexes; this approach uses the Kalman filter, a recursive, linear, digital filter, to model overlapped electrochemical peaks arising from parallel reduction paths, including the situation where two or more complexes undergo slow exchange. Stability constants are calculated from the individual electrochemical responses extracted by the filter.

This approach has been applied to studies of the complexation of NTA with various trace metals. A description of the model and results for the systems will be discussed.

SP618; 1981 November. 173-183. Crecelius, E. A.; Gurtisen, J. M.; Apts, C. W. Biological uptake and chemical speciation of copper in sea water determined by electrochemistry.

Key words: anodic stripping; bioavailability; complexing capacity; copper speciation.

The relationship between electroactive $\mathrm{Cu}$ concentrations and biological uptake of $\mathrm{Cu}$ was investigated to determine if electroactive $\mathrm{Cu}$ concentrations would better predict bioavailable $\mathrm{Cu}$ than total $\mathrm{Cu}$ concentrations. Measurements of electroactive $\mathrm{Cu}$ were made using differential-pulse anodic stripping voltammetry (DPASV) at $\mathrm{pH} 8$ with a mercury-coated glassy carbon electrode. The $\mathrm{Cu}$ complexing capacity of Pacific Northwest coastal waters averaged $18.7 \mu \mathrm{g} \mathrm{L}^{-1}$ (S.D.=5.5, $\mathrm{N}=57$ ). When $\mathrm{Cu}$ is added at concentrations that exceed the complexing capacity we observe both increased DPASV. measurable $\mathrm{Cu}$ and increased $\mathrm{Cu}$ body burdens in exposed animals. DPASV is a practical method for monitoring biologically active forms of $\mathrm{Cu}$ in contaminated waters.

SP618; 1981 November. 184-196. Uden, P. C. Specific element detection in chromatography by plasma emission spectroscopy.

Key words: chromatography; detection; metals; plasma emission; specific elements; spectroscopy.

The concept of direct interfacing of specific elemental and molecular identification devices with high-resolution gas chromatographs is established in such areas as GC/MS and GC/IR. Emission spectroscopic detection for element speciation has in recent times taken on new importance and this paper discusses some of the current trends in this field. Particular attention is paid to the interfacing of plasma emission spectrometers and the advantages of alternative systems are discussed. An atmospheric pressure helium microwave plasma is discussed for trace determinations in packed and open tubular column GC. Metal compounds and halogen compounds are covered with discussion of applications in the area of chlorinated organics in aqueous systems.

The atmospheric pressure DC argon plasma is applied for both gas chromatographic and high pressure liquid chromatographic studies involving various mobile phase combinations. An echelle spectrometer is incorporated in the system and applications include the determination of metal complexes and organometallics such as gasoline additives.

SP618; 1981 November. 197-216. Weiss, C. S.; Jewett, K. L.; Brinckman, F. E.; Fish, R. H. Application of molecular substituent parameters for the speciation of trace organometals in energyrelated process fluids by element-selective HPLC.

Key words: atomic absorption; energy-related process fluids; leachate; linear free energy; liquid chromatography; molecular substituent; oil shale; organoarsenic; organotin; retention index; speciation.

High performance liquid chromatographic separations coupled with element-selective detectors provide trace molecular speciation methods applicable to energy-related process fluids and associated waste leachates. These materials present a challenge because they contain complex matrices whose chemistries are not well understood, and the processes have the potential to generate compounds containing toxic metals and metalloids that are either unexpected or have not been previously identified. Consequently, the ability to reliably ascertain the molecular structures of such unknown substances by relatively simple measurements of their chromatographic retention properties is very desirable. Our present work shows a linear correlation of the logarithm of the chromatographic capacity factor with the linear sum of the appropriate structural substituent parameters for a number of organoarsenicals, separated by an ion-exchange mechanism, and a number of organotins, separated by a hydrophobic mechanism. An illustration of the application of this linear free energy relationship as a diagnostic in the separation of trace organoarsenicals, by ion-exchange chromatography, was performed on the analysis of process waters from oil shale retorting and on leachates from raw oil shale.

SP618; 1981 November. 217-225. Fowler, B. A. Relationships 
between trace element speciation and intracellular mechanisms of toxicity.

Key words: arsenicals; cadmium metallothionein; cellular toxicity; elemental interactions; lead inclusion bodies; methylmercury derivatives; methylation/demethylation.

The mechanisms and thresholds of trace element toxicity are highly dependent upon chemical form, differential binding to intracellular ligands, and interactive effects with other toxic or essential biological agents. In vivo methylation or demethylation conversions have been demonstrated for arsenic and mercury, respectively, but the precise influence of these processes on cellular toxicity is undefined. Major intracellular ligands, such as metallothionein for cadmium and inclusion bodies for lead, play a primary role in mediating toxicity by competing with target organelles for these elements. Interactive effects between lead and cadmium greatly reduce renal lead concentrations and formation of inclusions but not lead toxicity, thus indicating the importance of intracellular speciation to assessment under multielement exposure conditions.

SP618; 1981 November. 226-234. Packer, L.; Mehlhorn, R. J. Development of a methodology to assess organometallic effects on bioenergetic systems.

Key words: animal toxicity; breath analysis; endurance; freeradicals; iron; organometalloid toxicity; pentane; respiration.

A methodology for assessing the impact of subacute concentrations of organometallic agents on bioenergetic and oxidative damage processes in animals, cells and energy transducing subcellular organelles is described. Several of the assays are noninvasive and thus lend themselves to human tests. At the whole-animal level we utilize a treadmill chamber where physiological parameters of exercising animals are monitored. These include parameters of whole animals' work performance such as oxygen consumption, carbon dioxide evolution and endurance. Oxidative damage can be monitored in experiments by analyzing expired air of the animals for ethane and n-pentane. These alkanes correlate with lipid peroxidation in vivo. At the cellular and subcellular levels, respiratory activity, lipid peroxidation and free radical species are assayed. Respiratory activity is measured in muscle homogenates and isolated mitochondria using substrates which feed into different segments of the electron transport chain. To demonstrate how these assay procedures correlate, iron deficiency anemia in rats was analyzed. Physiologically, iron deficiency caused a $90 \%$ decrease in endurance which correlated with an $80 \%$ decrease in pyruvate-malate oxidation rates in muscle homogenates. Significant but smaller effects were seen in

hemoglobin/hematocrit levels (50\% decrease) and in maximal oxygen consumption (50\% decrease). Tissue free-radical signals observed by ESR at room temperature increased with exercise. Respiratory organelles have been shown to be a major source of endogenous free-radicals which initiate tissue oxidation; hence it is plausible that our exercise system will aggravate the effects of organometallics which act as pro-oxidants and that the proposed animal exercise system may be particularly appropriate for evaluating the potential impact of these substances on humans.

SP618; 1981 November. 235-243. Wolf, W. R. Duality of concern with trace metals in the environment-Toxic and/or essential.

Key words: environmental; essential elements; speciation; toxic; trace elements.

Both health science professionals and the general public express a high level of concern with exposure levels of "trace" metal containing substances in the environment. Although a great portion of this concern has been with the potential toxic or detrimental effects of exposure to these substances, a countering body of concern has developed regarding the nutritional or positive biological effects of a number of the "trace" elements. This duality of concern encompasses a widely overlapping list of elements. The same "trace" element can exhibit multiple biochemical roles from detrimental deficiency effects at very low levels of exposure, to beneficial optimal nutritional levels, to toxic effects at higher levels. These different biochemical roles can depend not only upon the absolute level of exposure, but also upon the interactions with varying levels of exposure of other nutrients or hazardous substances. These differing roles also depend strongly upon the differing forms or species of the element present. Levels of exposure, biotransformations, and interactions of these species must be understood, quantitated, and monitored before knowledgable recommendations on cleaning-up "toxic" elements or supplementing "nutritional" elements can be made, especially for these elements of dual concern.

SP618; 1981 November. 244-263. Irgolic, K. J.; Banks, C. H.; Bottino, N. R.; Chakraborti, D.; Gennity, J. M.; Hillman, D. C.; O'Brien, D. H.; Pyles, R. A.; Stockton, R. A.; Wheeler, A. E.; Zingaro, R. A. Analytical and biochemical aspects of the transformation of arsenic and selenium compounds into biomolecules.

Key words: arsenate; arsenic methylation; biological transformation; graphite furnace atomic absorption spectrometer; high pressure liquid chromatography; selenite.

Marine algae take up arsenic (selenium) when grown in a medium containing $10 \mathrm{ppm} \mathrm{As(arsenate)} \mathrm{[10} \mathrm{ppm} \mathrm{Se(selenite)].}$ Some of the arsenate is transformed into an arsenic-containing lipid. Selenium has been found in protein-cellulose, lipid and amino acid/carbohydrate fractions. The identification of organic arsenic and selenium compounds requires compound-specific, sensitive analytical techniques. High pressure liquid chromatography with a graphite furnace atomic absorption spectrometer as an element-specific detector is presently the most versatile technique for these analytical problems. NMR investigations of a model system for the methylation of arsenic compounds consisting of methylcobaloxime and methyldihaloarsines in chloroform solution showed that methyltransfer occurs easily and involves several steps. Work to elucidate the mechanism is in progress.

SP618; 1981 November. 264-273. Brierley, J. A. Bacterial processes for transformation of metals.

Key words: chemolithotrophic-bacteria; copper; iron; leaching; metal-reduction; metal-sulfides; mineral-oxidation; molybdenum; oil shale; Sulfolobus; Thiobacillus; uranium.

Microorganisms actively participate in the transformation of metals and metalloids by various processes including adsorption, absorption, alkylation, oxidation and reduction reactions. Bacteria of the genera Thiobacillus and Sulfolobus have a chemolithotrophic mode of metabolism and catalyze various metal transformations. The mixotrophic thiobacillus-like facultative thermophilic bacteria also participate in metal transformations. These bacteria are primarily involved in oxidation-reduction reactions of metals. Metal sulfides can be either directly oxidized by the microbes or oxidized by ferric iron, an end-product of microbial metabolism. These processes result in solubilization of iron, copper, molybdenum, uranium, and many other metals as well. In the absence of oxygen, iron and molybdenum may be reduced by microbes. Bacteria capable of these reactions are found, and they function in a variety of environments. Conditions which favor their growth are acidic $\mathrm{pH}$ (1.5 to 3.5$)$ and temperatures which can be as high as $75^{\circ} \mathrm{C}$ for Sulfolobus. The microbes have potential for mobilization of pollutants, or toxic elements, during resource extraction processes.

SP618; 1981 November. 274-286. Strandberg, G. W.; Shumate, S. 
E. II; Parrott, J. R., Jr.; North, S. E. Microbial accumulation of uranium, radium, and cesium.

Key words: cesium; metal accumulation; microbial; radium; uranium.

Diverse microbial species varied considerably in their ability to accumulate uranium, cesium, and radium. Mechanistic differences in uranium uptake by Saccharomyces cerevisiae and Pseudomonos aeruginosa were indicated. $S$. cerevisiae exhibited a slow (hours) surface accumulation of uranium which was subject to environmental factors, whereas $P$. aeruginosa accumulated uranium rapidly (minutes) as dense intracellular deposits and did not appear to be affected by environmental parameters. Metabolism was not required for uranium uptake by either organism. Cesium and radium were concentrated to a considerably lesser extent than uranium by the several species tested.

SP618; 1981 November. 286-300. Klein, D. A.; Hassler, R. A. Microbiological mobilization of arsenic from retorted oil shalesSpeciation and monitoring requirements.

Key words: arsenic; dimethylarsinic acid; microbial growth; mobilization; nutrients; oil shale; retorting; soil; speciation; volatilization.

Microbial effects on arsenic mobilization from retorted oil shales were investigated to better assess chemical speciation and analytical monitoring requirements for this type of study. Shake cultures were used for soluble arsenical monitoring while volatile products were recovered using gas flow trains with $\mathrm{KI} / \mathrm{I}_{2}$ trapping solutions. Increased microbial growth which occurred in the presence of balanced nutrients generally led to decreased supernatant arsenic levels, while nitrogen additions, which did not lead to higher microbial populations, resulted in distinct increases in supernatant arsenic. Low, but detectable, volatile arsenic release from retorted shale also occurred under aerobic conditions with added nutrients. With added dimethylarsinic acid (DMA), nutrient amendments resulted in increased As volatilization, which was highest under aerobic conditions and in the presence of retorted shale, in comparison with added soil. Monitoring and speciation requirements suggested by these studies include the need to better understand ammonium ion effects on arsenic solubilization, to develop criteria for abiotic controls, and to maintain species stability prior to sample analysis.

SP618; 1981 November. 301.324. Silver, S. Mechanisms of bacterial resistances to toxic heavy metals: Arsenic, antimony, silver, cadmium, and mercury.

Key words: antimony; arsenic; bacterial resistances; cadmium; detoxification; mercury; organomercury; plasmids; silver; toxic heavy metals.

Bacteria from many species and sources have genes that confer resistances to specific toxic heavy metals. These resistances often are determined by extrachromosomal DNA molecules (plasmids). The same resistances and mechanisms of resistances occur in bacteria from soil, water, industrial, and clinical sources. The mechanism of mercury and organomercurial resistance is the enzymatic detoxification of the mercurials into volatile species (methane, ethane, metallic $\mathrm{Hg}^{\circ}$ ) which are rapidly lost from the environment. The genetic control of this resistance has been studied and the enzymes reponsible have been purified and characterized. Cadmium resistance and arsenate resistance are due to separate blocks on the net accumulation of these toxic materials. Efficient efflux systems cause the rapid excretion of $\mathrm{Cd}^{2+}$ and $\mathrm{AsO}_{4}{ }^{3-}$. The mechanisms of arsenite and of antimony resistance, which are usually found associated with arsenate resistance, are not known. Silver resistance is due to lowered affinity of the cells for $\mathrm{Ag}^{+}$, which can be complexed with extracellular halides, SH groups, or organic compounds. Sensitivity is due to binding of $\mathrm{Ag}^{+}$more effectively to cells than to $\mathrm{Cl}^{-}$.

SP620. Bennett, H. E.; Glass, A. J.; Guenther, A. H.; Newnam, B. E., eds. Laser induced damage in optical materials: 1980 . Proceedings of a Symposium Sponsored by: National Bureau of Standards, American Society for Testing and Materials, Office of Naval Research, Department of Energy, Defense Advanced Research Project Agency, and Air Force Office of Scientific Research; 1980 September 30-October 1; Boulder, CO. Nat. Bur. Stand. (U.S.) Spec. Publ. 620; 1981 October. 483 p. SN003-003-02359-1.

Key words: laser damage; laser interaction; optical components; optical fabrication; optical materials and properties; thin film coatings.

The Twelfth Annual Symposium on Optical Materials for High Power Lasers (Boulder Damage Symposium) was held at the National Bureau of Standards in Boulder, Colorado, September $30-O c t o b e r$ 1, 1980. The Symposium was held under the auspices of ASTM Committee F-1, Subcommittee on Laser Standards, with the joint sponsorship of NBS, the Defense Advanced Research Project Agency, the Department of Energy, the Office of Naval Research, and the Air Force Office of Scientific Research. Over 150 scientists attended the Symposium, including representatives of the United Kingdom, France, Japan, and West Germany. The Symposium was divided into sessions concerning Materials and Measurements, Mirrors and Surfaces, Thin Films, and finally Fundamental Mechanisms. As in previous years, the emphasis of the papers presented at the Symposium was directed toward new frontiers and new developments. Particular emphasis was given to materials for high power systems. The wavelength range of prime interest was from 10.6 $\mu \mathrm{m}$ to the uv region. Highlights included surface characterization, thin film-substrate boundaries, and advances in fundamental lasermatter threshold interactions and mechanisms. The scaling of damage thresholds with pulse duration, focal area, and wavelength was discussed in detail. Harold E. Bennett of the Naval Weapons Center, Alexander J. Glass of the Lawrence Livermore Laboratories, Arthur H. Guenther of the Air Force Weapons Laboratory, and Brian E. Newnam of the Los Alamos Scientific Laboratory were co-chairmen of the Symposium. The Thirteenth Annual Symposium is scheduled for November 17.18, 1981 at the National Bureau of Standards, Boulder, Colorado. These proceedings include the following papers (indented):

SP620; 1981 October. 29-43. Braunstein, R.; Kim, R. K.; Braunstein, M. Infrared wavelength modulation spectroscopy of laser window materials.

Key words: $\mathrm{BaF}_{2} ; \mathrm{CaF}_{2} ;$ extrinsic and intrinsic infrared absorption; infrared wavelength modulation; $\mathrm{LaF}_{3}$; laser windows; $\mathrm{LiF} ; \mathrm{MgF}_{2} ; \mathrm{MgO} ; \mathrm{NaCl} ; \mathrm{NaF} ; \mathrm{SrF}_{2}$; volume and surface impurities.

We have previously shown that the infrared wavelength modulation system that we have developed is capable of measuring the complete spectral distribution of the extrinsic absorption in highly transparent solids at levels of $10^{-5} \mathrm{~cm}^{-1}$ in the spectral range from 2.5 to 12 microns. The previous measurement techniques that were employed for $\mathrm{KBr}$ and $\mathrm{KCl}$ have been extended to other crystalline systems which are of interest in light guiding applications, namely: $\mathrm{CaF}_{2}, \mathrm{LiF}, \mathrm{NaCl}, \mathrm{NaF}, \mathrm{LaF}_{3}$, $\mathrm{BaF}_{2}, \mathrm{MgF}_{2}, \mathrm{SrF}_{2}$, and $\mathrm{MgO}$. Rich and varied absorption structures were observed in all of these crystals enabling an identification of volume and surface absorption. Similar dominant bands are observed in many of these substances indicating the presence of common impurities regardless of the crystal and the origin of its growth. However, varied fine structures are observed in different crystals which are indicative of the individual characteristics of the chemistry of the crystal preparation. These measurements were performed in laboratory 
and dry $\mathrm{N}_{2}$ ambients and readily show the physisorption and desorption of surface contaminants. In appropriate materials, measurements were extended into the intrinsic multiphonon region.

SP620; 1981 October. 44.49. McDavid, J. M.; Yee, S. S. Photoacoustic spectroscopy of adsorbed surface impurities on alkali-halide laser windows.

Key words: alkali-halide laser windows; $\mathrm{KCl}$; $\mathrm{NaCl}$; optoacoustic spectroscopy; photoacoustic spectroscopy; surface impurities.

Significant infrared absorption can arise from vapors adsorbed on laser window surfaces during exposure to the atmosphere, during exposure to low-vapor-pressure system components, and during surface passivation or stabilization treatments. The photoacoustic effect has proved to be useful quantitative technique for the measurement of surface optical absorption and, when using a carbon dioxide laser source, provides a sensitivity of a few percent of one monolayer for vapor molecules chemisorbed on highly transparent substrates.

The work reported here involves an application of photoacoustic spectroscopy to surface absorption studies of high power laser window materials. Results are presented for the time dependence of surface absorption during controlled vapor exposure and for the wavelength dependence of surface absorption, in the 9-11 $\mu \mathrm{m}$ range, following atmospheric contamination. The application of these techniques to the determination of adsorption rate constants, activation energies, and the chemical identification of surface impurities is discussed.

SP620; 1981 October. 50.57. Van Stryland, E. W.; Woodall, M. A. Photoacoustic measurement of nonlinear absorption in solids.

Key words: absorption in solids; beam depletion; nonlinear absorption; photoacoustic technique.

The photoacoustic technique, using a piezoelectric transducer to detect acoustic signals induced in semiconductors by the absorption of light, has been used to measure two-photon absorption. The transducer signal amplitude is directly proportional to the absorbed energy from all absorption processes, nonlinear as well as linear. This signal was monitored as a function of the intensity of single, picosecond, $1.06 \mu \mathrm{m}$, pulses. At high intensities, beam depletion was observed, and a direct comparison was made with the sample transmission. The sensitivity of this technique allows nonlinear absorption to be measured at intensities significantly lower than when using transmission. In addition, the advantage of the nonlinear photoacoustic technique over the recently described calorimetric technique is that powerful signal averaging techniques can be used. This is because the absorption at a given intensity can be determined by a single pulse in a few microseconds while calorimetry requires at the least several seconds.

SP620; 1981 October. 58.74. Fernelius, N. C.; Dempsey, D. V.; O'Quinn, D. B. $1.3 \mu \mathrm{m}$ laser rate calorimetry and photoacoustic studies of the surface and bulk optical absorption in $\mathrm{CaF}_{2}$ single crystal samples.

Key words: $\mathrm{CaF}_{2}$; laser calorimetry; laser windows; optoacoustic; photoacoustic; surface-to-bulk optical absorption.

There are a variety of techniques for measuring the surface-tobulk optical absorption ratio most of which require specially prepared samples. In most works only one technique was employed on a given sample. In this work we tried three different techniques to measure the surface-to-bulk optical absorption ratio, $\mathrm{r}$, at $1.3 \mu \mathrm{m}$ on a group of similar $\mathrm{CaF}_{2}$ single crystal samples. Laser rate calorimetry results on a series of samples of varying thickness gave $\mathrm{r}=\beta, / \beta_{\mathrm{B}}=0.14 \mathrm{~cm}$ where $\beta_{\mathrm{s}}$ is the surface absorption and $\beta_{B}$ is the bulk optical absorption in $\mathrm{cm}^{-1}$. A second slope was not seen on the long bar samples which implies that $\mathbf{r}$ is less than $0.3 \mathrm{~cm}$. Analysis of photoacoustic chopping frequency variation studies using the Bennett-Forman theory gave $r=0.07 \mathrm{~cm}$ about half the multithickness result. Considering the experimental uncertainties all three techniques yielded consistent results.

SP620; 198I October. 75.87. Wu, S. T.; Bass, M. Intensity dependent absorption of alkali-halides at $10.6 \mu \mathrm{m}$.

Key words: alkali-halides; calorimetry; damage mechanisms; intensity dependent absorption; multipulse damage.

Repetitively pulsed laser calorimetry has been developed and applied to measure the intensity dependent absorption of $\mathrm{NaCl}$, $\mathrm{KCl}$ and $\mathrm{KBr}$ at $10.6 \mu \mathrm{m}$. The absorption increases as much as $70 \%$ at high laser intensity. Furthermore, multipulse damage data was obtained for $\mathrm{KBr}$ which indicates that the absorption increases very rapidly at intensities just below the single pulse damage threshold. The functional form of this phenomenon is still not well characterized. A simple model is suggested and the course of necessary additional investigations is discussed in this paper.

SP620; 1981 October. 88-93. Detrio, J. A.; Dempsey, D. A. Pulsed $\mathrm{CO}_{2}$ damage threshold measurements of $\mathrm{Rb}: \mathrm{KCl}$ and $\mathrm{NaCl}$.

Key words: alkali halides; bulk damage; $\mathrm{CO}_{2}$ laser; coating damage; laser damage; microsecond pulses; $\mathrm{NaCl}$; surface damage.

The I.on- $\mathrm{N}$ damage threshold of specimens of forged $\mathrm{NaCl}$, $\mathrm{Rb}: \mathrm{KCl}$ and antireflection coated $\mathrm{KCl}$ were measured at $10.6 \mu \mathrm{m}$ with a pulse duration of $10 \mu$ sec. An acid etch significantly improved the survivability of the $\mathrm{NaCl}$ specimens which normally received surface damage while the response of $\mathrm{KCl}$ was not influenced by the etch treatment. The coated $\mathrm{KCl}$ survived about as well as the uncoated specimens. The measured damage thresholds were: etched $\mathrm{NaCl}, 24.2 \mathrm{~J} / \mathrm{cm}^{2} ; \mathrm{NaCl}, 12.0 \mathrm{~J} / \mathrm{cm}^{2}$; etched $\mathrm{KCl}$, unetched $\mathrm{KCl}$, and coated $\mathrm{KCl}, 10 \mathrm{~J} / \mathrm{cm}^{2}$.

The damage morphology included entrance and exit surface damage on all types of specimens and bulk damage in the $\mathrm{KCl}$ specimens. The surface damage observed in some cases was an artifact produced by a plasma which formed at the specimen entrance face.

SP620; 1981 October. 94.101. Drexhage, M. G.; Bendow, B.; Lipson, H. G.; Moynihan, C. T. Infrared absorption in highly transparent glasses based on hafnium fluoride.

Key words: fluoride glasses; fluorohafnates; fluorozirconates; infrared absorption; infrared glasses; infrared materials; multispectral materials.

There has been considerable research activity recently in the preparation and characterization of multicomponent glasses based on heavy metal fluorides. These glasses offer excellent prospects for multispectral transmission (from $5.7 \mu \mathrm{m}$ to $0.2-0.3 \mu \mathrm{m}$ ), are amenable to the incorporation of a wide variety of atomic species, possess a moderately low refractive index $(\sim 1.5)$, and are readily forged into optical components. In previous work we reported investigations of the infrared edge of fluorozirconate glass. In this paper, we report investigations of the infrared absorption in the transparent regime of several new hafnium fluoride glasses developed recently at our laboratories. We find, as expected, that the infrared edge is shifted to longer wavelengths compared to $\mathrm{Zr}$-based glass, but with most other spectral features remaining similar for both families of glasses. One interesting feature, for example, is a shoulder in the vicinity of $1300-1400 \mathrm{~cm}^{-1}$ in the infrared edge spectrum of these glasses. We discuss the influence of processing conditions on the mid-IR absorption; it appears that at this time that RAP processing utilizing $\mathrm{CCl}_{4}$ yields the best glasses, with somewhat steeper IR edges and reduced absorption in the $3 \mu \mathrm{m}$ regime. An analysis of 
the infrared edge data in terms of multiphonon theory is utilized to estimate the intrinsic limiting absorption in the 3-5 $\mu \mathrm{m}$ regime. Finally, the infrared edge measurements are correlated with the fundamental vibrational spectra of the glasses deduced from Raman and reflectivity studies.

SP620; 1981 October. 102-109. Hack, H.; Neuroth, N. Internal damage to optical glasses with 3 ns-pulse laser.

Key words: damage types; nonlinear effect; optical glass; threshold values.

The beam of a $3 \mathrm{~ns}$ pulse laser $(1060 \mathrm{~nm})$ is focussed with a convex lense with $1,1 \mathrm{~m}$ focal length. The glass sample to be tested is $20 \mathrm{~mm}$ thick: its rear surface has a distance of $100 \mathrm{~mm}$ from the focus. The part of the sample which will be shot is illuminated with a Xenon lamp and inspected with a microscope with fourfold magnification before and after the shot. In each case a photo is taken.

Two types of internal damage are to be recognized: pointlike damage and threadlike damage. Some glass types show only pointlike damage, even at higher energies (applied energy densities up to $65 \mathrm{~J} / \mathrm{cm}^{2}$ ); other glass types show only threadlike damage caused by self-focussing (dependent to the $n_{2}$-value). In the most cases both damage types occur but at different levels of energy densities. In BK 1 and BK 7 glass no internal damage is to be seen when applying energy densities up to $65 \mathrm{~J} / \mathrm{cm}^{2}$.

SP620; 1981 October. 110-116. Vora, H.; Ready, J. F. Development of forging processes for large-scale deformation of lithium fluoride.

Key words: fluorides; forging; laser windows; lithium fluoride; mechanical properties; optical properties; ultraviolet transmission.

Development of forging processes that would permit largescale deformation of $\mathrm{LiF}$ without degradation of its optical properties is required for potential application of this material as an optical element in laser systems generating short intense pulses in the ultraviolet to heat targets for laser fusion. This paper describes forging processes that permit deformation of nearly 90\% without introducing internal cloudiness in LiF. Mechanical and optical properties of LiF forged using these processes are also discussed, including the microyield and microcreep at room temperature and the residual absorption in the visible and ultraviolet.

SP620; 1981 October. 117.128. Klein, C. A. Stress-induced birefringence, critical window orientation, and thermal lensing experiments.

Key words: elastic coefficients; high-energy laser; interferometric testing; optical distortion; photoelastic coefficients; polycrystalline aggregate; stress birefringence; thermal lensing; window materials.

A proper assessment of thermal lensing phenomena in high. average-power laser windows involves an evaluation of two optical distortion coefficients: (a) the coefficient $\chi_{+}$, which combines the effects of temperature-induced change in refractive index, surface bulging through constrained expansion, and photoelasticity averaged over the principal stress directions, and (b) the coefficient $\chi$, which exists only if there is stress birefringence. This evaluation can be performed for $<111\rangle$-oriented and for randomly-oriented aggregates of cubic single crystals. It is emphasized that the calculations require correct inputs in terms of elastic and photo-elastic coefficients. Among presently contemplated key laser-window material candidates, only $\mathrm{CaF}_{2}$ can exhibit significant stress-birefringence effects; this is not so in the $\langle 111\rangle$ orientation, which reflects a critical situation in the sense of Joiner, Marburger, and Steier. Windows made of $\mathrm{KCl}$ exhibit isotropic distortion patterns because the magnitude of the thermo-optic coefficient is such that the "small-birefringence condition," $\chi_{-} / \chi_{+}<<1$, is satisfied. The results of interferometric testing substantiate these considerations.

SP620; 1981 October. 129.143. Fernelius, N. C.; Dempsey, D. V.; Walsh, D. A.; O'Quinn, D. B.; Knecht, W. L. Survey of $1.3 \mu \mathrm{m}$ window materials.

Key words: infrared materials; iodine laser; laser calorimetry; optical absorption coefficients.

The purpose of this work is to screen candidate materials for use in the iodine laser which operates at $1.315 \mu \mathrm{m}$. Most of the results presented here are the effective optical absorption, $\boldsymbol{\beta}_{\text {eff }}$ measured by laser rate calorimetry using a Quantronix Nd:YAG laser modified to operate at $1.319 \mu \mathrm{m}$. Spectral transmission scans using the Beckman UV 5270 and Perkin-Elmer 180 spectrophotometers are presented on the less common materials. In the beginning we measured $\beta$ on materials obtained for $\mathrm{CO}_{2}$, $\mathrm{CO}$ and HF.DF laser studies. These included $\mathrm{NaCl}, \mathrm{KCl}, \mathrm{LiF}$, $\mathrm{CaF}_{2}, \mathrm{SrF}_{2}, \mathrm{Al}_{2} \mathrm{O}_{3}$ (sapphire), $\mathrm{ZnSe}, \mathrm{Si}$ and $\mathrm{GGG}\left(\mathrm{Gd}_{3} \mathrm{Ga}_{5} \mathrm{O}_{12}\right.$ ). At $1.3 \mu \mathrm{m}$ a number of oxides which were too absorbing to use at the longer wavelengths can be considered. We present results on a variety of commercially available fused silicas, $\mathrm{SiO}_{2}$. Additional results on $\mathrm{BaF}_{2}$, Raytran $\mathrm{ZnS}$, spinels (xMgO$\cdot \mathrm{Al}_{2} \mathrm{O}_{3}$ ), $\mathrm{MgO}$, Barr and Stroud BS37A and BS39B calcium aluminate glasses, CORTRAN 9753 and 9754 glasses, ZBT glass, undoped YLF $\left(\mathrm{LiYF}_{4}\right)$ and $\mathrm{As}_{2} \mathrm{~S}_{3}$ will be presented.

SP620; 1981 October. 144-158. Glassman, A. T. Material property requirements for laser windows.

Key words: absorption; laser windows; material properties; transmission.

Systems designers and theoreticians require reliable material properties-the designer to make informed selections of window materials, and the theoretician as input to his performance models and figure of merit calculations. The information is not always available, however, because (a) some measurements are difficult to make and exist for only a few selected materials; (b) stable lasers have not been readily available for use in making measurements, etc. at all wavelengths of interest. This paper indicates some gaps in material properties relevant to windows and gives directions for future experimental work, based on a literature search to compile data on candidate materials for infrared windows.

The types of gaps in material properties are illustrated by constructing a three-dimensional matrix of values, pointing out areas for future work. Data availability for many materials of interest is given.

Material properties for materials of interest are tabulated.

SP620; 1981 October. 159.169. Thomas, N.; Sonderman, J.; Stokowski, S.; Wallerstein, P.; Walmer, D. A new photographic technique for observing bulk laser damage.

Key words: bulk laser damage; fluorophosphate glass; inclusion density; KDP crystals; silicate glass.

A damage site camera was developed to record the onset of bulk laser damage in materials. The camera images and magnifies the damage track using forward-scattered laser light. Employing this camera we can detect the presence of very small $(<10 \mu \mathrm{m})$ damage sites with densities as low as $10 / \mathrm{cc}$. The camera is used at oblique incidence.

We have observed discrete damage sites generated within the bulk of some materials, such as silicate glass, fluorophosphate glass, and KDP crystals, by 1-ns, 1064-nm laser pulses. The energy fluxes at which bulk damage is initiated are in the range of 2 to $20 \mathrm{~J} / \mathrm{cm}^{2}$, much lower than the fluxes required to cause damage by intrinsic processes. Small foreign inclusions $(<1 \mu \mathrm{m}$ diameter) are the cause of these low bulk damage thresholds. The inclusion density varies from $10^{7} / \mathrm{cc}$ to less than $10 / \mathrm{cc}$. At 
threshold the damaged volumes are small ( $1-5 \mu \mathrm{m}$ in diameter) and thus, can be observed most easily by their forward-scattered light.

Several photographs are shown to illustrate the onset of bulk laser damage in fluorophosphate glass and KDP crystals.

SP620; 1981 October. 171.179. Namba, Y.; Tsuwa, H. Ultrafine finishing of ceramics and metals by float polishing.

Key words: crystallographic anisotropy; crystallographic perfection; initial permeability; metal mirror; polishing; polycrystalline material; residual stress; sapphire; specular reflectance; surface analysis; surface integrity; surface roughness.

This paper deals with a method of finishing ceramics and metals to obtain a high degree of smoothness, and also deals with geometrical, crystallographic, magnetic, optical and chemical properties of finished surfaces. Float polishing is developed to polish many electronic and optical materials ultrafinely without deformed layers. Samples were mechanically polished on a tin lap having fine grooves of concentric circles while in a polishing fluid mixed with the pure water and fine powder. The samples were suspended in the polishing fluid by hydrodynamic phenomena due to the specially shaped tin lap. Very good flatness, excellent retention of edge geometry and extremely small surface roughness of $10 \AA \mathrm{Rz}$ were obtained on the finished surfaces of amorphous materials and single crystals. The surface roughness of $20 \AA \mathrm{Rz}$ were obtained on polycrystalline materials by float polishing using special techniques in order to reduce crystallographic anisotropy.

SP620; 1981 October. 180.189. Temple, P. A.; Soileau, M. J. 1.06 $\mu \mathrm{m}$ laser-induced breakdown of $\mathrm{CO}_{2}$-laser-polished fused $\mathrm{SiO}_{2}$.

Key words: $\mathrm{CO}_{2}$-laser glazing; $\mathrm{CO}_{2}$-laser polishing; fused silica surface; laser damage; laser polishing; surface laser damage; 1.06$\mu \mathrm{m}$.

Fused silica surfaces have been treated with continuous wave $\mathrm{CO}_{2}$-laser radiation in an improved raster technique. Laser damage data for $1.06-\mu \mathrm{m}$, 9-nsec, small-spot irradiation are presented. It is shown that a reasonably sharp transition from laser damage prone to laser damage resistant surfaces takes place over a small $\mathrm{CO}_{2}$-laser power range. The transition to high damage resistance takes place at a silica surface temperature where material flow begins to take place, as evidenced by the onset of residual strain in the $\mathrm{CO}_{2}$-processed part. These data are taken as evidence that microcrack healing is an important mechanism in increased damage resistance in $\mathrm{CO}_{2}$-polished parts. Also presented are data which show that $\mathrm{CO}_{2}$-treated surfaces have a small-spot damage threshold at least as high as the bulk damage threshold of $\mathrm{SiO}_{2}$. In addition, these treated parts show no obvious change in surface appearance, as seen in total internal reflection microscopy. They also show little change in transmissive figure. It is suggested that uniform preheating be used to eliminate the strain presently seen in $\mathrm{CO}_{2}$-treated parts.

SP620; 1981 October. 190-200. Decker, D. L.; Hodgkin, V. A. Wavelength and temperature dependence of the absolute reflectance of metals at visible and infrared wavelengths.

Key words: aluminum; copper; Drude theory; infrared; reflectance; silver; surface roughness; temperature dependence.

This paper presents the wavelength dependence of $\mathrm{Ag}, \mathrm{Cu}$, and $\mathrm{Al}$ from $4000 \AA$ to $10 \mu \mathrm{m}$ wavelength. An intercomparison of data is made for surfaces prepared with a variety of techniques, including vacuum evaporation and diamond singlepoint machining. Briefly described, also, are experimental techniques and apparatus developed at the Naval Weapons Center for measuring temperature-dependent changes in the nearnormal incidence reflectance of a sample to a precision of a few parts in $10^{5}$. Reflectance results obtained from this instrument are presented for films and bulk material over the same wavelength range as above at temperatures from 270 to $450 \mathrm{~K}$. The sample and reference surfaces were protected in ultrahigh vacuum for these measurements.

A theoretical interpretation of results is presented, including both inter- and intraband electron transition effects, as well as extrinsic surface roughness effects. Of much practical interest for laser mirror applications, especially for pulsed mode operation, is the behavior of many metals upon temperature cycling, including hysteresis and time-dependent "annealing" behavior. Similar effects are also observed in both transport and thermal properties and are generally described under the name "premelting effects."

The data presented in this paper are of immediate engineering value in computing the performance of optical systems whose components necessarily operate at temperatures other than room temperature. It is also hoped that it will stimulate theoretical interest in examining some of the subtle and poorly understood dispersion effects such as those seen in the near-infrared in silver.

SP620; 1981 October. 201-209. Porteus, J. O.; Decker, D. L.; Seitel, S. C.; Soileau, M. J. Dependence of metal mirror damage thresholds on wavelength, material, pulse length, and preparation method.

Key words: cold work; crystalline disorder; defect damage; diamond-machined mirrors; Drude absorption; electron-beam melting; laser waveform; melt threshold; metal deposition; pulsed laser damage; slip threshold; thermal diffusion length; thermal stress.

Multithreshold pulsed laser-damage characteristics of precision diamond-machined $\mathrm{Cu}, \mathrm{Ag}$, and $\mathrm{Au}$ mirrors have been determined at 10.6, 3.8, 2.7, and $1.06 \mu \mathrm{m}$. The effect of pulse length has been examined at $10.6 \mu \mathrm{m}$ by comparing previously reported results obtained using a 100-nsec TEA pulse with new results using a $2-\mu \mathrm{sec}$ pulse from a hybrid TEA laser. The pulse lengths at 3.8 and $2.7 \mu \mathrm{m}$ are nominally $100 \mathrm{nsec}$, while that at $1.06 \mu \mathrm{m}$ is $9 \mathrm{nsec}$. Spatial intensity distributions are very nearly Gaussian at all wavelengths with $\mathrm{e}^{-2}$ focal spot diameters ranging approximately from $200 \mu \mathrm{m}$ at $10.6 \mu \mathrm{m}$ wavelength to $50 \mu \mathrm{m}$ at the shorter wavelengths. Melt thresholds were calculated from one-dimensional heat flow considerations with a correction for finite focal spot size. Laser waveforms are accurately modeled, and temperature-dependent absorption based on Drude theory is used. Calculated values for $\mathrm{Ag}$ are in excellent agreement with experiment, except at $1.06 \mu \mathrm{m}$, while calculated values for $\mathrm{Cu}$ and $\mathrm{Au}$ are in good agreement at $10.6 \mu \mathrm{m}$ only. An unusually large discrepancy at $3.8 \mu \mathrm{m}$ suggests a non-Drude-like absorption mechanism that is enhanced by the multipeaked nature of the waveform at this wavelength. Defect-related damage at submelting fluences has also been studied at the longer 10.6- $\mu \mathrm{m}$ pulse length on $\mathrm{Cu}$ mirrors representing a variety of preparation methods. Comparative evaluation of damage characteristics has resulted in identification of two new approaches to improving resistance to defect damage.

SP620; 1981 October. 210-221. Draper, C. W. Regrowth in laser irradiated elemental metals and alloys: surface nonuniformities and coupling phenomena.

Key words: laser irradiation; liquid phase regrowth; metal surfaces; nonuniformities.

The last half dozen years have seen a rapid growth in research on the effects of laser surface melting (LSM) and rapid self quenching on metallic systems. Modification of surface sensitive behavior has been demonstrated in a number of alloys systems. Even more recently there has been a renewed interest in laser surface alloying (LSA)-the melting/rapid quenching of predeposited film plus metal substrate.

The coupling process between metallic surface and focussed beam is sensitive to preirradiation surface quality. The resulting 
LSM or LSA area may be uniform as a result of controlled processing, or very nonuniform due to local variations in the coupling phenomena. Four categories are identified as the major causes for nonuniformities: topographical inhomogeneities, structural/chemical inhomogeneities, impurity effects and interference effects. If LSM or LSA processing is to move out of the laboratory and into the real world the significance of these differences will have to be addressed.

SP620; 1981 October. 222.226. Draper, C. W.; Buene, L.; Jacobson, D. C.; Poate, J. M.; Nakahara, S. Epitaxial regrowth and defects in laser irradiated single crystal $\mathrm{Ni}$.

Key words: defects; epitaxial regrowth; laser irradiation; liquid phase; single crystal.

We have used Q-switched Nd:YAG irradiation to melt surface layers of metallic single crystal nickel. The quality of the epitaxially regrown layers has been determined by Rutherford backscattering and channeling as well as by TEM. TEM shows a high density of extended defects (dislocation tangles and walls). We have also observed significant differences between irradiated $\langle 100\rangle$ and $\langle 111\rangle$ crystals cut from the same Ni boule. One explanation for the orientation dependence is that slip occurs more easily in $\langle 111\rangle$ planes of fcc structures and consequently there is a higher density of defect nucleation centers for $\langle 111\rangle$ liquid phase regrowth.

SP620; 1981 October. 227-238. Musal, H. M., Jr. Pulsed laser initiation of surface plasma on metal mirrors.

Key words: absorption waves; air breakdown; Cu mirrors; laserinduced electron emission; mirror surface damage; surface plasma.

Plasma formation at the surface of a metal mirror under intense pulsed laser irradiation in an ambient atmosphere usually leads to enhanced thermal and mechanical coupling with consequently increased surface damage.

Exo-electron emission associated with plastic micro-yielding of the metal surface under thermomechanical stress induced by a surface temperature rise $(\Delta T)$ is proposed here as a plasma initiation mechanism. Emission starts when $\Delta \mathrm{T}$ exceeds a relatively small critical value. Rapid buildup of the plasma proceeds via nonequilibrium ionization cascade in the ambient atmosphere when the incident laser flux exceeds a threshold value. Formation of a highly absorbing plasma layer requires delivery of a minimum fluence while the incident flux is higher than the above-mentioned flux threshold. At high values of incident flux, the required fluence decreases to an asymptotic minimum

The critical temperature rise, threshold flux, and asymptotic minimum fluence required for surface plasma formation are defined here in terms of the pertinent radiation, material, and atmosphere parameters.

SP620; 1981 October. 238.247. Thomas', S. J.; Phipps, C. R., Jr.; Harrison, R. F. Optical damage limitations for copper mirrors used in $\mathrm{CO}_{2}$-ICF laser systems.

Key words: copper mirrors; $\mathrm{CO}_{2}$ laser; multishot damage; nanosecond pulse; peak energy density; pulse distortion; singleshot damage threshold; vacuum chamber.

Because copper optics for $\mathrm{CO}_{2}$-ICF system applications will be highly stressed, the single-shot damage thresholds which have been reported, provide only part of the information needed for accurate performance predictions. We report here a performance comparison for copper mirrors finished by different techniques, including single-point diamond turning (SPDT), showing for the first time the relative decrease in $10-\mu \mathrm{m}$ damage thresholds corresponding to multiple shots, and the loss of reflected beam brightness arising at high fluence levels. We also report the intensity and energy loss due to plasma formation when $\mathrm{Cu}$ mirrors are used in air.

SP620; 1981 October. 248-255. Foley, J.; Sharma, S. K.; Wood, R. M. Absorption calorimetry and laser induced damage threshold measurements on germanium and zinc selenide.

Key words: absorptance; infra-red laser windows; pulsed $\mathrm{CO}_{2}$ laser damage; 10 micrometer optical components.

It has been shown that the absorptance of coated and uncoated germanium samples varies with surface state and ambient atmosphere. Absorption measurements made in air are up to $50 \%$ higher than identical measurements made under vacuum. The removable surface absorption on germanium has heen shown to be due to both hydrocarbon and water layers, depending on the previous cleaning and ambient conditions. The residual surface absorption depends on the surface polishing procedure. Bulk absorption levels as low as $0.007 \mathrm{~cm}^{-1}$ have been measured for freshly cleaned samples. Correlation between the surface condition and the laser induced damage thresholds at $10.6 \mu \mathrm{m}$ indicates that the damage threshold should be significantly higher in vacuum.

Absorption and laser induced damage threshold measurements have been made on a range of CVD grown $\mathrm{ZnSe}$ substrates. Correlation has been shown between the grain size and the direction of measurement and the bulk absorption coefficient.

SP620; 1981 October. 256-264. Bennett, H. E. Insensitivity of the catastrophic damage threshold of laser optics to dust and other surface defects.

Key words: coating imperfections; cw laser irradiation; digs; dust; laser damage; mirrors; optical components; scratches; surface defects.

When laser optics are exposed to continuous radiation or to pulse lengths long enough so that the damage level is determined simply by thermal heating, the mechanisms causing catastrophic damage are fairly clear. In particular, the role of surface defects should be predictable from an analysis of the standing wave pattern at the surface, the optical properties and shape of the defects, and simple heat transfer calculations. The conclusion drawn from such an analysis is that although dust and other surface defects may cause small isolated damage sites to occur, these sites will not grow under continued irradiation and produce catastrophic damage unless the defects exceed a critical diameter or are so closely spaced that they significantly affect the average absorption of the surface. Experimental evidence supports this hypothesis. Dust and other small surface defects are thus acceptable in reasonable concentrations on high power continuous wave laser optics provided that they do not exceed the critical size.

SP620; 1981 October. 265.276. Foltyn, S. R.; Newnam, B. E. Multiple-shot laser damage thresholds of ultraviolet reflectors at 248 and 308 nanometers.

Key words: excimer lasers; fluoride coatings; laser damage; multiple-shot damage; oxide coatings; pre-irradiation; thin films; ultraviolet reflectors.

Multiple-shot damage thresholds of dielectric reflectors have been measured at 248 and $308 \mathrm{~nm}$. Standard irradiation conditions were a 10 -ns pulsewidth, $0.6-\mathrm{mm}$ spot diameter and $35 \cdot \mathrm{Hz}$ pulse repetition frequency. The reflectors, from various sources, were composed of oxide and fluoride films.

Although damage was generally initiated at visible film defects, there was no correlation between damage susceptibility and the appearance of these defects. At levels near threshold, damage was most often observed as an increase in white-light scatter of a site with no growth upon continued irradiation; at higher levels, the damage site grew with successive shots. 
Test sites were subjected to at least $10^{3}$ shots and some sites received as many as $2.5 \times 10^{4}$ shots; however, with only one exception damage was found to occur within the first few shots or not at all.

Reflectors at $248 \mathrm{~nm}$ typically had damage thresholds in the $1.0-1.8 \mathrm{~J} / \mathrm{cm}^{2}$ range with two samples exhibiting unexpectedly high thresholds of 2.8 and $3.0 \mathrm{~J} / \mathrm{cm}^{2}$. In some cases, a subthreshold pre-irradiation treatment resulted in a 20-25\% enhancement in damage resistance.

SP620; 1981 October. 277.286. Wiggins, T. A.; Walker, T. W.; Guenther, A. H. A novel technique for investigating impurity initiated short pulse laser damage in thin films.

Key words: damage threshold; impurity-induced damage; laser damage; $\mathrm{ThF}_{4}$; thin films; $\mathrm{ZnS} ; \mathrm{ZrO}_{2}$.

A preliminary study has been conducted to investigate the effects of spot size on the laser induced damage threshold in thin films. Damage was produced by the interference fringes of two beams from a frequency-doubled Nd-YAG laser with a nearly Gaussian spatial profile. Fringes of spacing from 5 to $125 \mu \mathrm{m}$ were used on $\mathrm{ZnS}, \mathrm{ZrO}_{2}$ and $\mathrm{ThF}_{4} / \mathrm{ZnS}$ films. The damage threshold and morphology were observed and are reported.

Microscopic examination indicated that the appearance within a damaged region, which is as narrow as $1 \mu \mathrm{m}$, is not different from that produced by a single beam whose half-intensity diameter is a hundred times larger. Interesting results concerning the relative importance of scratches and other defects were revealed by Nomarski microscopic observation of the damage morphology.

It has been reported that laser damage to thin films is initiated primarily by small-size dielectric impurities and the dependence of the damage threshold on pulse length, film thickness, wavelength, and material investigated. In the present study it was observed that the damage threshold increased with decreasing film thickness in agreement with previous observations. However, there was no significant increase or decrease in threshold for the two-beam case over that for a single beam when the increased energy density due to interference effects is considered. In concert with previous observations it is concluded from the damage morphology and the absence of a spot size dependence that the source of damage initiation is impurities smaller than $1 \mu \mathrm{m}$ for these $5 \mathrm{~ns}$ pulse lengths.

SP620; 1981 October. 287-296. Wirtenson, G. R.; Willis, J. B.; Enemark, E. A. Evaluation of bichromatic coatings designed for pulsed laser fusion applications at $\mathbf{0 . 5 3}$ and $\mathbf{1 . 0 6}$ micrometers.

Key words: anti-reflection coatings; bichromatic; damage; high energy laser; laser damage; pulsed laser; reflectors; thin film.

Various bichromatic coatings designed to operate at both 0.53 and 1.06 micrometers have been evaluated for spectral performance and laser damage threshold to determine the suitability of these coatings for 1 nanosecond pulse laser fusion experiments and to establish baseline data. Anti-reflection, partially transmitting high reflection, and maximum reflection coatings, consisting of titania and silica layers, were deposited onto BK-7 substrates. For each type of coating, two different designs were examined. Spectral measurements indicate the coatings met performance goals. Laser damage threshold values at 1.06 micrometers were similar to those of previous monochromatic production coatings, while damage levels at 0.53 micrometers were about one-half these 1.06 micrometer values.

SP620; 1981 October. 297.299. Deaton, T. F.; Rainer, F.; Milam, D.; Smith, W. L. Survey of damage thresholds at $532 \mathrm{~nm}$ for production-run optical components.

Key words: laser-induced damage; pulsed lasers.

We report the results of a survey of 532-nm, 0.7-ns damage thresholds for a variety of optical components. The optics were all samples from current production runs, which included high. reflector and anti-reflector films, beam dumps, leached AR surfaces, and multiwavelength AR and HR films.

The experiment was similar to the usual 1064-nm damage measurement. The green light pulses were produced by frequency-doubling 1-ns pulses from a Nd:glass laser. The 532-nm beam was then focused to a spot size of about $2 \mathrm{~mm}$ diameter at the sample. Beam profiles were recorded on a vidicon and on $1-Z$ photographic plates.

Thresholds for the AR and HR films were typically $2-5 \mathrm{~J} / \mathrm{cm}^{2}$, somewhat lower than might have been expected. Some of the beam dumps damaged at $2 \mathrm{~J} / \mathrm{cm}^{2}$. Bare and graded-index surface thresholds were found to be comparable to $1-\mathrm{ns}$, 1064-nm thresholds.

SP620; 1981 October. 300-304. Soileau, M. J. Laser-induced damage in antireflection coatings for $\mathrm{LiNbO}_{3}$ crystals.

Key words: antireflection coatings; coating damage; laser damage; $\mathrm{LiNbO}_{3}$ coatings; Q-switch; surface damage.

Substrate preparation before coating and coating cleanliness are the dominant factors affecting laser-induced damage to antireflection-coated $\mathrm{LiNbO}_{3}$. Careful control of these factors resulted in an order-of-magnitude improvement in the laserinduced damage threshold.

SP620; 1981 October. 305-312. Donovan, T. M.; Porteus, J. O.; Seitel, S. C.; Kraatz, P. Multithreshold HF/DF pulsed laser damage measurements on evaporated and sputtered silicon films.

Key words: absorption; evaporation; hydrogen and oxygen impurity; magnetron sputtering; pulsed HF/DF laser damage thresholds; silicon films.

Previously, we reported a comparison of damage resistance of various coating materials deposited by vacuum evaporation and measured at 2.7 and $3.8 \mu \mathrm{m}$. It was found that Si films fail by a thermal process and, in agreement with other studies, that highindex materials, such as $\mathrm{Si}$, tend to have lower thresholds than low-index dielectric materials, such as $\mathrm{NaF}, \mathrm{Al}_{2} \mathrm{O}_{3}$, and $\mathrm{SiO}_{\mathrm{x}}$, which by comparison are relatively outstanding performers. In this paper, we report results of tests which show that the thresholds of magnetron-deposited sputtered $\mathrm{Si}$ films are enhanced over those deposited by vacuum evaporation. The sputtering process leads to lower hydrogen impurity content (100 ppm vs $200 \mathrm{ppm}$ ), lower absorption $\left(20 \mathrm{~cm}^{-1}\right.$ vs $\left.40 \mathrm{~cm}^{-1}\right)$, and a factor of two or more enhancement in damage threshold over vacuum-evaporated $\mathrm{Si}$ films. Further improvement may be possible by optimization of the sputter-deposition parameters.

SP620; 1981 October. 313-323. Aisenberg, S.; Stein, M. The use of ion-beam deposited diamond-like carbon for improved optical elements for high powered lasers.

Key words: barrier properties; diamond-like carbon; ion beam deposition; surface smoothness; thin films.

The use of thin films of ion-beam deposited diamond-like carbon (DLC) appears to provide a number of properties that could result in improved optical elements (windows, mirrors) for high powered lasers. Many of these properties have already been described.

Of particular importance for high powered lasers is the ultrasmooth nature of the ion-beam deposited DLC plus its transparency, chemical inertness, and barrier properties. Several mechanisms predict and explain why DLC films are observed to be smoother than the substrate, and can result in improved transmission and reflection coefficients. The problem of high power surface breakdown associated with microscopic irregularities in the substrate can be reduced as a result of the ionbeam deposition energy and the smoothing effects of a film of 
ion-beam diamond-like carbon. The relationship of DLC to high power breakdown at surface defects, as well as plasma breakdown will be discussed to show how the ion-beam diamondlike carbon coating can improve performance.

SP620; 1981 October. 324-334. Herrmann, W. C., Jr.; McNeil, J. $R$. Ion-beam deposited Ge-As-Se glass for applications in the $1 \mu$ to $16 \mu$ wavelength region.

Key words: chalcogenide glass; coating; ion beam; sputtering.

We have investigated application of Ge-As-Se glass as an optical coating material for use in the $1 \mu$ to $16 \mu$ wavelength region. Thin films of the material have demonstrated the following desirable characteristics: low absorption over the wavelength region of $1 \mu$ to $16 \mu$; low inherent stress; amorphous, homogeneous structure. Laser damage tests have been performed at $1.06 \mu$ and $10.6 \mu$. Interpretation and results of the damage tests are discussed. The films are robust and resistant to attack by HF.

The deposition technique employed ion beams to pre-clean the substrate and to deposit the Ge-As-Se material. Deposition was a hybrid process, with coating material initially being sputtered by the ion beam and subsequently being generated thermally due to target heating by the ion beam. Thus the advantages of sputter deposition are realized during initial stages of film growth, and faster deposition rates can then be achieved using thermal generation. Films of Ge-As-Se in excess of $100 \mu$ thick have been easily deposited, and a variety of materials has been used as substrates. This same sputter-thermal technique has been applied to other thin film materials.

SP620; 1981 October. 335-344. Anderson, W. J.; Hansen, W. N. Characterization of small absorptions in optical coatings.

Key words: absorption; coatings; internal reflection spectroscopy; silicon monoxide; thorium fluoride; zinc selenide; zinc sulfide.

Probably the main causes of laser damage in thin films used as optical coatings are impurities and non-stoichiometry, especially at interfaces. This paper considers the problems of identifying absorbing species, locating them in the optical structure and measuring the amount of absorbing species present. The ability to have large electromagnetic fields of the desired distribution in a multilayer system is used to optimize multiple reflection spectroscopy for the characterization of thin layers. The absorption coefficient caused by impurities in several $\mathrm{Th} \mathrm{F}_{4}$ films are calculated as a function of angle of incidence and polarization. The position of these impurities is predicted by comparing the absorption coefficients measured with several techniques. The method is general and can be applied to give accurate results on systems of multiple phases and varying substrate. While the method is valid for general stratified media, its accuracy depends upon the information content of the particular reflection spectrum.

SP620; 1981 October. 345-355. Leonard, T. A.; Loomis, J.; Harding, K. G.; Scott, M. Design and construction of three infrared ellipsometers for thin-film research.

Key words: diode laser; ellipsometer; infrared; polarizer; thin films.

The University of Dayton Research Institute (UDRI) has designed and is building three infrared ellipsometers in support of the Air Force high-energy laser program. One of these instruments is a conventional null type ellipsometer for operation in the 3.39 to $4.00 \mu \mathrm{m}$ wavelength range. Ellipsometric parameters are determined with a precision of $0.01^{\circ}$ at any angle of incidence from $20^{\circ}$ to $85^{\circ}$. Repeatability and absolute accuracy also approach $0.01^{\circ}$ which yields optical constants accurate to 0.1 percent in some cases. All components are mounted on removable carriers for easy interchange, although operation is normally in the PCSA (Polarizer, Compensator, Sample, Analyzer) configuration.

The system software has special provisions for the second instrument which is also a null type ellipsometer dedicated to monitoring vacuum deposited coatings in real time at $3.39 \mu \mathrm{m}$. The software can model non-ideal components in the optical path using Jones or Mueller matrices and provide completely corrected data with a single zone measurement after the instrument is characterized. The third instrument is a rotating polarizer, automated ellipsometer tunable from 1 to $12 \mu \mathrm{m}$. A dedicated computer operates the instrument and reduces data.

SP620; 1981 October. 356.368. Bennett, H. E.; Burge, D. K. Multilayer thickness uniformities required to meet wave front error tolerances in laser mirrors.

Key words: film thickness nonuniformity; high power laser mirrors; multilayer dielectric films; optical figure measurement; vacuum deposition techniques; wave front distortion.

The wave front error tolerance per mirror in typical laser optical trains is quite severe. Expressed in terms of an equivalent figure error in the visible region, values of an eighth wave are not uncommon for mirrors in infrared systems, and values of one hundredth of a wave have been suggested for mirrors in some ultraviolet laser optical trains. The effect of figure error, i.e., error in the geometrical shape of the mirror, is well known and for a metal-coated mirror is the only one of significance. However, if the mirror has a multilayer dielectric coating, an additional apparent figure error may be introduced by the physical thickness and phase change differences produced by coating thickness nonuniformities. This source of wave front error has been largely unrecognized but can be significant. For example, a $2 \%$ variation in thickness uniformity for a typical infrared multilayer coating may create a wave front error at the operating wavelength equal to that produced by a mirror whose optical figure is in error by an eighth wave in the visible. The entire wave front error tolerance for the mirror may thus be used up by a slightly nonuniform coating. Such wave front errors usually cannot be determined unambiguously by interferometric measurements made in the visible region. If multilayer dielectric mirrors are used, the wave front error should thus ideally be measured at the operating wavelength, often a difficult task. A discussion is given of thickness uniformity requirements for dielectric multilayer films and possible ways to achieve this uniformity.

SP620; 1981 October. 369-374. Danileiko, Y. K.; Manenkov, A. A.; Nechitailo, V. S. The role of absorbing defects in the laser damage of transparent materials.

Key words: absorbing defects; inclusions; laser damage.

In early studies of laser induced damage, it was found that absorbing defects, like platinum metallic inclusions in glasses considerably reduce the damage threshold. This fact has motivated numerous experimental and theoretical investigations of the role of absorbing defects in laser damage of transparent optical materials of different kinds (crystals, glasses, polymer materials). In a short paper it is impossible, of course, to present a review of all the experimental results and theoretical ideas concerning the influence of absorbing defects on laser damage processes. Therefore, in this paper, we restrict ourselves to only a brief review of research carried out in our laboratory at the Lebedev Physics Institute. For more complete and detailed information on this research, the reader should refer to the collection of papers in which a summary of research carried out by other groups is presented as well. Extensive information on the problem under discussion can also be found in the proceedings of the Laser Damage Symposium.

SP620; 1981 October. 375.384. Van Stryland, E. W.; Soileau, M. 
J.; Smirl, A. L.; Williams, W. E. Pulse-width and focal-volume dependence of laser-induced breakdown.

Key words: air breakdown; laser damage; $\mathrm{NaCl}$; picosecond phenomena; self-focusing; $\mathrm{SiO}_{2}$.

The laser-induced breakdown fields at $1.06 \mu \mathrm{m}$ of fused $\mathrm{SiO}_{2}$, single-crystal $\mathrm{NaCl}$, and air were measured as a function of focal volume and laser pulse width while keeping all other parameters, including the specimen, constant. The laser pulse width was varied from $40 \mathrm{psec}$ to $31 \mathrm{nsec}$, and the focal volume was varied by over two orders of magnitude. The dependence of the breakdown field for $\mathrm{NaCl}$ and $\mathrm{SiO}_{2}$ on the laser pulse width $\mathrm{t}_{\mathrm{p}}$ and the focal volume $V$ was empirically determined to be $E_{B}=$ $A V^{-1} t^{-1 / 4}+C$ and the dependence for air to be $E_{B}=\left(A V^{-1}+\right.$ C) $t_{p}^{-1 / 4}$, where $A$ and $C$ are material-dependent constants. Current theories of laser-induced breakdown are carefully compared with these present measurements and are found to be inconsistent.

SP620; 1981 October. 385-393. Soileau, M. J.; Franck, J. B. Veatch, T. C. On self-focusing and spot-size dependence of laserinduced breakdown.

Key words: Kerr liquids; laser damage; laser induced breakdown; $\mathrm{NaCl}$; self-focusing.

The laser-induced breakdown power $\left(\mathrm{P}_{\mathrm{B}}\right)$ was measured for various neat liquids and various combinations of miscible liquids. $P_{B}$ for the neat liquids and the mixtures was found to be proportional to $1 / n_{2}\left(n_{2}\right.$ is the nonlinear index of refraction). From this result, we conclude that self-focusing dominates the breakdown process for these liquids $\left(\mathrm{CS}_{2}\right.$, benzene, nitrobenzene, chlorobenzene, bromobenzene, and toulene). The dependence of $P_{B}$ for the neat liquids and mixtures on the f-number of the focusing optics was then measured and found to be in excellent agreement with the predictions of self-focusing theory for liquids with large $n_{2}$ 's. The liquids were then used as a "model system" to compare the results of similar measurements in single-crystal $\mathrm{NaCl}$. This comparison indicates that the observed focal spot-size dependence in $\mathrm{NaCl}$ is not due to self-focusing.

SP620; 1981 October. 394.402. Kelly, P.; Ritchie, D.; Braunlich, P.; Schmid, A.; Bryant, G. W. Deformation of intense laser beams tightly focused inside $\mathrm{NaCl}$ : A comparison of the multiphotonpolaron and avalanche models of optical breakdown.

Key words: avalanche breakdown; beam deformation; beam depletion; free carrier absorption; multiphoton absorption; polaron absorption.

Computer simulations of the interaction of intense picosecond laser pulses $(\lambda=532 \mathrm{~nm}$ ) with $\mathrm{NaCl}$ in the focal volume of a 2.54 $\mathrm{cm}$ focal length aberration-free lens indicate that severe beam deformation may take place at photon fluxes corresponding to the single-shot damage threshold measured under these experimental conditions. This deformation is the result of a change in the dielectric function which, in turn, is caused by free carriers generated via multiphoton-assisted avalanche ionization and/or multiphoton absorption. Strong self-defocusing prevents local photon fluxes from reaching values required to raise the lattice temperature sufficiently for damage to occur. We conclude that either both the avalanche ionization as well as the multiphoton-polaron absorption mechanisms of laser breakdown are invalid in their present form or that the experimentally determined damage thresholds do not pertain to the intrinsic properties of $\mathrm{NaCl}$.

SP620; 1981 October. 403-405. Braunlich, P.; Bryant, G.; Schmid, A. A correlation of laser damage vestige structure and spherical aberrations.

Key words: damage morphology; Gaussian beam; laser damage; spherical aberration.

Model calculations of spherical aberration effects on Gaussian beam propagation yield spatial intensity variations along the beam propagation direction. These intensity oscillations are compared with experimentally observed damage vestige sequences.

SP620; 1981 October. 406-415. Braunlich, P.; Brost, G.; Schmid, A.; Kelly, $P$. The role of laser-induced primary defect formation in optical breakdown of $\mathrm{NaCl}$.

Key words: damage threshold; excitons; $\mathrm{NaCl}$; optical breakdown; point defects; primary defects.

Rapid and efficient formation of primary defects $\left(V_{k}\right.$, , F., and $H$-centers, self-trapped excitons) influences the kinetic processes involved in laser-induced optical breakdown in alkali halides. These processes have been studied for the case of $\mathrm{NaCl}$ exposed to short (30 psec to $30 \mathrm{nsec}$ ) laser pulses at $\lambda=532 \mathrm{~nm}$ wavelength.

Rapid defect formation acts as a drain on the free electrons and holes, produced by multiphoton absorption in the laser photon field. As a consequence, the rate of temperature increase of the lattice by free or "dressed" carrier absorption is reduced and, thus the damage threshold is increased. This effect is compensated by additional channels of energy deposition to the lattice which are provided by formation of and photon absorption by the generated primary defects.

The net effect of the photochemical processes of primary defect formation is a slight reduction in the damage threshold and a marked decrease of the free carrier densities required for damage to occur. Beam deformation, caused by free-carrier induced changes of the dielectric function, should therefore be less pronounced than that predicted by model calculations of laser damage in the absence of primary defect formation.

SP620; 1981 October. 416.426. Jensen, B. The high frequency electron scattering rate and Drude Zener theory in compound semiconductors.

Key words: laser damage; optical constants; optical materials; semiconductors; stimulated bremsstrahlung absorption; wavelength dependence.

Recent advances in laser and semiconductor device technology have generated a growing concern over the validity of the standard transport theory in the limit of small length scales, high frequencies, and high field intensities. This has necessitated a reexamination of the foundations of transport theory and the necessary quantum extension in these limits. In this paper, the response of free carriers in a polar semiconductor to a high frequency electric field is examined. A frequency dependent relaxation time has been derived for free carriers in polar semiconducting compounds with the band structure of the Kane theory from a quantum extension of the Boltzmann transport equation. The expression obtained reduces to the usual quasiclassical Boltzmann result in the limit of low frequencies, elastic scattering mechanisms, and parabolic bands, and gives the quantum result at high frequencies when used in the Drude Zener formula for the optical conductivity. A high frequency extension of the Drude theory is thus obtained which gives the observed $\lambda^{3}$ dependence of the absorption coefficient characteristic of polar scattering in III.V and II-VI compounds in the near infrared, and reduces to the usual $\lambda^{2}$ dependence at sufficiently low frequencies. At high intensities, the scattering rate becomes a function of field intensity.

Numerical results for the electron scattering rate are calculated as functions of frequency and carrier concentration and compared with available experimental data for a number of III-V compounds.

SP620; 1981 October. 427.431. Bryant, G. W.; Schmid, A.; 
Braunlich, P.; Kelly, P.; Ritchie, D. Interactions of intense 2.7 $\mu \mathrm{m}$ picosecond laser pulses with germanium.

Key words: IR laser damage; photoexcited carriers; semiconductor plasma.

Computer simulations of the generation and temporal evolution of hot plasmas in germanium during the passage of intense picosecond laser pulses have been begun. At $2.7 \mu$ two-photon absorption is the generating mechanism for carriers. Carrier heating from free-carrier absorption and energy transfer to the lattice through phonon-carrier collisions are described with an electron-hole band model similar to one previously used to describe plasma dynamics in germanium driven by one-photon absorption. Details of this model will be presented and the results available to date will be discussed.

SP620; 1981 October. 432-437. Vaidyanathan, A.; Guenther, A. H.; Mitra, S. S. Band structure calculations of the two-photon absorption coefficients of $\mathrm{GaAs}$, InP, $\mathrm{CdTe}$, and $\mathrm{ZnSe}$.

Key words: band-structure; nonlinear absorption; oscillator strength; two-photon absorption.

The two-photon absorption coefficients of crystalline GaAs, InP, CdTe and $\mathrm{ZnSe}$ at $1.064 \mu \mathrm{m}$ and $0.694 \mu \mathrm{m}$ are calculated by means of a new model which combines the elements of second order perturbation theory and band structure calculations. A sufficient number of intermediate states are included to assure adequate convergence. This calculational procedure is shown to represent a significant improvement over currently available theoretical models, and the results are in good agreement with available experimental data.

SP620; 1981 October. 438-445. Vaidyanathan, A.; Guenther, A. H.; Mitra, S. S. Two-photon absorption in direct-gap crystals-An addendum.

Key words: band structure; effective mass; intraband-momentum matrix element; oscillator strength; two-photon absorption.

An error made in an earlier paper by the authors regarding the appropriate effective masses to be used in calculations employing the Basov formulation is corrected. The new results for twophoton absorption coefficient obtained from the Basov formula now agree quite well with those computed using Keldysh's treatment. Furthermore, it is shown that improved agreement between theoretical and experimental two-photon absorption coefficients is obtained by including nonparabolocity and degeneracy of the energy bands in the calculations.

SP620; 1981 October. 446-452. Judell, N.; Mitra, S. S.; Vaidyanathan, A.; Guenther, A. H. Three-photon absorption in direct-gap crystals.

Key words: direct gap semiconductors; non-linear optics; threephoton absorption.

Third-order time dependent perturbation theory is used to calculate the three-photon absorption coefficients of several direct-gap crystals. Both parabolic and nonparabolic energy band structures are employed. The intermediate states used in these calculations are the higher energy bands obtained from band structure calculations. The resulting three-photon absorption coefficients have the following frequency dependence. Initially the absorption coefficients increase with increasing energy, until a maximum is reached at a relatively low energy. On further increasing the photon energy the absorption coefficients decrease significantly. The behavior is qualitatively similar to the prediction of the Keldyoh formula, even though the magnitudes of the three-photon absorption coefficients obtained from the two calculations are different. The theoretical results for CdS at 1.06 $\mu \mathrm{m}$ agree well with the experimental data.

SP620; 1981 October. 453-458. Bendow, B.; Gianino, P. D.;
Tzoar, N. Theory of nonlinear beam propagation in optical waveguides.

Key words: fiber optics; nonlinear propagation; nonlinear pulses; self-focusing; solitons; waveguides.

We analyze the effect of transverse inhomogeneity on nonlinear beam propagation in a dielectric medium. Specifically, we consider the propagation of both $\mathrm{CW}$ beams and pulses in optical waveguides possessing a real nonlinear refractive index of the form $n=n_{1}(r, \omega)+n_{2}|E|^{2}$. The $C W$ problem is treated within the paraxial approximation, for the case of a Gaussian beam incident on-axis. For powers lower than the homogeneous medium critical power, waveguiding dominates, and the beam focal parameter, although altered quantitatively, continues to vary sinusoidally as a function of distance as in the linear waveguide case, with a spectral period independent of the nonlinearity. Above the critical power, however, waveguiding is superceded and nonlinearity dominates. The beam becomes unstable, and displays oscillatory focussing in a fashion which is very similar to self-focussing in homogeneous media. Our pulse propagation studies employ a rather different starting point, based on the slowly varying envelope approximation and involving an averaging over the transverse coordinates. Our principal objective is to determine the conditions for undistorted pulse propagation, i.e., the existence of optical solitons. We obtain the equations governing the existence of solitons and find that they differ significantly from those for the homogeneous medium case. In particular, while "bright" soliton propagation is restricted to the anomalous dispersion regime in homogeneous media, in waveguides it is possible to propagate "bright" solitons in regimes of normal dispersion as well.

SP622. Shives, T. R.; Willard, W. A., eds. Detection, diagnosis and prognosis: Contribution to the energy challenge. Proceedings of the 32d Meeting of the Mechanical Failures Prevention Group, held at the Inn at Santa Monica; 1980 October 7-9; Santa Monica, CA. Nat. Bur. Stand. (U.S.) Spec. Publ. 622; 1981 October. 329 p. SN003-003. 02361-2.

Key words: energy; energy conservation; failure detection; failure diagnosis; failure prevention; monitoring techniques; prognosis; sensors; wear; wear analysis.

These proceedings consist of a group of 21 submitted entries (19 papers and 2 abstracts) from the 32d meeting of the Mechanical Failures Prevention Group which was held at the Inn at Santa Monica, Santa Monica, CA, October 7-9, 1980. The subject of the symposium was the contribution of failure detection, diagnosis and prognosis to the energy challenge. Areas of special emphasis included energy management, techniques for failure detection in energy related systems, improved prognostic techniques for energy related systems, and opportunities for detection, diagnosis and prognosis in the energy field. These proceedings include the following papers (indented):

SP622; 1981 October. 3.10. Canada, R. G.; Jones, J. E. Statistical analysis of core barrel motion orbits.

Key words: core barrel motion; neutron detectors; reactor internals; surveillance; vibration.

Excore neutron detectors have been widely used to provide in. service monitoring of the mechanical motion of the reactor core support barrel in pressurized water reactors. The accepted procedure for determining core barrel motion (CBM) involves analyzing small fluctuations in the output signals of the excore neutron detectors and relating part of these fluctuations to the motion of the reactor core support barrel. This relationship between detector signal fluctuation and core barrel movement is non-trivial and statistical descriptors have been previously developed to make determination of CBM root-mean-squared magnitudes possible. This paper presents a new statistical descriptor, herein called Core Barrel Orbits, which provides 
information about both the magnitude and direction of core barrel motion. This analysis can be used to determine the most probable locations for CBM and to develop confidence limits on the maximum displacements which occur. Use of this detailed analysis can lead to detection of potential problems in the core barrel support or restraint mechanisms which would occur only at certain directions.

SP622; 1981 October. 11-30. Allen, J. W.; Hartman, W. F.; Robinson, J. C. Acoustic monitoring of power plant valves.

Key words: acoustic emissions; cavitation; feedwater control valve; pattern recognition; pressurized water reactor; remote surveillance.

Hydrodynamic related signals from pressurized water reactor (PWR) feedwater control valves (FCVs) measured by a minicomputer based monitoring system were able to predict and identify vibration levels and cavitation conditions during plant transients. These results came from a program sponsored by the Electric Power Research Institute (EPRI), the overall objective being to increase nuclear power plant availability by monitoring FCVs for failure mechanisms leading to plant shutdowns. Acoustic emission and accelerometer signals, inlet water pressure, feedwater temperature and signals necessary to calculate water velocity were analyzed. The analyses determined that cavitation and high vibration conditions occurred during certain operating conditions. These results can be used by power plant operators to avoid operating conditions where cavitation will occur.

SP622; 1981 October. 33-49. Tauber, T. Oil debris detection progress.

Key words: chip detectors; condition monitoring; failure detection; helicopter diagnostics; oil debris; wear monitoring.

For the last two years, the U.S. Army has been testing an Advanced Oil Debris Monitoring System on a fleet of 36 UH-1 helicopters at Fort Rucker, Alabama. To date, approximately 25,000 flight hours have been logged. The system has successfully detected all failures of oil-wetted engine and transmission components, most of which were missed by the conventional chip detectors and by SOAP. These include transmission input quill and mast bearing failures as well as engine No. 2, 3 and 21 bearing failures. In all cases, the system has given early, unambiguous and repeated failure alert. Component removal in early response to these alerts has resulted in very limited or no secondary damage. In some instances, only the affected component required replacement.

SP622; 1981 October. 50-61. Jones, G. W. Advanced radioactive engine wear analysis for filter design and evaluation.

Key words: effect of filtration; engine wear analysis; oil filter design; oil filter testing; particle size sensitivity; radioactive wear testing.

Tests were performed to evaluate the effect of filtration on the wear rates and particle size sensitivities of nine components within an engine. The wear measurement method used was a thin layer radioactive activation technique which had been proven in previous engine wear studies. In this test program, several advances were made in the wear analysis technique itself, which permitted measurements of one micrometer of wear on the components. These advances were primarily in the test technique and data analysis areas.

The tests performed yielded results significant to filter design and evaluation. The most important conclusion reached in regard to filtration was that particle wear sensitivity varied for different engine components. This variability included different particle size sensitivities and different effects of filtration on the engine components. This variation was used in the tests to demonstrate the different wear protection values of two filter media for several engine parts.

SP622; 1981 October. 62-73. Wertheimer, H. P.; Romine, D. J. Oxygen sensor for automobile combustion control.

Key words: air-fuel ratio; closed loop fuel metering system; exhaust emissions; exhaust gas oxygen sensor; exhaust gas recirculation; feedback; oxidation catalyst; oxygen partial pressure; stoichiometric; three way catalytic converters; zirconium dioxide.

"Three way" catalytic converters are effective in reducing automotive exhaust emissions of carbon monoxide, hydrocarbons, and oxides of nitrogen, but only when the exhaust chemistry is near stoichiometry. The exhaust gas oxygen sensor makes possible a closed loop fuel metering system to provide and maintain the required exhaust gas chemistry entering the three way converter. The oxygen sensor is a solid electrolyte galvanic cell which produces about one volt when it is exposed to a "rich" exhaust mixture and near zero volts when the exhaust is "lean." The sensor can signal the electronic control of the closed loop system whether the mixture is rich or lean, thereby permitting the system to control at or near stoichiometry. The system of three way converter together with closed loop fuel metering permits meeting stringent exhaust emission standards while maintaining better fuel economy than would be provided by known alternatives.

SP622; 1981 October. 74-81. McCord, R. M. Detection, diagnosis and prognosis of gas turbine engine health with the use of fibrescopes.

Key words: fibrescope; health condition; man-hour expenditure ratio; trend analysis; unlimited application.

The use of fibrescopes in determining the overall health condition of a turbine engine has experienced tremendous growth in the past three years. The maintenance technician can now monitor the performance of each engine, and if the trend analysis signifies structural degradation of gas path hardware, he no longer need remove the engine from service for expensive disassembly to verify suspect damage. With the new developments in the use of fibrescopes, he may now insert a fibrescope into the suspect area and verify that damage does or does not exist and to what extent. Man-hour expenditure ratio for the two methods averages about $160: 1$, and fuel savings per incident is normally 1000 gallons. This paper describes the growth of fibrescopes over the past five years and their practically unlimited application in transportation, marine, energy production and agricultural equipment in the future.

SP622; 1981 October. 85.96. Woody, M. W.; Hnatczuk, W. Integrated on-board detection diagnostic and prognostic system for military application.

Key words: bus controllers; control and distribution; crew station terminals; electrical power management; electronic components; microcomputers; MIL-STD.1553B multiplex data bus; multiplexing techniques; on-board diagnostics and prognostics; remote terminals; total tank subsystem integration.

Advanced electronic techniques are being applied by the U.S. Army to integrate on-board detection diagnosis and prognosis as an integral part of a total combat vehicle subsystem integration effort. The electronic system manages, controls and distributes electrical power in combat vehicles and provides the system integration functions of communications, data processing and integrated controls and displays to the subsystems. The subsystems include engine, electrical, fire control, stabilization, intercoms and safety. The system enhances the RAM-D of the combat vehicle by the inclusion of on-board diagnostics/prognostics, built-in-test and selected redundancy 
features. Electrical system design and modification flexibility is improved.

The system integration techniques are described which include a shared transmission medium called multiplex data bus, microcomputer control and solid state power control. The core element hardware and software design are discussed along with installation procedure for a baseline Army combat vehicle. The interfacing of off-board test equipment with an on-board multiplex bus system is discussed.

SP622; 1981 October. 97-112. Genot, A. Progress with Pielstick engine diagnostics and experimental results.

Key words: crankshaft bearing safety; medium speed diesel engine; piston ring wear; turbocharging monitoring.

In order to improve engine reliability and reduce maintenance expenses on ships, S.E.M.T. has developed a monitoring and diagnostic system for medium speed diesel engines used in marine propulsion or in power plants. The Pielstick Engine Diagnostic system has been presented at the 28th MFPG meeting. It automatically monitors wear of the piston top ring, main bearing alignment and shell wear, pressure charging circuit fouling condition and exhaust gas temperatures. A prototype system was fitted onto an 18 cylinder propulsion engine of the container ship M/V "RENOIR" which was commissioned in January 1978. This engine has now operated for 12,000 hours. The present paper reports the results obtained in the course of two years of operation of the system. The information given by the P.E.D. system is compared with that supplied by the servicing staffs routine inspections. This comparison has led S.E.M.T. to undertake new laboratory research which will concentrate on the problem of piston ring wear. The future improvements contemplated for the P.E.D. system as a consequence of this initial experience, as well as the necessity to have a better understanding of and a better monitoring system for the energy balance of the power stations, are explained.

SP622; 1981 October. 113-124. Envent, F.; Cohill, M.; Hegner, H. An energy conservation concept for operating machinery.

Key words: energy conservation; Engineering Maintenance Center; machinery monitoring; reliability centered maintenance.

Existing planned maintenance systems for complex machinery, such as those used aboard ships and aircrafts, in power plants, and processing plants, is viewed as time consuming and sometimes leads to unnecessary maintenance actions being performed. Moreover, such maintenance practices have become expensive and are not effective to keep complex machinery functioning with maximum efficiency.

In this paper, a conceptual approach to improving planned machinery maintenance as well as optimizing energy expenditures is described. This concept, making wide use of color displays and interactive techniques, represents a new practical solution to the implementation of an as-needed machinery maintenance, as opposed to the regularly scheduled open and fix procedure mostly performed. The implementation of this concept requires an on-line machinery monitoring system to detect equipment degradations. All performance monitoring and energy management tasks will be coordinated from a central location called the Engineering Maintenance Center (EMC).

This concept of machinery performance monitoring is discussed, highlighting such parameters as the functions to be performed by the EMC and the role of the maintenance personnel. Scenarios are also presented to illustrate the EMC functions such as energy management, machinery performance optimization, and detection, diagnosis and isolation of faults.

The immediate benefits to be obtained from this application of a reliability centered maintenance are improved energy utilization and machinery performance, and decreased maintenance costs.

SP622; 1981 October. 126-136. Fischer, J. P.; Schover, D. S.;
Board, D. Marine ball/roller bearing monitoring by shock pulse monitoring.

Key words: automatic alarm of bearing malfunction; marine machinery bearings; microprocessor based shock pulse analyzer; multiplexing bearing monitor channels; prediction of bearing failure; shock pulse bearing monitoring.

The American Steamship Company (ASC) (a subsidiary of GATX Corporation) operates a fleet of modern self-unloading bulk carriers on the Great Lakes. A group of these ships with similar drive trains have experienced bearing failures in a 1740 $\mathrm{Kw}$ generator. GARD, INC., was charged with the task of evaluating available bearing monitoring techniques and equipment for their ability to detect and predict bearing damage prior to destructive failure.

SKF Technology Services of SKF Industries, Inc. was called in as a consultant in the area of roller and ball bearing damage detection. They have developed a production instrument (Automatic Shock Pulse Analyzer, or ASPA) based on an advanced application of this technique. During the summer and fall of 1979 a number of critical bearings were monitored on three sister ships. Readings were correlated as to spectral energy content and prototype ASPA readings. In this set of evaluation tests, a bearing on one ship was classified as damaged. During maintenance and repair operations that winter, the suspect bearing was removed and found to have serious outer race damage.

During 1980, a production ASPA has been installed in one of three sister ships (THE MV H LEE WHITE) and a log of five crucial bearings is being kept.

When thoroughly proved out, further installations will be evaluated for similar machinery in the fleet.

SP622; 1981 October. 139-151. Gulbrandsen, G. E. Conservation: Loss prevention and risk management.

Key words: acoustic emission analysis; insurance; loss prevention; modal analysis; property conservation; risk management; shock pulse analysis; vibration analysis.

Many factors are involved in Risk Management and Property Conservation. Loss prevention, predictive-preventive maintenance, cost of money and insurance are just a few of the many. The subject of cost can be further subdivided into real and subjective costs; the real cost of unexpected losses and the subjective (though some feel real) cost of uncertainty. Today, the intelligent management of all factors is of prime importance. With the high costs of downtime and replacement parts, one can no longer rely on previous methods.

In the 1970's some insurance companies which deal in industrial property coverages, i.e., turbine insurance, pressure vessel insurance, compressor insurance, etc., have taken an active interest in the early detection of incipient faults. This interest has led some of these insurance companies to purchase sophisticated electronic devices (Acoustic Emission Analyzers, FFT Spectrum Analyzers, Shock Pulse Analyzers, etc.) and to use these instruments to help the Insureds conserve their assets. Property conservation, loss prevention, and risk management have now combined their collective capabilities to ensure the early detection of faults and maximum life of machines for the most economical cost of these procedures and insurance coverages.

SP622; 1981 October. 152.180. Lamping, G. A. Recent experiences with steam turbine disc cracking.

Key words: low alloy steels; steam turbine component failure; stress corrosion cracking; ultrasonic inspection; United States experiences.

Steam turbine disc cracking in large power generation turbines was essentially undetected until recently when utilities and turbine manufacturers started performing extensive 
nondestructive examination (NDE) programs. These were initiated in part due to the severe disc cracking problems experienced by British utilities during the 1970's. NDE being performed in the United States revealed significant disc bore and disc keyway cracking and erosion cutting previously undetected. Disc rim cracking in the blade attachment area also was experienced in some cases. The paper describes some typical areas where disc cracking has occurred and the NDE methods usually used to inspect for these defects. Metallurgical analysis work performed on several of the disc cracks shows intergranular stress corrosion cracking as the mode of failure. Repair methods presently employed to return the turbines to service and research programs under way to study the problem are also discussed.

SP622; 1981 October. 181-194. Leon, R. L. A Doppler technique for detecting and locating excessively vibrating blades in a running turbine.

Key words: blade vibration; Doppler signature; Doppler technique; high cycle fatigue; stress corrosion; turbine blade failures.

A method is presented for pinpointing those blades of a running turbine that might be vibrating excessively in resonance with either running speed, nozzle passing frequency, or one of their harmonics. Only one stationary sensor per stage is required, along with a once-per-rev phase reference signal. The method utilizes specific signal manipulations in both the time and frequency domains to uncover the telltale Doppler signature, which normally would be masked in the raw signal. It is shown in step-by-step fashion how each part of this manipulation process combines to achieve the desired goal. The technique appears to be well suited for eventual use in an on-line, microprocessor based, blade vibration monitoring system. However, since an experimental procedure to prove the technique has not yet been undertaken, it must still be considered as conceptual.

SP622; 1981 October. 195-219. Bishop, F. E. IMACS cost savings estimator-A trade-off analysis for a built-in monitoring system.

Key words: aircraft hydraulic systems; cost reduction; energy savings; failure detection; failure prevention; flight safety; fluid system components; maintenance effectiveness; on-board monitoring system.

Using aircraft as an example, the author presents the trade-off benefits achievable with a built-in, on-board monitoring system that instantaneously alerts users to malfunctions in their fluid system. The system, also applicable to naval vessels and military combat vehicles, is called IMACS (Instantaneous Maintenance and Analysis Checkout System).

Continuous in-system active sensors monitor fluid system flight-critical components for limit parameters such as temperature, leakage, flow, pressure, fluid level, presence of air, differential pressure, etc. The paper analyzes problems that can and do occur in aircraft hydraulic systems causing primary and secondary failures. An economic analysis is presented contrasting an aircraft with and without IMACS experiencing these problems. The effect of the problem is indicated and the necessary corrective action spelled out in terms of maintenance, man-hours, material and downtime.

Additional benefits derived from IMACS, such as reduced energy consumption, reduced logistic support, improved maintenance forecasting, reduced turnaround, improved scheduling and improved capability of personnel skill levels, are also discussed.

SP622; 1981 October. 220-221. Mohan, S. P. Operating experience with advanced computer based surveillance system.

Key words: base line signatures; frequency spectra; hot alignment; machinery parameters; temperatures; vibrations.

For a large grass roots ethylene plant, a computer system was developed and commissioned to provide continuous surveillance to its critical unspared as well as general purpose machinery. This system was justified on the basis of minimizing unplanned shutdowns and overall improvements in equipment availability factors. Based on utilization of the system during the plant startup and normal operation over the past year, the computer system is earning its anticipated credits. The data gathered by the computer system has been critical to (a) pinpointing the cause of events affecting machinery and (b) providing a basis for soundly engineered solutions.

SP622; 1981 October. 222-253. Boyce, M. P.; Norris, P. J. Mechanical and aerothermal diagnostics of turbomachinery.

Key words: aerothermal diagnostics; aerothermal parameters; performance; physical deterioration; reliability; turbomachinery.

To fully diagnose turbomachinery problems, both the mechanical and aerothermal parameters must be considered, since there is always a close interaction between them. The technique outlined in this paper deals with the complex aerothermal and mechanical problems in turbomachinery, and the interrelation of these parameters. Historically, the aerospace industry has been concerned with performance, while the petrochemical industry with reliability. Each of these industries looked at the two parameters exclusive of their interrelated relationships. The current trend is to use both mechanical and aerothermal parameters for monitoring and diagnostics.

This paper outlines techniques which can be used both for online diagnostics or offline problem solving. To determine deterioration in performance of a unit, it is necessary to first, determine whether the changes in performance are due to changes in ambient or process conditions, or whether these changes are due to an actual physical deterioration of the unit. Once it has been determined that the deterioration is due to physical deterioration of the system, the analysis must indicate the cause. This is done by examination of the various parameters and noting which of these parameters have caused the decay. Various groups of parameter changes indicate specific problems and these are correlated to diagnose problems.

SP622; 1981 October. 257-273. Neustadter, H. E. Data acquisition and analysis in the DOE/NASA wind energy program.

Key words: horizontal axis wind turbines; wind energy; wind turbine data systems; wind turbine generators; wind turbine performance.

The Lewis Research Center of NASA manages for the Department of Energy, the technology and engineering development of all large horizontal axis wind turbines. In support of this activity each wind turbine has various data systems used to acquire, process and analyze data. This report will identify four categories of data systems, each responding to a distinct information need. The categories are: Control, Technology, Engineering and Performance.

The focus of this report is on the Technology data system which consists of the following elements: (1) sensors which measure critical parameters such as wind speed and direction, output power, blade loads and strains, and tower vibrations; (2) remote multiplexing units (RMUs) mounted on each wind turbine which frequency modulate, multiplex and transmit sensor outputs; (3) the instrumentation available to record, process and display these signals; and (4) centralized computer analysis of data at the NASA-Lewis Research Center in Cleveland, Ohio.

RMU characteristics and multiplexing techniques are presented. Data processing is illustrated by following a typical signal through instruments such as the analog tape recorder, analog-to-digital converter, data compressor, digital tape recorder, video (CRT) display, and strip chart recorder. Sample 
output data from the $200 \mathrm{~kW}$ Mod-OA wind turbine at Clayton, New Mexico, are presented.

SP622; 1981 October. 274-288. McCabe, J. T. Periodic vibration monitoring at the SYNTHANE coal-gasification pilot plant.

Key words: coal conversion; mechanical components; SYNTHANE pilot plant; vibration monitoring.

Periodic vibration monitoring of mechanical equipment has proven to be an effective early-detection method for identifying and scheduling preventive maintenance. As a means of demonstrating this approach to the emerging U.S. synthetic fuel production industry, vibration monitoring was performed at the SYNTHANE pilot plant, one of several coal-gasification facilities sponsored by the U.S. Department of Energy (DOE). The procedures used in obtaining, analyzing and interpreting vibration data are described in this paper and, to provide a cost basis, the level of effort required to obtain, process, and report the data is given. The utility of the data as an aid to maintenance management in the pilot plant is evaluated and extrapolated to predict the general usefulness of periodic vibration monitoring as a data source for the management and operation of future, large, coal-conversion plants.

SP622; 1981 October. 289-309. Rabaud, J. Description of "La Rance" tidal power plant.

Key words: La Rance; tidal electricity production; tidal machinery.

A film in English on the Rance plant and its design features will be shown and references to an extensive bibliography will be given.

SP622; 1981 October. 310-320. Veneruso, A. F.; Chang, H. T. Detection, diagnosis and prognosis in geothermal well technology.

Key words: casing inspection; cement bond logging tool; geothermal technology.

For successful and safe operation of a geothermal well, the condition of the casing and cement must be accurately determined. Measurements on casing wall thickness, corrosion damage, holes, cracks, splits, etc., are needed to assess casing integrity. Cement bond logs are needed to detect channels or water pockets in cement behind pipe and to determine the state of the cement bond to the pipe and formation. Instrumentation for making such measurements is limited by the temperature capabilities $\left(<175^{\circ} \mathrm{C}\right)$ of existing logging equipment developed for the oil and gas industry. This paper reviews the instruments that are needed for geothermal casing and cementing inspection, identifies the principal deficiencies in their high temperature use, and describes Sandia's upgrade research program on multi-arm caliper and acoustic cement bond logging tool. The key electronic section in a multi-arm caliper will consist of $275^{\circ} \mathrm{C}$ circuits designed by Sandia. In an acoustic cement bond logging tool, a simple circuit with possibilities of using commercially available components for high temperature operation is being developed. These new tools will be field tested for operation at a minimum temperature of $275^{\circ} \mathrm{C}$ and pressure of 7000 psi for up to 1000 hours.

SP623. Pielert, J. H. Removing regulatory restraints to building rehabilitation: The Massachusetts experience. Nat. Bur. Stand. (U.S.) Spec. Publ. 623; 1981 October. 57 p. Available from: NTIS; PB 82 121047.

Key words: building code; building research; buildings; code enforcement; regulations; rehabilitation.

Throughout the United States, increasing concern is being expressed for the need to more fully utilize the existing building stock. The report documents a process that was initiated in late 1977 and continues to the present time in which particular regulatory problems impacting building rehabilitation were identified. a regulatory concept which responded to these problems was formulated, and the actual implementation of the new approach in the State of Massachusetts. Article 22 of the Massachusetts State Building Code is printed in the Appendix of the report and four case studies of actual buildings which were rehabilitated are included.

SP625. Taylor, L. S. X-ray measurements and protection 1913-1964: The role of the National Bureau of Standards and the National Radiological Organizations. Nat. Bur. Stand. (U.S.) Spec. Publ. 625; 1981 December. 386 p. SN003-003-02375-2.

Key words: dosage; dosimetry; health and safety; high voltage; ionization chamber; ionizing radiation; ionometer; radiation quantities and units; radiation standards and protection; radioactivity; radiology; radium; roentgen ray; tubes ( $x$ ray); $\mathrm{x}$-ray measurement; $\mathrm{x}$ rays.

An account of the initial U.S. concerns with, and subseyuent efforts to cope with, the safe use of ionizing radiation is given. National interest was focused in the National Bureau of Standards where radiation programs were established at the urging of, and with the close cooperation of, the radiological profession. The NBS ionizing radiation research programs, the first in the U.S. Government, were dedicated to establishing a sound scientific base for $\mathrm{x}$-ray measurement and protection. Along with the support and constant efforts of the national and international radiological organizations, the Bureau played a critically important role in the Nation's ability to use radiation effectively and safely. Based on widely scattered files assembled from both Government and non-government sources, this document represents a single source record of significant committee papers, correspondence, and decisions during the period 1913 to 1964, along with the author's summary of NBS work, and the interrelated efforts of many others. A listing of papers that contributed significantly to our understanding of ionizing radiation, and provides a complete record of the published output of the NBS radiation staff, is included.

\subsection{APPLIED MATHEMATICS SERIES}

Mathematical tables, manuals, and studies of special interest to physicists, engineers, chemists, biologists, mathematicians, computer programmers, and others engaged in scientific and technical work.

No publications issued in this series during this period. 


\subsection{NATIONAL STANDARD REFERENCE DATA SERIES}

Provides quantitative data on the physical and chemical properties of materials, compiled from the world's literature and critically evaluated. Develcped under a worldwide program coordinated by NBS. Program under the authority of National Standard Data Act (Public Law 90-396).

NSRDS-NBS61, Part IV. Janz, G. J.; Tomkins, R. P. T. Physical properties data compilations relevant to energy storage. IV. Molten salts: Data on additional single and multi-component salt systems. Nat. Stand. Ref. Data Ser., Nat. Bur. Stand. (U.S.) 61, Part IV; 1981 July. 870 p. SN003.003.02339.6.

Key words: corrosion; data compilations; electrochemical aluminum production; electrochemical energy storage materials; molten salts; physical properties; safety and hazards; thermal energy storage materials; thermal properties; thermodynamic properties; transport properties.

The present work provides selected data with value judgements for an additional set of 107 salt systems of interest as candidate materials for thermal energy storage sub-systems, for electrochemical energy storage systems, and in electrochemical aluminum production. The physical properties assessed are: melting points; phase diagrams; eutectic compositions; density; surface tension; viscosity; electrical conductivity; diffusion constants for ions; heat of fusion; heat capacity; volume change on fusion; vapor pressure; thermal conductivity (liquid and solid); and cryoscopic constant. The status of corrosion studies in the form of annotated bibliographic summaries, and salient observations on safety and hazards are also reported. A summarizing series of tables is provided as index to the data-gaps status for this set of candidate materials.

NSRDS.NBS68. Reader, J.; Corliss, C. H.; Wiese, W. I.; Martin, G. A. Wavelengths and transition probabilities for atoms and atomic ions. Part 1. Wavelengths. Part II. Transition probabilities. Nat. Stand. Ref. Data Ser., Nat. Bur. Stand. (U.S.) 68; 1980 December. 415 p. SN003-003-02288-8.

Key words: atom; atomic ion; intensities; line strengths; oscillator strengths; spectral lines; transition probabilities; wavelengths.
Wavelengths for about 47,000 spectral lines of atoms and atomic ions, as well as transition probabilities A for about 5000 lines, are tabulated. The data were selected in such a way as to include the prominent lines over a wide spectral region. Part I contains wavelengths of lines of neutral through quadruply ionized atoms in the range 40 to $40,000 \AA$. This information is presented in two different ways: 1) separate line lists grouped according to chemical element and further subdivided according to stage of ionization; and 2) a general table of wavelengths ordered numerically, with relative intensity, chemical element, and stage of ionization indicated for each line. Part II contains transition probability data for atoms in various stages of ionization, with emphasis on the neutral and singly ionized species. This table is arranged according to the chemical element and is further subdivided according to stage of ionization. Estimates of the accuracies of the A-values are provided. Wavelengths, energy levels, and statistical weights serve to identify the lines and to provide useful data for plasma spectroscopy applications.

NSRDS-NBS69. Hug, G. L. Optical spectra of nonmetallic inorganic transient species in aqueous solution. Nat. Stand. Ref. Data Ser., Nat. Bur. Stand. (U.S.) 69; 1981 May. 167 p. SN003-003-02322-1.

Key words: absorption spectra; aqueous solution; extinction coefficients; inorganic; radical ions; radicals; transients.

Optical absorption spectra are presented for 77 nonmetallic, inorganic radicals and radical ions, produced by radiolysis or photolysis in aqueous solution. This atlas results from the compilation, evaluation, and replotting of spectra from the literature. Additional information is included such as extinction coefficients, initial chemical conditions, methods of transient generation and spectral aquisition, references, mechanisms of transient formation, and notes on dosimetry. 


\subsection{BUILDING SCIENCE SERIES}

Disseminates technical information developed at the Bureau on building materials, components, systems, and whole structures. The series presents research results, test methods, and performance criteria related to the structural and environmental functions and the durability and safety characteristics of building elements and systems.

BSS130. Elder, J.; Tibbott, R. L. User acceptance of an energy efficient office building-A study of the Norris Cotton Federal Office Building. Nat. Bur. Stand. (U.S.) Bldg. Sci. Ser. 130; 1981 January. 122 p. SN003.003-02278-1.

Key words: energy conservation; lighting; man/environment research; noise; office building; post-occupancy evaluation; questionnaire; temperature; ventilation; windows.

The General Services Administration built the Norris Cotton Federal Office Building in Manchester, New Hampshire, and chose it as a "demonstration project for studying the effectiveness of energy conservation techniques in the design and operation of a contemporary office building." User acceptance of both the innovative and conventional design features in the building was measured by administering a questionnaire to employees shortly after occupancy of the building and again eight months later. The most positively rated feature overall was the lighting, but reaction to the high pressure sodium lighting system as installed in the Norris Cotton Building was strongly negative. Response to noise levels and disturbances was about evenly divided, but workers in open-plan offices were less satisfied with the noise climate than workers in partitioned offices. Most respondents were dissatisfied with the temperature and ventilation conditions and the small windows in the building. In general, the occupants rated the building much higher in appearance than their previous offices, slightly higher in terms of suitability for performance of their jobs, and slightly lower with respect to comfort. A literature review of recent survey studies of the office environment is included.

BSS131. Knab, L.; Mathey, R.; Jenkins, D. Laboratory evaluation of nondestructive methods to measure moisture in built-up roofing systems. Nat. Bur. Stand. (U.S.) Bldg. Sci. Ser. 131; 1981 January. 173 p. SN003.003-02281.1.

Key words: built-up roofing electrical capacitance; infrared thermography; insulation; moisture detection; moisture measurement; nondestructive evaluation; nondestructive testing; nuclear backscatter; roofing systems.

This laboratory study investigated the reliability and accuracy of three types of nondestructive evaluation (NDE) methods to quantitatively determine the moisture content of the insulation in built-up roofing specimens. These methods were electrical capacitance, nuclear backscatter, and infrared thermography. Thirtysix roofing specimens, which consisted of five types of rigid-board roof insulations with attached bituminous built-up membranes, were tested over both concrete and steel decks. A wide range of moisture contents was induced into the specimens by maintaining a constant water vapor pressure difference across them.

Two performance characteristics of the NDE methods were evaluated: (a) the minimum moisture content a method could detect, and (b) the relationship between NDE response and moisture content beyond the minimum detectable moisture content. The two performance characteristics were assessed through normalization parameters defined in terms of the NDE response and its scatter about a fitted curve. There were differences in the performance characteristics, the magnitude of which depend on the NDE method, the specimen composition, and the deck type used.

BSS132. Yokel, F. Y.; Yancey, C. W. C.; Mullen, C. L. A study of reaction forces on mobile home foundations caused by wind and flood loads. Nat. Bur. Stand. (U.S.) Bldg. Sci. Ser. 132; 1981 March. 84 p. SN003-003-02301-9.

Key words: buoyancy forces; flood forces; foundations; hurricane forces; mobile home; soil anchors; standards; tiedown; wind forces.

Forces acting on the foundations of mobile homes subjected to wind and flood loads were calculated and are presented in a series of computer-generated charts. The loading conditions considered are the two levels of wind loads presently stipulated in the Federal Mobile Home Construction and Safety Standard, a hurricane windload recommended by the National Bureau of Standards (NBSIR 77-1289), buoyancy forces, and draft forces resulting from flood water flow. The calculated forces are compared with present anchoring requirements in ANSI Standard 119.3 (NFPA No. 501 A). It is concluded that diagonal ties are instrumental in resisting wind forces, and that vertical ties are more effective than diagonal ties in resisting flood forces.

BSS133. Hill, J. E.; May, W. B., Jr.; Richtmyer, T. E.; Elder, J.; Tibbott, R. L.; Yonemura, G. T.; Hunt, C. M.; Chen, P. T. Performance of the Norris Cotton Federal Office Building for the first 3 years of operation. Nat. Bur. Stand. (U.S.) Bldg. Sci. Ser. 133; 1981 August. 140 p. SN003-003-02352-3.

Key words: building models, computer; energy conservation in commercial buildings; energy conservation, user acceptance; lighting measurements; performance data for commercial office buildings in New England; solar energy in commercial buildings.

The Norris Cotton Federal Office Building is a medium-size seven story Government office building of approximately $11,000 \mathrm{~m}^{2}$ $\left(117,000 \mathrm{ft}^{2}\right)$ total floor area. It is located in Manchester, New Hampshire, and was designed to demonstrate a number of energy saving concepts.

Some of the major energy conserving features of the building are the use of solar collectors; heavy masonry construction with exterior insulation; small overall window area; heat recovery from heat pumps, chillers, a natural gas-powered engine/generator, and the ventilation system; modular boilers; thermal storage tanks; and a variety of energy conserving lighting systems.

A team from the Center for Building Technology, National Bureau of Standards (NBS), has been monitoring the performance of the building since it was occupied in September 1976. The project has involved not only an analysis of building energy consumption, but also a study of the effectiveness of the various lighting systems, a determination of the response of the occupants to the building, and a cost analysis of the construction and operation of an energy conserving building. This report will describe the building's performance for the first 3 years of operation.

BSS134. Petersen, S. R.; Barnes, K. A.; Peavy, B. A. Determining cost-effective insulation levels for masonry and wood-frame walls in new single-family housing. Nat. Bur. Stand. (U.S.) Bldg. Sci. Ser. 134; 1981 August. 126 p. SN003-003-02354-0.

Key words: building design; building economics; energy conservation; exterior wall; HVAC calculations; insulation; lifecycle cost analysis; masonry; mass.

Economically optimal insulation methods and resistance levels for three different types of walls in a one-story, single-family residence are calculated for a wide range of geographic locations, energy prices, heating and cooling equipment efficiencies, and financial evaluation criteria. The three basic wall types examined are 8.in concrete block walls, brick and block walls, and wood-frame walls with lightweight siding. Changes in annual heating and cooling requirements for an $1176 \mathrm{ft}^{2}$ prototype house resulting from several different insulation resistances in each wall type are calculated using the NBS Load Determination program and Test Reference Year climate data for a number of geographic locations. Changes in heating requirements are correlated with heating degree days to provide estimates of energy savings in all geographic regions of the continental United States. Cooling requirements are not found to vary significantly with the thermal resistance of the walls under a typical operating profile except in the southwestern desert. An index number 
system is developed to quickly determine insulation levels based on the data generated in the report.

BSS135. Kovacs, W. D.; Salomone, L. A.; Yokel, F. Y. Energy measurement in the Standard Penetration Test. Nat. Bur. Stand. (U.S.) Bldg. Sci. Ser. 135; 1981 August. 99 p. SN003-003-02356-6.

Key words: energy measurement; field instrument force measurement; field testing; in-situ testing; soil mechanics; transducers.

Geotechnical engineers in the United States commonly use the Standard Penetration Test, SPT, in subsurface investigation for routine foundation designs. It has been said that perhaps up to 80 to 90 percent of the routine foundation designs are accomplished by the use of the SPT "N" value. Despite efforts to standardize more details of the SPT procedure, variability between tests is inherent under present guidelines.

A field measurement system and procedure which measures the energy delivered by a drill rig system were developed and successfully used to study the factors which affect delivered energy. Results are presented which indicate the energy delivered by certain drill rig systems used in engineering practice. Also, the transmission characteristics of certain hammer/anvil systems are examined. Guidance on the need to measure the actual fall height of the hammer during the Standard Penetration Test is provided based on the findings of the study.

BSS136. Harris, J. R.; Wright, R. N. Organization of building standards: Systematic techniques for scope and arrangement. Nat. Bur. Stand. (U.S.) Bldg. Sci. Ser. 136; 1981 September. 278 p. SN003.003-02363-9.

Key words: arrangement; building; classification; code; engineering; organization; provisions; scope; specification; standard; system analysis/engineering.

Standards should be organized so that they provide reliable and quick access to the provisions of the standard. Organization is considered to deal with both the scope and the arrangement of the provisions of a standard. It is found to have objective qualities that allow it to be treated formally. Necessary and desirable qualities for an organization are identified, verified, and adopted as objectives and guidelines. The basic element of the system for organizing standards is the classification of the provisions of a standard. A faceted structure, providing a clear division between those levels that are strictly logical and those that are not, is recommended for the classification system. A relevant basis is found for classifying requirements using an idealized model of the relation between syntax and semantics. Development of the classification constitutes a formal treatment of scope. The classification is easily transformed into an index. Development of an outline from the classifiers constitutes a formal treatment of the arrangement. Criteria for placement of provisions in outlines and for construction of outlines from the classification are proposed to promote the objectives of organization. A computer algorithm for interactive outline generation is developed and evaluated. Measures are defined for the comparison of alternate outlines for the same standard. 


\subsection{FEDERAL INFORMATION PROCESSING STANDARDS PUBLICATIONS}

Publications in this series collectively constitute the Federal Information Processing Standards Register. Register serves as the official source of information in the Federal Government regarding standards issued by NBS pursuant to the Federal Property and Administrative Services Act of 1949 as amended, Public Law 89.306 (79 Stat. 1127), and as implemented by Executive Order 11717 (38 FR 12315), dated May 11, 1973) and Part 6 of Title 15 CFR (Code of Federal Regulations). This series is available only from the National Technical Information Service, Springfield, VA 22161.

FIPS PUB 1-1. Little, J. C., Standards Coordinator. Code for information interchange. Nat. Bur. Stand.(U.S.) Fed. Info. Process. Stand. Publ. (FIPS PUB) 1-1; 1980 December. 4 p.

Key words: American National Standard Code for Information Interchange; ASCII; character set; coded character set; code for information interchange; collating sequence; data communication; data interchange; data processing; Federal Information Processing Standard; information interchange; information processing; standard.

This publication provides a standard code and character set for use in Federal information processing systems, communications systems, and associated equipment. It also provides a standard collating sequence. This Federal Information Processing Standard adopts in whole the American National Standard Code for Information Interchange (ASCII) X3.4-1977, a fundamental standard upon which many other standards are based. Twenty-six related standards are listed.

FIPS PUB 14-1. Little, J. L., Standards Coordinator. Hollerith punched card code. Nat. Bur. Stand. (U.S.) Fed. Info. Process. Stand. Publ. (FIPS PUB) 14-1; 1980 December. 4 p.

Key words: American National Standard Code for Information Interchange; ASCII; card punch; card reader; data communications; data interchange; data processing; EAM cards; electric accounting machine; Federal Information Processing Standard; Hollerith punched card code; Hollerith punched cards; information interchange; information processing; punched cards; standard.

This publication provides a standard set of 256 hole patterns which represent the 128 characters of the Federal Standard Code for Information Interchange (ASCII) FIPS 1-1, in 12-row, 80-column, rectangular hole, "Hollerith" punched cards, or the subsets of ASCII as specified in FIPS 15, Subsets of the Standard Code for Information Interchange, or supersets of ASCII as specified in FIPS 35, Code Extension Techniques in 7 or 8 Bits. It adopts all 256 hole patterns given in the American National Standard X3.26-1980, Hollerith Punched Card Code. The 128 hole patterns for ASCII are the same as in the original FIPS 14. Nine related standards are listed.

FIPS PUB 29-1. Vickers, M. V., Standards Coordinator Interpretation procedures for Federal Information Processing Standard Programming Languages. Nat. Bur. Stand. (U.S.) Fed. Info. Process. Stand. Publ. (FIPS PUB) 29-1; 1981 December 31. 4 p.

Key words: BASIC; COBOL; compilers; data processing; Federal Information Processing Standard; FORTRAN; information interchange; information processing; processor; programming language; software.

This FIPS PUB defines the procedures that will be followed in requesting interpretations of FIPS Programming Languages and in providing responses to those requests. The provisions of this document apply to all Federal departments and agencies and to vendors of processors in their dealings with the Federal Government.

FIPS PUB 74. Branstad, D. K., Standards Coordinator. Guidelines for implementing and using the NBS Data Encryption Standard. Nat. Bur. Stand. (U.S.) Fed. Info. Process. Stand. Publ. (FIPS PUB) 74; 1981 April $1.39 \mathrm{p}$.
Key words: computer security; cryptography; data integrity; encryption; Federal Information Processing Standards Publication; key distribution; network security; security.

The Data Encryption Standard (DES) was published as Federal Information Processing Standards Publication (FIPS PUB) 46 on January 15, 1977. The DES specifies a cryptographic algorithm for protecting computer data. FIPS PUB 81 defines four modes of operation for the DES which may be employed in a wide variety of applications. These guidelines are to be applied in conjunction with FIPS PUB 46 and FIPS PUB 81 when implementing and using the Data Encryption Standard. They provide information on what encryption is, general guidance on how encryption protects against certain vulnerabilities of computer networks, and specific guidance on the DES modes of operation in data communications applications. When used with the proper administrative procedures and when implemented in accordance with these guidelines, electronic devices performing the encryption and decryption operations of the standard can provide a high level of cryptographic protection to data in computer systems and networks.

FIPS PUB 75. Letmanyi, H.; Conti, D. M., Standards Coordinators. Guideline on constructing benchmarks for ADP system acquisitions. Nat. Bur. Stand. (U.S.) Fed. Info. Process. Stand. Publ. (FIPS PUB) 75; 1980 September. 43 p.

Key words: ADP acquisition; ADP procurement; benchmarking; Federal Information Processing Standards Publication; performance evaluation; workload analysis; workload characterization; workload representation.

This Guideline describes a step-by-step procedure for constructing benchmarks for use in the acquisition of ADP systems. Ten steps in the benchmark construction process are identified involving such areas as workload analysis and forecasting, construction of the benchmark mix, and documentation and testing of the benchmark package. Although the Guideline is directed to the technical staff who will actually be constructing the benchmark, portions of it should also be useful to management. In addition, the Guideline should be useful to those in private industry who are also involved in constructing benchmarks for use in the evaluation of alternative vendor systems.

FIPS PUB 78. Scace, E. L., Standards Coordinator. Guideline for implementing advanced data communication control procedures (ADCCP). Nat. Bur. Stand. (U.S.) Fed. Info. Process. Stand. Publ. (FIPS PUB) 78; 1980 September. 22 p.

Key words: advanced data communication control procedures; ANS X3.66; bit-oriented; code independence; data; data link control procedures; data transparency; Federal Information Processing Standards Publication; interoperability; teleprocessing; transmission.

This publication guides Federal agencies in the selection and implementation of Advanced Data Communication Control Procedures (ADCCP) contained in FIPS 71.

FIPS PUB 80. White, H. S., Jr., Standards Coordinator. Guide for the implementation of Federal Information Processing Standards (FIPS) in the acquisition and design of computer products and services. Nat. Bur. Stand. (U.S.) Fed. Info. Process. Stand. Publ. (FIPS PUB) 80; 1980 December. 96 p.

Key words: automated data processing (ADP); communications; Federal Information Processing Standard (FIPS); Federal Property Management Regulations (FPMR); procurement; standards; telecommunications.

This Guide provides information about the 67 FIPS publications currently in effect and identifies the computer products and services to which they may apply. This Guide is intended to serve as a 
supplemental reference in the use of approved standards and guidelines. It should prove useful in the preparation of specifications for computer systems, components, services and supplies.

This Guide serves as a checklist to assure that the proper standards are incorporated in planned acquisitions and in solicitation documents. Industry vendors and suppliers should find this Guide useful in the design of equipment and services that must conform to Federal Government procurement requirements.

FIPS PUB 81. Branstad, D., Standards Coordinator. DES modes of operation. Nat. Bur. Stand. (U.S.) Fed. Info. Process. Stand. Publ. (FIPS PUB) 81; 1980 December 2. 26 p.

Key words: computer security; cryptography; data security; DES; encryption; Federal Information Processing Standards; modes of operation.

The Federal Data Encryption Standard (DES) (FIPS 46) specifies a cryptographic algorithm to be used for the cryptographic protection of sensitive, but unclassified, computer data. This FIPS defines four modes of operation for the DES which may be used in a wide variety of applications. The modes specify how data will be encrypted (cryptographically protected) and decrypted (returned to original form). The modes included in this standard are the Electronic Codebook (ECB) mode, the Cipher Block Chaining (CBC) mode, the Cipher Feedback (CFB) mode, and the Output Feedback (OFB) mode.

FIPS PUB 82. Bagg, T. C., Standards Coordinator. Guideline for inspection and quality control for alphanumeric computer-output microforms. Nat. Bur. Stand. (U.S.) Fed. Info. Process. Stand. Publ. (FIPS PUB) 82; 1980 September. 4 p

Key words: automated data printing; computer-output microforms; Federal Information Processing Standards

Publication; form slide; microfilm; quality control.

This guideline provides basic information on problems associated with generating microforms by computers and describes test procedures to insure that the recorded output is of high quality.

FIPS PUB 83. Meissner, P., Standards Coordinator. Guideline on user authentication techniques for computer network access control. Nat. Bur. Stand. (U.S.) Fed. Info. Process. Stand. Publ. (FIPS PUB) 83; 1980 September. 38 p.

Key words: access control; authentication; authorization; computer network; computer security; encryption; Federal Information Processing Standards Publication; identification token; identity verification; password; personal attribute; personal identification.

This Guideline provides information and guidance to Federal agencies on techniques and practices which can be used to control acess to computer resources via remote terminals and networks. A variety of methods are described for verifying the identity of persons using remote terminals, as a safeguard against unauthorized usage. This Guideline discusses the three basic ways which may serve as a basis for verifying a person's identity: something the person KNOWS such as a password; something the person HAS, such as a key or access card; or something ABOUT the person, such as fingerprints, signature, voice, or other personal attribute. The ability to automatically verify a person's identity via a unique personal attribute offers the prospect of greater security, and equipment for accomplishing this is beginning to emerge. There are several promising laboratory developments, although such equipment has not yet been interfaced to computer terminals to any great extent. In view of the present dependence on authentication techniques other than personal attributes, this Guideline provides advice on the effective use of passwords. This Guideline also discusses a varity of cards and badges with various forms of machine-readable coding that may be used for access control. In order to protect information used for identity verification, encryption is recommended.
FIPS PUB 84. Bagg, T. C., Standards Coordinator. Microfilm readers. Nat. Bur. Stand. (U.S.) Fed. Info. Process. Stand. Publ. (FIPS PUB) 84; 1980 October. 4 p.

Key words: American National Standard; Computer Output Microfilm (COM); Federal Information Processing Standard (FIPS); illumination; image quality; microfilm; microform; readers.

The objective of this standard is to facilitate effective information interchange when the information is recorded on microforms generated by computer systems. The standard defines the minimum acceptable image quality for microfilm reading devices for the display of computer output microforms. It alse sets requirements for heat and noise factors associated with the safe use of such reading devices.

This standard adopts in whole the American National Standard for Microfilm Readers (ANSI/NMA MS20-1979).

FIPS PUB 85. Bagg, T. C., Standards Coordinator. Optical character recognition (OCR) inks. Nat. Bur. Stand. (U.S.) Fed. Info. Process. Stand. Publ. (FIPS PUB) 85; 1980 November. 4 p.

Key words: American National Standard; Federal Information Processing Standard (FIPS); interchange of machine readable information; nonread inks; Optical Character Recognition (OCR); OCR-A; OCR-B; read inks.

The objective of this standard is to define the spectral band for read inks and to provide spectrophotometric curves for red and blue nonread inks that are used by optical readers in order to facilitate the interchange of information among compatible OCR equipment.

This standard adopts in whole the American National Standard for Optical Character Recognition (OCR) Inks, ANSI X3.86-1980.

FIPS PUB 86. Little, J. L., Standards Coordinator. Additional controls for use with American National Standard Code for Information Interchange. Nat. Bur. Stand. (U.S.) Fed. Info. Process. Stand. Publ. (FIPS PUB) 86; 1981 January 29.4 p.

Key words: ADP terminals; ASCII; character-imaging devices; control functions; data interchange; display terminals; Federal Information Processing Standard; information interchange; printer terminals; standard; text communication; text interchange; typesetting composition.

This standard specifies a set of encoded control functions to facilitate data interchange between data processing equipment, data communication equipment, and two dimensional character-imaging input-output devices, such as interactive ADP terminals of the display or printer type, line printers, microfilm printers, typesetting composers, work processors, and related devices. These control functions augment the basic set of control functions prescribed by ASCII (FIPS PUB 1-1). These may be used in 7 or 8 bit environments in accordance with the standard for Code Extension Techniques (FIPS PUB 35). This standard adopts in whole American National Standard X3.64-1979, Additional Controls for Use with American National Standard Code for Information Interchange.

FIPS PUB 87. Shaw, J., Standards Coordinator. Guidelines for ADP contingency planning. Nat. Bur. Stand. (U.S.) Fed. Info. Process. Stand. Publ. (FIPS PUB) 87; 1981 March 27. 31 p.

Key words: ADP availability; ADP security; backup operations; computer applications; computer security; contingency planning; emergency response; Federal Information Processing Standards Publication; recovery actions.

This document provides guidelines to be used in the preparation of ADP contingency plans. The objective is to ensure that ADP personnel, and others who may be involved in the planning process, are aware of the types of information which should be included in such plans; to provide a recommended structure and a suggested 
format; and generally to make those responsible aware of the criticality of the contingency planning process.

FIPS PUB 88. Perry, W. E.; Goldfine, A., Standards Coordinators. Guideline on integrity assurance and control in database administration. Nat. Bur. Stand. (U.S.) Fed. Info. Process. Stand. Publ, (FIPS PUB) 88; 1981 August 14.71 p.

Key words: computer program; data administration; database audition; database controls; database integrity; database management; data dictionary system;

Federal Information Processing Standards Publication.

This guideline provides explicit direction to Federal database administration and database security personnel on how to improve database control. The document identifies integrity and security problems in the administration of database technology, and discusses those procedures and methods which have proven effective in addressing these problems. The guideline also provides an explicit, step-by-step procedure for examining and verifying the accuracy and completeness of a database.

FIPS PUB 89. Bagg, T., Standards Coordinator. Optical Character Recognition (OCR) Character Positioning. Nat. Bur. Stand. (U.S.) Fed. Info. Process. Stand. Publ. (FIPS PUB) 89; 1981 September 4. 4 p.

Key words: character positioning; computer systems; data entry (automatic); Federal Information Processing Standard; information processing systems; optical character recognition.

This FIPS PUB announces the adoption of American National Standard for Optical Character Recognition (OCR) Character Positioning, ANSI X3.93M-1981, as a Federal standard. This standard specifies the nominal position with allowable tolerances of OCR characters in relation to their location to other machine readable characters or sensed marks and to the document edges.

This standard is related to FIPS PUBS 32,40 , and 85.

\subsection{VOLUNTARY PRODUCT STANDARDS}

Developed under procedures published by the Department of Commerce in Part 10, Title 15, of the Code of Federal Regulations. The purpose of the standards is to establish nationally recognized requirements for products, and to provide all concerned interests with a basis for common understanding of the characteristics of the products. The National Bureau of Standards administers the Voluntary Product Standards program as a supplement to the activities of the private sector standardizing organizations.

No publications issued in this period. 


\subsection{TECHNICAL NOTES}

Studies or reports which are complete in themselves but restrictive in their treatment of a subject. Analogous to monographs but not so comprehensive in scope or definitive in treatment of the subject area. Often serve as a vehicle for final reports of work performed at NBS under the sponsorship of other Government agencies.

TN270-8. Wagman, D. D.; Evans, W. H.; Parker, V. B.; Schumm, R. H.; Nuttall, R. L. Selected values of chemical thermodynamic properties. Compounds of uranium, protactinium, thorium, actinium, and the alkali metals. Nat. Bur. Stand. (U.S.) Tech. Note 270-8; 1981 May. 149 p. SN003.003.02318-3.

Key words: actinium compounds; cesium compounds; enthalpy; entropy; francium compounds; Gibbs energy of formation; heat capacity; lithium compounds; potassium compounds; protactinium compounds; rubidium compounds; sodium compounds; thorium compounds; uranium compounds.

This publication contains tables of recommended values for the standard enthalpies (heats) of formation, Gibbs (free) energies of formation, entropies, enthalpy contents and heat capacities at 298.15 $\mathbf{K}$, and enthalpies of formation at $0 \mathrm{~K}$ for compounds of uranium, protactinium, thorium, actinium, lithium, sodium, potassium, rubidium, cesium, and francium.

TN1013. Crawford, M. L.; Workman, J. L. Using a TEM cell for EMC measurements of electronic equipment. Nat. Bur. Stand. (U.S.) Tech. Note 1013; 1981 July. 72 p. SN003-003-02350-7.

Key words: measurement procedures; susceptibility and emission measurements; transverse electromagnetic cell.

This publication describes the physical design and electrical evaluation of pertinent parameters which influence the use and operation of a transverse electromagnetic (TEM) cell. Detailed, stepby-step procedures are given for using a TEM cell for performing either radiated EM susceptibility testing or for measuring radiated EM emissions from electronic/electro-mechanical equipment. These measurement procedures provide guidelines to potential users and also indicate precautions to observe to minimize problems often encountered when performing EMC measurements and hence to enhance the cell's usefulness. Where available, a brief error analysis associated with the measurement technique is included.

TN1028. Kanda, M. Theoretical and experimental investigations of electromagnetic field distortion due to a perfectly conducting rectangular cylinder in a transverse electromagnetic cell. Nat. Bur. Stand. (U.S.) Tech. Note 1028; 1981 A pril. 48 p. SN003-003-02313.2.

Key words: electromagnetic compatibility (EMC); Green's function; integral equation; linear equation; method of moments; parallel plate waveguide; quasi electrostatic; TEM cell.

The study of electromagnetic compatibility (EMC), that is the electronic and biological effects due to electromagnetic (EM) radiation, and EM calibration require accurate EM measurement techniques for defining the EM interference (EM) characteristics. Thus, fully enclosed rectangular transverse electromagnetic (TEM) transmission lines with thin inner conductors are often used for generating standard known test fields. In all cases it is desirable that only the dominant TEM mode should propagate. In the EMC measurements, an object under test is placed inside of a TEM cell. The field from the TEM mode incident upon this scattering object is identical to that of a plane wave in a free space. However, the scattered field produced by the object in the TEM cell is different from the scattered field produced by the object in a free space, because of multiple reflections from the TEM cell walls, or equivalently, the mutual coupling between the object and the TEM cell. The purpose of this paper is to discuss the loading effects, i.e., the electromagnetic field distortion caused by an object under test in a TEM cell. In the theoretical analysis, the frequency domain integral equation for the magnetic field, or equivalently, the current density on the surface of a perfectly conducting cylinder in a parallel plate waveguide is solved by the method of moments to predict the degree of magnetic field distortion. The experimental investigations are performed by mounting a number of electrically small half loops on the surface of the perfectly conducting cylinder in a TEM cell. The loading effects in terms of magnetic field distortion are analyzed as the ratio of one of the object dimensions (height) to the separation distance between the inner conductor and the ground plane of the TEM cell. Also, the response of an electrically small loop to both the magnetic and electric components of the electromagnetic field is used to measure the phase relation between the magnetic and electric fields, which in turn can be used to determine the degree of degradation of the TEM mode due to the presence of the perfectly conducting cylinder. These theoretical and experimental results are compared with the available quasi electrostatic results.

TN1030. McCarty, R. D. Four mathematical models for the prediction of LNG densities. Nat. Bur. Stand. (U.S.) Tech. Note 1030; 1980 December. 84 p. SN003-003-02285-3.

Key words: cell model; comparisons; computer programs; corresponding states; equation of state; hard sphere; LNG; mixtures; PVTx data; revised Kosek and McKinley.

Four mathematical models of the equation of state for LNG like mixtures are presented. The four models include an extended corresponding states model, a cell model, a hard sphere model and a revised Klosek and McKinley model. Each of the models has been optimized to the same experimental data set which included data for pure nitrogen, methane, ethane, propane, iso and normal butane, iso and normal pentane and mixtures thereof. For LNG like mixtures (mixtures of the orthobaric liquid state at temperatures of $120 \mathrm{~K}$ or less and containing at least $60 \%$ methane, less than $4 \%$ nitrogen, less than $4 \%$ each of iso and normal butane and less than $2 \%$ total of iso and normal pentane), all of the models are estimated to predict densities to within $0.1 \%$ of the true value. The revised Klosek and McKinley model is valid only for mixtures within the range of temperature and composition specified above while the other three models are valid for a broader range of pressure, temperature and composition. The experimental PVTx data set used in the optimization together with comparisons are given and listings of computer programs for each of the models are included.

TN1031. Young, M. The use of LEDs to simulate weak YAG-laser beams. Nat. Bur. Stand. (U.S.) Tech. Note 1031; 1981 January. 48 p. SN003-003-02290-2.

Key words: laser simulator; light-emitting diodes; YAG laser.

The purpose of this report is to determine whether and under what conditions a light-emitting diode may be used to simulate a weak YAG-laser beam that has been scattered by a distant reflecting object. By examining the differences between laser radiation and LED radiation, we conclude that there is no theoretical reason that a LED may not be used in place of the laser beam.

TN1031, Revised. Young, M. The use of LEDs to simulate weak YAGlaser beams. Nat. Bur. Stand. (U.S.) Tech. Note 1031; 1981 January. 48 p. Available from: NTIS; PB 81-172470.

Key words: laser simulator; light-emitting diodes; YAG laser.

The purpose of this report is to determine whether and under what conditions a light-emitting diode may be used to simulate a weak YAG-laser beam that has been scattered by a distant reflecting object. By examining the differences between laser radiation and LED radiation, we conclude that there is no theoretical reason that a LED may not be used in place of the laser beam.

TN1032. Kim, E. M.; Franzen, D. L. Measurement of far-field and near-field radiation patterns from optical fibers. Nat. Bur. Stand. (U.S.) Tech. Note 1032; 1981 February. 48 p. SN003-003-02304-3.

Key words: attenuation; core diameter; far field; index profile; 
mode filter; numerical aperture; radiation angle; radiation patterns.

Systems are described for measuring the far- and near-field radiation patterns from optical fibers. Parameters which affect measurement precision, accuracy, resolution, and signal-to-noise ratio are discussed. Measurements utilizing radiation patterns are covered; this includes radiation angle (numerical aperture), attenuation using mode filters, index profile, core diameter, and mode volume transfer function. Experimental examples are given in many instances.

TN1033. Larsen, E. B.; Ries, F. X. Design and calibration of the NBS isotropic electric-field monitor (EFM-5), 02 to $100 \mathrm{MHz}$. Nat. Bur. Stand. (U.S.) Tech. Note 1033; 1981 March. 104 p. SN003-003-02311. 6.

Key words: electromagnetic field; electronic instrument design; field intensity meter; field strength measurement; isotropic antenna; radio frequency radiation; receiver calibration.

A broadband rf radiation monitor was developed at NBS for the frequency range of $200 \mathrm{kHz}$ to $1000 \mathrm{MHz}$. The isotropic antenna unit consists of three mutually-orthogonal dipoles, each $5 \mathrm{~cm}$ long by 2 $\mathrm{mm}$ wide. The receiver described in this paper has an accurate measurement range of 1 to $1000 \mathrm{~V} / \mathrm{m}$. The readout indication is in terms of the Hermitian or "total" magnitude of the electric (E) field strength, which is equal to the root-sum-square value of three perpendicular E-field components at the measurement point. Previous radiation monitors designed at NBS have demonstrated the accuracy of a crossed-dipole sensor for measuring the total E-field magnitude of complicated electromagnetic fields. Several prototype units of an advanced instrument have been fabricated and tested. This instrument, the EFM-5 Electric Field Monitor, has nearly perfect isotropy over a $60 \mathrm{~dB}$ dynamic range. The dial readout units are $\mathrm{dB}$ above $1 \mathrm{~V} / \mathrm{m}$ $(\mathrm{dBV} / \mathrm{m})$ and $\mathrm{V} / \mathrm{m}$. Three ranges cover 0 to $60 \mathrm{dBV} / \mathrm{m}$ (1 to 1000 $\mathrm{V} / \mathrm{m})$.

The electronic circuitry of the EFM-5 obtains the total magnitude of all field polarizations and all signals in the entire frequency band. The sensor response is isotropic; that is, there is no preferred field polarization or preferred direction of arrival of the incoming wave. Therefore this probe is well suited for measuring the near field of an emitter, including regions of multiple reflection, standing waves, etc. The EFM.5 can be used to monitor either the weak plane-wave fields in the far zone of a transmitting antenna, or the complicated fields only $5 \mathrm{~cm}$ from an of leakage source. The special features, electronic design, physical construction, and calibration are described in this paper.

TN1034. Danielson, B. L. Backscatter measurements on optical fibers. Nat. Bur. Stand. (U.S.) Tech. Note 1034; 1981 February. 52 p. SN003-003-02303-5.

Key words: backscattering; capture fractions; fiber scattering; optical time domain reflectometry; Rayleigh scattering.

An optical time domain reflectometer (OTDR) and its components are described in detail. The system performance for this device is examined. Experimental methods are described for the measurement of several parameters of interest in the characterization of optical fibers using the OTDR. These parameters include scattering loss and capture fractions for unperturbed fibers. Experimental capturefraction values are reported for several step and graded-index fibers and these results are compared with theoretical predictions. Rayleigh backscatter signatures are also presented for several fibers from different manufacturers. Fault signatures are shown for some intrinsic and extrinsic fiber perturbations.

TN1035. Siegwarth, J. D.; LaBrecque, J. F. A portable calibration densimeter for use in cryogenic liquids. Nat. Bur. Stand. (U.S.) Tech. Note 1035; 1981 March. 20 p. SN003-003-02308-6.

Key words: densimeter; density reference system; liquid methane;
LNG.

A portable densimeter designed specifically for calibrating liquefied natural gas densimeters but suitable for density measurement of a wide range of liquids, is described. The densimeter has been compared to the Density Reference System densimeter at the National Bureau of Standards and found to agree to well within the combined systematic error. The density results of this instrument and that of the DRS densimeter are statistically indistinguishable.

TN1036. Tryon, P. V. The bispectrum and higher-order spectra: A bibliography. Nat. Bur. Stand. (U.S.) Tech. Note 1036; 1981 April. 24 p. SN003-003-02312-4.

Key words: bispectrum; cumulant spectra; nonlinear time series; polyspectra; spectrum analysis; statistics; time series.

The bispectrum or Fourier transform of the 3d order moments of a time series is useful for the study of nonlinear or non-Gaussian phenomena. This bibliography cites 134 papers covering both theory and application. The entries are classified by content with special effort made to indicate papers that contain material on the computation, display and interpretation of the bispectrum.

TN1037. Wilson, P. F.; Chang, D. C.; Ma, M. T. Excitation of a TEM cell by a vertical electric Hertzian dipole. Nat. Bur. Stand. (U.S.) Tech. Note 1037;-1981 March. 44 p. SN003-003-02348-5.

Key words: characteristic impedance; field distribution; Hertzian dipole; integral equation; rectangular coaxial transmission line; TEM cell.

The excitation of a transverse electromagnetic (TEM) cell by a vertical electric Hertzian dipole is analyzed where the gap between the septum and side wall is assumed to be small. Approximate expressions for the field distribution and characteristic impedance are derived. These expressions are numerically evaluated for some typical geometries, and good agreement with previously published results is shown. The formation also allows a vertical offset for the septum position, thus offering more flexibility of increasing the size of the test area to accommodate larger pieces of test equipment.

TN1038. Young, M. Refracted-ray scanning (refracted near-field scanning) for measuring index profiles of optical fibers. Nat. Bur. Stand. (U.S.) Tech. Note 1038; 1981 May. 56 p. SN003-003-02342-6.

Key words: fiber index profile; index profile; near-field scanning; optical communications; optical fiber; optical waveguide; refracted near-field scanning; refracted-ray scanning; resolution limit.

The purpose of this work is twofold. First, it provides an elementary description and tutorial overview of the new refracted-ray method of measuring fiber-index profiles. Second, it presents new results concerning the theoretical foundation, the linearity and precision, resolution limit and edge response, and other aspects of the method. In particular, we find that index differences may be measured to 5 percent or better and spatial resolution is diffraction limited. We conclude by showing about 3 percent agreement with another laboratory and good agreement with numerical-aperture measurements performed by participants in a round-robin experiment.

TN1039. Ely, J. F.; Hanley, H. J. M. A computer program for the prediction of viscosity and thermal conductivity in hydrocarbon mixtures. Nat. Bur. Stand. (U.S.) Tech. Note 1039; 1981 April. 80 p. Available from: NTIS; PB 81-214165.

Key words: computer program; density; extended corresponding states; fluid mixtures; thermal conductivity; viscosity.

A model for the prediction of the density, viscosity and thermal conductivity of non-polar fluid mixtures over the entire range of pVT states is presented. The model is based on the extended corresponding states model and covers molecular weight ranges including $\mathrm{C}_{20}$. Only 
pure component equilibrium data such as the critical constants are required as input to the calculation procedure--no transport data are required. Extensive comparisons with experimental data for pure fluids and binary mixtures are presented. The average percentage deviation for both the viscosity and thermal conductivity was observed to be less than 8 percent. A computer program (TRAPP) which performs the calculations reported in this manuscript is described and listed in the Appendices.

TN1040. Sreenivasiah, I.; Chang, D. C.; Ma, M. T. A method of determining the emission and susceptibility levels of electrically small objects using a TEM cell. Nat. Bur. Stand. (U.S.) Tech. Note 1040; 1981 April. 52 p. SN003-003-02316-7.

Key words: analysis; dipole moments; electrically small objects; emission; measurements; quadrupoles; susceptibility; TEM cell.

An electrically small radiating source of arbitrary shape may, to a first order, be modeled by an equivalent dipole system consisting of three orthogonal electric dipoles and three orthogonal magnetic dipoles each excited with arbitrary amplitude and phase. Determination of the individual electric dipole moments and the cross-components of such an equivalent dipole system for the emission case by tests inside a TEM cell, was described in an earlier work by Sreenivasiah, Chang and $\mathrm{Ma}$, assuming a constant TEM modal field distribution in the space occupied by the test object. A method of accounting for the first-order variation in the TEM modal field distribution is presented in this report. This involves the inclusion of electric quadrupole terms in the modeling of the test object. Using the reciprocity principle, the same method is extended to the determination of susceptibility levels of electrically small test objects. Some experimental results for the susceptibility test, demonstrating the importance of the quadrupole terms, are presented.

TN1041. Siegwarth, J. D.; LaBrecque, J. F. Cryogenic fluids density reference system: Provisional accuracy statement (1980). Nat. Bur. Stand. (U.S.) Tech. Note 1041; 1981 A pril. 60 p. SN003-003-02346-9.

Key words: densimeter; density reference system; liquid methane; LNG.

The improved Density Reference System, the reference densimeter, and the method of determining sample density are described.

The uncertainty of the density reference system is $\pm 0.055 \%$. The contribution from the estimated systematic error in density was $\pm 0.022 \%$. The estimated uncertainty caused by random error is three times the standard deviation of $0.011 \%$ and is based on sixty-three measurements of the densities of saturated liquid methane. The total density uncertainty is taken to be the sum of the systematic and random errors. This applies to the density range of 400 to $480 \mathrm{~kg} / \mathrm{m}^{3}$ at pressures from 0.8 to $4 \mathrm{bar}$ absolute and temperatures between 109 and $128 \mathrm{~K}$. This accuracy statement is expected to apply over ranges of at least 400 to $1000 \mathrm{~kg} / \mathrm{m}^{3}$ in density, 77 to $300 \mathrm{~K}$ in temperature, and 0.8 to $7 \mathrm{bar}$ in pressure though the accuracy over these ranges has not been verified.

Compared to the original, this improved density reference system has greater accuracy, speed, reliability, and flexibility.

TN1042. Gans, W. L.; Nahman, N. S.. Shielded balanced and coaxial transmission lines-Parametric measurements and instrumentation relevant to signal waveform transmission in digital service. Nat. Bur. Stand. (U.S.) Tech. Note 1042; 1981 June. 260 p. Available from: NTIS; PB 82.105297.

Key words: bit error rate; coaxial transmission lines; digital communication impulse response; instrumentation; measurements; shielded balanced transmission lines; time domain; transmission line model; waveform.

A method is presented for determining the impulse and step responses of a shielded cable using time domain terminal measurements and a physically based mathematical model for the transmission line properties of the cable. The method requires a computer controlled time domain measurement system and was implemented using the NBS Automatic Pulse Measurement System (APMS). Data are also developed for the frequency domain complex propagation function (attenuation and its related minimum-phase shift). The method is applied to 12 shielded paired-conductor (balanced) cables and 5 coaxial cables. Time domain responses are presented for three nominal cable lengths, $60 \mathrm{~m}(200 \mathrm{ft}), 150 \mathrm{~m}(500$ $\mathrm{ft})$, and $300 \mathrm{~m}(1000 \mathrm{ft})$. The time domain responses are applied to the estimation of bit error rate increases due to the insertion of the cables into a digital signaling system employing a balanced polar NRZ waveform. Also discussed is the application of the time domain responses to time domain reflectometry techniques for cable acceptance tests and field-site testing of installed cables.

TN1043. Day, G. W., ed. The characterization of optical fiber waveguides. A bibliography with abstracts, 1970-1980. Nat. Bur. Stand. (U.S.) Tech. Note 1043; 1981 June. 72 p. SN003-003-02338.8.

Key words: bibliography; measurements; optical communications; optical fibers.

This bibliography contains approximately 450 citations of papers concerning the characterization of optical fiber waveguides. Papers from scientific journals, trade journals, and conferences are included along with book chapters. The citations are organized by parameter measured and measurement method. Where published abstracts are available, they are included.

TN1044. Weidman, M. P.; Hudson, P. A. WR 10 millimeter wave microcalorimeter. Nat. Bur. Stand. (U.S.) Tech. Note 1044; 1981 June. 16 p. Available from: NTIS; PB 81.243412.

Key words: calorimeter; millimeter wave; power; standard.

A microcalorimeter has been built in WR 10 waveguide, 75.110 $\mathrm{GHz}$, to serve as a power standard at the National Bureau of Standards (NBS). Included here is an evaluation of the errors in using the microcalorimeter for the measurement of effective efficiency of bolometer mounts.

The error analysis shows a systematic uncertainty of \pm .83 percent and a random uncertainty of .37 percent.

TN1045. McCarty, R. D.; Jacobsen, R. T. An equation of state for fluid ethylene. Nat. Bur. Stand. (U.S.) Tech. Note 1045; 1981 July. 164 p. SN003.003.02351.5.

Key words: computer programs; equation of state; ethylene thermodynamic properties; tabular values.

A thermodynamic property formulation for ethylene, developed as a part of a joint industry-government project, is presented. The formulation includes an equation of state, vapor pressure equation, and equation for the ideal gas heat capacity. The coefficients were determined by a least squares fit of selected experimental data. Comparisons of property values calculated using the equation of state with measured values are given. The equation of state is not valid in the critical region $\left(\rho_{c} \pm 0.3 \rho_{c}\right.$ for temperatures of $\left.T_{c} \pm 0.05 T_{c}\right)$. Errors on the order of 20 percent for derived properties and 10 percent for density may be encountered near the critical point. Tables of the thermodynamic properties of ethylene for the liquid and vapor phases for temperatures from the freezing line to $450 \mathrm{~K}$ with pressures to 40 $\mathrm{MPa}$ are presented. The equation of state and its derivative and integral functions for calculating thermodynamic properties are included. Estimates of the accuracy of calculated properties are given. A guide for use of computer programs for the calculation of thermodynamic properties of ethylene with listings of subprograms and a sample program to illustrate the use and results of the program are included.

TN1046. Day, G. W. Measurement of optical fiber bandwidth in the frequency domain. Nat. Bur. Stand. (U.S.) Tech. Note 1046; 1981 September. 48 p. SN003-003-02377-9.

Key words: fiber optics; optical communications; optical fiber 
bandwidth; optical fiber distortion; optical fibers.

The design, evaluation, and performance of a system for determining the magnitude of the transfer function (hence, bandwidth) of a multimode optical fiber are presented. The system operates to about $1450 \mathrm{MHz}$ using a tracking generator/spectrum analyzer combination for narrowband detection. It is constructed, almost entirely, from commercially available components. The system is less complex and easier to use than an equivalent time domain system and the measurement precision is comparable. Background information on time and frequency domain specifications, fiber bandwidth limitations, and alternate frequency domain techniques is also presented.

TN1047. Nahman, N. S.; Guillaume, M. E. Deconvolution of time domain waveforms in the presence of noise. Nat. Bur. Stand. (U.S.) Tech. Note 1047; 1981 October. 122 p. Available from: NTIS; PB $82-135153$

Key words: deconvolution; impulse response; inverse filtering; noise contamination; time domain; waveforms.

Deconvolution or inverse filtering was used to determine the impulse response of a system using noisy input and output time domain sequences (discrete data). Frequency and time domain methods were studied along with the synthesis of the filters required to obtain stable and smooth results. For the methods studied it was concluded that the superior technique was provided by an optimal frequency domain method implemented via the FFT. Also, it is pointed out that the time domain methods are only in their infancy and still retain the promise of avoiding transform domain filtering. Examples are presented in which the impulse responses are determined in the presence of varying degrees of noise for a coaxial transmission line, a wave-shaping filter, and a broadband antenna.

TN1133. Evans, D. D. Calculated operating temperatures of thermally insulated electric cables. Nat. Bur. Stand. (U.S.) Tech. Note 1133; 1981 January. 41 p. SN003.003.02279.9.

Key words: electrical fire; electric cable; models; thermal insulation; wiring system.

Steady-state operating temperatures of current-carrying electric cables buried axially in cylinders of thermal insulation were calculated. Combinations of six types of nonmetallic electric cable, two thermal insulation materials, five thermal insulation thicknesses and a range of currents both greater and less than the typical rated service currents for the cables were studied.

These calculations show that thermally insulated electric cables carrying the rated current may exceed the rated operating temperature limit for common cable jacket materials.

TN1135. Rossiter, W. J., Jr.; Mathey, R. G.; Busching, H. W.; Cullen, W. C. Cooling of bitumen during construction of built-up roofing systems-A mathematical model. Nat. Bur. Stand. (U.S.) Tech. Note 1135; 1981 March. 76 p. SN003-003-02302-7.

Key words: bitumen application temperatures; bituminous roofing; built-up roofing; cooling of roofing bitumens; mathematical model; roofing.

Construction of bituminous built-up roofing systems in the United States generally involves the application of hot bitumen to the roofing components, including deck, insulation, and felts, to adhere them to each other and to form a waterproof membrane. Adequate adhesion of the bitumen to the roofing component materials may be obtained only when the hot bitumen is applied at a viscosity sufficient to flow uniformly, to cover the component surfaces or substrate completely, and to provide the proper thickness. During construction, rapid cooling of hot bitumen increases its viscosity significantly. If the viscosity becomes too high, poor adhesion between components, voids within the bitumen, and an excessive and non-uniform thickness of the bitumen may result.
This report describes a mathematical model based on finite. difference equations for calculating transient heat flow to estimate the cooling time of hot roofing bitumen. Estimates of the time required for hot bitumen to cool from its application temperature to $300^{\circ} \mathrm{F}$ $\left(149^{\circ} \mathrm{C}\right)$ were computed as a function of material and environmental factors including: quantity of applied bitumen, bitumen application or contact temperature, air temperature, wind speed, and thermal properties of the bitumen and of the roofing components. The model was used to predict cooling times expected for hot asphalt applied to typical substrates with thermal properties representative of those of polyurethane foam and glass fiber insulation boards, insulating concrete, plywood, concrete, and steel decks, and roofing felt on decks or insulations. In addition, the model was used to predict cooling times for hot coal tar pitch applied to concrete and to felt adhered to glass fiber insulation. The results of the calculations demonstrate the widely-varying bitumen cooling times which depend upon the component material to which the bitumen is applied and the environmental conditions during application. Under certain environmental conditions, hot bitumen applied to some substrates cools extremely rapidly. In these cases, sufficient time for proper application may not be available.

TN1136. Waksman, D.; Streed, E.; Seiler, J. NBS solar collector durability/reliability test program plan. Nat. Bur. Stand. (U.S.) Tech. Note 1136; 1981 January. 85 p. SN003-003-02283-7.

Key words: accelerated aging; durability; environmental exposure; reliability; solar collectors; solar materials; stagnation testing.

The test program described in this plan is designed to evaluate both approved and proposed solar collector test procedures and to correlate laboratory, accelerated field and simulated operational exposures with actual field data.

The tests and exposure procedures described herein are intended to determine the influence of environmental exposure parameters that could affect the degradation of solar collectors and their materials. They are also intended, to the extent possible, to provide a correlation between changes that occur at the materials and the collector component levels. It is expected that the data obtained through their use will lead to more meaningful reliability/durability tests for solar collectors and their materials.

A wide variety of commercially available solar collectors and multiple material coupons of collector components are being tested at sites in different climatic regions. Appropriate laboratory tests are being conducted during the same time frame to determine physical and material properties for comparison with field test data and operational collector experience. The data obtained from these tests will be analyzed and correlated with that obtained from the current Government Demonstration Program and other related Governmentsponsored programs.

TN1137. Noyce, J. R. Evaluation of methods for the assay of radium228 in water. Nat. Bur. Stand. (U.S.) Tech. Note 1137; 1981 February. 18 p. SN003-003-02294-2.

Key words: analysis; assay; evaluation; radioactivity; radiochemistry; radium-228; reference method; water.

The technical literature from $196 ?$ to May 1980 was searched for methods for assaying radium-228 in water. These methods were evaluated for their suitability as potential EPA reference methods for drinking water assays. We suggest the present EPA reference method (Krieger, 1976) be retained but improved, and a second method (McCurdy and Mellor, 1979), which employs $\beta-\gamma$ coincidence counting, be added. Included in this report is a table that lists the principal features of 17 methods for radium-228 assays.

TN1138. Kusuda, T.; Bean, J. W. Savings in electric cooling energy by the use of a whole-house fan. Nat. Bur. Stand. (U.S.) Tech. Note 1138; 1981 May. 39 p. SN003-003-02317-5.

Key words: building thermal performance; energy calculation; 
energy conservation; thermal comfort; whole-house ventilation.

Hour-by-hour cooling performances of a typical ranch house, with and without the use of a whole-house fan, were compared for the climate conditions throughout the contiguous United States. The comparative analyses were made by the use of NBSWHF, a modified version of NBSLD, to simulate the complex thermal coupling of whole-house-fan ventilated attic space. The calculations were performed for two operational modes: a cyclic fan mode and a stepwise continuous mode. The calculation predicted a large cooling energy savings as compared to the house without the use of the whole-house fan, without significant deterioration of indoor thermal comfort.

TN1139. Collé, R.; Rubin, R. J.; Knab, L. 1.; Hutchinson, J. M. R. Radon transport through and exhalation from building materials: $A$ review and assessment. Nat. Bur. Stand. (U.S.) Tech. Note 1139; 1981 September. 101 p. SN003-003-02360-4.

Key words: buildings; concrete; diffusion; exhalation; materials; measurement; permeability; radon; transport.

This report was prepared, at the request of the U.S. Environmental Protection Agency, for the purpose of reviewing, assessing, and summarizing what is currently known about radon transport through and exhalation from building materials. In four chapters, the report 1) considers the routes of entry of radon into buildings, describes the basic models for radon transport through building materials, critically reviews the small number of existing values for the necessary transport coefficients, and summarizes the solutions of both steady. state and time-dependent transport cases; 2) reviews and considers how the microstructural properties and internal characteristics of building materials may affect the transport and exhalation of radon; 3) considers the exhalation process from a more macroscopic, phenomenological viewpoint, and summarizes selected experimental data on radium concentrations in building materials, radon flux and exhalation from soils and building materials, and the effects of meteorological variables on radon exhalation; and 4) reviews and assesses various measurement methodologies that are used for laboratory and in situ studies of radon transport and exhalation. Needs for further research in each area are also recommended.

TN1140. Streed, E.; Waksman, D. Uncertainty in determining thermal performance of liquid-heating flat-plate solar collectors. Nat. Bur. Stand. (U.S.) Tech. Note 1140; 1981 A pril. 97 p. SN003-003-02306-0.

Key words: collector rating; measurement; solar collector; standards; thermal performance; uncertainty.

Thermal performance measurements of eight liquid-heating flatplate solar collectors were conducted with two to four collectors of each type at four outdoor test sites. Tests were performed in accordance with the procedure prescribed by ASHRAE Standard 93 . 77. Statistical analysis of data sets for each collector type within test sites and between test sites was done using ASTM recommended methods to evaluate test method measurement uncertainty. Illustrations of the influence of thermal performance data uncertainty are presented for collector material degradation, collector rating and calculated system performance.

TN1141. Mighell, A. D.; Hubbard, C. R.; Stalick, J. K. NBS*AIDS80: A FORTRAN program for crystallographic data evaluation. Nat. Bur. Stand. (U.S.) Tech. Note 1141; 1981 April. 54 p. SN003.003. 02309.4.

Key words: computer evaluation; crystallographic data analysis; JCPDS-International Centre for Diffraction Data; metric symmetry; NBS Crystal Data Center; powder diffraction; unit cell.

Techniques for the computer-assisted evaluation of crystallographic data have been developed to improve the data compilations of the NBS Crystal Data Center and the JCPDS-International Centre for
Diffraction Data. The resulting computer program, NBS*AIDS80, can be used for the analysis of unit-cell and powder diffraction data by the general scientific community as well. Research and analysis components include calculation of standard cells and space groups, determination of metric symmetry, checking the data for consistency, generation of d-spacings and indices, and analysis of powder patterns with calculation of figures of merit. Detailed input and output specifications are given.

TN1142. Shorten, F. J., ed. NBS reactor: Summary of activities July 1979 to June 1980. Nat. Bur. Stand. (U.S.) Tech. Note 1142; 1981 May. 211 p. SN003.003-02314-1.

Key words: activation analysis; crystal structure; diffraction; isotopes; molecular dynamics; neutron; neutron radiography; nondestructive evaluation; nuclear reactor; radiation.

This report summarizes all those programs which depend on the NBS reactor. It covers the period from July 1979 through June 1980. The programs range from the use of neutron beams to study the structure and dynamics of materials through nuclear physics and neutron standards to sample irradiations for activation analysis, isotope production, radiation effects studies, neutron radiography, and nondestructive evaluation.

TN1143. Yonemura, G. T. Visual acuity testing of radiographic inspectors in nondestructive inspection. Nat. Bur. Stand. (U.S.) Tech. Note 1143; 1981 June. 29 p. SN003-003-02330-2.

Key words: acuity tests; nondestructive testing; quality testing; radiograph evaluation; visual inspection; visual testing.

Visual acuity tests for radiographic inspectors should be correlated with the type of tasks encountered in real world radiography. The testing procedures should be capable of assessing differences in day to day performance of a given inspector as well as the performance of one inspector relative to other inspectors. Single line targets with specific parametric values for contrast, width, and blur are recommended to provide a means for testing a radiographic inspector for visual acuity. These targets may be used for periodic tests by the employing organization or for more frequent self testing by the inspector. Statistics from the National Health Survey, procedures recommended by the NAS-NCR Committee on Vision and real world radiographs have been utilized in arriving at recommended test configurations.

TN1144. Turgel, R. S.; Oldham, N. M.; Stenbakken, G. N.; Kibalo, T. H. NBS phase angle calibration standard. Nat. Bur. Stand. (U.S.) Tech. Note 1144; 1981 July. 143 p. SN003-003-02344-2.

Key words: calibration; digital; microcomputer; phase angle; sinewave generator; standard; synthesis.

A detailed description is given of the construction and operation of a calibration standard designed for use with phase meters. The resolution of the calibrator is approximately 2 millidegrees and its accuracy is of the order of 5-10 millidegrees depending somewhat on frequency and amplitude ratio of the output.

The calibration standard is a source of two sinusoidal signals with an accurately known phase angle between them. The signals are generated using digital sinewave synthesis and are programmable in amplitude $(0-100 \mathrm{~V})$ and in frequency $(2 \mathrm{~Hz}-5 \mathrm{kHz})$. Operation is controlled from a front panel keyboard (or remotely with an adaptor) under control of a microprocessor. Selected parameters are displayed on an alpha-numeric readout.

To ensure accuracy, the system has an auto-zero feedback loop that compensates for residual differential phase shifts in the output amplifiers. The compensation scheme measures the departure of the output signals from true quadrature and applies a digital correction to the computing circuits of the sinewave generator.

TN1145. Souders, T. M.; Flach, D. R.; Bell, B. A. A calibration service for analog-to-digital and digital-to-analog converters. Nat. 
Bur. Siand. (U.S.) Tech. Note 1145; 1981 July. 73 p. SN003-00302352.4.

Key words: analog-to-digital converter; calibration service; data converter; differential linearity; digital-to-analog converter; error measurement; gain; input noise; linearity; offset.

An NBS calibration service for high performance 12- to 18-bit analog-to-digital converters (ADC's) and digital-to-analog converters (DAC's) is described. The service offers comprehensive measurements of linearity, differential linearity, gain, offset, and rms input noise (for ADC's), with systematic uncertainties as low as 3 ppm. Measurements are made at a minimum of 1024 different codewords. The measurement approach, design features, test programs and data reduction techniques are documented, as are the methods of error estimation and quality control. Representative reports of tests are included for both DAC's and ADC's.

TN1146. Jenkins, D. R.; Mathey, R. G.; Knab, L. I. Moisture detection in roofing by nondestructive means-A state-of-the-art survey. Nat. Bur. Stand. (U.S.) Tech. Note 1146; 1981 July. 82 p. SN003-003-02340-0.

Key words: built-up roofing; moisture; moisture detection; nondestructive evaluation; nondestructive testing; roofing; thermal resistance.

A literature survey is presented of nondestructive evaluation (NDE) methods for detection of moisture in roofing systems. The methods discussed include the use of capacitance-radio frequency instruments, capacitance-microwave instruments, nuclear meters, and thermal infrared scanners. For each method, the principles of operation are reviewed and the measured properties which are affected by moisture are identified. Factors other than moisture which may affect the response of the instruments are also described for each method. These factors produce responses which are similar to those due to moisture and include non-uniformities in the roofing system, roof construction details, and building equipment. The use of each NDE method in actual moisture surveys is reviewed.

It is emphasized in the report that the validity of roofing moisture surveys depends on both a knowledge of the factors noted above and a familiarity with roofing practice. Furthermore, cores of the roofing system at selected points are needed to confirm NDE observations.

To define operating conditions for infrared scanners, calculated temperatures of roof surfaces over dry and over wet insulation are presented for representative night and day conditions.

TN1147. Peavy, S. T.; Bremer, S. G. Test problems and results for OMITAB 80. Nat. Bur. Stand. (U.S.) Tech. Note 1147; 1981 November. 316 p. SN003-003-02379-5.

Key words: accuracy; ANS FORTRAN; computer system installation; examples; OMNITAB 80; software; test problems.

The lack of test problems and results for many computer software packages is a great hindrance to both the systems programmer and the general user. In this publication a set of 69 test problems and results for the OMNITAB 80 system is provided to assist individuals in checking the installation of the OMNITAB 80 system on their particular computer. The general user will also find these descriptive examples instructive in the use of the OMNITAB 80 system.

TN1148. Treado, S.; Kusuda, T. Solar radiation and illumination. Nat. Bur. Stand. (U.S.) Tech. Note 1148; 1981 November. 31 p. Available from: NTIS; PB 82-135823.

Key words: daylighting; illuminance; illumination; irradiance; solar radiation.

Experimental data were collected and analyzed under various cloud cover conditions to establish the relationship between solar irradiance and illuminance. Empirically derived equations are presented for estimating diffuse and total illuminance as a function of total and diffuse solar radiation.
TN1149. Campbell, P. G.; Martin, J. W.; McKnight, M. E. Short-term evaluation procedures for coatings on structural steel. Nat. Bur. Stand. (U.S.) Tech. Note 1149; 1981 September. 43 p. SN003-00302367.1.

Key words: accelerated aging tests; coatings; corrosion; predictability; reproducibility; time to failure.

This report presents the findings of the first of a two-phased study to aid the Federal Highway Administration in evaluating and selecting protective coatings for steel. The objectives of the study are to review existing short-term test procedures for selecting coatings, and to discuss analytical measurement techniques for characterizing coating systems and for monitoring coating degradation.

In assessing current accelerated aging testing procedures, several deficiencies became apparent. These included the reported lack of reproducibility in the rankings for different iterations of the same short-term test and the lack of correlatability between the rankings of short-term laboratory and long-term outdoor exposure tests.

It $w$ as concluded that, at the present time, coating manufacturers and users do not depend heavily on accelerated test results for making durability assessments. Instead durability assessments are based on outdoor exposure performance. Recommendations are made to design future short-term test procedures using reliability theory.

TN1152. Pommersheim, J. M.; Mathey, R. G. Mechanical performance of built-up roofing membranes. Nat. Bur. Stand. (U.S.) Tech. Note 1152; 1981 December. 60 p. Available from: NTIS; PB 82-151259.

Key words: adhesive; bitumen; bonding; built-up roofing membrane; complementary strain energy; felt; nonlinear; roofing membrane; splitting; strain energy; stress; substrate.

For built-up roofing membranes with either linear or non-linear stress-strain behavior, fully bonded to an underlying deck or substrate which undergoes displacement, it is the equality of the complementary strain energy of the fabric or felt layer with the strain energy of the bonding adhesive or bitumen layer, which governs both the conditions under which membrane integrity is lost and the mode of failure by either membrane splitting or adhesive bonding. The testing criteria developed are applied to a sample case.

\subsection{CONSUMER INFORMATION SERIES}

Practical information, based on NBS research and experience, covering areas of interest to the consumer. Easily understandable language and illustrations provide useful background knowledge for shopping in today's technological marketplace.

No publications issued in this series during this period. 
A special series of interim or final reports on work performed by NBS for outside sponsors (both government and non-government). In general, initial distribution is handled by the sponsor; public distribution by the National Technical Information Service (NTIS), Springfield, VA 22161 , in paper copy or microfiche form unless otherwise stated. When ordering this series from NTIS you must order it by the "COM, PB, or AD" number listed at the end of each entry.

NBSIR 77-1311. Kotter, F. R.; Misakian, M. AC transmission line field measurements. 1981 October. 83 p. Available from: NTIS; PB 82. 133554.

Key words: calibration; calibrators; electric fields; electric field strength; electric power transmission; field strength; magnetic fields.

The influence of temperature, relative humidity, frequency, waveform, proximity of the observer, as well as other parameters on the accuracy of a small number of two commercially available ac electric field meters is documented. Procedures for the calibration of these instruments are reviewed and guidance is given for the construction of a calibration facility suitable for use in achieving calibrations to an accuracy of 1 percent. Measurement errors which develop when these meters are used in a highly nonuniform singlephase field are investigated theoretically and experimentally. The performance of a magnetic field probe provided as an accessory with one of the field meters is described, and an analysis of the accuracy to be expected from the use of simple multi.turn rectangular loops for the calibration of such magnetic field meters is given.

NBSIR 78-869. Daywitt, W. C. Error equations used in the NBS Earth

Terminal Measurement System. 1977 December. 30 p. Available from: NTIS; PB 276253.

Key words: C/kT; EIRP; error analysis; G/T; precision measurements; radio star; satellite communications.

An outline for the derivation of equations employed in a measurement and error analysis of satellite EIRP using a calibrated radio star is presented. A table showing analysis results at $7.55 \mathrm{GHz}$ using Cassiopeia $\mathrm{A}$ for a satellite at $12^{\circ}$ elevation angle is given. The quadrature sum of the systematic errors appearing in the table is $10.1 \%$. Also presented is a curve of the systematic errors as a function of elevation angle showing a $7.3 \%$ minimum at high elevation angles. Included are working equations for the calculation of correction and errors.

NBSIR 78.871. Driver, L. D.; Ries, F. X.; Rebuldela, G. NBS rf voltage comparator. 1978 December. 25 p. Available from: NTIS; PB 292373.

Key words: comparator; rf voltage; rf voltmeters.

This manual describes a wideband of voltage comparator which covers the frequency range of $100 \mathrm{kHz}$ to $1 \mathrm{GHz}$ from $10 \mathrm{mV}$ to 20 volts. This device uses a pair of matched Schottky-barrier diodes in each independent channel of a 2 dual-channel configuration. The coaxial line sections are impedance compensated to assure a VSWR less than 1.03 up to $1 \mathrm{GHz}$. Applications covered are calibration of signal generators and if voltmeters. A troubleshooting and maintenance section is also included as well as illustrations and circuit diagrams to facilitate repair.

NBSIR 78-873. Tippet, J. C.; Chang, D. C. Higher order modes in rectangular coaxial line with infinitely thin inner conductor. 1978 March. 40 p. Available from: NTIS; PB 279054.

Key words: higher order modes; rectangular coaxial line; striplines.

The singular integral equation approach is used to derive the secular equations for both TE and TM waves in a rectangular coaxial line with zero thickness inner conductor. Approximations for the secular equations are found that reduce to simple expressions in terms of well-known special functions (elliptic integrals). When the strip width is exceedingly small or nearly equal to the width of the outer conductor, closed form expressions for the cut-off frequencies can be found by replacing the elliptic integrals by their asymptotic forms for modulus either near zero or one.

NBSIR 78-875. Voth, R. O. Producing liquid-solid mixtures of hydrogen using an Auger. 1978 February. 21 p. Available from: NTIS; PB 278723.

Key words: cryogenic; hydrogen; production efficiency; scraping Auger; slush; slush production.

An auger rotating inside a brass tube refrigerated with liquid helium was used to produce liquid-solid (slush) mixtures of hydrogen. The auger produced small particles of solid hydrogen so that the resulting mixture could be transferred and stored. The auger could produce slush continuously in an appropriate system; it could produce slush at pressures higher than the triple point pressure of the hydrogen; and the energy required to produce the slush was less than the energy required to produce slush hydrogen using the freeze-thaw process.

NBSIR 78-876. Kanda, M. Transients in resistively loaded antennas and their comparison with conical antennas and TEM horns. 1978 March. 35 p. Available from: NTIS; PB 279640.

Key words: conical antenna; EMP; FFT; loaded antenna; method of moments; TEM horn; time domain measurements; transients in resistivity.

The receiving and transmitting transient responses for a relatively short, linear antenna with continuous resistive loading are investigated theoretically and experimentally. The antenna considered is a nonconducting cylinder with continuously deposited, varying. conductivity, resistive loading.

The current distribution, the corresponding effective length, and the driving point impedance are calculated by use of the method of moments and compared with the Wu-King approximation. The receiving and transmitting transient responses are calculated in the frequency domain using the results of the effective length and driving point impedance. The use of FFT then allows the determination of transient fields for a known input waveform. The receiving and transmitting responses for a conical antenna and a TEM horn are also investigated theoretically to compare with the transient response of the resistively loaded antenna.

Time domain measurements were performed using a time domain antenna range with a time domain automatic network analyzer. The agreements between theory and experiments of the receiving and transmitting transient responses for the resistively loaded antenna, the conical antenna, and the TEM horn are very good.

The receiving transient response of the resistively loaded antenna indicates that an impulse shape of 70 ps duration is well preserved. This provides the unique capability of this antenna to measure fast, time-varying, transient fields with minimal pulse-shape distortion due to nonlinear amplitude or phase characteristics.

NBSIR 78-896. Jesch, R. L. Dielectric measurements of five different soil textural types as functions of frequency and moisture content. 1978 October. 26 p. Available from: NTIS; PB 291944.

Key words: attenuation; coaxial sample holder; density; dielectric properties; relative dielectric constant; soil textural types.

Open-circuit coaxial transmission-line sample holders were used to determine the electromagnetic dielectric properties of five soil textural types as functions of moisture level content and frequency. A brief outline of the theoretical basis is given for the open-circuit method along with the measurement system and the sample preparation. Experimental results are given for the different soil textural types for moisture levels ranging from 0 to 48 percent and for test frequencies ranging from 300 to $9300 \mathrm{MHz}$.

NBSIR 78-897. Jones, R. N.; Bussey, H. E.; Little, W. E.; Metzker, R. 
F. Electrical characteristics of corn, wheat, and soya in the 1-200 MHz range. 1978 October. 70 p. Available from: NTIS; PB 289812.

Key words: dielectric properties; grain; loss tangent; moisture.

A set of coaxial sample holders together with a measurement and data reduction technique has been developed and applied to the study of the dielectric properties $\left(\epsilon^{*}=\epsilon^{\prime}-\mathrm{j} \epsilon^{\prime \prime}\right)$ of wheat, corn, and soya over the 1 to $200 \mathrm{MHz}$ range. Particular attention was given to the behavior of the dielectric properties as a function of percent moisture content, frequency, and packing density. Data were also taken to evaluate the dependence of dielectric properties on temperature and sample holder configuration. Some study was also devoted to the correlation between dielectric constant $\left(\epsilon^{\prime}\right)$, loss factor $\left(\epsilon^{\prime \prime}\right)$, loss tangent $\left(\epsilon^{\prime \prime} / \epsilon^{\prime}\right)$, and percent moisture content. Particular emphasis was devoted to a study of high moisture corn (up to $40 \%$ ).

NBSIR 78-898. Kanda, M. Analytical and numerical techniques for analyzing an electrically short dipole with a nonlinear load. 1978 November. 21 p. Available from: NTIS; PB 289928.

Key words: Auger function; electrically-short dipole; iteration method; nonlinear differential equation; nonlinear load; timestepping finite difference equation technique.

An electrically short dipole with a nonlinear dipole load is analyzed theoretically using both the analytical and numerical techniques. The analytical solution is given in terms of the Auger function of imaginary order and imaginary argument, and is derived from the nonlinear differential equation for the Thévenin's equivalent circuit of a dipole with a diode. The numerical technique used was to solve the nodal equation using a time-stepping finite difference equation method. The nonlinear resistance of the diode is treated using an iteration technique. A comparison between the analytical and numerical solutions is given.

NBSIR 79.1591.4. Bullis, W. M., ed. Semiconductor technology program-Progress briefs. 1979 August. 12 p. Available from: NTIS; PB 81.154114.

Key words: electronics; integrated circuits; measurement technology; microelectronics; semiconductor devices; semiconductor materials; semiconductor process control; silicon.

This report provides information on the current status of NBS work on measurement technology for semiconductor materials, process control, and devices. Results of both in-house and contract research are covered. Highlighted activities include studies of: laser annealing of silicon, dopant density profiles, silicon dioxide film thickness standards, intrachip linewidth variation, photoresist sensitometry, optical linewidth measurements, optical imagery calculations, and moisture measurement by mass spectroscopy. In addition, brief descriptions of new and selected on-going projects are given. The report is not meant to be exhaustive; contacts for obtaining further information are listed. Compilations of recent publications and publications in press are also included.

NBSIR 79-1591-5. Bullis, W. M., ed. Semiconductor technology program-Progress briefs. 1979 December. 16 p. Available from: NTIS; PB 81-154122.

Key words: electronics; integrated circuits; measurement technology; microelectronics; semiconductor devices; semiconductor materials; semiconductor process control; silicon.

This report provides information on the current status of NBS work on measurement technology for semiconductor materials, process control, and devices. Results of both in-house and contract research are covered. Highlighted activities include: modeling of diffusion processes, analysis of model spreading resistance data, and studies of resonance ionization spectroscopy, resistivity-dopant density relationships in p-type silicon, deep level measurements, photoresist sensitometry, random fault measurements, power MOSFET thermal characteristics, power transistor switching characteristics, and gross leak testing. In addition, brief descriptions of new and selected on-going projects are given. The report is not meant to be exhaustive; contacts for obtaining further information are listed. Compilations of recent publications and publications in press are also included.

NBSIR 79-1600. Siegwarth, J. D. Radio frequency liquid level gauging in propane tank car safety tests-A feasibility study. 1979 January. 15 p. Available from: NTIS; PB 292978.

Key words: level gauging; propane; radio frequency.

Selected radio frequency (rf) resonances of an empty 30,300 liter $(8,000$ gallon) tank car have been measured to determine whether if can be used to gauge the propane liquid levels during tank car fire safety tests. The change of resonant frequencies of a small horizontal cylindrical tank as a function of liquid volume has been tested in order to estimate the precision to which the amount of propane in a tank car can be measured. The technique is applicable to routine tank car gauging.

NBSIR 79-1610. Belsher, D. R. Feasibility study on the use of a microwave system for the nondestructive evaluation of historic adobe structures. 1979 July. 32 p. Available from: NTIS; PB 298559.

Key words: adobe building materials; adobe soil; dielectric constant measurements; electromagnetic moisture measurements; nondestructive evaluation; radar soil measurements; soil moisture; underground radar; void detection.

A frequency-modulated continuous wave (FM-CW) radar system has been utilized at Tumacacori National Monument to provide usable on site information and to evaluate its potential for nondestructively measuring certain parameters associated with the soundness of historic adobe walls. The parameters of interest considered were layer thickness, presence, position, and thickness of voids or other inhomogeneities, and moisture content and its distribution. The results were generally favorable. The results indicate that an FM.CW system can nondestructively provide a major portion of the information needed to evaluate the soundness of adobe structures at a relatively low cost and in less time than present techniques. With some further work the FM.CW system can be developed into a useful archeological exploration and evaluation tool that should operate in rock and soils other than adobe.

NBSIR 79-1623. McHenry, H. I.; Rolfe, S. T. Fracture control practices for metal structures. 1980 January. 114 p. Available from: NTIS; PB 80-185648.

Key words: fracture control; fracture mechanics; mechanical properties; quality assurance; stress analysis; structural safety.

Fracture control practices are the engineering procedures and requirements that contribute to the prevention of fracture in metal structures. These practices are identified as the elements of fracture control and each element is defined and described. The systematic application of these practices to the prevention of fracture in a particular structure is accomplished by either the code approach or the performance specification approach to fracture control. In these two approaches, the consideration given to each of the elements of fracture control is discussed and illustrated with examples for specific types of structures. Selected practices that may be of use in Naval systems are discussed. An Appendix contains detailed summaries of the fracture control practices used in each of thirteen classes of metal structures, including ships, offshore structures, bridges, cryogenic tanks, pressure vessels, piping, power generation equipment, and aerospace structures.

NBSIR 79-1624. Tobler, R. L. Materials for cryogenic wind tunnel testing. 1980 May. 135 p. Available from: NTIS; N81-27120.

Key words: cryogenic properties; instrumentation; low temperature materials; materials properties; metals; nonmetallic materials; wind tunnels. 
A study was conducted to guide the evaluation and selection of materials and techniques to be used in construction of model aircraft for cryogenic wind tunnel testing. In this report, the mechanical, thermal, and electrical property behavior of materials at temperatures as low as $77 \mathrm{~K}$ is briefly reviewed. Metals, structural alloys, nonmetals, composites, joining methods, coatings, sealants, adhesives, contact agents, lubricants, transducers, and instrumentation for cryogenic applications are discussed. Acceptable structural materials, conductors, and insulators are discussed for service at temperatures in the range 367 to $77 \mathrm{~K}$. Numerous references to handbooks and other cryogenic data sources are cited as a guide to additional information.

NBSIR 79-1715. Waxman, M.; Davis, H. A.; Sengers, J. M. H. L.;

Klein, M. The equation of state of isobutane: An interim assessment.

1981 September. 44 p. Available from: NTIS; PB 82-120528.

Key words: critical region; density; equation of state; geothermal; isobutane; thermodynamic; vapor pressure.

New and very accurate measurements of the vapor pressure and two PVT isotherms of isobutane below critical are presented. The vapor pressure data have been correlated to within parts per 10,000 using a scaled vapor pressure equation. The Beattie data in the critical region has been correlated using a scaling law approach. This required a shift in the critical temperature of $0.3 \mathrm{~K}$ from that published by Beattie. The significance of this shift in an overall correlation is not yet clear. The two PV isotherms are compared with literature data and the results of Sage and Lacy shown to be inaccurate. Those of Connolly appear to be sufficiently accurate for including in future correlations. Two correlations of Starling et al. and that of Das et al. are intercompared and are compared with these new results. As might be expected, these correlations, being based on inaccurate data, are not of sufficient accuracy for use as representations of the properties of isobutane. A program is presented for the completion of a correlation of the properties of isobutane to engineering accuracy.

NBSIR 79-1752. Hocken, R. J.; Borchardt, B. R. On characterizing measuring machine geometry. 1979 June. 25 p. Available from: NTIS; PB 82-116377.

Key words: ball plate; grid plate; machine geometry; measuring machine; redundancy; 3-D metrology.

We present a simple method for removing axis nonorthogonality and checking for length dependent scale errors in two-dimensional measurements. Use of this method requires that a two-dimensional master gage (ball or grid plate, for example) be measured in two positions which differ by a rotation of the plate $90^{\circ}$ with respect to the measuring machine axes. The method is similar to that proposed by Reeve, but requires only linear least squares fitting on a small computer.

NBSIR 79-1904 (Navy). Kruger, J.; Carroll, J. J.; Melmed, A. J.;

Ritter, J. J.; Ambrose, J. R. Passive films, surface structure and stress corrosion and crevice corrosion susceptibility. 1979 November. 35 p. Available from: NTIS; ADA078-596.

Key words: aluminum; field ion microscopy; iron-chromium alloys; niobium; repassivation kinetics; stainless steel; triboellipsometry.

Field ion microscopy studies of the effects of hydrogen interactions with niobium, aluminum, 304 stainless steel and $\mathrm{Fe} .24 \% \mathrm{Cr}$ steel were carried out. Both $\mathrm{Nb}$ and $\mathrm{Al}$ exposed to hydrogen at $80 \mathrm{~K}$ and $30 \mathrm{~K}$ respectively showed significant surface disarray, but the effects were not deep or crystallographically selective. The 304 stainless steel show some indications of crystallographically selective attack Cathodically charged Fe-24\% $\mathrm{Cr}_{\mathrm{r}}$ showed a broad spectrum of effects.

A new triboellipsometric apparatus which is capable of measuring repassivation events occurring in times of the order of $0.1 \mathrm{~ms}$ is described.

NBSIR 79-1911. Maxwell, B. R. Procedures for testing, rating, and estimating the seasonal performance of engine-driven heat pump systems. 1979 September. 60 p. Available from: NTIS; PB 81123564.

Key words: building heating and cooling; engine-driven heat pump; heating and cooling equipment; heating, ventilating and air conditioning; heat pump.

A generic test and rating procedure is developed for heat enginedriven air-to-air heat pump systems. The procedures are classified according to whether the systems have single-speed, two-speed, or variable-speed capability, and whether they are operating in the heating or cooling mode. The test requirements generally consist of a series of steady-state tests to establish the impact of outdoor temperature on performance, two or more part-load (cyclic) tests to determine the effect of "on-off" cycling, two steady-state intermediate speed tests to determine part-speed performance, and a single frost accumulation test to estimate the effect of frost. A generalized calculation and rating procedure is developed. The system is rated in both modes based upon its steady-state performance at the ARI standard rating points, its seasonal performance factor, and its seasonal operating cost. A frost degradation coefficient is also established. The seasonal parameters are based upon either a residential or commercial/industrial building application which is located in either a generalized northern or southern climate.

NBSIR 79-1960 (DoE). Fields, R. J.; Tighe, N. J.; Fuller, E. R., Jr. Effect of strain rate on the fracture toughness of silicon nitride at $1400^{\circ} \mathrm{C}$. 1980 October. 24 p. Available from: NTIS; PB 81-166670.

Key words: crack growth; creep fracture; high temperature fracture; silicon nitride; strain rate effect; toughness.

The critical stress intensity factor for fracture, $\mathrm{K}_{\mathrm{IC}}$, was measured in $\mathrm{Si}_{3} \mathbf{N}_{4}$ at $1400^{\circ} \mathrm{C}$ as a function of strain rate. It was found that $\mathrm{K}_{1 \mathrm{C}}$ increased with decreasing rates of bending of notched bars. This effect could be approximately predicted using a time dependent plastic zone correction to $\mathrm{K}_{1 \mathrm{c}}$.

NBSIR 80-1627. Reed, R. P., ed. Materials studies for magnetic fusion energy applications at low temperatures-III. 1980 June. 387 p. Available from: NTIS; PB 80-191497.

Key words: composites; low temperature; mechanical and physical properties; structural alloys; welding.

The reports presented here summarize the work of the low temperature materials research project for the third year of the program. The various projects are outlined and the research results are presented. The major thrust of the measurements has been the evaluation of the low temperature mechanical and physical properties of stainless steel base metal and welds, with particular emphasis on the nitrogen-strengthened stainless steels. Aluminum alloys have received some consideration also. Work has also been done on the production and standardization of nonmetallics, primarily industrial laminates, for low temperature applications and on the measurement of their properties at cryogenic temperatures. The third NBS/DOE Vail workshop was held in October 1979. A brief description is given of that program.

NBSIR 80.1628. Johnson, E. G., Jr.; Phelan, R. J., Jr.; Boyle, D. R. Measuring features of the fluence at the far field of a $\mathrm{CO}_{2}$ pulsed laser-An issue study with suggestions on how to do it. 1980 April. 55 p. Available from: NTIS; PB 80-191141.

Key words: attenuators; beam profile; calorimetry; fluence; heat flow; irradiance; measurements of temperature; plasma effects; pulsed laser beams; $10.6 \mu \mathrm{m}$.

This study examines the problems for measuring the energy density 
incident on targets where the energy is from a pulse of high energy at $\mathrm{CO}_{2}$ wavelengths and where the targets are located at the far field. The analysis considers two targets-first, a ground-based target for testing and calibration of the measurement systems and second, a drone towed behind an airplane from which the energy distribution information is telemetered to the ground station. Although certain design limits are assumed, the results are general and therefore specific data about the laser sources is not supplied.

This study traces each stage of the measurement system from the reception of the incident laser pulse on the drone to the pulse-coded transmission of the sampled data to a ground-based computer.

The basic conclusion is that not all desired conditions of potential fluence can be studied. Plasma effects can prevent proper measurements. If the suggested $\mathbf{S F}_{6}$ gas can act as a calibrated attenuator and hence avoid the plasma effects, then the range of potential fluences can be increased substantially.

NBSIR 80-1630. Stubenrauch, C. F.; Yaghjian, A. D. Determination of mutual coupling between co-sited microwave antennas and calculation of near-zone electric field. 1981 June. 172 p. Available from: NTIS; PB 81-246217.

Key words: co-sited antennas; coupling; far fields; near fields; physical optics; planewave spectrum; reflector antennas.

The theory and computer programs which allow the efficient computation of coupling between co-sited antennas given their farfield patterns are developed. Coupling between two paraboloidal reflector antennas is computed using both measured far-field patterns and far-field patterns which were obtained from a physical optics (PO) model. These computed results are then compared to the coupling measured directly on an outdoor antenna range. Far fields calculated using the PO model are compared to those obtained from transformed near-field measurements for several reflector antennas. Theory and algorithms are also developed for calculating near-field patterns from far fields obtained from the PO model. Documentation of the near-field and coupling computer programs is presented in the appendices. Conclusions and recommendations for future work are included.

NBSIR 80-1631. Van Poolen, L. J. Analysis of liquid volume and liquid mass fractions at coexistence for pure fluids. 1980 May. 63 p. Available from: NTIS; PB 80-191133.

Key words: coexistence densities; critical density; critical point; liquid mass fraction; liquid volume fraction; phase equilibria; pure fluids.

An analysis of the behavior of liquid volume and liquid mass fractions at coexistence for pure fluids is made. Scaled equations for the saturation liquid and vapor densities are analyzed and relationships between various exponents and among constant coefficients are presented. Inequalities which exist among the saturation densities and their derivatives are developed. A procedure to correlate saturation data with the critical point is applied to ethylene. An experimental procedure to determine, simultaneously, saturated liquid and vapor densities at a given temperature is presented.

NBSIR 80-1634. Bowman, R. R. A temperature probe for radiofrequency heated material. 1981 January. 33 p. Available from: NTIS; PB 81-176125.

Key words: heating; probe; radio-frequency; temperature measurements.

One approach to measuring temperature in radio-frequency ( $\mathrm{rf}$ ) heated material employs thermocouple or thermistor sensors connected to suitable electronics by means of leads of such slight conductivity that they cause negligible field distortion and measurement artifacts. The particular type of probe discussed here uses a thermistor. Four leads are used to allow the thermistor resistance to be sensed despite the large and unstable lead resistances.
Though difficult to construct, small probes (about $1.1 \mathrm{~mm}$ in diameter) for use in biological tissue can be made that cause measurement artifacts so slight (about $0.01^{\circ} \mathrm{C}$ or less) that they are difficult to measure. Theory and experimental results are presented to show that these probes are very effective for use in materials with high water content. In most situations of interest, the short term stability is better than $0.01^{\circ} \mathrm{C}$, and the long term stability is about $0.1^{\circ} \mathrm{C}$.

NBSIR 80-1635. Miller, C. K. S., ed. A bibliography of publications in the NBS Electromagnetic Fields Division. 1980 November. 35 p. Available from: NTIS; PB 81-143158.

Key words: antennas; electromagnetic interference; electromagnetic metrology; em material properties; microwaves; noise; non-ionizing radiation; radiation hazards.

This bibliography lists the publications of the present personnel of the Electromagnetic Fields Division of NBS in the period from January 1970 through September 1979. A few earlier references that are directly related to the present work of the division are included.

NBSIR 80-1637. Daney, D. E., ed. Helium research in support of superconducting power transmission. Annual Report: October 1978 . September 1979. 1980 December. 58 p. Available from: NTIS; PB 81-156606.

Key words: cable cool-down; cable permeability; counter-flow cool-down; heat flux meters; liquid helium; permeability of composites; superconducting power transmission.

During FY 79, the NBS Thermophysical Properties Division program of research for superconducting power transmission line (SPTL) development focused on three tasks: 1) Analytical, numerical, and experimental modeling of counter-flow SPTL cool-down. 2) Measurement of radial permeability of a SPTL cable to gas flow. 3) Experimental evaluation of thermal flux meters as a possible technique for determining enclosure heat leak.

We have completed the first phase of our investigation of SPTL cool-down in which the aim was to develop a fundamental understanding of the counter-flow method of cool-down. The excellent agreement of our analytical, numerical, and experimental results gives us confidence that we can accurately model the cooldown of full scale SPTL's. The cable permeability measurements indicate that any significant rupture of the lead gas barrier of the cable will lead to an unacceptably high leak rate from the pressurized core.

During FY 80 we shall concentrate on developing cool-down strategies for full scale SPTL's using the detailed computer code previously developed at NBS. Field evaluation of thermal flux meters will also continue.

NBSIR 80-1642. Fickett, F. R.; Goodrich, L. F.; Clark, A. F Development of standards for superconductors. Annual report FY 80. 1980 December. 212 p. Available from: NTIS; PB 81.176141.

Key words: critical current; electrical property; magnetic property; stability; standards; superconductor.

Modern practical superconductors are complex materials and the determination of their critical parameters is a difficult task. Many approaches are possible for determining a given parameter and the results often depend on which one is chosen. The goal of this program, a cooperative venture involving DoE, NBS, and private industry, is to arrive at a set of useful voluntary standards for measurements on modern practical superconductors that will be acceptable to both manufacturers and users. Agreement on a set of standard definitions for the commonly used terms is also necessary. This report describes the status of the program at the end of the second year. The work in FY 80 has concentrated nearly exclusively on the critical current standard. Experimental determinations of the effect of various parameters on the measurement have been made by NBS and by the wire manufacturers. Significant progress has been 
made in the preparation of the actual critical current measurement standard and the definition standard. Draft copies of both are now ready for comment.

NBSIR 80-1971. Gass, S. I.; Hoffman, K. L.; Jackson, R. H. F.; Joel, L. S.; Saunders, P. B. Interim report on model assessment methodoiogy: Documentation assessment. 1980 January. $67 \mathrm{p}$. A vailable from: NTIS; PB 82.126673.

Key words: documentation assessment; documentation guidelines; large-scale models; model assessment.

This report describes the results of the documentation assessment phase of a project to assess the Department of Energy's Midterm Oil and Gas Supply Model. The objective here is not merely to record our conclusions about the documentation of that model, but also to present a methodological approach to documentation assessment. These methodological investigations have resulted in a set of guidelines which can be used both to assist project sponsors in determining their documentation needs, and as a standard against which to compare existing model reports. The documentation guidelines presented here amplify but do not alter substantively the DOE "Interim Model Guidelines" of December 1978 and are organized according to levels associated with the information needs of various phases of model operation. The documentation of the Midterm Oil and Gas Supply Model is discussed in light of these guidelines. The guidelines are recommended for incorporation into DOE model development projects.

NBSIR 80-1972. Huntoon, R. D. Needs and opportunitles in metrology of the Instituto de Pesqulsas Tecnologicas for effective support of Brazilian industry. 1980 February. 35 p. Available from: NTIS; PB 81.152860 .

Key words: Brazil; industrialization; international cooperation; measurement infrastructure; metrology; technologies; technology transfer.

IPT and NBS are now in the last year of a five-year AID-sponsored joint effort to help IPT accomplish its proper part in the "leapfrogging" industrialization of Brazil. This telescoping of conventional patterns of growth, to reach maturity more rapidly, injects dislocations into the balances between imported sophisticated technologies and supportive subordinate technologies such as, for example, the nation's measurement services. A four-week joint study of measurement services of IPT served as a basis for examining the dislocations in the Sao Paulo measurement infrastructure. By comparing the activities in IPT with those in a laboratory functioning in a more developed infrastructure, IPT, with its extensive knowledge of Brazilian technologies, was able to identify dislocations peculiar to Brazil and plan for itself appropriate corrective actions. The nature of the comparison and the corrective actions planned are presented in the report.

NBSIR 80-1975. Rubin, R. J. Analysis of mathematical models of Integrating monltorlng devlces. 1980 January. 37 p. Available from: NTIS; PB 81.164469.

Key words: diffusion; integrating device; permeation type; personal dosimeter; reaction; time-dependent.

Two mathematical models of a personal monitoring device are analyzed. The purpose of the device, which is to be worn by an individual, is to indicate the level of exposure to various criteria air pollutants such as $\mathrm{O}_{3}, \mathrm{SO}_{2}, \mathrm{NO}_{2}, \mathrm{CO}$ during the time period which might range from 24 hours to a week or longer. The goal of the analysis of the models is to determine how the quantity of pollutant which is collected is related to the average pollutant concentration and how material constants such as solubility and diffusion coefficients affect the operation of the device.

NBSIR 80-1979.1. Wright, R. N.; Fenves, S. J.; Harris, J. R. Modeling of standards: Technlcal aids for their formulation, expresslon, and use. 1980 December. 17 p. Available from: NTIS; PB 81.159600.

Key words: building standards; classification; decision tables; information networks; modeling; standards; standards-writers; systems analysis.

Standards are the primary communication and control mechanism used to describe building practices and products in communications between the various participants in the building process. Most prior research related to building standards has been concerned with understanding and improving the performance of building products. This work, in contrast, is concerned with improving the organization, expression and interpretation of the information contained in a standard. Techniques are described for objective and rigorous representation of the meaning of a standard. These allow it to be tested for aspects of clarity, completeness, consistency and correctness. Furthermore, the techniques allow alternative organizations and expressions to fit the needs of various users with assurance that meanings remain unchanged and that users will readily find and understand all provisions even in a new or unfamiliar standard.

NBSIR 80-2003. Lerner, N. D.; Collins, B. L. Workplace safety symbois: Current status and research needs. 1980 March. 62 p. Available from: NTIS; PB 81-157059.

Key words: communication; hazard; pictograms; safety; signs; standards; symbols; visual alerting; warnings.

Although written signs are a common means of conveying safety information in the workplace, pictographic symbols can be a more effective way of providing the same information. Symbols are independent of a particular written language, and can be more accurately and rapidly perceived than the comparable word message. Despite the many advantages of safety symbols, they can be ineffective or even dangerous if the intended meaning is not accurately communicated. As a result, there is a great need for careful evaluation, consistent application and eventual standardization for safety symbols.

This report documents an initial assessment of current symbol use and future requirements. It includes a review of the technical literature on symbol research; observation of safety sign and symbol use in the workplace; compilation of commercially available symbol referents; and review of national and international standards. Based upon these sources, an initial list of 40 symbol referents is presented along with research priorities for evaluating the effectiveness of symbols for these referents.

NBSIR 80.2006.3. Bullis, W. M., ed. Semiconductor technology program-Progress briefs. 1980 October. 12 p. Available from: NTIS; PB 81.154106.

Key words: electronics; integrated circuits; measurement technology; microelectronics; semiconductor devices; semiconductor materials; semiconductor process control; silicon.

This report provides information on the current status of NBS work on measurement technology for semiconductor materials, process control, and devices. Results of both in-house and contract research are covered. Highlighted activities include: optical linewidth and thermal resistance measurements, device modeling, dopant density profiles, resonance ionization spectroscopy, and deep-level measurements. Brief descriptions of selected on-going projects are included, and recent publications and publications in press are listed. Standardized oxide charge terminology developed by a joint IEEEECS committee is also described. The report is not meant to be exhaustive; contacts for obtaining further information are listed.

NBSIR 80-2006-4. Bullis, W. M., ed. Semiconductor technoiogy program-Progress briefs. 1980 December. 12 p. Available from: NTIS; PB 81-158602.

Key words: electronics; gallium arsenide; integrated circuits; 
measurement technology; microelectronics; semiconductor devices; semiconductor materials; semiconductor process control; silicon.

This report provides information on the current status of NBS work on measurement technology for semiconductor materials, process control, and devices. Emphasis is placed on silicon and silicon-based devices, but some work relating to gallium arsenide and other III.V compound semiconductors is included. Results of both inhouse and contract research are covered. Highlighted activities include: studies of the Raman spectra of boron-doped silicon, spreading resistance measurements on gallium arsenide, the annealing behavior of optical absorption of sulfur-doped silicon, aluminum-silica interlayers, and the time dependence of acoustic emission from seal cracks. Brief descriptions of the newly announced optical microscope linewidth measurement standard and a metallization step coverage random fault test structure are given. In addition, recent publications and publications in press are listed. The report is not meant to be exhaustive; contacts for obtaining further information are listed.

NBSIR 80-2018 (DOT). Churney, K. L.; Garvin, D. A third appraisal of methods for estimating self-reaction hazards. 1980 August. 326 p. Available from: NTIS; PB 81-188286.

Key words: adiabatic calorimetry; azidotriphenylmethane; decomposition reactions; differential scanning calorimetry; heat conduction calorimetry; kinetics of heat evolution; m-azidobenzoic acid; nitrocellulose; quantitative differential thermal analysis; thermal explosion theory; thermal hazards; thermal instability.

A study has been made of thermal explosion theory and thermal hazard tests. From this definitions have been developed, suitable for use in a transportation context, of thermally unstable materials. package configurations and thermal instability hazards. A thermally unstable materials package configuration is one: (1) composed of substances that can decompose or react with the evolution of heat, (2) for which a thermal mechanism of initiation of a temperature rise is present, and (3) in which a resulting rapid, large increase in the temperature of the material can occur. A thermal instability hazard exists if decomposition of the material-package configuration results in destruction of the package or neighboring objects or poses a safety threat. Typical destructions are explosion, rupture of package, and fire.

The properties of the material and package needed to predict the hazard are identified and methods for their measurement, using heat conduction and adiabatic calorimetry, are analyzed. It is concluded that, for least ambiguous scale-up of results to bulk shipping conditions, measurements should be made on large samples of the material under conditions such that heat transfer within the sample is large in comparison to heat transfer between the sample and its environment. Tests, such as the explosion temperature test, should be carried out to check the scaled-up hazard predictions.

An experimental study on three thermally unstable materials, nitrocellulose (I), m-azidobenzoic acid (II), and azidotriphenylmethane (III), was carried out using a differential scanning calorimeter (DSC). The procedure used is that of a proposed ASTM method of test for determining Arrhenius kinetic constants from the variation of the temperature of the maximum rate of heat evolution pre-exponential factors, $A$; are: $\Delta E=$ (I) $166.9 \pm 6.1$ (II) $139.9 \pm 5.9$, and (III) $\left.145.3 \pm 4.4 \mathrm{~kJ} \cdot \mathrm{mol}^{-1} ; \log _{10} \mathrm{~A}, \mathrm{~min}^{-1}\right)=(\mathrm{I})$ $18.0 \pm 0.7$, (II) $15.1 \pm 0.7$, and (III) $15.0 \pm 0.6$. A systematic error analysis clarifies and tends to support the test procedure. The main drawbacks to the test are its nonapplicability to complex systems and the small temperature range of the data, which makes the extrapolation of results to lower temperatures uncertain.

NBSIR 80-2020. Peiser, H. S.; Gutterman, B. M.; Louis, G. L.; Pring, J. M.; Taylor, J. K.; Tholen, A. D.; Smith, C. A., ed. Standardization and measurement services in the Sudan. 1980 August. 91 p. Available from: NTIS; PB 81-151425.

Key words: Agency for International Development; Arab
League Organization for Standardization and Metrology; chemical industry standards; cotton standards; food standards; measurement science and technology; standardization in developing countries; Sudan; weights and measures regulation.

A ten-member international team advisory to the Government of the Sudan's Ministry for Industry on Sudanese standardization and measurement services was organized by the U.S. National Bureau of Standards with financial support about equally from both the U.S. Agency for International Development and the Sudan. The team function was to survey Sudanese standardization and measurement services in support of development and consisted of the Secretary General of the Arab League Organization for Standardization and Metrology, the Assistant Secretary General of ASMO, senior representatives of the standardization communities of Indonesia, Morocco, the United Kingdom and four members of U.S. standards agencies. The Sudan provided a local counterpart survey team of fifteen senior officials under the chairmanship of the Deputy Minister for Industry and the organizational directorship of the Acting Director General of the Sudanese Department of Standardization and Quality Control. In the two weeks in which the combined team travelled in the Sudan, the international visitors put their collective knowledge and experience at the disposal of Sudanese colleagues. The team split up into smaller specialist groups to survey the application of standardization and measurement technology to the following sectors: food industries, weights and measures (in retail markets), chemical industries standards and quality control, and textile (cotton). Detailed notes on the visit to $\mathbf{4 8}$ Sudanese organizations in industry and Government are reported and generalized conclusions are recorded as presented to the Sudanese Government at the conclusion of the Survey.

NBSIR 80-2035. Toner, S. D.; Wakamiya, S. K. Bicycle wheel rim reflective materials test method development. 1980 April. 15 p. Available from: NTIS; PB 81-152365.

Key words: abrasion test development; bicycle compliance tests bicycle safety; reflective materials for bicycles; safety of bicycles; safety standards.

A prototype testing apparatus was designed and built for use in conducting abrasion tests on reflective materials applied to bicycle wheel rims. Data obtained on the effects of various arbitrarily selected test parameters on a pressure sensitive reflective tape, used as a model, indicate that the abrader and test procedures are suitable for use in compliance testing of rim-applied retroreflective materials. Materials of this type, intended for use in lieu of spoke reflectors or retroreflective tires, are not included in the current bicycle safety regulations promulgated by the Consumer Product Safety Commission.

NBSIR 80-2037. Berger, R. E.; Huckeba, J. A. Impact on human eyes by propelled objects. 1980 May. 14 p. Available from: NTIS; PB 81. 154130 .

Key words: contusion; eyeball deformation; impact; ocular injury; projectiles; safety.

High speed motion pictures were employed to observe experimental impacts on enucleated human eyeballs by low speed missiles which are representative of toy projectiles. A procedure was devised to adjust the intraocular pressure prior to each impact. For similar impact conditions, the gross deformation of eyeballs with normal pressure was not as severe as had been previously reported for eyeballs with near zero pressure. The maximum displacement was measured for several eyeballs with normal pressure. These displacements were shown to correlate with the predictions of a mathematical model which had been used to develop a test method for toy projectiles.

NBSIR 80-2053. Gass, S. I.; Joel, L. S. Concepts of model confidence. 1980 June. 41 p. Available from: NTIS; PB 81-158164.

Key words: decision making; model assessment; model 
confidence; model evaluation; model utility; model validation.

This report discusses the concept of confidence in results obtained from large-scale modeling systems. It is written in satisfaction of the "model confidence" tasks of a National Bureau of Standards project on "Energy Model Validation Procedure Development," funded by the Department of Energy. This report includes discussions of: our efforts to define model confidence; the workshop held for this purpose; a preliminary methodology to measure confidence; and, the survey conducted to obtain opinions on significant related issues.

NBSIR 80-2054, Vols. I and II. Quintiere, J. G.; McCaffrey, B. J. The burning of wood and plastic cribs in an enclosure. 1980 November. Vol. I, 204 p.; Vol. II, 572 p. Vol. I available from NTIS; PB 81. 182586.

Key words: crib fires; experiments; mathematical model; plastic; polyurethane; room fires; wood.

This study was designed to assess the fire hazard of a cellular plastic material which has comparable structural characteristics to wood. The study attempts to determine the relative fire risk of such materials in furniture. Rigid (high density) structural polyurethane foam and supar pine were selected for fuels and burned in the form of cribs in a room. The crib loading and door width were parameters experimentally varied. Twenty-one room fire experiments and eight free burn experiments were conducted. Measurements of mass loss, temperature, heat flux, $\mathrm{CO}_{2}$ and $\mathrm{O}_{2}$ concentration were recorded. These data were analyzed and empirical correlations were developed for air flow rate and upper gas temperature. A theoretical fire simulation model was developed and yielded results in fair to good agreement with the data. An extrapolation with the theoretical model was used to predict the critical (or minimum) fuel pyrolysis rate to cause flashover (as implied by $2 \mathrm{~W} / \mathrm{cm}^{2}$ of incident radiation to the room floor). This was done for various size rooms and door openings. It appears that to cause flashover, for a given room and door size, about twice as much wood must be involved in fire as the rigid polyurethane material. (Volume II is a summary and compilation of the room fire data referenced in volume I.)

NBSIR 80-2056. Lewis, J. G.; Rehm, R. G. The numerical solution of a nonseparable elliptic partial differential equation by preconditioned conjugate gradients. 1981 April. 66 p. A vailable from: NTIS; PB 82. 103318.

Key words: conjugate gradient algorithm; elliptic partial differential equations; iterative methods for linear algebraic equations; Neumann boundary conditions; space matrices.

In this report the combination of an iterative technique, the conjugate gradient algorithm, with a fast direct method, cyclic reduction, is used to solve the linear algebraic equations resulting from discretization of a nonseparable elliptic partial differential equation. An expository discussion of the conjugate gradient and preconditioned conjugate gradient algorithms and of their use in the solution of partial differential equations is presented. New results extending the use of the preconditioned conjugate gradients technique to singular linear equations which arise from discretized elliptic equations with Neumann boundary conditions are also given. The algorithms are applied to solve a specific elliptic equation which arises in the study of buoyant convection produced by a room fire. $A$ code was developed to implement the algorithms for this application. Numerical results obtained through testing and use of the code are discussed.

NBSIR 80-2069. Swartzendruber, L.; Boettinger, W.; Ives, L.; Coriell, S.; Ballard, D.; Laughlin, D.; Clough, R.; Biancaniello, F.; Blau, P.; Cahn, J.; Mehrabian, R.; Free, G.; Berger, H.; Mordfin, L. NBS: Nondestructive evaluation of nonuniformities in 2219 aluminum alloy plate-Relationship to processing. 1980 December. 244 p. A vailable from: NTIS; PB 81-172348.

Key words: aging; electrical conductivity; hardness; homogeneity; microstructure; precipitating; segregation; solidification; thermal mechanical treatment.

The compositional homogeneity, microstructure, hardness, electrical conductivity and mechanical properties of 2219 aluminum alloy plates are influenced by the process variables during casting, forming and thermomechanical treatment.

NBSIR 80-2080. Mordfin, L., ed. NDE publications: 1978. 1980 June. 26 p. Available from: NTIS; PB 81-197691.

Key words: abstracts; acoustic emission; bibliography; eddy currents; index; National Bureau of Standards; nondestructive evaluation; publications; radiography; ultrasonics.

This report is a supplement to NBSIR 78-1557, "NDE Publications: 1972-1977." It is a bibliography, with selected abstracts, of 72 National Bureau of Standards (NBS) publications on nondestructive evaluation (NDE) and related technologies, primarily for the calendar year 1978. A detailed subject index is included, as well as information on how copies of many of the publications may be obtained.

NBSIR 80-2081. Cooke, P. W. Comparison of selected codes and standards relating to existing residential buildings. 1980 July. $310 \mathrm{p}$. A vailable from: NTIS; PB 81-120842.

Key words: building rehabilitation; codes; comparative analysis; existing buildings; housing codes; maintenance; model codes; occupancy; performance levels; regulation; standards.

The performance levels of older residential buildings generally do not comply with the standards for safety or function that are required of new buildings. This report presents a comparative analysis of the specific provisions contained in seven codified documents that have been promulgated to regulate the health and safety aspects of existing residential buildings. The study examines and presents information on: (1) The extent to which codes vary among each other in establishing minimum requirements for life, health and safety in existing dwellings. (2) The differing approaches provided by traditional housing codes in contrast to more recently developed rehabilitation guideline documents regarding performance levels. (3) The degree to which each code meets its intended goals of providing and maintaining human shelter, protection, and privacy. The code provisions are compared and analyzed in sixteen major code areas (e.g., structural requirements, light and illumination, plumbing requirements, etc.). Various inconsistencies among code documents with respect to uniformity and irrational approaches in the historical development of code provisions are indicated.

NBSIR 80-2088. Lerner, N. D.; Collins, B. L. The assessment of safety symbol understandability by different testing methods. 1980 August. 60 p. Available from: NTIS; PB 81-185647.

Key words: communication; evaluation method; fire-safety; hazard warnings; meaningfulness; method; pictogram; response; symbol; understandability.

This paper reports an experiment on the understandability of pictorial symbols proposed for fire-safety alerting. The experiment was designed to determine the understandability of specific symbols and to assess the effects of variations in both presentation and response methods.

The symbols were presented as slides, booklets, or placards. Subjects indicated their understanding of each symbol's meaning either by writing down a brief definition or by selecting the correct answer from among four alternatives. For both methods, subjects rated their confidence in the correctness of the answers. In the second phase of the experiment, subjects were given fifteen different messages, and asked to draw a symbol for each idea.

Mode of symbol presentation had no effect on understandability, while the use of definition and multiple choice procedures led to generally similar conclusions. The confidence ratings provided additional information about discrepancies between the two response 
methods.

The understandability of the 25 symbols ranged from near zero to virtually total comprehension. These data underscore the need to determine the understandability of safety symbols prior to standardizing a symbol set.

NBSIR 80-2091. Branthover, B.; Veale, R.; Siu, C. Precision measurements on fibrous glass insulation. 1980 November. 36 p. Available from: NTIS; PB 81-239634.

Key words: fiber-glass; fibrous glass; insulation; measurement; $\mathbf{R}$ value; standard reference material; thermal resistance.

This paper is concerned with the technique in measuring the thickness of low density fibrous glass materials. The new method of measurement described provides a higher degree of accuracy than previously accomplished. The new procedure determined a specific force to be used for measuring. Also, a stacking technique was carried out to see what effect placing a number of test samples on top of each other would be. The results of the entire testing will aid in the development of a standard reference material to be used for thermal resistance measurement systems.

NBSIR 80-2094. Kelly, G. E.; Kuklewicz, M. E. A laboratory study of a gas-fired condensing boiler. 1980 November. 39 p. Available from: NTIS; PB 81-158230.

Key words: boilers; central heating; condensing boilers; efficiency, part load; fossil-fuel heating systems; gas-fired boilers; hydronic heating; pulse combustion.

As a part of the Department of Energy's energy conservation program for consumer products, the National Bureau of Standards (NBS) developed test procedures for conventional gas- and oil-fired furnaces and boilers. The Department of Energy (DoE) published their finalized version of these procedures in the Federal Register on May 10, 1978. In an effort to update and refine these test procedures, DoE directed NBS to develop a method of testing condensing furnaces and boilers which could be used to compare the annual performance of condensing and non-condensing residential heating systems. This report summarizes the laboratory tests of a gas-fired pulse-combustion condensing boiler that were carried out as a part of the development effort.

The performance of the pulse-combustion boiler was evaluated under both steady-state and part-load operating conditions. The efficiency of the unit was determined by the input/output method which measured the heat transferred to the circulating water and the energy input during each test period. Steady-state laboratory tests of the unit were conducted at constant return water temperatures of 100 , $110,120,130$ and $140^{\circ} \mathrm{F}\left(37.8,43.3,48.9,54.4\right.$ and $\left.60.0^{\circ} \mathrm{C}\right)$. Part-load performance tests were carried out at a number of these return water temperatures at on-times of approximately $5,15,22.5$ and 42 percent.

A modified version of the heat loss procedure for estimating the seasonal performance of a residential central furnace or boiler was also used to evaluate the boiler's steady-state efficiency and part-load efficiency at a 22.5 percent on-time. A cool-down test and heat-up test were performed to obtain dynamic information which was used to calculate the unit's cyclic performance. The predicted steady-state and part-load efficiencies from the heat loss method were found to be within three percent of the performance determined using the input/output method.

NBSIR 80-2099-4. Krause, R. F., Jr.; Fuller, E. R., Jr. Testing geothermal-well cements: Strength measurements following exposures to simulated geothermal fluids. 1981 December. 28 p. Available from: NTIS; PB 82-157009.

Key words: compressive strength; geothermal well; set-cement; simulated geothermal fluid; splitting tensile strength.

Compressive and splitting tensile strengths were measured for several set cements at room temperature after they had been exposed for periods of 1 week and 1 month to a $20 \mathrm{wt}$ percent salt solution pressurized to $20 \mathrm{MPa}$ and heated to $300^{\circ} \mathrm{C}$. The compressive strength was also measured following exposures for identical periods to distilled water pressurized to $20 \mathrm{MPa}$ and heated to $200^{\circ} \mathrm{C}$. Prior to the exposures, the cements had been set-cured for 2 days in molds immersed in water under the same pressure and temperature as the distilled water exposure. These measurements are part of a project being carried out to evaluate certain physical properties of cements which are candidates for use in finishing geothermal wells

NBSIR 80-2102. Dickens, B. User's manual for factor-jump thermogravimetry apparatus, and associated programs, including a general plotting program. 1981 March. 391 p. Available from: NTIS; PB 82.109851.

Key words: automated thermogravimetry; data analysis generalized plotting programs; interdata 7.16 computer; polynominal fitting; user manual.

The publication describes the operation of the computerized thermogravimetry apparatus. Detailed instructions are given to enable the user to bootstrap the computer and run the various programs. The items covered are 1) the thermogravimetry program TGRUNF; 2) the data file editing program TGEDIT; 3) estimation of trimmed means by TGTRIM; 4) general calculation of activation energy from rate data; probability plots by TGDEPG; 5) the generalized polynominal fitting program POLGEP; 6) publication tables by TABLEP; 7) the BASIC program BMPD which operates the computer interface in a general fashion; and 8) user-directed and automated plotting using the generalized plotting program PABS. Appendices on cautionary remarks and cable diagrams are included.

NBSIR 80-2104. Kao, J. Y.; Baker, D. W. A commentary on the instrumentation for building energy monitoring and control systems (EMCS). 1980 September. 28 p. Available from: NTIS; PB 81 185688 .

Key words: building energy monitoring; instrumentation; sensors.

This paper reviews sensors generally used for building energy monitoring and control systems (EMCS). The sensor operating principles, performances, calibration, maintenance, installation precautions, failure modes and their suitability for building EMCS use are discussed. Sensors covered in the paper include orifices, flow nozzles, Venturis, vortex shedding meters, and turbine meters for flow measurements, liquid-in-glass thermometers, resistance thermometers, and thermocouples for temperature measurements, and salt-phase transition hygrometers, impedance hygrometers, and dimensional change hygrometers for humidity measurements.

NBSIR 80-2106. Beine, W. B.; Sorrells, J. R. Soil impact attenuation performance: A field study. 1980 June. 38 p. Available from author only.

Key words: asphalt; impact attenuation; peak acceleration; playground safety; playground surfaces; soil; surfacing materials; test method.

Impact attenuation performance tests were conducted on the playing surface of selected public playgrounds in Montgomery County, Maryland, using a test method developed in an earlier laboratory investigation of surfacing materials. Controlled impacts were obtained by dropping an instrumented headform in guided freefall onto the test surfaces from various heights. The peak acceleration imparted to the headform during impact was recorded as the performance parameter.

At four playgrounds, the tests were performed on the undisturbed soil underlying existing play equipment. At a fifth location, the asphalt surface of an outdoor basketball court was tested. Soil samples from each playground were collected, analyzed and classified in accordance with standard methods prescribed by the ASTM. Separate tests were conducted following periods of dry and wet weather and on-site measurements of soil density and moisture content were recorded at the time of tests. 
Analysis of results indicated an apparent correlation between a soil's performance and its moisture content; correlation with other soil characteristics were not evident. The peak accelerations produced by the playground soils were much lower than those produced by the asphalt surface but higher than those produced by most loose materials previously tested in the laboratory.

NBSIR 80-2108. Wachtman, J. B., Jr.; Reneker, D. H. Technical activities 1980-Center for Materials Science. 1980 October. 483 p. Available from: NTIS; PB 81-245318.

Key words: Center for Materials Science; Ceramics Glass and Solid State Science Division; Chemical Stability and Corrosion Division; Fracture and Deformation Division; FY80 technical activities; Metallurgy Division; Polymers Science and Standards Division; Reactor Division.

The Center for Materials Science is part of the National Measurement Laboratory, one of the principal laboratories comprising the National Bureau of Standards. The Center is organized in six Divisions, each having responsibility in different areas of materials science appropriate to the major classes of materialsmetals, polymers, and ceramics and glass. These Divisions vary in their balance between theory and experiments, between direct standards work and research, and in their orientation toward industrial and Government needs and the needs of other components of the scientific and technical community. This volume summarizes the technical research activities and accomplishments of the six Divisions of the Center for Materials Science for Fiscal Year 1980.

NBSIR 80-2110. Kelly, G. E.; Kuklewicz, M. E. Recommended testing and calculation procedures for estimating the seasonal performance of residential condensing furnaces and boilers. 1981 April. $100 \mathrm{p}$. Available from: NTIS; PB 81-197030.

Key words: annual fuel utilization efficiency; annual operating costs; central heating equipment; condensing boilers; condensing furnaces; part-load performance; rating procedure; seasonal efficiency.

Procedures are developed for testing and rating the performance of residential central furnaces and boilers of the condensing type. $\mathbf{A}$ condensing furnace or boiler is a unit designed to condense part of the water vapor generated by the burning of hydrogen in the fuel and equipped with a means of draining this condensate. The test procedure is similar to one developed by the National Bureau of Standards for the Department of Energy covering noncondensing central heating equipment, except that it requires slightly tighter control of the laboratory temperature, a return water temperature of $120^{\circ} \mathrm{F}\left(48.9^{\circ} \mathrm{C}\right)$ with a 20 degree Fahrenheit (11.1 degree Celsius) water temperature rise for hot water boilers, and offers an optional test procedure for condensing units having no off-period losses. The rating procedure provides a method for estimating the steady-state, part-load and annual fuel utilization efficiencies of condensing furnaces and boilers. It accounts for the fact that the latent heat loss for a condensing unit is smaller than for a conventional furnace or boiler, since some of the water vapor generated from burning hydrogen in the fuel is condensed and thereby gives up part of its latent heat to the heat exchanger and jacket.

NBSIR 80-2111-1. Ellingwood, B. R. Review and refinement of ATC 3.06 tentative seismic provisions. Report of technical committee 1: Seismic risk maps. 1980 October. 30 p. Available from: NTIS; PB $81-187551$.

Key words: buildings; buildings (codes); buildings (design); earthquake; seismic risk maps; standards; structural engineering.

The Tentative Provisions for the Development of Seismic Regulations for Buildings were developed by the Applied Technology Council to present, in one comprehensive document, current state-of-knowledge pertaining to seismic engineering of buildings. The Tentative Provisions are in the process of being assessed by the building community. This report is one of a series of reports that documents the deliberations of a group of professionals jointly selected by the Building Seismic Safety Council and the National Bureau of Standards and charged with reviewing the Tentative Provisions prior to the conduct of trial designs. The report contains the recommendations and records of the committee charged with review of the seismic risk maps. The committee made 4 recommendations for revisions to the Tentative Provisions. These recommendations were made to the parent group, the Joint Committee on Review and Refinement, and their action on these recommendations is documented in a companion report.

NBSIR 80-2111-3. Salomone, L. A. Review and refinement of ATC 3.06 tentative seismic provisions. Report of technical committee 3: Foundations. 1980 October. 57 p. Available from: NTIS; PB 81. 187569.

Key words: buildings; design; earthquakes; engineering; foundations; professional practice; provisions; soil structure interaction; standards.

The Tentative Provisions for the Development of Seismic Regulations for Buildings were developed by the Applied Technology Council to present, in one comprehensive document, current state-of-knowledge pertaining to seismic engineering of buildings. The Tentative Provisions are in the process of being assessed by the building community. This report is one of a series of reports that documents the deliberations of a group of professionals jointly selected by the Building Seismic Safety Council and the National Bureau of Standards and charged with reviewing the Tentative Provisions prior to conducting trial designs.

This report documents the activities of Technical Committee 3: Foundations. Other committee reports are similarly available. The task of Technical Committee 3 was to review and refine Chapter 6, Soil-Structure Interaction and Chapter 7, Foundation Design Requirements in the ATC report (NBS SP.510) entitled, "Tentative Provisions for the Development of Seismic Regulations for Buildings." Two meetings were held. The opening meeting of the group was on December 11, 1979, and the concluding meeting was on February 5, 1980. The minutes of these meetings and the findings/recommendations of Technical Committee 3 are presented in this report. These recommendations were made to the parent group, the Joint Committee on Review and Refinement, and their action on these recommendations is documented in a companion report.

NBSIR 80-21114. Marshall, R. D.; Woodward, K. Review and refinement of ATC 3.06 tentative seismic provisions. Report of technical committee 4: Concrete. 1980 October. 254 p. Available from: NTIS; PB 82.130915.

Key words: building; building codes; building design; earthquakes; engineering; reinforced concrete; standards; structural engineering.

The Tentative Provisions for the Development of Seismic Regulations for Buildings were developed by the Applied Technology Council to present, in one comprehensive document, current state-of-knowledge pertaining to seismic engineering of buildings. The Tentative Provisions are in the process of being assessed by the building community. This report is one of a series of reports that documents the deliberations of a group of professionals jointly selected by the Building Seismic Safety Council and the National Bureau of Standards and charged with reviewing the Tentative Provisions prior to the conduct of trial designs. The report contains the recommendations and records of the committee charged with review of the reinforced concrete design provisions. The committee made 19 recommendations for revisions to the Tentative Provisions. These recommendations were made to the parent group, the Joint Committee on Review and Refinement, and their action on these recommendations is documented in a companion report.

NBSIR 80-2111-5. Leyendecker, E. V.; Cattaneo, L. E. Review and 
refinement of ATC 3.06 tentative seismic provisions. Report of technical committee 5: Masonry. 1980 October. 349 p. Available from: the author.

Key words: building; building codes; building design; earthquakes; engineering; masonry; standards; structural engineering.

The Tentative Provisions for the Development of Seismic Regulations for Buildings were developed by the Applied Technology Council to present, in one comprehensive document, the current state of knowledge pertaining to seismic engineering of buildings. The Tentative Provisions are in the process of being assessed by the building community. This report is one of a series of reports that documents the deliberations of a group of professionals jointly selected by the Building Seismic Safety Council and the National Bureau of Standards and charged with reviewing the Tentative Provisions prior to the conduct of trial designs. The report contains the recommendations and records of the committee charged with review of the masonry design provisions. The committee made 109 balloi recommendations for revisions to the Tentative Provisions. These recommendations were made to the parent group, the Joint Committee on Review and Refinement, and their action on these recommendations is documented in a companion report.

NBSIR 80-2111-6. Lew, H. S. Review and refinement of ATC 3-06 tentative seismic provisions. Report of technical committee 6: Steel. 1980 October. 37 p. Available from: NTIS; PB 81-187577.

Key words: building; building codes; building design; earthquakes; engineering; standards; steel; structural engineering.

The Tentative Provisions for the Development of Seismic Regulations for Buildings were developed by the Applied Technology Council to present, in one comprehensive document, current state-of-knowledge pertaining to seismic engineering of buildings. The Tentative Provisions are in the process of being assessed by the building community. This report is one of a series of reports that documents the deliberations of a group of professionals jointly selected by the Building Seismic Safety Council and the National Bureau of Standards and charged with reviewing the Tentative Provisions prior to the conduct of trial designs. The report contains the recommendations and records of the committee charged with review of the steel design provisions. The committee made 6 recommendations for revisions to the Tentative Provisions and three additional recommendations. These recommendations were made to the parent group, the Joint Committee on Review and Refinement, and their action on these recommendations is documented in a companion report.

NBSIR 80-2111-7. Yancey, C. W. C. Review and refinement of ATC 3.06 tentative seismic provisions. Report of technical committee 7: Wood. 1980 October. 41 p. Available from: NTIS; PB 81.187585.

Key words: building; building codes; building design; earthquakes; engineering; standards; structural engineering; wood.

The Tentative Provisions for the Development of Seismic Regulations for Buildings were developed by' the Applied Technology Council to present, in one comprehensive document, current state-of-knowledge pertaining to seismic engineering of buildings. The Tentative Provisions are in the process of being assessed by the building community. This report is one of a series of reports that documents the deliberations of a group of professionals jointly selected by the Building Seismic Safety Council and the National Bureau of Standards and charged with reviewing the Tentative Provisions prior to the conduct of trial designs. The report contains the recommendations and records of the committee charged with review of the provisions for the design and detailing of wood structures. The committee made 14 recommendations for revision to the Tentative Provisions. These recommendations were made to the parent group, the Joint Committee on Review and Refinement, and their action on these recommendations is documented in a companion report.

NBSIR 80-2111-8. Faison, T. K. Review and refinement of ATC 3-06 tentative seismic provisions. Report of technical committee 8: Architectural, mechanical and electrical. 1980 October. 43 p. Available from: NTIS; PB 81.187593.

Key words: architectural; electrical; elevators; mechanical; seismic coefficients.

The Tentative Provisions for the Development of Seismic Regulations for Buildings were developed by the Applied Technology Council to present, in one comprehensive document, current state-of-knowledge pertaining to seismic engineering of buildings. The Tentative Provisions are in the process of being assessed by the building community. This report is one of a series of reports that document the deliberations of a group of professionals jointly selected by the Building Seismic Safety Council and the National Bureau of Standards and charged with reviewing the Tentative Provisions prior to the conduct of trial designs. The report contains recommendations and records of the committee charged with the review of the material related to architectural, mechanical and electrical provisions. The committee made seven general recommendations for revision and one recommendation for the addition of a new section on elevator design requirements. These recommendations by Committee 8 were made to the parent group, the Joint Committee on Review and Refinement, and their action on these recommendations is documented in a companion report.

NBSIR 80-2111-11. Leyendecker, E. V.; Harris, J. R., eds. Review and
refinement of ATC 3-06 tentative seismic provisions. Report of joint
committee on review and refinement. 1980 October. 334 p. Only available from author.

Key words: building; building codes; building design; earthquakes; engineering; standards; structural engineering.

The Tentative Provisions for the Development of Seismic Regulations for Buildings were developed by the Applied Technology Council to present, in one comprehensive document, current state-of-knowledge pertaining to seismic engineering of buildings. The Tentative Provisions are in the process of being assessed by the building community. This report is one of a series of reports that documents the deliberations of a group of professionals jointly selected by the Building Seismic Safety Council and the National Bureau of Standards and charged with reviewing the Tentative Provisions prior to the conduct of trial designs. The group is divided into nine technical committees, each of which focused on a particular portion of the Tentative Provisions. The nine committees proposed recommendations for change to the parent group, the Joint Committee, through a Coordinating Committee. The Coordinating Committee made some modifications to the technical committees recommendations to ensure consistency among the recommendations. This report documents the actions of the Joint Committee on the 198 recommendations for change that were presented to it. The first part of the report is a summary of the results, and the appendices contain the full documentation for each recommended change. The actions of each of the nine technical committees is documented in a separate report.

NBSIR 80-2113. Kramer, S.; Olmert, M. ed. International activities. The Fiscal Year 1979 survey of international activities at NEL. 1980 September. 122 p. Available from: NTIS; PB 82.102146.

Key words: engineering; guest workers; international cooperation; international research; international standards; National Bureau of Standards (NBS); National Engineering Laboratory (NEL); technology transfer.

This report presents a survey of a major phase of the international activities of the National Engineering Laboratory (NEL), NBS, for the Fiscal Year 1979. 
There are a number of media through which international activities are conducted. The first of these is the formal visit by one or more NEL staffers to a foreign research organization or conference. The second category covers visits by foreign government scientists and research institutions to the NBS facilities. Other media include exchange visits and the hosting of overseas guest workers at NBS. Although this report covers only the first category and the listing of overseas guest workers at NBS, some significant and gratifying contributions to international technical progress are detailed here.

The report is organized by countries, international organizations and conferences. NEL professional staff involved in these activities are readily identified. Guest workers are listed with their research interests. The report is intended to serve as a directory and reference document for all those who seek information on the international activities of the National Engineering Laboratory, NBS.

NBSIR 80-2122. Campbell, P. G.; Sleater, G. A.; Post, M. A Development of guide specifications for the 1980 exterior restoration of the White House. 1980 October. 129 p. Available from: NTIS; PB 81-164535.

Key words: coatings; exterior restoration; field tests; guide specifications; laboratory tests; paint removal methods; White House.

At the request of the National Park Service, a study was performed to develop guide specifications for use in the 1980 exterior restoration of the White House. The study included: 1) an evaluation of historic practices and difficulties from painting of the White House, 2) an evaluation of technical literature on surface cleaning procedures and coating systems, 3 ) laboratory tests of selected coatings and field tests of selected surface cleaning procedures and coatings and 4) development of guide specifications. Four coating materials were selected for evaluation in laboratory and field tests and four surface cleaning methods were evaluated in a field test at the White House, of which one system was recommended for use in the 1980 restoration.

This report presents the findings of the study and includes the proposed guide specifications.

NBSIR 80-2127-1. Martinez, I. M.; Cherry, S. M., eds. Fourth annua conference on fire research. 1980 December. 70 p. Available from: NTIS; PB 81-158305.

Key words: chemistry of fire; combustion products; detection; fire hazards; fire modeling; fire protection; fire research; fire suppression; human behavior in fires; physics of fire; toxicity of combustion products

This report contains descriptions of the internal programs of the Center for Fire Research as well as extended abstracts of grants and contracts sponsored by the Center for Fire Research, National Bureau of Standards.

NBSIR 80-2131. Phillips, J. C. Performance of plastic packaging for hazardous materials transportation. Part VI. Mass loss and effective carbon atom number measurement. 1980 April. 32 p. Available from: NTIS; PB 81.239626.

Key words: effective carbon atom number; loss-rate; mass-loss n-alkanes; n-carboxylic esters; permeation; polyethylene.

This report describes mass loss experiments on two n-alkyl series (nalcohols and n-carboxylic esters) and a group of miscellaneous compounds. The experiments were performed at $50^{\circ} \mathrm{C}$ for low density and high density polyethylene bottles. The backbone chain length $\mathrm{N}_{\mathrm{A}}$, for each series was extended to 16 in order to characterize more fully the dependence of loss rate on chain length.

The results suggest rather strongly that the effective carbon atom number for a given permeant may be determined from a given density resin bottle using the n-alkane series as the standard lading. It is also shown that once the effective carbon atom number has been determined under chosen conditions, the permeability performance of the permeant for some other resin density may be predicted with reasonable accuracy. These results also seem to complement quite well the "permachor" method in minimizing the number of tests required for the determination of the performance of full-scale containers.

NBSIR 80-2136. Chang, Y. M. L.; Grot, R. A. Energy consumption and usage characteristics from field measurements of residential dishwashers, clothes washers and clothes dryers. 1980 October. $35 \mathrm{p}$. Available from: NTIS; PB 81-179160.

Key words: clothes dryer usage characteristics; clothes washer usage characteristic; data profiles; dishwasher usage characteristic; energy consumption; field measurements; usage patterns; water consumption.

The measured energy consumption and usage characteristics for household dishwashers, clothes washers, and clothes dryers for ten townhouses at Twin Rivers, N.J., are presented. Whenever the dishwashers and/or clothes washers were in use, the energy consumption, water consumption, frequency of usage, and water temperature were measured by a data acquisition system. The energy requirement for heating hot water could be established from the water heater's characteristic in a previous related report (NBSIR 78-1496). The electrical energy of electric clothes dryers and the gas consumption of gas clothes dryers were measured, as well as their frequency and duration of use, and exhaust temperature. Typical household usage patterns of these major appliances are also included.

It was found that, in general, the electrical energy required to operate dishwashers and clothes washers is about one tenth of the energy consumption for heating hot water. Cold water usage is about three times the hot water usage for each load of laundry. The energy loss from a pilot light burner of a gas dryer is about 50 percent of the total gas consumption of that dryer. As far as habits are concerned, the average family utilizes the dishwasher every other day and the laundry about five loads a week.

NBSIR 80-2138. Lozier, D. W. Multiprecision computing at NBS: Yesterday, today, and tomorrow. 1980 October. 54 p. Available from: NTIS; PB 81-243941.

Key words: AUGMENT and MP; Fortran arithmetic extensions; multiple precision; multiprecision Fortran software; multiprecision mathematical functions; precompilers for special arithmetic.

Multiprecision computing is a technique by which arithmetic operations may be performed on a computer to precision levels that are higher than the directly supported single and double precisions. The last ten years have seen the development of portable Fortran software of very high quality that essentially duplicates all the capabilities of standard Fortran, so that an existing standard Fortran program can be re-executed to arbitrarily high precision. In this paper some of the design techniques for such software, which have evolved at NBS and elsewhere, will be discussed. Methods for using the software presently available at NBS will be described, and a complete example will be given. Directions for further extensions and improvements will be indicated.

NBSIR 80-2141. Haight, W. C.; Hocken, R. J.; Borchardt, B. R.; Carroll, C. L.; Hartsock, R. G.; Reeve, C. P.; Scire, F. E.; Veale, R. C. Estimated accuracy of calibration of some membrane-type LNG transport tanks. 1981 January. 83 p. Available from: NTIS; PB 81-159907.

Key words: dimensions; laser; LNG; ships; tanks; volume.

The National Bureau of Standards has completed a study on the calibration uncertainty of ship cargo tanks used to transport Liquid Natural Gas (LNG). The data from measurements made on 18 such tanks is presented, along with a comparison to measurement determined from an independent technique. Calibration reports for each tank measured by NBS are presented, including tables that relate tank volume to liquid level in the tank. The measurement technique 
used was accurate to $\pm 0.05 \%$ of total volume and agreed with the independent measurement data to $\pm 0.2 \%$.

NBSIR 80-2142. Lashmore, D. S. Plating on aluminum. 1980 November. 65 p. A vailable from: NTIS; PB 81-239501.

Key words: adhesion; anodizing; coatings; electrodeposition; immersion deposition; plating; plating on anodized aluminum.

This report summarizes results on the "Plating on Aluminum" project, which was sponsored by the Aluminum Association. The program includes both anodizing and immersion pretreatments as well as a discussion of adhesion. In addition to the summary of results, some details of the last seven months of the project are presented including: (a) the morphology of the initial stages of anodic film formation; (b) the morphology of the interface of nickel coated anodized aluminum; and (c) a discussion of adhesion.

NBSIR 80-2147. Treado, S.; Kusuda, T. Daylighting, window management systems, and lighting controls. 1980 December. 80 p. Available from: NTIS; PB 81-152886.

Key words: daylight; energy conservation; fenestration design; illumination; lighting control; solar heat gain; window management.

This report investigates major factors concerning windows in buildings and their effect on visual conditions, thermal conditions, and energy requirements. Empirically obtained data are presented for daylight illumination as a function of solar radiation, sky condition, window size and orientation, and interior reflectance. The thermal and visual effects of several window management strategies are examined along with an analysis of automatic lighting controls. Daylight utilization is seen to offer great potential for minimizing lighting load in perimeter building areas, and careful determination of lighting needs and window management strategies can provide additional benefit.

NBSIR 80-2150. Thompson, B. E.; Chapman, R. E. Productivity in residential construction: An annotated bibliography. 1981 February. 59 p. A vailable from: NTIS; PB 81-163925.

Key words: building codes; building economics; construction; cost; economics; housing; productivity; regulation; renovation.

This report presents a state-of-the-art review of the technical literature related to one or more of the factors affecting productivity in residential construction. Particular emphasis is placed on identifying potential sources of variation between the level of productivity in new housing construction versus that in housing renovation. Although this report focuses on the residential sector, emphasis is also placed on topics such as construction management and cost control which perhaps more appropriately apply to the nonresidential sector. The references have been categorized so that articles dealing with specific productivity and construction topics can be easily identified. The categories emphasized in this report are: general productivity/productivity measurement; construction productivity; residential rehabilitation/renovation;

construction/housing costs; construction cost estimation and control; economics of construction; and building codes and regulations.

NBSIR 80-2151. Lechner, J. A.; Reeve, C. P.; Spiegelman, C. H. A new method of assigning uncertainty in volume calibration. 1980 December. 107 p. Available from: NTIS; PB 81-239519.

Key words: accountability; differential pressure; splines; statistics; volume calibration.

This paper presents a practical statistical overview of the pressure volume calibration curve for large nuclear materials processing tanks. It explains the appropriateness of applying splines (piecewise polynomials) to this curve, and it presents an overview of the associated statistical uncertainties. In order to implement these procedures a practical and portable FORTRAN IV program is provided along with its users' manual. Finally, the recommended procedure is demonstrated on actual tank data collected by NBS

NBSIR 80-2152. Wheatley, T.; Albus, J. S.; Nagel, R. N., eds. Proceedings of NBS/Air Force ICAM workshop on robot interfaces. 1980 January. 83 p. Available from: NTIS; PB 81.161119.

Key words: complex sensors; off-line programming; robot control; robot interfaces; robot standards; robot wrist; simple sensors.

This paper will describe the proceedings of a workshop on robot interfaces held at NBS from June 4-6, 1980. Five possible areas for standardization of interfaces were discussed: (1) Simple Sensors, the interface between simple peripheral devices and a robot control system; (2) the Wrist Interface, between the robot wrist and the end effector; (3) the Common Robot Control Interface, between the robot controller and the coordinate transformation module; (4) the Complex Sensory Interface, between the robot controller and vision, complex touch, and other sensors; and (5) Future Guidelines, covering data base, offline programming, and system integration interfaces. The goal was to define the areas ready for current standards, and those for which guidelines for future standards would be useful.

NBSIR 80-2154. Blanc, R.; Heafner, J.; Rosenthal, R.; Watkins, S. The NBS Computer Networking Program. 1980 October. 22 p. Available from: NTIS; PB 81-158255.

Key words: computer based office systems; computer networking; interfaces; local area networks; measurement techniques; protocols; standards.

This report provides a description of the NBS program in computer networking. The description includes activities in computer network protocols for large global networks, protocol interfaces, and measurement techniques for local area computer networks and work in computer based office systems. These efforts include the development of standards and the performance of the necessary research to support that standards development. Descriptions of the specific products being worked on are also included.

NBSIR 80-2155. Issen, L. A. Single-family residential fire and live loads survey. 1980 December. 179 p. Available from: NTIS; PB 81 . 166647.

Key words: field surveys; fire; fire loads; furnishings; household surveys; live loads; mobile homes; residential surveys; transfer functions.

A fire and live load survey of 359 residences, consisting of 61 single family attached (SFA), 200 single family detached (SFD), and 98 mobile homes $(\mathrm{MH})$, was made in the metropolitan Washington, DC area using an inventory technique rather than actual weighing of the room contents. The survey was performed to gain information on live and fire loads which would be used to develop a realistic fire test exposure curve for single family homes (SFA, SFD, and MH), and to gain experience in household surveys that would be useful in a similar nationwide survey. The fire load is reported in terms of composition and of an equivalent $8000 \mathrm{Btu} / \mathrm{lb}$ fuel. The live loads and movable contents fire loads were similar for the three occupancies, but the room finish fire load was higher in mobile homes due to the more extensive use of plywood in the interior wall finish. The weighted mean (according to floor area) of the live load observed in the survey for each of the three classes of housing was approximately $10 \mathrm{lb} / \mathrm{sq}$. $\mathrm{ft}$. The corresponding weighted mean movable contents fire loads were approximately: SFA, 7 lb/sq. ft.; SFD, 7 lb/sq. ft.; MH, 6 lb/sq. $\mathrm{ft}$. The total weighted mean fire loads (contents and finish) were approximately: SFA, $13 \mathrm{lb} / \mathrm{sq}$. ft.; SFD, $13 \mathrm{lb} / \mathrm{sq}$. ft.; MH, $18 \mathrm{lb} / \mathrm{sq}$. ft. The nominal 0.95 fractile live and fire loads were also calculated.

NBSIR 80-2157. Klote, J. H. A computer program for analysis of pressurized stairwells and pressurized elevator shafts. 1981 January. 87 p. Available from: NTIS; PB 81-159709. 
Key words: air movement; computer programs; egress; elevator shafts; escape means; pressurization; smoke control; stairwells.

Pressurized stairwells and pressurized elevators can be used as a means of providing a smoke free exit route during fire situations. This paper describes a computer program which analyzes systems intended to pressurize stairwells or elevator shafts. The basic assumptions and limitations of the program are also discussed. The appendices contain a program listing and examples.

NBSIR 80-2158. Ehrstein, J. R. Some considerations regarding film thickness standards for the semiconductor industry. 1980 November. 14 p. Available from: NTIS; PB 81.239535.

Key words: ellipsometry; polysilicon films; silicon dioxide films; silicon nitride films; standard reference materials; thin films.

Semiconductor integrated circuit manufacturing has witnessed a rapid evolution of processing techniques and a reduction of structural dimensions. This has placed a great burden on metrology for process development and for process monitoring, both because of the smallness of the dimensions involved and the variety of interferences encountered in measuring differing structural combinations of thin films. One possible means for improving the uniformity and control of semiconductor thin film measurement would be through the use of certified thickness standards, such as have been requested of the Standard Reference Material Program at the National Bureau of Standards.

This paper will first consider some of the requisite properties of Standard Reference Materials (SRMs) for effective use in improving the uniformity of measurements. It will then consider some of the limitations imposed by real-world thin film specimens and our state of understanding of their properties as well as by the different types of measurements a vailable. Finally, the need for improved measurement control will be related to the SRM program in light of these limitations.

NBSIR 80-2160. White, E.; Welch, V. M. J.; Hertz, H. S. The mass spectra and isotopic purity of compounds proposed for use in the "master analytical scheme for the analysis of organic compounds in water". 1980 November. 61 p. Available from: NTIS; PB 81. 238453.

Key words: deuterated compounds; isotopic purity; mass spectra; Master Analytical Scheme; organic compounds in water.

Mass spectra for 24 deuterated organic compounds are given in tabular and graphical form. The mole percent deuterium at labelled positions as determined by mass spectrometry for 23 compounds is given.

NBSIR 80-2163. Clifton, J. R.; Anderson, E. D. Nondestructive evaluation methods for quality acceptance of hardened concrete in structures. 1981 January. 54 p. Available from: NTIS; PB 81. 159618.

Key words: concrete; construction; nondestructive evaluation; quality assurance; steel reinforcing bars; test methods.

Nondestructive test methods which can be used in quality acceptance programs for hardened concrete have been critically reviewed and are described in this report. Methods have been identified which provide information on the strength, quality and uniformity, thickness, air content, stiffness, finish, density of concrete as well as the location and condition of steel reinforcement. Both commonly used methods and possible test methods are covered. In addition, the feasibility of combining two or more test methods for improving the prediction of the strength or quality of concrete is explored.

NBSIR 80-2165. Kessler, K. G. Technical activities 1980-Center for
Absolute Physical Quantities. 1980 October. 235 p. Available from: NTIS; PB 81-248478.

Key words: electrical measurements and standards; length and mass measurements and standards; quantum metrology; quantum physics; temperature and pressure measurements and standards; time and frequency.

This report summarizes research projects, measurement method development, calibration and data evaluation activities carried during Fiscal Year 1980 in the NBS Center for Absolute Physical Quantities. These activities fall in the areas of: quantum metrology; measurements and standards for the electrical units, temperature and pressure, length and mass, time and frequency; and quantum physics.

NBSIR 80-2168. Cassel, J. M.; Tesk, J. A.; Brauer, G. M.; Antonucci, J. M.; Wu, W.; McKinney, J. E.; Whitlock, R. P.; Hinman, R. Properties and interactions of oral structures and restorative materials. 1980 November. 110 p. Available from: NTIS; PB 81. 236895 .

Key words: accelerator; alloy; base metal; casting; composite; cyanoacrylate; dental alloy; initiator; resin; wear.

Development of more efficient, less toxic polymerization accelerators indicates much promise for the generically biocompatible $4,4, N$-diethylaminophenylacetic acid which is reactive at four fold reduced concentration relative to currently used amines. Raman, IR and ${ }^{13} \mathrm{C}$ NMR spectroscopy and DSC are being compared for investigation of dental resin polymerization. Solvent carrier and amine catalysts have been shown to influence stability of silane-silica bonding in composites. A state-of-the-art report on radiopaque plastics in dentistry was prepared. A silver staining technique developed to assist investigation of mechanisms of degradation and wear in composite restorations yields useful information on subsurface microdefects in in vivo and in vitro worn specimens. In vitro wear with a pin-on-disc machine indicates increased wear of a composite is generated by going from a very hard (apatite) pin to a softer (stainless steel) pin. A wear test placing greater emphasis on shear forces generated via food particle interplay with teeth has been developed. With thermal expansion measurements, significant differences in the excess volume retained on cooling porcelains fired as per manufacturers' recommendations were noted. Gap and shape changes in a porcelain veneered to alloy split ring offer promise for determining compatibility of porcelains for alloys.

NBSIR 80.2169. Hastie, J. W.; Bonnell, D. W. Molecular chemistry of inhibited combustion systems. 1980 December. 182 p. Available from: NTIS; PB 81.170375.

Key words: flame inhibition; $\mathrm{H}$-atoms; kinetics; mass spectrometry; phosphorus; radicals; Raman spectroscopy; temperature.

Virtually nothing is known about the high temperature chemistry of phosphorus in a flame environment. Phosphorus compounds are known flame retardants though the mechanistic evidence is empirically based. In the present study, high pressure sampling mass spectrometry and optical spectroscopic methods have been applied to the molecular characterization of P.containing flames. The key process controlling flame inhibition and enhancement is identified as: $\mathrm{HPO}_{2}+\mathrm{H} \rightleftharpoons \mathrm{H}_{2} \mathrm{O}+\mathrm{PO}$. New thermodynamic data are presented for $\mathrm{NaPO}_{2}$ and $\mathrm{LiPO}_{2}$ which are present in $\mathrm{Li} / \mathrm{Na}$-containing flames.

NBSIR 80-2170. Bukowski, R. W. Fire protection systems for rail transportation of Class A explosives: Interim report. 1980 November. 34 p. A vailable from: NTIS; PB 81-153975.

Key words: bombs (ordnance); boxcars; computer models; fire detection systems; fire suppression; full-scale fire tests; heat transfer; railroad accidents; small-scale fire tests; thermal protection.

As a result of several accidents involving fire induced detonation of 
military explosives during rail shipment, a research project, funded by the Federal Railroad Administration (FRA), was initiated at the Center for Fire Research (CFR) at the National Bureau of Standards (NBS). This project was initiated to evaluate various methods of protection of Class A explosives from fire, and to identify one or more cost-effective approaches which could be explored in greater detail in later studies.

Active systems (detection, notification, and extinguishment) and passive systems (thermal insulating barriers) were evaluated regarding cost, feasibility and level of protection provided for the major hazard scenarios involved in rail shipment of explosives. The passive, thermal barrier approach was selected as the most reliable and less costly of the options studied while providing an acceptable level of protection.

Small-scale and full-scale tests were conducted to obtain performance data on one specific thermal barrier material. Based on this data, a computer model was developed which can predict temperatures of the boxcar floor, top surface temperature of a thermal barrier, and casing/explosive interface temperature of a wood-pallet mounted bomb for a range of fire sizes. The model predictions compare favorably with measured results from a limited number of experiments. Further experimental data are needed to refine the model and establish an acceptable confidence level in the predicted values. The proposed work necessary to provide this refinement and verification is described.

NBSIR 80.2171. Lerchen, F. H.; Pielert, J. H.; Faison, T. K. Selected methods for condition assessment of structural, HVAC, plumbing and electrical systems in existing buildings. 1980 December. $111 \mathrm{p}$ Available from: NTIS; PB 81-186918.

Key words: building rehabilitation; concrete; electrical; evaluation; HVAC; masonry; plumbing; steel; structural systems; test methods; wood.

This report was developed with the intent of assisting government officials, designers, builders, code officials, and others involved with making technical decisions relative to building rehabilitation to evaluate the condition of existing buildings.

The report describes evaluation methods available specifically for use with the structural materials of concrete, steel, masonry, and wood, as well as, for use with the supportive systems of heating/ventilating/air conditioning, plumbing, and electrical. Both commonly used methods and other possible (but more technically complex) laboratory methods are described for the reader. Comparative tables are provided, where possible, to aid the reader in making a quick selection of the evaluation method most appropriate for the particular parameter to be tested. Considerably more information is included in the area of structural systems than in the rest of the report because of the fact that this report supplements a previous effort to develop a technical manual on structural strength for rehabilitation of existing buildings.

NBSIR 80.2172. Cooper L. Y. Estimating safe available egress time from fires. 1981 February. 73 p. Available from: NTIS; PB 81. 172355 .

Key words: combustion products; compartment fires; egress; fire detection; fire growth; hazard analysis; mathematical models room fires; smoke movement; tenability limits.

A general technique for estimating the time available for safe egress from a fire is presented. By introducing a qualitative and quantitative model of hazard development, the details of the technique are formulated for the room of fire origin problem. The inputs to the model are the area and ceiling height of the room, data from free burn tests of characteristic fuel assemblies likely to be found therein, the anticipated model of fire detection, and a criterion for the onset of untenability. The output is an estimate of the length of time between detection of a fire and the onset of untenable conditions. Results of applying the estimation technique are presented and discussed.

NBSIR 80.2173. Palla, R. L. Water usage characteristics of household appliances and the potential for water savings. 1980 December. $33 \mathrm{p}$. Available from: NTIS; PB 81-202178.

Key words: appliances; clothes washer; discharge; dishwasher; residential; supply; water conservation; water consumption.

A study has been undertaken to quantify appliance water consumption and the effectiveness of various consumer usage techniques and manufacturer design modifications for saving water Appliances considered are clothes washers and dishwashers. Through a comparison of estimated water savings, the study indicates that the most significant means of saving water are those which are already commercially available-front-loading machines and suds-saver options for clothes washers, and short-cycle settings for dishwashers. Water savings of about 20 to 30 percent estimated with these features or about 10 gallons for clothes washers and 3 gallons for dishwashers.

Several appliance design modifications are also evaluated but do not offer the same level of savings. Water usage characteristics such as supply and discharge flow rates and the effect of supply pressure and cycle selection are presented.

NBSIR 80-2174. Margulis, S. T. Building accessibility in relation to door hardware, door users, and door use. 1981 January. 54 p. Available from: NTIS; PB 81-159626.

Key words: architectural barriers; building accessibility; codes and standards; disability; door closers; door openers; doors; ergonomics; functional capacity; handicap; locks.

This report reviews the technical literature related to doors as architectural barriers. It examines the concept of disability and the associated concepts of impairment and handicap. It is concluded that these terms lack consensus of meaning. The concept of functional capacity is recommended as an alternative because of the more direct linkage between capacity and performance. A review of the conceptual literature on fuctional capacity and its measurement leads to the conclusion that functional capacities relevant to building accessibility generally have been identified, but more precise specifications and improved ergonomic procedures for testing capacities of the disabled are required. Furthermore, a distinction is drawn between functional capacity and door use patterns. The latter refers to how capacities are applied during actual door use. Last, door systems are examined, particularly locking and latching mechanisms, door openers and door closers. The existing literature on these raises questions about the adequacy of current accessibility codes and standards with regard to these components. Based on unresolved problems and current needs, research addressing accessibility relevant objectives is recommended.

NBSIR 80.2175. Cohen, E. R.; Birnbaum, G. Analysis of the shape of the far-infrared spectra of $\mathrm{H}_{2}-\mathrm{H}_{2}$ and $\mathrm{H}_{2}-\mathrm{He}$ collisions. 1981 April.

27 p. Available from: NTIS; PB 81-210395.

Key words: band shape analysis; collision-induced absorption; far infrared; $\mathrm{H}_{2}-\mathrm{H}_{2}$ collisions; $\mathrm{H}_{2}-\mathrm{He}$ collisions; planetary atmospheres; quadrupole-induced absorption; spectral moments.

The collision-induced far-infrared spectra due to $\mathrm{H}_{2}-\mathrm{H}_{2}$ and $\mathrm{H}_{2}-\mathrm{He}$ collisions have been previously measured in the range 20 to $900 \mathrm{~cm}^{-1}$ at $77.4,194.7$ and $292.4 \mathrm{~K}$. These spectra are fitted with a semiempirical line shape and the parameters in the shape function are evaluated. The accuracy of these fitting is discussed. The zeroth and first spectral moments for the isotropic overlap translational spectrum due to $\mathrm{H}_{2}-\mathrm{He}$ collisions are obtained and give a value for the range parameter in the induced-dipole function in good agreement with that given by an $a b$ initio calculation.

NBSIR 80-2177. Misakian, $M$. Generation and measurement of dc electric fields with space charge. 1981 January. 44 p. Available from: NTIS; PB 81-161929.

Key words: calibration; dc transmission lines; electric field; electric field meters; electric field strength; electric power 
transmission; measurements; space charge.

Characterization of the electrical environment in the vicinity of high voltage dc transmission lines requires measurement of a number of electrical parameters associated with the lines. These parameters include the electric field strength with significant space charge contributions. This report describes an experimental effort to generate known dc electric fields containing controlled amounts of space charge. An apparatus which has been built for this purpose is described and two types of field probes currently used for transmission line field measurements are examined with the apparatus. Limitations on the operation of one type of probe in the presence of very large current densities are identified and discussed.

NBSIR 80-2178. Becker, D. A.; Berke, J. G.; Matthews, R. T.; Yakowitz, H., eds. Technical activities 1980-Office of Recycled Materials. 1980 November. 74 p. Available from: NTIS; PB 81. 159899.

Key words: energy-from-waste; materials market; oil analysis; recycled materials; refused-derived-fuel; rerefined oil.

A review of recycled materials programs at NBS, for FY 1980 is presented in this annual report.

NBSIR 80-2178 (DoE). Hastie, J. W.; Bonnell, D. W.; Plante, E. R.; Horton, W. S. Vaporization and chemical transport under coal gasification conditions. 1980 December. 113 p. Available from: NTIS; PB 81-245839.

Key words: coal gasification; high temperature; mass spectrometry; slag; transpiration; vapor transport.

The present studies have resulted in the development of a new experimental technique, transpiration mass spectrometry, for the quantitative analysis of high vapor temperature gases and vapors. This technique has been applied to vapor transport and thermodynamic activity determinations for real and synthetic coal slag samples in reactive coal gas components at atmospheric pressure. The results indicate a highly non-ideal and non-monotonic (with temperature and composition variables) behavior for alkali metal vapor transport. Thus a priori predictions of alkali metal transport in coal gasifiers without actual activity data are virtually impossible at the present time. Surface segregation and diffusion limitations of alkali species in slags are also possible complicating effects.

NBSIR 80-2181. Sawyer, D. E.; Kessler, H. K.; Russell, T. J.; Lankford, W. F.; Schafft, H. A. Measurement techniques for solar cells, Annual Report: December 15, 1978 to December 14, 1979. 1981 January. 60 p. Available from: NTIS; PB 81-163875.

Key words: defect identification; laser scanner; measurement techniques; photovoltaics; solar cells; test pattern.

The NBS-developed laser scanner was used to examine a variety of devices: $\mathrm{Cu}_{2} \mathrm{~S} / \mathrm{CdS}$ cells, silicon tandem junction cells, $\mathrm{Zn}_{3} \mathrm{P}_{2}$ Schottky diode specimens, and edge-fed growth polycrystalline silicon cells. The results show that it is possible to detect cell designand processing-induced losses in conversion efficiency, areas of missing antireflection coating, lack of ohmic contact of the metallization to the cell, breaks in cell metallization fingers, fine cracks, scratches, and silicon carbide inclusions. Observation of photoresponse caused by point shunts in the junction and cell cracks is consistent with modeling work. A solar cell test pattern is described and the results of cell sheet resistance measurements are discussed. The test pattern includes four solar cells (three with intentional defects). It also includes microelectronic test structures to determine certain material and design characteristics of these cells. The test pattern serves as a vehicle for linking theoretical and experimental results and placing the scanner technique on a firm theoretical base. Modifications to the laser scanner system are described including an improved reflected-light detection system, a birefringence capability, temperature-controlled stages for heating (to $160^{\circ} \mathrm{C}$ ) and cooling (to $-60^{\circ}$ ), and a new light source for biasing large-diameter cells. Support to the Photovoltaic Program Office of the Solar Energy Research Institute through the conduct of workshops, consultations, and liaison activities is described.

NBSIR 80-2182. Marriott, A. R.; Skillman, G. H.; Salazar, S. B.; Hardgrave, W. T. M7: A general pattern matching facility users manual. 1981 January. 40 p. Available from: NTIS; PB 81-164758.

Key words: macroprocessor; pattern matching; query processor; software tool; text processor; translation; UNIX.

M7 is a general pattern matching and replacement facility. It is based on such macroprocessors as M6, MORTRAN, and STAGE 2, and has been implemented in the language $C$ on UNIX. By incorporating such features as stacks, counters, and tags, $\mathrm{M} 7$ is particularly useful for translating or reformatting queries, languages and data.

NBSIR 80-2184. Barnett, J. P. Energy analysis of a prototype singlefamily detached residence: The effects of climate, house size, orientation, internal heat release, and natural cooling. 1981 January. 46 p. Available from: NTIS; PB 81-166514.

Key words: building design; building energy performance standards; computer simulation of house energy requirements; degree days; energy analysis; single-family detached residence.

A computer study was done to determine how the annual heating and cooling requirements of a prototypical ranch-style house are affected by changes in four energy use parameters: climate (13 locations), floor area (nominal $800 \mathrm{ft}^{2}, 1200 \mathrm{ft}^{2}$, and $1800 \mathrm{ft}^{2}$ ), orientation (north, south, and east/west), and internal heat generation (two different levels in the $1200 \mathrm{ft}^{2}$ house). In addition, the effects of natural cooling on the annual cooling requirement were investigated.

The results are quantified such that the effects attributable to each variation are easily identified. Also, the heating and cooling requirements of the various sized houses are correlated to degree days.

Some of the more important findings regarding the prototypical house (as simulated in this study) are: (a) annual cooling requirements/unit area decreased with increasing floor area, while (b) annual heating requirements/unit area remained relatively constant regardless of floor area; (c) rotation of a house (with windows on only two facades) significantly affected the annual energy requirements (approximate range 20-50 percent); and (e) annual cooling requirements were significantly reduced (by as much as 48 percent) by the use of natural cooling.

NBSIR 80-2186. Babrauskas, V. Comhustion of mattresses exposed to flaming ignition sources Part II. Bench-scale tests and recommended standard test. 1981 February. 82 p. Available from: NTIS; PB 81 181919.

Key words: bedding; beds; compartment fires; fire tests; health care facilities; heat release rate; hospitals; mattresses; prisons; smoke production.

Ten mattress types were subjected to full-scale fire tests in the earlier part of this project. Burning behavior was determined and hazard was assessed by classifying into performance groups. In the present study bench-scale test procedures were examined for suitability for classifying mattress combustion behavior when exposed to flaming ignition sources. Several tests were examined, and a test protocol was developed based on two procedures-measurement of rate of heat release and smoke production. These procedures enable the performance classification, as established by full-scale tests, to be reproduced by convenient laboratory tests. Details are given for conducting the required tests and illustrative performance of some two dozen samples is recorded.

NBSIR 80-2187. Mink, A.; Silio, C. B., Jr. Local area network feasibility study for the Naval Sea Systems Command. 1981 January. 
55 p. Available from: NTIS; PB 81-170656.

Key words: carrier sense multiple access; environmental analysis; feasibility study; local area networks; requirements analysis.

The Naval Sea Systems Command (NAVSEA) has tasked the Institute for Computer Sciences and Technology (ICST) of the National Bureau of Standards (NBS) to perform a feasibility study of a Local Area Networking (LAN) capability to enhance NAVSEA's computing facilities. This report documents that feasibility study. In this report, NAVSEA's environment will first be established. This includes the physical and the computing environment. After establishing the environment, a representative set of application programs will be used to determine NAVSEA's LAN requirements. Then a discussion of LAN topologies is presented, and those applicable to NAVSEA are indicated. Implementations of these applicable topologies are discussed along with the technologies involved and their advantages and disadvantages.

NBSIR 80-2188. Levine, R. S., ed. Joint US-USSR seminar on mathematical methods for estimating the fire endurance of structural assemblies. 1980 December. 214 p. Available from: NTIS; PB 81 . 159956.

Key words: fire endurance; fires; mathematical models; reinforced concrete; steel structures; wood structures.

This publication is a compilation of papers presented May 14, 1980, at a Joint US.USSR Seminar on "Mathematical Methods for Estimating the Fire Resistance of Structural Assemblies." The seminar was arranged by the US.USSR Panel on Fire Resistance of Buildings and Structures as a part of their continuing protocol (agreement) to cooperate in this field. In turn, this agreement is part of a wider US.USSR agreement to cooperate in the field of Housing and Other Construction. The responsible U.S. government agency for the parent agreement is the Department of Housing and Urban Development (HUD). Mr. Lawrence P. Simons, Assistant Secretary for Housing and Federal Housing Commissioner is U.S. Deputy CoChairman for the Parent Agreement. Dr. Robert S. Levine of the National Bureau of Standards, Center for Fire Research and Dr. Igor G. Romanenkov, Laboratory Chief, Central Research Institute of Building Construction, GOSSTROY, are the Co-Chairmen of the Fire Panel. This is the first of a series of planned yearly joint seminars on specific applied fire safety research topics. The Soviet papers were translated into English by the Soviets, but editorial changes have been made by U.S. personnel who are expert in the particular subject. It is intended that the original authors' meanings have not been changed. In some cases, the U.S. presentations are not yet available as published papers, or were summaries of work that has already been published elsewhere. Hence, one of the U.S. presentations is in the form of slides and commentary. These presentations, in the form given, are intended to represent up-to-date U.S. technology.

NBSIR 81-1638. Crawford, M. L. Evaluation of a reverberation chamber facility for performing EM radiated fields susceptibility measurements. 1981 February. 44 p. Available from: NTIS; PB 81 . 181257.

Key words: electromagnetic radiated susceptibility measurements; mode tuned chamber; resonant cavity.

This report describes measurement procedures and results for evaluation of a large $2.44 \mathrm{~m} \times 3.05 \mathrm{~m} \times 7.62 \mathrm{~m}$ reverberating chamber facility in the frequency range $(100-1000) \mathrm{MHz}$. This facility, referred to as a translational electromagnetic environment chamber, "TEMEC," is a mode tuned chamber developed for use in measuring electronic equipment susceptibility to EM radiated fields. A brief description of mode tuned cavity theory is given along with a description of the TEMEC measurement setup and the National Bureau of Standards modification of this setup for analysis and evaluation purposes. Measurements described include: (1) evaluation of the chamber's transmitting and receiving antenna voltage standing wave ratio; (2) measurement of the chamber's insertion loss or coupling efficiency versus frequency; (3) determination of E-field uniformity in the chamber test zone versus frequency; and (4) determination of the absolute amplitude calibration accuracy of the test fields in the chamber based upon the receiving antenna received power measurements.

Conclusions given indicate the chamber may be useful for performing electromagnetic susceptibility measurements at frequencies down to $200 \mathrm{MHz}$. E-field variations (time averaged) in the chamber's test zone decrease from approximately $10 \mathrm{~dB}$ at $200-300 \mathrm{MHz}$ to less than $3.7 \mathrm{~dB}$ at $1000 \mathrm{MHz}$ and are anticipated to be less than $2 \mathrm{~dB}$ above $2 \mathrm{GHz}$. The uncertainty in establishing the absolute E-field amplitude in the chamber test zone is estimated to be less than $10 \mathrm{~dB}$

NBSIR 81-1639. Smith, D. R.; Hust, J. G. Effective thermal conductivity of a glass fiberboard standard reference material. 1981 February. 32 p. Available from: NTIS; PB 81-181240.

Key words: glass fiberboard; insulation; low-temperature; standard reference material; thermal conductivity.

This paper describes the results of thermal conductivity measurements on NBS SRM 1450 glass fiberboard insulation (1970 lot) at temperatures from $87 \mathrm{~K}$ to $340 \mathrm{~K}$. The measurements were performed in an atmosphere of dry nitrogen at a pressure near $84 \mathrm{kPa}$ $(630 \mathrm{~mm} \mathrm{Hg}$ ). The results are analyzed and compared to NBS certification data and to literature data for similar material. An annotated bibliography of literature sources is included.

NBSIR 81-1640. Smith, D. R.; Hust, J. G.; Van Poolen, L. J. Effective thermal conductivity of a glass fiberblanket standard reference material. 1981 February. 30 p. Available from: NTIS; PB 81 176133 .

Key words: glass fiberblanket; insulation; low temperature; standard reference material; thermal conductivity.

This paper describes the results of thermal conductivity measurements at temperatures from $84 \mathrm{~K}$ to $360 \mathrm{~K}$ on a glass fiberblanket insulation which is intended to be a Standard Reference Material (SRM). The measurements were performed in an atmosphere of dry nitrogen at a pressure near $84 \mathrm{kPa}(630 \mathrm{~mm} \mathrm{Hg})$. The results are analyzed and compared to literature data for similar material. An annotated bibliography of literature sources is included.

NBSIR 81-1641. Wakefield, J. P. Addendum to Earth Terminal Measurement System Maintenance Manual. 1981 October. 48 p. Available from: NTIS; PB 82-151994.

Key words: earth terminal; Earth Terminal Measurement System; figure of merit; noise temperature; satellite communication.

This addendum to the Earth Terminal Measurement System Maintenance Manual, NBSIR 78-895, describes the equipment and maintenance procedures required to support the retrofit package for the Earth Terminal Measurement System (ETMS) developed by the National Bureau of Standards. This retrofit adds a multi-input-port relay module which provides the capability of connecting three receiver channels to the ETMS, thereby enabling measurement of pertinent earth terminal parameters at as many as three frequencies in a single measurement pass. This manual does not include measurement theory nor measurement operating procedures that are described in the Earth Terminal Measurement System Operation Manual, NBSIR 78-879.

NBSIR 81-1643. Lewis, R. Efficient computation of the far field radiated by an arbitrary rectangular-aperture distribution. Computer program documentation. 1981 March. 45 p. Available from: NTIS; PB 81-200362.

Key words: algorithm; antenna; aperture; computer program; electromagnetic; far field; FFT; Fourier transform.

This report contains the computer documentation for calculating 
the far-zone electric field due to a user-prescribed electric-field distribution within a rectangular aperture. The far-field output is computed along two arbitrarily selected, perpendicular, spatialfrequency plane cuts. Program execution time is minimized by the use of fast Fourier transform (FFT) processing. The program was designed so that the required far-field output is obtained by processing only two, vector, one-dimensional FFTs. The far-field results are obtained in the form of elevation and azimuth vector components and electric-field-vector magnitude. A complete analytical discussion of the problem is presented, along with sample graphical output to illustrate how aliasing and output resolution limitations effect the graphical results.

NBSIR 81-1644. Yaghjian, A. D. An approximate expression for the principal beamwidths of directive antennas in terms of aperture fields. 1981 March. 14 p. Available from: NTIS; PB 81-200388.

Key words: antenna beamwidths; aperture fields; directive antennas.

An approximate, but general, formula for the half-power principal beamwidths of directive antennas is derived from the Fourier transform of the aperture electric field. The derivation, which can be accomplished by expanding the transform in a Taylor series, is greatly streamlined by introducing a Gaussian beam approximation. The formula which also relates beamwidth to directivity indirectly through simple integrations of the aperture field, reduces to a very compact expression in terms of the zero and second order moments of the aperture distribution for linearly polarized, uniformly phased aperture fields.

NBSIR 81-1645. Reed, R. P.; Simon, N. J., eds. Materials studies for magnetic fusion energy applications at low temperatures-IV. 1981 April. 604 p. Available from: NTIS; PB 81-222507.

Key words: alloys; aluminum alloys; castings; composites; cryogenic properties; low temperature; mechanical properties; nonmetallics; physical properties; stainless steels; structural alloys; welding.

This report contains results of a research program to produce material property data that will facilitate design and development of cryogenic structures for the superconducting magnets of magnetic fusion energy power plants and prototypes. The program was conceived and developed jointly by the staffs of the National Bureau of Standards and the Office of Fusion Energy of the Department of Energy; it is managed by NBS and sponsored by DoE. Research is conducted at NBS and at various other laboratories through subcontracts with NBS. The reports presented here summarize the fourth year of work on the low temperature materials research program. Highlights of the results are presented first. Research results are given for the four main program areas: structural alloys, weldings and castings, nonmetallics, and technology transfer. Objectives, approaches, and achievements are summarized in an introduction to each program area. The major portion of the program has been the evaluation of the low temperature mechanical and physical properties of stainless steel base metal and welds, with particular emphasis on the nitrogen-strengthened stainless steels. Aluminum alloys have received some consideration also. Work has been done on the production and standardization of nonmetallics, primarily industrial laminates, for low temperature applications and on the measurement of their properties at cryogenic temperatures. A brief description is given of the fourth NBS/DoE Vail workshop held in October 1980.

NBSIR 81-1646. Andrews, J. R.; Baldwin, E. E. Amplitude calibrator for oscilloscopes. 1981 April. 31 p. Available from: NTIS; PB 81. 211286.

Key words: calibration; calibrators; instrumentation; oscilloscope calibrator; oscilloscopes.

The amplitude calibrator is designed to provide known dc voltage levels or $1 \mathrm{kHz}$ square waves from $\pm 1 \mathrm{mV}$ to $\pm 5 \mathrm{~V}$. It features selectable output impedances of $<0.1 \Omega, 50 \Omega$, and $1 \mathrm{M} \Omega$. The instrument is designed with sufficient current capability to deliver its indicated voltage into a $50 \Omega$ termination. To protect delicate sampling oscilloscopes, a limiter circuit can also be activated to limit the output voltage to $\pm 1.8 \mathrm{~V}$.

NBSIR 81.1647. Bransford, J. W.; Clark, A. F. Laser-initiated combustion studies of selected aluminum, copper, iron, and nickel alloys. 1981 April. 89 p. Available from: NTIS; PB 81-215501.

Key words: alloys; combustion; combustion rate; ignition; metals; oxygen atmosphere; temperature measurement.

The results of combustion studies at atmospheric pressure on ten metal alloys are presented. The alloys studied were aluminum alloys $1100,2219,6061$, and tensile-50; 304, 347 and 21-6-9 stainless steel; inconel 600; beryllium-copper and a bronze. It was found that once ignition was achieved all alloys would generally burn to completion. The overall combustion process appears to obey a first-order rate process. Preliminary conclusions are presented along with recommendations for future work.

NBSIR 81-1648. Read, D. T.; McHenry, H. I. Interim progress report: J-integral method for fitness-for-service assessment. 1981 May. 52 p. Available from: NTIS; PB 82-135195.

Key words: crack opening displacement; design curve; elastic. plastic fracture mechanics; fitness-for-service; fracture mechanics; J-integral.

Progress to date on the J-integral fitness-for-service assessment project is reported. The goal of this project is to produce a method for evaluation of material toughness requirements, allowable defect sizes, and sustainable stresses and strains. Yielding fracture mechanics and, specifically, the J-integral are employed. The J-integral is regarded as the crack driving force; applied $\mathrm{J}$ values above some critical value are predictive of material tearing. The applied $\mathbf{J}$ value is a function of applied stress and strain, crack length, and material tensile properties. The critical J-integral value is measured in material fracture-toughness tests. The experimental focus of the present study is on measurement of the applied J-integral as a function of stress and strain in HY130 specimens with different crack lengths. Neither experimental data nor established predictive theories for the behavior of applied $\mathrm{J}$ values are available in the literature for short cracks, the type most likely to be found in a structural element. Results for relatively large cracks indicate that displacements imposed at the specimen ends are transmitted in full to the cracked cross section. The displacements add to the strain field of the crack and produce a large applied $\mathrm{J}$ value. This behavior is described as net section yielding. For relatively short cracks, displacements imposed at the specimen ends are absorbed as plastic strain all along the specimen length, add only slightly to the strain field of the crack, and produce small applied $\mathbf{J}$ values. This behavior is described as gross section yielding. It is important to note that cracks as small as $5 \%$ of the total cross section have been found to exhibit net section yielding.

NBSIR 81-1649. Daney, D. E., ed. Helium research in support of superconducting power transmission: Annual report for the period October 1, 1979-September 30, 1980. 1981 September. 44 p. Available from: NTIS; PB 82.122987.

Key words: cable cool-down; cool-down; heat flux meters; helium; superconducting power transmission.

During FY 80, the NBS Thermophysical Properties Division program of research for superconducting power transmission line (SPTL) development focused on two tasks: 1) Development of SPTL cool-down strategies; 2) Experimental evaluation of thermal flux meters as a possible technique for determining enclosure heat leak.

In developing SPTL cool-down strategies, we used a computer code (previously developed at NBS) to explore cool-down times for a wide variety of realistic boundary conditions. Cool-down times of 10 
days are feasible with only minor modification to previously proposed refrigeration systems if cooling channel lengths are reduced to two thirds or one half those dictated by steady-state considerations.

Evaluation of thermal flux meters was concluded. Below ground field tests revealed large effects due to seasonal variation in the soil heat flux. These effects can be largely cancelled by algebraic addition of the signals from a pair of horizontally opposed sensors. A brief above ground field evaluation indicated that diurnal variations in the heat flux completely mask heat fluxes typical of the anticipated enclosure heat flux.

NBSIR 81-1650. Weidman, M. WR-10 single six-port measurement system. 1981 September. 11 p. Available from: NTIS; PB 82-123696.

Key words: $\mathrm{mm}$ wave measurements; reflection coefficient; sixport measurement system; thermistor mount calibration.

A six-port system has been developed and used to measure effective efficiency $\left(n_{e}\right)$ and complex reflection coefficient $(\Gamma)$ in WR-10 $(75-110 \mathrm{GHz})$ waveguide at frequencies in the $93.5-96.5 \mathrm{GHz}$ range. The system is automated except for the control of the mm-wave klystron source. This report includes a brief description and background of the measurement system and a preliminary analysis of uncertainties.

NBSIR 81-1651. Sparks, L. L. Thermal conductivity of a concrete mortar from $95 \mathrm{~K}$ to $320 \mathrm{~K}$. 1981 October. $16 \mathrm{p}$. A vailable from: NTIS; PB 82-134370.

Key words: concrete; guarded hot plate; low temperature; moisture migration; mortar; porous solid; thermal conductivity.

The thermal conductivity of a single concrete mortar specimen with varying moisture content is reported in the temperature range from 95 to $320 \mathrm{~K}$. The measurements were made in a guarded-hotplate apparatus (ASTM C-177). Moisture migration caused by temperature gradients was minimized by studying the saturated specimen in the low.temperature region. Specimen moisture content and concomitant thermal conductivity were altered by imposing low. pressure, high-temperature conditions on the specimen. The effect of changing the moisture content is discussed.

NBSIR 81-1653. Chew, H. Modelling of oil shale retorts for electromagnetic sensing techniques. 1981 November. 47 p. Available from: NTIS; PB 82-153321.

Key words: oil shale retorts; remote sensing; scattering.

We report here some work on the modelling of oil shale retorts for electromagnetic sensing techniques. The aim is to obtain useful information about the contents of the retort (e.g., rubble size, void ratio, etc.) by means of electromagnetic probes. In this work, the retort is modelled by a spheroid with an average dielectric constant which depends on the void ratio. The near field due to a radiating dipole source in the vicinity of a spheroidal retort is computed using the Extended Boundary Condition Method due to Waterman, Barber, and Yeh. Numerical results are given at $4 \mathrm{MHz}$ for a retort with major axis $45.7(150 \mathrm{ft})$, minor axis $25.1 \mathrm{~m}(82.5 \mathrm{ft})$, bulk dielectric constant $8.8+3.7 \mathrm{j}$, and various void ratios. The results indicate feasibility of determining the void ratio by remote electromagnetic measurements. It is also believed that this work may be of interest beyond the immediate context of oil shale retort modelling.

NBSIR 81-1654. Arvidson, J. M.; Sparks, L. L. Low temperature mechanical properties of a polyurethane foam. 1981 November. $17 \mathrm{p}$. Available from: NTIS; PB 82-160441.

Key words: compressive strength; elongation; foam; insulation; low temperature; mechanical properties; proportional limit; shear strength; tensile strength; yield strength; Young's modulus.

Polyurethane foam, having a density of $32 \mathrm{~kg} / \mathrm{m}^{3}$, was tested at 295 , 111,76 , and $45 \mathrm{~K}$ in helium gas. The material properties reported are Young's modulus, proportional limit, yield strength (at $0.2 \%$ offset) tensile, shear, and compressive strengths, and elongation (elastic and plastic). To perform these tests, a unique apparatus was developed. This apparatus permits tension, compression, and shear testing of materials at any temperature ranging from 295 to $4 \mathrm{~K}$. Strain is measured with a concentric, overlapping-cylinder capacitance extensometer that is highly sensitive and linear in output.

NBSIR 81-2191 (DoE). Johnson, C. E.; Ugiansky, G. M. The use of the slow strain rate technique for the evaluation of structural materials for application in high-temperature gaseous environments. 1981 January. 92 p. A vailable from: NTIS; PB 81.179152

Key words: austenitic stainless steels; coal gasification; elevated temperatures; ferritic stainless steel; gaseous environments; nickel alloy 671 ; nickel alloy 800 ; slow strain rate test; structural materials.

The slow strain rate technique has been evaluated as an accelerated test for determining the performance of structural materials in simulated coal gasification environments. Seven alloys, namely Types 309, 310, 310S, 347 and 446 stainless steels; Incoloy 800; and Inconel 671 , were tested at temperatures from $370^{\circ} \mathrm{C}$ to $1040^{\circ} \mathrm{C}$ at strain rates from $10^{-4}$ to $10^{-7} / \mathrm{s}$ in $\mathrm{H}_{2} \mathrm{~S}$ plus water, gaseous mixtures of $\mathrm{CO}, \mathrm{CO}_{2}$, $\mathrm{H}_{2}, \mathrm{CH}_{4}, \mathrm{H}_{2} \mathrm{~S}$, and $\mathrm{H}_{2} \mathrm{O}$, and in nominally inert environments of $\mathrm{He}$ and Ar. Type 310 steel, the most extensively studied material, showed a marked reduction in mechanical properties at low strain rates $\left(<10^{-5} / \mathrm{s}\right)$ in $\mathrm{H}_{2} \mathrm{~S} / \mathrm{H}_{2} \mathrm{O}$ at $540^{\circ} \mathrm{C}$, and this was associated with the occurrence of a large degree of secondary intergranular cracking in addition to the main ductile fracture mode. The occurrence of the secondary cracking was taken as the primary indication of embrittlement in subsequent tests. Of particular significance, it occurred to some degree in all alloys tested in the simulated coal. gasification environments at $600^{\circ} \mathrm{C}$. Consequently, the mechanism(s) of the embrittlement phenomena remain uncertain; a number of possible causes including creep and several environmentally-induced fracture processes are outlined. It is shown that the overall results of the test program are in good agreement with in-plant experience. It is concluded that the slow strain rate technique is an effective method for evaluating the performance of structural materials in the elevated. temperature gaseous environments.

NBSIR 81-2195. Harris, J. R.; Leyendecker, E. V. Draft seismic standard for Federal buildings. 1981 January. 96 p. Available from: NTIS; PB 81-163842.

Key words: building; building design; earthquakes; engineering; Federal government; seismic safety; standards; structural engineering.

This standard has been prepared for uniform use by all Federal agencies as an adaptation of existing voluntary standards, model building codes, Federal regulations, and research reports. It is closely based on the seismic requirements of the Uniform Building Code (which, in turn, are based on the Recommended Lateral Force Requirements and Commentary published by the Structural Engineers Association of California). However, there are many instances of substantive differences from the UBC. Several important provisions, including the seismic zoning map, have been adapted from the Tentative Provisions for the Development of Seismic Regulations for Buildings developed by the Applied Technology Council. A number of provisions have been added to this standard that are based on the current practices and policies of various Federal agencies. Furthermore, this standard is organized considerably differently from the UBC and many provisions are phrased differently.

NBSIR 81.2197. Scace, R. I. Semiconductor technology for the nontechnologist. 1981 January. 29 p. A vailable from: NTIS; PB 81 166902 .

Key words: integrated circuits; semiconductor devices; semiconductor processes; semiconductors; semiconductor technology; silicon.

The properties of semiconductor materials, the methods of 
processing them, and the solid-state products made from them are described in terms intended to be understandable by the lay person. The semiconductor industry has grown at a rate of 21 percent per year compounded for the last twenty years, and its products have declined in unit cost by a factor of five in current dollars (a factor of ten in constant dollars) in the same period. This very satisfactory but anomalous behavior has attracted the interest of many who are not familiar with the technology of the industry, yet who need to have some understanding of it. This report is intended to help meet that need.

NBSIR 81-2199. Cattaneo, L.; Harris, J. R.; Reinhold, T. A.; Simiu, E.; Yancey, C. W. C. Wind, earthquake, snow, and hail loads on solar collectors. 1981 January. 97 p. Available from: NTIS; PB 81164550 .

Key words: earthquake loads; hail loads; snow loads; solar collectors; structural engineering; wind loads.

The report describes and interprets wind-tunnel, full-scale, and field studies of wind and snow loads on flat plate solar collectors, conducted under contract for the National Bureau of Standards, and uses results of these studies and other data available in the literature to develop information, guidelines, and criteria for the design of flat plate collectors subjected to the action of wind, snow, and earthquake loads. Also given in the report are data on hail loads, based on information and studies available in the literature.

NBSIR 81-2200. Braun, E.; Reyes-Virella, R. Evaluation of alternate mounting methods for the evaluation of brattice cloth on ASTM E-162, 1981 January. 27 p. Available from: NTIS; PB 81-172496.

Key words: ASTM E-162; brattice cloth; coal mining; fire test; flame spread; sample mounting; ventilation cloth.

Twenty-two brattice cloth samples representing a cross-section of materials available to the coal mining industry were tested using ASTM E.162, Surface Flammability of Materials Using a Radiant Heat Energy Source. The tests were conducted to evaluate alternative mounting methods that would improve test repeatability. Five mounting methods were studied. The study showed that the flame spread index was dependent on the mounting methods. The foil/spacer/backing method produced the highest values, while the backing only method produced the lowest values. However, measurement dispersion was unaffected by mounting method.

NBSIR 81-2202. Cooper, L. Y.; O'Neill, J. G. Fire tests of stairwellsprinkler systems. 1981 April. 83 p. Available from: NTIS; PB 81. 205288.

Key words: automatic sprinklers; cooling efficiency; evaporation cooling; smoke movement; spray nozzle; stairway protection; ventilated stair.

The effect of water curtain or spray nozzle fire protection of an open stairwell was studied experimentally. The experimental setup includes a three-story stairtower with a contiguous first story burn room. The instrumentation used to measure the flow phenomenology resulting from fire sizes up to $4 \mathrm{MW}\left[13.6\left(10^{6}\right) \mathrm{BTU} / \mathrm{hr}\right]$ with and without sprinkler operation is described. A model of evaporation cooling of the hot fire gases as they pass up through the first to second floor stairwell-sprinkler system component is introduced. Based on this model the data are analyzed and correlated for the purpose of identifying both a cooling and a water usage efficiency for each of the system components that were tested. Application of these measured performance characteristics and their extension to stairwellsprinkler components of different geometries and configurations are discussed.

NBSIR 81-2203. Vaishnav, M. P. Electrical aspects of the CSA/NBS weatherization study. 1981 January. 52 p. Available from: NTIS; PB 81.172512 .

Key words: branch circuit wiring problems; electrical problems; electrical wiring system; extension cord wiring; fuse/circuit breaker panel problems; overamping; residential structures; safety; thermal insulation.

Results of a study to determine the present condition of the electrical wiring system in existing houses at ten sites across the country are presented in this report. The aim of the investigation was to develop information to assess the potential safety impact that the addition of thermal insulation to residential structures could have on their electrical systems. A previous study indicated the nature of some of the technical problems found in selected homes as identified by the residents and by inspectors having no formal training in electrical inspections. The present study is based on the evaluation of electrical problems in 157 houses by technically qualified persons. It appears that a rather large percentage of the homes studied had one or more electrical problems related to overamping of circuits, service entrance conductors, fuse/circuit breaker panels, branch circuit wiring, extension cord wiring or lighting.

NBSIR 81.2204. Blackburn, D. L. Power transistor switching characterization, 1981 April. 30 p. Available from: NTIS; PB 81. 196347.

Key words: base current, reverse; degradation; reverse bias; second breakdown; switching; temperature; transistors, power.

The results of the first year of an experimental investigation of the switching characteristics of power transistors are discussed. The devices studied were housed in TO-3 cases and were of an $n^{+}-p-n^{-}-n^{+}$vertical dopant structure. The effects of the magnitude of the reverse-base current and temperature on the reverse-bias second breakdown characteristics are discussed. Brief discussions of device degradation due to second breakdown and of a constant voltage turn off circuit are included. A description of a vacuum tube voltage clamp circuit which reduces clamped collector voltage overshoot is given.

NBSIR 81-2205. Hoffman, J. D.; Yolken, H. T.; Reed, W. P.; Carpenter, B. S. National Measurement Laboratory Office of Measurements for Nuclear Technology 1980: Annual report. 1981 January. 57 p. Available from: NTIS; PB 81-171290.

Key words: accountability guide; bulk measurements; isotopic assay; leachability studies; nondestructive assay; nuclear safeguards; nuclear waste management; standard reference materials; statistical evaluation.

This annual report is a summary of the National Bureau of Standards (NBS) Measurements for Nuclear Technology (MNT) Program for Fiscal Year 1980. The MNT activities at NBS are divided into two programs: Nuclear Safeguards and Nuclear Waste Management.

The MNT Program is one of several matrix managed efforts at NBS. The programs provide a focus for the activity, both inside and outside NBS. The programs provide funds and directions for needed technical work that is done within NBS Centers. People remain attached to Centers but are funded for specific activities in a matrix management approach. A program provides central organization for outside inquiries and for distribution of results. Often programmatic focus can provide improved assistance to outside agencies with measurement problems. The MNT Program is operating in this manner.

NBSIR 81-2206. Fivozinsky, S. P., ed. Technical activities 1980Office of Standard Reference Data. 1981 February. 55 p. Available from: NTIS; PB 81-164527.

Key words: data compilation; energy and environmental data; evaluated data; materials data; standard reference data; technical activities 1980; thermochemical and thermophysical data.

The Office of Standard Reference Data is one of six program offices in the National Measurement Laboratory, National Bureau of 
Standards. The Standard Reference Data Program develops and disseminates data bases of critically evaluated physical/chemical properties of substances. These data bases are available through NBS and private publications, on magnetic tape, and from on-line retrieval systems.

The Office of Standard Reference Data is responsible for management and coordination of the program. Work is carried out through a decentralized network of data centers and projects referred to as the National Standard Reference Data System (NSRDS). This volume summarizes the activities of the Program for the year 1980.

NBSIR 81-2209 (FDA), McKenna, G. B.; Khoury, F. A.; Crissman, J. $M$. Relationship between morphology and mechanical properties of ultra high molecular weight polyethylene. 1981 April. 63 p. Available from: NTIS; PB 82-139072.

Key words: creep; failure; fatigue; mechanical properties; morphology; polymer; stress relaxation; UHMWPE; ultra high molecular weight polyethylene.

Aspects of the morphology of the constituent particles of the raw ultra high molecular weight polyethylene (UHMWPE) used in this study have been examined using scanning electron microscopy. In addition differential scanning calorimetry has been used to determine the melting point $(\sim 413 \mathrm{~K})$ and the crystallinity $(78 \%)$ of the raw polymer. Protocols have been established for the preparation of compression molded sheets of UHMWPE having crystallinities of $50 \%$ and $60 \%$. A memory of the particulate nature of the raw polymer is retained in the sheets. The following aspects of the mechanical behavior of the molded sheets have been examined: Stressstrain behavior at constant rate of clamp separation, creep and single step stress relaxation in uniaxial extension and failure under both static and sinusoidal loading conditions. The relaxation modulus of the lower density material (quenched) was found to be smaller by a factor or two than that of the higher density material (slow cooled). The short time creep of the quenched UHMWPE was significantly greater than that of the slow cooled polymer; however, the limiting creep strain prior to failure was nearly the same for both materials. Low cycle fatigue data obtained on the slow cooled UHMWPE suggests that it may be possible to characterize the failure behavior of this polymer using a cumulative damage rule. The lifetime of the UHMWPE under zero-tension sinusoidal loading is 6.7 times longer than the lifetime in static (creep) tests in agreement with the cumulative damage rule.

NBSIR 81-2210. Winter, F. Onsite wastewater systems-Current practices and a proposed basis for evaluation. 1981 March. $113 \mathrm{p}$. Available from: NTIS; PB 81-211393.

Key words: onsite wastewater systems; wastewater disposal; wastewater recirculation; wastewater reuse; wastewater treatment; water conservation.

A review of onsite wastewater systems and wastewater recirculation/reuse devices based on the literature and field inspections of systems in actual settings and usage is presented. Based upon the observations, an evaluation basis for onsite wastewater systems is proposed. Criteria and requirements for conducting and monitoring demonstration projects is presented. Wastewater systems identified as potentials for demonstration projects are suggested. Topics requiring further study are identified and recommended for specific research.

NBSIR 81-2212. Dehl, R. E.; Grant, W. H.; Cassel, J. M. Evaluation of methods of characterizing the porosity of porous polymeric implant materials: A review of the current status of porosity measurements. 1981 February. 49 p. Available from: NTIS; PB 81. 166928.

Key words: ossicular prosthesis; porosity measurement; porous biomaterials; porous polyethylene; PTFE-carbon composite; synthetic implants.

A search of the published literature pertaining to porous polymeric implant materials has been made. The two porous materials currently dominant in implant surgery, namely, porous high density polyethylene and a porous composite of polytetrafluoroethylene and carbon, have been reviewed with respect to the following criteria pertinent to their medical applications: (1) Information supplied by the manufacturers about their methods of characterizing the porosity of their materials, (2) recommendations of the ASTM for quantitative porosity characterization of these materials, and (3) clinical studies of the materials as replacement prostheses in middle ear surgery. A computer search for new quantitative methods of measuring pore size revealed nothing appropriate to these materials. However, disputes in the current literature suggest that current protocols for porosity characterization are inadequate for proper interlaboratory comparison of experimental data.

NBSIR 81-2213. Evans, D. D.; Davis, S. Technical support for the Consumer Product Safety Commission 1979 interim standards for cellulose insulation. 1981 March. 52 p. Available from: NTIS; PB 81.196396.

Key words: attic floor radiant panel test; cellulose insulation; fire test; flame spread; HH-I.515D; smoldering.

The attic floor radiant panel test (AFRPT) and smoldering combustion test are two fire performance tests required by the Consumer Product Safety Commission (CPSC) as part of a mandatory standard for loose fill cellulosic home insulation. In providing technical support to CPSC, the sensitivity to variations in parameters of each test method were studied. Specimen density was found to have no effect on critical radiant flux values when measured with the AFRPT for two paper-based cellulosic insulations tested at densities below $48 \mathrm{~kg} / \mathrm{m}^{3}$. A low flux exposure profile, ranging from 0.04 to $0.35 \mathrm{~W} / \mathrm{cm}^{2}$, was developed to extend the range of the AFRPT about the region of greatest interest. The critical radiant flux measured using the low flux profile was found to be sensitive to changes in preheat time from the prescribed two minute specimen preheat. Large-scale attic fire tests verified the predictive ability of AFRPT measurements. Results from the smoldering combustion test were shown to be sensitive to test room humidity conditions over the range of 39 to 84 percent $R H$.

NBSIR 81-2214. Gross, D. A review of measurements, calculations and specifications of air leakage through interior door assemblies. 1981 January. 25 p. Available from: NTIS; PB 81.179178.

Key words: air leakage; buildings; doors; fire doors; fire tests; infiltration; orifice flow; smoke control; smoke movement.

A review was made of measurements and calculations of air leakage through door assemblies in order to evaluate their effectiveness as barriers to smoke flow. It was noted that typical "fire doors" are not capable of meeting proposed limitations of air leakage at ambient temperatures and that significant air leakage may also occur through interior walls. It was concluded that first priority should be given to developing and validating a standardized test method for air leakage measurements.

NBSIR 81-2215. Yokel, F. Y.; Salomone, L. A.; Chung, R. M. Construction of housing in mine subsidence areas. 1981 January. 53 p. Available from: NTIS; PB 81.174690.

Key words: design criteria; foundation design; geotechnical engineering; housing construction; mine subsidence; mining; settlement; structural design; structural engineering.

Criteria for site exploration, risk assessment, site development and housing construction in actual and potential mine subsidence areas are recommended. Appendix A includes guidance for subsidence profile determination and a proposed mathematical model which may aid in predicting complex subsidence patterns. Appendix B includes a commentary and proposed equations and procedures for the design of rigid and flexible foundations.

NBSIR 81-2220. Beausoliel, R. W.; Clifton, J. R.; Meese, W. J. Effects 
of thermal insulations on electrical connections and outlet boxes. 1981 April. 52 p. Available from: NTIS; PB 81.197006.

Key words: cellulose thermal insulation; corrosion of electrical outlet boxes and devices; electrical devices; humidity, thermal insulation and corrosion of electrical wiring; shock hazards; ureaformaldehyde thermal insulation.

When residential walls are retrofitted with "foamed-in" ureaformaldehyde or "blown-in" cellulose thermal insulations, the insulation may enter electrical outlet and switch boxes. The effects of these thermal insulations on the durability of electrical components were studied. These studies were carried out at 44,75 , and 96 percent relative humidities with test periods between one and twelve months.

Laboratory test methods were developed and tests performed to determine the electrical and corrosive effects of urea-formaldehyde and cellulose thermal insulation contained in electrical outlet and switch boxes. The boxes were tested in humidity-controlled closedglass vessels at ambient temperatures. These tests were of an exploratory nature and did not cover all of the conditions that would exist in a residential wall. The testing methods are described in this report and the results are presented and interpreted.

Results indicate that these thermal insulations can cause significant corrosion of electrical components and can cause shock hazards and increased energy losses. It is concluded that these thermal insulations should be removed from electrical outlet and switch boxes.

NBSIR 81-2221. Beausoliel, R. W.; Meese, W. J. Experimentally determined performance of some residential circuit breakers. 1981 April. 49 p. Available from: NTIS; PB 81-197048.

Key words: branch circuits; circuit breaker; electrical fire; low ambient temperature; trip time.

Laboratory test results show that at low ambient temperatures some residential-type circuit breakers may not trip at currents up to 140 percent of rated currents. Under some environmental conditions this may lead to wiring temperatures that exceed the limitations specified in the National Electrical Code. The results also show that circuits sometimes open at the point of short circuits before circuit breakers operate. Ignition of combustibles proximate to the point of such short circuits sometimes occurs.

The results indicate the need for a more detailed study of overcurrent protection performance in the field and under laboratory conditions. Also needed are the development of more meaningful scientific/mathematical principles and models on the functional characteristics of circuit breakers.

NBSIR 81-2222. Spiegelman, C. H. Some conservative statistical approaches for presenting interlaboratory N.D.A enrichment measurements. 1981 March. 19 p. Available from: NTIS; PB 81 . 187973.

Key words: maxmin; minmax; multiple comparison; multivariate analysis; peak areas; principal components.

It is important to realistically evaluate interlaboratory measurements on prototype and primary standards. The emphasis in this paper is on nondestructive assay (NDA) measurements. These measurements are based on an estimate of the total number of counts from a target source. This estimate is called the peak area calculation. It is necessary to distinguish real differences between laboratory findings from artificial differences such as those caused by artifacts of individual peak area calculations. This report provides guidance for assessing the magnitude of these peak area artifacts. A wide variety of statistical tests for homogeneity of the peak areas for these standards is considered. For these tests, algorithms are provided for finding linear peak area computations which either make the standards appear most or least homogeneous. These tests include the usual $F$ statistics as well as the standardized range.

NBSIR 81-2223. Rawie, C. C. Estimating benefits and costs of building regulations: A step by step guide. 1981 June. 93 p. Available from: NTIS; PB 81.217812.

Key words: benefit-cost analysis; building economics; building regulation; codes; construction regulation; economics; energy conservation codes; fire safety codes; regulation.

This is a how-to guide intended to help building officials, elected officials, builders, architects, engineers, and others determine the benefits and costs of proposed building code changes. The guide describes seven steps in the benefit-cost analysis. They are: (1) define the problem, including selecting prototype buildings to analyze; (2) estimate impacts on building-related costs, including government costs; (3) estimate impacts on building safety and performance; (4) select a method of relating benefits and costs (the recommended measure is Net Monetary Benefits together with information on physical life safety impacts); (5) estimate aggregate impacts on the code jurisdiction as a whole; (6) perform a sensitivity analysis; and (7) write up the results, being careful to present information on nonmonetary as well as monetary effects. Worksheets are provided to assist in the analysis.

NBSIR 81-2224. Bullis, W. M. Advancement of reliability, processing and automation for integrated circuits with the National Bureau of Standards (ARPA/IC/NBS). 1981 March. 50 p. Available from: NTIS; PB 81-188203.

Key words: electronics; integrated circuits; metrology; microelectronics; semiconductors; silicon.

The program described in this report was undertaken in 1973 to develop the measurement technology necessary to enable device manufacturers to exert more effective control over the materials and processes they use to make integrated circuits with the ultimate aim of providing increased reliability at reasonable cost. Work was carried out in 12 technical areas: resistivity and dopant characterization; crystal defects and contaminants; oxides and other insulator films; physical analysis techniques; film and layer thickness; materials for infrared detector arrays; materials and procedures for wafer processing; photolithography; microelectronic test patterns; wafer inspection and test; die and interconnection bonding; and hermeticity. Many improved measurement technologies of considerable diversity were developed and documented during this program. The results have been widely and beneficially applied in other DoD projects and by the U.S. semiconductor industry. At the present time the work is being extended to accommodate the denser circuits, larger chips, and new processing techniques associated with very large scale integration

NBSIR 81-2226. Kingston, M. L., ed. NBS serial holdings 1981. 1981 March. 284 p. Available from: NTIS; PB 81-203739.

Key words: annual reports; diffusion in metals; fire; journals library holdings, NBS Library; NBS periodicals; periodicals; proceedings; serials; standards; transactions.

This publication contains holdings information for approximately 4600 titles representing current and noncurrent journals, periodicals, annuals, memoirs, proceedings, and transactions. The holdings of the NBS Library and 3 collections specializing in fire research, standards, and diffusion in metals are represented.

NBSIR 81-2228 (NBS). Gerhold, W. F.; Escalante, E.; Sanderson, B $T$. The corrosion behavior of selected stainless steels in soil environments. 1981 February. 80 p. Available from: NTIS; PB 81178154

Key words: coatings; corrosion behavior; field-tests; galvanic couples; soil environment; stainless steels; stress-corrosion behavior.

In order to obtain more definitive information regarding the corrosion and stress corrosion of stainless steels in soil environments, NBS in cooperation with the Committee of Stainless Steel Producers, 
AISI, initiated in 1970 a soil burial program in representative soil environments. Test materials included coated and uncoated sheet specimens in the annealed and sensitized condition, uncoated welded tubing specimens and galvanically coupled and uncoupled stressed and unstressed specimens. To date approximately 10,000 specimens have been buried at six soil test sites. This report contains the results obtained for specimens buried for up to approximately eight years.

NBSIR 81-2229. Snyder, R. L.; Hubbard, C. R.; Panagiotopoulos, N. C. Auto: A real time diffractometer control system. 1981 February. 108 p. Available from: NTIS; PB 81-179186.

Key words: automation; powder diffraction; quantitative analysis; $\mathrm{x}$ ray.

Two x-ray powder diffractometers are independently driven by the program. The program provides the capability to scan an entire spectrum and to carefully measure the intensities of individual lines for quantitative analysis. For intensity measurements, an optimization routine is used to either minimize data collection time, or to minimize the estimated standard deviation of the net intensity. The data file structures are designed to permit complete data analysis without further data input. The automation program also provides for all aspects of file handling including opening, writing, transferring and deleting.

NBSIR 81.2230. Bullis, W. M., ed. Semiconductor technology program-Progress briefs. 1981 March. 11 p. Available from: NTIS; PB 81-188120.

Key words: electronics; integrated circuits; measurement technology; microelectronics; semiconductor devices; semiconductor materials; semiconductor process control; silicon.

This report provides information on the current status of NBS work on measurement technology for semiconductor materials, process control, and devices. Emphasis is placed on silicon and silicon-based devices. Highlighted activities include an analysis of the cross-bridge sheet resistance test structure, observations of laserinduced patterns on semiconductor surfaces, a technique for analysis of data from test structures on process validation wafers, and advances in optical measurements of linewidth on wafers. Brief descriptions of upcoming linewidth measurement seminars and the second moisture measurement workshop are also given. In addition, recent publications and publications in press are listed. The report is not meant to be exhaustive; contacts for obtaining further information are listed.

NBSIR 81.2230.2. Bullis, W. M., ed. Semiconductor technology program-Progress briefs. 1981 July. 16 p. Available from: NTIS; PB 81-238529.

Key words: electronics; integrated circuits measurement technology; microelectronics; semiconductor devices; semiconductor materials; semiconductor process control; silicon.

This report provides information on the current status of NBS work on measurement technology for semiconductor materials, process control, and devices. Emphasis is placed on silicon and silicon-based devices. Highlighted activities include newly issued resistivity SRMs, characteristics of sulfur-related deep levels in silicon, photoluminescence of indium-doped silicon, effect of tertiary interferograms on Fourier transform spectroscopy, design information for a set of wafer optical linewidth standards, modeling of shortchannel MOS transistors, acoustic-emission testing of tape-bonded ICs, laser scanning of a solar cell test pattern, power loss of transistor leads during fast switching, and second breakdown and radiation effects in power MOS transistors. Brief descriptions of an upcoming linewidth measurement seminar and a survey of Federal IC processing facilities are given. In addition, recent publications and publications in press are listed. The report is not meant to be exhaustive; contacts for obtaining further information are listed.

NBSIR 81.2230-3. Galloway, K. F.; Scace, R. I.; Walters, E. J., eds.
Semiconductor technology program-Progress briefs. 1981 November. 12 p. Available from: NTIS; PB 82-135898.

Key words: electronics; integrated circuits; measurement technology; microelectronics; semiconductor devices; semiconductor materials; semiconductor process control; silicon.

This report provides information on the current status of NBS work on measurement technology for semiconductor materials, process control, and devices. Emphasis is placed on silicon and silicon-based devices. Highlighted activities include semiinsulating $\mathrm{GaAs}$ characterization, an automatic scanning spectroscopic ellipsometer, linewidth measurement and coherence, bandgap narrowing effects in silicon, the evaluation of electrical linewidth uniformity, and arsenic-implanted profiles in silicon. In addition, recent publications and publications in press are listed. The report is not meant to be exhaustive; contacts for obtaining further information are listed.

NBSIR 81-2231. Yonemura, G. T. Criteria for recommending lighting levels. 1981 March. 60 p. Available from: NTIS; PB 81-185126.

Key words: conspicuity; contrast; illumination; lighting; lighting levels; superthreshold visibility; vision.

The effect of lighting on behavior ranges from allowing simple detection of objects to creating moods and impressions. Lighting standards and recommendations for general applications should be based on the visibility (seeing) requirements where differences between individuals are minimal. Furthermore, lighting criteria or standards must evaluate the seeing process under stimulus conditions approximating those encountered in the real space. It is recommended that conspicuity, defined as: "how well the detail stands out from the background," or ease of seeing be the metric for visibility. Subjective visual response criteria cannot be universally applied where significant differences in interpretations and evaluations between individuals and/or groups of individuals occur. Instead they should be treated as design options to be applied when they are important aspects of the intended function of the space. In discussing the above issues, the paper identifies the major categories of variables included in the perception of the visual environment and organizes them logically with respect to their relationship in developing lighting criteria and standards. This analysis includes a breakdown of the visual processes into sensory and perceptual components.

NBSIR 81-2232. Masters, L. W.; Seiler, J. F.; Embree, E. J.; Roberts, W. E. Solar energy systems-Standards for absorber materials. 1981 January. 68 p. Available from: NTIS; PB 81-188278.

Key words: absorber materials; accelerated test methods; durability; solar energy; standards.

Absorber materials used in solar heating and cooling systems absorb energy from the sun and convert it to thermal energy. These materials must perform their intended functions both when first installed and after extended use. However, the environment in which absorber materials are exposed can cause degradation and loss of ability to function. Numerous problems with absorber materials in solar energy systems have demonstrated the need for standards to assess their performance and durability.

A study was performed to aid in the development of accelerated test methods needed for the evaluation of absorber materials and to incorporate the methods into draft standards for consideration as consensus standards by the American Society for Testing and Materials (ASTM).

After identifying the performance requirements for absorber materials, laboratory and field studies were performed to measure performance according to the requirements. The data obtained, using twelve absorber materials, were used as the technical basis for two draft standards.

This report presents the results of the research, including the proposed draft standards.

NBSIR 81-2234. Carver, G. P.; Mattis, R. L.; Buehler, M. G. 
Microelectronic test patterns NBS-12 and NBS-24. 1981 May. 40 p. Available from: NTIS; PB 81-214892.

Key words: cross-bridge sheet resistor; integrated gated-diode electrometer; microelectronic test pattern; microelectronic test structure; MOSFET dc profiler.

Microelectronic test patterns NBS-12 and NBS-24 are modular developmental patterns for experimentally evaluating improved designs of certain test structures. NBS-12 addresses geometric design considerations for the cross-bridge sheet resistor test structure. NBS 24 contains a variety of preliminary designs for the integrated gateddiode electrometer and a series of variations on the design of the MOSFET dc profiler. Both patterns also include assorted process parameter test structures. Tables of design parameters, functional descriptions of the test structures in each pattern, and computercomposed outline drawings showing all of the test structures are included.

NBSIR 81-2235. Anderson, W. E.; Ramboz, J. D. 1980 Annual

Report: Technical contributions to the development of incipient fault detection/location instrumentation. 1981 March. 134 p. Available from: NTIS; PB 81-188005.

Key words: aging; cables; dielectrics; electric insulation fault location; time-domain-reflectometry; underground transmission.

Technical barriers exist in the development of instrumentation to detect and locate incipient faults in underground transmission cables. Knowledge is required of the physical, chemical, and electromagnetic properties of cables which precede breakdown, of the manner in which characteristic rf signals propagate in cables, and of appropriate methods of coupling detection systems to operating transmission cables. A measurement program has been initiated that will provide data on the rf properties of cables and on the characteristics of some forms of incipient faults. Preliminary measurements demonstrate the limitations of frequency- or time-domain-reflectometry techniques in the detection of incipient faults. Software is presented which permits the Fourier transform of step-like waveforms.

NBSIR 81-2238. Yokel, F. Y.; Chung, R. M.; Yancey, C. W. C. NBS studies of mobile home foundations. 1981 March. 45 p. Available from: NTIS; PB 81-180978.

Key words: flood loads; mobile home foundations; mobile home standards; soil anchors; soil testing.

Two papers are presented which discuss the results of tests on soil anchors used to secure mobile homes and of an analytical study of wind and flood loads on soil anchors.

NBSIR 81-2239. Frohnsdorff, G.; Clifton, J. R. Fly ashes in cements and concretes: Technical needs and opportunities. 1981 March. $34 \mathrm{p}$. Available from: NTIS; PB 81-183428.

Key words: cement; concrete; fly ash.

Following a brief review of the nature of fly ashes and their levels of production and use in various countries, an estimate is made of the potentially achievable level of use of fly ash in cement and concrete in the United States. The estimate assumes that 20 percent of the mass of all the portland cement used in the United States could be replaced by the same mass of fly ash; it ignores possible competition from granulated blast-furnace slag as a finely-divided mineral admixture for concrete. It appears that about 16 million tonnes (18 million tons) per year of fly ash could be consumed in cement and concrete, provided there were sufficient ash of suitable quality and a general understanding of the technical requirements for satisfactory fly ash use. Present standards which affect the use of fly ashes are discussed. Steps which could be taken to improve knowledge of factors affecting fly ash performance in cement and concrete, and hence to improve standard test methods and specifications, are outlined.

NBSIR 81-2240. Hardgrave, W. T.; Salazar, S. B.; Beller, E. J. III
Design of information systems using scenario-driver techniques. 1981 March. 24 p. A vailable from: NTIS; PB 81-180986.

Key words: database design; data dictionary; design; flowchart; information flow; information systems; interactive systems; requirements; scenarios.

This paper describes a technique for developing information systems using a scenario-driven design approach. The approach emphasizes client (that is, the user who is purchasing the system) participation in the design process. The first step is to develop a collection of "scenarios" which document the interaction between the computer and the human user. Using the scenarios, information-flow diagrams and database designs may be constructed. After the client has approved these documents, they can be used to establish disk capacity requirements and transaction rates, and finally to specify all hardware and software requirements. The primary advantage of this approach is that the scenarios provide a good indication of the ultimate usefulness and cost of the system. The client can review these documents and approve, modify, or reject the system design before any software is generated. This paper describes the scenarios, the information flow technique, and the database design approach using, as an example, a small business application.

NBSIR 81-2241. Petersen, S. R.; Barnes, K. A.; Kelly, G. E. Engineering-economic analysis of improved heat pump performance for minimum standards development. 1981 July. 67 p. Available from: NTIS; PB 82.133810.

Key words: energy efficiency standards; engineering-economic analysis; heat pump; mechanical equipment efficiency ratings; minimum efficiency standards; space cooling requirements; space heating requirements.

This report provides a methodology and seasonal performance data that could be used in the development of a reference basis for minimum efficiency standards for heat pumps that are economically justified on a life-cycle basis. Criteria for economic optimization are outlined. The methodology used to compute seasonal heating and cooling performance ratings and the annual energy savings resulting from efficiency improvements, by climate region, is detailed. The interdependence between efficiency ratings in the heating and cooling modes is explored using statistical analysis. An example of the procedure for determining maximum cost-effective efficiency levels is demonstrated for a $36,000 \mathrm{Btu} / \mathrm{h}$ heat pump.

NBSIR 81-2242. Van Brunt, R. J.; Misakian, M.; Leep, D. A.; Moy, K. J.; Beaty, E. C. 1980 annual report: Technical assistance for future insulation systems research. 1981 April. 105 p. Available from: NTIS; PB 82-134354.

Key words: corona; decomposition; electrical insulation; gas chromatograph; inception voltages; mass spectrometer; partial discharge; sulfur hexafluoride; swarm data; water vapor.

An experimental technique has been developed to quantify the electrical characteristics of pulse-type partial discharges (corona) in compressed electronegative gases. By this method the pulse repetition rates, shapes, and height distributions are determined together with the average current. The technique has been applied to the investigation of corona behavior in compressed $\mathrm{SF}_{6}$ and $\mathrm{SF}_{6} / \mathrm{N}_{2}$ mixtures, and to measurements of corona inceptions in $\mathrm{SF}_{6}$ for both dc and $60 . \mathrm{Hz}$ ac voltages. A gas chromatograph-mass spectrometer has been employed to monitor production of trace contaminants by corona-induced degradation of $\mathrm{SF}_{6}$. The most abundant gaseous byproducts that were detected from dc corona were $\mathrm{H}_{2} \mathrm{O}, \mathrm{SOF}_{2}$, and $\mathrm{SO}_{2} \mathrm{~F}_{2}$. The results of chemical analysis were correlated with measurements of the electrical characteristics of the corona and it was discovered that the pulse height distributions and repetition rates were sensitive to the presence of trace contaminants at the ppm level. Experiments were also performed on the effect of trace levels of water vapor, introduced by heating a wire, on positive dc corona pulse burst behavior in $\mathbf{S F}_{6}$. It was noted that the burst activity was 
significantly inhibited by trace amounts of $\mathrm{H}_{2} \mathrm{O}$. These results indicate that measurement of pulse characteristics of positive corona can be used as a sensitive monitor of gaseous contamination levels. The effects of uv radiation and optical radiation from a tunable dye laser on electrical behavior of corona in $\mathrm{SF}_{6}$ and $\mathrm{N}_{2}$ were investigated. Ultra-violet light can have, under certain conditions, a large effect on initiation of electron avalanches in $\mathrm{SF}_{6}$ near onset. Optical radiation in the wavelength range 580 to $620 \mathrm{~nm}$ was found to produce a measurable effect on corona in $\mathrm{N}_{2}$ when it impinged on the electrode surfaces. A bibliography of electron swarm data needed to predict theoretically breakdown and to model discharge phenomena in gases was prepared.

NBSIR 81-2243. Gerhold, W. F.; Fink, J. L. Corrosion evaluation of underground telephone cable shielding materials. 1981 April. $77 \mathrm{p}$. Available from: NTIS; PB 82.135187.

Key words: alloys; corrosion; metallurgically-bonded; metals; plastic-bonded; soils; telephone cables; underground.

Corrosion data is given on the performance of base and plastic coated metals intended for use as cable shields for buried telephone cable. The materials investigated on specially prepared specimens were buried for periods up to six years in six different soil environments. Metals tested included homogeneous plastic-bonded and metallurgically.bonded laminates. Some specimens were exposed bare (uncoated), while others had plastic coatings or other types of coatings on either one or both sides. Metals studied included aluminum, copper, low carbon steel and stainless steel alloys.

NBSIR 81-2244. Carino, N. J. Temperature effects on the strengthmaturity relation of mortar. 1981 March. 99 p. Available from: NTIS; PB 81-183410.

Key words: compressive strength; concrete; curing temperature; early age; final set; hydration; initial set; laboratory testing; maturity; mortar; regression analysis; strength prediction; temperature effects.

A study was performed to gain a fundamental understanding of the traditional maturity method used to predict the in-place strength of concrete. Research was undertaken to answer two questions: 1) What are the quantitative effects of curing temperature on the compressive strength-maturity relation of concrete? 2) Is there an age beyond which temperature no longer affects the strength-maturity relation of concrete? To simplify testing, mortar cubes were used as specimens for compressive strength determinations. Penetration resistance measurements were performed to determine initial and final setting times. Phase I of the research addressed the first question and involved preparing and curing specimens at $5^{\circ}, 12^{\circ}, 23^{\circ}, 32^{\circ}$ and $43^{\circ} \mathrm{C}$. Phase II addressed the second question and involved curing specimens at $5^{\circ}$ and $32^{\circ} \mathrm{C}$ for short periods, followed by additional curing at $23^{\circ} \mathrm{C}$. It was found that initial set occurred at approximately the same maturity regardless of the curing temperature. A three. parameter hyperbolic equation was used to represent the strengthmaturity relation. The parameters, determined by regression analysis, were found to vary systematically with curing temperature. Theoretical justification for the hyperbolic equation is presented and a key assumption in the maturity method is identified. The strength versus age data were also analyzed and a new concept, effective age, is suggested as a possible alternative for representing the combined effects of time and temperature on the compressive strength development of concrete.

NBSIR 81-2245. Adler, S. C.; Pierman, B. C. Building accessibility for the disabled: A review of research needs. 1981 March. 46 p. A vailable from: NTIS; PB 81.184954.

Key words: accessibility; building accessibility; building research; fire safety; handicapped; life safety.

This report traces the evaluation of public policy on accessible environments; discusses the need for development of a research basis for the design of accessible buildings including accessibility standards for boih new and existing buildings, summarizes the results and research recommendations of both the Conference on Fire Safety for the Handicapped held at NBS on November 26-29, 1979 and the joint ATBCB/NBS Conference on Accessibility Guidelines held in Bethesda, Maryland on October 31-November 1, 1979, and presents an overview of current NBS accessibility research plans.

NBSIR 81-2246. Olson, G. J.; Iverson, W. P.; Brinckman, F. E. Biodeterioration of standard reference materials. 1981 April. 19 p. Available from: NTIS; PB 81.203408.

Key words: bacteria; biodeterioration; biotransformations; deterioration of materials; element volatilization; fungi; metals; microorganisms; standard reference materials.

Several National Bureau of Standards Standard Reference Materials have been examined for susceptibility to biodeterioration. Several of these materials were attacked by bacteria and fungi when stored at elevated humidity after exposure to outdoor air. Some SRMs underwent deterioration after following certificate instructions for handling. Suggestions for some certificate revisions are made.

NBSIR 81-2247. Loftus, J. J.; Juarez, N.; Maldonado, A.; Simenauer, J. A. Flammability measurements on fourteen different hydraulic fluids using a temperature-pressure spray ignition test. 1981 May. 30 p. Available from: NTIS; PB 81.214173.

Key words: flammability; heat flux; pressure; spray ignition; temperature rise.

This report describes a spray ignition flammability test procedure which was developed and designed to be used as an alternative to the spray ignition test used by the Mine Safety and Health Administration (MSHA) to qualify hydraulic fluids for use in underground coal mines. The test procedure allows for quantitative measurement of heat and energy developed by fluids which may ignite in the test and provides for rank ordering of fluids according to their flammability. The testing program included currently used fluids such as invert emulsions, synthetics, and water glycols. Studies showed that the water glycol fiuids were the only fluid type to resist ignition by spray testing. All other fluid types were ignited and in some cases produced considerable flaming.

NBSIR 81-2248. Ruegg, R. T.; Chapman, R. E. A regional economic assessment of selected window systems. $1981 \mathrm{July} .125 \mathrm{p}$. Available from: NTIS; PB 81.243255.

Key words: building economics; daylighting; energy conservation; engineering economics; life-cycle costs; passive solar; regional analysis; thermal efficiency; windows.

This study, the fifth in a series of reports from the National Bureau of Standards interdisciplinary project on windows, provides guidance in selecting and using windows in buildings for greater cost effectiveness. It presents the life-cycle costs of selected window systems used in a room of a representative residence and in an office module of a representative commercial office building for nine cities in the United States, representing five heating zones and four cooling zones. The cities covered are Miami, Florida; Atlanta, Georgia; Washington, D.C.; Portland, Maine; Indianapolis, Indiana; San Antonio, Texas; Los Angeles, California; Bismarck, North Dakota; and Seattle, Washington. The results of the regional analyses are summarized, and the implications of these results are considered, both for selecting windows in new buildings and for managing windows in existing buildings. The emphasis of this report is on conveying the research findings to builders, designers, and building owners and operators-those involved immediately with the building process. The research method is described in an earlier companion report, Economic Evaluation of Windows in Buildings: Methodology, NBS BSS 119.

NBSIR 81-2249. Mahajan, B. M. Unsteady water depth measurement in 
a partially filled $7.6 \mathrm{~cm}$ diameter horizontal pipe. 1981 April. $55 \mathrm{p}$. A vailable from: NTIS; PB 81.203416.

Key words: diameter; drain; flow; history; horizontal; length; partially-filled; pipe; slope; stream-depth; unsteady; volume; water.

A research program to investigate the wastewater solid transport in horizontal drains is under way. The objective of the program is to develop data base and establish correlations for selecting drain pipe diameter, length, and slope for an effective solid waste transport with reduced water usage.

The purposes of this portion of the research program, which is presented here, were: (1) to measure the stream depth histories of unsteady, non-uniform transient, partially-filled pipe flow that ensues when water from a plumbing fixture is discharged into the drain, and (2) to examine the effects of the presence of a cylindrical solid and other relevant variables on the stream depth. The variables selected for the study include: the water volume discharged from the fixture into the drain, drain slope, and the diameter and length of cylindrical solids.

The report contains a description of the experimental apparatus, instrumentation and procedures, and a summary of the stream depth data acquired from experiments in a $7.6 \mathrm{~cm}$ diameter drain.

The depth of water stream at any given cross-section of the drain rises rapidly to a peak value and then gradually falls off to zero. The peak value of stream depth at a pipe cross-section decreases as the distance from the drain entrance increases. At a given drain crosssection, the peak value of stream depth increases with an increase in the water volume used, a decrease in the pipe slope, and with the presence of a solid in the drain. The variations in the solid diameter influence the steam depth history more than the variation in its length.

NBSIR 81-2250. Chapman, R. E.; Thompson, B. E. Estimating area cost factors for military construction projects: A computerized approach. 1981 May. 77 p. Available from: NTIS; PB 81.215295.

Key words: applied economics; construction; cost; estimation; location factors; model building; statistical analysis.

This report describes a computerized procedure for estimating area cost factors for military construction projects. The empirical basis for this procedure rests upon the results of an econometric analysis of over 500 military construction projects. Technical and empirical evidence from a wide variety of published sources were also used to provide supplemental information on wage rates, material prices, and the level of construction activities in the localities where the projects were undertaken. This report is intended to serve as a user manual for military personnel concerned with the problem of periodically updating the area cost factors for each service's installations. A series of technical appendices are also included which describe the theoretical underpinnings of the econometric models which constitute the core of the computerized procedure as well as provide samples of computer output and a complete listing of the computer program.

NBSIR 81.2254. Westley, F. Tables of experimental rate constants for chemical reactions occurring in combustion (1971-1977). 1981 April. 302 p. Available from: NTIS; PB 81-205429.

Key words: Arrhenius parameters; chemical kinetics; combustion; decomposition; free radicals; gas phase; hydrocarbons; hydrogen; nitrogen; oxygen; rate of reaction; sulfur.

A table of experimental rate constants for gas phase chemical reactions occurring in combustion is presented. Specifically, it gives in tabular form the values of the parameters for the modified Arrhenius equation $\mathrm{k}=\mathrm{AT} \mathrm{T}^{\mathrm{\theta}} \exp (-\mathrm{E} / \mathrm{RT})$. The table covers reactions occurring in the combustion, oxidation and decomposition of aliphatic saturated or unsaturated $C_{1}$ to $C_{10}$ hydrocarbons, alcohols, aldehydes, ketones, thiols, ethers, peroxides, amines, amides and their free radicals, as well as the reactions of $\mathrm{O}, \mathrm{O}_{2}, \mathrm{H}, \mathrm{H}_{2}, \mathrm{OH}, \mathrm{H}_{2} \mathrm{O}, \mathrm{H}_{2} \mathrm{O}_{2}, \mathrm{~N}$, $\mathrm{N}_{2}, \mathrm{NO}, \mathrm{N}_{2} \mathrm{O}, \mathrm{NO}_{2}, \mathrm{~N}_{2} \mathrm{O}_{4}, \mathrm{~N}_{2} \mathrm{O}_{5}, \mathrm{~S}, \mathrm{~S}_{2}, \mathrm{SH}, \mathrm{SO}, \mathrm{SO}_{2}, \mathrm{SOH}, \mathrm{NS}$, with each other. The table includes 220 monomolecular, 1092 bimolecular, and 108 termolecular reactions totalling 1420 distinct chemical reactions. There are 2608 distinct entries, distributed as follows: 308 for first order reactions, 1984 for second order reactions and 316 for third order reactions. The kinetic data were compiled from 843 experimental papers published between 1971 and 1977.

NBSIR 81.2255. Chuang, T. A diffusive crack growth model for creep fracture. 1981 March. 58 p. Available from: NTIS; PB 81.196883.

Key words: crack growth model; creep cavitation; diffusive crack growth; energy release rate; high temperature fracture; J-integral; sialon; singular integral equation.

A grain boundary creep crack growth model is presented here based on the assumptions that the crack propagates along the grain boundary by a coupled process of surface and grain-boundary self. diffusion; the adjoining grains behave elastically; and steady state conditions prevail. Under the action of the applied stress, atoms on the crack surfaces are driven by surface diffusion toward the crack tip from where they are deposited non-uniformly by grain-boundary diffusion along the grain interface so that the grain boundary opens up in a wedge shape ahead of the advancing tip which in turn produces a misfit stress field. The total grain boundary stresses and opening displacements are solved from a system of integro-differential equations to give the following relating $\mathrm{K}$ to $\nu:(\mathrm{k} / \mathrm{k} \mathrm{min})=.1 / 2[(\nu / \nu$ min. $)^{1 / 12}+(v / v \text { min. })^{-1 / 12}$ ] where $K$ is stress intensity factor and $v$ the crack tip velocity. In terms of $v \propto \mathrm{k}^{\mathrm{n}}$, the equation predicts $\mathrm{n}$ varying from 12 to infinity. A comparison with data on Si-Al-O.N at $1400^{\circ} \mathrm{C}$ shows agreement between the theory and experiment.

A consideration of the energy balance for the present model is also presented which indicates $\mathrm{J}=\left(1-\nu^{2}\right) \mathrm{K}^{2} / \mathrm{E}$ is the correct energy release rate associated with the present crack growth model as is true in the theory of elastic fracture mechanics. However, the energy released in diffusion is in the form of work done by the normal stress along the grain boundary, not from the elastic strain energy loss of the adjoining grains.

NBSIR 81-2256. Braun, E.; Meade, R. E.; Smith, L. R. Analysis of fire data, large-scale tests, and small-scale tests for conveyor belts used in underground coal mines. 1981 May. 107 p. Available from: NTIS; PB 81-214884.

Key words: accident data; coal mines; conveyor belts; fire safety; fire tests; flame spread; ignition; test methods.

An investigation into the requirements and fire test performance of conveyor belts intended for use in underground coal mines was conducted. The aim of this study was to develop recommendations for the Mine Safety and Health Administration (MSHA) on a test method suitable for measuring the fire hazard potential of a conveyor belt in a coal mine environment. A review of incident data, large-scale tests, and small-scale tests was conducted to provide the necessary information. The incident data was analyzed with the goal of developing scenarios that could form the basis for a suitable test or to evaluate the appropriateness of the existing test.

Large-scale tests were reviewed to determine anticipated belt fire performance under "realistic" end-use conditions. The tests showed how geometry, input energy, and ventilation controlled belt fire performance. Small-scale tests were used to provide information on specific fire properties such as ease of ignition, flame spread, and smoke generating potential.

NBSIR 81-2258. Margulis, S. T. A methodology for evaluating housing in use: A case study approach. 1981 June. 224 p. Available from: NTIS; PB 82-104969.

Key words: dwelling units (residential); housing; Operation Breakthrough; post-occupancy housing evaluation; Project Feedback; questionnaires; research methods; survey research; user needs.

The National Bureau of Standards (NBS) has prepared a report on 
the methodology of Project Feedback, the evaluation of Operation Breakthrough housing in use (a post-occupancy housing evaluation). The report introduces housing evaluations and encourages their use by providing both housing questionnaires and a nontechnical, practical discussion of research methods in general and of survey research in particular. To increase the sophistication of housing evaluation research designs, the report includes a tested approach for selecting control group respondents for housing evaluations. In addition, it summarizes results of NBS's housing evaluations, principally to illustrate ways of categorizing (coding) occupants' answers, but also to introduce these studies and their results. The case study approach is meant to encourage readers to build on NBS's experiences. This book is suitable for research and instructional purposes.

NBSIR 81-2260. Lankford, W. F.; Kowalski, P.; Schafft, H. A. Laser scanning measurements on solar cell test pattern NBS-22. 1981 May. 55 p. Available from: NTIS; PB 81-214157.

Key words: laser scanner; model calculations; photovoltaic cells; sheet resistance; solar cell defects; solar cells; test structures.

The NBS laser scanner has been used to make measurements on the solar cell test pattern, NBS-22, to verify the theoretical calculations of Sawyer, Lehovec, and Fedotowski. The photovoltaic response from cells with controlled sheet resistance step changes and breaks in the metallization contact to the cell was measured and compared to the theoretical predictions. Laser scanner limits of resolution were identified and proposed improvements were discussed. Preliminary measurements indicate that it is possible to measure the sheet resistance of the emitter layer of solar cells with an accuracy of several percent, using the laser scanner.

NBSIR 81-2261. Braun, E. Review of literature, applicable test methods, and incident data for the evaluation of the fire performance of central telephone office equipment. 1981 May. 41 p. Available from: NTIS; PB 81-218455.

Key words: central office equipment; electronic equipment; fire test methods; incident data; telecommunications; telephone exchanges.

At the request of the Rural Electrification Administration, a program was initiated to evaluate the adequacy of current flammability specifications for electrical equipment used in telephone company central offices. The high reliance on telephone services for emergency communications necessitates that measures be considered to minimize the likelihood of fire related interruptions of service. This report provides a review of current test methods and specifications for central office equipment; a review of available fire test methods in various categories; a brief literature review of relevant fire research and fire testing of wiring, cables, and assemblies; and a review of fire incident data.

NBSIR 81-2262. Maximon, L. C.; de Miniac, A.; Aniel, T. Tagged photons. An analysis of the bremsstrahlung differential cross section in the range of interest for a tagged photon system. 1981 April. 147 p. Available from: NTIS; PB 81-227837.

Key words: Bethe-Heitler cross section; bremsstrahlung differential cross section; bremsstrahlung monochromator; photon beams; photonuclear research; tagged photon method.

We consider in detail the differential cross section for bremsstrahlung for angles and energies in the range of interest for a tagging system. We derive a high energy, small angle approximation for the differential cross section for bremsstrahlung. We use this approximation to determine the maxima and minimum of the cross section and to evaluate it at these extrema. It is shown that the differential cross section has a very sharp dip in the region of small momentum transfers. Coulomb corrections to the Born approximation are considered, and do not fill in this dip.

NBSIR 81-2263. Eby, R. K.; Broadhurst, M. G.; Cassel, J. M.;
Fanconi, B. M.; Sanchez, I. C.; Smith, L. E. Polymer Science and Standards Division-Annual Report 1980. 1981 April. 90 p. Available from: NTIS; PB 81.215287.

Key words: macromolecules; plastics; polymers; standards.

This publication outlines the organization and programs of the Polymer Science and Standards Division of the National Bureau of Standards. It contains summaries of all technical projects in the Division for the 1980 fiscal year.

NBSIR 81-2264. Smith, L. E.; Chang, S. S.; McCrackin, F. L.; Senich, G. A.; Wang, F. W. Models for the migration of low molecular weight additives in polyolefins. 1981 April. 141 p. Available from: NTIS; PB 81-202749.

Key words: antioxidants; diffusion; food packaging; inverse gas chromatography; migration; oil simulants; oligomers; polyolefins; radioactive tracer; spectrofluorimetry.

Food Packaging is an important encounter in the daily life. The low molecular weight components in the packaging materials may migrate into the foods. This program, sponsored by the Bureau of Foods of the Food and Drug Administration, tends to provide theoretical models, reliable data base, and methodology to study the migration phenomena and to provide reasonable worst-case estimates for the concentrations of the indirect additives in food. In this annual report we present a relationship of diffusion coefficients of gaseous diffusants in polyolefins based on free volume theory, procedures and results of inverse gas chromatography for migrant-polymer interaction parameters and diffusivities of oligomers in polyethylene, methods and preliminary results of spectrofluorimetry on the migration of antioxidants in polyethylene, results and correlations of extraction experiments with radioactive labeled migrants. It was found that anhydrous ethanol and lower numbers of pure triglycerides can successfully simulate the extractive behavior of food oils. The accelerating action of $n$-heptane over that of the food oil is quantified.

NBSIR 81-2265. Hunt, B. J.; Fattal, S. G. Review of technical information on scaffolds. 1981 May. 103 p. Available from: NTIS; PB 81-203754.

Key words: codes and standards; construction safety; design; finite element; loads; scaffolds; stability; stiffness; strength; structural safety; work surfaces.

This report presents a review of the available literature on scaffolds and is the thisd of several inter-related studies of a scaffolding research program at the National Bureau of Standards (NBS). This study was sponsored by the National Institute for Occupational Safety and Health (NIOSH) to improve scaffolding system performance and reduce the work related injuries and losses.

A computerized search of the published literature was performed and technical information that could serve to upgrade existing codes and standards for scaffolds or offer direction to future analytical research has been presented. This information concerned the design, erection, operation or maintenance of scaffolding systems. Appendix A presents the 22 types of scaffolds under study. In addition, U.S. scaffold patent claims and the manufacturers' literature was reviewed and discussed. Appendix B presents selective model analyses of scaffolds.

NBSIR 81-2266. Mahajan, B. M. Experimental investigation of transport of finite solids in a $76 \mathrm{~mm}$-diameter partially-filled pipe. 1981 April. 65 p. Available from: NTIS; PB 81-203176.

Key words: cylindrical; non-uniform; partially-filled; pitchedhorizontal drain; slope; solid; velocity; volume; water.

An exploratory experimental investigation of the hydro-transport mechanisms of finite solids with non-uniform, unsteady and transient water flow introduced into a pitched-horizontal drain ( $p \cdot h$ drain) pipe by discharging a plumbing fixture was carried out. The purpose of the investigation was to examine the effects of relevant variables on the 
velocity attained and the distance traversed by the solid in the p-h drain. The variables selected for the study include: the water volume used (i.e., the volume of water discharged from the plumbing fixture into the drain), diameter and length of cylindrical solid, and diameter and slope of the p-h drain.

This report contains a description of the experimental equipment and procedures, and a summary of the data acquired during the solid transport experiment in $7.6 \mathrm{~cm}$ diameter drain pipe at a pipe slope of $0.02,0.04$, and 0.06 .

The solid dragged on a thin film of water between the solid and drain wall when relatively small water volumes $\left(V_{w} \leqslant 1.9\right.$ liters or 0.5 gallons) were used, and the solid moved like a waterborne object when relatively large water volumes $\left(\mathrm{V}_{\mathrm{w}}=3.8\right.$ liters or 1.0 gallons) were used. The solid velocity and the distance traversed by the solid increased with an increase in the water volume used, an increase in the drain slope, a decrease in the solid diameter, and a decrease in the solid length.

NBSIR 81-2267. McKnight, R. H.; Kotter, F. R.; Misakian, M.; Ortiz, P. 1980 Annual Report: Electric and magnetic field measurements. 1981 May. 72 p. Available from: NTIS; PB 81-218182.

Key words: absolute filter; current density; high voltage dc transmission lines; ion counter; space charge density; Wilson plates.

The NBS program is concerned with developing methods for evaluating and calibrating instrumentation for use in measuring the electric field and various ion-related electrical quantities in the vicinity of high-voltage direct current (HVDC) transmission lines and in apparatus designed to simulate the transmission line environment.

A parallel plate apparatus designed to produce electric fields in the presence of space charge has been evaluated and used to determine the response of two vibrating plate electric field meters and a field mill.

Errors associated with lack of coplanarity of Wilson plate sensors with the ground plane have been investigated using both ac electric fields and dc electric fields with space charge in a parallel plate apparatus.

A low-speed-air-flow facility has been constructed to produce a volume containing space charge which can be used as a source of ions for evaluating and comparing instruments designed to measure various ion related properties.

NBSIR 81-2268. Lerner, N. D. Evaluation of exit directional symbols. 1981 May. 55 p. Available from: NTIS; PB 81-203671.

Key words: directional arrows; exit signs; fire safety; pictograms; symbols; understandability; visibility; visual alerting.

This paper discusses visibility considerations for exit symbols and the relationship between understandability and visibility concerns. Two experiments evaluated directional indicators (arrows) in the context of building exit signage. The first experiment compared the visibility of 32 arrows under degraded visual conditions that were comparable to a smoke environment. This experiment had two objectives: (1) the development of a methodology for assessing exit pictogram visibility; and (2) a comparison of the visibilities of the specific arrows tested. A second experiment obtained subjective rankings of the arrow types on the basis of several criteria of concern for exit signage. These criteria included connotative meaning, uniqueness from other directional indicators, and appropriateness.

The visibility procedure proved to be statistically sensitive and demonstrated differences in the visibility of different arrows. An analysis of the type of confusions that occurred in errors for each arrow suggested certain relations between graphic features and errors in detection. The second experiment indicated substantial agreement between participants in ranking the arrows and revealed strong relationships between the several criteria. Together the results of the two experiments were used to evaluate the set of arrows for appropriateness for use with exit designators.

Methodological issues in evaluating symbol visibility were considered and other issues of concern in testing exit signage, such as special user groups, were also discussed.

NBSIR 81-2271. Babrauskas, V. Will the second item ignite?. 1981 May. 35 p. Available from: NTIS; PB 81.214025.

Key words: burning rate; case goods; chairs; furniture fires; ignitability; room fires; upholstered furniture.

The burning of more than a single fuel item in a room fire has not been well characterized. The first step in describing multiple item burning is to determine if, in fact, it will occur. This question has been experimentally explored from two aspects. (1) The radiant heat fluxes from burning first-to-ignite objects have been measured, along with their mass loss rates. (2) The ignitability of exposed objects has been determined using a bench-scale uniform flux ignitability test. It is then suggested that whether the second item will ignite can best be determined analytically from considering these two sets of results.

NBSIR 81-2272. Jason, N. H. Fire research publications, 1980. 1981 April. 22 p. Available from: NTIS; PB 81-203317.

Key words: bibliographies; building fires; coal mines; combustion products; compartment fires; fabric flammability; fire research; fire tests; flame research; smoke.

"Fire Research Publications, 1980" is a supplement to the previous editions: 1969-72 NBSIR 73-246, NTIS Order No. COM-74-10989. 1973 NBSIR 74-511, NTIS Order No. COM-74-11448; 1974 NBSIR 75-736, NTIS Order No. COM-75-11018; 1975 NBSIR 76-1120, NTIS Order No. PB-257837; 1976 NBSIR 77.1277, NTIS Order No. PB269965; 1977 NBSIR 78-1504, NTIS Order No. PB-284462; 1978 NBSIR 79-1745, NTIS Order No. PB-295395; 1979 NBSIR 80-2114, NTIS Order No. PB80.103335.

Only publications prepared by members of the Center for Fire Research (CFR), by other National Bureau of Standards (NBS) personnel or external laboratories under contract or grant from the CFR are cited. Articles published in NBS house organs also are cited.

NBSIR 81-2273. Brown, P. W.; Clifton, J. R. Factors affecting the soundness of blended cements. 1981 June. 26 p. Available from: NTIS; PB 82-104746.

Key words: cement; expansion; fly ash; MgO; slag; soundness; strength.

Blended cements containing fly ash and blastfurnace slag were examined to assess their soundness in the presence of admixed $\mathrm{MgO}$. Measurements of linear expansion were combined with compressive strength determinations to evaluate the volume stabilities of samples cured under various conditions. The effects of magnesium silicate formation on volume stability were also examined.

NBSIR 81-2275. Hebner, R. E., Jr.; Kelley, E. F.; Thompson, J. E.; Sudarshan, T. S.; Jones, T. B. 1980 Annual Report: Optical measurements for interfacial conduction and breakdown. 1981 May. 87 p. Available from: NTIS; PB 81-216343.

Key words: dielectrics; electro-optics; gases; high voltage; insulation; interfaces; Kerr effect; liquids; Pockels effect; solids.

Techniques have been developed to measure the electric field distribution along an interface between insulating materials. Specifically, systems have been developed to measure the fields in transformer oil using the Kerr effect and to measure the surface charge at a gas-solid interface using the Pockels effect. Measurements are supplemented by calculations of the distortion produced by selected interfacial charge distributions. The relatively pure transformer oil used in these studies showed little space charge distortion of the electric field. There also was no statistically significant preference for breakdown in the vicinity of the interface. At vacuum-solid interfaces, however, interfacial breakdown was observed and surface charging was measured. As gas is introduced into the system, the surface charge is removed so that no effect of 
surface charging was detected at atmospheric pressure. At pressures above atmospheric, there is evidence that surface charging may again become important.

NBSIR 81-2276. Staples, B. R.; Wobbeking, T. F. A compilation of thermodynamic and transport properties of aqueous sulfuric acid. 1981 June. 26 p. Available from: NTIS; PB 81-229171.

Key words: activity coefficients; aqueous; compilation; conductivity; electrolytes; enthalpy; Gibbs energy; osmotic coefficients; solutions; sulfuric acid; thermodynamic properties; transport properties.

A detailed compilation of sources of data for the thermodynamic and transport properties of aqueous sulfuric acid is presented. All ranges of temperature, concentration, and pressure are included.

NBSIR 81-2277. Kao, J. Y.; Pierce, E. T. Control strategies for energy conservation-A case study of the Materials Building, National Bureau of Standards. 1981 May. 47 p. Available from: NTIS; PB 81 . 217804.

Key words: building energy analysis; computer modeling; controls; control strategies; energy conservation for nonresidential buildings; load calculations.

The BLAST.2 Computer Program is used to investigate various heating, ventilating and air conditioning control strategies and their combinations to reduce the energy consumption of a laboratory building located at the National Bureau of Standards, Gaithersburg site. The techniques of modeling the building load and air system performance are explained. The results are presented and discussed. Control strategies investigated include dry-bulb and enthalpy economizer cycles, resetting supply air temperatures by outside temperature and zone demand, shut-down of fan systems selectively, and converting interior systems to VAV systems. By combining the various control strategies, eight percent, twenty-nine percent and eight percent of heating, cooling and fan energy respectively may be saved.

NBSIR 81-2278. Kirklin, D. R.; Colbert, J.; Decker, P.; Abramowitz, S.; Domalski, E. S. Test procedures for the determination of the gross calorific value of refuse and refuse-derived-fuel by conventional bomb and large bomb calorimetry. 1981 June. 218 p. A vailable from: the author.

Key words: ASTM round robin testing; bomb calorimetry; higher heating value; refused-derived-fuel; sample characterization; sample processing effects; 2.5 and 25 gram capacity bomb calorimeters.

This report provides the results of investigations during fiscal year 1979 to develop test procedures for the determination of the calorific value of refuse and refuse-derived-fuel (RDF) by means of oxygen bomb calorimetry. The results of 138 calorimetric measurements are discussed, along with 32 calorimeter calibration measurements, for 20 different RDF samples. In addition, determinations have been carried out on these RDF samples for air dry loss, residual moisture, furnace ash, bomb ash, and sulfur content to correct the calorimetric data for their presence; the latter group of measurements amounts to 283 experiments. The calorimetric (and related) measurements can be categorized into five classes: (1) measurements carried out in conjunction with ASTM round robin testing of RDF-3, (2) measurements on an Americology RDF extracted using both selective and non-selective sampling methods, (3) measurements required to compare data obtained from the small ( 2.5 gram capacity) and large (25 gram capacity) bomb calorimeters, (4) measurements carried out in the large bomb calorimeter on seven processed samples of different particle size obtained from New Castle County Delaware MSW, and (5) measurements on some RDF's of special interest. Five appendixes include additional information on: the evaluation of data on higher heating values obtained during ASTM round robin testing of RDF-3, the large bomb calorimeter, spectrochemical analysis of bomb residues, conversion tables, and calculation of total moisture in an RDF sample.

An evaluation of data on higher heating values determined during the ASTM round robin testing of RDF-3 showed that the repeatability (within-lab agreement) and reproducibility (between-lab agreement) are 250 and $750 \mathrm{Btu} / \mathrm{lb}$ when values are converted to a dry basis. These variabilities are 5 and 7.5 times greater, respectively, than the precision levels accepted in ASTM protocols for determining the higher heating values of coal or coke on a dry basis (see Appendix A).

Measurements on three RDF samples in both small (2.5 gramcapacity) and large ( 25 gram-capacity) bomb calorimeters showed that equivalent high precision can be obtained in both calorimeters. The large bomb calorimeter has the advantage that it can accommodate RDF samples whose particle size is as large as $25 \mathrm{~mm}$ (1 inch).

The mean and standard deviation of the calorific values obtained for 20 different RDF samples give 9482 and $553 \mathrm{Btu} / \mathrm{b}$, respectively, on a moisture-ash free basis using the furnace ash method to determine the ash content. These results support the generally accepted value of $9500 \mathrm{Btu} / \mathrm{lb}$ as the higher heating value for RDF on a moisture-ash free basis.

NBSIR 81.2279. Hastie, J. W.; Plante, E. R.; Bonnell, D. W. Alkali vapor transport in coal conversion and combustion systems. 1981 May. 79 p. Available from: NTIS; PB 81.221319.

Key words: alkali vapor; coal conversion; combustion systems; vapor transport.

Alkali metal-containing vapor species are ubiquitous in coal conversion and combustion systems. These species originate from coal mineral and atmospheric impurities (organic and inorganic) and from ceramic construction materials. Alternatively, they are present as additives, such as with potassium seeding for MHD or with bulk glass as a particle absorbing medium, or with dolomite in fluidized bed systems. Alkali vapor transport over representative slag, glass, and simple halide, hydroxide, and sulfate systems is discussed in relation to materials and process limitations in coal-supported energy systems. Problems associated with molecular-level vapor transport measurements are also considered.

NBSIR 81-2280. Kirchhoff, W. H. Technical activities of the Office of Environmental Measurements-1980. 1981 May. 195 p. Available from: NTIS; PB 81-227860.

Key words: air; environmental measurements; methods; quality assurance; standards; water.

This report summarizes the technical activities of the National Bureau of Standards' Environmental Measurements Program during Fiscal Year 1980. These activities fall into four program categories: 1) Water Measurements-Standards Development; 2) Water Measurements-Methods - Development; 3) Air MeasurementsStandards Development; 4) Air Measurements-Methods Development.

NBSIR 81-2283. Hebner, R. E. Development of power system measurements: Quarterly Report, January 1, 1981 to March 31, 1981. 1981 April. 19 p. Available from: NTIS; PB 81-220261.

Key words: cables; dc fields; high voltage; incipient fault; insulation; $\mathrm{SF}_{6}$; space charge; transformer oil.

This report documents the progress on four technical investigations sponsored by the Department of Energy and performed by the Electrosystems Division, the National Bureau of Standards. The work described covers the period January 1, 1981 to March 31, 1981. This report emphasizes the errors associated with measurements of the vertical current density in an electrical environment consisting of a dc field with space charge, the measurement of If attenuation in distribution cables, the onset characteristics of positive and negative corona in compressed $\mathrm{SF}_{6}$ gas, and the measurement of the space 
charge density in transformer oil subjected to $60-\mathrm{Hz}$ excitation.

NBSIR 81.2286. Law Enforcement Standards Laboratory. The characterization of auto headlight glass by refractive index and density. 1981 October. 37 p. Available from: NTIS; PB 82-131723.

Key words: auto headlight glass; classification; density; forensic science; individualization; population statistics; refractive index.

By measurement of some physical property of headlight glass fragments found at the scene of an auto accident, forensic scientists have sometimes found it possible to infer that the glass was molded by a specific manufacturer, or even that it came from a broken headlamp on a specific car. The refractive index (RI) and density of the representative specimens are the most common parameters of interest to forensic scientists. In this study, performed in 1975, automotive headlights were obtained from several sources. From RI and density measurements, it is concluded that partial classification (by manufacturer and/or age) could be reliably achieved. There is less likelihood that an unknown fragment can be reliably individualized to a specific headlamp lens because of the inhomogeneity within each lens. The probability is not increased appreciably by taking repeated measurements, by annealing the specimens, by measuring RI at several wavelengths, or by measuring both RI and density.

NBSIR 81.2289. Lerchen, F. H.; Pielert, J. H.; Chen, P. T. Preliminary guidelines for condition assessment of buildings being considered for solar retrofit. 1981 July. 140 p. Available from: NTIS; PB 82.113259.

Key words: building rehabilitation; concrete; electrical; evaluation; HVAC; masonry; plumbing; solar; steel; structural systems; test methods; wood.

This report was developed to help Federal agencies determine if buildings in their inventory are suitable for solar energy retrofit. It describes evaluation methods which are available to the facilities engineer to assist in determining whether the building and its systems (structural, HVAC, plumbing, and electrical) can be retrofitted with solar energy equipment under the Solar Federal Buildings Program (SFBP). Techniques for preliminary on-site inspection are emphasized, while more detailed evaluation techniques are described briefly and are referenced further.

The report describes evaluation methods available specifically for use with the structural materials of concrete, steel, masonry, and wood, as well as for use with the heating/ventilating/air conditioning, plumbing, and electrical supportive systems.

Comparative tables are provided for each building material to aid the reader in making a quick selection of the evaluation method most appropriate for the parameter to be tested. In addition, checklists are provided which identify common problems associated with each building material, possible causes of the problem, and the potential impact of the problem on the existing building systems and the solar retrofit system.

NBSIR 81-2290. Swaffield, J. A. Entry transition water surface profile prediction in supercritical partially filled pipe flow. 1981 June. $107 \mathrm{p}$.

Available from the author.

Key words: building drainage; supercritical flow; transition length; vertical stack drain entry.

The criteria governing the development of steady partially filled supercritical pipe flow are presented together with the necessary techniques to determine the water surface profile in the pipe entry transition length.

The establishment of full bore flow is predicted for a range of flow rates and pipe design parameters. Based on the water surface profile calculation technique, pipe length predictions are presented to avoid the air pressure fluctuations in the drainage system that result from full bore flow establishment.

Tabular data is presented to allow design decisions to be made that link pipe slope, diameter and roughness to the need to avoid full bore flow. A graphical technique is also presented that removes the necessity to interpolate from the tabular data.

The effect of entry geometry loss coefficients is included in the techniques presented.

NBSIR 81-2291 (HUD). Gordon, C. C.; Palla, R. L., Jr. Performance aspects of water conservation techniques for appliances. 1981 May. 40 p. Available from: NTIS; PB 81.223448.

Key words: clothes washers; dishwashers; dishwasher subcycles; fill controls; grey water; performance; soil removal; water conservation; water discharge characteristics.

The performance of household clothes washers and dishwashers is evaluated assuming various water conservation methods. For clothes washers the effect of fill level setting on soil removal is presented. A guideline for setting fill level is suggested. For dishwashers the soil removal capabilities are evaluated to reduced fill volumes. An analysis of the percentage of soil removed for each wash/rinse subcycle shows the relative quantities of soil in the discharge for each subcycle.

NBSIR 81.2292. Braun, E. Review of fire test methods and incident data for portable electric cables in underground coal mines. 1981 June. 22 p. Available from: NTIS; PB 81.227878.

Key words: coal mines; electrical cables; fire incidents; fire tests; test methods; trailing cables.

Electrically powered underground coal mining machinery is connected to a load center or distribution box by electric cables. The connecting cables used on mobile machines are required to meet fire performance requirements defined in the Code of Federal Regulations. This report reviews Mine Safety and Health Administration's (MSHA) current test method and compares it to British practices. Incident data for fires caused by trailing cable failures and splice failures were also reviewed. It was found that the MSHA test method is more severe than the British but that neither evaluated grouped cable fire performance. The incident data indicated that the grouped configuration of cables on a reel accounted for a majority of the fires since 1970.

NBSIR 81-2293. Hastie, J. W.; Plante, E. R. Mass spectrometric studies of MHD slag thermochemistry. 1981 June. 33 p. Available from: NTIS; PB 81.221434.

Key words: chemical activity; magnetohydrodynamics; mass spectrometry; potassium slag; thermodynamics; vaporization.

The economic viability of open cycle coal-fired magnetohydrodynamics (MHD) is closely linked to the thermodynamic conditions of seed potassium, both in the plasma and slag phase. Previous attempts to model plasma conductivity and slag retention of seed have been highly inaccurate due to the limited thermodynamic data base available. These limitations have mainly included vapor pressure, enthalpy of formation, and solution activity data for slag phases. Results of an extensive systematic experimental program of measurements on these and related properties are presented. The following potassium-containing systems were studied: binary mixtures of $\mathrm{K}_{2} \mathrm{O}$ with $\mathrm{SiO}_{2}, \mathrm{Al}_{2} \mathrm{O}_{3}, \mathrm{Fe}_{2} \mathrm{O}_{3}$, and $\mathrm{ZrO}_{2}$; a series of more complex synthetic slags containing $\mathrm{K}_{2} \mathrm{O}, \mathrm{SiO}_{2}, \mathrm{CaO}, \mathrm{MgO}$, $\mathrm{Al}_{2} \mathrm{O}_{3}$, and $\mathrm{Fe}_{2} \mathrm{O}_{3}$; an MHD channel slag (Illinois No. 6 coal); and several slag. $\mathrm{NaCl}$ mixtures. Data were obtained over a sufficiently wide range of component-type, composition and temperature to reveal systematic trends in slag activities from which empirical predictions are possible. However, anomalous behavior was also noted, mainly in the form of nonequilibrium effects. Application of the vaporization and activity data to plasma-slag interaction and to the recovery of potassium seed from slag is also considered.

NBSIR 81-2294. Levine, R. S., ed. Survey of United States research projects publications in the field of fire safety of buildings and structures. 1981 June. 46 p. Available from: NTIS; PB 81-216988.

Key words: compartment fires; fire detection; fire endurance; fire 
modeling; fire tests; flammability; human behavior; smoke; suppression; toxicity.

This report is an annotated bibliography of some 166 U.S. papers on fire safety research, published 1978-1980. Prepared as part of a US.USSR agreement to cooperate in this field, it includes chapters on testing materials for flammability, smoke generation and toxicity, fire resistance of structures, compartment fire modeling, automatic detection and suppression, and human response in fires.

NBSIR 81.2295. Manning, J. R. NBS: Materials measurements. 1981 June. 149 p. Available from: NTIS; PB 81.238537.

Key words: adsorption; Auger spectroscopy; convection; ellipsometry; gallium; levitation calorimetry; specific heat surface tension; thermophysical properties; tungsten; wetting.

This report describes NBS work for NASA in support of NASA's Materials Processing in Space Program under NASA Government Order H-27954B (Properties of Electronic Materials) covering the period April 1, 1980 to March 31, 1981. The work emphasizes measurements of materials properties and thermophysical properties.

This work has been carried out in five tasks. These tasks have, as two of their focal points, the role of convection effects and the role of container effects, both of which would differ in space-based experiments from those found in ground-based experiments. The results obtained for each task are given in detailed summaries in the body of the report. The five tasks are Task 1-Surface Tensions and Their Variations with Temperature and Impurities; Task 2-Convection During Unidirectional Solidification; Task 3-Measurement of High Temperature Thermophysical Properties of Tungsten Liquid and Solid; Task 4-Thermodynamic Properties of Refractory Materials at High Temperatures; Task 5-Experimental and Theoretical Studies in Wetting and Multilayer Adsorption.

NBSIR 81-2296. Winter, F.; Galowin, L. S. Criteria and evaluation for two-step flush devices for water closets. 1981 June. 93 p. Available from: NTIS; PB 81-226839.

Key words: dual flush toilets; low flush toilets; water closets; water conservation.

Laboratory tests of two-step flush control devices for water closets were conducted to provide data and develop test methods for evaluating water saving devices for water closets. Criteria for performance and testing procedures for laboratory testing are recommended for evaluating two-step flush devices for installation in conventional water closets.

NBSIR 81-2297. Kelly, J. C.; Wolf, R.; Kennicott, P.; Nagel, R.; Wellington, J., eds. A technical briefing on the Initial Graphics Exchange Specification (IGES). 1981 July. 135 p. Available from: NTIS; PB 81-238719.

Key words: computer aided design (CAD); computer aided manufacturing (CAM); entity; exchange format; file; genmetry; graphics; interface.

This publication is a technical briefing on the Initial Graphics Exchange Specification (IGES) which was published as NBSIR 80. 1978 (R). The briefing serves two purposes: (1) to give the reader a broad overview of the Initial Graphics Exchange Specification and (2) to provide material for use in preparing briefings about IGES for presentation to technical groups.

The briefing is divided into two parts. Part 1 consists of a set of slides and associated text. The slides and text are organized in tandem. The intent of the text is to furnish associated information (for each slide) as opposed to furnishing specific text that is to be read verbatim. The information in this section is "soft" (not overly technical). Part 2 consists of more technical material. In this section text that can be read verbatim is provided for each slide.

NBSIR 81-2298. Moore, R. T. Results of fingerprint image quality experiments. 1981 June. 49 p. A vailable from: NTIS; PB 82-133539.

Key words: fingerprint cards; fingerprint images; fingerprint ink; image quality.

A series of experiments were conducted to determine the variation in the scores developed using a matching algorithm designated as the M.82 for mating fingerprints of different image quality that had been read by the Automatic Fingerprint Reader System (AFRS) of the FBI. The variations in image quality resulted from the use of a variety of card stocks and recording techniques to record the print of a single finger. For each image recording process, a sample of 56 finger-pairs were matched and mean single-finger score values were developed. These varied over a factor of more than 70 to one. The best scores were developed from images placed on very white, slick appearing, calendered card stock with the use of film strips that had been preinked and which could be separated to expose an ink film of predetermined thickness and uniformity. This combination had less resistance to smearing than fingerprints produced on the same stock from the use of a pre-inked porous pad. However, the latter still produced acceptably high scores.

NBSIR 81-2300. McCabe, M. E.; Ducas, W.; Orloski, M. J.; Decorte, K. N. Passive/hybrid solar components-An approach to standard thermal test methods. 1981 July. 86 p. Available from: NTIS; PB 81 227886 .

Key words: passive/hybrid components; solar energy; steady. state tests; thermal test procedures; transient thermal tests.

As part of a continuing program to develop standard test procedures to measure thermal performance of solar heating and cooling equipment, NBS has developed a plan and methodology that will be utilized for passive/hybrid solar components. A survey of passive solar products currently available or under development enabled the development of an interim classification system consisting of ten component classifications for purposes of thermal testing. A survey of currently available thermal test procedures was performed to assess the applicability of these test methods for passive/hybrid solar components. Existing test procedures that are useful for the Direct Gain Fenestration System classification are identified, and recommendations are made for evaluation of these laboratory-based procedures by comparison with field-based testing of components under controlled interior conditions. Recommendations are also made for the development of new test procedures for passive/hybrid components classifications for which existing test methods are not applicable. A proposed program to develop testing procedures for the Storage Wall Module classification is provided.

NBSIR 81-2303. Wan, C. A. NBS/ESDS-A computer model for the simulation of hot water systems. 1981 September. 64 p. Available from: NTIS; PB 82-139361.

Key words: computer model; distribution pipe; dynamic response; electricity; energy; explicit method; faucet temperature finite difference; heat loss; implicit method; insulation; water heater.

A computer model, entitled NBS/ESDS, has been developed using a finite difference method to simulate the generation, storage and distribution of a hot water system commonly found in many residences, commercial facilities or industrial facilities.

The conduction heat equation which governs the heat transfer in the water heater during all modes of operation, and the heat transfer in the distribution pipe network during its cooldown periods, is solved. A solution is also obtained for the pipe flow equation which governs the heat transfer in the distribution pipe network during the draw periods. Thermal stratification of water is modeled. A plug flow model is used to simulate the draw of heated water from the water heater.

A parametric study is also conducted on a given hot water system for a given draw schedule during a 24-hour period. The effects studied include insulating the hot water heater, insulating the 
distribution pipe, and incorporating a heat trap. Effects of concentrating the distributed draws to two short periods of time of the day on the hot water delivery and the heat loss with the same energy consumption are also studied.

NBSIR 81-2304. Weber, S. F.; Thompson, B. E.; Lippiatt, B. C Economic framework for cost-effective residential water conservation decisions. 1981 August. 65 p. Available from: NTIS; PB 81.246779.

Key words: benefits; cost-effective; costs; economic analysis; economic decision framework; energy conservation; marginal price; net present value; residential water conservation; wastewater treatment; water rate schedules; water-saving devices.

This report presents an economic framework for utilities to use in recommending water-saving devices that are cost-effective for homeowners. A variety of devices designed to save water used in water closets, showers, and indoor faucets are described in terms of their costs and benefits. The cost components considered are acquisition, installation, maintenance, and replacement costs. Benefits considered are water savings, avoidance of wastewater treatment, and energy savings in the case of reduced hot water use. A critical factor affecting the dollar value of benefits to homeowners is the price they pay for water. An analysis of water rate schedules, based on a national sample of 90 water utilities, indicates that homeowners' actual benefits from saving the last unit of water (as measured by its marginal price) are considerably lower than the average price paid for water. Thus, estimated water bill reductions will frequently be overstated if calculated on the basis of average price. The economic framework for selecting devices evaluates both mutually exclusive and compatible devices that are either modifications of existing plumbing fixtures or features of newly installed fixtures.

NBSIR 81.2305. Wachtman, J. B., Jr., ed. Proceedings, U.S.

Department of Commerce public workshop on critical materials needs in the aerospace industry, February 9.10, 1981. $1981 \mathrm{July} .701$ p. Available from: NTIS; PB 82.137266.

Key words: aerospace; chromium; cobalt; critical materials; tantalum; titanium.

On February 9 and 10,1981, the Department of Commerce held a Public Workshop to obtain the views of the American Public on three questions directly pertinent to the Department's responsibilities under the Materials and Minerals Policy, Research and Development Act of 1980, Public Law 96-479. These questions were: 1) What are the materials issues of primary concern to the American aerospace industry and its suppliers? 2) What recommendations do the American aerospace industry and its suppliers have for Federal action to address these issues? 3) Which specific materials should the Department of Commerce review in detail over the next few months in order to recommend the most urgently needed programs for Federal action?

The Workshop addressed these questions within three distinct areas: 1. Critical raw materials. Il. Critical en gineering materials. III. Substitution, conservation, specialized recycling, and higher performance.

This report includes the formal views presented to the plenary workshop sessions, the reports of the Workshop Task Forces in each of the three above areas, and the written submissions invited in the Federal Register notice of the Workshop.

NBSIR 81-2307. Swaffield, J. A. Dependence of model waste solid transport characteristics in drainage systems on solid geometry, mass and pipe system parameters. 1981 July. 45 p. Available from: NTIS; PB 81.229247.

Key words: building drainage; waste solid transportation; water conservation; w.c. efficiency.

Test results are presented for the transport characteristics of an extensive range of geometrically similar model solids in a $100 \mathrm{~mm}$ diameter UPVC drain pipe. Model solids were based on commercially available sanitary towels (napkins) discharged into the pipe system via a series of U.K. standard water closet (w.c.) types.

Following a data fit analysis, relationships are presented linking solid transport characteristics to solid, pipe and w.c. parameters.

These relationships, linked to observation of installed hospital drainage systems in the U.K., will allow laboratory test methods to be utilized in predicting the effect of design changes on system performance.

NBSIR 81-2308. Swaffield, J. A. An initial study of the application of the numerical method of characteristics to unsteady flow analysis in partially filled gravity drainage sized pipes. 1981 July. $64 \mathrm{p}$. Available from: NTIS; PB 81.229189.

Key words: building drainage; numerical solutions for transient depth; unsteady partially-filled pipe flow; waste solid transport.

The application of the numerical method of characteristics to the solution of the differential equations defining unsteady flow in partially filled drainage system sized pipes is outlined.

The derivation of the flow equations is presented, together with the necessary boundary equation formulation to represent variable inflow, system discharge and leakage flow past a stationary deposited solid.

A computer program, written in Fortran, is included, together with typical output, that establishes the applicability of this computational method to unsteady flow analysis in gravity flow drainage systems.

Proposals for the extension of the described techniques to the prediction of solid transport and flow attenuation in long pipes are also presented.

NBSIR 81-2310. Turner, G.; Collins, B. Pedestrian movement characteristics on building ramps. 1981 June. 59 p. Available from: NTIS; PB 81-229163.

Key words: building circulation; building ramps; pedestrian circulation; pedestrian flow; pedestrian movement; pedestrian ramps; ramps.

Knowledge of design for effective pedestrian circulation is one of the main requirements in building planning. While an understanding of pedestrian movement characteristics on the various component parts that make up a building circulation system is the key to effective planning, one component type, the ramp, has rarely been the subject of building circulation research.

The research described in this report is the result of an investigation of pedestrian movement characteristics on four specific building ramps during two different professional athletic contests. Variables on pedestrian movement such as speed, flow, and density were studied as well as the relationships between them. In addition, counts of pedestrians by sex were made. Results were compared for the specific ramps and with previously reported data obtained for ramps, stairs, and level surfaces.

NBSIR 81-2311. Richmond, J. C. Analysis of results of mini round robin reflectance test. 1981 August. 32 p. Available from: NTIS; PB 81-244873.

Key words: diffuse reflectance; directional-hemispherical reflectance; integrating spheres; laboratory intercomparisons; reflectance measurements; reflectance standards; solar properties of materials; spectral reflectance measurements.

A mini round robin test was conducted in which four national laboratories participated. The spectral directional-hemispherical reflectance for near-normal incidence of two samples, one black and one white, was measured in the wavelength range of 250 to $2500 \mathrm{~nm}$. The solar reflectance of the white sample and the solar absorptance of the black sample was then computed. Each laboratory used a different procedure for making the computations. The measured samples had previously been calibrated in the Radiometric Physics Division of the National Bureau of Standards for certification as reflectance standards. The average difference between the reported values and the calibration values varied from $+0.0116 \pm 0.0634$ to 0.0386 
\pm 0.0205 for the white samples and from $-0.00475 \pm 0.00366$ to + $0.00357 \pm 0.00433$ for the black samples. The differences for the white samples were ascribed primarily to errors in the reflectance value assigned to the reference standard used in the measurements. The differences for the black samples were ascribed primarily to zero line errors.

NBSIR 81-2312. Loftus, J. J. MSHA Wick Test for hydraulic fluids: A preliminary evaluation. 1981 July. 28 p. Available from: NTIS; PB 81.238511

Key words: coal mines; flammability; hydraulic fluids; ignition; Wick Test.

This report is an evaluation and analysis of "The Test to Determine the Effect of Evaporation on the Flammability of Hydraulic Fluids (Wick Test)." The Wick Test is used by the Mine Safety and Health Administration (MSHA) to qualify hydraulic fluids for use in underground coal mines. This report includes a review of the test method's operating characteristics as well as a comparison of test results from the Wick Test with two alternative test procedures-a hot wire coil test and a sustained flame contact test. The evaluation program included currently used fluids such as invert emulsions, synthetics, and water glycols. The Wick Test studies showed that the test method was not well defined and that reproducibility was poor.

NBSIR 81-2313. Baker, D. W.; Kao, J. Y.; Didion, D. A. Performance evaluation of a typical energy monitoring system for steam flow in buildings. 1981 July. 63 p. A vailable from: NTIS; PB 81-238545.

Key words: building energy monitoring; energy measurement; steam consumption measurement; uncertainty estimate.

Some important design features and measurement techniques are discussed for determining energy rates in building systems flowing steam. Emphasis is on use of differential pressure $(\Delta P)$ type flowmeter systems where $\Delta \mathrm{P}$, pressure and temperature instrumentation only can receive direct calibration. The role of systematic and random type errors in measurement of building energy is discussed and an appropriate method is given for estimating the uncertainty in the energy consumed. An example calculation of uncertainty in accumulated energy used for a 1 year period is given from typical operating data for one of the NBS laboratory buildings. The uncertainty is estimated to be $3.2 \%$ for a two-meter (series) configuration and $4.3 \%$ for a single meter (high range meter) configuration.

NBSIR 81-2315. Fry, J. P.; Chiang, W. P.; DeSmith, D. A.; Leder, D. S.; Nguyen, D. K.; Perreault, R. W. Draft specification for structured data interchange form. 1981 July. 126 p. Available from: NTIS; PB 81-247637.

Key words: database management; data conversion; data description; data interchange form; data translation; portability; software conversion.

This report defines the format of a self-describing data interchange file that is media and machine independent, and that would facilitate the transfer of structured data between dissimilar computing systems and software. The specification is intended for interchanging data that has been structured under the discipline of a database management system, although it may also prove applicable to interchanging data that has been structured under other software disciplines, such as the COBOL programming language. The specification covers the three principal types of database management systems, namely the hierarchical, network, and relational types, but it is believed to be sufficiently general to accommodate data from other database management systems. The data interchange file consists not only of the data occurrences or values to be transferred, but also a description of their logical and physical characteristics and the relationships within the data. This minimizes or eliminates the need for additional, non-standardized information transfer in order to successfully perform a database transfer.
The report is written in the form of a candidate standard, so that interested parties can review specific requirements, rather than simply the philosophy or concepts of the approach. However, at this time, this specification is not proposed as a Federal Information Processing Standard. This report is published in order to obtain independent views on the technical merits, efficacy, and potential costs of this avenue for resolving database transfer problems.

NBSIR 81-2316. Berke, J. G.; Hudson, C. H. Procurement of products containing recovered material: A summary of activities in seven states. 1981 July. 76 p. Available from: NTIS; PB 81-240475.

Key words: economic; institutional; procurement; purchasing; recovered/recycled materials; resource recovery; technical barriers.

This report summarizes the major study carried out in seven states dealing with the procurement of products containing recycled/recovered materials as specified in the Resource Conservation and Recovery Act (P.L. 94-580) and its amendments (P.L. 96-482). The report lists current state activities, regulations, statutes and policies, as well as technical, institutional and economic barriers to the public procurement of recycled products. A list of recommendations and suggested actions are also included.

NBSIR 81-2317. Pierce, E. T. Energy conservation by multiple glazing on heavy masonry buildings. 1981 June. 30 p. Available from: NTIS; PB 81-246977.

Key words: building energy analysis; energy conservation for nonresidential buildings; historic preservation and energy conservation; load calculation; masonry thermal mass; system simulation.

The BLAST-2 computer program is used to investigate multiple glazing as a means to reduce the energy consumption of two buildings of the Library of Congress in Washington, D.C. The Thomas Jefferson Building is of very heavy masonry construction, and the John Adams Building is of heavy masonry. The techniques of modeling the building load and air system performance are explained. The results are presented and discussed. Typical perimeter modules of various floors simulated with single, double, and triple glazing are faced in four compass directions. Similar results are obtained over a range of models. Application of double glazing will save up to ten percent of heating energy but not much cooling energy while maintaining present operating requirements.

NBSIR 81-2319. Carver, G. P. Development of test structures for characterization of the fabrication and performance of radiation. hardened charge-coupled device (CCD) imagers: Annual report, May 15,1980 to May 14, 1981. 1981 August. 26 p. Available from: NTIS; PB 81-244071.

Key words: CCD; cross-bridge sheet resistor; electrical alignment test structure; gated diode; integrated gated-diode electrometer; integrated test structure; MOSFET dc profiler; test structure.

The purpose of this project is to evaluate new test structures and test methods useful for the characterization of radiation-hardened CCD imagers. During the period covered by this report, consultation was provided to The Charles Stark Draper Laboratory, Inc. (CSDL) and to CSDL contractors on the implementation and use of test structures developed previously during this project. In addition, the results of measurements on CCD imager wafers using the surfacechannel and buried-channel, integrated gated-diode electrometers, before and after exposure to ${ }^{60} \mathrm{Co}$ radiation, and using the cross bridge/electrical alignment test structures are reported. Examples of cross sections of the CCDs made by beveling and staining are also presented.

NBSIR 81-2320. Palla, R. L., Jr. A heat transfer analysis of scald injury. 1981 July. 68 p. A vailable from: NTIS; PB 81-238503.

Key words: bioheat equation; burn; criterion; critical exposure; 
fluid; heat transfer; injury threshold; scald; thermal injury.

Numerical solutions for skin tissue temperature during scald injury events are obtained and utilized in conjunction with a thermal injury criterion, to predict critical exposure levels for various heated fluids. A one-dimensional tissue model of the type used by Love is employed to determine the initial tissue temperature distribution. The bio-heat equation for tissue heat transfer is then solved via an implicit finite difference technique, subject to convective heating and cooling at the surface.

The sensitivity of the critical exposure level to variations in tissue properties and convective heating coefficients is investigated. Thermal injury thresholds are presented for various fluids along with bounds to reflect uncertainty in assumed tissue properties. The results obtained are in good agreement with existing experimental scald injury data.

NBSIR 81-2321. Peavy, B. A. Single-room heat balance for building heat transfer. 1981 November. 22 p. Available from: NTIS; PB 82135609.

Key words: building heating/cooling loads; heat balance for a single room; heat transfer; radiosity shape factors.

A single-room heat balance has been developed to provide a more precise computational tool. The primary purpose for this tool is to evaluate the effects of approximations presently used in computer programs on the determination for building heating and cooling loads. Specific algorithms to be incorporated in the room heat balance concern radiosity shape factors, temperature difference dependent convection heat transfer coefficients, simulated room mass, and an iterative methodology for solution of room temperatures.

NBSIR 81-2325. Larrabee, R. C.; Phillips, W. E.; Thurber, W. R Measurement techniques for high power semiconductor materials and devices: Annual report, October 1, 1979 to September 30, 1980. 1981 September. 52 p. Available from: NTIS; PB 82-117706.

Key words: deep-level measurements; deep-level transient spectroscopy; lifetime; power-device grade silicon; thyristor material characterization; transient capacitance techniques.

The presence of deep-level impurities in semiconductor power devices is a consequence of their unintentional introduction during crystal growth and during the wafer fabrication procedure or their intentional introduction in order to adjust the switching properties of the device. Measurement techniques to detect, characterize, and identify such deep levels are required in order to monitor the presence of unintentional contamination or to characterize and understand the behavior of intentionally added impurities. The effective utilization of deep-level measurements for this purpose requires three things: (1) development of well-characterized measurement and data analysis procedures, (2) characterization of a variety of levels to establish the validity of the techniques and establishing a data bank of the properties of known defect levels, and (3) understanding the relationship between the presence of deep levels and the corresponding device parameters. Efforts in deep-level metrology during the present reporting period were concentrated specifically on the first and third of these basic requirements. This effort is divided into two ongoing tasks concerned with (1) the introduction of specific impurities into silicon wafers and the characterization of the resulting deep levels and (2) the correlation of the results of these deep-level characterization techniques with the electrical properties of devices. A third task concerned with the standardization of preferred procedures for specimen preparation for spreading resistance measurements on thyristor-grade silicon was essentially completed during the year.

NBSIR 81-2326. Walton, W. D. Investigation of solar collector fire incident. 1981 August. 46 p. Available from: NTIS; PB 82-118639.

Key words: building fires; fire safety; ignition; self-heating; solar collector fires; solar collectors; spontaneous ignition.

In May 1980, a fire involving a solar collector occurred in Boulder, Colorado in an unoccupied single family dwelling. Damage due to the fire was limited to a single solar collector and adjacent roofing and framing materials. Collectors of the same model on three other dwellings at the site showed signs of degradation which may have led to similar fires. The collector, installation, conditions leading to the fire, and events following the fire are described. The most likely point of ignition has been identified as the plywood collector backing. Results of a 30-day stagnation test and solar simulator testing are discussed. Recommendations to prevent future occurrences of this type of fire are presented.

NBSIR 81-2327. Garrity, S. D. Monitoring system development: A framework and application. 1981 September. 607 p. Available from: NTIS; PB 82.115817.

Key words: administrative experiment; evaluation systems; Experimental Technology Incentives Program; information systems; project management; system acquisition; system development.

Projects aimed at developing new systems where there are substantial uncertainties as to system requirements, development processes, and ultimate ownership can present project managers with a range of complex, unstructured problems. A process to help identify and solve these problems in a timely, controlled manner is of central importance to the successful conduct of a development with these circumstances.

This document describes a research project devoted to the examination of problems in complex system developments and to the development of a process managers can use to deal with them. Conducted within the Experimental Technology Incentives Program (ETIP), the research includes analysis of several ETIP projects, a review of the systems literature, presentation of a monitoring framework to help manage complex developments, and a brief application of the framework to one ETIP project.

The proposed monitoring process consists of a framework of thirty factors and a set of five functions which monitoring can serve. The factors are divided into three main categories-design, process, and user commitment characteristics-which reflect the general types of problems found to be important in the ETIP environment and the system literature. The functions of the framework, designed to serve both administrative and research purposes, include problem identification, strategy development, research, documentation, and dissemination. A model of how the framework might be implemented within a project is also described.

NBSIR 81-2328. Garrity, S. D. A manual for designing and implementing a process to monitor complex system developments. 1981 September. 65 p. Available from: NTIS; PB 82-104308.

Key words: administrative experiment; evaluation systems; Experimental Technology Incentives Program; information systems; project management; system acquisition; system development.

Projects aimed at developing new systems where there are substantial uncertainties as to system requirements, development processes, and ultimate ownership can present project managers with a range of complex, unstructured problems. A process to help identify and solve these problems in a timely, controlled manner is of central importance to the successful conduct of a development with these circumstances.

This document presents a manual for designing and implementing a process to monitor key areas of a complex system development. The manual is derived from an internal study of system developments in the Experimental Technology Incentives Program (ETIP) of the National Bureau of Standards.

The proposed monitoring process consists of a framework of thirty factors and a set of five functions which monitoring can serve. The factors are divided into three main categories-design, process, and 
user commitment characteristics-which reflect the general types of problems found to be important in the ETIP environment and the system literature. The functions of the framework, designed to serve both administrative and research purposes, include problem identification, strategy development, research, documentation, and dissemination. A model of how the framework might be implemented within a project is also described.

NBSIR 81-2329. Evans, D. D.; Madrzykowski, D. Characterizing the thermal response of fusible-link sprinklers. 1981 August. $40 \mathrm{p}$. Available from: NTIS; PB 82-107020.

Key words: fire extinguishment; fire models; fire protection; sprinkler heads; sprinkler systems; time constant.

Measurements of the thermal response of selected fusible-link sprinklers to sudden immersion into a hot gas flow are presented. Two methods of characterizing the response are detailed. One method explicitly accounts for phase change, the other does not. The methods are compared by using each to predict the response of a sprinkler to a selected mattress fire exposure.

NBSIR 81-2333. Klein, D. P. Reduced-scale modeling of mobile home fires: A progress report. 1981 December. 65 p. Available from: NTIS; PB 82.142076.

Key words: compartment fires; fire tests; flashover; interior finish; mobile homes; models; room fires.

A series of fire tests was conducted in the bedroom of a single-wide mobile home and in a quarter-scale model of that bedroom. The objectives of the tests were (1) to evaluate the relationship between fire buildup in the reduced-scale and full-scale enclosures and (2) to determine the feasibility of using a reduced-scale model test to assess the potential contribution of particular combinations of interior finish materials to fire growth in a mobile home.

The model tests simulated the phenomena of fire growth and flashover; however, the time to flashover occurred later in the model than in the full-scale bedroom. Flashover was taken as the time at which the level of radiation at the center of the floor reached 2 $\mathrm{W} / \mathrm{cm}^{2}$. Several modifications to the model were examined but none adequately corrected the time delay to flashover. However, by monitoring the upper air temperature in the model, the occurrence or nonoccurrence of flashover for corresponding full-scale tests could be reliably predicted. Therefore, the use of a quarter-scale model was judged to be feasible.

NBSIR 81-2334. Hebner, R. E., ed. Development of power system measurements-Quarterly report, April 1, 1981 to June 30, 1981. 1981 June. 20 p. Available from: NTIS; PB 81-214892.

Key words: cables; composite insulation; dc fields; high voltage; incipient fault; insulation; liquid breakdown; $\mathbf{S F}_{6}$; space charge; transformer oil.

This report documents the progress on four technical investigations sponsored by the Department of Energy and performed by the Electrosystems Division, the National Bureau of Standards. The work described covers the period April 1, 1981 to June 30, 1981. The report emphasizes measurements of ion density in air, the use of signals above $1 \mathrm{GHz}$ to detect incipient faults in cables, the measurement of the by-products which develop during partial discharge activity in $\mathbf{S F}_{6}$, and the determination of the breakdown behavior of an oil-paper interface.

NBSIR 81-2335. Clark, R. E. Effects of home weatherization on occupant comfort: First report of a field study. 1981 September. 84 p. A vailable from: NTIS; PB 81-245334.

Key words: Community Services Administration; field study; occupant comfort; optimal weatherization demonstration; thermal comfort; thermostat setting practices; weatherization; weatherization and thermal comfort.

This study reports preliminary examination of data testing the hypothesis that, when existing residences are treated with weatherization retrofitting measures intended primarily to save fuel, house occupants are likely to report improvement in wintertime comfort. Data were obtained through questionnaire-guided interviews with individuals in 108 experimental houses and 37 control houses. These houses, at nine sites representing a range of U.S. climates, were part of a three-year National Weatherization Demonstration, sponsored by the Community Services Administration and planned and managed by researchers at the Center for Building Technology of the National Bureau of Standards. The experimental houses had been weatherized to determine how much their fuel usage could be reduced by cost-effective retrofitting. The control houses had not been weatherized in the demonstration. Interview topics included: thermostat setting patterns, impressions of comparative comfort, amounts of clothing worn, and specific comfort and temperature ratings for the house as a whole and for individual rooms in the house. Preliminary examination of the data has focussed on: 1) a composite "comfort change" index, comprised of indicators derived from thermostat setting practices in unusually cold weather, impressions of change in comfort-related attributes of the indoor environment, amounts of clothing worn in winter, and comfort ratings, 2) the specific comfort ratings, and 3 ) the temperature ratings. The results presented offer strong indications of support for the hypothesis.

NBSIR 81-2336. Breitenberg, M. A.; Atkins, R. G. Consumer representation in standards development: Literature review and issue identification. 1981 September. 78 p. Available from: NTIS; PB 82135880.

Key words: bibliography; annotated, consumer representation; literature review; medical device standards; public participation programs; standards development; voluntary standards.

This report identifies for possible future study the major issues associated with the topic of consumer representation in the development of voluntary standards. The key terms (consumer and consumer respresentative) used in the report are defined, and background information on the Food and Drug Administration's medical device standards program is given. The report discusses important recent developments in consumer involvement in government and standards organization decisionmaking, and contains a review of Federal public participation programs. The literature on consumer representation is reviewed from the perspective of standards-developing groups and consumer organizations and the most salient documents are annotated. Based upon the available literature, supplemented by information obtained from personal contacts with affected parties, the major outstanding issues are summarized.

NBSIR 81-2337. Ramboz, J. D. Measurement and evaluation methods for an angular accelerometer. 1981 August. 75 p. Available from: NTIS; PB 82-115973.

Key words: angular acceleration; angular accelerometer calibration; instrumentation; measurement; shock and vibration.

A transducer which measures angular acceleration along one axis was investigated to assess three of its performance characteristics, viz., sensitivity factor, amplitude linearity, and response to linear (nonangular) input accelerations. Accelerometer specifications and response theory are presented. Test philosophy and methodology are discussed along with measurement results. Tests were conducted over a frequency range from 1.5 to $100 \mathrm{~Hz}$ and an acceleration range from 64 to $5000 \mathrm{rad} / \mathrm{s}^{2}$. Generally, the performance observed was in agreement with the manufacturer's preliminary specifications.

NBSIR 81-2342. Powell, J. W.; Rodgers, R. C., Jr. FEDSOL: Program user's manual and economic optimization guide for solar Federal buildings projects. 1981 August. 132 p. Available from: NTIS; PB 82-107012.

Key words: economic optimization; life-cycle costing; solar 
economics; solar energy.

This report provides a user's manual for the FEDSOL computer program and a guide for designing and sizing solar energy projects for Federal buildings. The life-cycle cost procedures implemented by the computer program and explained in the report are consistent with the Federal Rules for Life-Cycle Costing (10 CFR Part 436) as applied to solar energy projects.

The FEDSOL program determines the economically optimal size of a solar energy system for a user-specified building, location, system type, and set of economic conditions; it conducts numerous breakeven and sensitivity analyses; and it calculates measures of economic performance as required under the Federal Rules. The economic model in the program is linked with the SLR (Solar Load Ratio) design method developed at Los Alamos National Laboratory to predict the performance of active systems. The economics portion of the program can, however, be used apart from the SLR method, with performance data provided by the user. An environmental data file for 243 U.S. cities is included in the program. Highly user oriented, the FEDSOL program is intended as a design and sizing tool for use by architects, engineers, and facilities managers in developing plans for Federal solar energy projects.

NBSIR 81-2343. Schumm, R. H. Thermodynamic properties of solid alkali aluminosilicates at elevated temperatures. 1981 October. $51 \mathrm{p}$. Available from: NTIS; PB 82-131525.

Key words: enthalpy; entropy; equilibrium constant of formation; free energy of formation; Gibbs energy function; heat capacity; heat of formation; selected values; thermochemical tables.

This report presents selected heat capacities, entropies, enthalpies, standard enthalpies of formation, standard free energies of formation and the equilibrium formation constants for solid sodium aluminate, solid potassium aluminate and solid aluminosilicates of lithium, sodium, and potassium. Values are listed in 28 tables for 17 different compositions (13 pure compounds and their polymorphs; 4 mixtures, each in low. and high-temperature configurations) from $298 \mathrm{~K}$ to temperatures ranging up to $2000 \mathrm{~K}$.

NBSIR 81-2346. Dickinson, G. W. Energy management and control systems (EMCS)_User satisfaction study. 1981 August. 19 p. Available from: NTIS; PB 82-103656.

Key words: building energy conservation systems; EMCS; energy management; energy monitoring and control systems; HVAC controls; reliability study.

A study of 86 energy management and control systems (EMCS) was made to determine users' satisfaction and perceived system reliability. Many EMC systems reportedly were not meeting users' expectations for energy, dollar, or manpower savings. Nearly one. third of the EMCS users in this study were not satisfied with their system's performance. Undependability of the manufacturer and proprietary hardware and software were the most common problems reported. Factors found to have no apparent effect on user satisfaction were: (1) the type of facility (with the exception of hospitals) in which the EMCS is installed, (2) which manufacturer supplied the system, and (3) whether or not the system was owned by the Federal Government.

The data suggest that EMCS maintenance training, in-house maintenance capability, and reduced dependence on the system manufacturer may help improve user satisfaction.

NBSIR 81-2347. Faison, T. K. Field measurement of branch circuit wire temperatures. 1981 November. 44 p. Available from: NTIS; PB 82-140252.

Key words: branch circuit; electric wire; field measurements; temperature excursions; thermal insulation.

The National Bureau of Standards (NBS), under the sponsorship of the U.S. Department of Energy (DoE), planned and conducted a program to monitor temperature excursions of residential branch circuit wiring under field conditions. This program was conducted to develop a field data base needed to determine the prevailing conditions in the field and to respond to various assertions that the encapsulation of branch circuit wiring within thermal insulation is a potential hazard. It has been demonstrated through previous laboratory investigations that buried wires, when operated continuously at rated ampacity, will exceed the thermal limit established by the National Electrical Code (NEC). Approximately 2,800 circuits in 667 residences were monitored through a volunteer program with the utilities. The results of the study show that slightly more than one-third of the circuits monitored operated at or above the thermal limit established by the NEC, $60^{\circ} \mathrm{C}\left(140^{\circ} \mathrm{F}\right)$. Thermal distributions are shown for variables such as: region, season, and age.

NBSIR 81-2348. Frederikse, H. P. R.; Schenck, P. K.; Burns, G. W.; Dils, R. R.; Whetstone, J. R. Materials for instrumentation for fossil energy technologies. 1981 August. 135 p. Available from: NTIS; PB $82-116229$.

Key words: coal; control; fossil energy; materials; process parameters; research recommendations.

This report reviews the materials problems related to instrumentation for Fossil Energy Facilities. It discusses requirements for measurement of process parameters (flow, temperature, pressure, chemical composition), of construction material properties, and of fuel characteristics. The main part of the report is a suggested program plan for pertinent materials R\&D. Short descriptions of the various Fossil Energy Processes, listing of present research activities, and a set of 6 "Case Studies" are presented in an Appendix.

NBSIR 81-2350. Ohlemiller, T. J. Smoldering combustion hazards of thermal insulation materials. 1981 August. 64 p. Available from: NTIS; PB 82-107046.

Key words: boric acid; cellulosic insulation; ignition; propagation; smoldering combustion; test methods; wave structure.

The smoldering combustion hazards of cellulosic loose fill insulation materials fall into three categories: smolder initiation, smolder propagation and transition from smoldering into flaming. Our previous findings on the initiation problem are summarized briefly. They serve as the basis for recommendations on an improved smolder ignition test method which is designed to give ignition temperatures comparable to those in practice. The proposed test method requires checking against full-scale mock-up results before it can be considered for implementation.

Smolder propagation, driven by buoyant convection, through a thick $(18 \mathrm{~cm})$ layer of cellulosic insulation has been extensively examined. A heavy (25\% add-on) loading of boric acid cuts the propagation rate in half (from approx. 0.3 to $0.15 \mathrm{~cm} / \mathrm{min}$ ) but does not come close to stopping this process. Analysis of experimental profiles for temperature, oxygen level and remaining organic fraction strongly indicate that the smolder wave is oxygen-supply controlled and that it involves both first and second stages of oxidative heat release from the insulation material. The balance of involvement of the two stages varies with depth in the layer. It appears that efforts to develop improved means of suppressing smolder propagation must be directed at the entire oxidation process. However, since boric acid is fairly effective at slowing the second stage of oxidation, most new efforts should be aimed at the first stage of oxidation (which also is responsible for smolder initiation).

NBSIR 81.2353. Peavy, B. A. Documentation of program for determination of conduction transfer functions. 1981 November. 49 p. Available from: NTIS; PB 82-136284.

Key words: conduction heat transfer; conduction transfer functions; initialization of heat transfer problem; parallel heat flow; response factors; thick building construction.

Conduction transfer functions are used to predict the time. 
dependent one-dimensional conduction heat transfer at surfaces of single-or multi-layer building constructions based on heat flux and temperature history at each surface. By the use of conduction transfer functions, heat transfer problems employing non-linear boundary conditions such as thermal radiation and time-dependent changes in the surface film resistances can be solved.

Because conduction transfer functions are analytically derived with an initial time condition of zero temperature potential throughout the solid materials, it becomes necessary to initialize the computation by including exposure to a number of outdoor weather cycles such that satisfactory initial conditions of temperature and heat flux exist at the inside and outside surfaces.

The program is set up for the use of $1-, 2-$, or 3 -hour time intervals, depending on the thickness of the building construction. The program allows for the combination of two building constructions, e.g., the parallel heat flow paths found in wood-frame walls.

NBSIR 81.2354. Konig, P. A.; Newton, J. J.; Saltman, R. G. Federal requirements for a Federal Information Processing Standard Data Dictionary System. 1981 September. 80 p. Available from: NTIS; PB 82-117607.

Key words: computer program; database; database management system; data dictionary system; data inventory; data management; data standards; documentation; Federal Information Processing Standards Publication; requirements; software.

This report presents information and preliminary conclusions about Federal agencies' requirements for a Federal Information Processing Standard Data Dictionary System. Some initial requirements were identified through analysis of comments made on the "Prospectus for Data Dictionary System Standard" which describes NBS' efforts to develop a standard. Most of the data used to develop preliminary conclusions on Federal requirements was collected during interviews with Federal Government users and developers of Data Dictionary Systems. Comments received on the Prospectus and data collected during the interviews are summarized. Preliminary conclusions and issues being investigated also are presented.

NBSIR 81-2358. May, W. B., Jr. Analysis of data from the energy monitoring and control system at the Norris Cotton Federal Office Building. 1981 November. 98 p. Available from: NTIS; PB 82138744.

Key words: building envelope; building performance data; computer data acquisition; energy conservation in commercial buildings; energy monitoring and control systems; mechanical systems.

The Norris Cotton Federal Office Building (NCFOB) in Manchester, New Hampshire, is a medium-size office building, occupied in September 1976, designed to serve as a demonstration and a feasibility test for energy-conserving building features. A building energy monitoring and control system was operated as a data acquisition system over a 13-month period ending in September 1980. Experience encountered during the checkout and operation of the system is discussed. Results from data reduction procedures used to calculate approximately 160 parameters describing the energy performance of the building are presented on a monthly basis. Hourly data are also presented for daily building operation profiles, building envelope performance, and performance of the mechanical systems.

NBSIR 81-2361. Ventre, F. T.; Stahl, F. I.; Turner, G. E. Crowd ingress to places of assembly: Summary and proceedings of an experts' workshop. 1981 September. 147 p. Available from: NTIS; PB 82-138199.

Key words: auditorium; building standards; crowd ingress; design procedures; evaluation procedures; facility design; facility management; public assembly; public safety; stadiums.

The movement of large crowds into places of assembly has resulted in death and injury to facility patrons and staff. Facility designers and managers seeking guidance have found little relevant information in the technical literatures of architectural and crowd control. The Law Enforcement Assistance Administration and the National Bureau of Standards convened the most knowledgeable persons in North America in the topic of crowd ingress to places of assembly to: identify best current design practices; identify best current facilities management practices; and define research needed to support improved practices in design and management. The report documents the design and management practices suggested by the assembled experts, reports related activities of the International Association of Auditorium Managers and suggests a program of research leading to improved quantitative design and evaluation procedures for crowd ingress.

NBSIR 81-2363. Krasny, J. F.; Allen, P. J.; Maldonado, A.; Juarez, N Development of a candidate test method for the measurement of the propensity of cigarettes to cause smoldering ignition of upholstered furniture and mattresses. 1981 October. 43 p. Available from: NTIS; PB 82-135617.

Key words: cigarettes; flammability; ignition; mattresses; selfextinguishment; smoldering; upholstered furniture.

A candidate test method for the measurement of the propensity of cigarettes to cause smoldering ignition of upholstered furniture and mattresses was developed. It consists of placing burning cigarettes on pieces of a standard, alpha cellulose, chromatographic paper and measuring the weight loss rate of the paper/cigarette system. The results were compared to the propensity of cigarettes to ignite upholstered furniture substrates. The agreement was satisfactory. Of the approximately 30 cigarette brands investigated, most had a similar propensity to ignite the upholstered furniture substrates, but a few ignited fewer substrates, and exhibited longer times to ignition on those which ignited. Many upholstered furniture substrates resisted ignition by any cigarette, while others were ignited even by those commercial cigarettes which ranked lowest in ignition propensity in these tests. However, a substantial number of upholstered furniture types did ignite with some cigarettes but not with others. Selfextinguishment time does not define the propensity of a cigarette to ignite upholstered furniture.

NBSIR 81-2366. Weber, S. F.; Rudder, F. F., Jr.; Boehm, M. J. Method for assessing costs of noise control requirements in multifamily residential and educational buildings. 1981 December. 119 p. Available from: NTIS; PB 82-140047.

Key words: acoustical design; acoustics; architectural design; building codes; building economics; construction costs; cost minimization; economic impact; economics; energy; model code; noise control.

This report presents a methodology developed to measure the cost impacts of acoustical performance requirements for new buildings. The methodology can be applied to a wide range of noise control requirements. The cost items addressed by this methodology are expected changes in construction costs, the cost of acoustical testing to certify levels of performance, code administration costs, and energy savings due to modifications of the building envelope. The building components considered, which are those most commonly affected by noise control requirements, are doors, windows, interior walls, exterior walls, and floor/ceiling assemblies. The basic cost assessment method consists of linear cost estimation equations for most component designs commonly used in educational and multifamily residential buildings. Each equation relates the acoustical performance of the design to its construction cost so that construction costs associated with alternate levels of acoustical performance can be compared. The methodology also includes a cost minimization model useful for selecting the least-cost design for a particular level of acoustical performance.

NBSIR 81-2367. Swaffield, J. A. Prediction of the hydraulic jump 
location following a change of slope in a partially filled drainage pipe. 1981 November. 189 p. Available from: NTIS; PB 82-142134.

Key words: building drainage; gradually varied flow; hydraulic jump; partially filled pipe flow.

The criteria governing the formation of a hydraulic jump in a partially filled fluid conduit downstream of a slope change are presented together with the necessary techniques to enable water surface profiles and jump location to be predicted.

Computer programs designed to model the conditions leading to jump formation under flow and channel scale conditions compatible with current drainage system design are presented.

The results of a wide range of test conditions in terms of jump formation and position downstream of a change in channel slope are presented together with a set of criteria to be used in evaluating whether a jump will occur for a given set of design conditions.

NBSIR 81-2373. Loltus. J. J.: Maldonado-Rosado. A.: Allen. P. J. An evaluation of the MSHA temperature pressure spray ignition test for hydraulic fluids. 1981 September. $44 \mathrm{p}$. Available trom: NTIS: PB 82-131806.

Key words: coal mine; fire tests; hydraulic fluids; invert emulsions; pressure-temperature; spray; synthetics; water glycol.

The Mine Safety and Health Administration (MSHA) TemperaturePressure Spray Ignition Test for determining the fire resistance of hydraulic fluids was evaluated in spray ignition tests on 14 different hydraulic fluids including water glycols, synthetics, and invert emulsions. Results of these and of other tests designed to evaluate the test method and its procedures are discussed in this report.

NBSIR 81.2374. Lew, H. S.; Carino, N. J.; Fattal, S. G.; Batts, M. E. Investigation of construction failure of Harbour Cay condominum in Cocoa Beach, Florida. 1981 September. 138 p. Available from: NTIS; PB 82-117409.

Key words: building; collapse; concrete; concrete strength; construction; failure; flat plate; shear; strength.

The investigation of the collapse of a five-story reinforced concrete flat-plate structure under construction at Cocoa Beach, Florida is presented in this report. The investigation included onsite inspection, laboratory tests and analytical studies.

Based on the results of this investigation, it is concluded that the most probable cause of the failure was insufficient punching shear capacity in the fifth-floor slab to resist the applied construction loads.

Two factors contributed to the low punching shear capacity: one in the design and the other in the construction of the building. In the design, the omission of a check for punching shear resulted in a smaller slab thickness than needed to satisfy the Code requirements. In construction, the use of specified chairs having insufficient height to support the top reinforcing steel resulted in more than the cover specified in the structural drawings. Both factors contributed to reducing the effective depth of the slab such that it had insufficient strength to resist the construction loads.

The analysis showed that shear stresses in the slab at many column locations on the fifth floor exceeded the nominal shear strength. Thus, punching shear failure at one of the columns precipitated a progressive failure of the slab throughout the entire fifth floor. Collapse of the fifth floor, in turn, caused the successive collapse of the lower floor slabs. The failure of the fifth floor slab most likely initiated at column G.2, an interior column which supported the last bay of freshly placed roof concrete prior to the collapse.

NBSIR 81-2377. Yaniv, S. L.; Bauer, J. W.; Flynn, D. R.; Danner, W.

F. Effects of time-varying noise on annoyance: A review. 1981 October. 63 p. Available from: NTIS; PB 82.133513.

Key words: duration; general adverse effect of noise; intermittency; loudness; noise criteria; time-varying noise.

This report summarizes the literature dealing with the adverse response of people to time-varying noise, and identifies both the acoustical and nonacoustical factors that influence the relationship between time-varying noise and annoyance. An examination of the laboratory research concerned with the functional relationship between annoyance and the temporal and acoustic parameters of noise shows the tenuousness of such relationships. The adequacy of currently used and/or proposed rating procedures for predicting subjective response to time-varying noise is examined. Critical gaps in current knowledge are identified.

NBSIR 81-2378. Kusuda, T. Heat transfer analysis of underground heat and chilled-water distribution systems. 1981 November. $63 \mathrm{p}$. Available from: NTIS; PB 82-136235.

Key words: computer program; earth temperature; heat transfer; pipes; thermal insulation; thermal properties; underground systems.

Simplified calculation procedures for determining heat exchange between the earth and a multiplicity of buried pipes having different temperature and thermal insulation are presented. The procedures deal with cases where pipes are buried side by side, as well as those when several pipes are bundled in a conduit. The effects of seasonal variation of earth temperature are treated in a quasi-steady-state equation that includes the soil thermal properties, depth of burial, pipe sizes, and relative locations of pipes. Sample calculations are included, together with the Fortran program listing and thermal properties of earth to be used for the calculations.

NBSIR 81-2381. Rossiter, W. J., Jr.; Shipp, W. E. Solar industrial process heat systems-An assessment of standards for materials and components. 1981 September. 97 p. Available from: NTIS; PB 82. 133703.

Key words: field survey; industrial process heat; materials performance; solar collector; standards; test methods.

A study was conducted to obtain information on the performance of materials and components in operational solar industrial process heat (IPH) systems, and to provide recommendations for the development of standards including evaluative test procedures for materials and components. An assessment of the needs for standards for evaluating the long-term performance of materials and components of IPH systems was made. The assessment was based on the availability of existing standards, and information obtained from a field survey of operational systems, the literature, and discussions with individuals in the industry. Field inspections of 10 operational IPH systems were performed. The study did not address the thermal efficiencies and health and safety considerations of IPH systems.

The results of the study are presented in this report. It is concluded that standard test methods are needed for evaluating the long-term performance of materials and components used in IPH systems operating at high temperatures. Some standard test methods are available having applicability to materials and components in low temperature systems. However, in the latter case, data bases are lacking which demonstrate their applicability. Recommendations are made and priorities assigned for the development of standards for materials and components.

NBSIR 81-2382. Cherry, S. M., ed. Fifth annual conference on fire research. 1981 October. 187 p. Available from: NTIS; PB 82. 131459.

Key words: combustion; decision analysis; fire models; flame spread; human behavior; ignition; polymers; smoke; soot; toxicity; wood.

This report contains extended abstracts of grants and contracts for fire research sponsored by the Center for Fire Research, National Bureau of Standards, as well as descriptions of the internal programs of the Center for Fire Research.

NBSIR 81-2385. Baum, H. R.; Rehm, R. G.; Barnett, P. D.; Corley, 
D. M. Finite difference calculations of buoyant convection in an enclosure. Part I: The basic algorithm. 1981 December. $67 \mathrm{p}$. Available from: NTIS; PB 82-154386.

Key words: buoyant convection; computations-finite difference; Euler equations; finite difference equations; fire-enclosure; fluid flow; heat source-volumetric; partial differential equations.

A novel mathematical model of buoyant convection in an enclosure, developed earlier, is solved by finite difference techniques in the two-dimensional case. This model has been developed as a principal analytical tool for the prediction of the movement of smoke and not gases in fires. Effects of large density variations caused by substantial heating are retained while acoustic (high-frequency) waves, which are unimportant to buoyant convection, are analytically filtered out. No viscous or thermal conduction effects are included in the model. These two characteristics (filtering and no dissipative effects) distinguish the model from all others describing buoyant convection. The mathematical model consists of a mixed hyperbolic and elliptic set of nonlinear partial differential equations: the problem is a mixed initial, boundary value one. An explicit time-marching algorithm, second-order accurate in both space and time, is used to solve the equations. The computational procedure uses a software package for solving a nonseparable elliptic equation developed especially for this problem. The finite difference solutions have been carefully compared with analytical solutions obtained in special cases to determine the stability and accuracy of the numerical solutions The computer model has been used to compute the buoyant convection produced in an enclosure by a spatially distributed heat source simulating a fire. The computed results show qualitative agreement with experimentally observed buoyant convection in enclosure fires.

NBSIR 81-2386. Hibbard, B. B.; Krasny, J. F.; Braun, E.; Peacock, R. D. Airflow extinguishment of burning apparel fabrics. 1981 December. 40 p. Available from: NTIS; PB 82-165267.

Key words: airflow; burn (injuries); cotton; fabric flammability; fabrics; fire suppression; flame extinguishment; heat transfer; polyester; textiles.

The heat output of a burning fabric, subjected to a frontal airflow at various velocities, was measured behind the burning face of the fabric. Twelve different commercial fabrics were evaluated in this manner on the Apparel Fire Modeling Apparatus (AFMA). When the fabric burned to a given heat output, the selected level of airflow was applied to the face of the burning fabric in an attempt to extinguish the flame. Burn injury area, maximum total heat, and time to extinguishment were determined from AFMA data.

The burning fabrics quickly extinguished with an airflow of 213 meters per minute $(8 \mathrm{mph})$ or less in all but one of the fabrics studied. The one exception was an $85 / 15$ cotton/polyester double faced, terry cloth fabric where this and higher airflows only increased the rate of burning. For the eight fabrics investigated which contained cellulose, the maximum total heat and burn injury area increased as fabric weight increased. For most of the fabrics studied, the total heat transferred to the simulated body generally decreased with increasing air velocity. Fabrics were classified into three groups based upon these airflow extinguishment parameters.

NBSIR 81-2388. Hecht, $H$. Synopsis of interviews from a survey of software tool usage. 1981 November. 80 p. Available from: NTIS; PB 82-133521.

Key words: programming aids; software automation; software development; software engineering; software tools; software tools usage.

This report contains synopses of all interviews conducted as part of a survey of the development and use of software tools in industry and Government and a classification of the tools encountered. The purpose of the report is to document the detailed data in a form which may be helpful for further research.
NBSIR 81-2390. Wu, S. T.; Clifton, J. R. Analysis and modeling of corrosion of steel in prestressed concrete. 1981 November. $25 \mathrm{p}$. Available from: NTIS; PB 82-141771.

Key words: concrete; corrosion; general corrosion; mathematical modeling; prestressed concrete; prestressing steel; stress corrosion.

Development of conceptual and mathematical models describing the corrosion of steel in prestressed concrete is outlined. The application of the principles of stress corrosion and general corrosion to understanding the mechanisms involved in corrosion of steel in prestressed concrete is discussed. The first step in estimating the failure time of a prestressed concrete structural system because of corrosion of the reinforced steel is to estimate corrosion rates under various but realistic conditions. A simplified approach based on mathematical modeling of concretes properties for estimating corrosion rates is proposed.

Before the proposed mathematical model can be applied to practical problems information is needed on the specific mechanisms of corrosion cell processes of steel in prestressed concrete. In addition, well designed corrosion tests need to be performed in which all the important factors affecting the corrosion rates are considered.

NBSIR 81-2395. Loftus, J. J. An assessment of three different fire resistance tests for hydraulic fluids. 1981 October. $50 \mathrm{p}$. Available from: NTIS; PB 82-142217.

Key words: autoignition temperature; coal mines; fire point; fire resistance tests; fire tests; flammability; flash point; hydraulic fluids; ignition.

The Center for Fire Research (CFR) at the National Bureau of Standards (NBS) at the request of the Mine Safety and Health Administration (MSHA) and the Bureau of Mines (BOM) made an evaluation of assessment of the three different flammability tests used by MSHA for measuring the fire resistance of hydraulic fluids intended for use in underground coal mining operations. The methods described in the Code of Federal Regulations Schedule 30, Part 35, consist of the following: an Autogenous Ignition Temperature Test, a Temperature-Pressure Spray Ignition Test, and a Test to Determine the Effect of Evaporation on the Flammability of Hydraulic Fluids.

This report summarizes the various studies conducted in the evaluation and provides recommendations for improvement of the three test procedures.

NBSIR 81-2396 (ONR). Kruger, J.; Ritter, J. J.; Carroll, J. J.; Melmed, A. J. Passive films, surface structure and stress corrosion and crevice corrosion susceptibility. 1981 November. 67 p. Available from: NTIS; ADA078596.

Key words: alpha uranium; chloride ion; chromate ion electrochemistry; ellipsometry; field ion microscopy; inhibitors; organic coatings; passive film dissolution; pH measurements; surface hydride.

Part I: Transparent organic coatings on iron are used to simulate painted metal surfaces for simultaneous ellipsometric and electrochemical measurements. These studies show that significant changes occur both in the metal oxide film and in the subcoating environment during prolonged immersion in dilute $\mathrm{Cl}^{-}$media. The relationship of these changes to phenomena such as metal passivation, coating delamination, and inhibitor behavior are addressed.

Part II: The corrosion of metals protected by organic coatings is an incompletely understood phenomenon. Recent investigations by an in situ ellipsometric-electrochemical technique combined with microanalytical studies have provided new insights into the destructive processes. Events such as the roughening of the metal surface, chloride ion accumulation and the thickening of the surface oxide have been detected under the coating and are interpreted in terms of the chemistry which develops between the 
microenvironment and the substrate.

Part III: A description is given of a study of the dissolution of passive films formed by potentiostatic anodic oxidation of iron in sodium borate-boric acid solutions. Using an ellipsometricpotentiostatic technique, four different potential regions were identified where different dissolution behavior could be observed. Two different dissolution modes were found at potentials in the passive region; one dissolution mode at potentials at or slightly below the Flade potential, and one mode at potentials where cathodic reduction becomes possible.

Part IV: Field ion micrographs of alpha uranium show atomic details of many orthorhombic crystal planes. Images of the (010) and (001) plane edges are examined and discussed in particular. Micrographs indicate that a surface hydride phase is formed readily under hydrogen imaging conditions. Some micrographs indicate possible hydride particle precipitation at a major crystal defect boundary. No evidence was found, however, of hydrogen/stress induced surface cracks. Procedures used to prepare alpha uranium for field ion microscopy are described.

Part V: Qualitative ellipsometry was used in conjunction with electrochemical $\mathrm{pH}$ and potential measurements as a technique for the study of corrosion processes occurring under organic coatings. The objective of the work was to gain a deeper understanding of the mechanisms governing the corrosion protective actions of paints on metal surfaces. Computer modeling and experiments with collodion coatings on iron substrates indicated that changes in the ellipsometric parameter $\Delta$ could, for the most part, be safely interpreted as thickness alterations in the substrate oxide film and roughening of the substrate. Experiments with the Fe-collodion system in dilute chloride solutions exhibited three sequential stages of activity, two of which could be interpreted in terms of corrosion mechanisms using the optical and electrochemical measurements. Chromate ion as a corrosion inhibitor in coatings was also studied using this technique and was shown to have significant effects upon the development of the subcoating processes.

NBSIR 81-2397. Marshall, H. E.; Ruegg, R. T. Recommended practice for measuring benefit/cost and savings-to-investment ratios for buildings and building systems. 1981 November. 49 p. Available from: NTIS; PB 82-170648.

Key words: benefit-cost analysis; building economics; cost effective; economic analysis; energy conservation; investment analysis; life-cycle cost; recommended practice; savings-toinvestment ratio.

This report describes how to calculate a benefit-cost ratio $(B / C)$ and a savings-to-investment ratio (SIR) and how to use them in selecting building designs and building systems that will be cost effective in the long run. The $\mathrm{B} / \mathrm{C}$ relates positive benefits, such as revenues, to project costs in the form of a ratio. The SIR, a variation of the $\mathrm{B} / \mathrm{C}$, relates project savings (i.e., cost reductions) to project costs in a ratio. It is used when there are few if any positive cash flows from a project. The B/C and SIR can be used to help answer such questions as: "Is a project cost effective?" "Which size and/or design of a project is most cost effective?" "What priorities should be given individual projects competing for a limited budget?" The report addresses different formulations of the ratios and their implications for selecting cost-effective projects.

NBSIR 81-2398. Roitman, P.; Albers, J.; Ehrstein, J. R.; Myers, D. R. Investigation of the two-dimensional shape of ion-implanted regions. 1981 December. 71 p. Available from: NTIS; PB 82-154451.

Key words: arsenic ion implantation; electron-beam-induced current; etching; integrated circuit processing; ion channeling; ion implantation; lateral profiles; scanning electron microscopy; silicon technology; spreading resistance; two-dimensional profiles.

The two-dimensional shape of arsenic ion-implanted regions in single-crystal silicon was investigated both experimentally and theoretically. Experimentally, two techniques were shown to have the necessary submicron resolution: a junction etch process and an SEMinduced current collection method. A comparison of junction depths determined by the etch technique, the EBIC technique, and spreading resistance with the depths calculated using several amorphous target codes was made. For the case of low temperature $\left(600^{\circ} \mathrm{C}\right)$ anneals, the etch technique agrees very well with the junction depths predicted by the amorphous target code due to Winterbon. The lateral junction locations obtained from the etch technique are in good agreement with the predictions of a two-dimensional Monte-Carlo code (TRIM) which indicates that arsenic does not show any significant lateral scattering under mask edges. For the case of high temperature $\left(1000^{\circ} \mathrm{C}\right)$ anneals, the etch and EBIC techniques agree with each other, but show consistently deeper junction locations than does the spreading resistance technique. Comparison with arsenic-diffusion models shows that concentration-dependent effects are important. Presently available processing models do not appear to adequately forecast junction depths.

NBSIR 81-2402. Rawie, C. C. Estimating economic impacts of building codes. 1981 October. 163 p. Available from: NTIS; PB 82-139551.

Key words: benefit-cost analysis; building economics; building regulation; codes; construction economics; construction regulation; economics; fire safety codes; regulation.

This report describes a method for estimating the benefits and costs of proposed changes in building codes. A companion report by the author, Estimating Benefits and Costs of Building Regulations: A Stepby-Step Guide, published by the National Bureau of Standards as NBSIR 81-2223, provides a simplified description of the same basic method.

This report shows the reader how to set up the problem, discount impacts to their present value, estimate code impacts on building costs, estimate effects on building safety, compute aggregate impacts, and conduct a sensitivity analysis. One chapter discusses the problem of assigning a dollar value to life safety. Worksheets and an extensive list of references, including sources of data on building costs and hazards, are included.

NBSIR 81-2404. Walton, G. N. Calculation of inter-room air movement for multi-room building energy analysis. 1981 November. 38 p. Available from: NTIS; PB 82-142209.

Key words: building energy analysis; computer simulation; infiltration; natural ventilation.

A model is presented for computing the infiltration and air flow between rooms of a multi-room building in terms of basic principles of fluid mechanics. This model has been incorporated into a comprehensive loads-predicting computer program. Air flows, room temperatures, and heating loads for a typical townhouse under different conditions of environment and with various construction features are computed. These calculations show the feasibility of detailed multi-room air movement analysis. They also indicate that when the inter-room openings of a low-rise structure are large compared to the envelope openings, the infiltration and total load can be accurately, and more quickly, computed by assuming no resistance to air flow between rooms. This property will also allow simplified calculations for high-rise buildings with many rooms. Methods are proposed for handling more complex air flow phenomena.

NBSIR 81.2406. Wasik, S. P.; Tewari, Y. B.; Miller, M. M.; Martire, D. E. Octanol/water partition coefficients and aqueous solubilities of organic compounds. 1981 December. 69 p. Available from: NTIS; PB 82-141797.

Key words: activity coefficients; aqueous solubility; gas chromatography; generator column; high pressure liquid chromatography; octanol/water partition coefficients.

A generator column method for measuring the octanol/water partition coefficient, $K_{o / w}$, and the aqueous solubility, $C_{s}^{w}$, is described. When water is pumped through a generator column packed with solid 
support coated with an organic stationary phase, an aqueous solution is generated that is in equilibrium with the stationary phase. The solute concentration in the eluted aqueous phase was measured either by high pressure liquid chromatography or by solvent extraction followed by gas chromatographic analysis.

Aqueous solubilities and octanol/water partition coefficients of organic solutes, falling into 7 general chemical classes, have been systematically determined using the modified generator column method. From thermodynamics an equation is derived relating $\mathrm{K}_{\mathrm{o} / \mathrm{w}}$ to the volume-fraction-based solute activity coefficient in water $\left(\gamma_{\mathrm{s}}^{w}\right)$, the latter being determinable from $\mathrm{C}_{\mathrm{s}}^{\mathrm{w}}$. For each class of compounds, excellent linear correlations are found between $\log \mathrm{K}_{\mathrm{o} / \mathrm{w}}$ and $\log \gamma_{\mathrm{s}}^{\mathrm{w}}$, with slopes close to the theoretical value of unity.

NBSIR 81-2408. Hoffman, J. D.; Wachtman, J. B., Jr. National Measurement Laboratory Center for Materials Science, Annual report 1981. 1981 November. 553 p. Available from: NTIS; PB 82. 142126.

Key words: corrosion; fracture and deformation; glass and ceramics research; materials research; materials science; metallurgy; polymers research.

The Center for Materials Science is part of the National Measurement Laboratory, one of two principal laboratories comprising the National Bureau of Standards. The Center is organized in six Divisions, each having responsibility in different areas of materials science appropriate to the major classes of materialsmetals, polymers, and ceramics and glass. The Divisions vary in their balance between theory and experiment, between direct standards work and research, and in their orientation toward industrial and Government needs and the needs of other components of the scientific and technical community. This volume summarizes the technical research activities and accomplishments of the six Divisions of the Center for Materials Science for fiscal year 1981.

NBSIR 81-2410. McKnight, R. H.; Kotter, F. R.; Misakian, M. Measurement of ion current density at ground level in the vicinity of high voltage dc transmission lines. 1981 December. 28 p. Available from: NTIS; PB 82-156993.

Key words: high voltage dc; measurement errors; transmission lines; vertical current density; Wilson plates.

Sensors for measuring vertical current density at ground level near high voltage dc (HVDC) transmission lines are subject to error when the sensor is not in the ground plane. The magnitude of this error, for guarded and unguarded sensors, has been investigated using both dc electric fields with space charge and ac electric fields in a parallel plate facility. For conditions like those expected under HVDC transmission lines, the results obtained using ac and dc methods agreed to within experimental uncertainty. The measured errors are as large as 25 percent for guarded sensors and significantly larger for unguarded sensors. Data for various sensor elevations and guarding are presented in graphs to aid the designer.

NBSIR 81-2414. Moore, R. T. A discussion of GRIDNET simulation results. 1981 November. 16 p. Available from: NTIS; PB 82-142894.

Key words: alternate routing; communications networks; distributed control; message delay; network throughput; survivability.

This report is a review and evaluation of the results of computer simulation of GRIDNET as conducted under U.S. Department of Commerce contract NB80SBCA0477 during the period September 30, 1980 to September 4, 1981.

The objectives of the program were to test and modify algorithms which would permit messages to be routed from any source to any destination, in a network having thousands of nodes, and to accomplish this routing in an efficient manner using only limited local knowledge of network operability status. In addition, estimates were developed of the network's delay and throughput characteristics as a function of traffic loading.

Based on the simulation results, GRIDNET could be developed into a highly reliable and highly survivable data communications network that could support traffic between a large number of nodes in an effective and efficient manner.

NBSIR 81-2415. Steel, J. S. Furnace pressure probe investigation. 1981 November. 29 p. Available from: NTIS; PB 82-139999.

Key words: fire endurance; furnaces; pressure probes; static pressure.

Several pressure probes of types used in fire endurance furnaces were tested at room temperature and compared in a furnace. It is suggested that total pressure be measured instead of static pressure and that the most accurate probe is a small flush hole in the specimen.

NBSIR 81-2428. Collins, B. L.; Danner, W. F.; Tibbott, R. L. Communication systems for disabled users of buildings. 1981 December. 73 p. Available from: NTIS; PB 82-165283.

Key words: accessibility; alarms; blind; code requirements; communication; deaf; directional indicators; handicapped users; tactile warnings.

Communication systems in buildings are designed to provide both emergency and directional information to all building users. Yet such systems, which are typically comprised of visual signs and audible alarms, may fail to reach some of the estimated twenty-seven million hearing or vision impaired people in the United States. As a result, a number of alternative communication systems have been proposed for inclusion in accessibility guidelines. In the following pages the research base underlying communication provisions for each of three sensory modalities, vision, hearing and touch, are reviewed. In addition, various proposed code recommendations are presented and discussed. The adequacy of the research base for each provision is discussed along with the need for various code provisions. It is noted that code provisions for tactile warnings and exit markings are particularly inadequate. Recommendations for further research into tactile warnings, tactile signage, and visual alarms are suggested.

NBSIR 81-2433. Klote, J. H.; Zile, R. H. Smoke movement and smoke control on merchant ships. 1981 December. 68 p. Available from: NTIS; PB 82-165192.

Key words: ships; smoke control; smoke movement; stack effect; sulfur hexafluoride; tracer tests; ventilation systems.

In the past ten years considerable progress has been made in developing systems to control smoke movement in building fires. At present no such smoke control systems are in use on merchant ships. This paper discusses the basic concepts of smoke movement and smoke control with emphasis upon shipboard applicability. A report of simulated smoke movement tests performed on two merchant vessels is presented. Based upon these test results potential methods of smoke control for merchant vessels are discussed. Recommendations are made for future study. 


\subsection{GRANT/CONTRACT REPORTS AND NBS PATENTS}

Grant/contract reports are prepared by non-NBS persons or organizations working under grant or contract from the National Bureau of Standards. Those contract reports not incorporated into the formal NBS publication series are available directly from the National Technical Information Service (NTIS, Springfield, VA 22161) in paper copy or microfiche form unless otherwise stated. When ordering a report from NTIS you must order it by the "COM, PB, $A D$, or N" number as indicated.

Patents are legal documents which fully describe inventions in return for the right for 17 years to exclude others from making, using, or selling the inventions. They are obtained on NBS inventions of high commercial potential in order to establish Government ownership of the patent rights. The patents are then made available for the grant of nonexclusive licenses to all qualified applicants. A limited exclusive license may be granted under a particular patent, however, if it appears that some period of exclusivity is necessary as an incentive for the investment of risk capital. For information on licensing any of the following patents, write to the Office of the Legal Adviser, National Bureau of Standards, Washington, DC 20234. Copies of patents may be obtained from the U.S. Patent and Trademark Office, Washington, DC 20231 for 50 cents each.

NBS-GCR-76-81. Cornell, C. A., (NBS contact: Structures \&

Materials Division). A national office of live loads survey. 1976 October. 180 p. Available from: NTIS; PB 82-130436.

Key words: live loads; load survey; probability; statistics; structural engineering.

This report presents the final recommendations for a National Bureau of Standards program to survey, analyze, and model the gravity live loads in modern office buildings in the U.S. A basic premise is that a probabilistic model (or models) should be used as a basis for identifying the important parameters and thus for designing the experiment and for directing the analysis of the data.

Chapter 2 discusses these models. They include not only basic models for describing and predicting (in probabilistic terms) sustained and peak loads (and structural load effects), but also simple models for guiding design standards and an explanatory (room) sector load model (ESLM) to be used to study loads and to reduce sampling effort.

The other major chapters, Chapters 3 and 4, discuss the statistical aspects of the program: the survey design (by building, by room sector, and by bay) and the data reduction needed for the purposes of model parameter estimation, model checking, and possible model modification.

The final chapters summarize (1) the conduct of the survey (including a pilot survey and two stages of a main survey), (2) the computer program requirements, (3) the analytical and numerical studies to be carried out once the data reduction is complete, and (4) a set of reports that might represent the final product of the survey.

NBS-GCR-79-186-1. Griffith, J. W., (NBS contact: N. Jason). Life cycle cost workbook. 1981 September. 30 p. Available from: NTIS; PB 82-120510.

Key words: cash flow; economic analysis; fire safety; fire safety evaluation system; health care facilities; life cycle.

The workbook presents a methodology to compare total or relative (Life Cycle) cost of the alternative plans. It is designed to be useable by any person who has access to the necessary financial data and a rudimentary understanding of normal business financing and cost. The approach used is based on the completion of simple work sheets. All of the definitions and reference tables needed to execute the work sheets are included in the workbook. By use of this approach the health care provider, or other person responsible for making preliminary decisions among widely varying alternatives, can extend his information base beyond the traditional approach of first cost figures to consider the actual life cycle price.

NBS-GCR-79-188. You, H. Z.; Faeth, G. M., (NBS contact: N. Jason). An investigation of fire impingement on a horizontal ceiling. 1978 October. 112 p. Available from: NTIS; PB 82-130428.
Key words: ceilings; experiments; flame size; temperature distribution; turbulent burning.

This report discusses research completed under NBS Grant No. 7-9020, during the period September $-1,1977$ to August 31, 1978. The investigation considers the processes which occur when a turbulent fire impinges upon a horizontal ceiling. Measurements were conducted to determine free flame heights, impinging flame lengths along the ceiling, ceiling heat fluxes and mean temperature distributions in the flow. Both unconfined and confined ceilings were considered. Theoretical analysis was completed in order to suggest simple methods for correlating the data.

The fire source was simulated by burning wicks soaked with liquid fuel (methanol, ethanol, l-propanol and n-pentane). The range of test variables involved ceiling diameters of $610.660 \mathrm{~mm}$, ceiling heights of $58.940 \mathrm{~mm}$, wick diameters of $10.107 \mathrm{~mm}$ and curtain wall heights (for confined ceilings) as large as the ceiling height.

NBS-GCR-80-248. Ungar, E., (NBS contact: CBT X2693). Structureborne sound in buildings: Needed practical research in light of the current state of the art. 1980 June. 55 p. Available from: NTIS; PB 81.187064

Key words: acoustics; architectural acoustics; building acoustics; noise; noise control; sound transmission; structureborne noise.

An overview of the current state-of-the-art of structureborne sound in buildings is presented. A general introduction to the field of structureborne sound is included with a discussion of important phenomena. Summaries of recent investigations described in the technical literature are discussed relevant to excitation and local response, propagation, radiation, and control of structureborne sound in buildings. Topics for future research in structureborne sound in buildings are presented based upon this review. An annotated bibliography of recent investigations is appended.

NBS-GCR-80-249. Schultz, T. J. Impact noise testing and rating-1980. 1981 January. 363 p. Available from: NTIS; PB 81-158610.

Key words: acoustics; building acoustics; impact noise; noise control; noise isolation; noise rating

A state-of-the-art review of impact noise testing and rating is presented. A historical perspective of impact noise testing and rating covers the first efforts in the 1920's through the most recent research results. The current research is discussed, with the emphasis on the development of standardized test methods and impact noise rating methods. An outline for future research needs is presented in light of the current state-of-the-art. A bibliography containing over 200 references to impact noise testing and rating is included in the report.

NBS-GCR-80-250. Sharp, B. H.; Kasper, P. K.; Montroll, M. L., (NBS contact: S. Webber). Sound transmission through building structures-Review and recommendations for research. $1980 \mathrm{July}$. 144 p. Available from: NTIS; PB 81-187072.

Key words: acoustics; building acoustics; noise control; noise isolation; sound transmission; structure-borne noise.

This report presents a critical review of the status of technology in sound transmission through building structures, and identifies specific areas for further research. The approach taken in the review follows the steps involved in the design process, namely, prediction, measurement, and evaluation. Priorities for further research are based on the potential for achieving the following objectives: To develop new technology to reduce the cost of noise control in buildings; to increase confidence that designs will provide the required acoustical privacy; and to identify and apply sound isolation techniques that reduce energy consumption.

NBS-GCR-80-254. Thomas, W. C., (NBS contact: K. DeCorte). 
Effects of test fluid composition and flow rates on the thermal efficiency of solar collectors. 1980 August. $91 \mathrm{p}$. Available from: NTIS; PB 81.180267.

Key words: efficiency; flow rate; heat transfer fluid; solar collector; thermal performance.

Experimental and analytical investigations were carried out to determine the significance of the heat transfer fluid and mass flow rate on the thermal performance of two liquid-heating flat-plate solar collectors. The collector thermal performance was lowered significantly for high concentrations of glycol and mineral-base oil. Heat losses measured with oil were less than the losses measured from the ASHRAE prescribed procedures.

An analytical model and procedure were used to account for variations in the test environment and fluid parameters. Calculations show that the collector flow factor $\left(F_{R}\right)$ and efficiency factor $\left(F^{\prime}\right)$ depend on fluid parameters while the loss coefficient $\left(U_{L}\right)$ depends more strongly on fluid temperature.

NBS-GCR-80-255. Hunt, B., (NBS contact: S. Webber). Development of a research program for scaffolding standards. 1980 August. $276 \mathrm{p}$. Available from: NTIS; PB 81.180259.

Key words: accidents; codes and standards; construction loads; construction safety; finite element; scaffold failures; scaffolding practices; scaffolds; structural analysis.

Under the auspices of the National Institute for Occupational Safety and Health (NIOSH), the Construction Safety Group of the National Bureau of Standards (NBS) has embarked on a scaffolding research program to provide the necessary technical assistance for developing performance standards and design guidelines for scaffolds. This paper describes the various problem areas that exist with construction scaffolding and appendix $A$ presents the major types of scaffolds under study.

The first study is the analysis of scaffolding accidents and related employee casualties and is presented in appendix $B$. The second study is the review of current scaffolding codes and standards and is presented in appendix $C$. Two concurrent studies have been performed and are presented in the major portions of this paper. These are a review and evaluation of the technical literature on scaffolding and a study and analysis of in-field scaffold loading practices.

Using the results of these first four studies, this paper presents a newly developed analytically based scaffolding research plan. This plan consists of key topics of recommended research and an approach by which this plan can be implemented is demonstrated through selective modeling of scaffolding systems by a finite element analysis. Appendix D presents samples of the input and output data for the scaffold structural analysis. Final conclusions and recommendations are then presented as to the direction of future scaffolding research.

NBS-GCR-80-279. Patrick, S.; Giuntini, R., (NBS contact: E. Cohen) Terrestrial solar simulators for photovoltaic and combined photovoltaic and thermal device measurement and evaluation. 1980 October. 50 p. Available from: NTIS; PB 82-130063.

Key words: calibration; combined photovoltaic/thermal modules; measurements; photovoltaic modules; photovoltaics; reference cells; solar cells; terrestrial solar simulators; test methods.

The use of terrestrial solar simulators used for testing photovoltaic and combined photovoltaic/thermal devices by a representative cross section of the photovoltaics community is reviewed. Measurements. related needs identified by this community are also reviewed. Among the needs identified are major improvements in the area of standardized test and calibration procedures. Also identified is the need for a source dedicated to providing reference cells to the photovoltaics community and for workshops to address measurements-related problems and developments. Included are tables that review the type and use of terrestrial solar simulators used and that list data-sheet information about these simulators.
NBS-GCR-80-280. Achenbach, P. R. Functional performance requirements for the environmental and service systems in detached housing and their impact on building energy use. 1981 January. 168 p. Available from: NTIS; PB 81.157893.

Key words: building energy use; building service systems; energy budgets; housing research; housing standards; performance requirements; standards development.

The energy used for the environmental and service functions of space heating and cooling, domestic water heating, ventilation, lighting, and food and laundry services should all be taken into account in developing energy budgets for detached housing. The report states the functional performance requirements that should be met by these systems simultaneously with efficient energy utilization. It also identifies existing and proposed standards, guidelines, and criteria for the performance of these systems; shows how the performance criteria impact energy use; and summarizes the research needed to complete or produce consensus standards for the separate systems that can be introduced into energy performance standards for detached housing.

NBS-GCR-80-284. Nober, E. H.; Pierce, H.; Well, A.; Johnson, C. C.; Clifton, C. Waking effectiveness of household smoke and fire detection devices. 1980 October. 85 p. Available from: NTIS; PB 81 . 127565 .

Key words: adults; alarm responses; auditory perception; decibel levels; fire departments; frequency distribution; noise (sound) sleep; smoke detectors; wakefulness.

Normal-hearing, young adults were subjected to home smoke detector alarm signals of 85,70 , and $55 \mathrm{dBA}$ while asleep in their own bedrooms under quiet background conditions. In addition, other adults received 70 and $55 \mathrm{dBA}$ alarm signals masked by window air conditioner background noise. Each person, upon awakening from the alarm signal, was instructed to shut off the alarm and telephone the local fire department. The 85,70 , and $55 \mathrm{dBA}$ alarm levels were all sufficient to awaken the subjects at varying hours of the night and days of the week, under quiet background conditions. While there were statistically significant differences in waking times between 55 dBA and the other two alarm levels, the total times never exceeded 115 seconds for the combined alarm shutoff and the fire department telephone call at any alarm level. With background noise, waking times for the 70 and $55 \mathrm{dBA}$ alarm levels increased (85 dBA not tested). At $70 \mathrm{dBA}$, the total time for the alarm shutoff and the fire department telephone call ranged from 36 to 119 seconds. At $55 \mathrm{dBA}$, two persons failed to awaken and one person awakened after the fourminute test termination criteria. For the remaining seven persons, the total time for the combined alarm shutoff and the fire department telephone call ranged from 45 to 137 seconds.

NBS-GCR-80-285. Lehovec, K.; Fedotowsky, A., (NBS contact: Elaine Cohen). Solar cell mathematical analysis and computer simulation. 1981 January. 69 p. Available from: NTIS; PB 81. 163891.

Key words: computer simulations; fault detection; laser scanner; photovoltaics; solar cell analysis; solar cells.

Procedures have been established which permit the calculation of cell output by spot illumination for various boundary conditions imposed by faults, provided the attenuation length is independent of position. The attenuation length is the distance over which the photosignal from the illuminated point diminishes to $\mathrm{e}^{-1}$ of its value due to the back flow of current over the distributed junction network modeled for the solar cell. Faults explicitly treated so far are: cracks with and without leakage current across the exposed junction, point shorts, and partial electrode lift-offs. We also have established procedures for calculating the effect of variation of the attenuation length with distance from the grid electrodes on the cell output. The 
attenuation length may vary either due to sheet resistance variation, or else due to diode impedance variation. The results of these calculations provide guidance for the experimental techniques to be used in light spot scanning for solar cell analysis and fault detection.

NBS.GCR.80-291. Ryan, P.; De Vos, D., (NBS contact: J. Stroik). Building security demonstration: A survey of break-ins in two public housing sites. 1980 November. 100 p. Available from: NTIS; PB 81. 180549.

Key words: burglary; crime in public housing; household behavior patterns; housing characteristics (physical); housing security; public housing security; security hardware; victimization survey.

This report presents the findings of a baseline crime victimization survey conducted in two public housing sites. These findings are part of a test of the validity of security hardware standards developed by NBS. Included in this report is an explanation of the methodology used, demographic and behavior patterns, physical characteristics, and the crime victimization experienced at both sites. Conclusions include the observation that crime patterns indicate the usual complex variables at work and causal relationships are conjectured to be number and skill of criminals, access and exposure of entry portals, number of resident adults in the household, and quality of the building/site security system.

NBS-GCR-80-292. Maxwell, T. T.; Maples, G.; Dyer, D. F.; Burch, T., (NBS contact: Nora Jason). Fire safety of wood-burning appliances, Part II: Fireplace inserts-A design review. 1980 November. 19 p. Available from: NTIS; PB 81-161531.

Key words: fireplace inserts; fireplaces; fire prevention; fire safety; questionnaires; wood.

The objective of this review is to identify the generic types of fireplace inserts currently available. Three methods were used to gather information: 1) questionnaires were mailed out to manufacturers of woodburning equipment; 2) a literature review was conducted; 3 ) data was collected firsthand at an industry trade show. The information obtained by these methods was sorted and reviewed.

A precise definition of a fireplace insert was formulated in order to reduce the variety of units to be considered. The units which fit this definition were then categorized according to the following observable physical characteristics: 1) single-box or double-box construction, 2) glass doors or metal doors, 3) positive or non-positive flue connection, and 4) forced or natural convection.

NBS-GCR.80-294. Zukoski, E. E.; Kubota, T.; Cetegen, B., (NBS contact: Nora Jason). Entrainment in fire plumes. 1980 November. 53 p. A vailable from: NTIS; PB 81-181851.

Key words: diffusion flames; entrainment; fire plumes; flow rate; mass flow.

A new technique for measurement of mass flow rates in buoyant fire plumes is described. The characteristics of 10 to $200 \mathrm{kw}$ methane diffusion flames stabilized on porous-bed burners of $0.10-0.50 \mathrm{~m}$ diameter are described. A transition in the dependence of flame height on heat input and burner size was observed when the flame height was about four times the burner diameter. The mass flow rates in the buoyant plumes produced by the fires were measured for a range of elevations starting just below the time-averaged top of the flame and extending to six times this flame height. The mass flow rates in this region of the plume were correlated by the use of a simple plume model. Atmospheric and forced disturbances in the air being entrained increased the entrainment rate of the plume.

NBS-GCR-80-295. Tewarson, A., (NBS contact: Nora Jason). Physicochemical and combustion/pyrolysis properties of polymeric materials. 1980 December. 83 p. Available from: NTIS; PB 81-158651.

Key words: combustion products; fire modeling; foam (materials); heat release; plastics; polymers; polymethylmethacrylate; polyoxymethylene; polypropylene; polystyrene; polyurethane; polyvinyl chloride; pyrolysis; smoke; wood.

Data for the physico-chemical and combustion/pyrolysis properties of aromatic and nonaromatic granular and foamed polymeric materials are presented for use in fire modeling as well as for hazard evaluations and basic understanding of fires. The physico-chemical and combustion/pyrolysis properties include chemical formulae, stoichiometric air-to-fuel ratio, net heat of complete combustion, heat required to generate a unit mass of vapors, surface re-radiation loss, surface temperature, combustion efficiency including its convective and radiative components, product yields, light obscuration parameter, flame convective and radiative heat flux to the surface, Spalding's B-number modified for radiation and for combustion efficiency, normalized flame heights, ratio of $\mathrm{CO}_{2}$ concentration to gas temperature above ambient, ratio of optical density per unit path length to gas temperature above ambient, and energy required to generate a combustible vapor-air mixture.

The dependency of various fire properties such as heat release rate, product generation rates, light obscuration, etc., and fire detection, are analyzed in terms of the fire environment (thermal and over- or underventilated) and the properties of the materials.

NBS-GCR-80-296. Committee on Radiation Measurements, (NBS contact: Mr. Heaton). Directory of commercial calibration services for ionizing radiation survey instruments. 1981 April. $73 \mathrm{p}$. A vailable from: NTIS; PB 81.20641.

Key words: calibration; commercial services; directory; inaccuracy; ionizing radiation; radioactive sources; survey instruments.

This directory lists information provided by commercial services that calibrate survey instruments used to measure ionizing radiation, and information on the availability of calibrated radioactive sources. Companies included in this directory are cross-referenced. The first listing is by state, subdivided into the instrument calibration and source manufacturer categories. This listing includes the types of radiation services offered, calibration method(s) used, estimated calibration accuracies, radiation energy and intensity ranges, and types of instruments and radioactive sources manufactured or calibrated. The second list summarizes the services offered by radiation type. In the final list, each company is shown with its address, telephone number, and a contact person. Included in the appendices is information on possible sources of inaccuracy in calibrating survey instruments and on the properties of common calibration sources.

NBS-GCR-80-297. Bryan, J. L.; DiNenno, P. J.; Milke, J. A., (NBS contact: N. Jason). The determination of behavior response patterns in fire situations, project people II. Final Report-Incident reports, August 1977 to June 1980. 1980 December. 234 p. Available from: NTIS; PB 81-224545.

Key words: doors; evacuation; fire alarm systems; fire departments; fire extinguishers; fire investigations; hospitals; nursing homes; nursing staff; smoke; smoke detectors; sprinkler systems.

This report is a summary and initial analysis of the sixty-five fire incidents included in the study population of Project People II. The fire incidents have been analyzed to present in tabular form the descriptive characteristics of the facilities with the construction, interior finish, and fire zone features shown. Staff and fire department behavioral actions were summarized and are presented in another table, with the number of persons evacuated, the means of evacuation, the extinguishment bahavior, the closing of doors and the ventilation of smoke through the facility windows. The fire protection features of the facilities are presented in a third table. The sixty-five fire incidents included in this summary occurred between August 10, 1977 and June 25,1980 . The facilities involved in the incidents have primarily been 
health care facilities in accordance with the objectives of the research study, with twenty-five nursing home or convalescent center and thirty-three hospital incidents. In addition, two schools, two high rise apartments, two university dormitories and one correctional institution fire incidents were included due to the extensive evacuation behavior. The abstract of each fire incident report is presented with the diagrams of the maximum fire and smoke development in the realms and the movements of personnel in the behavioral episodes. The individual fire incidents were studied with a survey of the facility and interviews with critical fire department, staff and patient personnel.

NBS-GCR-80-298. Capozzi, D. M., (NBS contact: Nora Jason). An annotated bibliography for wood combustion activities in the residential sector. 1981 January. 141 p. Available from: NTIS; PB $81 \cdot 165490$.

Key words: bibliographies; chimneys; combustion; fire safety; heating equipment; literature reviews; stoves; wood.

The U.S. Department of Energy, as part of its conservation mission, is dedicated to increasing the use of renewable energy resources as a replacement for fossil fuels, particularly oil and natural gas. The increased use of wood energy for the space heating of residential and commercial buildings provides an important opportunity to replace the use of fuel oil.

This report cites over 500 recent publications within the United States and Canada related to wood combustion. A brief description of most articles is included. Where known, the availability of the various citations is annotated.

NBS-GCR-80-305. CAP GEMINI, INC., and J. G. Van Dyke and Associates, Inc. (NBS contact: E. Becker). Computer networking status, trends and utility for the Federal Government. 1980 November. 155 p. Available from: NTIS; PB 81-163859.

Key words: computer networking; technology assessment; technology forecast.

This report examines current computer networking offerings and, based upon a survey of sixty suppliers of computer networking products and services, projects technological and economic trends for the industry. In addition, the report evaluates the utility of computer networking for the Federal Government. The products and services of the sixty suppliers included in the survey typify the computer networking resources currently available to users. Summaries and assessments of those products and services surveyed are included in this report and constitute an examination of historical perspectives and trends, and of the current status of computer networking. The assessments made of current products and services form the basis for projections of trends in computer networking for the next ten years. Those projections are presented in terms of technological, marketplace, and economic factors. The report also provides a prognosis of the level of service and economic impact for future computer network users. The assessments of current products and services and the projections of trends are used to evaluate the utility of computer networking for the Federal Government. Management and procurement issues, as well as technological impact, are evaluated. The probable penetration of computer networking into the Federal Government environment is described, and the expected advantages and problems are presented.

NBS-GCR-80-306. Dickamore, W. G.; Dampier, I. K., (NBS contact Michael Hogan). Survey and market forecast of flexible disks. 1980 November. 110 p. Available from: NTIS; PB 81-171258.

Key words: cost/benefit analysis; flexible disk cartridge; flexible disk system; information interchange; marketed systems; standard.

This report includes the following information: division of a generic flexible disk system into its major addressable components, including the flexible disk cartridge, the drive, and the controller; identification of the system parameters affecting information interchange within each component; a survey of marketed systems to determine the most frequently occurring values within the identified parameters; compilation of parameters and associated values that should be considered in the design of a standard or family of standards for information interchange on the flexible disk cartridge; a survey of a future 5-year period identifying the volume of expected units that would support the proposed standards; a cost/benefit analysis of the Federal portion of the 5-year volume that would be influenced by the standards; and a description of positive and negative effects the proposed standards may have on flexible disk technology and the associated market.

NBS-GCR-80-307. Dickamore, W. G.; Kampier, I. K., (NBS contact Michael Hogan). Technology assessment of flexible disks as used in stand-alone text-processing systems. 1980 November. 86 p. Available from: NTIS; PB 81-171365.

Key words: costs and benefits; flexible disk cartridge; information interchange; label and file structure; standard; word processor.

This report includes the following information: identifying alternatives to the flexible disk cartridge for providing information interchange between stand-alone word processors; defining the technical problems associated with flexible disk cartridge information interchange; surveying the Federal Government to ascertain the volume and different types of stand-alone text processors currently being used and the 5-year projection of word-processing equipment needed; identifying any negative impacts from the establishment of a label format and file structure standard for flexible disk cartridges; and identifying all costs and benefits that would accrue to the Federal Government as a result of a label and file structure standard for flexible disk cartridges used in stand-alone word processors.

NBS-GCR-81-262. Fiorello, Shaw and Associates, and Aurora Associates, Inc. (NBS contact: P. Kay). Cost-benefit analysis of a Federal Information Processing Standard: COBOL (FIPS 21) Impacts on Air Force Data System Design Center functions. 1981 September. 58 p. Available from: NTIS; PB 82-140245.

Key words: automated data processing; computers; cost-benefit.

A cost-benefit case study of the Gunter Air Force Base experience in moving from assembly language to $\mathrm{COBOL}$, the report documents which costs and benefits are quantifiable and which can only be discussed qualitatively.

NBS-GCR-81-300. Pape, R., (NBS contact: Nora Jason). Preflashover room fire model: Parametric sensitivity analysis and development of a submodel for burning furniture items. 1981 January. $70 \mathrm{p}$. Available from: NTIS; PB 81-170664.

Key words: fire modeling; fire plumes; flame spread; furniture flammability; polyurethane foam combustion; room fires.

The focus of this project was to extend the Fire Modeling capabilities for furniture fires, both numerically and experimentally. Numerical work was based on the existing RFIRES computer code. A sensitivity analysis was performed for this program and a user manual compiled as part of this project. Two types of experiments were conducted-fires with real furniture, and idealized simple geometry fires, intended to study flame spread characteristics. Since the RFIRES code at present lacks a flame spread subroutine an extensive literature search was conducted to identify suitable flame spread models. Analysis of the available background here led to a formulation of the basic features required for a flame spread subroutine. Special emphasis was placed on the ability to treat nonstandard plume configurations.

NBS-GCR-81-301. Pape, R.; Waterman, T. E.; Eichler, T. V., (NBS contact: Nora Jason). Development of a fire in a room from ignition to full room involvement-RFIRES. 1981 January. $182 \mathrm{p}$. A vailable from: NTIS; PB 81.166639. 
Key words: burning rates; compartment fires; fire modeling; flashover; numerical methods; room fires.

This report collects in a unified manner documentation pertaining to the computer code RFIRES developed under the present contract, as well as under several earlier grants. RFIRES is a computer model developed to predict the response of a room to the burning of items within it. The model considers a rectangular room with a single door or window opening to a large reservoir of clean air; up to 10 rectangular box-shaped fuel items can be considered. The documentation includes flow charts, input and output instructions, code listing, summary of physical equations, results of comparisons to experimental fires, sensitivity analyses, and probabilistic descriptions of fuel item burning rates.

NBS-GCR-81-302. Shingleton, J. G.; Cassel, D. E.; Overton, R. L., (NBS contact: Mary Reppert). Air leakage in residential solar heating systems. 1981 February. 206 p. Available from: NTIS; PB $81-176380$.

Key words: air leakage; heat transfer; pebble-bed storage; solar collector; system efficiency; thermal performance.

A series of computer simulations was performed to evaluate the effects of component air leakage on system thermal performance for a typical residential solar heating system, located in Madison, Wisconsin. Auxiliary energy required to supplement solar energy for space heating was determined using the TRNSYS computer program, for a range of air leakage rates at the solar collector and pebble-bed storage unit. The air leakage model was based on field measurements of air flow in several residential solar air heating systems in the National Solar Demonstration Program for which leakage problems were reported. The study included the effects of heat transfer and mass transfer between the solar equipment room and the heated building, and also determined the effect of reduced air infiltration into the building due to pressurization by the solar air heating system. A simple method of estimating the effect of collector array air leakage on system thermal performance was evaluated, using the f CHART method.

NBS-GCR-81-308. Division of Purchasing, Dept. of Admin., State of Colorado (NBS contact: Kathy Hudson). Procurement of products containing recycled/recovered materials in the State of Colorado. 1981 March. 97 p. Available from: NTIS; PB 81-198236.

Key words: economic; institutional; procurement; purchasing; recovered/recycled materials; resource recovery; technical barriers.

The report examines the current State activities, regulations, statutes, and policies, as well as technical institutional and economic barriers relating to the public procurement of products containing recovered or recycled materials.

The report also lists the top ten commodities procured by the State in dollars plus a listing of products that, in the opinion of the State, have a high potential for the use of recycled or recovered material.

The report includes a list of recommendations or suggested actions that could be taken to potentially improve the manufacture, distribution, and procurement of products containing recovered or recycled materials.

NBS-GCR-81-309. Resource Recovery Section, Department of Environmental Regulation, (NBS contact: K. Hudson). State of Florida: The procurement of products containing recovered materials. 1981 May. 193 p. Available from: NTIS; PB 81-205445.

Key words: economic, institutional, technical barriers; procurement; purchasing; recovered/recycled materials; resource recovery.

The report examines the current State activities, regulations, statutes, and policies, as well as technical, institutional, and economic barriers relating to the public procurement of products containing recovered or recycled materials.

The report also lists the top ten commodities procured by the State in dollars plus a listing of products that, in the opinion of the State, have a high potential for the use of recycled or recovered material

The report includes a list of recommendations or suggested actions that could be taken to potentially improve the manufacture, distribution, and procurement of products containing recovered or recycled materials.

NBS-GCR-81-309, Supplement 1. (Resource Recovery Section, Dept of Environmental Regulation, State of Florida) (NBS contact: C. Hudson). An analysis of the procurement policies of: Dade County, The City of Miami, The Dade County School System, Miami Dade Community College. 1981 June. 34 p. Available from: NTIS; PB 81 . 225054.

Key words: econimic, institutional, technical barriers; procurement; purchasing; recovered/recycled materials; resource recovery.

The study examines the procurement policies of Dade County, City of Miami, Dade County School System, and the Miami Dade Community College on the procurement of products containing recycled materials. The study was to identify rules, regulations, policies, and barriers that affect such procurements. This report supplements the main study dealing with procurement policies at the state level (NBS-GCR-81-309).

NBS.GCR-81.310. Kittel, J., Michigan Energy and Resource Research Association, (NBS contact: K. Hudson). Procurement of products containing recovered materials: $A$ study of State of Michigan practices. 1981 March. 127 p. Available from: NTIS; PB 81-204448.

Key words: economic, institutional, technical barriers; procurement; purchasing; recovered/recycled materials; resource recovery.

The report examines the current State activities, regulations, statutes, and policies, as well as technical, institutional, and economic barriers relating to the public procurement of products containing recovered or recycled materials.

The report also lists the top ten commodities procured by the State in dollars plus a listing of products that, in the opinion of the State, have a high potential for the use of recycled or recovered material.

The report includes a list of recommendations or suggested actions that could be taken to potentially improve the manufacture, distribution, and procurement of products containing recovered or recycled materials.

NBS.GCR.81-311. Resource Conservation and Recovery Office, Minnesota Dept. of Administration (NBS contact: K. Hudson/J. Berke). A study of resource and energy conservation practices in the procurement and management of commodities. 1981 March. $63 \mathrm{p}$. Available from: NTIS; PB 81-187965.

Key words: economic; institutional; procurement; purchasing; recovered/recycled materials; resource recovery; technical barriers.

The report examines the current State activities, regulations, statutes, and policies, as well as technical institutional and economic barriers relating to the public procurement of products containing recovered or recycled materials.

The report also lists the top ten commodities procured by the State in dollars plus a listing of products that, in the opinion of the State, have a high potential for the use of recycled or recovered material.

The report includes a list of recommendations or suggested actions that could be taken to potentially improve the manufacture, distribution, and procurement of products containing recovered or recycled materials.

NBS.GCR-81-312. Josephson, E.; Bush, T.; Mazzone, G.; McElroy, 
G.; Galbraith, T., (NBS contact: K. Hudson/ J. Berke). Procurement of products containing recycled materials in the state of New Jersey. 1981 March. 76 p. Available from: NTIS; PB 81184335.

Key words: economic; institutional; procurement; purchasing; recovered/recycled materials; resource recovery; technical barriers.

The report examines the current State activities, regulations, statutes, and policies, as well as technical institutional and economic barriers relating to the public procurement of products containing recovered or recycled materials.

The report also lists the top ten commodities procured by the State in dollars plus a listing of products that, in the opinion of the State, have a high potential for the use of recycled or recovered material.

The report includes a list of recommendations or suggested actions that could be taken to potentially improve the manufacture, distribution, and procurement of products containing recovered or recycled materials.

NBS-GCR-81-313. Egan, J.; Higgins, R.; Spath, J.; Buchholz, A.; Wrzenski, S. C., (NBS contact: K. Hudson/ J. Berke). Procurement practices in New York State of products containing recycled materials. 1981 March. 25 p. Available from: NTIS; PB 81.187999.

Key words: economic; institutional; procurement; purchasing; recovered/recycled materials; resource recovery; technical barriers.

The report examines the current State activities, regulations, statutes, and policies, as well as technical institutional and economic barriers relating to the public procurement of products containing recovered or recycled materials.

The report also lists the top ten commodities procured by the State in dollars plus a listing of products that, in the opinion of the State, have a high potential for the use of recycled or recovered material.

The report includes a list of recommendations or suggested actions that could be taken to potentially improve the manufacture, distribution, and procurement of products containing recovered or recycled materials.

NBS-GCR-81-314. Energy Enterprises Northwest (NBS contact: Kathy Hudson). State of Washington: A study of procurement of products containing recycled materials. 1981 March. $116 \mathrm{p}$. Available from: NTIS; PB 81-197071.

Key words: economic; institutional; procurement; purchasing; recovered/recycled materials; resource recovery; technical barriers.

The report examines the current State activities, regulations, statutes, and policies, as well as technical institutional and economic barriers relating to the public procurement of products containing recovered or recycled materials.

The report also lists the top ten commodities procured by the State in dollars plus a listing of products that, in the opinion of the State, have a high potential for the use of recycled or recovered material.

The report includes a list of recommendations or suggested actions that could be taken to potentially improve the manufacture, distribution, and procurement of products containing recovered or recycled materials.

NBS-GCR-81.315. Waller, M. B.; Vreeland, R. G., (NBS contact: N. Jason). Report of a conference on fire emergency plans in group homes for the developmentally disabled. 1981 March. 32 p. Available from: NTIS; PB 81-180960.

Key words: developmentally disabled; evacuation; fire departments; fire emergency plan; fire protection; group homes; group home staff.

This report is the product of a conference held on June 1, 2, and 3,
1980 in Chapel Hill, North Carolina. The participants in the conference were brought together to discuss guidelines for fire emergency plans in group homes for the developmentally disabled. Participants included a broad cross section of those concerned about fire safety in group homes for the developmentally disabled, including fire protection engineers, group home owners and operators, fire service personnel, as well as experts in training group home staff and in developmental disabilities, insurance, and behavior in fire. This report contains some of the substance of their discussions. The guidelines provided in this report include suggestions regarding training programs and facility fire emergency plans. A checklist for both local fire service personnel and group home staff is provided to assist these groups in developing adequate fire emergency plans. Recommendations are made in the areas of information availability, incentive systems, and evaluation procedures.

NBS-GCR-81-316. Francis, R. L.; Chalmet, L. G., (NBS contact: N. Jason). Network models for building evacuation: A prototype primer. 1981 March. 251 p. Available from: NTIS; PB 81-197022.

Key words: buildings; egress; emergencies; evacuation; network models.

This document is a prototype primer on the network modeling of building evacuation. We call the document a primer because it is intended as an introduction to the subject. We call the document a prototype primer because, while students have successfully used the approaches we present we have not, as of this writing, has an opportunity to teach from the primer. If you think of a building evacuation as involving the flow of people through well defined passageways, it is natural to consider the evacuation problem to be a network flow problem. We believe you will discover, as compared to other approaches, that network approaches can be cheaper, more precise, can handle larger problems, and, because they can be used quickly and allow data to be changed easily, they greatly facilitate the comparison of many alternatives. A sequence of three successively more general and more detailed types of network models is presented, which we term for convenience the Graphical Model, the Intermediate Model, and the Dynamic Model respectively. Since the Intermediate and Dynamic Models share a number of common network modeling features, prior to considering the Intermediate Model we devote a chapter to basic network modeling ideas. The last two chapters contain a case study along with conclusions and a few words of warning.

NBS-GCR-81-317. Lin, T. D.; Abrams, M. S., (NBS contact: Nora Jason). Simulation of realistic thermal restraint during fire tests of floors and roofs. 1981 March. 178 p. Available from: NTIS; PB 81 179657.

Key words: buildings; concrete; fire endurance; fire tests; floors; reinforced concrete; roofs; thermal expansion; thermal restraint.

A five phase program was conducted to simulate and measure the thermal restraint of concrete floor and roof slabs during standard fire exposure tests. Phase I involved measuring temperature distributions, expansions and deflections of small size slabs. In Phases II and III, computer programs for heat flow calculations and stress analysis were developed. Six $14 \times 18 \mathrm{ft}$. floor slabs were tested for $4-\mathrm{hr}$. durations, during which expansions in both directions were controlled to follow computed time-expansion relationships. Differences were noted and are probably associated with creep strains not considered in the computer model. Four more large slabs were fire tested in Phases IV and V. Expansions and restraining forces were measured in tests with and without compressible pads. Reasonable agreement was obtained and it is therefore possible to use fixed frame furnaces to simulate realistic thermal restraints in fire tests of floor slabs. No spalling or structural damage was observed during any of the tests.

NBS-GCR-81-318. Vest, C. M., (NBS contact: G. Birnbaum). Holographic NDE: Status and future. 1981 May. 76 p. Available from: NTIS; PB 81-207409.

Key words: flaw detection; holography; inhomogeneities; light 
wave; nondestructive evaluation (NDE); optical holographic interferometry; technical literature.

This is the final report of a research program whose objective was to examine the current role and future potential of optical holographic interferometry for nondestructive evaluation of materials and components. The primary task carried out to meet this objective was a detailed evaluative review of the directly relevant technical literature. Our approach has been to attempt to delineate both technical and industrial criteria which must be met by industrially. viable NDE techniques, and then to render estimates of whether holographic NDE can meet these criteria. The technical literature was thoroughly reviewed in order to assess the current status of holographic NDE. This information together with recent developments in the techniques of holographic interferometry and in the equipment used for holographic NDE forms the basis of our response. The first section contains a description of the sources of data and information which formed the basis of the study, followed by a brief exposition of principles and techniques of holographic NDE. The technical literature is described and the cited references are categorized in a manner which is intended to assist the reader in locating those articles which are relevant to specific interests or applications. The next section is an analysis of the status and future of holographic NDE. Finally, conclusions are offered in the form of a response to the inquiries.

NBS-GCR-81-320. Zeizel, J.; Welch, P., (NBS contact: T. Faison). User needs research for family housing. 1981 May. 169 p. Available from: NTIS; PB 81.214074.

Key words: children; design; environmental psychology; families; housing; interiors; lifestyle; research; site planning; sociology; standards.

Research on the social, psychological and behavioral needs of families in housing is not always easily accessible to the designers and developers of housing. Housing Designed for Families compiles this "user needs" information in a handbook for people involved in regulation setting, home building, and housing design. The report's format reflects the types of decisions designers must make and the basic zones that a living environment comprises. Zones are defined in terms of privacy, formality, territory, and intergroup contact.

Housing regulations set health and safety standards for new homes, but to date have incorporated little available user needs research. Because of this, today's homes, particularly those built with government assistance, are safe; but they often lack the simple amenities of design that make them truly livable.

This report documents, zone by zone, design problems as they appear in the literature. Each section includes comments on related sections of HUD's Minimum Property Standards (MPS) and Manual of Acceptable Practices. Comments make clear which sections of the two documents are responsive to the needs of tenants, which sections seem to contradict each other, and what changes might enhance the standards responsiveness to the issues raised by the research.

NBS-GCR-81-321. Carlock, J. B. III, (NBS contact: M. McCabe). Use of FCHART version 3.0 to predict auxiliary energy use in residential solar energy systems. 1981 May. 101 p. Available from: NTIS; PB $81-217010$

Key words: auxiliary energy; FCHART computer program; National Solar Demonstration Program; solar energy systems; space heating load; thermal performance.

Thermal performance predictions for five residential buildings with solar space heating and hot water systems using the FCHART computer program are compared with measured thermal performance data from each site. The FCHART calculations are based on measured meteorological data and on space heating loads that are both measured and calculated using building thermal envelope data and the modified degree day method. Because of the imprecision inherent in the load measurements, comparisons with FCHART predictions based on measured loads could only determine that seasonal predictions fall within the tolerance limits of the measured performance. Comparisons with predictions based on the degree day method indicate that the degree day method is less accurate than the FCHART method itself.

NBS-GCR-81-322. Wedekind, D. R., (NBS contact: Mike McCabe) Multi-family solar domestic hot water analysis. 1981 May. $100 \mathrm{p}$. Available from: NTIS; PB 82-130139.

Key words: domestic hot water; recirculating hot water systems; solar collectors; system efficiency; thermal performance.

The National Bureau of Standards is presently reviewing the Definitive Performance Criteria for Residential Solar Heating, Cooling and Hot Water Systems. In support of that effort, this report describes a study designed to quantify the effect of daily domestic hot water loads and system design on the performance of multi-family solar domestic hot water systems.

Two multi-family solar domestic hot water systems judged representative of the systems funded by the HUD Residential Solar Demonstration Program, along with possible modifications to these systems, are modeled using the TRNSYS simulation computer program. The effects of collector array efficiency, storage capacity, and distribution heat losses were studied over a range of daily domestic hot water loads from 11 to $190 \mathrm{~L} /$ day-unit.

Results of simulations over representative climatic periods show that daily domestic hot water usage significantly affects solar system performance. Improved system designs are developed and modeled on an annual basis and comparison is made to system performance of the original design. Notable increases in performance can be obtained by the use of improved system configurations. Specific recommendations are made with direct emphasis on the definitive performance criteria.

NBS-GCR-81-323. Network Analysis Corporation Staff, (NBS contact: B. Wilson). 1980 November. 299 p. Available from: NTIS; PB 81.203622.

Key words: computer network protocols; computer networks; cost/benefit analysis; federal government networking; federal computer networking development; networking standards; penetration studies.

The National Bureau of Standards, Institute for Computer Sciences and Technology, has initiated a program to develop computer network protocol standards as Federal Information Processing Standards (FIPS). Cost/Benefit Analysis of High-Level Computer Network Protocol Class Standards is one of a series of reports being prepared under the network protocol standards program. This report evaluates six classes of high-level protocols as to the relative and aggregate costs and benefits associated with standardizing those protocols. Costs are defined to be just the costs of developing and adopting a standard, while benefits are the changes in acquisition and operating budgets due to the standard. The difference between cost and benefit yields the net economic benefit of a standard to the Government.

NBS-GCR-81-324. Shelton, C. B.; Moulton, J. R., (NBS contact: B. Wilson). Virtual terminal feature analysis-Draft report. 1981 March. 60 p. Available from: NTIS; PB 81-205387.

Key words: communication protocols; computer network protocols; feature analysis; network architecture; network virtual terminal protocols; virtual terminal protocols.

The National Bureau of Standards, Institute for Computer Sciences and Technology (ICST), has initiated a program to develop computer network protocol standards as Federal Information Processing Standards (FIPS). Virtual Terminal Feature Analysis is one of a series of draft reports being prepared under the network protocol standards program for distribution to government agencies, voluntary standards organizations, computer and communications equipment manufacturers, and other interested parties. This draft report analyses 
protocol features as a method of determining the kernel set of essential features of a protocol, along with the clusters of value-added features which will support various application categories

NBS-GCR-81-325. Wilson, R. G., (NBS contact: E. Cohen) Measurement technology for critical ion implantation parameters. 1981 June. 45 p. Available from: NTIS; PB 81-219628.

Key words: channeling angle; C.V profiling; dopant profiles; implantation-induced damage; ion channeling; ion dose measurement; ion implantation.

Practical information and experimental data are summarized on a research project on the control of ion-implantation doping to achieve accurate implantation doses and profiles. The studies and results reported in this and previous publications include: the dependence of implanted dopant distributions in silicon on implantation alignment angle, on implantation temperature, and on implantation fluence and energy; a profiling technique comparison study among differential C.V profiling, SIMS atom profiling, and spreading resistance profiling; range-energy results and straggle curves for column $V$ donor and column III acceptor impurities in the random equivalent orientation in silicon; channeling profiles for a number of ions in the $\langle 100\rangle,\langle 110\rangle$, or $\langle 111\rangle$ channeling orientations in silicon; and the nature of implantation damage in silicon. Knowledge and control of implantation dose, range, and straggle is important for the exploitation of the superior potential of implantation in the practical world of device and circuit fabrication.

NBS-GCR-81-327. McCarthy, D.; Acevedo, J.; Herman, D., (NBS contact: E. Cohen). Advanced planar silicon test structures. 1981 July. 92 p. Available from: NTIS; PB 81-229155.

Key words: gated diode; generation lifetime; integrated gated. diode electrometer; integrated test structure; leakage current; microelectronic test structures; surface recombination velocity; test pattern; test structure.

An integrated test structure that can be used to measure $p n$ junction reverse-bias leakage current has been implemented in modular test chips to investigate the effects of certain design parameters on the leakage measurements. The test structure is composed of a gated diode, a reset switch, and an integrally connected source-follower MOSFET which functions as an electrometer. The automated measurement procedure is discussed, and data obtained using the test structure are analyzed to determine the bulk generation lifetime and the surface recombination velocity in processed silicon wafers.

NBS-GCR-81-329. Martin, S. B.; Inman, L. B., (NBS contact: N. Jason). The effect of fire retardants on the heat-release rate of building materials. 1981 July. 39 p. Available from: NTIS; PB 81 . 238487.

Key words: calorimeters; cellulose; char; fire retardants; heat measurement; heat release calorimeters; heat release rate.

A method was developed for making up thick cellulosic specimens with thermocouples implanted at various depths to determine the time dependent temperature profiles when exposed to external radiant fluxes. Specimens of alpha cellulose, both untreated and treated with 2 percent by weight of $\mathrm{Na}_{2} \mathrm{~B}_{4} \mathrm{O}_{7}$, were exposed to an applied radiant flux of $3.7 \mathrm{~W} / \mathrm{cm}^{2}$ in the SRI heat release rate calorimeter in order to examine the effect of the fire retardants on the heat release rate. The results on a limited number of tests suggest that the char enhancement, produced by the presence of $\mathrm{Na}_{2} \mathrm{~B}_{4} \mathrm{O}_{7}$, reduces the heat release rate mainly by the reduction of the caloric value of the volatile products rather than by the insulating effect of the increased char layer thickness.

NBS-GCR-81-330. Berhinig, R. M., (NBS contact: N. Jason) Measurement of air flow around doors under standardized fire test conditions. 1981 July. 40 p. Available from: NTIS; PB 81.238495.

Key words: air flow; air leakage; ASTM E 152; doors; fire doors; fire tests.

Fire tests were conducted to obtain data for evaluating the reproducibility and accuracy of a test method being proposed by the International Standards Organization. The proposed test method is intended to provide for measurement of the air flow through fire door assemblies during a standardized fire exposure test.

The furnace used to conduct the fire tests was the vertical panel furnace of Underwriters Laboratories Inc. The furnace is ordinarily used to conduct tests in accordance with ASTM E 152 (UL 10B) Fire Test of Door Assemblies. The fire door assembly tested consisted of a nominal 3 by $7 \mathrm{ft}$. flush-type, hollow metal door attached to a steel frame mounted in a brick masonry wall.

During the tests, measurements were made of the air flow through the fire door assemblies, the deflection of the door assemblies and the temperature and pressure conditions within the furnace and test assemblies.

NBS-GCR-81-331. Alfeld, L. E., (NBS contact: N. Raufaste)

Research for building construction productivity-Report on the June 2, 1981 conference. 1981 August. 26 p. Available from: NTIS; PB 81.246803 .

Key words: building productivity; computer-integrated construction; conference; construction duration; productivity measurement; risks of failure; user productivity.

The conference was held to identify major research needs to improve commercial construction productivity. Productivity measures the relative values of the product of construction, the building, and the time, labor and capital used to produce, operate and maintain it. Therefore, both cost reduction and quality improvement are important in improvement of productivity. Twenty-six participants, from all sectors of the construction industry, met as a roundtable group. Five prepared papers served to stimulate wideranging discussion.

By the end of the day a consensus emerged around six primary research topics: 1. Develop a "family" of micro measures of construction productivity to assist individual firms in decision-making. 2. Improve macro measures of productivity to assist in understanding regional and aggregate industry trends. 3. Develop the methods needed to extend computer applications to all phases of construction decision-making. 4. Identify and develop methods to expedite the regulatory process. 5 . Measure the relationships between occupantuser productivity and building design. 6. Produce the knowledge of physical properties of buildings needed to reduce risks of building failures and lower costs of designing for building safety.

Conferees further agreed that the private sector, not government, must take the initiative to formulate and conduct research. However, government should support and conduct some research.

NBS-GCR-81-332. The Rice Center, (NBS contact: F. Ventre). A review of standards and common practices in building site regulation technical issues and research needs. 1981 September. $144 \mathrm{p}$. A vailable from: NTIS; PB 82.117631.

Key words: building site standards; regulation; site design practices; site engineering.

Widely used design and service standards are identified for five elements of site development subject to government regulation: streets, sitework, drainage, waste water and water supply. Standards in use are found to vary widely from minimums to contradict one another. The report suggests that local custom rather than site engineering research forms the basis for development regulation. The report also found that: 1) some standards do not protect health and safety; 2) many regulations do not incorporate by reference relevant standards; and 3) not all referenced standards are technically current The report lists areas of needed research and suggests methods for resolving conflicts among development standards.

NBS-GCR-81-333. White, E. T., (NBS contact: A. Rubin). Tracing 
lighting design decisions for new open office space: A pilot study. 1981 September. 196 p. Available from: NTIS; PB 82.132531

Key words: building program; lighting design; lighting research; lighting survey.

The research activity consisted of an exploration of the design decision making process used by architects, engineers, and lighting designers for the illumination needs in open office situations. Approximately 30 firms with extensive lighting design experience comprised the sample of organizations who participated in the study. They ranged in size from large $\mathrm{A} / \mathrm{E}$ and architectural companies to small lighting design and space planning offices. Personal interviews were conducted with the individual (or group) primarily responsible for making lighting design decisions. The object of the interview was to determine the criteria used (and their interrelationships) to make lighting decisions and to explore in detail how task lighting and ambient lighting requirements are established and put into practice to support necessary and desired activities. The data obtained during the interviews were used to formulate a lighting design process model, which explores the interactions among the many variables considered in lighting design. This design process model may be used as the first step in the formulation of a more generalized model which may be used for other design decisions-e.g., acoustics, thermal environment, space requirements, etc. This survey was co-sponsored by the llluminating Engineering Society of North America (IESNA), the American Institute of Architects (AIA) and the International Association of Lighting Designers (IALD).

NBS.GCR-81-334. Li, S. S., (NBS contact: E. Cohen). Conductivity mobility, Hall mobility, and resistivity in p-type silicon. 1981 September. 9 p. Available from: NTIS; PB 82.135864.

Key words: conductivity mobility; density of states; dopant (acceptor) density; effective mass; Hall mobility; hole density; resistivity; silicon

Detailed theoretical and experimental studies of the dopant density, hole density, Hall mobility, and resistivity in p-type silicon were carried out over a wide range of dopant density $\left(10^{14}\right.$ to $\left.3 \times 10^{18} \mathrm{~cm}^{-3}\right)$ and temperature (100 to $400 \mathrm{~K}$ ). Good agreement between theory and experiment was obtained, and the theoretical models appear to be adequate for interpreting the temperature and dopant density dependence of resistivity of boron-, gallium-, and indium-doped silicon over the ranges studied.

NBS.GCR.81.335. Reifer, D. J.; Montgomery, H. A., (NBS contact:

R. Houghton). Final report: Software tool taxonomy. 1981 June 1. 113 p. Available from: NTIS; PB 82-103961.

Key words: programming aids; software automation; software development; software engineering; software tools; software tools usage.

An initial set of software tool classifications are defined that can be used to: (1) categorize currently available tools, (2) standardize terminology associated with tools, and (3) ease the task of comparing and evaluating the utility of tools within a class. Example, classifications of 30 tools are provided.

NBS-GCR-81-336. Geer, N.; Graff, H. A.; Stromecky, P., (NBS contact: R. T. Moore). GRIDNET SIMULATION. Volume 1: System description and results. 1981 August. 61 p. Available from: NTIS; PB 82-116013.

Key words: alternate routing; communications networks CROSSFIRE; link protocols; local survivability; message delay; network simulation; network throughput; survivability.

This report is a summary of the results of a computer simulation of a novel, highly connected data communication network that was introduced by R. T. Moore as NBSIR 80-2149, GRIDNET, October 1980.

The objectives of the program were to test algorithms which would permit messages to be routed from any source to any destination, in a network having thousands of nodes, and to accomplish this routing in an efficient manner using only limited local knowledge of network operability status. In addition, estimates were developed of the network's delay and throughput characteristics as a function of traffic loading.

Based on the simulation results, GRIDNET could be developed into a highly reliable and highly survivable data communications network that could support traffic between a large number of nodes in an effective and efficient manner.

NBS-GCR-81-337. Manola, F.; Smith, J.; Smith, D., (NBS contact: L. Gallagher). Evaluation criteria for a CODASYL query language. 1980 March. 147 p. Available from: NTIS; PB 82.136748.

Key words: CODASYL; database management system; data model; DBMS; network data model; query language.

This report describes evaluation criteria which will be used in the development by CCA of a query language for CODASYL-type data structures. The evaluation criteria will be the basis for a specific set of capabilities to be provided by the query language. The capabilities to be provided are determined by choosing, for each evaluation criterion, a position describing a specific level of capability. The evaluation criteria are also useful in the evaluation of existing query languages. In addition to a discussion of the evaluation criteria and positions, the report includes a description of those database users for which a query language based on the proposed evaluation criteria is intended. The report also discusses related language facilities which might appear in the context of such a query language.

NBS-GCR-81.338. Manola, F., (NBS contact: L. Gallagher). Capabilities for a CODASYL query language. 1980 June. $126 \mathrm{p}$. Available from: NTIS; PB 82-136755.

Key words: CODASYL; DAPLEX; database management system; data model; DBMS; network data model; query language; relational model; SQL

This report describes proposed capabilities which are to be provided in a query language being designed by CCA which will operate on CODASYL-type database structures. In order to define the nature of these capabilities in a way sufficiently detailed to be the basis of language design, the capabilities are illustrated by examples using two prototype languages. These languages, while not complete, are currently under investigation. The languages are SNEQUEL, based on IBM's relational query language SQL, and CODAPLEX, based on Shipman's functional language DAPLEX. Proposed capabilities include those necessary for querying relational, as well as CODASYL, databases.

NBS-GCR-81.339. Manola, F.; Barnett, J., (NBS contact: L. Gallagher). A survey of CODASYL query languages. 1980 September. 201 p. Available from: NTIS; PB 82-136765.

Key words: CODASYL; database management system; data model; DBMS; network data model; query language.

This report describes capabilities provided by query languages designed for use with CODASYL-type database management systems. The languages surveyed include both commercially-available products and query languages which have been described in the research literature. The report first discusses criteria used to evaluate the various query languages studied, as well as capabilities which were considered of special interest in the study. The report then presents a separate section on each query language surveyed. The capabilities of each language are described with respect to the specified evaluation criteria, and a list of references for each language is provided.

NBS-GCR-81-342. Arthur D. Little, Inc. and General Systems Group, Inc. (NBS contact: P. Kay). The effects of future information processing technology on the Federal Government ADP situation. 1981 September. 110 p. Available from: NTIS; PB 82-138181 
Key words: automated data processing; computer utilization trends; Federal computers; hardware trends; information industry; software trends; technology forecast.

This report qualitatively forecasts the effects on the Federal ADP inventory of new and expected automated data processing technology, shifts in industry structure, and proposed changes in Federal ADP regulations. The report includes both forecasts jointly prepared by Arthur D. Little, Inc., and General Systems Group, Inc., and comments by ICST staff. Major sections of the document cover underlying technologies, changes in the information industry and market, future products and systems, the present Federal ADP situation, and proposed policy changes.

NBS-GCR-81-343. Bryan, J. L.; Milke, J. A., (NBS contact: N. Jason). The determination of behavior response patterns in fire situations, Project people II. Final report-Health care. 1981 October. 304 p. Available from: NTIS; PB 82-136771.

Key words: doors; evacuation; fire alarm systems; fire departments; fire extinguishers; fire investigations; hospitals; nursing homes; nursing staff; smoke; smoke detectors; sprinkler systems.

This study involved the detailed investigation of 59 fire incidents in Health Care Facilities located in the State of Maryland, with one facility in Philadelphia. A total of 150 staff participants, 9 patients and 53 fire department personnel were interviewed relative to the fire and smoke development during the fire incident, and the human behavior responses of the participants during the fire incident. The analysis and study of the fire incident and interview data enabled the examination of the parameters of the fire incident including: area of fire origin, ignition and fuel characteristics, and the fire protection design features of the building. The human behavior variables of the fire incidents relative to the means of becoming aware of the fire incident, and the first three actions of the participants were compared to the variables of the fire and smoke development, previous training and fire experience of the participants, and with their belief in the safety of the building. Statistical analyses were performed indicating the relationships among the variables. The evacuation behavior was studied with the sequences of the actions adopted by the personnel.

NBS-GCR-81-344. Mitler, H. E.; Emmons, H. W., (NBS contact: N. Jason). Documentation for CFC V, The fifth Harvard Computer Fire

Code. 1981 October. 187 p. Available from: NTIS; PB 82-139486.

Key words: compartment fires; computer programs; fire extinguishment; flashover; ignition; mathematical models; room fires.

This is a complete documentation of the fifth version of the Computer Fire Code (Mark 5). Mark 5 is of course a substantial improvement over Mark 4, which in turn had expanded and generalized Mark 3, etc. Although imposingly thick, this document has been written in a form which may be easily read, as each section begins with a brief outline of that section. The details are then given in the following paragraphs, for the interested reader. The Computer Fire Code permits the calculation of the evolution of a fire in an enclosure with a number of vents, and containing a number of objects (flammable or otherwise). The fire may be of several kinds, and the calculation will proceed for whatever time the user selects. Suggestions for the improvement of the program itself or of this document will be gratefully received. The tape containing the program itself is available at cost.

NBS-GCR-81-345. Helms, C. R.; Schwarz, S. A.; Spicer, W. E.; Taylor, N. J., (NBS contact: E. Cohen). Innovative measurement technology for the semiconductor device industry based on electron spectroscopy. 1981 November. 11 p. Available from: NTIS; PB 82 . 135922.

Key words: Auger-electron spectroscopy; electronics; MOS devices; semiconductors; silicon dioxide; sputter profiling.

This report summarizes the results of a 3-1/2 year research effort on electron spectroscopy techniques used in conjunction with ion etching for characterizing materials and structures used in semiconductor device technology. Results include both the establishment of the capabilities and limitations of these techniques and their application in studies of the chemistry, stoichiometry, and morphology of interfaces present in MOS device structures. A list of 27 publications which provide details of the various results is included in the report.

NBS-GCR-81-346. Zukoski, E. E.; Kubota, T.; Cetegen, B., (NBS contact: N. Jason). Entrainment in the near field of a fire plume. 1981 November. 66 p. Available from: NTIS; PB 82-136466.

Key words: diffusion flames; entrainment; fire models; fire plumes.

This report concerns entrainment measurements made in the near field of a fire plume. The flame geometry and entrainment rates of $10-80 \mathrm{~kW}$ methane diffusion flames stabilized on $0.1,0.19$ and $0.5 \mathrm{~m}$. dia. porous bed burners are described. Flame heights based on high speed photographs are presented and correlations are developed for several characteristics of the flame geometry. The entrainment process in a fire plume was found to occur in three distinct modes: a laminar flame, in the zone close to the burner surface; a turbulent flame, at larger heights; and a turbulent plume, in the region above the visible flame. A simple model based on these three processes is proposed and compared with entrainment measurements.

NBS-GCR-81-347. Swankin, D. A., (NBS contact: M. Brientenberg). Rationale statements for voluntary standards-Issues, techniques, and consequences. 1981 November. 90 p. Available from: NTIS; PB $82-135856$.

Key words: legislative history; product liability; rationale statemen ts; scope of standards; standards; standards writing.

This report provides an overview of the needs, practices and consequences of providing rationale statements for voluntary standards. The research consisted of a literature review, a review of relevant court cases, the collection and assessment of examples of rationale statements, and discussions with those involved in standards writing. Six examples of rationale are analyzed and a discussion of the most controversial issues associated with rationale statements follows. The report also contains a listing of product liability cases in which the issue of standards was raised and analyzes the legal consequences that rationale statements are likely to have on such cases.

NBS.GCR.ETIP 81-93. Foskett, W. H.; Jamieson, A.; Evans, J., (NBS contact: D. Fulmer). Innovation by regulation: The administration of control technology requirements under the Clean Air Act. 1981 March. 147 p. Available from: NTIS; PB 81-221616.

Key words: air pollution; Clean Air Act; EPA; ETIP; incentives; innovation; regulation.

This report, one of a series on factors affecting innovation through regulatory reform, presents case histories and analysis regarding the impact on innovation and technology of certain provisions of the Clean Air Act. Since new reforms are built on these requirements, their future is dependent on how they work. The report finds problems in the linkage between the Clean Air Act and industrial innovation.

NBS-GCR-ETIP 81.94. Foskett, W.; Olesen, T., (NBS contact: D.

Fulmer). Periodic review and technological escalation of performance standards: New source performance standards review process. 1980 December. 42 p. Available from: NTIS; PB 81-218117.

Key words: air pollution; Clean Air Act; EPA; ETIP; incentives; innovation; regulation.

This study is part of a research series by ETIP about the impacts of 
air pollution regulation on industrial innovation and creativity. It treats the results of periodic review of standards for new source performance. This review was mandated in order to incorporate technological advances that take place since the standards' original formulation. In fact, the study shows that revisions are seldom made raising the question as to whether technological advances are overlooked or are non-existent.

NBS-GCR-ETIP 81-95. Evans, J., (NBS contact: D. Fulmer). Controlled trading and site-specific SIP revisions: Competing for attention in a crowded administrative route. 1980 December. $111 \mathrm{p}$. Available from: NTIS; PB 81.218273.

Key words: air pollution; Clean Air Act; EPA; ETIP; incentives; innovation; regulation.

This report, one of a series on factors regarding the impacts on innovation of new regulatory incentives, deals with the administrative route being followed by "controlled trading" incentives. The report presents case studies and analysis showing how the success of these new reforms is endangered by the slowness of the administrative route for approval in a State Implementation Plan (SIP).

NBS-GCR-ETIP 81-96. Clarren, S.; Nalley, P.; Gregory, G.; Frederick, W.; Hausmann, R., (NBS contact: D. Fulmer). The evaluability assessment of the developing experiment in postmarketing surveillance of prescription drugs. 1981 March. $217 \mathrm{p}$. Available from: NTIS; PB 81.223232.

Key words: drug development; drug regulation; innovation; postmarketing surveillance; regulatory experiments; system design.

This report describes the context within which a regulatory administrative experiment is being developed by the Experimental Technology Incentives Program (ETIP) and the Food and Drug Administration (FDA). The purpose of the experiment is to determine the impact on drug development and approval of new methods of gathering and analyzing data after a drug is marketed. The report assesses the evaluability of the experiment, raising measurement considerations and proposing means for evaluation.

NBS-GCR-ETIP 81-97. Mulkey, M.; Timpane, K., (NBS contact: J. Green). Toward competitive provision of public record message services. 1981 October. 145 p. Available from: NTIS; PB 82-138819.

Key words: administrative experiment; communications; competition; ETIP; FCC; innovation incentives; regulatory reform.

This report describes the current status of a cooperative project involving the Experimental Technology Incentives Program (ETIP) of the National Bureau of Standards and the Federal Communications Commission. The partnership was initiated in 1977 as one of several similar ETIP efforts to develop knowledge useful to policymakers on the interaction between regulation and private sector innovation. This volume reports on market trends evolving in the wake of the FCC opening up public message communication services to competition. The report concludes that while a few firms have entered the market to offer the public record message telecommunications services on a nonsubscription basis, these services are oriented toward use by businesses, not the individual, occasional user. While development in public message services has been slow, the area of business-oriented subscription record and data telecommunications and teleprocessing services has been very active. The report calls out regulatory issues worth resolving in order to ensure public availability and affordability of new services.

U.S. Patent 4,243,763. Argentar, H. Tertiary aromatic amine accelerators in acrylic resin. 6 January $1981.13 \mathrm{p}$

Key words: acrylates; acrylic resins; aminoglutethimide or paraaminophenylacetic acid; cementing of bone; fillings; methacrylates; tertiary aromatic amines; unsaturated polyesters.

Tertiary aromatic amines derived from aminoglutethimide or para- aminophenylacetic acid act as accelerators for the peroxide catalyzed polymerization of acrylic resins, especially methacrylates, acrylates and unsaturated polyesters. The amines are characterized by good hardening, strength, color and toxicity characteristics, and are thereby particularly suited for use in the filling and restoration of human teeth and the cementing of bone.

U.S. Patent 4,245,169. Hamilton, C. Sampling circuit and method therefor. 13 January $1981.10 \mathrm{p}$.

Key words: analog signal; Josephson junction; sampling circuit; sampling pulses.

A sampling circuit and method therefor including a first Josephson junction for producing a series of sampling pulses for activating a second Josephson junction that is receptive of both the analog current signal being sampled and a bias current. The first Josephson junction is continually triggered at a fixed time relative to the repetitive analog signal to provide a series of sampling pulses and before each sampling pulse the bias level to the second Josephson junction is changed until the sum of the current from the sampling pulse, from the analog signal, and from the bias source exceeds the critical current value of the second Josephson junction causing it to switch from one voltage state to another. The value of the bias current at the point of switching is proportional to the current in the analog signal.

In one embodiment, the bias current is increased in a step-like fashion for each sampling pulse from the first Josephson junction until the critical value of the second Josephson junction is reached. The resolution of the analog signal for this embodiment is $1 / \mathrm{N}$ if there are N samples.

In another embodiment where the analog signal is known to exist between two extremes, the bias current for the first sample is initially positioned at a mid-range value between the extremes. If, at the sample time, the second Josephson junction is switched, the bias current for the next sample is reduced by $1 / 2$. If the second Josephson junction has not switched, the bias current is increased by $1 / 2$ for the next sample. This process continues for $\mathrm{N}$ samples, the resulting resolution being $1 / 2^{N}$.

U.S. Patent 4,251,565. Bowen, R. L. Use of a polyfunctional surfaceactive comonomer and other agents to improve adhesion between a resin or composite material and a substrate. 17 February $1981.14 \mathrm{p}$.

Key words: acrylate; adhesion; aminobenzoate; composite material; dentin; methacrylate; polyfunctional surface-active comonomers; reconstructive dental work; resin.

A family of polyfunctional surface-active comonomers which act to improve the adhesion between a resin or composite material and solid surfaces capable of binding polyvalent cations is described. These polyfunctional surface-active comonomers, or "PolySACs," are preferably the reaction product of an epoxy acrylate or methacrylate and an aminobenzoate. The adhesion of the resin or composite material to certain solid surfaces is also improved by the preparatory treatment of these surfaces with a monobasic acid of intermediate strength followed by treatment with a mordant.

In its most preferred form the invention is employed in reconstructive dental work to improve the adhesion between a resin or composite material and dentin. The dentin is cleansed with isotonic formic acid, treated with an isotonic ferric chloride mordant and then treated with a PolySAC which is the reaction product of an oligomeric diglycidyl ether, acrylic acid and lithium orthoaminobenzoate. The resin or composite material is then applied.

U.S. Patent 4,260,660. McCarter, R. J. Use of sulphur as an additive to inhibit the smoldering combustion of materials. 7 April $1981.6 \mathrm{p}$.

Key words: fibrous; polyurethane foam materials; smolder resistant upholstered furniture or mattress assembly; sulphur.

Disclosed is a smolder resistant upholstered furniture or mattress assembly and a method for rendering the composite materials of such assembly smolder resistant. Placement of a layer of sulphur 
immediately adjacent a normally smolder-prone material in the composite effectively prohibits the advances of smoldering combustion to a dangerous stage. The sulphur layer is advantageously applied by backcoating the fabric overlay, coating or impregnating the fibrous or polyurethane foam materials or separating layers of such materials with sulphur-containing films, fabrics and the like. An advantageously effective amount of sulphur is from about $25-50 \mathrm{~g} / \mathrm{m}^{2}$ to about $250-300 \mathrm{~g} / \mathrm{m}^{2}$ in a layer having a depth of about 1 to $3 \mathrm{~mm}$.

U.S. Patent 4,264,423. Negas, T.; Domingues, L. Fluidic thermistor/fugacity device. 28 April 1981.7 p.

Key words: device for measuring the fugacity of a material; E.M.F. measuring circuit; solid electrolyte probe.

A device for measuring the fugacity of a material, without requiring a separate device to measure temperature, is disclosed, wherein the device is a solid electrolyte probe, with the probe having a passageway therein, and metallic conductor leads on the outside of the probe and on the inside of the probe in the passageway. The metallic conductor leads are in contact with an E.M.F. measuring circuit, with the reference fluid being passed through the passageway. The passageway also includes a capillary restriction therein, and measuring devices are provided to measure the pressure drop of the reference fluid when flowing through the capillary, thereby permitting determination of temperature. The temperature determination combined with the E.M.F. measurement permit determination of the fugacity of the material.

U.S. Patent 4,281,246. White, E.; Hertz, H.; Christensen, R. Continuous-flow solution concentrator and liquid chromatograph/mass spectrometer interface and methods for using both. 28 July $1981.7 \mathrm{p}$.

Key words: elongated capillary tube; interface; liquid chromatograph; liquid stream; mass spectrometer; tapered concentrator wire.

An interface between a liquid chromatograph and a mass spectrometer is provided for conducting a liquid stream from the chromatograph to the spectrometer. The stream passes from the chromatograph continuously along a tapered concentrator wire toward the narrow end of the wire. The stream is heated in order to evaporate solvent therefrom and to increase the concentration of the solute therein. When the stream reaches the narrow end of the wire, the vacuum created by the mass spectrometer draws the stream through an elongated capillary tube which has a pointed carrier wire positioned therein. A gap is provided in the tube where the tube and the carrier wire intersect the concentrator wire. The capillary tube is either formed with a reduced diameter at one end adjacent the spectrometer or the carrier wire has a ball of solder at that end in order to partially restrict the flow through the tube. The carrier wire is longitudinally movable within the tube in order to control the flow of material into the spectrometer. Other uses for the tapered and heated wires are contemplated for removing solvent from a liquid stream in order to diminish the stream's flow rate or to increase the concentration of the solute of such a stream. Methods for conducting liquid from a liquid chromatograph to a mass spectrometer and for removing solvent from a liquid stream are also disclosed.

U.S. Patent 4,281,517. Zimmerman, J. E.; Sullivan, D. B. Single stage twin piston cryogenic refrigerator. 4 August $1981.8 \mathrm{p}$.

Key words: cylinder; helium; inner piston; outer piston; regenerator gaps; single stage, twin piston cryogenic refrigerator; two nested, concentric pistons.

A single stage, twin piston cryogenic refrigerator for cooling superconducting devices. The refrigerator uses helium as the heat transfer medium and is constructed with two nested, concentric pistons, an inner piston and an outer piston, mounted in a cylinder and driven $90^{\circ}$ out of phase by a common crankshaft. The inner piston extends through and below the outer piston and is received in a stationary insert mounted in the cylinder. The outer piston has a first piston face which forms a compression space with the stationary insert while the inner piston has a second piston face which forms an expansion space with the cylinder and the insert. The inner piston is formed with upper and lower piston halves joined by a flexible joint positioned in the compression space. Cylindrical regenerator gaps are present at the interface of the inner piston with the stationary insert and at the interface of the stationary insert with the cylinder.

\section{U.S. Patent 4,283,461. Edelman, S. Piezoelectric polymer antifouling} coating. 11 August $1981.6 \mathrm{p}$

Key words: antifouling coating; film; marine structures; piezoelectric polymer material; vegetable and animal life.

An antifouling coating for marine structures in the form of a film containing piezoelectric polymer material, which, when electrically activated vibrates at a selected frequency to present a surface interfacing with water which is inhospitable for attachment of vegetable and animal life including free-swimming organisms thereby discouraging their attachment and their subsequent growth thereon to the macrofoulant adult stage.

U.S. Patent 4,284,551. Argentar, H. Tertiary aromatic amine accelerators derived from para-aminophenethanol. 18 August 1981. $13 \mathrm{p}$.

Key words: accelerators; cementing of bone; high reactivity; low toxicity; peroxide catalyzed polymerization; tertiary aromatic amines; unsaturated polyesters; vinyl monomers.

Tertiary aromatic amines derived from para-aminophenethanol act as accelerators for the peroxide catalyzed polymerization of vinyl monomers, especially methacrylates and acrylates, and for the curing of unsaturated polyesters. The amines are characterized by generally high reactivity and low toxicity, and by their yielding polymers of high strength and low color, and are thereby particularly suited for use in polymerizable or curable formulations employed in the filling and restoration of human teeth, in the preparation of cold-curing denture base materials for fabricating or repairing dentures and in the cementing of bone.

U.S. Patent 4,287,473. Sawyer, D. Nondestructive method for detecting defects in photodetector and solar cell devices. 1 September 1981. 8 p.

Key words: forward-biasing; internal resistance; intrinsic distributed resistance; semiconductor device defects.

The invention described herein is a method for locating semiconductor device defects and for measuring the internal resistance of such devices by making use of the intrinsic distributed resistance nature of the devices. The method provides for forwardbiasing a solar cell or other device while it is scanning with an optical spot. The forward-biasing is achieved with either an illuminator light source or an external current source.

U.S. Patent 4,297,394. Edelman, S. Piezoelectric polymer antifouling coating and method of use and application. 27 October 1981. $5 \mathrm{p}$.

Key words: antifouling coating; film; marine structures; piezoelectric polymer material; vegetable and animal life.

An antifouling coating with method of use and method of application on marine structures in the form of a film containing piezoelectric polymer material, which, when electrically activated vibrates at a selected frequency to present a surface interfacing with water which is inhospitable for attachment of vegetable and animal life including free-swimming organisms thereby discouraging their attachment and their subsequent growth thereon to the macrofoulant adult stage.

U.S. Patent 4,302,345. McCarter, R. J. Flame retarding cellulosic 
materials with sodium or potassium thiocyanate. 24 November 1981. $6 \mathrm{p}$.

Key words: flame and smolder resistance; flame resistant cellulosic insulation material; thiocyanate compounds.

A flame resistant cellulosic insulation material, method of treating loosefill cellulosic material and compositions for imparting flame resistance and flame and smolder resistance to such materials are disclosed utilizing thiocyanate compounds interstitially distributed throughout the cellulosic insulation material as a flame retardant. Sodium and potassium thiocyanate are used in extremely small quantities in insulation materials and impart flame resistance due to the hygroscopic migration of the compounds into the insulation material. The effectiveness of such small quantities of thiocyanate make possible the addition and retention of effective amounts of powdered smolder inhibitors such as boric acid, and sulfur.

U.S. Patent 4,306,913. Mabie, C.; Menis, D. Method for preparing microporous glassy filler grains for dental resin composites. 22 December $1981.36 \mathrm{p}$.

Key words: dental composite resin restorations; finishability; microporous filler; system nontoxicity; $x$-ray opacification.

A microporous filler for dental composite resin restorations has been developed which gives greatly improved finishability, system non-toxicity and $x$-ray opacification. These fillers are prepared from frits obtained by the low temperature calcination of gelled inorganic "polymers" followed by a pulsed high-heat treatment. 


\section{TITLES AND ABSTRACTS OF PAPERS PUBLISHED IN NON-NBS MEDIA}

Reprints from the journals listed in this section may often be obtained from the authors. See page 2 for additional information.

19962. Hebner, R. E., Jr. Kerr effect, electro-optical, Article in Encyclopedia of Physics, R. G. Lerner and G. L. Trigg, eds., p. 483 (Addison-Wesley Publ. Co., Inc., Advanced Book Program, Reading, MA, 1981).

Key words: electric field; electro-optics; Kerr effect; measurement; optics; polarized light.

This paper is an introduction to the electro-optic Kerr effect for students, intelligent laymen, and scientists other than physicists. It contains a brief, phenomenological description of the effect, and typical applications are mentioned.

19963. Escalante, E. Soils and underground corrosion, Proc. 17th Annual Liberty Bell Corrosion Course, Philadelphia, PA, Sept. 19-21, 1979, 23 pages (1979).

Key words: earth resistivity; redox potential; soil corrosivity; soil $\mathrm{pH}$; soil resistivity; underground corrosion.

The effect of the soil environment on underground corrosion is often not given sufficient consideration in engineering design. This may be a consequence of the lack of soil data necessary for a soil corrosivity evaluation.

This paper will deal with measurements for soil characterization, galvanic currents, improper material selection and how these affect the corrosion of metals underground.

19964. Ackerson, B. J.; Hanley, H. J. M. Rayleigh scattering from a methane-ethane mixture, J. Chem. Phys. 73, No. 8, 3568-3572 (Oct. $15,1980)$.

Key words: concentration; diffusivity; methane-ethane; mixture one-fluid model; plait point; Rayleigh light scattering; thermal diffusivity.

The Rayleigh linewidth of a methane (71.07\%)—ethane mixture was measured by dynamic light scattering near the plait point. Results are reported for the concentration diffusivity and (preliminary only) for the thermal diffusivity. It was found that a large background contribution is present in the concentration diffusivity, in agreement with theoretical predictions. The thermal diffusivity is compared to a one-fluid model introduced previously. A critical temperature of $236.36 \pm 0.03 \mathrm{~K}$ and a critical pressure of $6.66 \pm 0.02 \mathrm{MPa}$ was determined for the mixture.

19965. Sieck, L. W. Reactions of $\left(\mathrm{CO}_{2}\right)_{2}{ }^{+}$and $(\mathrm{CO})_{2}{ }^{+}$association ions, Int. J. Chem. Kinet. 10, 335.366 (1978).

Key words: heats of formation; inorganic gases; ion-molecule reactions; mass spectrometry; reaction rates; reactor technology.

The reactions of $\left(\mathrm{CO}_{2}\right)_{2}{ }^{+}$and $(\mathrm{CO})_{2}{ }^{+}$with various additives have been investigated using the NBS high-pressure photoionization mass spectrometer at total pressures of 0.4-1.0 torr of either $\mathrm{CO}_{2}$ or $\mathrm{CO}$. The additives include $\mathrm{CH}_{4}, \mathrm{CD}_{4}, \mathrm{C}_{2} \mathrm{H}_{2}, \mathrm{O}_{2}, \mathrm{H}_{2} \mathrm{O},{ }^{15,14} \mathrm{~N}_{2} \mathrm{O}$, and $\mathrm{CO}$ in both $\mathrm{CO}_{2}$ and ${ }^{13} \mathrm{CO}_{2}$. Second- and third-order rate coefficients based on an ambipolar diffusion model are reported for 25 separate reaction pairs at $295^{\circ} \mathrm{K}$, as well as sequential cationic reaction mechanisms. An approximate value of $225 \pm 3 \mathrm{kcal} / \mathrm{mol}(941 \pm 13 \mathrm{~kJ} / \mathrm{mol})$ was derived for $\Delta H_{f}(\mathrm{CO})_{2}{ }^{+}$based on the kinetics observed in various $\mathrm{CO}$-additive mixtures. Some projections regarding the utility of the data under other conditions are also included.

19966. A yres, T. R.; Linsky, J. L. Outer atmospheres of cool stars. V. IUE observations of Capella: The rotation-activity connection, Astrophys. J. 241, No. 1, 279.299 (Oct. 1, 1980).

Key words: stars, binaries; stars, chromospheres; stars, emission line; stars, late-type; ultraviolet, spectra.

We present and analyze ultraviolet spectra of Capella (G6 III + F9 III) obtained with the International Ultraviolet Explorer. High. dispersion spectra in the $1150-2000 \AA$ region taken at orbital velocity crossing show no evidence for the increasing blueshifts with increasing temperature of formation previously inferred from Copernicus observations. We conclude that there is no discernible stellar wind from either component of the system. High-resolution emission-line profiles taken near the elongation at phase 78 days suggest that virtually all of the emission in transition-region (TR) lines $\left(2 \times 10^{4} \mathrm{~K} \leqslant T \leqslant 2 \times 10^{5} \mathrm{~K}\right)$ and most of the emission in chromospheric lines $\left(T \leqslant 10^{4} \mathrm{~K}\right)$ come from the late.F secondary of the system. This result is contrary to the assumption made in most previous studies that the slightly more massive Capella primary is the dominant ultraviolet emitter. We propose that the origin of the extraordinarily different activity levels on these otherwise very similar stars can be traced to the one property that is obviously different, namely, rotation: the Capella primary is a normal, sharp-lined, slow rotating giant, whereas the secondary has broader lines and is a rapid rotator for a late-type giant $\left(V \sin i \leqslant 30 \mathrm{~km} \mathrm{~s}^{-1}\right)$. Such a chromospheric rotation-activity connection has been demonstrated previously in the $\mathrm{Ca}$ II emission cores, and is very likely a consequence of enhanced surface magnetic fields produced by increased dynamo action in rapidly rotating, convective stars. We extend the rotation-activity connection to stellar transition regions and suggest, on the basis of published soft X-ray observations of a limited sample of stars, that it is valid for coronae as well.

19967. Ben-Reuven, A. Theory of two-atom coherence in gases. II Continuous-wave spectra, Phys. Rev. A 22, No. 6, 2585.2597 (Dec. 1980).

Key words: coherence; cooperative phenomena; spectral line shapes.

General expressions are derived for the spectral line shapes of resonance absorption and scattering of coherent radiation in collision. broadened gases, taking into account effects of coherent excitation of two or more atoms (or molecules), as steady-state solutions of a hierarchy of master equations described in a previous publication (paper I). Coupling between the coherent motions of the atoms, provided by a Bethe-Salpeter-type effective interaction, in the binary. collision approximation, forms the essential mechanism for introducing cooperative coherent effects into the steady-state spectra. Explicit expressions are given for the effects of two-atom coherence in the binary-collision approximation, in which the Bloch-type dressed-atom self-energy superoperator is modified by the presence of collisions in which both atoms retain memory of their coherent propagation before the collision. The self-energies include the effects of resonance exchange symmetrization in self-broadening, and are renormalized by the coincidence of radiative transitions during the collisions. The impact (near-resonance) and the quasistatic (line-wing) limits of the applied-frequency detunings are discussed. In the quasistatic limit, coherent many-atom excitations become irrelevant; however, interactions of both collision partners with the radiation during the collision accounts for such phenomena as collision-induced absorption or radiative collisions. In the impact limit, the inclusion of the Bethe-Salpeter interactions allows for the appearance of two-atom resonances. Magnitude estimates of these effects are discussed. Effects of higher-rank (many-body) coherences are formally discussed with the help of a diagrammatic method, leading into implicit bootstrap equations that can be solved by iterative or other procedures.

19968. Ben-Reuven, A. Theory of two-atom coherence in gases. I. Master equations, Phys. Rev. A 22, No. 6, 2572.2584 (Dec. 1980).

Key words: coherence; cooperative phenomena; spectral line shapes.

The response of a collision-broadened gas sample to driving coherent radiation is studied theoretically, taking into account effects of coherent excitations of two or more atoms (or molecules). In analogy to the Bloch-type master equation for one-atom coherences, describing the motion of a single atom "dressed" by the relevant (incident and detected) field modes, a master equation is derived for two-atom coherences, including an effective interaction of the BetheSalpeter-type, accounting for the mutual interaction of the coherently 
driven pair. The master equation includes also all symmetrization effects owing to resonance exchange between identical atoms, and is limited to nonreactive gas atoms (or molecules) undergoing binary collisions, including otherwise all (internal and translational) relaxation effects. The self-energy kernels are expressed in a nonperturbative fashion, in terms of binary-collision scattering amplitudes, and include renormalization effects due to coincidence of radiative couplings with the collisions (optical and radiative collisions). The concept of two-atom coherences is generalized to higher coherence ranks by constructing a hierarchy of master equations, including vertex operators that upgrade or downgrade the coherence rank, as a prelude to a diagrammatic method for calculating continuous-wave spectra. This hierarchy is compared with prevalent Agarwal-type master equations, based on the Dicke pseudospin method, and used in the study of two-level atoms.

19969. Brayman, H. C.; Fischell, D. R.; Cool, T. A. Spectroscopic studies of the products of reactions of yttrium and scandium atoms with halogen molecules. I. The origin of chemiluminescence, J. Chem. Phys. 73, No. 9, 4247.4259 (Nov. 1, 1980).

Key words: chemiluminescence; Franck-Condon factors; laserinduced fluorescence; radiative lifetimes; scandium; scandium halides; yttrium; yttrium halides.

Observations of laser induced fluorescence from the products of the reactions of yttrium and scandium atoms with halogen molecules have shown that chemiluminescence does not originate from electronically excited metal monohalide molecules, as previously suggested, but instead arises from electronically excited dihalide molecules, $\mathbf{M X}_{2}^{*}$. Production of the metal dihalides appears to require the formation of vibrationally excited metal monohalides, $\mathbf{M X}$, in a precursor reaction. Radiative lifetime measurements for chemiluminescent bands are presented.

19970. Celotta, R. J.; Pierce, D. T.; Wang, G. C.; Bader, S. D. Felcher, G. P. Surface magnetization in Ni(110) as studied by polarized electron scattering, (Proc. Fourth Int. Conf. on Solid Surfaces and the Third European Conf. on Surface Science, Cannes, France, Sept. 22-26, 1980), Supplement a la revue, Le Vide, les Couches Minces, No. 201, pp. 1045-1048 (1980).

Key words: electron scattering; magnetism; nickel; polarized electrons.

A new technique, capable of observing the surface magnetization of ferromagnetic single crystals, is described. Illustrative data is presented, giving the dependence of the magnetization of the $\mathrm{Ni}(110)$ surface on applied field, incident energy, absorbate and coverage.

19971. Davis, R. W.; Deutsch, S. A numerical-experimental study of Parshall flumes, J. Hydraul. Res. 18, No. 2, 135.152 (1980).

Key words: flumes, hydraulic models; hydraulics; numerical models; open-channel flow; Parshall flumes.

Parshall flumes are commonly used devices for the measurement of flow rates in open channels such as sewers and irrigation ditches. The operation of these flumes in "nonstandard" situations, such as when faced with distorted upstream velocity profiles or when placed in sloping channel beds, is poorly understood. This paper presents numerical solutions for the flow through Parshall flumes utilizing a three-dimensional, free-surface finite difference code. The effects on flume operation of channel slope, upstream velocity profile distortions, and flume geometry changes are assessed. Experimental results are obtained for a Parshall flume with a $15.2 \mathrm{~cm}$ (6 in.) throat width. Experimental and numerical data on important features of the flume flowfield are seen to compare well.

19972. Davis, R. W.; Moore, E. F. Numerical modeling of swirling laminar orifice flow, Proc. IMEKO Symp. on Flow Measurement and Control in Industry, Tokyo, Japan, Nov. 13-16, 1979, pp. 81-86 (Society of Instrument Control Engineers, Tokyo, Japan, 1980).

Key words: closed conduit meter; differential pressure meter; flow measurement; laminar flow; meter performance; numerical modeling; orifice meter; swirling flow.

The objective of this study is to investigate the effects of axisymmetric swirl on the performance of concentric orifice meters operating in the laminar flow regime. A computer code, TEACH, developed at Imperial College, London and modified at the National Bureau of Standards is used to predict the performance of a variety of these orifice meters under laminar flow conditions with inlet swirl numbers up to 50 . The parameters investigated are inlet swirl and axial velocity distributions, Reynolds number, and orifice plate thickness and hole diameter. It is seen that increasing swirl number results in decreasing values for the various orifice meter discharge coefficients. At the higher values of swirl number, a vortex breakdown zone forms at the inlet to the computational test section and, at the greatest swirl numbers, extends through the orifice meter itself. Salient features of the flowfield, such as the extent of the recirculation zone behind the orifice plate, are tabulated. In order to demonstrate the accuracy of the program under at least the simplest of circumstances, numerical predictions for swirl decay down a straight pipe are shown to compare well with an analytical solution. It is concluded that swirl effects on orifice meters can be substantial and that an investigation such as this for the more realistic situation of non-axisymmetric swirl at high Reynolds numbers would be very useful.

19973. Evans, D. J.; Hanley, H. J. M. Computer simulation of an $m \cdot 6 \cdot 8$ fluid under shear, Physica (Utrecht) A 103, No. 1.2, 343.353 (Sept. 1980).

Key words: argon; dynamics; $m-6-8$ potential; nonequilibrium molecular; shear; viscosity.

A homogeneous shear nonequilibrium molecular dynamics algorithm has been applied to a system of 108 particles interacting with an $m \cdot 6 \cdot 8$ potential. The viscosity was calculated at three densities corresponding approximately to the triple point, two-thirds melting and critical density. Comparing the results to argon data it appears that three body effects need to be considered at the higher densities. The variation of the viscosity and pressure with shear was noted. The viscosity behavior reinforces previous work with a Lennard-Jones fluid; the pressure behavior has not been reported before.

19974. Evans, D. J.; Hanley, H. J. M. Shear induced phase transitions in single fluids, Phys. Lett. 79A, No. 2-3, 178-180 (Sept. 29, 1980).

Key words: irreversible thermodynamics; Lennard-Jones fluid; nonequilibrium molecular dynamics; phase changes.

Computer simulation indicates that the pressure of a fluid under shear is a function of the strain rate. The phase diagram will be dependent on the shear and, in particular the critical point will be shifted. The effect is shown quantitatively for a Lennard-Jones fluid.

19975. Felcher, G. P.; Bader, S. D.; Celotta, R. J.; Pierce, D. T.; Wang, G. C. Magnetic state at the surface of a ferromagnet by polarized electron diffraction, Proc. Int. Conf., Lake Geneva, WI, May 28-30, 1980, S. K. Sinha, ed., pp. $107-111$ (Elsevier North Holland, Inc., New York, NY, 1980).

Key words: ferromagnetism; $\mathrm{Ni}$; nickel; polarized electrons; surface magnetism.

The results of the first experiment by polarized electron diffraction (PLEED) on a (110) surface of magnetized nickel are presented and discussed. The conclusion is that nickel is magnetically active at the surface; and further, that this new technique might become a powerful analytical tool for the study of surface magnetism.

19976. Giarratano, P. J.; Frederick, N. V. Transient pool boiling of liquid helium using a temperature-controlled heater surface, (Proc. Cryogenic Engineering Conf., Madison, WI, Aug. 21-24, 1979), Paper in Advances in Cryogenic Engineering, K. D. Timmerhaus and H. A. Snyder, eds., 25, $455-466$ (Plenum Press, New York, NY, 1980).

Key words: boiling; carbon film; heat transfer; helium; hysteresis; 
temperature control; transient.

The entire boiling curve of input heat flux versus temperature rise, $q$ vs. $\Delta T$, including transition boiling was obtained for various heating rates of $\mathrm{dT} / \mathrm{dt}$ ranging from 0 (steady state) to approximately 7500 $\mathrm{k} / \mathrm{s}$. This was achieved by using a specially designed electronic temperature controller to linearly vary the temperature versus time of a carbon film heater surface submerged in a liquid helium bath.

The boiling curve slope was positive, monotonic and without hysteresis during steady state $(\mathrm{dT} / \mathrm{dt}=0)$. However, as the heating rate was increased, the transition boiling region (negative slope region) began to emerge and became quite pronounced at a heating rate of $7500 \mathrm{k} / \mathrm{s}$. Also, the peak nucleate boiling heat flux, the minimum film boiling heat flux and the $\Delta T$ 's at which they occurred, all increased with increasing heating rate.

Hysteresis in heat flux during heating and cooling cycles increased with the heating rate and it also increased with increasing dwell time between heating and cooling.

The experimental results during the time to reach the minimum film boiling heat flux are qualitatively explained in terms of the additional heat input, in excess of that required in steady state for the same $\Delta T$, to heat and vaporize a liquid layer adjacent to the heater surface. The hysteresis observations are also qualitatively explained by the proposed model of the heat transfer process.

19977. Fuller, E. G. Giant resonances, Article in Encyclopedia of Physics, R. G. Lerner and G. L. Trigg, eds., pp. 882-884 (AddisonWesley Publ. Co., Inc., Advanced Book Program, Reading, MA, 1981).

Key words: giant resonances; inelastic electron scattering; inelastic hadron scattering; photonuclear reactions; sum rules.

Giant resonances are defined. Various sum rules used to measure their strengths as well as expressions for their energies are presented. Along with a brief summary of experimental systematics for electric dipole and quadrupole resonances, an indication of the problems associated with the determination of giant resonance strengths from experimental data is given. A short bibliography to sources of more detailed information is given.

19978. Fischell, D. R.; Brayman, H. C.; Cool, T. A. Spectroscopic studies of the products of reactions of yttrium and scandium atoms with halogen molecules. II. Laser induced fluorescence from yttrium and scandium monohalides, J. Chem. Phys. 73, No. 9, 4260-4272 (Nov. 1, 1980).

Key words: chemiluminescence; Franck-Condon factors; laserinduced fluorescence; radiative lifetimes; scandium; scandium halides; yttrium; yttrium halides.

Excitation spectra from the monohalides of yttrium and scandium were recorded with the laser induced fluorescence method. Spectroscopic constants and radiative lifetimes were determined for several previously unobserved electronic states. Computer generated spectral simulations were used for the determination of spectroscopic constants and Franck-Condon factors associated with the fluorescence band systems.

19979. Hall, J. T.; Holdeman, L. B.; Soulen, R. J., Jr. Microwave enhancement of superconductivity in aluminum tunnel junctions, Phys. Rev. Lett. 45, No. 12, $1011-1014$ (Sept. 22, 1980).

Key words: aluminum; critical current; energy gap; enhancement; microwaves; nonequilibrium superconductivity; photon-assisted tunneling; superconductivity; thin film; transition temperature; tunneling; tunnel junction.

Microwave radiation $(0.1$ to $12 \mathrm{GHz})$ was propagated in a microstrip transmission line formed by a superconducting $\mathrm{Al}$ film on a $\mathrm{BaF}_{2}$ substrate. Cross strips formed $\mathrm{Al}-\mathrm{Al}$ oxide- $\mathrm{Al}$ tunnel junctions that were used to study the effect of microwaves on the superconducting properties of the Al films. Large increases in the energy gap and transition temperature were observed for frequencies near $3.7 \mathrm{GHz}$. The enhancements were negligible below $1 \mathrm{GHz}$. Anomalous behavior of the features in the tunneling characteristics was observed above $5 \mathrm{GHz}$.

19980. Ditchek, B.; Ruff, A. W. Discussion of the paper "The study of fatigue mechanisms with electron channeling," by $D$. Davidson, (Proc. ASTM Symp., Kansas City, MO, May 22-23, 1978), Discussion in Am. Soc. Test. Mater. Spec. Tech. Publ. 675, 269-275 (1979).

Key words: deformation; electron channeling; fatigue; metals; SEM.

A publication by $\mathbf{D}$. Davidson on fatigue of alloys is reviewed. Comments are made on the selected area electron channeling method applied in this study, along with suggestions for further improvement in the application.

19981. Cahn, J. W. Thermodynamics of metastable equilibria, Proc. Second Rapid Solidification Processing: Principles and Technologies, Reston, VA, Mar. 23-26, 1980, pp. 24-34 (Claitor's Publ. Div., Baton Rouge, LA, Nov. 1980).

Key words: metastable phase equilibrium; partitionless solidification; phase diagrams; phase equilibrium hierarchy; rapid solidification; thermodynamics.

The thermodynamics of metastable phase equilibrium is closely related to that of stable phase equilibrium. Metastable phase equilibrium can often be represented by extensions of the curves on phase diagrams that represent stable phase equilibria. Thermodynamic principles rank phase equilibria but not phases in a hierarchy of increasing stability. Thermodynamics imposes precursor rules in multicomponent systems in which a prior reaction is required before a phase can appear. A given hierarchy persists over a domain on the phase diagram bounded by surfaces on which the ranking of two equilibria changes. Multicomponent phases per se do not form a hierarchy because a given phase can disappear and reappear in a sequence of spontaneous processes. Processing for producing metastable phases must place the system within a domain in which the desired phases can form spontaneously from the available phases.

19982. Carpenter, B. S.; Pilione, L. J. Determination of lithium and lithium-6 in solutions by the nuclear track technique, Anal. Chem. 52, 2452-2454 (1980).

Key words: abundances; alpha particles; cellulose nitrate detector; isotopic; lithium; nuclear track technique; triton particles.

This study demonstrates that the nuclear track technique coupled with absorbers can be used to determine the concentration or isotopic abundance of solutions containing lithium. These measurements are made by registering, in cellulose nitrate detectors, the triton particle produced in the ${ }^{6} \mathrm{Li}(\mathrm{n}, \alpha)^{3} \mathrm{H}$ nuclear reaction. In addition, this study illustrates that the use of the absorbers eliminates interferences from alpha particles produced from other nuclides during the irradiation of the samples.

19983. Sakurai, T.; Culbertson, R. J.; Melmed, A. J. Anomalous field evaporation of silicon, Surf. Sci. 78, L221-L226 (1978).

Key words: anomalous field evaporation; field evaporation; field ion microscopy.

When a sufficiently high electric field is applied to a metal surface, metal atoms desorb orderly as single ions from surface kink sites (field evaporation). We report here an anomaly found in the case of field evaporation of semiconductor emitters. When Si whiskers were fieldevaporated in vacuum at $78 \mathrm{~K}$, it was found that surface $\mathrm{Si}$ atoms evaporate randomly as clusters of $\mathrm{Si}$ atoms instead of single atoms. Field evaporation at room temperature proceeded in the normal fashion.

19984. Mathew, M.; Brown, W. E.; Austin, M.; Negas, T. Lead alkali apatites without hexad anion: The crystal structure of $\mathbf{P b}_{8} \mathbf{K}_{2}\left(\mathrm{PO}_{4}\right)_{6}$, J. Solid State Chem. 35, 69.76 (1980).

Key words: crystal structure; lead apatite; lead potassium apatite; 
substituted apatite.

The crystal structure of $\mathrm{Pb}_{8} \mathrm{~K}_{2}\left(\mathrm{PO}_{4}\right)_{6}$ has been determined by singlecrystal X-ray diffraction. The crystals are hexagonal, space group $P 6_{3} / m$, with $a=9.827(1)$ and $c=7.304$ (1) $\AA$. The structure was refined to a final $R=0.034$ for 551 observed reflections. The structure is that of apatite, $A_{6} B_{4}\left(X_{4}\right)_{6} Y_{2}$ with the hexad anion site $Y$ completely vacant. The $6 h$ sites are occupied by $\mathrm{Pb}$ alone whereas the $4 f$ sites contain equal amounts of $\mathrm{Pb}$ and $\mathrm{K}$. A short $\mathrm{Pb}-\mathrm{O}$ distance of $2.24 \AA$ indicates a covalent bond which may account for the incorporation of lead into bone mineral. The presence of lone pairs of electrons from the lead atoms in the vicinity of the hexad site may explain why this site remains empty.

19985. Leiss, J. E.; Norris, N. J.; Wilson, M. A. The design and performance of a long-pulse high-current linear induction accelerator at the National Bureau of Standards, Part. Accel. 10, 223-234 (1980).

Key words: high current relativistic electron beam generator; linear induction accelerator.

A prototype module of a unique long-pulse, low-cost, high-energy, high-current induction linear accelerator has been designed, constructed and tested at the National Bureau of Standards (NBS). Tests prove that such modules are capable of accelerating several kiloamperes at a voltage gradient of better than $0.25 \mathrm{MeV}$ per meter. Combined with a high-current injector, the prototype module has accelerated a $1 \cdot \mathrm{kA}, 2 \cdot \mu \mathrm{sec}(\mathrm{FWHM})$ electron beam pulse to over 0.8 $\mathrm{MeV}$ with a computed energy spread of less than 3\% (FWHM)

19986. Jacox, M. E. The reaction of $F$ atoms with $\mathrm{O}_{2}$ in an argon matrix, J. Mol. Spectrosc. 84, 74-88 (1980).

Key words: F-atom reactions; infrared spectrum; matrix isolation; normal coordinate analysis; photodissociation.

When $\mathrm{F}$ atoms produced by a microwave discharge through $\mathrm{NF}_{3}$ in argon carrier gas are codeposited with $\mathrm{O}_{2}$ in an argon matrix at $14 \mathrm{~K}$, prominent infrared absorptions-including several overtone and combination bands- of $\mathrm{FO}_{2}$ and of $\mathrm{O}_{2} \mathrm{~F}_{2}$ result. Also present is a peak at $1548 \mathrm{~cm}^{-1}$, contributed either by $\left(\mathrm{O}_{2}\right)_{2}$ or by $\mathrm{O}_{2}$ perturbed by a nearby $F_{2}$ molecule. Upon exposure of the sample to radiation of wavelength longer than $650 \mathrm{~nm}$, this peak disappears, with the concurrent appearance of absorptions assigned to $\mathrm{O}_{2} \mathrm{~F}_{2}$ trapped in a different type of site in the argon lattice. Detailed isotopic substitution studies support the previous vibrational assignments for $\mathrm{FO}_{2}$. The behavior of the OF-stretching overtone absorptions of $\mathrm{O}_{2} \mathrm{~F}_{2}$ suggests that the unobserved OO-stretching fundamental lies in the 1250-1300 $\mathrm{cm}^{-1}$ spectral region, resulting in Fermi resonance interaction between it and the two OF-stretching overtones. The normal coordinate analysis of $\mathrm{O}_{2} \mathrm{~F}_{2}$ is refined to include the vibrational data for ${ }^{16} \mathrm{O}^{18} \mathrm{OF}_{2}$. No discrete electronic absorptions are observed between 220 and 870 $\mathrm{nm}$. However, photodissociation of $\mathrm{FO}_{2}$ is observed with a threshold near $490 \mathrm{~nm}$, and photodissociation of $\mathrm{O}_{2} \mathrm{~F}_{2}$ occurs rapidly at wavelengths near $300 \mathrm{~nm}$.

19987. Hollis, J. M.; Snyder, L. E.; Lovas, F. J.; Ulich, B. L. A radio search for interstellar phosphorus compounds, Astrophys. J. 241, 158 160 (Oct. 1, 1980).

Key words: interstellar; nitric oxide; phosphine; phosphorus nitride; radio astronomy; sulfur dioxide.

The $J=1-0$ and 3-2 transitions of phosphorus nitride, PN, with resolvable hyperfine components at $46.99 \mathrm{GHz}$ and blended components at $140.97 \mathrm{GHz}$, and transitions of phosphine, $\mathrm{PH}_{3}$, at 47.39 and $46.94 \mathrm{GHz}$, arising from a small induced dipole moment, have been searched for but not found in interstellar molecular clouds. The $J=3 / 2-1 / 2, F=3 / 2-3 / 2$ transition of nitric oxide, NO, and the $J_{K-K+}=16_{4,12}-15_{5,11}$ transition of sulfur dioxide, $\mathrm{SO}_{2}$, have been detected in Orion and Sagittarius B2. An unidentified emission line, $\mathrm{U} 140921.8 \mathrm{MHz}$, has been observed in IRC+ 10216 .

19988. Speck, K. M.; Fraker, A. C. Anodic polarization behavior of Ti$\mathrm{Ni}$ and Ti-6A1-4V in simulated physiological solutions, J. Dent. Res. 59, No. 10, 1590.1595 (Oct. 1980).
Key words: Co-Cr-Mo; Co-Ni.Cr-Mo; corrosion; dental alloys; metal-amino acid reactions; stainless steel; titanium.

Anodic polarization measurements made in Hanks' physiological solution at $37^{\circ} \mathrm{C}$ and a $\mathrm{pH}$ of 7.4 show titanium materials to be the most passive of the following metals: titanium, Ti-6Al-4V, Ti-Ni (memory alloy), MP35N (Co-Ni-Cr-Mo), Co-Cr-Mo, 316L' stainless steel, and nickel. The influence of the amino acids, cysteine, and tryptophan on the corrosion behavior of $\mathrm{Ti}-\mathrm{Ni}$ and $\mathrm{Ti}-6 \mathrm{Al}-4 \mathrm{~V}$ was studied. Cysteine caused a lower breakdown potential for $\mathrm{Ti}-\mathrm{Ni}$, but it did not affect the breakdown of Ti-6Al-4V, although an increase in current density for Ti-6Al-4V was observed. Tryptophan produced no significant effects.

19989. Hertz, H. S. Certification of clinical and environmental trace organic RM's, (Proc. Int. Symp. on the Production and Use of Reference Materials, Bundesanstalt für Materialprüfung (BAM), Berlin, Germany, Nov. 13-16, 1979), Paper in Production and Use of Reference Materials, B. F. Schmitt, ed., pp. 393-398 (Bundesanstalt für Materialprüfung, Berlin, Federal Republic of Germany, 1980).

Key words: definitive methods; human serum; polynuclear aromatic hydrocarbons; reference materials; shale oil; SRM; trace organic analysis.

The United States National Bureau of Standards has recently issued its first Standard Reference Material (SRM) for trace organic analysis (an RM for anticonvulsant drugs in serum). During the coming year, three additional trace organic SRMs will be issued: generator columns for producing saturated aqueous solutions of polynuclear aromatic hydrocarbons, a shale oil certified for several toxic organic compounds, and human serum certified for normal clinical analytes. The methods used for the accurate and precise trace organic analyses required for the certifications of these materials form the basis for this paper. The methods of greatest current utility include capillary column gas chromatography, high performance liquid chromatography, and isotope dilution gas chromatography-mass spectrometry.

19990. Uriano, G. A. The certified reference materials program of the United States National Bureau of Standards, (Proc. Int. Symp. on the Production and Use of Reference Materials, Bundesanstalt fur Materialprüfung (BAM), Berlin, Germany, Nov. 13-16, 1979), Paper in Production and Use of Reference Materials, B. F. Schmitt, ed., pp. 7.24 (Bundesanstalt für Materialprüfung, Berlin, Federal Republic of Germany, 1980).

Key words: accuracy; certified reference material; chemical composition; physical property.

The United States National Bureau of Standards (NBS) has been producing and distributing certified reference materials (CRM's) since 1906. NBS currently issues nearly 1,000 different CRM's for use in a variety of measurement applications. Over 38,000 CRM units were distributed by NBS in the last year to approximately 10,000 users throughout the world. Approximately $25 \%$ of the units were distributed outside the United States. NBS CRM's serve four major user categories: (1) industrial research and quality control laboratories, (2) environmental analysis and monitoring laboratories, (3) laboratories performing clinical or health related measurements (including nutrition and toxicity studies) and (4) laboratories engaged in basic metrological research. This paper briefly reviews the past, recent and planned activities of the NBS CRM program. The role of CRM's in helping to establish accuracy and/or compatibility of measurements on a national or international scale is discussed with emphasis on the systems approach to measurement compatibility. Also presented are some of the personal views of the author concerning future opportunities for enhancing international cooperation with regard to the production, certification and use of reference materials.

19991. Hayward, R. W. CPT theorem, Article in Encyclopedia of Physics, R. G. Lerner and G. L. Trigg, eds., pp. 166-168 (AddisonWesley Publ. Co., Inc., Advanced Book Program, Reading, MA, 1981). 
Key words: charge conjugation; CPT theorem; parity; time reversal.

A short encyclopedic article on the foundations of the CPT theorem.

19992. Hayward, E. Photonuclear reactions, Article in Encyclopedia of Physics, R. G. Lerner and G. L. Trigg, eds., pp. 750-751 (AddisonWesley Publ. Co., Inc., Advanced Book Program, Reading, MA, 1981).

Key words: giant resonance; photonuclear; quasideuteron; resonance fluorescence.

The general features of photonuclear reactions are described for light and heavy nuclei, in the energy region up to $140 \mathrm{MeV}$.

19993. Maximon, L. C. Bremsstrahlung, Article in Encyclopedia of Physics, R. G. Lerner and G. L. Trigg, eds., pp. 89-91 (AddisonWesley Publ. Co., Inc., Advanced Book Program, Reading, MA, 1981).

Key words: astrophysics; bremsstrahlung; electromagnetic interactions; pair production; plasma diagnostics; radiation; synchrotron radiation.

We present an introductory survey of the subject of bremsstrahlung, relating it to other quantum electrodynamical processes. Its significance, both as a fundamental process in the framework of the theory and application of quantum mechanics and as a process which is central to a number of branches of physics (nuclear, plasma, and astrophysics, among others) is stressed.

19994. Jordan, T. H.; Dickens, B.; Schroeder, L. W.; Brown, W. E. Crystal structure of tritin (II) hydroxide oxide phosphate, $\mathrm{Sn}_{3} \mathrm{O}(\mathrm{OH}) \mathrm{PO}_{4}$, Inorg. Chem. 19, No. 9, 2551.2556 (Sept. 1980).

Key words: crystal structure; hydrogen bonding; hydroxyapatite; tin hydroxide oxide phosphate; tin phosphate; topical fluoride treatments.

$\mathrm{Sn}_{3} \mathrm{O}(\mathrm{OH}) \mathrm{PO}_{4}$ crystallizes in the monoclinic cell $a=4.8851$ (7) $\AA$ $b=11.465$ (2) $\AA, c=12.362$ (2) $\AA$, and $\beta=105.81$ (1) at $25^{\circ} \mathrm{C}$ with space group $P_{1} / c$ and $Z=4$. The structure has been solved and refined to $R_{\mathrm{w}}=0.047$ and $R=0.055$ by using 1938 observed X-ray diffraction data collected from a single crystal by a counter method $(\theta-2 \theta$ scans). Corrections for absorption and secondary extinction were not applied. The structure crystallizes in (010) layers. A lone pair of electrons on each $\mathrm{Sn}^{11}$ atom is directed into the interlayer space. Each $\mathrm{Sn}$ atom is coordinated to three oxygen atoms at $\sim 2.1 \AA$ and to other more distant ones at 2.5-3.3 $\AA$, with all the coordinated oxygen atoms in the same hemisphere about $\mathrm{Sn}$. The hydrogen bond in the structure appears to be between the hydroxide and oxide ions, forming an $(\mathrm{O}-\mathrm{H}$...O) grouping with $\mathrm{O} . . \mathrm{O}=2.602(9) \AA$.

19995. Jones, P. L.; Mead, R. D.; Kohler, B. E.; Rosner, S. D.; Lineberger, W. C. Photodetachment spectroscopy of $\mathbf{C}_{2}^{-}$ autodetaching resonances, $J$. Chem. Phys. 73, No. 9, $4419-4432$ (Nov. 1, 1980).

Key words: autoionization; electron affinity; molecular spectroscopic constants; negative ions; photodetachment; potential energy curves.

The cross section for photodetachment of $\mathrm{C}_{2}^{-}$is investigated in the photon energy range $14000-20000 \mathrm{~cm}^{-1}(1.75-2.5 \mathrm{eV})$. Sharp resonances due to autodetachment are observed at photon energies corresponding to transitions between high vibrational levels of the $\mathrm{C}_{2}^{-}$ $X^{2} \Sigma_{g}^{+}$state and high vibrational levels of the $C_{2}^{-} B{ }^{2} \Sigma_{u}^{+}$states. The resonances are narrower than $6 \mathrm{GHz}$, and those arising from the $v=5$ level of the $B$ state are about $1 / 10$ as strong as those arising from the $v=6$ and higher levels. In addition, the signal from the allowed direct photodetachment of $\mathrm{C}_{2}^{-} X$ state, which would produce a smooth background cross section, is not observed, indicating that it is less than the $10^{3}$ peak to background contrast ratio. A rotational analysis of the nine bands of the $\mathrm{C}_{2}^{-} B-X$ transition observed in this study, coupled with previous measurements provides a new, more precise set of spectroscopic constants for these states. These constants are then used to generate RKR potential curves for the $B$ and $X$ states of $\mathrm{C}_{2}^{-}$. Based on all $C_{2}^{-}$data, the only consistent interpretation of the observations is that the electron affinity of $\mathrm{C}_{2}$ is bounded by $3.374 \mathrm{eV}$ $\leqslant E A\left(\mathrm{C}_{2}\right) \leqslant 3.408 \mathrm{eV}$. The autodetachment rate into $\left(\mathrm{C}_{2} a^{3} \Pi_{u}+e\right)$ is deduced to be much faster than the rate into $\left(\mathrm{C}_{2} X^{1} \Sigma_{8}^{+}+e\right)$, probably as a result of strongly $R$ dependent configuration interaction in the $\mathrm{C}_{2}$ $B$ state. The weakness in the direct photodetachment process is attributed to poor vibrational overlap between the initial and final states.

19996. Celotta, R. J.; Pierce, D. T. Sources of polarized electrons, $A d v$. At. Mol. Phys. 16, 101-157 (1980).

Key words: electron gun; electron polarization; electron spin; polarized electrons; spin; spin polarization.

Sources of spin polarized electron beams are described and compared. Included are discussions of the basis of the polarizing effect, the operating characteristics, features, and applicability of each source. A discussion of relevant electron optical theory and a table comparing available sources are also presented.

19997. Yates, J. T., Jr.; Cavanagh, R. R. Vibrational spectroscopic studies of chemisorbed species on metal surfaces, (Proc. Fourth Int. Conf. on Solid Surfaces and the Third European Conf. on Surface Science, Cannes, France, Sept. 22-26, 1980), Supplement a la revue, Le Vide, les Couches Minces, No. 201, II, $750-753$ (1980).

Key words: carbon monoxide; chemisorption; isocyanide; rhodium; vibrational spectroscopy.

The use of transmission infrared spectroscopy to investigate the chemisorption of $\mathrm{CO}$ and an electronic analog to $\mathrm{CO}$ is described.

19998. Harris, J. R.; Fenves, S. J.; Wright, R. N. New tools for standards writers, ASTM Stand. News 8, $10-16$ (July 1980).

Key words: building standards; classification; decision tables; information networks; network; standards; standards-writers; systems analysis.

This paper describes rational methods intended to assist standards developers in the formulation and expression of standards. The methods provide ways to measure clarity, completeness, and consistency in the organization and content of a standard. Both guides to the organization of the text of a standard and a formal representation that can assist standards writers in determining whether their intent is correctly expressed are included. For illustrative purposes, the paper draws on a recent major application of the analysis to the Applied Technology Council's Tentative Provisions for the Development of Seismic Regulations for Buildings, and describes the experience gained through the interaction between the analysts and the team that developed the seismic provisions. Analysis of this interaction forms the basis for guidelines for the future role of analysts within the standards-writing groups.

19999. Radebaugh, R.; Fujii, G.; Read, D. T.; Clark, A. F. A standards program for ac losses in superconductors, (Proc. XV Int. Congress of Refrigeration, Venice, Italy, Sept. 23.29, 1979), Session A 1/2, Paper 10 in Progress in Refrigeration Science and Technology, 4 pages (International Institute of Refrigeration, Paris, France, 1980).

Key words: ac losses; calorimeter; cryogenics; multipliers; standards; superconductors.

The final goal of the program is to establish standard measurement practices and a standard reference material for calibration of apparatus. In this paper we discuss a comparison of several techniques for measuring ac losses in superconductors. The techniques are divided into either a calorimetric or electronic type. We have designed and are testing a sensitive calorimeter made of fiberglass. epoxy with vacuum-insulated walls. The present calorimeter is also 
designed to operate with a vacuum around the superconducting sample. In this mode the temperature rise gives the ac loss. Some of the electronic techniques which are being compared with each other and the calorimetric technique include analog and digital multipliers and a lock-in amplifier. Preliminary results show good agreement.

20000. Howard, C. P. Some fundamentals of desuperheaters, Proc. 1980 Conf. on Waste Heat Recovery for Energy Conservation, West Lafayette, IN, Sept. 15-17, 1980, 3 pages (Purdue University, West Lafayette, IN, 1981).

Key words: desuperheating heat exchangers; heat exchange design; heat recovery units.

A methodology of heat exchanger design for desuperheating heat exchangers (heat recovery units) is developed to utilize heat transfer and pressure loss characteristics for surfaces in counterflow arrangements with a non-condensing refrigerant and water as the working fluids. The analysis should provide a basis for testing noncondensing heat recovery units.

20001. Simon, T.; Linsky, J. L. IUE ultraviolet spectra and chromospheric models of HR 1099 and UX Arietis, Astrophys. J. 241, No. 2, $759-773$ (Oct. 15, 1980).

Key words: binary stars; late-type stars; stars, individual; stellar chromospheres; stellar coronae; ultraviolet spectra.

We present and analyze IUE spectra (1150-3200 $)$ of the RS CVntype systems HR 1099 and UX Ari obtained at three quiescent phases each. The emission line fluxes vary between exposures, but we find no apparent correlations with orbital phase and no enhancements during two radio flares. High-resolution $\mathrm{Mg}$ II spectra suggest, at least for UX Ari, that the strong emission in these lines originates with the $\mathrm{K}$ star rather than the $G$ star in these systems. Assuming that all of the emission originates with the $\mathrm{K}$ stars, surface fluxes are about 25 times those of the quiet Sun in the chromospheric lines ( $\mathrm{Mg}$ II, Si II) and 75-250 times those of the quiet Sun in the transition region lines $(T>20,000 \mathrm{~K})$. We have computed plane-parallel chromospheric models, extending from the temperature minimum to $40,000 \mathrm{~K}$, and synthesized line fluxes for $\mathrm{Mg}$ II, Si II, Si III, and $\mathrm{C}$ II for comparison with the observations. Using four atmospheric temperature structures and several different transition-region pressures, $P_{\text {TOP }}$, we conclude that $P_{\text {TOP }}$ probably lies in the range $0.18-1.0$ dynes $\mathrm{cm}^{-2}$. These pressures are consistent with the value of about 0.5 dynes $\mathrm{cm}^{-2}$ derived from the density diagnostic line ratios $I(\mathrm{C}$ III $\lambda 1909) / I(\mathrm{C}$ III $\lambda 1175)$ and $I(\mathrm{C}$ III $\lambda 1909) / I(\mathrm{Si}$ IV $\lambda$ 1403), and imply transition regions that are more extended than for the Sun, by a factor proportional to the pressure scale height, and not conductively heated. We point out that for close binary stars, the central reversals of the Ca II and $\mathrm{Mg}$ II resonance lines are probably not good diagnostics of $P_{\text {TOp. If coronal }}$ base pressures are the same as the transition region pressures we have derived, the Rosner-Tucker-Vaiana scaling law implies quiescent coronal loop dimensions $\sim 5-25 R_{0}$ for the $\mathrm{K}$ stars in these systems.

20002. Wyart, J. F.; Kaufman, V.; Sugar, J. Analysis of the spectrum of four-times-ionized uranium (UV), Phys. Scr. 22, 389-396 (1980).

Key words: energy levels; parametric calculations; uranium; wavelengths.

Wavelengths for 261 lines of four-times-ionized uranium between $443 \AA$ and $2325 \AA$ are reported. They are classified by 74 energy levels, most of which are attributed to the $6 p^{6} 5 f^{2}, 6 p^{6} 5 f 6 d, 6 p^{6} 5 f 7 s$, $6 p^{6} 6 d^{2}, 6 p^{6} 5 f 7 p$ and $6 p^{5} 5 f^{2}$ configurations. Energy parameters are derived for the two-electron configurations.

20003. Seltzer, S. M. X-ray source characteristics and detection efficiencies of prototype lixiscopes, (Proc. Lixiscope Conf., Greenbelt, MD, July 27.28, 1978), NASA Conf. Publ. 2112, pp. 11. 14 (NASA-Goddard Space Flight Center, Greenbelt, MD, July 1978).

Key words: CsI; detection efficiency; lixiscope; radioactive $\mathrm{x}$-ray source; rare-earth scintillator screen.

Important components used in the prototype Lixiscope (low- intensity $x$-ray-imaging scope) are the radioactive $x$-ray source and the scintillator screen. Calculated data are given pertinent to the spectra and intensity of $\mathrm{x}$-rays emitted by the commercially available ${ }^{125} \mathrm{I}$ and ${ }^{153} \mathrm{Gd}$ finite, encapsulated sources used. Detection efficiencies for a rare-earth and a CsI scintillator screen are compared.

20004. Baughcum, S. L.; Leone, S. R. Photofragmentation infrared emission studies of vibrationally excited free radicals $\mathbf{C H}_{3}$ and $\mathrm{CH}_{2} \mathrm{I}, \mathrm{J}$. Chem. Phys. 72, No. 12, 6531-6545 (June 15, 1980).

Key words: $\mathrm{CH}_{3} ; \mathrm{CH}_{2} \mathrm{I}$; infrared emission; laser; photofragmentation; radical.

Time and wavelength-resolved infrared fluorescence techniques are used to study the photofragmentation dynamics of $\mathrm{CH}_{2} \mathrm{I}_{2}$ and $\mathrm{CH}_{3} \mathrm{I}$ at the excimer laser wavelengths of 248 and $308 \mathrm{~nm}$. Emission is detected from vibrationally excited $\mathrm{CH}_{2} \mathrm{I}$ and $\mathrm{CH}_{3}$ radicals as well as from the excited iodine atoms $\left[I^{*}\left({ }^{2} P_{1 / 2} \rightarrow{ }^{2} P_{3 / 2}\right)\right]$ produced in the photolysis. A complete infrared fluorescence spectrum of the highly excited $\mathrm{CH}_{2} \mathrm{I}$ radical is obtained as a function of time after the $248 \mathrm{~nm}$ dissociating laser pulse, providing both spectroscopic and vibrational deactivation data for the radical. Significant $\mathrm{CH}_{2} \mathrm{I}$ emission is observed at all wavelengths, indicating that excitation occurs into a very high density of states, nearing the vibrational quasicontinuum. Stronger emission features are observed in the region of the $\mathrm{C}-\mathrm{H}$ stretching vibrations, the $\mathrm{CH}_{2}$ bending motion, and a combination band of these two modes. Deactivation rates for various spectral features of the highly excited $\mathrm{CH}_{2} \mathrm{I}$ radical with $\mathrm{CH}_{2} \mathrm{I}_{2}$ and argon are presented, along with a discussion concerning the relaxation of the highly excited radical spectrum. In the photolysis of $\mathrm{CH}_{3} \mathrm{I}$ at $248 \mathrm{~nm}$, infrared fluorescence is observed directly from the out-of-plane bend of the $\mathrm{CH}_{3}$ radical. In addition, at $248 \mathrm{~nm}$, the quantum yield $\left(\Phi_{1^{*}}\right)$ for $I^{*}\left({ }^{2} P_{1 / 2}\right)$ production is measured to be $0.81 \pm 0.03$ for $\mathrm{CH}_{3} \mathrm{I}$ and $0.46 \pm 0.04$ for $\mathrm{CH}_{2} \mathrm{I}_{2}$, while dissociation of $\mathrm{CH}_{2} \mathrm{I}_{2}$ at $308 \mathrm{~nm}$ yields $\Phi_{1^{*}}=0.25 \pm 0.02$. The $\mathrm{I}^{*}$ quenching rates with $\mathrm{CH}_{3} \mathrm{I}$ and $\mathrm{CH}_{2} \mathrm{I}_{2}$ are measured to be $(2.5 \pm 0.4) 10^{-13}$ and $(3.6 \pm 0.3) 10^{-13} \mathrm{~cm}^{3}$ molecule $\mathrm{sec}^{-1}$, respectively.

20005. Schäfer, L.; Sellers, H. L.; Lovas, F. J.; Suenram, R. D. Theory versus experiment: The case of glycine, J. Am. Chem. Soc. 102, 6566-6568 (1980).

Key words: ab initio calculations; amino acid; conformational energy; dipole moment; gas phase conformers; glycine; rotational constants; structure.

A comparison of theoretically calculated structures with experimentally determined rotational constants has led to excellent agreement of theory with experiment on the structures of two low energy conformers of gas phase glycine. The results have resolved an earlier apparent contradiction as to which conformer was the lowest energy in the gas phase. The lowest energy conformer is one which has bifurcated hydrogen bonds of the amine hydrogen to the carbonyl oxygen and a hydrogen bond between the hydroxyl hydrogen and the carbonyl oxygen. A second conformer which is $\sim 490 \mathrm{~cm}^{-1}$ higher in energy has a ring-like structure with the hydroxyl hydrogen forming a hydrogen bond to the lone pair of electrons on the amine nitrogen.

20006. Dumin, D. J. A low cost approach to modernize undergraduate electronic laboratory instruction, Proc. Third Biennial University/Industry/Government Microelectronics Symp., Lubbock, TX, May 21-23, 1979, pp. 168-170 (The Institute of Electrical and Electronics Engineers, Inc., New York, NY, May 1979).

Key words: electronic laboratory instruction; low cost approach.

A program for development of a low cost approach for modernizing microelectronic undergraduate education is presented. The plan is based on the use of integrated circuit test wafers as the instructional tools to be used in the laboratory portion of microelectronic courses. The use of integrated devices and circuits at the wafer probe level would assist students in attaining a feel for the size and limitations of modern microelectronic devices and would act as a physical basis for electronics instruction. The approach attempts to minimize the total cost of this program at the university level and, thus, should help assure that this program is adopted by a large 
number of colleges and universities. This approach should also be attractive to small universities and minority universities where equipment budgets have been traditionally low. This approach should also find extensive use in continuing education of electrical engineers.

20007. Rabolt, J. F.; Block, S.; Piermarini, G. J. The characterization of polymers under high pressure using Raman spectroscopy, (Proc. Sixth AIRAPT Conf. on High-Pressure Science and Technology, Boulder, CO, July 25.29, 1977), Paper in Physical Properties and Material Synthesis, K. D. Timmerhaus and M. S. Barber, eds., 1, 478.481 (Plenum Press, New York, NY, 1979).

Key words: characterization of polymers; polymers under high pressure; Raman spectroscopy.

20008. Girvin, S. M.; Penn, D. R. Theory of coherence effects in photoinduced Auger events: Resonant satellites in metals with filled $d$ bands, Phys. Rev. B 22, No. 8, $4081-4084$ (Oct. 15, 1980).

Key words: auger; core hole spectroscopy; metals with filled $d$-bands; photoemission; resonance; satellite.

This paper describes a theory of the Auger effect which treats the entire process of core-level excitation followed by decay of the core hole as a coherent sequence of events. We present an approximate analytic solution to the model recently introduced and solved numerically by Davis and Feldkamp. Their model offers an explanation of the resonance behavior of photoemission satellites in metals with filled $d$ bands. Our analytic results help clarify the physics of the resonant satellite phenomena.

20009. McClendon, L. T. A summary of oil shale activities at the National Bureau of Standards 1975-1979, EPA-600/7-80-125, 143 pages (United States Environmental Protection Agency, Office of Environmental Engineering and Technology, Washington, DC June 1980). (Available by purchase from the National Technical Information Service, Springfield, VA 22161.)

Key words: measurement methods; oil shale; standard reference materials; water pollution control.

This report provides a summary of NBS Oil Shale activities covering the period 1975 to 1979 . At the start of this period a Workshop on Standard Reference Materials (SRM's) needed for Oil Shale Processing was held at NBS and served to provide the priority guidance for the future of this program. A summary of the recommendations of that Workshop, the manuscripts presented during the Workshop, and the list of attendees is included in this report. The status of the Oil Shale Research at NBS is also presented consisting of developmental work on the feasibility of producing an Oil Shale and a Shale Oil Standard Reference Materials characterized for both trace inorganic and trace organic constituents. Additionally, information is given dealing with the development of measurement methods appropriate for Oil Shale and Shale Oil trace inorganic and trace organic analysis. Several papers are also included giving additional details on these matters. Other NBS Standard Reference Materials, which may be appropriate for the use by the Oil Shale community, are described briefly within this document. Finally, recommendations for future Oil Shale projects dealing with the development of measurement methods and Standard Reference Materials at NBS are presented.

20010. Hutchinson, J. M. R. Traceability, quality assurance, and standards, (Proc. Low-level Measurement Techniques Group Int. Committee for Radionuclide Metrology, Braunschweig, West Germany, June 18-19, 1979), Paper in Environment International 3, No. 5, 363.364 (Pergamon Press Limited, Oxford OX3, OBW, England, 1980).

Key words: environment; metrology; quality control; radioactivity; traceability.

The development of traceability in low-level radioactivity measurements is discussed. The role that the development of large quantities of natural matrix standards can play is also discussed.

20011. Menis, O.; Mackey, J. A.; Garn, P. D. A study of particulates of environmental interest by differential thermal analysis, Proc. Angewandte Chemische Thermodynamik und Thermoanalytik, Vortrage des Rapperswiler TA-Symp. (Experientia Supplementum; 37), Rapperswiler, Switzerland, Apr. 18-20, 1979, pp. $321-332$ (Birkhauser Verlag Basel, Switzerland, 1979).

Key words: alpha-quartz particulate; chrysotile asbestos particulate; DTA analysis of environmental particulates; DTA apparatus for rapid sample change and steam pressure control.

In this presentation we describe our current progress using thermoanalytical methods to study particulates of interest in environmental problems. The topics included are: (1) differential thermal analysis (DTA) of current NBS Standard Reference Material (SRM) 1645, River Sediment and NBS SRM 1648, Urban Particulate Matter; (2) studies of a suitable crystalline silica (ci-quartz and cristobalite) as a potential SRM; (3) preparation of mixtures of chrysotile asbestos and quartz in a suitable matrix, and (4) a modified DTA apparatus including a design of a DTA cell for rapid sample changing and operation under regulated steam pressure.

20012. Pierce, D. T.; Celotta, R. J.; Wang, G. C. Spin polarized electron scattering studies of W(100) (Extended Abstract), (Proc. Fourth Int. Conf. on Solid Surfaces and the Third European Conf on Surface Science, Cannes, France, Sept. 22-26, 1980), Supplement a la revue, Le Vide, les Couches Minces, No. 201, II, 1060-1063 (1980).

Key words: polarized LEED; spin detector; spin polarization; surface resonance; surface structure.

20013. Klein, R.; Fine, J. Desorption of cesium from molybdenum(111) Positive ion and neutral lifetime measurements (Extended Abstract), (Proc. Fourth Int. Conf. on Solid Surfaces and the Third European Conf. on Surface Science, Cannes, France, Sept. 22-26, 1980), Supplement a la revue, Le Vide, les Couches Minces, No. 201, II, 361-364 (1980).

Key words: cesium from molybdenum(111); ion and neutral lifetime; positive ion and neutral lifetime.

20014. Peterlin, A. Acoustic emission of polymers under tensile load, Chapter 2 in Advances in Chemistry Series No. 174, Probing Polymer Structures, J. L. Koenig, ed., pp. $15-23$ (American Chemical Society, Washington, DC, 1979).

Key words: acoustic emission of polymers; emission of polymers; polymers under tensile load.

The observation of acoustic emission from a crazing polymer depends very much on the plastic deformability of the polymer. The crazes in ductile materials propagate so smoothly by relatively slow plastic deformation at the tip of the craze that the acoustical signal is not strong enough to be unambiguously separated from the high noise level. It is only in very brittle material that the jumpwise craze propagation yields acoustic bursts strong enough to be recorded easily. Such materials are polyvinyltoluene with a strain-to-break of less than 1\%, low-molecular-weight polystyrene, and aged poly(methylmethacrylate). For brittle composite material it is better if the components differ greatly in their mechanical properties.

20015. Prosen, E. J. Microcalorimetry and its applications, (Proc. Symp. on Biological Effects, Imaging Techniques, and Dosimetry of Ionizing Radiations, Rockville, MD, June 6.8, 1979), Paper in HHS Publication (FDA) 80-8126, Radiological Health, pp. 147-153 (Department of Health and Human Services, Public Health Services, Food and Drug Administration, Washington, DC, 1980).

Key words: calorimetry; cardiac pacemakers; clinical chemistry; heat; metabolism; microcalorimetry; radiation.

The technique of microcalorimetry is illustrated briefly by the use of the NBS microcalorimeter for clinical analysis of substrates and enzymes in human serum, the identification of bacteria, the determination of heats of metabolism of living sea urchin eggs, and the determination of self-discharge of cardiac pacemaker batteries. A 
description is given of a special microcalorimeter-respirometer apparatus for the determination of the heat of metabolism per mole of oxygen utilized by living animal or cultured cells and its use in the study of stresses (such as ionizing and non-ionizing radiation, chemicals or drugs, and other stresses) on such living cells. Such measurements give an overall indication of the "health" or viability of such cell systems before and after the stress and perhaps will show recovery from stresses.

20016. Hsu, S. M.; Becker, D. A. Status report on the characterization of re-refined lubricating base oils, (Proc. Conf. on Fuels and Lubricants, Baltimore, MD, Oct. 20.23, 1980), Paper in $S A E$ Technical Paper Series No. 801382, pp. 1.10 (Society of Automotive Engineers, Inc., Warrendale, PA, 1980).

Key words: basestock; engine lubricants; lubricating oil; motor oil; petroleum oil; recycled oil; rerefined oil; test procedures.

The National Bureau of Standards (NBS) has been working on the development of test procedures for recycled petroleum oils since 1976. The first phase involved development and evaluation of tests for characterizing used oil recycled as burner fuel, and has been completed. The second phase, on re-refined motor oil, involves development of a set of test procedures capable of monitoring the quality, consistency, and additive response of re-refined lubricating oil basestocks.

Tests under investigation include physical property tests, chemical property tests, hydrocarbon type characterization methods, and bench scale performance tests primarily directed toward the measurement of additive response. Cooperative efforts include the ASTM/NBS Basestock Consistency Study (involving both re-refined and virgin lubricating oil basestocks), a study of engine deposits with the U.S Army Fuel and Lubricants Research Laboratory, and micro-oxidation test research at Pennsylvania State University. This paper describes the current status of this program, and includes representative data obtained on re-refined oils.

20017. Sindt, C. F. A system for inflating an air launched balloon, (Proc. 10th Air Force Geophysics Lab. Scientific Balloon Symp., Portsmouth, NH, Aug. 21.23, 1978), Report No. AFGL-TR-79-0053, C. L. Rice, ed., pp. 149-159 (National Technical Information Service, Springfield, VA, Mar. 1979).

Key words: balloon inflation; cryogenic helium balloon inflation; heat transfer; liquid helium; packed bed heat exchanger.

This paper describes a technique for inflating a balloon with helium as the balloon, its payload and the inflation system descend on a parachute. The helium used for inflation is stored in the liquid state and subsequently converted to gas using a hot bed heat exchanger. Two systems using this technique were tested. The first used a commercial liquid helium dewar and a heavy heat exchanger which was designed to prove the concept and not intended for flight. The second system which was to be taken to the launch altitude by a carrier balloon, provided $48.5 \mathrm{~kg}$ of helium gas at an average temperature of $248^{\circ} \mathrm{K}$ in five minutes. This system which weighed 363 $\mathrm{kg}$, including helium, was self contained and required a single electrical signal for activation.

20018. Sindt, C. F.; Ludtke, P. R. Thermal cycle tests of a modeled superconducting transmission line, (Proc. 1979 Cryogenic Engineering Conf., Madison, WI, Aug. 21.24, 1979), Paper in Advances in Cryogenic Engineering, K. D. Timmerhaus and H. A. Snyder, eds., 25, 69.80 (Plenum Press, New York, NY, 1980).

Key words: cable end fittings; pressure cycles; stress; superconducting power transmission line; terminals; thermal contraction.

The thermoelastic behavior of two sections of a model of a superconducting transmission cable was investigated by subjecting them to repeated thermal and pressure cycles between ambient and operating conditions. To simulate the installed condition of fixed ends, the 3.5 meter long cable section was held at a constant length.

A second test objective was to evaluate the end clamp assembly design. Two of these clamps were subjected to mechanical loads, to thermal cycles and assembly cycles and they maintained a gas tight seal to the lead gas jacket of the cable throughout the tests. This successful end clamp and the installation of the clamp are described.

Test results show tensile loads to $10000 \mathrm{~N}$ and compressive loads to $3000 \mathrm{~N}$ develop in the cable during a cycle where it is cooled to $77 \mathrm{~K}$ and pressurized to $1.5 \mathrm{MPa}$. Also photographs show several cable deformities which resulted during the test of the first cable; section. The test procedure for the second cable section was modified to more nearly represent the expected field conditions and this cable section showed no significant deterioration. Also, neither the cable section nor the end clamps leaked after 20 thermal and pressure cycles.

20019. Simiu, E.; Filliben, J. J. Weibull distributions and extreme wind speeds, Journal of the Structural Division 106, No. ST12, 2365-2374 (American Society of Civil Engineers, New York, NY, Dec. 1980).

Key words: climatology; loads (forces); probability distribution functions; reliability; statistical analysis; structural engineering; wind

An investigation is presented into the question as to whether or not it may be assumed that the extreme wind population at various stations in the U.S. is described by probabilistic models with shorter tails than the Type I distribution. Statistical evidence, based on the comparative analysis of extreme wind data and of data generated by Monte Carlo simulation, appears to support this assumption at a large number of stations in the United States.

20020. Radebaugh, R. Analysis of regenerator inefficiency for Stirlingcycle refrigerators with plastic displacers, (Proc. XV Int. Congress of Refrigeration, Venice, Italy, Sept. 23.29, 1979), Session A1/2, Paper 16 in Progress in Refrigeration Science and Technology, 6 pages (International Institute of Refrigeration, Paris, France, 1980).

Key words: calculations; cryogenics; helium; plastics; refrigerators; regenerators; Stirling-cycle.

The major source of heat flow in a Stirling-cycle refrigerator operating below about $15 \mathrm{~K}$ is due to regenerator inefficiency. All previous experiments and analyses of such inefficiencies have been for the case of negligible thermal resistance internal to the regenerator material. In the application of cooling a SQUID magnetometer, the low temperature parts of the refrigerator are made of plastic. In that case the internal thermal resistance is the dominant resistance. This paper analyzes the heat flow due to regenerator inefficiency when the materials have low thermal diffusivity. In such a case the heat flow is approximately proportional to $\dot{n}^{3 / 2} \mathbf{P}^{2}$, where $n$ is the speed and $P$ is the pressure of the helium gas. Since the refrigeration rate is proportional to $\dot{n} P$, a reduction in speed and pressure reduces the minimum temperature, until shuttle heat flow, proportional to $\dot{\mathbf{n}}^{1 / 2}$, and conduction heat flow become important. Comparison with experimental data at temperatures as low as $3 \mathrm{~K}$ will be given.

20021. Shier, D. R. On algorithms for finding the $\boldsymbol{k}$ shortest paths in a network, Networks 9, 195-214 (John Wiley \& Sons, Inc., Somerset, NJ, 1979).

Key words: algorithm; graph; $k$ shortest paths; network algorithms; shortest path; transportation networks.

This paper presents, within a unified framework, several new algorithms for computing $k$ shortest paths in a network. These algorithms utilize strategies which have proved to be efficient in solving shortest path problems. In addition, a computational study was conducted to assess the effects of the different "arc processing" orders which are characteristic of each algorithm. Testing was performed using generated classes of moderately large grid, complete and random networks. Two particular algorithms emerge as the most promising among those evaluated.

20022. Shier, D. R.; Witzgall, C. Arc tolerances in shortest path and network flow problems, Networks 10, 277.291 (John Wiley \& Sons, Inc., Somerset, NJ, 1980).

Key words: cutset; cycle; directed graph; network; network flow; 
optimization; retraction; robustness; rooted tree; sensitivity analysis; shortest path; tolerance.

This paper studies one aspect of the "robustness" of optimal solutions to shortest path and, more generally, network flow problems. Specifically, we characterize the maximum increase and the maximum decrease in an arc's cost that can be tolerated without changing optimality of the current solution. Calculation of these quantities is quite simple for nonbasic arcs, and somewhat more involved for basic arcs. When such tolerances are to be determined simultaneously for all arcs in the network, considerable duplication of effort can be avoided through the use of specialized algorithms. Several algorithms for calculating all arc tolerances are presented, one of which is shown to have complexity order $n^{2}$ for general networks with $n$ nodes.

20023. Goldman, D. T. Comments on the paper "NBS and Metrication," by A. A. Bartlett, Phys. Today Lett. pp. 69.72 (Dec. 1980).

Key words: AAPT vs. NBS; kilogram; mass/weight; metric controversy; newton.

The American Association of Physics Teachers Metrication Committee, under the Chairmanship of Professor Albert A. Bartlett of the University of Colorado, outlines a list of charges accusing the National Bureau of Standards of confusing the public about the meaning of the word weight which resulted in the censuring of the National Bureau of Standards by the AAPT. The NBS spokesman, Dr. David T. Goldman responds to each charge concluding that not to recognize the dual use of the term "weight" as both "mass" and "force" would mean that the Bureau was neglecting both important sectors of the economy and the experience of foreign countries who have been on the metric system for a long time. On the other hand, NBS has made the proper distinction between the International System of Units and the quantities to which they refer, using kilogram as the unit of mass and newton as the unit of force. Dr. Goldman also pointed out that a significant exchange of views between members of the education and technical professions and NBS had taken place on this subject.

20024. Barnes, I. L. The use of isotope dilution mass spectrometry for the certification of CRM's, (Proc. Int. Symp. on the Production and Use of Reference Materials, Bundesanstalt für Materialprüfung (BAM), Berlin, Germany, Nov. 13-16, 1979), Paper in Production and Use of Reference Materials, B. F. Schmitt, ed., pp. 161-164 (Bundesanstalt für Materialprüfung, Berlin, Federal Republic of Germany, 1980).

Key words: analysis; certification; isotope dilution mass spectrometry; mass spectrometry; standard reference materials.

The use of well characterized materials for the development of new analytical methodology is becoming ever more important as our knowledge of the effects of the concentration, both helpful and harmful, of a larger variety of trace elements increases. The requirement that these concentrations must be known in a standard with a level of precision three to ten times better than the expected use places a severe constraint on the analytical procedures used for certification. The further requirement that accuracy as well as precision is necessary so that all measurements fit into one measurement system, equally valid anywhere poses an even greater constraint

20025. Revesz, A. G.; Kruger, J. The role of noncrystalline films in passivation and breakdown of passivation, Proc. 7 th Int. Conf. on Metallic Corrosion, Rio de Janiero, Brazil, Oct. 4-11, 1978, pp. 330 340 (ABRACO, Rio de Janiero, Brazil, 1979).

Key words: noncrystalline films; passivation; passivation breakdown; structural flexibility.

Owing to the lack of grain boundaries, noncrystalline oxide films have better passivating properties than polycrystalline ones. Bond and/or structural flexibility is an important factor in determining the ability to form a noncrystalline structure and its stability: This point was demonstrated by the example of vitreous $\mathrm{SiO}_{2}$ and by ligand formation of transition metal ions. The flexibility of the noncrystalline structure ensures a good accommodation at the oxide/substrate interface without requiring an epitaxial relationship. Such an interface is less prone to chemical attack than an imperfect one. High degree of short range order in vitreous oxide films also contributes to their chemical resistance. The flexibility of the structure is responsible for the increased ductility of noncrystalline passivating films which is crucial from the viewpoint of pitting and stress corrosion.

The structural/bond flexibility and, hence, the tendency toward noncrystallinity can be increased by additives; the effect of $\mathrm{Cr}$ in the passivation behavior of stainless steel is an example. In this respect, hydrogen also plays a great role by forming $\mathrm{M}-\mathrm{OH}$ groups, hydrogen bonds, and $\mathrm{H}_{2} \mathrm{O}$ ligands.

The formation and stability of noncrystalline oxide films is a kinetic rather than thermodynamic problem as demonstrated, for instance, by the influence of substrate.

There is evidence in the literature that noncrystalline passive films are 1) more protective, forming films which exhibit lower limiting thicknesses and lower passive current densities; 2) more ductile, 3) less susceptible to breakdown by damaging species and 4) capable of reforming at greater repassivation rates than crystalline films.

20026. Ugiansky, G. M.; Kruger, J.; Staehle, R. W. An electrochemical study of an Al-Zn-Mg alloy, Proc. 7th Int. Conf. on Metallic Corrosion, Rio de Janiero, Brazil, Oct. 4-11, 1978, pp. 605.613 (ABRACO, Rio de Janiero, Brazil, 1979).

Key words: $\mathrm{Al}-\mathrm{Zn}-\mathrm{Mg}$; anodic polarization; corrosion phenomena; electrochemical polarization.

In the age hardened $\mathrm{Al}-\mathrm{Zn}-\mathrm{Mg}$ alloys, there are essentially three significant constituents that contribute to the various intergranular corrosion phenomena. These are: the equilibrium $\mathrm{MgZn}$, precipitate in the grain boundary, the precipitate free zone (PFZ) adjacent to the grain boundary, and the matrix. The technique used in this work is one of studying the localized corrosion of a multi-constituent alloy by examining the electrochemical polarization characteristics of separate electrodes that simulate these individual constituents in the grain boundary region.

The anodic polarization characteristics of the "constituents" were compared to determine the effect on the oxidation kinetics of: the $\mathrm{pH}$ the different anions in the environment $\left(\mathrm{Cl}^{-}, \mathrm{NO}_{3}^{-}, \mathrm{Cr}_{2} \mathrm{O}_{7}{ }^{-}\right)$and the different constituents. The results of the polarization studies are summarized as critical potentials and current densities and as potential-pH diagrams showing the regions of pitting, passive, and active behavior for the constituents and the aged alloy.

The polarization studies reveal certain general trends: 1) the regions of passive behavior for the constituents correlate with the known regions of potential for cathodic protection of aluminum against uniform corrosion, pitting corrosion, and stress corrosion cracking (SCC) in chloride-containing environments. 2) the regions of passivity and pitting for the $\mathrm{MgZn}_{2}$ in $\mathrm{Cl}^{-}$and in $\mathrm{NO}_{3}^{-}$are seen to be almost identical with respect to potential and $\mathrm{pH}$. With respect to SCC, the fact that the $\mathrm{MgZn}_{2}$ has virtually identical anodic behavior in both $\mathrm{Cl}$ and $\mathrm{NO}_{3}^{-}$must be taken into account in any proposed mechanism for SCC of Al.Zn.Mg alloys. 3) Pitting, activation and corrosion potentials for PFZ and matrix constituents are increasingly more noble in $\mathrm{Cl}^{-}, \mathrm{NO}_{3}^{-}$and $\mathrm{Cr}_{2} \mathrm{O}_{7}^{-}$solutions, respectively.

20027. Ugiansky, G. M.; Johnson, C. E. Stress corrosion testing at elevated temperatures in simulated coal conversion gases, Proc. 7 th Int. Conf. on Metallic Corrosion, Rio de Janiero, Brazil, Oct. 4-11, 1978, pp. $915-924$ (ABRACO, Rio de Janiero, Brazil, 1979).

Key words: coal gasification; elevated temperature stress corrosion; slow strain rate; stress corrosion.

A total of six different alloys-stainless steels (SS) Types $310,310 \mathrm{~S}$, 347 , and 446 , and nickel alloys 800 and 671 -were tested using the slow strain rate test technique in oxidizing/sulfidizing and in oxidizing/sulfidizing/carburizing simulated coal gasification environments, and in helium (and other inert environments) at both 450 and $600^{\circ} \mathrm{C}$ at a strain rate of $10^{-6} / \mathrm{s}$. Of the six alloys, four (Types 310 SS, 310 S SS, nickel alloy 800 , and nickel alloy 671) were found to 
be susceptible to cracking at $600^{\circ} \mathrm{C}$ by possibly different mechanisms, however, all were detected by the slow strain rate technique.

For cracking, the nickel alloy 671 required no reactive environment-it suffered internal cracking in helium and all other test environments used. However, the stainless steel Types $310,310 \mathrm{~S}$, and nickel alloy 800 , all of which failed through surface initiated cracking, required increasingly severe environments for cracking. The Type 310 SS required very little reactive environment-it cracked not only in the simulated coal gasification gases but also in low partial pressure $\mathrm{O}_{2}$ and/or $\mathrm{H}_{2} \mathrm{O}$ environments (for example, helium), as did the Type 310 SS, although with greater ductility than the Type 310 SS. The nickel alloy 800 required the rather reactive environment of the simulated coal gasification gases-it did not crack in the low partial pressure $\mathrm{O}_{2}$ and $\mathrm{H}_{2} \mathrm{O}$ environments.

The detection of the environmentally independent failure of alloy 671 shows that the use of the slow strain rate technique need not be limited to use for testing for susceptibility to cracking by stress corrosion cracking. The slow strain rate technique is shown to be a viable testing method for detecting an alloy's propensity to premature failure in simulated coal gasification environments.

20028. Wiederhorn, S. M. Erosion of ceramics, Proc. NACE Conf. on Corrosion/Erosion of Coal Conversion System Materials, Berkeley, CA, Jan. 24-26, 1979, pp. 444.479 (National Association of Corrosion Engineers, Houston, TX 77084, 1979).

Key words: ceramics; coal conversion; crack propagation; erosion; fracture toughness; hardness.

This paper reviews current research on the erosion of ceramic materials for engineering applications. Two types of erosion are described: that resulting from chipping and crack propagation, typical of dense homogeneous ceramics; and that resulting from crushing of porous bonding phases, typical of castable refractories. Significant recent scientific advances suggest that research on microstructure, dynamic hardness and fracture toughness may enhance our ability to predict the erosive behavior of homogeneous ceramics. Similarly, research on the strength of cement, on packing density, and on sizing of aggregate particles are recommended to improve the erosion resistance of castable refractories.

20029. Molino, J. A.; Zerdy, G. A.; Tremaine, S. G. Psychoacoustic evaluation of transmission line audible noise: Building attenuation effects, methodology comparison, and field study feasibility, $D O E / R A / 29323-1,84$ pages (U.S. Department of Energy, Office of Electric Energy Systems, Washington, DC, 1979). (Available by purchase from the National Technical Information Service, Springfield, VA 22161.)

Key words: corona noise; electric energy transmission; high voltage; human response to noise; noise pollution;

psychoacoustics; transmission line audible noise.

A behavioral preference procedure and a categorical rating scale were used to assess human aversion to samples of transmission line audible noise in two separate psychoacoustic experiments. Tape recorded samples of corona noise were played back to listeners in a realistic listening room. Both outdoor and indoor listening conditions were simulated. In addition, the feasibility of conducting social surveys of the responses of residents actually living near transmission lines was briefly investigated. Conclusions were drawn that have implications for the environmental impact of high-voltage transmission lines.

20030. Molino, J. A.; Zerdy, G. A.; Lerner, N. D.; Harwood, D. L. Preliminary tests of psychoacoustic facilities and techniques for studying the human response to transmission line audible noise, Energy Tech. HCP/T-6010/E2, 70 pages (U.S. Department of Energy, Division of Electric Energy Systems, Washington, DC, Dec. 1977). (Available by purchase from the National Technical Information Service, Springfield, VA 22161.)

Key words: corona noise; environmental noise; high voltage transmission lines; human response to noise; listening room.

Progress during the first year of the DOE-NBS project on transmission line audible noise is documented. Some overall background for the project is provided. Three pilot experiments are described in which techniques to measure the human response to high-voltage transmission line audible noise are evaluated. A new, DOE-NBS "realistic" listening room facility, to be employed in future psychoacoustic experiments, is also described.

20031. Rawie, C. C. Writing standards that meet regulatory needs, ASTM Stand. News 8, No. 9, 13-16 (Sept. 1980).

Key words: cost-benefit; economics; government regulation; safety regulation; standards.

This article urges discussion of certain issues arising from the use by regulatory agencies of standards developed by nongovernment standards-writing committees. The issues addressed are related to the technical and economic basis for nongovernment standards and documentation of such standards. Problems in these areas prevent regulatory agencies from taking full advantage of the expertise and resources that nongovernment standards-writing groups dedicate to writing standards. The aim of this paper is to promote discussion that will aid in resolving these problems and thus advance the regulatory use of nongovernment standards.

20032. Haber, S. A number-theoretic problem in numerical approximation of integrals, Proc. Approximations Theory III Symp., Austin, TX, Feb. 8-13, 1980, pp. 473.480 (Academic Press, New York, NY, 1980).

Key words: integrals; integration; irregularity of distribution; lattices; n-cube; periodic functions; quadrature; several variables.

Some time ago two mathematicians and a scientist devised a generalization of the simple trapezoid rule, for numerical integration of periodic functions of more than one variable. The method has been successful in some practical applications, but its use has been limited by our ignorance of certain parameters needed for its implementation. This paper describes the method and some remarkable problems-in the theory of numbers and in geometry-connected with it. The problems involve trigonometrical sums, affine lattices, and the most nearly uniform distribution of a finite set of points inside a cube. Solution of the problems could provide improvements to the method, and clues for determining the essential parameters.

20033. Murray, J. L.; Orser, D. J. Interactive computer graphics for storing phase diagrams, Bull. Alloy Phase Diagrams 1, No. 1, 19.22 (1980).

Key words: alloy phase diagram; computer graphics; critical evaluation; database management; thermodynamics.

Progress is described on an interactive graphics computer program needed for the creation of an automated phase diagram data base. Automation requires the ability to input literature data in various units and formats and to edit and update a phase diagram without unnecessary reentry of data. A relational data structure makes possible the necessary explicit representation of phase stability data. Examples of binary diagrams are given. Plans for global management of a large data base are described.

20034. Carlson, C. E.; Coyne, J. J.; Fishbane, P. M.; Gross, F.; Meshkov, S. E(1440): Glueball or quarkonium?, Phys. Lett. 98B, No. 1.2, 110-114 (Jan. 1, 1981).

Key words: $\mathrm{E}$ meson at $1440 \mathrm{MeV}$; flavor singlet; glueball; production process; quark model; strange meson.

The most likely assignment of the $\mathrm{E}(1440)$ is that of an ordinary $\overline{\mathrm{sS}}$, $L=1, J^{P C}=1^{++}$meson, rather than a flavor singlet meson or a $J^{P C}=$ $0^{-+}$or $1^{++}$glueball.

20035. Herron, J. T.; Huie, R. E. Rate constants at $298 \mathrm{~K}$ for the reactions $\mathrm{SO}+\mathrm{SO}+\mathrm{M} \rightarrow(\mathrm{SO})_{2}+\mathrm{M}$ and $\mathrm{SO}+(\mathrm{SO})_{2} \rightarrow \mathrm{SO}_{2}+\mathrm{S}_{2} \mathrm{O}$, Chem. Phys. Lett. 76, No. 2, 322-324 (Dec. 1, 1980).

Key words: chemical kinetics; disulfur monoxide; rate constant; 
sulfur dioxide; sulfur monoxide; sulfur monoxide dimer.

The decay of SO generated in the reaction $\mathrm{O}+\mathrm{OCS} \rightarrow \mathrm{CO}+\mathrm{SO}$ in the absence of $\mathrm{O}_{2}$ has been studied at $298 \mathrm{~K}$ from 2 to $8 \mathrm{Torr}$, using a tubular flow reactor coupled to a mass spectrometer. The products of the decay of $\mathrm{SO}$ are $\mathrm{S}, \mathrm{SO}_{2}, \mathrm{~S}_{2} \mathrm{O}$, and $(\mathrm{SO})_{2}$. The kinetics of the decay of $\mathrm{SO}$ could be accounted for on the basis of the mechanism: $\mathrm{SO}+$ $\mathrm{SO}+\mathrm{M} \rightarrow(\mathrm{SO})_{2}+\mathrm{M}$ (3), $\mathrm{SO}+\mathrm{SO} \rightarrow \mathrm{SO}_{2}+\mathrm{S}(4), \mathrm{SO}+(\mathrm{SO})_{2} \rightarrow \mathrm{SO}_{2}+$ $\mathrm{S}_{2} \mathrm{O}(5)$, and $\mathrm{SO} \rightarrow$ products (6), where the latter represents either a wall reaction or a slow bimolecular reaction with OCS. On the basis of computer modeling we derive $k_{3}=1.6 \times 10^{17} \mathrm{~cm}^{6} \mathrm{~mol}^{-2} \mathrm{~s}^{-1}$ and $k_{\mathrm{s}}=$ $2 \times 10^{10} \mathrm{~cm}^{3} \mathrm{~mol}^{-1} \mathrm{~s}^{-1}$.

20036. Jacox, M. E. The reaction of $F$ atoms with $\mathrm{C}_{2} \mathrm{H}_{2}$ vibrational spectrum of the $t$-2-fluorovinyl free radical isolated in solid argon, Chem. Phys. 53, $307-322$ (1980).

Key words: acetylene; F atoms; hydrogen bonding; infrared spectrum; matrix isolation; photodecomposition; reaction mechanism; $t$-2-fluorovinyl.

When the products of the reaction of $F$ atoms produced in a microwave discharge with acetylene are frozen in a large excess of argon at $14 \mathrm{~K}$, several new infrared absorptions appear which can be assigned to a product that photodecomposes at wavelengths shorter than $280 \mathrm{~nm}$. A reasonable fit of the isotopic data to a diagonal valence-force potential has been obtained using a molecular structure for $t$-2-fluorovinyl derived from theoretical calculations for closely related molecules, consistent with the conclusion from previous EPR studies that this radical is the principal product of the $\mathrm{F}+\mathrm{C}_{2} \mathrm{H}_{2}$ reaction. Some ambiguity remains in the assignment of the deformation fundamentals. Studies at higher F-atom concentrations support the conclusion from earlier mass spectrometric studies that HCCF results from the fragmentation of the excited $\mathrm{C}_{2} \mathrm{H}_{2} \mathrm{~F}_{2}$ formed on secondary $\mathrm{F}$-atom reaction. The $\mathrm{HF}$ stretching absorptions of the $\left[\mathrm{C}_{2} \mathrm{H}_{2}+\mathrm{HF}\right]$ and $[\mathrm{HCCF}+\mathrm{HF}]$ hydrogen-bonded complexes have been identified. Some evidence has also been obtained for the stabilization of the $\left[\mathrm{HC}_{2}+\mathrm{HF}\right]$ complex on photodecomposition of the t.2-fluorovinyl radical in solid argon.

20037. Shukla, R. C.; MacDonald, R. A. Thermal expansion of cubic crystals, High Temp. High Pressures 12, $291-296$ (1980).

Key words: anharmonic; Brillouin zone sums; central pair potential; fcc metals; free energy; high temperature nearestneighbor model; thermal expansion.

A method for an exact calculation of the thermal expansion $(\epsilon)$ of cubic crystals in the high-temperature limit is presented. The calculation is carried out for a nearest-neighbor centralforce (nncf) model of a monatomic fcc crystal by minimizing the Helmholtz free energy of the crystal as a function of temperature and volume. The vibrational part of the Helmholtz free energy is obtained from the harmonic and the two lowest-order (cubic and quartic) anharmonic terms of the perturbation theory. A parameter $\left(a_{1}\right)$ depending on the first and second derivatives of the pair potential $\phi(r)$ is introduced, and $\epsilon$ is calculated from the knowledge of $\phi(r)$ and of ten dimensionless Brillouin zone sums which are functions of $a_{1}$ alone. The numerical results for a number of materials $(\mathrm{Pb}, \mathrm{Al}, \mathrm{Ag}, \mathrm{Ni}, \mathrm{Cu}$, $\mathrm{Ca}$, and $\mathrm{Sr}$ ) are compared with their experimental values, and the validity of the nncf model is discussed in the context of thermal expansion.

20038. Manning, J. R. Effect of correlated jumps and vacancy wind interactions on atom jump frequencies and diffusion fluxes in a driving force, Scr. Metall. 15, 1-6 (1981).

Key words: atom jump frequencies; correlation factor for diffusion; diffusion coefficient; diffusion equations; drift velocity for diffusion; driving force for diffusion; vacancy flow; vacancy wind effect.

Relations between the actual, basic and effective atom jump frequencies resulting from diffusion in a driving force are derived. Here the basic jump frequencies are the product of an average vacancy concentration and the atom-vacancy exchange frequency.
The effective jump frequencies are the frequencies of independen: uncorrelated jumps. When the number of jumps to the left in the driving force does not equal the number to the right, both the basic and effective jump frequencies will differ from the actual frequencies of atom jumps to the left and right. Use of improper jump frequencies in kinetic diffusion expressions can lead to derivation of significantly incorrect equations for the atom flux.

20039. Mies, F. H.; Aryeh, Y. B. Kinetics and spectroscopy of nearresonant optical pumping in intense fields, J. Chem. Phys. 74, No. 1, 53-67 (Jan. 1, 1981).

Key words: damping theory; kinetics; lasers; lineshapes; optical pumping; resonance Raman; spectroscopy; strong fields.

The resonant Raman scattering in a three level system produces an optical pumping of molecules from the initial to the final states which are radiatively coupled to the intermediate resonant state. We consider the pumping that is produced by an intense, pulsed, monochromatic radiation field that is near-resonant with the initial resonance frequency. The Heitler-Ma damping theory is solved exactly for the case when the resonance fluorescence is weak compared to the Raman scattering, or any other processes which might cause irreversible decay of the resonant state. In particular, we examine the usefulness of dressed states to describe the intense field limit. The spectral lineshape for the Raman scattering is obtained. The transient behavior following the onset of the laser pulse is examined and a careful analysis of the pumping rate and its relationship to both weak and strong field theories is presented.

20040. Karl, G.; Meshkov, S.; Rosner, J. L. Quark magnetic moments and $\mathrm{E} 1$ radiative transitions in charmonium, Phys. Rev. Lett. 45, No. 4, 215-218 (July 28, 1980).

Key words: angular distributions; charmed mesons; dipole transitions; magnetic moments; photon decays; quarks.

In the long-wavelength limit, $\Gamma\left(\psi^{\prime} \rightarrow \gamma+\chi_{J}\right)=$ const $\times(2 J+1) p_{\gamma}{ }^{3}$ and $\Gamma\left(\chi_{r} \rightarrow \gamma+\psi\right)=$ const $\times p_{\gamma}{ }^{3}$. The corrections to these expressions of order $p_{\gamma} / m_{c}$ ( $m_{c}$ is the mass of the charmed quark) are calculated. These corrections are found to be proportional to $\langle\overrightarrow{\mathrm{L}} \cdot \overrightarrow{\mathrm{S}}\rangle_{\chi J} \times \kappa$, where $\langle\mathbf{L} \cdot \mathbf{S}\rangle_{\chi^{J}}=1,-1,-2$, for $J=2,1,0$, and $\kappa$ is the anomalous magnetic moment of the quark. Angular distributions of photons in the decays $\psi^{\prime} \rightarrow \gamma \chi$ and $\chi \rightarrow \gamma \psi$ also are predicted; small but probably measurable deviations from the pure $E 1$ limit are found.

20041. Ku, H. H. Discussion-Panel on the statistical evaluation in the course of certification, (Proc. Int. Symp. on the Production and Use of Reference Materials, Bundesanstalt für Materialprüfung (BAM), Berlin, Germany, Nov. 13-16, 1979), Paper in Production and Use of Reference Materials, B. F. Schmitt, ed., pp. $497-500$ (Bundesanstalt für Materialprüfung, Berlin, Federal Republic of Germany, 1980).

Key words: precision; reference material; stability of measurement process; statement of certainty.

Discussion on some statistical problems encountered in the course of certification of Reference Materials: (1) appropriate uncertainty statement on a certificate, (2) analysis leading to the value of uncertainty statement and the underlying assumptions, and (3) a "well-characterized" measurement system as basis of a "wellcharacterized" reference material.

20042. Okabe, H.; Kawasaki, M.; Tanaka, Y. The photodissociation of $\mathrm{CH}_{2} \mathrm{I}_{2}$ : Production of electronically excited $\mathrm{I}_{2}, J$. Chem. Phys. 73, No. $12,6162-6166$ (Dec. 15,1980 )

Key words: $I_{2}$ fluorescence absorption coefficient; $\mathrm{Kr}$ photosensitized reaction; methylene iodide; photodissociation; vacuum ultraviolet;

The primary photodissociation process of $\mathrm{CH}_{2} \mathrm{I}_{2}$ leading to the formation of electronically excited $\mathrm{I}_{2}\left({ }^{3} \Pi_{28}\right)$ has been studied. The process occurs with yields less than $1 \%$ for light of wavelengths below $1320 \pm 10 \AA$. The $I_{2}\left({ }^{3} I_{2 g}\right)$ initially produced by the $\operatorname{Kr}(1165$, $1236 \AA$ ) lines is vibrationally excited up to $v^{\prime}=35$. The addition of 
ar atmospheric Ar or $\mathrm{N}_{2}$ deactivated vibrational quanta to $v^{\prime}=0,1$ and 2 but little electronic quenching was observed. The quantum yield of $\mathrm{I}_{2}\left({ }^{3} \Pi_{2 \mathrm{~g}}\right)$ production is 0.006 at $1236 \AA$. At $1306 \AA$ little vibrational excitation of $\mathrm{I}_{2}\left({ }^{3} \Pi_{28}\right)$ was seen. In addition, very weak $\mathrm{I}_{2}$ emission bands in the $2500-2900$ and $4500-4900 \AA$ regions were found in $\mathrm{CH}_{2} \mathrm{I}_{2}$ photodissociation by the $\mathrm{Kr}$ lamp. The absorption cross sections of $\mathrm{CH}_{2} \mathrm{I}_{2}$ in the 1100 to $1500 \AA$ region have been measured and compared with the $I_{2}\left({ }^{3} \Pi_{28}\right)$ fluorescence excitation spectrum. The results show that the $\mathrm{I}_{2}$ emission bands arise mainly from the underlying absorption continua and not from structured features of $\mathrm{CH}_{2} \mathrm{I}_{2}$, suggesting that the $\sigma-\sigma^{*}$ transitions in the $\mathrm{C}$-I bond are responsible for yielding $\mathrm{I}_{2}\left({ }^{3} \Pi_{2 \mathrm{~g}}\right)$, while Rydberg transitions are ineffective in forming $\mathrm{I}_{2}\left({ }^{3} \Pi_{2 \mathrm{~g}}\right)$. The dissociation process. $\mathrm{CH}_{2} \mathrm{I}_{2} \rightarrow$ $\mathrm{CH}_{2}\left(X^{3} B_{1}\right)+\mathrm{I}_{2}\left({ }^{3} \Pi_{28}\right)$ involves an energy barrier of almost $1 \mathrm{eV}$, in agreement with other molecular detachment processes. The $\mathrm{Kr}$ photosensitized reaction of $\mathrm{CH}_{2} \mathrm{I}_{2}$ on the other hand gives rise to the $\mathrm{I}_{2}(H)$ emission in addition to emissions observed in direct photolysis. This state is likely to be ${ }^{1} \Sigma_{u}^{+}$rather than ${ }^{3} \Sigma_{u}^{+}$.

20043. Romanov, V. P.; Candela, G. A.; Roth, R. S.; Swartzendruber, L. J. Magnetic properties and hyperfine interactions in the $\beta^{\prime \prime \prime}$ phase of potassium ferrite, J. Appl. Phys. 50, No. 10, 6455.6458 (Oct. 1979).

Key words: conductivity; exchange interaction; ferrite; hyperfine field; magnetism; phase diagram; potassium.

The hyperfine field structure and magnetic susceptibility in the temperature range $5-295 \mathrm{~K}$ was measured for the $\beta^{\prime \prime \prime}$ phase of potassium ferrite. Results show that this new phase has an antiferromagnetic ordering similar to the $\beta$ phase. The hyperfineinteraction parameters have been determined and are compared with those found in magnetite, $\beta$-phase potassium ferrite, and barium hexaferrite.

20044. Parker, R. L. Crystal growth, Article in Encyclopedia of Physics, R. G. Lerner and G. L. Trigg, eds., pp. 175-176 (Addison-Wesley Publ. Co., Inc., Advanced Book Program, Reading, MA, 1981).

Key words: crystal; growth of crystals; mechanisms of growth.

A brief survey of the field of crystal growth is presented.

20045. Pradhan, A. K.; Norcross, D. W.; Hummer, D. G. Cross sections and excitation rates for electron collisions with heliumlike ions, Phys. Rev. A 23, No. 2, 619-631 (Feb. 1981).

Key words: autoionization resonance; electron collision cross sections; heliumlike ions.

We describe the techniques and the approximations used in extensive calculations for cross sections and reaction-rate parameters for electron-impact excitation of a number of heliumlike ions. All transitions involving the ground state and the $n=2$ states are considered. Calculations are made in the distorted-wave approximation using configuration-interaction wave functions to represent the target states. Autoionizing resonances in the scattering cross sections are included through bound-channel correlation-type functions and through quantum-defect-theory analysis of the reactance matrices. The resonances are shown to make considerable contributions to the cross sections and thereby, in many cases, to enhance the excitation-rate coefficients by a significant factor. This should have important consequences for practical applications in the analysis of laboratory and astrophysical plasmas. The accuracy of our approximations is also discussed.

20046. Roth, R. S. Thermal stability of long range order in oxides, Prog. Solid State Chem. 13, No. 2, $159-192$ (1980).

Key words: diffraction effects; long range order; modulated structures; oxides; phase equilibria.

Diffraction effects have been observed in many oxide phases which have led researchers to believe that unusual long range order may occur. Much of the confusion occurs because the diffraction effects are often non-intergral or non-rational by the classic crystallographic definitions. It is the purpose of this paper to examine some of the effects that are produced in oxides as a result of high temperature equilibration processes, how these effects are manifested by $\mathrm{x}$-ray, neutron and electron diffraction and how these effects change under changing thermal conditions.

20047. Muller, C. H. III; Phelps, A. V. Low-current electric discharges in $\mathrm{H}_{2}-\mathrm{He}$ mixtures, J. Appl. Phys. 51, No. 12, 6141.6148 (Dec. 1980).

Key words: ambipolar diffusion; electric discharges; helium Penning ionization; hydrogen.

Tests are made of the ability of currently available theory to predict experimental ratios of the electric field to gas density $E / N$ required for the steady-state operation of low-current, moderatepressure discharges in hydrogen-helium mixtures. In the experiments the fractional concentration of $\mathrm{H}_{2}$ in $\mathrm{He}$ was varied from $1 \%$ to $4 \%$ and the discharge current, i.e., $40 \pm 10 \mu \mathrm{A}$, and total gas density, i.e. $10^{23}-10^{25}$ atoms $/ \mathrm{m}^{3}$, were chosen to avoid gas heating and striations. The experimental $\mathrm{E} / \mathrm{N}$ values for the 4-mm-diam tube varied from $6 \times 10^{-21} \mathrm{~V} \mathrm{~m}^{2}$ for $1 \% \mathrm{H}_{2}$ in $\mathrm{He}$ at a gas density of $7 \times 10^{24} \mathrm{~m}^{-3}$ to about $4 \times 10^{-20} \mathrm{~V} \mathrm{~m}^{2}$ for $4 \% \mathrm{H}_{2}$ in $\mathrm{He}$ at $1.5 \times 10^{23} \mathrm{~m}^{-3}$. Theoretical steady. state $E / N$ values versus gas density were calculated using (a) solutions of the Boltzmann equation for He metastable excitation, He ionization, and $\mathrm{H}_{2}$ ionization rate coefficients; (b) published theoretical calculations of the effects of a finite Debye length and a finite ion mean free path on the electron loss rate; and (c) models for the ion-molecule conversion and Penning ionization processes. The differences between theoretical and experimental values of $E / N$ are less than $10 \%$ for total gas densities above $6 \times 10^{23} \mathrm{~m}^{-3}$, but are $20-30 \%$ for a gas density of $1.6 \times 10^{23} \mathrm{~m}^{-3}$. We conclude that except at low gas densities the simple theory gives a good description of lowcurrent discharges in this gas mixture.

20048. McMichael, J. M.; Klebanoff, P. S.; Mease, N. E. Experimental investigation of drag on a compliant surface, Chapter in Viscous Flow Drag Reduction. Progress in Astronautics and Aeronautics, G. R Hough, ed., 72, 410-438 (American Institute of Aeronautics and Astronautics, New York, NY 10104, 1980).

Key words: boundary layer; compliant surface; drag, experimental; flat plate, turbulent.

The feasibility of reducing turbulent skin.friction drag by means of surface compliance was studied experimentally in a fully developed, flat-plate turbulent boundary-layer in air, using a membranous surface backed by a thin cavity containing a layer of polyurethane foam. Surface motion characteristics, boundary-layer structure, and overall drag were measured over a range of free-stream speeds from 7 to 30 meters per second and a range of membrane tensions from 44 to 350 Newtons per meter. Low amplitude, long wavelength motions predominate, and no significant change from the rigid surface skin friction coefficients was observed.

20049. Mies, F. H. A scattering theory of diatomic molecules general formalism using the channel state representation, Mol. Phys. 41, No. 5, 953-972 (1980).

Key words: bound states; continuum states; diatomic spectroscopy; electronic-rotational states; predissociation; scattering theory.

We present a unified theory of diatomic molecules which reconciles bound state spectroscopy and atomic scattering theory. The total wavefunction is expanded in a complete set of atomic channel states which is entirely equivalent to an expansion in Hund's case $(e)$ electronic-rotational states. An analysis of the coupled radial, that is vibrational, functions places strong constraints on the asymptotic properties of the molecular wavefunctions. These are presented in terms of the reactance $\mathbf{K}$ and scattering $\mathbf{S}$ matrices of atomic scattering theory which offers a uniform treatment for open channels (inelastic scattering and continuum spectroscopy), closed channels (bound state spectroscopy) and mixtures of both (predissociation). The normalization of the total wavefunction is derived and related to the asymptotic boundary conditions both for continuum and bound states. 
20050. Molino, J. A.; Zerdy, G. A.; Lerner, N. D.; Harwood, D. L. Use of the "acoustic menu" in assessing human response to audible (corona) noise from electric transmission lines, J. Acoust. Soc. Am. 66, No. 5, 1435-1445 (Nov. 1979).

Key words: annoyance; audible noise; corona noise; EHV transmission lines; electric energy; environmental noise; human response; power line noise; psychoacoustics.

A behavioral preference procedure, the "acoustic menu," was used to assess human aversion to the sounds from two samples of transmission line audible noise and from samples of other environmental noises. The audible (corona) noise produced by extrahigh voltage (EHV), overhead transmission lines was tape-recorded during moist weather. Reproduced samples of corona noise, other common environmental noises, and artificial reference stimuli were compared in two experiments. The two corona noise samples were found to be equally preferred to: (1) a $1000 \mathrm{~Hz}$ octave band of noise about $11 \mathrm{~dB}$ SPL higher than the corona noises and (2) a collection of other environmental noises about $8 \mathrm{~dB}$ SPL higher than the corona noises. The corona noise samples were more aversive than the ambient sounds (including rainfall) occurring near rural transmission lines and were roughly equivalent in aversiveness to the noise from a room air conditioner (indoor recording). Knowledge of the source of the corona noise (from photographs and a brief description) did not affect its aversiveness. Of the simple frequency-weighting scales, the $A$-weighted sound level reduced the difference between the two corona noise samples and the set of other environmental sounds from about $8 \mathrm{~dB}$ SPL to about $3 \mathrm{~dB}$. The $D$-weighted sound level further reduced this discrepancy to about $2 \mathrm{~dB}$. These results were obtained with only two samples of corona noise; other samples may give different results.

20051. Parr, A. C.; Jason, A. J.; Stockbauer, R.; McCulloh, K. E. Photoionization and threshold photoelectron-photoion coincidence study of propyne from onset to $20 \mathrm{eV}$, Int. J. Mass Spectrom. Ion Phys. 30, 319.330 (1979).

Key words: breakdown curves; photoelectron-photoionization coincidence; photoionization; propyne; threshold photoelectron spectra.

Photoionization efficiency curves are obtained for propyne and its fragments $\mathrm{C}_{3} \mathrm{H}_{3}^{+}, \mathrm{C}_{3} \mathrm{H}_{2}^{+}$, and $\mathrm{C}_{3} \mathrm{H}^{+}$from threshold to $20 \mathrm{eV}$. Threshold photoelectron spectra and breakdown curves are given over the same energy range. The adiabatic ionization potential of the parent molecule, the appearance potentials of the fragments and the derived heats of formation of the ionic species are determined. The breakdown curve is almost identical to that obtained previously for allene when the different heats of formation of the neutral molecules are taken into account. This suggests that the propyne ion isomerizes to the allene ion before fragmentation.

20052. Codling, K.; West, J. B.; Parr, A. C.; Dehmer, J. L.; Stockbauer, R. L. Measurement of $\beta$ values and branching ratios in the region of the $3 s^{3} p^{6} 4 p^{1} \mathrm{P}^{\circ}{ }_{1}$ resonance in $A r$ and the $5 s 5 p^{6} 6 p^{1} \mathrm{P}^{\circ}$, resonance in Xe, J. Phys. B: Atom. Molec. Phys., Lett. to Ed. 13, L693.L697 (1980).

Key words: angular distribution; asymmetry parameter; branching ratios; resonance; xenon.

Variations in asymmetry parameter, $\beta$, and the ratio of partial photoionisation cross sections $\sigma\left({ }^{2} \mathbf{P}_{3 / 2}\right): \sigma\left({ }^{2} P_{1 / 2}\right)$ have been determined in the region of the $3 s^{3} \mathrm{p}^{6} 4 \mathrm{p}^{1} \mathrm{P}_{1}^{0}$ resonance in $\mathrm{Ar}$ and the $5 \mathrm{~s} 5 \mathrm{p}^{6} 6 \mathrm{p}{ }^{\prime} \mathrm{P}_{1}^{0}$ resonance in $\mathrm{Xe}$. In both cases there is a considerable variation in $\beta$ through the resonance. In $\mathrm{Ar}$ the $\beta$ values for the spin-orbit components are similar; in $\mathrm{Xe}$ they are significantly different. In $\mathrm{Xe}$, the branching ratio shows a modest variation through the resonance, whereas in Ar no noticeable change occurs.

20053. Sams, R. L.; Olson, W. B. Analysis of the high-resolution infrared spectrum of the $\nu_{2}$ bending mode of $\mathrm{HOCl}$ at $1238 \mathrm{~cm}^{-1}, J$.

Mol. Spectrosc. 84, 113-123 (1980).
Key words: diode laser; Ebert spectrometer; high resolution; hypochlorous acid $(\mathrm{HOCl})$; infrared spectrum; $\nu_{2}$ vibration.

The infrared spectrum of hypochlorous acid $(\mathrm{HOCl})$ is investigated in the $1238-\mathrm{cm}^{-1}\left(v_{2}\right)$ region with a recently completed $3.81 \cdot \mathrm{m}$ Ebert Spectrometer. A description of this instrument is included. Spectroscopic constants for $\nu_{2}$ are obtained.

20054. Santoro, A.; Marezio, M.; Roth, R. S.; Minor, D. Neutron powder diffraction study of the structures of $\mathrm{CeTaO}_{4}, \mathrm{CeNbO}_{4}$, and $\mathrm{NdTaO}_{4}$, J. Solid State Chem. 35, 167-175 (1980).

Key words: cerium neodymium tantalates; cerium niobate; neutron diffraction; oxidation mechanisms; profile refinement method; scattering amplitude of neodymium.

The crystal structures of $\mathrm{CeTaO}_{4}, \mathrm{CeNbO}_{4}$, and $\mathrm{NdTaO}_{4}$ have been refined with the Rietveld method using neutron powder diffraction data collected at room temperature. The results of these refinements show that the coordination of the $\mathrm{Ce}$ and $\mathrm{Nd}$ cations is 8 with an average $\mathrm{Ce}-\mathrm{O}$ distance of $2.520 \AA$ in $\mathrm{CeTaO}_{4}$ and $2.480 \AA$ in $\mathrm{CeNbO}_{4}$, and an average $\mathrm{Nd}-\mathrm{O}$ distance of $2.451 \AA$ in $\mathrm{NdTaO}_{4}$. The $\mathrm{Nb}$ and $\mathrm{Ta}$ cations in $\mathrm{CeNbO}_{4}$ and $\mathrm{NdTaO}_{4}$ are surrounded by six oxygen ions with $\mathrm{Nb}-\mathrm{O}$ distances varying between 1.851 and $2.482 \AA$, and $\mathrm{Ta}-\mathrm{O}$ distances varying between 1.861 and $2.353 \AA$. The structural arrangement of $\mathrm{CeTaO}_{4}$ can be regarded as that of an oxidized $\mathrm{ABO}_{3}$ perovskite. The difference in structure of $\mathrm{CeTaO}_{4}$ and $\mathrm{NdTaO}_{4}$ is most likely due to the difference in size of the rare-earth cations.

20055. Schmid, G. B.; Norcross, D. W.; Collins, L. A. VLAM, a program for computing the electron-molecule static interaction potential from a Legendre expansion of the molecular charge density, Comput. Phys. Commun. 21, $79-90$ (1980).

Key words: electron-molecule collisions; interaction potential; Legendre expansion; molecular potential; quantum chemistry; scattering.

The electron-molecule static interaction potential is of central importance to calculations of cross sections for electron-molecule collisions. This paper describes a program, VLAM, which enables the efficient numerical evaluation of this potential from a Legendre expansion of the molecular charge density. A complementary program for the calculation of the charge density is provided in an accompanying paper. When used together, these two programs provide for a more efficient and flexible evaluation of the static interaction potential than hitherto available. In section 2 , we describe the basic physics upon which our code rests. Section 3 outlines the program structure. A description of the test run is presented in section 4.

20056. Sengers, J. V.; Bedeaux, D.; Mazur, P.; Greer, S. C. Behavior of the dielectric constant of fluids near a critical point, Physica 104A, $573-594$ (1980).

Key words: binary liquids; critical mixing; critical phenomena; dielectric constant; fluids in an electric field; refractive index.

Thermodynamic and statistical mechanical arguments indicate that the temperature derivative of the dielectric constant of fluids diverges weakly at the critical point. Following a thermodynamic procedure proposed by Mistura, we derive an expansion for the static dielectric constant of fluids near the gas-liquid critical point and of binary liquids near the critical point of mixing, which includes correction terms to the asymptotic critical behavior. The relationship with the statistical mechanical derivation of the critical behavior of the dielectric constant is discussed. The paper concludes with an assessment of the current experimental situation.

20057. Stencel, R. E.; Mullan, D. J.; Linsky, J. L.; Basri, G. S.; Worden, S. P. The outer atmospheres of cool stars. VII. High resolution, absolute flux profiles of the $\mathrm{Mg}$ II $h$ and $k$ lines in stars of spectral types F8 to M5, Astrophys. J. Suppl. Ser. 44, 383-402 (Nov. 1980). 
Key words: circumstellar shells; late type stars; mass loss; stars, emission line; stellar chromospheres.

We present high-resolution IUE spectra of the emission cores of the $\mathrm{Mg}$ II resonance doublet at $280 \mathrm{~nm}$ in a selection of 54 stars covering a range of spectral type from $\mathrm{F} 8$ to $\mathrm{M} 5$ and of luminosity class from supergiant (Ia) to subgiant (IV). These spectra were obtained with the LWR echelle system onboard the IUE satellite, and have been calibrated in absolute flux units using $O A O 2$ photometry of Eta UMa as a standard, plus the Barnes and Evans relations for stellar angular diameters. The uncertainty in flux is probably of order $20 \%$. We discuss the qualitative line profile groupings, as determined by Basri and Linsky, and derive chromospheric radiative losses in the $h$ and $k$ lines; we discuss these loss rates as functions of effective temperature and luminosity class. We make further comparisons of these rates with rates derived for the $\mathrm{Ca}$ II $\mathrm{H}$ and $\mathrm{K}$ lines by Linsky and his colleagues. Chromospheric velocity fields and indicators of circumstellar envelopes are discussed in terms of profile asymmetries and other diagnostics. Line width measures and velocity shifts of the central reversals are tabulated, among other quantities, and several correlations noted. Finally, we discuss the relation of the Wilson $\mathrm{K}$ index and stellar coronae to $\mathrm{Mg}$ II emission, and note the occurrence of $\mathrm{Fe}$ II emission lines in the middle range of the UV of late-type stars.

20058. Taylor, B. N. Constants, fundamental, Article in Encyclopedia of Physics, R. G. Lerner and G. L. Trigg, eds., pp. 145.152 (Addison-Wesley Publ. Co., Inc., Advanced Book Program, Reading, MA, 1981).

Key words: basic standards; fine-structure constant; fundamental constants; Josephson effect; least-squares adjustments; quantum electrodynamics.

Four main topics will be touched on in this article: (1) The motivation for "the romance of the next decimal place," or why the fundamental physical constants are important and why their determination to ever greater levels of accuracy can have a profound effect on physics; (2) how a self-consistent set of "best values" of the fundamental constants is obtained, with emphasis on the 1973 leastsquares adjustment (the most recent comprehensive study carried out); (3) new developments in the fundamental constants field since the 1973 adjustment and their impact on the recommended set of best values resulting from that adjustment; and (4) future trends-where the field is heading over the next five to ten years.

20059. Walker, G. R.; Marshall, R. D. The development of a peak gust anemometer, Proc. 7th Australasian Hydraulics and Fluid Mechanics Conf., Brisbane, Queensland, Australia, Aug. 18-22, 1980, pp. 151-154 (The Institution of Engineers, Barton, A.C.T. 2600, Australia, Oct. 1980).

Key words: anemometer; drag sphere; gust speed; instrumentation; wind sensor; wind speed.

The development and field testing of a simple drag sphere device for recording peak wind gusts are described. Also presented are the performance criteria and rationale for selecting these criteria for tropical storm applications. Based on static and dynamic calibrations as well as limited field experience, recommendations are offered for improvements in future models of the peak gust anemometer.

20060. Mies, F. H. A scattering theory of diatomic molecules expansion in adiabatic electronic-rotational states, Mol. Phys. 41, No. 5, 973-986 (1980).

\section{Key words: adiabatic potentials; Born-Oppenheimer approximation; diatomic spectroscopy; Franck-Condon principle; rotational-electronic coupling; scattering theory.}

An exact theory of diatomic molecules must be consistent with atomic scattering theory. In general, scattering wavefunctions can, not be factored into electronic-rotational and radial terms. Yet the starting point of most diatomic analysis is the Born-Oppenheimer approximation (BOA) which factors the total molecule wavefunction into an electronic-rotational function and a radial or vibrational function which yields the Franck-Condon principle. This is a severe approximation which is at best valid for small amplitude vibrations, may be expected to fail near dissociation limits and is completely invalid in the dissociation continuum.

In the preceding paper we presented a complete theory of the total molecular wavefunction in terms of atomic channel states which gives a unified description of diatomic states throughout the entire spectrum. In this paper we perform an orthogonal transformation on the channel states and expand the total wavefunction in a complete set of adiabatic electronic-rotational states. These states are implicit, analytical functions of the interatomic coordinate $R$ and progressively change from one Hund's electronic-rotational coupling case to another as $R$ is varied. At infinity they approach pure Hund's case (e) which are, in fact, the proper atomic channel states we require to describe the scattering boundary conditions. At finite $R$ they transform to the usual Hund's cases appropriate to bound-state spectroscopy.

The coupled equations are developed, using the adiabatic representation, and the exact solutions are expressed in terms of the regular and irregular solutions of the adiabatic interaction potentials. Deviations from the BOA can be expressed in terms of an $R$-dependent reactance matrix and an $R$-dependent normalization matrix which can be obtained from solutions of the usual closecoupled equations of atomic scattering theory.

20061. Geltman, S. Approximate response of a two-level system to intense multimode radiation, Phys. Lett. 81A, No. 1, 27-30 (Jan. 5, 1981).

Key words: multimode laser; multiphoton ionization; $\mathrm{Na} 3 \mathrm{~s} \rightarrow 3 \mathrm{p}$; pulse shapes; Rabi oscillation; two-level system.

The rotating wave solution for a two-level atom in an intense monochromatic radiation field is extended by an approximation to the case of a multimode radiation field. Expressions for the resultant average excitation probability of the upper state are presented for rectangular and triangular pulses.

20062. Allan, D. W.; Weiss, M. A. Accurate time and frequency transfer during common-view of a GPS satellite, Proc. 34th Annual Symp. on Frequency Control, Ft. Monmouth, NJ, May 28-30, 1980, pp. 334-346 (Electronic Industries Association, Washington, DC, May 1980).

Key words: atomic clocks; automatic time comparisons; common-mode cancellation; frequency comparison; global positioning system; remote time measurement; time comparison; time receiver; worldwide time.

Even though the GPS is primarily a navigation system, if two clocks at known coordinates $\mathrm{A}$ and $\mathrm{B}$ are in common-view of a single GPS satellite, receivers at these two clock sites may coincidentally receive transmitted GPS clock times. By subtracting the received times of arrivals as measured by clocks $A$ and $B$ at the two sites while compensating for the propagation delays, one has an accurate measure of the time difference between clock A and clock B.

When all of the error contributions are assessed, it appears that $1 \mathrm{~ns}$ time stability and $10 \mathrm{~ns}$ of time accuracy should be achievable in measuring remote clocks-at distances of the order of a few thousand $\mathrm{km}$. The primary error sources are as follows: uncertainties in the satellite ephemeris, differential ionospheric delays, uncertainties in tropospheric delay estimation, and uncertainties in receiver delays.

We have chosen this common-view approach because it provides an opportunity for a high accuracy (10 ns) relatively low cost receiver due to the common-mode error cancellation achievable.

20063. Evans, D. J.; Hanley, H. J. M. A thermodynamics of steady homogeneous shear flow, Phys. Lett. 80A, No. 2-3, 175-177 (Nov. 24, 1980).

Key words: nonequilibrium molecular dynamics; nonlinear thermodynamics; shear flow; shear induced phase transition; soft sphere system; stability; steady state; turbulence.

Simulation data from model systems subjected to a shear can be 
described consistently by a thermodynamic formalism that includes the pressure and energy dependence on the strain rate. Stability criteria are examined for a system of soft spheres.

20064. Coyne, J. J. Kerma values by particle type, Proc. Int. Workshop on Ion Chambers for Neutron Dosimetry, Rijswijk. The Netherlands, Sept. 17-20, 1979, J. J. Broerse, ed., 195-207 (Harwood Academic Publishers, (EUR 6782 EN), The Netherlands, 1980).

Key words: kerma factors; neutron dosimetry; neutron radiation therapy; nuclear data; secondary charged particles; tissue equivalence.

The kerma values that were published in the ICRU Report 26 are compared with the values which have been submitted for publishing in Radiation Research. The differences in nuclear data which have prompted these small changes are discussed. Comparisons with other calculations and experiments are given. For tissue and tissueequivalent materials the percent of the total kerma due to various ions is also given.

20065. Mathew, M.; Mayer, I.; Dickens, B.; Schroeder, L. W. Substitution in barium-fluoride apatite: The crystal structures of $\mathrm{Ba}_{10}\left(\mathrm{PO}_{4}\right)_{6} \mathrm{~F}_{2}, \mathrm{Ba}_{6} \mathrm{La}_{2} \mathrm{Na}_{2}\left(\mathrm{PO}_{4}\right)_{6} \mathrm{~F}_{2}$ and $\mathrm{Ba}_{4} \mathrm{Nd}_{3} \mathrm{Na}_{3}\left(\mathrm{PO}_{4}\right)_{6} \mathrm{~F}_{2}, J$. Solid State Chem. 28, 79-95 (1979).

Key words: apatite; barium apatite; cation substitution; crystal structure; rare-earth apatite; substituted fluorapatite.

The crystal structures of the apatites $\mathrm{Ba}_{10}\left(\mathrm{PO}_{4}\right)_{6} \mathrm{~F}_{2}(\mathrm{I})$, $\mathrm{Ba}_{6} \mathrm{La}_{2} \mathrm{Na}_{2}\left(\mathrm{PO}_{4}\right)_{6} \mathrm{~F}_{2}$ (II) and $\mathrm{Ba}_{4} \mathrm{Nd}_{3} \mathrm{Na}_{3}\left(\mathrm{PO}_{4}\right)_{6} \mathrm{~F}_{2}$ (III) have been determined by single-crystal $\mathrm{X}$-ray diffraction. All three compounds crystallize in a hexagonal apatite-like structure. The unit cells and space groups are: I, $a=10.153$ (2), $c=7.733$ (1) $\AA, P 6_{3} / m ; a=9.9392$ (4), $c=7.4419$ (5) $\AA, P \overline{6}$; III, $a=9.786$ (2), $c=7.281$ (1) $\AA, P \overline{3}$. The structures were refined by normal full-matrix crystallographic least squares techniques. The final values of the refinement indicators $R_{\mathrm{w}}$ and $R$ are: I, $R_{\mathrm{w}}=0.026, R=0.027,613$ observed reflections; II, $R_{\mathrm{w}}=$ $0.081, R=0.074,579$ observed reflections; III, $R_{\mathrm{w}}=0.062, R=0.044$, 1262 observed reflections.

In $\mathrm{I}$, the $\mathrm{Ba}(1)$ atoms located in columns on threefold axes, are coordinated to nine oxygen atoms; the $\mathrm{Ba}(2)$ sites form triangles about the $\mathrm{F}$ site and are coordinated to six oxygen atoms and one fluoride ion. The fluoride ions are statistically displaced $\sim 0.25 \AA$ from the $\mathrm{Ba}(2)$ triangles. This displacement of the $\mathrm{F}$ ions is analogous to the displacement of $\mathrm{OH}$ ion in $\mathrm{Ca}_{10}\left(\mathrm{PO}_{4}\right)_{6}(\mathrm{OH})_{2}$.

The structures of II and III contain disordered cations. In II there is disorder between $\mathrm{La}$ and $\mathrm{Na}$ in the column cation sites as well as triangle sites. In III, Nd and $\mathrm{Na}$ ions are ordered in the column sites, but there is disorder among $\mathrm{Ba}$ and the remaining $\mathrm{Nd}$ and $\mathrm{Na}$ ions in the triangle sites to give an average site population of $\frac{2}{3} \mathrm{Ba}, \frac{1}{6} \mathrm{Nd}$, $\frac{1}{6}$ $\mathrm{Na}$. The coordination of the rare earth ions and $\mathrm{Na}$ ions in the ordered column sites are nine and six oxygens, respectively, in accord with the greater charge of the rare earth ions as compared with Na. The $F$ ions in both II and III suffer from considerable disorder in position, and their locations are not precisely known.

20066. Ekin, J. W. Strain scaling law for flux pinning in practical superconductors. Part 1: Basic relationship and application to $\mathrm{Nb}_{3} \mathrm{Sn}$ conductors, Cryogenics 20, No. 12, 611.624 (Nov. 1980).

Key words: critical current; mechanical properties; niobium-tin; scaling; strain; superconducting wire.

Critical current and flux pinning densities have been determined for a series of $\mathrm{Nb}_{3} \mathrm{Sn}, \mathrm{V}_{3} \mathrm{Ga}, \mathrm{Nb}_{3} \mathrm{Ge}$, and $\mathrm{NbTi}$ conductors as a function of uniaxial tensile strain in magnetic fields ranging from 4 to $19 \mathrm{~T}$. An empirical relationship has been found at $4.2 \mathrm{~K}$ that describes these data over the entire range of field under both compressive and tensile strain. The pinning force $F$ has been found to obey a scaling law of the form $\mathrm{F}=\left[B_{c 2}^{*}(\epsilon)\right]^{n} f(b)$, where $B_{c 2}^{*}$ is the strain-dependent uppercritical field determined from high-field critical-current measurements and $f(b)$ is a function only of the reduced magnetic field $b \equiv B / B_{\mathrm{c} 2}^{*}$. The detailed shape of $f(b)$ depends on the superconducting material and reaction conditions, but $n$ was found to be nearly constant for a given type of superconductor. For $\mathrm{Nb}_{3} \mathrm{Sn}$ conductors $n=1 \pm 0.3$, for multifilamentary $\mathrm{V}_{3} \mathrm{Ga} n \cong 1.3$, for CVD $\mathrm{Nb}_{3} \mathrm{Ge}$ tape $n \cong 1.6$, and for multifilamentary $\mathrm{NbTi} n \cong 3.3$. The importance of this relationship is that, for these conductors at least, it is possible to measure $F$ at one strain and then immediately be able to predict $F$ (and thus the critical current) at other strain levels simply by scaling the results by $\left[B_{c 2}^{*}(\epsilon)\right]^{n}$. Part 1 of this paper presents the basic uniaxial-strain scaling relationship and focuses on its application to $\mathrm{Nb}_{3} \mathrm{Sn}$ conductors. The strain scaling law with $n=1 \pm 0.3$ was found to hold for all $\mathrm{Nb}-\mathrm{Sn}$ based conductors examined thus far, including commercialmultifilamentary conductors, extremely fine-filament composites, partially-reacted specimens, 'insitu' conductors, and Nb-Hf/Cu-Sn-Ga conductors. The detailed dependence of $B_{\mathrm{c} 2}^{*}$ on strain was found to be nearly universal for highly-reacted commercial $\mathrm{Nb}_{3} \mathrm{Sn}$ specimens, greatly simplifying the application of the scaling law to this group of practical superconductors. These results are discussed within the context of flux pinning models and a general scaling relation is proposed which unifies the usual temperature-scaling relation with this strain-scaling relation.

20067. Engen, G. F. A least squares solution for use in the six-port measurement technique, IEEE Trans. Microwave Theory Tech. MTT. 28, No. 12, 1473-1477 (Dec. 1980)

Key words: automatic network analyzer; microwave; microwave measurements; six-port

Although based on the use of simple amplitude detectors, it is possible to obtain complex values of reflection coefficient, via the sixport technique, from the intersection of three circles in the complex plane. In a typical case, the circle centers are determined primarily by the six-port design and are nominally constant, while the radii are proportional to the square root of the ratio of the output of three of the detectors to a fourth one. As a practical matter, however, these circles will not intersect in a point because of noise or other errors in the detectors.

This paper develops a procedure for choosing $\Gamma$ in this context. Moreover, the question of what may be inferred about the system performance from the extent of this intersection failure is briefly considered.

20068. Flynn, T. M.; Timmerhaus, K. D. Cold utilization from liquefied natural gas (LNG), (Proc. XV Int. Congress of Refrigeration, Venice, Italy, Sept. 23-29, 1979), Session A 3/27 in Progress in Refrigeration Science and Technology, 7 pages (International Institute of Refrigeration, Paris, France, 1980).

Key words: chemical engineering; cold utilization; cryogenic energy conservation; liquefied natural gas; refrigeration recovery.

One of the most striking characteristics of liquefied natural gas (LNG) is the vast amount of cold, or potential refrigeration, available which is now almost completely wasted. LNG, at $-125^{\circ} \mathrm{C}$, when referenced to a $+15^{\circ} \mathrm{C}$ datum plane, has approximately the same potential thermodynamic credit as low pressure steam at about $160^{\circ} \mathrm{C}$. However, very little has actually been done in this field of energy conservation. There are over 100 large LNG facilities in the world. Of these, only about four use the refrigeration potential in LNG, and none of these are in the United States. The four commercial facilities known to use the available refrigeration in LNG are: 1) the LNG receiving facility in Barcelona, Spain, which uses an LNG cryogenic process to recover heavy hydrocarbons from Libyan LNG; 2) a similar operation at La Spezia, Italy; 3) a Japanese facility which produces liquid oxygen, liquid nitrogen and affords warehouse refrigeration for frozen food as well; and 4) an LNG assisted air separation plant in France.

The important aspects of these four ventures together with some other potential applications of this relatively unused energy source will be discussed in this paper.

20069. Fickett, F. R.; Clark, A. F. Development of standards for superconductors, Proc. 8th Int. Cryogenic Engineering Conf., Genoa, Italy, June 3-6, 1980, C. Rizzuto, ed., pp. $494-498$ (IPC Science and Technology Press, Ltd., Guildford, Surrey, England, 1980).

Key words: conductor; critical current; critical temperature; 
review; standard; superconductor.

The U.S. National Bureau of Standards is now working in conjunction with the manufacturers and users of superconducting wires to arrive at a set of voluntary standards for making and reporting measurements on these materials. The program involves the preparation of standard definitions, investigation of measurement practices, experimental determination of the effect of various apparatus parameters, and development of standard test methods. We present results from experimental work on critical current and critical temperature determinations as well as the results of a round-robin test on critical current measurement.

20070. McDonald, D. G.; Peterson, R. L.; Hamilton, C. A.; Harris, R. E.; Kautz, R. L. Picosecond applications of Josephson junctions, IEEE Trans. Electron Devices ED-27, No. 10, $1945-1965$ (Oct. 1980).

Key words: electronics; Josephson effect; measurements; superconductivity; time domain.

The behavior of simple superconducting circuits in the picosecond regime is described in a comprehensive way, with primary emphasis being given to the step function and pulse responses of these circuits. Topics receiving detailed discussion include Josephson-junction modeling with both the microscopic and shunted-junction models. Limitations of the shunted-junction model are explored by comparing it with experimental results and with the microscopic model. An approximate evaluation is given of the important dynamical properties of junctions made with the dominant fabrication technology ( $\mathrm{Pb}$-alloy systems), as a function of tunneling barrier thickness. Rounding out the device aspects of the discussion, we describe in detail the properties of superconducting microstrip transmission lines, with an emphasis on their high-speed behavior. Turning to simple circuits we review experimental results on the measurement of picosecond regime transient signals. The concept of turn-on delay is analyzed anew, providing simplified and extended results. Details of concepts for pulse height and pulsewidth measurements are explored, leading to the conclusion that the time resolution of superconducting circuits is limited to approximately the period of one plasma oscillation. With present $\mathrm{Pb}$-alloy fabrication technology this limit is $2 \mathrm{ps}$.

20071. Diller, D. E. Measurements of the viscosity of compressed gaseous and liquid methane, Physica (Utrecht) A 104, No. 3, 417-426 (Dec. 1980).

Key words: density dependence; gaseous and liquid methane; isothermal measurements; quartz crystal viscometer; shear viscosity coefficient.

The shear viscosity coefficient of compressed gaseous and liquid methane has been measured at temperatures between 100 and $300 \mathrm{~K}$ and at pressures up to $30 \mathrm{MPa}$ (4350 psia) with a torsionally oscillating quartz crystal viscometer. The estimated precision and accuracy of the measurements are about $0.5 \%$ and $2 \%$, respectively. The measurements have been compared with an equation previously proposed for calculating the viscosity of gaseous and liquid methane. Most of the differences between the equation and the measurements reported here are within our experimental error. Larger differences (up to $6 \%$ ) are reported at the lowest temperature $(100 \mathrm{~K}$ ) and high pressures, and along a supercritical isotherm at $200 \mathrm{~K}\left(T \approx 1.05 T_{\mathrm{c}}\right)$.

20072. Younglove, B. A.; McCarty, R. D. Speed-of-sound measurements for nitrogen gas at temperatures from 80 to $350 \mathrm{~K}$ and pressures to $1.5 \mathrm{MPa}, J$. Chem. Thermodyn. 12, No. 12, 1121-1128 (1980).

Key words: electrostatic transducers; gas; nitrogen; sonic cavity; thermodynamic properties; velocity of sound; virial coefficiénts.

Speeds of sound for nitrogen gas have been measured at pressures from 0.03 to $1.5 \mathrm{MPa}$ on 16 isotherms from 80 to $350 \mathrm{~K}$. Measurements were made in a fixed-path acoustic cavity using variable-frequency electrostatic transducers operating between 1 and $30 \mathrm{kHz}$. Correction for boundary-layer effects were made using existing values of thermodynamic properties.

20073. Loevinger, R. A formalism for calculation of absorbed dose to a medium from photon and electron beams, Med. Phys. 8, No. 1, 1.12 (Jan.-Feb. 1981).

Key words: absorbed dose; Bragg-Gray equation; calibration; cavity chamber; cavity theory; electron beam; in-phantom measurement; photon beam; radiation therapy.

A formalism is derived that relates the absorbed dose to a medium from photon and electron beams to the photon calibration factor of an ionization chamber. The formalism is applicable to the photon and electron beam energies that are currently of interest in radiation therapy. It is developed in terms of a cavity-gas calibration factor, a quantity characteristic of the chamber and independent of the energy of the calibration beam assuming the energy expended per ion pair is energy independent. The cavity-gas calibration factor can be obtained from a chamber calibration performed in terms of exposure, absorbed dose to water, or air kerma. The perturbation corrections due to replacement of the surrounding medium by the chamber wall and cavity are identified as ratios of the photon energy fluence, or the electron fluence, at the position of the chamber center. The unmanageable complexities of a theory that covers an ionization chamber made of several materials are avoided by limiting the development to a chamber made of a single material with the expectation that the inhomogeneities of real chambers can be treated as perturbations. Attention is called to certain theoretical aspects of this dosimetry development that do not appear to have been previously recognized.

20074. Sparks, L. L. Low-temperature properties of expanded polyurethane and polystyrene, (Proc. Symp. Thermal Insulation Performance, Tampa, FL, Oct. 23-25, 1978), Am. Soc. Test. Mater. Spec. Tech. Publ. 718, D. L. McElroy and R. P. Tye, eds., pp. 431. 452 (1981).

Key words: cellular plastic; low temperature; mechanical properties; polystyrene; polyurethane; thermal insulation; thermal properties.

Two materials being used extensively as cryogenic insulations are the expanded plastics polyurethane (PU) and polystyrene (PS). These cellular plastics are excellent thermal insulators with measured values of apparent thermal conductivity at a mean temperature of $112 \mathrm{~K}$ being 0.013 to $0.016 \mathrm{~W} / \mathrm{m} \cdot \mathrm{K}$. The effect of aging and conditions of aging are critical factors in determining the ultimate conductivity. The major component of the overall conductivity, gas conductivity, is affected by permeation of gases through the cell walls. The reasonably high strength-to-density ratio for PU and PS at room temperature, 8 to $9 \mathrm{kPa} / \mathrm{kg} / \mathrm{m}^{3}$ compared with $\sim 50$ for aluminum alloys and $\sim 25$ for stainless steels, allows these insulations to be self. supporting and to lend structural support to the system. The thermal, elastic, and mechanical properties are functions of several nonindependent parameters such as density, cell size, composition, and method of processing. The properties of these expanded plastics are also anisotropic. The large variations found in existing data reflect these complex dependencies. Expected behavior and experimentally determined data are discussed for the following properties: tensile, compressive, and shear strengths and the associated moduli, thermal conductivity, linear thermal expansion, specific heat, and thermal diffusivity. Temperatures below $300 \mathrm{~K}$ are considered.

20075. Zelkowitz, M. V. A case study in rapid prototyping, SoftwarePract. Exper. 10, 1037.1042 (1980).

Key words: applicative programming; design; functional programming; interpreters; prototypes.

A case study in rapid prototyping of a new language processor is described. A quick version of the system was developed in SNOBOL4, revised in SNOBOL4, and then rewritten in Pascal. This led to an operational system very early in the development cycle where operational characteristics of the system could be tested easily. The goals of this experiment as well as some of the results are described.

20076. Metcalf, H.; Phillips, W. Time-resolved subnatural-width 
Key words: high resolution spectroscopy; subnatural-line width spectroscopy; time-resolved spectroscopy.

Spectra that are narrower than the natural width of decaying states can be achieved by beginning the observation of signals at a fixed delay time after excitation rather than immediately afterward. These signals are weaker (and noisier) than the full, time-unresolved signals. Applications of this line-narrowing technique to precision spectroscopy are discussed, and the properties of the resulting signals are studied. Numerical simulations demonstrate that time-resolved line narrowing is highly desirable in a large number of cases.

20077. Hollis, J. M.; Snyder, L. E.; Suenram, R. D.; Lovas, F. J. A search for the lowest-energy conformer of interstellar glycine, Astrophys. J. 241, 1001-1006 (Nov. 1, 1980).

Key words: amino acid̨s; ethylene oxide; glycine; interstellar; methyl formate; radio astronomy.

We have conducted the first search for the lowest-energy conformation of interstellar glycine. We have detected an emission line in Sgr B2 which is coincident in frequency with the $J_{K-K+}=$ $14_{1,14}-13_{1,13}$ transition of conformer I glycine, but other transitions have not yet been found. While the carrier of the observed line is uncertain, we know of no other frequency-coincident species and, hence, cannot rule out glycine. We have identified several previously unidentified interstellar lines as methyl formate and discuss the evidence for the existence of the elusive interstellar ethylene oxidethe only reported interstellar ring-structured molecule. Five unidentified interstellar lines were found.

20078. Fanney, A. H.; Liu, S. T. Experimental system performance and comparison with computer predictions for six solar domestic hot water systems, (Proc. Int. Solar Energy Society Silver Jubilee Congress, Atlanta, GA, May 29-June 1, 1979), Paper in Sun II, K. W. Boer and B. H. Glen, eds., 1, $972-976$ (Pergamon Press, New York, NY, 1979).

Key words: computer codes-F.CHART, SOLCOST, TRNSYS; computer predictions for solar systems; NBS solar testing at Gaithersburg; solar hot water heating.

Currently three computer programs, F.CHART, SOLCOST, and TRNSYS, are being extensively utilized for the design and evaluation of solar space heating and domestic hot water systems. Although widely used, the accuracy of their predictions needs to be verified with experimental data. In order to provide data required for the validation of these computer codes for solar domestic hot water (SDHW) systems, the staff at the National Bureau of Standards has fabricated and instrumented a solar hot water test facility at its Gaithersburg, Maryland site. This paper describes the test facility, the experimental results, and compares the experimental results with the computer predictions for the first eight months of operation.

20079. Jenkins, J. P. A comparison of test results for flat-plate waterheating solar collectors using the BSE and ASHRAE procedures, (Proc. Int. Solar Energy Society Silver Jubilee Congress, Atlanta, GA, May 29-June 1, 1979), Paper in Sun II, K. W. Boer and B. H. Glen, eds., 1, 365-369 (Pergamon Press, New York, NY, 1979).

Key words: collector efficiency; comparison German BSE vs ASHRAE 93-77 procedures; flat-plate solar collectors; German Bundesverband Solarenergie; solar collector testing; waterheating solar collectors.

The German Bundesverband Solarenergie (BSE) Working Group recently adopted and published a procedure for testing solar collectors based on thermal performance. Research facilities for testing flat-plate waterheating collectors have been built at NBS in accordance with the BSE procedure and the existing ASHRAE Standard 93.77. The purpose of this paper is to describe the BSE test procedure and compare experimental test results with those obtained using the existing ASHRAE Standard 93-77. Included is a description of the collector test facilities at NBS and the results obtained from using both procedures.

20080. Simmons, J. D.; Maki, A. G.; Hougen, J. T. Spectroscopic analysis of the $d^{1} \Sigma^{+} \rightarrow a^{1} \Sigma^{+}$and $d^{1} \Sigma^{+} \rightarrow b^{1} \Pi$ green band systems of XeO, J. Mol. Spectrosc. 74, 70-101 (1979).

Key words: bond length; dissociation energies; electronic spectrum; RKR curves; rotational analysis; xenon oxide.

A comprehensive high resolution spectroscopic analysis has been made on the $\mathrm{XeO}$ green bands photographed in emission from an RF discharge source. Rotation-vibration constants derived from the analysis of the spectrum of the isotopically enriched species ${ }^{129} \mathrm{Xe}^{16} \mathrm{O}$ and ${ }^{129} \mathrm{Xe}^{18} \mathrm{O}$ were used to give RKR potential curves for the $d^{1} \Sigma^{+}$ and $b^{1} \Pi$ states. The bond distances and dissociation energies of the $d^{1} \Sigma^{+}$and $b^{1} \Pi$ states were respectively found to be $r_{e}=2.852 \pm 0.002$ $\AA, D_{\mathrm{c}}=693 \pm 10 \mathrm{~cm}^{-1}$ and $r_{\mathrm{e}}=2.548 \pm 0.002 \AA, D_{\mathrm{e}}=461 \pm 10 \mathrm{~cm}^{-1}$. For the $a^{1} \Sigma^{+}$state it was not possible to establish a unique vibrational numbering or to construct an RKR potential curve, since observed bands of the $d^{1} \Sigma^{+} \rightarrow a^{1} \Sigma^{+}$system involve only high vibrational levels of the $a^{1} \Sigma^{+}$state, which are severely predissociated. The observations are consistent with a fairly deep well, in agreement with the latest $a b$ initio calculations which give a well depth of $0.7 \mathrm{eV}$.

20081. Belanger, B. C.; Kieffer, L. J. Regional measurement assurance programs for physical measurements, (Proc. 8th IMEKO Congress, Moscow, USSR, May 21.27, 1979), Paper in Acto Imeko, pp. 649. 656 (Publishing House of the Hungarian Academy of Sciences, Budapest, Hungary, 1980).

Key words: accuracy; measurement quality assurance; traceability.

A Measurement Assurance Program (MAP) is a measurement quality assurance program that allows one to demonstrate that the total measurement uncertainty including both random error and systematic components of error relative to national or other designated standards is quantified, and sufficiently small to meet the requirements for the measurement process. In this paper a particularly effective approach to MAP's involving groups of participating laboratories in particular regions of the U.S. is described and compared to more traditional approaches to the achievement of traceability to national standards.

20082. Rosenkrantz, M. E.; Stevens, W. J.; Krauss, M.; Konowalow, D. D. Dipole polarizabilities of the group IIb atoms obtained from compact variational trial functions, $J$. Chem. Phys. 72, No. 4, 2525 . 2531 (Feb. 15, 1980)

Key words: compact variational trial functions; dipole polarizabilities; excited state polarizabilities; group IIb atoms; polarizability anisotropies.

In order to optimize basis sets for future molecular calculations, the energies and dipole polarizabilities of ${ }^{1} S,{ }^{3} P$, and ${ }^{1} P$ states of $\mathrm{Zn}, \mathrm{Cd}$, and $\mathrm{Hg}$ have been determined from Hartree-Fock (HF) and multiconfiguration calculations. These utilize either empirically fitted pseudopotentials or $a b$ initio effective core potentials (ECP). Our calculated polarizabilities for ground state atoms agree within $10 \%$ with recommended values so long as either the empirical pseudopotentials or the ECP based on relativistic HF calculations are used. Our calculations agree with the measured anisotropy of the polarizability of ${ }^{3} P_{1} \mathrm{Hg}$, but disagree with measured values of the ${ }^{3} P_{2}$ state anisotropy. Our calculated ${ }^{3} P_{2}$ anisotropy is consistent with both the measured and calculated ${ }^{3} P_{1} \mathrm{Hg}$ anisotropy; the measured ${ }^{3} P_{2}$ values are not. We find calculations based on nonrelativistic ECP to give incorrect polarizabilities.

20083. Mecherikunnel, A. T.; Richmond, J. C. Spectral distribution of solar radiation, NASA Tech. Memorandum No. 82021, pp. 1.86 (Goddard Space Flight Center, Greenbelt, MD 20771, Sept. 1980).

Key words: atmospheric absorption; solar constant; solar energy; solar irradiance; spectral atmospheric absorptance; spectral distribution of solar energy; spectral solar irradiance; terrestrial 
solar spectral irradiance.

Available quantitative data on solar total and spectral irradiance is examined in the context of utilization of solar irradiance for terrestrial applications of solar energy. A brief review is given on the extraterrestrial solar total and spectral irradiances values. Computed values of solar spectral irradiance at ground level for different air mass values and various levels of atmospheric pollution or turbidity are also presented. Wavelengths are given for computation of solar absorptance, transmittance and reflectance by the 100 -selectedordinate method and by the 50-selected-ordinate method from air mass 1.5 and 2 solar spectral irradiance for the four degrees of atmospheric pollution.

20084. Quade, C. R.; Suenram, R. D. The microwave spectrum of $\mathrm{CH}_{2}$ DOH, J. Chem. Phys. 73, No. 3, 1127-1131 (Aug. 1, 1980).

Key words: internal rotation; interstellar; methanol; microwave spectrum; molecular radioastronomy; rotational spectrum.

The torsional-rotational spectrum of $\mathrm{CH}_{2} \mathrm{DOH}$ has been investigated in order to gain fuller understanding of the internal rotation parameters as well as to provide molecular radio astronomers with a set of measured transition frequencies which can be used for an interstellar search for the molecule. $a$ - and $b$-dipole transitions have been assigned within the three substates of the torsional ground state and $c$-dipole transitions have been assigned from the $e_{1}$ to the $o_{1}$ substate. The potential energy coefficient $V_{1}=12 \pm 1 \mathrm{~cm}^{-1}$ has been determined with $V_{2}$ constrained to zero. Although the torsional wave functions are by no means sharp, the molecule does localize to symmetric and asymmetric forms.

20085. Maki, A. G.; Wells, J. S. High-resolution measurement and analysis of the infrared spectrum of nitric acid near $1700 \mathrm{~cm}^{-1}, J$. Mol. Spectrosc. 82, $427-434$ (1980).

Key words: infrared; molecular spectra; nitric acid; pollution; spectroscopy's tunable lasers.

The infrared spectrum of the $v_{2}$ band of nitric acid $\left(\mathrm{HNO}_{3}\right)$ has been measured with a tunable diode laser in the frequency interval from 1690 to $1727 \mathrm{~cm}^{-1}$. A total of 430 assigned transitions have been analyzed to yield a set of nine rovibrational constants for the upper state with a standard deviation of $0.0012 \mathrm{~cm}^{-1}$. The band is primarily $B$ type with a band center at $1709.568 \pm 0.005 \mathrm{~cm}^{-1}$. Because of the absence of perturbations, the band constants can be used to calculate transition frequencies and relative intensities with a high degree of accuracy.

20086. Mies, F. H.; Stevens, W. J.; Krauss, M. Model calculation of the electronic structure and spectroscopy of $\mathbf{H g}_{2}, J$. Mol. Spectrosc. 72, 303-331 (1978).

Key words: electronic structure; excited states; mercury; spectra; spin-orbit coupling; transition probability.

Energy curves and transition moments of the excited valence states of $\mathrm{Hg}_{2}$ were obtained in a model calculation based on calculated $\mathrm{Mg}_{2}$ energy levels and the assumption that the asymptotic spin-orbit matrix elements for the $\mathrm{Hg}$ atom are applicable to the molecular states. The spin-orbit and orbital-rotational interaction of the excited states of $\mathrm{Hg}_{2}$ is analyzed in both a Hund's case (c) and (a) representation. The intermediate $(a) \leftrightarrow(c)$ transition moments are obtained as a function of the internuclear distance. The effect of the orbital-rotational interaction which introduces Hund's case $(b)$ and $(e)$ couplings is found to be small for transitions among excited states under the conditions normally encountered for populating excimer states.

Using the energy level positions and transition moments, the observed spectra and predicted spectra are compared for both radiative transitions including the ground state and among the excited states. The lifetime of the $1_{u}\left({ }^{3} \Sigma_{u}{ }^{+}\right)$excimer state is calculated to be 1.4 $\mu \mathrm{sec}$ with the $335 \mathrm{~nm}$ band assigned to the $\mathrm{l}_{\mathrm{u}} \rightarrow X^{1} \Sigma_{\mathrm{g}}{ }^{+}$transition. The $485 \mathrm{~nm}$ bands cannot be assigned to any $\mathrm{Hg}_{2}$ transitions. Strong bound-continuum absorptions are predicted for the $485 \mathrm{~nm}$ bands. On the other hand, the $335 \mathrm{~nm}$ emission is predicted to be absorbed by bound-bound transitions only.

20087. Okabe, H. Photodissociation of nitric acid and water in the vacuum ultraviolet; vibrational and rotational distributions of $\mathrm{OH}^{2} \Sigma^{+}, J$. Chem. Phys. 72, No. 12, $6642-6650$ (June 15, 1980).

Key words: nitric acid; photodissociation; vacuum ultraviolet; vibrational and rotational distribution.

The absorption cross sections of nitric acid have been measured in the $1100-1900 \AA$ region. The process $\mathrm{HONO}_{2} \rightarrow \mathrm{OH}\left({ }^{2} \Sigma\right)+\mathrm{NO}_{2}$ occurs below $1475 \AA$, much shorter than the thermochemical threshold at $2040 \AA$. The $\mathrm{OH}\left({ }^{2} \Sigma\right)$ fluorescence yield is less than $2 \%$. The vibrational and rotational distributions of $\mathrm{OH}\left({ }^{2} \Sigma\right)$ from $\mathrm{HONO}_{2}$ photolysis at $1236 \AA$ have been measured and compared with those from $\mathrm{H}_{2} \mathrm{O}$ and $\mathrm{H}_{2} \mathrm{O}_{2}$ photolysis. The excess energy beyond that required to dissociate the molecule and to excite ground state $\mathrm{OH}$ to $\mathrm{OH}\left({ }^{2} \Sigma\right)$ is converted to rotation and much less to vibration of $\mathrm{OH}\left({ }^{2} \Sigma\right)$ in contrast with linear cyanogen molecules where the excess energy appears as vibration and much less as rotation of a CN product. The results of internal energy partitioning have been compared with calculations based on a simple quasidiatomic impulsive model. The deviation from the model is attributed to either a process involving a large change in bond angle or in bond length. The rotational distributions of $\mathrm{OH}\left({ }^{2} \Sigma\right)$ at $v^{\prime}=0$ show a narrow peak at $N^{\prime}=20$ for $\mathrm{H}_{2} \mathrm{O}$ photolysis and a broad peak at $N=10$ for $\mathrm{HONO}_{2}$ photolysis both at $1236 \AA$. The extent of rotational excitation is expressed in terms of an impact parameter. The large impact parameter is found for $\mathrm{H}_{2} \mathrm{O}$ and $\mathrm{H}_{2} \mathrm{O}_{2}$ photolysis while for $\mathrm{HONO}_{2}$ photolysis the impact parameter is small. The rotational distributions of $\mathrm{OH}\left({ }^{2} \Sigma\right)$ from $\mathrm{H}_{2} \mathrm{O}$ and $\mathrm{H}_{2} \mathrm{O}_{2}$ photolysis at $1236 \AA$ deviate completely from Boltzmann behavior while that from $\mathrm{HONO}_{2}$ approaches the Boltzmann distribution. The process to yield the electronically excited $\mathrm{NO}_{2}$ from $\mathrm{HONO}_{2}$ photolysis at $1236 \AA$ is less than $0.5 \%$.

20088. Todd, T. R.; Olson, W. B. The infrared spectra of ${ }^{12} \mathrm{C}^{32} \mathrm{~S}$,

${ }^{12} \mathrm{C}^{34} \mathrm{~S},{ }^{13} \mathrm{C}^{32} \mathrm{~S}$, and ${ }^{12} \mathrm{C}^{33} \mathrm{~S}$, J. Mol. Spectrosc. 74, 190-202 (1979).

Key words: carbon sulfur; diode laser; free radical; infrared absorption; vibration-rotation spectra.

Using a tunable diode laser spectrometer, the infrared absorption spectra of four isotopic species of carbon monosulfide have been observed in the positive column of a dc discharge of $\mathrm{CS}_{2}$ and Ar. The wavenumbers of 115 vibration-rotation transitions between 1180.5 and $1266.1 \mathrm{~cm}^{-1}$ have been measured. These lines were assigned to the $1-0$, $2-1,3-2$, and $4-3$ bands of ${ }^{12} \mathrm{C}^{32} \mathrm{~S}$, the 1.0 and $2-1$ bands of ${ }^{12} \mathrm{C}^{34} \mathrm{~S}$ and ${ }^{13} \mathrm{C}^{32} \mathrm{~S}$, and the 1.0 band of ${ }^{12} \mathrm{C}^{33} \mathrm{~S}$. These new data have been combined with the previous infrared and microwave results to determine Dunham coefficients $\left(Y_{i j}\right)$, the Dunham potential expansion constants $\left(a_{0}, a_{1}, a_{2}, a_{3}\right.$, and $\left.a_{4}\right)$, and the classical turning points by the RKR method.

20089. Siu, M. C. I. Fibrous glass board as a standard reference material for thermal resistance measurement system, (Proc. Symp. Thermal Insulation Performance, Tampa, FL, Oct. 23-25, 1978), Am. Soc. Test. Mater. Spec. Tech. Publ. 718, D. L. McElroy and R. P. Tye, eds., pp. 343-360 (1981).

Key words: calibration; fibrous glass board; guarded hot plate; heat flow meter; SRM; thermal conductivity; thermal resistance.

Results of thermal measurements on over 300 pairs of fibrous glass board specimens, and statistical analysis of the data, are presented. This material is available as a Standard Reference Material (SRM) from the Office of Standard Reference Materials, National Bureau of Standards, Washington, D.C. 20234. Considerations are discussed for effective use of this SRM in the calibration of measurement systems such as the guarded hot-plate and heat flow meter apparatuses.

20090. Sattler, J. P.; Worchesky, T. L.; Tobin, M. S.; Ritter, K, J.; Daley, T. W.; Lafferty, W. J. Submillimeter-wave emission assigmments for 1,1-difluoroethylene, Int. J. Infrared and Millimeter Waves 1, No. 1, 127-138 (1980).

Key words: difluoroethylene; diode laser spectra; heterodyne; 
optical pumping; submillimeter wave emissions.

Many of the known submillimeter wave emissions of 1,1. difluoroethylene are assigned by using new data from infrared diode laser heterodyne spectroscopy. Six new submillimeter lines are also reported and assigned.

20091. Penn, R. W.; McKenna, G. B. Fatigue effects in poly(methyl methacrylate), (Proc. 176th Meet. ACS Symp. Series, Miami, FL, Sept. 11-14, 1978), Chapter 23 in Durability of Macromolecular Materials, pp. 331.339 (American Chemical Society, Washington, DC, 1979).

Key words: additivity of damage; Bailey criterion; damage; failure, fatigue; poly(methyl methacrylate) (PMMA); time dependent failure.

The fatigue failure of polymeric materials is a function of, not only the number of cycles to which the material is subjected, but also of the frequency of test and the waveform of the test (e.g., square wave, sinusoidal, etc.). In this work, we have examined the fatigue response of poly(methyl methacrylate) (PMMA) in the framework of the concept of additive damage. That is, damage which the PMMA experiences as a result of a particular stress history is not recovered, but accumulates to some point in time at which material failure occurs. The equation which expresses this concept is: $\int_{0}^{t B}$ $(\mathrm{d} \xi) / \tau(\sigma(\xi))=1$, where $\tau(\sigma)$ is the time to fail at constant $\sigma$. In principle, knowing the proper form of $\tau(\sigma)$ it is possible to predict the time to fail under a complex loading history. We have determined the function, $\tau(\sigma)$ for commercial sheets of PMMA in uniaxial extension, using a servo-controlled hydraulic test machine. Constant rate of load failure times and fatigue failure times as predicted from the experimentally determined function $\tau(\sigma)$ and the equation are compared with experimental results. The applicability of the concept of additivity of damage to failure of PMMA is discussed.

20092. Wilson, C. L. Correction of differential capacitance profiles for Debye-length effects, IEEE Trans. Electron Devices ED.27, No. 12, 2262.2267 (Dec. 1980).

Key words: capacitance-voltage profiles; doping profiles; finiteelement analysis; numerical simulations; Shottky barrier diodes.

Conventional differential capacitance-voltage $(C-V)$ profiling methods are limited in resolution by the method used to convert $C-V$ data to profile data. A new method of numerical calculation of $C-V$ data, using cubic finite elements, is presented which allows a more exact relationship between $C-V$ data and profile data to be calculated. This new method of data reduction allows the resolution of differential $C-V$ profiling method to be extended to dimensions near the local extrinsic Debye length.

20093. Lentner, K. J.; Childers, C. B.; Tremaine, S. G. A semiautomatic system for ac/dc difference calibration, IEEE Trans. Instrum. Meas. IM-29, No. 4, 400-405 (Dec. 1980).

Key words: ac/dc difference; ac voltage measurement automation; calibration; metrology; thermal voltage connectors.

A semiautomatic ac/dc difference calibration system is described. The system operates over a frequency range of $20 \mathrm{~Hz}$ to $100 \mathrm{kHz}$, covering the voltage range from $0.5 \mathrm{~V}$ to $1 \mathrm{kV}$. For all voltages at frequencies in the range from $20 \mathrm{~Hz}$ to $20 \mathrm{kHz}$, the total uncertainty is $50 \mathrm{ppm}$ and $100 \mathrm{ppm}$ for voltages at frequencies between $20 \mathrm{kHz}$ and $100 \mathrm{kHz}$. In addition to ac/dc difference testing, the system can be readily adapted to calibrate precision ac digital voltmeters or ac calibrators. Results of extensive intercomparison testing of the new system against a manual test system, using a multirange thermal transfer instrument as a transport standard, are reported, and the results indicate that the differences obtained are well within the combined total uncertainty limits of the two systems.

20094. Souders, T. M.; Lechner, J. A. A technique for measuring the equivalent RMS input noise of $A / D$ converters, IEEE Trans. Instrum. Meas. IM-29, No. 4, 251-256 (Dec. 1980).

Key words: A/D converter noise; analog-to-digital converters; equivalent input noise; feedback loop; Markov chains; noise measurement.

A simple accurate technique is described for measuring the equivalent rms input noise of A/D converters. Noise can typically be measured with 10-percent accuracy in $1 \mathrm{~s}$, and the method has successfully been applied to converters with up to 16 bits of resolution. The measurements are made at input voltages corresponding to the test converter's decision levels, where the effects of noise are most pronounced. A feedback loop incorporating the unit under test locates and locks onto these levels. The method utilizes a theoretical relationship between the input noise and an expected number of counts derived digitally from the feedback loop response. A low-noise wideband operational amplifier is the only critical component required.

20095. Akins, D. L.; King, D. S.; Stephenson, J. C. Vibrational relaxation of $\tilde{\mathrm{A}} \mathrm{CF}_{2}\left(v_{2}=1-6\right)$ in non-reactive collisions, Chem. Phys.

Lett. 65, No. 2, 257-260 (Aug. 15, 1979).

Key words: energy transfer; laser excited fluorescence; multiphoton dissociation; vibrational relaxation.

Rate constants have been measured for collisional energy transfer as a function of vibrational quantum number in the first excited singlet state of the $\mathrm{CF}_{2}$ radical. Total vibrational deactivation rates at $T=295$ $\mathrm{K}$ are reported for the bending mode, $\tilde{\mathrm{A}} \mathrm{CF}_{2}\left(2^{n}\right)$, for $1 \leqslant n \leqslant 6$ in collisions with $\mathrm{He}, \mathrm{Ne}, \mathrm{N}_{2}$ and $\mathrm{SF}_{6}$ which do not electronically quench the $\tilde{\mathrm{A}} \mathrm{CF}_{2}$. The predominant pathway of vibrational deactivation is the single-quantum change $\tilde{\mathrm{A}} \mathrm{CF}_{2}\left(2^{\prime \prime}\right)+\mathrm{M} \rightarrow \tilde{\mathrm{A}} \mathrm{CF}_{2}$ $\left(2^{n-1}\right)+\mathrm{M}+\Delta E=494 \mathrm{~cm}^{-1}$. However, as $n$ increases, rates for two- and three-quantum changes become increasingly important. These results are generally consistent with the principles of vibrational energy transfer found previously for ground electronic state molecules.

20096. Layer, H. P. A portable iodine stabilized helium-neon laser, IEEE Trans. Instrum. Meas. IM-29, No. 4, 358.361 (Dec. 4, 1980).

Key words: helium-neon; iodine; length standards; stabilized laser.

A newly designed iodine stabilized helium-neon (He-Ne) laser is described which is stable to $3 \times 10^{-13}$ (1000-s sample time) but which exhibits an intensity dependent shift of about $8 \mathrm{kHz} / W \cdot \mathrm{cm}^{2}$. Closer agreement between dissimilar lasers is attained when the internal power densities are approximately equal.

20097. Christensen, R. G.; Hertz, H. S.; Meiselman, S.; White, E Liquid chromatograph/mass spectrometer interface with continuous sample preconcentration, Anal. Chem. 53, No. 2, 171.174 (Feb. 1981).

Key words: liquid chromatography; liquid chromatography/mass spectrometry; mass spectrometry; phenols; polynuclear aromatic hydrocarbons; quantitation; sample concentrator; trace organic analysis.

A liquid chromatograph/mass spectrometer system which performs enrichment of the sample in the effluent of a conventional liquid chromatograph prior to its being introduced into a differentially pumped quadrupole mass spectrometer is described. The effluent from the liquid chromatograph is concentrated by evaporation of most of the solvent. Solvent evaporation is accomplished by allowing the effluent to flow down an electrically heated wire with the current to the wire controlled by a feedback loop from a volume-sensing photocell. The concentrated effluent flows through a very small needle valve which regulates the flow into and thereby the pressure inside the mass spectrometer. The valve is constructed such that liquid is sprayed into the ion source of the mass spectrometer. The application of the system to polynuclear aromatic hydrocarbon characterization and to quantitation of phenolic compounds in alternate fuels is shown.

20098. Reeder, D. J. Place of reference materials and reference methods in the evaluation of drug effects, (Proc. Use of Laboratory Test Results. Variation Due to Drug Intake, Pont-à-Mousson, 
France, Dec. 17-19, 1979), Paper in Drug Effects on Laboratory Test Results. Developments in Clinical Biochemistry, G. Siest, ed., 2, 49-57 (Martinus Nijhoff Publishers, Boston, MA, 1980).

Key words: accuracy; drug effects; laboratory tests; precision; reference materials; reference methods; variability factors.

The presence of analytical errors in clinical laboratory testing may be the result of many factors. Even though most clinical tests are becoming very precise, reliance on precise tests for accurate analytical values is not sufficient because of potential bias caused by drug effects. Proper analytical techniques can minimize the sources of inaccuracies caused by variabilities and interferences but a true understanding of drug effects can only be obtained when measurements are placed on an accuracy base. Assurance that measurements are accurate can best be accomplished by use of reference materials and reference methods that are free of interferences and that can establish the accuracy of an analytical value. Development of new reference materials and reference methods will become increasingly important for proper health care.

20099. Hougen, J. T. Double group considerations, Jahn-Teller induced rovibronic effects, and the nuclear spin-electron spin hyperfine Hamiltonian for a molecule of symmetry $C_{3 v}$ in an electronic ${ }^{2} E$ state, J. Mol. Spectrosc. 81, 73.92 (1980).

Key words: degenerate electronic state; double groups; Hamiltonian; hyperfine; Jahn-Teller effect; methoxy; rotational levels.

Two different double groups of the point group $C_{3 v}$ are compared. These double groups arise for molecules in a ${ }^{2} E$ state, and are associated with the half-integral electron spin, and with a half-integral vibronic quantum number, respectively. For many aspects of molecular energy level calculations, the use of these double groups can be avoided if desired. Contributions to the effective rotational Hamiltonian for a $C_{3 v}$ molecule in a ${ }^{2} E$ electronic state are derived. These contributions correspond to large "l-type doubling" and " $(2$, -1) resonance" terms, and arise because of the Jahn-Teller distortion tendencies of the molecule. Similar contributions to the effective rotational Hamiltonian, which arise from interactions with other electronic states, are also briefly considered. A hyperfine Hamiltonian is derived, which describes the interaction of the three proton nuclear spins in a $C_{3 v}$ molecule of the type $\mathrm{CH}_{3} \mathrm{O}$ with the odd electron spin in a ${ }^{2} E$ electronic state. A prescription for determining hyperfine matrix elements in a Hund's case $\left(a_{\beta}\right)$ basis set is given.

20100. Julienne, P. S.; Konowalow, D. D.; Krauss, M.; Rosenkrantz, M. E.; Stevens, W. J. Photodissociation of $\mathbf{H g C l}$, Appl. Phys. Lett. 36, No. 2, 132-134 (Jan. 15, 1980).

Key words: ab initio calculations; cross section; $\mathrm{HgCl}$ laser; mercurous chloride; photodissociation; relativistic pseudopotentials.

Transition moments and energies have been calculated for the $X-A$, bound to continuum, transition in $\mathrm{HgCl}$. The photodissociation cross section at the peak $\left(-20000 \mathrm{~cm}^{-1}\right.$ or $\left.500 \mathrm{~nm}\right)$ is $1.2 \times 10^{-18} \mathrm{~cm}^{2}$ at 600 $\mathrm{K}$. The laser energy $17921 \mathrm{~cm}^{-1}$ or $558 \mathrm{~nm}$ ) for the $B \cdot X$ transition is close to the peak energy, and a significant absorption will result from a buildup in the concentration of $X$ state $\mathrm{HgCl}$ in the course of exciting the laser media. This absorption is suggested as an explanation for the low extraction efficiencies observed for $\mathrm{HgCl}$ lasers.

20101. King, D. S.; Stephenson, J. C. Intrinsic isotopic selectivity factors: $\mathrm{CO}_{2}$ TEA laser photolysis of $\mathrm{CF}_{2} \mathrm{Cl}_{2}, \mathrm{~J} . \mathrm{Am}$. Chem. Soc. 100, No. 23, $7151-7155$ (Nov. 8, 1978).

Key words: $\mathrm{CF}_{2} \mathrm{Cl}_{2}$ (difluoro dichloromethane, Freon-12); $\mathrm{CO}_{2}$ laser; collision free multiphoton dissociation; isotopic selectivity factors; laser excited fluorescence; laser isotope separation; multiphoton photochemistry.

The first real-time determination of isotopic selectivities in a pulsed
IR photolysis is reported. In situ detection of nascent $\mathrm{CF}_{2}$ photofragments by UV laser-excited fluorescence gave the probability of $\mathrm{CF}_{2}$ production from $\mathrm{CF}_{2} \mathrm{Cl}_{2}$ samples of natural $\left(98.9 \%{ }^{12} \mathrm{C}\right)$ and enriched $\left(92.0 \%{ }^{13} \mathrm{C}\right)$ carbon-13 abundance. These measurements gave the probability, ${ }^{12} \mathrm{P}$ or ${ }^{13} \mathrm{P}$, of a ${ }^{12} \mathrm{CF}_{2} \mathrm{Cl}_{2}$ or ${ }^{13} \mathrm{CF}_{2} \mathrm{Cl}_{2}$ reactant dissociating into a ${ }^{12} \mathrm{CF}_{2}$ or ${ }^{13} \mathrm{CF}_{2}$ photofragment, as a function of IR laser wavelength and fluence, prior to any energy exchange or scrambling processes. The maximum value for the selective collisionfree dissociation of ${ }^{12} \mathrm{CF}_{2} \mathrm{Cl}_{2}$ was $\alpha_{0}={ }^{12} P /{ }^{13} P=460$; for ${ }^{13} \mathrm{CF}_{2} \mathrm{Cl}_{2}$ the maximum value $1 / \alpha_{0}={ }^{13} P /{ }^{12} P=150$ was obtained

20102. Krauss, M.; Stevens, W. J.; Neumann, D. B. The dispersion damping functions and interaction energy curves for Xe-Xe, Chem. Phys. Lett. 71, No. 3, 500.502 (May 1, 1980).

Key words: dipole; dispersion; interaction energy; octupole; polarizabilities; quadrupole; Xe-Xe.

$A b$ initio potential energy curves have been calculated for the ground state of $\mathrm{Xe}_{2}$ including dispersion interactions. The total interaction energy has been approximated by a sum of the repulsive Hartree-Fock interaction plus the attractive dispersion interaction which is appropriately damped to take account of charge overlap.

20103. Fanney, A. H.; Liu, S. T. Performance of six solar domestic hot water systems in the mid-Atlantic region, Proc. Conf. Solar Heating and Cooling Systems Operational Results, Colorado Springs, CO, Nov. 27-30, 1979, pp. 25-31 (SERI/TP 245-430, DOE/Solar Energy Research Institute (SERI), Golden, CO, Nov. 1979).

Key words: energy; heat transfer; hot water; measurement; solar; testing.

In order to provide data required for the validation of computer programs for solar domestic hot water systems, the staff of the National Bureau of Standards (NBS) fabricated and instrumented six typical residential-size systems at its Gaithersburg, Maryland site. Three of the six systems utilize a single hot water tank and the other three utilize two tanks. Three different methods of freeze protection are being utilized among the systems: water-ethylene glycol mixture in the collector loop with an intermediate heat exchanger, drain-down using solenoid valves to dump the water from the collectors, and air. heating collectors in conjunction with an air-water heat exchanger. One system relies on the thermosyphon principle while the other five require electric power for fans or pumps. The systems have been operating since June 1978. This paper describes the testing done and gives the experimental results for the first year of operation.

20104. Ekin, J. W. Strain scaling law and the prediction of uniaxial and bending strain effects in multifilamentary superconductors, (Proc. Topical Conf., Upton, NY, May 28-29, 1980), Paper in Filamentary A15 Superconductors, M. Suenaga and A. F. Clark, ed., pp. 187.203 (Plenum Press, New York, NY, 1980).

Key words: critical current; multifilamentary $\mathrm{Nb}_{3} \mathrm{Sn} ; \mathrm{Nb}_{3} \mathrm{Sn}$; strain effects; strain scaling law; superconductors.

The strain-scaling law has been used to determine uniaxial-strain characteristics which describe the critical-current degradation in commercial multifilamentary $\mathrm{Nb}_{3} \mathrm{Sn}$ conductors from $4 \mathrm{~T}$ to $16 \mathrm{~T}$. Bending-strain degradation has then been calculated from the uniaxialstrain characteristics by taking an average appropriate for the twistpitch to current-transfer-length ratio of the conductor.

20105. Faulkner, D. J.; Freeman, K. C. Gas in globular clusters. 1 Time-independent now models, Astrophys. J. 211, No. 1, 77.90 (Jan. 1 1977).

Key words: globular clusters; hydrodynamics; interstellar matter; mass loss, stars; radiation radio sources; $21-\mathrm{cm}$.

A search for $\mathrm{Ha}$ emission in five globular clusters has yielded upper-limit ionized hydrogen masses within the core radii of $M_{H}+$ $<0.1-0.7 M_{0}$. This augments the findings of previous searches for ionized hydrogen (both radio and optical) that clusters are gas deficient with respect to the mass loss predictions of evolutionary 
theory, if the gas remains in the cluster.

Time-independent gas flow models have been constructed for globular clusters of $10^{5} \mathrm{M}_{\odot}$ and $10^{6} \mathrm{M}_{\odot}$, with physical assumptions chosen to maximize the predicted gas content. Ionization by electron impact was assumed, and the ionization state of both hydrogen and helium was calculated throughout the flow; the radiative cooling of the gas under these conditions was treated with considerable care. For the $10^{5} M_{0}$ cluster, steady-state flows were obtained for all gas input energies, $\beta$, down to $(2 \beta)^{1 / 2}=17 \mathrm{~km} \mathrm{~s}^{-1}$, and it seems certain that gas outflow will occur for all plausible stellar mass loss mechanisms. For the $10^{6} M_{\odot}$ cluster, radiative cooling prevented gas outflows for input energies less than $(2 \beta)^{1 / 2}<120 \mathrm{~km} \mathrm{~s}^{-1}$, and it appears that red giant-branch mass loss will result in the gradual accumulation of gas.

Integrated surface brightness and flux values for the flow models showed no clear conflict with the presently observed upper limits for the $21 \mathrm{~cm}$ line, the radio free-free, and $H \alpha$. In the $21 \mathrm{~cm}$ line case, however, the observations are on the threshold of the predicted emission for $10^{5} M_{\bullet}$ cluster flow models with no photoionization and with very low gas ejection energy, and further attempts to detect neutral hydrogen seem well worthwhile.

20106. Cutkosky, R. D. An automatic resistance thermometer bridge, IEEE Trans. Instrum. Meas. IM-29, No. 4, 330-333 (Dec. 1980).

Key words: automation; bridge; measurement; ohmmeter: resistance; thermometry; transformer.

A microprocessor-controlled bridge is described that utilizes a 15 $\mathrm{Hz}$ or $30-\mathrm{Hz}$ square waves to excite a shielded multistage transformer. Transformer taps are relay selected to null the detector. The reading, up to $32 \Omega$ in steps of $1 \mu \Omega$, is then displayed. An IEEE-488 bus provides remote operation.

20107. Bender, P. L. Improved methods for measuring present crustal movements, Paper in Dynamics of Plate Interiors, Geodynamics Series 1, 155-162 (American Geophysical Union, Washington, DC, 1980).

Key words: electromagnetic distance measurements; geodynamics; gravity; plate tectonics; space techniques.

Improvements in geodetic measurement techniques are likely to play an important role in a number of types of geodynamics studies during the next decade. Increased accuracy for horizontal distance and gravity measurements at many sites is expected using mutliplewavelength measurements and falling-retroreflector gravimeters. Improved tiltmeters and strainmeters are being developed, with attention being given to decreasing perturbations due to very local ground noise. Geodetic receivers using signals from the Global Positioning System satellites probably will make possible rapid relative position measurements with 1 to $3 \mathrm{~cm}$ accuracy in and around seismic zones. An international program of worldwide position measurements with about $3 \mathrm{~cm}$ accuracy is planned, using both laser range measurements to the LAGEOS satellite and long baseline radio interferometry.

20108. Chanmugam, G. Cyclotron absorption in accreting magnetic white dwarfs, Astrophys. J. 241, No. 3, 1122-1130 (Nov. 1, 1980).

Key words: binary stars; magnetic stars; ultraviolet sources; white dwarfs.

The cyclotron absorption coefficient is calculated using a threedimensional Maxwellian distribution for the electrons for a wide range of temperatures and frequencies. Numerical fits of the form $\alpha(\omega, \theta)=\exp \left[-\left(a_{0}+a_{1} \eta^{2}+a_{3} \eta^{6}\right)\right]$, where $\eta=[(\pi / 2)-\theta]$ are presented and compared with previous results, whose range of validity is clarified. The results are applied to a plasma slab which is perpendicular to the magnetic field, and it is shown that there are deviations from the Rayleigh-Jeans spectrum predicted in earlier works. Comparison with observations for AM Herculis suggests that its magnetic field is about $5 \times 10^{7}$ gauss (or less).

20109. Rafelski, J.; Danos, M. The importance of the reaction volume in hadronic collisions, Phys. Lett. 97B, No. 2, 279-282 (Dec. 1, 1980).

Key words: hadrons; heavy flavors; heavy ion collisions; statistical models; strangeness; strong interactions; thermodynamic models.

The pair production in the thermodynamic model is shown to depend sensitively on the (hadronic) reaction volume. Strangeness production in nucleus-nucleus collisions is treated as an example.

20110. Reader, J.; Luther, G.; Acquista, N. Revised $6 p^{2} P_{1 / 2}$ level of Mo XIV. J. Opt. Soc. Am. 71, No. 2, p. 204 (Feb. 1981).

Key words: ionized; molybdenum; spectrum; ultraviolet; vacuum; wavelengths.

New observations of the $4 s-6 p$ transitions of Mo xIV have led to a revised identification for the $4 d^{2} D_{3 / 2}-6 p^{2} P_{1 / 2}$ transition and a revised value for the $6 p^{2} P_{1 / 2}$ level.

20111. Maki, A. G.; Lovas, F. J. The infrared spectrum of ${ }^{31} \mathrm{P}^{14} \mathrm{~N}$ near $1300 \mathrm{~cm}^{-1}$, J. Mol. Spectrosc. 85, 368.374 (1981).

Key words: diode laser; high temperature; infrared spectrum molecular constants; phosphorus nitride; rotation-vibration spectrum.

The infrared absorption spectrum of the PN molecule has been measured at temperatures between 800 and $1050^{\circ} \mathrm{C}$ with a tunable diode laser. The transitions measured ranged from $J^{\prime \prime}=3$ to $J^{\prime \prime}=53$ and included the vibrational transitions $v=1 \leftarrow 0,2 \leftarrow 1,3 \leftarrow 2$, and $4 \leftarrow 3$. These measurements are combined with microwave measurements made by others to yield a consistent set of ten Dunham ro-vibrational constants and their uncertainties.

20112. Myers, D. R.; Wilson, R. G.; Comas, J. Considerations of ion channeling for semiconductor microstructure fabrication, J. Vac. Sci. Technol. 16, No. 6, 1893-1896 (Nov./Dec. 1979).

Key words: dopant profiles; ion channeling; ion implantation; randon equivalent direction; semiconductor device fabrication; semiconductor devices.

The performance of semiconductor devices becomes increasingly sensitive to details of the depth distribution of dopants as device dimensions are reduced. In this paper, limitations on the control of dopant distributions resulting from implantation into single-crystal silicon due to ion channeling are examined for a range of ion atomic numbers and energies characteristic of semiconductor device fabrication. Implantation profiles were obtained by $1 \mathrm{MHz}$ differential capacitance-voltage profiling and by secondary ion mass spectroscopy for silicon substrates implanted at angles from accurately channeled alignment to "random equivalent" orientation. The critical angle as calculated from an existing computer fit to the Moliere continuum potential was used to scale the angular dependence of the implantation profiles. Ion channeling is seen to have important consequences not only for the deeply penetrating regions of the dopant profile but for the near-surface profile as well. The results of this study indicate that to minimize the extent of unintentional channeling, alignment of the ion beam to the nearest low-index crystallographic direction must be at angles exceeding twice the critical angle and that the angle required can exceed the commonly used seven degree tilt angle, especially for ions of large atomic number at low energy.

20113. Linsky, J. L. Theory of stellar coronae: An interpretation of $x$-ray emission from non-degenerate stellar sources, (Proc. HEAD/AAS, Cambridge, MA, Jan. 28-30, 1980), Paper in Cool Stars, Stellar Systems, and the Sun, A. K. Dupree, ed., pp. 217-235 (Smithsonian Institute, Cambridge, MA, 1980).

Key words: stars, coronae; stars, winds; $\mathrm{x}$ rays, sources.

I present arguments to demonstrate that the acoustic wave heating theory of stellar coronae is inadequate to explain Einstein observations of stellar coronae as well as previous ultraviolet and X-ray observations of the Sun and other stars. I outline the various lines of evidence that imply that magnetic fields, stellar rotation rates, and to some extent convection zone parameters are the important quantities in determining coronal heating and thus X-ray emission. These 
general results and the recent Einstein Observatory stellar observations suggest a speculative scenario of stellar coronae which is described in detail.

20114. Nesbitt, D. J.; Leone, S. $\mathrm{R}$. $\mathrm{Br}^{*}\left({ }^{2} P_{1 / 2}\right)+\mathrm{H}_{2}(v=0,1)$ : Laser studies of the competition hetween reactive pathways and inelastic energy transfer channels, J. Chem. Phys. 73, No. 12, 6182-6190 (Dec. $15,1980)$.

Key words: electronic excitation; excited states; laser; reaction dynamics; spin-orbit; vibrational excitation.

A systematic experimental study has been made on inelastic and reactive processes in the $\operatorname{Br}^{*}\left({ }^{2} P_{1 / 2}\right)+\mathrm{H}_{2}(v=0,1)$ system. Laser photolysis and time-resolved infrared detection techniques allow direct observation of the previously unreported $E-V$ equilibrium: $\mathrm{Br}^{*}+\mathrm{H}_{2}(v=0) \rightleftharpoons \mathrm{Br}+\mathrm{H}_{2}(v=1)$. A nonlinear kinetic analysis is presented that permits an estimate of the quantum efficiencies for the forward and reverse $E-V$ processes, and provides an explanation for previous discrepancies in measured total $\mathrm{Br}^{*}$ quenching rates with $\mathrm{H}_{2}$. In this work the quenching rate constant for $\mathrm{Br}^{*}$ by $\mathrm{H}_{2}$ is determined at very low $\mathrm{Br}^{*}$ concentrations to be $6.3( \pm 1) \times 10^{-12} \mathrm{~cm}^{3}$ molecule $\mathrm{sec}^{-1}$. At higher concentrations of $\mathrm{Br}^{*}$, second order processes involving collisions of $\mathrm{Br}^{*}$ with $\mathrm{H}_{2}(v=1)$ have been investigated. A substantial fraction of the $\mathrm{Br}^{*}+\mathrm{H}_{2}(v=1)$ collisions react to form $\operatorname{HBr}(v=0)+\mathrm{H}\left({ }^{2} S_{1 / 2}\right)$. It is suggested that the resonant $E-V$ transfer pathway in the entrance channel $\mathrm{Br}^{*}+\mathrm{H}_{2}(\nu=1) \rightarrow \mathrm{Br}+\mathrm{H}_{2}(\nu=2)$ may provide an efficient mechanism for potential surface crossing thereby enhancing the reaction probability.

20115. Hougen, J. T.; Oka, T. Vihrationally induced nuclear quadrupole coupling in tetrahedral and octahedral molecules, $J$. Chem. Phys. 74, No. 3, 1830.1839 (Feb. 1, 1981).

Key words: degenerate vibrational effects; hyperfine energy levels; hyperfine Hamiltonian; quadrupole coupling theory; spherical top molecules; tetrahedral and octahedral molecules.

Hyperfine splittings arising from the presence of a quadrupolar nucleus at the center of a molecule belonging to the point group $T_{d}$ or $\mathrm{O}_{h}$ (e.g., ${ }^{189} \mathrm{OsO}_{4}$ or ${ }^{235} \mathrm{UF}_{6}$ ) are symmetry forbidden to a high degree of approximation. Nevertheless, quadrupole splittings can be induced by either vibrational or rotational distortions of the molecule, i.e., by distortions similar to those responsible for inducing electric dipole moments in $T_{d}$ molecules. Such hyperfine splittings have recently been observed in several laboratories using laser saturation spectroscopy. In this paper we investigate theoretically the quadrupole splittings induced by excitation of doubly and triply degenerate vibrations in $T_{d}$ and $O_{h}$ molecules. We find that much of the vibration-rotation formalism already present in the methane literature can be applied with only minor changes to the inducedquadrupole-coupling problem, and that rather simple theoretical relationships can be derived between the quadrupole splitting and the tetrahedral or octahedral vibration-rotation splitting of a given level. Values for the scalar and tensor contribution to the quadrupole coupling constant have been derived from the experimental data for ${ }^{189} \mathrm{OsO}_{4}$ reported by Bordé et al.

20116. Misra, D. N.; Bowen, R. L. Adsorptive bonding to hydroxyapatite I: Adsorption of anthranilic acid-the effect of solvents. Search for surface bonding groups for coupling agents to teeth, Biomater. 2, 28-32 (Jan. 1981).

Key words: adsorption; hydrogen-bonding; hydroxyapatite; Langmuir isotherm; reversibility.

A study was initiated to explore the nature of surface-active groups of model compounds that might cause chemisorption of comonomers used in the polymerization of dental resins on hydroxyapatite (the main constituent of bone) and thus effect a strong and durable bond between the resins and hard tooth tissues. In an effort to accomplish this objective, adsorption of anthranilic acid on hydroxyapatite was studied from three solvents: $95 \%$ aq. ethanol, dimethyl sulphoxide and methylene chloride. The adsorption is reversible from the first two solvents and the isotherms follow Langmuir plots. Adsorption is irreversible from methylene chloride; a constant amount of adsorbate is removed from different solutions above a certain threshold concentration but all adsorbate is removed from solutions below this threshold concentration. The saturation amounts obtained from the two Langmuir plots and the maximum irreversibly adsorbed amount in the above three solvents are $11.4,6.0$ and $17.2 \mathrm{mmol} / 100 \mathrm{~g}$, respectively. These, together with the surface area $\left(41.0 \mathrm{~m}^{2} / \mathrm{g} ; \mathrm{BET}\right.$, $\mathrm{N}_{2}$ ) of the hydroxyapatite, yield the effective molecular areas of 60 , 114 , and $40 \AA^{2}$, respectively. These areas can be matched, on geometrical considerations, with areas of 60,110 , and $40 \AA^{2}$ of the model adsorbate molecule rotating about: (i) its centre, lying flat on the surface, (ii) its carboxyl group at $45^{\circ}$ to the surface, and (iii) the carboxyl group in an upright position, respectively.

20117. Roth, $R$. S. Phase equilihria research in portions of the system $\mathrm{K}_{2} \mathrm{O}-\mathrm{MgO}-\mathrm{Fe}_{2} \mathrm{O}_{3}-\mathrm{Al}_{2} \mathrm{O}_{3}-\mathrm{SiO}_{2}$, (Proc. Symp. Solid State Chemistry: A Contemporary Overview, Laramie, WY, July 30. Aug. 4, 1978), Paper in Advances in Chemistry Series, No. 186, pp. 391-408 (American Chemical Society, Washington, DC, 1980).

Key words: crystal chemistry; crystal growth; phase equilibria; system $\mathrm{K}_{2} \mathrm{O} \cdot \mathrm{MgO} \cdot \mathrm{Fe}_{2} \mathrm{O}_{3} \cdot \mathrm{Al}_{2} \mathrm{O}_{3} \cdot \mathrm{SiO}_{2}$

Preliminary phase equilibria diagrams are given for some binary and ternary joins and cuts through the five-component system $\mathrm{K}_{2} \mathrm{O}$. $\mathrm{MgO} \cdot \mathrm{Fe}_{2} \mathrm{O}_{3} \cdot \mathrm{Al}_{2} \mathrm{O}_{3}-\mathrm{SiO}_{2}$. The diagrams are those that are most pertinent to the chemical reactions taking place between potassium seed and the four-component synthetic slag of importance in corrosion studies of a magnetohydrodynamics system. Although the systems $\mathrm{KAlO}_{2}-\mathrm{SiO}_{2}, \mathrm{KFeO}_{2}-\mathrm{SiO}_{2}$, and $\mathrm{K}_{2} \mathrm{MgSiO}_{4}-\mathrm{SiO}_{2}$ are apparently true binary joins, other pseudobinary cuts involving only kalsilite types of end members are generally not binary. The phase $\mathrm{K}_{2} \mathrm{MgSi}_{3} \mathrm{O}_{8}$ dissociates at low temperatures into a second kalsilite-like phase plus leucite. Thus leucite is a major component even just below liquidus temperatures of magnesia containing ternary and quaternary compositions that might be expected to exhibit only kalsilite-like phases.

20118. Mountain, R. D. Effective pair potentials from structure factors, (Proc. 4th Int. Conf. Liquid and Amorphous Metals, Grenoble, France, July 7-11, 1980), Paper in Journal de Physique C8, No. 41, Suppl. No. 8, C8.297-C8.300 (Les Editions de Physique, Orsay, France, Aug. 1980).

Key words: effective pair potential; liquid metal; liquid rubidium; Monte Carlo; pair distribution function; structure factor.

A procedure based on the Percus-Yevick equation for extracting pair potentials from structure factor data for liquid metals at elevated temperatures and reduced densities is described and applied to fluid rubidium. Monte Carlo results are used to establish the limits of the validity of the procedure.

20119. Wilson, W. K.; Parks, E. J. Comparison of accelerated aging of book papers in 1937 with 36 years natural aging, Restaurator 4, No. $1,1.55$ (1980).

Key words: accelerated; aging; alpha cellulose; comparison; copper number; fold; humid; natural; paper; $\mathrm{pH}$; tear; tensile strength.

In 1937 a group of 72 book papers, made in the National Bureau of Standards experimental paper mill, was tested before and after accelerated aging for 72 hours at $100^{\circ} \mathrm{C}$ in a circulating oven. In 1973, it was possible to retest 18 of these papers from three fiber sources, with six papers in each fiber group. Most of the papers were sized with rosin and alum, and some contained fillers.

Within the limitations of the significance of the accelerated aging procedure, the following conclusions may be drawn: (1) There are reasonably high correlations between changes in alpha cellulose and in copper number after accelerated aging in 1937 and after 36 years of natural aging. (2) Cold extract $\mathrm{pH}$ in 1937 correlates reasonably well with changes after natural aging in alpha cellulose, copper number, fold, tear and elongation. (3) Zero span tensile strength and wet strength as a percent of dry strength appear to be useful parameters in stability determinations.

Comparison with Parks' data on the accelerated aging of 
handsheets at $90^{\circ} \mathrm{C}$ at zero percent and at fifty percent relative humidity suggests that dry accelerated aging corresponds more nearly to natural aging than does accelerated aging at 50 percent relative humidity, but that accelerated aging should include some moisture. Graminski's recent data, obtained at various temperatures and relative humidities, support this conclusion.

The following tests appear to be useful in evaluating changes that occur in the aging of paper: alkali solubility, reducing power, $\mathrm{pH}$, internal tearing strength, elongation, zero span tensile strength, and strength when wet as percentage of strength when dry.

20120. Ledbetter, H. M.; Maerz, G. Temperature dependence of Young's modulus and internal friction of G-10CR and G-11CR epoxy resins, Cryogenics 20, No. 11, 655.658 (Nov. 1980).

Key words: composites; elastic constants; epoxy resin; internal friction; low temperatures; Young's modulus.

Young's moduli of the epoxy-resin matrix material used in NEMAdesignation G.10CR and G.11CR fibreglass-cloth-reinforced composites were measured dynamically and semicontinuously between ambient and liquid-nitrogen temperatures. Both materials exhibit regular temperature behaviour, showing large Young'smodulus changes, about 125 and $50 \%$, respectively. Internal friction decreased about $80 \%$ during cooling to liquid-nitrogen temperature $(76 \mathrm{~K})$. The different thermoelastic coefficients together with different internal friction reflect different internal structures in the two materials.

20121. McCabe, M. E. A collector sizing procedure for residential solar hot water systems with prescribed thermal performance requirements, (Proc. ASHRAE Semiannual Meet., Los Angeles, CA, Feb. 3.7, 1980), ASHRAE Trans. 86, Pt. 1, 420-439 (American Society of Heating, Refrigerating and Air Conditioning Engineers, Inc., New York, NY, 1980).

Key words: f-chart; HUD initiative; performance standards; solar collectors; solar water heaters; storage tanks; thermal performance.

A graphical procedure was developed based on the f-chart design method to determine whether solar domestic water heating systems proposed for use in the HUD Hot Water Initiative Program have sufficient collector area to provide $50 \%$ of the load as prescribed by the thermal performance requirements of the Program. The procedure was specifically developed for the 11 states participating in the HUD initiative (the New-England and Mid-Atlantic States and Florida), however, it should be applicable for the sizing of the collectors for solar water heaters in other locations with similar climates. In the procedure, the collector area is first determined for a reference solar water heating system located in one of 25 locations. Area adjustment factors are then determined for a number of possible differences between the proposed system and the reference system. The required collector area is calculated as the product of the reference area and the area adjustment factors. The maximum difference in collector area calculated by the procedure and the area calculated by the f-chart computer program is less than $10 \%$.

20122. Brown, W. E.; Tung, M. S.; Chow, L. C. Role of octacalcium phosphate in the incorporation of impurities into apatites, Proc. $2 d$ Int. Congress on Phosphorus Compounds, Boston, MA, Apr. 21-25, 1980, pp. 59-71 (Institut Mondial de Phosphate, Paris, France, Feb. 1981).

Key words: amorphous calcium phosphate; carbonate; defects; fluoride crystals; hydrolysis; hydroxyapatite; impurities; octacalcium phosphate.

Octacalcium phosphate (OCP) is structurally related to hydroxyapatite (OHAp) and is frequently a precursor in the formation of OHAp. We present evidence showing that hydrolysis of OCP to OHAp is a major mechanism for incorporation of impurities and structural defects which, in turn, greatly affect the chemical and physical properties of the resulting apatites.

Four sets of conditions were used to study the hydrolysis of OCP: $\mathrm{H}_{2} \mathrm{O}$ alone; $\mathrm{H}_{2} \mathrm{O}$ plus $\mathrm{F}^{-} ; \mathrm{H}_{2} \mathrm{O}$ plus $\mathrm{Na}_{2} \mathrm{CO}_{3}$; and $\mathrm{H}_{2} \mathrm{O}$ plus $\mathrm{F}^{-}$and
$\mathrm{Na}_{2} \mathrm{CO}_{3}$. The physical and chemical properties of the incorporated impurities closely resembled those of apatites found in nature, laboratory preparations, and commercial products, suggesting that the OCP-hydrolysis mechanism for impurity incorporation into the apatite lattice may be widespread.

It is proposed that a layer comprising two unit-cell thicknesses of OHAp bound by two 1/2-unit cells of OCP is the structural unit that accounts for $\mathrm{Ca} / \mathrm{P}$ molar ratios near 1.5 that are frequently encountered in amorphous calcium phosphates, cryptocrystalline apatites, and hydrolyzed OCP. We suggest the term "sesquiapatite" for this unit. Fissures between the sesquiapatite layers appear to be important sites for incorporation of impurity ions, and much of the water found in apatitic materials may reside in the fissures and as water of hydration in the adjacent half cells. It is proposed, also, that a second type of fissure, inclined to the (100), can form when hydrolysis occurs in the absence of $\mathrm{Ca}^{2+}$ ions in the solution.

20123. Parr, A. C.; Ederer, D. L.; Cole, B. E.; West, J. B.; Stockbauer, R.; Codling, K.; Dehmer, J. L. Triply-differential photoelectron studies of molecular autoionization profiles: The $\mathbf{7 1 0}$. $730 \AA$ region of the $\mathbf{N}_{2}$ spectrum, Phys. Rev. Lett. 46, No. 1, 22-25 (Jan. 5, 1981).

Key words: autoionization; Franck-Condon factors; ionization; nitrogen; $\mathrm{Nz}$; photoelectron spectra; photoionization; synchrotron radiation; vibrational intensity distribution.

Photoelectron studies of molecular autoionization resolved into position within the resonance profile, photoelectron ejection angle, and final vibrational state are reported. By using the first members of prominent window and absorption series converging to $\mathrm{N}_{2}{ }^{+} B^{2} \Sigma_{\mathrm{u}}{ }^{+}$as an example, striking variations of vibrational branching ratios and photoelectron asymmetry parameters within autoionizing profiles are demonstrated. Such triply differential data represent a very detailed characterization of the rovibronic interactions governing molecular autioionization.

20124. Haisch, B. M.; Linsky, J. L.; Harnden, F. R., Jr.; Rosner, R.; Seward, F. D.; Vaiana, G. S. Einstein x-ray observations of Proxima Centauri and the surrounding region, Astrophys. J. 242, No. 2, L99. L103 (Dec. 1, 1980).

Key words: flare stars; stars, individual; stellar coronae; x-ray sources; $\mathrm{x}$ rays, variable.

We report the first detection of both quiescent and flaring soft $X$-ray emission from a dMe flare star, Proxima Centauri (dM5e). The data are analyzed for temporal variability and spectral characteristics. The quiescent state is characterized by a mean X-ray luminosity of $\sim 1.5 \times 10^{27} \mathrm{ergs} \mathrm{s}^{-1}$, corresponding to a mean surface flux of $\sim 7 \times 10^{5}$ ergs $\mathrm{cm}^{-2} \mathrm{~s}^{-1}$, and an inferred temperature of $\sim 4 \times 10^{6} \mathrm{~K}$. The flare we have detected has a peak flux of $\sim 7.4 \times 10^{27} \mathrm{ergs} \mathrm{s}^{*-1}$ and a peak temperature of $\sim 17 \times 10^{6} \mathrm{~K}$. We discuss implications of these data for models of the quiescent and flare coronae of dMe stars.

20125. Shy, J. T.; Farley, J. W.; Lamb, W. E., Jr.; Wing, W. H. Observation of the infrared spectrum of the triatomic deuterium molecular ion $\mathrm{D}_{3}{ }^{+}$, Phys. Rev. Lett. 45, No. 7, 535-536 (Aug. 18, 1980).

Key words: infrared spectra; vibrational-rotational spectrum of $\mathrm{D}_{3}{ }^{+}$.

The infrared vibrational-rotational spectrum of the $\mathrm{D}_{3}{ }^{+}$isotope of the simplest polyatomic molecule is observed. Eight intervals between 1750 and $1850 \mathrm{~cm}^{-1}$ have been measured to an error of $0.0005 \mathrm{~cm}^{-1}$ or 0.3 pprin (at $70 \%$ confidence level). Four have been identified as members of the fundamental degenerate vibrational band. The rest apparently involve unidentified higher vibrational-rotational levels.

20126. Bernstein, B.; Zapas, L. J. Stability and cold drawing of viscoelastic bars, J. Rheol. 25, No. 1, 83.94 (1981).

Key words: BKZ material; cold drawing; constant rate of strain; creep; discontinuous deformations; finite strain; inhomogeneous deformations; multiphase deformations; necking; polyethylene; 
stability; stress relaxation; uniaxial strain; variational principles; viscoelasticity.

A theory of stability for deformation of $\mathrm{BKZ}$ viscoelastic bars is developed. This theory is an extension of the one given by Ericksen for elastic bars. For appropriate strain potentials under certain loads in uniaxial creep and constant rate of extension, the bar will deform homogeneously for some time, and then become unstable and neck. An example is given simulating the behavior of a linear polyethylene bar in creep and in constant rate of extension. For the sake of illustration, the data used at small deformations were obtained from actual experiments, but at higher stretches were hypothesized so as to give the points of instability as actually observed.

20127. Sengers, J. M. H. L.; Sengers, J. V. How close is "close to the critical point"?, Chapter 14 in Perspectives in Statistical Physics, H. J. Raveché, ed., pp. 241-271 (North-Holland Publ. Co., AE Amsterdam, The Netherlands, 1981).

Key words: asymptotic scaling; corrections to scaling; critical exponents; ethylene; Melville S. Green; steam; thermodynamic properties; Wegner expansion.

This chapter traces the path we walked with Melville S. Green towards an understanding of the static critical behavior of fluids. Particular attention is given to the range of asymptotic scaling behavior and the structure of the corrections to the asymptotic behavior of the thermodynamic properties of fluids near the critical point.

20128. Rendell, R. W.; Penn, D. R. Spin dependence of the electron mean free path in Fe, Co, and Ni, Phys. Rev. Lett. 45, No. 25, 2057. 2060 (Dec. 22, 1980).

Key words: electrons; ferromagnet; mean-free-path; spindependent.

Spin-dependent electron mean free paths as a function of the hot electron energy are calculated for $\mathrm{Fe}, \mathrm{Co}$, and $\mathrm{Ni}$. The difference in mean free paths of electrons for spin parallel and antiparallel to the majority spin direction of the ferromagnet is found to change sign and decrease rapidly in magnitude with increasing electron energy. Results for the mean free path with and without exchange included are also presented.

20129. McKenna, G. B.; Penn, R. W. Time-dependent failure of a polyolefin rubber candidate material for blood pump applications, $J$. Biomed. Mater. Res. 14, 689.703 (1980).

Key words: additivity of damage; biomaterials; blood pump; butyl rubber; carbon black filled rubber; circulatory assist devices; failure, time dependent failure; polyolefin rubber.

Failure behavior of a polyolefin elastomer which is a candidate material for blood pump applications has been studied under uniaxial and equibiaxial test conditions. Both static and dynamic (fatigue) testing were performed to study four aspects of material failure behavior as suggested by a cumulative damage failure model. Results from testing a standard formulation butyl rubber are presented for comparison. Our results show that the uniaxial failure behavior under static loads for the butyl rubber is superior to that of the polyolefin rubber at high loads but that the polyolefin is superior at low loads. Under fatigue loading conditions, the failure times for both rubbers decrease with increasing test frequency. The observed frequency dependence lies between that predicted by the cumulative damage model and that predicted by a cycle dependent fatigue model. The distribution of failure times for the polyolefin rubber is broader than that for the butyl rubber. For both uniaxial and equibiaxial testing, the distribution of failure times changes in going from the static testing to dynamic testing. This is true for both rubbers.

20130. McKenna, G. B.; Zapas, L. J. Viscoelastic behavior of poly(methyl methacrylate): Prediction of extensional response from torsional data, (Proc. VIII Int. Congress on Rheology, Naples, Italy, Sept. 1.5, 1980), Paper in Rheology, Applications (1980), G. Astarita, G. Marrucci, and L. Nicolais, eds., 3, $299-307$ (Plenum
Publishing Corp., New York, NY, 1980).

Key words: BKZ theory; poly(methyl methacrylate); simple extension; strain energy function; torsion; Valanis-Landel function; viscoelasticity.

Some years ago Rivlin showed that for certain deformations, one can treat isochronal data from single step stress relaxation experiments on viscoelastic materials in the same fashion as if the data were obtained for an elastic material. We have conducted single step stress relaxation experiments on cylinders of poly (methyl methacrylate) (PMMA) where we measured torque and normal force responses as functions of time and angle of twist. By assuming that torsion is an isochoric motion and that volume effects are separable, we obtained isochronal values for $\partial \mathrm{W} / \partial \mathrm{I}_{1}$ and $\partial \mathrm{W} / \partial \mathrm{I}_{2}$. Our results showed that $\partial W / \partial I_{1}$ is negative while $\partial W / \partial I_{2}$ is positive and greater in magnitude than $\partial \mathrm{W} / \partial \mathrm{I}_{1}$. These findings led to the possibility of explaining the phenomenon observed by Sternstein and Ho that the single step stress relaxation response of PMMA is different in torsion than in simple extension. Specifically, it was found that the rate of decay of the stress with respect to time is significantly higher in torsion than in extension. This phenomenon was observed at small strains where the stress responses in both torsion and extension were linear in the appropriate strain measure. The difference could not be accounted for either in terms of a time dependent Poisson's ratio or the material compressibility.

Using a form for viscoelastic potential functions which is similar to the Valanis-Landel form of the strain energy function for elastic materials, we calculated the response in simple extension from our torsion-normal force determined values of $\partial \mathrm{W} / \partial \mathrm{I}_{1}$ and $\partial \mathrm{W} / \partial \mathrm{I}_{2}$.

Although we obtained good agreement between our calculated and our observed behavior, we could not account fully for the observations of Sternstein and Ho.

20131. Kelley, E. F.; Hebner, R. E., Jr. Prebreakdown phenomena between sphere-sphere electrodes in transformer oil, Appl. Phys. Lett. 38, No. 4, 231-233 (Feb. 15, 1981).

Key words: electrical breakdown; liquid insulants; prebreakdown; propagation velocity; shockwave; streamers; transformer oil.

Measurements have been made of the propagation velocities of both cathode and anode streamers in transformer oil using sphere-sphere electrodes. Under the conditions of these experiments, the cathode streamer development was a three-step process, while the anode streamer development was a two-step process. The average propagation velocities for the three-steps of the cathode process were $2.4 \times 10^{4}, 1.5 \times 10^{5}$, and $3.9 \times 10^{6} \mathrm{~cm} / \mathrm{s}$. The two steps of the anode process propagated at average velocities of $3.8 \times 10^{5}$ and $4.4 \times 10^{6}$ $\mathrm{cm} / \mathrm{s}$.

20132. Boettinger, W. J.; Biancaniello, F. S.; Coriell, S. R. Solutal convection induced macrosegregation and the dendrite to composite transition in off-eutectic alloys, Metall. Trans. A 12A, 321-327 (Feb. 1981).

Key words: dendritic solidification; eutectic composite; eutectic solidification; macrosegregation; off-eutectic alloys; solutal convection.

The effect of solute gradient induced convection during vertical solidification on the macrosegregation of $\mathrm{Pb}$-rich $\mathrm{Pb}$-Sn off-eutectic alloys is determined experimentally as a function of composition and growth rate. In many cases macrosegregation is sufficient to prevent the plane from solidification of the alloy. The transition from dendritic to composite structure is found to occur when the composition of the solid is close enough to the eutectic composition to satisfy a stability criterion based on $G_{L} / V$ (liquid temperature gradient/growth rate). A vertical or horizontal magnetic field of 0.1 $\mathrm{T}$ (1 kilogauss) does not reduce macrosegregation, but downward solidification (liquid below solid) virtually eliminates macrosegregation in small $(\sim 3 \mathrm{~mm})$ diameter samples.

20133. Birnbaum, G.; Sutter, H. Collision-induced absorption in a 
highly symmetric molecule-SF 6 , Mol. Phys. 42, No. 1, 21.32 (1981).

Key words: band shape; collision induced absorption; hexadecapolar moment; liquid spectra; $\mathrm{SF}_{6}$.

The far-infrared absorption spectra of gaseous $(298 \mathrm{~K})$ and liquid $(273 \mathrm{~K}, 233 \mathrm{~K}) \mathrm{SF}_{6}$ obtained by Rosenberg and Birnbaum are analysed in terms of recent theoretical developments. Analysis of the band shape in the gas and liquid phases indicates that hexadecapoleinduced absorption accounts only for the low frequency portion of the collision-induced band. The major part of the absorption can be attributed to a much shorter range mechanism such as anisotropic overlap. The resulting induced dipole which varies very rapidly with intermolecular separation and is consistent with a (7-28) interaction energy gives a good description of the liquid spectra. Collisioninduced absorption and depolarized light scattering in $\mathbf{S F}_{6}$ are compared. From a decomposition of the collision-induced gaseous spectrum, the value of the hexadecapolar energy, $\Phi^{2} / \sigma^{9}$, is $2.7 \times 10^{-24}$ $\mathrm{J}(0.20 \mathrm{~K})$ which yields a value of the hexadecapole moment, $\Phi$, of $18 \times 10^{-60} \mathrm{C} \mathrm{m}^{4}$ for $\sigma=0.468 \mathrm{~nm}$.

20134. Unassigned.

20135. Haller, W. Surface derivatized silica-sol particles as immunospecific markers for high resolution electron microscopy, (Proc. Conf. Chemistry of Solid/Liquid Interfaces, Dubrovnik, Yugoslavia, June 25-July 3, 1979), Paper in Croatica Chemica Acto 53, No. 2, 373.379 (Croatica Chemica Acta, Zagreb, Yugoslavia, Feb. 1980).

Key words: electromicroscopy; immunospecific markers; labeling; markers; silica sol; silica spheres.

Colloidal silica spheres with $13.14 \mu \mathrm{m}$ diameter were derivatized by first introducing aldehyde surface functionality followed by covalent attachment of immunoglobulins specific to influenza virus. Size distribution width in the particle suspension was narrowed by chromatography on controlled pore glass.

Saturation labeling of virus surface antigens on infected human cells was carried out with suspensions having $10^{14}$ spheres per $\mathrm{cm}^{3}$. The visibility and the fine structure of the markers on cell surfaces are shown in transmission electromicroscopy with replicas and ultrathin sections.

20136. Berger, P. W.; Cerutti, E. The management of online reference search services in Federal libraries, Sci. Technol. Libr. 1, No. 1, 81. 107 (1980).

Key words: bibliographies; library management; online searches; online searching.

The various aspects of planning for online service are discussed from the viewpoint of sci-tech libraries operating in the Executive Branch of the Federal Government, not including the special cases of the three national (or quasi-national) libraries. The effects of statutory and geographic requirements on Federal libraries' online service are made clear, followed by such elements of planning as selling the program, integrating the service with others, selection of databases, staffing, equipment selection, and impact of the service.

20137. Cezairliyan, A.; Miiller, A. P. Specific heat capacity and electrical resistivity of a carbon-carbon composite in the range $1500-3000 \mathrm{~K}$ by a pulse heating method, Int. J. Thermophys. 1 , No. 3 , $317.330(1980)$

Key words: carbon-carbon; composites; dynamic measurements; electrical resistivity; graphite; heat capacity; high temperatures.

Measurements are described of specific heat capacity and electrical resistivity of a 2.2.3 T.50 carbon-carbon composite in the temperature range $1500-3000 \mathrm{~K}$ by a subsecond duration pulse heating technique. The results are represented by the relations: $c_{p}=1.691+2.598 \times 10^{-4}$ $T-2.691 \times 10^{-8} T^{2} ; p=733.0+6.594 \times 10^{-2} T$, where $c_{p}$ is in $\mathrm{J} \cdot \mathrm{g}^{-1} \cdot \mathrm{K}^{-1}, \rho$ is in $\mu \Omega \cdot \mathrm{cm}$, and $T$ is in $K$. Inaccuracy of specific heat capacity and electrical resistivity measurements is estimated to be not more than $\pm 3 \%$.
20138. Wang, G. C.; Celotta, R. J.; Pierce, D. T. Polarized lowenergy-electron diffraction from W(100), Phys. Rev. B 23, No. 4, 1761-1770 (Feb. 15, 1981).

Key words: polarized LEED; spin detector; spin polarization; surface structure.

A set of polarized low-energy-electron diffraction (PLEED) data from a $W(100)$ surface measured using a polarized electron beam is presented. The data include conventional LEED profiles $I(E, \theta)$ as well as $S(E, \theta)$ profiles which measure the spin dependence of the scattering. These profiles are obtained for specular beams at angles of incidence from $9^{\circ}-24^{\circ}$ and for five nonspecular beams at normal incidence. The potential usefulness of $S(E, \theta)$ profiles for structure determination is discussed and the use of PLEED for spin analysis is assessed.

20139. Straty, G. C.; Diller, D. E. $(p, V, T)$ of saturated and compressed fluid nitrogen, J. Chem. Thermodyn. 12, No. 10, $927-936$ (1980).

Key words: compressed liquid; density; nitrogen; PVT; saturated liquid.

The results of $(p, V, T)$ measurements on compressed fluid $\mathrm{N}_{2}$ at amount-of-substance densities from about $11 \mathrm{~mol} \mathrm{dm}^{-3}$ to greater than $28 \mathrm{~mol} \mathrm{dm}^{-1}$ at pressures to near $35.0 \mathrm{MPa}$ are reported. The motivation for these measurements was to provide single-source selfconsistent results for nitrogen, methane, and their mixtures. The results are to be used to formulate, test, and evaluate mathematical models for the prediction of $(p, V, T, x)$ of $\left\{(1-x) \mathrm{N}_{2}+x \mathrm{CH}_{4}\right\}$. Measurements on pure methane have been reported and measurements on selected gravimetrically prepared mixtures of nitrogen and methane are in progress.

20140. Sullivan, D. B.; Hamilton, C. A.; Kautz, R. L. Recent progress in cryoelectronics, IEEE Trans. Instrum. Meas. IM-29, No. 4, 319. 323 (Dec. 1980).

Key words: analog-to-digital conversion; electrical metrology; Josephson effect; quantum interference; sampling, standard volt; superconductivity.

Superconductivity has played a significant role in the advancement of electrical standards and measurement instrumentation. With the development of a superconducting integrated circuit technology for computer applications, new concepts and opportunities for advanced measurement systems are now emerging. In this paper we discuss some of these systems as well as the computer technology which has made them possible.

20141. Harvey, K. C. Shielded thermionic diode detector for precision spectroscopy, Rev. Sci. Instrum. 52, No. 2, 204.206 (Feb. 1981).

Key words: atomic detectors; Doppler-free spectroscopy; Rydberg states.

A thermionic diode with a shielded auxiliary compartment has been constructed to detect Doppler-free two-photon absorption. The shielded compartment prevents Zeeman and Stark broadening from external fields. The detector's construction and mode of operation are discussed.

20142. Kelley, E. F.; Hebner, R. E., Jr. The electric field distribution associated with prebreakdown phenomena in nitrobenzene, $J$. Appl. Phys. 52, No. 1, 191.195 (Jan. 1981).

Key words: breakdown; conduction; electric field; insulating liquid; Kerr effect; space charge; tree.

The electric field distribution between a prebreakdown tree and the electrode toward which it is propagating is determined. The electric field is measured in nitrobenzene, using the electro-optic Kerr effect. High-speed photography is used to provide a 10-ns resolution of the transient, prebreakdown events. The measurements show that to predict the Kerr fringe pattern, and thus the electric field distribution, 
it is sufficient to model the prebreakdown tree as a spherical conductor about the same size as the tree and at the potential of the electrode from which the tree is propagating.

20143. Metiu, H.; Gadzuk, J. W. Theory of rate processes at metal surfaces. II. The role of substrate electronic excitations, J. Chem. Phys. 74, No. 4, $2641-2653$ (Feb. 15, 1981).

Key words: electron-hole pairs; Franck-Condon factors; molecular processes; surface reactions.

A theoretical description of reactive molecular processes at metallic surfaces is formulated, following the Feschbach theory of nuclear reactions. Special attention is directed towards: (i) the role of substrate electronhole pair excitations, both as a heat bath and as an entity permitting access to reaction channels not open in the absence of an excitation continuum; (ii) the importance of nuclear FranckCondon factors; (iii) substrate induced quasiadiabatic electronic transitions on the incident atoms or molecules; and (iv) incorporation of substrate dynamic structure factors into the theory. Simple illustrative examples are presented which demonstrate the physical content of the formal theory and its relationship to various simplified models.

20144. Potzick, J. E.; Robertson, B. Voltage-controlled phase shifter for measuring transfer function in the presence of noise, Rev. Sci. Instrum. 52, No. 2, 280-286 (Feb. 1981).

Key words: amplitude detector; lock in amplifier; noise; phase detector; phase shifter; synchronous phase marker; transfer function; voltage controlled oscillator; voltage controlled phase shifter.

A linear voltage-controlled phase shifter has been constructed for use in a feedback loop to measure the amplitude and phase of a signal buried in noise. The feedback circuit has a phase marker output with an accuracy of 0.0025 rad while automatically following an arbitrarily large phase shift. In addition it has one output voltage proportional to the amplitude of the signal and another proportional to its phase with automatic translation in increments of $\pi$. A voltage-controlled oscillator is also described whose frequency and amplitude are proportional to their respective control voltages. The frequency control voltage for this oscillator is also used by the phase shifter circuit to make the proportionality constants for the outputs of the feedback circuit independent of frequency. Thus the circuits described make possible the highly accurate measurement of the transfer function of a two-port system that outputs more noise than signal.

20145. Rhyne, J. J. Magnetic inelastic neutron scattering studies of rare earth metals and Laves-phase compounds, Chapter 13 in Science and Technology of Rare Earth Materials, E. C. Subbarao and W. E. Wallace, eds., pp. 261.290 (Academic Press, Inc., New York, NY, 1980).

Key words: Laves-phase compounds; magnetic excitations; magnetism; neutron scattering; rare earth metals; spin waves.

An introduction will be given to the use of the inelastic thermal neutron scattering as a probe of the magnetic interactions in rare earth materials. As examples, the rare earth metals and cubic Laves-phase compounds will be emphasized.

For the rare earth metals, magnon dispersion will be discussed in terms of current theories which include effects of both q-dependent exchange and single and multi ion magnetic anisotropies. The details of the generalized susceptibility function obtained for the different heavy rare earth elements will be discussed in relation to their magnetic structure and electronic states. The magnetic field dependence of the $q=0$ anisotropy gap will be related to the magnetic-elastic properties of the elements.

Magnon dispersion in the light elements, for example $\mathrm{Nd}$, is complicated by the existence of more than one site and by the relatively large crystal field interactions. The interpretation of the data requires the use of Green's function random phase approximation methods.

The Laves-phase compounds with $\mathrm{Al}, \mathrm{Fe}$, and Co have recently been explored in some detail. The $\mathrm{Fe}$ and $\mathrm{Co}$ compounds are prototypical systems for the study of magnetic excitations involving two magnetic atoms and two sites. In contrast to the $\mathrm{Al}$ compounds, the $\mathrm{Fe}$ and $\mathrm{Co}$ materials show negligible rare earth-rare earth exchange interaction. At low temperature, linear spin wave theory adequately describes the excitation spectrum all temperatures for the Co and Al compounds, the RPA formalism is necessary to understand the observed inelastic scattering which involves transitions between nonadjacent magnetic states and excited state spin waves. Exchange constants and particularly crystal field parameters derived from the neutron studies on Laves-phase compounds will be compared to those obtained from magnetization data and the differences discussed.

20146. Deslattes, R. D.; Kessler, E. G.; Sauder, W. C.; Henins, A Remeasurement of $\gamma \cdot$.ray reference lines, Ann. Phys. 129, No. 2, 378 . 434 (Oct. 15, 1980).

Key words: crystal diffraction; gamma-ray wavelength; interferometry; $\gamma \cdot$ ray wavelength.

Over the last several years, we have established a new significantly improved measurement chain connecting $\gamma$-ray lines with visible reference standards. This chain has three links: First, a Si lattice repeat distance is determined by combined X-ray and optical interferometry. Second, this crystal calibration is transferred to other specimens suited to $\gamma$-ray diffraction. Finally, these crystals are used in a transmission double-crystal instrument to determine $\gamma$-ray wavelengths via the Bragg-Laue equation. To obtain the required precision and accuracy, the spectrometer is fitted with angle interferometers of considerable sensitivity $(\sim 0.05$ marcsec) and these are calibrated by summing to closure the external interfacial angles of a 72 -sided optical polygon. This report focuses on the apparatus and methodology of the third step but includes descriptions of the first two also. Results are presented from tests of closure using cascadecrossover relations and inter-order comparisons. Energies for $\gamma$-transitions in ${ }^{198} \mathrm{Au},{ }^{192} \mathrm{Ir},{ }^{170} \mathrm{Tm},{ }^{169} \mathrm{Yb}$, together with the $\mathrm{X}$-ray transition $W K \alpha_{1}$ are included. $\gamma$-Ray wavelength accuracies are estimated to be near $0.5 \mathrm{ppm}$.

20147. Mahajan, B. M.; Galowin, L. S. Water depth and solid velocity measurements in unsteady partially-filled horizontal pipe flow, Proc. Second Symp. Flow: Its Measurement and Control in Science and Industry, St. Louis, MO, Mar. 23-26, 1981, pp. 649.671 (Instrument Society of America, Research Triangle Park, NC, Mar. 1981)

Key words: depth-time history; horizontal; measurements; partially-filled; pipe flow; solid; unsteady; velocity; water.

Few investigations have been reported on the transport mechanisms of finite solids in time-dependent partially-filled pipe flows. Due to increased concern for water conservation, the effects of reduced water usage on maintaining adequate transport and sweeping velocities for waterborne solids in pitched horizontal drains of gravity drainage plumbing systems has become the subject of current research. Open channel flow analogues are not applicable since they are based upon steady flow conditions. Determination of the flow characteristics for the non-uniform, transient water flow with varying depth-time history and the waterborne solids requires experimental measurement methods which do not produce any interference with the transport mechanisms. The instrumentation methods adapted and developed for these tests were: (a) electrode pins flush mounted at uniform spaced intervals on the circumference of the pipe wall at selected cross sections to determine the depth as a function of time; and (b) light photocell detectors at prescribed spacing distances along the length of the pipe to detect the passage of the solid in order to determine the velocity. The experimental apparatus, instrumentation, and procedures are described. Typical results obtained from the tests are presented to illustrate the results derived from the tests. The ability of the methods adopted are shown to be suitable to derive the empirical data base required to develop mathematical correlations applicable to pipe sizes for effective low water drainage system performance.

20148. Oonk, R. L.; Jones, D. E.; Cole-Appel, B. E. Calculation of performance of $\boldsymbol{N}$ collectors in series from test data on a single collector, Sol. Energy, Tech. Note 23, No. 6, 535-536 (1979). 
Key words: collector slope and intercept; correction factor for in-series collectors; efficiency deterioration in series arrays; performance of collectors in series; solar collector arrays.

Solar collector arrays are often arranged so that fluid flows through the collectors in a combined series and parallel fashion. Often the data supplied by a collector manufacturer is the performance for a single collector. Because it is unreasonable to expect measured test data for all possible array arrangements, a method is needed for calculating the array slope and intercept from performance data on a single collector.

This paper presents a method of extrapolating the calculation from a single collector to an array, with a correction factor CFN to predict the performance of $N$ panels in series.

20149. Henderson, M. M.; MacNaughton, M. J., eds. Electronic communication: Technology and impacts, (Proc. AAAS Selected Symp. 52, Houston, TX, Jan. 3-8, 1979), Book: 191 pages (Westview Press Inc., Boulder, CO, 1980).

Key words: communications; communications network; computers; electronic technology.

Electronic communications technology and services permeate every aspect of national life. This book examines the current and expected states of the technology and considers the societal impact and policy issues arising from new technological developments. Particular attention is paid to evaluation of computerized conferencing for enhanced communication among researchers in specialized and interdisciplinary fields and to technology assessments of criminal justice and tax administration systems.

20150. McLaughlin, W. L.; Kosanić, M. M.; Marković, V. M.; Nenadovic, M. T.; Holcman, J.; Sehested, K. The kinetics of dye formation by pulse radiolysis of pararosaniline cyanide in aqueous or organic solution, Ris $\varnothing-M-2202,32$ pages (Ris $\varnothing$ National Laboratory, DK.4000 Roskilde, Denmark, Nov. 1979).

Key words: absorption spectra; aniline; aqueous solutions; chemical reaction kinetics; chemical reaction yield; cyanides; dose rates; dyes; organic solvents; pulsed irradiation; radiolysis; triphenylmethane dyes.

The radiation-induced conversion of the leucocyanide of pararosaniline dye, $\left[\mathrm{C}_{6} \mathrm{H}_{4} \mathrm{NH}_{2}\right]_{3} \equiv \mathrm{C}-\mathrm{CN}$, to the highly colored saltisomer of the dye, $\left[\mathrm{C}_{6} \mathrm{H}_{4} \mathrm{NH}_{2}\right]_{2}=\mathrm{C}=\mathrm{C}_{6} \mathrm{H}_{4}=\mathrm{NH}_{2}^{+}+\mathrm{CN}^{-}$, in acidic aqueous solution (wavelength of maximum absorption $\lambda_{\max }=540 \mathrm{~nm}$ ) or polar organis solution $\left(\lambda_{\max }=550 \mathrm{~nm}\right)$, takes place in two separate processes. The first is very fast (within $<50 \mathrm{~ns}$ ), and the second much slower following first-order kinetics with a rate constant that varies from $4 \times 10^{3} \mathrm{~s}^{-1}$ to $\sim 10^{6} \mathrm{~s}^{-1}$, as the acidity or concentration of an oxidizing agent increases. In oxygen-free acidic aqueous or organic solutions (argon saturated) there is an unstable transient species $\left(\lambda_{\max }=380 \mathrm{~nm}\right)$. When using $\mathrm{O}_{2}$ or $\mathrm{N}_{2} \mathrm{O}$-saturated aqueous or organic solution, there is no intermediate absorption band at $380 \mathrm{~nm}$, but the slow process of dye formation at 540 or $550 \mathrm{~nm}$ is still sequential to the initial fast process having somewhat faster kinetics than in Ar. saturated solution.

20151. Powell, J.; Ruegg, R. Life-cycle costing of solar energy investments, Chapter II in Solar Design Workbook, Solar Federal Buildings Program, Sec. II Solar System Evaluation, G. Franta, B. Glenn, S. Hogg, B. Hunn, S. Ternoey, and J. Yellott, eds., pp. 11 . 1-11-8 (SERI/SP-62-308, DOE/Solar Energy Research Institute (SERI), Golden, CO, Jan. 1981).

Key words: economics; Federal buildings; life-cycle costs; solar energy.

This chapter provides an overview of the life-cycle costing method of evaluating investments in solar energy. It describes the use of the method in determining the economically efficient design and size for a solar energy system, the cost effectiveness of a given system, and the establishment of project priorities.

20152. Deslattes, R. D. X-ray monochromator development for synchrotron radiation facilities, Nucl. Instrum. Methods $172,201.208$ (1980).

Key words: crystal; fluorescence; monochromators; scattering; synchrotron radiation.

This report contains some general comments about monochromators which include crystal dispersive elements. Systems considered include both those appropriate to production of monochromatic incident flux and those used for the analysis of fluorescent or scattered radiation. Results obtained to date with a particular fluorescence experiment using radiation from the SPEAR storage ring are briefly summarized.

20153. Eisenhauer, C. M.; Grund1, J. A.; Gilliam, D. M.; McGarry, E. D.; Spiegel, V. Benchmark referencing of neutron dosimetry measurements, (Proc. Third ASTM-Euratom Symp. Reactor Dosimetry, Ispra, Italy, Oct. 1-5, 1979), Paper in Dosimetry Methods for Fuels, Cladding and Structural Materials, EUR6813 EN-FR, II, pp. 919-927 (Commission of the European Communities, Luxemburg, 1980).

Key words: benchmark; Californium source; flux transfer; neutron flux; power reactor; pressure vessel.

The concept of benchmark referencing is illustrated by the determination of the neutron flux in the air cavity external to the pressure vessel of the Arkansas Power and Light Company. The value determined for this flux is traced by means of two flux transfer procedures and a source-strength intercomparison to the emission rate of the U.S. primary neutron source standard. Numerical results and estimates of uncertainty are included. The value of the full power flux of neutrons above $1 \mathrm{MeV}$ is $3.45 \times 10^{8} \pm 15 \% \mathrm{n} / \mathrm{cm}^{2} \mathrm{~s}$

20154. Harter, W. G.; Patterson, C. W. High resolution spectra of symmetric molecules: Understanding rotation and permutation dynamics, (Proc. Advances in Laser Chemistry, Pasadena, CA, Mar. 20.22, 1978), Paper in Advances in Laser Chemistry, A. Zewail, ed., pp. $455-463$ (Springer-Verlag, New York, NY, 1978).

Key words: $\mathrm{CF}_{4}$; high resolution; hyperfine structure; level clusters; molecular spectroscopy; spherical tops; symmetry analysis; Young tableaus.

Until recently, analysis of rovibronic fine structure of polyatomic molecules has involved complicated computations, and most researchers have ignored it or regarded it as something akin to the plague. However, rapidly increasing spectroscopic resolution has led to some surprising improvements in our understanding of molecular rovibronic dynamics for certain cases. It appears that new understanding of rotational effects in $X Y_{4}$ and $X Y_{6}$ spherical top molecules greatly simplifies the spectroscopic calculations, and may have simple applications for laser chemistry as well. We review here the elements of the recently developed quantum theory of level clusters, and speculate on what implications it might have for high resolution photochemistry.

20155. Mies, F. H.; Julienne, P. S. Oscillatory excimer emission: An analytic model, IEEE J. Quantum Electron. QE-15, No. 5, 272.280 (May 1979).

Key words: analytic quantum spectrum; continuum line shape; excimer emission spectra; gain cross sections; harmonic oscillator; stationary phase approximation.

An analytic quantum mechanical model is given for the line shape of the continuum emission from a model harmonic oscillator excimer state to a flat lower state. The model permits a qualitative characterization of the excimer emission profile for more realistic potentials and provides an estimate of bounds on excimer gain cross sections. The model is best on the red wavelength side of the spectrum, and it gives a good account of the McLennan bands of $I_{2}$. The Appendix discusses semiclassical approximations to the spectrum in a unified way. The stationary phase approximation is shown to lead to an expression very similar to the exact quantum result, and a new nondivergent approximation is derived for the red limit of the 
spectrum.

20156. Spence, D. On resonances in HF, J. Phys. B: At. Mol. Phys., Lett. to Ed. 14, No. 3, L107.L114 (1981).

Key words: electron transmission spectra; Feshbach resonances; HF.

We discuss a probable explanation of the totally inconsistent nature of some previously published Feshbach resonance spectra of hydrogen fluoride which has been provided by the recent work of Brion and Hitchcock. We have obtained new electron transmission spectra of HF in the energy range 12.8 to $16.0 \mathrm{eV}$, which confirm the earlier $\mathbf{H F}^{-}$resonance data of Spence and Noguchi and which reveal several previously unobserved resonance structures. These new structures are energetically coincident with the higher lying members of a series of peaks recently found in the $\mathrm{F}^{-} / \mathrm{HF}$ dissociative attachment cross section. We explain the anomalous scattering from the $\mathrm{HF}^{-2} \Pi(v=0)$ resonance level in terms of the mixing of the $b{ }^{3} \Pi$ and B ' $\Sigma$ parent Rydberg states which has been observed in optical absorption spectra. Finally, we point out a possible mechanism for a Lyman- $\alpha$ laser.

20157. Melmed, A. J.; Sakurai, T.; Kuk, Y.; Givargizov, E. I.

Feasibility of ToF atom-probe analysis of silicon, Surf. Sci. 103, No.

2-3, L139-L142 (1981).

Key words: atom-probe analysis; mass analysis; silicon; ToF mass spectroscopy.

Data are presented which establish that silicon with electrical resistivity up to at least $10 \Omega \mathrm{cm}$ can be field-evaporated and analyzed in a conventional energy-compensated ToF atom-probe instrument.

20158. Bartle, K. D.; Lee, M. L.; Wise, S. A. Factors affecting the retention of polycyclic aromatic hydrocarbons in gas chromatography, Chromatographia 14, No. 2, $69-72$ (Feb. 1981).

Key words: gas chromatography; polynuclear aromatic hydrocarbons; retention; vapour pressure.

The retention index of a planar polynuclear aromatic hydrocarbon on the GC phases OV-101, SE-52, and OV-17 is strictly related only to the logarithm of vapour pressure, and therefore indirectly to the boiling point, and less closely to the relative molecular mass. The very approximate relation with connectivity index is only a consequence of the latter. On a nematic liquid crystal phase, a variation of activity coefficient, expressed in terms of a shape factor, also influences the retention of PAH.

20159. Mordfin, L. Measurement of residual stresses: Problems and opportunities, Proc. Conf. Residual Stress for Designers and Metallurgists, Chicago, IL, Apr. 9-10, 1980, L. J. Vande Walle, ed., pp. 189-209 (American Society for Metals, Metal Park, OH, 1981).

Key words: Barkhausen noise; high-energy $\mathrm{x}$ rays; hole-drilling method; neutron diffraction; nondestructive evaluation; research needs; residual stress; stress measurement; ultrasonics; $\mathrm{x}$-ray diffraction.

Residual stresses are present in virtually every manufactured article and assembled structure although their effects are often not evident until the article or the structure is subjected to external loads or exposed to an adverse environment. Tensile residual stresses are generally detrimental, increasing the susceptiblility of a member to fatigue damage, stress corrosion and fracture. Compressive residual stresses are usually beneficial, tending to reduce these susceptibilities, but residual stresses of any kind can adversely affect dimensional stability. The ability to measure residual stresses accurately, quickly and easily, would enable their magnitudes to be controlled-to some extent, at least-by the tailoring of manufacturing and construction processes, and would also enable designers to properly account for their presence.

Existing methods for measuring residual stresses are limited in their capabilities and uncertain in their results. Problems exist in the interpretation of the measured data, and in the detection of sharp stress gradients in the interiors of members. Research is needed to clarify the confusing effects of microstructural features on nondestructive residual stress measurements, and there is need for dependable approaches to selecting the appropriate materials constants and conversion factors to be used. There is also a critical absence of adequate reference standards for calibrating or verifying the measurement techniques and equipment as well as an ever-present need for improvements in technique.

A research and development program addressing these needs will require a broad range of specialized and multidisciplinary competences which may well necessitate a collaborative or cooperative approach. Properly integrated and executed, the program would contribute to better measurement and control of residual stresses in manufactured articles and structures, and to the ability to monitor these stresses in service. Such an effort would promote increased productivity and resource conservation, and enhanced quality assurance in manufactured components and assemblies.

20160. Magerl, A.; Rowe, J. M.; Richter, D. Observation of lowenergy excitations in NbD: A simple lattice-dynamical model, Phys. Rev. $B$ 23, No. 4, 1605-1608 (Feb. 15, 1981).

Key words: lattice dynamics; metal hydride; niobium deuteride; phonons; $\beta$-phase; $20 \mathrm{meV}$ peak.

A tentative explanation of the $18-19 \mathrm{meV}$ peaks recently observed in $\mathrm{NbD}_{0.85}$ is presented. These peaks are attributed to the presence of flat optical modes in the lattice dynamics of $\beta$-phase NbD. These modes have significant structure factors over the whole pseudocubic zone in a multidomain crystal, mainly as a result of a large deuterium amplitude. Similar peaks in the $\alpha^{\prime}$ phase of $\mathrm{NbD}_{0.85}$ can also be explained as a "resonant interaction" where the broadening is due to the increased disorder.

20161. Guenther, F. R.; Parris, R. M.; Chesler, S. N.; Hilpert, L. R. Determination of phenolic compounds in alternate fuel matrices, $J$. Chromatog. 207, 256-261 (1981).

Key words: capillary column; gas chromatography; phenols; shale oil; solvent refined coal.

The gas-chromatographic determination of underivatized, methyl substituted phenols in shale oil and solvent refined coal is described. A simple acid/base extraction procedure was followed by injection onto a 20 meter Pluronic L64 capillary column. Fourteen phenols were separated and identified including phenol, the cresols, and all the dimethyl phenols. Identification was done on a GC/MS equipped with a Pluronic L64 capillary column. Isomers were further verified by comparison of pure standard retention times.

Quantitation was done utilizing an internal standard procedure. Quantitative data is given for seven phenols in the range of $168 \mu \mathrm{g} / \mathrm{g}$ to $29100 \mu \mathrm{g} / \mathrm{g}$.

20162. Breitenberg, M. A. Priority setting in standardization activities, Stand. Eng. 33, No. 1, 6-16 (Feb. 1981).

Key words: planning; priority ranking; priority setting; ranking; resource allocation standardization; work planning.

Setting workload priorities should be an important management function in any organization. This article discusses the need for a more formalized procedure for setting priorities and describes techniques a standards writing committee can use to select among possible standards activities.

20163. Johnson, R. G.; Behrens, J. W.; Bowman, C. D. Source imaging for FMIT using a neutron pin-hole camera, Proc. Symp. Neutron Cross-Sections from 10 to $50 \mathrm{MeV}$, Upton, NY, May 12-14, 1980, pp. 629-637 (National Nuclear Data Center, Upton, NY, July 1980).

Key words: associated particles; neutron imaging; neutron sources; pin-hole camera; position-sensitive proportional counter.

A pin-hole camera technique has been used to measure the variation in neutron emission intensity over the area of the neutron-producing 
target of the NBS Electron Linac. The method uses a linear positionsensitive proportional counter (PSPC) with an intrinsic spatial resolution of $1.0 \mathrm{~mm}$. The pin hole is made in a thick sheet of cadmium and neutron energy $(<0.3 \mathrm{eV})$ selection is achieved by timeof-flight. Both one-dimensional and two-dimensional detectors are now available for this work. In a completely separate experiment the neutron cone obtained from the $(d, t)$ reaction using the associatedparticle technique was imaged by a two-dimensional PSPC. This second measurement demonstrated the use of the two-dimensional detector for imaging high energy $(14 \mathrm{MeV})$ neutrons.

20164. Lucatorto, T. B.; McIlrath, T. J.; Mayo, S.; Furumoto, H. W. High-stability coaxial flashlamp-pumped dye laser, Appl. Opt. 19, No. 18, 3178-3180 (Sept. 15, 1980).

Key words: coaxial dye laser; dye laser; high power laser; high stability laser; quadraxial laser cell; tunable laser.

Measurements on the stability of a coaxial flashlamp-pumped dye laser demonstrated that the quality of the output can be dramatically improved by isolating the dye cell thermally from the flashlamp and ensuring uniform axially symmetric flow of the dye throughout the cell. A quadraxial laser tube in which the dye cell is surrounded by an evacuated annulus to provide thermal isolation and is terminated by specially designed end caps to provide uniform injection and removal of the dye solution was tested in a standard cavity with a 1200 . line/mm grating in Littrow configuration as the dispersive element. The performance characteristics were 1 -mrad divergence, $0.5 . \AA$ bandwidth of the spectral distribution, and 0.04 - $\AA$ jitter of wavelength at maximum intensity.

20165. Cavallo, L. M.; Mann, W. B. New National Bureau of Standards contemporary carbon-14 standards, (Proc. Radiocarbon Dating Conf., Heidelberg, West Germany, Aug. 19-26, 1979), Paper in Radiocarbon 22, No. 3, $962-963$ (American Journal of Science, New Haven, CT, 1980).

Key words: carbon-13 abundances; contemporary carbon-14 dating standards; international comparison; oxalic acid; radiocarbon; research material.

In 1957 the National Bureau of Standards (NBS) agreed, at the request of Dr. James R. Arnold, to store and distribute an oxalic acid contemporary carbon-14 standard. In 1978 stocks of this standard were practically gone so we approached Chas. Pfizer and Company, Inc., which had provided the original $1000 \mathrm{lb}$ of oxalic acid to Dr. Arnold to see if they could provide another one-batch lot of $1000 \mathrm{lb}$ of oxalic acid to replace the old standard. This they did and generously provided it at no cost to NBS. The oxalic acid was prepared by fermentation of French beet molasses from the 1977 spring, summer and autumn harvest, using Aspergillus niger variety. The acid was separated from the broth as the insoluble calcium salt and reconverted to free acid using sulfuric acid. The free acid was then crystallized, redissolved, filtered, recrystallized and dried. Samples of the old standard and the new material were sent to leading national and international laboratories for intercomparative massspectrometric and activity concentration measurements. The weighted average radioactivity-concentration ratio of the new to the old material (for $\delta^{13} \mathrm{C}=-19 \%$, relative to $\mathrm{PDB}$, for both) obtained from the data now available is $1.2894 \pm 0.0005$. The weighted activity concentration ratio based on the difference in isotopic fractionation between the two materials is $1.2933 \pm 0.0004$.

20166. Goldsmith, J. E. M.; Lawler, J. E. Optogalvanic spectroscopy, Contemp. Phys. 22, No. 2, 235.248 (1981).

Key words: discharge studies; optogalvanic; spectroscopy.

Applications of optogalvanic spectroscopy developed since the resurgence of interest in optogalvanic detection are reviewed. The optogalvanic effect is a change in the electrical properties of a discharge caused by illuminating the discharge with radiation having a wavelength corresponding to an atomic or molecular transition in the discharge. The general scheme of optogalvanic spectroscopy is presented, followed by a discussion of the gas discharge physics of the optogalvanic effect. Applications of optogalvanic detection in moderate resolution spectroscopy, in Doppler-free spectroscopy, and in analytic studies are discussed.

20167. Ruegg, R. T.; Sav, G. T. The microeconomics of solar energy, Chapter 28 in Solar Energy Handbook, J. F. Kreider and F. Kreith, eds., pp. 28-1-28.42 (McGraw-Hill Book Co., New York, NY, 1981).

Key words: cost effectiveness; energy conservation; energy economics; life-cycle costing; microeconomic analysis; solar energy economics.

The purpose of this chapter is to explain and illustrate how the techniques of microeconomic analysis can be used in the design, sizing, and evaluation of solar energy systems. For the purpose of exposition, the focus is on solar hot-water and spaceheating systems for residential and commercial buildings. However, the basic concepts and procedures will generally apply to the analysis of solar energy in diverse applications, e.g., industrial process heat systems, power production, and total energy systems.

The first part of this chapter presents basic concepts and methods of economic analysis, and illustrates their use in sample problems. An overview is given of the basic steps in life-cycle cost analysis, discounting cash flows, considerations important to making assumptions, and methods of dealing with uncertainty and inflation. The second part describes the major components of costs and savings associated with solar energy systems, including various types of system costs, energy costs and savings, taxes, and government incentives. The third part describes a methodology for optimizing a solar energy system for maximum net savings. The basic concepts of optimization are set forth, and an example optimization problem is solved.

20168. Kuczkowski, R. L.; Lovas, F. J.; Suenram, R. D.; Lattimer, R. P.; Hillig, K. W.; Ashe, A. J. The microwave spectrum, structure and dipole moment of 1,4-pentadiyne, J. Mol. Struct. 72, 143-152 (1981).

Key words: centrifugal distortion; dipole moment; microwave spectrum; molecular structure; pentadiyne; rotational spectra.

The microwave spectra of 1,4-pentadiyne and 1,4-pentadiyne-1,5-d, are assigned and rotational and centrifugal distortion constants obtained. A unique structure could not be determined. However, analysis of the moments of inertia indicates that the bond distances in $\mathrm{C}_{5} \mathrm{H}_{4}$ are close to typical values found in other related compounds. An interaction involving the acetylene moieties is evidenced by the derived bond angles. The data are consistent with either the central CCC angle being close to the tetrahedral value with the acetylene groups pushed away from linearity by approximately $3-4^{\circ}$ or opening of the central CCC angle to about $113^{\circ}$ along with linear acetylene groups. A range of structures between these two is also possible. The dipole moment is determined to be $0.516(5) \mathrm{D}$.

20169. Gills, T. E.; Bowen, R. L. Adhesive bonding of various materials to hard tooth tissues: Tracer study of mordant adsorption on enamel, (Proc. 1978 Int. Orthodontic Conf. Centennial Program, Philadelphia, PA, Apr. 10-12, 1978), Paper in Orthodontics: The State of the Art, H. G. Barrer, ed., pp. 163.171 (University of Pennsylvania Press, Philadelphia, PA, 1981).

Key words: adhesion; adsorption; caries prevention; composites dentistry; enamel; metal ions; orthodontics; radioactivity; tracer.

For improving adhesion between resins and hard tooth tissues, a mechanism has been proposed which calls for chelate bonding of polyfunctional monomers with surface calcium ions or, preferably, with cations that have higher chelate stability constants. For clinical utility, the substitution (ion exchange) must be very rapid and relatively irreversible. The present study evaluated selected metal ions simultaneously for their sorption (adsorption or absorption) on enamel surfaces. The test solution contained $2 M$ phosphoric acid and 1/6 M each of cupric, zinc, cobaltous, and ferric chloride, spiked with radioactive tracers. Eight noncarious premolar tooth crowns were cleaned, exposed to the solution for 60 seconds, rinsed with water, and then dried. The residual radioactivity was measured to quantify 
each element sorbed. The copper ion concentration was as high after a second, extensive washing as it was before, indicating irreversible adsorption. The copper was slightly higher than the zinc, and more than ten times higher than the retained cobalt ions. That these ions can be sorbed during an etching or "conditioning" treatment from a phosphoric acid solution is very important to the development of practical therapeutic measures for both restorative and preventive dentistry.

20170. DeReggi, A. S.; Roth, S. C.; Kenney, J. M.; Edelman, S.; Harris, G. R. Piezoelectric polymer probe for ultrasonic applications, J. Acoustic. Soc. Am. 69, No. 3, 853-859 (Mar. 1981).

Key words: arrays; broadband; miniature hydrophone; nonperturbing; piezoelectric polymer; ultrasonic probe.

Miniature piezoelectric polymer hydrophones for ultrasonic field characterization in the low megahertz region have been developed and tested. The principal advantages of these devices over conventional hydrophones are their uniform frequency response and minimal perturbation of the field. These characteristics are achieved by rendering a small central region of a thin sheet of the polymer polyvinylidene fluoride locally piezoelectric, and then supporting the sheet in the field by holding it taut in a metal hoop having dimensions larger than the field being probed. Both single elements having diameters less than $1 \mathrm{~mm}$ and multielement arrays have been formed on the polymer. Methods of construction, signal amplification, and, in one design, rf shielding are discussed, and data are presented on insertion loss, sensitivity, frequency response, and immunity to if interference.

20171. Humphreys, J. C.; McLaughlin, W. L. Dye film dosimetry for radiation processing, (Proc. Sixth Conf. Application of Accelerators in Research and Industry, Denton, TX, Nov. 3-5, 1980), IEEE Trans. Nucl. Sci. NS.28, No. 2, 1797-1801 (Apr. 1981).

Key words: calibration services; dosimetry standards; dye film dosimeters; isodose curves; measurement traceability; radiation processing; radiochromic dosimeters; transfer dosimeters.

Commercially available plastic films containing dyes or dye precursors are convenient dosimeters and imaging media for electron beams or photons used for industrial radiation processing. As "grainless" imaging systems having thicknesses down to a few micrometers, they provide high spatial resolution for determining detailed absorbed dose distributions through microdensitometric analysis. The radiation absorption properties of these systems are adjusted by changing film composition so that the dosimeter materials can be made to simulate the material of interest undergoing irradiation (e.g., tissue, bone, various insulating materials, semiconductor devices, and a wide assortment of polymers). Other advantages include long term stability, dose-rate independence, and ease of use and calibration. Radiochromic dye films with thicknesses varying from 0.005 to $1 \mathrm{~mm}$ are presently used to monitor electron-beam or gamma-ray doses from 10 to $10^{5} \mathrm{~Gy}\left(10^{3}\right.$ to $\left.10^{7} \mathrm{rad}\right)$, typical of those encountered in medical applications, radiation curing of polymeric composites, wire and cable insulation, shrinkable plastic tubing and film, as well as sterilization of medical supplies and treatment of municipal and industrial wastes. An NBS calibration service to industry involves the traceability of standard ${ }^{60} \mathrm{Co}$ gamma ray absorbed dose measurements by means of these films employed as transfer standards.

20172. Sockut, G. H. Comparison and mapping of the relational and CODASYL data models-An annotated bibliography, SIGMOD Record 11, No. 3, 55-68 (Apr. 1981).

Key words: CODASYL model; data base; data base management; data model; mapping; relational model.

Two of the best-known data models are the relational model and the CODASYL model (sometimes called the DBTG, network, or data structure set model). There have been many efforts at comparing the two models and at defining mappings between them. The publications listed in this bibliography describe some of these efforts and some related topics. I invite readers' suggestions for additional entries for possible inclusion in a future bibliography. I omit publications on the more general topic of data base conversion.

Following each reference, I describe the publication briefly, usually by quoting parts of the author's abstract and/or main text. Often I supplement or replace the author's text with my own words, enclosed in brackets ([]). I do not guarantee that an authors's abstract accurately reflects the content of the publication.

I do not evaluate the publications, nor do I guarantee the truth of their statements. Each publication may reflect its author's opinions. Also, because specifications have evolved over the last ten years, some statements may be out of date. The bibliography reveals that the authors have not reached consensus on equivalence of the models, nor on the models' relative merits, nor on the spelling of "data base".

20173. Schroeder, L. W.; Jordan, T. H.; Brown, W. E. Raman spectrum from a single crystal of $\mathrm{SnHPO}_{4}$, Spectrochim. Acta 37A, 21.29 (1981).

Key words: dimers; hydrogen bonds; lattice modes; polarization; Raman spectra; single crystal; tin phosphate.

The polarized Raman spectrum of a single crystal of $\mathrm{SnHPO}_{4}$ has been obtained in order to ascertain the vibrational characteristics of $\mathrm{HPO}_{4}^{2-}$ dimers in a known configuration. Bands due to hydroxyl, $\mathrm{OH}$, stretching, $\mathrm{POH}$ bending and the hydrogen bond were observed in addition to most of the predicted lattice modes.

The $\mathrm{OH}$ stretching mode was observed at $2730 \mathrm{~cm}^{-1}$, the in-plane POH bend at $1275 \mathrm{~cm}^{-1}$ and the out-of-plane POH bend at $818 \mathrm{~cm}^{-1}$. A band of $55 \mathrm{~cm}^{-1}$ is assigned, on the basis of its deuterium shift, to a deformation of the hydrogen bond. Very low frequency bands at 18 and $30 \mathrm{~cm}^{-1}$ reflect the layer structure of $\mathrm{SnHPO}_{4}$ in which intra-layer forces are strong and inter-layer (hydrogen bonds) forces are weak.

20174. McLaughlin, W. L. Dosimetry standards for industrial radiation processing, Chapter in National and International Standardization of Radiation Dosimetry 1, 89.106 (International Atomic Energy Agency, Vienna, Austria, 1978).

Key words: calorimetry; dose distributions; dosimetry; electron beams; gamma radiation; quality control; radiation dosimetry; radiation processing; radiation sterilization; radiochromic dyes; uniformity ratio.

The United States National Bureau of Standards (NBS) has recently made a series of calibration services available to the industrial radiation community and to users of large sources of gamma rays, $\mathrm{X}$-rays and electrons. These calibrations contribute to standardizing the measurement of large absorbed doses of ionizing radiation $\left(10^{1}-10^{6} \mathrm{~Gy}\right)$, over the energy range of interest in radiation processing $(0.1-10 \mathrm{MeV})$. Since, in practice, a wide variety of dosimetry systems are used for the many industrial applications, special problems have been encountered in developing proper calibration procedures and selecting transfer instruments that supply traceability to primary radiation measurement standards, for example, absorbed dose measurements by calorimetry. Standardized measurement procedures involve the use of a large calibrated cobalt 60 gamma-ray source at NBS, for which the dose rate has been determined calorimetrically. This source is used to calibrate the response of a relatively accurate and reproducible dose meter, namely, a radiochromic dye film covering the dose ranges of interest in radiation processing. Because of the ruggedness and stability of this dose meter and the absence of dose-rate dependence of its response, it can be used as a routine transfer instrument for postal dose intercomparisons and to determine absorbed dose rates from industrial radiation sources, to measure dose distributions, and to calibrate other dosimetry systems for use on a production line or in commissioning a new radiation process.

20175. Maximon, L. C.; Gimm, H. A. Pair production in the field of atomic electrons, Phys. Rev. $A$ 23, No. 1, 172-185 (Jan. 1981).

Key words: atomic screening; bremsstrahlung; incoherent scattering function; pair production; photon absorption cross section; triplet production.

The total cross section for pair production in the field of atomic 
electrons (triplet production) is considered in detail. A discussion of the relevant theoretical papers is presented; the more familiar cross section for pair production in the Coulomb field of the nucleus serves for comparison. The effect of exchange and $\gamma \cdot e$ interactions, atomic binding, and radiative corrections are all considered. Earlier expressions for the recoil distribution have been simplified considerably. Numerical values for screening corrections, exchange and $\gamma \cdot e$ contributions, and total cross sections are given. Comparison is made with experimental cross sections for pair production on hydrogen and deuterium.

20176. Miller, A.; McLaughlin, W. L. Evaluation of radiochromic dye films and other plastic dose meters under radiation processing conditions, Chapter in Tech. Report No. 205: High-Dose Measurements in Industrial Radiation Processing, pp. 119.138 (International Atomic Energy Agency, Vienna, Austria, 1981).

Key words: clear Perspex; dosimetry dyed plastic dosimeters; electron beams; gamma radiation; humidity dependence; plastic dosimeters; radiation processing; radiochromic dye films; red Perspex; temperature dependence.

Selection of dose meters for industrial irradiation purposes is mainly based on the specific dosimetry needs of the individual irradiation processes, weighed against knowledge of the well documented behaviour of the dose meters in question. These selection criteria are briefly discussed. A comprehensive study is made of radiochromic dye dose meters with respect to the data needed in making a meaningful evaluation of their merits and faults. Part of the programme has been carried out for several types of radiochromic dye films, as well as red, amber and clear Perspex dose meters, and the results of these measurements are given.

20177. Cool, T. A.; Koffend, J. B. Two-photon excitation of indium atoms by photodissociation of $\mathrm{InCl}$ and $\mathrm{InBr}, J$. Chem. Phys. 74, No. 4, 2287-2292 (Feb. 15, 1981).

Key words: cross sections; dissociative excitation; $\mathrm{InCl}, \mathrm{InBr}$ two-photon excitation.

Measurements are presented of two-photon dissociative excitation cross sections for $\mathrm{InCl}$ and $\mathrm{InBr}$ for photolysis with a $248 \mathrm{~nm} \mathrm{KrF}$ laser source. Values of the two-photon coupling parameter $\sigma / 1$ $\left(\mathrm{cm}^{4} / \mathrm{W}\right)$ of $4.9 \times 10^{-27}, 2.4 \times 10^{-28}, 7.9 \times 10^{-28}$, and $7.8 \times 10^{-28}$ are measured for the respective processes: $2 h v+\operatorname{InCl} \rightarrow \operatorname{In}\left(5 s^{2} 6 s\right)+\mathrm{Cl}$, $2 h v+\mathrm{InCl} \rightarrow \operatorname{In}\left(5 s^{2} 5 d\right)+\mathrm{Cl}, \quad 2 h v+\mathrm{InBr} \rightarrow \operatorname{In}\left(5 s^{2} 6 s\right)+\mathrm{Br}, \quad$ and $2 h v+$ $\mathrm{InBr} \rightarrow \operatorname{In}\left(5 s^{2} 5 d\right)+\mathrm{Br}$.

20178. Dillon, M. A.; Spence, D. The angular dependence of scattered electron spectra of neon and argon in the region of quasidiscrete autoionizing states, J. Chem. Phys. 74, No. 4, 2654-2655 (Feb. 15, 1981).

Key words: angular distributions; argon; autoionizing states; inelastic electron scattering; neon.

We have measured the angular dependence of inelastically scattered electrons in neon and argon in the energy region of quasi-discrete autoionizing states. Our spectra, obtained for incident electron energies of $200 \mathrm{eV}$, show that both singly and doubly excited autoionizing states exhibit angular distributions of the scattered electrons which are qualitatively as predicted by the Born approximation. Our results demonstrate that measured angular distributions of electrons scattered from quasi-discrete states can yield information on the symmetries and term symbols of these states, in a manner analogous to the well-known propensity rules for excitation of bound states.

20179. Walls, D. F.; Zoller, P.; Steyn-Ross, M. L. Optical bistability from three-level atoms, IEEE J. Quantum Electron. QE-17, No. 3, 380-384 (Mar. 1981).

Key words: Doppler-free transition; optical bistability; three level atoms.

A novel mechanism is proposed for optical bistability utilizing the population trapping which may occur in a coherent superposition of sublevels in a three-level system. The resulting bistability does not require atomic saturation and is insensitive to Doppler and laser bandwidth effects.

20180. Clarke, J. T.; Moos, H. W.; Atreya, S. K.; Lane, A. L. IUE detection of hursts of H Lya emission from Saturn, Nature 290, No. 5803, 226.227 (Mar. 19, 1981).

Key words: planetary atmospheres; Saturn; ultraviolet astronomy.

Potential sources of Lya emission have been investigated in a series of observations of the Saturnian system carried out between January and July 1980 using the short wavelength spectrograph of the IUE observatory. North-south maps of the Lya emission across the planet disk have shown pronounced spacial asymmetries in emission brightness. These asymmetries have been seen to vary markedly on a time scale of days and are interpreted as bursts of Lya emission of as much as $1 \mathrm{kR}$ brightness averaged over a $6 \times 10$ arc sec area, above a constant planetary emission level of 700-800 R.

20181. Hobbs, T. G. The health physics program at the National Bureau of Standards Linac, IEEE Trans. Nucl. Sci. NS-28, No. 2 , 1600-1602 (Apr. 1981).

Key words: accelerators; dosimetry; health physics; Linac; monitoring; radiation safety; safety; training.

A three-person Accelerator Health Physics team provides continuous radiation safety service for the National Bureau of Standards 150-MeV linear electron accelerator (Linac), which operates on a five days per week, 24 hours per day schedule, with one day per month down-time scheduled for maintenance. This team also serves the synchrotron ultraviolet light radiation facility (SURF), two Van de Graaffs, some other electrostatic accelerators, and several high-energy x-ray generators. Although the Linac is not licensed by a safety-regulating agency, as is the NBS Research Reactor, Health Physics makes no distinction between the two sources in providing coverage. Various regulations do impact on operations, e.g., the NRC dose totals and effluent releases and the OSHA requirements. Film, thermoluminescent dosimeters (TLD), and direct-reading pocket dosimeters are used for personnel dosimetry. The usual array of portable and fixed radiation survey meters and activation devices and some of the high-energy multi-particle activation techniques are used for area monitoring. Environmental surveillance is performed with TLD's, tissue-equivalent ion chamber, and a grass and soil assay program. Integrated Health Physics programs for all the NBS radiation facilities do instrument calibrations, training for Health Physics and operating staffs, administrative control of radioactive materials, waste management, and data compilations. The Linac began operations in 1965 and some workers have been affiliated with the facility since that time, but as at any facility, there have been personnel changes. Recent events have shown that a more emphatic training and retraining program is needed to ensure that all the staff are thoroughly familiar with safety interlocks, processes, and administrative controls.

20182. Younger, S. M. Distorted-wave electron-impact-ionization cross sections for highly ionized neonlike atoms, Phys. Rev. A 23, No. 3 , 1138.1146 (Mar. 1981).

Key words: distorted wave technique; electron impact ionization; neon isoelectronic sequence.

Electron-impact-ionization cross sections have been computed for several neonlike ions in a distorted-wave Born exchange approximation. For lower ionization stages the total cross section was found to be sensitive to the details of the distorted potential in which the partial waves were calculated. Ionization from the $25^{2}$ core subshell increased in importance for higher charge states. Isoelectronic curves are presented which allow the interpolation of nonrelativistic electron-impact-ionization cross sections for any ion in the neon sequence in the incident electron energy range of one to three times threshold. A comparison to available experimental and semiempirical formulas is made. 
20183. Kautz, R. L.; Costabile, G. A Josephson voltage standard using a series array of $\mathbf{1 0 0}$ junctions, IEEE Trans. Magn. MAG-17, No. 1, 780-783 (Jan. 1, 1981).

Key words: ac Josephson effect; Josephson junction; superconductivity; voltage standard.

Levinsen et al. have suggested that the voltage level of the Josephson-effect voltage standard might be increased by using a series array of highly hysteretic junctions which have rf-induced steps crossing the zero current axis. If the phase lock condition which yields quantization is stable then biasing the array at zero current insures that the voltage across each junction will be quantized, eliminating the need to individually bias the junctions. Here we describe the design and operation of a 100 junction array fabricated using photolithography. The array produced stable quantized voltages up to $27 \mathrm{mV}$ when operated at $20 \mathrm{GHz}$.

20184. Hamilton, C. A.; Lloyd, F. L.; Kautz, R. L. Analog measurement applications for high speed Josephson switches, IEEE Trans. Magn. MAG-17, No. 1, 577.582 (Jan. 1, 1981).

Key words: analog sampling; analog-to-digital; Josephson junctions; superconductivity.

This paper reviews high speed analog applications of Josephson switching devices. The design and performance of two different analog sampling circuits is described. A method is proposed for delivering room temperature signals to these samplers with $30 \mathrm{GHz}$ or more of bandwidth. An analog-to-digital converter based on quantum interference comparators is also described. This device has achieved conversion rates of $2 \times 10^{9}$ samples per second.

20185. McLaughlin, W. L. A national standardization programme for high-dose measurements, Tech. Reports Series No. 205: High-Dose Measurements in Industrial Radiation Processing, pp. 17.32 (International Atomic Energy Agency, Vienna, Austria, 1981).

Key words: calorimetry; dose distribution; dose mapping; dosimetry; electron beam; gamma radiation; radiation processing; radiation sterilization; radiochromic dyes; standardized radiation measurements; traceability; transfer dose meter.

A variety of dose meter calibration services is provided by NBS to industrial radiation users of intense radiation fields, in particular, large gamma-ray sources and electron accelerators at energies up to approximately $4 \mathrm{MeV}$. The services include the administering of known doses of ionizing photons and electrons to users' dose meters, supplying calibrated secondary-standard transfer dose meters and subsequent read-out and dose interpretation after irradiation, and special calibration services such as the determination of temperature dependence, rate dependence, or reproducibility of dose meter response and measurement of detailed dose distributions in specific irradiation geometries and in selected absorbing materials. The latter can, in fact, include dose profiles in heterogeneous absorbers and at surfaces and interfaces of different substances. Such information is important in research leading to the commissioning of a radiation process, in the commissioning dosimetry itself and, in some instances, in measurement assurance as it provides quality control of a given radiation treatment.

20186. Reader, J.; Ryabtsev, A. $3 p^{6} 3 d^{t}-3 p^{3} 3 d^{7}$ transitions in $\mathrm{Sr}$ XIII, Y Xiv, Zr XV, Nb Xv1, and Mo Xvi1, J. Opt. Soc. Am. 71, No. 3, 231237 (Mar. 1981).

Key words: ionized; molybdenum; niobium; spectrum; strontium; yttrium.

The $3 p^{6} 3 d^{4}-3 p^{5} 3 d^{4}$ transitions in Sr XIII, Y XIV, $\mathrm{Zr} \mathrm{XV}, \mathrm{Nb}$ xVI, and Mo XVII have been newly measured by means of a low-inductance vacuum spark and a $10.7-\mathrm{m}$ grazing-incidence spectrograph. The measurements have led to an improved analysis of this complex transition group in these ions. All levels of the combining configurations have been established. The energy parameters determined from least-squares fits to the observed levels are compared with Hartree-Fock calculations. The effective interaction $\alpha L(L+1)$ for the $3 p^{6} 3 d^{8}$ configuration decreases markedly with increasing ionization. The effective electrostatic interactions $D^{1}(3 p 3 d)$ and $X^{2}(3 p 3 d)$ for the $3 p^{5} 3 d^{9}$ configuration are practically constant through the sequence.

20187. Carlson, C. E.; Coyne, J. J.; Fishbane, P. M.; Gross, F.; Meshkov, S. Glueballs: Their experimental signature, Phys. Lett. 99B, No. 4, 353-357 (Feb. 26, 1981).

Key words: crow's foot diagram; experimental signature; oddball glueballs; photonic couplings; quantum chromodynamics; valence quarks.

We study hadrons with a 2-gluon valence component, emphasizing states not found in the quark model. We predict that $0^{++}, 0^{-+}, 1^{++}$, and the particularly interesting $1^{-+}$glueballs will be narrow, with widths ranging from a few $\mathrm{MeV}$ to less than one $\mathrm{MeV}$, and that spin 0 glueballs will mimic ss quarkonium states in their decay. We also comment on photonic couplings.

20188. Bialkowski, S. E. Simple parallel interface between an optical multichannel analyser detector controller and a microprocessor, $R e v$. Sci. Instrum. 51, No. 6, 850-851 (June 1980).

Key words: circuit diagram; control logic; interface; microprocessor; optical multichannel analyser; parallel line unit.

A simple circuit is given which will allow data transfer from a LSI. 11 DRV11 parallel interface unit to a PARC 1216 Optical Multichannel Detector Controller. The Circuit for OMA to LSI-11 data transfer is slightly more complicated. Data buffering is used so that data transfer can occur without time-out loss due to the dynamic memory refresh cycle of the LSI-11. The circuit may be constructed and mounted within the PARC 1216 unit with only minor, noncritical, modifications to the controller unit. The unit is able to transmit data from the 1216 controller running at dwell times as short as $40 \mu \mathrm{s} / \mathrm{channel}$.

20189. Dick, C. E.; Motz, J. W. Utilization of monoenergetic x-ray beams to examine the properties of radiographic intensifying screens, (Proc. Sixth Conf. Application of Accelerators in Research and Industry, Denton, TX, Nov. 3-5, 1980), IEEE Trans. Nucl. Sci. NS. 28, No. 2, 1554-1558 (Apr. 1981).

Key words: image information transfer; monoenergetic x-ray beams; number of light quanta; radiographic intensifying screens; single photon counting; $\mathrm{x}$ ray absorption.

Monoenergetic $\mathrm{x}$-ray beams, produced by the direct electron excitation of characteristic $\mathrm{K} \times$ rays in elemental targets, are important for measuring the response of different components of radiologic imaging systems. Previous studies have investigated the energy dependence of the scatter produced as an x-ray beam traverses the object to be radiographed and the efficacy of antiscatter grids in reducing this unwanted component of the image signal. Presently, these beams are being utilized to examine the image information transfer properties of $x$-ray intensifying screens conventionally used in screen-film imaging systems. The technique of single photon counting is being employed to determine the relative probability distribution of the number of optical photons emitted from the screen after absorption of an incident $x$ ray. These data can be used to determine the average number of light quanta emitted per absorbed $x$ ray. In addition, the shape of these distributions can be used to determine the image information transfer properties of a particular screen. Results of these measurements for a typical calcium tungstate screen and one of the new rare-earth phosphor screens are presented.

20190. Bartel, T. W. Effect of absorber geometry on apparent absorption coefficients as measured in a reverberation chamber, $J$. Acoust. Soc. Am. 69, No. 4, 1065-1074 (Apr. 1981).

Key words: absorption coefficient; acoustic impedance; acoustics; architectural acoustics; building acoustics; diffraction; reverberant field; reverberation room; sound absorption. 
Measurements were made in the NBS reverberation chamber to determine the apparent random incidence absorption coefficient as a function of the area, perimeter, and shape of the test specimens for three different materials arrayed as combinations of rectangular pieces. Measurements made with the specimen edges exposed and with the edges covered with reflecting material were compared. The experimental results were compared with a theoretical model [T. D. Northwood, J. Acoust. Soc. Am. 35, $1173-1177$ (1963)] using values of the acoustical admittance obtained from impedance tube measurements performed on the same materials. Both the experimental and theoretical results indicate that the random incidence absorption coefficient increases approximately linearly with $E$, the ratio of the perimeter to the area of the specimen for values of $E$ ranging from 1.3 to $3.3 \mathrm{~m}^{-1}$. The theoretical analysis indicates, however, that a linear extrapolation to the value $E=0$ is not necessarily valid. It was also found that if the specimen area and perimeter are kept constant, the values of the random incidence absorption coefficient decrease by a few percent as the specimen shape is changed to increase the number of vertices (inside plus outside right-angle corners) in the configuration.

20191. Cezairliyan, A. Electrical resistivity of molybdenum in the temperature range 1500 to $2650 \mathrm{~K}$, Int. J. Thermophys. 1, No. 4, 417 . 427 (1980)

Key words: dynamic measurements; electrical resistivity; high temperatures; molybdenum.

Measurement of the electrical resistivity of molybdenum in the temperature range 1500 to $2650 \mathrm{~K}$ by a subsecond-duration pulseheating technique is described. The specimens were of the National Bureau of Standards Molybdenum Standard Reference Material 781 for Enthalpy and Heat Capacity. The electrical resistivity of molybdenum in the temperature range 1500 to $2650 \mathrm{~K}$ is expressed by the following function (standard deviation $=0.2 \%$ ): $\rho=-6.7083+$ $2.8949 \times 10^{-2} T+5.2985 \times 10^{-7} T^{2}$, where $T$ is in $\mathrm{K}$ and $\rho$ is in $\mu \Omega \cdot \mathrm{cm}$. The estimated inaccuracy in the electrical resistivity data does not exceed $1 \%$.

20192. Geist, J.; Lowney, J. R. Effect of band-gap narrowing on the built-in electric field in $n$-type silicon, J. Appl. Phys. 52, No. 2, 11211123 (Feb. 1981).

Key words: band-gap narrowing; band tail; built-in electric field; silicon.

A relation is derived to describe the change in the built-in electric field in an $n$-type semiconductor due to band tailing and carrier freeze-out, as well as band-gap narrowing. Recent numerical models of these various phenomena were used to illustrate the effect on heavily doped $n$-type silicon. While neither band-gap narrowing, band tailing, nor deionization alone is sufficient to explain the large decrease in the built-in electric field that has been inferred from experimental measurements, the combination of all three effects may be sufficient.

20193. Wiese, W. L. Experimental and theoretical research on atomic data for highly ionized species at the National Bureau of Standards, Phys. Scr. 23, 194-196 (1981).

Key words: atomic data; highly ionized species; ionization cross sections; ionization rate coefficients; magnetic fusion research; spectral line identifications.

At NBS, a broad research effort is under way to produce, experimentally as well as theoretically, critically needed atomic data for magnetic fusion energy research. This program encompasses the determination of atomic energy levels, line wavelengths, transition probabilities, ionization and dielectronic recombination rates, and excitation and ionization cross sections. It also includes fundamental theoretical studies on ionization cross sections and on the density dependence of dielectronic recombination rates, as well as critical compilations of some of the above-mentioned atomic quantities.

20194. Krauss, M.; Stevens, W. J. Electronic structure of the negative
Key words: charge transfer; electronic structure; energy curve; $\mathrm{HCl}^{-}$; negative ion; polarization; transition moments.

The negative ion states of $\mathrm{HCl}^{-}$that go asymptotically to the $\mathrm{Cl}^{-}+$ $\mathrm{H}$ and $\mathrm{H}^{-}+\mathrm{Cl}$ asymptotes have been studied as a function of the internuclear distance using first-order configuration interaction wave functions. The $1 \Sigma^{2} \Sigma^{+}, 2 \Sigma^{2} \Sigma^{+}$, and $1 \Pi^{2} \Pi$ states all cross the neutral $\mathrm{HCl}$ energy curve. To the right of the crossing all three curves are bound relative to their asymptotes and exhibit energy minima. The crossings occur on the left hand limbs of the negative ion curves. To the left of the crossing the lowest negative ion curve mimics the neutral and the attached electron is in an orbital which is as diffuse as the basis set permits. Several roots of the FOCI were examined in the unstable region. A root that has a large component of the "tight" wave function appears in a number of the calculations and possibly can be attributed to a broad resonance. Transition and dipole moments of the bound energy curves exhibit the behavior expected of charge transfer interaction and polarization among these states.

20195. Hayward, E. Nuclear excitations using real and virtual photons, (Proc. Giant Multipole Resonance Topical Conf., Oak Ridge, TN, Oct. 15-17, 1979), Paper in Giant Multipole Resonance 1, 275.298 (Harwood Academic Publ., New York, NY, 1979).

Key words: electric quadrupole; electrodisintegration; giant resonance; size effects; sum rules; virtual photon.

The virtual photon technique is described along with its relationship to inelastic electron scattering and photonuclear physics. The results of a new evaluation of the experiment on the electrodisintegration of the nickel isotopes, which results in a much smaller E2 component in the $\alpha$ channel, are presented. The contributions of E0 and E3 excitations as well as nuclear size effects are discussed.

20196. Basch, H.; Stevens, W. J.; Krauss, M. The electronic structure and dipole moment function of $\mathrm{PbO}\left(X^{1} \Sigma^{+}\right), J$. Chem. Phys. 74, No. 4, 2416-2418 (Feb. 15, 1981).

Key words: dipole moment; effective core potentials; energy curve; $\mathrm{PbO}, \mathrm{CO}, \mathrm{X}^{\mathrm{i}} \Sigma^{+}$

Valence multiconfiguration self-consistent-field (MC-SCF) calculations of the energy and dipole moment function curves of $\mathrm{PbO}$ $\left(X^{1} \Sigma^{+}\right)$have been performed using effective core potentials (ECP) to replace the core electrons in the Hamiltonian. The present results are encouraging considering the small basis set and configuration list. This list is identical to that obtained in previous calculations on $\mathrm{CO}$, and should be adequate for all other Group IV/VI molecules. The agreement of the calculated dipole moment at $R_{e}$ for $\mathrm{PbO}$ with the experimental value for the ground vibrational state is good. The shape of the dipole moment function for a molecule with a heavy atom has been obtained and this shape is seen to be comparable to that for $\mathrm{CO}$.

20197. Hsu, N. N.; Breckenridge, F. R. Characterization and calibration of acoustic emission sensors, (Proc. Int. Conf. Acoustic Emission, Anaheim, CA, Sept. 10-13, 1979), Paper in Materials Evaluation 39, No. 1, 60-68 (ASNT, Columbus, OH, Jan. 1981).

Key words: acoustic emission; AE sensor calibration; AE sensor characterization; simulated acoustic emission; step-force calibration.

It is generally agreed that $\mathrm{AE}$ sensor calibration is necessary to the quantification of AE technology. However, how a sensor should be calibrated remains a question subject to argument.

In this paper, we first discuss conceptually how a sensor can be characterized, and what assumptions must be made to facilitate the actual calibration. The selection of a specific calibration technique depends on the particular application. A criterion for the selection is also discussed. Then various suggested calibration techniques are compared in terms of underlying principles and assumptions, specific methods and procedures, and limitations and advantages. The helium gas jet technique and the reciprocity technique are reviewed. 
The step-force calibration technique is described in detail. Sample calibration results of a commercial sensor are shown.

20198. Younger, S. M. Theoretical line strengths for the $4 d^{10}{ }^{1} S-4 d^{p} 4 f$ ${ }^{1} P^{\circ}$ resonance transition in the palladium isoelectronic sequence, Phys. Rev. A 22, No. 6, 2682-2689 (Dec. 1980).

Key words: atomic structure calculations; atomic transition probabilities; Hartree-Fock method; many-body perturbation theory; palladium isoelectronic sequence.

Theoretical line strengths for the $4 d^{10}{ }^{1} S-4 d^{9} 4 f^{1} P^{\circ}$ resonance transition in the palladium isoelectronic sequence have been computed in three approximations: configuration-averaged Hartree-Fock, term. dependent Hartree-Fock, and many-body perturbation theory. The Hartree-Fock $4 f$ state exhibits pronounced term dependence in the intermediate ionization stages $111 . \mathrm{XV}$, with the configuration-averaged radial orbital collapsing more rapidly with $Z$ than the $4 f^{1} P^{\circ}$ termdependent orbital due to the large repulsive exchange interaction with the $4 d^{9}$ subshell in the latter. The $4 d-4 f$ oscillator strength is small for low ionization stages, and does not reach a maximum until $\mathrm{Ba} \mathrm{XI}$. Contracted orbital many-body perturbation theory calculations confirm that, as expected for this closed-shell ground-state system, most of the correlation effects are concentrated in the $4 d^{8} 4 f^{2}{ }^{1} S$ configuration mixing with the ground state. As a comparison, Hartree-Fock data are also given for the transition $4 d^{10}{ }^{1} S-4 d^{9} 5 p{ }^{1} P^{\circ}$. For $\mathrm{Z}<60$ the $5 p^{1} P^{\circ}$ state is the lowest-lying ${ }^{1} P^{\circ}$ in the $P d$ I sequence.

20199. Rosenstock, H. M.; Stockbauer, R.; Parr, A. C. Photoelectronphotoion coincidence study of benzonitrile, J. Chim. Phys. 77, No. 7/8, 745.750 (1980).

Key words: benzonitrile ion; benzyne; coincidence; fragmentation; kinetics; photoelectron; photoionization; unimolecular.

The technique of photoelectron-photoion coincidence with variable ion source residence times has been applied to a study of benzonitrile ion fragmentation. The breakdown curve of the parent ions shows a strong dependence on residence time. Detailed analysis of this dependence shows that the ion fragments from its electronic ground state via a "tight" activated complex, in agreement with the conclusions of Chesnavich and Bowers. The rate-energy dependence agrees with that determined by Eland and Schulte, and the fragmentation threshold is in reasonable agreement with the threshold observed in electron impact at very long ion source residence times. The results lead to $\Delta \mathrm{H}_{0}^{\circ}\left(\mathrm{C}_{6} \mathrm{H}_{4}^{+}\right)=1321 \pm 10 \mathrm{~kJ} / \mathrm{mol}$. The energetics of additional neutral and ionic $\mathrm{C}_{6} \mathrm{H}_{4}$ isomers is estimated and discussed.

20200. Kingston, H.; Pella, P. A. Preconcentration of trace metals in environmental and biological samples by cation exchange resin filters for x-ray spectrometry, Anal. Chem. 53, No. 2, 223.227 (Feb. 1981).

Key words: cation exchange resin-loaded filters; environmental samples; ultra-trace analysis; $x$-ray spectrometry.

A preconcentration method is described for the X-ray spectrometric analysis of several trace elements in seawater, NBSSRM 1648 urban particulate, NBS-SRM 1632 trace elements in coal, and nickel in urine at concentrations as low as $1 \mathrm{ppb}$. The elements in the coal and urban particulate samples were loaded quantitatively on cation exchange resin filters and subsequently analyzed by energydispersive X-ray fluorescence spectrometry using secondary targets for monochromatic excitation of the filter sample. Prior to the analysis of seawater and urine the trace elements from the matrix were separated with a chelating resin. Comparison of the results obtained with NBS-SRM certificate values and/or those of other workers indicated agreement within $\pm 10 \%$. Detection and quantitation limits for this preconcentration method are also presented.

20201. Jensen, S.; Swyt, D. Sub-micrometer length metrology: Problems, techniques and solutions, Proc. Scanning Electron Microscopy, Chicago, IL, Apr. 21-25, 1980, pp. 393.406 (SEM Inc., Chicago, IL, 1980).

Key words: displacement; length measurement; linewidth; micro- measurement; micrometrology; optical microscopy; SEM; spacing; TEM.

The need for accurate dimensional measurements of features on micrometer-sized objects such as planar structures and spherical particles is rapidly expanding. This has fostered a number of metrological techniques utilizing the SEM, the CTEM, and optical microscopy. These techniques are reviewed and a comparison of their relative utility for sub-micrometer length metrology is made. Limitations inherent in each technique are explored. A review of presently available reference standards for both displacement and width measurements is made along with a discussion of the utility of each of these standards.

20202. Blau, P. J. Observations on the wear-in process during the sliding of several copper alloys against $\mathbf{5 2 1 0 0}$ steel, (Proc. 3d Int. Conf. Wear of Materials, San Francisco, CA, Mar. 30-Apr. 2, 1981), Paper in Wear of Materials-1981, S. K. Rhee, A. W. Ruff, and K. C. Ludema, eds., pp. 69-74 (The American Society of Mechanical Engineers, New York, NY, 1981).

Key words: break-in; copper alloys; friction; run-in; wear; wearin.

The changes in the surface and subsurface microstructures which occur in early stages of metal sliding contact may set the stage for longer term friction and wear behavior. The current paper presents the results of a study of the friction and wear break-in behavior of $\mathrm{Cu}$, $\mathrm{Cu}-7 \mathrm{wt} \% \mathrm{Al}$, and $\mathrm{Cu}-15 \mathrm{wt} \% \mathrm{Ag}$ rubbing against fixed 52100 steel ball bearing sliders in a stroke-by-stroke linear wear test device. Friction was measured at 81 locations on each wear track and plotted by computer to reveal the frictional "histories" of the given locations. The development of variations in the frictions traces was correlated with microstructure of the worn tracks to clarify the effects of previous sliding history on surface deterioration in the tested materials. Sliding behavior in air, flowing argon distilled water was reported for $\mathrm{Cu}$.

20203. Molino, J. A.; Lerner, N. D.; Zerdy, G. A. Audible noise from high-voltage transmission lines: Psychoacoustic findings, (Proc. U.S. Department of Energy Environmental Control Symp., Washington, DC, Nov. 28-30, 1978), Vol. 3. Solar Energy, Geothermal Energy. and Waste Heat Transmission, DOE/EV-0046, pp. 243.255 (U.S Department of Energy, Washington, DC, Sept. 1979).

Key words: audible noise; corona noise; extra high voltage (EHV) transmission lines.

The possible aversiveness of audible (corona) noise from extra-high voltage (EHV) transmission lines was investigated in a series of psychoacoustic experiments. Recordings of corona noise were made outdoors, generally at the edge of the property right-of-way. These recorded corona noise samples were then compared with other recorded environmental sounds, reference sounds, or spectrallymodified corona sounds, using a behavioral preference procedure. Results from three experiments, all employing the same general procedure, are presented here.

20204. Rakowsky, G. NBS-SURF II: A small, versatile synchrotron light source, (Proc. Sixth Conf. Application of Accelerators in Research and Industry, Denton, TX, Nov. 3.5, 1980), IEEE Trans. Nucl. Sci. NS-28, No. 2, 1519-1521 (Apr. 1981).

Key words: electron synchrotron; molecular physics; SURF II; synchrotron radiation; vacuum ultraviolet.

Synchrotron radiation sources do not have to be large multimegadollar installations. SURF II is based on a compact electron storage ring with a radius of only $0.84 \mathrm{~m}$, an operating energy of 250 $\mathrm{MeV}$, and useful light output down to $5 \mathrm{~nm}$. Small beam size, high brightness and wide-angle light ports give SURF II unique capabilities. Presently five beamlines are instrumented and operational, supporting experiments in atomic and molecular physics, surface science and materials studies, as well as providing optical calibration services. Nearing completion is a large facility for calibrating optical instruments, especially those intended for space 
flight. The capability of determining the absolute light flux emitted by SURF II has recently been improved and is now operational. The technique employs ultra-linear silicon photodiodes to detect and count individual electrons in the stored beam. Other user conveniences include close access to the machine, flexible scheduling and close interaction with the operations staff. The machine's simplicity contributes to reliability and a high ratio of beam time to downtime.

20205. Cotton, I. W. Perspective on trends in electronic communication, (Proc. AAAS Selected Symp. 52, Houston, TX Jan. 3.8, 1979), Paper in Electronic Communication: Technology and Impacts, M. M. Henderson and M. J. MacNaughton, eds., pp. 7.18 (Westview Press Inc., Boulder, CO, 1980).

Key words: communications; computers; data communications; electronic communications.

This review and perspective will concentrate on the area of electronic data communications, where major technological advances have been made in the past ten or fifteen years. Not only technological changes but relevant regulatory concerns are of interest to us, and will be covered here. Their impact on users will also be summarized.

20206. Penner, S.; Cutler, R. I.; Debenham, P. H.; Lindstrom, E. R.; Mohr, D. L.; Wilson, M. A. D.; Yoder, N. R.; Young, L. M.; Boyd, T. J.; Knapp, E. A.; Martin, R. E.; Potter, J. M.; Schneider, C. M.; Swenson, D. A.; Tallerico, P. J. The NBS-LASL CW Microtron, (Proc. Sixth Conf. Application of Accelerators in Research and Industry, Denton, TX, Nov. 3-5, 1980), IEEE Trans. Nucl. Sci. NS28, No. 2, 1526-1530 (Apr. 1981).

Key words: beam recirculation; electron accelerators; racetrack microtron; RTM.

The NBS-LASL racetrack microtron (RTM) is a joint research project of the National Bureau of Standards and the Los Alamos Scientific Laboratory. The project goals are to determine the feasibility of, and develop the necessary technology for building highenergy, high-current, continuous-beam (cw) electron accelerators using beam recirculation and room-temperature if accelerating structures. To achieve these goals, a demonstration accelerator will be designed, constructed, and tested. Parameters of the demonstration RTM are: injection energy-5 MeV; energy gain per pass-12 MeV; number of passes-15; final beam energy-185 MeV; maximum current $550 \mu \mathrm{A}$. One $450 \mathrm{~kW}$ cw klystron operating at $2380 \mathrm{MHz}$ will supply rf power to both the injector linac and the main accelerating section of the RTM. The disk and washer standing wave rf structure being developed at LASL will be used. SUPERFISH calculations indicate that an effective shunt impedance $\left(\mathrm{ZT}^{2}\right)$ of about $100 \mathrm{M} \Omega / \mathrm{m}$ can be obtained. Thus, if power dissipation of $25 \mathrm{~kW} / \mathrm{m}$ results in an energy gain of more than $1.5 \mathrm{MeV} / \mathrm{m}$. Accelerators of this type should be attractive for many applications. At beam energies above about $50 \mathrm{MeV}$, an RTM should be considerably cheaper to build and operate than a conventional pulsed if linac of the same maximum energy and time-average beam power. In addition, the RTM provides superior beam quality and a continuous beam which is essential for nuclear physics experiments requiring time-coincidence measurements between emitted particles.

20207. Quintiere, J. G. An approach to modeling wall fire spread in a room, Fire Saf. J. 3, 201-214 (1981).

Key words: burning rate; flame spread; modeling; room fires; wall fires.

An attempt is made to develop a framework for modeling wall fire spread in a room. The zone methodology for treating developing room fires is used, and its characteristic layer equations are discussed. A review was made of studies on the rate of burning and spread on vertical surfaces. Both of these phenomena are shown to depend on the incident radiative heat flux and the local oxygen concentration. Functional expressions are suggested, and a strategy is presented for incorporating these component analyses into a wall flame spread model. The goal of this model would be to assess the risk of rapid fire growth (flashover) relative to wall property data found through fundamental principles and through empirical fire test methods.

20208. Bennett, L. H.; Swartzendruber, L. J.; Reno, R. C. Nuclear hyperfine and positron annihilation methods for measurements of internal stress, (Proc. Joint Government-Industry Conf., Washington, DC, Feb. 26-27, 1979), Paper in Nondestructive Techniques for Measuring the Longitudinal Force in Rails, FRA/ORD-80/50, pp. 126-130 (U.S. Department of Transportation, Washington, DC, Dec. 1980). (Available from the National Technical Information Service, Springfield, VA 22161.)

Key words: hyperfine fields; internal stress; nondestructive evaluation; positron annihilation; steel.

A brief review of Mössbauer effect, nuclear magnetic resonance, perturbed angular correlation, and positron annihilation is given and their potential for measurements of residual stress in rails is assessed.

20209. Ruff, A. W.; Ives, L. K. Sliding wear behavior of electron beam surface melted 0-2 tool steel, (Proc. 3d Int. Conf. Wear of Materials, San Francisco, CA, Mar. 30-Apr. 2, 1981), Paper in Wear of Materials-1981, S. K. Rhee, A. W. Ruff, and K. C. Ludema, eds., pp. 326-334 (The American Society of Mechanical Engineers, New York, NY, 1981).

Key words: electron beam melting; electron microscopy; metal microstructure; steel; tool steel; wear.

Studies were carried out on the dry sliding wear behavior of electron beam melted surface layers on a type $0-2$ tool steel, and on annealed and conventionally hardened $0-2$ steel specimens for comparison. Wear tests were conducted in a flowing argon atmosphere at a sliding speed of $20 \mathrm{~cm} / \mathrm{s}$, a load of $10 \mathrm{~N}$, and against a 52100 bearing steel ring. Wear surface morphology was studied along with subsurface structure using optical and electron microscopy methods. The study concentrated on the wear of this steel after different processing treatments. Electron beam surface melting and subsequent rapid solidification in situ of the steel produced a highly refined martensitic microstructure having higher hardness values and better wear resistance then obtained using conventional quench hardening of that steel. Carbide distribution and martensite phase morphology were affected by this surface melting process; those microstructural characteristics influenced the wear behavior. Variations in electron beam power and surface speed during melting were explored in terms of their effect on the resulting surface layer. The wear test system used was computer interfaced and controlled, permitting continuous measurements of wear depth and friction force.

20210. Bennett, L. H. Nuclear magnetic resonance in alloys, (Proc. Materials Research Society Ann. Meet., Boston, MA, Nov. 17.20, 1980), Paper in Nuclear and Electron Resonance Spectroscopies Applied to Materials Science, Kaufman and Shenoy, eds., pp. 3.18 (Elsevier North Holland, Inc., New York, NY, 1981).

Key words: amorphous alloys; diffusion; intermetallic compounds; liquid alloys; magnetic materials; nuclear magnetic resonance; ordering; phase diagrams.

Many papers on NMR in alloys are addressed to NMR specialists rather than to metal or alloy specialists, and talk about the "potential" of NMR for application to alloys. This presentation emphasizes a review of some useful results of NMR experiments in alloys, including applications in diffusion, phase diagrams, magnetic materials, ordering in intermetallic compounds, liquid alloys and amorphous alloys.

20211. Rainwater, J. C. Delta-function expansion of Mayer function with application to virial coefficients, J. Stat. Phys. 19, No. 2, 177-189 (1978).

Key words: cluster expansion; exponential potential; inverse power potential; Mayer function; soft spheres; third virial coefficient.

The Mayer cluster integrals of a fluid with smooth, repulsive interactions are expanded in orders of a well-defined softness parameter. To first but not second order in softness, all virial 
coefficients are given by their hard-sphere forms with an effective diameter. A closed asymptotic expression is derived for the third virial coefficient which gives excellent results for the inverse power and exponential potentials.

20212. Koon, N. C.; Rhyne, J. J. Ground-state spin dynamics of $\mathrm{ErCo}_{2}$, Phys. Rev. B 23, No. 1, 207.214 (Jan. 1, 1981).

Key words: cobalt compounds; magnetism; neutron scattering; random phase approximation; rare earth compounds; spin waves.

We have studied the ground-state magnetic excitation spectrum of $\mathrm{ErCO}_{2}$ at $4.2 \mathrm{~K}$ using neutron inelastic scattering. Four modes were observed, two which are nondispersive and two which are weakly dispersive. Each of the weakly dispersive modes is degenerate with one of the nondispersive modes at the zone boundary, but falls lower in energy at the zone center. From the dynamic structure factor of the excitations we conclude that the nondispersive modes correspond to out-of-phase precession of the two rare-earth spins in the primitive cell, while the weakly dispersive modes correspond to in-phaseprecession. The results are described very well by a Green's-function random-phase-approximation theory using standard basis operators to account for the crystalline electric fields, which we find to be comparable in strength to the exchange interactions. The observed excitation modes are associated with transitions from the $\mathrm{Er}^{3+}$ ground state to the second and fourth excited states. By fitting the energies and intensities of the modes we determine $J_{\mathrm{Er} \cdot \mathrm{Er}}=0 \pm 0.01 \mathrm{meV}$, $J_{\text {Er.Co }}=-0.153 \mathrm{meV}, A_{4}^{0}=4.3 \mathrm{meV} / a_{0}^{4}$, and $A_{6}^{0}=-0.142 \mathrm{meV} / a_{0}^{6}$. We did not observe a predominantly Co spin-wave mode predicted by the theory, which may indicate a breakdown of the Heisenberg model when applied to the Co spins. Calculations for the observed modes, however, are nearly independent of the dynamics assumed for the Co sublattice.

20213. Blau, P. J. A simple method for cross-sectional examination of wear debris flakes, Wear (Short Commun.) 66, $257-258$ (1981).

Key words: brass; metallography; microscopy; particle analysis; wear debris; wear testing.

This brief note covers a procedure which can be used for mounting and cross-sectioning thin flakes of metallic wear debris $\mathbf{( 2 5 . 2 5 0}$ micrometers in size). The mounts can be used for metallographic studies of debris structure or for measurements of debris dimensions. The basic procedure involves the use of a glass coverslip "sandwich" mounted on edge in a standard, room temperature curing epoxy mount.

20214. Hardman, K.; Rhyne, J. J.; Smith, K.; Wallace, W. E. Structural investigation of $\mathbf{T h}_{6} \mathbf{M n}_{23} \mathrm{D}_{16}$ by neutron diffraction, $J$. Less-Common Met. 74, 97-102 (1980).

Key words: atomic positions of deuterium; crystal structure; deuterides; pressure isotherm; tetrahedral $\mathrm{D}$ sites; $\mathrm{Th}_{6} \mathrm{Mn}_{23} \mathrm{D}_{16}$.

The compound $\mathrm{Th}_{6} \mathrm{Mn}_{23} \mathrm{D}_{16}$ was studied at $295 \mathrm{~K}$ by neutron diffraction profile refinement methods. The pressure isotherm of $\mathrm{Th}_{6} \mathrm{Mn}_{23} \mathrm{D}_{\mathrm{x}}$ was measured at $295 \mathrm{~K}$ and exhibits a plateau between $\chi=17$ and $\chi=24$. The site occupancies and atomic positions were determined and are compared with results for $\mathrm{Y}_{6} \mathrm{Mn}_{23} \mathrm{D}_{x}$. In both the thorium and yttrium deuterides some of the deuterium atoms are located in the tetrahedral $\mathrm{f}$ sites of space group $\mathrm{Fm} 3 \mathrm{~m}$. However, the octahedral a site, which is fully occupied in the yttrium compound, is virtually empty in the thorium compound. The remaining deuterium atoms occupy the trigonal $i$ site in $T_{6} M_{23} D_{16}$, and not the tetrahedral $\mathrm{j}$ site as in $\mathrm{Y}_{6} \mathrm{Mn}_{23} \mathrm{D}_{18}$.

Low temperature scans $(4 \mathrm{~K})$ of $\mathrm{Th}_{6} \mathrm{Mn}_{23} \mathrm{D}_{16}$ revealed that the compound undergoes a crystallographic phase change from f.c.c. symmetry to orthorhombic symmetry.

20215. Rainwater, J. C. Temperature dependence of the magnetic susceptibility of a Fermi liquid, Phys. Lett. 71A, No. 2-3, 278-280 (Apr. 30, 1979).

Key words: Fermi fluids; liquid helium three; logarithmic temperature dependence; magnetic susceptibility.

The form of the temperature expansion for the magnetic susceptibility of a Fermi fluid is derived according to the extension by Ford, Mohling and Rainwater of Landau's Fermi liquid theory. The terms are proportional to, successively, a constant, $T^{2} \ln T, T^{2}, T^{4}(\ln T)^{2}$ $T^{4} \ln T$ and $T^{4}$. This result contrasts with several recent articles.

20216. Ederer, D. L.; Callcott, T. A.; Arakawa, E. T. In situ preparation of thin sodium films as an expendable filter in the vacuum ultraviolet, Rev. Sci. Instrum. 50, No. 12, 1618-1621 (Dec. 1979).

Key words: evaporation technique; $\mathrm{L}_{2,3}$ edge; second order suppression; sodium filters; soft $\mathrm{x}$ rays; thin film filters.

The use of a sodium film as a filter for wavelengths between 40 and $80 \mathrm{~nm}$ is discussed, and a technique to prepare these films is described. Sodium films of $230 \mathrm{~nm}$ thickness were demonstrated to have a transmission of $55 \%$ at $100 \mathrm{~K}$ at a wavelength $41 \mathrm{~nm}$. The transmission decreased to $40 \%$ after $18 \mathrm{~h}$ in a vacuum of $5 \times 10^{-8}$ Torr. Films of this thickness were shown to attenuate $20.5 \mathrm{~nm}$ radiation appearing in second order at $41 \mathrm{~nm}$ by at least a factor of 50 .

20217. Persensky, J. J.; Gagnon, J. L. Evaluation of a hand probe for use in a product safety regulation, Proc. Human Factors and Industrial Design in Consumer Products Symp., Medford, MA, May 28-30, 1980, pp. 155-172 (Tufts University, Medford, MA, 1980).

Key words: accident analysis; anthropometric probes; graphic modeling; product evaluation; product safety; safety test procedures.

An evaluation of a hand probe and test procedure was performed by the National Bureau of Standards (NBS) as part of a Consumer Product Safety Commission effort to establish a performance based safety standard for power lawn mowers. The evaluation involved analysis of in-depth accident reports, review and analysis of existing anthropometric data, and graphic modeling of hazard intrusion envelopes.

Analysis of mower-hand accident reports indicated that most mower accidents involved operators reaching into the discharge chute where the rotating blade was contacted. Data on hand position and orientation at the time of injury indicate a need for a test procedure which represents the dynamic nature of hand intrusion.

Evaluation of the proposed probe was made using published anthropometric data applied to a computer graphic model of the hand surrogate. The maximum height and area of the intrusion hazard envelope tested by the probe was found to be dependent on the validity of the measures incorporated in the design of the test probe itself. The computer graphic modeling technique allowed for the simulation of the effects of various anthropometric values on the probe profile and the resultant hazard intrusion envelope. The model allows several critical values including angle of joint rotation and digit and segment length to be varied and the envelope area and maximum intrusion height to be displayed and computed.

A program to utilize graphic modeling techniques as a means to design biomechanical surrogates is also discussed.

20218. Reader, J.; Acquista, N. $4 s^{2} 4 p^{3}-4 s 4 p^{4}$ and $4 s^{2} 4 p^{3}-4 s^{2} 4 p^{2} 5 s$ transitions in Y VII, Zr VIII, Nb IX, and Mo X, J. Opt. Soc. Am. 71, No. 4, 434-441 (Apr. 1981).

Key words: molybdenum; niobium; spectra; ultraviolet; yttrium; zirconium.

Spectra of ionized $\mathrm{Y}, \mathrm{Zr}, \mathrm{Nb}$, and Mo have been observed in sliding-spark and triggered-spark discharges on $10.7-\mathrm{m}$ normal- and grazing-incidence spectrographs at the National Bureau of Standards in Washington, D.C. From these observations the $4 s^{2} 4 p^{3}-4 s 4 p^{4}$ transitions in $\mathrm{Y}$ VII, $\mathrm{Zr}$ VIII, Nb IX, and Mo $\mathrm{x}$ have been identified. The $4 s^{2} 4 p^{3}-4 s^{2} 4 p^{2} 5 s$ transitions in Y VII-Mo X, previously identified by Rahimullah et al. [Phys. Scr. 14, 221.223 (1976); 18, 96.100 (1978)], have been confirmed. In $\mathrm{Y}$ VII the $4 s^{2} 4 p^{3}-4 s^{2} 4 p^{2} 6 s$ and $4 s 4 p^{4}-4 p^{5}$ transitions also have been found. The parameters obtained from leastsquares fits to the energy levels are compared with Hartree-Fock 
calculations. Preliminary values of the ionization energies have been determined as $110.02 \pm 0.15 \mathrm{eV}$ for $133.7 \pm 0.5 \mathrm{eV}$ for $\mathrm{Zr}$ VIII, 159.2 $\pm 0.7 \mathrm{eV}$ for $\mathrm{Nb} \mathrm{IX}$, and $186.4 \pm 1.2 \mathrm{eV}$ for Mo X.

20219. Cavanagh, R. R.; Yates, J. T. Site distribution studies of Rh supported on $\mathrm{Al}_{2} \mathrm{O}_{3}-\mathrm{An}$ infrared study of chemisorbed $\mathrm{CO}, \mathrm{J}$. Chem. Phys. 74, No. 7, 4150.4155 (Apr. 1, 1981).

Key words: chemisorption; $\mathrm{CO}$; dispersion; extinction coefficient; infrared spectroscopy; rhodium.

Using infrared spectroscopy, chemisorbed $\mathrm{CO}$ has been employed as a surface probe to observe differences in $\mathrm{Rh}$ site distributions on $\mathrm{Al}_{2} \mathrm{O}_{3}$-supported $\mathrm{Rh}$. It has been found that higher loadings of $\mathrm{Rh}$ lead to $R h$ site distributions resembling those found on $R h(111)$ single crystals, whereas low $\mathrm{Rh}$ loadings produce isolated $\mathrm{Rh}$ sites preferentially. The isolated $\mathrm{Rh}$ sites yield infrared spectra characteristic of a species $\mathrm{Rh}(\mathrm{CO})_{2}$. Chemisorption of oxygen onto the $\mathrm{CO}$-covered surface effectively eliminates crystalline $\mathrm{Rh}$ sites, leaving only the isolated $\mathrm{Rh}$ sites which maintain their bonding to $\mathrm{CO}$ as seen by infrared spectroscopy. Estimates of the infrared extinction coefficient for $\mathrm{Rh}(\mathrm{CO})$, have been made. The results are consistent with data from other laboratories which indicate that the $R h$ is in a $\mathrm{Rh}^{\delta+}$ oxidation state.

20220. Lyon, G. ANS FORTRAN: Past and near future, (Proc. Int. Computer Technology Conf. ASME Century 2-Emerging Technology Confs., San Francisco, CA, Aug. 12-15, 1980), Paper in Advances in Computer Technology-1980 2, $402-404$ (The American Society of Mechanical Engineers, New York, NY, 1980).

Key words: FORTRAN; higher level language; PASCAL; PL/I; standards.

Although the essential design of FORTRAN was made over twenty-five years ago, the language will persist. A large community supports its use and it is widely available. FORTRAN will remain viable because of this availability, because of an uncomplicated and malleable nature, and because of evolutionary improvements.

20221. Argentar, H.; Tesk, J. A.; Parry, E. E. An amine accelerator for colorfree curing of cold-curing methyl methacrylate denture systems, J. Am. Dent. Assoc. 102, 664-665 (May 1981).

Key words: amine; aniline derivative; butylaniline derivative; dental material; denture resin; methyl methacrylate;

polymerization; toluidine derivative.

The various methacrylate denture systems used in dentistry employ differing amounts of amine accelerators for producing a cured denture. The most generally used amine is $\mathrm{N}, \mathrm{N}$.dimethyl-p.toluidine (DMPT). Upon curing, this imparts an undesirable yellow color to the resin, thereby rendering it impossible to obtain a satisfactory match of some shades between systems.

A recently commercialized amine, N,N.dimethyl-p.t-butylaniline (DMBA), was tested and found to impart no color to specimens of denture resin as a result of the polymerization reaction. With the addition of a suitable quantity of a commercially available ultraviolet absorber, specimens containing DMBA exhibited satisfactory color stability when exposed to a sunlamp for 24 hours according to an American Dental Association specification for denture base resins.

If used in a denture base formulation, DMBA has the potential of allowing consistent shading from one system to another. This is of value from economic as well as esthetic consideration.

20222. Scarfe, C. M.; Cronin, D.; Wenzel, J.; Kauffman, D. Viscositytemperature relationships at $1 \mathrm{ATM}$ in the system diopside-anorthite, Paper in Carnegie Institution of Washington Year Book 79. Annual Report of the Director Geophysical Laboratory, No. 1825, pp. $315-318$ (Carnegie Institute of Washington, Washington, DC, Dec. 1980).

Key words: anorthite; Arrhenius equation; diopside; Fulcher equation; high-temperature; magma; viscosity.

The viscosity as a function of temperature has been measured for six melts in the diopside-anorthite system in the range $1175.1625^{\circ} \mathrm{C}$.
The results are fitted to Arrhenius and Fulcher equations.

20223. Mink, A.; Knoerdel, J.; Brooks, J. The design and implementation of an office application using existing high level tools, Proc. Trends and Applications 1981: Advances in Software Technology, Gaithersburg, $M D$, pp. 150-162 (IEEE Service Center, Piscataway, NJ, May 28, 1981).

Key words: accounting application; computer based office application; computer based office experiment; computer based office system; computer based office tools and office automation.

An experiment was conceived to provide insight into the problems of implementing computer based office application (CBOA) services using existing high level computer based office systems (CBOS) tools. The first step was to select a routine office application that was currently being performed in our environment, and an existing high level CBOS tool on which to build support for this application. Once the application and tool were selected, the office workers affected by this CBOA were encouraged to participate in the design and implementation process to gain the maximum feedback. The goals were to accomplish this implementation by using existing CBOS tools, doing no programming, and to have the users understand the implementation as well as the designers.

20224. Coriell, S. R.; Cordes, M. R.; Boettinger, W. J.; Sekerka, R. F. Effect of gravity on coupled convective and interfacial instabilities during directional solidification, (Proc. Twenty-Third COSPAR Plenary Meet., Budapest, Hungary, June 2-14, 1980), Paper in Adv. Space Res. 1, 5-11 (COSPAR, Great Britain, 1981).

Key words: binary alloy; convection; instability; lead-tin alloys; morphological; solidification.

We investigate the role of gravity in a linear stability analysis of the onset of coupled convective and morphological instabilities during directional solidification at constant velocity of a dilute alloy of tin in lead. For solidification vertically upwards, the temperature gradient alone would cause a negative density gradient and the solute gradient alone would cause a positive density gradient. Two types of instability are found, a convective type that occurs for long wavelengths and a morphological type that occurs for short wavelengths. In general, these are coupled but the morphological instabilities are practically independent of gravity and thus correspond to the predictions of previous morphological stability theory in which density changes and convection are neglected. The convective instabilities depend strongly on gravity; for a growth velocity of $V=1 \mu \mathrm{m} / \mathrm{s}$ and a temperature gradient in the liquid of $G_{L}=200 \mathrm{~K} / \mathrm{cm}$, the critical concentrations for convective instabilities are $3.1 \times 10^{-4}, 3.1 \times 10^{-2}$ and $2.39 \mathrm{wt} . \%$ for $\mathrm{g}_{\mathrm{e}}=980 \mathrm{~cm} / \mathrm{s}^{2}, 10^{-4} \mathrm{~g}_{\mathrm{e}}$ and $10^{-6} \mathrm{~g}_{\mathrm{e}}$, respectively. For low velocities, the convective instabilities occur at much lower solute concentrations than the morphological instabilities whereas at high velocities, the reverse is true. At intermediate velocities where the changeover takes place, there are oscillitory instabilities of mixed character whose periods range from $60 \mathrm{~s}$ at $\mathrm{g}_{\mathrm{e}}$ to $6 \times 10^{4} \mathrm{~s}$ at $10^{-6} \mathrm{~g}_{\mathrm{e}}$

20225. Thijsse, B. J. The dielectric constant of $\mathrm{SF}_{6}$ near the critical point, J. Chem. Phys. 74, No. 8, 4678-4692 (Apr. 15, 1981).

Key words: comparison with ${ }^{3} \mathrm{He} \mathrm{Ne}$, $\mathrm{Ne}$; critical anomaly; critical exponent $\beta$; dielectric constant; differential capacitance cell; gravity; $\mathrm{SF}_{6}$.

Measurements are reported of the dielectric constant of $\mathrm{SF}_{6}$ at the critical density $( \pm 1 \%)$ over a temperature range of $-10^{-3}<\left(T-T_{c}\right) / T_{c}<+10^{-2}$. The experimental cell was especially designed to suppress spurious effects resulting from temperature gradients and gravity. Temperature stability was better than $\pm 20 \mu \mathrm{K}$ and capacitance stability better than $0.5 \mathrm{ppm}$ over several days. The critical temperature was determined as $318.718 \pm 0.002 \mathrm{~K}$ and was completely free of drift. Capacitance data were reproduced within a few ppm over the duration of the experiment. No direct evidence of a $(T-T)^{1-a}$ anomaly was found; an upper bound of $5 \mathrm{ppm}$ is established. Effects of fluid stratification and phase separation were clearly seen and are well understood. We find a value of $0.325 \pm 0.005$ for the critical exponent $\beta$. 
20226. Misra, D. N.; Bowen, R. L. Adsorption of N-(2-hydroxy-3methacryloxypropyl)-N-phenylglycine (NPG-GMA) on cupric ionenriched hydroxyapatite surface to improve chemical bonding between dental resins and teeth, Biomater. 2, 78-82 (Apr. 1981).

Key words: adsorption; hydroxyapatite; irreversible; isotherm; polymer composites; reversible; tensile strength.

In continued studies of chemical bonding between a polymer and teeth through the mediation of coupling agents, the adsorption of N-(2-hydroxy-3-methacryloxypropyl)-N-phenylglycine (NPG-GMA) from ethanol solution was studied on cupric ion-treated synthetic hydroxyapatite in order to discriminate the adsorptive behaviour of the 'mordanted' as against the unmordanted (or pure) apatite. All features of the adsorption isotherm, however, agree with those of the isotherm obtained on pure hydroxyapatite. Also, polymer filled with cupric ion-treated hydroxyapatite coated with the irreversibly adsorbed NPG.GMA has a tensile strength comparable to that of the polymer filled with NPG-GMA coated pure hydroxyapatite.

20227. Hubbard, J. B.; Kayser, R. F. Dielectric friction and dielectric dispersion in electrolyte solutions with spin, J. Chem. Phys. 74, No. 6, 3535-3545 (Mar. 15, 1981).

Key words: angular momentum; dielectric decrement; dielectric dispersion; dielectric friction; electrolyte solutions; hydrodynamics with spin; ion mobility; rotational viscosity; Stokes law.

A self-consistent continuum model of dielectric friction on a moving ion is developed in which the only adjustable parameters are the hydrodynamic boundary conditions and a "rotational viscosity" $\eta_{R}$. Ion mobility and the solution dielectric properties are calculated as a function of ion size, ion charge, low and high frequency dielectric constants of the solvent, dielectric relaxation time of the solvent, rotational viscosity, and solvent shear viscosity. The consequences of varying $\eta_{R}$ are investigated: For $\eta_{R} \rightarrow \infty$ the continuum theory analyzed by Hubbard and Onsager (HO) is recovered; for small $\eta_{R}$, however, the polarization and "spin" fields in the solvent near the ion differ significantly from the HO model. While this has remarkably little effect on calculated ionic mobilities, the solution dielectric properties are noticeably altered. In particular, the kinetic dielectric decrement diverges as $\ln \eta_{R}$ as $\eta_{R}$ vanishes.

20228. Cavanagh, R. R.; Yates, J. T. Hydrogen spillover on aluminaA study by infrared spectroscopy, J. Catal. 68, 22-26 (1981).

Key words: aluminum oxide; carbon monoxide; chemisorption; infrared; spectra; supported rhodium.

Infrared spectroscopy has been used to monitor the exchange of $\mathrm{D}_{2}$ (g) with $\mathrm{OH}$ groups chemisorbed on $\mathrm{Al}_{2} \mathrm{O}_{3}$. It has been shown that near $300 \mathrm{~K}$, the rate of the exchange process is rapid in the presence of supported $\mathrm{Rh}$ particles on the $\mathrm{Al}_{2} \mathrm{O}_{3}$. A qualitative model for hydrogen "spillover" is presented in which dissociative adsorption of dihydrogen by the metal is a key step. It is shown that CO chemisorption on the supported $\mathrm{Rh}$ leads to a marked reduction in the "spillover" rate due to site blockage on the $\mathrm{R} h$. This is consistent with recent studies of behavior of the $\mathrm{CO}$ and $\mathrm{H}$ coadsorbed on $\mathrm{Rh}$ (111).

20229. Stevens, W. J.; Karo, A. M.; Hiskes, J. R. MCSCF pseudopotential calculations for the alkali hydrides and their anions, J. Chem. Phys. 74, No. 7, 3989-3998 (Apr. 1, 1981).

Key words: alkali hydrides; anions; core polarization; multiconfiguration; pseudopotentials; self-consistent-field.

Multiconfiguration self-consistent-field calculations have been carried out on the $X^{\prime} \Sigma^{+}$and $a^{3} \Sigma^{+}$states of $\mathrm{LiH}, \mathrm{NaH}, \mathrm{KH}, \mathrm{RbH}$, and $\mathrm{CsH}$, and on the $X^{2} \Sigma^{+}$states of their respective anions. Pseudopotentials, including core polarization terms, have been used to replace the core electrons, resulting in simple two- and three-electron calculations. Comparisons of the neutral potential curves with experiment and other $a b$ initio calculations (where available) show very good agreement. The agreement with $a b$ initio calculations on $\mathrm{LiH}^{-}$and $\mathrm{NaH}^{-}$is also very good. Adiabatic electron affinities have been calculated for $\mathrm{LiH}(0.293 \mathrm{eV}), \mathrm{NaH}(0.316 \mathrm{eV}), \mathrm{KH}(0.437 \mathrm{eV})$, $\mathrm{RbH}(0.422 \mathrm{eV})$, and $\mathrm{CsH}(0.438 \mathrm{eV})$.

20230. Bowen, R. L. Adhesive bonding of various materials to hard tooth tissues: XXIII. Isotonic monomer formulations, J. Dent. Res. 60, No. 5, 901.907 (May 1981).

Key words: adhesive resins; biomaterials; dental materials; monomers; osmotic pressure; polymers; solubility.

With regard to adhesion to dentin by chemical mechanisms, the vitality of odontoblasts and contiguous pulpal cells might require that materials influencing the dentinal tubules be maintained within certain limits of osmotic pressure. Resins for use in adhesive formulations can be made isotonic with vital tissues by adjusting the proportions of hydrophilic monomers and the water contents of the formulations.

20231. Mitchell, R. A.; Pontius, P. E. Force sensor-machine interaction, (Proc. 27th Int. Instrumentation Symp., Indianapolis, IN, Apr. 27.30, 1981), Part 1 in Instrumentation in the Aerospace Industry 2 and Advances in Test Measurement 18, 225.232 (Instrument Society of America, Research Triangle Park, NC, 1981).

Key words: force; force calibration; force measurement; load cell; machine-sensor interaction; proving ring; remainder plot.

Although a force sensor is designed to respond primarily to an axial component of applied force, the sensor may also produce a significant error signal in response to non-axial load components that result from small misalignments in the loading setup. There may also be significant time-dependent machine-sensor-interaction errors involving creep or mechanical oscillation. This paper describes techniques that are being used to reveal, identify, and quantify these sources of error. The techniques involve the use of high-resolution readout instruments and a dedicated microcomputer/graphics system to make rapid comparisons of the response of force sensors to different loading conditions.

20232. Deprit, A. The main problem in the theory of artificial satellites to order four, J. Guidance and Control 4, No. 2, 201-206 (Mar.-Apr. 1981).

Key words: celestial mechanics; Hamiltonian systems; nonlinear oscillation; satellite theory.

Software programs are designed to normalize the main problem in the theory of artificial satellites. At the onset, a canonical transformation of a new type, called the elimination of the parallax, reduces the system to a quasi-Keplerian one with varying angular momentum. As the first phase in a stepwise refinement, a separable radial intermediary is extracted from the simplified Hamiltonian; its normalization by a Poincaré transformation is executed by machine to the fourth order in closed form, that is to say, without developing the generator in powers of the eccentricity.

20233. Layer, H. P.; Rowley, W. R. C.; Marx, B. R. National Physical Laboratory-National Bureau of Standards iodine-stabilized heliumneon laser intercomparison, Opt. Lett. 6, No. 4, 188-190 (Apr. 1981).

Key words: helium-neon; intercomparison; iodine; length standards; stabilized laser.

The frequencies of two helium-neon lasers stabilized by intracavity saturated absorption of iodine $\left({ }^{127} I_{2}\right)$ built at the National Bureau of Standards (NBS) and the National Physical Laboratory have been compared by beat-frequency measurement. The NBS laser exhibited a power-dependent frequency shift that was reproducible and significant in size $\left(2 \times 10^{-19}\right)$ over the working range of the power output. The origin of the power shift has not been established, but several possible sources of the shift have been eliminated by our investigations. Specifically, the effect is not electronic in origin. No similar shift was observed in two lasers built at the National Physical Laboratory. 
20234. Hayward, E. Study of the giant resonances with virtual and real photons, (Proc. Int. Conf. Nuclear Physics with Electromagnetic Interactions, Mainz, Germany, June 5-9, 1979), Lecture Notes in Physics 108, pp. 300-310 (Springer-Verlag, New York, NY, 1979).

Key words: electric quadrupole; electrodisintegration; electrofission; giant resonances; virtual photons.

The relationship between inelastic electron scattering and electrodisintegration is discussed and the definition of the virtual photon spectrum introduced. Several tests of the E1 virtual photon analysis are cited. The results of the $(e, p)$ and $(e, \alpha)$ experiment on the nickel isotopes are discussed and evidence for the failure of the long wavelength approximation for E2 excitations presented. A short review of the electrofission experiments follows.

20235. Ramey-Smith, A. M.; Gagnon, J. L. Energy cost feedback: Consumer preferences, Proc. Human Factors and Industrial Design in Consumer Products, Medford, MA, May 28-30, 1980, pp. 96-116 (Tufts University, Medford, MA, 1981).

Key words: attitudes; consumer preference; energy; energy cost; feedback; feedback meters.

The present study addressed the issue of consumer preferences for various types of energy cost feedback for individual consumers. Its purpose was to provide human factors recommendations related to the performance characteristics of energy cost feedback devices for use in testing energy cost feedback meters. Simulation and interview techniques were used to provide consumer reaction to cumulative, instantaneous, and projected feedback presented as dollar and cent values. A majority of participants indicated a preference for cumulative feedback types. All types of cumulative feedback were easily understood, accurate in reflecting actual energy consumption, and suitable to several uses. Hourly instantaneous feedback was considered useful for monitoring energy use of individual appliances. All participants having two energy sources in their home expressed a preference for having feedback presented as separate cost figures rather than as a total cost. Recommendations for feedback types as well as some performance characteristics of energy cost feedback meters for further testing are discussed.

20236. Parr, A. C.; Rakowsky, G.; Ederer, D. L.; Stockbauer, R. L.; West, J. B.; Dehmer, J. L. Current research at NBS using synchrotron radiation at SURF-II, IEEE Trans. Nucl. Sci. NS-28, No. 2, 1210.1214 (Apr. 1981).

Key words: photoelectron; spectroscopy; synchrotron radiation.

The National Bureau of Standards (NBS) Synchrotron Ultraviolet Radiation Facility (SURF-II) is used in conjunction with a high flux normal incidence monochromator for angle resolved wavelength dependent photoelectron studies. The recent work has concentrated on studies of the effect of shape resonances on molecular vibrational intensity distributions as well as the effects of autoionization upon the vibrational intensity distributions over narrow wavelength regions. Results for $\mathrm{CO}, \mathrm{N}_{2}$, Ar and Xe will be discussed.

20237. Hayward, E.; Leicht, R. G. Elastic scattering of photons by ${ }^{12} \mathrm{C}$ and the E2 giant resonance, Ann. NY Acad. Sci. 40, 99-110 (Sept. 15, 1980).

Key words: dispersion relation; giant quadrupole resonance; photon scattering.

The elastic scattering of photons by the nucleus ${ }^{12} \mathrm{C}$ has been studied in the energy region of the Goldhaber-Teller oscillation and extending up to $40 \mathrm{MeV}$. Scattering cross sections were measured at 90 and 135 degrees, and the results were compared with predictions derived from the total photonuclear-absorption cross section by use of the optical theorem and the dispersion relation. The results show that for excitation energies below $25 \mathrm{MeV}$, only electric-dipole transitions need be considered but that in the energy range 30-40 MeV, the magnitude of the electric-quadrupole absorption is surprisingly large.
20238. Basch, H.; Julienne, P. S.; Krauss, M.; Rosenkrantz, M. E. Energy curves and moments for $\mathrm{PbHe}$ and $\mathrm{PbXe}, J$. Chem. Phys. 73, No. 12, 6247-6258 (Dec. 15, 1980).

Key words: collision induced adsorption; dipole moments; energy curves; $\mathrm{PbHe}$; $\mathrm{PbXe}$; transition moments.

Collision induced absorption at either the pump or Stokes wavelength could reduce the efficiency of Raman scattering of $\mathrm{XeCl}$ pump radiation in $\mathrm{Pb}$ vapor. Calculation of the collision induced absorption requires a knowledge of the different energy curves between the ground and upper states and the transition moment as a function of the internuclear distance. These curves were obtained in a first-order configuration interaction calculation of the $\mathrm{PbHe}, \mathrm{PbHe}^{+}$ $\mathrm{PbXe}$, and $\mathrm{PbXe}{ }^{+}$valence states. The atomic cores of $\mathrm{Pb}$ and $\mathrm{Xe}$ were replaced by relativistic effective core potentials so as to make the calculations tractable. The van der Waals interaction was also estimated by a variational perturbation calculation. These results were transformed to an $\Omega$ representation using an empirically determined spin-orbit operator. Using these theoretical data we estimate the collision induced absorption cross sections to be $10^{-23}$ and $10^{-21} \mathrm{~cm}^{2}$ per atmosphere of $\mathrm{Xe}$ for the first and second Stokes lines in the case of a $308.0 \mathrm{~nm}$ pump. Cross sections for $\mathrm{He}$ will be about an order of magnitude smaller due to the smaller transition moments. The energy defect for the pump wavelength is estimated to be so large that no significant absorption is expected for either $\mathrm{PbXe}$ or $\mathrm{PbHe}$ collision partners. There are also no calculated curve crossings at an energy lower than $0.25 \mathrm{eV}$, which indicates a very small probability for deactivation of $\mathrm{Pb}\left({ }^{3} P_{2}\right)$ by rare gas collision.

20239. Williams, E. S. A thermoelement comparator for automatic ac. dc difference measurements, IEEE Trans. Instrum. Meas. IM-29, No. 4, 405-409 (Dec. 4, 1980).

Key words: ac-dc comparator; ac-dc difference; automatic calibration; thermal converter; transfer voltmeter; voltage measurements.

Ac-dc differences in thermal voltage converters (TVC's) are determined relative to a similar instrument, from imbalance EMF's measured in a divider circuit to which both instrument outputs are connected. These EMF's are more stable than the input voltages to the converters, because input voltage fluctuations produce nearly equal proportional changes in the converter outputs. The divider balance is therefore affected only slightly. The divider and a Lindeck potentiometer for monitoring the test instrument output are driven by stepping motors and balanced automatically. Ac and dc voltages are applied by programmable power supplies whose outputs are adjusted, in response to imbalance EMF's in the potentiometer circuit, to produce equal output from the test instrument. The system automatically calibrates one voltage range at one or more frequencies, but requires an operator to change ranges and to enter the test parameter at a computer terminal.

20240. Galakhova, O. P.; Harkness, S.; Hermach, F. L.; Hirayama, H.; Martin, P.; Rozdestvenskaya, T. H.; Williams, E. S. An international comparison of thermal converters as ac-dc transfer standards, IEEE Trans. Instrum. Meas. IM-29, No. 4, 396-399 (Dec. $4,1980)$.

Key words: ac-dc transfer standards; comparisons, international; current measurements; international comparisons; measurements, ac-dc; thermal converters; voltage measurements.

The ac-dc differences of 4 sets of thermal current and voltage converters were determined at $40 \mathrm{~Hz}, 20 \mathrm{kHz}$, and $50 \mathrm{kHz}$ with reference to the standards of the national metrology laboratories of the United Kingdom, Japan, the Soviet Union, and the United States. For each voltage and current range and at each frequency, the average ac-dc difference determined by each laboratory for the 4 sets differed from the average of all laboratories by less than $10 \mathrm{ppm}$

20241. Whitlock, R. P.; Tesk, J. A.; Widera, G. E. O.; Holmes, A.; 
Parry, E. E. Consideration of some factors influencing compatibility of dental porcelains and alloys, Part I: Thermo-physical properties, Proc. 4th Int. Precious Metals Conf., Toronto, Canada, June 2-5, 1980, pp. 273-282 (Pergamon Canada, Toronto, Ontario, Canada, Apr. 1981).

Key words: bimaterial strips; bimetal strips; ceramic glasses; dental materials; dental porcelain; glass/metal seals; porcelain; porcelain/metal compatibility; stress in glass/metal composites; thermal expansion of alloys; thermal expansion of porcelain; thermal-mechanical stress.

Preliminary results on the determination of thermal expansion of several brands of dental porcelain and veneering alloys are presented. The porcelain specimens were cooled rapidly, similar to processing of porcelain veneered alloy prostheses. Large differences in expansion of up to $\sim 30$ percent were found to exist between some porcelains and alloys. These differences would virtually preclude the use of those combinations if expansion alone determined compatibility. Varying amounts of trapped excess volume were recovered during heating of porcelain expansion specimens. The trapping of this excess volume in rapidly cooled porcelain changes the thermal expansion relative to that for annealed procelain. The proposed thermal stress compatibility index of Ringle and others $(1978,1979)$ and Fairhurst, Harshinger, and Twiggs (1980) should employ the thermal expansion of rapidly cooled porcelain.

20242. Zajonc, A. G.; Phelps, A. V. Nonradiative transport of atomic excitation of Na vapor, Phys. Rev. A 23, No. 5, 2479.2487 (May 1981).

Key words: diffusion; experiment; fluorescence; laser; resonance radiation; sodium vapor.

Measurements are reported which show the effect of nonradiative losses at a gas-window interface on the backscattered fluorescence intensity for $\mathrm{Na}$ vapor at frequencies in the vicinity of the resonance lines near $589 \mathrm{~nm}$. The $\mathrm{Na} 3 P_{1 / 2,3 / 2}$ states are excited with a lowintensity single-mode tunable dye laser at high $\mathrm{Na}$ densities and the frequency integral of the backscattered fluorescence intensity in the $D 1$ and $D 2$ lines is measured. As the laser is tuned through resonance, the loss of atomic excitation to the window appears as a sharp decrease in the frequency-integrated fluorescence intensity. For example, at $7 \times 10^{20}$ atoms $\mathrm{m}^{-3}$ the fluorescence intensity decreases by a factor of 4 in a frequency interval of $4 \mathrm{GHz}$. Measured absolute fluorescence intensities versus laser frequency are compared with predictions made using the theory of Hummer and Kunasz which includes both radiative and nonradiative transport processes. The agreement between theory and experiment is remarkably good when one considers that the theory contains only one unknown coefficient, i.e., the reflection coefficient for excited atoms at the windows. In our case the excited atoms are assumed to be completely destroyed at the window.

20243. Katriel, J.; Hummer, D. G. Analytic solutions for three- and four-wave mixing via generalised Bose operators, J. Phys. A: Math. Gen. 14, 1211-1224 (1981).

Key words: Bose operators; four-wave mixing; nonlinear integral equations; nonlinear optics; second harmonic generation; threewave mixing.

New types of generalised Bose operators are constructed. They are applied to the linearisation of the equations of motion describing three-and four-wave mixing, resulting in integral equations for the temporal behaviour of the various fields. Some mathematical properties of these integral equations are studied, thus establishing the equivalence between approaches differing in the manner in which the linearisation is carried out. The integral equations are solved analytically in terms of Jacobian elliptic functions.

20244. Kennerly, R. E.; Van Brunt, R. J.; Gallagher, A. C. Highresolution measurement of the helium $1 s 2 s^{2} S$ resonance profile, Phys. Rev. A 23, No. 5, 2430-2442 (May 1981).

Key words: electron scattering; helium.
The 19.37-eV helium scattering resonance profile has been measured with an instrumental width less than the natural width and much less than that attained in any previously published study. The electron beam was produced by photoionization and scattered from a He beam from a supersonic nozzle. The instrumental width, including residual Doppler effects, was routinely around $5 \mathrm{meV}$ and the current was $5 \times 10^{-12} \mathrm{~A}$. The profile was measured at $22^{\circ}, 90^{\circ}$, and $135^{\circ}$ scattering angles. From the $90^{\circ}$ and $135^{\circ}$ data the $s$ - and p-wave phase shifts were found to be $1.813 \pm 0.017$ and $0.309 \pm 0.013$ radians, respectively. The natural width $\Gamma$ of the resonance was inferred by two methods: nonlinear least-squares fitting, and use of the integral of the differential cross section over the resonance. Both methods yield $11.0 \pm 0.5 \mathrm{meV}$, if it is assumed that there is no instrumental background in the scattered electron signal. Experimental evidence indicates that any such instrumental background must be less than $15 \%$ of the total signal, and is probably less than $5 \%$. If an extreme allowance is made for the larger figure, a $\Gamma$ as high as $13 \mathrm{meV}$ could be possible.

20245. Berger, H. Nondestructive testing in the 80's, Can. Soc. Nondestr. Test. 2, No. 7, 14-18 (Apr. 1981).

Key words: automated testing; continuous monitoring; nondestructive testing; radiography; signal processing; tomography; ultrasonics.

Factors such as inflation and the energy crisis have resulted in an increased use of NDT. This has stimulated new developments and the author discusses the possible variations or new methods which may be seen in the 1980's.

20246. Mordfin, L.; Payne, B. F.; Edelman, S. Enhanced reliability and reproducibility of measurements of machinery vibrations, Proc. Sixth Machinery Dynamics Seminar; Vibration Standards and Current Techniques for Flexible Rotor Balancing, Toronto, Canada, Sept. 2223, 1980, pp. 4-0-4-22 (National Research Council Canada, Ottawa, Ontario, Canada, Sept. 1980).

Key words: accelerometers; bearings; calibration; condition monitoring; damage criteria; inservice inspection; machinery dynamics; nondestructive evaluation; piezoelectric polymers; polymer gages; standards; vibration.

Using vibration measurements to monitor the condition of machinery and machine elements offers several advantages over traditional methods of nondestructive evaluation. This paper reviews some of the activities being carried out at the U.S. National Bureau of Standards (NBS) in support of this rapidly advancing measurement technology.

NBS has established and maintains a calibration service for vibration pickups over the frequency range from $2 \mathrm{~Hz}$ to $10,000 \mathrm{~Hz}$. The calibration procedures are based on the principle of reciprocity and on the interferometric measurement of displacement amplitude. These techniques are absolute, so that users of the service acquire both traceability and enhanced reproducibility in their vibration measurements.

NBS research and development studies on piezoelectric polymer gages have pointed the way toward enhanced reliability in vibration monitoring applications. One particular study, in which polymer gages helped to avoid inaccuracies that would be introduced by conventional accelerometers, is described.

The paper concludes with a brief discussion of the need for more comprehensive inservice inspection data which can facilitate the establishment of meaningful damage criteria for vibration monitoring programs.

20247. Schoonover, R. M.; Davis, R. S. Quick and accurate density determination of laboratory weights, (Proc. 8th Conf. IMEKO Technical Committee TC3 on Measurement of Force and Mass, Krakow, Poland, Sept. 9.11, 1980), Paper in Weighing Technology, pp. II23-II27 (Druk, Zaklad Poligraficzny Wydawnictwa SIGMA, Warszawa, Poland, 1980).

Key words: density measurement; density standard; electronic 
balance; hydrostatic balance; hydrostatic weighing; immersed balance; volume standard; volumetric measurement.

The assignment of mass values to laboratory weights or other objects of interest where the highest accuracy is desirable is normally accomplished by a difference measurement performed on a balance or mass comparator. The balance responds to gravitational force acting downward on the mass and the buoyant force of air acting in the opposite direction. When the objects being compared are of different densities, differences in the respective buoyant force must be accounted for in the mass value assignment algorithm. Mass determinations to $0.05 \mathrm{ppm}$ (parts per million) usually require density knowledge of the objects involved to 1 part in $10^{4}$. Thus a lengthy and tedious hydrostatic weighing is required to determine weight densities prior to mass calibration. To avoid the disadvantages of the classical hydrostatic weighing procedures, we have devised a method that utilizes a modern electronic top-loading balance completely immersed in a liquid of special properties. The balance response is calibrated with weights of known mass and volume and, in turn, the balance is used to determine the density of a unknown object, such as a standard weight. This technique has proved to be very successful in that in just a few minutes' time a density determination can be accomplished; accurate to better than 3 parts in $10^{5}$. Furthermore, sample degassing is not required and there is no delicate hydrostatic suspension demanding special treatment and protection. Our experience with this technique indicates that it is also capable of production-line density determinations, and hence useful in industrial metrology.

20248. Meshkov, S. Glueballs: Their spectra, production and decay, Paper in Recent Developments in High-Energy Physics, B. Kursunoglu, A. Perlmutter, and L. F. Scott, eds., pp. 43.59 (Plenum Publ. Corp., New York, NY, 1980).

Key words: configuration mixing; gauge theory; glueball; hyperball; quark; spectroscopy.

A systematic enumeration of the glueball spectrum of QCD and other $\mathrm{SU}(\mathrm{n})$ gauge theories yields $\mathrm{J}^{\mathrm{PC}}=\mathrm{odd}^{++}$, even ${ }^{+-}$, and $0^{--}$states in addition to the usual quark model states. Production and decay mechanisms for these new states are discussed. We obtain a significant radiative decay width of the $\psi / \mathrm{J}$ into a $0^{-+}$glueball via mixing with the $\eta_{c}$.

20249. Spence, D. Studies of the $\mathrm{O}_{2}{ }^{3} \Pi_{\mathrm{g}}(V)$ valence states and ${ }^{3} \Pi_{g}(R)$ Rydberg state in the Schumann-Runge continuum from ejected and scattered electron spectra, J. Chem. Phys. 74, No. 7, 3898-3904 (Apr. 1, 1981).

Key words: electron scattering; energy-loss spectra; Feshbach resonances; oxygen.

Electron-impact energy-loss spectra are obtained in $\mathrm{O}_{2}$ in the energy-loss range of 7 to $10 \mathrm{eV}$. These spectra contain some discrete features resulting from scattered electrons following excitation of Rydberg states, and other features from ejected electrons following decay of $\mathrm{O}_{2}^{-*}$ Feshbach resonances into valence electronic states in the continuum via the reaction $e+\mathrm{O}_{2}\left(X^{3} \Sigma_{g}^{-}\right) \rightarrow\left(\mathrm{O}_{2}^{-}\right) \longrightarrow \mathrm{O}_{2}^{3} \Pi_{g}(V)+e_{\text {ejected }}$ From the known $\mathrm{O}_{2}^{-*}$ energies and from measurements of the ejected electron energies, we find the ${ }^{3} \Pi_{g}(V)$ state to extend over an energy range of only about $0.20 \mathrm{eV}$ in the Franck-Condon region of the ground state, contrary to some previous experimental and theoretical results. From measurements of scattered electrons, effective excitation functions of several vibrational levels of the ${ }^{3} \Pi_{g}(R)$ Rydberg state are obtained. Of the many $\mathrm{O}_{2}^{-*}$ Feshbach resonances known to exist in this energy region, only one decays strongly into the ${ }^{3} \Pi_{g}(R)$ state.

20250. Ben-Reuven, A. Recent developments in line shape theory, (Proc. Fifth Int. Conf. Spectral Line Shapes, Berlin, Germany, July 7.11, 1980), Paper in Spectral Line Shapes, B. Wende, ed., pp. 867. 884 (Walter de Gruyter \& Co., New York, NY, 1981).

Key words: cooperative phenomena; multiphoton processes; spectral line shapes.

General explicit expressions for spectral line shapes are present assuming only (a) broadening by binary collisions (neglecting "recollisions," or triple-collision effects), involving (b) stable atoms or molecules (neglecting chemical reactions and ionization effects and considering, at most, (c) combined coherent propagation of two atoms or molecules (weak coherence coupling). This theory is otherwise valid for all field strengths, and incorporates both impact and quasistatic limits. Implicit equations evolving from the collisioninduced many-atom coherence effects are also discussed. The theory can be adapted to both the Doppler-limited domain, where the collision integrals can be presented as velocity-changing kernels, and the homogeneously-broadened domain, where the collision integrals may be approximately replaced by velocity-averaged relaxation parameters.

20251. Powell, C. J. Progress of the ASTM E-42 committee on surface analysis, Surf. Interface Anal. 3, No. 2, 94-98 (1981).

Key words: ASTM; standards; surface analysis.

The American Society for Testing and Materials (ASTM) formed Committee E-42 on Surface Analysis in 1976. Subcommittees have been established for the four surface-analysis techniques in common use (AES, XPS, ISS, and SIMS) and for terminology, ion-beam sputtering, standard reference materials, and editorial processing. The principal objective of the committee and its component groups is to advance the field of surface analysis and the quality of surface analyses through the development of appropriate standards, standard procedures, standard materials, roundrobins, symposia, workshops, and publications. A review is given of recent activities of the committee and of work in progress. The success of the committee will depend a great deal on adequate communication and coordination with other interested individuals and groups to ensure the adequacy and quality of proposed standards and to prevent unnecessary confusion or duplication of effort. Suggestions are made for this purpose.

20252. Olver, F. W. J. The general connection-formula problem for linear differential equations of the second order, Proc. Conf. Singular Perturbations and Asymptotics, Madison, WI, May 28-30, 1980, pp. 317-343 (Academic Press, Inc., New York, NY, 1980).

Key words: asymptotic approximation; connection formula; fractional transition point; irregular singularity; Liouville-Green approximation; Stokes line; turning point.

In the neighborhood of an irregular singularity $z_{\circ}$ of the differential equation $d^{2} w / d z^{2}=f(z) w$ asymptotic approximations to the solutions are supplied by the Liouville-Green functions

$\mathrm{f}^{-1 / 4}(\mathrm{z}) \exp \left\{ \pm \int \mathrm{f}^{1 / 2}(\mathrm{z}) \mathrm{dz}\right\}$. These approximations are valid only in certain sectors of the neighborhood of $z_{0}$, and to obtain asymptotic approximations to the solutions elsewhere in the neighborhood connection formulae are needed.

In the case in which the differential equation is of the form $d^{2} w / d z^{2}=\left\{u^{2} f(z)+g(z)\right\} w$, in which $u$ is a large parameter and $f(z)$ and $g(z)$ are independent of $u$, it is shown how asymptotic approximations may be constructed for the coefficients in the connection formulae when $u$ is large. The nature of these approximations depends on the nature of the other singularities of the differential equation in the complex plane, and also on the zeros of $\mathrm{f}(\mathrm{z})$.

20253. Antonucci, J. M. New monomers for use in dentistry, (Proc. Symp. Application of Polymers in Dentistry, Houston, TX, Mar. 23-28, 1980), Paper in Biomedical and Dental Applications of Polymers, C. G. Gebelein and F. F. Koblitz, eds., pp. 357-371 (Plenum Publ. Corp., New York, NY, 1981).

Key words: benzaldehyde methacrylates; bicyclic diacetal benzaldehyde dimethacrylates; diurea dimethacrylates; highly fluorinated thermosetting methacrylates; polymerization shrinkage; prepolymer monomers; water sorption.

There is a need to further enhance the durability of dental composite restorative and sealant materials. Dimethacrylates such as BIS-GMA (the diadduct of bisphenol $\mathbf{A}$ and glycidyl methacrylate) are now widely used to formulate the resin component of these 
materials. Some of the deficiencies of dental composites and sealants are traceable to impurities and inherent structural imperfections in the monomer systems. Structure-property studies are needed to explore ways of achieving minimal shrinkage on polymerization, reducing water sorption, promoting adhesion, generally optimizing the chemical, physical, and mechanical stabilities of the resulting polymers. The synthesis of such an ideal dental monomer system is not an easy task. This paper describes the synthesis of several alternate types of monomers that address some of these problem areas and hopefully have utility as components of dental monomer systems. One novel type monomer system of high purity is derived from isomeric crystalline monomers that can form liquid eutectics. Other types include functional monomers such as benzaldehyde methacrylates, bicyclic diacetal benzaldehyde dimethacrylates, diurea dimethacrylates, and highly fluorinated thermosetting methacrylates including comb type prepolymer monomers. The latter monomers are designed to minimize water sorption and polymerization shrinkage and maximize the chemical, physical, and mechanical properties of the resin binder.

20254. Brauer, G. M. Initiator-accelerator systems for acrylic resins and composites, (Proc. Symp. Application of Polymers in Dentistry, Houston, TX, Mar. 23-28, 1980), Paper in Biomedical and Dental Applications of Polymers, C. G. Gebelein and F. F. Koblitz, eds., pp. 395.409 (Plenum Publ. Corp., New York, NY, 1981).

Key words: accelerators; acrylic resin; benzoyl peroxide; composite; dental restorative; dialkylaminophenethanol; dialkylaminophenylalkanoic acid; initiator.

Acrylic resins, because of their desirable esthetics, ease of processing, optical clarity that can duplicate in appearance the oral tissues it replaces, satisfactory mechanical properties and excellent biocompatibility, are the materials of choice wherever plastics have found applications in dental practice. The ready acceptability of these materials is the result of the ease with which they can be converted into their final state even under clinical conditions. In practically all dental applications a liquid monomer-solid mixture is cured by a free radical initiated polymerization that is generated by heat, light, an initiator, or a redox initiator-accelerator system adapted to the constraints imposed by the oral environment.

20255. Purtell, L. P. Molecular diffusion in oscillating laminar flow in a pipe, Phys. Fluids 24, No. 5, $789-793$ (May 1981).

Key words: axial diffusion; mass transfer; oscillating flow; perturbation; pipe flow.

The effect of flow oscillations on the axial diffusion of a solute in a pipe is analyzed theoretically by a perturbation method for small oscillation Reynolds numbers. The specific case of an initial step distribution in concentration is solved to second order. Numerical results of diffusion enhancement are given for several values of the parameter involved and are found to be conveniently summarized in terms of an equivalent diffusion parameter.

20256. McGarry, E. D.; Fabry, A. Dosimetry characterization of a reactor pressure vessel simulator by fission chamber and foil activation measurements, (Proc. Third ASTM-Euratom Symp. Reactor Dosimetry, Ispra, Italy, Oct. 1-5, 1979), Paper in Dosimetry Methods for Fuels, Cladding and Structural Materials, EUR6813 EN$F R$, II, 1031-1041 (Commission of the European Communities, Luxembourg, 1980).

Key words: dosimetry; fission chamber measurements; foil activation measurements; pressure vessel simulator; reactor pressure vessel.

A summary is given of the significance and results of extensive dosimetry measurements in the low-power, Light-Water Reactor Pressure Vessel (RPV) Simulator at the Pool Critical Assembly. Calculated and measured neutron flux and spectral characteristics are compared for a RPV simulator that represents a clean-core calculational model with measured core-power distributions. The purpose of such a model is to validate transport theory computations needed to extrapolate into the pressure vessel dosimetry observations in the surveillance position. Also reported are investigations of the neutron field characteristics of other configurations, which were investigated to establish the design of a high-power RPV simulator to proof test the correlation of metallurgy and dosimetry results. The neutron-field characterization measurements include extensive investigation of flux level and spectrum changes, bucklings, reactorcore power linearity and stability, and neutron perturbations associated with dosimetry instrumentation, as well as those caused by insertion of the metallurgy and dosimetry surveillance capsule. Reproducibility of measurement techniques are verified by interlaboratory comparisons. The objective of high accuracy dosimetry is achieved by benchmark referencing all fission chambers and activation foils to their responses in existing standard neutron fields.

20257. Purtell, L. P.; Klebanoff, P. S.; Buckley, F. T. Turbulent boundary layer at low Reynolds number, Phys. Fluids 24, No. 5, 802 . 811 (May 1981).

Key words: boundary layer; low-Reynolds number; mean velocity; skin friction; turbulent; zero-pressure gradient.

The results of an experimental investigation of a turbulent boundary layer with zero-pressure gradient directed toward extending the data base at low Reynolds numbers are presented. The data obtained are concerned primarily with mean-velocity distributions, skin-friction coefficients, and distributions of intensity of the longitudinalcomponent of the turbulent-velocity fluctuations for Reynolds numbers based on momentum thickness as low as 465 . The validity, at low Reynolds numbers, of the semi-empirical laws characterizing the inner and outer regions of the boundary layer is examined.

20258. Mathew, M.; Takagi, S.; Waerstad, K. R.; Frazier, A. W. The crystal structure of synthetic chukhrovite,

$\mathrm{Ca}_{4} \mathrm{AlSi}\left(\mathrm{SO}_{4}\right) \mathrm{F}_{13} \bullet_{12 \mathrm{H}_{2}} \mathrm{O}$, Am. Mineral. 66, 392-397 (1981).

Key words: chukhrovite; crystal structure; sulphate ion hydration.

Synthetic chukhrovite, $\mathrm{Ca}_{4} \mathrm{AlSi}\left(\mathrm{SO}_{4}\right) \mathrm{F}_{13} \cdot 12 \mathrm{H}_{2} \mathrm{O}$ crystallizes in the cubic space group $F d 3$, with $a=16.710(2) \AA$ and $\mathrm{Z}=8$. The structure was refined $R_{w}=0.028$ and $R=0.025$, using 481 nonzero reflections.

$\mathrm{Al}$ and $\mathrm{Si}$ are statistically distributed at the same site, $\mathrm{M}[16(\mathrm{~d})]$, which is surrounded by six fluorine atoms to form the octahedral group $\mathrm{MF}_{6}$. The remaining fluoride ion is surrounded by a tetrahedron of $\mathrm{Ca}$ ions $[\mathrm{F}(1)-\mathrm{Ca}=2.411 \AA]$ as in fluorite. The overall coordination of $\mathrm{Ca}$ is seven with a geometry corresponding to that of a monocapped octahedron. Each corner of the $\mathrm{MF}_{6}$ octahedron is linked to a $\mathrm{Ca}$ coordination polyhedron and each $\mathrm{Ca}$ polyhedron shares three corners with three different $\mathrm{MF}_{6}$ octahedra. The $\mathrm{SO}_{4}$ ion is surrounded by 12 water molecules, all of which are hydrogenbonded to $\mathrm{SO}_{4}$ oxygens. The most unusual feature of this hydration is that all four faces of the $\mathrm{SO}_{4}$ ion are linked to faces of $\mathrm{Ca}$ polyhedra via hydrogen bonding.

20259. Rossiter, W. J., Jr.; Mathey, R. G. Guidelines for installation of solar components on low-sloped roofs-A summary, Proc. NBS/NRCA 6th Conf. on Roofing Technology, Gaithersburg, MD, Apr. 30-May 1, 1981, pp. 58-62 (National Roofing Contractors Assoc., Oak Park, IL, Apr. 1981).

Key words: collector installation; field survey; guidelines; lowsloped roofs; roofing performance; solar collectors.

This paper presents guidelines for installation of solar collectors and related equipment on low-sloped roofs of commercial, industrial, and multi-family type buildings. The guidelines are concerned primarily with the waterproofing integrity of the roofing system, access to the collectors and roofing, attachment of different types of collector support frames and rooftop safety. Technical information from the literature, building codes, roofing field surveys, and acceptable roofing practice provided the basis for the guidelines. The guidelines include recommendations for design of the solar installation with regard to roofing performance, workmanship during collector installation, and maintenance of roofs with solar components.

20260. Ekin, J. W. Strain scaling law for flux pinning in $\mathbf{N b T i}, \mathrm{Nb}_{3} \mathrm{Sn}$, 
$\mathbf{N b}-\mathrm{Hf} / \mathrm{Cu}-\mathrm{Sn}-\mathrm{Ga}, \mathrm{V}_{3} \mathbf{G a}$ and $\mathbf{N b}_{3} \mathrm{Ge}$, IEEE Trans. Magn. MAG-17, No. 1, 658-661 (Jan. 1, 1981).

Key words: flux pinning; Lorentz force; $\mathrm{Nb}_{3} \mathrm{Ge}$; $\mathrm{Nb} \cdot \mathrm{Hf} / \mathrm{Cu}-\mathrm{Sn}$. $\mathrm{Ga}$; $\mathrm{Nb}_{3} \mathrm{Sn}$; $\mathrm{NbTi}$; scaling; strain; strain scaling; superconductors; uniaxial strain.

Critical current and flux pinning densities have been determined for a series of practical conductors as a function of uniaxial tensile strain in magnetic fields ranging from $4 \mathrm{~T}$ to $19 \mathrm{~T}$. An empirical relation has been found at $4.2 \mathrm{~K}$ that accurately describes these data over the entire range of field under both compressive and tensile strain. The pinning force $F$ has been found to obey a scaling law of the form: $F=\left[B_{c 2}^{-}(\epsilon)\right]^{n} f(b)$ where $f(b)$ is a function only of the reduced magnetic field $\mathrm{b} \equiv \mathrm{B} / \mathrm{B}_{\mathrm{c} 2}^{*}$, and $\mathrm{B}_{\mathrm{c} 2}^{*}$ is the strain dependent upper-critical field determined from high-field critical-current measurements. This strain scaling law was found to hold for all superconductors examined thus far, including commercial multifilamentary wire, monofilamentary conductors, CVD tapes, extremely fine-filament conductors, partially. reacted specimens, and "in-situ" cast conductors. For $\mathrm{Nb}_{3} \mathrm{Sn}, \mathrm{n} \cong 1.0$, for $\mathrm{Nb}_{3} \mathrm{Sn}$ with $\mathrm{Hf}$ and $\mathrm{Ga}$ additions, $\mathrm{n} \cong 1.2$, for $\mathrm{V}_{3} \mathrm{Ga}, \mathrm{n} \cong 1.4$, for $\mathrm{Nb}_{3} \mathrm{Ge}, \mathrm{n} \cong 1.6$, and for $\mathrm{NbTi}, \mathrm{n} \cong 4$. The importance of this relationship is that, for these conductors at least, it is possible to measure $F$ at one strain and then immediately be able to predict $F$ (and thus $J_{c}$ ) at other strain levels simply by scaling the results by $\left[\mathrm{B}_{\mathrm{c} 2}^{*}(\epsilon)\right]^{\mathrm{n}}$. The relation between strain scaling and temperature scaling is discussed as it relates to flux pinning theories.

20261. Barger, R. L. Simple interferometric technique for alignment of segmented retroreflectors, Appl. Opt. 19, $2088-2089$ (July 1980).

Key words: interferometry; lasers; Michelson interferometry; optics; Ramsey fringes.

Optical systems are described for 1) obtaining multiple laser beams with wavefronts parallel to interferometric accuracy and with large separations, and 2) retro-reflecting laser beams with the same accuracy.

20262. Evans, D. J. Nonlinear viscous flow in two-dimensional systems, Phys. Rev. A 22, No. 1, 290.294 (July 1980).

Key words: asymptotic behavior; nonequilibrium molecular dynamics; shear viscosity; strain rate; two dimensions; viscous flow.

Nonequilibrium molecular dynamics calculations have been performed for soft disks. It was found that the strain-rate dependence $\gamma$ of shear viscosity $\eta$ and hydrostatic pressure $p$ could be described by the following functional forms: $\eta(\gamma)=A_{\eta} \log _{10}\left|\gamma T_{\eta}\right|$ and $\mathrm{p}(\gamma)=$ $p(O)+A_{p}\left|\gamma T_{p}\right| \log _{10}\left|\gamma T_{p}\right|$. These functional forms are the same as ones that have been predicted using asymptotic "long-time-tail" theories. The numerical values of the coefficients as determined from the simulations are several orders of magnitude greater than theory predicts. If the equation above for the effective shear viscosity is valid in the limit of small strain rates, then Navier-Stokes hydrodynamics does not exist in two-dimensional fluids.

20263. Rainwater, J. C. Softness expansion of gaseous transport properties. II. Moderately dense gases, J. Chem. Phys. 74, No. 7, 4130-4143 (Apr. 1, 1981).

Key words: collisional transfer; first density correction; inverse power potential; kinetic theory; modified Enskog theory; SniderCurtiss theory; thermal conductivity; three-body effects; viscosity.

The first density corrections to gaseous transport properties for the inverse power potential $\phi=\phi_{0}(\sigma / r)^{n}$ are evaluated according to the theory of Curtiss, Snider, and co-workers. A linear softness expansion predicts that the first density corrections are positive for large $n$ but negative for small $n$, and that the changes of sign occur at $n=32.75$ and $n=8.42$ for the viscosity and thermal conductivity, respectively. Direct numerical integration yields $n=29.60$ and $n=7.07$, respectively. These results explain the opposite signs of density corrections to the two transport properties seen in high temperature experimental data, where, effectively, $n=12$. Predictions of ti. modified Enskog theory are compared and contrasted with the above results. It is shown that some of the Snider-Curtiss (collisional transfer) collision integrals may be simplified, one of them being the usual dilute gas omega integral with Smith's collision lifetime included in the integrand.

20264. Weber, L. A. Measurements of the heat capacities $C_{v}$ of dense gaseous and liquid nitrogen and nitrogen trifluoride, J. Chem. Thermodyn. 13, 389-403 (1981).

Key words: calorimetry; nitrogen; nitrogen trifluoride; specific heats; thermophysical properties.

Heat capacities at constant volume are reported for nitrogen and nitrogen trifluoride in both the single-phase and two-phase regions. The measurements cover the range in temperature from 78 to $250 \mathrm{~K}$ for nitrogen and from 67 to $300 \mathrm{~K}$ for $\mathrm{NF}_{3}$. Pressures ranged up to about $30 \mathrm{MPa}$ and densities varied from somewhat less than the critical density to near the triple-point density. Comparisons are made with values calculated from the equation of state. The heat capacity of the saturated liquid is derived from the two-phase results and compared with earlier measurements.

20265. Drullinger, R. E.; Wineland, D. J.; Bergquist, J. C. Highresolution optical spectra of laser cooled ions, Appl. Phys. 22, 365.368 (1980).

\section{Key words: high resolution; laser cooling; optical spectra.}

We obtain essentially Doppler free spectra of the naturally occuring isotopes of $\mathrm{Mg}^{-}$, which are bound in a Penning trap, by using a frequency stabilized laser to continuously cool the ions, while the scatter rate from a second, frequency swept laser is monitored. We show that the magnetron motion as well as the cyclotron and axial motion can be minimized. Line position measurements yielding resonance transition energy isotope and hyperfine shifts are reported.

20266. Link, J.; Wintersgill, M. C.; Fontanella, J. J.; Bean, V. E.; Andeen, C. G. Pressure variation of the low.frequency dielectric constants of some anisotropic crystals, J. Appl. Phys. 52, No. 2, 936. 939 (Feb. 1981).

Key words: anisotropic crystals; calcite; dielectric constants; magnesium fluoride; pressure dependence; quartz; sapphire.

The low-frequency dielectric constant for quartz, sapphire, calcite, and magnesium fluoride has been measured both parallel and perpendicular to the optic axis at several temperatures over the pressure range $0-0.3 \mathrm{GPa}$. The results, when compared with the reported values for the effect of pressure on the high-frequency dielectric constant, indicate that pressure decreases the infrared contribution to the dielectric constant for all materials except calcite. Consequently, the infrared polarizability decreases with pressure for all but calcite. Further, it is found that the infrared polarizability decreases with pressure for calcite perpendicular to the optic axis, but increases with pressure parallel to the optic axis. In addition, it is shown that the volume-independent temperature derivative of the low-frequency dielectric constant is positive for sapphire, calcite, and magnesium fluoride showing that cubic terms in the lattice potential energy dominate, while for quartz, the derivative is negative implying that quartic terms are dominant.

20267. Hayward, E. The elastic photon scattering cross section for ${ }^{12} \mathrm{C}$ in the energy range 20-40 MeV, (Int. School of Intermediate Energy Nuclear Physics, Rome, Italy, June 15-29, 1979), Paper in Electron and Pion Interactions with Nuclei at Intermediate Energies 2, 667.675 (Harwood Academic Publ., New York, NY 1979).

Key words: absorption cross section; carbon 12; dispersion relation; photon scattering; quadrupole excitation.

The elastic scattering cross section for ${ }^{12} \mathrm{C}$ has been measured in the energy range $23.5-40 \mathrm{MeV}$. It has been found that this scattering results not only from electric dipole but also from electric quadrupole excitations. 
20268. Cummings, A. L.; Layer, H. P.; Hocken, R. J. Lasers and analytical polarimetry, Chapter 15 in Lasers in Chemical Analysis, G. M. Hieftje, J. C. Travis, and F. E. Lytle, eds., pp. 291-302 (The Humana Press, Clifton, NJ, 1981).

Key words: analytical polarimetry; conventional polarimetry; gas laser; polarized light; tunable dye laser.

Gas lasers and a tunable dye laser have been used to facilitate rapid and accurate optical rotation measurements with $3 \times 10^{-6}$ rad resolution. With the aid of the optogalvanic effect, a single frequency dye laser provided wavelength tunability with reproducibility to better than $3 \times 10^{-6} \mu \mathrm{m}(<3 \mathrm{GHz})$. The lasers have been shown to be superior to conventional light sources for precise polarimetry because of greater signal amplitude, higher signal to noise ratio, monochromacity and wavelength reproducibility. This has resulted in shorter measurement time, better reproducibility of results, and less sensitivity to sample dispersion. Furthermore, greater flexibility in polarimeter design is permissible. With lasers, the polarimeter can become a much more selective analytical tool than is presently the case.

20269. Ritter, J. J.; Kruger, J. A new ellipsometric-electrochemical approach to the study of corrosion under organic coatings, (Proc. Symp. Advances in Coating-Metals for Corrosion Protection, Las Vegas, NV, Aug. 27, 1980), Preprint Division of Polymer Chemistry, American Chemical Society 43, 575-580 (1980).

Key words: chromate inhibitors; corrosion mechanisms; organic coatings; painted metal; $\mathrm{pH}$ measurements; qualitative ellipsometry.

Qualitative ellipsometry was used in conjunction with electrochemical $\mathrm{pH}$ and potential measurements as a technique for the study of corrosion processes occurring under organic coatings. The objective of the work was to gain a deeper understanding of the mechanisms governing the corrosion protective actions of paints on metal surfaces. Computer modeling and experiments with collodion coatings on iron substrates indicated that changes in the ellipsometric parameter $\Delta$ could, for the most part, be safely interpreted as thickness alterations in the substrate oxide film and roughening of the substrate. Experiments with Fe-collodion system in dilute chloride solutions exhibited three sequential stages of activity, two of which could be interpreted in terms of corrosion mechanisms using the optical and electrochemical measurements. Chromate ion as a corrosion inhibitor in coatings was also studied using this technique, and was shown to have significant effects upon the development of the sub-coating processes.

20270. Weber, H.; Fraker, A. C. SEM studies of dental casting alloy corrosion behavior, Proc. 37th Annu. Electron Microscopy Society of America Meet., San Antonio, TX, Aug. 1979, G. W. Bailey, ed., pp. 280-281 (1979).

Key words: corrosion; dental castings; nickel-chromium; scanning electron microscopy.

Scanning electron microscope (SEM) studies were used to correlate surface film formation and local corrosion attack with the metal microstructure. The metals investigated were dental casting alloys based on the Ni-Cr system. By the use of anodic polarization measurements combined with SEM studies, it was found that there are significant differences in the corrosion behavior of the three commercial $\mathrm{Ni}-\mathrm{Cr}$ materials studied.

20271. Brown, W. E.; Chow, L. C. Thermodynamics of apatite crystal growth and dissolution, J. Cryst. Growth 53, 31.41 (1981).

Key words: crystallization; dissolution; hydroxyapatite; kinetics; phase diagrams; solubility isotherm; thermodynamics; tooth mineral.

The rates of dissolution and crystal growth of hydroxyapatite, $\mathrm{Ca}_{5}\left(\mathrm{PO}_{4}\right)_{3} \mathrm{OH}$, are subjects of considerable interest because of their involvement in many biological and commercial processes. An important factor governing these rates can be the composition of the quasi-saturated solution immediately adjacent to the crystal surface which acts as a boundary condition for diffusion, especially in unstirred systems. The composition of this saturated solution is shown to shift along the isotherm, depending on the relative rates of diffusion of $\mathrm{Ca}$ and $\mathrm{PO}_{4}$. Steady state is achieved when the $\mathrm{Ca} / \mathrm{P}$ rateof-diffusion ratio reaches $5 / 3$. These processes should be first studied in the simplest system, $\mathrm{Ca}(\mathrm{OH})_{2}-\mathrm{H}_{3} \mathrm{PO}_{4}-\mathrm{H}_{2} \mathrm{O}$, because this reduces the number of variables to a minimum, and in the $\mathrm{pH}$ range 3.5 to 6.0 where the $\mathrm{Ca} / \mathrm{P}$ concentration ratio is fixed at $\sim 1 / 2$ by electroneutrality. The complications resulting from the presence of additional components, such as strong and weak acids and a swamping electrolyte, are discussed.

20272. Shara, M. M.; Prialnik, D.; Shaviv, G. What determines the speed class of novae, Astrophys. J. 239, 586-591 (July 15, 1980)

Key words: CNO enrichment; novae; speed class.

Recent theoretical hydrodynamic models show that novae of different speed classes can be obtained by varying the CNO enrichment and envelope mass. Recent observations seem to indicate that various degrees of CNO enrichment are found in the ejected shells of slow novae. We propose a unified picture for novae of different speed classes as a combination of CNO enrichment and envelope mass. Several consequences of our scheme are discussed. The observational and theoretical results are explained in the context of our unified picture.

20273. Hamilton, J. C.; Jach, T. Structural phase transitions in nickel at the Curie temperature, Phys. Rev. Lett. 46, No. 11, 745.748 (Mar. $16,1981)$.

Key words: LEED; magnetism; phase transition; segregation.

It is observed that a reversible step period rearrangement on clean nickel single-crystal surfaces occurs in the immediate vicinity of the Curie temperature. Reversible carbon segregation is observed on the same crystal surfaces below the Curie point. The segregated carbon is carbidic, not graphitic, and indicates a change in the nickel surface electronic structure occurring at the ferromagnetic transition. Measured carbon coverages indicate a change greater than $0.2 \mathrm{eV}$ per carbon atom in the heat of segregation at the Curie point.

20274. Mehl, J. B.; Moldover, M. R. Precision acoustic measurements with a spherical resonator: $\mathrm{Ar}$ and $\mathrm{C}_{2} \mathrm{H}_{4}, J$. Chem. Phys. 74, No. 7, $4062-4077$ (Apr. 1, 1981).

Key words: acoustic relaxation; argon; ethylene; resonance; speed-of-sound; standing waves; virial coefficients.

The spherical acoustic resonator is a remarkably accurate and convenient tool for the measurement of thermophysical properties of gases at low and moderate densities. The speed of sound $(c)$ in a gas of interest can be measured with an accuracy of $0.02 \%$ merely by measuring the frequencies of the radial resonances when the resonator is filled with the gas of interest and then repeating the frequency measurements with a reference gas such as argon. The resonance frequencies of the radial modes are easily measured because these modes have very high $Q$ 's, typically 2000-10000. In this work the precision and accuracy of speed of sound measurements have been substantially improved by including a detailed acoustic model of the resonator in the analysis. Many of the important parameters of the model can be determined from acoustic measurements: Painstaking mechanical measurements are not required. We have used a spherical resonator to measure the speed and attenuation of sound in $\mathrm{C}_{2} \mathrm{H}_{4}$ in the temperature range $0-100^{\circ} \mathrm{C}$ and the pressure range $0.15-1.0 \mathrm{MPa}$. Our measured values of $c$ in $\mathrm{C}_{2} \mathrm{H}_{4}$ have a precision of $0.003 \%$ and agree with those of Gammon within the scatter of Gammon's data $( \pm 0.02 \%)$. This agreement is remarkable when one considers that our spherical resonator is operated in the frequency range $4-13 \mathrm{kHz}$ while Gammon has used a more conventional, cylindrical, variable path, acoustic interferometer operating at $500-2500 \mathrm{kHz}$. To attain this agreement, we did not have to make any highly accurate measurements other than frequency. Our measured values of the bulk 
relaxation frequency of $\mathrm{C}_{2} \mathrm{H}_{4}$ are within the scatter of the more recent values of the literature. In the course of our "calibration" measurements with argon we have redetermined the leading acoustic virial coefficient of argon. Our values for the virial are in satisfactory agreement with those in the literature. We include several practical suggestions for increasing the accuracy and/or versatility of spherical resonators.

20275. Wolynec, E.; Dodge, W. R.; Leicht, R. G.; Hayward E. Electrodisintegration of ${ }^{58} \mathrm{Ni},{ }^{60} \mathrm{Ni}$, and ${ }^{62} \mathrm{Ni}$, Phys. Rev. C 22, No. 3, 1012-1024 (Sept. 1980).

Key words: electric quadrupole; electrodisintegration; giant resonance; nickel isotopes; virtual photons.

The $(e, p)$ and $(e, \alpha)$ cross sections for targets of ${ }^{58} \mathrm{Ni},{ }^{60} \mathrm{Ni}$, and ${ }^{62} \mathrm{Ni}$ have been measured in the electron energy range 16-100 MeV. They have been analyzed using the distorted-wave Born approximation $E 1$ and $E 2$ virtual photon spectra. Protons are emitted primarily following $E 1$ absorption but $\alpha$-emission results from a combination of $E 1$ and $E 2$ absorption.

20276. Clough, R. B.; Chang, J. C.; Travis, J. P. Acoustic emission signatures and source microstructure using indentation fatigue and stress corrosion cracking in aluminum alloys, Scr. Metall. 15, 417.422 (1981).

Key words: acoustic emission; fatigue; indentation; nondestructive evaluation; stress corrosion cracking.

Acoustic emission due to identation is a new NDE technique for directly correlating acoustic emission behavior with the microstructure of a particular source. Previous work with this method demonstrates that reproducible acoustic emission signals can be generated in embrittled steels by incremental growth of cracks. Here the technique is illustrated for use in more ductile materials through the new techniques of indentation fatigue and indentation stress corrosion cracking. By these methods a direct correlation can be made between a particular source microstructure and the resulting acoustic emission behavior.

20277. Tesk, J. A.; Whitlock, R. P.; Widera, G. E. O.; Holmes, A.; Parry, E. E. Consideration of some factors influencing compatibility of dental porcelains and alloys, Part II: Porcelain/alloy stress, Proc. 4th Int. Precious Metals Conf., Toronto, Ontario, Canada, June 4-6, 1980, pp. 283-291 (Pergamon of Canada, Toronto, Ontario, Canada, Apr. 1981).

Key words: bonding, glass to metal; dental alloys; dental porcelain; finite element analysis, porcelain on metal; glass to metal seals; porcelain/alloy compatibility; porcelain/alloy composite structures; split ring compatibility test; stress analysis glasses; stress in dental alloys.

The change in gap of a porcelain veneered split metal ring has been previously proposed as an indication of compatibility (Whitlock and others, 1980). Gap changes were measured for several combinations of opaque and body porcelain fired on a dental alloy ring. Finite element and theoretical calculations of the gap change, when compared with experimental observations, indicate stress relief in porcelain-metal composite systems at temperatures well below the glass transition temperature, $\mathrm{T}_{\mathrm{g}}$. Extension of the bimaterial compatibility index to more complex (more than two layer) systems is shown to require further consideration. Stress analyses based on Timoshenko's equation for shape change of multimaterial thermostats leads to oversimplified states of stress and cannot yield the detailed information on shear and tensile stresses needed for viscous flow above $T_{g}$ and brittle fracture below $T_{g}$.

20278. Straty, G. C.; Diller, D. E. ( $p, V, T)$ of compressed and liquefied (nitrogen +methane), J. Chem. Thermodyn. 12, $937-953$ (1980).

Key words: compressed gas; density; liquid; methane; mixtures; nitrogen; PVT.

The results of $(p, V, T)$ measurements on 3 gravimetrically prepared mixtures of nitrogen + methane are reported. Results were obtained on a mixture with mole fraction $x \approx 0.5$ along 24 isochores at densities from about $8 \mathrm{~mol} \mathrm{dm}^{-3}$ to over $28 \mathrm{~mol} \mathrm{dm}^{-3}$ and at pressures to over $30 \mathrm{MPa}$. Results were also obtained along 6 selected isochores for each of 2 mixtures of nominally $\left(0.3 \mathrm{~N}_{2}+0.7 \mathrm{CH}_{4}\right)$ and $\left(0.7 \mathrm{~N}_{2}+\right.$ $0.3 \mathrm{CH}_{4}$ ). The temperatures ranged from about 75 to over $300 \mathrm{~K}$ at pressures to over $30 \mathrm{MPa}$.

20279. Madey, T. E.; Houston, J. E.; Dahlberg, S. C. Bonding geometry of $\mathrm{CO}$ on planar and stepped single crystal surfaces, (Proc. 4th Int. Conf. Solid Surfaces, Cannes, France, Sept. 22, 1980), J. Fr. Vac. Soc. 1, 205-208 (Sept. 1980).

Key words: adsorbed $\mathrm{CO}$; electron stimulated desorption; planar single crystal surface; stepped single crystal surface.

The structures of molecular CO adsorbed on a planar W(110) surface and on stepped surfaces containing W(110) terraces have been studied using the electron stimulated desorption ion angular distribution (ESDIAD) method. For $\mathrm{CO}$ adsorbed on the close packed $W(110)$ surface, the dominant bonding mode is via the carbon atom, with the $\mathrm{CO}$ molecular axis perpendicular to the plane of the surface. For $\mathrm{CO}$ adsorbed at step sites on 4 different surfaces vicinal to W(110), the axis of the molecule is "tilted" or inclined away from the normal to the surface. The ESDIAD method, in which ion desorption angles are related to surface bond angles, provides a direct determination of the structures of adsorbed molecules and molecular complexes on surfaces.

20280. Ledbetter, H. M. Sound velocities and elastic constants of steels 304, 310, and 316, Met. Sci. Tech. Note 14, No. 12, 595.596 (Dec. 1980).

Key words: bulk modulus; elastic constants; Poisson's ratio; shear modulus; sound velocity; stainless steel; Young's modulus.

Room temperature ultrasonic velocities, longitudinal and transverse, were measured in three AISI 300-series austenitic stainless steels. Elastic constants computed from these velocities show small but significant variations with composition.

20281. Weiss, A. W. Review of atomic structure calculations, Proc. Workshop Foundations of the Relativistic Theory of Atomic Structure, Argonne National Laboratory, Argonne, IL, Dec. 4-5, 1980, ANL-80. 125, pp. 166.178 (Mar. 1981).

Key words: atomic structure; electron correlation; relativistic corrections.

The current status of $a b$ initio atomic structure calculations is summarized for both the non-relativistic and relativistic cases. The relative advantages and disadvantages of the principal methods of calculation are described, and the prospects for further developments are discussed.

20282. Lynn, J. W.; Erwin, R. W.; Rhyne, J. J.; Chen, H. S. Spin glass-ferromagnetic phase transition in amorphous $\left(\mathrm{Fe}_{x} \mathrm{Ni}_{1-x}\right)_{0.75} \mathrm{P}_{0.16} \mathrm{~B}_{0.06} \mathrm{Al}_{0.03}$, J. Appl. Phys. 52, No. 3, 1738.1740 (Mar. 1981).

Key words: amorphous; ferromagnetic; neutron scattering; phasetransition; spin-glass; spin waves.

Inelastic neutron scattering studies have been carried out on the magnetically isotropic amorphous system $\left(\mathrm{Fe}_{\mathrm{x}} \mathrm{Ni}_{1-x}\right)_{0.75} \mathrm{P}_{0.16} \mathrm{~B}_{0.06} \mathrm{Al}_{0.03}$ in the concentration range $(x>0.17)$ where both ferromagnetic and spinglass behavior are observed. For $x=0.4$, well defined spin waves which obey the customary quadratic dispersion relation $E=D^{2}$ are observed below the Curie temperature $\left(T_{c}=365 \mathrm{~K}\right)$ and $D$ is found to increase with decreasing temperature as usual. Below $\sim 80 \mathrm{~K}$, however, $\mathrm{D}$ begins to decrease with decreasing temperature as the spin-glass state is approached. Accompanied by this decrease in D is an increase in the spin wave linewidths. Preliminary results for $x=0.3$ and $x=0.2$ show qualitatively similar behavior.

20283. Fernandez-Baca, J. A.; Lynn, J. W. Neutron scattering studies 
of the magnetic superconductor $\left(\mathbf{C e}_{1-x} \mathbf{T} \mathbf{T b}_{\mathbf{x}}\right) \mathbf{R} \mathbf{u}_{2}, J$. Appl. Phys. 52, No. 3, 2183.2185 (Mar. 1981).

Key words: (Ce-Tb)Ru $\mathrm{R}_{2}$; correlations; Laves-phase; magnetic; neutron scattering; superconductor.

Neutron scattering measurements have been carried out on polycrystalline specimens of $\left(\mathrm{Ce}_{1-\mathrm{x}} \mathrm{Tb}_{\mathrm{x}}\right) \mathrm{Ru}_{2}$, for $\mathrm{x}=0.07,0.18,0.44$, in order to study the inelastic magnetic scattering as well as the development of magnetic correlations at low temperatures. At small wavevectors the intensity in each sample increases with decreasing temperature indicating the development of ferromagnetic correlations at low temperatures, but the intensity saturates at low temperature with no peak in its temperature dependence. There is also no evidence of any magnetic Bragg peaks, which indicates that there is no long range ferromagnetic order. For $x=0.44$, inelastic scattering measurements show a distribution of magnetic scattering from low energies up to $4.5 \mathrm{meV}$, with a relatively well defined maximum at 3.3 $\mathrm{meV}$. At $\mathrm{T}=20 \mathrm{~K}$ additional scattering appears at $6.6 \mathrm{meV}$, while the scattering which peaked at $3.3 \mathrm{meV}$ appears to shift to lower energies.

20284. McDonald, D. G. Superconducting electronics, Phys. Today 34, No. 2, $37-47$ (Feb. 1981).

Key words: cryogenics; electronics; Josephson effect; superconductivity.

Scientific measurements using superconducting electronics are approaching fundamental limits, limits which no technology can surpass in some cases. Circuits with the same basic components have important advantages for large-scale integrated electronics. This combination of extraordinary performance and suitability for large scale integration presages the emergence of a broad new electronic technology.

20285. Newbury, D. E. Microanalysis of individual layered particles by secondary ion mass spectrometry, EPA-600/7-80-122, 68 pages (United States Environmental Agency, Office of Environmental Engineering and Technology, Washington, DC, July 1981). (Available from the National Technical Information Service, Springfield, VA 22161, July 1981).

Key words: depth profiling; ion microprobe; layered particles microanalysis; particulate analysis; secondary mass spectrometry.

Secondary ion mass spectrometry is evaluated for application to the determination of the composition and structure of individual particles Analyses of many elemental constituents at the ppm level can be obtained in individual particles as small as $2 \mu \mathrm{m}$ in diameter Molecular signals, both organic and inorganic, can be detected from particles above $20 \mu \mathrm{m}$ in diameter. Quantitative analyses of elemental constituents can be made with a relative accuracy of 25 percent by means of empirical relative sensitivity factors and within a factor of two by means of a physical model. Multielement depth profiles can be obtained from individual particles as small as $4 \mu \mathrm{m}$ in diameter. Depth profiles of individual particles from SRM 1648 Urban Air Particulate reveal pronounced surface concentrations of lead and barium. Implementation of SIMS depth profiling requires automation to make use of the full spectral information and to eliminate matrix effects by normalization.

20286. Ledbetter, H. M. Dynamic elastic modulus and internal friction in G-10CR and G-11CR fibreglass-cloth-epoxy composites, Cryogenics 20, No. 11, 637.640 (Nov. 1980)

Key words: composites; elastic constants; glass-epoxy; internal friction; Young's modulus

Young's moduli were determined dynamically for two fibreglasscloth-epoxy composites in the warp, fill, and normal directions between room temperature and liquid-nitrogen temperature. Dynamic internal friction relates inversely to dynamic modulus in the studied materials. The experimental arrangement consisted of a Marx threecomponent oscillator at frequencies between 40 and $90 \mathrm{kHz}$.

20287. Weidner, V. R.; Hsia, J. J. Reflection properties of pressed polytetrafluoroethylene powder, J. Opt. Soc. Am. 71, No. 7, 856-861 (July 1981).

Key words: absolute; bidirectional; diffuse; directional/hemispherical; hemispherical; integrating-sphere; powder; reflectance; tetrafluoroethylene.

The reflection properties of pressed polytetrafluoroethylene powder have been under investigation by the Radiometric Physics Division at the National Bureau of Standards for the past five years. This material has a great potential use, both as a standard of diffuse reflectance and as a coating for integrating spheres for applications in reflectance spectrophotometry and other signal-averaging devices. It possesses certain physical and optical properties that make it ideal for use in these applications. Techniques are given for preparing reflection standards and coating integrating spheres with the pressed powder. The effects of powder density and thickness on its reflectance are reported, and observations of possible problems with fluorescence that are due to the presence of contaminants in the powder are discussed. The absolute reflectance $\left(6^{\circ} /\right.$ hemispherical reflectance factor relative to a perfect diffuser) is reported for the spectral range of $200-2500 \mathrm{~nm}$. The directional/hemispherical reflectance factor relative to $6^{\circ}$ /hemispherical reflectance is given for several wavelengths in the ultraviolet and visible spectrum and for angles of incidence between 5 and $75^{\circ}$. The bidirectional reflectance factor is reported for 300,600 , and $1500 \mathrm{~nm}$ at angles of incidence of $-10,-30,-50$, and $-70^{\circ}$ and at viewing angles at $10^{\circ}$ intervals from -80 to $+80^{\circ}$.

20288. May, W. E. The development of an aqueous trace organic standard reference material for energy related applications: Investigation of the aqueous solubility behavior of polycyclic hydrocarbons, EPA-600/7-80-031, 87 pages (Available from the National Technical Information Service, Springfield, VA 22161, Feb. 1980).

Key words: polynuclear aromatic hydrocarbons; standard reference materials.

The development of a Standard Reference Material for aqueous solutions of known concentration of polynuclear aromatic hydrocarbons is an extremely difficult procedure. This paper is one of a series discussing the development of a generator column technique at NBS for the production of Standard Reference Materials for PAH's in water. In addition to providing the basis for SRM development the aqueous solubility is a fundamental parameter in assessing the extent and rate of the dissolution of energy based polynuclear aromatic hydrocarbons and their persistence in the aquatic environment.

20289. MacCrehan, W. A.; Durst, R. A. Assessing the environmental impact of fossil-fuel based energy sources, EPA-600/7-79-211, 63 pages (Available from the National Technical Information Service, Springfield, VA 22161, Nov. 1979).

Key words: column preconcentration; electrochemical detection in liquid chromatography; methyl-mercury; organometal speciation; tissue preparation; trimethyllead.

A new measurement approach for the determination of trace organometals in energy related environmental samples is described. The method is based on liquid chromatographic separation with electrochemical detection. A detailed description of the development of the electrochemical system, optimized for reducible analytes, is given. The conditions for the separation of methyl-, ethyl-, and phenylmercury in under 16 minutes by charge-neutralization reversephase chromatography are developed. Also, a separation of a number of organoleads, including trimethyl- and triethyllead, are described. The potential interferences in this type of organometal determination are investigated. Sample preparation methods and improvement in the detector selectivity are described that overcome these interferences A significant improvement in the selectivity of the detection system has been investigated using a differential pulse waveform.

Sample preparation methods for the determination of methylmercury in biological tissue are examined and measurements are made on two research materials: lyophilized tuna and shark meat 
A column preconcentration procedure for methyl- and ethylmercury in natural water samples is developed.

20290. Hodgeson, J. A.; Fatiadi, A. J. Survey on research needs on personal samplers for toxic organic compounds, EPA-600/4-80-042, 35 pages (Available from the National Technical Information Service, Springfield, VA 22161, Aug. 1980).

Key words: ambient atmosphere; benzene; chlorinated hydrocarbons.

A survey is presented on the research and development needs for personal monitoring devices for toxic organic compounds in the ambient atmosphere. This survey includes a description of organic compounds and their ambient concentrations, individual compounds of high priority, a summary of a literature survey, a description of commercially available samplers, a summary of recent developments in ambient personal monitoring and recommendations on major research needs. The high priority compounds identified were: methyl choloride, dichloromethane, benzene, carbon tetrachloride, chloroform, dichlorobenzenes, 1,2-dichloroethane, methyl chloroform, trichloroethylene and perchloroethylene. The literature survey covers the period, 1974-79. Commercially available personal samplers described are Dupont's Pro-Tek organic vapor badge, Abcor's gasbadge, 3-M's organic vapor monitor and the Minimonitor (P.W. West, Louisiana State University). Recent activities include a description of an EPA sponsored program at Monsanto Research Corporation on development of personal samplers for organics. A description is also given of several recent field studies on sampling and analysis for benzene and chlorinated hydrocarbons. The survey concludes with recommendations for research and development activities in the following areas: evaluation of sorbent materials, development of analytical techniques based on electron capture-gas chromatography, evaluation of available active and passive samplers, development of passive samplers and development of standard mixtures for evaluation of personal exposure devices.

20291. Streed, E. R.; Waksman, D. Uncertainty in determining thermal performance of liquid-heating flat-plate solar collectors, Trans. ASME 1103, 126-134 (May 1981).

Key words: collector rating; measurement; solar collector; standards; thermal performance; uncertainty.

Thermal performance measurements of eight types of liquid-heating flat-plate solar collectors were conducted with two to four collectors of each type at four outdoor test sites. Tests were performed in accordance with the procedure prescribed by ASHRAE Standard 93 77. Statistical analysis of data sets for each collector type within test sites and between test sites was done using ASTM recommended methods to evaluate test method measurement uncertainty.

20292. Swartzendruber, L. J. Magnetic leakage and force fields for artificial defects in magnetic particle test rings, Proc. 12th Symp. Nondestructive Evaluation. San Antonio, TX, Apr. 24-26, 1979, pp. 150.162 (American Society for Nondestructive Evaluation, Southwest Research Institute, San Antonio, TX, 1980).

Key words: magnetic leakage fields; magnetic particles; nondestructive evaluation; test rings.

Magnetic particle inspection systems are often checked for performance and sensitivity using a test sample with artificial defects. One test sample consists of a steel ring with artificial defects introduced by drilling a series of small holes at varying depths below the sample surface. The magnetic leakage fields caused by these artificial defects have been calculated for the case of a linear isotropic magnetic material, and determined approximately for a non-linear material near saturation. Of greater importance for magnetic particle inspection, the force fields acting on small magnetic particles have been calculated. Lines of constant force have the approximate shape of an ellipse with small eccentricity centered at the defect. Using these results, the magnetic particle surface density which evolves around the artificial defects from a uniform low density cloud of magnetic particles is estimated for the case of dry particles. The calculated fields and powder patterns are compared with those obtained on a typical test ring. Based on these results, the ability of such test rings to accurately gauge system performance is discussed.

20293. Simiu, E.; Shaver, J. R.; Filliben, J. J. Wind speed distributions and reliability estimates, J. Struct. Div. Am. Soc. Civ. Eng. Tech. Note 107, No. ST5, 1003-1007 (May 1981).

Key words: extreme value distributions; Rayleigh distributions; sampling errors; structural reliability; wind engineering; wind forces.

The purpose of this note is to present an investigation into the effect upon the estimation of safety levels for wind-sensitive structures of the model that describes the probabilistic behavior of the extreme wind speeds. Such an investigation is motivated by recent research results, according to which extreme wind speeds are in most cases best modeled by probability distributions with considerably shorter tails than the Type I Extreme Value distribution, in particular by the Rayleigh distribution. It is shown that safety levels estimated on the basis of the assumption that the Rayleigh distribution holds are significantly higher than those previously estimated in the literature.

20294. Wyckoff, J. M. Meeting State and local government needs by transfer of Federal laboratory technology, J. Technol. Transfer 5, No. 2, 1-21 (1981).

Key words: organizations; State and local government; technology transfer; trends.

The need of State and local governments to deal with an increasing range of technological problems has led to the development of relatively new ways to call on Federal laboratories. This paper discusses the structure of these mechanisms for technology transfer, organizations involved in the systems that have developed, trends that affect these organizations and current problem areas.

20295. Simiu, E.; Filliben, J. J. Wind direction effects on cladding and structural loads, Eng. Struct. 3, No. 3, 181-186 (July 1981).

Key words: buildings (codes); climatology; statistical analysis; structural engineering; wind engineering; wind (meteorology).

A simple procedure is proposed for estimating wind loads corresponding to various return periods, which takes into account directional information on both wind speeds and aerodynamic response. Examples of the application of the procedure are given, which show that cladding loads calculated without taking directional information on extreme wind speeds into account may in certain cases be larger than the actual loads by a factor of two or more. It is also shown that it is not appropriate, in general, to account for wind direction effects by multiplying loads determined without regard for these effects by a reduction factor of 0.8 , as has been suggested in the literature. In its present form, the procedure is applicable to cladding panels and to members of relatively rigid structures in regions not subjected to hurricane winds.

20296. Kweller, E. R.; Mullis, W. F. Determination of annual efficiency of vented heaters equipped with thermally activated vent dampers, (Proc. ASHRAE Semi-Annu. Meet. Symp. Efficiency of Combustion Systems, Chicago, IL, Jan. 25-29, 1981), ASHRAE Trans. 87, Pt. 1, 753-768 (June 1981).

Key words: heater efficiency (part-load); heating equipment; test procedures; thermal vent dampers; vent (stack) dampers.

Part-load space heater efficiency requires the determination of five flue losses. These are latent heat loss $\left(L_{L}\right)$, on-period sensible heat loss $\left(\mathrm{L}_{\mathrm{S}, \mathrm{ON}}\right)$, on-period infiltration loss $\left(\mathrm{L}_{1, \mathrm{ON}}\right)$, off-period sensible heat loss $\left(\mathrm{L}_{\mathrm{S}, \mathrm{OFF}}\right)$ and off-period infiltration loss $\left(\mathrm{L}_{\mathrm{1}, \mathrm{OFF}}\right)$.

We determined: Which of the part-load losses can be adequately measured using the current DOE procedure. Which part load losses cannot be measured using the DOE procedure. Seasonal efficiencies for three vented heaters using the current and a modified procedure.

All stack losses associated with the calculation of part-load utilization efficiency $\left(\eta_{\nu}\right)$ were reviewed in the development of a test method for heaters equipped with the thermally-activated vent 
damper. Annual fuel utilization efficiency and associated energy savings were determined for two thermal vent dampers tested with three types of gas-fueled heaters which operate in either the cycling mode or in the modulating fuel input mode.

Test results showed energy savings from 1 to 9 percent depending on the type of heater as well as the type of damper used.

20297. Ekin, J. W.; Schramm, R. E.; Superczynski, M. J. Training of epoxy-impregnated superconductor windings, (Proc. Int. Cryogenic Materials Conf., Madison, WI, Aug. 24-29, 1979), Paper J.5 in Advances in Cryogenic Engineering Materials, A. F. Clark and R. P. Reed, eds., 26, 677-683 (Plenum Press, New York, NY, 1980).

Key words: epoxy; epoxy-fiberglass composite; epoxy impregnation; fiberglass-epoxy composites; impregnation superconducting magnets; magnet training; superconducting magnets; training of superconductors.

A series of NbTi composite rings were constructed in which both the impregnant material and construction technique were varied. Preliminary results indicate that the training of potted superconducting magnets is associated with a process involving relief of stress concentration within epoxy impregnants. The degree of training is correlated with: 1) Level of operating current; 2) Presence of fiberglass in the coil structure; 3 ) Prestraining the superconductor prior to winding.

20298. Fujii, G.; Ekin, J. W.; Radebaugh, R.; Clark, A. F. Effect of thermal contraction of sample-holder material on critical current, (Proc. Int. Cryogenic Materials Conf., Madison, WI, Aug. 24.29, 1979), Paper I-1 in Advances in Cryogenic Engineering Materials, A. F. Clark and R. P. Reed, eds., 26, 589.598 (Plenum Press, New York, NY, 1980).

Key words: critical current $\left(I_{c}\right)$; fiber-glass epoxy (G-10); $\mathrm{Nb}_{3} \mathrm{Sn}$; phenolic; strain effect on $I_{c}$; superconducting standards; superconductors (multifilamentary); thermal expansion; $\mathrm{V}_{3} \mathrm{Ga}$.

As part of a program to develop standard practices for measuring critical current $\left(I_{c}\right)$, the $I_{c}$ characteristics of commercial multifilamentary $\mathrm{Nb}_{3} \mathrm{Sn}$ and $\mathrm{V}_{3} \mathrm{Ga}$ wires have been measured using phenolic, copper, and fiberglass epoxy (NEMA G-10) sample holders. For each of these materials, $I_{c}$ was measured with the sample in a hair-pin geometry supported in two different ways to prevent movement from Lorentz forces: first with the sample freely suspended in a slot machined in the support material, and second, with the sample frozen in place with vacuum grease. For the copper sample holder, no difference was found in the magnitude of $I_{c}$ between the grease and no grease method. For the composite materials, the $I_{c}$ depended on the fiber direction in the sample holder. For the phenolic and G.10 holder materials with the fiber direction parallel to the sample, no difference in $I_{c}$ was found between the grease and nogrease method. For the phenolic sample holder with the fiber direction perpendicular to the sample, however, the grease method produced a $25 \%$ degradation of $I_{c}$ at $7 \mathrm{~T}$ (for $\mathrm{Nb}_{3} \mathrm{Sn}$ ), and $11 \%$ (for $\mathrm{V}_{3} \mathrm{Ga}$ ) (compared with the no-grease method). This relatively large decrease in $I_{c}$ for the phenolic sample holder, with fiber direction perpendicular to the sample and with grease around the sample, is ascribed to compressive strain introduced by differential thermal contraction between the sample and the sample holder material. The data are correlated with thermal contraction measurements and $I_{c}$ data obtained on the same samples when subjected to controlled uniaxial strain.

20299. Zapas, L. J.; Crissman, J. M. Static fatigue of polyethylene in uniaxial creep in the presence of stress-cracking agents and solvent, (Proc. Symp. 178th Meet. American Chemical Society, Washington, DC, Sept. 10-14, 1979), Org. Coat. Plast. Chem. 40, 469-474 (American Chemical Society, Washington, DC, 1979).

Key words: cracking; fracture; polyethylene; stress-cracking; uniaxial creep.

Experiments on the static fatigue in uniaxial creep are described for two commercial polyethylenes having widely different stress-crack resistance. The behavior in air at two different temperatures is compared to that in stress-cracking agent or solvent at the same two temperatures. It is shown that the fracture can be categorized as one of two types depending upon the magnitude of the applied load. At relatively large loads, the specimen experiences an instability which eventually leads to failure by necking and subsequent fracture. At small loads, on the other hand, failure occurs by cracking. The region of applied loads for which the transition takes place from the one failure mode to the other depends strongly upon the chemical structure of the polymer. In the presence of a surface active type of stress-cracking agent only the failure mechanism associated with cracking is influenced, whereas in a solvent which swells the polymer both failure mechanisms are greatly affected. For the mechanism associated with cracking it is found that the curve for time to fail versus applied stress can be represented rather well, both in air and in stress-cracking agent, by an empirical relation determined some three decades ago to describe the fracture of inorganic glasses.

20300. Cole, B. E.; Cooper, J. W.; Ederer, D. L.; Mehlman, G.; Saloman, E. B. Stark effect on autoionizing resonances in the rare gases, J. Phys. B: Atom. Molec. Phys. Lett. Ed. 13, L175-L179 (1980).

Key words: autoionization; photoabsorption; Stark effect; synchrotron radiation.

The effects of electric fields of intensity up to about $10 \mathrm{kV} \mathrm{cm}^{-1}$ on the autoionizing resonances lying between the ${ }^{2} \mathrm{P}_{3 / 2}$ and ${ }^{2} \mathrm{P}_{1 / 2}$ ionization limits in Xe have been studied in absorption. Fields of this strength produce pronounced effects on series members for $n>11$. The major effects are a decrease in transmission in the neighbourhood of the resonances and a lowering of the classical field ionization potential.

20301. Ma, M. T. Synthesis of broadband antenna arrays as possible over-the-horizon radars, Chapter 9 in Research Topics in Electromagnetic Wave Theory, J. A. Kong, ed., pp. 188.209 (John Wiley \& Sons, New York, NY, 1981).

Key words: antenna array; broadband; high frequency; nonuniformly spaced; over-the-horizon radar; sea scatter synthesis.

Based on the requirements of broadband operation and other radiation characteristics, the side-terminated vertical half rhombic is selected as the antenna element in an array to be included as the central part of an over-the-horizon radar, which serves as a remote means for sea study. A nonuniformly spaced array of 25 elements is synthesized for the entire high-frequency band. The array has the capability of providing a maximum transmitting-receiving product gain in the order of $60 \mathrm{~dB}$ at low take-off angles, a product azimuth pattern with a nominal half-power beamwidth of $2^{\circ}$, a product sidelobe level of $-38 \mathrm{~dB}$, and a grating-lobe level of at least $-30 \mathrm{~dB}$, and of covering an approximate range of $3,000 \mathrm{~km}$ and a sector area of $40^{\circ}$.

20302. Kingston, H. M. Quantitative ultratrace transition metal analysis of high salinity waters utilizing chelating resin separation: Application to energy-related environmental samples, $E P A-600 / 7-79$ 174, 84 pages (Available from the National Technical Information Service, Springfield, VA 22161, Aug. 1979).

Key words: chelating resin separation; energy-related environmental samples; radiochemical tracers; transition metals; ultratrace analysis.

In order to accurately evaluate the impact of energy related activities, such as offshore drilling on the sea water, it is necessary to measure trace element concentrations in the presence of considerably higher levels of alkali and alkaline earth elements.

This report describes a technique which was developed for the elimination of the alkali and alkaline earth elements $\mathrm{Na}, \mathrm{K}, \mathrm{Ca}$ and $\mathrm{Mg}$ from the trace transition elements in sea water samples. This was accomplished by passing an ammonium acetate solution through a column of Chelex 100 resin after a sea water sample had been chelated. The alkali and alkaline earth elements were eluted from the column by ammonium acetate and the trace transition elements were then collected using nitric acid. 
The quantitative concentration, separation and removal of selected transition elements was tested using radiochemical tracers. The study revealed $>99.9$ percent recovery of $\mathrm{Cd}, \mathrm{Cu}, \mathrm{Mn}, \mathrm{Ni}$ and $\mathrm{Zn}$, using a $100 \mathrm{~mL}$ sample, and $>99$ percent with a 1 liter volume. Cobalt and $\mathrm{Pb}$ exhibited $>99$ percent and $>98$ percent recoveries, respectively, from a $100 \mathrm{~mL}$ sample, and $>97$ percent from a 1 liter volume. Iron was found to be recovered only approximately 92 percent in either volume.

The concentration and separation technique was applied to Chesapeake Bay and Alaskan sea water samples. The samples were introduced into a graphite furnace and were analyzed by graphite furnace atomic absorption spectrometry. Analysis of concentrations below $\mathrm{ng} / \mathrm{mL}$ for the trace elements mentioned was possible using this combination of sample preparation and instrumental analysis and no interelement interferences occurred. The combination of this sample preparation technique and the graphite furnace atomic absorption sensitivity enables extreme detection limits to be achieved from the elements mentioned, and gave measurements that were reliable and reproducible.

20303. Contini, M.; Kozlovsky, B. Z.; Shaviv, G. The calculation of the optical spectra of the Cygnus Loop, Astron. Astrophys. 92, No. 3, 273.280 (1980).

Key words: crab nebula; Cygnus Loop; optical spectra; supernova.

The optical spectra of the Cygnus Loop supernova remnant were calculated using a shock wave propagation model. Our purpose is to fit the observed line ratios of the single filaments of the Cygnus Loop and to investigate their structure. The best fitting parameters were obtained and discussed. Our most important results are: 1 . The model can be applied successfully to every filament in spite of the different kind of spectrum emitted by each of them. 2. The various filaments can be nicely fitted with a uniform abundance, where only the temperature is allowed to vary. 3. The relative abundances show an overabundance of hydrogen. (Nitrogen, oxygen and sulfur show an underabundance by a factor of ten relative to "normal" cosmic abundances.) 4. A consistent model for the X-ray and optical emission is presented for the Cygnus Loop, as well as the Crab Nebula. 3. The gas is fully ionized before entering the shock. 6 . In some filaments nonsteady state flow must be assumed in order to explain the high [O III]: $\mathrm{H} \beta$ line intensity ratio.

20304. Evans, D. J. Rheological properties of simple fluids by computer simulation, Phys. Rev. A 23, No. 4, 1988-1997 (Apr. 1981).

Key words: long time tail; molecular dynamics; nonequilibrium; non-Newtonian; Rheology; shear viscosity; viscoelasticity.

We present results of nonequilibrium molecular-dynamics calculations for the triple-point Lennard-Jones fluid undergoing shear flow. The calculations show that this simple fluid exhibits a wide variety of non-Newtonian behavior ranging from viscoelasticity, to shear dilatancy and flow birefringence. It is shown that the constitutive relations describing these phenomena are consistent with nonanalytic functional forms. For every property so far studied these functional forms agree with those predicted by long-time tail theories. However, the size of each effect is found to be several orders of magnitude greater than theoretical predictions.

20305. Wineland, D. J.; Itano, W. M. Spectroscopy of a single $\mathbf{M g}^{+}$ ion, Phys. Lett. 82A, No. 2, $75-78$ (Mar. 9, 1981).

Key words: atomic ions; atomic spectroscopy; double resonance spectroscopy; laser cooling; laser spectroscopy; stored ions.

A single ${ }^{24} \mathrm{Mg}^{+}$ion has been laser cooled in a Penning trap and its optical spectrum observed by a double resonance technique. Residual Doppler line broadening implies a cyclotron-magnetron temperature of $50 \pm 30 \mathrm{mK}$.

20306. Zimmerman, J. E. Cryogenics for SQUIDs, (Proc. Second Int. Conf. Superconducting Quantum Devices, Berlin, (West) Germany, May 6.9, 1980), Paper in Squid '80, H. D. Hahlbohm and H. Lübbig, eds., pp. $423-443$ (Walter de Gruyter \& Co., Berlin, (West)
Germany, 1980).

Key words: cryocooler; refrigeration; SQUID.

Liquid-helium cryostats continue to be the principal mechanism for cooling SQUIDs and SQUID instruments, but there is increasing interest in self-contained closed-cycle cryocoolers as an alternative. Mechanical gas refrigerators, in various configurations, and the JouleThomson effect seem likely to be the most practical mechanisms, but magnetic, electric, and other processes may be useful if suitable materials can be developed. While not much work on SQUID cryocoolers as such has been done yet, a large amount of work on higher temperature refrigerators for infrared sensors and for use in spacecraft provides a sophisticated technical base upon which to build. A low-power five-stage Stirling cryocooler with a singlecomponent SQUID gradiometer has begun operation in the author's laboratory. Although this machine requires only $20 \mathrm{~W}$ of mechanical power input to maintain a temperature of $7 \mathrm{~K}$, a large reduction of input power is theoretically possible.

20307. Peterson, R. L. Turn-on delays in single Josephson junction devices, (Proc. Second Int. Conf. Superconducting Quantum Devices, Berlin, (West) Germany, May 6-9, 1980), Paper in Squid '80, H. D. Hahlbohm and H. Lübbig, eds., pp. 685.702 (Walter de Gruyter \& Co., Berlin, (West) Germany, 1980).

Key words: electrical circuits; Josephson junctions; superconductivity; turn-on delay.

Previous calculations of turn-on delays in circuits containing Josephson junctions have assumed a current stepped up suddenly from the critical value for switching. We here examine the more general cases of the current starting below the critical current, as well as ramping through the critical value at a finite rate. We develop formulas for the ramp-rate dependence of turn-on delay where applicable and establish criteria for determining whether overdrive or ramp rate is the more important.

20308. Barnes, J. A. Clocks, atomic and molecular, Article in Encyclopedia of Physics 1, 124-125 (Addison-Wesley Publ. Co., Inc., Advanced Book Program, Reading, MA, 1980).

Key words: clocks; frequency standards; time.

An atomic (or molecular) clock is a device whose rate is controlled by an atomic (or molecular) resonance. A counting device counts the cycles of an oscillator whose frequency is controlled to be in agreement with an atomic (or molecular) resonance. The accumulated count is directly related to time. The definition of the unit of time (the second) is in terms of a resonance of cesium atoms.

20309. Weber, L. A. Measurements of the specific heat, $C_{v}$, of dense gaseous and liquid nitrogen, (Proc. XV Int. Institute of Refrigeration Conf., Venice, Italy, Sept. 23-29, 1979), Session B 1, Paper 7, 5 pages

(International Institute of Refrigeration, Paris $\left(17^{\circ}\right)$, France, 1980).

Key words: equation of state; gases; liquids; nitrogen; saturation properties; specific heat; vapor pressure.

The specific heats at constant volume have been measured for nitrogen in both the single-phase and two-phase regions. The data range in temperature from $78-250 \mathrm{~K}$ and in pressure up to 300 bar. Comparisons are made with values calculated via the equation of state. The two-phase measurements are used to make comparisons with the curvature of existing vapor pressure equations. Specific heats of the saturated liquid are derived and compared with earlier measurements.

20310. Evans, D. J. On the coupling of kinetic and potential contributions to transverse collective modes in fluids, Mol. Phys. Res. Notes 42, No. 1, $231-234$ (1981).

Key words: projection operators; shear viscosity; transverse current.

Conventional projection operator techniques are used to calculate 
exact expressions for the coupling of kinetic and potential terms in the Kubo-Green expressions for shear viscosity. The resulting expressions are valuable aids in the interpretation of non-equilibrium computer simulations of shear viscosity.

20311. Burnett, E. D. National Bureau of Standards hearing aid test procedures, Chapter III in Handbook of Hearing Aid Measurement 1981, pp. 9-176 (Veterans Administration, Washington, DC, 1981).

Key words: acoustic measurements; coherence function; free-field response; hearing aids; insertion response; random-noise response.

The methods used by the National Bureau of Standards for testing hearing aids for the Veterans Administration. Emphasis is placed on the measurement of the insertion response. New in the discussion this year are the measurement of telephone coil sensitivity, random-noise transfer functions, the coherence function, and the input-output characteristics.

20312. Nahman, N. Error criteria and the use of reference waveforms, Proc. ATE Seminar/Exhibit Automated Testing for Electronics Manufacturing, Pasadena Center, Pasadena, CA, Jan. 19-22, 1981, pp. IV-21_IV-28 (Benwill Publ. Corp., Boston, MA, 1981).

Key words: automatic test equipment; error criteria; reference waveform; waveform measurements.

For time dependent quantities such as pulses, ATE measurements require that a time resolved measured quantity be compared to some reference waveform at either selected points of the reference waveform or throughout the reference waveform's duration. The objective of the ATE measurement is to determine if the measured, quantity (waveform) lies within certain bounds. Consequently, error criteria must be defined to obtain quantitative error data. This paper discusses error criteria based upon the continuous difference between a measured waveform and some prescribed reference waveform over the duration of the reference waveform. After the underlying ideas of the error criteria and the reference waveform generator concept are explained, examples are given from actual measurement situations and simulation studies.

20313. Andrews, J. Pulse reference waveform standards development at NBS, Proc. ATE Seminar/Exhibit Automated Testing for Electronics Manufacturing, Pasadena Center, Pasadena, CA, Jan. 19-22, 1981, pp. IV.13-IV.19 (Benwill Publ. Corp., Boston, MA, 1981).

Key words: Debye dielectric; pulse; pulse generator; pulse standard; reference waveform; rise time; standard; transition duration; waveform.

NBS has developed a pulse reference waveform generator for use as a transfer standard for transition duration ( $10 \%$ to $\left.90 \% \mathrm{t}_{d}\right)$. The generator consists of a tunnel diode, step generator $\left(t_{d}=20 \mathrm{ps}\right)$ driving a low-pass filter. Three filters are available for $t_{d}=50,100$ and 200 ps. The output is a clean, gaussian-like step waveform. The low-pass filters, of NBS design, are $30 \mathrm{~cm}$ long, $7 \mathrm{~mm}$ diameter, coaxial lines filled with a lossy, Debye-type, liquid dielectric. The mathematical model describing these low-pass filters is quite accurate. The necessary parameters for the model can be obtained from independent measurements. The complete available output waveform into a matched load can be predicted with less than $1.5 \%$ uncertainty.

20314. Loevinger, R. NBS program for electron dosimetry, Proc. Symp. Electron Beam Therapy, Memorial Sloan-Kettering Cancer Center, New York, NY, Sept. 25-27, 1979, pp. 31.41 (June 1981).

Key words: calorimetry; dosimetry standards; electron beam; radiation therapy; radiation transport theory; stopping powers; traceability.

NBS has the responsibility to provide access to reliable national dosimetry standards in order that absorbed dose to tissue in radiation therapy be traceable to dosimetry standards. For high-energy electron beams the traceability is established by calibrating the beam with an instrument that has been assigned an NBS-traceable calibration factor in a cobalt 60 beam, and then using a calculated correction to the instrument response. For this purpose NBS provides updated values of energy-absorption coefficients and stopping-power ratios. The NBS radiation transport program is used to calculate electron dose distributions in water, electron spectra in air and in water, the angular distribution of scattered electrons, bremsstrahlung contamination of electron beams, and other properties of electron therapy beams, all as functions of a variety of physical factors.

The Fricke chemical dosimetry system has been used to provide measurement assurance for high-energy electron therapy since 1967, and a summary of the results to date shows a slow but definite improvement in the accuracy of electron beam calibration. A graphite calorimeter has been used for the experimental determination of electron stopping-power ratios at NBS, and has served as the NBS standard of absorbed dose. Work is underway at NBS to develop an absorbed-dose calorimeter that responds directly to temperature rise in water.

20315. Snyder, R. G.; Scherer, J. R.; Peterlin, A. Low-frequency

Raman study of drawn polyethylene, Macromolecules 14, 77.82 (1981).

Key words: acoustic vibration; drawn sample; polyethylene; Raman scattering.

The low-frequency Raman spectrum of unannealed, drawn $(20 x$, $60^{\circ} \mathrm{C}$ ) polyethylene displays a LAM-1 band whose shape and breadth are extraordinary relative to LAM-1 for other forms of crystalline polyethylene. From the shape of this band a distribution of lengths of straight-chain segments was derived. A value of $90 \AA$ was obtained for the most probable length of an extended segment. This value is about one-half that of the long period determined for similar drawn samples using small-angle X-ray scattering. Part of the difference between the Raman and SAXS values may be accounted for in terms of the breadth and asymmetry of the distribution of straight-chain segment lengths. The presence of a large concentration of straightchain segments having lengths less than $100 \AA$ and the unusual breadth of the distribution relative to the most probable length indicate a fundamental difference between the morphology of the unannealed drawn sample and that of solution- or melt-crystallized samples. The distribution of lengths displays a tail that is probably associated with tie chains. Annealing the sample moves the distribution curve to longer lengths and broadens it although its general shape and the high degree of alignment of chains parallel to the fiber axis are preserved.

20316. Carlson, C. E.; Coyne, J. J.; Fishbane, P. M.; Gross, F.; Meshkov, S. Glueball decay widths and glueball dominance of quarkonium decays, Phys. Rev. Brief Reports 23, No. II, 2765.2768 (June 1, 1981).

Key words: coupling constants; glueballs; OZI; QCD; quarkonium; $\phi$-meson.

We present a realization of quarkonium decay violating the OkuboZweig-lizuka (OZI) rule dominated by intermediate glueball resonances. This leads to glueball decay widths which interpolate OZI-rule allowed and forbidden widths, i.e., glueball widths of the order of tens of $\mathrm{MeV}$.

20317. Goldfarb, R. B.; Rao, K. V.; Fickett, F. R.; Chen, H. S. Magnetic susceptibility studies of amorphous Ni-Mn-P-B-Al alloys, $J$. Appl. Phys. 52, No. 3, 1744-1746 (Mar. 1981).

Key words: amorphous; manganese; nickel; spin glass; susceptibility.

Magnetic susceptibility studies have been made on amorphous $\left(\mathrm{Ni}_{1-x} \mathrm{Mn}_{x}\right)_{75} \mathrm{P}_{16} \mathrm{~B}_{6} \mathrm{Al}_{3}$ alloys in the temperature range $4-300 \mathrm{~K}$. In the case of the $x=0$ alloy, the mass susceptibility is quite small, $\sim 10^{-8}$ $\mathrm{m}^{3} / \mathrm{kg}$ at room temperature. With the addition of $\mathrm{Mn}$ to $\mathrm{x}=0.2$, the susceptibility increases to $\sim 5 \times 10^{-7} \mathrm{~m}^{3} / \mathrm{kg}$. The susceptibility does not change appreciably with the composition from $x=0.2$ to $x=0.4$. With the further addition of $\mathrm{Mn}$ to $\mathrm{x}=0.6$, the room temperature susceptibility increases to $\sim 3 \times 10^{-6} \mathrm{~m}^{3} / \mathrm{kg}$.

The $\mathrm{x}=0.2$ alloy shows a broad peak in susceptibility at $\sim 28 \mathrm{~K}$ and a shifted hysteresis loop at $4 \mathrm{~K}$ upon field cooling, behavior associated 
with spin glasses and mictomagnets. The shifted hysteresis loops are unstable at $4 \mathrm{~K}$ and shift back toward the origin upon cycling the field. Time dependent effects are also seen at $4 \mathrm{~K}$. These results are interpreted in terms of a continuous freezing of small paramagnetic clusters as the temperature is lowered.

20318. Suenram, R. D.; Lovas, F. J. Millimeter wave spectrum of glycine. A new conformer, J. Am. Chem. Soc. 102, No. 24, $7180-7184$ (Nov. 19, 1980).

Key words: amino acid; conformational energy; gas phase structure glycine; millimeter spectrum; structure.

More sensitive observations on the vapor of glycine, the smallest amino acid, have been made in the millimeter region. This work has led to the identification of a second conformer in the gas phase. This conformer has the amino hydrogens hydrogen bonded to the carbonyl oxygen and the hydroxyl hydrogen in the normal cis configuration with respect to the carbonyl group. This new conformer is $\approx 490$ (150) $\mathrm{cm}^{-1}$ lower in energy than the conformer originally reported. Its spectrum is weaker due to the smaller dipole moment $\left(\mu_{\mathrm{a}} \simeq 1.00\right.$ (15) D). The distortion fit of 37 transitions yield $A^{\prime \prime}=10341.76$ (17) $\mathrm{MHz}$, $B^{\prime \prime}=3876.195$ (9) $\mathrm{MHz}$, and $C^{\prime \prime}=2912.361$ (10) $\mathrm{MHz}$ for the ground state. The excellent agreement between experiment and theory is discussed.

20319. Kuczkowski, R. L.; Suenram, R. D.; Lovas, F. J. Microwave spectrum, structure, and dipole moment of sulfuric acid, J. Am. Chem. Soc. 103, 2561-2566 (1981).

Key words: dipole moment; gas phase spectrum; microwave spectrum; rotational spectrum; structure; sulfuric acid.

The microwave spectra of the normal, ${ }^{34} \mathrm{~S}, \mathrm{D}_{1}$, and $\mathrm{D}_{2}$ isotopic species of gaseous sulfuric acid have been assigned. Rotational and centrifugal distortion constants were obtained. A conformation with $C_{2}$ symmetry has been identified. Important structural parameters are $r(\mathrm{OH})=0.97$ (1) $\AA, r\left(\mathrm{SO}_{1}\right)=1.574(10) \AA, r\left(\mathrm{SO}_{2}\right)=1.422(10) \AA$, $<\mathrm{H}_{1} \mathrm{O}_{1} \mathrm{~S}=108.5(15),<\mathrm{O}_{1} \mathrm{SO}_{1^{\prime}}=101.3(10)^{\circ},<\mathrm{O}_{2} \mathrm{SO}_{2^{\prime}}=123.3(10)^{\circ}$. The torsional angle $\mathrm{O}_{1} \mathrm{SO}_{1} \mathrm{H}$ is $-90.9(10)^{\circ}$ and the two $\mathrm{SO}_{2}$ planes form an angle of $88.4(1)^{\circ}$. The dipole moment was determined to be 2.725 (15) D. A comparison of the derived structural parameters with related species is presented, and the possibility of the undetected existence of other stable conformations of sulfuric acid is discussed.

20320. Kovacs, W. D. What constitutes a turn?, Geotech. Test. J. Tech. Note 3, No. 3, 127-130 (Sept. 1981).

Key words: blow count; cathead; drilling rig; efficiency; energy; field test; in-situ test; standard penetration test (SPT).

There are wide variations in physical configuration and cathead equipment among the available drill rigs used to perform the standard penetration test. Such differences may be partly responsible for variations in blow count among different drill rigs. The paper draws attention to the fact that about half of the available drill rigs use clockwise rotation of the cathead while the others use counterclockwise rotation. Depending on which direction is used, differences in the actual number of turns could be off by as much as half a turn. This difference could result in a substantial variation in the energy delivered to the sampler and in the blow count for the same soil conditions.

20321. Buhl, D.; Clark, F. O.; Chin, G.; Glenar, D.; Kostiuk, T.; Mumma, M. J.; Lovas, F. J. Vibrationally excited silicon monoxide masers, (Proc. Symp. No. 87, Int. Astronomical Union, Mont Tremblant, Quebec, Canada, Aug. 6-10, 1979), Paper in Interstellar Molecules, B. H. Andrew, ed., pp. 537.538 (D. Reidel Publ. Co., Dordrecht, The Netherlands, 1980).

Key words: astronomy; infrared; maser; microwave spectra; silicon monoxide; stars.

Published data on a select group of SiO maser sources have been analyzed for velocity variations as a function of phase. No apparent correlation was found to a level of about $2 \mathrm{~km} / \mathrm{s}$. This places constraints on the location of the maser molecules. Such a correlation should be present at some level. The implications for future high resolution infrared measurements are discussed.

20322. Miller, C. K. S. Challenges of EMI measurements, (Proc. 1980 Workshop \& Symp. National Conference of Standards Laboratories, National Bureau of Standards, Gaithersburg, MD, Sept. 22-25, 1980), Paper in NCSL Newslett. 21, No. 1, 16-23 (Mar. 1981).

Key words: EM environment; electromagnetic interference; measurements; metrology.

In recent years there has been increasing publicity devoted to the proliferating problems associated with electromagnetic interference (EMI). The first step in understanding the nature of the EMI problem and ultimately controlling or regulating the sources is being able to quantify the problem through accurate measurement. EMI measurements are difficult to make because of the wide range of field strengths and frequencies, the complex waveforms, transient sources, field condition, etc. NBS has had an active program underway for some time to upgrade the state-of-the-art for EMI measurements and this program is producing important results. This paper reviews the EMI problem, describes the measurement challenges and NBS' response, and discusses the implications of EMI measurements for the metrology community

20323. Lindgren, R. A.; Flanz, J. B.; Hicks, R. S.; Parker, B.; Peterson, G. A.; Lawson, R. D.; Teeters, W.; Williamson, C. F.; Kowalski, S.; Maruyama, X. K. Comparison of inelastic electron scattering with $\left[g_{9 / 2}(8)\left(f_{7 / 2}^{-3}\right),\right]_{8-}$ shell-model calculations for the $T=1$ and $T=2,8^{-}$states in ${ }^{54} \mathrm{Fe}$, Phys. Rev. Lett. 46, No. 11, 706709 (Mar. 16, 1981).

Key words: electron scattering; isospin; $\mathrm{J}^{\pi}=8^{-}$transitions; shellmodel.

Inelastic electron scattering is used to identify $M 8$ transitions and assign $J^{\pi}=8^{-}$to states at $E_{\mathrm{x}}=8.314,8.949,9.974,10.677$, and 13.263 $\mathrm{MeV}$ excitations. Shell-model calculations within the model space $\left[g_{9 / 2} \otimes\left(f_{7 / 2}{ }^{-3}\right)\right]_{8}$ - suggest that the four lowest states are $T=1$ and the strongest excitation, to the state at $13.263 \mathrm{MeV}$, is $T=2$.

20324. Edgerly, D. E. Implications of United States participation in the International Organization of Legal Metrology (OIML), (Proc. 1980 Workshop \& Symp. National Conference of Standards Laboratories, National Bureau of Standards, Gaithersburg, MD, Sept. 22-25, 1980), Paper in NCSL Newslett. 21, No. 1, 32.37 (Mar. 1981).

Key words: economics; instruments; international; legal metrology; measurement; standards.

This paper examines the economics of the measurement instrument industry; provides an overview of domestic and international trends in standardization; and, finally, suggests the significance of the United States presence in the International Organization of Legal Metrology (OIML).

20325. Berger, $\mathbf{H}$. The role of calibration in nondestructive evaluation, (Proc. 1980 Workshop \& Symp. National Conference of Standards Laboratories, Gaithersburg, MD, Sept. 22-25, 1980), Paper in NCSL Newslett. 20, No. 4, 32-35 (Dec. 1980).

Key words: calibrations; nondestructive evaluation; quality; reliability; reproducibility; standards; testing.

Nondestructive evaluation (NDE) is used in industry to assure the quality of materials, components and assemblies. In these times of product liability issues, emphasis on conservation, and needs to improve productivity, industry interest in NDE is increasing. Improved procedures for NDE, involving broadly used methods such as radiography, ultrasonics, eddy currents, liquid penetrants and magnetic particles, will play a key role as we move toward improved product quality. These NDE methods are briefly reviewed in this paper, with emphasis placed on calibration methods that are now 
available, or to be available shortly, to aid industry to achieve NDE measurement reproducibility. There is a growing need for the metrologists in industry and government laboratories to provide calibration of NDE equipment and reference artifacts, in many cases with traceability to NBS. In this paper, the contributions to highreliability NDE that standards/metrology labs can provide are outlined.

20326. Rasberry, S. D.; Uriano, G. A. Standard reference materials for physical measurement traceability, (Proc. 1980 Workshop \& Symp. National Conference of Standards Laboratories, Gaithersburg, MD, Sept. 22-25, 1980), Paper in NCSL Newslett. 20, No. 4, 23-27 (Dec. 1980).

Key words: certified SRMs; measurement traceability; metrology; standard reference materials.

Standard Reference Materials are widely used to achieve measurement traceability to NBS in fields such as industrial analysis of steel, glass, plastics, cement and rubber; clinical chemistry; environmental measurements and many others. The metrology community is beginning to realize the benefits of SRMs in achieving physical measurement traceability for areas such as temperature and length (including linewidth). This paper describes the NBS Standard Reference Materials Program and explains how SRMs are certified. Examples are provided of how SRMs are currently being used by calibration laboratories to achieve traceability. Still other possible uses of SRMs in the future to support a wider spectrum of physical measurements will be discussed.

20327. Crissman, J. M.; Zapas, L. J. Lifetime behavior of polyethylene sheets under inflation in the presence of stress-cracking agent, (Proc. Symp. 178th Meet. American Chemical Society, Washington, DC, Sept. 10-14, 1979), Org. Coat. Plast. Chem. 40, $475-481$ (American Chemical Society, Washington, DC, 1979).

Key words: biaxial deformation; cracking; fracture; inflation; polyethylene; stress-cracking.

Experiments on the failure of polyethylene sheets under inflation at a constant applied pressure are described. The behavior in air at two different temperatures is compared to that in stress-cracking agent at the same two temperatures for two types of commercial polyethylene having widely different stress-crack resistance. As in uniaxial creep it is found that failure occurs by one of two modes, one necking and cold-drawing or the other crack initiation and growth, depending upon the magnitude of the applied constant pressure. The primary difference between uniaxial creep and the inflation experiment is that in the presence of a stress-cracking agent the mechanism associated with cracking (long times to fail) becomes very much more emphatic under biaxial deformations. Again, as in uniaxial creep, we find that the mechanism of failure by cracking can be represented rather well by an empirical relation developed long ago to describe the fracture of inorganic glasses. One interesting result of this work is the observation that, when compared on a relative basis to the behavior in air, the reduction in lifetime resulting from the presence of stresscracking agent is much greater for the polymer with superior stresscrack resistance, yet this polymer remains superior in overall performance because of its overwhelmingly better behavior in air.

20328. Ledbetter, H. M.; Moulder, J. C.; Austin, M. W. Young's modulus of a copper-stabilized niobium-titanium superconductive wire, Wire, 3 pages (Jan. 1981).

Key words: copper; elastic constants; niobium-titanium; rule-ofmixtures; sound velocity; superconductor; wire; Young's modulus.

Young's modulus was determined for an $\mathrm{Nb}$ - $\mathrm{Ti}$ superconductive wire. Two methods were used: continuous-wave-resonance and laser pulse-excitation. Young's moduli were also determined for the components copper and $\mathrm{Nb}-\mathrm{Ti}-$ in both wire and bulk forms. Some mechanical deformation effects on Young's modulus were also measured. From the components' elastic moduli, that of the composite was predicted accurately by a simple rule-of-mixtures relationship.

20329. Harris, J. R.; Wright, R. N. Unambiguous and complete:
Applying information science to specifications, Construct. Specifer 34 No. 4, 55-62 (Apr. 1981).

Key words: buildings; classification; construction specifications; decision tables; information networks; master specification; specification; specification writers; systems analysis.

Specifications are a primary means of technical communication in the construction community. This article is concerned with improving the organization, expression and interpretation of the information contained in construction specifications. The application to construction specifications of techniques previously developed for modeling standards is described. The techniques provide an objective and rigorous representation of the meaning of a specification and allow it to be tested for aspects of clarity, completeness, and consistency. Furthermore, the techniques allow alternative organizations and expressions to fit the needs of various users with assurance that meanings remain unchanged and that users will readily find and understand all provisions even in a new or unfamiliar standard.

20330. Phelan, R. J., Jr.; Larson, D. R.; Werner, P. E. Subnanosecond electrical modulation of light with hydrogenated amorphous silicon, Appl. Phys. Lett. 38, No. 8, 596.598 (Apr. 15, 1981).

Key words: amorphous silicon; electro-optic modulator; FranzKeldish effect; hydrogenated amorphous silicon; optical modulator; refractive index; silicon.

A silicon thin-film interferometer structure is used to demonstrate subnanosecond electrical modulation of light. Both thermally and electrically induced modulations are reported. An electrically induced change in the refractive index of $4.7 \times 10^{-4}$ is observed.

20331. Simpson, P. A. A system for characterizing detectors for measurement of power of $\mathrm{CO}_{2}$ TEA laser pulses, Proc. 11th ElectroOptics/Laser 79 Conf. \& Exposition, Anaheim, CA, Oct. 23-24, 1979, pp. 399-407 (Industrial and Scientific Conference Management, Inc., 222 West Adams St., Chicago, IL 60606, 1979).

Key words: IR detector; IR pulse measurement; IR pulse shaping; TEA laser pulse.

A system for characterizing detectors for the measurement of power of $\mathrm{CO}_{2}$ laser pulses in the range of $10 \mathrm{~kW}$ up to 1 to $2 \mathrm{MW}$ with a time resolution of several nanoseconds has been developed at the Boulder Laboratories of the National Bureau of Standards. This system measures the responsivity of the detectors. The dependence of responsivity on power density and optical signal risetime is studied. The basic principles involved are discussed and the results of measurements on some detectors are presented.

20332. Fine, J.; Hardy, S. C.; Andreadis, T. D. Ion bombardment cleaning of liquid gallium surfaces (Summary Abstract), J. Vac. Sci. Technol. 18, No. 3, 1310-1311 (Apr. 1981).

Key words: Auger spectroscopy; fluid flow; ion sputtering; liquid gallium surfaces; liquid surface; surface cleaning.

Although ion bombardment induced sputtering is an effective technique for producing clean surfaces on solids, there exist very few comparably convenient ultra-high vacuum methods for cleaning the surfaces of liquids. As a consequence of this very real need, modern surface science spectroscopies and techniques have only rarely been used to study liquid surfaces.

We have found that the preparation of clean surfaces on bulk liquid gallium can be accomplished by means of argon ion bombardment and that this process is accompanied by a newly observed type of surface phenomenon produced by ion impact-the rapid flow of impurity aggregates on a liquid metal surface. This unexpected mass flow, due entirely to the impact of the ion beam, is the mechanism that enables the ion beam to clean the entire liquid-vacuum interface. Because of this flow mechanism, ion bombardment may represent a new and significant method for cleaning other liquid metal surfaces and also could have application to the cleaning of nonmetallic liquids as well. 
20333. Gadzuk, J. W. Vibrational lineshapes of adsorbates: The role of substrate electronic excitations, Chem. Phys. Lett. 80, No. 1, 5-10 (May 15, 1981).

Key words: electron-phonon interactions; localized modes; vibrational spectroscopy.

The vibrational spectrum of an atom or molecule adsorbed on a metallic surface is influenced by the dynamic interaction between the localized oscillator and the continuum of electron-hole pair excitations in the solid. Features of an exactly soluble model for this interacting system are presented, the local spectral function is obtained in terms of calculable level shift and broadening functions, and some prototypical numerical results are displayed and discussed.

20334. Ogburn, F.; Schoonover, R. M.; Johnson, C. E. Density of autocatalytic nickel-phosphorus deposits, Plating Surf. Finish., 45 pages (Mar. 1981).

Key words: autocatalytic nickel-phosphorus; density; electroless nickel-phosphorus; nickel-phosphorus alloy.

The density and phosphorus content of 4 autocatalytic Ni.P deposits were determined: density, $8.048 \mathrm{~g} / \mathrm{cm}^{3}$; phosphorus content, 9.75\%; density, $8.056 \mathrm{~g} / \mathrm{cm}^{3}$; phosphorus content, $9.69 \%$; density, $8.087 \mathrm{~g} / \mathrm{cm}^{3}$; phosphorus content, $9.38 \%$; density, $8.204 \mathrm{~g} / \mathrm{cm}^{3}$; phosphorus content, $7.37 \%$.

20335. Santoro, R. J.; Semerjian, H. G.; Emmerman, P. J.; Goulard, R. Optical tomography for flow field diagnostics, Int. J. Heat Mass Transfer 24, No. 7, 1139-1150 (1981).

Key words: absorption measurements; combustion diagnostics; convolution algorithm; diffusion jet; laser diagnostics; reconstruction algorithm; tomography.

Optical tomography has been applied to an off-axis turbulent methane-air free jet to determine the mean methane concentration throughout the mixing region. Optical tomography is a multi-angular absorption technique which involves making $M$ line of sight absorption measurements (projections) at $N$ angles. These $M \times N$ measurements are then used to reconstruct the original twodimensional flow field. Absorption measurements were made on the methane using the near-resonance $3.39 \mu \mathrm{m}$ line of a $\mathrm{He}-\mathrm{Ne}$ laser. Mean concentration measurements were obtained at three positions downstream of the jet exit plane. Comparisons with the results of previous workers for the axial and radial mean concentration profiles show good agreement. Additionally, the sensitivity of the reconstructed results to the number of angles and scans used is briefly described. The results demonstrate the unique capabilities of optical tomography for flow field diagnostics.

20336. Vaiana, G. S.; Cassinelli, J. P.; Fabbiano, G.; Giacconi, R.; Golub, L.; Gorenstein, P.; Haisch, B. M.; Harnden, F. R., Jr.; Johnson, H. M.; Linsky, J. L.; Maxson, C. W.; Mewe, R.; Rosner, R.; Seward, F.; Topka, K.; Zwaan, C. Results from an extensive Einstein stellar survey, Astrophys. J. 244, 163.182 (Apr. 1, 1981).

Key words: coronae; stars; $\mathrm{x}$-ray sources.

We report the preliminary results of the Einstein Observatory stellar X-ray survey. To date, 143 soft X-ray sources have been identified with stellar counterparts, leaving no doubt that stars in general constitute a pervasive class of low-luminosity galactic X-ray sources. We have detected stars along the entire main sequence, of all luminosity classes, pre-main sequence stars as well as very evolved stars. Early type OB stars have X-ray luminosities in the range $\sim 10^{31}$ to $\sim 10^{34}$ ergs $\mathrm{s}^{-1}$. Late type main sequence stars show little dependence of X-ray emission levels upon stellar effective temperature; similarly, the observations suggest weak, if any, dependence of X-ray luminosity upon effective gravity. Instead, the data show a broad range of emission levels ( three orders of magnitude) throughout the main sequence later than FO. Comparison of the data with published theories of acoustically heated coronae shows that these models are inadequate to explain our results. The data are consistent with magnetically dominated coronae, as in the solar case.

20337. Cox, A. N.; Shaviv, G.; Hodson, S. W. On the ratio of mixing length to scale height in red dwarfs, Astrophys. J. 245, L37.L40 (Apr. 1981).

Key words: atmospheres; mass-luminosity relation; stars.

Previous completely convective theoretical models of low-mass stars, $M \leqslant 0.3 M_{\odot}$, predict a luminosity-mass relation which is below the observed one. We explain this disagreement by using the latest molecular opacities and by the consequent need to assume an $l / H_{p}$ ratio at $T<9000 \mathrm{~K}$ in the range $0.07-0.17$, rather than the more conventional values of $1.0-2.0$. When such a low surface layer $l / H$ ratio is assumed, we find significantly higher central temperatures (and hence luminosities) and quite large radiative cores, regardless of the deeper $l / H_{p}$ value and equation-of-state uncertainties. The low $l / H_{p}$ ratio is explained by the interaction of a magnetic field with convection. Several consequences of this result, including the interstellar abundance of ${ }^{3} \mathrm{He}^{+}$are discussed.

20338. Bergquist, J. C.; Barger, R. L.; Glaze, D. J. High resolution spectroscopy of calcium atoms, Proc. 4th Int. Conf. Laser Spectroscopy (FICOLS), Rottach-Egeren, West Germany, July 1979, pp. 120-129 (Springer-Verlag, New York, NY, 1979).

Key words: calcium; high resolution spectroscopy; laser spectroscopy; optical; Ramsey fringes; recoil; spectroscopy; wavelength frequency standard.

The recent results on saturated absorption optical interference spectroscopy of calcium are presented. The photon recoil splitting of the $\mathrm{Ca}{ }^{1} \mathrm{~S}_{0}-{ }^{3} \mathrm{P}_{1}$ intercombination line at $657 \mathrm{~nm}$ has been fully resolved. Linewidths as narrow as $3 \mathrm{kHz}$ half width half maximum intensity (HWHM) are reported for radiation beams spatially separated by up to $3.5 \mathrm{~cm}$. Second order Doppler is shown to be the present limitation to the accuracy of this technique. Methods are discussed which could lead to an optical wavelength/frequency standard with an accuracy of better than $10^{-14}$.

20339. Linsky, J. L. Evidence for chromospheres and coronae in stars: Recent observations, some unanswered theoretical questions, and a speculative scenario, (Proc. NATO Advanced Study Institute, Bonas, France, Aug. 25-Sept. 1980), Paper in Solar Phenomena in Stars and Stellar Systems, R. M. Bonnet and A. K. Dupree, eds., pp. 99.122 (D. Reidel Publ. Co., Dordrecht, The Netherlands, 1981).

Key words: stellar chromospheres; stellar coronae; ultraviolet spectra; $x \cdot$ ray sources.

Recent ultraviolet spectra and X-ray flux measurements by the International Ultraviolet Explorer (IUE) and Einstein X-ray Observatory have had a major impact on our knowledge of which regions of the HR diagram definitely contain stars with chromospheres and coronae and of the properties of these outer atmosphere layers. These observations are pointing to similarities and differences of these outer atmosphere layers compared with the solar atmosphere and the important roles played by magnetic fields. These data are also reorienting our thinking about physical processes occurring in the outer atmospheres of stars.

20340. Van Degrift, C. T.; Love, D. P. Modeling of tunnel diode oscillators, Rev. Sci. Instrum. 52, No. 5, 712.723 (May 1981).

Key words: circuit design; computer simulation; LC oscillators; pressure; temperature; transducers; tunnel diode; tunnel diode oscillator.

The detailed behavior of tunnel diode LC oscillators is calculated by three different methods and compared with experiment. We present both analytic formulas and numerical methods which give corrections to the oscillator frequency calculated using the expression $1 /(\mathrm{LC})^{1 / 2}$ and show that these corrections are often not negligible. The calculations also yield rf amplitude, bias current dependence, and 
other details necessary for the full realization of the performance of LC oscillators as transducers of pressure, temperature and other physical quantities.

20341. Marshak, H. Nuclear orientation of ${ }^{166 m} \mathrm{Ho}$ in ${ }^{165}$ Ho single crystal, Hyperfine Interact. 10, $1183-1188$ (1981).

Key words: gamma-ray anisotropy thermometry; gamma-ray transitions in ${ }^{166} \mathrm{Er}$; mixing ratios; nuclear magnetic moment; nuclear orientation; nuclear quadrupole moment.

Nuclear orientation measurements have been made on the system ${ }^{166 \mathrm{~m}} \mathrm{Ho}$ in single crystal ${ }^{165} \mathrm{Ho}$ metal. Our results for the nuclear magnetic moment, $\mu_{n m}^{\prime}=3.65(13) \mathrm{nm}$, is in excellent agreement with the recent results by the Oxford group in their work on $\mathrm{HoVO}_{4}$. We also report on the use of ${ }^{166 \mathrm{~m}} \mathrm{HoHo}$ as a $\gamma$-ray anisotropy thermometer.

20342. Placious, R. C.; Garrett, D. A.; Kasen, M. B.; Berger, H. Dimensioning flaws in pipeline girth welds by radiographic methods, Mater. Eval. 39, $755-759$ (July 1981).

Key words: defect size; girth welds; inspection quality; nondestructive evaluation; performance; pipeline; radiography; ultrasonics.

Methods for field radiographic inspection of pipeline girth welds are reviewed with an eye toward the need for obtaining accurate defect dimensional information from the radiographs. Several methods used to determine weld defect size, particularly the throughwall dimension, are discussed; potential sources of error are indicated. It is clear that there will be a need for nondestructive sizing of pipeline weld defects as greater use is made of fracture mechanics analysis, used in a precedent-setting decision in regard to several girth welds in the Trans-Alaska oil pipeline. For field radiography, this requirement for defect dimensions will demand increased attention to record keeping and radiographic variables such as $x$-ray energy, film type and screens, film processing and source location. However, it is pointed out that attention to these variables, even with present field radiographic requirements, can lead to a reduction in repeat radiographs and potential cost savings.

20343. Pantano, C. G.; Madey, T. E. Electron beam damage in Auger electron spectroscopy, Appl. Surf. Sci. 7, 115-141 (1981).

Key words: adsorption; Auger spectroscopy; decomposition; desorption; electromigration; electron beam damage.

The damaging effects of electronic excitation, charging and beam heating during Auger electron spectroscopy (AES) are treated. The fundamental origin, manifestation and practical consequences of the phenomena are reviewed. A damage threshold, or critical dose, for beam damage due to electronic excitation is derived and related to experimental parameters. The close correlation between the predicted thresholds and the critical doses for damage observed in typical AES analyses indicates that primary excitation processes dominate the beam damage mechanism. Charging and the electromigration of ions in glasses is also discussed in detail. It is suggested that AES analyses of materials with potential susceptibility to beam damage be executed and interpreted with caution.

20344. Peterlin, A.; McCrackin, F. L. Empirical relation for diffusion of gases in hydrocarbon polymers: Interpretation in terms of fractional free volume, J. Polym. Sci.: Polym. Phys. Ed. 19, 1003-1006 (1981).

Key words: crystallization; diffusion; diffusion coefficient; free volume; gas diffusion; polyethylene.

An empirical relationship between the diffusion coefficients of gases in three polyethylenes and their diffusion coefficient in natural rubber is given. The diffusion coefficients are given within a relative error of $4.7 \%$. This relationship is interpreted according to the fractional free volume theory. The ratios of the fractional free volumes in the polyethylenes to the fractional free volume in natural rubber are derived.

20345. Wasik, S. P. Partition of organoelements in octanol/water/air systems, (Proc. Symp. Division of Inorganic Chemistry 175th Meet. American Chemical Society, Anaheim, CA, Mar. 13-17, 1978), Chapter 19 in ACS Symp. Series 82. Organometals and Organometalloids. Occurrence and Fate in the Environment, F. E. Brinckman and J. M. Bellama, eds., pp. 314-326 (American Chemical Society, Washington, DC, 1978).

Key words: dimethylmercury; head-space; octanol/water partition coefficient; sea water; solubility.

A head-space method is presented for measuring the solubilities, the partition coefficients (air/water and octanol/water), and their temperature dependencies of organo-elements. A mixture of air and solute vapor is introduced into a closed-loop containing a stainless. steel bellows pump, a gas mixing chamber, and two gas sampling values. The first value is used to inject a small volume of head-space into a gas chromatograph for analysis. The second value connects the circulating gas phase with an equilibration cell containing a known amount of water or water-octanol mixture.

The air and solute vapors are circulated in the closed-loop until the solute concentrations are constant. The gas phase is then circulated through the equilibration cell and the head-space sample is analyzed after equilibration is reached. The partition coefficients are calculated from the ratio of the solute concentrations to the volume of the gas phase and of the liquid phase. The advantages of the method are that the measurements are taken at infinite solute dilution and that several solutes may be measured simultaneously. The values for the partition coefficients for $\left(\mathrm{CH}_{3}\right)_{2} \mathrm{Hg}$ in fresh and sea water are given.

20346. Leep, D. A.; Kotter, F. R. Discussion of "Measuring voltage and current harmonics on distribution systems" by $M$. F. McGranaghan, J. H. Shaw, and R. E. Owen, IEEE Trans. Power Appar. Syst. PAS-100, No. 7, 3599-3608 (July 1981).

Key words: harmonic components; high-impedance probes; voltage measurement.

Comments and questions concerning voltage-measurement techniques used by the authors.

20347. Day, G. W.; Franzen, D. L. Progress in fiber measurement. An LF meeting report from Boulder, Colorado. Laser Focus Mag., pp. 52.56 (Feb. 1981).

Key words: attenuation; bandwidth; index profile; measurements; optical fibers; single mode optical fibers.

This report summarizes the present status of optical fiber measurement technology based on papers and workshops at the Symposium on Optical Fiber Measurements held October 28-29, 1980 in Boulder, CO. Thirty-one papers covering the topics of attenuation, bandwidth, index profile and geometry, joint/defect characterization, single-mode fibers, applied measurements, and standards were presented.

20348. Escalante, E.; Bertocci, U.; Mullen, J.; Cohen, M.; Kruger, J. Development of in situ techniques for tbe detection of corrosion of copper concentric neutrals of electric cables in underground environments, Proc. IEEE Power Engineering Society 1981 Transmission and Distribution Conf. \& Exposition, Minneapolis, $M N$, Sept. 20-25, 1981, Paper No. 81 TD 623-8, pp. 1.7 (1981).

Key words: concentric neutrals; copper; corrosion; corrosion noise; polarization techniques; reference electrodes; underground corrosion.

The EPRI project described here is directed at the development of in situ techniques for measuring and detecting corrosion of copper concentric neutrals. Using a semiautomated experimental field site, measurements have been carried out on buried $50 \mathrm{~m}$ cables, that have demonstrated the utility of electrochemical polarization measurements in detecting and measuring the corrosion of buried $\mathrm{CCN}$. The progress in the development of microprocessor instrumentation for carrying out such measurements in a simple manner is described. It has also been demonstrated that the polarization measurements can be carried out on operating cables. Techniques for the measurement of 
the voltage and current fluctuations of corroding electrodes (corrosion noise) are described. These techniques are being investigated for their use in the detection of corrosion of $\mathrm{CCN}$ cables. They have been applied to copper electrodes in the laboratory, and preliminary tests have been carried out on buried cables. The results show that fluctuations can be detected on corroding copper in conditions similar to those occurring in the field.

20349. Lum, G. K.; Wiegand, C. E.; Kessler, E. G., Jr.; Deslattes, R. D.; Jacobs, L.; Schwitz, W.; Seki, R. Kaonic mass by critical absorption of kaonic-atom $x$ rays, Phys. Rev. D, 23, No. 11, 2522. 2532 (June 1, 1981).

Key words: critical absorption; erbium; exotic atom; kaon; potassium; $x$ ray.

The energy of $\mathrm{x}$ rays from the transition $6 h \rightarrow 5 g$ in kaonic atoms of potassium falls on the $K$ absorption edge of erbium. Measurement of the kaonic-x-ray attenuation in a precisely calibrated set of $\mathrm{Er}$ foils yields the $\mathrm{x}$-ray energy $57458.8 \pm 6.3 \mathrm{eV}$. The kaon mass is related to energy through the Klein.Gordon equation plus corrections for radiative effects, electron screening, and other effects. The negativekaon mass was found to be $493.640 \pm 0.054 \mathrm{MeV} / \mathrm{c}^{2}$ in agreement with the currently accepted value $493.669 \pm 0.018 \mathrm{MeV} / \mathrm{c}^{2}$ which was determined from $x$ rays emitted by high. $Z$ atoms where the corrections were larger than for $Z=19$.

20350. Kelley, R. D.; Goodman, D. W. Catalytic methanation over single crystal nickel and ruthenium: reaction kinetics on different crystal planes, Proc. American Chemical Society, Division of Fuel Chemistry, Houston, TX, Mar. 24-28, 1980, 25, No. 2, $43-52$ (American Chemical Society, Washington, DC, 1980).

Key words: catalysis; nickel; reaction rates; ruthenium; single crystal.

A specially designed ultrahigh vacuum system has been used to study the kinetics of the hydrogenation of $\mathrm{CO}$ over low surface area, single crystal catalysts. In a recent publication, we have reported reaction rate measurements for a $\mathrm{Ni}(100)$ catalyst and compared those results with kinetic data, derived from the literature, for small particle $\mathrm{Ni}$ supported on $\mathrm{Al}_{2} \mathrm{O}_{3}$. There was remarkable agreement between the two catalyst systems in regard to specific reaction rates (the rate normalized to the number of surface metal atoms), the activation energy, and the product distribution. In the present report, we compare reaction rates measured on two crystal planes of $\mathrm{Ni}$-the (100) and the close-packed (111) - and two crystal planes of Ru-the zig-zag, open (110) and the close packed (001). We also report the variation of the reaction rate and the surface carbon concentration with total pressure and with the $\mathrm{H}_{2}: \mathrm{CO}$ ratio. The surface carbon concentration (an active "carbidic" carbon species) varies with the total pressure and with the reactant gas ratio. A striking correlation has been found between the surface carbide level and the catalytic reaction rate.

20351. Kruger, J. Corrosion. Its character and consequences, $A m$. Soc. Test. Mater. Sid. News 9, No. 5, 21.23, 48 (May 1981).

Key words: causes of corrosion; corrosion; corrosion control; cultural impact of corrosion; economic impact of corrosion; health and safety impact of corrosion; strategic impact of corrosion; technological impact of corrosion.

The economic, health and safety, strategic, technological and cultural impacts of corrosion are described. A description is given of the causes of corrosion and ways that corrosion can be controlled.

20352. Wilson, M. A. D. Recirculation acceleration of high current relativistic electron beams-A feasibility study, (Proc. 1981 Particle Accelerator Conf., Accelerator Engineering and Technology, Washington, DC, Mar. 11.13, 1981), IEEE Trans. Nucl. Sci. NS.28, No. 3, 3375.3377 (June 1981)

Key words: accelerator; electron; high current; induction; recirculating.

One of the advanced accelerator concepts under study at NBS involves multiplying the energy gained by a long pulse, high current relativistic electron beam by directing the beam several times through the same induction accelerator during the time of one voltage pulse. Should this concept of the recirculation acceleration of intense electron beams be proven feasible, the savings in cost, size, and weight of a high current accelerator would be considerable. Energy gain by recirculation acceleration through a small-scale proof-of principle facility has been demonstrated at NBS. The study employs a $750 \mathrm{~A}, 750 \mathrm{keV}$ electron beam pulse, $2 \mu \mathrm{sec}$ long, generated by a linear induction accelerator of unique design which was also developed at NBS. Self-focusing beam transport through a chargeneutralizing low pressure gas is employed in the demonstration recirculator for simplicity. Success with gas-focus beam transport has been limited, due to beam-plasma instabilities and emittance limitations with the relatively low energy high current electron beam available at NBS. An alternate beam transport method for future high current recirculating accelerators is also presented.

20353. Penner, S. CW electron accelerators for nuclear physics, (Proc. 1981 Particle Accelerator Conf., Accelerator Engineering and Technology, Washington, DC, Mar. 11-13, 1981), IEEE Trans. Nucl. Sci. NS-28, No. 3, 2067.2073 (June 1981).

Key words: beam blowup; continuous duty; electron accelerator; microtron; multiple beams; pulse stretcher rings; race-track microtron.

The need for continuous duty electron accelerators for nuclear physics research is discussed. The various types of accelerators capable of satisfying the physics requirements are described and compared.

20354. Mattingly, G. E. Dynamic traceability of flow measurements, Proc. IMEKO Symp. Flow Measurement and Control in Industry, Tokyo, Japan, Nov. 13-16, 1979, pp. 0-4-1-0.4-11 (International Measurement Confederation, Tokyo, Japan, 1979).

Key words: calibration; fluid flow; measurement assurance; systematic error; traceability; transfer standards.

Given the present growth rate of the world's population and given the finite availability of the world's known material resources, it is obvious that custody transfer and equity in trade are becoming, and will continue to become, increasingly important concepts in affecting flow measurements in man's future. Consequently, the measurement systems that form the bases of such transactions are destined to become correspondingly crucial. Individual measurements will have to be made satisfactorily at all levels-in the "field," in the laboratories operated by the vendors of measurement instrumentation, in the independent measurement laboratories, and in the national laboratories in each and every country. Furthermore, it will become increasingly important that the quality of these measurements be satisfactorily assured to be as good as they are specified to be. Measurements, measurement standards, and their respective traceability networks will be required to "stand-up" to legal scrutiny and cross-examination in court.

To realistically assure that resource measurements-particularly fluid flow measurements are as good as specified, traceability systems which incorporate the dynamic nature of fluid flow measurements will have to be established and maintained. It is the purpose of this paper to put forth some ideas that are intended to describe how dynamic-traceability of flow measurements might be established and maintained.

20355. Shier, D. R. Selecting optimal product sizes, J. Undergraduate Math. Appl. 1, No. 4, $19-31$ (1980).

Key words: catalog; dynamic programming; iterative procedure; models; optimization; standards.

This article describes a practical problem that arises when only some of the possible sizes for an industrial product are to be stocked, and demands for unstocked sizes are met by using a larger stocked size (subsequently cut to the smaller desired size). The study was undertaken as the result of suggestions by colleagues in the Center for Building Technology, National Bureau of Standards, who were 
motivated by the possibility of simplifying and rationalizing the enormous array of "standard sizes" for products within the building industry.

Mathematical models can be identified that capture certain essential features of this problem, and associated solution methods can be developed as well. However, such models are not equally desirable in terms of their range of applicability and computational feasibility. The pitfalls and potentials of two particular approaches to selecting optimal sizes are illustrated here.

20356. Debenham, P. H. End magnet design for the NBS-LASL CW microtron, (Proc. 1981 Particle Accelerator Conf., Accelerator Engineering and Technology, Washington, DC, Mar. 11-13, 1981), IEEE Trans. Nucl. Sci. NS.28, No. 3, 2885-2887 (June 1981).

\section{Key words: end magnet; magnet design; racetrack microtron}

Beam quality and ease of operation in a racetrack microtron (RTM) are critically dependent on the properties of the end magnets. Good field uniformity is essential to maintain the microtron resonance condition, yet is difficult to achieve in a conventional C-magnet having the very large ratio of gap depth to height required in an end magnet. A novel magnet design has been developed for the NBS. LASL CW RTM which has a calculated field uniformity of better than two parts in $10^{4}$. The design incorporates a Purcell filter into a half picture-frame magnet and provides a $25 \%$ reduction in weight from an equivalent C-magnet. Dramatic improvement in the field uniformity of picture-frame magnets can be realized with this principle.

20357. Semerjian, H. G.; Santoro, R. J.; Goulard, R.; Emmerman, P. J. Optical tomography for diagnostics in combusting flows, Paper in Mechanical Properties of Bone, S. C. Cowin, ed., AMD-45, 119.128 (The American Society of Mechanical Engineers, New York, NY, 1981).

Key words: absorption measurements; combustion diagnostics; convolution algorithm; diffusion flame; diffusion jet; laser diagnostics; reconstruction algorithm; tomography.

The optical tomography technique has been used for diagnostics of the concentration field in a turbulent methane-air jet and laminar methane diffusion flame. Optical tomography is a multiangular absorption technique which involves making $M$ line of sight absorption measurements (projections) at $\mathrm{N}$ angles. These measurements are then used to reconstruct the original two dimensional flow field. Absorption measurements were made on methane using the $3.39 \mu \mathrm{m}$ line of a He-Ne laser. Mean concentration profiles were obtained in the turbulent jet at three axial positions. Comparisons with the results of previous workers for the axial and radial mean concentration profiles show excellent agreement. Absorption measurements of methane were also carried out in a laminar diffusion flame, at three axial positions within the reaction zone. Some of the concentration measurements have been compared with results of previous workers obtained using sampling techniques. Results indicate the need for simultaneous temperature measurements, and a more detailed study of methane absorption at elevated temperature.

20358. Blackburn, D. L.; Berning, D. W. An experimental study of reverse-bias second breakdown, Proc. International Electron Devices Meet., Washington, DC, Dec. 8-10, 1980, pp. 297-301 (The Institute of Electrical \& Electronics Engineer, Piscataway, NY, Dec. 1980).

Key words: avalanche injection; base current, reverse; current crowding; nondestructive testing; reverse bias; second breakdown; switching characterization; temperature; transistors, power.

Experimental results showing the influence of reverse-base current, case temperature, collector inductance, and peak collector current on the reverse-bias second breakdown (RBSB) behavior of high-voltage $n^{*}-p-n^{-}-n^{*}$ power transistors are presented. The results are in qualitative agreement with the theory that avalanche injection initiates RBSB. The inductance and peak collector current results are in conflict with the theory that RBSB is initiated at a critical temperature. It is concluded that for these devices for the condition studied, RBSB is not initiated at a critical temperature. It is shown that the theory of current focusing, in conjunction with the theory of avalanche injection, does not accurately predict the RBSB conditions during device sustaining. It is proposed that other mechanisms in addition to current focusing contribute to the nonuniformity of current during transistor turnoff.

20359. Schneider, S. J. Materials research for the clean utilization of coal. Quarterly progress report January.March 1979, DoE Report EA-6010-19, 34 pages (Available as FE6010-19 from the National Technical Information Service, Springfield, VA 22161, 1979).

Key words: ceramic corrosion; ceramic erosion; ceramic fracture chemical degradation; coal gasification material; failure avoidance; metal corrosion; metal erosion, and vaporization processes.

This report covers work on metal corrosion, metal erosion, ceramic deformation, fracture, erosion, and chemical degradation as related to coal gasification systems. This report also covers the failure avoidance program for DOE coal conversion pilot plants.

20360. Younglove, B.; McCarty, R. D. Thermodynamic properties of nitrogen gas derived from measurements of sound speed, NASA Reference Publ. 1051, 55 pages (Available as N80.14257 from the National Technical Information Service, Springfield, VA 22161, Dec. 1979).

Key words: equation of state; p- $\rho$-T data; speed of sound; thermodynamic properties.

A virial equation of state for nitrogen has been determined by use of newly measured speed-of-sound data and existing pressure-density temperature data in a multiproperty-fitting technique. The experimental data taken were chosen to optimize the equation of state for a pressure range of 0 to $10 \mathrm{~atm}$ and for a temperature range of 60 to $350 \mathrm{~K}$. Comparisons are made for thermodynamic properties calculated both from the new equation and from existing equations of state.

20361. Molino, J. A.; Zerdy, G. A.; Lerner, N. D.; Harwood, D. L. Initial psychoacoustic experiments on the human response to transmission line audible noise, January 1, 1978 to December 31, 1978, $D O E / E T / 6010-1,86$ pages (Available from the National Technical Information Services, Springfield, VA 22161, 1978).

Key words: aversiveness measures; corona noise; environmental noise impact; high-voltage transmission lines; human response to noise; modification of noise spectra.

A behavioral preference procedure, the "acoustic menu," was used to assess human aversion to the sounds from two samples of transmission line audible noise and from samples of other environmental noises. The audible (corona) noise produced by extrahigh voltage (EHV), overhead transmission lines was tape-recorded during moist weather. Reproduced samples of corona noise, other common environmental noises, and artificial reference stimuli were compared in two experiments. The two corona noise samples were found to be equally preferred to: (1) a $1000 \mathrm{~Hz}$ octave band of noise about $11 \mathrm{db}$ SPL higher than the corona noises and (2) a collection of other environmental noises about $8 \mathrm{db}$ SPL higher than the corona noises. The corona noise samples were more aversive than the ambient sounds (including rainfall) occuring near rural transmission lines and were roughly equivalent in aversiveness to the noise from a room air conditioner (indoor recording). Knowledge of the source of the corona noise (from photographs and a brief description) did not affect its aversiveness. Of the simple frequency-weighting scales, the A-weighted sound level reduced the difference between the two corona noise samples and the set of other environmental sounds from about $8 \mathrm{db}$ SPL to about $3 \mathrm{db}$ SPL. The D-weighted sound level further reduced this discrepancy to about $2 \mathrm{db}$. These results were obtained with only two samples of corona noise; other samples may give different results. A third experiment evaluated the aversiveness of audible noise from EHV electric transmission lines for spectral modification of 5 different tape-recorded samples. The relative 
contributions of low-frequency humming and buzzing and of highfrequency hissing and crackling components of the noise proved more aversive for all five samples to a group of 25 listeners. Therefore, for outdoor or open window listening, engineering efforts should be directed at reducing the high-frequency portion of the audible noise spectrum. A fourth experiment essentially replicated the above results with a different psychophysical method.

20362. Molino, J. A.; Zerdy, G. A.; Lerner, N. D.; Harwood, D. L. Psychoacoustic evaluation of the audible noise from EHV power lines, (Proc. 7th IEEE/PES Transmission and Distribution Conf., Atlanta, GA, Apr. 1-7, 1979), Paper 79CH1399-5-PWR, pp. 95-98 (Institute of Electrical and Electronics Engineers, Inc., New York, NY, 1979).

Key words: audible noise; EHV power lines; noise, audible.

A series of three psychoacoustic experiments was conducted with samples of audible (corona) noise tape recorded $15 \mathrm{~m}(50 \mathrm{ft})$ from extra-high-voltage transmission lines. Altogether 77 listeners responded to these and other sounds in a realistic listening room environment. The results showed that corona noise is more aversive than the ambient sounds (including rainfall) occuring near rural transmission lines and that knowledge of the source of the corona noise did not affect its aversiveness to the listeners. The results also showed that common acoustic measurement scales tend to underestimate the aversiveness of corona noise and that the highfrequency components of corona noise contribute most toward the aversive reactions of listeners.

20363. Bullis, W. M. Characterizing semiconducting materials, Semicond. Int., pp. $79-97$ (Oct. 1980)

Key words: characterizing semiconducting materials; packages, characterizing; process chemicals; semiconducting materials.

Materials characterization is needed to interpret device and circuit characteristics. Ancillary techniques associated with the analysis of process chemicals, control of process steps and characterizing packages will also assist in this.

20364. Hunt, C. M. Air infiltration: A review of some existing measurement techniques and data, (Proc. Symp. Building Air Change Rate and Infiltration Measurements, Washington, DC, Mar. 12, 1978), Am. Soc. Test. Mater. Spec. Tech. Publ. 719, Building Air Change Rate and Infiltration Measurements, C. M. Hunt, J. C. King, H. R. Trechsel, eds., pp. 3-24 (Oct. 1980).

Key words: air leakage measurement; infiltration review; ventilation analysis.

This paper reviews the state of the art in the measurement of ventilation and air infiltration. It considers tracer gas techniques and discusses some of the tracer gases used as well as some of the potential sources of error. It also discusses fan pressurization/evacuation procedures for measuring building tightness and compares fan and tracer measurements. It discusses the ASHRAE crack method. It also considers a number of factors influencing infiltration rates and finally reviews a few of the empirical equations which have been developed to correlate infiltration rate with wind velocity and inside-outside temperature difference.

20365. Grot, R. A. A low-cost method for measuring air infiltration rates in a large sample of buildings, (Proc. Symp. Building Air Change Rate and Infiltration Measurements, Washington, DC, Mar. 12, 1978), Am. Soc. Test. Mater. Spec. Tech. Publ. 719, Building Air Change Rate and Infiltration Measurements, C. M. Hunt, J. C. King, H. R. Trechsel, eds., pp. $50-59$ (Oct. 1980).

Key words: air infiltration; air sample bags; energy conservation; retrofit; sulfur hexafluoride; tracer gas; weatherization.

A method for collecting air infiltration data in a large sample of dwellings is presented. The method consists of a tracer gas dilution technique employing air sample bags which are analyzed in a central laboratory. The method will be applied to a Community Services
Administration optimal weatherization demonstration in approximately 300 dwellings at 16 sites throughout the United States. The method will yield air exchange rates under typical heating season conditions for each dwelling in the demonstration. Preliminary data on air infiltration rates in low-income housing in Portland, Maine are presented.

20366. Birnbaum, G.; Free, G., eds. Eddy-current characterization of materials and structures, (Proc. Symp. Eddy-Current Characterization of Materials and Structures, Gaithersburg, MD, Sept. 5-7, 1979), Am. Soc. Test. Mater. Spec. Tech. Publ. 722, 501 pages (American Society for Testing and Materials, Philadelphia, PA, Feb. 1981).

Key words: characterization of eddy currents; eddy current NDE; eddy currents; NDE; theoretical problems.

Eddy current testing in the industrial setting has been a common practice for many years. As industry has become more concerned about cost effectiveness, meaningful design criteria and the integrity of products, the role of eddy current testing has become more significant. In response to these concerns, there has been a virtual explosion of activity in all areas of eddy current NDE, including theory instrumentation, data analysis and applications.

20367. Coursey, B. M.; Moghissi, A. A. The preparation of counting samples, Part II in Monographie BIPM-3. The Application of LiquidScintillation Counting to Radionuclide Metrology, pp. 7.22 (Bureau International des Poids et Mesures, Sevres, France, 1980).

Key words: emulsion scintillators; liquid scintillators; radioactivity; radionuclide metrology; sample preparation

This section is intended as a guide to sample preparation for radionuclide metrology techniques employing liquid-scintillation (LS) counting. This material should also be useful to those engaged in applied-science studies, such as radioimmuno-assay or environmental monitoring, which generally involve comparative rather than direct activity measurements.

Examples are given for the preparation of samples for a number of radionuclides which differ in their chemical behavior as well as in their nuclear-decay schemes. A critical review of the literature in this field is also included (116 references). The results of this review are displayed in the form of a periodic chart, showing the 57 elements that have been incorporated into liquid-scintillation counting samples, and a table with literature references for 76 radionuclides which have been assayed by LS counting.

20368. Coursey, B. M.; Mann, W. B. Design of high-efficiency liquidscintillation counting systems, Part III in Monographie BIPM-3, The Application of Liquid-Scintillation Counting to Radionuclide Metrology, pp. 23.35 (Bureau International des Poids et Mesures, Sevres, France, 1980)

Key words: beta particle counting; figure of merit; high efficiency; light collection efficiency; liquid scintillation; phototubes.

In order to compare liquid-scintillation-counting systems we first refer to some figures of merit which have been used in this field. The most useful of these for design purposes is $\eta$, which is the number of photoelectrons arriving at the first dynode per kiloelectronvolt of energy deposited in the scintillator. In the second part of this chapter we consider methods of optimizing $\eta$, and finally a review is given on the liquid-scintillation systems that have been used in radionuclide metrology. Seventy-six references are given to the literature on design and construction of such systems. Several of the two-phototubes designs reported should give $\eta$ values, for $5 \cdot \mathrm{keV}$ monoenergetic electrons, in excess of 1 photoelectron $/ \mathrm{keV}$ for each phototube.

20369. Franzen, D. L.; Day, G. W.; Danielson, B. L.; Kim, E. M.; Chamberlain, G. E. Results of an interlaboratory measurement comparison among fiber manufacturers to determine attenuation, bandwidth, and N.A. of graded-index optical fibers, (Proc. 3d Int. Conf. Integrated Optics and Optical Fiber Communication, San Francisco, CA, Apr. 27-29, 1981), Digest of Technical Papers, pp. 74 - 
Key words: numerical aperture; optical fibers; radiation angle.

A procedure for determining the radiation angle (numerical aperture) of graded index optical fibers was tested by an interlaboratory measurement comparison among six fiber manufacturers and the National Bureau of Standards (NBS). Radiation Angle was determined using the five percent intensity points on an exit far-field radiation pattern. Measurements on five fibers representing low to high numerical aperture values show standard deviations of $2.9,2.4,2.0,1.5$ and 0.9 percent. The overall average, 2.0 percent, is close to the one standard deviation measurement precision reported by most participants.

20370. Wang, F. W.; Zimm, B. H. Novel cell for light scattering from solutions, Biopolymers 20, 1333-1335 (1981).

Key words: cell; laser; light scattering; polymers; Rayleigh ratio; solution purification; solutions; ultracentrifuge; water.

A novel cell for holding the sample in a light scattering apparatus is described. In this cell the usual glass window for the entrance of the incident beam is replaced by the free liquid surface. The cell is designed to be centrifuged in a swinging-bucket rotor. The Rayleigh ratio of water determined with this cell is given.

20371. Bullis, W. M. Metrology for submicrometer devices and structures, Proc. Microcircuit Engineering 1979, Microstructure Fabrication, Aachen, West Germany, Sept. 25-27, 1979, pp. 271-282 (German Section of IEEE, Aachen, West Germany, 1979).

Key words: dimensional metrology; electronics; integrated circuits; materials characterization; microelectronics; semiconductors.

The metrological requirements of semiconductor microelectronics, always challenging, are made even more stringent by the trend toward submicrometer devices and structures. This comes about not only because of the obvious demands associated with the smaller feature sizes of circuit elements but also because of the attendant requirements for more efficient design verification aids, computer simulations, and process validation and control techniques and because of the concurrent trend toward larger die and package sizes. This paper examines the types of metrological requirements associated with submicrometer devices and structures, summarizes the present state of the art in selected critical areas of metrology, and reviews current research and development efforts on advanced measurement technology, especially those at the National Bureau of Standards.

20372. Bullis, W. M. Role of Government in influencing the semiconductor industry-Technological aspects, (Proc.

Electrochemical Society Meet. Los Angeles, CA, Oct. 14-19, 1979),

J. Electro. Chem. Soc. Extended Abstracts 79-2, Abstr. No. 479, 12141215 (Oct. 1979).

Key words: cooperative technology; electronics; foreign competition; integrated circuits; semiconductor technology; VLSI.

Technological aspects of interactions between the U.S. semiconductor industry and the Federal Government will be reviewed. Perceived problems associated with research and development activities financed by foreign governments and basic changes in the nature and capitalization requirements of the industry will be considered from the point of view of the appropriateness of responsive technological efforts by the Federal Government. Various mechanisms for such efforts-both in place and projected-will be discussed.

20373. Madey, T. E. The use of angle-resolved electron and photon stimulated desorption for surface structural studies, Paper in Springer Series in Chemical Physics. Vol. 17: Inelastic Particle-Surface Collisions, E. Taglauer and W. Heiland, eds., pp. 80-103 (SpringerVerlag, Berlin, W. Germany, 1981).

Key words: chemisorption; electron stimulated desorption; ion
We review recent experiments and models related to desorption processes induced by electrons and photons incident on surfaces. The utility of angle-resolved electron and photon stimulated desorption of ions for studies of molecular structure at surfaces is emphasized.

20374. Burek, A. J.; Barrus, D. M.; Blake, R. L.; Fenimore, E. E. Analysis of solar X-ray emission line profiles, Astrophys. $J$. 243, No. 3, Pt. 1, 660-676 (Jan. 15, 1981).

Key words: active region; emission lines; line profiles; neon; solar; spectra; $x$ rays.

We report results of the analysis of the X-ray emission line profiles for the Ne $\mathrm{x} \mathrm{L} \alpha$ and $\mathrm{Fe} \mathrm{XVII} 4 d^{1} P_{1}$ lines produced in an active region that was undergoing a radio and $X$-ray gradual rise and fall (GRF) in intensity. The spectra were obtained with collimated Bragg spectrometers launched on a rocket from White Sands Missile Range on 1976 March 26. Using a crystal of ammonium acid phthalate, we have fully resolved the Ne x L $\alpha$ and Fe XVII $4 d{ }^{1} P_{1}$ lines, permitting an accurate determination of the $\mathrm{Ne} x \mathrm{~L} \alpha$ intensity and allowing Doppler broadened profiles for lines formed from ions having greatly different atomic mass and charge to be measured. An isothermal model derived from the $\mathrm{Ne} \mathrm{IX} / \mathrm{Ne} \mathrm{X}$ resonance line intensity ratio gives an electron temperature of $3.4 \times 10^{6} \mathrm{~K}$. An isothermal model, however, fails to account for the intensities of all lines and continuum observed. All multitemperature models that do reproduce the observed relative line intensities require the presence of a hot plasma component with an electron temperature in excess of $5 \times 10^{6} \mathrm{~K}$. The presence of a high temperature component is also suggested by the measured line to continuum ratio of 3.6 in the $12-15 \AA$ wavelength interval. Interpretation of the line profiles in terms of a multitemperature model requires an rms turbulence velocity of $48 \pm 15$ $\mathrm{km} \mathrm{s}^{-1}$ for $\mathrm{Fe} X \mathrm{XII}{ }^{1} P_{1}$ and $74 \pm 54 \mathrm{~km} \mathrm{~s}^{-1}$ for $\mathrm{Ne} \mathrm{X} \mathrm{L} \alpha$ at the $95 \%$ confidence level. Collimated scans across the active region show the presence of a compact source of intense $\mathrm{X}$-ray emission close to the magnetic neutral line, which is very probably the GRF plasma.

20375. Bertsch, D. L.; Dodge, W. R. Calibration of a gamma-ray telescope using tagged positron annihilation photons, Nucl. Instrum. Methods 185, $439-448$ (1981).

Key words: absolute efficiency; calibration of photon detectors; celestial gamma rays; cosmic radiation; monoenergetic photon source; positron annihilation; response function determination; wire chamber.

Measurements of detection efficiency, angular resolution, and energy resolution properties of a gamma-ray telescope used to study celestial gamma rays from balloon flight altitudes are described. Nearly monochromatic photons produced at the National Bureau of Standards tagged photon facility were used for the calibration. Details of the photon beam configuration and properties and results of the measurements made at photon energies of 15.1 and $31.1 \mathrm{MeV}$ are presented. Finally, the data are compared with a Monte Carlo analysis of the instrument properties.

20376. Danos, M. Cerenkov radiation, Article in Encyclopedia of Physics, pp. 106-107 (Addison-Wesley Publ. Co., Advanced Book Program, Reading, MA, 1980).

Key words: Cerenkov radiation; electrodynamics; particle detectors; relativistic particle; stopping power; tachyons.

The principles and users of Cerenkov radiation are described.

20377. Powell, R. L.; Fickett, F. R. Cryogenic properties of copper, I page wall chart (International Copper Research Assoc., 708 Third Ave., New York, NY 10017, Dec. 1979).

Key words: copper alloys; liquid helium temperature; room temperature; wall chart.

This publication is a wall chart describing the physical and mechanical properties of copper and selected copper alloys between room temperature and liquid helium temperature. The data presented 
is extracted from numerous compilations and recent publications.

20378. Ruthberg, Z. G. Risk assessment and managerial analysis, $E D P$ Auditor, pp. 31.43 (1980).

Key words: computer security; computer security audit; qualitative risk analysis; quantitative risk analysis; risk analysis; risk assessment; risk management.

Risk assessment (or analysis) is part of a growing set of concepts in the computer security and audit of computer security fields that are contributing to computer-based Generally Accepted Information Principles, Audit Practices, and Audit Standards for the information systems of the computer era. The National Bureau of Standards, the U.S. General Accounting Office, the American Institute of Certified Public Accountants, the Institute of Internal Auditors, and the Electronic Data Processing Auditors Association are contributing to these principles, practices, and standards. From a comprehensive management view, risk assessment is part of a larger activity which can be called a risk management program. Fifteen logical tasks properly belong to such a program. Risk analysis includes the first nine of these. The NBS method for risk analysis (a quantitative type) contains more tasks than the one defined here, but fewer than a risk management program. Although no risk assessment method in use today accomplishes all of its tasks well, many perform some in ways that are worth considering in future improved risk analyses. Since a quantitative risk analysis arrives at a conclusion with large uncertainties, qualitative methods are being used by some. SECURATE is such a method. More work needs to be done with both quantitative and qualitative methods before preferring one type over the other. The National Bureau of Standards has a current effort to produce a risk management standard that will specify a necessary minimum set of logical tasks.

20379. Barber, E. M.; Bettge, D. A.; Powell, F. J. Development of a modeling technique for evaluation of natural convection and ventilation in rooms, Proc. Semi-Annu. Meet. ASHRAE Survival Shelter Problems, San Francisco, CA, Jan. 19-22, 1970, pp. $36-39$ (American Society of Heating, Refrigerating and Air-Conditioning Engineers, Inc., New York, NY, 1970).

Key words: modeling technique; natural convection; photography; ventilation.

An experimental technique is being developed by which natural convection and ventilation in rooms and shelters can be studied. The technique relies on the use of a small-scale model to simulate the actual room or shelter, on the use of high temperature water in place of the room air to simulate the convective patterns, on time-exposure photographs made of trace particles suspended in the water to reveal the convection, and on temperature measurements made inside the model. The technique is discussed in terms of its potential for yielding useful information on free convection in spaces such as inside rooms.

20380. Bowen, R. L. Adhesive bonding of various materials to hard tooth tissues XXIV: Recent developments in adhesive resin formulations for dentin, Proc. Int. Congr. of Implantology and Biomaterials in Stomatology, Kyoto, Japan, June 9-11, 1980, pp. 66-73 (Osaka Dental University, Osaka 540, Japan, 1981).

Key words: adhesive resins; biomaterials; dental materials; monomers; osmotic pressure; polymers; solubility.

Strong and durable adhesive bonding of restorative materials to dentin could simplify cavity preparation and the repair of cervical and other lesions. Cut dentin has a disturbed or "smeared" surface layer that can be removed by physiological (isotonic) acidic solutions.

Bonding to this cleaned dentinal surface can be improved by the subsequent use of a "mordant," such as isotonic aqueous ferric chloride.

A third factor that can improve adhesion to dentin is the use of surface-active comonomers which can bond to these surface-held cations and can copolymerize with dental resins. Formulations containing these can be isotonic with tissue fluids by selecting monomers with respect to their water solubility and by using a mixture which will yield a solubility of 0.31 molar in water. Such a mixture saturated with water will be isotonic with a normal physiological saline solution or body fluids.

20381. Fiori, C. E.; Myklebust, R. L. A simplex method for fitting Gaussian profiles to $x$-ray spectra obtained with an energy-dispersive detector, (Proc. American Nuclear Society Topical Conf., Mayaguez, Puerto Rico, Apr. 30-May 4, 1978), Paper in Computers in Activation Analysis and Gamma-Ray Spectroscopy, B. S. Carpenter, M. D. D'Agostino, and H. P. Yule, eds., CONF-780421, pp. 139-149 (U.S. Department of Energy, Technical Information Center, Washington, DC, 1979).

Key words: computer program; energy-dispersive x-ray spectra; Gaussian profiles; peak fitting; peak overlap; sequential simplex.

A method is described for the fitting of Gaussian profiles to the pulse-height distributions of overlapping X-ray peaks measured with an energy-dispersive detector. The desired result is to know the areas under individual X-ray peaks in a spectrum in which the peaks are not resolved. A sequential simplex procedure for selection of the parameters in a mathematical expression that describes a spectral peak is used. Starting and stopping criteria for the procedure are discussed, and a computer program is outlined. Examples of the method applied to peaks obtained from a lithium-drifted silicon [ $\mathrm{Si}(\mathrm{Li})]$ detector are provided.

20382. Waterstrat, R. M. Structure relationships affecting the stability of A15- and $\mathrm{Ti}_{3}$ P-type compounds, J. Solid State Chem. 37, 370-382 (1981).

Key words: A15 compounds; coordination polyhedra; near. neighbor diagrams; structural stability; structure relationships; $\mathrm{Ti}_{3} \mathrm{P}$-type compounds.

Observed interatomic distances in $\mathrm{A} 15$ - and $\mathrm{Ti}_{3} \mathrm{P}$-type compounds are analyzed as deformations of ideal atomic rigid spheres. The analysis suggests that structural instabilities may develop in A15 compounds when the atomic size of the B element becomes approximately $10 \%$ smaller than that of the A element. These instabilities apparently originate from strong repulsive interactions along the atom chains. The internal strains associated with these interactions may be relieved by martensitic transformations, deviations from the ideal $\mathbf{A}_{3} \mathbf{B}$ stoichiometry, addition of ternary elements, and transformation to a $\mathrm{Ti}_{3} \mathrm{P}$-type structure. Instability apparently develops in the $\mathrm{Ti}_{3}$ P-type compounds when the $\mathrm{A}$ - and B-element atoms are nearly equal in size. The instability in this case would result primarily from repulsive forces associated with a strong compression of the B-element atoms.

20383. Wu, W.; Cobb, E. N. A silver staining technique for investigating wear of restorative dental composites, J. Biomed. Mater. Res. 15, 343.348 (1981).

Key words: dental composites; microdefects; restorative composite; staining technique; sublayer damages; wear.

A silver staining technique was developed to demonstrate microdefects in dental restorative composites. Fine silver particles were preferentially introduced into the damaged region to provide optical contrast between the damaged and the undamaged regions. The amount of silver deposition determined with an electron probe microanalyzer provided an indication of the extent of damage within the dental composites. Examples to demonstrate this technique were given with one clinically worn dental composite restoration and one in vitro worn composite sample.

20384. Casella, R. C. Neutron scattering from coherent admixtures of phonons with libronic excitations of diatomic impurities, Phys. Rev. $B$ 20, No. 12, 5318.5325 (Dec. 15, 1979).

Key words: coherent mixed-mode; diatomic impurities; neutron scattering; phonons; $S$-matrix; vector cubic harmonics.

The coherent mixed-mode states are represented in an $S$-matrix formalism. In the small-q quasicontinuum region, the phonon field is expanded in terms of vector cubic harmonics, analogues of vector 
spherical harmonics. Tunneling states of the diatoms in a cubic crystal field are analyzed via group-theoretically projected tight-binding local oscillator states developed by Dick. Plausible level schemes are constructed by joining the strong-coupling spectrum they describe to the weak-coupling (free-rotator) spectrum. This

semiphenomenological approach does not depend upon details of the generalized Devonshire hindering potential. It is found that the $T$-matrix elements connecting the phonons with the libronic or hindered-rotator excitations vanish in lowest nontrivial order unless the latter are of $E_{g}$ or $T_{2 g}$ symmetry. Expressions for estimating the relative strengths of these allowed $T$-matrix elements are derived. Results are applied to neutron scattering experiments on diluteimpurity $\mathrm{KCl}: \mathrm{CN}^{-}$and $\mathrm{KBr}: \mathrm{CN}^{-}$and comparisons made with optical experiments on these systems. Of the usually considered $\langle 100\rangle$ and (111) $\mathrm{CN}^{-}$impurity models for these crystals, our analysis shows that the $\langle 100\rangle$ model is eliminated by the neutron data, in agreement with earlier conclusions based upon optical and other experiments. The absence of a $T_{2 g}$ interaction as observed in the neutron experiments presents a difficulty for the $\langle 111\rangle$ model if the libronically excited observed $E_{g}$ and unobserved $T_{2 g}$ levels are split by an amount of the order of the ground-state tunnel splitting, i.e., $O\left(1 \mathrm{~cm}^{-1}\right)$. Under this assumption, the same difficulty exists for the $(110)$ model which we are also led to consider. The relative strengths of the $T_{2 g}$ to $E_{g}$ couplings of the phonon to the libronic excitations are estimated for both the $(111)$ and $(110)$ models. Whereas the estimation procedure is perhaps too crude to distinguish between these two models on the basis of the neutron data alone, the fact that for either model the computed ratio of $T_{2 g}$ to $E_{g}$ couplings is nearly equal to unity, under the assumption that the excited tunnel splitting are small, clearly suffices to rule out this latter assumption if either model is correct. It is concluded that the $T_{2 g}$ rotational excitations of the $\mathrm{CN}^{-}$ions lie considerably above the observed $E_{g}$ libronic excitations and that the former are lifetime broadened to the extent that formation of coherent mixed modes does not occur; hence the absence of neutron observed splitting of the phonon modes in the $T_{2 g}$ configuration. Our analysis of the Raman data lends support to this conclusion. Under the assumption of large excited-state splitting, our estimation procedure for the $T_{2 g} / E_{g}$ coupling ratio does not apply and the neutron data are compatible with either the $\langle 111\rangle$ or $\langle 110\rangle$ models. The earlier experiments favor the $\langle 111\rangle$ model.

20385. Linsky, J. L. Outer atmospheres of late-type stars, (Proc. Second Workshop for Physical Processes in Red Giants, Sicily, Italy, Sept. 3.13, 1980), Paper in Physical Processes in Red Giants, I. Iben, Jr. and A. Renzini, eds., pp. 247-261 (D. Reidel Publ. Co., Boston, MA, 1981).

Key words: late-type stars; stellar chromospheres; stellar coronae; ultraviolet spectra; $\mathrm{x}$-ray sources.

I will attempt to summarize our limited understanding of the outer atmospheres of red giants by describing recent important observational results concerning chromospheres and coronae in latetype stars and how red giants fit into the emerging picture. In particular, I will point out where in the cool half of the HR diagram chromospheres, transition regions, coronae, and large mass loss occur and suggest what the important parameters determining the energy balance of these layers might be. I will then summarize the chromospheric modelling process and describe in detail models of the late-type supergiants $\beta$ Dra (g2 II), $\epsilon$ Gem (G8 Ib), and $\alpha$ Ori (M2 Iab) recently computed by Basri and Linsky.

20386. Harris, J. R. Information flow in the development of earthquake provisions for building codes, Proc. Conf. $V$ Communicating Earthquake Hazard Reduction Information, Denver, CO, May 22-24, 1978, pp. 288-306 (Office of Earthquake Studies, U.S. Geological Survey, Menlo Park, CA, 1978).

Key words: building codes; building regulatory system; buildings; building standards; codes, building; earthquake; seismic risk; standards, building.

This report describes how information flows through the building regulatory system and how this flow is being taken into account in the development of new building code provisions for earthquake hazards. Many sectors of the construction community play a role in the complex flow of information in and out of building codes, each with its own set of decision makers, but the local building official is the key figure. This history of earthquake provisions in building codes shows a slow evolution that is typical in the building regulatory system, partially because of the complex pattern of communication and decision making. New provisions have recently been developed for consideration by the regulatory community, but it will be several years before their path through various standards and model codes into legal building codes is complete.

20387. Kratochvil, B. G.; Taylor, J. K. Sampling for chemical analysis, Anal. Chem. 53, No. 8, 924A-938A (July 1981).

Key words: chemical analysis; number of samples; particle size influence; sampling bulk materials; -sampling discrete lots; sampling plans; sampling segregated materials; size of sample.

A general discussion is given of the various factors that should be considered to provide valid samples for chemical analysis. Included are discussions of the common modes of sampling and the rationale behind the development of sampling plans for both bulk and stratified materials. Guidance is provided in determining the necessary number of sizes of samples required to reduce uncertainty due to sample variability to prescribed limits. Sampling of materials occurring in discrete units is also considered. While the discussion is directed primarily toward solid materials, the implications for other sampling situations are pointed out.

20388. Giampapa, M. S.; Bornmann, P. L.; Ayres, T. R.; Linsky, J. L.; Worden, S. P. The Mg II $h$ and $k$ lines in a sample of $\mathbf{d M e}$ and $\mathbf{d M}$ stars, Proc. Second Year of IUE: The Universe in Ultraviolet Wavelengths, Greenbelt, MD, May 7-9, 1980, pp. $279-286$ (NASA Goddard Space Flight Center, Greenbelt, MD, 1981).

Key words: flare stars; late-type stars; stellar chromospheres; ultraviolet spectra.

We present observed $\mathrm{Mg}$ II $\mathrm{h}$ and $\mathrm{k}$ line fluxes for a sample of 4 $\mathrm{dMe}$ and $3 \mathrm{dM}$ stars obtained with the IUE satellite in the long wavelength, low dispersion mode. The observed fluxes are converted to stellar surface flux units and the importance of chromospheric nonradiative heating in this sample of $m$ dwarf stars is intercompared. In addition, we compare the net chromospheric radiative losses due to the $\mathrm{Ca}$ II $\mathrm{H}$ and $\mathrm{K}$ lines in those stars in the sample for which calibrated $\mathrm{Ca}$ II $\mathrm{H}$ and $\mathrm{K}$ line data exists. Moreover, we estimate active region filling factors which likely give rise to the observed optical and ultraviolet chromospheric emission. Finally, we briefly discuss the implications of the results for homogeneous, singlecomponent stellar model chromospheres analyses.

20389. Girvin, S. M.; Penn, D. R. Resonant satellites in photoemission and Auger spectra of d-band metals, J. Appl. Phys. 52, No. 3, 1650 1653 (Mar. 1981).

Key words: Auger spectroscopy; d band metals; many body effects; photoemission; resonant satellite.

Photoemission and Auger electron spectroscopy are powerful tools in the study of the electronic and magnetic properties of $d$ band metals. In certain instances experimental spectra can be directly interpreted as a measure of some one body density of states. In other cases one must consider the many body dynamics of the measurement process in detail. Striking examples of the latter are the resonant satellites in several $\mathrm{d}$ band materials which have been observed in photoemission near the photon energy threshold for core hole production. An elementary introduction to the physics of this satellite phenomenon will be presented. A model recently introduced by Davis and Feldkamp to explain resonant satellites in filled $\mathrm{d}$ band materials will be discussed in some detail and the predictions of this model will be used to illustrate some general properties of resonant photoemission satellites.

20390. Dick, C. E.; Motz, J. W. Image information transfer properties of x-ray fluorescent screens, Med. Phys. 8, No. 3, $337-346$ (May/June 1981). 
Key words: detective quantum efficiency; image information transfer efficiency; light gain; statistical distribution of light photons; x-ray fluorescent screens.

The image information transfer efficiency for five $x$-ray fluorescent screens (calcium tungstate, barium halide, and three rare earth screens) has been experimentally determined with monoenergetic $\mathrm{x}$-ray beams at energies of $18,22,32,49,51,58$, and $69 \mathrm{keV}$. The transfer efficiency, which is defined by the ratio of the output to input signal-to-noise ratios, was determined from measurements of (a) the fraction of incident $x$ rays absorbed in the screen and $(b)$ the statistical distribution of the number of light photons emitted from the screen per absorbed $\mathrm{x}$ ray which was determined by light photon counting techniques. Comparisons of the information transfer efficiency, the average number of light photons emitted per absorbed $x$ ray, and the light output energy per Roentgen are given for the above screens and $\mathrm{x}$-ray energies.

20391. Fine, J. Absolute sputtering yield measurement methods: A review, (Invited Lectures \& Progress Reports of SPIG ' 80 , Dubrovnik, Yugoslavia, Aug. 25-30, 1980), Paper in The Physics of Ionized Gases, M. Matić, ed., pp. $379-420$ (Boris Kidric Institute of Nuclear Sciences, Beograd, Yugoslavia, 1980)

Key words: dynamic measurement methods; dynamic yield data; ion bombardment; sputtering yield measurement.

In this review of the determination of sputtering yields, the importance of characterizing the parameters of the target and ion is stressed together with the need for yield data of improved accuracy and sensitivity. Five categories of yield measurement methods are discussed together with a total of sixteen techniques which can be used to implement certain of those methods. Various advantages and disadvantages are cited for each of the sixteen techniques, many of which were chosen to demonstrate that static, low-sensitivity yield measurements can be significantly improved.

20392. Dulik, D.; Bernier, R.; Brauer, G. M. Effect of diluent monomer on the physical properties of Bis-GMA-based composites, $J$. Dent. Res. 60, No. 6, 983.989 (June 1981).

Key words: BIS.GMA diluent; composite; dental restorative; diluent; methacrylate monomer; physical properties of composite.

The high viscosity of "BIS.GMA," the major monomeric ingredient of dental composites, requires incorporation of a polymerizable diluent into the formulations of clinically useful restoratives. The objective of this study was to determine the effect of monomeric diluents on the properties of the liquid component of the resin and of the cured composite.

Viscosity and surface tension of the liquid increase with decreasing concentration and increasing molecular weight of the diluent. The setting time of the composite is lowered the larger the number of methacrylate groups in the diluent molecule. Strength of the cured material varies somewhat with the type of diluent employed. The coefficient of thermal expansion increases with the chain length of the polymethylene glycol dimethacrylate diluent. Composites containing diluents with more than one methacrylate group in the molecule have low water sorption, because of the highly crosslinked nature of the cured material. Addition of dimethacrylate diluents containing $-\left(\mathrm{CH}_{2}\right)_{\mathrm{n}}$ - recurring units generally yield composites with more desirable properties than those containing ethoxy ether $\left(\mathrm{CH}_{2} \mathrm{CH}_{2} \mathrm{O}\right)_{n}$ groups.

20393. Goodrich, L. F.; Ekin, J. W. Lap joint resistance and intrinsic critical current measurements on a $\mathrm{NbTi}$ superconducting wire, (Proc. 1980 Applied Superconductivity Conf., Santa Fe, NM, Sept. 29-Oct. 2, 1980), IEEE Trans. Mag. MAG-17, No. 1, 1.4 (Jan. 1981).

Key words: conductor; critical current; electrical property; magnet; niobium titanium; resistance; superconductor.

The lap joint resistance between two $\mathrm{Cu}: \mathrm{Nb}-\mathrm{Ti}$ wires (rectangular cross section, $0.53 \times 0.68 \mathrm{~mm}$ ) was measured at $4 \mathrm{~K}$ as a function of current, magnetic field and joint area $\left(0.5\right.$ to $\left.7 \mathrm{~mm}^{2}\right)$. A simple model, using the magnetoresistance and current dependence, allowed the joint interface resistance and the current transfer resistance to be separated. The critical current of the wire was also measured adjacent to the joint. These critical current data were compared with data taken on the control sample (no lap joint). From these comparisons operational checks were deduced for an intrinsic measurement of the critical current adjacent to a joint. The operational checks were on the reversibility of the V-I curves and on their current ramp-rate dependence. When these operational checks were applied to the critical current data of all the joints tested, the results agreed to $\pm 2 \%$.

20394. Jach, T.; Powell, C. J. $L_{3} V V$ Auger-electron line shapes and peak positions for near-threshold electron excitation in nickel and copper, Phys. Rev. Lett. 46, No. 14, 953.956 (Apr. 6, 1981).

Key words: Auger-electron spectra; band structure; copper; electron binding energies; nickel; post-collision interaction.

Measurements of the $L_{3} V V$ Auger-electron lineshapes for $\mathrm{Ni}$ and $\mathrm{Cu}$ at incident electron energies near the threshold for $L_{3}$-subshell ionization are shown to be substantially free of perturbing satellite effects. The $L_{3} V V$ Auger spectrum of Ni consists of two features, one of which is identified as due to atomic final states and the other as due to band effects. Shifts in the position of the $L_{3} V V$ features as a function of incident energy imply a systematic difference in coreelectron binding energies measured under adiabatic and sudden conditions.

20395. Acquista, N.; Reader, J. Spectrum and energy levels of ninetimes ionized strontium ( $\mathrm{Sr} \mathrm{X}$ ), J. Opt. Soc. Am. 71, No. 5, 569-573 (May 1981).

Key words: energy levels; spectra; strontium; ultraviolet; wavelengths.

The spectrum of the copper-like ion $\mathrm{Sr} x$ was observed with a low. inductance spark in the region $70-630 \AA$ on the $10.7-\mathrm{m}$ grazing. incidence spectrograph at the National Bureau of Standards. From the identification of 30 lines, a system of 23 energy levels of the type $3 d^{10} n l$ was determined. The level system includes the configurations $n s(n=4-7), n p(n=4-6)$, nd $(n=4-6), n f(n=4-6)$ and $n g(n=5)$. The $4 f^{2} F$ term is inverted. Also identified were 12 transitions of the type $3 d^{10} 4 s-3 d^{9} 4 s 4 p$ and $3 d^{10} 4 p-3 d^{9} 4 p^{2}$, permitting the determination of several $3 d^{9} 4 s 4 p$ and $3 d^{9} 4 p^{2}$ levels. The observed $3 d^{10} n l$ energy levels and parameters are compared with Hartree-Fock calculations. The ionization energy is determined from the $3 d^{10} n s$ and $n f$ series to be $1430000 \pm 500 \mathrm{~cm}^{-1}(177.30 \pm 0.06 \mathrm{eV})$. Data for $3 d-4 p$ transitions in $\mathrm{Sr} \mathrm{IX}$ and $\mathrm{Sr} \mathrm{XI}$ and $3 p-3 d$ transitions in $\mathrm{Sr} \mathrm{XII}$ are also presented.

20396. Benzinger, T. H.; Hammer, C. Unwinding the double helix: Complete equation for chemical equilibrium, Chapter 27 in Current Topics in Cellular Regulation. Vol. 18, Biological Cycles, R. W. Estabrook and P. Srere, eds., pp. 475-485 (Academic Press, New York, NY, 1981).

Key words: biopolymer thermodynamics; bond energy; Carnot engine; chemical equilibrium; fluctuations; heat capacities; membranes; midpoint temperature; polynucleotides; proteins; thermal content; thermal work function.

The energy that is required to unwind the double helix and to drive all other endothermic reactions in living matter is the "thermal work function."

According to the equation the energy required for unwinding the Poly A-Poly U double helix at the midpoint temperature $T_{m}=333 \mathrm{~K}$ would be $\Delta \mathrm{H}\left(\mathrm{T}_{\mathrm{m}}\right)=8000 \mathrm{cal} \mathrm{mol}^{-1}$, whereas $\Delta \mathrm{H}_{\mathrm{O}}=2000 \pm 500 \mathrm{cal}$ $\mathrm{mol}^{-1}$ is best estimate of the real driving energy. The difference of $6000 \pm 500 \mathrm{cal} \mathrm{mol}^{-1}$ is absorbed in "fluctuations" (Henry S. Frank, Rufus W. Lumry) of heat and entropy as in phase transitions, which do not contribute to the driving energies: (a) $\Delta W(T)$ and (b) $\Delta H_{O}$, the energy of chemical bonding.

20397. Bartle, K. D.; Lee, M. L.; Wise, S. A. Modern analytical 
methods for environmental polycyclic aromatic compounds, Chem. Soc. Rev. 10, No. 1, 113-158 (1981).

Key words: fluorescence; gas chromatography (GC); liquid chromatography (LC); luminescence; mass spectrometry (MS); nitrogen heterocycles; nuclear magnetic resonance (NMR); polycyclic aromatic compounds (PAC); polycyclic aromatic hydrocarbons (PAH); sample preparation; sulfur heterocycles.

Polycyclic aromatic compounds (PAC) are ubiquitous environmental pollutants from both natural and anthropogenic sources. PAC mixtures can be extremely complex since they contain numerous isomers. This complexity increases for samples which contain alkylated, multisubstituted, and partially hydrogenated compounds in addition to the parent PAC. In this paper, the most important analytical techniques for the analysis of complex mixtures of PAC are reviewed (over 400 references) with emphasis on the more recent studies involving advanced analytical techniques. Topics discussed include: sample preparation techniques, chromatographic methods (both high-performance liquid chromatography and glass capillary gas chromatography), mass spectrometric methods, and spectroscopic techniques (luminescence, nuclear magnetic resonance, and infrared).

20398. Casella, R. C. Isotopic shifts in complex crystals, Phys. Rev. $B$ 17, No. 8, $3381-3386$ (Apr. 15, 1978).

Key words: crystals; inequalities; isotopic shifts; little groups; phonons; Teller-Redlich rule.

We obtain relations between the phonon frequencies of crystals related by isotopic substitution when several ion types are involved in the mode being probed. Also, when the rigid molecular-ion approximation applies to extended subunits of the basic lattice cell, we consider the effects of substitution on combined translational and librational modes, involving more than one type of extended ion. The Teller-Redlich product rule is obtained for arbitrary wave vector $\vec{q}$ as are inequalities on the sums of eigenvalues $\omega^{2}(q r \gamma)$ associated with the $r$ h irreducible representation of the little group $G^{\text {? }}$.

20399. Codling, K.; Parr, A. C.; Ederer, D. L.; Stockbauer, R.; West, J. B.; Cole, B. E.; Dehmer, J. L. The effects of autoionisation on vibrational branching ratios and photoelectron angular distributions in molecular photoionization: The formation of the ground state of $\mathrm{O}_{2}^{+}$between 574 and 600 Å, J. Phys. B: At. Mol. Phys. 14, 657.666 (1981).

Key words: angular distribution; autoionization; differential photoelectron spectra; Franck-Condon factors; oxygen; photoelectron spectra; photoionization; synchrotron radiation; vibrational intensity distribution.

The partial cross sections (branching ratios) for leaving the $\mathrm{O}_{2}^{+}$ion in the ground $X^{2} \Pi_{g}$ state have been determined in the region of the $v=0$ and $v=1$ components of the neutral excited Rydberg state $\left(2 \sigma_{u}\right)^{-1} 3 \mathrm{~s} \sigma$ at 594.3 and $589.0 \AA$ by photoelectron spectroscopy. These cross sections have been further subdivided into partial cross sections for leaving the ion in a particular vibrational state. Moreover the asymmetry parameter, $\beta$, has been determined for each vibrational component in this limited spectral range. Considerable variations in both cross section and $\beta$ are observed.

20400. Daney, D. E.; Ludtke, P. R.; Eroshenko, V. M.; Yaskin, L. A. Cool down of cryogenic power transmission lines, (Proc. 15th Int. Congr. of Refrigeration, Venice, Italy, Sept. 23-29, 1979), Paper 19 in Progress in Refrigeration Science and Technology, Session A 1/2, 7 pages (International Institute of Refrigeration, Paris, France, 1980).

Key words: cool down; counter-flow heat exchanger cool down; counter-flow heat exchanger transient response; cryogenic power transmission line; cryogenic power transmission line cool down; superconducting power transmission line; transmission line cool down.

The time required to cool down a superconducting (or cryoresistive) power transmission line is critical to the success of such lines since excessive cool down times would result in unacceptably long interruptions of service when repairs are required.

We consider two cooling configurations: single stream (thermallyuncoupled return stream) and counterflow (thermally-coupled return stream). A review of the literature shows that for the thermallyuncoupled case the dimensionless cool down times is given by $\tau^{\prime} \sim N_{t u}$, where $N_{t u}$ is the number of heat exchanger transfer units. Our analytical, numerical and experimental work shows that for the thermally-coupled case, $\tau^{\prime} \sim N_{\mathrm{tu}}^{2}$. Thus, when the thermal coupling between the go and return streams is good $\left(N_{t u}>>1\right)$, the counter configuration gives much greater cool down times for the same flow rate.

20401. Brauer, G. M.; Stansbury, J. W.; Antonucci, J. M. 4-N,N. dialkylaminophenethanols, -alkanoic acids and esters: New accelerators for dental composites, J. Dent. Res. 60, No. 7, 1343.1348 (July 1981).

Key worcls: accelerator; acrylic resin; dental composites; initiatoraccelerator system; polymerization; tertiary amines; 4-N,N. dialkylaminophenalkanoic acid and ester; 4-N,Ndialkylaminophenethanol.

The newly synthesized 4-N,N-dialkylaminophenethanol, 4-N,Ndialkylaminophenalkanoic acid and ester are highly reactive accelerators for the polymerization of composites. With low amine concentration, nearly colorless restorations having good color stability are obtained.

20402. Martinez, R. I.; Herron, J. T.; Huie, R. E. The mechanism of ozone-alkene reactions in the gas phase. A mass spectrometric study of the reactions of eight linear and branched-chain alkenes, J. Am. Chem. Soc. 103, 3807-3820 (1981).

Key words: alkene; gas-phase; mechanism; ozone; reaction.

The stable products of the low-pressure (4-8 torr $(1$ torr $=133.33$ $\mathrm{Pa})$ gas-phase reactions of ozone with ethene, propene, 2-methylpropene, cis-2-butene, trans-2-butene, trans-2-pentene, 2,3dimethyl-2-butene, and 2-ethyl-1-butene have been identified by using a photoionization mass spectrometer coupled to a stirred-flow reactor. The products observed are characteristic of (i) a primary Criegee split to an oxoalkane (aldehyde or ketone) and a Criegee intermediate, (ii) reactions of the Criegee intermediates such as unimolecular decomposition, secondary ozonide formation, etc., and (iii) secondary alkene chemistry involving $\mathrm{OH}$ and other free-radical products formed by the unimolecular decomposition of the Criegee intermediates. The secondary $\mathrm{OH}$-alkene- $\mathrm{O}_{2}$ reactions account for a significant fraction of the alkene $\left(\mathrm{C}_{n} \mathrm{H}_{2 n}\right)$ consumed and lead to characteristic products such as $\mathrm{C}_{n}$ dioxoalkanes $\left[\mathrm{R}^{\prime} \mathrm{C}(\mathrm{O}) \mathrm{C}(\mathrm{O}) \mathrm{R}^{\prime \prime}\right.$, at $\left.m / e\left(\mathrm{C}_{n} \mathrm{H}_{2 n}+30\right)\right], \mathrm{C}_{n}$ acyloins [ $\mathrm{R}^{\prime} \mathrm{C}(\mathrm{O}) \mathrm{CH}(\mathrm{OH}) \mathrm{R}^{\prime \prime}$, at $m / e\left(\mathrm{C}_{n} \mathrm{H}_{2 n}+\right.$ 32)], and $\mathrm{C}_{n}$ alkanediols [ $\mathrm{R}^{\prime} \mathrm{CH}(\mathrm{OH}) \mathrm{CH}(\mathrm{OH}) \mathrm{R}^{\prime \prime}$, at $\left.m / e\left(\mathrm{C}_{n} \mathrm{H}_{2 n}+34\right)\right]$. $\mathrm{C}_{n}$ oxoalkanes and $\mathrm{C}_{n}$ epoxyalkanes observed at $m / e\left(\mathrm{C}_{n} \mathrm{H}_{2 n}+16\right)$ are probably formed primarily via epoxidation of the alkene by $\mathrm{O}_{3}$. A general mechanism has been proposed to account for the observations.

20403. Harris, J. R.; Wright, R. N. Computer aids for the organization of standards, Proc. Second Conf. Computing in Civil Engineering, Baltimore, MD, June 9-13, 1980, pp. 112-121 (American Society of Civil Engineering, New York, NY, 1980).

Key words: computer database; computer program; methodology; specifications; standards.

A decade of research has produced a systematic methodology for the analysis and representation of design standards. The methodology assists in the formulation, expression, and use of standards, specifications or legal code requirements. This paper reports on research for elements of that methodology for dealing with the scope and the arrangement of standards. Functional and structural descriptions of provisions provide rational bases for classification of each requirement of a standard. The classification is used as a database for computer software that produces an index and alternative outlines for the standard. A prototype program has been developed 
and implemented in FORTRAN on the UNIVAC 1108 at the National Bureau of Standards. Significant improvements over previous computer aids in the field include: flexibility in accommodating various classification schemes; the ability to generate an index based on meaningful and relevant terms; user selection from several logical criteria in the construction of outlines; the possibility of overriding the explicit logic of the classification; a routine for interactive generation of outlines that supports the creative talents of the user with the analytical and logical power of the computer. The program has been applied in recent projects to analyze and improve seismic design standards. The algorithms are being implemented in a general purpose, production-type computer aid for the analysis, formulation and expression of standards.

20404. Boisvert, R. F. Attainable accuracy of compact discretizations of the Poisson equation, Paper in Elliptic Problem Solvers, pp. 219. 223 (Academic Press, Inc., New York, NY, 1981).

Key words: compact formulas; finite differences; high order methods; nonrectangular domains; Poisson's equation.

In this paper we outline results on the maximum order of local truncation error attainable by compact as possible discretizations of Poisson's equation for a particular class of non-rectangular domains. We show, for instance, that although $\mathrm{O}\left(\mathrm{h}^{6}\right)$ accuracy is attainable for rectangular domains one cannot always achieve this even in very simple non-rectangular cases. A numerical example indicating the effect of using less accurate formulas for irregular elements is also given.

20405. Haisch, B.; Linsky, J. L.; Slee, O. B.; Siegman, B. C.; Nikoloff, I.; Candy, M.; Harwood, D.; Verveer, A.; Quinn, P. J.; Wilson, I.; Page, A. A.; Higson, P.; Deward, F. D. Simultaneous X-ray, ultraviolet, optical, and radio observations of the flare star Proxima Centauri, Astrophys. J. 245, 1009.1017 (May 1, 1981).

Key words: flare stars; stellar chromospheres; stellar coronae; ultraviolet sources; variable $\mathrm{x}$ rays; $\mathrm{x}$-ray sources.

We report on a coordinated program involving X-ray, ultraviolet, optical, and radio observations of the dM5e flare star Proxima Centauri. We detected one major $\mathrm{X}$-ray flare event with $\mathrm{L}_{\mathrm{x}}(0.2-4.0$ $\mathrm{keV}) 4.6 \times 10^{27}$ ergs s${ }^{-1}, \mathrm{~T}=1.7 \times 10^{7} \mathrm{~K}, \mathrm{EM}=5.8 \times 10^{50} \mathrm{~cm}^{-3}$ during the rise phase and $\mathrm{L}_{\mathrm{x}} \approx 6 \times 10^{27}$ ergs $\mathrm{s}^{-1}, \mathrm{~T}=1.2 \times 10^{7} \mathrm{~K}$, and $\mathrm{EM}=$ $9.4 \times 10^{50} \mathrm{~cm}^{-3}$ during the decay phase. We detected no ultraviolet, optical, or radio emission corresponding to this flare, but we did detect a total of 7 optical and 24 possible radio flares, including one event with simultaneous radio and optical emission. We interpret the absence of optical and ultraviolet emission at the time of the X-ray flare in terms of a model which the conductive flux to the lower atmosphere is inhibited geometrically, and the flare cools predominately by X-ray radiation. The observed $15 \mathrm{~min}$ exponential cooling time is consistent with an electron density of $1.8 \times 10^{11} \mathrm{~cm}^{-3}$ during the decay phase and a flare bubble radius of $1.9 \times 10^{9} \mathrm{~cm}=0.15$ $\mathrm{r}^{*}$. This dimension is not much larger than the $7 \times 10^{8} \mathrm{~cm}$ flux tube length predicted by the model of Rosner, Tucker and Vaiana. We conclude that we have observed an X-ray flare more like a typical moderate to strong solar flare than heretofore seen on a flare star.

20406. Antonucci, J. M.; Peckoo, R. J.; Schruhl, C.; Toth, E. E. Slowacting amine polymerization accelerators. Para-

dimethylaminobenzoic acid and its ethyl ester, J. Dent. Res. 60, No. 7, 1325-1330 (July 1981).

Key words: amine accelerators; biocompatibility; color stability; ethyl 4-dimethylaminobenzoate; mechanical strength; polymerization; slow-acting promoters; 4-dimethylaminobenzoic acid.

From theoretical considerations both paradimethylaminobenzoic acid and its ethyl ester would be expected to be slow-acting polymerization accelerators. Using these relatively slow-acting amine accelerators with a fast-polymerizing monomer system, it was possible to formulate composite materials having acceptable setting times at $37^{\circ} \mathrm{C}$ and excellent diametral tensile strengths.

20407. Brown, W. E.; Mathew, M.; Tung, M. S. Crystal chemistry of octacalcium phosphate, Prog. Cryst. Growth Charact. 4, 59.87 (1981).

Key words: amorphous calcium phosphate; biomineral; crystal growth; hydrolysis; hydroxyapatite; nonstoichiometry; octacalcium phosphate; sesquiapatite; tricalcium phosphate hydrate.

The chemical and crystallographic properties of octacalcium phosphate (OCP), $\mathrm{Ca}_{8} \mathrm{H}_{2}\left(\mathrm{PO}_{4}\right)_{6} \cdot 5 \mathrm{H}_{2} \mathrm{O}$, are reviewed. Special emphasis is placed on its structural relationship to hydroxyapatite, $\mathrm{Ca}_{5}\left(\mathrm{PO}_{4}\right)_{3} \mathrm{OH}$, and apatitic biominerals.

OCP is a well established phase in dental calculus, renal stones, and bone, and there is considerable evidence that it plays an important role as the initial phase in enamel and may participate in the crystal growth process throughout the formation of the enamel mineral. OCP provides a basis for visualizing structures of the materials referred to as "amorphous calcium phosphate," "tricalcium phosphate hydrate," and "cryptocrystalline apatite."

The formation of non-stoichiometric apatites and the incorporation of impurities are thought to be a consequence of the irreversibility of the hydrolysis of OCP to OHAp. Since three mechanisms have been proposed for the formation of OHAp, there should be three corresponding kinds of defect apatites and impure apatites. Several mechanisms involving OCP that could lead to non-stoichiometric OHAp are described.

20408. Senich, G. A. Measurements of diffusion in polymers by inverse gas chromatography, (Proc. ACS Division of Polymer Chemistry, New York, NY, Sept. 24-28, 1981), Am. Chem. Soc. Polymer Preprint 22, No. 2, 343.344 (Aug. 1981).

Key words: diffusion; diffusivity; gas chromatography; inverse gas chromatography; migration; octadecane; polyethylene; polyolefins.

The diffusivity of a volatile compound or probe in a polymer can be derived from nonequilibrium inverse gas chromatography (IGC) experiments conducted at rapid carrier gas flow rates. The van Deemter equation is commonly used to relate experimentally measured peak broadening to the probe-polymer diffusion coefficient. This relation neglects variations in the local carrier gas flow velocity and diffusive processes in the gas phase, two factors which can have a significant effect on the magnitude of the diffusivity found by IGC studies. Another significant parameter is the diffusion path length in the polymer phase. Two extremes in polymer geometry are possible within the column, a thin, uniformly distributed covering over the entire surface of the spherical column packing or annulus-like regions collected at the contact points between spheres, each with a different diffusion path length. The influence of these factors on probe-polymer diffusivity determinations is illustrated with results from an experimental IGC study of octadecane diffusion in high density polyethylene.

20409. Linsky, J. L.; Marstad, N. C. IUE spectra of F and late A stars, Proc. Second Year of IUE: The Universe in Ultraviolet Wavelengths, Greenbelt, MD, May 7-9, 1980, pp. 287-295 (NASA Goddard Space Flight Center, Greenbelt, MD, 1981).

Key words: chromospheres; stars, late-type stars; ultraviolet spectra.

We report on IUE spectra of $\alpha$ CMi (F5 IV-V), $\beta$ Cas (F2 IV), $\alpha$ Car (FO Ib), and $\gamma$ Boo (A7 III) in the context of the question as to whether chromospheres disappear in the early F. late A-portions of the HR diagram. Both $\alpha \mathrm{CMi}$ (Procyon) and $\beta$ Cas show bright emission line spectra indicative of chromospheres and transition regions, but neither a Car (Canopus) nor $\gamma$ Boo show any evidence of emission in their SWP spectra or at the Mg II lines, despite very deep exposures. These results are consistent with those recently published by Böhm-Vitense and Dettmann. We note that $\alpha \mathrm{CMi}$ has emission line fluxes roughly 6 times those of the quiet Sun, but the rapidly rotating $\delta$ Scutitype variable $\beta$ Cas has surface fluxes $10-50$ times those of the quiet Sun. Upper limits on emission line fluxes for $\alpha \mathrm{Car}$ are 4-20 times those of the quiet Sun and for $\gamma$ Boo are 15-80 times the quiet Sun. We conclude that the apparent absence of emission lines in the spectra of $a$ Car and $\gamma$ Boo should not be interpreted as 
due to the absence of nonradiatively heated outer atmospheres in stars hotter than spectral type FO, but rather to our inability to see emission lines with IUE against a background of scattered light and a bright photospheric absorption line spectrum either in low or high dispersion.

20410. Livio, M.; Shaviv, G. The stability of accretion disks to short wavelength perturbations, Astrophys. J. 244, 290-298 (Feb. 15, 1981).

Key words: accretion; stars, instabilities.

The stability of accretion disks against short wavelength perturbations is analyzed. The disk is shown to be unstable to slow thermal perturbations propagating in the z-direction, for sufficiently high values of the stress parameter $\alpha$ and sufficiently low values of the ratio of gas to total pressure. The acoustic flux from the "middle region" of the disk is estimated and discussed.

20411. Mordfin, L.; Berger, H. NDE standards for nuclear power systems: An NBS perspective, Proc. Third Int. Nondestructive Evaluation in the Nuclear Industry-1980, Salt Lake City, UT, Feb. 11-13, 1980, pp. 303-318 (American Society for Metals, Metals Park, OH, 1981).

Key words: acoustic emission; eddy currents; leak testing; mechanical signature analysis; nondestructive evaluation; nuclear pumps and valves; residual stresses; standards; ultrasonics; wear debris analysis.

The most effective approach toward achieving standardization in new NDE measurement methods, in order to enhance their reliability and reproducibility, is basically different from efforts to standardize well-established NDE measurement methods. This difference is described and illustrated by examples of recent and on-going NDE standardization activities at NBS.

Several needs for better NDE measurement capabilities in the nuclear industry are identified. These include needs for new or improved methods for measuring residual stresses and for assuring the operability of pumps and valves. Exploratory research at NBS on nuclear and high-energy $\mathrm{x}$-ray diffraction and on wear debris analysis, plus development work on polymer transducers and on leak testing standards, suggest approaches toward fulfilling these needs. The advantages of addressing the standardization requirements of a new NDE method as an integral part of the development of the measurement methodology are described.

Other NDE-related standardization needs in the nuclear industry, of a nonmethod-specific nature, are also identified. These pertain to the procedures by which defect-detection capabilities are assessed, and to the reporting of inservice inspection data.

20412. Langer, S. H.; Chanmugam, G.; Shaviv, G. Thermal instability in accretion flows onto degenerate stars, Astrophys. J. 245, L23-L26 (Apr. 1, 1981).

Key words: accretion flows; AM Her stars; cataclysmic variables; stellar atmospheres; thermal instability.

We report the discovery of a new kind of thermal instability in accretion flows onto stellar atmospheres. The instability results in periodic variations in the height of the standoff shock which forms above the stellar surface. The hard X-ray temperature and luminosity vary with this period, as does any other radiation produced in the hot gas between the shock and the surface. Conditions for the appearance of this instability are most favorable in accreting white dwarf stars, such as cataclysmic variables and AM Her stars. We suggest that a search for such variability be carried out in AM Her stars in both the hard X-ray UV-optical bands. The resulting period, which is of order 1-100 s for AM-Her stars, permits the determination of the accretion rate per unit area at the stellar surface.

20413. Bennett, L. H.; Watson, R. E. A database for enthalpies of formation of binary transition metal alloys, CALPHAD 5, No. 1, 19. 23 (1981).

Key words: alloys; database; enthalpies of formation; heats of formation; transition metal.

Experimental values of the enthalpy of formation are listed for the 61 of the 276 binary alloys formed from pairs of $\mathrm{Sc}$ to $\mathrm{Ni}, \mathrm{Y}$ to $\mathrm{Pd}$ and Lu to Pt for which data is available.

20414. Barger, R. L. Influence of second-order Doppler effect on optical Ramsey fringe profiles, Opt. Lett. 6, No. 3, 145.147 (Mar. 1981).

Key words: calcium; laser spectroscopy; optical Ramsey fringes; photon recoil; saturated absorption; second-order Doppler effect.

Resolution sufficient to resolve second-order Doppler broadening $(3.9 \mathrm{kHz})$ has been obtained for the Ca ${ }^{1} S_{0}-{ }^{3} P_{1}$ transition $(657 \mathrm{~nm})$ by using an atomic beam and optical Ramsey fringe techniques. Laserbeam separations up to $21 \mathrm{~cm}$ yield Ca linewidths (HWHM) as narrow as $1 \mathrm{kHz}$. As resolution is increased to approach, then exceed, the second-order linewidth, large asymmetries, shifts, and shape changes occur in the Ramsey fringe profile.

20415. Booker, R. L. Luminance.brightness comparisons of separated circular stimuli, J. Opt. Soc. Am. 71, No. 2, 139.144 (Feb. 1981)

Key words: brightness; color; human observers; luminance; photometry; psychophysical photometry; vision; visual signalling.

An experiment was conducted in which observers adjusted the luminance of a $2856 \mathrm{~K}$ white comparison source to appear equally as bright as a chromatic source of known luminance. Twenty different chromatic sources were used, each subtending a visual angle of $1^{\circ}$. The results show that, four sources having the same dominant wavelength, the amount of white light required to make a brightness match increases as saturation increases. Additional observations were made using four of the chromatic sources at viewing subtenses of $20^{\prime}$ and $6^{\prime}$. These results show that the amount of white light required to make a brightness match decreased as the source size decreased. The rate of decrease was most rapid for the red source.

20416. Chanmugam, G. Cyclotron emission from AM Herculis, Proc. Second Year of IUE: The Universe in Ultraviolet Wavelengths, Greenbelt, MD, May 7-9, 1980, pp. 515-519 (NASA Goddard Space Flight Center, Greenbelt, MD, 1981).

Key words: binary stars; cyclotron emission; magnetic; ultraviolet sources; white dwarfs.

The cyclotron absorption coefficients, in the ordinary and extraordinary modes, are calculated for the shock heated region of AM Her. The equations of radiative transfer are solved and the intensity of the emitted UV radiation determined as a function of angle. The average spectrum is shown to have deviations from the previously predicted Rayleigh-Jeans spectrum and the magnetic field of AM Her is deduced to be roughly $5 \times 10^{7}$ gauss.

20417. Chanmugam, G.; Dulk, G. A. Polarized radiation from hot plasmas and applications to AM Herculis binaries, Astrophys. J. 244, No. 2, 569.578 (Mar. 1, 1981).

Key words: cyclotron stars; magnetic polarization; optical; radiation theory; white dwarf stars.

Absorption coefficients for the ordinary and extraordinary modes are calculated for cyclotron radiation from hot plasmas with temperatures between 0.2 and $20 \mathrm{keV}$. The results are applied to the accretion columns of the AM Herculis binaries. The linear and circular polarization properties of the observed polarized light can be understood if the emission is at approximately the fifth harmonic of the cyclotron frequency. The magnetic field in these systems is deduced to be roughly $4 \times 10^{7}$ gauss.

20418. Stencel, R. E.; Mullan, D. J.; Basri, G. S.; Linsky, J. L. High resolution absolute flux profiles of the $\mathrm{Mg}$ II h \& $\mathbf{k}$ lines in evolved F8 to M5 stars, Proc. Second Year of IUE: The Universe in Ultraviolet Wavelengths, Greenbelt, MD, May 7-9, 1980, pp. 317-324 (NASA Goddard Space Flight Center, Greenbelt, MD, 1981).

Key words: late-type stars; stellar chromospheres; ultraviolet 
spectra.

We present the central results of a survey of the $\mathrm{Mg}$ II resonance line emission in a sample of over 50 evolved late-type stars, including spectral-luminosity types F8-M5 and Ia-IV. Observed and surface fluxes have been derived and correlations noted. The major findings include: a) $\mathrm{Mg}$ II $\mathrm{k}$ emission core asymmetry transition near Kl III, analogous to that known for $\mathrm{Ca}$ II $\mathrm{K}$; b) a small gravity and temperature dependence of the $\mathrm{Mg}$ II chromospheric radiative loss rate. These results and others are fully discussed in a report to appear in the Astrophysical Journal Supplement Series, encompassing IUE second year programs CCBDM, CMBRS and LFBSW.

20419. Madey, T. E.; Houston, J. E.; Seabury, C. W.; Rhodin, T. N. The structure of $\mathbf{N H}_{3}$ on $\mathbf{N i ( 1 1 1 ) , ~ J . ~ V a c . ~ S c i . ~ T e c h n o l . ~ 1 8 , ~ N o . ~ 2 , ~}$ 476-480 (Mar. 1981).

Key words: adsorption; ammonic; electron stimulated desorption; low energy electron diffraction; nickel; surface structure; thermal desorption.

In a recent study of the adsorption of $\mathrm{NH}_{3}$ on $\mathrm{Ni}(111)$ at $\mathrm{T} \sim 190 \mathrm{~K}$ using angle resolved UPS, it was concluded that $\mathrm{NH}_{3}$ is molecularly adsorbed, and is bonded to the surface via the $\mathrm{N}$ atom with the $\mathrm{H}$ atoms oriented away from the surface. To study the bonding configuration using a direct and independent technique, we have examined $\mathrm{NH}_{3}$ on $\mathrm{Ni}(111)$ using the electron stimulated desorption ion angular distribution (ESDIAD) method coupled with temperature programmed desorption (TPD) and low energy electron diffraction (LEED). For $\mathrm{NH}_{3}$ coverages achievable at $\mathrm{T}>150 \mathrm{~K}, \quad(\theta \leqslant 0.75$ monolayers) the ESDIAD patterns are dominated by a "halo" of ion emission with little ion yield normal to the surface; the "halo" pattern is consistent with molecular $\mathrm{NH}_{3}$ bonded to $\mathrm{Ni}$ via the $\mathrm{H}$ atom. Whereas angle-resolved UPS data indicate a specific azimuthal registry of $\mathrm{NH}_{3}$ with $\mathrm{Ni}(111)$ a well-defined azimuthal orientation is not evident from the ESDIAD results. Possible reasons for the differences between these results are examined, including final state effects in ESDIAD, and differences in sensitivity of the two methods to more than one possible configuration of the adsorbed $\mathrm{NH}_{3}$.

20420. Smid, M. E. Integrating the Data Encryption Standard into computer networks, IEEE Trans. Commun. COM-29, No. 6, 762-772 (June 1981).

Key words: cryptography; digital signatures; encryption; identifiers; key management; key notarization.

The NBS Data Encryption Standard may be integrated into computer networks to protect personal (nonshared) files, to communicate securely both on- and off-line with local and remote users, to protect against key substitution, to authenticate data, and to provide digital signatures using a nonpublic key encryption algorithm. Key notarization facilities give users the capability of exercising a set of commands for key management as well as for data encryption functions. The facilities perform notarization which, upon encryption, seals a key or password with the identities of the transmitter and intended receiver. Thus, in order to decrypt a message, the receiver must authenticate himself and supply the correct identity of the transmitter. This feature eliminates the threat of key substitution which must be protected against to attain a high level of security.

20421. Rowe, J. M.; Rush, J. J.; Pynn, R.; Heidemann, A.; Michel, K. $\mathrm{H}$. Observation of critical dynamics in $\mathrm{ND}_{4} \mathrm{Br}$ by high-resolution neutron spectroscopy, J. Chem. Phys. 74, No. 9, 5323.5324 (May 1, 1981).

Key words: critical scattering; high resolution; mean field theory; $\mathrm{ND}_{4} \mathrm{Br}$; neutron scattering; translation-rotation coupling.

The coherent quasielastic critical scattering in $\mathrm{ND}_{4} \mathrm{Br}$ has been measured by high resolution neutron scattering. The results do not agree with the predictions of simple theory.

20422. November, L. J.; Toomre, J.; Gebbie, K. B.; Simon, G. W. The detection of mesogranulation on the Sun, Astrophys. J. 245, L123. L126 (May 1, 1981).
Key words: mesogranulation; solar convection; velocities.

Time averages of velocity measurements at disk center on the quiet Sun reveal the presence of a fairly stationary pattern of cellular flow with a spatial scale of 5 to $10 \mathrm{Mm}$. Such mesogranulation has an rms vertical velocity amplitude of about $60 \mathrm{~ms}^{-1}$ superposed on the larger scale supergranular flows. The lifetimes of mesogranules appear to be at least two hours.

20423. Simon, T.; Linsky, J. L.; Schiffer, F. H. IUE spectra of a flare in HR 5110: A flaring RS CVn or ALGOL system?, Proc. Second Year of IUE: The Universe in Ultraviolet Wavelengths, Greenbelt, MD, May 7-9, 1980, pp. 435-441 (NASA Goddard Space Flight Center, Greenbelt, MD, 1981).

Key words: binary stars; stellar chromospheres; ultraviolet spectra.

U1traviolet spectra of the RS CVn-type binary system HR 5110 have been obtained with IUE on May 31, 1979 during a period of intense radio flaring of this star. High temperature transition region lines are present, but are not enhanced above observed quiescent strengths. The similarities of HR 5110 to the Algol system, AS Er, suggest that the 1979 May.June flare may involve mass exchange rather than annihilation of coronal magnetic fields.

20424. Wormsbecher, R. F.; Hessel, M. M.; Lovas, F. J. Microwave optical double resonance of $X^{1} \Sigma^{+}$of $\mathrm{NaK}, J$. Chem. Phys. 74, No. 12, 6983.6985 (June 15, 1981).

Key words: dipole moment; Dunham coefficients; heat pipe; laser spectroscopy; microwave optical double resonance; $\mathrm{NaK}$.

A microwave optical double resonance (MODR) study of the ground state, $X^{\prime} \Sigma^{+}$, of $\mathrm{NaK}$ is reported. The $v^{\prime \prime}=0 \mathrm{~J}=7 \rightarrow 8$ and $\mathrm{J}=8 \rightarrow 9$, and $v^{\prime \prime}=4 \mathrm{~J}=5 \leftarrow 6$ rotational transitions were observed as changes in the laser induced photoluminescence intensity with the $\mathrm{Ar}^{+}$laser coincidences $(12-0)$ band $\mathrm{P}(8)(4765 \AA)$ and $(7-4)$ band $\mathrm{Q}(5)$ $(4965 \AA)$, respectively of the $D^{1} \Pi-X^{1} \Sigma^{+}$system of $\mathrm{NaK}$. The improvement in sensitivity of the MODR technique over traditional microwave spectroscopy is discussed. Improved Dunham coefficients for the $X^{1} \Sigma^{+}$state are obtained by a weighted merged fit of the previous optical data with the three transitions obtained in the present work.

20425. O'Connell, J. S. Coincidence measurements with high energy electrons, (Proc. Workshop on Nuclear Physics with Real and Virtual Photons, Bologna, Italy, Nov. 25-28, 1980), Paper in From Collective States to Quarks in Nuclei, H. Arenhovel and A. M. Saruis, eds., pp. 286-295 (Springer-Verlag, Berlin, Germany, 1981).

Key words: electron beam; high energy electron scattering; nuclear reaction; nucleon.

This talk surveys reactions of the type $A\left(e, e^{\prime} x\right)$ where $x$ is a nucleon, two nucleons, pion, nucleon plus pion, or a photon. At high energy and momentum transfer, phenomena are studied that influence classical nuclear physics, but which have not yet been measured or theoretically understood. The interplay between measurement techniques, experimental feasibility and theoretical interpretability is stressed. A number of examples are given of counting rates for specific measurements with continuous duty factor electron beams with energies between 500 and $3000 \mathrm{MeV}$.

20426. West, J. B.; Codling, K.; Parr, A. C.; Ederer, D. L.; Cole, B. E.; Stockbauer, R.; Dehmer, J. L. Branching ratios and photoelectron angular distributions through the Hopfield bands in $\mathbf{N}_{2}$ between 650 and $730 \AA$ A. J. Phys. B: At. Mol. Phys. 14, 1791-1801 (1981).

Key words: angular distribution; autoionization; differential photoelectron spectra; Franck-Condon factors; nitrogen; photoelectron spectra; photoionization; synchrotron radiation; vibrational intensity distribution.

Vibrationally and angularly resolved photoelectron spectra have 
been recorded in the wavelength range $650-730 \AA$ for $N_{2}$, in the region of the autoionising resonances leading to the $\mathbf{B}^{2} \Sigma_{u}^{+}$state of the $\mathrm{N}_{2}^{+}$ion. The influence of these resonances on both the partial cross sections to the $A^{2} \Pi_{u}$ state and the $X^{2} \Sigma_{g}^{+}$state (the branching ratios) and the angular distribution of the photoemitted electrons is clearly demonstrated.

20427. Wells, J. S.; Petersen, F. R.; Maki, A. G.; Sukle, D. J. Heterodyne frequency measurements on the $11.6-\mu \mathrm{m}$ band of OCS: New frequency/wavelength calibration tables for 11.6 - and $5.8-\mu \mathrm{m}$ OCS bands, Appl. Opt. 20, No. 9, 1676-1684 (May 1, 1981).

Key words: carbonyl sulfide; heterodyne frequency measurements; high resolution spectroscopy; isotopic species of OCS; tunable diode laser; tunable laser spectroscopy.

Heterodyne frequency measurements between a ${ }^{13} \mathrm{CO}_{2}$ laser and tunable diode laser (either tuned or locked to various carbonyl sulfide absorption peaks) have been made on a number of $10^{\circ} 0-00^{\circ} 0$ band transitions in the isotopic species of carbonyl sulfide: $\mathrm{OC}^{34} \mathrm{~S}, \mathrm{O}^{13} \mathrm{CS}$, $\mathrm{OC}^{33} \mathrm{~S}$, ${ }^{18} \mathrm{OCS}$, and $\mathrm{O}^{13} \mathrm{C}^{34} \mathrm{~S}$. The ${ }^{13} \mathrm{CO}_{2}$ laser frequencies primarily resulted from either normal high-J or recently measured hot band $\left(01^{1} 1-11^{1} 0\right)_{1}$ transitions. These OCS frequency measurements have been combined with existing microwave data and new sets of molecular constants obtained. These constants will be used later to form part of a frequency calibration compendium based on OCS.

20428. Smith, E. W. Absorption and dispersion in the $\mathrm{O}_{2}$ microwave spectrum at atmospheric pressures, J. Chem. Phys. 74, No. 12, 6658 . 6673 (June 15, 1981).

Key words: absorption spectrum; dispersion spectrum; inelastic transition amplitudes; line coupling coefficients; $\mathrm{O}_{2}$ microwave spectrum; theoretical calculations.

Calculations are performed for absorption and phase dispersion at various frequencies within the $60 \mathrm{GHz}$ band of $\mathrm{O}_{2}$ for low pressures where the spectral lines are isolated, to atmospheric pressures where they merge to form a continuum band. A perturbation theory proposed by Rosenkranz was tested and found to be valid for pressures up to $100 \mathrm{kPa}$ (1 atm). The "line coupling coefficients," which describe the transfer of excitation from one radiating state to another, are also studied and various methods for evaluating these coefficients are analyzed and compared with experimental data. It is found that dispersion measurements are extremely sensitive to these coefficients and an experimental procedure for systematically measuring them is outlined; it is shown that such measurements can provide a very sensitive test for theoretical calculations of inelastic transition amplitudes.

\section{Unassigned.}

20430. Harris, J. R. Snow accumulation on and around solar collectors, Proc. 37th Eastern Snow Conf., Peterborough, Ontario, Canada, June 5-6, 1980, pp. 54-65 (Eastern Snow Conf., Atmospheric Environment Service, Downsview, Ontario, Canada, 1981).

Key words: building; load; roof; snow; snow load; solar collector; structural engineering.

Studies of the accumulation of snow and ice on buildings with flat plate solar collectors were carried out during the winter of 1979. Although the studies were generally qualitative, preliminary guidelines for snow load criteria were developed. Observations identified two significant phenomena: the sliding of snow from collector surfaces and the drifting of snow around protruding collectors. It was found that only those collectors which are relatively steep (more than $50^{\circ}$ from the horizontal) and are free of obstructions will shed snow reliably. The accumulation of snow sliding from collectors appears to be a significant load for roof structures. It was also found that collectors that protrude from roofs tend to create drifts, much as a snow fence. The common installation of several parallel rows of protruding collectors creates a situation somewhat like a "sawtooth" factory building roof and requires special attention in design of the roof structure. Further study of the problem is merited.
20431. Bender, P. L. Establishment of terrestrial reference frames by new observational techniques, (Proc. IAU Colloq. 56, Warsaw, Poland, Sept. 8-12, 1980), Paper in Reference Coordinate Systems for Earth Dynamics, E. M. Gaposchkin and B. Kolaczek, eds., pp. 23-36 (D. Reidel Publ. Co., Dordrecht, The Netherlands, 1981).

Key words: Earth rotation; geodesy; geodynamics; plate tectonics; space techniques.

The use of space techniques for establishing transcontinental and intercontinental distances is progressing very rapidly. We can think of the set of station locations used in either LAGEOS ranging of VLBI measurements as forming the vertices of a polyhedron. After correcting for tectonic plate motions using an adopted model, we expect the geometry of the polyhedron to be fairly stable over periods of the order of a year. However, after some period of time, a new set of station coordinates will be required because of improved data, unexpected station motions, etc. Methods for maintaining agreement with the previous set of station coordinates in some average sense are discussed in this paper. Some of the contributions expected from other new measurement method also are described.

20432. Franzen, D. L.; Kim, E. M. Interlaboratory measurement comparison to determine the radiation angle (N.A.) of graded-index optical fibers, Appl. Opt. 20, No. 7, 1218-1220 (Apr. 1, 1981).

Key words: numerical aperture; optical fibers; radiation angle.

A procedure for determining the radiation angle (N.A.) of graded. index optical fibers was tested by an interlaboratory measurement comparison among six fiber manufacturers and the National Bureau of Standards (NBS). Radiation angle was determined using the 5\% intensity points on an exit far-field radiation pattern. Measurements on five fibers representing low-to-high N.A. values show standard deviations of $2.9,2.4,2.0,1.5$, and $0.9 \%$. The overall average, $2.0 \%$, is close to the one standard deviation measurement precision reported by most participants.

20433. Goodman, D. A.; Cahn, J. W.; Bennett, L. H. The Centennial of the Gibbs-Konovalov rule for congruent points. Its underlying theoretical basis and its application to phase diagram evaluation, Bull. Alloy Phase Diagrams 2, No. 1, 29-34 (1981)

Key words: congruent melting; Gibbs-Konovalov rule; phase boundary; retrograde solubility; thermodynamics; Van't Hoff's equation.

One hundred years ago, Konovalov developed and tested the rule that at a congruent transformation the phase boundary reaches an extremum. An important equation for the slopes of phase boundaries, derived by Gibbs five years earlier, provides the underlying theoretical basis for the rule, which is still commonly ignored. Applications of the Gibbs-Konovalov equation to congruent transformations, retrograde solubility, phase boundary inflections, and solid-liquid equilibrium are discussed.

20434. Bossler, J. D.; Goad, C. D.; Bender, P. L. Using the global positioning system (GPS) for geodetic positioning, Bull. Geodesique 54, 553.563 (1980).

Key words: crustal movements; geodesy; geodynamics; geophysics; satellites; space techniques.

The development of relatively inexpensive satellite receivers in the early 1970's has resulted in cost-effective applications of satellites for a variety of geodetic surveying needs. Currently achievable accuracies range from 10 to 20 centimeters. The NAVSTAR Global Positioning System, now under development by the Department of Defense, incorporates advanced technology which has the potential capability of revolutionizing satellite geodesy.

Several concepts for utilizing GPS signals are briefly reviewed, and another concept, called the reconstructed carrier phase method, is described in some detail. This concept is being pursued by the Defense Mapping Agency, National Oceanic and Atmospheric 
Administration, and the U.S. Geological Survey. These agencies have numerous requirements for accurate positioning. Several prototype receivers are planned to be available for testing in mid-1982. These receivers should be highly portable, consume little power, and obtain base line accuracies of several centimeters in several hours of observation time. However, water vapor radiometers will be needed in order to achieve the full accuracy. Initial simulation results utilizing the reconstructed carrier phase method are included.

20435. Harris, J. R.; Fenves, S. J.; Wright, R. N. Logical analysis of tentative seismic provisions, J. Struc. Div. Proc. Am. Soc. Civil Eng. 107, No. ST8, 1629.1641 (Aug. 1981).

Key words: building; building codes; building standards; earthquake-resistant design; seismic design; systems analysis.

This paper describes a study of the format and expression of the Tentative Provisions for the Development of Seismic Regulations for Buildings developed by the Applied Technology Council. The methods of analysis employed provide objective measures of clarity, completeness, and consistency, and an alterative formal representation with which to examine the correctness of the provisions. The formal representation of the provisions and the findings of the analysis will assist those concerned with the future development of the provisions and their implementation within the various national standards and model codes.

20436. Strobridge, T. R.; Moulder, J. C.; Clark, A. F. Titanium combustion in turbine engines, Dept. Energy Report No. FAA-RD-79. 51, 133 pages (Available as ADA-075-657 to the U.S. public through the National Technical Information Service, Springfield, VA 22161, July 1979).

Key words: aircraft turbine engine; analytic models; combustion; compressor; correlation of experiments; dynamic combustion; ignition; jet engine; metal combustion; metal oxidation; oxidation; static combustion; thermodynamics; titanium alloys; titanium combustion.

Pure and alloyed titanium components are routinely used in aircraft turbine engines because of their uniquely high strength-to-weight ratios among structural metals, combined with excellent fatigue and corrosion resistance. Like most other metals, titanium is combustible under certain conditions of temperature, pressure and oxygen concentration and in fact there have been several instances of titanium combustion in service engines. Contemporary titanium use in aircraft turbine engines and the limits of that use are explored. Combustion incidents and their causes are discussed as well as the typical extent of damage. Current preventative measures are outlined. For better understanding, the fundamentals of metal combustion and the experimental research related to titanium combustion are thoroughly treated and analytical combustion models are presented. Finally, the relevant experimental combustion data are correlated as a function of static temperature and Reynolds number and compared to the model predictions.

20437. Phillips, J. C.; Peterlin, A.; Waters, P. F. Apparatus for infrared measurement of sorption/desorption in strained polymeric films, J. Polym. Sci. 19, 789.802 (1981).

Key words: apparatus; desorption; infrared absorption; mechanical properties; organic vapors; polyethylene; sorption; strained polymer film; transport.

A new apparatus has been developed for optical measurement of sorption/desorption in transparent polymer films at a given strain or stress. The technique utilizes a chosen infrared absorption frequency of the diffusing vapor in a spectral region where the film has negligible absorption. From the time dependence of the IR absorption at this frequency the sorption/desorption behavior of the film may be determined at any strain or stress. The simultaneous measurement of mechanical relaxation as a function of the amount of sorbed vapor is also possible. The results presented here show the applicability of the apparatus for determining the transport and mechanical properties of a low-density polyethylene film in ethyl acetate vapor at $30^{\circ} \mathrm{C}$

20438. Laufer, A. H. Kinetics of gas phase reactions of methylene, Rev.
Chem. Intermed. 4, 225-257 (1981).

Key words: abstraction; activation energies; addition; insertion; methylene; rate constants.

Kinetic parameters of methylene radicals in reaction with organic and inorganic substrates are reviewed. Preferred values for the parameters are discussed.

20439. Clark, F. O.; Johnson, D. R.; Troland, T. H.; Heiles, C. E. Polarized emission in the broad SiO feature from R Leo, (Proc. Int. Astronomical Union Symp. 87, Mont. Trenblant, Quebec, Canada, Aug. 6-10, 1979), Paper in Interstellar Molecules, B. H. Andrew, ed., pp. 543-544 (D. Reidel Publ. Co., Dordrecht, The Netherlands, 1980).

Key words: polarized emission; polarized SiO emission; R Leo; radiative transfer theory; $\mathrm{SiO}$ maser.

Linearly polarized $\mathrm{SiO}$ emission spread over $12 \mathrm{~km} / \mathrm{s}$ has been detected from the star $\mathrm{R}$ Leo. The position angle of polarized emission varies systematically with respect to the spectral line center. Interpreted in terms of radiative transfer theory, this change in position angle may be due to magnetorotation, which allows the determination of the magnetic field $\left(9 \times 10^{-3} / \cos \theta\right.$ Gauss), and the $\mathrm{SiO}$ systemic velocity $(-1 \pm 2 \mathrm{~km} / \mathrm{s})$.

20440. Metz, F. E. Two examples of passive solar retrofit for historic structures, (Proc. 5th Natl. Passive Solar Conf., Amherst, MA, Oct. 19-26, 1980), Extended Abstract in Passive Solar 1980, J. Hayes and R. Snyder, eds., 5.2, 1305.1306 (1980)

Key words: historic; passive solar; retrofit; solar greenhouse; Trombe wall.

Passive application for existing buildings is a multi-faceted challenge. The retrofit of structures in historic districts compounds this challenge. In spite of the many problems, the greatest potential for passive/hybrid applications may be within the vast abundance of our existing buildings.

20441. Pence, W. H.; Leone, S. R. Laser studies of electronic excitation transfer in atomic calcium: $\mathrm{Ca}\left(4 s 5 p{ }^{1} P_{1}\right)+\mathrm{Ar} \rightleftharpoons \mathrm{Ca}(4 s 5 p$ $\left.{ }^{3} P_{2,1,0}\right)+$ Ar, J. Chem. Phys. 74, No. 10, 5707.5717 (May 15, 1981).

Key words: calcium; collisional relaxation; energy transfer; laser; lifetimes; spin changes.

Time-resolved nsec kinetic methods are used to measure state specific electronic excitation transfer for the near-resonant process. In stimulated emission experiments it is found that the $\mathrm{Ca}\left(4 s s p{ }^{3} P_{2}\right)$ level is preferentially populated. The rate constant $k_{1}$ for the specific ${ }^{3} P_{2}$ channel is $5.2 \pm 0.5 \times 10^{-11} \mathrm{~cm}^{3}$ molecule $1 \mathrm{sec}^{-1}$. These results are discussed in terms of near-resonant spin changing collision mechanisms. Radiative lifetimes for $\mathrm{Ca}\left(4 s 5 p{ }^{1} P_{1}\right)$ and $\mathrm{Ca}\left(4 s 5 p^{3} P_{1}\right)$ are also determined to be $77 \pm 2 \mathrm{nsec}$ and $67 \pm 2 \mathrm{nsec}$, respectively.

20442. Grant, W. H.; Dehl, R. E. The adsorption of human serum albumin and $\gamma$-globulin on hydrophobic and hydrophilic surfaces, (Proc. Joint ACS \& CSJ Cong. Adhesion and Adsorption of Polymers, Honolulu, HI, Apr. 2-6, 1979), Paper in Adhesion and Adsorption of Polymers, L. H. Lee, ed., Pt. B, 827-835 (Plenum Publ. Corp., New York, NY, 1980).

Key words: desorption; gamma-globulin; human serum albumin (HSA); polyethylene; protein adsorption.

When a foreign surface is placed in contact with the blood there is little doubt that the initial event is the adsorption of plasma proteins onto the surface. The nature of the adsorbed protein, the absolute and relative amounts, the number and types of binding sites and the spatial distribution of the protein across the surface potentially affect the later stages of the interaction of the formed elements with the foreign surface. Since the initially deposited protein layer serves as the substrate for deposition of platelets, the other blood elements which can lead to formation of a thrombus, it seems likely that the detailed characteristics of the protein layer may determine the mechanism of 
platelets adhesion and aggregation. As an approach to the development of an understanding of this mechanism we are investigating the adsorption and desorption of an isolated blood protein on hydrophobic surfaces, since some of these surfaces have been accepted in relation to the amount of thrombus formation as good biomaterials and others as poor biomaterials. Thus, it has been shown that there is essentially no appreciable difference in the total adsorbance of human serum albumin (HSA) on a good or poor biomaterial but there is a tremendous difference in the amount of HSA retained on these surfaces after several days of prolonged desorption.

20443. DeVries, K. L.; Smith, R. H.; Fanconi, B. M. Free radicals and new end groups resulting from chain scission: 1, $\gamma$-irradiation of polyethylene, Polymer 21, 949.956 (Aug. 1980).

Key words: bond rupture; electron spin resonance; free radicals; infrared spectroscopy; irradiation; polyethylene.

Measurements of the concentrations of free radicals by electron spin resonance (ESR) and of new chemical species by Fourier transform infrared (FTIR) were carried out on polyethylene specimens exposed to $\gamma$-irradiation at dosages from 3 to 50 Mrads both in the presence and absence of oxygen. The improved signal-tonoise capability of the FTIR method permitted a direct comparison of the free radical concentration and the resultant concentration of new chemical groups. It was found that approximately 10 carbonyl groups and 2 carbon-carbon double bonds were formed per free radical. These results are comparable with previous estimates and form the basis for an investigation of chemical species formed during mechanical deformation and fracture which will be discussed in the second paper of this series.

20444. Velapoldi, R. A. Color test reagent/kits for preliminary identification of drugs of abuse, NIJ Standard-0605.00, 13 pages (Available by purchase as SN027-000-01116-1 from the Superintendent of Documents, U.S. Government Printing Office, Washington, DC 20402, July 1981).

Key words: chemical spot tests; color test reagents; color tests; drugs of abuse; field test kits; sensitivity; specificity.

This document is a performance standard for color test reagents and field kits used for preliminary tests to establish presumptive evidence of drugs of abuse for later verification in a forensic laboratory. Performance requirements are given for such attributes as specificity, sensitivity, color development, and labeling.

20445. Kelley, E. F.; Hebner, R. E., Jr. Electrical breakdown in composite insulating systems: Liquid-solid interface parallel to the field, IEEE Trans. Elec. Insul. EI-6, No. 4, 297.303 (1981).

Key words: electrical breakdown; electrical insulation; interface; paper; prebreakdown; transformer oil.

The location of electrical breakdown in a composite insulating system has been measured. In these experiments, a paper sample was mounted so that it connected the two electrodes. Electrode structures ranging from plane-plane to sphere-sphere were used. The electrodepaper system was tested in oil to determine the properties of an oil paper interface. The data indicated that, in a carefully prepared system, the breakdown will not necessarily occur at the interface. In addition, it was found that breakdown voltages were not significantly lower for those breakdowns which occurred at the interface than for those which did not. It was noted that if the paper were not dried or if many gaseous voids were left in or on the paper, the breakdown regularly occurred at the interface and at a lower voltage.

20446. Kaplan, A. E.; Weiss, E. R.; Byrne, S. T.; El-Torkey, N. M.; Margolis, S. A. Purified reduced nicotinamide adenine dinucleotide: Responses to lactate dehydrogenase isozymes from three cell sources, Science 212, 553-555 (May 1, 1981).

Key words: hepatoma; inhibitors; isozymes; kinetics; lactate dehydrogenase (LDH); nicotinamide adenine dinucleotide (NADH).
Lactate dehydrogenase (LDH, E.C. 1.1.1.27) isozymes from three single-cell sources reacted differently with reduced nicotinamide adenine dinucleotide (NADH) purified to published chromatographic and spectrophotometric specification and free of inhibitors of $\mathrm{LDH}$, when compared with a commercial preparation of NADH. The activity of LDH.1, purified from rabbit erythrocytes, increased the most with inhibitor-free NADH; the next most stimulated were the LDH isozymes from a control hepatocyte line; but hardly responsive at all were the same isozymes from chemically transformed cells. Thus isozyme composition alone did not account for the range of responses to purified NADH. The commercial preparation of NADH used in these studies contains the Strandjörd-Clayson inhibitors, the most potent group identified in NADH preparations relative to LDH activity. The results suggest that specific molecular differences in individual isozymes contribute to the differential response to the Strandjörd-Clayson inhibitors.

20447. Koffend, J. B.; Leone, S. R. Tunable laser photodissociation: Quantum yield of $I^{*}\left({ }^{2} \mathbf{P}_{1 / 2}\right)$ from $\mathrm{CH}_{2} \mathrm{I}_{2}$, Chem. Phys. Lett. 81, No. 1, 136-I4I (July 1981).

Key words: $\quad \mathrm{CH}_{2} \mathrm{I}_{2} ; \quad \mathbf{I}\left({ }^{2} \mathrm{P}_{1 / 2}\right) ;$ laser; methylene iodide; photodissociation; quantum yield.

Tunable laser $I^{*}\left({ }^{2} P_{1 / 2}\right)$ quantum yield measurements are presented for $\mathrm{CH}_{2} \mathrm{I}_{2}$ in the wavelength range $248-340 \mathrm{~nm}$. The results suggest that a curve crossing mechanism is operative in the dissociation.

20448. Coriell, S. R.; Boisvert, R. F.; Rehm, R. G.; Sekerka, R. F. Lateral solute segregation during unidirectional solidification of a binary alloy with a curved solid-liquid interface. II. Large departures from planarity, J. Cryst. Growth 54, No. 2, 167.175 (Aug. 1981).

Key words: binary alloy; diffusion; interface; segregation; solidification; solid-liquid.

The lateral solute segregation that results from a curved solid-liquid interface during steady state unidirectional solidification of a dilute binary alloy was previously calculated by perturbation theory for small departures of the interface shape from planarity. These calculations have been extended by other analytical and by numerical methods to the case where there are large departures of the interface from planarity. Numerical results are given for a sinusoidally shaped interface for various values of the distribution coefficient, $k$, and the parameter $\beta=V L / D$ where $V$ is the velocity of solidification, $L$ is the wavelength of the sinusoidal perturbation, and $D$ is the diffusivity of solute in the liquid. For both very large and very small values of $\beta$, the segregation is small and proportional to the amplitude of the sinusoidally shaped interface. For intermediate values of $\beta$, dependent on $\mathbf{k}$, the segregation is maximum and no longer proportional to the interface amplitude; the segregation profiles along the interface are distorted such as to be flat near projections of solid into the liquid and rapidly varying near projections of liquid into the solid.

20449. Wyart, J. F.; Reader, J.; Ryabtsev, A. 3d-4p transitions in the zinclike and copperlike ions $\mathrm{Y} \mathrm{X}, \mathrm{XI} ; \mathrm{Zr} \mathrm{XI}, \mathrm{XII}$; $\mathrm{Nb} \mathrm{XII}, \mathrm{XIII}$; and $\mathrm{Mo}$ XIII, XIV, J. Opt. Soc. Am. 71, No. 6, 692.698 (June 1981).

Key words: ionized; molybdenum; niobium; spectra; ultraviolet; vacuum; yttrium.

Lines occurring as satellites on the long-wavelength side of the $3 d^{10}-3 d^{9} 4 p$ resonance lines of Ni-like ions have been investigated with a low-inductance vacuum spark and a $10.7-\mathrm{m}$ spectrograph for the elements $\mathrm{Y}, \mathrm{Zr}, \mathrm{Nb}$, and $\mathrm{Mo}$. The lines are interpreted as $3 d^{10} 4 s-3 d^{9} 4 s 4 p$ and $3 d^{10} 4 p-3 d^{9} 4 p^{2}$ transitions in the Cu-like ions $\mathrm{Y} \times \mathrm{XI}$, $\mathrm{Zr}$ XII, Nb XIII, and Mo XIV and $3 d^{10} 4 s^{2}-3 d^{9} 4 s^{2} 4 p$ transitions in the $\mathrm{Zn}$ like ions $\mathrm{Y} \mathrm{X}, \mathrm{Zr} \mathrm{XI}, \mathrm{Nb}$ XII, and Mo XIII. The spectra of the Cu-like ions were interpreted by generalized least-squares fits for the energy levels of the sequence of four ions. Thirty-nine levels of $3 d^{9} 4 s 4 p$ were interpreted simultaneously with a root-mean-square deviation of 122 $\mathrm{cm}^{-1}$; forty-four levels of $3 d^{9} 4 p^{2}$ were interpreted in the same way with a root-mean-square deviation of $200 \mathrm{~cm}^{-1}$. Line identifications and energy levels were obtained for the $3 d^{10} 7 p$ configuration of the 
20450. Motz, J. W.; Dick, C. E.; Danos, M. Exposure limits imposed by screen-film systems on the transfer of image information, (Proc. Applications of Optical Instrumentation in Medicine IX, San Francisco, CA, Mar. 22.24, 1981), SPIE 273, 52.56 (The International Society for Optical Engineering, Bellingham, WA, 1981).

Key words: exposure limits for x-ray detectors; information detection efficiencies; information transfer; screen film signal-to. noise ratio; screen film systems; $x$-ray film; x-ray fluorescent screens.

Information transfer in a screen.film system occurs over a limited region of $x$-ray exposures because of the non-linear response of film to light emitted from the $x$-ray fluorescent screen. Based on screen light emission and film response data, the information transfer efficiency of a typical screen-film system is determined as a function of $x$-ray exposure for different $x$-ray energies. The method of determining this transfer efficiency is described, and the x-ray exposure regions over which information transfer can occur with this system is delineated for different $x$-ray energies.

20451. Decher, R.; Randall, J. L.; Bender, P. L.; Faller, J. E. Design aspects of a laser gravitational wave detector in space, (Proc. Active Optical Devices and Applications, Washington, DC, Apr. 10-11, 1981), SPIE 228, 149.153 (The International Society for Optical Engineering, Bellingham, WA, 1980).

Key words: astronomy; gravitational waves; gravity; laser interferometer; laser stability; space physics.

Some of the optical and mechanical aspects of a proposed laser gravitational wave antenna in space are discussed briefly. The proposed concept consists of a free-mass antenna with the test masses separated by $10^{6} \mathrm{~km}$. A laser heterodyne technique is used to measure the distance change between test masses resulting from gravitational wave interaction. The proposed scheme appears to offer the necessary sensitivity to detect gravitational radiation from binary stars predicted by General Relativity Theory.

20452. Lafferty, W. J.; Sattler, J. P.; Worchesky, T. L.; Ritter, K. J. Diode laser heterodyne spectroscopy on the $v_{4}$ and $\nu_{9}$ bands of 1,1 . difluoroethylene, J. Mol. Spectros. 87, $416-428$ (1981).

Key words: heterodyne measurements; infrared spectrum; molecular constants; spectral assignments; submillimeter laser transition assignments; 1,1-difluoroethylene.

Portions of the $v_{4}$ and $\nu_{9}$ bands of 1,1-difluoroethylene have been studied with a diode laser spectrometer. Line measurements were made by using markers produced by beating the output of the diode laser with the radiation from a $\mathrm{CO}_{2}$ laser. With a dual trace oscilloscope presentation, frequencies of infrared lines could be rapidly determined with a precision of better than $\pm 6 \mathrm{MHz}$. Ground state constants were derived by combining microwave data with 10 combination differences obtained in this work. Upper state constants were obtained for both bands by combining the infrared measurements of this work with excited state microwave data and heterodyne measured submillimeter laser frequencies.

20453. Scheeline, A.; Norris, J. A.; Travis, J. C.; DeVoe, J. R.; Walters, J. P. Particulates formed by a stabilized high voltage spark discharge, Spectrochim. Acta 36B, No. 4, 373.383 (1981).

Key words: discharge; emission spectroscopy; light scattering; particulates; spark; spectrochemical analysis.

Light scattering was used to demonstrate the presence of small $(\leqslant 1$ $\mu \mathrm{m})$ particles in the immediate surroundings of a stable spark discharge. Several parameters, including electrode composition and surface condition, which are of importance in emission spectrochemical analysis, were found to be of importance in affecting the scattering signal. Electron micrographs of the heaviest particles revealed two distinct types of particles. Analytical implications are
20454. Travis, J. C.; DeVoe, J. R. The optogalvanic effect, Chapter 5 in Lasers in Chemical Analysis, G. M. Hieftje, J. C. Travis, and F. E. Lytle, eds., pp. 93-124 (Humana Press, Clifton, NJ, 1981).

Key words: atomic spectroscopy; flame spectroscopy; hollow cathodes; laser enhanced ionization; optogalvanic effect.

This is a review article on the Optogalvanic Effect (OGE) which provides the reader with the necessary historical perspective to understand the basis of the technique. The OGE is compared with other methods which use lasers to produce selective ionization of atoms and molecules. The review concludes with an up-to-date description of laser enhanced ionization in flames including the mechanism, sources of error, and potential of this new quantitative analytical technique.

20455. Watson, R. E.; Bennett, L. H. Optimized prediction for heats of formation of transition metal alloys, CALPHAD 5, No. 1, 25.40 (1981).

Key words: alloys; band theory; d electrons; enthalpy of formation; heat of formation; optimized predictions; transition metals.

A simple electron band theory model of transition metal alloys is used to predict the heat of formation for 276 transitions metal alloys at equiatomic composition. The model employs a rectangular d-band electron density of states. Some of the input parameters, namely bandwidth, Fermi level position, and number of electrons in the band, are allowed to vary within certain constraints, to closely approximate any known value of the heat of formation. The resulting predictions are considered to have errors of the same order as the experiments.

20456. Duvall, K. C.; Wasson, O. A. The NBS $14 \mathrm{MeV}$ absolute neutron beam facility, (Proc. 1980 Conf. Application of Accelerators in Research and Industry, North Texas State Univ., Denton, TX, Nov. 3.5, 1980), IEEE Trans. Nucl. Sci. NS-28, No. 2, 1488-1489 (Apr. 1981).

Key words: associated particle; calibration; detection; facilities; fluence; monitor; sources; standardization; $14 \mathrm{MeV}$ neutrons.

A $14 \mathrm{MeV}$ absolute neutron beam has been established at the NBS 3 MV positive-ion Van de Graaff Accelerator Laboratory. The $T(d, n) \alpha$ reaction is used for the production of the $14.1 \mathrm{MeV}$ neutron flux with a source strength of $10^{8} \mathrm{n} / \mathrm{sec}$. The neutron flux is absolutely determined by the measurement of the associated alpha particle rate in a silicon surface barrier detector positioned at 84 degrees with respect to the beam axis. The facility may be used to measure the absolute response of active neutron monitoring devices with high accuracy. The neutron background contributions may be eliminated in the calibration measurements by utilizing the coincidence between the device and the solid state alpha detector. This method allows a neutron flux in a cone of 2 degree half angle to be placed at 90 degrees with respect to the beam axis.

20457. Bowman, C. D. Efficient neutron production using $12 \mathrm{MeV}$ electrons, (Proc. 1980 Conf. Application of Accelerators in Research and Industry, North Texas State Univ., Denton, TX, Nov. 3.5, 1980), IEEE Trans. Nucl. Sci. NS.28, No. 2, 1485-1487 (Apr. 1981).

Key words: accelerator; beryllium; bremsstrahlung; electrons; linac; neutrons; photonuclear; tungsten.

A comparison of $(\gamma, n)$ and atomic cross sections shows that neutron production with an electron beam can be as energy efficient with 12 . $\mathrm{MeV}$ electrons as with the conventionally used 30 to $100-\mathrm{MeV}$ electrons. Neutron production from tungsten using $100-\mathrm{MeV}$ electrons is compared with a thin tungsten converter followed by a deuteriumcontaining target using electrons near $12 \mathrm{MeV}$. For a $\mathrm{BeD}_{2}$ target a yield of $2 \times 10^{12}$ neutrons per kilojoule of beam power is predicted.

20458. Schrack, R. A.; Behrens, J. W.; Johnson, R.; Bowman, C. D. 
Resonance neutron radiography using an electron Linac, (Proc. 1980 Conf. Application of Accelerators in Research and Industry, North Texas State Univ., Denton, TX, Nov. 3-5, 1980), IEEE Trans. Nucl. Sci. NS-28, No. 2, 1640-1643 (Apr. 1981).

Key words: assay; imaging; neutron; nonlinear least squares; position-sensitive detectors; radiography; resonance.

The NBS electron LINAC is being used to implement a resonance neutron radiography system. Position-sensitive proportional counters indicate location and time of arrival of neutrons transmitted through the material being radiographed, this information is stored in an online computer system in a million word storage array for later analysis. Neutron transmission data is collected for a region from about 3 to $12 \mathrm{eV}$ neutron energy. Measurements have been made using silver, tungsten, gold, and the uranium isotopes. Onedimensional position-sensitive detectors having resolutions of $5 \mathrm{~mm}$ and $1 \mathrm{~mm}$ have been used to image objects from $1 \mathrm{~cm}$ to $13 \mathrm{~cm}$ in diameter. Two-dimensional position-sensitive detectors are now being developed to provide more rapid and accurate data accumulation. A 5 $\mathrm{cm} \times 5 \mathrm{~cm}$ folded wire proportional counter has been designed and built in collaboration with Oak Ridge National Laboratories. Measurements indicate a resolution on the order of $1 \mathrm{~mm}$. A detector using a multichannel plate image amplifier is being built to use a $1 \mathrm{~mm}$ thick, $25 \mathrm{~mm}$ diameter lithium glass disk for neutron detection. The ability of resonance neutron radiography to provide both assay and image of a specific isotope in a sample provides great advantages over conventional NDA and imaging systems. Applications to safeguarding of nuclear materials and industry will be demonstrated.

20459. Boisvert, R. F. High order compact difference formulas for elliptic problems with mixed boundary conditions, (Proc. Fourth Int. Symp. Computer Methods for Partial Differential Equations, Bethlehem, PA, June 30-July 2, 1981), Paper in Advances in Computer for Partial Differential Equations, R. Vichenevetsky and R. Stepleman, eds., IV, 193.199 (IMACS, Rutgers Univ., Dept. of Computer Science, New Brunswick, NJ, 1981)

Key words: elliptic boundary value problems; finite difference methods; high order accuracy; HODIE method; mixed boundary conditions; nonrectangular domains; partial differential equations; Poisson equation.

In this paper I describe how compact finite difference discretizations of the HODIE type may be derived for elliptic problems with mixed boundary conditions. Explicit formulas are given for the case of the Helmholtz equation on a rectangle and a proof yielding $\mathrm{O}\left(\mathrm{h}^{4}\right)$ convergence for this problem is outlined. In a second example compact difference formulas for the Poisson equation with normal derivative boundary conditions are presented for a class of non-rectangular domains with boundaries made up of straight lines. Numerical examples are also given.

20460. Jacox, M. E. The reaction of $F$ atoms with $\mathrm{C}_{2} \mathrm{H}_{4}$. Vibrational spectrum of the $\mathrm{C}_{2} \mathrm{H}_{4} \mathrm{~F}$ intermediate trapped in solid argon, Chem. Phys. 58, 289-302 (1981).

Key words: ethylene; F-atom reaction; infrared spectrum; matrix isolation; photodecomposition; tunneling; 2-fluoroethyl.

When the products of the reaction between $\mathrm{F}$ atoms produced in a microwave discharge and $\mathrm{C}_{2} \mathrm{H}_{4}$ are frozen in a large excess of argon at $14 \mathrm{~K}$, new infrared absorptions appear which can be assigned to the 2-fluoroethyl radical. Studies of the dependence of the product distribution on the $F$-atom concentration have confirmed that the stabilization of $\mathrm{C}_{2} \mathrm{H}_{4} \mathrm{~F}_{2}$ plays only a minor role under the sampling conditions typical of these experiments. Isotopic substitution experiments have demonstrated that the steric configuration about the $\mathrm{C}=\mathrm{C}$ bond is randomized as a result of the $\mathrm{F}$-atom reaction. Upon irradiation of the sample with the full light of a medium-pressure mercury arc, absorptions of vinyl fluoride and acetylene and of the acetylene-HF complex grow in intensity, while those of $\mathrm{FCD}_{2} \mathrm{CH}_{2}$ and of $\mathrm{FCD}_{2} \mathrm{CD}_{2}$ diminish in intensity and those of $\mathrm{FCH}_{2} \mathrm{CH}_{2}$ and of $\mathrm{FCH}_{2} \mathrm{CD}_{2}$ are unchanged. The $\mathrm{F}$-atom reactions and photolysis processes which occur in these experiments are discussed, and a tunneling mechanism is proposed to explain the isotopic selectivity in the 2-fluoroethyl photodecomposition. The vibrational spectrum of $\mathrm{FCH}_{2} \mathrm{CH}_{2}$ is compared with that derived in a recent $a b$ initio calculation.

20461. Sweger, D. M.; Sams, R. L. Diode laser spectra of the $v_{2}$ band of $\mathrm{H}_{2}{ }^{12} \mathrm{CO}$ and $\mathrm{H}_{2}{ }^{13} \mathrm{CO}, J$. Mol. Spectros. 87, 18-28 (1981).

Key words: diode laser; high resolution; $\mathrm{H}_{2}{ }^{13} \mathrm{CO}$; infrared; spectra; $v_{2}$.

The Q-branches of the $\nu_{2}$ (CO stretch) band of $\mathrm{H}_{2}{ }^{12} \mathrm{CO}$ and $\mathrm{H}_{2}{ }^{13} \mathrm{CO}$ have been studied in high resolution using an infrared diode laser. Accurate excited state constants, including A, B, C, $\Delta_{\mathrm{J}}, \Delta_{\mathrm{JK}}, \Delta_{\mathrm{K}}$, and $\mathrm{H}_{\mathrm{K}}$, were determined from an analysis of the data for 97 lines for $\mathrm{H}_{2}^{12} \mathrm{CO}$ and 79 lines for $\mathrm{H}_{2}{ }^{13} \mathrm{CO}$ and compared to previous reported values. Band centers were also determined and reported as 1746.0090 $\mathrm{cm}^{-1}$ for $\mathrm{H}_{2}{ }^{12} \mathrm{CO}$ and $1707.9814 \mathrm{~cm}^{-1}$ for $\mathrm{H}_{2}{ }^{13} \mathrm{CO}$

20462. Scheeline, A.; Travis, J. C.; DeVoe, J. R.; Walters, J. P. Discovery of an electrical post-pulse in the surroundings of a high voltage spark discharge, Spectrochim. Acta 36, No. 3, 153-161 (1981).

Key words: afterglow; discharge; electrostatic probe; emission spectroscopy; laser enhanced ionization; opto-galvanic effect; spark; spectrochemical analysis.

In the vicinity of a high voltage spark, an electrical pulse of negative polarity was observed, using a floating electrode. The pulse's behavior with respect to spark cathode composition, gas flow, spatial distribution, and related parameters was characterized. Suggestions as to the origin of the pulse are made. Attempts at observing laser enhanced ionization in the post-discharge time period are described.

20463. Rossiter, W. J., Jr.; Weidt, J. L.; Saxler, R. J. A field survey of the performance properties of insulation used to retrofit cavity walls of residences, (Proc. ASHRAE/DOE.ORNL Conf. Thermal Performance of the Exterior Envelopes of Buildings, Kissimmee, FL, Dec. 3-5, 1979), ASHRAE SP 28, pp. $901-915$ (ASHRAE, 345 E. 47th St., New York, NY, 1981).

Key words: conservation; energy; field survey; insulation; moisture content; residences; retrofit; thermal resistance.

A study was conducted to obtain information on the performance of retrofit insulations which had been installed in the sidewalls of existing residences. Most of the thirty-nine houses included in the study had wood-frame sidewall construction and were located in the mid-West, mid-Atlantic and Northeast.

The insulations represented those commonly used in the United States to retrofit side-walls of housing: urea-formaldehyde based foam, loose-fill cellulose, and loose-fill mineral fiber. With few exceptions, the insulations had been installed in the sidewalls at least 2 years prior to their examination and their ages ranged from about one and a half to ten years.

In the field phase of the study, small sections of sidewalls were opened in the late fall or early winter and the retrofit insulations were examined. Observations were made on performance factors such as: the completeness of filling the cavity, the condition of the insulation and wall components, and evidence of moisture accumulation such as water stains on sheathing, studs and other wall components.

Shrinkages of urea-formaldehyde based foams, and voids within loose-fill insulations were measured. Shrinkage was observed to have occurred for all urea-formaldehyde based foam specimens. For those seventeen cases in which the foam was not excessively cracked and the linear shrinkage was measurable, it was found to be within a range of 4 to 9 percent. For the six test houses containing loose-fill insulation which were opened at the top of the wall cavity, only one with cellulose contained a void at that location. It could not be determined whether the void was attributable to settling of the insulation or initial incomplete fill of the cavity.

In the laboratory phase of the study, insulation specimens removed from the walls of the residences were tested to determine their density, thermal resistivity and moisture content. In addition, the $\mathrm{pH}$ of the urea-formaldehyde based foam specimens was determined. Results of the laboratory measurements are discussed and compared with data and information obtained from other studies. Results 
indicated that the retrofitting of the inspected side-walls was for the most part accomplished without adverse effect upon them.

20464. Rendell, R. W.; Girvin, S. M. Hall voltage dependence on inversion-layer geometry in the quantum Hall-effect regime, Phys. Rev. $B$ 23, No. 12, 6610-6614 (June 15, 1981).

Key words: Hall voltage; inversion-layer; MOSFET; resistancestandard.

A calculation of the Hall voltage is presented within a model of a finite two-dimensional inversion layer. An explicit form for the electric field is obtained and this is found to have a power-law singularity in the corners of the inversion layer. This singularity is most pronounced in the quantum Hall-effect regime where the Hall angle approaches $\pi / 2$. The error in measuring the Hall voltage in this regime due to the shorting effect of the source and drain is calculated. This is found to be negligible at the level required for a new determination of the fine-structure constant and development of a new resistance standard using inversion-layer measurements in the quantum Hall-effect regime. Limitations of the model and other possible sources of error are briefly discussed.

20465. Fujii, G.; Ranney, M. A.; Clark, A. F. Thermal expansion of multifilamentary $\mathrm{NB}_{3} \mathrm{Sn}$ and $\mathrm{V}_{3} \mathrm{Ga}$ superconductive cables and fiberglass-epoxy and cotton-phenolic composite materials, Jpn. J. Appl. Phys. 20, No. 4, L267.L270 (Apr. 1981).

Key words: composite; fiberglass-epoxy; low temperature; $\mathrm{Nb}_{3} \mathrm{Sn}$; phenolic; superconductor; thermal expansion; $\mathrm{V}_{3} \mathrm{Ga}$.

The thermal contraction of multifilamentary $\mathrm{Nb}_{3} \mathrm{Sn}$ and $\mathrm{V}_{3} \mathrm{Ga}$ and the composite materials, fiberglass-epoxy and cotton-phenolic, was measured from robm temperature to $4 \mathrm{~K}$. Both the thermal contraction and the thermal expansion coefficient were tabulated as a function of temperature. The thermal contraction to $4 \mathrm{~K}$ of $\mathrm{V}_{3} \mathrm{Ga}$ was slightly greater than that of $\mathrm{Nb}_{3} \mathrm{Sn}$. For the composites, thermal contraction to $4 \mathrm{~K}$ was 2.8 times higher transverse to the fibers than parallel to them.

20466. Smyth, K. C.; Mallard, W. G. Laser-induced ionization and mobility measurements of very small particles in premixed flames at the sooting limit, Combust. Sci. Technol. 26, 35.41 (1981).

Key words: ionization; mobility; multiphoton; opto-galvanic spectroscopy; premixed flames; soot.

Laser-induced ionization signals are observed in a premixed $\mathrm{C}_{2} \mathrm{H}_{2}$ /air flame at the sooting limit and are attributed to thermoionization of very small particles. The mobilities of these species are derived from ion velocity measurements and compared with the mobility of $\mathrm{Na}^{+}$for identical flame conditions. From a series of measured mobilities estimates are made for the particle mass (2300-6100 amu) and diameter (1.8-2.2 nm). This experimental method is thus a new optical means for probing the early stages of soot formation.

20467. Popp, R. K.; Frantz, J. D.; Vogel, G. L. An electrode technique for measurement of chloride concentration in microsamples, $\mathrm{Am}$. Mineral. 65, 393-395 (1980).

Key words: chloride; electrode; microliter; rapid.

A simple device is described for adapting a chloride solid-state electrode for microliter volumes. The device, which consists of a nylon cylinder in which 4 holes are drilled, is waxed onto the surface of an inverted chloride electrode. Samples of 1-5 $\mu 1$ are deposited in the holes of the adapter and a $\mathrm{KNO}_{3}$-agar filled glass capillary is used to make contact between the samples and a reference electrode.

20468. Turk, G. C. Reduction of matrix ionization interference in laser-enhanced ionization spectrometry, Anal. Chem. 53, 1187-1190 (1981)

Key words: flame spectroscopy; ionization interference; laser enhanced ionization; laser spectroscopy; opto-galvanic spectroscopy; trace metal analysis.
Despite many attractive features, Laser Enhanced Ionization (LEI) Spectrometry has been difficult to apply to the analysis of many complex samples because of a susceptibility to severe ionization interference. The interference is a consequence of changes in the distribution of the electric field in the flame which occur when the concentration of charged species is increased by the unassisted thermal ionization of matrix elements. The effect of these changes can be minimized by aligning the laser beam as close to the surface of the cathode as possible. However, previously used LEI electrode configurations have utilized cathodes external to the flame, thus precluding the possibility of laser alignment near the cathode surface. A new water-cooled cathode has been designed which can be used directly inside the flame, very close to the laser beam. Tolerance of LEI signal collection to a matrix of sodium in an air-acetylene flame has been improved from less than $300 \mu \mathrm{g} / \mathrm{mL}$ to over $3000 \mu \mathrm{g} / \mathrm{mL}$.

20469. Rhyne, J. J. Magnetic materials, Article in Encyclopedia of Physics, pp. 547.550 (Addison-Wesley Publ. Co., Inc., Reading, MA, 1981).

Key words: amorphous magnetism; anisotropy; exchange; magnetic materials; magnetism; rare earth.

This is an introductory article on the subject of magnetic materials with particular emphasis on rare earth-based materials and amorphous magnetic alloys. It is intended as a general reference for nonspecialists in solid state physics or for workers in other scientific disciplines. It includes a number of general references in the field of magnetism and also to specific classes of magnetic materials.

20470. Metz, F. E. Health and safety criteria for passive solar systems, (Proc. 5th Natl. Passive Solar Conf., Amherst, MA, Oct. 19.26, 1980), Extended Abstract in Passive Solar 1980, J. Hayes and R. Snyder, eds., 5.2, $945-949$ (1980).

Key words: building codes; constraints; fire safety; glazing; hazards; passive solar.

Passive solar technologies may introduce new and unusual health and safety concerns within the building environment. Conversely, existing building code requirements may present constraints to the effective use of some passive technologies. Some of the health and safety topics to be covered include: fire safety, physical hazards, air quality, and environmental health factors. The solar industry will be confronted with even more restrictive health and safety requirements as passive construction becomes more prevalent and is extended to non-residential building types. This paper will present some of these constraints and identify possible hazards.

20471. Broadhurst, M. G.; Davis, G. T. Ferroelectric polarization in polymers, Ferroelectrics 32, 177.180 (1981).

Key words: cooperative model; ferroelectric polarization molecular dipoles; polarization; polyvinylidene fluoride; PVDF.

A cooperative model has been developed to describe ferroelectric polarization in polyvinylidene fluoride (PVDF). The molecular dipoles within the crystal are assumed to have two or more orientations available to them and the lattice energy of a given orientational site is assumed to be proportional to the fraction of molecules having that orientation. An analytical solution for the 2-site model predicts polarization hysteresis typical of ferroelectrics. However, a more complex 6-site model, which can be analyzed numerically, is needed to account for observed infrared hysteresis data and electric-field-induced $\mathrm{X}$-ray structural changes which have been reported for PVDF. Although the model is simple, rather complex behavior is observed including a gradual increase or decrease in the remnant polarization with number of cycles of electric field application. Though the agreements with various experimental data are good an obvious need to include kinetic effects in the model is indicated.

20472. Rosenstock, H. M.; Stockbauer, R.; Parr, A. C. Unimolecular 
kinetics of pyridine ion fragmentation, Int. J. Mass Spectrom. Ion Phys. 38, 323-331 (1981).

Key words: breakdown curve; fragmentation; heat of formation; ionization; mass spectrometry; photoionization; pyridine; quasiequilibrium theory; time dependent fragmentation; unimolecular decay kinetics.

The fragmentation of pyridine ion to form $\mathrm{C}_{4} \mathrm{H}_{4}^{+}$and $\mathrm{HCN}$ has been studied by means of photoelectron-photoion coincidence mass spectrometry with variable ion source residence time. A detailed analysis of the time-dependent breakdown curves leads to a $\mathrm{OK}$ fragmentation threshold of $12.15 \pm 0.02 \mathrm{eV}$, a tight transition state, and the energy dependence of the fragmentation rate. The results lead to $\Delta H_{\mathrm{f}}^{0}\left(\mathrm{C}_{4} \mathrm{H}_{4}^{+}\right)=1195 \pm 12 \mathrm{~kJ} / \mathrm{mol}$, somewhat higher than the value reported by Eland et al. There is some disagreement with earlier work on the energy dependence of the fragmentation rate. It is suggested that this may be due to distortion of the thermal vibrational population distribution of the molecule on vertical ionization.

20473. Lyon, G. E. Alternation-tree insertions for open-addressed hash buckets, Proc. 15th Annu. Conf. Information Sciences and Systems, Baltimore, MD, Mar. 25-27, 1981, pp. 398-401 (The Johns Hopkins University, Dept. Electrical Engineering, Baltimore, MD, 1981).

Key words: algorithm; buckets; efficiency; hashing; openaddressing; worst-case.

Open-addressing is a hashing method that uses no explicit table structures such as bit fields, pointers, or index linkages. It is a compact method of storing information that can later be retrieved, on the average, very quickly. However, sometimes the objective is not so much to achieve a fast average retrieval as it is to ensure that worst cases are short. It is known that for hashing into buckets, worst case searches can usually be limited to two probes, although known computational methods to do this can be slow. In attacking this problem of computation, principal focus will be upon a new, practical algorithm that constructs tables cheaply.

20474. Eitzen, D. G.; Berger, H.; Birnbaum, G. A basis for traceable NDE measurements, Part 1, Mater. Eval. 39, No. 9, 797.798 (Aug. 1981).

Key words: acoustic emission; calibration; eddy currents; magnetic particles; nondestructive evaluation; radiography; traceability; ultrasonics; visual testing.

The National Bureau of Standards is beginning to provide a mechanism for traceability for a number of NDE measurement procedures, an activity that is expected to have a significant, positive impact on the reproducibility and accuracy of NDE measurements. Much of the NDE standards activity has been in ultrasonics and acoustic emission; these efforts leading to calibration services for ultrasonic reference blocks and ultrasonic and acoustic emission transducers. Additional NDE standards are also available or are being developed in radiography, eddy currents, magnetic particles, liquid penetrants and visual testing. Part 1 deals with the standards activities in ultrasonics.

20475. Molino, J. A. Annoyance and noise, Chapter 16 in Handbook of Noise Control, Second Edition, C. M. Harris, ed., pp. 16-1-16-9 (McGraw-Hill Book Co., New York, NY, 1979).

Key words: acoustics; annoyance; environmental noise; noise measurements; psychoacoustics; psychology.

Psychophysical data are reviewed on the annoyance caused by noise. Annoyance varies with three primary acoustic factors: sound level, frequency, and duration. In addition, several secondary acoustic factors have an effect: spectral complexity, fluctuations in sound level, fluctuations in frequency, rise-time of the noise, and localization of the noise source. Non-acoustic factors also influence annoyance due to noise. These include physiology, adaptation, past experience, present activity, predictability, perceived necessity, individual differences, and personality.
20477. Molino, J. A. Auditory space perception, Article in International Encyclopedia of Psychiatry, Psychology, Psychoanalysis, and Neurology, pp. 222-227 (Aesculapius Publ. Inc., New York, NY, 1977).

Key words: audition; auditory localization; auditory space perception; experimental psychology; hearing; psychoacoustics.

Scientific data on human auditory space perception is presented. History and theories related to physical measurements are reviewed. Current methods and empirical results in auditory localization are given. A section on physiological models and practical applications is included.

20478. Egelhoff, W. F., Jr.; Tibbetts, G. G.; Lindau, I.; Hecht, M. H. Photoemission studies on thulium (Summary Abstract), J. Vac. Sci. Technol. 18, No. 2, p. 599 (Mar. 1981).

Key words: autoionization; photoelectron spectroscopy; potential energy surfaces; resonant photoemission; synchrotron radiation; thulium.

We have studied the autoionizing resonances found in the metal thulium. The type of resonance is $4 \mathrm{~d} \rightarrow 4 \mathrm{f}$ and its general character appears to be determined by the shape of the $4 f$ potential energy surface.

20479. Smith, E. W.; Talin, B.; Cooper, J. An analysis of Markovian Model Microfield Methods for Stark broadening, J. Quant. Spectrosc. Radiat. Transfer 26, No. 3, 229-242 (1981).

Key words: impact and unified theories; kangaroo process; Markovian model; model microfield method; plasma; Stark broadening.

Stark broadening theories which concentrate on the statistics of the plasma electric microfield rather than the dynamics of collisions have come to be known as Model Microfield Methods. In the present paper we present an analysis of Stark broadening problems based on Markovian model microfield statistics. Our derivation permits an easy comparison with traditional Stark broadening theories such as the impact and unified theories; this comparison is used to clarify the physical nature of the approximations employed by Markovian models. The strengths and weaknesses of various models are discussed, emphasizing the kangaroo process of Brissaud and Frisch, and methods are suggested for improving the current model microfield approach.

20480. Reite, M.; Zimmerman, J. T.; Zimmerman, J. E. Magnetic auditory evoked fields: Interhemispheric asymmetry, Electroencephalogr. Clin. Neurophysiol. 51, 388.392 (1981).

Key words: auditory evoked fields; biomagnetism; magnetoencephalography.

We recorded magnetic auditory evoked fields (AEF) from 10 normal adult human subjects using a SQUID gradiometer.

Stimuli were irregularly spaced $0.1 \mathrm{~ms}$ clicks with a modal interstimulus interval of $700 \mathrm{~ms}$ (range 500-1500). The magnetoencephalogram (MEG) was amplified, filtered with a 0.1-30 $\mathrm{Hz}$ bandpass, and averaged for $500 \mathrm{~ms}$ following 128 stimuli. The amplitude of the largest magnetic responses occurring within the first $400 \mathrm{~ms}$ were measured for bilateral, contralateral, and ipsilateral stimulation.

Recording from the right hemisphere, 8 of 10 subjects exhibited larger amplitude AEFs to contralateral stimulation, with contralateral responses being $134 \pm 37 \%$ of ipsilateral responses. Mean contralateral response amplitudes from the right hemisphere were $176 \pm 39$ femptotesla (fT) peak-to-peak; mean ipsilateral response amplitude was $134 \pm 33 \mathrm{fT}$. Recording from the left hemisphere, 7 of 7 subjects had higher amplitude contralateral AEF amplitudes, with contralateral responses being $147 \pm 52 \%$ of ipsilateral response amplitudes. Mean left hemisphere contralateral response amplitude was $129 \pm 43 \mathrm{fT}$. Contralateral and bilateral responses were similar in amplitude, with

20476. Unassigned. 
contralateral being of slightly greater magnitude over the left hemisphere.

These findings suggest that cortical current dipoles produced by contralateral auditory stimulation are of greater magnitude than those resulting from ipsilateral stimulation.

20481. Antonucci, J. M.; Misra, D. N.; Peckoo, R. J. The accelerative and adhesive bonding capabilities of surface-active accelerators, $J$. Dent. Res. 60, No. 7, 1332-1342 (July 1981).

Key words: adhesion; composites; coupling agents; dental resins; hydroxyapatite; polymerization; surface-active amine accelerators; tensile strength.

PolySAM-1, a multifunctional surface-active amine polymerization accelerator, and several simpler surface-active amine promoters were evaluated for their accelerative and adhesion-promoting potentials. Diametral tensile strength measurements, performed on composites prepared from a bis-GMA resin formulation with tribasic calcium phosphate as the filler, were used to assess the adhesive bonding capabilities of these amines.

20482. Han, C. C.; Akcasu, A. Z. On the dynamic structure factor, $S(q, \omega)$, of polymers in dilute solution, Polymer 22, 1019.1025 (1981).

Key words: characteristic frequency $\Omega(q)$; dynamic light scattering; dynamic neutron scattering; dynamic structure factor $S(q, \omega)$; hydrodynamic interaction; intermediate scattering function $S(q, t)$.

In principle, interpretation of dynamic scattering experiments requires theoretical calculation of either intermediate scattering functions $S(q, t)$ or dynamic structure factors $S(q, \omega)$. Although dynamic scattering experiments can be interpreted through the use of the 'characteristic frequency' (or 'first cumulant') $\Omega(q)$. The precision of extracting $\Omega(q)$ is greatly improved by using a normalized shape function. Due to the difficulty in obtaining analytical results, in this paper we present numerical calculations of the normalized shape function in the frequency domain. These results can be used to facilitate the extraction of $\Omega(q)$ from $S(q, \omega)$ directly both in light and neutron dynamic scattering experiments.

20483. Deslattes, R. D.; Kessler, E. G., Jr. Inner shell energies: Experimental problems, Proc. Workshop on Foundations of the Relativistic Theory of Atomic Structure, Argonne, IL, Dec. 4-5, 1980, pp. 215.235 (Argonne National Laboratory, Argonne, IL, Mar. 1981).

Key words: energy levels; relativistic effects; $x$ rays.

Progress in theoretical estimates of single vacancy atomic term values has challenged available experimental data. Our purpose in this brief survey of the experimental situation is to indicate both conceptual and technical problems which are currently faced. Although these interpretational difficulties are formidable, the emergence of systematic trends in the comparison of theory and experiment appears to invite a (possibly) simple explication.

20484. Misakian, M. Generation and measurement of dc electric fields with space charge, J. Appl. Phys. 52, No. 5, 3135.3144 (May 1981)

Key words: calibration; dc transmission lines; electric field; electric field meters; electric field strength; electric power transmission; measurements; space charge.

Characterization of the electrical environment in the vicinity of high voltage dc transmission lines requires measurement of a number of electrical parameters associated with the lines. These parameters include the electric field strength with significant space-charge contributions. This report describes an experimental effort to generate known dc electric fields containing controlled amounts of space charge. An apparatus which has been built for this purpose is described, and two types of field probes currently used for transmission line field measurements are examined with the apparatus. Limitations on the operation of one type of probe in the presence of very large current densities are identified and discussed.
20485. White, G. S. Feldman, A. Diffraction from a shallow rectangular groove, Appl. Opt. 20, No. 14, 2585-2589 (July 15, 1981).

Key words: alignment; diffraction; IR; NDE; optical; scattering; shallow groove; single slit; surface.

Infrared radiation scattered from a shallow groove is found to exhibit the same diffraction pattern as a single slit. A model based on a beam with a Gaussian intensity profile was found which explains the observed scattering patterns and which also describes the scattering pattern occurring when certain alignment errors are present in the system. The large scattering intensity from the shallow (depth $\sim \lambda / 20$ ) groove has implications in the use of optical scattering as a tool for nondestructive evaluation of surface flaws.

20486. Waterstrat, R. M. Analysis of selected alloys in the systems $\mathbf{C r}$ Pd, Cr-Ru, V.Pd and Ta-Pt, J. Less-Common Met. 80, P31-P36 (1981).

Key words: Al5 compounds; chromium alloys; noble-metal alloys; palladium alloys; phase diagrams; tantalum alloys; vanadium alloys.

Cr-Pd, Cr-Ru, V.Pd and Ta-Pt alloys were selected, appropriately annealed and subjected to electron microprobe analyses. The data enable a basis to be established for a more accurate location of phase boundaries which have not been accurately defined in previous studies.

20487. Manka, M. J.; Yeh, K. N.; Smith, B. F. A calorimetric study of flame-retardant-treated cotton/polyester blends, J. Fire Retard. Chem. 5, 144-159 (Aug. 1978).

Key words: blended fabrics; bromine; calorimetry cotton/polyester; flame retardance; phosphorus.

The effectiveness of three flame retardants containing phosphorus or phosphorus and bromine has been evaluated for cotton/polyester blended fabrics. The effectiveness was based on reducing the heat released from a burning fabric. The flame retardants of interest are triphenyl phosphate $\left((\phi \mathrm{O})_{3} \mathrm{PO}\right)$, triphenyl phosphite $\left((\phi \mathrm{O})_{3} \mathrm{P}\right)$ and tris(2,3-dibromopropyl) phosphate (T-2,3-P). The results are also compared with those previously obtained for phosphoric acid treated blends and are interpretable in terms of existing mechanisms of flameretardant action.

20488. Cromar, M. W.; Carelli, P. Low-noise tunnel junction dc SQUID's, Appl. Phys. Lett. 38, No. 9, 2723-2725 (May 1981).

Key words: integrated circuits; Josephson devices; magnetometers; SQUIDs; superconductivity; thin films.

We present the results of an investigation of thin-film dc superconducting quantum interference device (SQUID's) with very high energy resolution. The SQUID's were fabricated using integrated-circuit techniques appropriate for superconducting devices. A weakly coupled SQUID with very low inductance $(1 \mathrm{pH})$ had an intrinsic energy sensitivity $6 \times 10^{-34} \mathrm{~J} / \mathrm{Hz}=0.9 \mathrm{~h}$. A well-coupled SQUID (coupling constant $a=0.43$ ) had an energy sensitivity referred to the input coil of $4.7 \times 10^{-32} \mathrm{~J} / \mathrm{Hz}=71 \mathrm{~h}$

20489. Breckenridge, F. R.; Greenspan, M. Surface-wave displacement: Absolute measurements using a capacitive transducer, J. Acoust. Soc. Am. 69, No. 4, 1177.1185 (Apr. 1981)

Key words: capacitive transducer; displacement measurements; sensor, capacitive; surface waves; transducer calibration; transducer, capacitive.

We have constructed a capacitive transducer for the absolute measurement of the normal component of surface-wave motion on a flat solid, the direction of travel of the wave being known. The transducer backplate is a cylinder and has cylindrical extensions on each end that act as electrostatic guards to render calculable the sensitivity and capacitance of the transducer. Compliant support 
elements are incorporated, resulting in negligible mechanical loading to the surface and flat frequency response over the range of $10 \mathrm{kHz}$ to $1 \mathrm{MHz}$ or better. The amplifier noise floor is equivalent to about $4 \times 10^{-12} \mathrm{~m}$ rms with a $5 \cdot \mathrm{MHz}$ bandwidth. The upper limit to displacements measurable by this technique has not been established, but is large enough to encompass the range of interest. Estimated uncertainty of the sensitivity determination is less than $5 \%$.

20490. Nitz, D. E.; Smith, A. V.; Levenson, M. D.; Smith, S. J. Bandwidth-induced reversal of asymmetry in optical-doubleresonance amplitudes, Phys. Rev. A 24, No. 1, 288-293 (July 1981).

Key words: ac Stark effect; asymmetry; atom; double resonances; ionization; lasers; multiphoton; sodium; spectroscopy.

Optical-double-resonance measurements using ionization detection have been carried out in the $3 S_{1 / 2}-3 P_{1 / 2}-4 D$ atomic-sodium system. Asymmetries observed in production of $4 D$ atoms from the two components of the Stark-split $3 P_{1 / 2}$ state are found to be controlled by the far, very weak wings of the $17-\mathrm{MHz}$ full-width-at-half-maximum laser line which is used to drive the $3 S_{1 / 2}-3 P_{1 / 2}$ transition at detunings in the range $0-70 \mathrm{GHz}$. Suppression of the wings with a Fabry-Perot filter causes a pronounced reversal of the asymmetry.

20491. Wildman, D. W.; Schumann, L. W.; Gallagher, A. C. Electric discharge excited Tl1.Xe mixtures, J. Appl. Phys. 52, No. 5, 3264. 3268 (May 1981).

\section{Key words: electric discharges; thalium iodide.}

Pulsed, high-power, nonequilibrium discharges have been operated in TlI-Xe mixtures at typical TII densities of $10^{15}-10^{17} \mathrm{~cm}^{-3}, \mathrm{Xe}$ densities of $\sim 10^{19} \mathrm{~cm}^{-3}$, and power densities of $10^{5}-10^{6} \mathrm{~W} \mathrm{~cm} \mathrm{~cm}^{-3}$. The steady-state emission spectrum was measured for the wavelength region $275-850 \mathrm{~nm}$, and it was used to obtain the populations of the $\mathrm{Tl}$ and $\mathrm{Xe}$ excited states. The fraction of TII dissociated in the discharge is also reported as a function of TlI, Xe, and current density. A phenomenological model for the results is discussed.

20492. Pradhan, A. K.; Norcross, D. W.; Hummer, D. G. Rate coefficients for electron impact excitation of helium-like ions, Astrophys. J. 246, 1031-1039 (June 15, 1981).

Key words: collisional excitation; collisional rates; helium-like ions.

Accurate rate coefficients are presented for the excitation of all transitions involving the ground state and the $n=2$ levels in a number of helium-like ions: $\mathrm{Be}$ III, C V, O vil, Ne IX, Si xIII, Ca xIX, and Fe $x x v$. The results are tabulated in the temperature range $10^{4}-10^{9} \mathrm{~K}$, depending upon the ionization stage. Effects of autoionizing resonances in the inelastic scattering cross sections have been included, and it is shown that for most transitions these effects result in a very significant enhancement of the excitation rates. The present results, in many cases, are considerably different from previous calculations of collision strengths neglecting resonance effects. Some applications of the present data in the analysis of line intensities from laboratory and astrophysical plasmas are pointed out. General criteria for evaluating theoretical electron-ion scattering data and their accuracy are also briefly discussed.

20493. Quintiere, J. A simplified theory for generalizing results from a radiant panel rate of flame spread apparatus, Fire Mater. 5 , No. 2 , $52-60(1981)$

Key words: flame spread test method; ignition; radiant heating; theory; wall materials.

Experimental results on the rate of lateral flame spread and time for piloted ignition under an externally imposed radiant flux were analyzed with a simple theoretical model. The data were developed from a radiant panel apparatus that considers a wall mounted sample with a flux distribution $\left(\dot{q}_{t}{ }^{\prime \prime}\right)$ of $5 \mathrm{~W} \mathrm{~cm} \mathrm{~cm}^{-2}$ at the ignited end to $0.2 \mathrm{~W}$ $\mathrm{cm}^{-2}$ at the other end. It is shown that after an appropriate preheating time (flux exposure time before sample is ignited) the rate of flame spread $\left(V_{r}\right)$ results can be correlated by $V_{f}^{-1 / 2}=C\left(\dot{q}^{\prime \prime}{ }_{0, i g}-\dot{q}_{e}{ }^{\prime \prime}\right)$ where $C$ is a material 'constant' and $\dot{q}^{\prime \prime}$, is the minimum flux for piloted ignition-also a material (and configuration) constant. An extension of this model demonstrates that $V_{\mathrm{f}}$ can also be expressed in terms of an 'ignition temperature' and the surface temperature of the material. Both correlations are derivable from a single flame spread experiment. Results are presented for a number of typical wood and plastic materials.

20494. Hust, J. G.; Boscardin, R. Thermal conductivity of polyesteramide-imide film, Cryogenics 21, No. 5, 297.298 (May 1981).

Key words: insulation; low temperature; polyester-amide-imide film; thermal conductivity.

Thermal conductivity measurements were performed on polyesteramide-imide film from 4 to $323 \mathrm{~K}$. The specimen was in the form of a stack of aluminium discs coated on both sides with film. The results exhibit a temperature dependence similar to varnish, but are about half as large in conductivity. The results, based on nineteen data points, are estimated to be accurate to about $10 \%$.

20495. Evans, D. J. Equilibrium fluctuation expressions for the wavevector-and frequency-dependent shear viscosity, Phys. Rev. $A$ 23, No. 5, 2622-2626 (May 1981).

Key words: Kubo relations; shear viscosity; wavevector dependence.

We derive an expression for the wavevector and frequency dependent shear viscosity coefficient which involves equilibrium stress-stress time correlation functions. Except at zero wavevector the expression does not have the conventional Kubo form.

20496. Lovas, F. J.; Maki, A. G.; Olson, W. B. The infrared spectrum of $\mathrm{SiO}$ near $1240 \mathrm{~cm}^{-1}$ and its relation to the circumstellar $\mathrm{SiO}$ maser, J. Mol. Spectrosc. 87, $449-458$ (1981).

Key words: diode laser; high temperature; infrared spectrum; rotational constants; silicon monoxide; stellar $\mathrm{SiO}$ maser; vibrational constants.

The infrared absorption spectrum of the SiO molecule has been measured at temperatures between 1150 and $1350^{\circ} \mathrm{C}$ with a tunable diode laser. Transitions ranging from $J^{\prime \prime}=0$ to $J^{\prime \prime}=60$ and $v^{\prime \prime}=0$ and $v^{\prime \prime}=4$ have been measured. These measurements have been combined with microwave measurements made by other workers to yield a consistent set of Dunham ro-vibrational constants. The relation of the infrared absorption spectrum to inversion mechanisms for the circumstellar $\mathrm{SiO}$ maser is discussed.

20497. Bethin, J. R.; Chiang, C. K.; Franklin, A. D.; Snellgrove, R. A. An oxygen-sensitive electrode impedance in Sr-doped $\mathrm{LaCrO}_{3}, J$. Appl. Phys. 52, No. 6, $4115-4117$ (June 1981).

Key words: diffusion; electrode effect; lanthanum chromite; oxidation-reduction; surface properties.

Barrier layers are apparently formed on Sr-doped $\mathrm{LaCrO}_{3}$ that give rise to an appreciable electrode impedance with electrodes of $\mathrm{Au}$ or $\mathrm{Pt}$. The corresponding electrode resistance is quite sensitive to the oxygen activity in the atmosphere, following atmospheric changes at temperatures near room temperature with time constants of the order of minutes or days. The electrical properties of these barrier layers at room temperature do not conform to the models of simple Schottky layers on semiconductors, but exhibit a pattern of changing oxidation states at the interface region.

20498. Cavanagh, R. R.; Yates, J. T., Jr. Surface binding of an electronic analog to $\mathrm{CO}$ : Infrared evidence for $\mathrm{CH}_{3} \mathrm{NC}$ chemisorption on $\mathrm{Rh} / \mathrm{Al}_{2} \mathrm{O}_{3}$, J. Chem. Phys. 75, No. 3, 1551-1559 (Aug. 1, 1981).

Key words: chemisorption; CO; displacement; frequency shift; infrared; interaction; isocyanide; isomerization; Rhodium.

The chemisorption of methyl isocyanide by $\mathrm{Al}_{2} \mathrm{O}_{3}$ - supported $\mathrm{Rh}$ 
has been investigated using transmission infrared spectroscopy. Evidence for the absence of dissociation or isomerization upon chemisorption is presented. The identification of various surface binding sites is possible and is in agreement with the site distribution previously demonstrated for such samples using CO. Samples which are exposed to methyl isocyanide following saturation coverage with $\mathrm{CO}$ exhibit a $100 \mathrm{~cm}^{-1}$ decrease in the $\mathrm{C} \equiv \mathrm{O}$ stretching mode due to the presence of the isocyanide. This shift is interpreted in terms of a $\sigma$ donor- $\pi^{*}$ acceptor interaction between the isocyanide and $\mathrm{CO}$ adsorbates.

20499. Lippiatt, B. C.; Weber, S. F. Labor and materials cost of weatherizing low-income housing, (Proc. Natl. Conf. Optimal Weatherization, Washington, DC, Dec. 8.10, 1980), Paper in Optimal Weatherization, pp. 103-113 (Information Dynamics, Inc., Silver Spring, MD 20902, 1981).

Key words: building economics; data analysis; data collection; economic analysis; energy conservation; insulation; labor cost low-income housing; materials cost; statistics; unit costs; weatherization.

This paper presents the major results of a project involving the collection and analysis of field data from 12 U.S. cities on the costs of retrofitting low-income houses for energy conservation. The energy conservation techniques presented consist of a variety of architectural weatherization options designed to reduce heat losses due either to air infiltration or conduction. This project is part of the Community Services Administration Weatherization Demonstration Program being carried out with technical assistance from the National Bureau of Standards. The methods used to collect and synthesize the data on the major cost components of installing these architectural options are described. The results include the mean unit cost of installing each option by city, and the relative importance of labor and materials costs. The results suggest that if a primary national policy goal were to create jobs in weatherization, then the infiltration options should be emphasized since they are labor-intensive than the conduction options.

20500. Fong, J. T. Recommendations for future research sessions III \& IV on prediction of damage and failure, Proc. Natl. Science Foundation Workshop on a Continuum Mechanics Approach to Damage and Life Prediction, University of Cincinnati, Cincinatti, $\mathrm{OH}$, May 1980, pp. 180-183 (1980).

Key words: continuum mechanics; damage; fatigue; fracture; life prediction; mathematical modeling; measurement uncertainties; micromechanics; microstructure; nested modeling; quantitative microscopy; statistics.

This note is a brief summary of the contributions at a recent NSF workshop entitled "Continuum Mechanics Approach to Damage and Life Prediction." Emphasis is placed on the role of the uncertainties of experimental information in the formulation of a continuum mechanics approach to damage analysis. Promising areas of future research and critical issues for timely resolution are presented and discussed.

20501. Fong, J. T.; Dowling, N. E. Analysis of fatigue crack growth rate data from different laboratories, Proc. ASTM Symp. Fatigue Crack Growth Measurement and Data Analysis, Pittsburgh, PA, Oct. 1979, pp. 171-193 (American Society for Testing Materials, Philadelphia, PA, 1979).

Key words: applied regression analysis; data analysis; engineering judgment; fatigue; fatigue crack growth; fracture mechanics; interlaboratory data analysis; interlaboratory test program; linear regression analysis; mathematical modeling; statistics; steels.

A simple-minded yet quantitative approach to assessing interlaboratory fatigue crack growth rate data is proposed. Seven sets of $d a / d N$ versus $\Delta K$ data from six laboratories on nominally the same material and loading conditions in a cooperative test program sponsored by the Society of Automotive Engineers (SAE) are analyzed to illustrate this ad hoc approach. Each set of data is subjected to a standard first-order linear regression analysis based on the method of least squares. Three characteristics of the regression line [namely, the location of the "center" of the data, the slope, an the vertical half-width of the confidence band (for some specified level of confidence)l are used to define a composite measure of the closeness of one regression line to another. To illustrate the benefit of a statistically sound interlaboratory test program, the single-specimen SAE data are supplemented with fictitious replica data for the application of an interlaboratory data analysis procedure first proposed by Mandel (ASTM Standardization News, Vol. 5, No. 3 1977 , p. 17). "New" information based on the ad hoc approach of this paper and Mandel's method of interlaboratory data analysis is discussed in the context of other work on fatigue crack growth rate data analysis and the economic aspect of engineering testing.

20502. Hoffman, K. L.; Shier, D. R. A test problem generator for discrete linear $L_{1}$ approximation problems, $A C M$ Trans. Math. Software 6, No. 4, 587-593 (Dec. 1980).

Key words: $L_{1}$ approximation; least absolute deviation; problem generator; test data.

Described here are the theoretical development and computer implementation of a procedure that generates test problems for $L$ estimation of the linear model $\mathrm{y}=X \beta+\mathrm{u}$. The generation procedure allows the user flexibility in specifying the problem dimensions, the $L$ solution vector $\beta^{*}$, the distribution of the observed residuals $\epsilon$, as well as the column rank, row repetitions, and degree of degeneracy of the matrix $X$. The user can also specify the distributional form, mean, and variance for each independent variable. An important feature of the generator is that any problem it creates is guaranteed to have a unique solution $\beta$ whenever $X$ has full rank.

20503. Unassigned.

20504. Hellmuth, T.; Leuchs, G.; Smith, S. J.; Walther, H. Angular distribution of photoelectrons in multiphoton ionization, Proc. $2 d$ Int. Conf. Multiphoton Processes, Budapest, Hungary, Apr. 14-18, 1980, pp. 1.15 (Hungarian Academy of Science, Budapest, Hungary, 1981).

Key words: angular distribution; atoms; laser; multiphoton photoelectron; photoionization; resonant; sodium.

In resonant multiphoton ionization of sodium, with absorption of $\mathbf{n}$ successive photons, photoelectron angular distributions are characterized by polynomials in the even spherical harmonic up to $P_{2 n}$. Measurements of angular distributions for two- and three-photon ionization, using linearly polarized light from pulsed dye lasers, are reported. Three-photon ionization via the $3 \mathrm{P}$ and $\mathrm{nD}$ states clearly exhibits the characteristic $\mathrm{P}_{4}$ dependence (from the $3 \mathrm{P}_{1 / 2}$ intermediate state), and the $P_{6}$ dependence (from the $3 P_{3 / 2}$ intermediate state) Coefficients of $P_{0}, P_{2}, P_{4}$, and $P_{6}$ are in reasonable agreement with theory. No measurable difference is observed in the distributions for final bound states $6 \mathrm{D}$ and $10 \mathrm{D}$. Two-photon ionization via the $3 \mathrm{P}_{3 / 2}$ state is studied for parallel and for perpendicular orientations of linear laser polarization, which give different admixtures of $P_{4} \cdot P_{4}$ is also studied as a function of time delay of the second laser pulse. The time dependence of the coefficient of $\mathrm{P}_{4}$ exhibits quantum beats due to coherent excitation of the hyperfine components of the $3 \mathrm{P}_{3 / 2}$ state.

20505. Hiza, M. J. Liquid-vapor equilibria in binary systems containing ${ }^{4} \mathrm{He}$ or ${ }^{3} \mathrm{He}$ with $\mathrm{nH}_{2}$ or $\mathrm{nD}_{2}$, Fluid Phase Equilib. 6, 203.227 (1981).

Key words: binary systems; gas solubility; liquid-vapor equilibria; $\mathrm{nD}_{2}$ vapor pressures; $\mathrm{nH}_{2}$ vapor pressures

Equilibrium liquid and vapor compositions are reported at $2 \mathrm{~K}$ intervals for binary systems containing ${ }^{4} \mathrm{He}$ or ${ }^{3} \mathrm{He}$ with $\mathrm{nH}_{2}$ or $\mathrm{nD}_{2}$ between 20 and $30 \mathrm{~K}$ at pressures up to $20.3 \times 10^{5} \mathrm{~Pa}(20 \mathrm{~atm})$. Vapor pressures measured at $1 \mathrm{~K}$ intervals are also reported for $\mathrm{nH}_{2}$ from 20 to $30 \mathrm{~K}$ and for $\mathrm{nD}_{2}$ from 20 to $34 \mathrm{~K}$. A vapor-recirculation apparatus was used with equilibrium compositions determined by gas chromatographic analysis. The results show that, at the same temperature and partial pressure, (1) ${ }^{4} \mathrm{He}$ and ${ }^{3} \mathrm{He}$ are three to five times more soluble in liquid $\mathrm{nH}_{2}$ than in liquid $\mathrm{nD}_{2},(2){ }^{4} \mathrm{He}$ is slightly more soluble than ${ }^{3} \mathrm{He}$ in either liquid solvent, and (3) within the 
precision of measurement, vapor compositions are independent of the helium isotope present. Comparisons are made with corresponding liquid-vapor equilibria and vapor pressure data reported in the literature.

20506. Hellmuth, T.; Leuchs, G.; Smith, S. J.; Walther, H Multiphoton ionization of atoms, (Proc. Sergio Porto Memorial Symp., Rio de Janeiro, Brazil, June 29.July 3, 1980), Paper in Springer-Series in Optical Sciences, Lasers and Applications, W. O. N. Guimaraes, C. T. Lin, and A. Mooradian, eds., 26, pp. 194.203 (Springer-Verlag, Berlin, Germany, 1981).

Key words: angular distribution; atoms; laser; multiphoton; photoelectron; photoionization; resonant; sodium.

In resonant multiphoton ionization of sodium, with absorption of $\mathbf{n}$ successive photons, photoelectron angular distributions are characterized by polynomials in the even spherical harmonic up to $P_{2 n}$ : Measurements of angular distributions for two- and three-photon ionization, using linearly polarized light from pulsed dye lasers, are reported. Three-photon ionization via the $3 \mathrm{P}$ and $\mathrm{nD}$ states clearly exhibits the characteristic $\mathrm{P}_{4}$ dependence (from the $3 \mathrm{P}_{1 / 2}$ intermediate state), and the $\mathrm{P}_{6}$ dependence (from the $3 \mathrm{P}_{3 / 2}$ intermediate state) Coefficients of $\mathrm{P}_{0}, \mathrm{P}_{2}, \mathrm{P}_{4}$, and $\mathrm{P}_{6}$ and in reasonable agreement with theory. No measurable difference is observed in the distributions for final bound states $6 \mathrm{D}$ and $10 \mathrm{D}$. Two-photon ionization via the $3 \mathrm{P}_{3 / 2}$ state is studied for parallel and for perpendicular orientations of linear laser polarization, which give different admixtures of $\mathrm{P}_{4} \cdot \mathrm{P}_{4}$ is also studied as a function of time delay of the second laser pulse. The time dependence of the coefficient of $\mathrm{P}_{4}$ exhibits quantum beats due to coherent excitation of the hyperfine components of the $3 \mathrm{P}_{3 / 2}$ state.

20507. Daney, D. E.; Jones, M. C. Counterflow cool down of cryogenic power transmission lines, Cryogenics 21, No. 8, $463-473$ (Aug. 1981).

Key words: cable cool down; counterflow cool down; heat exchanger cool down; heat exchanger transient response; liquid helium; superconducting power transmission.

Cool down of cryogenic power transmission lines which use a counterflow cooling arrangement is investigated analytically, numerically, and experimentally. The results demonstrate that the cool down time, $t_{\text {cd }}$, is given by $t_{\mathrm{cd}} \sim U(l / \dot{m})^{2}$ where $U$ is the overall heat transfer coefficient between the go and return streams, $l$ the length, and $\dot{m}$ the mass flow rate. The accuracy and utility of a previously reported numerical cool down model are also confirmed by comparison with experiment.

20508. McCarty, R. D. LNG densities for custody transfer, Proc. 56th

Int. School of Hydrocarbon Measurement, Norman, OK, Apr. 14-16, 1981 pp. 515-517 (University of Oklahoma, Norman, OK, 1981).

Key words: custody transfer; densimeter; density; LNG; mathematical models; standard reference system.

Work has been carried out over the past eight years at the National Bureau of Standards to provide alternate methods for the accurate determination of the density of liquefied natural gas (LNG) that would serve as a basis for equitable custody transfer.

A magnetic suspension densimeter was used to obtain density data for LNG components and their mixtures with a total uncertainty in density of less than $0.1 \%$. These data were used to optimize and test mathematical models for LNG density calculations.

Four mathematical models for the calculation of LNG densities have been optimized, tested, and compared. These models are an extended corresponding states model, a cell model, a hard sphere model, and an empirical model due to Klosek and McKinley.

A density reference system (DRS) was constructed to determine the uncertainty of measurements made by several different types of densimeters proposed for use in the commercial trade of LNG. Several commercial densimeters have been tested and a transfer standard program implemented as a means of producing a calibration service for commercially built densimeters. The total uncertainty of the DRS is estimated to be $\pm 0.076 \%$.

A portable reference densimeter (PRD) has been developed specifically for calibrating LNG densimeters "in place." The measurements of density using the two systems, DRS and PRD, are statistically indistinguishable.

20509. Moos, H. W.; Clarke, J. T. Ultraviolet observations of the Io torus from the IUE observatory, Astrophys. J. 247, 354-361 (July 1, 1981).

Key words: International Ultraviolet Explorer; Io; Io torus; oxygen ions; sulphur ions; ultraviolet spectra.

The short wavelength spectrograph on the International Ultraviolet Explorer (IUE) has been used to obtain $11 \AA$ resolution spectra of the Io torus from $1175-1950 \AA$. The four spectra, obtained in the springs of 1979 and 1980, show emissions $(\sim 40 R)$ of $S$ II $\lambda 1256$ and $S$ III $\lambda 1199$. An unidentified feature is also present at $1729 \AA$; a tentative identification as an intercombination line of $S$ III is proposed. Weak features, probably due to $O$ III $\lambda 1664$ and $S$ IV $\lambda 1406$, appear in some of the spectra. Abundances of the ions are determined from the brightnesses of the observed features. Upper limits are also set for the abundances of a number of ionic and neutral species. An observation of lo itself does not show any additional or enhanced spectral features, permitting upper limits to be set on the injection rate for a number of species.

20510. Madey, T. E.; Stockbauer, R.; Flodström, S. A.; van der Veen, J. F.; Himpsel, F. J.; Eastman, D. E. Photon-stimulated desorption from covalently bonded species: $\mathrm{CO}$ absorbed on Ru(001), Phys. Rev. $B$ 23, No. 12, 6847.6850 (June 15, 1981).

Key words: carbon monoxide; chemisorption; photon stimulated desorption; ruthenium; surface; ultraviolet photoemission spectroscopy.

We report the first angle-resolved photon stimulated desorption (PSD) study of a covalent molecule bonded to a metal surface, $\mathrm{CO}$ on $\mathrm{Ru}(001)$. The mechanisms for ion desorption involve intramolecular excitations, not substrate atom excitations.

20511. Linsky, J. L. Winds in late-type stars: Mechanisms of mass outflow, (Proc. Effects of Mass Loss on Stellar Evolution Colloquim No. 59, Triests, Italy, Sept. 15.19, 1980), Paper in Effects of Mass Loss on Stellar Evolution, C. Chiosi and R. Stalio, eds., pp. 187-212 (D. Reidel Publishing Company, Dordrecht, Holland, 1981).

Key words: stars, atmospheres; stars, coronae; stars, late-type; stars, winds; ultraviolet, spectra; $x$ rays, sources.

Four basic mechanisms have been proposed to explain the acceleration ' of winds in late-type stars-thermal pressure gradients, radiation pressure on circumstellar dust grains, momentum addition by Alfvén waves, and momentum addition by periodic shock waves. In this review I describe recent work in applying these mechanisms to stars, and consider whether these mechanisms can work even in principle and whether they are consistent with recent ultraviolet and $\mathrm{X}$-ray data from the IUE and Einstein spacecraft. Thermally-driven winds are likely important for late-type dwarfs, where the mass loss rates are small, and perhaps also in G giants and supergiants, but they cannot operate alone in the $K$ and $M$ giants and supergiants. Radiatively-driven winds are probably unimportant for all cool stars, even the $M$ supergiants with dusty circumstellar envelopes. In principle, Alfvén waves can accelerate winds to high speeds provided the field lines are initially open or forced open by some mechanism but detailed calculations are needed. Magnetic reconnection is an interesting suggestion for an acceleration mechanism when the field lines are initially closed. For the Miras and semiregular variable supergiants, periodic shock waves provide a simple way of producing rapid mass loss. Thus we are making some progress in understanding mass loss mechanisms for the cool half of the H.R diagram.

20512. May, W. B., Jr.; Spielvogel, L. G. Analysis of computer- simulated thermal performance: The Norris Cotton Federal Office Building, (Proc. ASHRAE Semi-Annu. Meet., Chicago, IL, Jan. 25. 29, 1981), ASHRAE J. 87, Pt. 1, 43-50 (1981).

Key words: building models; building performance data; 
computer simulations, building; energy conservation in commercial buildings; heat pumps; validation of computer models, buildings.

Five computer-based simulations of the Norris Cotton Federal Office Building (NCFOB) in Manchester, New Hampshire, were performed using a state-of-the-art proprietary hour-by-hour building simulation program. Results of the simulations are compared with each other and with actual measured data at several levels of detail including total energy consumption, consumption by fuel type, and heating and cooling requirements. Good agreement between the simulation and actual data is demonstrated and consequences of design features are discussed.

20513. Durst, R. A.; Siggaard-Andersen, O.; Maas, A. H. J. Reference and quality control materials for $\mathrm{pH}$ and blood gases, (Proc. 5th Meet. IFCC Expert Panel on pH and Blood Gases, Copenhagen, Denmark, June 16.18, 1980), Paper in Blood pH, Carbon Dioxide, Oxygen, and Calcium-ion, O. Siggaard-Andersen, ed., pp. 89-100 (Private Press, Copenhagen, Denmark, 1981).

Key words: blood gases; blood $\mathrm{pH}$; carbon dioxide; oxygen; $\mathrm{pH}$; $\mathrm{pH}$ quality control; $\mathrm{pH}$ reference materials.

A review of clinical reference and quality control materials is presented. The hierarchical structure of the measurement chain from the basic measurement units of the SI via standards and measurement methods to the routine laboratory determinations is illustrated. The variety of reference materials available for blood $\mathrm{pH}$ and gases (carbon dioxide and oxygen) are discussed as well as future needs in this area. A brief description is given of the NBS pH certification procedures.

20514. Ehrlich, M. Choice of radiation protection quantities for a personnel dosimetry performance standard, (Proc. European Seminar on Radiation Protection Quantities for External Exposure, Braunschweig, Federal Republic of Germany, Oct. 13-15, 1980), Paper in European Seminar on Radiation Protection Quantities for External Exposure, G. Burger, H. G. Ebert, D. Harder, R. Kramer, S. Wagner, eds., pp. 239-242 (Harwood Academic Publishers, New York, NY, 1981).

Key words: conversion factors; deep and shallow dose equivalents; measurements with extrapolation chamber; Monte Carlo calculation; personnel dosimetry; standard.

This is a discussion of the difficulties encountered in the development of a personnel radiation dosimetry performance standard that were related to the choice of a suitable quantity for reporting the results and to the derivation of conversion factors to this quantity from the quantities in which personnel dosimeters are usually calibrated.

20515. Jacox, M. E. Hydrogen-atom abstraction by atomic fluorine. Vibrational spectrum of the $\mathbf{F}+\mathrm{CH}_{3} \mathrm{~F}$ reaction products trapped in solid argon, J. Chem. Phys. 59, 199.212 (1981).

Key words: $\mathrm{CF}$; $\mathrm{CH}_{2} \mathrm{~F} ; \mathrm{CH}_{2} \mathrm{~F}_{2} ; \mathrm{CH}_{3} \mathrm{~F}$; F-atom reactions; $\mathrm{HCF}$; HF complex; infrared spectrum; matrix isolation; photodecomposition.

When the products of the interaction between methyl fluoride and $F$ atoms, produced in a microwave discharge, are frozen in a large excess of argon at $14 \mathrm{~K}$, infrared absorptions previously assigned to $\mathrm{CH}_{2} \mathrm{~F}, \mathrm{HCF}$, and $\left[\mathrm{CH}_{3} \mathrm{~F}+\mathrm{HF}\right]$ appear. The reaction of $\mathrm{F}$ atoms in the solid also leads to the appearance of prominent absorptions of the $\left[\mathrm{CH}_{2} \mathrm{~F}+\mathrm{HF}\right]$ hydrogen-bonded complex. At higher F.atom concentrations, absorptions of $\mathrm{CH}_{2} \mathrm{~F}_{2}$ and of $\left[\mathrm{CH}_{2} \mathrm{~F}_{2}+\mathrm{HF}\right]$ become prominent. A revised assignment is proposed for $C_{2} F$. At wavelengths shorter than $280 \mathrm{~nm}, \mathrm{CH}_{2} \mathrm{~F}$ photodecomposes, producing $\mathrm{CF}$. The photodecomposition of $\left[\mathrm{CH}_{2} \mathrm{~F}+\mathrm{HF}\right]$, which has a somewhat higher energy threshold, leads to the appearance of $[\mathrm{CF}+\mathrm{HF}]$. A detailed discussion of the chemical reactions and photodecomposition processes characteristic of these experiments is presented.

20516. Danos, M. Nuclear forces from quark dynamics, (Workshop on
Nuclear Physics with Real and Virtual Photons, Bologna, Italy, Nov. 25-28, 1980), Paper in Collective States to Quarks in Nuclei, 1 page (Comitato Nazional Energia Nucleare, Bologna, Italy, 1980).

Key words: bag model; confinement; nuclear forces; OZI rule; QCD; quark dynamics.

In the quark picture the exchange mesons propagate as free off-themass-shell particles only in the asymptotic OPEP range. For closer separations they are manifested only as correlations in a deformed dibaryon system. The techniques of computing the model are available from the relativistic nuclear physics.

20517. Clark, F. O.; Johnson, D. R. The L134-L183-L1778 system of interstellar clouds, Astrophys. J. 247, 104-111 (July 1, 1981).

Key words: interstellar clouds; L134; L183; magnetic braking; radio astronomy; spatial map.

Microwave spectral lines of several different molecules have been used as probes of a group of interstellar clouds which are located some $36^{\circ}$ above the galactic plane. These data suggest that the group of clouds may represent fragments of an original larger cloud. At three identifiable scale sizes, the observed gas appears to have progressively less rotation than expected from simple conservation of angular momentum, implying the existence of a redistributing mechanism at an early epoch. Radial velocities for the inner $4^{\prime}$ of L134 and L183 are interpreted as retrograde rotation of the core region in each object. Recent magnetic braking calculations are employed to explain the observed effects.

20518. Lehman, D. R.; Parke, W. C.; Maximon, L. C. Numerical evaluation of integrals containing a spherical Bessel function by product integration, J. Math. Phys. 22, No. 7, 1399.1413 (July 1981).

Key words: numerical evaluation of integrals; oscillatory integrams; product integration; quadrature rules; spherical Bessel function; weights and abscissas.

A method is developed for numerical evaluation of integrals with $k$-integration range from 0 to $\infty$ that contain a spherical Bessel function $j(k r)$ explicitly. The required quadrature weights are easily calculated and the rate of convergence is rapid-only a relatively small number of quadrature points is needed-for an accurate evaluation even when $r$ is large. The quadrature rule is obtained by the method of product integration. With the abscissas chosen to be those of Clenshaw-Curtis and the Chebyshev polynomials as the interpolating polynomials, quadrature weights are obtained that depend on the spherical Bessel function. An inhomogenous recurrence relation is derived from which the weights can be calculated without accumulation of roundoff error. The procedure is summarized as an easily implementable algorithm. Questions of convergence are discussed and the rate of convergence demonstrated for several test integrals. Alternative procedures are given for generating the integration weights and an error analysis of the method is presented.

20519. Lide, D. R., Jr. Critical data for critical needs, Science 212 , 1343.1349 (June 19, 1981).

Key words: data centers; data evaluation; information dissemination; on-line networks; standard reference data.

Various types of scientific and technical data are required for the solution of key societal problems such as energy supply, environmental quality, and industrial productivity. Assurance of quality control of these data bases is essential. Modern computer and telecommunications technology offers opportunities for major improvements in the dissemination of factual data to users, but a higher level of consciousness regarding data management is needed from the scientific community, industry, and government.

20520. Turrell, B. G.; Marshak, H. Magnetic structure determined by nuclear orientation, Hyperfine Interact. 10, 1231-1236 (1981).

Key words: conical spin structures; gamma-ray anisotropy; 
In the last few years there has been an increased use of the nuclear orientation technique to study ordered magnetic structures. For ordered atomic magnets the spin structure can be inferred from measurements of the spatial distribution of nuclear radiations because the atomic spins define the nuclear quantization axes through the hyperfine interaction. In nuclear magnets the order is determined directly from the radiation pattern. Expressions for the spatial distributions of $\gamma$-radiation from conical ordered structures are given and applications to antiferromagnets and the rare-earth magnet holmium are discussed.

20521. Kusuda, T. A comparison of energy calculation procedures, ASHRAE J. 23, No. 8, 21.24 (Aug. 1981).

Key words: ASHRAE TC 4.7; computer analysis; energy calculation; simplified energy analysis.

ASHRAE's Technical Committee on Energy Calculations (TC 4.7) has developed a proposed simplified procedure suitable for manual or pocket calculators. The proposed simplified procedure was compared with seven detailed computer simulation energy analysis programsAXCESS, BLAST, BLDSIM, DOE-2, E-CUBE, ESAS, and TRACE. The comparative calculations were done on a Washington, DC office building, for four typical HVAC systems. Discrepancies between the results using the seven computer simulation methods involving seven separate analysts were generally greater than the differences between the simplified method and a single computer simulation method when both were used by the same analyst. Major reasons for discrepancies between the proposed TC 4.7 simplified method and the detailed computer simulation methods are discussed, as well as the difficulties in using the simplified procedure.

20522. Durst, R. A. Views on the current status of the pH scale, (Proc. 5th Meet. IFCC Expert Panel on $\mathrm{pH}$ and Blood Gases, Copenhagen, Denmark, June 16-18, 1980), Paper in Blood $p H$, Carbon Dioxide, Oxygen, and Calcium-ion, O. Siggaard-Andersen, ed., pp. 11-22 (Private Press, Copenhagen, Denmark, 1981).

Key words: blood pH; pH; pH scale; $\mathrm{pH}$ standards; practical $\mathrm{pH}$ scale.

Several international organizations, e.g., IUPAC, ISO, OIML, IEC, are currently reviewing and/or revising their definitions of the $\mathrm{pH}$ scale. This paper reviews the present status of the $\mathrm{pH}$ scale and proposals for changes in the definition. A compromise proposal for an international practical $\mathrm{pH}$ scale is presented which retains the features of the NBS multiple primary standard $\mathrm{pH}$ scale while permitting the introduction of a single primary standard defining point and an indefinite number of secondary standards.

20523. Haller, W.; Gschwender, H. G.; Peters, K. R. Application of a general equation for controlled pore glass permeation chromatography to an aggregating, spherical virus, $J$. Chromatogr. 211, 53.59 (1981).

Key words: chromatography; controlled pore glass; electronmicroscopy; LCM-virus; lymphocytic choriomeningitis virus; size determination.

A semi-empirical permeation chromatography equation relating pore size, species size and elution coefficient, which had previously been derived from chromatographic data of narrow-molecular-weight dextrans on controlled pore glass, was applied to the chromatography of an aggregating virus particle. A large number of chromatographic runs on columns of different pore sizes were combined and statistically evaluated. The resulting diameter distribution versus infectivity curve for the virus particle population shows distinct maxima at multiples of $50 \mathrm{~nm}$. This unit size agrees with electron microscopical observations and confirms the applicability of the chromatography equation.

20524. Simiu, E. Modern developments in wind engineering: Part 2, Eng. Struct. 3, 242.248 (Oct. 1981).

Key words: aerodynamics; building codes; meteorology; wind
This is the second paper in a series devoted to a review of the state of the art in wind engineering. The paper presents information on wind tunnel testing for both research and design purposes. Specific topics covered include: The influence of viscosity effects upon the reliability of test results for bodies with curved shapes and with sharp edges; the extent to which atmospheric turbulence needs to be simulated in laboratory tests; the effect of turbulence scale and turbulence intensity upon aerodynamic forces; and difficulties encountered in the simulation of point and area loads.

20525. Simiu, E. Modern developments in wind engineering: Part 1, Eng. Struct. 3, 233-241 (Oct. 1981).

Key words: atmospheric turbulence;. buildings; design (structural); extreme winds; hurricanes; micrometeorology; structures; tornadoes; wind engineering; wind profiles.

The paper presents a state of the art review of material applicable to structural design, from the following areas: meteorology, micrometeorology (mean wind profiles and atmospheric turbulence characterization), and extreme wind climatology in well-behaved climates, hurricane-prone regions, and tornado-prone regions.

20526. Smith, D. R.; Hust, J. G. Measurement of effective thermal conductivity of a glass fiberboard standard reference material, Cryogenics 21, No. 7, 408.410 (July 1981).

Key words: glass fiberboard; insulation; low-temperature; standard reference material; thermal conductivity.

This paper describes the results of thermal conductivity measurements on NBS SRM 1450 glass fiberboard insulation (1970 lot) at temperatures from $87 \mathrm{~K}$ to $340 \mathrm{~K}$. The measurements were performed in an atmosphere of dry nitrogen at a pressure near $84 \mathrm{kPa}$ $(630 \mathrm{~mm} \mathrm{Hg})$. The results are analysed and compared to NBS certification data and to literature data for similar material.

20527. Evans, D. J.; Hanley, H. J. M. Soft-disc melting in small systems, Phys. Lett. 83A, No. 7, 344.346 (June 15, 1981).

Key words: computer simulation; melting; molecular dynamics; phase transitions; soft discs.

The location of melting for a soft-disc system of 32,50 and 98 particles $(N)$, respectively, is determined by molecular dynamics.

20528. Whipple, T. A.; McHenry, H. I.; Read, D. T. Fracture behavior of ferrite-free stainless steel welds in liquid helium, (Proc. 61st AWS Annu. Meet., Los Angeles, CA, Apr. 13-18, 1980), Paper in Welding Research Supplement 60, No. 4, 72-s-78-s (Apr. 1981).

Key words: cryogenic properties; fatigue; fracture toughness; microstructure; stainless steel; welding.

Most research to date concerning the cryogenic toughness of stainless steel weldments has concentrated on the effects of delta ferrite content and nitrogen concentration. It has been shown that an increase in either of these leads to reduced cryogenic toughness. In this paper it has been shown that a wide variation in fracture toughness occurs in $316 \mathrm{~L}$ weldments that contain no delta ferrite, and only small differences in nitrogen concentration. This shows that welding parameters and minor compositional variability must also have significant influences. Optical microscopy has revealed a strong correlation between cryogenic fracture toughness and fusion zone grain width, which is controlled by welding parameters and weld. metal composition. An increase in grain size reduces the toughness. Microfissuring, which is a problem in low ferrite welds, was not observed to have an effect on the measured fracture toughness; but in one case it severely reduced the tensile ductility. Fatigue crack growth rate tests have shown little variation in the fatigue properties of $316 \mathrm{~L}$ SMA weldments, regardless of the variations in other properties.

20529. Bur, A. J.; Tsao, A. K. Depolarization of poled $\mathrm{PVF}_{2}$ samples 
with "thick" electrodes, Ferroelectrics 32, 185-189 (1981).

Key words: depolarization; films; melting; piezoelectricity; polyvinylidene fluoride; pyroelectricity; thick electrodes.

Twenty-six poled biaxially oriented $\mathrm{PVF}_{2}$ samples, which were prepared with electrode thickness ranging from $750 \AA$ to $3100 \AA$, were investigated in three experiments: (1) the hydrostatic piezoelectric coefficient $d_{p}$ and the pyroelectric coefficient $P y$ were measured for the original samples: (2) $d_{\rho}$ and $P y$ were measured after the thick electrodes were removed; and (3) the depolarization charge was measured by detecting the discharge as the samples were subjected to an abrupt temperature rise through the melting point. The results of these measurements are: (1) the values of $d_{p}$ and $P y$ for the as received samples were lower, the thicker the electrode; (2) with the thick electrodes removed from the samples, the values of $d_{p}$ and $P y$ were the same as those for the thick electrodes in place: and (3) the depolarization charge decreased as the original electrode thickness increased. We have concluded that the decrease in $d_{p}$ and $P y$ as a function of electrode thickness is essentially due to the decreases in polarization of the thick electroded specimens and that thick electrodes mechanically constrain the sample during poling so that optimum poling cannot be achieved. Previous measurements on these samples showed that the $d_{31}$ coefficient was inversely related to electrode thickness.

20530. Geist, J.; Liang, E.; Schaefer, A. R. Complete collection of minority carriers from the inversion layer in induced junction diodes, J. Appl. Phys. 52, No. 7, 4879.4881 (July 1981)

Key words: inversion layer; minority carrier collection; photodiodes; quantum efficiency; silicon photodiodes.

Mechanisms limiting the internal quantum efficiency in various types of oxide-passivated silicon photodiodes are discussed. It is argued that unit internal quantum efficiency is achievable in metallurgical junction, oxide- $n^{+}-p-p^{+}$photodiodes, if it is achievable in the inversion layer of induced junction diodes of the same type. Measurements of the variation in response of the latter type of photodiode under both oxide bias and reverse bias are described. The results indicate that $100 \%$ collection of the minority carriers generated in the inversion layer is achieved for sufficiently low flux levels. Implantation in the oxide of $\mathrm{Na}^{+}$ions to augment the trapped positive charge increases the maximum flux level at which $100 \%$ collection is observed.

20531. Netzer, F. P. Auger line shapes analysis for characterization of molecular surface reaction products, Appl. Surf. Sci. 7, 289.311 (1981).

Key words: Auger line shape analysis; Auger spectra of adsorbed molecules; carbon monoxide absorbed on transition metals; electron structure of absorbed molecules; high-resolution Auger spectroscopy; transition metal carbonyls.

The literature of Auger line shape analysis of gas-phase, condensedphase and adsorbed molecules is compiled and briefly reviewed, and the use of high-resolution Auger spectroscopy to probe the local electronic structure and the chemical environment of atoms involved in the Auger process is emphasized. Exemplifying results are presented and discussed for transition metal carbonyl complexes, as models of adsorbate-metal surface systems, and for KVV Auger spectra of several carbon, oxygen and nitrogen containing molecules adsorbed on metal surfaces.

20532. Gadzuk, J. W. Localized vibrational modes in Fermi liquids. General theory, Phys. Rev. B 24, No. 4, 1651-1663 (Aug. 15, 1981).

Key words: electron-phonon interactions; localized modes; vibrational spectroscopy.

There exist an abundance of physically realized situations in atomic, chemical, molecular, nuclear, and solid-state physics which are modeled as a discrete state interacting with a continuum. One of these situations, a localized oscillator coupled to be the particle-hole excitation continuum of a Fermi liquid, forms the area of inquiry here.
Special emphasis is placed on the electron-hole pair excitations of a metal, although the results are more general. In particular, an exactly soluble model, in which a localized harmonic oscillator is coupled to the pair-density fluctuations of a random-phase-approximation Fermi liquid by an interaction linear in both oscillator displacements and density operators, is considered. It is shown that this model is equivalent to a system of coupled harmonic oscillators, and, as such, the eigenstates are obtained via a straightforward normal-mode analysis. Expressions for the local spectral function of the oscillator are obtained in terms of level-shift and broadening functions which are evaluated in closed form in the density-of-states limit. Parametric calculations for vibrational line shapes are made, and the results are discussed in terms of "exponential-decay theory." The general results presented here have direct bearing in areas such as surface vibrational spectroscopy of adsorbed molecules, metal-hydride spectroscopy, and surface chemical-reactivity theory.

20533. Poliakoff, E. D.; Dehmer, P. M.; Dehmer, J. L.; Stockbauer, $\mathrm{R}$. The photoelectron spectrum of $\mathrm{Xe}_{3}$ by the photoelectron-photoion coincidence technique, J. Chem. Phys. 75, No. 3, 1568-1569 (Aug. 1, 1981).

Key words: clusters; coincidence; mass spectrometry; photoelectron spectroscopy; photoionization; $\mathrm{Xe}_{3}$.

The photoelectron spectrum of $\mathrm{Xe}_{3}$ found in a mixed beam of cluster molecules from a supersonic free jet expansion of Xe has been measured using photoelectron-photoion coincidence. The technique energy-analyzes the electron and mass-analyzes the ion from single photoionization events. The vertical ionization potential of $\mathrm{Xe}_{3}$ is $11.47(9)$ and the adiabatic ionization potential is $10.84(4) \mathrm{eV}$.

20534. Padial, N. T.; Norcross, D. W.; Collins, L. A. On the use of the unitarised Born approximation in electron collisions with polar molecules, J. Phys. B: At. Mol. Phys. 14, 2901.2909 (1981).

Key words: electron collisions; polar molecules; unitarized Born approximation.

The validity of the unitarised Born (B II) approximation for representing the $T$-matrix elements for electron-polar-molecule collisions is investigated for a number of polar systems. For intermediate and high values of the angular momentum (usually $l \geqslant 5$ ), the $B$ II results are in excellent agreement with those of a more elaborate close-coupling calculation. The method provides an efficient, economical means for calculating the higher angular momenta $T$-matrix elements that are needed in the completion formulae for polar-molecule cross sections. The methods have an advantage over such comparable schemes as the fixed-point-dipole technique in that contributions from potential moments above the dipole can be simply included.

20535. Ayres, T. R.; Marstad, N. C.; Linsky, J. L. Outer atmospheres of cool stars. IX. A survey of ultraviolet emission from F.K dwarfs and giants with IUE, Astrophys. J. 247, 545-559 (July 15, 1981).

Key words: stars, atmospheres; stars, chromospheres; stars, coronae; stars, late-type; ultraviolet, spectra.

We report preliminary results of an ultraviolet survey of cool-star emission properties with IUE. We present $1150-2000 \AA$ spectra of representative F-K dwarfs and giants and construct correlation diagrams that compare chromospheric $\left(T \leqslant 10^{4} \mathrm{~K}\right)$ and transition. region $\left(T \approx 10^{5} \mathrm{~K}\right)$ emission line strengths, and broad-band coronal $\left(T \geqslant 10^{6} \mathrm{~K}\right)$ soft-X-ray fluxes. We find that the transition-region (TR) and coronal emission in the G.K dwarfs and $G$ giants is well correlated with the $\mathrm{Mg} 11 \lambda 2800$ doublet emission strength, which in turn is symptomatic of chromospheric energy losses. However, the power-law slopes are steeper than unity, particularly for soft X-rays. We conclude that while the distinct atmospheric layers very likely are physically associated, the internal heating mechanisms must be quite different. Despite the apparent chromosphere-TR-corona correlations among the G.K dwarfs and $G$ giants, the earlier $(F)$ and later $(K)$ giants exhibit anomalous behavior. The former appear to have brighter TRs for a given chromospheric emission level, while the latter are systematically deficient in $T \geqslant 10^{5} \mathrm{~K}$ material. In fact, not 
only are TRs very weak among the red giants, but chromospheres and coronae are systematically weak as well (at least compared with the mean activity levels of F.G giants). We propose that the weakness of hot outer atmospheres in the red giants compared with the yellow giants can be understood as a consequence of stellar evolution since stars of slightly different spectral type in the giant branch likely have very different main-sequence progenitors.

20536. Feigerle, C. S.; Herman, Z.; Lineberger, W. C. Laser photoelectron spectrometry of $\mathrm{Sc}^{-}$and $\mathrm{Y}^{-}$: A determination of the order of electron filling in transition-metal anions, J. Electron Spectrosc. Relat. Phenom. 23, 441.450 (1981).

Key words: electron filling; negative ions; photodetachment; photoelectron spectrometry; $\mathrm{Sc}^{-}$; $\mathrm{Y}^{-}$.

The photoelectron spectra of $\mathrm{Sc}^{-}$and $\mathrm{Y}^{-}$have been obtained in a crossed ion- and laserbeam experiment. Analysis of the $\mathrm{Sc}^{-}$spectrum yields two bound terms of $3 d 4 s^{2} 4 p$ configuration $\left({ }^{1} D^{0}\right.$ and $\left.{ }^{3} D^{0}\right)$, with $\mathrm{EA}(\mathrm{Sc})=0.189 \pm 0.020 \mathrm{eV}$ and an excited-state binding energy of $0.042 \pm 0.020 \mathrm{eV}$. Similarly, the $\left(4 d 5 s^{2} s p\right)^{1} D^{0}$ ground state of $\mathrm{Y}^{-}$is bound by $0.308 \pm 0.012 \mathrm{eV}$ and a $\left(4 d 5 s^{2} 5 p\right)^{3} D^{0}$ excited term is bound by $0.165 \pm 0.025 \mathrm{eV}$. With the determination of the bound electronic configuration of $\mathrm{Sc}^{-}$as $3 d 4 s^{2} 4 p$, the order of filling of electron shells of the first transition series negative ions is found to be $4 s^{2} 4 p, 3 d 4 s^{2} 4 p$, followed by $3 d^{k} 4 s^{2}(k=3,4, \ldots, 10)$.

20537. Drummond, P. D.; Gardiner, C. W.; Walls, D. F Quasiprobability methods for nonlinear chemical and optical systems, Phys. Rev. A 24, No. 2, 914-926 (Aug. 1981).

Key words: aster equations; chemical processes; Fokker-Planck equations; quantum optics; quasiprobability.

A review of quasiprobability methods for transforming chemical and quantum-optical master equations into Fokker-Planck equations is presented. For cases where conventional representations lead to Fokker-Planck equations with non-positive-definite diffusion coefficients; e.g., sub-Poissonian statistics, a generalization of the representation involving an extension to the complex plane enables analytic results to be obtained for certain nonlinear chemical and optical processes. Alternatively, a different integration measure may be chosen which ensures a positive distribution and Fokker-Planck equation with positive-semidefite diffusion coefficients. This enables stochastic differential equations to be defined. These methods are applied to two-photon absorption and dispersive bistability in quantum optics where nonclassical photon statistics arise and to two models of nonlinear chemical reactions where sub.Poissonian statistics occur.

20538. Gujral, P. S.; Clark, R. J.; Burch, D. M. Transient thermal response of an intermittently cooled massive building, (Proc. ASHRAE/DOE/ORNL Conf. Thermal Performance Exterior Envelopes of Buildings, Orlando, FL, Dec. 3.5, 1979), ASHRAE SP 28. pp. 751.756 (American Society of Heating, Refrigeration and Air-Conditioning Engineers, New York, NY, 1981).

Key words: energy conservation; night cooling; night ventilation; thermal mass.

A single-room externally insulated masonry test house was built within the environmental chamber at the National Bureau of Standards. The structure was subjected to diurnal summer sol-air temperature cycles while two energy conservation schemes were monitored. Both schemes utilized the building thermal mass to store nighttime cooling energy; the first, through night mechanical cooling, and the second through the use of ambient air. The results indicate that mechanical cooling is effectively stored in the building mass during the night so that no additional daytime cooling is needed for even the most extreme summer conditions. The tests further indicate that circulation of night ambient air effectively cools the structure so that no mechanical cooling is needed for summer cycles typical of many regions in the United States.

The experimental test results are compared with those obtained from an analytical model, with excellent agreement.

20539. Richtmyer, T. E.; May, W. B.; Hunt, C. M.; Hill, J. E.
Thermal performance of the Norris Cotton Federal building in Manchester, New Hampshire, (Proc. ASHRAE/DOE/ORNL Conf. Thermal Performance Exterior Envelopes of Buildings, Orlando, FL, Dec. 3.5, 1979), ASHRAE SP 28, pp. 781.797 (American Society of Heating, Refrigeration and Air-Conditioning Engineers, New York, NY, 1981).

Key words: air-cooling; air leakage; energy; heat-recovery; insulation; measurement; office-building; radiant; solar; spaceheating.

The Norris Cotton Federal Office Building is a medium-size 7-story government office building of approximately $11,000 \mathrm{~m}^{2}\left(117,000 \mathrm{ft}^{2}\right)$ total floor space. It is located in Manchester, New Hampshire and has been designed to demonstrate a number of energy saving concepts.

Some of the major energy conserving features of the building are the use of solar collectors; heavy masonry construction with exterior insulation; small overall window area; heat recovery from heat pumps, chillers, a natural gas-powered engine/generator, and the ventilation system; modular boilers; thermal storage tanks; and a variety of energy conserving lighting systems.

The staff of the National Bureau of Standards (NBS) has been monitoring the performance of the building since it was occupied in September, 1976. This paper will describe the building's thermal performance for the first three years of operation. The energy consumption in the building is presented and compared to the original design goal of $625 \mathrm{MJ} /\left(\mathrm{m}^{2}\right.$.years) $\left(55 \mathrm{kBtu} /\left(\mathrm{ft}^{2}\right.\right.$.year $\left.)\right)$. The differences will be explained using the results of thermographic measurements and measurements of air exchange rates in the building along with analysis of the weather data and building operational problems that have occurred since 1976. It has been found that it is difficult to actually achieve designed-for performance in this building because its experimental mechanical system is unusually complex and its construction details are unconventional.

20540. Grot, R. A.; Clark, R. E. Air leakage characteristics and weatherization techniques for low-income housing, (Proc. ASHRAE/DOE/ORNL Conf. Thermal Performance Exterior Envelopes of Buildings, Orlando, FL, Dec. 3-5, 1979), ASHRAE SP 28. pp. 178-194 (American Society of Heating, Refrigeration and Air-Conditioning Engineers, New York, NY, 1981).

Key words: air infiltration; air sample bags; fan depressurization; low-income houses; weatherization.

Data are presented on the air leakage characteristics of approximately 250 dwellings occupied by low-income households in 14 cities, in all major climatic zones of the United States. Two types of measurements were used: a tracer-gas decay technique using air sample bags, which was developed at the National Bureau of Standards to measure natural air infiltration; and a fan depressurization test that measures induced air exchange rates. The data presented here show that for this group of dwellings natural air infiltration rates are distributed approximately lognormally.

The induced air exchange rates are a measure of the tightness of building envelopes. There is little correlation between the natural air infiltration rates and the induced air exchange rates in these dwellings, unless the buildings are divided into classes of similar buildings. The use of fan depressurization as a diagnostic tool to assist weatherization crews in tightening buildings is discussed. Preliminary estimates are presented of the reduction in induced air exchange rates that may be achieved by applying building weatherization techniques.

20541. Gillette, G. The energy potential of daylighting in a classroom, (Proc. ASHRAE/DOE/ORNL Conf. Thermal Performance Exterior Envelopes of Buildings, Orlando, FL, Dec. 3.5, 1979), ASHRAE SP 28, pp. $368-380$ (American Society of Heating, Refrigeration and Air-Conditioning Engineers, New York, NY, 1981).

Key words: daylighting; energy conservation; envelope design; instructional facilities; passive solar energy; windows.

An analysis is presented where a classroom of prescribed size and occupancy has various fenestration designs applied to it and the resulting thermal and daylighting energy performance calculated. An 
attempt is made to relate the heating/cooling requirements of a window opening with its potential as a source of natural light. The parameters glass area, glass type (double-pane, reflective, etc.), and ceiling height are evaluated for a classroom in Ann Arbor, Michigan operated over the course of a 9-month school year. Comparisons are made between the performance of a design based on ASHRAE Standard 90.75 and alternate fenestration designs. Although the computerized thermal analysis and the daylight analysis had to be done separately, actual weather data and corresponding daylight readings for Ann Arbor, Michigan are used for both. Results show a potential energy savings when daylighting is carefully integrated into the building's envelope design, especially for southern exposures, but such savings will be realized only if applied with the other energy variables in mind.

20542. Miiller, A. P.; Cezairliyan, A. The melting point of palladium by a pulse heating method, Int. J. Thermophys. 2, No. 1, $63-70$ (1981).

Key words: high temperatures; melting point; palladium; pulse heating.

The melting point of $99.95^{+} \%$ pure palladium was measured by means of a subsecond duration pulse heating technique. The average temperature at the melting point for three specimens was $1827 \mathrm{~K}$ with an average absolute deviation from the mean of $0.1 \mathrm{~K}$. The total error in temperature is estimated to be not more than $\pm 4 \mathrm{~K}$.

20543. Fortunko, C. M. Ultrasonic detection and sizing of twodimensional defects at long wavelengths, Appl. Phys. Lett. 38, No. 12, 980-982 (June 15, 1981).

Key words: elastic wave scattering; nondestructive evaluation; nondestructive testing; shear-horizontal waves; ultrasonic testing; ultrasonic wave scattering.

An ultrasonic technique is described for detecting and sizing of two-dimensional defects. The technique is particularly suitable for detecting two-dimensional defects in certain butt-weldments which cannot be fully inspected by conventional radiographic and ultrasonic methods. Use is made of noncontact, electromagnetic-acoustic transducers to excite and detect shear horizontal (SH) wave probing signals where wavelength is long compared to the defect depth dimensions. The new technique is demonstrated in conjunction with a weld fatigue testing investigation in which it is used to detect natural weld defects and to monitor crack initiation and growth under tension-tension fatigue loading. An experimental calibration curve is given for sizing two-dimensional surface defects, and the experimental results are explained in terms of a reciprocity relationship evaluated in the elastrostatic limit.

20544. Fortunko, C. M. Ultrasonic detection and sizing of twodimensional weld defects in the long-wavelength limit, Proc. 1980 Ultrasonics Symp., Boston, MA, Nov. 4-7, 1980, pp. $862-867$ (Institute of Electrical and Electronics Engineers, New York, NY, 1980).

Key words: electromagnetic transducers; nondestructive evaluation; ultrasonic testing; ultrasonic transducers; ultrasonic wave propagation; weld inspection.

A new ultrasonic inspection technique is described for detecting elongated defects in butt-weldments. The technique can be used to detect and size two-dimensional defects which can potentially impair the fitness-for-purpose condition of pipeline girth welds. The defect sizing is accomplished by inverting long-wavelength scattering data obtained with shear-horizontal (SH) waves. Noncoupling, electromagnetic-acoustic transducers (EMATs) are used to excite and detect the $450 \mathrm{kHz} \mathrm{SH}$-wave probing signals. The operation of a prototype system is demonstrated in conjunction with a weld fatigue investigation. The technique is used to detect an incomplete fusion defect with a through-wall depth of $0.5 \mathrm{~mm}(0.020$ inch) and to monitor crack growth in the heat affected zone (HAZ) under tensiontension fatigue loading. In addition, an experimental calibration curve is given for sizing two-dimensional surface defects. The experimental results are explained in terms of a reciprocity relationship evaluated in the elastostatic limit.

20545. Repjar, A. G.; Kremer, D. P. Results of planar near-field measurements on a compact range at 18 and $54 \mathrm{GHz}$, (Proc. Antennas and Propagation, Quebec, Canada, June 2.6, 1980), Paper in Antennas and Propagation 1, IEEE No. 80 CH 1557-8 AP, 256-259 (Institute of Electrical and Electronics Engineers, New York, NY, 1980).

Key words: compact range; millimeter measurements; near-field measurements; planar near-field measurements; precision reflector; scalar feeds; V.band measurements.

Significant progress in recent years has been made on planar nearfield measurements for antenna calibrations. Such measurements are also useful in the alignment and evaluation of compact ranges because they provide more information than a limited number of analogue plots in one dimension. Contour plots of amplitude and phase data obtained from more complete 2-dimensional measurements precisely and accurately locate sources of problems in the range reflector, with phase contour plots being more useful as diagnostic tools.

The goal of the compact range under test was to provide an approximate plane wave illumination over a measurement zone 120 $\mathrm{cm}$ square with less than $0.5 \mathrm{~dB}$ amplitude variation and less than 10 degree phase variation at any frequency in the 12 to $100 \mathrm{GHz}$ range. The results of the planar near-field measurements were to determine if the goal could be achieved.

20546. Read, D. T.; Reed, R. P. Fracture and strength properties of selected austenitic stainless steels at cryogenic temperatures, Cryogenics 21, No. 7, 415-418 (July 1981).

Key words: austenitic stainless steels; cryogenic temperatures fatigue crack growth rates; fracture toughness; nitrogen strengthening; tensile properties.

Austenitic stainless steels have an excellent combination of mechanical and physical properties for load-bearing structures of large superconducting magnets for plasma containment in magnetic fusion experiments. To assess their relative suitability fracture toughness, fatigue crack growth, and tensile properties data for five austenitic steels at 295,76 , and $4 \mathrm{~K}$ have been obtained. The steels were AISI 304, 316, 304LN, and 316LN, and an Fe.21cr.12Ni-5Mn alloy with a higher nitrogen content than the other four grades. The two principal findings were the systematic variation of yield strength with nitrogen content and a systematic inverse correlation between fracture toughness and yield strength. Data from previous studies are reviewed which confirm the trends of the present data

20547. Smith, D. R.; Hust, J. G.; Van Poolen, L. J. Measurement of effective thermal conductivity of glass fibre blanket standard reference material, Cryogenics 21, No. 8, 460.462 (Aug. 1981).

Key words: fiberblanket; insulation; low temperature; standard reference material; thermal conductivity.

This paper describes the results of thermal conductivity measurements at temperatures from $84 \mathrm{~K}$ to $360 \mathrm{~K}$ on a glass fibreblanket insulation which is intended to be a Standard Material (SRM). The measurements were performed in an atmosphere of dry nitrogen at a pressure near $84 \mathrm{kPa}(630 \mathrm{~mm} \mathrm{Hg})$. The results are analysed and compared to literature data for similar material.

20548. Read, D. T.; McHenry, H. I. Strain dependence of the J-contour integral in tensile panels, (Proc. Fifth Int. Conf. Fracture Advances in Fracture Research, Cannes, France, Mar. 29.Apr. 3, 1981), Paper in Advances in Fracture Research, D. Francois, ed., pp. 1715-1722 (Pergamon Press, New York, NY, 1980).

Key words: crack driving force; elastic-plastic; finite-elementanalysis; fracture mechanics; $J$ integral; tensile panels; theoretical models.

The $\mathbf{J}$ contour integral has been experimentally measured as a function of applied strain for single edge notch tensile panels under elastic-plastic loading conditions. The results have been compared to analytical predictions based on finite element analysis and to theoretical estimates based on models representing the two behavioral extremes, uniform strain and perfect plasticity. The experimental, 
analytical and theoretical results have the expected form: the J.integral initially increases as the square of the applied strain, and at strains above yield the J-integral is a linear function of strain. The experimental and analytical results are in reasonable agreement, while the uniform strain and perfect plasticity models under- and overestimate $J$ respectively. An extension of the perfect plasticity model is proposed to treat behavior between these two limiting cases.

20549. Yaghjian, A. D. Reply to criticism on "electric dyadic Green's functions in the source region", Proc. IEEE 69, No. 2, 282-285 (Feb. 1981).

Key words: delta function solutions; dyadic Green's functions; principal volume; source dyadic; volume integral equation.

A. D. Yaghjian wrote a paper, "Electric Dyadic Green's Functions in the Source Region," which was published in the February 1980 Issue of the Proceedings of the IEEE. Professor C. T. Tai subsequently wrote a letter to the editor of the Proceedings, in which he criticized some technical points in Yaghjian's paper. This letter contains Yaghjian's response to the issues raised by Tai. Both letters will be published together in a future issue of the Proceedings.

20550. Jacox, M. E. The reaction of excited argon atoms and of $F$ atoms with methanol. Vibrational spectrum of $\mathrm{CH}_{2} \mathrm{OH}$ isolated in solid argon, J. Chem. Phys. 59, 213-230 (1981).

Key words: $\mathrm{CH}_{2} \mathrm{OH}$; $\mathrm{CH}_{3} \mathrm{OH}$; DCO; F-atom reactions; HF complex; infrared spectrum; matrix isolation;

photodecomposition.

When the products of the interaction between methanol and either excited argon atoms or $\mathrm{F}$ atoms, produced in a discharge, are frozen in a large excess of argon at $14 \mathrm{~K}$, prominent infrared absorptions of $\mathrm{CH}_{2} \mathrm{OH}$ appear. Detailed isotopic substitution studies have permitted the derivation of a set of valence-force potential constants for this species. There is some evidence for the stabilization of $\mathrm{CH}_{3} \mathrm{O}$, previously identified as a product of these two reactions, but the reaction channel producing $\mathrm{CH}_{2} \mathrm{OH}$ predominates. Secondary $\mathrm{H}$-atoms abstraction and photodecomposition lead to the stabilization of $\mathrm{H}_{2} \mathrm{CO}$, with no evidence for an $\mathrm{HCOH}$ intermediate. $\mathrm{HCO}$ also appears. The highest frequency fundamental of DCO stabilized in solid argon agrees considerably more closely with the recently reported gas-phase band center than does the corresponding absorption in a carbon monoxide matrix. In the $F$-atom reaction studies the $\left[\mathrm{CH}_{2} \mathrm{OH}+\mathrm{HF}\right]$ hydrogen-bonded complex is also stabilized. The infrared absorption spectrum of this complex suggests that the HF is hydrogen-bonded to the lone pair electrons on the oxygen atom.

20551. Batts, M. E.; Changery, M. J. Wind speed averaging-time relationship: Experimental data, Proc. ASCE Annu. Fall Conv., Hollywood, FL, Oct. 27-31, 1980, 10 pages (American Society of Civil Engineers, New York, NY, Oct. 1980).

Key words: climatology; statistical analysis; wind (meteorology).

In the United States, the most common maximum windspeed measurements, for use in engineering calculations, are in units of fastest miles. Other measurements consist of averaged windspeed over longer intervals.

Due to the way current code procedures to determine dynamic wind loads involve mean wind speeds, some conversion relation is necessary in order to compare these velocities.

This paper deals with the problems associated with experimentally measuring the parameters of a conversion relation between windspeeds averaged over various time intervals.

20552. Senich, G. A. Migration to and from plastics, CHEMTECH 11 , No. 6, 360.365 (June 1981).

Key words: diffusion; gas chromatography; interaction parameter; inverse gas chromatography; migration; packaging; partition coefficient; polyethylene; polyolefin.

The extent of migration of an impurity or additive from a polymer container into its contents can be estimated from data readily obtained by inverse phase gas chromatography (IGC) experiments. The specific retention volume is used to determine the polymer-migrant interaction parameter which when combined with the solubility of the migrant in the solvent allows calculation of the equilibrium partition coefficient, a direct measure of the fraction of the additive which can be expected to migrate from the container to the product. If the shelf life is short, an equilibrium prediction may greatly exaggerate the degree of migration expected. A value for the diffusion coefficient of the migrant in the polymer can then be obtained from IGC experiments and employed to estimate the degree of migration which will occur over the expected shelf life. Results are given for IGC studies on high density polyethylene with decane and octadecane migrants.

20553. Purdy, J. A.; Harms, W. B.; Fivozinsky, S. A computer based file of $\mathrm{x}$-ray and electron beam central axis depth dose data for use in radiation therapy, Proc. Fourth Annu. Symp. Computer Applications in Medical Care, Washington, DC, Nov. 2-5, 1980, pp. 94-103 (Institute of Electrical \& Electronics Engineers, New York, NY, Nov. 1980).

Key words: computer; data compilation; depth dose; dosimetry; electron; radiation therapy; $x$ ray.

The central axis absorbed dose data for $\mathrm{x}$-ray and electron beams generated by linear accelerators in the energy range 4 thru $25 \mathrm{MV}$ are being compiled. The compilation includes specific $\mathrm{x}$-ray beam parameters (surface doses, output factors, percent depth doses, tissuephantom ratios, and wedge factors) as well as electron beam parameters (percent depth doses and output factors). The compilation includes published data sets of these parameters and those obtained directly from over 100 institutions participating in the study.

The data are grouped by accelerator model and input into computer files that provide a standard format suitable for intercomparisons. The software used to construct the computer files and to manipulate the data is discussed. Selected examples of the average values of parameters obtained to date with the standard deviations, the coefficients of variation, and the maximum and minimum values will be presented for several different linear accelerator models.

20554. Fivozinsky, S. P.; Purdy, J. A. Compilation and evaluation of radiation depth dose data from electron accelerators used for radiotherapy: Experiences of a pilot data project in a hospital environment, Proc. 7th Int. CODATA Conf. Data for Science and Technology, Kyoto, Japan, Oct. 8-11, 1980, pp. 50.54 (Pergamon Press, Oxford, United Kingdom, July 1981).

Key words: data compilation; depth dose; electron accelerator electrons; photon; radiation therapy.

The Office of Standard Reference Data of the U.S. National Bureau of Standards is supporting a pilot data project to provide evaluated compilations of central axis absorbed dose data for the use of the radiotherapy community. The project is presently concentrating on the compilation of measured photon and electron data from linear accelerators in the energy range 4 to $25 \mathrm{MeV}$. Data sets used in the study include published values and data obtained directly from over 100 participating institutions. This paper discusses the reasons for establishment of the project, methods of evaluation, formatting and dissemination, probable impact on the medical radiation physicist and radiation oncologist, and the unique aspects of a physics data center located in a clinical environment.

20555. Teague, E. C.; Vorburger, T. V.; Maystre, D. Light scattering from manufactured surfaces, Ann. CIRP 30, No. 2, 1-7 (Dec. 1981).

Key words: angular distributions; light scattering experiments; light scattering theory; manufactured surfaces; speckle; surface roughness.

We present an evaluation of light scattering theories and experimental techniques for measuring the roughness of manufactured surfaces. Our goal was to define the ultimate capabilities and regions of validity of these methods for deducing surface microtopography. 
Available theories are evaluated in terms of a heuristic interpretation of direct electromagnetic scattering from surfaces. Experimental methods using specular reflectance, total integrated scatter, angular scattering distribution, and speckle are reviewed in terms of their spatial-bandwidth sampling of surface wavelengths and their roughness amplitude sensitivity and accuracy. Graphs in a slope/relative-wavelength space are used to intercompare the regions of validity of theories, regions of applicability for experimental methods and regions occupied by typical manufactured surfaces. A relatively unknown theory, valid in regions occupied by many manufactured surfaces but previously unused for this purpose, is briefly described.

20556. Mosburg, E. R., Jr. Effect of ion current in the collisionless theory of floating ac probe measurements, Rev. Sci. Instrum. 52, No. 8, 1182-1186 (Aug. 1981).

Key words: electron temperature; floating probes; Langmuir probes; plasma diagnostics; probes.

Previous treatment of the theory of floating ac probes has considered only the electron current voltage dependence. In this paper the effect of including the voltage dependence of the ion current is examined, and equations are derived which allow the use of the calculations by Laframboise of the ion current to cylindrical probes having arbitrary ratios of probe diameter to Debye length. The error in electron temperature measurements introduced by neglecting the ion current variation, and the range of usefulness of the technique, is discussed. For example, in the normally expected range of floating potential, a measurement of the electron temperature could be in error by as much as $\sim 13 \%$ if the voltage dependence of ion current is ignored.

20557. Kasen, M. B. Mechanical properties of plastic composites under low temperature conditions, Composites, Lett. Ed., 12, No. 2, 107 (Apr. 1981).

Key words: composites; cryogenics; epoxy matrices; fiber content; flexibilized epoxies; mechanical properties.

A publication by K. Kaotani, "Mechanical Properties of Plastic Composites Under Low Temperature Conditions," Composites, 11, No. 2, April 1980, pp. 87-94, is interpreted for composite laminates having substantially higher fiber contents than that studied in the reported work. It is shown that the conclusion of the author as to the superiority of highly flexibilized epoxy matrices cannot be extrapolated to the general class of commercial laminates.

20558. Gann, R. G.; Earl, W. L.; Manka, M. J.; Miles, L. B. Mechanism of cellulose smoldering retardance by sulfur, Proc. Eighteenth Symp. Combustion, Pittsburgh, PA, Aug. 17-22, 1980, pp. 571-578 (The Combustion Institute, Pittsburgh, PA, 1981).

Key words: cellulose; combustion; fire; inhibition; smoldering; sulfur.

The addition of elemental sulfur is effective in preventing cotton smoldering, a process which leads to a major fraction of fire loss. The objective of this work is to determine the mechanism of the observed retardance. Double thickness samples of cotton terrycloth, approximately $18 \mathrm{~mm}$ by $100 \mathrm{~mm}$, were smoldered in a Pyrex cylinder. The samples contained $0,5,8$ and 10 percent sulfur by weight. The air velocity over the face of the sample was about 10 $\mathrm{cm} / \mathrm{s}$. The 10 percent treated samples extinguished. The smoldering velocities and peak temperatures of the others were determined. Differential scanning calorimetry indicated only minor thermal effects of the sulfur. Electron paramagnetic resonance data showed that a reduction in the number of free radicals in the char by a factor of two resulted from the addition of five percent sulfur. X-ray fluorescence measurements showed that the concentration of bound sulfur in the char was comparable to that of eliminated free radicals. Mass spectrometric studies indicated that little sulfur was oxidized, and then only to $\mathrm{SO}_{2}$. These results eliminate reaction of sulfur with oxygen as an important contributor to the retardancy. Rather, the sulfur vaporizes and reacts with the radical sites in the char. In the absence of sulfur, these sites would oxidize exothermically and regenerate to continue the smoldering. Being poisoned, less heat is released and at a slower rate and the resulting pyrolysis of further fuel is retarded. Thus the critical properties of sulfur are vaporization at the correct temperature, reactivity with the char, and stability of the resulting adduct.

20559. Lawn, B. R.; Hockey, B. J.; Wiederhorn, S. M. Atomically sharp cracks in brittle solids: An electron microscopy study, $J$. Mater. Sci. 15, 1207-1223 (1980)

Key words: brittle solids; electron; electron microscopy study; microscopy

The issue of bond rupture versus microplasticity as an essential mechanism of crack propagation in brittle solids is addressed. A detailed survey of existing theoretical and experimental evidence relating to this issue highlights the need for direct observations of events within the crack-tip "process zone," at a level approaching 10 $\mathrm{nm}$. Transmission electron microscopy is accordingly used to study arrested cracks about sharp-contact (Vickers indentation and particle impact) sites in $\mathrm{Si}, \mathrm{Ge}, \mathrm{SiC}$ and $\mathrm{Al}_{2} \mathrm{O}_{3}$. The nature of the deformation which accommodates the irreversible contact impression is first investigated, in the light of Marsh's proposal of an "equivalence" between indentation and crack-tip zone processes. Interfacial and tip regions of the surrounding cracks are then examined for any trace of a plasticity-controlled fracture process. Dislocation-like images are indeed evident at the crack planes; but these are shown to be totally inconsistent with any conventional slip mechanism. The close connection between the dislocation patterns and moiré fringe systems along the cracks points to "lattice mismatch" contrast in association with a partial closure and healing operation at the interface. Analysis of all other details in the crack patterns, e.g., the presence of a crackfront contrast band indicative of a residual strain field and the disposition of interfacial fracture steps relative to the dislocation/moiré system, reinforces this interpretation. It is concluded that the concept of an atomically sharp crack provides a sound basis for the theory of fracture of brittle solids.

20560. Kilmer, R. D. Assessment of locomotive crew in-cab occupational noise exposure, U.S. Department of Transportation Report No. FRA/ORD-80/91, 184 pages (Available as PB81-154395 from the National Technical Information Service, Springfield, VA 22161, Dec. 1980)

Key words: in-cab sound level; locomotive crew; simplified testing.

The railroad industry, unlike most other U.S. industries, is not subject to the safety regulations of the Occupational Safety and Health Administration. Instead, railroad workers are covered by the safety regulations of the Federal Railroad Administration (FRA). This report documents an extensive study designed to access the noise environment in locomotive cabs. Operational duty cycle and in-cab sound level data are presented for 18 test runs made on 16 different locomotives used in wide range of operational modes (e.g., through freight and local-transfer freights), varied terrains (mountainous, undulating and flat) and varied trip lengths (6 to 12 hours). The general conclusion of this study is that there does not appear to be a widespread problem of overexposure to noise based on the same type of evaluation as currently used by OSHA (only 1 out of 18 test runs exceeded the criteria). The noise exposure is within acceptable limits because the operational duty cycle is such that the sources which generate high sound levels (horn and brake) are operating only for short periods of time and because the locomotive spends a great deal of time in idle (diesel engine sound levels below $90 \mathrm{~dB}$ ). However, there was one test run for which an overexposure to noise was measured. To pinpoint such cases where overexposure to noise may occur, a simplified testing procedure is developed. This test consists of making in-cab sound level measurements of engine notch 8 (no load), horn sounding and brake application with the locomotive stationary. With these three sound level measurements and an estimate of the time that the locomotive is operating on-line, the in service noise dose can be estimated and a pass/fail assessment made of whether the noise exposure might exceed acceptable limits.

20561. Wlodawer, A.; Sjölin, L. Orientation of histidine residues in 
RNase A: Neutron diffraction study, Proc. Natl. Sci. USA 78, No. 5, pp. 2853-2855 (May 1981).

Key words: active site; histidine; hydrogen bonds; neutron diffraction; refinement; ribonuclease $\mathrm{A}$.

Difference Fourier maps have been calculated at $2.8-\AA$ resolution by using neutron diffraction data obtained from a single crystal of RNase A. The phases were derived from a model resulting from the joint refinement of $\mathrm{x}$-ray and neutron data at $2.0-\AA$ and $2.8-\AA$ resolution, respectively. The orientation of histidine-48 assumed during the refinement of the $\mathrm{x}$-ray model at $2.5 \AA$ was confirmed, whereas the other three histidines had to be rotated around $\mathrm{C}_{\beta}-\mathrm{C}_{\gamma}$ bonds in order to agree with the neutron difference Fourier maps. In the final model, histidine-12 is clearly hydrogen bonded to the carbonyl oxygen of threonine-45 and to the oxygen of the inorganic phosphate, and histidine-119 is bonded to another oxygen of the phosphate and to the oxygen OD1 of aspartic acid-121.

20562. Hummer, D. G. Expressions for the computer-evaluation of the four kernel functions for line formation with Doppler and Lorentz profiles, J. Quant. Spectrosc. Radiat. Transfer 26, No. 3, 187.195 (1981).

Key words: Doppler profiles; kernel functions; line formation; Lorentz profiles; Padé approximants.

Rational approximations for the kernel functions $K_{1}(\tau), K_{2}(\tau), M_{1}(\tau)$ and $M_{2}(\tau)$ that describe the transfer of radiation scattered with complete redistribution over Doppler and Lorentz profiles have been obtained from their series and asymptotic expressions by the techniques of Padé approximants with a maximum relative error of less than $10^{-4}$.

20563. Timmerhaus, K. D.; Flynn, T. M. Cryogenics, Paper in Encyclopedia of Chemical Processing and Design, J. J. McKetta and W. A. Cunningham, eds., 13, 261-381 (Marcel Dekker, New York, NY, 1981).

Key words: chemical engineering; chemical processing; cryogenic; instrumentation.

Cryogenic instrumentation as a discipline is primarily concerned with the condition or thermodynamic state of cryogenic fluids, such as pressure and temperature. Such information is typically required for chemical process optimization and control. In addition, as cryogenic fluids have become of greater commercial importance, there is the question of quantity transferred or delivered. Accordingly, the instrumentation system must also be able to provide information concerning liquid level, density, and flow rate.

This manuscript reviews the measurement tools and techniques that are currently used for the most common cryogenic measurements: pressure, temperature, liquid level, density and flow.

20564. Rendell, R. W.; Penn, D. R. Spin dependent attenuation lengths in ferromagnets, J. Appl. Phys. 52, No. 3, 1620-1621 (Mar. 1981).

Key words: attenuation lengths; electrons; ferromagnets; spindependent.

Spin dependent attenuation lengths (AL) as a function of the hot electron energy are calculated for Fe, Co, and $\mathrm{Ni}$. If $\lambda^{\dagger}\left(\lambda^{\dagger}\right)$ is the AL of an electron with spin parallel (antiparallel) to the majority spin direction of the ferromagnet then the quantity $A=\left(\lambda^{\downarrow}-\lambda^{i}\right) /\left(\lambda^{\downarrow}+\lambda^{i}\right)$ is found to change sign as a function of the electron energy.

20565. Mulholland, G.; Handa, T.; Sugawa, O.; Yamamoto, H. Smoke filling in an enclosure, Proc. 20th Joint ASME/AIChE Natl. Heat Transfer Conf., Milwaukee, WI, Aug. 2-5, 1981, Paper 81-HT-8, pp. 1.13 (The American Society of Mechanical Engineers, New York, NY 10017,1981$)$.

Key words: buoyant plumes; ceilings; compartment fires; diffusion flames; heat loss; smoke filling; smoke layers; smoke movement; time; walls.

The filling of an enclosure by smoke generated by a diffusion flame was studied by measuring the smoke extinction coefficient and temperature versus height during the filling process. An operational definition of the smoke front passage time is given and is shown to be useful in correlating experimental results. Semi-quantitative agreement is found between the Baines-Turner theory and experiments with propane and acetylene fuels with heat fluxes over the range 11 to 32 $\mathrm{kW}$. Smoke leakage near the floor of the enclosure and flow down the cool walls result in more rapid smoke filling than is predicted. Also, the filling process was found to be about $50 \%$ faster when the burner was moved off center. A thin smoke layer was found to broaden as it moved down suggesting either an effective diffusion from the layer or an enhanced oscillatory motion of the layer as it moves down. The temperature and smoke concentration profiles were found to have different shapes in part as a result of a large heat loss affecting the temperature profile. Approximately $80 \%$ of the total heat generated by combustion was lost to the ceiling and walls. The long time smoke concentration profile was qualitatively different from the Baines-Turner result for the asymptotic profile. It was found that the conversion ratio of fuel to particulate is a nonlinear function of fuel flow rate and that the shape of the curve is sigmoidal, which appears to be a common characteristic of sooting systems.

20566. Parobeck, P.; Tomb, T.; Ku, H.; Cameron, J. Measurement assurance program for weighings of respirable coal mine dust samples, J. Qual. Tech. 13, No. 3, 157-165 (July 1981).

Key words: control charts; dust filters; Federal Mine Safety and Health Act; measurement assurance program; measurement process; process parameters; respirable mine dust samples: statistical control; uncertainty; weighing.

A program is presented to control the weighing of respirable coal mine dust samples and to provide information on a continuing basis on the quality of the weighing operation. In addition to the description of such monitoring procedures, measurements are included to check the adequacy of assumptions made about the test items, such as, effects of aging, environmental and other factors related to the handling of the test items. The set of procedures presented in this article provides a measurement assurance program by which the uncertainty attached to each measurement can be scientifically demonstrated. An analysis of data giving the status of the process is presented.

20567. McCaffrey, B. J.; Quintiere, J. G.; Harkleroad, M. F Estimating room temperatures and the likelihood of flashover using fire test data correlations, Fire Technol. 17, No. 2, 98-119 (May 1981).

Key words: developing room fire; flashover; room geometry; temperature correlation; ventilation factor.

A simple procedure is presented for estimating room temperature and the likelihood of the occurrence of flashover in an enclosure. Upper room gas temperature from many fire test data sources is correlated with fire size, door opening, geometry and thermal properties of the enclosure. A cross plot of the data is then made at a prescribed hazardous temperature level yielding the flashover criteria. The fire safety engineer can use these results for quantitative estimations of the effects of building design and fire load, for example, on the tendency for flashover as defined by a temperature limit.

20568. Deprit, A. Intrinsic variational equations in three dimensions, Celestial Mech. 24, 185-193 (1981).

Key words: differential equations; Hill's equations; nonlinear dynamics; variations.

The variational equations along an orbit in a conservative dynamic system with three degrees of freedom may be separated into (i) a linear system of order four involving only the normal and binormal displacements and (ii) a quadrature to produce the tangential displacement.

20569. Yin, L. I.; Trombka, J. I.; Schmadebeck, R. L.; Seltzer, S. M.; Bielefeld, M. J. A hard $x-r a y$ and soft gamma-ray telescope spectrometer, SPIE J., Imaging Spectrosc. 268, $97-102$ (1981). 
Key words: gamma ray; imaging; multiple-pinhole mask; spectrometer; telescope; $x$ ray.

We propose a new design of a hard $\mathrm{X}$-ray and soft gamma-ray telescope spectrometer in the energy domain of $30 \mathrm{keV}$ to $200 \mathrm{keV}$ with reasonable spatial, temporal, and energy resolution for possible space flight missions. This design incorporates a Uniformly Redundant Array (URA) mask in the front end and the Low Intensity $\mathrm{X}$-ray Imaging Scope (Lixiscope) developed in our laboratory as the imaging spectrometer. Using a newly acquired intensifier tube with a digitizing anode, preliminary results indicate that such a complete hard X-ray and soft gamma-ray telescope spectrometer system is indeed feasible.

20570. Diller, D. E.; Haynes, W. M. Application of the torsionally oscillating quartz crystal viscometer to compressed gases and liquids, Prac. DoE Gas Miscible Displacement Research Seminar, Tulsa, OK, Jan. 8-9, 1981, pp. IX-1-IX-13 (Morgantown Energy Technology Center, Department of Energy, Morgantown, WV, 1981).

Key words: compressed gases and liquids; methane; propane; quartz crystal viscometer; saturated liquid; viscosity measurements.

The torsionally oscillating quartz crystal viscometer method is being used on compressed gases and liquids at the National Bureau of Standards, Boulder, Colorado. The method actually measures the viscosity-density product and requires independent density data to obtain the shear viscosity coefficient. Recent applications of the method to compressed gaseous and liquid methane and liquid propane are discussed. The claimed inaccuracy is less than 2 percent, based on the repeatability and on comparisons with accurate capillary tube and oscillating disk measurements. The method does not require calibration with fluids of known viscosity. The simplest form of the theory of the instrument appears to be satisfactory (within the claimed accuracy) for use on fluids where shear rate effects are negligible. Compared to most viscosity measuring techniques, the torsionally oscillating quartz crystal can be used with relative ease over wide ranges of temperature and pressure. The NBS apparatus has been used on seven pure fluids at temperatures between 15 and $320 \mathrm{~K}$ and at pressures to $35 \mathrm{MPa}$ (5075 psia).

20571. MacDonald, R. A.; MacDonald, W. M. Thermodynamic properties of fcc metals at high temperatures, Phys. Rev. B 24, No. 4, 1715.1724 (Aug. 15, 1981).

Key words: bulk moduli; fcc metals; free electron theory; Grüneisen parameter; Helmholtz free energy; high temperatures; lattice dynamics; nearest-neighbor, central force potential; specific heat; thermal expansion; thermodynamic properties.

We have carried out an exact and consistent calculation of the thermodynamic properties of monatomic fcc crystals at high temperatures. These properties are obtained from the Helmholtz free energy of the crystal $F(V, T)$ by means of the appropriate thermodynamic relations. It is crucial to the success of the calculation that we have been able to obtain the volume dependence of the free energy. $F(V, T)$ includes the static lattice energy and the vibrational contributions from the harmonic and lowest-order (cubic and quartic) anharmonic terms in perturbation theory evaluated in the hightemperature limit. The atoms interact via an effective nearest-neighbor central-force potential $\phi(r)$. We have calculated the specific heat at constant volume and at constant pressure, the thermal expansion, the coefficient of linear expansion, the isothermal and adiabatic bulk moduli, and the Grüneisen parameter for the following fcc metals: $\mathrm{Cu}, \mathrm{Ag}, \mathrm{Ca}, \mathrm{Sr}, \mathrm{Al}, \mathrm{Pb}$, and $\mathrm{Ni}$. Good agreement with experiment is obtained in all cases. We discuss the implications of these results for further studies of the properties of metals.

20572. Read, D. T.; Reed, R. P. Heating effects during tensile tests of AISI 304L stainless steel at $4 \mathrm{~K}$, (Proc. Cryogenic Engineering Conf., Madison, WI, Aug. 21-24, 1979), Paper B-2 in Advances in Cryogenic Engineering, A. F. Clark and R. P. Reed, eds., 26, 91-101 (Plenum Press, New York, NY, 1980).
Key words: adiabatic heating; cryogenic; load drop; martensitic transformation; serrated yielding; stainless steel; strain rate.

In structural alloy tensile specimens undergoing mechanical work in a liquid-helium bath, internal temperature increases are favored by their low specific heat and thermal conductivity at this temperature (4 $\mathrm{K}$ ). The well-known phenomenon of discontinuous yielding is commonly attributed to adiabatic heating of the specimen. The possible occurrence of such heating must be considered in testing structural materials for liquid-helium-temperature applications. Therefore, temperatures of small tensile specimens of AISI 304L stainless steel were measured during straining at $4 \mathrm{~K}$ at several strain rates. The number and magnitudes of the temperature rises and the number of load drops were used to calculate a specimen temperature profile during load drops. Examination of a specimen after one load drop occurred showed that only a small portion of the specimen deformed. The observed temperature rises and load drops were compared with calculated values. The possible effect of a latent heat of martensitic transformation on the specimen temperature was considered.

20573. Massoud Gasem, K. A.; Hiza, M. J.; Kidnay, A. J. Phase behavior in the nitrogen +ethylene system from 120 to $200 \mathrm{~K}$, Fluid Phase Equilib. 6, No. 3.4, 181.189 (June 1981).

Key words: liquid-liquid-vapor; liquid-vapor; phase equilibria; $\mathrm{N}_{2}+\mathrm{C}_{2} \mathrm{H}_{4} ; \mathrm{N}_{2}+\mathrm{C}_{2} \mathrm{H}_{6}$.

Liquid-vapor and liquid-liquid-vapor equilibria were measured for the $\mathrm{N}_{2}+\mathrm{C}_{2} \mathrm{H}_{4}$ system between 120 and $200 \mathrm{~K}$ and at pressures to 93 bar $\left(93 \times 10^{5} \mathrm{~Pa}\right)$. The data at $200 \mathrm{~K}$ are compared with existing measurements and show excellent agreement. At lower temperatures $(120-140 \mathrm{~K})$, the system exhibits a liquid phase separation. The pressures and temperatures corresponding to the appearance of two liquid phases were also determined from 105 to $135 \mathrm{~K}$ for the $\mathrm{N}_{2}+$ $\mathrm{C}_{2} \mathrm{H}_{6}$ system.

20574. Mazandarany, F. N.; Parker, D. M.; Koenig, R. F.; Read, D. T. A nitrogen-strengthened austenitic stainless steel for cryogenic magnet structures, (Proc. Cryogenic Engineering Conf., Madison, WI, Aug. 21-24, 1979), Paper B-9 in Advances in Cryogenic Engineering, A. F. Clark and R. P. Reed, eds., 26, 158-170 (Plenum Press, New York, NY, 1980).

Key words: cryogenic temperatures; fracture toughness; mechanical properties; nitrogen-strengthening; stainless steel; strength; welds.

The work described in this paper is part of a major program of developing, designing, and manufacturing a large superconducting magnet in support of the Large Coil Program. The oval-shaped toroidal magnet weighs approximately $397 \mathrm{kN}$, has bore diameters of $3.35 \mathrm{~m}$ and $2.35 \mathrm{~m}$, and is designed for a maximum magnetic field of 8 $\mathrm{T}$ at $10.65 \mathrm{kA}$. The limitations on magnet weight and available space for structural components coupled with extremely large magnetic forces presented a major challenge in the selection of a suitable structural material. After detailed considerations of the design and fabrication requirements of the coil, type $316 \mathrm{LN}$ stainless steel was selected for major structural components.

In support of the magnet development effort, an extensive test program was initiated to verify expected base-metal properties, to develop weld processes, and to establish weld and base-metal allowable design values. This paper presents the results of the testing and evaluation of $25-\mathrm{mm}$-thick type $316 \mathrm{LN}$ plate, shielded metal-arc weld (SMAW) deposits selected for structural welds and gas tungsten-arc weld (GTAW) and gas metal-arc weld (GMAW) deposits selected for seal welds. The welding development effort included measurements of impact properties at room temperature (RT) and $77 \mathrm{~K}$; chemical analysis; measurements of magnetic permeabilities and weld ferrite contents; metallographic and radiographic examinations of welds; and heat treatments, which were performed at the Materials and Processes Laboratory of General Electric in Schenectady, New York. Measurements of tensile, fracture toughness, and fatigue crack-growth rate (FCGR) properties from RT 
to $4 \mathrm{~K}$ were performed at the National Bureau of Standards in Boulder, Colorado.

20575. Rainwater, J. C.; Hanley, H. J. M. Prediction of the transport properties of natural gas and similar mixtures, (Proc. Cryogenic Engineering Conf., Boulder, CO, Aug. 2.5, 1977), Paper M.2 in Advances in Cryogenic Engineering, K. D. Timmerhaus, ed., 23, 561. 565 (Plenum Press, New York, NY, 1978).

Key words: corresponding states; hydrocarbons; mathematical model; methane; natural gas; thermal conductivity; transport properties; viscosity.

This paper presents a procedure to calculate the viscosity coefficient, $\mu$, and the thermal conductivity coefficient, $\lambda$, of a pure fluid or mixture from thermodynamic data. The procedure incorporates the extended corresponding states approach to the thermodynamic properties of nonconformal fluids. Details have been reported elsewhere.

20576. O'Neill, J. G. Brief status report on NBS/CFR sprinkler projects, Proc. Conf. Low Cost Residential Sprinkler System, Baltimore, MD, Nov. 29-30, 1977, pp. 1.12 (National Fire Prevention \& Control Admin., Washington, DC, June 1978).

Key words: carbon monoxide; health care; mattress; smoke obscuration; spray nozzle; sprinkler.

NBS/CFR, Program for Fire Detection and Control Systems is presently engaged in two major sprinkler research projects. One is a study of automatic sprinklers in health care facilities and, the other is a study of sprinkler and spray methods for protection of open or partially open stairways. The purpose of the first project is to determine engineering design parameters for automatic sprinklers in health care facilities. The impact of sprinklers is being measured against overall fire control, lead times necessary for evacuation and tenability conditions necessary for patients who must remain. Preliminary results from seven tests including smoke obscuration and carbon monoxide levels after sprinkler operation are presented. The results indicate that the presence of a privacy curtain between the patient's bed and the sprinkler delayed fire extinguishment and, subsequently, carbon monoxide levels were significantly higher.

The purpose of the second project is to develop alternative engineering design parameters for protection of open or partially open stairways by incorporating sprinkler or sprinkler-vent systems. A description of the test facility and an outline of the test plan are provided. Experimental work is scheduled to begin in early 1978 .

20577. Matthew, J. A. D.; Girvin, S. M. Breadths of resonant photoemission satellites and electron-excited direct-recombination emission, Phys. Rev. B 24, No. 4, 2249-2253 (Aug. 15, 1981).

Key words: Auger spectroscopy; coincidence experiment electron excited direct recombination; lifetime broadening; photoemission; resonant satellites.

Resonant photoemission involving core hole excitation and electron emission arising from direct recombination of electron excited resonant states are compared. At fixed photon energy the resonant emission has an energy distribution which is not broadened by the finite lifetime of the core hole and is therefore narrower than the corresponding Auger emission process. Electron excited resonant states give rise to emission which can be isolated only if it is at a different energy from that of the main Auger channel as in the rare earths, but this emission is core hole broadened. A coincidence experiment monitoring the energy loss of the incident electron and the energy distribution of the emitted electron is equivalent in resolution to resonant photon excitation.

20578. Cutkosky, R. D. Guarding techniques for resistance thermometers, IEEE Trans. Instrum. Meas. IM-30, No. 3, 217-220 (Sept. 1981).

Key words: bridges; guards; resistance measurement; temperature measurement; thermometers; thermometry.

Theoretical and practical aspects of the application of electrical guards to resistance thermometers are discussed, with a view toward suppressing errors caused by imperfect electrical insulation at high temperatures. Some simple techniques for modifying existing resistance thermometer bridges are described.

20579. Eitzen, D. G.; Breckenridge, F. R.; Clough, R. B.; Fuller, E. R.; Hsu, N. N.; Simmons, J. A. Summary of fundamental developments for quantitative acoustic emission measurements, Electric Power Research Institute Interim Report No. EPRI NP-1877, Project 608-1, 100 pages (Electric Power Research Institute, Palo Alto, CA, June 1981).

Key words: acoustic emission; calibration; deconvolution; NDE; nuclear safety; plate theory; transfer function.

The acoustic emission (AE) technique of surveying structural integrity is one of the newest methods of nondestructive evaluation. The technique uses passive surface-mounted sensors which detect surface disturbances caused by sources within the structure while the structure is under load. The AE signals contain potentially useful information about the location and identity of defects and about the criticality of the defects in a structure under load. While industrial applications of $\mathrm{AE}$ have progressed significantly in recent years, interpretation of the full AE signal content remains a problem and the full potential for using $\mathrm{AE}$ to determine and monitor structural integrity is yet to be exercised in field applications.

This report summarizes a research program supported jointly by the Electric Power Research Institute and the National Bureau of Standards. The intent of this report is to summarize the results and methods of the research. Another report with more details of the research has been prepared for the specialist.

The work under Phase 1 of the EPRI/NBS AE program has focused on: improved test standardization through the development of a calibration capability for AE sensors; improved sensor concepts and techniques for field and laboratory calibration; an improved basis for understanding and predicting $\mathrm{AE}$ behavior through the development of a mathematical framework for $\mathrm{AE}$ (transfer function formalism) through specific theoretical solutions to AE generation, transmission and inversion problems and the successful application of these theories to actual events in glass; an improved basis for assessing defect significance through the development of improved signal processing and inversion methods and through experimental results from $\mathrm{AE}$ in pressure vessel steels; the implementation of experiments to establish the feasibility of using causal methods, based on theoretical mechanics, to obtain source information in structural steels.

20580. Durst, R. A.; Bates, R. G. Hydrogen-ion activity, Paper in Encyclopedia Chemical Technology, 3d Edition, 13, 1-12 (1981).

Key words: acid-base indicators; activity, hydrogen-ion; buffer solutions; hydrogen-ion activity; $\mathrm{pH} ; \mathrm{pH}$ buffers; $\mathrm{pH}$ electrodes; pH scale; reference electrodes.

The concept of hydrogen-ion activity and its related parameter, $\mathrm{pH}$, is reviewed. Also discussed are the operational definition of $\mathrm{pH}$, the $\mathrm{pH}$ scale, and the method of assignment of $\mathrm{pH}$ values to reference buffer solutions. Practical information is included on $\mathrm{pH}$ measurement procedures, interpretation of measured $\mathrm{pH}$ values and characteristics of the instrumentation and electrodes used for laboratory and industrial $\mathrm{pH}$ measurements.

20581. Okabe, H. Photochemistry of acetylene at $1470 \AA$, J. Chem. Phys. 75, No. 6, 2772.2778 (Sept. 15, 1981).

Key words: absorption cross section of diacetylene; acetylene; $\mathrm{C}_{2} \mathrm{H}$; diacetylene; photochemistry; primary process; reactions of $\mathrm{C}_{2} \mathrm{H}$ with $\mathrm{H}_{2}$ and $\mathrm{CH}_{4}$.

The photolysis of acetylene at $1470 \AA$ has been studied over the pressure region from 13 to $1330 \mathrm{~N} \mathrm{~m}^{-2}$ (0.1 to 10 Torr $)$. The quantum yield of diacetylene formation has been measured as a function of $\mathrm{C}_{2} \mathrm{H}_{2}$ pressure and the partial pressures of $\mathrm{He}$ and $\mathrm{H}_{2}$. The quantum yields of acetylene disappearance and of ethylene and hydrogen formation were also briefly studied. From these results it was concluded that the major primary photochemical process is direct 
dissociation $\mathrm{C}_{2} \mathrm{H}_{2} \rightarrow \mathrm{C}_{2} \mathrm{H}+\mathrm{H}$ with a quantum yield of 0.3 and the process of $\mathrm{H}_{2}$ production $\mathrm{C}_{2} \mathrm{H}_{2} \rightarrow \mathrm{C}_{2}+\mathrm{H}_{2}$ is minor (quantum yield $\leqslant 0.1$ ). The remaining process is the formation of a metastable acetylene which reacts either with ground state acetylene to form $\mathrm{C}_{2} \mathrm{H}$ radicals or is deactivated by collisions with the walls or inert gases. The $\mathrm{C}_{2} \mathrm{H}$ radicals react with $\mathrm{H}_{2} 130 \pm 11$ times slower and with $\mathrm{CH}_{4} 31 \pm 1.7$ times slower than with $\mathrm{C}_{2} \mathrm{H}_{2}$. Absorption cross sections of diacetylene have been measured in the 1200 and $1800 \AA$ region. The role of acetylene photolysis in the Jovian atmosphere is briefly discussed.

20582. Ayres, T. R.; Moos, H. W.; Linsky, J. L. Far-ultraviolet fluorescence of carbon monoxide in the red giant Arcturus, Astrophys. J. 248, L137-L140 (Sept. 15, 1981).

Key words: chromosphere stars; late-type stars; molecular processes; ultraviolet spectra.

We present evidence that many of the weak features observed with the International Ultraviolet Explorer (IUE) in the far-ultraviolet (1150-2000 ̊) spectrum of the archetype red giant Arcturus (K2 III) are $A-X$ fourth positive bands of carbon monoxide excited by chromospheric emissions of $\mathrm{O} I, C \mathrm{I}$, and $\mathrm{H} I$. The appearance of fluorescent $\mathrm{CO}$ bands near the wavelengths of commonly used indicators of high-temperature $\left(T>2 \times 10^{4} \mathrm{~K}\right)$ plasma, such as $\mathrm{C}_{\text {II }}$ $\lambda 1335$ and $C$ IV $\lambda 1548$, introduces a serious ambiguity in diagnosing the presence of hot material in the outer atmospheres of the cool giants by means of low-dispersion IUE spectra.

20583. Hall, J. L. Stable lasers and optical frequency standards for testing the postulates of physics, Paper in Atomic Physics, D. Kleppner and F. M. Pipkin, eds., 7, 267.296 (Plenum Publ. Corp., New York, NY 10013, 1981).

Key words: optical frequency standards; special relativity, tests of; stable lasers.

We consider the application of precision laser frequency control and measurement techniques to interesting and fundamental experiments for physics. We discuss a recent high precision laser version of the Michelson-Morley experiment in terms of a test theory for (special) relativity due to Mansouri and Sexl. This experiment appears to provide the most stringent test yet of the Lorentz transformation. An improved time dilation experiment is considered.

20584. Falk, R. A.; Dunn, G. H.; Griffin, D. C.; Bottcher, C.; Gregory, D. C.; Crandall, D. H.; Pindzola, M. S. Excitationautoionization contributions to electron impact ionization, Phys. Rev. Lett. 47, No. 7, 494-497 (Aug. 17, 1981).

Key words: crossed beams; electron impact ionization; excitationautoionization; $\mathrm{Hf}^{3+}$; theory; $\mathrm{Ti}^{3+} ; \mathrm{Zr}^{3+}$.

Experimental measurements of electron impact ionization cross sections for the transition-series ions $\mathrm{Ti}^{+3}, \mathrm{Zr}^{+3}$, and $\mathrm{Hf}^{+3}$ demonstrate that excitation-autoionization increases the cross sections by more than an order of magnitude over that anticipated for direct ionization. Theoretical predictions of both the energies and approximate magnitudes of these excitation cross sections provide confidence that this important indirect contribution to the total ionization can be estimated for many cases.

20585. Konash, P. L.; Wise, S. A.; May, W. E. Selective quenchofluorometric detection of fluoranthenic polycyclic aromatic hydrocarbons in high-performance liquid chromatography, J. Liq. Chromatog. 4, No. 8, 1339-1349 (1981).

Key words: benzo[a]pyrene; benzofluoranthenes; coal tar; fluoranthenic $\mathbf{P A H}$; fluorescence detection; fluorescence quenching; liquid chromatography (LC); nitromethane; polycyclic aromatic hydrocarbons (PAH); shale oil.

The phenomenon of fluorescence quenching was used for selective HPLC detection of fluoranthenic polycyclic aromatic hydrocarbons (PAH). Termed a "Quenchofluorometric" detection systems, it employs a filter fluorimeter or spectrofluorimeter and nitromethane in the mobile phase as the fluorescence quenching reagent. Chromatograms obtained with and without the quenching reagent are compared for PAH standards, a coal tar extract, and a shale oil sample. The quenchofluorometric detection system provides an inexpensive method to achieve selective detection for fluoroanthenic PAH as a group.

20586. Brown, J. M.; Wise, S. A.; May, W. E. Determination of benzo [a] pyrene in recycled oils by a sequential HPLC method, $J$. Environ. Sci. Health. A15, No. 6, 613-623 (1980).

Key words: benzo[a]pyrene; liquid chromatography; recycled oils; residual oil; sequential HPLC; virgin oil.

Recently, several procedures have been developed at the National Bureau of Standards to measure some environmentally important contaminants in used lubricating oils. In this paper, a sequential highperformance liquid chromatographic method will be described which can be used to determine the concentration of benzo[a]pyrene in some virgin, used, and recycled oils.

20587. Blau, P. J. Interpretations of the friction and wear break-in behavior of metals in sliding contact, Wear 71, 29.43 (1981).

Key words: break-in; bronze; friction; run-in; steel; wear.

Studies of wear and friction transient behavior during early periods in metal sliding can lead to useful insights. Such break-in studies may help to identify the relative roles of competing processes which reach a balance during longer term sliding situations. Interpretations may be facilitated by considerations which compare friction curve shapes, changes in friction during break-in and the relative durations of friction and wear break-in periods. Data for dry sliding of 1015 steel, a high strength low alloy steel, $0-2$ tool steel and two $\mathrm{Cu}-\mathrm{Al}$ alloys are presented in several formats to emphasize differences and similarities in break-in behavior.

20588. Hougen, J. T. A rotational Hamiltonian for the ground vibrational state of hydrazine, J. Mol. Spectrosc. 89, 296-327 (1981).

Key words: group theory; Hamiltonian matrix; hydrazine; internal rotation; inversion; large amplitude motion; rotational levels; tunneling.

A Hamiltonian matrix suitable for fitting rotational energy levels of the hydrazine molecule in its ground vibrational and electronic states was obtained. This matrix is of dimension $16 \times 16$, where the 16 functions labeling the rows and columns consist of the two members of a near-prolate asymmetric rotor doublet (with given $\left|K_{a}\right|$ ) for the eight different, but chemically equivalent, conformers which the molecule can reach by various combinations of $-\mathrm{NH}_{2}$ inversion at either end of the molecule, and internal rotation about the $\mathrm{N}-\mathrm{N}$ bond. The matrix is derived in a phenomenological fashion, by applying group theoretical arguments to a model in which tunneling among the various frameworks is assumed to be very slow compared with the vibrational frequencies. A comprehensive treatment of the largeamplitude vibrational potential surfaces and associated tunneling pathways has not been carried out, nor have quartic $\left(f^{4}\right)$ centrifugal distortion effects been considered in a systematic fashion. Preliminary fits indicate that the model developed can be used to fit the hydrazine microwave data in a consistent fashion, and a full treatment of such data has been undertaken.

20589. Frommer, C.; Lovejoy, R. W.; Sams, R. L.; Olson, W. B. The infrared spectrum of the $\nu_{1}$ band of $\mathrm{SiD}_{3} \mathrm{H}, J$. Mol. Spectrosc. 89, 261-267 (1981).

Key words: high resolution infrared spectroscopy; rotational constants; $\mathrm{SiD}_{3} \mathrm{H} \mathrm{SiH}$ stretching vibration; $\mathrm{Si}$ isotopes.

High-resolution spectra of the $\nu_{1}$ stretching band of $\mathrm{SiD}_{3} \mathrm{H}$ were recorded and analyzed, yielding values for ground- and upper-state constants and the band center. For ${ }^{28} \mathrm{SiD}_{3} \mathrm{H}, B_{0}=1.777482(14)$ and $\nu_{1}=2187.2070(17) \mathrm{cm}^{-1}$. Similar analyses were performed on the less abundant species ${ }^{29} \mathrm{SiD}_{3} \mathrm{H}$ and ${ }^{30} \mathrm{SiD}_{3} \mathrm{H}$.

20590. Cooper, L. Y. Heat transfer from a buoyant plume to an 
unconfined ceiling, Proc. 20th Joint ASME/AIChE Natl. Heat Transfer Conf., Milwaukee, WI, August 2-5, 1981, Paper 81-HT-7, pp. 1.9 (The American Society of Mechanical Engineers, New York, NY 10017, 1981).

Key words: buoyant plume; ceiling; confined ceiling; fires; heat transfer.

The heat transfer to confined ceilings during enclosure fires is related to the heat transfer to unconfined ceiling surfaces from buoyant plume driven ceiling jets. This paper briefly discusses this relationship, and then focuses attention on the unconfined ceiling problem. Previously published theoretical and experimental studies dealing with interactions of unheated free jets and solid surfaces as well as literature which focuses directly on fire plume-unconfined ceiling interactions are brought to bear on the problem. A critical review of all of this literature results in easily applicable formulae for estimating the heat transfer in question.

20591. Cooper, L. Y.; Harkleroad, M.; Quintiere, J.; Rinkinen, W. An experimental study of upper hot layer stratification in full-scale multiroom fire scenarios, Proc. 20th Joint ASME/AIChE Natl. Heat Transfer Conf., Milwaukee, WI, Aug. 2-5, 1981, Paper 81-HT-9, pp.

1.12 (The American Society of Mechanical Engineers, New York, NY, 1981).

Key words: fire scenerios; full scale fire; multiroom fires; smoke dynamics; stratification.

This paper describes an experimental study of the dynamics of smoke filling in realistic, full-scale, multiroom fire scenarios. The test space involved 2 to 3 rooms, connected by open doorways. During, the course of the study these were partitioned to yield four different configurations ranging in total plan area from $40.6 \mathrm{~m}^{2}$ to $89.6 \mathrm{~m}^{2}$. One of the rooms was a burn room containing a methane burner which produced either a constant energy release rate of 25,100 , or $225 \mathrm{~kW}$ or a time-varying energy release rate which increased linearly with time from zero at ignition to $300 \mathrm{~kW}$ in 10 minutes. An artificial smoke source near the ceiling of the burn room provided a means for visualizing the descent of the hot layer and the dynamics of the smoke filling process in the various spaces. In addition to visual data, recorded on videotape, the development of the hot stratified layers in the various spaces was monitored by vertical arrays of thermocouples and photometers. A layer interface was identified from these data, and its position as a function of time was determined. An analysis and discussion of these results are presented.

20592. McCulloh, K. E.; Tilford, C. R. Nitrogen sensitivities of a sample of commercial hot cathode ionization gage tubes, J. Vac. Sci. Technol. 18, No. 3, 994-996 (Apr. 1981).

Key words: gage sensitivities; hot filament gages; ion gages; vacuum calibration; vacuum comparator; vacuum standards.

In order to help assess the magnitude of errors that might arise from the use of uncalibrated ionization gage tubes and help select the best type of tube when accurate measurements are required we have determined the nitrogen sensitivities from $10^{-5}$ to $10^{-2} \mathrm{~Pa}$ for lots of from two to four each of five different types of commercial hot cathode gage tubes. Included were conventional triodes and B-A structures of four distinct types. Two types with tungsten filamentsthe triodes and tubulated B-A structures-were markedly superior to the others with respect to agreement with manufacturer's sensitivity, linearity, and uniformity of sensitivity within a type. The least satisfactory results were obtained from the nude B-A structures, which showed significant nonlinearities, sensitivities ranging from $70 \%-110 \%$ of the manufacturer's value, and typical $25 \%$ differences in sensitivity between the two filaments of a dual-filament structure.

20593. Cezairliyan, A. A dynamic technique for measurements of thermophysical properties at high temperatures, High Temp. Sci. 13, 117.133 (1980).

Key words: dynamic techniques; high temperature; thermodynamics; thermophysical properties.

A technique is described for the dynamic measurement of selected thermophysical properties of electrically conducting substances in the range $1500 \mathrm{~K}$ to the melting temperature of the specimen. The technique is based on rapid resistive self-heating of the specimen from room temperature to any desired high temperature in less than one second by the passage of an electrical pulse through it and on measuring the pertinent quantities, such as current, voltage, and temperature, with millisecond resolution. The technique was applied to the measurement of heat capacity, electrical resistivity, hemispherical total emittance, normal spectral emittance, melting temperature, and temperature and energy of solid-solid phase transformations. Recent preliminary studies have indicated the applicability of the technique to the measurements of heat of fusion and thermal expansion.

20594. Pallavicini, R.; Golub, L.; Rosner, R.; Vaiana, G. S.; Ayres, T.; Linsky, J. L. Relations among stellar $x$-ray emission observed from Einstein, stellar rotation and bolometric luminosity, Astrophys. J. 248, 279.290 (Aug. 15, 1981).

Key words: early-type stars; late-type stars; stellar coronae; stellar rotation; $\mathrm{x}$-ray sources.

We have determined the correlation between observed stellar X-ray luminosities, bolometric luminosities, and projected rotational velocities $v \sin i$ for stars of various spectral types and luminosity classes observed by the Einstein Observatory. There are two clearly defined patterns of behavior observed. Early type stars (O3-AS) have $\mathrm{X}$-ray luminosities which are proportional to bolometric luminosity. The proportionality constant is $(1.4 \pm 0.3) \times 10^{-7}$ and is independent of luminosity class. In contrast, late type stars ( $G$ to $M$ ) have X-ray luminosities strongly dependent on rotation rate $\left[L_{x} \sim(v \sin i)^{i .9 \pm 0.5}\right]$ and independent of bolometric luminosity; this relation for late type stars is again found to be independent of luminosity class. This dependence is equivalent to a relation $f_{x} \sim \Omega^{2}$ between the X-ray surface flux and the stellar angular velocity. $F$ stars as a class are intermediate, having $\mathrm{X}$-ray luminosities substantially higher than would be predicted on the basis of the early type star $L_{x}-L_{\text {bol }}$ relation, but substantially lower than expected from the late type velocity dependence. The location of RS CVn stars as a class is discussed with respect to the dependence of $\mathrm{X}$-ray luminosity on rotation.

20595. Walls, D. F.; Kunasz, C. V.; Drummond, P. D.; Zoller, P. Bifurcations and multistability in two-photo processes, Phys. Rev. $A$ 24, No. 1, 627.630 (July 1981).

Key words: bifurcations; interferometer; laser; nonequilibrium phase transitions; nonlinear optics; optical bistability; two-photon processes.

We consider an intracavity nonlinear medium interacting with two modes of the electromagnetic field via a two photon process. The cavity is coherently driven at the frequencies of the two modes. We consider as the intracavity medium (a) a two photon absorber, (b) $\mathbf{N}$ three level atoms. These systems display a new class of bifurcations and in the case of the two photon absorber optical tristability is possible.

20596. Dunn, G. H. Electron-ion collisions, (Proc. The Physics of lonized Gases, Dubrovnik, Yugoslavia, Aug. 25-30, 1980), Paper in The Physics of Ionized Gases, M. Matic, ed., 49.95 (Boris Kidric Institute of Nuclear Sciences, Beograd, Yugoslavia, 1980).

Key words: electron-ion collisions; excitation; experiments; ionization; recombination; review.

Worldwide interest in and excitement over both controlled thermonuclear fusion and space physics coupled with advancing technology have generated significant efforts to determine cross sections for electron collisions with ions. Early work in various laboratories dates from the late 1950's, but it was not until 1961 that a successful measurement was reported. Since that time much work has been done and a number of reviews have been written on experimental techniques and results as well as on theory. This paper will emphasize experimental results and methods, with an attempt to update the excellent review of Dolder and Peart, which has a similar emphasis. Some overlap occurs with the Dolder and Peart paper; 
since some issues facing the field remain the same, and methods and examples for orientation and clarification may be the same. Processes of high interest in electron-ion collisions include: excitation, ionization, recombination, dissociation, and detachment. Only the first three will be discussed here. Rate measurements in plasmas also, of course, give valuable information about the processes in question and rate coefficients are-in many cases-what the user community ultimately needs for modelling and diagnostics. However, we confine the discussion here to cross sections from which rate coefficients may be calculated and which make a more meaningful test of theory. An extensive bibliography is included.

20597. Tachibana, K.; Phelps, A. V. Excitation of the $\mathrm{O}_{2}\left(a^{1} \Delta_{\mathrm{g}}\right)$ state by low energy electrons, J. Chem. Phys. 75, No. 7, 3315-3320 (Oct. 1, 1981).

Key words: argon; drift tube; electrons; excitation; metastables; oxygen; swarm.

Coefficients for the excitation of the $a^{1} \Delta_{g}$ metastable state of $\mathrm{O}_{2}$ by low energy electrons in $\mathrm{O}_{2}-\mathrm{Ar}$ mixtures have been measured using a drift tube technique. The time dependence of the absolute intensity of the $1.27 \mu \mathrm{m}$ band emission was measured for $1 \%$ and $5 \% \mathrm{O}_{2}$ in $\mathrm{Ar}$ at total gas densities of $(1-20) \times 10^{24} \mathrm{~m}^{-3}$. The electric field to gas density ratio $E / N$ was varied from $(0.3-10) \times 10^{-21} \mathrm{~V} \mathrm{~m}^{2}$, corresponding to calculated mean electron energies between about 0.8 and $4 \mathrm{eV}$. The measured decay constants yield a normalized diffusion coefficient for $\mathrm{O}_{2}\left(a \Delta_{\mathrm{g}}\right)$ in Ar of $(2.8 \pm 0.2) \times 10^{20} \mathrm{~m}^{-1} \mathrm{~s}^{-1}$ and a rate coefficient for quenching by $\mathrm{O}_{2}$ of $(1.57 \pm 0.1) \times 10^{-24} \mathrm{~m}^{3} \mathrm{~s}^{-1}$. Our experimental electron excitation coefficients agree satisfactorily with values calculated using previously derived sets of electron collision cross sections for $\mathrm{O}_{2}$ and $\mathrm{Ar}$.

20598. Stencel, R. E.; Linsky, J. L.; Brown, A.; Jordan, C.; Carpenter, K. G.; Wing, R. F.; Czyzak, S. Density sensitive C II lines in cool stars of low gravity, Mon. Not. R. Astron. Soc. Short Commun. 196, 47P.53P (1981).

Key words: late-type stars; stars, supergiants; stellar chromospheres; ultraviolet spectra.

It is shown that the relative intensities of emission lines within the multiplet uv 0.01 of $\mathrm{C}$ II, around $2325 \AA$, are sensitive to electron density in the range $10^{9}>N_{\mathrm{e}}>10^{7} \mathrm{~cm}^{-3}$.

The lines therefore offer a valuable method for measuring $N_{\mathrm{e}}$ in the chromospheres of late-type giants and supergiants. Calculated line ratios are compared with those observed in a range of objects.

20599. Yoder, C. F.; Peale, S. J. The tides of Io, Icarus 47, 1-35 (1981).

Key words: dissipation; Io; Jupiter satellite; resonances; tides.

The Galilean satellites Io, Europa, and Ganymede interact through several stable orbital resonances where $\lambda_{1}-2 \lambda_{2}+\widetilde{\omega}_{1}=0, \lambda_{1}-2 \lambda_{2}+\widetilde{\omega}_{2}=$ $180^{\circ}, \lambda_{2}-2 \lambda_{3}+\widetilde{\omega}_{2}=0$ and $\lambda_{1}-3 \lambda_{2}+2 \lambda_{3}=180^{\circ}$, with $\lambda_{i}$ being the mean longitude of the $i$ th satellite and $\widetilde{\sigma}_{\mathrm{i}}$ the longitude of the pericenter. The last relation involving all three bodies is known as the Laplace relation. A theory of origin and subsequent evolution of these resonances outlined earlier (C. F. Yoder, 1979b, Nature 279, 747-770) is described in detail. From an initially quasi-random distribution of the orbits the resonances are assembled through differential tidal expansion of the orbits. Io is driven out most rapidly and the first two resonance variables above are captured into libration about 0 and $180^{\circ}$ respectively with unit probability. The orbits of Io and Europa expand together maintaining the 2:1 orbital commensurability and Europa's mean angular velocity approaches a value which is twice that of Ganymede. The third resonance variable and simultaneously the Laplace angle are captured into libration with probability $\sim 0.9$. The tidal dissipation in Io is vital for the rapid damping of the libration amplitudes and for the establishment of a quasi-stationary orbital configuration. Here the eccentricity of Io's orbit is determined by a balance between the effects of tidal dissipation in Io and that in Jupiter, and its measured value leads to the relation $k_{1} f_{1} / Q_{1} \approx 900 k_{\mathrm{J}} / Q_{\mathrm{J}}$ with the $k$ 's being Love numbers, the $Q$ 's dissipation factors, and $f$ a factor to account for a molten core in Io. This relation and an upper bound on $Q_{1}$ deduced from Io's observed thermal activity establishes the bounds $6 \times 10^{4}<Q_{J}<2 \times 10^{6}$, where the lower bound follows from the limited expansion of the satellite orbits. The damping time for the Laplace libration and therefore a minimum lifetime of the resonance is $1600 Q_{\text {J }}$ years. Passage of the system through nearby three-body resonances excites free eccentricities. The remnant free eccentricity of Europa leads to the relation $Q_{2} / f_{2}>2 \times 10^{-4} Q_{1}$ for rigidity $\mu_{2}=5 \times 10^{11}$ dynes $/ \mathrm{cm}^{2}$. Probable capture into any of several stable $3: 1$ two-body resonances implies that the ratio of the orbital mean motions of any adjacent pair of satellites was never this large.

A generalized Hamiltonian theory of the resonances in which third. order terms in eccentricity are retained is developed to evaluate the hypothesis that the resonances were of primordial origin. The Laplace relation is unstable for values of Io's eccentricity $e_{1}>0.012$ showing that the theory which retains only the linear terms in $e_{1}$ is not valid for values of $e_{1}$ larger than about twice the current value. Processes by which the resonances can be established at the time of satellite formation are undefined, but even if primordial formation is conjectured, the bounds established above for $Q_{u}$ cannot be relaxed. Electromagnetic torques on Io are also not sufficient to relax the bounds on $Q_{J}$. Some ideas on processes for the dissipation of tidal energy in Jupiter yield values of $Q_{J}$ within the dynamical bounds, but

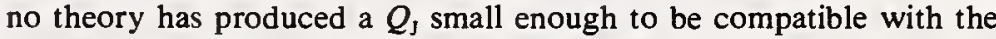
measurements of heat flow from Io given the above relation between $Q_{1}$ and $Q_{\text {s }}$. Tentative observational bounds on the secular acceleration of Io's mean motion are also shown not to be consistent with such low values of $Q_{\mathrm{J}}$. Io's heat flow may therefore be episodic. $Q_{\mathrm{J}}$ may actually be determined from improved analysis of 300 years of eclipse data.

20600. Prince, E. Comparison of profile and integrated-intensity methods in powder refinement, J. Appl. Cryst. 14, 157-159 (1981).

Key words: comparison of models; linear regression; neutron diffraction; powder refinement; significant differences; statistical analysis.

If a powder pattern consists of well resolved peaks, and if it is adequately described by a crystallographic model, as determined by statistical tests, the estimates of the parameters and their standard deviations will not be significantly different, in a statistical sense, whether the method of refinement uses peak profiles or integrated intensities. If the model does not fit adequately, the standard deviations cannot be calculated by statistical methods. If peaks overlap, there are correlations between the intensities of the individual peaks that are handled automatically in the profile method, but must be included explicitly in the integrated-intensity method. A background function should be included in the model, and its parameters refined. The background parameters have correlations with other parameters that become important if the pattern is complex, and therefore poorly resolved.

20601. Harrison, S. H.; Żeisler, R.; Wise, S. A. Pilot program for the National Environmental Specimen Bank-Phase 1, EPA-600/S1-81025, 6 pages (United States Environmental Protection Agency, Washington, DC, May 1981). (Available by purchase from the National Technical Information Service, Springfield, VA 22161.)

Key words: activation analysis; cleanroom design; human liver samples; liquid chromatography; sampling; specimen banking; storage; trace element analysis; trace organic analysis; voltammetry.

This work was performed under a joint NBS/EPA research program to develop state-of-the-art protocols for the sampling, storage, and analysis of biological and environmental-type matrices. This report summarizes the procedures used in the initial phase of a pilot program for determining the feasibility of the National Environmental Specimen Bank. A special clean-laboratory/storage facility has been designed and completed for use in this program. Detailed protocols for sampling, storage, and analysis of human liver samples have been designed for this study. The implementation of these protocols for human liver samples is described in this report.

20602. Chesler, S. N.; May, W. E.; White, P. A.; Parris, R. M.; Guenther, F. R. Determination of polychlorinated biphenyls in waste 
and lubricating oils, Proc. Joint Conf. on Measurements and Standards for Recycled Oil/Systems Performance and Durability, Gaithersburg, MD, Oct. 23-26, 1979, pp. $295-297$ (Nov. 1980).

Key words: gas chromatography; liquid chromatography; lubricating oils; PCB; polychlorinated biphenyls; waste oils.

A new method is reported for the determination of polychlorinated biphenyls (PCB) in waste and lubricating oils. The method utilizes high-performance preparative scale liquid chromatography for sample clean up and glass capillary gas chromatography with electron capture detection for quantitation. The method was found superior to prior existing methods.

20603. Traub, R. D.; Rains, T. C.; Garruto, R. M.; Gajdusek, D. C.; Gibbs, C. J., Jr. Brain destruction alone does not elevate brain aluminum, Neurology 31, No. 8, 986-990 (Aug. 1981).

Key words: aluminum; Alzheimer's disease; atomic absorption spectrometry; brain tissue; Creutzfeldt-Jakob disease; Huntington disease.

Graphite furnace atomic-absorption spectroscopy was used to measure aluminum concentrations in brain samples from 33 patients dying from a variety of neurologic diseases. Four samples from patients dying of nonneurologic causes also were studied. Nine samples (one from each of nine patients) of Creutzfeldt-Jakob disease brain contained normal amounts of aluminum. Aluminum was increased in 9 of 18 brain specimens with seven different pathologic processes. This included three of seven Alzheimer disease, two of three Huntington disease, two of two Parkinson disease, one of one progressive supranuclear palsy, one of one acoustic neuroma, one of two cerebrovascular disease, and one of two Guamanian amyotrophic lateral sclerosis (ALS). Aluminum was normal in the remaining samples (four normal, two ALS, one multiple sclerosis, one Pick disease, and two Guamanian parkinsonism-dementia). The significance of high aluminum values is not clear, but the normal values from the Creutzfeldt-Jakob cases imply that neuronal destruction per se need not lead to accumulation of aluminum in the brain.

20604. MacCrehan, W. A. Differential pulse detection in liquid chromatography and its application to the measurement of organometal cations, Anal. Chem. 53, No. 1, $74-77$ (Jan. 1981).

Key words: electrochemical detection in liquid chromatography; organomercury cations; organotin cations.

An examination is made of some advantages of differential pulse over amperometric electrochemical detection under certain measurement circumstances. The added selectivity, high sensitivity, and low base line drift of the differential mode are discussed. The separation and low level detection of organomercury and organotin cations are examined. Finally, the use of differential pulse detection for the elimination of electrode fouling problems is demonstrated.

20605. Hunt, C. M.; Treado, S. J. Air exchange measurements in a high-rise office building, (Proc. ASHRAE/DOE/ORNL Conf. on Thermal Performance of Exterior Envelope of Buildings, Orlando, FL, Dec. 3-5, 1979), ASHRAE SP 28, pp. 160-177 (American Society of Heating, Refrigeration and Air-Conditioning Engineers, New York, NY, 1981).

Key words: building air exchange; commercial building ventilation; energy consumption by buildings; office building ventilation; ventilation analysis; weather and building air exchange.

Air exchange rates were measured in the tower of an 11-story office building using $\mathrm{SF}_{6}$ as a tracer gas. Fall and winter air exchange rates, I $\left(\mathrm{hr}^{-1}\right)$, measured with make-up and main exhaust pathways blocked, could be represented as a function of wind spread, $\mathrm{W}(\mathrm{mi} / \mathrm{hr})$, by the equation $\mathbf{I}=1.08+0.036 \mathrm{~W}-0.0005 \mathrm{~W}^{2}, \sigma=0.15$.

Wind direction and temperature difference (stack effect) exerted little effect on air exchange rate. In this building, toilet exhausts and other weather-independent mechanisms were more important than natural infiltration in producing air exchange. Inside-outside pressure difference measurements were monitored as a function of temperature and wind speed. Also, a preliminary analysis of predicted air infiltration by the Shaw-Tamura model was undertaken. The results of this preliminary analysis were consistent with the observations that the stack effect was unimportant and that natural infiltration contributed only part of the total air exchange. However, the plot of calculated infiltration rate as a function of wind speed, using the Shaw-Tamura model, had a different form than the plot of measured data.

20606. Kasen, M. B. Cryogenic properties of filamentary-reinforced composites: An update, Cryogenics 21, 323-340 (June 1981).

Key words: advanced composites; cryogenics; elastic properties; fiber-reinforced composites; mat reinforcement; mechanical properties; structural composites; thermal properties; uniaxial reinforcement; woven-fabric.

Progress since 1975 in understanding the effect of cryogenic temperatures on the mechanical, elastic, thermal and electrical properties of fiber-reinforced structural composites is reviewed. The two categories considered are relatively inexpensive laminates reinforced with woven fabric or random mat and the more expensive uniaxial laminates often reinforced with high-performance, advanced fibers. The status of fundamental research and of test method development is reviewed and directions of effective future research are considered.

20607. Reed, R. P. Goal: Better standards for pipeline welds, Weld. Des. Fabr. 54, No. 8, 68.71 (Sept. 1981).

Key words: fitness-for-service; fracture mechanics; girth welds nondestructive inspection; oil and gas pipeline.

The NBS program to assist the Department of Transportation in establishing fitness-for-service alternative allowable flaw size standards is reviewed. The application of fracture mechanics technology to fitness-for-service standards is discussed. Past research is reviewed. NBS programs on fracture mechanics and nondestructive testing with respect to the Alaska Natural Gas Transportation System are presented.

20608. Datta, S. K.; Ledbetter, H. M. Anisotropic elastic constants of a fiber-reinforced boron-aluminum composite, Proc. Mechanics of Nondestructive Testing, Blacksburg, VA, Sept. 10-12, 1980, pp. 215 230 (Plenum Press, New York, NY, 1980).

Key words: boron-aluminum; composite; elastic constants; fiber reinforcement; sound velocity.

Elastic constants, both the $\mathrm{C}_{\mathrm{ij}}$ 's and the $\mathrm{S}_{\mathrm{ij}}$ 's, were measured and calculated for a laminated, uniaxially fiber-reinforced boron-aluminum composite. Three theoretical models were considered: square-array, hexagonal-array, and random-distribution. By combining several existing theoretical studies on randomly distributed fibers, a full set of elastic constants can be predicted for this model. The randomdistribution model agrees best with observation, especially for offdiagonal elastic constants. Considering all nine elastic constants, observation and theory differ on the average by six percent.

20609. Danos, M.; Zanon, D. Model for the hadron-hadron vertex: Dynamic origin of the OZI rule, Lett. Nuovo Cimento 31, No. 2, 61. 64 (1981).

Key words: hadron interactions; meson emission; OZI rule; quantum chromodynamics; strong interaction; vacuum polarization; $Z$ weigs rule.

It is shown that asymptotic freedom leads to a reaction mechanism which explains the experimental data of low energy hadron vertices, including the OZI rule.

20610. Cutkosky, R. D.; Davis, R. S. Simple control circuit for temperature regulation and other bridge applications, Rev. Sci. Instrum. 52, No. 9, 1403.1405 (Sept. 1981).

Key words: bridge reading circuit; electronic bridge; load cell; 
metrology; platinum resistance thermometer; resistance bridge; temperature control; temperature measurement.

A circuit is presented that makes use of low frequency square waves for exciting resistance bridges, detecting their departure from balance, and providing an output voltage for measurement or control purposes. Applications in several areas of precision metrology have been found.

20611. Wyart, J. F.; Kaufman, V.; Sugar, J. The $4 f^{13} 5 f$ configuration in the isoelectronic sequence of Yb III, Phys. Scr. 23, 1069-1078 (1981).

Key words: hafnium; lutetium; parametric theory; spectra; tantalum; tungsten.

Energy levels of the configuration $4 f^{3} 5 f$ have been observed for the first time in the spectra of Lu IV, Hf V, Ta VI, and W VII and have been interpreted by means of the Slater.Condon theory. The relative positions of two perturbing configurations $4 f^{2} 5 d^{2}$ and $4 f^{4} 5 p^{5} 5 f$ are discussed. The $\ddot{j}$ coupling scheme provides the best average purity for the labeling of the levels. Three hundred seventy-seven lines have been classified in these four spectra.

20612. Polvani, R. S.; Christ, B. W. Microcreep of instrument grade beryllium, SAMPE $Q$. 10, No. 4, 37-41 (July 1979).

Key words: beryllium; instrument grade; instrument grade beryllium; microcreep.

Inertial guidance system gyroscopes and optical mirrors are designed to be dimensionally stable to better than 1 microinch over long times. New gyroscope designs require still better stability, 0.01 microinch. Uniaxial microcreep experiments at elevated temperature show instrument grade beryllium deforms in excess of both design limitations. However, a microstrain hardening process was observed that seems a possibility for improving microcreep strength. Performing these experiments required development of a test system with better than 0.1 microinch resolution, less than 0.01 microinch thermal noise in the signal at $144^{\circ} \mathrm{F}$, and typically, sample misalignment of about $10^{-5}$ radians.

20613. Hanley, H. J. M.; Evans, D. J. Behavior of a nonconformal mixture via computer simulation, Int. J. Thermophys. 2, No. 1, 1-19 (1981).

Key words: conformal solution theory; local mole fraction; nonequilibrium molecular dynamics; radial distribution functions; soft spheres; Van der Waals one theory.

A binary $50 \%$ mixture of soft spheres is studied via nonequilibrium molecular dynamics, and the equilibrium and nonequilibrium radial distribution functions for a nonconformal mixture with a mass ratio of 10 and a size ratio of about 2 are examined. This model system is related to the real methane/decane mixture, and it is shown that apparently anomalous properties of this mixture, especially the viscosity, could perhaps be understood in terms of the local or ambient mole fraction. In addition, the postulates of the Van der Waals one fluid conformal solution theory are discussed, and a mixing rule for the mass is derived.

20614. Harvey, K. C. Lyman- $\beta$ lamp with cooled LiF window, Appl. Opt. 20, 2883-2885 (Sept. 1, 1981).

Key words: atomic beams; lamp; Lyman- $\beta$; optical pumping; vacuum ultraviolet.

The metastable state of atomic hydrogen has long been the object of precision measurements and many methods for its preparation in atomic beams have been developed. For some applications a slow dense beam of atomic hydrogen prepared in the metastable state by optical pumping with Lyman- $\beta$ radiation offers many advantages. Such a scheme is currently prohibited by the absence of an appropriate source of resonance radiation. The characteristics of this $1026 \AA$ lamp would include high intensity, narrow spectral width to match the beams absorption and low self-reversal of the line. No appropriate laser sources exist at this wavelength and, although resonance lamps exist in the visible and ultraviolet, no solid window material transmits at room temperature at 1026 Ä. Studies by Hunter and Malo of the transmission of various crystals as a function of temperature has shown that cooled $\mathrm{LiF}$ is a good transmitter at 1026 $\AA$. Indeed, a combination of a cooled crystal and a thin film of indium produced a filter for Lyman- $\beta$. An RF excited lamp cooled by liquid nitrogen with a $\mathrm{LiF}$ window is described here which provides an intense source of Lyman- $\beta$ with narrowed Doppler width.

20615. Seltzer, S. M. Calculated response of intrinsic germanium detectors to narrow beams of photons with energies up to $\sim 300 \mathrm{keV}$, Nucl. Instrum. Methods 188, 133.151 (1981).

Key words: calculation; formulae; Ge detector; low energy; Monte Carlo; x ray.

The response of small intrinsic Ge detectors has been calculated for photons incident with energies up to $\sim 300 \mathrm{keV}$. The results include the effects of scattering and escape from the detector of the photons and of $\mathrm{Ge}$ characteristic X-rays produced in photoelectric absorption. Included also are the effects of the backscattering of photons from material behind the detector. The results, based on Monte Carlo calculations for various photon energies and detector sizes, have been generalized on the basis of a single-scatter model and are given as a set of formulae that can be evaluated for any photon energy and detector size in the range of interest. The predictions obtained with the formulae are shown to agree well with available data from measurements and from Monte Carlo calculations made by other authors. The formulae are used to tabulate photopeak efficiencies for a large number of commercially available detectors, and to generate response functions for unfolding measured pulse-height distributions obtained for continuous incident spectra.

20616. Kasen, M. B.; MacDonald, G. R.; Beekman, D. H., Jr.; Schramm, R. E. Mechanical, electrical, and thermal characterization of G-10CR and G-11CR glass-cloth/epoxy laminates between room temperature and 4K, (Proc. Cryogenic Engineering Conf., Madison, WI, Aug. 21-24, 1979), Paper D-1 in Advances in Cryogenic Engineering Materials, A. F. Clark and R. P. Reed, eds., 26, 235-244 (Plenum Publ. Corp., New York, NY 10013, 1980).

Key words: composite; cryogenics; electrical properties; glass/epoxy; mechanical properties; thermal properties.

The U.S. magnetic fusion energy program will require large-scale use of industrial glass-reinforced/epoxy laminates for structural supports and for electrical and thermal insulation in superconducting magnets. There is an immediate need for a commercial supply of such materials having predictable cryogenic performance. NBS has cooperated with the laminating industry and the National Electrical Manufacturers' Association to establish and make available specifications for cryogenic grades of G-10 and G-11 glass. cloth/epoxy laminates. These materials are designated G-10CR and G-11CR. This paper presents mechanical, electrical, and thermal property characterization of these two materials from room temperature to $4 \mathrm{~K}$. A variant of these laminates made with boron-free glass was also tested.

20617. Younger, S. M. Cross sections and rates for direct electronimpact ionization of sodiumlike ions, Phys. Rev. $A$ 24, No. 3, 1272 1277 (Sept. 1981).

Key words: electron atom scattering; electron impact ionization sodium isoelectronic sequence.

Cross sections and rate coefficients for the direct electron-impact ionization of sodiumlike ions have been calculated in a distorted-wave Born exchange approximation. For higher charge states the cross section is dominated by inner-shell ionization of the $2 p^{6}$ subshell. Analytic fits to the data allow rapid calculation of cross sections and rate coefficients over a wide range of incident electron energies. The importance of electron-impact excitation to autoionizing states is discussed.

20618. Younger, S. M. Electron impact ionization cross sections and 
rates for highly ionized atoms, J. Quant. Spectrosc. Radiat. Transfer 26, No. 4, 329.337 (1981).

Key words: electron impact ionization; helium isoelectronic sequence; hydrogen isoelectronic sequence; lithium isoelectronic sequence.

Electron impact ionization cross sections and rates for hydrogen-, helium-and lithium.like ions are presented in simple analytical form, based on a set of parameters used in the formulae allow rigid generation of cross sections and rates for any ion in these sequences with a minimum of computational effort. The use of accurate Bethe slopes in the cross section-fitting algorithm yields good accuracy both in the near threshold and high energy regions. The rate coefficient is given in the form of a correction to the commonly used Seaton formula and is thus readily adaptable to existing plasma modeling computer codes. Comparisons of the present results with other theoretical and experimental data are made.

20619. Younger, S. M. Electron-impact-ionization cross sections and rates for highly ionized berylliumlike ions, Phys. Rev. $A$ 24, No. 3, 1278-1285 (Sept. 1981).

Key words: beryllium isoelectronic sequence; electron-atom scattering; electron impact ionization; metastable states.

Electron-impact-ionization cross sections and rates have been calculated for several berylliumlike ions in the $1 s^{2} 2 s^{2} S$ ground state and the $1 s^{2} 2 s 2 p{ }^{3} P$ metastable state using a distorted-wave Born exchange approximation. The ground-state cross section showed only minor deviations from classical scaling along the isoelectronic sequence. The $2 s 2 p{ }^{3} P$ cross section was more than twice that of the ground state at C III, decreasing to a factor of 1.35 greater at Ar XV. Dominant ground-state configuration interaction of the form $2 s^{2}+2 p^{2}$ changed the total cross section by only a few percent. An analytic fit to the distorted-wave results is given, and this allows the calculation of nonrelativistic electron-impact-ionization cross sections and rates for berylliumlike ions. A comparison with a vailable experimental data shows good agreement between measurements and distorted-wave calculations.

20620. Esfandiari, P.; Meijer, P. H. E.; Farrell, R. A.; Favin, S. New generating functions and results for the density polynomials of the lattice gas, Phys. Rev. B 24, No. 3, 1298-1311 (Aug. 1, 1981).

Key words: generating function; Helmholtz free energy; Ising model; lattice gas; renormalization; vertex.

This paper determines the density weight factors needed to evaluate the Helmholtz free energy for the Ising model of the lattice gas through tenth order in the coupling constants for arbitrary density and interactions. This is accomplished by developing new generating functions for the evaluation of the density factors that are associated with the articulated vertices which result from summing over the "hard-core" part of the interaction potential. These factors are polynomials in the density, and their coefficients are obtained from recurrence relations derived from the generating functions. This procedure is used to deduce general equations for articulation vertices of the first, second, and third kind. These general formulas are supplemented with a few specific cases of vertices of higher kind in order to evaluate the free energy through tenth order in the coupling constant. Application to the nearest neighbor model reproduces known results for the simple cubic (s.c.) and body-centered cubic (b.c.c.) lattices, and extends the known face-centered cubic (f.c.c.) results by two orders.

20621. Netzer, F. P.; Madey, T. E. The adsorption of NO on Ni(111): An ESDIAD/LEED study, Surf. Sci. 110, $251-260$ (1981).

Key words: chemisorption; electron stimulated desorption; nickel; nitric oxide; surface science; surface structure.

The ESDIAD method (electron stimulated desorption ion angular distributions) has been combined with LEED (low energy electron diffraction) in a study of the adsorption of NO on Ni(111). For adsorption at $80 \mathrm{~K}$, NO appears to be bonded with its molecular axis perpendicular to the $\mathrm{Ni(111)}$ surface at all coverages. Heating the 80 $\mathrm{K}$ layer leads to a striking structural change which we interpret as the formation of inclined or bent NO in the range $120 \leqslant T \leqslant 250 \mathrm{~K}$. Upon adsorption at $150 \mathrm{~K}$, only the bent form of NO is present at low coverages; at higher coverages at $150 \mathrm{~K}$, the perpendicular form appears, in agreement with recent electron energy loss spectroscopy (EELS) data of Lehwald, Yates, and Ibach. When NO is coadsorbed with $\mathrm{p}(2 \times 2)$ oxygen, the perpendicular form of NO dominates at all coverages and temperatures studied. Dissociated NO adsorbed at steps and defect sites on $\mathrm{Ni}(111)$ produces a well-defined hexagonal ESDIAD pattern.

20622. Bensema, W. D. Broadband orthogonal array antenna system microprocessor control and computation, Proc. 1981 IEEE Int. Symp. on Electromagnetic Compatibility, Boulder, CO, Aug. 18-20, 1981, pp. 43-45 (The Institute of Electrical and Electronics Engineers, Inc., New York, NY 10017, 1981).

Key words: antenna element switching; automatic peak finding and recording; CMOS microprocessor controller; effective omnidirectional antenna coverage; electromagnetic interference (EMI) measurement; field strength measurement; portable equipment; spectrum occupancy measurement.

A broadband orthogonal array antenna system and associated control circuitry have been designed to reduce the man hours required when making spectrum occupancy studies. Effective omnidirectional coverage is obtained by rapidly switching among arrays of conventional antennas; resultant field strengths are calculated and recorded using a microprocessor. The system is man pack portable, covers from $10 \mathrm{kHz}$ to $18 \mathrm{GHz}$ and is capable of recording fields up to $100 \mathrm{~V} /$ meter. The system has demonstrated a factor of 50 to 1 reduction in man hours required to carefully measure and record spectrum occupancy over conventional non-automated searching. This paper concentrates on the microprocessor computation, control, display, print out and convenience features. A companion paper discusses antenna systems and if switching.

20623. Sreenivasiah, I.; Chang, D. C.; Ma, M. T. A critical study of emission and susceptibility levels of electrically small objects from tests inside a TEM cell, Proc. 1981 IEEE Int. Symp. Electromagnetic Compatibility, Boulder, CO, Aug. 18-20, 1981, pp. 499.507 (The Institute of Electrical and Electronics Engineers, Inc., New York, NY 10017, 1981).

Key words: analysis; dipole moments; electrically small objects; emission; measurements; quadrupoles; susceptibility; TEM cell.

An electrically small radiating source of arbitrary shape may, to a first order, be modeled by an equivalent dipole system consisting of three orthogonal electric dipoles and three orthogonal magnetic dipoles each excited with arbitrary amplitude and phase. Determination of the individual electric dipole moments and the cross-components of such an equivalent dipole system for the emission case by tests inside a TEM cell, was described in an earlier work by Sreenivasiah, Chang and Ma, assuming a constant TEM modal field distribution in the space occupied by the test object. A method of accounting for the first-order variation in the TEM modal field distribution is presented in this report. This involves the inclusion of electric quadrupole terms in the modeling of the test object. Using the reciprocity principle, the same method is extended to the determination of susceptibility levels of electrically small test objects. Some experimental results for the susceptibility test, demonstrating the importance of the quadrupole terms, are presented.

20624. FitzGerrell, R. G. Free-space transmission loss for anechoic chamber performance evaluation, Proc. 1981 IEEE Int. Symp. Electromagnetic Compatibility, Boulder, CO, Aug. 18-20, 1981, pp 110-111 (The Institute of Electrical and Electronics Engineers, Inc., New York, NY 10017, 1981).

Key words: anechoic chamber; evaluation.

A longitudinal probe scan of an anechoic chamber yields a plot of measured transmission loss. Any deviation of this curve from calculated free-space transmission loss can be attributed to chamber 
reflections or, at small separation distances, to finite-range source antenna gain corrections or to source-probe interactions.

20625. Berger, H. A new look at the NBS programme in NDE, NDTITDS 14, No. 5, 292-293 (Oct. 1981).

Key words: acoustic emission; calibrations; eddy current conductivity; liquid penetrants; magnetic particle; nondestructive evaluation; radiography; standards; ultrasonics.

A brief review is presented of the NBS Nondestructive Evaluation (NDE) program. Emphasis is on progress in calibration and Standard Reference Material (SRM) activities; particularly as related to wellused methods for industrial NDE.

20626. Berger, A. E.; Solomon, J. M.; Ciment, M. An analysis of a uniformly accurate difference method for a singular perturbation problem, Math. Comput. 37, No. 155, 79.94 (July 1981).

Key words: difference methods; rates of convergence; singular perturbation problem; tridiagonal difference schemes; uniform accuracy; variable mesh schemes.

It will be proven that an exponential tridiagonal difference scheme, when applied with a uniform mesh of size $h$ to: $\epsilon u_{x x}+b(x) u_{x}=f(x)$ for $0<x<1, b>0, b$ and $f$ smooth, $\epsilon$ in $(0,1)$, and $u(0)$ and $u(1)$ given, is uniformly second-order accurate (i.e., the maximum of the errors at the grid points is bounded by $C h^{2}$ with the constant $C$ independent of $h$ and $\epsilon$ ). This scheme was derived by El-Mistikawy and Werle by a $C^{1}$ patching of a pair of piecewise constant coefficient approximate differential equations across a common grid point. The behavior of the approximate solution in between the grid points will be analyzed, and some numerical results will also be given.

20627. Ayres, T. R.; Linsky, J. L.; Vaiana, G. S.; Golub, L.; Rosner $\mathrm{R}$. The cool half of the H-R diagram in soft X-rays, Astrophys. J. 250, 293.299 (Nov. 1, 1981).

Key words: late-type stars; magnetic fields; stellar coronae; stellar rotation; stellar winds; $x$-ray sources.

We report results of an Einstein Guest Observing program to map the occurrence of soft X-ray emission, which is a signature of hot stellar coronae $\left(T>10^{6} \mathrm{~K}\right)$, in the cool half of the Hertzsprung Russell (H-R) diagram. We detect X-rays from F-M dwarfs and late $F$ through early $\mathrm{K}$ giants, but not from the cooler giants, other than the spectroscopic binary $\epsilon$ Car $(\mathrm{K} 0 \mathrm{II}+\mathrm{B})$, or from any supergiants, other than Canopus (F0 Ib-II). The empirical separation of the cool half of the H-R diagram into a region where stellar soft X-ray emission is a common phenomenon, and a region where hot coronae are rare, if present at all among single stars, is similar to that found previously by Linsky and Haisch for C IV $\lambda \lambda 1548,1551$ emission $\left(T \approx 10^{5} \mathrm{~K}\right)$ and by Stencel and Mullan for the onset of rapid mass loss in strong, cool $\left(T<10^{4} \mathrm{~K}\right)$ stellar winds. We discuss the energy balance in the outer atmospheres of the coronal stars, the likely absorption of X-ray emission by cool winds in the "hybrid-spectrum" supergiants, a rotation-activity connection among the $G$ dwarfs, and possible evolutionary origins of the structure seen in the cool half of the X-ray $\mathrm{H} \cdot \mathrm{R}$ diagram.

20628. McCrackin, F. L.; Mazur, J. Configuration properties of combbranched polymers, Macromolecules 14, No. 5, 1214-1220 (Sept.-Oct. 1981).

Key words: branched polymers; comb-branched polymers; expansion factor; Monte Carlo; radii of gyration; star-branched polymers.

Mean-square radii of gyration were computed for comb-branched polymers simulated by chains on a cubic lattice that incorporated both excluded volume and attractive energies between nonbounded segments of the polymer. The ratios $g$ of the radius of gyration of comb-branched polymer to that of a linear polymer of the same molecular weight at the $\theta$ point were found to be larger than the $g$ ratios calculated by the unrestricted random walk model of the polymer. The calculated $g$ ratios were compared with experimental measurements. For the comb-branched polymers, the radii of gyration of the backbones and the expansion factors were also calculated. The radii of gyration of the backbones at the $\theta$ conditions were greater than those of linear polymers, in contradiction to the random walk model, which requires that they be equal. The calculated expansion factors were less than those of linear polymers, also in contradiction to perturbation theories based on the random walk model, which predict greater expansion factors for branched polymers than for linear polymers.

20629. Girvin, S. M.; Rendell, R. W.; Bryant, G. W. New field theory formulation of localised states in disordered systems, J. Phys. C: Solid State Phys. Lett. Ed. 14, L881-L886 (1981).

Key words: density of states; disordered systems; field theory; localization; one dimension.

We present a new field theoretic formulation of the quantum mechanics of disordered systems. The problem is converted to an explicit field theory by changing variables in the functional integral over all random potentials to an integral over all possible wave functions. Unlike previous formulations this field theory has the nove feature of having the "right" sign for the coefficient of the "term. Thus the important excitations are kinks rather than instantons. In addition, it exhibits singular solutions with finite action. The method is illustrated with an exact calculation of the asymptotic density of states in a one dimensional gaussian white noise potential.

20630. Ebbesen, H. F.; Engen, G. F. Singularities in the calibration of six-port network analyzers, (Proc. 1981 IEEE MTT-S Int. Microwave Symp., Los Angeles, CA, June 15-19, 1981), Paper in 1981 IEEE MTT-S International Symposium Digest, IEEE Cat. No. $81 \mathrm{CH} 1592-5$, J. E. Raue, ed., pp. 149-150 (The Institute of Electrical and Electronics Engineers, Inc., 445 Hoes Lane, Piscataway, NJ 08854, 1981).

Key words: automatic network analyzer; microwave measurements; six-port

Numerical difficulties may be encountered when applying the TRL calibration algorithm to dual six-port analyzers.

The possible occurrence of singularities is explained, and means of avoiding these are proposed.

20631. Hoer, C. A. A high-power dual six-port automatic network analyzer for determining biological effects of $\mathbf{R F}$ and microwave radiation, (Proc. 1981 IEEE MTT-S Int. Microwave Symp., Los Angeles, CA, June 15-19, 1981), Paper in 1981 IEEE MTT-S International Microwave Symposium Digest, IEEE Cat. No. $81 \mathrm{CH} 1592-5$, J. E. Raue, ed., pp. $157-159$ (The Institute of Electrical and Electronics Engineers, Inc., 445 Hoes Lane, Piscataway, NJ 08854, 1981)

Key words: impedance; microwave radiation; network analyzer; power; reflection coefficient; scattering parameters; six-port.

The design, calibration and performance of a high-power (1 to $1000 \mathrm{~W}$ ) automatic network analyzer based on the six-port concept are described for the 10 to $100 \mathrm{MHz}$ range. Imprecision in measuring reflection coefficient $\Gamma$ is 0.0001 in magnitude and $0.005 /|\Gamma|$ degrees in phase. Corresponding estimated systematic errors are 0.001 and $0.1 /|\Gamma|$ degrees. Imprecision in measuring power is $0.01 \%$ of range $(20 \mathrm{~W}, 200 \mathrm{~W}$, or $1000 \mathrm{~W})$ with an estimated systematic error of $1.25 \%$ of reading.

20632. Lewis, R. L. Spherical-wave source-scattering-matrix analysis of the mutual coupling between two antennas, Proc. AP-S Int. Symp. 1981, Los Angeles, CA, June 16-19, 1981, pp. 261-264 (The Institute of Electrical and Electronics Engineers, Inc., 445 Hoes Lane, Piscataway, NJ 08854, 1981).

Key words: antennas; mutual antenna coupling; scattering matrix; spherical scanning.

Expressions are given for the mutual coupling between two antennas in terms of each antenna's spherical-wave source-scattering- 
matrix representation. A comparison to the "classical" scattering matrix definition is given in sufficient detail to permit conversion back and forth between source-scattering-matrix representation and the classical scattering matrix representation. The paper concludes with expressions for the transmission formulas, showing the different expressions corresponding to changes in direction of propagation. However, if the two antennas are both reciprocal, then the two-port scattering matrix is a symmetric matrix.

20633. Siegwarth, J. D.; Brennan, J. A. LNG instrumentation for custody transfer, Proc. American Gas Association 1981 Operating Section Distribution and Transmission Conf., (Distribution Conf. Anaheim, CA, May 18-20, 1981; Transmission Conf., Atlanta, GA, May 4-6, 1981), pp. T-119-T-123 (American Gas Assoc., Arlington, VA, 1981).

Key words: densimeter; flowmeter level gauge; LNG.

Recent work by NBS on measurement of density, liquid level, and liquid flow of Liquefied Natural Gas (LNG) is presented. The density work includes development methods and standards for calibrating densimeters and recent test work of some commercial densimeters. Commercial LNG level gages will be tested for accuracy in the liquid level gage program. The flow program involves testing of large (up to $81 \mathrm{~cm} \mathrm{dia)} \mathrm{flowmeters} \mathrm{for} \mathrm{LNG} \mathrm{service.}$

20634. Cruz, J. E. Design, construction, and calibration of the broadband electric field monitor (EFM-5), Proc. 1981 IEEE Int. Symp. Electromagnetic Compatibility, Boulder, CO, Aug. 18-20, 1981, pp. 439.444 (The Institute of Electrical and Electronics Engineers, Inc., 445 Hoes Lane, Piscataway, NJ 08854, 1981).

Key words: antenna unit; calibration; design; electronics unit; transmission line.

A broadband electric field monitor (EFM) in the frequency range of $200 \mathrm{kHz}$ to $1 \mathrm{GHz}$ has been developed with an isotropic response pattern and a $60 \mathrm{~dB}$ dynamic range (1 volt/meter to 1000 volts/meter). The readout is in terms of the total electric field magnitude. The EFM is designed to not perturb the field being measured and can measure average cw fields. This paper emphasizes the design and calibration of the EFM-5.

20635. Weeks, J. J.; Clark, E. S.; Eby, R. K. Crystal structure of the low temperature phase (II) of polytetrafluoroethylene, Polymer 22, 1480-1486 (Nov. 1981).

Key words: diffraction; electron; helix; incommensurable; polytetrafluoroethylene; structure; unit-cell; $x$ ray.

An electron diffraction pattern from polytetrafluoroethylene in Phase II was analysed to determine a chain conformation of 2.1598 $\mathrm{CF}_{2}$ units per turn of the helix. A structure was established from a combination of electron and X-ray diffraction data. The structure contains an ordered repeating pattern of a left- right-handed pair of chain stems. Although the structure is ordered in three dimensions, it cannot be described meaningfully in terms of classical space group nomenclature because very small changes in the structure result in very large changes in the cell parameters. The structure has a volume of $0.03542 \mathrm{~nm}^{3}$ per $\mathrm{CF}_{2}$ group, corresponding to a density of $2344 \mathrm{~kg}$ $\mathrm{m}^{-3}$.

20636. Baltes, H. P.; Geist, J. Kirchhoff's and Planck's radiation laws for small particles in scattering atmospheres or flames, J. Quant. Spectrosc. Radiat. Transfer 26, No. 6, $535-536$ (1981).

Key words: Kirchhoff's law; Mie scattering; Planck's law; small particle.

Size and shape effects that modify Planck's radiation law for small enclosures and the emission cross section for small particles do not affect the validity of Kirchhoffs radiation laws when applied to macroscopic collections of small particles such as in scattering atmospheres or flames.

20637. Day, R. H.; Lee, P.; Saloman, E. B.; Nagel, D. J. Photoelectric quantum efficiencies and filter window absorption coefficients from $20 \mathrm{eV}$ to $10 \mathrm{KeV}$, J. Appl. Phys. 52, No. 11, 6965-6973 (Nov. 1981).

Key words: fusion plasma diagnostic detectors; fusion plasma diagnostics; mass absorption coefficients of soft $\mathrm{x}$-ray windows; photoefficiency of practical cathodes; radiometry; soft $x$ ray.

Photodiodes with $\mathrm{x}$-ray sensitive photocathodes are commonly used as broadband $\mathrm{x}$-ray detectors in fusion plasma diagnostics. We have measured the photocathode quantum efficiency between 1-500 $\AA$ of common photocathode materials including aluminum, copper, nickel, gold, three forms of carbon, chromium, and cesium iodide. We have also studied the effects of the experimental environment and long. term cathode aging on the measured quantum efficiencies. In addition, we have measured the $\mathrm{x}$-ray mass-absorption coefficients of $\mathrm{x}$-ray filter windows of Kimfoil, aluminum, polypropylene, and Formvar in energy regions where data were previously unavailable. Measurements between 1-50 $\AA$ were performed at the Los Alamos Scientific Laboratory's low-energy x-ray calibration facility, while the measurements between $50-500 \AA$ were performed at the National Bureau of Standard's synchrotron ultraviolet radiation facility.

20638. Bur, A. J. Resistivity and piezoelectric measurements on oriented and unoriented thick PVF, films, Polymer 22, 1288-1289 (Sept. 1981).

Key words: electric breakdown; oriented films; piezoelectricity; polyvinylidene fluoride; pyroelectricity; resistivity.

Poling of unoriented specimens of $\mathrm{PVF}_{2}$ produced unsatisfactory results because the breakthrough $(0.5 \mathrm{MV} / \mathrm{cm})$ was too low to allow significant permanent polarization. For oriented specimens, however, it was observed that poling fields of $1 \mathrm{MV} / \mathrm{cm}$ and higher could be achieved without experiencing breakdown. With these higher fields oriented thick specimens of $\mathrm{PVF}_{2}$ could be made into piezoelectrically active specimens. In order to investigate the electrical properties of oriented and unoriented $\mathrm{PVF}_{2}$, resistivity measurements were carried out. It was observed that the resistivity for oriented $\mathrm{PVF}_{2}$ was at least an order of magnitude higher than that for unoriented $\mathrm{PVF}_{2}$.

20639. Broadhurst, M. G.; Davis, G. T.; DeReggi, A. S. Dielectric properties of PVDF: Low-field and high-field phenomena and time effects, Proc. Symp. High-Energy-Density Capacitors and Dielectric Materials, Boston, MA, Oct. 28, 1980, pp. 67-77 (National Academy Press, Washington, DC, 1981).

Key words: dielectric relaxation; electrical breakdown; electrode effects; ferroelectric transition; high energy density capacitors; polyvinylidene fluoride; relative permittivity; remnant polarization.

The electrical properties of polyvinylidene fluoride (PVDF) are reviewed in view of its consideration as a dielectric in high energy density capacitors. PVDF is a semicrystalline polymer which is known to crystallize in at least four different crystal phases. Electrical properties of this polymer depend upon the amount and type of crystals present as well as their orientation which makes the properties of PVDF much more dependent on processing conditions than is the case for completely amorphous polymers. Dielectric properties of the polymer as a function of temperature, frequency, and orientation are reviewed. Remnant polarization, ferroelectric switching, piezoelectric and pyroelectric activity, and electrode effects in PVDF and copolymers of vinylidene fluoride with trifluoroethylene or tetrafluoroethylene are discussed.

20640. Wells, J. S.; Petersen, F. R.; Maki, A. G.; Sukle, D. J. Heterodyne frequency measurements (at $11.6 \mu \mathrm{m}$ ) on isotopic species of carbonyl sulfide, $\mathrm{OC}^{34} \mathrm{~S}, \mathrm{O}^{13} \mathrm{CS}, \mathrm{OC}^{33} \mathrm{~S},{ }^{18} \mathrm{OCS}$, and $\mathrm{O}^{13} \mathrm{C}^{34} \mathrm{~S}$, J. Mol. Spectrosc. 89, 421.429 (1981).

Key words: carbonyl sulfide; heterodyne frequency measurements; high resolution spectroscopy; isotopic species of OCS; tunable diode laser; tunable laser spectroscopy.

Heterodyne frequency measurements between a ${ }^{13} \mathrm{CO}_{2}$ laser and 
tunable diode laser (either tuned or locked to various carbonyl sulfide absorption peaks) have been made on a number of $10^{\circ} \mathrm{O}-\mathrm{OO}^{\circ} \mathrm{O}$ band transitions in the isotopic species of carbonyl sulfide: $\mathrm{OC}^{34} \mathrm{~S}, \mathrm{O}^{13} \mathrm{CS}$, $\mathrm{OC}^{33} \mathrm{~S},{ }^{18} \mathrm{OCS}$, and $\mathrm{O}^{13} \mathrm{C}^{34} \mathrm{~S}$. The ${ }^{13} \mathrm{CO}_{2}$ laser frequencies primarily resulted from either normal high.J or recently measured hot band $\left(01^{\prime}-11^{1} 0\right)$, transitions. These OCS frequency measurements have been combined with existing microwave data and new sets of molecular constants obtained. These constants will be used later to form part of a frequency calibration compendium based on OCS.

20641. Petersen, F. R.; Wells, J. S.; Maki, A. G.; Siemsen, K. J. Heterodyne frequency measurements of ${ }^{13} \mathrm{CO}_{2}$ laser hot band transitions, Appl. Opt. 20, No. 20, 3635.3640 (Oct. 15, 1981).

Key words: band; improved ro-vibrational constants; laser frequency measurements.

The frequencies of twenty-eight lines in the $P$-branch of the $01^{1}-\left[11^{1} 0,03^{1} 0\right]_{1}$ band of ${ }^{13} \mathrm{C}^{16} \mathrm{O}_{2}$, observed in laser emission, and three lines in the $R$-branch, observed in absorption with a diode laser, have been carefully measured with stabilized $\mathrm{CO}_{2}$ lasers. A significant improvement in the ro-vibrational constants has been obtained from a least squares fit to these data. The laser lines stabilized by saturated absorption techniques provide convenient, accurate (uncertainty $<0.1$ $\mathrm{MHz}$ ) frequency references near $11.7 \mu \mathrm{m}$.

20642. Babrauskas, V. A laboratory flammability test for institutional mattresses, Fire J. 75, No. 6, 35-40, 93 (Nov. 1981).

Key words: beds; cell padding; correctional institutions; fire tests mattresses; prisons; rate of heat release tests; smoke production tests.

Minimum mattress fire safety is provided by the mandatory Federal cigarette ignition standard FF 4-72. This is generally considered not to be sufficient for the jail or prison environment where flaming ignitions can well be expected and egress may be restricted. Until now additional safety could only be provided by either explicitly banning certain materials or else requiring full-scale demonstration tests. The former discourages innovation while the latter is difficult to control and reproduce. To eliminate these difficulties, a set of benchscale laboratory test procedures has been developed for testing mattresses and classifying their performance. A program of full-scale fire testing identified four distinct performance groups. The laboratory test procedures correctly classify the performance into the same groups as the full-scale fire tests. For general use in correctional facilities only mattresses conforming to the highest group requirements (Group $A$ ) are recommended. The same test procedure is also applicable to cell padding materials.

20643. Pierce, D. T.; Celotta, R. J.; Wang, G. C.; McRae, E. G. Spin dependence of electronic surface resonance scattering from $W(100)$, Solid State Commun. 39, 1053-1055 (1981).

Key words: electronic resonance scattering; spin polarization; surface potential barrier; surface resonance.

In polarized low energy electron diffraction (PLEED) from $\mathrm{W}(100)$ at energies of $2-9 \mathrm{eV}$ and incident angles $15^{\circ}<\theta<45^{\circ}$, we observe a spin-splitting of the resonance sequence of peaks which converge on the (01) beam threshold. For $\theta>26^{\circ}$ the peak splitting to peak width ratio is approximately constant while for decreasing $\theta \leqslant 26^{\circ}$ it increases. An anomalous spin-up-only peak appears at small $\theta$. The nature of the spin dependent splitting is discussed within the four-beam layer multiple scattering approximation.

20644. Deutsch, D. R.; Clemons, E. K. DBMS standards: Current status and future directions, Proc. 6th Int. Conf. Very Large Databases, Montreal, Canada, Oct. 1-3, 1980, pp. 431.433 (The Institute of Electrical and Electronics Engineers Computer Society, 5855 Naples Plaza, Long Beach, CA 90803, 1980).

Key words: ANSI; CODASYL; database management; DBMS; IFIP; NBS; standards.

This paper surveys the database standardization arena; major organizations involved in developing database standards and their current activities are briefly described. These discussions provide a framework for comments and debate by panelists representing diverse parts of the DBMS community.

20645. Hebner, R. E.; Kelley, E. F.; Forster, E. O.; FitzPatrick, G. J. Observation of prebreakdown and breakdown phenomena in liquid hydrocarbons, Proc. 7th Int. Conf. Conduction and Breakdown in Dielectric Liquids, Berlin, West Germany. July 27-31, 1981, pp. 177. 181 (The Institute of Electrical and Electronics Engineers, 445 Hoes Lane, Piscataway, NJ 08854, 1981).

Key words: breakdown; dielectrics; high voltage; insulation liquids; shock waves.

Prebreakdown and breakdown events under quasi-uniform fields in n-hexane, isooctane, cyclohexane, and toluene have been photographed. For each liquid, two types of breakdown patterns are observed. One pattern is characteristic of growth from the cathode while the other is characteristic of growth from the anode. The measured postbreakdown phenomena were identical in all cases.

In addition to the optical studies, microscopic observations showed that organic films were produced on the electrode surface and that lead accumulates preferentially in the area of the electrode surface where breakdown occurs

20646. Zahn, M.; Forster, E. O.; Kelley, E. F.; Hebner, R. E., Jr Hydrodynamic shock wave propagation after electrical breakdown, Proc. 7th Int. Conf. Conduction and Breakdown in Dielectric Liquids, Berlin. West Germany, July 27-31, 1981, pp. $398-403$ (The Institute of Electrical and Electronic Engineers, 445 Hoes Lane, Piscataway, NJ 08854, 1981).

Key words: breakdown; dielectrics; high voltage; insulation; liquids; shock waves; transformer oil.

Laser schlieren measurements in liquid dielectrics have been used to examine the radial expansion of both the acoustic shock wave and the conductive breakdown channel during the first few microseconds after electrical breakdown under high voltage impulse conditions. It was found that the acoustic shock wave expands at a constant velocity while the expanding radius of the beakdown channel is proportional to the fourth root of the energy and the square root of time. These dependencies are predicted by modeling the breakdown channel as an expanding adiabatic ideal gas with an instantaneous input of energy. The Rankine-Hugoniot boundary conditions in the strong shock limit are used to relate discontinuities in velocity, pressure, and mass density across the cylindrical shock front using the same analysis which was used previously to describe exploding wires in air. When the expansion velocity of the gas column decreases below the acoustic wave velocity in the liquids, an acoustic wave propagates ahead of the electrohydrodynamic shock. Prior to this crossover time, acoustic waves cannot propagate away because the gas column expands faster than the acoustic velocity.

20647. Farley, J. W.; Wing, W. H. Accurate calculation of dynamic Stark shifts and depopulation rates of Rydberg energy levels induced by blackbody radiation. Hydrogen, helium, and alkali-metal atoms, Phys. Rev. A 23, No. 5, 2397-2424 (May 1981).

Key words: alkali metals; blackbody radiation; calculations; helium; hydrogen; induced transitions; Rydberg states; Stark shifts.

A highly excited (Rydberg) atom bathed in blackbody radiation is perturbed in two ways. A dynamic Stark shift is induced by the offresonant components of the blackbody radiation. Additional, electricdipole transitions to other atomic energy levels are induced by the resonant components of the blackbody radiation. This depopulation effect shortens the Rydberg-state lifetime, thereby broadening the energy level. Calculations of these two effects in many states of hydrogen, helium and the alkali-metal atoms $\mathrm{Li}, \mathrm{Na}, \mathrm{K}, \mathrm{Rb}$ and $\mathrm{Cs}$ are presented for $T=300 \mathrm{~K}$. Contributions from the entire blackbody spectrum and from both discrete and continuous perturbing states are included. The accuracy is considerably greater than that of previous estimates 
20648. Fanney, A. H.; Thomas, W. C. Simulation of thermal performance of solar collector arrays, Trans. ASME 103, 258.267 (Aug. 1981).

Key words: collector; indoor; outdoor; parallel; series; simulation; solar; testing.

An experimental method is described for simulating the useful energy supplied by collector arrays during tests of solar water heating systems. The method uses an electric heat source to simulate the absorbed solar energy in series with nonirradiated collectors to simulate the concurrent heat losses. This configuration maintains the collector-loop flow characteristics which are important for system tests.

Expressions are developed for programming the heat source for collector arrays connected in parallel and series combinations with the heat source located either upstream or downstream from the nonirradiated array. Thermal modelling of representative arrays is used to investigate the consequences of using linearized collector efficiency curves to program the heat source and of using nonirradiated collectors to simulate heat losses. The absence of absorbed solar radiation in collector covers indoors is shown to partially cancel the effects of generally higher windspeeds and increased longwave radiation from the outdoor environment.

The results of experiments to verify the performance of a nonirradiated array with a downstream electric heat source are presented. Day-long tests of a domestic solar hot water system with irradiated collectors were repeated using a nonirradiated array with a downstream heat source. The useful energy supplied in the two cases agreed to within 3.5 percent. The measured differences were consistent with the results of the analytical investigation. In representative situations, the use of nonirradiated collectors in series with an electric heat source may give up to 10 percent higher useful energy output as compared to an irradiated array. However, differences can be reduced by closely matching indoor and outdoor environmental conditions and by locating the heat source downstream from the nonirradiated collector array.

20649. Evans, D. J.; Hanley, H. J. M. Thermodynamic fluctuation theory for shear flow, Physica 108A, 567.574 (1981).

Key words: Couette flow; fluctuation theory; irreversible thermodynamics; nonequilibrium molecular dynamics; shear dilation effect.

A generalization of the Einstein relation for thermodynamic fluctuations is applied to a fluid undergoing Couette flow. It is shown that if the strain rate fluctuations are to reduce to the known equilibrium results, then the shear dilation effect, that is the variation of the pressure with shear at constant temperature and density, must be a nonanalytic function of strain rate. This conclusion is consistent with computer results obtained previously.

20650. Estin, A. J.; Stubenrauch, C. F.; Repjar, A. G.; Newell, A. C Optimized wavelength-sized scalar horns as antenna radiation standards, (Proc. Electrical and Electronic Measurement and Test Instrument Conf. and Expo., Ottawa, Canada, Sept. 22.24, 1981), EEMTIC 1981 Digest 81 CH1710-3, pp. 64-68 (The Institute of Electrical and Electronics Engineers, Inc., New York, NY, 1981).

Key words: antennas; beamwidth optimization; corrugated horn; directivity of optimization; scalar horn.

The properties of beamwidth, directivity, and polarization of wavelength-size scalar horns are analyzed and optimized theoretically and confirmed experimentally to determine the usefulness of such horns as standards. Agreement between theoretical predictions and measurements was good.

20651. Ely, J. F.; Hanley, H. J. M. Prediction of the viscosity and thermal conductivity in hydrocarbon mixtures-Computer program TRAPP, Proc. 60th Gas Processors Association Annu. Conv., San Antonio, TX, Mar. 23-25, 1981, pp. 20-29 (Gas Processors Association, Tulsa, OK, 1981).
Key words: computer program; density; extended corresponding states; fluid mixtures; thermal conductivity; viscosity.

A model for the prediction of the density, viscosity and thermal conductivity of non-polar fluid mixtures over the entire range of pVT states is presented. The model is based on the extended corresponding states model and covers molecular weight ranges including $\mathrm{C}_{20}$. Only pure component equilibrium data such as the critical constants are required as input to the calculation procedure-no transport data are required. Extensive comparisons with experimental data for pure fluids and binary mixtures are presented. The average percentage deviation for both the viscosity and thermal conductivity was observed to be less than $8 \%$. A computer program (TRAPP) which performs the calculations described in this manuscript is available from GPA.

20652. Eby, R. K.; Peterlin, A.; Khoury, F. A.; Broadhurst, M. G.; Fanconi, B. M. Crystalline polymers, Chapter 2 in Polymer Science and Engineering: Challenges, Needs, and Opportunities, pp. $49-57$ (National Research Council, Washington, DC, Nov. 1981).

Key words: crystalline; diffusion; electrical; mechanical; morphology; thermal.

The state of crystalline polymer science is reviewed briefly. Important unresolved problems are discussed, as are future research needs and trends. The future role of newly developed instrumentation is discussed. A list of important problems is presented in summary.

20653. Drake, M. C.; Rosasco, G. J.; Schneggenburger, R.; Nolen, R. L., Jr. Nondestructive analysis of laser fusion microsphere targets using rotational Raman spectroscopy, J. Appl. Phys. 50, No. 12, 7894. 7897 (Dec. 1979).

Key words: chemical analysis; hydrogen isotope ratios; laser fusion targets; nondestructive microanalysis; Raman spectroscopy; rotational Raman scattering.

The use of rotational Raman scattering for nondestructive analysis of laser fusion microsphere targets is reported. Typical hollow, glass microsphere sample characteristics are $100 \mu \mathrm{m}$ diameter, $1.0 \mu \mathrm{m}$ glass wall thickness, and $0.5 \mathrm{nl}$ volume containing a mixture of hydrogen, deuterium, and tritium gases at pressures of 10-70 atms (total gas sample weights of $5 \mathrm{ng}$ ). Relative species mole fraction measurements for all hydrogen isotope species are determined from spectral peak height analysis from rotational Raman spectra. Accuracies of \pm 0.01 (mole fraction) are obtained from spectra requiring 20 minutes scan time. Individual species detectability limits are less than $100 \mathrm{pg}$. Absolute partial pressure measurements (accurate to \pm 20 percent) are obtained using calibration spheres of known diameter and pressure.

20654. Christensen, R. G.; Hertz, H. S.; Reeder, D. J.; White, E. Developments in the analysis of priority pollutants by liquid chromatography/mass spectrometry and immunoassay procedures, (Proc. Advances in the Identification and Analysis of Organic Pollutants in Water, Las Vegas, NV, Aug. 24-29, 1980), Chapter 28 in Advances in the Identification and Analysis of Organic Pollutants in Water, L. H. Keith, ed., pp. 447.453 (Ann Arbor Sci. Pub. Inc., Ann Arbor, MI, 1980).

Key words: dinitrophenols; fluoroimmunoassay; liquid chromatograph-mass spectrometer; shale oil; trace organic analysis.

Due to the increasing demands for measurements of organic priority pollutants, several approaches to the rapid analysis of these compounds are being examined at the National Bureau of Standards. A liquid chromatograph-mass spectrometry (LC-MS) system which allows the use of conventional LC flow rates, a wide range of solvents, and a conventional differentially pumped quadrupole mass spectrometer has been developed. The effluent from a liquid chromatograph is concentrated by allowing it to flow down an electrically heated wire. The bottom of the concentrator wire crosses a gap in a capillary tube through which the concentrated effluent is 
drawn into the ion source of the mass spectrometer. Solution flow rates into the ion source can be adjusted, allowing spectra of either purely $\mathrm{CI}$ or mixed $\mathrm{EI} / \mathrm{CI}$ character to be obtained. In preliminary experiments with this system direct quantitative analysis of several phenolic compounds in complex alternate fuels has been possible.

In trying to develop a rapid screening procedure for priority pollutants in water, immunoassay techniques are being explored. A fluoroimmunoassay system for dinitrophenols in water is being developed. Antibodies have been covalently bound to slid phase polymeric beads. Dinitrophenols, added to an incubation mixture containing these beads, and fluorescent tracer compete for antibody sites. Binding curves are generated from data obtained by photon counting.

20655. Chiang, C. K. An all-polymeric solid state battery, Polymer 22, 1454-1456 (Nov. 1981).

Key words: battery; polyacetylene; polyacetylene-oxide; polymer; sodium; solid electrolyte.

An all-polymeric solid state battery was constructed using doped polyacetylene as the electrodes and polyethylene-oxide alkali metal complexes as the electrolyte. The experimental results indicated that this cell can produce a power density of at least $10-20 \mathrm{watt} / \mathrm{kg}$.

20656. Casella, R. C. Theory of neutron scattering from hydrogen in metals involving transitions to excited tunnel-split states, Phys. Rev. $B$ 24, No. 6, 2913-2920 (Sept. 15, 1981).

Key words: excited states; hydrogen impurities; metals; neutron scattering; niobium, theory; tunnel splittings.

A theory is developed for neutron-induced transitions to excited states of a proton trapped at a two-well potential near an interstitial impurity in a metal. The proton wave function is written as a linear combination of the states in each well (tight-binding approximation). Hence, in the main, the theory is limited to the low-lying excitations which lie below the central barrier. Otherwise, the theory is quite general and independent of the details of the potential. General expressions are derived for the differential cross section for transitions from the ground-state doublet to the excited states in terms of $\widetilde{F}(\vec{q})$, the transition form factor. A corresponding quantity, $F(\bar{q})$, appears in expressions for $d \sigma / d \Omega$ associated with transitions within the groundstate doublet. As in earlier work, concerned only with the ground. state doublet, the effects of asymmetric displacements of the well bottoms due to strains are considered. As is the case for the ground. state doublet, the effect of the asymmetry in the two-well potential is to introduce a general mixing angle for the excited-state doublet describing the admixture of the left-hand and right-hand single-well states. Whereas the effect of nonequal mixing on the inelastic differential cross section for transitions within the ground-state doublet is to diminish it by a factor $\left\langle\sin ^{2} 2 \theta\right\rangle,(\theta$ is the mixing angle), under suitable experimental conditions, I show that the corresponding cross section for neutron-induced transitions from the ground-state doublet to either component of the excited-state doublet is independent of both the latter cross section is independent of $\epsilon$, a measure of the asymmetry, provided $\epsilon / h \omega<<1$. (h $\omega=$ excitation energy.) Thus, the ratio $\Lambda$ of the higher energy to the lower energy inelastic cross section is, on the one hand, enhanced by a factor $1 /\left(\sin ^{2} 2 \theta\right)$ and, on the other, diminished by the quantity $|F(\mathrm{~d})|^{2}$. General expressions for $\Lambda$ and for the ratios of various other cross sections are derived in terms of $F(\vec{q})$ and $F(\vec{q})$. These are then estimated for the first excited state by making various simplifying approximations. $\Lambda$ is finite in the limit $\bar{q} \rightarrow 0, \bar{q}^{\prime} \rightarrow 0$, keeping their ratio fixed. If I assume that errors in making the small-q expansion tend to cancel in taking the ratio, then, utilizing the idealized oscillator model of Sussmann, I find the approximate relation, $\Lambda=\left(\rho^{2} / 8\right)\left(k_{F} / k_{1}\right)\left(h \omega / V_{0}\right)\left\langle\sin ^{2} 2 \theta\right\rangle$. Here $\rho$ is the actual experimental ratio of the high to low momentum transfers, $\left(\mathrm{k}_{F} / \mathrm{k}_{1}\right)$ is the ratio of the final to initial neutron momenta, and $V_{0}$ is the barrier height. Results are applied to existent and ongoing experiments on hydrogen and oxygen doped niobium.

20657. Kautz, R. L. Chaotic states of rf-biased Josephson junctions, $J$. Appl. Phys. 52, No. 10, 6241.6246 (Oct. 1981).
Key words: bifurcation cascade; chaos; Josephson effect; noise; period doubling; phase lock; strange attractor; superconductivity.

The existence of chaotic solutions to the rf driven StewartMcCumber model of Josephson junction has been demonstrated by Huberman et al. The present paper describes more fully the range of junction parameters and frequencies for which chaotic solutions occur, details the connection between chaotic states and instabilities in phase lock, and shows that chaotic noise can be much larger than the thermal noise of a junction.

20658. Ekin, J. W. Mechanical properties and strain effects in superconductors, (Proc. 1980 NATO Advanced Study Institute, Sintra, Portugal, Aug. 1980), Chapter 7 in Superconductor Materials Science, pp. 455-510 (Plenum Publ. Corp., New York, NY, 1981).

Key words: bending; bending strain; fatigue; mechanical properties; strain; stress; superconductors; training; uniaxial strain.

An introduction is given to the field of mechanical studies of practical superconductors. The literature is surveyed and the most important advances described. A treatment of current research efforts is presented along with an outline of future study areas.

20659. Ekin, J. W.; Sekine, H.; Tachikawa, T. Effect of strain on the critical current of Nb-Hf/Cu-Sn-Ga multifilamentary superconductors, J. Appl. Phys. 52, No. 10, 6252-6256 (Oct. 1981).

Key words: critical current; $\mathrm{Nb}-\mathrm{Hf} / \mathrm{Cu}-\mathrm{Sn}-\mathrm{Ga} ; \mathrm{Nb}_{3} \mathrm{Sn}$; strain; strain effect; superconductors.

The critical current of multifilamentary Nb-Hf/Cu-Sn-Ga has been determined as a function of both field and strain at fields from 4 to 19 $T$. The strain dependence of the critical current is significantly decreased compared with that of commercial multifilamentary $\mathrm{Nb}_{3} \mathrm{Sn}$ superconductors. This reduced strain sensitivity, coupled with an enhanced critical-current density above $12 \mathrm{~T}$, make this a candidate material for economic high-field magnet construction. An analysis of the data in terms of the strain scaling law indicates that the $\mathrm{Hf}$ and $\mathrm{Ga}$ additives do not produce this improvement through a decrease in the strain sensitivity of the bulk upper critical field $B_{c 2}^{*}$. Rather, they reduce the strain sensitivity of the critical current through a changed shape of the flux pinning curve and an enhancement in the strain-free maximum value of $B_{\mathrm{c} 2}^{\circ}\left(\mathrm{B}_{\mathrm{c} 2 \mathrm{~m}}^{*}=25 \mathrm{~T}\right.$ for $\mathrm{Nb}-\mathrm{Hf} / \mathrm{Cu}-\mathrm{Sn}-\mathrm{Ga}$ vs. $21 \mathrm{~T}$ for $\left.\mathrm{Nb}_{3} \mathrm{Sn}\right)$

20660. Lindgren, R. A.; Plum, M. A.; Gerace, W. J.; Hicks, R. S.; Parker, B.; Peterson, G. A.; Singhal, R.; Williamson, C. F.; Maruyama, X. K.; Petrovich, F. Isospin splitting of isovector highspin "stretched" particle-hole excitations in non-self-conjugate nuclei, Phys. Rev. Lett. 47, No. 18, 1266-1269 (Nov. 2, 1981).

Key words: electron scattering; isospin; isovector; $\mathrm{J}^{\pi}=8^{-}$; Lane model; magnetic states; stretched states; ${ }^{60} \mathrm{Ni}$.

A simple Lane model is used to parametrize the energy systematics of the isospin splitting of high spin magnetic states in non-selfconjugate nuclei. A strength parameter $V_{1}=106 \pm 10 \mathrm{MeV}$ is found.

20661. Yates, J. T., Jr.; McKee, D. W. Kinetic isotope effect in the heterogeneous reaction of graphite with $\mathrm{H}_{2} \mathrm{O}\left(\mathrm{D}_{2} \mathrm{O}\right), J$. Chem. Phys. 75, No. 6, 2711.2714 (Sept. 15, 1981).

Key words: catalytic; deuterium oxide; gasification; graphite; isotopic-effect.

Thermogravimetric studies have been carried out for the reaction of $\mathrm{H}_{2} \mathrm{O}\left(\mathrm{D}_{2} \mathrm{O}\right)$ with pure graphite surfaces and with graphite surfaces containing $\mathrm{BaCO}_{3}$ catalyst. At temperatures near $1200 \mathrm{~K}$ the kinetic isotope effect has been measured to be about a factor of 2 for both pure graphite and $\mathrm{BaCO}_{3}$-graphite. This value of the ratio of rates for $\mathrm{H}_{2} \mathrm{O}$ compared to $\mathrm{D}_{2} \mathrm{O}$ is consistent with zero point energy differences between the two isotopic reactants, and suggests that the transition state in the rate determining step involves a complex in 
which an $\mathrm{O}-\mathrm{H}$ bond is being broken. The similarity of the magnitude of the isotope effect for both the catalyzed and the uncatalyzed reaction suggests that in both cases a similar $\mathrm{O}-\mathrm{H}$ bond breaking mechanism is operative in the rate determining step.

20662. McCoy, W. H.; Colella, R. P.; Wallace, M. A. Assessing the performance of high-level computer network protocols, Proc. Protocol Testing-Towards Proof? INWG/NPL Workshop, Teddington, Middlesex, United Kingdom, May 27-29, 1981, 2, 25.53 (National Physical Laboratory, Teddington, Middlesex, United Kingdom, Sept. 1981).

Key words: computer networking; high-level protocols; open systems interconnection; protocol performance; protocol validation; standards.

There are two aspects to assessing the performance of high-level network protocols: 1) establishing correct performance and 2) determining and evaluating measurable parameters of performance. The first of these is strongly related to software verification, while the latter is more related to system performance evaluation. In this paper, both aspects are discussed with regard to techniques that are being employed by the Institute for Computer Sciences and Technology (ICST) of the National Bureau of Standards (NBS). The ICST is developing a laboratory for experimentally assessing performance of network protocols. The principal objective of this laboratory is to test protocols that have been proposed as draft standards for their viability as Federal Information Processing Standards (FIPS). Another goal is to determine certification methods and procedures for verifying compliance of a vendor's protocol to established FIPS. The protocols which are currently under development as prototype standards conform to the Open Systems Interconnection architecture of the International Standards Organization(ISO).

20663. Reeve, G. R. Synthesized isotropic pattern antennas for EM field measurements, Proc. IEEE Int. Symp. Electromagnetic Compatibility, Boulder, CO, Aug. 18-20, 1981, pp. 36.42 (The Institute of Electrical and Electronics Engineers, Inc., 445 Hoes Lane, Piscataway, NJ 08854, 1981).

Key words: active antenna; electrically short antenna; receiving monopole; tuned antenna.

This paper describes a set of EM field measurement antennas covering the range of $10 \mathrm{kHz}$ to $1 \mathrm{GHz}$ which, under microprocessor control, synthesize an isotropic response. Another antenna, covering the frequency range of $1 \mathrm{GHz}$ to $18 \mathrm{GHz}$, synthesizes a wide vertical angle, omnidirectional, response for signals of any linear, and one circular, polarization. These antennas together with an appropriate instrumentation system allow rapid acquisition of ambient field strength data.

20664. Forster, E. O.; FitzPatrick, G. J.; Hebner, R. E., Jr.; Kelley, E. F. Observations of prebreakdown and breakdown phenomena in liquid hydrocarbons II. Point-plane geometry, (Proc. Conf. Electrical Insulation and Dielectric Phenomena, Whitehaven, PA, Oct. 26-28, 1981), IEEE Ann. Report No. 81CH1668-3, pp. $377-389$ (The Institute of Electrical and Electronics Engineers, Inc., 445 Hoes Lane, Piscataway, NJ 08854, 1981).

Key words: breakdown; dielectrics; high voltage; insulation; liquids; shock waves.

Prebreakdown and breakdown phenomena have been measured optically in $n$-hexane, cyclohexane, isooctane and toluene using a point-plane electrode geometry. The voltage and electrode spacing were adjusted so that breakdown occurred either on the initial high voltage pulse or, in the case of a cathode point, on the positive overshoot following the initial negative pulse. The field strength for breakdown after voltage reversal is about one-third the strength determined using sphere-sphere electrodes and a unipolar pulse. The use of highly purified liquids and the point-plane electrode geometry serves to separate this study from previous ones.

20665. Fickett, F. R.; McHenry, H. I. Structural materials for large superconducting magnets, (Proc. 7th Int. Conf. Magnet Technology, Karlsruhe, West Germany, Mar. 30-Apr. 5, 1981), IEEE Trans. Mag. MAG-17, No. 5 2297.2304 (Sept. 1981).

Key words: alloys; aluminum; conductor; fiberglass; nonmetallics; review; stainless steel.

Insuring structural integrity of large superconducting magnet systems requires detailed knowledge of the low temperature behavior of many materials. This review describes recent research on alloys, nonmetallics and conductor composites related to their structural roles in these magnets. Special attention is paid to joining methods and their effect on the structure. It is encouraging to note that a reasonable amount of data is now available on a number of commercial alloys. It is somewhat disconcerting to see a rapid increase in design stress levels over the past several years, during which time very few large magnets have been put into operation.

20666. Bertotti, B. Statistics of narrow structures of the gravity field of the Earth. 1. General theory, J. Geophys. Res. 86, No. B11, 10835. 10842 (Nov. 10, 1981).

Key words: correlation functions; Earth; gravity field; narrow structures; statistical analysis.

With the increase in resolution and accuracy of gravity measurements it is becoming possible and increasingly important to concentrate attention on local analysis and on the role of elongated geophysical features. This paper deals with the statistical analysis of such features and develops for this purpose a new mathematical tool, the three- and four-point correlation function. Using an integration over the manifold of the rotation group, expressions are derived for these functions in terms of the spherical harmonic coefficients; I discuss also the corresponding quantities for a flat Earth and show how this approximation is recovered for small features. To gain an understanding of the structure of those functions, a simple, heuristic model is constructed in which the surface gravity field is expressed as a random superposition of elliptical elementary disturbances with random orientations. The three-point correlation function shows a characteristic behavior when the angle between its two vector arguments is of the order of the ratio of the axes of the ellipse. Other important information contained in this function is the skewness of the gravity field and a possible privileged screw sense.

20667. Blau, P. J. An investigation of the unlubricated friction and wear break-in behavior of a dual-phase steel, Wear 72, $67-80$ (1981).

Key words: break-in; dual-phase steel; friction; run-in; unlubricated wear; wear.

The friction and wear break-in behavior of a dual-phase steel (steel DP 80) was investigated using a stroke-by-stroke fixed ball-on-flat tribometer. The standard rider balls were steel 52100 ball bearings slid at a load of $4.9 \mathrm{~N}$ and a velocity of $0.5 \mathrm{~cm} \mathrm{~s}^{-1}$. The atmospheres used were air at a relative humidity of $(58 \pm 3) \%$ and flowing argon gas at a relative humidity of $30 \%-35 \%$. Surface finishes obtained by $1 \mu \mathrm{m}$ diamond lapping and by dry abrasion with 600 grit SiC cloth were tested. Frictional break-ins were shown to be reproducible on the $\mathrm{SiC}$ cloth samples and had a double peak in plots of the friction as a function of the number of strokes. Friction changes were less dramatic for diamond-polished flats. Argon environments reduced the friction and the wear track width. Freshly polished and cleaned surfaces broke in differently to those which had been stored in a desiccator for several weeks and then solvent cleaned before testing. The shapes of the friction break-in curves were altered in similar ways by changes in the surface finish and the test environment; this made if difficult to distinguish any unique effects of either of these variables.

20668. Blau, P. J. Mechanisms for transitional friction and wear behavior of sliding metals, Wear 72, 55-66 (1981).

Key words: friction; friction transition; microstructure; run-in; wear; wear transition.

Four processes which may cause transitions in the unlubricated 
sliding behavior of metals are described. These processes are (1) metal transfer, (2) film formation and removal, (3) debris generation and (4) cyclic surface deterioration. Although these are not the only processes which may cause unexpected sudden friction and wear transitions, they may contribute significantly to many frequently observed sliding phenomena. It is possible that some combination of these mechanisms may act to effect changes in the wear and friction behavior of a given tribological system.

The cyclic deterioration model involves four sequential stages in each cycle: (1) plastic deformation to obtain a highly deformed layer (HDL); (2) debris generation by crack initiation and growth in the HDL to produce flake-like debris; (3) debris removal through the shearing-off of load-bearing plateaus which slide along on their fracture surfaces to produce deep grooves; (4) plateau removal followed by a smoothing of the deep wear grooves (a process similar to the break-in of a rough surface with changes in the friction and the rate of wear). This process, when stages are occurring simultaneously on a large portion of the wear surface, could be observed as a cyclic variation in the friction and/or the wear rate.

20669. Brauer, G. M.; Dulik, D. M.; Hughes, H. N.; Dermann, K.; Rupp, N. W. Marginal adaptation of BIS-GMA-based composites containing various diluents, $J$. Dent. Res. 60, No. 12, 1966-1971 (Dec. 1981).

Key words: composite; curing shrinkage; dental restorative; diluent monomer; gap formation; marginal adaptation; marginal leạkage.

The objective of this study was to determine the gap formation and marginal leakage of dental restorative resins containing various diluents and diluent concentrations. Marginal adaptation of composites thermocycled for one week between $5^{\circ}$ and $55^{\circ} \mathrm{C}$ is dependent on the type and concentration of polymerizable monomer diluent present in the resin formulation. Curing shrinkage of the resin is a major contributor to breaking the marginal seal. Addition of dimethacrylate diluents containing $\left(\mathrm{CH}_{2}\right)_{n}$ recurring units generally yield composites with better marginal adaptation than those containing $\left(\mathrm{CH}_{2} \mathrm{CH}_{2} \mathrm{O}\right)_{7}$ groups. Marginal leakage is reduced on lowering the diluent concentration. Optimum adaptation will be obtained for compositions containing a minimum percentage of diluent with clinically acceptable working properties. Volume changes on temperature cycling resulting from differences in thermal expansion coefficients of composites do not affect the marginal integrity as much as does curing shrinkage.

20670. Caswell, R. S.; Coyne, J. J.; Goodman, L. J. Comparison of experimental and theoretical ionization yield spectra for neutrons, (Proc. Fourth Symp. Neutron Dosimetry, Munich-Neuherberg, Germany, June 1-5, 1981), Paper in Radiation Protection, G. Burger and H. G. Ebert, eds., 1, 201-212 (Commission of the European Communities, Munich, Germany, 1981)

Key words: energy deposition spectra; experimental spectra; ionization yield spectra; neutron dosimetry; quality of neutron dosimetry; TE proportional counter.

Measurements with tissue-equivalent proportional counters are used in neutron dosimetry to indicate the quality of the radiation. What is effectively measured is the ionization yield spectrum. However, the energy deposition spectrum is usually desired. These two spectra will differ if the ionization yield $(\mathrm{E} / \mathrm{W})$ varies as a function of secondary ion type and energy.

In the past we have calculated energy deposition spectra, and compared them to experimental ionization yield spectra. Our analytic energy deposition code has been modified to produce ionization yield spectra as well as energy deposition spectra.

These newly-calculated ionization yield spectra are compared with both walled and wall-less tissue-equivalent proportional counter spectra. Straggling effects are not included in this calculation, so representation of proton peaks for high neutron energies should not accurately reproduce the experimental spectra. The heavy-ion portion of the experimental spectra should be well reproduced.

Calculated ionization yield spectra are also compared with calculated energy deposition spectra. This permits evaluation of the differences which are useful in interpretation of the experimental proportional counter spectra. In general the effect is to increase the $\bar{y}_{D}$ as taken directly from the experimental spectra.

20671. Chen, H.; Kuriyama, M. A high-resolution X-ray facility, $J$. Appl. Cryst. Lab. Note 14, 280 (1981).

Key words: asymmetric crystals; high angular resolution; natural line width of $\mathrm{CU} \mathrm{Ka}_{1,2}$; small angle scattering; total reflection.

An $x$-ray diffraction system capable of producing and detecting $x$ rays with an angular resolution better than two seconds of arc has been developed.

20672. Chow, L. C.; Guo, M. K.; Hsieh, C. C.; Hong, Y. C. Apatitic fluoride increase in enamel from a topical treatment involving intermediate $\mathrm{CaHPO}_{4} \cdot 2 \mathrm{H}_{2} \mathrm{O}$ formation, an in vivo study, Caries Res. 15, 369-376 (1981).

Key words: acid pretreatment; acidulated phosphate fluoride; dental caries; dicalcium phosphate dihydrate; fluorapatite; topical fluoridation.

Previous laboratory and animal studies have reported high levels of apatitic fluoride uptake from a topical fluoride procedure in which teeth were pretreated with an acidic solution saturated with respect to $\mathrm{CaHPO}_{4} \cdot 2 \mathrm{H}_{2} \mathrm{O}$ (DCPD) prior to the fluoride treatment. A study was initiated to determine the fluoride uptake from this procedure under clinical conditions. A microbiopsy technique was used to measure enamel fluoride contents before, 1 month after, and 3 months after an application of an acidulated phosphate fluoride (APF) solution alone or in combination with a DCPD pretreatment on the maxillary incisors of 50 fourth-grade children in a nonfluoride area. 1 month after treatment, there was no significant difference in enamel fluoride concentration between APF and control groups. The DCPD/APF treatment produced mean fluoride uptakes of $1,011 \pm 134 \mathrm{ppm}(n=50)$ and $2,042 \pm 221 \mathrm{ppm}(\mathrm{n}=47)$ in the outer $3.5 \mu \mathrm{m}$ of enamel when measured 1 and 3 months, respectively, after treatment. The results indicate that the DCPD pretreatment can substantially increase permanently bound fluoride uptake under clinical conditions.

20673. Clough, R. B.; Simmons, J. A. Reproducible acoustic emission signatures by indentation in steels, Mater. Eval. 39, 1026-1031 (Oct. 1981).

Key words: acoustic emission; fracture; indentation; nondestructive evaluation.

Creating reproducible signals from defects is of great importance for quantitative studies of acoustic emission (AE). A method for doing so is presented here which consists of indentation of hardened steel plates. A survey was made of a variety of steels, including A533B pressure vessel steel with an embrittled weld, as well as embrittled W1, 01 and 02 tool steels. Regions in these steels with a hardness less than about 40 on the Rockwell $C$ scale produced no detectable AE, even with appreciable plastic deformation. Regions in the steels with a Rockwell $C$ hardness greater than about 50 produced detectable AE during indentation. In many cases, the signals were reproducible over an appreciable range and appeared to be similar, for epicenter measurements, to those produced by sudden unloading. However, monitoring on the same surface as the indentation in some cases produced sets of different but reproducible signals which gradually evolved with repeated loading. Examination with scanning electron microscopy indicated that the AE signals were produced by the nucleation and incremental growth of subsurface cracks, generally penny-shaped, less than a millimeter in size. A typical fracture toughness value for such cracks in an embrittled 02 tool steel was calculated to be about $19 \mathrm{MNm}^{-3 / 2}$. AE was also produced in asreceived A533B steel by indentation fatigue. The method suggests itself for materials studies of $\mathrm{AE}$ as well as a nondestructive method for in situ examination of structures for embrittlement.

20674. Cole, B. E.; Saloman, E. B.; Ederer, D. L. Ba II fluorescence from barium atoms resonantly photoionized by $584-\AA$ radiation, $J$. Opt. Soc. Am. 71, No. 12, 1458-1462 (Dec. 1981).

Key words: autoionization; barium vapor; fluorescence; heat 
pipe; photoabsorption; vacuum ultraviolet.

The first reported study of fluorescence spectrum of a metal vapor resonantly excited by vacuum-ultraviolet radiation has been made in $\mathrm{Ba}$ II. Seven $\mathrm{Ba}$ II fluorescent transitions have been identified for $\mathrm{Ba}$ vapor excited by $584 . \AA$ radiation. The intensities of these transitions are consistent with the population distribution of excited $\mathrm{Ba}$ II observed by using photoelectron spectroscopy. No detectable $\mathrm{Ba}$ II fluorescence was observed when 736-742 $\AA$ excitation was used.

20675. Coyne, J.; McDonald, J. C.; Menzel, H. G.; Schuhmacher, H. Detailed intercomparison of calculated and measured ionization yield spectra for $20 \mathrm{MeV}$ neutrons and the implications for high energy neutron dosimetry, (Proc. Fourth Symp. Neutron Dosimetry, Munich-Neuherberg, Germany, June 1-5, 1981), Paper in Radiation Protection, G. Burger and H. G. Ebert, eds., 1, 213.223 (Commission of the European Communities, Munich, Germany, 1981).

Key words: high energy neutrons; ionization yield spectra; neutron dosimetry; tissue-equivalent proportional counters; a-particles; $20 \mathrm{MeV}$ neutrons.

Tissue-equivalent proportional counters have been used to measure ionization yield spectra for approximately $20 \mathrm{MeV}$ neutrons at two different neutron facilities. The results of these measurements are compared with calculated ionization yield spectra which have been obtained by using the analytical method. The calculations have taken into account the variation of ionization yield with ion energy and ion type. The comparisons at $20 \mathrm{MeV}$ indicate that fewer $\alpha$ particles are observed than are predicted by the calculations, and call into question the nuclear data used in these calculations. These facts could have important implications for neutron kerma factors and the measurement of neutron dose at high energies. Re-examination of results at $14.5 \mathrm{MeV}$ indicate that there may already be problems at this energy. The need for better nuclear data in the energy region above $10 \mathrm{MeV}$ is evident.

20676. Ederer, D. L.; Parr, A. C.; Cole, B. E.; Stockbauer, R.; Dehmer, J. L.; West, J. B.; Codling, K. Vibrational-state dependence of partial cross sections and photoelectron angular distributions through autoionizing resonances: The $\mathbf{n}=\mathbf{3}$ Rydberg state converging to the $\mathrm{B}^{2} \Sigma^{+}$state of $\mathrm{CO}^{+}$, Proc. $R$. Soc. Lond. A378, 432-435 (1981).

Key words: angular distribution; autoionization; carbon monoxide; $\mathrm{CO}$; differential photoelectron spectra; FranckCondon factors; photoelectron spectra; photoionization; synchrotron radiation.

The branching ratios for leaving the $\mathrm{CO}^{+}$ion in a particular vibrational level of the ground $\mathrm{X}^{2} \Sigma^{+}$state have been determined as functions of photon energy through the $n=3$ autoionizing Rydberg state converging to the $\mathrm{B}^{2} \Sigma^{+}$state of $\mathrm{CO}^{+}$; there are substantial differences between theoretical and experimental Franck-Condon factors when "on" resonance. The branching ratios have been converted into absolute partial cross sections by normalizing to existing data obtained by using line sources. The asymmetry parameter $\beta$ has also been determined for each vibrational level in this spectral range. Considerable variations in both have been observed in the region of this resonance.

20677. Eisenhauer, C.; Schwartz, R. Difficulties in calculating spectrum-averaged values of neutron dose equivalent, Health Phys. 41, No. 5, 774-777 (Nov. 1981).

Key words: Cf fission spectrum; dose equivalent; heavy water; moderated Cf fission spectrum; neutron spectra; nuclear power reactor.

The problem of inconsistencies in standard tabulations of the flux to dose rate conversion factor for neutrons is examined. It is shown that such inconsistencies can lead to uncertainties of $25 \%$ in the dose equivalent calculated for neutron spectra in maintenance areas of nuclear power reactors.

20678. Friday, D. S. Dependence concepts for stochastic processes,
Paper in Statistical Distributions in Scientific Work, Vol. 5, Taillie, Patil, and Baldessari, eds., pp. 349-361 (D. Reidel Publ. Co., Dordrecht, The Netherlands, 1981).

Key words: dependence concepts; fractional Brownian motion; mixing; power law spectra; self-similarity; stochastic processes.

Dependence concepts are a relatively recent development in multivariate distribution theory. They allow dependence to be incorporated into a problem without requiring specific model assumptions, and define classes of multivariate distributions with useful properties. It is not apparent that related notions have also evolved in the theory and applications of stochastic processes. Multivariate concepts and stochastic process concepts, however, have had no influence on each other. In this paper an overview is presented of the work in stochastic processes that is analogous to multivariate dependence concepts.

20679. Heydemann, P. L. M. Determination and correction of quadrature fringe measurement errors in interferometers, Appl. Opt. 20, 3382-3384 (Oct. 1, 1981).

Key words: correction terms; interferometer; quadrature.

The precision and accuracy of interferometers using quadrature fringe detection is often limited not by the interferometer itself but by the detector system. There are three typical errors: unequal gain in the two channels, quadrature phase shift error, and zero offsets. This paper describes a simple method for determining the quadrature errors from experimental data obtained in the interferometer and correcting for them. A numerical example demonstrating the significant improvement in the precision of interferometer data is given.

20680. Itano, W. M.; Wineland, D. J. Precision measurement of the ground-state hyperfine constant of ${ }^{25} \mathrm{Mg}^{+}$, Phys. Rev. A 24, No. 3, 1364-1373 (Sept. 1981).

Key words: double resonance; frequency standards; $g$ factors; hyperfine structure; optical pumping; Ramsey resonance; ${ }^{25} \mathrm{Mg}^{+}$.

The ground-state hyperfine constant $A$ and the nuclear-to-electronic $g$-factor ratio $g_{/} / g_{J}$, of ${ }^{25} \mathrm{Mg}^{+}$have been measured by a laser opticalpumping double-resonance technique. The ions were stored in a Penning trap at a magnetic field of about $1 \mathrm{~T}$. The results are $A=-596.254376(54) \mathrm{MHz}$ and $g_{I} / g_{J}=9.299484(75) \times 10^{-5}$. The magnetic field at the ions was stabilized by servoing it to an $\left(\Delta m_{I}=0\right.$, $\left.\Delta m_{J}= \pm 1\right)$ electronic Zeeman transition. Other hyperfine $\left(\Delta m_{l}= \pm 1\right.$, $\left.\Delta m_{J}=0\right)$ transitions were detected while the field was thus stabilized. The derivative of the $\left(m_{l}, m_{j}\right)=(-3 / 2,1 / 2)$ to $(-1 / 2,1 / 2)$ transition frequency with respect to magnetic field $B_{0}$ goes to zero at $B_{0} \cong 1.24$ $T$. The corresponding resonance was observed at this field with linewidths as small as $0.012 \mathrm{~Hz}\left(Q=2.4 \times 10^{10}\right)$ by implementing the Ramsey interference method with two coherent rf pulses separated in time by up to $41.4 \mathrm{~s}$.

20681. Jones, F. E. Determination of water in solids by automatic Karl Fischer titration, Anal. Chem. 53, No. 12, 1955-1957 (Oct. 1981).

Key words: absolute determination of water; automatic titrators; Karl Fischer reagent; moisture in solids; uncertainty analysis; water determination.

Techniques have been developed to optimize the performance of automatic Karl Fischer reagent titrators in the determination of water in solids. Water is extracted from the material by methanol in a mixer/mill; the methanol-extracted $\mathrm{H}_{2} \mathrm{O}$ mixture is introduced volumetrically from a calibrated syringe into the titration vessel. The titrators (of the coulometric generation type and the motor-driven buret type) are standardized with distilled $\mathrm{H}_{2} \mathrm{O}$. An analysis has been made of the sources and estimated magnitudes of the uncertainties in the determination of $\mathrm{H}_{2} \mathrm{O}$. For solid material with a moisture content $\left(\% \mathrm{H}_{2} \mathrm{O}\right)$ of $15 \%$, the imprecision (standard deviation of the mean) is estimated to be $0.4 \%$ relative and the systematic uncertainty is estimated to be $\pm 0.4 \%$ relative.

20682. Jones, F. E.; Brickenkamp, C. S. Automatic Karl Fischer 
titration of moisture in grain, J. Assoc. Off. Anal. Chem. 64, No. 6, 1277.1283 (1981).

Key words: grain; Karl Fischer reagent; moisture content; titration; uncertainty of determination; water.

Automatic Karl Fischer titrators of the motor-driven buret type and the coulometric generation type were applied to the determination of moisture in grain. Techniques were developed to optimize the performance of the Karl Fischer titration method and to overcome disadvantages attributed to it. The imprecision (1 standard deviation) of a determination is typically $0.06 \%$ moisture content at a moisture content of $15 \%$; the systematic uncertainty from known sources is estimated to be $\pm 0.05 \%$ moisture content at a moisture content level of $15 \%$. It is suggested that the method be designated as the primary reference method for grain in general and for corn and soybeans in particular.

20683. Lettieri, T. R.; Jenkins, W. D.; Swyt, D. A. Sizing of individual optically levitated evaporating droplets by measurement of resonances in the polarization ratio, Appl. Opt. 20, 2799-2805 (Aug. $15,1981)$

Key words: evaporation; glycerol; light scattering; liquid droplets; Mie theory; optical levitation; particle size; polarization ratio; radiation pressure; resonances.

Resonances observed in the polarization ratio of light scattered at 90 degrees from single optically levitated evaporating droplets are shown to provide a means for continuous, high-resolution monitoring of droplet size. Due to the distinctive character of the individual features in the polarization ratio, each experimentally measured feature could be clearly identified with a specific calculated one. For evaporating droplets of glycerol from 6.6 to 11.5 micrometers in diameter, the sharp features which appeared in the calculations at approximately 0.03 micrometer intervals allowed measurement of droplet diameter to a resolution of 0.003 micrometers.

20684. Lucatorto, T. B.; McIlrath, T. J.; Sugar, J.; Younger, S. M. Radical redistribution of the $4 d$ oscillator strength observed in the photoabsorption of the $\mathrm{Ba}, \mathrm{Ba}^{+}$, and $\mathrm{Ba}^{++}$sequence, Phys. Rev.

Lett. 47, No. 16, 1124-1128 (Oct. 19, 1981).

Key words: laser-driven ionization technique; oscillator strength distribution; photoabsorption of ions; photoionization; sequential ionization; $4 d$-photoabsorption; $4 f$-wavefunction collapse.

The vacuum-ultraviolet absorption spectra of isolated $\mathrm{Ba}, \mathrm{Ba}^{+}$, and $\mathrm{Ba}^{++}$have been measured. In $\mathrm{Ba}$ and $\mathrm{Ba}^{+}$most of the $4 d$ absorption oscillator strength is in the continuum, but in $\mathrm{Ba}^{++}$several very strong discrete transitions are observed. Such behavior has never before been observed in the $4 d$ photoabsorption of vapors, solids, or compounds. An interpretation is given in terms of the partial collapse of the $n f$ bound states in $\mathrm{Ba}^{++}$.

20685. Magnotta, F.; Nesbitt, D. J.; Leone, S. R. Excimer laser photolysis studies of translational-to-vibrational energy transfer, Chem. Phys. Lett. 83, No. 1, 21.25 (Oct. 1981).

Key words: energy transfer; excimer laser; $\mathrm{HBr} ; \mathrm{HCl}$; photolysis; translation; vibration.

A new technique is presented to study translation-to-vibrational (T-V) energy transfer and translationally enhanced reactive events. The method utilizes excimer laser photolysis to produce translationally "hot" atoms and time-resolved infrared detection of specific excited states.

20686. Melmed, A. J.; Ceyer, S. T.; Tung, R. T.; Graham, W. R. A LEED inquiry into the question of reconstruction of $\{001\} \mathrm{Nb}$, Surf. Sci. Lett. 111, L701-L704 (1981).

Key words: low-energy-electron diffraction; niobium; reconstruction of surfaces; surfaces; surface structure.

We have examined the structure of the $\{001\}$ surface of $\mathrm{Nb}$ by
LEED and have found that a $(1 \times 1)$ diffraction pattern prevails over the entire range of temperature investigated, that is $\sim 15-500 \mathrm{~K}$. This is the first observation of an unreconstructed $\{001\}$ surface of a BCC metal over such a temperature range.

20687. Mountain, R. D. Time correlation functions and molecular motion in glasses, Ann. NY Acad. Sci. 371, 252-260 (1981).

Key words: dielectric relaxation; glass; infrared absorption; light scattering; supercooled liquid; time correlation function; velocity autocorrelation function.

The value of time correlation functions as a way to represent molecular motions in liquids and glasses will be illustrated by examining examples involving computer simulations, infrared absorption and dielectric relaxation and light scattering. A computer simulation is used to produce self-diffusion coefficient and the velocity autocorrelation function of a supercooled liquid state and a glassy state of a model of rubidium. With the accuracy of the simulation, in the self-diffusion coefficient of the glass is zero, indicating the need for special initial conditions to study the dynamics of diffusion in glass by molecular dynamics simulations. Various aspects of orientational motions are probed by infrared absorption, dielectric relaxation and depolarized light scattering. The connections of these three probes are examined with emphasis on depolarized light scattering. Experimental work on boron trioxide glass and supercooled benzel-benzoate are discussed within the framework of "local order" theory. This set of measurements illustrates the role of structural relaxation in governing the reorientation of molecules in supercooled liquids.

20688. Nesbitt, D. J.; Leone, S. R. Laser initiated chain reactions: A generalized extension to complex chemical chain systems, J. Chem. Phys. 75, No. 10, $4949-4959$ (Nov. 15, 1981).

Key words: chain reaction; $\mathrm{CH}_{3} \mathrm{SH} ; \mathrm{Cl}_{2}$; combustion; $\mathrm{H}_{2} ; \mathrm{HCl}$; laser.

We present a generalized extension of the detailed kinetic and experimental analysis for studying rapid gas phase chemical chain reactions initiated by short pulse laser photolysis. The key ingredients of the technique are pulsed laser initiation of the chain by dissociation of a small fraction of a precursor molecule to produce radicals $\left(\mathrm{Cl}_{2} \rightarrow\right.$ $\mathrm{Cl}+\mathrm{Cl}$ ), followed by time resolved, infrared chemiluminescence detection of product $\mathrm{HCl}(v)$ emission from the propagation steps $\left(\mathrm{Cl}+\mathrm{RH} \rightarrow \mathrm{R}+\mathrm{HCl}(v), \mathrm{R}+\mathrm{Cl}_{2} \rightarrow \mathrm{Cl}+\mathrm{RCl}\right)$. This laser initiated chain reaction technique is now extended to encompass a far wider range of chain kinetic rates, mechanisms, and product state distributions. Three prototypical systems are investigated which demonstrate distinctly different facets of the extended technique: (1) $\mathrm{Cl}_{2} / \mathrm{HBr}-\mathrm{a}$ slow chain reaction system, (2) $\mathrm{Cl}_{2} / \mathrm{CH}_{3} \mathrm{SH}$-a multiple chain reaction system, and (3) $\mathrm{Cl}_{2} / \mathrm{H}_{2}$-a slow starting chain reaction system in which the product emission appears after an initial induction period. Quantitative rate data are obtained for several chain propagation steps: $\mathrm{Br}+\mathrm{Cl}_{2} \rightarrow \mathrm{Cl}+\mathrm{BrCl}, k=2.4( \pm 0.2) \times 10^{-15} \mathrm{~cm}^{3}$ molecule $\mathrm{s}^{-1}$; $\mathrm{Cl}+\mathrm{CH}_{3} \mathrm{SH} \rightarrow \mathrm{CH}_{2} \mathrm{SH}+\mathrm{HCl}(v), k=4.3( \pm 1) \times 10^{-12} \mathrm{~cm}^{3}$ molecule ${ }^{-1} \mathrm{~s}^{-1}$; $\mathrm{CH}_{2} \mathrm{SH}+\mathrm{Cl}_{2} \rightarrow \mathrm{Cl}+\mathrm{ClCH}_{2} \mathrm{SH}, k=2.6( \pm \mathrm{l}) \times 10^{-13} \mathrm{~cm}^{3}$ molecule $\mathrm{s}^{-1} \mathrm{~s}^{-1}$. The results illuminate a more general approach to precise kinetic and mechanistic studies of complex chain reactions under nearly ideal pseudo-first-order laboratory conditions.

20689. Netzer, F. P.; Madey, T. E. Coadsorption-induced azimuthal ordering in molecular adsorbate layers: $\mathrm{H}_{2} \mathrm{O}$ and $\mathrm{NH}_{3}$ on oxygenprecovered Ni(111), Phys. Rev. Lett. 47, No. 13, $928-931$ (Sept. 28, 1981).

Key words: ammonia; chemisorption; coadsorption; electron stimulated desorption; nickel(111); structure; surface; water.

The structure and kinetics of $\mathrm{NH}_{3}$ and $\mathrm{H}_{2} \mathrm{O}$ interacting with oxygen predosed on a $\mathrm{Ni}(111)$ surface have been studied using ESDIAD (electron stimulated desorption ion angular distributions), LEED (low energy electron diffraction) and thermal desorption methods. The adsorption of either $\mathrm{NH}_{3}$ or $\mathrm{H}_{2} \mathrm{O}$ onto clean $\mathrm{Ni}(111)$ produces adsorption geometries in which the orientation of the $\mathrm{H}$ ligands in a fractional monolayer is random. When either $\mathrm{NH}_{3}$ or $\mathrm{H}_{2} \mathrm{O}$ is adsorbed on $\mathrm{Ni}(111)$ predosed with traces of oxygen, a three-fold symmetric 
ESDIAD pattern results, indicating a high degree of azimuthal ordering in the adsorbed $\mathrm{NH}_{3}$ and $\mathrm{H}_{2} \mathrm{O}$ in the absence of long-range order. The azimuths of the $\mathrm{H}$ ligands are in $[\overline{112}]$ directions.

20690. Parken, W. H.; Kelly, G. E. Estimating residential seasonal cooling requirements, (Proc. ASHRAE 1981 Semi-Annu. Meet., Chicago, IL, Jan. 25-29, 1981), ASHRAE Trans. 87, Pt. 1, 473-490 (1981).

Key words: airconditioning cooling load; cooling load; residential cooling; seasonal cooling; temperature bin.

A method is presented (referred to as the modified building load and temperature bin method, MBLTBM) for calculating residential building cooling loads and seasonal cooling requirements for various geographical regions. The method incorporates the effects of solar radiation, internal heat gains, outdoor temperature, infiltration, and thermal response on the cooling load for different periods of the day.

The MBLTBM can be used with improved precision over existing "simple" calculation procedures without the use of large, sophisticated and time consuming computer programs. The increased precision in determining seasonal cooling requirements can result in improved estimates of the cost of operating airconditioning equipment.

The method requires little additional data to what are presently required for calculating design cooling requirements. For the region of interest, temperature bin data (the number of hours during the cooling season the temperature, in $5^{\circ} \mathrm{F}\left(2.8^{\circ} \mathrm{C}\right)$ increments, occurs), the summer daily average temperature, and the average daily extreme temperatures are required and readily obtainable. Also needed for the calculation procedure are the solar heat gain factors for windows and incident solar radiation on vertical and horizontal opaque surfaces.

The modified building load and temperature bin method was compared to the more comprehensive method obtained by the hourby-hour cooling loads as determined by the BLAST computer program, and by results obtained by hand calculation presently in common use. The comparisons were made for a variety of residential structures, localities and orientations.

20691. Penn, D. R.; Rendell, R. W. Surface-enhanced photoabsorption and photoyield in small spheres, Phys. Rev. Lett. 47, No. 15, 1067. 1070 (Oct. 12, 1981).

Key words: photoabsorption; photoyield; small sphere; surface.

Calculations of the photoabsorption and photoyield of small metal spheres are reported for photon energies below the plasmon energy. It is found that the excitation of electron-hole pairs due to the presence of the surface results in (1) enhancements in the photoabsorption rates and photoyields that are typically $10-10^{2}$ and (2) similar enhancements in the threshold photoyields of small spheres relative to plane surfaces in agreement with recent experimental results.

20692. Peterson, R. L.; Van Vechten, D. Modeling the impedance of a Josephson-junction noise thermometer, Phys. Rev. B 24, No. 6, 3588 3591 (Sept. 15, 1981).

Key words: noise thermometer; rf SQUIDS; superconductivity.

Recent experiments on noise thermometers made with Josephson junctions [rf SQUID's (superconducting quantum interference devices) shunted by a small resistance $R \mid$ have shown that the SQUID impedance has an envelope which generally rises to the value of $R$ as the If power is increased, although the envelope can be distorted when the frequency is just above the rf circuit resonance. We summarize the results of a mathematical analysis which shows that in the absence of noise, and with no reactive feedback of the SQUID upon the rf circuit, the SQUID impedance will have a uniformly rising envelope because of the SQUID inductance. The calculated curves have the characteristics of the experimental observations in the rising-envelope cases.

20693. Pierce, D. T.; Celotta, R. J. Spin polarization in electron scattering from surfaces, Adv. Electron. Electron Phys. 56, 219-289 (1981).
Key words: electron polarization; electron scattering; PLEED; polarized LEED; surface magnetism; surface structure.

A summary is presented of modern experimental and theoretical treatments of electron spin polarization phenomena in low energy electron scattering from solids. Relativistic and exchange interactions are treated separately. The use of polarization measurements for surface structure determinations and the investigation of surface magnetism is emphasized. A total of 105 references are given.

20694. Pierce, D. T.; Girvin, S. M.; Unguris, J.; Celotta, R. J. Absorbed current electron spin polarization detector, Rev. Sci. Instrum. 52, No. 10, 1437.1444 (Oct. 1981).

Key words: polarized electrons; spin dependent absorption; spin detector.

The principle of spin analysis by means of measurement of the enhanced spin dependent absorption of a polarized electron beam at an energy near which the secondary yield is unity is presented. Both the collected charge method and the zero-crossing method are described for situations where the polarization can and cannot be reversed. A statistical analysis of the uncertainty in the polarization determination by each method is given. Annealed, evaporated Au films are shown to be suitable for the detecting surface of this spin detector. The figure of merit is derived and found for $\mathrm{Au}$ films to be comparable to the very best Mott detectors. The applications for which this simple compact spin detector are especially suited are discussed.

- 20695. Prialnik, D.; Shaviv, G.; Kovetz, A. The effect of diffusion on asymptotic branch evolution, Astrophys. J. 247, 225-235 (July 1, 1981).

Key words: asymptotic branch evolution; diffusion; planetary nebulae; shell flashes.

Evolution along the asymptotic branch leads to a configuration characterized by two burning shells that are close to each other and very thin. Under these circumstances, which involve steep composition gradients, encounters between charged particles of the stellar plasma lead to diffusion. We have studied the effect of diffusion on the asymptotic branch evolution of a $6 \mathrm{M}_{0}$ star model. Our most important conclusions are: (1) The timescale for diffusion is comparable to the nuclear timescale in each of the burning shells ( $\sim 10^{4} \mathrm{yr}$ for the $\mathrm{H}$ shell and $10^{6} \mathrm{yr}$ for the He shell). (2) A steady state develops with nuclear burning and diffusion in balance. (3) The formation of a steady state prevents He shell flashes. (4) In this steady state the stellar core ceases to grow. The nuclear fuel is supplied by diffusion which also carries away the burning products. (5) The steady state prevails until the whole envelope is lost through a stellar wind. Some consequences of the behavior of $\mathrm{L}_{\mathrm{BOL}}$ during and after the flash, the criteria for predicting shell flashes, as well as some conclusions in connection with observations of planetary nebulae and white dwarfs are discussed.

20696. Regev, O.; Shaviv, G. Formation of protostars in collapsing, rotating, turbulent clouds, Astrophys. J. 245, No. 3, $934-959$ (May 1, 1981).

Key words: star formation; turbulence in collapse.

Collapse and star formation processes in rotating turbulent interstellar gas clouds have been studied. For this purpose numerical collapse calculations have been performed for a number of representative cases. These calculations have been carried out by a two-dimensional hydrodynamical computer code, which solves the equations of hydrodynamics explicitly, coupled to the Poisson equation. The computer code has been written especially for this work and has been thoroughly tested. The calculations in this work have been performed with an effort to obtain physically reliable results (by repeating the same calculations with different numerical spatial resolutions). A physical mechanism for angular momentum transport by turbulent viscosity has been proposed and incorporated 
in new collapse calculations. The main results can be summarized as follows:

1. When there is no physical mechanism for angular momentum transport, the result of the collapse is a ringlike structure. If the rotation is fast enough, a quasi-static mild ringlike structure inside a flattened configuration forms. In the case of slower rotation a deep collapse with a ring ensues. The ring collapses onto itself, and its density grows up to the point where it becomes opaque. This result supports the findings of Bodenheimer and Tscharnuter.

2. The turbulent viscosity affects the nature of the collapse. The results of collapse calculations with turbulent viscosity are radically different from the respective "nonviscous" calculations. The angular momentum transport interferes with the ring formation and enables the formation of a central dense and opaque region. This region is surrounded by a flat disk.

3. For the two cases studied, the mass of the central object is a major fraction (30\%) of the total mass of the system.

4. The exact form of the central object and its ultimate fate depend on the parameters, especially $\beta$ ( $\beta \equiv$ rotational energy/gravitational energy) and $\operatorname{Re}$ (turbulent Reynolds number).

5. The present calculations cannot predict the future evolution of the central object because they have an insufficient spatial resolution at the center and the isothermal approximation breaks down. However, it seems likely that the collapse with turbulent viscosity leads to the formation of a central protostar surrounded by a disk (proto-planetary nebula) when the rotation is slow enough and the turbulence efficient. When the rotation is fast or the turbulent transport less efficient, the central opaque object is very flat. This raises the possibility of multiple system formation (e.g., binary).

6. The importance of this work lies in the fact that a new theoretical model is proposed, in which a central protostar forms as a result of the collapse of a protostellar rotating cloud.

20697. Reid, M.; McNeil, K. J.; Walls, D. F. Unified approach to multiphoton lasers and multiphoton bistability, Phys. Rev. $A$ 24, No. 4, 2029-2043 (Oct. 1981).

Key words: lasers; multiphoton lasers; optical bistability; unified theory.

This paper presents a unified theory of nonequilibrium transitions which occur in radiation interacting via an $n$-photon transition with atomic systems inside optical cavities. Incoherent pumping of the atoms and coherent driving of the cavity are included. Examples of such systems are the $n$-photon laser, the $n$-photon laser with injected signal, and $n$-photon optical bistability. The state equation and stability conditions for these phenomena are derived. Fluctuations are included via a Fokker-Planck equation in the constant-diffusion approximation for which potential solutions may be obtained in the steady state. When these solutions are used, moments of the photon distribution can be calculated for the above systems in the specific cases of one- and two-photon transitions.

20698. Rosman, K. J. R.; Barnes, I. L.; Moore, L. J.; Gramlich, J. W. Isotope composition of $\mathrm{Cd}, \mathrm{Ca}$ and $\mathrm{Mg}$ in the Brownfield chondrite, Geochem. J. 14, 269.277 (1980).

Key words: cadmium; isotope abundances; isotope fractionation; magnesium; meteorites.

The isotopic composition of cadmium, calcium and magnesium in the Brownfield chondrite have been measured. The measurements on cadmium show that this element is isotopically fractionated with the heavier isotopes relatively enriched to the extent of $2.7 \%$ per mass unit. This confirms earlier reports by Rosman and De Laeter (1976, 1978). Calcium and magnesium show no evidence of isotope fractionation, indicating that the process responsible for fractionating cadmium does not seem to have affected these more refractory elements.

20699. Sandle, W. J.; Gallagher, A. Optical bistability by an atomic vapor in a focusing Fabry.Perot cavity, Phys. Rev. A 24, No. 4, 2017. 2028 (Oct. 1981).

Key words: optical bistability.
We report experimental observations of dispersive- and absorptive. optical bistability due to saturation of the $D_{1}$ line of atomic sodium in a tight-focused Fabry.Perot cavity. Under the conditions of large optical power broadening and substantial collisional broadening by argon buffer gas used for part of the data, the sodium very nearly behaves as a two-level homogeneously broadened absorber. It thus yields a test of the two-level mean-field optical-bistability model. That model, including homogeneous broadening, fails to account quantitatively for the power levels and power dependencies of the cavity transmission. In particular, this model predicts too rapid an onset of atomic saturation with cavity power. In contrast, predictions of a two-level Gaussian-field optical-bistability model for the dependence of transmitted power as a function of laser power and frequency are confirmed by the experimental data. However, there is a remaining discrepancy in the absolute value of the cavity power.

20700. Scalabrin, A.; Saykally, R. J.; Evenson, K. M.; Radford, H. E.; Mizushima, $M$. Laser magnetic resonance measurement of rotational transitions in the metastable $a^{1} \Delta_{g}$ state of oxygen, $J$. Mol. Spectros. 89, 344-351 (1981).

Key words: far infrared; laser magnetic resonance; metastable oxygen molecule; rotational transitions.

Laser magnetic resonance (LMR) for five rotational transitions, $J=4 \leftarrow 3,5 \longleftarrow 4,7 \leftarrow 6,8 \leftarrow 7,9 \longleftarrow 8$, of the oxygen molecule ${ }^{16} \mathrm{O}^{16} \mathrm{O}$ in its metastable state, $a^{1} \Delta_{g} v=0$, are observed using six fir laser lines. Taking the known values of the $g$ factors, their zero-field frequencies are obtained as $340.0085(6), 424.9810(9), 594.870(1), 679.780(1)$, and $764.658(1) \mathrm{GHz}$, respectively. They are fit by $(E / h)=B_{0}[J(J+1)-4]+$ $D_{0}[J(J+1)-4]^{2}+(-1) \quad(1 / 2) q J \quad(J+1)[J(J+1)-2]$, where $B_{0}=$ $42.50457(10) \mathrm{GHz}, D_{0}=153.14(110) \mathrm{kHz}$, and $q=0.050(90) \mathrm{kHz}$.

20701. Simmons, J. A.; Clough, R. B. Theory of acoustic emission, Proc. Int. Conf. Dislocation Modelling of Physical Systems, Gainesville, FL, June 1980, pp. 464.497 (Pergamon Press, Oxford, England, 1981).

Key words: acoustic emission; crack motion; dislocations; fracture; inverse problem; nondestructive evaluation; plasticity; signal processing.

A theory of acoustic emission is presented based on a Green's function type of formalism, rather than on the conventional count rate concept. Sources are represented by stress drop rate tensors and conditions are derived from which the source can be considered small in terms of wavelength and distance to the transducer. These "pseudopoint" sources are examined over a restricted frequency bandwidth, called the "informative bandwidth." Such a bandlimited system may be described by a tensor transfer function type of formalism, facilitating the analysis and reducing the inverse problem-where the source is not known a priori-to a deconvolution operation. Due to the tensor nature of the source, multiple transducer measurements are necessary to reconstruct the source stress drop. The difficulty of using spectral techniques for data analysis in the presence of multiple sources is discussed. The forward problem for acoustic emission to be expected from plasticity, phase transformations, and cracks is solved within the pseudopoint approximation and the concept of retarded dipole density is introduced to deal with the problem of time delays from propagating inhomogeneity sources. In addition, the strong directionality of the signal with respect to source type and orientation is illustrated by calculating the acoustic emission signals generated by loop expansion of slip and climb (prismatic collapse) type dislocations. This directionality is shown to be the same for isotropic materials as that associated with Mode II/III or Mode I cracks and a comparison is provided between emission from expanding penney-shaped cracks and that from dislocation loops. Finally, the concepts of stress-controlled and materials-controlled acoustic emission are introduced and their significance is discussed in terms of relating AE source descriptions and material mechanisms.

20702. Soulen, R. J., Jr.; Van Vechten, D.; Costabile, G.; Jach, T.; Holdeman, L. B. The superconductive energy gap of $\mathbf{A u A l}_{2}$, Physica 108B, 823-824 (1981). 
Key words: $\mathrm{AuAl}_{2}$; energy gap; superconductivity; tunneling

We have prepared $200 \mathrm{~nm}$ films of $\mathrm{AuAl}_{2}$. The superconductive transition determined by resistive measurements was found to vary from film to film, from 177 to $184 \mathrm{mK}$, and the transition widths varied between 6 and $15 \mathrm{mK}$. Film surfaces were oxidized by the Greiner process and $\mathrm{Al}$ counter electrodes were deposited in order to produce tunnel junctions. The temperature dependence of the energy gap of $\mathrm{AuAl}_{2}$ was measured and found to agree well with BCS theory.

20703. Tobler, R. L. A review of antifriction materials and design for cryogenic environments, (Proc. Cryogenic Engineering Conf., Madison, WI, Aug. 21.24, 1979), Paper A.5 in Advances in Cryogenic Engineering, A. F. Clark and R. P. Reed, eds., 26, 66.77 (Plenum Press, New York, NY, 1980).

Key words: antifriction materials; friction; friction coefficients of materials; graphite; low temperature properties; lubrication; molybdenum disulfide; teflon; wear

Friction must be considered in the design of any low temperature apparatus incorporating shafts, pistons, cams, gears, or other parts with sliding interfaces. Friction is usually more severe at cryogenic temperatures, causing problems of wear and heating. The latter factor is of special consequence to the design of superconducting devices since frictional heating would increase steady state cryogen consumption and operating costs, or possibly cause superconductors to go normal. This paper reviews the low temperature friction behavior of important structural materials and sliding couple combinations. Design methods and materials selection with regard to environment are discussed. It is emphasized that metals exhibit higher self friction coefficients $(\mu \simeq 0.3$ to 1.0 ) than nonmetals $(\mu \simeq 0.04$ to 0.6 ) due to cold.welding. Common methods of overcoming friction at low temperatures include surface treatments, dry lubrication, and antifriction materials.

20704. Van Brunt, R. J.; Leep, D. Characterization of point-plane corona pulses in $\mathrm{SF}_{6}, J$. Appl. Phys. 52, No. 11, 6588-6600 (Nov. 1981).

Key words: corona; electron avalanches; inception voltage; partial discharges; pressure dependence; pulse-heightdistributions; pulses, $\mathrm{SF}_{6}$; sulfur hexafluoride.

The properties of streamer or electron avalanche pulses have been investigated for point-plane, positive and negative dc corona discharges in gaseous $\mathrm{SF}_{6}$ over the pressure range of 50 to $500 \mathrm{kPa}$. Measurements were made of the voltage and pressure dependence of electrically-detected corona pulse-height-distributions, pulse shapes and repetition rates. Positive corona pulses appear at onset as low level electron avalanches which, at higher voltages, develop into large streamers usually followed by a burst of many smaller pulses. The burst characteristics of positive corona show a definite dependence on pressure and voltage which is evident in the pulse. height-distribution data. Negative corona displays characteristics which are sensitive to cathode surface conditions. For a point electrode that is either exposed to uv radiation or has been conditioned by prior discharges, the negative corona appears predominately in a quasi-glow mode consisting of relatively low.level pulses of high repetition rate.

20705. Waksman, D. Solar collector certification activities and experiences, Proc. Commercial Photovoltaics Measurements Workshop. Vail, CO, July 27-29, 1981, pp. 279-289 (Available from the National Technical Information Service, Springfield, VA, Nov. 1981).

Key words: certification; experiences; issues and concerns; laboratory accreditation; solar collector; standards.

This paper is intended to provide organizations which may be involved in the development of certification programs for photovoltaic (PV) solar modules with the benefit of the experience gained to date in the development of certification programs for solar collectors. The various public and private sector certification activities that have been undertaken for solar collectors along with issues and concerns that have arisen from their use are presented.

20706. Wang, G. C.; Pierce, D. T.; Celotta, R. J. Study of temperature and hydrogen induced reconstruction and reordering of $W(100)$ by polarized electron scattering (Summary Abstract), J. Vac. Sci. Technol. 18, No. 2, 647-648 (Mar. 1981).

Key words: chemisorption; PLEED; polarized electrons; reconstruction; tungsten.

The intense polarized electron beam obtained by photoemission from negative electron affinity $\mathrm{GaAs}(100)$ was used to scatter electrons from the $W(100)$ high temperature $(1 \times 1)$ phase, the $(\sqrt{2} \times \sqrt{2}) \mathrm{R} 45^{\circ}$ phase (produced by cooling below $370 \mathrm{~K}$ ), and the room temperature hydrogen chemisorbed $C(2 \times 2)$ and $(1 \times 1)$ phases in order to study the structure changes due to hydrogen chemisorption and temperature variation.

20707. Waterstrat, R. M. Binary phase diagrams of transition elements, Bull. Alloy Phase Diagrams 2, No. 1, 35.38 (June 1981)

Key words: alloy phases; alloy theory; constitution diagrams intermetallic compounds; phase diagrams; transition elements.

A brief explanation is given for an accompanying chart of binary phase diagrams of the transition elements together with references and a commentary on the source of each reference.

20708. Weeks, J. J.; Eby, R. K.; Clark, E. S. Disorder in the crystal structures of phases I and II of copolymers of tetrafluoroethylene and hexafluoropropylene, Polymer 22, 1496.1499 (Nov. 1981).

Key words: copolymer; crystal; disorder; hexafluoropropylene; phases; tetrafluoroethylene; $x$ ray.

$X$-ray diffraction patterns for oriented and unoriented copolymers of tetrafluoroethylene and hexafluoropropylene are presented. They show a transition between 229 and $256 \mathrm{~K}$ for a copolymer with about 3.4 $\mathrm{CF}_{3}$ per hundred main chain carbon atoms. The lower temperature phase (II) appears to be similar to that of the homopolymer. A primary difference is the presence in the copolymer crystals of small random longitudinal displacements of the molecules caused by the $\mathrm{CF}_{3}$ groups. The displacements increase with increasing $\mathrm{CF}_{3}$ concentration. The higher temperature phase (I) is similar to that of the homopolymer with a difference again being the longitudinal disorder of the copolymer. The difference disappears at higher temperatures as longitudinal disorder is introduced thermally in the homopolymer. At $296 \mathrm{~K}$ (phase I) the molecular stems in a copolymer with about $3.4 \mathrm{CF}_{3}$ per hundred main chain carbon atoms are at an angle of $37.1 \mathrm{deg}$ with respect to the normal to the basal plane of the lamellas. In phase II, this number is the same within the limits of error

20709. Wise, S. A.; Bonnett, W. J.; Guenther, F. R.; May, W. E. A relationship between reversed-phase $C_{18}$ liquid chromatographic retention and the shape of polycyclic aromatic hydrocarbons, $J$. Chromatogr. Sci. 19, 457.465 (Sept. 1981).

Key words: aqueous solubility; gas chromatography; length-to breadth ratio; liquid chromatography; liquid crystals; polycyclic aromatic hydrocarbons (PAH); retention mechanism; reverse phase liquid chromatography.

A relationship between the shape, particularly the calculated length-to-breadth ratios, of polycyclic aromatic hydrocarbons (PAH) and their reversed-phase $\mathrm{C}_{18}$ liquid chromatographic (LC) retention is described. In general, when isomeric PAH are compared, the LC retention increases with increasing length-to-breadth ratio. Retention data and length-to-breadth ratios for $100 \mathrm{PAH}$ are compared. The elution orders for methyl-substituted fluorenes, anthracenes, phenanthrenes, pyrenes, fluoranthenes, benzo[c]phenanthrenes, benz[a]anthracenes, chrysenes, and benzo[a]pyrenes are reliably predicted from the calculated length-to-breadth ratios.

20710. Wiese, W. L.; Martin, G. A. Atomic spectroscopy, Chapter 5 in 
Physics Vade Mecum AIP 50th Anniversary, H. L. Anderson, ed., pp. 96-105 (American Institute of Physics, New York, NY, 1981).

Key words: atomic spectroscopy; lifetime; line shape; photon energy; selection rules; spectral continuum; spectral line; transition probability; wave function; wavelength.

A compendium of definitions of atomic spectroscopic quantities, along with formulae and tables of data which are frequently used by atomic spectroscopists and by scientists who are interested in applications of atomic spectroscopic data to other fields of physics, is presented. Topics covered include the following: Description of spectra, spectroscopic notation and electron coupling schemes; Photon energies and spectral line positions; Wavelength standards; Selection rules for discrete transitions; Atomic wave functions; Spectral line intensities, transition probabilities, f-values, and line strengths; Atomic lifetimes; Hydrogenic (1-electron) spectra; Atoms and ions with two or more electrons; Spectral line shapes, widths and shifts; Spectral continuum radiation.

20711. Yaghjian, A. D. A delta-distribution derivation of the electric field in the source region, (Proc. 1981 Int. IEEE Antennas and Propagation Symp., Los Angeles, CA, June 17-19, 1981), IEEE Dig., pp. 334-337 (Institute of Electronics and Electrical Engineers, Piscataway, NJ 08854, 1981).

Key words: delta-distribution; dyadic Green's function; electromagnetic theory.

The derivation of the expression appropriate for the dyadic Green's function in the source region is considered from the standpoint of the theory of distributions, in which the Green's function corresponding to a delta sequence is obtained for the special case of a spherical principal volume. The previously obtained result follows readily from this special case by circumscribing the arbitrary principal volume by the spherical principal volume and evaluating the resultant principalvalue integral.

20712. Yoo, K. C.; Roessler, B.; Armstrong, R. W.; Kuriyama, M. Reflection X-ray topography of hardness indentations in copper single crystals, Scr. Met. 15, 1245-1250 (1981).

Key words: copper single crystal; image contrast; indentation hardening; plastic deformation; $x$-ray topography.

The plastic deformation zones surrounding microhardness indentations put into the (110) surfaces of relatively soft copper and nickel single crystals have been studied by the asymmetrical crystal topography (ACT) method. The method gives valuable information about the importance of workhardening to determining the level of the microhardness pressure and to determining the magnitude of its anisotropy for different directions of the indenter axes for Knoop or diamond pyramid indentations. This is demonstrated especially for copper crystals by the very pronounced appearance of the strain patterns of diffraction contrast obtained at either type of hardness impression. Both the cumulative dislocation displacements (for extinction contrast) and their lattice rotations (for misorientation contrast) are employed in the analysis of the topographic strain patterns.

20713. Zwier, T. S.; Weisshaar, J. C.; Leone, S. R. Nascent product vibrational state distributions of ion-molecule reactions: The $\mathbf{H}+\mathbf{F} \rightarrow \mathbf{H F}(v)+e^{-}$associative detachment reaction, J. Chem. Phys. 75 , No. $10,4885-4892$ (Nov. 15,1981 ).

Key words: associative detachment; flowing afterglow; hydrogen fluoride; infrared chemiluminescence; ion-molecule; vibration.

The nascent product vibrational state distribution is obtained for the thermal energy associative detachment reaction $\mathrm{H}+\mathrm{F}^{-} \rightarrow$ $\mathrm{HF}(v \leqslant 5)+e^{-}, \quad \Delta H=-57.0 \mathrm{kcal} / \mathrm{mol}$. The relative vibrational populations are as follows: $N_{\mathrm{v}=1}^{0}=0.00 \pm 0.06 ; N_{\mathrm{v}=2}^{0}=0.23 \pm 0.04$; $N_{\mathrm{v}=3}^{0}=0.27 \pm 0.03 ; \quad N_{\mathrm{v}=4}^{0}=0.29 \pm 0.03 ; \quad$ and $\quad N_{\mathrm{v}=5}^{0}=0.21 \pm 0.03$.

Arguments are presented that suggest that $N_{\mathrm{v}=0}^{0}=0$. The average fraction of the total energy deposited in product vibration is $\left\langle f_{v}\right\rangle=$ $0.64 \pm 0.03$. The release of the very light electron as one of the reaction products places severe angular momentum constraints on the reaction system. A simple kinematic model predicts a highly non. Boltzmann $\mathrm{HF}$ rotational state distribution which increases with increasing $J$ up to some cutoff level. The high degree of vibrational excitation in the HF product has been accounted for by theoretical calculations of Gauyacq using the zero-range potential approximation.

20714. Covington, A. K.; Goldberg, R. N.; Sarbar, M. Computer simulation of titration curves with application to aqueous carbonate solutions, Anal. Chim. Acta 130, $103-109$ (1981).

Key words: analysis; carbonates; carbon dioxide; computer; $\mathrm{pH}$; simulation; titration.

A computer simulation calculation is used to generate theoretical acid-base titration curves for aqueous carbonate solutions. The calculation allows for the time delay in the conversion of aqueous $\mathrm{CO}_{2}$ to $\mathrm{H}_{2} \mathrm{CO}_{3}$ and permits the calculation of concentrations of the species in solution and their time derivatives during the course of a titration. Detailed calculations, which include the effect of the time delay, show that the existence of a peak in the differential titration curve corresponds to the protonation of a species in solution, and that no extra peaks are produced as a result of the time delay. The calculations also show the $\mathrm{pK}$ values obtained from such titrations are not affected by the time delay, and that a systematic error may be incurred when such titrations are used for analytical purposes when the rate of addition of titrant is too rapid.

20715. Farmer, B. L.; Eby, R. K. Energy calculations of the crystal structure of the low temperature phase (II) of polytetrafluoroethylene, Polymer 22, 1487.1495 (Nov. 1981).

Key words: calculation; crystal; disorder; energy; phase II; polytetrafluoroethylene; structure.

Energy analysis is used to determine the low energy crystal structure for polytetrafluoroethylene molecules in the $13 / 6$ and 54/25 conformations. The structure for the $54 / 25$ conformation contains an ordered repeating pattern of a left- and right-handed pair of molecules in a unit cell having projected parameters, $a^{\prime}, b^{\prime}$, and $\gamma^{\prime}$, of $9.60 \AA$, $5.62 \AA$, and 91.4 degrees, respectively. These results, the relative orientations of the molecules, and the relative translations of the molecules are in good agreement with results obtained in a concurrent and independent analysis of diffraction data. Energies calculated for rotational and translational displacements in various modes suggest that a range of structures and a fairly large amount of disorder may be present, also in agreement with experiment.

20716. Fowler, H. A.; Filliben, J. J. Dielectric function for ultraviolet plasmons in the short-lifetime limit, J. Appl. Phys. 52, No. 11, 6701. 6703 (Nov. 1981).

Key words: complex function; Drude formula; energy loss; free. electron gas; optical constants; plasmon.

The "inverted" Drude-Sellmeier formula, for a two-parameter freeelectron-gas spectrum of plasmon excitations, is evaluated for very large values of the line breadth. These function values for $1 / \epsilon(\omega)$ characterize a solid dominated by a free-electron plasma with short "damping time," over the ultraviolet range. A rounded energy.loss distribution is suggested at the critical damping limit.

20717. Gary, D. E.; Linsky, J. L. First detection of nonflare microwave emission from the coronae of single late-type dwarf stars, Astrophys. J. 250, 284-292 (Nov. 1, 1981).

Key words: radio sources, general; solar coronae; solar flares; stars, individual; stars, radio radiation; Sun, radio radiation.

We report on an observing program with the VLA in its C configuration to detect microwave radiation from the coronae of nearby late-type dwarf stars which are not members of close binary systems and do not have large winds. Six stars, chosen on the basis of strong apparent X-ray flux, were observed during a 24 hour period, and two stars were detected. $\chi^{1}$ Orionis $(\mathrm{G} 0 \mathrm{~V})$ was detected as a 0.6 $\mathrm{mJy}$ source $(\mathrm{S} / \mathrm{N} \approx 7)$ at $6 \mathrm{~cm}$, and we obtained an upper limit at 2 
$\mathrm{cm}$. The flare star UV Cet (dM 5.5e) was detected as a steady 1.55 mJy source $(\mathrm{S} / \mathrm{N} \approx 17)$ at $6 \mathrm{~cm}$ during a 2.5 hour observation. We obtained upper limits at $6 \mathrm{~cm}$ for the other stars observed: $\pi^{1} \mathrm{UMa}$ (G0 V), $\xi$ Boo A (G8 V), $70 \mathrm{Oph} A(\mathrm{~K} 0 \mathrm{~V})$, and $\epsilon$ Eri (K2 V). We believe that the most likely emission mechanism is gyro-resonance emission, i.e., cyclotron emission from nonrelativistic Maxwellian electrons. Assuming a coronal temperature of $0.5-1.0 \times 10^{7} \mathrm{~K}$ consistent with the X-ray data, we find that the observed $6 \mathrm{~cm}$ fluxes are consistent with emission in the sixth or lower harmonics with coronal magnetic fields $\approx 300$ gauss or larger covering a large fraction of the star. It is likely that $\chi^{1}$ Ori, solar active regions (plages), and perhaps UV Cet, are the first detected members of a new class of radio sources.

20718. Greer, S. C.; Moldover, M. R. Thermodynamic anomalies at critical points of fluids, Ann. Rev. Phys. Chem. 32, 233-265 (1981).

Key words: consolute point; critical exponents; critical point; equation of state; free energy; heat capacity; liquid and vapor density; phase transitions; scaling laws; thermodynamic properties.

Recent measurements of the thermodynamic properties of fluids near critical points are reviewed in the context of current theory. Measurements near liquid-vapor and liquid-liquid critical points (consolute points) are considered. The thermodynamic properties reviewed include: the density and composition of coexisting phases in equilibrium, thermal expansion, heat capacity, equation-of-state, and finally the dependence of the density of pure fluids upon height extremely near the critical temperature.

In general, the experimental determinations of the "universal" critical exponents and amplitude ratios which characterize the free energy anomaly asymptotically close to the critical point are consistent with the theoretical predictions for these quantities. Tests of the mutual consistency of fluid data and theory must consider the fact that we lack complete knowledge of the relationship between the laboratory thermodynamic variables and the abstract scaling variables which appear in the theory. The tests must also consider the possibility that real data are influenced by subdominant singularities in the free energy which are predicted to be confluent with the leading singularity at the critical point.

Some remarks are made concerning future directions for research. A list of 203 references to relevant literature is included.

20719. Groner, N. E.; Levin, B. M.; Nelson, H. E. Measuring evacuation difficulty in board and care homes, Fire J. 75, No. 5, 44. 50, 109 (Sept. 1981).

Key words: board and care homes; building codes; evacuation; fire safety; handicapped; life safety; residential care facilities.

The recent increase in multifatality fires in board and care homes can be attributed to the recent proliferation of these homes coupled with unavailability of an appropriate model code at the national level. Currently available model codes work poorly because they do not take into account the wide variations in the assistance needs of residents in an emergency evacuation and the availability of staff members to provide the needed assistance. The Center for Fire Research, National Bureau of Standards, has devised a fire safety evaluation system that bases the amount of fire protection features required in the building on a measure of the difficulty in evacuating the home. This measure, the Evacuation Difficulty Index, is computed from ratings of the residents on evacuation-relevant "risk factors" and on the availability of staff members to assist residents. The article outlines the rationale and development of the Evacuation Difficulty Index.

20720. Hall, J. L.; Hollberg, L.; Baer, T.; Robinson, H. G. Optical heterodyne saturation spectroscopy, Appl. Phys. Lett. 39, No. 9, 680682 (Nov. 1, 1981).

Key words: laser spectroscopy; optical heterodyne spectroscopy; saturated absorption.

We discuss a refined, hybrid rf/optical technique for studying sub-
Doppler saturated absorption/dispersion resonances with excellent precision and symmetry. Sensitivity is limited mainly by fundamental noise in the signal. Resonance profiles obtained in $\mathrm{I}_{2}$ are in remarkable agreement with theory. The method promises a new level of accuracy for laser locking to an optical resonance. Nonequilibrium response is also discussed.

20721. Han, C. C.; Akcasu, A. Z. Concentration dependence of diffusion coefficient at various molecular weights and temperatures, Polymer 22, 1165-1168 (Sept. 1981).

Key words: concentration dependence; diffusion coefficient; dynamic light scattering; polystyrene solutions; temperature dependence.

The effect of temperature on the concentration dependence of the diffusion coefficient $D(c)$ is investigated experimentally by light scattering using polystyrene with $\boldsymbol{M}_{w}=179,000$ and 900,000 in toluene and cyclohexane at several temperatures. It is found that $D(c)$ decreases with concentration under the theta condition, and increases in good solvents. The continuous transition from theta $\theta$ to good solvent behaviour is explored, and the results are compared with the existing theoretical predictions.

20722. Han, C. C.; Akcasu, A. Z. Dynamic light scattering of dilute polymer solutions in the nonasymptotic $q$ region, Macromolecules 14 , 1080-1084 (July-Aug. 1981).

Key words: dynamic light scattering; intermediate $q$-region; nonasymptotic $q$-region; polystyrene at $\theta$-temperature; polystyrene in good solvent.

Polystyrene solutions in the dilute region have been studied by dynamic light scattering experiments. The first cumulant, $\Omega(q)$, has been extracted consistently by either cumulant analysis or shape function analysis. It is found that $\Omega(q)$ approaches $q^{2}$ dependence for $q R_{g} \ll 1$ and $q^{3}$ dependence for $q R_{g} \gg 1$, with a broad transition region. It is also found that the magnitudes of the first cumulant in the $q^{3}$ region change as the solvent is changed from a $\theta$ solvent to a good solvent. In both cases, experimental results agree better with theoretical calculations which use a preaveraged Oseen tensor.

20723. Holdeman, L. B.; Hall, J. T.; VanVechten, D.; Soulen, R. J., Jr. Gap enhancement in aluminum tunnel junctions coupled to microstrip resonators, Physica 108B, $827-828$ (1981).

Key words: aluminum; energy gap; gap enhancement; microwaves; nonequilibrium; superconductivity; tunnel junction.

Irradiated $(9.7 \mathrm{GHz})$ Al films show gap enhancement that agrees with Eliashberg's theory.

20724. Johnson, R. G.; Behrens, J. W.; Bowman, C. D. Source imaging using neutron pinhole cameras based on position-sensitive proportional counters, Nucl. Technol. 55, 724-727 (Dec. 1981).

Key words: linear accelerator; neutron imaging techniques; position-sensitive proportional counters; Van de Graaff accelerator; $14 \mathrm{MeV}$ neutron energy.

A pinhole camera technique has been used to measure the variation in neutron emission intensity over the area of the neutron-producing target of the National Bureau of Standards Electron Linac. The method uses a one-dimensional position-sensitive proportional counter (PSPC) with an intrinsic spatial resolution of $1.0 \mathrm{~mm}$. The pinhole is made in a thick sheet of cadmium and neutron energy $(<0.3-\mathrm{eV})$ selection is achieved by time-of-flight. In a completely separate experiment, the neutron cone obtained from the $(d, t)$ reaction using the associated-particle technique was imaged by a two-dimensional PSPC. This second measurement demonstrated the use of the twodimensional detector for imaging high-energy (14-MeV) neutrons.

20725. Johnson, W. C.; Cahn, J. W. Comment on chemical potentials in solids, Script. Met. 15, 1259-1964 (1981).

Key words: chemical potential; diffusion; equilibrium; solid; 
stress, strain; thermodynamics.

In a pair of recent Scripta Metallurgica articles, Nolfi claims that chemical potentials can be defined for both solid (substitutional) and fluid (interstitial) components in nonhydrostatically stressed materials. This is accomplished assuming that at equilibrium the combined form of the first and second laws of thermodynamics for a closed stressed system can be extended directly to an open system. Unfortunately, the resulting equation is improper for it does not represent correctly the work done by the system. It is the intent of this note to clarify the basic postulates employed by Nolfi in defining equations for chemical potentials in stressed solids and to illustrate that extension from a closed to an open system is not necessarily straightforward.

20726. Ledbetter, H. M. Elastic constants of polycrystalline copper at low temperatures, Phys. Status Solidi (A) 66, 477.484 (1981).

Key words: copper; Debye temperature; elastic constants; low temperatures; polycrystals; sound velocities; tensor-property averaging.

Elastic constants of polycrystalline copper are determined between 4 and $295 \mathrm{~K}$ by measuring ultrasonic velocities, longitudinal and transverse. Five elastic constants are given: longitudinal modulus, shear modulus, Young's modulus, bulk modulus (reciprocal compressibility), and Poisson's ratio. Considering eight theories of single-crystal:polycrystal elastic constants, the Hershey-KrönerEshelby theory agrees best with observation, within $1 \%$ for the shear modulus, $G$. (Voigt and Reuss first-order bounds on $G$ differ by $29 \%$.) A problem exists concerning the calculation of the Debye temperature of polycrystalline aggregates.

20727. Levin, B. M.; Nelson, H. E. Fire safety and disabled persons, Fire J. 75, No. 5, 35-40 (Sept. 1981).

Key words: board and care homes; building codes; building fires; evacuation; fire alarm systems; fire safety; handicapped; life safety; refuge.

The scope and nature of the problem of disabled persons in fire emergencies are discussed. Although specific solutions may need to be developed for particular disability types and occupancy settings, there are some principles which should be generally applied. These principles relate to general safety, methods of enabling disabled persons to meet their own safety needs, the role of others in providing assistance, and fire emergency planning. The options of evacuation or remaining in areas of safety are considered. An evacuation problem can result from the circumstance where a mobility impaired person has accessed upper floors of a building through use of an elevator, but cannot safely use this elevator for egress in a fire emergency. Efforts are underway to modify the Life Safety Code of the National Fire Protection Association to address this problem.

Fire safety for disabled persons is discussed in the context of different occupancy types. There are currently no codes specifically established for one occupancy type, board and care homes. These homes may contain elderly, developmentally disabled, or emotionally impaired individuals. The National Bureau of Standards' effort to develop a fire safety evaluation system for this occupancy type is described.

20728. MacCrehan, W. A.; Durst, R. A. Dual-electrode, liquid chromatographic detector for the determination of analytes with high redox potential, Anal. Chem. 53, 1700-1704 (1981).

Key words: dichlone; dual-electrode detector; electrochemical detector; liquid chromatography; nitrobenzene; organotin cations.

An electrochemical detection approach for liquid chromatography is described utilizing two sequential, generator/detector electrodes. Analytes are first electrolyzed, and the reaction products are then detected electrochemically at a second electrode. In some cases, this allows the detection of analytes with high redox potentials, with good sensitivity and selectivity. The technique is tested on several organic analytes: dichlone, nitrobenzene, and three organotin cations.

20729. Misra, D. N.; Bowen, R. L. Interaction of zinc ions with hydroxylapatite, Paper in Adsorption from Aqueous Solutions, $\mathrm{P} \cdot$ Tewari, pp. 179-192 (Plenum Publ. Corp., New York, NY, ( 1981).

Key words: bonding with coupling agents; hydroxylapatite; interaction with $\mathrm{Zn}$ ions and enamel etching; reaction with $\mathrm{Zn}$ ions and rate laws; surface mordanting with $\mathrm{Zn}$ ions.

The interaction between zinc ions in aqueous nitrate solutions and hydroxylapatite, the structural prototype for the principal inorganic constituent of tooth and bone was studied. The nature of this interaction may be important in elucidating the role of zinc oxide commonly dissolved in the phosphoric acid solutions used to etch hard tooth tissues prior to the application of dental resins. This study also has a direct bearing on the suitability of zinc ions as candidate "mordants" for use with surface-active comonomers that promote adhesion between restorative resins and hard tooth tissues.

The reaction of zinc ions with hydroxylapatite diminishes with time even at dilute concentrations, and is not complete after several days at room temperature. X-ray analysis shows the formation of hopeite $\left.\left[\mathrm{Zn}_{3}\left(\mathrm{PO}_{4}\right)_{2} \cdot 4 \mathrm{H}_{2} \mathrm{O}\right)\right]$ and the zinc analogue $\left[\mathrm{Zn}_{2}(\mathrm{OH}) \mathrm{PO}_{4}\right]$ of libethenite. The initial phase of the interaction may be explained by two proposed rate laws occurring sequentially. One involves the exchange of the surface calcium ions with the zinc ions in solution and the other seems to depend inversely on the amount of adsorbed zinc ions. The rate constant of the second kinetic process is a linear function of the ionic strength of solution.

20730. Palmer, B. A.; Keller, R. A.; Kowalski, F. V.; Hall, J. L. Accurate wave-number measurements of uranium spectral lines, $J$. Opt. Soc. Am. 71, No. 8, 948-952 (Aug. 1981).

Key words: spectroscopic reference wavelengths; uranium spectrum; wavelength calibration.

Wave number measurements made on 10 uranium and 2 thorium transitions using the optogalvanic effect in a hollow cathode discharge to a position in a single-frequency, cw dye laser to the peak of a transition. The wavenumber of the dye laser was measured by comparing it to the wave number of an iodine-stabilized He-Ne laser. The accuracy of the wave number measurements was $\pm 0.002 \mathrm{~cm}^{-1}$ with previously reported uranium and thorium measurements whose estimated accuracy was $\pm 0.003 \mathrm{~cm}^{-1}$.

20731. Payne, B. F. Absolute calibration of back-to-back accelerometers, (Proc. 27th Int. Instrumentation Symp., Indianapolis, IN, Apr. 1981), Paper in Instrumentation in the Aerospace Industry 27, $483-488$ (Instrument Society of America, Research Triangle Park, NC, 1981).

Key words: acceleration; accelerometers; calibration; shakers; vibration exciters.

Back-to-back accelerometers are widely used as vibration standards. Some problems exist in accurate calibration of these accelerometers due to the effects of mass loading on the sensitivity. The previous calibration procedure was limited to comparison with a calibrated accelerometer. This was necessary in order to provide proper loading. A new method is presented here which describes a standard, absolute, interferometric calibration method using a dummy load. This method makes possible direct displacement measurements at the top mounting surface of the accelerometer under a loaded condition. In this paper experimental data are presented for three test conditions: (1) Comparison with a calibrated reference accelerometer, (2) absolute displacement measurements under a loaded condition, and (3) absolute displacement measurements under an unloaded condition. Data from these measurements are presented and compared for some typical back-to-back accelerometers.

20732. Peterlin, A. Tensile failure of crystalline polymers, $J$ Macromol. Sci. Phys. B19, No. 3, $401-419$ (1981).

Key words: chain rupture; crazing; crystalline polymers; electron spin resonance; microcrack; microfibril; shear deformation; tensile failure.

In the creep experiment the brittle fracture of the unoriented 
micrystalline polymers at very small and very high tensile load with the intermediate ductile region may be explained by the competition between the crazing and the shear band formation during the microcrack growth phase. The former type of microcrack growth leads to brittle fracture while the latter type yields the necking which transforms the original lamellar into the final fibrous structure. The actual fate of the strained sample depends on the growth time of the craze, $t_{g}$, and of the shear band formation time, $t_{s}$. If $T_{g}<t_{s}$ the material will break in a brittle manner and if $t_{g}>t_{s}$ the material will deform plastically. The failure of the fibrous material seems to occur when the ratio between the average distance and diameter of the microcracks reaches a value about 3 . The microcracks seem to form primarily at the defects of the microfibrillar structure, i.e., at the ends of microfibrils where the axial connection of subsequent crystal blocks through the amorphous layers by a great many taut tie molecules is either completely interrupted or at least drastically reduced. The stress concentration resulting from the opening of these defects into microcracks ruptures some of the adjacent microfibrils. Such nucleation and the subsequent lateral growth of the microcrack ruptures the taut tie molecules in its path. The ruptured molecules yield free radicals which can be monitored by the electron spin resonance.

20733. Richmond, J. C. Errors in passive infrared imaging systems due to reflected ambient flux, SPIE Infrared Imaging Syst. Technol. Proc. 226, 110-114 (1980).

Key words: ambient infrared radiation; blackbody cavity; corrections for reflectance; infrared imaging; infrared thermography; radiance temperature; reflected infrared radiation; temperature corrections; temperature measurements.

Passive infrared imaging systems produce a signal in which the amplitude at a particular spot is related to the radiance of the corresponding spot in the scene viewed. The differences in signal levels in different areas of the image is usually interpreted in terms of radiance temperature differences in the scene viewed, and may be converted to true radiance temperatures if the scene includes an object whose radiance temperature is known.

The radiance temperatures are usually converted to true temperatures by correcting for the emittance of objects in the scene. This would be correct in the absence of reflected ambient flux. However, for scenes at ambient temperatures, ambient flux is always present in significant amounts. Temperature errors due to reflected ambient flux are discussed from a theoretical standpoint, and a procedure for experimentally evaluating the ambient flux is suggested.

20734. Serbyn, M. R.; Penzes, W. B. A real-time active vibration controller, (Proc. 27th Int. Instrumentation Symp., Indianapolis, IN, Apr. 1981), Paper in Instrumentation in the Aerospace Industry 27, 489.494 (Instrument Society of America, Research Triangle Park, NC, 1981).

Key words: active vibration control; Michelson interferometer; optical path-length correction; phase comparator; real-time control; vibration control; vibration isolation.

The Michelson interferometer is viewed as a noisy system whose noise input consists of unwanted changes in its optical path length, and whose desired output is a constant optical path-length difference. A technique for maintaining this quantity at a value equal to a multiple of quarter wavelengths of the light is described. This is accomplished by periodically comparing the phase difference between the fundamental and the second-harmonic components of the photodetector output. Using this information, a logic unit causes positive or negative-going ramp signals to be generated and applied to the "fixed" mirror as a path-length correction.

20735. Siu, M. C. I.; Bulik, C. National Bureau of Standards line-heatsource guarded-hot-plate apparatus, Rev. Sci. Inst. 52, No. 11, 1709. 1716 (Nov. 1981).

Key words: edge heat loss; effective thermal conductivity; gaptemperature unbalance; guarded-hot-plate; heat source distribution; insulation; thermal conductivity.

A description is given of a line-heat-source guarded-hot-plate apparatus for determining the effective thermal conductivity of insulation materials from $250 \mathrm{~K}$ to $400 \mathrm{~K}$. Measurements made on fibrous glass boards are in good agreement with results obtained from the guarded-hot-plate apparatus in operation at the National Bureau of Standards for over 40 years.

20736. Swartzendruber, L. J.; Bennett, L. H.; Ives, L. K.; Shull, R. D. The Ti-Al phase diagram: The $\alpha-\alpha_{2}$ phase boundary, Mater. Sci. Eng. 51, P1-P9 (1981).

Key words: alloy phase diagram; aluminum; magnetic susceptibility; order-disorder; titanium; transmission electron microscopy.

Magnetic susceptibility measurements were used to determine the phase boundaries between the disordered hexagonal $\alpha$ and the ordered hexagonal $\boldsymbol{\alpha}_{2}$ phases in titanium.rich Ti-Al alloys

20737. Younger, S. M. Cross sections and rates for direct electronimpact ionization of sodiumlike ions, Phys. Rev. $A$ 24, No. 3, 1272. 1277 (Sept. 1981)

Key words: electron atom scattering; electron impact ionization; sodium isoelectronic sequence.

Cross sections and rate coefficients for the direct electron impact ionization of sodiumlike ions have been calculated in a distorted wave Born-exchange approximation. For higher charge states the cross section is dominated by inner shell ionization of the $2 \mathrm{p}^{6}$ subshell. Analytic fits to the data allow rapid calculation of cross sections and rate coefficients over a wide range of incident electron energies. The importance of electron impact excitation to autoionizing states is discussed.

20738. Younger, S. M. Electron impact ionization cross sections and rates for highly ionized atoms, $J$. Quant. Spectrosc. Radiat. Transfer 26, No. 4, 329-337 (1981).

Key words: electron impact ionization; helium isoelectronic sequence; hydrogen isoelectronic sequence; lithium isoelectronic sequence.

Electron impact ionization cross sections and rates for hydrogen-, helium-and lithium-like ions are presented in simple analytical form, based on a set of parameters derived from distorted wave exchange cross section calculations. Isoelectronic fits to the parameters used in the formulae allow rapid generation of cross sections and rates for any ion in these sequences with a minimum of computational effort. The use of accurate Bethe slopes in the cross section-fitting algorithm yields good accuracy both in the near threshold and high energy regions. The rate coefficient is given in the form of a correction to the commonly used Seaton formula and is thus readily adaptable to existing plasma modeling computer codes. Comparisons of the present results with other theoretical and experimental data are made.

20739. Younger, S. M. Electron-impact-ionization cross sections and rates for highly ionized berylliumlike ions, Phys. Rev. $A$ 24, No. 3, 1278.1285 (Sept. 1981)

Key words: beryllium isoelectronic sequence; electron-atom scattering; electron impact ionization; metastable states.

Electron impact ionization cross sections and rates have been calculated for several berylliumlike ions in the $1 \mathrm{~s}^{2} 2 \mathrm{~s}^{2} \mathrm{I} S$ ground state and the $1 s^{2} 2 s 2 p{ }^{3} \mathrm{P}$ metastable state using a distorted wave Bornexchange approximation. The ground state cross section showed only minor deviations from classical scaling along the isoelectronic sequence. The $2 \mathrm{~s} 2 \mathrm{p}{ }^{3} \mathrm{P}$ cross section was more than twice that of the ground state at carbon III, decreasing to a factor of 1.35 greater at Ar $\mathrm{XV}$. Dominant ground state configuration interaction of the form $2 s^{2}+2 p^{2}$ changed the total cross section by only a few percent. An analytic fit to the distorted wave data is given which allows calculation of non-relativistic electron impact ionization cross sections and rates for berylliumlike ions. A comparison to available experimental data shows good agreement between measurements and distorted wave calculations. 
20740. Quintiere, J.; Steckler, K.; McCaffrey, B. A model to predict the conditions in a room subject to crib fires, Proc. First Specialists Meet. Int. Combustion Institute, Talence, France, July 20-24, 1981, 12 pages (1981).

Key words: crib fire; plastic; room fire; wood; zone model.

A model is formulated and executed to predict the burning conditions of a crib fire in a room. The results are compared to data for wood or plastic crib fires. Selected results are presented on rate of mass loss, temperatures, concentrations, and mass flow rates. Both transient and peak fire conditions are reported. The overall accuracy of the model can be assessed.

20741. Ho, J. C.; Oberly, C. E.; Garrett, H. J.; Walker, M. S.; Zeitlin, B. A.; Ekin, J. W. Processing limits for ultrafine-multifilament $\mathbf{N b}_{3} \mathbf{S N}$, (Proc. Cryogenic Engineering Conf., Madison, WI, Aug. 21-24, 1979), Paper E.7 in Advances in Cryogenic Engineering, A. F. Clark and R. P. Reed, eds., 26, 358-366 (Plenum Press, New York, NY, 1980).

Key words: bronze process; $\mathrm{Nb}_{3} \mathrm{Sn}$; processing limits; ultrafine filament $\mathrm{Nb}_{3} \mathrm{Sn}$.

The high yield and fracture strengths of the ultrafine-filament bronze-process wire, combined with the demonstration of no fundamental processing limits in an extensive manufacturing methods evaluation effort, promise a bronze manufacturing process for highstrength ultrafine-filament $\mathrm{Nb}_{3} \mathrm{Sn}$ conductors. These bronze-process conductors can be well controlled geometrically so that the losses experienced by randomly close-spaced filaments of in situ-process $\mathrm{Nb}_{3} \mathrm{Sn}$ conductors can be tightly controlled.

Proper billet design and manufacturing methods may enable even smaller filaments to be produced. The economic value of an ultrafinefilament conductor produced by the bronze process must, however, be seriously considered because of the large number of draw-andanneal cycles that are required.

20742. Tobler, R. L.; Reed, R. P.; Burkhalter, D. S. Temperature dependence of yielding in austenitic stainless steels, (Proc. Cryogenic Engineering Conf., Madison, WI, Aug. 21-24, 1979), B-4 in Advances in Cryogenic Engineering, A. F. Clark and R. P. Reed, eds., 26, 107-119 (Plenum Press, New York, NY, 1980).

Key words: cryogenic properties of materials; iron alloys; low temperature mechanical properties; plastic deformation; temperature effects stainless steel alloys; yield stress.

The temperature dependence of yielding is discussed with specific reference to austenitic stainless steels. Tensile yield stress $\left(\sigma_{\mathrm{y}}\right)$ measurements at $0.2 \%$ plastic strain, a strain rate of $0.02 \mathrm{~mm} / \mathrm{s}$, and temperatures between 4 and $295 \mathrm{~K}$ are reported for a heat of AISI 316 stainless steel. The results are compared with previously published data for the same heat at elevated temperatures, and the yield stress is observed to decrease from $660 \mathrm{MPa}$ at $4 \mathrm{~K}$ to $102 \mathrm{MPa}$ at $922 \mathrm{~K}$. The decrease is regular, with no obvious anomalies, and apparently no low temperature martensitic transformations at $0.2 \%$ strain. The merits and limitations of various expressions for describing the temperature dependence of the yield stress are then evaluated, and an excellent data fit is obtained using the expression: $\sigma_{\mathrm{y}}=\sigma_{\mathrm{A}}+$ $\left(\sigma_{0}-\sigma_{A}\right) \exp \left(-C_{7} T\right)$ where $\sigma_{A}$ is an athermal stress, $\sigma_{0}$ is the yield stress at absolute zero, and $C_{7}$ is a constant related to thermodynamic parameters for thermally activated plastic flow.

20743. Vasconcellos, E. C. C.; Petersen, F. R.; Evenson, K. M. Frequencies and wavelengths from a new efficient FIR lasing gas: $\mathrm{CD}_{2} \mathrm{~F}_{2}$, Int. J. Infrared Millimeter Waves 2, No. 4, 705.711 (1981).

Key words: $\mathrm{CD}_{2} \mathrm{~F}_{2} ; \mathrm{CO}_{2}$ laser; FIR laser; laser frequency measurement; relative polarization; relative power; wave-lengths.

We report for the first time wavelength, relative polarization, and frequency measurements for 47 new $\mathrm{cw}$ FIR laser lines in the wavelength region from 120 to $1714 \mu \mathrm{m}$, all obtained by optically pumping $\mathrm{CD}_{2} \mathrm{~F}_{2}$ with a $\mathrm{CO}_{2}$ laser. Relative output powers were also measured. For comparison, the $189.8 \mu \mathrm{m}$ line pumped by $R_{1}(34)$ is nearly five times as efficient as the strong $118.8 \mu \mathrm{m}$ methyl alcohol line.

20744. Fortunko, C. M. Ultrasonic evaluation of austenitic stainless steel welds using shear horizontal waves, Appl. Phys. Lett. 39, No. 9, 699-700 (Nov. 1, 1981).

Key words: acoustic waves; elastic anisotropy; nondestructive evaluation; nondestructive testing; stainless steel; ultrasonic scattering; ultrasonic waves.

The propagation of horizontally polarized shear (SH) waves through an austenitic stainless steel weld metal-base metal interface is described. It is shown that SH wave probing signals are particularly suitable for nondestructive evaluation of those stainless steel weldments that are very difficult to evaluate using vertically polarized shear and longitudinal wave probing signals. The SH wave technique is demonstrated experimentally on a $304 \mathrm{~L} / 316 \mathrm{~L}$ stainless steel butt weldment using $454-\mathrm{kHz} \mathrm{SH}$ wave electromagnetic-acoustic transducers that excite SH wave probing signals at $70^{\circ}$ with respect to surface normal.

20745. Unassigned.

20746. Benjamin, I. A. The future of fire protection engineering, Proc. Ann. Fire Protection Seminar, Department of Fire Protection \& Safety Engineering, Illinois Institute of Technology, Chicago, IL, Mar. 14, 1981,7 pages (1981).

Key words: building codes; fire modeling; fire protection engineering; sprinkler systems; systems analysis.

The talk reviews the history and changes in fire protection engineering over the last 30 years and discusses areas where major changes will be occurring in the future in the profession.

20747. Conti, D. M. U.S. efforts to develop standard benchmark programs, (Proc. Int. Conf. Performance of Computer Installations, Lake Garda, Italy, June 22.23, 1978), Paper in Performance of Computer Installations, D. Ferrari, ed., pp. 55.66 (North Holland Publ. Co., Amsterdam, The Netherlands, 1978).

Key words: benchmarking; benchmark library; selection of ADP systems; standard benchmarks; synthetic benchmarks; workload characterization; workload definition.

This paper surveys attempts within the United States to develop and use standard benchmark programs. In addition, the paper describes several issues raised by a recent Government-industry study group which was organized to determine the technical feasibility of using standard benchmark programs on a Government-wide basis. Problems encountered by the study group as well as suggestions for future work are also presented.

20748. Goldfarb, R. B. Miniature multipin electrical feedthrough for vacuum use, Cryogenics 21, 746 (Dec. 1981).

Key words: electrical connector; electrical feedthrough; vacuum feed through.

A miniature multipin electrical feedthrough for room-temperature vacuum use is made from commercially available components and an easily machined bushing.

20749. Eby, R. K.; Broadhurst, M. G.; Chang, S. S.; Davis, G. T.; DiMarzio, E. A.; Fanconi, B. M.; Han, C. C.; Khoury, F. A.; Markovitz, H.; McKinney, J. E.; Mopsik, F. I.; Passaglia, E.; Sanchez, I. C.; VanderHart, D. L.; Zapas, I. J. High polymer physics, Chapter 13 in AIP 5th Anniversary Physics Vade Mecum, pp. 196-209 (Oct. 1981)

Key words: data; equations; handbook; physicists; physics; polymers.

This is the High Polymer Physics Chapter of a Physics Handbook 
which will be published by the American Institute of Physics in 1981 or 1982 to commemorate the 50th Anniversary of the AIP. Dr. Herbert Anderson of Los Alamos is the editor of the Handbook. In agreement with the other chapters, this chapter gives important formulas, equations, and numerical data on defined systems. It also gives data useful for experimental design. Some of the latter data are on less well designed systems. The sections of the chapter are Introduction, Polymer Molecules, Molecular Weight Averages, Chain Dimensions, Theta Solvents and Temperatures, Molecular Weight Characterization, Characterization in Solution by NMR, Crystal Structures, Bond Lengths and Bond Angles, Melting and Crystallization, Optical Properties, Heat Capacity, Glass Transition, Densification of Polymeric Glasses, Stress $\sigma_{i j}$ and Displacement $u_{j}$ at Crack Tips, Internal Friction Peaks in Semicrystalline Polymers, Mechanical Properties of Some Common Structural Polymers, Rheology, Electrical Properties, and Diffusion and Permeation. The chapter gives references to sources of more detailed information. The Handbook is aimed at physicists not engineers or non-physicist specialists. It is not intended to be instructional with derivations or explanations beyond those absolutely necessary to explain the formulas, data, and equations.

20750. May, W. E.; Brown-Thomas, J. M.; Hilpert, L. R.; Wise, S. A. The certification of selected polynuclear aromatic hydrocarbons in Standard Reference Material 1580, "Organics in Shale Oil," Proc. Polynuclear Aromatic Hydrocarbons: Fifth Int. Symp. Chemical Analysis and Biological Fate, Columbus, OH, Oct. 27-29, 1980, pp. 1-16 (Battelle Press, Columbus, OH, 1981).

Key words: benzo(a)pyrene; benzo(e)pyrene; fluoranthene; fluorescence detection; gas chromatography/mass spectrometry; high-performance liquid chromatography; perylene; pyrene; shale oil; standard reference materials.

Two independent analytical methods were developed and used to certify the concentrations of five polynuclear aromatic hydrocarbons (PAH) in a shale oil sample (SRM 1580). In the sequential highperformance liquid chromatographic method, PAH are isolated from the matrix by chromatography on an aminosilane column. The individual analyte species were separated, identified, and quantified by chromatography on an octadecylsilane column with spectrofluorimetric detection. Dilution of the sample in methylene chloride was the only sample preparation step necessary prior to analysis by gas chromatography/mass spectrometry (GC/MS). The GC/MS determinations were performed using selected ion monitoring (SIM) and a standard addition technique for quantification. The polynuclear aromatic hydrocarbons of interest were separated on a $30 \mathrm{~m}$ SE. 52 wall coated open tubular column interfaced to a quadrupole mass spectrometer through a $25 \mathrm{~cm}$ length of $\mathrm{Pt} / \mathrm{Ir}$ tubing $(0.15 \mathrm{~mm}$ i.d.). Excellent agreement was observed between the data obtained from these methods.

20751. Mann, D. B.; Brennan, J. A.; Kneebone, C. H. Gas mass flow reference system: A progress report, Proc. American Gas Association 1980 Operating Section Distribution and Transmission Conf., (Distribution Conf. Detroit, MI, Mar. 19-21, 1980; Transmission Conf. Salt Lake City, UT, May 5-7, 1980), pp. T-366-T.368 (American Gas Assoc., Arlington, VA, 1980).

Key words: fluid flow; gas; instrumentation; mass flow; measurement; metering; thermodynamics.

A new type of gas flow reference system under development at the National Bureau of Standards results in direct, accurate and precise mass flow rate measurements. The closed loop system allows continuous flow of nitrogen gas at line pressures of $4.1 \mathrm{MPa}$ (600 psia) and at ambient temperatures. The gas flow is then cooled to liquid nitrogen temperature, and weighed at low pressures and at a density of up to 17 times the density in the gas phase.

This new flow reference tool is currently undergoing extensive testing and evaluation to establish total system uncertainty relative to national standards of mass. The program sponsored by the Gas Research Institute and the American Gas Association, Inc., will be directed to improving accuracy and precision of natural gas flow measurements, such as new gas flow data on orifice meter discharge and expansion coefficients.

A description of the NBS process is provided as well as preliminary performance information. Present mass flow rates are from $3000 \mathrm{~kg} / \mathrm{h}$ $(6600 \mathrm{lb} / \mathrm{h})$ to $10000 \mathrm{~kg} / \mathrm{h}(22000 \mathrm{lb} / \mathrm{h})$ and plans for increasing this range are presented.

20752. Clark, R. E. Weatherization retrofitting and occupant comfort, (Proc. Nat. Conf. Optimal Weatherization, Washington, DC, Dec. 8-10, 1980), Paper in Optimal Weatherization, pp. 60-67 (Information Dynamics, Inc., 111 Claybrook Dr., Silver Spring, MD 20902, 1981).

Key words: comfort; Community Services Administration Optimal Weatherization Demonstration; energy savings; field study; thermal comfort; weatherization retrofitting

This report discusses preliminary findings of a field study of occupant comfort impressions in weatherized houses. The research was carried out as a part of the Community Services Administration National Optimal Weatherization Demonstration. Data were obtained from 145 households in nine of the Demonstration site cities by means of questionnaire-guided interviews. Approximately 25 percent of the surveyed households lived in control houses that were not weatherized. The data covered: 1) thermostat setting practices; 2) comparative amounts of clothing worn; 3) direct report of comparative comfort; 4) comfort ratings (on a five-point scale) and temperature ratings (on the ASHRAE seven-point scale). Twenty percent of the respondents were not sure about their recollections of comfort impressions. Preliminary analysis of the remaining 109 cases (again, 25 percent control houses) gave encouraging indications that occupants of houses that get retrofitted with weatherization measures are likely to notice improvement in wintertime comfort. Further, almost one-half of the households in weatherized houses having thermostats reported less propensity to turn up thermostats during unusually cold weather after their houses were weatherized than before, a change that should result in additional energy savings beyond those directly attributable to the weatherization retrofitting.

20753. Clark, A. F.; Fujii, G.; Ranney, M. A. The thermal expansion of several materials for superconducting magnets, (Proc. Int. Conf. Magnet Technology, Karlsruhe, West Germany, Mar. 30-Apr. 5, 1981), IEEE Trans. Magn. MAG-17, No. 5, 2316-2319 (The Institute of Electrical and Electronics Engineers, Inc., 345 East 47 th St., New York, NY 10017, Sept. 1981).

Key words: low temperature; nonmetallics; physical property; superconductor composites; thermal effect; thermal expansion.

The thermal expansion of several materials used in the construction of high field superconducting magnets has been measured from $4 \mathrm{~K}$ to room temperature. The materials were a $\mathrm{NbTi}$ and two Als multifilamentary conductors and several nonmetallic composites made from linen/phenolic, fiberglass/epoxy and superconducting wire/epoxy. The conductor expansions are typical of metals and the composite expansions are highly anisotropic. Both graphic and tabular values are provided by a computer fitting of the experimental data. The importance of thermal expansion differences in critical current measurement apparatus and superconducting magnet design are discussed.

20754. Kusuda, T.; Bean, J. W. Comparison of calculated hourly cooling load and indoor temperature with measured data for a high mass building tested in an environmental chamber, (Proc. ASHRAE Semi. Ann. Meet., Chicago, IL, Jan. 1981), ASHRAE Trans. 87, Pt. $1,1232 \cdot 1242(1981)$

Key words: desert climate; energy calculation; environmental chamber; heating/cooling load; nighttime cooling.

A one-room high mass test building designed for a hot and dry desert climate was tested in the NBS environmental chamber under simulated diurnal cycles representing typical summer sol-air temperature profiles. The measured nighttime cooling requirement as well as the daytime temperature drifts were compared against the calculated values obtained from the detailed hourly simulation computer program. The purpose of the tests was to prove the 
effectiveness of nighttime cooling when the cooling efficiency is high, and subsequent use of the stored coolness in the structure to eliminate daytime mechanical cooling when the cooling efficiency is low. Although the NBSLD calculated daytime temperature-rise profiles are very close to what was measured, the calculated cooling load profile differed from the measured data. The differences are, however, attributable to the large amount of latent cooling load resulting from the release of moisture from the structural concrete.

20755. Kusuda, T.; Pierce, E. T.; Bean, J. W. Comparison of calculated hourly cooling load and attic temperature with measured data for a Houston test house, (Proc. ASHRAE Semi Ann. Meet., Chicago, IL, Jan. 1981), ASHRAE Trans. 87, Pt. 1, 1185-1199 (1981).

Key words: attic temperature; BLAST; computer simulation; DoE.2; heating/cooling load calculation; indoor temperature; NBSLD.

During the summer and autumn of 1977, NBS conducted attic ventilation experiments in three test houses in Houston, Texas. Hourly cooling loads were measured, together with indoor/outdoor climatic data, to allow precise comparison between the computer-simulated values with those measured values. In this paper, measured hourly cooling loads as well as attic temperature of one of the test houses were compared with those determined by the detailed heat balance simulation calculation used in NBSLD, the National Bureau of Standards heating/cooling load determination program. Measured cooling loads for a steady three-day period were also compared with those determined by two other public domain energy simulation programs, DoE-2 and BLAST-2.

20756. Kusuda, T.; Sud, I.; Alereza, T. Comparison of DOE-2generated residential design energy budgets with those calculated by the degree-day and bin methods, (Proc. ASHRAE Semi Ann. Meet., Chicago, IL, Jan. 1981), ASHRAE Trans. 87, Pt. 1, 491.506 (1981).

Key words: bin method; building energy performance standards (BEPS); degree-day; DOE-2.

Residential design energy budgets for the buildings energy performance standards were generated by the use of the DOE-2 computer program for four different types of structures in 10 different localities throughout the U.S. The DOE-2 calculations for these conditions were repeated by simplified procedures using the degreeday and bin methods. By modifying the basis for the degree-day data from $65^{\circ} \mathrm{F}$ to building specific value, remarkably close agreements were obtained between the DOE-2 and the degree-day results. Also, good agreements were obtained between the DOE-2 results and the ASHRAE TC4.7 method.

20757. Eitzen, D. G.; Breckenridge, F. R.; Clough, R. B.; Fuller, E. R.; Hsu, N. N.; Simmons, J. A. Fundamental developments for quantitative acoustic emission measurements, Electric Power Research Institute Interim Report No. EPRI NP-2089, Project 608-1, 213 pages (Electric Power Research Institute, Palo Alto, CA, June 1981).

Key words: acoustic emission; calibration; development; transducer; waveform.

This report describes Phase I of a research program supported jointly by the Electric Power Research Institute and the National Bureau of Standards on the development of the acoustic emission (AE) technique of surveying technical integrity.

The work under Phase I of the EPRI/NBS AE program has focused on: improved test standardization through the development of a calibration capability for AE sensors; improved sensor concepts and techniques for field and laboratory calibration; an improved basis for understanding and predicting $\mathrm{AE}$ behavior through the development of a mathematical framework for AE (transfer function formalism) through specific theoretical solutions to $\mathrm{AE}$ generation, transmission and inversion problems and the successful application of these theories to actual events in glass; an improved basis for assessing defect significance through the development of improved signal processing and inversion methods and through experimental results from $\mathrm{AE}$ in pressure vessel steels; the implementation of experiments to establish the feasibility of using causal methods, based on theoretical mechanics, to obtain source information in structural steels.

20758. Kusuda, T. Standards criteria for HVAC systems and equipment performance simulation procedures, $A S H R A E J .23,25-28$ (Oct. 1981).

Key words: Building Energy Performance Standards (BEPS); energy calculations; HVAC systems and equipment; validation.

Systems simulations have been considered an essential part of the building energy calculations used for regulations such as the Building Energy Performance Standards. Regardless of whether or not the BEPS survive political changes with the current administration, these are still critical ingredients for HVAC equipment analysis and accurate energy calculations.

20759. Mehta, K. C.; Minor, J. E.; Marshall, R. D.; Reinhold, T. A. Wind speed-damage correlation in Hurricane Frederic, Proc. ASCE Convention and Exposition, St. Louis, MO, Oct. 26-30, 1981, 14 pages (American Society of Civil Engineers, New York, NY 10017, Nov. 1981).

Key words: building performance; hurricane winds; structural damage; structures; wind; wind speed.

Damage to buildings and other structures caused by Hurricane Frederic's winds is reviewed and classified by level of engineering design and by range of maximum wind speed. Observations concerning the performance of buildings and structures are grouped according to whether the buildings and structures are fully engineered, pre-engineered, marginally engineered, or non-engineered. A major emphasis is placed on estimating wind speeds, at sites of damage observations, from anemometer data. Wind speed data is converted to equivalent maximum fastest-mile wind speeds at elevations of 10 meters over open terrain. Performance observations are further correlated with fastest-mile wind speed ranges of $70-85$ $\mathrm{mph}, 85-100 \mathrm{mph}$, and $100-110 \mathrm{mph}$. These observations indicate that significant damage did not occur until wind speeds reached or exceeded design values.

20760. Yaghiian, A. D. Augmented electric- and magnetic-field integral equations which eliminate the spurious resonances, Proc. Int. URSISymp. Electromagnetic Waves, Munich, Germany, Aug. 26-29, 1980, 121 pages, B/1-121, B/4 (1980).

Key words: augmented electric- and magnetic-field integral equations; electric-field integral equation; electromagnetic theory; magnetic-field integral equation; scattering of electromagnetic waves; spurious resonances.

In 1949 Maue derived an electric-field integral equation (EFIE) and a magnetic-field integral equation (MFIE) for the surface current density $\overline{\mathbf{K}}_{\mathrm{s}}$ excited by arbitrary harmonic fields incident upon a perfectly conducting body in free space. A major flaw in these equations is that they fail to yield a unique solution for $\overline{\mathbf{K}}_{s}$ at frequencies equal to the resonant frequencies of the corresponding interior cavity. In this paper methods are presented for augmenting the EFIE and MFIE separately to eliminate the spurious resonant solutions from the exterior region without sacrificing the basic simplicity, solution capability and pure electric- and magnetic-field character of these two elegant equations. Included is an explanation of why the original EFIE and MFIE are deficient in the exterior region. The augmentation procedure transforms the EFIE from an integral equation of the first kind to an integral equation of the second kind which, like the MFIE, is amenable to a more stable numerical solution than the original EFIE. Finally, the problem of scattering from a perfectly conducting cube is solved numerically using the original and augmented equations, in order to demonstrate that the augmented equations indeed eliminate the spurious resonance at the cost of only an insignificant increase in computer programming, run time, and central memory requirements.

20761. Yaghjian, A. D. Augmented electric- and magnetic-field equations, Radio Sci. 16, No. 6, 987-1001 (Nov.-Dec. 1981).

Key words: augmented electric- and magnetic-field integral 
equations; electric-field integral equation; electromagnetic theory; magnetic-field integral equation; scattering of electromagnetic waves; spurious resonances.

In 1949 Maue derived an electric-field integral equation (EFIE) and a magnetic-field integral equation (MFIE) for the surface current density $K_{s}$ excited by arbitrary harmonic fields incident upon a perfectly conducting body in free space. A major flaw in these equations is that they fail to yield a unique solution for $K_{\mathrm{s}}$ at frequencies equal to the resonant frequencies of the corresponding interior cavity. In this paper methods are presented for augmenting the EFIE and MFIE separately to eliminate the spurious resonant solutions from the exterior region without sacrificing the basic simplicity, solution capability and pure electric- and magnetic-field character of these two elegant equations. Included is an explanation of why the original EFIE and MFIE are deficient in the exterior region. The augmentation procedure transforms the EFIE from an integral equation of the first kind to an integral equation of the second kind which, like the MFIE, is amenable to a more stable numerical solution than the original EFIE. Finally, the problem of scattering from a perfectly conducting cube is solved numerically using the original and augmented equations, in order to demonstrate that the augmented equations indeed eliminate the spurious resonance at the cost of only an insignificant increase in computer programming, run time, and central memory requirements.

20762. Berger, $\mathrm{H}$. An overview: New ideas in nondestructive evaluation,

(Proc. Meet. Rubber Division, American Chemical Society, Minneapolis, MN, June 2-5, 1981), Rubber Chem. Technol. 54, No. 5, 996-1002 (Nov./Dec. 1981).

Key words: acoustic emission; eddy currents; liquid penetrants; magnetic particles; microwaves; nondestructive evaluation; radiography; tire inspection; ultrasonics; visual-optical.

Nondestructive evaluation (NDE) normally brings to mind six major methods in use in industrial quality control, visual/optical, $x$-ray, ultrasonic, penetrant, magnetic and eddy current techniques. While it is true that most NDE involves these basic methods, it is also true that major changes are taking place in terms of modifications of these standard methods and in terms of new inspection approaches. The discussion includes descriptions of modifications such as ultrasonic imaging, $x$-ray tomography, pulsed eddy current techniques and signal processing. In addition, novel NDE approaches that appear to offer advantages of tire inspection are discussed; these include acoustic emission, pulsed eddy currents, microwaves and vibrothermography.

20763. Messman, J. D.; Rains, T. C. Determination of tetraalkyllead compounds in gasoline by liquid chromatography - atomic absorption spectrometry, Anal. Chem. 53, No. 11, $1632-1636$ (Sept. 1981).

Key words: atomic absorption spectrometry; gasoline analysis; high performance liquid chromatography; metal-selective detector; metal speciation; tetraalkyllead speciation.

A liquid chromatography-atomic absorption spectrometry (LC AAS) hybrid analytical technique is presented for metal speciation measurements on complex liquid samples. The versatility and inherent metal selectivity of the technique are illustrated by the rapid determination of five tetraalkyllead compounds in commercial gasoline. Separation of the individual tetraalkyllead species is achieved by reversed-phase liquid chromatography using an acetonitrile/water mobile phase. The effluent from the liquid chromatograph is introduced directly into the aspiration uptake capillary of the nebulizer of an air/acetylene flame atomic absorption spectrometer. Spectral interferences due to coeluting hydrocarbon matrix constituents were not observed at the $283.3 \mathrm{~nm}$ resonance line of lead used for analysis. Detection limits of this LC.AAS hybrid analytical technique, based on a $20-\mu$ l injection, are approximately 10 $\mathrm{ng} \mathrm{Pb}$ for each tetraalkyllead compound.

20764. Simiu, E.; Shaver, J. R.; Filliben, J. J. Extreme wind speeds and structural failure risks, (Proc. Conf. Climate and Risk, Arlington, VA, May 27-29, 1980), Paper MTR-80W322-01 in Climate and Risk, L. S. Pocinki and R. S. Greeley, eds., 1, 2-86-2-100 (The MITRE

\section{Corporation, McLean, VA 22102, Nov. 1980).}

Key words: building (codes); climatology; hurricanes; reliability; risk; structural engineering.

A first objective of the paper is to describe recent NBS research results in the field of extreme wind climatology. These results include the finding that at most locations in the U.S. extreme wind speeds are described by probabilistic models with considerably shorter tails than the Extreme Value Type I distribution. Estimates of hurricane wind speeds along the Gulf and East coasts are presented, based on Monte Carlo simulation techniques used in conjunction with statistical data on the climatological characteristics of hurricanes. Also presented in the paper are estimates of member reliabilities that take into account the various uncertainties concerning the behavior of the member during its anticipated life. The dependence of such estimates upon the assumed probability distribution of the extreme wind speeds is discussed, and it is shown that current reliability estimates based on an Extreme Value Type I model of the extreme wind speeds appear to be unduly pessimistic.

20765. Lewis, R. Spherical scanning data processing: An algorithm for halving the data processing effort when the radiation into the back hemisphere is negligible, (Proc. AP-S Int. Symp. Antennas and Propagation, Los Angeles, CA, June 16-19, 1981), 1981 Int. Symp. Digest, $81 \mathrm{CH}$ 1672-5, 1, 247-249 (Institute of Electronics and Electrical Engineers, 455 Hoes Lane, Piscataway, NJ 08554, 1981).

Key words: antennas; data processing; electromagnetic; spherical scanning.

An algorithm has been developed, for probe-corrected sphericalscanning data processing, that halves the computational effort involved when the field is negligible in the back hemisphere of the antenna under test. As the algorithm is described, it is compared to the algorithm for data processing over the entire sphere, thus demonstrating that the computational effort involved is just half of that required by full-sphere data processing.

20766. Radebaugh, R.; Linenberger, D.; Voth, R. O. Design of a regenerator test apparatus for temperatures down to $4 \mathrm{~K}, A F W A L$ TR-81-0350, 60 pages (For information contact the Flight Dynamics Laboratory, Air Force Wright Aeronautical Laboratories, Air Force Systems Command, Wright-Patterson Air Force Base, OH 45433, June 1981).

Key words: cryogenics; Gifford-McMahon cryocoolers; heat capacity; heat transfer; helium; refrigerators; regenerators; Stirling cryocoolers; thermal conductance; Vuilleumier cryocoolers.

The regenerator ineffectiveness is usually the dominant heat loss term in regenerative-cycle refrigerators which operate below $15 \mathrm{~K}$, but systematic measurements of regenerator ineffectiveness have never been done in this temperature range. This report introduces the proper definition of ineffectiveness, which is valid for non-ideal gases and systems where a pressure wave exists. Previous methods used and proposed for the measurement of regenerator ineffectiveness at cryogenic temperatures are reviewed and their limitations discussed. A new method, utilizing a variable expansion space, is presented which for the first time will allow measurements at all phase angles between the mass flow rate and the pressure wave. Temperatures emphasized are those between 4 and $35 \mathrm{~K}$.

20767. Diller, D. E.; Saber, J. M. Measurements of the viscosity of compressed gaseous and liquid ethane, Physica 108A, 143-152 (1981).

Key words: density dependence; gaseous and liquid ethane isothermal measurements; quartz crystal viscometer; saturated liquid; shear viscosity coefficient

The shear viscosity coefficient of compressed gaseous and liquid ethane has been measured at temperatures between 95 and $320 \mathrm{~K}$ and at pressures up to $30 \mathrm{MPa}$ (4350 psia) with a torsionally oscillating quartz crystal viscometer. The estimated precision and accuracy of the measurements are about 1 percent and 2 percent respectively. The 
measurements have been compared with an equation previously proposed for calculating the viscosity of gaseous and liquid ethane. Differences between the equation and the measurements reported here are less than 6 percent. The largest differences are found at low temperatures and high pressures, and along a supercritical isotherm at $320 \mathrm{~K}\left(\mathrm{~T} \approx 1.05 \mathrm{~T}_{\mathrm{c}}\right)$

20768. Ely, J. F.; Hanley, H. J. M. Prediction of transport properties. 1. Viscosity of fluids and mixtures, Ind. Eng. Chem. Fundam. 20, No. 4, 323-332 (Nov. 1981).

Key words: data evaluation; extended corresponding states; fluids; mixtures; one fluid model; prediction; Van der Waals theory; viscosity.

A model for the prediction of the viscosity of non-polar fluid mixtures over the entire range of PVT states is presented. The model is an extension of an earlier version (Hanley, 1976) to molecular weights which roughly correspond to that of $\mathrm{C}_{20}$. The proposed model is based on an extended corresponding states principle and requires only critical constants and Pitzer's acentric factor for each component as input. Extensive comparisons with experimental data for pure fluids and binary mixtures are presented. The average deviation between experiment and prediction is 8 percent for pure species and 7 percent for mixtures.

20769. Hands, B. A.; Arp, V. D. A correlation of thermal conductivity data for helium, Cryogenics 21, No. 12, 697.703 (Dec. 1981).

Key words: correlation; critical region; helium; thermal conductivity.

A correlation for the thermal conductivity of helium has been developed which covers the temperature range from temperatures just above the lambda line to $830 \mathrm{~K}$, and densities up to about 160 $\mathrm{kgm}^{-3}$. The data used incorporate some recent experimental results which cover the temperature range from $4 \mathrm{~K}$ to $20 \mathrm{~K}$ including the critical region. The correlation gives an equation which is probably correct to better than $\pm 6 \%$.

20770. Siegwarth, J. D. Gage installation can trim level-measurement, Oil Gas J. 79, No. 49, 142-144, 149, 152 (Dec. 1981).

Key words: custody transfer; gaging; liquid level; LNG tanks.

The systematic errors introduced into level measurements by the gage-mounting method and the tank environment are analyzed for the cable-type gage with emphasis on LNG level gaging. The cable-type gage consists of a movable surface sensor suspended by a cable, tape, or wire. Two gage-installation methods that eliminate most of the systematic errors are described. Though this study deals primarily with LNG gaging, it applies as well to the gaging of any liquid.

20771. Voth, R. O. Maximum practical efficiency of helium temperature refrigerators, Cryogenics 21, No. 11, $635-640$ (Nov. 1981).

Key words: component efficiency; cryogenics; efficiency; helium; maximum refrigerator efficiency; perfect gas analysis; refrigerator.

An ideal refrigerator using a perfect gas working fluid is defined which gives the efficiency of a refrigerator as a function of compressor and expander efficiency, heat exchanger temperature difference, and heat exchanger pressure drop. Although not suited to detailed hardware design, this approach clearly relates the overall cycle efficiency to component efficiencies. In contrast, computer studies of specific cycles using real fluid properties are usually such that the details tend to overshadow major trends. The results of the study show that in an efficient cycle the major losses are in the compressor and the cold end expansion device. For current compressor and expander efficiencies the maximum practical helium temperature refrigerator efficiency is about 37 percent of Carnot.

20772. Reed, R. P.; Tobler, R. L.; Elmer, J. W.; McHenry, H. I.; Yushchenko, K. A.; Paton, E. O. Strength and toughness of USSR Fe-20Cr-16Ni-6Mn-0.2N weldments at cryogenic temperatures, Paper in Proceedings of the Eighth International Cryogenic Engineering Conference, Genova, Italy, June 3-6, 1980, pp. $797-801$ (1980).

Key words: austenitic stainless steel; fracture toughness; low temperature; tensile properties.

The structural reinforcement of large superconducting magnets normally requires the use of a very strong tough alloy at liquid helium temperature. The base and weldment material of a Soviet austenitic stainless steel, a nitrogen-strengthened $\mathrm{Fe}-20 \mathrm{Cr}-16 \mathrm{Ni}-6 \mathrm{Mn}$ alloy, is evaluated and compared with conventional AISI 300 series stainless steels (Fe-18Cr-8Ni). Tensile and fracture toughness measurements were made on base, heat-affected zone and weld material. Both tungsten inert gas and submerged arc weld processes, using $20 \mathrm{~mm}$ thick plate, were used. Magnetic measurements were made after deformation and tensile flow strengths were compared with the temperature dependence of stable austenitic stainless steels to assess the occurrence and influence of martensitic phase transformations on low temperature properties. J.integral fracture toughness was measured and the base and weldment properties compared.

20773. Ledbetter, H. M. Predicted single-crystal elastic constants of stainless-steel 316, Br. J. Non Destr. Test. 23, No. 6, 286-287 (Nov. 1981).

Key words: acoustic waves; elastic anisotropy; elastic constants; Kroner method; sound velocities; ultrasonic inspection; ultrasonic waves.

For stainless-steel 316, the author gives an improved prediction of the three $\mathrm{C}_{\mathrm{ij}}$ elastic-stiffness constants, which determine the optimum direction for acoustic beams used to locate and size flaws. The predictive method applies to all cubic-crystal-structure anisotropic materials.

20774. Dickens, B. Pressure as an important variable in thermogravimetric studies of polymer degradations, Paper in Proceedings of the Second European Symposium on Thermal Analysis, University of Alberdeen, United Kingdom, Sept. 1-4, 1981, 219.222 (Heydon \& Son, Hillview Gardens, London, UK, 1981).

Key words: activation energy; bubbles; polymer degradation products; pressure; thermal degradation; thermogravimetry.

Application of the recently developed method of factor-jump thermogravimetry to the study of polymer degradation has provided monitoring of the apparent activation energy during the degradation of a single sample without the necessity of knowing the initial or final sample weight (except to relate the activation energy to extent of reaction).

Investigation of the degradation of several polymers in vacuo and under an atmosphere of $\mathrm{N}$, has shown that bubbles formed in the polymers in vacuum are supressed under $\mathrm{N}_{2}$. The phenomenon is related to that of normal boiling.

The volatilization of the degradation products is discussed, and the contributions of their latent heats to the observed activation energy is considered. Results will be most easily interpretated for unzipping polymers degrading at low to medium temperatures where the volatilization of molecules much larger than the monomer will not be important. For polymers degrading by random scission and by unzipping at higher temperatures (because of the stability of the polymer), volatilization of large molecules seriously effects the observed activation energy through the contribution of the latent heats. Further, they require the formation of additional surface area to allow their evaporation. This is the origin of the formation of the bubbles. Bursting bubbles make the sample weight intolerably unsteady and introduce great difficulties into the determination of the activation energy of degradation. 


\section{LISTING OF NBS PAPERS BY MAJOR SUBJECT AREAS}

This section provides a listing of papers organized by primary subject matter as follows:

Acoustics and Sound

Analytical Chemistry

Atomic and Molecular Studies

Building Technology

Computer Science and Technology

Consumer Information and Protection

Electromagnetic Metrology

Electronic Technology

Energy Conservation and Production

Engineering, Product and Information Standards

Environmental Studies: Pollution Measurement

Failure Analysis

Fire Research

Fluids: Liquids, Gases and Plasmas

General Theoretical Chemistry and Physics

Health and Safety

Instrumentation and Experimental Methods

Lasers and Their Applications

Low Temperature Science and Engineering

Mathematical and Statistical Methods

Measurement Science and Technology:

Policy and State-of-the-Art Surveys

Measurement Science and Technology:

Physical Standards and Fundamental Constants

Mechanics: Design, Testing and Measurement

Metrology: Physical Measurements
Nuclear Physics and Radiation Technology

Operations Analysis and Applications

Processing and Performance of Materials

Properties of Materials: Electronic, Magnetic and Optical

Properties of Materials: Structural and Mechanical

Properties of Materials: Thermodynamic and Transport

Standard Reference Data

Standard Reference Materials

Surfaces and Interfaces

Thermodynamics and Chemical Kinetics

Technology Incentives

Other Subjects of General Interest

It permits users of this catalog to scan the Bureau's output by major subject category. The user should bear in mind that a paper is listed once by major subject even though it might well contain other secondary subject matters of interest. The keyword index permits the reader to determine the overall context of a paper, and provides an excellent secondary reference source.

The categories currently in use for classifying NBS publications are listed below and are followed by a listing of each paper by category. Full citations (including key-words and abstracts) will be found under the appropriate publication series, which is included in the paper title. Also of use will be the key-word index (mentioned above) and the author index. 


\section{Acoustics and Sound}

Younglove, B. A. Velocity of sound in liquid propane. J. Res. Nat. Bur. Stand. (U.S.). 86(2): 165.170; 1981 March-April.

NBS-GCR-80-250. Sharp, B. H.; Kasper, P. K.; Montroll, M. L., (NBS contact: S. Webber). Sound transmission through building structures-Review and recommendations for research. $1980 \mathrm{July}$. 144 p. Available from: NTIS; PB 81-187072.

20050. Molino, J. A.; Zerdy, G. A.; Lerner, N. D.; Harwood, D. L. Use of the "acoustic menu" in assessing human response to audible (corona) noise from electric transmission lines, J. Acoust. Soc. Am. 66, No. 5, 1435-1445 (Nov. 1979).

20190. Bartel, T. W. Effect of absorber geometry on apparent absorption coefficients as measured in a reverberation chamber, $J$. Acoust. Soc. Am. 69, No. 4, 1065-1074 (Apr. 1981).

20197. Hsu, N. N.; Breckenridge, F. R. Characterization and calibration of acoustic emission sensors, (Proc. Int. Conf. Acoustic Emission, Anaheim, CA, Sept. 10-13, 1979), Paper in Materials Evaluation 39, No. 1, 60.68 (ASNT, Columbus, OH, Jan. 1981).

20489. Breckenridge, F. R.; Greenspan, M. Surface-wave displacement: Absolute measurements using a capacitive transducer, J. Acoust. Soc. Am. 69, No. 4, 1177.1185 (Apr. 1981).

\section{Analytical Chemistry}

DeVoe, H.; Miller, M. M.; Wasik, S. P. Generator columns and high pressure liquid chromatography for determining aqueous solubilities and octanol-water partition coefficients of hydrophobic substances. $J$. Res. Nat. Bur. Stand. (U.S.). 86(4): 361-366; 1981 July-August.

SP604. Heinrich, K. F. J.; Newbury, D. E.; Myklebust, R. L.; Fiori, C. E., eds. Energy dispersive $x$-ray spectrometry. Proceedings of a Workshop on Energy Dispersive X-Ray Spectrometry held at the National Bureau of Standards; 1979 April 23-25; Gaithersburg, MD. Nat. Bur. Stand. (U.S.) Spec. Publ. 604; 1981 June. 441 p. SN003. 003.02333.7.

SP604: 1981 June. 1-4. Heinrich, K. F. J. The development of energy-dispersive $x$-ray analysis.

SP604: 1981 June. 5-34. Fink, R. W. Properties of silicon and germanium semiconductor detectors for $\mathrm{x}$-ray spectrometry.

SP604: 1981 June. 35-44. Barbi, N. C.; Lister, D. B. A comparison of silicon and germanium $\mathrm{x}$-ray detectors.

SP604: 1981 June. 45-59. Dabrowski, A. J.; Singh, M.; Huth, G. C.; Iwanczyk., J. S. Room temperature energy dispersive $\mathrm{x}$-ray spectrometry with mercuric iodide detectors.

SP604: 1981 June. 61-70. Walter, F. J.; Stone, R.; Blackburn, D. H.; Pella, P. A. Standard techniques for measuring window absorption and other efficiency losses in semiconductor $x$-ray energy spectrometers.

SP604: 1981 June. 71-95. Russ, J. C.; Sandborg, A. O. Use of windowless detectors for energy dispersive light element $x$-ray analysis.

SP604: 1981 June. 97-126. Musket, R. G. Properties and applications of windowless $\mathrm{Si}(\mathrm{Li})$ detectors.

SP604: 1981 June. 127-139. Statham, P. J. The efficiency of Si(Li) detectors at very low photon energies.

SP604: 1981 June. 141-164. Statham, P. J. Electronic techniques for pulse-processing with solid-state $\mathrm{x}$-ray detectors.

SP604: 1981 June. 165.175. Lifshin, E.; Hayashi, S. R Understanding multichannel analyzers.

SP604: 1981 June. 177-191. Ryder, P. L. Statistical considerations of detectability limits and deconvolution in energy dispersive $x$-ray spectrometry.

SP604: 1981 June. 193-231. Schamber, F. H. Curve fitting techniques and their application to the analysis of energy dispersive spectra.

SP604: 1981 June. 233-272. Fiori, C. E.; Myklebust, R. L.; Gorlen, K. Sequential simplex: A procedure for resolving spectral interference in energy dispersive $\mathrm{x}$-ray spectrometry.

SP604: 1981 June. 273.296. McCarthy, J. J.; Schamber, F. H. Leastsquares fit with digital filter: A status report.

SP604: 1981 June. 297-313. Russ, J. C. Multiple least squares fitting for spectrum deconvolution.

SP604: 1981 June. 315.339. Fiori, C. E.; Newbury, D. E. Myklebust, R. L. Artifacts observed in energy dispersive $\mathbf{x} \cdot \mathbf{r a y}$ spectrometry in electron beam instruments-A cautionary guide.

SP604: 1981 June. 341.349. Williams, D. B.; Goldstein, J. I. Artifacts encountered in energy dispersive $x$-ray spectrometry in the analytical electron microscope.
SP604: 1981 June. 351-364. Zaluzec, N. J. Uncollimated fluorescing radiation in an analytical electron microscope (100-1000 keV): Sources and solutions.

SP604: 1981 June. 365-379. Myklebust, R. L.; Fiori, C. E.; Heinrich, K. F. J. Spectral processing techniques in a quantitative energy dispersive $\mathrm{x}$-ray microanalysis procedure (FRAME C).

SP604: 1981 June. 381-389. Brown, J. D. The effect of electron incidence angle on quantitative electron probe microanalysis.

SP604: 1981 June. 391.415. Short, M. A.; Bonfiglio, S. Energy and wavelength dispersive $x$-ray fluorescence analysis of heavy elements using radioactive, secondary fluorescence and $x$-ray tube sources, and with germanium, silicon, and scintillation detectors.

SP604: 1981 June. 417.439. Fiori, C. E.; Swyt, C. R. Energy dispersive detectors-A bibliography (1981).

U.S. Patent 4,281,246. White, E.; Hertz, H.; Christensen, R. Continuous-flow solution concentrator and liquid chromatograph/mass spectrometer interface and methods for using both. 28 July 1981.7 p.

19982. Carpenter, B. S.; Pilione, L. J. Determination of lithium and lithium.6 in solutions by the nuclear track technique, Anal. Chem. $\mathbf{5 2}$, 2452-2454 (1980).

20097. Christensen, R. G.; Hertz, H. S.; Meiselman, S.; White, E Liquid chromatograph/mass spectrometer interface with continuous sample preconcentration, Anal. Chem. 53, No. 2, 171.174 (Feb. 1981).

20158. Bartle, K. D.; Lee, M. L.; Wise, S. A. Factors affecting the retention of polycyclic aromatic hydrocarbons in gas chromatography, Chromatographia 14, No. 2, 69.72 (Feb. 1981).

20161. Guenther, F. R.; Parris, R. M.; Chesler, S. N.; Hilpert, L. R. Determination of phenolic compounds in alternate fuel matrices, $J$. Chromatog. 207, 256.261 (1981)

20200. Kingston, H.; Pella, P. A. Preconcentration of trace metals in environmental and biological samples by cation exchange resin filters for x-ray spectrometry, Anal. Chem. 53, No. 2, 223.227 (Feb. 1981).

20247. Schoonover, R. M.; Davis, R. S. Quick and accurate density determination of laboratory weights, (Proc. 8th Conf. IMEKO Technical Committee TC3 on Measurement of Force and Mass, Krakow, Poland, Sept. 9.11, 1980), Paper in Weighing Technology. pp. II23-II27 (Druk, Zaklad Poligraficzny Wydawnictwa SIGMA Warszawa, Poland, 1980).

20268. Cummings, A. L.; Layer, H. P.; Hocken, R. J. Lasers and analytical polarimetry, Chapter 15 in Lasers in Chemical Analysis, G. M. Hieftje, J. C. Travis, and F. E. Lytle, eds., pp. 291.302 (The Humana Press, Clifton, NJ, 1981)

20285. Newbury, D. E. Microanalysis of individual layered particles by secondary ion mass spectrometry, EPA-600/7-80-122, 68 pages (United States Environmental Agency, Office of Environmenta Engineering and Technology, Washington, DC, July 1981). (Available from the National Technical Information Service, Springfield, VA 22161, July 1981).

20370. Wang, F. W.; Zimm, B. H. Novel cell for light scattering from solutions, Biopolymers 20,1333.1335 (1981).

20387. Kratochvil, B. G.; Taylor, J. K. Sampling for chemical analysis, Anal. Chem. 53, No. 8, 924A-938A (July 1981).

20408. Senich, G. A. Measurements of diffusion in polymers by inverse gas chromatography, (Proc. ACS Division of Polymer Chemistry, New York, NY, Sept. 24-28, 1981), Am. Chem. Soc. Polymer Preprint 22, No. 2, 343-344 (Aug. 1981).

20444. Velapoldi, R. A. Color test reagent/kits for preliminary identification of drugs of abuse, NIJ Standard-0605.00, 13 pages (Available by purchase as SN027.000-01116-1 from the Superintendent of Documents, U.S. Government Printing Office, Washington, DC 20402, July 1981).

20446. Kaplan, A. E.; Weiss, E. R.; Byrne, S. T.; El-Torkey, N. M.; Margolis, S. A. Purified reduced nicotinamide adenine dinucleotide: Responses to lactate dehydrogenase isozymes from three cell sources, Science 212, 553.555 (May 1, 1981).

20453. Scheeline, A.; Norris, J. A.; Travis, J. C.; DeVoe, J. R. Walters, J. P. Particulates formed by a stabilized high voltage spark discharge, Spectrochim. Acta 36B, No. 4, 373.383 (1981).

20467. Popp, R. K.; Frantz, J. D.; Vogel, G. L. An electrode technique for measurement of chloride concentration in microsamples, $\mathrm{Am}$. Mineral. 65, 393.395 (1980).

20522. Durst, R. A. Views on the current status of the pH scale, (Proc. 5th Meet. IFCC Expert Panel on $\mathrm{pH}$ and Blood Gases, Copenhagen, Denmark, June 16.18, 1980), Paper in Blood pH, 
Carbon Dioxide, Oxygen, and Calcium-ion, O. Siggaard-Andersen, ed., pp. 11 -22 (Private Press, Copenhagen, Denmark, 1981).

20523. Haller, W.; Gschwender, H. G.; Peters, K. R. Application of a general equation for controlled pore glass permeation chromatography to an aggregating, spherical virus, J. Chromatogr. 211, $53-59$ (1981).

20580. Durst, R. A.; Bates, R. G. Hydrogen-ion activity, Paper in Encyclopedia Chemical Technology, 3d Edition, 13, 1-12 (1981).

20585. Konash, P. L.; Wise, S. A.; May, W. E. Selective quenchofluorometric detection of fluoranthenic polycyclic aromatic hydrocarbons in high-performance liquid chromatography, J. Liq. Chromatog. 4, No. 8, 1339.1349 (1981).

20603. Traub, R. D.; Rains, T. C.; Garruto, R. M.; Gajdusek, D. C.; Gibbs, C. J., Jr. Brain destruction alone does not elevate brain aluminum, Neurology 31, No. 8, 986-990 (Aug. 1981).

20604. MacCrehan, W. A. Differential pulse detection in liquid chromatography and its application to the measurement of organometal cations, Anal. Chem. 53, No. 1, 74.77 (Jan. 1981).

20681. Jones, F. E. Determination of water in solids by automatic Karl Fischer titration, Anal. Chem. 53, No. 12, 1955-1957 (Oct. 1981).

20682. Jones, F. E.; Brickenkamp, C. S. Automatic Karl Fischer titration of moisture in grain, J. Assoc. Off. Anal. Chem. 64, No. 6, 1277-1283 (1981).

20698. Rosman, K. J. R.; Barnes, I. L.; Moore, L. J.; Gramlich, J. W. Isotope composition of $\mathrm{Cd}, \mathrm{Ca}$ and $\mathrm{Mg}$ in the Brownfield chondrite, Geochem. J. 14, 269.277 (1980).

20709. Wise, S. A.; Bonnett, W. J.; Guenther, F. R.; May, W. E. A relationship between reversed-phase $C_{18}$ liquid chromatographic retention and the shape of polycyclic aromatic hydrocarbons, $J$. Chromalogr. Sci. 19, 457-465 (Sept. 1981).

20714. Covington, A. K.; Goldberg, R. N.; Sarbar, M. Computer simulation of titration curves with application to aqueous carbonate solutions, Anal. Chim. Acta 130, 103-109 (1981).

20728. MacCrehan, W. A.; Durst, R. A. Dual-electrode, liquid chromatographic detector for the determination of analytes with high redox potential, Anal. Chem. 53, 1700-1704 (1981).

20729. Misra, D. N.; Bowen, R. L. Interaction of zinc ions with hydroxylapatite, Paper in Adsorption from Aqueous Solutions, P. H. Tewari, pp. 179.192 (Plenum Publ. Corp., New York, NY, Oct. 1981).

20763. Messman, J. D.; Rains, T. C. Determination of tetraalkyllead compounds in gasoline by liquid chromatography - atomic absorption spectrometry, Anal. Chem. 53, No. 11, 1632.1636 (Sept. 1981).

\section{Atomic and Molecular Studies}

Roth, C. The configurations $3 d^{n} 4 p+3 d^{n-1} 4 s 4 p+3 d^{n-2} 4 s^{2} 4 p$ in the first spectra of the iron group. J. Res. Nat. Bur. Stand. (U.S.). 86(1): 33 77; 1981 January-February.

Brown, R. L. A method of calculating tunneling corrections for Eckart potential barriers. J. Res. Nat. Bur. Stand. (U.S.). 86(4): 357-359; 1981 July-August.

Lin, C. C. Turbulence, plasma containment, and galaxies. J. Res. Nat. Bur. Stand. (U.S.). 86(6): 557-563; 1981 November-December.

Brown, R. L. Rate constants for $\mathbf{H}$-atom transfer reactions by the BEBO method. J. Res. Nat. Bur. Stand. (U.S.). 86(6): 605.654; 1981 November-December.

SP593. Gallagher, J. W.; Van Blerkom, J.; Beaty, E. C.; Rumble, J.
R., Jr. Data index for energy transfer collisions of atoms and molecules-1970-1979. Nat. Bur. Stand. (U.S.) Spec. Publ. 593; 1981 April. 346 p. SN003-003-02315-9.

NBSIR 80-2175. Cohen, E. R.; Birnbaum, G. Analysis of the shape of the far-infrared spectra of $\mathbf{H}_{2}-\mathbf{H}_{2}$ and $\mathbf{H}_{2}-\mathrm{He}$ collisions. 1981 A pril. 27 p. Available from: NTIS; PB 81-210395.

19964. Ackerson, B. J.; Hanley, H. J. M. Rayleigh scattering from a methane-ethane mixture, $J$. Chem. Phys. 73, No. 8, 3568-3572 (Oct. $15,1980)$.

19966. Ayres, T. R.; Linsky, J. L. Outer atmospheres of cool stars. V. IUE observations of Capella: The rotation-activity connection, Astrophys. J. 241, No. 1, 279-299 (Oct. 1, 1980).

19967. Ben-Reuven, A. Theory of two-atom coherence in gases. II. Continuous-wave spectra, Phys. Rev. A 22, No. 6, 2585-2597 (Dec. 1980).

19968. Ben-Reuven, A. Theory of two-atom coherence in gases. I. Master equations, Phys. Rev. A 22, No. 6, 2572.2584 (Dec. 1980).

19969. Brayman, H. C.; Fischell, D. R.; Cool, T. A. Spectroscopic studies of the products of reactions of yttrium and scandium atoms with halogen molecules. I. The origin of chemiluminescence, $J$. Chem. Phys. 73, No. 9, 4247.4259 (Nov. 1, 1980).

19977. Fuller, E. G. Giant resonances, Article in Encyclopedia of Physics, R. G. Lerner and G. L. Trigg, eds., pp. 882-884 (AddisonWesley Publ. Co., Inc., Advanced Book Program, Reading, MA, 1981).

19978. Fischell, D. R.; Brayman, H. C.; Cool, T. A. Spectroscopic studies of the products of reactions of yttrium and scandium atoms with halogen molecules. II. Laser induced fluorescence from yttrium and scandium monohalides, J. Chem. Phys. 73, No. 9, 4260-4272 (Nov. 1, 1980).

19986. Jacox, M. E. The reaction of $F$ atoms with $\mathrm{O}_{2}$ in an argon matrix, J. Mol. Spectrosc. 84, 74-88 (1980).

19987. Hollis, J. M.; Snyder, L. E.; Lovas, F. J.; Ulich, B. L. A radio search for interstellar phosphorus compounds, Astrophys. J. 241, 158. 160 (Oct. 1, 1980).

19995. Jones, P. L.; Mead, R. D.; Kohler, B. E.; Rosner, S. D.; Lineberger, W. C. Photodetachment spectroscopy of $C_{2}^{-}$ autodetaching resonances, $J$. Chem. Phys. 73, No. 9, 4419.4432 (Nov. $1,1980)$.

19996. Celotta, R. J.; Pierce, D. T. Sources of polarized electrons, $A d v$. At. Mol. Phys. 16, 101-157 (1980).

20001. Simon, T.; Linsky, J. L. IUE ultraviolet spectra and chromospheric models of HR 1099 and UX Arietis, Astrophys. J. 241, No. 2, 759.773 (Oct. 15, 1980).

20002. Wyart, J. F.; Kaufman, V.; Sugar, J. Analysis of the spectrum of four-times-ionized uranium (UV), Phys. Scr. 22, 389-396 (1980).

20004. Baughcum, S. L.; Leone, S. R. Photofragmentation infrared emission studies of vibrationally excited free radicals $\mathrm{CH}_{3}$ and $\mathrm{CH}_{2} \mathrm{I}, J$. Chem. Phys. 72, No. 12, 6531-6545 (June 15, 1980).

20005. Schäfer, L.; Sellers, H. L.; Lovas, F. J.; Suenram, R. D Theory versus experiment: The case of glycine, J. Am. Chem. Soc. 102, 6566-6568 (1980).

20036. Jacox, M. E. The reaction of $F$ atoms with $\mathrm{C}_{2} \mathrm{H}_{2}$ vibrational spectrum of the $t$-2-fluorovinyl free radical isolated in solid argon, Chem. Phys. 53, 307.322 (1980).

20039. Mies, F. H.; Aryeh, Y. B. Kinetics and spectroscopy of nearresonant optical pumping in intense fields, $J$. Chem. Phys. 74, No. 1, 53-67 (Jan. 1, 1981).

20042. Okabe, H.; Kawasaki, M.; Tanaka, Y. The photodissociation of $\mathrm{CH}_{2} \mathrm{I}_{2}$ : Production of electronically excited $\mathrm{I}_{2}, J$. Chem. Phys. 73, No. 12, 6162.6166 (Dec. 15, 1980).

20045. Pradhan, A. K.; Norcross, D. W.; Hummer, D. G. Cross sections and excitation rates for electron collisions with heliumlike ions, Phys. Rev. A 23, No. 2, 619-631 (Feb. 1981).

20047. Muller, C. H. III; Phelps, A. V. Low-current electric discharges in $\mathrm{H}_{2}-\mathrm{He}$ mixtures, $J$. Appl. Phys. 51, No. 12, 6141-6148 (Dec. 1980).

20049. Mies, F. H. A scattering theory of diatomic molecules general formalism using the channel state representation, Mol. Phys. 41, No. 5, 953-972 (1980).

20051. Parr, A. C.; Jason, A. J.; Stockbauer, R.; McCulloh, K. E. 
Photoionization and threshold photoelectron-photoion coincidence study of propyne from onset to $20 \mathrm{eV}$, Int. J. Mass Spectrom. Ion Phys. 30, 319.330 (1979).

20052. Codling, K.; West, J. B.; Parr, A. C.; Dehmer, J. L.; Stockbauer, $R$. L. Measurement of $\beta$ values and branching ratios in the region of the $3 s 3 p^{6} 4 p{ }^{1} P^{\circ}{ }_{1}$ resonance in $A r$ and the $5 s 5 p^{6} 6 p{ }^{1} P^{\circ}{ }_{1}$ resonance in Xe, J. Phys. B: Atom. Molec. Phys., Lett. to Ed. 13, L693-L697 (1980).

20053. Sams, R. L.; Olson, W. B. Analysis of the high-resolution infrared spectrum of the $\nu_{2}$ bending mode of $\mathrm{HOCl}$ at $1238 \mathrm{~cm}^{-1}, J$. Mol. Spectrosc. 84, 113.123 (1980).

20057. Stencel, R. E.; Mullan, D. J.; Linsky, J. L.; Basri, G. S.; Worden, S. P. The outer atmospheres of cool stars. VII. High resolution, absolute flux profiles of the $\mathrm{Mg}$ II $h$ and $k$ lines in stars of spectral types F8 to M5, Astrophys. J. Suppl. Ser. 44, 383.402 (Nov. 1980).

20060. Mies, F. H. A scattering theory of diatomic molecules expansion in adiabatic electronic-rotational states, Mol. Phys. 41, No. 5, 973.986 (1980).

20061. Geltman, S. Approximate response of a two-level system to intense multimode radiation, Phys. Lett. 81A, No. 1, 27-30 (Jan. 5, 1981).

20076. Metcalf, H.; Phillips, W. Time-resolved subnatural-width spectroscopy, Opt. Lett. 5, No. 12, 540-542 (Dec. 1980).

20077. Hollis, J. M.; Snyder, L. E.; Suenram, R. D.; Lovas, F. J. A search for the lowest-energy conformer of interstellar glycine, Astrophys. J. 241, 1001-1006 (Nov. 1, 1980).

20080. Simmons, J. D.; Maki, A. G.; Hougen, J. T. Spectroscopic analysis of the $d^{1} \Sigma+\rightarrow a^{1} \Sigma+$ and $d^{1} \Sigma+\rightarrow b^{1} \Pi$ green band systems of XeO, J. Mol. Spectrosc. 74, 70-101 (1979).

20082. Rosenkrantz, M. E.; Stevens, W. J.; Krauss, M.; Konowalow, D. D. Dipole polarizabilities of the group IIb atoms obtained from compact variational trial functions, $J$. Chem. Phys. 72, No. 4, 2525. 2531 (Feb. 15, 1980).

20084. Quade, C. R.; Suenram, R. D. The microwave spectrum of $\mathrm{CH}_{2}$ DOH, J. Chem. Phys. 73, No. 3, 1127-1131 (Aug. 1, 1980).

20085. Maki, A. G.; Wells, J. S. High-resolution measurement and analysis of the infrared spectrum of nitric acid near $1700 \mathrm{~cm}^{-1}, J$. Mol. Spectrosc. 82, 427.434 (1980).

20086. Mies, F. H.; Stevens, W. J.; Krauss, M. Model calculation of the electronic structure and spectroscopy of $\mathbf{H g}_{2}, J$. Mol. Spectrosc. 72, 303.331 (1978).

20087. Okabe, H. Photodissociation of nitric acid and water in the vacuum ultraviolet; vibrational and rotational distributions of $\mathrm{OH}^{2} \Sigma^{+}, J$. Chem. Phys. 72, No. 12, $6642-6650$ (June 15, 1980).

20088. Todd, T. R.; Olson, W. B. The infrared spectra of ${ }^{12} \mathrm{C}^{32} \mathrm{~S}$, ${ }^{12} \mathrm{C}^{34} \mathrm{~S},{ }^{13} \mathrm{C}^{32} \mathrm{~S}$, and ${ }^{12} \mathrm{C}^{33} \mathrm{~S}, J$. Mol. Spectrosc. $74,190-202$ (1979).

20095. Akins, D. L.; King, D. S.; Stephenson, J. C. Vibrational relaxation of $\tilde{\mathbf{A}} \mathbf{C F}_{2}\left(\nu_{2}=1-6\right)$ in non-reactive collisions, Chem. Phys. Lett. 65, No. 2, 257.260 (Aug. 15, 1979).

20099. Hougen, J. T. Double group considerations, Jahn-Teller induced rovibronic effects, and the nuclear spin-electron spin hyperfine Hamiltonian for a molecule of symmetry $\mathrm{C}_{3 v}$ in an electronic ${ }^{2} \mathrm{E}$ state, J. Mol. Spectrosc. 81, 73.92 (1980).

20102. Krauss, M.; Stevens, W. J.; Neumann, D. B. The dispersion damping functions and interaction energy curves for $\mathrm{Xe}-\mathrm{Xe}$, Chem. Phys. Lett. 71, No. 3, 500-502 (May 1, 1980).

20105. Faulkner, D. J.; Freeman, K. C. Gas in globular clusters. I. Time-independent flow models, Astrophys. J. 211, No. 1, 77.90 (Jan. 1, 1977).

20108. Chanmugam, G. Cyclotron absorption in accreting magnetic white dwarfs, Astrophys. J. 241, No. 3, $1122-1130$ (Nov. 1, 1980).

20110. Reader, J.; Luther, G.; Acquista, N. Revised $6 p^{2} P_{1 / 2}$ level of Mo XIV, J. Opt. Soc. Am. 71, No. 2, p. 204 (Feb. 1981).

20111. Maki, A. G.; Lovas, F. J. The infrared spectrum of ${ }^{31} \mathrm{P}^{14} \mathrm{~N}$ near $1300 \mathrm{~cm}^{-1}, J$. Mol. Spectrosc. 85, 368-374 (1981).

20113. Linsky, J. L. Theory of stellar coronae: An interpretation of x-ray emission from non-degenerate stellar sources, (Proc. HEAD/AAS, Cambridge, MA, Jan. 28-30, 1980), Paper in Cool Stars, Stellar Systems, and the Sun. A. K. Dupree, ed., pp. 217.235 (Smithsonian Institute, Cambridge, MA, 1980).
20114. Nesbitt, D. J.; Leone, S. R. $B r^{*}\left({ }^{2} P_{1 / 2}\right)+\mathbf{H}_{2}(v=0,1)$ : Laser studies of the competition between reactive pathways and inelastic energy transfer channels, $J$. Chem. Phys. 73, No. 12, 6182-6190 (Dec. 15,1980 ).

20115. Hougen, J. T.; Oka, T. Vibrationally induced nuclear quadrupole coupling in tetrahedral and octahedral molecules, $J$. Chem. Phys. 74, No. 3, 1830-1839 (Feb. 1, 1981).

20123. Parr, A. C.; Ederer, D. L.; Cole, B. E.; West, J. B.; Stockbauer, R.; Codling, K.; Dehmer, J. L. Triply-differential photoelectron studies of molecular autoionization profiles: The $\mathbf{7 1 0}$ $730 \AA$ region of the $\mathrm{N}_{2}$ spectrum, Phys. Rev. Lett. 46, No. 1, 22.25 (Jan. 5, 1981).

20124. Haisch, B. M.; Linsky, J. L.; Harnden, F. R., Jr.; Rosner, R.; Seward, F. D.; Vaiana, G. S. Einstein x.ray observations of Proxima Centauri and the surrounding region, Astrophys. J. 242, No. 2, L99. L103 (Dec. 1, 1980).

20125. Shy, J. T.; Farley, J. W.; Lamb, W. E., Jr.; Wing, W. H Observation of the infrared spectrum of the triatomic deuterium molecular ion $\mathrm{D}_{3}{ }^{+}$, Phys. Rev. Lett. 45, No. 7, 535.536 (Aug. 18, 1980).

20133. Birnbaum, G.; Sutter, H. Collision-induced absorption in a highly symmetric molecule-SF , Mol. Phys. 42, No. 1, 21.32 (1981).

20155. Mies, F. H.; Julienne, P. S. Oscillatory excimer emission: An analytic model, IEEE J. Quantum Electron. QE-15, No. 5, 272.280 (May 1979).

20156. Spence, D. On resonances in HF, J. Phys. B: At. Mol. Phys., Lett. to Ed. 14, No. 3, L107-L114 (1981).

20168. Kuczkowski, R. L.; Lovas, F. J.; Suenram, R. D.; Lattimer, R. P.; Hillig, K. W.; Ashe, A. J. The microwave spectrum, structure and dipole moment of 1,4-pentadiyne, J. Mol. Struct. 72, 143-152 (1981).

20177. Cool, T. A.; Koffend, J. B. Two-photon excitation of indium atoms by photodissociation of $\mathrm{InCl}$ and $\mathrm{InBr}, J$. Chem. Phys. 74, No. 4, 2287.2292 (Feb. 15, 1981)

20178. Dillon, M. A.; Spence, D. The angular dependence of scattered electron spectra of neon and argon in the region of quasidiscrete autoionizing states, J. Chem. Phys. 74, No. 4, 2654-2655 (Feb. 15, 1981).

20179. Walls, D. F.; Zoller, P.; Steyn-Ross, M. L. Optical bistability from three-level atoms, IEEE J. Quantum Electron. QE-17, No. 3, 380-384 (Mar. 1981).

20180. Clarke, J. T.; Moos, H. W.; Atreya, S. K.; Lane, A. L. IUE detection of bursts of $\mathbf{H}$ Lya emission from Saturn, Nature 290, No. 5803, 226-227 (Mar. 19, 1981).

20182. Younger, S. M. Distorted-wave electron-impact-ionization cross sections for highly ionized neonlike atoms, Phys. Rev. $A$ 23, No. 3 1138.1146 (Mar. 1981).

20186. Reader, J.; Ryabtsev, A. $3 p^{6} 3 d^{8}-3 p^{5} 3 d^{9}$ transitions in $\mathrm{Sr} \mathrm{XIII}$, Y X IV, Zr XV, Nb XVI, and Mo XviI, J. Opt. Soc. Am. 71, No. 3, 231 237 (Mar. 1981)

20193. Wiese, W. L. Experimental and theoretical research on atomic data for highly ionized species at the National Bureau of Standards, Phys. Scr. 23, 194.196 (1981).

20194. Krauss, M.; Stevens, W. J. Electronic structure of the negative ion of $\mathbf{H C l}, J$. Chem. Phys. 74, No. 1, $570-577$ (Jan. 1, 1981).

20196. Basch, H.; Stevens, W. J.; Krauss, M. The electronic structure and dipole moment function of $\mathrm{PbO}\left(X^{1} \Sigma^{+}\right), J$. Chem. Phys. 74, No. 4, 2416-2418 (Feb. 15, 1981).

20198. Younger, S. M. Theoretical line strengths for the $4 d^{10}{ }^{1} S-4 d^{9} 4 f$ ${ }^{1} P^{\circ}$ reso nance transition in the palladium isoelectronic sequence, Phys. Rev. A 22, No. 6, 2682.2689 (Dec. 1980).

20218. Reader, J.; Acquista, N. $4 s^{2} 4 p^{3}-4 s 4 p^{4}$ and $4 s^{2} 4 p^{3}-4 s^{2} 4 p^{2} 5 s$ transitions in Y VII, Zr VIII, Nb IX, and Mo X, J. Opt. Soc. Am. 71, No. 4, 434.441 (Apr. 1981).

20229. Stevens, W. J.; Karo, A. M.; Hiskes, J. R. MCSCF pseudopotential calculations for the alkali hydrides and their anions, J. Chem. Phys. 74, No. 7, 3989.3998 (Apr. 1, 1981).

20238. Basch, H.; Julienne, P. S.; Krauss, M.; Rosenkrantz, M. E. Energy curves and moments for $\mathrm{PbHe}$ and $\mathrm{PbXe}, J$. Chem. Phys. 73, No. 12, 6247.6258 (Dec. 15, 1980).

20242. Zajonc, A. G.; Phelps, A. V. Nonradiative transport of atomic 
excitation of Na vapor, Phys. Rev. $A$ 23, No. 5, $2479-2487$ (May 1981).

20244. Kennerly, R. E.; Van Brunt, R. J.; Gallagher, A. C. Highresolution measurement of the helium $1 s 2 s^{2}{ }^{2} S$ resonance profile, Phys. Rev. $A$ 23, No. 5, 2430-2442 (May 1981).

20249. Spence, $D$. Studies of the $\mathrm{O}_{2}{ }^{3} \Pi_{\mathrm{g}}(V)$ valence states and ${ }^{3} \Pi_{\mathrm{g}}(R)$ Rydberg state in the Schumann-Runge continuum from ejected and scattered electron spectra, J. Chem. Phys. 74, No. 7, 3898-3904 (Apr. 1,1981 ).

20250. Ben-Reuven, A. Recent developments in line shape theory, (Proc. Fifth Int. Conf. Spectral Line Shapes, Berlin, Germany, July 7-11, 1980), Paper in Spectral Line Shapes, B. Wende, ed., pp. 867. 884 (Walter de Gruyter \& Co., New York, NY, 1981).

20272. Shara, M. M.; Prialnik, D.; Shaviv, G. What determines the speed class of novae, Astrophys. J. 239, 586-591 (July 15, 1980).

20274. Mehl, J. B.; Moldover, M. R. Precision acoustic measurements with a spherical resonator: $\mathrm{Ar}$ and $\mathrm{C}_{2} \mathrm{H}_{4}, J$. Chem. Phys. 74, No. 7, 4062-4077 (Apr. 1, 1981).

20281. Weiss, A. W. Review of atomic structure calculations, Proc. Workshop Foundations of the Relativistic Theory of Atomic Structure, Argonne National Laboratory, Argonne, IL, Dec. 4-5, 1980, ANL-80125, pp. 166-178 (Mar. 1981).

20300. Cole, B. E.; Cooper, J. W.; Ederer, D. L.; Mehlman, G.; Saloman, E. B. Stark effect on autoionizing resonances in the rare gases, J. Phys. B: Atom. Molec. Phys. Lett. Ed. 13, L175-L179 (1980).

20303. Contini, M.; Kozlovsky, B. Z.; Shaviv, G. The calculation of the optical spectra of the Cygnus Loop, Astron. Astrophys. 92, No. 3, 273-280 (1980).

20305. Wineland, D. J.; Itano, W. M. Spectroscopy of a single $\mathbf{M g}^{+}$ ion, Phys. Lett. 82A, No. 2, 75.78 (Mar. 9, 1981).

20319. Kuczkowski, R. L.; Suenram, R. D.; Lovas, F. J. Microwave spectrum, structure, and dipole moment of sulfuric acid, J. Am. Chem. Soc. 103, 2561-2566 (1981).

20321. Buhl, D.; Clark, F. O.; Chin, G.; Glenar, D.; Kostiuk, T.; Mumma, M. J.; Lovas, F. J. Vibrationally excited silicon monoxide masers, (Proc. Symp. No. 87, Int. Astronomical Union, Mont Tremblant, Quebec, Canada, Aug. 6-10, 1979), Paper in Interstellar Molecules, B. H. Andrew, ed., pp. 537.538 (D. Reidel Publ. Co., Dordrecht, The Netherlands, 1980).

20336. Vaiana, G. S.; Cassinelli, J. P.; Fabbiano, G.; Giacconi, R.; Golub, L.; Gorenstein, P.; Haisch, B. M.; Harnden, F. R., Jr.; Johnson, H. M.; Linsky, J. L.; Maxson, C. W.; Mewe, R.; Rosner, R.; Seward, F.; Topka, K.; Zwaan, C. Results from an extensive Einstein stellar survey, Astrophys. J. 244, 163-182 (Apr. 1, 1981).

20337. Cox, A. N.; Shaviv, G.; Hodson, S. W. On the ratio of mixing length to scale height in red dwarfs, Astrophys. J. 245, L37-L40 (Apr. 1981).

20338. Bergquist, J. C.; Barger, R. L.; Glaze, D. J. High resolution spectroscopy of calcium atoms, Proc. 4th Int. Conf. Laser Spectroscopy (FICOLS), Rottach-Egeren, West Germany, July 1979, pp. 120-129 (Springer-Verlag, New York, NY, 1979).

20339. Linsky, J. L. Evidence for chromospheres and coronae in stars: Recent observations, some unanswered theoretical questions, and a speculative scenario, (Proc. NATO Advanced Study Institute, Bonas, France, Aug. 25-Sept. 1980), Paper in Solar Phenomena in Stars and Stellar Systems, R. M. Bonnet and A. K. Dupree, eds., pp. 99.122 (D. Reidel Publ. Co., Dordrecht, The Netherlands, 1981).

20374. Burek, A. J.; Barrus, D. M.; Blake, R. L.; Fenimore, E. E. Analysis of solar X-ray emission line profiles, Astrophys. J. 243, No. 3, Pt. 1, 660-676 (Jan. 15, 1981).

20385. Linsky, J. L. Outer atmospheres of late-type stars, (Proc. Second Workshop for Physical Processes in Red Giants, Sicily, Italy, Sept. 3-13, 1980), Paper in Physical Processes in Red Giants, I. Iben, Jr. and A. Renzini, eds., pp. 247-261 (D. Reidel Publ. Co., Boston, MA, 1981).

20388. Giampapa, M. S.; Bornmann, P. L.; Ayres, T. R.; Linsky, J. L.; Worden, $\mathbf{S}$. P. The $\mathbf{M g}$ II $h$ and $k$ lines in a sample of $d M e$ and $d M$ stars, Proc. Second Year of IUE: The Universe in Ultraviolet Wavelengths, Greenbelt, MD, May 7.9, 1980, pp. 279-286 (NASA Goddard Space Flight Center, Greenbelt, MD, 1981).

20395. Acquista, N.; Reader, J. Spectrum and energy levels of nine- times ionized strontium (Sr x), J. Opt. Soc. Am. 71, No. 5, 569-573 (May 1981).

20399. Codling, K.; Parr, A. C.; Ederer, D. L.; Stockbauer, R.; West, J. B.; Cole, B. E.; Dehmer, J. L. The effects of autoionisation on vibrational branching ratios and photoelectron angular distributions in molecular photoionization: The formation of the ground state of $\mathrm{O}_{2}^{+}$between 574 and $600 \AA$, J. Phys. B. At. Mol. Phys. 14, 657-666 (1981).

20402. Martinez, R. I.; Herron, J. T.; Huie, R. E. The mechanism of ozone-alkene reactions in the gas phase. A mass spectrometric study of the reactions of eight linear and branched-chain alkenes, $\mathrm{J}$. $\mathrm{Am}$. Chem. Soc. 103, 3807-3820 (1981).

20405. Haisch, B.; Linsky, J. L.; Slee, O. B.; Siegman, B. C.; Nikoloff, I.; Candy, M.; Harwood, D.; Verveer, A.; Quinn, P. J.; Wilson, l.; Page, A. A.; Higson, P.; Deward, F. D. Simultaneous X-ray, ultraviolet, optical, and radio observations of the flare star Proxima Centauri, Astrophys. J. 245, 1009-1017 (May 1, 1981).

20409. Linsky, J. L.; Marstad, N. C. IUE spectra of F and late A stars, Proc. Second Year of IUE: The Universe in Ultraviolet Wavelengths, Greenbelt, MD, May 7-9, 1980, pp. 287-295 (NASA Goddard Space Flight Center, Greenbelt, MD, 1981)

20410. Livio, M.; Shaviv, G. The stability of accretion disks to short wavelength perturbations, Astrophys. J. 244, 290-298 (Feb. 15, 1981).

20412. Langer, S. H.; Chanmugam, G.; Shaviv, G. Thermal instability in accretion flows onto degenerate stars, Astrophys. $J$. 245, L23-L26 (Apr. 1, 1981).

20416. Chanmugam, G. Cyclotron emission from AM Herculis, Proc. Second Year of IUE: The Universe in Ultraviolet Wavelengths, Greenbelt, MD, May 7-9, 1980, pp. $515-519$ (NASA Goddard Space Flight Center, Greenbelt, MD, 1981).

20417. Chanmugam, G.; Dulk, G. A. Polarized radiation from hot plasmas and applications to AM Herculis binaries, Astrophys. $J$. 244, No. 2, 569.578 (Mar. 1, 1981).

20418. Stencel, R. E.; Mullan, D. J.; Basri, G. S.; Linsky, J. L. High resolution absolute flux profiles of the $\mathrm{Mg}$ II $\mathbf{h}$ \& $\mathbf{k}$ lines in evolved F8 to M5 stars, Proc. Second Year of IUE: The Universe in Ultraviolet Wavelengths, Greenbelt, MD, May 7-9, 1980, pp. $317-324$ (NASA Goddard Space Flight Center, Greenbelt, MD, 1981).

20421. Rowe, J. M.; Rush, J. J.; Pynn, R.; Heidemann, A.; Michel, K. $\mathrm{H}$. Observation of critical dynamics in $\mathbf{N D}_{\mathbf{4}} \mathbf{B r}$ by high-resolution neutron spectroscopy, J. Chem. Phys. 74, No. 9, 5323-5324 (May 1, 1981).

20422. November, L. J.; Toomre, J.; Gebbie, K. B.; Simon, G. W. The detection of mesogranulation on the Sun, Astrophys. J. 245, L123L126 (May 1, 1981).

20423. Simon, T.; Linsky, J. L.; Schiffer, F. H. IUE spectra of a flare in HR 5110: A flaring RS CVn or ALGOL system?, Proc. Second Year of IUE: The Universe in Ultraviolet Wavelengths, Greenbelt, MD, May 7-9, 1980, pp. 435-441 (NASA Goddard Space Flight Center, Greenbelt, MD, 1981).

20424. Wormsbecher, R. F.; Hessel, M. M.; Lovas, F. J. Microwave optical double resonance of $X^{1} \Sigma^{+}$of NaK, J. Chem. Phys. 74, No. 12, 6983-6985 (June 15, 1981).

20426. West, J. B.; Codling, K.; Parr, A. C.; Ederer, D. L.; Cole, B. E.; Stockbauer, R.; Dehmer, J. L. Branching ratios and photoelectron angular distributions through the Hopfield bands in $\mathbf{N}_{2}$ between 650 and $730 \AA$, J. Phys. B: At. Mol. Phys. 14, 1791-1801 (1981).

20427. Wells, J. S.; Petersen, F. R.; Maki, A. G.; Sukle, D. J. Heterodyne frequency measurements on the $11.6-\mu \mathrm{m}$ band of OCS: New frequency/wavelength calibration tables for 11.6 and $5.8-\mu \mathrm{m}$ OCS bands, Appl. Opt. 20, No. 9, 1676-1684 (May 1, 1981).

20428. Smith, E. W. Absorption and dispersion in the $\mathrm{O}_{2}$ microwave spectrum at atmospheric pressures, J. Chem. Phys. 74, No. 12, 6658 6673 (June 15, 1981).

20437. Phillips, J. C.; Peterlin, A.; Waters, P. F. Apparatus for infrared measurement of sorption/desorption in strained polymeric films, J. Polym. Sci. 19, 789-802 (1981).

20439. Clark, F. O.; Johnson, D. R.; Troland, T. H.; Heiles, C. E. Polarized emission in the broad SiO feature from $\mathrm{R}$ Leo, (Proc. Int. Astronomical Union Symp. 87, Mont. Trenblant, Quebec, Canada, 
Aug. 6-10, 1979), Paper in Interstellar Molecules, B. H. Andrew, ed., pp. 543-544 (D. Reidel Publ. Co., Dordrecht, The Netherlands, 1980).

20449. Wyart, J. F.; Reader, J.; Ryabtsev, A. $3 d-4 p$ transitions in the zinclike and copperlike ions $\mathrm{Y} X, \mathrm{XI} ; \mathrm{Zr} \mathrm{XI}, \mathrm{XII} ; \mathrm{Nb} \mathrm{XII}, \mathrm{XIII}$; and $\mathrm{Mo}$ XIII, XIV, J. Opt. Soc. Am. 71, No. 6, $692-698$ (June 1981).

20452. Lafferty, W. J.; Sattler, J. P.; Worchesky, T. L.; Ritter, K. J. Diode laser heterodyne spectroscopy on the $v_{4}$ and $v_{9}$ bands of 1,1 difluoroethylene, J. Mol. Spectros. 87, 416-428 (1981).

20460. Jacox, M. E. The reaction of $F$ atoms with $\mathrm{C}_{2} \mathrm{H}_{4}$. Vibrational spectrum of the $\mathrm{C}_{2} \mathrm{H}_{4} \mathrm{~F}$ intermediate trapped in solid argon, Chem. Phys. 58, 289.302 (1981).

20478. Egelhoff, W. F., Jr.; Tibbetts, G. G.; Lindau, I.; Hecht, M. H. Photoemission studies on thulium (Summary Abstract), J. Vac. Sci. Technol. 18, No. 2, p. 599 (Mar. 1981).

20479. Smith, E. W.; Talin, B.; Cooper, J. An analysis of Markovian Model Microfield Methods for Stark broadening, J. Quant. Spectrosc. Radiat. Transfer 26, No. 3, 229.242 (1981).

20490. Nitz, D. E.; Smith, A. V.; Levenson, M. D.; Smith, S. J. Bandwidth-induced reversal of asymmetry in optical-doubleresonance amplitudes, Phys. Rev. A 24, No. 1, 288-293 (July 1981).

20491. Wildman, D. W.; Schumann, L. W.; Gallagher, A. C. Electric discbarge excited TII-Xe mixtures, J. Appl. Phys. 52, No. 5, 3264 3268 (May 1981).

20492. Pradhan, A. K.; Norcross, D. W.; Hummer, D. G. Rate coefficients for electron impact excitation of helium-like ions, Astrophys. J. 246, 1031-1039 (June 15, 1981).

20496. Lovas, F. J.; Maki, A. G.; Olson, W. B. The infrared spectrum of $\mathrm{SiO}$ near $1240 \mathrm{~cm}^{-1}$ and its relation to the circumstellar $\mathrm{SiO}$ maser, J. Mol. Spectrosc. 87, $449-458$ (1981).

20504. Hellmuth, T.; Leuchs, G.; Smith, S. J.; Walther, H. Angular distribution of photoelectrons in multiphoton ionization, Proc. $2 d$ Int. Conf. Multiphoton Processes, Budapest, Hungary, Apr. 14-18, 1980, pp. 1.15 (Hungarian Academy of Science, Budapest, Hungary, 1981).

20506. Hellmuth, T.; Leuchs, G.; Smith, S. J.; Walther, H. Multiphoton ionization of atoms, (Proc. Sergio Porto Memorial Symp., Rio de Janeiro, Brazil, June 29-July 3, 1980), Paper in Springer-Series in Optical Sciences, Lasers and Applications, W. O. N. Guimaraes, C. T. Lin, and A. Mooradian, eds., 26, pp. 194-203 (Springer-Verlag, Berlin, Germany, 1981).

20509. Moos, H. W.; Clarke, J. T. Ultraviolet observations of the Io torus from the IUE observatory, Astrophys. J. 247, 354-361 (July 1, 1981).

20511. Linsky, J. L. Winds in late-type stars: Mechanisms of mass outflow, (Proc. Effects of Mass Loss on Stellar Evolution Colloquim No. 59, Triests, Italy, Sept. 15-19, 1980), Paper in Effects of Mass Loss on Stellar Evolution, C. Chiosi and R. Stalio, eds., pp. 187-212 (D. Reidel Publishing Company, Dordrecht, Holland, 1981).

20515. Jacox, M. E. Hydrogen-atom abstraction by atomic fluorine. Vibrational spectrum of the $\mathbf{F}+\mathrm{CH}_{3} \mathrm{~F}$ reaction products trapped in solid argon, J. Chem. Phys. 59, 199.212 (1981).

20517. Clark, F. O.; Johnson, D. R. The L134-L183-L1778 system of interstellar clouds, Astrophys. J. 247, 104-111 (July 1, 1981).

20534. Padial, N. T.; Norcross, D. W.; Collins, L. A. On the use of the unitarised Born approximation in electron collisions with polar molecules, J. Phys. B: At. Mol. Phys. 14, 2901.2909 (1981).

20535. Ayres, T. R.; Marstad, N. C.; Linsky, J. L. Outer atmospheres of cool stars. IX. A survey of ultraviolet emission from F-K dwarfs and giants witb IUE, Astrophys. J. 247, $545-559$ (July 15, 1981).

20536. Feigerle, C. S.; Herman, Z.; Lineberger, W. C. Laser photoelectron spectrometry of $\mathrm{Sc}^{-}$and $\mathrm{Y}^{-}$: A determination of the order of electron filling in transition-metal anions, $J$. Electron Spectrosc. Relat. Phenom. 23, $441-450$ (1981).

20550. Jacox, M. E. The reaction of excited argon atoms and of $F$ atoms with methanol. Vibrational spectrum of $\mathrm{CH}_{2} \mathrm{OH}$ isolated in solid argon, J. Chem. Phys. 59, 213.230 (1981).

20561. Wlodawer, A.; Sjölin, L. Orientation of histidine residues in RNase A: Neutron diffraction study, Proc. Natl. Sci. USA 78, No. 5 , pp. 2853-2855 (May 1981).
20562. Hummer, D. G. Expressions for the computer-evaluation of the four kernel functions for line formation with Doppler and Lorentz profiles, J. Quant. Spectrosc. Radiat. Transfer 26, No. 3, 187.195 (1981).

20577. Matthew, J. A. D.; Girvin, S. M. Breadths of resonant photoemission satellites and electron-excited direct-recombination emission, Phys. Rev. B 24, No. 4, 2249-2253 (Aug. 15, 1981).

20581. Okabe, H. Photochemistry of acetylene at $1470 \AA$, J. Chem. Phys. 75, No. 6, 2772-2778 (Sept. 15, 1981).

20582. Ayres, T. R.; Moos, H. W.; Linsky, J. L. Far-ultraviolet fluorescence of carbon monoxide in the red giant Arcturus, Astrophys. J. 248, L137-L140 (Sept. 15, 1981).

20584. Falk, R. A.; Dunn, G. H.; Griffin, D. C.; Bottcher, C.; Gregory, D. C.; Crandall, D. H.; Pindzola, M. S. Excitationautoionization contributions to electron impact ionization, Phys. Rev. Lett. 47, No. 7, $494-497$ (Aug. 17, 1981).

20588. Hougen, J. T. A rotational Hamiltonian for the ground vibrational state of hydrazine, J. Mol. Spectrosc. 89, 296.327 (1981).

20589. Frommer, C.; Lovejoy, R. W.; Sams, R. L.; Olson, W. B. The infrared spectrum of the $v_{1}$ band of $\mathrm{SiD}_{3} \mathrm{H}, J$. Mol. Spectrosc. 89, 261-267 (1981).

20594. Pallavicini, R.; Golub, L.; Rosner, R.; Vaiana, G. S.; Ayres, T.; Linsky, J. L. Relations among stellar x-ray emission observed from Einstein, stellar rotation and bolometric luminosity, Astrophys. J. 248, 279.290 (Aug. 15, 1981).

20596. Dunn, G. H. Electron-ion collisions, (Proc. The Physics of Ionized Gases, Dubrovnik, Yugoslavia, Aug. 25-30, 1980), Paper in The Physics of Ionized Gases, M. Matic, ed., 49.95 (Boris Kidric Institute of Nuclear Sciences, Beograd, Yugoslavia, 1980).

20597. Tachibana, K.; Phelps, A. V. Excitation of the $\mathbf{O}_{2}\left(a^{1} \Delta_{\mathrm{g}}\right)$ state by low energy electrons, $J$. Chem. Phys. 75, No. 7, 3315-3320 (Oct. 1, 1981).

20598. Stencel, R. E.; Linsky, J. L.; Brown, A.; Jordan, C.; Carpenter, K. G.; Wing, R. F.; Czyzak, S. Density sensitive C II lines in cool stars of low gravity, Mon. Not. R. Astron. Soc. Short Commun. 196, 47P.53P (1981).

20611. Wyart, J. F.; Kaufman, V.; Sugar, J. The $4 f^{13} 5 f$ configuration in the isoelectronic sequence of Yb III, Phys. Scr. 23, 1069.1078 (1981).

20617. Younger, S. M. Cross sections and rates for direct electronimpact ionization of sodiumlike ions, Phys. Rev. A 24, No. 3, 1272. 1277 (Sept. 1981).

20618. Younger, S. M. Electron impact ionization cross sections and rates for highly ionized atoms, J. Quant. Spectrosc. Radiat. Transfer 26, No. 4, 329-337 (1981).

20619. Younger, S. M. Electron-impact-ionization cross sections and rates for highly ionized berylliumlike ions, Phys. Rev. A 24, No. 3, 1278-1285 (Sept. 1981).

20627. Ayres, T. R.; Linsky, J. L.; Vaiana, G. S.; Golub, L.; Rosner, R. The cool balf of the H-R diagram in soft X-rays, Astrophys. J. 250, 293.299 (Nov. 1, 1981).

20628. McCrackin, F. L.; Mazur, J. Configuration properties of combbrancbed polymers, Macromolecules 14, No. 5, 1214-1220 (Sept.-Oct. 1981).

20640. Wells, J. S.; Petersen, F. R.; Maki, A. G.; Sukle, D. J. Heterodyne frequency measurements (at $11.6 \mu \mathrm{m}$ ) on isotopic species of carbonyl sulfide, $\mathrm{OC}^{34} \mathrm{~S}, \mathrm{O}^{13} \mathrm{CS}, \mathrm{OC}^{33} \mathrm{~S},{ }^{18} \mathrm{OCS}$, and $\mathrm{O}^{13} \mathrm{C}^{34} \mathrm{~S}$, J. Mol. Spectrosc. 89, $421-429$ (1981).

20641. Petersen, F. R.; Wells, J. S.; Maki, A. G.; Siemsen, K. J. Heterodyne frequency measurements of ${ }^{13} \mathrm{CO}_{2}$ laser hot band transitions, Appl. Opt. 20, No. 20, 3635-3640 (Oct. 15, 1981).

20647. Farley, J. W.; Wing, W. H. Accurate calculation of dynamic Stark shifts and depopulation rates of Rydberg energy levels induced by blackbody radiation. Hydrogen, helium, and alkali-metal atoms, Phys. Rev. A 23, No. 5, 2397.2424 (May 1981).

20674. Cole, B. E.; Saloman, E. B.; Ederer, D. L. Ba II fluorescence from barium atoms resonantly photoionized by $584-\AA$ radiation, $J$. Opt. Soc. Am. 71, No. 12, 1458-1462 (Dec. 1981).

20676. Ederer, D. L.; Parr, A. C.; Cole, B. E.; Stockbauer, R.; Dehmer, J. L.; West, J. B.; Codling, K. Vibrational-state dependence of partial cross sections and pbotoelectron angular distributions 
through autoionizing resonances: The $\mathbf{n}=\mathbf{3}$ Rydberg state converging to the $\mathrm{B}^{2} \Sigma^{+}$state of $\mathrm{CO}^{+}$, Proc. $R$. Soc. Lond. A378, 432.435 (1981).

20680. Itano, W. M.; Wineland, D. J. Precision measurement of the ground-state hyperfine constant of ${ }^{25} \mathrm{Mg}^{+}$, Phys. Rev. A 24, No. 3, 1364-1373 (Sept. 1981).

20684. Lucatorto, T. B.; McIlrath, T. J.; Sugar, J.; Younger, S. M. Radical redistribution of the $4 d$ oscillator strength observed in the photoabsorption of the $\mathrm{Ba}, \mathrm{Ba}^{+}$, and $\mathrm{Ba}^{++}$sequence, Phys. Rev. Lett. 47, No. 16, 1124-1128 (Oct. 19, 1981)

20685. Magnotta, F.; Nesbitt, D. J.; Leone, S. R. Excimer laser photolysis studies of translational-to-vibrational energy transfer, Chem. Phys. Lett. 83, No. 1, 21-25 (Oct. 1981)

20691. Penn, D. R.; Rendell, R. W. Surface-enhanced photoabsorption and photoyield in small spheres, Phys. Rev. Lett. 47, No. 15, 1067 1070 (Oct. 12, 1981)

20696. Regev, O.; Shaviv, G. Formation of protostars in collapsing, rotating, turbulent clouds, Astrophys. J. 245, No. 3, 934.959 (May 1 1981).

20710. Wiese, W. L.; Martin, G. A. Atomic spectroscopy, Chapter 5 in Physics Vade Mecum AIP 50th Anniversary, H. L. Anderson, ed., pp. 96-105 (American Institute of Physics, New York, NY, 1981).

20713. Zwier, T. S.; Weisshaar, J. C.; Leone, S. R. Nascent product vibrational state distributions of ion-molecule reactions: The $\mathrm{H}+\mathrm{F}^{-} \rightarrow \mathrm{HF}(v)+e^{-}$associative detachment reaction, J. Chem. Phys. 75, No. 10, 4885-4892 (Nov. 15, 1981).

20717. Gary, D. E.; Linsky, J. L. First detection of nonflare microwave emission from the coronae of single late-type dwarf stars, Astrophys. J. 250, 284.292 (Nov. 1, 1981).

20737. Younger, S. M. Cross sections and rates for direct electronimpact ionization of sodiumlike ions, Phys. Rev. $A$ 24, No. 3, 1272 1277 (Sept. 1981).

20738. Younger, S. M. Electron impact ionization cross sections and rates for highly ionized atoms, J. Quant. Spectrosc. Radiat. Transfer 26, No. 4, 329-337 (1981).

20739. Younger, S. M. Electron-impact-ionization cross sections and rates for highly ionized berylliumlike ions, Phys. Rev. $A$ 24, No. 3, 1278-1285 (Sept. 1981).

\section{Building Technology}

Mahajan, B. M.; Galowin, L. S.; Kopetka, P. A. Models of quasisteady and unsteady discharge from plumbing fixtures. J. Res. Nat. Bur. Stand. (U.S.). 86(2): 171-179; 1981 March-April.

SP446-5. Raufaste, N.; Olmert, M., eds. Building technology project summaries 1980-1981. Nat. Bur. Stand. (U.S.) Spec. Publ. 446-5; 1981 July. 82 p. SN003-003-02343-4.

SP457-5. Webber, S., ed. Building technology publications 1980Supplement 5. Nat. Bur. Stand. (U.S.) Spec. Publ. 457-5; 1981 June. 90 p. SN003-003-02332-9.

SP623. Pielert, J. H. Removing regulatory restraints to building rehabilitation: The Massachusetts experience. Nat. Bur. Stand. (U.S.) Spec. Publ. 623; 1981 October. 57 p. Available from: NTIS; PB 82 121047.

BSS131. Knab, L.; Mathey, R.; Jenkins, D. Laboratory evaluation of nondestructive methods to measure moisture in built-up roofing systems. Nat. Bur. Stand. (U.S.) Bldg. Sci. Ser. 131; 1981 January. 173 p. SN003-003-02281-1.

BSS132. Yokel, F. Y.; Yancey, C. W. C.; Mullen, C. L. A study of reaction forces on mobile home foundations caused by wind and flood loads. Nat. Bur. Stand. (U.S.) Bldg. Sci. Ser. 132; 1981 March. 84 p. SN003.003.02301.9.

BSS136. Harris, J. R.; Wright, R. N. Organization of building standards: Systematic techniques for scope and arrangement. Nat. Bur. Stand. (U.S.) Bldg. Sci. Ser. 136; 1981 September. 278 p. SN003-003-02363-9.

TN1135. Rossiter, W. J., Jr.; Mathey, R. G.; Busching, H. W.; Cullen, W. C. Cooling of bitumen during construction of built-up roofing systems-A mathematical model. Nat. Bur. Stand. (U.S.) Tech. Note 1135; 1981 March. 76 p. SN003-003-02302-7.

TN1146. Jenkins, D. R.; Mathey, R. G.; Knab, L. I. Moisture detection in roofing by nondestructive means-A state-of-the-art survey. Nat. Bur. Stand. (U.S.) Tech. Note 1146; 1981 July. 82 p. SN003-003-02340-0.

TN1152. Pommersheim, J. M.; Mathey, R. G. Mechanical performance of built-up roofing membranes. Nat. Bur. Stand. (U.S.) Tech. Note 1152; 1981 December. 60 p. Available from: NTIS; PB 82-151259.

NBSIR 79-1610. Belsher, D. R. Feasibility study on the use of a microwave system for the nondestructive evaluation of historic adobe structures. 1979 July. 32 p. Available from: NTIS; PB 298559.

NBSIR 80-2081. Cooke, P. W. Comparison of selected codes and standards relating to existing residential buildings. $1980 \mathrm{July} .310 \mathrm{p}$. Available from: NTIS; PB 81-120842.

NBSIR 80-2111-1. Ellingwood, B. R. Review and refinement of ATC 3-06 tentative seismic provisions. Report of technical committee 1: Seismic risk maps. 1980 October. 30 p. Available from: NTIS; PB $81-187551$.

NBSIR 80-2111-3. Salomone, L. A. Review and refinement of ATC 3-06 tentative seismic provisions. Report of technical committee 3: Foundations. 1980 October. 57 p. Available from: NTIS; PB 81. 187569.

NBSIR 80-2111-4. Marshall, R. D.; Woodward, K. Review and refinement of ATC 3-06 tentative seismic provisions. Report of technical committee 4: Concrete. 1980 October. 254 p. Available from: NTIS; PB 82-130915.

NBSIR 80-2111-5. Leyendecker, E. V.; Cattaneo, L. E. Review and refinement of ATC 3.06 tentative seismic provisions. Report of technical committee 5: Masonry. 1980 October. 349 p. Available from: the author.

NBSIR 80-2111-6. Lew, H. S. Review and refinement of ATC 3-06 tentative seismic provisions. Report of technical committee 6: Steel. 1980 October. 37 p. A vailable from: NTIS; PB 81.187577.

NBSIR 80-2111-7. Yancey, C. W. C. Review and refinement of ATC 3-06 tentative seismic provisions. Report of technical committee 7: Wood. 1980 October. 41 p. Available from: NTIS; PB 81-187585.

NBSIR 80-2111-8. Faison, T. K. Review and refinement of ATC 3-06 tentative seismic provisions. Report of technical committee 8: Architectural, mechanical and electrical. 1980 October. $43 \mathrm{p}$. A vailable from: NTIS; PB 81-187593.

NBSIR 80-2111-11. Leyendecker, E. V.; Harris, J. R., eds. Review and refinement of ATC 3.06 tentative seismic provisions. Report of joint committee on review and refinement. 1980 October. 334 p. Available from: author

NBSIR 80-2150. Thompson, B. E.; Chapman, R. E. Productivity in residential construction: An annotated bibliography. 1981 February. 59 p. A vailable from: NTIS; PB 81-163925.

NBSIR 80-2171. Lerchen, F. H.; Pielert, J. H.; Faison, T. K. Selected methods for condition assessment of structural, HVAC, plumbing and electrical systems in existing buildings. 1980 December. $111 \mathrm{p}$. Available from: NTIS; PB 81-186918.

NBSIR 80-2174. Margulis, S. T. Building accessibility in relation to door hardware, door users, and door use. 1981 January. $54 \mathrm{p}$. A vailable from: NTIS; PB 81.159626.

NBSIR 81-2195. Harris, J. R.; Leyendecker, E. V. Draft seismic standard for Federal buildings. 1981 January. 96 p. Available from: NTIS; PB 81-163842.

NBSIR 81-2210. Winter, F. Onsite wastewater systems-Current practices and a proposed basis for evaluation. 1981 March. $113 \mathrm{p}$. A vailable from: NTIS; PB 81-211393.

NBSIR 81-2215. Yokel, F. Y.; Salomone, L. A.; Chung, R. M. Construction of housing in mine subsidence areas. 1981 January. 53 p. Available from: NTIS; PB 81-174690.

NBSIR 81-2221. Beausoliel, R. W.; Meese, W. J. Experimentally determined performance of some residential circuit breakers. 1981 April. 49 p. A vailable from: NTIS; PB 81-197048.

NBSIR 81-2223. Rawie, C. C. Estimating benefits and costs of building regulations: A step by step guide. 1981 June. 93 p. Available from: NTIS; PB 81-217812.

NBSIR 81-2231. Yonemura, G. T. Criteria for recommending lighting levels. 1981 March. 60 p. Available from: NTIS; PB 81.185126.

NBSIR 81-2238. Yokel, F. Y.; Chung, R. M.; Yancey, C. W. C. NBS studies of mobile home foundations. 1981 March. 45 p. Available 
from: NTIS; PB 81-180978.

NBSIR 81.2239. Frohnsdorff, G.; Clifton, J. R. Fly ashes in cements and concretes: Technical needs and opportunities. 1981 March. 34 p. Available from: NTIS; PB 81.183428.

NBSIR 81-2245. Adler, S. C.; Pierman, B. C. Building accessibility for the disabled: A review of research needs. 1981 March. $46 \mathrm{p}$. A vailable from: NTIS; PB 81.184954.

NBSIR 81-2248. Ruegg, R. T.; Chapman, R. E. A regional economic assessment of selected window systems. $1981 \mathrm{July} .125$ p. Available from: NTIS; PB 81.243255.

NBSIR 81.2249. Mahajan, B. M. Unsteady water depth measurement in a partially filled $7.6 \mathrm{~cm}$ diameter horizontal pipe. 1981 April. $55 \mathrm{p}$. Available from: NTIS; PB 81.203416.

NBSIR 81-2250. Chapman, R. E.; Thompson, B. E. Estimating area cost factors for military construction projects: A computerized approach. 1981 May. 77 p. Available from: NTIS; PB 81-215295.

NBSIR 81-2258. Margulis, S. T. A methodology for evaluating housing in use: A case study approach. 1981 June. 224 p. Available from: NTIS; PB 82-104969.

NBSIR 81-2265. Hunt, B. J.; Fattal, S. G. Review of technical information on scaffolds. 1981 May. 103 p. Available from: NTIS; PB 81-203754.

NBSIR 81.2266. Mahajan, B. M. Experimental investigation of transport of finite solids in a $76 \mathrm{~mm}$-diameter partially-filled pipe. 1981 A pril. 65 p. Available from: NTIS; PB 81.203176.

NBSIR 81-2268. Lerner, N. D. Evaluation of exit directional symbols. 1981 May. 55 p. Available from: NTIS; PB 81-203671.

NBSIR 81-2290. Swaffield, J. A. Entry transition water surface profile prediction in supercritical partially filled pipe flow. $1981 \mathrm{June} .107 \mathrm{p}$. Available from: author.

NBSIR 81-2296. Winter, F.; Galowin, L. S. Criteria and evaluation for two-step flush devices for water closets. 1981 June. 93 p. Available from: NTIS; PB 81.226839.

NBSIR 81-2304. Weber, S. F.; Thompson, B. E.; Lippiatt, B. C. Economic framework for cost-effective residential water conservation decisions. 1981 August. 65 p. Available from: NTIS; PB 81-246779.

NBSIR 81-2307. Swaffield, J. A. Dependence of model waste solid transport characteristics in drainage systems on solid geometry, mass and pipe system parameters. 1981 July. 45 p. Available from: NTIS; PB 81.229247.

NBSIR 81-2308. Swaffield, J. A. An initial study of the application of the numerical method of characteristics to unsteady flow analysis in partially filled gravity drainage sized pipes. $1981 \mathrm{July} .64 \mathrm{p}$. Available from: NTIS; PB 81-229189.

NBSIR 81-2310. Turner, G.; Collins, B. Pedestrian movement characteristics on building ramps. 1981 June. 59 p. Available from: NTIS; PB 81-229163.

NBSIR 81-2335. Clark, R. E. Effects of home weatherization on occupant comfort: First report of a field study. 1981 September. 84 p. Available from: NTIS; PB 81.245334.

NBSIR 81-2367. Swaffield, J. A. Prediction of the hydraulic jump location following a change of slope in a partially filled drainage pipe. 1981 November. 189 p. Available from: NTIS; PB 82-142134.

NBSIR 81-2397. Marshall, H. E.; Ruegg, R. T. Recommended practice for measuring benefit/cost and savings-to-investment ratios for buildings and building systems. 1981 November. 49 p. Available from: NTIS; PB 82-170648.

NBSIR 81-2402. Rawie, C. C. Estimating economic impacts of building codes. 1981 October. 163 p. Available from: NTIS; PB 82-139551.

NBS-GCR-80-248. Ungar, E., (NBS contact: CBT X2693). Structureborne sound in buildings: Needed practical research in light of the current state of the art. 1980 June. 55 p. Available from: NTIS; PB 81-187064.

NBS-GCR-80-249. Schultz, T. J. Impact noise testing and rating-1980. 1981 January. 363 p. Available from: NTIS; PB 81.158610.

NBS-GCR-80-280. Achenbach, P. R. Functional performance requirements for the environmental and service systems in detached housing and their impact on building energy use. 1981 January. 168 p. Available from: NTIS; PB 81.157893.

NBS-GCR-81-320. Zeizel, J.; Welch, P., (NBS contact: T. Faison). User needs research for family housing. 1981 May. 169 p. Available from: NTIS; PB 81-214074.

NBS-GCR-81-331. Alfeld, L. E., (NBS contact: N. Raufaste) Research for building construction productivity-Report on the June 2, 1981 conference. 1981 August. 26 p. Available from: NTIS; PB 81.246803 .

NBS.GCR-81-332. The Rice Center, (NBS contact: F. Ventre). A review of standards and common practices in building site regulation technical issues and research needs. 1981 September. $144 \mathrm{p}$. Available from: NTIS; PB 82-117631.

NBS-GCR-81-333. White, E. T., (NBS contact: A. Rubin). Tracing lighting design decisions for new open office space: A pilot study. 1981 September. 196 p. Available from: NTIS; PB 82-132531.

20293. Simiu, E.; Shaver, J. R.; Filliben, J. J. Wind speed distributions and reliability estimates, J. Struct. Div. Am. Soc. Civ. Eng. Tech. Note 107, No. ST5, 1003-1007 (May 1981).

20295. Simiu, E.; Filliben, J. J. Wind direction effects on cladding and structural loads, Eng. Struct. 3, No. 3, 181.186 (July 1981).

20320. Kovacs, W. D. What constitutes a turn?, Geotech. Test. J. Tech. Note 3, No. 3, 127.130 (Sept. 1981).

20329. Harris, J. R.; Wright, R. N. Unambiguous and complete: Applying information science to specifications, Construct. Specifer 34 , No. 4, 55-62 (Apr. 1981).

20379. Barber, E. M.; Bettge, D. A.; Powell, F. J. Development of a modeling technique for evaluation of natural convection and ventilation in rooms, Proc. Semi-Annu. Meet. ASHRAE Survival Shelter Problems, San Francisco, CA, Jan. 19-22, 1970, pp. 36.39 (American Society of Heating, Refrigerating and Air.Conditioning Engineers, Inc., New York, NY, 1970).

20386. Harris, J. R. Information flow in the development of earthquake provisions for building codes, Proc. Conf. $V$ Communicating Earthquake Hazard Reduction Information, Denver, CO, May 22-24, 1978, pp. 288-306 (Office of Earthquake Studies, U.S. Geological Survey, Menlo Park, CA, 1978).

20435. Harris, J. R.; Fenves, S. J.; Wright, R. N. Logical analysis of tentative seismic provisions, J. Struc. Div. Proc. Am. Soc. Civil Eng. 107, No. ST8, 1629.1641 (Aug. 1981).

20499. Lippiatt, B. C.; Weber, S. F. Labor and materials cost of weatherizing low-income housing, (Proc. Natl. Conf. Optimal Weatherization, Washington, DC, Dec. 8.10, 1980), Paper in Optimal Weatherization, pp. 103-113 (Information Dynamics, Inc., Silver Spring, MD 20902, 1981).

20524. Simiu, E. Modern developments in wind engineering: Part 2, Eng. Struct. 3, 242.248 (Oct. 1981).

20525. Simiu, E. Modern developments in wind engineering: Part 1, Eng. Struct. 3, 233-241 (Oct. 1981).

20551. Batts, M. E.; Changery, M. J. Wind speed averaging-time relationship: Experimental data, Proc. ASCE Annu. Fall Conv., Hollywood, FL, Oct. 27-31, 1980, 10 pages (American Society of Civil Engineers, New York, NY, Oct. 1980).

20605. Hunt, C. M.; Treado, S. J. Air exchange measurements in a high-rise office building, (Proc. ASHRAE/DOE/ORNL Conf. on Thermal Performance of Exterior Envelope of Buildings, Orlando, FL, Dec. 3.5, 1979), ASHRAE SP 28, pp. $160-177$ (American Society of Heating, Refrigeration and Air-Conditioning Engineers, New York, NY, 1981).

20752. Clark, R. E. Weatherization retrofitting and occupant comfort, (Proc. Nat. Conf. Optimal Weatherization, Washington, DC, Dec. 8-10, 1980), Paper in Optimal Weatherization, pp. 60.67 (Information Dynamics, Inc., 111 Claybrook Dr., Silver Spring, MD 20902, 1981).

20759. Mehta, K. C.; Minor, J. E.; Marshall, R. D.; Reinhold, T. A Wind speed-damage correlation in Hurricane Frederic, Proc. ASCE Convention and Exposition, St. Louis, MO, Oct. 26-30, 1981, 14 pages (American Society of Civil Engineers, New York, NY 10017, Nov. 1981).

20764. Simiu, E.; Shaver, J. R.; Filliben, J. J. Extreme wind speeds and structural failure risks, (Proc. Conf. Climate and Risk, Arlington, VA, May 27-29, 1980), Paper MTR-80W322-01 in Climate and Risk, L. S. Pocinki and R. S. Greeley, eds., 1, 2-86-2-100 (The MITRE Corporation, McLean, VA 22102, Nov. 1980). 


\section{Computer Science and Technology}

Kuo, F. K. Computer networks-The ALOHA System. J. Res. Nat. Bur. Stand. (U.S.). 86(6): 591.595; 1981 November-December.

SP500-73. Federal Computer Performance Evaluation and Simulation Center. Computer science \& technology: Computer model documentation guide. Nat. Bur. Stand. (U.S.) Spec. Publ. 500-73; 1981 January. 56 p. SN003.003.02289.5.

SP500-74. Houghton, R. C., Jr. Computer science \& technology: Features of software development tools. Nat. Bur. Stand. (U.S.) Spec. Publ. 500-74; 1981 February. 24 p. SN003.003.02295-1.

SP500-75. Adrion, W. R.; Branstad, M. A.; Cherniavsky, J. C. Computer science \& technology: Validation, verification, and testing of computer software. Nat. Bur. Stand. (U.S.) Spec. Publ. 500-75; 1981 February. 62 p. SN003.003-02289.6.

SP500-76. Berg, J. L.; Graham, M.; Whitney, K., eds. Computer science \& technology: Database architectures-A feasibility worksbop report. Nat. Bur. Stand. (U.S.) Spec. Publ. 500-76; 1981 April. 64 p. SNO03.003.02305.1.

SP500-77. Cugini, J. V. Computer science \& tecbnology: Specifications and test methods for numeric accuracy in programming language standards. Nat. Bur. Stand. (U.S.) Spec. Publ. 500-77; 1981 June. 42 p. SN003-003-02324-8.

SP500-78. Branstad, M. A.; Adrion, W. R., eds. Computer science \& technology: NBS programming environment workshop report. Nat. Bur. Stand. (U.S.) Spec. Publ. S00-78; 1981 June. 106 p. SN003-003. 02334.5.

SP500-79. Gray, M. M. Computer science \& technology: An assessment and forecast of ADP in the Federal Government. Nat. Bur. Stand. (U.S.) Spec. Publ. 500-79; 1981 August. 151 p. SN003-003-02368-0.

SP500-80. Houghton, R. C., Jr., ed. Computer science \& technology: Proceedings of the NBS/IEEE/ACM software tool fair. Proceedings of the NBS/IEEE/ACM Software Tool Fair, held in conjunction with the 5th International Conference on Software Engineering; 1981 March 10-12; San Diego, CA. Nat. Bur. Stand. (U.S.) Spec. Publ. 500-80; 1981 October. 238 p. SN003-003-02362-1.

SP500-80: 1981 October. 1-6. Berild, S.; Nachmens, S. CS4: A tool for the design and generation of database systems.

SP500-80: 1981 October. 7-9. Wirth, N.; Ohran, R. LILITH: A modula macbine.

SP500-80: 1981 October. 10-14. Fenchel, R. S. SARA: A demonstration of the development of interactive software with integral help.

SP500-80: 1981 October. 15.20. Scherrer, D.; Martin, D.; Peterson, C. VIRTUAL OS: The software tools virtual operating system project.

SP500-80: 1981 October. 21-24. Resendez, D. L.; Winchester, J. W. SCG: Structure chart graphics system.

SP500-80: 1981 October. 25.28. Resendez, D. L.; Winchester, J. W. DQM: Design quality metrics system.

SP500-80: 1981 October. 29-32. Austell, W. P., Jr.; Willis, R. R. AISIM: Automated interactive simulation modelling system.

SP500-80: 1981 October. 33-37. Miller, J. C.; Lyons, M. J. THE ENGINE: COBOL structuring engine.

SP500-80: 1981 October. 38-43. Callender, E.; Sayani, H. H.; Teichroew, D. PSL/PSA: Problem statement language/problem statement analyzer.

SP500-80: 1981 October. 44-48. Callender, E.; Hartsough, C.; Kleine, H. SDDL: Software design and documentation language.

SP500-80: 1981 October. 49.57. Putnam, L. H. SLIM: A quantitative tool for software cost and schedule estimation.

SP500-80: 1981 October. 58-63. Levy, A. POD: Performance oriented design.

SP500-80: 1981 October. 64-68. Lycklama, H. PWB FOR VAX/VMS: Programmer workbench tools on VAX/VMS.

SP500-80: 1981 October. 69-75. Erickson, R.; Gerhart, S.; Lee, S.; Thompson, D. AFFIRM: A specification and verification system. SP500-80: 1981 October. 76-82. Penedo, M. H. SARA: SARA as a tool for software design: Building-block modelling and composition. SP500-80: 1981 October. 83.87. King, W. C. ARGUS/MICRO: ARGUS in the microcomputer environment.
SP500-80: 1981 October. 88-95. Stucki, L. G. DYNA: A tool from the ARGUS toolbox.

SP500-80: 1981 October. 96-102. Stucki, L. G. COMMAP: A tool from the ARGUS toolbox.

SP500-80: 1981 October. 103-110. Moy, S.; Nielsen, G.; Sanchez, R.; Wallace, T.; Mc Wethy, S. LOGICFLOW: A software design and analysis tool.

SP500-80: 1981 October. 111-116. Hoffman, R. H.; Loshbough, R. P.; Smith, R. W. SREM: Software requirements engineering methodology.

SP500-80: 1981 October. 117.121. Linden, N.; Yavne, M. SDP: A computerized tool for system design and maintenance.

SP500-80: 1981 October. 122-127. Hug, R.; Strelich, T.; Resnicow, M.; Ahola, R. SOFTOOL 80ß: A methodology and a comprehensive set of tools for software management, development, and maintenance. SP500-80: 1981 October. 128-135. Henderson, J. B.; Miller, E. F., Jr. ITB: Interactive test bed.

SP500-80: 198I October. 136-145. Henderson, J. B.; Miller, E. F., Jr.; Hirschberg, M. ISUS: Interactive semantic update system.

SP500-80: 1981 October. 146-151. Rosenbaum, J.; Early, C. FAME: Front-end analysis and modeling environment.

SP500-80: 1981 October. 152.158. Robillard, P. N.; Plamondon, R. SCHEMACODE: An interactive schematic pseudocode for program development, documentation, and structured coding.

SP500-80: 1981 October. 159-162. Huang, J. C. INSTRU: An automated software instrumentation system.

SP500-80: 1981 October. 163-166. Razouk, R. R. SARA: Controlflow analysis in SARA.

SP500-80: 1981 October. 167-173. Relles, N.; Price, L. A. ONLINE ASSIST: A user interface for online assistance.

SP500-80: 1981 October. 174-179. Barkley, J.; Powell, P. FTN-77 ANALYZER: FORTRAN 77 analyzer.

SP500-80: 1981 October. 180-185. Houghton, R. C., Jr.; Oakley, K. A. TOOLS DATABASE: NBS software tools database.

SP500.80: 1981 October. 186-189. White S. SRIMP: Software requirements integrated modeling program.

SP500-80: 1981 October. 190-193. Gilb, T.; Krzanik, L. AUTO DBO: Automated design by objectives.

SP500-80: 1981 October. 194-196. Overgaard, M.; Gianetta, J. UCSD P.SYSTEM: A portable software development system.

SP500-80: 1981 October. 197.199. Thall, R.; Anderson, G. MSEF: The microprocessor software engineering facility.

SP500-80: 1981 October. 200-207. Saib, S. H.; Benson, J. P.; Gannon, C.; DeHaan, W. R. IFTRAN@: A preprocessor for FORTRAN.

SP500-80: 1981 October. 208-217. Saib, S. H.; Benson, J. P.; Gannon, C.; DeHaan, W. R. RXVP80®: A software documentation, analysis, and test system.

SP500-81. Knoerdel, J. E. Computer science \& technology: A survey of standardization efforts of coded character sets for text processing. Nat. Bur. Stand. (U.S.) Spec. Publ. 500-81; 1981 September. 50 p. SN003-003.02355.8.

SP500-82. Hecht, H. Computer science \& technology: Final report: A survey of software tools usage. Nat. Bur. Stand. (U.S.) Spec. Publ. 500-82; 1981 November. 58 p. SN003-003-02378-7.

SP500-83. Potter, T. W., ed. Computer science \& technology: Proceedings of the Computer Performance Evaluation Users Group (CPEUG) 17tb meeting. "Increasing organizational productivity". Proceedings of the Computer Performance Evaluation Users Group (CPEUG) 17th Meeting; 1981 November 16.19; San Antonio, TX Nat. Bur. Stand. (U.S.) Spec. Publ. S00-83; 1981 November. 320 p. SN003-003-02374-4.

SP500-83: 1981 November. 7.14. Alexander, W.; Brice, R. Longrange prediction of network traffic.

SP500-83: 1981 November. 15.19. Chandler, P. Functional grouping of application programs in a timesharing environment.

SP500-83: 1981 November. 21-34. Wight, A. S. Cluster analysis in workload characterisation for VAX/VMS.

SP500-83: 1981 November. 39-43. Mullen, J. W. Capacity management considerations. 
SP500-83: 1981 November. 45-54. Penansky, S. G. Capacity considerations in disaster recovery planning.

SP500-83: 1981 November. 59.65. Wilson, C. B.; Mohr, J. M.; Chu, C. M. Service reporting for the bottom line.

SP500-83: 1981 November. 67-70. Elsen, L. C. Automating the automation process.

SP500-83: 1981 November. 71-80. Archer, N. P. Methodology for analyzing computer user productivity.

SP500.83: 1981 November. 85-91. Hardgrave W. T.; Salazar, S. B.; Beller, E. J. III. Design of information systems using scenario-driven techniques.

SP500-83: 1981 November. 93-99. Edwards, S. R. Containing the cost of software maintenance testing-An examination of the quality assurance role within U.S. Army Computer Systems Command.

SP500-83: 1981 November. 101-106. Pyper, W. R. Historical files for software quality assurance.

SP500-83: 1981 November. 109.118. Giese, K. W.; Halstead, D.; Wyrick, T. F. A step-by-step approach for developing and implementing a DP charging system.

SP500-83: 1981 November. 121. Kelly, J. C. Session overview: Control of the information system resource through performance management.

SP500-83: 1981 November. 123.138. Vincent, D. R. Increasing system productivity with operational standards.

SP500-83: 1981 November. 143-156. Bays, W. N.; Kincy, W. P., Jr. Universal skeleton for benchmarking batch and interactive workloads: UNISKEL BM.

SP500-83: 1981 November. 157.168. Cabrera, L. F.; Pâris, J. F. Comparing user response times on paged and swapped UNIX by the terminal probe method.

SP500-83: 1981 November. 169-177. Bennett, D. J. Practical application of remote terminal emulation in the Phase IV competitive system acquisition.

SP500-83: 1981 November. 181-189. Hodgins, B. D.; Cox, L. A., Jr. An evaluation technique for early selection of computer hardware.

SP500-83: 1981 November. 191-197. Rucks, A. C.; Ginter, P. M. Deficiencies in the process of procuring small computer systems: A case study.

SP500-83: 1981 November. 199-215. von Mayrhauser, A. K. Haftka, R. T. Cost/performance comparisons for structural analysis software on mini- and mainframe computers.

SP500-83: 1981 November. 223-230. Drayton, P. A. Experiences with DASD, tape, and MSS space management using DMS.

SP500-83: 1981 November. 231-238. Silliman, K. E. DASD capacity planning 3350 DASD.

SP500-83: 1981 November. 239-246. Atwood, J. W.; Yu, K. C. An analysis of a CDC844-41 disk subsystem.

SP500-83: 1981 November. 251-258. Kelly, J. C.; Blaylock, J. W. Tuning the facilities complex of UNIVAC 1100 OS.

SP500-83: 1981 November. 259-265. Lin, W. K.; Tonik, A. B Approximate evaluation of a bubble memory in a transaction driven system.

SP500-83: 1981 November. 271-276. Snow, A. P. Local integrated communication systems: The key to future productivity.

SP500-83: 1981 November. 277-290. Harrison, P. G. Performance analysis of a flow controlled communication network node.

SP500-83: 1981 November. 293-302. Jun, E. T. Resource management in a distributed processing environment-Computer resource selection guidelines.

SP500-83: 1981 November. 305-307. Cunningham, M. L. Office automation and productivity.

SP500-83: 1981 November. 311-312. Conti, D. M.; Giese, K. W. Kyriazi, C. N.; Trehan, R. ADP cost accounting \& chargeback.

SP500-83: 1981 November. 325. Artis, H. P. Measurement, modeling, and capacity planning: The secondary storage occupancy issue.

SP500-83: 1981 November. 327-328. Berry, W. W. Micro-computers in office automation.

SP500-83: 1981 November. 329. Sprung, J. G. Computer performance management in the ADP system life cycle.

SP500-83: 1981 November. 331-334. Neuse, D.; Chandy, K. M.; Misra, J.; Berry, R. Simulation tools in performance evaluation.
SP500-83: 1981 November. 335. Sauer, C. H. Computational methods for queueing network models.

SP500-83: 1981 November. 337 . Howard, P. C. Productivity in the development function.

SP500-83: 1981 November. 339. Conti, D. M. Acquisition benchmarking.

SP500-83: 1981 November. 341. Buschbach, T. R. Software conversion process and problems.

SP500-83: 1981 November. 343-348. Mullen, J. W. Resource measurement facility (RMF) data: A review of the basics.

SP500-84. Draper, J. M. Computer science \& technology: Costs and benefits of database management: Federal experience. Nat. Bur. Stand. (U.S.) Spec. Publ. 500-84; 1981 November. 106 p. SN003-00302376-1.

FIPS PUB 88. Perry, W. E.; Goldfine, A., Standards Coordinators. Guideline on integrity assurance and control in database administration. Nat. Bur. Stand. (U.S.) Fed. Info. Process. Stand. Publ. (FIPS PUB) 88; 1981 August 14. 71 p.

NBSIR 80-2138. Lozier, D. W. Multiprecision computing at NBS: Yesterday, today, and tomorrow. 1980 October. 54 p. Available from: NTIS; PB \$1-243941

NBSIR 80-2154. Blanc, R.; Heafner, J.; Rosenthal, R.; Watkins, S. The NBS Computer Networking Program. 1980 October. 22 p. Available from: NTIS; PB 81.158255.

NBSIR 80-2182. Marriott, A. R.; Skillman, G. H.; Salazar, S. B.; Hardgrave, W. T. M7: A general pattern matching facility users manual. 1981 January. 40 p. Available from: NTIS; PB 81-164758.

NBSIR 80-2187. Mink, A.; Silio, C. B., Jr. Local area network feasibility study for the Naval Sea Systems Command. 1981 January. 55 p. Available from: NTIS; PB 81-170656.

NBSIR 81-2240. Hardgrave, W. T.; Salazar, S. B.; Beller, E. J. III. Design of information systems using scenario-driver techniques. 1981 March. 24 p. Available from: NTIS; PB 81-180986.

NBSIR 81-2315. Fry, J. P.; Chiang, W. P.; DeSmith, D. A.; Leder, D. S.; Nguyen, D. K.; Perreault, R. W. Draft specification for structured data interchange form. $1981 \mathrm{July} .126 \mathrm{p}$. Available from: NTIS; PB 81.247637.

NBSIR 81-2388. Hecht, $H$. Synopsis of interviews from a survey of software tool usage. 1981 November. 80 p. Available from: NTIS; PB 82.133521.

NBS-GCR-80-306. Dickamore, W. G.; Dampier, I. K., (NBS contact: Michael Hogan). Survey and market forecast of flexible disks. 1980 November. 110 p. Available from: NTIS; PB 81-171258.

NBS-GCR-80-307. Dickamore, W. G.; Kampier, I. K., (NBS contact: Michael Hogan). Technology assessment of flexible disks as used in stand-alone text-processing systems. 1980 November. 86 p. Available from: NTIS; PB 81-171365.

NBS.GCR-81-323. Network Analysis Corporation Staff, (NBS contact: B. Wilson). 1980 November. 299 p. Available from: NTIS PB 81.203622.

NBS-GCR-81.324. Shelton, C. B.; Moulton, J. R., (NBS contact: B. Wilson). Virtual terminal feature analysis-Draft report. 1981 March. 60 p. Available from: NTIS; PB 81-205387.

NBS-GCR-81-335. Reifer, D. J.; Montgomery, H. A., (NBS contact: R. Houghton). Final report: Software tool taxonomy. 1981 June 1. 113 p. Available from: NTIS; PB 82-103961.

NBS-GCR-81-336. Geer, N.; Graff, H. A.; Stromecky, P., (NBS contact: R. T. Moore). GRIDNET SIMULATION. Volume 1: System description and results. 1981 August. 61 p. Available from: NTIS; PB 82-116013.

NBS-GCR-81-337. Manola, F.; Smith, J.; Smith, D., (NBS contact: L. Gallagher). Evaluation criteria for a CODASYL query language. 1980 March. 147 p. Available from: NTIS; PB 82-136748.

NBS-GCR-81-338. Manola, F., (NBS contact: L. Gallagher). Capabilities for a CODASYL query language. 1980 June. 126 p. Available from: NTIS; PB 82-136755.

NBS-GCR-81-339. Manola, F.; Barnett, J., (NBS contact: L. Gallagher). A survey of CODASYL query languages. 1980 September. 201 p. Available from: NTIS; PB 82-136765.

NBS-GCR-81-342. Arthur D. Little, Inc. and General Systems Group, Inc. (NBS contact: P. Kay). The effects of future information 
processing technology on the Federal Government ADP situation. 1981 September. 110 p. Available from: NTIS; PB 82-138181.

20055. Schmid, G. B.; Norcross, D. W.; Collins, L. A. VLAM, a program for computing the electron-molecule static interaction potential from a Legendre expansion of the molecular charge density, Comput. Phys. Commun. 21, $79-90$ (1980).

20075. Zelkowitz, M. V. A case study in rapid prototyping, SoftwarePract. Exper. 10, 1037.1042 (1980).

20172. Sockut, G. H. Comparison and mapping of the relational and CODASYL data models-An annotated bibliography, SIGMOD Record 11, No. 3, 55-68 (Apr. 1981).

20220. Lyon, G. ANS FORTRAN: Past and near future, (Proc. Int. Computer Technology Conf. ASME Century 2-Emerging Technology Confs., San Francisco, CA, Aug. 12-15, 1980), Paper in Advances in Computer Technology-1980 2, $402-404$ (The American Society of Mechanical Engineers, New York, NY, 1980).

20223. Mink, A.; Knoerdel, J.; Brooks, J. The design and implementation of an office application using existing high level tools, Proc. Trends and Applications 1981: Advances in Software Technology, Gaithersburg, $M D$, pp. 150-162 (IEEE Service Center, Piscataway, NJ, May 28, 1981).

20378. Ruthberg, Z. G. Risk assessment and managerial analysis, EDP Auditor, pp. 31-43 (1980).

20420. Smid, M. E. Integrating the Data Encryption Standard into computer networks, IEEE Trans. Commun. COM-29, No. 6, 762.772 (June 1981).

20473. Lyon, G. E. Alternation-tree insertions for open-addressed hash buckets, Proc. 15th Annu. Conf. Information Sciences and Systems, Baltimore, MD, Mar. 25-27, 1981, pp. 398-401 (The Johns Hopkins University, Dept. Electrical Engineering, Baltimore, MD, 1981).

20644. Deutsch, D. R.; Clemons, E. K. DBMS standards: Current status and future directions, Proc. 6th Int. Conf. Very Large Databases, Montreal, Canada, Oct. 1-3, 1980, pp. 431.433 (The Institute of Electrical and Electronics Engineers Computer Society, 5855 Naples Plaza, Long Beach, CA 90803, 1980).

20662. McCoy, W. H.; Colella, R. P.; Wallace, M. A. Assessing the performance of high-level computer network protocols, Proc. Protocol Testing-Towards Proof? INWG/NPL Workshop, Teddington, Middlesex, United Kingdom, May 27-29, 1981, 2, 25.53 (National Physical Laboratory, Teddington, Middlesex, United Kingdom, Sept. 1981).

20747. Conti, D. M. U.S. efforts to develop standard benchmark programs, (Proc. Int. Conf. Performance of Computer Installations, Lake Garda, Italy, June 22-23, 1978), Paper in Performance of Computer Installations, D. Ferrari, ed., pp. 55-66 (North Holland Publ. Co., Amsterdam, The Netherlands, 1978)

\section{Consumer Information and Protection}

H130, 1982 Edition. Brickenkamp, C. S., ed. Model state laws and regulations. Nat. Bur. Stand. (U.S.) Handb. 130, 1982 Edition; 1981 September. 104 p. SN003-003-02369-8.

H133. Brickenkamp, C. S.; Hasko, S.; Natrella, M. G. Checking the net contents of packaged goods. Nat. Bur. Stand. (U.S.) Handb. 133; 1981 June. 164 p. SN003-003-02331-1.

H44, 1982 Edition. Wollin, H. F., ed. Specifications, tolerances, and other technical requirements for weighing and measuring devices. Nat. Bur. Stand. (U.S.) Handb. 44, 1982 Edition; 1981 September. 205 p. SN003-003-02364-7.

NBSIR 81-2291 (HUD). Gordon, C. C.; Palla, R. I., Jr. Performance aspects of water conservation techniques for appliances. 1981 May. 40 p. Available from: NTIS; PB 81-223448.

NBSIR 81-2336. Breitenberg, M. A.; Atkins, R. G. Consumer representation in standards development: Literature review and issue identification. 1981 September. 78 p. Available from: NTIS; PB 82. 135880 .

\section{Electromagnetic Metrology}

Monogr. 162. Kerns, D. M. Plane-wave scattering-matrix theory of antennas and antenna-antenna interactions. Nat. Bur. Stand. (U.S.) Monogr. 162; 1981 June. 179 p. SN003-003-01995-0.

TN1013. Crawford, M. L.; Workman, J. L. Using a TEM cell for EMC measurements of electronic equipment. Nat. Bur. Stand. (U.S.) Tech. Note 1013; 1981 July. 72 p. SN003-003-02350-7.

TN1028. Kanda, M. Theoretical and experimental investigations of electromagnetic field distortion due to a perfectly conducting rectangular cylinder in a transverse electromagnetic cell. Nat. Bur. Stand. (U.S.) Tech. Note 1028; 1981 April. 48 p. SN003-003-02313-2.

TN1033. Larsen, E. B.; Ries, F. X. Design and calibration of the NBS isotropic electric-field monitor (EFM-5), 02 to 100 MHz. Nat. Bur. Stand. (U.S.) Tech. Note 1033; 1981 March. 104 p. SN003.003-02311. 6.

TN1037. Wilson, P. F.; Chang, D. C.; Ma, M. T. Excitation of a TEM cell by a vertical electric Hertzian dipole. Nat. Bur. Stand. (U.S.) Tech. Note 1037; 1981 March. 44 p. SN003-003-02348-5.

TN1040. Sreenivasiah, I.; Chang, D. C.; Ma, M. T. A method of determining the emission and susceptibility levels of electrically small objects using a TEM cell. Nat. Bur. Stand. (U.S.) Tech. Note 1040; 1981 April. 52 p. SN003-003-02316-7.

TN1042. Gans, W. L.; Nahman, N. S. Shielded balanced and coaxial transmission lines-Parametric measurements and instrumentation relevant to signal waveform transmission in digital service. Nat. Bur. Stand. (U.S.) Tech. Note 1042; 1981 June. 260 p. Available from: NTIS; PB 82-105297.

TN1044. Weidman, M. P.; Hudson, P. A. WR 10 millimeter wave microcalorimeter. Nat. Bur. Stand. (U.S.) Tech. Note 1044; 1981 June. 16 p. Available from: NTIS; PB 81-243412.

TN1047. Nahman, N. S.; Guillaume, M. E. Deconvolution of time domain waveforms in the presence of noise. Nat. Bur. Stand. (U.S.) Tech. Note 1047; 1981 October. 122 p. Available from: NTIS; PB $82-135153$

NBSIR 78-873. Tippet, J. C.; Chang, D. C. Higher order modes in rectangular coaxial line with infinitely thin inner conductor. 1978 March. 40 p. Available from: NTIS; PB 279054.

NBSIR 78-876. Kanda, $M$. Transients in resistively loaded antennas and their comparison with conical antennas and TEM borns. 1978 March. 35 p. Available from: NTIS; PB 279640.

NBSIR 78-898. Kanda, M. Analytical and numerical techniques for analyzing an electrically short dipole witb a nonlinear load. 1978 November. 21 p. Available frem: NTIS; PB 289928.

NBSIR 80-1630. Stubenrauch, C. F.; Yaghjian, A. D. Determination of mutual coupling between co-sited microwave antennas and calculation of near-zone electric field. 1981 June. 172 p. Available from: NTIS; PB 81-246217.

NBSIR 80-1635. Miller, C. K. S., ed. A bibliograpby of publications in the NBS Electromagnetic Fields Division. 1980 November. 35 p. Available from: NTIS; PB 81-143158.

NBSIR 81-1638. Crawford, M. L. Evaluation of a reverberation chamber facility for performing EM radiated fields susceptibility measurements. 1981 February. 44 p. Available from: NTIS; PB 81 181257.

NBSIR 81-1643. Lewis, R. Efficient computation of the far field radiated by an arbitrary rectangular-aperture distribution. Computer program documentation. 1981 March. 45 p. Available from: NTIS; PB 81-200362.

NBSIR 81-1644. Yaghjian, A. D. An approximate expression for the principal beamwidths of directive antennas in terms of aperture fields. 1981 March. 14 p. Available from: NTIS; PB 81-200388.

NBSIR 81-1646. Andrews, J. R.; Baldwin, E. E. Amplitude calibrator for oscilloscopes. 1981 April. 31 p. Available from: NTIS; PB 81211286.

NBSIR 81-1650. Weidman, M. WR-10 single six-port measurement system. 1981 September. 11 p. Available from: NTIS; PB 82.123696.

20067. Engen, G. F. A least squares solution for use in the six-port measurement technique, IEEE Trans. Microwave Theory Tech. MTT28, No. 12, 1473-1477 (Dec. 1980).

20094. Souders, T. M.; Lechner, J. A. A technique for measuring tbe equivalent RMS input noise of A/D converters, IEEE Trans. Instrum. Meas. IM-29, No. 4, 251-256 (Dec. 1980).

20149. Henderson, M. M.; MacNaughton, M. J., eds. Electronic 
communication: Technology and impacts, (Proc. AAAS Selected Symp. 52, Houston, TX, Jan. 3-8, 1979), Book: 191 pages (Westview Press Inc., Boulder, CO, 1980).

20239. Williams, E. S. A thermoelement comparator for automatic acdc difference measurements, IEEE Trans. Instrum. Meas. IM-29, No. 4, 405.409 (Dec. 4, 1980).

20240. Galakhova, O. P.; Harkness, S.; Hermach, F. L.; Hirayama, H.; Martin, P.; Rozdestvenskaya, T. H.; Williams, E. S. An international comparison of thermal converters as ac-dc transfer standards, IEEE Trans. Instrum. Meas. IM-29, No. 4, 396-399 (Dec. 4, 1980).

20301. Ma, M. T. Synthesis of broadband antenna arrays as possible over-the-horizon radars, Chapter 9 in Research Topics in Electromagnetic Wave Theory, J. A. Kong, ed., pp. 188-209 (John Wiley \& Sons, New York, NY, 1981).

20313. Andrews, J. Pulse reference waveform standards development at NBS, Proc. ATE Seminar/Exhibit Automated Testing for Electronics Manufacturing, Pasadena Center, Pasadena, CA, Jan. 19-22, 1981, pp. IV-13-IV-19 (Benwill Publ. Corp., Boston, MA, 1981).

20322. Miller, C. K. S. Challenges of EMI measurements, (Proc. 1980 Workshop \& Symp. National Conference of Standards Laboratories, National Bureau of Standards, Gaithersburg, MD, Sept. 22-25, 1980), Paper in NCSL Newslett. 21, No. 1, 16-23 (Mar. 1981).

20347. Day, G. W.; Franzen, D. L. Progress in fiber measurement. An LF meeting report from Boulder, Colorado. Laser Focus Mag., pp. 52.56 (Feb. 1981).

20545. Repjar, A. G.; Kremer, D. P. Results of planar near-field measurements on a compact range at 18 and $54 \mathrm{GHz}$, (Proc. Antennas and Propagation, Quebec, Canada, June 2-6, 1980), Paper in Antennas and Propagation 1, IEEE No. $80 \mathrm{CH} 1557-8$ AP, 256.259 (Institute of Electrical and Electronics Engineers, New York, NY, 1980).

20622. Bensema, W. D. Broadband orthogonal array antenna system microprocessor control and computation, Proc. 1981 IEEE Int. Symp. on Electromagnetic Compatibility, Boulder, CO, Aug. 18-20, 1981, pp. 43-45 (The Institute of Electrical and Electronics Engineers, Inc., New York, NY 10017, 1981).

20623. Sreenivasiah, I.; Chang, D. C.; Ma, M. T. A critical study of emission and susceptibility levels of electrically small objects from tests inside a TEM cell, Proc. 1981 IEEE Int. Symp. Electromagnetic Compatibility, Boulder, CO, Aug. 18-20, 1981, pp. $499-507$ (The Institute of Electrical and Electronics Engineers, Inc., New York, NY 10017, 1981).

20624. FitzGerrell, R. G. Free-space transmission loss for anechoic chamber performance evaluation, Proc. 1981 IEEE Int. Symp. Electromagnetic Compatibility, Boulder, CO. Aug. 18-20, 1981, pp. 110-111 (The Institute of Electrical and Electronics Engineers, Inc., New York, NY 10017, 1981).

20630. Ebbesen, H. F.; Engen, G. F. Singularities in the calibration of six-port network analyzers, (Proc. 1981 IEEE MTT-S Int. Microwave Symp., Los Angeles, CA, June 15.19, 1981), Paper in 1981 IEEE MTT-S International Symposium Digest, IEEE Cat. No. 81CH1592-5, J. E. Raue, ed., pp. 149.150 (The Institute of Electrical and Electronics Engineers, Inc., 445 Hoes Lane, Piscataway, NJ 08854, 1981).

20631. Hoer, C. A. A high-power dual six-port automatic network analyzer for determining biological effects of $R F$ and microwave radiation, (Proc. 1981 IEEE MTT.S Int. Microwave Symp., Los Angeles, CA, June 15-19, 1981), Paper in 1981 IEEE MTT-S International Microwave Symposium Digest, IEEE Cat. No. $81 \mathrm{CH} 1592-5$, J. E. Raue, ed., pp. 157.159 (The Institute of Electrical and Electronics Engineers, Inc., 445 Hoes Lane, Piscataway, NJ 08854, 1981).

20632. Lewis, R. L. Spherical-wave source-scattering-matrix analysis of the mutual coupling between two antennas, Proc. AP-S Int. Symp. 1981, Los Angeles, CA, June 16-19, 1981, pp. 261-264 (The Institute of Electrical and Electronics Engineers, Inc., 445 Hoes Lane, Piscataway, NJ 08854, 1981).

20634. Cruz, J. E. Design, construction, and calibration of the broadband electric field monitor (EFM-5), Proc. 1981 IEEE Int.
Symp. Electromagnetic Compatibility, Boulder, CO, Aug. 18-20, 1981, pp. 439.444 (The Institute of Electrical and Electronics Engineers, Inc., 445 Hoes Lane, Piscataway, NJ 08854, 1981).

20650. Estin, A. J.; Stubenrauch, C. F.; Repjar, A. G.; Newell, A. C. Optimized wavelength-sized scalar horns as antenna radiation standards, (Proc. Electrical and Electronic Measurement and Test Instrument Conf. and Expo., Ottawa, Canada, Sept. 22-24, 1981), EEMTIC 1981 Digest 81 CH1710-3, pp. 64-68 (The Institute of Electrical and Electronics Engineers, Inc., New York, NY, 1981).

20663. Reeve, G. R. Synthesized isotropic pattern antennas for EM field measurements, Proc. IEEE Int. Symp. Electromagnetic Compatibility, Boulder, CO, Aug. 18-20, 1981, pp. $36-42$ (The Institute of Electrical and Electronics Engineers, Inc., 445 Hoes Lane, Piscataway, NJ 08854, 1981).

20711. Yaghjian, A. D. A delta-distribution derivation of the electric field in the source region, (Proc. 1981 Int. IEEE Antennas and Propagation Symp., Los Angeles, CA, June 17-19, 1981), IEEE Dig., pp. 334-337 (Institute of Electronics and Electrical Engineers, Piscataway, NJ 08854, 1981).

20760. Yaghjian, A. D. Augmented electric- and magnetic-field integral equations which eliminate the spurious resonances, Proc. Int. URSISymp. Electromagnetic Waves, Munich, Germany, Aug. 26-29, 1980. 121 pages, $B / 1-121, B / 4(1980)$.

20761. Yaghjian, A. D. Augmented electric- and magnetic-field equations, Radio Sci. 16, No. 6, 987-1001 (Nov.-Dec. 1981).

\section{Electronic Technology}

Esaki, L. Highlights in semiconductor device development. J. Res. Nat. Bur. Stand. (U.S.) 86(6): 565-570; 1981 November-December.

SP400-64. Thurber, W. R.; Mattis, R. L.; Liu, Y. M.; Filliben, J. J. Semiconductor measurement technology: The relationship between resistivity and dopant density for phosphorusand boron-doped silicon. Nat. Bur. Stand. (U.S.) Spec. Publ. 400-64; 1981 May. 53 p. SN003003-02320-5.

SP400-65. Mitchell, M. A.; Linholm, L. W. Semiconductor measurement technology: Test patterns NBS-28 and NBS-28A: Random fault interconnect step coverage and other structures. Nat. Bur. Stand. (U.S.) Spec. Publ. 400-65; 1981 March. 35 p. SN003-00302297.7.

SP400-66. Linholm, L. W. Semiconductor measurement technology: The design, testing, and analysis of a comprehensive test pattern for measuring CMOS/SOS process performance and control. Nat. Bur. Stand. (U.S.) Spec. Publ. 400-66; 1981 August. 148 p. SN003-003. 02345-1.

SP400-67. Schwarz, S. A.; Helms, C. R.; Spicer, W. E.; Taylor, N. J. Semiconductor measurement technology: The capabilities and limitations of Auger sputter profiling for studies of semiconductors. Nat. Bur. Stand. (U.S.) Spec. Publ. 400-67; 1981 September. 53 p. Available from: NTIS; PB 82-120122.

SP400-68. Carver, G. P.; Cullins, W. A. Semiconductor measurement technology: A manual wafer probe station for an integrated circuit test system. Nat. Bur. Stand. (U.S.) Spec. Publ. 400-68; 1981 May. 19 p. SN003-003-02319-1.

SP400-69. Schafft, H. A.; Ruthberg, S.; Cohen, E. C., eds. Semiconductor measurement technology: ARPA/NBS workshop V. Moisture measurement technology for hermetic semiconductor devices. Proceedings of the ARPA/NBS Workshop V held at the National Bureau of Standards; 1978 March 22-23; Gaithersburg, MD. Nat. Bur. Stand. (U.S.) Spec. Publ. 400-69; 1981 May. 202 p. SN003-003-02326-4.

SP400-69: 1981 May. 3-23. Moore, B. A. Mass spectrometer measurements at RADC.

SP400-69: 1981 May. 24-32. Hartley, J. L. A method for measuring the PPM moisture-sensing limitations of mass spectrometers in testing small packages.

SP400-69: 1981 May. 33-41. Merrett, R. P. A dynamic method of calibrating a mass spectrometer used for measuring the water content of semiconductor encapsulations.

SP400-69: 1981 May. 42. Pernicka, J. C. Moisture measurement by mass spectrometer. 
SP400-69: 1981 May. 43-57. Haack, R. F.; Shumka, A. Microcircuit package gas analysis.

SP400-69: 1981 May. 58-68. Perkins, K. L. Comparison of mass spectrometric moisture measurements by different laboratories.

SP400-69: 1981 May. 69-74. Fong, V. The effects of assembly techniques upon the performance characteristics of $\mathrm{Al}_{2} \mathrm{O}_{3}$ in-situ moisture sensors.

SP400-69: 1981 May. 75-79. Bennewitz, P. F. Application considerations of solid state moisture chips for hybrids and associated calibration measurement techniques.

SP400-69: 1981 May. 80-81. Webster, H. L. Hysteresis and longterm repeatability of aluminum oxide humidity sensors.

SP400-69: 1981 May. 82-93. Messenger, C. G. Moisture standards and sensors.

SP400-69: 1981 May. 94-107. Merrett, R. P.; Sim, S. P. Assessment of the use of measurement of surface conductivity as a means of determining moisture content of hermetic semiconductor encapsulations.

SP400-69: 1981 May. 108-114. Senturia, S. D.; Huberman, M. G.; Van der Klott, R. Moisture sensing with the charge-flow transistor. SP400.69: 1981 May. 115-121. Knight, C. W. T.; Korgav, A.; Pierron, E. A surface conductance technique for the evaluation of internal package environments.

SP400-69: 1981 May. 126-127. Hasegawa, S. Moisture sensors.

SP400-69: 1981 May. 128-132. Hartley, J. L. Parts-per-million watervapor generating systen used to simulate moisture in small integrated circuit packages.

SP400-69: 1981 May. 133-142. Loucks, S. R.; Burnham, A. W. A total moisture analyzer for hermetic devices.

SP400-69: 1981 May. 143-152. Dun, H.; Mattes, B. L.; Stevenson, D. A. Analysis of water vapor in purified gas systems.

SP400-69: 1981 May. 153-158. Hickam, W. M.; Morgan, W. R. Mass spectrometric moisture analysis of hermetic semiconductor devices by acetylene conversion.

SP400-69: 1981 May. 159-178. Der Marderosian, A. Moisture in packages-A user's viewpoint.

SP400-69: 1981 May. 179-186. Thomas, R. W. Problems in specifying and measuring moisture content within electronic devices.

SP614. Meijer, P. H. E.; Mountain, R. D.; Soulen, R. J., Jr., eds. Sixth International Conference on Noise in Physical Systems. Proceedings of a Conference held at the National Bureau of Standards; 1981 April 6-10; Gaithersburg, MD. Nat. Bur. Stand. (U.S.) Spec. Publ. 614; 1981 September. 419 p. SN003-003-02353-1.

SP614: 1981 September. 3.11. van Vliet, K. M. Classification of noise phenomena.

SP614: 1981 September. 12-17. Landauer, R. Fundamental physical limitations of the computational process.

SP614: 1981 September. 18-21. Shlesinger, M. F.; Hughes, B. D.; Montroll, E. W. Fractal random walks.

SP614: 1981 September. 22-25. Frehland, E. Transport fluctuations around non-equilibrium steady states in discrete systems.

SP614: 1981 September. 26-29. Sancho, J. M.; San Miguel, M.; Katz, S.; Gunton, J. D. Multiplicative noise in stochastic differential equations: A numerical study.

SP614: 1981 September. 30-33. Mimaki, T.; Sato, H.; Tanabe, M. Level-crossing intervals of a Gaussian noise.

SP614: 1981 September. 34-37. Celasco, M.; Mazzetti, P.; Stepanescu, A. Study of disordered structures as a spatial noise problem.

SP614: 1981 September. 38-41. Buckingham, M. J. On the theory of ambient acoustic noise in the shallow ocean.

SP614: 1981 September. 42-46. Nougier, J. P.; Vaissiere, J. C. Gasquet, D. Mathematical formulation of the impedance field method. Application to the noise of the channel of field effect transistors.

SP614: 1981 September. 47-50. Sautereau, J. F.; Graffeuil, J.; Martin, J. C. Time domain large signal noise modeling in microwave oscillators.

SP614: 1981 September. 51-53. Walma, A. A. Spectral superposition and irreversibility.

SP614: 1981 September. 54-56. Walma, A. A. Classical noise and non-linearity.
SP614: 1981 September. 57-60. Bergman, D. J.; Ben-Jacob, E.; Schuss, Z. Fluctuations and transition rates in time dependent dissipative steady states.

SP614: 1981 September. 63-66. Sodini, D.; Rigaud, D. A new approach to model channel "thermal" noise of the JG. Fet considered as an active R.C line.

SP614: 1981 September. 67-70. Liu, S. T.; Huang, J. S. T. Noise and scaling properties of subthreshold MOSFETs.

SP614: 1981 September. 71.74. Cox, C. E.; Kandiah, K. The dependence of the low frequency noise of JFETs on device parameters and operating conditions.

SP614: 1981 September. 75.78. Kandiah, K.; Deighton, M. O. Whiting, F. B. The characteristics of noise due to individual defects in JFETs.

SP614: 1981 September. 79-83. Mehta, H.; van Vliet, K. M. Noise due to fast surface states in MOSFETs.

SP614: 1981 September. 84-87. Graffeuil, J.; Sautereau, J. F.; Tantrarongroj, K. Low frequency noise in GaAs FET's at low drain voltages.

SP614: 1981 September. 88-92. van der Ziel, A. Thermal noise in the semiconductor regime of double-injection diodes.

SP614: 1981 September. 93-96. Aoki, K.; Miyamae, K.; Kobayashi, T.; Yamamoto, K. Photocurrent noise caused by impact ionization of neutral donors, and free and bound excitons in n-GaAs.

SP614: 1981 September. 97-99. Knott, K. F. Low energy electron beam investigation of planar transistors using scanning electron microscopy with particular reference to burst noise.

SP614: 1981 September. 100-104. Sikula, J.; Sikulova, M.; Vasina P.; Koktavy, B. Burst noise in diodes.

SP614: 1981 September. 105-108. Alabedra, R.; Walma, A. A.; Maille, C.; Lecoy, G. Non-white multiplication noise in the dark current regime of $\mathrm{A} \mathrm{P}^{+}$IIPIIN ${ }^{+}$avalanche diode.

SP614: 1981 September. 109-112. Maille, C.; Alabedra, R.; Lecoy, G.; Savelli, M. Theory and experiment of avalanche noise in the weak multiplication regime.

SP614: 1981 September. 113-117. Gong, J.; van Vliet, K. M. Noise measurements on photo avalanche diodes.

SP614: 1981 September. 118-120. Blasquez, G.; Lavernhe, F.; Marty, A.; Bailbe, J. P. Noise in heterojunction transistors.

SP614: 1981 September. 121-124. Touboul, A.; Walma, A. A.; Lecoy, G. Digital analysis of superimposed fluctuations on a pulse train.

SP614: 1981 September. 125.129. Sikula, J.; Cermakova, A.; Cermak, M.; Vasina, P. Noise in dielectric materials.

SP614: 1981 September. 133-142. Weissman, M. B. Survey of recent $1 / f$ noise theories.

SP614: 1981 September. 143-146. Musha, T. 1/f fluctuations in biological systems.

SP614: 1981 September. 147-150. Scofield, J. H.; Darling, D. H.; Webb, W. W. 1/f noise in continuous metal films is not due to temperature fluctuations.

SP614: 1981 September. 151. Kilmer, J.; van Vliet, K. M.; Chenette, E. R.; Handel, P. H. Temperature response and correlation of $1 / f$ noise in transistors.

SP614: 1981 September. 152. Black, R. D.; Weissman, M. B.; Fliegle, F. M. Lack of spatial cross-correlation in $1 / \mathrm{f}$ noise in chrome films.

SP614: 1981 September. 153-156. Pavlin, I.; Engelsberg, S. Temperature fluctuations in a steady state.

SP614: 1981 September. 157-160. Machlup, S. Earthquakes, thunderstorms, and other $1 / \mathrm{f}$ noises.

SP614: 1981 September. 161-164. Ngai, K. L.; Liu, F. 1/f diffusion noise.

SP614: 1981 September. 165-168. Ngai, K. L. Temperature dependence of the excess $1 / \mathrm{f}$ noise in metals.

SP614: 1981 September. 169-172. Bell, D. A. The role of mobility in 1/f noise.

SP614: 1981 September. 173-177. Jindal, R. P.; van der Ziel, A. A model for 1 /f mobility fluctuations in elemental semiconductors.

SP614: 1981 September. 178-180. Zaklikiewicz, A. M. Common mechanism of $1 / \mathrm{f}$ noise and burst noise. 
SP614: 1981 September. 181-184. Bliek, L. M. 1/f voltage noise caused by scattering of electrons in metals.

SP614: 1981 September. 185-188. Pellegrini, B. Complements of $1 / \mathrm{f}$ noise theory.

SP614: 1981 September. 189-191. Uebersfeld, J.; Handel, P. H.; Gagnepain, J. J. Fluctuations of the relaxation time as a source of $1 / \mathrm{f}$ noise in macroscopic physical systems.

SP614: 1981 September. 192-195. Maccone, C. 1/f ${ }^{x}$ noises and the Riemann-Liouville fractional integral/derivative of the Brownian motion.

SP614: 1981 September. 196.198. Handel, P. H.; Wolf, D.; van Vliet, K. M. Non-Gaussian amplitude distribution of thermal noise in resistors with $1 / \mathrm{f}$ noise.

SP614: 1981 September. 199.201. D'Amico, A.; Cappuccio, G.; Petrocco, G.; Fotinos, C.; Stochino, G. $1 / \mathrm{f}^{a}$ noise behavior in infrared photoconductive polycrystalline thin films of $\mathbf{P b}_{x} \mathbf{S N}_{1-x} \mathrm{Te}$.

SP614: 1981 September. 202-205. Prudenziati, M.; Morten, B.; Masoero, A. Temperature dependence of $1 / \mathrm{f}$ noise in thick-film resistors.

SP614: 1981 September. 206-209. Jones, B. K. Excess conductance noise in silicon resistors.

SP614: 1981 September. 210-212. Amberiadis, K.; van der Ziel, A. Rucker, L. M. 1/f noise in gate-controlled implanted resistors.

SP614: 1981 September. 213-216. Krusin-Elbaum, L.; Voss, R. F. Low frequency voltage noise in small area Josephson junctions.

SP614: 1981 September. 217-220. van den Berg, R. J.; de Vos, A.; de Goede, J. Concentration fluctuations in small volumes of ionic solutions.

SP614: 1981 September. 221-225. Min, H. S. 1/f noise in MOS transistors in ohmic region under strong inversion.

SP614: 1981 September. 226-229. Park, H. S.; van der Ziel, A. 1/r noise models in MOSFETs.

SP614: 1981 September. 230-233. Gentil, P.; Mounib, A. 1/f noise in short channel MOSTs.

SP614: 1981 September. 234-235. Hiatt, C. F.; van der Ziel, A.; Zijlstra, R. J. J. Theoretical interpretation of low $1 / \mathrm{f}$ noise in JFETs. SP614: 1981 September. 236-239. Suh, C. H.; van der Ziel, A.; Jindal, R. P. 1/f noise in GaAs MESFETs.

SP614: 1981 September. 240-243. Kleinpenning, T. G. M. 1/f noise of hot carriers.

SP614: 1981 September. 244-247. Vandamme, L. K. J.; Dik, L. S. $\mathrm{H}$. Bias-temperature treatment, surface state density, and $1 / \mathrm{f}$ noise in MOSTs.

SP614: 1981 September. 248-250. Sikula, J.; Vasina, P.; Koktavy, B.; Chobola, Z. 1/f noise in Schottky barrier diodes.

SP614: 1981 September. 253-259. Ulrich, B. T. Josephson junctions, plasma physics, and electron temperatures.

SP614: 1981 September. 260-268. Blalock, T. V.; Shepard, R. L. Survey, applications, and prospects of Johnson noise thermometry.

SP614: 1981 September. 269-271. Klempt, G.; Storm, L. Determination of the thermodynamic temperature of fixed points by means of a highly accurate noise thermometer.

SP614: 1981 September. 272-275. Pickup, C. P. A precision noise thermometer for temperature scale studies.

SP614: 1981 September. 276-282. Zijlstra, R. J. J.; Westera, W. Electro-acoustical noise.

SP614: 1981 September. 283-287. Swinney, H. L.; Roux, J. C.; King, G. P. Experimental studies of noise in a chemical reaction and in a fluid flow.

SP614: 1981 September. 288-291. Gollub, J. P. Dynamical noise in tunnel diode oscillator systems.

SP614: 1981 September. 292-297. Giffard, R. P.; Michelson, P. F.; Taber, R. C. Noise in resonant gravitational wave detectors.

SP614: 1981 September. 298-301. Bonifazi, P.; Bordoni, F.; Pallottino, G. V.; Pizzella, G. Measurement of the Brownian noise of a harmonic oscillator with mass $M=389 \mathrm{Kg}$.

SP614: 1981 September. 302-304. Blasquez, G. Two-port noise characterization by means of a feedback method.

SP614: 1981 September. 305-308. Gasquet, D.; Vaissiere, J. C.; Nougier, J. P. New method for wide band measurement of noise temperature of one-port networks at high pulsed bias.
SP614: 1981 September. 309-313. Difilippo, F. C.; Pineyro, J. J. Application of the theory of random matrices to a reactor noise problem.

SP614: 1981 September. 314-316. D'Amico, A. Burst noise measurements on op amp's by R. F. SQUID.

SP614: 1981 September. 317.323. Grosse-Nobis, W. Domain walls in ferromagnetic material and irreversible magnetization processes.

SP614: 1981 September. 324-327. Bertotti, G.; Fiorillo, F.; Sassi, M. $\mathrm{P}$. Barkhausen noise and domain structure dynamics in $3 \% \mathrm{SiFe}$ single crystals.

SP614: 1981 September. 328-331. Bertocci, U. Noise in electrochemical systems.

SP614: 1981 September. 332-334. Haemmerli, A.; Janata, J.; Brophy, J. J. Ion noise in ISFETs.

SP614: 1981 September. 335-338. Corti, M. Quasielastic light scattering from macromolecules and micelles.

SP614: 1981 September. 339-342. Miraldi, E.; Trossi, L.; Valabrega, P. T.; Oldano, C. Frank elastic constant ratios evaluation in MBBA by intensity measurement of the scattered light.

SP614: 1981 September. 343-345. van der Meulen, J. P.; Zijlstra, R. J. J.; van Kooten, J. J. The determination of elastic constants of nematic liquid crystals from noise measurements of scattered laser light.

SP614: 1981 September. 349-354. Tucker, J. R. Quantum noise limited detection in tunneling devices at millimeter wavelengths.

SP614: 1981 September. 355-358. Leggett, A. J. Quantum tunnelling and noise in SQUIDs.

SP614: 1981 September. 359-363. Koch, R. H.; van Harlingen, D. $\mathrm{J}$.; Clarke, J. Quantum noise in Josephson junctions and SQUIDs.

SP614: 1981 September. 364.368. van der Ziel, A. Quantum noise effects at high frequencies and low temperatures.

SP614: 1981 September. 369-372. Tesche, C. D. A thermal activation model for the DC-SQUID.

SP614: 1981 September. 373-375. Kurkijarvi, J. Ultimate sensitivity of an AC-SQUID.

SP614: 1981 September. 376-380. Ben-Jacob, E.; Schuss, Z. The mean lifetimes of the meta-stable states of the DC-SQUID and its I-V characteristics.

SP614: 1981 September. 381-383. Ben-Jacob, E.; Bergman, D.; Carmeli, B.; Nitzan, A. External field effect on particle diffusion above a potential barrier.

SP614: 1981 September. 384-387. Dirks, H. M.; Beckstette, K. F.; Heiden, C. Flux flow noise during inhomogeneous vortex-motion. Dependence on DC-velocity field.

SP614: 1981 September. 388-390. Beckstette, K. F.; Dirks, H. M.; Heiden, C. Flux flow noise during inhomogeneous vortex-motion. Magnetic field and temperature dependence.

SP614: 1981 September. 391-394. Knoedler, C. M.; Voss, R. F.; Horn, P. M. Vortex noise in two dimensional superconducting films. SP614: 1981 September. 397.405. Nougier, J. P. Modeling noise of devices working under hot carrier conditions.

SP614: 1981 September. 406.408. Bareikis, V.; Galdikas, A.; Miliusyte, R.; Viktoravicius, V. Noise of hot holes in Ge due to predominant inelastic scattering.

SP614: 1981 September. 409.413. Bosman, G.; Zijlstra, R. J. J.; van Rheenen, A. Hot carrier transport noise in p-type silicon.

SP614: 1981 September. 414-416. Reggiani, L.; Brunetti, R.; Jacoboni, C. Monte Carlo calculation of hot-electron noise in $\mathrm{Si}$ at $77 \mathrm{~K}$.

NBSIR 79-1591.4. Bullis, W. M., ed. Semiconductor technology program-Progress briefs. 1979 August. 12 p. Available from: NTIS; PB 81.154114

NBSIR 79-1591-5. Bullis, W. M., ed. Semiconductor technology program-Progress briefs. 1979 December. 16 p. Available from: NTIS; PB 81.154122.

NBSIR 80-2006-3. Bullis, W. M., ed. Semiconductor technology program-Progress briefs. 1980 October. 12 p. Available from: NTIS; PB 81.154106

NBSIR 80-2006-4. Bullis, W. M., ed. Semiconductor technology program-Progress briefs. 1980 December. 12 p. Available from: 
NTIS; PB 81.158602

NBSIR 80-2158. Ehrstein, J. R. Some considerations regarding film thickness standards for the semiconductor industry. 1980 November. 14 p. A vailable from: NTIS; PB 81.239535.

NBSIR 81-2197. Scace, R. I. Semiconductor technology for the nontechnologist. 1981 January. 29 p. Available from: NTIS; PB 81 . 166902.

NBSIR 81-2204. Blackburn, D. L. Power transistor switching characterization. 1981 April. 30 p. Available from: NTIS; PB 81. 196347.

NBSIR 81-2224. Bullis, W. M. Advancement of reliability, processing and automation for integrated circuits with the National Bureau of Standards (ARPA/IC/NBS). 1981 March. 50 p. Available from: NTIS; PB 81.188203.

NBSIR 81-2230. Bullis, W. M., ed. Semiconductor technology program-Progress briefs. 1981 March. 11 p. Available from: NTIS; PB 81-188120.

NBSIR 81-2230-2. Bullis, W. M., ed. Semiconductor technology program-Progress briefs. 1981 July. 16 p. Available from: NTIS; PB 81.238529.

NBSIR 81-2230-3. Galloway, K. F.; Scace, R. I.; Walters, E. J., eds. Semiconductor technology program-Progress briefs. 198I November. 12 p. Available from: NTIS; PB 82.135898.

NBSIR 81-2234. Carver, G. P.; Mattis, R. L.; Buehler, M. G. Microelectronic test patterns NBS-12 and NBS-24. 1981 May. 40 p. Available from: NTIS; PB 81.214892.

NBSIR 81-2260. Lankford, W. F.; Kowalski, P.; Schafft, H. A. Laser scanning measurements on solar cell test pattern NBS-22. 1981 May. 55 p. Available from: NTIS; PB 81-214157.

NBSIR 81-2319. Carver, G. P. Development of test structures for characterization of the fabrication and performance of radiationhardened charge-coupled device (CCD) imagers: Annual report, May 15, 1980 to May 14, 1981. 1981 August. 26 p. Available from: NTIS; PB 81.244071.

NBSIR 81-2325. Larrabee, R. C.; Phillips, W. E.; Thurber, W. R. Measurement techniques for high power semiconductor materials and devices: Annual report, October 1, 1979 to September 30, 1980. 1981 September. 52 p. Available from: NTIS; PB 82.117706.

NBSIR 81-2398. Roitman, P.; Albers, J.; Ehrstein, J. R.; Myers, D. R. Investigation of the two-dimensional shape of ion-implanted regions. 1981 December. 71 p. Available from: NTIS; PB 82.154451.

NBS-GCR-80-279. Patrick, S.; Giuntini, R., (NBS contact: E. Cohen). Terrestrial solar simulators for photovoltaic and combined photovoltaic and thermal device measurement and evaluation. 1980 October. 50 p. Available from: NTIS; PB 82.130063.

NBS-GCR-81-325. Wilson, R. G., (NBS contact: E. Cohen). Measurement technology for critical ion implantation parameters. 1981 June. 45 p. Available from: NTIS; PB 81.219628.

NBS-GCR-81-327. McCarthy, D.; Acevedo, J.; Herman, D., (NBS contact: E. Cohen). Advanced planar silicon test structures. 1981 July. 92 p. A vailable from: NTIS; PB 8I-229155.

NBS-GCR-81-334. Li, S. S., (NBS contact: E. Cohen). Conductivity mobility, Hall mobility, and resistivity in p-type silicon. 1981 September. 9 p. Available from: NTIS; PB 82.135864.

NBS-GCR-81-345. Helms, C. R.; Schwarz, S. A.; Spicer, W. E.; Taylor, N. J., (NBS contact: E. Cohen). Innovative measurement technology for the semiconductor device industry based on electron spectroscopy. 1981 November. 11 p. Available from: NTIS; PB 82 135922.

U.S. Patent 4,287,473. Sawyer, D. Nondestructive method for detecting defects in photodetector and solar cell devices. 1 September 1981. 8 p.

20006. Dumin, D. J. A low cost approach to modernize undergraduate electronic laboratory instruction, Proc. Third Biennial University/Industry/Government Microelectronics Symp., Lubbock, TX, May 21-23, 1979, pp. 168.170 (The Institute of Electrical and Electronics Engineers, Inc., New York, NY, May 1979).

20092. Wilson, C. L. Correction of differential capacitance profiles for Debye-length effects, IEEE Trans. Electron Devices ED-27, No. 12, 2262-2267 (Dec. 1980).

20112. Myers, D. R.; Wilson, R. G.; Comas, J. Considerations of ion channeling for semiconductor microstructure fabrication, J. Vac. Sci. Technol. 16, No. 6, 1893-1896 (Nov./Dec. 1979).

20330. Phelan, R. J., Jr.; Larson, D. R.; Werner, P. E. Subnanosecond electrical modulation of light with hydrogenated amorphous silicon, Appl. Phys. Lett. 38, No. 8, 596.598 (Apr. 15, 1981).

20358. Blackburn, D. L.; Berning, D. W. An experimental study of reverse-bias second breakdown, Proc. International Electron Devices Meet., Washington, DC, Dec. 8-10, 1980, pp. 297-301 (The Institute of Electrical \& Electronics Engineer, Piscataway, NY, Dec. 1980).

20363. Bullis, W. M. Characterizing semiconducting materials, Semicond. Int., pp. 79.97 (Oct. 1980)

20371. Bullis, W. M. Metrology for submicrometer devices and structures, Proc. Microcircuit Engineering 1979, Microstructure Fabrication, Aachen, West Germany, Sept. 25-27, 1979, pp. 271-282 (German Section of IEEE, Aachen, West Germany, 1979).

20530. Geist, J.; Liang, E.; Schaefer, A. R. Complete collection of minority carriers from the inversion layer in induced junction diodes, J. Appl. Phys. 52, No. 7, 4879-4881 (July 1981).

\section{Energy Conservation and Production}

Monogr. 168. McCarty, R. D.; Hord, J.; Roder, H. M. Selected properties of hydrogen (engineering design data). Nat. Bur. Stand. (U.S.) Monogr. 168; 1981 February. 523 p. SN003-003.02296.9.

SP606. Trechsel, H. R.; Launey, S. J. Criteria for the installation of energy conservation measures. Nat. Bur. Stand. (U.S.) Spec. Publ. 606; 1981 July. 203 p. SN003-003-02337-0.

SP608. Berry, S. A., ed. Research and innovation in the building regulatory process. Proceedings of the Fifth Annual NBS/NCSBCS Joint Conference Technical Seminar on Solar Energy and Energy Conservation; 1980 August 6; Denver, CO. Nat. Bur. Stand. (U.S.) Spec. Publ. 608; 1981 May. 201 p. SN003-003-02321-3.

SP608: 1981 May. 21-35. Emery, A. F.; Dorri, B.; Kippenhan, C. J.; Heerwagen, D. R.; Banken, G. Alternate optimal control strategies for residential HVAC systems.

SP608: 1981 May. 37.56. Zackrison, H. B., Jr. Energy conscious electrical design.

SP608: 1981 May. 57-67. Kainlauri, E. O. Potential energy savings in buildings resulting from the NCSBCS code compared with BEPS.

SP608: 1981 May. 69.81. Loxley, T. E. Solar and geothermal housing innovations via inverted cave construction system.

SP608: 1981 May. 83-103. Forest, A. Basic human values and energy conserving lifestyles or the implications of California's innovative new Class $K$ code.

SP608: 1981 May. 105-112. Lord, D. Setting standards for building energy performance in Hawaii.

SP608: 1981 May. 113-122. Anderson, J. M.; Evans, P. M.; Smith, R. L. A simplified low cost technique for evaluating building energy performance standards subnittals.

SP608: 1981 May. 123-132. Glickman, D. S.; Terry, D. L. Energy audits of public buildings in Illinois.

SP608: 1981 May. 133-138. Kainlauri, E. O. A professional approach to class $A$ and school and hospital energy audits, with case studies. SP608: 1981 May. 139-157. McCarney, S. A simplified procedure for the use of solar energy in complying with the Colorado energy conservation standards for residential buildings.

SP608: 1981 May. 159-166. Gaines, R. S.; McLafferty, P. S. Solar systems facilitated by the proper application of existing building codes.

SP608: 1981 May. 167-172. Trant, B. S. Development of recommended requirements to code officials for solar heating, cooling and hot water systems.

SP608: 1981 May. 173-178. Williams, S. A method for retaining an experimental attitude in solar codes.

SP608: 1981 May. 179-181. Wrenn, P. State Government roles in national solar collector labeling, certification, and rating.

SP608: 1981 May. 183-194. Key, W. P.; Potter, T. Residential conservation service program solar model audit.

SP608: 1981 May. 195-200. Heinemeyer, R. R. Building codes vs. the design of passive solar heat storage. 
SP616. Gass, S. I., ed. Validation and assessment of energy models. Proceedings of a Symposium held at the National Bureau of Standards; 1980 May 19-21; Gaithersburg, MD. Nat. Bur. Stand. (U.S.) Spec. Publ. 616; 1981 October. 248 p. SN003-003-02373-6.

SP616: 1981 October. 25-41. Greenberger, M. Humanizing policy analysis: Confronting the paradox in energy modeling.

SP616: 1981 October. 43.47. Hoffman, K. Remarks on Wood and Greenberger papers.

SP616: 1981 October. 49-63. Richels, R. Building good models is not enough.

SP616: 1981 October. 65-77. Lady, G. M. Quality control for analysis.

SP616: 1981 October. 7-24. Wood, D. O. Energy model evaluation and analysis: Current practice.

SP616: 1981 October. 79-86. Hausman, J. A. Validation and assessment procedures for energy models.

SP616: 1981 October. 87.89. Jackson, R. H. F. Energy model assessment procedure development project.

SP616: 1981 October. 93-116. Freedman, D. Are energy models credible?

SP616: 1981 October. 117-120. Glassey, C. R.; Greenberg, H. J. Model credibility: How much does it depend upon data quality?

SP616: 1981 October. 139-147. Kydes, A. S.; Rubin, L. Workshop report 1-Composite model validation.

SP616: 1981 October. 149-158. Joel, L. S.; Maybee, J. S. Workshop report 2-The measurement of model confidence.

SP616: 1981 October. 159-169. Holloway, M. L. Workshop report 3-Model standards to aid assessment.

SP616: 1981 October. 171-181. Harris, C.; Hirshfeld, D. Workshop report 4-Sensitivity and statistical analysis of models.

SP616: 1981 October. 183-200. Gruhl, J.; Wood, D. O. Workshop report 5-Model assessment methodologies. Part A: Enumeration of validation methodologies.

SP616: 1981 October. 201-203. Woods, T. J. Workshop report 5-Model assessment methodologies. Part B: Model assessment methodologies.

SP616: 1981 October. 217-242. Goldman, A. J. Reflections on modeling and modeling assessment.

BSS130. Elder, J.; Tibbott, R. L. User acceptance of an energy efficient office building-A study of the Norris Cotton Federal Office Building. Nat. Bur. Stand. (U.S.) Bldg. Sci. Ser. 130; 1981 January. 122 p. SN003-003-02278-1.

BSS133. Hill, J. E.; May, W. B., Jr.; Richtmyer, T. E.; Elder, J.; Tibbott, R. L.; Yonemura, G. T.; Hunt, C. M.; Chen, P. T. Performance of the Norris Cotton Federal Office Building for the first 3 years of operation. Nat. Bur. Stand. (U.S.) Bldg. Sci. Ser. 133; 1981 August. 140 p. SN003-003-02352-3.

BSS134. Petersen, S. R.; Barnes, K. A.; Peavy, B. A. Determining cost-effective insulation levels for masonry and wood-frame walls in new single-family housing. Nat. Bur. Stand. (U.S.) Bldg. Sci. Ser. 134; 1981 August. 126 p. SN003-003-02354-0.

BSS135. Kovacs, W. D.; Salomone, L. A.; Yokel, F. Y. Energy measurement in the Standard Penetration Test. Nat. Bur. Stand. (U.S.) Bldg. Sci. Ser. 135; 1981 August. 99 p. SN003-003-02356-6.

TN1030. McCarty, R. D. Four mathematical models for the prediction of LNG densities. Nat. Bur. Stand. (U.S.) Tech. Note 1030; 1980 December. 84 p. SN003-003-02285-3.

TN1136. Waksman, D.; Streed, E.; Seiler, J. NBS solar collector durability/reliability test program plan. Nat. Bur. Stand. (U.S.) Tech. Note 1136; 1981 January. 85 p. SN003-003-02283-7.

TN1138. Kusuda, T.; Bean, J. W. Savings in electric cooling energy by the use of a whole-house fan. Nat. Bur. Stand. (U.S.) Tech. Note 1138; 1981 May. 39 p. SN003-003-02317-5.

TN1140. Streed, E.; Waksman, D. Uncertainty in determining thermal performance of liquid-heating flat-plate solar collectors. Nat. Bur. Stand. (U.S.) Tech. Note 1140; 1981 April. 97 p. SN003-003-02306-0.

TN1148. Treado, S.; Kusuda, T. Solar radiation and illumination. Nat. Bur. Stand. (U.S.) Tech. Note 1148; 1981 November. 31 p. Available from: NTIS; PB 82-135823.

NBSIR 77-1311. Kotter, F. R.; Misakian, M. AC transmission line field measurements. 1981 October. 83 p. Available from: NTIS; PB 82 . 133554.
NBSIR 79-1911. Maxwell, B. R. Procedures for testing, rating, and estimating the seasonal performance of engine-driven heat pump systems. 1979 September. 60 p. Available from: NTIS; PB 81. 123564.

NBSIR 80-1627. Reed, R. P., ed. Materials studies for magnetic fusion energy applications at low temperatures-III. 1980 June. 387 p. Available from: NTIS; PB 80-191497.

NBSIR 80-1971. Gass, S. I.; Hoffman, K. L.; Jackson, R. H. F.; Joel, L. S.; Saunders, P. B. Interim report on model assessment methodology: Documentation assessment. 1980 January. $67 \mathrm{p}$ Available from: NTIS; PB 82-126673.

NBSIR 80-2091. Branthover, B.; Veale, R.; Siu, C. Precision measurements on fibrous glass insulation. 1980 November. 36 p. Available from: NTIS; PB 81.239634.

NBSIR 80-2094. Kelly, G. E.; Kuklewicz, M. E. A laboratory study of a gas-fired condensing boiler. 1980 November. 39 p. Available from: NTIS; PB 81-158230.

NBSIR 80-2104. Kao, J. Y.; Baker, D. W. A commentary on the instrumentation for building energy monitoring and control systems (EMCS), 1980 September. 28 p. Available from: NTIS; PB 81 185688.

NBSIR 80-2110. Kelly, G. E.; Kuklewicz, M. E. Recommended testing and calculation procedures for estimating the seasonal performance of residential condensing furnaces and boilers. 1981 April. $100 \mathrm{p}$ Available from: NTIS; PB 81-197030.

NBSIR 80-2136. Chang, Y. M. L.; Grot, R. A. Energy consumption and usage characteristics from field measurements of residential dishwashers, clothes washers and clothes dryers. 1980 October. 35 p. Available from: NTIS; PB 81-179160.

NBSIR 80-2141. Haight, W. C.; Hocken, R. J.; Borchardt, B. R. Carroll, C. L.; Hartsock, R. G.; Reeve, C. P.; Scire, F. E.; Veale R. C. Estimated accuracy of calibration of some membrane-type LNG transport tanks. 1981 January. 83 p. Available from: NTIS; PB 81.159907.

NBSIR 80-2147. Treado, S.; Kusuda, T. Daylighting, window management systems, and lighting controls. 1980 December. $80 \mathrm{p}$ Available from: NTIS; PB 81-152886.

NBSIR 80-2178 (DoE). Hastie, J. W.; Bonnell, D. W.; Plante, E. R.; Horton, W. S. Vaporization and chemical transport under coal gasification conditions. 1980 December. 113 p. Available from: NTIS; PB 81-245839.

NBSIR 80-2181. Sawyer, D. E.; Kessler, H. K.; Russell, T. J. Lankford, W. F.; Schafft, H. A. Measurement techniques for solar cells, Annual Report: December 15, 1978 to December 14, 1979. 1981 January. 60 p. Available from: NTIS; PB 81-163875.

NBSIR 80-2184. Barnett, J. P. Energy analysis of a prototype singlefamily detached residence: The effects of climate, house size, orientation, internal heat release, and natural cooling. 1981 January. 46 p. Available from: NTIS; PB 81-166514.

NBSIR 81-1645. Reed, R. P.; Simon, N. J., eds. Materials studies for magnetic fusion energy applications at low temperatures-IV. 1981 April. 604 p. Available from: NTIS; PB 81.222507.

NBSIR 81-1653. Chew, H. Modelling of oil shale retorts for electromagnetic sensing techniques. 1981 November. 47 p. Available from: NTIS; PB 82.153321.

NBSIR 81-2199. Cattaneo, L.; Harris, J. R.; Reinhold, T. A.; Simiu, E.; Yancey, C. W. C. Wind, earthquake, snow, and hail loads on solar collectors. 1981 January. 97 p. Available from: NTIS; PB 81 . 164550 .

NBSIR 81-2232. Masters, L. W.; Seiler, J. F.; Embree, E. J.; Roberts, W. E. Solar energy systems-Standards for absorber materials. 1981 January. 68 p. Available from: NTIS; PB 81.188278.

NBSIR 81-2241. Petersen, S. R.; Barnes, K. A.; Kelly, G. E Engineering-economic analysis of improved heat pump performance for minimum standards development. 1981 July. 67 p. Available from: NTIS; PB 82-133810.

NBSIR 81-2277. Kao, J. Y.; Pierce, E. T. Control strategies for energy conservation-A case study of the Materials Building, National Bureau of Standards. 1981 May. 47 p. Available from: NTIS; PB 81. 217804.

NBSIR 81-2278. Kirklin, D. R.; Colbert, J.; Decker, P.; Abramowitz, 
S.; Domalski, E. S. Test procedures for the determination of the gross calorific value of refuse and refuse-derived-fuel by conventional bomb and large bomb calorimetry. 1981 June. 218 p. Available from: author.

NBSIR 81-2279. Hastie, J. W.; Plante, E. R.; Bonnell, D. W. Alkali vapor transport in coal conversion and combustion systems. 1981 May. 79 p. Available from: NTIS; PB 81-221319.

NBSIR 81-2289. Lerchen, F. H.; Pielert, J. H.; Chen, P. T. Preliminary guidelines for condition assessment of buildings being considered for solar retrofit. 1981 July. 140 p. Available from: NTIS; PB 82.113259.

NBSIR 81-2293. Hastie, J. W.; Plante, E. R. Mass spectrometric studies of MHD slag thermochemistry. 1981 June. 33 p. Available from: NTIS; PB 81.221434.

NBSIR 81-2300. McCabe, M. E.; Ducas, W.; Orloski, M. J.; Decorte, K. N. Passive/hybrid solar components-An approach to standard thermal test methods. 1981 July. 86 p. Available from: NTIS; PB 81 227886.

NBSIR 81-2303. Wan, C. A. NBS/ESDS-A computer model for the simulation of hot water systems. 1981 September. 64 p. Available from: NTIS; PB 82.139361.

NBSIR 81-2313. Baker, D. W.; Kao, J. Y.; Didion, D. A. Performance evaluation of a typical energy monitoring system for steam flow in buildings. 1981 July. 63 p. Available from: NTIS; PB 81.238545.

NBSIR 81-2317. Pierce, E. T. Energy conservation by multiple glazing on heavy masonry buildings. 1981 June. 30 p. Available from: NTIS PB 81.246977.

NBSIR 81-2321. Peavy, B. A. Single-room heat balance for building heat transfer. 1981 November. 22 p. Available from: NTIS; PB 82 135609.

NBSIR 81-2334. Hebner, R. E., ed. Development of power system measurements-Quarterly report, April 1, 1981 to June 30, 1981. 1981 June. 20 p. Available from: NTIS; PB 81.214892.

NBSIR 81-2342. Powell, J. W.; Rodgers, R. C., Jr. FEDSOL: Program user's manual and economic optimization guide for solar Federal buildings projects. 1981 August. 132 p. Available from: NTIS; PB 82-107012.

NBSIR 81-2343. Schumm, R. H. Thermodynamic properties of solid alkali aluminosilicates at elevated temperatures. 1981 October. $51 \mathrm{p}$. Available from: NTIS; PB 82-131525.

NBSIR 81-2346. Dickinson, G. W. Energy management and control systems (EMCS)—User satisfaction study. 1981 August. $19 \mathrm{p}$ Available from: NTIS; PB 82-103656.

NBSIR 81-2348. Frederikse, H. P. R.; Schenck, P. K.; Burns, G. W.; Dils, R. R.; Whetstone, J. R. Materials for instrumentation for fossil energy technologies. 1981 August. 135 p. Available from: NTIS; PB $82-116229$

NBSIR 81-2353. Peavy, B. A. Documentation of program for determination of conduction transfer functions. 1981 November. 49 p. Available from: NTIS; PB 82-136284.

NBSIR 81-2358. May, W. B., Jr. Analysis of data from the energy monitoring and control system at the Norris Cotton Federal Office Building. 1981 November. 98 p. Available from: NTIS; PB 82. 138744.

NBSIR 81-2378. Kusuda, T. Heat transfer analysis of underground heat and chilled-water distribution systems. 1981 November. $63 \mathrm{p}$. Available from: NTIS; PB 82-136235.

NBSIR 81-2381. Rossiter, W. J., Jr.; Shipp, W. E. Solar industrial process heat systems-An assessment of standards for materials and components. 1981 September. 97 p. Available from: NTIS; PB 82 133703.

NBSIR 81-2404. Walton, G. N. Calculation of inter-room air movement for multi-room building energy analysis. 1981 November. 38 p. Available from: NTIS; PB 82-142209.

NBS-GCR-80-254. Thomas, W. C., (NBS contact: K. DeCorte). Effects of test fluid composition and flow rates on the thermal efficiency of solar collectors. 1980 August. 91 p. Available from: NTIS; PB 81-180267.

NBS-GCR-80-285. Lehovec, K.; Fedotowsky, A., (NBS contact: Elaine Cohen). Solar cell mathematical analysis and computer simulation. 1981 January. 69 p. Available from: NTIS; PB 81 .
163891.

NBS-GCR-81-311. Resource Conservation and Recovery Office, Minnesota Dept. of Administration (NBS contact: K. Hudson/J. Berke). A study of resource and energy conservation practices in the procurement and management of commodities. 1981 March. 63 p. Available from: NTIS; PB 81.187965.

NBS-GCR-81-321. Carlock, J. B. Ill, (NBS contact: M. McCabe). Use of FCHART version 3.0 to predict auxiliary energy use in residential solar energy systems. 1981 May. 101 p. Available from: NTIS; PB 81.217010 .

NBS-GCR-81-322. Wedekind, D. R., (NBS contact: Mike McCabe). Multi-family solar domestic hot water analysis. 1981 May. 100 p. Available from: NTIS; PB 82-130139.

20000. Howard, C. P. Some fundamentals of desuperheaters, Proc. 1980 Conf. on Waste Heat Recovery for Energy Conservation, West Lafayette, IN, Sept. 15-17, 1980, 3 pages (Purdue University, West Lafayette, IN, 1981).

20028. Wiederhorn, S. M. Erosion of ceramics, Proc. NACE Conf. on Corrosion/Erosion of Coal Conversion System Materials, Berkeley, CA, Jan. 24-26, 1979, pp. 444-479 (National Association of Corrosion Engineers, Houston, TX 77084, 1979).

20078. Fanney, A. H.; Liu, S. T. Experimental system performance and comparison with computer predictions for six solar domestic hot water systems, (Proc. Int. Solar Energy Society Silver Jubilee Congress, Atlanta, GA, May 29-June 1, 1979), Paper in Sun II, K. W. Boer and B. H. Glen, eds., 1, 972.976 (Pergamon Press, New York, NY, 1979).

20079. Jenkins, J. P. A comparison of test results for flat-plate water. heating solar collectors using the BSE and ASHRAE procedures, (Proc. Int. Solar Energy Society Silver Jubilee Congress, Atlanta, GA, May 29.June 1, 1979), Paper in Sun II, K. W. Boer and B. H. Glen, eds., 1, 365-369 (Pergamon Press, New York, NY, 1979).

20089. Siu, M. C. I. Fibrous glass board as a standard reference material for thermal resistance measurement system, (Proc. Symp. Thermal Insulation Performance, Tampa, FL, Oct. 23-25, 1978), Am. Soc. Test. Mater. Spec. Tech. Publ. 718, D. L. McElroy and R. P. Tye, eds., pp. 343-360 (1981).

20103. Fanney, A. H.; Liu, S. T. Performance of six solar domestic hot water systems in the mid-Atlantic region, Proc. Conf. Solar Heating and Cooling Systems Operational Results, Colorado Springs, CO, Nov. 27-30, 1979, pp. 25-31 (SERI/TP 245-430, DOE/Solar Energy Research Institute (SERI), Golden, CO, Nov. 1979).

20121. McCabe, M. E. A collector sizing procedure for residential solar hot water systems with prescribed thermal performance requirements, (Proc. ASHRAE Semiannual Meet., Los Angeles, CA, Feb. 3-7, 1980), ASHRAE Trans. 86, Pt. 1, $420-439$ (American Society of Heating, Refrigerating and Air Conditioning Engineers, Inc., New York, NY, 1980).

20148. Oonk, R. L.; Jones, D. E.; Cole-Appel, B. E. Calculation of performance of $\boldsymbol{N}$ collectors in series from test data on a single collector, Sol. Energy, Tech. Note 23, No. 6, 535.536 (1979).

20151. Powell, J.; Ruegg, R. Life-cycle costing of solar energy investments, Chapter II in Solar Design Workbook, Solar Federal Buildings Program, Sec. II Solar System Evaluation, G. Franta, B. Glenn, S. Hogg, B. Hunn, S. Ternoey, and J. Yellott, eds., pp. 11 1-11-8 (SERI/SP.62-308, DOE/Solar Energy Research Institute (SERI), Golden, CO, Jan. 1981).

20167. Ruegg, R. T.; Sav, G. T. The microeconomics of solar energy, Chapter 28 in Solar Energy Handbook, J. F. Kreider and F. Kreith, eds., pp. 28-1-28-42 (McGraw-Hill Book Co., New York, NY, 1981).

20235. Ramey-Smith, A. M.; Gagnon, J. L. Energy cost feedback: Consumer preferences, Proc. Human Factors and Industrial Design in Consumer Products, Medford, MA, May 28-30, 1980, pp. 96-116 (Tufts University, Medford, MA, 1981).

20259. Rossiter, W. J., Jr.; Mathey, R. G. Guidelines for installation of solar components on low-sloped roofs-A summary, Proc. NBS/NRCA 6th Conf. on Roofing Technology, Gaithersburg, MD, Apr. 30-May 1, 1981, pp. 58-62 (National Roofing Contractors Assoc., Oak Park, IL, Apr. 1981).

20289. MacCrehan, W. A.; Durst, R. A. Assessing the environmental 
impact of fossil-fuel based energy sources, EPA-600/7-79-211, 63 pages (A vailable from the National Technical Information Service, Springfield, VA 22161, Nov. 1979).

20291. Streed, E. R.; Waksman, D. Uncertainty in determining thermal performance of liquid-heating flat-plate solar collectors, Trans. ASME 1103, 126-134 (May 1981).

20296. Kweller, E. R.; Mullis, W. F. Determination of annual efficiency of vented heaters equipped with thermally activated vent dampers, (Proc. ASHRAE Semi-Annu. Meet. Symp. Efficiency of Combustion Systems, Chicago, IL, Jan. 25-29, 1981), ASHRAE Trans. 87, Pt. 1, 753.768 (June 1981).

20359. Schneider, S. J. Materials research for the clean utilization of coal. Quarterly progress report January-March 1979, DoE Report $E A-6010-19,34$ pages (Available as FE6010-19 from the National Technical Information Service, Springfield, VA 22161, 1979).

20364. Hunt, C. M. Air infiltration: A review of some existing measurement techniques and data, (Proc. Symp. Building Air Change Rate and Infiltration Measurements, Washington, DC, Mar. 12, 1978), Am. Soc. Test. Mater. Spec. Tech. Publ. 719, Building Air Change Rate and Infiltration Measurements, C. M. Hunt, J. C. King, H. R. Trechsel, eds., pp. 3.24 (Oct. 1980).

20365. Grot, R. A. A low-cost method for measuring air infiltration rates in a large sample of buildings, (Proc. Symp. Building Air Change Rate and Infiltration Measurements, Washington, DC, Mar. 12, 1978), Am. Soc. Test. Mater. Spec. Tech. Publ. 719, Building Air Change Rate and Infiltration Measurements, C. M. Hunt, J. C. King, H. R. Trechsel, eds., pp. 50-59 (Oct. 1980).

20430. Harris, J. R. Snow accumulation on and around solar collectors, Proc. 37th Eastern Snow Conf., Peterborough, Ontario, Canada, June 5.6, 1980, pp. 54-65 (Eastern Snow Conf., Atmospheric Environment Service, Downsview, Ontario, Canada, 1981).

20440. Metz, F. E. Two examples of passive solar retrofit for historic structures, (Proc. 5th Natl. Passive Solar Conf., Amherst, MA, Oct. 19-26, 1980), Extended Abstract in Passive Solar 1980, J. Hayes and R. Snyder, eds., 5.2, 1305-1306 (1980).

20463. Rossiter, W. J., Jr.; Weidt, J. L.; Saxler, R. J. A field survey of the performance properties of insulation used to retrofit cavity walls of residences, (Proc. ASHRAE/DOE-ORNL Conf. Thermal Performance of the Exterior Envelopes of Buildings, Kissimmee, FL, Dec. 3-5, 1979), ASHRAE SP 28, pp. $901-915$ (ASHRAE, 345 E. 47th St., New York, NY, 1981).

20470. Metz, F. E. Health and safety criteria for passive solar systems, (Proc. 5th Natl. Passive Solar Conf., Amherst, MA, Oct. 19-26, 1980), Extended Abstract in Passive Solar 1980, J. Hayes and R. Snyder, eds., 5.2, 945-949 (1980).

20512. May, W. B., Jr.,; Spielvogel, L. G. Analysis of computersimulated thermal performance: The Norris Cotton Federal Office Building, (Proc. ASHRAE Semi-Annu. Meet., Chicago, IL, Jan. 25 29, 1981), ASHRAE J. 87, Pt. 1, 43-50 (1981).

20521. Kusuda, $T$. A comparison of energy calculation procedures, ASHRAE J. 23, No. 8, 21-24 (Aug. 1981).

20538. Gujral, P. S.; Clark, R. J.; Burch, D. M. Transient thermal response of an intermittently cooled massive building, (Proc. ASHRAE/DOE/ORNL Conf. Thermal Performance Exterior Envelopes of Buildings, Orlando, FL, Dec. 3-5, 1979), ASHRAE SP 28, pp. 751.756 (American Society of Heating, Refrigeration and Air-Conditioning Engineers, New York, NY, 1981).

20539. Richtmyer, T. E.; May, W. B.; Hunt, C. M.; Hill, J. E. Thermal performance of the Norris Cotton Federal building in Manchester, New Hampshire, (Proc. ASHRAE/DOE/ORNL Conf. Thermal Performance Exterior Envelopes of Buildings, Orlando, FL, Dec. 3.5, 1979), ASHRAE SP 28, pp. 781.797 (American Society of Heating, Refrigeration and Air-Conditioning Engineers, New York, NY, 1981).

20540. Grot, R. A.; Clark, R. E. Air leakage characteristics and weatherization techniques for low-income housing, (Proc. ASHRAE/DOE/ORNL Conf. Thermal Performance Exterior Envelopes of Buildings, Orlando, FL, Dec. 3.5, 1979), ASHRAE SP 28, pp. 178-194 (American Society of Heating, Refrigeration and Air.Conditioning Engineers, New York, NY, 1981).

20541. Gillette, G. The energy potential of daylighting in a classroom,
(Proc. ASHRAE/DOE/ORNL Conf. Thermal Performance Exterior Envelopes of Buildings, Orlando, FL, Dec. 3.5, 1979), ASHRAE SP 28, pp. $368-380$ (American Society of Heating, Refrigeration and Air-Conditioning Engineers, New York, NY, 1981).

20547. Smith, D. R.; Hust, J. G.; Van Poolen, L. J. Measurement of effective thermal conductivity of glass fibre blanket standard reference material, Cryogenics 21, No. 8, 460-462 (Aug. 1981).

20648. Fanney, A. H.; Thomas, W. C. Simulation of thermal performance of solar collector arrays, Trans. ASME 103, 258-267 (Aug. 1981).

20690. Parken, W. H.; Kelly, G. E. Estimating residential seasonal cooling requirements, (Proc. ASHRAE 1981 Semi-Annu. Meet., Chicago, IL, Jan. 25-29, 1981), ASHRAE Trans. 87, Pt. 1, 473-490 (1981).

20705. Waksman, D. Solar collector certification activities and experiences, Proc. Commercial Photovoltaics Measurements Workshop, Vail, CO, July 27-29, 1981, pp. 279-289 (A vailable from the National Technical Information Service, Springfield, VA, Nov. 1981).

20735. Siu, M. C. I.; Bulik, C. National Bureau of Standards line-heatsource guarded-hot-plate apparatus, Rev. Sci. Inst. 52, No. 11, 1709. 1716 (Nov. 1981)

20751. Mann, D. B.; Brennan, J. A.; Kneebone, C. H. Gas mass flow reference system: A progress report, Proc. American Gas Association 1980 Operating Section Distribution and Transmission Conf., (Distribution Conf. Detroit, MI, Mar. 19-21, 1980; Transmission Conf. Salt Lake City, UT, May 5-7, 1980), pp. T.366-T.368 (American Gas Assoc., Arlington, VA, 1980).

20754. Kusuda, T.; Bean, J. W. Comparison of calculated hourly cooling load and indoor temperature with measured data for a high mass building tested in an environmental chamber, (Proc. ASHRAE Semi. Ann. Meet., Chicago, IL, Jan. 1981), ASHRAE Trans. 87, Pt. 1, 1232-1242 (1981).

20755. Kusuda, T.; Pierce, E. T.; Bean, J. W. Comparison of calculated hourly cooling load and attic temperature with measured data for a Houston test house, (Proc. ASHRAE Semi Ann. Meet., Chicago, IL, Jan. 1981), ASHRAE Trans. 87, Pt. 1, 1185-1199 (1981).

20756. Kusuda, T.; Sud, I.; Alereza, T. Comparison of DOE-2generated residential design energy budgets with those calculated by the degree-day and bin methods, (Proc. ASHRAE Semi Ann. Meet., Chicago, IL, Jan. 1981), ASHRAE Trans. 87, Pt. 1, 491-506 (1981).

20758. Kusuda, T. Standards criteria for HVAC systems and equipment performance simulation procedures, $A S H R A E J .23,25-28$ (Oct. 1981).

\section{Engineering, Product, and Information Standards}

SP599. Wollin, H. F.; Barbrow, L. E.; Heffernan, A. P., eds. Report of the 65th National Conference on Weights and Measures 1980. Nat. Bur. Stand. (U.S.) Spec. Publ. 599; 1981 January. 319 p. SN003.003. 02286-1.

SP599: 1981 January. 1-5. Vincent, C. H. Expectations for the eighties.

SP599: 1981 January. 6-9. Ambler, E. 1980-A diamond anniversary, A golden opportunity.

SP599: 1981 January. 14-24. Birkeland, K. Societal and technological demands upon legal metrology -A strategy for meeting increased national and international needs.

SP599: 1981 January. 25-33. Page, W. J., Jr. Sharing power, responsibility, and resources in the Federal System.

SP599: 1981 January. 34-38. Jordan, B. A university looks at measurement science education.

SP599: 1981 January. 39-44. Elengo, J. J., Jr. The scale manufacturers association report on scale tolerances.

SP599: 1981 January. 45-53. Vastagh, S. A. The role of the U.S. Metric Board in the voluntary conversion of gas pumps to metric measurement.

SP599: 1981 January. 54-63. Simila, K. J.; Kloss, C. Report by the special study group on enforcement uniformity.

SP599: 1981 January. 64-68. Pierce, W. J. Mechanisms for unification of state and local laws and regulations. 
SP599: 1981 January. 69.84. Gerk, F. A. Western Weights and Measures Association.

SP599: 1981 January. 85-94. Young, W. M. Scale design and weighing trends in the eighties.

SP599: 1981 January. 95-101. Hodsman, G. F. Weights and measures regulations-The European scene in the eighties.

SP599: 1981 January. 102-109. Meyer, L. S. The consumer movement of the $1980 \mathrm{~s}-$ What we would like to see from weights and measures.

SP599: 1981 January. 110-114. Blasgen, M. W. Computers, microelectronics, and people.

SP599: 1981 January. 115-126. Muhe, W. Interdependence of metrology, standardization and quality assurance.

SP599: 1981 January. 127-137. Trask, R. P. Liquid hydrocarbon measurement in the decade of the eighties: The need for research and resourcefulness.

SP599: 1981 January. 138-146. Seiler, E. Type approval-A tool for measurement assurance in legal metrology.

SP599: 1981 January. 147.155. Parrott, M. W. Radiation measurement-A challenge that calls for cooperative federal and state action.

SP599: 1981 January. 156-164. Eagle, J. W.; Little, A. D. The 1980s food marketplace-Demographics, lifestyles, and technology.

SP599: 1981 January. 165-178. Bijloo, A. C. The system of control of prepackaged products in the Netherlands.

SP599: 1981 January. 180-201. Vincent, C. H. Report of the committee on national measurement policy and policy and coordination.

SP599: 1981 January. 202-211. Offner, D. I. Report of the committee on laws and regulations.

SP599: 1981 January. 212-240. Guensler, D. A. Report of the committee on specifications and tolerances.

SP599: 1981 January. 241-248. Darsey, S. J. Report of the committee on education, administration, and consumer affairs.

SP599: 1981 January. 249-262. Greene, C. H. Report of the committee on liaison.

SP599: 1981 January. 263.270. Vincent, C. H. Report of the executive committee.

SP599: 1981 January. 271-272. Scott, T. W. Report of the resolutions committee.

SP599: 1981 January. 273-275. Simila, K. J. Report of the nominations committee.

SP599: 1981 January. 276. Gerk, F. A. Report of the auditing committee.

SP599: 1981 January. 277.280. Nelson, A. M. Report of the conference treasurer.

SP605. Debelius, J. R. Standards committee activities of the National Bureau of Standards, 1980 highlights. Nat. Bur. Stand. (U.S.) Spec. Publ. 605; 1981 May. 102 p. SN003-003-02327-2.

FIPS PUB 1-1. Little, J. C., Standards Coordinator. Code for information interchange. Nat. Bur. Stand.(U.S.) Fed. Info. Process. Stand. Publ. (FIPS PUB) 1-1; 1980 December. 4 p.

FIPS PUB 14-1. Little, J. L., Standards Coordinator. Hollerith punched card code. Nat. Bur. Stand. (U.S.) Fed. Info. Process. Stand. Publ. (FIPS PUB) 14-1; 1980 December. 4 p.

FIPS PUB 29.1. Vickers, M. V., Standards Coordinator Interpretation procedures for Federal Information Processing Standard Programming Languages. Nat. Bur. Stand. (U.S.) Fed. Info. Process. Stand. Publ. (FIPS PUB) 29-1; 1981 December 31. 4 p.

FIPS PUB 74. Branstad, D. K., Standards Coordinator. Guidelines for implementing and using the NBS Data Encryption Standard. Nat. Bur. Stand. (U.S.) Fed. Info. Process. Stand. Publ. (FIPS PUB) 74; 1981 April 1. $39 \mathrm{p}$.

FIPS PUB 75. Letmanyi, H.; Conti, D. M., Standards Coordinators Guideline on constructing benchmarks for ADP system acquisitions. Nat. Bur. Stand. (U.S.) Fed. Info. Process. Stand. Publ. (FIPS PUB) 75; 1980 September. 43 p.

FIPS PUB 78. Scace, E. L., Standards Coordinator. Guideline for implementing advanced data communication control procedures (ADCCP). Nat. Bur. Stand. (U.S.) Fed. Info. Process. Stand. Publ. (FIPS PUB) 78; 1980 September. 22 p.
FIPS PUB 80. White, H. S., Jr., Standards Coordinator. Guide for the implementation of Federal Information Processing Standards (FIPS) in the acquisition and design of computer products and services. Nat. Bur. Stand. (U.S.) Fed. Info. Process. Stand. Publ. (FIPS PUB) 80; 1980 December. 96 p.

FIPS PUB 81. Branstad, D., Standards Coordinator. DES modes of operation. Nat. Bur. Stand. (U.S.) Fed. Info. Process. Stand. Publ. (FIPS PUB) 81; 1980 December 2. 26 p.

FIPS PUB 82. Bagg, T. C., Standards Coordinator. Guideline for inspection and quality control for alphanumeric computer-output microforms. Nat. Bur. Stand. (U.S.) Fed. Info. Process. Stand. Publ. (FIPS PUB) 82; 1980 September. 4 p

FIPS PUB 83. Meissner, P., Standards Coordinator. Guideline on user authentication techniques for computer network access control. Nat. Bur. Stand. (U.S.) Fed. Info. Process. Stand. Publ. (FIPS PUB) 83; 1980 September. $38 \mathrm{p}$.

FIPS PUB 84. Bagg, T. C., Standards Coordinator. Microfilm readers. Nat. Bur. Stand. (U.S.) Fed. Info. Process. Stand. Publ. (FIPS PUB) 84; 1980 October. $4 \mathrm{p}$

FIPS PUB 85. Bagg, T. C., Standards Coordinator. Optical character recognition (OCR) inks. Nat. Bur. Stand. (U.S.) Fed. Info. Process. Stand. Publ. (FIPS PUB) 85; 1980 November. 4 p.

FIPS PUB 86. Little, J. L., Standards Coordinator. Additional controls for use with American National Standard Code for Information Interchange. Nat. Bur. Stand. (U.S.) Fed. Info. Process. Stand. Publ. (FIPS PUB) 86; 1981 January 29.4 p.

FIPS PUB 87. Shaw, J., Standards Coordinator. Guidelines for ADP contingency planning. Nat. Bur. Stand. (U.S.) Fed. Info. Process. Stand. Publ. (FIPS PUB) 87; 1981 March 27. 31 p.

FIPS PUB 89. Bagg, T., Standards Coordinator. Optical Character Recognition (OCR) Character Positioning. Nat. Bur. Stand. (U.S.) Fed. Info. Process. Stand. Publ. (FIPS PUB) 89; 1981 September 4. 4 p.

NBSIR 80-1979-1. Wright, R. N.; Fenves, S. J.; Harris, J. R. Modeling of standards: Technical aids for their formulation, expression, and use. 1980 December. 17 p. Available from: NTIS; PB 81-159600.

NBSIR 81-2354. Konig, P. A.; Newton, J. J.; Saltman, R. G. Federal requirements for a Federal Information Processing Standard Data Dictionary System. 1981 September. 80 p. Available from: NTIS; PB 82-117607.

NBS-GCR-80-305. CAP GEMINI, INC., and J. G. Van Dyke and Associates, Inc. (NBS contact: E. Becker). Computer networking status, trends and utility for the Federal Government. 1980 November. 155 p. Available from: NTIS; PB 81-163859.

NBS-GCR-81-262. Fiorello, Shaw and Associates, and Aurora Associates, Inc. (NBS contact: P. Kay). Cost-benefit analysis of a Federal Information Processing Standard: COBOL (FIPS 21) Impacts on Air Force Data System Design Center functions. 1981 September. 58 p. Available from: NTIS; PB 82-140245.

NBS-GCR-81-347. Swankin, D. A., (NBS contact: M. Brientenberg) Rationale statements for voluntary standards-Issues, techniques, and consequences. 1981 November. 90 p. Available from: NTIS; PB $82-135856$

19998. Harris, J. R.; Fenves, S. J.; Wright, R. N. New tools for standards writers, ASTM Stand. News 8, 10-16 (July 1980).

20031. Rawie, C. C. Writing standards that meet regulatory needs, ASTM Stand. News 8, No. 9, 13-16 (Sept. 1980).

20162. Breitenberg, M. A. Priority setting in standardization activities, Stand. Eng. 33, No. 1, 6-16 (Feb. 1981).

20294. Wyckoff, J. M. Meeting State and local government needs by transfer of Federal laboratory technology, J. Technol. Transfer 5, No. 2, 1-21 (1981).

20324. Edgerly, D. E. Implications of United States participation in the International Organization of Legal Metrology (OIML), (Proc. 1980 Workshop \& Symp. National Conference of Standards Laboratories, National Bureau of Standards, Gaithersburg, MD, Sept. 22-25, 1980), Paper in NCSL Newslett. 21, No. 1, $32-37$ (Mar. 1981).

20403. Harris, J. R.; Wright, R. N. Computer aids for the organization of standards, Proc. Second Conf. Computing in Civil Engineering, Baltimore, MD, June 9-13, 1980, pp. 112-121 (American Society of 
Civil Engineering, New York, NY, 1980).

\section{Environmental Studies: Pollution Measurement}

SP618. Brinckman, F. E.; Fish, R. H., eds. Environmental speciation and monitoring needs for trace metal-containing substances from energy-related processes. Proceedings of the DoE/NBS Workshop held at the National Bureau of Standards; 1981 May 18-20; Gaithersburg, MD. Nat. Bur. Stand. (U.S.) Spec. Publ. 618; 1981 November. 336 p. Available from: NTIS; PB 82-132770.

SP618: 1981 November. 1-8. Stephens, R. D. Laboratory characterization of complex environmental contamination.

SP618: 1981 November. 21-38. Filby, R. H.; Sandstrom, D. R. Lytle, F. W.; Greegor, R. B.; Khalil, S. R.; Ekambaram, V.; Weiss, C. S.; Grimm, C. A. Chemistry of trace element species in coal liquefaction processes.

SP618: 1981 November. 39-53. Jenne, E. A. Speciation of aqueous contaminants-Role of the geochemical model.

SP618: 1981 November. 54-64. Craig, P. J.; Rapsomanikis, S. Biogeochemical cycles of metals and metalloids-The role of methylcobalamin and some other chemical routes to methylation in the environment.

SP618: 1981 November. 65-80. Chau, Y. K.; Wong, P. T. S. Some environmental aspects of organo-arsenic, lead, and tin.

SP618: 1981 November. 81-92. Manahan, S. E.; Fraley, D. M.; Vogt, C.; Yates, D. Environmental chemical investigations of trace element mobilization in synthetic fuels manufacture.

SP618: 1981 November. 9-20. Yen, T. F. The role of metal heteroatom complexes in fossil fuel production.

SP618: 1981 November. 93-104. Sievers, R. E.; Conditt, M. K Stanley, J. S. Speciation of organic compounds and trace elements in oil shale wastewaters.

SP618: 1981 November. 105.119. Olsen, K. B.; Wilkerson, C. L. Toste, A. P.; Hayes, D. J. Isolation of metallic complexes in shale oil and shale oil retort waters.

SP618: 1981 November. 120-133. Risby, T. H. The use of mass spectrometry for metal analysis.

SP618: 1981 November. 134-145. Braman, R. S.; Shelley, T. J. Diffusional discrimination and reversible chemisorption for speciation in air.

SP618: 1981 November. 146-162. Anderson, M. A. Kinetic and equilibrium control of interfacial reactions involving inorganic ionic solutes and hydrous oxide solids.

SP618: 1981 November. 163-172. Brown, T. F.; Caster, D. M Brown, S. D. Speciation of labile and quasi-labile metal complex systems using the Kalman filter.

SP618: 1981 November. 173-183. Crecelius, E. A.; Gurtisen, J. M.; Apts, C. W. Biological uptake and chemical speciation of copper in sea water determined by electrochemistry.

SP618: 1981 November. 184-196. Uden, P. C. Specific element detection in chromatography by plasma emission spectroscopy.

SP618: 1981 November. 197.216. Weiss, C. S.; Jewett, K. L. Brinckman, F. E.; Fish, R. H. Application of molecular substituent parameters for the speciation of trace organometals in energy-related process fluids by element-selective HPLC

SP618: 1981 November. 217.225. Fowler, B. A. Relationships between trace element speciation and intracellular mechanisms of toxicity.

SP618: 1981 November. 226-234. Packer, L.; Mehlhorn, R. J. Development of a methodology to assess organometallic effects on bioenergetic systems.

SP618: 1981 November. 235-243. Wolf, W. R. Duality of concern with trace metals in the environment-Toxic and/or essential.

SP618: 1981 November. 244-263. Irgolic, K. J.; Banks, C. H. Bottino, N. R.; Chakraborti, D.; Gennity, J. M.; Hillman, D. C. O'Brien, D. H.; Pyles, R. A.; Stockton, R. A.; Wheeler, A. E.; Zingaro, R. A. Analytical and biochemical aspects of the transformation of arsenic and selenium compounds into biomolecules. SP618: 1981 November. 264-273. Brierley, J. A. Bacterial processes for transformation of metals.

SP618: 1981 November. 274-286. Strandberg, G. W.; Shumate, S. E. II; Parrott, J. R., Jr.; North, S. E. Microbial accumulation of uranium, radium, and cesium.

SP618: 1981 November. 286-300. Klein, D. A.; Hassler, R. A. Microbiological mobilization of arsenic from retorted oil shalesSpeciation and monitoring requirements.

SP618: 1981 November. 301-324. Silver, S. Mechanisms of bacterial resistances to toxic heavy metals: Arsenic, antimony, silver, cadmium, and mercury.

TN1137. Noyce, J. R. Evaluation of methods for the assay of radium228 in water. Nat. Bur. Stand. (U.S.) Tech. Note 1137; 1981 February. 18 p. SN003-003-02294-2.

NBSIR 80-2160. White, E.; Welch, V. M. J.; Hertz, H. S. The mass spectra and isotopic purity of compounds proposed for use in the "master analytical scheme for the analysis of organic compounds in water". 1980 Noveniber. 61 p. Available from: NTIS; PB 81 . 238453.

NBSIR 80-2178. Becker, D. A.; Berke, J. G.; Matthews, R. T.; Yakowitz, H., eds. Technical activities 1980-Office of Recycled Materials. 1980 November. 74 p. Available from: NTIS; PB 81 . 159899.

NBSIR 81-2316. Berke, J. G.; Hudson, C. H. Procurement of products containing recovered material: A summary of activities in seven states. 1981 July. 76 p. Available from: NTIS; PB 81-240475.

NBSIR 81-2366. Weber, S. F.; Rudder, F. F., Jr.; Boehm, M. J. Method for assessing costs of noise control requirements in multifamily residential and educational buildings. 1981 December. 119 p. Available from: NTIS; PB 82-140047.

NBSIR 81-2377. Yaniv, S. L.; Bauer, J. W.; Flynn, D. R.; Danner, W. F. Effects of time-varying noise on annoyance: A review. 1981 October. 63 p. Available from: NTIS; PB 82.133513.

NBS-GCR-81-308. Division of Purchasing, Dept. of Admin., State of Colorado (NBS contact: Kathy Hudson). Procurement of products containing recycled/recovered materials in the State of Colorado. 1981 March. 97 p. Available from: NTIS; PB 81-198236.

NBS-GCR-81-314. Energy Enterprises Northwest (NBS contact: Kathy Hudson). State of Washington: A study of procurement of products containing recycled materials. 1981 March. $116 \mathrm{p}$. A vailable from: NTIS; PB 81-197071.

20009. McClendon, L. T. A summary of oil shale activities at the National Bureau of Standards 1975-1979, EPA-600/7-80-125, 143 pages (United States Environmental Protection Agency, Office of Environmental Engineering and Technology, Washington, DC June 1980). (Available by purchase from the National Technical Information Service, Springfield, VA 22161.)

20011. Menis, O.; Mackey, J. A.; Garn, P. D. A study of particulates of environmental interest by differential thermal analysis, Proc. Angewandte Chemische Thermodynamik und Thermoanalytik, Vortrage des Rapperswiler TA-Symp. (Experientia Supplementum; 37), Rapperswiler, Switzerland, Apr. 18-20, 1979, pp. 321.332 (Birkhauser Verlag Basel, Switzerland, 1979).

20029. Molino, J. A.; Zerdy, G. A.; Tremaine, S. G. Psychoacoustic evaluation of transmission line audible noise: Building attenuation effects, methodology comparison, and field study feasibility, $D O E / R A / 29323-1,84$ pages (U.S. Department of Energy, Office of Electric Energy Systems, Washington, DC, 1979). (Available by purchase from the National Technical Information Service, Springfield, VA 22161.)

20030. Molino, J. A.; Zerdy, G. A.; Lerner, N. D.; Harwood, D. L. Preliminary tests of psychoacoustic facilities and techniques for studying the human response to transmission line audible noise, Energy Tech. HCP/T-6010/E2, 70 pages (U.S. Department of Energy, Division of Electric Energy Systems, Washington, DC, Dec. 1977). (Available by purchase from the National Technical Information Service, Springfield, VA 22161.)

20203. Molino, J. A.; Lerner, N. D.; Zerdy, G. A. Audible noise from high-voltage transmission lines: Psychoacoustic findings, (Proc. U.S. Department of Energy Environmental Control Symp., Washington, DC, Nov. 28-30, 1978), Vol. 3. Solar Energy, Geothermal Energy. and Waste Heat Transmission, DOE/EV-0046, pp. 243.255 (U.S Department of Energy, Washington, DC, Sept. 1979).

20288. May, W. E. The development of an aqueous trace organic 
standard reference material for energy related applications: Investigation of the aqueous solubility behavior of polycyclic hydrocarbons, EPA-600/7-80-031, 87 pages (Available from the National Technical Information Service, Springfield, VA 22161, Feb. 1980).

20302. Kingston, H. M. Quantitative ultratrace transition metal analysis of high salinity waters utilizing chelating resin separation: Application to energy-related environmental samples, EPA-600/7-79174, 84 pages (Available from the National Technical Information Service, Springfield, VA 22161, Aug. 1979).

20345. Wasik, S. P. Partition of organoelements in octanol/water/air systems, (Proc. Symp. Division of Inorganic Chemistry 175th Meet. American Chemical Society, Anaheim, CA, Mar. 13-17, 1978), Chapter 19 in ACS Symp. Series 82. Organometals and Organometalloids. Occurrence and Fate in the Environment, F. E. Brinckman and J. M. Bellama, eds., pp. $314-326$ (American Chemical Society, Washington, DC, 1978).

20361. Molino, J. A.; Zerdy, G. A.; Lerner, N. D.; Harwood, D. L. Initial psychoacoustic experiments on the human response to transmission line audible noise, January 1, 1978 to December 31, 1978, DOE/ET/6010-1, 86 pages (Available from the National Technical Information Services, Springfield, VA 22161, 1978).

20362. Molino, J. A.; Zerdy, G. A.; Lerner, N. D.; Harwood, D. L. Psychoacoustic evaluation of the audible noise from EHV power lines, (Proc. 7th IEEE/PES Transmission and Distribution Conf., Atlanta, GA, Apr. 1-7, 1979), Paper 79CH1399-5-PWR, pp. 95-98 (Institute of Electrical and Electronics Engineers, Inc., New York, NY, 1979)

20397. Bartle, K. D.; Lee, M. L.; Wise, S. A. Modern analytical methods for environmental polycyclic aromatic compounds, Chem. Soc. Rev. 10, No. 1, 113-158 (1981).

20475. Molino, J. A. Annoyance and noise, Chapter 16 in Handbook of Noise Control, Second Edition, C. M. Harris, ed., pp. 16.1-16.9 (McGraw-Hill Book Co., New York, NY, 1979).

20586. Brown, J. M.; Wise, S. A.; May, W. E. Determination of benzo [a]pyrene in recycled oils by a sequential HPLC method, $J$. Environ. Sci. Health. A15, No. 6, 613-623 (1980).

20601. Harrison, S. H.; Zeisler, R.; Wise, S. A. Pilot program for the National Environmental Specimen Bank-Phase 1, EPA-600/S1-81025, 6 pages (United States Environmental Protection Agency, Washington, DC, May 1981). (Available by purchase from the National Technical Information Service, Springfield, VA 22161.)

20602. Chesler, S. N.; May, W. E.; White, P. A.; Parris, R. M.; Guenther, F. R. Determination of polychlorinated biphenyls in waste and lubricating oils, Proc. Joint Conf. on Measurements and Standards for Recycled Oil/Systems Performance and Durability, Gaithersburg, MD, Oct. 23-26, 1979, pp. 295.297 (Nov. 1980).

20654. Christensen, R. G.; Hertz, H. S.; Reeder, D. J.; White, E. Developments in the analysis of priority pollutants by liquid chromatography/mass spectrometry and immunoassay procedures, (Proc. Advances in the Identification and Analysis of Organic Pollutants in Water, Las Vegas, NV, Aug. 24-29, 1980), Chapter 28 in Advances in the Identification and Analysis of Organic Pollutants in Water, L. H. Keith, ed., pp. $447-453$ (Ann Arbor Sci. Pub. Inc., Ann Arbor, MI, 1980).

\section{Failure Analysis}

SP589. Free, G. M., ed. Eddy current nondestructive testing. Proceedings of the Workshop on Eddy Current Nondestructive Testing, held at the National Bureau of Standards; 1977 November 3.4; Gaithersburg, MD. Nat. Bur. Stand. (U.S.) Spec. Publ. 589; 1981 January. 153 p. SN003-003-02287-0.

SP589: 1981 January. 1-32. McMaster, R. C. The history, present status, and future developments of eddy current tests.

SP589: 1981 January. 33-37. Moyer, R. B. Eddy current testing: Present and future applications in the ferrous metal industry.

SP589: 1981 January. 39-44. Burley, C. E. Eddy current standards in nonferrous metals.

SP589: 1981 January. 45-48. Betz, R. A. Eddy current inspection of gas turbine blades.
SP589: 1981 January. 49-55. Wehrmeister, A. E. Eddy current examination in the nuclear industry.

SP589: 1981 January. 57-61. Denton, C. J. Eddy current inspection systems for stream generator tubing in nuclear power plants.

SP589: 1981 January. 63.76. Reinhart, E. R. Use of round robin tests to determine eddy current system performance.

SP589: 1981 January. 77.79. McEleney, P. C. Eddy current testing in government.

SP589: 1981 January. 81-85. McFarlan, T. W. Current status and future directions of eddy current instrumentation.

SP589: 1981 January. 87-105. Libby, H. L. Multifrequency eddy current inspection techniques.

SP589: 1981 January. 107-111. Waidelich, D. L. Pulsed eddy current techniques for nondestructive evaluation.

SP589: 1981 January. 113-119. Weismantel, E. E. The introduction of signal processing techniques to eddy current inspection.

SP589: 1981 January. 121-132. Jones, A. Development of non-ferrous conductivity standards at Boeing.

SP589: 1981 January. 133.136. Free, G. NBS eddy current standards program.

SP589: 1981 January. 137.141. Kahn, A. H.; Spal, R. D. Electromagnetic theory and its relationship to standards.

SP589: 1981 January. 143-147. Green, R. E. Discussion: New science directions and opportunities concluded from workshop sessions.

SP589: 1981 January. 149-154. Belecki, N. B. Discussion: New directions for standards concluded from workshop sessions.

SP622. Shives, T. R.; Willard, W. A., eds. Detection, diagnosis and prognosis: Contribution to the energy challenge. Proceedings of the 32d Meeting of the Mechanical Failures Prevention Group, held at the Inn at Santa Monica; 1980 October 7-9; Santa Monica, CA. Nat. Bur. Stand. (U.S.) Spec. Publ. 622; 1981 October. 329 p. SN003.003. 02361-2.

SP622: 1981 October. 3-10. Canada, R. G.; Jones, J. E. StatisticaI analysis of core barrel motion orbits.

SP622: 1981 October. 11-30. Allen, J. W.; Hartman, W. F.; Robinson, J. C. Acoustic monitoring of power plant valves.

SP622: 1981 October. 33-49. Tauber, T. Oil debris detection progress.

SP622: 1981 October. 50-61. Jones, G. W. Advanced radioactive engine wear analysis for filter design and evaluation.

SP622: 1981 October. 62.73. Wertheimer, H. P.; Romine, D. J. Oxygen sensor for automobile combustion control.

SP622: 1981 October. 74.81. McCord, R. M. Detection, diagnosis and prognosis of gas turbine engine health with the use of fibrescopes.

SP622: 1981 October. 85-96. Woody, M. W.; Hnatczuk, W. Integrated on-board detection diagnostic and prognostic system for military application.

SP622: 1981 October. 97.112. Genot, A. Progress with Pielstick engine diagnostics and experimental results.

SP622: 1981 October. 113-124. Envent, F.; Cohill, M.; Hegner, H. An energy conservation concept for operating machinery.

SP622: 1981 October. 126-136. Fischer, J. P.; Schover, D. S.; Board, D. Marine ball/roller bearing monitoring by shock pulse monitoring.

SP622: 1981 October. 139-151. Gulbrandsen, G. E. Conservation: Loss prevention and risk management.

SP622: 1981 October. 152-180. Lamping, G. A. Recent experiences with steam turbine disc cracking.

SP622: 1981 October. 181-194. Leon, R. L. A Doppler technique for detecting and locating excessively vibrating blades in a running turbine.

SP622: 1981 October. 195-219. Bishop, F. E. IMACS cost savings estimator-A trade-off analysis for a built-in monitoring system.

SP622: 1981 October. 220-221. Mohan, S. P. Operating experience with advanced computer based surveillance system.

SP622: 1981 October. 222-253. Boyce, M. P.; Norris, P. J. Mechanical and aerothermal diagnostics of turbomachinery.

SP622: 1981 October. 257.273. Neustadter, H. E. Data acquisition and analysis in the DOE/NASA wind energy program. 
SP622: 1981 October. 274-288. McCabe, J. T. Periodic vibration monitoring at the SYNTHANE coal-gasification pilot plant.

SP622: 1981 October. 289-309. Rabaud, J. Description of "La Rance" tidal power plant.

SP622: 1981 October. 310-320. Veneruso, A. F.; Chang, H. T. Detection, diagnosis and prognosis in geothermal well technology.

TN1143. Yonemura, G. T. Visual acuity testing of radiographic inspectors in nondestructive inspection. Nat. Bur. Stand. (U.S.) Tech. Note 1143; 1981 June. 29 p. SN003-003-02330-2.

NBSIR 80-2069. Swartzendruber, L.; Boettinger, W.; Ives, L.; Coriell, S.; Ballard, D.; Laughlin, D.; Clough, R.; Biancaniello, F.; Blau, P.; Cahn, J.; Mehrabian, R.; Free, G.; Berger, H.; Mordfin, L. NBS: Nondestructive evaluation of nonuniformities in 2219 aluminum alloy plate-Relationship to processing. 1980 December. 244 p. Available from: NTIS; PB 81.172348.

NBSIR 80-2080. Mordfin, L., ed. NDE publications: 1978. 1980 June. 26 p. Available from: NTIS; PB 81-197691.

NBSIR 81-1648. Read, D. T.; McHenry, H. I. Interim progress report: J-integral method for fitness-for-service assessment. 1981 May. 52 p. Available from: NTIS; PB 82-135195.

NBSIR 81-2255. Chuang, T. A diffusive crack growth model for creep fracture. 1981 March. 58 p. Available from: NTIS; PB 81-196883.

NBSIR 81.2374. Lew, H. S.; Carino, N. J.; Fattal, S. G.; Batts, M. E. Investigation of construction failure of Harbour Cay condominum in Cocoa Beach, Florida. 1981 September. 138 p. Available from: NTIS; PB 82-117409.

NBS-GCR-81-318. Vest, C. M., (NBS contact: G. Birnbaum). Holographic NDE: Status and future. 1981 May. 76 p. Available from: NTIS; PB 81-207409.

20208. Bennett, L. H.; Swartzendruber, L. J.; Reno, R. C. Nuclear hyperfine and positron annihilation methods for measurements of internal stress, (Proc. Joint Government-Industry Conf., Washington, DC, Feb. 26-27, 1979), Paper in Nondestructive Techniques for Measuring the Longitudinal Force in Rails, FRA/ORD-80/50, pp. 126-130 (U.S. Department of Transportation, Washington, DC, Dec. 1980). (Available from the National Technical Information Service, Springfield, VA 22161.)

20245. Berger, H. Nondestructive testing in the 80's, Can. Soc. Nondestr. Test. 2, No. 7, 14.18 (Apr. 1981).

20276. Clough, R. B.; Chang, J. C.; Travis, J. P. Acoustic emission signatures and source microstructure using indentation fatigue and stress corrosion cracking in aluminum alloys, Scr. Metall. 15, 417-422 (1981).

20292. Swartzendruber, L. J. Magnetic leakage and force fields for artificial defects in magnetic particle test rings, Proc. 12th Symp. Nondestructive Evaluation, San Antonio, TX, Apr. 24-26, 1979, pp. 150-162 (American Society for Nondestructive Evaluation, Southwest Research Institute, San Antonio, TX, 1980).

20325. Berger, $H$. The role of calibration in nondestructive evaluation, (Proc. 1980 Workshop \& Symp. National Conference of Standards Laboratories, Gaithersburg, MD, Sept. 22-25, 1980), Paper in NCSL Newslett. 20, No. 4, 32-35 (Dec. 1980).

20342. Placious, R. C.; Garrett, D. A.; Kasen, M. B.; Berger, H. Dimensioning flaws in pipeline girth welds by radiographic methods, Mater. Eval. 39, $755-759$ (July 1981).

20366. Birnbaum, G.; Free, G., eds. Eddy-current characterization of materials and structures, (Proc. Symp. Eddy-Current Characterization of Materials and Structures, Gaithersburg, MD, Sept. 5-7, 1979), Am. Soc. Test. Mater. Spec. Tech. Publ. 722, 501 pages (American Society for Testing and Materials, Philadelphia, PA, Feb. 1981).

20411. Mordfin, L.; Berger, H. NDE standards for nuclear power systems: An NBS perspective, Proc. Third Int. Nondestructive Evaluation in the Nuclear Industry-1980, Salt Lake City, UT, Feb. 11-13, 1980, pp. 303-318 (American Society for Metals, Metals Park, $\mathrm{OH}, 1981$ ).

20474. Eitzen, D. G.; Berger, H.; Birnbaun, G. A basis for traceable NDE measurements, Part 1, Mater. Eval. 39, No. 9, 797.798 (Aug. 1981).

20500. Fong, J. T. Recommendations for future research sessions III \& IV on prediction of damage and failure, Proc. Natl. Science Foundation Workshop on a Continuum Mechanics Approach to
Damage and Life Prediction, University of Cincinnati, Cincinatti, $\mathrm{OH}$, May 1980, pp. 180-183 (1980).

20543. Fortunko, C. M. Ultrasonic detection and sizing of twodimensional defects at long wavelengths, Appl. Phys. Lett. 38, No. 12, 980-982 (June 15, 1981).

20544. Fortunko, C. M. Ultrasonic detection and sizing of twodimensional weld defects in the long-wavelength limit, Proc. 1980 Ultrasonics Symp., Boston, MA, Nov. 4-7, 1980, pp. 862.867 (Institute of Electrical and Electronics Engineers, New York, NY, 1980).

20548. Read, D. T.; McHenry, H. I. Strain dependence of the J-contour integral in tensile panels, (Proc. Fifth Int. Conf. Fracture Advances in Fracture Research, Cannes, France, Mar. 29-Apr. 3, 1981), Paper in Advances in Fracture Research, D. Francois, ed., pp. 1715-1722 (Pergamon Press, New York, NY, 1980).

20559. Lawn, B. R.; Hockey, B. J.; Wiederhorn, S. M. Atomically sharp cracks in brittle solids: An electron microscopy study, $J$. Mater. Sci. 15, 1207-1223 (1980).

20579. Eitzen, D. G.; Breckenridge, F. R.; Clough, R. B.; Fuller, E. R.; Hsu, N. N.; Simmons, J. A. Summary of fundamental developments for quantitative acoustic emission measurements, Electric Power Research Institute Interim Report No. EPRI NP-1877, Project 608-1, 100 pages (Electric Power Research Institute, Palo Alto, CA, June 1981).

20607. Reed, R. P. Goal: Better standards for pipeline welds, Weld. Des. Fabr. 54, No. 8, 68.71 (Sept. 1981).

20625. Berger, H. A new look at the NBS programme in NDE, NDTITDS 14, No. 5, 292.293 (Oct. 1981).

20673. Clough, R. B.; Simmons, J. A. Reproducible acoustic emission signatures by indentation in steels, Mater. Eval. 39, 1026-1031 (Oct. 1981).

20701. Simmons, J. A.; Clough, R. B. Theory of acoustic emission, Proc. Int. Conf. Dislocation Modelling of Physical Systems, Gainesville, FL, June 1980, pp. $464-497$ (Pergamon Press, Oxford, England, 1981).

20744. Fortunko, C. M. Ultrasonic evaluation of austenitic stainless steel welds using shear horizontal waves, Appl. Phys. Lett. 39, No. 9, 699.700 (Nov. 1, 1981).

20757. Eitzen, D. G.; Breckenridge, F. R.; Clough, R. B.; Fuller, E. R.; Hsu, N. N.; Simmons, J. A. Fundamental developments for quantitative acoustic emission measurements, Electric Power Research Institute Interim Report No. EPRI NP-2089, Project 608-1, 213 pages (Electric Power Research Institute, Palo Alto, CA, June 1981).

20762. Berger, H. An overview: New ideas in nondestructive evaluation, (Proc. Meet. Rubber Division, American Chemical Society, Minneapolis, MN, June 2-5, 1981), Rubber Chem. Technol. 54, No. 5, 996-1002 (Nov./Dec. 1981).

\section{Fire Research}

SP480-43. Brown, J. E. Hydrocarbon vapor standards for performance evaluation of combustible gas detectors. Nat. Bur. Stand. (U.S.) Spec. Publ. 480-43; 1981 October. 15 p. SN003-003-02371-0.

TN1133. Evans, D. D. Calculated operating temperatures of thermally insulated electric cables. Nat. Bur. Stand. (U.S.) Tech. Note 1133; 1981 January. 41 p. SN003-003-02279.9.

NBSIR 80-2054, Vols. I and II. Quintiere, J. G.; McCaffrey, B. J. The burning of wood and plastic cribs in an enclosure. 1980 November. Vol. I, 204 p.; Vol. II, 572 p. Vol. I available from NTIS; PB 81. 182586

NBSIR 80-2122. Campbell, P. G.; Sleater, G. A.; Post, M. A. Development of guide specifications for the 1980 exterior restoration of the White House. 1980 October. 129 p. Available from: NTIS; PB 81-164535.

NBSIR 80-2127-1. Martinez, I. M.; Cherry, S. M., eds. Fourth annual conference on fire research. 1980 December. 70 p. Available from: NTIS; PB 81.158305.

NBSIR 80-2155. Issen, L. A. Single-family residential fire and live loads survey. 1980 December. 179 p. Available from: NTIS; PB 81. 166647.

NBSIR 80-2157. Klote, J. H. A computer program for analysis of pressurized stairwells and pressurized elevator shafts. 1981 January. 
87 p. Available from: NTIS; PB 81-159709.

NBSIR 80-2170. Bukowski, R. W. Fire protection systems for rail transportation of Class A explosives: Interim report. 1980 November. 34 p. Available from: NTIS; PB 81-153975.

NBSIR 80-2172. Cooper L. Y. Estimating safe available egress time from fires. 1981 February. 73 p. Available from: NTIS; PB 81. 172355.

NBSIR 80-2186. Babrauskas, V. Combustion of mattresses exposed to flaming ignition sources Part II. Bench-scale tests and recommended standard test. 1981 February. 82 p. Available from: NTIS; PB 81 . 181919.

NBSIR 80-2188. Levine, R. S., ed. Joint US-USSR seminar on mathematical methods for estimating the fire endurance of structural assemblies. 1980 December. 214 p. Available from: NTIS; PB 81159956.

NBSIR 81-2202. Cooper, L. Y.; O'Neill, J. G. Fire tests of stairwellsprinkler systems. 1981 April. 83 p. Available from: NTIS; PB 81205288.

NBSIR 81-2214. Gross, D. A review of measurements, calculations and specifications of air leakage through interior door assemblies. 1981 January. 25 p. Available from: NTIS; PB 81-179178.

NBSIR 81-2220. Beausoliel, R. W.; Clifton, J. R.; Meese, W. J. Effects of thermal insulations on electrical connections and outlet boxes. 1981 April. 52 p. Available from: NTIS; PB 81-197006.

NBSIR 81-2247. Loftus, J. J.; Juarez, N.; Maldonado, A.; Simenauer, J. A. Flammability measurements on fourteen different hydraulic fluids using a temperature-pressure spray ignition test. 1981 May. 30 p. Available from: NTIS; PB 81.214173.

NBSIR 81-2256. Braun, E.; Meade, R. E.; Smith, L. R. Analysis of fire data, large-scale tests, and small-scale tests for conveyor belts used in underground coal mines. 1981 May. 107 p. Available from: NTIS; PB 81-214884.

NBSIR 81-2261. Braun, E. Review of literature, applicable test methods, and incident data for the evaluation of the fire performance of central telephone office equipment. 1981 May. 41 p. Available from: NTIS; PB 81.218455.

NBSIR 81-2271. Babrauskas, V. Will the second item ignite?. 1981 May. 35 p. Available from: NTIS; PB 81.214025.

NBSIR 81-2272. Jason, N. H. Fire research publications, 1980. 1981 April. 22 p. Available from: NTIS; PB 81-203317.

NBSIR 81-2294. Levine, R. S., ed. Survey of United States research projects publications in the field of fire safety of buildings and structures. 1981 June. 46 p. Available from: NTIS; PB 81-216988.

NBSIR 81-2312. Loftus, J. J. MSHA Wick Test for hydraulic fluids: A preliminary evaluation. 1981 July. 28 p. Available from: NTIS; PB 81.238511.

NBSIR 81-2326. Walton, W. D. Investigation of solar collector fire incident. 1981 August. 46 p. Available from: NTIS; PB 82-118639.

NBSIR 81-2329. Evans, D. D.; Madrzykowski, D. Characterizing the thermal response of fusible-link sprinklers. 1981 August. 40 p. Available from: NTIS; PB 82-107020.

NBSIR 81-2333. Klein, D. P. Reduced-scale modeling of mobile home fires: A progress report. 1981 December. 65 p. Available from: NTIS; PB 82.142076.

NBSIR 81-2347. Faison, T. K. Field measurement of branch circuit wire temperatures. 1981 November. 44 p. Available from: NTIS; PB 82-140252.

NBSIR 81-2350. Ohlemiller, T. J. Smoldering combustion hazards of thermal insulation materials. 1981 August. 64 p. Available from: NTIS; PB 82-107046.

NBSIR 81-2363. Krasny, J. F.; Allen, P. J.; Maldonado, A.; Juarez, N. Development of a candidate test method for the measurement of the propensity of cigarettes to cause smoldering ignition of upholstered furniture and mattresses. 1981 October. 43 p. Available from: NTIS; PB 82.135617.

NBSIR 81-2373. Loftus, J. J.; Maldonado-Rosado, A.; Allen, P. J. An evaluation of the MSHA temperature-pressure spray ignition test for hydraulic fluids. 1981 September. 44 p. Available from: NTIS; PB 82-131806.

NBSIR 81-2382. Cherry, S. M., ed. Fifth annual conference on fire research. 1981 October. 187 p. Available from: NTIS; PB 82-
131459 .

NBSIR 81-2386. Hibbard, B. B.; Krasny, J. F.; Braun, E.; Peacock, R. D. Airflow extinguishment of burning apparel fabrics. 1981 December. 40 p. Available from: NTIS; PB 82-165267.

NBSIR 81-2395. Loftus, J. J. An assessment of three different fire resistance tests for hydraulic fluids. 1981 October. 50 p. Available from: NTIS; PB 82.142217.

NBSIR 81-2415. Steel, J. S. Furnace pressure probe investigation. 1981 November. 29 p. A vailable from: NTIS; PB 82.139999.

NBSIR 81-2433. Klote, J. H.; Zile, R. H. Smoke movement and smoke control on merchant ships. 1981 December. 68 p. Available from: NTIS; PB 82-165192.

NBS-GCR-76-81. Cornell, C. A., (NBS contact: Structures \& Materials Division). A national office of live loads survey. 1976 October. 180 p. Available from: NTIS; PB 82-130436.

NBS-GCR-79-186-1. Griffith, J. W., (NBS contact: N. Jason). Life cycle cost workbook. 1981 September. 30 p. Available from: NTIS; PB 82-120510.

NBS-GCR-79-188. You, H. Z.; Faeth, G. M., (NBS contact: N. Jason). An investigation of fire impingement on a horizontal ceiling. 1978 October. 112 p. Available from: NTIS; PB 82-130428.

NBS-GCR-80-284. Nober, E. H.; Pierce, H.; Well, A.; Johnson, C. C.; Clifton, C. Waking effectiveness of household smoke and fire detection devices. 1980 October. 85 p. Available from: NTIS; PB 81. 127565 .

NBS-GCR-80-292. Maxwell, T. T.; Maples, G.; Dyer, D. F.; Burch, T., (NBS contact: Nora Jason). Fire safety of wood-burning appliances, Part II: Fireplace inserts-A design review. 1980 November. 19 p. Available from: NTIS; PB 81-161531.

NBS-GCR-80-294. Zukoski, E. E.; Kubota, T.; Cetegen, B., (NBS contact: Nora Jason). Entrainment in fire plumes. 1980 November. 53 p. A vailable from: NTIS; PB 81-181851.

NBS-GCR-80-295. Tewarson, A., (NBS contact: Nora Jason). Physicochemical and combustion/pyrolysis properties of polymeric materials. 1980 December. 83 p. Available from: NTIS; PB 81-158651.

NBS-GCR-80-297. Bryan, J. L.; DiNenno, P. J.; Milke, J. A., (NBS contact: $\mathrm{N}$. Jason). The determination of behavior response patterns in fire situations, project people II. Final Report-Incident reports, August 1977 to June 1980. 1980 December. 234 p. Available from: NTIS; PB 81-224545.

NBS-GCR-80-298. Capozzi, D. M., (NBS contact: Nora Jason). An annotated bibliography for wood combustion activities in the residential sector. 1981 January. 141 p. Available from: NTIS; PB 81.165490 .

NBS-GCR-81-300. Pape, R., (NBS contact: Nora Jason). Preflashover room fire model: Parametric sensitivity analysis and development of a submodel for burning furniture items. 1981 January. 70 p. Available from: NTIS; PB 81.170664.

NBS-GCR-81-301. Pape, R.; Waterman, T. E.; Eichler, T. V., (NBS contact: Nora Jason). Development of a fire in a room from ignition to full room involvement-RFIRES. 1981 January. 182 p. Available from: NTIS; PB 81.166639.

NBS-GCR-81-302. Shingleton, J. G.; Cassel, D. E.; Overton, R. L., (NBS contact: Mary Reppert). Air leakage in residential solar heating systems. 1981 February. 206 p. Available from: NTIS; PB 81.176380 .

NBS-GCR-81-315. Waller, M. B.; Vreeland, R. G., (NBS contact: N. Jason). Report of a conference on fire emergency plans in group homes for the developmentally disabled. 1981 March. 32 p. Available from: NTIS; PB 81-180960.

NBS-GCR-81-316. Francis, R. L.; Chalmet, L. G., (NBS contact: N. Jason). Network models for building evacuation: A prototype primer. 1981 March. 251 p. Available from: NTIS; PB 81-197022.

NBS-GCR-81-317. Lin, T. D.; Abrams, M. S., (NBS contact: Nora Jason). Simulation of realistic thermal restraint during fire tests of floors and roofs. 1981 March. 178 p. Available from: NTIS; PB 81. 179657.

NBS-GCR-81-329. Martin, S. B.; Inman, L. B., (NBS contact: N. Jason). The effect of fire retardants on the heat-release rate of building materials. 1981 July. 39 p. Available from: NTIS; PB 81. 238487. 
NBS-GCR-81-330. Berhinig, R. M., (NBS contact: N. Jason). Measurement of air flow around doors under standardized fire test conditions. 1981 July. 40 p. Available from: NTIS; PB 81-238495.

NBS-GCR-81-343. Bryan, J. L.; Milke, J. A., (NBS contact: N. Jason). The determination of behavior response patterns in fire situations, Project people II. Final report-Health care. 1981 October. 304 p. Available from: NTIS; PB 82-136771.

NBS-GCR-81-344. Mitler, H. E.; Emmons, H. W., (NBS contact: N. Jason). Documentation for CFC V, The fifth Harvard Computer Fire Code. 1981 October. 187 p. Available from: NTIS; PB 82-139486.

NBS-GCR-81-346. Zukoski, E. E.; Kubota, T.; Cetegen, B., (NBS contact: N. Jason). Entrainment in the near field of a fire plume. 1981 November. 66 p. A vailable from: NTIS; PB 82-136466.

U.S. Patent $4,260,660$. McCarter, R. J. Use of sulphur as an additive to inhibit the smoldering combustion of materials. 7 April $1981.6 \mathrm{p}$.

U.S. Patent 4,302,345. McCarter, R. J. Flame retarding cellulosic materials with sodium or potassium thiocyanate. 24 November 1981. $6 \mathrm{p}$.

19972. Davis, R. W.; Moore, E. F. Numerical modeling of swirling laminar orifice flow, Proc. IMEKO Symp. on Flow Measurement and Control in Industry, Tokyo, Japan, Nov. 13-16, 1979, pp. 81-86 (Society of Instrument Control Engineers, Tokyo, Japan, 1980).

20207. Quintiere, J. G. An approach to modeling wall fire spread in a room, Fire Saf. J. 3, 201-214 (1981).

20466. Smyth, K. C.; Mallard, W. G. Laser-induced ionization and mobility measurements of very small particles in premixed flames at tbe sooting limit, Combust. Sci. Technol. 26, 35-41 (1981).

20487. Manka, M. J.; Yeh, K. N.; Smith, B. F. A calorimetric study of flame-retardant-treated cotton/polyester blends, J. Fire Retard. Chem. 5, 144-159 (Aug. 1978)

20493. Quintiere, J. A simplified theory for generalizing results from a radiant panel rate of flame spread apparatus, Fire Mater. 5, No. 2, $52-60$ (1981).

20558. Gann, R. G.; Earl, W. L.; Manka, M. J.; Miles, L. B Mechanism of cellulose smoldering retardance by sulfur, Proc. Eighteenth Symp. Combustion, Pittsburgh, PA, Aug. 17-22, 1980, pp. 571.578 (The Combustion Institute, Pittsburgh, PA, 1981).

20565. Mulholland, G.; Handa, T.; Sugawa, O.; Yamamoto, H. Smoke filling in an enclosure, Proc. 20th Joint ASME/AIChE Natl. Heat Transfer Conf., Milwaukee, WI, Aug. 2-5, 1981, Paper 81-HT-8, pp. 1.13 (The American Society of Mechanical Engineers, New York, NY 10017, 1981)

20567. McCaffrey, B. J.; Quintiere, J. G.; Harkleroad, M. F Estimating room temperatures and the likelihood of flashover using fire test data correlations, Fire Technol. 17, No. 2, 98-119 (May 1981).

20576. O'Neill, J. G. Brief status report on NBS/CFR sprinkler projects, Proc. Conf. Low Cost Residential Sprinkler System, Baltimore, MD, Nov. 29-30, 1977, pp. $1-12$ (National Fire Prevention \& Control Admin., Washington, DC, June 1978).

20590. Cooper, L. Y. Heat transfer from a buoyant plume to an unconfined ceiling, Proc. 20th Joint ASME/AIChE Natl. Heat Transfer Conf., Milwaukee, WI, August 2-5, 1981, Paper 81-HT-7, pp. 1.9 (The American Society of Mechanical Engineers, New York, NY 10017,1981$)$.

20591. Cooper, L. Y.; Harkleroad, M.; Quintiere, J.; Rinkinen, W. An experimental study of upper hot layer stratification in full-scale multiroom fire scenarios, Proc. 20th Joint ASME/AIChE Natl. Heat Transfer Conf., Milwaukee, WI, Aug. 2-5, 1981, Paper 81-HT-9, pp. 1.12 (The American Society of Mechanical Engineers, New York, NY, 1981).

20642. Babrauskas, V. A laboratory flammability test for institutional mattresses, Fire J. 75, No. 6, 35.40, 93 (Nov. 1981).

20727. Levin, B. M.; Nelson, H. E. Fire safety and disabled persons, Fire J. 75, No. 5, 35-40 (Sept. 1981)

20740. Quintiere, J.; Steckler, K.; McCaffrey, B. A model to predict the conditions in a room subject to crib fires, Proc. First Specialists Meet. Int. Combustion Institute, Talence, France, July 20-24, 1981, 12 pages (1981)

20746. Benjamin, I. A. The future of fire protection engineering, Proc. Ann. Fire Protection Seminar, Department of Fire Protection \& Safety
Engineering, Illinois Institute of Technology, Chicago, IL, Mar. 14, 1981,7 pages $(1981)$

\section{Fluids: Liquids, Gases, and Plasmas}

Rainwater, J. C. Propagation of density fluctuations in nonuniform fluids: Simple models. J. Res. Nat. Bur. Stand. (U.S.). 86(5): 429-455; 1981 September-October.

Roder, H. M. A transient hot wire thermal conductivity apparatus for fluids. J. Res. Nat. Bur. Stand. (U.S.). 86(5): 457.493; 1981 September-October.

Ely, J. F. An Enskog correction for size and mass difference effects in mixture viscosity prediction. J. Res. Nat. Bur. Stand. (U.S.). 86(6): 597-604; 1981 November-December.

SP260-73. Interrante, C. G.; Hicho, G. E. Standard reference materials: A standard reference material containing nominally fifteen percent austenite. Nat. Bur. Stand. (U.S.) Spec. Publ. 260-73; 1981 December. 34 p. SN003-003-02386-8.

NBSIR 80-1631. Van Poolen, L. J. Analysis of liquid volume and liquid mass fractions at coexistence for pure fluids. 1980 May. $63 \mathrm{p}$. Available from: NTIS; PB 80-191133.

19971. Davis, R. W.; Deutsch, S. A numerical-experimental study of Parshall flumes, J. Hydraul. Res. 18, No. 2, 135.152 (1980).

19973. Evans, D. J.; Hanley, H. J. M. Computer simulation of an $m \cdot 6-8$ fluid under shear, Physica (Utrecht) A 103, No. 1-2, 343-353 (Sept. 1980).

19974. Evans, D. J.; Hanley, H. J. M. Shear induced phase transitions in single fluids, Phys. Lett. 79A, No. 2-3, 178-180 (Sept. 29, 1980).

20048. McMichael, J. M.; Klebanoff, P. S.; Mease, N. E. Experimental investigation of drag on a compliant surface, Chapter in Viscous Flow Drag Reduction. Progress in Astronautics and Aeronautics, G. R. Hough, ed., 72, 410-438 (American Institute of Aeronautics and Astronautics, New York, NY 10104, 1980).

20059. Walker, G. R.; Marshall, R. D. The development of a peak gust anemometer, Proc. 7th Australasian Hydraulics and Fluid Mechanics Conf., Brisbane, Queensland, Australia, Aug. 18-22, 1980, pp. 151.154 (The Institution of Engineers, Barton, A.C.T. 2600, Australia, Oct. 1980).

20147. Mahajan, B. M.; Galowin, L. S. Water depth and solid velocity measurements in unsteady partially-filled horizontal pipe flow, Proc. Second Symp. Flow: Its Measurement and Control in Science and Industry, St. Louis, MO, Mar. 23-26, 1981, pp. $649-671$ (Instrument Society of America, Research Triangle Park, NC, Mar. 1981).

20255. Purtell, L. P. Molecular diffusion in oscillating laminar flow in a pipe, Phys. Fluids 24, No. 5, 789.793 (May 1981).

20257. Purtell, L. P.; Klebanoff, P. S.; Buckley, F. T. Turbulent boundary layer at low Reynolds number, Phys. Fluids 24, No. 5, 802. 811 (May 1981)

20262. Evans, D. J. Nonlinear viscous flow in two-dimensional systems, Phys. Rev. A 22, No. 1, 290-294 (July 1980).

20263. Rainwater, J. C. Softness expansion of gaseous transport properties. II. Moderately dense gases, J. Chem. Phys. 74, No. 7, 4130-4143 (Apr. 1, 1981).

20304. Evans, D. J. Rbeological properties of simple fluids by computer simulation, Phys. Rev. A 23, No. 4, 1988-1997 (Apr. 1981).

20310. Evans, D. J. On the coupling of kinetic and potential contributions to transverse collective modes in fluids, Mol. Phys. Res. Notes 42, No. 1, $231-234$ (1981).

20335. Santoro, R. J.; Semerjian, H. G.; Emmerman, P. J.; Goulard, R. Optical tomography for flow field diagnostics, Int. J. Heat Mass Transfer 24, No. 7, 1139-1150 (1981).

20354. Mattingly, G. E. Dynamic traceability of flow measurements, Proc. IMEKO Symp. Flow Measurement and Control in Industry, Tokyo, Japan, Nov. 13-16, 1979, pp. 0-4-1-0-4-11 (International Measurement Confederation, Tokyo, Japan, 1979).

20357. Semerjian, H. G.; Santoro, R. J.; Goulard, R.; Emmerman, P. J. Optical tomography for diagnostics in combusting flows, Paper in Mechanical Properties of Bone, S. C. Cowin, ed., AMD.45, 119.128 (The American Society of Mechanical Engineers, New York, NY, 1981).

20570. Diller, D. E.; Haynes, W. M. Application of the torsionally 
oscillating quartz crystal viscometer to compressed gases and liquids, Proc. DoE Gas Miscible Displacement Research Seminar, Tulsa, OK, Jan. 8-9, 1981, pp. 1X-1-1X-13 (Morgantown Energy Technology Center, Department of Energy, Morgantown, WV, 1981).

20613. Hanley, H. J. M.; Evans, D. J. Behavior of a nonconformal mixture via computer simulation, Int. J. Thermophys. 2, No. 1, 1.19 (1981).

20649. Evans, D. J.; Hanley, H. J. M. Thermodynamic fluctuation theory for shear flow, Physica 108A, 567-574 (1981).

20718. Greer, S. C.; Moldover, M. R. Thermodynamic anomalies at critical points of fluids, Ann. Rev. Phys. Chem. 32, 233-265 (1981).

\section{General Theoretical Chemistry and Physics}

Bennett, H. S. Upper limits for the number of bound states associated with the Yukawa potential. J. Res. Nat. Bur. Stand. (U.S.). 86(5). 503.508; 1981 September-October.

19991. Hayward, R. W. CPT theorem, Article in Encyclopedia of Physics, R. G. Lerner and G. L. Trigg, eds., pp. 166-168 (AddisonWesley Publ. Co., Inc., Advanced Book Program, Reading, MA, 1981).

19993. Maximon, L. C. Bremsstrahlung, Article in Encyclopedia of Physics, R. G. Lerner and G. L. Trigg, eds., pp. 89.91 (AddisonWesley Publ. Co., Inc., Advanced Book Program, Reading, MA, 1981).

20034. Carlson, C. E.; Coyne, J. J.; Fishbane, P. M.; Gross, F.; Meshkov, S. E(1440): Glueball or quarkonium?, Phys. Lett. 98B, No. 1-2, 110-114 (Jan. 1, 1981).

20040. Karl, G.; Meshkov, S.; Rosner, J. L. Quark magnetic moments and $\mathrm{E} 1$ radiative transitions in charmonium, Phys. Rev. Lett. 45, No. 4, 215.218 (July 28, 1980).

20109. Rafelski, J.; Danos, M. The importance of the reaction volume in hadronic collisions, Phys. Lett. 97B, No. 2, $279-282$ (Dec. 1, 1980).

20118. Mountain, R. D. Effective pair potentials from structure factors, (Proc. 4th Int. Conf. Liquid and Amorphous Metals, Grenoble, France, July 7-11, 1980), Paper in Journal de Physique C8, No. 41, Suppl. No. 8, C8-297_C8-300 (Les Editions de Physique, Orsay, France, Aug. 1980).

20127. Sengers, J. M. H. L.; Sengers, J. V. How close is "close to the critical point"?, Chapter 14 in Perspectives in Statistical Physics, H. J. Raveché, ed., pp. 241.271 (North-Holland Publ. Co., AE Amsterdam, The Netherlands, 1981).

20187. Carlson, C. E.; Coyne, J. J.; Fishbane, P. M.; Gross, F.; Meshkov, S. Glueballs: Their experimental signature, Phys. Lett. 99B, No. 4, 353-357 (Feb. 26, 1981).

20211. Rainwater, J. C. Delta-function expansion of Mayer function with application to virial coefficients, J. Stat. Phys. 19, No. 2, 177.189 (1978).

20215. Rainwater, J. C. Temperature dependence of the magnetic susceptibility of a Fermi liquid, Phys. Lett. 71A, No. 2-3, 278-280 (Apr. 30, 1979).

20243. Katriel, J.; Hummer, D. G. Analytic solutions for three- and four-wave mixing via generalised Bose operators, J. Phys. A: Math. Gen. 14, 1211.1224 (1981).

20248. Meshkov, S. Glueballs: Their spectra, production and decay, Paper in Recent Developments in High-Energy Physics, B Kursunoglu, A. Perlmutter, and L. F. Scott, eds., pp. 43-59 (Plenum Publ. Corp., New York, NY, 1980).

20316. Carlson, C. E.; Coyne, J. J.; Fishbane, P. M.; Gross, F.; Meshkov, S. Glueball decay widths and glueball dominance of quarkonium decays, Phys. Rev. Brief Reports 23, No. 11, 2765.2768 (June 1, 1981).

20384. Casella, R. C. Neutron scattering from coherent admixtures of phonons with libronic excitations of diatomic impurities, Phys. Rev. B 20, No. 12, 5318-5325 (Dec. 15, 1979).

20433. Goodman, D. A.; Cahn, J. W.; Bennett, L. H. The Centennial of the Gibbs-Konovalov rule for congruent points. Its underlying theoretical basis and its application to phase diagram evaluation, Bull. Alloy Phase Diagrams 2, No. 1, 29-34 (1981).

20483. Deslattes, R. D.; Kessler, E. G., Jr. Inner shell energies: Experimental problems, Proc. Workshop on Foundations of the
Relativistic Theory of Atomic Structure, Argonne, IL, Dec. 4-5, 1980. pp. 215.235 (Argonne National Laboratory, Argonne, IL, Mar. 1981).

20495. Evans, D. J. Equilibrium fluctuation expressions for the wavevector-and frequency-dependent shear viscosity, Phys. Rev. A 23, No. 5, 2622-2626 (May 1981).

20532. Gadzuk, J. W. Localized vibrational modes in Fermi liquids. General theory, Phys. Rev. B 24, No. 4, 1651.1663 (Aug. 15, 1981).

20537. Drummond, P. D.; Gardiner, C. W.; Walls, D. F. Quasiprobability methods for nonlinear chemical and optical systems, Phys. Rev. A 24, No. 2, 914.926 (Aug. 1981).

20549. Yaghjian, A. D. Reply to criticism on "electric dyadic Green's functions in the source region", Proc. IEEE 69, No. 2, 282-285 (Feb. 1981).

20599. Yoder, C. F.; Peale, S. J. The tides of Io, Icarus 47, 1-35 (1981).

20609. Danos, M.; Zanon, D. Model for the hadron-hadron vertex: Dynamic origin of the OZI rule, Lett. Nuovo Cimento 31, No. 2, 61 . 64 (1981).

20629. Girvin, S. M.; Rendell, R. W.; Bryant, G. W. New field theory formulation of localised states in disordered systems, J. Phys. C: Solid State Phys. Lett. Ed. 14, L881-L886 (1981).

20656. Casella, R. C. Theory of neutron scattering from hydrogen in metals involving transitions to excited tunnel-split states, Phys. Rev. $B$ 24, No. 6, 2913.2920 (Sept. 15, 1981).

20695. Prialnik, D.; Shaviv, G.; Kovetz, A. The effect of diffusion on asymptotic branch evolution, Astrophys. J. 247, 225.235 (July 1, 1981).

\section{Health and Safety}

Kilday, M. V. Enthalpies of solution of the nucleic acid bases. 6 . Guanine in aqueous $\mathrm{HCl}$ and $\mathrm{NaOH}$, and guanine hydrochlorides in aqueous HCl. J. Res. Nat. Bur. Stand. (U.S.). 86(4): 367.382; 1981 July-August.

SP480-37. Rubin, A. I.; Howett, G. L. Emergency vehicle warning systems. Nat. Bur. Stand. (U.S.) Spec. Publ. 480-37; 1981 May. 25 p. SNO03-003-02323-0.

SP480-40. Thornton, J. I.; Kraus, S.; Lerner, B.; Hendrickson, B. Paint solubility testing. Nat. Bur. Stand. (U.S.) Spec. Publ. 480-40; 1981 December. 21 p. Available from: NTIS; PB : 82-177049.

SP480-41. Flores, A.; Eliason, L. K.; Wu, Y. C. Breath alcohol sampling simulator (BASS) for qualification testing of breath alcohol measurement devices. Nat. Bur. Stand. (U.S.) Spec. Publ. 480-41; 1981 July. 31 p. SN003-003-02336-1.

SP480-44. Taggart, H. E. Methods of suppressing automotive interference. Nat. Bur. Stand. (U.S.) Spec. Publ. 480-44; 1981 December. 15 p. Available from: NTIS; PB 82-165259.

SP480-45. Clifton, J. R. Penetration resistance of concrete-A review. Nat. Bur. Stand. (U.S.) Spec. Publ. 480-45; 1981 December. 22 p. Available from: NTIS; PB 82-177932.

SP601. Weinstein, A.; Gibbons, D.; Brown, S.; Ruff, W., eds. Implant retrieval: Material and biological analysis. Proceedings of a Conference held at the National Bureau of Standards; 1980 May 1.3; Gaithersburg, MD. Nat. Bur. Stand. (U.S.) Spec. Publ. 601; 1981 January. 772 p. SN003-003-02292-6.

SP601: 1981 January. 3.28. Rose, R. M.; Crugnola, A. N.; Cimino, W. R.; Ries, M. D. The in-vivo performance of polyethylene components of total joint replacements.

SP601: 1981 January. 29-129. Guidoin, R.; King, M.; Blais, P.; Marois, M.; Gosselin, C.; Roy, P.; Courbier, R.; David, M.; Noël, H. P. A biological and structural evaluation of retrieved Dacron arterial prostheses.

SP601: 1981 January. 131-156. Plitz, W.; Griss. P. Clinical, histomorphological and material related observations on removed aluminaceramic hip joint components.

SP601: 1981 January. 157-174. Gibbons, D. F.; Buran, K. A. Microscopic analysis of retrieved polymethylmethacrylate (PMMA) bone cement.

SP601: 1981 January. 175-200. Yoganathan, A. P.; Harrison, E. C. Prosthetic heart valves: What have we learned from our clinical pathologic experience. 
SP601: 1981 January. 201-222. Taussig, L. M. Examination of orthopaedic implant failures.

SP601: 1981 January. 223.235. Wilkinson, R.; Shepherd, M. T. A study of inclusions in stainless steel implants and their effect on corrosion.

SP601: 1981 January. 239.267. Willert, H. G.; Buchhorn, G.; Buchhorn, U.; Semlitsch, M. Tissue response to wear debris in artificial joints.

SP601: 1981 January. 269-282. Schoen, F. J.; Titus, J. L.; Lawrie, G. M. Materials degeneration causing late failure of mechanical heart valve prostheses: Problems and promise.

SP601: 1981 January. 283-298. Anderson, J. M.; Barenberg, S.; Hiltner, A. Prosthetic valve wear products-Embolization and systemic presentation.

SP601: 1981 January. 299.321. Brown, S. A.; Merritt, K. Metal allergy and metallurgy.

SP601: 1981 January. 323-336. Williams, D. F.; McNamara, A.; Clark, G. C. F. Techniques for the examination of tissues in implant retrieval analysis.

SP601: 1981 January. 339-348. Levy, R. J.; Sanders, S. P.; Lian, J. B. Retrieval analysis of calcific degeneration of prosthetic tissue valves: The role of vitamin $\mathrm{K}$-dependent processes and other regulatory mechanisms.

SP601: 1981 January. 349.362. Parks, P. J. Prosthesis-related infections.

SP601: 1981 January. 363-393. Quijano, R. C. Study of 208 explanted bioprosthesis: Distribution of dysfunction patterns and assessment of durability at $\mathbf{1 0}$ years of use.

SP601: 1981 January. 395-422. Simpson, J. P.; Geret, V.; Brown, S. A.; Merritt, K. Retrieved fracture plates: Implant and tissue analysis. SP601: 1981 January. 423-447. Skinner, H.; Weinstein, A. M.; Clemow, A. J. T.; McPhillips-Meade, M.; Klawitter, J. J.; French, G. Corrosion, materials characteristics and local tissue reaction associated with osteosynthesis devices.

SP601: 1981 January. 449-469. Weinberg, S. The evaluation of explanted umbilical vein grafts.

SP601: 1981 January. 471-499. Clarke, I. C. Wear of polymeric prosthesis-Clinical reality, retrieved implants and laboratory predictions.

SP601: 1981 January. 503-508. Flink, R. C.; Malette, W Considerations concerning international standards for accumulating and reporting of implant experience with cardiac pacemakers.

SP601: 1981 January. 509-557. Hori, R. Y.; Lewis, J. L.; Wixson, R. L; Kramer, G. M. An analysis of $\mathbf{1 3 0}$ removed total hips and knees. SP601: 1981 January. 559.572. Wright, T. M; Burstein, A. H. The method of implant retrieval anlaysis at the Hospital for Special Surgery.

SP601: 1981 January. 573-613. Cook, S. D.; Weinstein, A. M. An interactive computer system for implant data retrieval/analysis.

SP601: 1981 January. 615.642. Schurman, D. J.; Burton, D. S.; Nagel, D. A.; McShane, D. A data retrieval system for implant failures in total joint replacement surgery.

SP601: 1981 January. 643-653. Dobbs, H. S. Prediction of femoral component performance through implant retrieval information. SP601: 1981 January. 655-666. Piehler, H. R.; Sloter, L. E. II. Legal and regulatory implications of implant retrieval and analysis.

SP613. Clark, H. E. Requirements for an effective national nonionizing radiation measurement system. Nat. Bur. Stand. (U.S.) Spec. Publ. 613; 1981 June. 41 p. SN003-003-02335-5.

TN1139. Collé, R.; Rubin, R. J.; Knab, L. I.; Hutchinson, J. M. R. Radon transport through and exhalation from building materials: $A$ review and assessment. Nat. Bur. Stand. (U.S.) Tech. Note 1139; 1981 September. 10l p. SN003-003-02360.4.

NBSIR 80-1975. Rubin, R. J. Analysis of mathematical models of integrating monitoring devices. 1980 January. 37 p. Available from: NTIS; PB 81.164469.

NBSIR 80-2003. Lerner, N. D.; Collins, B. L. Workplace safety symbols: Current status and research needs. 1980 March. $62 \mathrm{p}$. Available from: NTIS; PB 81.157059.

NBSIR 80-2018 (DOT). Churney, K. L.; Garvin, D. A third appraisal of methods for estimating self-reaction hazards. 1980 August. 326 p.
A vailable from: NTIS; PB 81.188286.

NBSIR 80-2035. Toner, S. D.; Wakamiya, S. K. Bicycle wheel rim reflective materials test method development. 1980 April. $15 \mathrm{p}$. Available from: NTIS; PB 81.152365.

NBSIR 80-2037. Berger, R. E.; Huckeba, J. A. Impact on human eyes by propelled objects. 1980 May. 14 p. Available from: NTIS; PB 81 . 154130 .

NBSIR 80-2088. Lerner, N. D.; Collins, B. L. The assessment of safety symbol understandability by different testing methods. 1980 August. 60 p. A vailable from: NTIS; PB 81.185647.

NBSIR 80-2106. Beine, W. B.; Sorrells, J. R. Soil impact attenuation performance: A field study. 1980 June. 38 p. Available from author only.

NBSIR 80.2131. Phillips, J. C. Performance of plastic packaging for hazardous materials transportation. Part VI. Mass loss and effective carbon atom number measurement. 1980 April. 32 p. Available from: NTIS; PB 81-239626.

NBSIR 80-2168. Cassel, J. M.; Tesk, J. A.; Brauer, G. M.; Antonucci, J. M.; Wu, W.; McKinney, J. E.; Whitlock, R. P.; Hinman, R. Properties and interactions of oral structures and restorative materials. 1980 November. 110 p. Available from: NTIS; PB 81. 236895.

NBSIR 81-2200. Braun, E.; Reyes-Virella, R. Evaluation of alternate mounting methods for the evaluation of brattice cloth on ASTM E-162. 1981 January. 27 p. A vailable from: NTIS; PB 81.172496.

NBSIR 81-2203. Vaishnav, M. P. Electrical aspects of the CSA/NBS weatherization study. 1981 January. 52 p. Available from: NTIS; PB 81.172512

NBSIR 81-2212. Dehl, R. E.; Grant, W. H.; Cassel, J. M. Evaluation of methods of characterizing the porosity of porous polymeric implant materials: A review of the current status of porosity measurements. 1981 February. 49 p. A vailable from: NTIS; PB 81. 166928.

NBSIR 81-2213. Evans, D. D.; Davis, S. Technical support for the Consumer Product Safety Commission 1979 interim standards for cellulose insulation. 1981 March. 52 p. Available from: NTIS; PB 81-196396.

NBSIR 81-2286. Law Enforcement Standards Laboratory. The characterization of auto headlight glass by refractive index and density. 1981 October. 37 p. A vailable from: NTIS; PB 82-131723.

NBSIR 81-2292. Braun, E. Review of fire test methods and incident data for portable electric cables in underground coal mines. 1981 June. 22 p. A vailable from: NTIS; PB 81-227878.

NBSIR 81-2298. Moore, R. T. Results of fingerprint image quality experiments. 1981 June. 49 p. Available from: NTIS; PB 82-133539.

NBSIR 81-2320. Palla, R. L., Jr. A heat transfer analysis of scald injury. 1981 July. 68 p. Available from: NTIS; PB 81.238503.

NBSIR 81-2361. Ventre, F. T.; Stahl, F. I.; Turner, G. E. Crowd ingress to places of assembly: Summary and proceedings of an experts' workshop. 1981 September. 147 p. Available from: NTIS; PB 82-138199.

NBSIR 81-2428. Collins, B. L.; Danner, W. F.; Tibbott, R. L. Communication systems for disabled users of buildings. 1981 December. 73 p. Available from: NTIS; PB 82-165283.

NBS-GCR-80-255. Hunt, B., (NBS contact: S. Webber). Development of a research program for scaffolding standards. 1980 August. $276 \mathrm{p}$. A vailable from: NTIS; PB 81.180259.

NBS-GCR-80-291. Ryan, P.; De Vos, D., (NBS contact: J. Stroik). Building security demonstration: A survey of break-ins in two public housing sites. 1980 November. 100 p. Available from: NTIS; PB 81. 180549 .

U.S. Patent 4,243,763. Argentar, H. Tertiary aromatic amine accelerators in acrylic resin. 6 January $1981.13 \mathrm{p}$.

U.S. Patent $4,251,565$. Bowen, R. L. Use of a polyfunctional surfaceactive comonomer and other agents to improve adhesion between a resin or composite material and a substrate. 17 February 1981.14 p.

U.S. Patent 4,284,551. Argentar, H. Tertiary aromatic amine accelerators derived from para-aminophenethanol. 18 August 1981. $13 \mathrm{p}$.

U.S. Patent 4,306,913. Mabie, C.; Menis, D. Method for preparing microporous glassy filler grains for dental resin composites. 22 
December $1981.36 \mathrm{p}$

19988. Speck, K. M.; Fraker, A. C. Anodic polarization behavior of Ti$\mathrm{Ni}$ and Ti-6A1-4V in simulated physiological solutions, $J$. Dent. Res. 59, No. 10, 1590-1595 (Oct. 1980).

20015. Prosen, E. J. Microcalorimetry and its applications, (Proc. Symp. on Biological Effects, Imaging Techniques, and Dosimetry of Ionizing Radiations, Rockville, MD, June 6-8, 1979), Paper in HHS Publication (FDA) 80-8126, Radiological Health, pp. 147.153 (Department of Health and Human Services, Public Health Services, Food and Drug Administration, Washington, DC, 1980).

20098. Reeder, D. J. Place of reference materials and reference methods in the evaluation of drug effects, (Proc. Use of Laboratory Test Results. Variation Due to Drug Intake, Pont-à-Mousson, France, Dec. 17-19, 1979), Paper in Drug Effects on Laboratory Test Results. Developments in Clinical Biochemistry, G. Siest, ed., 2, 49-57 (Martinus Nijhoff Publishers, Boston, MA, 1980).

20116. Misra, D. N.; Bowen, R. L. Adsorptive bonding to hydroxyapatite I: Adsorption of anthranilic acid-the effect of solvents. Search for surface bonding groups for coupling agents to teeth, Biomater. 2, 28.32 (Jan. 1981).

20122. Brown, W. E.; Tung, M. S.; Chow, L. C. Role of octacalcium phosphate in the incorporation of impurities into apatites, Proc. $2 d$ Int. Congress on Phosphorus Compounds, Boston, MA, Apr. 21-25, 1980, pp. $59-71$ (Institut Mondial de Phosphate, Paris, France, Feb. 1981).

20129. McKenna, G. B.; Penn, R. W. Time-dependent failure of a polyolefin rubber candidate material for blood pump applications, $J$. Biomed. Mater. Res. 14, 689.703 (1980)

20135. Haller, W. Surface derivatized silica-sol particles as immunospecific markers for high resolution electron microscopy, (Proc. Conf. Chemistry of Solid/Liquid Interfaces, Dubrovnik, Yugoslavia, June 25-July 3, 1979), Paper in Croatica Chemica Acta 53, No. 2, 373.379 (Croatica Chemica Acta, Zagreb, Yugoslavia, Feb. 1980).

20169. Gills, T. E.; Boweri, R. L. Adhesive bonding of various materials to hard tooth tissues: Tracer study of mordant adsorption on enamel, (Proc. 1978 Int. Orthodontic Conf. Centennial Program, Philadelphia, PA, Apr. 10.12, 1978), Paper in Orthodontics: The State of the Art, H. G. Barrer, ed., pp. 163.171 (University of Pennsylvania Press, Philadelphia, PA, 1981).

20181. Hobbs, T. G. The health physics program at the National Bureau of Standards Linac, IEEE Trans. Nucl. Sci. NS-28, No. 2, 1600-1602 (Apr. 1981).

20217. Persensky, J. J.; Gagnon, J. L. Evaluation of a hand probe for use in a product safety regulation, Proc. Human Factors and Industrial Design in Consumer Products Symp., Medford, MA. May 28-30, 1980, pp. 155-172 (Tufts University, Medford, MA, 1980).

20221. Argentar, H.; Tesk, J. A.; Parry, E. E. An amine accelerator for colorfree curing of cold-curing methyl methacrylate denture systems, J. Am. Dent. Assoc. 102, 664-665 (May 1981).

20226. Misra, D. N.; Bowen, R. L. Adsorption of N-(2-hydroxy-3methacryloxypropyl)-N-phenylglycine (NPG-GMA) on cupric ionenriched hydroxyapatite surface to improve chemical bonding between dental resins and teeth, Biomater. 2, 78-82 (Apr. 1981).

20230. Bowen, R. L. Adhesive bonding of various materials to hard tooth tissues: XXIII. Isotonic monomer formulations, J. Dent. Res. 60, No. 5, 901-907 (May 1981).

20241. Whitlock, R. P.; Tesk, J. A.; Widera, G. E. O.; Holmes, A.; Parry, E. E. Consideration of some factors influencing compatibility of dental porcelains and alloys, Part I: Thermo-physical properties, Proc. 4th Int. Precious Metals Conf., Toronto, Canada, June 2-5, 1980, pp. 273-282 (Pergamon Canada, Toronto, Ontario, Canada, Apr. 1981).

20253. Antonucci, J. M. New monomers for use in dentistry, (Proc. Symp. Application of Polymers in Dentistry, Houston, TX, Mar. 23.28, 1980), Paper in Biomedical and Dental Applications of Polymers, C. G. Gebelein and F. F. Koblitz, eds., pp. 357.371 (Plenum Publ. Corp., New York, NY, 1981).

20254. Brauer, G. M. Initiator-accelerator systems for acrylic resins and composites, (Proc. Symp. Application of Polymers in Dentistry, Houston, TX, Mar. 23.28, 1980), Paper in Biomedical and Dental
Applications of Polymers, C. G. Gebelein and F. F. Koblitz, eds., pp 395.409 (Plenum Publ. Corp., New York, NY, 1981).

20270. Weber, H.; Fraker, A. C. SEM studies of dental casting alloy corrosion behavior, Proc. 37th Annu. Electron Microscopy Society of America Meet., San Antonio, TX, Aug. 1979, G. W. Bailey, ed., pp. 280-281 (1979).

20277. Tesk, J. A.; Whitlock, R. P.; Widera, G. E. O.; Holmes, A.; Parry, E. E. Consideration of some factors influencing compatibility of dental porcelains and alloys, Part II: Porcelain/alloy stress, Proc. 4th Int. Precious Metals Conf., Toronto, Ontario, Canada, June 4-6, 1980, pp. 283-29l (Pergamon of Canada, Toronto, Ontario, Canada, Apr. 1981).

20290. Hodgeson, J. A.; Fatiadi, A. J. Survey on research needs on personal samplers for toxic organic compounds, EPA-600/4-80-042, 35 pages (Available from the National Technical Information Service, Springfield, VA 22161, Aug. 1980).

20311. Burnett, E. D. National Bureau of Standards hearing aid test procedures, Chapter III in Handbook of Hearing Aid Measurement 1981, pp. 9.176 (Veterans Administration, Washington, DC, 1981).

20314. Loevinger, R. NBS program for electron dosimetry, Proc. Symp. Electron Beam Therapy, Memorial Sloan-Kettering Cancer Center, New York, NY, Sept. 25-27, 1979, pp. 31.41 (June 1981).

20380. Bowen, R. L. Adhesive bonding of various materials to hard tooth tissues XXIV: Recent developments in adhesive resin formulations for dentin, Proc. Int. Congr. of Implantology and Biomaterials in Stomatology, Kyoto, Japan, June 9-11, 1980, pp. 66-73 (Osaka Dental University, Osaka 540, Japan, 1981).

20383. Wu, W.; Cobb, E. N. A silver staining technique for investigating wear of restorative dental composites, J. Biomed. Mater. Res. 15, 343-348 (1981).

20390. Dick, C. E.; Motz, J. W. Image information transfer properties of x-ray fluorescent screens, Med. Phys. 8, No. 3, 337.346 (May/June 1981).

20392. Dulik, D.; Bernier, R.; Brauer, G. M. Effect of diluent monomer on the physical properties of Bis-GMA-based composites, $J$. Dent. Res. 60, No. 6, 983-989 (June 1981).

20401. Brauer, G. M.; Stansbury, J. W.; Antonucci, J. M. 4-N,Ndialkylaminophenethanols, -alkanoic acids and esters: New accelerators for dental composites, J. Dent. Res. 60, No. 7, 1343.1348 (July 1981).

20406. Antonucci, J. M.; Peckoo, R. J.; Schruhl, C.; Toth, E. E. Slowacting amine polymerization accelerators. Paradimethylaminobenzoic acid and its ethyl ester, J. Dent. Res. 60, No. $7,1325.1330$ (July 1981)

20442. Grant, W. H.; Dehl, R. E. The adsorption of human serum albumin and $\gamma$-globulin on hydrophobic and hydrophilic surfaces, (Proc. Joint ACS \& CSJ Cong. Adhesion and Adsorption of Polymers, Honolulu, HI, Apr. 2-6, 1979), Paper in Adhesion and Adsorption of Polymers, L. H. Lee, ed., Pt. B, $827-835$ (Plenum Publ. Corp., New York, NY, 1980).

20450. Motz, J. W.; Dick, C. E.; Danos, M. Exposure limits imposed by screen-film systems on the transfer of image information, (Proc. Applications of Optical Instrumentation in Medicine IX, San Francisco, CA, Mar. 22-24, 1981), SPIE 273, 52.56 (The International Society for Optical Engineering, Bellingham, WA, 1981).

20480. Reite, M.; Zimmerman, J. T.; Zimmerman, J. E. Magnetic auditory evoked fields: Interhemispheric asymmetry, Electroencephalogr. Clin. Neurophysiol. 51, 388-392 (1981).

20481. Antonucci, J. M.; Misra, D. N.; Peckoo, R. J. The accelerative and adhesive bonding capabilities of surface-active accelerators, $J$. Dent. Res. 60, No. 7, 1332.1342 (July 1981)

20513. Durst, R. A.; Siggaard-Andersen, O.; Maas, A. H. J. Reference and quality control materials for $\mathrm{pH}$ and blood gases, (Proc. 5th Meet. IFCC Expert Panel on pH and Blood Gases, Copenhagen, Denmark, June 16-18, 1980), Paper in Blood pH, Carbon Dioxide, Oxygen, and Calcium-ion, O. Siggaard-Andersen, ed., pp. 89-100 (Private Press, Copenhagen, Denmark, 1981).

20514. Ehrlich, M. Choice of radiation protection quantities for a personnel dosimetry performance standard, (Proc. European Seminar on Radiation Protection Quantities for External Exposure, 
Braunschweig, Federal Republic of Germany, Oct. 13-15, 1980), Paper in European Seminar on Radiation Protection Quantities for External Exposure, G. Burger, H. G. Ebert, D. Harder, R. Kramer, S. Wagner, eds., pp. 239.242 (Harwood Academic Publishers, New York, NY, 1981).

20553. Purdy, J. A.; Harms, W. B.; Fivozinsky, S. A computer based file of $x$-ray and electron beam central axis depth dose data for use in radiation therapy, Proc. Fourth Annu. Symp. Computer Applications in Medical Care, Washington, DC, Nov. 2-5, 1980, pp. 94-103 (Institute of Electrical \& Electronics Engineers, New York, NY, Nov. 1980).

20554. Fivozinsky, S. P.; Purdy, J. A. Compilation and evaluation of radiation depth dose data from electron accelerators used for radiotherapy: Experiences of a pilot data project in a hospital environment, Proc. 7th Int. CODATA Conf. Data for Science and Technology, Kyoto, Japan, Oct. 8-11, 1980, pp. $50-54$ (Pergamon Press, Oxford, United Kingdom, July 1981).

20566. Parobeck, P.; Tomb, T.; Ku, H.; Cameron, J. Measurement assurance program for weighings of respirable coal mine dust samples, J. Qual. Tech. 13, No. 3, 157-165 (July 1981).

20669. Brauer, G. M.; Dulik, D. M.; Hughes, H. N.; Dermann, K.; Rupp, N. W. Marginal adaptation of BIS-GMA-based composites containing various diluents, J. Dent. Res. 60, No. 12, 1966-197I (Dec. 1981).

20672. Chow, L. C.; Guo, M. K.; Hsieh, C. C.; Hong, Y. C. Apatitic fluoride increase in enamel from a topical treatment involving intermediate $\mathrm{CaHPO}_{4} \cdot 2 \mathrm{H}_{2} \mathrm{O}$ formation, an in vivo study, Caries Res. 15, 369-376 (1981).

20677. Eisenhauer, C.; Schwartz, R. Difficulties in calculating spectrum-averaged values of neutron dose equivalent, Health Phys. 41, No. 5, $774-777$ (Nov. 1981).

\section{Instrumentation and Experimental Methods}

SP615. Lederer, P. S. Sensor handbook for automatic test, monitoring, diagnostic, and control systems applications to military vehicles and machinery. Nat. Bur. Stand. (U.S.) Spec. Publ. 615; 1981 October. 450 p. SN003-003-02372-8.

TN1035. Siegwarth, J. D.; LaBrecque, J. F. A portable calibration densimeter for use in cryogenic liquids. Nat. Bur. Stand. (U.S.) Tech. Note 1035; 1981 March. 20 p. SN003-003.02308-6.

NBSIR 80.2102. Dickens, B. User's manual for factor-jump thermogravimetry apparatus, and associated programs, including a general plotting program. 1981 March. 391 p. Available from: NTIS; PB 82-109851.

U.S. Patent 4,245,169. Hamilton, C. Sampling circuit and method therefor. 13 January $1981.10 \mathrm{p}$.

U.S. Patent 4,264,423. Negas, T.; Domingues, L. Fluidic thermistor/fugacity device. 28 April 1981. 7 p.

20003. Seltzer, S. M. X-ray source characteristics and detection efficiencies of prototype lixiscopes, (Proc. Lixiscope Conf. Greenbelt, MD, July 27.28, 1978), NASA Conf. Publ. 2112, pp. 11 14 (NASA - Goddard Space Flight Center, Greenbelt, MD, July 1978).

20017. Sindt, C. F. A system for inflating an air launched balloon, (Proc. 10th Air Force Geophysics Lab. Scientific Balloon Symp., Portsmouth, NH, Aug. 21-23, 1978), Report No. AFGL-TR-79-0053, C. L. Rice, ed., pp. 149.159 (National Technical Information Service, Springfield, VA, Mar. 1979).

20141. Harvey, K. C. Shielded thermionic diode detector for precision spectroscopy, Rev. Sci. Instrum. 52, No. 2, 204-206 (Feb. 1981).

20144. Potzick, J. E.; Robertson, B. Voltage-controlled phase shifter for measuring transfer function in the presence of noise, Rev. Sci. Instrum. 52, No. 2, 280-286 (Feb. 1981).

20170. DeReggi, A. S.; Roth, S. C.; Kenney, J. M.; Edelman, S.; Harris, G. R. Piezoelectric polymer probe for ultrasonic applications, J. Acoustic. Soc. Am. 69, No. 3, 853.859 (Mar. 198I).

20188. Bialkowski, S. E. Simple parallel interface between an optical multichannel analyser detector controller and a microprocessor, Rev. Sci. Instrum. 51, No. 6, 850.851 (June 1980).

20216. Ederer, D. L.; Callcott, T. A.; Arakawa, E. T. In situ preparation of thin sodium films as an expendable filter in the vacuum ultraviolet, Rev. Sci. Instrum. 50, No. 12, 1618-1621 (Dec. 1979).

20231. Mitchell, R. A.; Pontius, P. E. Force sensor-machine interaction, (Proc. 27th Int. Instrumentation Symp., Indianapolis, IN, Apr. 27.30, 1981), Part 1 in Instrumentation in the Aerospace Industry 2 and Advances in Test Measurement 18, 225.232 (Instrument Society of America, Research Triangle Park, NC, 1981).

20340. Van Degrift, C. T.; Love, D. P. Modeling of tunnel diode oscillators, Rev. Sci. Instrum. 52, No. 5, 712.723 (May 1981).

20556. Mosburg, E. R., Jr. Effect of ion current in the collisionless theory of floating ac probe measurements, Rev. Sci. Instrum. 52, No. 8, 1182-1186 (Aug. 1981).

20569. Yin, L. I.; Trombka, J. I.; Schmadebeck, R. L.; Seltzer, S. M.; Bielefeld, M. J. A hard $x$-ray and soft gamma-ray telescope spectrometer, SPIE J., Imaging Spectrosc. 268, 97-102 (1981).

20592. McCulloh, K. E.; Tilford, C. R. Nitrogen sensitivities of a sample of commercial hot cathode ionization gage tubes, J. Vac. Sci. Technol. 18, No. 3, 994-996 (Apr. 1981).

20610. Cutkosky, R. D.; Davis, R. S. Simple control circuit for temperature regulation and other bridge applications, Rev. Sci. Instrum. 52, No. 9, 1403-1405 (Sept. 1981).

20614. Harvey, K. C. Lyman- $\beta$ lamp with cooled LiF window, Appl. Opt. 20, 2883-2885 (Sept. 1, 1981).

20731. Payne, B. F. Absolute calibration of back-to-back accelerometers, (Proc. 27th Int. Instrumentation Symp., Indianapolis, IN, Apr. 1981), Paper in Instrumentation in the Aerospace Industry 27, 483.488 (Instrument Society of America, Research Triangle Park, NC, 1981).

20734. Serbyn, M. R.; Penzes, W. B. A real-time active vibration controller, (Proc. 27th Int. Instrumentation Symp., Indianapolis, IN, Apr. 1981), Paper in Instrumentation in the Aerospace Industry 27, 489-494 (Instrument Society of America, Research Triangle Park, NC, 1981).

20748. Goldfarb, R. B. Miniature multipin electrical feedthrough for vacuum use, Cryogenics 21, 746 (Dec. 1981).

20770. Siegwarth, J. D. Gage installation can trim level-measurement, Oil Gas J. 79, No. 49, 142-144, 149, 152 (Dec. 1981).

\section{Lasers and Their Applications}

SP620. Bennett, H. E.; Glass, A. J.; Guenther, A. H.; Newnam, B. E., eds. Laser induced damage in optical materials: 1980 . Proceedings of a Symposium Sponsored by: National Bureau of Standards, American Society for Testing and Materials, Office of Naval Research, Department of Energy, Defense Advanced Research Project Agency, and Air Force Office of Scientific Research; 1980 September 30-October I; Boulder, CO. Nat. Bur. Stand. (U.S.) Spec. Publ. 620; 1981 October. 483 p. SN003.003-02359.1.

SP620: 1981 October. 29-43. Braunstein, R.; Kim, R. K.; Braunstein, $M$. Infrared wavelength modulation spectroscopy of laser window materials.

SP620: 1981 October. 44.49. McDavid, J. M.; Yee, S. S. Photoacoustic spectroscopy of adsorbed surface impurities on alkali. halide laser windows.

SP620: 1981 October. 50-57. Van Stryland, E. W.; Woodall, M. A. Photoacoustic measurement of nonlinear absorption in solids.

SP620: 1981 October. 58-74. Fernelius, N. C.; Dempsey, D. V.; O'Quinn, D. B. $1.3 \mu \mathrm{m}$ laser rate calorimetry and photoacoustic studies of the surface and bulk optical absorption in $\mathrm{CaF}_{2}$ single crystal samples.

SP620: 1981 October. 75-87. Wu, S. T.; Bass, M. Intensity dependent absorption of alkali-halides at $10.6 \mu \mathrm{m}$.

SP620: 1981 October. 88-93. Detrio, J. A.; Dempsey, D. A. Pulsed $\mathrm{CO}_{2}$ damage threshold measurements of $\mathrm{Rb}: \mathrm{KCl}$ and $\mathrm{NaCl}$.

SP620: 1981 October. 94-101. Drexhage, M. G.; Bendow, B.; Lipson, H. G.; Moynihan, C. T. Infrared absorption in highly transparent glasses based on hafnium fluoride.

SP620: 1981 October. 102-109. Hack, H.; Neuroth, N. Internal damage to optical glasses with 3 ns-pulse laser. 
SP620: 1981 October. 110-116. Vora, H.; Ready, J. F. Development of forging processes for large-scale deformation of lithium fluoride. SP620: 1981 October. 117-128. Klein, C. A. Stress-induced birefringence, critical window orientation, and thermal lensing experiments.

SP620: 1981 October. 129.143. Fernelius, N. C.; Dempsey, D. V.; Walsh, D. A.; O'Quinn, D. B.; Knecht, W. L. Survey of $1.3 \mu \mathrm{m}$ window materials.

SP620: 1981 October. 144-158. Glassman, A. T. Material property requirements for laser windows.

SP620: 1981 October. 159-169. Thomas, N.; Sonderman, J.; Stokowski, S.; Wallerstein, P.; Walmer, D. A new pbotographic technique for observing bulk laser damage.

SP620: 1981 October. 171-179. Namba, Y.; Tsuwa, H. Ultrafine finishing of ceramics and metals by float polishing.

SP620: 1981 October. 180-189. Temple, P. A.; Soileau, M. J. 1.06 $\mu \mathrm{m}$ laser-induced breakdown of $\mathbf{C O}_{2}$-laser-polished fused $\mathrm{SiO}_{2}$.

SP620: 1981 October. 190-200. Decker, D. L.; Hodgkin, V. A. Wavelength and temperature dependence of the absolute reflectance of metals at visible and infrared wavelengths.

SP620: 1981 October. 201-209. Porteus, J. O.; Decker, D. L.; Seitel, S. C.; Soileau, M. J. Dependence of metal mirror damage thresholds on wavelength, material, pulse length, and preparation method.

SP620: 1981 October. 210-221. Draper, C. W. Regrowth in laser irradiated elemental metals and alloys: surface nonuniformities and coupling phenomena.

SP620: 1981 October. 222-226. Draper, C. W.; Buene, L.; Jacobson, D. C.; Poate, J. M.; Nakahara, S. Epitaxial regrowth and defects in laser irradiated single crystal Ni.

SP620: 1981 October. 227-238. Musal, H. M., Jr. Pulsed laser initiation of surface plasma on metal mirrors.

SP620: 1981 October. 238-247. Thomas, S. J.; Phipps, C. R., Jr.; Harrison, R. F. Optical damage limitations for copper mirrors used in $\mathrm{CO}_{2}$-ICF laser systems.

SP620: 1981 October. 248-255. Foley, J.; Sharma, S. K.; Wood, R M. Absorption calorimetry and laser induced damage threshold measurements on germanium and zinc selenide.

SP620: 1981 October. 256-264. Bennett, H. E. Insensitivity of the catastrophic damage threshold of laser optics to dust and other surface defects.

SP620: 1981 October. 265-276. Foltyn, S. R.; Newnam, B. E. Multiple-sbot laser damage tbresholds of ultraviolet reflectors at 248 and 308 nanometers.

SP620: 1981 October. 277-286. Wiggins, T. A.; Walker, T. W.; Guenther, A. H. A novel technique for investigating impurity initiated short pulse laser damage in thin films.

SP620: 1981 October. 287.296. Wirtenson, G. R.; Willis, J. B. Enemark, E. A. Evaluation of bichromatic coatings designed for pulsed laser fusion applications at $\mathbf{0 . 5 3}$ and $\mathbf{1 . 0 6}$ micrometers.

SP620: 1981 October. 297-299. Deaton, T. F.; Rainer, F.; Milam, D.; Smith, W. L. Survey of damage tbresbolds at $532 \mathrm{~nm}$ for production-run optical components.

SP620: 1981 October. 300-304. Soileau, M. J. Laser-induced damage in antireflection coatings for $\mathrm{LiNbO}_{3}$ crystals.

SP620: 1981 October. 305-312. Donovan, T: M.; Porteus, J. O.; Seitel, S. C.; Kraatz, P. Multithreshold HF/DF pulsed laser damage measurements on evaporated and sputtered silicon films.

SP620: 1981 October. 313-323. Aisenberg, S.; Stein, M. The use of ion-beam deposited diamond-like carbon for improved optical elements for high powered lasers.

SP620: 1981 October. 324-334. Herrmann, W. C., Jr.; McNeil, J. R. Ion-beam deposited Ge-As-Se glass for applications in tbe $1 \mu$ to $16 \mu$ wavelength region.

SP620: 1981 October. 335-344. Anderson, W. J.; Hansen, W. N. Characterization of small absorptions in optical coatings.

SP620: 1981 October. 345-355. Leonard, T. A.; Loomis, J.; Harding, K. G.; Scott, M. Design and construction of three infrared ellipsometers for thin-film research.

SP620: 1981 October. 356-368. Bennett, H. E.; Burge, D. K. Multilayer thickness uniformities required to meet wave front error tolerances in laser mirrors.
SP620: 1981 October. 369-374. Danileiko, Y. K.; Manenkov, A. A.; Nechitailo, V. S. The role of absorbing defects in the laser damage of transparent materials.

SP620: 1981 October. 375-384. Van Stryland, E. W.; Soileau, M. J.; Smirl, A. L.; Williams, W. E. Pulse-width and focal-volume dependence of laser-induced breakdown.

SP620: 1981 October. 385-393. Soileau, M. J.; Franck, J. B.; Veatch, T. C. On self-focusing and spot-size dependence of laser-induced breakdown.

SP620: 1981 October. 394.402. Kelly, P.; Ritchie, D.; Braunlich, P.; Schmid, A.; Bryant, G. W. Deformation of intense laser beams tightly focused inside $\mathrm{NaCl}$ : A comparison of the multiphoton-polaron and avalanche models of optical breakdown.

SP620: 1981 October. 403-405. Braunlich, P.; Bryant, G.; Schmid, A. A correlation of laser damage vestige structure and spherical aberrations.

SP620: 1981 October. 406-415. Braunlich, P.; Brost, G.; Schmid, A.; Kelly, $P$. The role of laser-induced primary defect formation in optical breakdown of $\mathrm{NaCl}$.

SP620: 1981 October. 416-426. Jensen, B. The high frequency electron scattering rate and Drude Zener theory in compound semiconductors.

SP620: 1981 October. 427-431. Bryant, G. W.; Schmid, A.; Braunlich, P.; Kelly, P.; Ritchie, D. Interactions of intense $2.7 \mu \mathrm{m}$ picosecond laser pulses with germanium.

SP620: 1981 October. 432-437. Vaidyanathan, A.; Guenther, A. H.; Mitra, S. S. Band structure calculations of the two-photon absorption coefficients of GaAs, InP, CdTe, and ZnSe.

SP620: 1981 October. 438-445. Vaidyanathan, A.; Guenther, A. H.; Mitra, S. S. Two-photon absorption in direct-gap crystals-An addendum.

SP620: 1981 October. 446-452. Judell, N.; Mitra, S. S.; Vaidyanathan, A.; Guenther, A. H. Three-photon absorption in direct-gap crystals.

SP620: 1981 October. 453-458. Bendow, B.; Gianino, P. D.; Tzoar $\mathrm{N}$. Theory of nonlinear beam propagation in optical waveguides.

TN1031, Revised. Young, M. The use of LEDs to simulate weak YAGlaser beams. Nat. Bur. Stand. (U.S.) Tech. Note 1031; 1981 January. 48 p. Available from: NTIS; PB 81.172470.

TN1031. Young, $M$. The use of LEDs to simulate weak YAG-laser beams. Nat. Bur. Stand. (U.S.) Tech. Note 1031; 1981 January. 48 p. SN003-003-02290-2.

NBSIR 80-1628. Johnson, E. G., Jr.; Phelan, R. J., Jr.; Boyle, D. R. Measuring features of the fluence at the far field of a $\mathrm{CO}_{2}$ pulsed laser-An issue study with suggestions on how to do it. 1980 April. 55 p. Available from: NTIS; PB 80-191141.

20090. Sattler, J. P.; Worchesky, T. L.; Tobin, M. S.; Ritter, K, J.; Daley, T. W.; Lafferty, W. J. Submillimeter-wave emission assignments for 1,1-difluoroetbylene, Int. J. Infrared and Millimeter Waves 1, No. 1, 127-138 (1980).

20100. Julienne, P. S.; Konowalow, D. D.; Krauss, M.; Rosenkrantz, M. E.; Stevens, W. J. Photodissociation of $\mathbf{H g C l}$, Appl. Phys. Lett. 36, No. 2, 132.134 (Jan. 15, 1980).

20101. King, D. S.; Stephenson, J. C. Intrinsic isotopic selectivity factors: $\mathrm{CO}_{2}$ TEA laser pbotolysis of $\mathrm{CF}_{2} \mathrm{Cl}_{2}, \mathrm{~J}$. Am. Chem. Soc. 100, No. 23, 7151.7155 (Nov. 8, 1978).

20154. Harter, W. G.; Patterson, C. W. High resolution spectra of symmetric molecules: Understanding rotation and permutation dynamics, (Proc. Advances in Laser Chemistry, Pasadena, CA, Mar. 20-22, 1978), Paper in Advances in Laser Chemistry, A. Zewail, ed., pp. $455-463$ (Springer-Verlag, New York, NY, 1978).

20164. Lucatorto, T. B.; McIlrath, T. J.; Mayo, S.; Furumoto, H. W. High-stability coaxial flashlamp-pumped dye laser, Appl. Opt. 19, No. 18, 3178-3180 (Sept. 15, 1980).

20166. Goldsmith, J. E. M.; Lawler, J. E. Optogalvanic spectroscopy, Contemp. Phys. 22, No. 2, 235-248 (1981).

20265. Drullinger, R. E.; Wineland, D. J.; Bergquist, J. C. Highresolution optical spectra of laser cooled ions, Appl. Phys. 22, 365.368 (1980).

20331. Simpson, P. A. A system for characterizing detectors for measurement of power of $\mathrm{CO}_{2}$ TEA laser pulses, Proc. 11th Electro- 
Optics/Laser 79 Conf. \& Exposition, Anaheim, CA, Oct. 23-24, 1979. pp. 399.407 (Industrial and Scientific Conference Management, Inc., 222 West Adams St., Chicago, IL 60606, 1979).

20414. Barger, R. L. Influence of second-order Doppler effect on optical Ramsey fringe profiles, Opt. Lett. 6, No. 3, 145.147 (Mar. 1981).

20441. Pence, W. H.; Leone, S. R. Laser studies of electronic excitation transfer in atomic calcium: $\mathrm{Ca}\left(4 s 5 p{ }^{1} P_{1}\right)+\mathrm{Ar} \rightleftharpoons \mathrm{Ca}(4 s 5 p$ ${ }^{3} P_{2,1,0}+$ Ar, $J$. Chem. Phys. 74, No. 10, $5707-5717$ (May 15, 1981).

20447. Koffend, J. B.; Leone, S. R. Tunable laser photodissociation: Quantum yield of $I^{*}\left({ }^{2} P_{1 / 2}\right)$ from $\mathbf{C H}_{2} I_{2}$, Chem. Phys. Lett. 81, No. $1,136.141$ (July 1981).

20451. Decher, R.; Randall, J. L.; Bender, P. L.; Faller, J. E. Design aspects of a laser gravitational wave detector in space, (Proc. Active Optical Devices and Applications, Washington, DC, Apr. 10-11, 1981), SPIE 228, 149.153 (The International Society for Optical Engineering, Bellingham, WA, 1980).

20454. Travis, J. C.; DeVoe, J. R. The optogalvanic effect, Chapter 5 in Lasers in Chemical Analysis, G. M. Hieftje, J. C. Travis, and F. E. Lytle, eds., pp. 93-124 (Humana Press, Clifton, NJ, 1981).

20461. Sweger, D. M.; Sams, R. L. Diode laser spectra of the $v_{2}$ band of $\mathrm{H}_{2}{ }^{12} \mathrm{CO}$ and $\mathrm{H}_{2}{ }^{13} \mathrm{CO}, J$. Mol. Spectros. 87, 18-28 (1981).

20468. Turk, G. C. Reduction of matrix ionization interference in laser-enhanced ionization spectrometry, Anal. Chem. 53, 1187-1190 (1981).

20595. Walls, D. F.; Kunasz, C. V.; Drummond, P. D.; Zoller, P. Bifurcations and multistability in two-photo processes, Phys. Rev. A 24, No. I, $627-630$ (July 1981).

20653. Drake, M. C.; Rosasco, G. J.; Schneggenburger, R.; Nolen, R. $\mathrm{L}$., Jr. Nondestructive analysis of laser fusion microsphere targets using rotational Raman spectroscopy, J. Appl. Phys. 50, No. 12, 7894 7897 (Dec. 1979).

20688. Nesbitt, D. J.; Leone, S. R. Laser initiated chain reactions: A generalized extension to complex chemical chain systems, J. Chem. Phys. 75, No. 10, $4949-4959$ (Nov. 15, 1981).

20697. Reid, M.; McNeil, K. J.; Walls, D. F. Unified approach to multiphoton lasers and multiphoton bistability, Phys. Rev. $A$ 24, No. 4, 2029-2043 (Oct. 1981)

20700. Scalabrin, A.; Saykally, R. J.; Evenson, K. M.; Radford, H. E.; Mizushima, M. Laser magnetic resonance measurement of rotational transitions in the metastable $a^{1} \Delta_{g}$ state of oxygen, $J$. Mol. Spectros. 89, 344-351 (1981).

20720. Hall, J. L.; Hollberg, L.; Baer, T.; Robinson, H. G. Optical heterodyne saturation spectroscopy, Appl. Phys. Lett. 39, No. 9, 680682 (Nov. 1, 1981)

20730. Palmer, B. A.; Keller, R. A.; Kowalski, F. V.; Hall, J. L. Accurate wave-number measurements of uranium spectral lines, $J$. Opt. Soc. Am. 71, No. 8, 948.952 (Aug. 1981).

20743. Vasconcellos, E. C. C.; Petersen, F. R.; Evenson, K. M. Frequencies and wavelengths from a new efficient FIR lasing gas: $\mathrm{CD}_{2} \mathbf{F}_{2}$, Int. J. Infrared Millimeter Waves 2, No. 4, 705.711 (1981).

\section{Low Temperature Science and Engineering}

SP607. Zimmerman, J. E.; Sullivan, D. B.; McCarthy, S. E., eds. Refrigeration for cryogenic sensors and electronic systems. Proceedings of a Conference held at the National Bureau of Standards; 1980 October 6.7; Boulder, CO. Nat. Bur. Stand. (U.S.) Spec. Publ. 607; 1981 May. 223 p. SN003-003-02310-8.

SP 607: 1981 May. 3-14. Roubeau, P. M. A preview of unconventional suggested cooling processes and some practical problems related to small refrigerators.

SP607: 1981 May. 15-20. Guernsey, R. W.; Flint, E. B. Refrigeration requirements for superconducting computers.

SP607: 1981 May. 21-29. White, R.; Haskin, W. Development approaches for long-life cryo-coolers.

SP607: 1981 May. 30-47. Horn, S. B.; Asher, M. S. Theoretical analysis of a 3-stage Stirling cycle cryocooler.

SP607: 1981 May. 48-56. Daney, D. E. Some thermodynamic considerations of helium temperature cryocoolers.
SP607: 1981 May. 57-69. Ackermann, R. A. Dynamic analysis of a small free-piston resonant cryorefrigerator.

SP607: 1981 May. 70-81. Radebaugh, R.; Linenberger, D.; Voth, R. $\mathrm{O}$. Methods for the measurement of regenerator ineffectiveness.

SP607: 1981 May. 82-92. Longsworth, R. C. Serviceable refrigerator system for small superconducting devices.

SP607: 1981 May. 93-102. Flint, E. B.; Jenkins, L. C.; Guernsey, R. $W$. Performance of a 1 watt $4 K$ cryosystem suitable for a superconducting computer.

SP607: 1981 May. 103-115. Gasser, M. G.; Sherman, A.; Beale, W. Developments toward achievement of a 3.5 year lifetime Stirling cycle refrigerator for space applications.

SP607: 1981 May. 116. Benson, G. M.; Vincent, R. J. Thor cryocooler.

SP607: 1981 May. 117-127. Urban, E. W.; Katz, L.; Hendricks, J. B.; Karr, G. R. A cryogenic system for the small infrared telescope for Spacelab 2.

SP607: 1981 May. 127-135. Hartwig, W. H. Requirements for and status of a $4.2^{\circ} \mathrm{K}$ adsorption refrigerator using zeolites.

SP607: 1981 May. 136-140. Byrd, E. A.; Hansen, R. G. Cooling of squid devices by means of liquid transfer techniques for reduced helium consumption and enhanced temperature stability.

SP607: 1981 May. 141-145. Daunt, J. G.; Heiden, C. A contamination free compressor for small scale Stirling refrigerators.

SP607: 1981 May. 146-153. Studer, P. A.; Gasser, M. G. A bidirectional linear motor/generator with integral magnetic bearings for long lifetime Stirling cycle refrigerators.

SP607: 1981 May. 154-159. Little, W. A. Design considerations for microminiature refrigerators using laminar flow heat exchangers. SP607: 1981 May. 160-163. Hollman, R.; Little, W. A. Progress in the development of microminiature refrigerators using photolithographic fabrication techniques.

SP607: 1981 May. 164-172. Wade, L. A. Low temperature regenerators.

SP607: 1981 May. 173-177. Zimmerman, J. E.; Sullivan, D. B.; Kautz, R. L.; Hobbs, R. D. Measurement of thermal properties of cryocooler materials.

SP607: 1981 May. 178-185. Tward, E. Gas heat switches.

SP607: 1981 May. 186-194. Sullivan, D. B.; Zimmerman, J. E.; Ives, J. T. Operation of a practical SQUID gradiometer in a low-power Stirling cryocooler.

SP607: 1981 May. 195-209. Nisenoff, M. Current status of high temperature Josephson device technology.

TN1041. Siegwarth, J. D.; LaBrecque, J. F. Cryogenic fluids density reference system: Provisional accuracy statement (1980). Nat. Bur. Stand. (U.S.) Tech. Note 1041; 1981 April. 60 p. SN003-003-02346-9.

NBSIR 78-875. Voth, R. O. Producing liquid-solid mixtures of hydrogen using an Auger. 1978 February. 21 p. Available from: NTIS; PB 278723.

NBSIR 79-1624. Tobler, R. L. Materials for cryogenic wind tunnel testing. 1980 May. 135 p. Available from: NTIS; N81.27120.

NBSIR 80-1637. Daney, D. E., ed. Helium research in support of superconducting power transmission. Annual Report: October 1978 . September 1979. 1980 December. 58 p. Available from: NTIS; PB 81.156606

NBSIR 80-1642. Fickett, F. R.; Goodrich, L. F.; Clark, A. F. Development of standards for superconductors. Annual report FY 80. 1980 December. 212 p. Available from: NTIS; PB 81-176141.

NBSIR 81-1647. Bransford, J. W.; Clark, A. F. Laser-initiated combustion studies of selected aluminum, copper, iron, and nickel alloys. 1981 April. 89 p. Available from: NTIS; PB 81-21550I.

NBSIR 81-1649. Daney, D. E., ed. Helium research in support of superconducting power transmission: Annual report for the period October 1, 1979-September 30, 1980. 1981 September. 44 p. Available from: NTIS; PB 82-122987.

NBSIR 81-1654. Arvidson, J. M.; Sparks, L. L. Low temperature mechanical properties of a polyurethane foam. 1981 November. $17 \mathrm{p}$. Available from: NTIS; PB 82-160441.

U.S. Patent 4,281,517. Zimmerman, J. E.; Sullivan, D. B. Single stage twin piston cryogenic refrigerator. 4 August $1981.8 \mathrm{p}$

19976. Giarratano, P. J.; Frederick, N. V. Transient pool boiling of liquid helium using a temperature-controlled heater surface, (Proc. 
Cryogenic Engineering Conf., Madison, WI, Aug. 21-24, 1979), Paper in Advances in Cryogenic Engineering, K. D. Timmerhaus and H. A. Snyder, eds., 25, 455.466 (Plenum Press, New York, NY, 1980).

19999. Radebaugh, R.; Fujii, G.; Read, D. T.; Clark, A. F. A standards program for ac losses in superconductors, (Proc. XV Int. Congress of Refrigeration, Venice, Italy, Sept. 23-29, 1979), Session A 1/2, Paper 10 in Progress in Refrigeration Science and Technology, 4 pages (International Institute of Refrigeration, Paris, France, 1980).

20018. Sindt, C. F.; Ludtke, P. R. Thermal cycle tests of a modeled superconducting transmission line, (Proc. 1979 Cryogenic Engineering Conf., Madison, WI, Aug. 21-24, 1979), Paper in Advances in Cryogenic Engineering, K. D. Timmerhaus and H. A. Snyder, eds., 25, 69-80 (Plenum Press, New York, NY, 1980).

20020. Radebaugh, R. Analysis of regenerator inefficiency for Stirlingcycle refrigerators with plastic displacers, (Proc. XV Int. Congress of Refrigeration, Venice, Italy, Sept. 23-29, 1979), Session A1/2, Paper 16 in Progress in Refrigeration Science and Technology, 6 pages (International Institute of Refrigeration, Paris, France, 1980).

20066. Ekin, J. W. Strain scaling law for flux pinning in practical superconductors. Part 1: Basic relationship and application to $\mathrm{Nb}_{3} \mathrm{Sn}$ conductors, Cryogenics 20, No. 12, 611-624 (Nov. 1980).

20068. Flynn, T. M.; Timmerhaus, K. D. Cold utilization from liquefied natural gas (LNG), (Proc. XV Int. Congress of Refrigeration, Venice, Italy, Sept. 23-29, 1979), Session A 3/27 in Progress in Refrigeration Science and Technology, 7 pages (International Institute of Refrigeration, Paris, France, 1980).

20069. Fickett, F. R.; Clark, A. F. Development of standards for superconductors, Proc. 8th Int. Cryogenic Engineering Conf., Genoa, Italy, June 3-6, 1980, C. Rizzuto, ed., pp. 494.498 (IPC Science and Technology Press, Ltd., Guildford, Surrey, England, 1980).

20071. Diller, D. E. Measurements of the viscosity of compressed gaseous and liquid methane, Physica (Utrecht) $A$ 104, No. 3, 417.426 (Dec. 1980).

20074. Sparks, L. L. Low-temperature properties of expanded polyurethane and polystyrene, (Proc. Symp. Thermal Insulation Performance, Tampa, FL, Oct. 23-25, 1978), Am. Soc. Test. Mater. Spec. Tech. Publ. 718, D. L. McElroy and R. P. Tye, eds., pp. 431. 452 (1981).

20120. Ledbetter, H. M.; Maerz, G. Temperature dependence of Young's modulus and internal friction of G-10CR and G-11CR epoxy resins, Cryogenics 20, No. 11, 655-658 (Nov. 1980).

20139. Straty, G. C.; Diller, D. E. $(p, V, T)$ of saturated and compressed fluid nitrogen, J. Chem. Thermodyn. 12, No. 10, 927.936 (1980).

20278. Straty, G. C.; Diller, D. E. $(p, V, T)$ of compressed and liquefied (nitrogen+methane), J. Chem. Thermodyn. 12, 937.953 (1980).

20286. Ledbetter, H. M. Dynamic elastic modulus and internal friction in G-10CR and G-11CR fibreglass-cloth-epoxy composites, Cryogenics 20, No. 11, 637.640 (Nov. 1980).

20297. Ekin, J. W.; Schramm, R. E.; Superczynski, M. J. Training of epoxy-impregnated superconductor windings, (Proc. Int. Cryogenic Materials Conf., Madison, WI, Aug. 24-29, 1979), Paper J.5 in Advances in Cryogenic Engineering Materials, A. F. Clark and R. P. Reed, eds., 26, 677.683 (Plenum Press, New York, NY, 1980).

20298. Fujii, G.; Ekin, J. W.; Radebaugh, R.; Clark, A. F. Effect of thermal contraction of sample-holder material on critical current, (Proc. Int. Cryogenic Materials Conf., Madison, WI, Aug. 24-29, 1979), Paper I-1 in Advances in Cryogenic Engineering Materials, A. F. Clark and R. P. Reed, eds., 26, 589.598 (Plenum Press, New York, NY, 1980).

20309. Weber, L. A. Measurements of the specific heat, $C_{v}$, of dense gaseous and liquid nitrogen, (Proc. XV Int. Institute of Refrigeration Conf., Venice, Italy, Sept. 23-29, 1979), Session B 1, Paper 7, 5 pages (International Institute of Refrigeration, Paris $\left(17^{\mathrm{e}}\right)$, France, 1980).

20328. Ledbetter, H. M.; Moulder, J. C.; Austin, M. W. Young's modulus of a copper-stabilized niobium-titanium superconductive wire, Wire, 3 pages (Jan. 1981).

20377. Powell, R. L.; Fickett, F. R. Cryogenic properties of copper, 1 page wall chart (International Copper Research Assoc., 708 Third Ave., New York, NY 10017, Dec. 1979).
20393. Goodrich, L. F.; Ekin, J. W. Lap joint resistance and intrinsic critical current measurements on a $\mathrm{NbTi}$ superconducting wire, (Proc. 1980 Applied Superconductivity Conf., Santa Fe, NM, Sept. 29-Oct. 2, 1980), IEEE Trans. Mag. MAG-17, No. 1, 1.4 (Jan. 1981).

20400. Daney, D. E.; Ludtke, P. R.; Eroshenko, V. M.; Yaskin, L. A Cool down of cryogenic power transmission lines, (Proc. 15th Int. Congr. of Refrigeration, Venice, Italy, Sept. 23-29, 1979), Paper 19 in Progress in Refrigeration Science and Technology, Session A 1/2, 7 pages (International Institute of Refrigeration, Paris, France, 1980).

20465. Fujii, G.; Ranney, M. A.; Clark, A. F. Thermal expansion of multifilamentary $\mathrm{NB}_{3} \mathrm{Sn}$ and $\mathrm{V}_{3} \mathrm{Ga}$ superconductive cables and fiberglass-epoxy and cotton-phenolic composite materials, Jpn. $J$. Appl. Phys. 20, No. 4, L267-L270 (Apr. 1981).

20505. Hiza, M. J. Liquid-vapor equilibria in binary systems containing ${ }^{4} \mathrm{He}$ or ${ }^{3} \mathrm{He}$ with $\mathrm{nH}_{2}$ or $\mathrm{nD}_{2}$, Fluid Phase Equilib. 6, 203.227 (1981)

20507. Daney, D. E.; Jones, M. C. Counterflow cool down of cryogenic power transmission lines, Cryogenics 21, No. 8, $463-473$ (Aug. 1981).

20508. McCarty, R. D. LNG densities for custody transfer, Proc. 56th Int. School of Hydrocarbon Measurement, Norman, OK, Apr. 14-16, 1981 pp. 515-517 (University of Oklahoma, Norman, OK, 1981).

20526. Smith, D. R.; Hust, J. G. Measurement of effective thermal conductivity of a glass fiberboard standard reference material, Cryogenics 21, No. 7, $408-410$ (July 1981).

20546. Read, D. T.; Reed, R. P. Fracture and strength properties of selected austenitic stainless steels at cryogenic temperatures, Cryogenics 21, No. 7, 415-418 (July 1981).

20563. Timmerhaus, K. D.; Flynn, T. M. Cryogenics, Paper in Encyclopedia of Chemical Processing and Design, J. J. McKetta and W. A. Cunningham, eds., 13, 261-381 (Marcel Dekker, New York, NY, 1981)

20572. Read, D. T.; Reed, R. P. Heating effects during tensile tests of AISI 304L stainless steel at $4 \mathrm{~K}$, (Proc. Cryogenic Engineering Conf., Madison, WI, Aug. 21-24, 1979), Paper B-2 in Advances in Cryogenic Engineering, A. F. Clark and R. P. Reed, eds., 26, 91-101 (Plenum Press, New York, NY, 1980).

20574. Mazandarany, F. N.; Parker, D. M.; Koenig, R. F.; Read, D T. A nitrogen-strengthened austenitic stainless steel for cryogenic magnet structures, (Proc. Cryogenic Engineering Conf., Madison, WI, Aug. 21.24, 1979), Paper B-9 in Advances in Cryogenic Engineering, A. F. Clark and R. P. Reed, eds., 26, 158-170 (Plenum Press, New York, NY, 1980).

20575. Rainwater, J. C.; Hanley, H. J. M. Prediction of the transport properties of natural gas and similar mixtures, (Proc. Cryogenic Engineering Conf., Boulder, CO, Aug. 2.5, 1977), Paper M.2 in Advances in Cryogenic Engineering, K. D. Timmerhaus, ed., 23, 561 565 (Plenum Press, New York, NY, 1978).

20606. Kasen, M. B. Cryogenic properties of filamentary-reinforced composites: An update, Cryogenics 21, 323-340 (June 1981).

20616. Kasen, M. B.; MacDonald, G. R.; Beekman, D. H., Jr.; Schramm, R. E. Mechanical, electrical, and thermal characterization of G-10CR and G-11CR glass-cloth/epoxy laminates between room temperature and 4K, (Proc. Cryogenic Engineering Conf., Madison, WI, Aug. 21-24, 1979), Paper D-1 in Advances in Cryogenic Engineering Materials, A. F. Clark and R. P. Reed, eds., 26, 235.244 (Plenum Publ. Corp., New York, NY 10013, 1980).

20633. Siegwarth, J. D.; Brennan, J. A. LNG instrumentation for custody transfer, Proc. American Gas Association 1981 Operating Section Distribution and Transmission Conf., (Distribution Conf. Anaheim, CA, May 18-20, 1981; Transmission Conf., Atlanta, GA, May 4-6, 1981), pp. T-119-T.123 (American Gas Assoc., Arlington, VA, 1981).

20658. Ekin, J. W. Mechanical properties and strain effects in superconductors, (Proc. 1980 NATO Advanced Study Institute, Sintra, Portugal, Aug. 1980), Chapter 7 in Superconductor Materials Science, pp. 455.510 (Plenum Publ. Corp., New York, NY, 1981).

20665. Fickett, F. R.; McHenry, H. I. Structural materials for large superconducting magnets, (Proc. 7th Int. Conf. Magnet Technology, Karlsruhe, West Germany, Mar. 30-Apr. 5, 1981), IEEE Trans. Mag. MAG-17, No. 5 2297.2304 (Sept. 1981).

20703. Tobler, R. L. A review of antifriction materials and design for 
cryogenic environments, (Proc. Cryogenic Engineering Conf., Madison, WI, Aug. 21-24, 1979), Paper A-5 in Advances in Cryogenic Engineering, A. F. Clark and R. P. Reed, eds., 26, 66-77 (Plenum Press, New York, NY, 1980).

20719. Groner, N. E.; Levin, B. M.; Nelson, H. E. Measuring evacuation difficulty in board and care homes, Fire $J .75$, No. 5, 44 . 50, 109 (Sept. 1981)

20741. Ho, J. C.; Oberly, C. E.; Garrett, H. J.; Walker, M. S.; Zeitlin, B. A.; Ekin, J. W. Processing limits for ultrafine-multifilament $\mathrm{Nb}_{3} \mathrm{SN}$, (Proc. Cryogenic Engineering Conf., Madison, WI, Aug. 21-24, 1979), Paper E-7 in Advances in Cryogenic Engineering, A. F. Clark and R. P. Reed, eds., 26, 358.366 (Plenum Press, New York, NY, 1980).

20742. Tobler, R. L.; Reed, R. P.; Burkhalter, D. S. Temperature dependence of yielding in austenitic stainless steels, (Proc. Cryogenic Engineering Conf., Madison, WI, Aug. 21-24, 1979), B-4 in Advances in Cryogenic Engineering, A. F. Clark and R. P. Reed, eds., 26, 107-119 (Plenum Press, New York, NY, 1980).

20753. Clark, A. F.; Fujii, G.; Ranney, M. A. The thermal expansion of several materials for superconducting magnets, (Proc. Int. Conf. Magnet Technology, Karlsruhe, West Germany, Mar. 30-Apr. 5, 1981), IEEE Trans. Magn. MAG-17, No. 5, 2316-2319 (The Institute of Electrical and Electronics Engineers, Inc., 345 East 47th St., New York, NY 10017, Sept. 1981).

20766. Radebaugh, R.; Linenberger, D.; Voth, R. O. Design of a regenerator test apparatus for temperatures down to $4 \mathrm{~K}, A F W A L-$ $T R-81-0350,60$ pages (For information contact the Flight Dynamics Laboratory, Air Force Wright Aeronautical Laboratories, Air Force Systems Command, Wright-Patterson Air Force Base, OH 45433, June 1981).

20767. Diller, D. E.; Saber, J. M. Measurements of the viscosity of compressed gaseous and liquid ethane, Physica 108A, 143-152 (1981).

20769. Hands, B. A.; Arp, V. D. A correlation of thermal conductivity data for helium, Cryogenics 21, No. 12, $697-703$ (Dec. 1981).

20771. Voth, R. O. Maximum practical efficiency of helium temperature refrigerators, Cryogenics 21, No. 11, 635.640 (Nov. 1981).

20772. Reed, R. P.; Tobler, R. L.; Elmer, J. W.; McHenry, H. I.; Yushchenko, K. A.; Paton, E. O. Strength and toughness of USSR Fe-20Cr-16Ni-6Mn-0.2N weldments at cryogenic temperatures, Paper in Proceedings of the Eighth International Cryogenic Engineering Conference, Genova, Italy, June 3-6, 1980, pp. $797-801$ (1980)

\section{Mathematical and Statistical Methods}

Mandel, J. Fitting curves and surfaces with monotonic and nonmonotonic four parameter equations. J. Res. Nat. Bur. Stand. (U.S.). 86(1): 1-25; 1981 January-February.

Pearl, M. H.; Goldman, A. J. A game-theoretic model of inspectionresource allocation. J. Res. Nat. Bur. Stand. (U.S.). 86(2): 193.215; 1981 March-April.

Shier, D. R.; Witzgall, C. Properties of labeling methods for determining shortest path trees. J. Res. Nat. Bur. Stand. (U.S.). 86(3): 317-330; 1981 May-June.

Goldman, A. J. Player aggregation in noncooperative games, II. J. Res. Nat. Bur. Stand. (U.S.). 86(4): 383-391; 1981 July-August.

Francis, R. L. A "uniformity principle" for evacuation route allocation. J. Res. Nat. Bur. Stand. (U.S.). 86(5): 509-513; 1981 September-October.

Stegun, I. A.; Zucker, R. Automatic computing methods for special functions. Part IV. Complex error function, Fresnel integrals, and other related functions. J. Res. Nat. Bur. Stand. (U.S.). 86(6): 661686; 1981 November-December.

TN1036. Tryon, P. V. The bispectrum and higher-order spectra: A bibliography. Nat. Bur. Stand. (U.S.) Tech. Note 1036; 1981 April. 24 p. SNO03-003-02312-4.

TN1147. Peavy, S. T.; Bremer, S. G. Test problems and results for OMITAB 80. Nat. Bur. Stand. (U.S.) Tech. Note 1147; 1981 November. 316 p. SN003-003.02379.5.
NBSIR 80-2053. Gass, S. I.; Joel, L. S. Concepts of model confidence. 1980 June. 41 p. Available from: NTIS; PB 81.158164.

NBSIR 80-2056. Lewis, J. G.; Rehm, R. G. The numerical solution of a nonseparable elliptic partial differential equation by preconditioned conjugate gradients. 1981 A pril. 66 p. A vailable from: NTIS; PB 82. 103318.

NBSIR 80-2151. Lechner, J. A.; Reeve, C. P.; Spiegelman, C. H. A new method of assigning uncertainty in volume calibration. 1980 December. 107 p. Available from: NTIS; PB 81.239519.

NBSIR 81-2222. Spiegelman, C. H. Some conservative statistical approaches for presenting interlaboratory N.D.A enrichment measurements. 1981 March. 19 p. A vailable from: NTIS; PB 81. 187973.

NBSIR 81-2385. Baum, H. R.; Rehm, R. G.; Barnett, P. D.; Corley, D. M. Finite difference calculations of buoyant convection in an enclosure. Part I: The basic algorithm. 1981 December. 67 p. Available from: NTIS; PB 82-154386.

NBSIR 81-2414. Moore, R. T. A discussion of GRIDNET simulation results. 1981 November. 16 p. Available from: NTIS; PB 82.142894 .

20019. Simiu, E.; Filliben, J. J. Weibull distributions and extreme wind speeds, Journal of the Structural Division 106, No. ST12, 2365-2374 (American Society of Civil Engineers, New York, NY, Dec. 1980).

20021. Shier, D. R. On algorithms for finding the $k$ shortest paths in a network, Networks 9, 195-214 (John Wiley \& Sons, Inc., Somerset, NJ, 1979).

20022. Shier, D. R.; Witzgall, C. Arc tolerances in shortest path and network flow problems, Networks 10, $277-291$ (John Wiley \& Sons, Inc., Somerset, NJ, 1980).

20032. Haber, S. A number-theoretic problem in numerical approximation of integrals, Proc. Approximations Theory III Symp., Austin, TX, Feb. 8-13, 1980, pp. 473.480 (Academic Press, New York, NY, 1980).

20232. Deprit, A. The main problem in the theory of artificial satellites to order four, J. Guidance and Control 4, No. 2, 201.206 (Mar.-Apr. 1981).

20252. Olver, F. W. J. The general connection-formula problem for linear differential equations of the second order, Proc. Conf. Singular Perturbations and Asymptotics, Madison, WI, May 28-30, 1980, pp. 317-343 (Academic Press, Inc., New York, NY, 1980).

20355. Shier, D. R. Selecting optimal product sizes, J. Undergraduate Math. Appl. 1, No. 4, 19-31 (1980).

20404. Boisvert, R. F. Attainable accuracy of compact discretizations of the Poisson equation, Paper in Elliptic Problem Solvers, pp. 219 223 (Academic Press, Inc., New York, NY, 1981).

20459. Boisvert, R. F. High order compact difference formulas for elliptic problems with mixed boundary conditions, (Proc. Fourth Int. Symp. Computer Methods for Partial Differential Equations, Bethlehem, PA, June 30-July 2, 1981), Paper in Advances in Computer for Partial Differential Equations, R. Vichenevetsky and R. Stepleman, eds., IV, 193.199 (IMACS, Rutgers Univ., Dept. of Computer Science, New Brunswick, NJ, 1981).

20501. Fong, J. T.; Dowling, N. E. Analysis of fatigue crack growth rate data from different laboratories, Proc. ASTM Symp. Fatigue Crack Growth Measurement and Data Analysis, Pittsburgh, PA, Oct. 1979, pp. 171.193 (American Society for Testing Materials, Philadelphia, PA, 1979).

20502. Hoffman, K. L.; Shier, D. R. A test problem generator for discrete linear $L_{1}$ approximation problems, $A C M$ Trans. Math. Software 6, No. 4, 587.593 (Dec. 1980).

20518. Lehman, D. R.; Parke, W. C.; Maximon, L. C. Numerical evaluation of integrals containing a spherical Bessel function by product integration, J. Math. Phys. 22, No. 7, 1399.1413 (July 1981).

20626. Berger, A. E.; Solomon, J. M.; Ciment, M. An analysis of a uniformly accurate difference method for a singular perturbation problem, Math. Comput. 37, No. 155, $79-94$ (July 1981).

20678. Friday, D. S. Dependence concepts for stochastic processes, Paper in Statistical Distributions in Scientific Work, Vol. 5, Taillie, Patil, and Baldessari, eds., pp. 349-361 (D. Reidel Publ. Co., Dordrecht, The Netherlands, 1981). 
Measurement Science and Technology: Physical Standards and Fundamental Constants

SP250, 1980 Edition. Kieffer, L. J., ed. Calibration and related measurement services of the National Bureau of Standards. Nat. Bur. Stand. (U.S.) Spec. Publ. 250, 1980 Edition; 1981 March. 105 p. SN003-003-02299-3.

SP330. Goldman, D. T.; Bell, R. J., eds. The International System of Units (SI). (Supersedes NBS Special Publication 330, 1977 Edition.) Nat. Bur. Stand. (U.S.) Spec. Publ. 330; 1981 December. 53 p. SN003-003.02380-9.

SP612. Sherwood, G. B. Standard reference data publications 19641980. Nat. Bur. Stand. (U.S.) Spec. Publ. 612; 1981 November. 121 p. A vailable from: NTIS; PB 82.134362.

TN1142. Shorten, F. J., ed. NBS reactor: Summary of activities July 1979 to June 1980. Nat. Bur. Stand. (U.S.) Tech. Note 1142; 1981 May. 211 p. SN003-003-02314.1.

NBSIR 81-2263. Eby, R. K.; Broadhurst, M. G.; Cassel, J. M.; Fanconi, B. M.; Sanchez, I. C.; Smith, L. E. Polymer Science and Standards Division-Annual Report 1980. 1981 April. 90 p. Available from: NTIS; PB 81-215287.

20183. Kautz, R. L.; Costabile, G. A Josephson voltage standard using a series array of 100 junctions, IEEE Trans. Magn. MAG-17, No. 1, 780.783 (Jan. I, 1981).

20233. Layer, H. P.; Rowley, W. R. C.; Marx, B. R. National Physical Laboratory - National Bureau of Standards iodine-stabilized heliumneon laser intercomparison, Opt. Lett. 6, No. 4, 188-190 (Apr. 1981).

20308. Barnes, J. A. Clocks, atomic and molecular, Article in Encyclopedia of Physics 1, 124-125 (Addison-Wesley Publ. Co., Inc., Advanced Book Program, Reading, MA, 1980).

\section{Measurement Science and Technology: Policy and State-of-the-Art Surveys}

Driver, R. G.; Houck, J. C.; Welch, B. E. An intercomparison of pressure standards between LNE and NBS. J. Res. Nat. Bur. Stand. (U.S.). 86(3): 277-279; 1981 May-June.

SP600. Porter, G. National Bureau of Standards 1980. Nat. Bur. Stand. (U.S.) Spec. Publ. 600; 1981 January. 40 p. SN003-003-023001.

SP611. French, J. C. New technology challenges metrology. Nat. Bur. Stand. (U.S.) Spec. Publ. 611; 1981 July. 90 p. SN003-003-02341-8.

NBSIR 80-1972. Huntoon, R. D. Needs and opportunities in metrology of the Instituto de Pesquisas Tecnologicas for effective support of Brazilian industry. 1980 February. 35 p. Available from: NTIS; PB $81-152860$.

NBSIR 80-2020. Peiser, H. S.; Gutterman, B. M.; Louis, G. L.; Pring, J. M.; Taylor, J. K.; Tholen, A. D.; Smith, C. A., ed. Standardization and measurement services in the Sudan. 1980 August. 91 p. Available from: NTIS; PB 81-151425.

NBSIR 80-2108. Wachtman, J. B., Jr.; Reneker, D. H. Technical activities 1980-Center for Materials Science. 1980 October. 483 p. Available from: NTIS; PB 81-245318.

NBSIR 80-2113. Kramer, S.; Olmert, M. ed. International activities. The Fiscal Year 1979 survey of international activities at NEL 1980 September. 122 p. Available from: NTIS; PB 82-102146.

NBSIR 80-2165. Kessler, K. G. Technical activities 1980-Center for Absolute Physical Quantities. 1980 October. 235 p. Available from: NTIS; PB 81.248478 .

NBSIR 81-2205. Hoffman, J. D.; Yolken, H. T.; Reed, W. P.; Carpenter, B. S. National Measurement Laboratory Office of Measurements for Nuclear Technology 1980: Annual report. 1981 January. 57 p. A vailable from: NTIS; PB 81.171290.

NBSIR 81-2280. Kirchhoff, W. H. Technical activities of the Office of Environmental Measurements-1980. 1981 May. 195 p. Available from: NTIS; PB 81.227860.

NBSIR 81-2305. Wachtman, J. B., Jr., ed. Proceedings, U.S. Department of Commerce public workshop on critical materials needs in the aerospace industry, February 9.10, 1981. 1981 July. 701 p. A vailable from: NTIS; PB 82-137266.

NBSIR 81-2408. Hoffman, J. D.; Wachtman, J. B., Jr. National Measurement Laboratory Center for Materials Science, Annual report 1981. 1981 November. 553 p. Available from: NTIS; PB 82. 142126

20058. Taylor, B. N. Constants, fundamental, Article in Encyclopedia of Physics, R. G. Lerner and G. L. Trigg, eds., pp. 145.152 (Addison-Wesley Publ. Co., Inc., Advanced Book Program, Reading, MA, 1981).

20372. Bullis, W. M. Role of Government in influencing the semiconductor industry-Technological aspects, (Proc.

Electrochemical Society Meet. Los Angeles, CA, Oct. 14-19, 1979), J. Electro. Chem. Soc. Extended Abstracts 79.2, Abstr. No. 479, 1214 1215 (Oct. 1979).

20583. Hall, J. L. Stable lasers and optical frequency standards for testing the postulates of physics, Paper in Alomic Physics, D. Kleppner and F. M. Pipkin, eds., 7, 267.296 (Plenum Publ. Corp., New York, NY 10013, 1981)

20749. Eby, R. K.; Broadhurst, M. G.; Chang, S. S.; Davis, G. T.; DiMarzio, E. A.; Fanconi, B. M.; Han, C. C.; Khoury, F. A.; Markovitz, H.; McKinney, J. E.; Mopsik, F. I.; Passaglia, E.; Sanchez, I. C.; VanderHart, D. L.; Zapas, I. J. High polymer physics, Chapter 13 in AIP 5th Anniversary Physics Vade Mecum, pp. 196.209 (Oct. 1981)

\section{Mechanics: Design, Testing, and Measurement}

SP602. Albus, J. S.; VanderBrug, G. J.; Barbera, A. J.; Fitzgerald, M. L.; Wheatley, T. E., eds. NBS/RIA robotics research workshop. Proceedings of a workshop held at the National Bureau of Standards; 1979 November 13.15; Gaithersburg, MD. Nat. Bur. Stand. (U.S.) Spec. Publ. 602; 1981 April. 54 p. SN003-003-02307-8.

NBSIR 80-2152. Wheatley, T.; Albus, J. S.; Nagel, R. N., eds. Proceedings of NBS/Air Force ICAM workshop on robot interfaces. I980 January. 83 p. Available from: NTIS; PB 81-161119.

NBSIR 80-2173. Palla, R. L. Water usage characteristics of household appliances and the potential for water savings. 1980 December. 33 p. A vailable from: NTIS; PB 81.202178.

NBSIR 81-2297. Kelly, J. C.; Wolf, R.; Kennicott, P.; Nagel, R.; Wellington, J., eds. A technical briefing on the Initial Graphics Exchange Specification (IGES). 1981 July. 135 p. Available from: NTIS; PB 81.238719.

20246. Mordfin, L.; Payne, B. F.; Edelman, S. Enhanced reliability and reproducibility of measurements of machinery vibrations, Proc. Sixth Machinery Dynamics Seminar; Vibration Standards and Current Techniques for Flexible Rotor Balancing, Toronto, Canada, Sept. 22. 23. 1980, pp. 4.0-4.22 (National Research Council Canada, Ottawa, Ontario, Canada, Sept. 1980).

20555. Teague, E. C.; Vorburger, T. V.; Maystre, D. Light scattering from manufactured surfaces, Ann. CIRP 30, No. 2, 1.7 (Dec. 1981).

\section{Metrology: Physical Measurements}

Jones, F. E. The refractivity of air. J. Res. Nat. Bur. Stand. (U.S.). 86(1): 27.32; 1981 January-February.

Magin, R. L.; Mangum, B. W.; Statler, J. A.; Thornton, D. D. Transition temperatures of the hydrates of $\mathrm{Na}_{2} \mathrm{SO}_{4}, \mathrm{Na}_{2} \mathrm{HPO}_{4}$, and $\mathrm{KF}$ as fixed points in biomedical thermometry. J. Res. Nal. Bur. Stand. (U.S.). 86(2): 181-192; 1981 March-April.

SP304A, Revised August 1981. Goldman, D. Brief history of measurement systems with a chart of the modernized metric system. Nat. Bur. Stand. (U.S.) Spec. Publ. 304A; 1981 August. 4 p. SN003 003-02366-3.

SP304, Revised August 1981. Goldman, D. The modernized metric system-The International System of Units (SI). Nat. Bur. Stand. (U.S.) Spec. Publ. 304; 1981 August. 1 chart. SN003-003.02365.5.

TN1032. Kim, E. M.; Franzen, D. L. Measurement of far.field and near-field radiation patterns from optical fibers. Nat. Bur. Stand. (U.S.) Tech. Note 1032; 1981 February. 48 p. SN003-003.02304-3.

TN1034. Danielson, B. L. Backscatter measurements on optical fibers. Nat. Bur. Stand. (U.S.) Tech. Note 1034; 1981 February. 52 p. SN003.003.02303.5

TN1038. Young, M. Refracted-ray scanning (refracted near-field 
scanning) for measuring index profiles of optical fibers. Nat. Bur. Stand. (U.S.) Tech. Note 1038; 1981 May. 56 p. SN003-003-02342-6.

TN1043. Day, G. W., ed. The characterization of optical fiber waveguides. A bibliography with abstracts, 1970-1980. Nat. Bur. Stand. (U.S.) Tech. Note 1043; 1981 June. 72 p. SN003-003-02338-8.

TN1046. Day, G. W. Measurement of optical fiber bandwidth in the frequency domain. Nat. Bur. Stand. (U.S.) Tech. Note 1046; 1981 September. 48 p. SN003-003-02377-9.

TN1144. Turgel, R. S.; Oldham, N. M.; Stenbakken, G. N.; Kibalo, T H. NBS phase angle calibration standard. Nat. Bur. Stand. (U.S.) Tech. Note 1144; 1981 July. 143 p. SN003-003-02344-2.

TN1145. Souders, T. M.; Flach, D. R.; Bell, B. A. A calibration service for analog-to-digital and digital-to-analog converters. Nat. Bur. Stand. (U.S.) Tech. Note 1145; 1981 July. 73 p. SN003-003 02352-4.

NBSIR 78-869. Daywitt, W. C. Error equations used in the NBS Earth Terminal Measurement System. 1977 December. 30 p. Available from: NTIS; PB 276253.

NBSIR 78-871. Driver, L. D.; Ries, F. X.; Rebuldela, G. NBS rf voltage comparator. 1978 December. 25 p. Available from: NTIS; PB 292373.

NBSIR 79-1600. Siegwarth, J. D. Radio frequency liquid level gauging in propane tank car safety tests-A feasibility study. 1979 January. 15 p. Available from: NTIS; PB 292978.

NBSIR 79-1752. Hocken, R. J.; Borchardt, B. R. On characterizing measuring machine geometry. 1979 June. 25 p. Available from: NTIS; PB 82-116377.

NBSIR 80-1634. Bowman, R. R. A temperature probe for radiofrequency heated material. 1981 January. 33 p. Available from: NTIS; PB 81-176125.

NBSIR 80-2177. Misakian, $M$. Generation and measurement of dc electric fields with space charge. 1981 January. 44 p. Available from: NTIS; PB 81-161929.

NBSIR 81-1641. Wakefield, J. P. Addendum to Earth Terminal Measurement System Maintenance Manual. 1981 October. 48 p. Available from: NTIS; PB 82-151994.

NBSIR 81-1651. Sparks, L. L. Thermal conductivity of a concrete mortar from $95 \mathrm{~K}$ to $320 \mathrm{~K}$. 1981 October. 16 p. Available from: NTIS; PB 82-134370.

NBSIR 81-2235. Anderson, W. E.; Ramboz, J. D. 1980 Annual Report: Technical contributions to the development of incipient fault detection/location instrumentation. 1981 March. 134 p. Available from: NTIS; PB 81-188005

NBSIR 81-2242. Van Brunt, R. J.; Misakian, M.; Leep, D. A.; Moy, K. J.; Beaty, E. C. 1980 annual report: Technical assistance for future insulation systems research. 1981 April. 105 p. Available from: NTIS; PB 82.134354.

NBSIR 81-2267. McKnight, R. H.; Kotter, F. R.; Misakian, M.; Ortiz, P. 1980 Annual Report: Electric and magnetic field measurements. 1981 May. 72 p. Available from: NTIS; PB 81-218182.

NBSIR 81-2275. Hebner, R. E., Jr.; Kelley, E. F.; Thompson, J. E. Sudarshan, T. S.; Jones, T. B. 1980 Annual Report: Optical measurements for interfacial conduction and breakdown. $1981 \mathrm{May}$. 87 p. Available from: NTIS; PB 81-216343.

NBSIR 81-2283. Hebner, R. E. Development of power system measurements: Quarterly Report, January 1, 1981 to March 31, 1981. 1981 April. 19 p. Available from: NTIS; PB 81-220261.

NBSIR 81-2311. Richmond, J. C. Analysis of results of mini round robin reflectance test. 1981 August. 32 p. Available from: NTIS; PB 81.244873

NBSIR 81-2337. Ramboz, J. D. Measurement and evaluation methods for an angular accelerometer. 1981 August. 75 p. Available from: NTIS; PB 82.115973.

NBSIR 81-2410. McKnight, R. H.; Kotter, F. R.; Misakian, M. Measurement of ion current density at ground level in the vicinity of high voltage dc transmission lines. 1981 December. 28 p. Available from: NTIS; PB 82-156993.

20062. Allan, D. W.; Weiss, M. A. Accurate time and frequency transfer during common-view of a GPS satellite, Proc. 34th Annual Symp. on Frequency Control, Ft. Monmouth, NJ, May 28-30, 1980, pp. 334-346 (Electronic Industries Association, Washington, DC,
May 1980).

20070. McDonald, D. G.; Peterson, R. L.; Hamilton, C. A.; Harris, R E.; Kautz, R. L. Picosecond applications of Josephson junctions, IEEE Trans. Electron Devices ED-27, No. 10, 1945-1965 (Oct. 1980).

20081. Belanger, B. C.; Kieffer, L. J. Regional measurement assurance programs for physical measurements, (Proc. 8th IMEKO Congress, Moscow, USSR, May 21-27, 1979), Paper in Acta Imeko, pp. 649. 656 (Publishing House of the Hungarian Academy of Sciences Budapest, Hungary, 1980).

20083. Mecherikunnel, A. T.; Richmond, J. C. Spectral distribution of solar radiation, NASA Tech. Memorandum No. 82021, pp. 1.86 (Goddard Space Flight Center, Greenbelt, MD 20771, Sept. 1980).

20093. Lentner, K. J.; Childers, C. B.; Tremaine, S. G. A semiautomatic system for ac/dc difference calibration, IEEE Trans. Instrum. Meas. IM-29, No. 4, 400-405 (Dec. 1980).

20096. Layer, H. P. A portable iodine stabilized helium-neon laser, IEEE. Trans. Instrum. Meas. IM-29, No. 4, 358-361 (Dec. 4, 1980).

20106. Cutkosky, R. D. An automatic resistance thermometer bridge, IEEE Trans. Instrum. Meas. IM-29, No. 4, $330-333$ (Dec. 1980).

20107. Bender, P. L. Improved methods for measuring present crustal movements, Paper in Dynamics of Plate Interiors, Geodynamics Series 1, 155-162 (American Geophysical Union, Washington, DC, 1980).

20131. Kelley, E. F.; Hebner, R. E., Jr. Prebreakdown phenomena between sphere-sphere electrodes in transformer oil, Appl. Phys. Lett. 38, No. 4, 231.233 (Feb. 15, 1981).

20142. Kelley, E. F.; Hebner, R. E., Jr. The electric field distribution associated with prebreakdown phenomena in nitrobenzene, J. Appl. Phys. 52, No. 1, 191-195 (Jan. 1981).

20184. Hamilton, C. A.; Lloyd, F. L.; Kautz, R. L. Analog measurement applications for high speed Josephson switches, IEEE Trans. Magn. MAG-17, No. 1, 577.582 (Jan. 1, 1981).

20201. Jensen, S.; Swyt, D. Sub-micrometer length metrology: Problems, techniques and solutions, Proc. Scanning Electron Microscopy, Chicago, IL, Apr. 21-25, 1980, pp. 393-406 (SEM Inc., Chicago, IL, 1980).

20204. Rakowsky, G. NBS-SURF II: A small, versatile synchrotron light source, (Proc. Sixth Conf. Application of Accelerators in Research and Industry, Denton, TX, Nov. 3-5, 1980), IEEE Trans. Nucl. Sci. NS-28, No. 2, 1519.1521 (Apr. 1981).

20236. Parr, A. C.; Rakowsky, G.; Ederer, D. L.; Stockbauer, R. L.; West, J. B.; Dehmer, J. L. Current research at NBS using synchrotron radiation at SURF-II, IEEE Trans. Nucl. Sci. NS-28, No. 2, 1210-1214 (Apr. 1981).

20261. Barger, R. L. Simple interferometric technique for alignment of segmented retroreflectors, Appl. Opt. 19, 2088-2089 (July 1980).

20287. Weidner, V. R.; Hsia, J. J. Reflection properties of pressed polytetrafluoroethylene powder, J. Opt. Soc. Am. 71, No. 7, 856-861 (July 1981).

20306. Zimmerman, J. E. Cryogenics for SQUIDs, (Proc. Second Int. Conf. Superconducting Quantum Devices, Berlin, (West) Germany, May 6-9, 1980), Paper in Squid '80, H. D. Hahlbohm and H. Lübbig, eds., pp. 423-443 (Walter de Gruyter \& Co., Berlin, (West) Germany, 1980).

20307. Peterson, R. L. Turn-on delays in single Josephson junction devices, (Proc. Second Int. Conf. Superconducting Quantum Devices, Berlin, (West) Germany, May 6-9, 1980), Paper in Squid '80, H. D. Hahlbohm and H. Lübbig, eds., pp. $685-702$ (Walter de Gruyter \& Co., Berlin, (West) Germany, 1980).

20312. Nahman, N. Error criteria and the use of reference waveforms, Proc. ATE Seminar/Exhibit Automated Testing for Electronics Manufacturing, Pasadena Center, Pasadena, CA, Jan. 19-22, 1981, pp. IV-21-IV-28 (Benwill Publ. Corp., Boston, MA, 1981).

20346. Leep, D. A.; Kotter, F. R. Discussion of "Measuring voltage and current harmonics on distribution systems" by M. F. McGranaghan, J. H. Shaw, and R. E. Owen, IEEE Trans. Power Appar. Syst. PAS-100, No. 7, $3599-3608$ (July 1981).

20369. Franzen, D. L.; Day, G. W.; Danielson, B. L.; Kim, E. M. Chamberlain, G. E. Results of an interlaboratory measurement comparison among fiber manufacturers to determine attenuation, bandwidth, and N.A. of graded-index optical fibers, (Proc. 3d Int. Conf. Integrated Optics and Optical Fiber Communication, San 
Francisco, CA, Apr. 27-29, 1981), Digest of Technical Papers, pp. 74 76 (OSA/IEEE, San Francisco, CA, 1981).

20415. Booker, R. L. Luminance-brightness comparisons of separated circular stimuli, J. Opt. Soc. Am. 71, No. 2, 139.144 (Feb. 1981).

20431. Bender, P. L. Establishment of terrestrial reference frames by new observational techniques, (Proc. IAU Colloq. 56, Warsaw, Poland, Sept. 8-12, 1980), Paper in Reference Coordinate Systems for Earth Dynamics, E. M. Gaposchkin and B. Kolaczek, eds., pp. 23-36 (D. Reidel Publ. Co., Dordrecht, The Netherlands, 1981).

20432. Franzen, D. L.; Kim, E. M. Interlaboratory measurement comparison to determine the radiation angle (N.A.) of graded-index optical fibers, Appl. Opt. 20, No. 7, 1218.1220 (Apr. 1, 1981).

20434. Bossler, J. D.; Goad, C. D.; Bender, P. L. Using the global positioning system (GPS) for geodetic positioning, Bull. Geodesique 54, 553.563 (1980).

20445. Kelley, E. F.; Hebner, R. E., Jr. Electrical breakdown in composite insulating systems: Liquid-solid interface parallel to the field, IEEE Trans. Elec. Insul. EI-6, No. 4, 297-303 (1981).

20462. Scheeline, A.; Travis, J. C.; DeVoe, J. R.; Walters, J. P. Discovery of an electrical post-pulse in the surroundings of a high voltage spark discharge, Spectrochim. Acta 36, No. 3, 153-161 (1981).

20484. Misakian, M. Generation and measurement of dc electric fields with space charge, J. Appl. Phys. 52, No. 5, 3135.3144 (May 1981).

20485. White, G. S.; Feldman, A. Diffraction from a shallow rectangular groove, Appl. Opt. 20, No. 14, 2585-2589 (July 15, 1981).

20488. Cromar, M. W.; Carelli, P. Low-noise tunnel junction dc SQUID's, Appl. Phys. Lett. 38, No. 9, 2723-2725 (May 1981).

20542. Miiller, A. P.; Cezairliyan, A. The melting point of palladium by a pulse heating method, Int. J. Thermophys. 2, No. 1, 63.70 (1981).

20560. Kilmer, R. D. Assessment of locomotive crew in-cab occupational noise exposure, U.S. Department of Transportation Report No. FRA/ORD-80/91, 184 pages (Available as PB81-154395 from the National Technical Information Service, Springfield, VA 22161, Dec. 1980).

20578. Cutkosky, R. D. Guarding techniques for resistance thermometers, IEEE Trans. Instrum. Meas. IM-30, No. 3, 217.220 (Sept. 1981).

20593. Cezairliyan, A. A dynamic technique for measurements of thermophysical properties at high temperatures, High Temp. Sci. 13, 117.133 (1980).

20636. Baltes, H. P.; Geist, J. Kirchhoff's and Planck's radiation laws for small particles in scattering atmospheres or flames, $J$. Quant. Spectrosc. Radiat. Transfer 26, No. 6, $535-536$ (1981).

20637. Day, R. H.; Lee, P.; Saloman, E. B.; Nagel, D. J. Photoelectric quantum efficiencies and filter window absorption coefficients from $20 \mathrm{eV}$ to $10 \mathrm{KeV}, J$. Appl. Phys. 52, No. 11, $6965-6973$ (Nov. 1981).

20645. Hebner, R. E.; Kelley, E. F.; Forster, E. O.; FitzPatrick, G. J. Observation of prebreakdown and breakdown phenomena in liquid hydrocarbons, Proc. 7th Int. Conf. Conduction and Breakdown in Dielectric Liquids, Berlin, West Germany, July 27-31, 1981, pp. 177. 181 (The Institute of Electrical and Electronics Engineers, 445 Hoes Lane, Piscataway, NJ 08854, 1981).

20646. Zahn, M.; Forster, E. O.; Kelley, E. F.; Hebner, R. E., Jr. Hydrodynamic shock wave propagation after electrical breakdown, Proc. 7th Int. Conf. Conduction and Breakdown in Dielectric Liquids, Berlin, West Germany, July 27-31, 1981, pp. $398-403$ (The Institute of Electrical and Electronic Engineers, 445 Hoes Lane, Piscataway, NJ 08854, 1981).

20666. Bertotti, B. Statistics of narrow structures of the gravity field of the Earth. 1. General theory, J. Geophys. Res. 86, No. B11, 10835 . 10842 (Nov. 10, 1981).

20671. Chen, H.; Kuriyama, M. A high-resolution X-ray facility, $J$. Appl. Cryst. Lab. Note 14, 280 (1981).

20679. Heydemann, P. L. M. Determination and correction of quadrature fringe measurement errors in interferometers, Appl. Opt. 20, 3382-3384 (Oct. 1, 1981).

20683. Lettieri, T. R.; Jenkins, W. D.; Swyt, D. A. Sizing of individual optically levitated evaporating droplets by measurement of resonances in the polarization ratio, Appl. Opt. 20, 2799-2805 (Aug. $15,1981)$.

20692. Peterson, R. L.; Van Vechten, D. Modeling the impedance of a
Josephson-junction noise thermometer, Phys. Rev. B 24, No. 6, 3588 3591 (Sept. 15, 1981).

20699. Sandle, W. J.; Gallagher, A. Optical bistability by an atomic vapor in a focusing Fabry-Perot cavity, Phys. Rev. A 24, No. 4, 2017. 2028 (Oct. 1981).

20704. Van Brunt, R. J.; Leep, D. Characterization of point-plane corona pulses in $\mathrm{SF}_{6}, J$. Appl. Phys. 52, No. 11, $6588-6600$ (Nov. 1981).

20712. Yoo, K. C.; Roessler, B.; Armstrong, R. W.; Kuriyama, M. Reflection X-ray topography of hardness indentations in copper single crystals, Scr. Met. 15, 1245.1250 (1981).

20722. Han, C. C.; Akcasu, A. Z. Dynamic light scattering of dilute polymer solutions in the nonasymptotic $q$ region, Macromolecules 14 , 1080-1084 (July-Aug. 1981).

20733. Richmond, J. C. Errors in passive infrared imaging systems due to reflected ambient flux, SPIE Infrared Imaging Syst. Technol. Proc. 226, 110-114 (1980).

20765. Lewis, R. Spherical scanning data processing: An algorithm for halving the data processing effort when the radiation into the back hemisphere is negligible, (Proc. AP.S Int. Symp. Antennas and Propagation, Los Angeles, CA, June 16-19, 1981), 1981 Int. Symp. Digest, $81 \mathrm{CH}$ 1672-5, 1, 247.249 (Institute of Electronics and Electrical Engineers, 455 Hoes Lane, Piscataway, NJ 08554, 1981).

\section{Nuclear Physics and Radiation Technology}

Pruitt, J. S.; Domen, S. R.; Loevinger, R. The graphite calorimeter as a standard of absorbed dose for cobalt-60 gamma radiation. J. Res. Nat. Bur. Stand. (U.S.). 86(5): 495-502; 1981 September-October.

H136. Eisenhower, E. H., Chairman, ANSI Subcommittee N43.3.5. American National Standard N432; Radiological safety for the design and construction of apparatus for gamma radiography. (ANSI N432. 1980). Nat. Bur. Stand. (U.S.) Handb. 136; 1981 January. 19 p. SN003-003.02293.4.

SP603. Eisenhower, E. H. Requirements for an effective national ionizing radiation measurements program. A report to the Congress by the National Bureau of Standards. Nat. Bur. Stand. (U.S.) Spec. Publ. 603; 1981 March. 139 p. SN003-003-02298-5.

SP625. Taylor, L. S. X-ray measurements and protection 1913-1964: The role of the National Bureau of Standards and the National Radiological Organizations. Nat. Bur. Stand. (U.S.) Spec. Publ. 625; 1981 December. 386 p. SN003-003-02375-2.

NBSIR 81-2262. Maximon, L. C.; de Miniac, A.; Aniel, T. Tagged photons. An analysis of the bremsstrahlung differential cross section in the range of interest for a tagged photon system. 1981 April. 147 p. Available from: NTIS; PB 81-227837.

NBS-GCR-80-296. Committee on Radiation Measurements, (NBS contact: Mr. Heaton). Directory of commercial calibration services for ionizing radiation survey instruments. 1981 April. 73 p. Available from: NTIS; PB 81.2064l.

19985. Leiss, J. E.; Norris, N. J.; Wilson, M. A. The design and performance of a long-pulse high-current linear induction accelerator at the National Bureau of Standards, Part. Accel. 10, 223.234 (1980).

19992. Hayward, E. Photonuclear reactions, Article in Encyclopedia of Physics, R. G. Lerner and G. L. Trigg, eds., pp. 750-751 (AddisonWesley Publ. Co., Inc., Advanced Book Program, Reading, MA, 1981).

20010. Hutchinson, J. M. R. Traceability, quality assurance, and standards, (Proc. Low-level Measurement Techniques Group Int. Committee for Radionuclide Metrology, Braunschweig, West Germany, June 18-19, 1979), Paper in Environment International 3, No. 5, 363-364 (Pergamon Press Limited, Oxford OX3, OBW, England, 1980).

20064. Coyne, J. J. Kerma values by particle type, Proc. Int. Workshop on Ion Chambers for Neutron Dosimetry, Rijswijk, The Netherlands, Sept. 17-20, 1979, J. J. Broerse, ed., 195-207 (Harwood Academic Publishers, (EUR 6782 EN), The Netherlands, 1980).

20073. Loevinger, R. A formalism for calculation of absorbed dose to a medium from photon and electron beams, Med. Phys. 8, No. 1, 1-12 (Jan.-Feb. 1981).

20146. Deslattes, R. D.; Kessler, E. G.; Sauder, W. C.; Henins, A. 
Remeasurement of $\gamma$-ray reference lines, Ann. Phys. 129, No. 2, 378 . 434 (Oct. 15, 1980).

20150. McLaughlin, W. L.; Kosanić, M. M.; Marković, V. M.; Nenadovic, M. T.; Holcman, J.; Sehested, K. The kinetics of dye formation by pulse radiolysis of pararosaniline cyanide in aqueous or organic solution, Ris $\varnothing-M-2202,32$ pages (Ris $\emptyset$ National Laboratory, DK.4000 Roskilde, Denmark, Nov. 1979).

20152. Deslattes, R. D. X-ray monochromator development for synchrotron radiation facilities, $\mathrm{Nucl}$. Instrum. Methods 172, 201-208 (1980).

20153. Eisenhauer, C. M.; Grundl, J. A.; Gilliam, D. M.; McGarry, E. D.; Spiegel, V. Benchmark referencing of neutron dosimetry measurements, (Proc. Third ASTM-Euratom Symp. Reactor Dosimetry, Ispra, Italy, Oct. 1-5, 1979), Paper in Dosimetry Methods for Fuels, Cladding and Structural Materials, EUR6813 EN-FR, II, pp. 919.927 (Commission of the European Communities, Luxemburg, 1980).

20163. Johnson, R. G.; Behrens, J. W.; Bowman, C. D. Source imaging for FMIT using a neutron pin-hole camera, Proc. Symp. Neutron Cross-Sections from 10 to $50 \mathrm{MeV}$, Upton, NY, May 12-14, 1980, pp. 629.637 (National Nuclear Data Center, Upton, NY, July 1980).

20165. Cavallo, L. M.; Mann, W. B. New National Bureau of Standards contemporary carbon-14 standards, (Proc. Radiocarbon Dating Conf., Heidelberg, West Germany, Aug. 19-26, 1979), Paper in Radiocarbon 22, No. 3, $962-963$ (American Journal of Science, New Haven, CT, 1980).

20171. Humphreys, J. C.; McLaughlin, W. L. Dye film dosimetry for radiation processing, (Proc. Sixth Conf. Application of Accelerators in Research and Industry, Denton, TX, Nov. 3-5, 1980), IEEE Trans. Nucl. Sci. NS-28, No. 2, 1797-1801 (Apr. 1981).

20174. McLaughlin, W. L. Dosimetry standards for industrial radiation processing, Chapter in National and International Standardization of Radiation Dosimetry 1, 89-106 (International Atomic Energy Agency, Vienna, Austria, 1978)

20175. Maximon, L. C.; Gimm, H. A. Pair production in the field of atomic electrons, Phys. Rev. A 23, No. 1, 172-185 (Jan. 1981).

20176. Miller, A.; McLaughlin, W. L. Evaluation of radiochromic dye films and other plastic dose meters under radiation processing conditions, Chapter in Tech. Report No. 205: High-Dose Measurements in Industrial Radiation Processing, pp. $119-138$ (International Atomic Energy Agency, Vienna, Austria, 1981).

20185. McLaughlin, W. L. A national standardization programme for high-dose measurements, Tech. Reports Series No. 205: High-Dose Measurements in Industrial Radiation Processing pp. 17.32 (International Atomic Energy Agency, Vienna, Austria, 1981).

20189. Dick, C. E.; Motz, J. W. Utilization of monoenergetic x-ray beams to examine the properties of radiographic intensifying screens, (Proc. Sixth Conf. Application of Accelerators in Research and Industry, Denton, TX, Nov. 3-5, 1980), IEEE Trans. Nucl. Sci. NS28, No. 2, 1554-1558 (Apr. 1981).

20195. Hayward, E. Nuclear excitations using real and virtual photons, (Proc. Giant Multipole Resonance Topical Conf., Oak Ridge, TN, Oct. 15.17, 1979), Paper in Giant Multipole Resonance 1, 275-298 (Harwood Academic Publ., New York, NY, 1979).

20206. Penner, S.; Cutler, R. I.; Debenham, P. H.; Lindstrom, E. R Mohr, D. L.; Wilson, M. A. D.; Yoder, N. R.; Young, L. M.; Boyd T. J.; Knapp, E. A.; Martin, R. E.; Potter, J. M.; Schneider, C. M.; Swenson, D. A.; Tallerico, P. J. The NBS-LASL CW Microtron, (Proc. Sixth Conf. Application of Accelerators in Research and Industry, Denton, TX, Nov. 3-5, 1980), IEEE Trans. Nucl. Sci. NS. 28, No. 2, 1526-1530 (Apr. 1981).

20234. Hayward, E. Study of the giant resonances with virtual and real photons, (Proc. Int. Conf. Nuclear Physics with Electromagnetic Interactions, Mainz, Germany, June 5-9, 1979), Lecture Notes in Physics 108, pp. 300-310 (Springer-Verlag, New York, NY, 1979).

20237. Hayward, E.; Leicht, R. G. Elastic scattering of photons by ${ }^{12} \mathrm{C}$ and the E2 giant resonance, Ann. NY Acad. Sci. 40, 99-110 (Sept. 15, 1980).

20256. McGarry, E. D.; Fabry, A. Dosimetry characterization of a reactor pressure vessel simulator by fission chamber and foil activation measurements, (Proc. Third ASTM-Euratom Symp. Reactor Dosimetry, Ispra, Italy, Oct. 1-5, 1979), Paper in Dosimetry Methods for Fuels, Cladding and Structural Materials, EUR6813 ENFR, II, 1031.1041 (Commission of the European Communities, Luxembourg, 1980).

20267. Hayward, E. The elastic photon scattering cross section for ${ }^{12} \mathrm{C}$ in the energy range 20-40 $\mathrm{MeV}$, (Int. School of Intermediate Energy Nuclear Physics, Rome, Italy, June 15.29, 1979), Paper in Electron and Pion Interactions with Nuclei at Intermediate Energies 2, 667-675 (Harwood Academic Publ., New York, NY 1979).

20275. Wolynec, E.; Dodge, W. R.; Leicht, R. G.; Hayward E. Electrodisintegration of ${ }^{58} \mathrm{Ni},{ }^{60} \mathrm{Ni}$, and ${ }^{62} \mathrm{Ni}$, Phys. Rev. C 22, No. 3, 1012-1024 (Sept. 1980).

20323. Lindgren, R. A.; Flanz, J. B.; Hicks, R. S.; Parker, B.; Peterson, G. A.; Lawson, R. D.; Teeters, W.; Williamson, C. F.; Kowalski, S.; Maruyama, X. K. Comparison of inelastic electron scattering with $\left[g_{9 / 2} \times\left(f_{7 / 2}{ }^{-3}\right)_{j}\right]_{8-}$ shell-model calculations for the $T=1$ and $T=2,8^{-}$states in ${ }^{34} \mathrm{Fe}$, Phys. Rev. Lett. 46, No. 11, 706 . 709 (Mar. 16, 1981)

20349. Lum, G. K.; Wiegand, C. E.; Kessler, E. G., Jr.; Deslattes, R. D.; Jacobs, L.; Schwitz, W.; Seki, R. Kaonic mass by critical absorption of kaonic-atom x rays, Phys. Rev. D, 23, No. 11, 2522. 2532 (June 1, 1981)

20352. Wilson, M. A. D. Recirculation acceleration of high current relativistic electron beams-A feasibility study, (Proc. 1981 Particle Accelerator Conf., Accelerator Engineering and Technology, Washington, DC, Mar. 11-13, 1981), IEEE Trans. Nucl. Sci. NS-28, No. 3, 3375-3377 (June 1981)

20353. Penner, S. CW electron accelerators for nuclear physics, (Proc. 1981 Particle Accelerator Conf., Accelerator Engineering and Technology, Washington, DC, Mar. 11.13, 1981), IEEE Trans. Nucl. Sci. NS-28, No. 3, 2067-2073 (June 1981).

20356. Debenham, P. H. End magnet design for the NBS-LASL CW microtron, (Proc. 1981 Particle Accelerator Conf., Accelerator Engineering and Technology, Washington, DC, Mar. 11-13, 1981), IEEE Trans. Nucl. Sci. NS-28, No. 3, 2885-2887 (June 1981)

20367. Coursey, B. M.; Moghissi, A. A. The preparation of counting samples, Part II in Monographie BIPM-3, The Application of LiquidScintillation Counting to Radionuclide Metrology, pp. 7.22 (Bureau International des Poids et Mesures, Sevres, France, 1980).

20368. Coursey, B. M.; Mann, W. B. Design of high-efficiency liquidscintillation counting systems, Part III in Monographie BIPM-3, The Application of Liquid-Scintillation Counting to Radionuclide Metrology, pp. 23-35 (Bureau International des Poids et Mesures, Sevres, France, 1980).

20375. Bertsch, D. L.; Dodge, W. R. Calibration of a gamma-ray telescope using tagged positron annihilation photons, Nucl. Instrum. Methods 185, $439-448$ (1981)

20376. Danos, M. Cerenkov radiation, Article in Encyclopedia of Physics, pp. 106-107 (Addison-Wesley Publ. Co., Advanced Book Program, Reading, MA, 1980).

20381. Fiori, C. E.; Myklebust, R. L. A simplex method for fitting Gaussian profiles to $\mathrm{x}$-ray spectra obtained with an energy-dispersive detector, (Proc. American Nuclear Society Topical Conf., Mayaguez, Puerto Rico, Apr. 30-May 4, 1978), Paper in Computers in Activation Analysis and Gamma-Ray Spectroscopy, B. S. Carpenter, M. D. D'Agostino, and H. P. Yule, eds., CONF-780421, pp. 139-149 (U.S. Department of Energy, Technical Information Center, Washington, DC, 1979).

20425. O'Connell, J. S. Coincidence measurements with high energy electrons, (Proc. Workshop on Nuclear Physics with Real and Virtual Photons, Bologna, Italy, Nov. 25-28, 1980), Paper in From Collective States to Quarks in Nuclei, H. Arenhovel and A. M. Saruis, eds., pp. 286-295 (Springer-Verlag, Berlin, Germany, 1981).

20456. Duvall, K. C.; Wasson, O. A. The NBS $14 \mathrm{MeV}$ absolute neutron beam facility, (Proc. 1980 Conf. Application of Accelerators in Research and Industry, North Texas State Univ., Denton, TX, Nov. 3-5, 1980), IEEE Trans. Nucl. Sci. NS-28, No. 2, 1488.1489 (Apr. 1981).

20457. Bowman, C. D. Efficient neutron production using $12 \mathrm{MeV}$ electrons, (Proc. 1980 Conf. Application of Accelerators in 
Research and Industry, North Texas State Univ., Denton, TX, Nov. 3-5, 1980), IEEE Trans. Nucl. Sci. NS-28, No. 2, 1485.1487 (Apr. 1981)

20458. Schrack, R. A.; Behrens, J. W.; Johnson, R.; Bowman, C. D. Resonance neutron radiography using an electron Linac, (Proc. 1980 Conf. Application of Accelerators in Research and Industry, North Texas State Univ., Denton, TX, Nov. 3-5, 1980), IEEE Trans. Nucl. Sci. NS.28, No. 2, 1640-1643 (Apr. 1981).

20516. Danos, M. Nuclear forces from quark dynamics, (Workshop on Nuclear Physics with Real and Virtual Photons, Bologna, Italy, Nov. 25-28, 1980), Paper in Collective States to Quarks in Nuclei, 1 page (Comitato Nazional Energia Nucleare, Bologna, Italy, 1980).

20615. Seltzer, S. M. Calculated response of intrinsic germanium detectors to narrow beams of photons with energies up to $\sim 300 \mathrm{keV}$, Nucl. Instrum. Methods 188, 133-151 (1981).

20660. Lindgren, R. A.; Plum, M. A.; Gerace, W. J.; Hicks, R. S.; Parker, B.; Peterson, G. A.; Singhal, R.; Williamson, C. F.; Maruyama, X. K.; Petrovich, F. Isospin splitting of isovector highspin "stretched" particle-hole excitations in non-self-conjugate nuclei, Phys. Rev. Lett. 47, No. 18, 1266-1269 (Nov. 2, 1981).

20670. Caswell, R. S.; Coyne, J. J.; Goodman, L. J. Comparison of experimental and theoretical ionization yield spectra for neutrons, (Proc. Fourth Symp. Neutron Dosimetry, Munich-Neuherberg, Germany, June 1-5, 1981), Paper in Radiation Protection, G. Burger and H. G. Ebert, eds., 1, 201-212 (Commission of the European Communities, Munich, Germany, 1981).

20675. Coyne, J.; McDonald, J. C.; Menzel, H. G.; Schuhmacher, H. Detailed intercomparison of calculated and measured ionization yield spectra for $20 \mathrm{MeV}$ neutrons and the implications for high energy neutron dosimetry, (Proc. Fourth Symp. Neutron Dosimetry, Munich-Neuherberg, Germany, June 1.5, 1981), Paper in Radiation Protection, G. Burger and H. G. Ebert, eds., 1, 213-223 (Commission of the European Communities, Munich, Germany, 1981).

20724. Johnson, R. G.; Behrens, J. W.; Bowman, C. D. Source imaging using neutron pinhole cameras based on position-sensitive proportional counters, Nucl. Technol. 55, 724-727 (Dec. 1981).

\section{Processing and Performance of Materials}

Graminski, E. L.; Parks, E. J. The effect of calcium carbonate on the stability of acid treated papers. J. Res. Nat. Bur. Stand. (U.S.). 86(3): 309-315; 1981 May.June.

TN1149. Campbell, P. G.; Martin, J. W.; McKnight, M. E. Short-term evaluation procedures for coatings on structural steel. Nat. Bur. Stand. (U.S.) Tech. Note 1149; 1981 September. 43 p. SN003.003. 02367.1.

NBSIR 79-1904 (Navy). Kruger, J.; Carroll, J. J.; Melmed, A. J.; Ritter, J. J.; Ambrose, J. R. Passive films, surface structure and stress corrosion and crevice corrosion susceptibility. 1979 November. 35 p. A vailable from: NTIS; ADA078.596.

NBSIR 80-2099-4. Krause, R. F., Jr.; Fuller, E. R., Jr. Testing geothermal-well cements: Strength measurements following exposures to simulated geothermal fluids. 1981 December. 28 p. Available from: NTIS; PB 82.157009.

NBSIR 80-2142. Lashmore, D. S. Plating on aluminum. 1980 November. 65 p. A vailable from: NTIS; PB 81-239501.

NBSIR 80-2163. Clifton, J. R.; Anderson, E. D. Nondestructive evaluation methods for quality acceptance of hardened concrete in structures. 1981 January. 54 p. Available from: NTIS; PB 81159618.

NBSIR 81-2191 (DoE). Johnson, C. E.; Ugiansky, G. M. The use of the slow strain rate technique for the evaluation of structural materials for application in high-temperature gaseous environments. 1981 January. 92 p. Available from: NTIS; PB 81-179152.

NBSIR 81-2228 (NBS). Gerhold, W. F.; Escalante, E.; Sanderson, B. $T$. The corrosion behavior of selected stainless steels in soil environments. 1981 February. 80 p. Available from: NTIS; PB 81.
178154.

NBSIR 81-2243. Gerhold, W. F.; Fink, J. L. Corrosion evaluation of underground telephone cable shielding materials. 1981 April. $77 \mathrm{p}$. Available from: NTIS; PB 82-135187.

NBSIR 81-2244. Carino, N. J. Temperature effects on the strengthmaturity relation of mortar. 1981 March. 99 p. Available from: NTIS; PB 81.183410.

NBSIR 81-2273. Brown, P. W.; Clifton, J. R. Factors affecting the soundness of blended cements. 1981 June. 26 p. Available from: NTIS; PB 82-104746.

NBSIR 81-2295. Manning, J. R. NBS: Materials measurements. 1981 June. 149 p. A vailable from: NTIS; PB 81.238537.

NBSIR 81-2390. Wu, S. T.; Clifton, J. R. Analysis and modeling of corrosion of steel in prestressed concrete. 1981 November. $25 \mathrm{p}$. Available from: NTIS; PB 82-141771.

NBSIR 81-2396 (ONR). Kruger, J.; Ritter, J. J.; Carroll, J. J.; Melmed, A. J. Passive films, surface structure and stress corrosion and crevice corrosion susceptibility. 1981 November. 67 p. A vailable from: NTIS; ADA078596.

U.S. Patent $4,283,461$. Edelman, S. Piezoelectric polymer antifouling coating. 11 August $1981.6 \mathrm{p}$.

U.S. Patent 4,297,394. Edelman, S. Piezoelectric polymer antifouling coating and method of use and application. 27 October 1981. 5 p.

19963. Escalante, E. Soils and underground corrosion, Proc. 17th Annual Liberty Bell Corrosion Course, Philadelphia, PA, Sept. 19-21, 1979, 23 pages (1979).

20016. Hsu, S. M.; Becker, D. A. Status report on the characterization of re-refined lubricating base oils, (Proc. Conf. on Fuels and Lubricants, Baltimore, MD, Oct. 20-23, 1980), Paper in $S A E$ Technical Paper Series No. 801382, pp. 1-10 (Society of Automotive Engineers, Inc., Warrendale, PA, 1980).

20025. Revesz, A. G.; Kruger, J. The role of noncrystalline films in passivation and breakdown of passivation, Proc. 7th Int. Conf. on Metallic Corrosion, Rio de Janiero, Brazil, Oct. 4-11, 1978, pp. 330340 (ABRACO, Rio de Janiero, Brazil, 1979).

20026. Ugiansky, G. M.; Kruger, J.; Staehle, R. W. An electrochemical study of an Al-Zn-Mg alloy, Proc. 7th Int. Conf. on Metallic Corrosion, Rio de Janiero, Brazil, Oct. 4-11, 1978, pp. $605-613$ (ABRACO, Rio de Janiero, Brazil, 1979).

20027. Ugiansky, G. M.; Johnson, C. E. Stress corrosion testing at elevated temperatures in simulated coal conversion gases, Proc. 7 th Int. Conf. on Metallic Corrosion, Rio de Janiero, Brazil, Oct. 4-11, 1978, pp. 915.924 (ABRACO, Rio de Janiero, Brazil, 1979).

20119. Wilson, W. K.; Parks, E. J. Comparison of accelerated aging of book papers in 1937 with 36 years natural aging, Restaurator 4, No. 1, 1.55 (1980).

20202. Blau, P. J. Observations on the wear-in process during the sliding of several copper alloys against $\mathbf{5 2 1 0 0}$ steel, (Proc. 3d Int. Conf. Wear of Materials, San Francisco, CA, Mar. 30-Apr. 2, 1981), Paper in Wear of Materials-1981, S. K. Rhee, A. W. Ruff, and K. C. Ludema, eds., pp. 69.74 (The American Society of Mechanical Engineers, New York, NY, 1981).

20209. Ruff, A. W.; Ives, L. K. Sliding wear behavior of electron beam surface melted 0.2 tool steel, (Proc. 3d Int. Conf. Wear of Materials, San Francisco, CA, Mar. 30-Apr. 2, 1981), Paper in Wear of Materials-1981, S. K. Rhee, A. W. Ruff, and K. C. Ludema, eds., pp. 326-334 (The American Society of Mechanical Engineers, New York, NY, 1981).

20213. Blau, P. J. A simple method for cross-sectional examination of wear debris flakes, Wear (Short Commun.) 66, 257.258 (1981).

20269. Ritter, J. J.; Kruger, J. A new ellipsometric-electrochemical approach to the study of corrosion under organic coatings, (Proc. Symp. Advances in Coating-Metals for Corrosion Protection, Las Vegas, NV, Aug. 27, 1980), Preprint Division of Polymer Chemistry, American Chemical Society 43, 575-580 (1980).

20334. Ogburn, F.; Schoonover, R. M.; Johnson, C. E. Density of autocatalytic nickel-phosphorus deposits, Plating Surf. Finish., 45 pages (Mar. 1981).

20348. Escalante, E.; Bertocci, U.; Mullen, J.; Cohen, M.; Kruger, J. Development of in situ techniques for the detection of corrosion of copper concentric neutrals of electric cables in underground 
environments, Proc. IEEE Power Engineering Society 1981 Transmission and Distribution Conf. \& Exposition, Minneapolis, MN, Sept. 20-25, 1981, Paper No. 81 TD 623-8, pp. 1.7 (1981).

20351. Kruger, J. Corrosion. Its character and consequences, $\mathrm{Am}$. Soc. Test. Mater. Std. News 9, No. 5, 21.23, 48 (May 1981).

20436. Strobridge, T. R.; Moulder, J. C.; Clark, A. F. Titanium combustion in turbine engines, Dept. Energy Report No. FAA-RD-79. 51, 133 pages (Available as ADA.075.657 to the U.S. public through the National Technical Information Service, Springfield, VA 22161, July 1979).

20552. Senich, G. A. Migration to and from plastics, $C H E M T E C H 11$, No. 6, 360-365 (June 1981).

20587. Blau, $P$. J. Interpretations of the friction and wear break-in behavior of metals in sliding contact, Wear 71, 29.43 (1981).

20612. Polvani, R. S.; Christ, B. W. Microcreep of instrument grade beryllium, SAMPE Q. 10, No. 4, 37.41 (July 1979).

20667. Blau, P. J. An investigation of the unlubricated friction and wear break-in behavior of a dual-phase steel, Wear 72, 67.80 (1981).

20668. Blau, P. J. Mechanisms for transitional friction and wear behavior of sliding metals, Wear 72, 55-66 (1981).

20774. Dickens, B. Pressure as an important variable in thermogravimetric studies of polymer degradations, Paper in Proceedings of the Second European Symposium on Thermal Analysis, University of Alberdeen, United Kingdom, Sept. 1-4, 1981, 219.222 (Heydon \& Son, Hillview Gardens, London, UK, 1981).

\section{Properties of Materials: Electronic, Magnetic, and Optical}

$\mathrm{Yu}, \mathrm{H}$. Application of light scattering to polymers, liquid crystals, and biological systems. J. Res. Nat. Bur. Stand. (U.S.). 86(6): 571.590; 1981 November-December.

NBSIR 78-896. Jesch, R. L. Dielectric measurements of five different soil textural types as functions of frequency and moisture content. 1978 October. 26 p. Available from: NTIS; PB 291944.

NBSIR 78-897. Jones, R. N.; Bussey, H. E.; Little, W. E.; Metzker, R. F. Electrical characteristics of corn, wheat, and soya in the 1-200 MHz range. 1978 October. 70 p. Available from: NTIS; PB 289812.

19962. Hebner, R. E., Jr. Kerr effect, electro-optical, Article in Encyclopedia of Physics, R. G. Lerner and G. L. Trigg, eds., p. 483 (Addison-Wesley Publ. Co., Inc., Advanced Book Program, Reading, MA, 1981).

19979. Hall, J. T.; Holdeman, L. B.; Soulen, R. J., Jr. Microwave enhancement of superconductivity in aluminum tunnel junctions, Phys. Rev. Lett. 45, No. 12, 1011-1014 (Sept. 22, 1980).

20008. Girvin, S. M.; Penn, D. R. Theory of coherence effects in photoinduced Auger events: Resonant satellites in metals with filled $d$ bands, Phys. Rev. B 22, No. 8, 4081.4084 (Oct. 15, 1980).

20043. Romanov, V. P.; Candela, G. A.; Roth, R. S.; Swartzendruber, L. J. Magnetic properties and hyperfine interactions in the $\beta^{\prime \prime \prime}$ phase of potassium ferrite, J. Appl. Phys. 50, No. 10, $6455-6458$ (Oct. 1979).

20056. Sengers, J. V.; Bedeaux, D.; Mazur, P.; Greer, S. C. Behavior of the dielectric constant of fluids near a critical point, Physica 104A, 573-594 (1980).

20104. Ekin, J. W. Strain scaling law and the prediction of uniaxial and bending strain effects in multifilamentary superconductors, (Proc. Topical Conf., Upton, NY, May 28-29, 1980), Paper in Filamentary A15 Superconductors, M. Suenaga and A. F. Clark, ed., pp. 187.203 (Plenum Press, New York, NY, 1980).

20128. Rendell, R. W.; Penn, D. R. Spin dependence of the electron mean free path in Fe, Co, and Ni, Phys. Rev. Lett. 45, No. 25, 2057. 2060 (Dec. 22, 1980).

20140. Sullivan, D. B.; Hamilton, C. A.; Kautz, R. L. Recent progress in cryoelectronics, IEEE Trans. Instrum. Meas. IM-29, No. 4, 319. 323 (Dec. 1980).

20145. Rhyne, J. J. Magnetic inelastic neutron scattering studies of rare earth metals and Laves-phase compounds, Chapter 13 in Science and Technology of Rare Earth Materials, E. C. Subbarao and W. E. Wallace, eds., pp. 261-290 (Academic Press, Inc., New York, NY, 1980).
20160. Magerl, A.; Rowe, J. M.; Richter, D. Observation of lowenergy excitations in NbD: A simple lattice-dynamical model, Phys. Rev. B 23, No. 4, 1605.1608 (Feb. 15, 1981).

20173. Schroeder, L. W.; Jordan, T. H.; Brown, W. E. Raman spectrum from a single crystal of $\mathrm{SnHPO}_{4}$, Spectrochim. Acta 37A, 21.29 (1981).

20191. Cezairliyan, A. Electrical resistivity of molybdenum in the temperature range 1500 to $2650 \mathrm{~K}$, Int. J. Thermophys. 1, No. 4, 417. 427 (1980).

20192. Geist, J.; Lowney, J. R. Effect of band-gap narrowing on the built-in electric field in $n$-type silicon, $J$. Appl. Phys. 52, No. 2, 1121 . 1123 (Feb. 1981).

20210. Bennett, L. H. Nuclear magnetic resonance in alloys, (Proc. Materials Research Society Ann. Meet., Boston, MA, Nov. 17.20, 1980), Paper in Nuclear and Electron Resonance Spectroscopies Applied to Materials Science, Kaufman and Shenoy, eds., pp. 3-18 (Elsevier North Holland, Inc., New York, NY, 1981)

20212. Koon, N. C.; Rhyne, J. J. Ground-state spin dynamics of ErCo $_{2}$, Phys. Rev. B 23, No. 1, 207.214 (Jan. 1, 1981).

20225. Thijsse, $B$. J. The dielectric constant of $\mathrm{SF}_{6}$ near the critical point, J. Chem. Phys. 74, No. 8, 4678-4692 (Apr. 15, 1981).

20260. Ekin, J. W. Strain scaling law for flux pinning in $\mathbf{N b T i}, \mathbf{N b}_{3} \mathrm{Sn}$, $\mathrm{Nb}-\mathrm{Hf} / \mathrm{Cu}-\mathrm{Sn}-\mathrm{Ga}, \mathrm{V}_{3} \mathrm{Ga}$ and $\mathrm{Nb}_{3} \mathrm{Ge}$, IEEE Trans. Magn. MAG-17, No. 1, 658.661 (Jan. 1, 1981).

20266. Link, J.; Wintersgill, M. C.; Fontanella, J. J.; Bean, V. E.; Andeen, C. G. Pressure variation of the low-frequency dielectric constants of some anisotropic crystals, J. Appl. Phys. 52, No. 2, 936. 939 (Feb. 1981).

20283. Fernandez-Baca, J. A.; Lynn, J. W. Neutron scattering studies of the magnetic superconductor $\left(\mathrm{Ce}_{1-\mathrm{x}} \mathrm{Tb}_{\mathrm{x}}\right) \mathrm{Ru}_{2}, J$. Appl. Phys. 52, No. 3, 2183.2185 (Mar. 1981).

20284. McDonald, D. G. Superconducting electronics, Phys. Today 34, No. 2, 37-47 (Feb. 1981).

20317. Goldfarb, R. B.; Rao, K. V.; Fickett, F. R.; Chen, H. S. Magnetic susceptibility studies of amorphous Ni-Mn-P.B-Al alloys, $J$. Appl. Phys. 52, No. 3, 1744-1746 (Mar. 1981)

20318. Suenram, R. D.; Lovas, F. J. Millimeter wave spectrum of glycine. A new conformer, J. Am. Chem. Soc. 102, No. 24, 7180.7184 (Nov. 19, 1980).

20341. Marshak, H. Nuclear orientation of ${ }^{166 m} \mathrm{Ho}_{0}$ in ${ }^{165} \mathrm{Ho}$ single crystal, Hyperfine Interact. 10, $1183-1188$ (1981).

20398. Casella, R. C. Isotopic shifts in complex crystals, Phys. Rev. $B$ 17 , No. 8, 3381.3386 (Apr. 15, 1978)

20464. Rendell, R. W.; Girvin, S. M. Hall voltage dependence on inversion-layer geometry in the quantum Hall-effect regime, Phys. Rev. B 23, No. 12, 6610-6614 (June 15, 1981).

20469. Rhyne, J. J. Magnetic materials, Article in Encyclopedia of Physics, pp. 547.550 (Addison-Wesley Publ. Co., Inc., Reading, MA, 1981).

20471. Broadhurst, M. G.; Davis, G. T. Ferroelectric polarization in polymers, Ferroelectrics 32, 177-180 (1981).

20497. Bethin, J. R.; Chiang, C. K.; Franklin, A. D.; Snellgrove, R. A. An oxygen-sensitive electrode impedance in Sr-doped $\mathrm{LaCrO}_{3}, J$. Appl. Phys. 52, No. 6, 4115-4117 (June 1981).

20520. Turrell, B. G.; Marshak, H. Magnetic structure determined by nuclear orientation, Hyperfine Interact. 10, 1231.1236 (1981).

20529. Bur, A. J.; Tsao, A. K. Depolarization of poled PVF, samples with "thick" electrodes, Ferroelectrics 32, $185-189$ (1981).

20564. Rendell, R. W.; Penn, D. R. Spin dependent attenuation lengths in ferromagnets, J. Appl. Phys. 52, No. 3, 1620-1621 (Mar. 1981).

20638. Bur, A. J. Resistivity and piezoelectric measurements on oriented and unoriented thick PVF, films, Polymer 22, 1288-1289 (Sept. 1981).

20639. Broadhurst, M. G.; Davis, G. T.; DeReggi, A. S. Dielectric properties of PVDF: Low-field and high-field phenomena and time effects, Proc. Symp. High-Energy-Density Capacitors and Dielectric Materials, Boston, MA, Oct. 28, 1980, pp. 67-77 (National Academy Press, Washington, DC, 1981).

20655. Chiang, C. K. An all-polymeric solid state battery, Polymer 22, 1454-1456 (Nov. 1981).

20657. Kautz, R. L. Chaotic states of rf-biased Josephson junctions, J. 
Appl. Phys. 52, No. 10, 6241.6246 (Oct. 1981).

20659. Ekin, J. W.; Sekine, H.; Tachikawa, T. Effect of strain on the critical current of Nb-Hf/Cu-Sn-Ga multifilamentary superconductors, J. Appl. Phys. 52, No. 10, 6252.6256 (Oct. 1981).

20664. Forster, E. O.; FitzPatrick, G. J.; Hebner, R. E., Jr.; Kelley, E F. Observations of prebreakdown and breakdown phenomena in liquid hydrocarbons II. Point-plane geometry, (Proc. Conf. Electrical Insulation and Dielectric Phenomena, Whitehaven, PA, Oct. 26-28, 1981), IEEE Ann. Report No. 81CH1668-3, pp. 377.389 (The Institute of Electrical and Electronics Engineers, Inc., 445 Hoes Lane, Piscataway, NJ 08854, 1981).

20702. Soulen, R. J., Jr.; Van Vechten, D.; Costabile, G.; Jach, T.; Holdeman, L. B. The superconductive energy gap of $\mathbf{A u A l}_{2}$, Physica 108B, 823.824 (1981).

20716. Fowler, H. A.; Filliben, J. J. Dielectric function for ultraviolet plasmons in the short-lifetime limit, J. Appl. Phys. 52, No. 11, 6701. 6703 (Nov. 1981).

20723. Holdeman, L. B.; Hall, J. T.; VanVechten, D.; Soulen, R. J., Jr. Gap enhancement in aluminum tunnel junctions coupled to microstrip resonators, Physica 108B, 827.828 (1981)

\section{Properties of Materials: Structural and Mechanical}

Malkus, D. S.; Fuller, E. R., Jr. An isoparametric finite element model for large-strain elastostatics. J. Res. Nat. Bur. Stand. (U.S.). 86(1): 79.109; 1981 January.February.

Monogr. 25, Section 18. Morris, M. C.; McMurdie, H. F.; Evans, E. H.; Paretzkin, B.; Parker, H. S.; Panagiotopoulos, N. C.; Hubbard C. R. Standard x-ray diffraction powder patterns. Section 18-Data for 58 substances. Nat. Bur. Stand. (U.S.) Monogr. 25, Sec. 18; 1981 October. 110 p. SN003-003-02370.1.

TN1141. Mighell, A. D.; Hubbard, C. R.; Stalick, J. K. NBS*AIDS80: A FORTRAN program for crystallographic data evaluation. Nat. Bur. Stand. (U.S.) Tech. Note 1141; 1981 April. 54 p. SN003-003. 02309.4.

NBSIR 79-1623. McHenry, H. 1.; Rolfe, S. T. Fracture control practices for metal structures. 1980 January. 114 p. Available from: NTIS; PB 80-185648.

NBSIR 79.1960 (DoE). Fields, R. J.; Tighe, N. J.; Fuller, E. R., Jr. Effect of strain rate on the fracture toughness of silicon nitride at $1400^{\circ}$ C. 1980 October. 24 p. Available from: NTIS; PB 81-166670.

NBSIR 81-2209 (FDA). McKenna, G. B.; Khoury, F. A.; Crissman, J. M. Relationship between morphology and mechanical properties of ultra high molecular weight polyethylene. 1981 April. 63 p. Available from: NTIS; PB 82.139072.

NBSIR 81-2229. Snyder, R. L.; Hubbard, C. R.; Panagiotopoulos, N. C. Auto: A real time diffractometer control system. 1981 February. 108 p. A vailable from: NTIS; PB 81.179186.

19980. Ditchek, B.; Ruff, A. W. Discussion of the paper "The study of fatigue mechanisms with electron channeling," by D. Davidson, (Proc. ASTM Symp., Kansas City, MO, May 22-23, 1978), Discussion in Am. Soc. Test. Mater. Spec. Tech. Publ. 675, 269-275 (1979).

19984. Mathew, M.; Brown, W. E.; Austin, M.; Negas, T. Lead alkali apatites without hexad anion: The crystal structure of $\mathrm{Pb}_{8} \mathrm{~K}_{2}\left(\mathrm{PO}_{4}\right)_{6}$, J. Solid State Chem. 35, 69-76 (1980).

19994. Jordan, T. H.; Dickens, B.; Schroeder, L. W.; Brown, W. E. Crystal structure of tritin (II) hydroxide oxide phosphate, $\mathrm{Sn}_{3} \mathrm{O}(\mathrm{OH}) \mathrm{PO}_{4}$, Inorg. Chem. 19, No. 9, 2551.2556 (Sept. 1980).

20007. Rabolt, J. F.; Block, S.; Piermarini, G. J. The characterization of polymers under high pressure using Raman spectroscopy, (Proc. Sixth AIRAPT Conf. on High-Pressure Science and Technology, Boulder, CO, July 25.29, 1977), Paper in Physical Properties and Material Synthesis, K. D. Timmerhaus and M. S. Barber, eds., 1, 478.481 (Plenum Press, New York, NY, 1979).

20014. Peterlin, A. Acoustic emission of polymers under tensile load, Chapter 2 in Advances in Chemistry Series No. 174, Probing Polymer Structures, J. L. Koenig, ed., pp. $15-23$ (American Chemical Society, Washington, DC, 1979).
20033. Murray, J. L.; Orser, D. J. Interactive computer graphics for storing phase diagrams, Bull. Alloy Phase Diagrams 1, No. 1, 19-22 (1980).

20037. Shukla, R. C.; MacDonald, R. A. Thermal expansion of cubic crystals, High Temp. High Pressures 12, 291.296 (1980).

20044. Parker, R. L. Crystal growth, Article in Encyclopedia of Physics, R. G. Lerner and G. L. Trigg, eds., pp. 175.176 (Addison-Wesley Publ. Co., Inc., Advanced Book Program, Reading, MA, 1981).

20046. Roth, R. S. Thermal stability of long range order in oxides, Prog. Solid State Chem. 13, No. 2, 159.192 (1980).

20054. Santoro, A.; Marezio, M.; Roth, R. S.; Minor, D. Neutron powder diffraction study of the structures of $\mathrm{CeTaO}_{4}, \mathrm{CeNbO}_{4}$, and $\mathrm{NdTaO}_{4}$, J. Solid State Chem. 35, 167.175 (1980).

20063. Evans, D. J.; Hanley, H. J. M. A thermodynamics of steady homogeneous shear flow, Phys. Lett. 80A, No. 2-3, $175-177$ (Nov. 24, 1980).

20065. Mathew, M.; Mayer, I.; Dickens, B.; Schroeder, L. W. Substitution in barium-fluoride apatite: The crystal structures of $\mathrm{Ba}_{10}\left(\mathrm{PO}_{4}\right)_{6} \mathrm{~F}_{2}, \mathrm{Ba}_{6} \mathrm{La}_{2} \mathrm{Na}_{2}\left(\mathrm{PO}_{4}\right)_{6} \mathrm{~F}_{2}$ and $\mathrm{Ba}_{4} \mathrm{Nd}_{3} \mathrm{Na}_{3}\left(\mathrm{PO}_{4}\right)_{6} \mathrm{~F}_{2}, J$. Solid State Chem. 28, 79.95 (1979).

20091. Penn, R. W.; McKenna, G. B. Fatigue effects in poly(methyl methacrylate), (Proc. 176th Meet. ACS Symp. Series, Miami, FL, Sept. 11.14, 1978), Chapter 23 in Durability of Macromolecular Materials, pp. 331.339 (American Chemical Society, Washington, DC, 1979).

20117. Roth, R. S. Phase equilibria research in portions of the system $\mathrm{K} 2 \mathrm{O} \cdot \mathrm{MgO} \cdot \mathrm{Fe}_{2} \mathrm{O}_{3} \cdot \mathrm{Al}_{2} \mathrm{O}_{3} \cdot \mathrm{SiO}_{2}$, (Proc. Symp. Solid State Chemistry: A Contemporary Overview, Laramie, WY, July 30. Aug. 4, 1978), Paper in Advances in Chemistry Series, No. 186, pp. 391.408 (American Chemical Society, Washington, DC, 1980).

20126. Bernstein, B.; Zapas, L. J. Stability and cold drawing of viscoelastic bars, J. Rheol. 25, No. 1, 83.94 (1981).

20130. McKenna, G. B.; Zapas, L. J. Viscoelastic behavior of poly(methyl methacrylate): Prediction of extensional response from torsional data, (Proc. VIII Int. Congress on Rheology, Naples, Italy, Sept. 1-5, 1980), Paper in Rheology, Applications (1980), G. Astarita, G. Marrucci, and L. Nicolais, eds., 3, 299-307 (Plenum Publishing Corp., New York, NY, 1980).

20132. Boettinger, W. J.; Biancaniello, F. S.; Coriell, S. R. Solutal convection induced macrosegregation and the dendrite to composite transition in off-eutectic alloys, Metall. Trans. A 12A, 321.327 (Feb. 1981).

20159. Mordfin, L. Measurement of residual stresses: Problems and opportunities, Proc. Conf. Residual Stress for Designers and Metallurgists, Chicago, IL, Apr. 9-10, 1980, L. J. Vande Walle, ed., pp. 189-209 (American Society for Metals, Metal Park, OH, 1981).

20214. Hardman, K.; Rhyne, J. J.; Smith, K.; Wallace, W. E. Structural investigation of $\mathbf{T h}_{6} \mathbf{M n}_{23} \mathrm{D}_{16}$ by neutron diffraction, $J$. Less-Common Met. 74, 97.102 (1980).

20222. Scarfe, C. M.; Cronin, D.; Wenzel, J.; Kauffman, D. Viscositytemperature relationships at $1 \mathrm{ATM}$ in the system diopside-anorthite, Paper in Carnegie Institution of Washington Year Book 79. Annual Report of the Director Geophysical Laboratory, No. 1825, pp. 315.318 (Carnegie Institute of Washington, Washington, DC, Dec. 1980).

20224. Coriell, S. R.; Cordes, M. R.; Boettinger, W. J.; Sekerka, R. F. Effect of gravity on coupled convective and interfacial instabilities during directional solidification, (Proc. Twenty-Third COSPAR Plenary Meet., Budapest, Hungary, June 2.14, 1980), Paper in $A d v$. Space Res. 1, $5-11$ (COSPAR, Great Britain, 1981).

20258. Mathew, M.; Takagi, S.; Waerstad, K. R.; Frazier, A. W. The crystal structure of synthetic chukhrovite, $\mathrm{Ca}_{4} \mathrm{AlSi}\left(\mathrm{SO}_{4}\right) \mathrm{F}_{13} \cdot 12 \mathrm{H}_{2} \mathrm{O}$, Am. Mineral. 66, 392.397 (1981).

20271. Brown, W. E.; Chow, L. C. Thermodynamics of apatite crystal growth and dissolution, J. Cryst. Growth 53, 31.41 (1981).

20273. Hamilton, J. C.; Jach, T. Structural phase transitions in nickel at the Curie temperature, Phys. Rev. Lett. 46, No. 11, 745.748 (Mar. 16, 1981).

20280. Ledbetter, H. M. Sound velocities and elastic constants of steels 304, 310, and 316, Met. Sci. Tech. Note 14, No. 12, 595.596 (Dec. 1980).

20282. Lynn, J. W.; Erwin, R. W.; Rhyne, J. J.; Chen, H. S. Spin 
glass-ferromagnetic phase transition in amorphous $\left(\mathrm{Fe}_{\mathbf{x}} \mathbf{N i}_{1-\mathrm{x}}\right)_{0.75} \mathbf{P}_{\mathbf{0 . 1 6}} \mathbf{B}_{0.06} \mathbf{A l}_{\mathbf{0 . 0 3}}$, J. Appl. Phys. 52, No. 3, 1738.1740 (Mar. 1981)

20299. Zapas, L. J.; Crissman, J. M. Static fatigue of polyethylene in uniaxial creep in the presence of stress-cracking agents and solvent, (Proc. Symp. 178th Meet. American Chemical Society, Washington, DC, Sept. 10-14, 1979), Org. Coat. Plast. Chem. 40, 469-474 (American Chemical Society, Washington, DC, 1979).

20315. Snyder, R. G.; Scherer, J. R.; Peterlin, A. Low-frequency Raman study of drawn polyethylene, Macromolecules 14, 77.82 (1981).

20327. Crissman, J. M.; Zapas, L. J. Lifetime behavior of polyethylene sheets under inflation in the presence of stress-cracking agent, (Proc. Symp. 178th Meet. American Chemical Society, Washington, DC, Sept. 10.14, 1979), Org. Coat. Plast. Chem. 40, 475.481 (American Chemical Society, Washington, DC, 1979).

20382. Waterstrat, R. M. Structure relationships affecting the stability of A15. and $\mathrm{Ti}_{3} \mathrm{P}$.type compounds, J. Solid State Chem. 37, 370-382 (1981).

20407. Brown, W. E.; Mathew, M.; Tung, M. S. Crystal chemistry of octacalcium phosphate, Prog. Cryst. Growth Charact. 4, $59-87$ (1981).

20443. DeVries, K. L.; Smith, R. H.; Fanconi, B. M. Free radicals and new end groups resulting from chain scission: 1. $\gamma$-irradiation of polyethylene, Polymer 21, 949-956 (Aug. 1980).

20448. Coriell, S. R.; Boisvert, R. F.; Rehm, R. G.; Sekerka, R. F. Lateral solute segregation during unidirectional solidification of a binary alloy with a curved solid-liquid interface. II. Large departures from planarity, J. Cryst. Growth 54, No. 2, 167.175 (Aug. 1981).

20482. Han, C. C.; Akcasu, A. Z. On the dynamic structure factor, $S(q, \omega)$, of polymers in dilute solution, Polymer 22, 1019-1025 (1981).

20486. Waterstrat, R. M. Analysis of selected alloys in the systems CrPd, Cr-Ru, V-Pd and Ta-Pt, J. Less-Common Met. 80, P31-P36 (1981).

20528. Whipple, T. A.; McHenry, H. I.; Read, D. T. Fracture behavior of ferrite-free stainless steel welds in liquid helium, (Proc. 61st AWS Annu. Meet., Los Angeles, CA, Apr. 13-18, 1980), Paper in Welding Research Supplement 60, No. 4, 72-s-78-s (Apr. 1981).

20557. Kasen, M. B. Mechanical properties of plastic composites unde low temperature conditions, Composites, Lett. Ed., 12, No. 2, 107 (Apr. 1981).

20600. Prince, E. Comparison of profile and integrated-intensity methods in powder refinement, J. Appl. Cryst. 14, 157-159 (1981).

20608. Datta, S. K.; Ledbetter, H. M. Anisotropic elastic constants of a fiber-reinforced boron-aluminum composite, Proc. Mechanics of Nondestructive Testing, Blacksburg, VA, Sept. 10-12, 1980, pp. 215 230 (Plenum Press, New York, NY, 1980).

20635. Weeks, J. J.; Clark, E. S.; Eby, R. K. Crystal structure of the low temperature phase (II) of polytetrafluoroethylene, Polymer 22, 1480-1486 (Nov. 1981).

20652. Eby, R. K.; Peterlin, A.; Khoury, F. A.; Broadhurst, M. G. Fanconi, B. M. Crystalline polymers, Chapter 2 in Polymer Science and Engineering: Challenges, Needs, and Opportunities, pp. 49.57 (National Research Council, Washington, DC, Nov. 1981).

20707. Waterstrat, R. M. Binary phase diagrams of transition elements, Bull. Alloy Phase Diagrams 2, No. 1, $35-38$ (June 1981).

20708. Weeks, J. J.; Eby, R. K.; Clark, E. S. Disorder in the crystal structures of phases I and II of copolymers of tetrafluoroethylene and hexafluoropropylene, Polymer 22, 1496-1499 (Nov. 1981).

20715. Farmer, B. L.; Eby, R. K. Energy calculations of the crystal structure of the low temperature phase (II) of polytetrafluoroethylene, Polymer 22, 1487-1495 (Nov. 1981)

20726. Ledbetter, H. M. Elastic constants of polycrystalline copper at low temperatures, Phys. Status Solidi (A) 66, $477-484$ (1981).

20732. Peterlin, A. Tensile failure of crystalline polymers, $J$. Macromol. Sci. Phys. B19, No. 3, 401.419 (1981).

20736. Swartzendruber, L. J.; Bennett, L. H.; Ives, L. K.; Shull, R. D. The Ti-Al phase diagram: The $\alpha-\alpha_{2}$ phase boundary, Mater. Sci. Eng. 51, PI-P9 (1981).

20773. Ledbetter, H. M. Predicted single-crystal elastic constants of stainless-steel 316, Br. J. Non Destr. Test. 23, No. 6, 286-287 (Nov. 1981).

\section{Properties of Materials: Thermodynamic and Transport}

deCastro, C. A. N.; Roder, H. M. Absolute determination of the thermal conductivity of argon at room temperature and pressures up to $68 \mathrm{MPa}$. J. Res. Nat. Bur. Stand. (U.S.). 86(3): 293-307; 1981 May-June.

NBSIR 81-2264. Smith, L. E.; Chang, S. S.; McCrackin, F. L.; Senich, G. A.; Wang, F. W. Models for the migration of low molecular weight additives in polyolefins. 1981 April. 141 p. Available from: NTIS; PB 81.202749

19981. Cahn, J. W. Thermodynamics of metastable equilibria, Proc. Second Rapid Solidification Processing: Principles and Technologies, Reston, VA, Mar. 23-26, 1980, pp. 24-34 (Claitor's Publ. Div., Baton Rouge, LA, Nov. 1980)

20038. Manning, J. R. Effect of correlated jumps and vacancy wind interactions on atom jump frequencies and diffusion fluxes in a driving force, Scr. Metall. 15, 1-6 (1981).

20137. Cezairliyan, A.; Miiller, A. P. Specific heat capacity and electrical resistivity of a carbon-carbon composite in the range 1500-3000 $\mathrm{K}$ by a pulse heating method, Int. J. Thermophys. 1, No. 3, 317-330 (1980).

20344. Peterlin, A.; McCrackin, F. L. Empirical relation for diffusion of gases in hydrocarbon polymers: Interpretation in terms of fractional free volume, J. Polym. Sci.: Polym. Phys. Ed. 19, 1003-1006 (1981).

20455. Watson, R. E.; Bennett, L. H. Optimized prediction for heats of formation of transition metal alloys, CALPHAD 5, No. $1,25.40$ (1981).

20494. Hust, J. G.; Boscardin, R. Thermal conductivity of polyesteramide-imide film, Cryogenics 21, No. 5, 297.298 (May 1981).

20527. Evans, D. J.; Hanley, H. J. M. Soft-disc melting in small systems, Phys. Lett. 83A, No. 7, 344-346 (June 15, 1981).

20571. MacDonald, R. A.; MacDonald, W. M. Thermodynamic properties of fcc metals at high temperatures, Phys. Rev. B 24, No. 4 , 1715-1724 (Aug. 15, 1981).

20651. Ely, J. F.; Hanley, H. J. M. Prediction of the viscosity and thermal conductivity in hydrocarbon mixtures-Computer program TRAPP, Proc. 60th Gas Processors Association Annu. Conv., San Antonio, TX, Mar. 23-25, 1981, pp. 20.29 (Gas Processors Association, Tulsa, OK, 1981)

20687. Mountain, R. D. Time correlation functions and molecular motion in glasses, Ann. NY Acad. Sci. 371, 252-260 (1981)

20721. Han, C. C.; Akcasu, A. Z. Concentration dependence of diffusion coefficient at various molecular weights and temperatures, Polymer 22, 1165-1168 (Sept. 1981).

\section{Standard Reference Data}

Goldberg, R. N. Evaluated activity and osmotic coefficients for aqueous solutions: Bi-univalent compounds of zinc, cadmium, and ethylene bis(trimethylammonium) chloride and iodide. J. Phys. Chem. Ref. Data. 10(1): 1.56; 1981.

Gaur, U.; Wunderlich, B. Heat capacity and other thermodynamic properties of linear macromolecules. II. Polyethylene. J. Phys. Chem. Ref. Data. 10(1): 119-152; 1981.

Martin, W. C.; Zalubas, R. Energy levels of sodium, Na I through Na XI. J. Phys. Chem. Ref. Data. 10(1): 153-196; 1981

Corliss, C.; Sugar, J. Energy levels of nickel, Ni I through Ni XXVIII. J. Phys. Chem. Ref. Data. 10(1): 197-290; 1981

Kestin, J.; Khalifa, H. E.; Correia, R. J. Tables of the dynamic and kinematic viscosity of aqueous $\mathrm{KCl}$ solutions in the temperature range $25-150^{\circ} \mathrm{C}$ and the pressure range 0.1-35 MPa. J. Phys. Chem. Ref. Data. 10(1): 57.70; 1981

Kestin, J.; Khalifa, H. E.; Correia, R. J. Tables of the dynamic and kinematic viscosity of aqueous $\mathrm{NaCl}$ solutions in the temperature range 20-150 ${ }^{\circ} \mathrm{C}$ and the pressure range 0.1-35 $\mathrm{MPa}, J$. Phys. Chem. Ref. Data. 10(1): 71-88; 1981

Gaur, U.; Shu, H. C.; Mehta, A.; Wunderlich, B. Heat capacity and 
other thermodynamic properties of linear macromolecules. I. Selenium. J. Phys. Chem. Ref. Data. 10(1): 89-118; 1981.

Marshall, W. L.; Franck, E. U. Ion product of water substance, $0-1000^{\circ} \mathrm{C}, 1-10,000$ bars. New international formulation and its background. J. Phys. Chem. Ref. Data. 10(2): 295-304; 1981.

Fuhr, J. R.; Martin, G. A.; Wiese, W. L.; Younger, S. M. Atomic transition probabilities for iron, cobalt, and nickel (A critical data compilation of allowed lines). J. Phys. Chem. Ref. Data. 10(2): 305. $566 ; 1981$.

Haas, J. L., Jr.; Robinson, G. R., Jr.; Hemingway, B. S. Thermodynamic tabulations for selected phases in the system $\mathrm{CaO}$ $\mathrm{Al}_{2} \mathrm{O}_{3}-\mathrm{SiO}_{2}-\mathrm{H}_{2} \mathrm{O}$ at $101.325 \mathrm{kPa}(1 \mathrm{~atm})$ between 273.15 and 1800 K. J. Phys. Chem. Ref. Data. 10(3): 575-670; 1981.

Goldberg, R. N. Evaluated activity and osmotic coefficients for aqueous solutions: Thirty-six uni-bivalent electrolytes. J. Phys. Chem. Ref. Data. 10(3): 671-766; 1981.

Staples, B. R. Activity and osmotic coefficients of aqueous alkali metal nitrites. J. Phys. Chem. Ref. Data. 10(3): 767-778; 1981.

Staples, B. R. Activity and osmotic coefficients of aqueous sulfuric acid at 298.15 K. J. Phys. Chem. Ref. Data. 10(3): 779-798; 1981.

Gaur, U.; Wunderlich, B. Heat capacity and other thermodynamic properties of linear macromolecules. III. Polyoxides. J. Phys. Chem. Ref. Data. 10(4): 1001-1049; 1981.

Gaur, U.; Wunderlich, B. Heat capacity and other thermodynamic properties of linear macromolecules. IV. Polypropylene. J. Phys. Chem. Ref. Data. 10(4): 1051-1064; 1981.

Olson, W. B.; Maki, A. G.; Lafferty, W. J. Tables of $\mathbf{N}_{2} \mathbf{O}$ absorption lines for the calibration of tunable infrared lasers from $522 \mathrm{~cm}^{-1}$ to $657 \mathrm{~cm}^{-1}$ and from $1115 \mathrm{~cm}^{-1}$ to $1340 \mathrm{~cm}^{-1}$.J. Phys. Chem. Ref. Data. 10(4): 1065-1084; 1981

Ozier, I.; Gerry, M. C. L.; Robiette, A. G. Microwave spectra of molecules of astrophysical interest. XX. Methane. J. Phys. Chem. Ref. Data. 10(4): 1085-1095; 1981.

Sugar, J.; Corliss, C. Energy levels of cobalt, Co I through Co XXVII. $J$. Phys. Chem. Ref. Data. 10(4): 1097-1174; 1981.

Mackay, D.; Shiu, W. Y. A critical review of Henry's law constants for chemicals of environmental interest. J. Phys. Chem. Ref. Data. 10(4): 1175-1199; 1981.

Wilkinson, F.; Brummer, J. G. Rate constants for the decay and reactions of the lowest electronically excited singlet state of molecular oxygen in solution. J. Phys. Chem. Ref. Data. 10(4): 809. 999; 1981.

Monogr. 167. Gevantman, L. H., ed. Physical properties data for rock salt. Nat. Bur. Stand. (U.S.) Monogr. 167; 1981 January. 288 p. SN003-003-02166-1.

NSRDS-NBS61, Part IV. Janz, G. J.; Tomkins, R. P. T. Physical properties data compilations relevant to energy storage. IV. Molten salts: Data on additional single and multi-component salt systems. Nat. Stand. Ref. Data Ser., Nat. Bur. Stand. (U.S.) 61, Part IV; 1981 July. 870 p. SN003-003-02339-6.

NSRDS-NBS68. Reader, J.; Corliss, C. H.; Wiese, W. L.; Martin, G. A. Wavelengths and transition probabilities for atoms and atomic ions. Part I. Wavelengths. Part II. Transition probabilities. Nat. Stand. Ref. Data Ser., Nat. Bur. Stand. (U.S.) 68; 1980 December. 415 p. SN003-003-02288-8.

NSRDS-NBS69. Hug, G. L. Optical spectra of nonmetallic inorganic transient species in aqueous solution. Nat. Stand. Ref. Data Ser., Nat. Bur. Stand. (U.S.) 69; 1981 May. 167 p. SN003-003-02322-1.

TN270-8. Wagman, D. D.; Evans, W. H.; Parker, V. B.; Schumm, R. H.; Nuttall, R. L. Selected values of chemical thermodynamic properties. Compounds of uranium, protactinium, thorium, actinium, and the alkali metals. Nat. Bur. Stand. (U.S.) Tech. Note 270-8; 1981 May. 149 p. SN003-003-02318-3.

NBSIR 81-2206. Fivozinsky, S. P., ed. Technical activities 1980Office of Standard Reference Data. 1981 February. 55 p. Available from: NTIS; PB 81-164527.

\section{Standard Reference Materials}

SP260-70. Marinenko, R. B.; Biancaniello, F.; DeRoberts, L.; Boyer, P. A.; Ruff, A. W. Standard reference materials: Preparation and characterization of an iron-chromium-nickel alloy for microanalysis: SRM 479a. Nat. Bur. Stand. (U.S.) Spec. Publ. 260-70; 1981 May. 36 p. SN003-003-02328-1.

SP260-71. Seward, R. W.; Mavrodineanu, R. Standard reference materials: Summary of the Clinical Laboratory Standards issued by the National Bureau of Standards. Nat. Bur. Stand. (U.S.) Spec. Publ. 260-71; 1981 November. 176 p. SN003-003-02381-7.

SP260-72. Reeder, D. J.; Coxon, B.; Enagonio, D.; Christensen, R. G.; Howell, B. F.; Paule, R. C.; Mandel, J.; Schaffer, R. Standard reference materials: SRM 900, antiepilepsy drug level assay standard. Nat. Bur. Stand. (U.S.) Spec. Publ. 260-72; 1981 June. 79 p. SN003-003-02329-9.

SP260, 1981-83 Edition. Seward, R. W. NBS Standard Reference Materials Catalog-1981-83 Edition. Nat. Bur. Stand. (U.S.) Spec. Publ. 260, 1981-83 Edition; 1981 November. 122 p. SN003-003. 02382-5.

NBSIR 81-1639. Smith, D. R.; Hust, J. G. Effective thermal conductivity of a glass fiberboard standard reference material. 1981 February. 32 p. Available from: NTIS; PB 81-181240.

NBSIR 81-1640. Smith, D. R.; Hust, J. G.; Van Poolen, L. J. Effective thermal conductivity of a glass fiberblanket standard reference material. 1981 February. 30 p. Available from: NTIS; PB 81. 176133

NBSIR 81-2246. Olson, G. J.; Iverson, W. P.; Brinckman, F. E. Biodeterioration of standard reference materials. 1981 April. $19 \mathrm{p}$. A vailable from: NTIS; PB 81-203408.

19989. Hertz, H. S. Certification of clinical and environmental trace organic RM's, (Proc. Int. Symp. on the Production and Use of Reference Materials, Bundesanstalt für Materialprüfung (BAM), Berlin, Germany, Nov. 13-16, 1979), Paper in Production and Use of Reference Materials, B. F. Schmitt, ed., pp. $393-398$ (Bundesanstalt für Materialprüfung, Berlin, Federal Republic of Germany, 1980).

19990. Uriano, G. A. The certified reference materials program of the United States National Bureau of Standards, (Proc. Int. Symp. on the Production and Use of Reference Materials, Bundesanstalt für Materialprüfung (BAM), Berlin, Germany, Nov. 13-16, 1979), Paper in Production and Use of Reference Materials, B. F. Schmitt, ed., pp. 7.24 (Bundesanstalt für Materialprüfung, Berlin, Federal Republic of Germany, 1980).

20024. Barnes, I. L. The use of isotope dilution mass spectrometry for the certification of CRM's, (Proc. Int. Symp. on the Production and Use of Reference Materials, Bundesanstalt für Materialprüfung (BAM), Berlin, Germany, Nov. 13-16, 1979), Paper in Production and Use of Reference Materials, B. F. Schmitt, ed., pp. 161-164 (Bundesanstalt für Materialprüfung, Berlin, Federal Republic of Germany, 1980)

20041. $\mathrm{Ku}, \mathrm{H}$. H. Discussion-Panel on the statistical evaluation in the course of certification, (Proc. Int. Symp. on the Production and Use of Reference Materials, Bundesanstalt für Materialprüfung (BAM), Berlin, Germany, Nov. 13-16, 1979), Paper in Production and Use of Reference Materials, B. F. Schmitt, ed., pp. 497-500 (Bundesanstalt für Materialprüfung, Berlin, Federal Republic of Germany, 1980).

20326. Rasberry, S. D.; Uriano, G. A. Standard reference materials for physical measurement traceability, (Proc. 1980 Workshop \& Symp. National Conference of Standards Laboratories, Gaithersburg, MD, Sept. 22-25, 1980), Paper in NCSL Newslett. 20, No. 4, 23-27 (Dec. 1980).

20519. Lide, D. R., Jr. Critical data for critical needs, Science 212, 1343-1349 (June 19, 1981).

20750. May, W. E.; Brown-Thomas, J. M.; Hilpert, L. R.; Wise, S. A. The certification of selected polynuclear aromatic hydrocarbons in Standard Reference Material 1580, "Organics in Shale Oil," Proc. Polynuclear Aromatic Hydrocarbons: Fifth Int. Symp. Chemical Analysis and Biological Fate, Columbus, OH, Oct. 27-29, 1980, pp. 1-16 (Battelle Press, Columbus, OH, 1981).

\section{Surfaces and Interfaces}

19970. Celotta, R. J.; Pierce, D. T.; Wang, G. C.; Bader, S. D.; Felcher, G. P. Surface magnetization in $\mathbf{N i ( 1 1 0 )}$ as studied by polarized electron scattering, (Proc. Fourth Int. Conf. on Solid 
Surfaces and the Third European Conf. on Surface Science, Cannes, France, Sept. 22-26, 1980), Supplement a la revue, Le Vide, les Couches Minces, No. 201, pp. 1045.1048 (1980).

19975. Felcher, G. P.; Bader, S. D.; Celotta, R. J.; Pierce, D. T.; Wang, G. C. Magnetic state at the surface of a ferromagnet by polarized electron diffraction, Proc. Int. Conf., Lake Geneva, WI, May 28-30, 1980, S. K. Sinha, ed., pp. 107.111 (Elsevier North Holland, Inc., New York, NY, 1980).

19983. Sakurai, T.; Culbertson, R. J.; Melmed, A. J. Anomalous field evaporation of silicon, Surf. Sci. 78, L221-L226 (1978).

19997. Yates, J. T., Jr.; Cavanagh, R. R. Vibrational spectroscopic studies of chemisorbed species on metal surfaces, (Proc. Fourth Int. Conf. on Solid Surfaces and the Third European Conf. on Surface Science, Cannes, France, Sept. 22-26, 1980), Supplement a la revue, Le Vide, les Couches Minces, No. 201, II, $750-753$ (1980).

20012. Pierce, D. T.; Celotta, R. J.; Wang, G. C. Spin polarized electron scattering studies of W(100) (Extended Abstract), (Proc. Fourth Int. Conf. on Solid Surfaces and the Third European Conf. on Surface Science, Cannes, France, Sept. 22-26, 1980), Supplement a la revue, Le Vide, les Couches Minces, No. 201, II, 1060-1063 (1980).

20013. Klein, R.; Fine, J. Desorption of cesium from molybdenum(111); Positive ion and neutral lifetime measurements (Extended Abstract), (Proc. Fourth Int. Conf. on Solid Surfaces and the Third European Conf. on Surface Science, Cannes, France, Sept. 22-26, 1980), Supplement a la revue, Le Vide, les Couches Minces, No. 201, II, 361-364 (1980).

20138. Wang, G. C.; Celotta, R. J.; Pierce, D. T. Polarized lowenergy-electron diffraction from W(100), Phys. Rev. B 23, No. 4, 1761-1770 (Feb. 15, 1981).

20143. Metiu, H.; Gadzuk, J. W. Theory of rate processes at metal surfaces. II. The role of substrate electronic excitations, J. Chem. Phys. 74, No. 4, $2641-2653$ (Feb. 15, 1981).

20157. Melmed, A. J.; Sakurai, T.; Kuk, Y.; Givargizov, E. I. Feasibility of ToF atom-probe analysis of silicon, Surf. Sci. 103, No. 2-3, L139-L142 (1981).

20219. Cavanagh, R. R.; Yates, J. T. Site distribution studies of Rh supported on $\mathrm{Al}_{2} \mathrm{O}_{3}-\mathrm{An}$ infrared study of chemisorbed $\mathrm{CO}, J$. Chem. Phys. 74, No. 7, 4150-4155 (Apr. 1, 1981).

20228. Cavanagh, R. R.; Yates, J. T. Hydrogen spillover on aluminaA study by infrared spectroscopy, J. Catal. 68, 22-26 (1981).

20251. Powell, C. J. Progress of the ASTM E-42 committee on surface analysis, Surf. Interface Anal. 3, No. 2, 94.98 (1981).

20279. Madey, T. E.; Houston, J. E.; Dahlberg, S. C. Bonding geometry of $\mathbf{C O}$ on planar and stepped single crystal surfaces, (Proc. 4th Int. Conf. Solid Surfaces, Cannes, France, Sept. 22, 1980), J. Fr. Vac. Soc. 1, 205-208 (Sept. 1980).

20332. Fine, J.; Hardy, S. C.; Andreadis, T. D. Ion bombardment cleaning of liquid gallium surfaces (Summary Abstract), J. Vac. Sci. Technol. 18, No. 3, 1310-1311 (Apr. 1981).

20333. Gadzuk, J. W. Vibrational lineshapes of adsorbates: The role of substrate electronic excitations, Chem. Phys. Lett. 80, No. 1, 5-10 (May 15, 1981)

20343. Pantano, C. G.; Madey, T. E. Electron beam damage in Auger electron spectroscopy, Appl. Surf. Sci. 7, 115-141 (1981).

20350. Kelley, R. D.; Goodman, D. W. Catalytic methanation over single crystal nickel and ruthenium: reaction kinetics on different crystal planes, Proc. American Chemical Society, Division of Fuel Chemistry, Houston, TX, Mar. 24-28, 1980, 25, No. 2, 43.52 (American Chemical Society, Washington, DC, 1980).

20373. Madey, T. E. The use of angle-resolved electron and photon stimulated desorption for surface structural studies, Paper in Springer Series in Chemical Physics. Vol. 17: Inelastic Particle-Surface Collisions, E. Taglauer and W. Heiland, eds., pp. 80-103 (SpringerVerlag, Berlin, W. Germany, 1981).

20389. Girvin, S. M.; Penn, D. R. Resonant satellites in photoemission and Auger spectra of d-band metals, J. Appl. Phys. 52, No. 3, 1650 . 1653 (Mar. 1981).

20391. Fine, J. Absolute sputtering yield measurement methods: A review, (Invited Lectures \& Progress Reports of SPIG '80, Dubrovnik, Yugoslavia, Aug. 25-30, 1980), Paper in The Physics of Ionized Gases, M. Matić, ed., pp. $379-420$ (Boris Kidric Institute of Nuclear Sciences, Beograd, Yugoslavia, 1980).

20394. Jach, T.; Powell, C. J. $L_{3} V V$ Auger-electron line shapes and peak positions for near-threshold electron excitation in nickel and copper, Phys. Rev. Lett. 46, No. 14, 953-956 (Apr. 6, 1981).

20419. Madey, T. E.; Houston, J. E.; Seabury, C. W.; Rhodin, T. N.
The structure of $\mathrm{NH}_{3}$ on Ni(111), J. Vac. Sci. Technol. 18, No. 2, 476-480 (Mar. 1981).

20498. Cavanagh, R. R.; Yates, J. T., Jr. Surface binding of an electronic analog to $\mathrm{CO}$ : Infrared evidence for $\mathrm{CH}_{3} \mathrm{NC}$ chemisorption on $\mathrm{Rh} / \mathrm{Al}_{2} \mathrm{O}_{3}, J$. Chem. Phys. 75, No. 3, 1551-1559 (Aug. 1, 1981).

20510. Madey, T. E.; Stockbauer, R.; Flodström, S. A.; van der Veen, J. F.; Himpsel, F. J.; Eastman, D. E. Photon-stimulated desorption from covalently bonded species: CO absorbed on Ru(001), Phys. Rev. $B$ 23, No. 12, 6847.6850 (June 15, 1981).

20531. Netzer, F. P. Auger line shapes analysis for characterization of molecular surface reaction products, Appl. Surf. Sci. 7, 289-311 (1981).

20533. Poliakoff, E. D.; Dehmer, P. M.; Dehmer, J. L.; Stockbauer, $R$. The photoelectron spectrum of $\mathrm{Xe}_{3}$ by the photoelectron-photoion coincidence technique, J. Chem. Phys. 75, No. 3, 1568-1569 (Aug. 1, 1981).

20621. Netzer, F. P.; Madey, T. E. The adsorption of NO on Ni(111): An ESDIAD/LEED study, Surf. Sci. 110, 251-260 (1981).

20643. Pierce, D. T.; Celotta, R. J.; Wang, G. C.; McRae, E. G. Spin dependence of electronic surface resonance scattering from $W(100)$, Solid State Commun. 39, 1053-1055 (1981).

20661. Yates, J. T., Jr.; McKee, D. W. Kinetic isotope effect in the heterogeneous reaction of graphite with $\mathrm{H}_{2} \mathrm{O}\left(\mathrm{D}_{2} \mathrm{O}\right), J$. Chem. Phys. 75, No. 6, $2711-2714$ (Sept. 15, 1981).

20686. Melmed, A. J.; Ceyer, S. T.; Tung, R. T.; Graham, W. R. A LEED inquiry into the question of reconstruction of $\{001\} \mathrm{Nb}$, Surf. Sci. Lett. 111, L701-L704 (1981).

20689. Netzer, F. P.; Madey, T. E. Coadsorption-induced azimuthal ordering in molecular adsorbate layers: $\mathrm{H}_{2} \mathrm{O}$ and $\mathrm{NH}_{3}$ on oxygenprecovered Ni(111), Phys. Rev. Lett. 47, No. 13, $928-931$ (Sept. 28, 1981).

20693. Pierce, D. T.; Celotta, R. J. Spin polarization in electron scattering from surfaces, Adv. Electron. Electron Phys. 56, $219-289$ (1981).

20694. Pierce, D. T.; Girvin, S. M.; Unguris, J.; Celotta, R. J. Absorbed current electron spin polarization detector, Rev. Sci. Instrum. 52, No. 10, 1437-1444 (Oct. 1981).

20706. Wang, G. C.; Pierce, D. T.; Celotta, R. J. Study of temperature and hydrogen induced reconstruction and reordering of $W(100)$ by polarized electron scattering (Summary Abstract), J. Vac. Sci. Technol. 18, No. 2, 647.648 (Mar. 1981).

\section{Technology Incentives}

NBSIR 81-2327. Garrity, S. D. Monitoring system development: A framework and application. 1981 September. 607 p. Available from: NTIS; PB 82-115817.

NBSIR 81.2328. Garrity, S. D. A manual for designing and implementing a process to monitor complex system developments. 1981 September. 65 p. Available from: NTIS; PB 82-104308.

NBS-GCR-ETIP 81-93. Foskett, W. H.; Jamieson, A.; Evans, J., (NBS contact: D. Fulmer). Innovation by regulation: The administration of control technology requirements under the Clean Air Act. 1981 March. 147 p. Available from: NTIS; PB 81-221616.

NBS-GCR-ETIP 81-94. Foskett, W.; Olesen, T., (NBS contact: D. Fulmer). Periodic review and technological escalation of performance standards: New source performance standards review process. 1980 December. 42 p. Available from: NTIS; PB 81-218117.

NBS-GCR-ETIP 81-95. Evans, J., (NBS contact: D. Fulmer). Controlled trading and site-specific SIP revisions: Competing for attention in a crowded administrative route. 1980 December. $111 \mathrm{p}$. Available from: NTIS; PB 81-218273.

NBS-GCR-ETIP 81.96. Clarren, S.; Nalley, P.; Gregory, G.; Frederick, W.; Hausmann, R., (NBS contact: D. Fulmer). The evaluability assessment of the developing experiment in postmarketing surveillance of prescription drugs. 1981 March. $217 \mathrm{p}$. Available from: NTIS; PB 81.223232.

NBS-GCR-ETIP 81-97. Mulkey, M.; Timpane, K., (NBS contact: J. Green). Toward competitive provision of public record message services. 1981 October. 145 p. Available from: NTIS; PB 82-138819.

NBS-GCR-81-309. Resource Recovery Section, Department of Environmental Regulation, (NBS contact: K. Hudson). State of Florida: The procurement of products containing recovered materials. 1981 May. 193 p. Available from: NTIS; PB 81-205445.

NBS-GCR.81.309, Supplement 1. (Resource Recovery Section, Dept. of Environmental Regulation, State of Florida) (NBS contact: C. 
Hudson). An analysis of the procurement policies of: Dade County, The City of Miami, The Dade County School System, Miami Dade Community College. 1981 June. 34 p. Available from: NTIS; PB 81. 225054.

NBS-GCR-81-310. Kittel, J., Michigan Energy and Resource Research Association, (NBS contact: K. Hudson). Procurement of products containing recovered materials: A study of State of Michigan practices. 1981 March. 127 p. Available from: NTIS; PB 81-204448.

NBS-GCR-81-312. Josephson, E.; Bush, T.; Mazzone, G.; McElroy, G.; Galbraith, T., (NBS contact: K. Hudson/ J. Berke) Procurement of products containing recycled materials in the state of New Jersey. 1981 March. 76 p. Available from: NTIS; PB 81. 184335.

NBS-GCR-81-313. Egan, J.; Higgins, R.; Spath, J.; Buchholz, A.; Wrzenski, S. C., (NBS contact: K. Hudson/ J. Berke). Procurement practices in New York State of products containing recycled materials. 1981 March. 25 p. Available from: NTIS; PB 81-187999.

20396. Benzinger, T. H.; Hammer, C. Unwinding the double helix: Complete equation for chemical equilibrium, Chapter 27 in Current Topics in Cellular Regulation. Vol. 18, Biological Cycles, R. W Estabrook and P. Srere, eds., pp. 475-485 (Academic Press, New York, NY, 1981).

\section{Thermodynamics and Chemical Kinetics}

Colbert, J. C.; Xiheng, H.; Kirklin, D. R. Enthalpy of combustion of microcrystalline cellulose. J. Res. Nat. Bur. Stand. (U.S.). 86(6): 655 . 660; 1981 November-December.

TN1039. Ely, J. F.; Hanley, H. J. M. A computer program for the prediction of viscosity and thermal conductivity in hydrocarbon mixtures. Nat. Bur. Stand. (U.S.) Tech. Note 1039; 1981 April. 80 p. Available from: NTIS; PB 81-214165.

TN1045. McCarty, R. D.; Jacobsen, R. T. An equation of state for fluid ethylene. Nat. Bur. Stand. (U.S.) Tech. Note 1045; 1981 July. 164 p. SN003-003-02351-5.

NBSIR 79.1715. Waxman, M.; Davis, H. A.; Sengers, J. M. H. L.; Klein, $M$. The equation of state of isobutane: An interim assessment. 1981 September. 44 p. Available from: NTIS; PB 82-120528.

NBSIR 80-2169. Hastie, J. W.; Bonnell, D. W. Molecular chemistry of inhibited combustion systems. 1980 December. 182 p. Available from: NTIS; PB 81-170375.

NBSIR 81-2254. Westley, F. Tables of experimental rate constants for chemical reactions occurring in combustion (1971-1977). 1981 April. 302 p. Available from: NTIS; PB 81-205429.

NBSIR 81.2276. Staples, B. R.; Wobbeking, T. F. A compilation of thermodynamic and transport properties of aqueous sulfuric acid. 1981 June. 26 p. Available from: NTIS; PB 81-229171.

NBSIR 81-2406. Wasik, S. P.; Tewari, Y. B.; Miller, M. M.; Martire, D. E. Octanol/water partition coefficients and aqueous solubilities of organic compounds. 1981 December. 69 p. Available from: NTIS PB 82-141797.

19965. Sieck, L. W. Reactions of $\left(\mathrm{CO}_{2}\right)_{2}{ }^{+}$and $(\mathrm{CO})_{2}{ }^{+}$association ions, Int. J. Chem. Kinet. 10, 335-366 (1978).

20035. Herron, J. T.; Huie, R. E. Rate constants at $298 \mathrm{~K}$ for the reactions $\mathrm{SO}+\mathrm{SO}+\mathrm{M} \rightarrow(\mathrm{SO})_{2}+\mathrm{M}$ and $\mathrm{SO}+(\mathrm{SO})_{2} \rightarrow \mathrm{SO}_{2}+\mathrm{S}_{2} \mathrm{O}$, Chem. Phys. Lett. 76, No. 2, 322-324 (Dec. 1, 1980).

20072. Younglove, B. A.; McCarty, R. D. Speed-of-sound measurements for nitrogen gas at temperatures from 80 to $350 \mathrm{~K}$ and pressures to $1.5 \mathrm{MPa}, J$. Chem. Thermodyn. 12, No. 12, 1121-1128 (1980).

20199. Rosenstock, H. M.; Stockbauer, R.; Parr, A. C. Photoelectronphotoion coincidence study of benzonitrile, J. Chim. Phys. 77, No. $7 / 8,745-750$ (1980).

20227. Hubbard, J. B.; Kayser, R. F. Dielectric friction and dielectric dispersion in electrolyte solutions with spin, J. Chem. Phys. 74, No. 6, 3535-3545 (Mar. 15, 1981)

20264. Weber, L. A. Measurements of the heat capacities $C_{y}$ of dense gaseous and liquid nitrogen and nitrogen trifluoride, J. Chem. Thermodyn. 13, 389.403 (1981).

20360. Younglove, B.; McCarty, R. D. Thermodynamic properties of nitrogen gas derived from measurements of sound speed, NASA Reference Publ. 1051, 55 pages (Available as N80-14257 from the National Technical Information Service, Springfield, VA 22161, Dec. 1979).

20413. Bennett, L. H.; Watson, R. E. A database for enthalpies of formation of binary transition metal alloys, CALPHAD 5, No. 1, 19 . $23(1981)$
20438. Laufer, A. H. Kinetics of gas phase reactions of methylene, Rev. Chem. Intermed. 4, 225-257 (1981).

20472. Rosenstock, H. M.; Stockbauer, R.; Parr, A. C. Unimolecular kinetics of pyridine ion fragmentation, Int. J. Mass Spectrom. Ion Phys. 38, 323.331 (1981).

20573. Massoud Gasem, K. A.; Hiza, M. J.; Kidnay, A. J. Phase behavior in the nitrogen + ethylene system from 120 to $200 \mathrm{~K}$, Fluid Phase Equilib. 6, No. 3.4, 181-189 (June 1981).

20620. Esfandiari, P.; Meijer, P. H. E.; Farrell, R. A.; Favin, S. New generating functions and results for the density polynomials of the lattice gas, Phys. Rev. B 24, No. 3, 1298-1311 (Aug. 1, 1981).

20725. Johnson, W. C.; Cahn, J. W. Comment on chemical potentials in solids, Script. Met. 15, 1259.1964 (1981).

20768. Ely, J. F.; Hanley, H. J. M. Prediction of transport properties. 1. Viscosity of fluids and mixtures, Ind. Eng. Chem. Fundam. 20, No. 4, 323.332 (Nov. 1981).

\section{Other Subjects of General Interest}

SP305. Supplement 12. Burris, B. L.; Morehouse, R. J., eds. Publications of the National Bureau of Standards 1980 Catalog. A compilation of abstracts and key word and author indexes. Nat. Bur. Stand. (U.S.) Spec. Publ. 305, Suppl. 12; 1981 June. 634 p. SN003. 003-02349-3.

SP610. Knight, N. H., ed. Telecommunication technologies, networking and libraries. Proceedings of a Conference on Telecommunication Technologies, Networking and Libraries, held at the National Bureau of Standards; 1977 June 3; Gaithersburg, MD. Nat. Bur. Stand. (U.S.) Spec. Publ. 610; 1981 December. 90 p. SN003-003. 02347-7.

SP610: 1981 December. 1-8. Cotton, I. W. Network architecture. SP610: 1981 December. 9-12. Darby, L. F. Competition vs. monopoly in telecommunications.

SP610: 1981 December. 13-16. Johnson, R. B. Competition vs. monopoly in telecommunications.

SP610: 1981 December. 17-27. Avram, H. D. The National Bibliographic Telecommunications Network.

SP610: 1981 December. 28.38. Little, J. L. Progress in the development of computer network protocols for library and information service applications.

SP610: 1981 December. 39-52. Meyer, S. L. Telecommunications and the expansion of information services.

SP610: 1981 December. 53-60. Belden, T. G. Uses of teleconferencing in crisis and warning situations.

SP610: 1981 December. 61-65. Henderson, E. Interactive telecommunications via satellite.

SP610: 1981 December. 66-73. Lee, R. W. H. Telecommunication R\&D-Implications and support for library programs.

SP610: 1981 December. 74-79. Warnar, R.; Henderson, M.; Berger, P. Videodiscs-A fast retrieval, mass storage media for libraries.

NBSIR 81-2226. Kingston, M. L., ed. NBS serial holdings 1981. 1981 March. 284 p. Available from: NTIS; PB 81.203739.

20023. Goldman, D. T. Comments on the paper "NBS and Metrication," by A. A. Bartlett, Phys. Today Lett. pp. $69-72$ (Dec. 1980).

20136. Berger, P. W.; Cerutti, E. The management of online reference search services in Federal libraries, Sci. Technol. Libr. 1, No. 1, 81. 107 (1980).

20205. Cotton, I. W. Perspective on trends in electronic communication, (Proc. AAAS Selected Symp. 52, Houston, TX, Jan. 3-8, 1979), Paper in Electronic Communication: Technology and Impacts, M. M. Henderson and M. J. MacNaughton, eds., pp. 7.18 (Westview Press Inc., Boulder, CO, 1980).

20477. Molino, J. A. Auditory space perception, Article in International Encyclopedia of Psychiatry, Psychology, Psychoanalysis, and Neurology, pp. 222-227 (Aesculapius Publ. Inc., New York, NY, 1977).

20568. Deprit, A. Intrinsic variational equations in three dimensions, Celestial Mech. 24, 185-193 (1981). 


\section{INDEXES}

\subsection{AUTHOR INDEX}

\section{A}

Abramowitz, S.; Domalski, E. S.; Kirklin, D. R.; Colbert, J.; Decker, P.; NBSIR 81-2278.

Abrams, M. S.,; Lin, T. D.; NBS-GCR-81-317.

Acevedo, J.; Herman, D.,; McCarthy, D.; NBS-GCR-81-327.

Achenbach, P. R.; NBS-GCR-80-280.

Ackermann, R. A.; SP607: 1981 May. 57.69.

Ackerson, B. J.; Hanley, H. J. M.; 19964.

Acquista, N.; Reader, J.; 20218.

Acquista, N.; Reader, J.; 20395

Acquista, N.; Reader, J.; Luther, G.; 20110.

Adler, S. C.; Pierman, B. C.; NBSIR 81-2245.

Adrion, W. R.; Branstad, M. A.; Cherniavsky, J. C.; SP500-75.

Adrion, W. R.,; Branstad, M. A.; SP500-78.

Ahola, R.; Hug, R.; Strelich, T.; Resnicow, M.; SP500-80: 1981 October. 122.127.

Aisenberg, S.; Stein, M.; SP620: 1981 October. 313.323.

Akcasu, A. Z.; Han, C. C.; 20482.

Akcasu, A. Z.; Han, C. C.; 20721.

Akcasu, A. Z.; Han, C. C.; 20722.

Akins, D. L.; King, D. S.; Stephenson, J. C.; 20095.

Alabedra, R.; Lecoy, G.; Savelli, M.; Maille, C.; SP614: 1981 September. 109-112.

Alabedra, R.; Walma, A. A.; Maille, C.; Lecoy, G.; SP614: 1981 September. 105-108.

Albers, J.; Ehrstein, J. R.; Myers, D. R.; Roitman, P.; NBSIR 81 2398.

Albus, J. S.; Nagel, R. N.,; Wheatley, T.; NBSIR 80-2152.

Albus, J. S.; VanderBrug, G. J.; Barbera, A. J.; Fitzgerald, M. L.; Wheatley, T. E.; SP602.

Alereza, T.; Kusuda, T.; Sud, I.; 20756.

Alexander, W.; Brice, R.; SP500-83: 1981 November. 7.14.

Alfeld, L. E.,; NBS-GCR-81-331.

Allan, D. W.; Weiss, M. A.; 20062.

Allen, J. W.; Hartman, W. F.; Robinson, J. C.; SP622: 1981 October. 11.30.

Allen, P. J.; Loftus; J. J.; Maldonado-Rosado, A.; NBSIR 81-2373.

Allen, P. J.; Maldonado, A.; Juarez, N.; Krasny, J. F.; NBSIR 81 2363.

Amberiadis, K.; van der Ziel, A.; Rucker, L. M.; SP614: 1981 September. 210-212.

Ambler, E.; SP599: 1981 January. 6.9.

Ambrose, J. R.; Kruger, J.; Carroll, J. J.; Melmed, A. J.; Ritter, J. J.; NBSIR 79-1904 (Navy).

Andeen, C. G.; Link, J.; Wintersgill, M. C.; Fontanella, J. J.; Bean, V. E.; 20266.

Anderson, E. D.; Clifton, J. R.; NBSIR 80-2163.

Anderson, G.; Thall, R.; SP500-80: 1981 October. 197-199.

Anderson, J. M.; Barenberg, S.; Hiltner, A.; SP601: 1981 January. 283-298.

Anderson, J. M.; Evans, P. M.; Smith, R. L.; SP608: 1981 May. 113. 122.

Anderson, M. A.; SP618: 1981 November. 146-162.

Anderson, W. E.; Ramboz, J. D.; NBSIR 81-2235.

Anderson, W. J.; Hansen, W. N.; SP620: 1981 October. 335-344.

Andreadis, T. D.; Fine, J.; Hardy, S. C.; 20332.

Andrews, J.; 20313

Andrews, J. R.; Baldwin, E. E.; NBSIR 81-1646.

Aniel, T.; Maximon, L. C.; de Miniac, A.; NBSIR 81-2262.

Antonucci, J. M.; 20253.

Antonucci, J. M.; Brauer, G. M.; Stansbury, J. W.; 20401.

Antonucci, J. M.; Misra, D. N.; Peckoo, R. J.; 20481.

Antonucci, J. M.; Peckoo, R. J.; Schruhl, C.; Toth, E. E.; 20406

Antonucci, J. M.; Wu, W.; McKinney, J. E.; Whitlock, R. P.; Hinman, R.; Cassel, J. M.; Tesk, J. A.; Brauer, G. M.; NBSIR 802168.

Aoki, K.; Miyamae, K.; Kobayashi, T.; Yamamoto, K.; SP614: 1981 September. 93-96.

Apts, C. W.; Crecelius, E. A.; Gurtisen, J. M.; SP618: 1981
November. 173-183.

Arakawa, E. T.; Ederer, D. L.; Callcott, T. A.; 20216.

Archer, N. P.; SP500-83: 1981 November. 71.80.

Argentar, H.; U.S. Patent 4,243, 763.

Argentar, H.; U.S. Patent 4,284,551.

Argentar, H.; Tesk, J. A.; Parry, E. E.; 20221.

Armstrong, R. W.; Kuriyama, M.; Yoo, K. C.; Roessler, B.; 20712.

Arp, V. D.; Hands, B. A.; 20769.

Artis, H. P.; SP500-83: 1981 November. 325

Arvidson, J. M.; Sparks, L. L.; NBSIR 81-1654.

Aryeh, Y. B.; Mies, F. H.; 20039.

Ashe, A. J.; Kuczkowski, R. L.; Lovas, F. J.; Suenram, R. D.; Lattimer, R. P.; Hillig, K. W.; 20168

Asher, M. S.; Horn, S. B.; SP607: 1981 May. 30-47.

Atkins, R. G.; Breitenberg, M. A.; NBSIR 81-2336.

Atreya, S. K.; Lane, A. L.; Clarke, J. T.; Moos, H. W.; 20180.

Atwood, J. W.; Yu, K. C.; SP500-83: 1981 November. 239-246.

Austell, W. P., Jr.; Willis, R. R.; SP500-80: 1981 October. 29-32.

Austin, M.; Negas, T.; Mathew, M.; Brown, W. E.; 19984.

Austin, M. W.; Ledbetter, H. M.; Moulder, J. C.; 20328.

Avram, H. D.; SP610: 1981 December. 17.27

Ayres, T.; Linsky, J. L.; Pallavicini, R.; Golub, L.; Rosner, R.; Vaiana, G. S. 20594.

Ayres, T. R.; Linsky, J. L.; 19966.

Ayres, T. R.; Linsky, J. L.; Vaiana, G. S.; Golub, L.; Rosner, R.; 20627.

Ayres, T. R.; Linsky, J. L.; Worden, S. P.; Giampapa, M. S.; Bornmann, P. L.; 20388.

Ayres, T. R.; Marstad, N. C.; Linsky, J. L.; 20535.

Ayres, T. R.; Moos, H. W.; Linsky, J. L.; 20582.

\section{B}

Babrauskas, V.; 20642.

Babrauskas, V.; NBSIR 81-2271.

Babrauskas, V.; NBSIR 80-2186.

Bader, S. D.; Celotta, R. J.; Pierce, D. T.; Wang, G. C.; Felcher, G. P.; 19975.

Bader, S. D.; Felcher, G. P.; Celotta, R. J.; Pierce, D. T.; Wang, G. C.; 19970

Baer, T.; Robinson, H. G.; Hall, J. L.; Hollberg, L.; 20720

Bagg, T.,; FIPS PUB 89.

Bagg, T. C.,; FIPS PUB 84.

Bagg, T. C.,; FIPS PUB 82.

Bagg, T. C.,; FIPS PUB 85.

Bailbe, J. P.; Blasquez, G.; Lavernhe, F.; Marty, A.; SP614: 1981 September. 118-120.

Baker, D. W.; Kao, J. Y.; NBSIR 80-2104.

Baker, D. W.; Kao, J. Y.; Didion, D. A.; NBSIR 81-2313.

Baldwin, E. E.; Andrews, J. R.; NBSIR 81-1646.

Ballard, D.; Laughlin, D.; Clough, R.; Biancaniello, F.; Blau, P.; Cahn, J.; Mehrabian, R.; Free, G.; Berger, H.; Mordfin, L.; Swartzendruber, L.; Boettinger, W.; Ives, L.; Coriell, S.; NBSIR 80-2069.

Baltes, H. P.; Geist, J.; 20636.

Banken, G.; Emery, A. F.; Dorri, B.; Kippenhan, C. J.; Heerwagen, D. R.; SP608: 1981 May. 21-35.

Banks, C. H.; Bottino, N. R.; Chakraborti, D.; Gennity, J. M.; Hillman, D. C.; O'Brien, D. H.; Pyles, R. A.; Stockton, R. A.; Wheeler, A. E.; Zingaro, R. A.; Irgolic, K. J.; SP618: 1981 November. 244.263

Barber, E. M.; Bettge, D. A.; Powell, F. J.; 20379

Barbera, A. J.; Fitzgerald, M. L.; Wheatley, T. E.; Albus, J. S.; VanderBrug, G. J.; SP602.

Barbi, N. C.; Lister, D. B.; SP604: 1981 June. 35-44

Barbrow, L. E.; Heffernan, A. P.,; Wollin, H. F.; SP599.

Bareikis, V.; Galdikas, A.; Miliusyte, R.; Viktoravicius, V.; SP614: 1981 September. 406-408.

Barenberg, S.; Hiltner, A.; Anderson, J. M.; SP601: 1981 January. 283.298.

Barger, R. L.; 20261. 
Barger, R. L.; 20414.

Barger, R. L.; Glaze, D. J.; Bergquist, J. C.; 20338.

Barkley, J.; Powell, P.; SP500-80: 1981 October. 174-179.

Barnes, I. L.; 20024.

Barnes, I. L.; Moore, L. J.; Gramlich, J. W.; Rosman, K. J. R.; 20698.

Barnes, J. A.; 20308.

Barnes, K. A.; Kelly, G. E.; Petersen, S. R.; NBSIR 81-2241.

Barnes, K. A.; Peavy, B. A.; Petersen, S. R.; BSS134.

Barnett, J.,; Manola, F.; NBS-GCR-81-339.

Barnett, J. P.; NBSIR 80-2184.

Barnett, P. D.; Corley, D. M.; Baum, H. R.; Rehm, R. G.; NBSIR 812385.

Barrus, D. M.; Blake, R. L.; Fenimore, E. E.; Burek, A. J.; 20374.

Bartel, T. W.; 20190.

Bartle, K. D.; Lee, M. L.; Wise, S. A.; 20158.

Bartle, K. D.; Lee, M. L.; Wise, S. A.; 20397.

Basch, H.; Julienne, P. S.; Krauss, M.; Rosenkrantz, M. E.; 20238.

Basch, H.; Stevens, W. J.; Krauss, M.; 20196.

Basri, G. S.; Linsky, J. L.; Stencel, R. E.; Mullan, D. J.; 20418.

Basri, G. S.; Worden, S. P.; Stencel, R. E.; Mullan, D. J.; Linsky, J. L.; 20057.

Bass, M.; Wu, S. T.; SP620: 1981 October. 75-87.

Bates, R. G.; Durst, R. A.; 20580.

Batts, M. E.; Changery, M. J.; 20551.

Batts, M. E.; Lew, H. S.; Carino, N. J.; Fattal, S. G.; NBSIR 81-2374.

Bauer, J. W.; Flynn, D. R.; Danner, W. F.; Yaniv, S. L.; NBSIR 81 2377.

Baughcum, S. L.; Leone, S. R.; 20004.

Baum, H. R.; Rehm, R. G.; Barnett, P. D.; Corley, D. M.; NBSIR 812385.

Bays, W. N.; Kincy, W. P., Jr.; SP500-83: 1981 November. 143-156.

Beale, W.; Gasser, M. G.; Sherman, A.; SP607: 1981 May. 103.115.

Bean, J. W.; Kusuda, T.; 20754.

Bean, J. W.; Kusuda, T.; TN1138.

Bean, J. W.; Kusuda, T.; Pierce, E. T.; 20755.

Bean, V. E.; Andeen, C. G.; Link, J.; Wintersgill, M. C.; Fontanella, J. J.; 20266.

Beaty, E. C.; Rumble, J. R., Jr.; Gallagher, J. W.; Van Blerkom, J.; SP593.

Beaty, E. C.; Van Brunt, R. J.; Misakian, M.; Leep, D. A.; Moy, K. J.; NBSIR 81-2242.

Beausoliel, R. W.; Clifton, J. R.; Meese, W. J.; NBSIR 81-2220.

Beausoliel, R. W.; Meese, W. J.; NBSIR 81-2221.

Becker, D. A.; Berke, J. G.; Matthews, R. T.; Yakowitz, H.; NBSIR 80-2178.

Becker, D. A.; Hsu, S. M.; 20016.

Beckstette, K. F.; Dirks, H. M.; Heiden, C.; SP614: 1981 September. 388.390.

Beckstette, K. F.; Heiden, C.; Dirks, H. M.; SP614: 1981 September. 384-387.

Bedeaux, D.; Mazur, P.; Greer, S. C.; Sengers, J. V.; 20056.

Beekman, D. H., Jr.; Schramm, R. E.; Kasen, M. B.; MacDonald, G. R.; 20616.

Behrens, J. W.; Bowman, C. D.; Johnson, R. G.; 20163.

Behrens, J. W.; Bowman, C. D.; Johnson, R. G.; 20724.

Behrens, J. W.; Johnson, R.; Bowman, C. D.; Schrack, R. A.; 20458.

Beine, W. B.; Sorrells, J. R.; NBSIR 80-2106.

Belanger, B. C.; Kieffer, L. J.; 20081.

Belden, T. G.; SP610: 1981 December. 53-60.

Belecki, N. B.; SP589: 1981 January. 149-154.

Bell, B. A.; Souders, T. M.; Flach, D. R.; TN1145.

Bell, D. A.; SP614: 1981 September. 169-172.

Bell, R. J.,; Goldman, D. T.; SP330.

Beller, E. J. III.; Hardgrave, W. T.; Salazar, S. B.; NBSIR 81-2240.

Beller, E. J. III.; Hardgrave W. T.; Salazar, S. B.; SP500-83: 1981

November. 85.91.

Belsher, D. R.; NBSIR 79.1610.

Bender, P. L.; 20107.

Bender, P. L.; 20431.

Bender, P. L.; Bossler, J. D.; Goad, C. D.; 20434.

Bender, P. L.; Faller, J. E.; Decher, R.; Randall, J. L.; 2045 I.
Bendow, B.; Gianino, P. D.; Tzoar, N.; SP620: 1981 October, 453. 458.

Bendow, B.; Lipson, H. G.; Moynihan, C. T.; Drexhage, M. G.; SP620: 1981 October. 94-101.

Ben-Jacob, E.; Bergman, D.; Carmeli, B.; Nitzan, A.; SP614: 1981 September, 381-383.

Ben-Jacob, E.; Schuss, Z.; SP614: 1981 September. 376-380.

Ben-Jacob, E.; Schuss, Z.; Bergman, D. J.; SP614: 1981 September. 57.60.

Benjamin, I. A.; 20746.

Bennett, D. J.; SP500-83: 1981 November. 169-177.

Bennett, H. E.; SP620: 1981 October. 256-264.

Bennett, H. E.; Burge, D. K.; SP620: 1981 October. 356-368.

Bennett, H. E.; Glass, A. J.; Guenther, A. H.; Newnam, B. E.,; SP620.

Bennett, H. S.; J. Res. 86(5): 503-508; 1981 September-October.

Bennett, L. H.; 20210.

Bennett, L. H.; Goodman, D. A.; Cahn, J. W.; 20433.

Bennett, L. H.; Ives, L. K.; Shull, R. D.; Swartzendruber, L. J.; 20736.

Bennett, L. H.; Swartzendruber, L. J.; Reno, R. C.; 20208.

Bennett, L. H.; Watson, R. E.; 20455.

Bennett, L. H.; Watson, R. E.; 20413.

Bennewitz, P. F.; SP400-69: 1981 May. 75-79.

Ben-Reuven, A.; 19968.

Ben-Reuven, A.; 19967.

Ben-Reuven, A.; 20250.

Bensema, W. D.; 20622.

Benson, G. M.; Vincent, R. J.; SP607: 1981 May. 116.

Benson, J. P.; Gannon, C.; DeHaan, W. R.; Saib, S. H.; SP500-80: 1981 October. 200-207.

Benson, J. P.; Gannon, C.; DeHaan, W. R.; Saib, S. H.; SP500-80: 1981 October. 208-217.

Benzinger, T. H.; Hammer, C.; 20396.

Berg, J. L.; Graham, M.; Whitney, K.,; SP500-76.

Berger, A. E.; Solomon, J. M.; Ciment, M.; 20626.

Berger, H.; 20245.

Berger, H.; 20325.

Berger, H.; 20625.

Berger, H.; 20762.

Berger, H.; Birnbaum, G.; Eitzen, D. G.; 20474.

Berger, H.; Mordfin, L.; 20411.

Berger, H.; Mordfin, L.; Swartzendruber, L.; Boettinger, W.; Ives, L.; Coriell, S.; Ballard, D.; Laughlin, D.; Clough, R.; Biancaniello, F.; Blau, P.; Cahn, J.; Mehrabian, R.; Free, G.; NBSIR 80-2069.

Berger, H.; Placious, R. C.; Garrett, D. A.; Kasen, M. B.; 20342.

Berger, P.; Warnar, R.; Henderson, M.; SP610: 1981 December. 74. 79.

Berger, P. W.; Cerutti, E.; 20136.

Berger, R. E.; Huckeba, J. A.; NBSIR 80-2037.

Bergman, D.; Carmeli, B.; Nitzan, A.; Ben-Jacob, E.; SP614: 1981 September. 381-383.

Bergman, D. J.; Ben-Jacob, E.; Schuss, Z.; SP614: 1981 September. 57.60 .

Bergquist, J. C.; Barger, R. L.; Glaze, D. J.; 20338.

Bergquist, J. C.; Drullinger, R. E.; Wineland, D. J.; 20265.

Berhinig, R. M.,; NBS-GCR-81-330.

Berild, S.; Nachmens, S.; SP500-80: 1981 October. 1-6.

Berke, J. G.; Hudson, C. H.; NBSIR 81-2316.

Berke, J. G.; Matthews, R. T.; Yakowitz, H.,; Becker, D. A.; NBSIR 80-2178.

Bernier, R.; Brauer, G. M.; Dulik, D.; 20392.

Berning, D. W.; Blackburn, D. L.; 20358.

Bernstein, B.; Zapas, L. J.; 20126.

Berry, R.; Neuse, D.; Chandy, K. M.; Misra, J.; SP500-83: 1981 November. 331-334.

Berry, S. A.,; SP608.

Berry, W. W.; SP500-83: 1981 November. 327-328.

Bertocci, U.; SP614: 1981 September. 328.331.

Bertocci, U.; Mullen, J.; Cohen, M.; Kruger, J.; Escalante, E.; 20348.

Bertotti, B.; 20666. 
Bertotti, G.; Fiorillo, F.; Sassi, M. P.; SP614: 1981 September. 324 327.

Bertsch, D. L.; Dodge, W. R.; 20375.

Bethin, J. R.; Chiang, C. K.; Franklin, A. D.; Snellgrove, R. A.; 20497.

Bettge, D. A.; Powell, F. J.; Barber, E. M.; 20379.

Betz, R. A.; SP589: 1981 January. 45-48.

Bialkowski, S. E.; 20188.

Biancaniello, F.; Blau, P.; Cahn, J.; Mehrabian, R.; Free, G.; Berger, H.; Mordfin, L.; Swartzendruber, L.; Boettinger, W.; Ives, L.; Coriell, S.; Ballard, D.; Laughlin, D.; Clough, R.; NBSIR 80-2069.

Biancaniello, F.; DeRoberts, L.; Boyer, P. A.; Ruff, A. W.; Marinenko, R. B.; SP260-70.

Biancaniello, F. S.; Coriell, S. R.; Boettinger, W. J.; 20132.

Bielefeld, M. J.; Yin, L. I.; Trombka, J. I.; Schmadebeck, R. L.; Seltzer, S. M.; 20569.

Bijloo, A. C.; SP599: 1981 January. 165-178.

Birkeland, K.; SP599: 1981 January. 14-24.

Birnbaum, G.; Cohen, E. R.; NBSIR 80-2175.

Birnbaum, G.; Eitzen, D. G.; Berger, H.; 20474.

Birnbaum, G.; Free, G.,; 20366.

Birnbaum, G.; Sutter, H.; 20133.

Bishop, F. E.; SP622: 1981 October. 195-219.

Black, R. D.; Weissman, M. B.; Fliegle, F. M.; SP614: 1981 September. 152.

Blackburn, D. H.; Pella, P. A.; Walter, F. J.; Stone, R.; SP604: 1981 June. 61-70.

Blackburn, D. L.; NBSIR 81-2204.

Blackburn, D. L.; Berning, D. W.; 20358.

Blais, P.; Marois, M.; Gosselin, C.; Roy, P.; Courbier, R.; David, M.;

Noël, H. P.; Guidoin, R.; King, M.; SP601: 1981 January. 29-129.

Blake, R. L.; Fenimore, E. E.; Burek, A. J.; Barrus, D. M.; 20374.

Blalock, T. V.; Shepard, R. L.; SP614: 1981 September. 260-268.

Blanc, R.; Heafner, J.; Rosenthal, R.; Watkins, S.; NBSIR 80-2154.

Blasgen, M. W.; SP599: 1981 January. 110-114.

Blasquez, G.; SP614: 1981 September. 302-304.

Blasquez, G.; Lavernhe, F.; Marty, A.; Bailbe, J. P.; SP614: 1981 September. 118-120.

Blau, P.; Cahn, J.; Mehrabian, R.; Free, G.; Berger, H.; Mordfin, L.;

Swartzendruber, L.; Boettinger, W.; Ives, L.; Coriell, S.; Ballard,

D.; Laughlin, D.; Clough, R.; Biancaniello, F.; NBSIR 80-2069.

Blau, P. J.; 20213.

Blau, P. J.; 20202.

Blau, P. J.; 20587.

Blau, P. J.; 20667

Blau, P. J.; 20668.

Blaylock, J. W.; Kelly, J. C.; SP500-83: 1981 November. 251-258.

Bliek, L. M.; SP614: 1981 September. 181-184.

Block, S.; Piermarini, G. J.; Rabolt, J. F.; 20007.

Board, D.; Fischer, J. P.; Schover, D. S.; SP622: 1981 October. 126. 136.

Boehm, M. J.; Weber, S. F.; Rudder, F. F., Jr.; NBSIR 81-2366.

Boettinger, W.; Ives, L.; Coriell, S.; Ballard, D.; Laughlin, D.; Clough, R.; Biancaniello, F.; Blau, P.; Cahn, J.; Mehrabian, R.; Free, G.; Berger, H.; Mordfin, L.; Swartzendruber, L.; NBSIR 80 2069.

Boettinger, W. J.; Biancaniello, F. S.; Coriell, S. R.; 20132.

Boettinger, W. J.; Sekerka, R. F.; Coriell, S. R.; Cordes, M. R.; 20224.

Boisvert, R. F.; 20459.

Boisvert, R. F.; 20404.

Boisvert, R. F.; Rehm, R. G.; Sekerka, R. F.; Coriell, S. R.; 20448.

Bonfiglio, S.; Short, M. A.; SP604: 1981 June. 391.415.

Bonifazi, P.; Bordoni, F.; Pallottino, G. V.; Pizzella, G.; SP614: 1981 September. 298-301.

Bonnell, D. W.; Hastie, J. W.; NBSIR 80-2169.

Bonnell, D. W.; Hastie, J. W.; Plante, E. R.; NBSIR 81-2279.

Bonnell, D. W.; Plante, E. R.; Horton, W. S.; Hastie, J. W.; NBSIR 80-2178 (DoE).

Bonnett, W. J.; Guenther, F. R.; May, W. E.; Wise, S. A.; 20709.

Booker, R. L.; 20415.
Borchardt, B. R.; Carroll, C. L.; Hartsock, R. G.; Reeve, C. P.; Scire, F. E.; Veale, R. C.; Haight, W. C.; Hocken, R. J.; NBSIR 80-2141. Borchardt, B. R.; Hocken, R. J.; NBSIR 79-1752.

Bordoni, F.; Pallottino, G. V.; Pizzella, G.; Bonifazi, P.; SP614: 1981 September. 298-301.

Bornmann, P. L.; Ayres, T. R.; Linsky, J. L.; Worden, S. P.; Giampapa, M. S.; 20388.

Boscardin, R.; Hust, J. G.; 20494.

Bosman, G.; Zijlstra, R. J. J.; van Rheenen, A.; SP614: 1981 September. 409-413.

Bossler, J. D.; Goad, C. D.; Bender, P. L.; 20434.

Bottcher, C.; Gregory, D. C.; Crandall, D. H.; Pindzola, M. S.; Falk, R. A.; Dunn, G. H.; Griffin, D. C.; 20584.

Bottino, N. R.; Chakraborti, D.; Gennity, J. M.; Hillman, D. C.; O'Brien, D. H.; Pyles, R. A.; Stockton, R. A.; Wheeler, A. E.; Zingaro, R. A.; Irgolic, K. J.; Banks, C. H.; SP618: 1981 November. 244-263.

Bowen, R. L.; 20230.

Bowen, R. L.; 20380

Bowen, R. L.; U.S. Patent 4,251,565.

Bowen, R. L.; Gills, T. E.; 20169.

Bowen, R. L.; Misra, D. N.; 20116.

Bowen, R. L.; Misra, D. N. 20226.

Bowen, R. L.; Misra, D. N.; 20729.

Bowman, C. D.; 20457.

Bowman, C. D.; Johnson, R. G.; Behrens, J. W.; 20163.

Bowman, C. D.; Johnson, R. G.; Behrens, J. W.; 20724

Bowman, C. D.; Schrack, R. A.; Behrens, J. W.; Johnson, R.; 20458.

Bowman, R. R.; NBSIR 80-1634.

Boyce, M. P.; Norris, P. J.; SP622: 1981 October. 222-253.

Boyd, T. J.; Knapp, E. A.; Martin, R. E.; Potter, J. M.; Schneider, C. M.; Swenson, D. A.; Tallerico, P. J.; Penner, S.; Cutler, R. I.; Debenham, P. H.; Lindstrom, E. R.; Mohr, D. L.; Wilson, M. A. D.; Yoder, N. R.; Young, L. M.; 20206.

Boyer, P. A.; Ruff, A. W.; Marinenko, R. B.; Biancaniello, F.; DeRoberts, L.; SP260-70.

Boyle, D. R.; Johnson, E. G., Jr.; Phelan, R. J., Jr.; NBSIR 80-1628.

Braman, R. S.; Shelley, T. J.; SP618: 1981 November. 134-145.

Bransford, J. W.; Clark, A. F.; NBSIR 81-1647.

Branstad, D.,; FIPS PUB 81.

Branstad, D. K.,; FIPS PUB 74

Branstad, M. A.; Adrion, W. R.,; SP500-78.

Branstad, M. A.; Cherniavsky, J. C.; Adrion, W. R.; SP500-75.

Branthover, B.; Veale, R.; Siu, C.; NBSIR 80-2091.

Brauer, G. M.; 20254.

Brauer, G. M.; Antonucci, J. M.; Wu, W.; McKinney, J. E.; Whitlock, R. P.; Hinman, R.; Cassel, J. M.; Tesk, J. A.; NBSIR 802168.

Brauer, G. M.; Dulik, D.; Bernier, R.; 20392.

Brauer, G. M.; Dulik, D. M.; Hughes, H. N.; Dermann, K.; Rupp, N. W.; 20669.

Brauer, G. M.; Stansbury, J. W.; Antonucci, J. M.; 20401.

Braun, E.; NBSIR 81-2292.

Braun, E.; NBSIR 81-2261.

Braun, E.; Meade, R. E.; Smith, L. R.; NBSIR 81-2256.

Braun, E.; Peacock, R. D.; Hibbard, B. B.; Krasny, J. F.; NBSIR 81 2386.

Braun, E.; Reyes-Virella, R.; NBSIR 81-2200.

Braunlich, P.; Brost, G.; Schmid, A.; Kelly, P.; SP620: 1981 October. 406-415.

Braunlich, P.; Bryant, G.; Schmid, A.; SP620: 1981 October. 403-405.

Braunlich, P.; Kelly, P.; Ritchie, D.; Bryant, G. W.; Schmid, A.; SP620: 1981 October. 427.431.

Braunlich, P.; Schmid, A.; Bryant, G. W.; Kelly, P.; Ritchie, D.; SP620: 1981 October. 394-402.

Braunstein, M.; Braunstein, R.; Kim, R. K.; SP620: 1981 October. 29. 43.

Braunstein, R.; Kim, R. K.; Braunstein, M.; SP620: 1981 October. 29. 43.

Brayman, H. C.; Cool, T. A.; Fischell, D. R.; 19978.

Brayman, H. C.; Fischell, D. R.; Cool, T. A.; 19969. 
Breckenridge, F. R.; Clough, R. B.; Fuller, E. R.; Hsu, N. N.; Simmons, J. A.; Eitzen, D. G.; 20579.

Breckenridge, F. R.; Clough, R. B.; Fuller, E. R.; Hsu, N. N.; Simmons, J. A.; Eitzen, D. G.; 20757.

Breckenridge, F. R.; Greenspan, M.; 20489.

Breckenridge, F. R.; Hsu, N. N.; 20197.

Breitenberg, M. A.; 20162.

Breitenberg, M. A.; Atkins, R. G.; NBSIR 81-2336.

Bremer, S. G.; Peavy, S. T.; TN1147.

Brennan, J. A.; Kneebone, C. H.; Mann, D. B.; 20751.

Brennan, J. A.; Siegwarth, J. D.; 20633.

Brice, R.; Alexander, W.; SP500-83: 1981 November. 7-14.

Brickenkamp, C. S.; Hasko, S.; Natrella, M. G.; H133.

Brickenkamp, C. S.; Jones, F. E.; 20682.

Brickenkamp, C. S.,; H130, 1982 Edition.

Brierley, J. A.; SP618: 1981 November. 264-273.

Brinckman, F. E.; Fish, R. H.; Weiss, C. S.; Jewett, K. L.; SP618: 1981 November. 197.216.

Brinckman, F. E.; Fish, R. H.,; SP618.

Brinckman, F. E.; Olson, G. J.; Iverson, W. P.; NBSIR 81-2246.

Broadhurst, M. G.; Cassel, J. M.; Fanconi, B. M.; Sanchez, I. C.; Smith, L. E.; Eby, R. K.; NBSIR 81-2263.

Broadhurst, M. G.; Chang, S. S.; Davis, G. T.; DiMarzio, E. A.; Fanconi, B. M.; Han, C. C.; Khoury, F. A.; Markovitz, H.; McKinney, J. E.; Mopsik, F. I.; Passaglia, E.; Sanchez, I. C.; VanderHart, D. L.; Zapas, I. J.; Eby, R. K.; 20749.

Broadhurst, M. G.; Davis, G. T.; 20471.

Broadhurst, M. G.; Davis, G. T.; DeReggi, A. S.; 20639.

Broadhurst, M. G.; Fanconi, B. M.; Eby, R. K.; Peterlin, A.; Khoury, F. A.; 20652.

Brooks, J.; Mink, A.; Knoerdel, J.; 20223.

Brophy, J. J.; Haemmerli, A.; Janata, J.; SP614: 1981 September. 332. 334.

Brost, G.; Schmid, A.; Kelly, P.; Braunlich, P.; SP620: 1981 October. 406-415.

Brown, A.; Jordan, C.; Carpenter, K. G.; Wing, R. F.; Czyzak, S.; Stencel, R. E.; Linsky, J. L.; 20598.

Brown, J. D.; SP604: 1981 June. 381-389.

Brown, J. E.; SP480-43.

Brown, J. M.; Wise, S. A.; May, W. E.; 20586.

Brown, P. W.; Clifton, J. R.; NBSIR 81-2273.

Brown, R. L.; J. Res. 86(6): 605-654; 1981 November-December.

Brown, R. L.; J. Res. 86(4): 357.359; 1981 July-August.

Brown, S.; Ruff, W.,; Weinstein, A.; Gibbons, D.; SP601.

Brown, S. A.; Merritt, K.; SP601: 1981 January. 299-321.

Brown, S. A.; Merritt, K.; Simpson, J. P.; Geret, V.; SP601: 1981 January. 395-422.

Brown, S. D.; Brown, T. F.; Caster, D. M.; SP618: 1981 November. 163.172.

Brown, T. F.; Caster, D. M.; Brown, S. D.; SP618: 1981 November. 163.172.

Brown, W. E.; Austin, M.; Negas, T.; Mathew, M.; 19984.

Brown, W. E.; Chow, L. C.; 20271.

Brown, W. E.; Jordan, T. H.; Dickens, B.; Schroeder, L. W.; 19994.

Brown, W. E.; Mathew, M.; Tung, M. S.; 20407.

Brown, W. E.; Schroeder, L. W.; Jordan, T. H.; 20173.

Brown, W. E.; Tung, M. S.; Chow, L. C.; 20122.

Brown-Thomas, J. M.; Hilpert, L. R.; Wise, S. A.; May, W. E.; 20750.

Brummer, J. G.; Wilkinson, F.; JPCRD 10(4): 809-999; 1981.

Brunetti, R.; Jacoboni, C.; Reggiani, L.; SP614: 1981 September. 414. 416.

Bryan, J. L.; DiNenno, P. J.; Milke, J. A.,; NBS-GCR-80-297.

Bryan, J. L.; Milke, J. A.,; NBS-GCR-81-343.

Bryant, G.; Schmid, A.; Braunlich, P.; SP620: 1981 October. 403-405.

Bryant, G. W.; Girvin, S. M.; Rendell, R. W.; 20629.

Bryant, G. W.; Kelly, P.; Ritchie, D.; Braunlich, P.; Schmid, A.; SP620: 1981 October. 394.402.

Bryant, G. W.; Schmid, A.; Braunlich, P.; Kelly, P.; Ritchie, D.; SP620: 1981 October. 427.431.

Buchholz, A.; Wrzenski, S. C.,; Egan, J.; Higgins, R.; Spath, J.; NBS-
GCR $81 \cdot 313$.

Buchhorn, G.; Buchhorn, U.; Semlitsch, M.; Willert, H. G.; SP601: 1981 January. 239-267.

Buchhorn, U.; Semlitsch, M.; Willert, H. G.; Buchhorn, G.; SP60I: 1981 January. 239-267.

Buckingham, M. J.; SP614: 1981 September. 38-41.

Buckley, F. T.; Purtell, L. P.; Klebanoff, P. S.; 20257.

Buehler, M. G.; Carver, G. P.; Mattis, R. L.; NBSIR 81-2234.

Buene, L.; Jacobson, D. C.; Poate, J. M.; Nakahara, S.; Draper, C. W.; SP620: 1981 October. 222-226.

Buhl, D.; Clark, F. O.; Chin, G.; Glenar, D.; Kostiuk, T.; Mumma, M. J.; Lovas, F. J.; 20321.

Bukowski, R. W.; NBSIR 80-2170.

Bulik, C.; Siu, M. C. I.; 20735.

Bullis, W. M.; 20363.

Bullis, W. M.; 20371

Bullis, W. M.; 20372.

Bullis, W. M.; NBSIR 81-2224.

Bullis, W. M.,; NBSIR 80-2006-3.

Bullis, W. M.,; NBSIR 79-1591-4.

Bullis, W. M.,; NBSIR 79-1591-5.

Bullis, W. M.,; NBSIR 80-2006-4.

Bullis, W. M.,; NBSIR 81-2230.

Bullis, W. M.,; NBSIR 81-2230-2.

Bur, A. J.; 20638.

Bur, A. J.; Tsao, A. K.; 20529.

Buran, K. A.; Gibbons, D. F.; SP601: 1981 January. 157-174.

Burch, D. M.; Gujral, P. S.; Clark, R. J.; 20538.

Burch, T.,; Maxwell, T. T.; Maples, G.; Dyer, D. F.; NBS-GCR-80292.

Burek, A. J.; Barrus, D. M.; Blake, R. L.; Fenimore, E. E.; 20374.

Burge, D. K.; Bennett, H. E.; SP620: 1981 October. 356.368.

Burkhalter, D. S.; Tobler, R. L.; Reed, R. P.; 20742.

Burley, C. E.; SP589: 1981 January. 39-44.

Burnett, E. D.; 20311.

Burnham, A. W.; Loucks, S. R.; SP400-69: 1981 May. 133-142.

Burns, G. W.; Dils, R. R.; Whetstone, J. R.; Frederikse, H. P. R.; Schenck, P. K.; NBSIR 81-2348.

Burris, B. L.; Morehouse, R. J.,; SP305. Supplement 12.

Burstein, A. H.; Wright, T. M; SP601: 1981 January. 559.572.

Burton, D. S.; Nagel, D. A.; McShane, D.; Schurman, D. J.; SP601: 1981 January. 615-642.

Buschbach, T. R.; SP500-83: 1981 November. 341.

Busching, H. W.; Cullen, W. C.; Rossiter, W. J., Jr.; Mathey, R. G.; TN1135.

Bush, T.; Mazzone, G.; McElroy, G.; Galbraith, T.,; Josephson, E.; NBS-GCR-81-312.

Bussey, H. E.; Little, W. E.; Metzker, R. F.; Jones, R. N.; NBSIR 78897.

Byrd, E. A.; Hansen, R. G.; SP607: 1981 May. 136-140.

Byrne, S. T.; El-Torkey, N. M.; Margolis, S. A.; Kaplan, A. E.; Weiss, E. R.; 20446.

\section{C}

Cabrera, L. F.; Pâris, J. F.; SP500-83: 1981 November. 157-168.

Cahn, J.; Mehrabian, R.; Free, G.; Berger, H.; Mordfin, L.; Swartzendruber, L.; Boettinger, W.; Ives, L.; Coriell, S.; Ballard, D.; Laughlin, D.; Clough, R.; Biancaniello, F.; Blau, P.; NBSIR 802069.

Cahn, J. W.; 19981.

Cahn, J. W.; Bennett, L. H.; Goodman, D. A.; 20433.

Cahn, J. W.; Johnson, W. C.; 20725.

Callcott, T. A.; Arakawa, E. T.; Ederer, D. L.; 20216.

Callender, E.; Hartsoıgh, C.; Kleine, H.; SP500-80: 1981 October. 44. 48.

Callender, E.; Sayani, H. H.; Teichroew, D.; SP500-80: 1981 October. $38-43$.

Cameron, J.; Parobeck, P.; Tomb, T.; Ku, H.; 20566.

Campbell, P. G.; Martin, J. W.; McKnight, M. E.; TN1149.

Campbell, P. G.; Sleater, G. A.; Post, M. A.; NBSIR 80-2122. 
Canada, R. G.; Jones, J. E.; SP622: 1981 October. 3-10.

Candela, G. A.; Roth, R. S.; Swartzendruber, L. J.; Romanov, V. P.; 20043.

Candy, M.; Harwood, D.; Verveer, A.; Quinn, P. J.; Wilson, I.; Page, A. A.; Higson, P.; Deward, F. D.; Haisch, B.; Linsky, J. L.; Slee, O. B.; Siegman, B. C.; Nikoloff, I.; 20405.

Capozzi, D. M.,; NBS-GCR-80-298.

Cappuccio, G.; Petrocco, G.; Fotinos, C.; Stochino, G.; D'Amico, A.; SP614: 1981 September. 199-201.

Carelli, P.; Cromar, M. W.; 20488.

Carino, N. J.; NBSIR 81-2244.

Carino, N. J.; Fattal, S. G.; Batts, M. E.; Lew, H. S.; NBSIR 81-2374.

Carlock, J. B. III,; NBS-GCR-81-321.

Carlson, C. E.; Coyne, J. J.; Fishbane, P. M.; Gross, F.; Meshkov, S.; 20034.

Carlson, C. E.; Coyne, J. J.; Fishbane, P. M.; Gross, F.; Meshkov, S.; 20187.

Carlson, C. E.; Coyne, J. J.; Fishbane, P. M.; Gross, F.; Meshkov, S.; 20316.

Carmeli, B.; Nitzan, A.; Ben-Jacob, E.; Bergman, D.; SP614: 1981 September. 381.383.

Carpenter, B. S.; Hoffman, J. D.; Yolken, H. T.; Reed, W. P.; NBSIR 81-2205.

Carpenter, B. S.; Pilione, L. J.; 19982.

Carpenter, K. G.; Wing, R. F.; Czyzak, S.; Stencel, R. E.; Linsky, J. L.; Brown, A.; Jordan, C.; 20598.

Carroll, C. L.; Hartsock, R. G.; Reeve, C. P.; Scire, F. E.; Veale, R. C.; Haight, W. C.; Hocken, R. J.; Borchardt, B. R.; NBSIR 802141.

Carroll, J. J.; Melmed, A. J.; Kruger, J.; Ritter, J. J.; NBSIR 81-2396 (ONR).

Carroll, J. J.; Melmed, A. J.; Ritter, J. J.; Ambrose, J. R.; Kruger, J.; NBSIR 79-1904 (Navy).

Carver, G. P.; NBSIR 81-2319.

Carver, G. P.; Cullins, W. A.; SP400-68.

Carver, G. P.; Mattis, R. L.; Buehler, M. G.; NBSIR 81-2234.

Casella, R. C.; 20398.

Casella, R. C.; 20384.

Casella, R. C.; 20656.

Cassel, D. E.; Overton, R. L.; Shingleton, J. G.; NBS-GCR-81-302.

Cassel, J. M.; Dehl, R. E.; Grant, W. H.; NBSIR 81-2212.

Cassel, J. M.; Fanconi, B. M.; Sanchez, I. C.; Smith, L. E.; Eby, R. K.; Broadhurst, M. G.; NBSIR 81-2263.

Cassel, J. M.; Tesk, J. A.; Brauer, G. M.; Antonucci, J. M.; Wu, W.; McKinney, J. E.; Whitlock, R. P.; Hinman, R.; NBSIR 80-2168.

Cassinelli, J. P.; Fabbiano, G.; Giacconi, R.; Golub, L.; Gorenstein, P.; Haisch, B. M.; Harnden, F. R., Jr.; Johnson, H. M.; Linsky, J. L.; Maxson, C. W.; Mewe, R.; Rosner, R.; Seward, F.; Topka, K.; Zwaan, C.; Vaiana, G. S.; 20336.

Caster, D. M.; Brown, S. D.; Brown, T. F.; SP618: 1981 November. 163-172.

Caswell, R. S.; Coyne, J. J.; Goodman, L. J.; 20670.

Cattaneo, L.; Harris, J. R.; Reinhold, T. A.; Simiu, E.; Yancey, C. W. C.; NBSIR 81-2199.

Cattaneo, L. E.; Leyendecker, E. V.; NBSIR 80-2111-5.

Cavallo, L. M.; Mann, W. B.; 20165.

Cavanagh, R. R.; Yates, J. T.; 20219.

Cavanagh, R. R.; Yates, J. T.; 20228.

Cavanagh, R. R.; Yates, J. T., Jr.; 19997.

Cavanagh, R. R.; Yates, J. T., Jr.; 20498.

Celasco, M.; Mazzetti, P.; Stepanescu, A.; SP614: 1981 September. 34. 37.

Celotta, R. J.; Pierce, D. T.; 19996.

Celotta, R. J.; Pierce, D. T.; 20693.

Celotta, R. J.; Pierce, D. T.; Girvin, S. M.; Unguris, J.; 20694.

Celotta, R. J.; Pierce, D. T.; Wang, G. C.; 20138.

Celotta, R. J.; Pierce, D. T.; Wang, G. C.; Bader, S. D.; Felcher, G. P.; 19970.

Celotta, R. J.; Pierce, D. T.; Wang, G. C.; Felcher, G. P.; Bader, S. D.; 19975.

Celotta, R. J.; Wang, G. C.; McRae, E. G.; Pierce, D. T.; 20643.
Celotta, R. J.; Wang, G. C.; Pierce, D. T.; 20012.

Celotta, R. J.; Wang, G. C.; Pierce, D. T.; 20706.

Cermak, M.; Vasina, P.; Sikula, J.; Cermakova, A.; SP614: 1981 September. 125-129.

Cermakova, A.; Cermak, M.; Vasina, P.; Sikula, J.; SP614: 1981 September. 125-129.

Cerutti, E.; Berger, P. W.; 20136.

Cetegen, B.,; Zukoski, E. E.; Kubota, T.; NBS-GCR-80-294.

Cetegen, B.,; Zukoski, E. E.; Kubota, T.; NBS-GCR-81-346.

Ceyer, S. T.; Tung, R. T.; Graham, W. R.; Melmed, A. J.; 20686.

Cezairliyan, A.; 20191.

Cezairliyan, A.; 20593.

Cezairliyan, A.; Miiller, A. P.; 20137.

Cezairliyan, A.; Miiller, A. P.; 20542.

Chakraborti, D.; Gennity, J. M.; Hillman, D. C.; O'Brien, D. H.; Pyles, R. A.; Stockton, R. A.; Wheeler, A. E.; Zingaro, R. A.; Irgolic, K. J.; Banks, C. H.; Bottino, N. R.; SP618: 1981 November. 244-263.

Chalmet, L. G.,; Francis, R. L.; NBS-GCR-81-316.

Chamberlain, G. E.; Franzen, D. L.; Day, G. W.; Danielson, B. L.; Kim, E. M.; 20369.

Chandler, P.; SP500-83: 1981 November. 15-19.

Chandy, K. M.; Misra, J.; Berry, R.; Neuse, D.; SP500-83: 1981 November. 331-334.

Chang, D. C.; Ma, M. T.; Sreenivasiah, I.; 20623.

Chang, D. C.; Ma, M. T.; Sreenivasiah, I.; TN1040.

Chang, D. C.; Ma, M. T.; Wilson, P. F.; TN1037.

Chang, D. C.; Tippet, J. C.; NBSIR 78-873.

Chang, H. T.; Veneruso, A. F.; SP622: 1981 October. 310.320

Chang, J. C.; Travis, J. P.; Clough, R. B.; 20276.

Chang, S. S.; Davis, G. T.; DiMarzio, E. A.; Fanconi, B. M.; Han, C. C.; Khoury, F. A.; Markovitz, H.; McKinney, J. E.; Mopsik, F. I.; Passaglia, E.; Sanchez, I. C.; VanderHart, D. L.; Zapas, I. J.; Eby, R. K.; Broadhurst, M. G.; 20749.

Chang, S. S.; McCrackin, F. L.; Senich, G. A.; Wang, F. W.; Smith, L. E.; NBSIR 81-2264.

Chang, Y. M. L.; Grot, R. A.; NBSIR 80-2136.

Changery, M. J.; Batts, M. E.; 20551.

Chanmugam, G.; 20108.

Chanmugam, G.; 20416.

Chanmugam, G.; Dulk, G. A.; 20417.

Chanmugam, G.; Shaviv, G.; Langer, S. H.; 20412.

Chapman, R. E.; Ruegg, R. T.; NBSIR 81-2248.

Chapman, R. E.; Thompson, B. E.; NBSIR 80-2150.

Chapman, R. E.; Thompson, B. E.; NBSIR 81-2250.

Chau, Y. K.; Wong, P. T. S.; SP618: 1981 November. 65-80.

Chen, H.; Kuriyama, M.; 20671.

Chen, H. S.; Goldfarb, R. B.; Rao, K. V.; Fickett, F. R.; 20317.

Chen, H. S.; Lynn, J. W.; Erwin, R. W.; Rhyne, J. J.; 20282.

Chen, P. T.; Hill, J. E.; May, W. B., Jr.; Richtmyer, T. E.; Elder, J.;

Tibbott, R. L.; Yonemura, G. T.; Hunt, C. M.; BSS133.

Chen, P. T.; Lerchen, F. H.; Pielert, J. H.; NBSIR 81-2289.

Chenette, E. R.; Handel, P. H.; Kilmer, J.; van Vliet, K. M.; SP614: 1981 September. 151.

Cherniavsky, J. C.; Adrion, W. R.; Branstad, M. A.; SP500-75.

Cherry, S. M.,; NBSIR 81-2382.

Cherry, S. M.; Martinez, I. M.; NBSIR 80-2127-1.

Chesler, S. N.; Hilpert, L. R.; Guenther, F. R.; Parris, R. M.; 20161.

Chesler, S. N.; May, W. E.; White, P. A.; Parris, R. M.; Guenther, F. R.; 20602.

Chew, H.; NBSIR 81-1653.

Chiang, C. K.; 20655.

Chiang, C. K.; Franklin, A. D.; Snellgrove, R. A.; Bethin, J. R.; 20497.

Chiang, W. P.; DeSmith, D. A.; Leder, D. S.; Nguyen, D. K.; Perreault, R. W.; Fry, J. P.; NBSIR 81-2315.

Childers, C. B.; Tremaine, S. G.; Lentner, K. J.; 20093.

Chin, G.; Glenar, D.; Kostiuk, T.; Mumma, M. J.; Lovas, F. J.; Buhl, D.; Clark, F. O.; 20321.

Chobola, Z.; Sikula, J.; Vasina, P.; Koktavy, B.; SP614: 1981 September. 248-250. 
Chow, L. C.; Brown, W. E.; 20271.

Chow, L. C.; Brown, W. E.; Tung, M. S.; 20122.

Chow, L. C.; Guo, M. K.; Hsieh, C. C.; Hong, Y. C.; 20672.

Christ, B. W.; Polvani, R. S.; 20612.

Christensen, R.; White, E.; Hertz, H.; U.S. Patent 4,281,246.

Christensen, R. G.; Hertz, H. S.; Meiselman, S.; White, E.; 20097.

Christensen, R. G.; Hertz, H. S.; Reeder, D. J.; White, E.; 20654.

Christensen, R. G.; Howell, B. F.; Paule, R. C.; Mandel, J.; Schaffer,

R.; Reeder, D. J.; Coxon, B.; Enagonio, D.; SP260-72.

Chu, C. M.; Wilson, C. B.; Mohr, J. M.; SP500-83: 1981 November. 59.65 .

Chuang, T.; NBSIR 81-2255.

Chung, R. M.; Yancey, C. W. C.; Yokel, F. Y.; NBSIR 81-2238.

Chung, R. M.; Yokel, F. Y.; Salomone, L. A.; NBSIR 81-2215.

Churney, K. L.; Garvin, D.; NBSIR 80-2018 (DOT).

Ciment, M.; Berger, A. E.; Solomon, J. M.; 20626.

Cimino, W. R.; Ries, M. D.; Rose, R. M.; Crugnola, A. N.; SP601: 1981 January. 3-28.

Clark, A. F.; Bransford, J. W.; NBSIR 81-1647.

Clark, A. F.; Fickett, F. R.; 20069.

Clark, A. F.; Fickett, F. R.; Goodrich, L. F.; NBSIR 80-1642.

Clark, A. F.; Fujii, G.; Ekin, J. W.; Radebaugh, R.; 20298.

Clark, A. F.; Fujii, G.; Ranney, M. A.; 20465.

Clark, A. F.; Fujii, G.; Ranney, M. A.; 20753.

Clark, A. F.; Radebaugh, R.; Fujii, G.; Read, D. T.; 19999.

Clark, A. F.; Strobridge, T. R.; Moulder, J. C.; 20436.

Clark, E. S.; Eby, R. K.; Weeks, J. J.; 20635.

Clark, E. S.; Weeks, J. J.; Eby, R. K.; 20708.

Clark, F. O.; Chin, G.; Glenar, D.; Kostiuk, T.; Mumma, M. J.;

Lovas, F. J.; Buhl, D.; 20321.

Clark, F. O.; Johnson, D. R.; 20517.

Clark, F. O.; Johnson, D. R.; Troland, T. H.; Heiles, C. E.; 20439.

Clark, G. C. F.; Williams, D. F.; McNamara, A.; SP601: 1981 January. 323-336.

Clark, H. E.; SP613.

Clark, R. E.; 20752.

Clark, R. E.; NBSIR 81-2335.

Clark, R. E.; Grot, R. A.; 20540.

Clark, R. J.; Burch, D. M.; Gujral, P. S.; 20538.

Clarke, I. C.; SP601: 1981 January. 471.499.

Clarke, J.; Koch, R. H.; van Harlingen, D. J.; SP614: 1981 September. 359-363.

Clarke, J. T.; Moos, H. W.; 20509.

Clarke, J. T.; Moos, H. W.; Atreya, S. K.; Lane, A. L.; 20180.

Clarren, S.; Nalley, P.; Gregory, G.; Frederick, W.; Hausmann, R.,; NBS-GCR-ETIP 81-96.

Clemons, E. K.; Deutsch, D. R.; 20644.

Clemow, A. J. T.; McPhillips-Meade, M.; Klawitter, J. J.; French, G.; Skinner, H.; Weinstein, A. M.; SP601: 1981 January. 423-447.

Clifton, C.; Nober, E. H.; Pierce, H.; Well, A.; Johnson, C. C.; NBSGCR-80-284.

Clifton, J. R.; SP480-45.

Clifton, J. R.; Anderson, E. D.; NBSIR 80-2163.

Clifton, J. R.; Brown, P. W.; NBSIR 81-2273.

Clifton, J. R.; Frohnsdorff, G.; NBSIR 81-2239.

Clifton, J. R.; Meese, W. J.; Beausoliel, R. W.; NBSIR 81-2220.

Clifton, J. R.; Wu, S. T.; NBSIR 81-2390.

Clough, R.; Biancaniello, F.; Blau, P.; Cahn, J.; Mehrabian, R.; Free, G.; Berger, H.; Mordfin, L.; Swartzendruber, L.; Boettinger, W.; Ives, L.; Coriell, S.; Ballard, D.; Laughlin, D.; NBSIR 80-2069.

Clough, R. B.; Chang, J. C.; Travis, J. P.; 20276.

Clough, R. B.; Fuller, E. R.; Hsu, N. N.; Simmons, J. A.; Eitzen, D. G.; Breckenridge, F. R.; 20579.

Clough, R. B.; Fuller, E. R.; Hsu, N. N.; Simmons, J. A.; Eitzen, D. G.; Breckenridge, F. R.; 20757.

Clough, R. B.; Simmons, J. A.; 20701.

Clough, R. B.; Simmons, J. A.; 20673.

Cobb, E. N.; Wu, W.; 20383.

Codling, K.; Dehmer, J. L.; Parr, A. C.; Ederer, D. L.; Cole, B. E.; West, J. B.; Stockbauer, R.; 20123.

Codling, K.; Ederer, D. L.; Parr, A. C.; Cole, B. E.; Stockbauer, R.;
Dehmer, J. L.; West, J. B.; 20676.

Codling, K.; Parr, A. C.; Ederer, D. L.; Cole, B. E.; Stockbauer, R.; Dehmer, J. L.; West, J. B.; 20426.

Codling, K.; Parr, A. C.; Ederer, D. L.; Stockbauer, R.; West, J. B.; Cole, B. E.; Dehmer, J. L.; 20399.

Codling, K.; West, J. B.; Parr, A. C.; Dehmer, J. L.; Stockbauer, R. L.; 20052.

Cohen, E. C.,; Schafft, H. A.; Ruthberg, S.; SP400-69.

Cohen, E. R.; Birnbaum, G.; NBSIR 80-2175.

Cohen, M.; Kruger, J.; Escalante, E.; Bertocci, U.; Mullen, J.; 20348.

Cohill, M.; Hegner, H.; Envent, F.; SP622: 1981 October. 113.124.

Colbert, J.; Decker, P.; Abramowitz, S.; Domalski, E. S.; Kirklin, D. R.; NBSIR 81-2278.

Colbert, J. C.; Xiheng, H.; Kirklin, D. R.; J. Res. 86(6): 655-660; 1981 November-December.

Cole, B. E.; Cooper, J. W.; Ederer, D. L.; Mehlman, G.; Saloman, E. B.; 20300.

Cole, B. E.; Dehmer, J. L.; Codling, K.; Parr, A. C.; Ederer, D. L.; Stockbauer, R.; West, J. B.; 20399.

Cole, B. E.; Saloman, E. B.; Ederer, D. L.; 20674

Cole, B. E.; Stockbauer, R.; Dehmer, J. L.; West, J. B.; Codling, K.; Ederer, D. L.; Parr, A. C.; 20676.

Cole, B. E.; Stockbauer, R.; Dehmer, J. L.; West, J. B.; Codling, K.; Parr, A. C.; Ederer, D. L.; 20426.

Cole, B. E.; West, J. B.; Stockbauer, R.; Codling, K.; Dehmer, J. L.; Parr, A. C.; Ederer, D. L.; 20123.

Cole-Appel, B. E.; Oonk, R. L.; Jones, D. E.; 20148.

Colella, R. P.; Wallace, M. A.; McCoy, W. H.; 20662.

Collé, R.; Rubin, R. J.; Knab, L. I.; Hutchinson, J. M. R.; TN1139.

Collins, B.; Turner, G.; NBSIR 81-2310.

Collins, B. L.; Danner, W. F.; Tibbott, R. L.; NBSIR 81-2428.

Collins, B. L.; Lerner, N. D.; NBSIR 80-2003.

Collins, B. L.; Lerner, N. D.; NBSIR 80-2088.

Collins, L. A.; Padial, N. T.; Norcross, D. W.; 20534.

Collins, L. A.; Schmid, G. B.; Norcross, D. W.; 20055.

Comas, J.; Myers, D. R.; Wilson, R. G.; 20112.

Conditt, M. K.; Stanley, J. S.; Sievers, R. E.; SP618: 1981 November. 93.104.

Conti, D. M.; 20747.

Conti, D. M.; SP500-83: 1981 November. 339.

Conti, D. M.; Giese, K. W.; Kyriazi, C. N.; Trehan, R.; SP500-83: 1981 November. 311-312.

Conti, D. M.,; Letmanyi, H.; FIPS PUB 75.

Contini, M.; Kozlovsky, B. Z.; Shaviv, G.; 20303.

Cook, S. D.; Weinstein, A. M.; SP601: 1981 January. 573.613.

Cooke, P. W.; NBSIR 80-2081.

Cool, T. A.; Brayman, H. C.; Fischell, D. R.; 19969.

Cool, T. A.; Fischell, D. R.; Brayman, H. C.; 19978.

Cool, T. A.; Koffend, J. B.; 20177.

Cooper, J.; Smith, E. W.; Talin, B.; 20479.

Cooper, J. W.; Ederer, D. L.; Mehlman, G.; Saloman, E. B.; Cole, B. E.; 20300

Cooper, L. Y.; 20590.

Cooper, L. Y.; Harkleroad, M.; Quintiere, J.; Rinkinen, W.; 20591.

Cooper, L. Y.; O'Neill, J. G.; NBSIR 81-2202.

Cooper L. Y.; NBSIR 80-2172.

Cordes, M. R.; Boettinger, W. J.; Sekerka, R. F.; Coriell, S. R.; 20224.

Coriell, S.; Ballard, D.; Laughlin, D.; Clough, R.; Biancaniello, F.; Blau, P.; Cahn, J.; Mehrabian, R.; Free, G.; Berger, H.; Mordfin, L.; Swartzendruber, L.; Boettinger, W.; Ives, L.; NBSIR 80-2069.

Coriell, S. R.; Boettinger, W. J.; Biancaniello, F. S.; 20132.

Coriell, S. R.; Boisvert, R. F.; Rehm, R. G.; Sekerka, R. F.; 20448.

Coriell, S. R.; Cordes, M. R.; Boettinger, W. J.; Sekerka, R. F.; 20224.

Corley, D. M.; Baum, H. R.; Rehm, R. G.; Barnett, P. D.; NBSIR 81 2385.

Corliss, C.; Sugar, J.; JPCRD 1O(1): 197-290; 1981.

Corliss, C.; Sugar, J.; JPCRD 10(4): 1097-1174; 1981.

Corliss, C. H.; Wiese, W. L.; Martin, G. A.; Reader, J.; NSRDSNBS68. 
Cornell, C. A.,; NBS-GCR-76-81.

Correia, R. J.; Kestin, J.; Khalifa, H. E.; JPCRD 10(1): 71.88; 1981. Correia, R. J.; Kestin, J.; Khalifa, H. E.; JPCRD 10(1): 57.70; 1981. Corti, M.; SP614: 1981 September. 335-338.

Costabile, G.; Jach, T.; Holdeman, L. B.; Soulen, R. J., Jr.; Van Vechten, D.; 20702.

Costabile, G.; Kautz, R. L.; 20183.

Cotton, I. W.; 20205.

Cotton, I. W.; SP610: 1981 December. 1.8.

Courbier, R.; David, M.; Noël, H. P.; Guidoin, R.; King, M.; Blais, P.; Marois, M.; Gosselin, C.; Roy, P.; SP601: 1981 January. 29-129.

Coursey, B. M.; Mann, W. B.; 20368.

Coursey, B. M.; Moghissi, A. A.; 20367.

Covington, A. K.; Goldberg, R. N.; Sarbar, M.; 20714.

Cox, A. N.; Shaviv, G.; Hodson, S. W.; 20337.

Cox, C. E.; Kandiah, K.; SP614: 1981 September. 71.74.

Cox, L. A., Jr.; Hodgins, B. D.; SP500-83: 1981 November. 181-189.

Coxon, B.; Enagonio, D.; Christensen, R. G.; Howell, B. F.; Paule, R.

C.; Mandel, J.; Schaffer, R.; Reeder, D. J.; SP260-72.

Coyne, J.; McDonald, J. C.; Menzel, H. G.; Schuhmacher, H.; 20675.

Coyne, J. J.; 20064.

Coyne, J. J.; Fishbane, P. M.; Gross, F.; Meshkov, S.; Carlson, C. E.; 20034.

Coyne, J. J.; Fishbane, P. M.; Gross, F.; Meshkov, S.; Carlson, C. E.; 20187.

Coyne, J. J.; Fishbane, P. M.; Gross, F.; Meshkov, S.; Carlson, C. E.; 20316.

Coyne, J. J.; Goodman, L. J.; Caswell, R. S.; 20670.

Craig, P. J.; Rapsomanikis, S.; SP618: 1981 November. 54.64.

Crandall, D. H.; Pindzola, M. S.; Falk, R. A.; Dunn, G. H.; Griffin, D. C.; Bottcher, C.; Gregory, D. C.; 20584.

Crawford, M. L.; NBSIR 81-1638.

Crawford, M. L.; Workman, J. L.; TN1013.

Crecelius, E. A.; Gurtisen, J. M.; Apts, C. W.; SP618: 1981 November. 173-183.

Crissman, J. M.; McKenna, G. B.; Khoury, F. A.; NBSIR 81.2209 (FDA).

Crissman, J. M.; Zapas, L. J.; 20327.

Crissman, J. M.; Zapas, L. J.; 20299.

Cromar, M. W.; Carelli, P.; 20488.

Cronin, D.; Wenzel, J.; Kauffman, D.; Scarfe, C. M.; 20222.

Crugnola, A. N.; Cimino, W. R.; Ries, M. D.; Rose, R. M.; SP601: 1981 January. 3-28.

Cruz, J. E.; 20634.

Cugini, J. V.; SP500-77.

Culbertson, R. J.; Melmed, A. J.; Sakurai, T.; 19983.

Cullen, W. C.; Rossiter, W. J., Jr.; Mathey, R. G.; Busching, H. W.; TN1135.

Cullins, W. A.; Carver, G. P.; SP400-68.

Cummings, A. L.; Layer, H. P.; Hocken, R. J.; 20268.

Cunningham, M. L.; SP500-83: 1981 November. 305-307.

Cutkosky, R. D.; 20106.

Cutkosky, R. D.; 20578.

Cutkosky, R. D.; Davis, R. S.; 20610.

Cutler, R. I.; Debenham, P. H.; Lindstrom, E. R.; Mohr, D. L.; Wilson, M. A. D.; Yoder, N. R.; Young, L. M.; Boyd, T. J.; Knapp, E. A.; Martin, R. E.; Potter, J. M.; Schneider, C. M.; Swenson, D. A.; Tallerico, P. J.; Penner, S.; 20206.

Czyzak, S.; Stencel, R. E.; Linsky, J. L.; Brown, A.; Jordan, C.; Carpenter, K. G.; Wing, R. F.; 20598.

\section{D}

Dabrowski, A. J.; Singh, M.; Huth, G. C.; Iwanczyk., J. S.; SP604: 1981 June. 45-59.

Dahlberg, S. C.; Madey, T. E.; Houston, J. E.; 20279.

Daley, T. W.; Lafferty, W. J.; Sattler, J. P.; Worchesky, T. L.; Tobin, M. S.; Ritter, K, J.; 20090.

D'Amico, A.; SP614: 1981 September. 314-316.

D'Amico, A.; Cappuccio, G.; Petrocco, G.; Fotinos, C.; Stochino, G.; SP614: 1981 September. 199-201.
Dampier, I. K.,; Dickamore, W. G.; NBS-GCR-80-306.

Daney, D. E.; SP607: 1981 May. 48-56.

Daney, D. E.; Jones, M. C.; 20507.

Daney, D. E.; Ludtke, P. R.; Eroshenko, V. M.; Yaskin, L. A.; 20400.

Daney, D. E.,; NBSIR 80-1637.

Daney, D. E.,; NBSIR 81-1649.

Danielson, B. L.; TN1034.

Danielson, B. L.; Kim, E. M.; Chamberlain, G. E.; Franzen, D. L.; Day, G. W.; 20369.

Danileiko, Y. K.; Manenkov, A. A.; Nechitailo, V. S.; SP620: 1981 October. 369-374.

Danner, W. F.; Tibbott, R. L.; Collins, B. L.; NBSIR 81-2428.

Danner, W. F.; Yaniv, S. L.; Bauer, J. W.; Flynn, D. R.; NBSIR 81 2377.

Danos, M.; 20376.

Danos, M.; 20516.

Danos, M.; Motz, J. W.; Dick, C. E.; 20450.

Danos, M.; Rafelski, J.; 20109.

Danos, M.; Zanon, D.; 20609.

Darby, L. F.; SP610: 1981 December. 9.12.

Darling, D. H.; Webb, W. W.; Scofield, J. H.; SP614: 1981 September. 147-150.

Darsey, S. J.; SP599: 1981 January. 241-248.

Datta, S. K.; Ledbetter, H. M.; 20608.

Daunt, J. G.; Heiden, C.; SP607: 1981 May. 141-145.

David, M.; Noël, H. P.; Guidoin, R.; King, M.; Blais, P.; Marois, M.; Gosselin, C.; Roy, P.; Courbier, R.; SP601: 1981 January. 29.129.

Davis, G. T.; Broadhurst, M. G.; 20471.

Davis, G. T.; DeReggi, A. S.; Broadhurst, M. G.; 20639.

Davis, G. T.; DiMarzio, E. A.; Fanconi, B. M.; Han, C. C.; Khoury, F. A.; Markovitz, H.; McKinney, J. E.; Mopsik, F. I.; Passaglia, E.; Sanchez, I. C.; VanderHart, D. L.; Zapas, I. J.; Eby, R. K.; Broadhurst, M. G.; Chang, S. S.; 20749.

Davis, H. A.; Sengers, J. M. H. L.; Klein, M.; Waxman, M.; NBSIR 79-1715.

Davis, R. S.; Cutkosky, R. D.; 20610.

Davis, R. S.; Schoonover, R. M.; 20247.

Davis, R. W.; Deutsch, S.; 19971.

Davis, R. W.; Moore, E. F.; 19972.

Davis, S.; Evans, D. D.; NBSIR 81-2213.

Day, G. W.; TN1046.

Day, G. W.; Danielson, B. L.; Kim, E. M.; Chamberlain, G. E.; Franzen, D. L.; 20369.

Day, G. W.; Franzen, D. L.; 20347.

Day, G. W.,; TN1043.

Day, R. H.; Lee, P.; Saloman, E. B.; Nagel, D. J.; 20637.

Daywitt, W. C.; NBSIR 78-869.

Deaton, T. F.; Rainer, F.; Milam, D.; Smith, W. L.; SP620: 1981 October. 297-299.

Debelius, J. R.; SP605.

Debenham, P. H.; 20356.

Debenham, P. H.; Lindstrom, E. R.; Mohr, D. L.; Wilson, M. A. D.; Yoder, N. R.; Young, L. M.; Boyd, T. J.; Knapp, E. A.; Martin, R. E.; Potter, J. M.; Schneider, C. M.; Swenson, D. A.; Tallerico, P. J.; Penner, S.; Cutler, R. I.; 20206.

deCastro, C. A. N.; Roder, H. M.; J. Res. 86(3): 293-307; 1981 May. June.

Decher, R.; Randall, J. L.; Bender, P. L.; Faller, J. E.; 20451.

Decker, D. L.; Hodgkin, V. A.; SP620: 1981 October. 190-200.

Decker, D. L.; Seitel, S. C.; Soileau, M. J.; Porteus, J. O.; SP620: 1981 October. 201-209.

Decker, P.; Abramowitz, S.; Domalski, E. S.; Kirklin, D. R.; Colbert, J.; NBSIR 81-2278.

Decorte, K. N.; McCabe, M. E.; Ducas, W.; Orloski, M. J.; NBSIR $81-2300$.

de Goede, J.; van den Berg, R. J.; de Vos, A.; SP614: 1981 September. 217.220.

DeHaan, W. R.; Saib, S. H.; Benson, J. P.; Gannon, C.; SP500-80: 1981 October. 200-207.

DeHaan, W. R.; Saib, S. H.; Benson, J. P.; Gannon, C.; SP500-80: 1981 October. 208-217. 
Dehl, R. E.; Grant, W. H.; 20442.

Dehl, R. E.; Grant, W. H.; Cassel, J. M.; NBSIR 81-2212.

Dehmer, J. L.; Codling, K.; Parr, A. C.; Ederer, D. L.; Stockbauer, R.; West, J. B.; Cole, B. E.; 20399.

Dehmer, J. L.; Parr, A. C.; Ederer, D. L.; Cole, B. E.; West, J. B.; Stockbauer, R.; Codling, K.; 20123.

Dehmer, J. L.; Parr, A. C.; Rakowsky, G.; Ederer, D. L.; Stockbauer, R. L.; West, J. B.; 20236.

Dehmer, J. L.; Stockbauer, R.; Poliakoff, E. D.; Dehmer, P. M.; 20533.

Dehmer, J. L.; Stockbauer, R. L.; Codling, K.; West, J. B.; Parr, A. C.; 20052.

Dehmer, J. L.; West, J. B.; Codling, K.; Ederer, D. L.; Parr, A. C.; Cole, B. E.; Stockbauer, R.; 20676.

Dehmer, J. L.; West, J. B.; Codling, K.; Parr, A. C.; Ederer, D. L.; Cole, B. E.; Stockbauer, R.; 20426.

Dehmer, P. M.; Dehmer, J. L.; Stockbauer, R.; Poliakoff, E. D.; 20533.

Deighton, M. O.; Whiting, F. B.; Kandiah, K.; SP614: 1981 September. 75.78.

de Miniac, A.; Aniel, T.; Maximon, L. C.; NBSIR 81-2262.

Dempsey, D. A.; Detrio, J. A.; SP620: 1981 October. 88-93.

Dempsey, D. V.; O'Quinn, D. B.; Fernelius, N. C.; SP620: 1981 October. 58.74.

Dempsey, D. V.; Walsh, D. A.; O'Quinn, D. B.; Knecht, W. L.; Fernelius, N. C.; SP620: 1981 October. 129.143.

Denton, C. J.; SP589: 1981 January. 57.61.

Deprit, A.; 20232.

Deprit, A.; 20568.

DeReggi, A. S.; Broadhurst, M. G.; Davis, G. T.; 20639.

DeReggi, A. S.; Roth, S. C.; Kenney, J. M.; Edelman, S.; Harris, G. R.; 20170.

Dermann, K.; Rupp, N. W.; Brauer, G. M.; Dulik, D. M.; Hughes, H. N.; 20669.

Der Marderosian, A.; SP400-69: 1981 May. 159.178.

DeRoberts, L.; Boyer, P. A.; Ruff, A. W.; Marinenko, R. B.; Biancaniello, F.; SP260-70.

Deslattes, R. D.; 20152.

Deslattes, R. D.; Jacobs, L.; Schwitz, W.; Seki, R.; Lum, G. K.; Wiegand, C. E.; Kessler, E. G., Jr.; 20349.

Deslattes, R. D.; Kessler, E. G.; Sauder, W. C.; Henins, A.; 20146.

Deslattes, R. D.; Kessler, E. G., Jr.; 20483.

DeSmith, D. A.; Leder, D. S.; Nguyen, D. K.; Perreault, R. W.; Fry, J. P.; Chiang, W. P.; NBSIR 81-2315.

Detrio, J. A.; Dempsey, D. A.; SP620: 1981 October. 88.93.

Deutsch, D. R.; Clemons, E. K.; 20644.

Deutsch, S.; Davis, R. W.; 19971.

DeVoe, H.; Miller, M. M.; Wasik, S. P.; J. Res. 86(4): 361.366; 1981 July-August.

DeVoe, J. R.; Travis, J. C.; 20454.

DeVoe, J. R.; Walters, J. P.; Scheeline, A.; Norris, J. A.; Travis, J. C.; 20453.

DeVoe, J. R.; Walters, J. P.; Scheeline, A.; Travis, J. C.; 20462.

de Vos, A.; de Goede, J.; van den Berg, R. J.; SP614: 1981 September. 217.220.

De Vos, D.,; Ryan, P.; NBS-GCR-80-291.

DeVries, K. L.; Smith, R. H.; Fanconi, B. M.; 20443.

Deward, F. D.; Haisch, B.; Linsky, J. L.; Slee, O. B.; Siegman, B. C.; Nikoloff, I.; Candy, M.; Harwood, D.; Verveer, A.; Quinn, P. J.; Wilson, 1.; Page, A. A.; Higson, P.; 20405.

Dick, C. E.; Danos, M.; Motz, J. W.; 20450.

Dick, C. E.; Motz, J. W.; 20189.

Dick, C. E.; Motz, J. W.; 20390

Dickamore, W. G.; Dampier, I. K.,; NBS-GCR-80-306.

Dickamore, W. G.; Kampier, I. K.,; NBS-GCR-80-307.

Dickens, B.; 20774.

Dickens, B.; NBSIR 80-2102.

Dickens, B.; Schroeder, L. W.; Brown, W. E.; Jordan, T. H.; 19994.

Dickens, B.; Schroeder, L. W.; Mathew, M.; Mayer, I.; 20065.

Dickinson, G. W.; NBSIR 81-2346.

Didion, D. A.; Baker, D. W.; Kao, J. Y.; NBSIR 81-2313.
Difilippo, F. C.; Pineyro, J. J.; SP614: 1981 September. 309.313.

Dik, L. S. H.; Vandamme, L. K. J.; SP614: 1981 September. 244-247.

Diller, D. E.; 20071

Diller, D. E.; Haynes, W. M.; 20570.

Diller, D. E.; Saber, J. M.; 20767.

Diller, D. E.; Straty, G. C.; 20139.

Diller, D. E.; Straty, G. C.; 20278.

Dillon, M. A.; Spence, D.; 20178.

Dils, R. R.; Whetstone, J. R.; Frederikse, H. P. R.; Schenck, P. K.; Burns, G. W.; NBSIR 81-2348.

DiMarzio, E. A.; Fanconi, B. M.; Han, C. C.; Khoury, F. A.; Markovitz, H.; McKinney, J. E.; Mopsik, F. I.; Passaglia, E.; Sanchez, I. C.; VanderHart, D. L.; Zapas, I. J.; Eby, R. K.; Broadhurst, M. G.; Chang, S. S.; Davis, G. T.; 20749.

DiNenno, P. J.; Milke, J. A.,; Bryan, J. L.; NBS-GCR-80-297.

Dirks, H. M.; Beckstette, K. F.; Heiden, C.; SP614: 1981 September. 384.387.

Dirks, H. M.; Heiden, C.; Beckstette, K. F.; SP614: 1981 September. 388-390.

Ditchek, B.; Ruff, A. W.; 19980.

Dobbs, H. S.; SP601: 1981 January. 643-653.

Dodge, W. R.; Bertsch, D. L.; 20375.

Dodge, W. R.; Leicht, R. G.; Hayward E.; Wolynec, E.; 20275.

Domalski, E. S.; Kirklin, D. R.; Colbert, J.; Decker, P.; Abramowitz, S.; NBSIR 81-2278.

Domen, S. R.; Loevinger, R.; Pruitt, J. S.; J. Res. 86(5): 495.502; 1981 September-October.

Domingues, L.; Negas, T.; U.S. Patent 4,264,423.

Donovan, T. M.; Porteus, J. O.; Seitel, S. C.; Kraatz, P.; SP620: 1981 October. 305-312.

Dorri, B.; Kippenhan, C. J.; Heerwagen, D. R.; Banken, G.; Emery, A. F.; SP608: 1981 May. 21-35.

Dowling, N. E.; Fong, J. T.; 20501.

Drake, M. C.; Rosasco, G. J.; Schneggenburger, R.; Nolen, R. L., Jr.; 20653.

Draper, C. W.; SP620: 1981 October. 210.221.

Draper, C. W.; Buene, L.; Jacobson, D. C.; Poate, J. M.; Nakahara, S.; SP620: 1981 October. 222.226.

Draper, J. M.; SP500-84.

Drayton, P. A.; SP500-83: 1981 November. 223-230.

Drexhage, M. G.; Bendow, B.; Lipson, H. G.; Moynihan, C. T.; SP620: 1981 October. 94.101.

Driver, L. D.; Ries, F. X.; Rebuldela, G.; NBSIR 78-871.

Driver, R. G.; Houck, J. C.; Welch, B. E.; J. Res. 86(3): 277.279; 1981 May-June.

Drullinger, R. E.; Wineland, D. J.; Bergquist, J. C.; 20265.

Drummond, P. D.; Gardiner, C. W.; Walls, D. F.; 20537.

Drummond, P. D.; Zoller, P.; Walls, D. F.; Kunasz, C. V.; 20595.

Ducas, W.; Orloski, M. J.; Decorte, K. N.; McCabe, M. E.; NBSIR 81-2300.

Dulik, D.; Bernier, R.; Brauer, G. M.; 20392.

Dulik, D. M.; Hughes, H. N.; Dermann, K.; Rupp, N. W.; Brauer, G. M.; 20669.

Dulk, G. A.; Chanmugam, G.; 20417.

Dumin, D. J.; 20006.

Dun, H.; Mattes, B. L.; Stevenson, D. A.; SP400-69: 1981 May. 143. 152.

Dunn, G. H.; 20596

Dunn, G. H.; Griffin, D. C.; Bottcher, C.; Gregory, D. C.; Crandall, D. H.; Pindzola, M. S.; Falk, R. A.; 20584.

Durst, R. A.; 20522.

Durst, R. A.; Bates, R. G.; 20580

Durst, R. A.; MacCrehan, W. A.; 20289

Durst, R. A.; MacCrehan, W. A.; 20728.

Durst, R. A.; Siggaard-Andersen, O.; Maas, A. H. J.; 20513.

Duvall, K. C.; Wasson, O. A.; 20456.

Dyer, D. F.; Burch, T.,; Maxwell, T. T.; Maples, G.; NBS-GCR-80292. 
Eagle, J. W.; Little, A. D.; SP599: 1981 January. 156-164.

Earl, W. L.; Manka, M. J.; Miles, L. B.; Gann, R. G.; 20558.

Early, C.; Rosenbaum, J.; SP500-80: 1981 October. 146-151.

Eastman, D. E.; Madey, T. E.; Stockbauer, R.; Flodström, S. A.; van der Veen, J. F.; Himpsel, F. J.; 20510.

Ebbesen, H. F.; Engen, G. F.; 20630.

Eby, R. K.; Broadhurst, M. G.; Cassel, J. M.; Fanconi, B. M.; Sanchez, I. C.; Smith, L. E.; NBSIR 81-2263.

Eby, R. K.; Broadhurst, M. G.; Chang, S. S.; Davis, G. T.; DiMarzio, E. A.; Fanconi, B. M.; Han, C. C.; Khoury, F. A.; Markovitz, H.; McKinney, J. E.; Mopsik, F. I.; Passaglia, E.; Sanchez, I. C.; VanderHart, D. L.; Zapas, I. J.; 20749.

Eby, R. K.; Clark, E. S.; Weeks, J. J.; 20708.

Eby, R. K.; Farmer, B. L.; 20715.

Eby, R. K.; Peterlin, A.; Khoury, F. A.; Broadhurst, M. G.; Fanconi, B. M.; 20652.

Eby, R. K.; Weeks, J. J.; Clark, E. S.; 20635.

Edelman, S.; U.S. Patent 4,297,394.

Edelman, S.; U.S. Patent 4,283,461.

Edelman, S.; Harris, G. R.; DeReggi, A. S.; Roth, S. C.; Kenney, J. M.; 20170.

Edelman, S.; Mordfin, L.; Payne, B. F.; 20246.

Ederer, D. L.; Callcott, T. A.; Arakawa, E. T.; 20216.

Ederer, D. L.; Cole, B. E.; Saloman, E. B.; 20674.

Ederer, D. L.; Cole, B. E.; Stockbauer, R.; Dehmer, J. L.; West, J. B.; Codling, K.; Parr, A. C.; 20426.

Ederer, D. L.; Cole, B. E.; West, J. B.; Stockbauer, R.; Codling, K.; Dehmer, J. L.; Parr, A. C.; 20123.

Ederer, D. L.; Mehlman, G.; Saloman, E. B.; Cole, B. E.; Cooper, J. W.; 20300

Ederer, D. L.; Parr, A. C.; Cole, B. E.; Stockbauer, R.; Dehmer, J. L.; West, J. B.; Codling, K.; 20676.

Ederer, D. L.; Stockbauer, R.; West, J. B.; Cole, B. E.; Dehmer, J. L.; Codling, K.; Parr, A. C.; 20399.

Ederer, D. L.; Stockbauer, R. L.; West, J. B.; Dehmer, J. L.; Parr, A. C.; Rakowsky, G.; 20236.

Edgerly, D. E.; 20324.

Edwards, S. R.; SP500-83: 1981 November. 93.99.

Egan, J.; Higgins, R.; Spath, J.; Buchholz, A.; Wrzenski, S. C.,; NBSGCR-81-313.

Egelhoff, W. F., Jr.; Tibbetts, G. G.; Lindau, I.; Hecht, M. H.; 20478.

Ehrlich, M.; 20514.

Ehrstein, J. R.; NBSIR 80-2158.

Ehrstein, J. R.; Myers, D. R.; Roitman, P.; Albers, J.; NBSIR 812398.

Eichler, T. V.,; Pape, R.; Waterman, T. E.; NBS-GCR-81-301.

Eisenhauer, C.; Schwartz, R.; 20677.

Eisenhauer, C. M.; Grundl, J. A.; Gilliam, D. M.; McGarry, E. D.; Spiegel, V.; 20153.

Eisenhower, E. H.; SP603.

Eisenhower, E. H.,; H136.

Eitzen, D. G.; Berger, H.; Birnbaum, G.; 20474.

Eitzen, D. G.; Breckenridge, F. R.; Clough, R. B.; Fuller, E. R.; Hsu, N. N.; Simmons, J. A.; 20579.

Eitzen, D. G.; Breckenridge, F. R.; Clough, R. B.; Fuller, E. R.; Hsu, N. N.; Simmons, J. A.; 20757.

Ekambaram, V.; Weiss, C. S.; Grimm, C. A.; Filby, R. H.; Sandstrom, D. R.; Lytle, F. W.; Greegor, R. B.; Khalil, S. R.; SP618: 1981 November. 21-38.

Ekin, J. W.; 20066.

Ekin, J. W.; 20104.

Ekin, J. W.; 20260.

Ekin, J. W.; 20658.

Ekin, J. W.; Goodrich, L. F.; 20393.

Ekin, J. W.; Ho, J. C.; Oberly, C. E.; Garrett, H. J.; Walker, M. S.; Zeitlin, B. A.; 20741 .

Ekin, J. W.; Radebaugh, R.; Clark, A. F.; Fujii, G.; 20298

Ekin, J. W.; Schramm, R. E.; Superczynski, M. J.; 20297.

Ekin, J. W.; Sekine, H.; Tachikawa, T.; 20659.

Elder, J.; Tibbott, R. L.; BSSI30.
Elder, J.; Tibbott, R. L.; Yonemura, G. T.; Hunt, C. M.; Chen, P. T.; Hill, J. E.; May, W. B., Jr.; Richtmyer, T. E.; BSS133.

Elengo, J. J., Jr.; SP599: 1981 January. 39-44.

Eliason, L. K.; Wu, Y. C.; Flores, A.; SP480-41.

Ellingwood, B. R.; NBSIR 80-2111-1.

Elmer, J. W.; McHenry, H. I.; Yushchenko, K. A.; Paton, E. O. Reed, R. P.; Tobler, R. L.; 20772.

Elsen, L. C.; SP500-83: 1981 November. 67.70.

El-Torkey, N. M.; Margolis, S. A.; Kaplan, A. E.; Weiss, E. R.; Byrne, S. T.; 20446

Ely, J. F.; J. Res. 86(6): 597.604; 1981 November-December.

Ely, J. F.; Hanley, H. J. M.; 20651.

Ely, J. F.; Hanley, H. J. M.; 20768.

Ely, J. F.; Hanley, H. J. M.; TN1039.

Embree, E. J.; Roberts, W. E.; Masters, L. W.; Seiler, J. F.; NBSIR $81-2232$.

Emery, A. F.; Dorri, B.; Kippenhan, C. J.; Heerwagen, D. R.; Banken, G.; SP608: 1981 May. 21-35.

Emmerman, P. J.; Goulard, R.; Santoro, R. J.; Semerjian, H. G.; 20335 .

Emmerman, P. J.; Semerjian, H. G.; Santoro, R. J.; Goulard, R.; 20357.

Emmons, H. W., Mitler, H. E.; NBS-GCR-81-344.

Enagonio, D.; Christensen, R. G.; Howell, B. F.; Paule, R. C.; Mandel, J.; Schaffer, R.; Reeder, D. J.; Coxon, B.; SP260-72.

Enemark, E. A.; Wirtenson, G. R.; Willis, J. B.; SP620: 1981 October 287.296.

Engelsberg, S.; Pavlin, I.; SP614: 1981 September. 153.156.

Engen, G. F.; 20067

Engen, G. F.; Ebbesen, H. F.; 20630.

Envent, F.; Cohill, M.; Hegner, H.; SP622: 1981 October. 113-124.

Erickson, R.; Gerhart, S.; Lee, S.; Thompson, D.; SP500-80: 1981 October. 69.75

Eroshenko, V. M.; Yaskin, L. A.; Daney, D. E.; Ludtke, P. R.; 20400.

Erwin, R. W.; Rhyne, J. J.; Chen, H. S.; Lynn, J. W.; 20282.

Esaki, L.; J. Res. 86(6): 565-570; 1981 November-December.

Escalante, E.; 19963.

Escalante, E.; Bertocci, U.; Mullen, J.; Cohen, M.; Kruger, J.; 20348.

Escalante, E.; Sanderson, B. T.; Gerhold, W. F.; NBSIR 81-2228 (NBS).

Esfandiari, P.; Meijer, P. H. E.; Farrell, R. A.; Favin, S.; 20620

Estin, A. J.; Stubenrauch, C. F.; Repjar, A. G.; Newell, A. C.; 20650.

Evans, D. D.; TN1133.

Evans, D. D.; Davis, S.; NBSIR 81-2213.

Evans, D. D.; Madrzykowski, D.; NBSIR 81-2329.

Evans, D. J.; 20262.

Evans, D. J.; 20304

Evans, D. J.; 20310.

Evans, D. J.; 20495.

Evans, D. J.; Hanley, H. J. M.; 19974

Evans, D. J.; Hanley, H. J. M.; 19973.

Evans, D. J.; Hanley, H. J. M.; 20063

Evans, D. J.; Hanley, H. J. M.; 20527.

Evans, D. J.; Hanley, H. J. M.; 20613.

Evans, D. J.; Hanley, H. J. M.; 20649.

Evans, E. H.; Paretzkin, B.; Parker, H. S.; Panagiotopoulos, N. C. Hubbard, C. R.; Morris, M. C.; McMurdie, H. F.; Monogr. 25, Section 18.

Evans, J.,; NBS-GCR-ETIP $81-95$

Evans, J.,; Foskett, W. H.; Jamieson, A.; NBS-GCR-ETIP 81-93.

Evans, P. M.; Smith, R. L.; Anderson, J. M.; SP608: 1981 May. 113. 122.

Evans, W. H.; Parker, V. B.; Schumm, R. H.; Nuttall, R. L.; Wagman, D. D.; TN270-8.

Evenson, K. M.; Radford, H. E.; Mizushima, M.; Scalabrill, A.; Saykally, R. J.; 20700

Evenson, K. M.; Vasconcellos, E. C. C.; Petersen, F. R.; 20743.

\section{F}

Fabbiano, G.; Giacconi, R.; Golub, L.; Gorenstein, P.; Haisch, B. M.; 
Harnden, F. R., Jr.; Johnson, H. M.; Linsky, J. L.; Maxson, C. W.; Mewe, R.; Rosner, R.; Seward, F.; Topka, K.; Zwaan, C.; Vaiana, G. S.; Cassinelli, J. P.; 20336.

Fabry, A.; McGarry, E. D.; 20256.

Faeth, G. M.,; You, H. Z.; NBS-GCR-79-188

Faison, T. K.; NBSIR 80.2111-8.

Faison, T. K.; NBSIR 81-2347.

Faison, T. K.; Lerchen, F. H.; Pielert, J. H.; NBSIR 80-2171.

Falk, R. A.; Dunn, G. H.; Griffin, D. C.; Bottcher, C.; Gregory, D. C.; Crandall, D. H.; Pindzola, M. S.; 20584.

Faller, J. E.; Decher, R.; Randall, J. L.; Bender, P. L.; 20451.

Fanconi, B. M.; DeVries, K. L.; Smith, R. H.; 20443.

Fanconi, B. M.; Eby, R. K.; Peterlin, A.; Khoury, F. A.; Broadhurst, M. G.; 20652.

Fanconi, B. M.; Han, C. C.; Khoury, F. A.; Markovitz, H.; McKinney, J. E.; Mopsik, F. I.; Passaglia, E.; Sanchez, I. C.; VanderHart, D. L.; Zapas, I. J.; Eby, R. K.; Broadhurst, M. G.; Chang, S. S.; Davis, G. T.; DiMarzio, E. A.; 20749.

Fanconi, B. M.; Sanchez, I. C.; Smith, L. E.; Eby, R. K.; Broadhurst, M. G.; Cassel, J. M.; NBSIR 81-2263.

Fanney, A. H.; Liu, S. T.; 20078.

Fanney, A. H.; Liu, S. T.; 20103.

Fanney, A. H.; Thomas, W. C.; 20648.

Farley, J. W.; Lamb, W. E., Jr.; Wing, W. H.; Shy, J. T.; 20125.

Farley, J. W.; Wing, W. H.; 20647.

Farmer, B. L.; Eby, R. K.; 20715.

Farrell, R. A.; Favin, S.; Esfandiari, P.; Meijer, P. H. E.; 20620.

Fatiadi, A. J.; Hodgeson, J. A.; 20290.

Fattal, S. G.; Batts, M. E.; Lew, H. S.; Carino, N. J.; NBSIR 81-2374.

Fattal, S. G.; Hunt, B. J.; NBSIR 81-2265.

Faulkner, D. J.; Freeman, K. C.; 20105.

Favin, S.; Esfandiari, P.; Meijer, P. H. E.; Farrell, R. A.; 20620.

Fedotowsky, A.,; Lehovec, K.; NBS-GCR-80-285.

Feigerle, C. S.; Herman, Z.; Lineberger, W. C.; 20536.

Felcher, G. P.; Bader, S. D.; Celotta, R. J.; Pierce, D. T.; Wang, G. C.; 19975.

Felcher, G. P.; Celotta, R. J.; Pierce, D. T.; Wang, G. C.; Bader, S. D.; 19970.

Feldman, A.; White, G. S.; 20485.

Fenchel, R. S.; SP500-80: 1981 October. 10-14

Fenimore, E. E.; Burek, A. J.; Barrus, D. M.; Blake, R. L.; 20374.

Fenves, S. J.; Harris, J. R.; Wright, R. N.; NBSIR 80-1979-1.

Fenves, S. J.; Wright, R. N.; Harris, J. R.; 19998.

Fenves, S. J.; Wright, R. N.; Harris, J. R.; 20435.

Fernandez-Baca, J. A.; Lynn, J. W.; 20283.

Fernelius, N. C.; Dempsey, D. V.; O'Quinn, D. B.; SP620: 1981 October. 58-74.

Fernelius, N. C.; Dempsey, D. V.; Walsh, D. A.; O'Quinn, D. B.; Knecht, W. L.; SP620: 1981 October. 129-143.

Fickett, F. R.; Chen, H. S.; Goldfarb, R. B.; Rao, K. V.; 20317.

Fickett, F. R.; Clark, A. F.; 20069.

Fickett, F. R.; Goodrich, L. F.; Clark, A. F.; NBSIR 80-1642.

Fickett, F. R.; McHenry, H. I.; 20665.

Fickett, F. R.; Powell, R. L.; 20377.

Fields, R. J.; Tighe, N. J.; Fuller, E. R., Jr.; NBSIR 79.1960 (DoE).

Filby, R. H.; Sandstrom, D. R.; Lytle, F. W.; Greegor, R. B.; Khalil, S. R.; Ekambaram, V.; Weiss, C. S.; Grimm, C. A.; SP618: 1981 November. 21-38.

Filliben, J. J.; Fowler, H. A.; 20716.

Filliben, J. J.; Simiu, E.; 20019.

Filliben, J. J.; Simiu, E.; 20295.

Filliben, J. J.; Simiu, E.; Shaver, J. R.; 20293.

Filliben, J. J.; Simiu, E.; Shaver, J. R.; 20764.

Filliben, J. J.; Thurber, W. R.; Mattis, R. L.; Liu, Y. M.; SP400-64.

Fine, J.; 20391.

Fine, J.; Hardy, S. C.; Andreadis, T. D.; 20332.

Fine, J.; Klein, R.; 20013.

Fink, J. L.; Gerhold, W. F.; NBSIR 81-2243.

Fink, R. W.; SP604: 1981 June. 5-34.

Fiori, C. E.; Heinrich, K. F. J.; Myklebust, R. L.; SP604: 1981 June. 365-379.
Fiori, C. E.; Myklebust, R. L.; 20381.

Fiori, C. E.; Myklebust, R. L.; Gorlen, K.; SP604: 1981 June. 233-272.

Fiori, C. E.; Newbury, D. E.; Myklebust, R. L.; SP604: 1981 June. 315-339.

Fiori, C. E.; Swyt, C. R.; SP604: 1981 June. 417-439.

Fiori, C. E.,; Heinrich, K. F. J.; Newbury, D. E.; Myklebust, R. L.; SP604.

Fiorillo, F.; Sassi, M. P.; Bertotti, G.; SP614: 1981 September. 324 327.

Fischell, D. R.; Brayman, H. C.; Cool, T. A.; 19978.

Fischell, D. R.; Cool, T. A.; Brayman, H. C.; 19969.

Fischer, J. P.; Schover, D. S.; Board, D.; SP622: 1981 October. 126136.

Fish, R. H.; Weiss, C. S.; Jewett, K. L.; Brinckman, F. E.; SP618: 1981 November. 197-216.

Fish, R. H.,; Brinckman, F. E.; SP618.

Fishbane, P. M.; Gross, F.; Meshkov, S.; Carlson, C. E.; Coyne, J. J.; 20034.

Fishbane, P. M.; Gross, F.; Meshkov, S.; Carlson, C. E.; Coyne, J. J.; 20187.

Fishbane, P. M.; Gross, F.; Meshkov, S.; Carlson, C. E.; Coyne, J. J.; 20316.

Fitzgerald, M. L.; Wheatley, T. E.; Albus, J. S.; VanderBrug, G. J.; Barbera, A. J.; SP602.

FitzGerrell, R. G.; 20624.

FitzPatrick, G. J.; Hebner, R. E.; Kelley, E. F.; Forster, E. O.; 20645.

FitzPatrick, G. J.; Hebner, R. E., Jr.; Kelley, E. F.; Forster, E. O.; 20664.

Fivozinsky, S.; Purdy, J. A.; Harms, W. B.; 20553.

Fivozinsky, S. P.; Purdy, J. A.; 20554.

Fivozinsky, S. P.,; NBSIR 81-2206.

Flach, D. R.; Bell, B. A.; Souders, T. M.; TN1145.

Flanz, J. B.; Hicks, R. S.; Parker, B.; Peterson, G. A.; Lawson, R. D.; Teeters, W.; Williamson, C. F.; Kowalski, S.; Maruyama, X. K.; Lindgren, R. A.; 20323.

Fliegle, F. M.; Black, R. D.; Weissman, M. B.; SP614: 1981 September. 152.

Flink, R. C.; Malette, W.; SP601: 1981 January. 503-508

Flint, E. B.; Guernsey, R. W.; SP607: 1981 May. 15-20.

Flint, E. B.; Jenkins, L. C.; Guernsey, R. W.; SP607: 1981 May. 93. 102.

Flodström, S. A.; van der Veen, J. F.; Himpsel, F. J.; Eastman, D. E.; Madey, T. E.; Stockbauer, R.; 20510.

Flores, A.; Eliason, L. K.; Wu, Y. C.; SP480-41.

Flynn, D. R.; Danner, W. F.; Yaniv, S. L.; Bauer, J. W.; NBSIR 81 2377.

Flynn, T. M.; Timmerhaus, K. D.; 20068.

Flynn, T. M.; Timmerhaus, K. D.; 20563.

Foley, J.; Sharma, S. K.; Wood, R. M.; SP620: 1981 October. 248-255.

Foltyn, S. R.; Newnam, B. E.; SP620: 1981 October. 265-276.

Fong, J. T.; 20500.

Fong, J. T.; Dowling, N. E.; 20501.

Fong, V.; SP400-69: 1981 May. 69-74.

Fontanella, J. J.; Bean, V. E.; Andeen, C. G.; Link, J.; Wintersgill, M. C.; 20266.

Forest, A.; SP608: 1981 May. 83-103.

Forster, E. O.; FitzPatrick, G. J.; Hebner, R. E.; Kelley, E. F.; 20645.

Forster, E. O.; FitzPatrick, G. J.; Hebner, R. E., Jr.; Kelley, E. F.; 20664.

Forster, E. O.; Kelley, E. F.; Hebner, R. E., Jr.; Zahn, M.; 20646.

Fortunko, C. M.; 20543.

Fortunko, C. M.; 20544.

Fortunko, C. M.; 20744.

Foskett, W.; Olesen, T.; NBS-GCR-ETIP 81-94.

Foskett, W. H.; Jamieson, A.; Evans, J.,; NBS-GCR-ETIP 81 -93.

Fotinos, C.; Stochino, G.; D'Amico, A.; Cappuccio, G.; Petrocco, G.; SP614: 1981 September. 199-201.

Fowler, B. A.; SP618: 1981 November. 217-225.

Fowler, H. A.; Filliben, J. J.; 20716.

Fraker, A. C.; Speck, K. M.; 19988.

Fraker, A. C.; Weber, H.; 20270. 
Fraley, D. M.; Vogt, C.; Yates, D.; Manahan, S. E.; SP618: 1981 November. 81-92.

Francis, R. L.; J. Res. 86(5): 509.513; 1981 September-October.

Francis, R. L.; Chalmet, L. G.,; NBS-GCR-81-316.

Franck, E. U.; Marshall, W. L.; JPCRD 10(2): 295-304; 1981.

Franck, J. B.; Veatch, T. C.; Soileau, M. J.; SP620: 1981 October. 385-393.

Franklin, A. D.; Snellgrove, R. A.; Bethin, J. R.; Chiang, C. K.; 20497.

Frantz, J. D.; Vogel, G. L.; Popp, R. K.; 20467.

Franzen, D. L.; Day, G. W.; 20347.

Franzen, D. L.; Day, G. W.; Danielson, B. L.; Kim, E. M.; Chamberlain, G. E.; 20369.

Franzen, D. L.; Kim, E. M.; 20432.

Franzen, D. L.; Kim, E. M.; TN1032.

Frazier, A. W.; Mathew, M.; Takagi, S.; Waerstad, K. R.; 20258.

Frederick, N. V.; Giarratano, P. J.; 19976.

Frederick, W.; Hausmann, R.,; Clarren, S.; Nalley, P.; Gregory, G.; NBS-GCR-ETIP 81-96.

Frederikse, H. P. R.; Schenck, P. K.; Burns, G. W.; Dils, R. R.; Whetstone, J. R.; NBSIR 81-2348.

Free, G.; SP589: 1981 January. 133-136.

Free, G.; Berger, H.; Mordfin, L.; Swartzendruber, L.; Boettinger, W.; Ives, L.; Coriell, S.; Ballard, D.; Laughlin, D.; Clough, R.; Biancaniello, F.; Blau, P.; Cahn, J.; Mehrabian, R.; NBSIR 80-2069.

Free, G.,; Birnbaum, G.; 20366.

Free, G. M.,; SP589.

Freedman, D.; SP616: 198i October. 93-116.

Freeman, K. C.; Faulkner, D. J.; 20105.

Frehland, E.; SP614: 1981 September. 22-25.

French, G.; Skinner, H.; Weinstein, A. M.; Clemow, A. J. T.; McPhillips-Meade, M.; Klawitter, J. J.; SP601: 1981 January. 423. 447.

French, J. C.; SP611.

Friday, D. S.; 20678.

Frohnsdorff, G.; Clifton, J. R.; NBSIR 81-2239.

Frommer, C.; Lovejoy, R. W.; Sams, R. L.; Olson, W. B.; 20589.

Fry, J. P.; Chiang, W. P.; DeSmith, D. A.; Leder, D. S.; Nguyen, D. K.; Perreault, R. W.; NBSIR 81-2315.

Fuhr, J. R.; Martin, G. A.; Wiese, W. L.; Younger, S. M.; JPCRD 10(2): 305.566; 1981.

Fujii, G.; Ekin, J. W.; Radebaugh, R.; Clark, A. F.; 20298.

Fujii, G.; Ranney, M. A.; Clark, A. F.; 20465.

Fujii, G.; Ranney, M. A.; Clark, A. F.; 20753.

Fujii, G.; Read, D. T.; Clark, A. F.; Radebaugh, R.; 19999.

Fuller, E. G.; 19977.

Fuller, E. R.; Hsu, N. N.; Simmons, J. A.; Eitzen, D. G.; Breckenridge, F. R.; Clough, R. B.; 20579.

Fuller, E. R.; Hsu, N. N.; Simmons, J. A.; Eitzen, D. G.; Breckenridge, F. R.; Clough, R. B.; 20757.

Fuller, E. R., Jr.; Fields, R. J.; Tighe, N. J.; NBSIR 79-1960 (DoE).

Fuller, E. R., Jr.; Krause, R. F., Jr.; NBSIR 80-2099-4.

Fuller, E. R., Jr.; Malkus, D. S.; J. Res. 86(1): 79-109; 1981 JanuaryFebruary.

Furumoto, H. W.; Lucatorto, T. B.; McIlrath, T. J.; Mayo, S.; 20164.

\section{$\mathbf{G}$}

Gadzuk, J. W.; 20333.

Gadzuk, J. W.; 20532.

Gadzuk, J. W.; Metiu, H.; 20143.

Gagnepain, J. J.; Uebersfeld, J.; Handel, P. H.; SP614: 1981 September. 189-191.

Gagnon, J. L.; Persensky, J. J.; 20217.

Gagnon, J. L.; Ramey-Smith, A. M.; 20235.

Gaines, R. S.; McLafferty, P. S.; SP608: 1981 May. 159.166.

Gajdusek, D. C.; Gibbs, C. J., Jr.; Traub, R. D.; Rains, T. C.; Garruto, R. M.; 20603.

Galakhova, O. P.; Harkness, S.; Hermach, F. L.; Hirayama, H.; Martin, P.; Rozdestvenskaya, T. H.; Williams, E. S.; 20240.

Galbraith, T.,; Josephson, E.; Bush, T.; Mazzone, G.; McElroy, G.;
NBS-GCR-81-312.

Galdikas, A.; Miliusyte, R.; Viktoravicius, V.; Bareikis, V.; SP614: 1981 September. 406-408.

Gallagher, A.; Sandle, W. J.; 20699.

Gallagher, A. C.; Kennerly, R. E.; Van Brunt, R. J.; 20244

Gallagher, A. C.; Wildman, D. W.; Schumann, L. W.; 20491

Gallagher, J. W.; Van Blerkom, J.; Beaty, E. C.; Rumble, J. R., Jr.; $S P 593$.

Galloway, K. F.; Scace, R. I.; Walters, E. J.; NBSIR 81-2230-3.

Galowin, L. S.; Kopetka, P. A.; Mahajan, B. M.; J. Res. 86(2): 171 . 179; 1981 March-April.

Galowin, L. S.; Mahajan, B. M.; 20147.

Galowin, L. S.; Winter, F.; NBSIR 81-2296.

Gann, R. G.; Earl, W. L.; Manka, M. J.; Miles, L. B.; 20558.

Gannon, C.; DeHaan, W. R.; Saib, S. H.; Benson, J. P.; SP500-80: 1981 October. 208-217.

Gannon, C.; DeHaan, W. R.; Saib, S. H.; Benson, J. P.; SP500-80: 1981 October. 200-207.

Gans, W. L.; Nahman, N. S.; TN1042.

Gardiner, C. W.; Walls, D. F.; Drummond, P. D.; 20537.

Garn, P. D.; Menis, O.; Mackey, J. A.; 20011.

Garrett, D. A.; Kasen, M. B.; Berger, H.; Placious, R. C.; 20342.

Garrett, H. J.; Walker, M. S.; Zeitlin, B. A.; Ekin, J. W.; Ho, J. C.; Oberly, C. E.; 20741.

Garrity, S. D.; NBSIR 81-2328.

Garrity, S. D.; NBSIR 81-2327.

Garruto, R. M.; Gajdusek, D. C.; Gibbs, C. J., Jr.; Traub, R. D.; Rains, T. C.; 20603.

Garvin, D.; Churney, K. L.; NBSIR 80-2018 (DOT).

Gary, D. E.; Linsky, J. L.; 20717.

Gasquet, D.; Nougier, J. P.; Vaissiere, J. C.; SP614: 1981 September. 42.46.

Gasquet, D.; Vaissiere, J. C.; Nougier, J. P.; SP614: 1981 September. 305.308.

Gass, S. I.; Hoffman, K. L.; Jackson, R. H. F.; Joel, L. S.; Saunders, P. B.; NBSIR 80-1971.

Gass, S. I.; Joel, L. S.; NBSIR 80-2053.

Gass, S. I.,; $S P 616$.

Gasser, M. G.; Sherman, A.; Beale, W.; SP607: 1981 May. 103-115.

Gasser, M. G.; Studer, P. A.; SP607: 1981 May. 146-153.

Gaur, U.; Shu, H. C.; Mehta, A.; Wunderlich, B.; JPCRD 10(1): 89. $118 ; 1981$.

Gaur, U.; Wunderlich, B.; JPCRD 10(1): 119-152; 1981.

Gaur, U.; Wunderlich, B.; JPCRD 10(4): 1051-1064; 1981.

Gaur, U.; Wunderlich, B.; JPCRD 10(4): 1001-1049; 1981.

Gebbie, K. B.; Simon, G. W.; November, L. J.; Toomre, J.; 20422.

Geer, N.; Graff, H. A.; Stromecky, P.; NBS-GCR-81-336.

Geist, J.; Baltes, H. P.; 20636.

Geist, J.; Liang, E.; Schaefer, A. R.; 20530

Geist, J.; Lowney, J. R.; 20192.

Geltman, S.; 20061.

Gennity, J. M.; Hillman, D. C.; O'Brien, D. H.; Pyles, R. A.; Stockton, R. A.; Wheeler, A. E.; Zingaro, R. A.; Irgolic, K. J.; Banks, C. H.; Bottino, N. R.; Chakraborti, D.; SP618: 1981 November. 244.263.

Genot, A.; SP622: 1981 October. 97.112.

Gentil, P.; Mounib, A.; SP614: 1981 September. 230-233.

Gerace, W. J.; Hicks, R. S.; Parker, B.; Peterson, G. A.; Singhal, R.; Williamson, C. F.; Maruyama, X. K.; Petrovich, F.; Lindgren, R. A.; Plum, M. A.; 20660.

Geret, V.; Brown, S. A.; Merritt, K.; Simpson, J. P.; SP601: 1981 January. 395.422.

Gerhart, S.; Lee, S.; Thompson, D.; Erickson, R.; SP500-80: 1981 October. 69-75.

Gerhold, W. F.; Escalante, E.; Sanderson, B. T.; NBSIR 81-2228 (NBS).

Gerhold, W. F.; Fink, J. L.; NBSIR 81-2243.

Gerk, F. A.; SP599: 1981 January. 69-84.

Gerk, F. A.; SP599: 1981 January. 276.

Gerry, M. C. L.; Robiette, A. G.; Ozier, I.; JPCRD 10(4): 1085-1095; 1981. 
Gevantman, L. H.,; Monogr. 167.

Giacconi, R.; Golub, L.; Gorenstein, P.; Haisch, B. M.; Harnden, F. R., Jr.; Johnson, H. M.; Linsky, J. L.; Maxson, C. W.; Mewe, R.; Rosner, R.; Seward, F.; Topka, K.; Zwaan, C.; Vaiana, G. S.; Cassinelli, J. P.; Fabbiano, G.; 20336.

Giampapa, M. S.; Bornmann, P. L.; Ayres, T. R.; Linsky, J. L.; Worden, S. P.; 20388.

Gianetta, J.; Overgaard, M.; SPSO0-80: 1981 October. 194-196.

Gianino, P. D.; Tzoar, N.; Bendow, B.; SP620: 1981 October. 453. 458.

Giarratano, P. J.; Frederick, N. V.; 19976.

Gibbons, D.; Brown, S.; Ruff, W.,; Weinstein, A.; SP601.

Gibbons, D. F.; Buran, K. A.; SP601: 1981 January. 157-174.

Gibbs, C. J., Jr.; Traub, R. D.; Rains, T. C.; Garruto, R. M.; Gajdusek, D. C.; 20603.

Giese, K. W.; Halstead, D.; Wyrick, T. F.; SP500-83: 1981 November. 109.118.

Giese, K. W.; Kyriazi, C. N.; Trehan, R.; Conti, D. M.; SP500.83: 1981 November. 311.312.

Giffard, R. P.; Michelson, P. F.; Taber, R. C.; SP614: 1981 September. 292-297.

Gilb, T.; Krzanik, L.; SP500-80: 1981 October. 190-193.

Gillette, G.; 20541.

Gilliam, D. M.; McGarry, E. D.; Spiegel, V.; Eisenhauer, C. M.; Grundl, J. A.; 20153.

Gills, T. E.; Bowen, R. L.; 20169.

Gimm, H. A.; Maximon, L. C.; 20175.

Ginter, P. M.; Rucks, A. C.; SP500-83: 1981 November. 191-197.

Girvin, S. M.; Matthew, J. A. D.; 20577.

Girvin, S. M.; Penn, D. R.; 20008.

Girvin, S. M.; Penn, D. R.; 20389.

Girvin, S. M.; Rendell, R. W.; 20464.

Girvin, S. M.; Rendell, R. W.; Bryant, G. W.; 20629.

Girvin, S. M.; Unguris, J.; Celotta, R. J.; Pierce, D. T.; 20694.

Giuntini, R.,; Patrick, S.; NBS-GCR-80-279.

Givargizov, E. I.; Melmed, A. J.; Sakurai, T.; Kuk, Y.; 20157.

Glass, A. J.; Guenther, A. H.; Newnam, B. E.; Bennett, H. E.; SP620.

Glassey, C. R.; Greenberg, H. J.; SP616: 1981 October. 117.120.

Glassman, A. T.; SP620: 1981 October. 144-158.

Glaze, D. J.; Bergquist, J. C.; Barger, R. L.; 20338.

Glenar, D.; Kostiuk, T.; Mumma, M. J.; Lovas, F. J.; Buhl, D.; Clark, F. O.; Chin, G.; 20321.

Glickman, D. S.; Terry, D. L.; SP608: 1981 May. 123.132.

Goad, C. D.; Bender, P. L.; Bossler, J. D.; 20434.

Goldberg, R. N.; JPCRD 10(3): 671-766.

Goldberg, R. N.; JPCRD 1O(1): 1.56; 1981.

Goldberg, R. N.; Sarbar, M.; Covington, A. K.; 20714.

Goldfarb, R. B.; 20748.

Goldfarb, R. B.; Rao, K. V.; Fickett, F. R.; Chen, H. S.; 20317.

Goldfine, A.; Perry, W. E.; FIPS PUB 88.

Goldman, A. J.; J. Res. 86(4): 383-391; 1981 July-August.

Goldman, A. J.; SP616: 1981 October. 217-242.

Goldman, A. J.; Pearl, M. H.; J. Res. 86(2): 193.215; 1981 MarchApril.

Goldman, D.; SP304A, Revised August 1981.

Goldman, D.; SP304, Revised August 1981.

Goldman, D. T.; 20023.

Goldman, D. T.; Bell, R. J.,; SP330.

Goldsmith, J. E. M.; Lawler, J. E.; 20166.

Goldstein, J. I.; Williams, D. B.; SP604: 1981 June. 341-349.

Gollub, J. P.; SP614: 1981 September. 288-291.

Golub, L.; Gorenstein, P.; Haisch, B. M.; Harnden, F. R., Jr.; Johnson, H. M.; Linsky, J. L.; Maxson, C. W.; Mewe, R.; Rosner, R.; Seward, F.; Topka, K.; Zwaan, C.; Vaiana, G. S.; Cassinelli, J. P.; Fabbiano, G.; Giacconi, R.; 20336.

Golub, L.; Rosner, R.; Ayres, T. R.; Linsky, J. L.; Vaiana, G. S.; 20627.

Golub, L.; Rosner, R.; Vaiana, G. S.; Ayres, T.; Linsky, J. L.; Pallavicini, R.; 20594.

Gong, J.; van Vliet, K. M.; SP614: 1981 September. 113-117.
Goodman, D. A.; Cahn, J. W.; Bennett, L. H.; 20433.

Goodman, D. W.; Kelley, R. D.; 20350.

Goodman, L. J.; Caswell, R. S.; Coyne, J. J.; 20670.

Goodrich, L. F.; Clark, A. F.; Fickett, F. R.; NBSIR 80-1642.

Goodrich, L. F.; Ekin, J. W.; 20393.

Gordon, C. C.; Palla, R. L., Jr.; NBSIR 81-2291 (HUD).

Gorenstein, P.; Haisch, B. M.; Harnden, F. R., Jr.; Johnson, H. M.; Linsky, J. L.; Maxson, C. W.; Mewe, R.; Rosner, R.; Seward, F.; Topka, K.; Zwaan, C.; Vaiana, G. S.; Cassinelli, J. P.; Fabbiano, G.; Giacconi, R.; Golub, L.; 20336.

Gorlen, K.; Fiori, C. E.; Myklebust, R. L.; SP604: 1981 June. 233.272.

Gosselin, C.; Roy, P.; Courbier, R.; David, M.; Noël, H. P.; Guidoin, R.; King, M.; Blais, P.; Marois, M.; SP601: 1981 January. 29-129.

Goulard, R.; Emmerman, P. J.; Semerjian, H. G.; Santoro, R. J.; 20357.

Goulard, R.; Santoro, R. J.; Semerjian, H. G.; Emmerman, P. J.; 2033.5.

Graff, H. A.; Stromecky, P.,; Geer, N.; NBS-GCR-81-336.

Graffeuil, J.; Martin, J. C.; Sautereau, J. F.; SP614: 1981 September. 47-50.

Graffeuil, J.; Sautereau, J. F.; Tantrarongroj, K.; SP614: 1981 September. 84.87.

Graham, M.; Whitney, K.,; Berg, J. L.; SP500-76.

Graham, W. R.; Melmed, A. J.; Ceyer, S. T.; Tung, R. T.; 20686.

Graminski, E. L.; Parks, E. J.; J. Res. 86(3): 309-315; 1981 May.June.

Gramlich, J. W.; Rosman, K. J. R.; Barnes, I. L.; Moore, L. J.; 20698.

Grant, W. H.; Cassel, J. M.; Deh1, R. E.; NBSIR 81-2212.

Grant, W. H.; Dehl, R. E.; 20442.

Gray, M. M.; SP500-79.

Greegor, R. B.; Khalil, S. R.; Ekambaram, V.; Weiss, C. S.; Grimm, C. A.; Filby, R. H.; Sandstrom, D. R.; Lytle, F. W.; SP618: 1981 November. 21.38.

Green, R. E.; SP589: 1981 January. 143-147.

Greenberg, H. J.; Glassey, C. R.; SP616: 1981 October. 117.120.

Greenberger, M.; SP616: 1981 October. 25-41.

Greene, C. H.; SP599: 1981 January. 249.262.

Greenspan, M.; Breckenridge, F. R.; 20489.

Greer, S. C.; Moldover, M. R.; 20718.

Greer, S. C.; Sengers, J. V.; Bedeaux, D.; Mazur, P.; 20056.

Gregory, D. C.; Crandall, D. H.; Pindzola, M. S.; Falk, R. A.; Dunn, G. H.; Griffin, D. C.; Bottcher, C.; 20584.

Gregory, G.; Frederick, W.; Hausmann, R.,; Clarren, S.; Nalley, P.; NBS-GCR-ETIP 81-96.

Griffin, D. C.; Bottcher, C.; Gregory, D. C.; Crandall, D. H.; Pindzola, M. S.; Falk, R. A.; Dunn, G. H.; 20584.

Griffith, J. W.,; NBS-GCR-79-186-1.

Grimm, C. A.; Filby, R. H.; Sandstrom, D. R.; Lytle, F. W.; Greegor, R. B.; Khalil, S. R.; Ekambaram, V.; Weiss, C. S.; SP618: 1981 November. 21-38.

Griss. P.; Plitz, W.; SP601: 1981 January. 131-156.

Groner, N. E.; Levin, B. M.; Nelson, H. E.; 20719.

Gross, D.; NBSIR 81-2214.

Gross, F.; Meshkov, S.; Carlson, C. E.; Coyne, J. J.; Fishbane, P. M.; 20034.

Gross, F.; Meshkov, S.; Carlson, C. E.; Coyne, J. J.; Fishbane, P. M.; 20187.

Gross, F.; Meshkov, S.; Carlson, C. E.; Coyne, J. J.; Fishbane, P. M.; 20316.

Grosse-Nobis, W.; SP614: 1981 September. 317-323.

Grot, R. A.; 20365.

Grot, R. A.; Chang, Y. M. L.; NBSIR 80-2136.

Grot, R. A.; Clark, R. E.; 20540.

Gruhl, J.; Wood, D. O.; SP616: 1981 October. 183.200.

Grundl, J. A.; Gilliam, D. M.; McGarry, E. D.; Spiegel, V.; Eisenhauer, C. M.; 20153.

Gschwender, H. G.; Peters, K. R.; Haller, W.; 20523.

Guensler, D. A.; SP599: 1981 January. 212-240.

Guenther, A. H.; Judell, N.; Mitra, S. S.; Vaidyanathan, A.; SP620: 1981 October. 446-452.

Guenther, A. H.; Mitra, S. S.; Vaidyanathan, A.; SP620: 1981 October. 438-445. 
Guenther, A. H.; Mitra, S. S.; Vaidyanathan, A.; SP620: 1981 October. 432-437.

Guenther, A. H.; Newnam, B. E.,; Bennett, H. E.; Glass, A. J.; SP620.

Guenther, A. H.; Wiggins, T. A.; Walker, T. W.; SP620: 1981 October. 277-286.

Guenther, F. R.; Chesler, S. N.; May, W. E.; White, P. A.; Parris, R. M.; 20602.

Guenther, F. R.; May, W. E.; Wise, S. A.; Bonnett, W. J.; 20709.

Guenther, F. R.; Parris, R. M.; Chesler, S. N.; Hilpert, L. R.; 20161.

Guernsey, R. W.; Flint, E. B.; SP607: 1981 May. 15-20.

Guernsey, R. W.; Flint, E. B.; Jenkins, L. C.; SP607: 1981 May. 93. 102.

Guidoin, R.; King, M.; Blais, P.; Marois, M.; Gosselin, C.; Roy, P.; Courbier, R.; David, M.; Noël, H. P.; SP601: 1981 January. 29-129.

Guillaume, M. E.; Nahman, N. S.; TN1047.

Gujral, P. S.; Clark, R. J.; Burch, D. M.; 20538.

Gulbrandsen, G. E.; SP622: 1981 October. 139-151.

Gunton, J. D.; Sancho, J. M.; San Miguel, M.; Katz, S.; SP614: 1981 September. 26-29.

Guo, M. K.; Hsieh, C. C.; Hong, Y. C.; Chow, L. C.; 20672.

Gurtisen, J. M.; Apts, C. W.; Crecelius, E. A.; SP618: 1981 November. 173-183.

Gutterman, B. M.; Louis, G. L.; Pring, J. M.; Taylor, J. K.; Tholen, A. D.; Smith, C. A., ; Peiser, H. S.; NBSIR 80-2020.

\section{H}

Haack, R. F.; Shumka, A.; SP400-69: 1981 May. 43-57.

Haas, J. L., Jr.; Robinson, G. R., Jr.; Hemingway, B. S.; JPCRD 10(3): 575-670; 1981.

Haber, S.; 20032.

Hack, H.; Neuroth, N.; SP620: 1981 October. 102-109.

Haemmerli, A.; Janata, J.; Brophy, J. J.; SP614: 1981 September. 332. 334.

Haftka, R. T.; von Mayrhauser, A. K.; SP500-83: 1981 November. 199-215.

Haight, W. C.; Hocken, R. J.; Borchardt, B. R.; Carroll, C. L.; Hartsock, R. G.; Reeve, C. P.; Scire, F. E.; Veale, R. C.; NBSIR 80-2141.

Haisch, B.; Linsky, J. L.; Slee, O. B.; Siegman, B. C.; Nikoloff, I.; Candy, M.; Harwood, D.; Verveer, A.; Quinn, P. J.; Wilson, I.; Page, A. A.; Higson, P.; Deward, F. D.; 20405.

Haisch, B. M.; Harnden, F. R., Jr.; Johnson, H. M.; Linsky, J. L.; Maxson, C. W.; Mewe, R.; Rosner, R.; Seward, F.; Topka, K.; Zwaan, C.; Vaiana, G. S.; Cassinelli, J. P.; Fabbiano, G.; Giacconi, R.; Golub, L.; Gorenstein, P.; 20336.

Haisch, B. M.; Linsky, J. L.; Harnden, F. R., Jr.; Rosner, R.; Seward, F. D.; Vaiana, G. S.; 20124.

Hall, J. L.; 20583.

Hall, J. L.; Hollberg, L.; Baer, T.; Robinson, H. G.; 20720.

Hall, J. L.; Palmer, B. A.; Keller, R. A.; Kowalski, F. V.; 20730.

Hall, J. T.; Holdeman, L. B.; Soulen, R. J., Jr.; 19979.

Hall, J. T.; VanVechten, D.; Soulen, R. J., Jr.; Holdeman, L. B.; 20723.

Haller, W.; 20135.

Haller, W.; Gschwender, H. G.; Peters, K. R.; 20523.

Halstead, D.; Wyrick, T. F.; Giese, K. W.; SP500-83: 1981 November. 109-118.

Hamilton, C.; U.S. Patent 4,245,169.

Hamilton, C. A.; Harris, R. E.; Kautz, R. L.; McDonald, D. G.; Peterson, R. L.; 20070.

Hamilton, C. A.; Kautz, R. L.; Sullivan, D. B.; 20140.

Hamilton, C. A.; Lloyd, F. L.; Kautz, R. L.; 20184.

Hamilton, J. C.; Jach, T.; 20273.

Hammer, C.; Benzinger, T. H.; 20396.

Han, C. C.; Akcasu, A. Z.; 20482.

Han, C. C.; Akcasu, A. Z.; 20722.

Han, C. C.; Akcasu, A. Z.; 20721.

Han, C. C.; Khoury, F. A.; Markovitz, H.; McKinney, J. E.; Mopsik, F. I.; Passaglia, E.; Sanchez, I. C.; VanderHart, D. L.; Zapas, I. J.;
Eby, R. K.; Broadhurst, M. G.; Chang, S. S.; Davis, G. T DiMarzio, E. A.; Fanconi, B. M.; 20749.

Handa, T.; Sugawa, O.; Yamamoto, H.; Mulholland, G.; 20565.

Handel, P. H.; Gagnepain, J. J.; Uebersfeld, J.; SP614: 1981 September. 189.191.

Handel, P. H.; Kilmer, J.; van Vliet, K. M.; Chenette, E. R.; SP614: 1981 September. 151

Handel, P. H.; Wolf, D.; van Vliet, K. M.; SP614: 1981 September. 196.198.

Hands, B. A.; Arp, V. D.; 20769.

Hanley, H. J. M.; Ackerson, B. J.; 19964.

Hanley, H. J. M.; Ely, J. F.; 20651.

Hanley, H. J. M.; Ely, J. F.; 20768.

Hanley, H. J. M.; Ely, J. F.; TN1039.

Hanley, H. J. M.; Evans, D. J.; 19973.

Hanley, H. J. M.; Evans, D. J.; 19974.

Hanley, H. J. M.; Evans, D. J.; 20063.

Hanley, H. J. M.; Evans, D. J.; 20613.

Hanley, H. J. M.; Evans, D. J.; 20527.

Hanley, H. J. M.; Evans, D. J.; 20649.

Hanley, H. J. M.; Rainwater, J. C.; 20575.

Hansen, R. G.; Byrd, E. A.; SP607: 1981 May. 136-140.

Hansen, W. N.; Anderson, W. J.; SP620: 1981 October. 335.344.

Hardgrave, W. T.; Marriott, A. R.; Skillman, G. H.; Salazar, S. B.; NBSIR 80-2182.

Hardgrave, W. T.; Salazar, S. B.; Beller, E. J. III.; NBSIR 81-2240.

Hardgrave W. T.; Salazar, S. B.; Beller, E. J. III.; SP500-83: 1981 November. 85-91.

Harding, K. G.; Scott, M.; Leonard, T. A.; Loomis, J.; SP620: 1981 October. 345.355.

Hardman, K.; Rhyne, J. J.; Smith, K.; Wallace, W. E.; 20214.

Hardy, S. C.; Andreadis, T. D.; Fine, J.; 20332.

Harkleroad, M.; Quintiere, J.; Rinkinen, W.; Cooper, L. Y.; 20591.

Harkleroad, M. F.; McCaffrey, B. J.; Quintiere, J. G.; 20567.

Harkness, S.; Hermach, F. L.; Hirayama, H.; Martin, P.; Rozdestvenskaya, T. H.; Williams, E. S.; Galakhova, O. P.; 20240.

Harms, W. B.; Fivozinsky, S.; Purdy, J. A.; 20553.

Harnden, F. R., Jr.; Johnson, H. M.; Linsky, J. L.; Maxson, C. W.; Mewe, R.; Rosner, R.; Seward, F.; Topka, K.; Zwaan, C.; Vaiana, G. S.; Cassinelli, J. P.; Fabbiano, G.; Giacconi, R.; Golub, L.; Gorenstein, P.; Haisch, B. M.; 20336.

Harnden, F. R., Jr.; Rosner, R.; Seward, F. D.; Vaiana, G. S.; Haisch, B. M.; Linsky, J. L.; 20124.

Harris, C.; Hirshfeld, D.; SP616: 1981 October. 171-181.

Harris, G. R.; DeReggi, A. S.; Roth, S. C.; Kenney, J. M.; Edelman, S.; 20170.

Harris, J. R.; 20386.

Harris, J. R.; 20430.

Harris, J. R.; Fenves, S. J.; Wright, R. N.; 19998.

Harris, J. R.; Fenves, S. J.; Wright, R. N.; 20435.

Harris, J. R.; Leyendecker, E. V.; NBSIR 81-2195.

Harris, J. R.; Reinhold, T. A.; Simiu, E.; Yancey, C. W. C.; Cattaneo, L.; NBSIR 81-2199.

Harris, J. R.; Wright, R. N.; 20329.

Harris, J. R.; Wright, R. N.; 20403.

Harris, J. R.; Wright, R. N.; BSS136.

Harris, J. R.; Wright, R. N.; Fenves, S. J.; NBSIR 80-1979-1.

Harris, J. R.,; Leyendecker, E. V.; NBSIR 80-2111-11.

Harris, R. E.; Kautz, R. L.; McDonald, D. G.; Peterson, R. L.; Hamilton, C. A.; 20070.

Harrison, E. C.; Yoganathan, A. P.; SP601: 1981 January. 175-200.

Harrison, P. G.; SP500-83: 1981 November. 277-290.

Harrison, R. F.; Thomas, S. J.; Phipps, C. R., Jr.; SP620: 1981 October. 238-247.

Harrison, S. H.; Zeisler, R.; Wise, S. A.; 20601.

Harter, W. G.; Patterson, C. W.; 20154.

Hartley, J. L.; SP400-69: 1981 May. 128-132.

Hartley, J. L.; SP400-69: 1981 May. 24-32.

Hartman, W. F.; Robinson, J. C.; Allen, J. W.; SP622: 1981 October. 11-30.

Hartsock, R. G.; Reeve, C. P.; Scire, F. E.; Veale, R. C.; Haight, W. 
C.; Hocken, R. J.; Borchardt, B. R.; Carroll, C. L.; NBSIR 802141.

Hartsough, C.; Kleine, H.; Callender, E.; SP500-80: 1981 October. 44. 48.

Hartwig, W. H.; SP607: 1981 May. 127-135.

Harvey, K. C.; 20141

Harvey, K. C.; 20614.

Harwood, D.; Verveer, A.; Quinn, P. J.; Wilson, I.; Page, A. A.; Higson, P.; Deward, F. D.; Haisch, B.; Linsky, J. L.; Slee, O. B.; Siegman, B. C.; Nikoloff, I.; Candy, M.; 20405.

Harwood, D. L.; Molino, J. A.; Zerdy, G. A.; Lerner, N. D.; 20030.

Harwood, D. L.; Molino, J. A.; Zerdy, G. A.; Lerner, N. D.; 20050.

Harwood, D. L.; Molino, J. A.; Zerdy, G. A.; Lerner, N. D.; 20362.

Harwood, D. L.; Molino, J. A.; Zerdy, G. A.; Lerner, N. D.; 20361.

Hasegawa, S.; SP400-69: 1981 May. 126-127.

Haskin, W.; White, R.; SP607: 1981 May. 21-29.

Hasko, S.; Natrella, M. G.; Brickenkamp, C. S.; H133.

Hassler, R. A.; Klein, D. A.; SP618: 1981 November. 286-300.

Hastie, J. W.; Bonnell, D. W.; NBSIR 80-2169.

Hastie, J. W.; Bonnell, D. W.; Plante, E. R.; Horton, W. S.; NBSIR 80-2178 (DoE).

Hastie, J. W.; Plante, E. R.; NBSIR 81-2293.

Hastie, J. W.; Plante, E. R.; Bonnell, D. W.; NBSIR 81-2279.

Hausman, J. A.; SP616: 1981 October. 79.86.

Hausmann, R.,; Clarren, S.; Nalley, P.; Gregory, G.; Frederick, W.; NBS-GCR-ETIP 8I-96.

Hayashi, S. R.; Lifshin, E.; SP604: 1981 June. 165-175.

Hayes, D. J.; Olsen, K. B.; Wilkerson, C. L.; Toste, A. P.; SP618: 1981 November. 105-119.

Haynes, W. M.; Diller, D. E.; 20570

Hayward, E.; 19992.

Hayward, E.; 20267.

Hayward, E.; 20234.

Hayward, E.; 20195.

Hayward, E.; Leicht, R. G.; 20237.

Hayward, R. W.; 19991.

Hayward E.; Wolynec, E.; Dodge, W. R.; Leicht, R. G.; 20275.

Heafner, J.; Rosenthal, R.; Watkins, S.; Blanc, R.; NBSIR 80-2154.

Hebner, R. E.; NBSIR 81-2283.

Hebner, R. E.; Kelley, E. F.; Forster, E. O.; FitzPatrick, G. J.; 20645.

Hebner, R. E.,; NBSIR 81-2334.

Hebner, R. E., Jr.; 19962.

Hebner, R. E., Jr.; Kelley, E. F.; 20142.

Hebner, R. E., Jr.; Kelley, E. F.; 20131.

Hebner, R. E., Jr.; Kelley, E. F.; 20445.

Hebner, R. E., Jr.; Kelley, E. F.; Forster, E. O.; FitzPatrick, G. J.; 20664.

Hebner, R. E., Jr.; Kelley, E. F.; Thompson, J. E.; Sudarshan, T. S.; Jones, T. B.; NBSIR 81-2275.

Hebner, R. E., Jr.; Zahn, M.; Forster, E. O.; Kelley, E. F.; 20646.

Hecht, H.; SP500-82.

Hecht, H.; NBSIR 81-2388.

Hecht, M. H.; Egelhoff, W. F., Jr.; Tibbetts, G. G.; Lindau, I.; 20478.

Heerwagen, D. R.; Banken, G.; Emery, A. F.; Dorri, B.; Kippenhan, C. J.; SP608: 1981 May. 21.35.

Heffernan, A. P.,; Wollin, H. F.; Barbrow, L. E.; SP599.

Hegner, H.; Envent, F.; Cohill, M.; SP622: 1981 October. 113-124.

Heidemann, A.; Michel, K. H.; Rowe, J. M.; Rush, J. J.; Pynn, R.; 20421 .

Heiden, C.; Beckstette, K. F.; Dirks, H. M.; SP614: 1981 September. 388.390 .

Heiden, C.; Daunt, J. G.; SP607: 1981 May. 141-145.

Heiden, C.; Dirks, H. M.; Beckstette, K. F.; SP614: 1981 September. 384-387.

Heiles, C. E.; Clark, F. O.; Johnson, D. R.; Troland, T. H.; 20439

Heinemeyer, R. R.; SP608: 1981 May. 195-200.

Heinrich, K. F. J.; SP604: 1981 June. 1-4.

Heinrich, K. F. J.; Myklebust, R. L.; Fiori, C. E.; SP604: 1981 June. 365-379.

Heinrich, K. F. J.; Newbury, D. E.; Myklebust, R. L.; Fiori, C. E.; SP604.
Hellmuth, T.; Leuchs, G.; Smith, S. J.; Walther, H.; 20504.

Hellmuth, T.; Leuchs, G.; Smith, S. J.; Walther, H.; 20506.

Helms, C. R.; Schwarz, S. A.; Spicer, W. E.; Taylor, N. J.,; NBSGCR $81-345$.

Helms, C. R.; Spicer, W. E.; Taylor, N. J.; Schwarz, S. A.; SP400-67.

Hemingway, B. S.; Haas, J. L., Jr.; Robinson, G. R., Jr.; JPCRD 10(3): 575-670; 1981.

Henderson, E.; SP610: 1981 December. 61-65.

Henderson, J. B.; Miller, E. F., Jr.; SP500-80: 1981 October. 128-135.

Henderson, J. B.; Miller, E. F., Jr.; Hirschberg, M.; SP500-80: 1981 October. 136-145.

Henderson, M.; Berger, P.; Warnar, R.; SP610: 1981 December. 74 79.

Henderson, M. M.; MacNaughton, M. J.,; 20149.

Hendricks, J. B.; Karr, G. R.; Urban, E. W.; Katz, L.; SP607: 1981 May. 117-127.

Hendrickson, B.; Thornton, J. I.; Kraus, S.; Lerner, B.; SP480-40.

Henins, A.; Deslattes, R. D.; Kessler, E. G.; Sauder, W. C.; 20146.

Hermach, F. L.; Hirayama, H.; Martin, P.; Rozdestvenskaya, T. H.; Williams, E. S.; Galakhova, O. P.; Harkness, S.; 20240.

Herman, D.,; McCarthy, D.; Acevedo, J.; NBS-GCR-81-327.

Herman, Z.; Lineberger, W. C.; Feigerle, C. S.; 20536.

Herrmann, W. C., Jr.; McNeil, J. R.; SP620: 1981 October. 324-334.

Herron, J. T.; Huie, R. E.; 20035.

Herron, J. T.; Huie, R. E.; Martinez, R. I.; 20402.

Hertz, H.; Christensen, R.; White, E.; U.S. Patent 4,281,246

Hertz, H. S.; 19989.

Hertz, H. S.; Meiselman, S.; White, E.; Christensen, R. G.; 20097.

Hertz, H. S.; Reeder, D. J.; White, E.; Christensen, R. G.; 20654.

Hertz, H. S.; White, E.; Welch, V. M. J.; NBSIR 80-2160

Hessel, M. M.; Lovas, F. J.; Wormsbecher, R. F.; 20424.

Heydemann, P. L. M.; 20679.

Hiatt, C. F.; van der Ziel, A.; Zijlstra, R. J. J.; SP614: 1981 September. 234.235.

Hibbard, B. B.; Krasny, J. F.; Braun, E.; Peacock, R. D.; NBSIR 81 2386

Hicho, G. E.; Interrante, C. G.; SP260-73.

Hickam, W. M.; Morgan, W. R.; SP400-69: 1981 May. 153-158.

Hicks, R. S.; Parker, B.; Peterson, G. A.; Lawson, R. D.; Teeters, W.; Williamson, C. F.; Kowalski, S.; Maruyama, X. K.; Lindgren, R. A.; Flanz, J. B.; 20323.

Hicks, R. S.; Parker, B.; Peterson, G. A.; Singhal, R.; Williamson, C. F.; Maruyama, X. K.; Petrovich, F.; Lindgren, R. A.; Plum, M. A.; Gerace, W. J.; 20660

Higgins, R.; Spath, J.; Buchholz, A.; Wrzenski, S. C.,; Egan, J.; NBSGCR $-81-313$.

Higson, P.; Deward, F. D.; Haisch, B.; Linsky, J. L.; Slee, O. B.; Siegman, B. C.; Nikoloff, I.; Candy, M.; Harwood, D.; Verveer, A.; Quinn, P. J.; Wilson, I.; Page, A. A.; 20405.

Hill, J. E.; May, W. B., Jr.; Richtmyer, T. E.; Elder, J.; Tibbott, R. L.; Yonemura, G. T.; Hunt, C. M.; Chen, P. T.; BSS133.

Hill, J. E.; Richtmyer, T. E.; May, W. B.; Hunt, C. M.; 20539

Hillig, K. W.; Ashe, A. J.; Kuczkowski, R. L.; Lovas, F. J.; Suenram R. D.; Lattimer, R. P.; 20168.

Hillman, D. C.; O'Brien, D. H.; Pyles, R. A.; Stockton, R. A.; Wheeler, A. E.; Zingaro, R. A.; Irgolic, K. J.; Banks, C. H.; Bottino, N. R.; Chakraborti, D.; Gennity, J. M.; SP618: 1981 November. 244-263.

Hilpert, L. R.; Guenther, F. R.; Parris, R. M.; Chesler, S. N.; 20161.

Hilpert, L. R.; Wise, S. A.; May, W. E.; Brown-Thomas, J. M.; 20750.

Hiltner, A.; Anderson, J. M.; Barenberg, S.; SP601: 1981 January. 283.298.

Himpsel, F. J.; Eastman, D. E.; Madey, T. E.; Stockbauer, R.; Flodström, S. A.; van der Veen, J. F.; 20510

Hinman, R.; Cassel, J. M.; Tesk, J. A.; Brauer, G. M.; Antonucci, J. M.; Wu, W.; McKinney, J. E.; Whitlock, R. P.; NBSIR 80-2168.

Hirayama, H.; Martin, P.; Rozdestvenskaya, T. H.; Williams, E. S.; Galakhova, O. P.; Harkness, S.; Hermach, F. L.; 20240.

Hirschberg, M.; Henderson, J. B.; Miller, E. F., Jr.; SP500-80: 1981 October. 136-145. 
Hirshfeld, D.; Harris, C.; SP616: 1981 October. 171-181.

Hiskes, J. R.; Stevens, W. J.; Karo, A. M.; 20229.

Hiza, M. J.; 20505.

Hiza, M. J.; Kidnay, A. J.; Massoud Gasem, K. A.; 20573.

Hnatczuk, W.; Woody, M. W.; SP622: 1981 October. 85-96.

Ho, J. C.; Oberly, C. E.; Garrett, H. J.; Walker, M. S.; Zeitlin, B. A. Ekin, J. W.; 20741.

Hobbs, R. D.; Zimmerman, J. E.; Sullivan, D. B.; Kautz, R. L.; SP607: 1981 May. 173-177.

Hobbs, T. G.; 20181.

Hocken, R. J.; Borchardt, B. R.; NBSIR 79-1752.

Hocken, R. J.; Borchardt, B. R.; Carroll, C. L.; Hartsock, R. G.; Reeve, C. P.; Scire, F. E.; Veale, R. C.; Haight, W. C.; NBSIR 802141.

Hocken, R. J.; Cummings, A. L.; Layer, H. P.; 20268.

Hockey, B. J.; Wiederhorn, S. M.; Lawn, B. R.; 20559.

Hodgeson, J. A.; Fatiadi, A. J.; 20290.

Hodgins, B. D.; Cox, L. A., Jr.; SP500-83: 1981 November. 181-189.

Hodgkin, V. A.; Decker, D. L.; SP620: 1981 October. 190-200.

Hodsman, G. F.; SP599: 1981 January. 95-101.

Hodson, S. W.; Cox, A. N.; Shaviv, G.; 20337.

Hoer, C. A.; 20631.

Hoffman, J. D.; Wachtman, J. B., Jr.; NBSIR 81-2408.

Hoffman, J. D.; Yolken, H. T.; Reed, W. P.; Carpenter, B. S.; NBSIR 81-2205.

Hoffman, K.; SP616: 1981 October. 43-47.

Hoffman, K. L.; Jackson, R. H. F.; Joel, L. S.; Saunders, P. B.; Gass, S. I.; NBSIR 80-1971.

Hoffman, K. L.; Shier, D. R.; 20502.

Hoffman, R. H.; Loshbough, R. P.; Smith, R. W.; SP500-80: 1981 October. 111-116.

Holcman, J.; Sehested, K.; McLaughlin, W. L.; Kosanić, M. M.; Marković, V. M.; Nenadovic, M. T.; 20150.

Holdeman, L. B.; Hall, J. T.; VanVechten, D.; Soulen, R. J., Jr.; 20723.

Holdeman, L. B.; Soulen, R. J., Jr.; Hall, J. T.; 19979.

Holdeman, L. B.; Soulen, R. J., Jr.; Van Vechten, D.; Costabile, G.; Jach, T.; 20702.

Hollberg, L.; Baer, T.; Robinson, H. G.; Hall, J. L.; 20720.

Hollis, J. M.; Snyder, L. E.; Lovas, F. J.; Ulich, B. L.; 19987.

Hollis, J. M.; Snyder, L. E.; Suenram, R. D.; Lovas, F. J.; 20077.

Hollman, R.; Little, W. A.; SP607: 1981 May. 160-163.

Holloway, M. L.; SP616: 1981 October. 159-169.

Holmes, A.; Parry, E. E.; Tesk, J. A.; Whitlock, R. P.; Widera, G. E. O.; 20277.

Holmes, A.; Parry, E. E.; Whitlock, R. P.; Tesk, J. A.; Widera, G. E. O.; 20241.

Hong, Y. C.; Chow, L. C.; Guo, M. K.; Hsieh, C. C.; 20672.

Hord, J.; Roder, H. M.; McCarty, R. D.; Monogr. 168.

Hori, R. Y.; Lewis, J. L.; Wixson, R. L; Kramer, G. M.; SP601: 1981 January. 509-557.

Horn, P. M.; Knoedler, C. M.; Voss, R. F.; SP614: 1981 September. 391-394.

Horn, S. B.; Asher, M. S.; SP607: 1981 May. 30-47.

Horton, W. S.; Hastie, J. W.; Bonnell, D. W.; Plante, E. R.; NBSIR 80-2178 (DoE).

Houck, J. C.; Welch, B. E.; Driver, R. G.; J. Res. 86(3): 277-279; 1981 May-June.

Hougen, J. T.; 20099.

Hougen, J. T.; 20588.

Hougen, J. T.; Oka, T.; 20115.

Hougen, J. T.; Simmons, J. D.; Maki, A. G.; 20080.

Houghton, R. C., Jr.; SP500-74.

Houghton, R. C., Jr.; Oakley, K. A.; SP500-80: 1981 October. 180 185.

Houghton, R. C., Jr.,; SP500-80.

Houston, J. E.; Dahlberg, S. C.; Madey, T. E.; 20279.

Houston, J. E.; Seabury, C. W.; Rhodin, T. N.; Madey, T. E.; 20419.

Howard, C. P.; 20000

Howard, P. C.; SP500-83: 1981 November. 337.

Howell, B. F.; Paule, R. C.; Mandel, J.; Schaffer, R.; Reeder, D. J.;
Coxon, B.; Enagonio, D.; Christensen, R. G.; SP260-72.

Howett, G. L.; Rubin, A. I.; $S P 480-37$.

Hsia, J. J.; Weidner, V. R.; 20287.

Hsieh, C. C.; Hong, Y. C.; Chow, L. C.; Guo, M. K.; 20672.

Hsu, N. N.; Breckenridge, F. R.; 20197.

Hsu, N. N.; Simmons, J. A.; Eitzen, D. G.; Breckenridge, F. R.; Clough, R. B.; Fuller, E. R.; 20579.

Hsu, N. N.; Simmons, J. A.; Eitzen, D. G.; Breckenridge, F. R.; Clough, R. B.; Fuller, E. R.; 20757.

Hsu, S. M.; Becker, D. A.; 20016.

Huang, J. C.; SP500-80: 1981 October. 159-162.

Huang, J. S. T.; Liu, S. T.; SP614: 1981 September. 67-70.

Hubbard, C. R.; Morris, M. C.; McMurdie, H. F.; Evans, E. H.; Paretzkin, B.; Parker, H. S.; Panagiotopoulos, N. C.; Monogr. 25, Section 18.

Hubbard, C. R.; Panagiotopoulos, N. C.; Snyder, R. L.; NBSIR 81 2229.

Hubbard, C. R.; Stalick, J. K.; Mighell, A. D.; TN1141.

Hubbard, J. B.; Kayser, R. F.; 20227.

Huberman, M. G.; Van der Klott, R.; Senturia, S. D.; SP400-69: 1981 May. 108-114.

Huckeba, J. A.; Berger, R. E.; NBSIR 80-2037.

Hudson, C. H.; Berke, J. G.; NBSIR 81-2316.

Hudson, P. A.; Weidman, M. P.; TN1044.

Hug, G. L.; NSRDS-NBS69.

Hug, R.; Strelich, T.; Resnicow, M.; Ahola, R.; SP500-80: 1981 October. 122-127.

Hughes, B. D.; Montroll, E. W.; Shlesinger, M. F.; SP614: 1981 September. 18-21.

Hughes, H. N.; Dermann, K.; Rupp, N. W.; Brauer, G. M.; Dulik, D. M.; 20669

Huie, R. E.; Herron, J. T.; 20035.

Huie, R. E.; Martinez, R. I.; Herron, J. T.; 20402.

Hummer, D. G.; 20562.

Hummer, D. G.; Katriel, J.; 20243.

Hummer, D. G.; Pradhan, A. K.; Norcross, D. W.; 20045.

Hummer, D. G.; Pradhan, A. K.; Norcross, D. W.; 20492.

Humphreys, J. C.; McLaughlin, W. L.; 20171.

Hunt, B.,; NBS-GCR-80-255.

Hunt, B. J.; Fattal, S. G.; NBSIR 81-2265.

Hunt, C. M.; 20364

Hunt, C. M.; Chen, P. T.; Hill, J. E.; May, W. B., Jr.; Richtmyer, T E.; Elder, J.; Tibbott, R. L.; Yonemura, G. T.; BSS133.

Hunt, C. M.; Hill, J. E.; Richtmyer, T. E.; May, W. B.; 20539.

Hunt, C. M.; Treado, S. J.; 20605.

Huntoon, R. D.; NBSIR 80-1972.

Hust, J. G.; Boscardin, R.; 20494.

Hust, J. G.; Smith, D. R.; 20526.

Hust, J. G.; Smith, D. R.; NBSIR 81-1639.

Hust, J. G.; Van Poolen, L. J.; Smith, D. R.; 20547.

Hust, J. G.; Van Poolen, L. J.; Smith, D. R.; NBSIR 81-1640.

Hutchinson, J. M. R.; 20010

Hutchinson, J. M. R.; Collé, R.; Rubin, R. J.; Knab, L. I.; TN1139.

Huth, G. C.; Iwanczyk., J. S.; Dabrowski, A. J.; Singh, M.; SP604: 1981 June. 45-59.

I

Inman, L. B.,; Martin, S. B.; NBS-GCR-81-329.

Interrante, C. G.; Hicho, G. E.; SP260-73.

Irgolic, K. J.; Banks, C. H.; Bottino, N. R.; Chakraborti, D.; Gennity, J. M.; Hillman, D. C.; O'Brien, D. H.; Pyles, R. A.; Stockton, R. A.; Wheeler, A. E.; Zingaro, R. A.; SP618: 1981 November. 244. 263.

Issen, L. A.; NBSIR 80-2155.

Itano, W. M.; Wineland, D. J.; 20305.

Itano, W. M.; Wineland, D. J.; 20680.

Iverson, W. P.; Brinckman, F. E.; Olson, G. J.; NBSIR 81-2246.

Ives, J. T.; Sullivan, D. B.; Zimmerman, J. E.; SP607: 1981 May. 186194.

Ives, L.; Coriell, S.; Ballard, D.; Laughlin, D.; Clough, R.; 
Biancaniello, F.; Blau, P.; Cahn, J.; Mehrabian, R.; Free, G.; Berger, H.; Mordfin, L.; Swartzendruber, L.; Boettinger, W.; NBSIR 80-2069.

Ives, L. K.; Ruff, A. W.; 20209.

Ives, L. K.; Shull, R. D.; Swartzendruber, L. J.; Bennett, L. H.; 20736.

Iwanczyk., J. S.; Dabrowski, A. J.; Singh, M.; Huth, G. C.; SP604: 1981 June. 45-59.

\section{J}

Jach, T.; Hamilton, J. C.; 20273.

Jach, T.; Holdeman, L. B.; Soulen, R. J., Jr.; Van Vechten, D.; Costabile, G.; 20702.

Jach, T.; Powell, C. J.; 20394.

Jackson, R. H. F.; SP616: 1981 October. 87.89.

Jackson, R. H. F.; Joel, L. S.; Saunders, P. B.; Gass, S. I.; Hoffman,

K. L.; NBSIR 80-1971.

Jacoboni, C.; Reggiani, L.; Brunetti, R.; SP614: 1981 September. 414. 416.

Jacobs, L.; Schwitz, W.; Seki, R.; Lum, G. K.; Wiegand, C. E.; Kessler, E. G., Jr.; Deslattes, R. D.; 20349.

Jacobsen, R. T.; McCarty, R. D.; TN1045.

Jacobson, D. C.; Poate, J. M.; Nakahara, S.; Draper, C. W.; Buene, L.; SP620: 1981 October. 222-226.

Jacox, M. E.; 19986.

Jacox, M. E.; 20036

Jacox, M. E.; 20515.

Jacox, M. E.; 20460

Jacox, M. E.; 20550.

Jamieson, A.; Evans, J.,; Foskett, W. H.; NBS-GCR-ETIP 81-93.

Janata, J.; Brophy, J. J.; Haemmerli, A.; SP614: 1981 September. 332. 334.

Janz, G. J.; Tomkins, R. P. T.; NSRDS-NBS61, Part IV.

Jason, A. J.; Stockbauer, R.; McCulloh, K. E.; Parr, A. C.; 20051.

Jason, N. H.; NBSIR 81-2272.

Jenkins, D.; Knab, L.; Mathey, R.; BSS131.

Jenkins, D. R.; Mathey, R. G.; Knab, L. I.; TN1146.

Jenkins, J. P.; 20079.

Jenkins, L. C.; Guernsey, R. W.; Flint, E. B.; SP607: 1981 May. 93. 102

Jenkins, W. D.; Swyt, D. A.; Lettieri, T. R.; 20683.

Jenne, E. A.; SP618: 1981 November. 39.53.

Jensen, B.; SP620: 1981 October. 416.426.

Jensen, S.; Swyt, D.; 20201.

Jesch, R. L.; NBSIR 78-896.

Jewett, K. L.; Brinckman, F. E.; Fish, R. H.; Weiss, C. S.; SP618: 1981 November. 197.216.

Jindal, R. P.; Suh, C. H.; van der Ziel, A.; SP614: 1981 September. 236-239.

Jindal, R. P.; van der Ziel, A.; SP614: 1981 September. 173-177.

Joel, L. S.; Gass, S. I.; NBSIR 80-2053.

Joel, L. S.; Maybee, J. S.; SP616: 1981 October. 149-158.

Joel, L. S.; Saunders, P. B.; Gass, S. I.; Hoffman, K. L.; Jackson, R. H. F.; NBSIR 80-1971.

Johnson, C. C.; Clifton, C.; Nober, E. H.; Pierce, H.; Well, A.; NBSGCR-80-284.

Johnson, C. E.; Ogburn, F.; Schoonover, R. M.; 20334.

Johnson, C. E.; Ugiansky, G. M.; 20027.

Johnson, C. E.; Ugiansky, G. M.; NBSIR 81-2191 (DoE).

Johnson, D. R.; Clark, F. O.; 20517.

Johnson, D. R.; Troland, T. H.; Heiles, C. E.; Clark, F. O.; 20439.

Johnson, E. G., Jr.; Phelan, R. J., Jr.; Boyle, D. R.; NBSIR 80-1628.

Johnson, H. M.; Linsky, J. L.; Maxson, C. W.; Mewe, R.; Rosner, R.; Seward, F.; Topka, K.; Zwaan, C.; Vaiana, G. S.; Cassinelli, J. P.; Fabbiano, G.; Giacconi, R.; Golub, L.; Gorenstein, P.; Haisch, B. M.; Harnden, F. R., Jr.; 20336.

Johnson, R.; Bowman, C. D.; Schrack, R. A.; Behrens, J. W.; 20458.

Johnson, R. B.; SP610: 1981 December. 13-16.

Johnson, R. G.; Behrens, J. W.; Bowman, C. D.; 20163.

Johnson, R. G.; Behrens, J. W.; Bowman, C. D.; 20724.
Johnson, W. C.; Cahn, J. W.; 20725.

Jones, A.; SP589: 1981 January. 121.132.

Jones, B. K.; SP614: 1981 September. 206-209.

Jones, D. E.; Cole-Appel, B. E.; Oonk, R. L.; 20148.

Jones, F. E.; 20681

Jones, F. E.; J. Res. 86(1): 27-32; 1981 January-February.

Jones, F. E.; Brickenkamp, C. S.; 20682.

Jones, G. W.; SP622: 1981 October. 50-61.

Jones, J. E.; Canada, R. G.; SP622: 1981 October. 3.10.

Jones, M. C.; Daney, D. E.; 20507.

Jones, P. L.; Mead, R. D.; Kohler, B. E.; Rosner, S. D.; Lineberger, W. C.; 19995.

Jones, R. N.; Bussey, H. E.; Little, W. E.; Metzker, R. F.; NBSIR 78897.

Jones, T. B.; Hebner, R. E., Jr.; Kelley, E. F.; Thompson, J. E.; Sudarshan, T. S.; NBSIR 81-2275.

Jordan, B.; SP599: 1981 January. 34-38.

Jordan, C.; Carpenter, K. G.; Wing, R. F.; Czyzak, S.; Stencel, R. E.; Linsky, J. L.; Brown, A.; 20598.

Jordan, T. H.; Brown, W. E.; Schroeder, L. W.; 20173.

Jordan, T. H.; Dickens, B.; Schroeder, L. W.; Brown, W. E.; 19994

Josephson, E.; Bush, T.; Mazzone, G.; McElroy, G.; Galbraith, T., NBS-GCR-81-312.

Juarez, N.; Krasny, J. F.; Allen, P. J.; Maldonado, A.; NBSIR 812363.

Juarez, N.; Maldonado, A.; Simenauer, J. A.; Loftus, J. J.; NBSIR 81 2247.

Judell, N.; Mitra, S. S.; Vaidyanathan, A.; Guenther, A. H.; SP620: 1981 October. 446.452.

Julienne, P. S.; Konowalow, D. D.; Krauss, M.; Rosenkrantz, M. E.; Stevens, W. J.; 20100.

Julienne, P. S.; Krauss, M.; Rosenkrantz, M. E.; Basch, H.; 20238.

Julienne, P. S.; Mies, F. H.; 20155.

Jun, E. T.; SP500-83: 1981 November. 293.302.

\section{$\mathbf{K}$}

Kahn, A. H.; Spal, R. D.; SP589: 1981 January. 137.141.

Kainlauri, E. O.; SP608: 1981 May. 133-138.

Kainlauri, E. O.; SP608: 1981 May. 57.67.

Kampier, I. K.,; Dickamore, W. G.; NBS-GCR-80-307.

Kanda, M.; NBSIR 78-876.

Kanda, M.; NBSIR 78-898.

Kanda, M.; TN1028.

Kandiah, K.; Cox, C. E.; SP614: 1981 September. 71.74.

Kandiah, K.; Deighton, M. O.; Whiting, F. B.; SP614: 1981 September. 75-78.

Kao, J. Y.; Baker, D. W.; NBSIR 80-2104.

Kao, J. Y.; Didion, D. A.; Baker, D. W.; NBSIR 81-2313.

Kao, J. Y.; Pierce, E. T.; NBSIR 81-2277.

Kaplan, A. E.; Weiss, E. R.; Byrne, S. T.; El-Torkey, N. M.; Margolis, S. A.; 20446.

Karl, G.; Meshkov, S.; Rosner, J. L.; 20040.

Karo, A. M.; Hiskes, J. R.; Stevens, W. J.; 20229.

Karr, G. R.; Urban, E. W.; Katz, L.; Hendricks, J. B.; SP607: 1981 May. 117.127.

Kasen, M. B.; 20606.

Kasen, M. B.; 20557

Kasen, M. B.; Berger, H.; Placious, R. C.; Garrett, D. A.; 20342.

Kasen, M. B.; MacDonald, G. R.; Beekman, D. H., Jr.; Schramm, R. E.; 20616

Kasper, P. K.; Montroll, M. L.,; Sharp, B. H.; NBS-GCR-80-250.

Katriel, J.; Hummer, D. G.; 20243.

Katz, L.; Hendricks, J. B.; Karr, G. R.; Urban, E. W.; SP607: 1981 May. 117.127.

Katz, S.; Gunton, J. D.; Sancho, J. M.; San Miguel, M.; SP614: 1981 September. 26-29.

Kauffman, D.; Scarfe, C. M.; Cronin, D.; Wenzel, J.; 20222

Kaufman, V.; Sugar, J.; Wyart, J. F.; 20002.

Kaufman, V.; Sugar, J.; Wyart, J. F.; 20611.

Kautz, R. L.; 20657. 
Kautz, R. L.; Costabile, G.; 20183.

Kautz, R. L.; Hamilton, C. A.; Lloyd, F. L.; 20184.

Kautz, R. L.; Hobbs, R. D.; Zimmerman, J. E.; Sullivan, D. B.; SP607: 1981 May. 173-177.

Kautz, R. L.; McDonald, D. G.; Peterson, R. L.; Hamilton, C. A.; Harris, R. E.; 20070.

Kautz, R. L.; Sullivan, D. B.; Hamilton, C. A.; 20140.

Kawasaki, M.; Tanaka, Y.; Okabe, H.; 20042.

Kayser, R. F.; Hubbard, J. B.; 20227.

Keller, R. A.; Kowalski, F. V.; Hall, J. L.; Palmer, B. A.; 20730

Kelley, E. F.; Forster, E. O.; FitzPatrick, G. J.; Hebner, R. E.; 20645.

Kelley, E. F.; Forster, E. O.; FitzPatrick, G. J.; Hebner, R. E., Jr.; 20664.

Kelley, E. F.; Hebner, R. E., Jr.; 20131

Kelley, E. F.; Hebner, R. E., Jr.; 20142.

Kelley, E. F.; Hebner, R. E., Jr.; 20445.

Kelley, E. F.; Hebner, R. E., Jr.; Zahn, M.; Forster, E. O.; 20646.

Kelley, E. F.; Thompson, J. E.; Sudarshan, T. S.; Jones, T. B.; Hebner, R. E., Jr.; NBSIR 81-2275.

Kelley, R. D.; Goodman, D. W.; 20350.

Kelly, G. E.; Kuklewicz, M. E.; NBSIR 80-2094.

Kelly, G. E.; Kuklewicz, M. E.; NBSIR 80-2110.

Kelly, G. E.; Parken, W. H.; 20690.

Kelly, G. E.; Petersen, S. R.; Barnes, K. A.; NBSIR 81-2241.

Kelly, J. C.; SP500-83: 1981 November. 121.

Kelly, J. C.; Blaylock, J. W.; SP500-83: 1981 November. 251-258.

Kelly, J. C.; Wolf, R.; Kennicott, P.; Nagel, R.; Wellington, J.,; NBSIR 81-2297.

Kelly, P.; Braunlich, P.; Brost, G.; Schmid, A.; SP620: 1981 October 406-415.

Kelly, P.; Ritchie, D.; Braunlich, P.; Schmid, A.; Bryant, G. W.; SP620: 1981 October. 394-402.

Kelly, P.; Ritchie, D.; Bryant, G. W.; Schmid, A.; Braunlich, P.; SP620: 1981 October. 427.431.

Kennerly, R. E.; Van Brunt, R. J.; Gallagher, A. C.; 20244.

Kenney, J. M.; Edelman, S.; Harris, G. R.; DeReggi, A. S.; Roth, S. C.; 20170.

Kennicott, P.; Nagel, R.; Wellington, J.,; Kelly, J. C.; Wolf, R.; NBSIR 81-2297.

Kerns, D. M.; Monogr. 162.

Kessler, E. G.; Sauder, W. C.; Henins, A.; Deslattes, R. D.; 20146.

Kessler, E. G., Jr.; Deslattes, R. D.; 20483.

Kessler, E. G., Jr.; Deslattes, R. D.; Jacobs, L.; Schwitz, W.; Seki, R.; Lum, G. K.; Wiegand, C. E.; 20349.

Kessler, H. K.; Russell, T. J.; Lankford, W. F.; Schafft, H. A.; Sawyer, D. E.; NBSIR 80-2181.

Kessler, K. G.; NBSIR 80-2165.

Kestin, J.; Khalifa, H. E.; Correia, R. J.; JPCRD 10(1): 57-70; 1981.

Kestin, J.; Khalifa, H. E.; Correia, R. J.; JPCRD 10(1): 71-88; 1981.

Key, W. P.; Potter, T.; SP608: 1981 May. 183-194.

Khalifa, H. E.; Correia, R. J.; Kestin, J.; JPCRD 10(1): 57-70; 1981.

Khalifa, H. E.; Correia, R. J.; Kestin, J.; JPCRD 10(1): 71-88; 1981.

Khalil, S. R.; Ekambaram, V.; Weiss, C. S.; Grimm, C. A.; Filby, R. H.; Sandstrom, D. R.; Lytle, F. W.; Greegor, R. B.; SP618: 1981 November. 21-38.

Khoury, F. A.; Broadhurst, M. G.; Fanconi, B. M.; Eby, R. K.; Peterlin, A.; 20652.

Khoury, F. A.; Crissman, J. M.; McKenna, G. B.; NBSIR 81-2209 (FDA).

Khoury, F. A.; Markovitz, H.; McKinney, J. E.; Mopsik, F. I.; Passaglia, E.; Sanchez, I. C.; VanderHart, D. L.; Zapas, I. J.; Eby, R. K.; Broadhurst, M. G.; Chang, S. S.; Davis, G. T.; DiMarzio, E. A.; Fanconi, B. M.; Han, C. C.; 20749.

Kibalo, T. H.; Turgel, R. S.; Oldham, N. M.; Stenbakken, G. N.; TN1144.

Kidnay, A. J.; Massoud Gasem, K. A.; Hiza, M. J.; 20573.

Kieffer, L. J.; Belanger, B. C.; 20081.

Kieffer, L. J.,; SP250, 1980 Edition.

Kilday, M. V.; J. Res. 86(4): 367.382; 1981 July-August.

Kilmer, J.; van Vliet, K. M.; Chenette, E. R.; Handel, P. H.; SP614: 1981 September. 151.
Kilmer, R. D.; 20560

Kim, E. M.; Chamberlain, G. E.; Franzen, D. L.; Day, G. W.; Danielson, B. L.; 20369.

Kim, E. M.; Franzen, D. L.; 20432.

Kim, E. M.; Franzen, D. L.; TN1032.

Kim, R. K.; Braunstein, M.; Braunstein, R.; SP620: 1981 October. 29. 43.

Kincy, W. P., Jr.; Bays, W. N.; SP500-83: 1981 November. $143-156$

King, D. S.; Stephenson, J. C.; 20101.

King, D. S.; Stephenson, J. C.; Akins, D. L.; 20095.

King, G. P.; Swinney, H. L.; Roux, J. C.; SP614: 1981 September. 283-287.

King, M.; Blais, P.; Marois, M.; Gosselin, C.; Roy, P.; Courbier, R.; David, M.; Noël, H. P.; Guidoin, R.; SP601: 1981 January. 29.129.

King, W. C.; SP500-80: 1981 October. 83-87.

Kingston, H.; Pella, P. A.; 20200

Kingston, H. M.; 20302.

Kingston, M. L.,; NBSIR 81-2226.

Kippenhan, C. J.; Heerwagen, D. R.; Banken, G.; Emery, A. F.; Dorri, B.; SP608: 1981 May, 21-35.

Kirchhoff, W. H.; NBSIR 81-2280.

Kirklin, D. R.; Colbert, J.; Decker, P.; Abramowitz, S.; Domalski, E. S.; NBSIR $81-2278$

Kirklin, D. R.; Colbert, J. C.; Xiheng, H.; J. Res. 86(6): 655.660; 1981 November-December.

Kittel, J.,; NBS.GCR-81-310.

Klawitter, J. J.; French, G.; Skinner, H.; Weinstein, A. M.; Clemow, A. J. T.; McPhillips-Meade, M.; SP601: 1981 January. 423-447.

Klebanoff, P. S.; Buckley, F. T.; Purtell, L. P.; 20257.

Klebanoff, P. S.; Mease, N. E.; McMichael, J. M.; 20048.

Klein, C. A.; SP620: 1981 October. 117-128.

Klein, D. A.; Hassler, R. A.; SP618: 1981 November. 286-300.

Klein, D. P.; NBSIR 81-2333.

Klein, M.; Waxman, M.; Davis, H. A.; Sengers, J. M. H. L.; NBSIR 79-1715.

Klein, R.; Fine, J.; 20013.

Kleine, H.; Callender, E.; Hartsough, C.; SP500-80: 1981 October. 44.

Kleinpenning, T. G. M.; SP614: 1981 September. 240-243.

Klempt, G.; Storm, L.; SP614: 1981 September. 269-271.

Kloss, C.; Simila, K. J.; SP599: 1981 January. 54-63.

Klote, J. H.; NBSIR 80-2157.

Klote, J. H.; Zile, R. H.; NBSIR 81-2433.

Knab, L.; Mathey, R.; Jenkins, D.; BSS131.

Knab, L. I.; Hutchinson, J. M. R.; Collé, R.; Rubin, R. J.; TN1139.

Knab, L. I.; Jenkins, D. R.; Mathey, R. G.; TN1146.

Knapp, E. A.; Martin, R. E.; Potter, J. M.; Schneider, C. M.; Swenson, D. A.; Tallerico, P. J.; Penner, S.; Cutler, R. I.; Debenham, P. H.; Lindstrom, E. R.; Mohr, D. L.; Wilson, M. A. D.; Yoder, N. R.; Young, L. M.; Boyd, T. J.; 20206

Knecht, W. L.; Fernelius, N. C.; Dempsey, D. V.; Walsh, D. A.; O'Quinn, D. B.; SP620: 1981 October. 129.143.

Kneebone, C. H.; Mann, D. B.; Brennan, J. A.; 20751.

Knight, C. W. T.; Korgav, A.; Pierron, E.; SP400-69: 1981 May. 115. 121.

Knight, N. H.,; SP610.

Knoedler, C. M.; Voss, R. F.; Horn, P. M.; SP614: 1981 September. $391-394$.

Knoerdel, J.; Brooks, J.; Mink, A.; 20223.

Knoerdel, J. E.; SP500-81.

Knott, K. F.; SP614: 1981 September. 97-99.

Kobayashi, T.; Yamamoto, K.; Aoki, K.; Miyamae, K.; SP614: 1981 September. 93-96.

Koch, R. H.; van Harlingen, D. J.; Clarke, J.; SP614: 1981 September. 359-363.

Koenig, R. F.; Read, D. T.; Mazandarany, F. N.; Parker, D. M.; 20574.

Koffend, J. B.; Cool, T. A.; 20177.

Koffend, J. B.; Leone, S. R.; 20447.

Kohler, B. E.; Rosner, S. D.; Lineberger, W. C.; Jones, P. L.; Mead, R. D.; 19995. 
Koktavy, B.; Chobola, Z.; Sikula, J.; Vasina, P.; SP614: 1981 September. 248-250.

Koktavy, B.; Sikula, J.; Sikulova, M.; Vasina, P.; SP614: 1981 September. 100.104.

Konash, P. L.; Wise, S. A.; May, W. E.; 20585.

Konig, P. A.; Newton, J. J.; Saltman, R. G.; NBSIR 81-2354.

Konowalow, D. D.; Krauss, M.; Rosenkrantz, M. E.; Stevens, W. J.; Julienne, P. S.; 20100.

Konowalow, D. D.; Rosenkrantz, M. E.; Stevens, W. J.; Krauss, M.; 20082.

Koon, N. C.; Rhyne, J. J.; 20212.

Kopetka, P. A.; Mahajan, B. M.; Galowin, L. S.; J. Res. 86(2): 171. 179; 1981 March-April.

Korgav, A.; Pierron, E.; Knight, C. W. T.; SP400-69: 1981 May. 115. 121.

Kosanić, M. M.; Marković, V. M.; Nenadovic, M. T.; Holcman, J.; Sehested, K.; McLaughlin, W. L.; 20150.

Kostiuk, T.; Mumma, M. J.; Lovas, F. J.; Buhl, D.; Clark, F. O.; Chin, G.; Glenar, D.; 20321.

Kotter, F. R.; Leep, D. A.; 20346.

Kotter, F. R.; Misakian, M.; NBSIR 77-1311.

Kotter, F. R.; Misakian, M.; McKnight, R. H.; NBSIR 81-2410.

Kotter, F. R.; Misakian, M.; Ortiz, P.; McKnight, R. H.; NBSIR 812267.

Kovacs, W. D.; 20320.

Kovacs, W. D.; Salomone, L. A.; Yokel, F. Y.; BSS135.

Kovetz, A.; Prialnik, D.; Shaviv, G.; 20695.

Kowalski, F. V.; Hall, J. L.; Palmer, B. A.; Keller, R. A.; 20730.

Kowalski, P.; Schafft, H. A.; Lankford, W. F.; NBSIR 81-2260.

Kowalski, S.; Maruyama, X. K.; Lindgren, R. A.; Flanz, J. B.; Hicks, R. S.; Parker, B.; Peterson, G. A.; Lawson, R. D.; Teeters, W.; Williamson, C. F.; 20323.

Kozlovsky, B. Z.; Shaviv, G.; Contini, M.; 20303.

Kraatz, P.; Donovan, T. M.; Porteus, J. O.; Seitel, S. C.; SP620: 1981 October. 305.312.

Kramer, G. M.; Hori, R. Y.; Lewis, J. L.; Wixson, R. L; SP60I: 1981 January. 509-557.

Kramer, S.; Olmert, M.; NBSIR 80-2113.

Krasny, J. F.; Allen, P. J.; Maldonado, A.; Juarez, N.; NBSIR 812363.

Krasny, J. F.; Braun, E.; Peacock, R. D.; Hibbard, B. B.; NBSIR 81 2386.

Kratochvil, B. G.; Taylor, J. K.; 20387.

Kraus, S.; Lerner, B.; Hendrickson, B.; Thornton, J. I.; SP480-40.

Krause, R. F., Jr.; Fuller, E. R., Jr.; NBSIR 80-2099-4.

Krauss, M.; Basch, H.; Stevens, W. J.; 20196.

Krauss, M.; Konowalow, D. D.; Rosenkrantz, M. E.; Stevens, W. J.; 20082.

Krauss, M.; Mies, F. H.; Stevens, W. J.; 20086.

Krauss, M.; Rosenkrantz, M. E.; Basch, H.; Julienne, P. S.; 20238.

Krauss, M.; Rosenkrantz, M. E.; Stevens, W. J.; Julienne, P. S.; Konowalow, D. D.; 20100.

Krauss, M.; Stevens, W. J.; 20194.

Krauss, M.; Stevens, W. J.; Neumann, D. B.; 20102.

Kremer, D. P.; Repjar, A. G.; 20545.

Kruger, J.; 20351.

Kruger, J.; Carroll, J. J.; Melmed, A. J.; Ritter, J. J.; Ambrose, J. R.; NBSIR 79-1904 (Navy).

Kruger, J.; Escalante, E.; Bertocci, U.; Mullen, J.; Cohen, M.; 20348.

Kruger, J.; Revesz, A. G.; 20025.

Kruger, J.; Ritter, J. J.; 20269.

Kruger, J.; Ritter, J. J.; Carroll, J. J.; Melmed, A. J.; NBSIR 81-2396 (ONR).

Kruger, J.; Staehle, R. W.; Ugiansky, G. M.; 20026.

Krusin-Elbaum, L.; Voss, R. F.; SP614: 1981 September. 213-216.

Krzanik, L.; Gilb, T.; SP500-80: 1981 October. 190-193.

Ku, H.; Cameron, J.; Parobeck, P.; Tomb, T.; 20566.

Ku, H. H.; 20041.

Kubota, T.; Cetegen, B.,; Zukoski, E. E.; NBS-GCR-80-294.

Kubota, T.; Cetegen, B.,; Zukoski, E. E.; NBS-GCR-81-346.

Kuczkowski, R. L.; Lovas, F. J.; Suenram, R. D.; Lattimer, R. P.;
Hillig, K. W.; Ashe, A. J.; 20168.

Kuczkowski, R. L.; Suenram, R. D.; Lovas, F. J.; 20319.

Kuk, Y.; Givargizov, E. I.; Melmed, A. J.; Sakurai, T.; 20157.

Kuklewicz, M. E.; Kelly, G. E.; NBSIR 80-2110.

Kuklewicz, M. E.; Kelly, G. E.; NBSIR 80-2094.

Kunasz, C. V.; Drummond, P. D.; Zoller, P.; Walls, D. F.; 20595.

Kuo, F. K.; J. Res. 86(6): 591-595; 1981 November-December.

Kuriyama, M.; Chen, H.; 20671.

Kuriyama, M.; Yoo, K. C.; Roessler, B.; Armstrong, R. W.; 20712.

Kurkijarvi, J.; SP614: 1981 September. 373-375.

Kusuda, T.; 20521.

Kusuda, T.; 20758

Kusuda, T.; NBSIR 81-2378.

Kusuda, T.; Bean, J. W.; 20754.

Kusuda, T.; Bean, J. W.; TN1138.

Kusuda, T.; Pierce, E. T.; Bean, J. W.; 20755.

Kusuda, T.; Sud, I.; Alereza, T.; 20756.

Kusuda, T.; Treado, S.; TN1148.

Kusuda, T.; Treado, S.; NBSIR 80-2147.

Kweller, E. R.; Mullis, W. F.; 20296.

Kydes, A. S.; Rubin, L.; SP616: 1981 October. 139-147.

Kyriazi, C. N.; Trehan, R.; Conti, D. M.; Giese, K. W.; SP500-83: 1981 November. 311-312.

\section{$\mathbf{L}$}

LaBrecque, J. F.; Siegwarth, J. D.; TN1035.

LaBrecque, J. F.; Siegwarth, J. D.; TN1041.

Lady, G. M.; SP616: 1981 October. 65-77.

Lafferty, W. J.; Olson, W. B.; Maki, A. G.; JPCRD 10(4): 1065-1084; 1981.

Lafferty, W. J.; Sattler, J. P.; Worchesky, T. L.; Ritter, K. J.; 20452.

Lafferty, W. J.; Sattler, J. P.; Worchesky, T. L.; Tobin, M. S.; Ritter, K, J.; Daley, T. W.; 20090.

Lamb, W. E., Jr.; Wing, W. H.; Shy, J. T.; Farley, J. W.; 20125.

Lamping, G. A.; SP622: 1981 October. 152-180.

Landauer, R.; SP614: 1981 September. 12.17.

Lane, A. L.; Clarke, J. T.; Moos, H. W.; Atreya, S. K.; 20180.

Langer, S. H.; Chanmugam, G.; Shaviv, G.; 20412.

Lankford, W. F.; Kowalski, P.; Schafft, H. A.; NBSIR 81-2260.

Lankford, W. F.; Schafft, H. A.; Sawyer, D. E.; Kessler, H. K.; Russell, T. J.; NBSIR 80-2181.

Larrabee, R. C.; Phillips, W. E.; Thurber, W. R.; NBSIR 81-2325.

Larsen, E. B.; Ries, F. X.; TNI033.

Larson, D. R.; Werner, P. E.; Phelan, R. J., Jr.; 20330.

Lashmore, D. S.; NBSIR 80-2142.

Lattimer, R. P.; Hillig, K. W.; Ashe, A. J.; Kuczkowski, R. L.; Lovas, F. J.; Suenram, R. D.; 20168.

Laufer, A. H.; 20438.

Laughlin, D.; Clough, R.; Biancaniello, F.; Blau, P.; Cahn, J.; Mehrabian, R.; Free, G.; Berger, H.; Mordfin, L.; Swartzendruber, L.; Boettinger, W.; Ives, L.; Coriell, S.; Ballard, D.; NBSIR 802069.

Launey, S. J.; Trechsel, H. R.; SP606.

Lavernhe, F.; Marty, A.; Bailbe, J. P.; Blasquez, G.; SP614: 1981 September. 118-120.

Lawler, J. E.; Goldsmith, J. E. M.; 20166.

Lawn, B. R.; Hockey, B. J.; Wiederhorn, S. M.; 20559.

Lawrie, G. M.; Schoen, F. J.; Titus, J. L.; SP601: 1981 January. 269. 282.

Lawson, R. D.; Teeters, W.; Williamson, C. F.; Kowalski, S.; Maruyama, X. K.; Lindgren, R. A.; Flanz, J. B.; Hicks, R. S.; Parker, B.; Peterson, G. A.; 20323.

Layer, H. P.; 20096.

Layer, H. P.; Hocken, R. J.; Cummings, A. L.; 20268.

Layer, H. P.; Rowley, W. R. C.; Marx, B. R.; 20233.

Lechner, J. A.; Reeve, C. P.; Spiegelman, C. H.; NBSIR 80-2151.

Lechner, J. A.; Souders, T. M.; 20094.

Lecoy, G.; Alabedra, R.; Walma, A. A.; Maille, C.; SP614: 1981 September. 105-108.

Lecoy, G.; Savelli, M.; Maille, C.; Alabedra, R.; SP614: 1981 
September. 109.112.

Lecoy, G.; Touboul, A.; Walma, A. A.; SP614: 1981 September. 121. 124.

Ledbetter, H. M.; 20280.

Ledbetter, H. M.; 20286.

Ledbetter, H. M.; 20726

Ledbetter, H. M.; 20773.

Ledbetter, H. M.; Datta, S. K.; 20608.

Ledbetter, H. M.; Maerz, G.; 20120.

Ledbetter, H. M.; Moulder, J. C.; Austin, M. W.; 20328.

Leder, D. S.; Nguyen, D. K.; Perreault, R. W.; Fry, J. P.; Chiang, W. P.; DeSmith, D. A.; NBSIR 81-2315.

Lederer, P. S.; SP615.

Lee, M. L.; Wise, S. A.; Bartle, K. D.; 20158.

Lee, M. L.; Wise, S. A.; Bartle, K. D.; 20397.

Lee, P.; Saloman, E. B.; Nagel, D. J.; Day, R. H.; 20637.

Lee, R. W. H.; SP610: 1981 December. 66-73.

Lee, S.; Thompson, D.; Erickson, R.; Gerhart, S.; SP500-80: 1981 October. 69.75.

Leep, D.; Van Brunt, R. J.; 20704

Leep, D. A.; Kotter, F. R.; 20346.

Leep, D. A.; Moy, K. J.; Beaty, E. C.; Van Brunt, R. J.; Misakian, M.; NBSIR 81-2242.

Leggett, A. J.; SP614: 1981 September. 355.358.

Lehman, D. R.; Parke, W. C.; Maximon, L. C.; 20518.

Lehovec, K.; Fedotowsky, A.,; NBS-GCR-80-285.

Leicht, R. G.; Hayward, E.; 20237.

Leicht, R. G.; Hayward E.; Wolynec, E.; Dodge, W. R.; 20275.

Leiss, J. E.; Norris, N. J.; Wilson, M. A.; 19985.

Lentner, K. J.; Childers, C. B.; Tremaine, S. G.; 20093.

Leon, R. L.; SP622: 1981 October. 181-194.

Leonard, T. A.; Loomis, J.; Harding, K. G.; Scott, M.; SP620: 1981 October. 345.355.

Leone, S. R.; Baughcum, S. L.; 20004.

Leone, S. R.; Koffend, J. B.; 20447.

Leone, S. R.; Magnotta, F.; Nesbitt, D. J.; 20685.

Leone, S. R.; Nesbitt, D. J.; 20114.

Leone, S. R.; Nesbitt, D. J.; 20688.

Leone, S. R.; Pence, W. H.; 20441.

Leone, S. R.; Zwier, T. S.; Weisshaar, J. C.; 20713.

Lerchen, F. H.; Pielert, J. H.; Chen, P. T.; NBSIR 81-2289.

Lerchen, F. H.; Pielert, J. H.; Faison, T. K.; NBSIR 80-2171.

Lerner, B.; Hendrickson, B.; Thornton, J. I.; Kraus, S.; SP480-40.

Lerner, N. D.; NBSIR 81-2268.

Lerner, N. D.; Collins, B. L.; NBSIR 80-2003.

Lerner, N. D.; Collins, B. L.; NBSIR 80-2088.

Lerner, N. D.; Harwood, D. L.; Molino, J. A.; Zerdy, G. A.; 20050.

Lerner, N. D.; Harwood, D. L.; Molino, J. A.; Zerdy, G. A.; 20030.

Lerner, N. D.; Harwood, D. L.; Molino, J. A.; Zerdy, G. A.; 20362.

Lerner, N. D.; Harwood, D. L.; Molino, J. A.; Zerdy, G. A.; 20361.

Lerner, N. D.; Zerdy, G. A.; Molino, J. A.; 20203.

Letmanyi, H.; Conti, D. M.,; FIPS PUB 75.

Lettieri, T. R.; Jenkins, W. D.; Swyt, D. A.; 20683.

Leuchs, G.; Smith, S. J.; Walther, H.; Hellmuth, T.; 20504.

Leuchs, G.; Smith, S. J.; Walther, H.; Hellmuth, T.; 20506.

Levenson, M. D.; Smith, S. J.; Nitz, D. E.; Smith, A. V.; 20490.

Levin, B. M.; Nelson, H. E.; 20727.

Levin, B. M.; Nelson, H. E.; Groner, N. E.; 20719.

Levine, R. S.,; NBSIR 80-2188.

Levine, R. S.; NBSIR 81-2294.

Levy, A.; SP500.80: 1981 October. 58.63.

Levy, R. J.; Sanders, S. P.; Lian, J. B.; SP601: 1981 January. 339-348.

Lew, H. S.; NBSIR 80-2111-6.

Lew, H. S.; Carino, N. J.; Fattal, S. G.; Batts, M. E.; NBSIR 81-2374.

Lewis, J. G.; Rehm, R. G.; NBSIR 80-2056.

Lewis, J. L.; Wixson, R. L; Kramer, G. M.; Hori, R. Y.; SP601: 1981 January. 509-557.

Lewis, R.; 20765.

Lewis, R.; NBSIR 81-1643.

Lewis, R. L.; 20632.

Leyendecker, E. V.; Cattaneo, L. E.; NBSIR 80-2111-5.
Leyendecker, E. V.; Harris, J. R.; NBSIR 81-2195.

Leyendecker, E. V.; Harris, J. R.,; NBSIR 80-2111-11.

Li, S. S.,; NBS-GCR-81-334.

Lian, J. B.; Levy, R. J.; Sanders, S. P.; SP601: 1981 January. 339-348.

Liang, E.; Schaefer, A. R.; Geist, J.; 20530.

Libby, H. L.; SP589: 1981 January. 87-105.

Lide, D. R., Jr.; 20519.

Lifshin, E.; Hayashi, S. R.; SP604: 1981 June. 165.175.

Lin, C. C.; J. Res. 86(6): 557.563; 1981 November-December.

Lin, T. D.; Abrams, M. S.,; NBS-GCR-81-317.

Lin, W. K.; Tonik, A. B.; SP500-83: 1981 November. 259-265.

Lindau, I.; Hecht, M. H.; Egelhoff, W. F., Jr.; Tibbetts, G. G.; 20478.

Linden, N.; Yavne, M.; SP500-80: 1981 October. 117-121.

Lindgren, R. A.; Flanz, J. B.; Hicks, R. S.; Parker, B.; Peterson, G. A.; Lawson, R. D.; Teeters, W.; Williamson, C. F.; Kowalski, S.; Maruyama, X. K.; 20323.

Lindgren, R. A.; Plum, M. A.; Gerace, W. J.; Hicks, R. S.; Parker, B.; Peterson, G. A.; Singhal, R.; Williamson, C. F.; Maruyama, X. K.; Petrovich, F.; 20660.

Lindstrom, E. R.; Mohr, D. L.; Wilson, M. A. D.; Yoder, N. R.; Young, L. M.; Boyd, T. J.; Knapp, E. A.; Martin, R. E.; Potter, J. M.; Schneider, C. M.; Swenson, D. A.; Tallerico, P. J.; Penner, S.; Cutler, R. I.; Debenham, P. H.; 20206.

Lineberger, W. C.; Feigerle, C. S.; Herman, Z.; 20536.

Lineberger, W. C.; Jones, P. L.; Mead, R. D.; Kohler, B. E.; Rosner, S. D.; 19995.

Linenberger, D.; Voth, R. O.; Radebaugh, R.; 20766.

Linenberger, D.; Voth, R. O.; Radebaugh, R.; SP607: 1981 May. 70. 81.

Linholm, L. W.; SP400-66.

Linholm, L. W.; Mitchell, M. A.; SP400-65.

Link, J.; Wintersgill, M. C.; Fontanella, J. J.; Bean, V. E.; Andeen, C. G.; 20266.

Linsky, J. L.; 20113.

Linsky, J. L.; 20385.

Linsky, J. L.; 20339.

Linsky, J. L.; 20511.

Linsky, J. L.; Ayres, T. R.; 19966

Linsky, J. L.; Ayres, T. R.; Marstad, N. C.; 20535

Linsky, J. L.; Ayres, T. R.; Moos, H. W.; 20582.

Linsky, J. L.; Basri, G. S.; Worden, S. P.; Stencel, R. E.; Mullan, D. J.; 20057.

Linsky, J. L.; Brown, A.; Jordan, C.; Carpenter, K. G.; Wing, R. F.; Czyzak, S.; Stencel, R. E.; 20598.

Linsky, J. L.; Gary, D. E.; 20717.

Linsky, J. L.; Harnden, F. R., Jr.; Rosner, R.; Seward, F. D.; Vaiana, G. S.; Haisch, B. M.; 20124.

Linsky, J. L.; Marstad, N. C.; 20409.

Linsky, J. L.; Maxson, C. W.; Mewe, R.; Rosner, R.; Seward, F.; Topka, K.; Zwaan, C.; Vaiana, G. S.; Cassinelli, J. P.; Fabbiano, G.; Giacconi, R.; Golub, L.; Gorenstein, P.; Haisch, B. M.; Harnden, F. R., Jr.; Johnson, H. M.; 20336.

Linsky, J. L.; Pallavicini, R.; Golub, L.; Rosner, R.; Vaiana, G. S.; Ayres, T.; 20594.

Linsky, J. L.; Schiffer, F. H.; Simon, T.; 20423.

Linsky, J. L.; Simon, T.; 20001.

Linsky, J. L.; Slee, O. B.; Siegman, B. C.; Nikoloff, I.; Candy, M.; Harwood, D.; Verveer, A.; Quinn, P. J.; Wilson, I.; Page, A. A.; Higson, P.; Deward, F. D.; Haisch, B.; 20405.

Linsky, J. L.; Stencel, R. E.; Mullan, D. J.; Basri, G. S.; 20418.

Linsky, J. L.; Vaiana, G. S.; Golub, L.; Rosner, R.; Ayres, T. R.; 20627.

Linsky, J. L.; Worden, S. P.; Giampapa, M. S.; Bornmann, P. L.; Ayres, T. R.; 20388.

Lippiatt, B. C.; Weber, S. F.; 20499.

Lippiatt, B. C.; Weber, S. F.; Thompson, B. E.; NBSIR 81-2304.

Lipson, H. G.; Moynihan, C. T.; Drexhage, M. G.; Bendow, B.; SP620: 1981 October. 94-101.

Lister, D. B.; Barbi, N. C.; SP604: 1981 June. 35.44.

Little, A. D.; Eagle, J. W.; SP599: 1981 January. 156-164.

Little, J. C., FIPS PUB l-1. 
Little, J. L.; SP610: 1981 December. 28-38.

Little, J. L.,; FIPS PUB 14-1.

Little, J. L.,; FIPS PUB 86.

Little, W. A.; SP607: 1981 May. 154-159.

Little, W. A.; Hollman, R.; SP607: 1981 May. 160-163.

Little, W. E.; Metzker, R. F.; Jones, R. N.; Bussey, H. E.; NBSIR 78897.

Liu, F.; Ngai, K. L.; SP614: 1981 September. 161-164.

Liu, S. T.; Fanney, A. H.; 20078.

Liu, S. T.; Fanney, A. H.; 20103.

Liu, S. T.; Huang, J. S. T.; SP614: 1981 September. 67.70.

Liu, Y. M.; Filliben, J. J.; Thurber, W. R.; Mattis, R. L.; SP400-64.

Livio, M.; Shaviv, G.; 20410.

Lloyd, F. L.; Kautz, R. L.; Hamilton, C. A.; 20184.

Loevinger, R.; 20073.

Loevinger, R.; 20314.

Loevinger, R.; Pruitt, J. S.; Domen, S. R.; J. Res. 86(5): 495.502; 1981

September-October.

Loftus, J. J.; NBSIR 81-2395.

Loftus, J. J.; NBSIR 81-2312.

Loftus, J. J.; Juarez, N.; Maldonado, A.; Simenauer, J. A.; NBSIR 812247.

Loftus, J. J.; Maldonado-Rosado, A.; Allen, P. J.; NBSIR 81-2373.

Longsworth, R. C.; SP607: 1981 May. 82-92.

Loomis, J.; Harding, K. G.; Scott, M.; Leonard, T. A.; SP620: 1981 October. 345-355.

Lord, D.; SP608: 1981 May. 105.112.

Loshbough, R. P.; Smith, R. W.; Hoffman, R. H.; SP500-80: 1981 October. 111.116

Loucks, S. R.; Burnham, A. W.; SP400-69: 1981 May. 133-142.

Louis, G. L.; Pring, J. M.; Taylor, J. K.; Tholen, A. D.; Smith, C. A., ; Peiser, H. S.; Gutterman, B. M.; NBSIR 80-2020.

Lovas, F. J.; Buhl, D.; Clark, F. O.; Chin, G.; Glenar, D.; Kostiuk, T.; Mumma, M. J.; 20321.

Lovas, F. J.; Hollis, J. M.; Snyder, L. E.; Suenram, R. D.; 20077.

Lovas, F. J.; Kuczkowski, R. L.; Suenram, R. D.; 20319.

Lovas, F. J.; Maki, A. G.; 20111.

Lovas, F. J.; Maki, A. G.; Olson, W. B.; 20496.

Lovas, F. J.; Suenram, R. D.; 20318.

Lovas, F. J.; Suenram, R. D.; Lattimer, R. P.; Hillig, K. W.; Ashe, A. J.; Kuczkowski, R. L.; 20168.

Lovas, F. J.; Suenram, R. D.; Schäfer, L.; Sellers, H. L.; 20005.

Lovas, F. J.; Ulich, B. L.; Hollis, J. M.; Snyder, L. E.; 19987.

Lovas, F. J.; Wormsbecher, R. F.; Hessel, M. M.; 20424.

Love, D. P.; Van Degrift, C. T.; 20340.

Lovejoy, R. W.; Sams, R. L.; Olson, W. B.; Frommer, C.; 20589.

Lowney, J. R.; Geist, J.; 20192.

Loxley, T. E.; SP608: 1981 May. 69.81.

Lozier, D. W.; NBSIR 80-2138.

Lucatorto, T. B.; McIlrath, T. J.; Mayo, S.; Furumoto, H. W.; 20164.

Lucatorto, T. B.; Mcllrath, T. J.; Sugar, J.; Younger, S. M.; 20684.

Ludtke, P. R.; Eroshenko, V. M.; Yaskin, L. A.; Daney, D. E.; 20400.

Ludtke, P. R.; Sindt, C. F.; 20018.

Lum, G. K.; Wiegand, C. E.; Kessler, E. G., Jr.; Deslattes, R. D.; Jacobs, L.; Schwitz, W.; Seki, R.; 20349.

Luther, G.; Acquista, N.; Reader, J.; 20110.

Lycklama, H.; SP500-80: 1981 October. 64.68.

Lynn, J. W.; Erwin, R. W.; Rhyne, J. J.; Chen, H. S.; 20282.

Lynn, J. W.; Fernandez-Baca, J. A.; 20283.

Lyon, G.; 20220.

Lyon, G. E.; 20473.

Lyons, M. J.; Miller, J. C.; SP500-80: 1981 October. 33-37.

Lytle, F. W.; Greegor, R. B.; Khalil, S. R.; Ekambaram, V.; Weiss, C. S.; Grimm, C. A.; Filby, R. H.; Sandstrom, D. R.; SP618: 1981 November. 21-38.

\section{$\mathbf{M}$}

Ma, M. T.; 20301.

Ma, M. T.; Sreenivasiah, I.; Chang, D. C.; 20623.

Ma, M. T.; Sreenivasiah, I.; Chang, D. C.; TN1040.
Ma, M. T.; Wilson, P. F.; Chang, D. C.; TN1037.

Maas, A. H. J.; Durst, R. A.; Siggaard-Andersen, O.; 20513.

Mabie, C.; Menis, D.; U.S. Patent 4,306, 913.

Maccone, C.; SP614: 1981 September. 192-195.

MacCrehan, W. A.; 20604.

MacCrehan, W. A.; Durst, R. A.; 20289.

MacCrehan, W. A.; Durst, R. A.; 20728.

MacDonald, G. R.; Beekman, D. H., Jr.; Schramm, R. E.; Kasen, M. B.; 20616.

MacDonald, R. A.; MacDonald, W. M.; 20571.

MacDonald, R. A.; Shukla, R. C.; 20037.

MacDonald, W. M.; MacDonald, R. A.; 20571

Machlup, S.; SP614: 1981 September. 157.160.

Mackay, D.; Shiu, W. Y.; JPCRD 10(4): 1175.1199; 1981.

Mackey, J. A.; Garn, P. D.; Menis, O.; 20011.

MacNaughton, M. J.,; Henderson, M. M.; 20149.

Madey, T. E.; 20373.

Madey, T. E.; Houston, J. E.; Dahlberg, S. C.; 20279.

Madey, T. E.; Houston, J. E.; Seabury, C. W.; Rhodin, T. N.; 20419.

Madey, T. E.; Netzer, F. P.; 20621.

Madey, T. E.; Netzer, F. P.; 20689.

Madey, T. E.; Pantano, C. G.; 20343.

Madey, T. E.; Stockbauer, R.; Flodström, S. A.; van der Veen, J. F.; Himpsel, F. J.; Eastman, D. E.; 20510

Madrzykowski, D.; Evans, D. D.; NBSIR 81-2329.

Maerz, G.; Ledbetter, H. M.; 20120.

Magerl, A.; Rowe, J. M.; Richter, D.; 20160.

Magin, R. L.; Mangum, B. W.; Statler, J. A.; Thornton, D. D.; J. Res. 86(2): 181.192; 1981 March-April.

Magnotta, F.; Nesbitt, D. J.; Leone, S. R.; 20685.

Mahajan, B. M.; NBSIR 81-2249.

Mahajan, B. M.; NBSIR 81-2266.

Mahajan, B. M.; Galowin, L. S.; 20147.

Mahajan, B. M.; Galowin, L. S.; Kopetka, P. A.; J. Res. 86(2): 171. 179; 1981 March-April.

Maille, C.; Alabedra, R.; Lecoy, G.; Savelli, M.; SP614: 1981 September. 109-112.

Maille, C.; Lecoy, G.; Alabedra, R.; Walma, A. A.; SP614: 1981 September. 105-108.

Maki, A. G.; Hougen, J. T.; Simmons, J. D.; 20080.

Maki, A. G.; Lafferty, W. J.; Olson, W. B.; JPCRD 10(4): 1065-1084; 1981.

Maki, A. G.; Lovas, F. J.; 20111.

Maki, A. G.; Olson, W. B.; Lovas, F. J.; 20496.

Maki, A. G.; Siemsen, K. J.; Petersen, F. R.; Wells, J. S.; 20641.

Maki, A. G.; Sukle, D. J.; Wells, J. S.; Petersen, F. R.; 20427.

Maki, A. G.; Sukle, D. J.; Wells, J. S.; Petersen, F. R.; 20640.

Maki, A. G.; Wells, J. S.; 20085.

Maldonado, A.; Juarez, N.; Krasny, J. F.; Allen, P. J.; NBSIR 812363.

Maldonado, A.; Simenauer, J. A.; Loftus, J. J.; Juarez, N.; NBSIR 812247.

Maldonado-Rosado, A.; Allen, P. J.; Loftus, J. J.; NBSIR 81-2373.

Malette, W.; Flink, R. C.; SP601: 1981 January. 503-508.

Malkus, D. S.; Fuller, E. R., Jr.; J. Res. 86(1): 79-109; 1981 January. February.

Mallard, W. G.; Smyth, K. C.; 20466.

Manahan, S. E.; Fraley, D. M.; Vogt, C.; Yates, D.; SP618: 1981 November. 81.92.

Mandel, J.; J. Res. 86(1): 1.25; 1981 January-February.

Mandel, J.; Schaffer, R.; Reeder, D. J.; Coxon, B.; Enagonio, D.; Christensen, R. G.; Howell, B. F.; Paule, R. C.; SP260-72.

Manenkov, A. A.; Nechitailo, V. S.; Danileiko, Y. K.; SP620: 1981 October. 369-374.

Mangum, B. W.; Statler, J. A.; Thornton, D. D.; Magin, R. L.; J. Res. 86(2): 181-192; 1981 March-April.

Manka, M. J.; Miles, L. B.; Gann, R. G.; Earl, W. L.; 20558.

Manka, M. J.; Yeh, K. N.; Smith, B. F.; 20487.

Mann, D. B.; Brennan, J. A.; Kneebone, C. H.; 20751.

Mann, W. B.; Cavallo, L. M.; 20165.

Mann, W. B.; Coursey, B. M.; 20368. 
Manning, J. R.; 20038.

Manning, J. R.; NBSIR 81-2295.

Manola, F.; Barnett, J.,; NBS-GCR-81-339.

Manola, F.; Smith, J.; Smith, D.,; NBS-GCR-81-337.

Manola, F.,; NBS-GCR-81-338.

Maples, G.; Dyer, D. F.; Burch, T.,; Maxwell, T. T.; NBS-GCR-80292.

Marezio, M.; Roth, R. S.; Minor, D.; Santoro, A.; 20054.

Margolis, S. A.; Kaplan, A. E.; Weiss, E. R.; Byrne, S. T.; El-Torkey, N. M.; 20446.

Margulis, S. T.; NBSIR 80-2174.

Margulis, S. T.; NBSIR 81-2258.

Marinenko, R. B.; Biancaniello, F.; DeRoberts, L.; Boyer, P. A.; Ruff, A. W.; SP260-70.

Marković, V. M.; Nenadovic, M. T.; Holcman, J.; Sehested, K.; McLaughlin, W. L.; Kosanić, M. M.; 20150.

Markovitz, H.; McKinney, J. E.; Mopsik, F. I.; Passaglia, E.; Sanchez, I. C.; VanderHart, D. L.; Zapas, I. J.; Eby, R. K.; Broadhurst, M. G.; Chang, S. S.; Davis, G. T.; DiMarzio, E. A.; Fanconi, B. M.; Han, C. C.; Khoury, F. A.; 20749.

Marois, M.; Gosselin, C.; Roy, P.; Courbier, R.; David, M.; Noël, H. P.; Guidoin, R.; King, M.; Blais, P.; SP601: 1981 January. 29-129.

Marriott, A. R.; Skillman, G. H.; Salazar, S. B.; Hardgrave, W. T.; NBSIR 80-2182.

Marshak, H.; 20341.

Marshak, H.; Turrell, B. G.; 20520.

Marshall, H. E.; Ruegg, R. T.; NBSIR 81-2397.

Marshall, R. D.; Reinhold, T. A.; Mehta, K. C.; Minor, J. E.; 20759.

Marshall, R. D.; Walker, G. R.; 20059.

Marshall, R. D.; Woodward, K.; NBSIR 80-2111-4.

Marshall, W. L.; Franck, E. U.; JPCRD 10(2): 295-304; 1981.

Marstad, N. C.; Linsky, J. L.; 20409.

Marstad, N. C.; Linsky, J. L.; Ayres, T. R.; 20535.

Martin, D.; Peterson, C.; Scherrer, D.; SP500-80: 1981 October. 15 20.

Martin, G. A.; Reader, J.; Corliss, C. H.; Wiese, W. L.; NSRDSNBS68.

Martin, G. A.; Wiese, W. L.; 20710.

Martin, G. A.; Wiese, W. L.; Younger, S. M.; Fuhr, J. R.; JPCRD 10(2): 305-566; 1981.

Martin, J. C.; Sautereau, J. F.; Graffeuil, J.; SP614: 1981 September. 47.50.

Martin, J. W.; McKnight, M. E.; Campbell, P. G.; TN1149.

Martin, P.; Rozdestvenskaya, T. H.; Williams, E. S.; Galakhova, O. P.; Harkness, S.; Hermach, F. L.; Hirayama, H.; 20240.

Martin, R. E.; Potter, J. M.; Schneider, C. M.; Swenson, D. A.; Tallerico, P. J.; Penner, S.; Cutler, R. I.; Debenham, P. H.; Lindstrom, E. R.; Mohr, D. L.; Wilson, M. A. D.; Yoder, N. R.; Young, L. M.; Boyd, T. J.; Knapp, E. A.; 20206.

Martin, S. B.; Inman, L. B.; NBS-GCR-81-329.

Martin, W. C.; Zalubas, R.; JPCRD 1O(1): 153-196; 1981.

Martinez, I. M.; Cherry, S. M.,; NBSIR 80-2127-1.

Martinez, R. I.; Herron, J. T.; Huie, R. E.; 20402.

Martire, D. E.; Wasik, S. P.; Tewari, Y. B.; Miller, M. M.; NBSIR 81-2406.

Marty, A.; Bailbe, J. P.; Blasquez, G.; Lavernhe, F.; SP614: 1981 September. 118-120.

Maruyama, X. K.; Lindgren, R. A.; Flanz, J. B.; Hicks, R. S.; Parker, B.; Peterson, G. A.; Lawson, R. D.; Teeters, W.; Williamson, C. F.; Kowalski, S.; 20323.

Maruyama, X. K.; Petrovich, F.; Lindgren, R. A.; Plum, M. A.; Gerace, W. J.; Hicks, R. S.; Parker, B.; Peterson, G. A.; Singhal, R.; Williamson, C. F.; 20660.

Marx, B. R.; Layer, H. P.; Rowley, W. R. C.; 20233.

Masoero, A.; Prudenziati, M.; Morten, B.; SP614: 1981 September. 202-205.

Massoud Gasem, K. A.; Hiza, M. J.; Kidnay, A. J.; 20573.

Masters, L. W.; Seiler, J. F.; Embree, E. J.; Roberts, W. E.; NBSIR 81-2232.

Mathew, M.; Brown, W. E.; Austin, M.; Negas, T.; 19984.

Mathew, M.; Mayer, I.; Dickens, B.; Schroeder, L. W.; 20065.
Mathew, M.; Takagi, S.; Waerstad, K. R.; Frazier, A. W.; 20258.

Mathew, M.; Tung, M. S.; Brown, W. E.; 20407.

Mathey, R.; Jenkins, D.; Knab, L.; BSS131.

Mathey, R. G.; Busching, H. W.; Cullen, W. C.; Rossiter, W. J., Jr.; TN1135.

Mathey, R. G.; Knab, L. I.; Jenkins, D. R.; TN1146.

Mathey, R. G.; Pommersheim, J. M.; TN1152.

Mathey, R. G.; Rossiter, W. J., Jr.; 20259.

Mattes, B. L.; Stevenson, D. A.; Dun, H.; SP400-69: 1981 May. 143. 152.

Matthew, J. A. D.; Girvin, S. M.; 20577.

Matthews, R. T.; Yakowitz, H.,; Becker, D. A.; Berke, J. G.; NBSIR 80-2178.

Mattingly, G. E.; 20354.

Mattis, R. L.; Buehler, M. G.; Carver, G. P.; NBSIR 81-2234.

Mattis, R. L.; Liu, Y. M.; Filliben, J. J.; Thurber, W. R.; SP400-64.

Mavrodineanu, R.; Seward, R. W.; SP260-71.

Maximon, L. C.; 19993.

Maximon, L. C.; de Miniac, A.; Aniel, T.; NBSIR 81-2262.

Maximon, L. C.; Gimm, H. A.; 20175.

Maximon, L. C.; Lehman, D. R.; Parke, W. C.; 20518.

Maxson, C. W.; Mewe, R.; Rosner, R.; Seward, F.; Topka, K.; Zwaan, C.; Vaiana, G. S.; Cassinelli, J. P.; Fabbiano, G.; Giacconi, R.; Golub, L.; Gorenstein, P.; Haisch, B. M.; Harnden, F. R., Jr.; Johnson, H. M.; Linsky, J. L.; 20336.

Maxwell, B. R.; NBSIR 79-1911.

Maxwell, T. T.; Maples, G.; Dyer, D. F.; Burch, T.; NBS-GCR-80292.

May, W. B.; Hunt, C. M.; Hill, J. E.; Richtmyer, T. E.; 20539.

May, W. B., Jr.; NBSIR 81-2358.

May, W. B., Jr.; Richtmyer, T. E.; Elder, J.; Tibbott, R. L.; Yonemura, G. T.; Hunt, C. M.; Chen, P. T.; Hill, J. E.; BSS133.

May, W. B., Jr.,; Spielvogel, L. G.; 20512.

May, W. E.; 20288.

May, W. E.; Brown, J. M.; Wise, S. A.; 20586.

May, W. E.; Brown-Thomas, J. M.; Hilpert, L. R.; Wise, S. A.; 20750.

May, W. E.; Konash, P. L.; Wise, S. A.; 20585.

May, W. E.; White, P. A.; Parris, R. M.; Guenther, F. R.; Chesler, S. N.; 20602.

May, W. E.; Wise, S. A.; Bonnett, W. J.; Guenther, F. R.; 20709.

Maybee, J. S.; Joel, L. S.; SP616: 1981 October. 149.158.

Mayer, I.; Dickens, B.; Schroeder, L. W.; Mathew, M.; 20065.

Mayo, S.; Furumoto, H. W.; Lucatorto, T. B.; McIlrath, T. J.; 20164.

Maystre, D.; Teague, E. C.; Vorburger, T. V.; 20555.

Mazandarany, F. N.; Parker, D. M.; Koenig, R. F.; Read, D. T.; 20574.

Mazur, J.; McCrackin, F. L.; 20628.

Mazur, P.; Greer, S. C.; Sengers, J. V.; Bedeaux, D.; 20056.

Mazzetti, P.; Stepanescu, A.; Celasco, M.; SP614: 1981 September. 34. 37.

Mazzone, G.; McElroy, G.; Galbraith, T.,; Josephson, E.; Bush, T.; NBS-GCR-81-312.

McCabe, J. T.; SP622: 1981 October. 274-288.

McCabe, M. E.; 20121.

McCabe, M. E.; Ducas, W.; Orloski, M. J.; Decorte, K. N.; NBSIR $81-2300$.

McCaffrey, B.; Quintiere, J.; Steckler, K.; 20740.

McCaffrey, B. J.; Quintiere, J. G.; NBSIR 80-2054, Vols. I and II.

McCaffrey, B. J.; Quintiere, J. G.; Harkleroad, M. F.; 20567.

McCarney, S.; SP608: 1981 May. 139-157.

McCarter, R. J.; U.S. Patent 4,302,345.

McCarter, R. J.; U.S. Patent 4,260,660.

McCarthy, D.; Acevedo, J.; Herman, D.,; NBS-GCR-81-327.

McCarthy, J. J.; Schamber, F. H.; SP604: 1981 June. 273-296.

McCarthy, S. E.,; Zimmerman, J. E.; Sullivan, D. B.; SP607.

McCarty, R. D.; 20508.

McCarty, R. D.; TN1030.

McCarty, R. D.; Hord, J.; Roder, H. M.; Monogr. 168.

McCarty, R. D.; Jacobsen, R. T.; TN1045.

McCarty, R. D.; Younglove, B.; 20360. 
McCarty, R. D.; Younglove, B. A.; 20072.

McClendon, L. T.; 20009.

McCord, R. M.; SP622: 1981 October. 74-81.

McCoy, W. H.; Colella, R. P.; Wallace, M. A.; 20662.

McCrackin, F. L.; Mazur, J.; 20628.

McCrackin, F. L.; Peterlin, A.; 20344

McCrackin, F. L.; Senich, G. A.; Wang, F. W.; Smith, L. E.; Chang, S. S.; NBSIR 81-2264.

McCulloh, K. E.; Parr, A. C.; Jason, A. J.; Stockbauer, R.; 20051.

McCulloh, K. E.; Tilford, C. R.; 20592.

McDavid, J. M.; Yee, S. S.; SP620: 1981 October. 44.49.

McDonald, D. G.; 20284.

McDonald, D. G.; Peterson, R. L.; Hamilton, C. A.; Harris, R. E.;

Kautz, R. L.; 20070

McDonald, J. C.; Menzel, H. G.; Schuhmacher, H.; Coyne, J.; 20675. McEleney, P. C.; SP589: 1981 January. 77.79.

McElroy, G.; Galbraith, T.,; Josephson, E.; Bush, T.; Mazzone, G.; NBS-GCR-81-312.

McFarlan, T. W.; SP589: 1981 January. 81.85.

McGarry, E. D.; Fabry, A.; 20256.

McGarry, E. D.; Spiegel, V.; Eisenhauer, C. M.; Grundl, J. A.;

Gilliam, D. M.; 20153.

McHenry, H. I.; Fickett, F. R.; 20665.

McHenry, H. I.; Read, D. T.; 20548.

McHenry, H. I.; Read, D. T.; NBSIR 81-1648.

McHenry, H. I.; Read, D. T.; Whipple, T. A.; 20528.

McHenry, H. I.; Rolfe, S. T.; NBSIR 79-1623.

McHenry, H. I.; Yushchenko, K. A.; Paton, E. O.; Reed, R. P.; Tobler, R. L.; Elmer, J. W.; 20772.

McIlrath, T. J.; Mayo, S.; Furumoto, H. W.; Lucatorto, T. B.; 20164.

McIlrath, T. J.; Sugar, J.; Younger, S. M.; Lucatorto, T. B.; 20684.

McKee, D. W.; Yates, J. T., Jr.; 20661.

McKenna, G. B.; Khoury, F. A.; Crissman, J. M.; NBSIR 81-2209 (FDA).

McKenna, G. B.; Penn, R. W.; 20129.

McKenna, G. B.; Penn, R. W.; 20091.

McKenna, G. B.; Zapas, L. J.; 20130.

McKinney, J. E.; Mopsik, F. I.; Passaglia, E.; Sanchez, I. C.; VanderHart, D. L.; Zapas, I. J.; Eby, R. K.; Broadhurst, M. G.; Chang, S. S.; Davis, G. T.; DiMarzio, E. A.; Fanconi, B. M.; Han, C. C.; Khoury, F. A.; Markovitz, H.; 20749.

McKinney, J. E.; Whitlock, R. P.; Hinman, R.; Cassel, J. M.; Tesk, J. A.; Brauer, G. M.; Antonucci, J. M.; Wu, W.; NBSIR 80-2168.

McKnight, M. E.; Campbell, P. G.; Martin, J. W.; TN1149.

McKnight, R. H.; Kotter, F. R.; Misakian, M.; NBSIR 81-2410.

McKnight, R. H.; Kotter, F. R.; Misakian, M.; Ortiz, P.; NBSIR 812267.

McLafferty, P. S.; Gaines, R. S.; SP608: 1981 May. 159-166.

McLaughlin, W. L.; 20174.

McLaughlin, W. L.; 20185.

McLaughlin, W. L.; Humphreys, J. C.; 20171.

McLaughlin, W. L.; Kosanić, M. M.; Marković, V. M.; Nenadovic,

M. T.; Holcman, J.; Sehested, K.; 20150.

McLaughlin, W. L.; Miller, A.; 20176.

McMaster, R. C.; SP589: 1981 January. 1-32.

McMichael, J. M.; Klebanoff, P. S.; Mease, N. E.; 20048.

McMurdie, H. F.; Evans, E. H.; Paretzkin, B.; Parker, H. S.; Panagiotopoulos, N. C.; Hubbard, C. R.; Morris, M. C.; Monogr. 25, Section 18.

McNamara, A.; Clark, G. C. F.; Williams, D. F.; SP601: 1981 January. 323-336.

McNeil, J. R.; Herrmann, W. C., Jr.; SP620: 1981 October. 324-334.

McNeil, K. J.; Walls, D. F.; Reid, M.; 20697.

McPhillips-Meade, M.; Klawitter, J. J.; French, G.; Skinner, H.; Weinstein, A. M.; Clemow, A. J. T.; SP601: 1981 January. 423-447.

McRae, E. G.; Pierce, D. T.; Celotta, R. J.; Wang, G. C.; 20643.

McShane, D.; Schurman, D. J.; Burton, D. S.; Nagel, D. A.; SP601: 1981 January. 615.642.

Mc Wethy, S.; Moy, S.; Nielsen, G.; Sanchez, R.; Wallace, T.; SP50080: 1981 October. 103-110.

Mead, R. D.; Kohler, B. E.; Rosner, S. D.; Lineberger, W. C.; Jones,
P. L.; 19995.

Meade, R. E.; Smith, L. R.; Braun, E.; NBSIR 81-2256.

Mease, N. E.; McMichael, J. M.; Klebanoff, P. S.; 20048.

Mecherikunnel, A. T.; Richmond, J. C.; 20083.

Meese, W. J.; Beausoliel, R. W.; NBSIR 81-2221.

Meese, W. J.; Beausoliel, R. W.; Clifton, J. R.; NBSIR 81-2220.

Mehl, J. B.; Moldover, M. R.; 20274.

Mehlhorn, R. J.; Packer, L.; SP618: 1981 November. 226-234.

Mehlman, G.; Saloman, E. B.; Cole, B. E.; Cooper, J. W.; Ederer, D. L.; 20300.

Mehrabian, R.; Free, G.; Berger, H.; Mordfin, L.; Swartzendruber, L.; Boettinger, W.; Ives, L.; Coriell, S.; Ballard, D.; Laughlin, D.; Clough, R.; Biancaniello, F.; Blau, P.; Cahn, J.; NBSIR 80-2069.

Mehta, A.; Wunderlich, B.; Gaur, U.; Shu, H. C.; JPCRD 10(1): 89. $118 ; 1981$.

Mehta, H.; van Vliet, K. M.; SP614: 1981 September. 79.83

Mehta, K. C.; Minor, J. E.; Marshall, R. D.; Reinhold, T. A.; 20759.

Meijer, P. H. E.; Farrell, R. A.; Favin, S.; Esfandiari, P.; 20620.

Meijer, P. H. E.; Mountain, R. D.; Soulen, R. J., Jr.,; SP614.

Meiselman, S.; White, E.; Christensen, R. G.; Hertz, H. S.; 20097.

Meissner, P.,; FIPS PUB 83.

Melmed, A. J.; Ceyer, S. T.; Tung, R. T.; Graham, W. R.; 20686.

Melmed, A. J.; Kruger, J.; Ritter, J. J.; Carroll, J. J.; NBSIR 81-2396 (ONR).

Melmed, A. J.; Ritter, J. J.; Ambrose, J. R.; Kruger, J.; Carroll, J. J.; NBSIR 79-1904 (Navy).

Melmed, A. J.; Sakurai, T.; Culbertson, R. J.; 19983.

Melmed, A. J.; Sakurai, T.; Kuk, Y.; Givargizov, E. I.; 20157.

Menis, D.; Mabie, C.; U.S. Patent 4,306,913.

Menis, O.; Mackey, J. A.; Garn, P. D.; 20011.

Menzel, H. G.; Schuhmacher, H.; Coyne, J.; McDonald, J. C.; 20675.

Merrett, R. P.; SP400-69: 1981 May. 33.41.

Merrett, R. P.; Sim, S. P.; SP400-69: 1981 May. 94-107.

Merritt, K.; Brown, S. A.; SP601: 1981 January. 299-321.

Merritt, K.; Simpson, J. P.; Geret, V.; Brown, S. A.; SP60I: 1981 January. 395-422.

Meshkov, S.; 20248.

Meshkov, S.; Carlson, C. E.; Coyne, J. J.; Fishbane, P. M.; Gross, F.; 20034.

Meshkov, S.; Carlson, C. E.; Coyne, J. J.; Fishbane, P. M.; Gross, F.; 20187.

Meshkov, S.; Carlson, C. E.; Coyne, J. J.; Fishbane, P. M.; Gross, F.; 20316.

Meshkov, S.; Rosner, J. L.; Karl, G.; 20040.

Messenger, C. G.; SP400-69: 1981 May. 82-93.

Messman, J. D.; Rains, T. C.; 20763.

Metcalf, H.; Phillips, W.; 20076.

Metiu, H.; Gadzuk, J. W.; 20143.

Metz, F. E.; 20470.

Metz, F. E.; 20440

Metzker, R. F.; Jones, R. N.; Bussey, H. E.; Little, W. E.; NBSIR 78897.

Mewe, R.; Rosner, R.; Seward, F.; Topka, K.; Zwaan, C.; Vaiana, G. S.; Cassinelli, J. P.; Fabbiano, G.; Giacconi, R.; Golub, L.; Gorenstein, P.; Haisch, B. M.; Harnden, F. R., Jr.; Johnson, H. M.;

Linsky, J. L.; Maxson, C. W.; 20336.

Meyer, L. S.; SP599: 1981 January. 102-109.

Meyer, S. L.; SP610: 1981 December. 39-52.

Michel, K. H.; Rowe, J. M.; Rush, J. J.; Pynn, R.; Heidemann, A.; 20421.

Michelson, P. F.; Taber, R. C.; Giffard, R. P.; SP614: 1981 September. 292-297.

Mies, F. H.; 20060.

Mies, F. H.; 20049.

Mies, F. H.; Aryeh, Y. B.; 20039.

Mies, F. H.; Julienne, P. S.; 20155.

Mies, F. H.; Stevens, W. J.; Krauss, M.; 20086.

Mighell, A. D.; Hubbard, C. R.; Stalick, J. K.; TN1141.

Miiller, A. P.; Cezairliyan, A.; 20137.

Miiller, A. P.; Cezairliyan, A.; 20542

Milam, D.; Smith, W. L.; Deaton, T. F.; Rainer, F.; SP620: 1981 
October. 297-299.

Miles, L. B.; Gann, R. G.; Earl, W. L.; Manka, M. J.; 20558.

Miliusyte, R.; Viktoravicius, V.; Bareikis, V.; Galdikas, A.; SP614: 1981 September. 406-408.

Milke, J. A.,; Bryan, J. L.; NBS-GCR-81-343.

Milke, J. A.,; Bryan, J. L.; DiNenno, P. J.; NBS-GCR-80-297.

Miller, A.; McLaughlin, W. L.; 20176.

Miller, C. K. S.; 20322.

Miller, C. K. S.,; NBSIR 80-1635.

Miller, E. F., Jr.; Henderson, J. B.; SP500-80: 1981 October. 128-135.

Miller, E. F., Jr.; Hirschberg, M.; Henderson, J. B.; SP500-80: 1981 October. 136-145.

Miller, J. C.; Lyons, M. J.; SP500-80: 1981 October. 33-37.

Miller, M. M.; Martire, D. E.; Wasik, S. P.; Tewari, Y. B.; NBSIR 81-2406.

Miller, M. M.; Wasik, S. P.; DeVoe, H.; J. Res. 86(4): 361-366; 1981 July-August.

Mimaki, T.; Sato, H.; Tanabe, M.; SP614: 1981 September. 30-33.

Min, H. S.; SP614: 1981 September. 221-225.

Mink, A.; Knoerdel, J.; Brooks, J.; 20223.

Mink, A.; Silio, C. B., Jr.; NBSIR 80-2187.

Minor, D.; Santoro, A.; Marezio, M.; Roth, R. S.; 20054.

Minor, J. E.; Marshall, R. D.; Reinhold, T. A.; Mehta, K. C.; 20759.

Miraldi, E.; Trossi, L.; Valabrega, P. T.; Oldano, C.; SP614: 1981 September. 339-342.

Misakian, M.; 20484.

Misakian, M.; NBSIR 80-2177.

Misakian, M.; Kotter, F. R.; NBSIR 77-1311.

Misakian, M.; Leep, D. A.; Moy, K. J.; Beaty, E. C.; Van Brunt, R. J.; NBSIR 81-2242.

Misakian, M.; McKnight, R. H.; Kotter, F. R.; NBSIR 81-2410.

Misakian, M.; Ortiz, P.; McKnight, R. H.; Kotter, F. R.; NBSIR 812267.

Misra, D. N.; Bowen, R. L.; 20116.

Misra, D. N.; Bowen, R. L.; 20226.

Misra, D. N.; Bowen, R. L.; 20729.

Misra, D. N.; Peckoo, R. J.; Antonucci, J. M.; 20481.

Misra, J.; Berry, R.; Neuse, D.; Chandy, K. M.; SP500-83: 1981 November. 331-334.

Mitchell, M. A.; Linholm, L. W.; SP400-65.

Mitchell, R. A.; Pontius, P. E.; 20231.

Mitler, H. E.; Emmons, H. W.,; NBS-GCR-81-344.

Mitra, S. S.; Vaidyanathan, A.; Guenther, A. H.; SP620: 1981 October. 432-437.

Mitra, S. S.; Vaidyanathan, A.; Guenther, A. H.; SP620: 1981 October. 438-445.

Mitra, S. S.; Vaidyanathan, A.; Guenther, A. H.; Judell, N.; SP620: 1981 October. 446-452.

Miyamae, K.; Kobayashi, T.; Yamamoto, K.; Aoki, K.; SP614: 1981 September. 93-96.

Mizushima, M.; Scalabrin, A.; Saykally, R. J.; Evenson, K. M.; Radford, H. E.; 20700.

Moghissi, A. A.; Coursey, B. M.; 20367.

Mohan, S. P.; SP622: 1981 October. 220-221.

Mohr, D. L.; Wilson, M. A. D.; Yoder, N. R.; Young, L. M.; Boyd, T. J.; Knapp, E. A.; Martin, R. E.; Potter, J. M.; Schneider, C. M.; Swenson, D. A.; Tallerico, P. J.; Penner, S.; Cutler, R. I.; Debenham, P. H.; Lindstrom, E. R.; 20206.

Mohr, J. M.; Chu, C. M.; Wilson, C. B.; SP500-83: 1981 November. 59.65.

Moldover, M. R.; Greer, S. C.; 20718.

Moldover, M. R.; Mehl, J. B.; 20274.

Molino, J. A.; 20477.

Molino, J. A.; 20475.

Molino, J. A.; Lerner, N. D.; Zerdy, G. A.; 20203.

Molino, J. A.; Zerdy, G. A.; Lerner, N. D.; Harwood, D. L.; 20050.

Molino, J. A.; Zerdy, G. A.; Lerner, N. D.; Harwood, D. L.; 20030.

Molino, J. A.; Zerdy, G. A.; Lerner; N. D.; Harwood, D. L.; 20361

Molino, J. A.; Zerdy, G. A.; Lerner, N. D.; Harwood, D. L.; 20362.

Molino, J. A.; Zerdy, G. A.; Tremaine, S. G.; 20029.

Montgomery, H. A.,; Reifer, D. J.; NBS-GCR-81-335.
Montroll, E. W.; Shlesinger, M. F.; Hughes, B. D.; SP614: 1981 September. 18-21.

Montroll, M. L.,; Sharp, B. H.; Kasper, P. K.; NBS-GCR-80-250

Moore, B. A.; SP400-69: 1981 May. 3-23.

Moore, E. F.; Davis, R. W.; 19972.

Moore, L. J.; Gramlich, J. W.; Rosman, K. J. R.; Barnes, I. L.; 20698.

Moore, R. T.; NBSIR 81-2414.

Moore, R. T.; NBSIR 81-2298.

Moos, H. W.; Atreya, S. K.; Lane, A. L.; Clarke, J. T.; 20180.

Moos, H. W.; Clarke, J. T.; 20509.

Moos, H. W.; Linsky, J. L.; Ayres, T. R.; 20582.

Mopsik, F. I.; Passaglia, E.; Sanchez, I. C.; VanderHart, D. L.; Zapas, I. J.; Eby, R. K.; Broadhurst, M. G.; Chang, S. S.; Davis, G. T. DiMarzio, E. A.; Fanconi, B. M.; Han, C. C.; Khoury, F. A.; Markovitz, H.; McKinney, J. E.; 20749.

Mordfin, L.; 20159.

Mordfin, L.; Berger, H.; $204 i 1$.

Mordfin, L.; Payne, B. F.; Edelman, S.; 20246.

Mordfin, L.; Swartzendruber, L.; Boettinger, W.; Ives, L.; Coriell, S.; Ballard, D.; Laughlin, D.; Clough, R.; Biancaniello, F.; Blau, P.; Cahn, J.; Mehrabian, R.; Free, G.; Berger, H.; NBSIR 80-2069.

Mordfin, L.,; NBSIR 80-2080.

Morehouse, R. J.,; Burris, B. L.; SP305. Supplement 12.

Morgan, W. R.; Hickam, W. M.; SP400-69: 1981 May. 153-158.

Morris, M. C.; McMurdie, H. F.; Evans, E. H.; Paretzkin, B.; Parker, H. S.; Panagiotopoulos, N. C.; Hubbard, C. R.; Monogr. 25, Section 18.

Morten, B.; Masoero, A.; Prudenziati, M.; SP614: 1981 September 202.205.

Mosburg, E. R., Jr.; 20556.

Motz, J. W.; Dick, C. E.; 20189.

Motz, J. W.; Dick, C. E.; 20390

Motz, J. W.; Dick, C. E.; Danos, M.; 20450

Moulder, J. C.; Austin, M. W.; Ledbetter, H. M.; 20328.

Moulder, J. C.; Clark, A. F.; Strobridge, T. R.; 20436.

Moulton, J. R.,; Shelton, C. B.; NBS-GCR-81-324.

Mounib, A.; Gentil, P.; SP614: 1981 September. 230-233.

Mountain, R. D.; 20118.

Mountain, R. D.; 20687.

Mountain, R. D.; Soulen, R. J., Jr.,; Meijer, P. H. E.; SP614.

Moy, K. J.; Beaty, E. C.; Van Brunt, R. J.; Misakian, M.; Leep, D. A.; NBSIR 81-2242.

Moy, S.; Nielsen, G.; Sanchez, R.; Wallace, T.; Mc Wethy, S.; SP50080: 1981 October. 103-110.

Moyer, R. B.; SP589: 1981 January. 33-37.

Moynihan, C. T.; Drexhage, M. G.; Bendow, B.; Lipson, H. G.; SP620: 1981 October. 94-101.

Muhe, W.; SP599: 1981 January. 115-126.

Mulholland, G.; Handa, T.; Sugawa, O.; Yamamoto, H.; 20565.

Mulkey, M.; Timpane, K.,; NBS-GCR-ETIP 81-97.

Mullan, D. J.; Basri, G. S.; Linsky, J. L.; Stencel, R. E.; 20418.

Mullan, D. J.; Linsky, J. L.; Basri, G. S.; Worden, S. P.; Stencel, R. E.; 20057.

Mullen, C. L.; Yokel, F. Y.; Yancey, C. W. C.; BSS132.

Mullen, J.; Cohen, M.; Kruger, J.; Escalante, E.; Bertocci, U.; 20348.

Mullen, J. W.; SP500-83: 1981 November. 39-43.

Mullen, J. W.; SP500-83: 1981 November. 343-348.

Muller, C. H. III; Phelps, A. V.; 20047.

Mullis, W. F.; Kweller, E. R.; 20296.

Mumma, M. J.; Lovas, F. J.; Buhl, D.; Clark, F. O.; Chin, G.; Glenar, D.; Kostiuk, T.; 20321.

Murray, J. L.; Orser, D. J.; 20033.

Musal, H. M., Jr.; SP620: 1981 October. 227-238.

Musha, T.; SP614: 1981 September. 143-146.

Musket, R. G.; SP604: 1981 June. 97-126.

Myers, D. R.; Roitman, P.; Albers, J.; Ehrstein, J. R.; NBSIR 81 2398.

Myers, D. R.; Wilson, R. G.; Comas, J.; 20112.

Myklebust, R. L.; Fiori, C. E.; 20381.

Myklebust, R. L.; Fiori, C. E.; Heinrich, K. F. J.; SP604: 1981 June. 365-379. 
Myklebust, R. L.; Fiori, C. E.; Newbury, D. E.; SP604: 1981 June. 315-339.

Myklebust, R. L.; Fiori, C. E.,; Heinrich, K. F. J.; Newbury, D. E.; SP604.

Myklebust, R. L.; Gorlen, K.; Fiori, C. E.; SP604: 1981 June. 233.272.

\section{$\mathbf{N}$}

Nachmens, S.; Berild, S.; SP500-80: 1981 October. 1.6.

Nagel, D. A.; McShane, D.; Schurman, D. J.; Burton, D. S.; SP601: 1981 January. 615-642.

Nagel, D. J.; Day, R. H.; Lee, P.; Saloman, E. B.; 20637.

Nagel, R.; Wellington, J.,; Kelly, J. C.; Wolf, R.; Kennicott, P.; NBSIR 81-2297.

Nagel, R. N.,; Wheatley, T.; Albus, J. S.; NBSIR 80-2152.

Nahman, N.; 20312.

Nahman, N. S.; Gans, W. L.; TN1042.

Nahman, N. S.; Guillaume, M. E.; TN1047.

Nakahara, S.; Draper, C. W.; Buene, L.; Jacobson, D. C.; Poate, J. M.; SP620: 1981 October. 222-226.

Nalley, P.; Gregory, G.; Frederick, W.; Hausmann, R.,; Clarren, S.; NBS-GCR-ETIP 81-96.

Namba, Y.; Tsuwa, H.; SP620: 1981 October. 171-179.

Natrella, M. G.; Brickenkamp, C. S.; Hasko, S.; H133.

Nechitailo, V. S.; Danileiko, Y. K.; Manenkov, A. A.; SP620: 1981 October. 369-374.

Negas, T.; Domingues, L.; U.S. Patent 4,264.423.

Negas, T.; Mathew, M.; Brown, W. E.; Austin, M.; 19984.

Nelson, A. M.; SP599: 1981 January. 277.280.

Nelson, H. E.; Groner, N. E.; Levin, B. M.; 20719.

Nelson, H. E.; Levin, B. M.; 20727.

Nenadovic, M. T.; Holcman, J.; Sehested, K.; McLaughlin, W. L.; Kosanić, M. M.; Marković, V. M.; 20150.

Nesbitt, D. J.; Leone, S. R.; 20114.

Nesbitt, D. J.; Leone, S. R.; 20688.

Nesbitt, D. J.; Leone, S. R.; Magnotta, F.; 20685.

Netzer, F. P.; 20531.

Netzer, F. P.; Madey, T. E.; 20621.

Netzer, F. P.; Madey, T. E.; 20689.

Neumann, D. B.; Krauss, M.; Stevens, W. J.; 20102.

Neuroth, N.; Hack, H.; SP620: 1981 October. 102-109.

Neuse, D.; Chandy, K. M.; Misra, J.; Berry, R.; SP500-83: 1981 November. 331.334.

Neustadter, H. E.; SP622: 1981 October. 257.273.

Newbury, D. E.; 20285.

Newbury, D. E.; Myklebust, R. L.; Fiori, C. E.; SP604: 1981 June. 315-339.

Newbury, D. E.; Myklebust, R. L.; Fiori, C. E.,; Heinrich, K. F. J.; SP604.

Newell, A. C.; Estin, A. J.; Stubenrauch, C. F.; Repjar, A. G.; 20650.

Newnam, B. E.; Foltyn, S. R.; SP620: 1981 October. 265.276.

Newnam, B. E.,; Bennett, H. E.; Glass, A. J.; Guenther, A. H.; SP620.

Newton, J. J.; Saltman, R. G.; Konig, P. A.; NBSIR 81-2354.

Ngai, K. L.; SP614: 1981 September. 165.168.

Ngai, K. L.; Liu, F.; SP614: 1981 September. 161-164.

Nguyen, D. K.; Perreault, R. W.; Fry, J. P.; Chiang, W. P.; DeSmith, D. A.; Leder, D. S.; NBSIR 81-2315.

Nielsen, G.; Sanchez, R.; Wallace, T.; Mc Wethy, S.; Moy, S.; SP50080: 1981 October. 103.110.

Nikoloff, I.; Candy, M.; Harwood, D.; Verveer, A.; Quinn, P. J.; Wilson, I.; Page, A. A.; Higson, P.; Deward, F. D.; Haisch, B.; Linsky, J. L.; Slee, O. B.; Siegman, B. C.; 20405.

Nisenoff, M.; SP607: 1981 May. 195.209.

Nitz, D. E.; Smith, A. V.; Levenson, M. D.; Smith, S. J.; 20490.

Nitzan, A.; Ben-Jacob, E.; Bergman, D.; Carmeli, B.; SP614: 1981 September. 381-383.

Nober, E. H.; Pierce, H.; Well, A.; Johnson, C. C.; Clifton, C.; NBSGCR-80-284.

Noël, H. P.; Guidoin, R.; King, M.; Blais, P.; Marois, M.; Gosselin, C.; Roy, P.; Courbier, R.; David, M.; SP601: 1981 January. 29-129.
Nolen, R. L., Jr.; Drake, M. C.; Rosasco, G. J.; Schneggenburger, R.; 20653.

Norcross, D. W.; Collins, L. A.; Padial, N. T.; 20534.

Norcross, D. W.; Collins, L. A.; Schmid, G. B.; 20055.

Norcross, D. W.; Hummer, D. G.; Pradhan, A. K.; 20045.

Norcross, D. W.; Hummer, D. G.; Pradhan, A. K.; 20492.

Norris, J. A.; Travis, J. C.; DeVoe, J. R.; Walters, J. P.; Scheeline, A.; 20453

Norris, N. J.; Wilson, M. A.; Leiss, J. E.; 19985.

Norris, P. J.; Boyce, M. P.; SP622: 1981 October. 222.253.

North, S. E.; Strandberg, G. W.; Shumate, S. E. II; Parrott, J. R., Jr.; SP618: 1981 November. 274-286.

Nougier, J. P.; SP614: 1981 September. 397.405.

Nougier, J. P.; Gasquet, D.; Vaissiere, J. C.; SP614: 1981 September. 305.308.

Nougier, J. P.; Vaissiere, J. C.; Gasquet, D.; SP614: 1981 September. $42-46$

November, L. J.; Toomre, J.; Gebbie, K. B.; Simon, G. W.; 20422

Noyce, J. R.; TN1137.

Nuttall, R. L.; Wagman, D. D.; Evans, W. H.; Parker, V. B.; Schumm, R. H.; TN270-8.

O

Oakley, K. A.; Houghton, R. C., Jr.; SP500-80: 1981 October. 180. 185.

Oberly, C. E.; Garrett, H. J.; Walker, M. S.; Zeitlin, B. A.; Ekin, J. W.; Ho, J. C.; 20741.

O'Brien, D. H.; Pyles, R. A.; Stockton, R. A.; Wheeler, A. E.; Zingaro, R. A.; Irgolic, K. J.; Banks, C. H.; Bottino, N. R.; Chakraborti, D.; Gennity, J. M.; Hillman, D. C.; SP618: 1981 November. 244-263.

O'Connell, J. S.; 20425.

Offner, D. I.; SP599: 1981 January. 202.211.

Ogburn, F.; Schoonover, R. M.; Johnson, C. E.; 20334.

Ohlemiller, T. J.; NBSIR 81-2350.

Ohran, R.; Wirth, N.; SP500-80: 1981 October. 7.9.

Oka, T.; Hougen, J. T.; 20115.

Okabe, H.; 20087.

Okabe, H.; 20581.

Okabe, H.; Kawasaki, M.; Tanaka, Y.; 20042.

Oldano, C.; Miraldi, E.; Trossi, L.; Valabrega, P. T.; SP614: 1981 September. 339-342.

Oldham, N. M.; Stenbakken, G. N.; Kibalo, T. H.; Turgel, R. S.; TN1144.

Olesen, T.,; Foskett, W.; NBS-GCR-ETIP 81-94.

Olmert, M.; Kramer, S.; NBSIR 80-2113.

Olmert, M.,; Raufaste, N.; SP446-5.

Olsen, K. B.; Wilkerson, C. L.; Toste, A. P.; Hayes, D. J.; SP618: 1981 November. 105.119.

Olson, G. J.; Iverson, W. P.; Brinckman, F. E.; NBSIR 81-2246.

Olson, W. B.; Frommer, C.; Lovejoy, R. W.; Sams, R. L.; 20589.

Olson, W. B.; Lovas, F. J.; Maki, A. G.; 20496.

Olson, W. B.; Maki, A. G.; Lafferty, W. J.; JPCRD 10(4): 1065-1084; 1981.

Olson, W. B.; Sams, R. L.; 20053.

Olson, W. B.; Todd, T. R.; 20088.

Olver, F. W. J.; 20252

O'Neill, J. G.; 20576.

O'Neill, J. G.; Cooper, L. Y.; NBSIR 81-2202.

Oonk, R. L.; Jones, D. E.; Cole-Appel, B. E.; 20148.

O'Quinn, D. B.; Fernelius, N. C.; Dempsey, D. V.; SP620: 1981 October. 58-74.

O'Quinn, D. B.; Knecht, W. L.; Fernelius, N. C.; Dempsey, D. V.; Walsh, D. A.; SP620: 1981 October. 129.143.

Orloski, M. J.; Decorte, K. N.; McCabe, M. E.; Ducas, W.; NBSIR 81-2300.

Orser, D. J.; Murray, J. L.; 20033.

Ortiz, P.; McKnight, R. H.; Kotter, F. R.; Misakian, M.; NBSIR 812267.

Overgaard, M.; Gianetta, J.; SP500-80: 1981 October. 194-196. 
Overton, R. L.,; Shingleton, J. G.; Cassel, D. E.; NBS-GCR-81-302.

Ozier, I.; Gerry, M. C. L.; Robiette, A. G.; JPCRD 10(4): 1085-1095; 1981.

\section{$\mathbf{P}$}

Packer, L.; Mehlhorn, R. J.; SP618: 1981 November. 226-234.

Padial, N. T.; Norcross, D. W.; Collins, L. A.; 20534.

Page, A. A.; Higson, P.; Deward, F. D.; Haisch, B.; Linsky, J. L.; Slee, O. B.; Siegman, B. C.; Nikoloff, I.; Candy, M.; Harwood, D.; Verveer, A.; Quinn, P. J.; Wilson, I.; 20405.

Page, W. J., Jr.; SP599: 1981 January. 25.33.

Palla, R. L.; NBSIR 80-2173.

Palla, R. L., Jr.; NBSIR 81-2320.

Palla, R. L., Jr.; Gordon, C. C.; NBSIR 81-2291 (HUD).

Pallavicini, R.; Golub, L.; Rosner, R.; Vaiana, G. S.; Ayres, T.; Linsky, J. L.; 20594.

Pallottino, G. V.; Pizzella, G.; Bonifazi, P.; Bordoni, F.; SP614: 1981 September. 298-301.

Palmer, B. A.; Keller, R. A.; Kowalski, F. V.; Hall, J. L.; 20730.

Panagiotopoulos, N. C.; Hubbard, C. R.; Morris, M. C.; McMurdie, H. F.; Evans, E. H.; Paretzkin, B.; Parker, H. S.; Monogr. 25, Section 18.

Panagiotopoulos, N. C.; Snyder, R. L.; Hubbard, C. R.; NBSIR 812229.

Pantano, C. G.; Madey, T. E.; 20343.

Pape, R.; Waterman, T. E.; Eichler, T. V.,; NBS-GCR-81-301.

Pape, R.,; NBS-GCR-81-300.

Paretzkin, B.; Parker, H. S.; Panagiotopoulos, N. C.; Hubbard, C. R.; Morris, M. C.; McMurdie, H. F.; Evans, E. H.; Monogr. 25, Section 18.

Pâris, J. F.; Cabrera, L. F.; SP500-83: 1981 November. 157-168.

Park, H. S.; van der Ziel, A.; SP614: 1981 September. 226.229.

Parke, W. C.; Maximon, L. C.; Lehman, D. R.; 20518.

Parken, W. H.; Kelly, G. E.; 20690.

Parker, B.; Peterson, G. A.; Lawson, R. D.; Teeters, W.; Williamson, C. F.; Kowalski, S.; Maruyama, X. K.; Lindgren, R. A.; Flanz, J. B.; Hicks, R. S.; 20323.

Parker, B.; Peterson, G. A.; Singhal, R.; Williamson, C. F.; Maruyama, X. K.; Petrovich, F.; Lindgren, R. A.; Plum, M. A.; Gerace, W. J.; Hicks, R. S.; 20660.

Parker, D. M.; Koenig, R. F.; Read, D. T.; Mazandarany, F. N.; 20574.

Parker, H. S.; Panagiotopoulos, N. C.; Hubbard, C. R.; Morris, M. C.; McMurdie, H. F.; Evans, E. H.; Paretzkin, B.; Monogr. 25, Section 18.

Parker, R. L.; 20044.

Parker, V. B.; Schumm, R. H.; Nuttall, R. L.; Wagman, D. D.; Evans, W. H.; TN270-8.

Parks, E. J.; Graminski, E. L.; J. Res. 86(3): 309-315; 1981 May-June. Parks, E. J.; Wilson, W. K.; 20119.

Parks, P. J.; SP601: 1981 January. 349-362.

Parobeck, P.; Tomb, T.; Ku, H.; Cameron, J.; 20566.

Parr, A. C.; Cole, B. E.; Stockbauer, R.; Dehmer, J. L.; West, J. B.; Codling, K.; Ederer, D. L.; 20676.

Parr, A. C.; Dehmer, J. L.; Stockbauer, R. L.; Codling, K.; West, J. B.; 20052.

Parr, A. C.; Ederer, D. L.; Cole, B. E.; Stockbauer, R.; Dehmer, J. L.; West, J. B.; Codling, K.; 20426.

Parr, A. C.; Ederer, D. L.; Cole, B. E.; West, J. B.; Stockbauer, R.; Codling, K.; Dehmer, J. L.; 20123.

Parr, A. C.; Ederer, D. L.; Stockbauer, R.; West, J. B.; Cole, B. E.; Dehmer, J. L.; Codling, K.; 20399.

Parr, A. C.; Jason, A. J.; Stockbauer, R.; McCulloh, K. E.; 20051.

Parr, A. C.; Rakowsky, G.; Ederer, D. L.; Stockbauer, R. L.; West, J. B.; Dehmer, J. L.; 20236.

Parr, A. C.; Rosenstock, H. M.; Stockbauer, R.; 20199.

Parr, A. C.; Rosenstock, H. M.; Stockbauer, R.; 20472.

Parris, R. M.; Chesler, S. N.; Hilpert, L. R.; Guenther, F. R.; 20161.

Parris, R. M.; Guenther, F. R.; Chesler, S. N.; May, W. E.; White, P. A.; 20602.
Parrott, J. R., Jr.; North, S. E.; Strandberg, G. W.; Shumate, S. E. II; SP618: 1981 November. 274-286.

Parrott, M. W.; SP599: 1981 January. 147.155.

Parry, E. E.; Argentar, H.; Tesk, J. A.; 20221.

Parry, E. E.; Tesk, J. A.; Whitlock, R. P.; Widera, G. E. O.; Holmes, A.; 20277.

Parry, E. E.; Whitlock, R. P.; Tesk, J. A.; Widera, G. E. O.; Holmes, A.; 20241 .

Passaglia, E.; Sanchez, I. C.; VanderHart, D. L.; Zapas, I. J.; Eby, R. K.; Broadhurst, M. G.; Chang, S. S.; Davis, G. T.; DiMarzio, E. A.; Fanconi, B. M.; Han, C. C.; Khoury, F. A.; Markovitz, H.; McKinney, J. E.; Mopsik, F. I.; 20749.

Paton, E. O.; Reed, R. P.; Toblegr, R. L.; Elmer, J. W.; McHenry, H. I.; Yushchenko, K. A.; 20772.

Patrick, S.; Giuntini, R.,; NBS-GCR-80-279.

Patterson, C. W.; Harter, W. G.; 20154.

Paule, R. C.; Mandel, J.; Schaffer, R.; Reeder, D. J.; Coxon, B.; Enagonio, D.; Christensen, R. G.; Howell, B. F.; SP260-72.

Pavlin, I.; Engelsberg, S.; SP614: 1981 September. 153-156.

Payne, B. F.; 20731.

Payne, B. F.; Edelman, S.; Mordfin, L.; 20246.

Peacock, R. D.; Hibbard, B. B.; Krasny, J. F.; Braun, E.; NBSIR 812386.

Peale, S. J.; Yoder, C. F.; 20599.

Pearl, M. H.; Goldman, A. J.; J. Res. 86(2): 193-215; 1981 MarchApril.

Peavy, B. A.; NBSIR 81-2321.

Peavy, B. A.; NBSIR 81-2353.

Peavy, B. A.; Petersen, S. R.; Barnes, K. A.; BSS134.

Peavy, S. T.; Bremer, S. G.; TN1147.

Peckoo, R. J.; Antonucci, J. M.; Misra, D. N.; 20481.

Peckoo, R. J.; Schruhl, C.; Toth, E. E.; Antonucci, J. M.; 20406.

Peiser, H. S.; Gutterman, B. M.; Louis, G. L.; Pring, J. M.; Taylor, J.

K.; Tholen, A. D.; Smith, C. A., ; NBSIR 80-2020.

Pella, P. A.; Kingston, H.; 20200.

Pella, P. A.; Walter, F. J.; Stone, R.; Blackburn, D. H.; SP604: 1981 June. 61.70 .

Pellegrini, B.; SP614: 1981 September. 185.188.

Penansky, S. G.; SP500-83: 1981 November. 45.54.

Pence, W. H.; Leone, S. R.; 20441.

Penedo, M. H.; SP500-80: 1981 October. 76-82.

Penn, D. R.; Girvin, S. M.; 20008.

Penn, D. R.; Girvin, S. M.; 20389.

Penn, D. R.; Rendell, R. W.; 20128.

Penn, D. R.; Rendell, R. W.; 20564.

Penn, D. R.; Rendell, R. W.; 20691.

Penn, R. W.; McKenna, G. B.; 20091.

Penn, R. W.; McKenna, G. B.; 20129.

Penner, S.; 20353.

Penner, S.; Cutler, R. I.; Debenham, P. H.; Lindstrom, E. R.; Mohr, D. L.; Wilson, M. A. D.; Yoder, N. R.; Young, L. M.; Boyd, T. J.; Knapp, E. A.; Martin, R. E.; Potter, J. M.; Schneider, C. M.; Swenson, D. A.; Tallerico, P. J.; 20206.

Penzes, W. B.; Serbyn, M. R.; 20734.

Perkins, K. L.; SP400-69: 1981 May. 58-68.

Pernicka, J. C.; SP400-69: 1981 May. 42.

Perreault, R. W.; Fry, J. P.; Chiang, W. P.; DeSmith, D. A.; Leder, D. S.; Nguyen, D. K.; NBSIR 81-2315.

Perry, W. E.; Goldfine, A.,; FIPS PUB 88.

Persensky, J. J.; Gagnon, J. L.; 20217.

Peterlin, A.; 20014.

Peterlin, A.; 20732.

Peterlin, A.; Khoury, F. A.; Broadhurst, M. G.; Fanconi, B. M.; Eby, R. K.; 20652.

Peterlin, A.; McCrackin, F. L.; 20344.

Peterlin, A.; Snyder, R. G.; Scherer, J. R.; 20315.

Peterlin, A.; Waters, P. F.; Phillips, J. C.; 20437.

Peters, K. R.; Haller, W.; Gschwender, H. G.; 20523.

Petersen, F. R.; Evenson, K. M.; Vasconcellos, E. C. C.; 20743.

Petersen, F. R.; Maki, A. G.; Sukle, D. J.; Wells, J. S.; 20427.

Petersen, F. R.; Maki, A. G.; Sukle, D. J.; Wells, J. S.; 20640. 
Petersen, F. R.; Wells, J. S.; Maki, A. G.; Siemsen, K. J.; 20641.

Petersen, S. R.; Barnes, K. A.; Kelly, G. E.; NBSIR 81-2241.

Petersen, S. R.; Barnes, K. A.; Peavy, B. A.; BSS134.

Peterson, C.; Scherrer, D.; Martin, D.; SP500-80: 1981 October. 15. 20.

Peterson, G. A.; Lawson, R. D.; Teeters, W.; Williamson, C. F.; Kowalski, S.; Maruyama, X. K.; Lindgren, R. A.; Flanz, J. B.; Hicks, R. S.; Parker, B.; 20323.

Peterson, G. A.; Singhal, R.; Williamson, C. F.; Maruyama, X. K.; Petrovich, F.; Lindgren, R. A.; Plum, M. A.; Gerace, W. J.; Hicks, R. S.; Parker, B.; 20660.

Peterson, R. L.; 20307.

Peterson, R. L.; Hamilton, C. A.; Harris, R. E.; Kautz, R. L.; McDonald, D. G.; 20070.

Peterson, R. L.; Van Vechten, D.; 20692

Petrocco, G.; Fotinos, C.; Stochino, G.; D'Amico, A.; Cappuccio, G.; SP614: 1981 September. 199.201.

Petrovich, F.; Lindgren, R. A.; Plum, M. A.; Gerace, W. J.; Hicks, R. S.; Parker, B.; Peterson, G. A.; Singhal, R.; Williamson, C. F.; Maruyama, X. K.; 20660

Phelan, R. J., Jr.; Boyle, D. R.; Johnson, E. G., Jr.; NBSIR 80-1628.

Phelan, R. J., Jr.; Larson, D. R.; Werner, P. E.; 20330.

Phelps, A. V.; Muller, C. H. III; 20047.

Phelps, A. V.; Tachibana, K.; 20597.

Phelps, A. V.; Zajonc, A. G.; 20242.

Phillips, J. C.; NBSIR 80-2131.

Phillips, J. C.; Peterlin, A.; Waters, P. F.; 20437.

Phillips, W.; Metcalf, H.; 20076.

Phillips, W. E.; Thurber, W. R.; Larrabee, R. C.; NBSIR 81.2325.

Phipps, C. R., Jr.; Harrison, R. F.; Thomas, S. J.; SP620: 1981 October. 238.247.

Pickup, C. P.; SP614: 1981 September. 272.275.

Piehler, H. R.; Sloter, L. E. II.; SP60I: 1981 January. 655-666.

Pielert, J. H.; SP623.

Pielert, J. H.; Chen, P. T.; Lerchen, F. H.; NBSIR 81-2289.

Pielert, J. H.; Faison, T. K.; Lerchen, F. H.; NBSIR 80-2171.

Pierce, D. T.; Celotta, R. J.; 19996

Pierce, D. T.; Celotta, R. J.; 20693.

Pierce, D. T.; Celotta, R. J.; Wang, G. C.; 20012

Pierce, D. T.; Celotta, R. J.; Wang, G. C.; 20706.

Pierce, D. T.; Celotta, R. J.; Wang, G. C.; McRae, E. G.; 20643.

Pierce, D. T.; Girvin, S. M.; Unguris, J.; Celotta, R. J.; 20694.

Pierce, D. T.; Wang, G. C.; Bader, S. D.; Felcher, G. P.; Celotta, R. J.; 19970.

Pierce, D. T.; Wang, G. C.; Celotta, R. J.; 20138.

Pierce, D. T.; Wang, G. C.; Felcher, G. P.; Bader, S. D.; Celotta, R J.; 19975.

Pierce, E. T.; NBSIR 81.2317.

Pierce, E. T.; Bean, J. W.; Kusuda, T.; 20755

Pierce, E. T.; Kao, J. Y.; NBSIR 81-2277.

Pierce, H.; Well, A.; Johnson, C. C.; Clifton, C.; Nober, E. H.; NBSGCR-80-284.

Pierce, W. J.; SP599: 1981 January. 64.68.

Pierman, B. C.; Adler, S. C.; NBSIR 81-2245.

Piermarini, G. J.; Rabolt, J. F.; Block, S.; 20007.

Pierron, E.; Knight, C. W. T.; Korgav, A.; SP400-69: 1981 May. 115. 121.

Pilione, L. J.; Carpenter, B. S.; 19982.

Pindzola, M. S.; Falk, R. A.; Dunn, G. H.; Griffin, D. C.; Bottcher C.; Gregory, D. C.; Crandall, D. H.; 20584

Pineyro, J. J.; Difilippo, F. C.; SP614: 1981 September. 309.313.

Pizzella, G.; Bonifazi, P.; Bordoni, F.; Pallottino, G. V.; SP614: 1981 September. 298-301.

Placious, R. C.; Garrett, D. A.; Kasen, M. B.; Berger, H.; 20342.

Plamondon, R.; Robillard, P. N.; SP500-80: 1981 October. 152.158.

Plante, E. R.; Bonnell, D. W.; Hastie, J. W.; NBSIR 81-2279.

Plante, E. R.; Hastie, J. W.; NBSIR 81-2293.

Plante, E. R.; Horton, W. S.; Hastie, J. W.; Bonnell, D. W.; NBSIR 80.-2178 (DoE)

Plitz, W.; Griss. P.; SP601: 1981 January. 131.156.

Plum, M. A.; Gerace, W. J.; Hicks, R. S.; Parker, B.; Peterson, G. A.;
Singhal, R.; Williamson, C. F.; Maruyama, X. K.; Petrovich, F.; Lindgren, R. A.; 20660.

Poate, J. M.; Nakahara, S.; Draper, C. W.; Buene, L.; Jacobson, D. C.; SP620: 1981 October. 222.226.

Poliakoff, E. D.; Dehmer, P. M.; Dehmer, J. L.; Stockbauer, R.; 20533.

Polvani, R. S.; Christ, B. W.; 20612.

Pommersheim, J. M.; Mathey, R. G.; TN1152.

Pontius, P. E.; Mitchell, R. A.; 20231.

Popp, R. K.; Frantz, J. D.; Vogel, G. L.; 20467

Porter, G.; SP600.

Porteus, J. O.; Decker, D. L.; Seitel, S. C.; Soileau, M. J.; SP620 1981 October. 201-209.

Porteus, J. O.; Seitel, S. C.; Kraatz, P.; Donovan, T. M.; SP620: 1981 October. 305.312.

Post, M. A.; Campbell, P. G.; Sleater, G. A.; NBSIR 80-2122.

Potter, J. M.; Schneider, C. M.; Swenson, D. A.; Tallerico, P. J.; Penner, S.; Cutler, R. I.; Debenham, P. H.; Lindstrom, E. R.; Mohr, D. L.; Wilson, M. A. D.; Yoder, N. R.; Young, L. M.; Boyd, T. J.; Knapp, E. A.; Martin, R. E.; 20206.

Potter, T.; Key, W. P.; SP608: 1981 May. 183.194.

Potter, T. W.; SP500-83.

Potzick, J. E.; Robertson, B.; 20144

Powell, C. J.; 20251.

Powell, C. J.; Jach, T.; 20394.

Powell, F. J.; Barber, E. M.; Bettge, D. A.; 20379.

Powell, J.; Ruegg, R.; 20151.

Powell, J. W.; Rodgers, R. C., Jr.; NBSIR 81-2342.

Powell, P.; Barkley, J.; SP500-80: 1981 October. 174-179.

Powell, R. L.; Fickett, F. R.; 20377.

Pradhan, A. K.; Norcross, D. W.; Hummer, D. G.; 20045

Pradhan, A. K.; Norcross, D. W.; Hummer, D. G.; 20492.

Prialnik, D.; Shaviv, G.; Kovetz, A.; 20695.

Prialnik, D.; Shaviv, G.; Shara, M. M.; 20272.

Price, L. A.; Relles, N.; SP500-80: 1981 October. 167-173.

Prince, E.; 20600.

Pring, J. M.; Taylor, J. K.; Tholen, A. D.; Smith, C. A., ; Peiser, H S.; Gutterman, B. M.; Louis, G. L.; NBSIR 80-2020.

Prosen, E. J.; 20015.

Prudenziati, M.; Morten, B.; Masoero, A.; SP614: 1981 September 202.205.

Pruitt, J. S.; Domen, S. R.; Loevinger, R.; J. Res. 86(5): 495-502; 1981 September-October.

Purdy, J. A.; Fivozinsky, S. P.; 20554.

Purdy, J. A.; Harms, W. B.; Fivozinsky, S.; 20553.

Purtell, L. P.; 20255.

Purtell, L. P.; Klebanoff, P. S.; Buckley, F. T.; 20257.

Putnam, L. H.; SP500-80: 1981 October. 49-57.

Pyles, R. A.; Stockton, R. A.; Wheeler, A. E.; Zingaro, R. A.; Irgolic K. J.; Banks, C. H.; Bottino, N. R.; Chakraborti, D.; Gennity, J. M.; Hillman, D. C.; O'Brien, D. H.; SP618: 1981 November. 244-263.

Pynn, R.; Heidemann, A.; Michel, K. H.; Rowe, J. M.; Rush, J. J.; 20421.

Pyper, W. R.; SP500-83: 1981 November. 101-106.

\section{Q}

Quade, C. R.; Suenram, R. D.; 20084

Quijano, R. C.; SP601: 1981 January. 363-393.

Quinn, P. J.; Wilson, I.; Page, A. A.; Higson, P.; Deward, F. D.;

Haisch, B.; Linsky, J. L.; Slee, O. B.; Siegman, B. C.; Nikoloff, I.; Candy, M.; Harwood, D.; Verveer, A.; 20405.

Quintiere, J.; 20493.

Quintiere, J.; Rinkinen, W.; Cooper, L. Y.; Harkleroad, M.; 20591.

Quintiere, J.; Steckler, K.; McCaffrey, B.; 20740.

Quintiere, J. G.; 20207.

Quintiere, J. G.; Harkleroad, M. F.; McCaffrey, B. J.; 20567.

Quintiere, J. G.; McCaffrey, B. J.; NBSIR 80-2054, Vols. I and II. 


\section{$\mathbf{R}$}

Rabaud, J.; SP622: 1981 October. 289-309.

Rabolt, J. F.; Block, S.; Piermarini, G. J.; 20007.

Radebaugh, R.; 20020.

Radebaugh, R.; Clark, A. F.; Fujii, G.; Ekin, J. W.; 20298.

Radebaugh, R.; Fujii, G.; Read, D. T.; Clark, A. F.; 19999.

Radebaugh, R.; Linenberger, D.; Voth, R. O.; 20766.

Radebaugh, R.; Linenberger, D.; Voth, R. O.; SP607: 1981 May. 70. 81 .

Radford, H. E.; Mizushima, M.; Scalabrin, A.; Saykally, R. J.; Evenson, K. M.; 20700.

Rafelski, J.; Danos, M.; 20109.

Rainer, F.; Milam, D.; Smith, W. L.; Deaton, T. F.; SP620: 1981 October. 297-299.

Rains, T. C.; Garruto, R. M.; Gajdusek, D. C.; Gibbs, C. J., Jr.; Traub, R. D.; 20603.

Rains, T. C.; Messman, J. D.; 20763.

Rainwater, J. C.; 20211.

Rainwater, J. C.; 20215.

Rainwater, J. C.; 20263.

Rainwater, J. C.; J. Res. 86(5): 429.455; 1981 September-October.

Rainwater, J. C.; Hanley, H. J. M.; 20575.

Rakowsky, G.; 20204.

Rakowsky, G.; Ederer, D. L.; Stockbauer, R. L.; West, J. B.; Dehmer, J. L.; Parr, A. C.; 20236

Ramboz, J. D.; NBSIR 81-2337.

Ramboz, J. D.; Anderson, W. E.; NBSIR 81-2235.

Ramey-Smith, A. M.; Gagnon, J. L.; 20235.

Randall, J. L.; Bender, P. L.; Faller, J. E.; Decher, R.; 20451.

Ranney, M. A.; Clark, A. F.; Fujii, G.; 20465.

Ranney, M. A.; Clark, A. F.; Fujii, G.; 20753.

Rao, K. V.; Fickett, F. R.; Chen, H. S.; Goldfarb, R. B.; 20317.

Rapsomanikis, S.; Craig, P. J.; SP618: 1981 November. 54-64.

Rasberry, S. D.; Uriano, G. A.; 20326.

Raufaste, N.; Olmert, M.,; SP446-5.

Rawie, C. C.; 20031.

Rawie, C. C.; NBSIR 81-2223.

Rawie, C. C.; NBSIR 81-2402.

Razouk, R. R.; SP500-80: 1981 October. 163-166.

Read, D. T.; Clark, A. F.; Radebaugh, R.; Fujii, G.; 19999.

Read, D. T.; Mazandarany, F. N.; Parker, D. M.; Koenig, R. F.; 20574.

Read, D. T.; McHenry, H. I.; 20548.

Read, D. T.; McHenry, H. I.; NBSIR 81-1648.

Read, D. T.; Reed, R. P.; 20546.

Read, D. T.; Reed, R. P.; 20572.

Read, D. T.; Whipple, T. A.; McHenry, H. I.; 20528.

Reader, J.; Acquista, N.; 20218.

Reader, J.; Acquista, N.; 20395.

Reader, J.; Corliss, C. H.; Wiese, W. L.; Martin, G. A.; NSRDSNBS68.

Reader, J.; Luther, G.; Acquista, N.; 20110.

Reader, J.; Ryabtsev, A.; 20186

Reader, J.; Ryabtsev, A.; Wyart, J. F.; 20449.

Ready, J. F.; Vora, H.; SP620: 1981 October. 110-116.

Rebuldela, G.; Driver, L. D.; Ries, F. X.; NBSIR 78-871.

Reed, R. P.; 20607.

Reed, R. P.; Burkhalter, D. S.; Tobler, R. L.; 20742.

Reed, R. P.; Read, D. T.; 20546.

Reed, R. P.; Read, D. T.; 20572.

Reed, R. P.; Simon, N. J.,; NBSIR 81-1645.

Reed, R. P.; Tobler, R. L.; Elmer, J. W.; McHenry, H. 1.; Yushchenko, K. A.; Paton, E. O.; 20772.

Reed, R. P.,; NBSIR 80-1627.

Reed, W. P.; Carpenter, B. S.; Hoffman, J. D.; Yolken, H. T.; NBSIR 81-2205.

Reeder, D. J.; 20098.

Reeder, D. J.; Coxon, B.; Enagonio, D.; Christensen, R. G.; Howell, B. F.; Paule, R. C.; Mandel, J.; Schaffer, R.; SP260-72.

Reeder, D. J.; White, E.; Christensen, R. G.; Hertz, H. S.; 20654.

Reeve, C. P.; Scire, F. E.; Veale, R. C.; Haight, W. C.; Hocken, R. J.;
Borchardt, B. R.; Carroll, C. L.; Hartsock, R. G.; NBSIR 80-2141.

Reeve, C. P.; Spiegelman, C. H.; Lechner, J. A.; NBSIR 80-2151.

Reeve, G. R.; 20663.

Regev, O.; Shaviv, G.; 20696.

Reggiani, L.; Brunetti, R.; Jacoboni, C.; SP614: 1981 September. 414. 416.

Rehm, R. G.; Barnett, P. D.; Corley, D. M.; Baum, H. R.; NBSIR 812385.

Rehm, R. G.; Lewis, J. G.; NBSIR 80-2056

Rehm, R. G.; Sekerka, R. F.; Coriell, S. R.; Boisvert, R. F.; 20448.

Reid, M.; McNeil, K. J.; Walls, D. F.; 20697.

Reifer, D. J.; Montgomery, H. A.,; NBS-GCR-81-335.

Reinhart, E. R.; SP589: 1981 January. 63-76.

Reinhold, T. A.; Mehta, K. C.; Minor, J. E.; Marshall, R. D.; 20759.

Reinhold, T. A.; Simiu, E.; Yancey, C. W. C.; Cattaneo, L.; Harris, J. R.; NBSIR 81-2199.

Reite, M.; Zimmerman, J. T.; Zimmerman, J. E.; 20480.

Relles, N.; Price, L. A.; SP500-80: 1981 October. 167-173.

Rendell, R. W.; Bryant, G. W.; Girvin, S. M.; 20629.

Rendell, R. W.; Girvin, S. M.; 20464

Rendell, R. W.; Penn, D. R.; 20128.

Rendell, R. W.; Penn, D. R.; 20564.

Rendell, R. W.; Penn, D. R.; 20691.

Reneker, D. H.; Wachtman, J. B., Jr.; NBSIR 80-2108.

Reno, R. C.; Bennett, L. H.; Swartzendruber, L. J.; 20208.

Repjar, A. G.; Kremer, D. P.; 20545.

Repjar, A. G.; Newell, A. C.; Estin, A. J.; Stubenrauch, C. F.; 20650.

Resendez, D. L.; Winchester, J. W.; SP500-80: 1981 October. 21.24.

Resendez, D. L.; Winchester, J. W.; SP500-80: 1981 October. 25.28.

Resnicow, M.; Ahola, R.; Hug, R.; Strelich, T.; SP500-80: 1981 October. 122-127.

Revesz, A. G.; Kruger, J.; 20025.

Reyes-Virella, R.; Braun, E.; NBSIR 81-2200.

Rhodin, T. N.; Madey, T. E.; Houston, J. E.; Seabury, C. W.; 20419.

Rhyne, J. J.; 20145.

Rhyne, J. J.; 20469.

Rhyne, J. J.; Chen, H. S.; Lynn, J. W.; Erwin, R. W.; 20282.

Rhyne, J. J.; Koon, N. C.; 20212.

Rhyne, J. J.; Smith, K.; Wallace, W. E.; Hardman, K.; 20214.

Richels, R.; SP616: 1981 October. 49-63.

Richmond, J. C.; 20733.

Richmond, J. C.; NBSIR 81-2311.

Richmond, J. C.; Mecherikunnel, A. T.; 20083.

Richter, D.; Magerl, A.; Rowe, J. M.; 20160.

Richtmyer, T. E.; Elder, J.; Tibbott, R. L.; Yonemura, G. T.; Hunt, C. M.; Chen, P. T.; Hill, J. E.; May, W. B., Jr.; BSS133.

Richtmyer, T. E.; May, W. B.; Hunt, C. M.; Hill, J. E.; 20539.

Ries, F. X.; Larsen, E. B.; TN1033.

Ries, F. X.; Rebuldela, G.; Driver, L. D.; NBSIR 78-871.

Ries, M. D.; Rose, R. M.; Crugnola, A. N.; Cimino, W. R.; SP601 1981 January. 3.28.

Rigaud, D.; Sodini, D.; SP614: 1981 September. 63-66.

Rinkinen, W.; Cooper, L. Y.; Harkleroad, M.; Quintiere, J.; 20591.

Risby, T. H.; SP618: 1981 November. 120-133.

Ritchie, D.; Braunlich, P.; Schmid, A.; Bryant, G. W.; Kelly, P.; SP620: 1981 October. 394.402

Ritchie, D.; Bryant, G. W.; Schmid, A.; Braunlich, P.; Kelly, P.; SP620: 1981 October. 427.431.

Ritter, J. J.; Ambrose, J. R.; Kruger, J.; Carroll, J. J.; Melmed, A. J.; NBSIR $79-1904$ (Navy).

Ritter, J. J.; Carroll, J. J.; Melmed, A. J.; Kruger, J.; NBSIR 81-2396 (ONR).

Ritter, J. J.; Kruger, J.; 20269.

Ritter, K, J.; Daley, T. W.; Lafferty, W. J.; Sattler, J. P.; Worchesky, T. L.; Tobin, M. S.; 20090

Ritter, K. J.; Lafferty, W. J.; Sattler, J. P.; Worchesky, T. L.; 20452.

Roberts, W. E.; Masters, L. W.; Seiler, J. F.; Embree, E. J.; NBSIR 81-2232.

Robertson, B.; Potzick, J. E.; 20144.

Robiette, A. G.; Ozier, I.; Gerry, M. C. L.; JPCRD 10(4): 1085-1095; 1981. 
Robillard, P. N.; Plamondon, R.; SP500-80: 1981 October. 152-158.

Robinson, G. R., Jr.; Hemingway, B. S.; Haas, J. L., Jr.; JPCRD 10(3): $575-670 ; 1981$

Robinson, H. G.; Hall, J. L.; Hollberg, L.; Baer, T.; 20720

Robinson, J. C.; Allen, J. W.; Hartman, W. F.; SP622: 1981 October. 11-30.

Roder, H. M.; J. Res. 86(5): 457.493; 1981 September-October.

Roder, H. M.; deCastro, C. A. N.; J. Res. 86(3): 293-307; 1981 May. June.

Roder, H. M.; McCarty, R. D.; Hord, J.; Monogr. 168.

Rodgers, R. C., Jr.; Powell, J. W.; NBSIR 81-2342.

Roessler, B.; Armstrong, R. W.; Kuriyama, M.; Yoo, K. C.; 20712.

Roitman, P.; Albers, J.; Ehrstein, J. R.; Myers, D. R.; NBSIR 81 . 2398.

Rolfe, S. T.; McHenry, H. I.; NBSIR 79-1623.

Romanov, V. P.; Candela, G. A.; Roth, R. S.; Swartzendruber, L. J.; 20043.

Romine, D. J.; Wertheimer, H. P.; SP622: 1981 October. $62-73$.

Rosasco, G. J.; Schneggenburger, R.; Nolen, R. L., Jr.; Drake, M. C.; 20653.

Rose, R. M.; Crugnola, A. N.; Cimino, W. R.; Ries, M. D.; SP601: 1981 January. 3-28.

Rosenbaum, J.; Early, C.; SP500-80: 1981 October. 146-151.

Rosenkrantz, M. E.; Basch, H.; Julienne, P. S.; Krauss, M.; 20238.

Rosenkrantz, M. E.; Stevens, W. J.; Julienne, P. S.; Konowalow, D. D.; Krauss, M.; 20100

Rosenkrantz, M. E.; Stevens, W. J.; Krauss, M.; Konowalow, D. D.; 20082.

Rosenstock, H. M.; Stockbauer, R.; Parr, A. C.; 20199.

Rosenstock, H. M.; Stockbauer, R.; Parr, A. C.; 20472.

Rosenthal, R.; Watkins, S.; Blanc, R.; Heafner, J.; NBSIR 80-2154.

Rosman, K. J. R.; Barnes, I. L.; Moore, L. J.; Gramlich, J. W.; 20698.

Rosner, J. L.; Karl, G.; Meshkov, S.; 20040.

Rosner, R.; Ayres, T. R.; Linsky, J. L.; Vaiana, G. S.; Golub, L.; 20627.

Rosner, R.; Seward, F.; Topka, K.; Zwaan, C.; Vaiana, G. S.; Cassinelli, J. P.; Fabbiano, G.; Giacconi, R.; Golub, L.; Gorenstein, P.; Haisch, B. M.; Harnden, F. R., Jr.; Johnson, H. M.; Linsky, J. L.; Maxson, C. W.; Mewe, R.; 20336.

Rosner, R.; Seward, F. D.; Vaiana, G. S.; Haisch, B. M.; Linsky, J. L.; Harnden, F. R., Jr.; 20124.

Rosner, R.; Vaiana, G. S.; Ayres, T.; Linsky, J. L.; Pallavicini, R.; Golub, L.; 20594.

Rosner, S. D.; Lineberger, W. C.; Jones, P. L.; Mead, R. D.; Kohler, B. E.; 19995.

Rossiter, W. J., Jr.; Mathey, R. G.; 20259.

Rossiter, W. J., Jr.; Mathey, R. G.; Busching, H. W.; Cullen, W. C.; TN1135.

Rossiter, W. J., Jr.; Shipp, W. E.; NBSIR 81-2381.

Rossiter, W. J., Jr.; Weidt, J. L.; Saxler, R. J.; 20463.

Roth, C.; J. Res. 86(1): 33-77; 1981 January-February.

Roth, R. S.; 20046.

Roth, R. S.; 20117

Roth, R. S.; Minor, D.; Santoro, A.; Marezio, M.; 20054

Roth, R. S.; Swartzendruber, L. J.; Romanov, V. P.; Candela, G. A.; 20043.

Roth, S. C.; Kenney, J. M.; Edelman, S.; Harris, G. R.; DeReggi, A. S.; 20170

Roubeau, P. M.; SP607: 1981 May. 3-14.

Roux, J. C.; King, G. P.; Swinney, H. L.; SP614: 1981 September 283.287.

Rowe, J. M.; Richter, D.; Magerl, A.; 20160

Rowe, J. M.; Rush, J. J.; Pynn, R.; Heidemann, A.; Michel, K. H.; 20421.

Rowley, W. R. C.; Marx, B. R.; Layer, H. P.; 20233.

Roy, P.; Courbier, R.; David, M.; Noël, H. P.; Guidoin, R.; King, M.;

Blais, P.; Marois, M.; Gosselin, C.; SP601: 1981 January. 29-129.

Rozdestvenskaya, T. H.; Williams, E. S.; Galakhova, O. P.; Harkness,

S.; Hermach, F. L.; Hirayama, H.; Martin, P.; 20240.

Rubin, A. I.; Howett, G. L.; SP480-37.

Rubin, L.; Kydes, A. S.; SP616: 1981 October. 139.147.
Rubin, R. J.; NBSIR 80-1975.

Rubin, R. J.; Knab, L. I.; Hutchinson, J. M. R.; Collé, R.; TN1139.

Rucker, L. M.; Amberiadis, K.; van der Ziel, A.; SP614: 1981 September. 210-212.

Rucks, A. C.; Ginter, P. M.; SP500-83: 1981 November. 191-197.

Rudder, F. F., Jr.; Boehm, M. J.; Weber, S. F.; NBSIR 81-2366.

Ruegg, R.; Powell, J.; 20151.

Ruegg, R. T.; Chapman, R. E.; NBSIR 81-2248.

Ruegg, R. T.; Marshall, H. E.; NBSIR 81-2397.

Ruegg, R. T.; Sav, G. T.; 20167.

Ruff, A. W.; Ditchek, B.; 19980.

Ruff, A. W.; Ives, L. K.; 20209.

Ruff, A. W.; Marinenko, R. B.; Biancaniello, F.; DeRoberts, L.; Boyer, P. A.; SP260-70.

Ruff, W.,; Weinstein, A.; Gibbons, D.; Brown, S.; SP601.

Rumble, J. R., Jr.; Gallagher, J. W.; Van Blerkom, J.; Beaty, E. C.; SP593.

Rupp, N. W.; Brauer, G. M.; Dulik, D. M.; Hughes, H. N.; Dermann, K.; 20669.

Rush, J. J.; Pynn, R.; Heidemann, A.; Michel, K. H.; Rowe, J. M.; 20421.

Russ, J. C.; SP604: 1981 June. 297-313.

Russ, J. C.; Sandborg, A. O.; SP604: 1981 June. 71-95.

Russell, T. J.; Lankford, W. F.; Schafft, H. A.; Sawyer, D. E.; Kessler, H. K.; NBSIR 80-2181.

Ruthberg, S.; Cohen, E. C.,; Schafft, H. A.; SP400-69.

Ruthberg, Z. G.; 20378.

Ryabtsev, A.; Reader, J.; 20186.

Ryabtsev, A.; Wyart, J. F.; Reader, J.; 20449

Ryan, P.; De Vos, D.,; NBS-GCR-80-291.

Ryder, P. L.; SP604: 1981 June. 177-191.

\section{S}

Saber, J. M.; Diller, D. E.; 20767.

Saib, S. H.; Benson, J. P.; Gannon, C.; DeHaan, W. R.; SP500-80: 1981 October. 208-217.

Saib, S. H.; Benson, J. P.; Gannon, C.; DeHaan, W. R.; SP500-80: 1981 October. 200-207.

Sakurai, T.; Culbertson, R. J.; Melmed, A. J.; 19983.

Sakurai, T.; Kuk, Y.; Givargizov, E. I.; Melmed, A. J.; 20157.

Salazar, S. B.; Beller, E. J. III.; Hardgrave, W. T.; NBSIR 81-2240

Salazar, S. B.; Beller, E. J. III.; Hardgrave W. T.; SP500-83: 1981 November. 85.91.

Salazar, S. B.; Hardgrave, W. T.; Marriott, A. R.; Skillman, G. H.; NBSIR 80-2182.

Saloman, E. B.; Cole, B. E.; Cooper, J. W.; Ederer, D. L.; Mehlman, G.; 20300

Saloman, E. B.; Ederer, D. L.; Cole, B. E.; 20674.

Saloman, E. B.; Nagel, D. J.; Day, R. H.; Lee, P.; 20637.

Salomone, L. A.; NBSIR 80-2111-3.

Salomone, L. A.; Chung, R. M.; Yokel, F. Y.; NBSIR 81-2215.

Salomone, L. A.; Yokel, F. Y.; Kovacs, W. D.; BSS135.

Saltman, R. G.; Konig, P. A.; Newton, J. J.; NBSIR 81-2354.

Sams, R. L.; Olson, W. B.; 20053.

Sams, R. L.; Olson, W. B.; Frommer, C.; Lovejoy, R. W.; 20589.

Sams, R. L.; Sweger, D. M.; 20461.

Sanchez, I. C.; Smith, L. E.; Eby, R. K.; Broadhurst, M. G.; Cassel, J. M.; Fanconi, B. M.; NBSIR 81-2263.

Sanchez, I. C.; VanderHart, D. L.; Zapas, I. J.; Eby, R. K.; Broadhurst, M. G.; Chang, S. S.; Davis, G. T.; DiMarzio, E. A.; Fanconi, B. M.; Han, C. C.; Khoury, F. A.; Markovitz, H.; McKinney, J. E.; Mopsik, F. I.; Passaglia, E.; 20749.

Sanchez, R.; Wallace, T.; Mc Wethy, S.; Moy, S.; Nielsen, G.; SP500 80: 1981 October. 103-110.

Sancho, J. M.; San Miguel, M.; Katz, S.; Gunton, J. D.; SP614: 1981 September. 26-29.

Sandborg, A. O.; Russ, J. C.; SP604: 1981 June. 71-95.

Sanders, S. P.; Lian, J. B.; Levy, R. J.; SP601: 1981 January. 339-348.

Sanderson, B. T.; Gerhold, W. F.; Escalante, E.; NBSIR 81-2228 (NBS). 
Sandle, W. J.; Gallagher, A.; 20699.

Sandstrom, D. R.; Lytle, F. W.; Greegor, R. B.; Khalil, S. R.; Ekambaram, V.; Weiss, C. S.; Grimm, C. A.; Filby, R. H.; SP618: 1981 November. 21-38.

San Miguel, M.; Katz, S.; Gunton, J. D.; Sancho, J. M.; SP614: 1981 September. 26-29.

Santoro, A.; Marezio, M.; Roth, R. S.; Minor, D.; 20054.

Santoro, R. J.; Goulard, R.; Emmerman, P. J.; Semerjian, H. G.; 20357.

Santoro, R. J.; Semerjian, H. G.; Emmerman, P. J.; Goulard, R.; 20335.

Sarbar, M.; Covington, A. K.; Goldberg, R. N.; 20714.

Sassi, M. P.; Bertotti, G.; Fiorillo, F.; SP614: 1981 September. 324 327.

Sato, H.; Tanabe, M.; Mimaki, T.; SP614: 1981 September. 30-33.

Sattler, J. P.; Worchesky, T. L.; Ritter, K. J.; Lafferty, W. J.; 20452.

Sattler, J. P.; Worchesky, T. L.; Tobin, M. S.; Ritter, K, J.; Daley, T. W.; Lafferty, W. J.; 20090.

Sauder, W. C.; Henins, A.; Deslattes, R. D.; Kessler, E. G.; 20146.

Sauer, C. H.; SP500-83: 1981 November. 335.

Saunders, P. B.; Gass, S. I.; Hoffman, K. L.; Jackson, R. H. F.; Joel, L. S.; NBSIR 80-1971.

Sautereau, J. F.; Graffeuil, J.; Martin, J. C.; SP614: 1981 September. 47-50.

Sautereau, J. F.; Tantrarongroj, K.; Graffeuil, J.; SP614: 1981 September. 84-87.

Sav, G. T.; Ruegg, R. T.; 20167.

Savelli, M.; Maille, C.; Alabedra, R.; Lecoy, G.; SP614: 1981 September. 109-112.

Sawyer, D.; U.S. Patent 4,287,473.

Sawyer, D. E.; Kessler, H. K.; Russell, T. J.; Lankford, W. F.; Schafft, H. A.; NBSIR 80-2181.

Saxler, R. J.; Rossiter, W. J., Jr.; Weidt, J. L.; 20463.

Sayani, H. H.; Teichroew, D.; Callender, E.; SP500-80: 1981 October. $38-43$.

Saykally, R. J.; Evenson, K. M.; Radford, H. E.; Mizushima, M.; Scalabrin, A.; 20700.

Scace, E. L.,; FIPS PUB 78.

Scace, R. I.; NBSIR 81-2197.

Scace, R. I.; Walters, E. J.,; Galloway, K. F.; NBSIR 81-2230-3.

Scalabrin, A.; Saykally, R. J.; Evenson, K. M.; Radford, H. E.; Mizushima, M.; 20700.

Scarfe, C. M.; Cronin, D.; Wenzel, J.; Kauffman, D.; 20222.

Schaefer, A. R.; Geist, J.; Liang, E.; 20530.

Schäfer, L.; Sellers, H. L.; Lovas, F. J.; Suenram, R. D.; 20005.

Schaffer, R.; Reeder, D. J.; Coxon, B.; Enagonio, D.; Christensen, R. G.; Howell, B. F.; Paule, R. C.; Mandel, J.; SP260-72.

Schafft, H. A.; Lankford, W. F.; Kowalski, P.; NBSIR 81-2260.

Schafft, H. A.; Ruthberg, S.; Cohen, E. C.,; SP400-69.

Schafft, H. A.; Sawyer, D. E.; Kessler, H. K.; Russell, T. J.; Lankford, W. F.; NBSIR 80-2181.

Schamber, F. H.; SP604: 1981 June. 193-231

Schamber, F. H.; McCarthy, J. J.; SP604: 1981 June. 273-296

Scheeline, A.; Norris, J. A.; Travis, J. C.; DeVoe, J. R.; Walters, J. P.; 20453.

Scheeline, A.; Travis, J. C.; DeVoe, J. R.; Walters, J. P.; 20462.

Schenck, P. K.; Burns, G. W.; Dils, R. R.; Whetstone, J. R.; Frederikse, H. P. R.; NBSIR 81-2348.

Scherer, J. R.; Peterlin, A.; Snyder, R. G.; 20315.

Scherrer, D.; Martin, D.; Peterson, C.; SP500-80: 1981 October. 15 20.

Schiffer, F. H.; Simon, T.; Linsky, J. L.; 20423.

Schmadebeck, R. L.; Seltzer, S. M.; Bielefeld, M. J.; Yin, L. I.; Trombka, J. I.; 20569.

Schmid, A.; Braunlich, P.; Bryant, G.; SP620: 1981 October. 403-405.

Schmid, A.; Braunlich, P.; Kelly, P.; Ritchie, D.; Bryant, G. W.; SP620: 1981 October. 427-431.

Schmid, A.; Bryant, G. W.; Kelly, P.; Ritchie, D.; Braunlich, P.; SP620: 1981 October. 394-402.

Schmid, A.; Kelly, P.; Braunlich, P.; Brost, G.; SP620: 1981 October. 406-415.
Schmid, G. B.; Norcross, D. W.; Collins, L. A.; 20055.

Schneggenburger, R.; Nolen, R. L., Jr.; Drake, M. C.; Rosasco, G. J. 20653.

Schneider, C. M.; Swenson, D. A.; Tallerico, P. J.; Penner, S.; Cutler, R. I.; Debenham, P. H.; Lindstrom, E. R.; Mohr, D. L.; Wilson, M. A. D.; Yoder, N. R.; Young, L. M.; Boyd, T. J.; Knapp, E. A.; Martin, R. E.; Potter, J. M.; 20206.

Schneider, S. J.; 20359.

Schoen, F. J.; Titus, J. L.; Lawrie, G. M.; SP601: 1981 January. 269 282.

Schoonover, R. M.; Davis, R. S.; 20247

Schoonover, R. M.; Johnson, C. E.; Ogburn, F.; 20334.

Schover, D. S.; Board, D.; Fischer, J. P.; SP622: 1981 October. 126 136.

Schrack, R. A.; Behrens, J. W.; Johnson, R.; Bowman, C. D.; 20458

Schramm, R. E.; Kasen, M. B.; MacDonald, G. R.; Beekman, D. H., Jr.; 20616.

Schramm, R. E.; Superczynski, M. J.; Ekin, J. W.; 20297.

Schroeder, L. W.; Brown, W. E.; Jordan, T. H.; Dickens, B.; 19994.

Schroeder, L. W.; Jordan, T. H.; Brown, W. E.; 20173.

Schroeder, L. W.; Mathew, M.; Mayer, I.; Dickens, B.; 20065.

Schruhl, C.; Toth, E. E.; Antonucci, J. M.; Peckoo, R. J.; 20406.

Schuhmacher, H.; Coyne, J.; McDonald, J. C.; Menzel, H. G.; 20675.

Schultz, T. J.; NBS-GCR-80-249.

Schumann, L. W.; Gallagher, A. C.; Wildman, D. W.; 20491.

Schumm, R. H.; NBSIR 81-2343.

Schumm, R. H.; Nuttall, R. L.; Wagman, D. D.; Evans, W. H. Parker, V. B.; TN270-8.

Schurman, D. J.; Burton, D. S.; Nagel, D. A.; McShane, D.; SP601: 1981 January. 615-642.

Schuss, Z.; Ben-Jacob, E.; SP614: 1981 September. 376-380.

Schuss, Z.; Bergman, D. J.; Ben-Jacob, E.; SP614: 1981 September 57.60 .

Schwartz, R.; Eisenhauer, C.; 20677.

Schwarz, S. A.; Helms, C. R.; Spicer, W. E.; Taylor, N. J.; SP400-67.

Schwarz, S. A.; Spicer, W. E.; Taylor, N. J.,; Helms, C. R.; NBS GCR $-81-345$

Schwitz, W.; Seki, R.; Lum, G. K.; Wiegand, C. E.; Kessler, E. G. Jr.; Deslattes, R. D.; Jacobs, L.; 20349.

Scire, F. E.; Veale, R. C.; Haight, W. C.; Hocken, R. J.; Borchardt, B. R.; Carroll, C. L.; Hartsock, R. G.; Reeve, C. P.; NBSIR 802141.

Scofield, J. H.; Darling, D. H.; Webb, W. W.; SP614: 1981 September. 147.150 .

Scott, M.; Leonard, T. A.; Loomis, J.; Harding, K. G.; SP620: 1981 October. 345-355.

Scott, T. W.; SP599: 1981 January. 271-272.

Seabury, C. W.; Rhodin, T. N.; Madey, T. E.; Houston, J. E.; 20419

Sehested, K.; McLaughlin, W. L.; Kosanić, M. M.; Marković, V. M.; Nenadovic, M. T.; Holcman, J.; 20150

Seiler, E.; SP599: 1981 January. 138-146.

Seiler, J.; Waksman, D.; Streed, E.; TN1136.

Seiler, J. F.; Embree, E. J.; Roberts, W. E.; Masters, L. W.; NBSIR 81-2232.

Seitel, S. C.; Kraatz, P.; Donovan, T. M.; Porteus, J. O.; SP620: 1981 October. 305-312.

Seitel, S. C.; Soileau, M. J.; Porteus, J. O.; Decker, D. L.; SP620: 1981 October. 201-209.

Sekerka, R. F.; Coriell, S. R.; Boisvert, R. F.; Rehm, R. G.; 20448

Sekerka, R. F.; Coriell, S. R.; Cordes, M. R.; Boettinger, W. J.; 20224.

Seki, R.; Lum, G. K.; Wiegand, C. E.; Kessler, E. G., Jr.; Deslattes, R. D.; Jacobs, L.; Schwitz, W.; 20349.

Sekine, H.; Tachikawa, T.; Ekin, J. W.; 20659.

Sellers, H. L.; Lovas, F. J.; Suenram, R. D.; Schäfer, L.; 20005.

Seltzer, S. M.; 20003.

Seltzer, S. M.; 20615.

Seltzer, S. M.; Bielefeld, M. J.; Yin, L. I.; Trombka, J. I.; Schmadebeck, R. L.; 20569.

Semerjian, H. G.; Emmerman, P. J.; Goulard, R.; Santoro, R. J.; 20335. 
Semerjian, H. G.; Santoro, R. J.; Goulard, R.; Emmerman, P. J.; 20357.

Semlitsch, M.; Willert, H. G.; Buchhorn, G.; Buchhorn, U.; SP601: 1981 January. 239-267.

Sengers, J. M. H. L.; Klein, M.; Waxman, M.; Davis, H. A.; NBSIR 79.1715 .

Sengers, J. M. H. L.; Sengers, J. V.; 20127.

Sengers, J. V.; Bedeaux, D.; Mazur, P.; Greer, S. C.; 20056.

Sengers, J. V.; Sengers, J. M. H. L.; 20127.

Senich, G. A.; 20408.

Senich, G. A.; 20552.

Senich, G. A.; Wang, F. W.; Smith, L. E.; Chang, S. S.; McCrackin, F. L.; NBSIR 81.2264.

Senturia, S. D.; Huberman, M. G.; Van der Klott, R.; SP400-69: 1981 May. 108-114.

Serbyn, M. R.; Penzes, W. B.; 20734.

Seward, F.; Topka, K.; Zwaan, C.; Vaiana, G. S.; Cassinelli, J. P.; Fabbiano, G.; Giacconi, R.; Golub, L.; Gorenstein, P.; Haisch, B. M.; Harnden, F. R., Jr.; Johnson, H. M.; Linsky, J. L.; Maxson, C. W.; Mewe, R.; Rosner, R.; 20336.

Seward, F. D.; Vaiana, G. S.; Haisch, B. M.; Linsky, J. L.; Harnden, F. R., Jr.; Rosner, R.; 20124.

Seward, R. W.; SP260, 1981-83 Edition.

Seward, R. W.; Mavrodineanu, R.; SP260-71.

Shara, M. M.; Prialnik, D.; Shaviv, G.; 20272.

Sharma, S. K.; Wood, R. M.; Foley, J.; SP620: 1981 October. 248.255.

Sharp, B. H.; Kasper, P. K.; Montroll, M. L.,; NBS-GCR-80-250.

Shaver, J. R.; Filliben, J. J.; Simiu, E.; 20293.

Shaver, J. R.; Filliben, J. J.; Simiu, E.; 20764.

Shaviv, G.; Contini, M.; Kozlovsky, B. Z.; 20303.

Shaviv, G.; Hodson, S. W.; Cox, A. N.; 20337.

Shaviv, G.; Kovetz, A.; Prialnik, D.; 20695.

Shaviv, G.; Langer, S. H.; Chanmugam, G.; 20412.

Shaviv, G.; Livio, M.; 20410.

Shaviv, G.; Regev, O.; 20696.

Shaviv, G.; Shara, M. M.; Prialnik, D.; 20272.

Shaw, J.,; FIPS PUB 87.

Shelley, T. J.; Braman, R. S.; SP618: 1981 November. 134-145.

Shelton, C. B.; Moulton, J. R.,; NBS-GCR-81-324.

Shepard, R. L.; Blalock, T. V.; SP614: 1981 September. 260-268.

Shepherd, M. T.; Wilkinson, R.; SP601: 1981 January. 223.235.

Sherman, A.; Beale, W.; Gasser, M. G.; SP607: 1981 May. 103-115.

Sherwood, G. B.; SP612.

Shier, D. R.; 20021.

Shier, D. R.; 20355.

Shier, D. R.; Hoffman, K. L.; 20502.

Shier, D. R.; Witzgall, C.; 20022.

Shier, D. R.; Witzgall, C.; J. Res. 86(3): 317.330; 1981 May-June.

Shingleton, J. G.; Cassel, D. E.; Overton, R. L.,; NBS-GCR-81-302.

Shipp, W. E.; Rossiter, W. J., Jr.; NBSIR 81-2381.

Shiu, W. Y.; Mackay, D.; JPCRD 10(4): 1175-1199; 1981.

Shives, T. R.; Willard, W. A.,; SP622.

Shlesinger, M. F.; Hughes, B. D.; Montroll, E. W.; SP614: 1981 September. 18-21.

Short, M. A.; Bonfiglio, S.; SP604: 1981 June. 391-415.

Shorten, F. J.,; TN1142.

Shu, H. C.; Mehta, A.; Wunderlich, B.; Gaur, U.; JPCRD 10(1): 89. $118 ; 1981$.

Shukla, R. C.; MacDonald, R. A.; 20037.

Shull, R. D.; Swartzendruber, L. J.; Bennett, L. H.; Ives, L. K.; 20736.

Shumate, S. E. II; Parrott, J. R., Jr.; North, S. E.; Strandberg, G. W.; SP618: 1981 November. 274-286.

Shumka, A.; Haack, R. F.; SP400-69: 1981 May. 43-57.

Shy, J. T.; Farley, J. W.; Lamb, W. E., Jr.; Wing, W. H.; 20125.

Sieck, L. W.; 19965.

Siegman, B. C.; Nikoloff, I.; Candy, M.; Harwood, D.; Verveer, A.; Quinn, P. J.; Wilson, I.; Page, A. A.; Higson, P.; Deward, F. D.; Haisch, B.; Linsky, J. L.; Slee, O. B.; 20405.

Siegwarth, J. D.; 20770.

Siegwarth, J. D.; NBSIR 79-1600.
Siegwarth, J. D.; Brennan, J. A.; 20633.

Siegwarth, J. D.; LaBrecque, J. F.; TN1035.

Siegwarth, J. D.; LaBrecque, J. F.; TN1041.

Siemsen, K. J.; Petersen, F. R.; Wells, J. S.; Maki, A. G.; 20641

Sievers, R. E.; Conditt, M. K.; Stanley, J. S.; SP618: 1981 November. 93-104.

Siggaard-Andersen, O.; Maas, A. H. J.; Durst, R. A.; 20513.

Sikula, J.; Cermakova, A.; Cermak, M.; Vasina, P.; SP614: 1981 September. 125.129.

Sikula, J.; Sikulova, M.; Vasina, P.; Koktavy, B.; SP614: 1981 September. 100-104.

Sikula, J.; Vasina, P.; Koktavy, B.; Chobola, Z.; SP614: 1981 September. 248.250.

Sikulova, M.; Vasina, P.; Koktavy, B.; Sikula, J.; SP614: 1981 September. 100-104.

Silio, C. B., Jr.; Mink, A.; NBSIR 80-2187.

Silliman, K. E.; SP500-83: 1981 November. 231.238.

Silver, S.; SP618: 1981 November. 301.324.

Sim, S. P.; Merrett, R. P.; SP400-69: 1981 May. 94-107.

Simenauer, J. A.; Loftus, J. J.; Juarez, N.; Maldonado, A.; NBSIR 81 2247.

Simila, K. J.; SP599: 1981 January. 273-275.

Simila, K. J.; Kloss, C.; SP599: 1981 January. 54-63.

Simiu, E.; 20525.

Simiu, E.; 20524.

Simiu, E.; Filliben, J. J.; 20019.

Simiu, E.; Filliben, J. J.; 20295.

Simiu, E.; Shaver, J. R.; Filliben, J. J.; 20293.

Simiu, E.; Shaver, J. R.; Filliben, J. J.; 20764.

Simiu, E.; Yancey, C. W. C.; Cattaneo, L.; Harris, J. R.; Reinhold, T. A.; NBSIR 81-2199.

Simmons, J. A.; Clough, R. B.; 20673.

Simmons, J. A.; Clough, R. B.; 20701.

Simmons, J. A.; Eitzen, D. G.; Breckenridge, F. R.; Clough, R. B.; Fuller, E. R.; Hsu, N. N.; 20579.

Simmons, J. A.; Eitzen, D. G.; Breckenridge, F. R.; Clough, R. B.; Fuller, E. R.; Hsu, N. N.; 20757.

Simmons, J. D.; Maki, A. G.; Hougen, J. T.; 20080.

Simon, G. W.; November, L. J.; Toomre, J.; Gebbie, K. B.; 20422.

Simon, N. J.,; Reed, R. P.; NBSIR 81-1645.

Simon, T.; Linsky, J. L.; 20001.

Simon, T.; Linsky, J. L.; Schiffer, F. H.; 20423.

Simpson, J. P.; Geret, V.; Brown, S. A.; Merritt, K.; SP601: 1981 January. 395.422.

Simpson, P. A.; 20331.

Sindt, C. F.; 20017.

Sindt, C. F.; Ludtke, P. R.; 20018.

Singh, M.; Huth, G. C.; Iwanczyk., J. S.; Dabrowski, A. J.; SP604: 1981 June. 45.59.

Singhal, R.; Williamson, C. F.; Maruyama, X. K.; Petrovich, F.; Lindgren, R. A.; Plum, M. A.; Gerace, W. J.; Hicks, R. S.; Parker, B.; Peterson, G. A.; 20660.

Siu, C.; Branthover, B.; Veale, R.; NBSIR 80-2091.

Siu, M. C. I.; 20089.

Siu, M. C. I.; Bulik, C.; 20735.

Sjölin, L.; Wlodawer, A.; 20561.

Skillman, G. H.; Salazar, S. B.; Hardgrave, W. T.; Marriott, A. R.; NBSIR 80-2182.

Skinner, H.; Weinstein, A. M.; Clemow, A. J. T.; McPhillips-Meade, M.; Klawitter, J. J.; French, G.; SP601: 1981 January. 423-447.

Sleater, G. A.; Post, M. A.; Campbell, P. G.; NBSIR 80-2122.

Slee, O. B.; Siegman, B. C.; Nikoloff, I.; Candy, M.; Harwood, D.; Verveer, A.; Quinn, P. J.; Wilson, I.; Page, A. A.; Higson, P.; Deward, F. D.; Haisch, B.; Linsky, J. L.; 20405.

Sloter, L. E. II.; Piehler, H. R.; SP601: 1981 January. 655-666.

Smid, M. E.; 20420

Smirl, A. L.; Williams, W. E.; Van Stryland, E. W.; Soileau, M. J.; SP620: 1981 October. 375-384.

Smith, A. V.; Levenson, M. D.; Smith, S. J.; Nitz, D. E.; 20490.

Smith, B. F.; Manka, M. J.; Yeh, K. N.; 20487.

Smith, C. A., ; Peiser, H. S.; Gutterman, B. M.; Louis, G. L.; Pring, J. 
M.; Taylor, J. K.; Tholen, A. D.; NBSIR 80-2020.

Smith, D.,; Manola, F.; Smith, J.; NBS-GCR-81-337.

Smith, D. R.; Hust, J. G.; 20526.

Smith, D. R.; Hust, J. G.; NBSIR 81-1639.

Smith, D. R.; Hust, J. G.; Van Poolen, L. J.; 20547.

Smith, D. R.; Hust, J. G.; Van Poolen, L. J.; NBSIR 81-1640.

Smith, E. W; 20428.

Smith, E. W.; Talin, B.; Cooper, J.; 20479.

Smith, J.; Smith, D.,; Manola, F.; NBS-GCR-81-337.

Smith, K.; Wallace, W. E.; Hardman, K.; Rhyne, J. J.; 20214

Smith, L. E.; Chang, S. S.; McCrackin, F. L.; Senich, G. A.; Wang, F. W.; NBSIR 81-2264.

Smith, L. E.; Eby, R. K.; Broadhurst, M. G.; Cassel, J. M.; Fanconi, B. M.; Sanchez, I. C.; NBSIR 81-2263.

Smith, L. R.; Braun, E.; Meade, R. E.; NBSIR 81-2256.

Smith, R. H.; Fanconi, B. M.; DeVries, K. L.; 20443.

Smith, R. L.; Anderson, J. M.; Evans, P. M.; SP608: 1981 May. 113. !22.

Smith, R. W.; Hoffman, R. H.; Loshbough, R. P.; SPS00-80: 1981 October. 111-116.

Smith, S. J.; Nitz, D. E.; Smith, A. V.; Levenson, M. D.; 20490.

Smith, S. J.; Walther, H.; Hellmuth, T.; Leuchs, G.; 20504.

Smith, S. J.; Walther, H.; Hellmuth, T.; Leuchs, G.; 20506.

Smith, W. L.; Deaton, T. F.; Rainer, F.; Milam, D.; SP620: 1981 October. 297-299.

Smyth, K. C.; Mallard, W. G.; 20466.

Snellgrove, R. A.; Bethin, J. R.; Chiang, C. K.; Franklin, A. D.; 20497.

Snow, A. P.; SP500-83: 1981 November. 271-276.

Snyder, L. E.; Lovas, F. J.; Ulich, B. L.; Hollis, J. M.; 19987.

Snyder, L. E.; Suenram, R. D.; Lovas, F. J.; Hollis, J. M.; 20077.

Snyder, R. G.; Scherer, J. R.; Peterlin, A.; 20315.

Snyder, R. L.; Hubbard, C. R.; Panagiotopoulos, N. C.; NBSIR 812229.

Sockut, G. H.; 20172.

Sodini, D.; Rigaud, D.; SP614: 1981 September. 63-66.

Soileau, M. J.; SP620: 1981 October. 300.304.

Soileau, M. J.; Franck, J. B.; Veatch, T. C.; SP620: 1981 October. 385-393.

Soileau, M. J.; Porteus, J. O.; Decker, D. L.; Seitel, S. C.; SP620: 1981 October. 201-209.

Soileau, M. J.; Smirl, A. L.; Williams, W. E.; Van Stryland, E. W.; SP620: 1981 October. 375-384.

Soileau, M. J.; Temple, P. A.; SP620: 1981 October. 180-189.

Solomon, J. M.; Ciment, M.; Berger, A. E.; 20626.

Sonderman, J.; Stokowski, S.; Wallerstein, P.; Walmer, D.; Thomas, N.; SP620: 1981 October. 159-169.

Sorrells, J. R.; Beine, W. B.; NBSIR 80-2106.

Souders, T. M.; Flach, D. R.; Bell, B. A.; TN1145.

Souders, T. M.; Lechner, J. A.; 20094.

Soulen, R. J., Jr.; Hall, J. T.; Holdeman, L. B.; 19979.

Soulen, R. J., Jr.; Holdeman, L. B.; Hall, J. T.; VanVechten, D.; 20723.

Soulen, R. J., Jr.; Van Vechten, D.; Costabile, G.; Jach, T.; Holdeman, L. B.; 20702.

Soulen, R. J., Jr.,; Meijer, P. H. E.; Mountain, R. D.; SP614.

Spal, R. D.; Kahn, A. H.; SP589: 1981 January. 137-141.

Sparks, L. L.; 20074.

Sparks, L. L.; NBSIR 81-1651.

Sparks, L. L.; Arvidson, J. M.; NBSIR 81-1654.

Spath, J.; Buchholz, A.; Wrzenski, S. C.,; Egan, J.; Higgins, R.; NBSGCR-81-313.

Speck, K. M.; Fraker, A. C.; 19988.

Spence, D.; 20156.

Spence, D.; 20249.

Spence, D.; Dillon, M. A.; 20178.

Spicer, W. E.; Taylor, N. J.; Schwarz, S. A.; Helms, C. R.; SP400-67.

Spicer, W. E.; Taylor, N. J.,; Helms, C. R.; Schwarz, S. A.; NBSGCR $-81-345$.

Spiegel, V.; Eisenhauer, C. M.; Grundl, J. A.; Gilliam, D. M.; McGarry, E. D.; 20153.
Spiegelman, C. H.; NBSIR 81-2222.

Spiegelman, C. H.; Lechner, J. A.; Reeve, C. P.; NBSIR 80-2151.

Spielvogel, L. G.; May, W. B., Jr.,; 20512.

Sprung, J. G.; SP500-83: 1981 November. 329.

Sreenivasiah, I.; Chang, D. C.; Ma, M. T.; 20623.

Sreenivasiah, I.; Chang, D. C.; Ma, M. T.; TN1040.

Staehle, R. W.; Ugiansky, G. M.; Kruger, J.; 20026.

Stahl, F. 1.; Turner, G. E.; Ventre, F. T.; NBSIR 81-2361.

Stalick, J. K.; Mighell, A. D.; Hubbard, C. R.; TN1141.

Stanley, J. S.; Sievers, R. E.; Conditt, M. K.; SP618: 1981 November. 93.104.

Stansbury, J. W.; Antonucci, J. M.; Brauer, G. M.; 20401.

Staples, B. R.; JPCRD 10(3): 767.778; 1981.

Staples, B. R.; JPCRD 10(3): 779.798; 1981.

Staples, B. R.; Wobbeking, T. F.; NBSIR 81-2276.

Statham, P. J.; SP604: 1981 June. 141-164.

Statham, P. J.; SP604: 1981 June. 127-139.

Statler, J. A.; Thornton, D. D.; Magin, R. L.; Mangum, B. W.; J. Res. 86(2): 181-192; 1981 March-April.

Steckler, K.; McCaffrey, B.; Quintiere, J.; 20740.

Steel, J. S.; NBSIR 81-2415.

Stegun, I. A.; Zucker, R.; J. Res. 86(6): 661-686; 1981 NovemberDecember.

Stein, M.; Aisenberg, S.; SP620: 1981 October. 313.323.

Stenbakken, G. N.; Kibalo, T. H.; Turgel, R. S.; Oldham, N. M.; TNI144.

Stencel, R. E.; Linsky, J. L.; Brown, A.; Jordan, C.; Carpenter, K. G.; Wing, R. F.; Czyzak, S.; 20598.

Stencel, R. E.; Mullan, D. J.; Basri, G. S.; Linsky, J. L.; 20418.

Stencel, R. E.; Mullan, D. J.; Linsky, J. L.; Basri, G. S.; Worden, S. P.; 20057.

Stepanescu, A.; Celasco, M.; Mazzetti, P.; SP614: 1981 September. 34 37.

Stephens, R. D.; SP618: 1981 November. 1.8.

Stephenson, J. C.; Akins, D. L.; King, D. S.; 20095.

Stephenson, J. C.; King, D. S.; 20101.

Stevens, W. J.; Julienne, P. S.; Konowalow, D. D.; Krauss, M.; Rosenkrantz, M. E.; 20100.

Stevens, W. J.; Karo, A. M.; Hiskes, J. R.; 20229.

Stevens, W. J.; Krauss, M.; 20194.

Stevens, W. J.; Krauss, M.; Basch, H.; 20196.

Stevens, W. J.; Krauss, M.; Konowalow, D. D.; Rosenkrantz, M. E.; 20082.

Stevens, W. J.; Krauss, M.; Mies, F. H.; 20086.

Stevens, W. J.; Neumann, D. B.; Krauss, M.; 20102.

Stevenson, D. A.; Dun, H.; Mattes, B. L.; SP400-69: 1981 May. 143. 152.

Steyn-Ross, M. L.; Walls, D. F.; Zoller, P.; 20179.

Stochino, G.; D'Amico, A.; Cappuccio, G.; Petrocco, G.; Fotinos, C.; SP614: 1981 September. 199-201.

Stockbauer, R.; Codling, K.; Dehmer, J. L.; Parr, A. C.; Ederer, D. L.; Cole, B. E.; West, J. B.; 20123.

Stockbauer, R.; Dehmer, J. L.; West, J. B.; Codling, K.; Ederer, D. L.; Parr, A. C.; Cole, B. E.; 20676.

Stockbauer, R.; Dehmer, J. L.; West, J. B.; Codling, K.; Parr, A. C.; Ederer, D. L.; Cole, B. E.; 20426.

Stockbauer, R.; Flodström, S. A.; van der Veen, J. F.; Himpsel, F. J.; Eastman, D. E.; Madey, T. E.; 20510.

Stockbauer, R.; McCulloh, K. E.; Parr, A. C.; Jason, A. J.; 20051.

Stockbauer, R.; Parr, A. C.; Rosenstock, H. M.; 20199.

Stockbauer, R.; Parr, A. C.; Rosenstock, H. M.; 20472.

Stockbauer, R.; Poliakoff, E. D.; Dehmer, P. M.; Dehmer, J. L.; 20533.

Stockbauer, R.; West, J. B.; Cole, B. E.; Dehmer, J. L.; Codling, K.; Parr, A. C.; Ederer, D. L.; 20399.

Stockbauer, R. L.; Codling, K.; West, J. B.; Parr, A. C.; Dehmer, J. L.; 20052.

Stockbauer, R. L.; West, J. B.; Dehmer, J. L.; Parr, A. C.; Rakowsky, G.; Ederer, D. L.; 20236.

Stockton, R. A.; Wheeler, A. E.; Zingaro, R. A.; Irgolic, K. J.; Banks, C. H.; Bottino, N. R.; Chakraborti, D.; Gennity, J. M.; Hillman, D. 
C.; O'Brien, D. H.; Pyles, R. A.; SP618: 1981 November. 244.263.

Stokowski, S.; Wallerstein, P.; Walmer, D.; Thomas, N.; Sonderman, J.; SP620: 1981 October. 159-169.

Stone, R.; Blackburn, D. H.; Pella, P. A.; Walter, F. J.; SP604: 1981 June. 61-70.

Storm, L.; Klempt, G.; SP614: 1981 September. 269-271.

Strandberg, G. W.; Shumate, S. E. II; Parrott, J. R., Jr.; North, S. E.; SP618: 1981 November. 274-286.

Straty, G. C.; Diller, D. E.; 20139.

Straty, G. C.; Diller, D. E.; 20278.

Streed, E.; Seiler, J.; Waksman, D.; TN1136.

Streed, E.; Waksman, D.; TN1140.

Streed, E. R.; Waksman, D.; 20291.

Strelich, T.; Resnicow, M.; Ahola, R.; Hug, R.; SP500-80: 1981 October. 122-127.

Strobridge, T. R.; Moulder, J. C.; Clark, A. F.; 20436.

Stromecky, P.,; Geer, N.; Graff, H. A.; NBS.GCR-81-336.

Stubenrauch, C. F.; Repjar, A. G.; Newell, A. C.; Estin, A. J.; 20650.

Stubenrauch, C. F.; Yaghjian, A. D.; NBSIR 80-1630.

Stucki, L. G.; SP500-80: 1981 October. 96-102.

Stucki, L. G.; SP500-80: 1981 October. 88-95.

Studer, P. A.; Gasser, M. G.; SP607: 1981 May. 146-153.

Sud, I.; Alereza, T.; Kusuda, T.; 20756.

Sudarshan, T. S.; Jones, T. B.; Hebner, R. E., Jr.; Kelley, E. F.; Thompson, J. E.; NBSIR 81-2275.

Suenram, R. D.; Lattimer, R. P.; Hillig, K. W.; Ashe, A. J.; Kuczkowski, R. L.; Lovas, F. J.; 20168.

Suenram, R. D.; Lovas, F. J.; 20318.

Suenram, R. D.; Lovas, F. J.; Hollis, J. M.; Snyder, L. E.; 20077.

Suenram, R. D.; Lovas, F. J.; Kuczkowski, R. L.; 20319.

Suenram, R. D.; Quade, C. R.; 20084.

Suenram, R. D.; Schäfer, L.; Sellers, H. L.; Lovas, F. J.; 20005.

Sugar, J.; Corliss, C.; JPCRD 10(1): 197.290; 1981.

Sugar, J.; Corliss, C.; JPCRD 10(4): 1097-1174; 1981.

Sugar, J.; Wyart, J. F.; Kaufman, V.; 20002.

Sugar, J.; Wyart, J. F.; Kaufman, V.; 20611.

Sugar, J.; Younger, S. M.; Lucatorto, T. B.; McIlrath, T. J.; 20684.

Sugawa, O.; Yamamoto, H.; Mulholland, G.; Handa, T.; 20565.

Suh, C. H.; van der Ziel, A.; Jindal, R. P.; SP614: 1981 September. 236-239.

Sukle, D. J.; Wells, J. S.; Petersen, F. R.; Maki, A. G.; 20427

Sukle, D. J.; Wells, J. S.; Petersen, F. R.; Maki, A. G.; 20640.

Sullivan, D. B.; Hamilton, C. A.; Kautz, R. L.; 20140.

Sullivan, D. B.; Kautz, R. L.; Hobbs, R. D.; Zimmerman, J. E.; SP607: 1981 May. 173-177.

Sullivan, D. B.; McCarthy, S. E.,; Zimmerman, J. E.; SP607.

Sullivan, D. B.; Zimmerman, J. E.; U.S. Patent 4,281,517.

Sullivan, D. B.; Zimmerman, J. E.; Ives, J. T.; SP607: 1981 May. 186. 194.

Superczynski, M. J.; Ekin, J. W.; Schramm, R. E.; 20297.

Sutter, H.; Birnbaum, G.; 20133.

Swaffield, J. A.; NBSIR 81-2290.

Swaffield, J. A.; NBSIR 81-2307.

Swaffield, J. A.; NBSIR 81-2308.

Swaffield, J. A.; NBSIR 81-2367.

Swankin, D. A.,; NBS-GCR-81-347.

Swartzendruber, L.; Boettinger, W.; Ives, L.; Coriell, S.; Ballard, D.; Laughlin, D.; Clough, R.; Biancaniello, F.; Blau, P.; Cahn, J.;

Mehrabian, R.; Free, G.; Berger, H.; Mordfin, L.; NBSIR 80-2069.

Swartzendruber, L. J.; 20292.

Swartzendruber, L. J.; Bennett, L. H.; Ives, L. K.; Shull, R. D.; 20736.

Swartzendruber, L. J.; Reno, R. C.; Bennett, L. H.; 20208.

Swartzendruber, L. J.; Romanov, V. P.; Candela, G. A.; Roth, R. S.; 20043.

Sweger, D. M.; Sams, R. L.; 20461.

Swenson, D. A.; Tallerico, P. J.; Penner, S.; Cutler, R. I.; Debenham, P. H.; Lindstrom, E. R.; Mohr, D. L.; Wilson, M. A. D.; Yoder, N. R.; Young, L. M.; Boyd, T. J.; Knapp, E. A.; Martin, R. E.; Potter, J. M.; Schneider, C. M.; 20206.

Swinney, H. L.; Roux, J. C.; King, G. P.; SP614: 1981 September.
283.287.

Swyt, C. R.; Fiori, C. E.; SP604: 1981 June. 417.439.

Swyt, D.; Jensen, S.; 20201.

Swyt, D. A.; Lettieri, T. R.; Jenkins, W. D.; 20683.

\section{$\mathbf{T}$}

Taber, R. C.; Giffard, R. P.; Michelson, P. F.; SP614: 1981 September. 292-297.

Tachibana, K.; Phelps, A. V.; 20597.

Tachikawa, T.; Ekin, J. W.; Sekine, H.; 20659.

Taggart, H. E.; SP480-44.

Takagi, S.; Waerstad, K. R.; Frazier, A. W.; Mathew, M.; 20258.

Talin, B.; Cooper, J.; Smith, E. W.; 20479.

Tallerico, P. J.; Penner, S.; Cutler, R. I.; Debenham, P. H.; Lindstrom, E. R.; Mohr, D. L.; Wilson, M. A. D.; Yoder, N. R.; Young, L. M.; Boyd, T. J.; Knapp, E. A.; Martin, R. E.; Potter, J. M.; Schneider, C. M.; Swenson, D. A.; 20206.

Tanabe, M.; Mimaki, T.; Sato, H.; SP614: 1981 September. 30-33.

Tanaka, Y.; Okabe, H.; Kawasaki, M.; 20042.

Tantrarongroj, K.; Graffeuil, J.; Sautereau, J. F.; SP614: 1981 September. 84-87.

Tauber, T.; SP622: 1981 October. 33-49.

Taussig, L. M.; SP601: 1981 January. 201-222.

Taylor, B. N.; 20058.

Taylor, J. K.; Kratochvil, B. G.; 20387.

Taylor, J. K.; Tholen, A. D.; Smith, C. A., ; Peiser, H. S.; Gutterman, B. M.; Louis, G. L.; Pring, J. M.; NBSIR 80-2020.

Taylor, L. S.; SP625.

Taylor, N. J.; Schwarz, S. A.; Helms, C. R.; Spicer, W. E.; SP400-67.

Taylor, N. J.,; Helms, C. R.; Schwarz, S. A.; Spicer, W. E.; NBSGCR $-81-345$.

Teague, E. C.; Vorburger, T. V.; Maystre, D.; 20555.

Teeters, W.; Williamson, C. F.; Kowalski, S.; Maruyama, X. K.; Lindgren, R. A.; Flanz, J. B.; Hicks, R. S.; Parker, B.; Peterson, G. A.; Lawson, R. D.; 20323.

Teichroew, D.; Callender, E.; Sayani, H. H.; SP500-80: 1981 October. 38-43.

Temple, P. A.; Soileau, M. J.; SP620: 1981 October. 180-189.

Terry, D. L.; Glickman, D. S.; SP608: 1981 May. 123-132.

Tesche, C. D.; SP614: 1981 September. 369-372.

Tesk, J. A.; Brauer, G. M.; Antonucci, J. M.; Wu, W.; McKinney, J. E.; Whitlock, R. P.; Hinman, R.; Cassel, J. M.; NBSIR 80-2168.

Tesk, J. A.; Parry, E. E.; Argentar, H.; 20221.

Tesk, J. A.; Whitlock, R. P.; Widera, G. E. O.; Holmes, A.; Parry, E. E.; 20277.

Tesk, J. A.; Widera, G. E. O.; Holmes, A.; Parry, E. E.; Whitlock, R. P.; 20241.

Tewari, Y. B.; Miller, M. M.; Martire, D. E.; Wasik, S. P.; NBSIR $81-2406$

Tewarson, A.,; NBS-GCR-80-295.

Thall, R.; Anderson, G.; SP500-80: 1981 October. 197.199.

Thijsse, B. J.; 20225.

Tholen, A. D.; Smith, C. A., ; Peiser, H. S.; Gutterman, B. M.; Louis, G. L.; Pring, J. M.; Taylor, J. K.; NBSIR 80-2020.

Thomas, N.; Sonderman, J.; Stokowski, S.; Wallerstein, P.; Walmer, D.; SP620: 1981 October. 159-169.

Thomas, R. W.; SP400-69: 1981 May. 179-186.

Thomas, S. J.; Phipps, C. R., Jr.; Harrison, R. F.; SP620: 1981 October. 238-247.

Thomas, W. C.; Fanney, A. H.; 20648.

Thomas, W. C.,; NBS-GCR-80-254.

Thompson, B. E.; Chapman, R. E.; NBSIR 80-2150.

Thompson, B. E.; Chapman, R. E.; NBSIR 81-2250.

Thompson, B. E.; Lippiatt, B. C.; Weber, S. F.; NBSIR 81-2304.

Thompson, D.; Erickson, R.; Gerhart, S.; Lee, S.; SP500-80: 1981 October. 69.75.

Thompson, J. E.; Sudarshan, T. S.; Jones, T. B.; Hebner, R. E., Jr.; Kelley, E. F.; NBSIR 81-2275.

Thornton, D. D.; Magin, R. L.; Mangum, B. W.; Statler, J. A.; J. Res. 86(2): 181-192; 1981 March-A pril. 
Thornton, J. I.; Kraus, S.; Lerner, B.; Hendrickson, B.; SP480-40. Thurber, W. R.; Larrabee, R. C.; Phillips, W. E.; NBSIR 81-2325. Thurber, W. R.; Mattis, R. L.; Liu, Y. M.; Filliben, J. J.; SP400-64.

Tibbetts, G. G.; Lindau, I.; Hecht, M. H.; Egelhoff, W. F., Jr.; 20478.

Tibbott, R. L.; Collins, B. L.; Danner, W. F.; NBSIR 81-2428.

Tibbott, R. L.; Elder, J.; BSS130.

Tibbott, R. L.; Yonemura, G. T.; Hunt, C. M.; Chen, P. T.; Hill, J. E.; May, W. B., Jr.; Richtmyer, T. E.; Elder, J.; BSS133.

Tighe, N. J.; Fuller, E. R., Jr.; Fields, R. J.; NBSIR 79-1960 (DoE).

Tilford, C. R.; McCulloh, K. E.; 20592.

Timmerhaus, K. D.; Flynn, T. M.; 20068.

Timmerhaus, K. D.; Flynn, T. M.; 20563.

Timpane, K.,; Mulkey, M.; NBS-GCR-ETIP 81-97.

Tippet, J. C.; Chang, D. C.; NBSIR 78-873.

Titus, J. L.; Lawrie, G. M.; Schoen, F. J.; SP601: 1981 January. 269. 282.

Tobin, M. S.; Ritter, K, J.; Daley, T. W.; Lafferty, W. J.; Sattler, J. P.; Worchesky, T. L.; 20090.

Tobler, R. L.; 20703.

Tobler, R. L.; NBSIR 79-1624.

Tobler, R. L.; Elmer, J. W.; McHenry, H. I.; Yushchenko, K. A.; Paton, E. O.; Reed, R. P.; 20772.

Tobler, R. L.; Reed, R. P.; Burkhalter, D. S.; 20742.

Todd, T. R.; Olson, W. B.; 20088.

Tomb, T.; Ku, H.; Cameron, J.; Parobeck, P.; 20566.

Tomkins, R. P. T.; Janz, G. J.; NSRDS-NBS61, Part IV.

Toner, S. D.; Wakamiya, S. K.; NBSIR 80-2035.

Tonik, A. B.; Lin, W. K.; SP500-83: 1981 November. 259-265.

Toomre, J.; Gebbie, K. B.; Simon, G. W.; November, L. J.; 20422.

Topka, K.; Zwaan, C.; Vaiana, G. S.; Cassinelli, J. P.; Fabbiano, G.; Giacconi, R.; Golub, L.; Gorenstein, P.; Haisch, B. M.; Harnden, F. R., Jr.; Johnson, H. M.; Linsky, J. L.; Maxson, C. W.; Mewe, R.; Rosner, R.; Seward, F.; 20336.

Toste, A. P.; Hayes, D. J.; Olsen, K. B.; Wilkerson, C. L.; SP618: 1981 November. 105.119.

Toth, E. E.; Antonucci, J. M.; Peckoo, R. J.; Schruhl, C.; 20406.

Touboul, A.; Walma, A. A.; Lecoy, G.; SP614: 1981 September. 121. 124.

Trant, B. S.; SP608: 1981 May. 167.172.

Trask, R. P.; SP599: 1981 January. 127-137.

Traub, R. D.; Rains, T. C.; Garruto, R. M.; Gajdusek, D. C.; Gibbs, C. J., Jr.; 20603.

Travis, J. C.; DeVoe, J. R.; 20454.

Travis, J. C.; DeVoe, J. R.; Walters, J. P.; Scheeline, A.; 20462.

Travis, J. C.; DeVoe, J. R.; Walters, J. P.; Scheeline, A.; Norris, J. A.; 20453.

Travis, J. P.; Clough, R. B.; Chang, J. C.; 20276.

Treado, S.; Kusuda, T.; NBSIR 80-2147.

Treado, S.; Kusuda, T.; TN1148.

Treado, S. J.; Hunt, C. M.; 20605.

Trechsel, H. R.; Launey, S. J.; SP606.

Trehan, R.; Conti, D. M.; Giese, K. W.; Kyriazi, C. N.; SP500-83: 1981 November. 311-312.

Tremaine, S. G.; Lentner, K. J.; Childers, C. B.; 20093.

Tremaine, S. G.; Molino, J. A.; Zerdy, G. A.; 20029.

Troland, T. H.; Heiles, C. E.; Clark, F. O.; Johnson, D. R.; 20439.

Trombka, J. I.; Schmadebeck, R. L.; Seltzer, S. M.; Bielefeld, M. J.; Yin, L. I.; 20569.

Trossi, L.; Valabrega, P. T.; Oldano, C.; Miraldi, E.; SP614: 1981 September. 339.342.

Tryon, P. V.; TN1036.

Tsao, A. K.; Bur, A. J.; 20529.

Tsuwa, H.; Namba, Y.; SP620: 1981 October. 171-179.

Tucker, J. R.; SP614: 1981 September. 349-354.

Tung, M. S.; Brown, W. E.; Mathew, M.; 20407.

Tung, M. S.; Chow, L. C.; Brown, W. E.; 20122.

Tung, R. T.; Graham, W. R.; Melmed, A. J.; Ceyer, S. T.; 20686.

Turgel, R. S.; Oldham, N. M.; Stenbakken, G. N.; Kibalo, T. H.; TN1144.

Turk, G. C.; 20468.

Turner, G.; Collins, B.; NBSIR 81-2310.
Turner, G. E.; Ventre, F. T.; Stahl, F. I.; NBSIR 81-2361.

Turrell, B. G.; Marshak, H.; 20520.

Tward, E.; SP607: 1981 May. 178-185.

Tzoar, N.; Bendow, B.; Gianino, P. D.; SP620: 1981 October. 453. 458.

$\mathbf{U}$

Uden, P. C.; SP618: 1981 November. 184-196.

Uebersfeld, J.; Handel, P. H.; Gagnepain, J. J.; SP614: 1981 September. 189-191.

Ugiansky, G. M.; Johnson, C. E.; 20027.

Ugiansky, G. M.; Johnson, C. E.; NBSIR 81-2191 (DoE).

Ugiansky, G. M.; Kruger, J.; Staehle, R. W.; 20026.

Ulich, B. L.; Hollis, J. M.; Snyder, L. E.; Lovas, F. J.; 19987.

Ulrich, B. T.; SP614: 1981 September. 253-259.

Ungar, E.,; NBS-GCR-80-248.

Unguris, J.; Celotta, R. J.; Pierce, D. T.; Girvin, S. M.; 20694.

Urban, E. W.; Katz, L.; Hendricks, J. B.; Karr, G. R.; SP607: 1981

May. 117-127.

Uriano, G. A.; 19990.

Uriano, G. A.; Rasberry, S. D.; 20326.

\section{V}

Vaiana, G. S.; Ayres, T.; Linsky, J. L.; Pallavicini, R.; Golub, L.; Rosner, R.; 20594

Vaiana, G. S.; Cassinelli, J. P.; Fabbiano, G.; Giacconi, R.; Golub, L.; Gorenstein, P.; Haisch, B. M.; Harnden, F. R., Jr.; Johnson, H. M.; Linsky, J. L.; Maxson, C. W.; Mewe, R.; Rosner, R.; Seward, F.; Topka, K.; Zwaan, C.; 20336.

Vaiana, G. S.; Golub, L.; Rosner, R.; Ayres, T. R.; Linsky, J. L.; 20627.

Vaiana, G. S.; Haisch, B. M.; Linsky, J. L.; Harnden, F. R., Jr.; Rosner, R.; Seward, F. D.; 20124.

Vaidyanathan, A.; Guenther, A. H.; Judell, N.; Mitra, S. S.; SP620: 1981 October. 446-452.

Vaidyanathan, A.; Guenther, A. H.; Mitra, S. S.; SP620: 1981 October. 432.437.

Vaidyanathan, A.; Guenther, A. H.; Mitra, S. S.; SP620: 1981 October. 438-445.

Vaishnav, M. P.; NBSIR 81-2203.

Vaissiere, J. C.; Gasquet, D.; Nougier, J. P.; SP614: 1981 September. 42-46.

Vaissiere, J. C.; Nougier, J. P.; Gasquet, D.; SP614: 1981 September. 305-308.

Valabrega, P. T.; Oldano, C.; Miraldi, E.; Trossi, L.; SP614: 1981 September. 339-342.

Van Blerkom, J.; Beaty, E. C.; Rumble, J. R., Jr.; Gallagher, J. W.; SP593.

Van Brunt, R. J.; Gallagher, A. C.; Kennerly, R. E.; 20244.

Van Brunt, R. J.; Leep, D.; 20704.

Van Brunt, R. J.; Misakian, M.; Leep, D. A.; Moy, K. J.; Beaty, E. C.; NBSIR 81-2242.

Vandamme, L. K. J.; Dik, L. S. H.; SP614: 1981 September. 244-247.

Van Degrift, C. T.; Love, D. P.; 20340.

van den Berg, R. J.; de Vos, A.; de Goede, J.; SP614: 1981 September. 217.220.

VanderBrug, G. J.; Barbera, A. J.; Fitzgerald, M. L.; Wheatley, T. E.; Albus, J. S.; SP602.

VanderHart, D. L.; Zapas, I. J.; Eby, R. K.; Broadhurst, M. G.; Chang, S. S.; Davis, G. T.; DiMarzio, E. A.; Fanconi, B. M.; Han, C. C.; Khoury, F. A.; Markovitz, H.; McKinney, J. E.; Mopsik, F. I.; Passaglia, E.; Sanchez, I. C.; 20749.

Van der Klott, R.; Senturia, S. D.; Huberman, M. G.; SP400-69: 1981 May. 108.114.

van der Meulen, J. P.; Zijlstra, R. J. J.; van Kooten, J. J.; SP614: 1981 September. 343-345.

van der Veen, J. F.; Himpsel, F. J.; Eastman, D. E.; Madey, T. E.; Stockbauer, R.; Flodström, S. A.; 20510. 
van der Ziel, A.; SP614: 1981 September. 88-92.

van der Ziel, A.; SP614: 1981 September. 364.368.

van der Ziel, A.; Jindal, R. P.; SP614: 1981 September. 173-177.

van der Ziel, A.; Jindal, R. P.; Suh, C. H.; SP614: 1981 September. 236-239.

van der Ziel, A.; Park, H. S.; SP614: 1981 September. 226-229.

van der Ziel, A.; Rucker, L. M.; Amberiadis, K.; SP614: 1981 September. 210-212.

van der Ziel, A.; Zijlstra, R. J. J.; Hiatt, C. F.; SP614: 1981 September. 234-235.

van Harlingen, D. J.; Clarke, J.; Koch, R. H.; SP614: 1981 September. 359-363.

van Kooten, J. J.; van der Meulen, J. P.; Zijlstra, R. J. J.; SP614: 1981 September. 343-345.

Van Poolen, L. J.; NBSIR 80-1631.

Van Poolen, L. J.; Smith, D. R.; Hust, J. G.; 20547.

Van Poolen, L. J.; Smith, D. R.; Hust, J. G.; NBSIR 81-1640.

van Rheenen, A.; Bosman, G.; Zijlstra, R. J. J.; SP614: 1981 September. 409-413.

Van Stryland, E. W.; Soileau, M. J.; Smirl, A. L.; Williams, W. E.; SP620: 1981 October. 375-384.

Van Stryland, E. W.; Woodall, M. A.; SP620: 1981 October. 50.57.

Van Vechten, D.; Costabile, G.; Jach, T.; Holdeman, L. B.; Soulen, R. J., Jr.; 20702.

Van Vechten, D.; Peterson, R. L.; 20692.

VanVechten, D.; Soulen, R. J., Jr.; Holdeman, L. B.; Hall, J. T.; 20723.

van Vliet, K. M.; SP614: 1981 September. 3.11.

van Vliet, K. M.; Chenette, E. R.; Handel, P. H.; Kilmer, J.; SP614: 1981 September. 151.

van Vliet, K. M.; Gong, J.; SP614: 1981 September. 113-117.

van Vliet, K. M.; Handel, P. H.; Wolf, D.; SP614: 1981 September. 196-198.

van Vliet, K. M.; Mehta, H.; SP614: 1981 September. 79.83.

Vasconcellos, E. C. C.; Petersen, F. R.; Evenson, K. M.; 20743.

Vasina, P.; Koktavy, B.; Chobola, Z.; Sikula, J.; SP614: 1981 September. 248.250.

Vasina, P.; Koktavy, B.; Sikula, J.; Sikulova, M.; SP614: 1981 September. 100-104.

Vasina, P.; Sikula, J.; Cermakova, A.; Cermak, M.; SP614: 1981 September. 125.129.

Vastagh, S. A.; SP599: 1981 January. 45-53.

Veale, R.; Siu, C.; Branthover, B.; NBSIR 80-2091.

Veale, R. C.; Haight, W. C.; Hocken, R. J.; Borchardt, B. R.; Carroll, C. L.; Hartsock, R. G.; Reeve, C. P.; Scire, F. E.; NBSIR 80-2141.

Veatch, T. C.; Soileau, M. J.; Franck, J. B.; SP620: 1981 October. 385.393.

Velapoldi, R. A.; 20444

Veneruso, A. F.; Chang, H. T.; SP622: 1981 October. 310-320.

Ventre, F. T.; Stahl, F. I.; Turner, G. E.; NBSIR 81-2361.

Verveer, A.; Quinn, P. J.; Wilson, I.; Page, A. A.; Higson, P.; Deward, F. D.; Haisch, B.; Linsky, J. L.; Slee, O. B.; Siegman, B. C.; Nikoloff, I.; Candy, M.; Harwood, D.; 20405.

Vest, C. M.,; NBS-GCR-81-318.

Vickers, M. V.,; FIPS PUB 29-1.

Viktoravicius, V.; Bareikis, V.; Galdikas, A.; Miliusyte, R.; SP614: 1981 September. 406-408.

Vincent, C. H.; SP599: 1981 January. 263-270.

Vincent, C. H.; SP599: 1981 January. 180-201.

Vincent, C. H.; SP599: 1981 January. 1-5.

Vincent, D. R.; SP500-83: 1981 November. 123.138.

Vincent, R. J.; Benson, G. M.; SP607: 1981 May. 116.

Vogel, G. L.; Popp, R. K.; Frantz, J. D.; 20467.

Vogt, C.; Yates, D.; Manahan, S. E.; Fraley, D. M.; SP618: 1981 November. 81.92.

von Mayrhauser, A. K.; Haftka, R. T.; SP500-83: 1981 November. 199-215.

Vora, H.; Ready, J. F.; SP620: 1981 October. 110.116.

Vorburger, T. V.; Maystre, D.; Teague, E. C.; 20555.

Voss, R. F.; Horn, P. M.; Knoedler, C. M.; SP614: 1981 September. 391.394.
Voss, R. F.; Krusin-Elbaum, L.; SP614: 1981 September. 213-216.

Voth, R. O.; 20771.

Voth, R. O.; NBSIR 78-875.

Voth, R. O.; Radebaugh, R.; Linenberger, D.; 20766.

Voth, R. O.; Radebaugh, R.; Linenberger, D.; SP607: 1981 May. 70. 81.

Vreeland, R. G.,; Waller, M. B.; NBS-GCR-81-315.

\section{$\mathbf{W}$}

Wachtman, J. B., Jr.; Hoffman, J. D.; NBSIR 81-2408.

Wachtman, J. B., Jr.; Reneker, D. H.; NBSIR 80-2108.

Wachtman, J. B., Jr.,; NBSIR 81-2305.

Wade, L. A.; SP607: 1981 May. 164.172.

Waerstad, K. R.; Frazier, A. W.; Mathew, M.; Takagi, S.; 20258.

Wagman, D. D.; Evans, W. H.; Parker, V. B.; Schumm, R. H.; Nuttall, R. L.; TN270-8.

Waidelich, D. L.; SP589: 1981 January. 107.111.

Wakamiya, S. K.; Toner, S. D.; NBSIR 80-2035.

Wakefield, J. P.; NBSIR 81-1641.

Waksman, D.; 20705.

Waksman, D.; Streed, E.; TN1140.

Waksman, D.; Streed, E.; Seiler, J.; TN1136.

Waksman, D.; Streed, E. R.; 20291.

Walker, G. R.; Marshall, R. D.; 20059.

Walker, M. S.; Zeitlin, B. A.; Ekin, J. W.; Ho, J. C.; Oberly, C. E.; Garrett, H. J.; 20741.

Walker, T. W.; Guenther, A. H.; Wiggins, T. A.; SP620: 1981 October. 277.286.

Wallace, M. A.; McCoy, W. H.; Colella, R. P.; 20662.

Wallace, T.; Mc Wethy, S.; Moy, S.; Nielsen, G.; Sanchez, R.; SP50080: 1981 October. 103-110.

Wallace, W. E.; Hardman, K.; Rhyne, J. J.; Smith, K.; 20214.

Waller, M. B.; Vreeland, R. G.,; NBS-GCR-81-315.

Wallerstein, P.; Walmer, D.; Thomas, N.; Sonderman, J.; Stokowski, S.; SP620: 1981 October. 159-169.

Walls, D. F.; Drummond, P. D.; Gardiner, C. W.; 20537.

Walls, D. F.; Kunasz, C. V.; Drummond, P. D.; Zoller, P.; 20595.

Walls, D. F.; Reid, M.; McNeil, K. J.; 20697.

Walls, D. F.; Zoller, P.; Steyn-Ross, M. L.; 20179.

Walma, A. A.; SP614: 1981 September. 54-56.

Walma, A. A.; SP614: 1981 September. 51.53.

Walma, A. A.; Lecoy, G.; Touboul, A.; SP614: 1981 September. 121. 124.

Walma, A. A.; Maille, C.; Lecoy, G.; Alabedra, R.; SP614: 1981 September. 105-108.

Walmer, D.; Thomas, N.; Sonderman, J.; Stokowski, S.; Wallerstein, P.; SP620: 1981 October. 159-169.

Walsh, D. A.; O'Quinn, D. B.; Knecht, W. L.; Fernelius, N. C.; Dempsey, D. V.; SP620: 1981 October. 129.143.

Walter, F. J.; Stone, R.; Blackburn, D. H.; Pella, P. A.; SP604: 1981 June. 61-70.

Walters, E. J.,; Galloway, K. F.; Scace, R. I.; NBSIR 81-2230-3.

Walters, J. P.; Scheeline, A.; Norris, J. A.; Travis, J. C.; DeVoe, J. R.; 20453.

Walters, J. P.; Scheeline, A.; Travis, J. C.; DeVoe, J. R.; 20462.

Walther, H.; Hellmuth, T.; Leuchs, G.; Smith, S. J.; 20506.

Walther, H.; Hellmuth, T.; Leuchs, G.; Smith, S. J.; 20504.

Walton, G. N.; NBSIR 81-2404.

Walton, W. D.; NBSIR 81-2326.

Wan, C. A.; NBSIR 81-2303.

Wang, F. W.; Smith, L. E.; Chang, S. S.; McCrackin, F. L.; Senich G. A.; NBSIR 81-2264.

Wang, F. W.; Zimm, B. H.; 20370.

Wang, G. C.; Bader, S. D.; Felcher, G. P.; Celotta, R. J.; Pierce, D. T.; 19970 .

Wang, G. C.; Celotta, R. J.; Pierce, D. T.; 20138.

Wang, G. C.; Felcher, G. P.; Bader, S. D.; Celotta, R. J.; Pierce, D. T.; 19975.

Wang, G. C.; McRae, E. G.; Pierce, D. T.; Celotta, R. J.; 20643.

Wang, G. C.; Pierce, D. T.; Celotta, R. J.; 20012. 
Wang, G. C.; Pierce, D. T.; Celotta, R. J.; 20706.

Warnar, R.; Henderson, M.; Berger, P.; SP610: 1981 December. 74. 79.

Wasik, S. P.; 20345.

Wasik, S. P.; DeVoe, H.; Miller, M. M.; J. Res. 86(4): 361-366; 1981 July-August.

Wasik, S. P.; Tewari, Y. B.; Miller, M. M.; Martire, D. E.; NBSIR 81-2406.

Wasson, O. A.; Duvall, K. C.; 20456

Waterman, T. E.; Eichler, T. V.,; Pape, R.; NBS-GCR-81-301.

Waters, P. F.; Phillips, J. C.; Peterlin, A.; 20437.

Waterstrat, R. M.; 20382.

Waterstrat, R. M.; 20486

Waterstrat, R. M.; 20707.

Watkins, S.; Blanc, R.; Heafner, J.; Rosenthal, R.; NBSIR 80-2154.

Watson, R. E.; Bennett, L. H.; 20455.

Watson, R. E.; Bennett, L. H.; 20413.

Waxman, M.; Davis, H. A.; Sengers, J. M. H. L.; Klein, M.; NBSIR 79-1715.

Webb, W. W.; Scofield, J. H.; Darling, D. H.; SP614: 1981 September. 147-150.

Webber, S.,; SP457-5.

Weber, H.; Fraker, A. C.; 20270.

Weber, L. A.; 20264

Weber, L. A.; 20309.

Weber, S. F.; Lippiatt, B. C.; 20499.

Weber, S. F.; Rudder, F. F., Jr.; Boehm, M. J.; NBSIR 81-2366.

Weber, S. F.; Thompson, B. E.; Lippiatt, B. C.; NBSIR 81-2304.

Webster, H. L.; SP400-69: 1981 May. 80-81.

Wedekind, D. R.,; NBS-GCR-81-322.

Weeks, J. J.; Clark, E. S.; Eby, R. K.; 20635.

Weeks, J. J.; Eby, R. K.; Clark, E. S.; 20708

Wehrmeister, A. E.; SP589: 1981 January. 49-55.

Weidman, M.; NBSIR 81-1650.

Weidman, M. P.; Hudson, P. A.; TN1044.

Weidner, V. R.; Hsia, J. J.; 20287.

Weidt, J. L.; Saxler, R. J.; Rossiter, W. J., Jr.; 20463

Weinberg, S.; SP601: 1981 January. 449-469.

Weinstein, A.; Gibbons, D.; Brown, S.; Ruff, W.,; SP601.

Weinstein, A. M.; Clemow, A. J. T.; McPhillips-Meade, M.; Klawitter, J. J.; French, G.; Skinner, H.; SP601: 1981 January. 423 447.

Weinstein, A. M.; Cook, S. D.; SP601: 1981 January. 573-613.

Weismantel, E. E.; SP589: 1981 January. 113-119.

Weiss, A. W.; 20281.

Weiss, C. S.; Grimm, C. A.; Filby, R. H.; Sandstrom, D. R.; Lytle, F. W.; Greegor, R. B.; Khalil, S. R.; Ekambaram, V.; SP618: 1981 November. 21-38.

Weiss, C. S.; Jewett, K. L.; Brinckman, F. E.; Fish, R. H.; SP618: 1981 November. 197-216.

Weiss, E. R.; Byrne, S. T.; El-Torkey, N. M.; Margolis, S. A.; Kaplan, A. E.; 20446

Weiss, M. A.; Allan, D. W.; 20062.

Weisshaar, J. C.; Leone, S. R.; Zwier, T. S.; 20713.

Weissman, M. B.; SP614: 1981 September. 133-142.

Weissman, M. B.; Fliegle, F. M.; Black, R. D.; SP614: 1981 September. 152.

Welch, B. E.; Driver, R. G.; Houck, J. C.; J. Res. 86(3): 277-279; 1981 May-June.

Welch, P.,; Zeizel, J.; NBS-GCR-81-320.

Welch, V. M. J.; Hertz, H. S.; White, E.; NBSIR 80-2160

Well, A.; Johnson, C. C.; Clifton, C.; Nober, E. H.; Pierce, H.; NBSGCR-80-284.

Wellington, J.,; Kelly, J. C.; Wolf, R.; Kennicott, P.; Nagel, R.; NBSIR 81-2297.

Wells, J. S.; Maki, A. G.; 20085.

Wells, J. S.; Maki, A. G.; Siemsen, K. J.; Petersen, F. R.; 20641.

Wells, J. S.; Petersen, F. R.; Maki, A. G.; Sukle, D. J.; 20427.

Wells, J. S.; Petersen, F. R.; Maki, A. G.; Sukle, D. J.; 20640.

Wenzel, J.; Kauffman, D.; Scarfe, C. M.; Cronin, D.; 20222.

Werner, P. E.; Phelan, R. J., Jr.; Larson, D. R.; 20330.
Wertheimer, H. P.; Romine, D. J.; SP622: 1981 October. $62-73$.

West, J. B.; Codling, K.; Ederer, D. L.; Parr, A. C.; Cole, B. E.; Stockbauer, R.; Dehmer, J. L.; 20676.

West, J. B.; Codling, K.; Parr, A. C.; Ederer, D. L.; Cole, B. E.; Stockbauer, R.; Dehmer, J. L.; 20426.

West, J. B.; Cole, B. E.; Dehmer, J. L.; Codling, K.; Parr, A. C.; Ederer, D. L.; Stockbauer, R.; 20399.

West, J. B.; Dehmer, J. L.; Parr, A. C.; Rakowsky, G.; Ederer, D. L.; Stockbauer, R. L.; 20236.

West, J. B.; Parr, A. C.; Dehmer, J. L.; Stockbauer, R. L.; Codling, $\mathrm{K} ; 20052$.

West, J. B.; Stockbauer, R.; Codling, K.; Dehmer, J. L.; Parr, A. C.; Ederer, D. L.; Cole, B. E.; 20123.

Westera, W.; Zijlstra, R. J. J.; SP614: 1981 September. 276-282.

Westley, F.; NBSIR 81-2254.

Wheatley, T.; Albus, J. S.; Nagel, R. N.,; NBSIR 80-2152.

Wheatley, T. E.; Albus, J. S.; VanderBrug, G. J.; Barbera, A. J.; Fitzgerald, M. L.; SP602.

Wheeler, A. E.; Zingaro, R. A.; Irgolic, K. J.; Banks, C. H.; Bottino, N. R.; Chakraborti, D.; Gennity, J. M.; Hillman, D. C.; O'Brien, D. H.; Pyles, R. A.; Stockton, R. A.; SP618: 1981 November. 244.263.

Whetstone, J. R.; Frederikse, H. P. R.; Schenck, P. K.; Burns, G. W.; Dils, R. R.; NBSIR 81-2348.

Whipple, T. A.; McHenry, H. I.; Read, D. T.; 20528.

White, E.; Christensen, R. G.; Hertz, H. S.; Meiselman, S.; 20097.

White, E.; Christensen, R. G.; Hertz, H. S.; Reeder, D. J.; 20654

White, E.; Hertz, H.; Christensen, R.; U.S. Patent 4,281,246.

White, E.; Welch, V. M. J.; Hertz, H. S.; NBSIR 80-2160.

White, E. T.,; NBS-GCR-81-333.

White, G. S.; Feldman, A.; 20485.

White, H. S., Jr.,; FIPS PUB 80.

White, P. A.; Parris, R. M.; Guenther, F. R.; Chesler, S. N.; May, W. E.; 20602.

White, R.; Haskin, W.; SP607: 1981 May. 21.29.

White S.; SP500-80: 1981 October. 186-189.

Whiting, F. B.; Kandiah, K.; Deighton, M. O.; SP614: 1981 September. 75.78.

Whitlock, R. P.; Hinman, R.; Cassel, J. M.; Tesk, J. A.; Brauer, G. M.; Antonucci, J. M.; Wu, W.; McKinney, J. E.; NBSIR 80-2168.

Whitlock, R. P.; Tesk, J. A.; Widera, G. E. O.; Holmes, A.; Parry, E. E.; 20241.

Whitlock, R. P.; Widera, G. E. O.; Holmes, A.; Parry, E. E.; Tesk, J. A.; 20277.

Whitney, K.,; Berg, J. L.; Graham, M.; SP500-76.

Widera, G. E. O.; Holmes, A.; Parry, E. E.; Tesk, J. A.; Whitlock, R. P.; 20277.

Widera, G. E. O.; Holmes, A.; Parry, E. E.; Whitlock, R. P.; Tesk, J. A.; 20241.

Wiederhorn, S. M.; 20028.

Wiederhorn, S. M.; Lawn, B. R.; Hockey, B. J.; 20559.

Wiegand, C. E.; Kessler, E. G., Jr.; Deslattes, R. D.; Jacobs, L.; Schwitz, W.; Seki, R.; Lum, G. K.; 20349.

Wiese, W. L.; 20193.

Wiese, W. L.; Martin, G. A.; 20710

Wiese, W. L.; Martin, G. A.; Reader, J.; Corliss, C. H.; NSRDS NBS68.

Wiese, W. L.; Younger, S. M.; Fuhr, J. R.; Martin, G. A.; JPCRD 10(2): 305-566; 1981

Wiggins, T. A.; Walker, T. W.; Guenther, A. H.; SP620: 1981 October. 277-286.

Wight, A. S.; SP500-83: 1981 November. 21-34

Wildman, D. W.; Schumann, L. W.; Gallagher, A. C.; 20491.

Wilkerson, C. L.; Toste, A. P.; Hayes, D. J.; Olsen, K. B.; SP618: 1981 November. 105-119.

Wilkinson, F.; Brummer, J. G.; JPCRD 10(4): 809-999; 1981.

Wilkinson, R.; Shepherd, M. T.; SP601: 1981 January. 223-235.

Willard, W. A.,; Shives, T. R.; SP622.

Willert, H. G.; Buchhorn, G.; Buchhorn, U.; Semlitsch, M.; SP601: 1981 January. 239-267.

Williams, D. B.; Goldstein, J. I.; SP604: 1981 June. 341-349.

Williams, D. F.; McNamara, A.; Clark, G. C. F.; SP60I: 1981 
January. 323-336.

Williams, E. S.; 20239.

Williams, E. S.; Galakhova, O. P.; Harkness, S.; Hermach, F. L. Hirayama, H.; Martin, P.; Rozdestvenskaya, T. H.; 20240.

Williams, S.; SP608: 1981 May. 173.178.

Williams, W. E.; Van Stryland, E. W.; Soileau, M. J.; Smirl, A. L.; SP620: 1981 October. 375.384.

Williamson, C. F.; Kowalski, S.; Maruyama, X. K.; Lindgren, R. A.; Flanz, J. B.; Hicks, R. S.; Parker, B.; Peterson, G. A.; Lawson, R. D.; Teeters, W.; 20323.

Williamson, C. F.; Maruyama, X. K.; Petrovich, F.; Lindgren, R. A.; Plum, M. A.; Gerace, W. J.; Hicks, R. S.; Parker, B.; Peterson, G. A.; Singhal, R.; 20660.

Willis, J. B.; Enemark, E. A.; Wirtenson, G. R.; SP620: 1981 October. 287-296.

Willis, R. R.; Austell, W. P., Jr.; SP500-80: 1981 October. 29.32.

Wilson, C. B.; Mohr, J. M.; Chu, C. M.; SP500-83: 1981 November. 59.65 .

Wilson, C. L.; 20092.

Wilson, I.; Page, A. A.; Higson, P.; Deward, F. D.; Haisch, B.; Linsky, J. L.; Slee, O. B.; Siegman, B. C.; Nikoloff, I.; Candy, M.; Harwood, D.; Verveer, A.; Quinn, P. J.; 20405.

Wilson, M. A.; Leiss, J. E.; Norris, N. J.; 19985.

Wilson, M. A. D.; 20352.

Wilson, M. A. D.; Yoder, N. R.; Young, L. M.; Boyd, T. J.; Knapp, E. A.; Martin, R. E.; Potter, J. M.; Schneider, C. M.; Swenson, D. A.; Tallerico, P. J.; Penner, S.; Cutler, R. I.; Debenham, P. H.; Lindstrom, E. R.; Mohr, D. L.; 20206.

Wilson, P. F.; Chang, D. C.; Ma, M. T.; TN1037.

Wilson, R. G.; Comas, J.; Myers, D. R.; 20112.

Wilson, R. G.,; NBS-GCR-81-325.

Wilson, W. K.; Parks, E. J.; 20119.

Winchester, J. W.; Resendez, D. L.; SP500-80: 1981 October. 25-28.

Winchester, J. W.; Resendez, D. L.; SP500-80: 1981 October. 21-24.

Wineland, D. J.; Bergquist, J. C.; Drullinger, R. E.; 20265.

Wineland, D. J.; Itano, W. M.; 20305.

Wineland, D. J.; Itano, W. M.; 20680.

Wing, R. F.; Czyzak, S.; Stencel, R. E.; Linsky, J. L.; Brown, A.; Jordan, C.; Carpenter, K. G.; 20598.

Wing, W. H.; Farley, J. W.; 20647.

Wing, W. H.; Shy, J. T.; Farley, J. W.; Lamb, W. E., Jr.; 20125.

Winter, F.; NBSIR 81-2210.

Winter, F.; Galowin, L. S.; NBSIR 81-2296.

Wintersgill, M. C.; Fontanella, J. J.; Bean, V. E.; Andeen, C. G.; Link, J.; 20266.

Wirtenson, G. R.; Willis, J. B.; Enemark, E. A.; SP620: 1981 October. 287.296.

Wirth, N.; Ohran, R.; SP500-80: 1981 October. 7.9.

Wise, S. A.; Bartle, K. D.; Lee, M. L.; 20158.

Wise, S. A.; Bartle, K. D.; Lee, M. L.; 20397.

Wise, S. A.; Bonnett, W. J.; Guenther, F. R.; May, W. E.; 20709.

Wise, S. A.; Harrison, S. H.; Zeisler, R.; 20601.

Wise, S. A.; May, W. E.; Brown, J. M.; 20586.

Wise, S. A.; May, W. E.; Brown-Thomas, J. M.; Hilpert, L. R.; 20750.

Wise, S. A.; May, W. E.; Konash, P. L.; 20585.

Witzgall, C.; Shier, D. R.; 20022.

Witzgall, C.; Shier, D. R.; J. Res. 86(3): 317-330; 1981 May-June.

Wixson, R. L; Kramer, G. M.; Hori, R. Y.; Lewis, J. L.; SP601: 1981 January. 509-557.

Wlodawer, A.; Sjölin, L.; 20561.

Wobbeking, T. F.; Staples, B. R.; NBSIR 81-2276.

Wolf, D.; van Vliet, K. M.; Handel, P. H.; SP614: 1981 September. 196.198.

Wolf, R.; Kennicott, P.; Nagel, R.; Wellington, J.,; Kelly, J. C.; NBSIR 81-2297.

Wolf, W. R.; SP618: 1981 November. 235-243.

Wollin, H. F.; Barbrow, L. E.; Heffernan, A. P.,; SP599.

Wollin, H. F.,; H44, 1982 Edition.

Wolynec, E.; Dodge, W. R.; Leicht, R. G.; Hayward E.; 20275.

Wong, P. T. S.; Chau, Y. K.; SP618: 1981 November. 65.80
Wood, D. O.; SP616: 1981 October. 7.24.

Wood, D. O.; Gruhl, J.; SP616: 1981 October. 183-200.

Wood, R. M.; Foley, J.; Sharma, S. K.; SP620: 1981 October. 248-255.

Woodall, M. A.; Van Stryland, E. W.; SP620: 1981 October. 50-57.

Woods, T. J.; SP616: 1981 October. 201-203.

Woodward, K.; Marshall, R. D.; NBSIR 80-2111-4.

Woody, M. W.; Hnatczuk, W.; SP622: 1981 October. 85.96.

Worchesky, T. L.; Ritter, K. J.; Lafferty, W. J.; Sattler, J. P.; 20452.

Worchesky, T. L.; Tobin, M. S.; Ritter, K, J.; Daley, T. W.; Lafferty, W. J.; Sattler, J. P.; 20090.

Worden, S. P.; Giampapa, M. S.; Bornmann, P. L.; Ayres, T. R.; Linsky, J. L.; 20388.

Worden, S. P.; Stencel, R. E.; Mullan, D. J.; Linsky, J. L.; Basri, G. S.; 20057.

Workman, J. L.; Crawford, M. L.; TN1013.

Wormsbecher, R. F.; Hessel, M. M.; Lovas, F. J.; 20424.

Wrenn, P.; SP608: 1981 May. 179-181.

Wright, R. N.; Fenves, S. J.; Harris, J. R.; NBSIR 80-1979-1.

Wright, R. N.; Harris, J. R.; 20329.

Wright, R. N.; Harris, J. R.; 20403.

Wright, R. N.; Harris, J. R.; BSS136.

Wright, R. N.; Harris, J. R.; Fenves, S. J.; 19998.

Wright, R. N.; Harris, J. R.; Fenves, S. J.; 20435.

Wright, T. M; Burstein, A. H.; SP601: 1981 January. 559.572.

Wrzenski, S. C.,; Egan, J.; Higgins, R.; Spath, J.; Buchholz, A.; NBSGCR-81-313.

Wu, S. T.; Bass, M.; SP620: 1981 October. 75-87.

Wu, S. T.; Clifton, J. R.; NBSIR 81-2390.

Wu, W.; Cobb, E. N.; 20383.

Wu, W.; McKinney, J. E.; Whitlock, R. P.; Hinman, R.; Cassel, J. M.;

Tesk, J. A.; Brauer, G. M.; Antonucci, J. M.; NBSIR 80-2168.

Wu, Y. C.; Flores, A.; Eliason, L. K.; SP480-41.

Wunderlich, B.; Gaur, U.; JPCRD 10(4): 1001-1049; 1981

Wunderlich, B.; Gaur, U.; JPCRD 10(1): 119-152; 1981.

Wunderlich, B.; Gaur, U.; JPCRD 10(4): 1051-1064; 1981.

Wunderlich, B.; Gaur, U.; Shu, H. C.; Mehta, A.; JPCRD 10(1): 89. $118 ; 1981$

Wyart, J. F.; Kaufman, V.; Sugar, J.; 20002.

Wyart, J. F.; Kaufman, V.; Sugar, J.; 20611.

Wyart, J. F.; Reader, J.; Ryabtsev, A.; 20449.

Wyckoff, J. M.; 20294.

Wyrick, T. F.; Giese, K. W.; Halstead, D.; SP500-83: 1981 November. 109-118.

\section{$\mathbf{X}$}

Xiheng, H.; Kirklin, D. R.; Colbert, J. C.; J. Res. 86(6): 655.660; 1981 November-December.

\section{$\mathbf{Y}$}

Yaghjian, A. D.; 20549

Yaghjian, A. D.; 20711.

Yaghjian, A. D.; 20761.

Yaghjian, A. D.; 20760.

Yaghjian, A. D.; NBSIR 81-1644.

Yaghjian, A. D.; Stubenrauch, C. F.; NBSIR 80-1630.

Yakowitz, H.,; Becker, D. A.; Berke, J. G.; Matthews, R. T.; NBSIR 80-2178.

Yamamoto, H.; Mulholland, G.; Handa, T.; Sugawa, O.; 20565.

Yamamoto, K.; Aoki, K.; Miyamae, K.; Kobayashi, T.; SP614: 1981 September. 93-96.

Yancey, C. W. C.; NBSIR 80-2111-7.

Yancey, C. W. C.; Cattaneo, L.; Harris, J. R.; Reinhold, T. A.; Simiu, E.; NBSIR 81-2199.

Yancey, C. W. C.; Mullen, C. L.; Yokel, F. Y.; BSS132.

Yancey, C. W. C.; Yokel, F. Y.; Chung, R. M.; NBSIR 81-2238.

Yaniv, S. L.; Bauer, J. W.; Flynn, D. R.; Danner, W. F.; NBSIR 812377.

Yaskin, L. A.; Daney, D. E.; Ludtke, P. R.; Eroshenko, V. M.; 20400. 
Yates, D.; Manahan, S. E.; Fraley, D. M.; Vogt, C.; SP618: 1981 November. 81.92.

Yates, J. T.; Cavanagh, R. R.; 20228.

Yates, J. T.; Cavanagh, R. R.; 20219.

Yates, J. T., Jr.; Cavanagh, R. R.; 19997

Yates, J. T., Jr.; Cavanagh, R. R.; 20498.

Yates, J. T., Jr.; McKee, D. W.; 20661.

Yavne, M.; Linden, N.; SP500-80: 1981 October. 117.121.

Yee, S. S.; McDavid, J. M.; SP620: 1981 October. 44-49.

Yeh, K. N.; Smith, B. F.; Manka, M. J.; 20487.

Yen, T. F.; SP618: 1981 November. 9.20.

Yin, L. I.; Trombka, J. I.; Schmadebeck, R. L.; Seltzer, S. M.; Bielefeld, M. J.; 20569.

Yoder, C. F.; Peale, S. J.; 20599.

Yoder, N. R.; Young, L. M.; Boyd, T. J.; Knapp, E. A.; Martin, R E.; Potter, J. M.; Schneider, C. M.; Swenson, D. A.; Tallerico, P. J.; Penner, S.; Cutler, R. I.; Debenham, P. H.; Lindstrom, E. R.; Mohr, D. L.; Wilson, M. A. D.; 20206.

Yoganathan, A. P.; Harrison, E. C.; SP601: 1981 January. 175.200.

Yokel, F. Y.; Chung, R. M.; Yancey, C. W. C.; NBSIR 81-2238.

Yokel, F. Y.; Kovacs, W. D.; Salomone, L. A.; BSS135.

Yokel, F. Y.; Salomone, L. A.; Chung, R. M.; NBSIR 81-2215.

Yokel, F. Y.; Yancey, C. W. C.; Mullen, C. L.; BSS132.

Yolken, H. T.; Reed, W. P.; Carpenter, B. S.; Hoffman, J. D.; NBSIR 81-2205.

Yonemura, G. T.; TN1143.

Yonemura, G. T.; NBSIR 81-2231.

Yonemura, G. T.; Hunt, C. M.; Chen, P. T.; Hill, J. E.; May, W. B., Jr.; Richtmyer, T. E.; Elder, J.; Tibbott, R. L.; BSS133.

Yoo, K. C.; Roessler, B.; Armstrong, R. W.; Kuriyama, M.; 20712.

You, H. Z.; Faeth, G. M.,; NBS-GCR-79-188.

Young, L. M.; Boyd, T. J.; Knapp, E. A.; Martin, R. E.; Potter, J. M.; Schneider, C. M.; Swenson, D. A.; Tallerico, P. J.; Penner, S.; Cutler, R. I.; Debenham, P. H.; Lindstrom, E. R.; Mohr, D. L.; Wilson, M. A. D.; Yoder, N. R.; 20206.

Young, M.; TN1031, Revised.

Young, M.; TN1038.

Young, M.; TN1031.

Young, W. M.; SP599: 1981 January. 85-94.

Younger, S. M.; 20182.

Younger, S. M.; 20198.

Younger, S. M.; 20617.

Younger, S. M.; 20618.

Younger, S. M.; 20619.

Younger, S. M.; 20737.

Younger, S. M.; 20739.

Younger, S. M.; 20738.

Younger, S. M.; Fuhr, J. R.; Martin, G. A.; Wiese, W. L.; JPCRD 10(2): 305-566; 1981.

Younger, S. M.; Lucatorto, T. B.; Mcllrath, T. J.; Sugar, J.; 20684.

Younglove, B.; McCarty, R. D.; 20360.

Younglove, B. A.; J. Res. 86(2): 165.170; 1981 March.April.

Younglove, B. A.; McCarty, R. D.; 20072.

Yu, H.; J. Res. 86(6): 571-590; 1981 November.December.

Yu, K. C.; Atwood, J. W.; SP500-83: 1981 November. 239.246.

Yushchenko, K. A.; Paton, E. O.; Reed, R. P.; Tobler, R. L.; Elmer, J. W.; McHenry, H. I.; 20772.

\section{$\mathbf{Z}$}

Zackrison, H. B., Jr.; SP608: 1981 May. 37.56.

Zahn, M.; Forster, E. O.; Kelley, E. F.; Hebner, R. E., Jr.; 20646.

Zajonc, A. G.; Phelps, A. V.; 20242.

Zaklikiewicz, A. M.; SP614: 1981 September. 178-180.

Zalubas, R.; Martin, W. C.; JPCRD 10(1): 153-196; 1981.

Zaluzec, N. J.; SP604: 1981 June. 351-364.

Zanon, D.; Danos, M.; 20609.

Zapas, I. J.; Eby, R. K.; Broadhurst, M. G.; Chang, S. S.; Davis, G. T.; DiMarzio, E. A.; Fanconi, B. M.; Han, C. C.; Khoury, F. A.; Markovitz, H.; McKinney, J. E.; Mopsik, F. I.; Passaglia, E.;

Sanchez, I. C.; VanderHart, D. L.; 20749.
Zapas, L. J.; Bernstein, B.; 20126.

Zapas, L. J.; Crissman, J. M.; 20299.

Zapas, L. J.; Crissman, J. M.; 20327.

Zapas, L. J.; McKenna, G. B.; 20130.

Zeisler, R.; Wise, S. A.; Harrison, S. H.; 20601.

Zeitlin, B. A.; Ekin, J. W.; Ho, J. C.; Oberly, C. E.; Garrett, H. J.; Walker, M. S.; 20741

Zeizel, J.; Welch, P.,; NBS-GCR-81-320.

Zelkowitz, M. V.; 20075.

Zerdy, G. A.; Lerner, N. D.; Harwood, D. L.; Molino, J. A.; 20030.

Zerdy, G. A.; Lerner, N. D.; Harwood, D. L.; Molino, J. A.; 20050.

Zerdy, G. A.; Lerner, N. D.; Harwood, D. L.; Molino, J. A.; 20362.

Zerdy, G. A.; Lerner, N. D.; Harwood, D. L.; Molino, J. A.; 20361.

Zerdy, G. A.; Molino, J. A.; Lerner, N. D.; 20203.

Zerdy, G. A.; Tremaine, S. G.; Molino, J. A.; 20029.

Zijlstra, R. J. J.; Hiatt, C. F.; van der Ziel, A.; SP614: 1981 September. 234.235.

Zijlstra, R. J. J.; van Kooten, J. J.; van der Meulen, J. P.; SP614: 1981 September. 343-345.

Zijlstra, R. J. J.; van Rheenen, A.; Bosman, G.; SP614: 1981 September. 409.413.

Zijlstra, R. J. J.; Westera, W.; SP614: 1981 September. 276-282.

Zile, R. H.; Klote, J. H.; NBSIR 81-2433.

Zimm, B. H.; Wang, F. W.; 20370

Zimmerman, J. E.; 20306

Zimmerman, J. E.; Ives, J. T.; Sullivan, D. B.; SP607: 1981 May. 186. 194.

Zimmerman, J. E.; Reite, M.; Zimmerman, J. T.; 20480.

Zimmerman, J. E.; Sullivan, D. B.; U.S. Patent 4,281,517.

Zimmerman, J. E.; Sullivan, D. B.; Kautz, R. L.; Hobbs, R. D.; SP607: 1981 May. 173.177.

Zimmerman, J. E.; Sullivan, D. B.; McCarthy, S. E.,; SP607.

Zimmerman, J. T.; Zimmerman, J. E.; Reite, M.; 20480.

Zingaro, R. A.; Irgolic, K. J.; Banks, C. H.; Bottino, N. R.; Chakraborti, D.; Gennity, J. M.; Hillman, D. C.; O'Brien, D. H.; Pyles, R. A.; Stockton, R. A.; Wheeler, A. E.; SP618: 1981 November. 244.263.

Zoller, P.; Steyn.Ross, M. L.; Walls, D. F.; 20179.

Zoller, P.; Walls, D. F.; Kunasz, C. V.; Drummond, P. D.; 20595.

Zucker, R.; Stegun, I. A.; J. Res. 86(6): 661-686; 1981 NovemberDecember.

Zukoski, E. E.; Kubota, T.; Cetegen, B.,; NBS-GCR-80-294.

Zukoski, E. E.; Kubota, T.; Cetegen, B.,; NBS-GCR-81-346.

Zwaan, C.; Vaiana, G. S.; Cassinelli, J. P.; Fabbiano, G.; Giacconi, R.; Golub, L.; Gorenstein, P.; Haisch, B. M.; Harnden, F. R., Jr.; Johnson, H. M.; Linsky, J. L.; Maxson, C. W.; Mewe, R.; Rosner, R.; Seward, F.; Topka, K.; 20336.

Zwier, T. S.; Weisshaar, J. C.; Leone, S. R.; 20713. 


\subsection{KEY WORD INDEX}

\section{A}

AAPT vs. NBS; kilogram; mass/weight; metric controversy; newton; 20023.

ab initio calculations; amino acid; conformational energy; dipole moment; gas phase conformers; glycine; rotational constants; structure; 20005

abrasion test development; bicycle compliance tests; bicycle safety; reflective materials for bicycles; safety of bicycles; safety standards; NBSIR 80-2035.

absolute chemical rate; BEBO activation energy; bond-order-bondenergy; chemical rate constants; computer program; hydrogen atom transfer; J. Res. 86(6): 605-654; 1981 November-December.

absolute filter; current density; high voltage dc transmission lines; ion counter; space charge density; Wilson plates; NBSIR 81-2267.

absorbed dose; Bragg-Gray equation; calibration; cavity chamber; cavity theory; electron beam; in-phantom measurement; photon beam; radiation therapy; 20073.

absorbed dose standard; cobalt-60 gamma rays; electron density; graphite calorimeter; ionization chamber; scaling theorem; water phantom; J. Res. 86(5): 495-502; 1981 September-October.

absorber materials; accelerated test methods; durability; solar energy; standards; NBSIR 81-2232.

absorbing defects; inclusions; laser damage; SP620: 1981 October. 369. 374.

absorptance; infra-red laser windows; pulsed $\mathrm{CO}_{2}$ laser damage; 10 micrometer optical components; SP620: 1981 October. 248-255.

absorption; coatings; internal reflection spectroscopy; silicon monoxide; thorium fluoride; zinc selenide; zinc sulfide; SP620: 1981 October. 335.344.

absorption; evaporation; hydrogen and oxygen impurity; magnetron sputtering; pulsed HF/DF laser damage thresholds; silicon films; SP620: 1981 October. 305-312.

absorption; laser windows; material properties; transmission; SP620: 1981 October. 144-158.

absorption in solids; beam depletion; nonlinear absorption; photoacoustic technique; SP620: 1981 October. 50.57.

absorption spectra; aqueous solution; extinction coefficients; inorganic; radical ions; radicals; transients; NSRDS-NBS69.

absorption waves; air breakdown; $\mathrm{Cu}$ mirrors; laser-induced electron emission; mirror surface damage; surface plasma; SP620: 1981 October. 227.238.

abstracts; acoustic emission; bibliography; eddy currents; index; National Bureau of Standards; nondestructive evaluation; publications; radiography; ultrasonics; NBSIR 80-2080.

abstracts; building technology; Center for Building Technology; key words; publications; SP457-5.

abstracts, NBS publications; key words; publications; SP305. Supplement 12.

abundances; alpha particles; cellulose nitrate detector; isotopic; lithium; nuclear track technique; triton particles; 19982.

accelerants; arson; combustible gas; detectors; diffusion rates; diffusion tubes; gas chromatography; hydrocarbons; vapor detector; vapor generator; vapor standards; $S P 480-43$.

accelerated aging; durability; environmental exposure; reliability; solar collectors; solar materials; stagnation testing; TN1136.

accelerated aging tests; coatings; corrosion; predictability; reproducibility; time to failure; TN1149.

accelerated test methods; durability; solar energy; standards; absorber materials; NBSIR 81-2232.

acceleration; accelerometers; calibration; shakers; vibration exciters; 20731.

accelerator; alloy; base metal; casting; composite; cyanoacrylate; dental alloy; initiator; resin; wear; NBSIR 80-2168.

accelerators; cementing of bone; high reactivity; low toxicity; peroxide catalyzed polymerization; tertiary aromatic amines unsaturated polyesters; vinyl monomers; U.S. Patent 4,284,551.

accelerometers; calibration; shakers; vibration exciters; acceleration; 20731.

access control; authentication; authorization; computer network computer security; encryption; Federal Information Processing Standards Publication; identification token; identity verification; password; personal attribute; personal identification; FIPS PUB 83.

accessibility; alarms; blind; code requirements; communication; deaf; directional indicators; handicapped users; tactile warnings; NBSIR 81-2428.

accessibility; building accessibility; building research; fire safety; handicapped; life safety; NBSIR 81-2245.

accident data; coal mines; conveyor belts; fire safety; fire tests; flame spread; ignition; test methods; NBSIR 81-2256.

accidents; codes and standards; construction loads; construction safety; finite element; scaffold failures; scaffolding practices; scaffolds; structural analysis; NBS-GCR-80-255.

accountability; differential pressure; splines; statistics; volume calibration; NBSIR 80-2151.

accountability guide; bulk measurements; isotopic assay; leachability studies; nondestructive assay; nuclear safeguards; nuclear waste management; standard reference materials; statistical evaluation; NBSIR 81-2205.

accuracy; ANS FORTRAN; computer system installation; examples; OMNITAB 80; software; test problems; TN1147.

accuracy; certified reference material; chemical composition; physical property; 19990

acetylene; F atoms; hydrogen bonding; infrared spectrum; matrix isolation; photodecomposition; reaction mechanism; $t$-2-fluorovinyl 20036.

ac losses; calorimeter; cryogenics; multipliers; standards superconductors; 19999.

acoustical design; acoustics; architectural design; building codes; building economics; construction costs; cost minimization; economic impact; economics; energy; model code; noise control; NBSIR 81-2366

acoustic emission; bibliography; eddy currents; index; National Bureau of Standards; nondestructive evaluation; publications; radiography; ultrasonics; abstracts; NBSIR 80-2080.

acoustic emission; calibration; development; transducer; waveform 20757.

acoustic emission; eddy currents; liquid penetrants; magnetic particles; microwaves; nondestructive evaluation; radiography; tire inspection; ultrasonics; visual-optical; 20762.

acoustic emission analysis; insurance; loss prevention; modal analysis property conservation; risk management; shock pulse analysis; vibration analysis; SP622: 1981 October. 139.151.

acoustic emission of polymers; emission of polymers; polymers under tensile load; 20014.

acoustic emissions; cavitation; feedwater control valve; pattern recognition; pressurized water reactor; remote surveillance; SP622 1981 October. 11.30

acoustics; architectural acoustics; building acoustics; noise; noise control; sound transmission; structureborne noise; NBS-GCR-80248.

acoustics; architectural design; building codes; building economics; construction costs; cost minimization; economic impact; economics; energy; model code; noise control; acoustical design; NBSIR 81 2366.

acoustics; building acoustics; impact noise; noise control; noise isolation; noise rating; $N B S-G C R-80-249$.

acoustics; building acoustics; noise control; noise isolation; sound transmission; structure-borne noise; NBS-GCR-80-250.

acoustic waves; elastic anisotropy; elastic constants; Kroner method; sound velocities; ultrasonic inspection; ultrasonic waves; 20773.

acoustic waves; elastic anisotropy; nondestructive evaluation; nondestructive testing; stainless steel; ultrasonic scattering ultrasonic waves; 20744.

acrylate; adhesion; aminobenzoate; composite material; dentin methacrylate; polyfunctional surface-active comonomers; reconstructive dental work; resin; U.S. Patent 4,251,565.

acrylates; acrylic resins; aminoglutethimide or para-aminophenylacetic acid; cementing of bone; fillings; methacrylates; tertiary aromatic amines; unsaturated polyesters; U.S. Patent $4,243,763$.

acrylic cement; joint replacements; polyethylene components; SP601: 
1981 January. 3-28.

acrylic resins; aminoglutethimide or para-aminophenylacetic acid; cementing of bone; fillings; methacrylates; tertiary aromatic amines; unsaturated polyesters; acrylates; U.S. Patent 4,243,763.

actinium compounds; cesium compounds; enthalpy; entropy; francium compounds; Gibbs energy of formation; heat capacity; lithium compounds; potassium compounds; protactinium compounds; rubidium compounds; sodium compounds; thorium compounds; uranium compounds; TN270-8.

activation analysis; crystal structure; diffraction; isotopes; molecular dynamics; neutron; neutron radiography; nondestructive evaluation; nuclear reactor; radiation; $T N 1142$.

activation energy; bubbles; polymer degradation products; pressure; thermal degradation; thermogravimetry; 20774.

active vibration control; Michelson interferometer; optical path-length correction; phase comparator; real-time control; vibration control; vibration isolation; 20734.

activity coefficient; cadmium; critical evaluation; electrolyte; ethylene bis(trimethylammonium) chloride; ethylene

bis(trimethylammonium) iodide; excess Gibbs energy; osmotic coefficient; solutions; thermodynamic properties; zinc; JPCRD 10(1): 1-56; 1981

activity coefficient; cesium; critical evaluation; electrolyte; excess Gibbs energy; lithium; osmotic coefficient; potassium; rubidium; sodium; solutions; thermodynamic properties; JPCRD 10(3): 671. 766.

activity coefficients; alkali metal nitrite salts; aqueous; critical evaluation; $\mathrm{CsNO}_{2}$; electrolytes; excess Gibbs energy; $\mathrm{KNO}_{2}$; $\mathrm{LiNO}_{2} ; \mathrm{NaNO}_{2}$; osmotic coefficients; $\mathrm{RbNO}_{2}$; solutions; standard reference data; thermodynamic properties; JPCRD 10(3): 767.778; 1981.

activity coefficients; aqueous; compilation; conductivity; electrolytes; enthalpy; Gibbs energy; osmotic coefficients; solutions; sulfuric acid; thermodynamic properties; transport properties; NBSIR 812276.

activity coefficients; aqueous; critical evaluation; electrolytes; excess free energy; free energy; ionic; osmotic coefficients; solutions; sulfuric acid; standard reference data; thermodynamic properties; JPCRD 10(3): 779-798; 1981

activity coefficients; aqueous solubility; gas chromatography; generator column; high pressure liquid chromatography; octanol/water partition coefficients; NBSIR 81-2406.

acuity tests; nondestructive testing; quality testing; radiograph evaluation; visual inspection; visual testing; TN1143.

adhesion; aminobenzoate; composite material; dentin; methacrylate; polyfunctional surface-active comonomers; reconstructive dental work; resin; acrylate; U.S. Patent 4,251,565.

adhesion; anodizing; coatings; electrodeposition; immersion

deposition; plating; plating on anodized aluminum; NBSIR 80-2142.

adhesive; bitumen; bonding; built-up roofing membrane;

complementary strain energy; felt; nonlinear; roofing membrane; splitting; strain energy; stress; substrate; TN1152.

adiabatic calorimetry; azidotriphenylmethane; decomposition reactions; differential scanning calorimetry; heat conduction calorimetry; kinetics of heat evolution; m-azidobenzoic acid; nitrocellulose; quantitative differential thermal analysis; thermal explosion theory; thermal hazards; thermal instability; NBSIR 80 2018 (DOT).

adiabatic compressibility; liquid; pressure; propane; sound; specific heat ratios; speed; temperature; velocity; J. Res. 86(2): 165-170; 1981 March-April.

adiabatic potentials; Born-Oppenheimer approximation; diatomic spectroscopy; Franck-Condon principle; rotational-electronic coupling; scattering theory; 20060.

administrative experiment; communications; competition; ETIP; FCC; innovation incentives; regulatory reform; NBS-GCR-ETIP 81-97.

administrative experiment; evaluation systems; Experimental Technology Incentives Program; information systems; project management; system acquisition; system development; NBSIR 81 2328.

administrative experiment; evaluation systems; Experimental
Technology Incentives Program; information systems; project management; system acquisition; system development; NBSIR 812327.

adobe building materials; adobe soil; dielectric constant measurements; electromagnetic moisture measurements; nondestructive evaluation; radar soil measurements; soil moisture; underground radar; void detection; NBSIR 79-1610.

adobe soil; dielectric constant measurements; electromagnetic moisture measurements; nondestructive evaluation; radar soil measurements; soil moisture; underground radar; void detection; adobe building materials; NBSIR 79-1610.

ADP acquisition; ADP procurement; benchmarking; Federal Information Processing Standards Publication; performance evaluation; workload analysis; workload characterization; workload representation; FIPS PUB 75.

ADP availability; ADP security; backup operations; computer applications; computer security; contingency planning; emergency response; Federal Information Processing Standards Publication; recovery actions; FIPS PUB 87.

ADP installation management; cost-benefit; performance management; productivity; user service reporting; SP500-83: 1981 November. 59.65.

ADP procurement; benchmarking; Federal Information Processing Standards Publication; performance evaluation; workload analysis; workload characterization; workload representation; ADP acquisition; FIPS PUB 75.

ADP security; backup operations; computer applications; computer security; contingency planning; emergency response; Federal Information Processing Standards Publication; recovery actions; ADP availability; FIPS PUB 87.

ADP terminals; ASCII; character-imaging devices; control functions; data interchange; display terminals; Federal Information Processing Standard; information interchange; printer terminals; standard; text communication; text interchange; typesetting composition; FIPS PUB 86 .

adsorption; Auger spectroscopy; convection; ellipsometry; gallium; levitation calorimetry; specific heat; surface tension; thermophysical properties; tungsten; wetting; NBSIR 81-2295.

adsorption; compressor; cryogenics; molecular adsorption; refrigeration; zeolites; SP607: 1981 May. 127-135.

adsorption; cryocoolers; cryostats; helium; refrigeration; Stirling cycle; SP607: 1981 May. 3-14.

adsorption; desorption; hydrolyzable cations; hydrous oxides; kinetics; protolyzable anions; thermodynamics; SP618: 1981 November. 146. 162.

adults; alarm responses; auditory perception; decibel levels; fire departments; frequency distribution; noise (sound); sleep; smoke detectors; wakefulness; NBS-GCR-80-284.

advanced data communication control procedures; ANS X3.66; bitoriented; code independence; data; data link control procedures; data transparency; Federal Information Processing Standards Publication; interoperability; teleprocessing; transmission; FIPS PUB 78 .

aerospace; chromium; cobalt; critical materials; tantalum; titanium; NBSIR 81-2305.

aerothermal diagnostics; aerothermal parameters; performance; physical deterioration; reliability; turbomachinery; SP622: 1981 October. 222-253.

aerothermal parameters; performance; physical deterioration; reliability; turbomachinery; aerothermal diagnostics; SP622: 1981 October. 222-253.

Agency for International Development; Arab League Organization for Standardization and Metrology; chemical industry standards; cotton standards; food standards; measurement science and technology; standardization in developing countries; Sudan; weights and measures regulation; NBSIR 80-2020.

aggregation; equilibrium; game theory; mathematical economics; noncooperative games; total orders; J. Res. 86(4): 383-391; 1981 July.August.

aging; cables; dielectrics; electric insulation fault location; timedomain-reflectometry; underground transmission; NBSIR 81-2235. 
aging; electrical conductivity; hardness; homogeneity; microstructure; precipitating; segregation; solidification; thermal mechanical treatment; NBSIR 80-2069.

air; environmental measurements; methods; quality assurance; standards; water; NBSIR 81-2280.

air analysis; diffusion; hollow tubes; mercury; preconcentration; SP618: 1981 November. 134-145.

air breakdown; $\mathrm{Cu}$ mirrors; laser-induced electron emission; mirror surface damage; surface plasma; absorption waves; SP620: 1981 October. 227.238.

air breakdown; laser damage; $\mathrm{NaCl}$; picosecond phenomena; self-

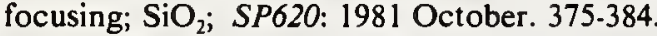

aircraft hydraulic systems; cost reduction; energy savings; failure detection; failure prevention; flight safety; fluid system components; maintenance effectiveness; on-board monitoring system; SP622: 1981 October. 195-219.

air density; index of refraction of air; refractivity of air; wavelength of light in air; J. Res. 86(1): 27.32; 1981 January-February.

air flow; air leakage; ASTM E 152; doors; fire doors; fire tests; NBSGCR-81-330

airflow; burn (injuries); cotton; fabric flammability; fabrics; fire suppression; flame extinguishment; heat transfer; polyester; textiles; NBSIR 81-2386.

air-fuel ratio; closed loop fuel metering system; exhaust emissions; exhaust gas oxygen sensor; exhaust gas recirculation; feedback; oxidation catalyst; oxygen partial pressure; stoichiometric; three way catalytic converters; zirconium dioxide; SP622: 1981 October. 62.73 .

air leakage; ASTM E 152; doors; fire doors; fire tests; air flow; NBS. GCR-81-330.

air leakage; buildings; doors; fire doors; fire tests; infiltration; orifice flow; smoke control; smoke movement; NBSIR 81-2214.

air leakage; heat transfer; pebble-bed storage; solar collector; system efficiency; thermal performance; NBS-GCR-81-302.

air movement; computer programs; egress; elevator shafts; escape means; pressurization; smoke control; stairwells; NBSIR 80-2157.

air pollutants; antenna coupling; electrical distribution; glass-liquidus; insulation; monitors; neutron diffraction, samplers; SRM's; timedomain calibrations; weights and measures; $\mathrm{x}$-ray diffraction; $D I M / N B S$ 65(1): 1-28; 1981.

air pollution; automation; buildings; certified reference materials; computers; construction; data converter; mass comparator; radiation; radiocarbon; soft aluminum; windows; $D I M / N B S$ 65(4): $1.28 ; 1981$.

air pollution; Clean Air Act; EPA; ETIP; incentives; innovation; regulation; NBS-GCR-ETIP 81-93.

air pollution; Clean Air Act; EPA; ETIP; incentives; innovation; regulation; NBS-GCR-ETIP 81-94.

air pollution; Clean Air Act; EPA; ETIP; incentives; innovation; regulation; NBS-GCR-ETIP 81-95.

alarm responses; auditory perception; decibel levels; fire departments; frequency distribution; noise (sound); sleep; smoke detectors; wakefulness; adults; $N B S-G C R-80-284$.

alarms; blind; code requirements; communication; deaf; directional indicators; handicapped users; tactile warnings; accessibility; NBSIR 81-2428.

algorithm; antenna; aperture; computer program; electromagnetic; far field; FFT; Fourier transform; NBSIR 81-1643.

algorithm; graph; $k$ shortest paths; network algorithms; shortest path; transportation networks; 20021

alkali-halide laser windows; $\mathrm{KCl} ; \mathrm{NaCl}$; optoacoustic spectroscopy; photoacoustic spectroscopy; surface impurities; SP620: 1981 October. 44-49.

alkali halides; bulk damage; $\mathrm{CO}_{2}$ laser; coating damage; laser damage; microsecond pulses; $\mathrm{NaCl}$; surface damage; SP620: 1981 October. 88.93.

alkali-halides; calorimetry; damage mechanisms; intensity dependent absorption; multipulse damage; SP620: 1981 October. 75-87.

alkali metal nitrite salts; aqueous; critical evaluation; $\mathrm{CsNO}_{2}$; electrolytes; excess Gibbs energy; $\mathrm{KNO}_{2} ; \mathrm{LiNO}_{2} ; \mathrm{NaNO}_{2}$; osmotic coefficients; $\mathrm{RbNO}_{2}$; solutions; standard reference data; thermodynamic properties; activity coefficients; JPCRD 10(3): 767 $778 ; 1981$

alkaline reserve; cellulose stabilizers; paper deacidification; paper destabilization; paper permanence; $\mathrm{pH}$ history; J. Res. 86(3): 309. 315; 1981 May-June.

alkali vapor; coal conversion; combustion systems; vapor transport; NBSIR 81-2279.

alkanes; aromatics; critical review, evaluated data; gases; halogenated hydrocarbons; Henry's law constants; liquids; pesticides, solids; solubility; vapor pressure; JPCRD 10(4): 1175-1199; 1981

alkylleads; alkyltins; chemical speciation; GC.AAS techniques; LC. AAS techniques; organoarsenics; organometals; partition coefficients; toxicity; SP618: 1981 November. 65-80.

alkylpyridines; dissolved organic compounds; gas chromatography/mass spectrometry; hydrophobic bases; metal complexation; nitrogen bases; oil shale; retort water; speciation; trace metals; SP618: 1981 November. 93-104.

alkyltins; chemical speciation; GC-AAS techniques; LC-AAS techniques; organoarsenics; organometals; partition coefficients; toxicity; alkylleads; SP618: 1981 November. 65-80.

allowed transitions; cobalt; $f$-values; iron; isoelectronic sequence; line strengths; nickel; oscillator strengths; systematic trends; transition probabilities; JPCRD 10(2): 305.566; 1981.

alloy; base metal; casting; composite; cyanoacrylate; dental alloy; initiator; resin; wear; accelerator; NBSIR 80-2168.

alloy; carbon; computers; energy; fine structure; interface; materials; measurement; office building; phase diagrams; radioactivity; research; sediment; soil; DIM/NBS 65(8): 1.28; 1981.

alloy phase diagram; aluminum; magnetic susceptibility; orderdisorder; titanium; transmission electron microscopy; 20736

alloy phase diagram; computer graphics; critical evaluation; database management; thermodynamics; 20033

alloys; aluminum alloys; castings; composites; cryogenic properties; low temperature; mechanical properties; nonmetallics; physical properties; stainless steels; structural alloys; welding; NBSIR 81 1645.

alloys; combustion; combustion rate; ignition; metals; oxygen atmosphere; temperature measurement; NBSIR 81-1647.

alloys; corrosion; metallurgically-bonded; metals; plastic-bonded; soils; telephone cables; underground; NBSIR 81-2243.

ALOHANET; ALOHA system; computer network; packet broadcasting; radio computer communication network; satellite communication; J. Res. 86(6): 591-595; 1981 November-December.

ALOHA system; computer network; packet broadcasting; radio computer communication network; satellite communication; ALOHANET; J. Res. 86(6): 591-595; 1981 November-December.

alpha particles; cellulose nitrate detector; isotopic; lithium; nuclear track technique; triton particles; abundances; 19982.

alpha-quartz particulate; chrysotile asbestos particulate; DTA analysis of environmental particulates; DTA apparatus for rapid sample change and steam pressure control; 20011.

alpha uranium; chloride ion; chromate ion; electrochemistry; ellipsometry; field ion microscopy; inhibitors; organic coatings; passive film dissolution; $\mathrm{pH}$ measurements; surface hydride; NBSIR 81-2396 (ONR).

alternate routing; communications networks; CROSSFIRE; link protocols; local survivability; message delay; network simulation; network throughput; survivability; NBS-GCR-81-336.

alternate routing; communications networks; distributed control; message delay; network throughput; survivability; NBSIR 81-2414.

alternative; California; Class $\mathrm{K}$ code; conservation; discrimination; energy; United Stand; SP608: 1981 May. 83-103.

alternative fuel; bomb calorimetry; cellulose; enthalpy of combustion; refuse-derived-fuel; test substance; J. Res. 86(6): 655-660; 1981 November-December.

alumina-ceramic; artificial hip joints; endoprostheses; histomorphological; SP601: 1981 January. 131-156.

aluminum; copper; Drude theory; infrared; reflectance; silver; surface roughness; temperature dependence; SP620: 1981 October. 190-200.

aluminum; critical current; energy gap; enhancement; microwaves; nonequilibrium superconductivity; photon-assisted tunneling; 
superconductivity; thin film; transition temperature; tunneling; tunnel junction; 19979.

aluminum; energy gap; gap enhancement; microwaves; nonequilibrium; superconductivity; tunnel junction; 20723.

aluminum; field ion microscopy; iron-chromium alloys; niobium; repassivation kinetics; stainless steel; triboellipsometry; NBSIR 791904 (Navy).

aluminum; magnetic susceptibility; order-disorder; titanium; transmission electron microscopy; alloy phase diagram; 20736.

aluminum alloys; castings; composites; cryogenic properties; low temperature; mechanical properties; nonmetallics; physical properties; stainless steels; structural alloys; welding; alloys; NBSIR 81-1645.

$\mathrm{Al} \cdot \mathrm{Zn} \cdot \mathrm{Mg}$; anodic polarization; corrosion phenomena; electrochemical polarization; 20026.

ambient infrared radiation; blackbody cavity; corrections for reflectance; infrared imaging; infrared thermography; radiance temperature; reflected infrared radiation; temperature corrections; temperature measurements; 20733.

ambient temperature; argon; critical enhancement; hard sphere; hot wire; thermal conductivity; transient; J. Res. 86(3): 293-307; 1981 May-June.

ambipolar diffusion; electric discharges; helium Penning ionization; hydrogen; 20047.

American National Standard; Computer Output Microfilm (COM); Federal Information Processing Standard (FIPS); illumination; image quality; microfilm; microform; readers; FIPS PUB 84.

American National Standard; Federal Information Processing Standard (FIPS); interchange of machine readable information; nonread inks; Optical Character Recognition (OCR); OCR.A; OCR.B; read inks; FIPS PUB 85.

American National Standard Code for Information Interchange; ASCII; card punch; card reader; data communications; data interchange; data processing; EAM cards; electric accounting machine; Federal Information Processing Standard; Hollerith punched card code; Hollerith punched cards; information interchange; information processing; punched cards; standard; FIPS PUB 14-1.

American National Standard Code for Information Interchange; ASCII; character set; coded character set; code for information interchange; collating sequence; data communication; data interchange; data processing; Federal Information Processing Standard; information interchange; information processing; standard; FIPS PUB 1-1.

amino acid; conformational energy; dipole moment; gas phase conformers; glycine; rotational constants; structure; ab initio calculations; 20005.

amino acids; ethylene oxide; glycine; interstellar; methyl formate; radio astronomy; 20077.

aminobenzoate; composite material; dentin; methacrylate; polyfunctional surface-active comonomers; reconstructive dental work; resin; acrylate; adhesion; U.S. Patent 4,251,565.

aminoglutethimide or para-aminophenylacetic acid; cementing of bone; fillings; methacrylates; tertiary aromatic amines; unsaturated polyesters; acrylates; acrylic resins; U.S. Patent 4,243,763.

amphoteric latex; dynamic light scattering; light scattering; nematic liquid crystal; photo-receptor membrane vesicles; random coil polymers; J. Res. 86(6): 571.590; 1981 November-December.

analog signal; Josephson junction; sampling circuit; sampling pulses; U.S. Patent 4,245,169.

analog-to-digital converter; calibration service; data converter; differential linearity; digital-to-analog converter; error measurement; gain; input noise; linearity; offset; TN1145.

analysis; assay; evaluation; radioactivity; radiochemistry; radium-228; reference method; water; TN1137.

analysis; biological; cardiovascular; implants; metals; orthopaedic; polymers; retrieval; SP601.

analysis; certification; isotope dilution mass spectrometry; mass spectrometry; standard reference materials; 20024.

analysis; characterization; composition; materials; properties; research materials; standard reference materials; SP260, 1981-83 Edition. analysis; data elements; I/O; logical swapping; measurement; MVS; paging/swapping; TSO; SP500-83: 1981 November. 343-348.

analysis; dipole moments; electrically small objects; emission; measurements; quadrupoles; susceptibility; TEM cell; TN1040.

analysis; performance evaluation; pictures; simulation; tools; SP50083: 1981 November. 331.334.

analysis of moisture content; mass spectrometer measurement; moisture; moisture generators; moisture sensors; quality control; reliability of semiconductor devices; semiconductor devices; SP400-69.

analytical electron microscope; artifacts; energy dispersive $\mathrm{x}$-ray spectrometry; scanning transmission electron microscope; SP604: 1981 June. 341.349.

analytical electron microscope; electron-optical column; energy dispersive spectrometer; uncollimated fluorescing radiation; SP604: 1981 June. 351.364.

analytic models; communication networks; flow control; Markov processes; performance prediction; queueing models; simulation; validation; SP500-83: 1981 November. 277.290.

anemometer; drag sphere; gust speed; instrumentation; wind sensor; wind speed; 20059.

angular acceleration; angular accelerometer; calibration; instrumentation; measurement; shock and vibration; NBSIR 812337.

angular accelerometer; calibration; instrumentation; measurement; shock and vibration; angular acceleration; NBSIR 81-2337.

angular distribution; asymmetry parameter; branching ratios; resonance; xenon; 20052.

angular distributions; charmed mesons; dipole transitions; magnetic moments; photon decays; quarks; 20040.

anharmonic; Brillouin zone sums; central pair potential; fcc metals; free energy; high temperature nearest-neighbor model; thermal expansion; 20037.

animal toxicity; breath analysis; endurance; free-radicals; iron; organometalloid toxicity; pentane; respiration; SP618: 1981 November. 226-234.

annotated, consumer representation; literature review; medical device standards; public participation programs; standards development; voluntary standards; bibliography; NBSIR 81-2336.

annoyance; audible noise; corona noise; EHV transmission lines; electric energy; environmental noise; human response; power line noise; psychoacoustics; 20050.

annual fuel utilization efficiency; annual operating costs; central heating equipment; condensing boilers; condensing furnaces; partload performance; rating procedure; seasonal efficiency; NBSIR 80-2110.

annual operating costs; central heating equipment; condensing boilers; condensing furnaces; part-load performance; rating procedure; seasonal efficiency; annual fuel utilization efficiency; NBSIR 802110.

annual report; committee participation; standards committees; SP605.

annual reports; diffusion in metals; fire; journals; library holdings, NBS Library; NBS periodicals; periodicals; proceedings; serials; standards; transactions; NBSIR 81-2226.

anodic polarization; corrosion phenomena; electrochemical polarization; Al-Zn-Mg; 20026.

anodic stripping; bioavailability; complexing capacity; copper speciation; SP618: 1981 November. 173-183.

anodizing; coatings; electrodeposition; immersion deposition; plating; plating on anodized aluminum; adhesion; NBSIR 80-2142.

anomalous field evaporation; field evaporation; field ion microscopy; 19983.

ANS FORTRAN; computer system installation; examples; OMNITAB 80; software; test problems; accuracy; TN1147.

ANS X3.66; bit-oriented; code independence; data; data link control procedures; data transparency; Federal Information Processing Standards Publication; interoperability; teleprocessing; transmission; advanced data communication control procedures; FIPS PUB 78.

antenna; aperture; computer program; electromagnetic; far field; FFT; Fourier transform; algorithm; NBSIR 81-1643.

antenna-antenna systems; antenna measurements; antenna-scatterer 
systems; antenna theory; microwave antenna theory; scattering matrix description of antennas; Monogr. 162.

antenna beamwidths; aperture fields; directive antennas; NBSIR 81 1644.

antenna coupling; electrical distribution; glass-liquidus; insulation; monitors; neutron diffraction, samplers; SRM's; time-domain calibrations; weights and measures; x-ray diffraction; air pollutants; $D I M / N B S$ 65(1): 1.28; 1981 .

antenna measurements; antenna-scatterer systems; antenna theory; microwave antenna theory; scattering-matrix description of antennas; antenna-antenna systems; Monogr. 162.

antennas; data processing; electromagnetic; spherical scanning; 20765.

antennas; electromagnetic interference; electromagnetic metrology em material properties; microwaves; noise; non-ionizing radiation radiation hazards; $N B S I R \quad 80-1635$.

antenna-scatterer systems; antenna theory; microwave antenna theory; scattering-matrix description of antennas; antenna-antenna systems; antenna measurements; Monogr. 162.

antenna theory; microwave antenna theory; scattering-matrix description of antennas; antenna-antenna systems; antenna measurements; antenna-scatterer systems; Monogr. 162.

anticonvulsants; antiepilepsy drugs; blood analysis; chromatography, gas; chromatography, liquid; drug standards; enzyme immunoassay; epilepsy; ethosuximide; mass spectrometry; nuclear magnetic resonance spectroscopy; phenobarbital; phenytoin; primidone; reference material; serum base modification; serum matrix; SP26072.

antiepilepsy drugs; blood analysis; chromatography, gas; chromatography, liquid; drug standards; enzyme immunoassay; epilepsy; ethosuximide; mass spectrometry; nuclear magnetic resonance spectroscopy; phenobarbital; phenytoin; primidone; reference material; serum base modification; serum matrix; anticonvulsants; SP260-72.

antifouling coating; film; marine structures; piezoelectric polymer material; vegetable and animal life; U.S. Patent 4,297,394.

antifouling coating; film; marine structures; piezoelectric polymer material; vegetable and animal life; U.S. Patent 4,283,461.

antimony; arsenic; bacterial resistances; cadmium; detoxification; mercury; organomercury; plasmids; silver; toxic heavy metals; SP618: 1981 November. 301.324.

antioxidants; diffusion; food packaging; inverse gas chromatography; migration; oil simulants; oligomers; polyolefins; radioactive tracer; spectrofluorimetry; NBSIR 81-2264.

anti-reflection coatings; bichromatic; damage; high energy laser; laser damage; pulsed laser; reflectors; thin film; SP620: 1981 October. 287.296.

antireflection coatings; coating damage; laser damage; LiNbO coatings; Q-switch; surface damage; SP620: 1981 October. 300-304.

aortic prosthesis; embolization; prosthetic heart valve failure; SP601: 1981 January. 283-298.

apatite; barium apatite; cation substitution; crystal structure; rare-earth apatite; substituted fluorapatite; 20065.

aperture; computer program; electromagnetic; far field; FFT; Fourier transform; algorithm; antenna; NBSIR 81-1643.

aperture fields; directive antennas; antenna beamwidths; NBSIR 81 1644.

appliances; clothes washer; discharge; dishwasher; residential; supply; water conservation; water consumption; NBSIR 80-2173.

application development; computer software; cost/benefit analysis; database management systems; data management; program maintenance; requirements studies; software procurement; SP50084.

applicative programming; design; functional programming; interpreters; prototypes; 20075.

applied economics; construction; cost; estimation; location factors; model building; statistical analysis; NBSIR 81-2250.

aqueous; compilation; conductivity; electrolytes; enthalpy; Gibbs energy; osmotic coefficients; solutions; sulfuric acid; thermodynamic properties; transport properties; activity coefficients; NBSIR 81-2276.

aqueous; critical evaluation; $\mathrm{CsNO}_{2}$; electrolytes; excess Gibbs energy;
$\mathrm{KNO}_{2} ; \mathrm{LiNO}_{2} ; \mathrm{NaNO}_{2} ;$ osmotic coefficients; $\mathrm{RbNO}_{2}$; solutions; standard reference data; thermodynamic properties; activity coefficients; alkali metal nitrite salts; JPCRD 10(3): 767-778; 1981.

aqueous; critical evaluation; electrolytes; excess free energy; free energy; ionic; osmotic coefficients; solutions; sulfuric acid; standard reference data; thermodynamic properties; activity coefficients; JPCRD 10(3): 779-798; 1981.

aqueous solubility; gas chromatography; generator column; high pressure liquid chromatography; octanol/water partition coefficients; activity coefficients; NBSIR 81-2406.

aqueous solution; extinction coefficients; inorganic; radical ions; radicals; transients; absorption spectra; NSRDS-NBS69.

aqueous solutions; compilation; potassium chloride; reference data; viscosity; JPCRD 10(1): 57-70; 1981.

aqueous solutions; compilation; reference data; sodium chloride; viscosity; JPCRD 10(1): 71.88; 1981.

aqueous speciation; computerized; geochemical model; heavy metals; redox couples; trace elements; SP618: 1981 November. 39.53.

Arab League Organization for Standardization and Metrology; chemical industry standards; cotton standards; food standards; measurement science and technology; standardization in developing countries; Sudan; weights and measures regulation; Agency for International Development; NBSIR 80-2020.

architectural; electrical; elevators; mechanical; seismic coefficients; NBSIR 80-2111-8.

architectural acoustics; building acoustics; noise; noise control; sound transmission; structureborne noise; acoustics; NBS-GCR-80-248.

architectural barriers; building accessibility; codes and standards; disability; door closers; door openers; doors; ergonomics; functional capacity; handicap; locks; NBSIR 80-2174.

architectural design; building codes; building economics; construction costs; cost minimization; economic impact; economics; energy; model code; noise control; acoustical design; acoustics; NBSIR 81 2366.

argand diagram; complex error function; continued fraction; Dawson's function; FORTRAN subroutine; Fresnel integrals; key values; line broadening function; plasma dispersion function; Voigt function; J. Res. 86(6): 661-686; 1981 November-December.

argon; critical enhancement; hard sphere; hot wire; thermal conductivity; transient; ambient temperature; J. Res. 86(3): 293-307; 1981 May.June.

argon; dynamics; $m \cdot 6.8$ potential; nonequlibrium molecular; shear; viscosity; 19973.

aromatics; critical review, evaluated data; gases; halogenated hydrocarbons; Henry's law constants; liquids; pesticides, solids; solubility; vapor pressure; alkanes; JPCRD 10(4): 1175-1199; 1981.

arrangement; building; classification; code; engineering; organization; provisions; scope; specification; standard; system analysis/engineering; BSS136.

Arrhenius parameters; chemical kinetics; combustion; decomposition free radicals; gas phase; hydrocarbons; hydrogen; nitrogen; oxygen; rate of reaction; sulfur; NBSIR 81-2254.

arsenate; arsenic methylation; biological transformation; graphite furnace atomic absorption spectrometer; high pressure liquid chromatography; selenite; SP618: 1981 November. 244-263.

arsenic; bacterial resistances; cadmium; detoxification; mercury; organomercury; plasmids; silver; toxic heavy metals; antimony; SP618: 1981 November. 301-324.

arsenic; chromium; coal hydrogenation; cobalt; liquefaction; mercury; nickel; process waters; selenium; solvent refined coal; titanium; trace elements; SP618: 1981 November. 21-38.

arsenic; dimethylarsinic acid; microbial growth; mobilization; nutrients; oil shale; retorting; soil; speciation; volatilization; $S P 618$ 1981 November. 286-300.

arsenicals; cadmium metallothionein; cellular toxicity; elemental interactions; lead inclusion bodies; methylmercury derivatives; methylation/demethylation; SP618: 1981 November. 217-225.

arsenic ion implantation; electron-beam-induced current; etching; integrated circuit processing; ion channeling; ion implantation; lateral profiles; scanning electron microscopy; silicon technology; spreading resistance; two-dimensional profiles; NBSIR 81-2398. 
arsenic methylation; biological transformation; graphite furnace atomic absorption spectrometer; high pressure liquid chromatography; selenite; arsenate; SP618: 1981 November. 244. 263.

arson; combustible gas; detectors; diffusion rates; diffusion tubes; gas chromatography; hydrocarbons; vapor detector; vapor generator; vapor standards; accelerants; SP480-43.

arterial prostheses; biodeterioration; polyethylene terephthalate (Dacron); SP601: 1981 January. 29-129.

artifacts; energy dispersive $\mathrm{x}$-ray spectrometry; scanning transmission electron microscope; analytical electron microscope; SP604: 1981 June. 341-349.

artificial hip joints; endoprostheses; histo-morphological; aluminaceramic; SP601: 1981 January. 131-156.

artificial joints; biomaterials; wear debris; SP601: 1981 January. 239. 267.

ASCII; card punch; card reader; data communications; data interchange; data processing; EAM cards; electric accounting machine; Federal Information Processing Standard; Hollerith punched card code; Hollerith punched cards; information interchange; information processing; punched cards; standard; American National Standard Code for Information Interchange; FIPS PUB 14-1.

ASCII; character-imaging devices; control functions; data interchange; display terminals; Federal Information Processing Standard; information interchange; printer terminals; standard; text communication; text interchange; typesetting composition; ADP terminals; FIPS PUB 86.

ASCII; character set; coded character set; code for information interchange; collating sequence; data communication; data interchange; data processing; Federal Information Processing Standard; information interchange; information processing; standard; American National Standard Code for Information Interchange; FIPS PUB 1-1.

ASHRAE 90.75; class $\mathrm{K}$ code; computer modeling; electrical design; energy audit; energy conservation; HVAC systems; performance standards; solar collector; solar energy; space heating and cooling; thermal storage; SP608.

ASP (Arthritis Surgery Proforma); data retrieval system; implant failures; SP601: 1981 January. 615.642.

asphalt; impact attenuation; peak acceleration; playground safety; playground surfaces; soil; surfacing materials; test method; NBSIR 80-2106.

assay; evaluation; radioactivity; radiochemistry; radium-228; reference method; water; analysis; TN1137.

assessment; composite models; data quality; energy models; mathematical models; model confidence; model credibility; policy models; sensitivity analysis; validation; SP616.

ASTM E-162; brattice cloth; coal mining; fire test; flame spread; sample mounting; ventilation cloth; NBSIR 81-2200.

ASTM E 152; doors; fire doors; fire tests; air flow; air leakage; NBS. GCR $-81-330$

ASTM round robin testing; bomb calorimetry; higher heating value; refused-derived-fuel; sample characterization; sample processing effects; 2.5 and 25 gram capacity bomb calorimeters; NBSIR 812278.

astrophysics; bremsstrahlung; electromagnetic interactions; pair production; plasma diagnostics; radiation; synchrotron radiation; 19993.

asymmetry parameter; branching ratios; resonance; xenon; angular distribution; 20052.

atactic; crystal; crystallinity; density; enthalpy; entropy; fusion; Gibbs energy; glass transition; heat capacity; isotactic; linear macromolecule; melt; polypropylene; syndiotactic; JPCRD 10(4): $1051-1064 ; 1981$

ATE; automatic test equipment; calibration; data acquisition; durability; dynamic calibration; environmental testing; evaluation; measurand; reliability; sensor; transducer; SP615.

atom; atomic ion; intensities; line strengths; oscillator strengths; spectral lines; transition probabilities; wavelengths; NSRDSNBS68. atom; charge transfer; chemi-ionization; collisional ionization; cross section; detachment; energy transfer; excitation; ion; molecule; reaction rate; relaxation; SP593.

atomic absorption; energy-related process fluids; leachate; linear free energy; liquid chromatography; molecular substituent; oil shale organoarsenic; organotin; retention index; speciation; SP618: 1981 November. 197.216.

atomic absorption spectrometry; gasoline analysis; high performance liquid chromatography; metal-selective detector; metal speciation; tetraalkyllead speciation; 20763.

atomic absorption spectrophotometry; EDAX analysis; enzyme histochemistry; histology; implant retrieval analysis; polarography; tissue response; SP601: 1981 January. 323-336.

atomic and molecular properties; bibliographies; chemical kinetics; evaluated data; fluid properties; indexes; publication list; solid state; thermodynamic and transport properties; SP612.

atomic clocks; automatic time comparisons; common-mode cancellation; frequency comparison; global positioning system; remote time measurement; time comparison; time receiver worldwide time; 20062.

atomic energy levels; atomic ions; atomic spectra; electron configurations; ionization potentials; sodium; JPCRD 10(1): 153 $196 ; 1981$.

atomic energy levels; atomic spectra; cobalt; cobalt energy levels; spectra; JPCRD 10(4): 1097.1174; 1981.

atomic energy levels; atomic spectra; nickel energy levels; JPCRD 10(1): 197.290; 1981.

atomic ion; intensities; line strengths; oscillator strengths; spectral lines; transition probabilities; wavelengths; atom; NSRDS-NBS68.

atomic ions; atomic spectra; electron configurations; ionization potentials; sodium; atomic energy levels; JPCRD 10(1): 153.196; 1981.

atomic ions; calibration; clock synchronization; Columbia River model; computer; insulation; lasers; powder data; progress; R-values; single-crystal; SRM's; DIM/NBS 65(2): 1-28; 1981.

atomic spectra; cobalt; cobalt energy levels; spectra; atomic energy levels; JPCRD 10(4): 1097.1174; 1981.

atomic spectra; electron configurations; ionization potentials; sodium atomic energy levels; atomic ions; JPCRD 10(1): 153-196; 1981.

atomic spectra; nickel energy levels; atomic energy levels; JPCRD 10(1): $197-290 ; 1981$

atomic weight; coal; computers; drill failure; Faraday; firefighting; hot atom; industrial innovation; measurements; oils, PCB's; standards $D I M / N B S$ 65(6): 1.24; 1981 .

atom jump frequencies; correlation factor for diffusion; diffusion coefficient; diffusion equations; drift velocity for diffusion; driving force for diffusion; vacancy flow; vacancy wind effect; 20038.

attenuation; coaxial sample holder; density; dielectric properties; relative dielectric constant; soil textural types; NBSIR 78-896.

attenuation; core diameter; far field; index profile; mode filter; numerical aperture; radiation angle; radiation patterns; TN1032.

attenuators; beam profile; calorimetry; fluence; heat flow; irradiance; measurements of temperature; plasma effects; pulsed laser beams $10.6 \mu \mathrm{m} ;$ NBSIR 80-1628.

attic floor radiant panel test; cellulose insulation; fire test; flame spread; HH.I.515D; smoldering; NBSIR 81-2213.

attic temperature; BLAST; computer simulation; DoE-2; heating/cooling load calculation; indoor temperature; NBSLD 20755.

audible noise; corona noise; EHV transmission lines; electric energy; environmental noise; human response; power line noise; psychoacoustics; annoyance; 20050.

auditorium; building standards; crowd ingress; design procedures evaluation procedures; facility design; facility management; public assembly; public safety; stadiums; NBSIR $81-2361$.

auditor training; energy conservation; program measure; Residential Conservation Service; Solar Model Audit; SP608: 1981 May. 183. 194.

auditory perception; decibel levels; fire departments; frequency distribution; noise (sound); sleep; smoke detectors; wakefulness adults; alarm responses; NBS-GCR-80-284. 
auger; core hole spectroscopy; metals with filled $d$-bands; photoemission; resonance; satellite; 20008.

Auger-electron spectroscopy; electronics; MOS devices; semiconductors; silicon dioxide; sputter profiling; NBS-GCR-8I345 .

Auger electron spectroscopy (AES); metal-oxide-semiconductor (MOS); secondary ion mass spectrometry (SIMS); semiconductor devices; sputtering; $x \cdot$ ray photoelectron spectroscopy (XPS, ESCA); SP400-67.

Auger function; electrically-short dipole; iteration method; nonlinear differential equation; nonlinear load; time-stepping finite difference equation technique; NBSIR 78-898.

Auger spectroscopy; convection; ellipsometry; gallium; levitation calorimetry; specific heat; surface tension; thermophysical properties; tungsten; wetting; adsorption; NBSIR 81-2295.

AUGMENT and MP; Fortran arithmetic extensions; multiple precision; multiprecision Fortran software; multiprecision mathematical functions; precompilers for special arithmetic; NBSIR 80-2138.

augmented electric- and magnetic-field integral equations; electricfield integral equation; electromagnetic theory; magnetic-field integral equation; scattering of electromagnetic waves; spurious resonances; 20761.

augmented electric- and magnetic-field integral equations; electricfield integral equation; electromagnetic theory; magnetic-field integral equation; scattering of electromagnetic waves; spurious resonances; 20760.

austenite in ferrite; austenite standard; electron microprobe; powder metallurgy; quantitative microscopy; standard reference material; $x$-ray diffraction; $x$-ray fluorescence analysis; SP260-73.

austenite standard; electron microprobe; powder metallurgy; quantitative microscopy; standard reference material; x-ray diffraction; $x$-ray fluorescence analysis; austenite in ferrite; SP26073.

austenitic stainless steel; electron probe microanalysis; $\mathrm{Fe} \cdot \mathrm{Cr} \cdot \mathrm{Ni}$ alloy; homogeneity testing; standard reference material; SP260-70.

austenitic stainless steel; fracture toughness; low temperature; tensile properties; 20772.

austenitic stainless steels; coal gasification; elevated temperatures; ferritic stainless steel; gaseous environments; nickel alloy 671; nickel alloy 800 ; slow strain rate test; structural materials; NBSIR 81-2191 (DoE).

authentication; authorization; computer network; computer security; encryption; Federal Information Processing Standards Publication; identification token; identity verification; password; personal attribute; personal identification; access control; FIPS PUB 83.

authorization; computer network; computer security; encryption; Federal Information Processing Standards Publication; identification token; identity verification; password; personal attribute; personal identification; access control; authentication; FIPS PUB 83.

auto headlight glass; classification; density; forensic science; individualization; population statistics; refractive index; NBSIR 812286.

autoignition temperature; coal mines; fire point; fire resistance tests; fire tests; flammability; flash point; hydraulic fluids; ignition; NBSIR 81-2395.

autoionization; electron affinity; molecular spectroscopic constants; negative ions; photodetachment; potential energy curves; 19995.

autoionization resonance; electron collision cross sections; heliumlike ions; 20045 .

automated automation; computer performance management; SP50083: 1981 November. 67.70 .

automated data printing; computer-output microforms; Federal Information Processing Standards Publication; form slide; microfilm; quality control; FIPS PUB 82.

automated data processing; computers; cost-benefit; NBS-GCR-81262.

automated data processing; computer utilization trends; Federal computers; hardware trends; information industry; software trends; technology forecast; NBS-GCR-81-342. automated data processing (ADP); communications; Federal Information Processing Standard (FIPS): Federal Property Management Regulations (FPMR); procurement; standards; telecommunications; FIPS PUB 80.

automated software tools; software lifecycle; software testing; software verification; test coverage; test data generation; validation; verification; SPSO0-75.

automated thermogravimetry; data analysis; generalized plotting programs; interdata 7.16 computer; polynominal fitting; user manual; NBSIR 80-2102.

automatic alarm of bearing malfunction; marine machinery bearings; microprocessor based shock pulse analyzer; multiplexing bearing monitor channels; prediction of bearing failure; shock pulse bearing monitoring; SP622: 1981 October. 126.136.

automatic ignition devices; caulks; effectiveness; energy conservation; energy conservation measures; installation; insulation; oil burners; practices; safety; sealants; standards; storm doors; storm windows; vent dampers; water heaters; windows; SP606.

automatic network analyzer; microwave; microwave measurements; six-port; 20067.

automatic sprinklers; cooling efficiency; evaporation cooling; smoke movement; spray nozzle; stairway protection; ventilated stair; NBSIR 81-2202.

automatic test equipment; calibration; data acquisition; durability; dynamic calibration; environmental testing; evaluation; measurand; reliability; sensor; transducer; ATE; SP615.

automatic time comparisons; common-mode cancellation; frequency comparison; global positioning system; remote time measurement; time comparison; time receiver; worldwide time; atomic clocks; 20062.

automation; buildings; certified reference materials; computers; construction; data converter; mass comparator; radiation; radiocarbon; soft aluminum; windows; air pollution; DIM/NBS 65(4): $1.28 ; 1981$

automation; information management; information technology; productivity; word processing; SP500-83: 1981 November. 305-307. automation; powder diffraction; quantitative analysis; $\mathrm{x}$ ray; NBSIR 81.2229

automotive; EMI; ignition interference; suppression; SP480-44.

automotive paints; household paints; sequential tests; solubility tests; $S P 480-40$.

auxiliary energy; FCHART computer program; National Solar Demonstration Program; solar energy systems; space heating load; thermal performance; NBS-GCR-81-321.

availability; environmental contamination; extraction tests; fossil fuels; laboratory characterization; metals; mining industry; public health impacts; residues; toxic elements; SP618: 1981 November. 1-8.

avalanche breakdown; beam deformation; beam depletion; free carrier absorption; multiphoton absorption; polaron absorption; SP620: 1981 October. 394-402.

azidotriphenylmethane; decomposition reactions; differential scanning calorimetry; heat conduction calorimetry; kinetics of heat evolution; m-azidobenzoic acid; nitrocellulose; quantitative differential thermal analysis; thermal explosion theory; thermal hazards; thermal instability; adiabatic calorimetry; NBSIR 80-2018 (DOT).

backscattering; capture fractions; fiber scattering; optical time domain reflectometry; Rayleigh scattering; TNI034.

backup operations; computer applications; computer security; contingency planning; emergency response; Federal Information Processing Standards Publication; recovery actions; ADP availability; ADP security; FIPS PUB 87.

bacteria; biodeterioration; biotransformations; deterioration of materials; element volatilization; fungi; metals; microorganisms; standard reference materials; NBSIR 81-2246.

bacterial mobilization; biogeochemical cycles; biological uptake; cellular toxicity-essentiality mechanisms; chromatography; elementselective speciation; environmental monitoring; fossil fuels; 
metal(loid) complexes; organometallic compounds; trace element standards; waste cycling; SP618.

bacterial resistances; cadmium; detoxification; mercury; organomercury; plasmids; silver; toxic heavy metals; antimony; arsenic; SP618: 1981 November. 301-324.

$\mathrm{BaF}_{2} ; \mathrm{CaF}_{2} ;$ extrinsic and intrinsic infrared absorption; infrared wavelength modulation; $\mathrm{LaF}_{3}$; laser windows; $\mathrm{LiF} ; \mathrm{MgF}_{2} ; \mathrm{MgO}$; $\mathrm{NaCl} ; \mathrm{NaF} ; \mathrm{SrF}_{2} ;$ volume and surface impurities; SP620: 1981 October. 29-43.

balloon inflation; cryogenic helium balloon inflation; heat transfer; liquid helium; packed bed heat exchanger; 20017.

ball plate; grid plate; machine geometry; measuring machine; redundancy; 3-D metrology; NBSIR 79-1752.

band shape analysis; collision-induced absorption; far infrared; $\mathrm{H}_{2}-\mathrm{H}_{2}$ collisions; $\mathrm{H}_{2}-\mathrm{He}$ collisions; planetary atmospheres; quadrupoleinduced absorption; spectral moments; NBSIR 80-2175.

band structure; effective mass; intraband-momentum matrix element; oscillator strength; two-photon absorption; SP620: 1981 October. 438.445.

band-structure; nonlinear absorption; oscillator strength; two-photon absorption; SP620: 1981 October. 432.437.

Bargmann limits; bound-state estimates; Lieb limits; number of bound states; Schwinger limits; screened Coulomb potential; Sobolev's inequality; Yukawa potential; J. Res. 86(5): 503.508; 1981 September-October.

barium apatite; cation substitution; crystal structure; rare-earth apatite; substituted fluorapatite; apatite; 20065.

barrier properties; diamond-like carbon; ion beam deposition; surface smoothness; thin films; SP620: 1981 October. 313-323.

base current, reverse; degradation; reverse bias; second breakdown; switching; temperature; transistors, power; NBSIR 81-2204.

base line signatures; frequency spectra; hot alignment; machinery parameters; temperatures; vibrations; SP622: 1981 October. 220-221.

base metal; casting; composite; cyanoacrylate; dental alloy; initiator; resin; wear; accelerator; alloy; NBSIR 80-2168.

basestock; engine lubricants; lubricating oil; motor oil; petroleum oil; recycled oil; rerefined oil; test procedures; 20016.

BASIC; COBOL; compilers; data processing; Federal Information Processing Standard; FORTRAN; information interchange; information processing; processor; programming language; software; FIPS PUB 29-1.

basic measurements and standards; building technology; calibration methods; computer standards and guidelines; energy research; environmental measurements; fire safety; industrial productivity; materials properties; research; standards; technology transfer; SP600.

basic standards; fine-structure constant; fundamental constants; Josephson effect; least-squares adjustments; quantum electrodynamics; 20058.

basic weights and measures law; method of sale of commodities; open dating; packaging and labeling; registration of service persons; unit pricing; weighmaster law; H130, 1982 Edition.

beam deformation; beam depletion; free carrier absorption; multiphoton absorption; polaron absorption; avalanche breakdown; SP620: 1981 October. 394-402.

beam depletion; free carrier absorption; multiphoton absorption; polaron absorption; avalanche breakdown; beam deformation; SP620: 1981 October. 394-402.

beam depletion; nonlinear absorption; photoacoustic technique; absorption in solids; SP620: 1981 October. 50-57.

beam profile; calorimetry; fluence; heat flow; irradiance; measurements of temperature; plasma effects; pulsed laser beams; $10.6 \mu \mathrm{m}$; attenuators; NBSIR 80-1628.

BEBO activation energy; bond-order-bond-energy; chemical rate constants; computer program; hydrogen atom transfer; absolute chemical rate; J. Res. 86(6): 605-654; 1981 November-December.

bedding; beds; compartment fires; fire tests; health care facilities; heat release rate; hospitals; mattresses; prisons; smoke production; NBSIR 80-2186.

beds; compartment fires; fire tests; health care facilities; heat release rate; hospitals; mattresses; prisons; smoke production; bedding;
NBSIR 80-2186.

bellows; compressor; contamination; continuous operation; cryocooler; low power refrigerator; Stirling refrigerator; SP607: 1981 May. 141-145.

benchmarking; benchmark library; selection of ADP systems; standard benchmarks; synthetic benchmarks; workload characterization; workload definition; 20747.

benchmarking; capacity planning; chargeback systems; computer performance management; data base machines; end user productivity; human factors evaluation; information system management; office automation; performance management systems; resource measurement facility; simulation; supercomputers; SPSOO83.

benchmarking; Federal Information Processing Standards Publication; performance evaluation; workload analysis; workload characterization; workload representation; ADP acquisition; ADP procurement; FIPS PUB 75.

benchmarking; FIPS PUB 75; vendor performance; SP500-83: 1981 November. 339.

benchmark library; selection of ADP systems; standard benchmarks; synthetic benchmarks; workload characterization; workload definition; benchmarking; 20747.

benchmarks; interactive workloads; UNISKEL; universal skeleton; SP500-83: 1981 November. 143-156.

benchmark testing; Phase IV; remote terminal emulation; response time; terminal networks; workload sizing; SP500-83: 1981 November. 169-177.

benefit-cost analysis; building economics; building regulation; codes; construction regulation; economics; energy conservation codes; fire safety codes; regulation; NBSIR 81-2223.

benefit-cost analysis; building economics; building regulation; codes; construction economics; construction regulation; economics; fire safety codes; regulation; NBSIR 81-2402.

benefit-cost analysis; building economics; cost effective; economic analysis; energy conservation; investment analysis; life-cycle cost; recommended practice; savings-to-investment ratio; NBSIR 81 2397.

benefits; cost-effective; costs; economic analysis; economic decision framework; energy conservation; marginal price; net present value; residential water conservation; wastewater treatment; water rate schedules; water-saving devices; NBSIR 81-2304.

benzo(a)pyrene; benzo(e)pyrene; fluoranthene; fluorescence detection; gas chromatography/mass spectrometry; high-performance liquid chromatography; perylene; pyrene; shale oil; standard reference materials; 20750.

benzo(e)pyrene; fluoranthene; fluorescence detection; gas chromatography/mass spectrometry; high-performance liquid chromatography; perylene; pyrene; shale oil; standard reference materials; benzo(a)pyrene; 20750.

BEPS; code compliance; code conflicts; energy conservation standards; solar energy; trainee performance testing; SP608: 1981 May. 139-157.

beryllium isoelectronic sequence; electron-atom scattering; electron impact ionization; metastable states; 20739.

beta rays; Federal and State laboratories; gamma rays; instrument calibrations; intermediate calibration laboratories; ionizing radiation measurements; measurement accuracies; neutrons; radiation dosimetry; radiation protection; radiation standards; $x$ rays; SP603.

Bethe-Heitler cross section; bremsstrahlung differential cross section; bremsstrahlung monochromator; photon beams; photonuclear research; tagged photon method; NBSIR 81-2262.

bibliographies; building fires; coal mines; combustion products; compartment fires; fabric flammability; fire research; fire tests; flame research; smoke; NBSIR 81-2272.

bibliographies; chemical kinetics; evaluated data; fluid properties; indexes; publication list; solid state; thermodynamic and transport properties; atomic and molecular properties; SP612.

bibliographies; chimneys; combustion; fire safety; heating equipment; literature reviews; stoves; wood; NBS-GCR-80-298.

bibliography; annotated, consumer representation; literature review; medical device standards; public participation programs; standards 
development; voluntary standards; NBSIR 81-2336.

bibliography; eddy currents; index; National Bureau of Standards; nondestructive evaluation; publications; radiography; ultrasonics; abstracts; acoustic emission; NBSIR 80-2080.

bibliography; electron column instruments; energy dispersive detectors; scanning electron microscopy; $x$-ray spectrometry, review of scientific instruments; SP604: 1981 June. 417-439.

bibliography; measurements; optical communications; optical fibers; TN1043.

bichromatic; damage; high energy laser; laser damage; pulsed laser; reflectors; thin film; anti-reflection coatings; SP620: 1981 October 287-296.

bicycle compliance tests; bicycle safety; reflective materials for bicycles; safety of bicycles; safety standards; abrasion test development; NBSIR 80-2035.

bicycle safety; reflective materials for bicycles; safety of bicycles; safety standards; abrasion test development; bicycle compliance tests; NBSIR 80-2035.

binary liquids; critical mixing; critical phenomena; dielectric constant; fluids in an electric field; refractive index; 20056.

binary stars; late-type stars; stars, individual; stellar chromospheres; stellar coronae; ultraviolet spectra; 20001.

bin method; building energy performance standards (BEPS); degreeday; DOE-2; 20756.

bioavailability; complexing capacity; copper speciation; anodic stripping; SP618: 1981 November. 173-183.

bioavailability; element speciation; gas chromatography; ionization processes; liquid chromatography; mass spectrometry; SP618: 1981 November. 120-133.

biodeterioration; biotransformations; deterioration of materials; element volatilization; fungi; metals; microorganisms; standard reference materials; bacteria; NBSIR 81-2246.

biodeterioration; polyethylene terephthalate (Dacron); arterial prostheses; SP601: 1981 January. 29-129.

biogeochemical cycles; biological uptake; cellular toxicity-essentiality mechanisms; chromatography; element-selective speciation; environmental monitoring; fossil fuels; metal(loid) complexes; organometallic compounds; trace element standards; waste cycling; bacterial mobilization; SP618.

bioheat equation; burn; criterion; critical exposure; fluid; heat transfer; injury threshold; scald; thermal injury; NBSIR 81-2320.

biological; cardiovascular; implants; metals; orthopaedic; polymers; retrieval; analysis; SP601.

biological transformation; graphite furnace atomic absorption spectrometer; high pressure liquid chromatography; selenite; arsenate; arsenic methylation; SP618: 1981 November. 244-263.

biological uptake; cellular toxicity-essentiality mechanisms; chromatography; element-selective speciation; environmental monitoring; fossil fuels; metal(loid) complexes; organometallic compounds; trace element standards; waste cycling; bacterial mobilization; biogeochemical cycles; SP618.

biomaterials; wear debris; artificial joints; SP601: 1981 January. 239 267.

biomedical thermometry; disodium hydrogen phosphate; fixed point; hydration temperature; potassium fluoride; salt hydrate; sodium sulfate; temperature calibration; temperature standard; thermistor; thermometer; transition temperature; J. Res. 86(2): 181-192; 1981 March-April.

bio-prostheses; prosthetic heart valves; thromboembolism; SP601: 1981 January. 175-200.

bioprosthesis; dysfunction patterns; valvular substitute; SP601: 1981 January. 363-393.

biotransformations; deterioration of materials; element volatilization; fungi; metals; microorganisms; standard reference materials; bacteria; biodeterioration; NBSIR 81-2246.

BIPM; CCU; CGPM; CIPM; metric; SI; translation; SP330.

bispectrum; cumulant spectra; nonlinear time series; polyspectra; spectrum analysis; statistics; time series; TN1036.

bit error rate; coaxial transmission lines; digital communication impulse response; instrumentation; measurements; shielded balanced transmission lines; time domain; transmission line model; waveform;
TN1042.

bit-oriented; code independence; data; data link control procedures; data transparency; Federal Information Processing Standards Publication; interoperability; teleprocessing; transmission; advanced data communication control procedures; ANS X3.66; FIPS PUB 78.

bitumen; bonding; built-up roofing membrane; complementary strain energy; felt; nonlinear; roofing membrane; splitting; strain energy; stress; substrate; adhesive; TN1152.

bitumen application temperatures; bituminous roofing; built-up roofing; cooling of roofing bitumens; mathematical model; roofing; TN1135.

bituminous roofing; built-up roofing; cooling of roofing bitumens; mathematical model; roofing; bitumen application temperatures TN1135.

blackbody cavity; corrections for reflectance; infrared imaging; infrared thermography; radiance temperature; reflected infrared radiation; temperature corrections; temperature measurements ambient infrared radiation; 20733.

blade vibration; Doppler signature; Doppler technique; high cycle fatigue; stress corrosion; turbine blade failures; SP622: 198 October. 181-194.

BLAST; building energy performance standards; computer modeling; maintain accuracy; reduce cost; reduce input; standard evaluation technique; SP608: 1981 May. 113-122.

BLAST; computer simulation; DoE-2; heating/cooling load calculation; indoor temperature; NBSLD; attic temperature; 20755.

blind; code requirements; communication; deaf; directional indicators handicapped users; tactile warnings; accessibility; alarms; NBSIR $81-2428$.

Blodgett-Langmuir pseudo-crystals; Bragg crystal diffraction; energydispersive; x-ray spectrochemical analysis; SP604: 1981 June. 1-4.

blood alcohol analysis; blood alcohol content; breath alcohol content; breath sampling; evidential breath testers; human respiratory characteristics; intoxication; SP480-41.

blood alcohol content; breath alcohol content; breath sampling evidential breath testers; human respiratory characteristics; intoxication; blood alcohol analysis; SP480-41.

blood analysis; chromatography, gas; chromatography, liquid; drug standards; enzyme immunoassay; epilepsy; ethosuximide; mass spectrometry; nuclear magnetic resonance spectroscopy; phenobarbital; phenytoin; primidone; reference material; serum base modification; serum matrix; anticonvulsants; antiepilepsy drugs; SP260-72.

board and care homes; building codes; building fires; evacuation; fire alarm systems; fire safety; handicapped; life safety; refuge; 20727.

boilers; central heating; condensing boilers; efficiency, part load; fossil-fuel heating systems; gas-fired boilers; hydronic heating; pulse combustion; NBSIR 80-2094.

boiling; carbon film; heat transfer; helium; hysteresis; temperature control; transient; 19976.

bomb calorimetry; cellulose; enthalpy of combustion; refuse-derived. fuel; test substance; alternative fuel; J. Res. 86(6): 655-660; 1981 November-December.

bomb calorimetry; higher heating value; refused-derived-fuel; sample characterization; sample processing effects; 2.5 and 25 gram capacity bomb calorimeters; ASTM round robin testing; NBSIR $81-2278$.

bombs (ordnance); boxcars; computer models; fire detection systems; fire suppression; full-scale fire tests; heat transfer; railroad accidents; small-scale fire tests; thermal protection; NBSIR 80-2170.

bonding; built-up roofing membrane; complementary strain energy; felt; nonlinear; roofing membrane; splitting; strain energy; stress; substrate; adhesive; bitumen; TN1152.

bonding with coupling agents; hydroxylapatite; interaction with $\mathrm{Zn}$ ions and enamel etching; reaction with $\mathrm{Zn}$ ions and rate laws; surface mordanting with $\mathrm{Zn}$ ions; 20729.

bond-order-bond-energy; chemical rate constants; computer program hydrogen atom transfer; absolute chemical rate; BEBO activation energy; J. Res. 86(6): 605-654; 1981 November-December.

bone plates; cobalt-chrome-nickel-tungsten-iron-alloy; fretting 
corrosion; histologic analysis; stainless steel 316 LVM; titanium, titanium alloy; SP601: 1981 January. 395-422.

boric acid; cellulosic insulation; ignition; propagation; smoldering combustion; test methods; wave structure; NBSIR 81-2350.

Born-Oppenheimer approximation; diatomic spectroscopy; FranckCondon principle; rotational-electronic coupling; scattering theory; adiabatic potentials; 20060

boron; capacitance-voltage technique; dopant density; electron mobility; Hall effect; hole mobility; Irvin curves; phosphorus; resistivity; semiconductor; silicon; SP400-64.

boundary layer; compliant surface; drag, experimental; flat plate, turbulent; 20048.

bound-state estimates; Lieb limits; number of bound states; Schwinger limits; screened Coulomb potential; Sobolev's inequality; Yukawa potential; Bargmann limits; J. Res. 86(5): 503-508; 1981 SeptemberOctober.

bound states; continuum states; diatomic spectroscopy; electronicrotational states; predissociation; scattering theory; 20049.

boxcars; computer models; fire detection systems; fire suppression; full-scale fire tests; heat transfer; railroad accidents; small-scale fire tests; thermal protection; bombs (ordnance); NBSIR 80-2170.

Bragg crystal diffraction; energy-dispersive; x-ray spectrochemical analysis; Blodgett-Langmuir pseudo-crystals; SP604: 1981 June. 1.4.

Bragg-Gray equation; calibration; cavity chamber; cavity theory; electron beam; in-phantom measurement; photon beam; radiation therapy; absorbed dose; 20073.

branch circuit; electric wire; field measurements; temperature excursions; thermal insulation; NBSIR 81-2347.

branch circuits; circuit breaker; electrical fire; low ambient temperature; trip time; NBSIR 81-2221.

branch circuit wiring problems; electrical problems; electrical wiring system; extension cord wiring; fuse/circuit breaker panel problems; overamping; residential structures; safety; thermal insulation; NBSIR 81-2203.

branching ratios; resonance; xenon; angular distribution; asymmetry parameter; 20052.

brattice cloth; coal mining; fire test; flame spread; sample mounting; ventilation cloth; ASTM E-162; NBSIR 81-2200.

Brazil; industrialization; international cooperation; measurement infrastructure; metrology; technologies; technology transfer; NBSIR 80-1972.

breakdown curves; photoelectron-photoionization coincidence; photoionization; propyne; threshold photoelectron spectra; 20051.

breath alcohol content; breath sampling; evidential breath testers; human respiratory characteristics; intoxication; blood alcohol analysis; blood alcohol content; $S P 480-41$.

breath analysis; endurance; free-radicals; iron; organometalloid toxicity; pentane; respiration; animal toxicity; SP618: 1981 November. 226-234.

breath sampling; evidential breath testers; human respiratory characteristics; intoxication; blood alcohol analysis; blood alcohol content; breath alcohol content; SP480-41.

bremsstrahlung; electromagnetic interactions; pair production; plasma diagnostics; radiation; synchrotron radiation; astrophysics; 19993.

bremsstrahlung differential cross section; bremsstrahlung monochromator; photon beams; photonuclear research; tagged photon method; Bethe-Heitler cross section; NBSIR 81-2262.

bremsstrahlung monochromator; photon beams; photonuclear research; tagged photon method; Bethe-Heitler cross section; bremsstrahlung differential cross section; NBSIR 81-2262.

Brillouin zone sums; central pair potential; fcc metals; free energy; high temperature nearest-neighbor model; thermal expansion anharmonic; 20037.

bronze process; $\mathrm{Nb}_{3} \mathrm{Sn}$; processing limits; ultrafine filament $\mathrm{Nb}_{3} \mathrm{Sn}$; 20741.

bubble memory; mathematical model; standard transaction; transaction driven system; SP500-83: 1981 November. 259-265.

bubbles; polymer degradation products; pressure; thermal degradation; thermogravimetry; activation energy; 20774.

building; building codes; building design; earthquakes; engineering; masonry; standards; structural engineering; NBSIR 80-2111-5. building; building codes; building design; earthquakes; engineering; reinforced concrete; standards; structural engineering; NBSIR 80 2111-4.

building; building codes; building design; earthquakes; engineering; standards; structural engineering; NBSIR 80-2111-11.

building; building codes; building design; earthquakes; engineering; standards; structural engineering; wood; NBSIR 80-2111-7.

building; building codes; building design; earthquakes; engineering; standards; steel; structural engineering; NBSIR 80-2111-6.

building; building design; earthquakes; engineering; Federal government; seismic safety; standards; structural engineering; NBSIR 81-2195.

building; classification; code; engineering; organization; provisions; scope; specification; standard; system analysis/engineering; arrangement; BSS136.

building; collapse; concrete; concrete strength; construction; failure; flat plate; shear; strength; NBSIR 81-2374.

building accessibility; building research; fire safety; handicapped; life safety; accessibility; NBSIR 81-2245.

building accessibility; codes and standards; disability; door closers; door openers; doors; ergonomics; functional capacity; handicap; locks; architectural barriers; NBSIR 80-2174.

building acoustics; impact noise; noise control; noise isolation; noise rating; acoustics; NBS-GCR-80-249.

building acoustics; noise; noise control; sound transmission; structureborne noise; acoustics; architectural acoustics; NBS-GCR80-248.

building acoustics; noise control; noise isolation; sound transmission; structure-borne noise; acoustics; NBS-GCR-80-250.

building circulation; building ramps; pedestrian circulation; pedestrian flow; pedestrian movement; pedestrian ramps; ramps; NBSIR 81 2310.

building code; building envelope; comparison; compliance; energy budget; energy conservation; performance standards; Statement of Review; verification; SP608: 1981 May. 57-67.

building code; building research; buildings; code enforcement; regulations; rehabilitation; $S P 623$.

building codes; building design; earthquakes; engineering; masonry; standards; structural engineering; building; NBSIR 80-2111-5.

building codes; building design; earthquakes; engineering; reinforced concrete; standards; structural engineering; building; NBSIR 80 2111-4.

building codes; building design; earthquakes; engineering; standards; steel; structural engineering; building; NBSIR 80-2111-6.

building codes; building design; earthquakes; engineering; standards; structural engineering; building; NBSIR 80-2111-11.

building codes; building design; earthquakes; engineering; standards; structural engineering; wood; building; NBSIR 80-2111-7.

building codes; building economics; construction; cost; economics; housing; productivity; regulation; renovation; NBSIR 80-2150.

building codes; building economics; construction costs; cost minimization; economic impact; economics; energy; model code; noise control; acoustical design; acoustics; architectural design; NBSIR 81-2366.

building codes; building fires; evacuation; fire alarm systems; fire safety; handicapped; life safety; refuge; board and care homes; 20727.

building codes; building officials; codes and standards; convective loops; greenhouses; heat exchangers; light and ventilation; solar systems; space heating and cooling; training programs; SP608: 1981 May. 159-166.

building codes; building officials; consensus; construction; document; installation; recommended requirements; solar energy; solar systems; SP608: 1981 May. 167-172.

building (codes); climatology; hurricanes; reliability; risk; structural engineering; 20764.

building codes; fire modeling; fire protection engineering; sprinkler systems; systems analysis; 20746.

building design; building economics; energy conservation; exterior wall; HVAC calculations; insulation; life-cycle cost analysis; masonry; mass; BSS134. 
dilding design; building energy performance standards; computer simulation of house energy requirements; degree days; energy analysis; single-family detached residence; NBSIR 80-2184.

building design; earthquakes; engineering; Federal government; seismic safety; standards; structural engineering; building; NBSIR 81-2195.

building design; earthquakes; engineering; masonry; standards; structural engineering; building; building codes; NBSIR 80-2111-5.

building design; earthquakes; engineering; reinforced concrete; standards; structural engineering; building; building codes; NBSIR 80-2111-4.

building design; earthquakes; engineering; standards; steel; structural engineering; building; building codes; NBSIR 80-2111-6.

building design; earthquakes; engineering; standards; structural engineering; building; building codes; NBSIR 80-2111-11.

building design; earthquakes; engineering; standards; structural engineering; wood; building; building codes; NBSIR 80-2111-7.

building drainage; gradually varied flow; hydraulic jump; partially filled pipe flow; NBSIR 81-2367.

building drainage; numerical solutions for transient depth; unsteady partially-filled pipe flow; waste solid transport; NBSIR 81-2308.

building drainage; supercritical flow; transition length; vertical stack drain entry; NBSIR 81-2290.

building drainage; waste solid transportation; water conservation; w.c. efficiency; NBSIR 81-2307.

building economics; building regulation; codes; construction economics; construction regulation; economics; fire safety codes; regulation; benefit-cost analysis; NBSIR 81-2402.

building economics; building regulation; codes; construction regulation; economics; energy conservation codes; fire safety codes; regulation; benefit-cost analysis; NBSIR 81-2223.

building economics; construction; cost; economics; housing; productivity; regulation; renovation; building codes; NBSIR 80 2150.

building economics; construction costs; cost minimization; economic impact; economics; energy; model code; noise control; acoustical design; acoustics; architectural design; building codes; NBSIR 81 2366.

building economics; cost effective; economic analysis; energy conservation; investment analysis; life-cycle cost; recommended practice; savings-to-investment ratio; benefit-cost analysis; NBSIR 81-2397.

building economics; daylighting; energy conservation; engineering economics; life-cycle costs; passive solar; regional analysis; thermal efficiency; windows; NBSIR 81-2248.

building economics; energy conservation; exterior wall; HVAC calculations; insulation; life-cycle cost analysis; masonry; mass; building design; BSS134.

building energy analysis; computer modeling; controls; control strategies; energy conservation for nonresidential buildings; load calculations; NBSIR 81-2277.

building energy analysis; computer simulation; infiltration; natural ventilation; NBSIR 81-2404.

building energy analysis; energy conservation for nonresidential buildings; historic preservation and energy conservation; load calculation; masonry thermal mass; system simulation; NBSIR 81 2317.

building energy conservation systems; EMCS; energy management; energy monitoring and control systems; HVAC controls; reliability study; NBSIR 81-2346.

building energy monitoring; energy measurement; steam consumption measurement; uncertainty estimate; NBSIR 81-2313.

building energy monitoring; instrumentation; sensors; NBSIR 80 2104.

building energy performance; codes; energy conservation; energy policy; Hawaii; performance standards; SP608: 1981 May. 105-112.

building energy performance standards; computer modeling; maintain accuracy; reduce cost; reduce input; standard evaluation technique; BLAST; SP608: 1981 May. 113-122.

building energy performance standards; computer simulation of house energy requirements; degree days; energy analysis; single-family detached residence; building design; NBSIR 80-2184.

building energy performance standards (BEPS); degree-day; DOE-2; bin method; 20756.

Building Energy Performance Standards (BEPS); energy calculations; HVAC systems and equipment; validation; 20758.

building energy use; building service systems; energy budgets; housing research; housing standards; performance requirements; standards development; $N B S-G C R-80-280$

building envelope; building performance data; computer data acquisition; energy conservation in commercial buildings; energy monitoring and control systems; mechanical systems; NBSIR 81 2358.

building envelope; comparison; compliance; energy budget; energy conservation; performance standards; Statement of Review; verification; building code; SP608: 1981 Maý. 57.67.

building evacuation; mathematical optimization; network flow; J. Res. 86(5): 509.513; 1981 September-October.

building fires; coal mines; combustion products; compartment fires; fabric flammability; fire research; fire tests; flame research; smoke; bibliographies; NBSIR 81-2272.

building fires; evacuation; fire alarm systems; fire safety; handicapped; life safety; refuge; board and care homes; building codes; 20727.

building fires; fire safety; ignition; self-heating; solar collector fires; solar collectors; spontaneous ignition; NBSIR 81-2326.

building heating and cooling; engine-driven heat pump; heating and cooling equipment; heating, ventilating and air conditioning; heat pump; NBSIR 79-1911.

building heating/cooling loads; heat balance for a single room; heat transfer; radiosity shape factors; NBSIR 81 -2321.

building models, computer; energy conservation in commercial buildings; energy conservation, user acceptance; lighting measurements; performance data for commercial office buildings in New England; solar energy in commercial buildings; BSS133.

building officials; codes and standards; convective loops; greenhouses; heat exchangers; light and ventilation; solar systems; space heating and cooling; training programs; building codes; SP608: 1981 May. 159.166 .

building officials; consensus; construction; document; installation; recommended requirements; solar energy; solar systems; building codes; SP608: 1981 May. 167-172.

building performance; hurricane winds; structural damage; structures; wind; wind speed; 20759.

building performance data; computer data acquisition; energy conservation in commercial buildings; energy monitoring and control systems; mechanical systems; building envelope; NBSIR 81 2358.

building productivity; computer-integrated construction; conference; construction duration; productivity measurement; risks of failure; user productivity; NBS-GCR-81-331.

building program; lighting design; lighting research; lighting survey; NBS-GCR-81-333.

building ramps; pedestrian circulation; pedestrian flow; pedestrian movement; pedestrian ramps; ramps; building circulation; NBSIR 81-2310.

building regulation; codes; construction economics; construction regulation; economics; fire safety codes; regulation; benefit-cost analysis; building economics; NBSIR 81-2402.

building regulation; codes; construction regulation; economics; energy conservation codes; fire safety codes; regulation; benefit-cost analysis; building economics; NBSIR 81-2223.

building rehabilitation; codes; comparative analysis; existing buildings; housing codes; maintenance; model codes; occupancy; performance levels; regulation; standards; NBSIR 80-2081.

building rehabilitation; concrete; electrical; evaluation; HVAC; masonry; plumbing; steel; structural systems; test methods; wood; NBSIR 80-2171.

building rehabilitation; concrete; electrical; evaluation; HVAC; masonry; plumbing; solar; steel; structural systems; test methods; wood; NBSIR 81-2289.

building research; buildings; code enforcement; regulations; rehabilitation; building code; SP623. 
building research; building technology; codes; criteria; measurement methods; performance criteria; project summaries; technical bases; SP446-5.

building research; fire safety; handicapped; life safety; accessibility; building accessibility; NBSIR 81-2245.

buildings; buildings (codes); buildings (design); earthquake; seismic risk maps; standards; structural engineering; NBSIR 80-2111-1.

buildings; certified reference materials; computers; construction; data converter; mass comparator; radiation; radiocarbon; soft aluminum; windows; air pollution; automation; DIM/NBS 65(4): 1-28; 1981.

buildings; code enforcement; regulations; rehabilitation; building code; building research; SP623.

buildings; concrete; diffusion; exhalation; materials; measurement; permeability; radon; transport; TN1139.

buildings; concrete; fire endurance; fire tests; floors; reinforced concrete; roofs; thermal expansion; thermal restraint; NBS-GCR81-317.

buildings; construction; energy conservation; ground coupled; hybrid solar energy system; inverted cave; low grade geothermal; radiant floor heating; space heating and cooling; thermal mass; walls; water source heat pump; SP608: 1981 May. 69.81.

buildings; design; earthquakes; engineering; foundations; professional practice; provisions; soil structure interaction; standards; NBSIR 80-2111-3.

buildings; doors; fire doors; fire tests; infiltration; orifice flow; smoke control; smoke movement; air leakage; NBSIR 81-2214.

buildings; egress; emergencies; evacuation; network models; NBS. GCR -81-316.

buildings (codes); buildings (design); earthquake; seismic risk maps; standards; structural engineering; buildings; NBSIR 80-2111-1.

buildings (design); earthquake; seismic risk maps; standards; structural engineering; buildings; buildings (codes); NBSIR 80-2111-1.

building service systems; energy budgets; housing research; housing standards; performance requirements; standards development; building energy use; NBS-GCR-80-280.

building site standards; regulation; site design practices; site engineering; NBS-GCR-81-332.

building standards; classification; decision tables; information networks; modeling; standards; standards-writers; systems analysis; NBSIR 80-1979-1.

building standards; classification; decision tables; information networks; network; standards; standards-writers; systems analysis 19998.

building standards; crowd ingress; design procedures; evaluation procedures; facility design; facility management; public assembly public safety; stadiums; auditorium; NBSIR 81-2361.

building technology; calibration methods; computer standards and guidelines; energy research; environmental measurements; fire safety; industrial productivity; materials properties; research; standards; technology transfer; basic measurements and standards; SP600.

building technology; Center for Building Technology; key words; publications; abstracts; SP457-5.

building technology; codes; criteria; measurement methods; performance criteria; project summaries; technical bases; building research; SP446-5.

building thermal performance; energy calculation; energy conservation; thermal comfort; whole-house ventilation; TN1138.

built-up roofing; cooling of roofing bitumens; mathematical model; roofing; bitumen application temperatures; bituminous roofing; TN1135.

built-up roofing; moisture; moisture detection; nondestructive evaluation; nondestructive testing; roofing; thermal resistance; TN1146.

built-up roofing electrical capacitance; infrared thermography; insulation; moisture detection; moisture measurement; nondestructive evaluation; nondestructive testing; nuclear backscatter; roofing systems; BSS131.

built-up roofing membrane; complementary strain energy; felt; nonlinear; roofing membrane; splitting; strain energy; stress; substrate; adhesive; bitumen; bonding; TN1152. bulk damage; $\mathrm{CO}_{2}$ laser; coating damage; laser damage; microsecond pulses; $\mathrm{NaCl}$; surface damage; alkali halides; SP620: 1981 October. 88.93

bulk laser damage; fluorophosphate glass; inclusion density; KDP crystals; silicate glass; SP620: 1981 October. 159.169.

bulk measurements; isotopic assay; leachability studies; nondestructive assay; nuclear safeguards; nuclear waste management; standard reference materials; statistical evaluation; accountability guide; NBSIR 81-2205.

buoyancy forces; flood forces; foundations; hurricane forces; mobile home; soil anchors; standards; tiedown; wind forces; BSS132.

buoyant convection; computations-finite difference; Euler equations; finite difference equations; fire-enclosure; fluid flow; heat sourcevolumetric; partial differential equations; NBSIR 81-2385.

burglary; crime in public housing; household behavior patterns; housing characteristics (physical); housing security; public housing security; security hardware; victimization survey; NBS-GCR-80291.

burn; criterion; critical exposure; fluid; heat transfer; injury threshold; scald; thermal injury; bioheat equation; NBSIR 81-2320.

burning rate; case goods; chairs; furniture fires; ignitability; room fires; upholstered furniture; NBSIR 81-2271.

burning rates; compartment fires; fire modeling; flashover; numerical methods; room fires; NBS-GCR-81-301

burn (injuries); cotton; fabric flammability; fabrics; fire suppression; flame extinguishment; heat transfer; polyester; textiles; airflow; NBSIR 81-2386.

bus controllers; control and distribution; crew station terminals; electrical power management; electronic components; microcomputers; MIL-STD-1553B multiplex data bus; multiplexing techniques; on-board diagnostics and prognostics; remote terminals; total tank subsystem integration; SP622: 1981 October. 85-96.

business case; business elements; business problem; capacity management; capacity planning; disaster recovery planning; SP50083: 1981 November. 39-43.

business elements; business problem; capacity management; capacity planning; disaster recovery planning; business case; SP500-83: 1981 November. 39-43.

business problem; capacity management; capacity planning; disaster recovery planning; business case; business elements; SP500-83: 1981 November. 39-43.

\section{C}

cable cool-down; cable permeability; counter-flow cool-down; heat flux meters; liquid helium; permeability of composites; superconducting power transmission; NBSIR 80-1637.

cable cool-down; cool-down; heat flux meters; helium; superconducting power transmission; NBSIR 81-1649.

cable end fittings; pressure cycles; stress; superconducting power transmission line; terminals; thermal contraction; 20018.

cable permeability; counter-flow cool-down; heat flux meters; liquid helium; permeability of composites; superconducting power transmission; cable cool-down; NBSIR 80-1637.

cables; composite insulation; dc fields; high voltage; incipient fault; insulation; liquid breakdown; $\mathrm{SF}_{6}$; space charge; transformer oil; NBSIR 81-2334.

cables; dc fields; high voltage; incipient fault; insulation; $\mathrm{SF}_{6}$; space charge; transformer oil; NBSIR 81-2283.

cables; dielectrics; electric insulation fault location; time-domainreflectometry; underground transmission; aging; NBSIR 81-2235.

cadmium; critical evaluation; electrolyte; ethylene bis(trimethylammonium) chloride; ethylene bis(trimethylammonium) iodide; excess Gibbs energy; osmotic coefficient; solutions; thermodynamic properties; zinc; activity coefficient; JPCRD 10(1): 1.56; 1981.

cadmium; detoxification; mercury; organomercury; plasmids; silver; toxic heavy metals; antimony; arsenic; bacterial resistances; SP618: 1981 November. 301-324.

cadmium metallothionein; cellular toxicity; elemental interactions; lead inclusion bodies; methylmercury derivatives; 
methylation/demethylation; arsenicals; SP618: 1981 November. 217-225.

cadmium-NTA; convolution voltammetry; electrochemistry; Kalman filter; metal speciation; modeling; semiderivative; stability constants; voltammetry; zinc-NTA; SP618: 1981 November. 163-172.

$\mathrm{CaF}_{2}$; extrinsic and intrinsic infrared absorption; infrared wavelength modulation; $\mathrm{LaF}_{3}$; laser windows; $\mathrm{LiF} ; \mathrm{MgF}_{2} ; \mathrm{MgO} ; \mathrm{NaCl} ; \mathrm{NaF}$; $\mathrm{SrF}_{2} ;$ volume and surface impurities; $\mathrm{BaF}_{2} ; S P 620$ : 1981 October. 29.43.

$\mathrm{CaF}_{2}$; laser calorimetry; laser windows; optoacoustic; photoacoustic; surface-to-bulk optical absorption; SP620: 1981 October. 58-74.

calcification; calcific degeneration; porcine xenograft valves; prosthesis failure; vitamin K-dependent; SP601: 1981 January. 339 348.

calcific degeneration; porcine xenograft valves; prosthesis failure; vitamin K-dependent; calcification; SP601: 1981 January. 339-348.

calculations; cryogenics; helium; plastics; refrigerators; regenerators; Stirling-cycle; 20020.

calibration; calibrators; electric fields; electric field strength; electric power transmission; field strength; magnetic fields; NBSIR 77-1311. calibration; calibrators; instrumentation; oscilloscope calibrator; oscilloscopes; NBSIR 81-1646.

calibration; cavity chamber; cavity theory; electron beam; in-phantom measurement; photon beam; radiation therapy; absorbed dose; Bragg-Gray equation; 20073.

calibration; clock synchronization; Columbia River model; computer; insulation; lasers; powder data; progress; R-values; single-crystal; SRM's; atomic ions; DIM/NBS 65(2): 1-28; 1981.

calibration; combined photovoltaic/thermal modules; measurements; photovoltaic modules; photovoltaics; reference cells; solar cells; terrestrial solar simulators; test methods; NBS-GCR-80-279.

calibration; commercial services; directory; inaccuracy; ionizing radiation; radioactive sources; survey instruments; NBS-GCR-80. 296.

calibration; data acquisition; durability; dynamic calibration; environmental testing; evaluation; measurand; reliability; sensor; transducer; ATE; automatic test equipment; $S P 615$.

calibration; dc transmission lines; electric field; electric field meters; electric field strength; electric power transmission; measurements; space charge; NBSIR 80-2177.

calibration; development; transducer; waveform; acoustic emission; 20757.

calibration; digital; microcomputer; phase angle; sinewave generator; standard; synthesis; TN1144.

calibration; dosimetry; electric fields; electromagnetic interference; exposure history; instruments; magnetic fields; nonionizing radiation; regional calibration laboratories; standards; techniques; SP613.

calibration; electrical; electronics; engineering; hazards; measurement; metrology; National Bureau of Standards; power; radiation; semiconductor; technology; SP611.

calibration; instrumentation; measurement; shock and vibration; angular acceleration; angular accelerometer; NBSIR 81-2337.

calibration; measurement assurance; measurement services; standards; traceability; SP250, 1980 Edition.

calibration; shakers; vibration exciters; acceleration; accelerometers; 20731.

calibration methods; computer standards and guidelines; energy research; environmental measurements; fire safety; industrial productivity; materials properties; research; standards; technology transfer; basic measurements and standards; building technology; SPGON.

calibration service; data converter; differential linearity; digital-toanalog converter; error measurement; gain; input noise; linearity; offset; analog-to-digital converter; TN1145.

calibrators; electric fields; electric field strength; electric power transmission; field strength; magnetic fields; calibration; NBSIR 771311.

calibrators; instrumentation; oscilloscope calibrator; oscilloscopes; calibration; NBSIR 81-1646.

California; Class $\mathrm{K}$ code; conservation; discrimination; energy; United
Stand; alternative; SP608: 1981 May. 83-103.

calorimeter; cryogenics; multipliers; standards; superconductors; ac losses; 19999.

calorimeter; millimeter wave; power; standard; TN1044.

calorimeters; cellulose; char; fire retardants; heat measurement; heat release calorimeters; heat release rate; NBS-GCR-81-329.

calorimetry; cardiac pacemakers; clinical chemistry; heat; metabolism; microcalorimetry; radiation; 20015.

calorimetry; crystal; enthalpy; entropy; floor temperature; fusion; Gibbs energy; glass transition; heat capacity; linear macromolecule; melt; selenium; JPCRD 10(1): 89-118; 1981.

calorimetry; $\mathrm{C}_{5} \mathrm{H}_{5} \mathrm{~N}_{5} \mathrm{O}$; density; enthalpy of solution; Gua. $\mathrm{HCl} \cdot 1 \mathrm{H}_{2} \mathrm{O}$; Gua. $\mathrm{HCl} \cdot 2 \quad \mathrm{H}_{2} \mathrm{O} ; \quad \mathrm{Gua} \cdot 2 \mathrm{HCl}$; guanine (Gua); guanine hydrochlorides; heat capacity; nucleic acid base; solution calorimetry; thermochemistry; $\Delta C_{\rho}$ of reaction; J. Res. 86(4): 367 382; 1981 July-August.

calorimetry; damage mechanisms; intensity dependent absorption; multipulse damage; alkali-halides; SP620: 1981 October. 75-87.

calorimetry; fluence; heat flow; irradiance; measurements of temperature; plasma effects; pulsed laser beams; $10.6 \mu \mathrm{m}$; attenuators; beam profile; NBSIR 80-1628.

capacitance-voltage technique; dopant density; electron mobility; Hall effect; hole mobility; Irvin curves; phosphorus; resistivity; semiconductor; silicon; boron; SP400-64.

capacity management; capacity planning; disaster recovery planning; business case; business elements; business problem; SP500-83: 1981 November. 39-43.

capacity planning; chargeback systems; computer performance management; data base machines; end user productivity; human factors evaluation; information system management; office automation; performance management systems; resource measurement facility; simulation; supercomputers; benchmarking; SP500-83.

capacity planning; contingency planning; disaster recovery planning; SP500-83: 1981 November. 45-54.

capacity planning; device architecture; secondary storage; space management; SP500-83: 1981 November. 325.

capacity planning; disaster recovery planning; business case; business elements; business problem; capacity management; SP500-83: 1981 November. 39-43.

capture fractions; fiber scattering; optical time domain reflectometry; Rayleigh scattering; backscattering; TN1034.

carbon; computers; energy; fine structure; interface; materials; measurement; office building; phase diagrams; radioactivity; research; sediment; soil; alloy; $D I M / N B S$ 65(8): 1-28; 1981.

carbon film; heat transfer; helium; hysteresis; temperature control; transient; boiling; 19976.

carbon monoxide; chemisorption; isocyanide; rhodium; vibrational spectroscopy; 19997.

cardiac pacemakers; clinical chemistry; heat; metabolism; microcalorimetry; radiation; calorimetry; 20015.

cardiac pacemakers; international standards; reporting of implant experience; SP601: 1981 January. 503-508.

cardiovascular; implants; metals; orthopaedic; polymers; retrieval; analysis; biological; SP601.

card punch; card reader; data communications; data interchange; data processing; EAM cards; electric accounting machine; Federal Information Processing Standard; Hollerith punched card code; Hollerith punched cards; information interchange; information processing; punched cards; standard; American National Standard Code for Information Interchange; ASCII; FIPS PUB 14-1.

card reader; data communications; data interchange; data processing; EAM cards; electric accounting machine; Federal Information Processing Standard; Hollerith punched card code; Hollerith punched cards; information interchange; information processing; punched cards; standard; American National Standard Code for Information Interchange; ASCII; card punch; FIPS PUB 14-1.

carrier sense multiple access; environmental analysis; feasibility study; local area networks; requirements analysis; NBSIR 80-2187.

case goods; chairs; furniture fires; ignitability; room fires; upholstered furniture; burning rate; NBSIR 81-2271. 
cash flow; economic analysis; fire safety; fire safety evaluation system; health care facilities; life cycle; NBS-GCR-79-186-1.

casing inspection; cement bond logging tool; geothermal technology; SP622: 1981 October. 310-320.

casting; composite; cyanoacrylate; dental alloy; initiator; resin; wear; accelerator; alloy; base metal; NBSIR 80-2168.

castings; composites; cryogenic properties; low temperature mechanical properties; nonmetallics; physical properties; stainless steels; structural alloys; welding; alloys; aluminum alloys; NBSIR 81-1645.

catalysts automotive; energy dispersive analysis; heavy element fluorescence analysis; intrinsic germanium detectors; secondary target fluorescence analysis; source excited fluorescence analysis; $\mathrm{x}$-ray fluorescence analysis; SP604: 1981 June. 391.415.

cation substitution; crystal structure; rare-earth apatite; substituted fluorapatite; apatite; barium apatite; 20065.

CATV systems; coaxial cable; data communications; fiber optics information systems; integrated communications; local area networks, microwave; video teleconferencing; SP500-83: 1981 November. 271-276.

caulks; effectiveness; energy conservation; energy conservation measures; installation; insulation; oil burners; practices; safety; sealants; standards; storm doors; storm windows; vent dampers; water heaters; windows; automatic ignition devices; SP606.

cavitation; feedwater control valve; pattern recognition; pressurized water reactor; remote surveillance; acoustic emissions; SP622: 1981 October. 11-30.

cavity chamber; cavity theory; electron beam; in-phantom measurement; photon beam; radiation therapy; absorbed dose; Bragg.Gray equation; calibration; 20073.

cavity theory; electron beam; in-phantom measurement; photon beam; radiation therapy; absorbed dose; Bragg-Gray equation; calibration; cavity chamber; 20073.

CCD; cross-bridge sheet resistor; electrical alignment test structure; gated diode; integrated gated-diode electrometer; integrated test structure; MOSFET dc profiler; test structure; NBSIR 81-2319.

CCU; CGPM; CIPM; metric; SI; translation; BIPM; SP330.

CDC 844.41; computer system evaluation; disk subsystem configurations; input/output; modeling; rollin/rollout files; simulation; SP500-83: 1981 November. 239-246.

$\mathrm{CD}_{2} \mathrm{~F}_{2} ; \mathrm{CO}_{2}$ laser; FIR laser; laser frequency measurement; relative polarization; relative power; wave-lengths; 20743.

ceilings; experiments; flame size; temperature distribution; turbulent burning; NBS-GCR-79-188.

cell model; comparisons; computer programs; corresponding states; equation of state; hard sphere; LNG; mixtures; PVTx data; revised Kosek and McKinley; TNIO3O.

cellular plastic; low temperature; mechanical properties; polystyrene; polyurethane; thermal insulation; thermal properties; 20074.

cellular toxicity; elemental interactions; lead inclusion bodies; methylmercury derivatives; methylation/demethylation; arsenicals; cadmium metallothionein; SP618: 1981 November. 217.225.

cellular toxicity-essentiality mechanisms; chromatography; elementselective speciation; environmental monitoring; fossil fuels; metal(loid) complexes; organometallic compounds; trace element standards; waste cycling; bacterial mobilization; biogeochemical cycles; biological uptake; SP618.

cellulose; char; fire retardants; heat measurement; heat release calorimeters; heat release rate; calorimeters; NBS-GCR-81-329.

cellulose; enthalpy of combustion; refuse-derived-fuel; test substance; alternative fuel; bomb calorimetry; J. Res. 86(6): 655.660; 1981 November-December.

cellulose insulation; fire test; flame spread; HH-I-515D; smoldering; attic floor radiant panel test; NBSIR 81-2213.

cellulose nitrate detector; isotopic; lithium; nuclear track technique; triton particles; abundances; alpha particles; 19982.

cellulose stabilizers; paper deacidification; paper destabilization; paper permanence; $\mathrm{pH}$ history; alkaline reserve; J. Res. 86(3): 309-315; 1981 May-June.

cellulose thermal insulation; corrosion of electrical outlet boxes and devices; electrical devices; humidity, thermal insulation and corrosion of electrical wiring; shock hazards; urea-formaldehyde thermal insulation; NBSIR 81-2220.

cellulosic insulation; ignition; propagation; smoldering combustion; test methods; wave structure; boric acid; NBSIR 81-2350.

cement; concrete; fly ash; NBSIR 81-2239.

cement; expansion; fly ash; MgO; slag; soundness; strength; NBSIR 81-2273.

cement and concrete; cover plates; earth's tilt; electric; fire; fracture toughness; measurement; parameter; physics; solar collector; space charges; technical competence; DIM/NBS 65(3): 1.24; 1981.

cement bond logging tool; geothermal technology; casing inspection; SP622: 1981 October. 310.320.

cementing of bone; fillings; methacrylates; tertiary aromatic amines; unsaturated polyesters; acrylates; acrylic resins; aminoglutethimide or para-aminophenylacetic acid; U.S. Patent 4,243,763.

cementing of bone; high reactivity; low toxicity; peroxide catalyzed polymerization; tertiary aromatic amines; unsaturated polyesters; vinyl monomers; accelerators; U.S. Patent 4,284,551.

Center for Building Technology; key words; publications; abstracts; building technology; SP457-5.

Center for Materials Science; Ceramics Glass and Solid State Science Division; Chemical Stability and Corrosion Division; Fracture and Deformation Division; FY80 technical activities; Metallurgy Division; Polymers Science and Standards Division; Reactor Division; NBSIR 80-2108.

central heating; condensing boilers; efficiency, part load; fossil-fuel heating systems; gas-fired boilers; hydronic heating; pulse combustion; boilers; NBSIR 80-2094.

central heating equipment; condensing boilers; condensing furnaces; part-load performance; rating procedure; seasonal efficiency; annual fuel utilization efficiency; annual operating costs; NBSIR 80-2110.

central office equipment; electronic equipment; fire test methods; incident data; telecommunications; telephone exchanges; NBSIR 81-2261.

central pair potential; fcc metals; free energy; high temperature nearest-neighbor model; thermal expansion; anharmonic; Brillouin zone sums; 20037.

ceramics; coal conversion; crack propagation; erosion; fracture toughness; hardness; 20028.

Ceramics Glass and Solid State Science Division; Chemical Stability and Corrosion Division; Fracture and Deformation Division; FY80 technical activities; Metallurgy Division; Polymers Science and Standards Division; Reactor Division; Center for Materials Science; NBSIR 80-2108.

cerium neodymium tantalates; cerium niobate; neutron diffraction; oxidation mechanisms; profile refinement method; scattering amplitude of neodymium; 20054.

cerium niobate; neutron diffraction; oxidation mechanisms; profile refinement method; scattering amplitude of neodymium; cerium neodymium tantalates; 20054.

certification; Class A energy audit; economic analysis; energy analysis; energy conservation; Federal programs; government buildings; hospitals; rate of return; schools; technical assistance; SP608: 1981 May. 133-138.

certification; consumer protection; Interstate Solar Coordination Council; labeling procedures; national testing program; regulation; solar collector; standards; voluntary program; SP608: 1981 May. 179.181 .

certification; isotope dilution mass spectrometry; mass spectrometry; standard reference materials; analysis; 20024.

certified reference material; chemical composition; physical property; accuracy; 19990

certified reference materials; computers; construction; data converter; mass comparator; radiation; radiocarbon; soft aluminum; windows; air pollution; automation; buildings; $D I M / N B S$ 65(4): 1.28; 1981 .

cesium; critical evaluation; electrolyte; excess Gibbs energy; lithium; osmotic coefficient; potassium; rubidium; sodium; solutions; thermodynamic properties; activity coefficient; JPCRD 10(3): 671. 766.

cesium; metal accumulation; microbial; radium; uranium; SP618: 1981 November. 274-286. 
cesium compounds; enthalpy; entropy; francium compounds; Gibbs energy of formation; heat capacity; lithium compounds; potassium compounds; protactinium compounds; rubidium compounds; sodium compounds; thorium compounds; uranium compounds; actinium compounds; $T N 270-8$.

cesium from molybdenum(111); ion and neutral lifetime; positive ion and neutral lifetime; 20013.

CGPM; CIPM; metric; SI; translation; BIPM; CCU; SP330.

chain rupture; crazing; crystalline polymers; electron spin resonance; microcrack; microfibril; shear deformation; tensile failure; 20732.

chairs; furniture fires; ignitability; room fires; upholstered furniture; burning rate; case goods; NBSIR 81-2271.

chalcogenide glass; coating; ion beam; sputtering; SP620: 1981 October. 324-334.

channeling angle; C.V profiling; dopant profiles; implantation-induced damage; ion channeling; ion dose measurement; ion implantation; NBS-GCR-81-325.

char; fire retardants; heat measurement; heat release calorimeters; heat release rate; calorimeters; cellulose; NBS-GCR-81-329.

character-imaging devices; control functions; data interchange; display terminals; Federal Information Processing Standard; information interchange; printer terminals; standard; text communication; text interchange; typesetting composition; ADP terminals; ASCII; FIPS PUB 86.

characteristic impedance; field distribution; Hertzian dipole; integral equation; rectangular coaxial transmission line; TEM cell; TN1037.

characteristics; discharge; drainage; flow; model; plumbing; quasisteady; unsteady; J. Res. 86(2): 171-179; 1981 March-April.

characterization; composition; materials; properties; research materials; standard reference materials; analysis; SP260, 1981-83 Edition.

characterization of polymers; polymers under high pressure; Raman spectroscopy; 20007.

character positioning; computer systems; data entry (automatic); Federal Information Processing Standard; information processing systems; optical character recognition; FIPS PUB 89.

character set; coded character set; code for information interchange; collating sequence; data communication; data interchange; data processing; Federal Information Processing Standard; information interchange; information processing; standard; American National Standard Code for Information Interchange; ASCII; FIPS PUB 1-1.

chargeback; chargeout; charging; charging system; DP cost accounting; DP cost allocation; pricing; standard costing; SP5OO83: 1981 November. 109-118.

chargeback; cost accounting; cost recovery; SP500-83: 1981 November. $311-312$.

chargeback systems; computer performance management; data base machines; end user productivity; human factors evaluation; information system management; office automation; performance management systems; resource measurement facility; simulation; supercomputers; benchmarking; capacity planning; SP500-83.

charge conjugation; CPT theorem; parity; time reversal; 19991.

chargeout; charging; charging system; DP cost accounting; DP cost allocation; pricing; standard costing; chargeback; SP500-83: 1981 November. 109-118.

charge transfer; chemi-ionization; collisional ionization; cross section; detachment; energy transfer; excitation; ion; molecule; reaction rate; relaxation; atom; SP593.

charging; charging system; DP cost accounting; DP cost allocation; pricing; standard costing; chargeback; chargeout; SP500-83: 1981 November. 109-118.

charging system; DP cost accounting; DP cost allocation; pricing; standard costing; chargeback; chargeout; charging; SP500-83: 1981 November. 109-118.

charmed mesons; dipole transitions; magnetic moments; photon decays; quarks; angular distributions; 20040.

checkerboard pressure; elastostatics; finite elements; in-core solver; isoparametrics; Mooney-Rivlin material; nonlinear equation-solvers; penalty method; plane-stress; strain invariants; tensile-test specimen; J. Res. 86(1): 79-109; 1981 January-February. chemical; electrical; geological; magnetic; mechanical; monograph; optical; physical; properties data; radiation damage; rock salt; sodium chloride; Monogr. 167.

chemical activity; magnetohydrodynamics; mass spectrometry; potassium slag; thermodynamics; vaporization; NBSIR 81-2293.

chemical composition; clinical materials; standard reference materials; SP260-71.

chemical composition; physical property; accuracy; certified reference material; 19990.

chemical engineering; cold utilization; cryogenic energy conservation; liquefied natural gas; refrigeration recovery; 20068.

chemical industry standards; cotton standards; food standards; measurement science and technology; standardization in developing countries; Sudan; weights and measures regulation; Agency for International Development; Arab League Organization for Standardization and Metrology; NBSIR 80-2020.

chemical kinetics; combustion; decomposition; free radicals; gas phase; hydrocarbons; hydrogen; nitrogen; oxygen; rate of reaction; sulfur; Arrhenius parameters; NBSIR 81-2254.

chemical kinetics; data compilation; oxidation; photochemistry; rates; review; singlet oxygen; solution; JPCRD 10(4): 809-999; 1981.

chemical kinetics; disulfur monoxide; rate constant; sulfur dioxide; sulfur monoxide; sulfur monoxide dimer; 20035.

chemical kinetics; evaluated data; fluid properties; indexes; publication list; solid state; thermodynamic and transport properties; atomic and molecular properties; bibliographies; SP612.

chemical potential; diffusion; equilibrium; solid; stress, strain; thermodynamics; 20725.

chemical rate constants; computer program; hydrogen atom transfer; absolute chemical rate; BEBO activation energy; bond-order-bondenergy; J. Res. 86(6): 605-654; 1981 November-December.

chemical rate constants; Eckart potential; potential barriers; quantum mechanical tunneling calculations; tunneling corrections; J. Res. 86(4): 357-359; 1981 July.August.

chemical speciation; GC-AAS techniques; LC.AAS techniques; organoarsenics; organometals; partition coefficients; toxicity; alkylleads; alkyltins; SP618: 1981 November. 65-80.

Chemical Stability and Corrosion Division; Fracture and Deformation Division; FY80 technical activities; Metallurgy Division; Polymers Science and Standards Division; Reactor Division; Center for Materials Science; Ceramics Glass and Solid State Science Division; NBSIR 80-2108.

chemi-ionization; collisional ionization; cross section; detachment; energy transfer; excitation; ion; molecule; reaction rate; relaxation; atom; charge transfer; SP593.

chemiluminescence; Franck-Condon factors; laser-induced fluorescence; radiative lifetimes; scandium; scandium halides; yttrium; yttrium halides; 19969.

chemiluminescence; Franck-Condon factors; laser-induced fluorescence; radiative lifetimes; scandium; scandium halides; yttrium; yttrium halides; 19978.

chemisorption; isocyanide; rhodium; vibrational spectroscopy; carbon monoxide; 19997.

chemistry of fire; combustion products; detection; fire hazards; fire modeling; fire protection; fire research; fire suppression; human behavior in fires; physics of fire; toxicity of combustion products; NBSIR 80-2127-1.

chemolithotrophic-bacteria; copper; iron; leaching; metal-reduction; metal-sulfides; mineral-oxidation; molybdenum; oil shale;

Sulfolobus; Thiobacillus; uranium; SP618: 1981 November. 264-273.

children; design; environmental psychology; families; housing; interiors; lifestyle; research; site planning; sociology; standards; NBS-GCR-81-320.

chimneys; combustion; fire safety; heating equipment; literature reviews; stoves; wood; bibliographies; NBS-GCR-80-298.

chip detectors; condition monitoring; failure detection; helicopter diagnostics; oil debris; wear monitoring; SP622: 1981 October. 3349.

chloride ion; chromate ion; electrochemistry; ellipsometry; field ion microscopy; inhibitors; organic coatings; passive film dissolution; pH measurements; surface hydride; alpha uranium; NBSIR 81-2396 
(ONR).

chromate ion; electrochemistry; ellipsometry; field ion microscopy; inhibitors; organic coatings; passive film dissolution; $\mathrm{pH}$ measurements; surface hydride; alpha uranium; chloride ion; NBSIR 81-2396 (ONR).

chromatography; detection; metals; plasma emission; specific elements; spectroscopy; SP618: 1981 November. 184-196.

chromatography; direct current plasma; hydrophilic; hydrophobic; metal complex; porphyrin; shale oil; speciation; trace metals; SP618: 1981 November. 105-119.

chromatography; element-selective speciation; environmental monitoring; fossil fuels; metal(loid) complexes; organometallic compounds; trace element standards; waste cycling; bacterial mobilization; biogeochemical cycles; biological uptake; cellular toxicity-essentiality mechanisms; SP618.

chromatography, gas; chromatography, liquid; drug standards; enzyme immunoassay; epilepsy; ethosuximide; mass spectrometry; nuclear magnetic resonance spectroscopy; phenobarbital; phenytoin; primidone; reference material; serum base modification; serum matrix; anticonvulsants; antiepilepsy drugs; blood analysis; SP260-72.

chromatography, liquid; drug standards; enzyme immunoassay; epilepsy; ethosuximide; mass spectrometry; nuclear magnetic resonance spectroscopy; phenobarbital; phenytoin; primidone; reference material; serum base modification; serum matrix; anticonvulsants; antiepilepsy drugs; blood analysis; chromatography, gas; SP260-72.

chromium; coal hydrogenation; cobalt; liquefaction; mercury; nickel; process waters; selenium; solvent refined coal; titanium; trace elements; arsenic; SP618: 1981 November. 21-38.

chromium; cobalt; critical materials; tantalum; titanium; aerospace; NBSIR $81-2305$.

chrysotile asbestos particulate; DTA analysis of environmental particulates; DTA apparatus for rapid sample change and steam pressure control; alpha-quartz particulate; 20011.

$\mathrm{CH}_{2} \mathrm{I}$; infrared emission; laser; photofragmentation; radical; $\mathrm{CH}_{3}$ 20004.

$\mathrm{CH}_{3} ; \mathrm{CH}_{2} \mathrm{I}$; infrared emission; laser; photofragmentation; radical; 20004 .

cigarettes; flammability; ignition; mattresses; self-extinguishment smoldering; upholstered furniture; NBSIR 81-2363.

CIPM; metric; SI; translation; BIPM; CCU; CGPM; SP330.

circuit breaker; electrical fire; low ambient temperature; trip time branch circuits; NBSIR $81-2221$.

circumstellar shells; late type stars; mass loss; stars, emission line; stellar chromospheres; 20057.

C/kT; EIRP; error analysis; G/T; precision measurements; radio star; satellite communications; NBSIR 78-869.

Class A energy audit; economic analysis; energy analysis; energy conservation; Federal programs; government buildings; hospitals; rate of return; schools; technical assistance; certification; SP608: 1981 May. 133-138.

classification; code; engineering; organization; provisions; scope; specification; standard; system analysis/engineering; arrangement; building; BSS136.

classification; decision tables; information networks; modeling; standards; standards-writers; systems analysis; building standards; NBSIR 80-1979-1.

classification; decision tables; information networks; network; standards; standards-writers; systems analysis; building standards; 19998.

classification; density; forensic science; individualization; population statistics; refractive index; auto headlight glass; NBSIR 81-2286.

classification criteria; gamma radiography; national standard; performance tests; radiation source; safety standard; H136.

class $\mathrm{K}$ code; computer modeling; electrical design; energy audit; energy conservation; HVAC systems; performance standards; solar collector; solar energy; space heating and cooling; thermal storage; ASHRAE 90.75; SP608.

Class K code; conservation; discrimination; energy; United Stand; alternative; California; SP608: 1981 May. 83-103.
Clean Air Act; EPA; ETIP; incentives; innovation; regulation; air pollution; NBS-GCR-ETIP 81-94.

Clean Air Act; EPA; ETIP; incentives; innovation; regulation; air pollution; NBS-GCR-ETIP $81-93$.

Clean Air Act; EPA; ETIP; incentives; innovation; regulation; air pollution; NBS-GCR-ETIP 81-95

clearance seal; gas compressor; linear motor/generator; magnetic bearings; Stirling Cycle; SP607: 1981 May. 146-153.

clearance seals; cryogenics; hydrodynamic gas bearing; linear magnetic suspension; long lifetime; space application; Stirling cycle; SP607: 1981 May. 103-115.

climatology; hurricanes; reliability; risk; structural engineering; building (codes); 20764.

climatology; loads (forces); probability distribution functions; reliability; statistical analysis; structural engineering; wind; 20019

clinical chemistry; heat; metabolism; microcalorimetry; radiation; calorimetry; cardiac pacemakers; 20015.

clinical materials; standard reference materials; chemical composition; SP260-71.

clock synchronization; Columbia River model; computer; insulation; lasers; powder data; progress; R-values; single-crystal; SRM's; atomic ions; calibration; DIM/NBS 65(2): 1-28; 1981

closed conduit meter; differential pressure meter; flow measurement laminar flow; meter performance; numerical modeling; orifice meter; swirling flow; 19972.

closed cycle refrigeration systems; Josephson effects; proximity effect devices; superconducting devices; tunnel devices; weak links; SP607: 1981 May. 195-209.

closed loop fuel metering system; exhaust emissions; exhaust gas oxygen sensor; exhaust gas recirculation; feedback; oxidation catalyst; oxygen partial pressure; stoichiometric; three way catalytic converters; zirconium dioxide; air-fuel ratio; SP622: 1981 October. $62-73$.

clothes dryer usage characteristics; clothes washer usage characteristic; data profiles; dishwasher usage characteristic; energy consumption; field measurements; usage patterns; water consumption; NBSIR 80-2136.

clothes washer; discharge; dishwasher; residential; supply; water conservation; water consumption; appliances; NBSIR 80-2173.

clothes washers; dishwashers; dishwasher subcycles; fill controls; grey water; performance; soil removal; water conservation; water discharge characteristics; NBSIR 81-2291 (HUD).

clothes washer usage characteristic; data profiles; dishwasher usage characteristic; energy consumption; field measurements; usage patterns; water consumption; clothes dryer usage characteristics; NBSIR 80-2136.

cluster analysis; workload characterisation; SP500-83: 198 November. 21.34.

coal; computers; drill failure; Faraday; firefighting; hot atom; industrial innovation; measurements; oils, PCB's; standards; atomic weight; $D I M / N B S$ 65(6): 1-24; 1981.

coal; control; fossil energy; materials; process parameters; research recommendations; NBSIR 81-2348

coal; environmental chemistry; shale oil; synthetic fuels; trace elements; SP618: 1981 November. 81-92.

coal conversion; combustion systems; vapor transport; alkali vapor; NBSIR 81-2279.

coal conversion; crack propagation; erosion; fracture toughness; hardness; ceramics; 20028.

coal conversion; mechanical components; SYNTHANE pilot plant; vibration monitoring; SP622: 1981 October. 274-288.

coal gasification; elevated temperatures; ferritic stainless steel; gaseous environments; nickel alloy 671; nickel alloy 800 ; slow strain rate test; structural materials; austenitic stainless steels; NBSIR 81-2191 (DoE).

coal gasification; elevated temperature stress corrosion; slow strain rate; stress corrosion; 20027.

coal gasification; high temperature; mass spectrometry; slag; transpiration; vapor transport; NBSIR 80-2178 (DoE).

coal hydrogenation; cobalt; liquefaction; mercury; nickel; process waters; selenium; solvent refined coal; titanium; trace elements; 
arsenic; chromium; SP618: 1981 November. 21.38.

coal mine; fire tests; hydraulic fluids; invert emulsions; pressuretemperature; spray; synthetics; water glycol; NBSIR 81-2373.

coal mines; combustion products; compartment fires; fabric flammability; fire research; fire tests; flame research; smoke; bibliographies; building fires; NBSIR 81-2272.

coal mines; conveyor belts; fire safety; fire tests; flame spread; ignition; test methods; accident data; NBSIR 81-2256.

coal mines; electrical cables; fire incidents; fire tests; test methods; trailing cables; NBSIR 81-2292.

coal mines; fire point; fire resistance tests; fire tests; flammability; flash point; hydraulic fluids; ignition; autoignition temperature; $N B S I R$ 81-2395.

coal mines; flammability; hydraulic fluids; ignition; Wick Test; NBSIR 81-2312.

coal mining; fire test; flame spread; sample mounting; ventilation cloth; ASTM E-162; brattice cloth; NBSIR 81-2200.

coating; ion beam; sputtering; chalcogenide glass; SP620: 1981 October. 324-334.

coating damage; laser damage; $\mathrm{LiNbO}_{3}$ coatings; Q-switch; surface damage; antireflection coatings; SP620: 1981 October. 300.304.

coating damage; laser damage; microsecond pulses; $\mathrm{NaCl}$; surface damage; alkali halides; bulk damage; $\mathrm{CO}_{2}$ laser; SP620: 1981 October. 88-93.

coating imperfections; cw laser irradiation; digs; dust; laser damage; mirrors; optical components; scratches; surface defects; SP620: 1981 October. 256-264.

coatings; corrosion; predictability; reproducibility; time to failure; accelerated aging tests; TN1149.

coatings; corrosion behavior; field-tests; galvanic couples; soil environment; stainless steels; stress-corrosion behavior; NBSIR 812228 (NBS).

coatings; electrodeposition; immersion deposition; plating; plating on anodized aluminum; adhesion; anodizing; NBSIR 80-2142.

coatings; exterior restoration; field tests; guide specifications; laboratory tests; paint removal methods; White House; NBSIR 80 2122.

coatings; internal reflection spectroscopy; silicon monoxide; thorium fluoride; zinc selenide; zinc sulfide; absorption; SP620: 1981 October. 335-344.

coaxial cable; data communications; fiber optics information systems; integrated communications; local area networks, microwave; video teleconferencing; CATV systems; SP500-83: 1981 November. 271. 276.

coaxial sample holder; density; dielectric properties; relative dielectric constant; soil textural types; attenuation; NBSIR 78-896.

coaxial transmission lines; digital communication impulse response; instrumentation; measurements; shielded balanced transmission lines; time domain; transmission line model; waveform; bit error rate; TN1042.

cobalt; cobalt energy levels; spectra; atomic energy levels; atomic spectra; JPCRD 10(4): 1097-1174; 1981.

cobalt; critical materials; tantalum; titanium; aerospace; chromium; NBSIR 81-2305.

cobalt; $f$-values; iron; isoelectronic sequence; line strengths; nickel; oscillator strengths; systematic trends; transition probabilities; allowed transitions; JPCRD 10(2): 305.566; 1981.

cobalt; liquefaction; mercury; nickel; process waters; selenium; solvent refined coal; titanium; trace elements; arsenic; chromium; coal hydrogenation; SP618: 1981 November. 21-38.

cobalt-chrome-nickel-tungsten-iron-alloy; fretting corrosion; histologic analysis; stainless steel $316 \mathrm{LVM}$; titanium, titanium alloy; bone plates; SP601: 1981 January. 395-422.

cobalt energy levels; spectra; atomic energy levels; atomic spectra; cobalt; JPCRD 10(4): 1097.1174; 1981.

cobalt.60 gamma rays; electron density; graphite calorimeter; ionization chamber; scaling theorem; water phantom; absorbed dose standard; J. Res. 86(5): 495.502; 1981 September-October.

COBOL; compilers; data processing; Federal Information Processing Standard; FORTRAN; information interchange; information processing; processor; programming language; software; BASIC;
FIPS PUB 29-1.

Co-Cr-Mo; Co-Ni-Cr-Mo; corrosion; dental alloys; metal-amino acid reactions; stainless steel; titanium; 19988.

CODASYL; DAPLEX; database management system; data model; DBMS; network data model; query language; relational model; SQL; NBS-GCR-81-338.

CODASYL; database management system; data model; DBMS; network data model; query language; NBS-GCR-81-337.

CODASYL; database management system; data model; DBMS; network data model; query language; NBS-GCR-81-339.

code; engineering; organization; provisions; scope; specification; standard; system analysis/engineering; arrangement; building; classification; BSS136.

code compliance; code conflicts; energy conservation standards; solar energy; trainee performance testing; BEPS; SP608: 1981 May. 139. 157.

code conflicts; energy conservation standards; solar energy; trainee performance testing; BEPS; code compliance; SP608: 1981 May. 139-157.

coded character set; code for information interchange; collating sequence; data communication; data interchange; data processing; Federal Information Processing Standard; information interchange; information processing; standard; American National Standard Code for Information Interchange; ASCII; character set; FIPS PUB 1-1.

coded character sets; code extension techniques; code standards; control functions; information interchange; text communications; text processor; SP500-81.

code enforcement; regulations; rehabilitation; building code; building research; buildings; SP623.

code extension techniques; code standards; control functions; information interchange; text communications; text processor; coded character sets; SP500-81.

code for information interchange; collating sequence; data communication; data interchange; data processing; Federal Information Processing Standard; information interchange; information processing; standard; American National Standard Code for Information Interchange; ASCII; character set; coded character set; FIPS PUB 1-1.

code independence; data; data link control procedures; data transparency; Federal Information Processing Standards Publication; interoperability; teleprocessing; transmission; advanced data communication control procedures; ANS X3.66; bit-oriented; FIPS PUB 78

code requirements; communication; deaf; directional indicators; handicapped users; tactile warnings; accessibility; alarms; blind; NBSIR 81-2428.

codes; comparative analysis; existing buildings; housing codes; maintenance; model codes; occupancy; performance levels; regulation; standards; building rehabilitation; NBSIR 80-2081.

codes; concrete; masonry; prohibitions; re-examination; thermal storage; wood structure; SP608: 1981 May. 195-200.

codes; construction economics; construction regulation; economics; fire safety codes; regulation; benefit-cost analysis; building economics; building regulation; NBSIR 81-2402.

codes; construction regulation; economics; energy conservation codes; fire safety codes; regulation; benefit-cost analysis; building economics; building regulation; NBSIR 81-2223.

codes; criteria; measurement methods; performance criteria; project summaries; technical bases; building research; building technology; SP446-5.

codes; energy conservation; energy policy; Hawaii; performance standards; building energy performance; SP608: 1981 May. 105.112.

codes and standards; construction loads; construction safety; finite element; scaffold failures; scaffolding practices; scaffolds; structural analysis; accidents; NBS-GCR-80-255.

codes and standards; construction safety; design; finite element; loads; scaffolds; stability; stiffness; strength; structural safety; work surfaces; NBSIR 81-2265.

codes and standards; convective loops; greenhouses; heat exchangers; light and ventilation; solar systems; space heating and cooling; 
training programs; building codes; building officials; SP608: 1981 May. 159-166.

codes and standards; disability; door closers; door openers; doors; ergonomics; functional capacity; handicap; locks; architectural barriers; building accessibility; NBSIR 80-2174.

code standards; control functions; information interchange; text communications; text processor; coded character sets; code extension techniques; SP500-81.

coexistence densities; critical density; critical point; liquid mass fraction; liquid volume fraction; phase equilibria; pure fluids; NBSIR 80-1631.

coherence; cooperative phenomena; spectral line shapes; 19968

coherence; cooperative phenomena; spectral line shapes; 19967.

cold utilization; cryogenic energy conservation; liquefied natural gas; refrigeration recovery; chemical engineering; 20068.

cold work; crystalline disorder; defect damage; diamond-machined mirrors; Drude absorption; electron-beam melting; laser waveform; melt threshold; metal deposition; pulsed laser damage; slip threshold; thermal diffusion length; thermal stress; SP620: 1981 October. 201-209.

collapse; concrete; concrete strength; construction; failure; flat plate; shear; strength; building; NBSIR 81-2374.

collating sequence; data communication; data interchange; data processing; Federal Information Processing Standard; information interchange; information processing; standard; American National Standard Code for Information Interchange; ASCII; character set; coded character set; code for information interchange; FIPS PUB 1-1.

collector efficiency; comparison German BSE vs ASHRAE 93-77 procedures; flat-plate solar collectors; German Bundesverband Solarenergie; solar collector testing; water-heating solar collectors; 20079.

collector rating; measurement; solar collector; standards; thermal performance; uncertainty; TN1140.

collimation; deadlayer determinations; germanium; monoenergetic photons; partly-resolved doublets; silicon; tailing; $x$-ray detectors; SP604: 1981 June. 5.34.

collisional ionization; cross section; detachment; energy transfer; excitation; ion; molecule; reaction rate; relaxation; atom; charge transfer; chemi-ionization; SP593.

collision-induced absorption; far infrared; $\mathrm{H}_{2}-\mathrm{H}_{2}$ collisions; $\mathrm{H}_{2}-\mathrm{He}$ collisions; planetary atmospheres; quadrupole-induced absorption; spectral moments; band shape analysis; NBSIR 80-2175.

Columbia River model; computer; insulation; lasers; powder data; progress; R-values; single-crystal; SRM's; atomic ions; calibration; clock synchronization; DIM/NBS 65(2): 1-28; 1981.

combined photovoltaic/thermal modules; measurements; photovoltaic modules; photovoltaics; reference cells; solar cells; terrestrial solar simulators; test methods; calibration; NBS-GCR-80-279.

combustible gas; detectors; diffusion rates; diffusion tubes; gas chromatography; hydrocarbons; vapor detector; vapor generator; vapor standards; accelerants; arson; SP480-43.

combustion; combustion rate; ignition; metals; oxygen atmosphere; temperature measurement; alloys; NBSIR 81-1647.

combustion; decision analysis; fire models; flame spread; human behavior; ignition; polymers; smoke; soot; toxicity; wood; NBSIR 81-2382.

combustion; decomposition; free radicals; gas phase; hydrocarbons; hydrogen; nitrogen; oxygen; rate of reaction; sulfur; Arrhenius parameters; chemical kinetics; NBSIR 81-2254.

combustion; fire safety; heating equipment; literature reviews; stoves; wood; bibliographies; chimneys; NBS.GCR-80-298.

combustion products; compartment fires; egress; fire detection; fire growth; hazard analysis; mathematical models; room fires; smoke movement; tenability limits; NBSIR 80-2172.

combustion products; compartment fires; fabric flammability; fire research; fire tests; flame research; smoke; bibliographies; building fires; coal mines; NBSIR 81-2272.

combustion products; detection; fire hazards; fire modeling; fire protection; fire research; fire suppression; human behavior in fires; physics of fire; toxicity of combustion products; chemistry of fire;
NBSIR 80-2127-1.

combustion products; fire modeling; foam (materials); heat release plastics; polymers; polymethylmethacrylate; polyoxymethylene; polypropylene; polystyrene; polyurethane; polyvinyl chloride pyrolysis; smoke; wood; NBS-GCR-80-295.

combustion rate; ignition; metals; oxygen atmosphere; temperature measurement; alloys; combustion; NBSIR 81-1647.

combustion systems; vapor transport; alkali vapor; coal conversion; NBSIR 81-2279.

comfort; Community Services Administration Optimal

Weatherization Demonstration; energy savings; field study; thermal comfort; weatherization retrofitting; 20752

commercial services; directory; inaccuracy; ionizing radiation; radioactive sources; survey instruments; calibration; NBS-GCR-80. 296.

committee participation; standards committees; annual report; SP605.

commodity inspection; compliance testing; net weight; package checking; packaged product inspection; prepackaged consumer goods; statistical sampling; $H 133$.

common-mode cancellation; frequency comparison; global positioning system; remote time measurement; time comparison; time receiver; worldwide time; atomic clocks; automatic time comparisons; 20062.

communication; deaf; directional indicators; handicapped users; tactile warnings; accessibility; alarms; blind; code requirements; NBSIR 81.2428.

communication; evaluation method; fire-safety; hazard warnings; meaningfulness; method; pictogram; response; symbol; understandability; NBSIR 80-2088.

communication; hazard; pictograms; safety; signs; standards; symbols; visual alerting; warnings; NBSIR 80-2003.

communication networks; flow control; Markov processes; performance prediction; queueing models; simulation; validation; analytic models; SP500-83: 1981 November. 277-290.

communication protocols; computer network protocols; feature analysis; network architecture; network virtual terminal protocols; virtual terminal protocols; NBS-GCR-81-324.

communications; competition; ETIP; FCC; innovation incentives; regulatory reform; administrative experiment; NBS-GCR-ETIP 81 97.

communications; Federal Information Processing Standard (FIPS); Federal Property Management Regulations (FPMR); procurement; standards; telecommunications; automated data processing (ADP); FIPS PUB 80

communications networks; CROSSFIRE; link protocols; local survivability; message delay; network simulation; network throughput; survivability; alternate routing; NBS-GCR-81-336.

communications networks; distributed controi; message delay; network throughput; survivability; alternate routing; NBSIR 812414.

Community Services Administration; field study; occupant comfort; optimal weatherization demonstration; thermal comfort; thermostat setting practices; weatherization; weatherization and thermal comfort; NBSIR 81-2335.

Community Services Administration Optimal Weatherization Demonstration; energy savings; field study; thermal comfort weatherization retrofitting; comfort; 20752.

comparative analysis; existing buildings; housing codes; maintenance; model codes; occupancy; performance levels; regulation; standards; building rehabilitation; codes; NBSIR $80-2081$.

comparator; if voltage; if voltmeters; NBSIR 78-871.

comparison; compliance; energy budget; energy conservation; performance standards; Statement of Review; verification; building code; building envelope; SP608: 1981 May. 57.67.

comparison German BSE vs ASHRAE 93.77 procedures; flat-plate solar collectors; German Bundesverband Solarenergie; solar collector testing; water-heating solar collectors; collector efficiency; 20079.

comparisons; computer programs; corresponding states; equation of state; hard sphere; LNG; mixtures; PVTx data; revised Kosek and McKinley; cell model; TN1030.

compartment fires; computer programs; fire extinguishment; 
flashover; ignition; mathematical models; room fires; NBS-GCR-81344.

compartment fires; egress; fire detection; fire growth; hazard analysis; mathematical models; room fires; smoke movement; tenability limits; combustion products; NBSIR 80-2172.

compartment fires; fabric flammability; fire research; fire tests; flame research; smoke; bibliographies; building fires; coal mines; combustion products; NBSIR 81-2272.

compartment fires; fire detection; fire endurance; fire modeling; fire tests; flammability; human behavior; smoke; suppression; toxicity; NBSIR 81-2294.

compartment fires; fire modeling; flashover; numerical methods; room fires; burning rates; $N B S-G C R-81-301$.

compartment fires; fire tests; flashover; interior finish; mobile homes; models; room fires; NBSIR 81-2333.

compartment fires; fire tests; health care facilities; heat release rate; hospitals; mattresses; prisons; smoke production; bedding; beds; NBSIR 80-2186.

competition; ETIP; FCC; innovation incentives; regulatory reform; administrative experiment; communications; NBS-GCR-ETIP 8197.

compilation; conductivity; electrolytes; enthalpy; Gibbs energy; osmotic coefficients; solutions; sulfuric acid; thermodynamic properties; transport properties; activity coefficients; aqueous; NBSIR 81-2276.

compilation; potassium chloride; reference data; viscosity; aqueous solutions; JPCRD 10(1): 57.70; 1981

compilation; reference data; sodium chloride; viscosity; aqueous solutions; JPCRD 10(1): 71-88; 1981 .

compilers; data processing; Federal Information Processing Standard; FORTRAN; information interchange; information processing; processor; programming language; software; BASIC; COBOL; FIPS PUB 29-1.

complementary strain energy; felt; nonlinear; roofing membrane; splitting; strain energy; stress; substrate; adhesive; bitumen; bonding; built-up roofing membrane; TNI152.

complex error function; continued fraction; Dawson's function; FORTRAN subroutine; Fresnel integrals; key values; line broadening function; plasma dispersion function; Voigt function; argand diagram; J. Res. 86(6): 661-686; 1981 November-December.

complexing capacity; copper speciation; anodic stripping; bioavailability; SP618: 1981 November. 173.183.

complexity; labeling; network; sequence list; shortest path; tree; $J$. Res. 86(3): 317-330; 1981 May-June.

complex sensors; off-line programming; robot control; robot interfaces; robot standards; robot wrist; simple sensors; NBSIR 80 2152.

compliance; energy budget; energy conservation; performance standards; Statement of Review; verification; building code; building envelope; comparison; SP608: 1981 May. 57-67.

compliance testing; net weight; package checking; packaged product inspection; prepackaged consumer goods; statistical sampling; commodity inspection; $H 133$.

compliant surface; drag, experimental; flat plate, turbulent; boundary layer; 20048 .

component development; contamination control; cryogenics; helium retention; long-life; refrigerators; reliability; rotary-reciprocating; turbo-Brayton; Vuilleumier; SP607: 1981 May. 21-29.

component efficiency; cryogenics; efficiency; helium; maximum refrigerator efficiency; perfect gas analysis; refrigerator; 20771.

component loosening; debonding joint replacements; knee replacements; SP601: 1981 January. 509.557.

composite; cyanoacrylate; dental alloy; initiator; resin; wear; accelerator; alloy; base metal; casting; NBSIR 80-2168.

composite insulation; dc fields; high voltage; incipient fault; insulation; liquid breakdown; $\mathrm{SF}_{6}$; space charge; transformer oil; cables; NBSIR 81-2334.

composite material; dentin; methacrylate; polyfunctional surfaceactive comonomers; reconstructive dental work; resin; acrylate; adhesion; aminobenzoate; U.S. Patent 4,251,565.

composite models; data quality; energy models; mathematical models; model confidence; model credibility; policy models; sensitivity analysis; validation; assessment; SP616.

composites; cryocoolers; plastics; refrigeration; regenerators; specific heat; thermal conductivity; SP607: 1981 May. 173-177.

composites; cryogenic properties; low temperature; mechanical properties; nonmetallics; physical properties; stainless steels; structural alloys; welding; alloys; aluminum alloys; castings; NBSIR 81-1645.

composites; low temperature; mechanical and physical properties; structural alloys; welding; NBSIR 80-1627.

composition; materials; properties; research materials; standard reference materials; analysis; characterization; SP260, 1981-83 Edition.

compressive strength; concrete; curing temperature; early age; final set; hydration; initial set; laboratory testing; maturity; mortar; regression analysis; strength prediction; temperature effects; NBSIR 81-2244.

compressive strength; elongation; foam; insulation; low temperature; mechanical properties; proportional limit; shear strength; tensile strength; yield strength; Young's modulus; NBSIR 81-1654.

compressive strength; geothermal well; set-cement; simulated geothermal fluid; splitting tensile strength; NBSIR 80-2099-4.

compressor; contamination; continuous operation; cryocooler; low power refrigerator; Stirling refrigerator; bellows; SP607: 1981 May 141.145.

compressor; cryogenics; molecular adsorption; refrigeration; zeolites; adsorption; SP607: 1981 May. 127.135.

computations-finite difference; Euler equations; finite difference equations; fire-enclosure; fluid flow; heat source-volumetric; partial differential equations; buoyant convection; NBSIR 81-2385.

computer; insulation; lasers; powder data; progress; R-values; singlecrystal; SRM's; atomic ions; calibration; clock synchronization; Columbia River model; $D I M / N B S$ 65(2): 1-28; 1981.

computer aided design (CAD); computer aided manufacturing (CAM); entity; exchange format; file; geometry; graphics; interface; NBSIR 81-2297.

computer aided manufacturing (CAM); entity; exchange format; file; geometry; graphics; interface; computer aided design (CAD); NBSIR 81-2297.

computer applications; computer security; contingency planning; emergency response; Federal Information Processing Standards Publication; recovery actions; ADP availability; ADP security; backup operations; FIPS PUB 87.

computer arithmetic; conformance testing; numeric accuracy; programming language standards; SP500-77.

computer based office systems; computer networking; interfaces; local area networks; measurement techniques; protocols; standards; NBSIR 80-2154.

computer codes-F.CHART, SOLCOST, TRNSYS; computer predictions for solar systems; NBS solar testing at Gaithersburg; solar hot water heating; 20078.

computer data acquisition; energy conservation in commercial buildings; energy monitoring and control systems; mechanical systems; building envelope; building performance data; NBSIR 81 2358 .

computer evaluation; crystallographic data analysis; JCPDSInternational Centre for Diffraction Data; metric symmetry; NBS Crystal Data Center; powder diffraction; unit cell; TN1141.

computer graphics; critical evaluation; database management; thermodynamics; alloy phase diagram; 20033.

computer-integrated construction; conference; construction duration; productivity measurement; risks of failure; user productivity; building productivity; NBS-GCR-81-331.

computerized; geochemical model; heavy metals; redox couples; trace elements; aqueous speciation; SP618: 1981 November. 39-53.

computer model; distribution pipe; dynamic response; electricity; energy; explicit method; faucet temperature; finite difference; heat loss; implicit method; insulation; water heater; NBSIR 81-2303.

computer modeling; controls; control strategies; energy conservation for nonresidential buildings; load calculations; building energy analysis; NBSIR 81-2277. 
computer modeling; electrical design; energy audit; energy conservation; HVAC systems; performance standards; solar collector; solar energy; space heating and cooling; thermal storage; ASHRAE 90-75; class K code; SP608.

computer modeling; maintain accuracy; reduce cost; reduce input; standard evaluation technique; BLAST; building energy performance standards; SP608: 1981 May. 113-122.

computer models; fire detection systems; fire suppression; full-scale fire tests; heat transfer; railroad accidents; small-scale fire tests; thermal protection; bombs (ordnance); boxcars; NBSIR 80-2170.

computer network; computer security; encryption; Federal Information Processing Standards Publication; identification token; identity verification; password; personal attribute; personal identification; access control; authentication; authorization; FIPS PUB 83.

computer network; packet broadcasting; radio computer communication network; satellite communication; ALOHANET; ALOHA system; J. Res. 86(6): 591-595; 1981 November-December.

computer networking; interfaces; local area networks; measurement techniques; protocols; standards; computer based office systems; NBSIR 80-2154.

computer networking; technology assessment; technology forecast; NBS-GCR-80-305.

computer network interconnection; computer network protocol; digital transmission of information; electronic message transmission image transmission; information networks; national bibliographic network; satellite communications; slow-scan television; telecommunications policy; teleconferencing; videodiscs; SP610.

computer network protocol; digital transmission of information; electronic message transmission; image transmission; information networks; national bibliographic network; satellite communications; slow-scan television; telecommunications policy; teleconferencing; videodiscs; computer network interconnection; SP610.

computer network protocols; computer networks; cost/benefit analysis; federal government networking; federal computer networking development; networking standards; penetration studies; NBS-GCR-81-323.

computer network protocols; feature analysis; network architecture; network virtual terminal protocols; virtual terminal protocols; communication protocols; NBS-GCR-81-324.

computer networks; cost/benefit analysis; federal government networking; federal computer networking development; networking standards; penetration studies; computer network protocols; NBS-GCR-81-323.

computer networks; long-range forecasting; user behavior; workload forecasting; SP500-83: 1981 November. 7-14.

Computer Output Microfilm (COM); Federal Information Processing Standard (FIPS); illumination; image quality; microfilm; microform; readers; American National Standard; FIPS PUB 84.

computer-output microforms; Federal Information Processing Standards Publication; form slide; microfilm; quality control; automated data printing; FIPS PUB 82.

computer performance; performance tools; resource availability data; SP500-83: 1981 November. 329.

computer performance management; automated automation; SP500. 83: 1981 November. 67-70.

computer performance management; data base machines; end user productivity; human factors evaluation; information system management; office automation; performance management systems; resource measurement facility; simulation; supercomputers; benchmarking; capacity planning; chargeback systems; SP500-83.

computer predictions for solar systems; NBS solar testing at Gaithersburg; solar hot water heating; computer codes-F. CHART, SOLCOST, TRNSYS; 20078.

computer procurement; proposal evaluation; RFP preparation; small systems; SP500-83: 1981 November. 191-197.

computer program; data administration; database audition; database controls; database integrity; database management; data dictionary system; Federal Information Processing Standards Publication; FIPS PUB 88.

computer program; database; database management system; data dictionary system; data inventory; data management; data standards; documentation; Federal Information Processing Standards Publication; requirements; software; NBSIR 81-2354.

computer program; density; extended corresponding states; fluid mixtures; thermal conductivity; viscosity; TN1039.

computer program; earth temperature; heat transfer; pipes; thermal insulation; thermal properties; underground systems; NBSIR 81 2378.

computer program; electromagnetic; far field; FFT; Fourier transform; algorithm; antenna; aperture; NBSIR 81-1643.

computer program; hydrogen atom transfer; absolute chemical rate; BEBO activation energy; bond-order-bond-energy; chemical rate constants; J. Res. 86(6): 605-654; 1981 November-December.

computer programs; corresponding states; equation of state; hard sphere; LNG; mixtures; PVTx data; revised Kosek and McKinley; cell model; comparisons; TN1030.

computer programs; egress; elevator shafts; escape means; pressurization; smoke control; stairwells; air movement; NBSIR 802157.

computer programs; equation of state; ethylene thermodynamic properties; tabular values; TN1045.

computer programs; fire extinguishment; flashover; ignition mathematical models; room fires; compartment fires; NBS-GCR-81344.

computer resource selection; cost; long range plan; system requirements; user requirements; SP500-83: 1981 November. 293. 302.

computers; construction; data converter; mass comparator; radiation; radiocarbon; soft aluminum; windows; air pollution; automation; buildings; certified reference materials; DIM/NBS 65(4): 1-28; 1981.

computers; cost-benefit; automated data processing; NBS-GCR-81262.

computers; drill failure; Faraday; firefighting; hot atom; industrial innovation; measurements; oils, PCB's; standards; atomic weight; coal; DIM/NBS 65(6): 1-24; 1981

computers; energy; fine structure; interface; materials; measurement; office building; phase diagrams; radioactivity; research; sediment; soil; alloy; carbon; DIM/NBS 65(8): 1.28; 1981.

computer security; contingency planning; emergency response; Federal Information Processing Standards Publication; recovery actions; ADP availability; ADP security; backup operations; computer applications; FIPS PUB 87.

computer security; cryptography; data integrity; encryption; Federal Information Processing Standards Publication; key distribution; network security; security; FIPS PUB 74.

computer security; cryptography; data security; DES; encryption; Federal Information Processing Standards; modes of operation FIPS PUB 81 .

computer security; encryption; Federal Information Processing Standards Publication; identification token; identity verification; password; personal attribute; personal identification; access control; authentication; authorization; computer network; FIPS PUB 83.

computer simulation; DoE-2; heating/cooling load calculation; indoor temperature; NBSLD; attic temperature; BLAST; 20755.

computer simulation; infiltration; natural ventilation; building energy analysis; NBSIR 81-2404.

computer simulation of house energy requirements; degree days; energy analysis; single-family detached residence; building design; building energy performance standards; NBSIR 80-2184.

computer simulations; fault detection; laser scanner; photovoltaics; solar cell analysis; solar cells; NBS-GCR-80-285.

computer software; cost/benefit analysis; database management systems; data management; program maintenance; requirements studies; software procurement; application development; SP500-84.

computer standards and guidelines; energy research; environmental measurements; fire safety; industrial productivity; materials properties; research; standards; technology transfer; basic measurements and standards; building technology; calibration methods; SP600.

computer system; data processing function; performance evaluation; performance management; productivity; system tuning; SP500-83: 
1981 November. 121

computer system evaluation; disk subsystem configurations; input/output; modeling; rollin/rollout files; simulation; CDC 844 . 41; SP500-83: 1981 November. 239-246.

computer system installation; examples; OMNITAB 80; software; test problems; accuracy; ANS FORTRAN; TN1147.

computer systems; data entry (automatic); Federal Information Processing Standard; information processing systems; optical character recognition; character positioning; FIPS PUB 89.

computer utilization trends; Federal computers; hardware trends; information industry; software trends; technology forecast; automated data processing; NBS-GCR-81-342.

concentration; diffusivity; methane-ethane; mixture; one-fluid model; plait point; Rayleigh light scattering; thermal diffusivity; 19964.

concrete; concrete strength; construction; failure; flat plate; shear; strength; building; collapse; NBSIR 81-2374.

concrete; construction; nondestructive evaluation; quality assurance; steel reinforcing bars; test methods; NBSIR 80-2163.

concrete; corrosion; general corrosion; mathematical modeling; prestressed concrete; prestressing steel; stress corrosion; NBSIR 812390.

concrete; curing temperature; early age; final set; hydration; initial set; laboratory testing; maturity; mortar; regression analysis; strength prediction; temperature effects; compressive strength; NBSIR 81 . 2244.

concrete; diffusion; exhalation; materials; measurement; permeability; radon; transport; buildings; TN1139.

concrete; dynamic properties; fiber-reinforcement; impact; impulsive loading; penetration resistance; reinforcing bars; stress waves; SP480-45.

concrete; electrical; evaluation; HVAC; masonry; plumbing; solar; steel; structural systems; test methods; wood; building rehabilitation; NBSIR 81-2289.

concrete; electrical; evaluation; HVAC; masonry; plumbing; steel; structural systems; test methods; wood; building rehabilitation; NBSIR 80-2171.

concrete; fire endurance; fire tests; floors; reinforced concrete; roofs; thermal expansion; thermal restraint; buildings; NBS-GCR-81-317.

concrete; fly ash; cement; NBSIR 81-2239.

concrete; guarded hot plate; low temperature; moisture migration; mortar; porous solid; thermal conductivity; NBSIR 81-1651.

concrete; masonry; prohibitions; re-examination; thermal storage; wood structure; codes; SP608: 1981 May. 195-200

concrete strength; construction; failure; flat plate; shear; strength; building; collapse; concrete; NBSIR 81-2374.

condensing boilers; condensing furnaces; part-load performance; rating procedure; seasonal efficiency; annual fuel utilization efficiency; annual operating costs; central heating equipment; NBSIR 80-2110.

condensing boilers; efficiency, part load; fossil-fuel heating systems; gas-fired boilers; hydronic heating; pulse combustion; boilers; central heating; NBSIR 80-2094.

condensing furnaces; part-load performance; rating procedure; seasonal efficiency; annual fuel utilization efficiency; annual operating costs; central heating equipment; condensing boilers; NBSIR 80-2110.

condition monitoring; failure detection; helicopter diagnostics; oil debris; wear monitoring; chip detectors; SP622: 1981 October. 3349.

conduction heat transfer; conduction transfer functions; initialization of heat transfer problem; parallel heat flow; response factors; thick building construction; NBSIR 81-2353.

conduction transfer functions; initialization of heat transfer problem; parallel heat flow; response factors; thick building construction; conduction heat transfer; NBSIR 81-2353.

conductivity; defect detection; eddy current test; multifrequency; nondestructive testing; SP589.

conductivity; electrolytes; enthalpy; Gibbs energy; osmotic coefficients; solutions; sulfuric acid; thermodynamic properties; transport properties; activity coefficients; aqueous; compilation; NBSIR 81-2276. conductivity; exchange interaction; ferrite; hyperfine field; magnetism; phase diagram; potassium; 20043.

conductivity mobility; density of states; dopant (acceptor) density; effective mass; Hall mobility; hole density; resistivity; silicon; $N B S$ GCR-81-334.

conductor; critical current; critical temperature; review; standard; superconductor; 20069.

conference; construction duration; productivity measurement; risks of failure; user productivity; building productivity; computerintegrated construction; NBS-GCR-81-331.

configuration management $(\mathrm{CM})$; cross reference; data structure; design, documentation; functional; historical file; keyword; life cycle management; requirements; software quality assurance (SQA); specification; SP500-83: 1981 November. 101-106.

conformance testing; numeric accuracy; programming language standards; computer arithmetic; SP500-77.

conformational energy; dipole moment; gas phase conformers; glycine; rotational constants; structure; ab initio calculations; amino acid; 20005 .

conical antenna; EMP; FFT; loaded antenna; method of moments TEM horn; time domain measurements; transients in resistivity; NBSIR 78-876.

Co-Ni-Cr-Mo; corrosion; dental alloys; metal-amino acid reactions stainless steel; titanium; Co-Cr-Mo; 19988.

conjugate gradient algorithm; elliptic partial differential equations iterative methods for linear algebraic equations; Neumann boundary conditions; space matrices; NBSIR 80-2056.

consensus; construction; document; installation; recommended requirements; solar energy; solar systems; building codes; building officials; SP608: 1981 May. 167.172.

conservation; discrimination; energy; United Stand; alternative; California; Class K code; SP608: 1981 May. 83-103.

conspicuity; contrast; illumination; lighting; lighting levels; superthreshold visibility; vision; NBSIR 81-2231.

conspicuity; effective intensity; emergency warning lights; flashing lights; lights, warning; sirens; sound level; warning light; warning signals; SP480-37.

construction; cost; economics; housing; productivity; regulation; renovation; building codes; building economics; NBSIR 80-2150.

construction; cost; estimation; location factors; model building; statistical analysis; applied economics; NBSIR 81-2250.

construction; data converter; mass comparator; radiation; radiocarbon; soft aluminum; windows; air pollution; automation; buildings; certified reference materials; computers; $D I M / N B S$ 65(4): 1.28; 1981.

construction; document; installation; recommended requirements; solar energy; solar systems; building codes; building officials; consensus; SP608: 1981 May. 167-172.

construction; energy audit; energy conservation; Illinois; maintenance; public buildings; state government; cost; SP608: 1981 May. 123-132.

construction; energy conservation; ground coupled; hybrid solar energy system; inverted cave; low grade geothermal; radiant floor heating; space heating and cooling; thermal mass; walls; water source heat pump; buildings; SP608: 1981 May. 69-81.

construction; failure; flat plate; shear; strength; building; collapse; concrete; concrete strength; NBSIR 81-2374.

construction; nondestructive evaluation; quality assurance; steel reinforcing bars; test methods; concrete; NBSIR 80-2163.

construction costs; cost minimization; economic impact; economics; energy; model code; noise control; acoustical design; acoustics; architectural design; building codes; building economics; NBSIR 81-2366.

construction duration; productivity measurement; risks of failure; user productivity; building productivity; computer-integrated construction; conference; NBS-GCR-81-331.

construction economics; construction regulation; economics; fire safety codes; regulation; benefit-cost analysis; building economics; building regulation; codes; NBSIR 81-2402.

construction loads; construction safety; finite element; scaffold failures; scaffolding practices; scaffolds; structural analysis; accidents; codes and standards; NBS-GCR-80-255. 
construction regulation; economics; energy conservation codes; fire safety codes; regulation; benefit-cost analysis; building economics; building regulation; codes; NBSIR 81-2223.

construction regulation; economics; fire safety codes; regulation; benefit-cost analysis; building economics; building regulation; codes; construction economics; NBSIR 81-2402.

construction safety; design; finite element; loads; scaffolds; stability; stiffness; strength; structural safety; work surfaces; codes and standards; NBSIR 81-2265.

construction safety; finite element; scaffold failures; scaffolding practices; scaffolds; structural analysis; accidents; codes and standards; construction loads; NBS-GCR-80-255.

consumer protection; Interstate Solar Coordination Council; labeling procedures; national testing program; regulation; solar collector; standards; voluntary program; certification; SP608: 1981 May. 179. 181.

contamination; continuous operation; cryocooler; low power refrigerator; Stirling refrigerator; bellows; compressor; SP607: 1981 May. 141-145.

contamination control; cryogenics; helium retention; long-life refrigerators; reliability; rotary-reciprocating; turbo-Brayton; Vuilleumier; component development; SP607: 1981 May. 21.29.

contingency planning; disaster recovery planning; capacity planning; SP500-83: 1981 November. 45-54.

contingency planning; emergency response; Federal Information Processing Standards Publication; recovery actions; ADP availability; ADP security; backup operations; computer applications; computer security; FIPS PUB 87.

continued fraction; Dawson's function; FORTRAN subroutine; Fresnel integrals; key values; line broadening function; plasma dispersion function; Voigt function; argand diagram; complex error function; J. Res. 86(6): 661.686; 1981 November-December.

continuous operation; cryocooler; low power refrigerator; Stirling refrigerator; bellows; compressor; contamination; SP607: 1981 May. 141.145.

continuum states; diatomic spectroscopy; electronic-rotational states; predissociation; scattering theory; bound states; 20049.

contrast; illumination; lighting; lighting levels; superthreshold visibility; vision; conspicuity; NBSIR 81-2231.

control; fossil energy; materials; process parameters; research recommendations; coal; NBSIR 81-2348.

control and distribution; crew station terminals; electrical power management; electronic components; microcomputers; MIL-STD. 1553B multiplex data bus; multiplexing techniques; on-board diagnostics and prognostics; remote terminals; total tank subsystem integration; bus controllers; SP622: 1981 October. 85-96.

control functions; data interchange; display terminals; Federal Information Processing Standard; information interchange; printer terminals; standard; text communication; text interchange; typesetting composition; ADP terminals; ASCII; character-imaging devices; FIPS PUB 86.

control functions; information interchange; text communications; text processor; coded character sets; code extension techniques; code standards; SP500-81.

controls; control strategies; energy conservation for nonresidential buildings; load calculations; building energy analysis; computer modeling; NBSIR 81-2277.

control strategies; energy conservation for nonresidential buildings; load calculations; building energy analysis; computer modeling; controls; NBSIR 81-2277.

contusion; eyeball deformation; impact; ocular injury; projectiles; safety; NBSIR 80-2037.

convection; ellipsometry; gallium; levitation calorimetry; specific heat; surface tension; thermophysical properties; tungsten; wetting; adsorption; Auger spectroscopy; NBSIR 81-2295.

convective heat transfer; cryocooler; heat transfer by evaporating and condensing helium; helium refrigerator; superconducting computer; thermal couplings; thermal isolation; SP607: 1981 May. 93-102.

convective loops; greenhouses; heat exchangers; light and ventilation; solar systems; space heating and cooling; training programs; building codes; building officials; codes and standards; SP608: 1981
May. 159.166.

conversion; database; data description; data dictionary; data directory; data manipulation; DBMS; language; query; standards; SP500-76.

conveyor belts; fire safety; fire tests; flame spread; ignition; test methods; accident data; coal mines; NBSIR 81-2256.

convolution voltammetry; electrochemistry; Kalman filter; metal speciation; modeling; semiderivative; stability constants; voltammetry; zinc-NTA; cadmium-NTA; SP618: 1981 November. 163-172.

cool-down; heat flux meters; helium; superconducting power transmission; cable cool-down; NBSIR 81-1649.

cooling efficiency; evaporation cooling; smoke movement; spray nozzle; stairway protection; ventilated stair; automatic sprinklers; NBSIR 81-2202.

cooling of roofing bitumens; mathematical model; roofing; bitumen application temperatures; bituminous roofing; built-up roofing; TN1135.

cooperative phenomena; spectral line shapes; coherence; 19967. cooperative phenomena; spectral line shapes; coherence; 19968.

copper; Debye temperature; elastic constants; low temperatures; polycrystals; sound velocities; tensor-property averaging; 20726.

copper; deterioration; electromagnetic radiation; film; industry; metals; productivity; research associate; scanning technique; spin dependent; SRM's; standards; tape; DIM/NBS 65(5): 1-24; 1981.

copper; Drude theory; infrared; reflectance; silver; surface roughness; temperature dependence; aluminum; SP620: 1981 October. 190-200.

copper; iron; leaching; metal-reduction; metal-sulfides; mineraloxidation; molybdenum; oil shale; Sulfolobus; Thiobacillus; uranium; chemolithotrophic-bacteria; SP618: 1981 November. 264 273.

copper mirrors; $\mathrm{CO}_{2}$ laser; multishot damage; nanosecond pulse; peak energy density; pulse distortion; single-shot damage threshold; vacuum chamber; SP620: 1981 October. 238-247.

copper speciation; anodic stripping; bioavailability; complexing capacity; SP618: 1981 November. 173-183.

core barrel motion; neutron detectors; reactor internals; surveillance; vibration; SP622: 1981 October. 3-10.

core diameter; far field; index profile; mode filter; numerical aperture; radiation angle; radiation patterns; attenuation; TN1032.

core hole spectroscopy; metals with filled $d$-bands; photoemission; resonance; satellite; auger; 20008.

corona; decomposition; electrical insulation; gas chromatograph; inception voltages; mass spectrometer; partial discharge; sulfur hexafluoride; swarm data; water vapor; NBSIR 81-2242.

corona noise; EHV transmission lines; electric energy; environmental noise; human response; power line noise; psychoacoustics; annoyance; audible noise; 20050.

corona noise; electric energy transmission; high voltage; human response to noise; noise pollution; psychoacoustics; transmission line audible noise; 20029.

corona noise; environmental noise; high voltage transmission lines; human response to noise; listening room; 20030.

corrections for reflectance; infrared imaging; infrared thermography; radiance temperature; reflected infrared radiation; temperature corrections; temperature measurements; ambient infrared radiation; blackbody cavity; 20733.

correlation; critical region; helium; thermal conductivity; 20769.

correlation factor for diffusion; diffusion coefficient; diffusion equations; drift velocity for diffusion; driving force for diffusion; vacancy flow; vacancy wind effect; atom jump frequencies; 20038.

corresponding states; Enskog theory; mass difference effects; mixture viscosity; size difference effects; J. Res. 86(6): 597.604; 1981 November-December.

corresponding states; equation of state; hard sphere; LNG; mixtures; PVTx data; revised Kosek and McKinley; cell model; comparisons; computer programs; TNIO3O.

corrosion; data compilations; electrochemical aluminum production; electrochemical energy storage materials; molten salts; physical properties; safety and hazards; thermal energy storage materials; thermal properties; thermodynamic properties; transport properties; NSRDS-NBS61, Part IV. 
corrosion; dental alloys; metal-amino acid reactions; stainless steel; titanium; Co-Cr-Mo; Co-Ni-Cr-Mo; 19988.

corrosion; fracture and deformation; glass and ceramics research; materials research; materials science; metallurgy; polymers research; NBSIR 81 -2408.

corrosion; general corrosion; mathematical modeling; prestressed concrete; prestressing steel; stress corrosion; concrete; NBSIR 812390.

corrosion; metallurgically-bonded; metals; plastic-bonded; soils; telephone cables; underground; alloys; NBSIR 81-2243.

corrosion; osteosynthesis devices; tissue reaction; SP601: 1981 January. 423.447.

corrosion; predictability; reproducibility; time to failure; accelerated aging tests; coatings; TN1149.

corrosion; stainless steel implants; wavelength dispersive $\mathbf{x}$-ray analysis; SP601: 1981 January. 223.235.

corrosion behavior; field-tests; galvanic couples; soil environment; stainless steels; stress-corrosion behavior; coatings; NBSIR 81-2228 (NBS).

corrosion of electrical outlet boxes and devices; electrical devices; humidity, thermal insulation and corrosion of electrical wiring; shock hazards; urea-formaldehyde thermal insulation; cellulose thermal insulation; NBSIR 81-2220.

corrosion phenomena; electrochemical polarization; $\mathrm{Al} \cdot \mathrm{Zn} \cdot \mathrm{Mg}$; anodic polarization; 20026.

co-sited antennas; coupling; far fields; near fields; physical optics; planewave spectrum; reflector antennas; NBSIR 80-1630.

cost; construction; energy audit; energy conservation; Illinois; maintenance; public buildings; state government; SP608: 1981 May. 123-132.

cost; economics; housing; productivity; regulation; renovation; building codes; building economics; construction; NBSIR 80-2150.

cost; estimation; location factors; model building; statistical analysis; applied economics; construction; NBSIR 81-2250.

cost; long range plan; system requirements; user requirements; computer resource selection; SP500-83: 1981 November. 293-302.

cost accounting; cost recovery; chargeback; SP500-83: 1981 November. 311-312.

cost-benefit; automated data processing; computers; NBS-GCR-81262.

cost-benefit; economics; government regulation; safety regulation; standards; 20031.

cost-benefit; performance management; productivity; user service reporting; ADP installation management; SP500-83: 1981 November. 59-65.

cost/benefit analysis; database management systems; data management; program maintenance; requirements studies; software procurement; application development; computer software; SP50084.

cost/benefit analysis; federal government networking; federal computer networking development; networking standards; penetration studies; computer network protocols; computer networks; NBS-GCR-81-323.

cost/benefit analysis; flexible disk cartridge; flexible disk system; information interchange; marketed systems; standard; NBS-GCR80-306.

cost-effective; costs; economic analysis; economic decision framework; energy conservation; marginal price; net present value; residential water conservation; wastewater treatment; water rate schedules; water-saving devices; benefits; NBSIR 81-2304.

cost effective; economic analysis; energy conservation; investment analysis; life-cycle cost; recommended practice; savings-toinvestment ratio; benefit-cost analysis; building economics; NBSIR 81-2397.

cost minimization; economic impact; economics; energy; model code; noise control; acoustical design; acoustics; architectural design; building codes; building economics; construction costs; NBSIR 812366.

cost recovery; chargeback; cost accounting; SP500-83: 1981 November. 311-312.

cost reduction; energy savings; failure detection; failure prevention; flight safety; fluid system components; maintenance effectiveness; on-board monitoring system; aircraft hydraulic systems; SP622 1981 October. 195-219.

costs; economic analysis; economic decision framework; energy conservation; marginal price; net present value; residential water conservation; wastewater treatment; water rate schedules; water. saving devices; benefits; cost-effective; NBSIR 81-2304.

costs and benefits; flexible disk cartridge; information interchange; label and file structure; standard; word processor; NBS-GCR-80307.

cost savings; energy conservation; HVAC systems; innovation; mathematical model; optimization; SP608: 1981 May. 21-35.

cotton; fabric flammability; fabrics; fire suppression; flame extinguishment; heat transfer; polyester; textiles; airflow; burn (injuries); NBSIR 81-2386.

cotton standards; food standards; measurement science and technology; standardization in developing countries; Sudan; weights and measures regulation; Agency for International Development; Arab League Organization for Standardization and Metrology; chemical industry standards; NBSIR 80-2020.

counter-flow cool-down; heat flux meters; liquid helium; permeability of composites; superconducting power transmission; cable cool. down; cable permeability; NBSIR 80-1637.

coupling; far fields; near fields; physical optics; planewave spectrum; reflector antennas; co-sited antennas; NBSIR 80-1630.

cover plates; earth's tilt; electric; fire; fracture toughness; measurement; parameter; physics; solar collector; space charges; technical competence; cement and concrete; DIM/NBS 65(3): 1.24; 1981 .

$\mathrm{CO}_{2}$ laser; coating damage; laser damage; microsecond pulses; $\mathrm{NaCl}$; surface damage; alkali halides; bulk damage; SP620: 1981 October. 88.93.

$\mathrm{CO}_{2}$ laser; FIR laser; laser frequency measurement; relative polarization; relative power; wave-lengths; $\mathrm{CD}_{2} \mathrm{~F}_{2} ; 20743$.

$\mathrm{CO}_{2}$ laser; multishot damage; nanosecond pulse; peak energy density; pulse distortion; single-shot damage threshold; vacuum chamber; copper mirrors; SP620: 1981 October. 238.247.

$\mathrm{CO}_{2}$-laser glazing; $\mathrm{CO}_{2}$-laser polishing; fused silica surface; laser damage; laser polishing; surface laser damage; $1.06 . \mu \mathrm{m} ; S P 620$ : 1981 October. 180.189.

$\mathrm{CO}_{2}$-laser polishing; fused silica surface; laser damage; laser polishing; surface laser damage; $1.06 \cdot \mu \mathrm{m} ; \mathrm{CO}_{2}$-laser glazing; SP620: 1981 October. 180-189.

$C P T$ theorem; parity; time reversal; charge conjugation; 19991.

crack growth; creep fracture; high temperature fracture; silicon nitride; strain rate effect; toughness; NBSIR 79-1960 (DoE).

crack growth model; creep cavitation; diffusive crack growth; energy release rate; high temperature fracture; J-integral; sialon; singular integral equation; NBSIR 81-2255.

crack opening displacement; design curve; elastic-plastic fracture mechanics; fitness-for-service; fracture mechanics; J-integral; NBSIR 81-1648.

crack propagation; erosion; fracture toughness; hardness; ceramics; coal conversion; 20028.

crankshaft bearing safety; medium speed diesel engine; piston ring wear; turbocharging monitoring; SP622: 1981 October. 97-112.

crazing; crystalline polymers; electron spin resonance; microcrack; microfibril; shear deformation; tensile failure; chain rupture; 20732.

creep; failure; fatigue; mechanical properties; morphology; polymer; stress relaxation; UHMWPE; ultra high molecular weight polyethylene; NBSIR 81-2209 (FDA).

creep cavitation; diffusive crack growth; energy release rate; high temperature fracture; J-integral; sialon; singular integral equation; crack growth model; NBSIR 81-2255.

creep fracture; high temperature fracture; silicon nitride; strain rate effect; toughness; crack growth; NBSIR 79-1960 (DoE).

crew station terminals; electrical power management; electronic components; microcomputers; MIL-STD-1553B multiplex data bus; multiplexing techniques; on-board diagnostics and prognostics; remote terminals; total tank subsystem integration; bus controllers; control and distribution; SP622: 1981 October. 85-96. 
crib fire; plastic; room fire; wood; zone model; 20740

crib fires; experiments; mathematical model; plastic; polyurethane; room fires; wood; NBSIR 80-2054, Vols. I and II.

crime in public housing; household behavior patterns; housing characteristics (physical); housing security; public housing security; security hardware; victimization survey; burglary; NBS-GCR-80291.

criteria; measurement methods; performance criteria; project summaries; technical bases; building research; building technology; codes; SP446-5.

criterion; critical exposure; fluid; heat transfer; injury threshold; scald; thermal injury; bioheat equation; burn; NBSIR 81-2320.

critical current; critical temperature; review; standard; superconductor; conductor; 20069.

critical current; electrical property; magnetic property; stability; standards; superconductor; NBSIR 80-1642.

critical current; energy gap; enhancement; microwaves; nonequilibrium superconductivity; photon-assisted tunneling; superconductivity; thin film; transition temperature; tunneling; tunnel junction; aluminum; 19979.

critical current; mechanical properties; niobium-tin; scaling; strain; superconducting wire; 20066.

critical density; critical point; liquid mass fraction; liquid volume fraction; phase equilibria; pure fluids; coexistence densities; $N B S I R$ 80-1631.

critical enhancement; hard sphere; hot wire; thermal conductivity; transient; ambient temperature; argon; J. Res. 86(3): 293-307; 1981 May-June.

critical evaluation; $\mathrm{CsNO}_{2}$; electrolytes; excess Gibbs energy; $\mathrm{KNO}_{2}$; $\mathrm{LiNO}_{2} ; \mathrm{NaNO}_{2}$; osmotic coefficients; $\mathrm{RbNO}_{2}$; solutions; standard reference data; thermodynamic properties; activity coefficients; alkali metal nitrite salts; aqueous; JPCRD 10(3): 767.778; 1981.

critical evaluation; database management; thermodynamics; alloy phase diagram; computer graphics; 20033.

critical evaluation; electrolyte; ethylene bis(trimethylammonium) chloride; ethylene bis(trimethylammonium) iodide; excess Gibbs energy; osmotic coefficient; solutions; thermodynamic properties; zinc; activity coefficient; cadmium; JPCRD 10(1): 1-56; 1981.

critical evaluation; electrolyte; excess Gibbs energy; lithium; osmotic coefficient; potassium; rubidium; sodium; solutions; thermodynamic properties; activity coefficient; cesium; JPCRD 10(3): 671-766.

critical evaluation; electrolytes; excess free energy; free energy; ionic; osmotic coefficients; solutions; sulfuric acid; standard reference data; thermodynamic properties; activity coefficients; aqueous; JPCRD 10(3): 779-798; 1981.

critical exposure; fluid; heat transfer; injury threshold; scald; thermal injury; bioheat equation; burn; criterion; NBSIR 81-2320.

critical materials; tantalum; titanium; aerospace; chromium; cobalt; NBSIR 81-2305.

critical mixing; critical phenomena; dielectric constant; fluids in an electric field; refractive index; binary liquids; 20056.

critical phenomena; dielectric constant; fluids in an electric field; refractive index; binary liquids; critical mixing; 20056.

critical point; liquid mass fraction; liquid volume fraction; phase equilibria; pure fluids; coexistence densities; critical density; NBSIR 80-1631.

critical region; density; equation of state; geothermal; isobutane; thermodynamic; vapor pressure; NBSIR 79-1715.

critical region; helium; thermal conductivity; correlation; 20769.

critical review, evaluated data; gases; halogenated hydrocarbons; Henry's law constants; liquids; pesticides, solids; solubility; vapor pressure; alkanes; aromatics; JPCRD 10(4): 1175-1199; 1981.

critical temperature; review; standard; superconductor; conductor; critical current; 20069.

cross-bridge sheet resistor; electrical alignment test structure; gated diode; integrated gated-diode electrometer; integrated test structure; MOSFET dc profiler; test structure; CCD; NBSIR 81-2319.

cross-bridge sheet resistor; integrated gated-diode electrometer; microelectronic test pattern; microelectronic test structure; MOSFET dc profiler; NBSIR 81-2234.

CROSSFIRE; link protocols; local survivability; message delay; network simulation; network throughput; survivability; alternate routing; communications networks; NBS-GCR-81-336.

cross reference; data structure; design, documentation; functional; historical file; keyword; life cycle management; requirements; software quality assurance (SQA); specification; configuration management (CM); SP500-83: 1981 November. 101-106.

cross-referencing capabilities; interactive computer system; orthopaedic implants; SP601: 1981 January. 573-613.

cross section; detachment; energy transfer; excitaticn; ion; molecule; reaction rate; relaxation; atom; charge transfer; chemi-ionization; collisional ionization; SP593.

crowd ingress; design procedures; evaluation procedures; facility design; facility management; public assembly; public safety; stadiums; auditorium; building standards; NBSIR 81-2361.

cryocooler; cryogenic; helium; low temperature; refrigerator; Stirling cycle; SP607: 1981 May. 30-47.

cryocooler; gas bearings; refrigeration; regenerator; resonant free piston; Stirling cycle; SP607: 1981 May. 116.

cryocooler; heat transfer by evaporating and condensing helium; helium refrigerator; superconducting computer; thermal couplings; thermal isolation; convective heat transfer; SP607: 1981 May. 93102.

cryocooler; hybrid cryocoolers; refrigeration; regenerative cooler; regenerator; Stirling cooler; Stirling cycle; SP607: 1981 May. 48.56.

cryocooler; low power refrigerator; Stirling refrigerator; bellows; compressor; contamination; continuous operation; SP607: 1981 May. 141-145.

cryocoolers; cryogenic sensors; helium; refrigeration; superconducting devices; SP607.

cryocoolers; cryostats; helium; refrigeration; Stirling cycle; adsorption; SP607: 1981 May. 3.14.

cryocoolers; low temperature; magnetic gradiometer; refrigeration; SQUID; Stirling cycle; superconductivity; SP607: 1981 May. 186194.

cryocoolers; plastics; refrigeration; regenerators; specific heat; thermal conductivity; composites; SP607: 1981 May. 173-177.

cryogenic; helium; low temperature; refrigerator; Stirling cycle; cryocooler; SP607: 1981 May. 30-47.

cryogenic; hydrogen; production efficiency; scraping Auger; slush; slush production; NBSIR 78-875.

cryogenic; Joule-Thomson; microminiature devices; photolithographic fabrication; refrigerators; SP607: 1981 May. 160-163.

cryogenic energy conservation; liquefied natural gas; refrigeration recovery; chemical engineering; cold utilization; 20068.

cryogenic equipment; Josephson junction devices; superconducting computers; thermal contact; 4K refrigeration; SP607: 1981 May. 15. 20.

cryogenic helium balloon inflation; heat transfer; liquid helium; packed bed heat exchanger; balloon inflation; 20017.

cryogenic properties; instrumentation; low temperature materials; materials properties; metals; nonmetallic materials; wind tunnels; NBSIR 79-1624.

cryogenic properties; low temperature; mechanical properties; nonmetallics; physical properties; stainless steels; structural alloys; welding; alloys; aluminum alloys; castings; composites; NBSIR 811645.

cryogenic properties of materials; iron alloys; low temperature mechanical properties; plastic deformation; temperature effects stainless steel alloys; yield stress; 20742.

cryogenic refrigerator; Josephson technology; liquid helium; serviceable; superconducting device; thermal coupling; SP607: 1981 May. 82-92.

cryogenics; efficiency; helium; maximum refrigerator efficiency; perfect gas analysis; refrigerator; component efficiency; 20771.

cryogenics; gas adsorption; heat switch; refrigerators; SP607: 1981 May. 178-185.

cryogenics; Gifford-McMahon cryocoolers; heat capacity; heat transfer; helium; refrigerators; regenerators; Stirling cryocoolers; thermal conductance; Vuilleumier cryocoolers; 20766.

cryogenics; Gifford-McMahon cryocoolers; heat capacity; heat transfer; helium; refrigerators; regenerators; Stirling cryocoolers; 
thermal conductance; Vuilleumier cryocoolers; SP607: 1981 May. 70-81.

cryogenics; heat capacity; refrigeration; regenerators; specific heat; SP607: 1981 May. 164-172.

cryogenics; helium; infrared; SQUID; temperature sensors; SP607: 1981 May. 136-140.

cryogenics; helium; plastics; refrigerators; regenerators; Stirling-cycle; calculations; 20020.

cryogenics; helium retention; long-life; refrigerators; reliability; rotaryreciprocating; turbo-Brayton; Vuilleumier; component development; contamination control; SP607: 1981 May. 21.29.

cryogenics; hydrodynamic gas bearing; linear magnetic suspension; long lifetime; space application; Stirling cycle; clearance seals; SP607: 1981 May. 103.115.

cryogenics; infrared telescope; Spacelab; superfluid helium; SP607: 1981 May. 117-127.

cryogenics; molecular adsorption; refrigeration; zeolites; adsorption; compressor; SP607: 1981 May. 127-135.

cryogenics; multipliers; standards; superconductors; ac losses; calorimeter; 19999.

cryogenic sensors; helium; refrigeration; superconducting devices; cryocoolers; SP607.

cryostats; helium; refrigeration; Stirling cycle; adsorption; cryocoolers; SP607: 1981 May. 3-14.

cryptography; data integrity; encryption; Federal Information Processing Standards Publication; key distribution; network security; security; computer security; FIPS PUB 74.

cryptography; data security; DES; encryption; Federal Information Processing Standards; modes of operation; computer security; FIPS PUB 81 .

crystal; crystallinity; density; enthalpy; entropy; fusion; Gibbs energy; glass transition; heat capacity; linear macromolecule; melt polyethylene; JPCRD 1O(I): 119-152; 1981.

crystal; crystallinity; density; enthalpy; entropy; fusion; Gibbs energy; glass transition; heat capacity; linear macromolecule; poly(oxymethylene)s; poly(oxyphenylene)s; poly(oxypropylene); JPCRD 10(4): 1001-1049; 1981.

crystal; crystallinity; density; enthalpy; entropy; fusion; Gibbs energy; glass transition; heat capacity; isotactic; linear macromolecule; melt; polypropylene; syndiotactic; atactic; JPCRD 10(4): 1051.1064 1981

crystal; enthalpy; entropy; floor temperature; fusion; Gibbs energy; glass transition; heat capacity; linear macromolecule; melt selenium; calorimetry; JPCRD 10(1): 89-118; 1981

crystal; growth of crystals; mechanisms of growth; 20044.

crystalline disorder; defect damage; diamond-machined mirrors; Drude absorption; electron-beam melting; laser waveform; melt threshold; metal deposition; pulsed laser damage; slip threshold; thermal diffusion length; thermal stress; cold work; SP620: 1981 October. 201-209.

crystalline polymers; electron spin resonance; microcrack; microfibril shear deformation; tensile failure; chain rupture; crazing; 20732.

crystallinity; density; enthalpy; entropy; fusion; Gibbs energy; glass transition; heat capacity; linear macromolecule; melt; polyethylene; crystal; JPCRD 1O(1): 119-152; 1981

crystallinity; density; enthalpy; entropy; fusion; Gibbs energy; glass transition; heat capacity; isotactic; linear macromolecule; melt; polypropylene; syndiotactic; atactic; crystal; JPCRD 10(4): 1051. 1064; 1981.

crystallinity; density; enthalpy; entropy; fusion; Gibbs energy; glass transition; heat capacity; linear macromolecule;

poly(oxymethylene)s; poly(oxyphenylene)s; poly(oxypropylene); crystal; JPCRD 10(4): 1001-1049; 1981.

crystallographic anisotropy; crystallographic perfection; initial permeability; metal mirror; polishing; polycrystalline material; residual stress; sapphire; specular reflectance; surface analysis; surface integrity; surface roughness; SP620: 1981 October. 171.179.

crystallographic data analysis; JCPDS-International Centre for Diffraction Data; metric symmetry; NBS Crystal Data Center; powder diffraction; unit cell; computer evaluation; TN1141.

crystallographic perfection; initial permeability; metal mirror; polishing; polycrystalline material; residual stress; sapphire; specular reflectance; surface analysis; surface integrity; surface roughness; crystallographic anisotropy; SP620: 1981 October. 171-179.

crystal structure; densities; lattice constants; powder patterns; reference intensities; standard; x-ray diffraction; Monogr. 25, Section 18.

crystal structure; diffraction; isotopes; molecular dynamics; neutron; neutron radiography; nondestructive evaluation; nuclear reactor; radiation; activation analysis; $T N 1142$.

crystal structure; hydrogen bonding; hydroxyapatite; tin hydroxide oxide phosphate; tin phosphate; topical fluoride treatments; 19994.

crystal structure; lead apatite; lead potassium apatite; substituted apatite; 19984.

crystal structure; rare-earth apatite; substituted fluorapatite; apatite; barium apatite; cation substitution; 20065.

CsI; detection efficiency; lixiscope; radioactive $x$-ray source; rareearth scintillator screen; 20003

$\mathrm{CsNO}_{2}$; electrolytes; excess Gibbs energy; $\mathrm{KNO}_{2} ; \mathrm{LiNO}_{2} ; \mathrm{NaNO}_{2}$; osmotic coefficients; $\mathrm{RbNO}_{2}$; solutions; standard reference data; thermodynamic properties; activity coefficients; alkali metal nitrite salts; aqueous; critical evaluation; JPCRD 10(3): 767.778; 1981.

Cu mirrors; laser-induced electron emission; mirror surface damage; surface plasma; absorption waves; air breakdown; SP620: 1981 October. 227.238.

cumulant spectra; nonlinear time series; polyspectra; spectrum analysis; statistics; time series; bispectrum; TNI036.

curing temperature; early age; final set; hydration; initial set; laboratory testing; maturity; mortar; regression analysis; strength prediction; temperature effects; compressive strength; concrete; NBSIR 81-2244.

current density; high voltage dc transmission lines; ion counter; space charge density; Wilson plates; absolute filter; NBSIR 81-2267.

curve fitting; empirical fitting; surface fitting; J. Res. 86(I): 1-25; 1981 January-February.

curve fitting; energy dispersive spectra; SP604: 1981 June. 193-231.

custody transfer; gaging; liquid level; LNG tanks; 20770.

cutset; cycle; directed graph; network; network flow; optimization; retraction; robustness; rooted tree; sensitivity analysis; shortest path; tolerance; 20022.

C.V profiling; dopant profiles; implantation-induced damage; ion channeling; ion dose measurement; ion implantation; channeling angle; NBS-GCR-81-325.

cw laser irradiation; digs; dust; laser damage; mirrors; optical components; scratches; surface defects; coating imperfections; SP620: 1981 October. 256.264.

cyanoacrylate; dental alloy; initiator; resin; wear; accelerator; alloy; base metal; casting; composite; NBSIR 80-2168.

cycle; directed graph; network; network flow; optimization; retraction; robustness; rooted tree; sensitivity analysis; shortest path; tolerance; cutset; 20022

cylinder; helium; inner piston; outer piston; regenerator gaps; single stage, twin piston cryogenic refrigerator; two nested, concentric pistons; U.S. Patent 4,281,517.

cylindrical; non-uniform; partially-filled; pitched-horizontal drain; slope; solid; velocity; volume; water; NBSIR 81-2266.

$\mathrm{C}_{5} \mathrm{H}_{5} \mathrm{~N}_{5} \mathrm{O}$; density; enthalpy of solution; Gua. $\mathrm{HCl} \cdot 1 \mathrm{H}_{2} \mathrm{O}$; Gua. $\mathrm{HCl} \cdot 2$ $\mathrm{H}_{2} \mathrm{O}$; Gua.2 $\mathrm{HCl}$; guanine (Gua); guanine hydrochlorides; heat capacity; nucleic acid base; solution calorimetry; thermochemistry; $\Delta C_{\rho}$ of reaction; calorimetry; J. Res. 86(4): 367-382; 1981 JulyAugust.

\section{D}

damage; high energy laser; laser damage; pulsed laser; reflectors; thin film; anti-reflection coatings; bichromatic; SP620: 1981 October. 287.296.

damage mechanisms; intensity dependent absorption; multipulse damage; alkali-halides; calorimetry; SP620: 1981 October. 75-87.

damage morphology; Gaussian beam; laser damage; spherical aberration; SP620: 1981 October. 403-405.

damage threshold; excitons; $\mathrm{NaCl}$; optical breakdown; point defects; 
primary defects; SP620: 1981 October. 406-415.

damage threshold; impurity-induced damage; laser damage; $\mathrm{ThF}_{4}$; thin films; $\mathrm{ZnS} ; \mathrm{ZrO}_{2} ;$ SP620: 1981 October. 277-286.

damage types; nonlinear effect; optical glass; threshold values; SP620: 1981 October. 102.109.

damped wave motion; density fluctuations; diffusion; dynamic structure factor; initial value problem; nonuniform systems; onedimensional models; Rayleigh-Brillouin scattering; J. Res. 86(5): 429-455; 1981 September-October.

damping theory; kinetics; lasers; lineshapes; optical pumping; resonance Raman; spectroscopy; strong fields; 20039.

DAPLEX; database management system; data model; DBMS; network data model; query language; relational model; SQL; CODASYL; NBS-GCR-81-338.

DASD; data set; disk management system; migration; MSS; performance; space management; SP500-83: 1981 November. 223230.

DASD 1/O capacity; density of data reference; I/O capacity algorithms; 3350 DASD; SP500-83: 1981 November. 231-238.

data; data link control procedures; data transparency; Federal Information Processing Standards Publication; interoperability; teleprocessing; transmission; advanced data communication control procedures; ANS X3.66; bit-oriented; code independence; FIPS PUB 78.

data; equations; handbook; physicists; physics; polymers; 20749.

data acquisition; durability; dynamic calibration; environmental testing; evaluation; measurand; reliability; sensor; transducer; ATE; automatic test equipment; calibration; SP615.

data administration; database audition; database controls; database integrity; database management; data dictionary system; Federal Information Processing Standards Publication; computer program; FIPS PUB 88.

data analysis; generalized plotting programs; interdata 7.16 computer; polynominal fitting; user manual; automated thermogravimetry; NBSIR 80-2102.

database; database management system; data dictionary system; data inventory; data management; data standards; documentation; Federal Information Processing Standards Publication; requirements; software; computer program; NBSIR 81-2354.

database; data description; data dictionary; data directory; data manipulation; DBMS; language; query; standards; conversion; SP500-76.

database audition; database controls; database integrity; database management; data dictionary system; Federal Information Processing Standards Publication; computer program; data administration; FIPS PUB 88.

database controls; database integrity; database management; data dictionary system; Federal Information Processing Standards Publication; computer program; data administration; database audition; FIPS PUB 88.

database design; data dictionary; design; flowchart; information flow; information systems; interactive systems; requirements; scenarios; SP500-83: 1981 November. 85-91.

database design; data dictionary; design; flowchart; information flow; information systems; interactive systems; requirements; scenarios; NBSIR 81-2240.

database integrity; database management; data dictionary system; Federal Information Processing Standards Publication; computer program; data administration; database audition; database controls; FIPS PUB 88

data base machines; end user productivity; human factors evaluation; information system management; office automation; performance management systems; resource measurement facility; simulation; supercomputers; benchmarking; capacity planning; chargeback systems; computer performance management; SP500-83.

database management; data conversion; data description; data interchange form; data translation; portability; software conversion; NBSIR 81-2315.

database management; data dictionary system; Federal Information Processing Standards Publication; computer program; data administration; database audition; database controls; database integrity; FIPS PUB 88.

database management; thermodynamics; alloy phase diagram; computer graphics; critical evaluation; 20033.

database management system; data dictionary system; data inventory; data management; data standards; documentation; Federal Information Processing Standards Publication; requirements; software; computer program; database; NBSIR 81-2354.

database management system; data model; DBMS; network data model; query language; CODASYL; NBS-GCR-81-337.

database management system; data model; DBMS; network data model; query language; relational model; SQL; CODASYL; DAPLEX; NBS-GCR-81-338.

database management system; data model; DBMS; network data model; query language; CODASYL; NBS-GCR-81-339.

database management systems; data management; program maintenance; requirements studies; software procurement; application development; computer software; cost/benefit analysis; SP500-84.

data base management systems; Federal contracts services; Federal Government computers; programming languages; software expenditures; software program life-cycle analysis; SP500-79.

data communication; data interchange; data processing; Federal Information Processing Standard; information interchange; information processing; standard; American National Standard Code for Information Interchange; ASCII; character set; coded character set; code for information interchange; collating sequence; FIPS PUB $1-1$.

data communications; data interchange; data processing; EAM cards; electric accounting machine; Federal Information Processing Standard; Hollerith punched card code; Hollerith punched cards; information interchange; information processing; punched cards; standard; American National Standard Code for Information Interchange; ASCII; card punch; card reader; FIPS PUB 14-1.

data communications; fiber optics information systems; integrated communications; local area networks, microwave; video teleconferencing; CATV systems; coaxial cable; SP500-83: 1981 November. 271-276.

data compilation; energy and environmental data; evaluated data; materials data; standard reference data; technical activities 1980; thermochemical and thermophysical data; NBSIR 81-2206.

data compilation; oxidation; photochemistry; rates; review; singlet oxygen; solution; chemical kinetics; JPCRD 10(4): 809-999; 1981 .

data compilations; electrochemical aluminum production; electrochemical energy storage materials; molten salts; physical properties; safety and hazards; thermal energy storage materials; thermal properties; thermodynamic properties; transport properties; corrosion; NSRDS-NBS61, Part IV.

data conversion; data description; data interchange form; data translation; portability; software conversion; database management; NBSIR 81-2315.

data converter; differential linearity; digital-to-analog converter; error measurement; gain; input noise; linearity; offset; analog-to-digital converter; calibration service; TN1145.

data converter; mass comparator; radiation; radiocarbon; soft aluminum; windows; air pollution; automation; buildings; certified reference materials; computers; construction; DIM/NBS 65(4): $1.28 ; 1981$.

data description; data dictionary; data directory; data manipulation; DBMS; language; query; standards; conversion; database; SP50076.

data description; data interchange form; data translation; portability; software conversion; database management; data conversion; NBSIR 81-2315.

data dictionary; data directory; data manipulation; DBMS; language; query; standards; conversion; database; data description; SP500-76.

data dictionary; design; flowchart; information flow; information systems; interactive systems; requirements; scenarios; database design; SP500-83: 1981 November. 85-91.

data dictionary; design; flowchart; information flow; information systems; interactive systems; requirements; scenarios; database design; NBSIR 81-2240. 
data dictionary system; data inventory; data management; data standards; documentation; Federal Information Processing Standards Publication; requirements; software; computer program; database; database management system; NBSIR 81-2354.

data dictionary system; Federal Information Processing Standards Publication; computer program; data administration; database audition; database controls; database integrity; database management; FIPS PUB 88.

data directory; data manipulation; DBMS; language; query; standards; conversion; database; data description; data dictionary; SP500-76.

data elements; I/O; logical swapping; measurement; MVS; paging/swapping; TSO; analysis; SP500-83: 1981 November. 343. 348

data entry (automatic); Federal Information Processing Standard; information processing systems; optical character recognition; character positioning; computer systems; FIPS PUB 89.

data evaluation; extended corresponding states; fluids; mixtures; one fluid model; prediction; Van der Waals theory; viscosity; 20768.

data integrity; encryption; Federal Information Processing Standards Publication; key distribution; network security; security; computer security; cryptography; FIPS PUB 74.

data interchange; data processing; EAM cards; electric accounting machine; Federal Information Processing Standard; Hollerith punched card code; Hollerith punched cards; information interchange; information processing; punched cards; standard; American National Standard Code for Information Interchange; ASCII; card punch; card reader; data communications; FIPS PUB 14-1.

data interchange; data processing; Federal Information Processing Standard; information interchange; information processing; standard; American National Standard Code for Information Interchange; ASCII; character set; coded character set; code for information interchange; collating sequence; data communication; FIPS PUB 1-1.

data interchange; display terminals; Federal Information Processing Standard; information interchange; printer terminals; standard; text communication; text interchange; typesetting composition; ADP terminals; ASCII; character-imaging devices; control functions; FIPS PUB 86.

data interchange form; data translation; portability; software conversion; database management; data conversion; data description; NBSIR 81-2315.

data inventory; data management; data standards; documentation; Federal Information Processing Standards Publication; requirements; software; computer program; database; database management system; data dictionary system; NBSIR 81-2354.

data link control procedures; data transparency; Federal Information Processing Standards Publication; interoperability; teleprocessing; transmission; advanced data communication control procedures; ANS X3.66; bit-oriented; code independence; data; FIPS PUB 78.

data management; data standards; documentation; Federal Information Processing Standards Publication; requirements; software; computer program; database; database management system; data dictionary system; data inventory; NBSIR 81-2354.

data management; program maintenance; requirements studies; software procurement; application development; computer software; cost/benefit analysis; database management systems; SP500-84.

data manipulation; DBMS; language; query; standards; conversion; database; data description; data dictionary; data directory; SP5OO76.

data model; DBMS; network data model; query language; CODASYL; database management system; NBS-GCR-81-339.

data model; DBMS; network data model; query language; CODASYL; database management system; NBS-GCR-81-337.

data model; DBMS; network data model; query language; relational model; SQL; CODASYL; DAPLEX; database management system; NBS-GCR-81-338.

data processing; EAM cards; electric accounting machine; Federal Information Processing Standard; Hollerith punched card code; Hollerith punched cards; information interchange; information processing; punched cards; standard; American National Standard Code for Information Interchange; ASCII; card punch; card reader; data communications; data interchange; FIPS PUB 14-1.

data processing; electromagnetic; spherical scanning; antennas; 20765. data processing; Federal Information Processing Standard; FORTRAN; information interchange; information processing; processor; programming language; software; BASIC; COBOL; compilers; FIPS PUB 29-1.

data processing; Federal Information Processing Standard information interchange; information processing; standard; American National Standard Code for Information Interchange; ASCII; character set; coded character set; code for information interchange; collating sequence; data communication; data interchange; FIPS PUB 1-1.

data processing function; performance evaluation; performance management; productivity; system tuning; computer system; SP50083: 1981 November. 121.

data profiles; dishwasher usage characteristic; energy consumption; field measurements; usage patterns; water consumption; clothes dryer usage characteristics; clothes washer usage characteristic; NBSIR 80-2136.

data quality; energy models; mathematical models; model confidence; model credibility; policy models; sensitivity analysis; validation assessment; composite models; SP616.

data retrieval system; implant failures; ASP (Arthritis Surgery Proforma); SP601: 1981 January. 615.642.

data security; DES; encryption; Federal Information Processing Standards; modes of operation; computer security; cryptography; FIPS PUB 81.

data set; disk management system; migration; MSS; performance; space management; DASD; SP500-83: 1981 November. 223-230.

data standards; documentation; Federal Information Processing Standards Publication; requirements; software; computer program; database; database management system; data dictionary system; data inventory; data management; NBSIR 81-2354.

data structure; design, documentation; functional; historical file; keyword; life cycle management; requirements; software quality assurance (SQA); specification; configuration management (CM); cross reference; SP500-83: 1981 November. 101-106.

data translation; portability; software conversion; database management; data conversion; data description; data interchange form; NBSIR 81-2315.

data transparency; Federal Information Processing Standards Publication; interoperability; teleprocessing; transmission; advanced data communication control procedures; ANS X3.66; bit-oriented; code independence; data; data link control procedures; FIPS PUB 78.

Dawson's function; FORTRAN subroutine; Fresnel integrals; key values; line broadening function; plasma dispersion function; Voigt function; argand diagram; complex error function; continued fraction; J. Res. 86(6): 661-686; 1981 November-December.

daylight; energy conservation; fenestration design; illumination; lighting control; solar heat gain; window management; NBSIR 802147.

daylighting; energy conservation; engineering economics; life-cycle costs; passive solar; regional analysis; thermal efficiency; windows; building economics; NBSIR 81-2248.

daylighting; illuminance; illumination; irradiance; solar radiation; TN1148.

DBMS; language; query; standards; conversion; database; data description; data dictionary; data directory; data manipulation; SP500-76.

DBMS; network data model; query language; CODASYL; database management system; data model; NBS-GCR-81-339.

DBMS; network data model; query language; CODASYL; database management system; data model; NBS-GCR-81-337.

DBMS; network data model; query language; relational model; SQL; CODASYL; DAPLEX; database management system; data model; NBS-GCR-81-338.

dc fields; high voltage; incipient fault; insulation; liquid breakdown; $\mathrm{SF}_{6}$; space charge; transformer oil; cables; composite insulation; 
NBSIR 81-2334

dc fields; high voltage; incipient fault; insulation; $\mathrm{SF}_{6}$; space charge; transformer oil; cables; NBSIR 81-2283.

dc transmission lines; electric field; electric field meters; electric field strength; electric power transmission; measurements; space charge; calibration; NBSIR 80-2177.

dead layer; low x-ray energies; $\mathrm{Si}(\mathrm{Li})$ detectors; SP604: 1981 June. 127.139.

dead-layer; SRM-477; window absorption; window thickness; window transmission; $\mathrm{x}$-ray detectors; $\mathrm{x}$-ray energy spectrometers; SP604: 1981 June. 61-70.

deadlayer determinations; germanium; monoenergetic photons; partlyresolved doublets; silicon; tailing; $\mathrm{x}$-ray detectors; collimation; SP604: 1981 June. 5-34.

deaf; directional indicators; handicapped users; tactile warnings; accessibility; alarms; blind; code requirements; communication; NBSIR 81-2428.

debonding joint replacements; knee replacements; component loosening; SP601: 1981 January. 509.557.

Debye temperature; elastic constants; low temperatures; polycrystals; sound velocities; tensor-property averaging; copper; 20726.

decibel levels; fire departments; frequency distribution; noise (sound); sleep; smoke detectors; wakefulness; adults; alarm responses; auditory perception; NBS-GCR-80-284.

decision analysis; fire models; flame spread; human behavior; ignition; polymers; smoke; soot; toxicity; wood; combustion; NBSIR 812382.

decision making; model assessment; model confidence; model evaluation; model utility; model validation; NBSIR 80-2053.

decision tables; information networks; modeling; standards; standardswriters; systems analysis; building standards; classification; NBSIR 80-1979-1.

decision tables; information networks; network; standards; standardswriters; systems analysis; building standards; classification; 19998.

decomposition; electrical insulation; gas chromatograph; inception voltages; mass spectrometer; partial discharge; sulfur hexafluoride; swarm data; water vapor; corona; NBSIR 81-2242.

decomposition; free radicals; gas phase; hydrocarbons; hydrogen; nitrogen; oxygen; rate of reaction; sulfur; Arrhenius parameters; chemical kinetics; combustion; NBSIR 81-2254.

decomposition reactions; differential scanning calorimetry; heat conduction calorimetry; kinetics of heat evolution; $m$-azidobenzoic acid; nitrocellulose; quantitative differential thermal analysis; thermal explosion theory; thermal hazards; thermal instability; adiabatic calorimetry; azidotriphenylmethane; NBSIR 80-2018 (DOT).

deconvolution; energy dispersive $\mathrm{x}$-ray spectrometry; Fourier methods; Gaussian curves; SP604: 1981 June. 177-191.

deconvolution; impulse response; inverse filtering; noise contamination; time domain; waveforms; TN1047.

deed; developers; energy wars; experimental code; field test; guideline; low income; non-code; policymaking; protestors; solar code; victim role; SP608: 1981 May. 173-178.

deep-level measurements; deep-level transient spectroscopy; lifetime; power-device grade silicon; thyristor material characterization; transient capacitance techniques; NBSIR 81-2325.

deep-level transient spectroscopy; lifetime; power-device grade silicon; thyristor material characterization; transient capacitance techniques; deep-level measurements; NBSIR 81-2325.

defect damage; diamond-machined mirrors; Drude absorption; electron-beam melting; laser waveform; melt threshold; metal deposition; pulsed laser damage; slip threshold; thermal diffusion length; thermal stress; cold work; crystalline disorder; SP620: 1981 October. 201.209.

defect detection; eddy current test; multifrequency; nondestructive testing; conductivity; SP589.

defect identification; laser scanner; measurement techniques; photovoltaics; solar cells; test pattern; NBSIR 80-2181.

defects; epitaxial regrowth; laser irradiation; liquid phase; single crystal; SP620: 1981 October. 222-226.

definitive methods; human serum; polynuclear aromatic hydrocarbons; reference materials; shale oil; SRM; trace organic analysis; 19989.

deformation; electron channeling; fatigue; metals; SEM; 1998.

degradation; reverse bias; second breakdown; switching; temperature; transistors, power; base current, reverse; NBSIR 81-2204.

degree-day; DOE-2; bin method; building energy performance standards (BEPS); 20756.

degree days; energy analysis; single-family detached residence; building design; building energy performance standards; computer simulation of house energy requirements; NBSIR 80-2184.

Delphi Forecast; robot control; robotic assembly; robotics research; robotic standards; robot vision; SP602.

densimeter; density reference system; liquid methane; LNG; TN1041. densimeter; density reference system; liquid methane; LNG; TN1035. densities; lattice constants; powder patterns; reference intensities; standard; x-ray diffraction; crystal structure; Monogr. 25, Section 18. density; dielectric properties; relative dielectric constant; soil textural types; attenuation; coaxial sample holder; NBSIR 78-896.

density; enthalpy; entropy; fusion; Gibbs energy; glass transition; heat capacity; linear macromolecule; melt; polyethylene; crystal; crystallinity; JPCRD 10(1): 119-152; 1981.

density; enthalpy; entropy; fusion; Gibbs energy; glass transition; heat capacity; linear macromolecule; poly(oxymethylene)s; poly(oxyphenylene)s; poly(oxypropylene); crystal; crystallinity; JPCRD 10(4): 1001-1049; 1981.

density; enthalpy; entropy; fusion; Gibbs energy; glass transition; heat capacity; isotactic; linear macromolecule; melt; polypropylene; syndiotactic; atactic; crystal; crystallinity; JPCRD 10(4): 1051-1064; 1981.

density; enthalpy of solution; Gua. $\mathrm{HCl} \cdot 1 \mathrm{H}_{2} \mathrm{O}$; Gua. $\mathrm{HCl} \cdot 2 \mathrm{H}_{2} \mathrm{O}$; Gua.2 $\mathrm{HCl}$; guanine (Gua); guanine hydrochlorides; heat capacity; nucleic acid base; solution calorimetry; thermochemistry; $\Delta C_{p}$ of reaction; calorimetry; $\mathrm{C}_{5} \mathrm{H}_{5} \mathrm{~N}_{5} \mathrm{O}$; J. Res. 86(4): 367-382; 1981 July-August.

density; equation of state; geothermal; isobutane; thermodynamic; vapor pressure; critical region; NBSIR 79-1715.

density; extended corresponding states; fluid mixtures; thermal conductivity; viscosity; computer program; TN1039.

density; forensic science; individualization; population statistics; refractive index; auto headlight glass; classification; NBSIR 812286.

density dependence; gaseous and liquid ethane; isothermal measurements; quartz crystal viscometer; saturated liquid; shear viscosity coefficient; 20767.

density dependence; gaseous and liquid methane; isothermal measurements; quartz crystal viscometer; shear viscosity coefficient; 20071.

density fluctuations; diffusion; dynamic structure factor; initial value problem; nonuniform systems; one-dimensional models; RayleighBrillouin scattering; damped wave motion; J. Res. 86(5): 429-455; 1981 September-October.

density of data reference; I/O capacity algorithms; 3350 DASD; DASD 1/O capacity; SP500-83: 1981 November. 231.238.

density of states; dopant (acceptor) density; effective mass; Hall mobility; hole density; resistivity; silicon; conductivity mobility; NBS-GCR-81-334.

density reference system; liquid methane; LNG; densimeter; TN1035. density reference system; liquid methane; LNG; densimeter; TN1041. density wave; galactic spirals; hydrodynamic instability and turbulence; plasma dynamics; spiral grand design; WASER; winding dilemma; J. Res. 86(6): 557.563; 1981 November. December.

dental alloy; initiator; resin; wear; accelerator; alloy; base metal; casting; composite; cyanoacrylate; NBSIR 80-2168.

dental alloys; metal-amino acid reactions; stainless steel; titanium; Co-Cr-Mo; Co-Ni-Cr-Mo; corrosion; 19988.

dental composite resin restorations; finishability; microporous filler; system nontoxicity; x-ray opacification; U.S. Patent 4,306,913.

dentin; methacrylate; polyfunctional surface-active comonomers; reconstructive dental work; resin; acrylate; adhesion; aminobenzoate; composite material; U.S. Patent 4,251,565.

DES; encryption; Federal Information Processing Standards; modes 
of operation; computer security; cryptography; data security; FIPS PUB 81 .

desert climate; energy calculation; environmental chamber; heating/cooling load; nighttime cooling; 20754.

design; earthquakes; engineering; foundations; professional practice; provisions; soil structure interaction; standards; buildings; NBSIR 80-2111-3.

design; environmental psychology; families; housing; interiors; lifestyle; research; site planning; sociology; standards; children; NBS-GCR-81-320.

design; finite element; loads; scaffolds; stability; stiffness; strength; structural safety; work surfaces; codes and standards; construction safety; NBSIR 81-2265.

design; flowchart; information flow; information systems; interactive systems; requirements; scenarios; database design; data dictionary; SP500-83: 1981 November. 85-91.

design; flowchart; information flow; information systems; interactive systems; requirements; scenarios; database design; data dictionary; NBSIR 81-2240.

design; functional programming; interpreters; prototypes; applicative programming; 20075.

design criteria; foundation design; geotechnical engineering; housing construction; mine subsidence; mining; settlement; structural design; structural engineering; NBSIR 81-2215.

design curve; elastic-plastic fracture mechanics; fitness-for-service; fracture mechanics; J-integral; crack opening displacement; $N B S I R$ $81-1648$.

design, documentation; functional; historical file; keyword; life cycle management; requirements; software quality assurance (SQA); specification; configuration management $(\mathrm{CM})$; cross reference; data structure; SP500-83: 1981 November. 101-106.

design procedures; evaluation procedures; facility design; facility management; public assembly; public safety; stadiums; auditorium; building standards; crowd ingress; NBSIR 81-2361.

desorption; hydrolyzable cations; hydrous oxides; kinetics; protolyzable anions; thermodynamics; adsorption; SP618: 1981 November. 146-162.

desuperheating heat exchangers; heat exchange design; heat recovery units; 20000.

detachment; energy transfer; excitation; ion; molecule; reaction rate; relaxation; atom; charge transfer; chemi-ionization; collisional ionization; cross section; SP593.

detection; fire hazards; fire modeling; fire protection; fire research; fire suppression; human behavior in fires; physics of fire; toxicity of combustion products; chemistry of fire; combustion products; NBSIR 80-2127-1.

detection; metals; plasma emission; specific elements; spectroscopy; chromatography; SP618: 1981 November. 184-196.

detection efficiency; lixiscope; radioactive $x$-ray source; rare-earth scintillator screen; CsI; 20003.

detector; energy dispersive $\mathrm{x}$-ray spectrometry; Gaussian profiles; lithium-drifted silicon ( $\mathrm{Si}(\mathrm{Li})$ ) detector; $\mathrm{x}$-ray microanalysis; SP604: 1981 June. 233-272.

detectors; diffusion rates; diffusion tubes; gas chromatography; hydrocarbons; vapor detector; vapor generator; vapor standards; accelerants; arson; combustible gas; SP480-43.

deterioration; electromagnetic radiation; film; industry; metals; productivity; research associate; scanning technique; spin dependent; SRM's; standards; tape; copper; DIM/NBS 65(5): 1.24; 1981.

deterioration of materials; element volatilization; fungi; metals; microorganisms; standard reference materials; bacteria; biodeterioration; biotransformations; NBSIR 81-2246.

detoxification; mercury; organomercury; plasmids; silver; toxic heavy metals; antimony; arsenic; bacterial resistances; cadmium; SP618: 1981 November. 301-324.

deuterated compounds; isotopic purity; mass spectra; Master Analytical Scheme; organic compounds in water; NBSIR 80-2160.

developers; energy wars; experimental code; field test; guideline; low income; non-code; policymaking; protestors; solar code; victim role; deed; SP608: 1981 May. 173-178. development; transducer; waveform; acoustic emission; calibration; 20757.

developmental center test; environmental test; field validation test; independent testing; quality assurance program; software change package; software maintenance testing; SPS00-83: 1981 November. 93-99.

developmentally disabled; evacuation; fire departments; fire emergency plan; fire protection; group homes; group home staff; NBS-GCR-81-315.

development environment; development function; productivity; SP500-83: 1981 November. 337.

development function; productivity; development environment; SP500-83: 1981 November. 337.

development support systems; programming environments; software development; software tools; toolboxes; SP500-78.

device architecture; secondary storage; space management; capacity planning; SP500-83: 1981 November. 325.

device for measuring the fugacity of a material; E.M.F. measuring circuit; solid electrolyte probe; U.S. Patent 4,264,423.

diameter; drain; flow; history; horizontal; length; partially-filled; pipe; slope; stream-depth; unsteady; volume; water; NBSIR 81-2249.

diamond-like carbon; ion beam deposition; surface smoothness; thin films; barrier properties; SP620: 1981 October. 313.323.

diamond-machined mirrors; Drude absorption; electron-beam melting; laser waveform; melt threshold; metal deposition; pulsed laser damage; slip threshold; thermal diffusion length; thermal stress; cold work; crystalline disorder; defect damage; SP620: 1981 October. 201-209.

diatomic spectroscopy; electronic-rotational states; predissociation scattering theory; bound states; continuum states; 20049.

diatomic spectroscopy; Franck-Condon principle; rotational-electronic coupling; scattering theory; adiabatic potentials; Born-Oppenheimer approximation; 20060.

dichlone; dual-electrode detector; electrochemical detector; liquid chromatography; nitrobenzene; organotin cations; 20728.

dielectric constant; fluids in an electric field; refractive index; binary liquids; critical mixing; critical phenomena; 20056.

dielectric constant measurements; electromagnetic moisture measurements; nondestructive evaluation; radar soil measurements; soil moisture; underground radar; void detection; adobe building materials; adobe soil; NBSIR 79-1610.

dielectric properties; grain; loss tangent; moisture; NBSIR 78-897.

dielectric properties; relative dielectric constant; soil textural types; attenuation; coaxial sample holder; density; NBSIR 78-896.

dielectrics; electric insulation fault location; time-domainreflectometry; underground transmission; aging; cables; NBSIR 81 2235.

dielectrics; electro-optics; gases; high voltage; insulation; interfaces; Kerr effect; liquids; Pockels effect; solids; NBSIR 81-2275.

differential linearity; digital-to-analog converter; error measurement; gain; input noise; linearity; offset; analog-to-digital converter; calibration service; data converter; TN1145.

differential pressure; splines; statistics; volume calibration; accountability; NBSIR 80-2151.

differential pressure meter; flow measurement; laminar flow; meter performance; numerical modeling; orifice meter; swirling flow; closed conduit meter; 19972.

differential scanning calorimetry; heat conduction calorimetry kinetics of heat evolution; $\mathrm{m}$-azidobenzoic acid; nitrocellulose; quantitative differential thermal analysis; thermal explosion theory; thermal hazards; thermal instability; adiabatic calorimetry; azidotriphenylmethane; decomposition reactions; NBSIR 80-2018 (DOT).

diffraction; isotopes; molecular dynamics; neutron; neutron radiography; nondestructive evaluation; nuclear reactor; radiation; activation analysis; crystal structure; TN1142.

diffraction effects; long range order; modulated structures; oxides; phase equilibria; 20046.

diffuse reflectance; directional-hemispherical reflectance; integrating spheres; laboratory intercomparisons; reflectance measurements; reflectance standards; solar properties of materials; spectral 
reflectance measurements; NBSIR 81-2311.

diffusion; dynamic structure factor; initial value problem; nonuniform systems; one-dimensional models; Rayleigh-Brillouin scattering; damped wave motion; density fluctuations; J. Res. 86(5): 429.455; 1981 September-October.

diffusion; equilibrium; solid; stress, strain; thermodynamics; chemical potential; 20725 .

diffusion; exhalation; materials; measurement; permeability; radon transport; buildings; concrete; TN1139.

diffusion; food packaging; inverse gas chromatography; migration; oil simulants; oligomers; polyolefins; radioactive tracer; spectrofluorimetry; antioxidants; NBSIR 8I-2264.

diffusion; hollow tubes; mercury; preconcentration; air analysis; SP618: 1981 November. 134.145.

diffusion; integrating device; permeation type; personal dosimeter; reaction; time-dependent; NBSIR 80-1975.

diffusion coefficient; diffusion equations; drift velocity for diffusion; driving force for diffusion; vacancy flow; vacancy wind effect; atom jump frequencies; correlation factor for diffusion; 20038.

diffusion equations; drift velocity for diffusion; driving force for diffusion; vacancy flow; vacancy wind effect; atom jump frequencies; correlation factor for diffusion; diffusion coefficient; 20038 .

diffusion flames; entrainment; fire models; fire plumes; NBS-GCR-81346.

diffusion flames; entrainment; fire plumes; flow rate; mass flow; NBS GCR-80-294.

diffusion in metals; fire; journals; library holdings, NBS Library; NBS periodicals; periodicals; proceedings; serials; standards; transactions; annual reports; NBSIR 81-2226.

diffusion rates; diffusion tubes; gas chromatography; hydrocarbons; vapor detector; vapor generator; vapor standards; accelerants; arson; combustible gas; detectors; SP480-43.

diffusion tubes; gas chromatography; hydrocarbons; vapor detector vapor generator; vapor standards; accelerants; arson; combustible gas; detectors; diffusion rates; SP480-43.

diffusive crack growth; energy release rate; high temperature fracture; J-integral; sialon; singular integral equation; crack growth model; creep cavitation; NBSIR 81-2255.

diffusivity; methane-ethane; mixture; one-fluid model; plait point; Rayleigh light scattering; thermal diffusivity; concentration; 19964.

digital; microcomputer; phase angle; sinewave generator; standard; synthesis; calibration; TNII44.

digital communication impulse response; instrumentation; measurements; shielded balanced transmission lines; time domain; transmission line model; waveform; bit error rate; coaxial transmission lines; $T N 1042$.

digital filter; electron excited $x$-ray spectra; energy-dispersive $x$-ray spectrum deconvolution; multiple least squares fitting; nuclear spectroscopy; SP604: 1981 June. 297.313.

digital filter; filter-fit method; linear least-squares; $x$-ray analysis; SP604: 1981 June. 273-296.

digital-to-analog converter; error measurement; gain; input noise; linearity; offset; analog-to-digital converter; calibration service; data converter; differential linearity; $T N 1145$.

digital transmission of information; electronic message transmission; image transmission; information networks; national bibliographic network; satellite communications; slow-scan television; telecommunications policy; teleconferencing; videodiscs; computer network interconnection; computer network protocol; SP610.

digs; dust; laser damage; mirrors; optical components; scratches; surface defects; coating imperfections; cw laser irradiation; SP620: 1981 October. 256-264.

dimensions; laser; LNG; ships; tanks; volume; NBSIR 80-214l.

dimethylarsinic acid; microbial growth; mobilization; nutrients; oil shale; retorting; soil; speciation; volatilization; arsenic; SP6I8: 1981 November. 286.300.

diode; field-effect transistor; III-V compounds; IMPATT diode; integrated circuit; LASER; LED; photocell; solar cell; solid state; transistor; tunnel diode; J. Res. 86(6): 565.570; 1981 NovemberDecember. diode laser; Ebert spectrometer; high resolution; hypochlorous acid ( $\mathrm{HOCl}$ ); infrared spectrum; $v_{2}$ vibration; 20053.

diode laser; ellipsometer; infrared; polarizer; thin films; SP620: 1981 October. 345-355.

dipole moment; gas phase conformers; glycine; rotational constants; structure; ab initio calculations; amino acid; conformational energy; 20005 .

dipole moments; electrically small objects; emission; measurements; quadrupoles; susceptibility; TEM cell; analysis; TN1040.

dipole transitions; magnetic moments; photon decays; quarks; angular distributions; charmed mesons; 20040.

direct current plasma; hydrophilic; hydrophobic; metal complex; porphyrin; shale oil; speciation; trace metals; chromatography; SP6I8: 1981 November. 105.119.

directed graph; network; network flow; optimization; retraction; robustness; rooted tree; sensitivity analysis; shortest path; tolerance; cutset; cycle; 20022.

direct gap semiconductors; non-linear optics; three-photon absorption; SP620: 1981 October. 446-452.

directional arrows; exit signs; fire safety; pictograms; symbols; understandability; visibility; visual alerting; NBSIR 81-2268.

directional-hemispherical reflectance; integrating spheres; laboratory intercomparisons; reflectance measurements; reflectance standards; solar properties of materials; spectral reflectance measurements; diffuse reflectance; NBSIR 81-2311.

directional indicators; handicapped users; tactile warnings; accessibility; alarms; blind; code requirements; communication; deaf; NBSIR 81-2428.

directive antennas; antenna beamwidths; aperture fields; NBSIR $8 I$ 1644.

directory; inaccuracy; ionizing radiation; radioactive sources; survey instruments; calibration; commercial services; NBS-GCR-80-296.

disability; door closers; door openers; doors; ergonomics; functional capacity; handicap; locks; architectural barriers; building accessibility; codes and standards; NBSIR 80-2174.

disaster recovery planning; business case; business elements; business problem; capacity management; capacity planning; SP500-83: 1981 November. 39-43.

disaster recovery planning; capacity planning; contingency planning; SP500-83: 1981 November. 45-54.

discharge; dishwasher; residential; supply; water conservation; water consumption; appliances; clothes washer; NBSIR 80-2173.

discharge; drainage; flow; model; plumbing; quasi-steady; unsteady; characteristics; J. Res. 86(2): 171-179; 1981 March-April.

discrimination; energy; United Stand; alternative; California; Class $\mathbf{K}$ code; conservation; SP608: 1981 May. 83-103.

dishwasher; residential; supply; water conservation; water consumption; appliances; clothes washer; discharge; NBSIR 802173.

dishwashers; dishwasher subcycles; fill controls; grey water; performance; soil removal; water conservation; water discharge characteristics; clothes washers; NBSIR 81-2291 (HUD)

dishwasher subcycles; fill controls; grey water; performance; soil removal; water conservation; water discharge characteristics; clothes washers; dishwashers; NBSIR 81-2291 (HUD).

dishwasher usage characteristic; energy consumption; field measurements; usage patterns; water consumption; clothes dryer usage characteristics; clothes washer usage characteristic; data profiles; NBSIR $80-2136$.

disk management system; migration; MSS; performance; space management; DASD; data set; SP500-83: 1981 November. 223-230.

disk subsystem configurations; input/output; modeling; rollin/rollout files; simulation; CDC 844.41; computer system evaluation; SP50083: 1981 November. 239.246.

disodium hydrogen phosphate; fixed point; hydration temperature; potassium fluoride; salt hydrate; sodium sulfate; temperature calibration; temperature standard; thermistor; thermometer; transition temperature; biomedical thermometry; J. Res. 86(2): 181 192; 1981 March-April.

displacer and piston drives; linear electric motors; linear free-displacer cryorefrigerator; linear resonant machinery; phasor notation; 
thermodynamic and dynamic analyses; SP607: 1981 May. 57.69.

display terminals; Federal Information Processing Standard; information interchange; printer terminals; standard; text communication; text interchange; typesetting composition; ADP terminals; ASCII; character.imaging devices; control functions; data interchange; FIPS PUB 86.

dissolved organic compounds; gas chromatography/mass spectrometry; hydrophobic bases; metal complexation; nitrogen bases; oil shale; retort water; speciation; trace metals; alkylpyridines; SP618: 1981 November. 93-104.

distortion dipole transitions; interstellar molecules; methane; microwave spectra; molecular parameters; radio astronomy; rotational transitions; JPCRD 10(4): 1085-1095; 1981.

distributed control; message delay; network throughput; survivability; alternate routing; communications networks; NBSIR 81-2414.

distribution pipe; dynamic response; electricity; energy; explicit method; faucet temperature; finite difference; heat loss; implicit method; insulation; water heater; computer model; NBSIR 81-2303.

disulfur monoxide; rate constant; sulfur dioxide; sulfur monoxide; sulfur monoxide dimer; chemical kinetics; 20035.

document; installation; recommended requirements; solar energy; solar systems; building codes; building officials; consensus; construction; SP608: 1981 May. 167.172.

documentation; Federal Information Processing Standards Publication; requirements; software; computer program; database; database management system; data dictionary system; data inventory; data management; data standards; NBSIR 81-2354.

documentation; manuals; models; simulation; SP500-73.

documentation assessment; documentation guidelines; large-scale models; model assessment; NBSIR 80-1971.

documentation guidelines; large-scale models; model assessment; documentation assessment; NBSIR 80-1971.

DOE-2; bin method; building energy performance standards (BEPS); degree-day; 20756.

DoE-2; heating/cooling load calculation; indoor temperature; NBSLD; attic temperature; BLAST; computer simulation; 20755

domestic hot water; recirculating hot water systems; solar collectors; system efficiency; thermal performance; NBS-GCR-81-322.

domestic standards; education programs; electronic devices; international recommendations; legal metrology; measurement assurance; metrication; model laws and regulations; packing and labeling; specifications and tolerances; technology transfer; weights and measures; SP599.

door closers; door openers; doors; ergonomics; functional capacity; handicap; locks; architectural barriers; building accessibility; codes and standards; disability; NBSIR 80-2174.

door openers; doors; ergonomics; functional capacity; handicap; locks; architectural barriers; building accessibility; codes and standards; disability; door closers; NBSIR 80-2174

doors; ergonomics; functional capacity; handicap; locks; architectural barriers; building accessibility; codes and standards; disability; door closers; door openers; NBSIR 80-2174.

doors; evacuation; fire alarm systems; fire departments; fire extinguishers; fire investigations; hospitals; nursing homes; nursing staff; smoke; smoke detectors; sprinkler systems; NBS-GCR-80-297.

doors; evacuation; fire alarm systems; fire departments; fire extinguishers; fire investigations; hospitals; nursing homes; nursing staff; smoke; smoke detectors; sprinkler systems; NBS-GCR-81-343.

doors; fire doors; fire tests; air flow; air leakage; ASTM E 152; NBSGCR-81-330

doors; fire doors; fire tests; infiltration; orifice flow; smoke control; smoke movement; air leakage; buildings; NBSIR 81-2214.

dopant (acceptor) density; effective mass; Hall mobility; hole density; resistivity; silicon; conductivity mobility; density of states; NBSGCR-81-334.

dopant density; electron mobility; Hall effect; hole mobility; Irvin curves; phosphorus; resistivity; semiconductor; silicon; boron; capacitance-voltage technique; SP4(0)-64.

dopant profiles; implantation-induced damage; ion channeling; ion dose measurement; ion implantation; channeling angle; C.V profiling; NBS-GCR-81-325.
Doppler signature; Doppler technique; high cycle fatigue; stress corrosion; turbine blade failures; blade vibration; SP622: 1981 October. 181-194.

Doppler technique; high cycle fatigue; stress corrosion; turbine blade failures; blade vibration; Doppler signature; SP622: 1981 October 181.194 .

dosage; dosimetry; health and safety; high voltage; ionization chamber; ionizing radiation; ionometer; radiation quantities and units; radiation standards and protection; radioactivity; radiology; radium; roentgen ray; tubes ( $\mathrm{x}$ ray); $\mathrm{x}$-ray measurement; $\mathrm{x}$ rays; $S P 625$

dosimetry; electric fields; electromagnetic interference; exposure history; instruments; magnetic fields; nonionizing radiation; regional calibration laboratories; standards; techniques; calibration; SP613.

dosimetry; health and safety; high voltage; ionization chamber; ionizing radiation; ionometer; radiation quantities and units; radiation standards and protection; radioactivity; radiology; radium; roentgen ray; tubes ( $x$ ray); $x$-ray measurement; $x$ rays; dosage; $S P 625$.

DP cost accounting; DP cost allocation; pricing; standard costing; chargeback; chargeout; charging; charging system; SP500-83: 1981 November. 109-118.

DP cost allocation; pricing; standard costing; chargeback; chargeout; charging; charging system; DP cost accounting; SP500-83: 1981 November. 109-118.

drag, experimental; flat plate, turbulent; boundary layer; compliant surface; 20048.

drag sphere; gust speed; instrumentation; wind sensor; wind speed; anemometer; 20059.

drain; flow; history; horizontal; length; partially-filled; pipe; slope; stream-depth; unsteady; volume; water; diameter; NBSIR 81-2249.

drainage; flow; model; plumbing; quasi-steady; unsteady; characteristics; discharge; J. Res. 86(2): 171.179; 1981 March-April.

drift velocity for diffusion; driving force for diffusion; vacancy flow; vacancy wind effect; atom jump frequencies; correlation factor for diffusion; diffusion coefficient; diffusion equations; 20038.

drill failure; Faraday; firefighting; hot atom; industrial innovation; measurements; oils, PCB's; standards; atomic weight; coal; computers; $D I M / N B S$ 65(6): 1.24; 1981

driving force for diffusion; vacancy flow; vacancy wind effect; atom jump frequencies; correlation factor for diffusion; diffusion coefficient; diffusion equations; drift velocity for diffusion; 20038.

Drude absorption; electron-beam melting; laser waveform; melt threshold; metal deposition; pulsed laser damage; slip threshold; thermal diffusion length; thermal stress; cold work; crystalline disorder; defect damage; diamond-machined mirrors; SP620: 1981 October. 201-209.

Drude theory; infrared; reflectance; silver; surface roughness; temperature dependence; aluminum; copper; SP620: 1981 October. 190.200.

drug development; drug regulation; innovation; post-marketing surveillance; regulatory experiments; system design; NBS-GCRETIP 81.96

drug regulation; innovation; post-marketing surveillance; regulatory experiments; system design; drug development; NBS-GCR-ETIP 81-96.

drug standards; enzyme immunoassay; epilepsy; ethosuximide; mass spectrometry; nuclear magnetic resonance spectroscopy; phenobarbital; phenytoin; primidone; reference material; serum base modification; serum matrix; anticonvulsants; antiepilepsy drugs; blood analysis; chromatography, gas; chromatography, liquid; SP260-72.

DTA analysis of environmental particulates; DTA apparatus for rapid sample change and steam pressure control; alpha-quartz particulate; chrysotile asbestos particulate; 20011.

DTA apparatus for rapid sample change and steam pressure control; alpha-quartz particulate; chrysotile asbestos particulate; DTA analysis of environmental particulates; 20011.

dual-electrode detector; electrochemical detector; liquid chromatography; nitrobenzene; organotin cations; dichlone; 20728. dual flush toilets; low flush toilets; water closets; water conservation; 
NBSIR 81-2296.

durability; dynamic calibration; environmental testing; evaluation; measurand; reliability; sensor; transducer; ATE; automatic test equipment; calibration; data acquisition; SP615.

durability; environmental exposure; reliability; solar collectors; solar materials; stagnation testing; accelerated aging; TN1136.

durability; solar energy; standards; absorber materials; accelerated test methods; NBSIR 81-2232.

duration; general adverse effect of noise; intermittency; loudness; noise criteria; time-varying noise; NBSIR 81-2377.

dust; laser damage; mirrors; optical components; scratches; surface defects; coating imperfections; cw laser irradiation; digs; SP620: 1981 October. 256.264.

dwelling units (residential); housing; Operation Breakthrough; postoccupancy housing evaluation; Project Feedback; questionnaires; research methods; survey research; user needs; NBSIR 81-2258.

dynamic analysis; programming aids; software development; software engineering; software tools; static analysis; taxonomy; SP500-74.

dynamic calibration; environmental testing; evaluation; measurand; reliability; sensor; transducer; ATE; automatic test equipment; calibration; data acquisition; durability; SP615.

dynamic light scattering; light scattering; nematic liquid crystal; photo-receptor membrane vesicles; random coil polymers; amphoteric latex; J. Res. 86(6): 571-590; 1981 November-December.

dynamic properties; fiber-reinforcement; impact; impulsive loading; penetration resistance; reinforcing bars; stress waves; concrete; $S P 480-45$.

dynamic response; electricity; energy; explicit method; faucet temperature; finite difference; heat loss; implicit method; insulation; water heater; computer model; distribution pipe; NBSIR 81-2303.

dynamics; $m \cdot 6.8$ potential; nonequlibrium molecular; shear; viscosity; argon; 19973.

dynamic structure factor; initial value problem; nonuniform systems; one-dimensional models; Rayleigh-Brillouin scattering; damped wave motion; density fluctuations; diffusion; J. Res. 86(5): 429-455; 1981 September-October.

dysfunction patterns; valvular substitute; bioprosthesis; SP601: 1981 January. 363.393.

\section{$\mathbf{E}$}

EAM cards; electric accounting machine; Federal Information Processing Standard; Hollerith punched card code; Hollerith punched cards; information interchange; information processing; punched cards; standard; American National Standard Code for Information Interchange; ASCII; card punch; card reader; data communications; data interchange; data processing; FIPS PUB 141 .

early age; final set; hydration; initial set; laboratory testing; maturity; mortar; regression analysis; strength prediction; temperature effects; compressive strength; concrete; curing temperature; NBSIR 81. 2244.

earthquake; seismic risk maps; standards; structural engineering; buildings; buildings (codes); buildings (design); NBSIR 80-2111-1.

earthquake loads; hail loads; snow loads; solar collectors; structural engineering; wind loads; NBSIR 81-2199.

earthquakes; engineering; Federal government; seismic safety; standards; structural engineering; building; building design; NBSIR 81-2195.

earthquakes; engineering; foundations; professional practice; provisions; soil structure interaction; standards; buildings; design; NBSIR 80-2111-3.

earthquakes; engineering; masonry; standards; structural engineering; building; building codes; building design; NBSIR 80-2111-5.

earthquakes; engineering; reinforced concrete; standards; structural engineering; building; building codes; building design; NBSIR 802111-4.

earthquakes; engineering; standards; steel; structural engineering; building; building codes; building design; NBSIR 80-2111-6.

earthquakes; engineering; standards; structural engineering; building; building codes; building design; NBSIR 80-2111-11. earthquakes; engineering; standards; structural engineering; wood; building; building codes; building design; NBSIR 80-2111-7.

earth resistivity; redox potential; soil corrosivity; soil $\mathrm{pH}$; soil resistivity; underground corrosion; 19963.

earth's tilt; electric; fire; fracture toughness; measurement; parameter; physics; solar collector; space charges; technical competence; cement and concrete; cover plates; DIM/NBS 65(3): 1-24; 1981.

earth temperature; heat transfer; pipes; thermal insulation; thermal properties; underground systems; computer program; NBSIR 812378.

earth terminal; Earth Terminal Measurement System; figure of merit; noise temperature; satellite communication; NBSIR 81-1641.

Earth Terminal Measurement System; figure of merit; noise temperature; satellite communication; earth terminal; NBSIR 81 1641.

Ebert spectrometer; high resolution; hypochlorous acid $(\mathrm{HOCl})$; infrared spectrum; $v_{2}$ vibration; diode laser; 20053.

Eckart potential; potential barriers; quantum mechanical tunneling calculations; tunneling corrections; chemical rate constants; J. Res. 86(4): 357-359; 1981 July-August.

econimic, institutional, technical barriers; procurement; purchasing; recovered/recycled materials; resource recovery; NBS-GCR-81309, Supplement 1.

economic; institutional; procurement; purchasing; recovered/recycled materials; resource recovery; technical barriers; NBSIR 81-2316.

economic; institutional; procurement; purchasing; recovered/recycled materials; resource recovery; technical barriers; NBS-GCR-81-313.

economic; institutional; procurement; purchasing; recovered/recycled materials; resource recovery; technical barriers; NBS-GCR-81-308.

economic; institutional; procurement; purchasing; recovered/recycled materials; resource recovery; technical barriers; NBS-GCR-81-311.

economic; institutional; procurement; purchasing; recovered/recycled materials; resource recovery; technical barriers; NBS-GCR-81-312.

economic; institutional; procurement; purchasing; recovered/recycled materials; resource recovery; technical barriers; NBS-GCR-81-314.

economic analysis; economic decision framework; energy conservation; marginal price; net present value; residential water conservation; wastewater treatment; water rate schedules; watersaving devices; benefits; cost-effective; costs; NBSIR 81-2304.

economic analysis; energy analysis; energy conservation; Federal programs; government buildings; hospitals; rate of return; schools; technical assistance; certification; Class A energy audit; SP608: 1981 May. 133-138.

economic analysis; energy conservation; investment analysis; life-cycle cost; recommended practice; savings-to-investment ratio; benefitcost analysis; building economics; cost effective; NBSIR 81-2397.

economic analysis; fire safety; fire safety evaluation system; health care facilities; life cycle; cash flow; NBS-GCR-79-186-1.

economic decision framework; energy conservation; marginal price; net present value; residential water conservation; wastewater treatment; water rate schedules; water-saving devices; benefits; costeffective; costs; economic analysis; NBSIR 81-2304.

economic impact; economics; energy; model code; noise control; acoustical design; acoustics; architectural design; building codes; building economics; construction costs; cost minimization; NBSIR 81-2366.

economic, institutional, technical barriers; procurement; purchasing; recovered/recycled materials; resource recovery; NBS-GCR-81309.

economic, institutional, technical barriers; procurement; purchasing; recovered/recycled materials; resource recovery; NBS-GCR-81310.

economic optimization; life-cycle costing; solar economics; solar energy; NBSIR 81-2342.

economics; energy; model code; noise control; acoustical design; acoustics; architectural design; building codes; building economics; construction costs; cost minimization; economic impact; NBSIR 81 2366.

economics; energy conservation codes; fire safety codes; regulation; benefit-cost analysis; building economics; building regulation; codes; construction regulation; NBSIR 81-2223. 
economics; fire safety codes; regulation; benefit-cost analysis; building economics; building regulation; codes; construction economics; construction regulation; NBSIR 81-2402.

economics; government regulation; safety regulation; standards; costbenefit; 20031.

economics; housing; productivity; regulation; renovation; building codes; building economics; construction; cost; NBSIR 80-2150.

EDAX analysis; enzyme histochemistry; histology; implant retrieval analysis; polarography; tissue response; atomic absorption spectrophotometry; SP601: 1981 January. 323-336.

eddy currents; index; National Bureau of Standards; nondestructive evaluation; publications; radiography; ultrasonics; abstracts; acoustic emission; bibliography; NBSIR 80-2080.

eddy currents; liquid penetrants; magnetic particles; microwaves; nondestructive evaluation; radiography; tire inspection; ultrasonics; visual-optical; acoustic emission; 20762.

eddy current test; multifrequency; nondestructive testing. conductivity; defect detection; SP589.

edge heat loss; effective thermal conductivity; gap-temperature unbalance; guarded-hot-plate; heat source distribution; insulation; thermal conductivity; 20735.

education programs; electronic devices; international recommendations; legal metrology; measurement assurance; metrication; model laws and regulations; packing and labeling; specifications and tolerances; technology transfer; weights and measures; domestic standards; SP599.

effective area; intercomparison; piston gage; pressure; primary standard; transfer standard; J. Res. 86(3): 277.279; 1981 May.June.

effective carbon atom number; loss-rate; mass-loss; n-alkanes; n-carboxylic esters; permeation; polyethylene; NBSIR 80-2131.

effective intensity; emergency warning lights; flashing lights; lights, warning; sirens; sound level; warning light; warning signals; conspicuity; SP480-37.

effective mass; Hall mobility; hole density; resistivity; silicon; conductivity mobility; density of states; dopant (acceptor) density; NBS-GCR-81-334.

effective mass; intraband-momentum matrix element; oscillator strength; two-photon absorption; band structure; SP620: 1981 October. 438-445.

effectiveness; energy conservation; energy conservation measures; installation; insulation; oil burners; practices; safety; sealants; standards; storm doors; storm windows; vent dampers; water heaters; windows; automatic ignition devices; caulks; SP606.

effective thermal conductivity; gap-temperature unbalance; guarded. hot-plate; heat source distribution; insulation; thermal conductivity; edge heat loss; 20735.

effect of filtration; engine wear analysis; oil filter design; oil filter testing; particle size sensitivity; radioactive wear testing; SP622: 1981 October. 50-61.

efficiency; flow rate; heat transfer fluid; solar collector; thermal performance; NBS-GCR-80-254.

efficiency; helium; maximum refrigerator efficiency; perfect gas analysis; refrigerator; component efficiency; cryogenics; 20771.

efficiency, part load; fossil-fuel heating systems; gas-fired boilers; hydronic heating; pulse combustion; boilers; central heating; condensing boilers; NBSIR 80-2094.

egress; elevator shafts; escape means; pressurization; smoke control stairwells; air movement; computer programs; NBSIR 80-2157.

egress; emergencies; evacuation; network models; buildings; NBSGCR $-81-316$

egress; fire detection; fire growth; hazard analysis; mathematical models; room fires; smoke movement; tenability limits; combustion products; compartment fires; NBSIR 80-2172.

EHV transmission lines; electric energy; environmental noise; human response; power line noise; psychoacoustics; annoyance; audible noise; corona noise; 20050

EIRP; error analysis; G/T; precision measurements; radio star; satellite communications; $\mathrm{C} / \mathrm{kT}$; NBSIR 78-869.

elastic anisotropy; elastic constants; Kroner method; sound velocities; ultrasonic inspection; ultrasonic waves; acoustic waves; 20773.

elastic anisotropy; nondestructive evaluation; nondestructive testing; stainless steel; ultrasonic scattering; ultrasonic waves; acoustic waves; 20744

elastic coefficients; high-energy laser; interferometric testing; optical distortion; photoelastic coefficients; polycrystalline aggregate; stress birefringence; thermal lensing; window materials; SP620: 1981 October. 117.128.

elastic constants; Kroner method; sound velocities; ultrasonic inspection; ultrasonic waves; acoustic waves; elastic anisotropy; 20773.

elastic constants; low temperatures; polycrystals; sound velocities; tensor-property averaging; copper; Debye temperature; 20726.

elastic-plastic fracture mechanics; fitness-for-service; fracture mechanics; J-integral; crack opening displacement; design curve; NBSIR 81-1648.

elastostatics; finite elements; in-core solver; isoparametrics; Mooney. Rivlin material; nonlinear equation-solvers; penalty method; planestress; strain invariants; tensile-test specimen; checkerboard pressure; J. Res. 86(1): 79-109; 1981 January-February.

electric; fire; fracture toughness; measurement; parameter; physics; solar collector; space charges; technical competence; cement and concrete; cover plates; earth's tilt; DIM/NBS 65(3): 1.24; 1981.

electric accounting machine; Federal Information Processing Standard; Hollerith punched card code; Hollerith punched cards; information interchange; information processing; punched cards; standard; American National Standard Code for Information Interchange; ASCII; card punch; card reader; data communications; data interchange; data processing; EAM cards; FIPS PUB 14-1.

electrical; electronics; engineering; hazards; measurement; metrology; National Bureau of Standards; power; radiation; semiconductor; technology; calibration; SP611.

electrical; elevators; mechanical; seismic coefficients; architectural; NBSIR 80-2111-8.

electrical; evaluation; HVAC; masonry; plumbing; solar; steel; structural systems; test methods; wood; building rehabilitation; concrete; NBSIR 81-2289.

electrical; evaluation; HVAC; masonry; plumbing; steel; structural systems; test methods; wood; building rehabilitation; concrete; NBSIR 80-2171.

electrical; geological; magnetic; mechanical; monograph; optical; physical; properties data; radiation damage; rock salt; sodium chloride; chemical; Monogr. 167.

electrical alignment test structure; gated diode; integrated gated-diode electrometer; integrated test structure; MOSFET dc profiler; test structure; CCD; cross-bridge sheet resistor; NBSIR 81-2319.

electrical cables; fire incidents; fire tests; test methods; trailing cables; coal mines; NBSIR 81.2292.

electrical conductivity; hardness; homogeneity; microstructure; precipitating; segregation; solidification; thermal mechanical treatment; aging; NBSIR 80-2069.

electrical connector; electrical feedthrough; vacuum feedthrough; 20748.

electrical design; energy audit; energy conservation; HVAC systems; performance standards; solar collector; solar energy; space heating and cooling; thermal storage; ASHRAE 90-75; class $\mathrm{K}$ code; computer modeling; SP608.

electrical design; energy distribution; National Electrical Code; power factor correction; source energy; transformers; SP608: 1981 May. 37.56 .

electrical devices; humidity, thermal insulation and corrosion of electrical wiring; shock hazards; urea-formaldehyde thermal insulation; cellulose thermal insulation; corrosion of electrical outlet boxes and devices; NBSIR 81-2220.

electrical distribution; glass-liquidus; insulation; monitors; neutron diffraction, samplers; SRM's; time-domain calibrations; weights and measures; $\mathrm{x}$-ray diffraction; air pollutants; antenna coupling; DIM/NBS 65(1): 1.28; 1981.

electrical feedthrough; vacuum feedthrough; electrical connector; 20748.

electrical fire; electric cable; models; thermal insulation; wiring system; TN1133. 
electrical fire; low ambient temperature; trip time; branch circuits; circuit breaker; NBSIR 81-2221.

electrical insulation; gas chromatograph; inception voltages; mass spectrometer; partial discharge; sulfur hexafluoride; swarm data; water vapor; corona; decomposition; NBSIR 81-2242.

electrically.short dipole; iteration method; nonlinear differential equation; nonlinear load; time-stepping finite difference equation technique; Auger function; NBSIR 78-898.

electrically small objects; emission; measurements; quadrupoles; susceptibility; TEM cell; analysis; dipole moments; TN104O.

electrical measurements and standards; length and mass measurements and standards; quantum metrology; quantum physics; temperature and pressure measurements and standards; time and frequency; NBSIR 80-2165.

electrical power management; electronic components; microcomputers; MIL-STD-1553B multiplex data bus; multiplexing techniques; on-board diagnostics and prognostics; remote terminals; total tank subsystem integration; bus controllers; control and distribution; crew station terminals; SP622: 1981 October. 85-96.

electrical problems; electrical wiring system; extension cord wiring; fuse/circuit breaker panel problems; overamping; residential structures; safety; thermal insulation; branch circuit wiring problems; NBSIR 81-2203.

electrical property; magnetic property; stability; standards; superconductor; critical current; NBSIR 80-1642.

electrical wiring system; extension cord wiring; fuse/circuit breaker panel problems; overamping; residential structures; safety; thermal insulation; branch circuit wiring problems; electrical problems; NBSIR 81-2203.

electric cable; models; thermal insulation; wiring system; electrical fire; TN1133.

electric discharges; helium Penning ionization; hydrogen; ambipolar diffusion; 20047.

electric energy; environmental noise; human response; power line noise; psychoacoustics; annoyance; audible noise; corona noise; EHV transmission lines; 20050.

electric energy transmission; high voltage; human response to noise; noise pollution; psychoacoustics; transmission line audible noise; corona noise; 20029.

electric field; electric field meters; electric field strength; electric power transmission; measurements; space charge; calibration; dc transmission lines; NBSIR 80-2177.

electric field; electro-optics; Kerr effect; measurement; optics; polarized light; 19962.

electric-field integral equation; electromagnetic theory; magnetic-field integral equation; scattering of electromagnetic waves; spurious resonances; augmented electric- and magnetic-field integral equations; 20761.

electric-field integral equation; electromagnetic theory; magnetic-field integral equation; scattering of electromagnetic waves; spurious resonances; augmented electric- and magnetic-field integral equations; 20760.

electric field meters; electric field strength; electric power transmission; measurements; space charge; calibration; dc transmission lines; electric field; NBSIR 80-2177.

electric fields; electric field strength; electric power transmission; field strength; magnetic fields; calibration; calibrators; NBSIR 77-1311.

electric fields; electromagnetic interference; exposure history; instruments; magnetic fields; nonionizing radiation; regional calibration laboratories; standards; techniques; calibration; dosimetry; SP613.

electric field strength; electric power transmission; field strength; magnetic fields; calibration; calibrators; electric fields; NBSIR 771311 .

electric field strength; electric power transmission; measurements; space charge; calibration; dc transmission lines; electric field; electric field meters; NBSIR 80-2177.

electric insulation fault location; time-domain-reflectometry; underground transmission; aging; cables; dielectrics; NBSIR 81 2235.

electricity; energy; explicit method; faucet temperature; finite difference; heat loss; implicit method; insulation; water heater; computer model; distribution pipe; dynamic response; NBSIR 81 2303.

electric power transmission; field strength; magnetic fields; calibration; calibrators; electric fields; electric field strength; NBSIR 77-1311.

electric power transmission; measurements; space charge; calibration; dc transmission lines; electric field; electric field meters; electric field strength; NBSIR 80-2177.

electric wire; field measurements; temperature excursions; thermal insulation; branch circuit; NBSIR 81-2347.

electrochemical aluminum production; electrochemical energy storage materials; molten salts; physical properties; safety and hazards; thermal energy storage materials; thermal properties; thermodynamic properties; transport properties; corrosion; data compilations; NSRDS-NBS61, Part IV.

electrochemical detector; liquid chromatography; nitrobenzene; organotin cations; dichlone; dual-electrode detector; 20728.

electrochemical energy storage materials; molten salts; physical properties; safety and hazards; thermal energy storage materials; thermal properties; thermodynamic properties; transport properties; corrosion; data compilations; electrochemical aluminum production; NSRDS-NBS61, Part IV.

electrochemical polarization; $\mathrm{Al}-\mathrm{Zn}-\mathrm{Mg}$; anodic polarization; corrosion phenomena; 20026.

electrochemistry; ellipsometry; field ion microscopy; inhibitors; organic coatings; passive film dissolution; $\mathrm{pH}$ measurements; surface hydride; alpha uranium; chloride ion; chromate ion; NBSIR $81-2396$ (ONR).

electrochemistry; Kalman filter; metal speciation; modeling semiderivative; stability constants; voltammetry; zinc-NTA; cadmium-NTA; convolution voltammetry; SP618: 1981 November. 163.172.

electrodeposition; immersion deposition; plating; plating on anodized aluminum; adhesion; anodizing; coatings; NBSIR 80-2142.

electrolyte; ethylene bis(trimethylammonium) chloride; ethylene bis(trimethylammonium) iodide; excess Gibbs energy; osmotic coefficient; solutions; thermodynamic properties; zinc; activity coefficient; cadmium; critical evaluation; JPCRD 1O(1): 1-56; 1981.

electrolyte; excess Gibbs energy; lithium; osmotic coefficient; potassium; rubidium; sodium; solutions; thermodynamic properties; activity coefficient; cesium; critical evaluation; JPCRD 10(3): 671 . 766.

electrolytes; enthalpy; Gibbs energy; osmotic coefficients; solutions; sulfuric acid; thermodynamic properties; transport properties; activity coefficients; aqueous; compilation; conductivity; NBSIR $81-2276$.

electrolytes; excess free energy; free energy; ionic; osmotic coefficients; solutions; sulfuric acid; standard reference data; thermodynamic properties; activity coefficients; aqueous; critical evaluation; JPCRD 10(3): 779-798; 1981.

electrolytes; excess Gibbs energy; $\mathrm{KNO}_{2} ; \mathrm{LiNO}_{2} ; \mathrm{NaNO}_{2}$; osmotic coefficients; $\mathrm{RbNO}_{2}$; solutions; standard reference data; thermodynamic properties; activity coefficients; alkali metal nitrite salts; aqueous; critical evaluation; $\mathrm{CsNO}_{2} ; J P C R D$ 10(3): 767.778; 1981 .

electromagnetic; far field; FFT; Fourier transform; algorithm; antenna; aperture; computer program; NBSIR 81-1643.

electromagnetic; spherical scanning; antennas; data processing; 20765. electromagnetic compatibility (EMC); Green's function; integral equation; linear equation; method of moments; parallel plate waveguide; quasi electrostatic; TEM cell; TN1028.

electromagnetic field; electronic instrument design; field intensity meter; field strength measurement; isotropic antenna; radio frequency radiation; receiver calibration; TN1033.

electromagnetic interactions; pair production; plasma diagnostics; radiation; synchrotron radiation; astrophysics; bremsstrahlung; 19993.

electromagnetic interference; electromagnetic metrology; em material properties; microwaves; noise; non-ionizing radiation; radiation hazards; antennas; NBSIR 80-1635. 
electromagnetic interference; exposure history; instruments; magnetic fields; nonionizing radiation; regional calibration laboratories; standards; techniques; calibration; dosimetry; electric fields; SP613.

electromagnetic metrology; em material properties; microwaves; noise; non-ionizing radiation; radiation hazards; antennas; electromagnetic interference; NBSIR 80-1635.

electromagnetic moisture measurements; nondestructive evaluation; radar soil measurements; soil moisture; underground radar; void detection; adobe building materials; adobe soil; dielectric constant measurements; NBSIR 79-1610.

electromagnetic radiated susceptibility measurements; mode tuned chamber; resonant cavity; NBSIR $81-1638$.

electromagnetic radiation; film; industry; metals; productivity; research associate; scanning technique; spin dependent; SRM's; standards; tape; copper; deterioration; DIM/NBS 65(5): 1-24; 1981.

electromagnetic theory; magnetic-field integral equation; scattering of electromagnetic waves; spurious resonances; augmented electric. and magnetic-field integral equations; electric-field integral equation; 20761.

electromagnetic theory; magnetic-field integral equation; scattering of electromagnetic waves; spurious resonances; augmented electricand magnetic-field integral equations; electric-field integral equation; 20760.

electron affinity; molecular spectroscopic constants; negative ions; photodetachment; potential energy curves; autoionization; 19995.

electron-atom scattering; electron impact ionization; metastable states; beryllium isoelectronic sequence; 20739.

electron atom scattering; electron impact ionization; sodium isoelectronic sequence; 20737.

electron beam; in-phantom measurement; photon beam; radiation therapy; absorbed dose; Bragg-Gray equation; calibration; cavity chamber; cavity theory; 20073.

electron-beam-induced current; etching; integrated circuit processing; ion channeling; ion implantation; lateral profiles; scanning electron microscopy; silicon technology; spreading resistance; twodimensional profiles; arsenic ion implantation; NBSIR 81-2398.

electron-beam melting; laser waveform; melt threshold; metal deposition; pulsed laser damage; slip threshold; thermal diffusion length; thermal stress; cold work; crystalline disorder; defect damage; diamond-machined mirrors; Drude absorption; SP620: 1981 October. 201-209.

electron channeling; fatigue; metals; SEM; deformation; 19980.

electron collision cross sections; heliumlike ions; autoionization resonance; 20045

electron column instruments; energy dispersive detectors; scanning electron microscopy; $\mathrm{x}$-ray spectrometry, review of scientific instruments; bibliography; SP604: 1981 June. 417-439.

electron configurations; ionization potentials; sodium; atomic energy levels; atomic ions; atomic spectra; JPCRD 10(1): 153-196; 1981.

electron density; graphite calorimeter; ionization chamber; scaling theorem; water phantom; absorbed dose standard; cobalt 60 gamma rays; J. Res. 86(5): 495.502; 1981 September-October.

electron excitation; energy-dispersive spectrometry; energy resolution; peak shapes; surface-layer analyses; ultra-thin-windowed (UTW) $\mathrm{Si}(\mathrm{Li})$ detectors; windowless detectors; $\mathrm{x}$-ray transmission; SP604: 1981 June. 97.126.

electron excited $\mathrm{x}$-ray spectra; energy-dispersive $\mathrm{x}$-ray spectrum deconvolution; multiple least squares fitting; nuclear spectroscopy; digital filter; SP604: 1981 June. 297-313.

electron gun; electron polarization; electron spin; polarized electrons; spin; spin polarization; 19996.

electronic components; microcomputers; MIL-STD-1553B multiplex data bus; multiplexing techniques; on-board diagnostics and prognostics; remote terminals; total tank subsystem integration; bus controllers; control and distribution; crew station terminals; electrical power management; SP622: 1981 October. 85-96.

electronic devices; international recommendations; legal metrology; measurement assurance; metrication; model laws and regulations; packing and labeling; specifications and tolerances; technology transfer; weights and measures; domestic standards; education programs; SP599. electronic equipment; fire test methods; incident data; telecommunications; telephone exchanges; central office equipment NBSIR 81-2261.

electronic instrument design; field intensity meter; field strength measurement; isotropic antenna; radio frequency radiation; receiver calibration; electromagnetic field; TN1033.

electronic laboratory instruction; low cost approach; 20006.

electronic message transmission; image transmission; information networks; national bibliographic network; satellite communications; slow-scan television; telecommunications policy; teleconferencing; videodiscs; computer network interconnection; computer network protocol; digital transmission of information; SP610.

electronic-rotational states; predissociation; scattering theory; bound states; continuum states; diatomic spectroscopy; 20049.

electronics; engineering; hazards; measurement; metrology; National Bureau of Standards; power; radiation; semiconductor; technology calibration; electrical; SP611.

electronics; gallium arsenide; integrated circuits; measurement technology; microelectronics; semiconductor devices; semiconductor materials; semiconductor process control; silicon; NBSIR 80-2006-4.

electronics; integrated circuits; measurement technology; microelectronics; semiconductor devices; semiconductor materials; semiconductor process control; silicon; NBSIR 79-1591-5.

electronics; integrated circuits; measurement technology; microelectronics; semiconductor devices; semiconductor materials semiconductor process control; silicon; NBSIR 79-1591-4.

electronics; integrated circuits; measurement technology; microelectronics; semiconductor devices; semiconductor materials; semiconductor process control; silicon; NBSIR 80-2006-3.

electronics; integrated circuits; measurement technology; microelectronics; semiconductor devices; semiconductor materials semiconductor process control; silicon; NBSIR 81-2230.

electronics; integrated circuits; measurement technology; microelectronics; semiconductor devices; semiconductor materials; semiconductor process control; silicon; NBSIR 81-2230-3.

electronics; integrated circuits; metrology; microelectronics; semiconductors; silicon; NBSIR 81-2224.

electronics; integrated circuits measurement technology; microelectronics; semiconductor devices; semiconductor materials; semiconductor process control; silicon; NBSIR 81-2230-2.

electronics; interconnect; microelectronics; random fault; step coverage; test structures; SP400-65.

electronics; Josephson effect; measurements; superconductivity; time domain; 20070

electronics; MOS devices; semiconductors; silicon dioxide; sputter profiling; Auger-electron spectroscopy; NBS-GCR-81-345.

electron impact ionization; helium isoelectronic sequence; hydrogen isoelectronic sequence; lithium isoelectronic sequence; 20738.

electron impact ionization; metastable states; beryllium isoelectronic sequence; electron-atom scattering; 20739.

electron impact ionization; sodium isoelectronic sequence; electron atom scattering; 20737.

electron incidence angle; electron probe microanalysis; SP604: 1981 June. 381-389.

electron microprobe; powder metallurgy; quantitative microscopy; standard reference material; $x$-ray diffraction; $x$-ray fluorescence analysis; austenite in ferrite; austenite standard; SP260-73.

electron mobility; Hall effect; hole mobility; Irvin curves; phosphorus; resistivity; semiconductor; silicon; boron; capacitance-voltage technique; dopant density; SP400-64.

electron-molecule collisions; interaction potential; Legendre expansion; molecular potential; quantum chemistry; scattering; 20055 .

electron-optical column; energy dispersive spectrometer; uncollimated fluorescing radiation; analytical electron microscope; SP604: 1981 June. 351-364.

electron polarization; electron spin; polarized electrons; spin; spin polarization; electron gun; 19996.

electron probe microanalysis; electron incidence angle; SP604: 1981 June. 381-389. 
electron probe microanalysis; energy dispersive x-ray spectrometry; germanium (lithium) detector; scanning electron microscopy; silicon (lithium) detector; spectral deconvolution; $\mathrm{x}$-ray analysis; $\mathrm{x}$-ray fluorescence; SP604.

electron probe microanalysis; energy dispersive $x$-ray spectrometry; microanalysis; qualitative analysis; quantitative analysis; scanning electron microscopy; spectral artifacts; transmission electron microscopy; SP604: 1981 June. 315-339.

electron probe microanalysis; $\mathrm{Fe}-\mathrm{Cr}-\mathrm{Ni}$ alloy; homogeneity testing; standard reference material; austenitic stainless steel; SP260-70.

electron scattering; magnetism; nickel; polarized electrons; 19970.

electron spin; polarized electrons; spin; spin polarization; electron gun; electron polarization; 19996.

electron spin resonance; microcrack; microfibril; shear deformation; tensile failure; chain rupture; crazing; crystalline polymers; 20732.

electro-optics; gases; high voltage; insulation; interfaces; Kerr effect; liquids; Pockels effect; solids; dielectrics; NBSIR 81-2275.

electro-optics; Kerr effect; measurement; optics; polarized light; electric field; 19962.

electrostatic transducers; gas; nitrogen; sonic cavity; thermodynamic properties; velocity of sound; virial coefficients; 20072.

elemental interactions; lead inclusion bodies; methylmercury derivatives; methylation/demethylation; arsenicals; cadmium metallothionein; cellular toxicity; SP618: 1981 November. 217.225.

element-selective speciation; environmental monitoring; fossil fuels; metal(loid) complexes; organometallic compounds; trace element standards; waste cycling; bacterial mobilization; biogeochemical cycles; biological uptake; cellular toxicity-essentiality mechanisms; chromatography; SP618.

element speciation; gas chromatography; ionization processes; liquid chromatography; mass spectrometry; bioavailability; SP618: 1981 November. 120-133.

element volatilization; fungi; metals; microorganisms; standard reference materials; bacteria; biodeterioration; biotransformations; deterioration of materials; NBSIR 81-2246.

elevated temperatures; ferritic stainless steel; gaseous environments; nickel alloy 671 ; nickel alloy 800 ; slow strain rate test; structural materials; austenitic stainless steels; coal gasification; NBSIR 812191 (DoE)

elevated temperature stress corrosion; slow strain rate; stress corrosion; coal gasification; 20027.

elevators; mechanical; seismic coefficients; architectural; electrical; NBSIR 80-2111-8.

elevator shafts; escape means; pressurization; smoke control; stairwells; air movement; computer programs; egress; NBSIR 802157.

ellipsometer; infrared; polarizer; thin films; diode laser; SP620: 1981 October. 345.355.

ellipsometry; field ion microscopy; inhibitors; organic coatings; passive film dissolution; $\mathrm{pH}$ measurements; surface hydride; alpha uranium; chloride ion; chromate ion; electrochemistry; NBSIR 812396 (ONR).

ellipsometry; gallium; levitation calorimetry; specific heat; surface tension; thermophysical properties; tungsten; wetting; adsorption; Auger spectroscopy; convection; NBSIR 81-2295.

ellipsometry; polysilicon films; silicon dioxide films; silicon nitride films; standard reference materials; thin films; NBSIR 8G-2158.

elliptic partial differential equations; iterative methods for linear algebraic equations; Neumann boundary conditions; space matrices; conjugate gradient algorithm; NBSIR 80-2056.

elongated capillary tube; interface; liquid chromatograph; liquid stream; mass spectrometer; tapered concentrator wire; U.S. Patent $4,281,246$.

elongation; foam; insulation; low temperature; mechanical properties; proportional limit; shear strength; tensile strength; yield strength; Young's modulus; compressive strength; NBSIR 81-1654.

embolization; prosthetic heart valve failure; aortic prosthesis; SP601: 1981 January. 283.298.

EMCS; energy management; energy monitoring and control systems; HVAC controls; reliability study; building energy conservation systems; NBSIR 81-2346. emergencies; evacuation; network models; buildings; egress; NBSGCR-81-316.

emergency response; Federal Information Processing Standards Publication; recovery actions; ADP availability; ADP security; backup operations; computer applications; computer security; contingency planning; FIPS PUB 87.

emergency warning lights; flashing lights; lights, warning; sirens; sound level; warning light; warning signals; conspicuity; effective intensity; SP480-37.

E meson at $1440 \mathrm{MeV}$; flavor singlet; glueball; production process; quark model; strange meson; 20034.

E.M.F. measuring circuit; solid electrolyte probe; device for measuring the fugacity of a material; U.S. Patent 4,264,423.

EMI; ignition interference; suppression; automotive; SP480-44.

emission; measurements; quadrupoles; susceptibility; TEM cell; analysis; dipole moments; electrically small objects; TN1040.

emission of polymers; polymers under tensile load; acoustic emission of polymers; 20014.

em material properties; microwaves; noise; non-ionizing radiation; radiation hazards; antennas; electromagnetic interference; electromagnetic metrology; NBSIR 80-1635.

EMP; FFT; loaded antenna; method of moments; TEM horn; time domain measurements; transients in resistivity; conical antenna; NBSIR 78-876.

empirical fitting; surface fitting; curve fitting; J. Res. 86(1): 1.25; 1981 January-February.

encryption; Federal Information Processing Standards; modes of operation; computer security; cryptography; data security; DES; FIPS PUB 81.

encryption; Federal Information Processing Standards Publication; identification token; identity verification; password; personal attribute; personal identification; access control; authentication; authorization; computer network; computer security; FIPS PUB 83.

encryption; Federal Information Processing Standards Publication; key distribution; network security; security; computer security; cryptography; data integrity; FIPS PUB 74.

endoprostheses; histo-morphological; alumina-ceramic; artificial hip joints; SP601: 1981 January. 131-156.

endurance; free-radicals; iron; organometalloid toxicity; pentane; respiration; animal toxicity; breath analysis; SP618: 1981 November. 226-234.

end user performance; interactive systems; performance analysis; productivity analysis; text editors; SP500-83: 1981 November. 71 . 80.

end user productivity; human factors evaluation; information system management; office automation; performance management systems; resource measurement facility; simulation; supercomputers; benchmarking; capacity planning; chargeback systems; computer performance management; data base machines; SP500-83.

energy; energy conservation; failure detection; failure diagnosis; failure prevention; monitoring techniques; prognosis; sensors; wear; wear analysis; SP622.

energy; explicit method; faucet temperature; finite difference; heat loss; implicit method; insulation; water heater; computer model; distribution pipe; dynamic response; electricity; NBSIR 81-2303.

energy; fine structure; interface; materials; measurement; office building; phase diagrams; radioactivity; research; sediment; soil; alloy; carbon; computers; DIM/NBS 65(8): 1.28; 1981.

energy; model code; noise control; acoustical design; acoustics; architectural design; building codes; building economics; construction costs; cost minimization; economic impact; economics; NBSIR 81-2366.

energy; United Stand; alternative; California; Class $K$ code; conservation; discrimination; SP608: 1981 May. 83-103.

energy analysis; energy conservation; Federal programs; government buildings; hospitals; rate of return;. schools; technical assistance; certification; Class A energy audit; economic analysis; SP608: 1981 May. 133.138.

energy analysis; single-family detached residence; building design; building energy performance standards; computer simulation of house energy requirements; degree days; NBSIR 80-2184. 
energy and environmental data; evaluated data; materials data; standard reference data; technical activities 1980; thermochemical and thermophysical data; data compilation; NBSIR 81-2206.

energy audit; energy conservation; HVAC systems; performance standards; solar collector; solar energy; space heating and cooling; thermal storage; ASHRAE 90-75; class $\mathrm{K}$ code; computer modeling; electrical design; SP608.

energy audit; energy conservation; Illinois; maintenance; public buildings; state government; cost; construction; SP608: 1981 May. 123-132.

energy budget; energy conservation; performance standards; Statement of Review; verification; building code; building envelope; comparison; compliance; SP608: 1981 May. 57.67.

energy budgets; housing research; housing standards; performance requirements; standards development; building energy use; building service systems; NBS-GCR-80-280.

energy calculation; energy conservation; thermal comfort; wholehouse ventilation; building thermal performance; TN1138.

energy calculation; environmental chamber; heating/cooling load; nighttime cooling; desert climate; 20754.

energy calculations; HVAC systems and equipment; validation; Building Energy Performance Standards (BEPS); 20758.

energy conservation; energy conservation measures; installation; insulation; oil burners; practices; safety; sealants; standards; storm doors; storm windows; vent dampers; water heaters; windows; automatic ignition devices; caulks; effectiveness; SP606.

energy conservation; energy policy; Hawaii; performance standards; building energy performance; codes; SP608: 1981 May. 105-112.

energy conservation; engineering economics; life-cycle costs; passive solar; regional analysis; thermal efficiency; windows; building economics; daylighting; NBSIR 81-2248.

energy conservation; Engineering Maintenance Center; machinery monitoring; reliability centered maintenance; SP622: 1981 October. 113.124.

energy conservation; exterior wall; HVAC calculations; insulation; life-cycle cost analysis; masonry; mass; building design; building economics; BSS134.

energy conservation; failure detection; failure diagnosis; failure prevention; monitoring techniques; prognosis; sensors; wear; wear analysis; energy; SP622.

energy conservation; Federal programs; government buildings; hospitals; rate of return; schools; technical assistance; certification; Class A energy audit; economic analysis; energy analysis; SP608: 1981 May. 133.138.

energy conservation; fenestration design; illumination; lighting control; solar heat gain; window management; daylight; NBSIR 802147.

energy conservation; ground coupled; hybrid solar energy system; inverted cave; low grade geothermal; radiant floor heating; space heating and cooling; thermal mass; walls; water source heat pump; buildings; construction; SP608: 1981 May. 69-81.

energy conservation; HVAC systems; innovation; mathematical model; optimization; cost savings; SP608: 1981 May. 21-35.

energy conservation; HVAC systems; performance standards; solar collector; solar energy; space heating and cooling; thermal storage; ASHRAE 90-75; class $\mathrm{K}$ code; computer modeling; electrical design; energy audit; SP 608 .

energy conservation; Illinois; maintenance; public buildings; state government; cost; construction; energy audit; SP608: 1981 May. 123-132.

energy conservation; investment analysis; life-cycle cost; recommended practice; savings-to-investment ratio; benefit-cost analysis; building economics; cost effective; economic analysis; NBSIR 81-2397.

energy conservation; lighting; man/environment research; noise; office building; post-occupancy evaluation; questionnaire; temperature; ventilation; windows; BSS 130 .

energy conservation; marginal price; net present value; residential water conservation; wastewater treatment; water rate schedules water-saving devices; benefits; cost-effective; costs; economic analysis; economic decision framework; NBSIR 81-2304. energy conservation; performance standards; Statement of Review; verification; building code; building envelope; comparison; compliance; energy budget; SP608: 1981 May. 57.67.

energy conservation; program measure; Residential Conservation Service; Solar Model Audit; auditor training; SP608: 1981 May. 183.194.

energy conservation; thermal comfort; whole-house ventilation; building thermal performance; energy calculation; TN1138.

energy conservation codes; fire safety codes; regulation; benefit-cost analysis; building economics; building regulation; codes; construction regulation; economics; NBSIR 81-2223.

energy conservation for nonresidential buildings; historic preservation and energy conservation; load calculation; masonry thermal mass; system simulation; building energy analysis; NBSIR 81-2317.

energy conservation for nonresidential buildings; load calculations; building energy analysis; computer modeling; controls; control strategies; NBSIR 81-2277.

energy conservation in commercial buildings; energy conservation, user acceptance; lighting measurements; performance data for commercial office buildings in New England; solar energy in commercial buildings; building models, computer; BSS133.

energy conservation in commercial buildings; energy monitoring and control systems; mechanical systems; building envelope; building performance data; computer data acquisition; NBSIR 81-2358.

energy conservation measures; installation; insulation; oil burners; practices; safety; sealants; standards; storm doors; storm windows; vent dampers; water heaters; windows; automatic ignition devices; caulks; effectiveness; energy conservation; SP606.

energy conservation standards; solar energy; trainee performance testing; BEPS; code compliance; code conflicts; SP608: 1981 May. 139.157.

energy conservation, user acceptance; lighting measurements; performance data for commercial office buildings in New England; solar energy in commercial buildings; building models, computer; energy conservation in commercial buildings; BSS 133 .

energy consumption; field measurements; usage patterns; water consumption; clothes dryer usage characteristics; clothes washer usage characteristic; data profiles; dishwasher usage characteristic; NBSIR 80-2136

energy dispersive; light elements; soft $\mathrm{x}$ rays; spectral distortion; windowless detectors; SP604: 1981 June. 71.95

energy-dispersive; $x$-ray spectrochemical analysis; Blodgett-Langmuir pseudo-crystals; Bragg crystal diffraction; SP604: 1981 June. 1.4.

energy dispersive analysis; heavy element fluorescence analysis; intrinsic germanium detectors; secondary target fluorescence analysis; source excited fluorescence analysis; $x$-ray fluorescence analysis; catalysts automotive; SP604: 1981 June. 391-415.

energy dispersive detectors; scanning electron microscopy; $\mathbf{x}$-ray spectrometry, review of scientific instruments; bibliography; electron column instruments; SP604: 1981 June. 417.439.

energy dispersive spectra; curve fitting; SP604: 1981 June. 193-231.

energy dispersive spectrometer; uncollimated fluorescing radiation; analytical electron microscope; electron-optical column; SP604: 1981 June. 351-364.

energy-dispersive spectrometry; energy resolution; peak shapes; surface-layer analyses; ultra-thin-windowed (UTW) $\mathrm{Si}(\mathrm{Li})$ detectors; windowless detectors; $x$-ray transmission; electron excitation; SP604: 1981 June. 97-126.

energy dispersive $\mathrm{x}$-ray microanalysis; NBS theoretical matrix correction; $x$-ray continuum; SP604: 1981 June. 365.379.

energy dispersive $\mathrm{x}$-ray spectrometry; Fourier methods; Gaussian curves; deconvolution; SP604: 1981 June. 177-191.

energy dispersive $x$-ray spectrometry; Gaussian profiles; lithium. drifted silicon $(\mathrm{Si}(\mathrm{Li}))$ detector; $x$-ray microanalysis; detector; SP604: 1981 June. 233-272.

energy dispersive $\mathrm{x}$-ray spectrometry; germanium (lithium) detector; scanning electron microscopy; silicon (lithium) detector; spectral deconvolution; $\mathbf{x}$-ray analysis; $\mathbf{x}$-ray fluorescence; electron probe microanalysis; SP604.

energy dispersive $\mathrm{x}$-ray spectrometry; microanalysis; qualitative analysis; quantitative analysis; scanning electron microscopy 
spectral artifacts; transmission electron microscopy; electron probe microanalysis; SP604: 1981 June. 315-339.

energy dispersive $x$-ray spectrometry; scanning transmission electron microscope; analytical electron microscope; artifacts; SP604: 1981 June. 341-349.

energy-dispersive $\mathrm{x}$-ray spectrum deconvolution; multiple least squares fitting; nuclear spectroscopy; digital filter; electron excited $x$-ray spectra; SP604: 1981 June. 297-313.

energy distribution; National Electrical Code; power factor correction; source energy; transformers; electrical design; SP608: 1981 May. 37.56.

energy efficiency standards; engineering-economic analysis; heat pump; mechanical equipment efficiency ratings; minimum efficiency standards; space cooling requirements; space heating requirements; NBSIR 81-2241.

energy-from-waste; materials market; oil analysis; recycled materials; refused-derived-fuel; rerefined oil; NBSIR 80-2178.

energy gap; enhancement; microwaves; nonequilibrium superconductivity; photon-assisted tunneling; superconductivity; thin film; transition temperature; tunneling; tunnel junction; aluminum; critical current; 19979.

energy gap; gap enhancement; microwaves; nonequilibrium; superconductivity; tunnel junction; aluminum; 20723.

energy levels; parametric calculations; uranium; wavelengths; 20002.

energy management; energy monitoring and control systems; HVAC controls; reliability study; building energy conservation systems; EMCS; NBSIR 81-2346.

energy measurement; field instrument force measurement; field testing; in-situ testing; soil mechanics; transducers; BSS135.

energy measurement; steam consumption measurement; uncertainty estimate; building energy monitoring; NBSIR 81-2313.

energy models; mathematical models; model confidence; model credibility; policy models; sensitivity analysis; validation; assessment; composite models; data quality; SP616.

energy monitoring and control systems; HVAC controls; reliability study; building energy conservation systems; EMCS; energy management; NBSIR 81-2346.

energy monitoring and control systems; mechanical systems; building envelope; building performance data; computer data acquisition; energy conservation in commercial buildings; NBSIR $81-2358$.

energy policy; Hawaii; performance standards; building energy performance; codes; energy conservation; SP608: 1981 May. 105. 112.

energy-related process fluids; leachate; linear free energy; liquid chromatography; molecular substituent; oil shale; organoarsenic; organotin; retention index; speciation; atomic absorption; SP618: 1981 November. 197.216.

energy release rate; high temperature fracture; J-integral; sialon; singular integral equation; crack growth model; creep cavitation; diffusive crack growth; NBSIR 81-2255.

energy research; environmental measurements; fire safety; industrial productivity; materials properties; research; standards; technology transfer; basic measurements and standards; building technology; calibration methods; computer standards and guidelines; SP600.

energy resolution; mercuric iodide detectors; $x$-ray spectra; $x$-ray spectrometry; SP604: 1981 June. 45-59.

energy resolution; peak shapes; surface-layer analyses; ultra-thinwindowed (UTW) $\mathrm{Si}(\mathrm{Li})$ detectors; windowless detectors; $\mathrm{x}$-ray transmission; electron excitation; energy-dispersive spectrometry; SP604: 1981 June. 97.126.

energy savings; failure detection; failure prevention; flight safety; fluid system components; maintenance effectiveness; on-board monitoring system; aircraft hydraulic systems; cost reduction; SP622: 1981 October. 195-219.

energy savings; field study; thermal comfort; weatherization retrofitting; comfort; Community Services Administration Optimal Weatherization Demonstration; 20752.

energy transfer; excitation; ion; molecule; reaction rate; relaxation; atom; charge transfer; chemi-ionization; collisional ionization; cross section; detachment; SP593.

energy wars; experimental code; field test; guideline; low income; non-code; policymaking; protestors; solar code; victim role; deed; developers; SP608: 1981 May. 173-178.

engine-driven heat pump; heating and cooling equipment; heating, ventilating and air conditioning; heat pump; building heating and cooling; NBSIR 79-1911.

engineering; Federal government; seismic safety; standards; structural engineering; building; building design; earthquakes; NBSIR 81 2195.

engineering; foundations; professional practice; provisions; soil structure interaction; standards; buildings; design; earthquakes; NBSIR 80-2111-3.

engineering; guest workers; international cooperation; international research; international standards; National Bureau of Standards (NBS); National Engineering Laboratory (NEL); technology transfer; NBSIR 80-2113.

engineering; hazards; measurement; metrology; National Bureau of Standards; power; radiation; semiconductor; technology; calibration; electrical; electronics; SP611.

engineering; masonry; standards; structural engineering; building; building codes; building design; earthquakes; NBSIR 80-2111-5.

engineering; organization; provisions; scope; specification; standard; system analysis/engineering; arrangement; building; classification; code; $B S S 136$.

engineering; reinforced concrete; standards; structural engineering; building; building codes; building design; earthquakes; NBSIR 80 2111-4.

engineering; standards; steel; structural engineering; building; building codes; building design; earthquakes; NBSIR 80-2111-6.

engineering; standards; structural engineering; building; building codes; building design; earthquakes; NBSIR 80-2111-11.

engineering; standards; structural engineering; wood; building; building codes; building design; earthquakes; NBSIR 80-2111-7.

engineering-economic analysis; heat pump; mechanical equipment efficiency ratings; minimum efficiency standards; space cooling requirements; space heating requirements; energy efficiency standards; NBSIR 81-2241.

engineering economics; life-cycle costs; passive solar; regional analysis; thermal efficiency; windows; building economics; daylighting; energy conservation; NBSIR 81-2248.

Engineering Maintenance Center; machinery monitoring; reliability centered maintenance; energy conservation; SP622: 1981 October. 113-124.

engine lubricants; lubricating oil; motor oil; petroleum oil; recycled oil; rerefined oil; test procedures; basestock; 20016.

engine wear analysis; oil filter design; oil filter testing; particle size sensitivity; radioactive wear testing; effect of filtration; SP622: 1981 October. 50-61.

enhancement; microwaves; nonequilibrium superconductivity; photonassisted tunneling; superconductivity; thin film; transition temperature; tunneling; tunnel junction; aluminum; critical current; energy gap; 19979.

Enskog theory; mass difference effects; mixture viscosity; size difference effects; corresponding states; J. Res. 86(6): 597.604; 1981 November-December.

enthalpy; enthalpy of formation; entropy; equilibrium constant for formation; Gibbs energy function; Gibbs energy of formation; heat capacity; lime-alumina-silica-water; minerals; thermodynamic data; JPCRD 10(3): 575-670; 1981.

enthalpy; entropy; equilibrium constant of formation; free energy of formation; Gibbs energy function; heat capacity; heat of formation; selected values; thermochemical tables; NBSIR 81-2343.

enthalpy; entropy; floor temperature; fusion; Gibbs energy; glass transition; heat capacity; linear macromolecule; melt; selenium calorimetry; crystal; JPCRD 1O(1): 89.118; 1981.

enthalpy; entropy; francium compounds; Gibbs energy of formation; heat capacity; lithium compounds; potassium compounds; protactinium compounds; rubidium compounds; sodium compounds; thorium compounds; uranium compounds; actinium compounds; cesium compounds; TN270-8.

enthalpy; entropy; fusion; Gibbs energy; glass transition; heat capacity; isotactic; linear macromolecule; melt; polypropylene; 
syndiotactic; atactic; crystal; crystallinity; density; JPCRD 10(4): 1051-1064; 1981.

enthalpy; entropy; fusion; Gibbs energy; glass transition; heat capacity; linear macromolecule; melt; polyethylene; crystal; crystallinity; density; JPCRD 10(1): 119-152; 1981.

enthalpy; entropy; fusion; Gibbs energy; glass transition; heat capacity; linear macromolecule; poly(oxymethylene)s; poly(oxyphenylene)s; poly(oxypropylene); crystal; crystallinity; density; JPCRD 10(4): 1001-1049; 1981.

enthalpy; Gibbs energy; osmotic coefficients; solutions; sulfuric acid; thermodynamic properties; transport properties; activity coefficients; aqueous; compilation; conductivity; electrolytes; NBSIR 81-2276.

enthalpy of combustion; refuse-derived-fuel; test substance; alternative fuel; bomb calorimetry; cellulose; J. Res. 86(6): 655-660; 1981 November-December.

enthalpy of formation; entropy; equilibrium constant for formation; Gibbs energy function; Gibbs energy of formation; heat capacity; lime-alumina-silica-water; minerals; thermodynamic data; enthalpy; JPCRD 10(3): 575-670; 1981.

enthalpy of solution; Gua. $\mathrm{HCl} \cdot 1 \mathrm{H}_{2} \mathrm{O}$; Gua. $\mathrm{HCl} \cdot 2 \mathrm{H}_{2} \mathrm{O}$; Gua.2 $\mathrm{HCl}$; guanine (Gua); guanine hydrochlorides; heat capacity; nucleic acid base; solution calorimetry; thermochemistry; $\Delta C_{p}$ of reaction; calorimetry; $\mathrm{C}_{5} \mathrm{H}_{5} \mathrm{~N}_{5} \mathrm{O}$; density; J. Res. 86(4): 367.382; 1981 July. August.

entity; exchange format; file; geometry; graphics; interface; computer aided design (CAD); computer aided manufacturing (CAM); NBSIR 81-2297.

entrainment; fire models; fire plumes; diffusion flames; $N B S-G C R-81$ 346.

entrainment; fire plumes; flow rate; mass flow; diffusion flames; $N B S$. GCR-80-294.

entropy; equilibrium constant for formation; Gibbs energy function; Gibbs energy of formation; heat capacity; lime-alumina-silica-water; minerals; thermodynamic data; enthalpy; enthalpy of formation; JPCRD 10(3): 575-670; 1981.

entropy; equilibrium constant of formation; free energy of formation; Gibbs energy function; heat capacity; heat of formation; selected values; thermochemical tables; enthalpy; NBSIR 81-2343.

entropy; floor temperature; fusion; Gibbs energy; glass transition; heat capacity; linear macromolecule; melt; selenium; calorimetry; crystal; enthalpy; JPCRD 10(1): 89.118; 1981.

entropy; francium compounds; Gibbs energy of formation; heat capacity; lithium compounds; potassium compounds; protactinium compounds; rubidium compounds; sodium compounds; thorium compounds; uranium compounds; actinium compounds; cesium compounds; enthalpy; TN270-8.

entropy; fusion; Gibbs energy; glass transition; heat capacity; isotactic; linear macromolecule; melt; polypropylene; syndiotactic; atactic; crystal; crystallinity; density; enthalpy; JPCRD 10(4): 1051-1064; 1981.

entropy; fusion; Gibbs energy; glass transition; heat capacity; linear macromolecule; melt; polyethylene; crystal; crystallinity; density; enthalpy; JPCRD 10(1): 119.152; 1981.

entropy; fusion; Gibbs energy; glass transition; heat capacity; linear macromolecule; poly(oxymethylene)s; poly(oxyphenylene)s; poly(oxypropylene); crystal; crystallinity; density; enthalpy; JPCRD 10(4): 1001-1049; 1981.

environment; GC.MS; heavy metals; lead; mass spectrometry; mercury; methylation; SP618: 1981 November. 54-64.

environment; metrology; quality control; radioactivity; traceability; 20010.

environmental; essential elements; speciation; toxic; trace elements; SP618: 1981 November. 235-243.

environmental analysis; feasibility study; local area networks; requirements analysis; carrier sense multiple access; NBSIR 80 2187.

environmental chamber; heating/cooling load; nighttime cooling; desert climate; energy calculation; 20754.

environmental chemistry; shale oil; synthetic fuels; trace elements; coal; SP618: 1981 November. 81.92. environmental contamination; extraction tests; fossil fuels; laboratory characterization; metals; mining industry; public health impacts; residues; toxic elements; availability; SP618: 1981 November. 1-8. environmental exposure; reliability; solar collectors; solar materials; stagnation testing; accelerated aging; durability; TN1136.

environmental measurements; fire safety; industrial productivity; materials properties; research; standards; technology transfer; basic measurements and standards; building technology; calibration methods; computer standards and guidelines; energy research; SP600.

environmental measurements; methods; quality assurance; standards; water; air; NBSIR 81-2280.

environmental monitoring; fossil fuels; metal(loid) complexes; organometallic compounds; trace element standards; waste cycling; bacterial mobilization; biogeochemical cycles; biological uptake cellular toxicity-essentiality mechanisms; chromatography; elementselective speciation; SP618.

environmental noise; high voltage transmission lines; human response to noise; listening room; corona noise; 20030.

environmental noise; human response; power line noise; psychoacoustics; annoyance; audible noise; corona noise; EHV transmission lines; electric energy; 20050.

environmental psychology; families; housing; interiors; lifestyle; research; site planning; sociology; standards; children; design; NBS. GCR-81-320.

environmental test; field validation test; independent testing; quality assurance program; software change package; software maintenance testing; developmental center test; SP500-83: 1981 November. 93.99.

environmental testing; evaluation; measurand; reliability; sensor; transducer; ATE; automatic test equipment; calibration; data acquisition; durability; dynamic calibration; SP615.

enzyme histochemistry; histology; implant retrieval analysis; polarography; tissue response; atomic absorption spectrophotometry; EDAX analysis; SP601: 1981 January. 323-336.

enzyme immunoassay; epilepsy; ethosuximide; mass spectrometry; nuclear magnetic resonance spectroscopy; phenobarbital; phenytoin; primidone; reference material; serum base modification; serum matrix; anticonvulsants; antiepilepsy drugs; blood analysis; chromatography, gas; chromatography, liquid; drug standards; SP260-72.

EPA; ETIP; incentives; innovation; regulation; air pollution; Clean Air Act; NBS-GCR-ETIP 81-95.

EPA; ETIP; incentives; innovation; regulation; air pollution; Clean Air Act; NBS-GCR-ETIP 81.94.

EPA; ETIP; incentives; innovation; regulation; air pollution; Clean Air Act; NBS-GCR-ETIP 81-93.

epilepsy; ethosuximide; mass spectrometry; nuclear magnetic resonance spectroscopy; phenobarbital; phenytoin; primidone; reference material; serum base modification; serum matrix; anticonvulsants; antiepilepsy drugs; blood analysis; chromatography, gas; chromatography, liquid; drug standards; enzyme immunoassay; SP260-72.

epitaxial regrowth; laser irradiation; liquid phase; single crystal; defects; SP620: 1981 October. 222-226.

equation; formulation; high pressure; high temperature; IAPS; International Association for the Properties of Steam; ionization; ionization constant; ion product; review; water; water substance; JPCRD 10(2): 295-304; 1981.

equation of state; ethylene thermodynamic properties; tabular values; computer programs; TN1045.

equation of state; geothermal; isobutane; thermodynamic; vapor pressure; critical region; density; NBSIR 79-1715.

equation of state; hard sphere; LNG; mixtures; PVTx data; revised Kosek and McKinley; cell model; comparisons; computer programs; corresponding states; TN1030.

equations; handbook; physicists; physics; polymers; data; 20749.

equilibrium; game theory; mathematical economics; noncooperative games; total orders; aggregation; J. Res. 86(4): 383-391; 1981 July. August.

equilibrium; solid; stress, strain; thermodynamics; chemical potential; 
diffusion; 20725.

equilibrium constant for formation; Gibbs energy function; Gibbs energy of formation; heat capacity; lime-alumina-silica-water; minerals; thermodynamic data; enthalpy; enthalpy of formation; entropy; JPCRD 10(3): 575.670; 1981.

equilibrium constant of formation; free energy of formation; Gibbs energy function; heat capacity; heat of formation; selected values; thermochemical tables; enthalpy; entropy; NBSIR 81-2343.

ergonomics; functional capacity; handicap; locks; architectural barriers; building accessibility; codes and standards; disability; door closers; door openers; doors; NBSIR 80-2174.

erosion; fracture toughness; hardness; ceramics; coal conversion; crack propagation; 20028.

error analysis; $\mathrm{G} / \mathrm{T}$; precision measurements; radio star; satellite communications; C/kT; EIRP; NBSIR 78-869.

error measurement; gain; input noise; linearity; offset; analog-to-digital converter; calibration service; data converter; differential linearity; digital-to-analog converter; TN1145.

escape means; pressurization; smoke control; stairwells; air movement; computer programs; egress; elevator shafts; NBSIR 80-2157.

essential elements; speciation; toxic; trace elements; environmental; SP618: 1981 November. 235-243.

estimation; location factors; model building; statistical analysis; applied economics; construction; cost; NBSIR 81-2250.

etching; integrated circuit processing; ion channeling; ion implantation; lateral profiles; scanning electron microscopy; silicon technology; spreading resistance; two-dimensional profiles; arsenic ion implantation; electron-beam-induced current; NBSIR 81-2398.

ethosuximide; mass spectrometry; nuclear magnetic resonance spectroscopy; phenobarbital; phenytoin; primidone; reference material; serum base modification; serum matrix; anticonvulsants; antiepilepsy drugs; blood analysis; chromatography, gas; chromatography, liquid; drug standards; enzyme immunoassay; epilepsy; SP260-72.

ethylene bis(trimethylammonium) chloride; ethylene bis(trimethylammonium) iodide; excess Gibbs energy; osmotic coefficient; solutions; thermodynamic properties; zinc; activity coefficient; cadmium; critical evaluation; electrolyte; JPCRD 10(1): $1.56 ; 1981$.

ethylene bis(trimethylammonium) iodide; excess Gibbs energy; osmotic coefficient; solutions; thermodynamic properties; zinc; activity coefficient; cadmium; critical evaluation; electrolyte; ethylene bis(trimethylammonium) chloride; JPCRD 10(1): 1.56; 1981.

ethylene oxide; glycine; interstellar; methyl formate; radio astronomy; amino acids; 20077.

ethylene thermodynamic properties; tabular values; computer programs; equation of state; TN1045.

ETIP; FCC; innovation incentives; regulatory reform; administrative experiment; communications; competition; NBS-GCR-ETIP 81-97.

ETIP; incentives; innovation; regulation; air pollution; Clean Air Act; EPA; NBS-GCR-ETIP 81-93.

ETIP; incentives; innovation; regulation; air pollution; Clean Air Act; EPA; NBS-GCR-ETIP 81-95.

ETIP; incentives; innovation; regulation; air pollution; Clean Air Act; EPA; NBS-GCR-ETIP 81-94.

Euler equations; finite difference equations; fire-enclosure; fluid flow; heat source-volumetric; partial differential equations; buoyant convection; computations-finite difference; NBSIR 81-2385.

evacuation; fire alarm systems; fire departments; fire extinguishers; fire investigations; hospitals; nursing homes; nursing staff; smoke; smoke detectors; sprinkler systems; doors; NBS-GCR-80-297.

evacuation; fire alarm systems; fire departments; fire extinguishers; fire investigations; hospitals; nursing homes; nursing staff; smoke; smoke detectors; sprinkler systems; doors; NBS-GCR-81-343.

evacuation; fire alarm systems; fire safety; handicapped; life safety; refuge; board and care homes; building codes; building fires; 20727.

evacuation; fire departments; fire emergency plan; fire protection; group homes; group home staff; developmentally disabled; NBSGCR $-81-315$.

evacuation; network models; buildings; egress; emergencies; NBS-
GCR $-81-316$.

evaluated data; fluid properties; indexes; publication list; solid state; thermodynamic and transport properties; atomic and molecular properties; bibliographies; chemical kinetics; SP612.

evaluated data; materials data; standard reference data; technical activities 1980; thermochemical and thermophysical data; data compilation; energy and environmental data; NBSIR 81-2206.

evaluation; HVAC; masonry; plumbing; solar; steel; structural systems; test methods; wood; building rehabilitation; concrete; electrical; NBSIR 81-2289.

evaluation; HVAC; masonry; plumbing; steel; structural systems; test methods; wood; building rehabilitation; concrete; electrical; NBSIR 80-2171.

evaluation; measurand; reliability; sensor; transducer; ATE; automatic test equipment; calibration; data acquisition; durability; dynamic calibration; environmental testing; SP615.

evaluation; radioactivity; radiochemistry; radium-228; reference method; water; analysis; assay; TN1137.

evaluation method; fire-safety; hazard warnings; meaningfulness; method; pictogram; response; symbol; understandability; communication; NBSIR 80-2088.

evaluation procedures; facility design; facility management; public assembly; public safety; stadiums; auditorium; building standards; crowd ingress; design procedures; NBSIR 81-2361.

evaluation systems; Experimental Technology Incentives Program; information systems; project management; system acquisition; system development; administrative experiment; NBSIR 81-2327.

evaluation systems; Experimental Technology Incentives Program; information systems; project management; system acquisition; system development; administrative experiment; NBSIR 81-2328.

evaporation; hydrogen and oxygen impurity; magnetron sputtering; pulsed HF/DF laser damage thresholds; silicon films; absorption; SP620: 1981 October. 305-312.

evaporation cooling; smoke movement; spray nozzle; stairway protection; ventilated stair; automatic sprinklers; cooling efficiency; NBSIR 81-2202.

evidential breath testers; human respiratory characteristics; intoxication; blood alcohol analysis; blood alcohol content; breath alcohol content; breath sampling; SP480-41.

evolution; fracture mechanics; gamma rays; inventors; molecular interactions; molecular processes; neomycin; neutron dosimeters; polymers; radiography; SRM's; stars; visual acuity; DIM/NBS 65(7): 1-24; 1981.

examples; OMNITAB 80; software; test problems; accuracy; ANS FORTRAN; computer system installation; TN1147.

excess free energy; free energy; ionic; osmotic coefficients; solutions; sulfuric acid; standard reference data; thermodynamic properties; activity coefficients; aqueous; critical evaluation; electrolytes; JPCRD 10(3): 779-798; 1981

excess Gibbs energy; $\mathrm{KNO}_{2} ; \mathrm{LiNO}_{2} ; \mathrm{NaNO}_{2} ;$ osmotic coefficients; $\mathrm{RbNO}_{2}$; solutions; standard reference data; thermodynamic properties; activity coefficients; alkali metal nitrite salts; aqueous; critical evaluation; $\mathrm{CsNO}_{2}$; electrolytes; JPCRD 10(3): 767-778; 1981.

excess Gibbs energy; lithium; osmotic coefficient; potassium; rubidium; sodium; solutions; thermodynamic properties; activity coefficient; cesium; critical evaluation; electrolyte; JPCRD 10(3): 671.766 .

excess Gibbs energy; osmotic coefficient; solutions; thermodynamic properties; zinc; activity coefficient; cadmium; critical evaluation; electrolyte; ethylene bis(trimethylammonium) chloride; ethylene bis(trimethylammonium) iodide; JPCRD 10(1): 1.56; 1981 .

exchange format; file; geometry; graphics; interface; computer aided design (CAD); computer aided manufacturing (CAM); entity; NBSIR 81-2297.

exchange interaction; ferrite; hyperfine field; magnetism; phase diagram; potassium; conductivity; 20043.

excimer lasers; fluoride coatings; laser damage; multiple-shot damage; oxide coatings; pre-irradiation; thin films; ultraviolet reflectors; SP620: 1981 October. 265-276.

excitation; ion; molecule; reaction rate; relaxation; atom; charge 
transfer; chemi-ionization; collisional ionization; cross section; detachment; energy transfer; SP593.

excitons; $\mathrm{NaCl}$; optical breakdown; point defects; primary defects; damage threshold; SP620: 1981 October. 406-415.

exhalation; materials; measurement; permeability; radon; transport; buildings; concrete; diffusion; TN1139.

exhaust emissions; exhaust gas oxygen sensor; exhaust gas recirculation; feedback; oxidation catalyst; oxygen partial pressure; stoichiometric; three way catalytic converters; zirconium dioxide; air-fuel ratio; closed loop fuel metering system; SP622: 1981 October. 62-73.

exhaust gas oxygen sensor; exhaust gas recirculation; feedback; oxidation catalyst; oxygen partial pressure; stoichiometric; three way catalytic converters; zirconium dioxide; air-fuel ratio; closed loop fuel metering system; exhaust emissions; SP622: 1981 October. 62.73 .

exhaust gas recirculation; feedback; oxidation catalyst; oxygen partial pressure; stoichiometric; three way catalytic converters; zirconium dioxide; air-fuel ratio; closed loop fuel metering system; exhaust emissions; exhaust gas oxygen sensor; SP622: 1981 October. 62-73.

existing buildings; housing codes; maintenance; model codes; occupancy; performance levels; regulation; standards; building rehabilitation; codes; comparative analysis; NBSIR 80-2081.

exit signs; fire safety; pictograms; symbols; understandability; visibility; visual alerting; directional arrows; $N B S I R$ 81-2268.

expansion; fly ash; MgO; slag; soundness; strength; cement; NBSIR $81-2273$.

experimental code; field test; guideline; low income; non-code; policymaking; protestors; solar code; victim role; deed; developers; energy wars; SP608: 1981 May. 173-178.

Experimental Technology Incentives Program; information systems project management; system acquisition; system development; administrative experiment; evaluation systems; NBSIR 81-2328.

Experimental Technology Incentives Program; information systems; project management; system acquisition; system development; administrative experiment; evaluation systems; NBSIR 81-2327.

experiments; flame size; temperature distribution; turbulent burning; ceilings; NBS-GCR-79-188.

experiments; mathematical model; plastic; polyurethane; room fires; wood; crib fires; NBSIR 80-2054, Vols. I and II.

explicit method; faucet temperature; finite difference; heat loss; implicit method; insulation; water heater; computer model; distribution pipe; dynamic response; electricity; energy; NBSIR 81 2303.

exposure history; instruments; magnetic fields; nonionizing radiation; regional calibration laboratories; standards; techniques; calibration; dosimetry; electric fields; electromagnetic interference; SP613.

extended corresponding states; fluid mixtures; thermal conductivity; viscosity; computer program; density; TN1039.

extended corresponding states; fluids; mixtures; one fluid model; prediction; Van der Waals theory; viscosity; data evaluation; 20768.

extension cord wiring; fuse/circuit breaker panel problems; overamping; residential structures; safety; thermal insulation; branch circuit wiring problems; electrical problems; electrical wiring system; NBSIR 81-2203.

exterior restoration; field tests; guide specifications; laboratory tests; paint removal methods; White House; coatings; NBSIR 80-2122.

exterior wall; HVAC calculations; insulation; life-cycle cost analysis; masonry; mass; building design; building economics; energy conservation; BSS134.

extinction coefficients; inorganic; radical ions; radicals; transients; absorption spectra; aqueous solution; NSRDS-NBS69.

extraction tests; fossil fuels; laboratory characterization; metals; mining industry; public health impacts; residues; toxic elements; availability; environmental contamination; SP618: 1981 November. 1.8 .

extrinsic and intrinsic infrared absorption; infrared wavelength modulation; $\mathrm{LaF}_{3}$; laser windows; LiF; $\mathrm{MgF}_{2} ; \mathrm{MgO} ; \mathrm{NaCl} ; \mathrm{NaF}$; $\mathrm{SrF}_{2} ;$ volume and surface impurities; $\mathrm{BaF}_{2} ; \mathrm{CaF}_{2} ; \mathrm{SP620}: 1981$ October. 29-43.

eyeball deformation; impact; ocular injury; projectiles; safety; contusion; NBSIR 80-2037.

fabric flammability; fabrics; fire suppression; flame extinguishment; heat transfer; polyester; textiles; airflow; burn (injuries); cotton; NBSIR 81-2386

fabric flammability; fire research; fire tests; flame research; smoke; bibliographies; building fires; coal mines; combustion products; compartment fires; NBSIR 81-2272.

fabrics; fire suppression; flame extinguishment; heat transfer; polyester; textiles; airflow; burn (injuries); cotton; fabric flammability; NBSIR 81-2386.

facilities complex; performance parameters;. UNIVAC; UNIVAC 1100 OS; SP500-83: 1981 November. 251-258.

facility design; facility management; public assembly; public safety; stadiums; auditorium; building standards; crowd ingress; design procedures; evaluation procedures; NBSIR 81-2361.

facility management; public assembly; public safety; stadiums; auditorium; building standards; crowd ingress; design procedures; evaluation procedures; facility design; NBSIR 81-2361.

failure; fatigue; mechanical properties; morphology; polymer; stress relaxation; UHMWPE; ultra high molecular weight polyethylene; creep; NBSIR 81-2209 (FDA).

failure; flat plate; shear; strength; building; collapse; concrete; concrete strength; construction; NBSIR 81-2374.

failure detection; failure diagnosis; failure prevention; monitoring techniques; prognosis; sensors; wear; wear analysis; energy; energy conservation; SP622.

failure detection; failure prevention; flight safety; fluid system components; maintenance effectiveness; on-board monitoring system; aircraft hydraulic systems; cost reduction; energy savings; SP622: 1981 October. 195-219.

failure detection; helicopter diagnostics; oil debris; wear monitoring; chip detectors; condition monitoring; SP622: 1981 October. 33-49.

failure diagnosis; failure prevention; monitoring techniques; prognosis sensors; wear; wear analysis; energy; energy conservation; failure detection; SP622.

failure prevention; flight safety; fluid system components; maintenance effectiveness; on-board monitoring system; aircraft hydraulic systems; cost reduction; energy savings; failure detection; SP622: 1981 October. 195.219.

failure prevention; monitoring techniques; prognosis; sensors; wear wear analysis; energy; energy conservation; failure detection; failure diagnosis; SP622.

families; housing; interiors; lifestyle; research; site planning; sociology; standards; children; design; environmental psychology; NBS-GCR 81-320.

Faraday; firefighting; hot atom; industrial innovation; measurements; oils, PCB's; standards; atomic weight; coal; computers; drill failure; $D I M / N B S$ 65(6): 1-24; 1981

far field; FFT; Fourier transform; algorithm; antenna; aperture; computer program; electromagnetic; NBSIR 81-1643.

far field; index profile; mode filter; numerical aperture; radiation angle; radiation patterns; attenuation; core diameter; TN1032.

far fields; near fields; physical optics; planewave spectrum; reflector antennas; co-sited antennas; coupling; NBSIR 80-1630.

far infrared; $\mathrm{H}_{2}-\mathrm{H}_{2}$ collisions; $\mathrm{H}_{2}-\mathrm{He}$ collisions; planetary atmospheres; quadrupole-induced absorption; spectral moments; band shape analysis; collision-induced absorption; NBSIR 80-2175.

fatigue; mechanical properties; morphology; polymer; stress relaxation; UHMWPE; ultra high molecular weight polyethylene; creep; failure; NBSIR 81-2209 (FDA).

fatigue; metals; SEM; deformation; electron channeling; 19980.

fatigue corrosion; internal fixation devices; mechanical fatigue; metal prostheses; stress corrosion; SP601: 1981 January. 201-222.

F-atom reactions; infrared spectrum; matrix isolation; normal coordinate analysis; photodissociation; 19986.

F atoms; hydrogen bonding; infrared spectrum; matrix isolation; photodecomposition; reaction mechanism; $t$-2-fluorovinyl; acetylene; 20036 
faucet temperature; finite difference; heat loss; implicit method; insulation; water heater; computer model; distribution pipe; dynamic response; electricity; energy; explicit method; NBSIR 812303.

fault detection; laser scanner; photovoltaics; solar cell analysis; solar cells; computer simulations; NBS-GCR-80-285.

FCC; innovation incentives; regulatory reform; administrative experiment; communications; competition; ETIP; NBS-GCR-ETIP 81-97.

fcc metals; free energy; high temperature nearest-neighbor model; thermal expansion; anharmonic; Brillouin zone sums; central pair potential; 20037.

FCHART computer program; National Solar Demonstration Program; solar energy systems; space heating load; thermal performance; auxiliary energy; NBS-GCR-81-321.

feasibility study; local area networks; requirements analysis; carrier sense multiple access; environmental analysis; NBSIR 80-2187.

feature analysis; network architecture; network virtual terminal protocols; virtual terminal protocols; communication protocols; computer network protocols; NBS-GCR-81-324.

$\mathrm{Fe}-\mathrm{Cr}-\mathrm{Ni}$ alloy; homogeneity testing; standard reference material; austenitic stainless steel; electron probe microanalysis; SP260-70.

Federal and State laboratories; gamma rays; instrument calibrations; intermediate calibration laboratories; ionizing radiation measurements; measurement accuracies; neutrons; radiation dosimetry; radiation protection; radiation standards; $\mathrm{x}$ rays; beta rays; $S P 603$

federal computer networking development; networking standards; penetration studies; computer network protocols; computer networks; cost/benefit analysis; federal government networking; NBS-GCR-81-323.

Federal computers; hardware trends; information industry; software trends; technology forecast; automated data processing; computer utilization trends; NBS-GCR-81-342.

Federal contracts services; Federal Government computers; programming languages; software expenditures; software program life-cycle analysis; data base management systems; SP500-79.

Federal Conversion Support Center; reimbursable services; software conversion; SP500-83: 1981 November. 341.

Federal government; seismic safety; standards; structural engineering; building; building design; earthquakes; engineering; NBSIR 812195.

Federal Government computers; programming languages; software expenditures; software program life-cycle analysis; data base management systems; Federal contracts services; SP500-79.

federal government networking; federal computer networking development; networking standards; penetration studies; computer network protocols; computer networks; cost/benefit analysis; NBSGCR-81-323.

Federal Information Processing Standard; FORTRAN; information interchange; information processing; processor; programming language; software; BASIC; COBOL; compilers; data processing; FIPS PUB 29-1.

Federal Information Processing Standard; Hollerith punched card code; Hollerith punched cards; information interchange; information processing; punched cards; standard; American National Standard Code for Information Interchange; ASCII; card punch; card reader; data communications; data interchange; data processing; EAM cards; electric accounting machine; FIPS PUB 14-1.

Federal Information Processing Standard; information interchange; information processing; standard; American National Standard Code for Information Interchange; ASCII; character set; coded character set; code for information interchange; collating sequence; data communication; data interchange; data processing; FIPS PUB $1-1$.

Federal Information Processing Standard; information interchange; printer terminals; standard; text communication; text interchange; typesetting composition; ADP terminals; ASCII; character-imaging devices; control functions; data interchange; display terminals; FIPS PUB 86.
Federal Information Processing Standard; information processing systems; optical character recognition; character positioning; computer systems; data entry (automatic); FIPS PUB 89.

Federal Information Processing Standard (FIPS); Federal Property Management Regulations (FPMR); procurement; standards; telecommunications; automated data processing (ADP); communications; FIPS PUB 80.

Federal Information Processing Standard (FIPS); illumination; image quality; microfilm; microform; readers; American National Standard; Computer Output Microfilm (COM); FIPS PUB 84.

Federal Information Processing Standard (FIPS); interchange of machine readable information; nonread inks; Optical Character Recognition (OCR); OCR-A; OCR-B; read inks; American National Standard; FIPS PUB 85.

Federal Information Processing Standards; modes of operation; computer security; cryptography; data security; DES; encryption; FIPS PUB 81.

Federal Information Processing Standards Publication; computer program; data administration; database audition; database controls; database integrity; database management; data dictionary system; FIPS PUB 88.

Federal Information Processing Standards Publication; form slide; microfilm; quality control; automated data printing; computeroutput microforms; FIPS PUB 82.

Federal Information Processing Standards Publication; identification token; identity verification; password; personal attribute; personal identification; access control; authentication; authorization; computer network; computer security; encryption; FIPS PUB 83.

Federal Information Processing Standards Publication; interoperability; teleprocessing; transmission; advanced data communication control procedures; ANS X3.66; bit-oriented; code independence; data; data link control procedures; data transparency; FIPS PUB 78.

Federal Information Processing Standards Publication; key distribution; network security; security; computer security; cryptography; data integrity; encryption; FIPS PUB 74.

Federal Information Processing Standards Publication; performance evaluation; workload analysis; workload characterization; workload representation; ADP acquisition; ADP procurement; benchmarking; FIPS PUB 75.

Federal Information Processing Standards Publication; recovery actions; ADP availability; ADP security; backup operations; computer applications; computer security; contingency planning; emergency response; FIPS PUB 87.

Federal Information Processing Standards Publication; requirements; software; computer program; database; database management system; data dictionary system; data inventory; data management; data standards; documentation; NBSIR 81-2354.

Federal programs; government buildings; hospitals; rate of return; schools; technical assistance; certification; Class A energy audit; economic analysis; energy analysis; energy conservation; SP608: 1981 May. 133-138.

Federal Property Management Regulations (FPMR); procurement; standards; telecommunications; automated data processing (ADP); communications; Federal Information Processing Standard (FIPS); FIPS PUB 80.

feedback; oxidation catalyst; oxygen partial pressure; stoichiometric; three way catalytic converters; zirconium dioxide; air.fuel ratio; closed loop fuel metering system; exhaust emissions; exhaust gas oxygen sensor; exhaust gas recirculation; SP622: 1981 October. 6273.

feedwater control valve; pattern recognition; pressurized water reactor; remote surveillance; acoustic emissions; cavitation; SP622: 1981 October. 11-30.

felt; nonlinear; roofing membrane; splitting; strain energy; stress; substrate; adhesive; bitumen; bonding; built-up roofing membrane; complementary strain energy; TN1152.

femoral component; loosening; metal-on-metal prostheses; metal-onplastic prostheses; SP601: 1981 January. 643-653.

femoral component loosening; polymethylmethacrylate (PMMA); prosthesis interface; SP601: 1981 January. 157.174. 
- alestration design; illumination; lighting control; solar heat gain; window management; daylight; energy conservation; NBSIR 80 2147.

ferrite; hyperfine field; magnetism; phase diagram; potassium; conductivity; exchange interaction; 20043.

ferritic stainless steel; gaseous environments; nickel alloy 671; nickel alloy 800 ; slow strain rate test; structural materials; austenitic stainless steels; coal gasification; elevated temperatures; NBSIR 81 . $2191(\mathrm{DoE})$.

ferromagnetism; Ni; nickel; polarized electrons; surface magnetism; 19975.

FFT; Fourier transform; algorithm; antenna; aperture; computer program; electromagnetic; far field; NBSIR 81-1643.

FFT; loaded antenna; method of moments; TEM horn; time domain measurements; transients in resistivity; conical antenna; EMP; NBSIR $78-876$

fiber-glass; fibrous glass; insulation; measurement; $R$ value; standard reference material; thermal resistance; NBSIR 80-2091.

fiber index profile; index profile; near-field scanning; optical communications; optical fiber; optical waveguide; refracted nearfield scanning; refracted.ray scanning; resolution limit; TN1038.

fiber optics; nonlinear propagation; nonlinear pulses; self-focusing; solitons; waveguides; SP620: 1981 October. 453-458.

fiber optics; optical communications; optical fiber bandwidth; optical fiber distortion; optical fibers; TN1046.

fiber optics information systems; integrated communications; local area networks, microwave; video teleconferencing; CATV systems coaxial cable; data communications; SP500-83: 1981 November 271.276 .

fiber-reinforcement; impact; impulsive loading; penetration resistance; reinforcing bars; stress waves; concrete; dynamic properties; SP480-45.

fiber scattering; optical time domain reflectometry; Rayleigh scattering; backscattering; capture fractions; TN1034.

fibrescope; health condition; man-hour expenditure ratio; trend analysis; unlimited application; SP622: 1981 October. 74-81.

fibrous; polyurethane foam materials; smolder resistant upholstered furniture or mattress assembly; sulphur; U.S. Patent 4,260,660.

fibrous glass; insulation; measurement; $R$ value; standard reference material; thermal resistance; fiber-glass; NBSIR 80-2091.

field distribution; Hertzian dipole; integral equation; rectangular coaxial transmission line; TEM cell; characteristic impedance; TN1037.

field-effect transistor; III-V compounds; IMPATT diode; integrated circuit; LASER; LED; photocell; solar cell; solid state; transistor; tunnel diode; diode; J. Res. 86(6): 565-570; 1981 NovemberDecember.

field evaporation; field ion microscopy; anomalous field evaporation; 19983.

field instrument force measurement; field testing; in-situ testing; soil mechanics; transducers; energy measurement; BSS135.

field intensity meter; field strength measurement; isotropic antenna; radio frequency radiation; receiver calibration; electromagnetic field; electronic instrument design; TN1033.

field ion microscopy; anomalous field evaporation; field evaporation; 19983

field ion microscopy; inhibitors; organic coatings; passive film dissolution; $\mathrm{pH}$ measurements; surface hydride; alpha uranium; chloride ion; chromate ion; electrochemistry; ellipsometry; NBSIR $81-2396(O N R)$.

field ion microscopy; iron-chromium alloys; niobium; repassivation kinetics; stainless steel; triboellipsometry; aluminum; NBSIR 79. 1904 (Navy).

field measurements; temperature excursions; thermal insulation; branch circuit; electric wire; NBSIR 81-2347.

field measurements; usage patterns; water consumption; clothes dryer usage characteristics; clothes washer usage characteristic; data profiles; dishwasher usage characteristic; energy consumption; NBSIR 80.2136

field strength; magnetic fields; calibration; calibrators; electric fields; electric field strength; electric power transmission; NBSIR 77-1311. field strength measurement; isotropic antenna; radio frequency radiation; receiver calibration; electromagnetic field; electronic instrument design; field intensity meter; TN1033

field study; occupant comfort; optimal weatherization demonstration; thermal comfort; thermostat setting practices; weatherization; weatherization and thermal comfort; Community Services Administration; NBSIR 81-2335.

field study; thermal comfort; weatherization retrofitting; comfort; Community Services Administration Optimal Weatherization Demonstration; energy savings; 20752.

field survey; industrial process heat; materials performance; solar collector; standards; test methods; NBSIR 81-2381.

field surveys; fire; fire loads; furnishings; household surveys; live loads; mobile homes; residential surveys; transfer functions; NBSIR 80-2155.

field test; guideline; low income; non-code; policymaking; protestors; solar code; victim role; deed; developers; energy wars; experimental code; SP608: 1981 May. 173.178.

field testing; in-situ testing; soil mechanics; transducers; energy measurement; field instrument force measurement; BSS135.

field-tests; galvanic couples; soil environment; stainless steels; stresscorrosion behavior; coatings; corrosion behavior; NBSIR 81-2228 (NBS).

field tests; guide specifications; laboratory tests; paint removal methods; White House; coatings; exterior restoration; NBSIR 802122.

field validation test; independent testing; quality assurance program; software change package; software maintenance testing; developmental center test; environmental test; SP500-83: 1981 November. 93-99.

figure of merit; noise temperature; satellite communication; earth terminal; Earth Terminal Measurement System; NBSIR 81-1641.

file; geometry; graphics; interface; computer aided design (CAD); computer aided manufacturing (CAM); entity; exchange format; NBSIR 81.2297.

fill controls; grey water; performance; soil removal; water conservation; water discharge characteristics; clothes washers; dishwashers; dishwasher subcycles; NBSIR 81-2291 (HUD).

fillings; methacrylates; tertiary aromatic amines; unsaturated polyesters; acrylates; acrylic resins; aminoglutethimide or paraaminophenylacetic acid; cementing of bone; U.S. Patent 4,243, 763.

film; industry; metals; productivity; research associate; scanning technique; spin dependent; SRM's; standards; tape; copper; deterioration; electromagnetic radiation; $D I M / N B S$ 65(5): 1-24; 1981.

film; marine structures; piezoelectric polymer material; vegetable and animal life; antifouling coating; U.S. Patent 4,283,461.

film; marine structures; piezoelectric polymer material; vegetable and animal life; antifouling coating; U.S. Patent 4,297,394.

film thickness nonuniformity; high power laser mirrors; multilayer dielectric films; optical figure measurement; vacuum deposition techniques; wave front distortion; SP620: 1981 October. 356-368.

filter-fit method; linear least-squares; $x$-ray analysis; digital filter; SP604: 1981 June. 273.296.

final set; hydration; initial set; laboratory testing; maturity; mortar; regression analysis; strength prediction; temperature effects; compressive strength; concrete; curing temperature; early age; NBSIR 81-2244.

fine structure; interface; materials; measurement; office building; phase diagrams; radioactivity; research; sediment; soil; alloy; carbon; computers; energy; DIM/NBS 65(8): 1-28; 1981 .

fine-structure constant; fundamental constants; Josephson effect; leastsquares adjustments; quantum electrodynamics; basic standards; 20058.

fingerprint cards; fingerprint images; fingerprint ink; image quality; NBSIR $81-2298$

fingerprint images; fingerprint ink; image quality; fingerprint cards; NBSIR 81-2298.

fingerprint ink; image quality; fingerprint cards; fingerprint images; NBSIR 81-2298.

finishability; microporous filler; system nontoxicity; x-ray 
opacification; dental composite resin restorations; U.S. Patent $4,306,913$

finite difference; heat loss; implicit method; insulation; water heater; computer model; distribution pipe; dynamic response; electricity; energy; explicit method; faucet temperature; NBSIR 81-2303.

finite difference equations; fire-enclosure; fluid flow; heat sourcevolumetric; partial differential equations; buoyant convection; computations-finite difference; Euler equations; NBSIR 81-2385.

finite element; loads; scaffolds; stability; stiffness; strength; structural safety; work surfaces; codes and standards; construction safety; design; NBSIR 81-2265.

finite element; scaffold failures; scaffolding practices; scaffolds; structural analysis; accidents; codes and standards; construction loads; construction saíety; NBS-GCR-80-255.

finite elements; in-core solver; isoparametrics; Mooney-Rivlin material; nonlinear equation-solvers; penalty method; plane-stress; strain invariants; tensile-test specimen; checkerboard pressure; elastostatics; J. Res. 86(1): 79-109; 1981 January-February

FIPS PUB 75; vendor performance; benchmarking; SP500-83: 1981 November. 339.

fire; fire loads; furnishings; household surveys; live loads; mobile homes; residential surveys; transfer functions; field surveys; NBSIR $80-2155$.

fire; fracture toughness; measurement; parameter; physics; solar collector; space charges; technical competence; cement and concrete; cover plates; earth's tilt; electric; DIM/NBS 65(3): 1-24; 1981.

fire; journals; library holdings, NBS Library; NBS periodicals; periodicals; proceedings; serials; standards; transactions; annual reports; diffusion in metals; NBSIR 81 -2226.

fire alarm systems; fire departments; fire extinguishers; fire investigations; hospitals; nursing homes; nursing staff; smoke; smoke detectors; sprinkler systems; doors; evacuation; NBS-GCR-80-297.

fire alarm systems; fire departments; fire extinguishers; fire investigations; hospitals; nursing homes; nursing staff; smoke; smoke detectors; sprinkler systems; doors; evacuation; NBS-GCR-81-343.

fire alarm systems; fire safety; handicapped; life safety; refuge; board and care homes; building codes; building fires; evacuation; 20727.

fire departments; fire emergency plan; fire protection; group homes; group home staff; developmentally disabled; evacuation; NBSGCR $-81-315$.

fire departments; fire extinguishers; fire investigations; hospitals; nursing homes; nursing staff; smoke; smoke detectors; sprinkler systems; doors; evacuation; fire alarm systems; NBS-GCR-80-297.

fire departments; fire extinguishers; fire investigations; hospitals; nursing homes; nursing staff; smoke; smoke detectors; sprinkler systems; doors; evacuation; fire alarm systems; NBS-GCR-81-343.

fire departments; frequency distribution; noise (sound); sleep; smoke detectors; wakefulness; adults; alarm responses; auditory perception; decibel levels; NBS-GCR-80-284.

fire detection; fire endurance; fire modeling; fire tests; flammability; human behavior; smoke; suppression; toxicity; compartment fires; NBSIR 81-2294.

fire detection; fire growth; hazard analysis; mathematical models; room fires; smoke movement; tenability limits; combustion products; compartment fires; egress; NBSIR 80-2172.

fire detection systems; fire suppression; full-scale fire tests; heat transfer; railroad accidents; small-scale fire tests; thermal protection; bombs (ordnance); boxcars; computer models; NBSIR 80-2170.

fire doors; fire tests; air flow; air leakage; ASTM E 152; doors; NBSGCR-81-330.

fire doors; fire tests; infiltration; orifice flow; smoke control; smoke movement; air leakage; buildings; doors; NBSIR 81-2214.

fire emergency plan; fire protection; group homes; group home staff; developmentally disabled; evacuation; fire departments; NBS-GCR81-315.

fire-enclosure; fluid flow; heat source-volumetric; partial differential equations; buoyant convection; computations-finite difference; Euler equations; finite difference equations; NBSIR 81-2385.

fire endurance; fire modeling; fire tests; flammability; human behavior; smoke; suppression; toxicity; compartment fires; fire detection;
NBSIR 81-2294.

fire endurance; fires; mathematical models; reinforced concrete; steel structures; wood structures; NBSIR 80-2188.

fire endurance; fire tests; floors; reinforced concrete; roofs; thermal expansion; thermal restraint; buildings; concrete; NBS-GCR-81-317.

fire endurance; furnaces; pressure probes; static pressure; NBSIR 81 2415.

fire extinguishers; fire investigations; hospitals; nursing homes; nursing staff; smoke; smoke detectors; sprinkler systems; doors; evacuation; fire alarm systems; fire departments; NBS-GCR-80-297.

fire extinguishers; fire investigations; hospitals; nursing homes; nursing staff; smoke; smoke detectors; sprinkler systems; doors; evacuation; fire alarm systems; fire departments; NBS-GCR-81-343.

fire extinguishment; fire models; fire protection; sprinkler heads; sprinkler systems; time constant; NBSIR 81-2329.

fire extinguishment; flashover; ignition; mathematical models; room fires; compartment fires; computer programs; NBS-GCR-81-344.

firefighting; hot atom; industrial innovation; measurements; oils, PCB's; standards; atomic weight; coal; computers; drill failure; Faraday; DIM/NBS 65(6): 1-24; 1981.

fire growth; hazard analysis; mathematical models; room fires; smoke movement; tenability limits; combustion products; compartment fires; egress; fire detection; NBSIR 80-2172.

fire hazards; fire modeling; fire protection; fire research; fire suppression; human behavior in fires; physics of fire; toxicity of combustion products; chemistry of fire; combustion products; detection; NBSIR 80-2127-1.

fire incidents; fire tests; test methods; trailing cables; coal mines; electrical cables; NBSIR 81-2292.

fire investigations; hospitals; nursing homes; nursing staff; smoke; smoke detectors; sprinkler systems; doors; evacuation; fire alarm systems; fire departments; fire extinguishers; NBS-GCR-80-297.

fire investigations; hospitals; nursing homes; nursing staff; smoke; smoke detectors; sprinkler systems; doors; evacuation; fire alarm systems; fire departments; fire extinguishers; NBS-GCR-81-343.

fire loads; furnishings; household surveys; live loads; mobile homes; residential surveys; transfer functions; field surveys; fire; NBSIR 80-2155.

fire modeling; fire plumes; flame spread; furniture flammability; polyurethane foam combustion; room fires; NBS-GCR-81-300.

fire modeling; fire protection; fire research; fire suppression; human behavior in fires; physics of fire; toxicity of combustion products; chemistry of fire; combustion products; detection; fire hazards; NBSIR 80-2127-1.

fire modeling; fire protection engineering; sprinkler systems; systems analysis; building codes; 20746.

fire modeling; fire tests; flammability; human behavior; smoke; suppression; toxicity; compartment fires; fire detection; fire endurance; NBSIR 81-2294.

fire modeling; flashover; numerical methods; room fires; burning rates; compartment fires; NBS-GCR-81-301.

fire modeling; foam (materials); heat release; plastics; polymers; polymethylmethacrylate; polyoxymethylene; polypropylene; polystyrene; polyurethane; polyvinyl chloride; pyrolysis; smoke; wood; combustion products; NBS-GCR-80-295.

fire models; fire plumes; diffusion flames; entrainment; NBS-GCR-81346.

fire models; fire protection; sprinkler heads; sprinkler systems; time constant; fire extinguishment; NBSIR 81-2329.

fire models; flame spread; human behavior; ignition; polymers; smoke; soot; toxicity; wood; combustion; decision analysis; NBSIR 81 2382.

fireplace inserts; fireplaces; fire prevention; fire safety; questionnaires; wood; NBS-GCR-80-292.

fireplaces; fire prevention; fire safety; questionnaires; wood; fireplace inserts; NBS-GCR-80-292.

fire plumes; diffusion flames; entrainment; fire models; NBS-GCR-81. 346.

fire plumes; flame spread; furniture flammability; polyurethane foam combustion; room fires; fire modeling; NBS-GCR-81-300.

fire plumes; flow rate; mass flow; diffusion flames; entrainment; $N B S$ - 
GCR-80-294.

fire point; fire resistance tests; fire tests; flammability; flash point; hydraulic fluids; ignition; autoignition temperature; coal mines; NBSIR 81-2395.

fire prevention; fire safety; questionnaires; wood; fireplace inserts; fireplaces; NBS-GCR-80-292.

fire protection; fire research; fire suppression; human behavior in fires; physics of fire; toxicity of combustion products; chemistry of fire; combustion products; detection; fire hazards; fire modeling; $N B S I R$ 80-2127-1.

fire protection; group homes; group home staff; developmentally disabled; evacuation; fire departments; fire emergency plan; $N B S$ GCR-81-315.

fire protection; sprinkler heads; sprinkler systems; time constant; fire extinguishment; fire models; NBSIR 81-2329.

fire protection engineering; sprinkler systems; systems analysis; building codes; fire modeling; 20746.

fire research; fire suppression; human behavior in fires; physics of fire; toxicity of combustion products; chemistry of fire; combustion products; detection; fire hazards; fire modeling; fire protection; NBSIR 80-2127-1.

fire research; fire tests; flame research; smoke; bibliographies; building fires; coal mines; combustion products; compartment fires; fabric flammability; NBSIR 81-2272.

fire resistance tests; fire tests; flammability; flash point; hydraulic fluids; ignition; autoignition temperature; coal mines; fire point; NBSIR 81-2395.

fire retardants; heat measurement; heat release calorimeters; heat release rate; calorimeters; cellulose; char; NBS-GCR-81-329.

fires; mathematical models; reinforced concrete; steel structures; wood structures; fire endurance; NBSIR 80-2188.

fire safety; fire safety evaluation system; health care facilities; life cycle; cash flow; economic analysis; NBS-GCR-79-186-1.

fire safety; fire tests; flame spread; ignition; test methods; accident data; coal mines; conveyor belts; NBSIR 81-2256.

fire safety; handicapped; life safety; accessibility; building accessibility; building research; NBSIR 81-2245.

fire safety; handicapped; life safety; refuge; board and care homes; building codes; building fires; evacuation; fire alarm systems; 20727.

fire-safety; hazard warnings; meaningfulness; method; pictogram; response; symbol; understandability; communication; evaluation method; NBSIR 80-2088.

fire safety; heating equipment; literature reviews; stoves; wood; bibliographies; chimneys; combustion; NBS-GCR-80-298.

fire safety; ignition; self-heating; solar collector fires; solar collectors; spontaneous ignition; building fires; NBSIR 81-2326.

fire safety; industrial productivity; materials properties; research; standards; technology transfer; basic measurements and standards; building technology; calibration methods; computer standards and guidelines; energy research; environmental measurements; SP600.

fire safety; pictograms; symbols; understandability; visibility; visual alerting; directional arrows; exit signs; NBSIR 8I-2268.

fire safety; questionnaires; wood; fireplace inserts; fireplaces; fire prevention; NBS-GCR-80-292.

fire safety codes; regulation; benefit-cost analysis; building economics; building regulation; codes; construction regulation; economics; energy conservation codes; NBSIR 81-2223.

fire safety codes; regulation; benefit-cost analysis; building economics; building regulation; codes; construction economics; construction regulation; economics; NBSIR 81-2402.

fire safety evaluation system; health care facilities; life cycle; cash flow; economic analysis; fire safety; $N B S-G C R-79-186-1$.

fire suppression; flame extinguishment; heat transfer; polyester; textiles; airflow; burn (injuries); cotton; fabric flammability; fabrics; NBSIR 81-2386.

fire suppression; full-scale fire tests; heat transfer; railroad accidents; small-scale fire tests; thermal protection; bombs (ordnance); boxcars; computer models; fire detection systems; NBSIR 80-2170.

fire suppression; human behavior in fires; physics of fire; toxicity of combustion products; chemistry of fire; combustion products; detection; fire hazards; fire modeling; fire protection; fire research;
NBSIR 80-2127-1.

fire test; flame spread; HH.I-515D; smoldering; attic floor radiant panel test; cellulose insulation; NBSIR 81-2213.

fire test; flame spread; sample mounting; ventilation cloth; ASTM E-162; brattice cloth; coal mining; NBSIR 81-2200.

fire test methods; incident data; telecommunications; telephone exchanges; central office equipment; electronic equipment; NBSIR 81-2261.

fire tests; air flow; air leakage; ASTM E 152; doors; fire doors; NBSGCR-81-330.

fire tests; flame research; smoke; bibliographies; building fires; coal mines; combustion products; compartment fires; fabric flammability; fire research; NBSIR 81-2272.

fire tests; flame spread; ignition; test methods; accident data; coal mines; conveyor belts; fire safety; NBSIR 81-2256.

fire tests; flammability; flash point; hydraulic fluids; ignition; autoignition temperature; coal mines; fire point; fire resistance tests; NBSIR 81-2395.

fire tests; flammability; human behavior; smoke; suppression; toxicity; compartment fires; fire detection; fire endurance; fire modeling; NBSIR 81-2294.

fire tests; flashover; interior finish; mobile homes; models; room fires; compartment fires; NBSIR 81-2333.

fire tests; floors; reinforced concrete; roofs; thermal expansion; thermal restraint; buildings; concrete; fire endurance; $N B S-G C R$ $81-317$.

fire tests; health care facilities; heat release rate; hospitals; mattresses; prisons; smoke production; bedding; beds; compartment fires; NBSIR 80-2186.

fire tests; hydraulic fluids; invert emulsions; pressure-temperature; spray; synthetics; water glycol; coal mine; NBSIR 81-2373.

fire tests; infiltration; orifice flow; smoke control; smoke movement; air leakage; buildings; doors; fire doors; NBSIR 81-2214.

fire tests; test methods; trailing cables; coal mines; electrical cables; fire incidents; NBSIR 81-2292.

FIR laser; laser frequency measurement; relative polarization; relative power; wave-lengths; $\mathrm{CD}_{2} \mathrm{~F}_{2} ; \mathrm{CO}_{2}$ laser; 20743.

fitness-for-service; fracture mechanics; J-integral; crack opening displacement; design curve; elastic-plastic fracture mechanics; NBSIR 81-1648.

fixed point; hydration temperature; potassium fluoride; salt hydrate; sodium sulfate; temperature calibration; temperature standard; thermistor; thermometer; transition temperature; biomedical thermometry; disodium hydrogen phosphate; J. Res. 86(2): 181-192; 1981 March-April.

flame and smolder resistance; flame resistant cellulosic insulation material; thiocyanate compounds; U.S. Patent 4,302,345.

flame extinguishment; heat transfer; polyester; textiles; airflow; burn (injuries); cotton; fabric flammability; fabrics; fire suppression; NBSIR 81-2386.

flame inhibition; H-atoms; kinetics; mass spectrometry; phosphorus; radicals; Raman spectroscopy; temperature; NBSIR 80-2169.

flame research; smoke; bibliographies; building fires; coal mines; combustion products; compartment fires; fabric flammability; fire research; fire tests; NBSIR 81-2272.

flame resistant cellulosic insulation material; thiocyanate compounds; flame and smolder resistance; U.S. Patent 4,302,345.

flame size; temperature distribution; turbulent burning; ceilings; experiments; $N B S-G C R-79-188$.

flame spread; furniture flammability; polyurethane foam combustion; room fires; fire modeling; fire plumes; NBS-GCR-81-300.

flame spread; HH-I-515D; smoldering; attic floor radiant panel test; cellulose insulation; fire test; NBSIR 81-2213.

flame spread; human behavior; ignition; polymers; smoke; soot; toxicity; wood; combustion; decision analysis; fire models; NBSIR 81-2382.

flame spread; ignition; test methods; accident data; coal mines; conveyor belts; fire safety; fire tests; NBSIR 81-2256.

flame spread; sample mounting; ventilation cloth; ASTM E-162; brattice cloth; coal mining; fire test; NBSIR 81-2200.

flammability; flash point; hydraulic fluids; ignition; autoignition 
temperature; coal mines; fire point; fire resistance tests; fire tests; NBSIR 81-2395.

flammability; heat flux; pressure; spray ignition; temperature rise; NBSIR 81-2247.

flammability; human behavior; smoke; suppression; toxicity; compartment fires; fire detection; fire endurance; fire modeling; fire tests; NBSIR 81-2294.

flammability; hydraulic fluids; ignition; Wick Test; coal mines; NBSIR 81-2312.

flammability; ignition; mattresses; self-extinguishment; smoldering; upholstered furniture; cigarettes; NBSIR 81-2363.

flashing lights; lights, warning; sirens; sound level; warning light; warning signals; conspicuity; effective intensity; emergency warning lights; SP480-37.

flashover; ignition; mathematical models; room fires; compartment fires; computer programs; fire extinguishment; NBS-GCR-81-344.

flashover; interior finish; mobile homes; models; room fires; compartment fires; fire tests; NBSIR 81-2333.

flashover; numerical methods; room fires; burning rates; compartment fires; fire modeling; $N B S-G C R-81-301$.

flash point; hydraulic fluids; ignition; autoignition temperature; coal mines; fire point; fire resistance tests; fire tests; flammability; NBSIR 81-2395.

flat plate; shear; strength; building; collapse; concrete; concrete strength; construction; failure; NBSIR 81-2374.

flat-plate solar collectors; German Bundesverband Solarenergie; solar collector testing; water-heating solar collectors; collector efficiency; comparison German BSE vs ASHRAE 93.77 procedures; 20079.

flat plate, turbulent; boundary layer; compliant surface; drag, experimental; 20048.

flavor singlet; glueball; production process; quark model; strange meson; E meson at $1440 \mathrm{MeV} ; 20034$.

flaw detection; holography; inhomogeneities; light wave; nondestructive evaluation (NDE); optical holographic interferometry; technical literature; NBS-GCR-81-318.

flexible disk cartridge; flexible disk system; information interchange; marketed systems; standard; cost/benefit analysis; NBS-GCR-80306.

flexible disk cartridge; information interchange; label and file structure; standard; word processor; costs and benefits; NBS-GCR80-307.

flexible disk system; information interchange; marketed systems; standard; cost/benefit analysis; flexible disk cartridge; NBS-GCR80-306.

flight safety; fluid system components; maintenance effectiveness; onboard monitoring system; aircraft hydraulic systems; cost reduction; energy savings; failure detection; failure prevention; SP622: 1981 October. 195-219.

flood forces; foundations; hurricane forces; mobile home; soil anchors; standards; tiedown; wind forces; buoyancy forces; BSS132.

flood loads; mobile home foundations; mobile home standards; soil anchors; soil testing; NBSIR 81-2238.

floors; reinforced concrete; roofs; thermal expansion; thermal restraint; buildings; concrete; fire endurance; fire tests; NBS-GCR $81-317$.

floor temperature; fusion; Gibbs energy; glass transition; heat capacity; linear macromolecule; melt; selenium; calorimetry; crystal; enthalpy; entropy; JPCRD 1O(1): 89-118; 1981.

flow; history; horizontal; length; partially-filled; pipe; slope; streamdepth; unsteady; volume; water; diameter; drain; NBSIR 81-2249.

flow; model; plumbing; quasi-steady; unsteady; characteristics; discharge; drainage; J. Res. 86(2): 171-179; 1981 March-April.

flowchart; information flow; information systems; interactive systems; requirements; scenarios; database design; data dictionary; design; SP500-83: 1981 November. 85-91.

flowchart; information flow; information systems; interactive systems; requirements; scenarios; database design; data dictionary; design; NBSIR 81-2240.

flow control; Markov processes; performance prediction; queueing models; simulation; validation; analytic models; communication networks; SP500-83: 1981 November. 277-290. flow measurement; laminar flow; meter performance; numerical modeling; orifice meter; swirling flow; closed conduit meter; differential pressure meter; 19972.

flow rate; heat transfer fluid; solar collector; thermal performance; efficiency; NBS-GCR-80-254.

flow rate; mass flow; diffusion flames; entrainment; fire plumes; NBS. GCR-80-294.

fluence; heat flow; irradiance; measurements of temperature; plasma effects; pulsed laser beams; $10.6 \mu \mathrm{m}$; attenuators; beam profile; calorimetry; NBSIR 80-1628.

fluid; heat transfer; injury threshold; scald; thermal injury; bioheat equation; burn; criterion; critical exposure; NBSIR 81-2320.

fluid flow; gas; instrumentation; mass flow; measurement; metering; thermodynamics; 20751.

fluid flow; heat source-volumetric; partial differential equations; buoyant convection; computations-finite difference; Euler equations; finite difference equations; fire-enclosure; NBSIR 81 2385.

fluid mixtures; thermal conductivity; viscosity; computer program; density; extended corresponding states; TNIO39.

fluid properties; indexes; publication list; solid state; thermodynamic and transport properties; atomic and molecular properties; bibliographies; chemical kinetics; evaluated data; SP612.

fluids; mixtures; one fluid model; prediction; Van der Waals theory; viscosity; data evaluation; extended corresponding states; 20768.

fluids in an electric field; refractive index; binary liquids; critical mixing; critical phenomena; dielectric constant; 20056.

fluid system components; maintenance effectiveness; on-board monitoring system; aircraft hydraulic systems; cost reduction; energy savings; failure detection; failure prevention; flight safety; SP622: 1981 October. 195-219.

flumes, hydraulic models; hydraulics; numerical models; open-channel flow; Parshall flumes; 19971.

fluoranthene; fluorescence detection; gas chromatography/mass spectrometry; high-performance liquid chromatography; perylene; pyrene; shale oil; standard reference materials; benzo(a)pyrene; benzo(e)pyrene; 20750.

fluorescence detection; gas chromatography/mass spectrometry; highperformance liquid chromatography; perylene; pyrene; shale oil; standard reference materials; benzo(a)pyrene; benzo(e)pyrene; fluoranthene; 20750.

fluoride coatings; laser damage; multiple-shot damage; oxide coatings; pre-irradiation; thin films; ultraviolet reflectors; excimer lasers; SP620: 1981 October. 265-276.

fluoride glasses; fluorohafnates; fluorozirconates; infrared absorption; infrared glasses; infrared materials; multispectral materials; SP620: 1981 October. 94-101.

fluorides; forging; laser windows; lithium fluoride; mechanical properties; optical properties; ultraviolet transmission; SP620: 1981 October. 110.116.

fluorohafnates; fluorozirconates; infrared absorption; infrared glasses; infrared materials; multispectral materials; fluoride glasses; SP620: 1981 October. 94-101.

fluorophosphate glass; inclusion density; KDP crystals; silicate glass; bulk laser damage; SP620: 1981 October. 159-169.

fluorozirconates; infrared absorption; infrared glasses; infrared materials; multispectral materials; fluoride glasses; fluorohafnates; SP620: 1981 October. 94-101.

fly ash; cement; concrete; NBSIR 81-2239.

fly ash; MgO; slag; soundness; strength; cement; expansion; NBSIR $81-2273$.

foam; insulation; low temperature; mechanical properties; proportional limit; shear strength; tensile strength; yield strength; Young's modulus; compressive strength; elongation; NBSIR 811654.

foam (materials); heat release; plastics; polymers; polymethylmethacrylate; polyoxymethylene; polypropylene; polystyrene; polyurethane; polyvinyl chloride; pyrolysis; smoke; wood; combustion products; fire modeling; NBS-GCR-80-295.

food packaging; inverse gas chromatography; migration; oil simulants; oligomers; polyolefins; radioactive tracer; spectrofluorimetry; 
antioxidants; diffusion; NBSIR 81-2264.

food standards; measurement science and technology; standardization in developing countries; Sudan; weights and measures regulation; Agency for International Development; Arab League Organization for Standardization and Metrology; chemical industry standards; cotton standards; NBSIR 80-2020.

forensic science; individualization; population statistics; refractive index; auto headlight glass; classification; density; NBSIR 81-2286.

forging; laser windows; lithium fluoride; mechanical properties; optical properties; ultraviolet transmission; fluorides; SP620: 1981 October. 110.116.

form slide; microfilm; quality control; automated data printing; computer-output microforms; Federal Information Processing Standards Publication; FIPS PUB 82.

formulation; high pressure; high temperature; IAPS; International Association for the Properties of Steam; ionization; ionization constant; ion product; review; water; water substance; equation; JPCRD 10(2): 295-304; 1981.

FORTRAN; information interchange; information processing; processor; programming language; software; BASIC; COBOL; compilers; data processing; Federal Information Processing Standard; FIPS PUB 29-1.

Fortran arithmetic extensions; multiple precision; multiprecision Fortran software; multiprecision mathematical functions; precompilers for special arithmetic; AUGMENT and MP; NBSIR 80.2138.

FORTRAN subroutine; Fresnel integrals; key values; line broadening function; plasma dispersion function; Voigt function; argand diagram; complex error function; continued fraction; Dawson's function; J. Res. 86(6): 661-686; 1981 November-December.

forward.biasing; internal resistance; intrinsic distributed resistance; semiconductor device defects; U.S. Patent 4,287,473.

fossil energy; materials; process parameters; research recommendations; coal; control; NBSIR 81-2348.

fossil-fuel heating systems; gas-fired boilers; hydronic heating; pulse combustion; boilers; central heating; condensing boilers; efficiency, part load; NBSIR 80-2094.

fossil fuels; laboratory characterization; metals; mining industry; public health impacts; residues; toxic elements; availability; environmental contamination; extraction tests; SP618: 1981 November. 1.8.

fossil fuels; metal(loid) complexes; organometallic compounds; trace element standards; waste cycling; bacterial mobilization; biogeochemical cycles; biological uptake; cellular toxicity. essentiality mechanisms; chromatography; element-selective speciation; environmental monitoring; SP618.

fossil porphyrins; fuel conversion; fuel extraction; heterocyclics; metal chelates; metallo-complexes; origin of pollutants; pollutant properties; synfuel; SP618: 1981 November. 9-20.

foundation design; geotechnical engineering; housing construction; mine subsidence; mining; settlement; structural design; structural engineering; design criteria; NBSIR 81-2215.

foundations; hurricane forces; mobile home; soil anchors; standards; tiedown; wind forces; buoyancy forces; flood forces; BSS132.

foundations; professional practice; provisions; soil structure interaction; standards; buildings; design; earthquakes; engineering; NBSIR 80-2111-3.

Fourier methods; Gaussian curves; deconvolution; energy dispersive x-ray spectrometry; SP604: 1981 June. 177-191.

Fourier transform; algorithm; antenna; aperture; computer program; electromagnetic; far field; FFT; NBSIR 81-1643.

fracture and deformation; glass and ceramics research; materials research; materials science; metallurgy; polymers research; corrosion; NBSIR 81-2408.

Fracture and Deformation Division; FY80 technical activities; Metallurgy Division; Polymers Science and Standards Division; Reactor Division; Center for Materials Science; Ceramics Glass and Solid State Science Division; Chemical Stability and Corrosion Division; NBSIR 80-2108.

fracture control; fracture mechanics; mechanical properties; quality assurance; stress analysis; structural safety; NBSIR 79-1623. fracture mechanics; gamma rays; inventors; molecular interactions; molecular processes; neomycin; neutron dosimeters; polymers; radiography; SRM's; stars; visual acuity; evolution; DIM/NBS 65(7): $1.24 ; 1981$.

fracture mechanics; J-integral; crack opening displacement; design curve; elastic-plastic fracture mechanics; fitness-for-service; NBSIR 81-1648.

fracture mechanics; mechanical properties; quality assurance; stress analysis; structural safety; fracture control; NBSIR 79-1623.

fracture toughness; hardness; ceramics; coal conversion; crack propagation; erosion; 20028.

fracture toughness; low temperature; tensile properties; austenitic stainless steel; 20772.

fracture toughness; measurement; parameter; physics; solar collector; space charges; technical competence; cement and concrete; cover plates; earth's tilt; electric; fire; $D I M / N B S$ 65(3): 1-24; 1981 .

francium compounds; Gibbs energy of formation; heat capacity; lithium compounds; potassium compounds; protactinium compounds; rubidium compounds; sodium compounds; thorium compounds; uranium compounds; actinium compounds; cesium compounds; enthalpy; entropy; TN270-8.

Franck-Condon factors; laser-induced fluorescence; radiative lifetimes; scandium; scandium halides; yttrium; yttrium halides; chemiluminescence; 19969.

Franck-Condon factors; laser-induced fluorescence; radiative lifetimes; scandium; scandium halides; yttrium; yttrium halides; chemiluminescence; 19978.

Franck-Condon principle; rotational-electronic coupling; scattering theory; adiabatic potentials; Born-Oppenheimer approximation; diatomic spectroscopy; 20060.

free carrier absorption; multiphoton absorption; polaron absorption; avalanche breakdown; beam deformation; beam depletion; SP620: 1981 October. 394-402.

free energy; high temperature nearest-neighbor model; thermal expansion; anharmonic; Brillouin zone sums; central pair potential; fcc metals; 20037.

free energy; ionic; osmotic coefficients; solutions; sulfuric acid; standard reference data; thermodynamic properties; activity coefficients; aqueous; critical evaluation; electrolytes; excess free energy; JPCRD 10(3): 779-798; 1981.

free energy of formation; Gibbs energy function; heat capacity; heat of formation; selected values; thermochemical tables; enthalpy; entropy; equilibrium constant of formation; NBSIR 81-2343.

free radicals; gas phase; hydrocarbons; hydrogen; nitrogen; oxygen; rate of reaction; sulfur; Arrhenius parameters; chemical kinetics; combustion; decomposition; NBSIR 81-2254.

free-radicals; iron; organometalloid toxicity; pentane; respiration; animal toxicity; breath analysis; endurance; SP618: 1981 November. 226-234.

frequency comparison; global positioning system; remote time measurement; time comparison; time receiver; worldwide time; atomic clocks; automatic time comparisons; common-mode cancellation; 20062.

frequency distribution; noise (sound); sleep; smoke detectors; wakefulness; adults; alarm responses; auditory perception; decibel levels; fire departments; NBS-GCR-80-284.

frequency spectra; hot alignment; machinery parameters; temperatures; vibrations; base line signatures; SP622: 1981 October. 220-221.

Fresnel integrals; key values; line broadening function; plasma dispersion function; Voigt function; argand diagram; complex error function; continued fraction; Dawson's function; FORTRAN subroutine; J. Res. 86(6): 661-686; 1981 November-December.

fretting corrosion; histologic analysis; stainless steel 316 LVM; titanium, titanium alloy; bone plates; cobalt-chrome-nickel-tungsteniron-alloy; SP601: 1981 January. 395-422.

fuel conversion; fuel extraction; heterocyclics; metal chelates; metallo. complexes; origin of pollutants; pollutant properties; synfuel; fossil porphyrins; SP618: 1981 November. 9-20.

fuel extraction; heterocyclics; metal chelates; metallo-complexes; origin of pollutants; pollutant properties; synfuel; fossil porphyrins; 
fuel conversion; SP618: 1981 November. 9.20.

full-scale fire tests; heat transfer; railroad accidents; small-scale fire tests; thermal protection; bombs (ordnance); boxcars; computer models; fire detection systems; fire suppression; NBSIR 80-2170.

functional; historical file; keyword; life cycle management; requirements; software quality assurance (SQA); specification; configuration management (CM); cross reference; data structure; design, documentation; SP500-83: 1981 November. 101-106.

functional capacity; handicap; locks; architectural barriers; building accessibility; codes and standards; disability; door closers; door openers; doors; ergonomics; NBSIR 80-2174.

functional categories; resource oriented groupings; timesharing; SP500-83: 1981 November. 15-19.

functional programming; interpreters; prototypes; applicative programming; design; 20075.

fundamental constants; Josephson effect; least-squares adjustments; quantum electrodynamics; basic standards; fine-structure constant; 20058.

fungi; metals; microorganisms; standard reference materials; bacteria; biodeterioration; biotransformations; deterioration of materials; element volatilization; NBSIR 81-2246.

furnaces; pressure probes; static pressure; fire endurance; NBSIR 81 2415.

furnishings; household surveys; live loads; mobile homes; residential surveys; transfer functions; field surveys; fire; fire loads; NBSIR 80 2155.

furniture fires; ignitability; room fires; upholstered furniture; burning rate; case goods; chairs; NBSIR 81-2271.

furniture flammability; polyurethane foam combustion; room fires; fire modeling; fire plumes; flame spread; NBS-GCR-81-300.

fuse/circuit breaker panel problems; overamping; residential structures; safety; thermal insulation; branch circuit wiring problems; electrical problems; electrical wiring system; extension cord wiring; NBSIR 81-2203.

fused silica surface; laser damage; laser polishing; surface laser damage; 1.06- $\mu \mathrm{m} ; \mathrm{CO}_{2}$-laser glazing; $\mathrm{CO}_{2}$-laser polishing; SP620: 1981 October. 180.189.

fusion; Gibbs energy; glass transition; heat capacity; isotactic; linear macromolecule; melt; polypropylene; syndiotactic; atactic; crystal; crystallinity; density; enthalpy; entropy; JPCRD 10(4): 1051-1064; 1981.

fusion; Gibbs energy; glass transition; heat capacity; linear macromolecule; melt; selenium; calorimetry; crystal; enthalpy; entropy; floor temperature; JPCRD 10(1): 89-118; 1981.

fusion; Gibbs energy; glass transition; heat capacity; linear macromolecule; melt; polyethylene; crystal; crystallinity; density; enthalpy; entropy; JPCRD 10(1): 119-152; 1981.

fusion; Gibbs energy; glass transition; heat capacity; linear macromolecule; poly(oxymethylene)s; poly(oxyphenylene)s; poly(oxypropylene); crystal; crystallinity; density; enthalpy; entropy; JPCRD 10(4): 1001-1049; 1981.

$f$-values; iron; isoelectronic sequence; line strengths; nickel; oscillator strengths; systematic trends; transition probabilities; allowed transitions; cobalt; JPCRD 10(2): 305-566; 1981.

FY80 technical activities; Metallurgy Division; Polymers Science and Standards Division; Reactor Division; Center for Materials Science; Ceramics Glass and Solid State Science Division; Chemical Stability and Corrosion Division; Fracture and Deformation Division; NBSIR 80-2108.

\section{G}

gaging; liquid level; LNG tanks; custody transfer; 20770.

gain; input noise; linearity; offset; analog-to-digital converter; calibration service; data converter; differential linearity; digital-toanalog converter; error measurement; TN1145.

galactic spirals; hydrodynamic instability and turbulence; plasma dynamics; spiral grand design; WASER; winding dilemma; density wave; J. Res. 86(6): 557-563; 1981 November-December.

gallium; levitation calorimetry; specific heat; surface tension; thermophysical properties; tungsten; wetting; adsorption; Auger spectroscopy; convection; ellipsometry; NBSIR 81-2295.

gallium arsenide; integrated circuits; measurement technology; microelectronics; semiconductor devices; semiconductor materials; semiconductor process control; silicon; electronics; NBSIR 80)2006-4.

galvanic couples; soil environment; stainless steels; stress-corrosion behavior; coatings; corrosion behavior; field-tests; NBSIR 81-2228 (NBS).

game theory; mathematical economics; noncooperative games; total orders; aggregation; equilibrium; J. Res. 86(4): 383-391; 1981 JulyA ugust.

gamma radiography; national standard; performance tests; radiation source; safety standard; classification criteria; $H 136$.

gamma rays; instrument calibrations; intermediate calibration laboratories; ionizing radiation measurements; measurement accuracies; neutrons; radiation dosimetry; radiation protection; radiation standards; $x$ rays; beta rays; Federal and State laboratories; SP603.

gamma rays; inventors; molecular interactions; molecular processes; neomycin; neutron dosimeters; polymers; radiography; SRM's; stars; visual acuity; evolution; fracture mechanics; DIM/NBS 65(7): $1-24 ; 1981$

gap enhancement; microwaves; nonequilibrium; superconductivity; tunnel junction; aluminum; energy gap; 20723.

gap-temperature unbalance; guarded-hot-plate; heat source distribution; insulation; thermal conductivity; edge heat loss; effective thermal conductivity; 20735.

gas; instrumentation; mass flow; measurement; metering; thermodynamics; fluid flow; 20751.

gas; nitrogen; sonic cavity; thermodynamic properties; velocity of sound; virial coefficients; electrostatic transducers; 20072.

gas adsorption; heat switch; refrigerators; cryogenics; SP607: 1981 May. 178-185.

gas bearings; refrigeration; regenerator; resonant free piston; Stirling cycle; cryocooler; SP607: 1981 May. 116.

gas chromatograph; inception voltages; mass spectrometer; partial discharge; sulfur hexafluoride; swarm data; water vapor; corona; decomposition; electrical insulation; NBSIR 81-2242.

gas chromatography; generator column; high pressure liquid chromatography; octanol/water partition coefficients; activity coefficients; aqueous solubility; NBSIR 81-2406.

gas chromatography; hydrocarbons; vapor detector; vapor generator; vapor standards; accelerants; arson; combustible gas; detectors; diffusion rates; diffusion tubes; SP480-43.

gas chromatography; ionization processes; liquid chromatography; mass spectrometry; bioavailability; element speciation; SP618: 1981 November. 120.133.

gas chromatography/mass spectrometry; high-performance liquid chromatography; perylene; pyrene; shale oil; standard reference materials; benzo(a)pyrene; benzo(e)pyrene; fluoranthene; fluorescence detection; 20750.

gas chromatography/mass spectrometry; hydrophobic bases; metal complexation; nitrogen bases; oil shale; retort water; speciation; trace metals; alkylpyridines; dissolved organic compounds; SP618: 1981 November. 93-104.

gas compressor; linear motor/generator; magnetic bearings; Stirling Cycle; clearance seal; SP607: 1981 May. 146-153.

gaseous and liquid ethane; isothermal measurements; quartz crystal viscometer; saturated liquid; shear viscosity coefficient; density dependence; 20767.

gaseous and liquid methane; isothermal measurements; quartz crystal viscometer; shear viscosity coefficient; density dependence; 20071.

gaseous environments; nickel alloy 671; nickel alloy 800 ; slow strain rate test; structural materials; austenitic stainless steels; coal gasification; elevated temperatures; ferritic stainless steel; $N B S I R$ $81-2191$ (DoE).

gases; halogenated hydrocarbons; Henry's law constants; liquids; pesticides, solids; solubility; vapor pressure; alkanes; aromatics; critical review, evaluated data; JPCRD 10(4): 1175-1199; 1981.

gases; high voltage; insulation; interfaces; Kerr effect; liquids; Pockels effect; solids; dielectrics; electro-optics; NBSIR 81-2275. 
gas-fired boilers; hydronic heating; pulse combustion; boilers; central heating; condensing boilers; efficiency, part load; fossil-fuel heating systems; NBSIR 80-2094.

gasoline analysis; high performance liquid chromatography; metalselective detector; metal speciation; tetraalkyllead speciation; atomic absorption spectrometry; 20763.

gas phase; hydrocarbons; hydrogen; nitrogen; oxygen; rate of reaction; sulfur; Arrhenius parameters; chemical kinetics; combustion; decomposition; free radicals; NBSIR 81-2254.

gas phase conformers; glycine; rotational constants; structure; ab initio calculations; amino acid; conformational energy; dipole moment; 20005.

gated diode; generation lifetime; integrated gated-diode electrometer; integrated test structure; leakage current; microelectronic test structures; surface recombination velocity; test pattern; test structure; NBS-GCR-81-327.

gated diode; integrated gated-diode electrometer; integrated test structure; MOSFET dc profiler; test structure; CCD; cross-bridge sheet resistor; electrical alignment test structure; NBSIR 81-2319.

Gaussian beam; laser damage; spherical aberration; damage morphology; SP620: 1981 October. 403.405.

Gaussian curves; deconvolution; energy dispersive $x$-ray spectrometry; Fourier methods; SP604: 1981 June. 177.191.

Gaussian profiles; lithium-drifted silicon ( $\mathrm{Si}(\mathrm{Li})$ ) detector; $x$-ray microanalysis; detector; energy dispersive x-ray spectrometry; SP604: 1981 June. 233-272.

GC.AAS techniques; LC.AAS techniques; organoarsenics; organometals; partition coefficients; toxicity; alkylleads; alkyltins; chemical speciation; SP618: 1981 November. 65-80.

GC-MS; heavy metals; lead; mass spectrometry; mercury; methylation; environment; SP618: 1981 November. 54-64.

general adverse effect of noise; intermittency; loudness; noise criteria; time-varying noise; duration; NBSIR 81-2377.

general corrosion; mathematical modeling; prestressed concrete; prestressing steel; stress corrosion; concrete; corrosion; NBSIR 812390.

generalized plotting programs; interdata 7.16 computer; polynominal fitting; user manual; automated thermogravimetry; data analysis; NBSIR 80-2102.

generation lifetime; integrated gated-diode electrometer; integrated test structure; leakage current; microelectronic test structures; surface recombination velocity; test pattern; test structure; gated diode; NBS-GCR-81-327.

generator column; high pressure liquid chromatography; octanol/water partition coefficients; activity coefficients; aqueous solubility; gas chromatography; NBSIR 81-2406.

generator column; hydrophobic; octanol; partition coefficient; propylbenzene; solubility; solute; water; J. Res. 86(4): 361-366; 1981 July-August.

geochemical model; heavy metals; redox couples; trace elements; aqueous speciation; computerized; SP618: 1981 November. 39-53.

geological; magnetic; mechanical; monograph; optical; physical; properties data; radiation damage; rock salt; sodium chloride; chemical; electrical; Monogr. 167.

geometry; graphics; interface; computer aided design (CAD); computer aided manufacturing (CAM); entity; exchange format; file; NBSIR 81-2297.

geotechnical engineering; housing construction; mine subsidence; mining; settlement; structural design; structural engineering; design criteria; foundation design; NBSIR 81-2215.

geothermal; isobutane; thermodynamic; vapor pressure; critical region; density; equation of state; NBSIR 79-1715.

geothermal technology; casing inspection; cement bond logging tool; SP622: 1981 October. 310.320.

geothermal well; set-cement; simulated geothermal fluid; splitting tensile strength; compressive strength; NBSIR 80-2099-4.

German Bundesverband Solarenergie; solar collector testing; waterheating solar collectors; collector efficiency; comparison German BSE vs ASHRAE 93-77 procedures; flat-plate solar collectors; 20079.

germanium; monoenergetic photons; partly-resolved doublets; silicon; tailing; x-ray detectors; collimation; deadlayer determinations; SP604: 1981 June. 5-34.

germanium; semiconductor detectors; silicon; tailing; $\mathrm{x}$-ray spectroscopy; SP604: 1981 June. 35-44.

germanium (lithium) detector; scanning electron microscopy; silicon (lithium) detector; spectral deconvolution; $\mathrm{x}$-ray analysis; $\mathrm{x}$-ray fluorescence; electron probe microanalysis; energy dispersive $\mathrm{x}$-ray spectrometry; SP604.

giant resonance; photonuclear; quasideuteron; resonance fluorescence; 19992.

giant resonances; inelastic electron scattering; inelastic hadron scattering; photonuclear reactions; sum rules; 19977.

Gibbs energy; glass transition; heat capacity; isotactic; linear macromolecule; melt; polypropylene; syndiotactic; atactic; crystal; crystallinity; density; enthalpy; entropy; fusion; JPCRD 10(4): 1051. $1064 ; 1981$.

Gibbs energy; glass transition; heat capacity; linear macromolecule; melt; polyethylene; crystal; crystallinity; density; enthalpy; entropy; fusion; JPCRD 10(1): 119.152; 1981.

Gibbs energy; glass transition; heat capacity; linear macromolecule; melt; selenium; calorimetry; crystal; enthalpy; entropy; floor temperature; fusion; JPCRD 10(1): 89.118; 1981.

Gibbs energy; glass transition; heat capacity; linear macromolecule; poly(oxymethylene)s; poly(oxyphenylene)s; poly(oxypropylene) crystal; crystallinity; density; enthalpy; entropy; fusion; JPCRD 10(4): 1001-1049; 1981

Gibbs energy; osmotic coefficients; solutions; sulfuric acid; thermodynamic properties; transport properties; activity coefficients; aqueous; compilation; conductivity; electrolytes; enthalpy; NBSIR 81-2276.

Gibbs energy function; Gibbs energy of formation; heat capacity lime-alumina-silica-water; minerals; thermodynamic data; enthalpy, enthalpy of formation; entropy; equilibrium constant for formation; JPCRD 10(3): 575.670; 1981

Gibbs energy function; heat capacity; heat of formation; selected values; thermochemical tables; enthalpy; entropy; equilibrium constant of formation; free energy of formation; NBSIR 81-2343.

Gibbs energy of formation; heat capacity; lime-alumina-silica-water; minerals; thermodynamic data; enthalpy; enthalpy of formation entropy; equilibrium constant for formation; Gibbs energy function; JPCRD 10(3): 575.670; 1981.

Gibbs energy of formation; heat capacity; lithium compounds; potassium compounds; protactinium compounds; rubidium compounds; sodium compounds; thorium compounds; uranium compounds; actinium compounds; cesium compounds; enthalpy; entropy; francium compounds; TN270-8.

Gifford-McMahon cryocoolers; heat capacity; heat transfer; helium refrigerators; regenerators; Stirling cryocoolers; thermal conductance; Vuilleumier cryocoolers; cryogenics; 20766.

Gifford-McMahon cryocoolers; heat capacity; heat transfer; helium; refrigerators; regenerators; Stirling cryocoolers; thermal conductance; Vuilleumier cryocoolers; cryogenics; SP607: 1981 May. 70.81 .

glass and ceramics research; materials research; materials science; metallurgy; polymers research; corrosion; fracture and deformation; NBSIR 81-2408.

glass fiberblanket; insulation; low temperature; standard reference material; thermal conductivity; NBSIR 81-1640.

glass fiberboard; insulation; low-temperature; standard reference material; thermal conductivity; NBSIR 81-1639.

glass-liquidus; insulation; monitors; neutron diffraction, samplers; SRM's; time-domain calibrations; weights and measures; x-ray diffraction; air pollutants; antenna coupling; electrical distribution; $D I M / N B S$ 65(1): 1-28; 1981.

glass transition; heat capacity; isotactic; linear macromolecule; melt; polypropylene; syndiotactic; atactic; crystal; crystallinity; density; enthalpy; entropy; fusion; Gibbs energy; JPCRD 10(4): 1051-1064; 1981.

glass transition; heat capacity; linear macromolecule; melt; polyethylene; crystal; crystallinity; density; enthalpy; entropy; fusion; Gibbs energy; JPCRD 10(1): 119.152; 1981. 
glass transition; heat capacity; linear macromolecule; melt; selenium; calorimetry; crystal; enthalpy; entropy; floor temperature; fusion; Gibbs energy; JPCRD 10(1): 89-118; 1981.

glass transition; heat capacity; linear macromolecule; poly(oxymethylene)s; poly(oxyphenylene)s; poly(oxypropylene); crystal; crystallinity; density; enthalpy; entropy; fusion; Gibbs energy; JPCRD 10(4): 1001.1049; 1981.

global positioning system; remote time measurement; time comparison; time receiver; worldwide time; atomic clocks; automatic time comparisons; common-mode cancellation; frequency comparison; 20062.

glueball; production process; quark model; strange meson; E meson at $1440 \mathrm{MeV}$; flavor singlet; 20034.

glycine; interstellar; methyl formate; radio astronomy; amino acids; ethylene oxide; 20077.

glycine; rotational constants; structure; ab initio calculations; amino acid; conformational energy; dipole moment; gas phase conformers; 20005.

government buildings; hospitals; rate of return; schools; technical assistance; certification; Class $\mathrm{A}$ energy audit; economic analysis; energy analysis; energy conservation; Federal programs; SP608: 1981 May. 133-138.

government regulation; safety regulation; standards; cost-benefit; economics; 20031.

gradually varied flow; hydraulic jump; partially filled pipe flow; building drainage; NBSIR 81-2367.

grain; loss tangent; moisture; dielectric properties; NBSIR 78-897.

graph; $k$ shortest paths; network algorithms; shortest path; transportation networks; algorithm; 20021.

graphics; interface; computer aided design (CAD); computer aided manufacturing (CAM); entity; exchange format; file; geometry; NBSIR 81-2297.

graphite calorimeter; ionization chamber; scaling theorem; water phantom; absorbed dose standard; cobalt 60 gamma rays; electron density; J. Res. 86(5): 495-502; 1981 September-October.

graphite furnace atomic absorption spectrometer; high pressure liquid chromatography; selenite; arsenate; arsenic methylation; biological transformation; SP618: 1981 November. 244-263.

greenhouses; heat exchangers; light and ventilation; solar systems; space heating and cooling; training programs; building codes; building officials; codes and standards; convective loops; SP608: 1981 May. 159-166.

Green's function; integral equation; linear equation; method of moments; parallel plate waveguide; quasi electrostatic; TEM cell; electromagnetic compatibility (EMC); TN1028.

grey water; performance; soil removal; water conservation; water discharge characteristics; clothes washers; dishwashers; dishwasher subcycles; fill controls; NBSIR 81-2291 (HUD).

grid plate; machine geometry; measuring machine; redundancy; 3-D metrology; ball plate; NBSIR 79-1752.

ground coupled; hybrid solar energy system; inverted cave; low grade geothermal; radiant floor heating; space heating and cooling; thermal mass; walls; water source heat pump; buildings; construction; energy conservation; SP608: 1981 May. 69.81.

group homes; group home staff; developmentally disabled; evacuation; fire departments; fire emergency plan; fire protection; NBS-GCR-81-315.

group home staff; developmentally disabled; evacuation; fire departments; fire emergency plan; fire protection; group homes; NBS-GCR-81-315.

growth of crystals; mechanisms of growth; crystal; 20044.

$\mathrm{G} / \mathrm{T}$; precision measurements; radio star; satellite communications; $\mathrm{C} / \mathrm{kT}$; EIRP; error analysis; NBSIR 78-869.

$\mathrm{Gua} \cdot \mathrm{HCl} \cdot 1 \mathrm{H}_{2} \mathrm{O}$; Gua. $\mathrm{HCl} \cdot 2 \mathrm{H}_{2} \mathrm{O}$; Gua.2 $\mathrm{HCl}$; guanine (Gua); guanine hydrochlorides; heat capacity; nucleic acid base; solution calorimetry; thermochemistry; $\Delta C_{p}$ of reaction; calorimetry; $\mathrm{C}_{5} \mathrm{H}_{5} \mathrm{~N}_{5} \mathrm{O}$; density; enthalpy of solution; J. Res. 86(4): 367-382; 1981 July-August.

$\mathrm{Gua} \cdot \mathrm{HCl} \cdot 2 \mathrm{H}_{2} \mathrm{O}$; Gua.2 $\mathrm{HCl}$; guanine (Gua); guanine hydrochlorides; heat capacity; nucleic acid base; solution calorimetry; thermochemistry; $\Delta C_{p}$ of reaction; calorimetry; $\mathrm{C}_{s} \mathrm{H}_{s} \mathrm{~N}_{s} \mathrm{O}$; density; enthalpy of solution; Gua. $\mathrm{HCl} \cdot 1 \mathrm{H}_{2} \mathrm{O} ;$ J. Res. 86(4): 367-382; 1981 July-August.

guanine (Gua); guanine hydrochlorides; heat capacity; nucleic acid base; solution calorimetry; thermochemistry; $\Delta C_{p}$ of reaction; calorimetry; $\mathrm{C}_{5} \mathrm{H}_{5} \mathrm{~N}_{5} \mathrm{O}$; density; enthalpy of solution; Gua. $\mathrm{HCl} \cdot 1$ $\mathrm{H}_{2} \mathrm{O}$; Gua.HCl.2 H $\mathrm{H}_{2} \mathrm{O}$; Gua.2 HCl; J. Res. 86(4): 367-382; 1981 JulyAugust.

guanine hydrochlorides; heat capacity; nucleic acid base; solution calorimetry; thermochemistry; $\Delta C_{p}$ of reaction; calorimetry; $\mathrm{C}_{5} \mathrm{H}_{5} \mathrm{~N}_{5} \mathrm{O}$; density; enthalpy of solution; Gua. $\mathrm{HCl} \cdot 1 \mathrm{H}_{2} \mathrm{O}$; Gua. $\mathrm{HCl} \cdot 2$ $\mathrm{H}_{2} \mathrm{O}$; Gua.2 HCl; guanine (Gua); J. Res. 86(4): 367.382; 1981 July. August.

guarded-hot-plate; heat source distribution; insulation; thermal conductivity; edge heat loss; effective thermal conductivity; gaptemperature unbalance; 20735.

guarded hot plate; low temperature; moisture migration; mortar; porous solid; thermal conductivity; concrete; NBSIR 81-1651.

$\mathrm{Gua} \cdot 2 \mathrm{HCl}$; guanine (Gua); guanine hydrochlorides; heat capacity; nucleic acid base; solution calorimetry; thermochemistry; $\Delta C_{\rho}$ of reaction; calorimetry; $\mathrm{C}_{5} \mathrm{H}_{5} \mathrm{~N}_{5} \mathrm{O}$; density; enthalpy of solution; $\mathrm{Gua} \cdot \mathrm{HCl} \cdot 1 \mathrm{H} \mathrm{H}_{2} \mathrm{O}$; Gua.HCl.2 $\mathrm{H}_{2} \mathrm{O}$; J. Res. 86(4): 367.382; 1981 JulyAugust.

guest workers; international cooperation; international research; international standards; National Bureau of Standards (NBS); National Engineering Laboratory (NEL); technology transfer; engineering; NBSIR 80-2113.

guideline; low income; non-code; policymaking; protestors; solar code; victim role; deed; developers; energy wars; experimental code; field test; SP608: 1981 May. 173-178.

guide specifications; laboratory tests; paint removal methods; White House; coatings; exterior restoration; field tests; NBSIR 80-2122.

gust speed; instrumentation; wind sensor; wind speed; anemometer; drag sphere; 20059.

\section{H}

hail loads; snow loads; solar collectors; structural engineering; wind loads; earthquake loads; NBSIR 81-2199.

Hall effect; hole mobility; Irvin curves; phosphorus; resistivity; semiconductor; silicon; boron; capacitance-voltage technique; dopant density; electron mobility; SP400-64.

Hall mobility; hole density; resistivity; silicon; conductivity mobility; density of states; dopant (acceptor) density; effective mass; NBS. GCR-81-334.

halogenated hydrocarbons; Henry's law constants; liquids; pesticides, solids; solubility; vapor pressure; alkanes; aromatics; critical review, evaluated data; gases; JPCRD 10(4): 1175-1199; 1981.

handbook; physicists; physics; polymers; data; equations; 20749.

handicap; locks; architectural barriers; building accessibility; codes and standards; disability; door closers; door openers; doors; ergonomics; functional capacity; NBSIR 80-2174.

handicapped; life safety; accessibility; building accessibility; building research; fire safety; NBSIR 81-2245.

handicapped; life safety; refuge; board and care homes; building codes; building fires; evacuation; fire alarm systems; fire safety; 20727.

handicapped users; tactile warnings; accessibility; alarms; blind; code requirements; communication; deaf; directional indicators; NBSIR 81-2428.

hardness; ceramics; coal conversion; crack propagation; erosion; fracture toughness; 20028.

hardness; homogeneity; microstructure; precipitating; segregation; solidification; thermal mechanical treatment; aging; electrical conductivity; NBSIR 80-2069.

hard sphere; hot wire; thermal conductivity; transient; ambient temperature; argon; critical enhancement; J. Res. 86(3): 293-307; 1981 May-June.

hard sphere; LNG; mixtures; PVTx data; revised Kosek and McKinley; cell model; comparisons; computer programs; corresponding states; equation of state; TN1030.

hardware trends; information industry; software trends; technology 
forecast; automated data processing; computer utilization trends; Federal computers; NBS-GCR-81-342.

H-atoms; kinetics; mass spectrometry; phosphorus; radicals; Raman spectroscopy; temperature; flame inhibition; NBSIR 80-2169.

Hawaii; performance standards; building energy performance; codes; energy conservation; energy policy; SP608: 1981 May. 105-112.

hazard; pictograms; safety; signs; standards; symbols; visual alerting; warnings; communication; NBSIR 80-2003.

hazard analysis; mathematical models; room fires; smoke movement; tenability limits; combustion products; compartment fires; egress; fire detection; fire growth; NBSIR 80-2172.

hazards; measurement; metrology; National Bureau of Standards; power; radiation; semiconductor; technology; calibration; electrical; electronics; engineering; SP611.

hazard warnings; meaningfulness; method; pictogram; response; symbol; understandability; communication; evaluation method; firesafety; NBSIR 80-2088.

health and safety; high voltage; ionization chamber; ionizing radiation; ionometer; radiation quantities and units; radiation standards and protection; radioactivity; radiology; radium; roentgen ray; tubes ( $\mathrm{x}$ ray); $x$-ray measurement; $x$ rays; dosage; dosimetry; SP625.

health care facilities; heat release rate; hospitals; mattresses; prisons; smoke production; bedding; beds; compartment fires; fire tests; NBSIR 80-2186.

health care facilities; life cycle; cash flow; economic analysis; fire safety; fire safety evaluation system; NBS-GCR-79-186-1.

health condition; man-hour expenditure ratio; trend analysis; unlimited application; fibrescope; SP622: 1981 October. 74-81.

heat; metabolism; microcalorimetry; radiation; calorimetry; cardiac pacemakers; clinical chemistry; 20015.

heat balance for a single room; heat transfer; radiosity shape factors; building heating/cooling loads; NBSIR 81-2321.

heat capacity; heat of formation; selected values; thermochemical tables; enthalpy; entropy; equilibrium constant of formation; free energy of formation; Gibbs energy function; NBSIR 81-2343.

heat capacity; heat transfer; helium; refrigerators; regenerators; Stirling cryocoolers; thermal conductance; Vuilleumier cryocoolers; cryogenics; Gifford-McMahon cryocoolers; 20766

heat capacity; heat transfer; helium; refrigerators; regenerators; Stirling cryocoolers; thermal conductance; Vuilleumier cryocoolers; cryogenics; Gifford-McMahon cryocoolers; SP607: 1981 May. 70-81.

heat capacity; isotactic; linear macromolecule; melt; polypropylene; syndiotactic; atactic; crystal; crystallinity; density; enthalpy; entropy; fusion; Gibbs energy; glass transition; JPCRD 10(4): 1051$1064 ; 1981$.

heat capacity; lime-alumina-silica-water; minerals; thermodynamic data; enthalpy; enthalpy of formation; entropy; equilibrium constant for formation; Gibbs energy function; Gibbs energy of formation; JPCRD 10(3): 575-670; 1981.

heat capacity; linear macromolecule; melt; polyetliylene; crystal; crystallinity; density; enthalpy; entropy; fusion; Gibbs energy; glass transition; JPCRD 10(1): 119-152; 1981.

heat capacity; linear macromolecule; melt; selenium; calorimetry; crystal; enthalpy; entropy; floor temperature; fusion; Gibbs energy; glass transition; JPCRD 10(1): 89-118; 1981.

heat capacity; linear macromolecule; poly(oxymethylene)s; poly(oxyphenylene)s; poly(oxypropylene); crystal; crystallinity; density; enthalpy; entropy; fusion; Gibbs energy; glass transition; JPCRD 10(4): 1001-1049; 1981.

heat capacity; lithium compounds; potassium compounds; protactinium compounds; rubidium compounds; sodium compounds; thorium compounds; uranium compounds; actinium compounds; cesium compounds; enthalpy; entropy; francium compounds; Gibbs energy of formation; TN270-8.

heat capacity; nucleic acid base; solution calorimetry; thermochemistry; $\Delta C_{p}$ of reaction; calorimetry; $\mathrm{C}_{5} \mathrm{H}_{5} \mathrm{~N}_{5} \mathrm{O}$; density; enthalpy of solution; Gua. $\mathrm{HCl} \cdot 1 \mathrm{H}, \mathrm{O}$; Gua. $\mathrm{HCl} \cdot 2 \mathrm{H} \mathrm{O}$; Gua. $2 \mathrm{HCl}$; guanine (Gua); guanine hydrochlorides; J. Res. 86(4): 367.382; 1981 July-August.

heat capacity; refrigeration; regenerators; specific heat; cryogenics;
SP607: 1981 May. 164-172.

heat conduction calorimetry; kinetics of heat evolution; $\mathrm{m}$-azidobenzoic acid; nitrocellulose; quantitative differential thermal analysis; thermal explosion theory; thermal hazards; thermal instability; adiabatic calorimetry; azidotriphenylmethane; decomposition reactions; differential scanning calorimetry; NBSIR 80-2018 (DOT).

heat exchange design; heat recovery units; desuperheating heat exchangers; 20000 .

heat exchangers; light and ventilation; solar systems; space heating and cooling; training programs; building codes; building officials; codes and standards; convective loops; greenhouses; SP608: 1981 May. 159-166.

heat flow; irradiance; measurements of temperature; plasma effects; pulsed laser beams; $10.6 \mu \mathrm{m}$; attenuators; beam profile; calorimetry; fluence; NBSIR 80.1628.

heat flux; pressure; spray ignition; temperature rise; flammability; NBSIR 81-2247.

heat flux meters; helium; superconducting power transmission; cable cool-down; cool-down; NBSIR 81-1649.

heat flux meters; liquid helium; permeability of composites; superconducting power transmission; cable cool-down; cable permeability; counter-flow cool-down; NBSIR 80-1637.

heating; probe; radio-frequency; temperature measurements; NBSIR 80-1634.

heating and cooling equipment; heating, ventilating and air conditioning; heat pump; building heating and cooling; enginedriven heat pump; NBSIR 79-1911.

heating/cooling load; nighttime cooling; desert climate; energy calculation; environmental chamber; 20754.

heating/cooling load calculation; indoor temperature; NBSLD; attic temperature; BLAST; computer simulation; DoE-2; 20755.

heating equipment; literature reviews; stoves; wood; bibliographies; chimneys; combustion; fire safety; NBS-GCR-80-298.

heating, ventilating and air conditioning; heat pump; building heating and cooling; engine-driven heat pump; heating and cooling equipment; NBSIR 79-1911.

heat loss; implicit method; insulation; water heater; computer model; distribution pipe; dynamic response; electricity; energy; explicit method; faucet temperature; finite difference; NBSIR 81-2303.

heat measurement; heat release calorimeters; heat release rate; calorimeters; cellulose; char; fire retardants; NBS-GCR-81-329.

heat of formation; selected values; thermochemical tables; enthalpy; entropy; equilibrium constant of formation; free energy of formation; Gibbs energy function; heat capacity; NBSIR 81-2343.

heat pump; building heating and cooling; engine-driven heat pump; heating and cooling equipment; heating, ventilating and air conditioning; NBSIR 79-1911.

heat pump; mechanical equipment efficiency ratings; minimum efficiency standards; space cooling requirements; space heating requirements; energy efficiency standards; engineering-economic analysis; NBSIR 81 -2241.

heat recovery units; desuperheating heat exchangers; heat exchange design; 20000.

heat release; plastics; polymers; polymethylmethacrylate; polyoxymethylene; polypropylene; polystyrene; polyurethane; polyvinyl chloride; pyrolysis; smoke; wood; combustion products; fire modeling; foam (materials); NBS-GCR-80-295.

heat release calorimeters; heat release rate; calorimeters; cellulose; char; fire retardants; heat measurement; NBS-GCR-81-329.

heat release rate; calorimeters; cellulose; char; fire retardants; heat measurement; heat release calorimeters; NBS-GCR-81-329.

heat release rate; hospitals; mattresses; prisons; smoke production; bedding; beds; compartment fires; fire tests; health care facilities; NBSIR 80-2186.

heats of formation; inorganic gases; ion-molecule reactions; mass spectrometry; reaction rates; reactor technology; 19965.

heat source distribution; insulation; thermal conductivity; edge heat loss; effective thermal conductivity; gap-temperature unbalance; guarded-hot-plate; 20735.

heat source-volumetric; partial differential equations; buoyant 
convection; computations-finite difference; Euler equations; finite difference equations; fire-enclosure; fluid flow; NBSIR 81-2385.

heat switch; refrigerators; cryogenics; gas adsorption; SP607: 1981 May. 178-185.

heat transfer; helium; hysteresis; temperature control; transient; boiling; carbon film; 19976.

heat transfer; helium; refrigerators; regenerators; Stirling cryocoolers; thermal conductance; Vuilleumier cryocoolers; cryogenics; GiffordMcMahon cryocoolers; heat capacity; 20766.

heat transfer; helium; refrigerators; regenerators; Stirling cryocoolers; thermal conductance; Vuilleumier cryocoolers; cryogenics; GiffordMcMahon cryocoolers; heat capacity; SP607: 1981 May. 70-81.

heat transfer; injury threshold; scald; thermal injury; bioheat equation; burn; criterion; critical exposure; fluid; NBSIR 81-2320.

heat transfer; liquid helium; packed bed heat exchanger; balloon inflation; cryogenic helium balloon inflation; 20017.

heat transfer; pebble-bed storage; solar collector; system efficiency; thermal performance; air leakage; NBS-GCR-81-302.

heat transfer; pipes; thermal insulation; thermal properties; underground systems; computer program; earth temperature; NBSIR 81-2378.

heat transfer; polyester; textiles; airflow; burn (injuries); cotton; fabric flammability; fabrics; fire suppression; flame extinguishment; NBSIR 81-2386.

heat transfer; radiosity shape factors; building heating/cooling loads; heat balance for a single room; NBSIR 81-2321.

heat transfer; railroad accidents; small-scale fire tests; thermal protection; bombs (ordnance); boxcars; computer models; fire detection systems; fire suppression; full-scale fire tests; NBSIR 80 2170.

heat transfer by evaporating and condensing helium; helium refrigerator; superconducting computer; thermal couplings; thermal isolation; convective heat transfer; cryocooler; SP607: 1981 May. 93.102.

heat transfer fluid; solar collector; thermal performance; efficiency; flow rate; $N B S-G C R-80-254$.

heavy element fluorescence analysis; intrinsic germanium detectors; secondary target fluorescence analysis; source excited fluorescence analysis; $x$-ray fluorescence analysis; catalysts automotive; energy dispersive analysis; SP604: 1981 June. 391.415.

heavy metals; lead; mass spectrometry; mercury; methylation; environment; GC-MS; SP618: 1981 November. 54-64.

heavy metals; redox couples; trace elements; aqueous speciation; computerized; geochemical model; SP618: 1981 November. 39.53.

helicopter diagnostics; oil debris; wear monitoring; chip detectors; condition monitoring; failure detection; SP622: 1981 October. 3349.

helium; hot wire; nitrogen; pressure; temperature; thermal conductivity; transient; J. Res. 86(5): 457.493; 1981 SeptemberOctober.

helium; hysteresis; temperature control; transient; boiling; carbon film; heat transfer; 19976.

helium; infrared; SQUID; temperature sensors; cryogenics; SP607: 1981 May. 136-140.

helium; inner piston; outer piston; regenerator gaps; single stage, twin piston cryogenic refrigerator; two nested, concentric pistons; cylinder; U.S. Patent 4,281,517.

helium; low temperature; refrigerator; Stirling cycle; cryocooler; cryogenic; SP607: 1981 May. 30-47.

helium; maximum refrigerator efficiency; perfect gas analysis; refrigerator; component efficiency; cryogenics; efficiency; 20771.

helium; plastics; refrigerators; regenerators; Stirling-cycle; calculations; cryogenics; 20020.

helium; refrigeration; Stirling cycle; adsorption; cryocoolers; cryostats; SP607: 1981 May. 3-14.

helium; refrigeration; superconducting devices; cryocoolers; cryogenic sensors; SP607.

helium; refrigerators; regenerators; Stirling cryocoolers; thermal conductance; Vuilleumier cryocoolers; cryogenics; GiffordMcMahon cryocoolers; heat capacity; heat transfer; 20766.

helium; refrigerators; regenerators; Stirling cryocoolers; thermal conductance; Vuilleumier cryocoolers; cryogenics; GiffordMcMahon cryocoolers; heat capacity; heat transfer; SP607: 1981 May. 70.81 .

helium; superconducting power transmission; cable cool-down; cooldown; heat flux meters; NBSIR 81-1649.

helium; thermal conductivity; correlation; critical region; 20769.

helium isoelectronic sequence; hydrogen isoelectronic sequence; lithium isoelectronic sequence; electron impact ionization; 20738.

heliumlike ions; autoionization resonance; electron collision cross sections; 20045.

helium Penning ionization; hydrogen; ambipolar diffusion; electric discharges; 20047.

helium refrigerator; superconducting computer; thermal couplings; thermal isolation; convective heat transfer; cryocooler; heat transfer by evaporating and condensing helium; SP607: 1981 May: 93-102.

helium retention; long-life; refrigerators; reliability; rotary. reciprocating; turbo.Brayton; Vuilleumier; component development; contamination control; cryogenics; SP607: 1981 May. 21.29.

Henry's law constants; liquids; pesticides, solids; solubility; vapor pressure; alkanes; aromatics; critical review, evaluated data; gases; halogenated hydrocarbons; JPCRD 10(4): 1175-1199; 1981.

Hertzian dipole; integral equation; rectangular coaxial transmission line; TEM cell; characteristic impedance; field distribution; TN1037.

heterocyclics; metal chelates; metallo-complexes; origin of pollutants; pollutant properties; synfuel; fossil porphyrins; fuel conversion; fuel extraction; SP618: 1981 November. 9.20.

HH.I.515D; smoldering; attic floor radiant panel test; cellulose insulation; fire test; flame spread; NBSIR 81-2213.

high current relativistic electron beam generator; linear induction accelerator; 19985.

high cycle fatigue; stress corrosion; turbine blade failures; blade vibration; Doppler signature; Doppler technique; SP622: 1981 October. 181-194.

high-energy laser; interferometric testing; optical distortion; photoelastic coefficients; polycrystalline aggregate; stress birefringence; thermal lensing; window materials; elastic coefficients; SP620: 1981 October. 117-128.

high energy laser; laser damage; pulsed laser; reflectors; thin film; anti-reflection coatings; bichromatic; damage; SP620: 1981 October. 287.296.

higher heating value; refused-derived-fuel; sample characterization; sample processing effects; 2.5 and 25 gram capacity bomb calorimeters; ASTM round robin testing; bomb calorimetry; NBSIR 81-2278.

higher order modes; rectangular coaxial line; striplines; NBSIR 78 873.

high performance liquid chromatography; metal-selective detector; metal speciation; tetraalkyllead speciation; atomic absorption spectrometry; gasoline analysis; 20763.

high-performance liquid chromatography; perylene; pyrene; shale oil; standard reference materials; benzo(a)pyrene; benzo(e)pyrene; fluoranthene; fluorescence detection; gas chromatography/mass spectrometry; 20750.

high power laser mirrors; multilayer dielectric films; optical figure measurement; vacuum deposition techniques; wave front distortion; film thickness nonuniformity; SP620: 1981 October. 356-368.

high pressure; high temperature; IAPS; International Association for the Properties of Steam; ionization; ionization constant; ion product; review; water; water substance; equation; formulation; JPCRD 10(2): 295-304; 1981.

high pressure liquid chromatography; octanol/water partition coefficients; activity coefficients; aqueous solubility; gas chromatography; generator column; NBSIR 81-2406.

high pressure liquid chromatography; selenite; arsenate; arsenic methylation; biological transformation; graphite furnace atomic absorption spectrometer; SP618: 1981 November. 244-263.

high reactivity; low toxicity; peroxide catalyzed polymerization; tertiary aromatic amines; unsaturated polyesters; vinyl monomers; accelerators; cementing of bone; U.S. Patent 4,284,551. 
high resolution; hypochlorous acid $(\mathrm{HOCl})$; infrared spectrum; $v_{2}$ vibration; diode laser; Ebert spectrometer; 20053.

high resolution spectroscopy; subnatural-line width spectroscopy; time-resolved spectroscopy; 20076

high temperature; IAPS; International Association for the Properties of Steam; ionization; ionization constant; ion product; review; water; water substance; equation; formulation; high pressure; JPCRD 10(2): 295-304; 1981 .

high temperature; mass spectrometry; slag; transpiration; vapor transport; coal gasification; NBSIR 80-2178 (DoE).

high temperature fracture; J.integral; sialon; singular integral equation; crack growth model; creep cavitation; diffusive crack growth; energy release rate; NBSIR 81-2255.

high temperature fracture; silicon nitride; strain rate effect; toughness; crack growth; creep fracture; NBSIR 79-1960 (DoE).

high temperature nearest-neighbor model; thermal expansion; anharmonic; Brillouin zone sums; central pair potential; fcc metals; free energy; 20037.

high voltage; human response to noise; noise pollution; psychoacoustics; transmission line audible noise; corona noise; electric energy transmission; 20029.

high voltage; incipient fault; insulation; liquid breakdown; $\mathrm{SF}_{6}$; space charge; transformer oil; cables; composite insulation; dc fields; NBSIR 81-2334.

high voltage; incipient fault; insulation; $\mathrm{SF}_{6}$; space charge; transformer oil; cables; dc fields; NBSIR 81-2283.

high voltage; insulation; interfaces; Kerr effect; liquids; Pockels effect; solids; dielectrics; electro-optics; gases; NBSIR 81-2275.

high voltage; ionization chamber; ionizing radiation; ionometer; radiation quantities and units; radiation standards and protection radioactivity; radiology; radium; roentgen ray; tubes ( $x$ ray); $x$-ray measurement; $x$ rays; dosage; dosimetry; health and safety; SP625.

high voltage $\mathrm{dc}$; measurement errors; transmission lines; vertical current density; Wilson plates; NBSIR 81-2410.

high voltage dc transmission lines; ion counter; space charge density Wilson plates; absolute filter; current density; NBSIR 81-2267.

high voltage transmission lines; human response to noise; listening room; corona noise; environmental noise; 20030.

hip hemiarthroplasties; metal allergy; metal sensitivity; reactions to implants; SP601: 1981 January. 299-321.

histological examinations; hydrocephalus; prosthesis related infections; SP601: 1981 January. 349-362

histologic analysis; stainless steel 316 LVM; titanium, titanium alloy; bone plates; cobalt-chrome-nickel-tungsten-iron-alloy; fretting corrosion; SP601: 1981 January. 395-422.

histology; implant retrieval analysis; polarography; tissue response; atomic absorption spectrophotometry; EDAX analysis; enzyme histochemistry; SP601: 1981 January. 323-336.

histo-morphological; alumina-ceramic; artificial hip joints; endoprostheses; SP601: 1981 January. 131-156.

historical file; keyword; life cycle management; requirements software quality assurance (SQA); specification; configuration management (CM); cross reference; data structure; design, documentation; functional; SP500-83: 1981 November. 101-106.

historic preservation and energy conservation; load calculation; masonry thermal mass; system simulation; building energy analysis; energy conservation for nonresidential buildings; NBSIR 81-2317.

history; horizontal; length; partially-filled; pipe; slope; stream-depth; unsteady; volume; water; diameter; drain; flow; NBSIR 81-2249.

hole density; resistivity; silicon; conductivity mobility; density of states; dopant (acceptor) density; effective mass; Hall mobility; NBS-GCR-81-334.

hole mobility; Irvin curves; phosphorus; resistivity; semiconductor; silicon; boron; capacitance-voltage technique; dopant density; electron mobility; Hall effect; SP400-64.

Hollerith punched card code; Hollerith punched cards; information interchange; information processing; punched cards; standard American National Standard Code for Information Interchange; ASCII; card punch; card reader; data communications; data interchange; data processing; EAM cards; electric accounting machine; Federal Information Processing Standard; FIPS PUB 14-
1.

Hollerith punched cards; information interchange; information processing; punched cards; standard; American National Standard Code for Information Interchange; ASCII; card punch; card reader; data communications; data interchange; data processing; EAM cards; electric accounting machine; Federal Information Processing Standard; Hollerith punched card code; FIPS PUB 14-1.

hollow tubes; mercury; preconcentration; air analysis; diffusion; SP618: 1981 November. 134-145.

holography; inhomogeneities; light wave; nondestructive evaluation (NDE); optical holographic interferometry; technical literature; flaw detection; NBS-GCR-81-318.

homogeneity; microstructure; precipitating; segregation; solidification; thermal mechanical treatment; aging; electrical conductivity; hardness; NBSIR 80-2069.

homogeneity testing; standard reference material; austenitic stainless steel; electron probe microanalysis; $\mathrm{Fe}-\mathrm{Cr}$-Ni alloy; SP260-70.

horizontal; length; partially-filled; pipe; slope; stream-depth; unsteady; volume; water; diameter; drain; flow; history; NBSIR 81-2249.

horizontal axis wind turbines; wind energy; wind turbine data systems; wind turbine generators; wind turbine performance; SP622: 1981 October. 257.273.

hospitals; mattresses; prisons; smoke production; bedding; beds; compartment fires; fire tests; health care facilities; heat release rate; NBSIR 80-2186.

hospitals; nursing homes; nursing staff; smoke; smoke detectors; sprinkler systems; doors; evacuation; fire alarm systems; fire departments; fire extinguishers; fire investigations; NBS-GCR-80297.

hospitals; nursing homes; nursing staff; smoke; smoke detectors; sprinkler systems; doors; evacuation; fire alarm systems; fire departments; fire extinguishers; fire investigations; NBS-GCR-81. 343.

hospitals; rate of return; schools; technical assistance; certification; Class A energy audit; economic analysis; energy analysis; energy conservation; Federal programs; government buildings; SP608: 1981 May. 133-138.

hot alignment; machinery parameters; temperatures; vibrations; base line signatures; frequency spectra; SP622: 1981 October. 220-221.

hot atom; industrial innovation; measurements; oils, PCB's; standards; atomic weight; coal; computers; drill failure; Faraday; firefighting; DIM/NBS 65(6): 1-24; 1981.

hot carrier noise; noise; noise in devices; noise measurement; quantum noise; $1 / \mathrm{f}$ noise; SP614.

hot wire; nitrogen; pressure; temperature; thermal conductivity; transient; helium; J. Res. 86(5): 457-493; 1981 September-October.

hot wire; thermal conductivity; transient; ambient temperature; argon; critical enhancement; hard sphere; J. Res. 86(3): 293-307; 1981 MayJune.

household behavior patterns; housing characteristics (physical); housing security; public housing security; security hardware; victimization survey; burglary; crime in public housing; NBS-GCR$80-291$.

household paints; sequential tests; solubility tests; automotive paints; SP480-40.

household surveys; live loads; mobile homes; residential surveys; transfer functions; field surveys; fire; fire loads; furnishings; NBSIR $80-2155$.

housing; interiors; lifestyle; research; site planning; sociology; standards; children; design; environmental psychology; families; NBS-GCR-81-320.

housing; Operation Breakthrough; post-occupancy housing evaluation; Project Feedback; questionnaires; research methods; survey research; user needs; dwelling units (residential); NBSIR 812258.

housing; productivity; regulation; renovation; building codes; building economics; construction; cost; economics; NBSIR 80-2150.

housing characteristics (physical); housing security; public housing security; security hardware; victimization survey; burglary; crime in public housing; household behavior patterns; NBS-GCR-80-291.

housing codes; maintenance; model codes; occupancy; performance 
levels; regulation; standards; building rehabilitation; codes; comparative analysis; existing buildings; NBSIR 80-2081.

housing construction; mine subsidence; mining; settlement; structural design; structural engineering; design criteria; foundation design; geotechnical engineering; NBSIR 81-2215.

housing research; housing standards; performance requirements; standards development; building energy use; building service systems; energy budgets; NBS-GCR-80-280.

housing security; public housing security; security hardware; victimization survey; burglary; crime in public housing; household behavior patterns; housing characteristics (physical); NBS-GCR-80291.

housing standards; performance requirements; standards development; building energy use; building service systems; energy budgets; housing research; NBS-GCR-80-280.

human behavior; ignition; polymers; smoke; soot; toxicity; wood; combustion; decision analysis; fire models; flame spread; NBSIR $81-2382$.

human behavior; smoke; suppression; toxicity; compartment fires; fire detection; fire endurance; fire modeling; fire tests; flammability; NBSIR 81-2294.

human behavior in fires; physics of fire; toxicity of combustion products; chemistry of fire; combustion products; detection; fire hazards; fire modeling; fire protection; fire research; fire suppression; NBSIR 80-2127-1.

human factors evaluation; information system management; office automation; performance management systems; resource measurement facility; simulation; supercomputers; benchmarking; capacity planning; chargeback systems; computer performance management; data base machines; end user productivity; SP500-83.

human respiratory characteristics; intoxication; blood alcohol analysis; blood alcohol content; breath alcohol content; breath sampling; evidential breath testers; SP480-41.

human response; power line noise; psychoacoustics; annoyance; audible noise; corona noise; EHV transmission lines; electric energy; environmental noise; 20050.

human response to noise; listening room; corona noise; environmental noise; high voltage transmission lines; 20030.

human response to noise; noise pollution; psychoacoustics; transmission line audible noise; corona noise; electric energy transmission; high voltage; 20029.

human serum; polynuclear aromatic hydrocarbons; reference materials; shale oil; SRM; trace organic analysis; definitive methods: 19989.

humidity, thermal insulation and corrosion of electrical wiring; shock hazards; urea-formaldehyde thermal insulation; cellulose thermal insulation; corrosion of electrical outlet boxes and devices; electrical devices; NBSIR 81-2220.

hurricane forces; mobile home; soil anchors; standards; tiedown; wind forces; buoyancy forces; flood forces; foundations; BSS 132.

hurricanes; reliability; risk; structural engineering; building (codes); climatology; 20764.

hurricane winds; structural damage; structures; wind; wind speed; building performance; 20759.

HVAC; masonry; plumbing; solar; steel; structural systems; test methods; wood; building rehabilitation; concrete; electrical; evaluation; NBSIR 81 -2289.

HVAC; masonry; plumbing; steel; structural systems; test methods; wood; building rehabilitation; concrete; electrical; evaluation; NBSIR 80-2171.

HVAC calculations; insulation; life-cycle cost analysis; masonry; mass; building design; building economics; energy conservation; exterior wall; BSS134.

HVAC controls; reliability study; building energy conservation systems; EMCS; energy management; energy monitoring and control systems; NBSIR 81-2346.

HVAC systems; innovation; mathematical model; optimization; cost savings; energy conservation; SP608: 1981 May. 21-35.

HVAC systems; performance standards; solar collector; solar energy; space heating and cooling; thermal storage; ASHRAE 90-75; class $\mathrm{K}$ code; computer modeling; electrical design; energy audit; energy conservation; SP608.

HVAC systems and equipment; validation; Building Energy Performance Standards (BEPS); energy calculations; 20758.

hybrid cryocoolers; refrigeration; regenerative cooler; regenerator; Stirling cooler; Stirling cycle; cryocooler; SP607: 1981 May. 48.56.

hybrid solar energy system; inverted cave; low grade geothermal; radiant floor heating; space heating and cooling; thermal mass; walls; water source heat pump; buildings; construction; energy conservation; ground coupled; SP608: 1981 May. 69-81.

hydration; initial set; laboratory testing; maturity; mortar; regression analysis; strength prediction; temperature effects; compressive strength; concrete; curing temperature; early age; final set; $N B S I R$ 81.2244.

hydration temperature; potassium fluoride; salt hydrate; sodium sulfate; temperature calibration; temperature standard; thermistor; thermometer; transition temperature; biomedical thermometry; disodium hydrogen phosphate; fixed point; J. Res. 86(2): 181-192; 1981 March-April.

hydraulic fluids; ignition; autoignition temperature; coal mines; fire point; fire resistance tests; fire tests; flammability; flash point; NBSIR 81-2395.

hydraulic fluids; ignition; Wick Test; coal mines; flammability; NBSIR 81-2312.

hydraulic fluids; invert emulsions; pressure-temperature; spray; synthetics; water glycol; coal mine; fire tests; NBSIR 81-2373.

hydraulic jump; partially filled pipe flow; building drainage; gradually varied flow; NBSIR 81.2367 .

hydraulics; numerical models; open-channel flow; Parshall flumes; flumes, hydraulic models; 19971.

hydrocarbons; hydrogen; nitrogen; oxygen; rate of reaction; sulfur; Arrhenius parameters; chemical kinetics; combustion; decomposition; free radicals; gas phase; NBSIR 81-2254.

hydrocarbons; vapor detector; vapor generator; vapor standards; accelerants; arson; combustible gas; detectors; diffusion rates; diffusion tubes; gas chromatography; SP480-43.

hydrocephalus; prosthesis related infections; histological examinations; SP601: 1981 January. 349-362.

hydrodynamic gas bearing; linear magnetic suspension; long lifetime; space application; Stirling cycle; clearance seals; cryogenics; SP607: 1981 May. 103-115.

hydrodynamic instability and turbulence; plasma dynamics; spiral grand design; WASER; winding dilemma; density wave; galactic spirals; J. Res. 86(6): 557-563; 1981 November-December.

hydrogen; ambipolar diffusion; electric discharges; helium Penning ionization; 20047.

hydrogen; hydrogen computer codes; hydrogen design data; hydrogen gas; hydrogen graphs; hydrogen handbook; hydrogen liquid; hydrogen properties; hydrogen safety; hydrogen solid; hydrogen tables; hydrogen thermophysical properties; Monogr. 168.

hydrogen; nitrogen; oxygen; rate of reaction; sulfur; Arrhenius parameters; chemical kinetics; combustion; decomposition; free radicals; gas phase; hydrocarbons; NBSIR 81-2254.

hydrogen; production efficiency; scraping Auger; slush; slush production; cryogenic; NBSIR 78-875.

hydrogen and oxygen impurity; magnetron sputtering; pulsed HF/DF laser damage thresholds; silicon films; absorption; evaporation; SP620: 1981 October. 305-312.

hydrogen atom transfer; absolute chemical rate; BEBO activation energy; bond-order-bond-energy; chemical rate constants; computer program; J. Res. 86(6): 605.654; 1981 November-December.

hydrogen bonding; hydroxyapatite; tin hydroxide oxide phosphate; tin phosphate; topical fluoride treatments; crystal structure; 19994.

hydrogen bonding; infrared spectrum; matrix isolation; photodecomposition; reaction mechanism; $t$-2-fluorovinyl; acetylene; $\mathrm{F}$ atoms; 20036.

hydrogen computer codes; hydrogen design data; hydrogen gas; hydrogen graphs; hydrogen handbook; hydrogen liquid; hydrogen properties; hydrogen safety; hydrogen solid; hydrogen tables; hydrogen thermophysical properties; hydrogen; Monogr. 168.

hydrogen design data; hydrogen gas; hydrogen graphs; hydrogen handbook; hydrogen liquid; hydrogen properties; hydrogen safety; 
hydrogen solid; hydrogen tables; hydrogen thermophysical properties; hydrogen; hydrogen computer codes; Monogr. 168.

hydrogen gas; hydrogen graphs; hydrogen handbook; hydrogen liquid; hydrogen properties; hydrogen safety; hydrogen solid; hydrogen tables; hydrogen thermophysical properties; hydrogen; hydrogen computer codes; hydrogen design data; Monogr. 168.

hydrogen graphs; hydrogen handbook; hydrogen liquid; hydrogen properties; hydrogen safety; hydrogen solid; hydrogen tables; hydrogen thermophysical properties; hydrogen; hydrogen computer codes; hydrogen design data; hydrogen gas; Monogr. 168. hydrogen handbook; hydrogen liquid; hydrogen properties; hydrogen safety; hydrogen solid; hydrogen tables; hydrogen thermophysical properties; hydrogen; hydrogen computer codes; hydrogen design data; hydrogen gas; hydrogen graphs; Monogr. 168.

hydrogen isoelectronic sequence; lithium isoelectronic sequence; electron impact ionization; helium isoelectronic sequence; 20738.

hydrogen liquid; hydrogen properties; hydrogen safety; hydrogen solid; hydrogen tables; hydrogen thermophysical properties; hydrogen; hydrogen computer codes; hydrogen design data; hydrogen gas; hydrogen graphs; hydrogen handbook; Monogr. 168.

hydrogen properties; hydrogen safety; hydrogen solid; hydrogen tables; hydrogen thermophysical properties; hydrogen; hydrogen computer codes; hydrogen design data; hydrogen gas; hydrogen graphs; hydrogen handbook; hydrogen liquid; Monogr. 168.

hydrogen safety; hydrogen solid; hydrogen tables; hydrogen thermophysical properties; hydrogen; hydrogen computer codes; hydrogen design data; hydrogen gas; hydrogen graphs; hydrogen handbook; hydrogen liquid; hydrogen properties; Monogr. 168.

hydrogen solid; hydrogen tables; hydrogen thermophysical properties; hydrogen; hydrogen computer codes; hydrogen design data; hydrogen gas; hydrogen graphs; hydrogen handbook; hydrogen liquid; hydrogen properties; hydrogen safety; Monogr. 168.

hydrogen tables; hydrogen thermophysical properties; hydrogen; hydrogen computer codes; hydrogen design data; hydrogen gas; hydrogen graphs; hydrogen handbook; hydrogen liquid; hydrogen properties; hydrogen safety; hydrogen solid; Monogr. 168.

hydrogen thermophysical properties; hydrogen; hydrogen computer codes; hydrogen design data; hydrogen gas; hydrogen graphs; hydrogen handbook; hydrogen liquid; hydrogen properties; hydrogen safety; hydrogen solid; hydrogen tables; Monogr. 168.

hydrolyzable cations; hydrous oxides; kinetics; protolyzable anions; thermodynamics; adsorption; desorption; SP618: 1981 November. 146-162.

hydronic heating; pulse combustion; boilers; central heating; condensing boilers; efficiency, part load; fossil-fuel heating systems; gas-fired boilers; NBSIR 80-2094.

hydrophilic; hydrophobic; metal complex; porphyrin; shale oil; speciation; trace metals; chromatography; direct current plasma; SP618: 1981 November. 105.119.

hydrophobic; metal complex; porphyrin; shale oil; speciation; trace metals; chromatography; direct current plasma; hydrophilic; SP618: 1981 November. 105.119.

hydrophobic; octanol; partition coefficient; propylbenzene; solubility; solute; water; generator column; J. Res. 86(4): 361-366; 1981 July. August.

hydrophobic bases; metal complexation; nitrogen bases; oil shale; retort water; speciation; trace metals; alkylpyridines; dissolved organic compounds; gas chromatography/mass spectrometry; SP618: 1981 November. 93-104.

hydrous oxides; kinetics; protolyzable anions; thermodynamics; adsorption; desorption; hydrolyzable cations; SP618: 1981 November. 146-162.

hydroxyapatite; tin hydroxide oxide phosphate; tin phosphate; topical fluoride treatments; crystal structure; hydrogen bonding; 19994.

hydroxylapatite; interaction with $\mathrm{Zn}_{\mathrm{n}}$ ions and enamel etching; reaction with $\mathrm{Zn}$ ions and rate laws; surface mordanting with $\mathrm{Zn}$ ions; bonding with coupling agents; 20729.

hyperfine field; magnetism; phase diagram; potassium; conductivity; exchange interaction; ferrite; $20 \times 43$.

hypochlorous acid (HOCl); infrared spectrum; $v_{2}$ vibration; diode laser; Ebert spectrometer; high resolution; 20053. hysteresis; temperature control; transient; boiling; carbon film; heat transfer; helium; 19976.

$\mathrm{H}_{2}-\mathrm{He}$ collisions; planetary atmospheres; quadrupole-induced absorption; spectral moments; band shape analysis; collision-induced absorption; far infrared; $\mathrm{H}_{2}-\mathrm{H}_{2}$ collisions; NBSIR 80-2175.

$\mathrm{H}_{2}-\mathrm{H}_{2}$ collisions; $\mathrm{H}_{2}-\mathrm{He}$ collisions; planetary atmospheres; quadrupole-induced absorption; spectral moments; band shape analysis; collision-induced absorption; far infrared; NBSIR 80-2175.

\section{I}

IAPS; International Association for the Properties of Steam; ionization; ionization constant; ion product; review; water; water substance; equation; formulation; high pressure; high temperature; JPCRD 10(2): 295-304; 1981.

identification token; identity verification; password; personal attribute; personal identification; access control; authentication; authorization; computer network; computer security; encryption; Federal Information Processing Standards Publication; FIPS PUB 83.

identity verification; password; personal attribute; personal identification; access control; authentication; authorization; computer network; computer security; encryption; Federal Information Processing Standards Publication; identification token; FIPS PUB 83.

ignitability; room fires; upholstered furniture; burning rate; case goods; chairs; furniture fires; NBSIR 81-2271.

ignition; autoignition temperature; coal mines; fire point; fire resistance tests; fire tests; flammability; flash point; hydraulic fluids; NBSIR 81 -2395.

ignition; mathematical models; room fires; compartment fires; computer programs; fire extinguishment; flashover; NBS.GCR-81344.

ignition; mattresses; self-extinguishment; smoldering; upholstered furniture; cigarettes; flammability; NBSIR 81-2363.

ignition; metals; oxygen atmosphere; temperature measurement; alloys; combustion; combustion rate; NBSIR 81-1647.

ignition; polymers; smoke; soot; toxicity; wood; combustion; decision analysis; fire models; flame spread; human behavior; NBSIR 81 2382.

ignition; propagation; smoldering combustion; test methods; wave structure; boric acid; cellulosic insulation; NBSIR 81-2350.

ignition; self-heating; solar collector fires; solar collectors; spontaneous ignition; building fires; fire safety; NBSIR 81-2326.

ignition; test methods; accident data; coal mines; conveyor belts; fire safety; fire tests; flame spread; NBSIR 81-2256.

ignition; Wick Test; coal mines; flammability; hydraulic fluids; NBSIR 81-2312.

ignition interference; suppression; automotive; EMI; SP480-44.

III.V compounds; IMPATT diode; integrated circuit; LASER; LED; photocell; solar cell; solid state; transistor; tunnel diode; diode; field.effect transistor; J. Res. 86(6): 565-570; 1981 NovemberDecember.

Illinois; maintenance; public buildings; state government; cost construction; energy audit; energy conservation; SP608: 1981 May. 123-132.

illuminance; illumination; irradiance; solar radiation; daylighting; TN1148.

illumination; image quality; microfilm; microform; readers; American National Standard; Computer Output Microfilm (COM); Federal Information Processing Standard (FIPS); FIPS PUB 84.

illumination; irradiance; solar radiation; daylighting; illuminance; TN1148.

illumination; lighting; lighting levels; superthreshold visibility; vision; conspicuity; contrast; NBSIR 81-2231.

illumination; lighting control; solar heat gain; window management; daylight; energy conservation; fenestration design; NBSIR 80-2147. image quality; fingerprint cards; fingerprint images; fingerprint ink; NBSIR 81-2298.

image quality; microfilm; microform; readers; American National Standard; Computer Output Microfilm (COM); Federal Information Processing Standard (FIPS); illumination; FIPS PUB 
image transmission; information networks; national bibliographic network; satellite communications; slow-scan television; telecommunications policy; teleconferencing; videodiscs; computer network interconnection; computer network protocol; digital transmission of information; electronic message transmission; SP610.

immersion deposition; plating; plating on anodized aluminum; adhesion; anodizing; coatings; electrodeposition; NBSIR 80-2142.

impact; impulsive loading; penetration resistance; reinforcing bars; stress waves; concrete; dynamic properties; fiber-reinforcement; SP480-45.

impact; ocular injury; projectiles; safety; contusion; eyeball deformation; NBSIR 80-2037.

impact attenuation; peak acceleration; playground safety; playground surfaces; soil; surfacing materials; test method; asphalt; NBSIR 802106.

impact noise; noise control; noise isolation; noise rating; acoustics; building acoustics; NBS-GCR-80-249.

IMPATT diode; integrated circuit; LASER; LED; photocell; solar cell; solid state; transistor; tunnel diode; diode; field-effect transistor; III.V compounds; J. Res. 86(6): 565.570; 1981 November-December.

implantation-induced damage; ion channeling; ion dose measurement; ion implantation; channeling angle; C.V profiling; dopant profiles; NBS-GCR-81-325.

implant failures; ASP (Arthritis Surgery Proforma); data retrieval system; SP601: 1981 January. 615-642.

implant retrieval analysis; joint prostheses; metallurgical analysis; prosthetic devices; SP601: 1981 January. 559-572.

implant retrieval analysis; polarography; tissue response; atomic absorption spectrophotometry; EDAX analysis; enzyme histochemistry; histology; SP601: 1981 January. 323-336.

implant retrieval and analysis; liability trials; performance testing; regulatory enforcement; SP601: 1981 January. 655-666.

implants; metals; orthopaedic; polymers; retrieval; analysis; biological; cardiovascular; SP601.

implicit method; insulation; water heater; computer model; distribution pipe; dynamic response; electricity; energy; explicit method; faucet temperature; finite difference; heat loss; NBSIR 81 2303.

impulse response; inverse filtering; noise contamination; time domain; waveforms; deconvolution; TN1047.

impulsive loading; penetration resistance; reinforcing bars; stress waves; concrete; dynamic properties; fiber-reinforcement; impact; $S P 480-45$.

impurity-induced damage; laser damage; $\mathrm{ThF}_{4}$; thin films; $\mathrm{ZnS} ; \mathrm{ZrO}_{2}$; damage threshold; SP620: 1981 October. 277-286.

inaccuracy; ionizing radiation; radioactive sources; survey instruments; calibration; commercial services; directory; NBSGCR-80-296.

incentives; innovation; regulation; air pollution; Clean Air Act; EPA; ETIP; NBS-GCR-ETIP 81-93.

incentives; innovation; regulation; air pollution; Clean Air Act; EPA; ETIP; NBS-GCR-ETIP 81-94.

incentives; innovation; regulation; air pollution; Clean Air Act; EPA; ETIP; NBS-GCR-ETIP 81-95.

inception voltages; mass spectrometer; partial discharge; sulfur hexafluoride; swarm data; water vapor; corona; decomposition; electrical insulation; gas chromatograph; NBSIR 81-2242.

incident data; telecommunications; telephone exchanges; central office equipment; electronic equipment; fire test methods; NBSIR 812261.

incipient fault; insulation; liquid breakdown; $\mathrm{SF}_{6}$; space charge; transformer oil; cables; composite insulation; dc fields; high voltage; NBSIR 81-2334.

incipient fault; insulation; $\mathrm{SF}_{6}$; space charge; transformer oil; cables; dc fields; high voltage; NBSIR 81-2283.

inclusion density; KDP crystals; silicate glass; bulk laser damage; fluorophosphate glass; SP620: 1981 October. 159-169.

inclusions; laser damage; absorbing defects; SP620: 1981 October. 369.
374.

in-core solver; isoparametrics; Mooney-Rivlin material; nonlinear equation-solvers; penalty method; plane-stress; strain invariants; tensile-test specimen; checkerboard pressure; elastostatics; finite elements; J. Res. 86(1): 79-109; 1981 January-February.

independent testing; quality assurance program; software change package; software maintenance testing; developmental center test; environmental test; field validation test; SP500-83: 1981 November. 93-99.

index; National Bureau of Standards; nondestructive evaluation; publications; radiography; ultrasonics; abstracts; acoustic emission; bibliography; eddy currents; NBSIR 80-2080.

indexes; publication list; solid state; thermodynamic and transport properties; atomic and molecular properties; bibliographies; chemical kinetics; evaluated data; fluid properties; SP612.

index of refraction of air; refractivity of air; wavelength of light in air; air density; J. Res. 86(1): 27-32; 1981 January-February.

index profile; mode filter; numerical aperture; radiation angle; radiation patterns; attenuation; core diameter; far field; TN1032.

index profile; near-field scanning; optical communications; optical fiber; optical waveguide; refracted near-field scanning; refracted-ray scanning; resolution limit; fiber index profile; TN1038.

individualization; population statistics; refractive index; auto headlight glass; classification; density; forensic science; NBSIR 81-2286.

indoor temperature; NBSLD; attic temperature; BLAST; computer simulation; DoE-2; heating/cooling load calculation; 20755.

industrial innovation; measurements; oils, PCB's; standards; atomic weight; coal; computers; drill failure; Faraday; firefighting; hot atom; DIM/NBS 65(6): 1-24; 1981.

industrialization; international cooperation; measurement infrastructure; metrology; technologies; technology transfer; Brazil; NBSIR 80-1972.

industrial process heat; materials performance; solar collector; standards; test methods; field survey; NBSIR 81-2381.

industrial productivity; materials properties; research; standards; technology transfer; basic measurements and standards; building technology; calibration methods; computer standards and guidelines; energy research; environmental measurements; fire safety; SP600.

industry; metals; productivity; research associate; scanning technique; spin dependent; SRM's; standards; tape; copper; deterioration; electromagnetic radiation; film; DIM/NBS 65(5): 1.24; 1981.

inelastic electron scattering; inelastic hadron scattering; photonuclear reactions; sum rules; giant resonances; 19977.

inelastic hadron scattering; photonuclear reactions; sum rules; giant resonances; inelastic electron scattering; 19977.

infiltration; natural ventilation; building energy analysis; computer simulation; NBSIR 81-2404.

infiltration; orifice flow; smoke control; smoke movement; air leakage; buildings; doors; fire doors; fire tests; NBSIR 81-2214.

information flow; information systems; interactive systems; requirements; scenarios; database design; data dictionary; design; flowchart; SP500-83: 1981 November. 85-91.

information flow; information systems; interactive systems; requirements; scenarios; database design; data dictionary; design; flowchart; NBSIR 81-2240.

information industry; software trends; technology forecast; automated data processing; computer utilization trends; Federal computers; hardware trends; NBS-GCR-81-342.

information interchange; information processing; processor; programming language; software; BASIC; COBOL; compilers; data processing; Federal Information Processing Standard; FORTRAN; FIPS PUB 29-1.

information interchange; information processing; punched cards; standard; American National Standard Code for Information Interchange; ASCII; card punch; card reader; data communications; data interchange; data processing; EAM cards; electric accounting machine; Federal Information Processing Standard; Hollerith punched card code; Hollerith punched cards; FIPS PUB 14-1.

information interchange; information processing; standard; American 
National Standard Code for Information Interchange; ASCII; character set; coded character set; code for information interchange; collating sequence; data communication; data interchange; data processing; Federal Information Processing Standard; FIPS PUB 1-1.

information interchange; label and file structure; standard; word processor; costs and benefits; flexible disk cartridge; NBS-GCR-80307.

information interchange; marketed systems; standard; cost/benefit analysis; flexible disk cartridge; flexible disk system; NBS-GCR-80306.

information interchange; printer terminals; standard; text communication; text interchange; typesetting composition; ADP terminals; ASCII; character-imaging devices; control functions; data interchange; display terminals; Federal Information Processing Standard; FIPS PUB 86.

information interchange; text communications; text processor; coded character sets; code extension techniques; code standards; control functions; SP500-81.

information management; information technology; productivity; word processing; automation; SP500-83: 1981 November. 305-307.

information networks; modeling; standards; standards-writers; systems analysis; building standards; classification; decision tables; NBSIR 80-1979-1.

information networks; national bibliographic network; satellite communications; slow-scan television; telecommunications policy; teleconferencing; videodiscs; computer network interconnection; computer network protocol; digital transmission of information; electronic message transmission; image transmission; SP610.

information networks; network; standards; standards-writers; systems analysis; building standards; classification; decision tables; 19998.

information processing; processor; programming language; software; BASIC; COBOL; compilers; data processing; Federal Information Processing Standard; FORTRAN; information interchange; FIPS PUB 29-1.

information processing; punched cards; standard; American National Standard Code for Information Interchange; ASCII; card punch; card reader; data communications; data interchange; data processing; EAM cards; electric accounting machine; Federal Information Processing Standard; Hollerith punched card code; Hollerith punched cards; information interchange; FIPS PUB 14-1.

information processing; standard; American National Standard Code for Information Interchange; ASCII; character set; coded character set; code for information interchange; collating sequence; data communication; data interchange; data processing; Federal Information Processing Standard; information interchange; FIPS PUB 1-1.

information processing systems; optical character recognition; character positioning; computer systems; data entry (automatic); Federal Information Processing Standard; FIPS PUB 89.

information system management; office automation; performance management systems; resource measurement facility; simulation; supercomputers; benchmarking; capacity planning; chargeback systems; computer performance management; data base machines; end user productivity; human factors evaluation; SP500-83.

information systems; interactive systems; requirements; scenarios; database design; data dictionary; design; flowchart; information flow; SP500-83: 1981 November. 85.91.

information systems; interactive systems; requirements; scenarios; database design; data dictionary; design; flowchart; information flow; NBSIR 81-2240.

information systems; project management; system acquisition; system development; administrative experiment; evaluation systems; Experimental Technology Incentives Program; NBSIR 81-2328.

information systems; project management; system acquisition; system development; administrative experiment; evaluation systems; Experimental Technology Incentives Program; NBSIR 81-2327.

information technology; productivity; word processing; automation; information management; SP500-83: 1981 November. 305.307.

infrared; polarizer; thin films; diode laser; ellipsometer; SP620: 1981 October. 345-355. infrared; reflectance; silver; surface roughness; temperature dependence; aluminum; copper; Drude theory; SP620: 1981 October. 190-200.

infrared; SQUID; temperature sensors; cryogenics; helium; SP607: 1981 May. 136.140.

infrared absorption; infrared glasses; infrared materials; multispectral materials; fluoride glasses; fluorohafnates; fluorozirconates; SP620: 1981 October. 94-101.

infrared emission; laser; photofragmentation; radical; $\mathrm{CH}_{3} ; \mathrm{CH}_{2} \mathbf{I}$; 20004.

infrared glasses; infrared materials; multispectral materials; fluoride glasses; fluorohafnates; fluorozirconates; infrared absorption; SP620: 1981 October. 94-101.

infrared imaging; infrared thermography; radiance temperature; reflected infrared radiation; temperature corrections; temperature measurements; ambient infrared radiation; blackbody cavity; corrections for reflectance; 20733.

infra-red laser windows; pulsed $\mathrm{CO}_{2}$ laser damage; 10 micrometer optical components; absorptance; SP620: 1981 October. 248.255.

infrared materials; iodine laser; laser calorimetry; optical absorption coefficients; SP620: 1981 October. 129.143.

infrared materials; multispectral materials; fluoride glasses; fluorohafnates; fluorozirconates; infrared absorption; infrared glasses; SP620: 1981 October. 94-101.

infrared spectrum; matrix isolation; normal coordinate analysis; photodissociation; F-atom reactions; 19986.

infrared spectrum; matrix isolation; photodecomposition; reaction mechanism; $t$-2-fluorovinyl; acetylene; $F$ atoms; hydrogen bonding; 20036.

infrared spectrum; $\nu_{2}$ vibration; diode laser; Ebert spectrometer; high resolution; hypochlorous acid $(\mathrm{HOCl}) ; 20053$.

infrared standards from $1115 \mathrm{~cm}^{-1}$ to $1340 \mathrm{~cm}^{-1}$; infrared wavelength calibration; $\mathrm{N}_{2} \mathrm{O}$; nitrous oxide; spectroscopic constants of $\mathrm{N}_{2} \mathrm{O}$; infrared standards from $522 \mathrm{~cm}^{-1}$ to $657 \mathrm{~cm}^{-1}$; JPCRD 10(4): 1065 . 1084; 1981.

infrared standards from $522 \mathrm{~cm}^{-1}$ to $657 \mathrm{~cm}^{-1}$; infrared standards from $1115 \mathrm{~cm}^{-1}$ to $1340 \mathrm{~cm}^{-1}$; infrared wavelength calibration; $\mathrm{N}_{2} \mathrm{O}$; nitrous oxide; spectroscopic constants of $\mathrm{N}_{2} \mathrm{O}$; JPCRD 10(4): 1065 . $1084 ; 1981$.

infrared telescope; Spacelab; superfluid helium; cryogenics; SP607: 1981 May. 117.127.

infrared thermography; insulation; moisture detection; moisture measurement; nondestructive evaluation; nondestructive testing; nuclear backscatter; roofing systems; built-up roofing electrical capacitance; BSS131.

infrared thermography; radiance temperature; reflected infrared radiation; temperature corrections; temperature measurements; ambient infrared radiation; blackbody cavity; corrections for reflectance; infrared imaging; 20733.

infrared wavelength calibration; $\mathrm{N}_{2} \mathrm{O}$; nitrous oxide; spectroscopic constants of $\mathrm{N}_{2} \mathrm{O}$; infrared standards from $522 \mathrm{~cm}^{-1}$ to $657 \mathrm{~cm}^{-1}$. infrared standards from $1115 \mathrm{~cm}^{-1}$ to $1340 \mathrm{~cm}^{-1}$; JPCRD 10(4): 1065-1084; 1981.

infrared wavelength modulation; $\mathrm{LaF}_{3}$; laser windows; $\mathrm{LiF} ; \mathrm{MgF}_{2}$; $\mathrm{MgO} ; \mathrm{NaCl} ; \mathrm{NaF} ; \mathrm{SrF}_{2}$; volume and surface impurities; $\mathrm{BaF}_{2} ; \mathrm{CaF}_{2}$; extrinsic and intrinsic infrared absorption; SP620: 1981 October. 29. 43.

inhibitors; organic coatings; passive film dissolution; $\mathrm{pH}$ measurements; surface hydride; alpha uranium; chloride ion; chromate ion; electrochemistry; ellipsometry; field ion microscopy; NBSIR 81-2396 (ONR).

inhomogeneities; light wave; nondestructive evaluation (NDE); optical holographic interferometry; technical literature; flaw detection; holography; NBS-GCR-81-318.

initialization of heat transfer problem; parallel heat flow; response factors; thick building construction; conduction heat transfer; conduction transfer functions; NBSIR 81-2353.

initial permeability; metal mirror; polishing; polycrystalline material; residual stress; sapphire; specular reflectance; surface analysis; surface integrity; surface roughness; crystallographic anisotropy; crystallographic perfection; SP620: 1981 October. 171-179. 
initial set; laboratory testing; maturity; mortar; regression analysis; strength prediction; temperature effects; compressive strength; concrete; curing temperature; early age; final set; hydration; NBSIR 81-2244.

initial value problem; nonuniform systems; one-dimensional models; Rayleigh.Brillouin scattering; damped wave motion; density fluctuations; diffusion; dynamic structure factor; J. Res. 86(5): 429. 455; 1981 September-October.

initiator; resin; wear; accelerator; alloy; base metal; casting; composite; cyanoacrylate; dental alloy; NBSIR 80-2168.

injury threshold; scald; thermal injury; bioheat equation; burn; criterion; critical exposure; fluid; heat transfer; NBSIR 81-2320.

inner piston; outer piston; regenerator gaps; single stage, twin piston cryogenic refrigerator; two nested, concentric pistons; cylinder; helium; U.S. Patent 4,281,517.

innovation; mathematical model; optimization; cost savings; energy conservation; HVAC systems; SP608: 1981 May. 21-35.

innovation; post-marketing surveillance; regulatory experiments; system design; drug development; drug regulation; NBS-GCR. ETIP 81-96.

innovation; regulation; air pollution; Clean Air Act; EPA; ETIP; incentives; NBS-GCR-ETIP 81-94.

innovation; regulation; air pollution; Clean Air Act; EPA; ETIP; incentives; NBS-GCR-ETIP 81-95.

innovation; regulation; air pollution; Clean Air Act; EPA; ETIP; incentives; NBS-GCR-ETIP 81-93.

innovation incentives; regulatory reform; administrative experiment; communications; competition; ETIP; FCC; NBS-GCR-ETIP 81-97.

inorganic; radical ions; radicals; transients; absorption spectra; aqueous solution; extinction coefficients; NSRDS-NBS69.

inorganic gases; ion-molecule reactions; mass spectrometry; reaction rates; reactor technology; heats of formation; 19965.

in-phantom measurement; photon beam; radiation therapy; absorbed dose; Bragg.Gray equation; calibration; cavity chamber; cavity theory; electron beam; 20073.

input noise; linearity; offset; analog-to-digital converter; calibration service; data converter; differential linearity; digital-to-analog converter; error measurement; gain; TN1145.

input/output; modeling; rollin/rollout files; simulation; CDC 844-41; computer system evaluation; disk subsystem configurations; SP50083: 1981 November. 239-246.

in-situ testing; soil mechanics; transducers; energy measurement; field instrument force measurement; field testing; BSS135.

inspection; mathematical model; regulation; theory of games; J. Res. 86(2): 193.215; 1981 March-April.

installation; insulation; oil burners; practices; safety; sealants; standards; storm doors; storm windows; vent dampers; water heaters; windows; automatic ignition devices; caulks; effectiveness; energy conservation; energy conservation measures; SP606.

installation; recommended requirements; solar energy; solar systems; building codes; building officials; consensus; construction; document; SP608: 1981 May. 167-172.

institutional; procurement; purchasing; recovered/recycled materials; resource recovery; technical barriers; economic; NBSIR 81-2316.

institutional; procurement; purchasing; recovered/recycled materials; resource recovery; technical barriers; economic; NBS-GCR-81-311.

institutional; procurement; purchasing; recovered/recycled materials; resource recovery; technical barriers; economic; NBS-GCR-81-314. institutional; procurement; purchasing; recovered/recycled materials; resource recovery; technical barriers; economic; NBS-GCR-81-312. institutional; procurement; purchasing; recovered/recycled materials; resource recovery; technical barriers; economic; NBS-GCR-81-313.

institutional; procurement; purchasing; recovered/recycled materials; resource recovery; technical barriers; economic; NBS-GCR-81-308.

instruction mix technique; performance prediction; sensitivity technique; SP500-83: 1981 November. 181-189.

instrumentation; low temperature materials; materials properties; metals; nonmetallic materials; wind tunnels; cryogenic properties; NBSIR 79-1624.

instrumentation; mass flow; measurement; metering; thermodynamics; fluid flow; gas; 20751. instrumentation; measurement; shock and vibration; angular acceleration; angular accelerometer; calibration; NBSIR 81-2337.

instrumentation; measurements; shielded balanced transmission lines; time domain; transmission line model; waveform; bit error rate; coaxial transmission lines; digital communication impulse response; TN1042.

instrumentation; oscilloscope calibrator; oscilloscopes; calibration; calibrators; NBSIR 81-1646.

instrumentation; sensors; building energy monitoring; NBSIR 80 2104.

instrumentation; wind sensor; wind speed; anemometer; drag sphere; gust speed; 20059.

instrument calibrations; intermediate calibration laboratories; ionizing radiation measurements; measurement accuracies; neutrons; radiation dosimetry; radiation protection; radiation standards; $\mathrm{x}$ rays; beta rays; Federal and State laboratories; gamma rays; SP603.

instruments; magnetic fields; nonionizing radiation; regional calibration laboratories; standards; techniques; calibration; dosimetry; electric fields; electromagnetic interference; exposure history; SP613.

insulation; interfaces; Kerr effect; liquids; Pockels effect; solids; dielectrics; electro-optics; gases; high voltage; NBSIR 81-2275.

insulation; lasers; powder data; progress; R-values; single-crystal; SRM's; atomic ions; calibration; clock synchronization; Columbia River model; computer; DIM/NBS 65(2): 1.28; 1981.

insulation; life-cycle cost analysis; masonry; mass; building design; building economics; energy conservation; exterior wall; HVAC calculations; BSS134.

insulation; liquid breakdown; $\mathrm{SF}_{6}$; space charge; transformer oil; cables; composite insulation; dc fields; high voltage; incipient fault; NBSIR 81-2334.

insulation; low temperature; mechanical properties; proportional limit; shear strength; tensile strength; yield strength; Young's modulus; compressive strength; elongation; foam; NBSIR 81-1654.

insulation; low-temperature; standard reference material; thermal conductivity; glass fiberboard; NBSIR 81-1639.

insulation; low temperature; standard reference material; thermal conductivity; glass fiberblanket; NBSIR 81-1640.

insulation; measurement; $R$ value; standard reference material; thermal resistance; fiber-glass; fibrous glass; NBSIR 80-2091.

insulation; moisture detection; moisture measurement; nondestructive evaluation; nondestructive testing; nuclear backscatter; roofing systems; built-up roofing electrical capacitance; infrared thermography; BSS131.

insulation; monitors; neutron diffraction, samplers; SRM's; timedomain calibrations; weights and measures; x-ray diffraction; air pollutants; antenna coupling; electrical distribution; glass-liquidus; DIM/NBS 65(1): 1.28; 1981.

insulation; oil burners; practices; safety; sealants; standards; storm doors; storm windows; vent dampers; water heaters; windows; automatic ignition devices; caulks; effectiveness; energy conservation; energy conservation measures; installation; SP606.

insulation; $\mathrm{SF}_{6}$; space charge; transformer oil; cables; dc fields; high voltage; incipient fault; NBSIR 81-2283.

insulation; thermal conductivity; edge heat loss; effective thermal conductivity; gap-temperature unbalance; guarded-hot-plate; heat source distribution; 20735.

insulation; water heater; computer model; distribution pipe; dynamic response; electricity; energy; explicit method; faucet temperature; finite difference; heat loss; implicit method; NBSIR 81-2303.

insurance; loss prevention; modal analysis; property conservation; risk management; shock pulse analysis; vibration analysis; acoustic emission analysis; SP622: 1981 October. 139.151.

integral equation; linear equation; method of moments; parallel plate waveguide; quasi electrostatic; TEM cell; electromagnetic compatibility (EMC); Green's function; TN1028.

integral equation; rectangular coaxial transmission line; TEM cell; characteristic impedance; field distribution; Hertzian dipole; TN1037.

integrals; integration; irregularity of distribution; lattices; n-cube; periodic functions; quadrature; several variables; 20032. 
integrated circuit; LASER; LED; photocell; solar cell; solid state; transistor; tunnel diode; diode; field-effect transistor; III-V compounds; IMPATT diode; J. Res. 86(6): 565-570; 1981 November-December.

integrated circuit processing; ion channeling; ion implantation; lateral profiles; scanning electron microscopy; silicon technology; spreading resistance; two-dimensional profiles; arsenic ion implantation; electron-beam-induced current; etching; NBSIR 81 2398.

integrated circuits; measurement technology; microelectronics; semiconductor devices; semiconductor materials; semiconductor process control; silicon; electronics; NBSIR 79-1591-4.

integrated circuits; measurement technology; microelectronics; semiconductor devices; semiconductor materials; semiconductor process control; silicon; electronics; NBSIR 79-1591-5.

integrated circuits; measurement technology; microelectronics; semiconductor devices; semiconductor materials; semiconductor process control; silicon; electronics; NBSIR 80-2006-3.

integrated circuits; measurement technology; microelectronics; semiconductor devices; semiconductor materials; semiconductor process control; silicon; electronics; gallium arsenide; NBSIR 802006-4.

integrated circuits; measurement technology; microelectronics; semiconductor devices; semiconductor materials; semiconductor process control; silicon; electronics; NBSIR 81-2230-3.

integrated circuits; measurement technology; microelectronics semiconductor devices; semiconductor materials; semiconductor process control; silicon; electronics; NBSIR 81-2230.

integrated circuits; metrology; microelectronics; semiconductors; silicon; electronics; NBSIR 81-2224.

integrated circuits; microelectronics; process validation wafer; random faults; silicon-on-sapphire; test pattern; test structure; yield; SP400-66.

integrated circuits; semiconductor devices; semiconductor processes; semiconductors; semiconductor technology; silicon; NBSIR 81 2197.

integrated circuits measurement technology; microelectronics; semiconductor devices; semiconductor materials; semiconductor process control; silicon; electronics; NBSIR 81-2230-2.

integrated circuit test methods; microelectronic test structure; probe station; semiconductor process control; test pattern; test structure; wafer chuck; wafer probe station; SP400-68.

integrated communications; local area networks, microwave; video teleconferencing; CATV systems; coaxial cable; data communications; fiber optics information systems; SP500-83: 198 November. 271.276.

integrated gated-diode electrometer; integrated test structure; leakage current; microelectronic test structures; surface recombination velocity; test pattern; test structure; gated diode; generation lifetime; NBS-GCR-81-327.

integrated gated-diode electrometer; integrated test structure; MOSFET dc profiler; test structure; CCD; cross-bridge sheet resistor; electrical alignment test structure; gated diode; NBSIR 812319.

integrated gated-diode electrometer; microelectronic test pattern; microelectronic test structure; MOSFET dc profiler; cross-bridge sheet resistor; NBSIR 81-2234.

integrated test structure; leakage current; microelectronic test structures; surface recombination velocity; test pattern; test structure; gated diode; generation lifetime; integrated gated-diode electrometer; NBS-GCR-81-327.

integrated test structure; MOSFET dc profiler; test structure; CCD; cross-bridge sheet resistor; electrical alignment test structure; gated diode; integrated gated-diode electrometer; NBSIR 81-2319.

integrating device; permeation type; personal dosimeter; reaction; time-dependent; diffusion; NBSIR 80-1975.

integrating spheres; laboratory intercomparisons; reflectance measurements; reflectance standards; solar properties of materials; spectral reflectance measurements; diffuse reflectance; directionalhemispherical reflectance; NBSIR 81-2311.

integration; irregularity of distribution; lattices; n-cube; periodic functions; quadrature; several variables; integrals; 20032.

intensities; line strengths; oscillator strengths; spectral lines; transition probabilities; wavelengths; atom; atomic ion; NSRDS-NBS68.

intensity dependent absorption; multipulse damage; alkali-halides; calorimetry; damage mechanisms; SP620: 1981 October. 75.87.

interaction potential; Legendre expansion; molecular potential; quantum chemistry; scattering; electron-molecule collisions; 20055.

interaction with $\mathrm{Zn}$ ions and enamel etching; reaction with $\mathrm{Zn}$ ions and rate laws; surface mordanting with $\mathrm{Zn}$ ions; bonding with coupling agents; hydroxylapatite; 20729.

interactive computer system; orthopaedic implants; cross-referencing capabilities; SP601: 1981 January. 573.613.

interactive systems; performance analysis; productivity analysis; text editors; end user performance; SP500-83: 1981 November. 71-80.

interactive systems; requirements; scenarios; database design; data dictionary; design; flowchart; information flow; information systems; SP500-83: 1981 November. 85-91.

interactive systems; requirements; scenarios; database design; data dictionary; design; flowchart; information flow; information systems; NBSIR 81-2240.

interactive workloads; UNISKEL; universal skeleton; benchmarks; SP500-83: 1981 November. 143-156.

interchange of machine readable information; nonread inks; Optical Character Recognition (OCR); OCR-A; OCR-B; read inks; American National Standard; Federal Information Processing Standard (FIPS); FIPS PUB 85.

intercomparison; piston gage; pressure; primary standard; transfer standard; effective area; J. Res. 86(3): 277.279; 1981 May.June.

interconnect; microelectronics; random fault; step coverage; test structures; electronics; SP400-65.

interdata 7.16 computer; polynominal fitting; user manual; automated thermogravimetry; data analysis; generalized plotting programs; NBSIR 80-2102.

interface; computer aided design (CAD); computer aided manufacturing (CAM); entity; exchange format; file; geometry; graphics; NBSIR 81-2297.

interface; liquid chromatograph; liquid stream; mass spectrometer; tapered concentrator wire; elongated capillary tube; U.S. Patent $4,281,246$.

interface; materials; measurement; office building; phase diagrams; radioactivity; research; sediment; soil; alloy; carbon; computers; energy; fine structure; $D I M / N B S$ 65(8): 1.28; 1981.

interfaces; Kerr effect; liquids; Pockels effect; solids; dielectrics; electro-optics; gases; high voltage; insulation; NBSIR 81-2275.

interfaces; local area networks; measurement techniques; protocols; standards; computer based office systems; computer networking; NBSIR 80-2154.

interferometric testing; optical distortion; photoelastic coefficients; polycrystalline aggregate; stress birefringence; thermal lensing; window materials; elastic coefficients; high-energy laser; SP620: 1981 October. 117.128.

interior finish; mobile homes; models; room fires; compartment fires; fire tests; flashover; NBSIR 81-2333.

interiors; lifestyle; research; site planning; sociology; standards; children; design; environmental psychology; families; housing; NBS-GCR-81-320.

intermediate calibration laboratories; ionizing radiation measurements; measurement accuracies; neutrons; radiation dosimetry; radiation protection; radiation standards; $x$ rays; beta rays; Federal and State laboratories; gamma rays; instrument calibrations; SP603.

intermittency; loudness; noise criteria; time-varying noise; duration; general adverse effect of noise; NBSIR 81-2377.

internal fixation devices; mechanical fatigue; metal prostheses; stress corrosion; fatigue corrosion; SP601: 1981 January. 201-222.

internal reflection spectroscopy; silicon monoxide; thorium fluoride; zinc selenide; zinc sulfide; absorption; coatings; SP620: 1981 October. 335.344.

internal resistance; intrinsic distributed resistance; semiconductor device defects; forward-biasing; U.S. Patent 4,287,473.

International Association for the Properties of Steam; ionization; ionization constant; ion product; review; water; water substance; 
equation; formulation; high pressure; high temperature; IAPS; JPCRD 10(2): 295-304; 1981.

international cooperation; international research; international standards; National Bureau of Standards (NBS); National Engineering Laboratory (NEL); technology transfer; engineering; guest workers; NBSIR 80-2113.

international cooperation; measurement infrastructure; metrology; technologies; technology transfer; Brazil; industrialization; NBSIR 80-1972.

international recommendations; legal metrology; measurement assurance; metrication; model laws and regulations; packing and labeling; specifications and tolerances; technology transfer; weights and measures; domestic standards; education programs; electronic devices; SP599.

international research; international standards; National Bureau of Standards (NBS); National Engineering Laboratory (NEL); technology transfer; engineering; guest workers; international cooperation; NBSIR 80-2113.

international standards; National Bureau of Standards (NBS); National Engineering Laboratory (NEL); technology transfer; engineering; guest workers; international cooperation; international research; NBSIR 80-2113.

international standards; reporting of implant experience; cardiac pacemakers; SP601: 1981 January. 503.508.

International System of Units; measurement systems; metric system; modernized metric system; SI; weights and measures; SP304A, Revised August 1981.

International System of Units; measurement systems; metric system; modernized metric system; SI; weights and measures; SP304, Revised August 1981.

interoperability; teleprocessing; transmission; advanced data communication control procedures; ANS X3.66; bit-oriented; code independence; data; data link control procedures; data transparency; Federal Information Processing Standards Publication; FIPS PUB 78.

interpreters; prototypes; applicative programming; design; functional programming; 20075.

Interstate Solar Coordination Council; labeling procedures; national testing program; regulation; solar collector; standards; voluntary program; certification; consumer protection; SP608: 1981 May. 179. 181.

interstellar; methyl formate; radio astronomy; amino acids; ethylene oxide; glycine; 20077.

interstellar; nitric oxide; phosphine; phosphorus nitride; radio astronomy; sulfur dioxide; 19987.

interstellar molecules; methane; microwave spectra; molecular parameters; radio astronomy; rotational transitions; distortion dipole transitions; JPCRD 10(4): 1085-1095; 1981.

intoxication; blood alcohol analysis; blood alcohol content; breath alcohol content; breath sampling; evidential breath testers; human respiratory characteristics; SP480-41.

intraband-momentum matrix element; oscillator strength; two-photon absorption; band structure; effective mass; SP620: 1981 October. 438.445.

intrinsic distributed resistance; semiconductor device defects; forwardbiasing; internal resistance; U.S. Patent 4,287,473.

intrinsic germanium detectors; secondary target fluorescence analysis; source excited fluorescence analysis; x-ray fluorescence analysis; catalysts automotive; energy dispersive analysis; heavy element fluorescence analysis; SP604: 1981 June. 391-415.

inventors; molecular interactions; molecular processes; neomycin; neutron dosimeters; polymers; radiography; SRM's; stars; visual acuity; evolution; fracture mechanics; gamma rays; $D I M / N B S$ 65(7): 1.24; 1981

inverse filtering; noise contamination; time domain; waveforms; deconvolution; impulse response; TN1047.

inverse gas chromatography; migration; oil simulants; oligomers; polyolefins; radioactive tracer; spectrofluorimetry; antioxidants; diffusion; food packaging; NBSIR 81-2264.

inverted cave; low grade geothermal; radiant floor heating; space heating and cooling; thermal mass; walls; water source heat pump; buildings; construction; energy conservation; ground coupled; hybrid solar energy system; SP608: 1981 May. 69.81.

invert emulsions; pressure-temperature; spray; synthetics; water glycol; coal mine; fire tests; hydraulic fluids; NBSIR 81-2373.

investment analysis; life-cycle cost; recommended practice; savings-toinvestment ratio; benefit-cost analysis; building economics; cost effective; economic analysis; energy conservation; NBSIR 81-2397.

I/O; logical swapping; measurement; MVS; paging/swapping; TSO; analysis; data elements; SP5O0-83: 1981 November. 343-348.

I/O capacity algorithms; 3350 DASD; DASD I/O capacity; density of data reference; SP5D0-83: 1981 November. 231.238.

iodine laser; laser calorimetry; optical absorption coefficients; infrared materials; SP620: 1981 October. 129-143.

ion; molecule; reaction rate; relaxation; atom; charge transfer; chemiionization; collisional ionization; cross section; detachment; energy transfer; excitation; SP593.

ion and neutral lifetime; positive ion and neutral lifetime; cesium from molybdenum(111); 20013.

ion beam; sputtering; chalcogenide glass; coating; SP620: 1981 October. 324-334.

ion beam deposition; surface smoothness; thin films; barrier properties; diamond-like carbon; SP620: 1981 October. 313.323

ion channeling; ion dose measurement; ion implantation; channeling angle; C.V profiling; dopant profiles; implantation-induced damage; NBS-GCR-81-325.

ion channeling; ion implantation; lateral profiles; scanning electron microscopy; silicon technology; spreading resistance; twodimensional profiles; arsenic jon implantation; electron-beaminduced current; etching; integrated circuit processing; NBSIR 81 2398.

ion counter; space charge density; Wilson plates; absolute filter; current density; high voltage dc transmission lines; NBSIR 81-2267.

ion dose measurement; ion implantation; channeling angle; C.V profiling; dopant profiles; implantation-induced damage; ion channeling; NBS-GCR-81-325.

ionic; osmotic coefficients; solutions; sulfuric acid; standard reference data; thermodynamic properties; activity coefficients; aqueous; critical evaluation; electrolytes; excess free energy; free energy; JPCRD 10(3): 779.798; 1981.

ion implantation; channeling angle; C.V profiling; dopant profiles; implantation-induced damage; jon channeling; ion dose measurement; NBS-GCR-81-325

ion implantation; lateral profiles; scanning electron microscopy; silicon technology; spreading resistance; two-dimensional profiles; arsenic ion implantation; electron-beam-induced current; etching; integrated circuit processing; jon channeling; NBSIR 81-2398.

ionization; ionization constant; ion product; review; water; water substance; equation; formulation; high pressure; high temperature; IAPS; International Association for the Properties of Steam; JPCRD 10(2): 295-304; 1981.

ionization chamber; ionizing radiation; ionometer; radiation quantities and units; radiation standards and protection; radioactivity; radiology; radium; roentgen ray; tubes ( $x$ ray); $x$-ray measurement; $x$ rays; dosage; dosimetry; health and safety; high voltage; SP625.

ionization chamber; scaling theorem; water phantom; absorbed dose standard; cobalt-60 gamma rays; electron density; graphite calorimeter; J. Res. 86(5): 495.502; 1981 September-October.

ionization constant; ion product; review; water; water substance; equation; formulation; high pressure; high temperature; IAPS International Association for the Properties of Steam; ionization, JPCRD 10(2): 295-304; 1981.

ionization potentials; sodium; atomic energy levels; atomic ions atomic spectra; electron configurations; JPCRD 10(1): 153-196; 1981.

ionization processes; liquid chromatography; mass spectrometry; bioavailability; element speciation; gas chromatography; SP618: 1981 November. 120-133.

ionizing radiation; ionometer; radiation quantities and units; radiation standards and protection; radioactivity; radiology; radium; roentgen ray; tubes ( $x$ ray); $x$-ray measurement; $x$ rays; dosage; dosimetry; health and safety; high voltage; ionization chamber; SP625. 
ionizing radiation; radioactive sources; survey instruments; calibration; commercial services; directory; inaccuracy; $N B S-G C R$ 80-296.

ionizing radiation measurements; measurement accuracies; neutrons; radiation dosimetry; radiation protection; radiation standards; $\mathbf{x}$ rays; beta rays; Federal and State laboratories; gamma rays; instrument calibrations; intermediate calibration laboratories; SP603.

ion-molecule reactions; mass spectrometry; reaction rates; reactor technology; heats of formation; inorganic gases; 19965.

ionometer; radiation quantities and units; radiation standards and protection; radioactivity; radiology; radium; roentgen ray; tubes ( $x$ ray); $x$-ray measurement; $x$ rays; dosage; dosimetry; health and safety; high voltage; ionization chamber; ionizing radiation; SP625.

ion product; review; water; water substance; equation; formulation; high pressure; high temperature; IAPS; International Association for the Properties of Steam; ionization; ionization constant; JPCRD 10(2): 295.304; 1981.

IR laser damage; photoexcited carriers; semiconductor plasma; SP620: 1981 October. 427.431.

iron; isoelectronic sequence; line strengths; nickel; oscillator strengths; systematic trends; transition probabilities; allowed transitions; cobalt; f-values; JPCRD 10(2): 305.566; 1981.

iron; leaching; metal-reduction; metal-sulfides; mineral-oxidation; molybdenum; oil shale; Sulfolobus; Thiobacillus; uranium; chemolithotrophic-bacteria; copper; SP618: 1981 November. 264. 273.

iron; organometalloid toxicity; pentane; respiration; animal toxicity; breath analysis; endurance; free-radicals; SP618: 1981 November. 226-234.

iron alloys; low temperature mechanical properties; plastic deformation; temperature effects stainless steel alloys; yield stress cryogenic properties of materials; 20742.

iron-chromium alloys; niobium; repassivation kinetics; stainless steel; triboellipsometry; aluminum; field ion microscopy; NBSIR 79-1904 (Navy).

iron group elements; least squares optimization; theoretical spectroscopy; J. Res. 86(1): 33-77; 1981 January-February.

irradiance; measurements of temperature; plasma effects; pulsed laser beams; $10.6 \mu \mathrm{m}$; attenuators; beam profile; calorimetry; fluence heat flow; NBSIR 80-1628.

irradiance; solar radiation; daylighting; illuminance; illumination; $T N 1148$.

irregularity of distribution; lattices; n.cube; periodic functions quadrature; several variables; integrals; integration; 20032.

irreversible thermodynamics; Lennard-Jones fluid; nonequilibrium molecular dynamics; phase changes; 19974.

Irvin curves; phosphorus; resistivity; semiconductor; silicon; boron capacitance-voltage technique; dopant density; electron mobility; Hall effect; hole mobility; SP4O0-64.

isobutane; thermodynamic; vapor pressure; critical region; density equation of state; geothermal; NBSIR 79-1715.

isocyanide; rhodium; vibrational spectroscopy; carbon monoxide; chemisorption; 19997.

isoelectronic sequence; line strengths; nickel; oscillator strengths; systematic trends; transition probabilities; allowed transitions; cobalt; $f$-values; iron; JPCRD 10(2): 305-566; 1981.

isoparametrics; Mooney-Rivlin material; nonlinear equation-solvers penalty method; plane-stress; strain invariants; tensile-test specimen; checkerboard pressure; elastostatics; finite elements; in-core solver; J. Re.s. 86(1): 79.109; 1981 January-February.

isotactic; linear macromolecule; melt; polypropylene; syndiotactic atactic; crystal; crystallinity; density; enthalpy; entropy; fusion; Gibbs energy; glass transition; heat capacity; JPCRD 10(4): 1051 . 1064; 1981.

isothermal measurements; quartz crystal viscometer; saturated liquid; shear viscosity coefficient; density dependence; gaseous and liquid ethane; 20767.

isothermal measurements; quartz crystal viscometer; shear viscosity coefficient; density dependence; gaseous and liquid methane; 20071 .

isotope dilution mass spectrometry; mass spectrometry; standard reference materials; analysis; certification; 20024.

isotopes; molecular dynamics; neutron; neutron radiography; nondestructive evaluation; nuclear reactor; radiation; activation analysis; crystal structure; diffraction; TN1142.

isotopic; lithium; nuclear track technique; triton particles; abundances; alpha particles; cellulose nitrate detector; 19982.

isotopic assay; leachability studies; nondestructive assay; nuclear safeguards; nuclear waste management; standard reference materials; statistical evaluation; accountability guide; bulk measurements; NBSIR 81-2205.

isotopic purity; mass spectra; Master Analytical Scheme; organic compounds in water; deuterated compounds; NBSIR 80-2160.

isotropic antenna; radio frequency radiation; receiver calibration; electromagnetic field; electronic instrument design; field intensity meter; field strength measurement; $T N 1033$.

iteration method; nonlinear differential equation; nonlinear load; timestepping finite difference equation technique; Auger function; electrically-short dipole; NBSIR 78-898.

iterative methods for linear algebraic equations; Neumann boundary conditions; space matrices; conjugate gradient algorithm; elliptic partial differential equations; NBSIR 80-2056.

$l_{2}$ fluorescence absorption coefficient; $\mathrm{Kr}$ photosensitized reaction; methylene iodide; photodissociation; vacuum ultraviolet; 20042.

\section{$\mathbf{J}$}

JCPDS-International Centre for Diffraction Data; metric symmetry; NBS Crystal Data Center; powder diffraction; unit cell; computer evaluation; crystallographic data analysis; TN1141.

J.integral; crack opening displacement; design curve; elastic-plastic fracture mechanics; fitness-for-service; fracture mechanics; NBSIR 81-1648.

J-integral; sialon; singular integral equation; crack growth model; creep cavitation; diffusive crack growth; energy release rate; high temperature fracture; NBSIR 81-2255.

joint prostheses; metallurgical analysis; prosthetic devices; implant retrieval analysis; SP601: 1981 January. 559.572.

joint replacements; polyethylene components; acrylic cement; SP601: 1981 January. 3-28.

Josephson effect; least-squares adjustments; quantum electrodynamics; basic standards; fine-structure constant; fundamental constants; 20058.

Josephson effect; measurements; superconductivity; time domain; electronics; 20070.

Josephson effects; proximity effect devices; superconducting devices; tunnel devices; weak links; closed cycle refrigeration systems; SP607: 1981 May. 195-209.

Josephson junction; sampling circuit; sampling pulses; analog signal; U.S. Patent 4,245, 169.

Josephson junction devices; superconducting computers; thermal contact; 4K refrigeration; cryogenic equipment; SP607: 1981 May. 15.20.

Josephson technology; liquid helium; serviceable; superconducting device; thermal coupling; cryogenic refrigerator; SP607: 1981 May. 82.92

Joule-Thomson; microminiature devices; photolithographic fabrication; refrigerators; cryogenic; SP607: 1981 May. 160-163.

Joule-Thomson refrigerators; laminar flow heat exchanger; microminiature refrigerators; SP607: 1981 May. 154-159.

journals; library holdings, NBS Library; NBS periodicals; periodicals; proceedings; serials; standards; transactions; annual reports; diffusion in metals; fire; NBSIR 81-2226.

\section{$\mathbf{K}$}

Kalman filter; metal speciation; modeling; semiderivative; stability constants; voltammetry; zinc-NTA; cadmium-NTA; convolution voltammetry; electrochemistry; SP618: 1981 November. 163-172.

$\mathrm{KCl}$; NaCl; optoacoustic spectroscopy; photoacoustic spectroscopy; surface impurities; alkali-halide laser windows; SP620: 198] 
October. 44-49.

KDP crystals; silicate glass; bulk laser damage; fluorophosphate glass; inclusion density; SP620: 1981 October. 159-169.

kerma factors; neutron dosimetry; neutron radiation therapy; nuclear data; secondary charged particles; tissue equivalence; 20064.

Kerr effect; liquids; Pockels effect; solids; dielectrics; electro-optics; gases; high voltage; insulation; interfaces; NBSIR 81-2275.

Kerr effect; measurement; optics; polarized light; electric field; electro-optics; 19962.

Kerr liquids; laser damage; laser induced breakdown; $\mathrm{NaCl}$; selffocusing; SP620: 1981 October. 385-393.

key distribution; network security; security; computer security; cryptography; data integrity; encryption; Federal Information Processing Standards Publication; FIPS PUB 74.

key values; line broadening function; plasma dispersion function; Voigt function; argand diagram; complex error function; continued fraction; Dawson's function; FORTRAN subroutine; Fresnel integrals; J. Res. 86(6): 661.686; 1981 November-December.

keyword; life cycle management; requirements; software quality assurance (SQA); specification; configuration management (CM); cross reference; data structure; design, documentation; functional historical file; SP500-83: 1981 November. 101-106.

key words; publications; abstracts; building technology; Center for Building Technology; SP457-5.

key words; publications; abstracts, NBS publications; SP305. Supplement 12.

kilogram; mass/weight; metric controversy; newton; AAPT vs. NBS 20023.

kinetics; lasers; lineshapes; optical pumping; resonance Raman; spectroscopy; strong fields; damping theory; 20039.

kinetics; mass spectrometry; phosphorus; radicals; Raman spectroscopy; temperature; flame inhibition; H-atoms; NBSIR 80 2169.

kinetics; protolyzable anions; thermodynamics; adsorption; desorption; hydrolyzable cations; hydrous oxides; SP618: 1981 November. 146 162.

kinetics of heat evolution; m-azidobenzoic acid; nitrocellulose; quantitative differential thermal analysis; thermal explosion theory; thermal hazards; thermal instability; adiabatic calorimetry azidotriphenylmethane; decomposition reactions; differential scanning calorimetry; heat conduction calorimetry; NBSIR 80-2018 (DOT).

knee replacements; component loosening; debonding joint replacements; SP601: 1981 January. 509.557.

$\mathrm{KNO}_{2} ; \mathrm{LiNO}_{2} ; \mathrm{NaNO}_{2}$; osmotic coefficients; $\mathrm{RbNO}_{2}$; solutions standard reference data; thermodynamic properties; activity coefficients; alkali metal nitrite salts; aqueous; critical evaluation; $\mathrm{CsNO}_{2}$; electrolytes; excess Gibbs energy; JPCRD 10(3): 767-778; 1981.

Kroner method; sound velocities; ultrasonic inspection; ultrasonic waves; acoustic waves; elastic anisotropy; elastic constants; 20773.

$\mathrm{Kr}$ photosensitized reaction; methylene iodide; photodissociation; vacuum ultraviolet; $I_{2}$ fluorescence absorption coefficient; 20042.

$k$ shortest paths; network algorithms; shortest path; transportation networks; algorithm; graph; 20021.

\section{$\mathbf{L}$}

label and file structure; standard; word processor; costs and benefits; flexible disk cartridge; information interchange; NBS-GCR-80-307.

labeling; network; sequence list; shortest path; tree; complexity; $J$. Res. 86(3): 317-330; 1981 May-June.

labeling procedures; national testing program; regulation; solar collector; standards; voluntary program; certification; consumer protection; Interstate Solar Coordination Council; SP608: 1981 May. 179-181.

laboratory characterization; metals; mining industry; public health impacts; residues; toxic elements; availability; environmental contamination; extraction tests; fossil fuels; SP618: 1981 November $1-8$.

laboratory intercomparisons; reflectance measurements; reflectance standards; solar properties of materials; spectral reflectance measurements; diffuse reflectance; directional-hemispherical reflectance; integrating spheres; NBSIR 81-2311.

laboratory testing; maturity; mortar; regression analysis; strength prediction; temperature effects; compressive strength; concrete; curing temperature; early age; final set; hydration; initial set; NBSIR 81-2244.

laboratory tests; paint removal methods; White House; coatings; exterior restoration; field tests; guide specifications; NBSIR 802122.

$\mathrm{LaF}_{3}$; laser windows; $\mathrm{LiF} ; \mathrm{MgF}_{2} ; \mathrm{MgO} ; \mathrm{NaCl} ; \mathrm{NaF} ; \mathrm{SrF}_{2}$; volume and surface impurities; $\mathrm{BaF}_{2} ; \mathrm{CaF}_{2}$; extrinsic and intrinsic infrared absorption; infrared wavelength modulation; SP620: 1981 October 29-43.

laminar flow; meter performance; numerical modeling; orifice meter; swirling flow; closed conduit meter; differential pressure meter; flow measurement; 19972.

laminar flow heat exchanger; microminiature refrigerators; JouleThomson refrigerators; SP607: 1981 May. 154-159.

language; query; standards; conversion; database; data description; data dictionary; data directory; data manipulation; DBMS; SP500 76.

La Rance; tidal electricity production; tidal machinery; SP622: 1981 October. 289-309.

large-scale models; model assessment; documentation assessment; documentation guidelines; NBSIR 80-1971.

LASER; LED; photocell; solar cell; solid state; transistor; tunnel diode; diode; field-effect transistor; III.V compounds; IMPATT diode; integrated circuit; J. Res. 86(6): 565-570; 1981 November December.

laser; LNG; ships; tanks; volume; dimensions; NBSIR 80-2141.

laser; photofragmentation; radical; $\mathrm{CH}_{3} ; \mathrm{CH}_{2} \mathbf{I}$; infrared emission; 20004.

laser. calorimetry; laser windows; optoacoustic; photoacoustic; surface-to-bulk optical absorption; $\mathrm{CaF}_{2} ;$ SP620: 1981 October. 58 74.

laser calorimetry; optical absorption coefficients; infrared materials; iodine laser; SP620: 1981 October. 129-143.

laser damage; absorbing defects; inclusions; SP620: 1981 October. 369 374.

laser damage; laser induced breakdown; $\mathrm{NaCl}$; selfffocusing; Kerr liquids; SP620: 1981 October. 385-393.

laser damage; laser interaction; optical components; optical fabrication; optical materials and properties; thin film coatings; SP620.

laser damage; laser polishing; surface laser damage; $1.06-\mu \mathrm{m} ; \mathrm{CO}_{2}$ laser glazing; $\mathrm{CO}_{2}$-laser polishing; fused silica surface; SP620: 198 October. 180-189.

laser damage; $\mathrm{LiNbO}_{3}$ coatings; Q-switch; surface damage; antireflection coatings; coating damage; SP620: 1981 October. 300 304.

laser damage; microsecond pulses; $\mathrm{NaCl}$; surface damage; alkali halides; bulk damage; $\mathrm{CO}_{2}$ laser; coating damage; SP620: 1981 October. 88-93.

laser damage; mirrors; optical components; scratches; surface defects; coating imperfections; cw laser irradiation; digs; dust; SP620: 1981 October. 256.264.

laser damage; multiple-shot damage; oxide coatings; pre-irradiation; thin films; ultraviolet reflectors; excimer lasers; fluoride coatings; SP620: 1981 October. 265-276.

laser damage; $\mathrm{NaCl}$; picosecond phenomena; self-focusing; $\mathrm{SiO}_{2}$; air breakdown; SP620: 1981 October. 375-384.

laser damage; optical constants; optical materials; semiconductors; stimulated bremsstrahlung absorption; wavelength dependence; SP620: 1981 October. 416-426.

laser damage; pulsed laser; reflectors; thin film; anti-reflection coatings; bichromatic; damage; high energy laser; SP620: 1981 October. 287-296.

laser damage; spherical aberration; damage morphology; Gaussian beam; SP620: 1981 October. 403-405.

laser damage; $\mathrm{ThF}_{4}$; thin films; $\mathrm{ZnS} ; \mathrm{ZrO}_{2}$; damage threshold; 
impurity-induced damage; SP620: 1981 October. 277-286.

laser frequency measurement; relative polarization; relative power; wave-lengths; $\mathrm{CD}_{2} \mathrm{~F}_{2} ; \mathrm{CO}_{2}$ laser; FIR laser; 20743.

laser induced breakdown; $\mathrm{NaCl}$; self-focusing; Kerr liquids; laser damage; SP620: 1981 October. 385.393.

laser-induced damage; pulsed lasers; SP620: 1981 October. 297.299.

laser-induced electron emission; mirror surface damage; surface plasma; absorption waves; air breakdown; Cu mirrors; SP620: 1981 October. 227.238.

laser-induced fluorescence; radiative lifetimes; scandium; scandium halides; yttrium; yttrium halides; chemiluminescence; FranckCondon factors; 19978.

laser-induced fluorescence; radiative lifetimes; scandium; scandium halides; yttrium; yttrium halides; chemiluminescence; Franck. Condon factors; 19969.

laser interaction; optical components; optical fabrication; optical materials and properties; thin film coatings; laser damage; SP620.

laser irradiation; liquid phase; single crystal; defects; epitaxial regrowth; SP620: 1981 October. 222-226.

laser irradiation; liquid phase regrowth; metal surfaces; nonuniformities; SP620: 1981 October. 210.221.

laser polishing; surface laser damage; $1.06-\mu \mathrm{m} ; \mathrm{CO}_{2}$-laser glazing; $\mathrm{CO}_{2}$ laser polishing; fused silica surface; laser damage; SP620: 1981 October. 180-189.

lasers; lineshapes; optical pumping; resonance Raman; spectroscopy; strong fields; damping theory; kinetics; 20039.

lasers; powder data; progress; R-values; single-crystal; SRM's; atomic ions; calibration; clock synchronization; Columbia River model; computer; insulation; $D I M / N B S$ 65(2): 1.28; 1981.

laser scanner; measurement techniques; photovoltaics; solar cells; test pattern; defect identification; NBSIR 80-2181.

laser scanner; model calculations; photovoltaic cells; sheet resistance; solar cell defects; solar cells; test structures; NBSIR 81-2260.

laser scanner; photovoltaics; solar cell analysis; solar cells; computer simulations; fault detection; NBS-GCR -80-285.

laser simulator; light-emitting diodes; YAG laser; TN1031, Revised.

laser simulator; light-emitting diodes; YAG laser; TN1031.

laser waveform; melt threshold; metal deposition; pulsed laser damage; slip threshold; thermal diffusion length; thermal stress; cold work; crystalline disorder; defect damage; diamond-machined mirrors; Drude absorption; electron-beam melting; SP620: 1981 October. 201.209.

laser windows; $\mathrm{LiF} ; \mathrm{MgF}_{2} ; \mathrm{MgO} ; \mathrm{NaCl} ; \mathrm{NaF} ; \mathrm{SrF}_{2} ;$ volume and surface impurities; $\mathrm{BaF}_{2} ; \mathrm{CaF}_{2}$; extrinsic and intrinsic infrared absorption; infrared wavelength modulation; $\mathrm{LaF}_{3} ;$ SP620: 1981 October. 29.43.

laser windows; lithium fluoride; mechanical properties; optical properties; ultraviolet transmission; fluorides; forging; SP620: 1981 October. 110-116.

laser windows; material properties; transmission; absorption; SP620: 1981 October. 144.158.

laser windows; optoacoustic; photoacoustic; surface-to-bulk optical absorption; $\mathrm{CaF}_{2}$; laser calorimetry; SP620: 1981 October. 58.74.

lateral profiles; scanning electron microscopy; silicon technology; spreading resistance; two-dimensional profiles; arsenic ion implantation; electron-beam-induced current; etching; integrated circuit processing; ion channeling; ion implantation; NBSIR 81 2398.

late type stars; mass loss; stars, emission line; stellar chromospheres; circumstellar shells; 20057.

late-type stars; stars, individual; stellar chromospheres; stellar coronae; ultraviolet spectra; binary stars; 20001.

lattice constants; powder patterns; reference intensities; standard; x-ray diffraction; crystal structure; densities; Monogr. 25, Section 18.

lattices; n-cube; periodic functions; quadrature; several variables; integrals; integration; irregularity of distribution; 20032.

LC.AAS techniques; organoarsenics; organometals; partition coefficients; toxicity; alkylleads; alkyltins; chemical speciation; GC. AAS techniques; SP618: 1981 November. $65-80$.

leachability studies; nondestructive assay; nuclear safeguards; nuclear waste management; standard reference materials; statistical evaluation; accountability guide; bulk measurements; isotopic assay; NBSIR 81-2205.

leachate; linear free energy; liquid chromatography; molecular substituent; oil shale; organoarsenic; organotin; retention index; speciation; atomic absorption; energy-related process fluids; SP618 1981 November. 197.216

leaching; metal-reduction; metal-sulfides; mineral-oxidation; molybdenum; oil shale; Sulfolobus; Thiobacillus; uranium chemolithotrophic-bacteria; copper; iron; SP618: 1981 November 264-273.

lead; mass spectrometry; mercury; methylation; environment; GC-MS; heavy metals; SP618: 1981 November. 54-64.

lead apatite; lead potassium apatite; substituted apatite; crystal structure; 19984.

lead inclusion bodies; methylmercury derivatives; methylation/demethylation; arsenicals; cadmium metallothionein; cellular toxicity; elemental interactions; SP618: 1981 November 217.225.

lead potassium apatite; substituted apatite; crystal structure; lead apatite; 19984.

leakage current; microelectronic test structures; surface recombination velocity; test pattern; test structure; gated diode; generation lifetime; integrated gated-diode electrometer; integrated test structure; NBS-GCR-81-327.

least-squares adjustments; quantum electrodynamics; basic standards; fine-structure constant; fundamental constants; Josephson effect; 20058.

least squares optimization; theoretical spectroscopy; iron group elements; J. Res. 86(1): 33-77; 1981 January-February.

LED; photocell; solar cell; solid state; transistor; tunnel diode; diode; field-effect transistor; III-V compounds; IMPATT diode; integrated circuit; LASER; J. Res. 86(6): 565.570; 1981 November-December.

legal metrology; measurement assurance; metrication; model laws and regulations; packing and labeling; specifications and tolerances; technology transfer; weights and measures; domestic standards; education programs; electronic devices; international recommendations; SP599.

Legendre expansion; molecular potential; quantum chemistry; scattering; electron-molecule collisions; interaction potential; 20055.

legislative history; product liability; rationale statements; scope of standards; standards; standards writing; NBS-GCR-81-347.

length; partially-filled; pipe; slope; stream-depth; unsteady; volume; water; diameter; drain; flow; history; horizontal; NBSIR 81-2249.

length and mass measurements and standards; quantum metrology; quantum physics; temperature and pressure measurements and standards; time and frequency; electrical measurements and standards; NBSIR 80-2165.

length-measuring devices; liquid-measuring devices; measures; scales; specifications; taximeters; tolerances; volume-measuring devices; weighing devices; weights; $H 44,1982$ Edition.

Lennard-Jones fluid; nonequilibrium molecular dynamics; phase changes; irreversible thermodynamics; 19974.

level gauging; propane; radio frequency; NBSIR 79-1600.

levitation calorimetry; specific heat; surface tension; thermophysical properties; tungsten; wetting; adsorption; Auger spectroscopy; convection; ellipsometry; gallium; NBSIR 81-2295.

liability trials; performance testing; regulatory enforcement; implant retrieval and analysis; SP601: 1981 January. 655-666.

library holdings, NBS Library; NBS periodicals; periodicals; proceedings; serials; standards; transactions; annual reports; diffusion in metals; fire; journals; NBSIR 81-2226.

Lieb limits; number of bound states; Schwinger limits; screened Coulomb potential; Sobolev's inequality; Yukawa potential; Bargmann limits; bound-state estimates; J. Res. 86(5): 503-508; 1981 September-October.

$\mathrm{LiF} ; \mathrm{MgF}_{2} ; \mathrm{MgO} ; \mathrm{NaCl} ; \mathrm{NaF} ; \mathrm{SrF}_{2} ;$ volume and surface impurities; $\mathrm{BaF}_{2} ; \mathrm{CaF}_{2}$; extrinsic and intrinsic infrared absorption; infrared wavelength modulation; $\mathrm{LaF}_{3}$; laser windows; SP620: 1981 October. 29.43.

life cycle; cash flow; economic analysis; fire safety; fire safety evaluation system; health care facilities; NBS-GCR-79-186-1. 
life-cycle cost; recommended practice; savings-to-investment ratio; benefit-cost analysis; building economics; cost effective; economic analysis; energy conservation; investment analysis; NBSIR 81-2397.

life-cycle cost analysis; masonry; mass; building design; building economics; energy conservation; exterior wall; HVAC calculations; insulation; BSS134.

life-cycle costing; solar economics; solar energy; economic optimization; NBSIR 81-2342.

life-cycle costs; passive solar; regional analysis; thermal efficiency; windows; building economics; daylighting; energy conservation; engineering economics; NBSIR 81-2248.

life cycle management; requirements; software quality assurance (SQA); specification; configuration management (CM); cross reference; data structure; design, documentation; functional; historical file; keyword; SPS00-83: 1981 November. 101-106.

life safety; accessibility; building accessibility; building research; fire safety; handicapped; NBSIR 81-2245.

life safety; refuge; board and care homes; building codes; building fires; evacuation; fire alarm systems; fire safety; handicapped; 20727.

lifestyle; research; site planning; sociology; standards; children; design; environmental psychology; families; housing; interiors; NBS-GCR-81-320.

lifetime; power-device grade silicon; thyristor material characterization; transient capacitance techniques; deep-level measurements; deep-level transient spectroscopy; NBSIR 81-2325.

light and ventilation; solar systems; space heating and cooling; training programs; building codes; building officials; codes and standards; convective loops; greenhouses; heat exchangers; SP608: 1981 May. 159-166.

light elements; soft $\mathrm{x}$ rays; spectral distortion; windowless detectors; energy dispersive; SP604: 1981 June. 71-95.

light-emitting diodes; YAG laser; laser simulator; TN1031, Revised.

light-emitting diodes; YAG laser; laser simulator; TN1031.

lighting; lighting levels; superthreshold visibility; vision; conspicuity; contrast; illumination; NBSIR 81-2231.

lighting; man/environment research; noise; office building; postoccupancy evaluation; questionnaire; temperature; ventilation; windows; energy conservation; BSS130.

lighting control; solar heat gain; window management; daylight; energy conservation; fenestration design; illumination; NBSIR 80 2147.

lighting design; lighting research; lighting survey; building program; NBS-GCR-81-333.

lighting levels; superthreshold visibility; vision; conspicuity; contrast; illumination; lighting; NBSIR 81-2231.

lighting measurements; performance data for commercial office buildings in New England; solar energy in commercial buildings; building models, computer; energy conservation in commercial buildings; energy conservation, user acceptance; $B S S 133$.

lighting research; lighting survey; building program; lighting design; $N B S-G C R-81-333$.

lighting survey; building program; lighting design; lighting research; NBS-GCR-81-333.

light scattering; nematic liquid crystal; photo-receptor membrane vesicles; random coil polymers; amphoteric latex; dynamic light scattering; J. Res. 86(6): 571-590; 1981 November-December.

lights, warning; sirens; sound level; warning light; warning signals; conspicuity; effective intensity; emergency warning lights; flashing lights; SP480-37.

light wave; nondestructive evaluation (NDE); optical holographic interferometry; technical literature; flaw detection; holography; inhomogeneities; NBS-GCR-81-318.

lime-alumina-silica-water; minerals; thermodynamic data; enthalpy; enthalpy of formation; entropy; equilibrium constant for formation; Gibbs energy function; Gibbs energy of formation; heat capacity; JPCRD 10(3): 575-670; 1981.

$\mathrm{LiNbO}_{3}$ coatings; Q-switch; surface damage; antireflection coatings; coating damage; laser damage; SP620: 1981 October. 300-304.

linear accelerator; neutron imaging techniques; position-sensitive proportional counters; Van de Graaff accelerator; $14 \mathrm{MeV}$ neutron energy; 20724.

linear electric motors; linear free-displacer cryorefrigerator; linear resonant machinery; phasor notation; thermodynamic and dynamic analyses; displacer and piston drives; SP607: 1981 May. 57-69.

linear equation; method of moments; parallel plate waveguide; quasi electrostatic; TEM cell; electromagnetic compatibility (EMC); Green's function; integral equation; TN1028.

linear free-displacer cryorefrigerator; linear resonant machinery; phasor notation; thermodynamic and dynamic analyses; displacer and piston drives; linear electric motors; SP607: 1981 May. 57-69.

linear free energy; liquid chromatography; molecular substituent; oil shale; organoarsenic; organotin; retention index; speciation; atomic absorption; energy-related process fluids; leachate; SP618: 1981 November. 197-216.

linear induction accelerator; high current relativistic electron beam generator; 19985

linearity; offset; analog-to-digital converter; calibration service; data converter; differential linearity; digital-to-analog converter; error measurement; gain; input noise; TNI145.

linear least-squares; $\mathrm{x}$-ray analysis; digital filter; filter-fit method; SP604: 1981 June. 273-296.

linear macromolecule; melt; polyethylene; crystal; crystallinity; density; enthalpy; entropy; fusion; Gibbs energy; glass transition; heat capacity; JPCRD 10(1): 119.152; 1981.

linear macromolecule; melt; polypropylene; syndiotactic; atactic; crystal; crystallinity; density; enthalpy; entropy; fusion; Gibbs energy; glass transition; heat capacity; isotactic; JPCRD 10(4): $1051.1064 ; 1981$

linear macromolecule; melt; selenium; calorimetry; crystal; enthalpy; entropy; floor temperature; fusion; Gibbs energy; glass transition; heat capacity; JPCRD 10(1): 89-118; 1981.

linear macromolecule; poly(oxymethylene)s; poly(oxyphenylene)s; poly(oxypropylene); crystal; crystallinity; density; enthalpy; entropy; fusion; Gibbs energy; glass transition; heat capacity; JPCRD 10(4): 1001-1049; 1981 .

linear magnetic suspension; long lifetime; space application; Stirling cycle; clearance seals; cryogenics; hydrodynamic gas bearing; SP607: 1981 May. 103-115.

linear motor/generator; magnetic bearings; Stirling Cycle; clearance seal; gas compressor; SP607: 1981 May. 146-153.

linear resonant machinery; phasor notation; thermodynamic and dynamic analyses; displacer and piston drives; linear electric motors; linear free-displacer cryorefrigerator; SP607: 1981 May. 57. 69.

line broadening function; plasma dispersion function; Voigt function; argand diagram; complex error function; continued fraction; Dawson's function; FORTRAN subroutine; Fresnel integrals; key values; J. Res. 86(6): 661-686; 1981 November-December.

lineshapes; optical pumping; resonance Raman; spectroscopy; strong fields; damping theory; kinetics; lasers; 20039.

line strengths; nickel; oscillator strengths; systematic trends; transition probabilities; allowed transitions; cobalt; $f$-values; iron; isoelectronic sequence; JPCRD 10(2): 305-566; 1981.

line strengths; oscillator strengths; spectral lines; transition probabilities; wavelengths; atom; atomic ion; intensities; NSRDS. NBS68.

link protocols; local survivability; message delay; network simulation; network throughput; survivability; alternate routing; communications networks; CROSSFIRE; NBS-GCR-81-336.

$\mathrm{LiNO}_{2} ; \mathrm{NaNO}_{2}$; osmotic coefficients; $\mathrm{RbNO}_{2}$; solutions; standard reference data; thermodynamic properties; activity coefficients; alkali metal nitrite salts; aqueous; critical evaluation; $\mathrm{CsNO}_{2}$; electrolytes; excess Gibbs energy; $\mathrm{KNO}_{2} ; J P C R D$ 10(3): 767-778; 1981.

liquefaction; mercury; nickel; process waters; selenium; solvent refined coal; titanium; trace elements; arsenic; chromium; coal hydrogenation; cobalt; SP618: 1981 November. 21-38.

liquefied natural gas; refrigeration recovery; chemical engineering; cold utilization; cryogenic energy conservation; 20068.

liquid; pressure; propane; sound; specific heat ratios; speed; temperature; velocity; adiabatic compressibility; J. Res. 86(2): 165 . 
170; 1981 March-April.

liquid breakdown; $\mathrm{SF}_{6}$; space charge; transformer oil; cables; composite insulation; dc fields; high voltage; incipient fault; insulation; NBSIR 81-2334.

liquid chromatograph; liquid stream; mass spectrometer; tapered concentrator wire; elongated capillary tube; interface; U.S. Patent 4.281 .246$.

liquid chromatography; mass spectrometry; bioavailability; element speciation; gas chromatography; ionization processes; SP618: 1981 November. 120-133.

liquid chromatography; molecular substituent; oil shale; organoarsenic; organotin; retention index; speciation; atomic absorption; energy-related process fluids; leachate; linear free energy; SP618: 1981 November. 197-216.

liquid chromatography; nitrobenzene; organotin cations; dichlone; dual-electrode detector; electrochemical detector; 20728.

liquid helium; packed bed heat exchanger; balloon inflation; cryogenic helium balloon inflation; heat transfer; 20017.

liquid helium; permeability of composites; superconducting power transmission; cable cool-down; cable permeability; counter-flow cool-down; heat flux meters; NBSIR 80-1637.

liquid helium; serviceable; superconducting device; thermal coupling; cryogenic refrigerator; Josephson technology; SP607: 1981 May. 82-92.

liquid level; LNG tanks; custody transfer; gaging; 20770.

liquid mass fraction; liquid volume fraction; phase equilibria; pure fluids; coexistence densities; critical density; critical point; NBSIR 80-1631.

liquid-measuring devices; measures; scales; specifications; taximeters; tolerances; volume-measuring devices; weighing devices; weights; length-measuring devices; H44, 1982 Edition.

liquid methane; LNG; densimeter; density reference system; TN104I.

liquid methane; LNG; densimeter; density reference system; TN1035.

liquid penetrants; magnetic particles; microwaves; nondestructive evaluation; radiography; tire inspection; ultrasonics; visual-optical; acoustic emission; eddy currents; 20762.

liquid phase; single crystal; defects; epitaxial regrowth; laser irradiation; SP620: 1981 October. 222-226.

liquid phase regrowth; metal surfaces; nonuniformities; laser irradiation; SP620: 1981 October. 210-221.

liquids; pesticides, solids; solubility; vapor pressure; alkanes; aromatics; critical review, evaluated data; gases; halogenated hydrocarbons; Henry's law constants; JPCRD 10(4): 1175-1199; 1981.

liquids; Pockels effect; solids; dielectrics; electro-optics; gases; high voltage; insulation; interfaces; Kerr effect; NBSIR 81-2275.

liquid stream; mass spectrometer; tapered concentrator wire elongated capillary tube; interface; liquid chromatograph; U.S. Patent 4,281,246.

liquid volume fraction; phase equilibria; pure fluids; coexistence densities; critical density; critical point; liquid mass fraction; NBSIR 80-1631.

listening room; corona noise; environmental noise; high voltage transmission lines; human response to noise; 20030.

literature review; medical device standards; public participation programs; standards development; voluntary standards; bibliography; annotated, consumer representation; NBSIR 81-2336.

literature reviews; stoves; wood; bibliographies; chimneys; combustion; fire safety; heating equipment; NBS-GCR-80-298.

lithium; nuclear track technique; triton particles; abundances; alpha particles; cellulose nitrate detector; isotopic; 19982.

lithium; osmotic coefficient; potassium; rubidium; sodium; solutions; thermodynamic properties; activity coefficient; cesium; critical evaluation; electrolyte; excess Gibbs energy; JPCRD 10(3): 671 . 766.

lithium compounds; potassium compounds; protactinium compounds; rubidium compounds; sodium compounds; thorium compounds; uranium compounds; actinium compounds; cesium compounds; enthalpy; entropy; francium compounds; Gibbs energy of formation; heat capacity; TN270-8.

lithium-drifted silicon ( $\mathrm{Si}(\mathrm{Li})$ ) detector; x-ray microanalysis; detector; energy dispersive x-ray spectrometry; Gaussian profiles; SP604: 1981 June. 233-272.

lithium fluoride; mechanical properties; optical properties; ultraviolet transmission; fluorides; forging; laser windows; SP620: 1981 October. 110-116.

lithium isoelectronic sequence; electron impact ionization; helium isoelectronic sequence; hydrogen isoelectronic sequence; 20738.

live loads; load survey; probability; statistics; structural engineering; NBS-GCR-76-81.

live loads; mobile homes; residential surveys; transfer functions; field surveys; fire; fire loads; furnishings; household surveys; NBSIR 80 . 2155.

lixiscope; radioactive $\mathrm{x}$-ray source; rare-earth scintillator screen; CsI; detection efficiency; 20003.

LNG; densimeter; density reference system; liquid methane; TNI04I.

LNG; densimeter; density reference system; liquid methane; TN1035.

LNG; mixtures; PVTx data; revised Kosek and McKinley; cell model; comparisons; computer programs; corresponding states; equation of state; hard sphere; TN1030.

LNG; ships; tanks; volume; dimensions; laser; NBSIR 80-2141.

LNG tanks; custody transfer; gaging; liquid level; 20770.

load calculation; masonry thermal mass; system simulation; building energy analysis; energy conservation for nonresidential buildings; historic preservation and energy conservation; NBSIR 81-2317.

load calculations; building energy analysis; computer modeling; controls; control strategies; energy conservation for nonresidential buildings; NBSIR 81-2277.

loaded antenna; method of moments; TEM horn; time domain measurements; transients in resistivity; conical antenna; EMP; FFT; NBSIR 78-876.

loads; scaffolds; stability; stiffness; strength; structural safety; work surfaces; codes and standards; construction safety; design; finite element; NBSIR 81-2265.

loads (forces); probability distribution functions; reliability; statistical analysis; structural engineering; wind; climatology; 20019.

load survey; probability; statistics; structural engineering; live loads; NBS-GCR-76-81.

local area networks; measurement techniques; protocols; standards; computer based office systems; computer networking; interfaces; NBSIR 80-2154.

local area networks; requirements analysis; carrier sense multiple access; environmental analysis; feasibility study; NBSIR 80-2187.

local area networks, microwave; video teleconferencing; CATV systems; coaxial cable; data communications; fiber optics information systems; integrated communications; SP500-83: 1981 November. 271-276.

local survivability; message delay; network simulation; network throughput; survivability; alternate routing; communications networks; CROSSFIRE; link protocols; NBS-GCR-81-336.

location factors; model building; statistical analysis; applied economics; construction; cost; estimation; NBSIR 81-2250.

locks; architectural barriers; building accessibility; codes and standards; disability; door closers; door openers; doors; ergonomics; functional capacity; handicap; NBSIR 80-2174.

logical swapping; measurement; MVS; paging/swapping; TSO; analysis; data elements; I/O; SP500-83: 1981 November. 343-348.

long-life; refrigerators; reliability; rotary-reciprocating; turbo-Brayton; Vuilleumier; component development; contamination control; cryogenics; helium retention; SP607: 1981 May. 21-29.

long lifetime; space application; Stirling cycle; clearance seals; cryogenics; hydrodynamic gas bearing; linear magnetic suspension; SP607: 1981 May. 103.115.

long-range forecasting; user behavior; workload forecasting; computer networks; SP500-83: 1981 November. 7-14.

long range order; modulated structures; oxides; phase equilibria; diffraction effects; 20046.

long range plan; system requirements; user requirements; computer resource selection; cost; SP500-83: 1981 November. 293-302.

loosening; metal-on-metal prostheses; metal-on-plastic prostheses; femoral component; SP601: 1981 January. 643-653.

loss prevention; modal analysis; property conservation; risk 
management; shock pulse analysis; vibration analysis; acoustic emission analysis; insurance; SP622: 1981 October. 139-151.

loss-rate; mass-loss; n-alkanes; n-carboxylic esters; permeation; polyethylene; effective carbon atom number; NBSIR 80-2131.

loss tangent; moisture; dielectric properties; grain; NBSIR 78-897.

loudness; noise criteria; time-varying noise; duration; general adverse effect of noise; intermittency; NBSIR 81-2377.

low alloy steels; steam turbine component failure; stress corrosion cracking; ultrasonic inspection; United States experiences; SP622: 1981 October. 152.180

low ambient temperature; trip time; branch circuits; circuit breaker; electrical fire; NBSIR 81-2221.

low cost approach; electronic laboratory instruction; 20006

low flush toilets; water closets; water conservation; dual flush toilets; NBSIR 81-2296.

low grade geothermal; radiant floor heating; space heating and cooling; thermal mass; walls; water source heat pump; buildings; construction; energy conservation; ground coupled; hybrid solar energy system; inverted cave; SP608: 1981 May. 69-81.

low income; non-code; policymaking; protestors; solar code; victim role; deed; developers; energy wars; experimental code; field test; guideline; SP608: 1981 May. 173-178.

low power refrigerator; Stirling refrigerator; bellows; compressor; contamination; continuous operation; cryocooler; SP607: 1981 May. 141.145 .

low temperature; magnetic gradiometer; refrigeration; SQUID; Stirling cycle; superconductivity; cryocoolers; SP607: 1981 May. 186.194.

low temperature; mechanical and physical properties; structural alloys; welding; composites; NBSIR 80-1627.

low temperature; mechanical properties; nonmetallics; physical properties; stainless steels; structural alloys; welding; alloys; aluminum alloys; castings; composites; cryogenic properties; NBSIR 81-1645.

low temperature; mechanical properties; polystyrene; polyurethane; thermal insulation; thermal properties; cellular plastic; 20074.

low temperature; mechanical properties; proportional limit; shear strength; tensile strength; yield strength; Young's modulus; compressive strength; elongation; foam; insulation; NBSIR 81-1654.

low temperature; moisture migration; mortar; porous solid; thermal conductivity; concrete; guarded hot plate; NBSIR 81-1651.

low temperature; nonmetallics; physical property; superconductor composites; thermal effect; thermal expansion; 20753.

low temperature; refrigerator; Stirling cycle; cryocooler; cryogenic; helium; SP607: 1981 May. 30-47.

low-temperature; standard reference material; thermal conductivity; glass fiberboard; insulation; NBSIR 81-1639.

low temperature; standard reference material; thermal conductivity; glass fiberblanket; insulation; NBSIR 81-1640.

low temperature; tensile properties; austenitic stainless steel; fracture toughness; 20772.

low temperature materials; materials properties; metals; nonmetallic materials; wind tunnels; cryogenic properties; instrumentation; NBSIR 79-1624.

low temperature mechanical properties; plastic deformation; temperature effects stainless steel alloys; yield stress; cryogenic properties of materials; iron alloys; 20742.

low temperatures; polycrystals; sound velocities; tensor-property averaging; copper; Debye temperature; elastic constants; 20726.

low toxicity; peroxide catalyzed polymerization; tertiary aromatic amines; unsaturated polyesters; vinyl monomers; accelerators; cementing of bone; high reactivity; U.S. Patent 4,284,551.

low x-ray energies; $\mathrm{Si}(\mathrm{Li})$ detectors; dead layer; SP604: 1981 June. 127-139.

lubricating oil; motor oil; petroleum oil; recycled oil; rerefined oil; test procedures; basestock; engine lubricants; 20016.

\section{$\mathbf{M}$}

machine geometry; measuring machine; redundancy; 3-D metrology; ball plate; grid plate; NBSIR 79-1752. machinery monitoring; reliability centered maintenance; energy conservation; Engineering Maintenance Center; SP622: 1981 October. 113-124.

machinery parameters; temperatures; vibrations; base line signatures; frequency spectra; hot alignment; SP622: 1981 October. 220-221.

macromolecules; plastics; polymers; standards; NBSIR 81-2263.

macroprocessor; pattern matching; query processor; software tool; text processor; translation; UNIX; NBSIR 80.2182.

magnetic; mechanical; monograph; optical; physical; properties data; radiation damage; rock salt; sodium chloride; chemical; electrical; geological; Monogr. 167.

magnetic bearings; Stirling Cycle; clearance seal; gas compressor; linear motor/generator; SP607: 1981 May. 146-153.

magnetic-field integral equation; scattering of electromagnetic waves; spurious resonances; augmented electric- and magnetic-field integral equations; electric-field integral equation; electromagnetic theory; 20761.

magnetic-field integral equation; scattering of electromagnetic waves; spurious resonances; augmented electric- and magnetic-field integral equations; electric-field integral equation; electromagnetic theory; 20760.

magnetic fields; calibration; calibrators; electric fields; electric field strength; electric power transmission; field strength; NBSIR 77 . 1311.

magnetic fields; nonionizing radiation; regional calibration laboratories; standards; techniques; calibration; dosimetry; electric fields; electromagnetic interference; exposure history; instruments; SP613.

magnetic gradiometer; refrigeration; SQUID; Stirling cycle; superconductivity; cryocoolers; low temperature; SP607: 1981 May. 186-194.

magnetic moments; photon decays; quarks; angular distributions; charmed mesons; dipole transitions; 20040.

magnetic particles; microwaves; nondestructive evaluation; radiography; tire inspection; ultrasonics; visual-optical; acoustic emission; eddy currents; liquid penetrants; 20762.

magnetic property; stability; standards; superconductor; critical current; electrical property; NBSIR 80-1642.

magnetic susceptibility; order-disorder; titanium; transmission electron microscopy; alloy phase diagram; aluminum; 20736

magnetism; nickel; polarized electrons; electron scattering; 19970

magnetism; phase diagram; potassium; conductivity; exchange interaction; ferrite; hyperfine field; 20043.

magnetohydrodynamics; mass spectrometry; potassium slag; thermod ynamics; vaporization; chemical activity; NBSIR 81-2293.

magnetron sputtering; pulsed $\mathrm{HF} / \mathrm{DF}$ laser damage thresholds; silicon films; absorption; evaporation; hydrogen and oxygen impurity; SP620: 1981 October. 305-312.

maintain accuracy; reduce cost; reduce input; standard evaluation technique; BLAST; building energy performance standards; computer modeling; SP608: 1981 May. 113-122.

maintenance; model codes; occupancy; performance levels; regulation; standards; building rehabilitation; codes; comparative analysis; existing buildings; housing codes; NBSIR 80-2081.

maintenance; public buildings; state government; cost; construction; energy audit; energy conservation; Illinois; SP608: 1981 May. 123. 132.

maintenance effectiveness; on-board monitoring system; aircraft hydraulic systems; cost reduction; energy savings; failure detection; failure prevention; flight safety; fluid system components; SP622: 1981 October. 195-219.

man/environment research; noise; office building; post-occupancy evaluation; questionnaire; temperature; ventilation; windows; energy conservation; lighting; $B S S 130$.

man-hour expenditure ratio; trend analysis; unlimited application; fibrescope; health condition; SP622: 1981 October. 74-81

manuals; models; simulation; documentation; SP500-73.

marginal price; net present value; residential water conservation; wastewater treatment; water rate schedules; water-saving devices; benefits; cost-effective; costs; economic analysis; economic decision framework; energy conservation; NBSIR 81-2304. 
marine machinery bearings; microprocessor based shock pulse analyzer; multiplexing bearing monitor channels; prediction of bearing failure; shock pulse bearing monitoring; automatic alarm of bearing malfunction; SP622: 1981 October. 126.136.

marine structures; piezoelectric polymer material; vegetable and animal life; antifouling coating; film; U.S. Patent 4,283,461.

marine structures; piezoelectric polymer material; vegetable and animal life; antifouling coating; film; U.S. Patent 4,297,394.

marketed systems; standard; cost/benefit analysis; flexible disk cartridge; flexible disk system; information interchange; NBS.GCR80-306.

Markov processes; performance prediction; queueing models; simulation; validation; analytic models; communication networks; flow control; SPS00-83: 1981 November. 277.290.

masonry; mass; building design; building economics; energy conservation; exterior wall; HVAC calculations; insulation; lifecycle cost analysis; BSSI34.

masonry; plumbing; solar; steel; structural systems; test methods; wood; building rehabilitation; concrete; electrical; evaluation; HVAC; NBSIR 81-2289.

masonry; plumbing; steel; structural systems; test methods; wood; building rehabilitation; concrete; electrical; evaluation; HVAC; NBSIR 80-2171.

masonry; prohibitions; re-examination; thermal storage; wood structure; codes; concrete; SP608: 1981 May. 195.200.

masonry; standards; structural engineering; building; building codes; building design; earthquakes; engineering; NBSIR 80-2111-5.

masonry thermal mass; system simulation; building energy analysis; energy conservation for nonresidential buildings; historic preservation and energy conservation; load calculation; NBSIR 81 . 2317.

mass; building design; building economics; energy conservation; exterior wall; HVAC calculations; insulation; life-cycle cost analysis; masonry; BSS134.

mass comparator; radiation; radiocarbon; soft aluminum; windows; air pollution; automation; buildings; certified reference materials; computers; construction; data converter; DIM/NBS 65(4): 1.28; 1981 .

mass difference effects; mixture viscosity; size difference effects; corresponding states; Enskog theory; J. Res. 86(6): 597.604; 1981 November-December.

mass flow; diffusion flames; entrainment; fire plumes; flow rate; $N B S$ GCR-80-294.

mass flow; measurement; metering; thermodynamics; fluid flow; gas; instrumentation; 20751.

mass-loss; n-alkanes; n-carboxylic esters; permeation; polyethylene; effective carbon atom number; loss-rate; NBSIR 80-2131.

mass loss; stars, emission line; stellar chromospheres; circumstellar shells; late type stars; 20057.

mass spectra; Master Analytical Scheme; organic compounds in water; deuterated compounds; isotopic purity; NBSIR 80-2160.

mass spectrometer; partial discharge; sulfur hexafluoride; swarm data; water vapor; corona; decomposition; electrical insulation; gas chromatograph; inception voltages; NBSIR 81-2242.

mass spectrometer; tapered concentrator wire; elongated capillary tube; interface; liquid chromatograph; liquid stream; U.S. Patent $4,281,246$.

mass spectrometer measurement; moisture; moisture generators; moisture sensors; quality control; reliability of semiconductor devices; semiconductor devices; analysis of moisture content; SP4(X)-69.

mass spectrometry; bioavailability; element speciation; gas chromatography; ionization processes; liquid chromatography; SP618: 1981 November. 120.133.

mass spectrometry; mercury; methylation; environment; GC.MS; heavy metals; lead; SP618: 1981 November. 54-64.

mass spectrometry; nuclear magnetic resonance spectroscopy; phenobarbital; phenytoin; primidone; reference material; serum base modification; serum matrix; anticonvulsants; antiepilepsy drugs; blood analysis; chromatography, gas; chromatography, liquid; drug standards; enzyme immunoassay; epilepsy; ethosuximide; SP260-72. mass spectrometry; phosphorus; radicals; Raman spectroscopy; temperature; flame inhibition; H-atoms; kinetics; NBSIR 80-2169.

mass spectrometry; potassium slag; thermodynamics; vaporization; chemical activity; magnetohydrodynamics; NBSIR 81-2293

mass spectrometry; reaction rates; reactor technology; heats of formation; inorganic gases; ion-molecule reactions; 19965.

mass spectrometry; slag; transpiration; vapor transport; coal gasification; high temperature; NBSIR 80-2178 (DoE).

mass spectrometry; standard reference materials; analysis; certification; isotope dilution mass spectrometry; 20024.

mass/weight; metric controversy; newton; AAPT vs. NBS; kilogram 20023.

Master Analytical Scheme; organic compounds in water; deuterated compounds; isotopic purity; mass spectra; NBSIR 80-2160.

material properties; transmission; absorption; laser windows; SP620: 1981 October. 144-158.

materials; measurement; office building; phase diagrams; radioactivity; research; sediment; soil; alloy; carbon; computers; energy; fine structure; interface; DIM/NBS 65(8): 1.28; 1981.

materials; measurement; permeability; radon; transport; buildings; concrete; diffusion; exhalation; TN1139.

materials; process parameters; research recommendations; coal; control; fossil energy; NBSIR 81-2348.

materials; properties; research materials; standard reference materials; analysis; characterization; composition; SP260, 1981-83 Edition.

materials data; standard reference data; technical activities 1980; thermochemical and thermophysical data; data compilation; energy and environmental data; evaluated data; NBSIR 81-2206.

materials market; oil analysis; recycled materials; refused-derived-fuel; rerefined oil; energy-from-waste; NBSIR 80-2178.

materials performance; solar collector; standards; test methods; field survey; industrial process heat; NBSIR 81-2381.

materials properties; metals; nonmetallic materials; wind tunnels; cryogenic properties; instrumentation; low temperature materials; NBSIR 79-1624.

materials properties; research; standards; technology transfer; basic measurements and standards; building technology; calibration methods; computer standards and guidelines; energy research; environmental measurements; fire safety; industrial productivity; SP600.

materials research; materials science; metallurgy; polymers research; corrosion; fracture and deformation; glass and ceramics research; NBSIR $81-2408$.

materials science; metallurgy; polymers research; corrosion; fracture and deformation; glass and ceramics research; materials : research; NBSIR $81-2408$.

mathematical economics; noncooperative games; total orders aggregation; equilibrium; game theory; J. Res. 86(4): 383-391; 198 July-August.

mathematical model; optimization; cost savings; energy conservation; HVAC systems; innovation; SP608: 1981 May. 21.35.

mathematical model; plastic; polyurethane; room fires; wood; crib fires; experiments; NBSIR 80-2054, Vols. I and II.

mathematical model; regulation; theory of games; inspection; J. Res. 86(2): 193-215; 1981 March-April.

mathematical model; roofing; bitumen application temperatures; bituminous roofing; built-up roofing; cooling of roofing bitumens; TN1135.

mathematical model; standard transaction; transaction driven system; bubble memory; SPS00-83: 1981 November. 259-265.

mathematical modeling; prestressed concrete; prestressing steel; stress corrosion; concrete; corrosion; general corrosion; NBSIR 81-2390.

mathematical models; model confidence; model credibility; policy models; sensitivity analysis; validation; assessment; composite models; data quality; energy models; SP616.

mathematical models; reinforced concrete; steel structures; wood structures; fire endurance; fires; NBSIR 80-2188.

mathematical models; room fires; compartment fires; computer programs; fire extinguishment; flashover; ignition; NBS-GCR-81344.

mathematical models; room fires; smoke movement; tenability limits; 
combustion products; compartment fires; egress; fire detection; fire growth; hazard analysis; NBSIR 80-2172.

mathematical optimization; network flow; building evacuation; J. Res. 86(5): 509.513; 1981 September-October.

matrix isolation; normal coordinate analysis; photodissociation; F-atom reactions; infrared spectrum; 19986.

matrix isolation; photodecomposition; reaction mechanism; $t$-2 fluorovinyl; acetylene; F atoms; hydrogen bonding; infrared spectrum; 20036.

mattresses; prisons; smoke production; bedding; beds; compartment fires; fire tests; health care facilities; heat release rate; hospitals: NBSIR 80-2186.

mattresses; self-extinguishment; smoldering; upholstered furniture; cigarettes; flammability; ignition; NBSIR 81-2363.

maturity; mortar; regression analysis; strength prediction; temperature effects; compressive strength; concrete; curing temperature; early age; final set; hydration; initial set; laboratory testing; NBSIR 81 2244.

maximum refrigerator efficiency; perfect gas analysis; refrigerator; component efficiency; cryogenics; efficiency; helium; 20771.

maxmin; minmax; multiple comparison; multivariate analysis; peak areas; principal components; NBSIR 81-2222.

$\mathrm{m}$-azidobenzoic acid; nitrocellulose; quantitative differential thermal analysis; thermal explosion theory; thermal hazards; thermal instability; adiabatic calorimetry; azidotriphenylmethane; decomposition reactions; differential scanning calorimetry; heat conduction calorimetry; kinetics of heat evolution; NBSIR 80-2018 (DOT).

meaningfulness; method; pictogram; response; symbol; understandability; communication; evaluation method; fire-safety; hazard warnings; NBSIR 80-2088.

measurand; reliability; sensor; transducer; ATE; automatic test equipment; calibration; data acquisition; durability; dynamic calibration; environmental testing; evaluation; SP615.

measurement; metering; thermodynamics; fluid flow; gas; instrumentation; mass flow; 20751.

measurement; metrology; National Bureau of Standards; power radiation; semiconductor; technology; calibration; electrical; electronics; engineering; hazards; SP611.

measurement; MVS; paging/swapping; TSO; analysis; data elements; I/O; logical swapping; SP500-83: 1981 November. 343-348.

measurement; office building; phase diagrams; radioactivity; research; sediment; soil; alloy; carbon; computers; energy; fine structure interface; materials; DIM/NBS 65(8): 1-28; 1981.

measurement; optics; polarized light; electric field; electro-optics; Kerr effect; 19962.

measurement; parameter; physics; solar collector; space charges; technical competence; cement and concrete; cover plates; earth's tilt; electric; fire; fracture toughness; $D I M / N B S$ 65(3): 1-24; 1981

measurement; permeability; radon; transport; buildings; concrete; diffusion; exhalation; materials; TN1139.

measurement; $\mathrm{R}$ value; standard reference material; therma resistance; fiber-glass; fibrous glass; insulation; NBSIR 80-2091.

measurement; shock and vibration; angular acceleration; angular accelerometer; calibration; instrumentation; NBSIR 81-2337.

measurement; solar collector; standards; thermal performance; uncertainty; collector rating; TN1140.

measurement accuracies; neutrons; radiation dosimetry; radiation protection; radiation standards; $\mathbf{x}$ rays; beta rays; Federal and State laboratories; gamma rays; instrument calibrations; intermediate calibration laboratories; ionizing radiation measurements; SP603.

measurement assurance; measurement services; standards; traceability; calibration; SP250, 1980 Edition.

measurement assurance; metrication; model laws and regulations; packing and labeling; specifications and tolerances; technology transfer; weights and measures; domestic standards; education programs; electronic devices; international recommendations; legal metrology; SP599.

measurement errors; transmission lines; vertical current density; Wilson plates; high voltage dc; NBSIR 81-2410.

measurement infrastructure; metrology; technologies; technology transfer; Brazil; industrialization; international cooperation; NBSIR 80-1972.

measurement methods; oil shale; standard reference materials; water pollution control; 20009.

measurement methods; performance criteria; project summaries; technical bases; building research; building technology; codes; criteria; SP446-5.

measurement procedures; susceptibility and emission measurements; transverse electromagnetic cell; TN1013.

measurements; oils, PCB's; standards; atomic weight; coal; computers; drill failure; Faraday; firefighting; hot atom; industrial innovation; DIM/NBS 65(6): 1.24; 1981 .

measurements; optical communications; optical fibers; bibliography TN1043.

measurements; photovoltaic modules; photovoltaics; reference cells; solar cells; terrestrial solar simulators; test methods; calibration; combined photovoltaic/thermal modules; NBS-GCR-80-279.

measurements; quadrupoles; susceptibility; TEM cell; analysis; dipole moments; electrically small objects; emission; TN1040.

measurements; shielded balanced transmission lines; time domain; transmission line model; waveform; bit error rate; coaxial transmission lines; digital communication impulse response; instrumentation; TN1042.

measurements; space charge; calibration; dc transmission lines; electric field; electric field meters; electric field strength; electric power transmission; NBSIR 80-2177.

measurements; superconductivity; time domain; electronics; Josephson effect; 20070.

measurement science and technology; standardization in developing countries; Sudan; weights and measures regulation; Agency for International Development; Arab League Organization for Standardization and Metrology; chemical industry standards; cotton standards; food standards; NBSIR 80-2020.

measurement services; standards; traceability; calibration measurement assurance; SP250, 1980 Edition.

measurements of temperature; plasma effects; pulsed laser beams; 10.6 $\mu \mathrm{m}$; attenuators; beam profile; calorimetry; fluence; heat flow; irradiance; NBSIR 80-1628.

measurement systems; metric system; modernized metric system; SI; weights and measures; International System of Units; SP304, Revised August 1981

measurement systems; metric system; modernized metric system; SI weights and measures; International System of Units; SP304A, Revised August 1981.

measurement techniques; photovoltaics; solar cells; test pattern; defect identification; laser scanner; NBSIR 80-2181.

measurement techniques; protocols; standards; computer based office systems; computer networking; interfaces; local area networks; NBSIR 80-2154.

measurement technology; microelectronics; semiconductor devices; semiconductor materials; semiconductor process control; silicon; electronics; integrated circuits; NBSIR 79-1591-5.

measurement technology; microelectronics; semiconductor devices; semiconductor materials; semiconductor process control; silicon, electronics; integrated circuits; NBSIR 79-1591-4.

measurement technology; microelectronics; semiconductor devices; semiconductor materials; semiconductor process control; silicon; electronics; integrated circuits; NBSIR 80-2006-3.

measurement technology; microelectronics; semiconductor devices; semiconductor materials; semiconductor process control; silicon; electronics; gallium arsenide; integrated circuits; NBSIR 80-2006-4.

measurement technology; microelectronics; semiconductor devices; semiconductor materials; semiconductor process control; silicon; electronics; integrated circuits; NBSIR 81-2230.

measurement technology; microelectronics; semiconductor devices; semiconductor materials; semiconductor process control; silicon electronics; integrated circuits; NBSIR 81-2230-3.

measures; scales; specifications; taximeters; tolerances; volumemeasuring devices; weighing devices; weights; length-measuring devices; liquid-measuring devices; H44, 1982 Edition.

measuring machine; redundancy; 3-D metrology; ball plate; grid plate; 
machine geometry; NBSIR 79-1752.

mechanical; monograph; optical; physical; properties data; radiation damage; rock salt; sodium chloride; chemical; electrical; geological; magnetic; Monogr. 167.

mechanical; seismic coefficients; architectural; electrical; elevators; NBSIR 80-2111-8.

mechanical and physical properties; structural alloys; welding; composites; low temperature; NBSIR 80-1627.

mechanical components; SYNTHANE pilot plant; vibration monitoring; coal conversion; SP622: 1981 October. 274-288.

mechanical equipment efficiency ratings; minimum efficiency standards; space cooling requirements; space heating requirements; energy efficiency standards; engineering-economic analysis; heat pump; NBSIR 81-2241.

mechanical fatigue; metal prostheses; stress corrosion; fatigue corrosion; internal fixation devices; SP601: 1981 January. 201-222.

mechanical heart; valve prostheses; valvular dysfunction; SP601: 1981 January. 269-282.

mechanical properties; morphology; polymer; stress relaxation; UHMWPE; ultra high molecular weight polyethylene; creep; failure; fatigue; NBSIR 81-2209 (FDA).

mechanical properties; niobium-tin; scaling; strain; superconducting wire; critical current; 20066.

mechanical properties; nonmetallics; physical properties; stainless steels; structural alloys; welding; alloys; aluminum alloys; castings; composites; cryogenic properties; low temperature; NBSIR 811645 .

mechanical properties; optical properties; ultraviolet transmission; fluorides; forging; laser windows; lithium fluoride; SP620: 1981 October. 110-116.

mechanical properties; polystyrene; polyurethane; thermal insulation; thermal properties; cellular plastic; low temperature; 20074.

mechanical properties; proportional limit; shear strength; tensile strength; yield strength; Young's modulus; compressive strength; elongation; foam; insulation; low temperature; NBSIR 81-1654.

mechanical properties; quality assurance; stress analysis; structural safety; fracture control; fracture mechanics; NBSIR 79-1623.

mechanical systems; building envelope; building performance data; computer data acquisition; energy conservation in commercial buildings; energy monitoring and control systems; 'NBSIR 81-2358. mechanisms of growth; crystal; growth of crystals; 20044.

medical device standards; public participation programs; standards development; voluntary standards; bibliography; annotated, consumer representation; literature review; NBSIR 81-2336.

medium speed diesel engine; piston ring wear; turbocharging monitoring; crankshaft bearing safety; SP622: 1981 October. 97-112.

melt; polyethylene; crystal; crystallinity; density; enthalpy; entropy; fusion; Gibbs energy; glass transition; heat capacity; linear macromolecule; JPCRD 10(1): 119.152; 1981.

melt; polypropylene; syndiotactic; atactic; crystal; crystallinity; density; enthalpy; entropy; fusion; Gibbs energy; glass transition; heat capacity; isotactic; linear macromolecule; JPCRD 10(4): 1051 1064; 1981.

melt; selenium; calorimetry; crystal; enthalpy; entropy; floor temperature; fusion; Gibbs energy; glass transition; heat capacity; linear macromolecule; JPCRD 10(1): 89.118; 1981 .

melt threshold; metal deposition; pulsed laser damage; slip threshold; thermal diffusion length; thermal stress; cold work; crystalline disorder; defect damage; diamond-machined mirrors; Drude absorption; electron-beam melting; laser waveform; SP620: 1981 October. 201-209.

mercuric iodide detectors; $x$-ray spectra; $x$-ray spectrometry; energy resolution; SP604: 1981 June. 45-59.

mercury; methylation; environment; GC-MS; heavy metals; lead; mass spectrometry; SP618: 1981 November. 54-64.

mercury; nickel; process waters; selenium; solvent refined coal; titanium; trace elements; arsenic; chromium; coal hydrogenation; cobalt; liquefaction; SP618: 1981 November. 21-38.

mercury; organomercury; plasmids; silver; toxic heavy metals; antimony; arsenic; bacterial resistances; cadmium; detoxification; SP618: 1981 November. 301-324. mercury; preconcentration; air analysis; diffusion; hollow tubes; SP618: 1981 November. $134-145$.

message delay; network simulation; network throughput; survivability; alternate routing; communications networks; CROSSFIRE; link protocols; local survivability; NBS-GCR-81336.

message delay; network throughput; survivability; alternate routing; communications networks; distributed control; NBSIR 81-2414.

metabolism; microcalorimetry; radiation; calorimetry; cardiac pacemakers; clinical chemistry; heat; 20015.

metal accumulation; microbial; radium; uranium; cesium; SP618: 1981 November. 274.286.

metal allergy; metal sensitivity; reactions to implants; hip hemiarthroplasties; SP601: 1981 January. 299-321.

metal-amino acid reactions; stainless steel; titanium; Co-Cr.Mo; CoNi-Cr-Mo; corrosion; dental alloys; 19988.

metal chelates; metallo-complexes; origin of pollutants; pollutant properties; synfuel; fossil porphyrins; fuel conversion; fuel extraction; heterocyclics; SP618: 1981 November. 9.20.

metal complex; porphyrin; shale oil; speciation; trace metals; chromatography; direct current plasma; hydrophilic; hydrophobic; SP618: 1981 November. 105-119.

metal complexation; nitrogen bases; oil shale; retort water; speciation; trace metals; alkylpyridines; dissolved organic compounds; gas chromatography/mass spectrometry; hydrophobic bases; SP618: 1981 November. 93-104.

metal deposition; pulsed laser damage; slip threshold; thermal diffusion length; thermal stress; cold work; crystalline disorder; defect damage; diamond-machined mirrors; Drude absorption; electron-beam melting; laser waveform; melt threshold; SP620: 1981 October. 201.209.

metallo-complexes; origin of pollutants; pollutant properties; synfuel; fossil porphyrins; fuel conversion; fuel extraction; heterocyclics; metal chelates; SP618: 1981 November. 9-20.

metal(loid) complexes; organometallic compounds; trace element standards; waste cycling; bacterial mobilization; biogeochemical cycles; biological uptake; cellular toxicity.essentiality mechanisms; chromatography; element-selective speciation; environmental monitoring; fossil fuels; SP618.

metallurgical analysis; prosthetic devices; implant retrieval analysis; joint prostheses; SP601: 1981 January. 559-572.

metallurgically-bonded; metals; plastic-bonded; soils; telephone cables; underground; alloys; corrosion; NBSIR 81-2243.

metallurgy; polymers research; corrosion; fracture and deformation; glass and ceramics research; materials research; materials science; NBSIR 81-2408.

Metallurgy Division; Polymers Science and Standards Division; Reactor Division; Center for Materials Science; Ceramics Glass and Solid State Science Division; Chemical Stability and Corrosion Division; Fracture and Deformation Division; FY80 technical activities; NBSIR 80-2108.

metal mirror; polishing; polycrystalline material; residual stress; sapphire; specular reflectance; surface analysis; surface integrity; surface roughness; crystallographic anisotropy; crystallographic perfection; initial permeability; SP620: 1981 October. 171-179.

metal-on-metal prostheses; metal-on-plastic prostheses; femoral component; loosening; SP601: 1981 January. 643.653.

metal-on-plastic prostheses; femoral component; loosening; metal-onmetal prostheses; SP601: 1981 January. 643.653.

metal-oxide-semiconductor (MOS); secondary ion mass spectrometry (SIMS); semiconductor devices; sputtering; $\mathrm{x}$-ray photoelectron spectroscopy (XPS, ESCA); Auger electron spectroscopy (AES); SP400-67.

metal prostheses; stress corrosion; fatigue corrosion; internal fixation devices; mechanical fatigue; SP601: 1981 January. 201-222.

metal-reduction; metal-sulfides; mineral-oxidation; molybdenum; oil shale; Sulfolobus; Thiobacillus; uranium; chemolithotrophicbacteria; copper; iron; leaching; SP618: 1981 November. 264-273.

metals; microorganisms; standard reference materials; bacteria; biodeterioration; biotransformations; deterioration of materials; element volatilization; fungi; NBSIR 81-2246. 
metals; mining industry; public health impacts; residues; toxic elements; availability; environmental contamination; extraction tests; fossil fuels; laboratory characterization; SP618: 1981 November. 1.8.

metals; nonmetallic materials; wind tunnels; cryogenic properties; instrumentation; low temperature materials; materials properties; NBSIR 79-1624.

metals; orthopaedic; polymers; retrieval; analysis; biological; cardiovascular; implants; SP601.

metals; oxygen atmosphere; temperature measurement; alloys; combustion; combustion rate; ignition; NBSIR 81-1647.

metals; plasma emission; specific elements; spectroscopy; chromatography; detection; SP618: 1981 November. 184-196.

metals; plastic-bonded; soils; telephone cables; underground; alloys; corrosion; metallurgically-bonded; NBSIR 81-2243.

metals; productivity; research associate; scanning technique; spin dependent; SRM's; standards; tape; copper; deterioration; electromagnetic radiation; film; industry; $D I M / N B S$ 65(5): 1.24; 1981.

metals; SEM; deformation; electron channeling; fatigue; 19980.

metal-selective detector; metal speciation; tetraalkyllead speciation; atomic absorption spectrometry; gasoline analysis; high performance liquid chromatography; 20763.

metal sensitivity; reactions to implants; hip hemiarthroplasties; metal allergy; SP601: 1981 January. 299.321.

metal speciation; modeling; semiderivative; stability constants; voltammetry; zinc.NTA; cadmium-NTA; convolution voltammetry; electrochemistry; Kalman filter; SP618: 1981 November. 163-172.

metal speciation; tetraalkyllead speciation; atomic absorption spectrometry; gasoline analysis; high performance liquid chromatography; metal-selective detector; 20763.

metal-sulfides; mineral-oxidation; molybdenum; oil shale; Sulfolobus; Thiobacillus; uranium; chemolithotrophic-bacteria; copper; iron; leaching; metal-reduction; SP618: 1981 November. 264-273.

metal surfaces; nonuniformities; laser irradiation; liquid phase regrowth; SP620: 1981 October. 210-221.

metals with filled $d$-bands; photoemission; resonance; satellite; auger; core hole spectroscopy; 20008 .

metastable phase equilibrium; partitionless solidification; phase diagrams; phase equilibrium hierarchy; rapid solidification; thermodynamics; 19981.

metastable states; beryllium isoelectronic sequence; electron-atom scattering; electron impact ionization; 20739.

metering; thermodynamics; fluid flow; gas; instrumentation; mass flow; measurement; 20751.

meter performance; numerical modeling; orifice meter; swirling flow; closed conduit meter; differential pressure meter; flow measurement; laminar flow; 19972.

methacrylate; polyfunctional surface-active comonomers; reconstructive dental work; resin; acrylate; adhesion; aminobenzoate; composite material; dentin; U.S. Patent 4,251,565.

methacrylates; tertiary aromatic amines; unsaturated polyesters; acrylates; acrylic resins; aminoglutethimide or paraaminophenylacetic acid; cementing of bone; fillings; U.S. Patent $4,243,763$.

methane; microwave spectra; molecular parameters; radio astronomy; rotational transitions; distortion dipole transitions; interstellar molecules; JPCRD 10(4): 1085-1095; 1981.

methane-ethane; mixture; one-fluid model; plait point; Rayleigh light scattering; thermal diffusivity; concentration; diffusivity; 19964.

method; pictogram; response; symbol; understandability; communication; evaluation method; fire-safety; hazard warnings; meaningfulness; NBSIR 80-2088.

method of moments; parallel plate waveguide; quasi electrostatic; TEM cell; electromagnetic compatibility (EMC); Green's function; integral equation; linear equation; TN1028.

method of moments; TEM horn; time domain measurements; transients in resistivity; conical antenna; EMP; FFT; loaded antenna; NBSIR 78-876.

method of sale of commodities; open dating; packaging and labeling; registration of service persons; unit pricing; weighmaster law; basic weights and measures law; H130, 1982 Edition.

methods; quality assurance; standards; water; air; environmental measurements; NBSIR 81-2280.

methylation; environment; GC-MS; heavy metals; lead; mass spectrometry; mercury; SP618: 1981 November. 54-64.

methylation/demethylation; arsenicals; cadmium metallothionein; cellular toxicity; elemental interactions; lead inclusion bodies; methylmercury derivatives; SP618: 1981 November. 217.225.

methylene iodide; photodissociation; vacuum ultraviolet; $\mathbf{I}_{2}$ fluorescence absorption coefficient; $\mathrm{Kr}$ photosensitized reaction; 20042.

methyl formate; radio astronomy; amino acids; ethylene oxide; glycine; interstellar; 20077.

methylmercury derivatives; methylation/demethylation; arsenicals; cadmium metallothionein; cellular toxicity; elemental interactions; lead inclusion bodies; SP618: 1981 November. 217.225.

metric; SI; translation; BIPM; CCU; CGPM; CIPM; SP330.

metrication; model laws and regulations; packing and labeling; specifications and tolerances; technology transfer; weights and measures; domestic standards; education programs; electronic devices; international recommendations; legal metrology; measurement assurance; SP599.

metric controversy; newton; AAPT vs. NBS; kilogram; mass/weight; 20023.

metric symmetry; NBS Crystal Data Center; powder diffraction; unit cell; computer evaluation; crystallographic data analysis; JCPDSInternational Centre for Diffraction Data; TN1141.

metric system; modernized metric system; SI; weights and measures; International System of Units; measurement systems; SP304, Revised August 1981.

metric system; modernized metric system; SI; weights and measures; International System of Units; measurement systems; SP304A, Revised August 1981.

metrology; microelectronics; semiconductors; silicon; electronics; integrated circuits; NBSIR 81-2224.

metrology; National Bureau of Standards; power; radiation; semiconductor; technology; calibration; electrical; electronics; engineering; hazards; measurement; SP611.

metrology; quality control; radioactivity; traceability; environment; 20010.

metrology; technologies; technology transfer; Brazil; industrialization; international cooperation; measurement infrastructure; NBSIR 801972.

$\mathrm{MgF}_{2} ; \mathrm{MgO} ; \mathrm{NaCl} ; \mathrm{NaF} ; \mathrm{SrF}_{2} ;$ volume and surface impurities; $\mathrm{BaF}_{2}$; $\mathrm{CaF}_{2}$; extrinsic and intrinsic infrared absorption; infrared wavelength modulation; $\mathrm{LaF}_{3}$; laser windows; LiF; SP620: 1981 October. 29-43

$\mathrm{MgO} ; \mathrm{NaCl} ; \mathrm{NaF} ; \mathrm{SrF}_{2} ;$ volume and surface impurities; $\mathrm{BaF}_{2} ; \mathrm{CaF}_{2} ;$ extrinsic and intrinsic infrared absorption; infrared wavelength modulation; $\mathrm{LaF}_{3}$; laser windows; $\mathrm{LiF} ; \mathrm{MgF}_{2} ; \quad S P 620$ : 1981 October. 29.43.

$\mathrm{MgO}$; slag; soundness; strength; cement; expansion; fly ash; NBSIR 81-2273.

Michelson interferometer; optical path-length correction; phase comparator; real-time control; vibration control; vibration isolation; active vibration control; 20734.

microanalysis; qualitative analysis; quantitative analysis; scanning electron microscopy; spectral artifacts; transmission electron microscopy; electron probe microanalysis; energy dispersive $x$-ray spectrometry; SP604: 1981 June. 315-339.

microbial; radium; uranium; cesium; metal accumulation; SP618: 1981 November. 274-286.

microbial growth; mobilization; nutrients; oil shale; retorting; soil; speciation; volatilization; arsenic; dimethylarsinic acid; SP618: 1981 November. 286-300.

microcalorimetry; radiation; calorimetry; cardiac pacemakers; clinical chemistry; heat; metabolism; 20015.

microcomputer; phase angle; sinewave generator; standard; synthesis; calibration; digital; TN1144.

microcomputers; MIL-STD-1553B multiplex data bus; multiplexing techniques; on-board diagnostics and prognostics; remote terminals; 
total tank subsystem integration; bus controllers; control and distribution; crew station terminals; electrical power management; electronic components; SP622: 1981 October. 85.96.

micro-computers; office automation; SPS00-83: 1981 November. 327. 328.

microcrack; microfibril; shear deformation; tensile failure; chain rupture; crazing; crystalline polymers; electron spin resonance; 20732.

microelectronics; process validation wafer; random faults; silicon-onsapphire; test pattern; test structure; yield; integrated circuits; SP400-66.

microelectronics; random fault; step coverage; test structures; electronics; interconnect; SP400-65.

microelectronics; semiconductor devices; semiconductor materials; semiconductor process control; silicon; electronics; integrated circuits; measurement technology; NBSIR 79-1591-4.

microelectronics; semiconductor devices; semiconductor materials; semiconductor process control; silicon; electronics; integrated circuits; measurement technology; NBSIR 79-1591-5.

microelectronics; semiconductor devices; semiconductor materials; semiconductor process control; silicon; electronics; gallium arsenide; integrated circuits; measurement technology; NBSIR 802006-4.

microelectronics; semiconductor devices; semiconductor materials; semiconductor process control; silicon; electronics; integrated circuits; measurement technology; NBSIR 80-2006-3.

microelectronics; semiconductor devices; semiconductor materials; semiconductor process control; silicon; electronics; integrated circuits; measurement technology; NBSIR 81-2230.

microelectronics; semiconductor devices; semiconductor materials; semiconductor process control; silicon; electronics; integrated circuits; measurement technology; NBSIR 81-2230-3.

microelectronics; semiconductor devices; semiconductor materials; semiconductor process control; silicon; electronics; integrated circuits measurement technology; NBSIR 81-2230-2.

microelectronics; semiconductors; silicon; electronics; integrated circuits; metrology; NBSIR 81-2224.

microelectronic test pattern; microelectronic test structure; MOSFET dc profiler; cross-bridge sheet resistor; integrated gated-diode electrometer; NBSIR 81-2234.

microelectronic test structure; MOSFET dc profiler; cross-bridge sheet resistor; integrated gated-diode electrometer; microelectronic test pattern; NBSIR 81-2234.

microelectronic test structure; probe station; semiconductor process control; test pattern; test structure; wafer chuck; wafer probe station; integrated circuit test methods; SP400-68.

microelectronic test structures; surface recombination velocity; test pattern; test structure; gated diode; generation lifetime; integrated gated-diode electrometer; integrated test structure; leakage current; NBS-GCR-81-327.

microfibril; shear deformation; tensile failure; chain rupture; crazing; crystalline polymers; electron spin resonance; microcrack; 20732.

microfilm; microform; readers; American National Standard; Computer Output Microfilm (COM); Federal Information Processing Standard (FIPS); illumination; image quality; FIPS PUB 84.

microfilm; quality control; automated data printing; computer-output microforms; Federal Information Processing Standards Publication; form slide; FIPS PUB 82.

microform; readers; American National Standard; Computer Output Microfilm (COM); Federal Information Processing Standard (FIPS); illumination; image quality; microfilm; FIPS PUB 84.

microminiature devices; photolithographic fabrication; refrigerators; cryogenic; Joule-Thomson; SP607: 1981 May. 160-163.

microminiature refrigerators; Joule-Thomson refrigerators; laminar flow heat exchanger; SP607: 1981 May. 154-159.

microorganisms; standard reference materials; bacteria; biodeterioration; biotransformations; deterioration of materials; element volatilization; fungi; metals; NBSIR 81-2246.

microporous filler; system nontoxicity; $x$-ray opacification; dental composite resin restorations; finishability; U.S. Patent 4,306,913. microprocessor based shock pulse analyzer; multiplexing bearing monitor channels; prediction of bearing failure; shock pulse bearing monitoring; automatic alarm of bearing malfunction; marine machinery bearings; SP622: 1981 October. 126-136.

microsecond pulses; $\mathrm{NaCl}$; surface damage; alkali halides; bulk damage; $\mathrm{CO}_{2}$ laser; coating damage; laser damage; SP620: 1981 October. 88.93.

microstructure; precipitating; segregation; solidification; thermal mechanical treatment; aging; electrical conductivity; hardness; homogeneity; NBSIR 80-2069.

microwave; microwave measurements; six.port; automatic network analyzer; 20067.

microwave antenna theory; scattering-matrix description of antennas; antenna-antenna systems; antenna measurements; antenna-scatterer systems; antenna theory; Monogr. 162.

microwave measurements; six-port; automatic network analyzer; microwave; 20067.

microwaves; noise; non-ionizing radiation; radiation hazards; antennas; electromagnetic interference; electromagnetic metrology; em material properties; NBSIR 80-1635.

microwaves; nondestructive evaluation; radiography; tire inspection; ultrasonics; visual-optical; acoustic emission; eddy currents; liquid penetrants; magnetic particles; 20762.

microwaves; nonequilibrium; superconductivity; tunnel junction; aluminum; energy gap; gap enhancement; 20723.

microwaves; nonequilibrium superconductivity; photon-assisted tunneling; superconductivity; thin film; transition temperature; tunneling; tunnel junction; aluminum; critical current; energy gap; enhancement; 19979.

microwave spectra; molecular parameters; radio astronomy; rotational transitions; distortion dipole transitions; interstellar molecules; methane; JPCRD 10(4): 1085.1095; 1981.

migration; MSS; performance; space management; DASD; data set; disk management system; SP500-83: 1981 November. 223-230.

migration; oil simulants; oligomers; polyolefins; radioactive tracer; spectrofluorimetry; antioxidants; diffusion; food packaging; inverse gas chromatography; NBSIR 81-2264.

millimeter wave; power; standard; calorimeter; TN1044.

MIL-STD-1553B multiplex data bus; multiplexing techniques; onboard diagnostics and prognostics; remote terminals; total tank subsystem integration; bus controllers; control and distribution; crew station terminals; electrical power management; electronic components; microcomputers; SP622: 1981 October. 85-96.

mineral-oxidation; molybdenum; oil shale; Sulfolobus; Thiobacillus; uranium; chemolithotrophic.bacteria; copper; iron; leaching; metalreduction; metal-sulfides; SP618: 1981 November. 264-273.

minerals; thermodynamic data; enthalpy; enthalpy of formation; entropy; equilibrium constant for formation; Gibbs energy function; Gibbs energy of formation; heat capacity; lime-alumina-silica-water; JPCRD 10(3): 575-670; 1981.

mine subsidence; mining; settlement; structural design; structural engineering; design criteria; foundation design; geotechnical engineering; housing construction; NBSIR 81-2215.

minimum efficiency standards; space cooling requirements; space heating requirements; energy efficiency standards; engineeringeconomic analysis; heat pump; mechanical equipment efficiency ratings; NBSIR $81-2241$.

mining; settlemeni; structural design; structural engineering; design criteria; foundation design; geotechnical engineering; housing construction; mine subsidence; NBSIR 81-2215.

mining industry; public health impacts; residues; toxic elements; availability; environmental contamination; extraction tests; fossil fuels; laboratory characterization; metals; SP618: 1981 November. 1.8 .

minmax; multiple comparison; multivariate analysis; peak areas; principal components; maxmin; NBSIR 81-2222.

mirrors; optical components; scratches; surface defects; coating imperfections; cw laser irradiation; digs; dust; laser damage; SP620: 1981 October. 256-264.

mirror surface damage; surface plasma; absorption waves; air breakdown; Cu mirrors; laser-induced electron emission; SP620: 
1981 October. 227.238.

mixture; one-fluid model; plait point; Rayleigh light scattering thermal diffusivity; concentration; diffusivity; methane-ethane; 19964.

mixtures; one fluid model; prediction; Van der Waals theory; viscosity; data evaluation; extended corresponding states; fluids; 20768.

mixtures; PVTx data; revised Kosek and McKinley; cell model; comparisons; computer programs; corresponding states; equation of state; hard sphere; LNG; TN1030.

mixture viscosity; size difference effects; corresponding states; Enskog theory; mass difference effects; J. Res. 86(6): 597.604; 1981 November.December.

$\mathrm{mm}$ wave measurements; reflection coefficient; six-port measurement system; thermistor mount calibration; NBSIR 81-1650.

mobile home; soil anchors; standards; tiedown; wind forces; buoyancy forces; flood forces; foundations; hurricane forces; BSS132.

mobile home foundations; mobile home standards; soil anchors; soil testing; flood loads; NBSIR 81-2238.

mobile homes; models; room fires; compartment fires; fire tests; flashover; interior finish; NBSIR 81-2333.

mobile homes; residential surveys; transfer functions; field surveys; fire; fire loads; furnishings; household surveys; live loads; NBSIR 80-2155.

mobile home standards; soil anchors; soil testing; flood loads; mobile home foundations; NBSIR 81-2238.

mobilization; nutrients; oil shale; retorting; soil; speciation; volatilization; arsenic; dimethylarsinic acid; microbial growth; SP618: 1981 November. 286-300.

modal analysis; property conservation; risk management; shock pulse analysis; vibration analysis; acoustic emission analysis; insurance; loss prevention; SP622: 1981 October. 139-151.

mode filter; numerical aperture; radiation angle; radiation patterns; attenuation; core diameter; far field; index profile; TN1032.

model; plumbing; quasi-steady; unsteady; characteristics; discharge; drainage; flow; J. Res. 86(2): 171.179; 1981 March-A pril.

model assessment; documentation assessment; documentation guidelines; large-scale models; NBSIR 80-1971.

model assessment; model confidence; model evaluation; model utility; model validation; decision making; NBSIR 80-2053.

model building; statistical analysis; applied economics; construction; cost; estimation; location factors; NBSIR 81-2250.

model calculations; photovoltaic cells; sheet resistance; solar cell defects; solar cells; test structures; laser scanner; NBSIR 81-2260.

model code; noise control; acoustical design; acoustics; architectural design; building codes; building economics; construction costs; cost minimization; economic impact; economics; energy; NBSIR 81. 2366.

model codes; occupancy; performance levels; regulation; standards; building rehabilitation; codes; comparative analysis; existing buildings; housing codes; maintenance; NBSIR 80-2081.

model confidence; model credibility; policy models; sensitivity analysis; validation; assessment; composite models; data quality; energy models; mathematical models; SP616.

model confidence; model evaluation; model utility; model validation; decision making; model assessment; NBSIR 80-2053.

model credibility; policy models; sensitivity analysis; validation; assessment; composite models; data quality; energy models; mathematical models; model confidence; SP616.

model evaluation; model utility; model validation; decision making; model assessment; model confidence; NBSIR 80-2053.

modeling; rollin/rollout files; simulation; CDC 844.41; computer system evaluation; disk subsystem configurations; input/output; SP500-83: 1981 November. 239-246.

modeling; semiderivative; stability constants; voltammetry; zinc-NTA; cadmium-NTA; convolution voltammetry; electrochemistry; Kalman filter; metal speciation; SP618: 1981 November. 163-172.

modeling; standards; standards-writers; systems analysis; building standards; classification; decision tables; information networks; NBSIR 80-1979-1.

model laws and regulations; packing and labeling; specifications and tolerances; technology transfer; weights and measures; domestic standards; education programs; electronic devices; international recommendations; legal metrology; measurement assurance; metrication; SP599.

models; room fires; compartment fires; fire tests; flashover; interior finish; mobile homes; NBSIR 81-2333.

models; simulation; documentation; manuals; SP500-73.

models; thermal insulation; wiring system; electrical fire; electric cable; TN1133.

model utility; model validation; decision making; model assessment; model confidence; model evaluation; NBSIR 80-2053.

model validation; decision making; model assessment; model confidence; model evaluation; model utility; NBSIR 80-2053.

modernized metric sy'stem; Sl; weights and measures; International System of Units; measurement systems; metric system; SP?04A, Revised August 1981.

modernized metric system; SI; weights and measures; International System of Units; measurement systems; metric system; SP304, Revised August 1981.

modes of operation; computer security; cryptography; data security; DES; encryption; Federal Information Processing Standards; FIPS PUB 81.

mode tuned chamber; resonant cavity; electromagnetic radiated susceptibility measurements; NBSIR 81-1638.

modulated structures; oxides; phase equilibria; diffraction effects; long range order; 20046.

moisture; dielectric properties; grain; loss tangent; NBSIR 78-897.

moisture; moisture detection; nondestructive evaluation; nondestructive testing; roofing; thermal resistance; built-up roofing; TN1146.

moisture; moisture generators; moisture sensors; quality control; reliability of semiconductor devices; semiconductor devices; analysis of moisture content; mass spectrometer measurement; SP400-69.

moisture detection; moisture measurement; nondestructive evaluation; nondestructive testing; nuclear backscatter; roofing systems; built. up roofing electrical capacitance; infrared thermography; insulation; BSS131.

moisture detection; nondestructive evaluation; nondestructive testing; roofing; thermal resistance; built-up roofing; moisture; TN1146.

moisture generators; moisture sensors; quality control; reliability of semiconductor devices; semiconductor devices; analysis of moisture content; mass spectrometer measurement; moisture; SP400-69.

moisture measurement; nondestructive evaluation; nondestructive testing; nuclear backscatter; roofing systems; built-up roofing electrical capacitance; infrared thermography; insulation; moisture detection; BSS131.

moisture migration; mortar; porous solid; thermal conductivity; concrete; guarded hot plate; low temperature; NBSIR 81-1651.

moisture sensors; quality control; reliability of semiconductor devices; semiconductor devices; analysis of moisture content; mass spectrometer measurement; moisture; moisture generators; SP40069.

molecular adsorption; refrigeration; zeolites; adsorption; compressor; cryogenics; SP607: 1981 May. 127-135.

molecular dynamics; neutron; neutron radiography; nondestructive evaluation; nuclear reactor; radiation; activation analysis; crystal structure; diffraction; isotopes; TN1142.

molecular interactions; molecular processes; neomycin; neutron dosimeters; polymers; radiography; SRM's; stars; visual acuity; evolution; fracture mechanics; gamma rays; inventors; $D I M / N B S$ 65(7): $1-24 ; 1981$.

molecular parameters; radio astronomy; rotational transitions; distortion dipole transitions; interstellar molecules; methane; microwave spectra; JPCRD 10(4): 1085-1095; 1981.

molecular potential; quantum chemistry; scattering; electron-molecule collisions; interaction potential; Legendre expansion; 20055.

molecular processes; neomycin; neutron dosimeters; polymers; radiography; SRM's; stars; visual acuity; evolution; fracture mechanics; gamma rays; inventors; molecular interactions; $D I M / N B S$ 65(7): 1.24; 1981 
molecular spectroscopic constants; negative ions; photodetachment; potential energy curves; autoionization; electron affinity; 19995.

molecular substituent; oil shale; organoarsenic; organotin; retention index; speciation; atomic absorption; energy-related process fluids; leachate; linear free energy; liquid chromatography; SP618: 1981 November. 197.216.

molecule; reaction rate; relaxation; atom; charge transfer; chemi. ionization; collisional ionization; cross section; detachment; energy transfer; excitation; ion; SP593.

molten salts; physical properties; safety and hazards; thermal energy storage materials; thermal properties; thermodynamic properties; transport properties; corrosion; data compilations; electrochemical aluminum production; electrochemical energy storage materials; NSRDS-NBS6], Part IV.

molybdenum; oil shale; Sulfolobus; Thiobacillus; uranium; chemolithotrophic-bacteria; copper; iron; leaching; metal-reduction; metal-sulfides; mineral-oxidation; SP618: 1981 November. 264-273.

monitoring techniques; prognosis; sensors; wear; wear analysis; energy; energy conservation; failure detection; failure diagnosis; failure prevention; SP622.

monitors; neutron diffraction, samplers; SRM's; time-domain calibrations; weights and measures; $x$-ray diffraction; air pollutants; antenna coupling; electrical distribution; glass-liquidus; insulation; DIM/NBS 65(J): 1.28; 1981.

monoenergetic photons; partly-resolved doublets; silicon; tailing; $x$-ray detectors; collimation; deadlayer determinations; germanium; SP604: 1981 June. 5.34.

monograph; optical; physical; properties data; radiation damage; rock salt; sodium chloride; chemical; electrical; geological; magnetic; mechanical; Monogr. 167.

Mooney-Rivlin material; nonlinear equation-solvers; penalty method plane-stress; strain invariants; tensile-test specimen; checkerboard pressure; elastostatics; finite elements; in-core solver; isoparametrics; J. Res. 86(1): 79-109; 1981 January.February.

morphology; polymer; stress relaxation; UHMWPE; ultra high molecular weight polyethylene; creep; failure; fatigue; mechanical properties; NBSIR 81-2209 (FDA)

mortar; porous solid; thermal conductivity; concrete; guarded hot plate; low temperature; moisture migration; NBSIR 81-1651.

mortar; regression analysis; strength prediction; temperature effects; compressive strength; concrete; curing temperature; early age; final set; hydration; initial set; laboratory testing; maturity; NBSIR 81 2244.

MOS devices; semiconductors; silicon dioxide; sputter profiling. Auger-electron spectroscopy; electronics; NBS-GCR-81-345.

MOSFET dc profiler; cross.bridge sheet resistor; integrated gated. diode electrometer; microelectronic test pattern; microelectronic test structure; NBSIR 81-2234.

MOSFET dc profiler; test structure; CCD; cross-bridge sheet resistor; electrical alignment test structure; gated diode; integrated gateddiode electrometer; integrated test structure; NBSIR 81-2319.

motor oil; petroleum oil; recycled oil; rerefined oil; test procedures; basestock; engine lubricants; lubricating oil; 20016.

MSS; performance; space management; DASD; data set; disk management system; migration; SP5(K)-83: 1981 November. 223 230.

multichannel analyzers; solid state detector; SP604: 1981 June. 165. 175

multifrequency; nondestructive testing; conductivity; defect detection; eddy current test; SP589.

multilayer dielectric films; optical figure measurement; vacuum deposition techniques; wave front distortion; film thickness nonuniformity; high power laser mirrors; SP620: 1981 October. 356-368.

multimode laser; multiphoton ionization; $\mathrm{Na} 3 \mathrm{~s} \rightarrow 3 \mathrm{p}$; pulse shapes; Rabi oscillation; two-level system; 20061.

multiphoton absorption; polaron absorption; avalanche breakdown; beam deformation; beam depletion; free carrier absorption; SP620: 1981 October. 394.40)2

multiphoton ionization; $\mathrm{Na} 3 \mathrm{~s} \rightarrow 3 \mathrm{p}$; pulse shapes; Rabi oscillation; twolevel system; multimode laser; 200161. multiple comparison; multivariate analysis; peak areas; principal components; maxmin; minmax; NBSIR 81-2222.

multiple least squares fitting; nuclear spectroscopy; digital filter electron excited $x$-ray spectra; energy-dispersive $x$-ray spectrum deconvolution; SP604: 1981 June. 297-313.

multiple precision; multiprecision Fortran software; multiprecision mathematical functions; precompilers for special arithmetic; AUGMENT and MP; Fortran arithmetic extensions; NBSIR 802138 .

multiple-shot damage; oxide coatings; pre-irradiation; thin films; ultraviolet reflectors; excimer lasers; fluoride coatings; laser damage; SP620: 1981 October. 265.276.

multiplexing bearing monitor channels; prediction of bearing failure shock pulse bearing monitoring; automatic alarm of bearing malfunction; marine machinery bearings; microprocessor based shock pulse analyzer; SP622: 1981 October. 126-136.

multiplexing techniques; on-board diagnostics and prognostics; remote terminals; total tank subsystem integration; bus controllers; control and distribution; crew station terminals; electrical power management; electronic components; microcomputers; MIL-STD 1553B multiplex data bus; SP622: 1981 October. 85.96.

multipliers; standards; superconductors; ac losses; calorimeter cryogenics; 19999.

multiprecision Fortran software; multiprecision mathematical functions; precompilers for special arithmetic; AUGMENT and MP; Fortran arithmetic extensions; multiple precision; NBSIR 80 2138 .

multiprecision mathematical functions; precompilers for special arithmetic; AUGMENT and MP; Fortran arithmetic extensions; multiple precision; multiprecision Fortran software; NBSIR 80 2138.

multipulse damage; alkali-halides; calorimetry; damage mechanisms; intensity dependent absorption; SP620: 1981 October. 75.87.

multishot damage; nanosecond pulse; peak energy density; pulse distortion; single-shot damage threshold; vacuum chamber; copper mirrors; $\mathrm{CO}_{2}$ laser; SP620: 1981 October. 238-247.

multispectral materials; fluoride glasses; fluorohafnates; fluorozirconates; infrared absorption; infrared glasses; infrared materials; SP620: 1981 October, 94-101.

multivariate analysis; peak areas; principal components; maxmin minmax; multiple comparison; NBSIR 81-2222.

MVS; paging/swapping; TSO; analysis; data elements; I/O; logical swapping; measurement; SP500-83: 1981 November. 343-348.

m-6.8 potential; nonequlibrium molecular; shear; viscosity; argon; dynamics; 19973.

\section{$\mathbf{N}$}

$\mathrm{NaCl} ; \mathrm{NaF} ; \mathrm{SrF}_{2} ;$ volume and surface impurities; $\mathrm{BaF}_{2} ; \mathrm{CaF}_{2}$; extrinsic and intrinsic infrared absorption; infrared wavelength modulation; $\mathrm{LaF}_{3}$; laser windows; LiF; $\mathrm{MgF}_{2} ; \mathrm{MgO} ;$ SP620: 1981 October. 29 43.

$\mathrm{NaCl}$; optical breakdown; point defects; primary defects; damage threshold; excitons; SP620: 1981 October. 406.415.

$\mathrm{NaCl}$; optoacoustic spectroscopy; photoacoustic spectroscopy; surface impurities; alkali-halide laser windows; KCl; SP620: 1981 October 44.49.

$\mathrm{NaCl}$; picosecond phenomena; self-focusing; $\mathrm{SiO}_{2}$; air breakdown; laser damage; SP620: 1981 October. 375-384.

$\mathrm{NaCl}$; self-focusing; Kerr liquids; laser damage; laser induced breakdown; SP620: 1981 October. 385-393.

$\mathrm{NaCl}$; surface damage; alkali halides; bulk damage; $\mathrm{CO}_{2}$ laser; coating damage; laser damage; microsecond pulses; SP620: 1981 October. 88.93.

$\mathrm{NaF} ; \mathrm{SrF}_{2}$; volume and surface impurities; $\mathrm{BaF}_{2} ; \mathrm{CaF}_{2}$; extrinsic and intrinsic infrared absorption; infrared wavelength modulation; $\mathrm{LaF}_{3}$ laser windows; $\mathrm{LiF} ; \mathrm{MgF}_{2} ; \mathrm{MgO} ; \mathrm{NaCl} ;$ SP620: 1981 October. 29 43.

n-alkanes; n-carboxylic esters; permeation; polyethylene; effective carbon atom number; loss-rate; mass-loss; NBSIR 80-2131

nanosecond pulse; peak energy density; pulse distortion; single-shot 
damage threshold; vacuum chamber; copper mirrors; $\mathrm{CO}_{2}$ laser; multishot damage; SP620: 1981 October. 238-247.

$\mathrm{NaNO}_{2}$; osmotic coefficients; $\mathrm{RbNO}_{2}$; solutions; standard reference data; thermodynamic properties; activity coefficients; alkali metal nitrite salts; aqueous; critical evaluation; $\mathrm{CsNO}_{2}$; electrolytes; excess Gibbs energy; $\mathrm{KNO}_{2} ; \mathrm{LiNO}_{2} ;$ JPCRD 10(3): 767.778; 1981.

national bibliographic network; satellite communications; slow-scan television; telecommunications policy; teleconferencing; videodiscs; computer network interconnection; computer network protocol; digital transmission of information; electronic message transmission; image transmission; information networks; SP610.

National Bureau of Standards; nondestructive evaluation; publications; radiography; ultrasonics; abstracts; acoustic emission; bibliography; eddy currents; index; NBSIR 80-2080.

National Bureau of Standards; power; radiation; semiconductor; technology; calibration; electrical; electronics; engineering; hazards; measurement; metrology; SP611.

National Bureau of Standards (NBS); National Engineering Laboratory (NEL); technology transfer; engineering; guest workers; international cooperation; international research; international standards; NBSIR 80-2113.

National Electrical Code; power factor correction; source energy; transformers; electrical design; energy distribution; SP608: 1981 May. 37-56.

National Engineering Laboratory (NEL); technology transfer: engineering; guest workers; international cooperation; international research; international standards; National Bureau of Standards (NBS); NBSIR 80-2113.

National Solar Demonstration Program; solar energy systems; space heating load; thermal performance; auxiliary energy; FCHART computer program; NBS-GCR-81-321.

national standard; performance tests; radiation source; safety standard; classification criteria; gamma radiography; $H 136$.

national testing program; regulation; solar collector; standards; voluntary program; certification; consumer protection; Interstate Solar Coordination Council; labeling procedures; SP608: 1981 May. 179.181.

natural ventilation; building energy analysis; computer simulation; infiltration; NBSIR 81-2404.

$\mathrm{Na} 3 \mathrm{~s} \rightarrow 3 \mathrm{p}$; pulse shapes; Rabi oscillation; two-level system; multimode laser; multiphoton ionization; 20061.

NBS Crystal Data Center; powder diffraction; unit cell; computer evaluation; crystallographic data analysis; JCPDS-International Centre for Diffraction Data; metric symmetry; TN1141.

NBSLD; attic temperature; BLAST; computer simulation; DoE-2; heating/cooling load calculation; indoor temperature; 20755.

NBS periodicals; periodicals; proceedings; serials; standards; transactions; annual reports; diffusion in metals; fire; journals library holdings, NBS Library; NBSIR 81-2226.

NBS solar testing at Gaithersburg; solar hot water heating; computer codes-F-CHART, SOLCOST, TRNSYS; computer predictions for solar systems; 20078 .

NBS theoretical matrix correction; x-ray continuum; energy dispersive x-ray microanalysis; SP604: 1981 June. 365-379.

$\mathrm{Nb}_{3} \mathrm{Sn}$; processing limits; ultrafine filament $\mathrm{Nb}_{3} \mathrm{Sn}$; bronze process; 20741.

n-carboxylic esters; permeation; polyethylene; effective carbon atom number; loss-rate; mass-loss; n-alkanes; NBSIR 80-2131.

n-cube; periodic functions; quadrature; several variables; integrals; integration; irregularity of distribution; lattices; 20032.

near fields; physical optics; planewave spectrum; reflector antennas; co-sited antennas; coupling; far fields; NBSIR 80-1630.

near-field scanning; optical communications; optical fiber; optical waveguide; refracted near-field scanning; refracted-ray scanning, resolution limit; fiber index profile; index profile; TN1038.

negative ions; photodetachment; potential energy curves; autoionization; electron affinity; molecular spectroscopic constants; 19995.

nematic liquid crystal; photo-receptor membrane vesicles; random coil polymers; amphoteric latex; dynamic light scattering; light scattering; J. Res. 86(6): 571-590; 1981 November-December. neomycin; neutron dosimeters; polymers; radiography; SRM's; star visual acuity; evolution; fracture mechanics; gamma rays; inventors; molecular interactions; molecular processes; DIM/NBS 65(7): 1.24; 1981.

net present value; residential water conservation; wastewater treatment; water rate schedules; water-saving devices; benefits; costeffective; costs; economic analysis; economic decision framework; energy conservation; marginal price; NBSIR 81-2304.

net weight; package checking; packaged product inspection; prepackaged consumer goods; statistical sampling; commodity inspection; compliance testing; H133.

network; network flow; optimization; retraction; robustness; rooted tree; sensitivity analysis; shortest path; tolerance; cutset; cycle; directed graph; 20022.

network; sequence list; shortest path; tree; complexity; labeling; $J$. Res. 86(3): 317-330; 1981 May-June.

network; standards; standards-writers; systems analysis; building standards; classification; decision tables; information networks; 19998

network algorithms; shortest path; transportation networks; algorithm; graph; $k$ shortest paths; 20021.

network architecture; network virtual terminal protocols; virtual terminal protocols; communication protocols; computer network protocols; feature analysis; NBS-GCR-81-324

network data model; query language; CODASYL; database management system; data model; DBMS; NBS-GCR-81-339.

network data model; query language; CODASYL; database management system; data model; DBMS; NBS-GCR-81-337.

network data model; query language; relational model; SQL CODASYL; DAPLEX; database management system; data model; DBMS; NBS-GCR-81-338.

network flow; building evacuation; mathematical optimization; J. Res 86(5): 509-513; 1981 September-October.

network flow; optimization; retraction; robustness; rooted tree; sensitivity analysis; shortest path; tolerance; cutset; cycle; directed graph; network; 20022.

networking standards; penetration studies; computer network protocols; computer networks; cost/benefit analysis; federal government networking; federal computer networking development; NBS-GCR-81-323.

network models; buildings; egress; emergencies; evacuation; NBSGCR-81-316.

network models; product form; queueing models; system resources; SP500-83: 1981 November. 335 .

network security; security; computer security; cryptography; data integrity; encryption; Federal Information Processing Standards Publication; key distribution; FIPS PUB 74.

network simulation; network throughput; survivability; alternate routing; communications networks; CROSSFIRE; link protocols; local survivability; message delay; NBS-GCR-81-336.

network throughput; survivability; alternate routing; communications networks; CROSSFIRE; link protocols; local survivability; message delay; network simulation; NBS-GCR-81-336.

network throughput; survivability; alternate routing; communications networks; distributed control; message delay; NBSIR 81-2414.

network virtual terminal protocols; virtual terminal protocols; communication protocols; computer network protocols; feature analysis; network architecture; NBS-GCR-81-324.

Neumann boundary conditions; space matrices; conjugate gradient algorithm; elliptic partial differential equations; iterative methods for linear algebraic equations; NBSIR 80-2056.

neutron; neutron radiography; nondestructive evaluation; nuclear reactor; radiation; activation analysis; crystal structure; diffraction; isotopes; molecular dynamics; TN1142.

neutron detectors; reactor internals; surveillance; vibration; core barrel motion; SP622: 1981 October. 3-10.

neutron diffraction; oxidation mechanisms; profile refinement method; scattering amplitude of neodymium; cerium neodymium tantalates; cerium niobate; 20054.

neutron diffraction, samplers; SRM's; time-domain calibrations; weights and measures; $\mathrm{x}$-ray diffraction; air pollutants; antenna 
coupling; electrical distribution; glass-liquidus; insulation; monitors; $D I M / N B S$ 65(1): 1.28; 1981.

neutron dosimeters; polymers; radiography; SRM's; stars; visual acuity; evolution; fracture mechanics; gamma rays; inventors; molecular interactions; molecular processes; neomycin; $D I M / N B S$ 65(7): 1-24; 1981

neutron dosimetry; neutron radiation therapy; nuclear data; secondary charged particles; tissue equivalence; kerma factors; 20064.

neutron imaging techniques; position-sensitive proportional counters; Van de Graaff accelerator; $14 \mathrm{MeV}$ neutron energy; linear accelerator; 20724.

neutron radiation therapy; nuclear data; secondary charged particles; tissue equivalence; kerma factors; neutron dosimetry; 20064.

neutron radiography; nondestructive evaluation; nuclear reactor; radiation; activation analysis; crystal structure; diffraction; isotopes; molecular dynamics; neutron; TN1142.

neutrons; radiation dosimetry; radiation protection; radiation standards; $x$ rays; beta rays; Federal and State laboratories; gamma rays; instrument calibrations; intermediate calibration laboratories; ionizing radiation measurements; measurement accuracies; SP603.

newton; AAPT vs. NBS; kilogram; mass/weight; metric controversy; 20023.

$\mathrm{Ni}$; nickel; polarized electrons; surface magnetism; ferromagnetism; 19975.

nickel; oscillator strengths; systematic trends; transition probabilities; allowed transitions; cobalt; $f$-values; iron; isoelectronic sequence; line strengths; JPCRD 10(2): 305-566; 1981.

nickel; polarized electrons; electron scattering; magnetism; 19970.

nickel; polarized electrons; surface magnetism; ferromagnetism; $\mathrm{Ni}$; 19975.

nickel; process waters; selenium; solvent refined coal; titanium; trace elements; arsenic; chromium; coal hydrogenation; cobalt; liquefaction; mercury; SP618: 1981 November. 21-38.

nickel alloy 671 ; nickel alloy 800 ; slow strain rate test; structural materials; austenitic stainless steels; coal gasification; elevated temperatures; ferritic stainless steel; gaseous environments; NBSIR 81-2191 (DoE).

nickel alloy 800; slow strain rate test; structural materials; austenitic stainless steels; coal gasification; elevated temperatures; ferritic stainless steel; gaseous environments; nickel alloy 671; NBSIR 812191 (DoE).

nickel energy levels; atomic energy levels; atomic spectra; JPCRD 10(I): 197.290; 1981 .

nighttime cooling; desert climate; energy calculation; environmental chamber; heating/cooling load; 20754.

niobium; repassivation kinetics; stainless steel; triboellipsometry; aluminum; field ion microscopy; iron-chromium alloys; NBSIR 791904 (Navy).

niobium-tin; scaling; strain; superconducting wire; critical current; mechanical properties; 20066.

nitric oxide; phosphine; phosphorus nitride; radio astronomy; sulfur dioxide; interstellar; 19987.

nitrobenzene; organotin cations; dichlone; dual-electrode detector; electrochemical detector; liquid chromatography; 20728.

nitrocellulose; quantitative differential thermal analysis; thermal explosion theory; thermal hazards; thermal instability; adiabatic calorimetry; azidotriphenylmethane; decomposition reactions; differential scanning calorimetry; heat conduction calorimetry; kinetics of heat evolution; m-azidobenzoic acid; NBSIR 80-2018 (DOT).

nitrogen; oxygen; rate of reaction; sulfur; Arrhenius parameters; chemical kinetics; combustion; decomposition; free radicals; gas phase; hydrocarbons; hydrogen; NBSIR 81-2254.

nitrogen; pressure; temperature; thermal conductivity; transient; helium; hot wire; J. Res. 86(5): 457.493; 1981 September-October.

nitrogen; sonic cavity; thermodynamic properties; velocity of sound; virial coefficients; electrostatic transducers; gas; 20072.

nitrogen bases; oil shale; retort water; speciation; trace metals; alkylpyridines; dissolved organic compounds; gas

chromatography/mass spectrometry; hydrophobic bases; metal complexation; SP618: 1981 November. 93-104. nitrous oxide; spectroscopic constants of $\mathrm{N}_{2} \mathrm{O}$; infrared standards from $522 \mathrm{~cm}^{-1}$ to $657 \mathrm{~cm}^{-1}$; infrared standards from $1115 \mathrm{~cm}^{-1}$ to 1340 $\mathrm{cm}^{-1}$; infrared wavelength calibration; $\mathrm{N}_{2} \mathrm{O} ; J P C R D$ 10(4): 1065 . $1084 ; 1981$.

noise; noise control; sound transmission; structureborne noise; acoustics; architectural acoustics; building acoustics; NBS-GCR-80 248.

noise; noise in devices; noise measurement; quantum noise; $1 / \mathrm{f}$ noise; hot carrier noise; SP614.

noise; non-ionizing radiation; radiation hazards; antennas; electromagnetic interference; electromagnetic metrology; em material properties; microwaves; NBSIR 80-1635.

noise; office building; post-occupancy evaluation; questionnaire; temperature; ventilation; windows; energy conservation; lighting; man/environment research; BSS130.

noise contamination; time domain; waveforms; deconvolution; impulse response; inverse filtering; TN1047.

noise control; acoustical design; acoustics; architectural design; building codes; building economics; construction costs; cost minimization; economic impact; economics; energy; model code; NBSIR 81-2366.

noise control; noise isolation; noise rating; acoustics; building acoustics; impact noise; NBS-GCR-80-249.

noise control; noise isolation; sound transmission; structure-borne noise; acoustics; building acoustics; NBS-GCR-80-250.

noise control; sound transmission; structureborne noise; acoustics; architectural acoustics; building acoustics; noise; NBS-GCR-80-248.

noise criteria; time-varying noise; duration; general adverse effect of noise; intermittency; loudness; NBSIR 81-2377.

noise in devices; noise measurement; quantum noise; $1 / \mathrm{f}$ noise; hot carrier noise; noise; SP614.

noise isolation; noise rating; acoustics; building acoustics; impact noise; noise control; NBS-GCR-80-249.

noise isolation; sound transmission; structure-borne noise; acoustics; building acoustics; noise control; NBS-GCR-80-250.

noise measurement; quantum noise; $1 / \mathrm{f}$ noise; hot carrier noise; noise; noise in devices; SP614.

noise pollution; psychoacoustics; transmission line audible noise; corona noise; electric energy transmission; high voltage; human response to noise; 20029.

noise rating; acoustics; building acoustics; impact noise; noise control; noise isolation; $N B S-G C R-80-249$.

noise (sound); sleep; smoke detectors; wakefulness; adults; alarm responses; auditory perception; decibel levels; fire departments; frequency distribution; NBS-GCR-80-284.

noise temperature; satellite communication; earth terminal; Earth Terminal Measurement System; figure of merit; NBSIR 81-1641.

non-code; policymaking; protestors; solar code; victim role; deed; developers; energy wars; experimental code; field test; guideline; low income; SP608: 1981 May. 173-178.

noncooperative games; total orders; aggregation; equilibrium; game theory; mathematical economics; J. Res. 86(4): 383-391; 1981 JulyAugust.

noncrystalline films; passivation; passivation breakdown; structural flexibility; 20025 .

nondestructive assay; nuclear safeguards; nuclear waste management; standard reference materials; statistical evaluation; accountability guide; bulk measurements; isotopic assay; leachability studies; NBSIR 81-2205.

nondestructive evaluation; nondestructive testing; nuclear backscatter; roofing systems; built-up roofing electrical capacitance; infrared thermography; insulation; moisture detection; moisture measurement; BSS131.

nondestructive evaluation; nondestructive testing; roofing; thermal resistance; built-up roofing; moisture; moisture detection; TN1146.

nondestructive evaluation; nondestructive testing; stainless steel; ultrasonic scattering; ultrasonic waves; acoustic waves; elastic anisotropy; 20744.

nondestructive evaluation; nuclear reactor; radiation; activation analysis; crystal structure; diffraction; isotopes; molecular dynamics; neutron; neutron radiography; TN1142. 
nondestructive evaluation; publications; radiography; ultrasonics; abstracts; acoustic emission; bibliography; eddy currents; index; National Bureau of Standards; NBSIR 80-2080.

nondestructive evaluation; quality assurance; steel reinforcing bars; test methods; concrete; construction; NBSIR 80-2163.

nondestructive evaluation; radar soil measurements; soil moisture; underground radar; void detection; adobe building materials; adobe soil; dielectric constant measurements; electromagnetic moisture measurements; NBSIR 79-1610.

nondestructive evaluation; radiography; tire inspection; ultrasonics; visual-optical; acoustic emission; eddy currents; liquid penetrants; magnetic particles; microwaves; 20762.

nondestructive evaluation (NDE); optical holographic interferometry; technical literature; flaw detection; holography; inhomogeneities; light wave; NBS-GCR-81-318.

nondestructive testing; conductivity; defect detection; eddy current test; multifrequency; SP589.

nondestructive testing; nuclear backscatter; roofing systems; built-up roofing electrical capacitance; infrared thermography; insulation; moisture detection; moisture measurement; nondestructive evaluation; BSS131.

nondestructive testing; quality testing; radiograph evaluation; visual inspection; visual testing; acuity tests; TN1143.

nondestructive testing; roofing; thermal resistance; built-up roofing; moisture; moisture detection; nondestructive evaluation; TN1146.

nondestructive testing; stainless steel; ultrasonic scattering; ultrasonic waves; acoustic waves; elastic anisotropy; nondestructive evaluation; 20744 .

nonequilibrium; superconductivity; tunnel junction; aluminum; energy gap; gap enhancement; microwaves; 20723.

nonequilibrium molecular dynamics; nonlinear thermodynamics; shear flow; shear induced phase transition; soft sphere system; stability; steady state; turbulence; 20063.

nonequilibrium molecular dynamics; phase changes; irreversible thermodynamics; Lennard-Jones fluid; 19974.

nonequilibrium superconductivity; photon-assisted tunneling; superconductivity; thin film; transition temperature; tunneling; tunnel junction; aluminum; critical current; energy gap; enhancement; microwaves; 19979.

nonequlibrium molecular; shear; viscosity; argon; dynamics; $m \cdot 6.8$ potential; 19973.

non-jonizing radiation; radiation hazards; antennas; electromagnetic interference; electromagnetic metrology; em material properties; microwaves; noise; NBSIR 80-1635.

nonionizing radiation; regional calibration laboratories; standards; techniques; calibration; dosimetry; electric fields; electromagnetic interference; exposure history; instruments; magnetic fields; SP613.

nonlinear; roofing membrane; splitting; strain energy; stress; substrate; adhesive; bitumen; bonding; built-up roofing membrane; complementary strain energy; felt; TN1152.

nonlinear absorption; oscillator strength; two-photon absorption; band-structure; SP620: 1981 October. 432.437.

nonlinear absorption; photoacoustic technique; absorption in solids; beam depletion; SP620: 1981 October. 50-57.

nonlinear differential equation; nonlinear load; time-stepping finite difference equation technique; Auger function; electrically-short dipole; iteration method; NBSIR 78-898.

nonlinear effect; optical glass; threshold values; damage types; SP620: 1981 October. 102-109.

nonlinear equation-solvers; penalty method; plane-stress; strain invariants; tensile-test specimen; checkerboard pressure; elastostatics; finite elements; in-core solver; isoparametrics; Mooney-Rivlin material; J. Res. 86(1): 79-109; 1981 JanuaryFebruary.

nonlinear load; time-stepping finite difference equation technique; Auger function; electrically-short dipole; iteration method; nonlinear differential equation; NBSIR 78-898.

non-linear optics; three-photon absorption; direct gap semiconductors; SP620: 1981 October. 446-452.

nonlinear propagation; nonlinear pulses; self-focusing; solitons; waveguides; fiber optics; SP620: 1981 October. 453-458. nonlinear pulses; self-focusing; solitons; waveguides; fiber optics; nonlinear propagation; SP620: 1981 October. 453-458.

nonlinear thermodynamics; shear flow; shear induced phase transition; soft sphere system; stability; steady state; turbulence; nonequilibrium molecular dynamics; 20063.

nonlinear time series; polyspectra; spectrum analysis; statistics; time series; bispectrum; cumulant spectra; TN1036.

nonmetallic materials; wind tunnels; cryogenic properties; instrumentation; low temperature materials; materials properties; metals; NBSIR 79-1624.

nonmetallics; physical properties; stainless steels; structural alloys; welding; alloys; aluminum alloys; castings; composites; cryogenic properties; low temperature; mechanical properties; NBSIR 811645.

nonmetallics; physical property; superconductor composites; thermal effect; thermal expansion; low temperature; 20753.

nonread inks; Optical Character Recognition (OCR); OCR-A; OCR. B; read inks; American National Standard; Federal Information Processing Standard (FIPS); interchange of machine readable information; FIPS PUB 85.

non-uniform; partially-filled; pitched-horizontal drain; slope; solid; velocity; volume; water; cylindrical; NBSIR 81-2266.

nonuniformities; laser irradiation; liquid phase regrowth; metal surfaces; SP620: 1981 October. 210-221.

nonuniform systems; one-dimensional models; Rayleigh-Brillouin scattering; damped wave motion; density fluctuations; diffusion; dynamic structure factor; initial value problem; J. Res. 86(5): 429 . 455; 1981 September-October.

normal coordinate analysis; photodissociation; F-atom reactions; infrared spectrum; matrix isolation; 19986.

nuclear backscatter; roofing systems; built-up roofing electrical capacitance; infrared thermography; insulation; moisture detection; moisture measurement; nondestructive evaluation; nondestructive testing; BSS131.

nuclear data; secondary charged particles; tissue equivalence; kerma factors; neutron dosimetry; neutron radiation therapy; 20064.

nuclear magnetic resonance spectroscopy; phenobarbital; phenytoin; primidone; reference material; serum base modification; serum matrix; anticonvulsants; antiepilepsy drugs; blood analysis; chromatography, gas; chromatography, liquid; drug standards; enzyme immunoassay; epilepsy; ethosuximide; mass spectrometry; SP260-72.

nuclear reactor; radiation; activation analysis; crystal structure; diffraction; isotopes; molecular dynamics; neutron; neutron radiography; nondestructive evaluation; TN1142.

nuclear safeguards; nuclear waste management; standard reference materials; statistical evaluation; accountability guide; bulk measurements; isotopic assay; leachability studies; nondestructive assay; NBSIR $81-2205$.

nuclear spectroscopy; digital filter; electron excited x-ray spectra; energy-dispersive $\mathrm{x}$-ray spectrum deconvolution; multiple least squares fitting; SP604: 1981 June. 297-313.

nuclear track technique; triton particles; abundances; alpha particles; cellulose nitrate detector; isotopic; lithium; 19982.

nuclear waste management; standard reference materials; statistical evaluation; accountability guide; bulk measurements; isotopic assay; leachability studies; nondestructive assay; nuclear safeguards; NBSIR 81-2205.

nucleic acid base; solution calorimetry; thermochemistry; $\Delta C_{\rho}$ of reaction; calorimetry; $\mathrm{C}_{S} \mathrm{H}_{5} \mathrm{~N}_{5} \mathrm{O}$; density; enthalpy of solution; Gua.HCl.l $\quad \mathrm{H}_{2} \mathrm{O}$; Gua.HCl.2 $\mathrm{H}_{2} \mathrm{O}$; Gua.2 $\mathrm{HCl}$; guanine (Gua); guanine hydrochlorides; heat capacity; J. Res. 86(4): 367-382; 1981 July-August.

number of bound states; Schwinger limits; screened Coulomb potential; Sobolev's inequality; Yukawa potential; Bargmann limits; bound-state estimates; Lieb limits; J. Res. 86(5): 503.508; 1981 September-October.

numeric accuracy; programming language standards; computer arithmetic; conformance testing; SP500-77.

numerical aperture; radiation angle; radiation patterns; attenuation; core diameter; far field; index profile; mode filter; TNIO32. 
numerical methods; room fires; burning rates; compartment fires; fire modeling; flashover; NBS-GCR-81-301.

numerical modeling; orifice meter; swirling flow; closed conduit meter; differential pressure meter; flow measurement; laminar flow; meter performance; 19972.

numerical models; open-channel flow; Parshall flumes; flumes, hydraulic models; hydraulics; 19971.

numerical solutions for transient depth; unsteady partially-filled pipe flow; waste solid transport; building drainage; NBSIR 81-2308.

nursing homes; nursing staff; smoke; smoke detectors; sprinkler systems; doors; evacuation; fire alarm systems; fire departments; fire extinguishers; fire investigations; hospitals; NBS-GCR-80-297.

nursing homes; nursing staff; smoke; smoke detectors; sprinkler systems; doors; evacuation; fire alarm systems; fire departments; fire extinguishers; fire investigations; hospitals; $N B S-G C R-81-343$.

nursing staff; smoke; smoke detectors; sprinkler systems; doors; evacuation; fire alarm systems; fire departments; fire extinguishers fire investigations; hospitals; nursing homes; NBS-GCR-80-297.

nursing staff; smoke; smoke detectors; sprinkler systems; doors evacuation; fire alarm systems; fire departments; fire extinguishers: fire investigations; hospitals; nursing homes; $N B S-G C R-81-343$.

nutrients; oil shale; retorting; soil; speciation; volatilization; arsenic dimethylarsinic acid; microbial growth; mobilization; SP618: 1981 November. 286-300.

$\mathrm{N}_{2} \mathrm{O}$; nitrous oxide; spectroscopic constants of $\mathrm{N}_{2} \mathrm{O}$; infrared standards from $522 \mathrm{~cm}^{-1}$ to $657 \mathrm{~cm}^{-1}$; infrared standards from $1115 \mathrm{~cm}^{-1}$ to $1340 \mathrm{~cm}^{-1}$; infrared wavelength calibration; JPCRD 10(4): 1065 . $1084 ; 1981$.

\section{O}

occupancy; performance levels; regulation; standards; building rehabilitation; codes; comparative analysis; existing buildings housing codes; maintenance; model codes; NBSIR 80-2081.

occupant comfort; optimal weatherization demonstration; thermal comfort; thermostat setting practices; weatherization; weatherization and thermal comfort; Community Services Administration; field study; NBSIR 81-2335.

OCR-A; OCR-B; read inks; American National Standard; Federal Information Processing Standard (FIPS); interchange of machine readable information; nonread inks; Optical Character Recognition (OCR); FIPS PUB 85

OCR-B; read inks; American National Standard; Federal Information Processing Standard (FIPS); interchange of machine readable information; nonread inks; Optical Character Recognition (OCR); OCR-A; FIPS PUB 85 .

octanol; partition coefficient; propylbenzene; solubility; solute; water; generator column; hydrophobic; J. Res. 86(4): 361-366; 1981 JulyAugust.

octanol/water partition coefficients; activity coefficients; aqueous solubility; gas chromatography; generator column; high pressure liquid chromatography; NBSIR 81-2406.

ocular injury; projectiles; safety; contusion; eyeball deformation; impact; NBSIR 80-2037.

office automation; micro-computers; SP500-83: 1981 November. 327. 328.

office automation; performance management systems; resource measurement facility; simulation; supercomputers; benchmarking; capacity planning; chargeback systems; computer performance management; data base machines; end user productivity; human factors evaluation; information system management; SP500-83.

office building; phase diagrams; radioactivity; research; sediment; soil; alloy; carbon; computers; energy; fine structure; interface materials; measurement; $D I M / N B S$ 65(8): 1.28; 1981.

office building; post-occupancy evaluation; questionnaire; temperature; ventilation; windows; energy conservation; lighting; man/environment research; noise; BSS130.

off-line programming; robot control; robot interfaces; robot standards robot wrist; simple sensors; complex sensors; NBSIR 80-2152.

offset; analog-to-digital converter; calibration service; data converter; differential linearity; digital-to-analog converter; error measurement; gain; input noise; linearity; $T N 1145$

oil analysis; recycled materials; refused-derived-fuel; rerefined oil; energy-from-waste; materials market; NBSIR 80-2178.

oil burners; practices; safety; sealants; standards; storm doors; storm windows; vent dampers; water heaters; windows; automatic ignition devices; caulks; effectiveness; energy conservation; energy conservation measures; installation; insulation; SP606.

oil debris; wear monitoring; chip detectors; condition monitoring; failure detection; helicopter diagnostics; SP622: 1981 October. 33 49.

oil filter design; oil filter testing; particle size sensitivity; radioactive wear testing; effect of filtration; engine wear analysis; SP622: 1981 October. 50-61.

oil filter testing; particle size sensitivity; radioactive wear testing; effect of filtration; engine wear analysis; oil filter design; SP622 1981 October. 50-61.

oil shale; organoarsenic; organotin; retention index; speciation; atomic absorption; energy-related process fluids; leachate; linear free energy; liquid chromatography; molecular substituent; SP618: 1981 November. 197-216.

oil shale; retorting; soil; speciation; volatilization; arsenic; dimethylarsinic acid; microbial growth; mobilization; nutrients SP618: 1981 November. 286-300.

oil shale; retort water; speciation; trace metals; alkylpyridines; dissolved organic compounds; gas chromatography/mass spectrometry; hydrophobic bases; metal complexation; nitrogen bases; SP618: 1981 November. 93-104.

oil shale; standard reference materials; water pollution control measurement methods; 20009.

oil shale; Sulfolobus; Thiobacillus; uranium; chemolithotrophicbacteria; copper; iron; leaching; metal-reduction; metal-sulfides mineral-oxidation; molybdenum; SP618: 1981 November. 264-273.

oil shale retorts; remote sensing; scattering; NBSIR 81-1653.

oil simulants; oligomers; polyolefins; radioactive tracer; spectrofluorimetry; antioxidants; diffusion; food packaging; inverse gas chromatography; migration; NBSIR 81-2264.

oils, PCB's; standards; atomic weight; coal; computers; drill failure; Faraday; firefighting; hot atom; industrial innovation; measurements; DIM/NBS 65(6): 1.24; 1981.

oligomers; polyolefins; radioactive tracer; spectrofluorimetry; antioxidants; diffusion; food packaging; inverse gas chromatography; migration; oil simulants; NBSIR 81-2264.

OMNITAB 80; software; test problems; accuracy; ANS FORTRAN; computer system installation; examples; TN1147.

on-board diagnostics and prognostics; remote terminals; total tank subsystem integration; bus controllers; control and distribution; crew station terminals; electrical power management; electronic components; microcomputers; MIL-STD-1553B multiplex data bus; multiplexing techniques; SP622: 1981 October. 85-96.

on-board monitoring system; aircraft hydraulic systems; cost reduction; energy savings; failure detection; failure prevention; flight safety; fluid system components; maintenance effectiveness; SP622: 1981 October. 195-219.

one-dimensional models; Rayleigh-Brillouin scattering; damped wave motion; density fluctuations; diffusion; dynamic structure factor; initial value problem; nonuniform systems; J. Res. 86(5): 429-455; 1981 September-October.

one-fluid model; plait point; Rayleigh light scattering; thermal diffusivity; concentration; diffusivity; methane-ethane; mixture; 19964.

one fluid model; prediction; Van der Waals theory; viscosity; data evaluation; extended corresponding states; fluids; mixtures; 20768.

onsite wastewater systems; wastewater disposal; wastewater recirculation; wastewater reuse; wastewater treatment; water conservation; NBSIR 81-2210.

open-channel flow; Parshall flumes; flumes, hydraulic models; hydraulics; numerical models; 19971.

open dating; packaging and labeling; registration of service persons; unit pricing; weighmaster law; basic weights and measures law; method of sale of commodities; H130, 1982 Edition.

Operation Breakthrough; post-occupancy housing evaluation; Project 
Feedback; questionnaires; research methods; survey research; user needs; dwelling units (residential); housing; NBSIR 81-2258.

sptical; physical; properties data; radiation damage; rock salt; sodium chloride; chemical; electrical; geological; magnetic; mechanical; monograph; Monogr. 167.

optical absorption coefficients; infrared materials; iodine laser; laser calorimetry; SP620: 1981 October. 129.143.

optical breakdown; point defects; primary defects; damage threshold; excitons; $\mathrm{NaCl}$; SP620: 1981 October. 406-415.

optical character recognition; character positioning; computer systems; data entry (automatic); Federal Information Processing Standard; information processing systems; FIPS PUB 89.

Optical Character Recognition (OCR); OCR-A; OCR.B; read inks; American National Standard; Federal Information Processing Standard (FIPS); interchange of machine readable information; nonread inks; FIPS PUB 85 .

optical communications; optical fiber; optical waveguide; refracted near-field scanning; refracted-ray scanning; resolution limit; fiber index profile; index profile; near-field scanning; TN1038.

optical communications; optical fiber bandwidth; optical fiber distortion; optical fibers; fiber optics; TN1046.

optical communications; optical fibers; bibliography; measurements; TN1043.

optical components; optical fabrication; optical materials and properties; thin film coatings; laser damage; laser interaction; SP620.

optical components; scratches; surface defects; coating imperfections; cw laser irradiation; digs; dust; laser damage; mirrors; SP620: 1981 October. 256-264.

optical constants; optical materials; semiconductors; stimulated bremsstrahlung absorption; wavelength dependence; laser damage; SP620: 1981 October. 416-426.

optical distortion; photoelastic coefficients; polycrystalline aggregate; stress birefringence; thermal lensing; window materials; elastic coefficients; high-energy laser; interferometric testing; SP620: 1981 October. 117.128.

optical fabrication; optical materials and properties; thin film coatings; laser damage; laser interaction; optical components; SP620.

optical fiber; optical waveguide; refracted near-field scanning; refracted-ray scanning; resolution limit; fiber index profile; index profile; near-field scanning; optical communications; TN1038.

optical fiber bandwidth; optical fiber distortion; optical fibers; fiber optics; optical communications; TN1046.

optical fiber distortion; optical fibers; fiber optics; optical communications; optical fiber bandwidth; TN1046.

optical fibers; bibliography; measurements; optical communications; TN1043.

optical fibers; fiber optics; optical communications; optical fiber bandwidth; optical fiber distortion; TN1046.

optical figure measurement; vacuum deposition techniques; wave front distortion; film thickness nonuniformity; high power laser mirrors; multilayer dielectric films; SP620: 1981 October. 356.368.

optical glass; threshold values; damage types; nonlinear effect; SP620: 1981 October. 102-109.

optical holographic interferometry; technical literature; flaw detection; holography; inhomogeneities; light wave; nondestructive evaluation (NDE); NBS-GCR-81-318.

optical materials; semiconductors; stimulated bremsstrahlung absorption; wavelength dependence; laser damage; optical constants; SP620: 1981 October. 416-426.

optical materials and properties; thin film coatings; laser damage; laser interaction; optical components; optical fabrication; SP620.

optical path-length correction; phase comparator; real-time control; vibration control; vibration isolation; active vibration control; Michelson interferometer; 20734.

optical properties; ultraviolet transmission; fluorides; forging; laser windows; lithium fluoride; mechanical properties; SP620: 1981 October. 110-116.

optical pumping; resonance Raman; spectroscopy; strong fields; damping theory; kinetics; lasers; lineshapes; 20039.

optical time domain reflectometry; Rayleigh scattering; backscattering; capture fractions; fiber scattering; TN1034.

optical waveguide; refracted near-field scanning; refracted-ray scanning; resolution limit; fiber index profile; index profile; nearfield scanning; optical communications; optical fiber; TNIO38.

optics; polarized light; electric field; electro-optics; Kerr effect; measurement; 19962.

optimal weatherization demonstration; thermal comfort; thermostat setting practices; weatherization; weatherization and thermal comfort; Community Services Administration; field study; occupant comfort; NBSIR 81-2335.

optimization; cost savings; energy conservation; HVAC systems; innovation; mathematical model; SP608: 1981 May. 21.35.

optimization; retraction; robustness; rooted tree; sensitivity analysis; shortest path; tolerance; cutset; cycle; directed graph; network; network flow; 20022.

optoacoustic; photoacoustic; surface-to-bulk optical absorption; $\mathrm{CaF}_{2}$; laser calorimetry; laser windows; SP620: 1981 October. 58.74.

optoacoustic spectroscopy; photoacoustic spectroscopy; surface impurities; alkali-halide laser windows; $\mathrm{KCl}$; NaCl; SP620: 1981 October. 44.49.

order-disorder; titanium; transmission electron microscopy; alloy phase diagram; aluminum; magnetic susceptibility; 20736.

organic coatings; passive film dissolution; $\mathrm{pH}$ measurements; surface hydride; alpha uranium; chloride ion; chromate ion; electrochemistry; ellipsometry; field ion microscopy; inhibitors; NBSIR 81.2396 (ONR).

organic compounds in water; deuterated compounds; isotopic purity; mass spectra; Master Analytical Scheme; NBSIR 80-2160.

organization; provisions; scope; specification; standard; system analysis/engineering; arrangement; building; classification; code; engineering; BSS136.

organoarsenic; organotin; retention index; speciation; atomic absorption; energy-related process fluids; leachate; linear free energy; liquid chromatography; molecular substituent; oil shale; SP618: 1981 November. 197-216.

organoarsenics; organometals; partition coefficients; toxicity; alkylleads; alkyltins; chemical speciation; GC-AAS techniques; LC AAS techniques; SP618: 1981 November. 65.80.

organomercury; plasmids; silver; toxic heavy metals; antimony; arsenic; bacterial resistances; cadmium; detoxification; mercury; SP618: 1981 November. 301-324.

organometallic compounds; trace element standards; waste cycling; bacterial mobilization; biogeochemical cycles; biological uptake; cellular toxicity-essentiality mechanisms; chromatography; elementselective speciation; environmental monitoring; fossil fuels; metal(loid) complexes; SP618.

organometalloid toxicity; pentane; respiration; animal toxicity; breath analysis; endurance; free-radicals; iron; SP618: 1981 November. 226-234.

organometals; partition coefficients; toxicity; alkylleads; alkyltins; chemical speciation; GC-AAS techniques; LC-AAS techniques; organoarsenics; SP618: 1981 November. 65-80.

organotin; retention index; speciation; atomic absorption; energyrelated process fluids; leachate; linear free energy; liquid chromatography; molecular substituent; oil shale; organoarsenic; SP618: 1981 November. 197.216.

organotin cations; dichlone; dual-electrode detector; electrochemical detector; liquid chromatography; nitrobenzene; 20728.

orifice flow; smoke control; smoke movement; air leakage; buildings; doors; fire doors; fire tests; infiltration; NBSIR 81-2214.

orifice meter; swirling flow; closed conduit meter; differential pressure meter; flow measurement; laminar flow; meter performance; numerical modeling; 19972.

origin of pollutants; pollutant properties; synfuel; fossil porphyrins; fuel conversion; fuel extraction; heterocyclics; metal chelates; metallo-complexes; SP618: 1981 November. 9.20.

orthopaedic; polymers; retrieval; analysis; biological; cardiovascular; implants; metals; SP601.

orthopaedic implants; cross-referencing capabilities; interactive computer system; SP601: 1981 January. 573-613.

oscillator strength; two-photon absorption; band structure; effective 
mass; intraband-momentum matrix element; SP620: 1981 October. 438.445.

oscillator strength; two-photon absorption; band-structure; nonlinear absorption; SP620: 1981 October. 432.437.

oscillator strengths; spectral lines; transition probabilities; wavelengths; atom; atomic ion; intensities; line strengths; NSRDSNBS68.

oscillator strengths; systematic trends; transition probabilities; allowed transitions; cobalt; $f$-values; iron; isoelectronic sequence; line strengths; nickel; JPCRD 10(2): 305-566; 1981

oscilloscope calibrator; oscilloscopes; calibration; calibrators; instrumentation; NBSIR 81-1646.

oscilloscopes; calibration; calibrators; instrumentation; oscilloscope calibrator; NBSIR 81-1646.

osmotic coefficient; potassium; rubidium; sodium; solutions; thermodynamic properties; activity coefficient; cesium; critical evaluation; electrolyte; excess Gibbs energy; lithium; JPCRD 10(3): 671.766.

osmotic coefficient; solutions; thermodynamic properties; zinc; activity coefficient; cadmium; critical evaluation; electrolyte; ethylene bis(trimethylammonium) chloride; ethylene bis(trimethylammonium) iodide; excess Gibbs energy; JPCRD 10(1): 1.56; 1981 .

osmotic coefficients; $\mathrm{RbNO}_{2}$; solutions; standard reference data; thermodynamic properties; activity coefficients; alkali metal nitrite salts; aqueous; critical evaluation; $\mathrm{CsNO}_{2}$; electrolytes; excess Gibbs energy; $\mathrm{KNO}_{2} ; \mathrm{LiNO}_{2} ; \mathrm{NaNO}_{2} ; J P C R D$ 10(3): 767.778; 1981.

osmotic coefficients; solutions; sulfuric acid; standard reference data; thermodynamic properties; activity coefficients; aqueous; critical evaluation; electrolytes; excess free energy; free energy; ionic; JPCRD 10(3): 779.798; 1981.

osmotic coefficients; solutions; sulfuric acid; thermodynamic properties; transport properties; activity coefficients; aqueous; compilation; conductivity; electrolytes; enthalpy; Gibbs energy; NBSIR 81.2276.

ossicular prosthesis; porosity measurement; porous biomaterials; porous polyethylene; PTFE-carbon composite; synthetic implants; NBSIR 81-2212.

osteosynthesis devices; tissue reaction; corrosion; SP601: 1981 January. 423.447 .

outer piston; regenerator gaps; single stage, twin piston cryogenic refrigerator; two nested, concentric pistons; cylinder; helium; inner piston; U.S. Patent 4,281,517.

overamping; residential structures; safety; thermal insulation; branch circuit wiring problems; electrical problems; electrical wiring system; extension cord wiring; fuse/circuit breaker panel problems; $N B S I R$ 81-2203.

oxidation; photochemistry; rates; review; singlet oxygen; solution; chemical kinetics; data compilation; JPCRD 10(4): 809-999; 1981.

oxidation catalyst; oxygen partial pressure; stoichiometric; three way catalytic converters; zirconium dioxide; air-fuel ratio; closed loop fuel metering system; exhaust emissions; exhaust gas oxygen sensor; exhaust gas recirculation; feedback; SP622: 1981 October. 62-73.

oxidation mechanisms; profile refinement method; scattering amplitude of neodymium; cerium neodymium tantalates; cerium niobate; neutron diffraction; $2(x) 54$.

oxide coatings; pre-irradiation; thin films; ultraviolet reflectors; excimer lasers; fluoride coatings; laser damage; multiple-shot damage; SP620: 1981 October. 265-276.

oxides; phase equilibria; diffraction effects; long range order; modulated structures; $2(0) 46$.

oxygen; rate of reaction; sulfur; Arrhenius parameters; chemical kinetics; combustion; decomposition; free radicals; gas phase; hydrocarbons; hydrogen; nitrogen; NBSIR 81-2254.

oxygen atmosphere; temperature measurement; alloys; combustion; combustion rate; ignition; metals; NBSIR 81-1647.

oxygen partial pressure; stoichiometric; three way catalytic converters; zirconium dioxide; air-fuel ratio; closed loop fuel metering system; exhaust emissions; exhaust gas oxygen sensor; exhaust gas recirculation; feedback; oxidation catalyst; SP622: 1981 October. 62-73. package checking; packaged product inspection; prepackaged consumer goods; statistical sampling; commodity inspection; compliance testing; net weight; H133.

packaged product inspection; prepackaged consumer goods; statistical sampling; commodity inspection; compliance testing; net weight; package checking; H133.

packaging and labeling; registration of service persons; unit pricing weighmaster law; basic weights and measures law; method of sale of commodities; open dating; H130, 1982 Edition.

packed bed heat exchanger; balloon inflation; cryogenic helium balloon inflation; heat transfer; liquid helium; 20017.

packet broadcasting; radio computer communication network; satellite communication; ALOHANET; ALOHA system; computer network; J. Res. 86(6): 591-595; 1981 November-December.

packing and labeling; specifications and tolerances; technology transfer; weights and measures; domestic standards; education programs; electronic devices; international recommendations; legal metrology; measurement assurance; metrication; model laws and regulations; SP599.

paging/swapping; TSO; analysis; data elements; 1/O; logical swapping; measurement; MVS; SP500-83: 1981 November. 343-348.

paint removal methods; White House; coatings; exterior restoration; field tests; guide specifications; laboratory tests; NBSIR 80-2122.

pair production; plasma diagnostics; radiation; synchrotron radiation; astrophysics; bremsstrahlung; electromagnetic interactions; 19993.

paper deacidification; paper destabilization; paper permanence; $\mathrm{pH}$ history; alkaline reserve; cellulose stabilizers; J. Res. 86(3): 309.315; 1981 May-June.

paper destabilization; paper permanence; $\mathrm{pH}$ history; alkaline reserve; cellulose stabilizers; paper deacidification; J. Res. 86(3): 309.315; 1981 May-June.

paper permanence; $\mathrm{pH}$ history; alkaline reserve; cellulose stabilizers; paper deacidification; paper destabilization; J. Res. 86(3): 309-315; 1981 May-June.

parallel heat flow; response factors; thick building construction; conduction heat transfer; conduction transfer functions; initialization of heat transfer problem; NBSIR 81-2353.

parallel plate waveguide; quasi electrostatic; TEM cell; electromagnetic compatibility (EMC); Green's function; integral equation; linear equation; method of moments; TN1028.

parameter; physics; solar collector; space charges; technical competence; cement and concrete; cover plates; earth's tilt; electric; fire; fracture toughness; measurement; DIM/NBS 65(3): 1.24; 1981. parametric calculations; uranium; wavelengths; energy levels; 20002. parity; time reversal; charge conjugation; CPT theorem; 19991.

Parshall flumes; flumes, hydraulic models; hydraulics; numerical models; open-channel flow; 19971.

partial differential equations; buoyant convection; computations-finite difference; Euler equations; finite difference equations; fireenclosure; fluid flow; heat source-volumetric; NBSIR 81-2385.

partial discharge; sulfur hexafluoride; swarm data; water vapor; corona; decomposition; electrical insulation; gas chromatograph; inception voltages; mass spectrometer; NBSIR 81-2242.

partially-filled; pipe; slope; stream-depth; unsteady; volume; water; diameter; drain; flow; history; horizontal; length; NBSIR 81-2249.

partially-filled; pitched-horizontal drain; slope; solid; velocity; volume; water; cylindrical; non-uniform; NBSIR 81-2266

partially filled pipe flow; building drainage; gradually varied flow; hydraulic jump; NBSIR 81-2367.

particle size sensitivity; radioactive wear testing; effect of filtration; engine wear analysis; oil filter design; oil filter testing; SP622: 1981 October. 50-61.

partition coefficient; propylbenzene; solubility; solute; water; generator column; hydrophobic; octanol; J. Res. 86(4): 361-366; 1981 July-August.

partition coefficients; toxicity; alkylleads; alkyltins; chemical speciation; GC.AAS techniques; LC-AAS techniques; organoarsenics; organometals; SP618: 1981 November. 65.80. 
partitionless solidification; phase diagrams; phase equilibrium hierarchy; rapid solidification; thermodynamics; metastable phase equilibrium; 19981.

part-load performance; rating procedure; seasonal efficiency; annual fuel utilization efficiency; annual operating costs; central heating equipment; condensing boilers; condensing furnaces; NBSIR 80 2110.

partly-resolved doublets; silicon; tailing; $x$-ray detectors; collimation; deadlayer determinations; germanium; monoenergetic photons; SP604: 1981 June. 5-34.

passivation; passivation breakdown; structural flexibility; noncrystalline films; 20025.

passivation breakdown; structural flexibility; noncrystalline films; passivation; 20025.

passive film dissolution; $\mathrm{pH}$ measurements; surface hydride; alpha uranium; chloride ion; chromate ion; electrochemistry; ellipsometry; field ion microscopy; inhibitors; organic coatings; NBSIR 81-2396 (ONR).

passive/hybrid components; solar energy; steady-state tests; thermal test procedures; transient thermal tests; NBSIR 81-2300.

passive solar; regional analysis; thermal efficiency; windows; building economics; daylighting; energy conservation; engineering economics; life-cycle costs; NBSIR 81-2248.

password; personal attribute; personal identification; access control; authentication; authorization; computer network; computer security; encryption; Federal Information Processing Standards Publication; identification token; identity verification; FIPS PUB 83.

pattern matching; query processor; software tool; text processor; translation; UNIX; macroprocessor; NBSIR 80-2182.

pattern recognition; pressurized water reactor; remote surveillance; acoustic emissions; cavitation; feedwater control valve; SP622: 1981 October. 11-30.

peak acceleration; playground safety; playground surfaces; soil; surfacing materials; test method; asphalt; impact attenuation; NBSIR 80-2106.

peak areas; principal components; maxmin; minmax; multiple comparison; multivariate analysis; NBSIR 81-2222.

peak energy density; pulse distortion; single-shot damage threshold; vacuum chamber; copper mirrors; $\mathrm{CO}_{2}$ laser; multishot damage; nanosecond pulse; SP620: 1981 October. 238-247.

peak shapes; surface-layer analyses; ultra-thin-windowed (UTW) $\mathrm{Si}(\mathrm{Li})$ detectors; windowless detectors; $\mathrm{x}$-ray transmission; electron excitation; energy-dispersive spectrometry; energy resolution; SP604: 1981 June. 97-126.

pebble-bed storage; solar collector; system efficiency; thermal performance; air leakage; heat transfer; NBS-GCR-81-302.

pedestrian circulation; pedestrian flow; pedestrian movement; pedestrian ramps; ramps; building circulation; building ramps; NBSIR 81-2310.

pedestrian flow; pedestrian movement; pedestrian ramps; ramps; building circulation; building ramps; pedestrian circulation; NBSIR $81-2310$.

pedestrian movement; pedestrian ramps; ramps; building circulation; building ramps; pedestrian circulation; pedestrian flow; NBSIR 812310.

pedestrian ramps; ramps; building circulation; building ramps; pedestrian circulation; pedestrian flow; pedestrian movement; NBSIR 81-2310.

penalty method; plane-stress; strain invariants; tensile-test specimen; checkerboard pressure; elastostatics; finite elements; in-core solver; isoparametrics; Mooney-Rivlin material; nonlinear equation-solvers; J. Res. 86(1): 79-109; 1981 January-February.

penetration resistance; reinforcing bars; stress waves; concrete; dynamic properties; fiber-reinforcement; impact; impulsive loading; SP480-45.

penetration studies; computer network protocols; computer networks; cost/benefit analysis; federal government networking; federal computer networking development; networking standards; NBSGCR-81-323.

pentane; respiration; animal toxicity; breath analysis; endurance; free- radicals; iron; organometalloid toxicity; SP618: 1981 November. 226-234.

perfect gas analysis; refrigerator; component efficiency; cryogenics efficiency; helium; maximum refrigerator efficiency; 20771.

performance; physical deterioration; reliability; turbomachinery; aerothermal diagnostics; aerothermal parameters; SP622: 1981 October. 222-253.

performance; soil removal; water conservation; water discharge characteristics; clothes washers; dishwashers; dishwasher subcycles; fill controls; grey water; NBSIR 81-2291 (HUD).

performance; space management; DASD; data set; disk management system; migration; MSS; SP500-83: 1981 November. 223-230.

performance analysis; productivity analysis; text editors; end user performance; interactive systems; SP500-83: 1981 November. 71-80. performance comparison; program behavior; structural analysis; SP500-83: 1981 November. 199-215.

performance criteria; project summaries; technical bases; building research; building technology; codes; criteria; measurement methods; SP446-5.

performance data for commercial office buildings in New England; solar energy in commercial buildings; building models, computer; energy conservation in commercial buildings; energy conservation, user acceptance; lighting measurements; BSS 133 .

performance evaluation; performance management; productivity; system tuning; computer system; data processing function; SP5O083: 1981 November. 121

performance evaluation; pictures; simulation; tools; analysis; SP50083: 1981 November. 331-334.

performance evaluation; workload analysis; workload characterization; workload representation; ADP acquisition; ADP procurement; benchmarking; Federal Information Processing Standards Publication; FIPS PUB 75.

performance levels; regulation; standards; building rehabilitation; codes; comparative analysis; existing buildings; housing codes; maintenance; model codes; occupancy; NBSIR 80-2081.

performance management; productivity; system tuning; computer system; data processing function; performance evaluation; SP50083: 1981 November. 121

performance management; productivity; user service reporting; ADP installation management; cost-benefit; SP500-83: 1981 November. 59.65 .

performance management systems; resource measurement facility; simulation; supercomputers; benchmarking; capacity planning; chargeback systems; computer performance management; data base machines; end user productivity; human factors evaluation; information system management; office automation; SP500-83.

performance parameters; UNIVAC; UNIVAC 1100 OS; facilities complex; SP500-83: 1981 November. 251-258.

performance prediction; queueing models; simulation; validation; analytic models; communication networks; flow control; Markov processes; SP500-83: 1981 November. 277-290.

performance prediction; sensitivity technique; instruction mix technique; SP500-83: 1981 November. 181-189.

performance requirements; standards development; building energy use; building service systems; energy budgets; housing research; housing standards; NBS-GCR-80-280.

performance standards; building energy performance; codes; energy conservation; energy policy; Hawaii; SP608: 1981 May. 105-112.

performance standards; solar collector; solar energy; space heating and cooling; thermal storage; ASHRAE 90-75; class $\mathrm{K}$ code; computer modeling; electrical design; energy audit; energy conservation; HVAC systems; SP608.

performance standards; Statement of Review; verification; building code; building envelope; comparison; compliance; energy budget; energy conservation; SP608: 1981 May. 57-67.

performance testing; regulatory enforcement; implant retrieval and analysis; liability trials; SP601: 1981 January. 655-666.

performance tests; radiation source; safety standard; classification criteria; gamma radiography; national standard; H136.

performance tools; resource availability data; computer performance; SP500-83: 1981 November. 329. 
periodicals; proceedings; serials; standards; transactions; annual reports; diffusion in metals; fire; journals; library holdings, NBS Library; NBS periodicals; NBSIR 81-2226.

periodic functions; quadrature; several variables; integrals; integration; irregularity of distribution; lattices; n-cube; 20032.

permeability; radon; transport; buildings; concrete; diffusion; exhalation; materials; measurement; $T N 1139$.

permeability of composites; superconducting power transmission cable cool-down; cable permeability; counter-flow cool-down; heat flux meters; liquid helium; NBSIR 80-1637.

permeation; polyethylene; effective carbon atom number; loss-rate; mass-loss; n-alkanes; n-carboxylic esters; NBSIR 80-2131.

permeation type; personal dosimeter; reaction; time-dependent; diffusion; integrating device; NBSIR 80-1975.

peroxide catalyzed polymerization; tertiary aromatic amines; unsaturated polyesters; vinyl monomers; accelerators; cementing of bone; high reactivity; low toxicity; U.S. Patent 4,284,551.

personal attribute; personal identification; access control; authentication; authorization; computer network; computer security; encryption; Federal Information Processing Standards Publication; identification token; identity verification; password FIPS PUB 83

personal dosimeter; reaction; time-dependent; diffusion; integrating device; permeation type; NBSIR 80-1975.

personal identification; access control; authentication; authorization computer network; computer security; encryption; Federal Information Processing Standards Publication; identification token; identity verification; password; personal attribute; FIPS PUB 83.

perylene; pyrene; shale oil; standard reference materials; benzo(a)pyrene; benzo(e)pyrene; fluoranthene; fluorescence detection; gas chromatography/mass spectrometry; highperformance liquid chromatography; 20750.

pesticides, solids; solubility; vapor pressure; alkanes; aromatics; critical review, evaluated data; gases; halogenated hydrocarbons; Henry's law constants; liquids; JPCRD 10(4): 1175.1199; 1981.

petroleum oil; recycled oil; rerefined oil; test procedures; basestock engine lubricants; lubricating oil; motor oil; 20016

phase angle; sinewave generator; standard; synthesis; calibration; digital; microcomputer; $T N 1144$.

phase changes; irreversible thermodynamics; Lennard-Jones fluid; nonequilibrium molecular dynamics; 19974.

phase comparator; real-time control; vibration control; vibration isolation; active vibration control; Michelson interferometer; optical path-length correction; 20734 .

phase diagram; potassium; conductivity; exchange interaction; ferrite; hyperfine field; magnetism; 20043.

phase diagrams; phase equilibrium hierarchy; rapid solidification; thermodynamics; metastable phase equilibrium; partitionless solidification; 19981.

phase diagrams; radioactivity; research; sediment; soil; alloy; carbon; computers; energy; fine structure; interface; materials; measurement; office building; $D I M / N B S 65(8)$ : 1-28; 1981.

phase equilibria; diffraction effects; long range order; modulated structures; oxides; 20046

phase equilibria; pure fluids; coexistence densities; critical density; critical point; liquid mass fraction; liquid volume fraction; NBSIR 80-1631.

phase equilibrium hierarchy; rapid solidification; thermodynamics; metastable phase equilibrium; partitionless solidification; phase diagrams; 19981.

Phase IV; remote terminal emulation; response time; terminal networks; workload sizing; benchmark testing; SP500-83: 1981 November. 169-177.

phasor notation; thermodynamic and dynamic analyses; displacer and piston drives; linear electric motors; linear free-displacer cryorefrigerator; linear resonant machinery; SP607: 1981 May. 57 69.

phenobarbital; phenytoin; primidone; reference material; serum base modification; serum matrix; anticonvulsants; antiepilepsy drugs; blood analysis; chromatography, gas; chromatography, liquid; drug standards; enzyme immunoassay; epilepsy; ethosuximide; mass spectrometry; nuclear magnetic resonance spectroscopy; $S P 260-72$.

phenytoin; primidone; reference material; serum base modification serum matrix; anticonvulsants; antiepilepsy drugs; blood analysis; chromatography, gas; chromatography, liquid; drug standards; enzyme immunoassay; epilepsy; ethosuximide; mass spectrometry; nuclear magnetic resonance spectroscopy; phenobarbital; SP260-72.

pH history; alkaline reserve; cellulose stabilizers; paper deacidification; paper destabilization; paper permanence; J. Res. 86(3): 309-315; 1981 May-June.

pH measurements; surface hydride; alpha uranium; chloride ion; chromate ion; electrochemistry; ellipsometry; field ion microscopy; inhibitors; organic coatings; passive film dissolution; NBSIR 81 . 2396 (ONR).

phosphine; phosphorus nitride; radio astronomy; sulfur dioxide; interstellar; nitric oxide; 19987.

phosphorus; radicals; Raman spectroscopy; temperature; flame inhibition; H-atoms; kinetics; mass spectrometry; NBSIR 80-2169.

phosphorus; resistivity; semiconductor; silicon; boron; capacitancevoltage technique; dopant density; electron mobility; Hall effect; hole mobility; Irvin curves; SP400-64.

phosphorus nitride; radio astronomy; sulfur dioxide; interstellar; nitric oxide; phosphine; 19987.

photoacoustic; surface-to-bulk optical absorption; $\mathrm{CaF}_{2}$; laser calorimetry; laser windows; optoacoustic; SP620: 1981 October. 58. 74.

photoacoustic spectroscopy; surface impurities; alkali-halide laser windows; $\mathrm{KCl} ; \mathrm{NaCl}$; optoacoustic spectroscopy; SP620: 1981 October. 44-49.

photoacoustic technique; absorption in solids; beam depletion; nonlinear absorption; SP620: 1981 October. 50.57.

photocell; solar cell; solid state; transistor; tunnel diode; diode; fieldeffect transistor; III.V compounds; IMPATT diode; integrated circuit; LASER; LED; J. Res. 86(6): 565-570; 1981 NovemberDecember.

photochemistry; rates; review; singlet oxygen; solution; chemical kinetics; data compilation; oxidation; JPCRD 10(4): 809-999; 1981.

photodecomposition; reaction mechanism; t-2-fluorovinyl; acetylene; F atoms; hydrogen bonding; infrared spectrum; matrix isolation; 20036.

photodetachment; potential energy curves; autoionization; electron affinity; molecular spectroscopic constants; negative ions; 19995.

photodissociation; F-atom reactions; infrared spectrum; matrix isolation; normal coordinate analysis; 19986.

photodissociation; vacuum ultraviolet; $I_{2}$ fluorescence absorption coefficient; $\mathrm{Kr}$ photosensitized reaction; methylene iodide; 20042.

photoelastic coefficients; polycrystalline aggregate; stress birefringence; thermal lensing; window materials; elastic coefficients; high-energy laser; interferometric testing; optical distortion; SP620: 1981 October. 117-128.

photoelectron-photoionization coincidence; photoionization; propyne; threshold photoelectron spectra; breakdown curves; 20051.

photoemission; resonance; satellite; auger; core hole spectroscopy; metals with filled $d$-bands; 20008 .

photoexcited carriers; semiconductor plasma; IR laser damage SP620: 1981 October. 427-431.

photofragmentation; radical; $\mathrm{CH}_{3} ; \mathrm{CH}_{2} \mathrm{I}$; infrared emission; laser; 20004.

photoionization; propyne; threshold photoelectron spectra; breakdown curves; photoelectron-photoionization coincidence; 20051.

photolithographic fabrication; refrigerators; cryogenic; JouleThomson; microminiature devices; SP607: 1981 May. 160-163.

photon-assisted tunneling; superconductivity; thin film; transition temperature; tunneling; tunnel junction; aluminum; critical current; energy gap; enhancement; microwaves; nonequilibrium superconductivity; 19979.

photon beam; radiation therapy; absorbed dose; Bragg-Gray equation; calibration; cavity chamber; cavity theory; electron beam; inphantom measurement; 20073.

photon beams; photonuclear research; tagged photon method; BetheHeitler cross section; bremsstrahlung differential cross section; 
bremsstrahlung monochromator; NBSIR 81-2262.

photon decays; quarks; angular distributions; charmed mesons; dipole transitions; magnetic moments; 20040.

photonuclear; quasideuteron; resonance fluorescence; giant resonance; 19992.

photonuclear reactions; sum rules; giant resonances; inelastic electron scattering; inelastic hadron scattering; 19977.

photonuclear research; tagged photon method; Bethe-Heitler cross section; bremsstrahlung differential cross section; bremsstrahlung monochromator; photon beams; NBSIR 81-2262.

photo-receptor membrane vesicles; random coil polymers; amphoteric latex; dynamic light scattering; light scattering; nematic liquid crystal; J. Res. 86(6): 571.590; 1981 November-December.

photovoltaic cells; sheet resistance; solar cell defects; solar cells; test structures; laser scanner; model calculations; NBSIR 81-2260.

photovoltaic modules; photovoltaics; reference cells; solar cells; terrestrial solar simulators; test methods; calibration; combined photovoltaic/thermal modules; measurements; NBS-GCR-80-279.

photovoltaics; reference cells; solar cells; terrestrial solar simulators; test methods; calibration; combined photovoltaic/thermal modules; measurements; photovoltaic modules; NBS-GCR-80-279.

photovoltaics; solar cell analysis; solar cells; computer simulations; fault detection; laser scanner; NBS-GCR-80-285.

photovoltaics; solar cells; test pattern; defect identification; laser scanner; measurement techniques; NBSIR 80-2181.

physical; properties data; radiation damage; rock salt; sodium chloride; chemical; electrical; geological; magnetic; mechanical; monograph; optical; Monogr. 167.

physical deterioration; reliability; turbomachinery; aerothermal diagnostics; aerothermal parameters; performance; SP622: 1981 October. 222-253.

physical optics; planewave spectrum; reflector antennas; co-sited antennas; coupling; far fields; near fields; NBSIR 80-1630.

physical properties; safety and hazards; thermal energy storage materials; thermal properties; thermodynamic properties; transport properties; corrosion; data compilations; electrochemical aluminum production; electrochemical energy storage materials; molten salts; NSRDS-NBS61, Part IV.

physical properties; stainless steels; structural alloys; welding; alloys; aluminum alloys; castings; composites; cryogenic properties; low temperature; mechanical properties; nonmetallics; NBSIR 81-1645.

physical property; accuracy; certified reference material; chemical composition; 19990.

physical property; superconductor composites; thermal effect; thermal expansion; low temperature; nonmetallics; 20753.

physicists; physics; polymers; data; equations; handbook; 20749.

physics; polymers; data; equations; handbook; physicists; 20749.

physics; solar collector; space charges; technical competence; cement and concrete; cover plates; earth's tilt; electric; fire; fracture toughness; measurement; parameter; DIM/NBS 65(3): 1.24; 1981.

physics of fire; toxicity of combustion products; chemistry of fire; combustion products; detection; fire hazards; fire modeling; fire protection; fire research; fire suppression; human behavior in fires; NBSIR 80-2127-1.

picosecond phenomena; self-focusing; $\mathrm{SiO}_{2}$; air breakdown; laser damage; $\mathrm{NaCl}$; SP620: 1981 October. 375-384.

pictogram; response; symbol; understandability; communication; evaluation method; fire-safety; hazard warnings; meaningfulness; method; NBSIR 80-2088.

pictograms; safety; signs; standards; symbols; visual alerting; warnings; communication; hazard; NBSIR 80-2003.

pictograms; symbols; understandability; visibility; visual alerting; directional arrows; exit signs; fire safety; NBSIR 81-2268.

pictures; simulation; tools; analysis; performance evaluation; SP50083: 1981 November. 331-334.

piezoelectric polymer material; vegetable and animal life; antifouling coating; film; marine structures; U.S. Patent 4,297,394.

piezoelectric polymer material; vegetable and animal life; antifouling coating; film; marine structures; U.S. Patent 4,283,461.

pipe; slope; stream-depth; unsteady; volume; water; diameter; drain; flow; history; horizontal; length; partially-filled; NBSIR 81-2249. pipes; thermal insulation; thermal properties; underground systems; computer program; earth temperature; heat transfer; NBSIR 812378 .

piston gage; pressure; primary standard; transfer standard; effective area; intercomparison; J. Res. 86(3): 277.279; 1981 May-June.

piston ring wear; turbocharging monitoring; crankshaft bearing safety; medium speed diesel engine; SP622: 1981 October. 97-112.

pitched-horizontal drain; slope; solid; velocity; volume; water; cylindrical; non-uniform; partially-filled; NBSIR 81-2266.

plait point; Rayleigh light scattering; thermal diffusivity; concentration; diffusivity; methane-ethane; mixture; one-fluid model; 19964.

plane-stress; strain invariants; tensile-test specimen; checkerboard pressure; elastostatics; finite elements; in-core solver; isoparametrics; Mooney-Rivlin material; nonlinear equation-solvers; penalty method; J. Res. 86(1): 79-109; 1981 January-February.

planetary atmospheres; quadrupole-induced absorption; spectral moments; band shape analysis; collision-induced absorption; far infrared; $\mathrm{H}_{2}-\mathrm{H}_{2}$ collisions; $\mathrm{H}_{2}-\mathrm{He}$ collisions; NBSIR 80-2175.

planewave spectrum; reflector antennas; co-sited antennas; coupling; far fields; near fields; physical optics; NBSIR 80-1630.

plasma diagnostics; radiation; synchrotron radiation; astrophysics; bremsstrahlung; electromagnetic interactions; pair production; 19993.

plasma dispersion function; Voigt function; argand diagram; complex error function; continued fraction; Dawson's function; FORTRAN subroutine; Fresnel integrals; key values; line broadening function; J. Res. 86(6): 661-686; 1981 November-December.

plasma dynamics; spiral grand design; WASER; winding dilemma; density wave; galactic spirals; hydrodynamic instability and turbulence; J. Res. 86(6): 557-563; 1981 November-December.

plasma effects; pulsed laser beams; $10.6 \mu \mathrm{m}$; attenuators; beam profile; calorimetry; fluence; heat flow; irradiance; measurements of temperature; NBSIR 80-1628.

plasma emission; specific elements; spectroscopy; chromatography; detection; metals; SP618: 1981 November. 184-196.

plasmids; silver; toxic heavy metals; antimony; arsenic; bacterial resistances; cadmium; detoxification; mercury; organomercury; SP618: 1981 November. 301-324.

plastic; polyurethane; room fires; wood; crib fires; experiments; mathematical model; NBSIR 80-2054, Vols. I and II.

plastic; room fire; wood; zone model; crib fire; 20740.

plastic-bonded; soils; telephone cables; underground; alloys; corrosion; metallurgically-bonded; metals; NBSIR 81-2243.

plastic deformation; temperature effects stainless steel alloys; yield stress; cryogenic properties of materials; iron alloys; low temperature mechanical properties; 20742.

plastics; polymers; polymethylmethacrylate; polyoxymethylene; polypropylene; polystyrene; polyurethane; polyvinyl chloride; pyrolysis; smoke; wood; combustion products; fire modeling; foam (materials); heat release; NBS-GCR-80-295.

plastics; polymers; standards; macromolecules; NBSIR 81-2263.

plastics; refrigeration; regenerators; specific heat; thermal conductivity; composites; cryocoolers; SP607: 1981 May. 173-177.

plastics; refrigerators; regenerators; Stirling-cycle; calculations; cryogenics; helium; 20020.

plating; plating on anodized aluminum; adhesion; anodizing; coatings; electrodeposition; immersion deposition; NBSIR 80-2142.

plating on anodized aluminum; adhesion; anodizing; coatings; electrodeposition; immersion deposition; plating; NBSIR 80-2142.

playground safety; playground surfaces; soil; surfacing materials; test method; asphalt; impact attenuation; peak acceleration; NBSIR 802106.

playground surfaces; soil; surfacing materials; test method; asphalt; impact attenuation; peak acceleration; playground safety; NBSIR 80-2106.

plumbing; quasi-steady; unsteady; characteristics; discharge; drainage; flow; model; J. Res. 86(2): 171-179; 1981 March-April.

plumbing; solar; steel; structural systems; test methods; wood; building rehabilitation; concrete; electrical; evaluation; HVAC; masonry; NBSIR 81-2289. 
plumbing; steel; structural systems; test methods; wood; building rehabilitation; concrete; electrical; evaluation; HVAC; masonry; NBSIR 80-2171.

Pockels effect; solids; dielectrics; electro-optics; gases; high voltage; insulation; interfaces; Kerr effect; liquids; NBSIR 81-2275.

point defects; primary defects; damage threshold; excitons; $\mathrm{NaCl}$; optical breakdown; SP620: 1981 October. 406-415.

polarized electrons; electron scattering; magnetism; nickel; 19970.

polarized electrons; spin; spin polarization; electron gun; electron polarization; electron spin; 19996.

polarized electrons; surface magnetism; ferromagnetism; $\mathrm{Ni}$; nickel; 19975.

polarized LEED; spin detector; spin polarization; surface resonance; surface structure; 20012.

polarized light; electric field; electro-optics; Kerr effect; measurement; optics; 19962.

polarizer; thin films; diode laser; ellipsometer; infrared; SP620: 1981 October. 345.355.

polarography; tissue response; atomic absorption spectrophotometry; EDAX analysis; enzyme histochemistry; histology; implant retrieval analysis; SP601: 1981 January. 323-336.

polaron absorption; avalanche breakdown; beam deformation; beam depletion; free carrier absorption; multiphoton absorption; SP620: 1981 October. 394-402.

policymaking; protestors; solar code; victim role; deed; developers; energy wars; experimental code; field test; guideline; low income; non-code; SP608: 1981 May. 173-178.

policy models; sensitivity analysis; validation; assessment; composite models; data quality; energy models; mathematical models; model confidence; model credibility; SP616.

polishing; polycrystalline material; residual stress; sapphire; specular reflectance; surface analysis; surface integrity; surface roughness; crystallographic anisotropy; crystallographic perfection; initial permeability; metal mirror; SP620: 1981 October. 171-179.

pollutant properties; synfuel; fossil porphyrins; fuel conversion; fuel extraction; heterocyclics; metal chelates; metallo-complexes; origin of pollutants; SP618: 1981 November. 9-20.

polycrystalline aggregate; stress birefringence; thermal lensing; window materials; elastic coefficients; high-energy laser; interferometric testing; optical distortion; photoelastic coefficients; SP620: 1981 October. 117-128.

polycrystalline material; residual stress; sapphire; specular reflectance; surface analysis; surface integrity; surface roughness; crystallographic anisotropy; crystallographic perfection; initial permeability; metal mirror; polishing; SP620: 1981 October. 171. 179.

polycrystals; sound velocities; tensor-property averaging; copper; Debye temperature; elastic constants; low temperatures; 20726.

polyester; textiles; airflow; burn (injuries); cotton; fabric flammability; fabrics; fire suppression; flame extinguishment; heat transfer; NBSIR 81-2386.

polyethylene; crystal; crystallinity; density; enthalpy; entropy; fusion; Gibbs energy; glass transition; heat capacity; linear macromolecule; melt; JPCRD 10(1): 119-152; 1981.

polyethylene; effective carbon atom number; loss-rate; mass-loss; n-alkanes; n-carboxylic esters; permeation; NBSIR 80-2131.

polyethylene acetabulae; polymeric prosthesis; wear; SP601: 1981 January. 471.499.

polyethylene components; acrylic cement; joint replacements; SP601: 1981 January. 3-28.

polyethylene terephthalate (Dacron); arterial prostheses; biodeterioration; SP601: 1981 January. 29-129.

polyfunctional surface-active comonomers; reconstructive dental work; resin; acrylate; adhesion; aminobenzoate; composite material; dentin; methacrylate; U.S. Patent 4,251,565.

polymer; stress relaxation; UHMWPE; ultra high molecular weight polyethylene; creep; failure; fatigue; mechanical properties; morphology; NBSIR 81-2209 (FDA).

polymer degradation products; pressure; thermal degradation; thermogravimetry; activation energy; bubbles; 20774.

polymeric prosthesis; wear; polyethylene acetabulae; SP601: 1981
January. 471-499.

polymers; data; equations; handbook; physicists; physics; 20749.

polymers; polymethylmethacrylate; polyoxymethylene;

polypropylene; polystyrene; polyurethane; polyvinyl chloride; pyrolysis; smoke; wood; combustion products; fire modeling; foam (materials); heat release; plastics; NBS-GCR-80-295.

polymers; radiography; SRM's; stars; visual acuity; evolution; fracture mechanics; gamma rays; inventors; molecular interactions; molecular processes; neomycin; neutron dosimeters; DIM/NBS 65(7): 1.24; 1981.

polymers; retrieval; analysis; biological; cardiovascular; implants; metals; orthopaedic; SP601.

polymers; smoke; soot; toxicity; wood; combustion; decision analysis; fire models; flame spread; human behavior; ignition; NBSIR 81 . 2382.

polymers; standards; macromolecules; plastics; NBSIR 81-2263.

polymers research; corrosion; fracture and deformation; glass and ceramics research; materials research; materials science; metallurgy; NBSIR 81-2408.

Polymers Science and Standards Division; Reactor Division; Center for Materials Science; Ceramics Glass and Solid State Science Division; Chemical Stability and Corrosion Division; Fracture and Deformation Division; FY80 technical activities; Metallurgy Division; NBSIR 80-2108.

polymers under high pressure; Raman spectroscopy; characterization of polymers; 20007.

polymers under tensile load; acoustic emission of polymers; emission of polymers; 20014.

polymethylmethacrylate; polyoxymethylene; polypropylene; polystyrene; polyurethane; polyvinyl chloride; pyrolysis; smoke wood; combustion products; fire modeling; foam (materials); heat release; plastics; polymers; NBS-GCR-80-295.

polymethylmethacrylate (PMMA); prosthesis interface; femoral component loosening; SP601: 1981 January. 157-174.

polynominal fitting; user manual; automated thermogravimetry; data analysis; generalized plotting programs; interdata 7.16 computer; NBSIR 80-2102.

polynuclear aromatic hydrocarbons; reference materials; shale oil SRM; trace organic analysis; definitive methods; human serum 19989.

polyolefins; radioactive tracer; spectrofluorimetry; antioxidants; diffusion; food packaging; inverse gas chromatography; migration oil simulants; oligomers; NBSIR 81-2264.

polyoxymethylene; polypropylene; polystyrene; polyurethane; polyvinyl chloride; pyrolysis; smoke; wood; combustion products fire modeling; foam (materials); heat release; plastics; polymers polymethylmethacrylate; NBS-GCR-80-295.

poly(oxymethylene)s; poly(oxyphenylene)s; poly(oxypropylene); crystal; crystallinity; density; enthalpy; entropy; fusion; Gibbs energy; glass transition; heat capacity; linear macromolecule JPCRD 10(4): 1001.1049; 1981.

poly(oxyphenylene)s; poly(oxypropylene); crystal; crystallinity; density; enthalpy; entropy; fusion; Gibbs energy; glass transition; heat capacity; linear macromolecule; poly(oxymethylene)s; JPCRD 10(4): 1001-1049; 1981.

poly(oxypropylene); crystal; crystallinity; density; enthalpy; entropy; fusion; Gibbs energy; glass transition; heat capacity; linear macromolecule; poly(oxymethylene)s; poly(oxyphenylene)s; JPCRD 10(4): 1001-1049; 1981.

polypropylene; polystyrene; polyurethane; polyvinyl chloride; pyrolysis; smoke; wood; combustion products; fire modeling; foam (materials); heat release; plastics; polymers; polymethylmethacrylate; polyoxymethylene; NBS-GCR-80-295.

polypropylene; syndiotactic; atactic; crystal; crystallinity; density; enthalpy; entropy; fusion; Gibbs energy; glass transition; heat capacity; isotactic; linear macromolecule; melt; JPCRD 10(4): 1051 1064; 1981.

polysilicon films; silicon dioxide films; silicon nitride films; standard reference materials; thin films; ellipsometry; NBSIR 80-2158.

polyspectra; spectrum analysis; statistics; time series; bispectrum; cumulant spectra; nonlinear time series; TN1036. 
polystyrene; polyurethane; polyvinyl chloride; pyrolysis; smoke wood; combustion products; fire modeling; foam (materials); heat release; plastics; polymers; polymethylmethacrylate; polyoxymethylene; polypropylene; NBS-GCR-80-295.

polystyrene; polyurethane; thermal insulation; thermal properties; cellular plastic; low temperature; mechanical properties; 20074.

polyurethane; polyvinyl chloride; pyrolysis; smoke; wood; combustion products; fire modeling; foam (materials); heat release; plastics; polymers; polymethylmethacrylate; polyoxymethylene; polypropylene; polystyrene; NBS-GCR-80-295.

polyurethane; room fires; wood; crib fires; experiments; mathematical model; plastic; NBSIR 80-2054, Vols. I and II.

polyurethane; thermal insulation; thermal properties; cellular plastic low temperature; mechanical properties; polystyrene; 20074.

polyurethane foam combustion; room fires; fire modeling; fire plumes; flame spread; furniture flammability; NBS-GCR-81-300

polyurethane foam materials; smolder resistant upholstered furniture or mattress assembly; sulphur; fibrous; U.S. Patent 4,260,660.

polyvinyl chloride; pyrolysis; smoke; wood; combustion products; fire modeling; foam (materials); heat release; plastics; polymers polymethylmethacrylate; polyoxymethylene; polypropylene; polystyrene; polyurethane; NBS-GCR-80-295.

population statistics; refractive index; auto headlight glass; classification; density; forensic science; individualization; NBSIR $81-2286$.

porcine xenograft valves; prosthesis failure; vitamin K-dependent calcification; calcific degeneration; SP601: 1981 January. 339-348.

porosity measurement; porous biomaterials; porous polyethylene; PTFE-carbon composite; synthetic implants; ossicular prosthesis; NBSIR 81-2212.

porous biomaterials; porous polyethylene; PTFE-carbon composite; synthetic implants; ossicular prosthesis; porosity measurement NBSIR 81-2212.

porous polyethylene; PTFE-carbon composite; synthetic implants; ossicular prosthesis; porosity measurement; porous biomaterials; NBSIR 81-2212.

porous solid; thermal conductivity; concrete; guarded hot plate; low temperature; moisture migration; mortar; NBSIR 81-1651.

porphyrin; shale oil; speciation; trace metals; chromatography; direct current plasma; hydrophilic; hydrophobic; metal complex; SP618 1981 November. 105.119.

portability; software conversion; database management; data conversion; data description; data interchange form; data translation; NBSIR 81-2315.

position-sensitive proportional counters; Van de Graaff accelerator; 14 $\mathrm{MeV}$ neutron energy; linear accelerator; neutron imaging techniques; 20724.

positive ion and neutral lifetime; cesium from molybdenum(111); ion and neutral lifetime; 20013.

post-marketing surveillance; regulatory experiments; system design; drug development; drug regulation; innovation; NBS-GCR-ETIP 81-96.

post-occupancy evaluation; questionnaire; temperature; ventilation; windows; energy conservation; lighting; man/environment research; noise; office building; BSS130.

post-occupancy housing evaluation; Project Feedback; questionnaires; research methods; survey research; user needs; dwelling units (residential); housing; Operation Breakthrough; NBSIR 81-2258.

potassium; conductivity; exchange interaction; ferrite; hyperfine field; magnetism; phase diagram; 20043.

potassium; rubidium; sodium; solutions; thermodynamic properties; activity coefficient; cesium; critical evaluation; electrolyte; exces Gibbs energy; lithium; osmotic coefficient; JPCRD 10(3): 671.766.

potassium chloride; reference data; viscosity; aqueous solutions; compilation; JPCRD 10(1): 57-70; 1981.

potassium compounds; protactinium compounds; rubidium compounds; sodium compounds; thorium compounds; uranium compounds; actinium compounds; cesium compounds; enthalpy entropy; francium compounds; Gibbs energy of formation; heat capacity; lithium compounds; TN270-8.

potassium fluoride; salt hydrate; sodium sulfate; temperature calibration; temperature standard; thermistor; thermometer transition temperature; biomedical thermometry; disodium hydrogen phosphate; fixed point; hydration temperature; J. Res. 86(2): 181.192; 1981 March.April.

potassium slag; thermodynamics; vaporization; chemical activity; magnetohydrodynamics; mass spectrometry; NBSIR 81-2293.

potential barriers; quantum mechanical tunneling calculations; tunneling corrections; chemical rate constants; Eckart potential; $J$. Res. 86(4): 357.359; 1981 July-August.

potential energy curves; autoionization; electron affinity; molecular spectroscopic constants; negative ions; photodetachment; 19995.

powder data; progress; R-values; single-crystal; SRM's; atomic ions; calibration; clock synchronization; Columbia River model; computer; insulation; lasers; DIM/NBS 65(2): 1-28; 1981.

powder diffraction; quantitative analysis; $\mathrm{x}$ ray; automation; NBSIR 81-2229.

powder diffraction; unit cell; computer evaluation; crystallographic data analysis; JCPDS-International Centre for Diffraction Data metric symmetry; NBS Crystal Data Center; TN1141.

powder metallurgy; quantitative microscopy; standard reference material; $x$-ray diffraction; $x$-ray fluorescence analysis; austenite in ferrite; austenite standard; electron microprobe; SP260-73.

powder patterns; reference intensities; standard; $x$-ray diffraction; crystal structure; densities; lattice constants; Monogr. 25, Section 18.

power; radiation; semiconductor; technology; calibration; electrical electronics; engineering; hazards; measurement; metrology; National Bureau of Standards; SP611.

power; standard; calorimeter; millimeter wave; TN1044.

power-device grade silicon; thyristor material characterization; transient capacitance techniques; deep-level measurements; deeplevel transient spectroscopy; lifetime; NBSIR 81-2325.

power factor correction; source energy; transformers; electrical design; energy distribution; National Electrical Code; SP608: 1981 May. 37.56.

power line noise; psychoacoustics; annoyance; audible noise; corona noise; EHV transmission lines; electric energy; environmental noise; human response; 20050.

practices; safety; sealants; standards; storm doors; storm windows; vent dampers; water heaters; windows; automatic ignition devices; caulks; effectiveness; energy conservation; energy conservation measures; installation; insulation; oil burners; SP606.

precipitating; segregation; solidification; thermal mechanical treatment; aging; electrical conductivity; hardness; homogeneity; microstructure; NBSIR 80-2069.

precision; reference material; stability of measurement process; statement of certainty; 20041.

precision measurements; radio star; satellite communications; $\mathrm{C} / \mathrm{kT}$; EIRP; error analysis; G/T; NBSIR 78.869.

precompilers for special arithmetic; AUGMENT and MP; Fortran arithmetic extensions; multiple precision; multiprecision Fortran software; multiprecision mathematical functions; NBSIR 80-2138.

preconcentration; air analysis; diffusion; hollow tubes; mercury; SP618: 1981 November. 134-145.

predictability; reproducibility; time to failure; accelerated aging tests; coatings; corrosion; TN1149.

prediction; Van der Waals theory; viscosity; data evaluation; extended corresponding states; fluids; mixtures; one fluid model; 20768.

prediction of bearing failure; shock pulse bearing monitoring; automatic alarm of bearing malfunction; marine machinery bearings; microprocessor based shock pulse analyzer; multiplexing bearing monitor channels; SP622: 1981 October. 126-136.

predissociation; scattering theory; bound states; continuum states diatomic spectroscopy; electronic-rotational states; 20049.

pre-irradiation; thin films; ultraviolet reflectors; excimer lasers; fluoride coatings; laser damage; multiple-shot damage; oxide coatings; SP620: 1981 October. 265-276.

prepackaged consumer goods; statistical sampling; commodity inspection; compliance testing; net weight; package checking; packaged product inspection; $H 133$.

pressure; primary standard; transfer standard; effective area intercomparison; piston gage; J. Res. 86(3): 277.279; 1981 May. 
June

pressure; propane; sound; specific heat ratios; speed; temperature; velocity; adiabatic compressibility; liquid; J. Res. 86(2): 165.170; 1981 March-April.

pressure; spray ignition; temperature rise; flammability; heat flux; NBSIR 81-2247.

pressure; temperature; thermal conductivity; transient; helium; hot wire; nitrogen; J. Res. 86(5): 457.493; 1981 September-October.

pressure; thermal degradation; thermogravimetry; activation energy; bubbles; polymer degradation products; 20774.

pressure cycles; stress; superconducting power transmission line; terminals; thermal contraction; cable end fittings; 20018.

pressure probes; static pressure; fire endurance; furnaces; NBSIR 81 2415.

pressure-temperature; spray; synthetics; water glycol; coal mine; fire tests; hydraulic fluids; invert emulsions; NBSIR 81-2373.

pressurization; smoke control; stairwells; air movement; computer programs; egress; elevator shafts; escape means; NBSIR 80-2157.

pressurized water reactor; remote surveillance; acoustic emissions; cavitation; feedwater control valve; pattern recognition; SP622: 1981 October. 11-30.

prestressed concrete; prestressing steel; stress corrosion; concrete; corrosion; general corrosion; mathematical modeling; NBSIR 81 . 2390.

prestressing steel; stress corrosion; concrete; corrosion; general corrosion; mathematical modeling; prestressed concrete; NBSIR 81-2390.

pricing; standard costing; chargeback; chargeout; charging; charging system; DP cost accounting; DP cost allocation; SP500-83: 1981 November. 109-118.

primary defects; damage threshold; excitons; $\mathrm{NaCl}$; optical breakdown; point defects; SP620: 1981 October. 406-415.

primary standard; transfer standard; effective area; intercomparison; piston gage; pressure; J. Res. 86(3): 277.279; 1981 May-June.

primidone; reference material; serum base modification; serum matrix anticonvulsants; antiepilepsy drugs; blood analysis; chromatography, gas; chromatography, liquid; drug standards; enzyme immunoassay; epilepsy; ethosuximide; mass spectrometry; nuclear magnetic resonance spectroscopy; phenobarbital; phenytoin; SP260-72.

principal components; maxmin; minmax; multiple comparison; multivariate analysis; peak areas; NBSIR 81-2222.

printer terminals; standard; text communication; text interchange; typesetting composition; ADP terminals; ASCII; character-imaging devices; control functions; data interchange; display terminals; Federal Information Processing Standard; information interchange FIPS PUB 86

prisons; smoke production; bedding; beds; compartment fires; fire tests; health care facilities; heat release rate; hospitals; mattresses; NBSIR 80-2186.

probability; statistics; structural engineering; live loads; load survey; NBS-GCR-76-81.

probability distribution functions; reliability; statistical analysis; structural engineering; wind; climatology; loads (forces); 20019.

probe; radio-frequency; temperature measurements; heating; NBSIR 80-1634.

probe station; semiconductor process control; test pattern; test structure; wafer chuck; wafer probe station; integrated circuit test methods; microelectronic test structure; SP400-68.

proceedings; serials; standards; transactions; annual reports; diffusion in metals; fire; journals; library holdings, NBS Library; NBS periodicals; periodicals; NBSIR $81-2226$

processing limits; ultrafine filament $\mathrm{Nb}_{3} \mathrm{Sn}$; bronze process; $\mathrm{Nb}_{3} \mathrm{Sn}$; 20741

processor; programming language; software; BASIC; COBOL; compilers; data processing; Federal Information Processing Standard; FORTRAN; information interchange; information processing; FIPS PUB 29-1.

process parameters; research recommendations; coal; control; fossil energy; materials; NBSIR $81-2348$.

process validation wafer; random faults; silicon-on-sapphire; test pattern; test structure; yield; integrated circuits; microelectronics; SP400-66.

process waters; selenium; solvent refined coal; titanium; trace elements; arsenic; chromium; coal hydrogenation; cobalt; liquefaction; mercury; nickel; SP618: 1981 November. 21-38.

procurement; purchasing; recovered/recycled materials; resource recovery; econimic, institutional, technical barriers; NBS-GCR-81309, Supplement 1 .

procurement; purchasing; recovered/recycled materials; resource recovery; economic, institutional, technical barriers; NBS-GCR-81310.

procurement; purchasing; recovered/recycled materials; resource recovery; economic, institutional, technical barriers; NBS-GCR-81309.

procurement; purchasing; recovered/recycled materials; resource recovery; technical barriers; economic; institutional; NBSIR 81 2316.

procurement; purchasing; recovered/recycled materials; resource recovery; technical barriers; economic; institutional; NBS-GCR-81314.

procurement; purchasing; recovered/recycled materials; resource recovery; technical barriers; economic; institutional; NBS-GCR-81312 .

procurement; purchasing; recovered/recycled materials; resource recovery; technical barriers; economic; institutional; NBS-GCR-81308.

procurement; purchasing; recovered/recycled materials; resource recovery; technical barriers; economic; institutional; NBS-GCR-81311 .

procurement; purchasing; recovered/recycled materials; resource recovery; technical barriers; economic; institutional; NBS-GCR-81313.

procurement; standards; telecommunications; automated data processing (ADP); communications; Federal Information Processing Standard (FIPS); Federal Property Management Regulations (FPMR); FIPS PUB 80.

product form; queueing models; system resources; network models; SP500-83: 1981 November. 335.

production efficiency; scraping Auger; slush; slush production; cryogenic; hydrogen; NBSIR 78-875.

production process; quark model; strange meson; $E$ meson at 1440 $\mathrm{MeV}$; flavor singlet; glueball; 20034.

productivity; development environment; development function; SP500-83: 1981 November. 337.

productivity; regulation; renovation; building codes; building economics; construction; cost; economics; housing; NBSIR 802150.

productivity; research associate; scanning technique; spin dependent; SRM's; standards; tape; copper; deterioration; electromagnetic radiation; film; industry; metals; DIM/NBS 65(5): 1.24; 1981.

productivity; system tuning; computer system; data processing function; performance evaluation; performance management; SP500-83: 1981 November. 121.

productivity; user service reporting; ADP installation management; cost-benefit; performance management; SP500-83: 1981 November. 59-65.

productivity; word processing; automation; information management; information technology; SP500-83: 1981 November. 305-307.

productivity analysis; text editors; end user performance; interactive systems; performance analysis; SP500-83: 1981 November. 71-80.

productivity measurement; risks of failure; user productivity; building productivity; computer-integrated construction; conference; construction duration; NBS-GCR-81-331.

product liability; rationale statements; scope of standards; standards standards writing; legislative history; NBS-GCR-81-347.

professional practice; provisions; soil structure interaction; standards; buildings; design; earthquakes; engineering; foundations; NBSIR 80-2111-3.

profile refinement method; scattering amplitude of neodymium cerium neodymium tantalates; cerium niobate; neutron diffraction oxidation mechanisms; 20054. 
prognosis; sensors; wear; wear analysis; energy; energy conservation; failure detection; failure diagnosis; failure prevention; monitoring techniques; SP622.

program behavior; structural analysis; performance comparison; SP500-83: 1981 November. 199-215.

program maintenance; requirements studies; software procurement; application development; computer software; cost/benefit analysis; database management systems; data management; SP500-84.

program measure; Residential Conservation Service; Solar Model Audit; auditor training; energy conservation; SP608: 1981 May. 183-194.

programming aids; software automation; software development; software engineering; software tools; software tools usage; SP50082.

programming aids; software automation; software development; software engineering; software testing; software tools; SP500-80.

programming aids; software automation; software development; software engineering; software tools; software tools usage; NBSIR 81-2388.

programming aids; software automation; software development; software engineering; software tools; software tools usage; NBSGCR-81-335.

programming aids; software development; software engineering; software tools; static analysis; taxonomy; dynamic analysis; $S P 500$ 74.

programming environments; software development; software tools; toolboxes; development support systems; SP500-78.

programming language; software; BASIC; COBOL; compilers; data processing; Federal Information Processing Standard; FORTRAN; information interchange; information processing; processor; FIPS $P U B$ 29-1.

programming languages; software expenditures; software program life-cycle analysis; data base management systems; Federal contracts services; Federal Government computers; SP500-79.

programming language standards; computer arithmetic; conformance testing; numeric accuracy; SP500-77.

progress; R-values; single-crystal; SRM's; atomic ions; calibration; clock synchronization; Columbia River model; computer; insulation; lasers; powder data; DIM/NBS 65(2): 1-28; 1981.

prohibitions; re-examination; thermal storage; wood structure; codes; concrete; masonry; SP608: 1981 May. 195-200.

Project Feedback; questionnaires; research methods; survey research; user needs; dwelling units (residential); housing; Operation Breakthrough; post-occupancy housing evaluation; NBSIR 81-2258.

projectiles; safety; contusion; eyeball deformation; impact; ocular injury; NBSIR 80-2037.

project management; system acquisition; system development; administrative experiment; evaluation systems; Experimental Technology Incentives Program; information systems; NBSIR 812327.

project management; system acquisition; system development; administrative experiment; evaluation systems; Experimental Technology Incentives Program; information systems; NBSIR 812328.

project summaries; technical bases; building research; building technology; codes; criteria; measurement methods; performance criteria; SP446-5.

propagation; smoldering combustion; test methods; wave structure; boric acid; cellulosic insulation; ignition; NBSIR 81-2350.

propane; radio frequency; level gauging; NBSIR 79-1600.

propane; sound; specific heat ratios; speed; temperature; velocity; adiabatic compressibility; liquid; pressure; J. Res. 86(2): 165-170; 1981 March-April.

properties; research materials; standard reference materials; analysis; characterization; composition; materials; SP260, 1981-83 Edition.

properties data; radiation damage; rock salt; sodium chloride; chemical; electrical; geological; magnetic; mechanical; monograph; optical; physical; Monogr. 167.

property conservation; risk management; shock pulse analysis; vibration analysis; acoustic emission analysis; insurance; loss prevention; modal analysis; SP622: 1981 October. 139-151. proportional limit; shear strength; tensile strength; yield strength; Young's modulus; compressive strength; elongation; foam; insulation; low temperature; mechanical properties; NBSIR 811654.

proposal evaluation; RFP preparation; small systems; computer procurement; SP500-83: 1981 November. 191-197.

propylbenzene; solubility; solute; water; generator column; hydrophobic; octanol; partition coefficient; J. Res. 86(4): 361-366; 1981 July-August.

propyne; threshold photoelectron spectra; breakdown curves; photoelectron-photoionization coincidence; photoionization; 20051.

prosthesis failure; vitamin K-dependent; calcification; calcific degeneration; porcine xenograft valves; SP601: 1981 January. 339. 348.

prosthesis interface; femoral component loosening; polymethylmethacrylate (PMMA); SP601: 1981 January. 157.174.

prosthesis related infections; histological examinations; hydrocephalus; SP601: 1981 January. 349-362.

prosthetic devices; implant retrieval analysis; joint prostheses; metallurgical analysis; SP601: 1981 January. 559-572.

prosthetic heart valve failure; aortic prosthesis; embolization; SP601: 1981 January. 283-298.

prosthetic heart valves; thromboembolism; bio-prostheses; SP601: 1981 January. 175-200.

protactinium compounds; rubidium compounds; sodium compounds; thorium compounds; uranium compounds; actinium compounds; cesium compounds; enthalpy; entropy; francium compounds; Gibbs energy of formation; heat capacity; lithium compounds; potassium compounds; TN270-8.

protestors; solar code; victim role; deed; developers; energy wars; experimental code; field test; guideline; low income; non-code; policymaking; SP608: 1981 May. 173-178.

protocols; standards; computer based office systems; computer networking; interfaces; local area networks; measurement techniques; NBSIR 80-2154.

protolyzable anions; thermodynamics; adsorption; desorption; hydrolyzable cations; hydrous oxides; kinetics; SP618: 1981 November. 146-162.

prototypes; applicative programming; design; functional programming; interpreters; 20075.

provisions; scope; specification; standard; system analysis/engineering; arrangement; building; classification; code; engineering; organization; BSS136.

provisions; soil structure interaction; standards; buildings; design; earthquakes; engineering; foundations; professional practice; NBSIR 80-2111-3.

proximity effect devices; superconducting devices; tunnel devices; weak links; closed cycle refrigeration systems; Josephson effects; SP607: 1981 May. 195-209.

psychoacoustics; annoyance; audible noise; corona noise; EHV transmission lines; electric energy; environmental noise; human response; power line noise; 20050.

psychoacoustics; transmission line audible noise; corona noise; electric energy transmission; high voltage; human response to noise; noise pollution; 20029.

PTFE-carbon composite; synthetic implants; ossicular prosthesis; porosity measurement; porous biomaterials; porous polyethylene; NBSIR 81-2212.

public assembly; public safety; stadiums; auditorium; building standards; crowd ingress; design procedures; evaluation procedures; facility design; facility management; NBSIR 81-2361.

publication list; solid state; thermodynamic and transport properties; atomic and molecular properties; bibliographies; chemical kinetics; evaluated data; fluid properties; indexes; SP612.

publications; abstracts; building technology; Center for Building Technology; key words; SP457-5.

publications; abstracts, NBS publications; key words; SP305. Supplement 12.

publications; radiography; ultrasonics; abstracts; acoustic emission; bibliography; eddy currents; index; National Bureau of Standards; nondestructive evaluation; NBSIR 80-2080. 
public buildings; state government; cost; construction; energy audit; energy conservation; Illinois; maintenance; SP608: 1981 May. 123 132.

public health impacts; residues; toxic elements; availability; environmental contamination; extraction tests; fossil fuels; laboratory characterization; metals; mining industry; SP618: 1981 November. 1-8.

public housing security; security hardware; victimization survey; burglary; crime in public housing; household behavior patterns; housing characteristics (physical); housing security; NBS-GCR-80291.

public participation programs; standards development; voluntary standards; bibliography; annotated, consumer representation; literature review; medical device standards; NBSIR 81-2336.

public safety; stadiums; auditorium; building standards; crowd ingress; design procedures; evaluation procedures; facility design; facility management; public assembly; NBSIR 81-2361.

pulse combustion; boilers; central heating; condensing boilers; efficiency, part load; fossil-fuel heating systems; gas-fired boilers; hydronic heating; NBSIR 80-2094.

pulsed $\mathrm{CO}_{2}$ laser damage; 10 micrometer optical components; absorptance; infra-red laser windows; SP620: 1981 October. 248 255.

pulsed HF/DF laser damage thresholds; silicon films; absorption; evaporation; hydrogen and oxygen impurity; magnetron sputtering; SP620: 1981 October. 305-312.

pulse distortion; single-shot damage threshold; vacuum chamber; copper mirrors; $\mathrm{CO}_{2}$ laser; multishot damage; nanosecond pulse; peak energy density; SP620: 1981 October. 238-247.

pulsed laser; reflectors; thin film; anti-reflection coatings; bichromatic; damage; high energy laser; laser damage; SP620: 1981 October. 287.296.

pulsed laser beams; $10.6 \mu \mathrm{m}$; attenuators; beam profile; calorimetry; fluence; heat flow; irradiance; measurements of temperature; plasma effects; NBSIR 80-1628.

pulsed laser damage; slip threshold; thermal diffusion length; thermal stress; cold work; crystalline disorder; defect damage; diamondmachined mirrors; Drude absorption; electron-beam melting; laser waveform; melt threshold; metal deposition; SP620: 1981 October. 201-209.

pulsed lasers; laser-induced damage; SP620: 1981 October. 297-299.

pulse-processing; solid-state x-ray detectors; $x$-ray energy spectrometry; SP604: 1981 June. 141-164.

pulse shapes; Rabi oscillation; two-level system; multimode laser; multiphoton ionization; $\mathrm{Na} 3 \mathrm{~s} \rightarrow 3 \mathrm{p} ; 20061$.

punched cards; standard; American National Standard Code for Information Interchange; ASCII; card punch; card reader; data communications; data interchange; data processing; EAM cards; electric accounting machine; Federal Information Processing Standard; Hollerith punched card code; Hollerith punched cards; information interchange; information processing; FIPS PUB 14-1.

purchasing; recovered/recycled materials; resource recovery; econimic, institutional, technical barriers; procurement; $N B S-G C R$ 81-309, Supplement 1.

purchasing; recovered/recycled materials; resource recovery; economic, institutional, technical barriers; procurement; NBSGCR-81-310.

purchasing; recovered/recycled materials; resource recovery; economic, institutional, technical barriers; procurement; NBSGCR-81-309.

purchasing; recovered/recycled materials; resource recovery; technical barriers; economic; institutional; procurement; NBSIR 812316.

purchasing; recovered/recycled materials; resource recovery; technical barriers; economic; institutional; procurement; NBSGCR-81-312.

purchasing; recovered/recycled materials; resource recovery; technical barriers; economic; institutional; procurement; NBS. GCR-81-308.

purchasing; recovered/recycled materials; resource recovery; technical barriers; economic; institutional; procurement; NBS-
GCR-81-314.

purchasing; recovered/recycled materials; resource recovery; technical barriers; economic; institutional; procurement; NBS. GCR-81-311.

purchasing; recovered/recycled materials; resource recovery; technical barriers; economic; institutional; procurement; NBS GCR-81-313.

pure fluids; coexistence densities; critical density; critical point; liquid mass fraction; liquid volume fraction; phase equilibria; NBSIR 80 1631.

PVTx data; revised Kosek and McKinley; cell model; comparisons; computer programs; corresponding states; equation of state; hard sphere; LNG; mixtures; TN1030.

pyrene; shale oil; standard reference materials; benzo(a)pyrene; benzo(e)pyrene; fluoranthene; fluorescence detection; gas chromatography/mass spectrometry; high-performance liquid chromatography; perylene; 20750.

pyrolysis; smoke; wood; combustion products; fire modeling; foam (materials); heat release; plastics; polymers; polymethylmethacrylate; polyoxymethylene; polypropylene; polystyrene; polyurethane; polyvinyl chloride; NBS-GCR-80-295.

\section{Q}

Q-switch; surface damage; antireflection coatings; coating damage; laser damage; $\mathrm{LiNbO}_{3}$ coatings; SP620: 1981 October. 300-304.

quadrature; several variables; integrals; integration; irregularity of distribution; lattices; $n$-cube; periodic functions; 20032 .

quadrupole-induced absorption; spectral moments; band shape analysis; collision-induced absorption; far infrared; $\mathrm{H}_{2}-\mathrm{H}_{2}$ collisions; $\mathrm{H}_{2}$-He collisions; planetary atmospheres; NBSIR 80-2175.

quadrupoles; susceptibility; TEM cell; analysis; dipole moments; electrically small objects; emission; measurements; TN1040.

qualitative analysis; quantitative analysis; scanning electron microscopy; spectral artifacts; transmission electron microscopy; electron probe microanalysis; energy dispersive $x$-ray spectrometry; microanalysis; SP604: 1981 June. 315-339.

quality assurance; standards; water; air; environmental measurements; methods; NBSIR 81-2280.

quality assurance; steel reinforcing bars; test methods; concrete; construction; nondestructive evaluation; NBSIR 80-2163.

quality assurance; stress analysis; structural safety; fracture control; fracture mechanics; mechanical properties; NBSIR 79-1623.

quality assurance program; software change package; software maintenance testing; developmental center test; environmental test; field validation test; independent testing; SP500-83: 1981 November. 93-99.

quality control; automated data printing; computer-output microforms; Federal Information Processing Standards Publication; form slide; microfilm; FIPS PUB 82.

quality control; radioactivity; traceability; environment; metrology; 20010.

quality control; reliability of semiconductor devices; semiconductor devices; analysis of moisture content; mass spectrometer measurement; moisture; moisture generators; moisture sensors; SP400-69.

quality testing; radiograph evaluation; visual inspection; visual testing; acuity tests; nondestructive testing; $T N / 143$.

quantitative analysis; scanning electron microscopy; spectral artifacts; transmission electron microscopy; electron probe microanalysis; energy dispersive $x$-ray spectrometry; microanalysis; qualitative analysis; SP604: 1981 June. 315-339.

quantitative analysis; $\mathrm{x}$ ray; automation; powder diffraction; NBSIR 81-2229.

quantitative differential thermal analysis; thermal explosion theory; thermal hazards; thermal instability; adiabatic calorimetry; azidotriphenylmethane; decomposition reactions; differential scanning calorimetry; heat conduction calorimetry; kinetics of heat evolution; m-azidobenzoic acid; nitrocellulose; NBSIR 80-2018 (DOT).

quantitative microscopy; standard reference material; $\mathrm{x}$-ray 
diffraction; x-ray fluorescence analysis; austenite in ferrite; austenite standard; electron microprobe; powder metallurgy; SP260-73.

quantum chemistry; scattering; electron-molecule collisions; interaction potential; Legendre expansion; molecular potential; 20055.

quantum electrodynamics; basic standards; fine-structure constant; fundamental constants; Josephson effect; least-squares adjustments; 20058.

quantum mechanical tunneling calculations; tunneling corrections; chemical rate constants; Eckart potential; potential barriers; J. Res. 86(4): 357-359; 1981 July-August.

quantum metrology; quantum physics; temperature and pressure measurements and standards; time and frequency; electrical measurements and standards; length and mass measurements and standards; NBSIR 80-2165.

quantum noise; $1 / \mathrm{f}$ noise; hot carrier noise; noise; noise in devices; noise measurement; SP614.

quantum physics; temperature and pressure measurements and standards; time and frequency; electrical measurements and standards; length and mass measurements and standards; quantum metrology; NBSIR 80-2165.

quark model; strange meson; E meson at $1440 \mathrm{MeV}$; flavor singlet; glueball; production process; 20034.

quarks; angular distributions; charmed mesons; dipole transitions; magnetic moments; photon decays; 20040.

quartz crystal viscometer; saturated liquid; shear viscosity coefficient; density dependence; gaseous and liquid ethane; isothermal measurements; 20767.

quartz crystal viscometer; shear viscosity coefficient; density dependence; gaseous and liquid methane; isothermal measurements; 20071.

quasideuteron; resonance fluorescence; giant resonance; photonuclear; 19992.

quasi electrostatic; TEM cell; electromagnetic compatibility (EMC); Green's function; integral equation; linear equation; method of moments; parallel plate waveguide; TN1028.

quasi-steady; unsteady; characteristics; discharge; drainage; flow; model; plumbing; J. Res. 86(2): 171-179; 1981 March-April.

query; standards; conversion; database; data description; data dictionary; data directory; data manipulation; DBMS; language; $S P 500-76$.

query language; CODASYL; database management system; data model; DBMS; network data model; NBS-GCR-81-339.

query language; CODASYL; database management system; data model; DBMS; network data model; NBS-GCR-81-337.

query language; relational model; SQL; CODASYL; DAPLEX; database management system; data model; DBMS; network data model; NBS-GCR-81-338.

query processor; software tool; text processor; translation; UNIX; macroprocessor; pattern matching; NBSIR 80-2182.

questionnaire; temperature; ventilation; windows; energy conservation; lighting; man/environment research; noise; office building; post-occupancy evaluation; BSS130.

questionnaires; research methods; survey research; user, needs; dwelling units (residential); housing; Operation Breakthrough; postoccupancy housing evaluation; Project Feedback; NBSIR 81-2258.

questionnaires; wood; fireplace inserts; fireplaces; fire prevention; fire safety; NBS-GCR-80-292.

queueing models; simulation; validation; analytic models; communication networks; flow control; Markov processes; performance prediction; SP500-83: 1981 November. 277.290.

queueing models; system resources; network models; product form; SP500-83: 1981 November. 335.

\section{$\mathbf{R}$}

Rabi oscillation; two-level system; multimode laser; multiphoton ionization; $\mathrm{Na} \mathrm{3s \rightarrow 3p;} \mathrm{pulse} \mathrm{shapes;} 20061$.

radar soil measurements; soil moisture; underground radar; void detection; adobe building materials; adobe soil; dielectric constant measurements; electromagnetic moisture measurements; nondestructive evaluation; NBSIR 79-1610.

radiance temperature; reflected infrared radiation; temperature corrections; temperature measurements; ambient infrared radiation; blackbody cavity; corrections for reflectance; infrared imaging; infrared thermography; 20733.

radiant floor heating; space heating and cooling; thermal mass; walls; water source heat pump; buildings; construction; energy conservation; ground coupled; hybrid solar energy system; inverted cave; low grade geothermal; SP608: 1981 May. 69-81.

radiation; activation analysis; crystal structure; diffraction; isotopes; molecular dynamics; neutron; neutron radiography; nondestructive evaluation; nuclear reactor; TN1142.

radiation; calorimetry; cardiac pacemakers; clinical chemistry; heat; metabolism; microcalorimetry; 20015.

radiation; radiocarbon; soft aluminum; windows; air pollution; automation; buildings; certified reference materials; computers; construction; data converter; mass comparator; DIM/NBS 65(4): $1.28 ; 1981$.

radiation; semiconductor; technology; calibration; electrical; electronics; engineering; hazards; measurement; metrology; National Bureau of Standards; power; SP611.

radiation; synchrotron radiation; astrophysics; bremsstrahlung; electromagnetic interactions; pair production; plasma diagnostics; 19993.

radiation angle; radiation patterns; attenuation; core diameter; far field; index profile; mode filter; numerical aperture; TN1032.

radiation damage; rock salt; sodium chloride; chemical; electrical; geological; magnetic; mechanical; monograph; optical; physical; properties data; Monogr. 167.

radiation dosimetry; radiation protection; radiation standards; $\mathrm{x}$ rays; beta rays; Federal and State laboratories; gamma rays; instrument calibrations; intermediate calibration laboratories; ionizing radiation measurements; measurement accuracies; neutrons; SP603.

radiation hazards; antennas; electromagnetic interference; electromagnetic metrology; em material properties; microwaves; noise; non-ionizing radiation; NBSIR 80-1635.

radiation patterns; attenuation; core diameter; far field; index profile; mode filter; numerical aperture; radiation angle; TN1032.

radiation protection; radiation standards; $x$ rays; beta rays; Federal and State laboratories; gamma rays; instrument calibrations; intermediate calibration laboratories; ionizing radiation measurements; measurement accuracies; neutrons; radiation dosimetry; SP603.

radiation quantities and units; radiation standards and protection; radioactivity; radiology; radium; roentgen ray; tubes ( $x$ ray); $x$-ray measurement; $x$ rays; dosage; dosimetry; health and safety; high voltage; ionization chamber; ionizing radiation; ionometer; SP625.

radiation source; safety standard; classification criteria; gamma radiography; national standard; performance tests; H136.

radiation standards; $\mathrm{x}$ rays; beta rays; Federal and State laboratories; gamma rays; instrument calibrations; intermediate calibration laboratories; ionizing radiation measurements; measurement accuracies; neutrons; radiation dosimetry; radiation protection SP603.

radiation standards and protection; radioactivity; radiology; radium; roentgen ray; tubes ( $x$ ray); $x$-ray measurement; $x$ rays; dosage; dosimetry; health and safety; high voltage; ionization chamber; ionizing radiation; ionometer; radiation quantities and units; SP625.

radiation therapy; absorbed dose; Bragg.Gray equation; calibration; cavity chamber; cavity theory; electron beam; in-phantom measurement; photon beam; 20073.

radiative lifetimes; scandium; scandium halides; yttrium; yttrium halides; chemiluminescence; Franck-Condon factors; laser-induced fluorescence; 19978.

radiative lifetimes; scandium; scandium halides; yttrium; yttrium halides; chemiluminescence; Franck-Condon factors; laser-induced fluorescence; 19969.

radical; $\mathrm{CH}_{3} ; \mathrm{CH}_{2} \mathrm{I}$; infrared emission; laser; photofragmentation; 20004.

radical ions; radicals; transients; absorption spectra; aqueous solution; extinction coefficients; inorganic; NSRDS-NBS69. 
radicals; Raman spectroscopy; temperature; flame inhibition; H-atoms; kinetics; mass spectrometry; phosphorus; NBSIR 80.2169.

radicals; transients; absorption spectra; aqueous solution; extinction coefficients; inorganic; radical ions; NSRDS-NBS69.

radioactive sources; survey instruments; calibration; commercial services; directory; inaccuracy; ionizing radiation; NBS-GCR.80 296.

radioactive tracer; spectrofluorimetry; antioxidants; diffusion; food packaging; inverse gas chromatography; migration; oil simulants; oligomers; polyolefins; NBSIR 81-2264.

radioactive wear testing; effect of filtration; engine wear analysis; oil filter design; oil filter testing; particle size sensitivity; SP622: 1981 October. 50-61.

radioactive $\mathrm{x}$-ray source; rare-earth scintillator screen; CsI; detection efficiency; lixiscope; 20003.

radioactivity; radiochemistry; radium.228; reference method; water; analysis; assay; evaluation; TN1137.

radioactivity; radiology; radium; roentgen ray; tubes ( $x$ ray); $x$-ray measurement; $x$ rays; dosage; dosimetry; health and safety; high voltage; ionization chamber; ionizing radiation; ionometer; radiation quantities and units; radiation standards and protection; SP625.

radioactivity; research; sediment; soil; alloy; carbon; computers; energy; fine structure; interface; materials; measurement; office building; phase diagrams; DIM/NBS 65(8): 1-28; 1981.

radioactivity; traceability; environment; metrology; quality control; 20010.

radio astronomy; amino acids; ethylene oxide; glycine; interstellar; methyl formate; 20077.

radio astronomy; rotational transitions; distortion dipole transitions; interstellar molecules; methane; microwave spectra; molecular parameters; JPCRD 10(4): 1085.1095; 1981.

radio astronomy; sulfur dioxide; interstellar; nitric oxide; phosphine; phosphorus nitride; 19987.

radiocarbon; soft aluminum; windows; air pollution; automation; buildings; certified reference materials; computers; construction; data converter; mass comparator; radiation; DIM/NBS 65(4): 1.28; 1981.

radiochemistry; radium-228; reference method; water; analysis; assay; evaluation; radioactivity; $T N 1137$.

radio computer communication network; satellite communication; ALOHANET; ALOHA system; computer network; packet broadcasting; J. Res. 86(6): 591.595; 1981 November-December.

radio frequency; level gauging; propane; NBSIR 79-1600.

radio-frequency; temperature measurements; heating; probe; NBSIR 80.1634.

radio frequency radiation; receiver calibration; electromagnetic field; electronic instrument design; field intensity meter; field strength measurement; isotropic antenna; TNIO33.

radiograph evaluation; visual inspection; visual testing; acuity tests; nondestructive testing; quality testing; TN1143.

radiography; SRM's; stars; visual acuity; evolution; fracture mechanics; gamma rays; inventors; molecular interactions; molecular processes; neomycin; neutron dosimeters; polymers; $D I M / N B S$ 65(7): 1-24; 1981.

radiography; tire inspection; ultrasonics; visual-optical; acoustic emission; eddy currents; liquid penetrants; magnetic particles; microwaves; nondestructive evaluation; 20762.

radiography; ultrasonics; abstracts; acoustic emission; bibliography; eddy currents; index; National Bureau of Standards; nondestructive evaluation; publications; NBSIR 80.2080.

radiology; radium; roentgen ray; tubes ( $x$ ray); $x$-ray measurement; $x$ rays; dosage; dosimetry; health and safety; high voltage; ionization chamber; ionizing radiation; ionometer; radiation quantities and units; radiation standards and protection; radioactivity; SP625.

radiosity shape factors; building heating/cooling loads; heat balance for a single room; heat transfer; NBSIR 81-2321.

radio star; satellite communications; $\mathrm{C} / \mathrm{kT}$; EIRP; error analysis; $\mathrm{G} / \mathrm{T}$; precision measurements; NBSIR 78-869.

radium; roentgen ray; tubes ( $x$ ray); $x$-ray measurement; $x$ rays; dosage; dosimetry; health and safety; high voltage; ionization chamber; ionizing radiation; ionometer; radiation quantities and units; radiation standards and protection; radioactivity; radiology; SP625.

radium; uranium; cesium; metal accumulation; microbial; SP618: 1981 November. 274-286.

radium-228; reference method; water; analysis; assay; evaluation; radioactivity; radiochemistry; $T N 1137$.

radon; transport; buildings; concrete; diffusion; exhalation; materials; measurement; permeability; TN1139.

railroad accidents; small-scale fire tests; thermal protection; bombs (ordnance); boxcars; computer models; fire detection systems; fire suppression; full-scale fire tests; heat transfer; NBSIR 80-2170.

Raman spectroscopy; characterization of polymers; polymers under high pressure; 20007.

Raman spectroscopy; temperature; flame inhibition; $\mathrm{H}$-atoms; kinetics; mass spectrometry; phosphorus; radicals; NBSIR 80-2169.

ramps; building circulation; building ramps; pedestrian circulation; pedestrian flow; pedestrian movement; pedestrian ramps; NBSIR $81-2310$.

random coil polymers; amphoteric latex; dynamic light scattering; light scattering; nematic liquid crystal; photo-receptor membrane vesicles; J. Res. 86(6): 571-590; 1981 November-December.

random fault; step coverage; test structures; electronics; interconnect; microelectronics; SP400-65.

random faults; silicon-on-sapphire; test pattern; test structure; yield; integrated circuits; microelectronics; process validation wafer; $S P 400-66$

rapid solidification; thermodynamics; metastable phase equilibrium; partitionless solidification; phase diagrams; phase equilibrium hierarchy; 19981.

rare-earth apatite; substituted fluorapatite; apatite; barium apatite; cation substitution; crystal structure; 20065.

rare-earth scintillator screen; CsI; detection efficiency; lixiscope; radioactive $\mathrm{x}$-ray source; 20003.

rate constant; sulfur dioxide; sulfur monoxide; sulfur monoxide dimer; chemical kinetics; disulfur monoxide; 20035.

rate of reaction; sulfur; Arrhenius parameters; chemical kinetics; combustion; decomposition; free radicals; gas phase; hydrocarbons; hydrogen; nitrogen; oxygen; NBSIR 81-2254.

rate of return; schools; technical assistance; certification; Class $A$ energy audit; economic analysis; energy analysis; energy conservation; Federal programs; government buildings; hospitals; SP608: 1981 May. 133-138.

rates; review; singlet oxygen; solution; chemical kinetics; data compilation; oxidation; photochemistry; JPCRD 10(4): 809-999; 1981 .

rating procedure; seasonal efficiency; annual fuel utilization efficiency; annual operating costs; central heating equipment; condensing boilers; condensing furnaces; part-load performance; NBSIR 80 2110.

rationale statements; scope of standards; standards; standards writing legislative history; product liability; NBS-GCR-81-347.

Rayleigh-Brillouin scattering; damped wave motion; density fluctuations; diffusion; dynamic structure factor; initial value problem; nonuniform systems; one-dimensional models; J. Res. 86(5): 429.455; 1981 September-October.

Rayleigh light scattering; thermal diffusivity; concentration; diffusivity; methane-ethane; mixture; one-fluid model; plait point; 19964.

Rayleigh scattering; backscattering; capture fractions; fiber scattering; optical time domain reflectometry; TNI034.

$\mathrm{RbNO}_{2}$; solutions; standard reference data; thermodynamic properties; activity coefficients; alkali metal nitrite salts; aqueous; critical evaluation; $\mathrm{CsNO}_{2}$; electrolytes; excess Gibbs energy; $\mathrm{KNO}_{2}$ $\mathrm{LiNO}_{2} ; \mathrm{NaNO}_{2}$; osmotic coefficients; JPCRD 10(3): 767.778; 1981.

reaction; time-dependent; diffusion; integrating device; permeation type; personal dosimeter; NBSIR 80-1975.

reaction mechanism; t-2.fluorovinyl; acetylene; $F$ atoms; hydrogen bonding; infrared spectrum; matrix isolation; photodecomposition; 20036.

reaction rate; relaxation; atom; charge transfer; chemi-ionization collisional ionization; cross section; detachment; energy transfer; 
excitation; ion; molecule; SP593.

reaction rates; reactor technology; heats of formation; inorganic gases; ion-molecule reactions; mass spectrometry; 19965.

reactions to implants; hip hemiarthroplasties; metal allergy; metal sensitivity; SP601: 1981 January. 299-321.

reaction with $\mathrm{Zn}$ ions and rate laws; surface mordanting with $\mathrm{Zn}$ ions; bonding with coupling agents; hydroxylapatite; interaction with $\mathrm{Zn}$ ions and enamel etching; 20729.

Reactor Division; Center for Materials Science; Ceramics Glass and Solid State Science Division; Chemical Stability and Corrosion Division; Fracture and Deformation Division; FY80 technical activities; Metallurgy Division; Polymers Science and Standards Division; NBSIR 80-2108.

reactor internals; surveillance; vibration; core barrel motion; neutron detectors; SP622: 1981 October. 3.10.

reactor technology; heats of formation; inorganic gases; ion-molecule reactions; mass spectrometry; reaction rates; 19965.

readers; American National Standard; Computer Output Microfilm (COM); Federal Information Processing Standard (FIPS); illumination; image quality; microfilm; microform; FIPS PUB 84

read inks; American National Standard; Federal Information Processing Standard (FIPS); interchange of machine readable inforınation; nonread inks; Optical Character Recognition (OCR); OCR.A; OCR-B; FIPS PUB 85

real-time control; vibration control; vibration isolation; active vibration control; Michelson interferometer; optical path.length correction; phase comparator; 20734.

receiver calibration; electromagnetic field; electronic instrument design; field intensity meter; field strength measurement; isotropic antenna; radio frequency radiation; TN1033.

recirculating hot water systems; solar collectors; system efficiency; thermal performance; domestic hot water; NBS-GCR-81-322.

recommended practice; savings-to-investment ratio; benefit-cost analysis; building economics; cost effective; economic analysis; energy conservation; investment analysis; life-cycle cost; NBSIR 81-2397.

recommended requirements; solar energy; solar systems; building codes; building officials; consensus; construction; document installation; SP608: 1981 May. 167-172.

reconstructive dental work; resin; acrylate; adhesion; aminobenzoate; composite material; dentin; methacrylate; polyfunctional surfaceactive comonomers; U.S. Patent 4,251,565.

recovered/recycled materials; resource recovery; econimic, institutional, technical barriers; procurement; purchasing; NBS GCR-81-309, Supplement 1.

recovered/recycled materials; resource recovery; economic, institutional, technical barriers; procurement; purchasing; NBS. GCR $-81-310$.

recovered/recycled materials; resource recovery; economic, institutional, technical barriers; procurement; purchasing; NBS GCR-81-309.

recovered/recycled materials; resource recovery; technical barriers; economic; institutional; procurement; purchasing; NBSIR 81-2316.

recovered/recycled materials; resource recovery; technical barriers; economic; institutional; procurement; purchasing; NBS-GCR-81308.

recovered/recycled materials; resource recovery; technical barriers; economic; institutional; procurement; purchasing; NBS-GCR-81. 313.

recovered/recycled materials; resource recovery; technical barriers; economic; institutional; procurement; purchasing; NBS-GCR-81 314.

recovered/recycled materials; resource recovery; technical barriers; economic; institutional; procurement; purchasing; $N B S-G C R-81$. 311.

recovered/recycled materials; resource recovery; technical barriers; economic; institutional; procurement; purchasing; NBS-GCR-81 312.

recovery actions; ADP availability; ADP security; backup operations; computer applications; computer security; contingency planning; emergency response; Federal Information Processing Standards
Publication; FIPS PUB 87

rectangular coaxial line; striplines; higher order modes; NBSIR $7 \%$. 873.

rectangular coaxial transmission line; TEM cell; characteristic impedance; field distribution; Hertzian dipole; integral equation; TN1037.

recycled materials; refused-derived-fuel; rerefined oil; energy-from waste; materials market; oil analysis; NBSIR 80-2178.

recycled oil; rerefined oil; test procedures; basestock; engine lubricants; lubricating oil; motor oil; petroleum oil; 20016.

redox couples; trace elements; aqueous speciation; computerized geochemical model; heavy metals; SP618: 1981 November. 39.53.

redox potential; soil corrosivity; soil pH; soil resistivity; underground corrosion; earth resistivity; 19963.

reduce cost; reduce input; standard evaluation technique; BLAST; building energy performance standards; computer modeling; maintain accuracy; SP608: 1981 May. 113-122.

reduce input; standard evaluation technique; BLAST; building energy performance standards; computer modeling; maintain accuracy; reduce cost; SP608: 1981 May. 113-122.

redundancy; 3.D metrology; ball plate; grid plate; machine geometry; measuring machine; NBSIR 79-1752.

re-examination; thermal storage; wood structure; codes; concrete; masonry; prohibitions; SP608: 1981 May. 195.200.

reference cells; solar cells; terrestrial solar simulators; test methods; calibration; combined photovoltaic/thermal modules; measurements; photovoltaic modules; photovoltaics; NBS-GCR-80 279.

reference data; sodium chloride; viscosity; aqueous solutions; compilation; JPCRD 10(1): 71.88; 1981.

reference data; viscosity; aqueous solutions; compilation; potassium chloride; JPCRD 10(1): 57.70; 1981

reference intensities; standard; $x$-ray diffraction; crystal structure; densities; lattice constants; powder patterns; Monogr. 25, Section 18.

reference material; serum base modification; serum matrix anticonvulsants; antiepilepsy drugs; blood analysis;

chromatography, gas; chromatography, liquid; drug standards; enzyme immunoassay; epilepsy; ethosuximide; mass spectrometry; nuclear magnetic resonance spectroscopy; phenobarbital; phenytoin; primidone; SP260-72.

reference material; stability of measurement process; statement of certainty; precision; 20041.

reference materials; shale oil; SRM; trace organic analysis; definitive methods; human serum; polynuclear aromatic hydrocarbons; 19989.

reference method; water; analysis; assay; evaluation; radioactivity; radiochemistry; radium.228; TN1137.

reflectance; silver; surface roughness; temperature dependence; aluminum; copper; Drude theory; infrared; SP620: 1981 October. 190.200 .

reflectance measurements; reflectance standards; solar properties of materials; spectral reflectance measurements; diffuse reflectance; directional-hemispherical reflectance; integrating spheres; laboratory intercomparisons; NBSIR 81-2311.

reflectance standards; solar properties of materials; spectral reflectance measurements; diffuse reflectance; directionalhemispherical reflectance; integrating spheres; laboratory intercomparisons; reflectance measurements; NBSIR 81-2311.

reflected infrared radiation; temperature corrections; temperature measurements; ambient infrared radiation; blackbody cavity; corrections for reflectance; infrared imaging; infrared thermography; radiance temperature; 20733

reflection coefficient; six-port measurement system; thermistor mount calibration; mm wave measurements; NBSIR 81-1650.

reflective materials for bicycles; safety of bicycles; safety standards; abrasion test development; bicycle compliance tests; bicycle safety; NBSIR 80-2035.

reflector antennas; co-sited antennas; coupling; far fields; near fields; physical optics; planewave spectrum; NBSIR 80-1630.

reflectors; thin film; anti-reflection coatings; bichromatic; damage; high energy laser; laser damage; pulsed laser; SP620: 1981 October. 287.296. 
refracted near-field scanning; refracted-ray scanning; resolution limit; fiber index profile; index profile; near-field scanning; optical communications; optical fiber; optical waveguide; TN1038.

refracted-ray scanning; resolution limit; fiber index profile; index profile; near-field scanning; optical communications; optical fiber; optical waveguide; refracted near-field scanning; TN1038.

refractive index; auto headlight glass; classification; density; forensic science; individualization; population statistics; NBSIR 81-2286.

refractive index; binary liquids; critical mixing; critical phenomena; dielectric constant; fluids in an electric field; 20056.

refractivity of air; wavelength of light in air; air density; index of refraction of air; J. Res. 86(1): 27.32; 1981 January-February.

refrigeration; regenerative cooler; regenerator; Stirling cooler; Stirling cycle; cryocooler; hybrid cryocoolers; SP607: 1981 May. 48.56.

refrigeration; regenerator; resonant free piston; Stirling cycle; cryocooler; gas bearings; SP607: 1981 May. 116.

refrigeration; regenerators; specific heat; cryogenics; heat capacity; SP607: 1981 May. 164.172.

refrigeration; regenerators; specific heat; thermal conductivity; composites; cryocoolers; plastics; SP607: 1981 May. 173.177.

refrigeration; SQUID; Stirling cycle; superconductivity; cryocoolers; low temperature; magnetic gradiometer; SP607: 1981 May. 186-194.

refrigeration; Stirling cycle; adsorption; cryocoolers; cryostats; helium; SP607: 1981 May. 3-14.

refrigeration; superconducting devices; cryocoolers; cryogenic sensors; helium; SP607.

refrigeration; zeolites; adsorption; compressor; cryogenics; molecular adsorption; SP607: 1981 May. 127-135.

refrigeration recovery; chemical engineering; cold utilization; cryogenic energy conservation; liquefied natural gas; 20068 .

refrigerator; component efficiency; cryogenics; efficiency; helium; maximum refrigerator efficiency; perfect gas analysis; 20771.

refrigerator; Stirling cycle; cryocooler; cryogenic; helium; low temperature; SP607: 1981 May. 30-47.

refrigerators; cryogenic; Joule-Thomson; microminiature devices; photolithographic fabrication; SP607: 1981 May. 160-163.

refrigerators; cryogenics; gas adsorption; heat switch; SP607: 1981 May. 178.185.

refrigerators; regenerators; Stirling cryocoolers; thermal conductance; Vuilleumier cryocoolers; cryogenics; Gifford-McMahon cryocoolers; heat capacity; heat transfer; helium; 20766.

refrigerators; regenerators; Stirling cryocoolers; thermal conductance Vuilleumier cryocoolers; cryogenics; Gifford-McMahon cryocoolers; heat capacity; heat transfer; helium; SD607: 1981 May. $70-81$

refrigerators; regenerators; Stirling-cycle; calculations; cryogenics; helium; plastics; 20020.

refrigerators; reliability; rotary-reciprocating; turbo-Brayton; Vuilleumier; component development; contamination control; cryogenics; helium retention; long-life; SP607: 1981 May. 21-29.

refuge; board and care homes; building codes; building fires; evacuation; fire alarm systems; fire safety; handicapped; life safety; 20727.

refused-derived-fuel; rerefined oil; energy-from-waste; materials market; oil analysis; recycled materials; NBSIR 80-2178.

refused-derived-fuel; sample characterization; sample processing effects; 2.5 and 25 gram capacity bomb calorimeters; ASTM round robin testing; bomb calorimetry; higher heating value; NBSIR 81 2278.

refuse-derived-fuel; test substance; alternative fuel; bomb calorimetry; cellulose; enthalpy of combustion; J. Res. 86(6): 655-660; 1981 November-December.

regenerative cooler; regenerator; Stirling cooler; Stirling cycle; cryocooler; hybrid cryocoolers; refrigeration; SP607: 1981 May. 48 56.

regenerator; resonant free piston; Stirling cycle; cryocooler; gas bearings; refrigeration; SP607: 1981 May. 116.

regenerator; Stirling cooler; Stirling cycle; cryocooler; hybrid cryocoolers; refrigeration; regenerative cooler; SP607: 1981 May $48-56$.

regenerator gaps; single stage, twin piston cryogenic refrigerator; two nested, concentric pistons; cylinder; helium; inner piston; outer piston; U.S. Patent 4,281,517.

regenerators; specific heat; cryogenics; heat capacity; refrigeration SP607: 1981 May. 164-172.

regenerators; specific heat; thermal conductivity; composites; cryocoolers; plastics; refrigeration; SP607: 1981 May. 173-177.

regenerators; Stirling cryocoolers; thermal conductance; Vuilleumier cryocoolers; cryogenics; Gifford-McMahon cryocoolers; heat capacity; heat transfer; helium; refrigerators; 20766.

regenerators; Stirling cryocoolers; thermal conductance; Vuilleumier cryocoolers; cryogenics; Gifford-McMahon cryocoolers; heat capacity; heat transfer; helium; refrigerators; SP607: 1981 May. 7081.

regenerators; Stirling-cycle; calculations; cryogenics; helium; plastics; refrigerators; 20020.

regional analysis; thermal efficiency; windows; building economics; daylighting; energy conservation; engineering economics; life-cycle costs; passive solar; NBSIR $81-2248$.

regional calibration laboratories; standards; techniques; calibration; dosimetry; electric fields; electromagnetic interference; exposure history; instruments; magnetic fields; nonionizing radiation; SP613.

registration of service persons; unit pricing; weighmaster law; basic weights and measures law; method of sale of commodities; open dating; packaging and labeling; H130, 1982 Edition.

regression analysis; strength prediction; temperature effects compressive strength; concrete; curing temperature; early age; final set; hydration; initial set; laboratory testing; maturity; mortar NBSIR 81-2244.

regulation; air pollution; Clean Air Act; EPA; ETIP; incentives; innovation; NBS-GCR-ETIP 81-94.

regulation; air pollution; Clean Air Act; EPA; ETIP; incentives; innovation; NBS-GCR-ETIP 81-95.

regulation; air pollution; Clean Air Act; EPA; ETIP; incentives; innovation; NBS-GCR-ETIP 81-93.

regulation; benefit-cost analysis; building economics; building regulation; codes; construction regulation; economics; energy conservation codes; fire safety codes; NBSIR 81-2223.

regulation; benefit-cost analysis; building economics; building regulation; codes; construction economics; construction regulation; economics; fire safety codes; NBSIR 81-2402.

regulation; renovation; building codes; building economics; construction; cost; economics; housing; productivity; NBSIR 80 2150.

regulation; site design practices; site engineering; building site standards; NBS-GCR-81-332.

regulation; solar collector; standards; voluntary program; certification; consumer protection; Interstate Solar Coordination Council; labeling procedures; national testing program; SP608: 1981 May. 179.181.

regulation; standards; building rehabilitation; codes; comparative analysis; existing buildings; housing codes; maintenance; model codes; occupancy; performance levels; NBSIR 80-2081.

regulation; theory of games; inspection; mathematical model; J. Res. 86(2): 193.215; 1981 March-April.

regulations; rehabilitation; building code; building research; buildings; code enforcement; SP623.

regulatory enforcement; implant retrieval and analysis; liability trials; performance testing; SP601: 1981 January. 655.666.

regulatory experiments; system design; drug development; drug regulation; innovation; post-marketing surveillance; NBS-GCR ETIP $81-96$

regulatory reform; administrative experiment; communications; competition; ETIP; FCC; innovation incentives; NBS-GCR-ETIP 81-97.

rehabilitation; building code; building research; buildings; code enforcement; regulations; SP623.

reimbursable services; software conversion; Federal Conversion Support Center; SP500-83: 1981 November. 341.

reinforced concrete; roofs; thermal expansion; thermal restraint; buildings; concrete; fire endurance; fire tests; floors; NBS-GCR-81317. 
reinforced concrete; standards; structural engineering; building building codes; building design; earthquakes; engineering; NBSIR 80-2111-4.

reinforced concrete; steel structures; wood structures; fire endurance; fires; mathematical models; NBSIR 80-2188.

reinforcing bars; stress waves; concrete; dynamic properties; fiberreinforcement; impact; impulsive loading; penetration resistance; SP480-45.

relational model; SQL; CODASYL; DAPLEX; database management system; data model; DBMS; network data model; query language; NBS-GCR-81-338.

relative dielectric constant; soil textural types; attenuation; coaxial sample holder; density; dielectric properties; NBSIR 78-896.

relative polarization; relative power; wave-lengths; $\mathrm{CD}_{2} \mathrm{~F}_{2} ; \mathrm{CO}_{2}$ laser; FIR laser; laser frequency measurement; 20743.

relative power; wave-lengths; $\mathrm{CD}_{2} \mathrm{~F}_{2} ; \mathrm{CO}_{2}$ laser; FIR laser; laser frequency measurement; relative polarization; 20743.

relaxation; atom; charge transfer; chemi-ionization; collisional ionization; cross section; detachment; energy transfer; excitation; ion; molecule; reaction rate; SP593.

reliability; risk; structural engineering; building (codes); climatology; hurricanes; 20764.

reliability; rotary-reciprocating; turbo-Brayton; Vuilleumier; component development; contamination control; cryogenics; helium retention; long.life; refrigerators; SP607: 1981 May. 21.29.

reliability; sensor; transducer; ATE; automatic test equipment; calibration; data acquisition; durability; dynamic calibration; environmental testing; evaluation; measurand; SP615.

reliability; solar collectors; solar materials; stagnation testing; accelerated aging; durability; environmental exposure; TN1136.

reliability; statistical analysis; structural engineering; wind; climatology; loads (forces); probability distribution functions; 20019.

reliability; turbomachinery; aerothermal diagnostics; aerothermal parameters; performance; physical deterioration; SP622: 1981 October. 222-253.

reliability centered maintenance; energy conservation; Engineering Maintenance Center; machinery monitoring; SP622: 1981 October. 113.124.

reliability of semiconductor devices; semiconductor devices; analysis of moisture content; mass spectrometer measurement; moisture; moisture generators; moisture sensors; quality control; SP400-69.

reliability study; building energy conservation systems; EMCS; energy management; energy monitoring and control systems; HVAC controls; NBSIR 81-2346.

remote sensing; scattering; oil shale retorts; NBSIR 81-1653.

remote surveillance; acoustic emissions; cavitation; feedwater control valve; pattern recognition; pressurized water reactor; SP622: 1981 October. 11.30.

remote terminal emulation; response time; terminal networks; workload sizing; benchmark testing; Phase IV; SP500-83: 1981 November. 169-177.

remote terminals; total tank subsystem integration; bus controllers; control and distribution; crew station terminals; electrical power management; electronic components; microcomputers; MIL-STD. 1553B multiplex data bus; multiplexing techniques; on-board diagnostics and prognostics; SP622: 1981 October. 85-96.

remote time measurement; time comparison; time receiver; world wide time; atomic clocks; automatic time comparisons; common-mode cancellation; frequency comparison; global positioning system; 20062.

renovation; building codes; building economics; construction; cost; economics; housing; productivity; regulation; NBSIR 80-2150.

repassivation kinetics; stainless steel; triboellipsometry; aluminum; field ion microscopy; iron-chromium alloys; niobium; NBSIR 791904 (Navy).

reporting of implant experience; cardiac pacemakers; international standards; SP601: 1981 January. 503-508.

reproducibility; time to failure; accelerated aging tests; coatings; corrosion; predictability; TN1149.

requirements; scenarios; database design; data dictionary; design; flowchart; information flow; information systems; interactive systems; SP500-83: 1981 November. 85-91.

requirements; scenarios; database design; data dictionary; design; flowchart; information flow; information systems; interactive systems; NBSIR 81-2240.

requirements; software; computer program; database; database management system; data dictionary system; data inventory; data management; data standards; documentation; Federal Information Processing Standards Publication; NBSIR 81-2354.

requirements; software quality assurance (SQA); specification; configuration management (CM); cross reference; data structure; design, documentation; functional; historical file; keyword; life cycle management; SP500-83: 1981 November. 101-106.

requirements analysis; carrier sense multiple access; environmental analysis; feasibility study; local area networks; NBSIR 80-2187.

requirements studies; software procurement; application development; computer software; cost/benefit analysis; database management systems; data management; program maintenance; SP500-84.

rerefined oil; energy-from-waste; materials market; oil analysis; recycled materials; refused-derived-fuel; NBSIR 80-2178.

rerefined oil; test procedures; basestock; engine lubricants; lubricating oil; motor oil; petroleum oil; recycled oil; 20016.

research; sediment; soil; alloy; carbon; computers; energy; fine structure; interface; materials; measurement; office building; phase diagrams; radioactivity; $D I M / N B S$ 65(8): 1.28; 1981.

research; site planning; sociology; standards; children; design; environmental psychology; families; housing; interiors; lifestyle; NBS-GCR-81-320.

research; standards; technology transfer; basic measurements and standards; building technology; calibration methods; computer standards and guidelines; energy research; environmental measurements; fire safety; industrial productivity; materials properties; SP600.

research associate; scanning technique; spin dependent; SRM's; standards; tape; copper; deterioration; electromagnetic radiation; film; industry; metals; productivity; DIM/NBS 65(5): 1.24; 1981.

research materials; standard reference materials; analysis; characterization; composition; materials; properties; SP260, 1981-83 Edition.

research methods; survey research; user needs; dwelling units (residential); housing; Operation Breakthrough; post-occupancy housing evaluation; Project Feedback; questionnaires; NBSIR 81 . 2258.

research recommendations; coal; control; fossil energy; materials; process parameters; NBSIR 81-2348.

residential; supply; water conservation; water consumption; appliances; clothes washer; discharge; dishwasher; NBSIR 80-2173.

Residential Conservation Service; Solar Model Audit; auditor training; energy conservation; program measure; SP608: 1981 May. 183.194.

residential structures; safety; thermal insulation; branch circuit wiring problems; electrical problems; electrical wiring system; extension cord wiring; fuse/circuit breaker panel problems; overamping; NBSIR 81-2203.

residential surveys; transfer functions; field surveys; fire; fire loads; furnishings; household surveys; live loads; mobile homes; NBSIR $80-2155$

residential water conservation; wastewater treatment; water rate schedules; water-saving devices; benefits; cost-effective; costs; economic analysis; economic decision framework; energy conservation; marginal price; net present value; NBSIR 81-2304.

residual stress; sapphire; specular reflectance; surface analysis; surface integrity; surface roughness; crystallographic anisotropy; crystallographic perfection; initial permeability; metal mirror; polishing; polycrystalline material; SP620: 1981 October. 171-179.

residues; toxic elements; availability; environmental contamination; extraction tests; fossil fuels; laboratory characterization; metals; mining industry; public health impacts; SP618: 1981 November. 1-8.

resin; acrylate; adhesion; aminobenzoate; composite material; dentin; methacrylate; polyfunctional surface-active comonomers; reconstructive dental work; U.S. Patent 4,251,565. 
resin; wear; accelerator; alloy; base metal; casting; composite; cyanoacrylate; dental alloy; initiator; NBSIR 80-2168.

resistivity; semiconductor; silicon; boron; capacitance-voltage technique; dopant density; electron mobility; Hall effect; hole mobility; Irvin curves; phosphorus; SP400-64.

resistivity; silicon; conductivity mobility; density of states; dopant (acceptor) density; effective mass; Hall mobility; hole density; NBS-GCR-81-334.

resolution limit; fiber index profile; index profile; near-field scanning; optical communications; optical fiber; optical waveguide; refracted near-field scanning; refracted-ray scanning; TN1038.

resonance; satellite; auger; core hole spectroscopy; metals with filled $d$-bands; photoemission; 20008.

resonance; xenon; angular distribution; asymmetry parameter; branching ratios; 20052.

resonance fluorescence; giant resonance; photonuclear; quasideuteron; 19992.

resonance Raman; spectroscopy; strong fields; damping theory; kinetics; lasers; lineshapes; optical pumping; 20039.

resonant cavity; electromagnetic radiated susceptibility measurements; mode tuned chamber; NBSIR 81-1638.

resonant free piston; Stirling cycle; cryocooler; gas bearings; refrigeration; regenerator; SP607: 1981 May. 116.

resource availability data; computer performance; performance tools; SP500-83: 1981 November. 329.

resource measurement facility; simulation; supercomputers; benchmarking; capacity planning; chargeback systems; computer performance management; data base machines; end user productivity; human factors evaluation; information system management; office automation; performance management systems; SP500-83.

resource oriented groupings; timesharing; functional categories; SP500-83: 1981 November. 15-19.

resource recovery; econimic, institutional, technical barriers; procurement; purchasing; recovered/recycled materials; NBSGCR-81-309, Supplement 1 .

resource recovery; economic, institutional, technical barriers; procurement; purchasing; recovered/recycled materials; NBSGCR-81-309.

resource recovery; economic, institutional, technical barriers; procurement; purchasing; recovered/recycled materials; NBSGCR-81-310.

resource recovery; technical barriers; economic; institutional; procurement; purchasing; recovered/recycled materials; NBSIR 81-2316.

resource recovery; technical barriers; economic; institutional; procurement; purchasing; recovered/recycled materials; NBSGCR $-81-311$.

resource recovery; technical barriers; economic; institutional; procurement; purchasing; recovered/recycled materials; NBSGCR-81-312.

resource recovery; technical barriers; economic; institutional; procurement; purchasing; recovered/recycled materials; NBSGCR-81-313.

resource recovery; technical barriers; economic; institutional; procurement; purchasing; recovered/recycled materials; NBSGCR $-81-314$

resource recovery; technical barriers; economic; institutional; procurement; purchasing; recovered/recycled materials; NBSGCR-81-308

respiration; animal toxicity; breath analysis; endurance; free-radicals; iron; organometalloid toxicity; pentane; SP618: 1981 November. 226-234.

response; symbol; understandability; communication; evaluation method; fire-safety; hazard warnings; meaningfulness; method; pictogram; NBSIR 80-2088.

response factors; thick building construction; conduction heat transfer; conduction transfer functions; initialization of heat transfer problem; parallel heat flow; NBSIR 81-2353.

response time; terminal networks; workload sizing; benchmark testing; Phase IV; remote terminal emulation; SP500-83: 1981 November.
169-177.

retention index; speciation; atomic absorption; energy-related process fluids; leachate; linear free energy; liquid chromatography; molecular substituent; oil shale; organoarsenic; organotin; SP618 1981 November. 197.216.

retorting; soil; speciation; volatilization; arsenic; dimethylarsinic acid; microbial growth; mobilization; nutrients; oil shale; SP618: 1981 November. 286.300.

retort water; speciation; trace metals; alkylpyridines; dissolved organic compounds; gas chromatography/mass spectrometry; hydrophobic bases; metal complexation; nitrogen bases; oil shale; SP618: 1981 November. 93-104.

retraction; robustness; rooted tree; sensitivity analysis; shortest path; tolerance; cutset; cycle; directed graph; network; network flow; optimization; 20022.

retrieval; analysis; biological; cardiovascular; implants; metals; orthopaedic; polymers; SP601.

reverse bias; second breakdown; switching; temperature; transistors, power; base current, reverse; degradation; NBSIR 81-2204.

review; singlet oxygen; solution; chemical kinetics; data compilation; oxidation; photochemistry; rates; JPCRD 10(4): 809.999; 1981.

review; standard; superconductor; conductor; critical current; critical temperature; 20069.

review; water; water substance; equation; formulation; high pressure; high temperature; IAPS; International Association for the Properties of Steam; ionization; ionization constant; ion product; JPCRD 10(2): 295-304; 1981.

revised Kosek and McKinley; cell model; comparisons; computer programs; corresponding states; equation of state; hard sphere; LNG; mixtures; PVTx data; TN1030.

RFP preparation; small systems; computer procurement; proposal evaluation; SP500-83: 1981 November. 191-197.

If voltage; rf voltmeters; comparator; NBSIR 78-871.

rf voltmeters; comparator; rf voltage; $N B S I R$ 78-871.

rhodium; vibrational spectroscopy; carbon monoxide; chemisorption; isocyanide; 19997.

risk; structural engineering; building (codes); climatology; hurricanes; reliability; 20764.

risk management; shock pulse analysis; vibration analysis; acoustic emission analysis; insurance; loss prevention; modal analysis; property conservation; SP622: 1981 October. 139.151.

risks of failure; user productivity; building productivity; computerintegrated construction; conference; construction duration; productivity measurement; NBS-GCR-81-331.

robot control; robotic assembly; robotics research; robotic standards; robot vision; Delphi Forecast; SP602.

robot control; robot interfaces; robot standards; robot wrist; simple sensors; complex sensors; off-line programming; NBSIR 80-2152.

robotic assembly; robotics research; robotic standards; robot vision; Delphi Forecast; robot control; SP602.

robotics research; robotic standards; robot vision; Delphi Forecast; robot control; robotic assembly; SP602.

robotic standards; robot vision; Delphi Forecast; robot control; robotic assembly; robotics research; SP602.

robot interfaces; robot standards; robot wrist; simple sensors; complex sensors; off-line programming; robot control; NBSIR 80-2152.

robot standards; robot wrist; simple sensors; complex sensors; off-line programming; robot control; robot interfaces; NBSIR 80-2152.

robot vision; Delphi Forecast; robot control; robotic assembly; robotics research; robotic standards; SP602.

robot wrist; simple sensors; complex sensors; off-line programming; robot control; robot interfaces; robot standards; NBSIR 80-2152.

robustness; rooted tree; sensitivity analysis; shortest path; tolerance; cutset; cycle; directed graph; network; network flow; optimization; retraction; 20022

rock salt; sodium chloride; chemical; electrical; geological; magnetic; mechanical; monograph; optical; physical; properties data; radiation damage; Monogr. 167

roentgen ray; tubes ( $\mathrm{x}$ ray); $\mathrm{x}$-ray measurement; $\mathrm{x}$ rays; dosage; dosimetry; health and safety; high voltage; ionization chamber; ionizing radiation; ionometer; radiation quantities and units; 
radiation standards and protection; radioactivity; radiology; radium; SP625.

rollin/rollout files; simulation; CDC 844-41; computer system evaluation; disk subsystem configurations; input/output; modeling; SP500-83: 1981 November. 239-246.

roofing; bitumen application temperatures; bituminous roofing; builtup roofing; cooling of roofing bitumens; mathematical model; TN1135.

roofing; thermal resistance; built-up roofing; moisture; moisture detection; nondestructive evaluation; nondestructive testing; TN1146.

roofing membrane; splitting; strain energy; stress; substrate; adhesive; bitumen; bonding; built-up roofing membrane; complementary strain energy; felt; nonlinear; TN1152.

roofing systems; built-up roofing electrical capacitance; infrared thermography; insulation; moisture detection; moisture measurement; nondestructive evaluation; nondestructive testing; nuclear backscatter; BSS131.

roofs; thermal expansion; thermal restraint; buildings; concrete; fire endurance; fire tests; floors; reinforced concrete; NBS-GCR-81-317.

room fire; wood; zone model; crib fire; plastic; 20740.

room fires; burning rates; compartment fires; fire modeling; flashover; numerical methods; NBS-GCR-81-301.

room fires; compartment fires; computer programs; fire extinguishment; flashover; ignition; mathematical models; NBSGCR-81-344.

room fires; compartment fires; fire tests; flashover; interior finish; mobile homes; models; NBSIR 81-2333.

room fires; fire modeling; fire plumes; flame spread; furniture flammability; polyurethane foam combustion; NBS-GCR-81-300.

room fires; smoke movement; tenability limits; combustion products; compartment fires; egress; fire detection; fire growth; hazard analysis; mathematical models; NBSIR 80-2172.

room fires; upholstered furniture; burning rate; case goods; chairs; furniture fires; ignitability; NBSIR 81-2271.

room fires; wood; crib fires; experiments; mathematical model; plastic; polyurethane; NBSIR 80-2054, Vols. I and II.

rooted tree; sensitivity analysis; shortest path; tolerance; cutset; cycle; directed graph; network; network flow; optimization; retraction; robustness; 20022.

rotary-reciprocating; turbo-Brayton; Vuilleumier; component development; contamination control; cryogenics; helium retention; long-life; refrigerators; reliability; SP607: 1981 May. 21-29.

rotational constants; structure; ab initio calculations; amino acid; conformational energy; dipole moment; gas phase conformers; glycine; 20005.

rotational-electronic coupling; scattering theory; adiabatic potentials; Born-Oppenheimer approximation; diatomic spectroscopy; FranckCondon principle; 20060.

rotational transitions; distortion dipole transitions; interstellar molecules; methane; microwave spectra; molecular parameters; radio astronomy; JPCRD 10(4): 1085-1095; 1981.

rubidium; sodium; solutions; thermodynamic properties; activity coefficient; cesium; critical evaluation; electrolyte; excess Gibbs energy; lithium; osmotic coefficient; potassium; JPCRD 10(3): 671. 766.

rubidium compounds; sodium compounds; thorium compounds; uranium compounds; actinium compounds; cesium compounds; enthalpy; entropy; francium compounds; Gibbs energy of formation; heat capacity; lithium compounds; potassium compounds; protactinium compounds; TN270-8.

$\mathrm{R}$ value; standard reference material; thermal resistance; fiber-glass; fibrous glass; insulation; measurement; NBSIR 80-2091.

R-values; single-crystal; SRM's; atomic ions; calibration; clock synchronization; Columbia River model; computer; insulation; lasers; powder data; progress; $D I M / N B S$ 65(2): 1-28; 1981.

\section{S}

safety; contusion; eyeball deformation; impact; ocular injury; projectiles; NBSIR 80-2037. safety; sealants; standards; storm doors; storm windows; vent dampers; water heaters; windows; automatic ignition devices; caulks; effectiveness; energy conservation; energy conservation measures; installation; insulation; oil burners; practices; SP606.

safety; signs; standards; symbols; visual alerting; warnings; communication; hazard; pictograms; NBSIR 80-2003.

safety; thermal insulation; branch circuit wiring problems; electrical problems; electrical wiring system; extension cord wiring; fuse/circuit breaker panel problems; overamping; residential structures; NBSIR 81-2203.

safety and hazards; thermal energy storage materials; thermal properties; thermodynamic properties; transport properties; corrosion; data compilations; electrochemical aluminum production; electrochemical energy storage materials; molten salts; physical properties; NSRDS-NBS61, Part IV.

safety of bicycles; safety standards; abrasion test development; bicycle compliance tests; bicycle safety; reflective materials for bicycles; NBSIR 80-2035.

safety regulation; standards; cost-benefit; economics; government regulation; 20031.

safety standard; classification criteria; gamma radiography; national standard; performance tests; radiation source; $H 136$.

safety standards; abrasion test development; bicycle compliance tests; bicycle safety; reflective materials for bicycles; safety of bicycles; NBSIR 80-2035.

salt hydrate; sodium sulfate; temperature calibration; temperature standard; thermistor; thermometer; transition temperature; biomedical thermometry; disodium hydrogen phosphate; fixed point; hydration temperature; potassium fluoride; J. Res. 86(2): 181 . 192; 1981 March-April.

sample characterization; sample processing effects; 2.5 and 25 gram capacity bomb calorimeters; ASTM round robin testing; bomb calorimetry; higher heating value; refused-derived-fuel; NBSIR 81 . 2278.

sample mounting; ventilation cloth; ASTM E-162; brattice cloth; coal mining; fire test; flame spread; NBSIR 81-2200.

sample processing effects; 2.5 and 25 gram capacity bomb calorimeters; ASTM round robin testing; bomb calorimetry; higher heating value; refused-derived-fuel; sample characterization; NBSIR 81-2278.

sampling circuit; sampling pulses; analog signal; Josephson junction; U.S. Patent 4,245,169.

sampling pulses; analog signal; Josephson junction; sampling circuit; U.S. Patent 4,245, 169.

sapphire; specular reflectance; surface analysis; surface integrity; surface roughness; crystallographic anisotropy; crystallographic perfection; initial permeability; metal mirror; polishing; polycrystalline material; residual stress; SP620: 1981 October. 171. 179.

satellite; auger; core hole spectroscopy; metals with filled $d$-bands; photoemission; resonance; 20008.

satellite communication; ALOHANET; ALOHA system; computer network; packet broadcasting; radio computer communication network; J. Res. 86(6): 591-595; 1981 November-December.

satellite communication; earth terminal; Earth Terminal Measurement System; figure of merit; noise temperature; NBSIR 81-1641.

satellite communications; C/kT; EIRP; error analysis; $\mathrm{G} / \mathrm{T}$; precision measurements; radio star; NBSIR 78-869.

satellite communications; slow-scan television; telecommunications policy; teleconferencing; videodiscs; computer network interconnection; computer network protocol; digital transmission of information; electronic message transmission; image transmission; information networks; national bibliographic network; SP610.

saturated liquid; shear viscosity coefficient; density dependence; gaseous and liquid ethane; isothermal measurements; quartz crystal viscometer; 20767.

savings-to-investment ratio; benefit-cost analysis; building economics; cost effective; economic analysis; energy conservation; investment analysis; life-cycle cost; recommended practice; NBSIR 81-2397.

scaffold failures; scaffolding practices; scaffolds; structural analysis; accidents; codes and standards; construction loads; construction 
safety; finite element; NBS-GCR-80-255.

scaffolding practices; scaffolds; structural analysis; accidents; codes and standards; construction loads; construction safety; finite element; scaffold failures; NBS-GCR-80-255.

scaffolds; stability; stiffness; strength; structural safety; work surfaces; codes and standards; construction safety; design; finite element; loads; NBSIR 81-2265.

scaffolds; structural analysis; accidents; codes and standards: construction loads; construction safety; finite element; scaffold failures; scaffolding practices; NBS-GCR-80-255.

scald; thermal injury; bioheat equation; burn; criterion; critical exposure; fluid; heat transfer; injury threshold; NBSIR 81-2320.

scales; specifications; taximeters; tolerances; volume-measuring devices; weighing devices; weights; length-measuring devices; liquid-measuring devices; measures; H44, 1982 Edition.

scaling; strain; superconducting wire; critical current; mechanical properties; niobium-tin; 20066.

scaling theorem; water phantom; absorbed dose standard; cobalt- 60 gamma rays; electron density; graphite calorimeter; ionization chamber; J. Res. 86(5): 495.502; 1981 September-October.

scandium; scandium halides; yttrium; yttrium halides; chemiluminescence; Franck-Condon factors; laser-induced fluorescence; radiative lifetimes; 19969.

scandium; scandium halides; yttrium; yttrium halides; chemiluminescence; Franck-Condon factors; laser-induced fluorescence; radiative lifetimes; 19978.

scandium halides; yttrium; yttrium halides; chemiluminescence; Franck-Condon factors; laser-induced fluorescence; radiative lifetimes; scandium; 19978.

scandium halides; yttrium; yttrium halides; chemiluminescence; Franck-Condon factors; laser-induced fluorescence; radiative lifetimes; scandium; 19969.

scanning electron microscopy; silicon (lithium) detector; spectral deconvolution; $x$-ray analysis; $x$-ray fluorescence; electron probe microanalysis; energy dispersive $\mathrm{x}$-ray spectrometry; germanium (lithium) detector; SP604.

scanning electron microscopy; silicon technology; spreading resistance; two-dimensional profiles; arsenic ion implantation; electron-beam-induced current; etching; integrated circuit processing; ion channeling; ion implantation; lateral profiles; NBSIR 81-2398.

scanning electron microscopy; spectral artifacts; transmission electron microscopy; electron probe microanalysis; energy dispersive $\mathrm{x}$-ray spectrometry; microanalysis; qualitative analysis; quantitative analysis; SP604: 1981 June. 315-339.

scanning electron microscopy; $x$-ray spectrometry, review of scientific instruments; bibliography; electron column instruments; energy dispersive detectors; SP604: 1981 June. 417.439.

scanning technique; spin dependent; SRM's; standards; tape; copper; deterioration; electromagnetic radiation; film; industry; metals; productivity; research associate; DIM/NBS 65(5): 1-24; 1981

scanning transmission electron microscope; analytical electron microscope; artifacts; energy dispersive x-ray spectrometry; SP604: 1981 June. 341-349.

scattering; electron-molecule collisions; interaction potential; Legendre expansion; molecular potential; quantum chemistry; 20055.

scattering; oil shale retorts; remote sensing; NBSIR 81-1653.

scattering amplitude of neodymium; cerium neodymium tantalates; cerium niobate; neutron diffraction; oxidation mechanisms; profile refinement method; 20054.

scattering-matrix description of antennas; antenna-antenna systems; antenna measurements; antenna-scatterer systems; antenna theory; microwave antenna theory; Monogr. 162.

scattering of electromagnetic waves; spurious resonances; augmented electric-and magnetic-field integral equations; electric-field integral equation; electromagnetic theory; magnetic-field integral equation; 20761.

scattering of electromagnetic waves; spurious resonances; augmented electric-and magnetic-field integral equations; electric-field integral equation; electromagnetic theory; magnetic-field integral equation; scattering theory; adiabatic potentials; Born-Oppenheimer approximation; diatomic spectroscopy; Franck-Condon principle; rotational-electronic coupling; 20060.

scattering theory; bound states; continuum states; diatomic spectroscopy; electronic-rotational states; predissociation; 20049.

scenarios; database design; data dictionary; design; flowchart; information flow; information systems; interactive systems; requirements; SP500-83: 1981 November. 85-91.

scenarios; database design; data dictionary; design; flowchart; information flow; information systems; interactive systems; requirements; NBSIR 81-2240.

schools; technical assistance; certification; Class A energy audit; economic analysis; energy analysis; energy conservation; Federal programs; government buildings; hospitals; rate of return; SP608: 1981 May. 133-138.

Schwinger limits; screened Coulomb potential; Sobolev's inequality; Yukawa potential; Bargmann limits; bound-state estimates; Lieb limits; number of bound states; J. Res. 86(5): 503.508; 1981 September-October.

scope; specification; standard; system analysis/engineering; arrangement; building; classification; code; engineering; organization; provisions; BSS136.

scope of standards; standards; standards writing; legislative history; product liability; rationale statements; NBS-GCR-81-347.

scraping Auger; slush; slush production; cryogenic; hydrogen; production efficiency; NBSIR 78-875.

scratches; surface defects; coating imperfections; cw laser irradiation; digs; dust; laser damage; mirrors; optical components; SP620: 1981 October. 256.264.

screened Coulomb potential; Sobolev's inequality; Yukawa potential; Bargmann limits; bound-state estimates; Lieb limits; number of bound states; Schwinger limits; J. Res. 86(5): 503.508; 1981 September-October.

sealants; standards; storm doors; storm windows; vent dampers; water heaters; windows; automatic ignition devices; caulks; effectiveness; energy conservation; energy conservation measures; installation; insulation; oil burners; practices; safety; SP606.

seasonal efficiency; annual fuel utilization efficiency; annual operating costs; central heating equipment; condensing boilers; condensing furnaces; part-load performance; rating procedure; NBSIR 80-2110.

secondary charged particles; tissue equivalence; kerma factors; neutron dosimetry; neutron radiation therapy; nuclear data; 20064.

secondary ion mass spectrometry (SIMS); semiconductor devices; sputtering; x-ray photoelectron spectroscopy (XPS, ESCA); Auger electron spectroscopy (AES); metal-oxide-semiconductor (MOS); SP400-67.

secondary storage; space management; capacity planning; device architecture; SP500-83: 1981 November. 325.

secondary target fluorescence analysis; source excited fluorescence analysis; $x$-ray fluorescence analysis; catalysts automotive; energy dispersive analysis; heavy element fluorescence analysis; intrinsic germanium detectors; SP604: 1981 June. 391.415.

second breakdown; switching; temperature; transistors, power; base current, reverse; degradation; reverse bias; NBSIR 81-2204.

security; computer security; cryptography; data integrity; encryption; Federal Information Processing Standards Publication; key distribution; network security; FIPS PUB 74.

security hardware; victimization survey; burglary; crime in public housing; household behavior patterns; housing characteristics (physical); housing security; public housing security; NBS-GCR-80 291.

sediment; soil; alloy; carbon; computers; energy; fine structure; interface; materials; measurement; office building; phase diagrams; radioactivity; research; $D I M / N B S$ 65(8): 1-28; 1981.

segregation; solidification; thermal mechanical treatment; aging; electrical conductivity; hardness; homogeneity; microstructure; precipitating; NBSIR 80-2069.

seismic coefficients; architectural; electrical; elevators; mechanical; NBSIR 80-2111-8.

seismic risk maps; standards; structural engineering; buildings; 
buildings (codes); buildings (design); earthquake; NBSIR 80-2111-1. seismic safety; standards; structural engineering; building; building design; earthquakes; engineering; Federal government; NBSIR 812195.

selected values; thermochemical tables; enthalpy; entropy; equilibrium constant of formation; free energy of formation; Gibbs energy function; heat capacity; heat of formation; NBSIR 81-2343.

selection of ADP systems; standard benchmarks; synthetic benchmarks; workload characterization; workload definition; benchmarking; benchmark library; 20747.

selenite; arsenate; arsenic methylation; biological transformation; graphite furnace atomic absorption spectrometer; high pressure liquid chromatography; SP618: 1981 November. 244-263.

selenium; calorimetry; crystal; enthalpy; entropy; floor temperature; fusion; Gibbs energy; glass transition; heat capacity; linear macromolecule; melt; JPCRD 1O(1): 89-118; 1981

selenium; solvent refined coal; titanium; trace elements; arsenic; chromium; coal hydrogenation; cobalt; liquefaction; mercury; nickel; process waters; SP618: 1981 November. 21.38.

self-extinguishment; smoldering; upholstered furniture; cigarettes; flammability; ignition; mattresses; NBSIR 81-2363.

self-focusing; Kerr liquids; laser damage; laser induced breakdown; $\mathrm{NaCl}$; SP620: 1981 October. 385-393.

self-focusing; $\mathrm{SiO}_{2}$; air breakdown; laser damage; $\mathrm{NaCl}$; picosecond phenomena; SP620: 1981 October. 375-384.

self-focusing; solitons; waveguides; fiber optics; nonlinear propagation; nonlinear pulses; SP620: 1981 October. 453-458.

self-heating; solar collector fires; solar collectors; spontaneous ignition; building fires; fire safety; ignition; NBSIR 81-2326.

SEM; deformation; electron channeling; fatigue; metals; 19980.

semiconductor; silicon; boron; capacitance-voltage technique; dopant density; electron mobility; Hall effect; hole mobility; Irvin curves; phosphorus; resistivity; SP400-64.

semiconductor; technology; calibration; electrical; electronics; engineering; hazards; measurement; metrology; National Bureau of Standards; power; radiation; SP611.

semiconductor detectors; silicon; tailing; $x$-ray spectroscopy; germanium; SP604: 1981 June. 35.44.

semiconductor device defects; forward-biasing; internal resistance; intrinsic distributed resistance; U.S. Patent 4,287,473.

semiconductor devices; analysis of moisture content; mass spectrometer measurement; moisture; moisture generators; moisture sensors; quality control; reliability of semiconductor devices; SP400-69.

semiconductor devices; semiconductor materials; semiconductor process control; silicon; electronics; integrated circuits: measurement technology; microelectronics; NBSIR 79-1591-4.

semiconductor devices; semiconductor materials; semiconductor process control; silicon; electronics; integrated circuits; measurement technology; microelectronics; NBSIR 79-1591-5.

semiconductor devices; semiconductor materials; semiconductor process control; silicon; electronics; integrated circuits; measurement technology; microelectronics; NBSIR 80-2006-3.

semiconductor devices; semiconductor materials; semiconductor process control; silicon; electronics; gallium arsenide; integrated circuits; measurement technology; microelectronics; NBSIR 802006-4.

semiconductor devices; semiconductor materials; semiconductor process control; silicon; electronics; integrated circuits measurement technology; microelectronics; NBSIR 81-2230-2.

semiconductor devices; semiconductor materials; semiconductor process control; silicon; electronics; integrated circuits; measurement technology; microelectronics; NBSIR 81-2230.

semiconductor devices; semiconductor materials; semiconductor process control; silicon; electronics; integrated circuits; measurement technology; microelectronics; NBSIR 81-2230-3.

semiconductor devices; semiconductor processes; semiconductors; semiconductor technology; silicon; integrated circuits; NBSIR 812197.

semiconductor devices; sputtering; $x$-ray photoelectron spectroscopy (XPS, ESCA); Auger electron spectroscopy (AES); metal-oxide- semiconductor (MOS); secondary ion mass spectrometry (SIMS); SP4O0-67.

semiconductor materials; semiconductor process control; silicon; electronics; gallium arsenide; integrated circuits; measurement technology; microelectronics; semiconductor devices; NBSIR 802006-4.

semiconductor materials; semiconductor process control; silicon; electronics; integrated circuits; measurement technology; microelectronics; semiconductor devices; NBSIR 79-1591-4.

semiconductor materials; semiconductor process control; silicon; electronics; integrated circuits; measurement technology; microelectronics; semiconductor devices; NBSIR 79-1591-5.

semiconductor materials; semiconductor process control; silicon; electronics; integrated circuits; measurement technology; microelectronics; semiconductor devices; NBSIR 80-2006-3.

semiconductor materials; semiconductor process control; silicon; electronics; integrated circuits; measurement technology; microelectronics; semiconductor devices; NBSIR 81-2230-3.

semiconductor materials; semiconductor process control; silicon; electronics; integrated circuits; measurement technology; microelectronics; semiconductor devices; NBSIR 81-2230.

semiconductor materials; semiconductor process control; silicon; electronics; integrated circuits measurement technology; microelectronics; semiconductor devices; NBSIR 81-2230-2.

semiconductor plasma; IR laser damage; photoexcited carriers; SP620: 1981 October. 427-431.

semiconductor process control; silicon; electronics; gallium arsenide; integrated circuits; measurement technology; microelectronics; semiconductor devices; semiconductor materials; NBSIR 80-20064.

semiconductor process control; silicon; electronics; integrated circuits; measurement technology; microelectronics; semiconductor devices; semiconductor materials; NBSIR 79-1591-4.

semiconductor process control; silicon; electronics; integrated circuits; measurement technology; microelectronics; semiconductor devices; semiconductor materials; NBSIR 79-1591-5.

semiconductor process control; silicon; electronics; integrated circuits; measurement technology; microelectronics; semiconductor devices; semiconductor materials; NBSIR 80-2006-3.

semiconductor process control; silicon; electronics; integrated circuits; measurement technology; microelectronics; semiconductor devices; semiconductor materials; NBSIR 81-2230-3.

semiconductor process control; silicon; electronics; integrated circuits; measurement technology; microelectronics; semiconductor devices; semiconductor materials; NBSIR 81-2230.

semiconductor process control; silicon; electronics; integrated circuits measurement technology; microelectronics; semiconductor devices; semiconductor materials; NBSIR 81-2230-2.

semiconductor process control; test pattern; test structure; wafer chuck; wafer probe station; integrated circuit test methods; microelectronic test structure; probe station; SP400-68.

semiconductor processes; semiconductors; semiconductor technology; silicon; integrated circuits; semiconductor devices; NBSIR 81-2197.

semiconductors; semiconductor technology; silicon; integrated circuits; semiconductor devices; semiconductor processes; NBSIR 81.2197.

semiconductors; silicon; electronics; integrated circuits; metrology; microelectronics; NBSIR 81-2224.

semiconductors; silicon dioxide; sputter profiling; Auger-electron spectroscopy; electronics; MOS devices; NBS-GCR-81-345.

semiconductors; stimulated bremsstrahlung absorption; wavelength dependence; laser damage; optical constants; optical materials SP620: 1981 October. 416-426.

semiconductor technology; silicon; integrated circuits; semiconductor devices; semiconductor processes; semiconductors; NBSIR 81 2197.

semiderivative; stability constants; voltammetry; zinc-NTA; cadmium. NTA; convolution voltammetry; electrochemistry; Kalman filter; metal speciation; modeling; SP618: 1981 November. 163-172.

sensitivity analysis; shortest path; tolerance; cutset; cycle; directed graph; network; network flow; optimization; retraction; robustness; 
rooted tree; 20022.

sensitivity analysis; validation; assessment; composite models; data quality; energy models; mathematical models; model confidence; model credibility; policy models; SP616.

sensitivity technique; instruction $\mathrm{mix}$ technique; performance prediction; SP500-83: 1981 November. 181-189.

sensor; transducer; ATE; automatic test equipment; calibration; data acquisition; durability; dynamic calibration; environmental testing; evaluation; measurand; reliability; SP615.

sensors; building energy monitoring; instrumentation; NBSIR 80 2104.

sensors; wear; wear analysis; energy; energy conservation; failure detection; failure diagnosis; failure prevention; monitoring techniques; prognosis; SP622.

sequence list; shortest path; tree; complexity; labeling; network; $J$. Res. 86(3): 317-330; 1981 May-June.

sequential tests; solubility tests; automotive paints; household paints; SP480-40.

serials; standards; transactions; annual reports; diffusion in metals; fire; journals; library holdings, NBS Library; NBS periodicals; periodicals; proceedings; NBSIR 81-2226.

serum base modification; serum matrix; anticonvulsants; antiepilepsy drugs; blood analysis; chromatography, gas; chromatography, liquid; drug standards; enzyme immunoassay; epilepsy; ethosuximide; mass spectrometry; nuclear magnetic resonance spectroscopy; phenobarbital; phenytoin; primidone; reference material; SP260-72.

serum matrix; anticonvulsants; antiepilepsy drugs; blood analysis; chromatography, gas; chromatography, liquid; drug standards; enzyme immunoassay; epilepsy; ethosuximide; mass spectrometry; nuclear magnetic resonance spectroscopy; phenobarbital; phenytoin; primidone; reference material; serum base modification; $S P 260-72$.

serviceable; superconducting device; thermal coupling; cryogenic refrigerator; Josephson technology; liquid helium; SP607: 1981 May. 82-92.

set-cement; simulated geothermal fluid; splitting tensile strength; compressive strength; geothermal well; NBSIR 80-2099-4.

settlement; structural design; structural engineering; design criteria; foundation design; geotechnical engineering; housing construction; mine subsidence; mining; NBSIR $81-2215$.

several variables; integrals; integration; irregularity of distribution; lattices; n-cube; periodic functions; quadrature; 20032.

$\mathrm{SF}_{b}$; space charge; transformer oil; cables; composite insulation; dc fields; high voltage; incipient fault; insulation; liquid breakdown; NBSIR 81-2334.

$\mathrm{SF}_{6}$; space charge; transformer oil; cables; dc fields; high voltage; incipient fault; insulation; NBSIR 81-2283.

shakers; vibration exciters; acceleration; accelerometers; calibration; 20731.

shale oil; speciation; trace metals; chromatography; direct current plasma; hydrophilic; hydrophobic; metal complex; porphyrin; SP618: 1981 November. 105-119.

shale oil; SRM; trace organic analysis; definitive methods; human serum; polynuclear aromatic hydrocarbons; reference materials; 19989.

shale oil; standard reference materials; benzo(a)pyrene; benzo(e)pyrene; fluoranthene; fluorescence detection; gas chromatography/mass spectrometry; high-performance liquid chromatography; perylene; pyrene; 20750.

shale oil; synthetic fuels; trace elements; coal; environmental chemistry; SP618: 1981 November. 81.92.

shear; strength; building; collapse; concrete; concrete strength; construction; failure; flat plate; NBSIR 81-2374.

shear; viscosity; argon; dynamics; $m \cdot 6.8$ potential; nonequlibrium molecular; 19973.

shear deformation; tensile failure; chain rupture; crazing; crystalline polymers; electron spin resonance; microcrack; microfibril; 20732.

shear flow; shear induced phase transition; soft sphere system; stability; steady state; turbulence; nonequilibrium molecular dynamics; nonlinear thermodynamics; $2(x) 63$. shear induced phase transition; soft sphere system; stability; steady state; turbulence; nonequilibrium molecular dynamics; nonlinear thermodynamics; shear flow; 20063.

shear strength; tensile strength; yield strength; Young's modulus; compressive strength; elongation; foam; insulation; low temperature; mechanical properties; proportional limit; NBSIR 81 1654.

shear viscosity coefficient; density dependence; gaseous and liquid ethane; isothermal measurements; quartz crystal viscometer; saturated liquid; 20767.

shear viscosity coefficient; density dependence; gaseous and liquid methane; isothermal measurements; quartz crystal viscometer; 20071.

sheet resistance; solar cell defects; solar cells; test structures; laser scanner; model calculations; photovoltaic cells; NBSIR 81-2260

shielded balanced transmission lines; time domain; transmission line model; waveform; bit error rate; coaxial transmission lines; digital communication impulse response; instrumentation; measurements; TN1042.

ships; smoke control; smoke movement; stack effect; sulfur hexafluoride; tracer tests; ventilation systems; NBSIR 81-2433.

ships; tanks; volume; dimensions; laser; LNG; NBSIR 80-2141.

shock and vibration; angular acceleration; angular accelerometer; calibration; instrumentation; measurement; NBSIR 81-2337.

shock hazards; urea-formaldehyde thermal insulation; cellulose thermal insulation; corrosion of electrical outlet boxes and devices; electrical devices; humidity, thermal insulation and corrosion of electrical wiring; NBSIR 81-2220.

shock pulse analysis; vibration analysis; acoustic emission analysis; insurance; loss prevention; modal analysis; property conservation; risk management; SP622: 1981 October. 139.151.

shock pulse bearing monitoring; automatic alarm of bearing malfunction; marine machinery bearings; microprocessor based shock pulse analyzer; multiplexing bearing monitor channels; prediction of bearing failure; SP622: 1981 October. 126.136.

shortest path; tolerance; cutset; cycle; directed graph; network; network flow; optimization; retraction; robustness; rooted tree; sensitivity analysis; 20022.

shortest path; transportation networks; algorithm; graph; $k$ shortest paths; network algorithms; 20021.

shortest path; tree; complexity; labeling; network; sequence list; $J$. Res. 86(3): 317.330; 1981 May.June.

SI; translation; BIPM; CCU; CGPM; CIPM; metric; SP330

SI; weights and measures; International System of Units; measurement systems; metric system; modernized metric system; SP304, Revised August 1981.

SI; weights and measures; International System of Units; measurement systems; metric system; modernized metric system; SP304A, Revised August 1981.

sialon; singular integral equation; crack growth model; creep cavitation; diffusive crack growth; energy release rate; high temperature fracture; J-integral; NBSIR 81-2255.

signs; standards; symbols; visual alerting; warnings; communication; hazard; pictograms; safety; NBSIR 80-2003.

silicate glass; bulk laser damage; fluorophosphate glass; inclusion density; KDP crystals; SP620: 1981 October. 159-169.

silicon; boron; capacitance-voltage technique; dopant density; electron mobility; Hall effect; hole mobility; Irvin curves; phosphorus; resistivity; semiconductor; SP400-64.

silicon; conductivity mobility; density of states; dopant (acceptor) density; effective mass; Hall mobility; hole density; resistivity; NBS-GCR-81-334.

silicon; electronics; gallium arsenide; integrated circuits; measurement technology; microelectronics; semiconductor devices; semiconductor materials; semiconductor process control; NBSIR 80-2006-4.

silicon; electronics; integrated circuits; measurement technology; microelectronics; semiconductor devices; semiconductor materials; semiconductor process control; NBSIR 79-1591-5.

silicon; electronics; integrated circuits; measurement technology; microelectronics; semiconductor devices; semiconductor materials 
semiconductor process control; NBSIR 79-1591-4.

silicon; electronics; integrated circuits; measurement technology; microelectronics; semiconductor devices; semiconductor materials; semiconductor process control; NBSIR 80-2006-3.

silicon; electronics; integrated circuits; measurement technology; microelectronics; semiconductor devices; semiconductor materials; semiconductor process control; NBSIR 81-2230.

silicon; electronics; integrated circuits; measurement technology; microelectronics; semiconductor devices; semiconductor materials; semiconductor process control; NBSIR 81-2230-3.

silicon; electronics; integrated circuits; metrology; microelectronics; semiconductors; NBSIR 81-2224.

silicon; electronics; integrated circuits measurement technology; microelectronics; semiconductor devices; semiconductor materials; semiconductor process control; NBSIR 81-2230-2.

silicon; integrated circuits; semiconductor devices; semiconductor processes; semiconductors; semiconductor technology; NBSIR 81 2197.

silicon; tailing; x-ray detectors; collimation; deadlayer determinations; germanium; monoenergetic photons; partly-resolved doublets; SP604: 1981 June. 5-34.

silicon; tailing; x-ray spectroscopy; germanium; semiconductor detectors; SP604: 1981 June. 35-44.

silicon dioxide; sputter profiling; Auger-electron spectroscopy; electronics; MOS devices; semiconductors; NBS-GCR-81-345.

silicon dioxide films; silicon nitride films; standard reference materials; thin films; ellipsometry; polysilicon films; NBSIR 80-2158.

silicon films; absorption; evaporation; hydrogen and oxygen impurity; magnetron sputtering; pulsed HF/DF laser damage thresholds; SP620: 1981 October. 305.312.

silicon (lithium) detector; spectral deconvolution; $x$-ray analysis; $x$-ray fluorescence; electron probe microanalysis; energy dispersive x-ray spectrometry; germanium (lithium) detector; scanning electron microscopy; SP604.

silicon monoxide; thorium fluoride; zinc selenide; zinc sulfide; absorption; coatings; internal reflection spectroscopy; SP620: 1981 October. 335.344.

silicon nitride; strain rate effect; toughness; crack growth; creep fracture; high temperature fracture; NBSIR 79-1960 (DoE).

silicon nitride films; standard reference materials; thin films; ellipsometry; polysilicon films; silicon dioxide films; NBSIR 802158.

silicon-on-sapphire; test pattern; test structure; yield; integrated circuits; microelectronics; process validation wafer; random faults; SP400-66.

silicon technology; spreading resistance; two-dimensional profiles; arsenic ion implantation; electron-beam-induced current; etching; integrated circuit processing; ion channeling; ion implantation; lateral profiles; scanning electron microscopy; NBSIR 81-2398.

$\mathrm{Si}(\mathrm{Li})$ detectors; dead layer; low x-ray energies; SP604: 1981 June. 127.139.

silver; surface roughness; temperature dependence; aluminum; copper; Drude theory; infrared; reflectance; SP620: 1981 October. 190-200.

silver; toxic heavy metals; antimony; arsenic; bacterial resistances; cadmium; detoxification; mercury; organomercury; plasmids; SP618: 1981 November. 301-324.

simple sensors; complex sensors; off-line programming; robot control; robot interfaces; robot standards; robot wrist; NBSIR 80-2152.

simulated geothermal fluid; splitting tensile strength; compressive strength; geothermal well; set-cement; NBSIR 80-2099-4.

simulation; CDC 844-41; computer system evaluation; disk subsystem configurations; in put/output; modeling; rollin/rollout files; SP50083: 1981 November. 239.246.

simulation; documentation; manuals; models; SP500-73.

simulation; supercomputers; benchmarking; capacity planning; chargeback systems; computer performance management; data base machines; end user productivity; human factors evaluation; information system management; office automation; performance management systems; resource measurement facility; SP500-83.

simulation; tools; analysis; performance evaluation; pictures; SP50083: 1981 November. 331-334. simulation; validation; analytic models; communication networks; flow control; Markov processes; performance prediction; queueing models; SP500-83: 1981 November. 277.290

sinewave generator; standard; synthesis; calibration; digital; microcomputer; phase angle; TN1144.

single crystal; defects; epitaxial regrowth; laser irradiation; liquid phase; SP620: 1981 October. 222-226.

single-crystal; SRM's; atomic ions; calibration; clock synchronization; Columbia River model; computer; insulation; lasers; powder data; progress; R-values; DIM/NBS 65(2): 1.28; 1981.

single-family detached residence; building design; building energy performance standards; computer simulation of house energy requirements; degree days; energy analysis; NBSIR 80-2184.

single-shot damage threshold; vacuum chamber; copper mirrors; $\mathrm{CO}_{2}$ laser; multishot damage; nanosecond pulse; peak energy density; pulse distortion; SP620: 1981 October. 238-247.

single stage, twin piston cryogenic refrigerator; two nested, concentric pistons; cylinder; helium; inner piston; outer piston; regenerator gaps; U.S. Patent 4,281,517.

singlet oxygen; solution; chemical kinetics; data compilation; oxidation; photochemistry; rates; review; JPCRD 10(4): 809-999; 1981.

singular integral equation; crack growth model; creep cavitation; diffusive crack growth; energy release rate; high temperature fracture; J-integral; sialon; NBSIR 81-2255.

$\mathrm{SiO}_{2}$; air breakdown; laser damage; $\mathrm{NaCl}$; picosecond phenomena; self-focusing; SP620: 1981 October. 375-384.

sirens; sound level; warning light; warning signals; conspicuity; effective intensity; emergency warning lights; flashing lights; lights, warning; SP480-37.

site design practices; site engineering; building site standards; regulation; NBS-GCR-81-332.

site engineering; building site standards; regulation; site design practices; NBS-GCR-81-332.

site planning; sociology; standards; children; design; environmental psychology; families; housing; interiors; lifestyle; research; NBS. GCR-81-320.

six-port; automatic network analyzer; microwave; microwave measurements; 20067.

six-port measurement system; thermistor mount calibration; $\mathrm{mm}$ wave measurements; reflection coefficient; NBSIR 81-1650.

size difference effects; corresponding states; Enskog theory; mass difference effects; mixture viscosity; J. Res. 86(6): 597-604; 1981 November-December.

slag; soundness; strength; cement; expansion; fly ash; $\mathrm{MgO} ; N B S I R$ 81-2273.

slag; transpiration; vapor transport; coal gasification; high temperature; mass spectrometry; NBSIR 80-2178 (DoE).

sleep; smoke detectors; wakefulness; adults; alarm responses; auditory perception; decibel levels; fire departments; frequency distribution; noise (sound); NBS-GCR-80-284.

slip threshold; thermal diffusion length; thermal stress; cold work; crystalline disorder; defect damage; diamond-machined mirrors; Drude absorption; electron-beam melting; laser waveform; melt threshold; metal deposition; pulsed laser damage; SP620: 1981 October. 201-209.

slope; solid; velocity; volume; water; cylindrical; non-uniform; partially-filled; pitched-horizontal drain; NBSIR 81-2266.

slope; stream-depth; unsteady; volume; water; diameter; drain; flow; history; horizontal; length; partially-filled; pipe; NBSIR 81-2249.

slow-scan television; telecommunications policy; teleconferencing; videodiscs; computer network interconnection; computer network protocol; digital transmission of information; electronic message transmission; image transmission; information networks; national bibliographic network; satellite communications; SP610.

slow strain rate; stress corrosion; coal gasification; elevated temperature stress corrosion; 20027.

slow strain rate test; structural materials; austenitic stainless steels; coal gasification; elevated temperatures; ferritic stainless steel; gaseous environments; nickel alloy 671; nickel alloy $800 ; N B S I R$ 81-2191 (DoE). 
slush; slush production; cryogenic; hydrogen; production efficiency; scraping Auger; NBSIR 78-875.

slush production; cryogenic; hydrogen; production efficiency; scraping Auger; slush; NBSIR 78-875.

small-scale fire tests; thermal protection; bombs (ordnance); boxcars; computer models; fire detection systems; fire suppression; full-scale fire tests; heat transfer; railroad accidents; NBSIR 80-2170.

small systems; computer procurement; proposal evaluation; RFP preparation; SP500-83: 1981 November. 191-197.

smoke; bibliographies; building fires; coal mines; combustion products; compartment fires; fabric flammability; fire research; fire tests: flame research; NBSIR 81-2272.

smoke; smoke detectors; sprinkler systems; doors; evacuation; fire alarm systems; fire departments; fire extinguishers; fire investigations; hospitals; nursing homes; nursing staff; $N B S-G C R$ 80-297.

smoke; smoke detectors; sprinkler systems; doors; evacuation; fire alarm systems; fire departments; fire extinguishers; fire investigations; hospitals; nursing homes; nursing staff; NBS-GCR$81-343$.

smoke; soot; toxicity; wood; combustion; decision analysis; fire models; flame spread; human behavior; ignition; polymers; NBSIR $81-2382$.

smoke; suppression; toxicity; compartment fires; fire detection; fire endurance; fire modeling; fire tests; flammability; human behavior; NBSIR 81-2294.

smoke; wood; combustion products; fire modeling; foam (materials); heat release; plastics; polymers; polymethylmethacrylate; polyoxymethylene; polypropylene; polystyrene; polyurethane; polyvinyl chloride; pyrolysis; NBS-GCR-80-295.

smoke control; smoke movement; air leakage; buildings; doors; fire doors; fire tests; infiltration; orifice flow; NBSIR 81-2214.

smoke control; smoke movement; stack effect; sulfur hexafluoride; tracer tests; ventilation systems; ships; NBSIR 81-2433.

smoke control; stairwells; air movement; computer programs; egress; elevator shafts; escape means; pressurization; NBSIR 80-2157.

smoke detectors; sprinkler systems; doors; evacuation; fire alarm systems; fire departments; fire extinguishers; fire investigations; hospitals; nursing homes; nursing staff; smoke; NBS-GCR-80-297.

smoke detectors; sprinkler systems; doors; evacuation; fire alarm systems; fire departments; fire extinguishers; fire investigations; hospitals; nursing homes; nursing staff; smoke; NBS-GCR-81-343.

smoke detectors; wakefulness; adults; alarm responses; auditory perception; decibel levels; fire departments; frequency distribution; noise (sound); sleep; NBS-GCR-80-284.

smoke movement; air leakage; buildings; doors; fire doors; fire tests; infiltration; orifice flow; smoke control; NBSIR 81-2214.

smoke movement; spray nozzle; stairway protection; ventilated stair; automatic sprinklers; cooling efficiency; evaporation cooling; NBSIR 81-2202.

smoke movement; stack effect; sulfur hexafluoride; tracer tests; ventilation systems; ships; smoke control; NBSIR 81-2433.

smoke movement; tenability limits; combustion products; compartment fires; egress; fire detection; fire growth; hazard analysis; mathematical models; room fires; NBSIR 80-2172.

smoke production; bedding; beds; compartment fires; fire tests; health care facilities; heat release rate; hospitals; mattresses; prisons; NBSIR 80-2186.

smoldering; attic floor radiant panel test; cellulose insulation; fire test; flame spread; HH.I-515D; NBSIR 81-2213.

smoldering; upholstered furniture; cigarettes; flammability; ignition; mattresses; self-extinguishment; NBSIR 81-2363.

smoldering combustion; test methods; wave structure; boric acid; cellulosic insulation; ignition; propagation; NBSIR 81-2350.

smolder resistant upholstered furniture or mattress assembly; sulphur; fibrous; polyurethane foam materials; U.S. Patent 4,260,660.

snow loads; solar collectors; structural engineering; wind loads; earthquake loads; hail loads; NBSIR 81 -2199.

Sobolev's inequality; Yukawa potential; Bargmann limits; bound-state estimates; Lieb limits; number of bound states; Schwinger limits; screened Coulomb potential; J. Res. 86(5): 503-508; 1981
September-October

sociology; standards; children; design; environmental psychology; families; housing; interiors; lifestyle; research; site planning; NBSGCR-81-320.

sodium; atomic energy levels; atomic ions; atomic spectra; electron configurations; ionization potentials; JPCRD 10(1): 153-196; 1981.

sodium; solutions; thermodynamic properties; activity coefficient; cesium; critical evaluation; electrolyte; excess Gibbs energy; lithium; osmotic coefficient; potassium; rubidium; JPCRD 10(3): 671.766 .

sodium chloride; chemical; electrical; geological; magnetic; mechanical; monograph; optical; physical; properties data; radiation damage; rock salt; Monogr. 167.

sodium chloride; viscosity; aqueous solutions; compilation; reference data; JPCRD 10(1): 71-88; 1981.

sodium compounds; thorium compounds; uranium compounds; actinium compounds; cesium compounds; enthalpy; entropy; francium compounds; Gibbs energy of formation; heat capacity; lithium compounds; potassium compounds; protactinium compounds; rubidium compounds; TN270-8.

sodium isoelectronic sequence; electron atom scattering; electron impact ionization; 20737.

sodium sulfate; temperature calibration; temperature standard; thermistor; thermometer; transition temperature; biomedical thermometry; disodium hydrogen phosphate; fixed point; hydration temperature; potassium fluoride; salt hydrate; J. Res. 86(2): 181.192; 1981 March-April.

soft aluminum; windows; air pollution; automation; buildings; certified reference materials; computers; construction; data converter; mass comparator; radiation; radiocarbon; DIM/NBS 65(4): 1-28; 1981.

soft sphere system; stability; steady state; turbulence; nonequilibrium molecular dynamics; nonlinear thermodynamics; shear flow; shear induced phase transition; 20063.

software; BASIC; COBOL; compilers; data processing; Federal Information Processing Standard; FORTRAN; information interchange; information processing; processor; programming language; FIPS PUB 29-1.

software; computer program; database; database management system; data dictionary system; data inventory; data management; data standards; documentation; Federal Information Processing Standards Publication; requirements; NBSIR 81-2354.

software; test problems; accuracy; ANS FORTRAN; computer system installation; examples; OMNITAB 80; TN1147.

software automation; software development; software engineering; software testing; software tools; programming aids; SP500-80.

software automation; software development; software engineering; software tools; software tools usage; programming aids; SP500-82.

software automation; software development; software engineering; software tools; software tools usage; programming aids; NBSIR 81 2388 .

software automation; software development; software engineering; software tools; software tools usage; programming aids; NBS-GCR$81-335$.

software change package; software maintenance testing; developmental center test; environmental test; field validation test; independent testing; quality assurance program; SP500-83: 1981 November. 93-99.

software conversion; database management; data conversion; data description; data interchange form; data translation; portability; NBSIR 81-2315.

software conversion; Federal Conversion Support Center; reimbursable services; SP500-83: 1981 November. 341.

software development; software engineering; software testing; software tools; programming aids; software automation; SP500-80.

software development; software engineering; software tools; software tools usage; programming aids; software automation; SP500-82.

software development; software engineering; software tools; software tools usage; programming aids; software automation; NBSIR 81 2388.

software development; software engineering; software tools; software tools usage; programming aids; software automation; NBS-GCR - 
81-335.

software development; software engineering; software tools; static analysis; taxonomy; dynamic analysis; programming aids; SP500-74.

software development; software tools; toolboxes; development support systems; programming environments; SP500-78.

software engineering; software testing; software tools; programming aids; software automation; software development; SP500-80.

software engineering; software tools; software tools usage; programming aids; software automation; software development; SP500-82.

software engineering; software tools; software tools usage; programming aids; software automation; software development; NBSIR 81-2388.

software engineering; software tools; software tools usage; programming aids; software automation; software development; NBS-GCR-81-335.

software engineering; software tools; static analysis; taxonomy; dynamic analysis; programming aids; software development; SP500-74.

software expenditures; software program life-cycle analysis; data base management systems; Federal contracts services; Federal Government computers; programming languages; SP500-79.

software lifecycle; software testing; software verification; test coverage; test data generation; validation; verification; automated software tools; SP500-75.

software maintenance testing; developmental center test; environmental test; field validation test; independent testing; quality assurance program; software change package; SP500-83: 1981 November. 93.99.

software procurement; application development; computer software; cost/benefit analysis; database management systems; data management; program maintenance; requirements studies; SP50084.

software program life-cycle analysis; data base management systems; Federal contracts services; Federal Government computers; programming languages; software expenditures; SP500-79.

software quality assurance (SQA); specification; configuration management (CM); cross reference; data structure; design, documentation; functional; historical file; keyword; life cycle management; requirements; SP500-83: 1981 November. 101-106.

software testing; software tools; programming aids; software automation; software development; software engineering; SP500-80.

software testing; software verification; test coverage; test data generation; validation; verification; automated software tools; software lifecycle; SP500-75.

software tool; text processor; translation; UNIX; macroprocessor; pattern matching; query processor; NBSIR 80-2182.

software tools; programming aids; software automation; software development; software engineering; software testing; SP500-80.

software tools; software tools usage; programming aids; software automation; software development; software engineering; SP500-82.

software tools; software tools usage; programming aids; software automation; software development; software engineering; NBSIR $81-2388$.

software tools; software tools usage; programming aids; software automation; software development; software engineering; $N B S$ GCR-81-335.

software tools; static analysis; taxonomy; dynamic analysis; programming aids; software development; software engineering; SP500-74.

software tools; toolboxes; development support systems; programming environments; software development; SP500-78.

software tools usage; programming aids; software automation; software development; software engineering; software tools; SP500-82.

software tools usage; programming aids; software automation; software development; software engineering; software tools; NBSIR 81-2388.

software tools usage; programming aids; software automation; software development; software engineering; software tools; $N B S$. GCR-81-335.

software trends; technology forecast; automated data processing; computer utilization trends; Federal computers; hardware trends; information industry; NBS-GCR-81-342.

software verification; test coverage; test data generation; validation; verification; automated software tools; software lifecycle; software testing; SP500-75.

soft $\mathrm{x}$ rays; spectral distortion; windowless detectors; energy dispersive; light elements; SP604: 1981 June. 71-95.

soil; alloy; carbon; computers; energy; fine structure; - interface; materials; measurement; office building; phase diagrams; radioactivity; research; sediment; $D I M / N B S$ 65(8): 1-28; 1981.

soil; speciation; volatilization; arsenic; dimethylarsinic acid; microbial growth; mobilization; nutrients; oil shale; retorting; SP618: 1981 November. 286-300.

soil; surfacing materials; test method; asphalt; impact attenuation; peak acceleration; playground safety; playground surfaces; NBSIR 80 . 2106.

soil anchors; soil testing; flood loads; mobile home foundations; mobile home standards; NBSIR 81-2238.

soil anchors; standards; tiedown; wind forces; buoyancy forces; flood forces; foundations; hurricane forces; mobile home; BSS132.

soil corrosivity; soil $\mathrm{pH}$; soil resistivity; underground corrosion; earth resistivity; redox potential; 19963.

soil environment; stainless steels; stress-corrosion behavior; coatings; corrosion behavior; field-tests; galvanic couples; NBSIR 81-2228 (NBS).

soil mechanics; transducers; energy measurement; field instrument force measurement; field testing; in-situ testing; BSS135.

soil moisture; underground radar; void detection; adobe building materials; adobe soil; dielectric constant measurements; electromagnetic moisture measurements; nondestructive evaluation radar soil measurements; NBSIR 79-1610.

soil pH; soil resistivity; underground corrosion; earth resistivity; redox potential; soil corrosivity; 19963.

soil removal; water conservation; water discharge characteristics; clothes washers; dishwashers; dishwasher subcycles; fill controls; grey water; performance; NBSIR 81-2291 (HUD).

soil resistivity; underground corrosion; earth resistivity; redox potential; soil corrosivity; soil pH; 19963.

soils; telephone cables; underground; alloys; corrosion; metallurgically-bonded; metals; plastic-bonded; NBSIR 81-2243.

soil structure interaction; standards; buildings; design; earthquakes; engineering; foundations; professional practice; provisions; NBSIR 80-2111-3.

soil testing; flood loads; mobile home foundations; mobile home standards; soil anchors; NBSIR 81-2238.

soil textural types; attenuation; coaxial sample holder; density; dielectric properties; relative dielectric constant; NBSIR 78-896.

solar; steel; structural systems; test methods; wood; building rehabilitation; concrete; electrical; evaluation; HVAC; masonry; plumbing; NBSIR 81-2289.

solar cell; solid state; transistor; tunnel diode; diode; field-effect transistor; III-V compounds; IMPATT diode; integrated circuit; LASER; LED; photocell; J. Res. 86(6): 565.570; 1981 November. December.

solar cell analysis; solar cells; computer simulations; fault detection; laser scanner; photovoltaics; NBS-GCR-80-285.

solar cell defects; solar cells; test structures; laser scanner; model calculations; photovoltaic cells; sheet resistance; NBSIR 81-2260.

solar cells; computer simulations; fault detection; laser scanner; photovoltaics; solar cell analysis; NBS-GCR-80-285.

solar cells; terrestrial solar simulators; test methods; calibration; combined photovoltaic/thermal modules; measurements; photovoltaic modules; photovoltaics; reference cells; NBS-GCR80-279.

solar cells; test pattern; defect identification; laser scanner; measurement techniques; photovoltaics; NBSIR 80-2181.

solar cells; test structures; laser scanner; model calculations; photovoltaic cells; sheet resistance; solar cell defects; NBSIR 81- 
2260.

solar code; victim role; deed; developers; energy wars; experimental code; field test; guideline; low income; non-code; policymaking; protestors; SP608: 1981 May. 173-178.

solar collector; solar energy; space heating and cooling; thermal storage; ASHRAE 90-75; class $\mathrm{K}$ code; computer modeling; electrical design; energy audit; energy conservation; HVAC systems; performance standards; SP608.

solar collector; space charges; technical competence; cement and concrete; cover plates; earth's tilt; electric; fire; fracture toughness; measurement; parameter; physics; DIM/NBS 65(3): 1.24; 1981.

solar collector; standards; test methods; field survey; industrial process heat; materials performance; NBSIR 81-2381.

solar collector; standards; thermal performance; uncertainty; collector rating; measurement; $T N 1140$.

solar collector; standards; voluntary program; certification; consumer protection; Interstate Solar Coordination Council; labeling procedures; national testing program; regulation; SP608: 1981 May. 179-181.

solar collector; system efficiency; thermal performance; air leakage; heat transfer; pebble-bed storage; NBS-GCR-81-302.

solar collector; thermal performance; efficiency; flow rate; heat transfer fluid; NBS-GCR-80-254.

solar collector fires; solar collectors; spontaneous ignition; building fires; fire safety; ignition; self-heating; NBSIR 81-2326.

solar collectors; solar materials; stagnation testing; accelerated aging; durability; environmental exposure; reliability; TN1136.

solar collectors; spontaneous ignition; building fires; fire safety; ignition; self-heating; solar collector fires; NBSIR 81-2326.

solar collectors; structural engineering; wind loads; earthquake loads; hail loads; snow loads; NBSIR 81-2199.

solar collectors; system efficiency; thermal performance; domestic hot water; recirculating hot water systems; NBS-GCR-81-322.

solar collector testing; water-heating solar collectors; collector efficiency; comparison German BSE vs ASHRAE 93.77 procedures; flat-plate solar collectors; German Bundesverband Solarenergie; 20079.

solar economics; solar energy; economic optimization; life-cycle costing; NBSIR 81-2342.

solar energy; economic optimization; life-cycle costing; solar economics; NBSIR 81-2342.

solar energy; solar systems; building codes; building officials; consensus; construction; document; installation; recommended requirements; SP608: 1981 May. 167.172.

solar energy; space heating and cooling; thermal storage; ASHRAE 90.75; class $\mathrm{K}$ code; computer modeling; electrical design; energy audit; energy conservation; HVAC systems; performance standards; solar collector; SP608.

solar energy; standards; absorber materials; accelerated test methods; durability; NBSIR 81-2232.

solar energy; steady-state tests; thermal test procedures; transient thermal tests; passive/hybrid components; NBSIR 81-2300.

solar energy; trainee performance testing; BEPS; code compliance; code conflicts; energy conservation standards; SP608: 1981 May. 139-157.

solar energy in commercial buildings; building models, computer; energy conservation in commercial buildings; energy conservation, user acceptance; lighting measurements; performance data for commercial office buildings in New England; BSS133.

solar energy systems; space heating load; thermal performance; auxiliary energy; FCHART computer program; National Solar Demonstration Program; NBS-GCR-81-321.

solar heat gain; window management; daylight; energy conservation; fenestration design; illumination; lighting control; NBSIR 80-2147.

solar hot water heating; computer codes-F-CHART, SOLCOST, TRNSYS; computer predictions for solar systems; NBS solar testing at Gaithersburg; 20078.

solar materials; stagnation testing; accelerated aging; durability; environmental exposure; reliability; solar collectors; TN1136.

Solar Model Audit; auditor training; energy conservation; program measure; Residential Conservation Service; SP608: 1981 May. 183-
194

solar properties of materials; spectral reflectance measurements; diffuse reflectance; directional-hemispherical reflectance; integrating spheres; laboratory intercomparisons; reflectance measurements; reflectance standards; NBSIR 81-2311.

solar radiation; daylighting; illuminance; illumination; irradiance TN1148.

solar systems; building codes; building officials; consensus; construction; document; installation; recommended requirements solar energy; SP608: 1981 May. 167-172.

solar systems; space heating and cooling; training programs; building codes; building officials; codes and standards; convective loops; greenhouses; heat exchangers; light and ventilation; SP608: 1981 May. 159-166.

solid; stress, strain; thermodynamics; chemical potential; diffusion; equilibrium; 20725.

solid; velocity; volume; water; cylindrical; non-uniform; partially. filled; pitched-horizontal drain; slope; NBSIR 81-2266.

solid electrolyte probe; device for measuring the fugacity of a material; E.M.F. measuring circuit; U.S. Patent 4,264,423.

solidification; thermal mechanical treatment; aging; electrical conductivity; hardness; homogeneity; microstructure; precipitating; segregation; NBSIR 80-2069.

solids; dielectrics; electro-optics; gases; high voltage; insulation; interfaces; Kerr effect; liquids; Pockels effect; NBSIR 81-2275.

solid state; thermodynamic and transport properties; atomic and molecular properties; bibliographies; chemical kinetics; evaluated data; fluid properties; indexes; publication list; SP612.

solid state; transistor; tunnel diode; diode; field.effect transistor; III-V compounds; IMPATT diode; integrated circuit; LASER; LED; photocell; solar cell; J. Res. 86(6): 565-570; 1981 NovemberDecember.

solid state detector; multichannel analyzers; SP604: 1981 June. 165. 175.

solid-state $\mathrm{x}$-ray detectors; $\mathrm{x}$-ray energy spectrometry; pulseprocessing; SP604: 1981 June. 141-164.

solitons; waveguides; fiber optics; nonlinear propagation; nonlinear pulses; self-focusing; SP620: 1981 October. 453-458.

solubility; solute; water; generator column; hydrophobic; octanol; partition coefficient; propylbenzene; J. Res. 86(4): 361.366; 1981 July.August.

solubility; vapor pressure; alkanes; aromatics; critical review, evaluated data; gases; halogenated hydrocarbons; Henry's law constants; liquids; pesticides, solids; JPCRD 10(4): 1175-1199; 1981.

solubility tests; automotive paints; household paints; sequential tests; SP480-40.

solute; water; generator column; hydrophobic; octanol; partition coefficient; propylbenzene; solubility; J. Res. 86(4): 361-366; 1981 July-August.

solution; chemical kinetics; data compilation; oxidation; photochemistry; rates; review; singlet oxygen; JPCRD 10(4): 809 999; 1981.

solution calorimetry; thermochemistry; $\Delta C_{p}$ of reaction; calorimetry; $\mathrm{C}_{5} \mathrm{H}_{5} \mathrm{~N}_{5} \mathrm{O}$; density; enthalpy of solution; Gua. $\mathrm{HCl} \cdot 1 \mathrm{H} \mathrm{H}_{2} \mathrm{O}$; Gua. $\mathrm{HCl} \cdot 2$ $\mathrm{H}_{2} \mathrm{O}$; Gua.2 $\mathrm{HCl}$; guanine (Gua); guanine hydrochlorides; heat capacity; nucleic acid base; J. Res. 86(4): 367-382; 1981 July August.

solutions; standard reference data; thermodynamic properties; activity coefficients; alkali metal nitrite salts; aqueous; critical evaluation; $\mathrm{CsNO}_{2}$; electrolytes; excess Gibbs energy; $\mathrm{KNO}_{2} ; \mathrm{LiNO}_{2} ; \mathrm{NaNO}_{2}$; osmotic coefficients; $\mathrm{RbNO}_{2}$; JPCRD 10(3): 767.778; 1981 .

solutions; sulfuric acid; standard reference data; thermodynamic properties; activity coefficients; aqueous; critical evaluation; electrolytes; excess free energy; free energy; ionic; osmotic coefficients; JPCRD 10(3): 779-798; 1981.

solutions; sulfuric acid; thermodynamic properties; transport properties; activity coefficients; aqueous; compilation; conductivity; electrolytes; enthalpy; Gibbs energy; osmotic coefficients; NBSIR 81-2276.

solutions; thermodynamic properties; activity coefficient; cesium; critical evaluation; electrolyte; excess Gibbs energy; lithium; 
osmotic coefficient; potassium; rubidium; sodium; JPCRD 10(3): 671.766.

solutions; thermodynamic properties; zinc; activity coefficient; cadmium; critical evaluation; electrolyte; ethylene bis(trimethylammonium) chloride; ethylene bis(trimethylammonium) iodide; excess Gibbs energy; osmotic coefficient; JPCRD 10(1): 1-56; 1981.

solvent refined coal; titanium; trace elements; arsenic; chromium; coal hydrogenation; cobalt; liquefaction; mercury; nickel; process waters; selenium; SP618: 1981 November. 21.38.

sonic cavity; thermodynamic properties; velocity of sound; virial coefficients; electrostatic transducers; gas; nitrogen; 20072.

soot; toxicity; wood; combustion; decision analysis; fire models; flame spread; human behavior; ignition; polymers; smoke; NBSIR 812382.

sound; specific heat ratios; speed; temperature; velocity; adiabatic compressibility; liquid; pressure; propane; J. Res. 86(2): 165-170; 1981 March-April.

sound level; warning light; warning signals; conspicuity; effective intensity; emergency warning lights; flashing lights; lights, warning; sirens; SP480-37.

soundness; strength; cement; expansion; fly ash; MgO; slag; NBSIR 81-2273.

sound transmission; structureborne noise; acoustics; architectural acoustics; building acoustics; noise; noise control; NBS-GCR-80248.

sound transmission; structure-borne noise; acoustics; building acoustics; noise control; noise isolation; NBS-GCR-80-250.

sound velocities; tensor-property averaging; copper; Debye temperature; elastic constants; low temperatures; polycrystals; 20726.

sound velocities; ultrasonic inspection; ultrasonic waves; acoustic waves; elastic anisotropy; elastic constants; Kroner method; 20773.

source energy; transformers; electrical design; energy distribution; National Electrical Code; power factor correction; SP608: 1981 May. 37.56.

source excited fluorescence analysis; $x$-ray fluorescence analysis; catalysts automotive; energy dispersive analysis; heavy element fluorescence analysis; intrinsic germanium detectors; secondary target fluorescence analysis; SP604: 1981 June. 391-415.

space application; Stirling cycle; clearance seals; cryogenics; hydrodynamic gas bearing; linear magnetic suspension; long lifetime; SP607: 1981 May. 103-115.

space charge; calibration; dc transmission lines; electric field; electric field meters; electric field strength; electric power transmission; measurements; NBSIR 80-2177.

space charge; transformer oil; cables; composite insulation; dc fields; high voltage; incipient fault; insulation; liquid breakdown; $\mathbf{S F}_{6}$; NBSIR 81-2334.

space charge; transformer oil; cables; dc fields; high voltage; incipient fault; insulation; $\mathrm{SF}_{6} ; \mathrm{NBSIR}$ 81-2283.

space charge density; Wilson plates; absolute filter; current density; high voltage dc transmission lines; ion counter; NBSIR 81-2267.

space charges; technical competence; cement and concrete; cover plates; earth's tilt; electric; fire; fracture toughness; measurement; parameter; physics; solar collector; DIM/NBS 65(3): 1.24; 1981.

space cooling requirements; space heating requirements; energy efficiency standards; engineering-economic analysis; heat pump; mechanical equipment efficiency ratings; minimum efficiency standards; NBSIR 81-2241.

space heating and cooling; thermal mass; walls; water source heat pump; buildings; construction; energy conservation; ground coupled; hybrid solar energy system; inverted cave; low grade geothermal; radiant floor heating; SP608: 1981 May. 69.81.

space heating and cooling; thermal storage; ASHRAE 90-75; class $\mathrm{K}$ code; computer modeling; electrical design; energy audit; energy conservation; HVAC systems; performance standards; solar collector; solar energy; SP608.

space heating and cooling; training programs; building codes; building officials; codes and standards; convective loops; greenhouses; heat exchangers; light and ventilation; solar systems; SP608: 1981 May.
159-166.

space heating load; thermal performance; auxiliary energy; FCHART computer program; National Solar Demonstration Program; solar energy systems; NBS-GCR-81-321.

space heating requirements; energy efficiency standards; engineering. economic analysis; heat pump; mechanical equipment efficiency ratings; minimum efficiency standards; space cooling requirements; NBSIR 81-2241.

Spacelab; superfluid helium; cryogenics; infrared telescope; SP607: 1981 May. 117-127.

space management; capacity planning; device architecture; secondary storage; SP500-83: 1981 November. 325.

space management; DASD; data set; disk management system; migration; MSS; performance; SP5DD-83: 1981 November. 223-230.

space matrices; conjugate gradient algorithm; elliptic partial differential equations; iterative methods for linear algebraic equations; Neumann boundary conditions; NBSIR 80-2056

speciation; atomic absorption; energy-related process fluids; leachate; linear free energy; liquid chromatography; molecular substituent; oil shale; organoarsenic; organotin; retention index; SP618: 1981 November. 197.216.

speciation; toxic; trace elements; environmental; essential elements; SP618: 1981 November. 235.243.

speciation; trace metals; alkylpyridines; dissolved organic compounds; gas chromatography/mass spectrometry; hydrophobic bases; metal complexation; nitrogen bases; oil shale; retort water; SP618: 1981 November. 93-104.

speciation; trace metals; chromatography; direct current plasma; hydrophilic; hydrophobic; metal complex; porphyrin; shale oil; SP618: 1981 November. 105.119.

speciation; volatilization; arsenic; dimethylarsinic acid; microbial growth; mobilization; nutrients; oil shale; retorting; soil; SP618 1981 November. 286.300.

specification; configuration management (CM); cross reference; data structure; design, documentation; functional; historical file; keyword; life cycle management; requirements; software quality assurance (SQA); SP500-83: 1981 November. 101.106.

specification; standard; system analysis/engineering; arrangement; building; classification; code; engineering; organization; provisions; scope; BSS136.

specifications; taximeters; tolerances; volume-measuring devices; weighing devices; weights; length-measuring devices; liquid measuring devices; measures; scales; H44, 1982 Edition.

specifications and tolerances; technology transfer; weights and measures; domestic standards; education programs; electronic devices; international recommendations; legal metrology; measurement assurance; metrication; model laws and regulations packing and labeling; SP599.

specific elements; spectroscopy; chromatography; detection; metals; plasma emission; SP618: 1981 November. 184.196.

specific heat; cryogenics; heat capacity; refrigeration; regenerators; SP607: 1981 May. 164-172.

specific heat; surface tension; thermophysical properties; tungsten wetting; adsorption; Auger spectroscopy; convection; ellipsometry; gallium; levitation calorimetry; NBSIR 81-2295.

specific heat; thermal conductivity; composites; cryocoolers; plastics; refrigeration; regenerators; SP607: 1981 May. 173-177.

specific heat ratios; speed; temperature; velocity; adiabatic compressibility; liquid; pressure; propane; sound; J. Res. 86(2): 165. 170; 1981 March.April.

spectra; atomic energy levels; atomic spectra; cobalt; cobalt energy levels; JPCRD 10(4): 1097.1174; 1981.

spectral artifacts; transmission electron microscopy; electron probe microanalysis; energy dispersive $x$-ray spectrometry; microanalysis; qualitative analysis; quantitative analysis; scanning electron microscopy; SP604: 1981 June. 315-339.

spectral deconvolution; $x$-ray analysis; $x$-ray fluorescence; electron probe microanalysis; energy dispersive $x$-ray spectrometry; germanium (lithium) detector; scanning electron microscopy; silicon (lithium) detector; SP604.

spectral distortion; windowless detectors; energy dispersive; light 
elements; soft x rays; SP604: 1981 June. 71-95.

spectral lines; transition probabilities; wavelengths; atom; atomic ion; intensities; line strengths; oscillator strengths; NSRDS-NBS68. spectral line shapes; coherence; cooperative phenomena; 19968. spectral line shapes; coherence; cooperative phenomena; 19967. spectral moments; band shape analysis; collision-induced absorption; far infrared; $\mathrm{H}_{2}-\mathrm{H}_{2}$ collisions; $\mathrm{H}_{2}-\mathrm{He}$ collisions; planetary atmospheres; quadrupole-induced absorption; NBSIR 80-2175.

spectral reflectance measurements; diffuse reflectance; directionalhemispherical reflectance; integrating spheres; laboratory intercomparisons; reflectance measurements; reflectance standards; solar properties of materials; NBSIR $81-2311$.

spectrofluorimetry; antioxidants; diffusion; food packaging; inverse gas chromatography; migration; oil simulants; oligomers; polyolefins; radioactive tracer; NBSIR 81-2264.

spectroscopic constants of $\mathrm{N}_{2} \mathrm{O}$; infrared standards from $522 \mathrm{~cm}^{-1}$ to $657 \mathrm{~cm}^{-1}$; infrared standards from $1115 \mathrm{~cm}^{-1}$ to $1340 \mathrm{~cm}^{-1}$; infrared wavelength calibration; $\mathrm{N}_{2} \mathrm{O}$; nitrous oxide; JPCRD IO(4): 1065 . $1084 ; 1981$.

spectroscopic reference wavelengths; uranium spectrum; wavelength calibration; 20730.

spectroscopy; chromatography; detection; metals; plasma emission; specific elements; SP618: 1981 November. 184-196.

spectroscopy; strong fields; damping theory; kinetics; lasers; lineshapes; optical pumping; resonance Raman; 20039.

spectrum analysis; statistics; time series; bispectrum; cumulant spectra; nonlinear time series; polyspectra; TN1036.

specular reflectance; surface analysis; surface integrity; surface roughness; crystallographic anisotropy; crystallographic perfection; initial permeability; metal mirror; polishing; polycrystalline material; residual stress; sapphire; SP620: 1981 October. 171.179.

speed; temperature; velocity; adiabatic compressibility; liquid; pressure; propane; sound; specific heat ratios; J. Res. 86(2): 165-170; 1981 March-A pril.

spherical aberration; damage morphology; Gaussian beam; laser damage; SP620: 1981 October. 403-405.

spherical scanning; antennas; data processing; electromagnetic; 20765.

spin; spin polarization; electron gun; electron polarization; electron spin; polarized electrons; 19996.

spin dependent; SRM's; standards; tape; copper; deterioration; electromagnetic radiation; film; industry; metals; productivity; research associate; scanning technique; $D I M / N B S$ 65(5): 1.24; 1981

spin detector; spin polarization; surface resonance; surface structure; polarized LEED; 20012.

spin polarization; electron gun; electron polarization; electron spin; polarized electrons; spin; 19996.

spin polarization; surface resonance; surface structure; polarized LEED; spin detector; 20012.

spiral grand design; WASER; winding dilemma; density wave; galactic spirals; hydrodynamic instability and turbulence; plasma dynamics; J. Res. 86(6): 557.563; 1981 November-December.

splines; statistics; volume calibration; accountability; differential pressure; NBSIR 80-2151.

splitting; strain energy; stress; substrate; adhesive; bitumen; bonding; built-up roofing membrane; complementary strain energy; felt; nonlinear; roofing membrane; TN1152.

splitting tensile strength; compressive strength; geothermal well; setcement; simulated geothermal fluid; NBSIR 80-2099-4.

spontaneous ignition; building fires; fire safety; ignition; self-heating; solar collector fires; solar collectors; NBSIR 81-2326.

spray; synthetics; water glycol; coal mine; fire tests; hydraulic fluids; invert emulsions; pressure-temperature; NBSIR 81-2373.

spray ignition; temperature rise; flammability; heat flux; pressure; NBSIR 81-2247.

spray nozzle; stairway protection; ventilated stair; automatic sprinklers; cooling efficiency; evaporation cooling; smoke movement; NBSIR 81-2202.

spreading resistance; two-dimensional profiles; arsenic ion implantation; electron-beam-induced current; etching; integrated circuit processing; ion channeling; ion implantation; lateral profiles; scanning electron microscopy; silicon technology; NBSIR 81-2398. sprinkler heads; sprinkler systems; time constant; fire extinguishment; fire models; fire protection; NBSIR 81-2329.

sprinkler systems; doors; evacuation; fire alarm systems; fire departments; fire extinguishers; fire investigations; hospitals; nursing homes; nursing staff; smoke; smoke detectors; NBS-GCR-80-297.

sprinkler systems; doors; evacuation; fire alarm systems; fire departments; fire extinguishers; fire investigations; hospitals; nursing homes; nursing staff; smoke; smoke detectors; NBS-GCR-81-343.

sprinkler systems; systems analysis; building codes; fire modeling; fire protection engineering; 20746.

sprinkler systems; time constant; fire extinguishment; fire models; fire protection; sprinkler heads; NBSIR 81-2329.

spurious resonances; augmented electric- and magnetic-field integral equations; electric-field integral equation; electromagnetic theory; magnetic-field integral equation; scattering of electromagnetic waves; 20761.

spurious resonances; augmented electric- and magnetic-field integral equations; electric-field integral equation; electromagnetic theory; magnetic-field integral equation; scattering of electromagnetic waves; 20760.

sputtering; chalcogenide glass; coating; ion beam; SP620: 1981 October. 324.334.

sputtering; $x \cdot$ ray photoelectron spectroscopy (XPS, ESCA); Auger electron spectroscopy (AES); metal-oxide-semiconductor (MOS); secondary ion mass spectrometry (SIMS); semiconductor devices; SP400-67.

sputter profiling; Auger-electron spectroscopy; electronics; MOS devices; semiconductors; silicon dioxide; NBS-GCR-81-345.

SQL; CODASYL; DAPLEX; database management system; data model; DBMS; network data model; query language; relational model; NBS-GCR-81-338.

SQUID; Stirling cycle; superconductivity; cryocoolers; low temperature; magnetic gradiometer; refrigeration; SP607: 1981 May. 186-194.

SQUID; temperature sensors; cryogenics; helium; infrared; SP607: 1981 May. 136.140.

$\mathrm{SrF}_{2} ;$ volume and surface impurities; $\mathrm{BaF}_{2} ; \mathrm{CaF}_{2} ;$ extrinsic and intrinsic infrared absorption; infrared wavelength modulation; $\mathrm{LaF}_{3}$; laser windows; $\mathrm{LiF} ; \mathrm{MgF}_{2} ; \mathrm{MgO} ; \mathrm{NaCl} ; \mathrm{NaF} ; \quad S P 620: 198$ October. 29.43.

SRM; trace organic analysis; definitive methods; human serum; polynuclear aromatic hydrocarbons; reference materials; shale oil; 19989.

SRM's; atomic ions; calibration; clock synchronization; Columbia River model; computer; insulation; lasers; powder data; progress; R.values; single-crystal; DIM/NBS 65(2): 1.28; 1981.

SRM's; standards; tape; copper; deterioration; electromagnetic radiation; film; industry; metals; productivity; research associate; scanning technique; spin dependent; DIM/NBS 65(5): 1-24; 1981.

SRM's; stars; visual acuity; evolution; fracture mechanics; gamma rays; inventors; molecular interactions; molecular processes; neomycin; neutron dosimeters; polymers; radiography; $D I M / N B S$ 65(7): $1.24 ; 1981$

SRM's; time-domain calibrations; weights and measures; $\mathrm{x}$-ray diffraction; air pollutants; antenna coupling; electrical distribution; glass-liquidus; insulation; monitors; neutron diffraction, samplers; DIM/NBS 65(1): 1.28; 1981 .

SRM-477; window absorption; window thickness; window transmission; $x$-ray detectors; $x$-ray energy spectrometers; dead. layer; SP604: 1981 June. 61-70.

stability; standards; superconductor; critical current; electrical property; magnetic property; NBSIR 80-1642.

stability; steady state; turbulence; nonequilibrium molecular dynamics; nonlinear thermodynamics; shear flow; shear induced phase transition; soft sphere system; 20063.

stability; stiffness; strength; structural safety; work surfaces; codes and standards; construction safety; design; finite element; loads; scaffolds; NBSIR 81-2265.

stability constants; voltammetry; zinc-NTA; cadmium.NTA; convolution voltammetry; electrochemistry; Kalman filter; metal speciation; modeling; semiderivative; SP618: 1981 November. 163 - 
172.

stability of measurement process; statement of certainty; precision; reference material; 20041.

stack effect; sulfur hexafluoride; tracer tests; ventilation systems; ships; smoke control; smoke movement; NBSIR 81-2433.

stadiums; auditorium; building standards; crowd ingress; design procedures; evaluation procedures; facility design; facility management; public assembly; public safety; NBSIR 81-2361.

stagnation testing; accelerated aging; durability; environmental exposure; reliability; solar collectors; solar materials; TN1136.

stainless steel; titanium; Co-Cr-Mo; Co-Ni-Cr-Mo; corrosion; dental alloys; metal-amino acid reactions; 19988

stainless steel; triboellipsometry; aluminum; field ion microscopy; ironchromium alloys; niobium; repassivation kinetics; NBSIR 79-1904 (Navy).

stainless steel; ultrasonic scattering; ultrasonic waves; acoustic waves; elastic anisotropy; nondestructive evaluation; nondestructive testing; 20744.

stainless steel implants; wavelength dispersive $x$-ray analysis; corrosion; SP601: 1981 January. 223-235.

stainless steels; stress-corrosion behavior; coatings; corrosion behavior; field-tests; galvanic couples; soil environment; NBSIR 812228 (NBS).

stainless steels; structural alloys; welding; alloys; aluminum alloys; castings; composites; cryogenic properties; low temperature; mechanical properties; nonmetallics; physical properties; NBSIR $81-1645$.

stainless steel 316 LVM; titanium, titanium alloy; bone plates; cobaltchrome-nickel-tungsten-iron-alloy; fretting corrosion; histologic analysis; SP601: 1981 January. 395-422.

stairway protection; ventilated stair; automatic sprinklers; cooling efficiency; evaporation cooling; smoke movement; spray nozzle; NBSIR 81-2202.

stairwells; air movement; computer programs; egress; elevator shafts; escape means; pressurization; smoke control; NBSIR 80-2157.

standard; American National Standard Code for Information Interchange; ASCII; card punch; card reader; data communications; data interchange; data processing; EAM cards; electric accounting machine; Federal Information Processing Standard; Hollerith punched card code; Hollerith punched cards; information interchange; information processing; punched cards; FIPS PUB 14-1.

standard; American National Standard Code for Information Interchange; ASCII; character set; coded character set; code for information interchange; collating sequence; data communication; data interchange; data processing; Federal Information Processing Standard; information interchange; information processing; FIPS PUB 1-1.

standard; calorimeter; millimeter wave; power; TN1044.

standard; cost/benefit analysis; flexible disk cartridge; flexible disk system; information interchange; marketed systems; NBS-GCR-80306.

standard; superconductor; conductor; critical current; critical temperature; review; 20069.

standard; synthesis; calibration; digital; microcomputer; phase angle; sinewave generator; TN1144.

standard; system analysis/engineering; arrangement; building; classification; code; engineering; organization; provisions; scope; specification; BSS136.

standard; text communication; text interchange; typesetting composition; ADP terminals; ASCII; character-imaging devices; control functions; data interchange; display terminals; Federal Information Processing Standard; information interchange; printer terminals; FIPS PUB 86 .

standard; word processor; costs and benefits; flexible disk cartridge; information interchange; label and file structure; NBS-GCR-80-307.

standard; $x$-ray diffraction; crystal structure; densities; lattice constants; powder patterns; reference intensities; Monogr. 25, Section 18.

standard benchmarks; synthetic benchmarks; workload characterization; workload definition; benchmarking; benchmark library; selection of ADP systems; 20747.

standard costing; chargeback; chargeout; charging; charging system; DP cost accounting; DP cost allocation; pricing; SP500-83: 1981 November. 109-118.

standard evaluation technique; BLAST; building energy performance standards; computer modeling; maintain accuracy; reduce cost; reduce input; SP608: 1981 May. 113-122.

standardization in developing countries; Sudan; weights and measures regulation; Agency for International Development; Arab League Organization for Standardization and Metrology; chemical industry standards; cotton standards; food standards; measurement science and technology; NBSIR 80-2020.

standard reference data; technical activities 1980; thermochemical and thermophysical data; data compilation; energy and environmental data; evaluated data; materials data; NBSIR 81-2206.

standard reference data; thermodynamic properties; activity coefficients; alkali metal nitrite salts; aqueous; critical evaluation; $\mathrm{CsNO}_{2}$; electrolytes; excess Gibbs energy; $\mathrm{KNO}_{2} ; \mathrm{LiNO}_{2} ; \mathrm{NaNO}_{2}$; osmotic coefficients; $\mathrm{RbNO}_{2}$; solutions; JPCRD 10(3): 767-778; 1981.

standard reference data; thermodynamic properties; activity coefficients; aqueous; critical evaluation; electrolytes; excess free energy; free energy; ionic; osmotic coefficients; solutions; sulfuric acid; JPCRD 10(3): 779.798; 1981.

standard reference material; austenitic stainless steel; electron probe microanalysis; Fe-Cr-Ni alloy; homogeneity testing; SP260-70.

standard reference material; thermal conductivity; glass fiberblanket; insulation; low temperature; NBSIR 81-1640.

standard reference material; thermal conductivity; glass fiberboard; insulation; low-temperature; NBSIR 81-1639.

standard reference material; thermal resistance; fiber-glass; fibrous glass; insulation; measurement; $\mathrm{R}$ value; NBSIR 80-2091.

standard reference material; x-ray diffraction; x-ray fluorescence analysis; austenite in ferrite; austenite standard; electron microprobe; powder metallurgy; quantitative microscopy; SP26073.

standard reference materials; analysis; certification; isotope dilution mass spectrometry; mass spectrometry; 20024.

standard reference materials; analysis; characterization; composition; materials; properties; research materials; SP260, 1981-83 Edition.

standard reference materials; bacteria; biodeterioration; biotransformations; deterioration of materials; element volatilization; fungi; metals; microorganisms; NBSIR 81-2246.

standard reference materials; benzo(a)pyrene; benzo(e)pyrene; fluoranthene; fluorescence detection; gas chromatography/mass spectrometry; high-performance liquid chromatography; perylene; pyrene; shale oil; 20750.

standard reference materials; chemical composition; clinical materials; SP260-71.

standard reference materials; statistical evaluation; accountability guide; bulk measurements; isotopic assay; leachability studies; nondestructive assay; nuclear safeguards; nuclear waste management; NBSIR 81-2205.

standard reference materials; thin films; ellipsometry; polysilicon films; silicon dioxide films; silicon nitride films; NBSIR 80-2158.

standard reference materials; water pollution control; measurement methods; oil shale; 20009.

standards; absorber materials; accelerated test methods; durability; solar energy; NBSIR 81-2232.

standards; atomic weight; coal; computers; drill failure; Faraday; firefighting; hot atom; industrial innovation; measurements; oils, PCB's; DIM/NBS 65(6): 1.24; 1981.

standards; building rehabilitation; codes; comparative analysis; existing buildings; housing codes; maintenance; model codes; occupancy; performance levels; regulation; NBSIR 80-2081.

standards; buildings; design; earthquakes; engineering; foundations; professional practice; provisions; soil structure interaction; NBSIR 80-2111-3.

standards; children; design; environmental psychology; families; housing; interiors; lifestyle; research; site planning; sociology; NBSGCR-81-320. 
standards; computer based office systems; computer networking; interfaces; local area networks; measurement techniques; protocols; NBSIR 80-2154.

standards; conversion; database; data description; data dictionary; data directory; data manipulation; DBMS; language; query; SP500-76.

standards; cost-benefit; economics; government regulation; safety regulation; 20031.

standards; macromolecules; plastics; polymers; NBSIR 81-2263.

standards; standards-writers; systems analysis; building standards; classification; decision tables; information networks; network; 19998.

standards; standards-writers; systems analysis; building standards; classification; decision tables; information networks; modeling; NBSIR 80-1979-1.

standards; standards writing; legislative history; product liability; rationale statements; scope of standards; NBS-GCR-81-347.

standards; steel; structural engineering; building; building codes; building design; earthquakes; engineering; NBSIR 80-2111-6.

standards; storm doors; storm windows; vent dampers; water heaters; windows; automatic ignition devices; caulks; effectiveness; energy conservation; energy conservation measures; installation; insulation; oil burners; practices; safety; sealants; SP606.

standards; structural engineering; building; building codes; building design; earthquakes; engineering; masonry; NBSIR 80-2111-5.

standards; structural engineering; building; building codes; building design; earthquakes; engineering; NBSIR 80-2111-11.

standards; structural engineering; building; building codes; building design; earthquakes; engineering; reinforced concrete; NBSIR 802111-4.

standards; structural engineering; building; building design; earthquakes; engineering; Federal government; seismic safety; NBSIR 81-2195.

standards; structural engineering; buildings; buildings (codes); buildings (design); earthquake; seismic risk maps; NBSIR 80-2111-1.

standards; structural engineering; wood; building; building codes; building design; earthquakes; engineering; NBSIR 80-2111-7.

standards; superconductor; critical current; electrical property; magnetic property; stability; NBSIR 80-1642.

standards; superconductors; ac losses; calorimeter; cryogenics; multipliers; 19999.

standards; symbols; visual alerting; warnings; communication; hazard; pictograms; safety; signs; NBSIR 80-2003.

standards; tape; copper; deterioration; electromagnetic radiation; film; industry; metals; productivity; research associate; scanning technique; spin dependent; SRM's; DIM/NBS 65(5): 1.24; 1981.

standards; techniques; calibration; dosimetry; electric fields; electromagnetic interference; exposure history; instruments; magnetic fields; nonionizing radiation; regional calibration laboratories; SP613.

standards; technology transfer; basic measurements and standards; building technology; calibration methods; computer standards and guidelines; energy research; environmental measurements; fire safety; industrial productivity; materials properties; research; SP600.

standards; telecommunications; automated data processing (ADP); communications; Federal Information Processing Standard (FIPS); Federal Property Management Regulations (FPMR); procurement; FIPS PUB 80.

standards; test methods; field survey; industrial process heat; materials performance; solar collector; NBSIR 81.2381.

standards; thermal performance; uncertainty; collector rating; measurement; solar collector; TN1140.

standards; tiedown; wind forces; buoyancy forces; flood forces; foundations; hurricane forces; mobile home; soil anchors; BSS132.

standards; traceability; calibration; measurement assurance; measurement services; SP250, 1980 Edition.

standards; transactions; annual reports; diffusion in metals; fire; journals; library holdings, NBS Library; NBS periodicals; periodicals; proceedings; serials; NBSIR 81-2226.

standards; voluntary program; certification; consumer protection; Interstate Solar Coordination Council; labeling procedures; national testing program; regulation; solar collector; SP608: 1981 May. 179. 181.

standards; water; air; environmental measurements; methods; quality assurance; NBSIR 81-2280.

standards committees; annual report; committee participation; SP605.

standards development; building energy use; building service systems; energy budgets; housing research; housing standards; performance requirements; NBS-GCR-80-280.

standards development; voluntary standards; bibliography; annotated, consumer representation; literature review; medical device standards; public participation programs; NBSIR 81-2336.

standards-writers; systems analysis; building standards; classification; decision tables; information networks; network; standards; 19998.

standards-writers; systems analysis; building standards; classification; decision tables; information networks; modeling; standards; NBSIR 80-1979-1.

standards writing; legislative history; product liability; rationale statements; scope of standards; standards; NBS-GCR-81-347.

standard transaction; transaction driven system; bubble memory; mathematical model; SP500-83: 1981 November. 259-265.

stars; visual acuity; evolution; fracture mechanics; gamma rays; inventors; molecular interactions; molecular processes; neomycin; neutron dosimeters; polymers; radiography; SRM's; $D I M / N B S$ 65(7): 1.24; 1981 .

stars, binaries; stars, chromospheres; stars, emission line; stars, late. type; ultraviolet, spectra; 19966.

stars, chromospheres; stars, emission line; stars, late-type; ultraviolet, spectra; stars, binaries; 19966.

stars, emission line; stars, late-type; ultraviolet, spectra; stars, binaries; stars, chromospheres; 19966.

stars, emission line; stellar chromospheres; circumstellar shells; late type stars; mass loss; 20057.

stars, individual; stellar chromospheres; stellar coronae; ultraviolet spectra; binary stars; late-type stars; 20001.

stars, late-type; ultraviolet, spectra; stars, binaries; stars, chromospheres; stars, emission line; 19966.

state government; cost; construction; energy audit; energy conservation; Illinois; maintenance; public buildings; SP608: 1981 May. 123-132.

statement of certainty; precision; reference material; stability of measurement process; 20041.

Statement of Review; verification; building code; building envelope; comparison; compliance; energy budget; energy conservation; performance standards; SP608: 1981 May. 57-67.

static analysis; taxonomy; dynamic analysis; programming aids; software development; software engineering; software tools; SP500-74.

static pressure; fire endurance; furnaces; pressure probes; NBSIR 812415.

statistical analysis; applied economics; construction; cost; estimation; location factors; model building; NBSIR 81-2250.

statistical analysis; structural engineering; wind; climatology; loads (forces); probability distribution functions; reliability; 20019.

statistical evaluation; accountability guide; bulk measurements; isotopic assay; leachability studies; nondestructive assay; nuclear safeguards; nuclear waste management; standard reference materials; NBSIR 81-2205.

statistical sampling; commodity inspection; compliance testing; net weight; package checking; packaged product inspection; prepackaged consumer goods; H133.

statistics; structural engineering; live loads; load survey; probability; NBS-GCR-76-81.

statistics; time series; bispectrum; cumulant spectra; nonlinear time series; polyspectra; spectrum analysis; TN1036.

statistics; volume calibration; accountability; differential pressure; splines; NBSIR 80-2151.

steady state; turbulence; nonequilibrium molecular dynamics; nonlinear thermodynamics; shear flow; shear induced phase transition; soft sphere system; stability; 20063.

steady-state tests; thermal test procedures; transient thermal tests; passive/hybrid components; solar energy; NBSIR $81-2300$. 
steam consumption measurement; uncertainty estimate; building energy monitoring; energy measurement; NBSIR 81-2313.

steam turbine component failure; stress corrosion cracking; ultrasonic inspection; United States experiences; low alloy steels; SP622: 1981 October. 152-180.

steel; structural engineering; building; building codes; building design; earthquakes; engineering; standards; NBSIR 80-2111-6.

steel; structural systems; test methods; wood; building rehabilitation; concrete; electrical; evaluation; HVAC; masonry; plumbing; NBSIR 80-2171.

steel; structural systems; test methods; wood; building rehabilitation; concrete; electrical; evaluation; HVAC; masonry; plumbing; solar; NBSIR 81-2289.

steel reinforcing bars; test methods; concrete; construction; nondestructive evaluation; quality assurance; NBSIR 80-2163.

steel structures; wood structures; fire endurance; fires; mathematical models; reinforced concrete; NBSIR 80-2188.

stellar chromospheres; circumstellar shells; late type stars; mass loss; stars, emission line; 20057.

stellar chromospheres; stellar coronae; ultraviolet spectra; binary stars; late-type stars; stars, individual; 20001.

stellar coronae; ultraviolet spectra; binary stars; late-type stars; stars, individual; stellar chromospheres; 20001.

step coverage; test structures; electronics; interconnect; microelectronics; random fault; SP400-65.

stiffness; strength; structural safety; work surfaces; codes and standards; construction safety; design; finite element; loads; scaffolds; stability; NBSIR 81-2265.

stimulated bremsstrahlung absorption; wavelength dependence; laser damage; optical constants; optical materials; semiconductors; SP620: 1981 October. 416.426.

Stirling cooler; Stirling cycle; cryocooler; hybrid cryocoolers; refrigeration; regenerative cooler; regenerator; SP607: 1981 May. 48.56.

Stirling cryocoolers; thermal conductance; Vuilleumier cryocoolers; cryogenics; Gifford-McMahon cryocoolers; heat capacity; heat transfer; helium; refrigerators; regenerators; 20766.

Stirling cryocoolers; thermal conductance; Vuilleumier cryocoolers; cryogenics; Gifford-McMahon cryocoolers; heat capacity; heat transfer; helium; refrigerators; regenerators; SP607: 1981 May. 70. 81.

Stirling cycle; adsorption; cryocoolers; cryostats; helium; refrigeration; SP607: 1981 May. 3-14.

Stirling-cycle; calculations; cryogenics; helium; plastics; refrigerators; regenerators; 20020.

Stirling Cycle; clearance seal; gas compressor; linear motor/generator; magnetic bearings; SP607: 1981 May. 146-153.

Stirling cycle; clearance seals; cryogenics; hydrodynamic gas bearing; linear magnetic suspension; long lifetime; space application; SP607: 1981 May. 103-115.

Stirling cycle; cryocooler; cryogenic; helium; low temperature; refrigerator; SP607: 1981 May. 30-47.

Stirling cycle; cryocooler; gas bearings; refrigeration; regenerator; resonant free piston; SP607: 1981 May. 116.

Stirling cycle; cryocooler; hybrid cryocoolers; refrigeration; regenerative cooler; regenerator; Stirling cooler; SP607: 1981 May. 48-56.

Stirling cycle; superconductivity; cryocoolers; low temperature; magnetic gradiometer; refrigeration; SQUID; SP607: 1981 May. 186-194.

Stirling refrigerator; bellows; compressor; contamination; continuous operation; cryocooler; low power refrigerator; SP607: 1981 May. 141.145 .

stoichiometric; three way catalytic converters; zirconium dioxide; airfuel ratio; closed loop fuel metering system; exhaust emissions; exhaust gas oxygen sensor; exhaust gas recirculation; feedback; oxidation catalyst; oxygen partial pressure; SP622: 1981 October. 62.73 .

storm doors; storm windows; vent dampers; water heaters; windows; automatic ignition devices; caulks; effectiveness; energy conservation; energy conservation measures; installation; insulation; oil burners; practices; safety; sealants; standards; SP606.

storm windows; vent dampers; water heaters; windows; automatic ignition devices; caulks; effectiveness; energy conservation; energy conservation measures; installation; insulation; oil burners; practices; safety; sealants; standards; storm doors; SP606.

stoves; wood; bibliographies; chimneys; combustion; fire safety; heating equipment; literature reviews; NBS-GCR-80-298.

strain; superconducting wire; critical current; mechanical properties; niobium-tin; scaling; 20066.

strain energy; stress; substrate; adhesive; bitumen; bonding; built-up roofing membrane; complementary strain energy; felt; nonlinear; roofing membrane; splitting; TN1152.

strain invariants; tensile-test specimen; checkerboard pressure; elastostatics; finite elements; in-core solver; isoparametrics; Mooney-Rivlin material; nonlinear equation-solvers; penalty method; plane-stress; J. Res. 86(1): 79.109; 1981 January-February.

strain rate effect; toughness; crack growth; creep fracture; high temperature fracture; silicon nitride; NBSIR 79-1960 (DoE).

strange meson; E meson at $1440 \mathrm{MeV}$; flavor singlet; glueball; production process; quark model; 20034.

stream-depth; unsteady; volume; water; diameter; drain; flow; history; horizontal; length; partially-filled; pipe; slope; NBSIR 81-2249.

strength; building; collapse; concrete; concrete strength; construction; failure; flat plate; shear; NBSIR 81-2374.

strength; cement; expansion; fly ash; MgO; slag; soundness; NBSIR 81-2273.

strength; structural safety; work surfaces; codes and standards; construction safety; design; finite element; loads; scaffolds; stability; stiffness; NBSIR 81-2265.

strength prediction; temperature effects; compressive strength; concrete; curing temperature; early age; final set; hydration; initial set; laboratory testing; maturity; mortar; regression analysis; NBSIR 81-2244.

stress; substrate; adhesive; bitumen; bonding; built-up roofing membrane; complementary strain energy; felt; nonlinear; roofing membrane; splitting; strain energy; TN1152.

stress; superconducting power transmission line; terminals; thermal contraction; cable end fittings; pressure cycles; 20018.

stress analysis; structural safety; fracture control; fracture mechanics; mechanical properties; quality assurance; NBSIR 79-1623.

stress birefringence; thermal lensing; window materials; elastic coefficients; high-energy laser; interferometric testing; optical distortion; photoelastic coefficients; polycrystalline aggregate SP620: 1981 October. 117.128.

stress corrosion; coal gasification; elevated temperature stress corrosion; slow strain rate; 20027.

stress corrosion; concrete; corrosion; general corrosion; mathematical modeling; prestressed concrete; prestressing steel; NBSIR 81-2390.

stress corrosion; fatigue corrosion; internal fixation devices; mechanical fatigue; metal prostheses; SP601: 1981 January. 201-222.

stress corrosion; turbine blade failures; blade vibration; Doppler signature; Doppler technique; high cycle fatigue; SP622: 1981 October. 181.194.

stress-corrosion behavior; coatings; corrosion behavior; field-tests; galvanic couples; soil environment; stainless steels; NBSIR 81-2228 (NBS).

stress corrosion cracking; ultrasonic inspection; United States experiences; low alloy steels; steam turbine component failure; SP622: 1981 October. 152-180.

stress relaxation; UHMWPE; ultra high molecular weight polyethylene; creep; failure; fatigue; mechanical properties; morphology; polymer; NBSIR 81-2209 (FDA).

stress, strain; thermodynamics; chemical potential; diffusion; equilibrium; solid; 20725.

stress waves; concrete; dynamic properties; fiber-reinforcement; impact; impulsive loading; penetration resistance; reinforcing bars; SP480-45.

striplines; higher order modes; rectangular coaxial line; NBSIR 78873.

strong fields; damping theory; kinetics; lasers; lineshapes; optical pumping; resonance Raman; spectroscopy; 20039. 
structural alloys; welding; alloys; aluminum alloys; castings; composites; cryogenic properties; low temperature; mechanical properties; nonmetallics; physical properties; stainless steels; NBSIR 81-1645.

structural alloys; welding; composites; low temperature; mechanical and physical properties; NBSIR 80-1627.

structural analysis; accidents; codes and standards; construction loads; construction safety; finite element; scaffold failures; scaffolding practices; scaffolds; NBS-GCR-80-255.

structural analysis; performance comparison; program behavior; SP500-83: 1981 November. 199-215.

structural damage; structures; wind; wind speed; building performance; hurricane winds; 20759.

structural design; structural engineering; design criteria; foundation design; geotechnical engineering; housing construction; mine subsidence; mining; settlement; NBSIR 81-2215.

structural engineering; building; building codes; building design; earthquakes; engineering; reinforced concrete; standards; NBSIR 80-2111-4.

structural engineering; building; building codes; building design; earthquakes; engineering; standards; stee1; NBSIR 80-2111-6.

structural engineering; building; building codes; building design; earthquakes; engineering; masonry; standards; NBSIR 80-2111-5.

structural engineering; building; building codes; building design; earthquakes; engineering; standards; NBSIR 80-2111-11.

structural engineering; building; building design; earthquakes; engineering; Federal government; seismic safety; standards; NBSIR 81-2195.

structural engineering; building (codes); climatology; hurricanes; reliability; risk; 20764.

structural engineering; buildings; buildings (codes); buildings (design); earthquake; seismic risk maps; standards; NBSIR 80-2111-1.

structural engineering; design criteria; foundation design; geotechnical engineering; housing construction; mine subsidence; mining; settlement; structural design; NBSIR 81-2215.

structural engineering; live loads; load survey; probability; statistics; NBS-GCR-76-81.

structural engineering; wind; climatology; loads (forces); probability distribution functions; reliability; statistical analysis; 20019.

structural engineering; wind loads; earthquake loads; hail loads; snow loads; solar collectors; NBSIR 81-2199.

structural engineering; wood; building; building codes; building design; earthquakes; engineering; standards; NBSIR 80-2111-7.

structural flexibility; noncrystalline films; passivation; passivation breakdown; 20025.

structural materials; austenitic stainless steels; coal gasification; elevated temperatures; ferritic stainless steel; gaseous environments; nickel alloy 671; nickel alloy 800 ; slow strain rate test; NBSIR 81 2191 (DoE).

structural safety; fracture control; fracture mechanics; mechanical properties; quality assurance; stress analysis; NBSIR 79-1623.

structural safety; work surfaces; codes and standards; construction safety; design; finite element; loads; scaffolds; stability; stiffness; strength; NBSIR 81-2265.

structural systems; test methods; wood; building rehabilitation; concrete; electrical; evaluation; HVAC; masonry; plumbing; steel; NBSIR 80-2171.

structural systems; test methods; wood; building rehabilitation; concrete; electrical; evaluation; HVAC; masonry; plumbing; solar; steel; NBSIR 81.2289 .

structure; ab initio calculations; amino acid; conformational energy; dipole moment; gas phase conformers; glycine; rotational constants; 20005.

structureborne noise; acoustics; architectural acoustics; building acoustics; noise; noise control; sound transmission; NBS-GCR-80248.

structure-borne noise; acoustics; building acoustics; noise control; noise isolation; sound transmission; $N B S-G C R-80-250$.

structures; wind; wind speed; building performance; hurricane wincis; structural damage; 20759.

subnatural-line width spectroscopy; time-resolved spectroscopy; high resolution spectroscopy; 20076

substituted apatite; crystal structure; lead apatite; lead potassium apatite; 19984.

substituted fluorapatite; apatite; barium apatite; cation substitution; crystal structure; rare-earth apatite; 20065.

substrate; adhesive; bitumen; bonding; built-up roofing membrane; complementary strain energy; felt; nonlinear; roofing membrane; splitting; strain energy; stress; TN1152.

Sudan; weights and measures regulation; Agency for International Development; Arab League Organization for Standardization and Metrology; chemical industry standards; cotton standards; food standards; measurement science and technology; standardization in developing countries; NBSIR 80-2020.

Sulfolobus; Thiobacillus; uranium; chemolithotrophic-bacteria; copper; iron; leaching; metal-reduction; metal-sulfides; mineraloxidation; molybdenum; oil shale; SP618: 1981 November. 264-273.

sulfur; Arrhenius parameters; chemical kinetics; combustion; decomposition; free radicals; gas phase; hydrocarbons; hydrogen nitrogen; oxygen; rate of reaction; NBSIR 81-2254.

sulfur dioxide; interstellar; nitric oxide; phosphine; phosphorus nitride; radio astronomy; 19987.

sulfur dioxide; sulfur monoxide; sulfur monoxide dimer; chemical kinetics; disulfur monoxide; rate constant; 20035.

sulfur hexafluoride; swarm data; water vapor; corona; decomposition; electrical insulation; gas chromatograph; inception voltages; mass spectrometer; partial discharge; NBSIR 81-2242.

sulfur hexafluoride; tracer tests; ventilation systems; ships; smoke control; smoke movement; stack effect; NBSIR 81-2433.

sulfuric acid; standard reference data; thermodynamic properties; activity coefficients; aqueous; critical evaluation; electrolytes; excess free energy; free energy; ionic; osmotic coefficients; solutions; JPCRD 10(3): 779.798; 1981.

sulfuric acid; thermodynamic properties; transport properties; activity coefficients; aqueous; compilation; conductivity; electrolytes; enthalpy; Gibbs energy; osmotic coefficients; solutions; NBSIR 81 2276.

sulfur monoxide; sulfur monoxide dimer; chemical kinetics; disulfur monoxide; rate constant; sulfur dioxide; 20035.

sulfur monoxide dimer; chemical kinetics; disulfur monoxide; rate constant; sulfur dioxide; sulfur monoxide; 20035.

sulphur; fibrous; polyurethane foam materials; smolder resistant upholstered furniture or mattress assembly; U.S. Patent 4,260,660.

sum rules; giant resonances; inelastic electron scattering; inelastic hadron scattering; photonuclear reactions; 19977.

supercomputers; benchmarking; capacity planning; chargeback systems; computer performance management; data base machines; end user productivity; human factors evaluation; information system management; office automation; performance management systems; resource measurement facility; simulation; SP500-83.

superconducting computer; thermal couplings; thermal isolation convective heat transfer; cryocooler; heat transfer by evaporating and condensing helium; helium refrigerator; SP607: 1981 May. 93102.

superconducting computers; thermal contact; $4 \mathrm{~K}$ refrigeration; cryogenic equipment; Josephson junction devices; SP607: 1981 May. 15-20.

superconducting device; thermal coupling; cryogenic refrigerator; Josephson technology; liquid helium; serviceable; SP607: 1981 May. 82-92.

superconducting devices; cryocoolers; cryogenic sensors; helium; refrigeration; SP607.

superconducting devices; tunnel devices; weak links; closed cycle refrigeration systems; Josephson effects; proximity effect devices; SP607: 1981 May. 195-209.

superconducting power transmission; cable cool-down; cable permeability; counter-flow cool-down; heat flux meters; liquid helium; permeability of composites; NBSIR 80-1637.

superconducting power transmission; cable cool-down; cool-down; heat flux meters; helium; NBSIR 81-1649.

superconducting power transmission line; terminals; thermal contraction; cable end fittings; pressure cycles; stress; 20018. 
superconducting wire; critical current; mechanical properties; niobium-tin; scaling; strain; 20066.

superconductivity; cryocoolers; low temperature; magnetic gradiometer; refrigeration; SQUID; Stirling cycle; SP607: 1981 May. 186-194.

superconductivity; thin film; transition temperature; tunneling; tunnel junction; aluminum; critical current; energy gap; enhancement; microwaves; nonequilibrium superconductivity; photon-assisted tunneling; 19979.

superconductivity; time domain; electronics; Josephson effect; measurements; 20070.

superconductivity; tunnel junction; aluminum; energy gap; gap enhancement; microwaves; nonequilibrium; 20723.

superconductor; conductor; critical current; critical temperature; review; standard; 20069.

superconductor; critical current; electrical property; magnetic property; stability; standards; NBSIR 80-1642.

superconductor composites; thermal effect; thermal expansion; low temperature; nonmetallics; physical property; 20753.

superconductors; ac losses; calorimeter; cryogenics; multipliers; standards; 19999.

supercritical flow; transition length; vertical stack drain entry; building drainage; NBSIR $81-2290$.

superfluid helium; cryogenics; infrared telescope; Spacelab; SP607: 1981 May. 117.127.

superthreshold visibility; vision; conspicuity; contrast; illumination lighting; lighting levels; NBSIR 81-2231.

supply; water conservation; water consumption; appliances; clothes washer; discharge; dishwasher; residential; NBSIR 80-2173.

suppression; automotive; EMI; ignition interference; SP480-44.

suppression; toxicity; compartment fires; fire detection; fire endurance; fire modeling; fire tests; flammability; human behavior; smoke; NBSIR 81-2294.

surface analysis; surface integrity; surface roughness; crystallographic anisotropy; crystallographic perfection; initial permeability; metal mirror; polishing; polycrystalline material; residual stress; sapphire; specular reflectance; SP620: 1981 October. 171-179.

surface damage; alkali halides; bulk damage; $\mathrm{CO}_{2}$ laser; coating damage; laser damage; microsecond pulses; $\mathrm{NaCl}$; SP620: 1981 October. 88.93.

surface damage; antireflection coatings; coating damage; laser damage; $\mathrm{LiNbO}_{3}$ coatings; Q-switch; SP620: 1981 October. 300-304.

surface defects; coating imperfections; cw laser irradiation; digs; dust; laser damage; mirrors; optical components; scratches; SP620: 1981 October. 256.264.

surface fitting; curve fitting; empirical fitting; J. Res. 86(1): 1.25; 1981 January-February.

surface hydride; alpha uranium; chloride ion; chromate ion; electrochemistry; ellipsometry; field ion microscopy; inhibitors; organic coatings; passive film dissolution; $\mathrm{pH}$ measurements; NBSIR 81-2396 (ONR).

surface impurities; alkali-halide laser windows; $\mathrm{KCl} ; \mathrm{NaCl}$; optoacoustic spectroscopy; photoacoustic spectroscopy; SP620: 1981 October. 44.49.

surface integrity; surface roughness; crystallographic anisotropy; crystallographic perfection; initial permeability; metal mirror; polishing; polycrystalline material; residual stress; sapphire; specular reflectance; surface analysis; SP620: 1981 October. 171-179.

surface laser damage; $1.06 \cdot \mu \mathrm{m} ; \mathrm{CO}_{2}$-laser glazing; $\mathrm{CO}_{2}$-laser polishing; fused silica surface; laser damage; laser polishing; SP620: 1981 October. 180-189.

surface-layer analyses; ultra-thin-windowed (UTW) $\mathrm{Si}(\mathrm{Li})$ detectors; windowless detectors; $\mathrm{x}$-ray transmission; electron excitation; energy-dispersive spectrometry; energy resolution; peak shapes; SP604: 1981 June. 97-126.

surface magnetism; ferromagnetism; Ni; nickel; polarized electrons; 19975.

surface mordanting with $\mathrm{Zn}$ ions; bonding with coupling agents; hydroxylapatite; interaction with $\mathrm{Zn}$ ions and enamel etching; reaction with $\mathrm{Zn}$ ions and rate laws; 20729.

surface plasma; absorption waves; air breakdown; $\mathrm{Cu}$ mirrors; laser- induced electron emission; mirror surface damage; SP620: 1981 October. 227.238.

surface recombination velocity; test pattern; test structure; gated diode; generation lifetime; integrated gated-diode electrometer; integrated test structure; leakage current; microelectronic test structures; NBS-GCR-81-327.

surface resonance; surface structure; polarized LEED; spin detector; spin polarization; 20012.

surface roughness; crystallographic anisotropy; crystallographic perfection; initial permeability; metal mirror; polishing; polycrystalline material; residual stress; sapphire; specular reflectance; surface analysis; surface integrity; SP620: 1981 October. 171-179.

surface roughness; temperature dependence; aluminum; copper; Drude theory; infrared; reflectance; silver; SP620: 1981 October. 190-200.

surface smoothness; thin films; barrier properties; diamond-like carbon; ion beam deposition; SP620: 1981 October. 313-323.

surface structure; polarized LEED; spin detector; spin polarization; surface resonance; 20012.

surface tension; thermophysical properties; tungsten; wetting; adsorption; Auger spectroscopy; convection; ellipsometry; gallium; levitation calorimetry; specific heat; NBSIR 81-2295.

surface-to-bulk optical absorption; $\mathrm{CaF}_{2}$; laser calorimetry; laser windows; optoacoustic; photoacoustic; SP620: 1981 October. 58-74.

surfacing materials; test method; asphalt; impact attenuation; peak acceleration; playground safety; playground surfaces; soil; NBSIR 80-2106.

surveillance; vibration; core barrel motion; neutron detectors; reactor internals; SP622: 1981 October. 3-10.

survey instruments; calibration; commercial services; directory; inaccuracy; ionizing radiation; radioactive sources; NBS-GCR-80 296.

survey research; user needs; dwelling units (residential); housing; Operation Breakthrough; post-occupancy housing evaluation; Project Feedback; questionnaires; research methods; NBSIR 81 . 2258.

survivability; alternate routing; communications networks; CROSSFIRE; link protocols; local survivability; message delay; network simulation; network throughput; NBS-GCR-81-336.

survivability; alternate routing; communications networks; distributed control; message delay; network throughput; NBSIR 81-2414.

susceptibility; TEM cell; analysis; dipole moments; electrically small objects; emission; measurements; quadrupoles; TN1040.

susceptibility and emission measurements; transverse electromagnetic cell; measurement procedures; TN1013.

swarm data; water vapor; corona; decomposition; electrical insulation; gas chromatograph; inception voltages; mass spectrometer; partial discharge; sulfur hexafluoride; NBSIR 81-2242.

swirling flow; closed conduit meter; differential pressure meter; flow measurement; laminar flow; meter performance; numerical modeling; orifice meter; 19972.

switching; temperature; transistors, power; base current, reverse; degradation; reverse bias; second breakdown; NBSIR 81-2204.

symbol; understandability; communication; evaluation method; firesafety; hazard warnings; meaningfulness; method; pictogram; response; NBSIR 80-2088.

symbols; understandability; visibility; visual alerting; directional arrows; exit signs; fire safety; pictograms; NBSIR 81-2268.

symbols; visual alerting; warnings; communication; hazard; pictograms; safety; signs; standards; NBSIR 80-2003.

synchrotron radiation; astrophysics; bremsstrahlung; electromagnetic interactions; pair production; plasma diagnostics; radiation; 19993.

syndiotactic; atactic; crystal; crystallinity; density; enthalpy; entropy; fusion; Gibbs energy; glass transition; heat capacity; isotactic; linear macromolecule; melt; polypropylene; JPCRD 10(4): 1051-1064; 1981.

synfuel; fossil porphyrins; fuel conversion; fuel extraction; heterocyclics; metal chelates; metallo-complexes; origin of pollutants; pollutant properties; SP618: 1981 November. 9.20.

SYNTHANE pilot plant; vibration monitoring; coal conversion; 
mechanical components; SP622: 1981 October. 274-288.

synthesis; calibration; digital; microcomputer; phase angle; sinewave generator; standard; TN1144.

synthetic benchmarks; workload characterization; workload definition; benchmarking; benchmark library; selection of ADP systems; standard benchmarks; 20747.

synthetic fuels; trace elements; coal; environmental chemistry; shale oil; SP618: 1981 November. 81-92.

synthetic implants; ossicular prosthesis; porosity measurement; porous biomaterials; porous polyethylene; PTFE-carbon composite; NBSIR 81-2212.

synthetics; water glycol; coal mine; fire tests; hydraulic fluids; invert emulsions; pressure-temperature; spray; NBSIR 81-2373.

system acquisition; system development; administrative experiment; evaluation systems; Experimental Technology Incentives Program; information systems; project management; NBSIR 81-2328.

system acquisition; system development; administrative experiment; evaluation systems; Experimental Technology Incentives Program; information systems; project management; NBSIR 81-2327.

system analysis/engineering; arrangement; building; classification; code; engineering; organization; provisions; scope; specification; standard; $B S S 136$.

systematic trends; transition probabilities; allowed transitions; cobalt; $f$-values; iron; isoelectronic sequence; line strengths; nickel; oscillator strengths; JPCRD 10(2): 305-566; 1981.

system design; drug development; drug regulation; innovation; postmarketing surveillance; regulatory experiments; NBS-GCR-ETIP 81-96.

system development; administrative experiment; evaluation systems; Experimental Technology Incentives Program; information systems; project management; system acquisition; NBSIR 81-2327.

system development; administrative experiment; evaluation systems; Experimental Technology Incentives Program; information systems; project management; system acquisition; NBSIR 81-2328.

system efficiency; thermal performance; air leakage; heat transfer; pebble bed storage; solar collector; NBS-GCR-81-302.

system efficiency; thermal performance; domestic hot water; recirculating hot water systems; solar collectors; NBS-GCR-81-322.

system inefficiencies; system productivity; SP5O0-83: 1981 November. 123-138.

system nontoxicity; x-ray opacification; dental composite resin restorations; finishability; microporous filler; U.S. Patent 4,306,913.

system productivity; system inefficiencies; SP5O0-83: 1981 November. 123.138.

system requirements; user requirements; computer resource selection; cost; long range plan; SP5OD-83: 1981 November. 293-302.

system resources; network models; product form; queueing models; SP5(N)-83: 1981 November. 335.

systems analysis; building codes; fire modeling; fire protection engineering; sprinkler systems; 20746.

systems analysis; building standards; classification; decision tables; information networks; network; standards; standards-writers; 19998.

systems analysis; building standards; classification; decision tables; information networks; modeling; standards; standards-writers; NBSIR 80-1979-1.

system simulation; building energy analysis; energy conservation for nonresidential buildings; historic preservation and energy conservation; load calculation; masonry thermal mass; NBSIR 81 . 2317.

system tuning; computer system; data processing function; performance evaluation; performance management; productivity; SP5(N)-83: 1981 November. 121.

\section{$\mathbf{T}$}

tabular values; computer programs; equation of state; ethylene thermodynamic properties; $T N 1045$.

tactile warnings; accessibility; alarms; blind; code requirements; communication; deaf; directional indicators; handicapped users; NBSIR 81-2428.

tagged photon method; Bethe-Heitler cross section; bremsstrahlung differential cross section; bremsstrahlung monochromator; photon beams; photonuclear research; NBSIR 81-2262.

tailing; x-ray detectors; collimation; deadlayer determinations; germanium; monoenergetic photons; partly-resolved doublets; silicon; SP604: 1981 June. 5-34.

tailing; x-ray spectroscopy; germanium; semiconductor detectors; silicon; SP604: 1981 June. 35.44.

tanks; volume; dimensions; laser; LNG; ships; NBSIR 80-2141.

tantalum; titanium; aerospace; chromium; cobalt; critical materials; NBSIR 81-2305.

tape; copper; deterioration; electromagnetic radiation; film; industry; metals; productivity; research associate; scanning technique; spin dependent; SRM's; standards; DIM/NBS 65(5): 1-24; 1981.

tapered concentrator wire; elongated capillary tube; interface; liquid chromatograph; liquid stream; mass spectrometer; U.S. Patent $4,281,246$.

taximeters; tolerances; volume-measuring devices; weighing devices; weights; length-measuring devices; liquid-measuring devices; measures; scales; specifications; H44, 1982 Edition.

taxonomy; dynamic analysis; programming aids; software development; software engineering; software tools; static analysis; SP500-74.

technical activities 1980; thermochemical and thermophysical data; data compilation; energy and environmental data; evaluated data; materials data; standard reference data; NBSIR 81-2206.

technical assistance; certification; Class $\mathbf{A}$ energy audit; economic analysis; energy analysis; energy conservation; Federal programs; government buildings; hospitals; rate of return; schools; SP608: 1981 May. 133-138.

technical barriers; economic; institutional; procurement; purchasing; recovered/recycled materials; resource recovery; NBSIR 81-2316.

technical barriers; economic; institutional; procurement; purchasing; recovered/recycled materials; resource recovery; NBS-GCR-81314.

technical barriers; economic; institutional; procurement; purchasing; recovered/recycled materials; resource recovery; NBS-GCR-81312.

technical barriers; economic; institutional; procurement; purchasing; recovered/recycled materials; resource recovery; NBS-GCR-81. 313.

technical barriers; economic; institutional; procurement; purchasing; recovered/recycled materials; resource recovery; NBS-GCR-81308.

technical barriers; economic; institutional; procurement; purchasing; recovered/recycled materials; resource recovery; NBS-GCR-81311.

technical bases; building research; building technology; codes; criteria; measurement methods; performance criteria; project summaries; SP446-5.

technical competence; cement and concrete; cover plates; earth's tilt; electric; fire; fracture toughness; measurement; parameter; physics; solar collector; space charges; DIM/NBS 65(3): 1.24; 1981.

technical literature; flaw detection; holography; inhomogeneities; light wave; nondestructive evaluation (NDE); optical holographic interferometry; NBS.GCR-81-318.

techniques; calibration; dosimetry; electric fields; electromagnetic interference; exposure history; instruments; magnetic fields; nonionizing radiation; regional calibration laboratories; standards; SP613.

technologies; technology transfer; Brazil; industrialization; international cooperation; measurement infrastructure; metrology; NBSIR 80-1972.

technology; calibration; electrical; electronics; engineering; hazards; measurement; metrology; National Bureau of Standards; power; radiation; semiconductor; $S P 611$.

technology assessment; technology forecast; computer networking; NBS-GCR-80-305.

technology forecast; automated data processing; computer utilization trends; Federal computers; hardware trends; information industry; software trends; NBS-GCR-81-342.

technology forecast; computer networking; technology assessment; 
NBS-GCR-80-305.

technology transfer; basic measurements and standards; building technology; calibration methods; computer standards and guidelines; energy research; environmental measurements; fire safety; industrial productivity; materials properties; research; standards; SP600.

technology transfer; Brazil; industrialization; international cooperation; measurement infrastructure; metrology; technologies; NBSIR 80-1972.

technology transfer; engineering; guest workers; international cooperation; international research; international standards; National Bureau of Standards (NBS); National Engineering Laboratory (NEL); NBSIR 80-2113.

technology transfer; weights and measures; domestic standards; education programs; electronic devices; international recommendations; legal metrology; measurement assurance; metrication; model laws and regulations; packing and labeling; specifications and tolerances; SP599.

telecommunications; automated data processing (ADP); communications; Federal Information Processing Standard (FIPS); Federal Property Management Regulations (FPMR); procurement; standards; FIPS PUB 80.

telecommunications; telephone exchanges; central office equipment; electronic equipment; fire test methods; incident data; NBSIR 812261.

telecommunications policy; teleconferencing; videodiscs; computer network interconnection; computer network protocol; digital transmission of information; electronic message transmission; image transmission; information networks; national bibliographic network; satellite communications; slow-scan television; SP610.

teleconferencing; videodiscs; computer network interconnection; computer network protocol; digital transmission of information; electronic message transmission; image transmission; information networks; national bibliographic network; satellite communications; slow-scan television; telecommunications policy; SP610.

telephone cables; underground; alloys; corrosion; metallurgicallybonded; metals; plastic-bonded; soils; NBSIR 81-2243.

telephone exchanges; central office equipment; electronic equipment; fire test methods; incident data; telecommunications; NBSIR 812261.

teleprocessing; transmission; advanced data communication control procedures; ANS X3.66; bit-oriented; code independence; data; data link control procedures; data transparency; Federal Information Processing Standards Publication; interoperability; FIPS PUB 78.

TEM cell; analysis; dipole moments; electrically small objects; emission; measurements; quadrupoles; susceptibility; TN1040.

TEM cell; characteristic impedance; field distribution; Hertzian dipole; integral equation; rectangular coaxial transmission line; TN1037.

TEM cell; electromagnetic compatibility (EMC); Green's function; integral equation; linear equation; method of moments; parallel plate waveguide; quasi electrostatic; TN1028.

TEM horn; time domain measurements; transients in resistivity; conical antenna; EMP; FFT; loaded antenna; method of moments; NBSIR $78-876$

temperature; flame inhibition; H-atoms; kinetics; mass spectrometry; phosphorus; radicals; Raman spectroscopy; NBSIR 80-2169.

temperature; thermal conductivity; transient; helium; hot wire; nitrogen; pressure; J. Res. 86(5): 457-493; 1981 September-October.

temperature; transistors, power; base current, reverse; degradation; reverse bias; second breakdown; switching; NBSIR 81-2204.

temperature; velocity; adiabatic compressibility; liquid; pressure; propane; sound; specific heat ratios; speed; J. Res. 86(2): 165-170; 1981 March-April.

temperature; ventilation; windows; energy conservation; lighting; man/environment research; noise; office building; post-occupancy evaluation; questionnaire; BSS130.

temperature and pressure measurements and standards; time and frequency; electrical measurements and standards; length and mass measurements and standards; quantum metrology; quantum physics; NBSIR 80-2165. temperature calibration; temperature standard; thermistor; thermometer; transition temperature; biomedical thermometry; disodium hydrogen phosphate; fixed point; hydration temperature; potassium fluoride; salt hydrate; sodium sulfate; J. Res. 86(2): 181192; 1981 March-April.

temperature control; transient; boiling; carbon film; heat transfer; helium; hysteresis; 19976.

temperature corrections; temperature measurements; ambient infrared radiation; blackbody cavity; corrections for reflectance; infrared imaging; infrared thermography; radiance temperature; reflected infrared radiation; 20733.

temperature dependence; aluminum; copper; Drude theory; infrared; reflectance; silver; surface roughness; SP620: 1981 October. 190200 .

temperature distribution; turbulent burning; ceilings; experiments; flame size; NBS-GCR-79-188.

temperature effects; compressive strength; concrete; curing temperature; early age; final set; hydration; initial set; laboratory testing; maturity; mortar; regression analysis; strength prediction NBSIR 81-2244.

temperature effects stainless steel alloys; yield stress; cryogenic properties of materials; iron alloys; low temperature mechanical properties; plastic deformation; 20742.

temperature excursions; thermal insulation; branch circuit; electric wire; field measurements; NBSIR 81-2347.

temperature measurement; alloys; combustion; combustion rate; ignition; metals; oxygen atmosphere; NBSIR 81-1647.

temperature measurements; ambient infrared radiation; blackbody cavity; corrections for reflectance; infrared imaging; infrared thermography; radiance temperature; reflected infrared radiation; temperature corrections; 20733.

temperature measurements; heating; probe; radio-frequency; NBSIR 80-1634.

temperature rise; flammability; heat flux; pressure; spray ignition; NBSIR 81-2247.

temperatures; vibrations; base line signatures; frequency spectra; hot alignment; machinery parameters; SP622: 1981 October. 220-221.

temperature sensors; cryogenics; helium; infrared; SQUID; SP607: 1981 May. 136-140.

temperature standard; thermistor; thermometer; transition temperature; biomedical thermometry; disodium hydrogen phosphate; fixed point; hydration temperature; potassium fluoride salt hydrate; sodium sulfate; temperature calibration; J. Res. 86(2): 181-192; 1981 March-April.

tenability limits; combustion products; compartment fires; egress; fire detection; fire growth; hazard analysis; mathematical models; room fires; smoke movement; NBSIR 80-2172.

tensile failure; chain rupture; crazing; crystalline polymers; electron spin resonance; microcrack; microfibril; shear deformation; 20732.

tensile properties; austenitic stainless steel; fracture toughness; low temperature; 20772.

tensile strength; yield strength; Young's modulus; compressive strength; elongation; foam; insulation; low temperature; mechanical properties; proportional limit; shear strength; NBSIR 81-1654.

tensile-test specimen; checkerboard pressure; elastostatics; finite elements; in-core solver; isoparametrics; Mooney-Rivlin material nonlinear equation-solvers; penalty method; plane-stress; strain invariants; J. Res. 86(1): 79-109; 1981 January-February.

tensor-property averaging; copper; Debye temperature; elastic constants; low temperatures; polycrystals; sound velocities; 20726

terminal networks; workload sizing; benchmark testing; Phase IV; remote terminal emulation; response time; SP500-83: 1981 November. 169-177.

terminal-probe method; UNIX; SP500-83: 1981 November. 157-168. terminals; thermal contraction; cable end fittings; pressure cycles; stress; superconducting power transmission line; 20018.

terrestrial solar simulators; test methods; calibration; combined photovoltaic/thermal modules; measurements; photovoltaic modules; photovoltaics; reference cells; solar cells; NBS-GCR-80279.

tertiary aromatic amines; unsaturated polyesters; acrylates; acrylic 
resins; aminoglutethimide or para-aminophenylacetic acid; cementing of bone; fillings; methacrylates; U.S. Patent 4,243, 763.

tertiary aromatic amines; unsaturated polyesters; vinyl monomers; accelerators; cementing of bone; high reactivity; low toxicity; peroxide catalyzed polymerization; U.S. Patent 4,284,551.

test coverage; test data generation; validation; verification; automated software tools; software lifecycle; software testing; software verification; SP500-75.

test data generation; validation; verification; automated software tools; software lifecycle; software testing; software verification; test coverage; SP500-75.

test method; asphalt; impact attenuation; peak acceleration; playground safety; playground surfaces; soil; surfacing materials; NBSIR 80-2106.

test methods; accident data; coal mines; conveyor belts; fire safety; fire tests; flame spread; ignition; NBSIR 81-2256.

test methods; calibration; combined photovoltaic/thermal modules; measurements; photovoltaic modules; photovoltaics; reference cells; solar cells; terrestrial solar simulators; NBS-GCR-80-279.

test methods; concrete; construction; nondestructive evaluation; quality assurance; steel reinforcing bars; NBSIR 80-2163.

test methods; field survey; industrial process heat; materials performance; solar collector; standards; NBSIR 81-2381.

test methods; trailing cables; coal mines; electrical cables; fire incidents; fire tests; NBSIR 81-2292.

test methods; wave structure; boric acid; cellulosic insulation; ignition; propagation; smoldering combustion; NBSIR 81-2350.

test methods; wood; building rehabilitation; concrete; electrical; evaluation; HVAC; masonry; plumbing; steel; structural systems; NBSIR 80-2171.

test methods; wood; building rehabilitation; concrete; electrical; evaluation; HVAC; masonry; plumbing; solar; steel; structural systems; NBSIR 81-2289.

test pattern; defect identification; laser scanner; measurement techniques; photovoltaics; solar cells; NBSIR 80-2181.

test pattern; test structure; gated diode; generation lifetime; integrated gated.diode electrometer; integrated test structure; leakage current; microelectronic test structures; surface recombination velocity; NBS-GCR-81-327.

test pattern; test structure; wafer chuck; wafer probe station; integrated circuit test methods; microelectronic test structure; probe station; semiconductor process control; SP400-68.

test pattern; test structure; yield; integrated circuits; microelectronics; process validation wafer; random faults; silicon-on-sapphire; SP4OO66.

test problems; accuracy; ANS FORTRAN; computer system installation; examples; OMNITAB 80; software; TN1147.

test procedures; basestock; engine lubricants; lubricating oil; motor oil; petroleum oil; recycled oil; rerefined oil; 20016.

test structure; CCD; cross-bridge sheet resistor; electrical alignment test structure; gated diode; integrated gated-diode electrometer; integrated test structure; MOSFET dc profiler; NBSIR 81-2319.

test structure; gated diode; generation lifetime; integrated gated-diode electrometer; integrated test structure; leakage current; microelectronic test structures; surface recombination velocity; test pattern; NBS-GCR-81-327.

test structure; wafer chuck; wafer probe station; integrated circuit test methods; microelectronic test structure; probe station; semiconductor process control; test pattern; SP400-68.

test structure; yield; integrated circuits; microelectronics; process validation wafer; random faults; silicon-on-sapphire; test pattern; SP400-66.

test structures; electronics; interconnect; microelectronics; random fault; step coverage; SP400-65.

test structures; laser scanner; model calculations; photovoltaic cells; sheet resistance; solar cell defects; solar cells; NBSIR 81-2260.

test substance; alternative fuel; bomb calorimetry; cellulose; enthalpy of combustion; refuse-derived-fuel; J. Res. 86(6): 655-660; 1981 November-December.

tetraalkyllead speciation; atomic absorption spectrometry; gasoline analysis; high performance liquid chromatography; metal-selective detector; metal speciation; 20763.

text communication; text interchange; typesetting composition; ADP terminals; ASCII; character-imaging devices; control functions; data interchange; display terminals; Federal Information Processing Standard; information interchange; printer terminals; standard FIPS PUB 86

text communications; text processor; coded character sets; code extension techniques; code standards; control functions; information interchange; SP500-81.

text editors; end user performance; interactive systems; performance analysis; productivity analysis; SP500-83: 1981 November. 71.80.

textiles; airflow; burn (injuries); cotton; fabric flammability; fabrics; fire suppression; flame extinguishment; heat transfer; polyester; NBSIR 81-2386.

text interchange; typesetting composition; ADP terminals; ASCII character-imaging devices; control functions; data interchange; display terminals; Federal Information Processing Standard; information interchange; printer terminals; standard; text communication; FIPS PUB 86.

text processor; coded character sets; code extension techniques; code standards; control functions; information interchange; text communications; SP500-81.

text processor; translation; UNIX; macroprocessor; pattern matching; query processor; software tool; NBSIR 80-2182.

theoretical spectroscopy; iron group elements; least squares optimization; J. Res. 86(1): 33-77; 1981 January-February.

theory of games; inspection; mathematical model; regulation; $J$. Res. 86(2): 193-215; 1981 March-April.

thermal comfort; thermostat setting practices; weatherization; weatherization and thermal comfort; Community Services Administration; field study; occupant comfort; optimal weatherization demonstration; NBSIR 81-2335.

thermal comfort; weatherization retrofitting; comfort; Community Services Administration Optimal Weatherization Demonstration; energy savings; field study; 20752.

thermal comfort; whole-house ventilation; building thermal performance; energy calculation; energy conservation; TN1138.

thermal conductance; Vuilleumier cryocoolers; cryogenics; GiffordMcMahon cryocoolers; heat capacity; heat transfer; helium; refrigerators; regenerators; Stirling cryocoolers; 20766.

thermal conductance; Vuilleumier cryococlers; cryogenics; GiffordMcMahon cryocoolers; heat capacity; heat transfer; helium; refrigerators; regenerators; Stirling cryocoolers; SP607: 1981 May. 70.81 .

thermal conductivity; composites; cryocoolers; plastics; refrigeration; regenerators; specific heat; SP607: 1981 May. 173.177.

thermal conductivity; concrete; guarded hot plate; low temperature; moisture migration; mortar; porous solid; NBSIR 81-1651.

thermal conductivity; correlation; critical region; helium; 20769.

thermal conductivity; edge heat loss; effective thermal conductivity; gap-temperature unbalance; guarded-hot-plate; heat source distribution; insulation; 20735.

thermal conductivity; glass fiberblanket; insulation; low temperature; standard reference material; NBSIR 81-1640.

thermal conductivity; glass fiberboard; insulation; low-temperature; standard reference material; NBSIR 81-1639.

thermal conductivity; transient; ambient temperature; argon; critical enhancement; hard sphere; hot wire; J. Res. 86(3): 293.307; 1981 May-June.

thermal conductivity; transient; helium; hot wire; nitrogen; pressure; temperature; J. Res. 86(5): 457.493; 1981 September-October.

thermal conductivity; viscosity; computer program; density; extended corresponding states; fluid mixtures; $T N 1039$.

thermal contact; $4 \mathrm{~K}$ refrigeration; cryogenic equipment; Josephson junction devices; superconducting computers; SP607: 1981 May. $15-20$.

thermal contraction; cable end fittings; pressure cycles; stress; superconducting power transmission line; terminals; 20018.

thermal coupling; cryogenic refrigerator; Josephson technology; liquid helium; serviceable; superconducting device; SP607: 1981 May. 82-92. 
thermal couplings; thermal isolation; convective heat transfer; cryocooler; heat transfer by evaporating and condensing helium; helium refrigerator; superconducting computer; SP607: 1981 May. 93-102.

thermal degradation; thermogravimetry; activation energy; bubbles; polymer degradation products; pressure; 20774.

thermal diffusion length; thermal stress; cold work; crystalline disorder; defect damage; diamond-machined mirrors; Drude absorption; electron-beam melting; laser waveform; melt threshold; metal deposition; pulsed laser damage; slip threshold; SP620: 1981 October. 201-209.

thermal diffusivity; concentration; diffusivity; methane-ethane; mixture; one-fluid model; plait point; Rayleigh light scattering; 19964.

thermal effect; thermal expansion; low temperature; nonmetallics; physical property; superconductor composites; 20753.

thermal efficiency; windows; building economics; daylighting; energy conservation; engineering economics; life-cycle costs; passive solar; regional analysis; $N B S I R \quad 81-2248$.

thermal energy storage materials; thermal properties; thermodynamic properties; transport properties; corrosion; data compilations; electrochemical aluminum production; electrochemical energy storage materials; molten salts; physical properties; safety and hazards; NSRDS-NBS61, Part IV.

thermal expansion; anharmonic; Brillouin zone sums; central pair potential; fcc metals; free energy; high temperature nearestneighbor model; 20037.

thermal expansion; low temperature; nonmetallics; physical property; superconductor composites; thermal effect; 20753.

thermal expansion; thermal restraint; buildings; concrete; fire endurance; fire tests; floors; reinforced concrete; roofs; NBS-GCR81-317.

thermal explosion theory; thermal hazards; thermal instability; adiabatic calorimetry; azidotriphenylmethane; decomposition reactions; differential scanning calorimetry; heat conduction calorimetry; kinetics of heat evolution; m-azidobenzoic acid; nitrocellulose; quantitative differential thermal analysis; NBSIR 80 2018 (DOT).

thermal hazards; thermal instability; adiabatic calorimetry; azidotriphenylmethane; decomposition reactions; differential scanning calorimetry; heat conduction calorimetry; kinetics of heat evolution; m-azidobenzoic acid; nitrocellulose; quantitative differential thermal analysis; thermal explosion theory; NBSIR 80 2018 (DOT).

thermal injury; bioheat equation; burn; criterion; critical exposure; fluid; heat transfer; injury threshold; scald; NBSIR 81-2320.

thermal instability; adiabatic calorimetry; azidotriphenylmethane; decomposition reactions; differential scanning calorimetry; heat conduction calorimetry; kinetics of heat evolution; m-azidobenzoic acid; nitrocellulose; quantitative differential thermal analysis; thermal explosion theory; thermal hazards; NBSIR 80-2018 (DOT).

thermal insulation; branch circuit; electric wire; field measurements; temperature excursions; NBSIR 81-2347.

thermal insulation; branch circuit wiring problems; electrical problems; electrical wiring system; extension cord wiring; fuse/circuit breaker panel problems; overamping; residential structures; safety; NBSIR 81-2203.

thermal insulation; thermal properties; cellular plastic; low temperature; mechanical properties; polystyrene; polyurethane; 20074.

thermal insulation; thermal properties; underground systems; computer program; earth temperature; heat transfer; pipes; NBSIR 81-2378.

thermal insulation; wiring system; electrical fire; electric cable; models; TN1133.

thermal isolation; convective heat transfer; cryocooler; heat transfer by evaporating and condensing helium; helium refrigerator; superconducting computer; thermal couplings; SP607: 1981 May. 93.102.

thermal lensing; window materials; elastic coefficients; high-energy laser; interferometric testing; optical distortion; photoelastic coefficients; polycrystalline aggregate; stress birefringence; SP620: 1981 October. 117-128.

thermal mass; walls; water source heat pump; buildings; construction; energy conservation; ground coupled; hybrid solar energy system; inverted cave; low grade geothermal; radiant floor heating; space heating and cooling; SP608: 1981 May. 69.81.

thermal mechanical treatment; aging; electrical conductivity; hardness; homogeneity; microstructure; precipitating; segregation; solidification; NBSIR 80-2069.

thermal performance; air leakage; heat transfer; pebble-bed storage; solar collector; system efficiency; NBS-GCR-81-302.

thermal performance; auxiliary energy; FCHART computer program; National Solar Demonstration Program; solar energy systems; space heating load; NBS-GCR-81-321.

thermal performance; domestic hot water; recirculating hot water systems; solar collectors; system efficiency; NBS-GCR-81-322.

thermal performance; efficiency; flow rate; heat transfer fluid; solar collector; NBS-GCR-80-254.

thermal performance; uncertainty; collector rating; measurement; solar collector; standards; TN1140.

thermal properties; cellular plastic; low temperature; mechanical properties; polystyrene; polyurethane; thermal insulation; 20074.

thermal properties; thermodynamic properties; transport properties; corrosion; data compilations; electrochemical aluminum production; electrochemical energy storage materials; molten salts; physical properties; safety and hazards; thermal energy storage materials; NSRDS-NBS61, Part IV.

thermal properties; underground systems; computer program; earth temperature; heat transfer; pipes; thermal insulation; NBSIR 81 . 2378.

thermal protection; bombs (ordnance); boxcars; computer models; fire detection systems; fire suppression; full-scale fire tests; heat transfer; railroad accidents; small-scale fire tests; NBSIR 80-2170.

thermal resistance; built-up roofing; moisture; moisture detection; nondestructive evaluation; nondestructive testing; roofing; $T N 1146$.

thermal resistance; fiber-glass; fibrous glass; insulation; measurement; $\mathrm{R}$ value; standard reference material; NBSIR 80-2091.

thermal restraint; buildings; concrete; fire endurance; fire tests; floors; reinforced concrete; roofs; thermal expansion; NBS-GCR-81-317.

thermal storage; ASHRAE 90-75; class $\mathrm{K}$ code; computer modeling; electrical design; energy audit; energy conservation; HVAC systems; performance standards; solar collector; solar energy; space heating and cooling; SP608.

thermal storage; wood structure; codes; concrete; masonry; prohibitions; re-examination; SP608: 1981 May. 195-200.

thermal stress; cold work; crystalline disorder; defect damage; diamond-machined mirrors; Drude absorption; electron-beam melting; laser waveform; melt threshold; metal deposition; pulsed laser damage; slip threshold; thermal diffusion length; SP620: 1981 October. 201-209.

thermal test procedures; transient thermal tests; passive/hybrid components; solar energy; steady-state tests; NBSIR 81-2300.

thermistor; thermometer; transition temperature; biomedical thermometry; disodium hydrogen phosphate; fixed point; hydration temperature; potassium fluoride; salt hydrate; sodium sulfate; temperature calibration; temperature standard; J. Res. 86(2): 181. 192; 1981 March-April.

thermistor mount calibration; $\mathrm{mm}$ wave measurements; reflection coefficient; six-port measurement system; NBSIR 81-1650.

thermochemical and thermophysical data; data compilation; energy and environmental data; evaluated data; materials data; standard reference data; technical activities 1980; NBSIR 81-2206.

thermochemical tables; enthalpy; entropy; equilibrium constant of formation; free energy of formation; Gibbs energy function; heat capacity; heat of formation; selected values; NBSIR 81-2343.

thermochemistry; $\Delta C_{p}$ of reaction; calorimetry; $\mathrm{C}_{5} \mathrm{H}_{5} \mathrm{~N}_{5} \mathrm{O}$; density; enthalpy of solution; Gua. $\mathrm{HCl} \cdot 1 \mathrm{H}_{2} \mathrm{O}$; Gua. $\mathrm{HCl} \cdot 2 \mathrm{H}_{2} \mathrm{O}$; Gua.2 $\mathrm{HCl}_{\text {; }}$ guanine (Gua); guanine hydrochlorides; heat capacity; nucleic acid base; solution calorimetry; J. Res. 86(4): 367.382; 1981 July-August.

thermodynamic; vapor pressure; critical region; density; equation of state; geothermal; isobutane; NBSIR 79-1715. 
thermodynamic and dynamic analyses; displacer and piston drives; linear electric motors; linear free-displacer cryorefrigerator; linear resonant machinery; phasor notation; SP607: 1981 May. 57.69.

thermodynamic and transport properties; atomic and molecular properties; bibliographies; chemical kinetics; evaluated data; fluid properties; indexes; publication list; solid state; SP612.

thermodynamic data; enthalpy; enthalpy of formation; entropy; equilibrium constant for formation; Gibbs energy function; Gibbs energy of formation; heat capacity; lime-alumina-silica-water; minerals; JPCRD 10(3): 575-670; 1981.

thermodynamic properties; activity coefficient; cesium; critical evaluation; electrolyte; excess Gibbs energy; lithium; osmotic coefficient; potassium; rubidium; sodium; solutions; JPCRD 10(3): 671-766.

thermodynamic properties; activity coefficients; alkali metal nitrite salts; aqueous; critical evaluation; $\mathrm{CsNO}_{2}$; electrolytes; excess Gibbs energy; $\mathrm{KNO}_{2} ; \mathrm{LiNO}_{2} ; \mathrm{NaNO}_{2} ;$ osmotic coefficients; $\mathrm{RbNO}_{2}$ solutions; standard reference data; JPCRD 10(3): 767.778; 1981.

thermodynamic properties; activity coefficients; aqueous; critical evaluation; electrolytes; excess free energy; free energy; ionic; osmotic coefficients; solutions; sulfuric acid; standard reference data; JPCRD 10(3): 779.798; 1981.

thermodynamic properties; transport properties; activity coefficients aqueous; compilation; conductivity; electrolytes; enthalpy; Gibbs energy; osmotic coefficients; solutions; sulfuric acid; NBSIR 812276.

thermodynamic properties; transport properties; corrosion; data compilations; electrochemical aluminum production;

electrochemical energy storage materials; molten salts; physical properties; safety and hazards; thermal energy storage materials; thermal properties; NSRDS-NBS61, Part IV.

thermodynamic properties; velocity of sound; virial coefficients electrostatic transducers; gas; nitrogen; sonic cavity; 20072.

thermodynamic properties; zinc; activity coefficient; cadmium; critical evaluation; electrolyte; ethylene bis(trimethylammonium) chloride; ethylene bis(trimethylammonium) iodide; excess Gibbs energy; osmotic coefficient; solutions; JPCRD 10(1): 1-56; 1981.

thermodynamics; adsorption; desorption; hydrolyzable cations; hydrous oxides; kinetics; protolyzable anions; SP618: 1981 November. 146-162.

thermodynamics; alloy phase diagram; computer graphics; critical evaluation; database management; 20033.

thermodynamics; chemical potential; diffusion; equilibrium; solid; stress, strain; 20725.

thermodynamics; fluid flow; gas; instrumentation; mass flow; measurement; metering; 20751.

thermodynamics; metastable phase equilibrium; partitionless solidification; phase diagrams; phase equilibrium hierarchy; rapid solidification; 19981.

thermodynamics; vaporization; chemical activity; magnetohydrodynamics; mass spectrometry; potassium slag; NBSIR 81-2293.

thermogravimetry; activation energy; bubbles; polymer degradation products; pressure; thermal degradation; 20774.

thermometer; transition temperature; biomedical thermometry; disodium hydrogen phosphate; fixed point; hydration temperature; potassium fluoride; salt hydrate; sodium sulfate; temperature calibration; temperature standard; thermistor; J. Res. 86(2): 181.192; 1981 March-April.

thermophysical properties; tungsten; wetting; adsorption; Auger spectroscopy; convection; ellipsometry; gallium; levitation calorimetry; specific heat; surface tension; NBSIR 81-2295.

thermostat setting practices; weatherization; weatherization and thermal comfort; Community Services Administration; field study; occupant comfort; optimal weatherization demonstration; thermal comfort; NBSIR 81-2335.

$\mathrm{ThF}_{4}$; thin films; $\mathrm{ZnS} ; \mathrm{ZrO}_{2}$; damage threshold; impurity-induced damage; laser damage; SP620: 1981 October. 277.286.

thick building construction; conduction heat transfer; conduction transfer functions; initialization of heat transfer problem; parallel heat flow; response factors; NBSIR 81-2353. thin film; anti-reflection coatings; bichromatic; damage; high energy laser; laser damage; pulsed laser; reflectors; SP620: 1981 October. 287.296.

thin film; transition temperature; tunneling; tunnel junction; aluminum; critical current; energy gap; enhancement; microwaves; nonequilibrium superconductivity; photon-assisted tunneling; superconductivity; 19979.

thin film coatings; laser damage; laser interaction; optical components; optical fabrication; optical materials and properties; SP620.

thin films; barrier properties; diamond-like carbon; ion beam deposition; surface smoothness; SP620: 1981 October. 313.323.

thin films; diode laser; ellipsometer; infrared; polarizer; SP620: 1981 October. 345-355.

thin films; ellipsometry; polysilicon films; silicon dioxide films; silicon nitride films; standard reference materials; NBSIR 80-2158.

thin films; ultraviolet reflectors; excimer lasers; fluoride coatings; laser damage; multiple-shot damage; oxide coatings; pre-irradiation; SP620: 1981 October. 265-276.

thin films; $\mathrm{ZnS} ; \mathrm{ZrO}_{2}$; damage threshold; impurity-induced damage; laser damage; $\mathrm{ThF}_{4} ;$ SP620: 1981 October. 277-286.

Thiobacillus; uranium; chemolithotrophic-bacteria; copper; iron; leaching; metal-reduction; metal-sulfides; mineral-oxidation; molybdenum; oil shale; Sulfolobus; SP618: 1981 November. 264. 273.

thiocyanate compounds; flame and smolder resistance; flame resistant cellulosic insulation material; U.S. Patent 4,302,345.

thorium compounds; uranium compounds; actinium compounds; cesium compounds; enthalpy; entropy; francium compounds; Gibbs energy of formation; heat capacity; lithium compounds; potassium compounds; protactinium compounds; rubidium compounds; sodium compounds; TN270-8.

thorium fluoride; zinc selenide; zinc sulfide; absorption; coatings; internal reflection spectroscopy; silicon monoxide; SP620: 1981 October. 335-344.

three-photon absorption; direct gap semiconductors; non-linear optics; SP620: 1981 October. 446-452.

three way catalytic converters; zirconium dioxide; air-fuel ratio; closed loop fuel metering system; exhaust emissions; exhaust gas oxygen sensor; exhaust gas recirculation; feedback; oxidation catalyst; oxygen partial pressure; stoichiometric; SP622: 1981 October. 62.73.

threshold photoelectron spectra; breakdown curves; photoelectronphotoionization coincidence; photoionization; propyne; 20051.

threshold values; damage types; nonlinear effect; optical glass; SP620: 1981 October. 102-109.

thromboembolism; bio-prostheses; prosthetic heart valves; SP601: 1981 January. 175-200.

thyristor material characterization; transient capacitance techniques; deep-level measurements; deep-level transient spectroscopy; lifetime; power-device grade silicon; NBSIR 81-2325.

tidal electricity production; tidal machinery; La Rance; SP622: 1981 October. 289-309.

tidal machinery; La Rance; tidal electricity production; SP622: 1981 October. 289-309.

tiedown; wind forces; buoyancy forces; flood forces; foundations; hurricane forces; mobile home; soil anchors; standards; BSS132.

time and frequency; electrical measurements and standards; length and mass measurements and standards; quantum metrology; quantum physics; temperature and pressure measurements and standards; NBSIR 80-2165.

time comparison; time receiver; worldwide time; atomic clocks; automatic time comparisons; common-mode cancellation; frequency comparison; global positioning system; remote time measurement; 20062.

time constant; fire extinguishment; fire models; fire protection; sprinkler heads; sprinkler systems; NBSIR 81-2329.

time-dependent; diffusion; integrating device; permeation type; personal dosimeter; reaction; NBSIR 80-1975.

time domain; electronics; Josephson effect; measurements; superconductivity; 20070.

time domain; transmission line model; waveform; bit error rate; 
coaxial transmission lines; digital communication impulse response; instrumentation; measurements; shielded balanced transmission lines; $T$ N 1042.

time domain; waveforms; deconvolution; impulse response; inverse filtering; noise contamination; TN1047.

time-domain calibrations; weights and measures; x-ray diffraction; air pollutants; antenna coupling; electrical distribution; glass-liquidus insulation; monitors; neutron diffraction, samplers; SRM's; DIM/NBS 65(1): 1-28; 1981.

time domain measurements; transients in resistivity; conical antenna; EMP; FFT; loaded antenna; method of moments; TEM horn; NBSIR 78-876.

time-domain-reflectometry; underground transmission; aging; cables; dielectrics; electric insulation fault location; NBSIR 81-2235.

time receiver; worldwide time; atomic clocks; automatic time comparisons; common-mode cancellation; frequency comparison; global positioning system; remote time measurement; time comparison; 20062.

time-resolved spectroscopy; high resolution spectroscopy; subnaturalline width spectroscopy; 20076.

time reversal; charge conjugation; CPT theorem; parity; 19991.

time series; bispectrum; cumulant spectra; nonlinear time series; polyspectra; spectrum analysis; statistics; TN1036.

timesharing; functional categories; resource oriented groupings; SP500-83: 1981 November. 15-19.

time-stepping finite difference equation technique; Auger function; electrically-short dipole; iteration method; nonlinear differential equation; nonlinear load; NBSIR 78-898.

time to failure; accelerated aging tests; coatings; corrosion; predictability; reproducibility; TN/149.

time-varying noise; duration; general adverse effect of noise; intermittency; loudness; noise criteria; NBSIR 81-2377.

tin hydroxide oxide phosphate; tin phosphate; topical fluoride treatments; crystal structure; hydrogen bonding; hydroxyapatite 19994.

tin phosphate; topical fluoride treatments; crystal structure; hydrogen bonding; hydroxyapatite; tin hydroxide oxide phosphate; 19994.

tire inspection; ultrasonics; visual-optical; acoustic emission; eddy currents; liquid penetrants; magnetic particles; microwaves; nondestructive evaluation; radiography; 20762.

tissue equivalence; kerma factors; neutron dosimetry; neutron radiation therapy; nuclear data; secondary charged particles; 20064.

tissue reaction; corrosion; osteosynthesis devices; SP601: 1981 January. 423.447.

tissue response; atomic absorption spectrophotometry; EDAX analysis; enzyme histochemistry; histology; implant retrieval analysis; polarography; SP601: 1981 January. 323-336.

titanium; aerospace; chromium; cobalt; critical materials; tantalum; NBSIR 81-2305.

titanium; Co-Cr-Mo; Co-Ni-Cr-Mo; corrosion; dental alloys; metalamino acid reactions; stainless steel; 19988.

titanium; trace elements; arsenic; chromium; coal hydrogenation; cobalt; liquefaction; mercury; nickel; process waters; selenium; solvent refined coal; SP618: 1981 November. 21-38.

titanium; transmission electron microscopy; alloy phase diagram; aluminum; magnetic susceptibility; order-disorder; 20736.

titanium, titanium alloy; bone plates; cobalt-chrome-nickel-tungsteniron-alloy; fretting corrosion; histologic analysis; stainless steel 316 LVM; SP601: 1981 January. 395-422.

tolerance; cutset; cycle; directed graph; network; network flow; optimization; retraction; robustness; rooted tree; sensitivity analysis; shortest path; 20022.

tolerances; volume-measuring devices; weighing devices; weights; length-measuring devices; liquid-measuring devices; measures; scales; specifications; taximeters; H44, 1982 Edition.

toolboxes; development support systems; programming environments; software development; software tools; SP500-78.

tools; analysis; performance evaluation; pictures; simulation; SP5OO83: 1981 November. 331-334.

topical fluoride treatments; crystal structure; hydrogen bonding; hydroxyapatite; tin hydroxide oxide phosphate; tin phosphate;
19994.

total orders; aggregation; equilibrium; game theory; mathematical economics; noncooperative games; J. Res: 86(4): 383-391; $1981 \mathrm{July}$. August.

total tank subsystem integration; bus controllers; control and distribution; crew station terminals; electrical power management; electronic components; microcomputers; MIL-STD.1553B multiplex data bus; multiplexing techniques; on-board diagnostics and prognostics; remote terminals; SP622: 1981 October. 85-96.

toughness; crack growth; creep fracture; high temperature fracture; silicon nitride; strain rate effect; NBSIR 79-1960 (DoE).

toxic; trace elements; environmental; essential elements; speciation; SP618: 1981 November. 235-243.

toxic elements; availability; environmental contamination; extraction tests; fossil fuels; laboratory characterization; metals; mining industry; public health impacts; residues; SP618: 1981 Novennber. $1-8$.

toxic heavy metals; antimony; arsenic; bacterial resistances; cadmium; detoxification; mercury; organomercury; plasmids; silver; SP618: 1981 November. 301-324.

toxicity; alkylleads; alkyltins; chemical speciation; GC.AAS techniques; LC-AAS techniques; organoarsenics; organometals; partition coefficients; SP618: 1981 November. 65-80.

toxicity; compartment fires; fire detection; fire endurance; fire modeling; fire tests; flammability; human behavior; smoke; suppression; NBSIR 81-2294.

toxicity; wood; combustion; decision analysis; fire models; flame spread; human behavior; ignition; polymers; smoke; soot; NBSIR $81-2382$

toxicity of combustion products; chemistry of fire; combustion products; detection; fire hazards; fire modeling; fire protection; fire research; fire suppression; human behavior in fires; physics of fire; NBSIR 80.2127.I.

traceability; calibration; measurement assurance; measurement services; standards; SP250, 1980 Edition.

traceability; environment; metrology; quality control; radioactivity; 20010.

trace elements; aqueous speciation; computerized; geochemical model; heavy metals; redox couples; SP618: 1981 November. 39-53.

trace elements; arsenic; chromium; coal hydrogenation; cobalt; liquefaction; mercury; nickel; process waters; selenium; solvent refined coal; titanium; SP618: 1981 November. 21-38.

trace elements; coal; environmental chemistry; shale oil; synthetic fuels; SP618: 1981 November. 81-92.

trace elements; environmental; essential elements; speciation; toxic; SP618: 1981 November. 235-243.

trace element standards; waste cycling; bacterial mobilization; biogeochemical cycles; biological uptake; cellular toxicityessentiality mechanisms; chromatography; element-selective speciation; environmental monitoring; fossil fuels; metal(loid) complexes; organometallic compounds; SP618.

trace metals; alkylpyridines; dissolved organic compounds; gas chromatography/mass spectrometry; hydrophobic bases; metal complexation; nitrogen bases; oil shale; retort water; speciation; SP618: 1981 November. 93-104.

trace metals; chromatography; direct current plasma; hydrophilic; hydrophobic; metal complex; porphyrin; shale oil; speciation; SP618: 1981 November. 105-119.

trace organic analysis; definitive methods; human serum; polynuclear aromatic hydrocarbons; reference materials; shale oil; SRM; 19989.

tracer tests; ventilation systems; ships; smoke control; smoke movement; stack effect; sulfur hexafluoride; NBSIR 81-2433.

trailing cables; coal mines; electrical cables; fire incidents; fire tests; test methods; NBSIR 81-2292.

trainee performance testing; BEPS; code compliance; code conflicts; energy conservation standards; solar energy; SP608: 1981 May. 139 157.

training programs; building codes; building officials; codes and standards; convective loops; greenhouses; heat exchangers; light and ventilation; solar systems; space heating and cooling; SP608: 1981 May. 159.166 
transaction driven system; bubble memory; mathematical model standard transaction; SP500-83: 1981 November. 259.265.

transactions; annual reports; diffusion in metals; fire; journals; library holdings, NBS Library; NBS periodicals; periodicals; proceedings; serials; standards; NBSIR 81-2226.

transducer; ATE; automatic test equipment; calibration; data acquisition; durability; dynamic calibration; environmental testing; evaluation; measurand; reliability; sensor; SP615.

transducer; waveform; acoustic emission; calibration; development; 20757.

transducers; energy measurement; field instrument force measurement; field testing; in-situ testing; soil mechanics; BSS135.

transfer functions; field surveys; fire; fire loads; furnishings; household surveys; live loads; mobile homes; residential surveys; NBSIR 80 2155.

transfer standard; effective area; intercomparison; piston gage pressure; primary standard; J. Res. 86(3): 277-279; 1981 May-June.

transformer oil; cables; composite insulation; dc fields; high voltage; incipient fault; insulation; liquid breakdown; $\mathrm{SF}_{6} ;$ space charge; NBSIR 81-2334.

transformer oil; cables; dc fields; high voltage; incipient fault; insulation; $\mathrm{SF}_{6}$; space charge; NBSIR 81-2283.

transformers; electrical design; energy distribution; National Electrical Code; power factor correction; source energy; SP608: 1981 May. 37.56.

transient; ambient temperature; argon; critical enhancement; hard sphere; hot wire; thermal conductivity; J. Res. 86(3): 293-307; 1981 May-June.

transient; boiling; carbon film; heat transfer; helium; hysteresis; temperature control; 19976.

transient; helium; hot wire; nitrogen; pressure; temperature; thermal conductivity; J. Res. 86(5): 457-493; 1981 September-October.

transient capacitance techniques; deep-level measurements; deep-level transient spectroscopy; lifetime; power-device grade silicon; thyristor material characterization; NBSIR 81-2325.

transients; absorption spectra; aqueous solution; extinction coefficients; inorganic; radical ions; radicals; NSRDS-NBS69.

transients in resistivity; conical antenna; EMP; FFT; loaded antenna; method of moments; TEM horn; time domain measurements; NBSIR $78-876$

transient thermal tests; passive/hybrid components; solar energy; steady-state tests; thermal test procedures; NBSIR 81-2300.

transistor; tunnel diode; diode; field-effect transistor; III-V compounds; IMPATT diode; integrated circuit; LASER; LED photocell; solar cell; solid state; J. Res. 86(6): 565-570; 1981 November-December.

transistors, power; base current, reverse; degradation; reverse bias; second breakdown; switching; temperature; NBSIR 81-2204.

transition length; vertical stack drain entry; building drainage; supercritical flow; NBSIR 81-2290.

transition probabilities; allowed transitions; cobalt; $f$-values; iron isoelectronic sequence; line strengths; nickel; oscillator strengths; systematic trends; JPCRD 10(2): 305-566; 1981.

transition probabilities; wavelengths; atom; atomic ion; intensities; line strengths; oscillator strengths; spectral lines; NSRDS-NBS68.

transition temperature; biomedical thermometry; disodium hydrogen phosphate; fixed point; hydration temperature; potassium fluoride; salt hydrate; sodium sulfate; temperature calibration; temperature standard; thermistor; thermometer; J. Res. 86(2): 181.192; 1981 March-April

transition temperature; tunneling; tunnel junction; aluminum; critical current; energy gap; enhancement; microwaves; nonequilibrium superconductivity; photon-assisted tunneling; superconductivity; thin film; 19979 .

translation; BIPM; CCU; CGPM; CIPM; metric; SI; SP330

translation; UNIX; macroprocessor; pattern matching; query processor; software tool; text processor; NBSIR 80-2182.

transmission; absorption; laser windows; material properties; SP620: 1981 October. 144-158.

transmission; advanced data communication control procedures; ANS X3.66; bit-oriented; code independence; data; data link control procedures; data transparency; Federal Information Processing Standards Publication; interoperability; teleprocessing; FIPS PUB 78.

transmission electron microscopy; alloy phase diagram; aluminum; magnetic susceptibility; order-disorder; titanium; 20736.

transmission electron microscopy; electron probe microanalysis; energy dispersive $\mathrm{x}$-ray spectrometry; microanalysis; qualitative analysis; quantitative analysis; scanning electron microscopy; spectral artifacts; SP604: 1981 June. 315-339.

transmission line audible noise; corona noise; electric energy transmission; high voltage; human response to noise; noise pollution; psychoacoustics; 20029.

transmission line model; waveform; bit error rate; coaxial transmission lines; digital communication impulse response; instrumentation; measurements; shielded balanced transmission lines; time domain; TN1042.

transmission lines; vertical current density; Wilson plates; high voltage dc; measurement errors; NBSIR 81-2410.

transpiration; vapor transport; coal gasification; high temperature; mass spectrometry; slag; NBSIR 80-2178 (DoE).

transport; buildings; concrete; diffusion; exhalation; materials; measurement; permeability; radon; TN1139.

transportation networks; algorithm; graph; $k$ shortest paths; network algorithms; shortest path; 20021.

transport properties; activity coefficients; aqueous; compilation; conductivity; electrolytes; enthalpy; Gibbs energy; osmotic coefficients; solutions; sulfuric acid; thermodynamic properties NBSIR 81-2276.

transport properties; corrosion; data compilations; electrochemical aluminum production; electrochemical energy storage materials; molten salts; physical properties; safety and hazards; thermal energy storage materials; thermal properties; thermodynamic properties; NSRDS-NBS61, Part IV.

transverse electromagnetic cell; measurement procedures; susceptibility and emission measurements; TN1013.

tree; complexity; labeling; network; sequence list; shortest path; $J$. Res. 86(3): 317-330; 1981 May-June.

trend analysis; unlimited application; fibrescope; health condition; man-hour expenditure ratio; SP622: 1981 October. 74-81.

triboellipsometry; aluminum; field ion microscopy; iron-chromium alloys; niobium; repassivation kinetics; stainless steel; NBSIR 791904 (Navy).

trip time; branch circuits; circuit breaker; electrical fire; low ambient temperature; $N B S I R$ 81-2221.

triton particles; abundances; alpha particles; cellulose nitrate detector; isotopic; lithium; nuclear track technique; 19982.

TSO; analysis; data elements; I/O; logical swapping; measurement; MVS; paging/swapping; SP500-83: 1981 November. 343-348.

tubes ( $\mathrm{x}$ ray); $\mathrm{x}$-ray measurement; $\mathrm{x}$ rays; dosage; dosimetry; health and safety; high voltage; ionization chamber; ionizing radiation; ionometer; radiation quantities and units; radiation standards and protection; radioactivity; radiology; radium; roentgen ray; SP625.

tungsten; wetting; adsorption; Auger spectroscopy; convection; ellipsometry; gallium; levitation calorimetry; specific heat; surface tension; thermophysical properties; NBSIR 81-2295.

tunnel devices; weak links; closed cycle refrigeration systems; Josephson effects; proximity effect devices; superconducting devices; SP607: 1981 May. 195-209.

tunnel diode; diode; field-effect transistor; III-V compounds; IMPATT diode; integrated circuit; LASER; LED; photocell; solar cell; solid state; transistor; J. Res. 86(6): 565-570; 1981 NovemberDecember.

tunneling; tunnel junction; aluminum; critical current; energy gap; enhancement; microwaves; nonequilibrium superconductivity; photon-assisted tunneling; superconductivity; thin film; transition temperature; 19979.

tunneling corrections; chemical rate constants; Eckart potential; potential barriers; quantum mechanical tunneling calculations; $J$. Res. 86(4): 357-359; 1981 July-A ugust.

tunnel junction; aluminum; critical current; energy gap; enhancement; microwaves; nonequilibrium superconductivity; photon-assisted 
tunneling; superconductivity; thin film; transition temperature; tunneling; 19979.

tunnel junction; aluminum; energy gap; gap enhancement; microwaves; nonequilibrium; superconductivity; 20723.

turbine blade failures; blade vibration; Doppler signature; Doppler technique; high cycle fatigue; stress corrosion; SP622: 1981 October. 181-194.

turbo-Brayton; Vuilleumier; component development; contamination control; cryogenics; helium retention; long-life; refrigerators; reliability; rotary-reciprocating; SP607: 1981 May. 21-29.

turbocharging monitoring; crankshaft bearing safety; medium speed diesel engine; piston ring wear; SP622: 1981 October. 97.112.

turbomachinery; aerothermal diagnostics; aerothermal parameters; performance; physical deterioration; reliability; SP622: 1981 October. 222-253.

turbulence; nonequilibrium molecular dynamics; nonlinear thermodynamics; shear flow; shear induced phase transition; soft sphere system; stability; steady state; 20063.

turbulent burning; ceilings; experiments; flame size; temperature distribution; NBS-GCR-79-188.

two-dimensional profiles; arsenic ion implantation; electron-beaminduced current; etching; integrated circuit processing; ion channeling; ion implantation; lateral profiles; scanning electron microscopy; silicon technology; spreading resistance; NBSIR 812398.

two-level system; multimode laser; multiphoton ionization; $\mathrm{Na} 3 \mathrm{~s} \rightarrow 3 \mathrm{p}$; pulse shapes; Rabi oscillation; 20061.

two nested, concentric pistons; cylinder; helium; inner piston; outer piston; regenerator gaps; single stage, twin piston cryogenic refrigerator; U.S. Patent 4,281,517.

two-photon absorption; band structure; effective mass; intraband momentum matrix element; oscillator strength; SP620: 1981 October. 438.445.

two-photon absorption; band-structure; nonlinear absorption; oscillator strength; SP620: 1981 October. 432.437.

typesetting composition; ADP terminals; ASCII; character-imaging devices; control functions; data interchange; display terminals Federal Information Processing Standard; information interchange; printer terminals; standard; text communication; text interchange; FIPS PUB 86.

$t$-2-fluorovinyl; acetylene; $\mathrm{F}$ atoms; hydrogen bonding; infrared spectrum; matrix isolation; photodecomposition; reaction mechanism; 20036.

\section{U}

UHMWPE; ultra high molecular weight polyethylene; creep; failure; fatigue; mechanical properties; morphology; polymer; stress relaxation; NBSIR 81-2209 (FDA).

ultrafine filament $\mathrm{Nb}_{3} \mathrm{Sn}$; bronze process; $\mathrm{Nb}_{3} \mathrm{Sn}$; processing limits; 20741.

ultra high molecular weight polyethylene; creep; failure; fatigue; mechanical properties; morphology; polymer; stress relaxation UHMWPE; NBSIR 81-2209 (FDA).

ultrasonic inspection; ultrasonic waves; acoustic waves; elastic anisotropy; elastic constants; Kroner method; sound velocities; 20773.

ultrasonic inspection; United States experiences; low alloy steels; steam turbine component failure; stress corrosion cracking; SP622 1981 October. 152-180.

ultrasonics; abstracts; acoustic emission; bibliography; eddy currents; index; National Bureau of Standards; nondestructive evaluation publications; radiography; NBSIR 80-2080.

ultrasonics; visual-optical; acoustic emission; eddy currents; liquid penetrants; magnetic particles; microwaves; nondestructive evaluation; radiography; tire inspection; 20762

ultrasonic scattering; ultrasonic waves; acoustic waves; elastic anisotropy; nondestructive evaluation; nondestructive testing; stainless steel; 20744.

ultrasonic waves; acoustic waves; elastic anisotropy; elastic constants Kroner method; sound velocities; ultrasonic inspection; 20773. ultrasonic waves; acoustic waves; elastic anisotropy; nondestructive evaluation; nondestructive testing; stainless steel; ultrasonic scattering; 20744.

ultra-thin-windowed (UTW) Si(Li) detectors; windowless detectors; $\mathrm{x}$-ray transmission; electron excitation; energy-dispersive spectrometry; energy rcsolution; peak shapes; surface-layer analyses; SP604: 1981 June. 97-126.

ultraviolet reflectors; excimer lasers; fluoride coatings; laser damage; multiple-shot damage; oxide coatings; pre-irradiation; thin films; SP620: 1981 October. 265-276.

ultraviolet spectra; binary stars; late-type stars; stars, individual; stellar chromospheres; stellar coronae; 20001.

ultraviolet, spectra; stars, binaries; stars, chromospheres; stars, emission line; stars, late-type; 19966.

ultraviolet transmission; fluorides; forging; laser windows; lithium fluoride; mechanical properties; optical properties; SP620: 1981 October. 110-116.

umbilical vein grafts; vascular prosthesis; SP601: 1981 January. 449. 469.

uncertainty; collector rating; measurement; solar collector; standards thermal performance; TN1140.

uncertainty estimate; building energy monitoring; energy measurement; steam consumption measurement; NBSIR 81-2313.

uncollimated fluorescing radiation; analytical electron microscope; electron-optical column; energy dispersive spectrometer; SP604: 1981 June. 351-364.

underground; alloys; corrosion; metallurgically-bonded; metals; plastic-bonded; soils; telephone cables; NBSIR 81-2243.

underground corrosion; earth resistivity; redox potential; soil corrosivity; soil pH; soil resistivity; 19963.

underground radar; void detection; adobe building materials; adobe soil; dielectric constant measurements; electromagnetic moisture measurements; nondestructive evaluation; radar soil measurements; soil moisture; NBSIR 79-1610.

underground systems; computer program; earth temperature; heat transfer; pipes; thermal insulation; thermal properties; NBSIR 81 . 2378

underground transmission; aging; cables; dielectrics; electric insulation fault location; time-domain-reflectometry; NBSIR 81-2235.

understandability; communication; evaluation method; fire-safety hazard warnings; meaningfulness; method; pictogram; response symbol; NBSIR 80-2088.

understandability; visibility; visual alerting; directional arrows; exit signs; fire safety; pictograms; symbols; NBSIR 81-2268.

UNISKEL; universal skeleton; benchmarks; interactive workloads; SP500-83: 1981 November. 143.156.

unit cell; computer evaluation; crystallographic data analysis; JCPDS-International Centre for Diffraction Data; metric symmetry; NBS Crystal Data Center; powder diffraction; TN1141.

United Stand; alternative; California; Class K code; conservation; discrimination; energy; SP608: 1981 May. 83.103.

United States experiences; low alloy steels; steam turbine component failure; stress corrosion cracking; ultrasonic inspection; SP622: 1981 October. 152-180.

unit pricing; weighmaster law; basic weights and measures law; method of sale of commodities; open dating; packaging and labeling; registration of service persons; H130, 1982 Edition.

UNIVAC; UNIVAC 1100 OS; facilities complex; performance parameters; SP500-83: 1981 November. 251-258.

UNIVAC 1100 OS; facilities complex; performance parameters; UNIVAC; SP500-83: 1981 November. 251.258

universal skeleton; benchmarks; interactive workloads; UNISKEL; SP500-83: 1981 November. 143-156.

UNIX; macroprocessor; pattern matching; query processor; software tool; text processor; translation; NBSIR 80-2182.

UNIX; terminal-probe method; SP500-83: 1981 November. 157.168.

unlimited application; fibrescope; health condition; man-hour expenditure ratio; trend analysis; SP622: 1981 October. 74-81

unsaturated polyesters; acrylates; acrylic resins; aminoglutethimide or para-aminophenylacetic acid; cementing of bone; fillings; methacrylates; tertiary aromatic amines; U.S. Patent $4,243,763$. 
unsaturated polyesters; vinyl monomers; accelerators; cementing of bone; high reactivity; low toxicity; peroxide catalyzed polymerization; tertiary aromatic amines; U.S. Patent 4,284,551.

unsteady; characteristics; discharge; drainage; flow; model; plumbing; quasi-steady; J. Res. 86(2): 171-179; 1981 March-April.

unsteady; volume; water; diameter; drain; flow; history; horizontal; length; partially-filled; pipe; slope; stream-depth; NBSIR 81-2249.

unsteady partially-filled pipe flow; waste solid transport; building drainage; numerical solutions for transient depth; NBSIR 81-2308.

upholstered furniture; burning rate; case goods; chairs; furniture fires; ignitability; room fires; NBSIR 81-2271.

upholstered furniture; cigarettes; flammability; ignition; mattresses; self-extinguishment; smoldering; NBSIR 81-2363.

uranium; cesium; metal accumulation; microbial; radium; SP618: 1981 November. 274-286.

uranium; chemolithotrophic-bacteria; copper; iron; leaching; metalreduction; metal-sulfides; mineral-oxidation; molybdenum; oil shale; Sulfolobus; Thiobacillus; SP618: 1981 November. 264-273.

uranium; wavelengths; energy levels; parametric calculations; 20002.

uranium compounds; actinium compounds; cesium compounds; enthalpy; entropy; francium compounds; Gibbs energy of formation; heat capacity; lithium compounds; potassium compounds; protactinium compounds; rubidium compounds; sodium compounds; thorium compounds; TN270-8.

uranium spectrum; wavelength calibration; spectroscopic reference wavelengths; 20730.

urea-formaldehyde thermal insulation; cellulose thermal insulation; corrosion of electrical outlet boxes and devices; electrical devices; humidity, thermal insulation and corrosion of electrical wiring; shock hazards; NBSIR 81-2220.

usage patterns; water consumption; clothes dryer usage characteristics; clothes washer usage characteristic; data profiles; dishwasher usage characteristic; energy consumption; field measurements; NBSIR 80-2136.

user behavior; workload forecasting; computer networks; long-range forecasting; SP500-83: 1981 November. 7-14.

user manual; automated thermogravimetry; data analysis; generalized plotting programs; interdata 7-16 computer; polynominal fitting; NBSIR 80-2102.

user needs; dwelling units (residential); housing; Operation Breakthrough; post-occupancy housing evaluation; Project Feedback; questionnaires; research methods; survey research; NBSIR 81-2258.

user productivity; building productivity; computer-integrated construction; conference; construction duration; productivity measurement; risks of failure; NBS-GCR-81-331.

user requirements; computer resource selection; cost; long range plan; system requirements; SP500-83: 1981 November. 293-302.

user service reporting; ADP installation management; cost-benefit; performance management; productivity; SP500-83: 1981 November. 59.65.

\section{V}

vacancy flow; vacancy wind effect; atom jump frequencies; correlation factor for diffusion; diffusion coefficient; diffusion equations; drift velocity for diffusion; driving force for diffusion; 20038.

vacancy wind effect; atom jump frequencies; correlation factor for diffusion; diffusion coefficient; diffusion equations; drift velocity for diffusion; driving force for diffusion; vacancy flow; 20038.

vacuum chamber; copper mirrors; $\mathrm{CO}_{2}$ laser; multishot damage; nanosecond pulse; peak energy density; pulse distortion; single-shot damage threshold; SP620: 1981 October. 238.247.

vacuum deposition techniques; wave front distortion; film thickness nonuniformity; high power laser mirrors; multilayer dielectric films; optical figure measurement; SP620: 1981 October. 356-368.

vacuum feedthrough; electrical connector; electrical feedthrough; 20748.

vacuum ultraviolet; $I_{2}$ fluorescence absorption coefficient; $\mathrm{Kr}$ photosensitized reaction; methylene iodide; photodissociation;
20042.

validation; analytic models; communication networks; flow control; Markov processes; performance prediction; queueing models; simulation; SP500-83: 1981 November. 277-290.

validation; assessment; composite models; data quality; energy models; mathematical models; model confidence; model credibility; policy models; sensitivity analysis; SP616.

validation; Building Energy Performance Standards (BEPS); energy calculations; HVAC systems and equipment; 20758.

validation; verification; automated software tools; software lifecycle; software testing; software verification; test coverage; test data generation; SP500-75.

valve prostheses; valvular dysfunction; mechanical heart; SP601: 1981 January. 269-282.

valvular dysfunction; mechanical heart; valve prostheses; SP601: 1981 January. 269-282.

valvular substitute; bioprosthesis; dysfunction patterns; SP601: 1981 January. 363-393.

Van de Graaff accelerator; $14 \mathrm{MeV}$ neutron energy; linear accelerator;. neutron imaging techniques; position-sensitive proportional counters; 20724.

Van der Waals theory; viscosity; data evaluation; extended corresponding states; fluids; mixtures; one fluid model; prediction; 20768 .

vapor detector; vapor generator; vapor standards; accelerants; arson; combustible gas; detectors; diffusion rates; diffusion tubes; gas chromatography; hydrocarbons; SP480-43.

vapor generator; vapor standards; accelerants; arson; combustible gas; detectors; diffusion rates; diffusion tubes; gas chromatography; hydrocarbons; vapor detector; SP480-43.

vaporization; chemical activity; magnetohydrodynamics; mass spectrometry; potassium slag; thermodynamics; NBSIR 81-2293.

vapor pressure; alkanes; aromatics; critical review, evaluated data; gases; halogenated hydrocarbons; Henry's law constants; liquids; pesticides, solids; solubility; JPCRD 10(4): 1175-1199; 1981.

vapor pressure; critical region; density; equation of state; geothermal; isobutane; thermodynamic; NBSIR 79-1715.

vapor standards; accelerants; arson; combustible gas; detectors; diffusion rates; diffusion tubes; gas chromatography; hydrocarbons; vapor detector; vapor generator; SP480-43.

vapor transport; alkali vapor; coal conversion; combustion systems; NBSIR 81-2279.

vapor transport; coal gasification; high temperature; mass spectrometry; slag; transpiration; NBSIR 80-2178 (DoE).

vascular prosthesis; umbilical vein grafts; SP601: 1981 January. 449. 469.

vegetable and animal life; antifouling coating; film; marine structures; piezoelectric polymer material; U.S. Patent 4, 283,461.

vegetable and animal life; antifouling coating; film; marine structures; piezoelectric polymer material; U.S. Patent 4,297,394.

velocity; adiabatic compressibility; liquid; pressure; propane; sound; specific heat ratios; speed; temperature; J. Res. 86(2): 165-170; 1981 March-April.

velocity; volume; water; cylindrical; non-uniform; partially-filled; pitched-horizontal drain; slope; solid; NBSIR 81-2266.

velocity of sound; virial coefficients; electrostatic transducers; gas; nitrogen; sonic cavity; thermodynamic properties; 20072.

vendor performance; benchmarking; FIPS PUB 75; SP500-83: 1981 November. 339.

vent dampers; water heaters; windows; automatic ignition devices; caulks; effectiveness; energy conservation; energy conservation measures; installation; insulation; oil burners; practices; safety; sealants; standards; storm doors; storm windows; SP606.

ventilated stair; automatic sprinklers; cooling efficiency; evaporation cooling; smoke movement; spray nozzle; stairway protection; NBSIR 81-2202.

ventilation; windows; energy conservation; lighting; man/environment research; noise; office building; post-occupancy evaluation; questionnaire; temperature; $B S S 130$.

ventilation cloth; ASTM E-162; brattice cloth; coal mining; fire test; flame spread; sample mounting; NBSIR 81-2200. 
ventilation systems; ships; smoke control; smoke movement; stack effect; sulfur hexafluoride; tracer tests; NBSIR 81-2433.

verification; automated software tools; software lifecycle; software testing; software verification; test coverage; test data generation; validation; SP500-75.

verification; building code; building envelope; comparison; compliance; energy budget; energy conservation; performance standards; Statement of Review; SP608: 1981 May. 57-67.

vertical current density; Wilson plates; high voltage dc; measurement errors; transmission lines; NBSIR 81-2410.

vertical stack drain entry; building drainage; supercritical flow; transition length; NBSIR 81-2290.

vibration; core barrel motion; neutron detectors; reactor internals; surveillance; SP622: 1981 October. 3-10.

vibrational spectroscopy; carbon monoxide; chemisorption; isocyanide; rhodium; 19997.

vibration analysis; acoustic emission analysis; insurance; loss prevention; modal analysis; property conservation; risk management; shock pulse analysis; SP622: 198I October. 139-151.

vibration control; vibration isolation; active vibration control; Michelson interferometer; optical path-length correction; phase comparator; real-time control; 20734.

vibration exciters; acceleration; accelerometers; calibration; shakers; 20731.

vibration isolation; active vibration control; Michelson interferometer; optical path-length correction; phase comparator; real-time control vibration control; 20734.

vibration monitoring; coal conversion; mechanical components; SYNTHANE pilot plant; SP622: 1981 October. 274-288.

vibrations; base line signatures; frequency spectra; hot alignment machinery parameters; temperatures; SP622: 1981 October. 220-221

victimization survey; burglary; crime in public housing; household behavior patterns; housing characteristics (physical); housing security; public housing security; security hardware; NBS-GCR-80291.

victim role; deed; developers; energy wars; experimental code; field test; guideline; low income; non-code; policymaking; protestors; solar code; SP608: 1981 May. 173-178.

videodiscs; computer network interconnection; computer network protocol; digital transmission of information; electronic message transmission; image transmission; information networks; national bibliographic network; satellite communications; slow-scan television; telecommunications policy; teleconferencing; SP610.

video teleconferencing; CATV systems; coaxial cable; data communications; fiber optics information systems; integrated communications; local area networks, microwave; SP500-83: 198 November. 271.276.

vinyl monomers; accelerators; cementing of bone; high reactivity; low toxicity; peroxide catalyzed polymerization; tertiary aromatic amines; unsaturated polyesters; U.S. Patent 4,284,551.

virial coefficients; electrostatic transducers; gas; nitrogen; sonic cavity; thermodynamic properties; velocity of sound; 20072.

virtual terminal protocols; communication protocols; computer network protocols; feature analysis; network architecture; network virtual terminal protocols; NBS-GCR-81-324.

viscosity; aqueous solutions; compilation; potassium chloride; reference data; JPCRD 10(1): 57.70; 1981 .

viscosity; aqueous solutions; compilation; reference data; sodium chloride; JPCRD 10(1): 71-88; 1981.

viscosity; argon; dynamics; $m-6-8$ potential; nonequlibrium molecular; shear; 19973

viscosity; computer program; density; extended corresponding states; fluid mixtures; thermal conductivity; TN1039.

viscosity; data evaluation; extended corresponding states; fluids; mixtures; one fluid model; prediction; Van der Waals theory; 20768.

visibility; visual alerting; directional arrows; exit signs; fire safety; pictograms; symbols; understandability; NBSIR 81-2268.

vision; conspicuity; contrast; illumination; lighting; lighting levels; superthreshold visibility; NBSIR 81-2231.

visual acuity; evolution; fracture mechanics; gamma rays; inventors; molecular interactions; molecular proccsses; neomycin; neutron dosimeters; polymers; radiography; SRM's; stars; DIM/NBS 65(7), $1-24 ; 1981$

visual alerting; directional arrows; exit signs; fire safety; pictograms; symbols; understandability; visibility; NBSIR 81-2268.

visual alerting; warnings; communication; hazard; pictograms; safety; signs; standards; symbols; NBSIR 80-2003.

visual inspection; visual testing; acuity tests; nondestructive testing; quality testing; radiograph evaluation; TN1143.

visual-optical; acoustic emission; eddy currents; liquid penetrants; magnetic particles; microwaves; nondestructive evaluation; radiography; tire inspection; ultrasonics; 20762.

visual testing; acuity tests; nondestructive testing; quality testing; radiograph evaluation; visual inspection; TN1143.

vitamin K-dependent; calcification; calcific degeneration; porcine xenograft valves; prosthesis failure; SP601: 1981 January. 339-348.

void detection; adobe building materials; adobe soil; dielectric constant measurements; electromagnetic moisture measurements; nondestructive evaluation; radar soil measurements; soil moisture; underground radar; NBSIR 79-1610.

Voigt function; argand diagram; complex error function; continued fraction; Dawson's function; FORTRAN subroutine; Fresnel integrals; key values; line broadening function; plasma dispersion function; J. Res. 86(6): 661-686; 1981 November-December

volatilization; arsenic; dimethylarsinic acid; microbial growth; mobilization; nutrients; oil shale; retorting; soil; speciation; SP618 1981 November. 286-300.

voltammetry; zinc-NTA; cadmium-NTA; convolution voltammetry; electrochemistry; Kalman filter; metal speciation; modeling semiderivative; stability constants; SP618: 1981 November. 163-172. volume; dimensions; laser; LNG; ships; tanks; NBSIR 80-2141.

volume; water; cylindrical; non-uniform; partially-filled; pitched horizontal drain; slope; solid; velocity; NBSIR 81-2266.

volume; water; diameter; drain; flow; history; horizontal; length; partially-filled; pipe; slope; stream-depth; unsteady; NBSIR 81-2249.

volume and surface impurities; $\mathrm{BaF}_{2} ; \mathrm{CaF}_{2}$; extrinsic and intrinsic infrared absorption; infrared wavelength modulation; $\mathrm{LaF}_{3}$; laser windows; $\mathrm{LiF} ; \mathrm{MgF}_{2} ; \mathrm{MgO} ; \mathrm{NaCl} ; \mathrm{NaF} ; \mathrm{SrF}_{2} ; \quad S P 620: 1981$ October. 29.43.

volume calibration; accountability; differential pressure; splines statistics; NBSIR 80-2151.

volume-measuring devices; weighing devices; weights; lengthmeasuring devices; liquid-measuring devices; measures; scales; specifications; taximeters; tolerances; H44, 1982 Edition.

voluntary program; certification; consumer protection; Interstate Solar Coordination Council; labeling procedures; national testing program; regulation; solar collector; standards; SP608: 1981 May 179-181.

voluntary standards; bibliography; annotated, consumer representation; literature review; medical device standards; public participation programs; standards development; NBSIR 81-2336.

Vuilleumier; component development; contamination control; cryogenics; helium retention; long-life; refrigerators; reliability rotary-reciprocating; turbo-Brayton; SP607: 1981 May. 21.29.

Vuilleumier cryocoolers; cryogenics; Gifford-McMahon cryocoolers; heat capacity; heat transfer; helium; refrigerators; regenerators; Stirling cryocoolers; thermal conductance; 20766.

Vuilleumier cryocoolers; cryogenics; Gifford-McMahon cryocoolers; heat capacity; heat transfer; helium; refrigerators; regenerators; Stirling cryocoolers; thermal conductance; SP607: 1981 May. 70-81.

\section{W}

wafer chuck; wafer probe station; integrated circuit test methods; microelectronic test structure; probe station; semiconductor process control; test pattern; test structure; SP400-68.

wafer probe station; integrated circuit test methods; microelectronic test structure; probe station; semiconductor process control; test pattern; test structure; wafer chuck; SP400-68.

wakefulness; adults; alarm responses; auditory perception; decibel levels; fire departments; frequency distribution; noise (sound); sleep; 
smoke detectors; NBS-GCR-80-284.

walls; water source heat pump; buildings; construction; energy conservation; ground coupled; hybrid solar energy system; inverted cave; low grade geothermal; radiant floor heating; space heating and cooling; thermal mass; SP608: 1981 May. 69-81.

warning light; warning signals; conspicuity; effective intensity; emergency warning lights; flashing lights; lights, warning; sirens; sound level; SP480-37.

warnings; communication; hazard; pictograms; safety; signs; standards; symbols; visual alerting; NBSIR 80-2003.

warning signals; conspicuity; effective intensity; emergency warning lights; flashing lights; lights, warning; sirens; sound level; warning light; $S P 480-37$.

WASER; winding dilemma; density wave; galactic spirals; hydrodynamic instability and turbulence; plasma dynamics; spiral grand design; J. Res. 86(6): 557.563; 1981 November-December.

waste cycling; bacterial mobilization; biogeochemical cycles; biological uptake; cellular toxicity-essentiality mechanisms; chromatography; element-selective speciation; environmental monitoring; fossil fuels; metal(loid) complexes; organometallic compounds; trace element standards; SP618.

waste solid transport; building drainage; numerical solutions for transient depth; unsteady partially-filled pipe flow; NBSIR 81-2308.

waste solid transportation; water conservation; w.c. efficiency; building drainage; NBSIR $81-2307$.

wastewater disposal; wastewater recirculation; wastewater reuse; wastewater treatment; water conservation; onsite wastewater systems; NBSIR 81-2210.

wastewater recirculation; wastewater reuse; wastewater treatment; water conservation; onsite wastewater systems; wastewater disposal; NBSIR 81-2210.

wastewater reuse; wastewater treatment; water conservation; onsite wastewater systems; wastewater disposal; wastewater recirculation; NBSIR 81-2210.

wastewater treatment; water conservation; onsite wastewater systems; wastewater disposal; wastewater recirculation; wastewater reuse; NBSIR 81-2210.

wastewater treatment; water rate schedules; water-saving devices; benefits; cost-effective; costs; economic analysis; economic decision framework; energy conservation; marginal price; net present value; residential water conservation; NBSIR 81-2304.

water; air; environmental measurements; methods; quality assurance; standards; NBSIR 81-2280

water; analysis; assay; evaluation; radioactivity; radiochemistry; radium-228; reference method; $T N 1137$.

water; cylindrical; non-uniform; partially-filled; pitched-horizontal drain; slope; solid; velocity; volume; NBSIR 81-2266.

water; diameter; drain; flow; history; horizontal; length; partially. filled; pipe; slope; stream-depth; unsteady; volume; NBSIR 81-2249.

water; generator column; hydrophobic; octanol; partition coefficient; propylbenzene; solubility; solute; J. Res. 86(4): 361-366; 1981 JulyAugust.

water; water substance; equation; formulation; high pressure; high temperature; IAPS; International Association for the Properties of Steam; ionization; ionization constant; ion product; review; JPCRD 10(2): 295-304; 1981.

water closets; water conservation; dual flush toilets; low flush toilets; NBSIR 81-2296.

water conservation; dual flush toilets; low flush toilets; water closets; NBSIR 81-2296.

water conservation; onsite wastewater systems; wastewater disposal; wastewater recirculation; wastewater reuse; wastewater treatment; NBSIR 81-2210.

water conservation; water consumption; appliances; clothes washer; discharge; dishwasher; residential; supply; NBSIR 80-2173.

water conservation; water discharge characteristics; clothes washers; dishwashers; dishwasher subcycles; fill controls; grey water; performance; soil removal; NBSIR 81-2291 (HUD).

water conservation; w.c. efficiency; building drainage; waste solid transportation; NBSIR 81-2307.

water consumption; appliances; clothes washer; discharge; dishwasher; residential; supply; water conservation; NBSIR 802173.

water consumption; clothes dryer usage characteristics; clothes washer usage characteristic; data profiles; dishwasher usage characteristic; energy consumption; field measurements; usage patterns; NBSIR 80-2136.

water discharge characteristics; clothes washers; dishwashers; dishwasher subcycles; fill controls; grey water; performance; soil removal; water conservation; NBSIR 81-2291 (HUD).

water glycol; coal mine; fire tests; hydraulic fluids; invert emulsions; pressure-temperature; spray; synthetics; NBSIR 81-2373.

water heater; computer model; distribution pipe; dynamic response; electricity; energy; explicit method; faucet temperature; finite difference; heat loss; implicit method; insulation; NBSIR 81-2303.

water heaters; windows; automatic ignition devices; caulks; effectiveness; energy conservation; energy conservation measures; installation; insulation; oil burners; practices; safety; sealants; standards; storm doors; storm windows; vent dampers; SP606.

water-heating solar collectors; collector efficiency; comparison German BSE vs ASHRAE 93-77 procedures; flat-plate solar collectors; German Bundesverband Solarenergie; solar collector testing; 20079.

water phantom; absorbed dose standard; cobalt-60 gamma rays; electron density; graphite calorimeter; ionization chamber; scaling theorem; J. Res. 86(5): 495.502; 1981 September-October.

water pollution control; measurement methods; oil shale; standard reference materials; 20009.

water rate schedules; water-saving devices; benefits; cost-effective; costs; economic analysis; economic decision framework; energy conservation; marginal price; net present value; residential water conservation; wastewater treatment; NBSIR 81-2304.

water-saving devices; benefits; cost-effective; costs; economic analysis; economic decision framework: energy conservation; marginal price; net present value; residential water conservation; wastewater treatment; water rate schedules; NBSIR 81-2304.

water source heat pump; buildings; construction; energy conservation; ground coupled; hybrid solar energy system; inverted cave; low grade geothermal; radiant floor heating; space heating and cooling; thermal mass; walls; SP608: 1981 May. 69.81.

water substance; equation; formulation; high pressure; high temperature; IAPS; International Association for the Properties of Steam; ionization; ionization constant; ion product; review; water; JPCRD 10(2): 295-304; 1981.

water vapor; corona; decomposition; electrical insulation; gas chromatograph; inception voltages; mass spectrometer; partial discharge; sulfur hexafluoride; swarm data; NBSIR 81-2242.

waveform; acoustic emission; calibration; development; transducer; 20757.

waveform; bit error rate; coaxial transmission lines; digital communication impulse response; instrumentation; measurements; shielded balanced transmission lines; time domain; transmission line model; TN1042.

waveforms; deconvolution; impulse response; inverse filtering; noise contamination; time domain; TN1047.

wave front distortion; film thickness nonuniformity; high power laser mirrors; multilayer dielectric films; optical figure measurement; vacuum deposition techniques; SP620: 1981 October. 356.368.

waveguides; fiber optics; nonlinear propagation; nonlinear pulses; selffocusing; solitons; SP620: 1981 October. 453-458.

wavelength calibration; spectroscopic reference wavelengths; uranium spectrum; 20730.

wavelength dependence; laser damage; optical constants; optical materials; semiconductors; stimulated bremsstrahlung absorption; SP620: 1981 October. 416-426.

wavelength dispersive $\mathrm{x}$-ray analysis; corrosion; stainless steel implants; SP601: 1981 January. 223-235.

wavelength of light in air; air density; index of refraction of air; refractivity of air; J. Res. 86(1): 27-32; 1981 January-February.

wavelengths; atom; atomic ion; intensities; line strengths; oscillator strengths; spectral lines; transition probabilities; NSRDS-NBS68. wave-lengths; $\mathrm{CD}_{2} \mathrm{~F}_{2} ; \quad \mathrm{CO}_{2}$ laser; FIR laser; laser frequency 
measurement; relative polarization; relative power; 20743.

wavelengths; energy levels; parametric calculations; uranium; 20002.

wave structure; boric acid; cellulosic insulation; ignition; propagation; smoldering combustion; test methods; NBSIR 81-2350.

w.c. efficiency; building drainage; waste solid transportation; water conservation; NBSIR 81-2307.

weak links; closed cycle refrigeration systems; Josephson effects; proximity effect devices; superconducting devices; tunnel devices; SP607: 1981 May. 195-209.

wear; accelerator; alloy; base metal; casting; composite; cyanoacrylate; dental alloy; initiator; resin; NBSIR 80-2168.

wear; polyethylene acetabulae; polymeric prosthesis; SP601: 1981 January. 471-499.

wear; wear analysis; energy; energy conservation; failure detection; failure diagnosis; failure prevention; monitoring techniques; prognosis; sensors; SP622.

wear analysis; energy; energy conservation; failure detection; failure diagnosis; failure prevention; monitoring techniques; prognosis; sensors; wear; SP622.

wear debris; artificial joints; biomaterials; SP601: 1981 January. 239. 267.

wear monitoring; chip detectors; condition monitoring; failure detection; helicopter diagnostics; oil debris; SP622: 1981 October. 33.49 .

weatherization; weatherization and thermal comfort; Community Services Administration; field study; occupant comfort; optimal weatherization demonstration; thermal comfort; thermostat setting practices; NBSIR 81-2335.

weatherization and thermal comfort; Community Services Administration; field study; occupant comfort; optimal weatherization demonstration; thermal comfort; thermostat setting practices; weatherization; NBSIR 81-2335.

weatherization retrofitting; comfort; Community Services Administration Optimal Weatherization Demonstration; energy savings; field study; thermal comfort; 20752.

weighing devices; weights; length-measuring devices; liquid-measuring devices; measures; scales; specifications; taximeters; tolerances; volume-measuring devices; H44, 1982 Edition.

weighmaster law; basic weights and measures law; method of sale of commodities; open dating; packaging and labeling; registration of service persons; unit pricing; H130, 1982 Edition.

weights; length-measuring devices; liquid-measuring devices; measures; scales; specifications; taximeters; tolerances; volumemeasuring devices; weighing devices; H44, 1982 Edition.

weights and measures; domestic standards; education programs; electronic devices; international recommendations; legal metrology; measurement assurance; metrication; model laws and regulations; packing and labeling; specifications and tolerances; technology transfer; SP599.

weights and measures; International System of Units; measurement systems; metric system; modernized metric system; SI; SP304, Revised August 1981.

weights and measures; International System of Units; measurement systems; metric system; modernized metric system; SI; SP304A, Revised August 1981.

weights and measures; x-ray diffraction; air pollutants; antenna coupling; electrical distribution; glass-liquidus; insulation; monitors; neutron diffraction, samplers; SRM's; time-domain calibrations; $D I M / N B S$ 65(1): 1-28; 1981.

weights and measures regulation; Agency for International Development; Arab League Organization for Standardization and Metrology; chemical industry standards; cotton standards; food standards; measurement science and technology; standardization in developing countries; Sudan; NBSIR 80-2020.

welding; alloys; aluminum alloys; castings; composites; cryogenic properties; low temperature; mechanical properties; nonmetallics; physical properties; stainless steels; structural alloys; NBSIR 811645.

welding; composites; low temperature; mechanical and physical properties; structural alloys; NBSIR 80-1627.

wetting; adsorption; Auger spectroscopy; convection; ellipsometry; gallium; levitation calorimetry; specific heat; surface tension; thermophysical properties; tungsten; NBSIR 81-2295.

White House; coatings; exterior restoration; field tests; guide specifications; laboratory tests; paint removal methods; NBSIR 8(). 2122.

whole-house ventilation; building thermal performance; energy calculation; energy conservation; thermal comfort; TN1138.

Wick Test; coal mines; flammability; hydraulic fluids; ignition; NBSIR 81-2312.

Wilson plates; absolute filter; current density; high voltage dc transmission lines; ion counter; space charge density; NBSIR 81 2267.

Wilson plates; high voltage dc; measurement errors; transmission lines; vertical current density; NBSIR 81-2410.

wind; climatclogy; loads (forces); probability distribution functions; reliability; statistical analysis; structural engineering; 20019.

wind; wind speed; building performance; hurricane winds; structural damage; structures; 20759.

wind energy; wind turbine data systems; wind turbine generators; wind turbine performance; horizontal axis wind turbines; SP622: 1981 October. 257-273.

wind forces; buoyancy forces; flood forces; foundations; hurricane forces; mobile home; soil anchors; standards; tiedown; BSS 132.

winding dilemma; density wave; galactic spirals; hydrodynamic instability and turbulence; plasma dynamics; spiral grand design; WASER; J. Res. 86(6): 557.563; 1981 November-December.

wind loads; earthquake loads; hail loads; snow loads; solar collectors; structural engineering; NBSIR 81-2199.

window absorption; window thickness; window transmission; x-ray detectors; x-ray energy spectrometers; dead-layer; SRM-477; SP604: 1981 June. 61.70.

windowless detectors; energy dispersive; light elements; soft $\mathrm{x}$ rays; spectral distortion; SP604: 1981 June. 71-95.

windowless detectors; x-ray transmission; electron excitation; energydispersive spectrometry; energy resolution; peak shapes; surfacelayer analyses; ultra-thin-windowed (UTW) Si(Li) detectors; SP604: 1981 June. 97.126.

window management; daylight; energy conservation; fenestration design; illumination; lighting control; solar heat gain; NBSIR 802147.

window materials; elastic coefficients; high-energy laser; interferometric testing; optical distortion; photoelastic coefficients; polycrystalline aggregate; stress birefringence; thermal lensing; SP620: 1981 October. 117.128.

windows; air pollution; automation; buildings; celtified reference materials; computers; construction; data converter; mass comparator; radiation; radiocarbon; soft aluminum; $D I M / N B S$ 65(4): 1-28; 1981.

windows; automatic ignition devices; caulks; effectiveness; energy conservation; energy conservation measures; installation; insulation; oil burners; practices; safety; sealants; standards; storm doors; storm windows; vent dampers; water heaters; SP606.

windows; building economics; daylighting; energy conservation; engineering economics; life-cycle costs; passive solar; regional analysis; thermal efficiency; NBSIR 81-2248.

windows; energy conservation; lighting; man/environment research; noise; office building; post-occupancy evaluation; questionnaire; temperature; ventilation; $B S S 130$.

window thickness; window transmission; x-ray detectors; $x$-ray energy spectrometers; dead-layer; SRM.477; window absorption; SP604: 1981 June. 61-70.

window transmission; $\mathrm{x}$-ray detectors; $\mathrm{x}$-ray energy spectrometers; dead-layer; SRM.477; window absorption; window thickness; SP604: 1981 June. 61-70.

wind sensor; wind speed; anemometer; drag sphere; gust speed; instrumentation; 20059.

wind speed; anemometer; drag sphere; gust speed; instrumentation; wind sensor; 20059.

wind speed; building performance; hurricane winds; structural damage; structures; wind; 20759.

wind tunnels; cryogenic properties; instrumentation; low temperature 
materials; materials properties; metals; nonmetallic materials; NBSIR 79-1624.

wind turbine data systems; wind turbine generators; wind turbine performance; horizontal axis wind turbines; wind energy; SP622: 1981 October. 257-273.

wind turbine generators; wind turbine performance; horizontal axis wind turbines; wind energy; wind turbine data systems; SP622: 1981 October. $257-273$.

wind turbine performance; horizontal axis wind turbines; wind energy; wind turbine data systems; wind turbine generators; SP622. 1981 October. 257.273.

wiring system; electrical fire; electric cable; models; thermal insulation; TN1133.

wood; bibliographies; chimneys; combustion; fire safety; heating equipment; literature reviews; stoves; NBS-GCR-80-298.

wood; building; building codes; building design; earthquakes; engineering; standards; structural engineering; NBSIR 80-2111-7.

wood; building rehabilitation; concrete; electrical; evaluation; HVAC; masonry; plumbing; steel; structural systems; test methods; NBSIR $80-2171$.

wood; building rehabilitation; concrete; electrical; evaluation; HVAC; masonry; plumbing; solar; steel; structural systems; test methods; NBSIR 81-2289.

wood; combustion; decision analysis; fire models; flame spread; human behavior; ignition; polymers; smoke; soot; toxicity; NBSIR 81-2382

wood; combustion products; fire modeling; foam (materials); heat release; plastics; polymers; polymethylmethacrylate; polyoxymethylene; polypropylene; polystyrene; polyurethane polyvinyl chloride; pyrolysis; smoke; NBS-GCR-80-295.

wood; crib fires; experiments; mathematical model; plastic; polyurethane; room fires; NBSIR 80-2054, Vols. I and II.

wood; fireplace inserts; fireplaces; fire prevention; fire safety questionnaires; NBS-GCR-80-292.

wood; zone model; crib fire; plastic; room fire; 20740.

wood structure; codes; concrete; masonry; prohibitions; re examination; thermal storage; SP608: 1981 May. 195-200.

wood structures; fire endurance; fires; mathematical models; reinforced concrete; steel structures; NBSIR 80-2188.

word processing; automation; information management; information technology; productivity; SP5D0-83: 1981 November. 305-307.

word processor; costs and benefits; flexible disk cartridge; information interchange; label and file structure; standard; NBS-GCR-80-307.

workload analysis; workload characterization; workload representation; ADP acquisition; ADP procurement; benchmarking; Federal Information Processing Standards Publication; performance evaluation; FIPS PUB 75.

workload characterisation; cluster analysis; SP500-83: 1981 November. 21.34

workload characterization; workload definition; benchmarking benchmark library; selection of ADP systems; standard benchmarks; synthetic benchmarks; 20747.

workload characterization; workload representation; ADP acquisition; ADP procurement; benchmarking; Federal Information Processing Standards Publication; performance evaluation; workload analysis; FIPS PUB 75.

workload definition; benchmarking; benchmark library; selection of ADP systems; standard benchmarks; synthetic benchmarks; workload characterization; 20747.

workload forecasting; computer networks; long-range forecasting; user behavior; SP5(K)-83: 1981 November. 7-14.

workload representation; ADP acquisition; ADP procurement; benchmarking; Federal Information Processing Standards Publication; performance evaluation; workload analysis; workload characterization; FIPS PUB 75.

workload sizing; benchmark testing; Phase IV; remote terminal emulation; response ume; terminal networks; SPS(1)-83: 1981 November. 169-177.

work surfaces; codes and standards; construction safety; design; finite element; loads; scaffolds; stability; stiffness; strength; structural safety; NBSIR 81-2265. worldwide time; atomic clocks; automatic time comparisons; common-mode cancellation; frequency comparison; global positioning system; remote time measurement; time comparison; time receiver; 20062.

\section{$\mathrm{X}$}

xenon; angular distribution; asymmetry parameter; branching ratios; resonance; 20052.

$x$ ray; automation; powder diffraction; quantitative analysis; $N B S I R$ $81-2229$.

$x$-ray analysis; digital filter; filter-fit method; linear least-squares; SP604: 1981 June. 273.296.

$\mathrm{x}$-ray analysis; $\mathrm{x}$-ray fluorescence; electron probe microanalysis; energy dispersive $x$-ray spectrometry; germanium (lithium) detector; scanning electron microscopy; silicon (lithium) detector; spectral deconvolution; SP604.

$\mathrm{x}$-ray continuum; energy dispersive $\mathrm{x}$-ray microanalysis; NBS theoretical matrix correction; SP604: 1981 June. 365-379.

$\mathrm{x}$-ray detectors; collimation; deadlayer determinations; germanium; monoenergetic photons; partly.resolved doublets; silicon; tailing; SP604: 1981 June. 5-34.

x-ray detectors; x-ray energy spectrometers; dead-layer; SRM-477; window absorption; window thickness; window transmission; SP604: 1981 June. 61-70.

$x$-ray diffraction; air pollutants; antenna coupling; electrical distribution; glass-liquidus; insulation; monitors; neutron diffraction, samplers; SRM's; time-domain calibrations; weights and measures; DIM/NBS 65(1): 1.28; 1981.

X-ray diffraction; crystal structure; densities; lattice constants; powder patterns; reference intensities; standard; Monogr. 25. Section 18.

$\mathrm{x}$-ray diffraction; $\mathrm{x}$-ray fluorescence analysis; austenite in ferrite; austenite standard; electron microprobe; powder metallurgy; quantitative microscopy; standard reference material; SP260-73.

X-ray energy spectrometers; dead-layer; SRM-477; window absorption; window thickness; window transmission; $x$-ray detectors; SP604: 1981 June. 61-70.

$\mathrm{x}$-ray energy spectrometry; pulse-processing; solid-state $\mathrm{x}$-ray detectors; SP604: 1981 June. 141-164.

$\mathrm{x}$-ray fluorescence; electron probe microanalysis; energy dispersive x-ray spectrometry; germanium (lithium) detector; scanning electron microscopy; silicon (lithium) detector; spectral deconvolution; x-ray analysis; SP604.

$x$-ray fluorescence analysis; austenite in ferrite; austenite standard; electron microprobe; powder metallurgy; quantitative microscopy; standard reference material; x-ray diffraction; SP260-73.

$\mathrm{x}$-ray fluorescence analysis; catalysts automotive; energy dispersive analysis; heavy element fluorescence analysis; intrinsic germanium detectors; secondary target fluorescence analysis; source excited fluorescence analysis; SP604: 1981 June. 391-415.

$\mathrm{x}$-ray measurement; $\mathrm{x}$ rays; dosage; dosimetry; health and safety; high voltage; ionization chamber; ionizing radiation; ionometer; radiation quantities and units; radiation standards and protection; radioactivity; radiology; radium; roentgen ray; tubes (x ray); SP625.

$\mathrm{x}$-ray microanalysis; detector; energy dispersive $\mathrm{x}$-ray spectrometry; Gaussian profiles; lithium.drifted silicon (Si(Li)) detector; SP604: 1981 June. 233-272.

x-ray opacification; dental composite resin restorations; finishability; microporous filler; system nontoxicity; U.S. Patent 4,306,913.

$\mathrm{x}$-ray photoelectron spectroscopy (XPS, ESCA); Auger electron spectroscopy (AES); metal-oxide-semiconductor (MOS); secondary ion mass spectrometry (SIMS); semiconductor devices; sputtering; SP4O0-67.

$x$ rays; beta rays; Federal and State laboratories; gamma rays; instrument calibrations; intermediate calibration laboratories; ionizing radiation measurements; measurement accuracies; neutrons; radiation dosimetry; radiation protection; radiation standards; SP603.

$\mathrm{x}$ rays; dosage; dosimetry; health and safety; high voltage; ionization chamber; ionizing radiation; ionometer; radiation quantities and units; radiation standards and protection; radioactivity; radiology; 
radium; roentgen ray; tubes (x ray); x-ray measurement; SP625.

$x$-ray spectra; $x$-ray spectrometry; energy resolution; mercuric iodide detectors; SP604: 1981 June. 45-59.

$\mathrm{x}$-ray spectrochemical analysis; Blodgett-Langmuir pseudo-crystals; Bragg crystal diffraction; energy-dispersive; SP604: 1981 June. 1-4.

$\mathrm{x}$-ray spectrometry; energy resolution; mercuric iodide detectors; $\mathrm{x}$-ray spectra; SP604: 1981 June. 45-59.

$\mathrm{x}$-ray spectrometry, review of scientific instruments; bibliography; electron column instruments; energy dispersive detectors; scanning electron microscopy; SP604: 1981 June. 417.439.

$\mathrm{x}$-ray spectroscopy; germanium; semiconductor detectors; silicon; tailing; SP604: 1981 June. 35-44.

$x$-ray transmission; electron excitation; energy-dispersive spectrometry; energy resolution; peak shapes; surface-layer analyses; ultra-thin-windowed (UTW) Si(Li) detectors; windowless detectors; SP604: 1981 June. 97-126.

\section{Y}

YAG laser; laser simulator; light-emitting diodes; TN103I.

YAG laser; laser simulator; light-emitting diodes; TN1031, Revised.

yield; integrated circuits; microelectronics; process validation wafer; random faults; silicon-on-sapphire; test pattern; test structure; SP400-66.

yield strength; Young's modulus; compressive strength; elongation foam; insulation; low temperature; mechanical properties; proportional limit; shear strength; tensile strength; NBSIR 81-1654.

yield stress; cryogenic properties of materials; iron alloys; low temperature mechanical properties; plastic deformation; temperature effects stainless steel alloys; 20742.

Young's modulus; compressive strength; elongation; foam; insulation; low temperature; mechanical properties; proportional limit; shear strength; tensile strength; yield strength; NBSIR 81-1654.

yttrium; yttrium halides; chemiluminescence; Franck-Condon factors; laser-induced fluorescence; radiative lifetimes; scandium; scandium halides; 19969.

yttrium; yttrium halides; chemiluminescence; Franck-Condon factors; laser-induced fluorescence; radiative lifetimes; scandium; scandium halides; 19978.

yttrium halides; chemiluminescence; Franck-Condon factors; laserinduced fluorescence; radiative lifetimes; scandium; scandium halides; yttrium; 19978.

yttrium halides; chemiluminescence; Franck-Condon factors; laserinduced fluorescence; radiative lifetimes; scandium; scandium halides; yttrium; 19969.

Yukawa potential; Bargmann limits; bound-state estimates; Lieb limits; number of bound states; Schwinger limits; screened Coulomb potential; Sobolev's inequality; J. Res. 86(5): 503-508; 1981 September-October.

\section{$\mathbf{Z}$}

zeolites; adsorption; compressor; cryogenics; molecular adsorption; refrigeration; SP607: 1981 May. 127.135.

zinc; activity coefficient; cadmium; critical evaluation; electrolyte; ethylene bis(trimethylammonium) chloride; ethylene

bis(trimethylammonium) iodide; excess Gibbs energy; osmotic coefficient; solutions; thermodynamic properties; JPCRD 1O(1): $1-56 ; 1981$.

zinc.NTA; cadmium.NTA; convolution voltammetry; electrochemistry; Kalman filter; metal speciation; modeling; semiderivative; stability constants; voltammetry; SP618: 1981 November. 163.172.

zinc selenide; zinc sulfide; absorption; coatings; internal reflection spectroscopy; silicon monoxide; thorium fluoride; SP620: 1981 October. 335.344.

zinc sulfide; absorption; coatings; internal reflection spectroscopy; silicon monoxide; thorium fluoride; zinc selenide; SP620: 1981 October. 335-344.

zirconium dioxide; air-fuel ratio; closed loop fuel metering system; exhaust emissions; exhaust gas oxygen sensor; exhaust gas recirculation; feedback; oxidation catalyst; oxygen partial pressure; stoichiometric; three way catalytic converters; SP622: 1981 October. 62-73.

$\mathrm{ZnS} ; \mathrm{ZrO}_{2} ;$ damage threshold; impurity-induced damage; laser damage; $\mathrm{ThF}_{4}$; thin films; SP620: 1981 October. 277.286.

zone model; crib fire; plastic; room fire; wood; 20740.

$\mathrm{ZrO}_{2}$; damage threshold; impurity-induced damage; laser damage; $\mathrm{ThF}_{4}$; thin films; ZnS; SP620: 1981 October. 277-286.

$1 / \mathrm{f}$ noise; hot carrier noise; noise; noise in devices; noise measurement; quantum noise; SP6I4.

10 micrometer optical components; absorptance; infra-red laser windows; pulsed $\mathrm{CO}_{2}$ laser damage; SP620: 1981 October. 248-255.

$10.6 \mu \mathrm{m}$; attenuators; beam profile; calorimetry; fluence; heat flow; irradiance; measurements of temperature; plasma effects; pulsed laser beams; NBSIR 80-1628.

1.06- $\mu \mathrm{m} ; \mathrm{CO}_{2}$-laser glazing; $\mathrm{CO}_{2}$-laser polishing; fused silica surface; laser damage; laser polishing; surface laser damage; SP620: 1981 October. 180-189.

$14 \mathrm{MeV}$ neutron energy; linear accelerator; neutron imaging techniques; position-sensitive proportional counters; Van de Graaff accelerator; 20724.

2.5 and 25 gram capacity bomb calorimeters; ASTM round robin testing; bomb calorimetry; higher heating value; refused-derivedfuel; sample characterization; sample processing effects; NBSIR 81 2278 .

3-D metrology; ball plate; grid plate; machine geometry; measuring machine; redundancy; NBSIR 79-1752.

3350 DASD; DASD I/O capacity; density of data reference; I/O capacity algorithms; SPS00-83: 1981 November. 231-238.

$4 \mathrm{~K}$ refrigeration; cryogenic equipment; Josephson junction devices; superconducting computers; thermal contact; SP607: 1981 May. 15. 20.

$\Delta C_{p}$ of reaction; calorimetry; $\mathrm{C}_{5} \mathrm{H}_{5} \mathrm{~N}_{5} \mathrm{O}$; density; enthalpy of solution; Gua.HCl.l $\mathrm{H}_{2} \mathrm{O}$; Gua.HCl.2 $\mathrm{H}_{2} \mathrm{O} ;$ Gua.2 $\mathrm{HCl}$; guanine (Gua) guanine hydrochlorides; heat capacity; nucleic acid base; solution calorimetry; thermochemisiry; J. Res. 86(4): 367-382; 1981 July. August.

$v_{2}$ vibration; diode laser; Ebert spectrometer; high resolution; hypochlorous acid ( $\mathrm{HOCl}$ ); infrared spectrum; 20053. 


\section{APPENDIX A. LIST OF DEPOSITORY LIBRARIES IN THE UNITED STATES}

\section{ALABAMA}

Alexander City: Alexander City State Junior College, Thomas D. Russell Library (1967).

Auburn: Auburn University, Ralph Brown Draughon Library (1907).

Birmingham:

Birmingham Public Library (1895).

Birmingham Southern College Library (1932).

Jefferson State Junior College, James B. Allen Library (1970).

Samford University Library (1884).

Enterprise: Enterprise State Junior College, Learning Resources Center (1967).

Florence: University of North Alabama, Collier Library (1932).

Gadsden: Gadsden Public Library (1963).

Huntsville: University of Alabama in Huntsville Library (1964).

Jacksonville: Jacksonville State University Library (1929).

Mobile:

Mobile Public Library (1963).

Spring Hill College, Thomas Byrne Memorial Library (1937).

University of South Alabama Library (1968).

Montgomery:

Alabama State Department of Archives and History Library (1884).

Alabama Supreme Court and State Law Library (1884)

Auburn University at Montgomery Library (1971)-REGIONAL.

Maxwell A.F. Base: Air University Library (1963).

Normal: Alabama Agricultural and Mechanical College, J. F. Drake Memorial Library (1963).

Troy: Troy State University, Lurleen B. Wallace Educational Resources Center (1963)

Tuskegee Institute: Tuskegee Institute, Hollis Burke Frissell Library (1907).

University:

University of Alabama Library (1860)-REGIONAL.

University of Alabama, School of Law Library (1967).

\section{ALASKA}

Anchorage:

Anchorage Municipal Libraries, Z. J. Loussac Public Library (1978).

Supreme Court of Alaska Library (1973).

University of Alaska at Anchorage Library (1961).

Fairbanks: University of Alaska, Elmer E. Rasmuson Library (1922).

Juneau: Alaska State Library (1900).

Ketchikan: Ketchikan Community College Libraty (1970).

\section{ARIZONA}

Coolidge: Central Arizona College, Instructional Materials Center (1973).

Flagstaff: Northern Arizona University Library (1937).

Phoenix:

Department of Library, Archives, and Public Records (unknown)-REGIONAL.

Grand Canyon College, Fleming Library (1978).

Phoenix Public Library (1917).

Prescott: Yavapai College Librarv (1976).

Tempe:

Arizona State University, College of Law Library (1977).

Arizona State University Library (1944).

Thatcher: Eastern Arizona College Library (1963).

Tucson:

Tucson Public Library (1970).

University of Arizona Library (1907)-REGIONAL.

Yuma: Yuma City-County Library (1963).

\section{ARKANSAS}

Arkadelphia: Ouachita Baptist University, Riley Library (1963).

Batesville: Arkansas College, Mabee Learning Resources Center (1963).

Clarksville: College of the Ozarks, Dobson Memorial Library (1925).

Conway: Hendrix College, Olin C. Bailey Library (1903).

Fayet teville:

University of Arkansas Library (1907).

University of Arkansas, School of Law Library (1978).

Little Rock:

Arkansas Library Commission (1978).

Arkansas Supreme Court Library (1962).

Little Rock Public Library (1953).

University of Arkansas at Little Rock Library (1973).

Magnolia: Southern Arkansas University, Magale Library (1956).

Monticello: University of Arkansas at Monticello Library (1956).

Pine Bluff: University of Arkansas at Pine Bluff, Watson Memorial Library (1976).

Russellville: Arkansas Tech University, Tomlinson Library (1925).

Searcy: Harding College, Beaumont Memorial Library (1963).

State University: Arkansas State University, Dean B. Ellis Library (1913).

Walnut Ridge: Southern Baptist College, Felix Goodson Library (1967).

\section{CALIFORNIA}

Anaheim: Anaheim Public Library (1963).

Arcadia: Arcadia Public Library (1975).

Arcata: Humboldt State College Library (1963).

Bakersfield:

California State College at Bakersfield Library (1974)

Kern County Library (1943).

Berkeley:

University of California, Earl Warren Legal Center, Law Library (1963).

University of California, General Library (1907). Carson:

California State University, Dominguez Hill Education Resources Center (1973).

Carson Regional Library (1973).

Chico: California State University at Chico Library (1962).

Claremont: Claremont Colleges' Libraries, Honnold Library (1913)

Coalinga: West Hills Community College Library (1978).

Compton: Compton Library (1972).

Culver City: Culver City Library (1966)

Davis:

University of California at Davis Library (1953).

University of California at Davis, School of Law Library (1972).

Downey: Downey City Library (1963).

Fresno:

California State University at Fresno Library (1962).

Fresno County Free Library (1920).

Fullerton: California State University at Fullerton Library (1963).

Garden Grove: Garden Grove Regional Library (1963).

Gardena: Gardena Public Library (1966).

Hayward: California State University at Hayward Library (1963).

Huntington Park: Huntington Park Library, San Antonio Region (1970).

Inglewood: Inglewood Public Library (1963).

Irvine: University of California at Irvine, General Library (1963).

La Jolla: University of California, San Diego, University Library (1963).

Lakewood: Angelo Iacoboni Public Library (1970). 
Lancaster: Lancaster Regional Library (1967).

La Verne: University of La Verne, School of Law Library (1979).

Long Beach

California State University at Long Beach Library (1962).

Long Beach Public Library (1933).

Los Angeles:

California State University at Los Angeles, John F. Kennedy Memorial Library (1956).

Los Angeles County Law Library (1963).

Los Angeles Public Library (1891).

Loyola University of Los Angeles Library (1933).

Loyola University, School of Law Library (1979).

Occidental College Library (1941).

Pepperdine University Library (1963).

Southwestern University, School of Law Library (1975).

University of California at Los Angeles Library (1932).

University of California at Los Angeles, Law Library (1958).

University of Southern California Library (1933).

University of Southern California, Law Center Library (1978).

Whittier College, School of Law Library (1978).

Menlo Park: Department of the Interior, Geological Survey Library (1962).

Montebello: Montebello Library (1966).

Monterey: U.S. Naval Postgraduate School, Dudley Knox Library (1963).

Monterey Park: Bruggemeyer Memorial Library (1964).

Northridge: California State University at Northridge Library (1958).

Norwalk: Norwalk Public Library (1973).

Oakland:

Mills College Library (1966).

Oakland Public Library (1923).

Ontario: Ontario City Library (1974).

Pasadena:

California Institute of Technology, Millikan Memorial Library (1933).

Pasadena Public Library (1963).

Pleasant Hill: Contra Costa County Library (1964).

Redding: Shasta County Library (1956).

Redlands: University of Redlands, Armacost Library (1933).

Redwood City: Redwood City Public Library (1966).

Reseda: West Valley Regional Branch Library (1966).

Richmond: Richmond Public Library (1943).

Riverside:

Riverside Public Library (1947).

University of California at Riverside Library (1963).

Sacramento:

California State Library (1895)-REGIONAL.

California State University at Sacramento Library (1963).

Sacramento County Law Library (1963).

Sacramento Public Library (1880).

University of the Pacific, McGeorge School of Law Library (1978).

San Bernardino: San Bernardino County Free Library (1964).

San Diego:

San Diego County Law Library (1973).

San Diego County Library (1966).

San Diego Public Library (1895).

San Diego State University, Malcoln A. Love Library (1962).

University of San Diego, School of Law Library (1967).

San Francisco:

Golden Gate University, School of Law Library (1979).

Hastings College of Law Library (1972).

Mechanics' Institute Library (1889).

San Francisco Public Library (1889).

San Francisco State College, Paul Leonard Library (1955).

Supreme Court of California Library (1979).

U.S. Court of Appeals, Ninth Circuit Library (1971).

University of San Francisco, Richard A. Gleeson Library (1963).

San Jose: San Jose State University Library (1962).

San Leandro: San Leandro Community Library Center (1961).

San Luis Obispo: California State Polytechnic University Li- brary (1969)

San Rafael: Marin County Free Library (1975).

Santa Ana:

Orange County Law Library (1975)

Santa Ana Public Library (1959).

Santa Barbara: University of California at Santa Barbara Library (1960).

Santa Clara: University of Santa Clara, Orradre Library (1963).

Santa Cruz: University of California at Santa Cruz Library (1963).

Santa Rosa: Santa Rosa-Sonoma County Public Library (1896).

Stanford

Stanford University Libraries (1895).

Stanford University, Robert Crown Law Library (1978).

Stockton: Public Library of Stockton and San Joaquin County (1884).

Thousand Oaks: California Lutheran College Library (1964).

Torrance: Torrance Civic Center Library (1969).

Turlock: California State University at Stanislaus Library (1964).

Valencia: Valencia Regional Library (1972).

Ventura: Ventura County Library Services Agency (1975).

Visalia: Tulare County Free Library (1967).

Walnut: Mount San Antonio College Library (1966).

West Covina: West Covina Library (1966).

Whittier: Whittier College, Wardman Library (1963).

\section{CANAL ZONE}

Balboa Heights: Canal Zone Library-Museum (1963).

\section{COLORADO}

Alamosa: Adams State College, Learning Resources Center (1963).

Boulder: University of Colorado Libraries (1879)-REGIONAL. Colorado Springs:

Colorado College, Tutt Library (1880).

University of Colorado, Colorado Springs Library (1974).

Denvèr:

Auraria Libraries (1978)

Colorado State Library (unknown).

Colorado Supreme Court Library (1978).

Denver Public Library (1884)-REGIONAL.

Department of the Interior, Bureau of Reclamation Library (1962).

Regis College, Dayton Memorial Library (1915).

University of Denver, Penrose Library (1909).

University of Denver, School of Law Library (1978).

U.S. Court of Appeals, Tenth Circuit Library (1973).

Fort Collins: Colorado State University Libraries (1907)

Golden: Colorado School of Mines, Arthur Lakes Library (1939).

Grand Junction: Mesa County Public Library (1975).

Greeley: University of Northern Colorado Library (1966).

Gunnison: Western State College, Leslie J. Savage Library (1932)

La Junta: Otero Junior College, Wheeler Library (1963).

Lakewood: Jefferson County Public Library, Lakewood Regional Library (1968).

Pueblo:

Pueblo Regional Library (1893).

University of Southern Colorado Library (1965).

U.S. Air Force Academy: Academy Library (1956).

\section{CONNECTICUT}

Bridgeport: Bridgeport Public Library (1884).

Danbury: Western Connecticut State College, Ruth A. Haas Library (1967).

Danielson: Quinebaug Valley Community College (1975).

Enfield: Enfield Public Library (1967).

Hartford:

Connecticut State Library (unknown)-REGIONAL.

Hartford Public Library (1945).

Trinity College Library (1895). 
Middletown: Wesleyan University, Olin Library (1906).

Mystic: Marine Historical Association, Inc., G. W. Blunt White Library (1964).

New Britain: Central Connecticut State College, Elihu Burritt Library (1973).

New Haven:

Southern Connecticut State College, Hilton C. Buley Library (1968).

Yale University Library (1859).

New London:

Connecticut College Library (1926)

U.S. Coast Guard Academy Library (1939).

Stamford: The Ferguson Library (1973).

Storrs: University of Connecticut Library (1907).

Waterbury:

Post College, Traurig Library (1977).

Silas Bronson Public Library (1869).

West Hartford: University of Connecticut, School of Law Library (1978).

West Haven: University of New Haven Library (1971).

\section{DELAWARE}

Dover:

Delaware State College, William C. Jason Library (1962).

Department of Community Affairs and Economic Development, Division of Libraries (1972).

State Law Library in Kent County (unknown).

Georgetown:

Delaware Technical and Community College Library (1968)

Sussex County Law Library (1976)

Newark: University of Delaware, Morris Library (1907).

Wilmington:

Delaware Law School Library (1976).

New Castle County Law Library (1974).

Wilmington Institute and New Castle County Library (1861).

\section{DISTRICT OF COLUMBIA}

Washington:

Administrative Conference of the United States Library (1977).

Advisory Commission on Intergovernmental Relations Library (1972)

Catholic University of America, Columbus School of Law, Robert J. White Law Library (1979)

Civil Aeronautics Board Library (1974).

Department of the Army Library (1969).

Department of Commerce Library (1955).

Department of Energy Library (1963).

Department of Health, Education, and Welfare Library (1954).

Department of Housing and Urban Development Library (1969).

Department of the Interior Library (1895).

Department of Justice Main Library (1895).

Department of Labor Library (1976).

Department of the Navy Library (1895).

Department of the Navy, Office of the Judge Advocate General Library (1963).

Department of State Library (1895).

Department of State Law Library (1966).

Department of Transportation, National Highway Traffic Safety Administration Library (1968).

Department of the Treasury Library (1895)

District of Columbia Public Library (1943).

Executive Office of the President, Office of Management and Budget Library (1965).

Federal Deposit Insurance Corporation Library (1972).

Federal Election Commission Library (1975).

Federal Reserve System, Board of Governors Research Library (1978).

Federal Reserve System Law Library (1976).

General Accounting Office Library (1974).
General Services Administration Library (1975).

Georgetown University Library (1969).

Georgetown University Law Center, Fred O. Dennis Law Library (1978)

George Washington University, National Law Center (1978)

Library of Congress, Congressional Research Service (1978).

Library of Congress, Exchange and Gift Division (1977).

Merit Systems Protection Board Library (1979).

National Defense University Library (1895).

University of the District of Columbia, Mount Vernon Campus Library and Media Center (1970).

U.S. Court of Appeals, Judges' Library (1975).

U.S. Office of Personnel Management Library (1963)

U.S. Postal Service Library (1895).

U.S. Supreme Court Library (1978).

Veterans' Administration, Central Office Library (1967).

\section{FLORIDA}

Boca Raton: Florida Atlantic University Library (1963).

Clearwater: Clearwater Public Library (1972).

Coral Gables: University of Miami Library (1939).

Crestview: Robert F. L. Sikes Public Library (1978)

Daytona Beach: Volusia County Public Libraries (1963).

De Land: Stetson University, duPont-Ball Library (1887).

Fort Lauderdale:

Broward County Library System (1967).

Nova University Law Library (1967).

Fort Pierce: Indian River Community College Library (1975).

Gainesville:

University of Florida, Holland Law Center, School of Law Library (1978).

University of Florida Libraries (1907)-REGIONAL.

Jacksonville:

Haydon Burnes Library (1914).

Jacksonville University, Swisher Library (1962).

University of North Florida Library (1972).

Lakeland: Lakeland Public Library (1928).

Leesburg: Lake-Sumter Community College Library (1963).

Melbourne: Florida Institute of Technology Library (1963).

Miami:

Florida International University Library (1970).

Miami Public Library (1952).

North Miami: Florida International University, North Miami Campus Library (1977).

Opa Locka: Biscayne College Library (1966)

Orlando: Florida Technological University Library (1966).

Palatka: St. Johns River Junior College Library (1963).

Pensacola: University of West Florida, John C. Pace Library (1966)

Punta Gorda: Charlotte County Library System (1973).

St. Petersburg:

St. Petersburg Public Library (1965).

Stetson University, College of Law Library (1975).

Sarasota: Selby Public Library (1970).

Tallahassee:

Florida Agricultural and Mechanical University, Coleman Memorial Library (1936)

Florida State University, College of Law Library (1978).

Florida State University, Robert M. Stozier Library (1941). (1941).

Florida Supreme Court Library (1974).

State Library of Florida (1929).

Tampa:

Tampa-Hillsborough County Public Library (1965)

University of South Florida Library (1962).

University of Tampa, Merl Kelce Library (1953).

Winter Park: Rollins College, Mills Memorial Library (1909),

\section{GEORGIA}

Albany: Albany-Dougherty Public Library (1964).

Americus: Georgia Southwestern College, James Earl Carter Library (1966). 
Athens:

University of Georgia Libraries (1907)-REGIONAL.

University of Georgia, School of Law Library (1979)

Atlanta:

Atlanta Public Library (1880).

Atlanta University, Trevor Arnett Library (1962).

Emory University, Robert W. Woodruff Library (1928).

Emory University, School of Law Library (1968).

Georgia Institute of Technology Library (1963).

Georgia State Library (unknown).

Georgia State University, William Russell Pullen Library (1970)

Augusta: Augusta College, Reese Library (1962).

Brunswick: Brunswick-Glynn County Regional Library (1965).

Carrollton: West Georgia College Library (1962).

Columbus: Columbus College, Simon Schwob Memorial Library (1975).

Dahlonega: North Georgia College, Stewart Library (1939).

Dalton: Dalton Junior College Library (1978).

Decatur: DeKalb Community College-South Campus, Learning Resources Center (1973).

Macon:

Mercer University, Stetson Memorial Library (1964)

Mercer University, Walter F. George School of Law Li. brary (1978).

Marietta: Kennesaw College, Memorial Library (1968).

Milledgeville: Georgia College at Milledgeville, Ina Dillard Russell Library (1950)

Mount Berry: Berry College, Memorial Library (1970).

Savannah: Savannah Public and Chatham-Effingham-Liberty Regional Library (1857).

Statesboro: Georgia Southern College Library (1939).

Valdosta: Valdosta State College Library (1956)

\section{GUAM}

Agana:

Nieves M. Flores Memorial Library (1962).

University of Guam, Robert F. Kennedy Memorial Library (1978).

\section{HAWAII}

Hilo: University of Hawaii, Hilo Campus Library (1962).

Honolulu:

Hawaii Medical Library, Inc. (1968).

Hawaii State Library (1929).

Municipal Reference Library of the City and County of Honolulu (1965)

Supreme Court Law Library (1973).

University of Hawaii Library (1907)-REGIONAL.

University of Hawaii, School of Law Library (1978).

Laie: Brigham Young University, Hawaii Campus, Joseph F. Smith Library (1964).

Lihue: Kauai Public Library (1967).

Pearl City: Leeward Community College Library (1967).

Wailuku: Maui Public Library (1962).

\section{IDAHO}

Boise:

Boise Public Library and Information Center (1929).

Boise State University Library (1966).

Idaho State Law Library (unknown).

Idaho State Library (1971).

Caldwell: College of Idaho, Terteling Library (1930).

Moscow:

University of Idaho, College of Law Library (1978).

University of Idaho Library (1907)-REGIONAL.

Pocatello: Idaho State University Library (1908).

Rexburg: Ricks College, David O. McKay Library (1946).

Twin Falls: College of Southern Idaho Library (1970).

\section{ILLINOIS}

Bloomington: Illinois Wesleyan University Libraries (1964).

Carbondale:

Southern Illinois University, Morris Library (1932)

Southern Illinois University, School of Law Library (1978).

Carlinville: Blackburn College, Lumpkin Library (1954).

Carterville: Shawnee Library System (1971).

Champaign: University of Illinois, College of Law Library (1965).

Charleston: Eastern Illinois University, Booth Library (1962).

Chicago:

Chicago Public Library (1876).

Chicago Staie University, Paul and Emily Douglas Library (1954).

DePaul University, Lincoln Park Campus Library (1975).

DePaul University, School of Law Library (1979).

Field Museum of Natural History Library (1963).

Illinois Institute of Technology, Chicago-Kent Law Library (1978).

John Crerar Library (1909).

Loyola University of Chicago, E. M. Cudahy Memorial Li brary (1966).

Loyola University, School of Law Library (1979).

Northeastern Illinois University Library (1961).

Northwestern University, School of Law Library (1978)

University of Chicago, Law Library (1964).

University of Chicago Library (1897)

University of Illinois at Chicago Circle Library (1957).

William J. Campbell Library of the U.S. Courts (1979)

Decatur: Decatur Public Library (1954)

De Kalb: Northern Illinois University, Founders' Memorial Library (1960)

Edwardsville: Southern Illinois University, Lovejoy Memorial Library (1959).

Elsah: Principia College, Marshall Brooks Library (1957).

Evanston: Northwestern University Library (1876).

Freeport: Freeport Public Library (1905).

Galesburg: Galesburg Public Library (1896).

Glen Ellyn: Lewis University, College of Law Library (1978).

Jacksonville: MacMurray College, Henry Pfeiffer Library (1957).

Kankakee: Olivet Nazarene College, Benner Library and Resources Center (1946).

Lake Forest: Lake Forest College, Donnelley Library (1962).

Lebanon: McKendree College, Holman Library (1968).

Lisle: Illinois Benedictine College, Theodore F. Lownik Library (1911).

Lockport: Lewis University Library (1952).

Macomb: Western Illinois University, Memorial Library (1962).

Moline: Black Hawk College, Learning Resources Center (1970).

Monmouth: Monmouth College Library (1860).

Morton Grove: Oakton Community College Library (1976).

Mt. Carmel: Wabash Valley College, Bauer Media Center (1975)

Mt. Prospect: Mt. Prospect Public Library (1977).

Normal: Illinois State University, Milner Library (1877).

Oak Park: Oak Park Public Library (1963).

Oglesby: Illinois Valley Community College, Jacobs Memorial Library (1976).

Palos Hills: Moraine Valley Community College Library (1972).

Park Forest South: Governors' State University Library (1974).

Peoria:

Bradley University, Cullom Davis Library (1963).

Peoria Public Library (1883)

River Forest: Rosary College Library (1966).

Rockford: Rockford Public Library (unknown).

Springfield: Illinois State Library (unknown)-REGIONAL.

Urbana: University of Illinois Library (1907).

Wheaton: Wheaton College Library (1964).

Woodstock: Woodstock Public Library (1963)

\section{INDIANA}

Anderson: Anderson College, Charles E. Wilson Library (1959) 
Bloomington:

Indiana University Library (1881).

Indiana University, School of Law Library (1978)

Crawfordsville: Wabash College, Lilly Library (1906).

Evansville:

Evansville and Vanderburgh County Public Library (1928).

Indiana State University, Evansville Campus Library (1969).

Fort Wayne:

Indiana-Purdue Universities, Regional Campus Library (1965).

Public Library of Fort Wayne and Allen County (1896).

Franklin: Franklin College Library (1976).

Gary:

Gary Public Library (1943).

Indiana University, Northwest Campus Library (1966).

Greencastle: De Pauw University, Roy O. West Library (1879).

Hammond: Hammond Public Library (1964).

Hanover: Hanover College Library (1892).

Huntington: Huntington College, Loew-Alumni Library (1964).

Indianapolis:

Butler University (1965).

Indianapolis-Marion County Public Library (1906).

Indiana State Library (unknown)-REGIONAL.

Indiana Supreme Court Law Library (1975).

Indiana University, School of Law Library (1967).

Kokomo: Indiana University, Kokomo Regional Campus Library (1969).

Lafayette: Purdue University Libraries and Audio.Visual Center (1907)

Muncie:

Ball State University Library (1959).

Muncie Public Library (1906).

New Albany: Indiana University, Southeastern Campus Library (1965).

Notre Dame: University of Notre Dame, Memorial Library (1883).

Rensselaer: St. Joseph's College Library (1964).

Richmond:

Earlham College, Lilly Library (1964)

Morrison-Reeves Library (1906).

South Bend: Indiana University at South Bend Library (1965)

Terre Haute: Indiana State University, Cunningham Memorial Library (1906).

Valparaiso:

Valparaiso University, Moellering Memorial Library (1930).

Valparaiso University, School of Law Library (1978)

\section{IOWA}

Ames: Iowa State University of Science and Technology Library (1907).

Cedar Falls: University of Northern Iowa Library (1946).

Council Bluffs:

Free Public Library (1885).

Iowa Western Community College, Herbert Hoover Media Library (1972).

Davenport: Davenport Public Library (1973).

Des Moines:

Drake University, Cowles Library (1966)

Drake University Law Library (1972).

Public Library of Des Moines (1888).

State Library Commission of Iowa (unknown).

Dubuque:

Carnegie-Stout Public Library (unknown).

Loras College, Wahlert Memorial Library (1967).

Fayette: Upper Iowa College, Henderson-Wilder Library (1974).

Grinnell: Grinnell College Library (1874).

Iowa City:

University of Iowa, College of Law Library (1968).

University of Iowa Libraries (1884)-REGIONAL.

Lamoni: Graceland College, Frederick Madison Smith Library (1927).

Mason City: North Iowa Area Community College Library (1976).

Mount Vernon: Cornell College, Russell D. Cole Library (1896).

Orange City: Northwestern College, Ramaker Library (1970).

Sioux City: Sioux City Public Library (1894).

\section{KANSAS}

Atchison: Benedictine College, North Campus Library (1965)

Baldwin City: Baker University Library (1908)

Colby: Colby Community Junior College, H. F. Davis Memorial Library (1968).

Emporia: Emporia State University, William Allen White Library (1909).

Hays: Fort Hays State University, Forsyth Library (1926).

Hutchinson: Hutchinson Public Library (1963).

Lawrence:

University of Kansas, School of Law Library (1971).

University of Kansas, Watson Library (1869)-REGIONAL.

Manhattan: Kansas State University, Farrell Library (1907).

Pittsburg: Pittsburg State University Library (1952).

Salina: Kansas Wesleyan University, Memorial Library (1930).

Shawnee Mission: Johnson County Library (1979).

Topeka:

Kansas State Historical Society Library (1877).

Kansas State Library (unknown).

Kansas Supreme Court Law Library (1975)

Washburn University of Topeka, Law Library (1971).

Wichita: Wichita State University Library (1901).

\section{KENTUCKY}

Ashland: Ashland Public Library (1946).

Barbourville: Union College, Abigail E. Weeks Memorial Library (1958).

Bowling Green: Western Kentucky University, Helm-Cravens Graduate Center and Library (1934).

Covington: Thomas More College Library (1970).

Danville: Centre College, Grace Doherty Library (1884).

Frankfort:

Kentucky Department of Libraries (1967).

Kentucky State Law Library (unknown).

Kentucky State University, Blazer Library (1972).

Highland Heights: Northern Kentucky University, W. Frank Steely Library (1973)

Hopkinsville: Hopkinsville Community College Library (1976).

Lexington:

University of Kentucky, Law Library (1968).

University of Kentucky Libraries (1907)-REGIONAL.

Louisville:

Louisville Free Public Library (1904).

University of Louisville, Belknap Campus Library (1925)

University of Louisville, School of Law Library (1975).

Morehead: Morehead State University, Johnson Camden Library (1955).

Murray: Murray State University Library (1924).

Owensboro: Kentucky Wesleyan College Library (1966).

Richmond: Eastern Kentucky University, John Grant Crabbe Library (1966)

\section{LOUISIANA}

Baton Rouge:

Louisiana State Library (1976).

Louisiana State University, School of Law Library (1929).

Louisiana State University Library (1907)-REGIONAL.

Southern University Library (1952).

Eunice: Louisiana State University at Eunice, LeDoux Library (1969).

Hammond: Southeastern Louisiana University, Sims Memorial Library (1966).

Lafayette: University of Southwestern Louisiana Libraries (1938).

Lake Charles: McNeese State University, Lether E. Frazar Memorial Library (1941).

Monroe: Northeast Louisiana University, Sandel Library (1963).

Natchitoches: Northwestern State University, Watson Library (1887)

New Orleans:

Isaac Delgado College, Moss Memorial Library (1968).

Law Library of Louisiana (unknown). 
Loyola University Library (1942).

Loyola University, School of Law Library (1978).

New Orleans Public Library (1883).

Southern University in New Orleans Library (1962).

Tulane University, Howard-Tilton Memorial Library (1942).

Tulane University, Law Library (1976).

University of New Orleans Library (1963).

U.S. Court of Appeals, Fifth Circuit Library (1973).

Pineville: Louisiana College, Richard W. Norton Memorial Library (1969).

Ruston: Louisiana Technical University Library (1896)-RE GIONAL.

Shreveport:

Louisiana State University at Shreveport Library (1967).

Shreve Memorial Library (1923).

Thibodaux: Nicholls State University Library (1962).

\section{MAINE}

Augusta:

Maine Law and Legislative Reference Library (1973).

Maine State Library (unknown).

Bangor: Bangor Public Library (1884).

Brunswick: Bowdoin College Library (1884).

Castine: Maine Maritime Academy, Nutting Memorial Library (1969).

Lewiston: Bates College Library (1883).

Orono: University of Maine, Raymond H. Fogler Library (1907)-REGIONAL.

Portland:

Portland Public Library (1884)

University of Maine, School of Law Library (1964).

Presque Isle: University of Maine at Presque Isle Library (1979).

Springvale: Nasson College Library (1961).

Waterville: Colby College, Miller Library (1884).

\section{MARYLAND}

Annapolis:

Maryland State Law Library (unknown).

U.S. Naval Academy, Nimitz Library (1895).

Baltimore:

Enoch Pratt Free Library (1887).

Johns Hopkins University, Milton S. Eisenhower Library (1882).

Morgan State College, Soper Library (1940).

University of Baltimore, Langsdale Library (1973).

University of Maryland, Baltimore County Library (1971).

University of Maryland, School of Law Library (1969).

Bel Air: Harford Community College Library (1967).

Beltsville: Department of Agriculture, National Agricultural Library (1895).

Bethesda: Department of Health, Education, and Welfare, National Library of Medicine (1978).

Chestertown: Washington College, Chester M. Miller Library (1891).

College Park: University of Maryland, McKeldin Library (1925)-REGIONAL.

Cumberland: Allegany Community College Library (1974).

Frostburg: Frostburg State College Library (1967).

Patuxent River: U.S. Naval Air Station Library (1968)

Rockville: Montgomery County Department of Public Libraries (1951)

Salisbury: Salisbury State College, Blackwell Library (1965).

Towson: Goucher College, Julia Roger Library (1966).

Westminster: Western Maryland College, Hoover Library (1886)

\section{MASSACHUSETTS}

Amherst:

Amherst College Library (1884)

University of Massachusetts Library (1907).

Belmont: Belmont Memorial Library (1968).
Boston:

Boston Athenaeum Library (unknown).

Boston Public Library (1859)-REGIONAL.

Boston University, School of Law, Pappas Library (1979).

Northeastern University Libraries (1962).

State Library of Massachusetts (unknown).

Supreme Judicial Court, Social Law Library (1979).

U.S. Court of Appeals, First Circuit Library (1978).

Brookline: Public Library of Brookline (1925).

Cambridge:

Harvard College Library (1860)

Massachusetts Institute of Technology Libraries (1946)

Middlesex County Law Library (1978).

Chestnut Hill: Boston College, Bapst Library (1963).

Chicopee: Our Lady of the Elms College Library (1969).

Lowell: University of Lowell, Alumni/Lydon Library (1952).

Lynn: Lynn Public Library (1953).

Marlborough: Marlborough Public Library (1971).

Medford: Tufts University Library (1899).

Milton: Curry College Library (1972).

New Bedford: New Bedford Free Public Library (1858).

Newton Centre: Boston College, School of Law Library (1979).

North Dartmouth: Southeastern Massachusetts University Li brary (1965).

North Easton: Stonehill College, Cushing-Martin Library (1962) Springfield:

Springfield City Library (1966).

Western New England College, School of Law Library (1978).

Waltham: Brandeis University Library (1965)

Wellesley: Wellesley College Library (1943).

Wenham: Gordon College, Winn Library (1963).

Williamstown: Williams College Library (unknown).

Worcester:

American Antiquarian Society Library (1814).

University of Massachusetts, Medical Center Library (1972)

Worcester Public Library (1859).

\section{MICHIGAN}

Albion: Albion College, Stockwell Memorial Library (1966).

Allendale: Grand Valley State College Library (1963).

Alma: Alma College, Monteith Library (1963).

Ann Arbor:

Great Lakes Basin Commission Library (1971).

University of Michigan, Harlan Hatcher Library (1884).

University of Michigan, School of Law Library (1978).

Benton Harbor: Benton Harbor Public Library (1907).

Bloomfield Hills: Cranbrook Institute of Science Library (1940).

Dearborn:

Henry Ford Centennial Library (1969).

Henry Ford Community College Library (1957).

Detroit:

Detroit College of Law Library (1979).

Detroit Public Library (1868)-REGIONAL.

Marygrove College Library (1965).

Mercy College of Detroit Library (1965).

University of Detroit Library (1884).

University of Detroit, School of Law Library

Wayne State University, G. Flint Purdy Library (1937).

Wayne State University, Law Library (1971).

Dowagiac: Southwestern Michigan College, Mathews Library (1971).

East Lansing: Michigan State University Library (1907).

Escanaba: Michigan State Library, Upper Peninsula Branch (1964).

Farmington: Oakland Community College, Martin Luther King Learning Resources Center, (1968).

Flint:

Flint Public Library (1967)

University of Michigan at Flint Library (1959).

Grand Rapids:

Calvin College Library (1967)

Grand Rapids Public Library (1876).

Houghton: Michigan Technological University Library (1876). 
Jackson: Jackson Public Library (1965).

Kalamazoo:

Kalamazoo Public Library (1907).

Western Michigan University Library (1963).

Lansing:

Michigan State Library (unknown)-REGIONAL.

Thomas M. Cooley Law School Library (1978).

Livonia: Schoolcraft College Library (1962).

Marquette: Northern Michigan University, Olsen Library (1963).

Monroe: Monroe County Library System (1974).

Mt. Clemens: Macomb County Library (1968).

Mt. Pleasant: Central Michigan University Library (1958).

Muskegon: Hackley Public Library (1894).

Olivet: Olivet College Library (1974).

Petoskey: North Central Michigan College Library (1962).

Port Huron: Saint Clair County Library (1876).

Rochester: Oakland University, Kresge Library (1964).

Saginaw: Hoyt Public Library (1890).

Traverse City: Northwestern Michigan College, Mark Osterlin Library (1964).

University Center: Delta College Library (1963).

Warren: Warren Public Library, Arthur J. Miller Branch (1973).

Wayne: Wayne Oakland Federated Library System (1957).

Ypsilanti: Eastern Michigan University Library (1965).

\section{MINNESOTA}

Bemidji: Bemidji State College, A. C. Clark Library (1963). Collegeville: St. John's University, Alcuin Library (1954).

Duluth: Duluth Public Library (1909).

Mankato: Mankato State College, Memorial Library (1962).

Minneapolis:

Anoka County Library (1971).

Hennepin County Libraries (1971).

Minneapolis Public Library (1893).

University of Minnesota, School of Law Library (1978).

University of Minnesota, Wilson Library (1907)-REGIONAL.

Moorhead: Moorhead State College Library (1956).

Morris: University of Minnesota at Morris Library (1963).

Northfield:

Carleton College Library (1930).

St. Olaf College, Rolvaag Memorial Library (1930).

St. Cloud: St. Cloud State College Library (1962).

St. Paul:

Hamline University, School of Law Library (1978).

Minnesota Historical Society Library (1867).

Minnesota State Law Library (unknown).

St. Paul Public Library (1914).

University of Minnesota, St. Paul Campus Library (1974).

William Mitchell College of Law Library (1979).

Saint Peter: Gustavus Adolphus College Library (1941).

Stillwater: Stillwater Public Library (1893).

Willmar: Crow River Regional Library (1958).

Winona: Winona State University, Maxwell Library (1969).

\section{MISSISSIPPI}

Cleveland: Delta State University, W. B. Roberts Library (1975). Clinton: Mississippi College, School of Law Library (1977).

Columbus: Mississippi State University for Women, John Clayton Fant Memorial Library (1929).

Hattiesburg: University of Southern Mississippi, Joe Cook Memorial Library (1935).

Jackson:

Jackson State University, Henry Thomas Sampson Library (1968).

Millsaps College, Millsaps-Wilson Library (1963).

Mississippi Library Commission (1947).

Mississippi State Law Library (unknown).

Lorman: Alcorn State University Library (1970).

Mississippi State: Mississippi State University, Mitchell Memorial Library (1907).

University:

University of Mississippi Library (1833)-REGIONAL.

University of Mississippi, School of Law Library (1967).

\section{MISSOURI}

Cape Girardeau: Southeast Missouri State University, Kent LiColumbia: brary (1916).

University of Missouri at Columbia Library (1862).

University of Missouri at Columbia, School of Law Library (1978).

Fayette: Central Methodist College, George M. Smiley Library (1962).

Fulton: Westminster College, Reeves Library (1875).

Jefferson City:

Lincoln University, Inman E. Page Library (1944).

Missouri State Library (1963).

Missouri Supreme Court Library (unknown).

Joplin: Missouri Southern State College Library (1966).

Kansas City:

Kansas City Public Library (1881).

Rockhurst College, Greenlease Library (1917).

University of Missouri at Kansas City, General Library (1938).

University of Missouri at Kansas City, Leon E. Bloch School of Law Library (1978).

Kirksville: Northeast Missouri State University, Pickler Memorial Library (1966).

Liberty: William Jewell College, Charles F. Curry Library (1900).

Rolla: University of Missouri at Rolla Library (1907).

St. Charles: Lindenwood College, Margaret Leggat Butler Library (1973).

St. Joseph: St. Joseph Public Library (1891).

St. Louis:

Maryville College Library (1976)

St. Louis County Library (1970).

St. Louis Public Library (1866).

St. Louis University, Law Library (1967)

St. Louis University, Pius XII Memorial Library (1966).

University of Missouri at St. Louis, Thomas Jefferson Library (1966).

U.S. Court of Appeals, Eighth Circuit Library (1972).

Washington University, John M. Olin Library (1906).

Washington University, School of Law Library (1978).

Springfield:

Drury College, Walker Library (1874).

Southwest Missouri State University Library (1963).

Warrensburg: Central Missouri State University, Ward Edwards Library (1914).

\section{MONTANA}

Billings: Eastern Montana College Library (1924).

Bozeman: Montana State University Library (1907).

Butte: Montana College of Mineral Science and Technology Library (1901)

Helena:

Carroll College Library (1974).

Montana Historical Society Library (unknown).

Montana State Library (1966).

State Law Library of Montana (1977).

Missoula: University of Montana Library (1909)-REGIONAL.

\section{NEBRASKA}

Blair: Dana College, Dana-LIFE Library (1924).

Crete: Doane College, Perkins Library (1944).

Fremont: Midland Lutheran College Library (1924).

Kearney: Kearney State College, Calvin T. Ryan Library (1962). Lincoln:

Nebraska Publications Clearinghouse, Nebraska Library Commission (1972)-REGIONAL.

Nebraska State Library (unknown).

University of Nebraska at Lincoln, D. L. Love Memorial Library (1907)-JOINT REGIONAL.

Omaha:

Creighton University, Alumni Memorial Library (1964).

Omaha Public Library, W. Dale Clark Library (1880). 
University of Nebraska at Omaha, University Library (1939).

Scottsbluff: Scottsbluff Public Library (1925).

Wayne: Wayne State College, U.S. Connecticut Library (1970).

\section{NEVADA}

Carson City:

Nevada State Library (unknown).

Nevada Supreme Court Library (1973).

Las Vegas:

Clark County Library District (1974).

Reno:

University of Nevada, James Dickinson Library (1959).

National Judicial College, Law Library (1979).

Nevada State Historical Society Library (1974).

University of Nevada Library (1907)-REGIONAL

\section{NEW HAMPSHIRE}

Concord:

Franklin Pierce Law Center Library (1973).

New Hampshire State Library (unknown).

Durham: University of New Hampshire Library (1907).

Hanover: Dartmouth College Library (1884).

Henniker: New England College Library (1966).

Manchester:

Manchester City Library (1884).

New Hampshire College, H. A. B. Shapiro Memorial Library (1976).

St. Anselm's College, Geisel Library (1963).

Nashua: Nashua Public Library (1971).

\section{NEW JERSEY}

Bayonne: Bayonne Free Public Library (1909).

Bloomfield: Free Public Library of Bloomfield (1965).

Bridgeton: Cumberland County Library (1966).

Camden:

Rutgers University, Camden Library (1966).

Rutgers University, School of Law Library (1979).

Convent Station: College of St. Elizabeth, Mahoney Library (1938).

Dover: County College of Morris, Library Learning Resources Center (1975).

East Brunswick: East Brunswick Public Library (1977).

East Orange: East Orange Public Library (1966).

Elizabeth: Free Public Library of Elizabeth (1895).

Glassboro: Glassboro State College, Savitz Learning Resource Center (1963).

Hackensack: Johnson Free Public Library (1966).

Irvington: Free Public Library of Irvington (1966).

Jersey City:

Free City Public Library (1879).

Jersey City State College, Forrest A. Irwin Library (1963).

Lawrenceville: Rider College Library (1975).

Madison: Drew University, Rose Memorial Library (1939).

Mahwah: Ramapo College Library (1971).

Mount Holly: Burlington County Library (1966).

New Brunswick:

New Brunswick Free Public Library (1908).

Rutgers University Library (1907).

Newark:

Newark Public Library (1906)-REGIONAL.

Rutgers, The State University, John Cotton Dana Library (1966).

Rutgers University, School of Law Library (1979).

Passaic: Passaic Public Library (1964).

Phillipsburg: Phillipsburg Free Public Library (1976).

Plainfield: Plainfield Public Library (1971).

Pomona: Stockton State College Library (1972).

Princeton: Princeton University Library (1884).

Rutherford: Fairleigh Dickinson University Library (1953).

Shrewsbury: Monmouth County Library (1968).

South Orange: Seton Hall University, McLaughlin Library (1947).
Teaneck: Fairleigh Dickinson University, Teaneck Campus Library (1963).

Toms River: Ocean County College, Learning Resources Center (1966).

Trenton:

New Jersey State Library, Law and Reference Bureau (unknown)

Trenton Free Public Library (1902).

Union: Kean College of New Jersey, Nancy Thompson Library (1973).

Upper Montclair: Montclair State College, Harry S. Sprague Library (1967).

Wayne: Wayne Public Library (1972).

West Long Branch: Monmouth College, Guggenheim Memorial Library (1963).

Woodbridge: Free Public Library of Woodbridge (1965).

\section{NEW MEXICO}

Albuquerque:

University of New Mexico, Medical Center Library (1973).

University of New Mexico, School of Law Library (1973).

University of New Mexico, Zimmerman Library (1896)-RE. GIONAL.

Hobbs: New Mexico Junior College, Pannell Library (1969).

Las Cruces: New Mexico State University Library (1907).

Las Vegas: New Mexico Highlands University, Donnelly Library (1913).

Portales: Eastern New Mexico University Library (1962).

Santa Fe:

New Mexico State Library (1960)-REGIONAL.

Supreme Court Law Library (unknown).

Silver City: Western New Mexico University, Miller Library (1972).

\section{NEW YORK}

Albany:

New York State Library (unknown)-REGIONAL.

State University of New York at Albany, University Li brary (1964).

Auburn: Seymour Library (1972).

Bayside: Queensborough Community College Library (1972).

Binghamton: State University of New York at Binghamton, University Library (1962).

Brockport: State University of New York at Brockport, Drake Memorial Library (1967)

Bronx:

Fordham University Library (1937).

Herbert H. Lehman College Library (1967).

New York Public Library, Mott Haven Branch (1973).

State University of New York, Maritime College Library (1947).

Brooklyn:

Brooklyn College Library (1936).

Brooklyn Law School Library (1974).

Brooklyn Public Library (1908).

Polytechnic Institute of Brooklyn Libraries (1963).

Pratt Institute Library (1891).

State University of New York, Downstate Medical Research Library (1958).

Buffalo:

Buffalo and Erie County Public Library (1895).

State University of New York at Buffalo, School of Law, Charles B. Sears Law Library (1978).

State University of New York at Buffalo, Lockwood Memorial Library (1963).

Canton: St. Lawrence University, Owen D. Young Library (1920).

Cheektowago: Cheektowago Public Library (1978).

Corning: Corning Community College, Arthur A. Houghton, Jr. Library (1963).

Cortland: State University of New York at Cortland, Memorial Library (1964).

Delhi: State University Agricultural and Technical College Library (1970). 
East Islip: East Islip Public Library (1973).

Elmira: Elmira College, Gannett-Tripp Learning Center (1956).

Farmingdale: State University of New York at Farmington Library (1917).

Flushing:

Cathedral College Library (1971).

Queens College, Paul Klapper Library (1939).

Garden City: Adelphi University Library (1966).

Geneseo: State University of New York at Geneseo, Milne Library (1967).

Greenvale: Long Island University, B. Davis Schwartz Memorial Library (1964).

Hamilton: Colgate University Library (1902).

Hempstead:

Hofstra University Library (1964).

Hofstra University, School of Law Library (1979).

Ithaca:

Cornell University Libraries (1907).

Cornell University, School of Law Library (1978).

New York State College of Agriculture and Home Economics, Albert R. Mann Library (1943).

Jamaica:

Queens Borough Public Library (1926).

St. John's University Library (1956).

St. John's University, School of Law Library (1978).

Kings Point: U.S. Merchant Marine Academy, Academy Library (1962).

Mount Vernon: Mount Vernon Public Library (1962).

New Paltz: State University College at New Paltz, Sojourner Truth Library (1965).

New York City:

City University of New York, City College Library (1884).

Columbia University Libraries (1882).

Cooper Union for the Advancement of Science and Arts Li. brary (1930).

Medical Library Center of New York (1976).

New York Law Institute Library (1909).

New York Public Library, Astor Branch (1907).

New York Public Library, Lenox Branch (1884).

New York University, Law Library (1973).

New York University Libraries (1967).

The College of Insurance Library (1965).

U.S. Court of Appeals, Second Circuit Library (1976).

Néwburgh: Newburgh Free Library (1909).

Niagara Falls: Niagara Falls Public Library (1976).

Oakdale: Dowling College (1965).

Oneonta: State University College at Oneonta, James M. Milne Library (1966).

Oswego: State University of New York at Oswego, Penfield Library (1966).

Plattsburgh: State University College at Plattsburgh, Benjamin F. Feinberg Library (1967).

Potsdam:

Clarkson College of Technology Library (1938).

State University College at Potsdam, Frederick W. Crumb Memorial Library (1964).

Poughkeepsie: Vassar College Library (1943).

Purchase: State University of New York at Purchase Library (1969)

Rochester:

Rochester Public Library (1963).

University of Rochester Library (1880).

St. Bonaventure: St. Bonaventure University, Friedsam Memorial Library (1938).

Saratoga Springs: Skidmore College Library (1964).

Schenectady: Union College, Schaffer Library (1901).

Southampton: Southampton College Library (1973).

Staten Island: Wagner College, Horrmann Library, Grymes Hill (1953).

Stony Brook: State University of New York at Stony Brook, Main Library (1963).

Syracuse:

Onondaga County Public Library (1978).

Syracuse University Library (1878).

Syracuse University, William C. Ruger Law Library (1978).

Troy: Troy Public Library (1869).

Uniondale: Nassau Library System (1965).
Utica:

Utica Public Library (1885).

State University College at Utica/Rome Library (1977).

West Point: U.S. Military Academy Library (unknown).

White Plains: Pace University, School of Law Library (1978).

Yonkers:

Sarah Lawrence College Library (1969).

Yonkers Public Library (1910).

Yorktown Heights: Mercy College Library (1976).

\section{NORTH CAROLINA}

Asheville: University of North Carolina at Asheville, D. Hiden Ramsey Library (1965).

Boiling Springs: Gardner-Webb College Library (1974).

Boone: Appalachian State University Library (1963).

Buies Creek: Campbell College, Carrie Rich Memorial Library (1965)

Chapel Hill:

University of North Carolina at Chapel Hill Library (1884)-REGIONAL.

University of North Carolina at Chapel Hill, School of Law Library (1978).

Charlotte:

Public Library of Charlotte and Mecklenburg County (1964).

Queens College, Everett Library (1927).

University of North Carolina at Charlotte, Atkins Library (1964).

Cullowhee: Western Carolina University, Hunter Library (1953).

Davidson: Davidson College Library (1893).

Durham:

Duke University, School of Law Library (1978).

Duke University, William R. Perkins Library (1890).

North Carolina Central University, James E. Shepard Memorial Library (1973).

Elon College: Iris Holt McEwen Library (1971).

Fayetteville: Fayetteville State University, Charles W. Chestnutt Library (1971)

Greensboro:

North Carolina Agricultural and Technical State University, F. D. Bluford Library (1937).

University of North Carolina at Greensboro, Walter Clinton Jackson Library (1963).

Greenville: East Carolina University Library (1951).

Laurinburg: St. Andrews Presbyterian College, DeTamble Library (1969)

Lexington: Davidson County Public Library (1971).

Mount Olive: Mount Olive College, Moye Library (1971).

Murfreesboro: Chowan College, Whitaker Library (1963).

Pembroke: Pembroke State University, Mary H. Livermore Library (1956).

Raleigh:

Department of Cultural Resources, Division of State Library (unknown).

North Carolina State University, D. H. Hill Library (1923).

North Carolina Supreme Court Library (1972).

Wake County Public Library (1969).

Rocky Mount: North Carolina Wesleyan College Library (1969).

Salisbury: Catawba College Library (1925).

Wilmington: University of North Carolina at Wilmington, William M. Randall Library (1965).

Wilson: Atlantic Christian College, Clarence L. Hardy Library (1930).

Winston-Salem:

Forsyth County Public Library (1954).

Wake Forest University, Z. Smith Reynolds Library (1902).

\section{NORTH DAKOTA}

Bismarck:

North Dakota State Historical Society Library (1907).

North Dakota State Library (1971).

North Dakota Supreme Court Law Library (unknown).

Veterans' Memorial Public Library (1967).

Dickinson: Dickinson State College, Stoxen Library (1968). 
Fargo:

Fargo Public Library (1964)

North Dakota State University Library (1907)-REGION. $A L$, in cooperation with University of North Dakota, Chester Fritz Library.

Grand Forks: University of North Dakota, Chester Fritz Library (1890).

Minot: Minot State College, Memorial Library (1925).

Valley City: Valley City State College Library (1913)

\section{OHIO}

Ada: Ohio Northern University, J. P. Taggart Law Library (1965).

Akron:

Akron-Summit Public Library (1952).

University of Akron, Bierce Library (1963).

University of Akron, C. Blake McDowell Law Center, School of Law Library (1978)

Alliance: Mount Union College Library (1888).

Ashland: Ashland College Library (1938).

A thens: Ohio University Library (1886).

Batavia: University of Cincinnati at Batavia, Clermont General and Technical College Library (1973).

Bluffton: Bluffton College, Musselman Library (1951).

Bowling Green: Bowling Green State University Library (1933).

Canton: Malone College, Everett L. Cattell Library (1970).

Chardon: Geauga County Public Library (1971).

Cincinnati:

Public Library of Cincinnati and Hamilton County (1884).

University of Cincinnati, Central Library (1929).

University of Cincinnati, College of Law, Marx Law Library (1978).

Cleveland:

Case Western Reserve University, Freiberger Library (1913)

Case Western Reserve University, School of Law Library (1979)

Cleveland Heights-University Heights Public Library (1970).

Cleveland Public Library (1886).

Cleveland State University, Cleveland-Marshall College of Law, Joseph W. Bartunek III Law Library (1978).

Cleveland State University Library (1966).

John Carroll University, Grasselli Library (1963).

Municipal Reference Library (1970).

Columbus:

Capital University Library (1968).

Ohio State Library (unknown)-REGIONAL.

Ohio State University Libraries (1907).

Ohio Supreme Court Law Library (1973).

The Public Library of Columbus and Franklin County (1885).

Dayton:

Dayton and Montgomery County Public Library (1909).

University of Dayton, Albert Emanuel Library (1969).

Wright State University Library (1965).

Delaware: Ohio Wesleyan University, L. A. Beeghly Library (1845)

Elyria: Elyria Public Library (1966)

Findlay: Findlay College, Shafer Library (1969).

Gambier: Kenyon College Library (1873).

Granville: Denison University Library (1884).

Hiram: Hiram College, Teachout-Price Memorial Library (1874).

Kent: Kent State University Libraries (1962).

Marietta: Marietta College, Dawes Memorial Library (1884).

Middletown: Miami University at Middletown, Gardner-Harvey Library (1970).

New Concord: Muskingum College Library (1966).

Oberlin: Oberlin College Library (1858).

Oxford: Miami University at Oxford, Alumni Library (1909).

Portsmouth: Portsmouth Public Library (unknown).

Rio Grande: Rio Grande College, Jeanette Albiez Davis Library (1966).

Springfield: Warder Public Library (1884).
Steubenville

Public Library of Steubenville and Jefferson County (1950).

The College of Steubenville, Starvaggi Memorial Library (1971).

Tiffin: Heidelberg College, Beeghly Library (1964).

Toledo:

Toledo-Lucas County Public Library (1884).

University of Toledo Library (1963)

Westerville: Otterbein College Library (1967).

Wooster: College of Wooster, Andrews Library (1966).

Youngstown:

Public Library of Youngstown and Mahoning County (1923).

Youngstown State University, William F. Maag Library (1971)

\section{OKLAHOMA}

Ada: East Central University, Linscheid Library (1914).

Alva: Northwestern Oklahoma State University Library (1907).

Bartlesville: U.S. Department of Energy, Bartlesville Energy Research Center Library (1962)

Bethany: Bethany Nazarene College, R. T. Williams Library (1971)

Durant: Southeastern Oklahoma State University Library (1929).

Edmond: Central State University Library (1934).

Enid: Public Library of Enid and Garfield County (1908).

Langston: Langston University, G. Lamar Harrison Library (1941).

Muskogee: Muskogee Public Library (1971)

Norman

University of Oklahoma Library (1893)

University of Oklahoma, School of Law Library (1978).

Oklahoma City:

Metropolitan Library System (1974)

Oklahoma City University Library (1963)

Oklahoma Department of Libraries (1893)-REGIONAL.

Shawnee: Oklahoma Baptist University Library (1933).

Stillwater: Oklahoma State University Library (1907).

Tahlequah: Northeastern Oklahoma State University, John Vaughan Library (1923).

Tulsa:

Tulsa City-County Library (1963).

University of Tulsa, College of Law Library (1979).

University of Tulsa, McFarlin Library (1929).

Weatherford: Southwestern Oklahoma State University, Al Harris Library (1958).

\section{OREGON}

Ashland: Southern Oregon State College Library (1953).

Corvallis: Oregon State University Library (1907).

Eugene: University of Oregon Library (1883).

Forest Grove: Pacific University Library (1897).

La Grande: Eastern Oregon College, Walter M. Pierce Library (1954).

McMinnville: Linfield College, Northup Library (1965).

Monmouth: Oregon College of Education Library (1967).

Portland:

Lewis and Clark College, Aubrey R. Watzek Library (1967).

Library Association of Portland (1884).

Portland State University Library (1963)-REGIONAL.

Reed College Library (1912).

U.S. Department of Energy, Bonneville Power Administration Library (1962)

Salem:

Oregon State Library (unknown)

Oregon Supreme Court Library (1974).

Willamette University, College of Law Library (1979).

Willamette University, Main Library (1969).

\section{PENNSYLVANIA}

Allentown: Muhlenberg College, Haas Library (1939).

Altoona: Altoona Public Library (1969). 
Bethlehem: Lehigh University, Linderman Library (1876).

Blue Bell: Montgomery County Community College, Learning Resources Center (1975).

Carlisle:

Dickinson College, Boyd Lee Spahr Library (1947).

Dickinson College, School of Law, Sheeley-Lee Law Li. brary (1978).

Cheyney: Cheyney State College, Leslie Pickney Hill Library (1967).

Collegeville: Ursinus College, Myrin Library (1963).

Coraopolis: Robert Morris College Library (1978).

Doylestown: Bucks County Free Library (1970).

East Stroudsburg: East Stroudsburg State College, Kemp Library (1966).

Erie: Erie City and County Library (1897).

Greenville: Thiel College, Langenheim Memorial Library (1963).

Harrisburg: State Library of Pennsylvania (unknown)-REGION. AL.

Haverford: Haverford College, Magill Library (1897).

Hazleton: Hazleton Area Public Library (1964).

Indiana: Indiana University of Pennsylvania, Rhodes R. Stabley Library (1962).

Johnstown: Cambria County Library System (1965).

Lancaster: Franklin and Marshall College, Fackenthal Library (1895).

Lewisburg: Bucknell University, Ellen Clarke Bertrand Library (1963).

Mansfield: Mansfield State College Library (1968).

Meadville: Allegheny College, Lawrence Lee Pelletier Library (1907).

MillersvUniversity, School of Law Library (1978).

La Roche College, John J. Wright Library (1974).

University of Pittsburgh, Hillman Library (1910).

University of Pittsburgh, School of Law Library (1979).

U.S. Department of Interior, Bureau of Mines Library (1962).

Pottsville: Pottsville Free Public Library (1967).

Reading: Reading Public Library (1901).

Scranton: Scranton Public Library (1895).

Shippensburg: Shippensburg State College, Ezra Lehman Memorial Library (1973).

Slippery Rock: Slippery Rock State College Library (1965).

Swarthmore: Swarthmore College Library (1923).

University Park: Pennsylvania State University Library (1907).

Villanova: Villanova University, Pulling Law Library (1964).

Warren: Warren Library Association (1885).

Washington: Washington and Jefferson College, Memorial Library (1884)

Waynesburg: Waynesburg College Library (1964).

West Chester: West Chester State College, Francis Harvey Green Library (1967).

Wilkes-Barre: King's College, D. Leonard Corgan Library (1949).

Williamsport: Lycoming College Library (1970).

York: York College of Pennsylvania Library (1963).

Youngwood: Westmoreland County Community College, Learning Resources Center (1972).

\section{PUERTO RICO}

Mayaguez: University of Puerto Rico, Mayaguez Campus Library (1928).

Ponce:

Catholic University of Puerto Rico, Encarnacion Valdes Library (1966).

Catholic University of Puerto Rico, School of Law Library (1978).

Rio Piedras: University of Puerto Rico, General Library (1928).

\section{RHODE ISLAND}

Kingston: University of Rhode Island Library (1907).

Newport: U.S. Naval War College Library (1963).
Providence:

Brown University, John D. Rockefeller, Jr. Library (unknown).

Providence College, Phillips Memorial Library (1969).

Providence Public Library (1884).

Rhode Island College, James P. Adams Library (1965).

Rhode Island State Library (before 1895).

Warwick: Warwick Public Library (1966).

Westerly: Westerly Public Library (1909).

Woonsocket: Woonsocket Harris Public Library (1977).

\section{SOUTH CAROLINA}

Charleston:

Baptist College at Charleston, L. Mendel Rivers Library (1967).

The Citadel, Daniel Library (1962).

The College of Charleston, Robert Scott Small Library (1869).

Clemson: Clemson University Library (1893).

Columbia:

Benedict College, Learning Resources Center (1969).

Richland County Public Library (1978).

South Carolina State Library (before 1895).

University of South Carolina, Thomas Cooper Library (1884).

Conway: University of South Carolina, Coastal Carolina College Library (1974).

Due West: Erskine College, McCain Library (1968).

Florence:

Florence County Library (1967).

Francis Marion College, James A. Rogers Library (1970).

Greenville:

Furman University Library (1962).

Greenville County Library (1966).

Greenwood: Lander College, Larry A. Jackson Library (1967).

Orangeburg: South Carolina State College, Miller F. Whittaker Library (1953).

Rock Hill: Winthrop College, Dacus Library (1896).

Spartanburg: Spartanburg County Public Library (1967).

\section{SOUTH DAKOTA}

Aberdeen: Northern State College Library (1963).

Brookings: South Dakota State University, H. M. Briggs Library (1889).

Pierre:

South Dakota State Library (1973).

South Dakota Supreme Court Library (1978)

Rapid City:

Rapid City Public Library (1963).

South Dakota School of Mines and Technology (1963).

Sioux Falls:

Augustana College, Mikkelsen Library and Learning Resources Center (1969).

Sioux Falls Public Library (1903).

Spearfish: Black Hills State College Library (1942).

Vermillion: University of South Dakota, I. D. Weeks Library (1889).

Yankton: Yankton College, James Lloyd Library (1904).

\section{TENNESSEE}

Bristol: King College, E. W. King Library (1970).

Chattanooga:

Chattanooga-Hamilton County Bicentennial Library (1908).

U.S. Tennessee Valley Authority Technical Library (1976).

Clarksville: Austin Peay State University, Felix G. Woodward Library (1945).

Cleveland: Cleveland State Community College Library (1973).

Columbia: Columbia State Community College, John W. Finney Memorial Library (1973).

Cookeville: Tennessee Technological University, Jere Whitson Memorial Library (1969).

Jackson: Lambuth College, Luther L. Gobbel Library (1967). 
Jefferson City: Carson-Newman College Library (1964).

Johnson City: East Tennessee State University, Sherrod Library (1942).

Knoxville:

Knoxville-Knox County Public Library (1973).

University of Tennessee at Knoxville, James D. Hoskins Library (1907).

University of Tennessee at Knoxville, Law Library (1971).

Martin: University of Tennessee at Martin, Paul Meek Library (1957).

Memphis:

Memphis-Shelby County Public Library and Information Center (1896).

Memphis State University, John W. Brister Library (1966).

Murfreesboro: Middle Tennessee State University Library (1912).

Nashville:

Fisk University Library (1965).

Joint University Libraries (1884).

Public Library of Nashville and Davidson County (1884).

Tennessee State Law Library (1976).

Tennessee State Library and Archives (unknown).

Tennessee State University Library (1972).

Vanderbilt University Law Library (1976).

Sewance: University of the South, Jesse Ball duPont Library (1873).

\section{TEXAS}

Abilene:

Abilene Christian University, Margarett and Herman Brown Library (1978).

Hardin-Simmons University, Rupert and Pauline Richardson Library (1940).

Arlington:

Arlington Public Library (1970)

University of Texas at Arlington Library (1963).

Austin:

Texas State Law Library (1972).

Texas State Library (unknown)-REGIONAL.

University of Texas at Austin Library (1884).

University of Texas at Austin, Lyndon B. Johnson School of Public Affairs Library (1966).

University of Texas at Austin, Tarlton Law Library (1965).

Baytown: Lee College Library (1970).

Beaumont: Lamar University, Mary and John Gray Library (1957).

Brownwood: Howard Payne College, Walker Memorial Library (1964).

Canyon: West Texas State University Library (1928).

College Station: Texas Agricultural and Mechanical University Library (1907).

Commerce: East Texas State University Library (1937).

Corpus Christi: Corpus Christi University Library (1976)

Corsicana: Navarro College, Gaston T. Gooch Library (1965).

Dallas:

Bishop College, Zale Library (1966).

Dallas Baptist College Library (1967).

Dallas Public Library (1900)

Southern Methodist University, Fondren Library (1925).

University of Texas Health Science Center Library at Dallas (1975).

Denton: North Texas State University Library (1948)

Edinburg: Pan American University Library (1959).

El Paso:

El Paso Public Library (1906)

University of Texas at El Paso Library (1966).

Fort Worth:

Fort Worth Public Library (1905).

Texas Christian University, Mary Couts Burnett Library (1916).

Galveston: Rosenberg Library (1909).

Houston:

Houston Public Library (1884).

North Harris County College, Learning Resources Center (1974).
Rice University, Fondren Library (1967)

University of Houston Library (1957).

University of Houston, School of Law Library (1979)

Huntsville: Sam Houston State University Library (1949).

Irving: Irving Public Library System (1974).

Kingsville: Texas Arts and Industries University Library (1944).

Lake Jackson: Brazosport College Library (1969).

Laredo: Laredo Junior College, Yeary Library (1970).

Longview: Nicholson Memorial Public Library (1961).

Lubbock:

Texas Tech University Library (1935)-REGIONAL.

Texas Tech University, School of Law Library (1978).

Marshall: Wiley College, Thomas Winston Cole, Sr. Library (1962).

Mesquite: Mesquite Public Library (1975).

Nacogdoches: Stephen F. Austin State University, Steen Library (1965).

Plainview: Wayland Baptist College, Van Howeling Memorial Library (1963).

Richardson: University of Texas at Dallas Library (1972).

San Angelo: Angelo State University Library (1964).

San Antonio:

San Antonio College Library (1972)

San Antonio Public Library, (1899).

St. Mary's University, Academic Library (1964).

Trinity University Library (1964).

University of Texas at San Antonio Library (1973).

San Marcos: Southwest Texas State University Library (1955).

Seguin: Texas Lutheran College, Blumberg Memorial Library (1970).

Sherman: Austin College, Arthur Hopkins Library (1963).

Texarkana: Texarkana Community College, Palmer Memorial Library (1963)

Victoria: University of Houston at Victoria Campus Library (1973).

Waco: Baylor University, Moody Memorial Library (1905).

Wichita Falls: Midwestern University, Moffett Library (1963).

\section{UTAH}

Cedar City: Southern Utah State College Library (1964)

Ephraim: Snow College, Lucy A. Phillips Library (1963)

Logan: Utah State University, Merrill Library and Learning Resources Center (1907)-REGIONAL.

Ogden: Weber State College, Stewart Library (1962).

Provo:

Brigham Young University, Harold B. Lee Library (1908).

Brigham Young University, Law Library (1972).

Salt Lake City:

University of Utah, Eccles Health Sciences Library (1970).

University of Utah, Law Library (1966).

University of Utah, Marriott Library (1893).

Utah State Library Commission, (unknown).

Utah State Supreme Court, Law Library (1975).

\section{VERMONT}

Burlington: University of Vermont, Bailey Library (1907).

Castleton: Castleton State College, Calvin Coolidge Library (1969).

Johnson: Johnson State College, John Dewey Library (1955).

Lyndonville: Lyndon State College, Samuel Reed Hall Library (1969).

Middlebury: Middlebury College, Egbert Starr Library (1884).

Montpelier: Vermont Department of Libraries (before 1895).

Northfield: Norwich University Library (1908).

South Royalton: Vermont School of Law Library (1978).

\section{VIRGIN ISLANDS}

St. Croix: Florence Williams Public Library (1974).

St. Thomas:

College of the Virgin Islands, Ralph M. Paiewonsky Library (1973).

Enid M. Baa Public Library (1968). 


\section{VIRGINIA}

Blacksburg: Virginia Polytechnic Institute and State University, Carol M. Newman Library (1907).

Bridgewater: Bridgewater College, Alexander Mack Memorial Library (1902).

Charlottesville:

University of Virginia, Alderman Library (1910)-REGIONAL.

University of Virginia Law School, Arthur J. Morris Law Library (1964).

Chesapeake: Chesapeake Public Library (1970).

Danville: Danville Community College Library (1969).

Emory: Emory and Henry College, Kelly Library (1884).

Fairfax: George Mason University Library (1960).

Fredericksburg: Mary Washington College, E. Lee Trinkle Library (1940)

Hampden-Sydney: Hampden-Sydney College, Eggleston Library (1891).

Hampton: Hampton Institute, Huntington Memorial Library (1977).

Harrisonburg: James Madison University, Madison Memorial Library (1973).

Hollins College: Hollins College, Fishburn Library (1967).

Lexington:

Virginia Military Institute, Preston Library (1874).

Washington and Lee University, Cyrus Hall McCormick Library (1910).

Washington and Lee University, Wilbur C. Hall School of Law Library (1978).

Martinsville: Patrick Henry Community College Library (1971). Norfolk:

Norfolk Public Library (1895).

Old Dominion University Library (1963).

U.S. Armed Forces Staff College Library (1963).

Petersburg: Virginia State College, Johnston Memorial Library (1907).

Quantico:

Federal Bureau of Investigation, Academy Library (1970).

U.S. Marine Corps Schools, James Carson Breckinridge Library (1967).

Reston: Department of the Interior, Geological Survey, National Center Library (1962).

Richmond:

University of Richmond, Boatwright Memorial Library (1900).

U.S. Court of Appeals, Fourth Circuit Library (1973)

Virginia Commonwealth University, James Branch Cabell Library (1971).

Virginia State Law Library (1973).

Virginia State Library (unknown).

Roanoke: Roanoke Public Library (1964).

Salem: Roanoke College Library (1886).

Williamsburg:

College of William and Mary, Marshall-Wythe Law Library (1978).

College of William and Mary, Swem Library (1936).

Wise: Clinch Valley College, John Cook Wyllie Library (1971).

\section{WASHINGTON}

Bellingham: Western Washington University, Mabel Zoe Wilson Library (1963).

Cheney: Eastern Washington University Library (1966).

Ellensberg: Central Washington University Library (1962).

Everett: Everett Public Library (1914).

Olympia:

Evergreen State College, Daniel J. Evans Library (1972)

Washington State Library (unknown)-REGIONAL.

Port Angeles: North Olympic Library System (1965).

Pullman: Washington State University Library (1907).

Seattle:

Seattle Public Library (1908).

University of Washington Libraries (1890).

University of Washington, Law Library (1965).
Spokane:

Gonzaga University, School of Law Library (1979)

Spokane Public Library (1910)

Tacoma:

Tacoma Public Library (1894).

University of Puget Sound, Collins Memorial Library (1938).

University of Puget Sound, School of Law Library (1978).

Vancouver: Fort Vancouver Regional Library (1962).

Walla Walla: Whitman College, Penrose Memorial Library (1890).

\section{WEST VIRGINIA}

Athens: Concord College Library (1924).

Bluefield: Bluefield State College Library (1972).

Charleston:

Kanawha County Public Library (1952).

West Virginia College of Graduate Studies Library (1977).

West Virginia Library Commission (unknown).

West Virginia Supreme Court Law Library (1977).

Elkins: Davis and Elkins College Library (1913).

Fairmont: Fairmont State College Library (1884).

Glenville: Glenville State College, Robert F. Kidd Library (1966).

Huntington: Marshall University, James E. Morrrow Library (1925).

Institute: West Virginia State College, Drain-Jordan Library (1907).

Morgantown: West Virginia University Library (1907)-RE. GIONAL.

Salem: Salem College Library (1921).

Shepherdstown: Shepherd College Library (1971).

Weirton: Mary H. Weir Public Library (1963).

\section{WISCONSIN}

Appleton: Lawrence University, Seeley G. Mudd Library (1869).

Beloit: Beloit College Libraries (1888).

Eau Claire: University of Wisconsin-Eau Claire, William D. McIntyre Library (1951).

Fond du Lac: Fond du Lac Public Library (1966).

Green Bay: University of Wisconsin-Green Bay Library (1968).

La Crosse:

La Crosse Public Library (1883).

University of Wisconsin-La Crosse, Murphy Library (1965).

Madison:

Madison Public Library (1965).

State Historical Society Library (1870)-REGIONAL, in cooperation with University of Wisconsin-Madison, Memorial Library.

University of Wisconsin-Madison, Memorial Library (1939).

Wisconsin State Law Library (unknown).

Milwaukee:

Alverno College Library (1971).

Milwaukee County Law Library (1934).

Milwaukee Public Library (1861)-REGIONAL.

Mount Mary College Library (1964).

University of Wisconsin-Milwaukee Library (1960).

Oshkosh: University of Wisconsin-Oshkosh, Forrest R. Polk Library (1956).

Platteville: University of Wisconsin-Platteville, Karrmann Library (1964).

Racine: Racine Public Library (1898).

River Falls: University of Wisconsin-River Falls, Chalmer Davee Library (1962)

Stevens Point: University of Wisconsin-Stevens Point, Learning Resources Center (1951).

Superior:

Superior Public Library (1908)

University of Wisconsin-Superior, Jim Dan Hill Library (1935).

Waukesha: Waukesha Public Library (1966)

Wausau: Marathon County Public Library (1971).

Whitewater: University of Wisconsin-Whitewater, Harold Andersen Library (1963). 


\section{WYOMING}

Casper: Natrona County Public Library (1929).

\section{Cheyenne:}

Wyoming State Law Library (1977).

Wyoming State Library (unknown)-REGIONAL.

\section{Laramie:}

University of Wyoming, Coe Library (1907).

University of Wyoming, School of Law Library (1978).

Powell: Northwest Community College Library (1967).

Riverton: Central Wyoming College Library (1969).

Rock Springs: Western Wyoming Community College Library (1969).

Sheridan: Sheridan College, Kooi Library (1963). 


\section{APPENDIX B. LIST OF DISTRICT OFFICES OF THE U.S. DEPARTMENT OF COMMERCE}

\section{ALABAMA}

Blrmingham-Gayle C. Shelton, Jr., Director, Suite 200-201,908 South 20th Street, 35205, Area Code 205 Tel 254. 1331, FTS 229-1331

\section{ALASKA}

Anchorage-Blaine D. Porter, Director, 701 C Street. P.O. Box 32, 99513. Area Code 907 Tel 271-5041, FTS Dial 8399 0150. Ask for 271-5041

\section{ARIZONA}

Phoenlx -Donald W. Fry Director, Suite 2950 Valley Bank Center, 201 North Central Avenue 85073, Area Code 602 Tel 261-3285, FTS 261-3285

\section{ARKANSAS}

Little Rock-Robert E. Kistler, Director Suite 635, Savers Federal Building, 320 W. Capitol Avenue, 72201. Area Code 501 Tel 378-5794, FTS 740-5794

\section{CALIFORNIA}

Los Angeles-Paul W. Leinenbach, Director Room 800, 11777 San Vicente Boulevard 90049. Area Code $213 \mathrm{Te}$ 824-7591, FTS 799-7591

-San Dlego-110 West C Street 92101. Area Code 714 Tel 293-5395

San Franclsco-Betty D. Neuhart Direc tor Federal Building, Box 36013, 450 Golden Gate Avenue 94102, Area Code 415 Tel 556-5860. FTS 556-5868

\section{COLORADO}

Denver-Donald L. Schilke, Director Room 177, U.S. Customhouse, 721-19th Street, 80202, Area Code 303 Tel 837 . 3246, FTS 327-3246

\section{CONNECTICUT}

Hartford-Director (Vacant), Room 610B. Federal Office Building, 450 Main Street 06103, Area Code 203 Tel 2443530, FTS 244-3530

\section{FLORIDA}

Mlaml-Ivan A. Cosimi, Director, Room 821. City National Bank Building, 25 Wes Flagler Street 33130, Area Code $305 \mathrm{Tel}$ 350-5267, FTS 350-5267

- Clearwater-128 North Osceola Avenue 33515, Area Code 813 Tel 461-0011

-Jacksonvllle-815 S. Maine Street. Suite 100, 32207, Area Code 904 Tel 791-2796, FTS 946-2796

-Tallahassee-Collins Bldg., Rm. G20 32304, Area Code 904 Tel 4886469 , FTS $946-4320$

\section{GEORGIA}

Atlanta-Daniel M. Paul, Director, Suite 600, 1365 Peachtree Street, N.E. 30309 Area Code 404 Tel 881-7000, FTS 2577000

Savannah-James W. Mclntire, Director 222 U.S. Courthouse \& P.O. Box 9746 125-29 Bull Street, 31412, Area Code 912 Tel 944-4204, FTS 248-4204

\section{HAW All}

Honolulu-H. Tucker Gratz, Director, 4106 Federal Building, P.O. Box 50026, 300 Ala Moana Boulevard 96850. Area Code 808 Tel 546-8694, FTS 8 808-5468694

\section{ILLINOIS}

Chlcago-Gerald M. Marks, Director, 1406 Mid Continental Plaza Building, 55 East Monroe Street 60603, Area Code 312 Tel 353-4450, FTS 353-4450

- Commerce BusIness Dally

Room 1304, 433 West Van Buren Street 60607, Area Code 312 Tel 3532950

\section{INDIANA}

Indlanapolls-Mel R. Sherar, Director 357 U.S. Courthouse \& Federal Office Building 46 East Ohio Street 46204. Area Code 317 Tel 269-6214, FTS 331-6214

\section{IOWA}

Des Molnes-Jesse N. Durden, Director 817 Federal Building, 210 Walnut Street 50309. Area Code 515 Tel 284-4222 FTS 862-4222

\section{KENTUCKY}

Loulsullle-Donald R. Henderson, Director, Room 636B, U.S. Post Office and Court House Building 40202, Area Code 502 Tel 582-5066, FTS 352-5066

\section{LOUISIANA}

New Orleans-Raymond E. Eveland, Director, 432 International Trade Mart, No. 2 Canal Street 70130, Area Code 504 Tel 589-6546, FTS 682-6546

\section{MAINE}

- Augusta (Boston, Massachusetts Dlstrlct)-1 Memorial Circle, Casco Bank Bldg., Area Code 207 Tel 6232239, FTS $833-6249$

\section{MARYLAND}

Baltimore-Carroll F. Hopkins, Director 415 U.S. Customhouse, Gay and Lombard Streets 21202. Area Code 301 Tel 962-3560, FTS 922-3560

\section{MASSACHUSETTS}

Boston-Francis J. O'Connor, Director 10th Floor, 441 Stuart Street 02116, Area Code 617 Tel 223-2312, FTS 223-2312

\section{MICHIGAN}

Detrolt-Raymond R. Riesgo, Director 445 Federal Building, 231 Wes Layfayette 48226, Area Code $313 \mathrm{Tel}$ 226-3650, FTS 226-3650

-Grand Raplds-300 Monroe N.W. Rm. 40949503 Area Code $616 \mathrm{Te}$ 456-2411/33 FTS 372-2411

\section{MINNESOTA}

MInneapolls-Glenn A. Matson, Director, 218 Federal Building, 110 South Fourth Street 55401. Area Code 612 Tel 725-2133, FTS 725-2133

\section{MISSISSIPPI}

Jackson-Mark E. Spinney, Director, City Center Plaza, Suite 550, 200 East Pascagoula 39201, Area Code $601 \mathrm{Tel}$ 960-4388, FTS 490-4388

\section{MISSOURI}

St. Louls-Donald R. Loso, Director, 120 South Central Avenue 63105, Area Code 314 Tel 425-3302-4, FTS 279-3302

Kansas Clty-James D. Cook, Director Room 1840, 601 East 12th Street 64106 Area Code 816 Tel 374-3142, FTS 758 3142

\section{NEBRASKA}

Omaha-George H. Payne, Director, Empire State Bldg., 1st Floor, 300 South 19th Street, 68102, Area Code 402 Tel 2213664, FTS 864-3664

\section{NEVADA}

Reno-Joseph J. Jeremy, Director, 777 W. 2nd Street, Room 120, 89503, Area Code 702 Tel $784-5203$, FTS 470-5203

\section{NEW JERSEY}

Newark-Thomas J Murray, Director 4th Floor, Gateway Building, Markel Street \& Penn Plaza 07102, Area Code 201 Tel 645-6214, FTS 341-6214

\section{NEW MEXICO}

Albuquerque-William E. Dwyer, Director, 505 Marquette Ave., NW, Suite 1015 87102. Area Code 505 Tel 766-2386, FTS 474-2386

\section{NEW YORK}

Buffalo-Robert F. Magee, Director, 1312 Federal Building, 111 West Huron Street 14202. Area Code 716 Tel 8464191. FTS 437-4191

New York-Arthur C. Rutzen, Director, Room 3718, Federal Office Building, 26 Federal Plaza, Foley Square 10278, Area Code 212 Tel 264-0634, FTS 264-0600 
NORTH CAROLINA

Greensboro-Joel B. New, Director, 203 Federal Building. West Market Street P.O. Box 195027402 , Area Code $919 \mathrm{Te}$ 378-5345, FTS 699-5345

\section{OHIO}

CIncinnatl-Gordon B. Thomas, Director, 10504 Federal Office Building, 550 Main Street 45202, Area Code $513 \mathrm{Tel}$ 684-2944, FTS 684-2944

Cleveland-Zelda W. Milner, Director, Room 600, 666 Euclid Avenue 44114, Area Code 216 Tel 522-4750, FTS 2934750

\section{OKLAHOMA}

- Oklahoma Clity (Dallas, Texas Distrlct)-4024 Lincoln Boulevard 73105, Area Code 405 Tel 231-5302. FTS $736-5302$

\section{OREGON}

Portland-Lloyd R. Porter, Director, Room 618, 1220 S.W. 3rd Avenue 97204. Area Code 503 Tel 221-3001. FTS 423-3001

\section{PENNSYLVANIA}

Phlladelphla-Patrick P. McCabe, Director, 9448 Federal Building, 600 Arch Street 19106 Area Code 215 Tel 5972866, FTS 597-2866

PIttsburgh-William M. Bradiey, Director, 2002 Federal Building, 1000 Liberty Avenue 15222, Area Code 412 Tel 6442850, FTS $722-2850$
San Juan (Hato Rey) - J. Enrique Vilella Director, Room 659-Federal Building 00918, Area Code 809 Tel 753-4555, Ext 555, FTS 8-809-7533

\section{RHODE ISLAND}

-Provldence (Boston, Massachusetts

Dlstrlct)-7 Jackson Walkway 02903 , Area Code $401 \mathrm{Tel}$ 277-2605, ext. 22, FTS $838-4482$

\section{SOUTH CAROLINA}

Columbla-Margaret A. Patrick Director Strom Thurmond Fed. Bldg., Suite 172 1835 Assembly Street 29201 Area Code 803 Tel 765-5345, FTS 677-5345

- Charleston-505 Federal Building 334 Meeting Street 29403, Area Code 803 Tel 677-4361, FTS677-4361

- Greenville-P.O. Box 5823, Station B, 29606, Area Code 803 235-5919

\section{TENNESSEE}

Memphls-Bradford H. Rice, Director, Room 710, 147 Jefferson Avenue 38103 , Area Code 901 Tel 521-3213, FTS 222 3213

-Nashvllle-Room 1020, Andrew Jackson Office Building 37219, Area Code 615 Tel 251-5161 FTS 852-5161

\section{TEXAS}

Dallas-C. Carmon Stiles, Director, Room 7A5, 1100 Commerce Street 75242 Area Code 214 Tel 767-0542, FTS 729-0542

Houston-Felicito C. Guerrero, Director 2625 Federal Bldg., Courthouse, 515 Rusk Street 77002, Area Code $713 \mathrm{Te}$ 226-4231, FTS 527-4231
Salt Lake Clty-Stephen P. Smoot, Director, U.S. Courthouse, $350 \mathrm{~S}$. Main Street 84101, Area Code 801 Tel 524 5116, FTS 588-5116

\section{VIRGINIA}

Richmond-Philip A Ouzts, Director, 8010 Federal Bldg., 400 North 8ith Street, 23240, Area Code 804 Tel 771-2246 FTS $925-2246$

- Falrfax-8550 Arlington Blvd. 22031. Area Code 703 Tel 56C-6460. FTS 235- 1519

\section{WASHINGTON}

Seattle-Eric C. Silbersiein, Director, Room 706, Lake Union Building, 1700 Westlake Avenue North 98109. Area Code 206 Tel 442-5616. FTS 399-5615

\section{WEST VIRGINIA}

Charleston-Roger L. Fortner, Director 3000 New Federal Building, 500 Quarrier Street 25301, Area Code $304 \mathrm{Tel} 343$ 6181 , ext. 375 , FTS 924-1375

\section{WISCONSIN}

Mllwaukee-Russell H. Leitch, Director Federal Bldg/US. Courthouse, 517 Eas Wisconsin Avenue 53202, Area Code 414 Tel 29I-3473, FTS 362-3473

\section{WYOMING}

Cheyenne-Lowell O. Burns, Director 6022 O'Mahoney Federal Center, 2120 Capitol Avenue 82001, Area Code 307 Tel 778-2220, ext. 2151, FTS 328-2151 

NBS-114A (REV. 2-8C)

1. PUBLICATION OR REPORT NO.

BIBLIOGRAPHIC DATA

SHEET (See instructions)

4. TITLE AND SUBTITLE

Publications of the National Bureau of Standards

1981 Catalog

\section{5. $\operatorname{AUTHOR}(S)$}

Betty L. Burris and Rebecca J. Morehouse, Editors

6. PERFORMING ORGANIZATION (If joint or other than NBS, see instructions)

NATIONAL BUREAU OF STANDARDS

DEPARTMENT OF COMMERCE

WASHINGTON, D.C. 20234

7. Contract/Grant No.

8. Type of Report \& Period Covered

9. SPONSORING ORGANIZATION NAME AND COMPLETE ADDRESS (Street, City, State, ZIP)

Same as item 6 .

10. SUPPLEMENTARY NOTES

Library of Congress Catalog Card No. 48-47112.

[D Document describes a computer program; SF-185, FIPS Software Summary, is attached.

11. ABSTRACT (A 200-word or less factual summary of most significant information. If document includes a significant tibliography or literature survey, mention it here)

The 13th Supplement to Special Publication 305 lists the 1981 papers which reflect the results of the National Bureau of Standards programs. Also included are those NBS papers published prior to 1981 but not reported in previous supplements of SP305. In addition to bibliographic data, key words, and abstracts for each publication and/or paper, the catalog provides an author and key word index.

12. KEY WORDS (Six to twelve entries; alphabetical order: capitalize only proper names; and separate key words by semicolons)

abstracts, NBS publications; key words; publications, NBS .

\section{AVAILABILITY}

X] Unlimited

[ For Official Distribution. Do Not Release to NTIS

X] Order From Superintendent of Documents, U.S. Government Printing Office, Washington. D.C. 20402.

— Order From National Technical Information Service (NTIS), Springfield, VA. 2216I
14. NO. OF PRINTED PAGES

474

15. Price

$\$ 11.00$ 



\section{Announcement of New Publications}

of the

National Bureau of Standards

Superintendent of Documents, Government Printing Office,

Washington, DC 20402

\section{Dear Sir:}

Please add my name to the announcement list of new publications as issued by the National Bureau of Standards.

Name

Company

Address.

City ..State . Zip code.......

(Notification Key N519) 



ORDER FORM To: Superintendent of Documents, U.S. Government Printing Office, Washington, D.C. 20402
Cnclosed is $\mathbf{C}$ Crodit Card Orders Oniy
$\square$ money order, or charge to my
Deposit Account No.

Company or personal name

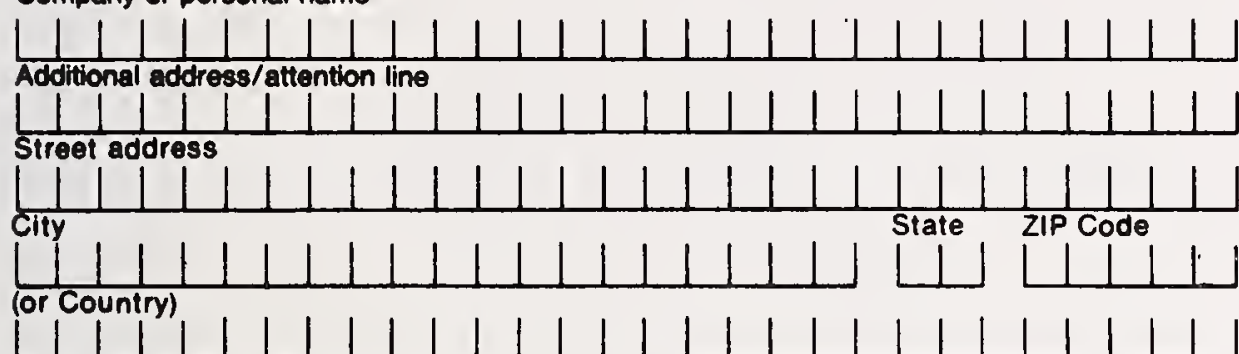

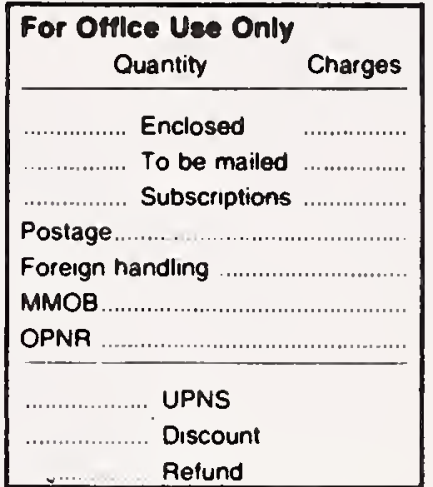

\section{PLEASE PRINT OR TYPE}

ORDER FORM To: Superintendent of Documents, U.S. Government Printing Office, Washington, D.C. 20402
Enclosed is $\$$ Credit Card Orders Only
$\begin{aligned} & \text { Tonoy order, or charge to my } \\ & \text { Deposit Account No. }\end{aligned}$
Order No.

Company or personal name

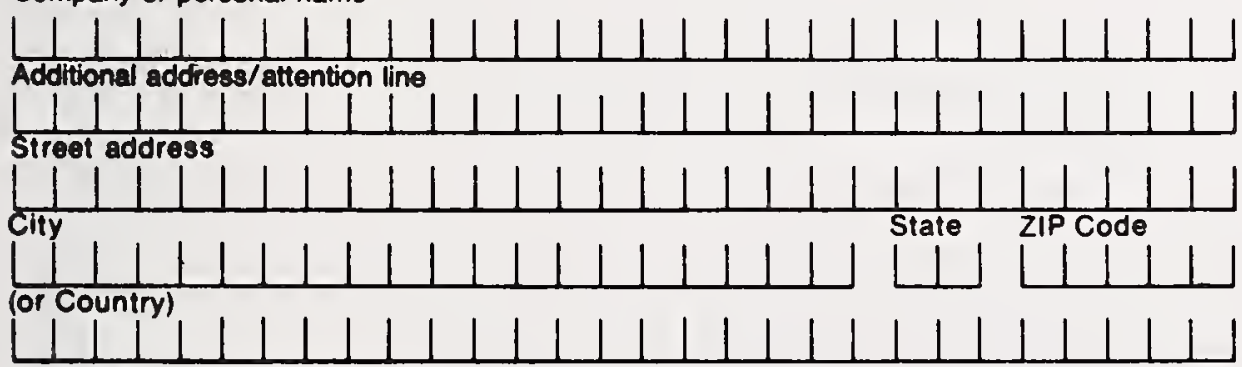

\begin{tabular}{|c|c|}
\hline $\begin{array}{c}\text { For Offlce Use Only } \\
\text { Ouantity }\end{array}$ & Charges \\
\hline 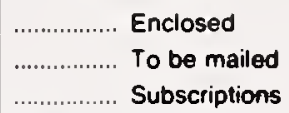 & \\
\hline Postage & 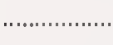 \\
\hline Foreign nandling ... & \\
\hline MMOB $\ldots \ldots \ldots \ldots$ & \\
\hline OPNR .......... & \\
\hline UPNS & \\
\hline $\begin{array}{c}\text { Discount } \\
\text { Refund }\end{array}$ & \\
\hline
\end{tabular}

PLEASE PRINT OR TYPE 

PURCHASER:

Telephone:

Attention:

-

For DTIC Users Only

DTIC User Code

Contract Number

(last 6 characters only)

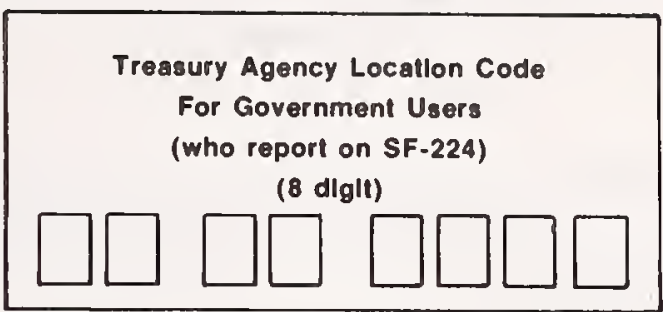

For Government Users

(8 dlglt)

Date
SHIP TO:

(Enter if different from address at left)

Name

Organization

Address

City, State, ZIP

\section{ORDER OPTIONS}

$\square$ Charge my NTIS deposit account no.

$\square$ Purchase order no.

$\square$ Check enclosed for $\$$

$\square$ Ship \& Bill See reverse (not applicable outside North America).

$\square$ Charge to my $\square$ American Express $\square$ Visa $\square$ Master Card

Account no.

Card expiration date

Signature

(Required to validate order)
It is vital that you order by NTIS order number or your order will be manually filled, causing a delay. You can opt for airmail/first class delivery as indicated below. Just check the Priority Mail Services box. If you're really pressed for time, call the NTIS Rush Handling Service (703) $487-4700$ or $(800) 336-4700$. For a $\$ 10$ per copy charge your order will be mailed within 8 working hours. Or, you can pick up your order in the Washington Information Center \& Bookstore or at our Springfield Operations Center within 24 hours for a $\$ 6$ per copy charge.

\begin{tabular}{|c|c|c|c|c|c|c|c|c|}
\hline \multirow[b]{2}{*}{ NTIS ORDER NUMBER *** } & \multirow{2}{*}{$\begin{array}{l}\text { USER ROUTING } \\
\text { CODE (see reverse) }\end{array}$} & \multicolumn{3}{|c|}{ QUANTITY } & \multirow{2}{*}{$\begin{array}{l}\text { UNIT * } \\
\text { PRICE }\end{array}$} & \multirow{2}{*}{\multicolumn{2}{|c|}{$\begin{array}{l}\text { PRIORITY* } \\
\text { MAIL } \\
\text { SERVICES }\end{array}$}} & \multirow{2}{*}{$\begin{array}{l}\text { TOTAL } \\
\text { PRICE }\end{array}$} \\
\hline & & $\begin{array}{l}\text { Paper } \\
\text { Copy }\end{array}$ & $\begin{array}{l}\text { Micro- } \\
\text { fiche }\end{array}$ & $\begin{array}{c}\text { Other } \\
\text { (specify) }\end{array}$ & & & & \\
\hline & & & & & & & & \\
\hline & & & & & & & & \\
\hline & & & & & & & & \\
\hline & & & & & & & & \\
\hline & & & & & & & & \\
\hline & & & & & & & & \\
\hline & & & & & & & & \\
\hline & & & & & & & & \\
\hline & & & & & & & & \\
\hline $\begin{array}{l}\text {-Add } \$ 3 \text { per item for First Class Deliv } \\
\text { Add } \$ 4 \text { for each paper copy Airmail } \\
\text { " ALL PRIIES SUBJECT TO CHANGE. } \\
\text { "If ordering by title or if item ordered }\end{array}$ & $\begin{array}{l}\text { nerica; } \\
\text { e North America. } \\
\text { tape, please see revers }\end{array}$ & side. & & & & & $\$$ & \\
\hline
\end{tabular}


USER ROUTING CODE:

SHIP \& BILL SERVICE:

ORDERING MAGNETIC TAPE: (check model)
NTIS can label each item for routıng withın your organizatıon. If you want this service put your routing code in the box marked USER ROUTING CODE (LImıt eight characters).

Prepayment helps 10 expedite your order and can be accomplished through the use of an NTIS Deposit Account. check. money order, or charge card account number - For "Ship and BIll," NTIS charges $\$ 5$ extra for each order (regardless of the number of items; $\$ 5$ extra for each NTISearch: • NTIS does not "Ship and Bill" for orders outside North America
$\square 7$ track
$\square 800 \mathrm{BPI}$
$\square$ odd parity
$\square 556 \mathrm{BPI}$
$\square$ even parity
$\square 9$ track
$\square 1600 \mathrm{BPI}$
$\square 800 \mathrm{BP}$

(odd parity)
ORDERING BY TITLE:

If ordering without an NTIS order number (by tıtle only) allow an additional two weeks.

\section{TITLE \#1}

\begin{tabular}{|l|l|l|}
\hline Sponsor's Series \# & Contract or Grant Number of Report & Date Published \\
\hline Originator (Give specific laboratory, or division and location.) & Personal Author
\end{tabular}

Originator (Give specific laboratory, or division and localion.)

Turn to other side. Write " 1 " in the NTIS Order Number block and complete the rest of the line

\section{TITLE \#2}

Sponsor's Serles \#

\section{TITLE \#3}

Sponsor's Series \#

Turn to other side. Write " 3 " in the NTIS Order Number block and complete the rest of the line

\section{TITLE \#4}

Sponsor's Series \#

\section{TITLE \#5}

Sponsor's Series \# 
PURCHASER:

Telephone:

Attention:
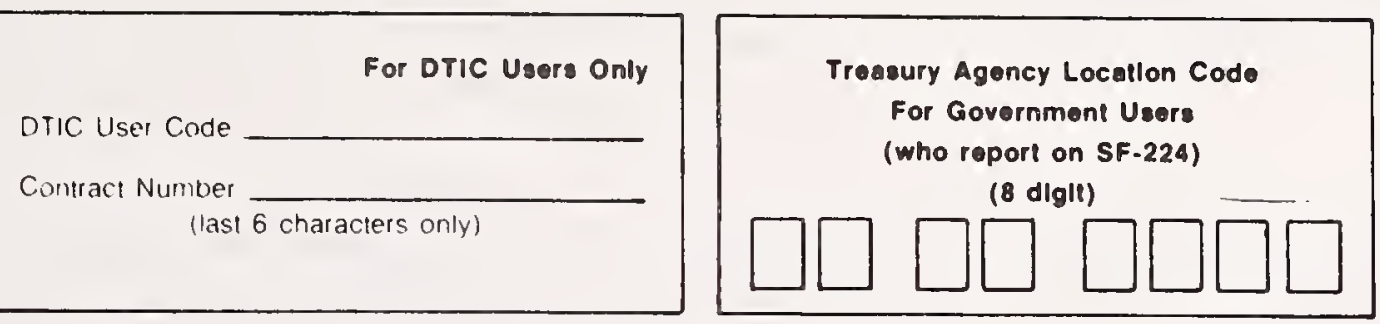

SHIP TO:

(Enter if different from address at left)

Date

Name

Organizatıon

Address

City. State, ZIP

\section{Method of Payment}

$\square$ Charge my NTIS deposit account no

$\square$ Purchase order no

$\square$ Check enclosed for $\$$

$\square$ Ship \& Bill See reverse (not applicable outside North America)

$\square$ Charge to my $\square$ American Express $\square$ Visa $\square$ Master cirrd

Account no

Card expiration dare

Signature

(Required 10 validate order)

\section{ORDER OPTIONS}

It is vital that you order by NTIS order number or your order will be manually filled. causing a delay. You can opt for airmail/first class delivery as indicated below. Just check the Priority Mail Services box. If you're really pressed for time, call the NTIS Rush Handling Service (703) 487.4700 or $(800) 336.4700$. For a $\$ 10$ per copy charge your order will be malled within 8 working hours. Or, you can pick up your order in the Washington Information Center \& Bookstore or at our Springfield Operations Center within 24 hours for a $\$ 6$ per copy charge.

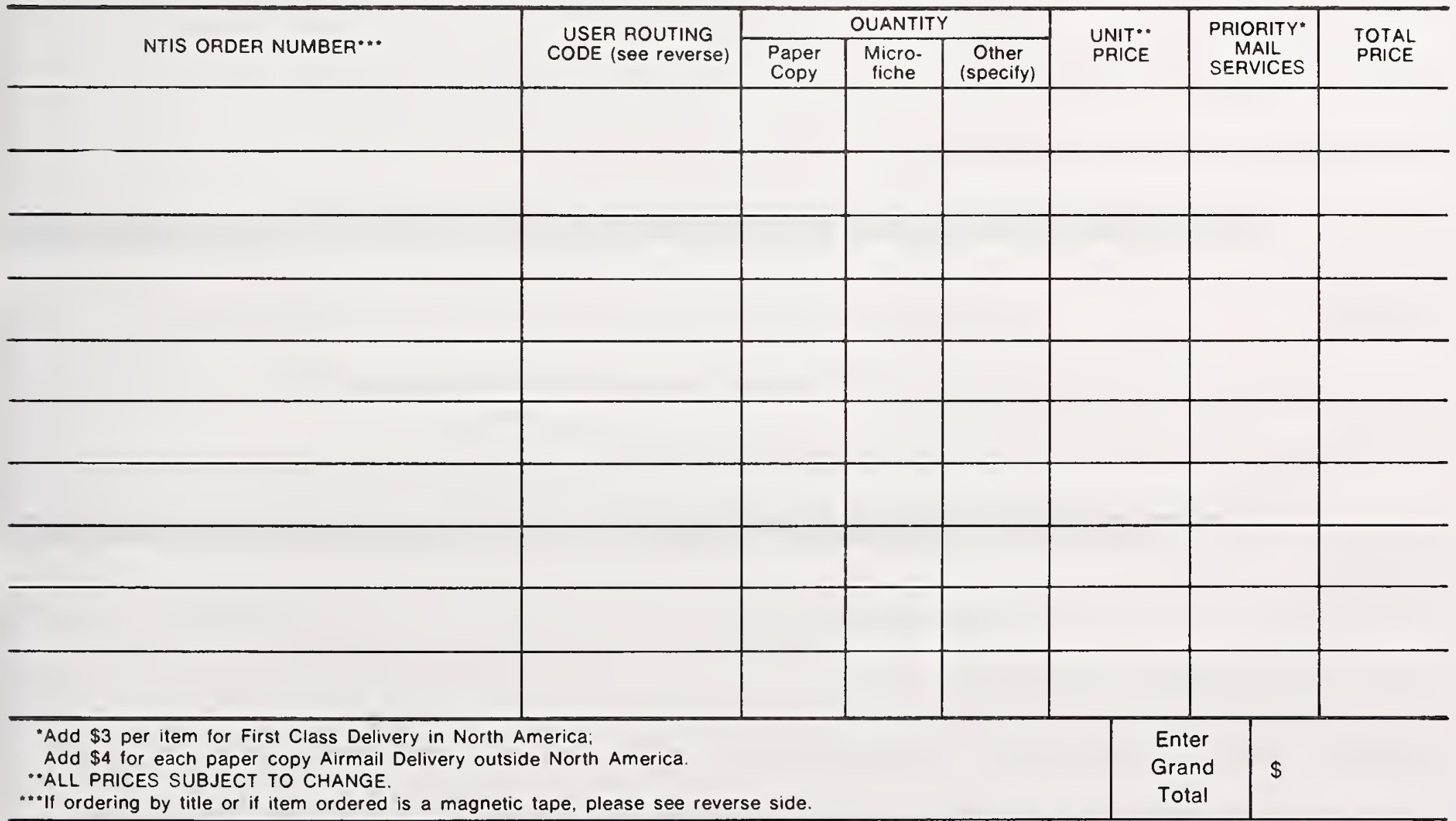


USER ROUTING CODE:

SHIP \& BILL SERVICE:

ORDERING MAGNETIC TAPE: (check model)
NTIS can label each item for routing within your organization. If you want this service put your routing code in the box marked USER ROUTING CODE (LIm) eight characters).

Prepayment helps 10 expedite your order and can be accomplished through the use of an NTIS Deposit Account. check. money order, or charge card account number - For "Ship and BIll," NTIS charges $\$ 5$ extra for each order (regardless of the number of ttems; $\$ 5$ extra for each NTISearch: - NTIS does not "Ship and Bill" for orders outside North America
$\square 7$ track
$\square 800 \mathrm{BPI}$
$\square$ odd parity
$\square 556 \mathrm{BPI}$
$\square$ even parity
$\square 9$ track
$\square 1600 \mathrm{BP}$
$\square 800 \mathrm{BPI}$

(odd parity)

ORDE.RING BY TITLE:

If ordering without an NTIS order number (by title only) allow an additional two weeks.

\section{TITLE \#1}

\begin{tabular}{|l|l|l|}
\hline Sponsor's Series \# & Contract or Grant Number of Report & Date Published \\
\hline \multicolumn{2}{|l|}{ Originator (Give specific laboratory, or division and location.) } & Personal Author
\end{tabular}

Turn to other side. Write " 1 " in the NTIS Order Number block and complete the rest of the line.

\section{TITLE \#2}

Sponsor's Serles \# Contract or Grant Number of Report

Turn to other side. Write "2" in the NTIS Order Number block and complete the rest of the line.

\section{TITLE \#3}

Sponsor's Series \# Contract or Grant Number of Report

Originator (Give specific laboratory, or division and location.)

Turn to other side. Write " 3 " in the NTIS Order Number block and complete the rest of the line.

\section{TITLE \#4}

Sponsor's Series \# Contract or Grant Number of Report

\section{TITLE \#5}

Sponsor's Series \#

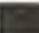

\begin{tabular}{l|l}
\hline & \\
\hline
\end{tabular}

Originator (Give specitic laboratory, or division and location.) 

5 




\section{NBS TECHNICAL PUBLICATIONS}

\section{PERIODICALS}

JOURNAL OF RESEARCH-The Journal of Research of the National Bureau of Standards reports NBS research and development in those disciplines of the physical and engineering sciences in which the Bureau is active. These include physics, chemistry, engineering, nathematics, and computer sciences. Papers cover al broad range of subjects, with major emphasis on measurement methodology and the basic technology underlying standardization Also included from time to time are survey articles on topics closely related to the Bureau's technical and scientific programs As a special service to subscribers each issue contains complete citations to all recent Bureau publications in both NBS and nonNBS media. Issued six times a year. Annual subscription: domestic \$18; forcign \$22.50. Single copy, \$4.25 domcstic; $\$ 5.35$ foreign.

\section{NONPERIODICALS}

Monographs-Major contributions to the technical literature on various subjects related to the Bureau's scientific and technical activities.

Handbooks-Recommended codes ol engineering and industrial practice (including safety codes) developed in cosperation with interested industries, prolessional organizations, and regulatory bodies.

Special Publications-Include proceedings of conferences sponsored by NBS, NBS annual reports, and other special publications appropriate to this grouping such as wall charts, pocket cards, and bibliographies.

Applied Mathematics Series-Mathematical tables, manuals, and studies ol special interest to physicists, engineers, chemists, biologists, mathematicians, conputer programmers, and others engaged in scientilic and technical work.

National Standard Reference Data Series-Provides quantitative datil on the physical and chemical propertics of milterials, contpiled lrom the world's literature and critically evaluated. Developed under a worldwide program coordinated by NBS under the aluthority of the Nattonal Standird Data Act (Public Law 90-396).

NOTE: The principal publication outlet lor the foregoing data is the Journal of Physical and Chemical Reference Datal (JPCRD) published quarterly lor NBS by the Americin Chemical Society (ACS) and the American Institute ol Physics (AIP). Subscriptions. reprints. and supplements ilvailiable l'rom ACS, 1155 Sixteenth St.. NW, Washington, DC 20056.
Building Science Series-Disseminates technical in Tormation developed at the Bureau on building materials, components, systems, and whole structures. The series presents research results, test methods, and performance criteria related to the structural and environmental functions and the durability and safety characteristics of building elements and systems.

Technical Notes-Studies or reports which are complete in themselves but restrictive in their treatment of a subject. Analogous to monographs but not so comprehensive in scope or definitive in treatment of the subject area. Often serve as a vehicle for final reports of work performed at NBS under the sponsurship of other government agencies:

Voluntary Product Standards-Developed under procedures published by the Department of Commerce in Part 10. Title 15. of the Code of Federal Regulations. The standards establish nationally recognized requirements for products, and provide all concerned interests with a basis for common understanding of the characteristics of the products. NBS administers this program as a supplement to the activities ol the private sector standardizing organizations

Consumer Information Series-Practical information. based on NBS research and experience, covering areas of interest to the consumer. Easily understandable language and illustrations provide useful background knowledge for shopping in today's technological marketplace.

Order the above NBS publications from: Superimendent of Dorkments. Government Primting Office, Hashington. DC 2040)?

Order the following NBS putlications-FIPS and NBSIR:s-from the National Terhnical Information Services. Springfield. I.A ?2I6I.

Federal Information Processing Standards Publications (FIPS PUB)-Publicitions in this series collectively constitute the Federal Information Processing Standards Register. The Register serves als the official source ol information in the Federal Governmont regarding standards issued by NBS pursuant to the federal Property and Administrative Services Act ol 1949 as amended, Public Lalu 89-306 (79 Stat. 1127), and als implemented by Executive Order 11717 (38 FR 12315, dated May 11, 1973) and Palrt 6 ol Title 15 CFR (Code ol Federal Regulations)

NBS Interagency Reports (NBSIR) - A special series ol' interim or final reports on work performed by NBS for outside sponsors (both government and non-government). In general, initial distribution is handled by the sponsor: pubitic distribution is by the National Technical Information Services. Springtield, VA 22161. in paper copy or microliche form. 


\section{EDGE INDEX}

A Guide to Users of This Publication

Descriptive

NBS Periodical and Non-Periodical Publications

Purchase Procedures and Document Availability

Citations (index code is shown within parentheses)

Journal of Research

Journal of Physical and Chemical Reference Data (JPCRD)

DIMENSIONS/NBS (DIM/NBS)

Monographs (Monogr.)

Handbooks $(\mathrm{H})$

Special Publications (SP)

Applied Mathematics Series (AMS)

National Standard Reference Dała Series (NSRDS)

Building Science Series (BSS)

Federal Information Processing Standards Publications (FIPS PUBS)

Voluntary Product Standards (VPS)

Technical Notes (TN)

Consumer Information Series (CIS)

NBS Interagency Reports (NBSIR)

Grant/Contract Reports and Patents (GCR and/or NBS Patents)

NBS Papers Published in Non-NBS Media [5-digit arabic number]

Listing of NBS Papers by Major Subject Areas

Indexes

Author Index

Key Word Index

Depository Libraries in the United States

District Offices of the U.S. Department of Commerce 